\title{
Proceedings of the 2009 World Molecular Imaging Congress Montreal, Canada, September 23-26
}

The abstract ID nomenclature used in this journal supplement does not correspond to the nomenclature used in the WMIC 2009 Program Book and browsable, electronic data provided at the conference and online via the congress website (http://www.wmicmeeting.org). This issue and the Program Book are arranged differently. For additional information like author email addresses and disclosures of corporate relationships, please browse or search the abstracts via the WMIC website: http://wmicmeeting.org/2009searchabstracts.

\section{INDEX}

\section{Development/Novel use of Imaging Probes}

\section{Nanoparticle Probes}

Novel MRI Probes Including Hyperpolarization

Novel Multi-Modality Probes

Novel Optical Probes

Novel PET/SPECT Probes

Ultrasound Probes

\section{Imaging Disease/Organ Processes}

Cancer

Cardiovascular and MSK

Central Nervous System

Immune System

\section{Imaging Methodology}

CT

Hybrid

MRI

Optics

PET

SPECT

Ultrasound

\section{Imaging Molecular and/or Cellular Processes}

$\begin{array}{ll}\text { Antibody } & \mathrm{J} 682 \\ \text { Apoptosis, Autophagy, Necrosis } & \mathrm{J} 687 \\ \text { Energy Metabolism } & \mathrm{J} 696 \\ \text { Gene Expression } & \mathrm{J} 703 \\ \text { Immune Cells } & \mathrm{J} 725 \\ \text { Infection } & \mathrm{J} 734 \\ \text { Inflammation } & \mathrm{J} 739 \\ \text { None of the above } & \mathrm{J} 756 \\ \text { Signal Transduction } & \mathrm{J} 758 \\ \text { Stem Cells and Tissue Regeneration } & \mathrm{J} 768 \\ \text { Transport Systems } & \mathrm{J} 795\end{array}$

\section{In Vivo Work}

$\begin{array}{ll}\text { Animals } & \text { J801 } \\ \text { Humans } & \text { J872 } \\ \text { Translational Studies } & \text { J878 }\end{array}$

\section{Therapy Including Drug Therapy}

$\begin{array}{lc}\text { As Part of Drug Development } & \text { J883 } \\ \text { Image Guided Therapy } & \text { J903 } \\ \text { Monitoring Drug Therapy } & \text { J924 } \\ \text { Treatment efficacy } & \text { J952 }\end{array}$




\section{Development/Novel Use of Imaging Probes}

\section{Nanoparticle Probes}

J001

\section{ULTRA HIGH SENSITIVITY TARGETED PHOTOACOUSTIC IMAGING AGENTS FOR CANCER EARLY DETECTION IN LIVING MICE}

Adam de la Zerda ${ }^{1,2}$, Zhuang Liu $^{3}$, Sunil Bodapati ${ }^{2}$, Robert Teed ${ }^{2}$, ${\text { Cristina } \text { Zavaleta }^{2} \text {, Srikant Vaithilingam }}^{1}$, Xiaoyuan Chen $^{2}$, Butrus Khuri-Yakub $^{1}$, Hongjie Dai ${ }^{3}$, Sanjiv S. Gambhir ${ }^{2,4}$

${ }^{1}$ Electrical Engineering, Stanford University, Stanford, CA, USA, ${ }^{2}$ Radiology, Stanford University, Stanford, CA, USA, ${ }^{3}$ Chemistry, Stanford University, Stanford, CA, USA, ${ }^{4}$ Bioengineering, Stanford University, Stanford, CA, USA.

Photoacoustic molecular imaging of living subjects offers high spatial resolution at increased tissue depths compared to optical imaging strategies. We have recently demonstrated single walled carbon nanotubes (SWNTs) conjugated to Indocyanine Green (SWNT-ICG) as targeted photoacoustic imaging agents in-vitro. In the current work, we created a significantly improved SWNTICG particle with over 1000-times better sensitivity than plain SWNT and demonstrated their ability to target tumors when injected intravenously to a living mouse. The targeted SWNTICG particles were synthesized by coupling of ICG molecules to the surface of SWNT-RGD particles through pi-pi stacking interactions. Control SWNT-ICG particles were created using the untargeted SWNT-RAD instead. We verified the particles are stable in serum and target $\alpha_{v} \beta_{3}$ integrin through cell uptake studies with U87 cells. We found the photoacoustic signal produced by the particles to be highly linear to their concentration both in phantom studies $\left(R^{2}=0.99\right)$ as well as in living mice injected with the particles subcutaneously $\left(R^{2}=0.971\right)$. We further measured the detection sensitivity of SWNT-ICG in living mice ( $n=3$ mice) and found it to be $30 \mathrm{pM}$. This represents more than 3 orders of magnitude improvement compared to plain SWNTs sensitivity in living mice $(p<0.05)$. Furthermore, xenograft-bearing mice were tail-vein injected with RGD-targeted SWNT-ICG. At $2 \mathrm{~h}$ post-injection, mice injected with the RGD-targeted particles showed 2.1-times higher photoacoustic signal in the tumor compared to mice injected with control particles $(p<0.05, n=4$ mice). Finally, we demonstrated the superiority of the SWNT-ICG-RGD particles by incubating them with U87 cells and detecting in living mice 1000times such cells than if the cells were incubated with plain SWNTRGD. This is the first photoacoustic imaging agent tested and targeted in living animals that we know of that can reach such a high sensitivity of $30 \mathrm{pM}$.

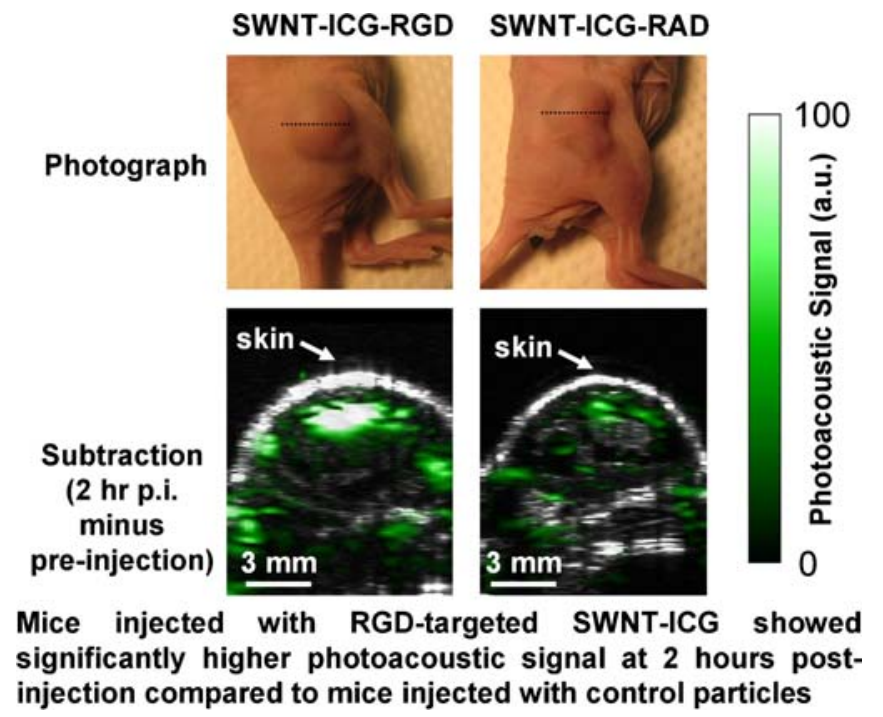

J002

TIME AS A FACTOR IN HUMAN PANCREATIC ISLETS LABELING

$\underline{\text { Daniel Jirak }^{1}}{ }^{1}$, Monika Dezortova ${ }^{1}$, Vit Herynek ${ }^{1}$, Jan Kriz ${ }^{2}$, Peter Girman $^{2}$, Frantisek Saudek ${ }^{2}$, Milan Hajek ${ }^{1}$

${ }^{1}$ MR-Unit, Dept. Diagnostic and Interventional Radiology, Institute for Clinical and Experimental Medicine, Prague, Czech Republic, ${ }^{2}$ Lab. of Langerhans Islets, Dept. Diabetology, Institute for Clinical and Experimental Medicine, Prague, Czech Republic.

Introduction In vitro labeling of pancreatic islets (PI) with iron nanoparticles enables their direct posttransplant visualization by magnetic resonance (MR); superparamagnetic iron oxide (SPIO) contrast agents enable the PI visualization on MR images as hypointense areas. However, it is important to ensure proper conditions for their efficient labeling, especially iron concentration in the medium and the labeling time. Here, we present data obtained in phantoms and patients that show that we cannot assure clear visualization of human pancreatic islets on MR images if the labeling is shorter than $10 \mathrm{~h}$. Methods Phantom study: Isolated human PI were labeled with clinically approved SPIO contrast agent Resovist ${ }^{\circledR}$ for $6-10 \mathrm{~h}, 16$, 24 , and 48 hrs. $50-500$ labeled PI were placed in a $4 \%$ gelatin. Human patient study $(n=2)$ : PI were labeled 7 or $44 \mathrm{hrs}$ with Resovist $\AA$. 220000 labeled PI or more were transplanted into the liver. Iron concentration was $140 \mu \mathrm{g} \mathrm{Fe} / \mathrm{mL}$ according to [1-2]. A 3T whole-body scanner, equipped with a commercial array coil, was used. Images were 
acquired using a FLASH sequence with $0.78 \times 0.78 \times 3 \mathrm{~mm} 3$ resolution with breatholding and with $1.25 \times 1.25 \times 2 \mathrm{~mm} 3$ resolution and triggering. Results Phantom study: On the contrary to PI labeled 6-10 hrs, regions of signal void were clearly detected in phantoms only if the islet labeling lasted 16 or more hours. Patient study: PI were clearly recognized in case of $44 \mathrm{hrs}$ labeling. On the contrary, only ill-defined hypointense areas were observed in MR images in a patient with transplanted PI labeled for $7 \mathrm{hrs}$ (see Figure). Conclusion Our data agree with the fact that iron loading with SPIO contrast agent is a timedependent process [3]. Longer time of labeling is preferable for the ability to confirm the technical success of transplantation and to monitor the graft loss of transplanted PI by MR imaging. The study was supported by grants MZ0IKEM2005, GACR 203/09/1242 and EU/ ENCITE. 1) Jirak D et al. Magn Reson Med 2004; 52:1228 2) Toso C et al. Am J Transplant 2008; 8:701 3) Berkova Z et al. Transplantation 2008; 85:155
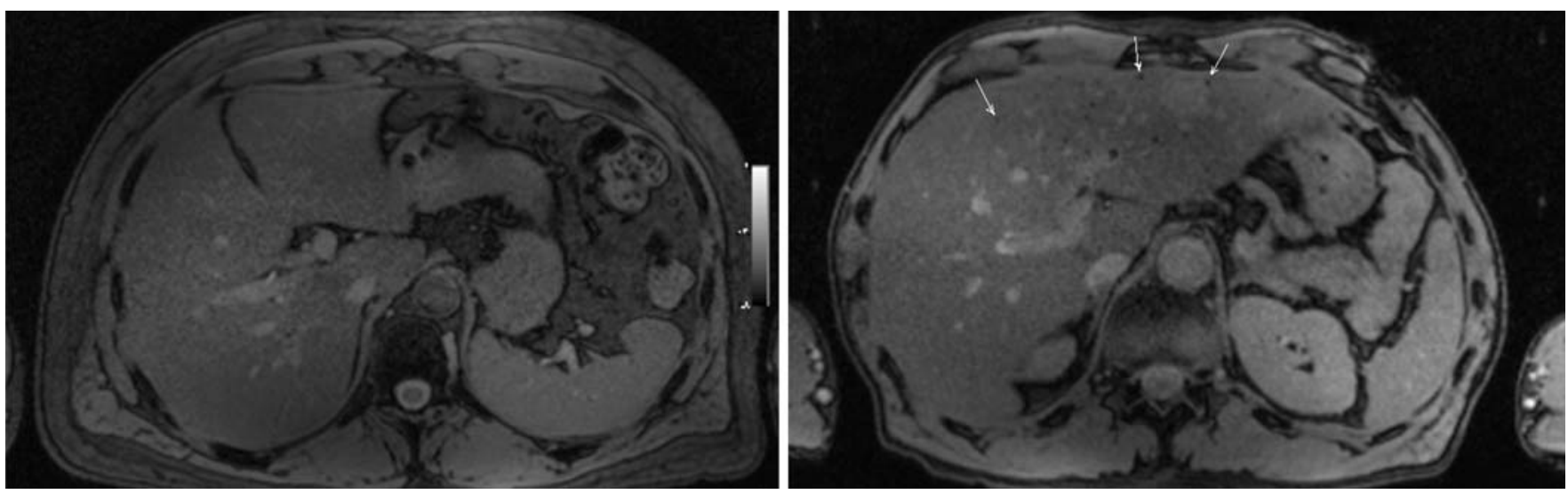

Figure. Liver with transplanted PI labeled 7 (left) and $44 \mathrm{~h}$ (right). Arrows point out some of visible PI.

J003

\section{REAL-TIME IMAGING OF NANOPARTICLE TRAFFICKING FROM THE LUNGS TO THE BODY}

Hak Soo Choi ${ }^{1}$, Aya Matsui ${ }^{3,1}$, Yoshitomo Ashitate ${ }^{1,3}$, Jeong Heon Lee $^{1}$, Rafiou Oketokoun ${ }^{1}$, Numpon $\operatorname{Insin}^{4}$, Moungi G. Bawendi ${ }^{4}$, Akira G. Tsuda ${ }^{5}$, John V. Frangioni ${ }^{1,2}$

${ }^{1}$ Medicine, Beth Israel Deaconess Medical Center, Boston, MA, USA, ${ }^{2}$ Radiology, Beth Israel Deaconess Medical Center, Boston, MA, USA, ${ }^{3}$ Surgical Oncology, Hokkaido University Graduate School of Medicine, Hokkaido, Japan, ${ }^{4}$ Chemistry, Massachusetts Institute of Technology, Cambridge, MA, USA, ${ }^{5}$ Molecular and Integrative Physiological Sciences, Harvard School of Public Health, Boston, MA, USA.

Background: The uptake, biodistribution, and clearance of inhaled nanoparticles (NPs) are of great importance in determining the toxic effects of inhaled air pollutants and viral pathogens. However, the translocation and elimination processes for NPs after inhalation are largely unknown. The focus of this study was to define the physicochemical parameters that mediate uptake of NPs from the lung into the body. Materials and Methods: A series of NIR fluorescent NPs were engineered with varying composition, shape, size, and surface charge. Silica and quantum dots (QDs) with various core(shell) and organic coating ligands were used to produce different sized and charged inorganic/organic hybrid NPs (INPs). Organic NPs (ONPs) were prepared using HSA, PEG, and block copolymers. The overall hydrodynamic diameter (HD) of NPs ranged from 5 to $300 \mathrm{~nm}$ in $100 \%$ rat serum. The optical properties of engineered NPs were analyzed in terms of absorbance and fluorescence. INPs and ONPs were designed to have maximum fluorescence emission at $800 \mathrm{~nm}$ and $700 \mathrm{~nm}$, respectively. NPs were administered to the lungs of SpragueDawley rats, and animal imaging was performed using the FLARE ${ }^{\mathrm{TM}}$ imaging system. Results: In vivo translocation of NPs was primarily governed by the size of NPs irrespective of their composition. The size threshold for translocation from lungs to lymph nodes was found to be $\approx 30 \mathrm{~nm}$ for the INP series and $\approx 34 \mathrm{~nm}$ for the ONP series. In NPs under this size threshold, surface charge played a critical role in controlling translocation from the lung to the body. Anionic- and cationic-coated NPs showed similar HDs in serum because of partial adsorption to proteins, but cationic charged INPs were completely trapped in the lungs. Moreover, the HD of NPs determined the extent of translocation and clearance from the lymph nodes to the bloodstream. The smallest NPs $(5 \mathrm{~nm} \mathrm{HD})$ rapidly translocated to the lymph nodes, then within minutes appeared in the systemic circulation and urine. Conclusion: In this study, we have systematically engineered various NPs to demonstrate the translocation threshold from the lungs into the lymph nodes and bloodstream. Although NPs are stable and show narrow size distribution in PBS, this study suggests that the final HD in serum and surface charge are critical parameters for the ultimate migration and clearance of NPs from the lungs. Taken together, our results provide a platform based on NIR fluorescent NPs for developing toxicological and pharmacokinetic models for air pollution, inhaled viruses, and drug delivery. 


\section{J004}

UPCONVERTING LUMINESCENT NANOMATERIALS: APPLICATION TO IN VIVO OPTICAL IMAGING

Scott Hilderbrand $^{1}$, Fangwei Shao ${ }^{1}$, Christopher D. Salthouse ${ }^{1}$, Umar Mahmood $^{2}$, Ralph Weissleder ${ }^{1}$

${ }^{1}$ Center for Systems Biology, Massachusetts General Hospital/Harvard Medical School, Boston, MA, USA, ${ }^{2}$ Radiology/Nuclear Medicine, Massachusetts General Hospital/Harvard Medical School, Boston, MA, USA.

Upconverting luminescent lanthanide-based materials are an attractive platform for use in diagnostic imaging. These materials can be engineered to have excitation and emission in the far-red to near infrared (NIR), a regime where biological tissue has increased optical transparency. The anti-Stokes emission displayed by upconverting materials opens the possibility for significantly improved target to background levels since few, if any, biological components display strong upconversion emission. In this report, the development of yttrium oxide nanoparticles doped with erbium and ytterbium for in vivo optical imaging is detailed. Coating the particles with a polyacrylic acid (PAA) and polyethylene glycol (PEG) shell imparts long-term aqueous stability and allows for their application in vivo. The nanoparticles have a hydrodynamic diameter of $101 \mathrm{~nm}$ and show minimal cytotoxicity. Strong upconversion emission at $660 \mathrm{~nm}$ is observed after excitation of the particles with an inexpensive $980 \mathrm{~nm}$ diode laser. The nanoparticles are easily modified with additional near-infrared emitting organic reporters. As a result of their anti-Stokes excitation, there is no interference between the upconversion emission signal from the nanoparticle cores and the emission from the appended organic NIR fluorophores. This opens the possiblity for use of these new nanomaterials for multi-channel imaging applications. The utility of the upconverting nanoparticles is demonstrated by their in vivo use as a real time vascular imaging agent.

\section{J005}

PEPTIDE-TARGETED MULTIMODAL CORE-SHELL SILICA NANOPARTICLES FOR PET IMAGING OF INTEGRIN EXPRESSION

$\underline{\text { Oula Penate-Medina }}{ }^{1}$, Miriam Benezra ${ }^{1}$, Hooisweng $\mathrm{Ow}^{3}$, Andrew Burns $^{4}$, Valerie A. Longo ${ }^{1}$, Erik $\mathrm{Herz}^{2}$, Steven Larson ${ }^{1}$, Ulrich Wiesner $^{2}$, Michelle S. Bradbury ${ }^{1}$

${ }^{1}$ Radiology, Sloan Kettering Institute for Cancer Research, New York, NY, USA, ${ }^{2}$ Materials Science and Engineering, Cornell-Ithaca, Ithaca, NY, USA, ${ }^{3}$ Hybrid SIlica Technologies, Ithaca, NY, USA, ${ }^{4}$ GE Global Research, Niskayuna, NY, USA.

A clinically translatable, fluorescent nanoparticle probe exhibiting high biostability, efficient renal clearance, enhanced photophysical properties, and the possibility of radiotargeting, creates an intriguing dualmodality probe for active tumor targeting and theragnostics. These features are key to optimizing biodistribution and transport across biological barriers, while maintaining high contrast. We have synthesized a new generation of clinically promising, non-toxic, and biocompatible near infrared fluorescence (NIRF) $\mathrm{C}$ dots, tuned to hydrodynamic diameters as small as 3.3 and $6.0 \mathrm{~nm}$, which can be used as positron emission tomography (PET) tracers for imaging integrin expression on activated endothelial cell surfaces. Multimodal C dots, PEG-coated to prevent protein adsorption and facilitate urinary excretion, were modified to target $\alpha v \beta 3$-integrin-expressing tumors by covalently coupling integrin-binding peptides (RGD). PEGylated dots served as controls. In-vitro binding characteristics of targeted and non- targeted dots to a $\alpha v \beta 3$-integrin expressing melanoma cell line were investigated using flow cytometry and radioactive cell binding assays (iodine-124). Significant concentration- and time-dependent differences in cell-surface binding were observed between the RGD-dot and control dots. RGD-dot specificity was demonstrated using antibodies to $\alpha v \beta 3$ integrin, as well as by blocking integrin receptors with excess RGD. In vivo PET biodistribution studies revealed no significant differences in renal clearance when compared with the non-targeted formulation. Following intravenous injection of the 6-nm targeted probe into mice bearing hindleg melanoma xenografts, rapid and enhanced receptormediated tumor uptake of intravenously injected 124I-labeled RGDdot was observed by serial PET imaging over a 96-hr imaging period. Region-of-interest measurements over the tumor site demonstrated accumulation of the RGD-dot within the first hour after injection; peak uptake was observed at 4-hrs, with $4 \% \mathrm{ID} / \mathrm{g}$ values determined. Enhanced tumor retention and renal clearance were additionally found relative to that seen after injection of the PEG-coated 124I-labeled dot, with maximum tumor-to-muscle ratios found at 24-hr after injection. Subcutaneous hindleg tumors were clearly visualized at all post-injection time points $(0.5-96 \mathrm{~h})$ by multimodal NIRF-PET imaging, and confirmed by ex-vivo gamma counting. These results suggest that $\mathrm{C}$ dots constitute a translatable materials platform, and pave the way for this technology to address important biological issues.

\section{J006}

A PEPTIDE TARGETED NANOGLOBULAR CONTRAST AGENT FOR CANCER MOLECULAR IMAGING WITH MRI Mingqian $\mathrm{Tan}^{1}$, Xueming $\mathrm{Wu}^{1}$, Furong $\mathrm{Ye}^{1}$, Eun-Kee Jeong ${ }^{2}$, Zheng-Rong $\mathrm{Lu}^{3,1}$

${ }^{1}$ Pharmaceutics, University of Utah, Salt Lake City, UT, USA, ${ }^{2}$ Radiology, University of Utah, Salt Lake City, UT, USA, ${ }^{3}$ Biomedical Engineering, Case Western Reserve University, Cleveland, OH, USA.

Magnetic resonance imaging (MRI) is one of the most powerful imaging techniques for visualizing the internal structure and function of the body. However, MRI has not been effectively used for molecular imaging due to its low sensitivity. In most of cases, the local concentration of molecular targets is often too low to generate significant contrast enhancement for effective MR molecular imaging. To achieve effective molecular imaging with MRI, an MRI contrast agent specific to a molecular target with abundant presence in the target of interest is desirable to increase local concentration of the contrast agent via specific binding. A recent work has shown that a cyclic CGLIIQKNEC (CLT1) peptide can specifically bind to fibrin-fibronectin clots in various tumor tissues with little non-specific binding to normal tissues. The incorporation of the peptide to nanoglobular dendrimer-Gd(III) complexes with well-defined nanosizes may result in more efficient targeted contrast agents for cancer molecular imaging with MRI due to increased relaxivity and high Gd(III) chelate to peptide ratio. Here, we synthesized a targeted nanoglobular contrast agent by conjugating peptide CLT1 to a G3 nanoglobular Gd(III) complex with click chemistry for cancer MR molecular imaging. The $\mathrm{T} 1$ and $\mathrm{T} 2$ relaxivities of the peptide targeted contrast agent were 7.9 and $16.2 \mathrm{mM}-1 \mathrm{sec}-1$ at $3 \mathrm{~T}$, respectively. Approximately 3 peptides and 42 Gd-DO3A chelates were conjugated to the surface of 64 amine groups of nanoglobules. The peptide-targeted nanoglobular contrast agent showed significantly higher contrast enhancement than the nontargeted agent in tumor (Fig. 1, $* p<0.05, * * p<0.01$ ) for at least $60 \mathrm{~min}$ at a dose of $0.03 \mathrm{mmol}-\mathrm{Gd} / \mathrm{kg}$ in female athymic mice bearing MDAMB-231 human breast carcinoma xenografts. The peptide targeted contrast agent has a potential for specific cancer molecular imaging with MRI. 


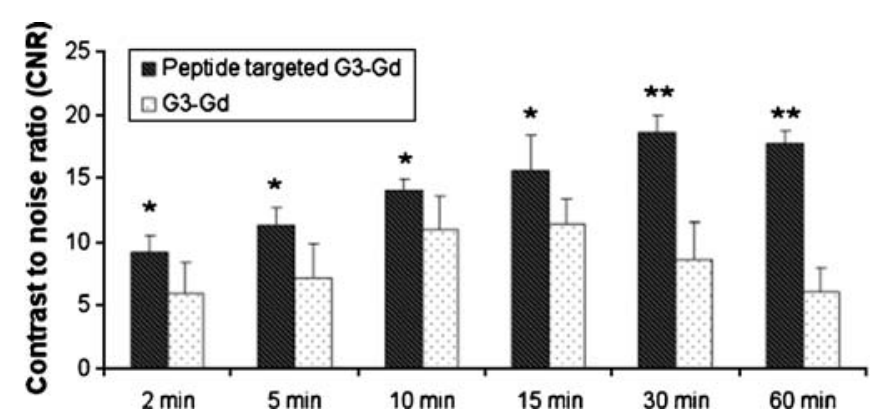

Figure 1. Contrast to noise ratio of peptide targeted and non-targeted G3 nanoglobular contrast agents within tumor tissue.

J007

\section{INTRAVITAL IMAGING OF HUMAN PROSTATE CANCER USING BOMBESIN-TARGETED VIRAL NANOPARTICLES}

John D. Lewis

${ }^{1}$ Medical Biophysics, University of Western Ontario, London, ON, Canada, ${ }^{2}$ Surgery, London Regional Cancer Center, London, ON, Canada.

Viral nanoparticles offer an attractive multivalent platform for diagnostic in vivo imaging of prostate and other cancers. We have developed a nanoparticle platform based on the cowpea mosaic virus (CPMV) that offers discrete control over the conjugation of detection moieties, solubilization polymers and targeting ligands to the viral capsid. We report here the specific targeting and imaging of human PC-3 prostate tumors in vivo with PEGylated fluorescent viral nanoparticles conjugated to a pan-bombesin peptide. The amphibian tetradecapeptide, bombesin, selectively interacts with the gastrin-releasing peptide (GRP) receptor family that is over-expressed on human prostate cancer cells. Bombesin peptides were conjugated to CPMV particles functionalized with a near-infrared (NIR) dye (Alexa Fluor 647) and polyethylene glycol (PEG) using the copper(I)-catalyzed azide-alkyne cycloaddition reaction. Absorbance measurements indicated that each nanoparticle contained 90 NIR dyes and 80-95 PEG or bombesin-PEG units. The integrity of CPMV particles was verified by FPLC, SDS PAGE and transmission electron microscopy. The bombesin-targeted CPMV particles showed a marked increase in uptake by PC-3 cells compared to a non-targeted control as measured by flow cytometry, and specificity was confirmed by successful blocking with an excess of soluble bombesin peptide. Targeting of PC-3 cells in vitro was confirmed by confocal microscopy. Bombesin conjugated CPMV showed significant targeting and uptake in human prostate tumors in vivo, using a shell-less avian embryo tumor model. Taken together, we have shown here that bombesin-targeted viral nanoparticles offer a highly selective imaging tool for human prostate tumors, using a platform with future potential for clinical non-invasive imaging strategies and drug delivery.

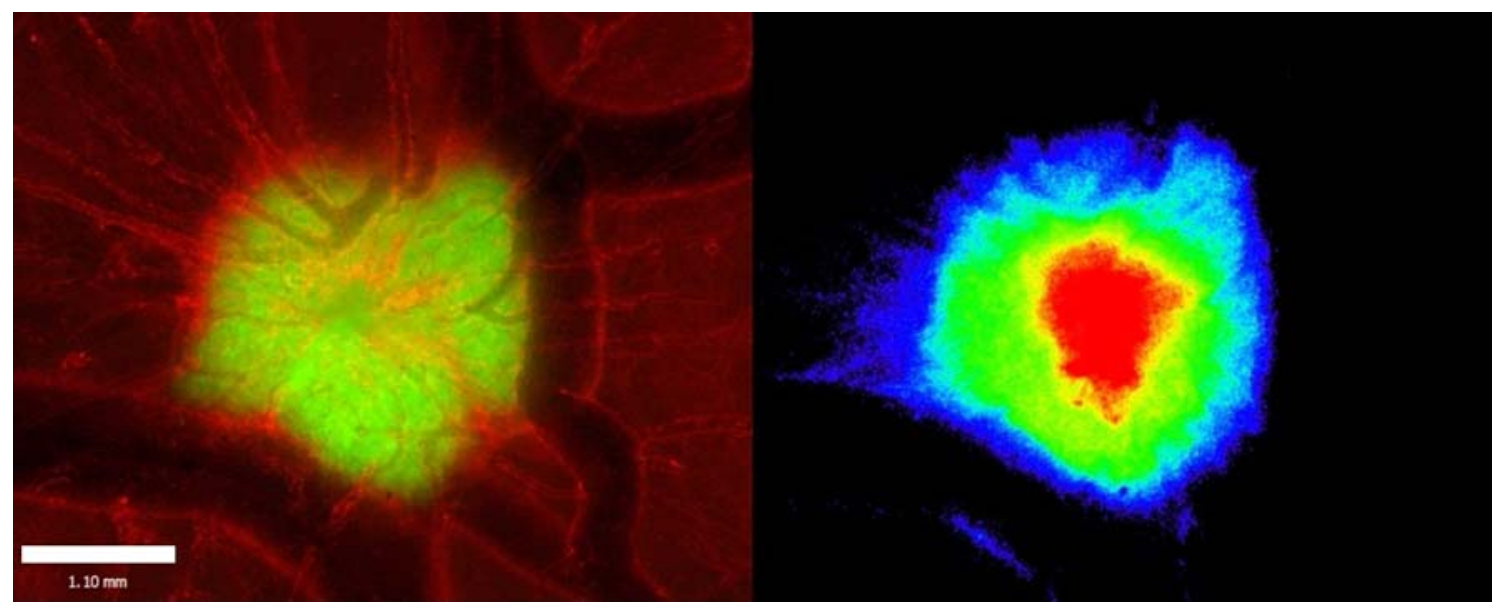

\section{J008}

\section{BIODISTRIBUTION OF A RADIOLABELED CT CONTRAST AGENT QUANTIFIED BY DUAL MODALITY SPECT AND SPECTRAL CT}

Anke de Vries ${ }^{1}$, Ewald Roessl ${ }^{2}$, Jens-Peter Schlomka ${ }^{2}$, Roland Proksa ${ }^{2}$, $\overline{\text { Klaas Nicolay }}^{1}$, Holger Gruell ${ }^{1,3}$

${ }^{1}$ Biomedical Engineering, Eindhoven University of Technology, Eindhoven, Netherlands, ${ }^{2}$ Imaging Systems and Interventions, Philips Research Europe, Hamburg, Germany, ${ }^{3}$ Biomolecular Engineering, Philips Research Europe, Eindhoven, Netherlands.

CT is a frequently used clinical imaging modality often requiring the use of radiopaque contrast agents. The observed contrast in conventional CT depends on the absorption coëfficient per imaging voxel and is thus a function of contrast agent concentration and tissue properties. Based on conventional $\mathrm{CT}$ it is therefore not possible to distinguish between absorption coming for example bones or calcifications and contrast agents, nor to quantify the contrast agent concentration in a tissue. Spectral CT is a new imaging technique which is able to quantify concentrations of high Z-elements such as iodine. The technique makes use of an energy resolved detector to quantify the k-edge absorption of high $\mathrm{Z}$ elements and to distinguish different elements (1). Here, we present the results of a multimodal imaging study, where we quantify the biodistribution of a new type of radiolabeled iodinated blood pool agent with Spectral CT and compare it to biodistribution data obtained with SPECT. The CT blood pool agent is based on an emulsified iodinated oil, stabilized in aqueous solution using an amphiphilic di-block copolymer. $5 \mathrm{~mol} \%$ of DOTA functionalized diblock-polymer was incorporated to allow radiochemical labeling. Swiss mice were injected with ca. $160 \mu \mathrm{L}$ of radiolabeled iodinated emulsion (16 $\mathrm{MBq}{ }^{111}$ Indium). Biodistribution 1 hour post-injection was investigated with conventional CT as well as with SPECT and spectral CT. A clear correlation could be observed between the spectral CT imaging technique and SPECT as demonstrated in the included figure, indicating that spectral $\mathrm{CT}$ is able to quantify the 
iodine concentration in-vivo. Furthermore, the presented long-circulating iodinated nanoparticles are shown to be promising as a CT contrast agent in future diagnostics. Reference 1) E. Rössl, R. Proksa, Phys. Med. Biol. 52, 4679 (2007)
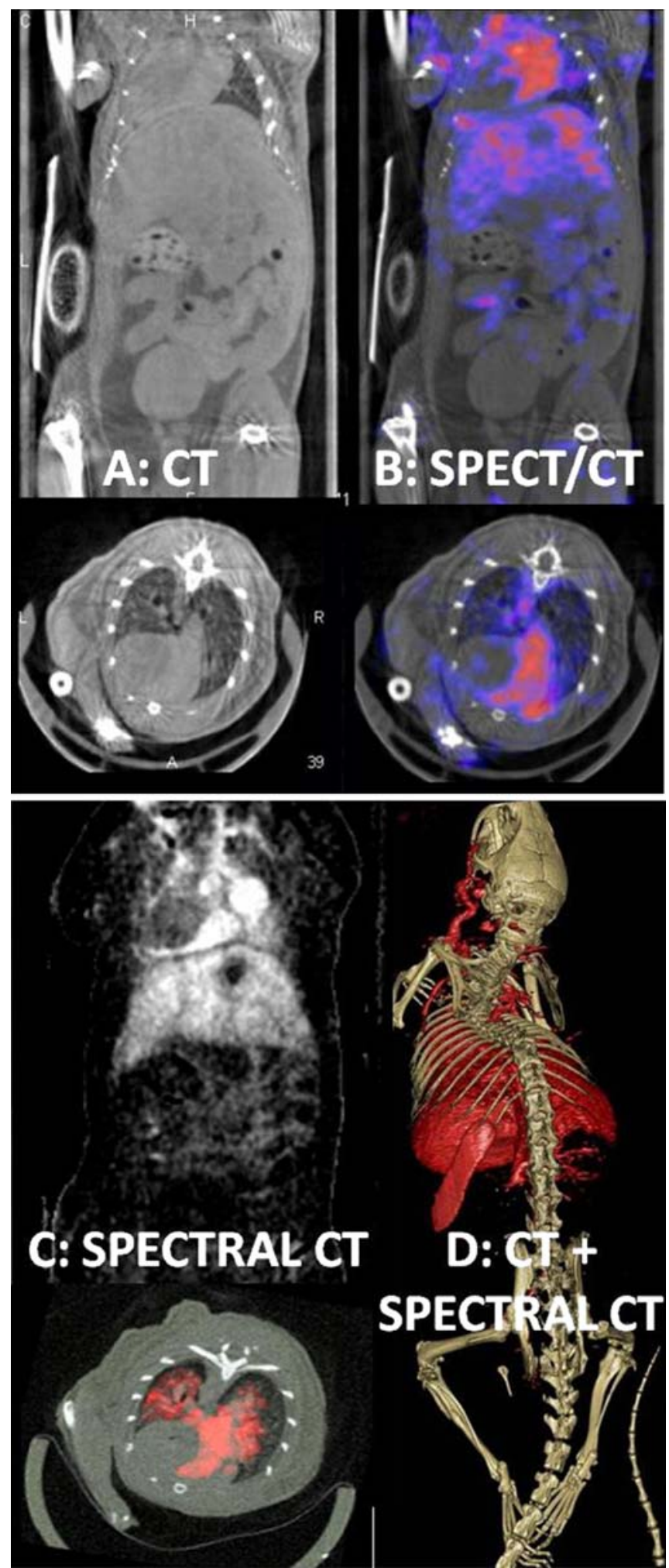

Figure 1: Multimodal imaging of a mouse post-injection of iodinated nanoparticles A) CT scan B) CT combined with SPECT showing the distribution of ${ }^{111}$ Indium C) Spectral CT scan showing the distribution of iodine D) Conventional CT (bone structure) combined with Spectral CT, showing the distribution of iodine.
J009

SYNTHESIS OF AMPHIPHILIC TRIBLOCK COPOLYMERS WITH MULTIDENTATE LIGANDS FOR SURFACE COATING OF QUANTUM DOTS

Tongxin Wang ${ }^{1}$, Rajagopalan Sridhar ${ }^{2}$, Paul C. Wang ${ }^{1}$

${ }^{1}$ Radiology, Howard University, Washington, DC, USA, ${ }^{2}$ Radiation Oncology, Howard university, Washington, DC, USA.

In order to develop biocompatible optical imaging agents, a novel triblock copolymer, designed as a multidentate ligand, was synthesized to coat quantum dot nanocrystals (QDs). The copolymer consists of a polycarboxylic acid block at one end and a polythiol block at the other end with an intervening cross-linked poly(styrene-co-divinylbenzene) block bridging the ends. The multiple mercapto groups from the polythiol block act as multidentate ligands to stabilize QDs, while the polycarboxylic acid block improves the water solubility of QDs and offers reaction sites for surface modification or conjugation with biomolecules. The cross-linked poly(styrene-co-divinylbenzene) block provides a densely compacted hydrophobic shell outside QDs. This will prohibit the diffusion of other molecules or ions through the macromolecular shell and protect quantum dots from degradation. By using this new multidentate polymer coating, QDs can be transferred from organic solvent into aqueous phase. Because the QDs are directly bound to multidentate mercapto groups instead of TOPO, they are less likely to be affected by the mercapto or disulfide groups within proteins or other biomolecules. Therefore, it is expected that QDs stabilized with such multidentate mercapto polymer have a greater potential for In vivo use under complex physiological conditions.
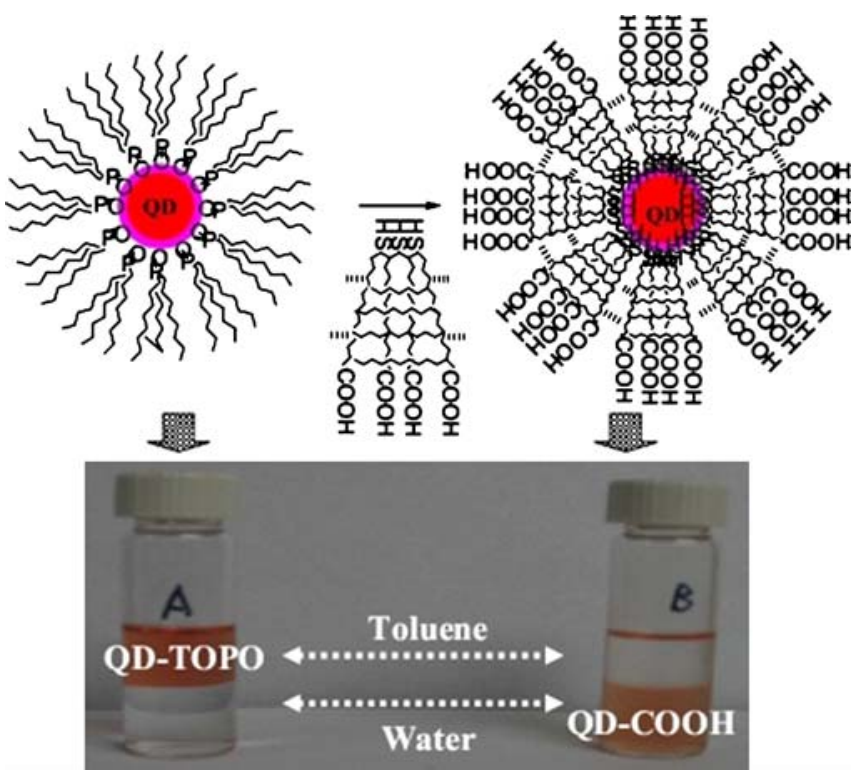

J010

PRECLINICAL EVALUATION OF RAMAN NANOPARTICLES FOR THEIR POTENTIAL USE IN CLINICAL ENDOSCOPIC IMAGING

Cristina Zavaleta, Keith B. Hartman, Zheng Miao, Zhen Cheng, Sanjiv S. Gambhir

Radiology, Stanford University, Stanford, CA, USA. 
Raman spectroscopy continues to prove itself as a powerful non-invasive molecular imaging tool to evaluate nanoparticle delivery in preclinical models. Its pM sensitivity and multiplexing capabilities are unsurpassed. However, its limited depth of light penetration hinders direct clinical translation. Therefore, a more suitable way to harness its attributes in a clinical setting would be to couple Raman spectroscopy with endoscopy. It was recently reported that flat lesions in the colon were five times more likely to contain cancerous tissue than polyps detected by conventional colonoscopy. The use of an accessory Raman endoscope in conjunction with locally administered tumor targeting Raman nanoparticles during a routine colonoscopy could offer a new way to sensitively detect these dysplastic flat lesions. In this study we evaluated the natural biodistribution of gold surface enhanced Raman scattering (SERS) nanoparticles by radiolabeling them with ${ }^{64} \mathrm{Cu}$ and imaging their localization over time using microPET. Mice were injected either intravenously (IV) or intrarectally (IR) with approximately $100 \mu \mathrm{Ci}$ of ${ }^{64} \mathrm{Cu}$-SERS nano- particles and imaged with microPET at various time points: immediately, $30 \mathrm{~m}, 2,5$, and $24 \mathrm{~h}$ post injection. Three mice from each group (IV and IR) were sacrificed at 2, 5 and $24 \mathrm{~h}$ and their organs were collected, weighed and counted in a gamma counter to determine $\%$ injected dose per gram $(\% \mathrm{ID} / \mathrm{g})$. Quantitative biodistribution data obtained from each organ correlated well with the corresponding microPET images, revealing that mice injected IV had significantly higher uptake $(p<0.05)$ in the liver $(5 \mathrm{~h}=18.9 \% \mathrm{ID} / \mathrm{g})(24 \mathrm{~h}=4.8 \% \mathrm{ID} / \mathrm{g})$, as opposed to mice injected IR $(5 \mathrm{~h}=1.27 \% \mathrm{ID} / \mathrm{g})(24 \mathrm{~h}=0.3 \% \mathrm{ID} / \mathrm{g})$. Mice injected IR showed localized uptake in the large intestine $(5 \mathrm{~h}=9 \% \mathrm{ID} / \mathrm{g})$ $(24 \mathrm{~h}=4.3 \% \mathrm{ID} / \mathrm{g}$ ) with minimal uptake in other organs. Raman imaging of the excised tissues confirmed the presence of SERS nanoparticles within tissues of interest. These results suggest that topical application of SERS nanoparticles in the colon appears to minimize their systemic distribution, thus avoiding potential toxicity and supporting the clinical translation of Raman spectroscopy as an endoscopic imaging tool.

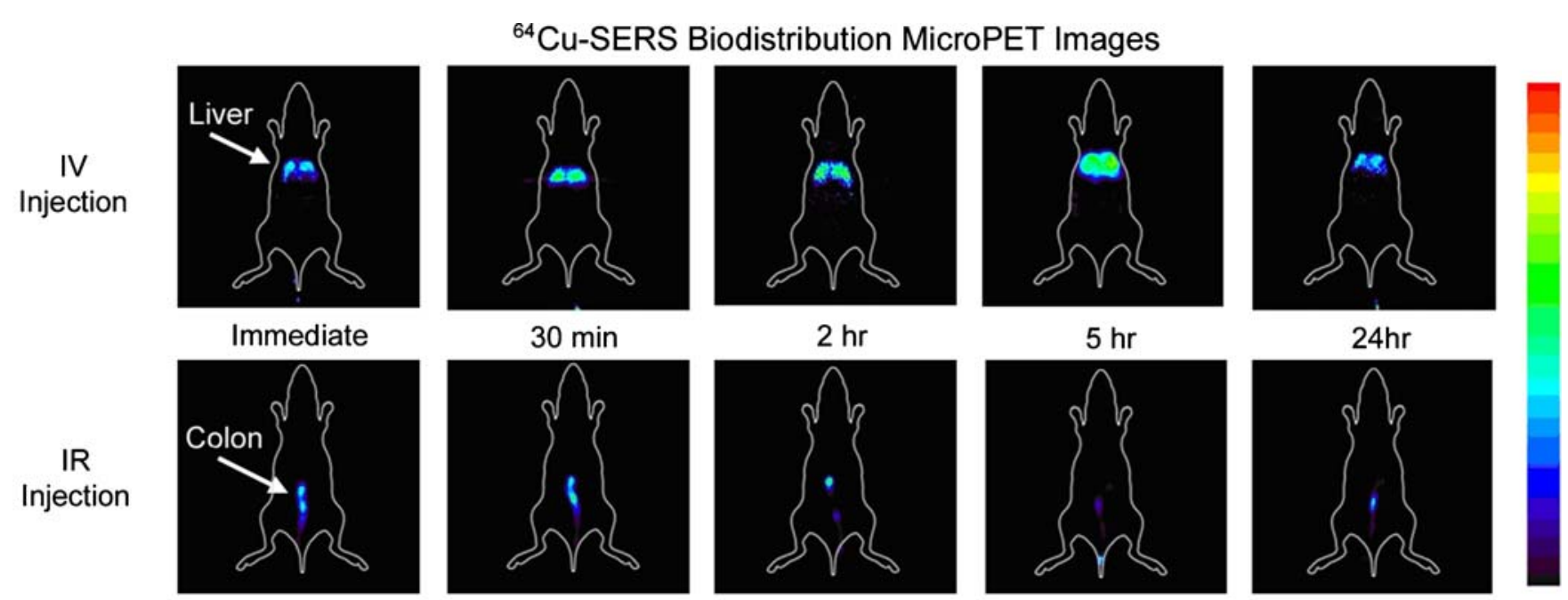

Figure Legend: MicroPET imaging in mice after either intravenous (IV) or intrarectal (IR) administration of Cu-64 labeled SERS nanoparticles at various time points post injection. The SERS nanoparticles appear to localize in the liver immediately after IV injection and remain in the liver over 24 hours whereas the SERS nanoparticles injected IR remain localized in the colon over 24 hours. Colored scale bar to right represents ${ }^{64} \mathrm{Cu}$-SERS uptake where red represents most uptake and black represents no uptake.

J011

\section{BIO-DISTRIBUTION OF ULTRA-FINE POLLUTANTS IN NORMAL AND ASTHMATIC MICE USING PET} Heather A. Palko ${ }^{1}$, Angelique Louie ${ }^{2}$

${ }^{\mathrm{T}}$ Chemistry, UC Davis, Davis, CA, USA, ${ }^{2}$ Biomedical Engineering, UC Davis, Davis, CA, USA.

Air pollution of all types is now understood to be detrimental to human health. It has recently been shown that there is a connection between air pollution and serious health effects. These effects include problems with lung function, blood coagulation and cardiovascular complications. Several studies have shown that particles with a diameter less than $0.1 \mu \mathrm{m}$ have increased toxicity compared with larger particles. Trans-epithelial movement of inhaled particulate matter (PM) can result in PM accumulation in peripheral organs through the hypothesized mechanisms of endothelial cell-facilitated transport and macrophage uptake. The aim of this work is to use Positron Emission Tomography and other radiotracer techniques to track the deposition and fate of model ultra-fine particles In vivo after delivery to the lungs. Amine terminated polystyrene nanoparticles $(78 \mathrm{~nm})$ conjugated to DOTA$64 \mathrm{Cu}$ were used as a model of ultra-fine particles. In these studies it has been shown that the polystyrene nanoparticles are capable of translocating out of the lungs in both of the mouse models. Current work includes utilizing carboxylic acid terminated nanoparticles to determine how surface charge affects translocation. Future work will focus on how size and composition affect translocation out of the lung.

\section{J012}

VALIDATION OF TARGETED LOCALIZATION OF ADENOMATOUS COLON POLYPS IN APCMIN/+ MICE BY MICROCT COLONOGRAPHY, OCT AND MULTISPECTRAL OPTICAL IMAGING

Celeste A. Roney ${ }^{1}$, Biying $\mathrm{Xu}^{2}$, Shuai Yuan ${ }^{3}$, Yu Chen ${ }^{3}$, Jianwu Xie ${ }^{1}$, Gary L. Griffiths $^{2}$, Ronald M. Summers ${ }^{1}$

${ }^{1}$ Radiology and Imaging Sciences, National Institutes of Health, Bethesda, MD, USA, ${ }^{2}$ Imaging Probe Development Center, National Institutes of Health, Bethesda, MD, USA, ${ }^{3}$ Fischell Department of Bioengineering, University of Maryland, College Park, MD, USA. 
Malignant adenomatous polyposis coli (APC) genes predispose transformations to neoplasia in the progression to colorectal carcinoma (CRC). Early detection facilitates clinical management and therapy. Novel lectinmediated polymerized targeted liposomes (Rh-I-UEA-1), with polyp specificity and incorporated imaging agents, were fabricated to locate and image APCMin/+ adenomatous polyps. The biomarker $\alpha$-L-fucose covalently joins the liposomal conjugated lectin ulex euroapeus agglutinin (UEA-1), via glycosidic linkage to the polyp mucin layer. Encapsulated contrast agent Omnipaque validated surface targeting by computed tomography (CT). Multispectral optical imaging (MSI) corroborated a global perspective of specific binding (Rhodamine B $532 \mathrm{~nm}$ emission, 590-620 nm excitation) of targeted Rh-I-UEA-1 polymerized liposomes to polyps with good nonspecific binding to normal mucosa. High-resolution co-registered optical coherence tomography (OCT) and fluorescence molecular imaging (FMI) reveal the spatial correlation of contrast distribution and tissue morphology. Freshly excised $(N=10)$ APCMin $+/$ bowels were incubated with targeted liposomes (UEA-1 lectin), control liposomes (no lectin), or Omnipaque and imaged by the three techniques. CT Hounsfield unit (HU) quantitative analyses confirmed targeted liposomes more strongly bind polyps (406.70 $\pm 70.31 \mathrm{HU}$ versus normal tissue, $37.14 \pm 19.46 \mathrm{HU})$ than do nontargeted liposomes $(152.12 \pm 17.84 \mathrm{HU}$ versus normal mucosa $50.81 \pm 27.61 \mathrm{HU})$ or Omnipaque alone $(270.62 \pm$ $38.84 \mathrm{HU}$ versus normal mucosa $193.66 \pm 15.65 \mathrm{HU}$ ). OCT, with anatomical depth capabilities, along with the co-registered FMI, substantiated Rh-I-UEA-1 liposome binding along the mucinous polyp surface. UEA-1 lectin denotes $\alpha$-L-fucose biomarker carbohydrate expression at the mucin glycoprotein layer; Rh-I-UEA-1 polymerized liposomes target and image adenomatous polyps in APCMin/+ mice. Thus, lay rich opportunities for improved sensitivity and specificity of diagnostic accuracy in CRC detection.

\section{Computed Tomography (CT)}
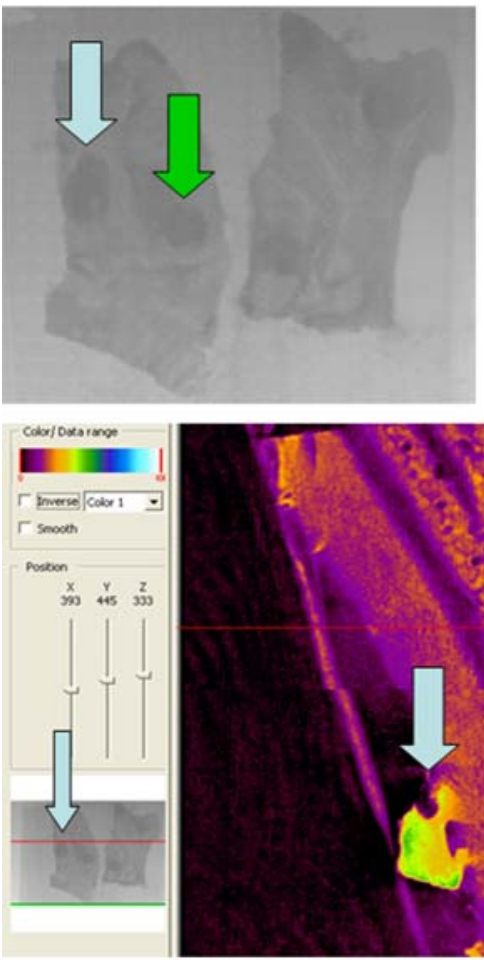

(A) Multispectral Optical
Imaging (MSI)

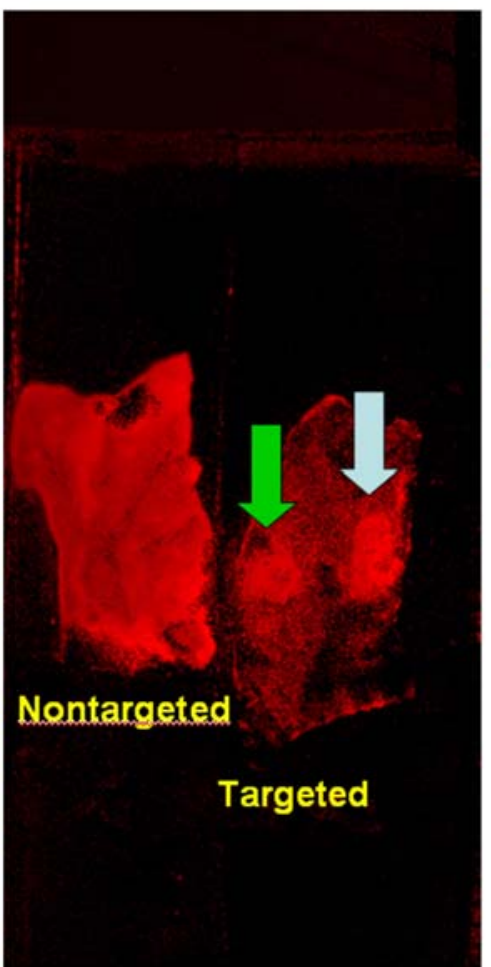

(B)

\section{Optical Coherence Tomography (OCT) with Fluorescence Molecular Imaging} (FMI)
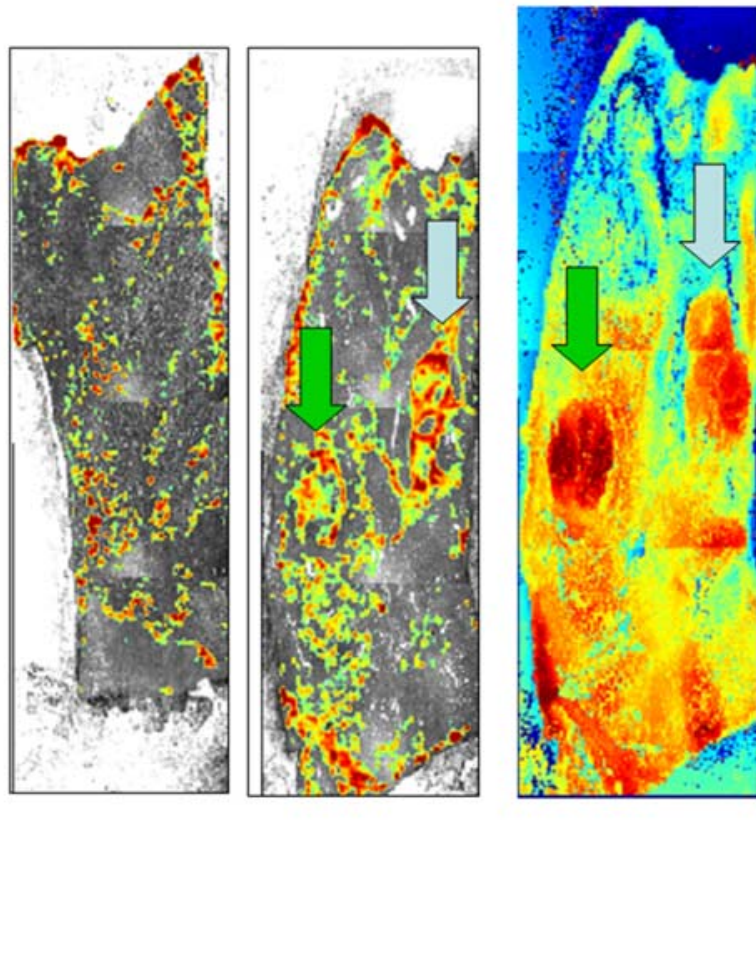

Fig. 1. Novel Rh-I-UEA-1 targeted liposomes bind APCMin/+ polyps along the mucin surface. Multimodality validation is: microCT (A), MSI (B) and OCT + FMI (C). Polyps are indicated by arrows.

\section{J013}

\section{IMAGING OF VASCULAR TARGETED LAPO4 NANOPARTICLES DOPED WITH ACTINIUM-225}

Stephen J. Kennel ${ }^{1}$, Jonathan Woodward ${ }^{3}$, Alan Stuckey ${ }^{1}$, Jonathan Wall $^{1}$, Saed Mirzadeh ${ }^{2}$

${ }^{1}$ Department of Medicine, University of Tennessee Graduate School of Medicine, Knoxville, TN, USA, ${ }^{2}$ Nuclear Science and Techology Division, Oak Ridge National Laboratory, Oak Ridge, TN, USA, ${ }^{3}$ Chemical Sciences Division, Oak Ridge National Laboratory, Oak Ridge, TN, USA.
Nanoparticles (NP) of diverse composition have been touted as potential carriers for various therapeutic drugs including radioisotopes. Targeting of relatively large constructs such as antibody or peptide coated NP has been shown to be efficient only when the target is a circulating lymphocyte or endothelial surface protein. In this application, the $\alpha$ particle emitter, Ac-225 (t1/2=10 d), has been incorporated into lanthanum phosphate NP with the intent that the decay cascade of $225 \mathrm{Ac}$ via emission of four $\alpha$-particles with release of $\sim 28 \mathrm{MeV}$ of energy can provide a potent and site specific radioimmunotherapy. When $225 \mathrm{Ac}$ is attached to targeting agents with standard chelate conjugation methods, the daughter radionuclides are released after the initial $\alpha$-decay 
occurs. The major advantage of the use of NP incorporation of Ac-255 is to sequester the daughter radionuclides $221 \mathrm{Fr}(\mathrm{t} 1 / 2=4 \mathrm{~min}), 217 \mathrm{At}(\mathrm{t} 1 / 2=$ $0.03 \mathrm{sec})$ and $213 \mathrm{Bi}(\mathrm{t} 1 / 2=45 \mathrm{~min})$ at the site of targeting. We show here by using microSPECT/CT imaging that 225Ac-LaPO4-NP coupled with MAb 201B, in a model of vascular targeting, accumulated rapidly in mouse lung after iv injection due to binding to the thrombomodulin expressed preferentially on lung endothelial cells. Specific targeting was demonstrated by showing that competition with excess unlabeled MAb or with NP targeted with control MAb resulted in NP being deposited mainly in liver and spleen. Although most of the $\gamma$ rays from these radionuclides are too high in energy to be detected efficiently, appropriate energy windows were found that provided dramatic microSPECT images of the NP distribution in vivo. Standard biodistribution techniques showed that $\sim 32 \%$ of the total injected dose of 225Ac-LaPO4-NP was bound in lung $1 \mathrm{~h}$ post-injection yielding a value of $\% \mathrm{ID} / \mathrm{g}>200$. Simultaneous analyses of the biodistribution of $225 \mathrm{Ac}$ and $213 \mathrm{Bi}$ demonstrated that more than half of the daughter radionuclides were retained in the NP. We conclude that Ac225 incorporated in LaPO4 NP can be a potent radioimmunotherapeutic when targeted efficiently to the appropriate site and that specific delivery can be monitored by SPECT imaging. Research supported in part by the DOE under contract DE-AC05-00OR22725 with UT-Battelle, LLC.

J014

CADMIUM-FREE HIGHLY LUMINESCENT SEMICONDUCTOR NANOCRYSTALS FOR IN VIVO FLUORESCENCE IMAGING

Grégory Beaune ${ }^{1}$, Sudarsan Tamang ${ }^{2}$, T. Jean Daou ${ }^{1}$, Liang $\mathrm{Li}^{2}$, Véronique Josserand $^{3}$, Peter Reiss ${ }^{2}$, Isabelle Texier ${ }^{1}$

${ }^{1}$ LETI DTBS, CEA, Grenoble, France, ${ }^{2}$ INAC SPrAM (UMR 5819), CEA, Grenoble, France, ${ }^{3}$ IAB, INSERM U823/UJF, La Tronche, France.
Fluorescence imaging provides a better insight of biological mechanisms in vivo and is an acknowledged tool for drug discovery and development. Optical methods have some advantages in comparison to other existing techniques: low cost, no radioactivity, short acquisition times and high sensitivity. However, their limits lie in the optical properties (diffusion and absorption) of the tissues. Therefore near infrared (650-900 nm) highly fluorescent probes are needed for imaging within a few centimeters deep. The design of high-quality NIR-emitting quantum dots, with outstanding optical properties in comparison to organic dyes, should lead to novel contrast agents with improved performance (higher fluorescence quantum yields and photo-stability). While certain types of cadmium and lead chalcogenide quantum dots show appropriate optical properties for in vivo imaging, they suffer from the low acceptability due to their intrinsic toxicity. Indium phosphide nanocrystals (InP) could be an interesting alternative. Other compositions, such as I-IIIVI2-type chalcopyrites (CuInSe2-CISe, CuInS2 - CIS) are also very promising as semi-conducting core materials for the design of NIR emitting quantum dots. In this communication, new cadmium-free quantum dot compositions will be presented. The obtained samples exhibit size-dependent fluorescence in the near infrared spectral range, with high luminescence quantum yields and excellent photo-stability (Fig. left). At the same time, the core particle diameter is small (3$6 \mathrm{~nm}$ ), which is of crucial importance for their bio-distribution. The chemical coating of these materials is also of tremendous importance. A suitable zwitterionic coating ensures a homogeneous biodistribution of the particles (Fig. middle and right), provided the hydrodynamic diameter of the probes remains below $\approx 15 \mathrm{~nm}$. With these improved compositions and coatings, the use of quantum dots for preclinical studies becomes relevant.
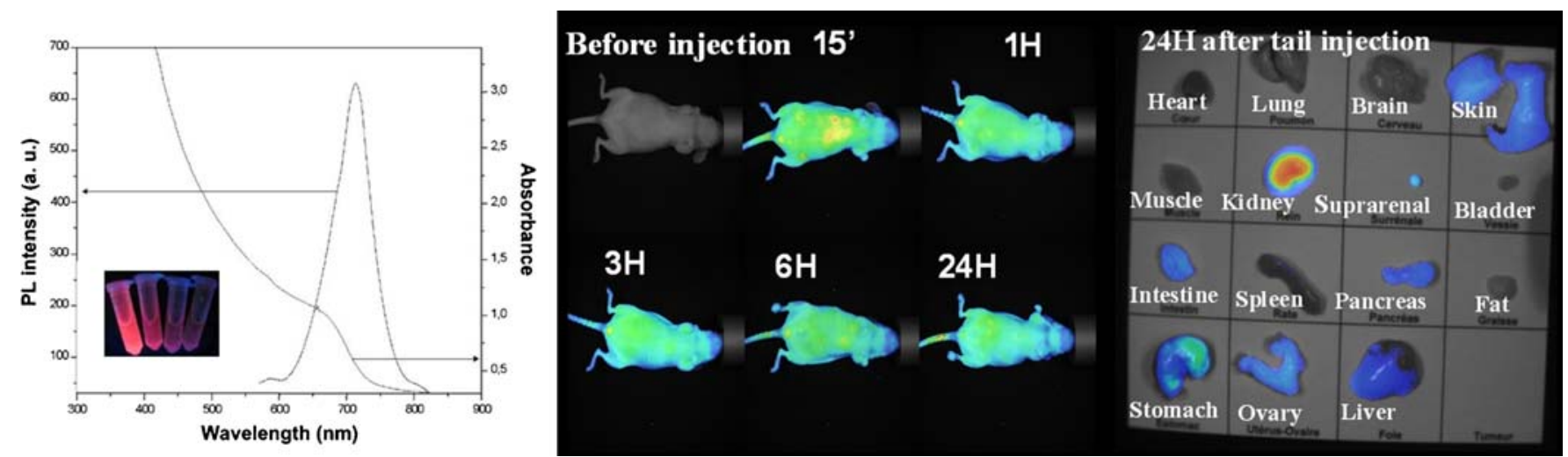

J015

\section{NEW INDOCYANINE GREEN LOADED NANOPARTICLES: A STEP TOWARDS CLINICAL TRANSLATION OF FLUORESCENCE IMAGING TECHNIQUES}

Fabrice P. Navarro ${ }^{1}$, Michel Berger ${ }^{1}$, Stéphanie Guillermet ${ }^{1}$, Véronique Josserand $^{2}$, Laurent Guyon ${ }^{1}$, Mathieu Goutayer ${ }^{1}$, Françoise Vinet ${ }^{1}$, Isabelle Texier $^{1}$

${ }^{1}$ LETI DTBS, CEA, Grenoble, France, ${ }^{2}$ IAB, INSERM U823/UJF, La Tronche, France.

Fluorescence imaging is powerful for in vivo visualization of biological processes, potentially within a few centimeters deep. Nowadays, numerous aqueous soluble near infrared organic dyes are available commercially, but only Indocyanine Green (ICG) has been approved by the FDA for human parenteral administration. There- fore, this organic dye appears as the label of choice for designing fluorescent probes dedicated to human applications. However, its limited aqueous solubility and optical properties, as well as the absence in the dye structure of functional group for covalent coupling to targeting moieties such as antibodies, limit the present potentialities of ICG. ICG encapsulation in nanoparticle-based systems has been explored in order to overcome these limitations. However, the most relevant systems still present several drawbacks, such as important leakage of the dye outside the nanoparticles, and low ICG-loading capacity without fluorescence auto-quenching. In this study, the encapsulation of ICG in new $30 \mathrm{~nm}$ diameter lipid nanoparticles (LNP) is described. ICG-loaded lipid nanoparticles (LNP-ICG) display numerous advantages: the formulation ingredients are cheap lipids and biocompatible surfactants already approved for human use; the manufacturing process is very robust and solvent-free; LNP-ICG display high chemical stability and 
improved optical properties (Fig. left). The biodistribution of ICG in mice when delivered through LNP in comparison to free ICG has been studied in healthy and tumor-bearing animals ( $N=20 /$ group). Efficient accumulation of LNP-ICG in tumor site has been observed (Fig. middle). In the future, even more efficient targeting should be achieved through LNP functionalization. The efficiency of LNP-ICG for lymph node labeling has also been demonstrated (Fig. right). For all these reasons, LNP-ICG, which all ingredients are already separately approved for human use, pave the way for clinical translation to human of new fluorescence imaging techniques.

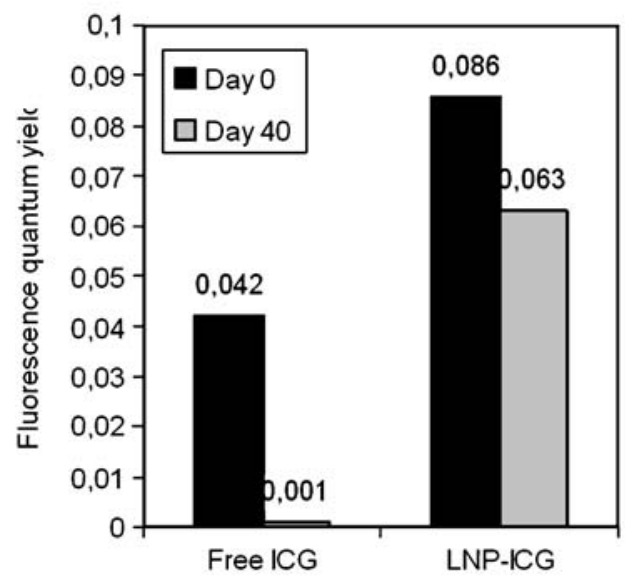

J016

\section{LIPID NANOPARTICLES (LNP): A NEW TECHNOLOGY OF NANOCARGOS FOR DRUG AND CONTRAST AGENT DELIVERY}

Mathieu Goutayer ${ }^{1}$, Sandrine Dufort ${ }^{2}$, Thomas Delmas ${ }^{1}$, Anne-Claude Couffin $^{1}$, Fabrice P. Navarro ${ }^{1}$, Emilie Heinrich ${ }^{1}$, Jean-Luc Coll ${ }^{2}$, Françoise Vinet ${ }^{1}$, Isabelle Texier ${ }^{1}$

${ }^{1}$ LETI DTBS, CEA, Grenoble, France, ${ }^{2}$ INSERM U823/UJF, IAB, La Tronche, France.

Paul Ehrlich introduced in 1904 the "magic bullet" concept, a drug aimed at a disease site and not harmful for healthy tissues. It was the birth of the "drug delivery system". In parallel, the development of molecular imaging pointed out the necessity of highly specific probes for improved image contrast and accurate diagnostic. Both drug delivery and molecular imaging seek for more efficient targeting of drugs and contrast agents in pathological area, especially through the help of nanocargos. The nanometer size (30-200 nm) seems particularly attractive: for instance, passive targeting of nanoparticles to tumor sites is favored by the leaky vasculature of tumors along with the low lymphatic drainage in the tumor interstitium (EPR effect). Among a wide variety of nanocargos, lipid-based particles present several advantages: their ingredients are mostly low cost lipids and biocompatible surfactants, their manufacturing process is versatile and upscalable. Liposomes have been widely studied. However, they are not optimized for the encapsulation of lipophilic molecules and cannot be stored in injection-ready formulations for long duration. A new technology for the encapsulation of lipophilic molecules -both drugs and contrast agents- has been developed. Lipid nanoparticles (LNP, 25$200 \mathrm{~nm}$ diameter) are composed of a lipid core stabilized by phospholipids and PEGylated surfactants (Fig. left). They can incorporate a wide range of lipophilic molecules. The PEG coating ensures the LNP a good in vivo stealthiness, as assessed by the biodistribution recorded using fluorescence imaging. LNP can moreover be functionalized by tumor-targeting ligands, such as the cRGD peptide. cRGDLNP exhibit specific adhesion to $\alpha v \beta 3$ integrins expressing cells in vitro (Fig. middle). In vivo fluorescence imaging shows an improved tumor accumulation of cRGD-LNP, compared to non functionalized LNP (Fig. right). LNP, with ingredients already approved for human use, appear as promising cargos for imaging and drug delivery applications.

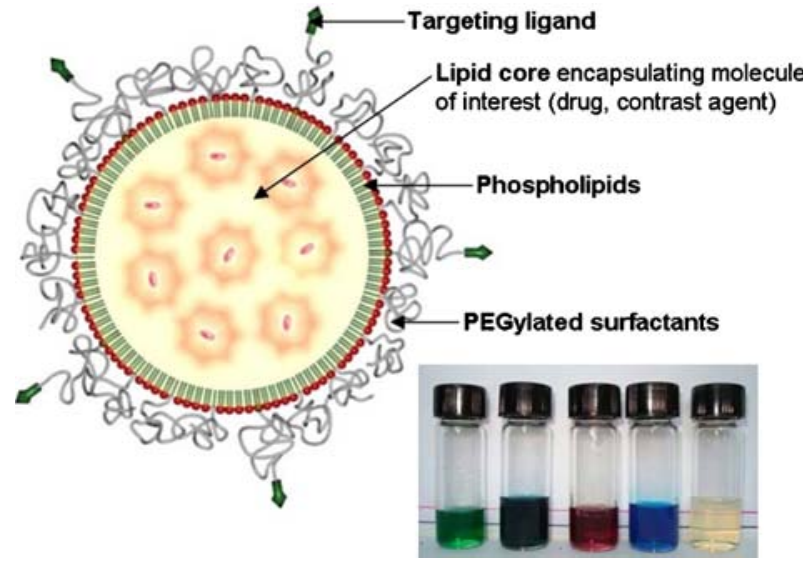

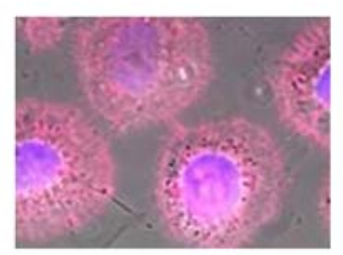

CRGD-LNP

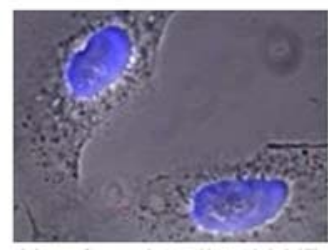

Non-functionalized LNP

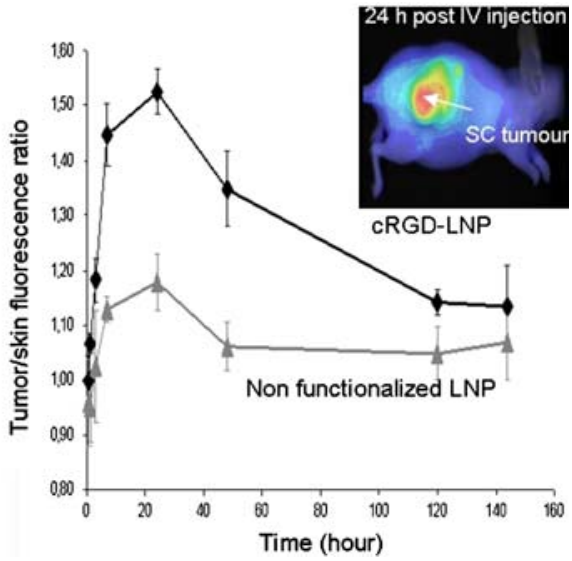


J017

DEVELOPMENT OF STABILIZED GOLD NANOPARTICLES AND NANORODS AS CONTRAST AGENTS USING CHEMICALLY MODIFIED GLYCOL CHITOSAN

Dae Kyung Eun ${ }^{1}$, Eui-Joon $\mathrm{Cha}^{1}$, In-Cheol $\mathrm{Sun}^{2}$, Dong Kee $\mathrm{Yi}^{3}$, Kwangmeyung $\mathrm{Kim}^{2}$, Ick Chan Kwon ${ }^{2}$, Cheol-Hee Ahn ${ }^{1}$

${ }^{1}$ Materials science and engineering, Seoul National University, Seoul, Korea, South, ${ }^{2}$ Biomedical research center, Korea Institute of Science and Technology, Seoul, Korea, South, ${ }^{3}$ Bionanotechnology, Kyungwon University, Seongnam, Korea, South.

Gold nanomaterials such as nanoparticles and nanorods are well-known candidate for biomedical application due to their intrinsic properties. Gold nanomaterials have been widely applied to various medical applications due to distinguished multi functionality. However gold nanoparticles and nanorods are extremely unstable in physiological conditions and form aggregate. For the reason of stability problem, the surface of these gold nanomaterials should be modified with anti-fouling and biocompatible materials. Therefore, a number of strategies for surface coating have been developed and are under development. Glycol chitosan (GC) is one of the excellent candidates to introduce stability to gold nanoparticles and nanorods. It is well-known that chitosan has biocompatibility. However, the usage of chitosan is limited because of its poor water solubility. GC, one of the derivatives of chitosan which has the enhanced water solubility, has been widely used for biological application. In this study, we described strategies for stabilized gold nanoparticles and nanorods using glycol chitosan and verified their potential for biomedical application. Stabilized nanomaterials are prepared using two different methods and obtained successfully. Gold nanoparticles were synthesized by reducing gold chloride using GC and gold nanorods were stabilized by cathecol conjugated GC. Gold nanoparticles were used as CT agents. Attenuation value of GC coated gold nanoparticles is as high as commercially available CT imaging agent. These results show that GC coated gold nanoparticles can be good candidates for CT agents. Gold nanorods were used as photothermal therapeutic agent. Gold nanorods show photothermal effect associated with electromagnetic radiation. This is very useful for disease treatment. Prepared GC coated nanorods show significantly increased stability, compared with non-coated gold nanorods. This result shows that gold nanorods can be potential candidates for photothermal therapeutic agent.
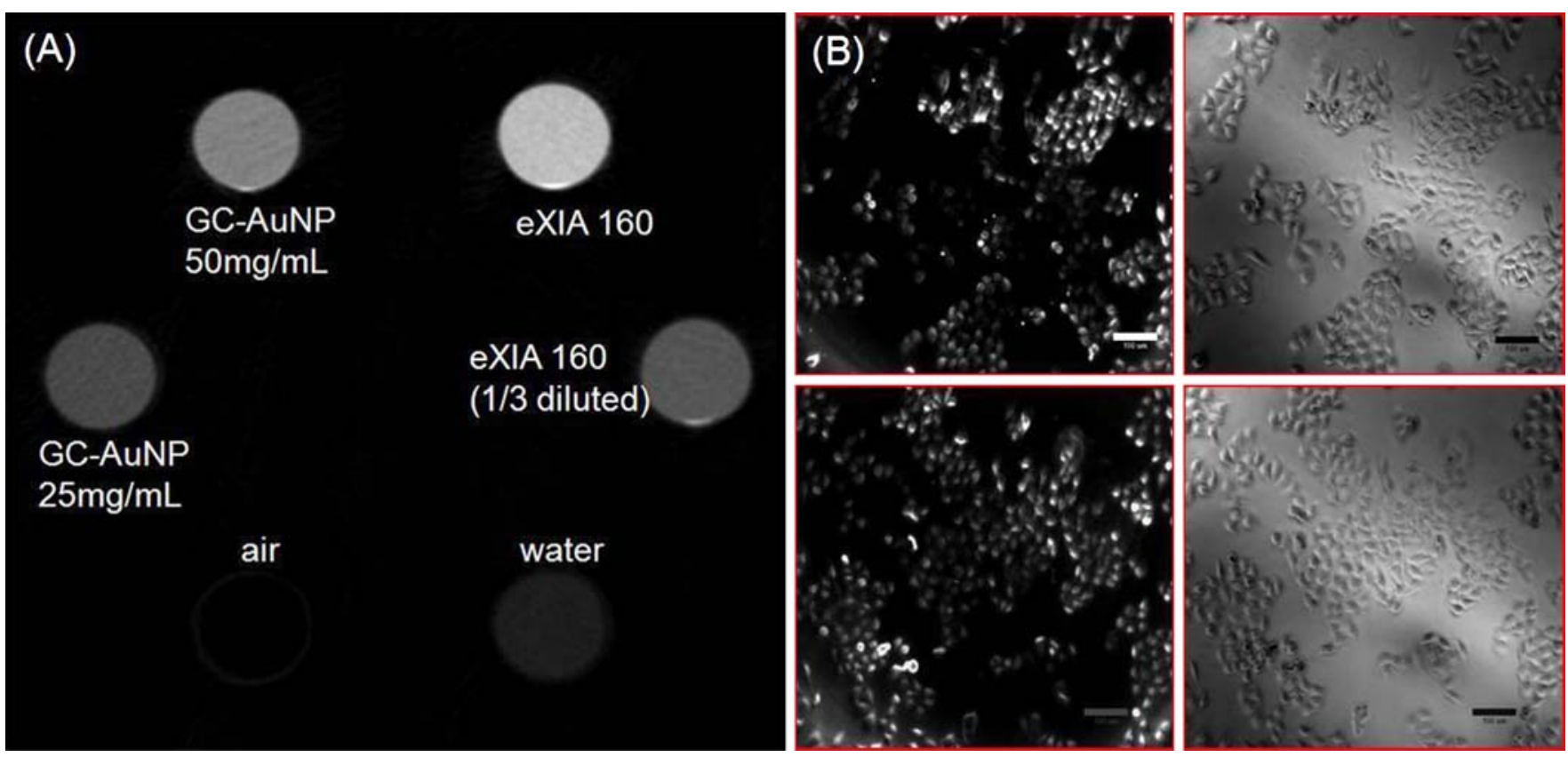

(A) Micro CT images of gold nanoparticles reduced by GC (GC-AuNP), commercially available CT agent (eXIA 160) and negative controls (water and air). (B) Dark (left)/Bright (right) field microscope images of Hela cells after $1 \mathrm{~h}$ (upper images) and $2 \mathrm{~h}$ (lower images) incubation with GCAuNPs. Scale bar: $100 \mu \mathrm{m}$.

J018

\section{MONITORING THE EFFICACY OF B CELL DEPLETION THERAPY BY DUAL SPIO-ENHANCED MR AND NIR CELL TRACKING}

Daniel L. Thorek ${ }^{1}$, Vaishali Arora $^{2}$, Patricia Y. Tsao ${ }^{2}$, Robert Eisenberg $^{2}$, Andrew Tsourkas ${ }^{1}$

${ }^{1}$ Bioengineering, University of Pennsylvania, Philadelphia, PA, USA,

${ }^{2}$ School of Medicine, University of Pennsylvania, Philadelphia, PA, USA.

Targeted depletion of B cells through the use of monoclonal antibodies is an FDA approved therapy for non-Hodgkin's lymphoma. The antibody depletion approach has recently been extended to treat other B cell associated diseases, in particular autoimmune disorders such as systemic lupus erythematosus. Peripheral blood lymphocyte (PBL) counts are used to monitor the pharmacodynamics of treatment both in drug development and clinical applications. Using this approach, depletion is assessed by the degree to which the global B cell population changes as a percentage of total PBL. However, it is probable that systemic measures of the distribution and quantity of B cells in the spleen and other lymphoid organs would more accurately reflect the therapeutic potential and physiological impact of depletion therapy. We have therefore investigated the use of superparamagnetic iron oxide (SPIO) nanoparticles, in conjunction with near infrared (NIR) dyes, to noninvasively track $\mathrm{B}$ cells by magnetic resonance (MR) and in vivo fluorescence imaging in recipient $\mathrm{C} 57 \mathrm{BL} / 6$ mice. Results showed rapid homing of tail vein injected SPIO- and NIR-labeled B cells (20 Mcells) to the spleen. Spleen signal relative to background muscle was reduced by approximately $40 \%$ on $\mathrm{T} 2 *$-w images $24 \mathrm{~h}$ following introduction. The signal recovered as cells were 
cleared from the spleen over the course of 15 days. B cell depleting monoclonal antibodies, anti-CD79 $\alpha / \beta(200 \mu \mathrm{g})$, administered 1 day after injection of exogenous labeled B cells, reduced the global B cell population. Furthermore, the effect on the distribution of SPIO- and NIR-labeled cells in the spleen was detected by MR and fluorescent imaging and verified by immunohistochemistry. This work provides the basis for a means of monitoring the longitudinal cellular dynamics associated with immunotherapeutic agents relevant to autoimmune disease treatment.

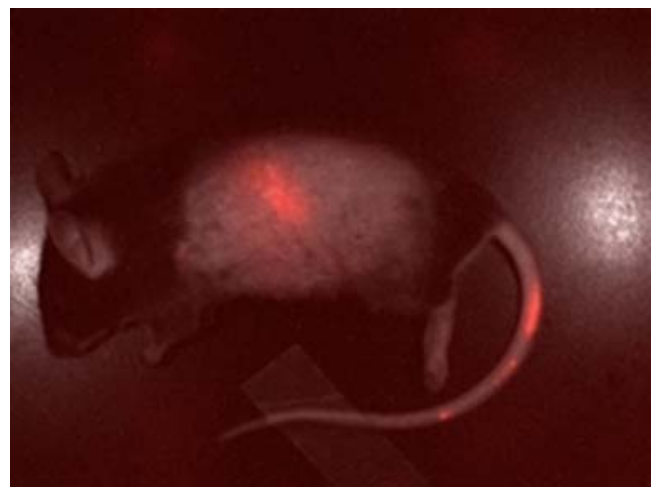

J019

\section{CU-64 LIPOSOMAL LABELING METHODS USING BIFUNCTIONAL CHELATORS (CB-TE2A VS TETA) AND IN VIVO STABILITY}

Jai Woong Seo ${ }^{1}$, Hua Zhang ${ }^{1}$, Lisa M. Mahakian ${ }^{1}$, Claude F. Meares ${ }^{2}$, Riccardo Ferdani $^{3}$, Carolyn Anderson ${ }^{3}$, Katherine Ferrara ${ }^{1}$

${ }^{1}$ Biomedical Engineering, UC Davis, Davis, CA, USA, ${ }^{2}$ Chemistry, UC Davis, Davis, CA, USA, ${ }^{3}$ Radiology, Washington University School of Medicine, St. Louis, MO, USA.
Stable chelation of radio-labeled liposomes is essential for accurate pharmacokinetic modeling. We developed liposomal $\mathrm{Cu}-64$ labeling methods using a 6-BAT lipid to incorporate the chelator during particle formation (1). However, when high concentrations of lipopeptide are incorporated within the particle, the labeling yield decreases. In addition, the in vivo stability of CB-TE2A has been reported to be higher than TETA for labeling small molecules (2). As a result, we have designed a novel liposomal Cu-64 labeling method using CBTE2A, and evaluated the stability of long circulating liposomes each labeled with one of two bifunctional chelators (CB-TE2A-PDEA vs TETA-PDP, figure 1). Both CB-TE2A and TETA bifunctional chelators were first complexed with $\mathrm{Cu}-64$ within $1.5 \mathrm{~h}$ at $90^{\circ} \mathrm{C}$ and $30^{\circ} \mathrm{C}$, respectively. After treatment with TCEP, both activated thiolchelators were incorporated onto liposomes bearing $1 \%$ maleimidePEG2k lipids (Figure 1). The labeling yield exceeded $80 \%$ with both $\mathrm{Cu}-64$ bifunctional chelators. $\mathrm{Cu}-64$-labeled CB-TE2A and TETA liposomes were injected intravenously into FVB mice (two groups, $n=3$ ) and scanned at $0,6,18,30$, and $48 \mathrm{~h}$. Blood pool data from reconstructed images showed a similar clearance of CB-TE2A and TETA labeled liposomes. Distribution of CB-TE2A- and TETA-labeled liposomes at $48 \mathrm{~h}$ was $6.83 \pm 1.67$ and $7.75 \pm 0.93 \% \mathrm{ID} / \mathrm{g}$ in blood, $26.71 \pm 5.26 \%$ and $26.54 \pm 5.48 \%$ in spleen, $19.28 \pm 4.42 \%$ and $19.67 \pm$ $5.29 \%$ in liver, respectively. The results indicate that both CB-TE2A and TETA bifunctional chelators on liposomes have similar stability in blood and could be exploited as useful tools for $\mathrm{Cu}-64$ liposomal labeling methods. (1) Seo, J. W., Zhang, H., Kukis, D. L., Meares, C. F., and Ferrara, K. W. (2008) A novel method to label preformed liposomes with ${ }^{64} \mathrm{Cu}$ for positron emission tomography (PET) imaging. Bioconjug Chem 19, 2577-2584. (2) Boswell, C. A., Sun, X., Niu, W., Weisman, G. R., Wong, E. H., Rheingold, A. L., and Anderson, C. J. (2004) Comparative in vivo stability of copper-64-labeled cross-bridged and conventional tetraazamacrocyclic complexes. J Med Chem 47, $1465-1474$.<smiles>O=C(O)CN1CCN(CCc2ccc(NC(=O)CCCCCN(CC(=O)O)CC(=O)O)cc2)CCN(CC(=O)O)CC1</smiles>

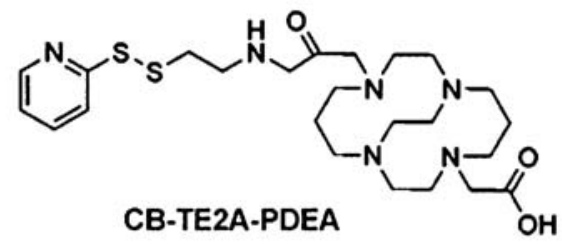

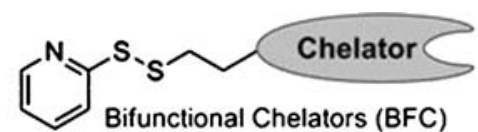

Bifunctional Chelators (BFC)

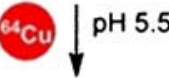

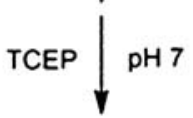

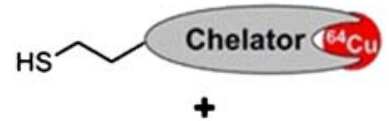

Preformed maleimide liposome

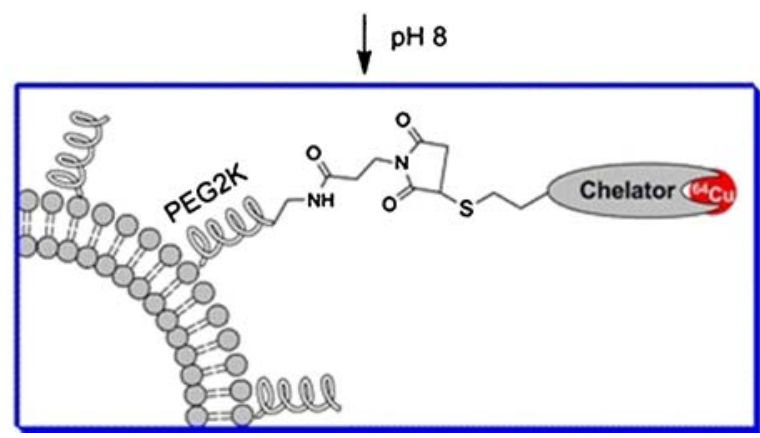

Figure 1. Bifunctional chelators and liposomal Cu-64 labeling method 
J020

\section{FUNCTIONALIZED GOLD NANOPARTICLES AS MRI CONTRAST AGENTS: CHEMICAL AND PHYSICAL CHARACTERIZATION AND IMAGING STUDIES}

Maria Isabel Prata ${ }^{1}$, José A. Martins ${ }^{2}$, Miguel F. Ferreira ${ }^{2}$, Paula Ferreira $^{2}$, Ana C. Santos ${ }^{1}$, André F. Martins ${ }^{3}$, Carlos F. Geraldes ${ }^{3}$ ${ }^{1}$ IBILI, University of Coimbra, Coimbra, Portugal, ${ }^{2}$ Centro de Química, University of Minho, Braga, Portugal, ${ }^{3} \mathrm{CNC}$ and Biochemistry Department, University of Coimbra, Coimbra, Portugal.

The new imaging paradigm Molecular Imaging - the in vivo characterization and measurement of biological processes at cellular and molecular level aims at quantifying molecular changes associated with the onset and development of pathologic states, providing therefore early diagnosis and prognosis of disease. The imaging of cells and cellular and sub-cellular structures, requires imaging agents of high relaxivity density endowed with targeting ability to specific cellular receptors. Nanoparticles, are ideal platforms for the development of molecular imaging and therapeutic agents as they provide for a high density surface clustering of reports and targeting vectors. Gold nanoparticles are especially attractive for imaging and therapy due to their surface plasmon resonance (SPR) enhanced light scattering and absortion, which allows efficient convertion of absorbed light into localized heat. Selective laser photothermal therapy is a promising new cancer therapy. The use of $\omega$-functionalized thiols for stabilizing and functionalize gold nanoparticles is well established due to the stability of the Au-S bond and to the amenable synthesis of bifunctional thiols. Lipoic acid is a convenient moiety for the synthesis of bifunctional $\omega$ funtionalized thiols for the stabilization and functionalization of gold nanoparticles due to its inherant biocompatibility (biological origin), strength of the Au-S bond and easy conjugation chemistry. Metal chelators of the DO3A monoamide type, known to complex $\mathrm{Ln}^{3+}$ ions with high thermodynamic and kinetic stability, were used for complexation of $\mathrm{Gd}^{3+}$ ions as reporters for MR detection and gamma imaging and biodistribution $\left({ }^{153} \mathrm{Sm}^{3+}\right)$ studies. In this work we will report the synthesis and full chemical and physical characterization of the coated gold paramagnetic (complexed with gadolinium) nanoparticles specific functionalized to target the neovasculature. We will present also the results of biodistribution and scintigraphic studies obtained with the ${ }^{153} \mathrm{Sm}$ labelled nanoparticles.

J021

LABELLING HUMAN MONONUCLEAR CELLS WITH SUPERPARAMAGNETIC IRON OXIDE DOES NOT AFFECT CELL VIABILITY AND MIGRATORY CAPACITY: VALIDATION OF A GOOD MANUFACTURING PRACTICE COMPLIANT METHOD

Catherine A. Shaw ${ }^{1}$, Jennifer M. Richards ${ }^{1}$, Kevin Dhaliwal ${ }^{2}$, Anne P. Atkinson $^{3}$, A. J. Simpson ${ }^{2}$, Thomas M. Connolly ${ }^{4}$, Giora Z. Feuerstein ${ }^{4}$, George R. Barclay ${ }^{3,5}$, Marc L. Turner, ${ }^{3,5}$, David E. Newby ${ }^{1}$

${ }^{1}$ Centre for Cardiovascular Science, University of Edinburgh, Edinburgh, United Kingdom, ${ }^{2}$ Centre for Inflammation Research, University of Edinburgh, Edinburgh, United Kingdom, ${ }^{3}$ Scottish National Blood Transfusion Service, Edinburgh, United Kingdom, ${ }^{4}$ Translational Medicine Research Collaboration, Edinburgh, United Kingdom, ${ }^{5}$ Scottish Centre for Regenerative Medicine, University of Edinburgh, Edinburgh, United Kingdom.

Background: Several studies have used superparamagnetic iron oxide (SPIO) as a labelling agent to track inflammatory cells in vivo using magnetic resonance imaging (MRI). However, no such method currently exists for human studies. We have recently established a
Good Manufacturing Practice (GMP) compliant protocol for the successful ex vivo labelling of autologous human peripheral blood mononuclear cells (PBMCs) suitable for re-infusion into the donor. In order to track SPIO labelled cells to inflammatory sites it is essential that they remain viable and maintain their migratory capacity. Here we validate our GMP compliant protocol by investigating the uptake of SPIO by human mononuclear cells and characterising the effect of SPIO loading on cell viability and migratory function. Methods: Human peripheral blood mononuclear cells (PBMCs) were isolated from healthy volunteers and labelled with SPIO (Endorem; $100 \mu \mathrm{g} / \mathrm{mL}$ ) and Protamine sulphate $(4 \mu \mathrm{g} / \mathrm{mL})$ at room temperature for $2 \mathrm{~h}$ on a roller mixer. SPIO up-take by cells was confirmed by Prussian Blue staining of cytospin preparations and quantified by the Ferrozine colorimetric assay for iron. Cell viability was assessed by flow cytometric analysis of annexin $\mathrm{V}$ binding in combination with propidium iodide staining, and by lactate dehydrogenase (LDH) release. A modified Boyden Chamber assay was used to determine the migratory capacity of labelled cells towards the chemokine, monocyte chemoattractant protein 1 (MCP-1). Results: Prussian Blue staining of cytospin preparations and the Ferrozine assay confirmed uptake of SPIO by PBMCs following a $2 \mathrm{~h}$ labelling period. Cell viability was unaffected compared to unlabelled cells up to $72 \mathrm{~h}$ postlabelling. SPIO loading did not affect the in vitro migratory capacity of PBMCs relative to unlabelled cells. Conclusions: We conclude that ex vivo labelling of human mononuclear cells with SPIO does not adversely affect cell viability or migratory function, and that cells labelled in this manner may be used as a method to track cells using MRI in inflammatory conditions in humans. This work was supported by an award from the Translational Medicine Research Collaboration.

\section{J022}

IRON OXIDE MICELLE-ASSEMBLED NANOPARTICLES ("IRONMAN"): A BOTTOM-UP APPROACH TO BUILDING MULTIMODAL IMAGING PROBES

Benjamin Scott ${ }^{1,2}$, Weiguo Cao ${ }^{1,3}$, Ian Corbin ${ }^{1}$, Gang Zheng ${ }^{1,2}$

${ }^{1}$ Division of Biophysics and Bioimaging, Ontario Cancer Institute, Toronto, ON, Canada, ${ }^{2}$ Department of Medical Biophysics, University of Toronto, Toronto, ON, Canada, ${ }^{3}$ Department of Chemistry, Shanghai University, Shanghai, China.

Magnetic iron oxide nanoparticles (IONP's) are used extensively in biomedical research due to the pronounced effect they have on magnetic fields and in turn on MR images. Consequently, an area of intense research is the development of stable, biocompatible IONP's probes possessing targeting moieties. As such, having a reliable strategy in place to develop these stable, biocompatible, targeting nanoprobes is vital to advancing the field and one we are currently pursuing. Aside from the desire for straight forward production, a key factor in building suitable probes is the overall particle size and distribution. It is well known that in order for IONP's to be useful in a range of technological and biological applications, a small size $(<20 \mathrm{~nm})$ and narrow particle distribution is necessary [1]. We have developed a novel bottom-up approach in which oleyl stabilised, 'ultrasmall' ( $\sim 4 \mathrm{~nm})$, monodispersed IONP's are encapsulated within a phospholipid monolayer coating. The resulting 'micelle-assembly' provides the desired stability and biocompatibility sought, without jeopardising nanoparticle size or distribution (particles increase to $\sim 15 \mathrm{~nm}$ in size with the phospholipid coating). Furthermore, through subtle modifications to the phospholipid head groups, an azide functionality has been successfully introduced. This modification has allowed for "click" chemistry to be utilised to conjugate a targeting peptide sequence (containing the complementary ethynyl site) to the surface. This is a novel approach toward the synthesis of targeting 
nanoparticles and having such a reliable "clickable" precursor provides a platform from which a library of multimodal, targeting devices can be built. In addition, a fluorescent marker has been appended to the peptide moiety allowing for the conjugation reaction to be easily monitored, as well as provide an additional level of tumour detection to the device. The design, synthesis, conjugation and stability studies of this novel "IronMAN" probe are reported. [1] Sun et al. J. Am. Chem. Soc, 2002, 124, 8204

\section{J023}

\section{RAMAN ENCODED TARGETING FLUORESCENT GOLD} NANOPARTICLES FOR CANCER IMAGING

Benjamin Scott ${ }^{1,2}$, Patrick Z. McVeigh ${ }^{1,2}$, Gilbert Walker ${ }^{3}$, Brian Wilson $^{1,2}$, Gang Zheng ${ }^{1,2}$

${ }^{1}$ Division of Biophysics and Bioimaging, Ontario Cancer Institute, Toronto, ON, Canada, ${ }^{2}$ Department of Medical Biophysics, University of Toronto, Toronto, ON, Canada, ${ }^{3}$ Department of Chemistry, University of Toronto, Toronto, ON, Canada.

The implementation of gold nanoparticles for cancer detection is an extremely valuable tool in biomedical imaging and an area we are currently investigating. A Raman active dye (crystal violet) has been successfully anchored to the surface of $60 \mathrm{~nm}$ gold nanoparticles, imparting surface-enhanced Raman scattering (SERS) properties. Subsequent PEG coating of this metal-dye complex markedly improves the biocompatibility making it suitable for in vivo analysis. Furthermore, PEG ligands with azide functional groups have been utilised, peptide ligands possessing the complementary ethynyl functionality can be effectively "clicked" to the surface of the gold nanoparticles allowing for the development of potentially targeted diagnostic devices. Additionally, a fluorophore has been appended to the peptide sequence allowing the nanoparticles to be monitored via optical imaging in parallel with Raman monitoring. The resultant 'dual' properties of these metal based nanoparticles can work in concert to enhance tumour detection. Moreover, with the effective "click" conjugation strategy employed, a wide range of "clickable" targeting ligands can be appended to the surface allowing for a library of probes to be developed. What is also worth noting is that subtle modifications to the stabilising ligands could allow for large concentrations of gold to congregate in a specific targeted area, which could potentially lead to the use of gold nanoparticles as an effective CT contrast agent. The design and synthesis of the targeting nanoparticles and subsequent targeting studies are reported.

\section{J024}

\section{LANTHANIDE-DOPED UPCONVERTING NANO-PHOSPHORS FOR NIR LIVE CELL IMAGING \\ Jiefu Jin, Wingtak Wong}

Department of Chemistry, The University of Hong Kong, Hong Kong, China.

Live cell fluorescent imaging has aroused growing attention due to its essential application in visualizing cellular content, such as bioregulating cations, vital small molecules, and specific organelles. NIR bio-imaging technique is an emerging non-invasive imaging modality characteristic by high contrast and deep penetration. ${ }^{1}$ However, inherent drawbacks of traditional NIR dyes, such as poor photostability of organic fluophors and severe cytotoxicity of quantum dots, largely limit their functionality as powerful biological probes. ${ }^{2,3}$ Therefore we are recently engaged in the development of novel versatile lanthanide-based upconverting nano-phosphors for achieving NIR bio-imaging. The core materials are $\mathrm{NaYF}_{4}$ mono-dispersed nanocrystals doped by various lanthanide metal ions synthesized via surfactant-mediated hydrothermal approach with controlled morphology, uniform size, desired phases and composition (Fig.1). Delicate modification is carried out on the nanoparticle surface to induce watersolubility and active targeting. Typical up-conversion photo-properties of as-synthesized nanocrystals are observed in both solid-state and water solutions with high quantum efficiency. Cell viability assay preformed on nano-phosphors shows no apparent cytotoxicity towards HeLa cell line, even at extremely high concentration elevating to $200 \mu \mathrm{g} / \mathrm{ml}$. Moreover, cellular localization of nano-phosphors are investigated by confocal microscopy equipped with Ti:Sapphire laser. We believe this work should be pioneered to the systematic synthesis of bio-functional lanthanide-doped nanocrystals and their development into luminescent probes for NIR live cell imaging. References 1. Frangioni, J. V. Curr. Opin. Chem. Biol.2003, 7, 626. 2. Hardman, R. Environ. Health Perspect.2006, 114, 165. 3. Derfus,M.; Chan, W. C. W.; Bhatia, S. N. Nano Lett.2004, 4, 11.

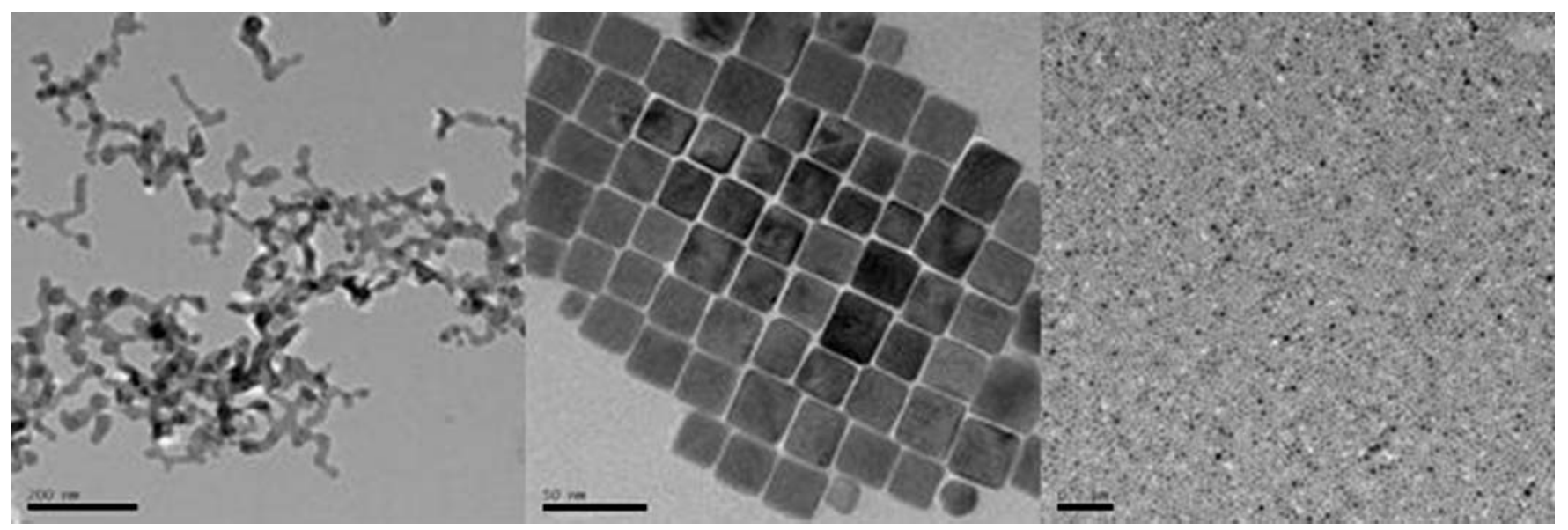

Figure 1. TEM images of cubic phase lanthanide ion doped $\mathrm{NaYF}_{4}$ nanocrystals with different morphologies. Left: branched-structure nanoparticles; Middle: nanocubes; Right: spherical nanoparticles. 
J025

PREPARATION OF FLUORESCENT NANODIAMOND STABLY DISPERSED UNDER PHYSIOLOGICAL ENVIRONMENT AND ITS APPLICATION TO CELLULAR IMAGING

Naoki Komatsu $^{1}$, Tatsuya Takimoto ${ }^{1}$, Sawako Shimizu ${ }^{2,1}$, Masahito Morita $^{3,4}$, Takahide Kimura ${ }^{1}$, Tokuhiro Chano ${ }^{2}$, Toshiro Inubushi ${ }^{3}$

${ }^{1}$ Chemistry, Shiga University of Medical Science, Otsu, Japan, ${ }^{2}$ Clinical Laboratory Medicine, Shiga University of Medical Science, Otsu, Japan, ${ }^{3}$ Biomedical MR Science Center, Shiga University of Medical Science, Otsu, Japan, ${ }^{4}$ Immunology Frontier Research Center, Osaka University, Suita, Japan.

Although quantum dots and carbon nanotubes have been developed as promising fluorescent probes in modern biotechnology, they always have considerable concerns about cytotoxicity. Herium- or proton-implanted nanodiamond (ND) powder, however, has been reported to be successfully employed as a fluorescent probe with no photobleaching and low cytotoxicity. This clearly shows the potential of ND powder as a molecular imaging agent. In continuation of our effort to develop an ND-based imaging probe, we found that a fluorescent tag (fluorescein) was covalently bound to the surface of ND through a long alkyl chain and the resulting fluorescent $\mathrm{ND}$ (f-ND) was successfully introduced into cell and observed by fluorescent microscopy. At the first attempt, fluorescein was intended to be immobilized onto the aminated ND (ND-NH2) prepared by the reported method. However, almost no luminescence was observed in a fluorescent image. These results clearly indicate the failure in immobilization of the fluorescent tag on the ND-NH2. Therefore, we changed the strategy to introduce the fluorescent tag through long alkyl chain covalently bound on the ND surface. For attaching the linker, we used a very reliable radical reaction found by Tsubota; the hydrogenated ND having $30 \mathrm{~nm}$ average size was functionalized with BOC (tert-butoxycarbonyl)-protected aminododecanoic acid in the presence of benzoyl peroxide. After deprotection under acidic conditions, the amine was allowed to react with succinimidyl ester of fluorescein to give f-ND. Importantly, the f-ND prepared via BOC-protected aminoacid is found to be much brighter than those prepared through direct functionalization with aminododecanoic acid without any protection. The uptake of the f-ND dispersed under physiological environment was achieved using HVJ vector, which was confirmed by fluorescent images as shown in the Figure.

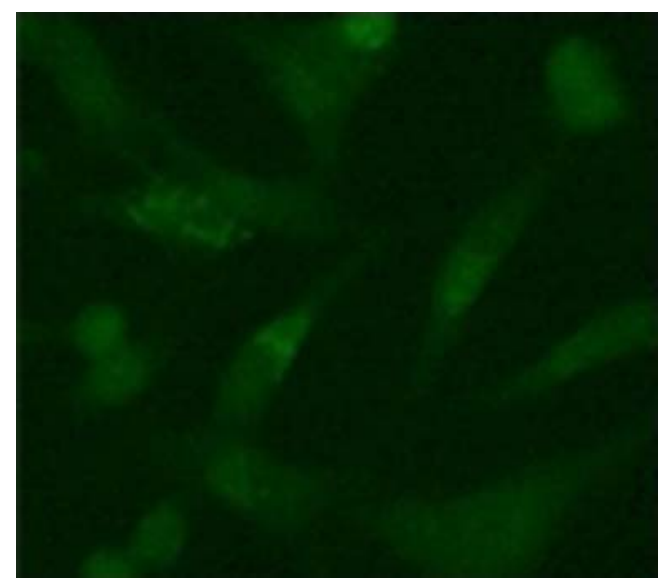

J026

MAGNETIC RESONANCE IMAGING OF ATHEROSCLEROTIC LESIONS USING TARGETED IRON OXIDE NANOPARTICLES WITH HUMAN ANTIBODY AGAINST OXIDIZED LDL

Yuyu Yao $^{1}$, Rui Zhao ${ }^{2}$, Yu Zhang ${ }^{3}$, Naifeng Liu' ${ }^{1}$, Gaojun Teng ${ }^{2}$ ${ }^{\top}$ The Department of Cardiology, Nanjing, China, ${ }^{2}$ Laboratory of Molecular Imaging, Department of Radiology, Nanjing, China, ${ }^{3}$ Laboratory of Molecular and Biomolecular Electronics, Nanjing, China.
Background: Oxidized low-density lipoprotein (OxLDL) is a major component of the plaque lipid pool and contributes significantly to the sudden rupture of vulnerable plaques. This study was synthesize, characterize and test the efficacy of superparamagnetic iron oxide nanoparticles labeled antibodies against OxLDL (OxLDL -SPIO) may detect of plaque vulnerability in apoE-/- mice. Methods and Results: We have developed the monoclone antibody against OxLDL, which immunostain advanced human atherosclerotic lesions with a distinct pattern, suggesting predilection for the necrotic core, the anti- OxLDL SPIO was prepared by grafting anti-human OxLDL antibody on the surface of DMSA-coated maghemite nanoparticles using the linker of EDC (1-ethyl-3-[3-dimethylaminopropyl] carbodimide) hydrochloride. The conjunction amount of antibodies and the activity of OxLDL SPIO were evaluated by enzyme linked immunosorbent assay (ELISA) and immunofluorescence. THP-1 macrophages were incubated with OxLDL $(30 \mu \mathrm{g} / \mathrm{ml}$ for $72 \mathrm{~h})$ and resulted in foam cell formation, incubated with these labeled antibodies and the $\mathrm{T} 2 *$ relaxivity of centrifuged pellets of these cells was determined by using an inversion recovery sequence on a $1.5 \mathrm{~T}$ scanner. Measured T2* relaxation times of the cells incubated the anti- OxLDL -SPIO ( $402 \pm 22 \mathrm{~ms}$ ) was significantly different from the $\mathrm{T} 2 *$ relaxivity obtained from cells with untargeted-SPIO ( $560 \pm 37 \mathrm{~ms}$ ). MR imaging was performed on 18 monthes apoE-/- mice at 7.0T prior to and 1, $24 \mathrm{~h}$ after injection of $1 \mathrm{mg} \mathrm{Fe} / \mathrm{kg}$ dose. Images were obtained using conventional SE sequences and FLASH T1 sequences. Significant modulation of the MR signal was observed with anti- OxLDL -SPIO (16.3 \pm 1.6 ,vs $9.4 \pm 4$, $P<0.01)$, minor MR signal modulation was observed following administration of untargeted-SPIO $(16.0 \pm 4$, vs $11.7 \pm 2.2, P>0.05)$. Aortas were excised and stained for iron. Histology showed iron deposition within intraplaque macrophage after OxLDL -SPIO administration, histomorphologic studies was correlated with the MR imaging. Conclusion: OxLDL -targeted SPIO may allow detection of atherosclerotic lesions.

\section{J027}

\section{GD-ENCAPSULATED POROUS POLYMERSOMES AS HIGHLY EFFICIENT MRI CONTRAST AGENTS} Zhiliang Cheng, Daniel L. Thorek, Andrew Tsourkas

Department of Bioengineering, University of Pennsylvania, Philadelphia, PA, USA.

The use of Gd-encapsulated nanovesicles as MR contrast agents has largely been ignored due to the detrimental effects of the slow water exchange rate through the vesicle bilayer on the relaxivity of encapsulated Gd. Here, we describe the facile synthesis of porous polymer nanovesicles that exhibit improved permeability to water flux, high structural stability, and a large capacity to store chelated Gd within the aqueous core. The porous polymersomes, $130 \mathrm{~nm}$ in diameter, were produced through the aqueous assembly of the polymers, $\mathrm{PEO}(1300)$-b$\operatorname{PBD}(2500)$ (PBdEO) and PEO(2000)-b-PCL(2700). Subsequent hydrolysis of the caprolactone (PCL) block resulted in a highly permeable outer membrane. To prevent the leakage of small Gd-chelate through the pores, Gd was conjugated to PAMAM dendrimer via DTPA dianhydride prior to encapsulation. As a result of the slower rotational correlation time of Gd-labeled dendrimers, the porous outer membrane of the nanovesicle, and the high Gd payload, the nanovesicles were found to exhibit a relaxivity (R1) of 292,109 mM-1s-1 per particle. The polymersomes were also found to exhibit low cellular toxicity and a long circulation half-life. Here, the design, assembly, characterization and application of the paramagnetic polymersomes will be presented. 


\section{J028}

PREPARATION OF MANNAN-COATED SUPERPARAMAGNETIC IRON OXIDE NANOPARTICLES FOR LYMPH NODE TARGETING

In-Kyu Park $^{1,4}$, Sangjoon Lee ${ }^{1,6}$, Jin-Sook Kwon ${ }^{2}$, Yong Yeon Jeong ${ }^{3,4}$, Mi-Kyong Yoo ${ }^{5}$, Chong-Su Cho ${ }^{5}$

${ }^{1}$ Biomedical Sciences, Chonnam National University Medical School, Gwangju, Korea, South, ${ }^{2}$ Heart Research Center, Chonnam National University Hospital, Gwangju, Korea, South, ${ }^{3}$ Department of Radiology, Chonnam National University Medical School, Gwangju, Korea, South, ${ }^{4}$ The Brain Korea 21 Project, Centre for Biomedical Human Resources at Chonnam National University, Chonnam National University Medical School, Gwangju, Korea, South, ${ }^{5}$ Department of Agricultural Biotechnology, Seoul National University, Seoul, Korea, South, ${ }^{6}$ Bio Imaging Research Center, Gwangju Institute of Science and Technology, Gwangju, Korea, South.

The functionalized superparamagnetic iron oxide nanoparticles (SPIONs) have been used as magnetic resonance imaging agents, site-specific releasing agents, labeling and sorting agents of the cells, and separation agents because they are superparamagnetic, non-toxic, and biocompatible. However, the SPIONs have still several problems such as aggregation and non-specific intracellular uptake in vivo. Amphiphilic polymers with targeting moieties were simply coated to the SPION surface to prevent the particles from aggregation and to enhance their specific cellular targeting. Mannan as a specific ligand can be recognized by the macrophages and dendritic cells through the specific interaction. Mannan as a major structural component of some yeasts are mainly composed of Dmannose. It was alreadly reported that the mannan of Saccharomyces cereviae (S. cereviae) is recognized by mannose receptors on macrophages. Lymph nodes are components of the lymphatic system. The lymph nodes filter the lymphatic fluid and store special cells that can capture cancer cells or bacteria that are traveling through the body in the lymph fluid. Also, the lymph nodes are very critical for the immune systems and principal places where many immune reactions are initiated. The easy detection of lymph node metastases in patients is very important because various cancer diseases are involved in the lymph node metastases. In this study, we aimed to prepare SPIONs for lymph node targeting by simple coating of mannan to the SPION surface because the mannan is amphiphilic natural polymer and it is recognized by mannose receptors on macrophages, It can be expected that capacities to be taken up by the macrophages within the lymph node will be applicable specifically for the diagnosis of lymph node metastases without agglomeration in vivo. Mannan-coated SPIONs (mannan-SPIONs) were characterized by TEM, ELS, XRD and FT-IR.
The cellular uptake of the mannan-SPIONs was assessed by FACS and their macrophage-targeted delivery was also investigated through in vivo lymph node MR imaging.

\section{J029}

\section{IN VIVO REAL-TIME, MULTICOLOR, OPTICAL LYMPHATIC IMAGING USING QUANTUM-DOTS}

Nobuyuki Kosaka, Mikako Ogawa, Noriko Sato, Peter Choyke, Hisataka Kobayashi; Molecular Imaging Program, National Cancer Institute / NIH, Bethesda, MD, USA.

Purpose: The lymphatic network is complex and difficult to visualize in real-time. Since each lymph node can receive lymphatic fluid from multiple afferent lymphatic channels, simultaneous visualization of each lymphatic drainage pattern is needed to map the lymphatic network. Optical imaging is unique in that each fluorophore emits a unique spectral wavelength thus permitting simultaneous depiction of multiple lymphatics at the same time. However, spectral imaging usually requires lengthy acquisitions and post processing to unmix background autofluorescence from target signal and this prevents real-time display. Herein, we describe real-time in vivo multicolor optical lymphatic imaging using cadmium-selenium-based quantum-dots (Qdots) that enable the simultaneous visualization of up to 5 distinct lymphatic basins in real-time without the need for complex camera systems. Materials and Methods: Five visible-light Qdots (carboxyl-Qdot 545, 565, 585, 605, and 655) were injected intradermally in five different sites in anesthetized mice, and in vivo real-time and spectral fluorescence multicolor imaging of the lymphatics were performed. After obtaining in vivo, in situ, and ex vivo lymphatic images, histology was performed on all lymph nodes. Results: In all 7 mice, in vivo lymphatic images successfully resolved all 5 lymphatic basins with different colors in real-time even through the skin. Moreover, multicolor optical imaging clearly demonstrated the lymph node that received drainage from two different afferent lymphatics without image processing. Longitudinal in vivo experiments showed that the enhancement persisted for at least 7 days. Immunohistochemistry analysis revealed that the Qdots were mainly taken up by macrophages/dendritic cells in the lymphatic sinuses, while the lymphoid follicles and lymphatic cords were devoid of Qdots. Conclusion: This method has potential for studying the anatomy and flow within the lymphatic system without complex camera systems or image processing. Moreover, this real time multicolor optical lymphatic imaging can potentially facilitate surgical or endoscopic resection of sentinel lymph nodes and map out the lymphatic drainage of particular body regions in real time using direct visualization assuming that such agents are non-toxic.

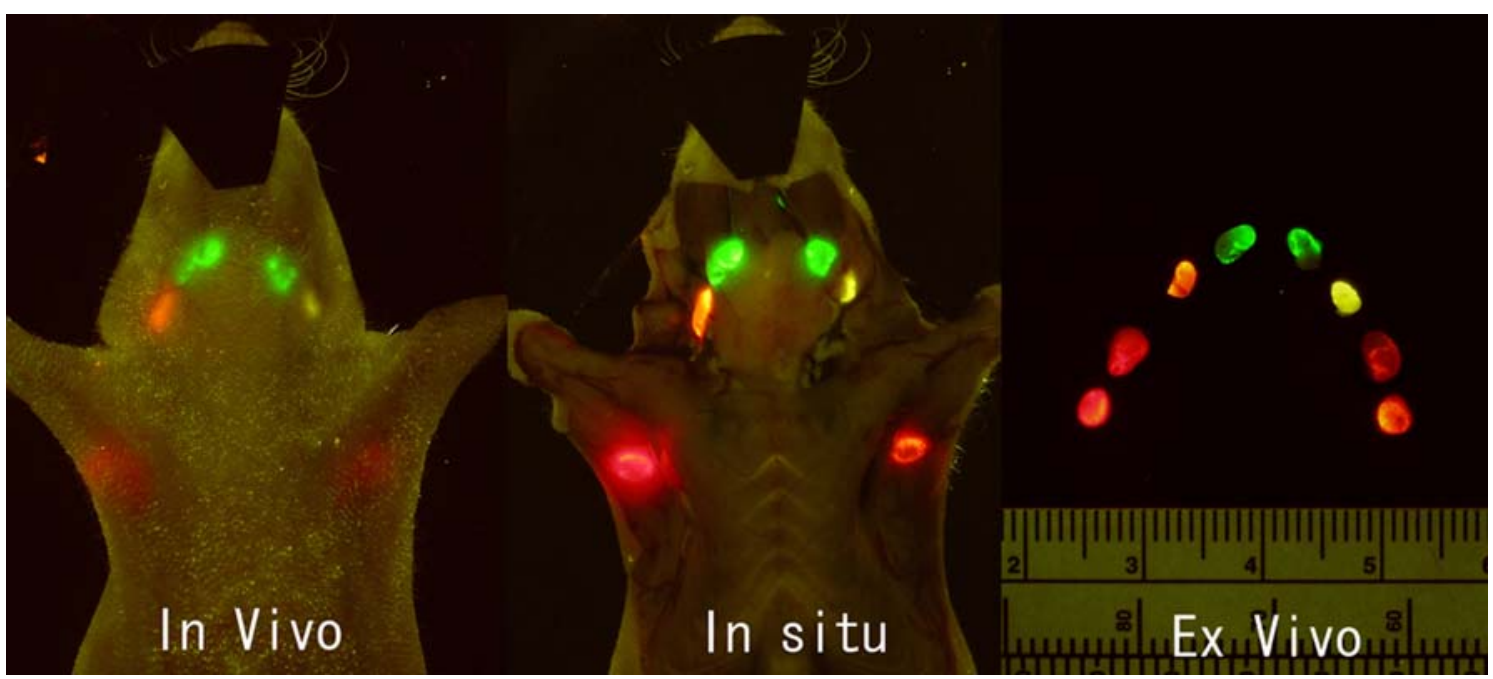


J030

THE EXPERIMENTAL STUDY OF LYMPH NODE IMAGE WITH ULTRASMALL SUPERPARAMAGNETIC IRON OXIDE: COMPARISON WITH PATHOLOGICAL RESULT

Hua dan Xue, Jing Lei, Zhengyu Jin

Department of Radiology, Peking Union Medical College Hospital, Beijing, China.

Objective:To assess USPIO enhanced Magnetic Resonance image characteristics of lymph nodes on lymphadenitis and metastatic animal models, and to explore its relevance with microstructural findings. Methods: $36 \mathrm{New}$-Zealand white rabbits were randomly divided into two groups. Complete Freund's adjuvant and VX2 cell suspension were injected subcutaneously in the sighs to set up lymphadenitis and metastatic lymph node model respectively. MR scan were performed before and $24 \mathrm{~h}$ after USPIO (90umolFe/kg, Guerbet) injection. iso-layer HE staining,
Prussian blue staining and electronic microscope were performed for investigation of pathological microstructure changes and iron particle distributions in lymph node. Relationship between lymph nodes USPIO enhancement and its microstructures were further analyzed. Results:36 lymph nodes in lymphadenitis group showed different types of reactive hyperplasia. 32 nodes showed a ring-like USPIO enhancement in the medulla region, and pathological methods indicated USPIO particles located mainly inside the macrophages at inflammatory lymphatic medulla other than the paracortical and cortical areas,where are much less macrophages inside. 26 lymph nodes in metastatic group were invaded by tumor cells. Four with completed structrual destruction showed a homogenous no enhancement character; 19 lymph nodes with partially structrual destruction showed heterogenous enhancement, and there were 3 lymph nodes with sub-capsular focal metastasis showed ring-like enhancment similarly to inflammatory ones. Conclusion: USPIO enhancement pattern of different lymph nodes is closely related to distribution and functional status of the intra-node macrophages. Thus may affect accuracy of the lymph node property diagnosis based on USPIO enhanced image.

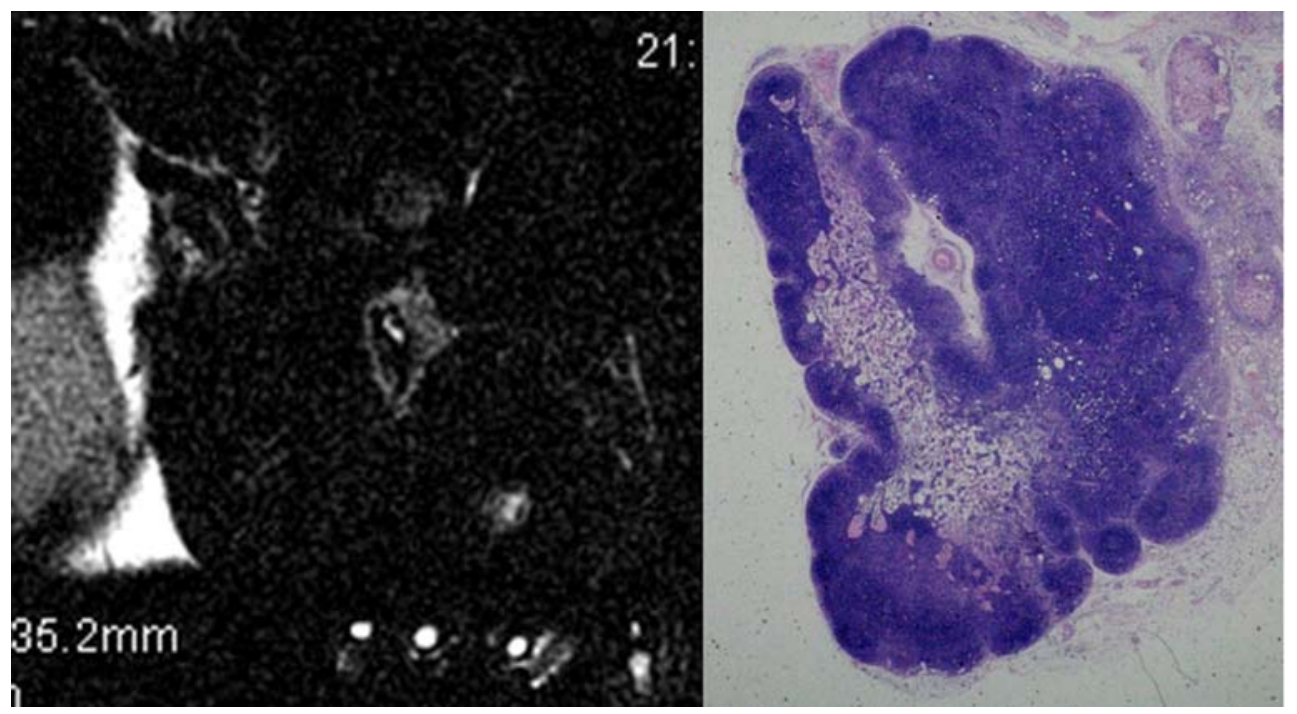

Lymph node was ring-like enhanced after USPIO injection, also noted a high signal dot at the center (left). Compared with HE result (right), overt enhanced area correlated with lymphatic medulla and peripheral area correlated with the paracortical and cortical area. Center high signal dot was vascular structures at the hilum.

J031

\section{VISUALIZATION OF DRUG ACCUMULATION \\ AND RELEASE IN A DISSEMINATED MOUSE TUMOR MODEL USING MULTIMODAL THERMO-SENSITIVE POLYMER-MODIFIED LIPOSOME}

$\underline{\text { Daisuke Kokuryo }^{1}}{ }^{,}$Seiji Nakashima ${ }^{2}$, Kenji Kono $^{2}$, Iwao Kanno ${ }^{1}$, Ichio Aoki ${ }^{1}$

${ }^{1}$ Biophysics Group, Molecular Imaging Center, National Institute of Radiological Sciences, Chiba, Japan, ${ }^{2}$ Graduate School of Engineering, Osaka Prefecture University, Sakai, Japan.

Introduction: The purpose of this study is to visualize drug delivery in deep tissue using a multimodal thermo-sensitive 'polymer-modified' liposome (MTPL). The temperature-sensitive liposome containing doxorubicin (DOX) has previously been developed to increase the treatment efficacy in the heated area with minimum side-effects. In our previous work, a polymer-modified liposome containing $\mathrm{MnSO}_{4}$, rhodamine and DOX allowed more accurate thermo-triggered drug-release in the subcutaneous tumor model (Kono K: Bioconjug Chem., 2005, Aoki I: WMIC 2008). As the next step, the MTPL was applied to an advance-stage model consisting of disseminated/metastatic tumor in deeper tissue. In this paper, we observed the dynamics of MTPL accumulation and visualized the drug release using both MRI and optical imaging. Methods: Our MTPL was composed of EYPC/DOPE/Cholesterol/PEG2000-PE/Et-EO87-OD/Rhodamine$\mathrm{PE}\left(23.4 / 15 / 54.6 / 2 / 4 / 1 \mathrm{~mol} \%\right.$ ) enclosing $\mathrm{MnSO}_{4}(300 \mathrm{mM})$ and DOX. The structure of the MTPL changed from hydrophilic to hydrophobic at around $42.0^{\circ} \mathrm{C}$. For ex vivo and in vivo experiments, Colon- 26 cancer cells $(1.0 \times$ $10^{6}$ cells) were intraperioneally transplanted into nude female BALB/c mice. In order to observe the MTPL accumulation after IV administration, ex vivo optical images were obtained using an IVIS-Lumina System (Caliper Life Science, CA). The model mice were sacrificed 4,8 or $16 \mathrm{~h}$ after the IV administration ( $0.2 \mathrm{ml}$ per mouse), and the liver, kidney and tumors were extracted for optical imaging measurement. The kinetics and 
drug-release of the MTPL was visualized by acquiring $\mathrm{T}_{1}$-weighted MR images before and after heating using a 7.0 Tesla horizontal animal MRI (Magnet: Kobelco + JASTEC, Japan, Console: Bruker Biospin, Germany) with a $35 \mathrm{~mm}$ diameter volume coil (RAPID biomedical $\mathrm{GmbH}$, Germany). The MTPL ( $0.3 \mathrm{ml}$ per mouse) was intravenously administered, and the body was heated to a rectal temperature of around $42.0^{\circ} \mathrm{C}$ for $3-$ 5 min. Results and discussion: The fluorescence intensity at 8-hours after administration in the tumor area was higher than that at 4-hours. On the other hand, the signal intensity in the liver at 8-hours decreased in comparison with the intensity at 4-hours. Therefore, applying the heatingtrigger at 8-hours was more suitable for minimising side-effects. An alteration of MR signal intensity in the tumor and kidney was observed after heating. Kinetics and drug-release of the MTPL were visualized and assessed in deeper tissue using both MRI and optical imaging, although the anticancer benefit has not yet been thoroughly evaluated.

\section{$\mathbf{J 0 3 2}$}

\section{BOMBESIN-TAGGED POLYMERIC NANOPARTICLES FOR PROSTATE CANCER IMAGING IN VIVO}

Chang-Moon Lee, Hwan-Jeong Jeong, Eun-Mi Kim, Su-Jin Cheong, Dong Wook Kim, Seok Tae Lim, Myung-Hee Sohn

Department of Nuclear Medicine, Chonbuk National University Medical School, Jeonju, Korea, South.
Overexpression of gastrin-releasing peptide receptor (GRPR) has been discovered primary in androgen-independent human prostate tissues, thus, provides a potential target for prostate cancer diagnosis and therapy. The GRPR-targeted peptide (bombesin, BBN)-tagged magneto-polymeric nanoparticles were prepared for prostate cancer imaging in vivo. We evaluated the binding characteristics of $\mathrm{BBN}$ tagged magneto-polymeric nanoparticles to prostate cancer cells, PC3 in vitro. Further, we progress to in vivo cancer imaging in a prostate cancer mouse model. Hydrophobically-modified glycol chitosan was synthesized to form self-assembled nanoparticles. BBN was coupled to the polymer using a bifunctional linker and the near-infrared (NIR) fluorophore, Cy5.5 was labeled on the nanoparticles. Iron oxide nanoprticles were loaded into the core of the polymeric nanoparticles. In cell binding studies, BBN-tagged nanoparticles were mostly located in the membranes of PC3 cells. We confirmed that BBN-tagged magneto-polymeric nanoparticles selectively bind to $\mathrm{PC} 3$ cells through a competition binding assay. In vivo optical imaging showed that $\mathrm{BBN}$-tagged polymeric nanoparticles accumulate into tumor lesions with 5.5 of tumor to normal muscle ratio. Comparing to the accumulation of non-tagged nanoparticles to tumor lesions, the tumor uptake of BBN-tagged nanoparticles was higher three times. In conclusion, BBN-tagged magneto-polymeric nanoprticles may be potential as a dual probe for prostate cancer imaging, and may be employed as a therapeutic carrier to deliver an anticancer drug.

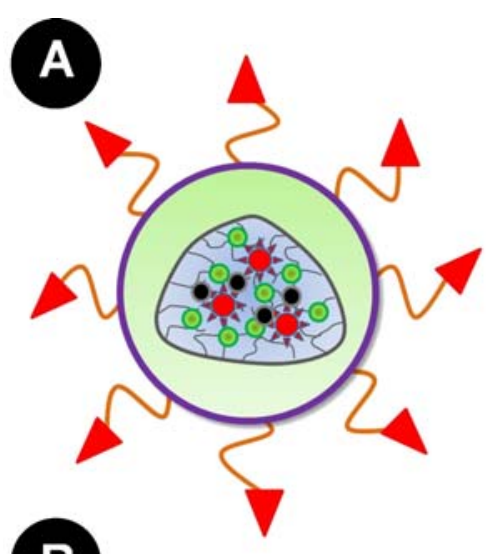

B

J $\mathcal{L}_{\text {Glycol chitosan chain }}$

N-acetyl histidine

Bombesin

Cy5.5

Iron oxide nanoparticle

Polymeric nanopaticles

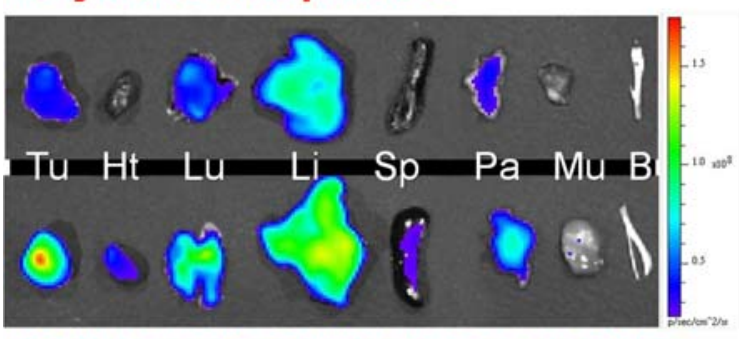

BBN-tagged polymeric nanopaticles

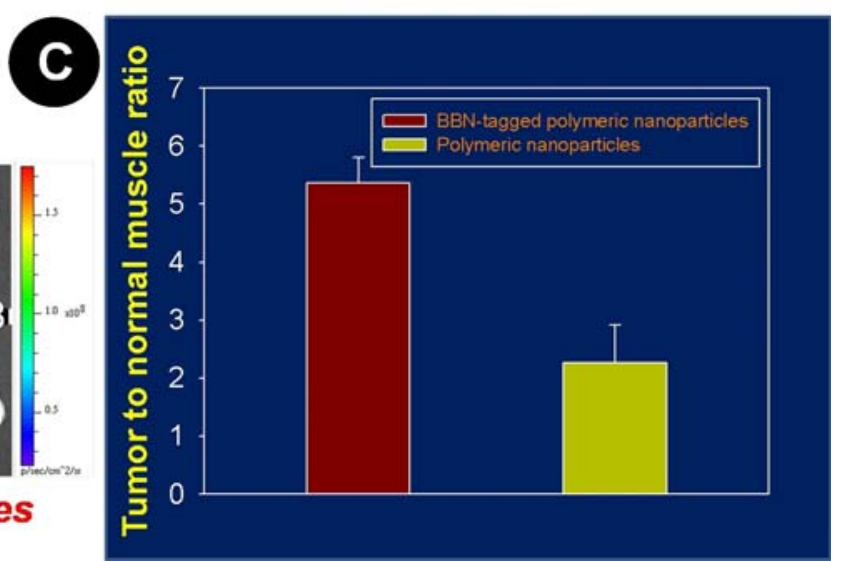

(A) The diagram illustrates the BBN-tagged magneto-polymeric nanoparticles. (B) Ex vivo optical imaging for biodistribution of BBN-tagged polymeric nano- particles in prostate cancer mice. After $6 \mathrm{~h}$ of injection via the tail vein, the organs were collected from mice, and images were obtained using IVIS in vivo optical imag- ing system. Tu: Tumor; Ht: Heart; Lu: Lung; Li: Liver; Sp: Spleen; Pa: Pancreas; Mu: Muscle; B: Bone. (c)The graph shows the tumor to muscle ratio of BBN-tagged polymeric nanoparticles $(n=3$ or 4$)$. Data are means \pm SDs. 
J033

\section{IN VIVO MRI MULTICONTRAST KINETIC ANALYSIS FOR THE VISUALIZATION OF THE UPTAKE \\ AND INTRACELLULAR TRAFFICKING \\ OF PARAMAGNETICALLY LABELED NANOVESICLES}

Silvio Aime, Daniela Delli Castelli, Enzo Terreno, Walter Dastrù, Evelina Cittadino, Francesco Mainini, Elena Torres

Chemistry I.F.M., University of Torino, Turin, Italy.

Nanovesicles are supramolecular aggregates characterized by membranes made of naturally occurring or synthetic amphiphiles. Such vesicles are already used in the pharmaceutical field mainly as drug delivery systems. Despite their large use, there is still a lack of informations about the interaction between the nanovesicles and cells in tumor environment and their intracellular fate after the cellular uptake. Most of the information on this topic comes out from cellular studies that are not always reliable models for mimicking the in vivo system. The aim of this work is the visualisation of the metabolic pathway of these vesicles directly in vivo by means of MRI. Since MRI does not provide enough spatial resolution to observe directly events at subcellular level, we have developed a multicontrast analysis that provides indirect evidence about the uptake and the intracellular trafficking of the nanovesicles. The method relies on the peculiarity of nanovesicles encapsulating paramagnetic Ln(III)-based complexes that may act as T1, T2, and CEST agents. Upon injecting the nanoparticles directly into the tumor mass and by acquiring T1, T2, and CEST MR-images, it is possible to follow the evolution of liposomes at the tumor region (intravascular/extravascular localisation; macrophage/ tumour uptake; intracellular de-assembly; washing out etc...)(see Fig. Below). In order to account for the observe MRI data, a kinetic model able to describe the underlying biological processes have been developed. The interpolation of the data provides a rough estimation of the kinetic constants for each process considered in the model (figure below, right). The analysis revealed an unexpected fast cellular uptake in spite of the PEG coating of the vesicles, and a very slow release of the encapsulated complexes from liposomes to the cytosol. In conclusion MRI multicontrast analysis appears as a powerful tool for visualizing liposomes' delivery process at the tumor site.
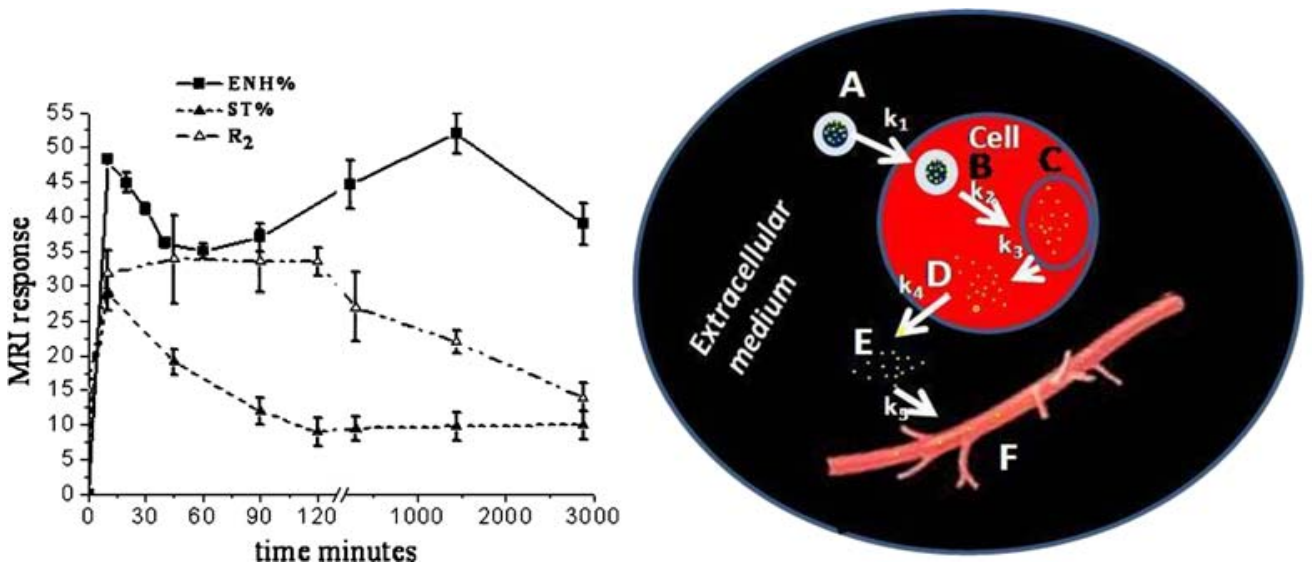

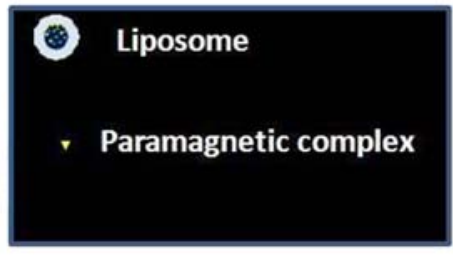

J034

\section{QUANTIFICATION OF THE R1 AND R2 RELAXIVITIES OF RAT MESENCHYMAL STEM CELLS LABELED} WITH GD- DTPA-LIPOSOMES FOR CELL TRACKING AT 3.0T Jamal Guenoun, Gyula Kotek, Piotr A. Wielopolski, Gabriela N. Doeswijk, Gabriel P. Krestin, Monique R. Bernsen Erasmus MC, Rotterdam, Netherlands.

Purpose: To determine the $\mathrm{r} 1$ and $\mathrm{r} 2$ relaxivities of Gd-DTPA-liposome labeled rat mesenchymal stem cells in vitro to enable cell number quantification and tracking in future stem cell transplantation studies. Material and methods: Gd-DTPA was incorporated in the water phase of cationic liposomes using lipid-film hydration of cholesterol, DOTAP and DPPC at molar ratios 2.35:1.65:1.0. Liposomes were analyzed for phosphate content and size by spectrophotometer analysis and dynamic light scattering respectively. The average diameter of the liposomes was $134 \mathrm{~nm}$ with a polydispersity $<0.1$. Cells were labelled by incubation with Gd-DTPA liposomes for $24 \mathrm{~h}$ using $53 \mathrm{~nm}$ lipids $/ \mathrm{cm} 2$ growth surface at $70 \%$ confluency. Average incorporated Gd-amount per cell was measured by ICP-OES. After assessing the viability of cells by trypan blue exclusion, a dilution series of 10 homogenous samples in the range of 16-8192 cells per $0.125 \mu \mathrm{l}$ agar $(0,3 \%)$ was made and scanned with an corresponding isotropic voxel size $(500 \times 500 \times 500 \mu \mathrm{m} 3=$ $0.125 \mu \mathrm{l})$ at $3.0 \mathrm{~T}$. T1 mapping was performed using both a rf-spoiled GRE sequence with multiple flip angles (range $1^{\circ}-70^{\circ}$, TR $40 \mathrm{~ms}$., TE $4 \mathrm{~ms}$.) and a SE sequence with multiple TRs (range 200-1400 ms., TE $10 \mathrm{~ms}$.). T2 was mapped using a SE sequence (TR $35 \mathrm{~ms} . / \alpha 15^{\circ}$ ) with increasing TE (TE 3-23 ms.). Longitudinal and transverse cellular relaxivities were measured as $\mathrm{r} 1=\mathrm{R} 1 /[$ cell concentration] and $\mathrm{r} 2=\mathrm{R} 2 /[$ cell concentration] respectively, with $\mathrm{R} 1=1 / \mathrm{T} 1$ and $\mathrm{R} 2=1 / \mathrm{T} 2$. Results: By measuring the actual $\mathrm{T} 1$ and $\mathrm{T} 2$ relaxation times on a voxelbasis, $\mathrm{R} 1$ and R2 were calculated for the 10 different cell concentrations. Both relaxation rates were found to be linearly dependent on cell concentration for described $\mathrm{T} 1$ and $\mathrm{T} 2$ mapping methods. The $\mathrm{r} 1$ and $\mathrm{r} 2$ relaxivity per cell were determined from the slopes of the regression lines of the R1 and $\mathrm{R} 2$ relaxation rates against cell concentration. This yielded a cellular $\mathrm{r} 1$ of $1,1 * 10^{-3} * \mathrm{~s}^{-1} *$ cell $^{-1}$ and a cellular $\mathrm{r} 2$ of $75,2 * 10^{-3} * \mathrm{~s}^{-1} *$ cell $^{-1}$. Conclusion: The longitudinal (r1) and transverse ( 2 2) cellular relaxivities of cells loaded with Gd-DTPA liposomes was calculated in vitro with the described methodology. This calibration is important for future MRI cellular studies to provide non-invasive measures of cell concentration. 


\section{J035}

\section{HYDROTROPIC MICELLES FOR MAGNETIC RESONANCE IMAGING AND CANCER THERAPY}

Hong Yeol Yoon ${ }^{1}$, Ki Young Choi ${ }^{1,2}$, Saravanakumar Gurusamy ${ }^{1}$, Ah Young $\mathrm{Kim}^{1,2}$, Kwangmeyung $\mathrm{Kim}^{2}$, Jaehyung Park ${ }^{1,4}$, Ick Chan Kwon $^{2}$, Kinam Park ${ }^{3}$

${ }^{1}$ Departments of Advanced Polymer and Nanopharmaceutical Sciences, Kyung Hee University, Seoul, Korea, South, ${ }^{2}$ Biomedical Research Center, Korea Institute of Science and Technology, Seoul, Korea, South, ${ }^{3}$ Departments of Pharmaceutics and Biomedical Engineering, Purdue University, Seoul, IN, USA, ${ }^{4}$ BioImaging Research Center, Gwangju Institute of Science and Technology, Gwangju, Korea, South.

Multifunctional polymeric micelles that enable to deliver imaging agents and therapeutic drugs have gained much attention because of their potential applications for diagnosis and therapy of cancer. Their inner cores allow encapsulation of hydrophobic agents and, when systemically administrated, they are known to be passively accumulated at the tumor site via EPR (enhanced permeability and retention) effect. In recent years, a novel type of hydrotropic polymer micelles, composed of poly(ethylene glycol)-block-poly(2-(4-(vinyl benzyloxy)$\mathrm{N}, \mathrm{N}$-diethylnicotinamide), have been developed to deliver poorly water-soluble anticancer drug, paclitaxel (PTX), to the cancer site. In this study, we investigated the potential of hydrotropic micelles as the carrier of PTX and magnetic resonance imaging (MRI) agent, which can be used for both of diagnosis and therapy of cancer. The oleic acidcoated superparamagnetic iron oxides (OA-SPIONs) were prepared by the thermal decomposition method as the MRI agent. PTX and OASPIONs were effectively encapsulated into the hydrotropic inner cores of the micelles by using the dialysis method. It was found that the mean diameter of nanoparticles and encapsulation efficiency of the drug were significantly dependent on the feed ratio of polymer micelle, PTX, and OA-SPION. The resulting nanoparticles could release PTX in a sustained manner in the physiological solution. The MRI data showed that hydrotropic polymer micelles containing PTX and OA-SPION were selectively accumulated at the tumor site in vivo, when systemically administrated into the tumor-bearing mice. In conclusion, hydrotropic polymer micelle loaded with PTX and OA-SPION might be useful for cancer imaging and therapy.

\section{J036}

\section{DUAL MODAL CONTRAST AGENT WITH NOVEL} NON-POLYMERIC FLUORESCENT COATING (FLUSPIOS) Jabadurai Jayapaul $^{1,4}$, Michael A. Hodenius ${ }^{2}$, Alexandra Buhl ${ }^{3}$, Peter Comba $^{4}$, Fabian Kiessling ${ }^{1}$, Jessica Gätjens ${ }^{1}$

${ }^{1}$ Department of Experimental Molecular Imaging: Medical Faculty, RWTH Aachen University, Aachen, Germany, ${ }^{2}$ Applied Medical Engineering, Medical Faculty, Helmholtz Institute, RWTH Aachen University, Aachen, Germany, ${ }^{3}$ Department of Diagnostic Radiology: Medical Faculty, RWTH Aachen University, Aachen, Germany, ${ }^{4}$ Institute of Inorganic Chemistry, University of Heidelberg, Heidelberg, Germany.

We report here the first successful nonpolymeric fluorescence coating of iron oxide nanoparticles without any chemical surface modification. In this project, we have developed novel ultrasmall, fluorescent and superparamagnetic iron oxide particles (FLUSPIOs) as dual-mode contrast agents for magnetic resonance and optical imaging. We have focused on flavin mononucleotide (FMN) as fluorescent, nonpolymeric coating molecule, which binds strongly to the iron oxide surface due to the inherent phosphate groups. The USPIOs were synthesized by the chemical co-precipitation method. Nonpolymeric coating was achieved by incubating nanoparticles and $\mathrm{FMN}$ at $20^{\circ} \mathrm{C}$ for $1 \mathrm{~h}$. Removal of excess FMN was performed by dialysis against water at $4^{\circ} \mathrm{C}$ or by high gradient magnetophoresis. The average size and fluorescence of the FLUSPIOs were evaluated by Scanning Electron Microscopy (SEM), fluorescence spectrometry and fluorescence microscopy, respectively. The relaxivities $\mathrm{r} 2$ and $\mathrm{r} 1$ were determined at room temperature using a clinical Philips Achieva 3T MR scanner. The Vaskovsky phosphate estimation method was carried out for determining the amount of phosphonic acid coupled to the iron oxide surface. The cytotoxicity of the FLUSPIOs was evaluated by trypan blue staining in which A431 cells were incubated with our particles $(\mathrm{c}(\mathrm{Fe})=3.0 \mu \mathrm{mol} / \mathrm{mL})$ for $3 \mathrm{~h}$. Preliminary SEM measurements showed an average particle size of $30 \pm 5 \mathrm{~nm}$. The FLUSPIOs green luminescence was evaluated by fluorescence microscopy. Fluorescence spectrometry of FMN displayed emission at $530 \mathrm{~nm}$ in buffer solution, independent of $\mathrm{pH}$. The drop of emission intensity is negliglible after 3 days. The Vaskovsky phosphate determination method showed that $9 \mu \mathrm{mol} / \mathrm{mL}$ of FMN was bound to $143 \mu \mathrm{mol} / \mathrm{mL}$ of the iron core. MR measurements showed the expected relaxitiviy values $\mathrm{r} 2$ and $\mathrm{r} 1$ for FLUSPIOs as $27 \pm 2 \mathrm{~s}-1 \mathrm{mM}-1$ and $0.37 \mathrm{~s}-$ $1 \mathrm{mM}-1$. No toxic effects of FLUSPIOs on A431 cells could be detected by trypan blue staining. The physicochemical characterization of FLUSPIOs is in progress. Further specific targeting of the PC-3 cells by FLUSPIOs will be discussed. FMN coated USPIOs displayed excellent stability at physiological $\mathrm{pH}$ and provide the possibility of further functionalization with specific antibodies. This novel fluorescent nonpolymeric coating allows us to tailor the properties of magnetofluorescent nanoparticles in a simple way without any chemical surface modification and it offers an excellent opportunity for various biomedical applications

\section{J037}

\section{POLY(AMINO ACID) MICELLE-MEDIATED AGGREGATION OF MAGNETITE NANOPARTICLES FOR EFFECTIVE MAGNETIC RESONANCE IMAGING OF CANCER} Hyun Jin Lee ${ }^{1,2}$, Yong Yeon Jeong ${ }^{2}$

${ }^{\mathrm{D}}$ Department of Chemical and Biomolecular Engineering, KAIST, Daejeon, Korea, South, ${ }^{2}$ Department of Diagnostic Radiology, Chonnam National University Medical School, Gwangju, Korea, South.

We demonstrate simple assembly of magnetite (Fe3O4) nanoparticles enclosed by micelles from a poly(amino acid)s derivative and its display as an efficient MRI contrast agent for tumor imaging. $6 \mathrm{~nm}$ magnetite nanoparticles with hydrophobic surface were preprared by thermal decomposition method. Synthetic cationic poly(amino acid) (Poly- $\alpha, \beta$-(N-2-dimethylaminoethyl L-aspartamide) grafted with octadecyl chains (PDMAEA-g-C18) which can encapsulate the magnetite nanoparticles inside the core and offer a functional outer surface was prepared. Through swollen micelles obtained via emulsion formation, PDMAEA-g-C18 micelles were loaded with magnetite nanoparticles (P-SPION). Good aqueous colloidal stability and appropriate surface properties from the PDMAEA shell and high $\mathrm{T} 2$ relaxivity by the tight aggregation of magnetite nanoparticles in core were observed and expected to be advantageous for the practical in vitro/in vivo application. P-SPION was applied as MRI contrast agent and showed very successful in vitro/in vivo MR imaging of CT26 tumor without help of any targeting ligands or magnetic field guidance. The appropriate size of particulates less than $100 \mathrm{~nm}$ enabled the targeted accumulation to tumor by the enhanced permeability and retention (EPR) effect and the positively charged poly(amino acid)s shell containing tertiary amine groups enabled localization near and internalization to cancer cells and the clustered magnetic nanoparticles highly enhanced the MRI sensitivity. P-SPION could be utilized as a molecular marker for cancer diagnosis or cell labeling. In addition, 
multifunctional nanoparticles could be easily developed with introduction of other bioactive agents such as an anticancer drug, genes, a label for optical imaging, or an active tumor-targeting moiety for further biomedical explorations.
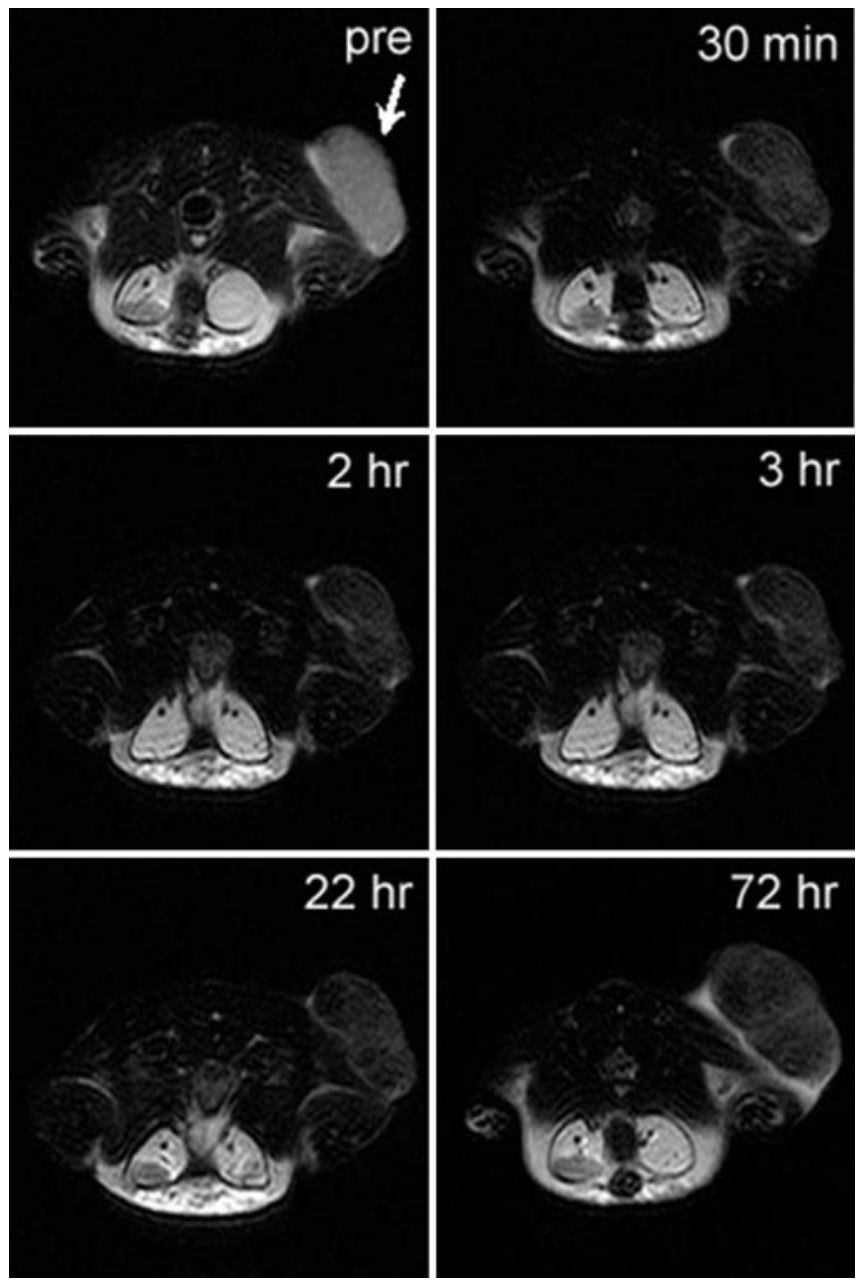

T2-weighted MR images of a CT26 tumor-bearing mouse taken at 0 , $0.5,2,3,22$, and $72 \mathrm{~h}$ postinjection of P-SPION

\section{J038}

\section{A NOVEL MAGNETOFLUORESCENT NANOPARTICLE PLATFORM FOR CELL LABELING AND TRACKING} Suelin Chen ${ }^{1,2}$, Ralph Weissleder ${ }^{3}$, Lee Josephson ${ }^{2}$

${ }^{1}$ Department of Materials Science and Engineering, MIT, Cambridge, MA, USA, ${ }^{2}$ Center for Molecular Imaging Research, Massachusetts General Hospital/Harvard Medical School, Charlestown, MA, USA, ${ }^{3}$ Center for Systems Biology, Massachusetts General Hospital/Harvard Medical School, Boston, MA, USA.

The ability to track cells in vivo is important both for learning more about biological mechanisms such as cellular migratory capacity as well as for the evaluation and development of cell-based therapies. We have developed a novel carboxymethyl dextran-coated superparamagnetic iron oxide nanoparticle that has high potency and excellent stability without crosslinking. The polymer carboxymethyl dextran was chosen as an optimal coating after performing a series of screens that tested a panel of polymers' ability to bind to iron, determined the optimal synthetic conditions to maximize transverse relaxivity (R2), and assessed nanoparticle stability under highly stringent conditions. Protamine labeled with rhodamine has been conjugated to the carboxymethyl dextran coating, facilitating cell internalization and allowing the nanoparticle to be characterized and tracked by fluorescence in addition to MR. This protamine-nanoparticle conjugate is evaluated as a cell labeling material in mouse mesenchymal stem cells as a model for tracking cell therapies and in human glioblastoma cells as a model for monitoring tumor growth in xenografts. Here we have developed a robust and thoroughly characterized nanoparticle that can be generalized to many cell labeling applications and that has the potential for clinical/translational use.

\section{J039}

\section{IDENTIFICATION AND VISUALIZATION \\ OF SLOW-PROLIFERATING MELANOMA CELLS}

Sergey Magnitsky ${ }^{1}$, Alexander Roesch ${ }^{2}$, Meenhard Herlyn ${ }^{2}$, Jerry D. Glickson $^{1}$

${ }^{1}$ Radiology, University of Pennsylvania, Philadelphia, NJ, USA, ${ }^{2}$ Wistar Institute, Philadelphia, PA, USA.

Introduction: Small subpopulations of tumor cells exhibiting high tumorogenicity, self-renewal capacity and resistance to treatments have been described in breast, prostate, lung and bladder cancers. Proliferation studies of these "tumor-initiating" cells indicate that they are residing in the G0/G1 state of cell cycle. A chromatin remodeling factor JARID1B was identified as a molecular marker for slow-cycling cells in melanoma. The goal of this study was to develop a technique to identify and track slow cycling/dormant cells in vitro and in vivo. Results: 95-98\% of WM3734 melanoma cells internalized iron oxide particles after 12 hours of incubation with SPIO (Super Paramagnetic Iron Oxide) particles, with an average iron concentration of $16 \mathrm{pg}$ of $\mathrm{Fe} / \mathrm{cell}$. Cell sorting experiments detected a distinct iron-retaining subcell population after 20 days of proliferation of iron labeled cells. Semiquantitative RT-PCR test of iron-retaining cells revealed the highest expression of JARID1B protein in the most iron-positive cells, while iron-free cells expressed lower amounts of JARID1B $(p<0.05)$. One million of iron-labeled cells were implanted subcutaneously into NODSCID mice. In vivo MR images of tumor xinografts grown for 8 weeks from labeled cells exhibited regions of hypointensity, while control tumors (grown from unlabeled cells) did not show a similar pattern. Ex vivo MR images of tumor tissue grown from iron labeled cells exhibited a different contrast compare to control tumors: more hypointense regions were detected in the iron positive tumor, and the presence of iron-oxide particles was confirmed by Prussian Blue staining. Discussion and Conclusion: Dilution of iron oxide particles following cell proliferation has been reported. We used this property of MR contrast agent to identify slow-cycling cells and image them in vivo and ex vivo. Uniform cell labeling with MRI detectable iron can be achieved after $12 \mathrm{~h}$ of melanoma cells incubation with iron oxide particles. The iron-retaining subcell population was detected after 20 days of the proliferation. QPCR analysis indicated a good correlation between iron positive cells and expression of JARID1B protein. The iron-retaining cells were detectable by in vivo and ex vivo MR imaging. These findings provide a simple practical method for investigating properties of slow-cycling cells, which are probably 
playing an essential role in drug resistance, metastasis dissemination and tumor recurrence.

\section{J040}

\section{MAGNETIC SPECTROSCOPY OF NANOPARTICLE VIBRATION (MSNV) MEASUREMENT OF NANOPARTICLE BINDING}

John B. Weaver ${ }^{1,2}$, Adam M. Rauwerdink ${ }^{2}$

${ }^{1}$ Radiology, Dartmouth College Medical School, Hanover, NH, USA,

${ }^{2}$ Thayer School of Engineering, Dartmouth College, Hanover, NH, USA.

Magnetic spectroscopy of nanoparticle vibration (MSNV) uses the harmonics induced in the nanoparticle magnetization by a sinusoidal applied field to measure the Brownian motion of the nanoparticles. Brownian motion reflects the nanoparticle temperature, viscosity of the media and binding. MSNV has been used to measure temperature very accurately. We report the results of two experiments that demonstrate that MSNV reflects nanoparticle binding as well. It should be possible to measure binding in vivo dynamically without waiting for the free nanoparticles to clear. In the first experiment, a thin slab of meat was soaked in Concanavalin-A after which dextran coated iron oxide nanoparticles were added. The Con-A bound the nanoparticles to the extracellular matrix of the meat. The MSNV signal was 0.201 prior to being added to the meat and 0.199 following addition to the meat. When the unbound nanoparticles were washed off leaving only the bound nanoparticles, the MSNV signal dropped $6 \%$ to 0.189 . Addition of $50 \mu \mathrm{L}$ of glucose caused the dextran coated nanoparticles to be released because the Con-A preferentially binds glucose and the MSNV signal returned to 0.199 . The standard deviation of the measurements was 0.0003 so the $6 \%$ drop in MSNV signal was highly significant; the change was more than thirty standard deviations. The second experiment used nonspecific binding between iron oxide nanoparticles and polystyrene spheres. $10 \mu \mathrm{L}$ of streptavidin labeled $25 \mathrm{~nm}$ diameter iron oxide nanoparticles diluted with $200 \mu \mathrm{L}$ of PBS had an MSNV signal of 0.261 . Mixing of the nanoparticles with $2 \mu \mathrm{m}$ microspheres resulted in nonspecific binding and a significant increase in the MSNV signal to 0.295 . The nanoparticles could be seen to precipitate out with the microspheres over time in this solution but not in the controls. The control used BSA to occupy the microsphere binding sites prior to the addition of the nanoparticles and the MSV signal dropped only slightly to 0.245 which was the same value obtained when BSA was mixed with the nanoparticles without any spheres. The standard deviation was again 0.0003 so the changes were also highly significant. The total MSNV signal is the sum from the bound and unbound nanoparticles so the bound fraction can be observed as a function of time. Because magnetic particle imaging (MPI) uses the same harmonics used by MSNV, it should be possible to incorporate MSNV methods into MPI producing images from nanogram concentrations where the contrast reflects the parameters determining Brownian motion, including binding.

J041

\section{MRI RELAXOMETRY OF $\mathrm{MN}_{0.5} \mathrm{ZN}_{0.5} \mathrm{GD}_{\mathrm{X}} \mathrm{FE}_{(2-\mathrm{X})} \mathrm{O}_{4}$ NANOPARTICLES}

Bashar Issa ${ }^{1}$, Shahnaz Qadri², Yousef Haik ${ }^{2}$

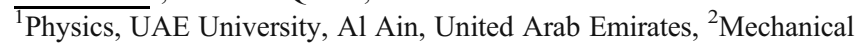
Engineering, UAE University, Al-Ain, United Arab Emirates.

We studied the temperature behaviour of $1 / \mathrm{T} 1$ and $1 / \mathrm{T} 2$ for both uncoated and coated new class of magnetic nanoparticles (MNPs) composed of biocompatible elements $\mathrm{Zn}, \mathrm{Mn}$, Gd and iron oxides. Samples of Gd substituted Mn-Zn Ferrite MNPs were synthesized using chemical co-precipitation method and ferritization.Measurement of $\mathrm{T} 1$ and $\mathrm{T} 2$ at $1.5 \mathrm{~T}$ was performed at temperatures: $8-58$ degrees $\mathrm{C}$ using FSE sequence. The concentration of the elements were Gd:Mn: Fe: $\mathrm{Zn}=1.84: 24.88: 57.57: 15.71 \%$. The concentration in units of mmol $\mathrm{Fe} / \mathrm{kg}$ ranged from control to 0.775 . The average diameter for the uncoated and coated MNPs was 45 and $85 \mathrm{~nm}$. The measured 1/T2 (fig. 1) is shown as a function of temperature for different concentrations. The increase in $1 / \mathrm{T} 2$ with temperature is more monotonic than that of $1 / \mathrm{T} 1$ (not shown). 1/T2 increases also with concentration at all temperatures. The variation of $1 / \mathrm{T} 1$ and $1 / \mathrm{T} 2$ with MNPs concentration is bigger for the uncoated particles than for the coated ones. Relaxation is mostly diffusion modulated. The values of $1 / \mathrm{T} 2$ begin to decrease above $50^{\circ} \mathrm{C}$ closer to the melting point of the agar $\left(70^{\circ} \mathrm{C}\right)$. Relaxivities ( $\mathrm{r} 1$ and r2) defined as the linear slopes of $1 / \mathrm{T} 1$ and $1 / \mathrm{T} 2$ with concentration in units $\mathrm{s}^{-1} \mathrm{mM}^{-1}$ are shown at all temperatures in table 1 . The increase of $r_{2}$ with temperature was relatively large and linear for the uncoated MNPS $\left(83.6-131.1 \mathrm{~s}^{-1} \mathrm{mM}^{-1}\right)$ with a slope of approximately $0.989 \mathrm{~s}^{-1} \mathrm{mM}^{-1} /{ }^{\circ} \mathrm{C}$ and $\mathrm{R} 2=0.983$. This is larger than for the coated particles. $\mathrm{r}_{1}$ values are also shown in the table. Conclusions:Results obtained here indicate a more significant change in R2 than R1 with temperature for this class of MNPs. This is expected due to the large magnetic moment of the MNPs making it act as a negative contrast agent. The size of the magnetic moment can be controlled through the size of particle and its composition. These particles have already been used as hyperthermia agents and this study investigates extending their use as MRI contrast agents. relaxivity vs. temperature.

\begin{tabular}{|c|c|c|c|c|c|c|}
\hline $\mathrm{T}\left({ }^{\circ} \mathrm{C}\right.$ & 8 & 18 & 31 & 39 & 49 & 58 \\
\hline $\mathrm{r} 1(\mathrm{u})$ & 1.34 & 1.36 & 1.73 & 1.78 & 1.60 & 0.35 \\
\hline $\mathrm{r} 1(\mathrm{c})$ & 0.19 & 0.26 & 0.35 & 0.39 & 1.66 & 0.35 \\
\hline $\mathrm{r} 2(\mathrm{u})$ & 83.6 & 93.3 & 109.5 & 118.9 & 39.5 & 131.1 \\
\hline $\mathrm{r} 2(\mathrm{c})$ & 30.8 & 28.6 & 32.8 & 39.3 & 39.3 & 41.0 \\
\hline
\end{tabular}




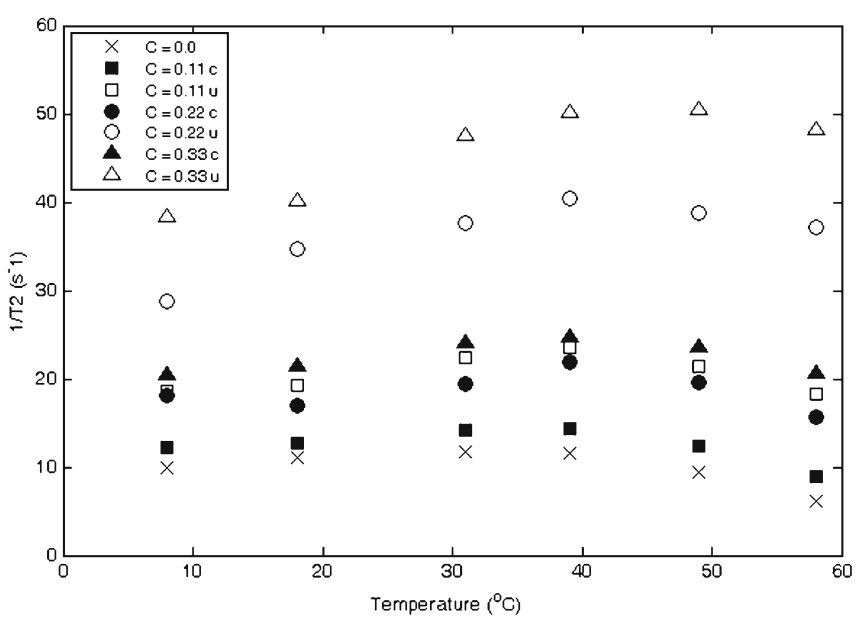

J042

IMAGING THE WARBURG EFFECT IN TUMORS WITH A PH SENSITIVE MR CONTRAST AGENT

Samuel Crayton ${ }^{1}$, Andrew Tsourkas ${ }^{1,2}$

${ }^{7}$ Bioengineering, University of Pennsylvania, Philadelphia, PA, USA,

${ }^{2}$ Radiology, University of Pennsylvania, Philadelphia, PA, USA.

Current radiological contrast agents provide tumor contrast largely due to the non-specific enhanced permeability and retention effect. Contrast agents with inherent specificity for the tumor microenvironment have the potential to improve diagnostic sensitivity, detection of metastatic disease, and monitoring of treatment response, while reducing falsepositive results. Although there is variance among tumors and individual patients, it is known that many human solid tumors reside in environments with an extracellular $\mathrm{pH}$ that is both sub-physiologic and lower than surrounding healthy tissue, typically in the range of 6.5 7.0. This acidification of the tumor microenvironment is commonly called the Warburg effect. As such, we have developed an MRI contrast agent capable of selective retention in these sub-physiologic $\mathrm{pH}$ tumor microenvironments. Since the Warburg effect has been noted in a very wide range of malignancies, we believe our contrast agent possess a unique advantage over receptor/ligand approaches for tumor specific imaging. Currently, we have demonstrated that out fluorescently labeled construct is capable of clearly labeling MCF-7 cells incubated at $\mathrm{pH}$ 6.5. Less intense labeling is exhibited with incubation at $\mathrm{pH} 7.0$, and no appreciable labeling is found with incubation at $\mathrm{pH}$ 7.5.

\section{J043}

\section{BRIGHT AND PHOTOSTABLE FLUORESCENT IMAGING AGENTS: LATEX NANOSPHERES CONJUGATED TO SECONDARY ANTIBODY}

$\underline{\text { Tao Ji}}$, Catherine Muenker, Douglas Vizard, Rao V. Papineni, William McLaughlin

Carestream Health Inc, New Haven, CT, USA.
Secondary antibodies were conjugated to fluorescent latex nanospheres via two different synthetic routes: DTT Reduction and Traut's Reagent Modification. These two conjugation methods give different conjugation efficiency and different performance in the binding to the primary antibodies. Western blotting results show that the nanosphere-antibody conjugates synthesized using the DTT Reduction route outperform the conjugates synthesized using the Traut's Reagent Modification route and other commercially available fluorescent antibodies. These extremely bright fluorescent imaging agents significantly increase detection sensitivity and can be used for a variety of applications, such as western blotting, cell staining, and flow cytometry etc.

J044

\section{DESIGN OF FLUOROPHORES FOR THE CONSTRUCTION OF PHOTONIC PH SENSITIVE SHELL-CROSSLINKED (SCK) NANOPARTICLES FOR OPTICAL IMAGING AND MONITORING}

John Freskos ${ }^{1}$, Richard B. Dorshow ${ }^{1}$, William Neumann ${ }^{3}$, Gurong $\overline{\text { Sun }}^{2}$, Karen L. Wooley ${ }^{2}$, Nam S. Lee ${ }^{2}$, Jeng-Jong Shieh ${ }^{1}$

${ }^{1}$ Biophotonics, Covidien, Hazelwood, MO, USA, ${ }^{2}$ Chemistry and Radiology, Washington University in St. Louis, St. Louis, MO, USA, ${ }^{3}$ Depart of Pharmaceutical Sciences, Southern Illinois University Edwardsville, Edwardsville, IL, USA.

This poster will describe the elaboration of the 2,5-diaminopyrazine3,6-dicarboxylic acid scaffold for the efficient design of denselyfunctionalized small molecule fluorophores that possess surprisingly bright emission in the yellow to red regions of the electromagnetic spectrum. These molecules have typical absorption and emission maxima of $450 \mathrm{~nm}$ and $550 \mathrm{~nm}$, respectively, due to the push-pull orientation of the substitution pattern. The small compact nature of these monoheterocyclic fluorophores is perfectly suited for their desired use as reporter systems within nanostructural platforms. In order to realize this potential, we have developed robust synthetic methodologies that have made it possible to efficiently append functionality onto the pyrazine scaffold, which is useful for studying the interaction of the fluorophore with block copolymer derived micelles and shell-crosslinked (SCK) nanoparticles having distinct core-shell morphology. The interactions of the fluorophore within the nanostructures ranges from physical association based upon molecular recognition governed by the hydrophobic core (polyphenol) and hydrophilic shell (poly(acrylic acid) to covalent crosslinking of the shell domain. Further, we have incorporated auxiliary oligoarginine functionality which can interact with shell-domain carboxylates before or after covalent crosslinking to both effect and respond to changes in nanostructure morphology as a function of environmental conditions. We have found that the physical association of oligoarginine-containing pyrazine crosslinkers is substantially enhanced over non-arginine containing linkers due to the electrostatic interaction of the cationic centers with the anionic nanoparticle shell. The design and synthesis of these highly functionalized crosslinkable fluorophores and their incorporation into SCK nanoparticles will be described. 


\section{J045}

\section{NEAR-INFRARED FLUORESCENCE IMAGING USING POLYMERIC NANOPARTICLE-BASED MMP-ACTIVATABLE PROBE IN COLON CANCER}

Soon Man Yoon $^{1,2}$, Seung-Jae Myung ${ }^{1,2}$, In-Wha Kim ${ }^{3}$, Eun Ju Do ${ }^{3}$, Yeon $\mathrm{Mi} \mathrm{Ryu}^{3}$, Soo Jung Park ${ }^{1,2}$, Yi Rang Kim ${ }^{1,2}$, Ju Hee Ryu ${ }^{4}$, Kwangmeyung Kim ${ }^{4}$, Ick Chan Kwon ${ }^{4}$, Mi Jung Kim ${ }^{5}$, Dae Hyuk Moon $^{6}$, Dong-Hoon Yang ${ }^{1}$, Kyung-Jo Kim ${ }^{1}$, Byong Duk Ye ${ }^{1}$, JeongSik Byeon ${ }^{1}$, Suk-Kyun Yang ${ }^{1}$, Jin-Ho Kim ${ }^{1,2}$

${ }^{1}$ Department of Internal Medicine, Asan Medcial Center, University of Ulsan College of Medicine, Seoul, Korea, South, ${ }^{2}$ Asan Digestive Disease Research Institute, Seoul, Korea, South, ${ }^{3}$ Molecular Imaging Center, Asan Institute for Life Sciences, Seoul, Korea, South, ${ }^{4}$ Biomedical Research Center, Korea Institute of Science and Technology, Seoul, Korea, South, ${ }^{5}$ Department of Pathology, Asan Medical Center, University of Ulsan College of Medicine, Seoul, Korea, South, ${ }^{6}$ Department of Nuclear Medicine, Asan Medcial Center, University of Ulsan College of Medicine, Seoul, Korea, South.

Background \& Aims: Near-infrared fluorescence (NIRF) imaging using an activatable probe allows more sensitive and specific diagnosis of tumors. We aim to investigate the effectiveness of a novel reporter, polymeric nanoparticle-based MMP-activatable probe, in the colon cancer model. Methods: We used a mouse colon cancer model resembling human sporadic colon cancer established with azoxymethane. A histologic analysis of colonic lesions was carried out and the MMP expression was evaluated by western blot, and immunohistochemical stain. NIRF imaging was performed with a novel MMPactivatable probe, an MMP-inactivatable probe, and saline. In addition, we observed the change of NIRF signal intensity with intratumoral adminstration of an MMP-inhibitor followed by injection of a novel MMP-activatable probe. Results: Variable-sized multiple tumors developed in mice colon and showed progressive stages from adenomas to adenocarcinomas. MMPs expression progressively increased through normal, adenoma, and adenocarcinoma. NIRF signal increased with each tumor stage from adenoma to adenocarcinoma after injection of a novel MMP-activatable probe. Tumorbackground-ratios (TBRs) of adenomas and adenocarcinomas to adjacent normal mucosa by a novel probe were significantly higher than those found with an MMP-inactivatable probe (7.9 vs 2.5 in adenomas, 15.8 vs 4.2 in adenocarcinomas, respectively, $p<0.05$ ). NIRF signal intensity of the tumor injected with an MMP-inhibitor was decreased prominently. Conclusions: A novel polymeric nanoparticle-based probe showing enhanced tumor targeting could be used as an effective tool for NIRF imaging in tumor detection. This technology may allow more accurate diagnosis and also enable to monitor the response of cancer therapy.

\section{J046}

\section{A NOVEL "BEADS ON A BEAD" APPROACH ENABLES HIGH THROUGHPUT DISCOVERY OF LIGANDS FOR NON-INVASIVE MOLECULAR IMAGING}

Choi-Fong Cho ${ }^{1}$, Giulio Amadei ${ }^{2}$, Leonard G. Luyt ${ }^{2}$, John D. Lewis ${ }^{1}$ ${ }^{\mathrm{T}}$ Medical Biophysics, University of Western Ontario, Guelph, ON, Canada, ${ }^{2}$ Chemistry, University of Western Ontario, London, ON, Canada.

Metastasis contributes to greater than $90 \%$ of cancer mortalities. Non-invasive detection of angiogenic factors, which are expressed during the earliest stages of tumour development may aid in the diagnosis of neoplasms before they metastasize. EGFL7 is a protein that is known to accumulate at sites of tumour angiogenesis. We hypothesize that molecular imaging using peptides targeted to EGFL7 will allow the detection of nascent tumours. Here, we describe a novel "beads on a bead" screening approach that significantly accelerates the identification and isolation of positive peptide hits from random combinatorial libraries. A one bead one compound (OBOC) library (the "bait") was synthesized on $90 \mu \mathrm{m}$ Tentagel beads so that each bead was coated with a single octapeptide species. We then coated micron-sized magnetic "prey" beads with the full-length EGFL7 protein that were purified using the baculovirus expression system and incubated these with the peptide library. Positive hits consisted of bait library beads that became coated with prey magnetic beads, and these were isolated from the negative library beads using a magnetic separator device that was constructed for this task. The hit peptides were cleaved and sequenced "on bead" using a novel MALDI-TOF/MS technique and the relative binding affinity of each of these peptides will be determined using surface plasmon resonance. The described strategy allows one to construct and screen a combinatorial peptide library and obtain peptide sequence from positive hits in less than a week. We have demonstrated that the target-coated prey magnetic beads associated with the bait library beads in an affinity-dependent fashion with an accuracy of greater than $98 \%$. The most promising antiEGFL7 peptide candidates will be conjugated to viral nanoparticles based on the cowpea mosaic virus (CPMV) and evaluated in vivo in a xenograft human prostate cancer model in the chick embryo. These studies could provide a basis for a new generation of "smart" imaging agents for the non-invasive diagnosis of cancers.

\section{J047}

\section{MAGNETIC POLY(LACTIDE-CO-GLYCOLIDE) NANOPARTICLES FOR EFFICIENT CELL LABELING Michael Nkansah $^{1}$, Erik Shapiro ${ }^{2,1}$}

Biomedical Engineering, Yale University, New Haven, CT, USA, ${ }^{2}$ Diagnostic Radiology, Yale School of Medicine, New Haven, CT, USA.

Cells magnetically labeled with iron oxide are useful for MRI tracking of processes like cell migration and tumor metastases in vivo. While dextran-coated iron oxide particles have been the primary contrast agent used in the last 20 years, a serious drawback to their use is the low iron content per particle. To address this, micron-sized, iron oxideloaded, poly(divinylbenzene) beads (Bangs Labs; Fishers, IN) containing $60 \%$ iron have been used to label cells. Poly(divinylbenzene), however, is non-degradable and not FDA-approved, limiting any potential clinical utility of this contrast agent. Our goal in this study, therefore, was to fabricate iron oxide-containing, biodegradable nanoparticles of poly(lactide-co-glycolide) (PLGA) capable of labeling cells for MRI-based cell tracking. First, 10-nm iron oxide nanocrystals were synthesized by thermal decomposition of iron oleate. Next, magnetic PLGA nanoparticles with an average size of $130 \pm 35 \mathrm{~nm}$ were fabricated using an oil-in-water single emulsion method. Monodisperse, superparamagnetic iron oxide nanocrystals were optimally loaded at an encapsulation efficiency of $50 \% . \mathrm{R}_{2}{ }^{*}$ molar relaxivity was measured to be $592 \pm 93 \mathrm{mM}^{-1} \mathrm{~s}^{-1}$, three times that of Feridex $\AA$, the current FDAapproved contrast agent commonly used for cell labeling. Furthermore, the resulting biodegradable nanoparticles were $100 \%$ more effective at labeling human mammary tumor cells than Feridex ${ }^{\circledR}$. Lastly, an in vitro degradation study showed no change in transverse relaxivity of the PLGA nanoparticles after one week under physiological conditions. In this study, we synthesized biodegradable iron oxide contrast agents and demonstrated their effectiveness in magnetically labeling cells. These versatile nanoparticles combine the biocompatibility of Feridex ${ }^{\circledR}$ with the high iron loading capacity of the Bangs beads. 

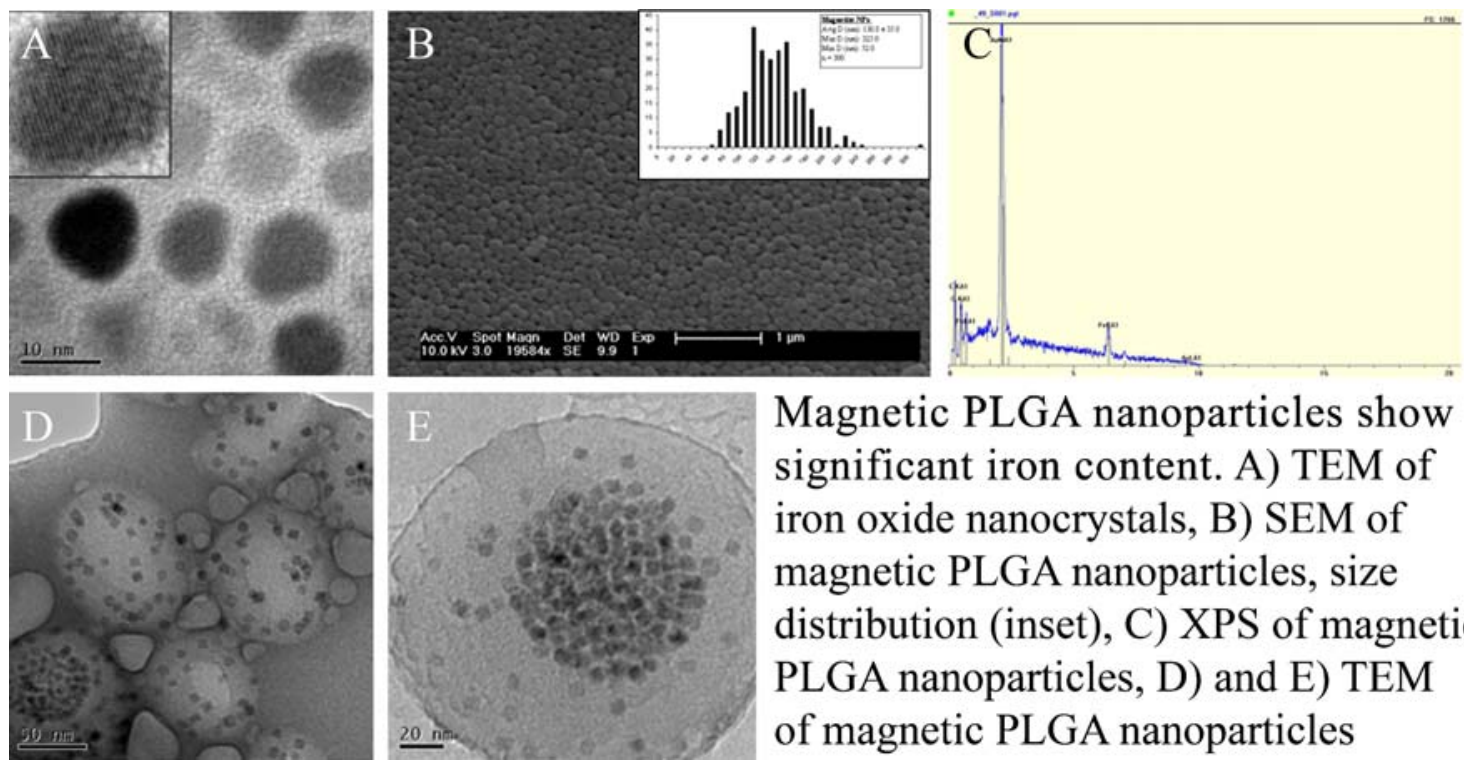

Magnetic PLGA nanoparticles show significant iron content. A) TEM of iron oxide nanocrystals, B) SEM of magnetic PLGA nanoparticles, size distribution (inset), C) XPS of magnetic PLGA nanoparticles, D) and E) TEM of magnetic PLGA nanoparticles

\section{J048}

\section{POSITIVELY CHARGED MPIOS ENHANCE MAGNETIC CELL LABELING EFFICIENCY}

Kevin Tang

Yale University, New Haven, NJ, USA.

MRI offers the potential to noninvasively track cells in vivo using innovative approaches to cell labeling and image acquisition. Cells loaded with iron oxide particles generate a pronounced contrast in T2and $\mathrm{T} 2 *$-weighted images. We have developed strategies for labeling cells with micron sized iron oxide particles (MPIOs) for in vivo visualization by MRI. Although this approach is well established and has a variety of applications, current protocols employ long labeling times. This is problematic for cells which do not fare well in culture, such as neutrophils and hepatocytes. It has been demonstrated that incubation of iron oxide nanoparticles with positively charged transfection agents, such as poly-l-lysine (PLL) increases labeling efficiency. Therefore, we sought to ascertain whether pre-incubating
MPIOs with various quantities of PLL would produce similar enhancements. We compared the short-term labeling efficiency and toxicity of different MPIO/PLL formulations and $\mathrm{COOH}$ and $\mathrm{NH} 2$ modified MPIOs, in mouse embryonic fibroblasts. MPIOs used were $1.63 \mu \mathrm{m}$ diameter, polystyrene/divinyl benzene coated, and were characterized by measuring zeta potential using Phase Analysis Light Scattering. The labeling efficiency of each particle was assessed by simple incubation for $1-4 \mathrm{~h}$ and counting labeled cells via flow cytometry. The toxicity of each particle formulation was simultaneously assessed using Sytox Blue Dead Cell Stain. A subpopulation of well labeled cells $(\geq 4$ MPIOs/cell) was distinguished by counting number of MPIOs internalized in each labeled cell using a confocal microscope. The results, shown in Figure 1, indicate that positively charged MPIOs have better labeling efficiency than negatively charged MPIOs. Furthermore, positively charged MPIOs also have a higher percentage of well labeled cells than negatively charged MPIOs. The NH2 MPIOs also show exceptionally high labeling, suggesting that the $\mathrm{NH} 2$ groups may have a positive influence on cellular uptake despite the net negative charge on the particle.

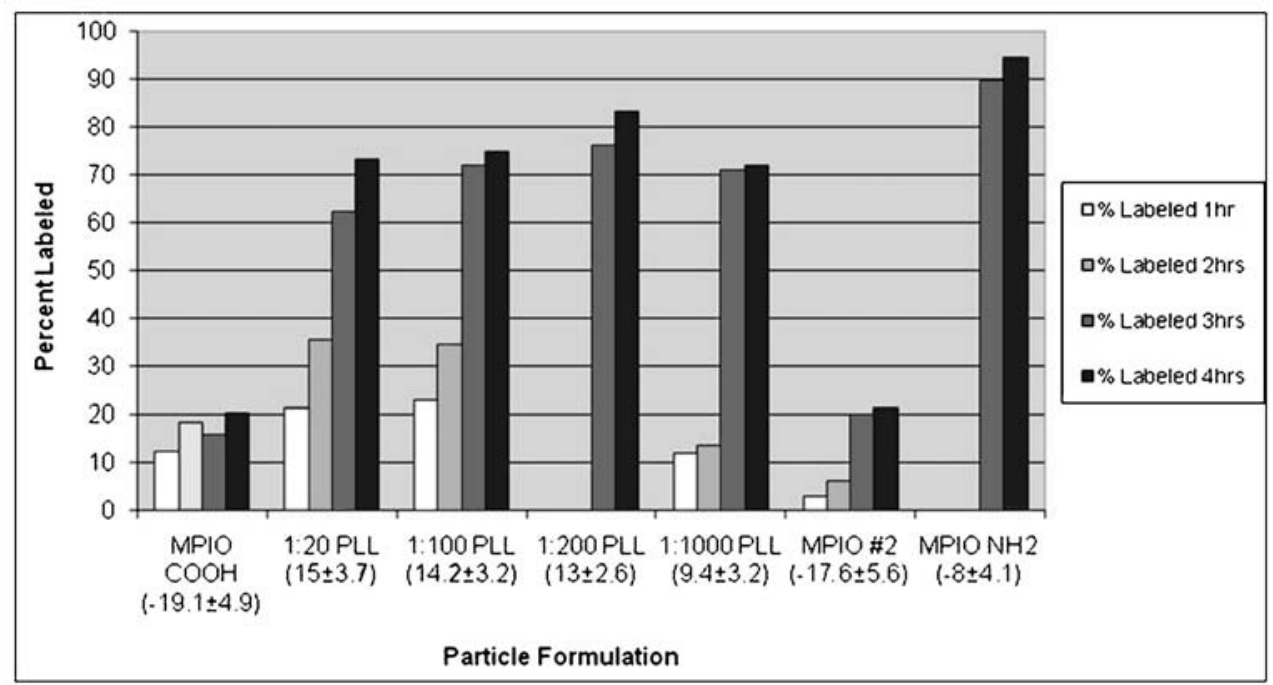

Figure 1: Labeling efficiency versus particle formulation. Zeta potential is shown in $\mathrm{mV} .1$ and 2 hour time points not available for 1:200 PLL and MPIO-NH2. Clearly visible is the trend that labeling efficiency increases with increasing zeta potential, both for early and later time points. 
J049

TARGETING OF TUMOR CELL BY VEGFR3-CONJUGATED IRON OXIDE NANOPARTICLES IN VITRO USING MR Jing Lei ${ }^{1}$, Hua dan Xue ${ }^{1}$, Zhuo $\mathrm{Li}^{1}$, Jie Meng ${ }^{2}$, Zhengyu Jin ${ }^{1}$ ${ }^{1}$ Peking Union Medical College Hospital, BeiJing, China, ${ }^{2}$ Biomedical Engineering, Institute of Basic Medical Sciences Chinses Academy of Medical Sciences, Beijing, China.

BACKGROUND: Reports indicate that vascular endothelial growth factor receptor type 3 (VEGFR3) is involved in invasion, proliferation, and lymphangiogenesis of tumors,so it is a hopeful target for tumor diagnosis. Objective: In this study VEGFR3-Conjugated USPIO was synthesized, and their ability to label MDA-MB-231 cell for magnetic resonance imaging (MRI) was compared.Methods: USPIO was coated by VEGFR3, and detected the signal differences of USPIO and VEGFR3-USPIO in different concentrations level. The expression level of VEGFR3 of MDA-MB-231 cell was detected by immunofluorescence. Label to cell and was evaluated using Prussian blue staining and MR imaging.Results:VEGFR3-USPIO were proven to be suitable for MR imaging due to small size,excellent stability in ferrofluid,and low cytotoxicity. There was no significant signal difference with USPIO and VEGFR3-USPIO in five concentrations by MRI. In addition VEGFR3 was higher expessed in MDA-MB-231 cell, and it decreased in $\mathrm{T} 2$ relaxation exhibited enhanced targeted delivery efficiency to tumor cell than USPIO in vitro.Distribution of the iron particles around cells was confirmed by Prussian blue staining. Conclusion: VEGFR3-USPIO particle provides the higher R2 and most effectively reduce the T2 relaxation time than USPIO in MDA-MB-231 cell. This suggests VEGFR3-Conjugated USPIO particles are suited for tumor cell labeling approaches in MRI.

T2,R2,T2* and R2* value after VEGFR3-USPIO and USPIO label tumor cell

\begin{tabular}{|c|c|c|c|c|}
\hline & T2value & R2value & T2*value & R2*value \\
\hline VEGFR3-USPIO label cell & 41.592 & 24.150 & 3.62 & 303.44 \\
\hline USPIO label cell & 59.560 & 16.886 & 4.26 & 245.26 \\
\hline
\end{tabular}

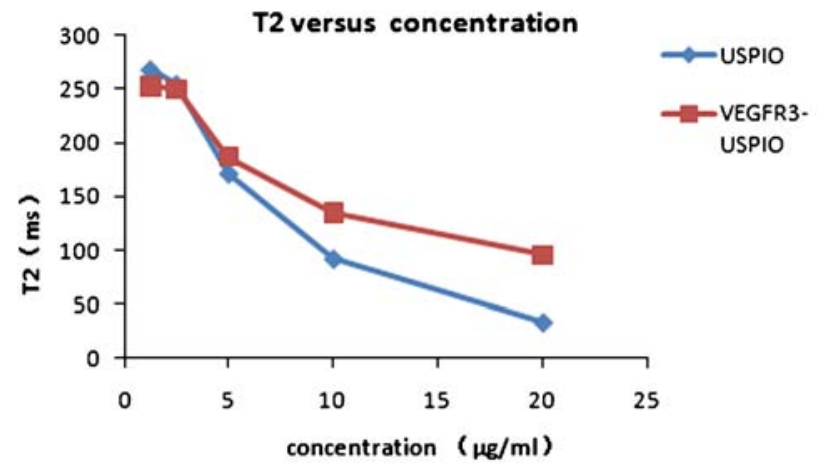

Fig. T2 versus concentrations of USIPO and VEGFR3-USPIO. T2 was reduced with concenration increaced of both USPIO and VEGFR3usPIO.

\section{J050}

\section{NANOPARTICLE-AUGMENTED PHOTOACOUSTICS TO IMAGE MOLECULAR SIGNATURE OF CANCER IN-VIVO}

Srivalleesha Mallidi ${ }^{1}$, Pratixa P. Joshi ${ }^{1}$, Seungsoo Kim ${ }^{1}$, Andrei B. Karpiouk $^{1}$, Konstantin Sokolov ${ }^{1,2}$, Stanislav Y. Emelianov ${ }^{1}$

${ }^{1}$ Biomedical Engineering, The University of Texas at Austin, Austin, TX, USA, ${ }^{2}$ Imaging Physics and Biomedical Engineering, University of Texas M.D. Anderson Cancer Center, Houston, TX, USA.

Recently gold nanoparticles have been introduced as optical contrast agents because of their strong optical absorption and scattering properties at visible and near-infrared wavelengths. When gold nanoparticles are functionalized to specifically target cancer biomarkers such as epidermal growth factor receptor (EGFR), they undergo molecular specific aggregation. This receptor mediated clustering of gold nanoparticles leads to an optical red-shift of the plasmon resonance frequency and an increase of optical absorption in the red region. Taking advantage of this phenomenon, we previously demonstrated on
3-D tissue models and ex-vivo murine tissue that highly selective and sensitive detection of cancer cells is feasible using combined ultrasound and photoacoustic imaging and biconjugated gold nanoparticles. The combined ultrasound and photoacoustic images provided information regarding the biodistribution of the molecularly targeted nanoparticles within the anatomical map of tissue. In this study, we evaluated the feasibility of molecular specific, multi-wavelength photoacoustic imaging technique in detecting plasmon resonance coupling of gold nanoparticles in subcutaneous tumor xenografts in-vivo. Subcutaneous tumor implantation of human epithelial cancer cells (A431 cells that overexpress EGFR) were performed in immunodeficient mice. Tumors were allowed to grow up to $\sim 68 \mathrm{~mm}$ in diameter. EGFR targeted or PEGylated gold nanoparticles were intravenously injected via tail vein. Three-dimensional ultrasound and photoacoustic imaging of the tumor region was performed at various time points to observe the accumulation of gold nanoparticles in the tumor. Inductively coupled plasma mass spectroscopy (ICP MS) was used to confirm the presence and biodistribution of gold nanoparticles in the tumor and other organs. Histological analysis was performed on the tissues to further determine the presence of gold nanoparticles. The photoacoustic images obtained after the injection of gold nanoparticles clearly showed contrast enhancement in the tumor region. The histological analysis of the tissues and the ICP-MS technique confirmed the presence of gold nanoparticles in the tumor region. Moreover, the change in the optical absorption of the tissue due to molecular interactions of EGFR targeted and PEGylated gold nanoparticles were observed by analyzing the multi-wavelength photoacoustic images of tumor. In conclusion, combined ultrasound and photoacoustic imaging technique has the ability to image fine molecular changes that can aid in early detection of cancer.

\section{J051}

\section{GRP RECEPTOR-AVID GOLD NANOPARTICLES FOR IMAGING AND THERAPY OF CANCER}

Raghuraman Kannan, Nripen Chanda, Ajit Zambre, Ravi Shukla, Evan J. Boote, Kavita K. Katti, Kattesh V. Katti

Radiology, University of Missouri-Columbia, Columbia, MO, USA. 
High affinity of gold nanoparticles to tumor vasculature coupled with their imaging and therapeutic properties make them attractive vectors for the development of theranostic agents for accurate diagnosis and therapy various types of human cancers. Creation of tumor avid and site specific gold nanoparticles for molecular imaging and therapy of cancer will provide realistic prospects for their utility the design and development of nanomedical products. Our research efforts are focused on breast, lung and prostate tumor avidity of bombesin peptide (BBN) as these tumors over express receptors for Gastrin Releasing Peptide (GRP) for which BBN peptide analogs have been shown to be highly specific with optimal receptor affinity via agonistic /antagonistic interactions. We have recently developed a vast library of gold nanoparticle conjugated BBN peptides (AuNP-BBN). Our in vitro cell specific binding affinity studies of AuNPBBNs have demonstrated excellent avidity of gold nanoparticulate peptides with IC50s in the nano to picomolar ranges. Cellular interactions of AuNP-BBN have provided important insights on the ability of gold nanoparticle-conjugated peptides to internalize via endocytosis and thus provide a realistic means accumulating diagnostic/therapeutic payloads of gold nanoparticles cell specifically for diagnosis and breast, colon and prostate tumors. This presentation includes (i) new design concepts for the development of AuNP-BBN; (ii) binding affinity and tumor specific avidity of library of AuNP-BBN and iii) in vivo molecular imaging and therapy studies which demonstrate the tremendous clinical potential of this new generation of tumor specific gold nanoparticles for future applications in early and accurate diagnosis and therapy of breast, colon and prostate cancer patients. This work has been supported by the National Institute of Health/National Cancer Institute under the Cancer Nanotechnology Platform program and Nanoscience and Nanotechnology program (grant numbers: 5R01CA119412-04 and 1R21CA128460-02).

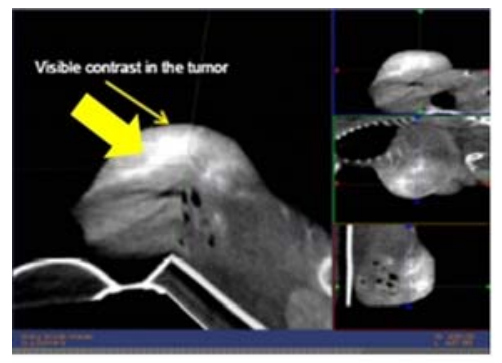

Breast Cancer

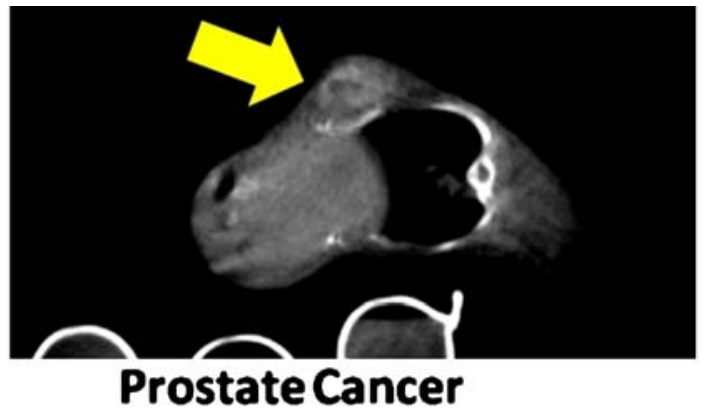

Prostate Cancer

\section{Gold nanoparticles as X-ray contrast agent for imaging breast and prostate cancer}

\section{J052}

\section{PARAMETRIC MODELING OF RAMAN SPECTRA \\ OF NANOPARTICLES FOR QUANTITATIVE UNMIXING}

Kranthi K. Kode ${ }^{1}$, Catherine Shachaf ${ }^{3}$, Sailaja V. Elchuri ${ }^{3}$, Garry Nolan $^{3}$, David Paik ${ }^{2}$

${ }^{1}$ Computational \& Mathematical Engineering, Stanford University, Stanford, CA, USA, ${ }^{2}$ Radiology, Stanford University, Stanford, CA, USA, ${ }^{3}$ Microbiology and Immunology, Stanford University, Stanford, CA, USA.

Purpose: Raman spectroscopy can differentiate the spectral fingerprint of many molecules, resulting in very high multiplexing capabilities. Even with surface enhancement, one of the problems in quantitative unmixing of Raman spectra is related to the presence of a relatively high fluorescence background. Also, changes in experimental conditions can result in subtle widening and shifting of the very narrow Raman peaks which make quantitative unmixing very difficult. The objective of our study was to apply mathematical techniques on parametric models of the Raman spectra of nanoparticles to unmix the contributions from multiple nanoparticles to allow for simultaneous multiplexed quantitation of nanoparticle concentrations for tumor cell characterization as part of an integrated in vitro diagnostics and in vivo imaging cancer nanotechnology project. Materials and Methods: A Gaussian is used to model the individual Raman peaks of each COIN (Composite Organic Inorganic Nanoparticle) in solution. Instead of using preprocessing techniques such as baseline separation to remove the broad fluorescent components, Raman peaks were extracted from the spectra. During modeling, parameters such as the peak height, peak location and full width at half maximum (FWHM) of Raman peaks were extracted from the reference spectra of individual COINs using numerical optimization. These Raman peaks are then subtracted from the combined spectra in different proportions to minimize the Raman nature of the remainder spectrum. Results: We modeled individual Raman peaks to accurately represent the Raman spectra of COINs. We successfully unmixed up to 7 COINS using the algorithm and achieved considerable improvement over the results obtained using direct linear least squares. The errors in estimation of the individual contributions of each nanoparticle using least squares were in the range of $20-70 \%$ while the error based on results from our optimization algorithm are in the range of $10-20 \%$. Conclusion: With the accurate Raman peak separation as opposed to blind baseline correction methods, quantitative unmixing of Raman spectra will be much more reliable resulting in huge potential for application of Raman spectroscopy in the analysis of nanoparticles.

\section{$\mathbf{J 0 5 3}$}

\section{NANOTECHNOLOGY BASED CT CONTRAST AGENT} FOR FUNCTIONAL CANCER IMAGING

Bulent Aydogan ${ }^{2,4}$, Ji Li ${ }^{1}$, Tijana Rajh ${ }^{5}$, Steve Chmura $^{2}$, Charles Pelizzari $^{2}$, Christian Wietholt ${ }^{3,1}$, Ahmed Chaudhary ${ }^{2}$

${ }^{1}$ Department of Radiology, University of Chicago, Chicago, IL, USA, ${ }^{2}$ Department of Radiation and Cellular Oncology, University of Chicago, Chicago, IL, USA, ${ }^{3}$ Department of Medicine/Cardiology, University of Chicago, Chicago, IL, USA, ${ }^{4}$ Department of Radiation Oncology, University of Illinois at Chicago, Chicago, IL, USA, ${ }^{5}$ Center for Nanoscale Materials, Argonne National Laboratory, Argonne, IL, USA. 
Purpose: To study the feasibility of using 2-Deoxy-D-Glucose (2-DG) labeled gold nanoparticle (AuNP-DG) as a metabolic functional CT contrast agent through in vitro experiments. Material and Methods: The gold nanoparticles (AuNP) were fabricated using a citrate acid reduction method. The size of the AuNP was determined from Transmission Electron Microscopy images to be $4 \mathrm{~nm}$ in diameter. The conjugation of the 1-DG and 2-DG with the AuNP core was accomplished using mercapto group in the 1 and 2-carbon positions, respectively. The A549 cells used in these experiments are derived from human lung cancers where PET is commonly employed. Three groups of cell samples $(\sim 1 \times$ 105 cells per sample) were incubated with the AuNP-1DG, AuNP-2DG and the unlabeled AuNP, respectively, for $30 \min \left(37^{\circ} \mathrm{C}, 7 \% \mathrm{CO} 2\right)$. Following the incubation, the cells were washed with sterile PBS six times to remove the excess gold nanoparticles. The cells were then spun to cell pellets using a centrifuge. The cell pellets were imaged using a microCT scanner immediately after centrifuging $(75 \mathrm{kVp}, 135 \mu \mathrm{A}$, $1184 \times 1120$ matrix size, 360 views, averaging 5 frames per view). The reconstructed CT images were analyzed using a commercial software package. Results: Significant contrast enhancement in the cell samples incubated with the AuNP-2DG with respect to the cell samples incubated with the unlabeled AuNP and AuNP-1DG was observed in multiple CT slices. Conclusions: Results from these experiments strongly suggest enhanced uptake of the AuNP-DG over the unlabeled AuNP and AuNP$1 \mathrm{DG}$ by the highly glycolytic cancer cells in vitro and indicate that AuNP-DG could serve as a metabolic functional CT contrast agent with tumor targeting capability. In vivo experiments are underway to determine the feasibility of using AuNP-DG in preclinical cancer research.

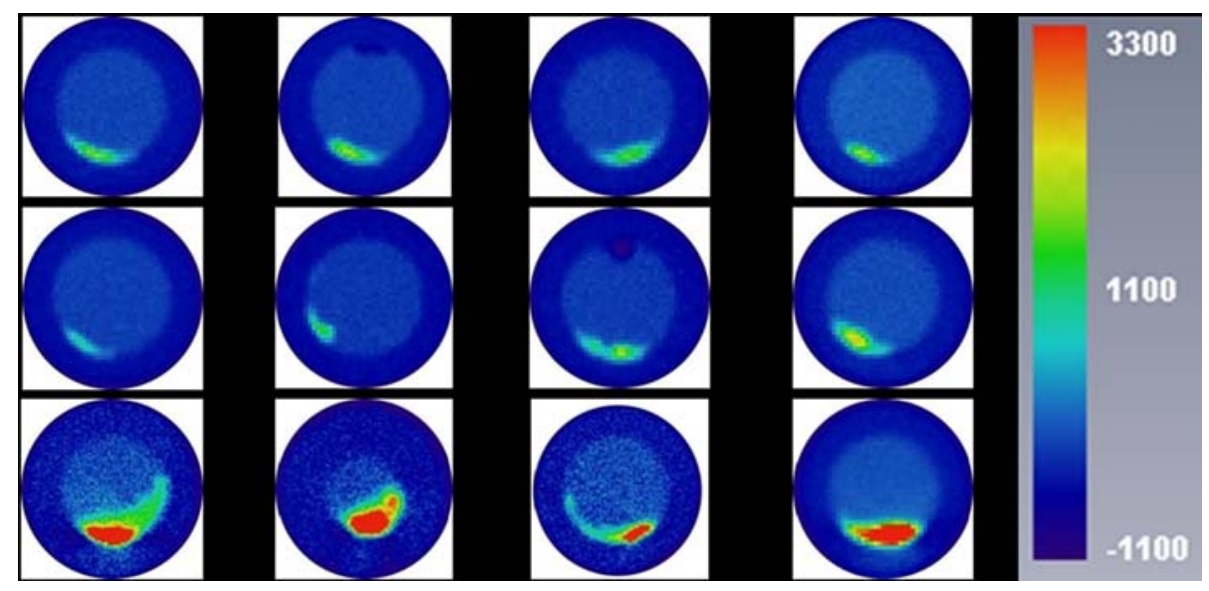

Axial CT slices of three groups of cell samples. (A) Cells incubated with AuNP and (B) with AuNP-1DG and (C) with AuNP-2DG. The intensity is expressed in Hounsfield Units (HU) with color-coding shown in the legend on the right. The contrast enhancement in Group C is about three times higher than that of other groups. The dark blue annuli are the walls of the plastic vials.

J054

\section{SYNTHESIS AND SPECTROSCOPIC ANALYSIS OF SUPER-PARAMAGNETIC NANOPARTICLES FOR MAGNETIC PARTICLE IMAGING}

Kerstin Luedtke-Buzug, Sven Biederer, Timo F. Sattel, Tobias Knopp, Thorsten M. Buzug

University of Luebeck, Institute of Medical Engineering, Luebeck, Germany.

Nanosized materials, especially super-paramagnetic particles based on iron-oxide (SPIO), have advanced to one of the most interesting materials for medical applications from cancer therapy to imaging methods. SPIOs like Resovist (Bayer Schering Pharma), play a key role in a recently proposed imaging technique called magnetic particle imaging (MPI). In this imaging method, the particles are not used as contrast agents, they rather act as tracer. Unfortunately, however, Resovist does not meet all expectations for MPI. For optimal imaging results, a narrow particle size distribution and a high magnetization response is required. Therefore, we started to design new tracer particles, which are more adapted to the demands of MPI. In the case of Resovist, the magnetite core is covered with carboxydextran to prevent the particles from agglomeration. In this contribution, it will be shown that dextran-covered iron-oxide nanoparticles are acceptable candidates as a surrogate for the technical optimization of the MPI system. Furthermore, we prepared tracer with other biocompatible coating materials like chitosan or PEG. Preparation optimization strategies for our new MPI tracer will be presented. A crucial step for optimization is the particle purification strategy, which shows great affects onto the spectral distribution of the SPIOs. The spectral results obtained with magnetic particle spectroscopy (MPS) and photon crosscorrelation spectroscopy (PCCS) have been analyzed and will be 
presented. The hydrodynamic parameter measured with PCCS is important for metabolism prediction, whereas with MPS, a prediction of the particle core diameter and with that the MPI quality of the synthesized particles is possible. Particles with a large metal-core diameter show superior performance compared to those with a small core diameter.
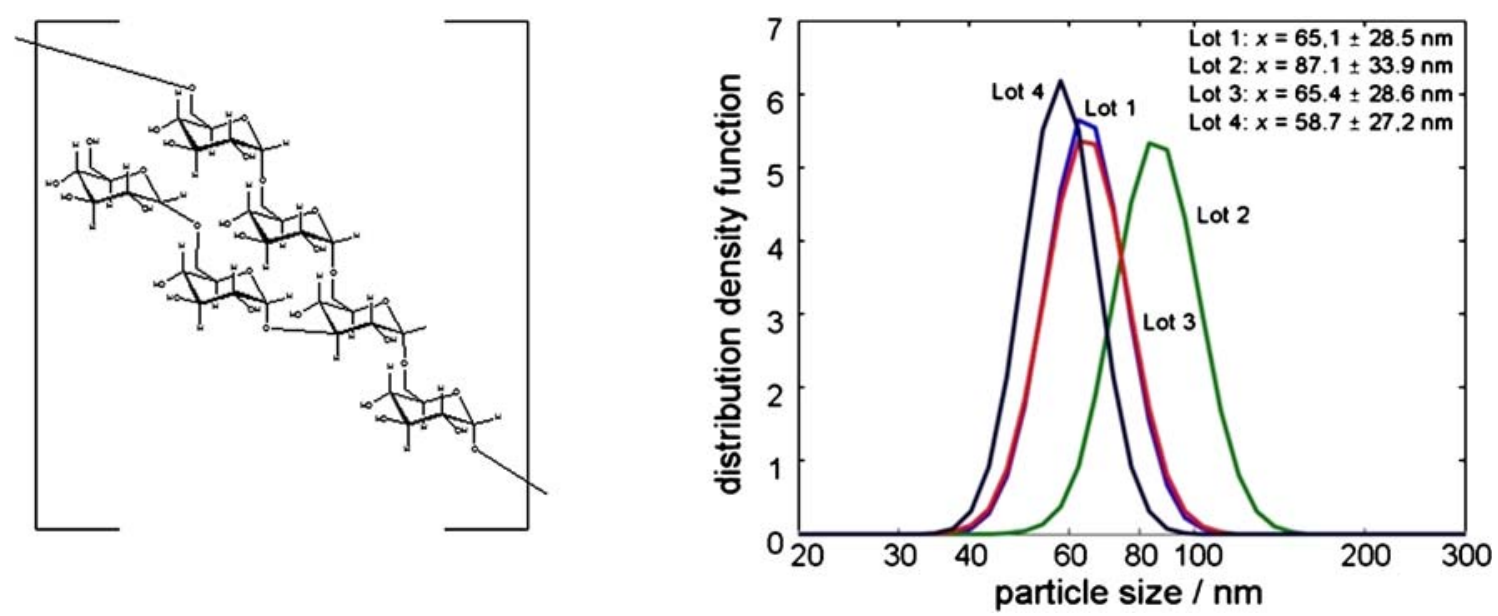

Left: Schematic representation of the structure of a dextran unit. Right: Density distribution of Resovist. It has been found that different lots show different hydrodynamic particle size distributions.

J055

\section{EFFECT OF DEGREE OF SUBSTITUTION OF HIGH MOLECULAR WEIGHT GLYCOL CHITOSAN NANOPARTICLES ON TUMOR TARGETING CHARACTERISTICS}

Seung Young Lee ${ }^{1}$, YoungSoo $\mathrm{Kim}^{2}$, Hyung Jun $\mathrm{Ahn}^{1}$, In-Chan Youn $^{1}$, Youngro Byun ${ }^{3}$, Dong Jin $\mathrm{Kim}^{2}$, Sang Yoon Kim ${ }^{4}$, Kuiwon $\mathrm{Choi}^{1}$, Kwangmeyung $\mathrm{Kim}^{1}$

${ }^{1}$ Biomedical Research Center, Korea Institute of Science and Technology, Seoul, Korea, South, ${ }^{2}$ Center for Chemoinformatics Research, Korea Institute of Science and Technology, Seoul, Korea, South, ${ }^{3}$ College of Pharmacy, Seoul National University, Seoul, Korea, South, ${ }^{4}$ Department of Otolaryngology, Asan Medical Center, University of Ulsan College of Medicine, Seoul, Korea, South.

Several drug delivery carriers containing nanoparticles, liposomes, and micelles have attracted a great deal of attention as potential agents for cancer treatment and diagnosis. Their tumor targeting specificity, however, is still relatively low, and this limits their application to cancer diagnosis and therapy. In this study, in order to evaluate and optimize the tumor targeting characteristics of high molecular weight chitosan nanoparticles (CNPs) with $250 \mathrm{kDa}$, four CNPs with different degree of substitution (CNPs-1, 2, 3, and 4) were prepared by changing the mole ratio of cholanic acid. Although their physicochemical properties such as polydispersity, size and zeta-potential in aqueous condition were not significantly different, their deformability and stability in physiological conditions greatly depend on the degree of substitution (DS) of cholanic acid. As increased DS, the more stable and rigid CNPs were formed. In vivo tissue distribution and tumor accumulation of four Cy5.5-CNPs, Cy5.5-linear glycol chitosan, and Cy5.5 were monitored in SCC7 tumor-bearing mice by using NIR fluorescence imaging systems. Linear glycol chitosan and Cy5.5 molecules were rapidly excreted from the body, whereas four Cy5.5CNPs displayed higher tumor accumulation than linear glycol chitosan and Cy5.5 via the EPR effect. Among them, CNPs-3 showed the prolonged blood circulation time, decreased time-dependent excretion from the body, and elevated tumor accumulation compared to other CNPs. The reasons for the highest tumor accumulation of CNPs-3 might be considered as follows: in vivo stability of CNPs-3 is better than those of CNPs- 1 and -2 , and its tissue uptake by liver and spleen was lower than CNPs-4 which had stiffness property. We proposed that CNPs-3 with $250 \mathrm{kDa}$ is the best for the drug delivery carriers because of long blood circulation time and the highest tumor accumulation than other CNPs. 
A

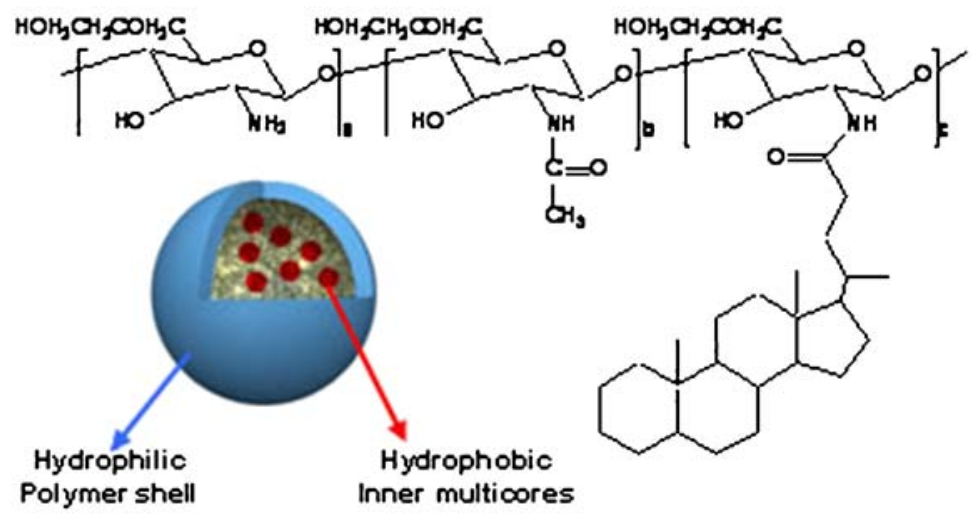

Chitosan based nanoparticles (CNPs)

(DS of $5 \beta$-Cholanic Acid: $4.2 \%, 10.4 \%, 17.4 \%, 34 \%$ )
B

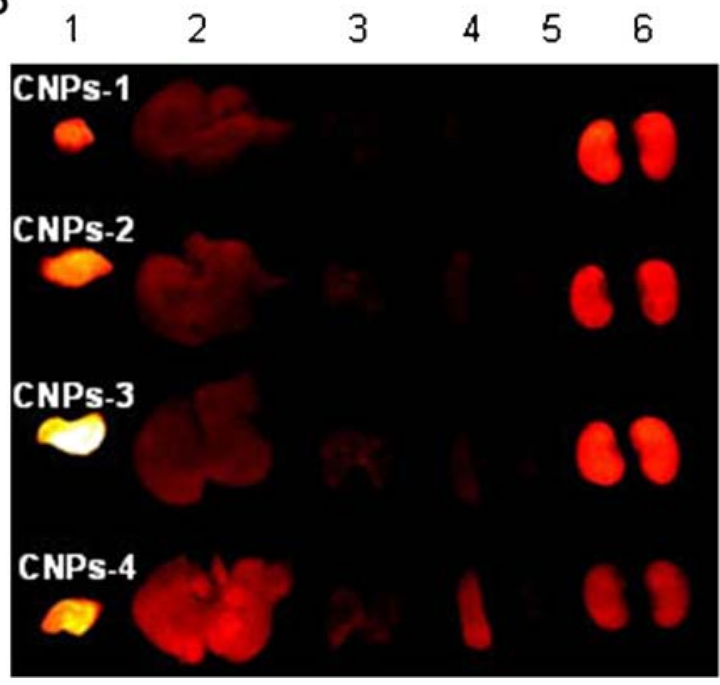

Figure. (A) The representative structure of chitosan based nanoparticles (CNPs) with different degree of substitution (B) ex vivo NIR fluorescence images of dissected organs of mice bearing SCC7 tumors sacrificed at 2 day, after intravenous injection of four Cy5.5-CNPs. 1:tumor; 2:liver; 3: lung; 4:spleen; 5:heart; 6:kidney.

J056

THE IMAGING PROBE DEVELOPMENT CENTER AT THE NATIONAL INSTITUTES OF HEALTH: PRODUCTION OF DIVERSE NANOPARTICLE-BASED MOLECULAR IMAGING PROBES

Biying Xu, Haitao Wu, Agnieszka Sulima, Colin M. Wilson, Nalini Shenoy, Sarah Cheal, Brittany Aicher, Shannon Cofiell, Zhen-Dan Shi, Andres E. Dulcey, Olga Vasalatiy, Valerie Smith, Falguni Bhattacharyya, Yulin Hu, Changhui Li, Kyle Barbacow, Jeffrey Lamb, Courtney Baglino, David Boone, Gary L. Griffiths

IPDC, NHLBI/NIH, Rockville, MD, USA.

Nanoparticle-based molecular imaging is emerging as an interdisciplinary area that shows great promise in helping to understand the components, processes, dynamics and therapies of all types of diseases at molecular levels. The Imaging Probe Development Center (IPDC) was established as part of the National Institutes of Health (NIH) Roadmap for Medical Research Initiative (http://nihroadmap.nih.gov/), and is dedicated to producing and providing molecular imaging probes for $\mathrm{NIH}$ and collaborating scientists. Such probes include optical, radionuclide, ultrasound and magnetic resonance agents, and also multi-modality imaging agents. With respect to nanoparticle-like compositions, in the past couple of years, we have successfully produced several types of agents. These include: 1) UEA-1conjugated polymerized liposomes for targeting and specific binding of colon polyps for microCT colonography, optical coherence tomography and multispectral optical imaging; 2) Doxorubicin-conjugated DTPA-Gd-functionalized PAMAM dendrimers for MRI and drug delivery to glioma; 3) Reduced dextran-coated ultrasmall superparamagnetic iron oxide nanoparticles for fMRI. 4) Pegylated functionalized gold nanoparticles with various core sizes for targeted cancer imaging. The preparation, testing and application of these agents will be described. To learn more, readers are also encouraged to visit the IPDC website at http://www.ipdc.nih.gov
J057

LIPOSOME CONTRAST AGENT FOR CT-BASED DETECTION AND LOCALIZATION OF NEOPLASTIC AND INFLAMMATORY LESIONS IN RABBITS: VALIDATION WITH FDG-PET AND HISTOLOGY

Jinzi Zheng $^{2,1}$, Christine Allen ${ }^{4,1}$, Douglass C. Vines ${ }^{3,1}$, Stefano Serra ${ }^{5}$, Martin Charron ${ }^{6}$, David A. Jaffray ${ }^{3,1}$

${ }^{1}$ STTARR Innovation Centre, Princess Margaret Hospital, Toronto, ON, Canada, ${ }^{2}$ Medical Biophysics, University of Toronto, Toronto, ON, Canada, ${ }^{3}$ Radiation Oncology, University of Toronto, Toronto, ON, Canada, ${ }^{4}$ Pharmaceutical Sciences, University of Toronto, Toronto, ON, Canada, ${ }^{5}$ Pathology, Toronto General Hospital, Toronto, ON, Canada, ${ }^{6}$ Radiology, Hospital for Sick Children, Toronto, ON, Canada.

Nano-sized colloidal particles accumulate at sites of tumor and inflammation through a passive process that selects for vascular fenestrationsknown as the enhanced permeation and retention (EPR) effect. An $80 \mathrm{~nm}$ liposome-based CT contrast agent was successfully developed to provide significant contrast enhancement in these abnormal lesions. In this study, a rabbit model of combined VX2-carcinoma and immune myositis was used to test the performance of the CT liposome agent relative to FDG-PET. Both liposome-CT and FDG-PET identified all nine primary tumors (volumes $=70-7008 \mathrm{~mm} 3$, SUVmax $=1.5-10.9$, HUmean=103.0-140.6). However, liposome-CT exhibited greater sensitivity for detection of inflammatory lesions. Specifically, it detected 25 inflammatory lesions (volumes $=14-2732 \mathrm{~mm} 3$, HUmean $=114.5-268.6$ ), while FDG-PET identified 7 (volumes $=54-1044 \mathrm{~mm} 3, \mathrm{SUVmax}=2.7-7.1$ ). Furthermore, as illustrated in Figure 1, using single time point imaging at 5 days postliposome administration ( $1 \mathrm{~h}$ post FDG injection and 12 days post tumor inoculation), the CT data set allowed for statistically significant differences in the mean $\mathrm{HU}$ to be measured from lesions that make up the two disease groups $(p<0.0005)$. On the other hand, partial volume adjusted SUVmax 
values for the two lesion types calculated from the FDG-PET data set did not yield a significant difference $(p>0.25)$. These results suggest that this liposome contrast agent may play a potential role in increasing the sensitivity and specificity of CT-based disease screening and subsequent image-guided biopsy.

(a)

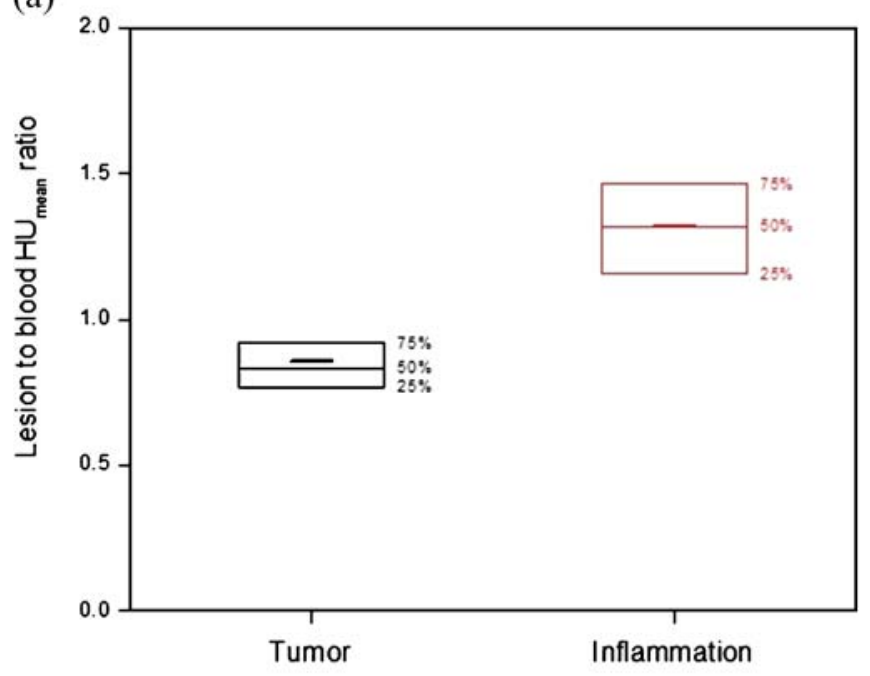

(b)

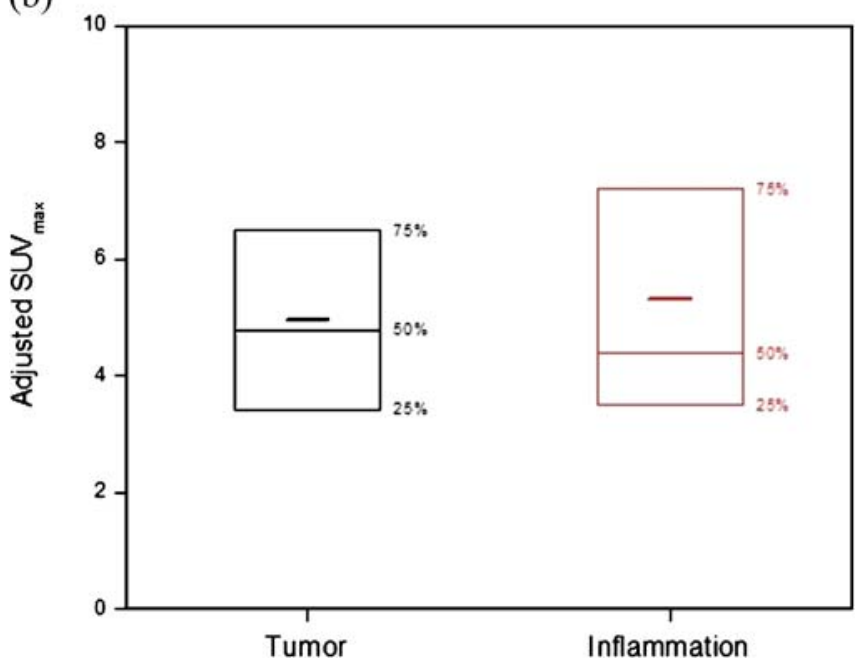

Figure 1: Imaging signal intensities of neoplastic and inflammatory lesions a) from the liposome-CT data set; and b) measured in the FDGPET data set for the two lesion types. The upper, middle and lower bounds of the boxes indicate the 75th, 50th and 25th percentile, and the dash inside each box marks the mean value of each sample population.

\section{J058}

\section{SEMICONDUCTING ORGANIC POLYMER DOTS FOR SENTINEL LYMPH NODE MAPPING BY FLUORESCENCE IMAGING}

Sehoon Kim $^{1}$, Kwangmeyung Kim ${ }^{1}$, Kuiwon $\mathrm{Choi}^{1}$, James F. Leary ${ }^{2}$, Ick Chan Kwon $^{1}$

${ }^{1}$ Biomedical Research Center, Korea Institute of Science and Technology, Seoul, Korea, South, ${ }^{2}$ Basic Medical Sciences and Biomedical Engineering, Bindley Bioscience Center and Birck Nanotechnology Center, Purdue University, West Lafayette, IN, USA.

Fluorescence and its related techniques have made significant contributions to the advances of modern life sciences, as powerful tools allowing a closer access to various biological processes at the molecular and cellular levels. Oncological studies through in vivo molecular imaging are a representative example of recently developed fluorescence-based applications. As a fluorescent probe, semiconductor quantum dots used in vivo has shown great potential to revolutionize biomedical imaging owing to their outstanding optical properties compared to organic counterparts. However, there exist concerns regarding potential toxicity by their heavy metal composition, which can be a roadblock to clinical translation. Here we present all-organic semiconductor dots as a toxic element-free alternative, i.e., surfactant-coated $\pi$-conjugated polymer nanoparticles whose properties such as bright near-infrared (NIR) emission, stable water dispersion, $\mathrm{CHNO}$-only chemical constitution and mesoscopic size range $(<50 \mathrm{~nm})$ are favorable for in vivo applications. We demonstrate that these semiconducting polymer dots can be produced in a single step into a ready-to-use formulation via solvent-free colloidal polymerization. By virtue of a proper molecular design of the polymeric backbone structure, the fluorescence emitting capability of the dots was retained in the chain-aggregated nanoparticulate solid state, as opposed to the aggregation-quenched fluorescence of common red/NIR-emissive conjugated polymers. Fluorescence tracking of their lymphatic drainage in a mouse model enables real-time sentinel lymph node mapping that allows a major cancer surgery, sentinel lymph node biopsy, to be performed in a fast and precise way. These results demonstrate the potential of nanostructured molecular semiconductors as a new generation of semiconducting nanomaterials for biomedical research.

\section{J059}

\section{MAGNETIC RESONANCE IMAGING OF ORTHOTROPIC HEPATOCELLULAR CARCINOMA IN MICE WITH DIFFERENT IRON OXIDE NANOPARTICLES}

Lihong $\mathrm{Bu}^{1,2}$, Kai Chen ${ }^{1}$, Jin $\mathrm{Xie}^{1}$, Jing Huang ${ }^{1}$, Kin Wai Sun ${ }^{1}$, Y. A. Wang ${ }^{1}$, Mei-Sze Chua ${ }^{1}$, Samuel So ${ }^{1}$, Baozhong Shen ${ }^{2}$, Xiaoyuan Chen ${ }^{1}$ ${ }^{1}$ MIPS, Department of Radiology, Stanford University, Stanford, CA, USA, ${ }^{2}$ Department of Radiology, The Fourth Hospital of Harbin Medical University, Harbin, China.

Objective: The purpose of this study was to evaluate the capability of two groups of triblock copolymer coated iron oxide nanoparticles to image hepatocellular carcinoma (HCC). Materials and Methods: Iron oxide nanoparticles with core diameter of $10,20,30$ and $50 \mathrm{~nm}$ were synthesized from pyrolysis and rendered water-soluble by amphiphilic triblock copolymer coating, which consists of a polybutylacrylate segment, a polyethylacrylate segment, a polymethacrylic acid segment and a hydrophobic hydrocarbon side chain. These particles were then PEGylated with either NH2- $\mathrm{PEG}_{2000}-\mathrm{COOH}$ or $\mathrm{NH} 2-\mathrm{PEG}_{2000}-\mathrm{OMe}$ to further improve water solubility and biocompatibility. In vitro uptake experiment was performed with Huh7 hepatocellular carcinoma (HCC) and RAW 264.7 murine macrophage cell lines. To evaluate the hepatic uptake and clearance of the probes, all eight different particles (4 with methoxy end groups and 4 with carboxylate end groups), together with Feridex, were intravenously injected into $\mathrm{Balb} / \mathrm{C}$ mice at various concentrations $\left(15,5,2.5\right.$, and $1 \mathrm{mg} \mathrm{Fe} / \mathrm{kg}$ ) and $\mathrm{T}_{2}$-weighted MRI was performed at $1 \mathrm{~h}$ postinjection (p.i.). To detect orthotopic Huh7 tumor grown in athymic nude mice, the particles were administered at a dose of $2.5 \mathrm{mg} / \mathrm{kg} \mathrm{Fe}$ and $\mathrm{T}_{2}$ MR scans were performed ( $1 \mathrm{~h}$ dynamic scan and static scans at $4 \mathrm{~h}, 24 \mathrm{~h}, 72 \mathrm{~h}, 1 \mathrm{wk}, 2 \mathrm{wk}$, and $3 \mathrm{wk}$ ). Histology studies and ICP analysis were performed to consolidate the MRI observation. Results: The ICP analysis indicated that all the 8 nanoparticles, as well as Feridex, can be intracellularly internalized by macrophage but not Huh7 cells. The polymer coated particles of different sizes and Feridex led to similar signal reduction in the liver of normal mice. In the orthotropic Huh7 model, all 4 methoxy capped nanoparticles showed similar tumor/ liver ratio to that of Feridex. The carboxylate capped IONPs, however, 
had much improved tumor/liver ratios and faster clearance than Feridex at all time points examined. Prussian blue staining confirmed that all the particles were trapped in liver Kupffer cells but not tumor cells. Conclusion: Triblock copolymer coated large core iron oxide nanoparticles with PEGylate carboxylate ending groups is superior to FDA approved Feridex in hepatocellular carcinoma detection.

\section{J060}

CyLoP-1, A NEW TOOL FOR THE CYTOSOLIC DELIVERY OF INTRACELLULAR TARGETED PROBES

Deepti Jha ${ }^{1}$, Ritu Mishra ${ }^{1}$, Sven Gottschalk ${ }^{1}$, Karl-Heinz Wiesmueller ${ }^{2}$, Kamil Ugurbil $^{1}$, Joern Engelmann ${ }^{1}$

${ }^{1}$ High Field magnetic Resonance Center, Max planck institute for Biological Cybernetics, Tuebingen, Germany, ${ }^{2}$ EMC microcollections $\mathrm{GmbH}$, Tuebingen, Germany.

Cell Penetrating Peptides (CPPs) have gained attention in biomedical field because of their ability to deliver a variety of hydrophobic moieties through the membrane barrier. CPPs are generally taken up by endocytotic pathways and vesicular encapsulation limits the interaction of the attached cargo with targets present in the cytosol. Here we present the systematic development of a cysteine rich CPP (derived from polypeptide Crotamine [1]) by Structure Activity Relationship (SAR) studies. Various peptides were synthesized by $\mathrm{Fmoc} / \mathrm{tBu}$ strategy and labeled with fluorescein isothiocyanate at the N-terminus. After purification by RP-HPLC, the products were lyophilized and characterized by ESI-MS. Uptake of fluorescently labeled peptides was assessed in NIH-3T3 mouse fibroblasts by fluorescence spectroscopy and microscopy. Our studies identified a novel CPP, CyLoP-1, which is efficiently delivered into the entire cytoplasm of cells besides being encapsulated in vesicles at low concentrations of $2.5 \mu \mathrm{M}$ in a non-cytotoxic and temperature independent fashion. It proved to be most efficient in its natural L-form as SAR studies indicated a clear negative effect of chiral inversion of the residues on cellular uptake and distribution of CyLoP-1. The additional feature of cytosolic gain was further explored by the covalent attachment of different cargoes to CyLoP-1. Payload consisted of fluorophore, a Magnetic Resonance Imaging agent (Gd)-DOTA, a bio-active peptide (SmacN7), the larger peptide Penetratin, or a PNA molecule. The regular decrease in the uptake and localization pattern concomitant with the increase in the cargo size was observed. This indicates the dependence of internalization and distribution profile of $\mathrm{CyLoP}-1$ constructs on the nature, charge as well as size of the cargo. Efficient intracellular delivery into the entire cytosol and a low cytotoxicity makes CyLoP-1 a promising candidate for cytosolic delivery of cargoes of small sizes like intracellular targeted probes. [1] Kerkis A. et al, FASEB J. 2004, 18, 1407.

\section{J061}

\section{IMAGE-BASED EVALUATION OF THE EFFECTS OF PARTICLE SIZE AND MOLECULAR TARGETING ON THE INTRATUMORAL DISTRIBUTION OF POLYMERIC NANOPARTICLES}

Helen Lee ${ }^{1}$, Humphrey Fonge ${ }^{1}$, Bryan D. Hoang ${ }^{1}$, Raymond M. Reilly $^{1,2}$, Christine Allen ${ }^{1,3}$

${ }^{1}$ Department of Pharmaceutical Sciences, University of Toronto, Toronto, ON, Canada, ${ }^{2}$ Department of Medical Imaging, University of Toronto, Toronto, ON, Canada, ${ }^{3}$ Department of Chemical Engineering and Applied Chemistry, University of Toronto, Toronto, ON, Canada.

INTRODUCTION: Nano-sized polymeric micelles (PMs) are primarily developed for delivering drugs to tumors via passive and active targeting. However, the intratumoral distribution of passively targeted
PMs versus actively targeted PMs remains relatively unexplored. It has also been speculated that smaller particle size may lead to enhanced tumor penetration, resulting in a more homogeneous distribution within solid tumors. In this study, $27 \mathrm{~nm}$ and $60 \mathrm{~nm}$ PMs (EGF+/EGF-) are labeled with 111 indium $(111 \mathrm{In})$ for studying their penetration and distribution in EGFR-overexpressed tumors via autoradiographic imaging. The PMs were also fluorescently tagged (AF647) for visualization under confocal microscopy in order to evaluate their micro-distribution with respect to blood vessels. METHODS: 111InPMs were administered intravenously to tumor-bearing mice. Correlation between the localization of $111 \mathrm{In}-\mathrm{PMs}$ (autoradiography) and microvessel density (CD-31-stained histological images) within tumors were obtained via ROI analyses. Tumors excised from mice injected with AF647-PMs were sectioned, stained with FITC-anti-CD-31 antibodies and DAPI for visualization of blood vessels and cell nuclei, respectively. RESULTS: The intratumoral distribution of $111 \mathrm{In}-\mathrm{PMs}$ (EGF+/EGF-) was heterogeneous, but positively correlated with the microvessel density $(r=0.7, p<0.001)$. Overlay of the autoradiography image with the corresponding CD-31-stained histology image revealed that tumor regions with high radioactivity accumulation are mostly associated with intense CD-31-staining. In addition, the attachment of EGF as a targeting moiety did not influence the intratumoral distribution of $111 \mathrm{In}-\mathrm{PMs}$ as no significant difference in the correlation coefficients was observed $(p>0.96)$. Microscopic evaluation revealed that the AF647-60 nm-PMs (EGF-/EGF+) mainly reside in close proximity to blood vessels (mostly within $50 \mu \mathrm{m}$ ). While, the AF647$27 \mathrm{~nm}$-PMs seem to afford a more homogeneous distribution within the imaged area $(<500 \times 500 \mu \mathrm{m})$. Some of the AF647-27 nm-PMs were found to localize as far as $300 \mu \mathrm{m}$ from the nearest blood vessels. CONCLUSION: Tumors with well-developed vasculature networks are likely to result in increased PM extravasation into tumors via passive targeting, a characteristic that is independent of the presence of a targeting ligand. In this study, smaller PMs were found to travel further away from blood vessels than larger PMs, allowing for more cell-PM interaction which is essential for effective active targeting.

\section{J062}

\section{CLATHRIN NANOPARTICLES AS POTENTIAL} HIGH-RELAXIVITY MAGNETIC RESONANCE NANOPROBES FOR MOLECULAR BRAIN IMAGING Gordana D. Vitaliano $^{1,2}$, David Rios ${ }^{1,2}$, Franco Vitaliano ${ }^{1}$, Martin H. Teicher $^{1,2}$

${ }^{1}$ McLean Hospital, Belmont, MA, USA, ${ }^{2}$ Department of Psychiatry, Harvard Medical School, Boston, MA, USA.

Background: Magnetic Resonance Imaging is a noninvasive visualization technique with high spatial resolution, but low sensitivity for visualization of molecular targets. In order to improve MRI sensitivity for molecular brain imaging, our goal was to develop clathrin-based nanoprobes with high molecular relaxivity that incorporate high payloads of Gadolinium (Gd) contrast agents, which can be delivered non-invasively and target specific receptors in the rat brain. Methods: Gadolinium-DTPA-ITC was conjugated to clathrin cages through reactive lysine residues. We determined the chelate to protein molar ratio by using a standard spectrophotometric method based on the reaction between a DTPA-ITC ligand protein conjugate and an yttrium (III) complex of Arsenazo III. Relaxivity for each sample was calculated using T1 data and gadolinium concentration as determined by NMR analysis. Maleimide-PEG-Dopamine-3 Antibody (D3Ab) and Maleimide-PEG-rhodamine conjugates were prepared, clathrin-nanoparticles were PEGylated and delivered intranasally. Animals were sacrificed 3 hours after intranasal administration of D3Ab-labeled fluorescent Clathrin nanoparticles. Results: Electron Microscopy has shown a large proportion of Gd-DTPA-clathrin cages that form hexagonal 
barrels $52 \mathrm{~nm}$ in size. The mean Ligand/Protein molar ratio was $27 \pm 2.4$. At 0.47T, Gd-DTPA-ITC-Clathrin-Cages displayed relaxivity of 283,176 $\mathrm{mM}^{-1} \mathrm{~s}^{-1}$ per particle, and $97 \mathrm{mM}^{-1} \mathrm{~s}^{-1}$ per Gd ion. Three hours after intranasal administration D3Ab-labeled Clathrin nanoparticles were found only in D3 related brain regions in rats. Fluorescent and light microscopic examination of the D3 brain regions confirmed specific targeting of the D3 receptors with D3Ab-nanoprobes. Confocal laser microscopy confirmed integrity of the nanoparticles in the rat brain. Clathrin and $\mathrm{D} 3 \mathrm{Ab}$ florescence co-localized in the D3 brain regions. Conclusions: New Clathrin nanoparticles displayed an unusually high molecular relaxivity, were successfully delivered non-invasively nasally into the rat brain, were able to target specific receptors, and remained stable in the rat brain. They showed over 50,000-fold greater molecular relaxivity than any currently approved Gd-MRI contrast agent. These preliminary results should encourage further investigations into the use of clathrin cages as a new nanoplatform for MR contrast enhanced molecular brain imaging.

\section{Novel MRI Probes Including Hyperpolarization}

\section{J063}

\section{CAN GENE PROBES DETECT ANGIOGENESIS IN LIVING BRAINS?}

Philip K. Liu ${ }^{1}$, Zerong You ${ }^{2}$, Christina Liu ${ }^{1}$

${ }^{1}$ Radiology, Massachusetts General Hospital, Charlestown, MA, USA, ${ }^{2}$ Pediatrics, Massachusetts General Hospital, Charlestown, MA, USA.

Gliogenesis, revascularization (angiogenesis), and neurogenesis are three major events thought to contribute to brain repair. The interaction of these three processes after global cerebral ischemia (GCI) in the brain is not totally understood. Gene expression at the transcription level during brain repair that follows GCI may be related to metabolic response and plasticity, and all involve the presence of neural progenitor cells (NPC); therefore, understanding the changes that occur throughout the repair process will aid translation of gene targeting for therapies. However, detection of de novo NPC in the brain is not routinely performed clinically because the techniques rely on the use of biopsy or autopsy samples. The biopsy procedure to obtain brain tissue severely limits the utility of these methods because they remove the same cells that we wish to save in vivo, and often clearly precludes longitudinal therapeutic evaluation. To overcome these problems we developed an alternative method that uses brain probes for magnetic resonance imaging (MRI); this novel method provides a powerful and less invasive means of in vivo detection of gene action in brain cells. Superparamagnetic iron oxide nanoparticles (SPION, a T2 susceptibility MR contrast agent) are linked to phosphorothioate-modified antisense oligodeoxynucleotides (sODN) to target endogenous gene transcripts of astroglia in live C57black6 mouse brains. Because sODN are charged molecules, they are taken up by the brain cells along with SPION; sODN retention is determined by sequence homology with cellular mRNA targets. By virtue of its coupling with the complementary sODN, SPION retention in living brains can thus imaged by MRI. We induced GCI by transient bilateral carotid occlusion for 60 minutes. We detected abnormal water diffusion by way of hyperintense diffusionweighted imaging and calculated significant reduction using the apparent diffusion coefficient (rADC) at one day of reperfusion. BBB leakage was detected in the region of $\mathrm{rADC}$ at four weeks after GCI. We detected angiogenesis using MR probe targeting cells with high actin gene transcript in the 8th week, and gliosis using MR probe targeting astroglia in the 9th week post GCI. We found gliosis and angiogenesis in close and adjacent regions, but not in the same cells. The MRI data were confirmed by immunohistology in the 10th week. MRI diagnosis using gene probes has applications for cell typing based on specific mRNA in living cells in vivo. Supported by NIH (R01NS45845; R21NS057556, R21DA024235, RO1DA026108), NCRR (P41RR14075).

\section{J064}

\section{NADH RESPONSIVE MRI CONTRAST AGENT: A REVERSIBLY ACTIVATABLE SENSOR FOR OXIDATIVE STRESS}

Chuqiao Tu, Angelique Louie; Department of Biomedical Engineering, University of California, Davis, Davis, CA, USA.

Disruption of redox homoeostasis in living systems may lead to oxidative stress and can induce many pathological conditions, e.g., Alzheimer's disease, Parkinson'sdisease, atherosclerosis, and cancer etc. Therefore, noninvasive monitoring of tissue redox status could have great utility in clinical applications. Herein we describe the synthesis and characterization of an activatable, T1-weighted magnetic resonance imaging (MRI) contrast agent (also detectable in optical imaging) triggered by NADH, an indicator of superoxide in living systems. The agent is generated by coupling a spironaphthoxazine molecule to a macrocycle-based gadolinium complex, GdDO3A. In the dark the agent in water forms the MC (open) isomer, which has a r1relaxivity of $5.58 \pm 0.36 \mathrm{mM}-1 \mathrm{~s}-1$ and emits strong fluorescence at $539 \mathrm{~nm}$ with a quantum yield of $22 \%$. Visible light irradiation does not influence the magnetic and optical properties of the agent. However, in the presence of NADH the agent undergoes an isomerization to its $\mathrm{SO}$ (closed) isomer. Removal of the phenoxide oxygen atom leads to an r1relaxivityincrease of $54 \%$ to $8.60 \pm 0.74 \mathrm{mM}-1 \mathrm{~s}-1$, while the strong fluorescence is quenched gradually and disappears in $20 \mathrm{~min}$. The intensity change is clearly seen in MRI and optical imaging in both aqueous solutions and cells in culture. Both MC and SO isomers of the agent are non-toxic to cells. The reaction of the agent and NADH was found to be reversible. After the T1 measurement of the reaction betweenthe agent and NADH finished, hydrogen peroxide was applied to the system and it was found that the T1 value of the agent was fully restored.Currently, most MRI contrast agents sensitive to redox are nitroxides, which have some limitations because of their relatively low relaxivity and their short life span. Gadolinium-based contrast agents sensitive to redox would open MRI to a host of new applications in functional imaging, particularly in evaluation ofthe role of reactive oxygen species (ROS) in oxidative diseases such as Alzheimer's disease, stroke, and other neurologic disorders.

\section{$\mathbf{J 0 6 5}$}

\section{NOVEL PARAMAGNETIC GD(III) BASED LABELS TARGETED} TO CELL SURFACE THIOLS FOR CELLULAR IMAGING

Giuseppe Digilio $^{2}$, Valeria Menchise ${ }^{3,1}$, Eliana Gianolio ${ }^{1}$, Valeria Catanzaro ${ }^{1}$, Franco Fedeli ${ }^{1}$, Roberta Napolitano ${ }^{1}$, Concetta Gringeri ${ }^{3}$, Silvio Aime ${ }^{1}$

${ }^{1}$ Center for Molecular Imaging, University of Turin, Torino, Italy, ${ }^{2}$ Dept. of Environmental and Life Sciences, Università del Piemonte Orientale, Alessandria, Italy, ${ }^{3}$ Institute of Biostructures and Bioimages, CNR, Napoli, Italy.

We have very recently described a new method for labeling cells with Gd(III) based CAs by exploiting the chemical reactivity of protein thiols exposed on the outer surface of cell membranes [1,2]. The imaging probe we synthesized (Gd-L1, Figure 1) is composed of a contrastographic unit based on a Gd(III) chelate, a reactive moiety for the recognition of thiols, and a flexible linker connecting them. This probe can bind to Exofacial Protein Thiols (EPTs) through a covalent disulfide bridge, leading to a very satisfactory level of cell labeling. Here we investigate the structural determinants of the paramagnetic labels that are at the basis of a high labeling efficiency. Besides compound Gd-L1, three novel Gd(III)-complexes based either on the Gd-DO3A or the Gd-DTPA chelating cage have been synthesized and evaluated for their ability to label human myeloid leukemia K562 cells. All probes were found to promptly react with EPTs. However, the DO3A-based probes could efficiently label cells, whereas the DTPA-based ones could not. The extent of uptake of the DO3A-based probes was found to be proportional to the 
concentration of EPTs on the cell surface. Chemical blocking of EPTs resulted into the inhibition of the uptake. Lactate, that can coordinate the $\mathrm{Gd}$ (III) ion in Gd-DO3A-like probes thus making a ternary complex, was found to strongly inhibit the uptake of the DO3A-based probes. These results suggest that the labelling efficacy is related to the internalization of the probe occurring after the formation of the probe/EPTs disulfide bridged covalent adduct. Such internalization is likely triggered by the coordination of the GdDO3A metal ion by cell surface carboxylates. Finally, the proposed labeling procedure has been extended to cells other than K562, initially chosen as a model system, to demonstrate its general applicability. 1. C. Carrera et al., Dalton Trans. 2007, 4980-4987. 2. G. Digilio et al. , Chem. Commun., 2009, 893-895.

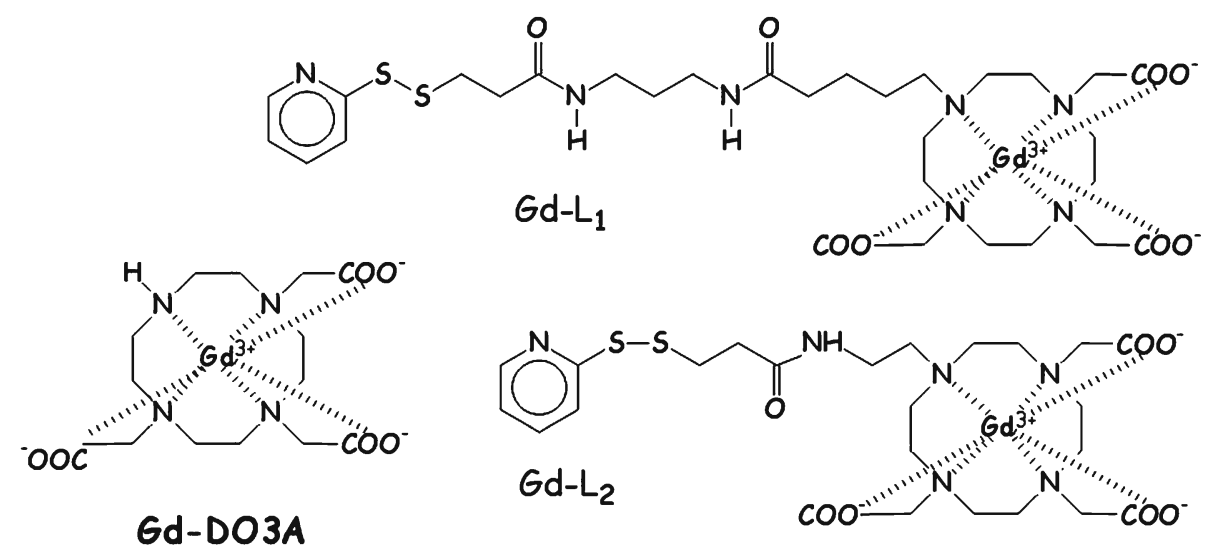

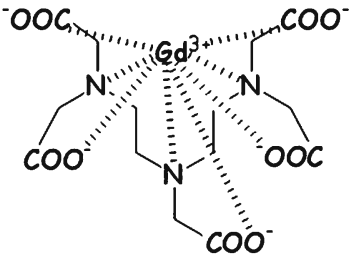

Gd-DTPA

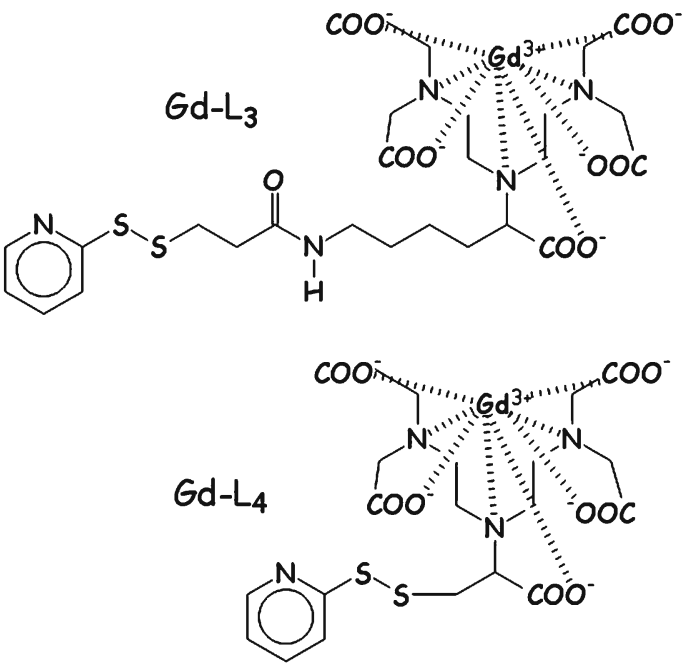

Structures of the paramagnetic labels targeted to EPTs

\section{J066}

\section{VECTORIZATION OF IRON OXIDE NANOPARTICLES WITH A NEURON-PENETRATING RVG PEPTIDE:} AN ATTEMPT TO CROSS THE BLOOD BRAIN BARRIER Jean-Marie Colet ${ }^{2}$, Sophie Laurent ${ }^{1}$, Sebastien Boutry ${ }^{1}$, Zehra Kahvecioglu ${ }^{2}$, Luce Vander Elst ${ }^{1}$, Robert N. Muller ${ }^{1}$

${ }^{1}$ NMR and Molecular Imaging Laboratory, University of Mons, Mons, Belgium, ${ }^{2}$ Human Biology and Toxicology, University of Mons, Mons, Belgium.

Introduction: Neurotropic viruses cross the BBB to infect brain cells. Amongst those, rabies virus shows a high degree of neurotropism in vivo through very well characterized cellular mechanisms. As recently demonstrated by Kumar et al. (Nature, 448, 39, 2007), a short 29-aminoacid peptide from rabies virus glycoprotein (RVG) interacts specifically with the nicotinic acetylcholine receptor (AchR) on endothelial and neuronal cells to enable viral entry into the brain. USPIO are ultrasmall superparamagnetic iron oxide nanoparticles presenting a prolonged plasma half-life. In the brain, they are restricted to the vascular space due to their physico-chemical properties that prevent them to cross the BBB. In this study, USPIO were vectorized with the 29-amino-acid peptide from RVG to promote their way through the BBB. Materials and Methods: USPIO were functionalized with the short RVG peptide and then with an aminoPEG 750 to avoid rapid elimination. The particles were fully characterized by relaxometry and magnetometry. Imaging of anaesthetized mice brain was performed on a Bruker Avance machine at 4.7T. Images were acquired with the TURBO-RARE sequence $(\mathrm{TR}=3090 \mathrm{~ms} / \mathrm{TE}=$ $42.1 \mathrm{~ms} / \mathrm{Tacq}=13.1 \mathrm{~min}$.) before and 0.5, 4, and $22 \mathrm{~h}$ after i.v. administration of either USPIO at a single dose of $300 \mu \mathrm{mol}$ of iron $/ \mathrm{kg}$ of body weight. Results: $30 \mathrm{~min}$ after the administration of RVG-USPIO, a $37 \%$ reduction in signal intensity was reproducibly observed in brain subregions. $22 \mathrm{~h}$ post-RGV-USPIO injection, a signal reduction of $18 \%$ was still measured, as compared to a $8 \%$ signal reduction with naked USPIO. Conclusions: In this study, we have successfully developed new vectorized nanoparticles with the RVG peptide known to allow crossing 
of BBB. Preliminary in vivo imaging assessment of mouse brain showed sustained $(>22 \mathrm{~h})$ reduction of signal intensity, suggesting that a fraction of the injected particles effectively crossed the BBB. Pharmacokinetics properties of the RVG-coated particles and brain distribution using histochemistry and FITC-RVG-particles are under investigations.

\section{J067}

\section{IMAGING DOPAMINE IN THE BRAIN WITH A NOVEL MRI} CONTRAST AGENT

Gil G. Westmeyer ${ }^{1}$, Mikhail G. Shapiro ${ }^{2}$, Philip Romero ${ }^{4}$, Jerzy Szablowski $^{2}$, Christopher R. Otey ${ }^{4}$, Francer Arnold ${ }^{4}$, Alan Jasanoff ${ }^{2,3}$

${ }^{1}$ Brain and Cognitive Sciences, M.I.T., Cambridge, MA, USA, ${ }^{2}$ Biological Engineering, M.I.T., Cambridge, MA, USA, ${ }^{3}$ Nuclear Science and Engineering, M.I.T., Cambridge, MA, USA, ${ }^{4}$ Division of Chemistry \& Chemical Engineering, CALTECH, Pasadena, CA, USA.

Dopaminergic signaling in the mammalian brain is critical for control of motor function and reinforcement learning and is pathologically altered in Parkinson's disease and drug addiction. To further our understanding of the complex spatiotemporal patterns of dopamine neurotransmission, molecular imaging methods would be of great help. Here we report in vivo experiments using the first molecular imaging agent that senses dopamine concentrations in conjunction with magnetic resonance imaging (MRI). The novel dopamine sensor is based on the metalloprotein P450-BM3 (BM3h), a genetically encodable MRI contrast agent whose sensitivity and specificity for dopamine was greatly increased using directed evolution techniques. Binding of dopamine to a site close to the mutant BM3h's paramagnetic heme iron lowers the protein's T1 relaxation rate and leads to a relative MRI signal decrease. The sensor quantitatively detects dopamine release from PC12 cells in culture. Coinjection of the sensor with exogenous dopamine into rodent brain results in a clearly observable hypointense MRI signal, compared to areas injected with sensor alone. In addition, potassium challenge of the rat forebrain leads to a significant MRI signal decrease mediated by the sensor. Ongoing experiments in the lab seek to apply the sensor for imaging phasic dopamine release during reward-related sensory paradigms and behavior.

\section{$\mathbf{J 0 6 8}$}

\section{NOVEL REDOX-SENSITIVE MRI CONTRAST AGENTS AS BIOMARKERS FOR OXIDATIVE STRESS IN A PRE-CLINICAL TUMOR XENOGRAFT MODEL} Bhumasamudram Jagadish ${ }^{2}$, Gerald P. Guntle ${ }^{3}$, Vijay Gokhale ${ }^{4}$, Amanda F. Baker ${ }^{3}$, Eugene A. Mash ${ }^{2}$, Natarajan Raghunand ${ }^{1,3}$

${ }^{1}$ Radiology, The University of Arizona, Tucson, AZ, USA, ${ }^{2}$ Chemistry, The University of Arizona, Tucson, AZ, USA, ${ }^{3}$ Cancer Center Division, The University of Arizona, Tucson, AZ, USA, ${ }^{4}$ Pharmacology \& Toxicology, The University of Arizona, Tucson, AZ, USA.

The disulfide/thiol redox status of the extracellular milieu is known to affect tumor sensitivity to radiotherapy and platinum-based drugs, and may impact the effectiveness of all drugs which produce oxidative stress. We present the development of DOTA- and DO3A-based thiol complexes of gadolinium designed to be responsive to the disulfide/thiol ratio. These complexes covalently bind to human serum albumin (HSA) at Cys-34 under normal oxidizing conditions, but are competed off by endogenous thiols in highly reducing microenvironments. Binding to a macromolecule such as HSA typically increases the longitudinal water-proton relaxivity (r1) of gadolinium complexes. We report strategies to reduce the $\mathrm{r} 1$ when the complex is bound to HSA ("off'), relative to the r1 when the complex is not bound to HSA ("on"). The rationale is that MRI contrast produced by such molecules would highlight tissue microenvironments that are highly reducing. Highly reducing microenvironments would be protective of the tumor against drugs such as 2-deoxyglucose, and conversely, post- treatment changes in tumor redox would be indicative of drug pharmacodynamics. Gadolinium complexes of the general form Gd-DO3A-X-SH or Gd-DOTA-Y-SH were synthesized and characterized, where $\mathrm{X}$ is alkyl, aromatic or PEG, and $\mathrm{Y}$ is alkyl. Binding affinities of the Gd complexes for HSA were determined in vitro in the presence of varying amounts of Eu analogs or homocysteine. In addition, $r 1$ relaxivities of the Gd complexes were measured in the presence and absence of HSA. In preliminary in vivo MRI studies, Gd-DOTA-(CH2)6-SH and Gd-DO3A-(CH2-CH2-O)2$(\mathrm{CH} 2) 3-\mathrm{SH}$ were used for imaging PANC-1 and MIA-PaCa-2 tumors grown in SCID mice. DCE-MRI was performed on a $7 \mathrm{~T}$ scanner using a GRE sequence $\left(\mathrm{TR}=80 \mathrm{~ms}\right.$, TE $=4 \mathrm{~ms}, \alpha=23^{\circ}$, matrix $\left.=128 \times 128\right)$. Data were fitted to a 2-compartment model for Ktrans, vP and ve. Tumor T1 maps were acquired before and $60 \mathrm{~min}$ after injection of $0.05 \mathrm{mmole} / \mathrm{Kg}$ of Gd. In PANC-1 tumors, both Gd complexes exhibited a significantly slower Ktrans than Gd-DTPA, indicative of spontaneous in vivo binding to HSA; this difference could be abolished by co-injecting homocysteine with the $\mathrm{Gd}$ complex. Preliminary results in MIA-PaCa-2 tumors indicate that the change in tumor $\mathrm{T} 1$ at $60 \mathrm{~min}$ post-injection of Gd-DO3A-(CH2-CH2-O)2-(CH2)3SH was significantly smaller in mice treated $24 \mathrm{~h}$ earlier with $500 \mathrm{mg} / \mathrm{Kg}$ of 2DG, relative to untreated mice bearing size-matched control tumors. Experiments are underway to correlate these MRI findings with histopathological measures of treatment-related changes in tumor redox status.

\section{J069}

\section{OF MOLECULES AND MAGNETS, MAKING SENSORS WITH XENON}

Ivan Dmochowski

Chemistry, UPenn, Philadelphia, PA, USA.

Ivan J. Dmochowski, Ph.D. University of Pennsylvania, Department of Chemistry, Philadelphia, PA 19104-6323 Our lab has developed design principles for a new class of xenon-based magnetic resonance imaging contrast agents. In the process, we have developed molecules with the highest known affinity for xenon, and shown how to engineer interactions with biomedically relevant targets, such as carbonic anhydrase, folate receptor, and integrins. Xe-129 exhibits several ppm changes in NMR chemical shift when bound to these targets. We have also shown that xenon biosensors can be introduced into cells and animals, and are nontoxic. This technology provides new avenues for early disease detection.

\section{J070}

MAKING OF CYTOTOXIC T-CELLS FOR IN VIVO MRI PROBES Ali S. Arbab, Branislava Janic, Kourosh Jafari-Khouzani, Robert A. Knight, Steve Brown

Cellular and Molecular Imaging Laboratory, Henry Ford Hospital, Detroit, MI, USA.

Tumor immunology has long been a research focus for developing cell-based vaccine therapies. Dendritic (DCs), as well as T-cells, that can be isolated from lymphatic organs or peripheral blood, are considered to be the best candidates for developing such therapeutic approaches. The purposes of this study were to determine whether cord blood derived T-cells can be sensitized effectively against human glioma cell line U251 using primed DCs and to use these cells as probes for detecting implanted glioma in rat model. Cord blood CD2+/ $\mathrm{CD} 3+$ and $\mathrm{CD} 14+$ cells were collected from cord blood under an approved IRB protocol. CD14+ cells were converted to immature DCs by appropriate cytokine stimulation. During the procedure to make immature DCs, U251 cell lysate was added to the media to prime the DCs. At the end of priming TNF- $\alpha$ was added to the media to make mature DCs. Cell morphology was evaluated by an inverted microscope. Expression of specific cell markers (CD14, CD83, CD86, HLA-DR, and CD31) was analyzed by flow cytometry. The mature DCs (irradiated with $35 \mathrm{~Gy}$ ) were co-cultured with T-cells at ratio of 1:10 in 
RPMI-1640 media containing 10\% FBS and IL-2 (10 ng/ml). Proliferation of the T-cells was analyzed by MTT assay and compared with the proliferation of control T-cells that were cultured without DCs, using the same media. Different markers of T-cell (both normal and activated) were also analyzed. To be used as cellular MRI probes, sensitized T-cells (CTLs) were magnetically labeled with ferumoxides-protamine sulfate. Specificity of magnetically labeled and unlabeled CTLs to recognize U251 cells were studies both in in vitro and in vivo experiments (implanted U251 glioma). The level of expression of immature DCs' markers (such as CD86, HLA-DR) gradually increased over time. After subsequent incubation of immature DCs in media containing TNF- $\alpha$, there was a dramatic increase in the number of CD83 positive cells that indicated maturation of DCs. T-cells, in the presence of cell-lysate primed irradiated mature DCs, showed increase in proliferation as assessed by MTT assay and activation detected by flowcytometer. Both labeled and unlabeled CTLs showed similar specificity towards U251 cells. Intravenously injected magnetically labeled CTLs showed migration and accumulation to the tumor sites. The low signal intensity areas (or R2* values) seen on MRI was significantly higher in tumors that received CTLs compared to magnetically labeled control T-cells. T-cells can be sensitized against glioma cells and cab be used as cellular probes for MRI.

\section{J071}

\section{ACIDIC PH-RESPONSIVE MPEG-PAE AS CARRIERS FOR PATHOLOGIC THERANOSTICS}

GuangHui Gao ${ }^{1}$, Jaewon Lee ${ }^{2,3}$, Geun Ho $\operatorname{Im}^{2,3}$, Jung Hee Lee ${ }^{2,3}$, Doo Sung Lee $^{1}$

${ }^{1}$ Department of Polymer Science and Engineering, Sungkyunkwan University, Suwon, Korea, South, ${ }^{2}$ Department of Radiology, Samsung Medical Center, School of Medicine, Sungkyunkwan University, Seoul, Korea, South, ${ }^{3}$ The Center for Molecular and Cellular Imaging, Samsung Biomedical Research Institute, Seoul, Korea, South.
Nanometer-scale particles are becoming extensive in biomedical applications, especially polymeric micelles as carriers of hydrophobic agents (drugs or contrast agents) for cancer diagnosis and therapy. Because pathologic tissues have more acidic environment as a result of lactic acid produced by hypoxia and acidic extracellular organelles, here, we designed a pH-responsive micelles as carriers for pathologic therapy and diagnosis. In our system, the $\mathrm{pH}-$ responsive polymeric micelles were composed of hydrophilic methyl ether poly(ethylene glycol) (MPEG) and $\mathrm{pH}$-responsive degradable poly $(\beta$-amino ester) segments. MPEG-poly $(\beta$-amino ester) could be efficiently loaded with doxorubicin (DOX) using a solvent evaporation method. In an in vitro drug release study, these DOX-loaded polymeric micelles with rapid release of DOX from the micelles in weakly acidic environments ( $\mathrm{pH}$ 6.4) but very slow release under physiological conditions $(\mathrm{pH}$ 7.4). When in vivo anti-tumor activity of $\mathrm{pH}$-responsive polymeric micelles was evaluated by injecting the DOX-loaded polymeric micelles into B16F10 tumor-bearing mice, these micelles notably suppressed tumor growth and also prolonged survival of the tumor-bearing mice, compared with mice treated with free DOX. Monodispersed magnetite nanoparticles, soluble in organic solvents, are synthesized by the modified thermal decomposition method in the high boiling solvent. After loading magnetite nanopartilces into the polymeric micelles, it can be used for rapid acidic $\mathrm{pH}$-triggered magnetic nanoparticles release and aggregation at tumor site for MRI diagnostic applications. By using 3.0T MRI scanner, the relaxation property of the magnetite-loaded polymeric micelles was calculated, indicating a dominating $\mathrm{T} 2$ relaxation shortening effect that shows dark signal voids in T2-weighted MRI. From the in vivo result, this kind of $\mathrm{pH}$-responsive can be used for the brain pathologic diagnosis. It would improve the possibility to provide a new way to pathological diagnosis and therapy in the future.

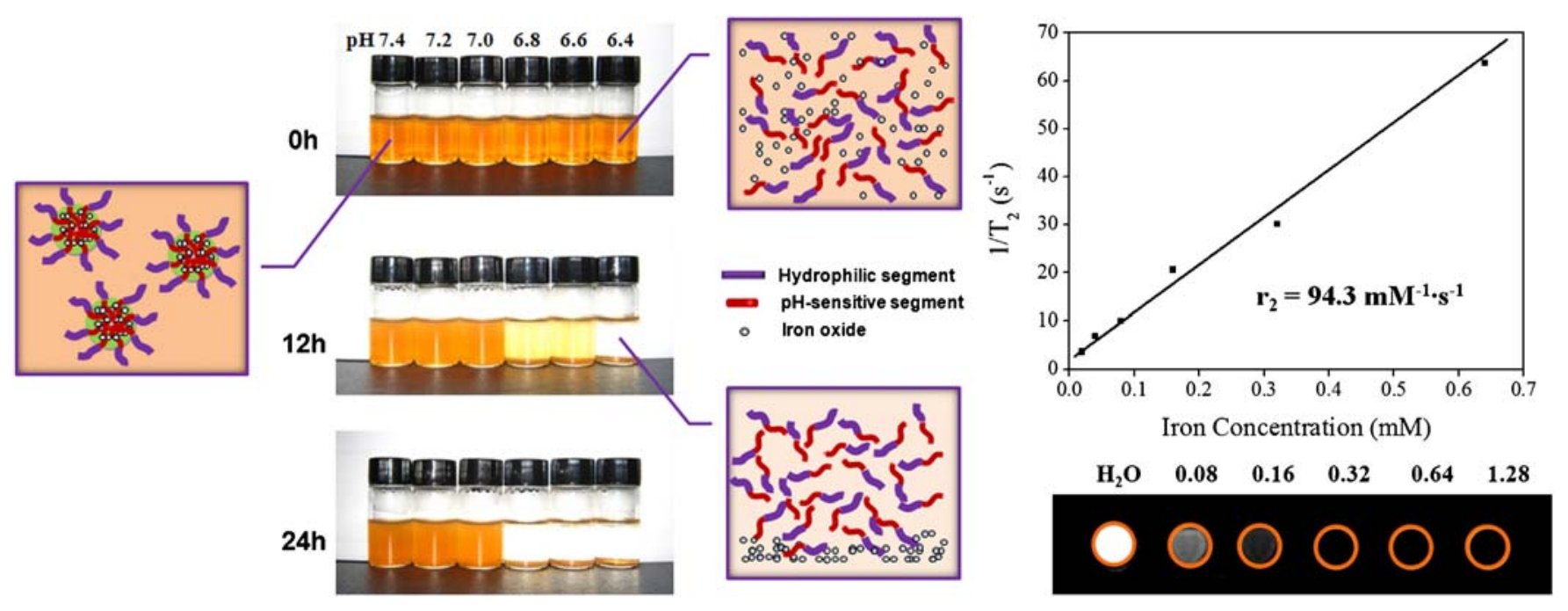

J072

\section{REGULATION SYSTEMS OF 19F NMR SIGNAL INTENSITY USING CUBIC SILSESQUIOXANES FOR MONITORING ENZYMATIC ACTIVITY}

Kazuo Tanaka, Yoshiki Chujo; Department of polymerchemistry, Graduate school of engineering, Kyoto University, Kyoto, Japan.

We have paid attention to cubic octameric polyhedral oligomeric silsesquioxanes (POSS, Figure 1) as a scaffold of the imaging probes with
19F MRI. POSS are water-soluble rigid nanoblocks, and POSS-based compounds showed superior biocompatibility such as less toxicity and good stability. In addition, POSS has the compact structure compared to the same generation of poly(amido)amine dendrimers which are known as a typical water-soluble dendrimer. These characteristics can be particularly beneficial for the accumulation of fluorine atoms with high density to improve sensitivity. Thus, POSS are promised to be a good scaffold to assemble functional molecules for the construction of the functional imaging probes. In the presentation, we report several regulation systems of 19F NMR signals for monitoring bioreactions based on POSS. The 19F 
NMR signal intensity of water-soluble perfluorinated POSS can be switched via the change of the relaxation time of 19F NMR signals due to the alteration of the motion of the probe molecule or the paramagnetic relaxation enhancement by the neighbor metal complex. We demonstrate a series of imaging probes for monitoring enzymatic reactions, microenvironmental polarity, and redox reactions with 19F MRI.<smiles>[R][Si]1([R])O[Si]2([R])O[Si]3([R])O[Si]([R])(O1)O[Si]1([R])O[Si]([R])(O2)O[Si]([R])(O3)O1</smiles>

Figure 1. The chemical structure of POSS.

\section{J073}

\section{POLY- $\beta$-CYCLDEXTRIN BASED PLATFORM FOR PH} MAPPING VIA A RATIOMETRIC 19F/1H MRI METHOD Eliana Gianolio, Roberta Napolitano, Franco Fedeli, Francesca Arena, Silvio Aime

Chemistry IFM, University of Torino, Torino, Italy.

Mapping $\mathrm{pH}$ is an important task in Medical Imaging as changes in $\mathrm{pH}$ usually accompany the development of various pathologies including tumors, stroke, infection,... Several paramagnetic metal complexes whose relaxivity is $\mathrm{pH}$-dependent have been reported. However none of them have been succesfully applied in vivo because in order to have images reporting the $\mathrm{pH}$ map it is necessary to transform the observed changes in relaxation rates (R1) in changes of relaxivities ( $\mathrm{r} 1$ ). This transformation requires the knowledge of the local concentration of the metal complex. A route to acquire this information may be pursued through the acquisition of the MR image of a heteronuclear signal originated from a molecule that displays the same "in vivo" biodistribution of the paramagnetic complex. Herein we report a supramolecular construct formed by: i) a Polycyclodextrin substrate that hosts ii) a suitably functionalized $\mathrm{pH}$ responsive $\mathrm{Gd}(\mathrm{III})$ complex and iii) an analogously functionalized 19F-containing molecule (Fig 1). The binding to the PolyCD substrate is pursued through the introduction of an adamantane group on both $\mathrm{Gd}$ and $\mathrm{F}$ containing systems. Adamantane is known to have a high binding affinity to $\beta-C D$ cavities. The proof of concept of this approach has been obtained by acquiring the $1 \mathrm{H}$ and 19F-MRI images of a phantom consisting of four tubes filled with solutions of $\mathrm{Gd} / \mathrm{F} / \mathrm{PolyCD}$ adduct at different values of concentration and $\mathrm{pH}$. As shown in Figure 1A the 1H-MR image does not account for a proportional change in contrast with $\mathrm{pH}$ because the observed R1 is dependent on both $\mathrm{pH}$ and concentration. Through the acquisition of the 19F-MR image it has been possible to assess the concentration of the adduct in the four tubes thus allowing the $\mathrm{R} 1 \rightarrow \mathrm{r} 1$ transformation (Fig. 1B). The method proved to work well with a small $(1-2 \%)$ error in the $\mathrm{pH}$ assessment. Finally the Poly-CD/F/Gd adduct can be edowed with targeting properties by hosting in one of the empty $\beta$-CD cavities an adamantane functionalized moiety able to provide the system with the proper recognition towards the target of interest.

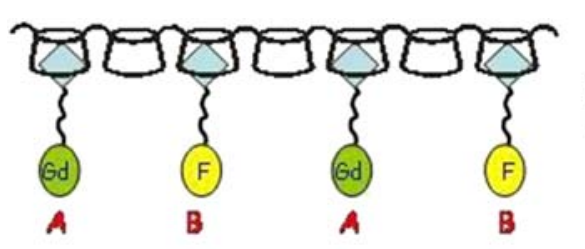

Unknown Gd(III) concentrations

A

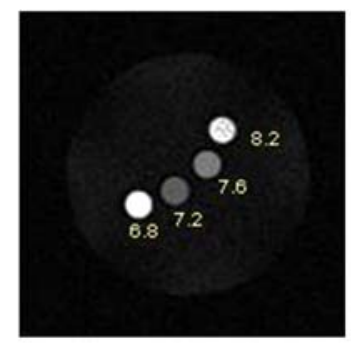

$T_{1}$-weighted 'H-MR Image at 7.1T
$A \rightarrow{ }^{1} \mathrm{H}-\mathrm{MRI}$ responsive agent

B $\rightarrow{ }^{19}$ F-MRI quantitation reporting unit

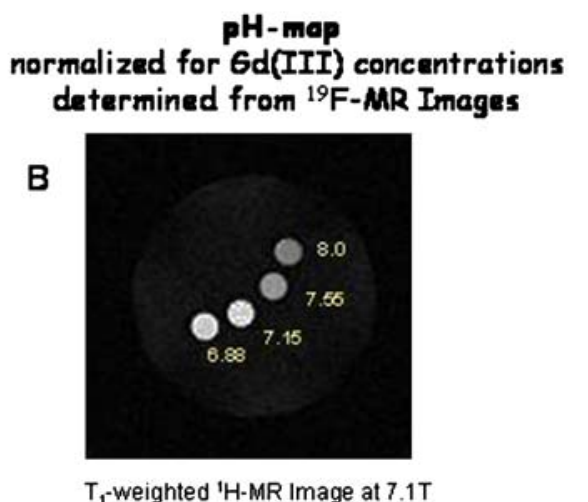

Iopamidol is an extensively used non-ionic X-Ray contrast agent with a very-good clinical acceptance. Iopamidol contains two amide groups that may be exploited for the generation of CEST effect (Chemical Exchange Saturation Transfer). The exchange rate of the two pools of exchangeable protons is strongly $\mathrm{pH}$-dependent. Thus a ratiometric method for $\mathrm{pH}$ assessment has been set-up based on the comparison of the saturation transfer effects induced by selective irradation of the two resonances. This ratiometric approach allows to get rid of the knowledge of the local concentration of the contrast agent. The aim of this study was to evaluate the ability of Iopamidol to report about $\mathrm{pH}$ - 
changes both in "in vitro" and in "in vivo" conditions. Saturation transfer effects were measured in a phantom containing seven vials of a $30 \mathrm{mM}$ phosphate buffer solution of Iopamidol in the $\mathrm{pH}$ range between 5.5 and 7.9. CEST spectra were acquired on a Bruker Avance $300(7 \mathrm{~T})$ at $37^{\circ} \mathrm{C}$ using a fast spin-echo sequence preceeded by a $3 \mu \mathrm{T}$ saturation pulse applied for $5 \mathrm{sec}$ and interpolated, on a voxel-by-voxel basis, by smoothing splines. The ratio of the saturation transfer effects upon irradiating the two amide absorptions was used for the calculation of the corresponding $\mathrm{pH}$ values. The $\mathrm{pH}$ dependence of the ratiometric method shows a linear relationship in the range 5.5-7.4 (Fig. 1A). Healthy mice were used to assess the potential of Iopamidol as $\mathrm{pH}-$ reporter in "in vivo" conditions. A dose of $0.75 \mathrm{mgI} / \mathrm{g}$ body weight (clinically accepted) was injected via tail vein and pre- and postcontrast images were acquired as described above. Iopamidol quickly accumulates in the kidneys and therefore two ROIs were drawn over the kidneys and the $\mathrm{pH}$ map was assessed by the ratiometric method. In post-contrast image, the $\mathrm{pH}$ map, overimposed to the anatomical image (Fig. 1B), is displayed over the kidneys region (Fig. 1C). A mean renal cortical $\mathrm{pH}$ of $7.0 \pm 0.11$, a mean medullary $\mathrm{pH}$ of $6.85 \pm 0.15$ and a mean $\mathrm{pH}$ in the calyx of $6.6 \pm 0.2$ were measured. This study demonstrates the feasibility of imaging tissue $\mathrm{pH}$ by CEST spectra using Iopamidol as $\mathrm{pH}$-responsive contrast agent.
A

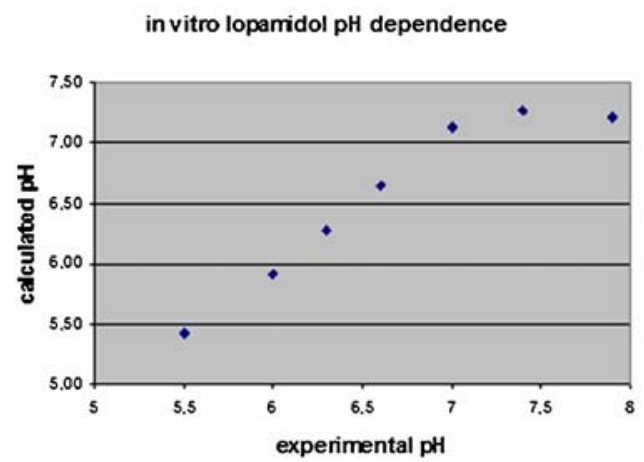

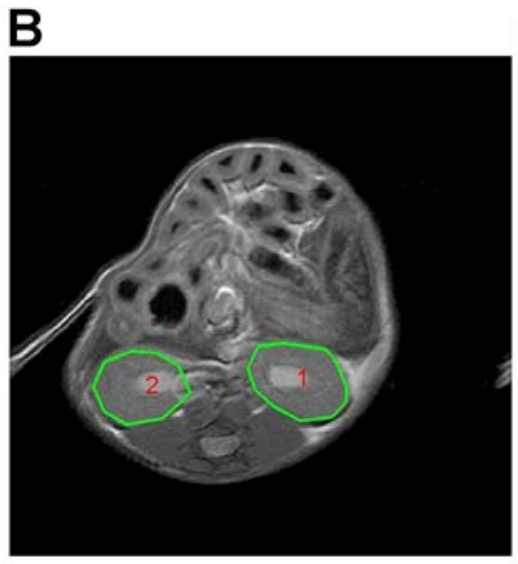

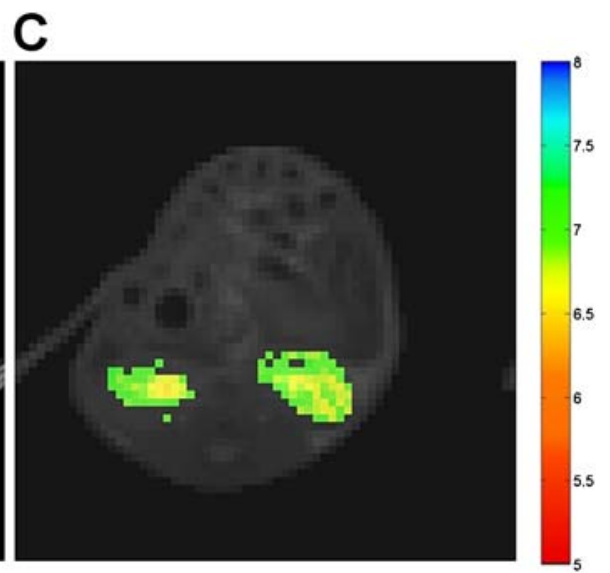

J075

PREPARATION OF A PROTEIN NANOCARRIER

ENCAPSULATING A CATIONIC GADOLINIUM COMPLEX AS A MAGNETIC RESONANCE IMAGING (MRI) CONTRAST AGENT FOR TUMOR IMAGING

Akira Makino, Shunsaku Kimura; Department of Material Chemistry, Kyoto University, Kyoto, Japan.

Apoferritin is a class of iron storage proteins composed of 24 polypeptide subunits to form a hollow cage-like self-assembled architecture (apoferritin), which external and internal diameters are ca. $13 \mathrm{~nm}$ and $8 \mathrm{~nm}$, respectively. The inner side of apoferritin is known to be negatively charged and cationic iron ions pass in and out of the apoferritin cavity through $0.7-1.0 \mathrm{~nm}$ pores at the outer shell. To take an advantage of the ionic interaction between a Gd-complex and the inner surface of apoferritin at the encapsulation step, a newly designed cationic Gd complex, Gd-Me2DO2A, was synthesized and encapsulated in apoferritin. The loading efficiency of the cationic Gd complex in apoferritin was improved better than the commercially available anionic Gd-DOTA complex (Figure 1). The resulting Gd-loaded apoferritin showed high $\mathrm{T} 1$ relaxivity due to the favorable Gdapoferritin interaction and the increased water coordination number. Further, the Gd-loaded apoferritin was easily functionalized by chemical modification using lysine residues on apoferritin outer surface. On this presentation, the preparation method of the Gd-loaded apoferritin MRI contrast agent and in vivo imaging using a tumorbearing mouse are discussed.

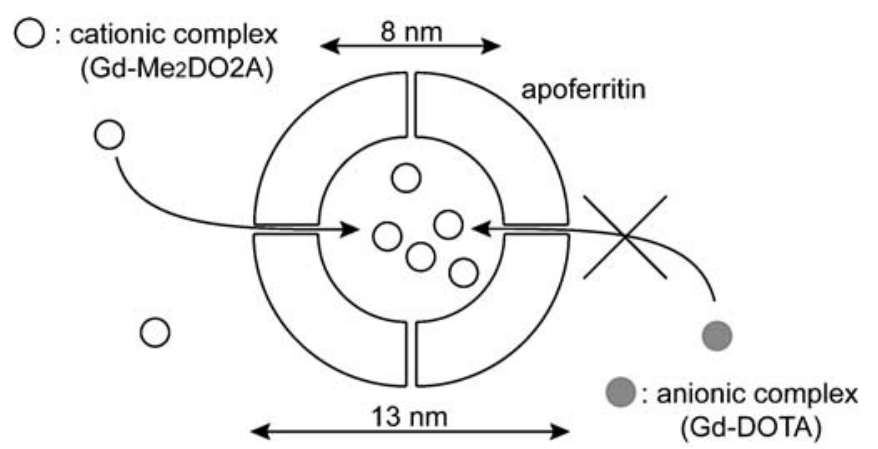

Figure 1. Schematic illustration of the encapsulation process of Gd-complex into apoferritin

\section{J076}

\section{DEVELOPMENT OF NOVEL FE-BASED 1H MRI LACZ GENE REPORTERS FOR IN VIVO ASSESSMENT}

Jian-Xin Yu, Dawen Zhao, Ralph Mason

Radiology, The University of Texas Southwestern Medical Center at Dallas, Dallas, TX, USA.

The lacZ gene encoding $\beta$-gal is recognized as the most widely used reporter gene, and its introduction has become a standard means of assaying clonal insertion, transcriptional activation, protein expression, and protein interaction. Therefore, the development of non-invasive 
lacZ gene reporter detection techniques based on appropriate molecules and imaging modalities is important. A variety of lacZ gene reporters has been developed, though most have only been utilized in vitro, with a very few successful applications in vivo so far. We now report the development of a new class of Fe-based 1H MRI lacZ gene reporter based on clinically applied iron-depletion agents. The great promise of iron depletion as a therapeutic strategy for various diseases has stimulated extensive research on the development of iron chelators as iron depletion agents. The FDA already approved deferasirox as the first oral drug for treatment of chronic iron overload in 2005. So far, five iron chelators have been approved for use in iron overload, anticancer or antifungal therapy. Some others are being evaluated in preclinical settings. Based on the structural similarities between the iron complexes with the clinically applied chelators and the known Fe-based $1 \mathrm{H}$ MRI contrast agents, we believe the clinically applied irondepletion agents can act as Fe-based 1H MRI contrast agents to produce strong contrast effects. We will present the design, synthesis and evaluation of series of lacZ reporter molecules based on the $\beta$-gal catalyzed release of clinically applied iron depletion agents: many are active with the enzyme, producing obvious contrast, here NS-A4 as a representative in Figure 1 [Conditions: T1-weighted $1 \mathrm{H}$ MRI, $200 \mathrm{MHz}, \mathrm{TR}=300 \mathrm{~ms}, \mathrm{TE}=20 \mathrm{~ms}, 1.5 \mathrm{~mm}$ slice, $128 \times 64,40 \times$ $40 \mathrm{~mm} 2$. (A) Control, NS-A4 (5 mmol), FAC (2.5 mmol), PBS $(650 \mathrm{~mL})$; (B) NS-A4 (5 mmol), FAC $(2.5 \mathrm{mmol}), \beta$-galactosidase $\mathrm{G}$ 5160 (5units), PBS (650 mL)]. Acknowledgements Supported by NCI R21 CA120774, SAIRP U24 CA126608, and BTRP \#P41-RR02584.

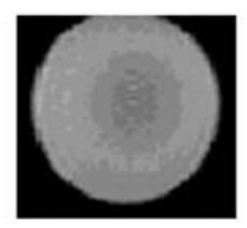

\section{Figure 1}
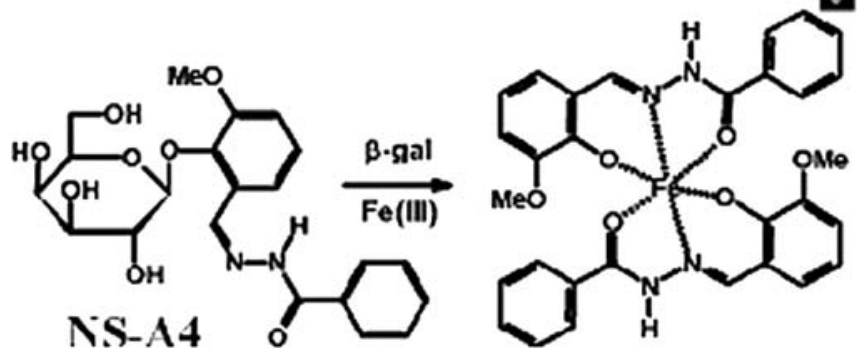

J077

UNUSUAL CALCIUM SENSITIVITY

OF AMINOBIS(METHYLENEPHOSPHONATE) -CONTAINING MRI CONTRAST AGENTS

$\underline{\text { Ilgar Mamedov }}{ }^{1}$, Jörg Henig ${ }^{2}$, Goran Angelovski ${ }^{1}$, Éva Tóth ${ }^{3}$, Nikos ${ }_{\text {K. Logothetis }}{ }^{1}$, Hermann A. Mayer ${ }^{2}$

${ }^{1}$ Max Planck Institute for Biological Cybernetics, Tuebingen, Germany, ${ }^{2}$ Institute for Inorganic Chemistry, University of Tübingen, Tuebingen, Germany, ${ }^{3}$ Centre de Biophysique Moléculaire, CNRS rue Charles Sadron, Orléans, France.

Calcium plays an important role in regulating a great variety of neuronal processes. A strong interest exists to generate gadolinium complexes which can act as calcium-sensors, in particular for magnetic resonance imaging (MRI). Here we report a new series of potential contrast agents based on DO3A, having an alkylaminobis(methylenephosphonate) side chain (propyl L1, butyl L2, pentyl L3 or hexyl L4). The complexation efficiency of the aminopolyphosphonic acid towards $\mathrm{Ca} 2+$, along with the ability to increase the presence of water in the secondary sphere and thus the longitudinal relaxivities (r1) of Gd3+-based CAs, was combined for potential use in sensing the extracellular calcium in the brain, especially at high field magnets. The paramagnetic response of the complexes GdL1GdL4 was studied by means of relaxometric titrations at different $\mathrm{Ca} 2+$ concentrations. The initial relaxivities of the complexes were found to be higher than usually reported for the DO3A type ligands at high field magnets (9.4T). The r1 values of 6.92, 7.43, 6.70 and $5.76 \mathrm{mM}-1 \mathrm{~s}-1$ were determined for GdL1, GdL2, GdL3, and GdL4 respectively. Interestingly the complexes exhibit unusual properties in the presence of $\mathrm{Ca} 2+$. The sensitivity of the complexes towards $\mathrm{Ca} 2+$ increases with the extension of the aliphatic side chain. In contrast to previously reported probes $\mathrm{rl}$ decreases upon addition of $\mathrm{Ca} 2+$. No changes in $\mathrm{r} 1$ of GdL1 were found over the whole span of $\mathrm{Ca} 2+$ concentration. In the case of GdL2, a moderate decrease of $\mathrm{r} 1$ was observed upon addition of $\mathrm{Ca} 2+$, whereas $\mathrm{r} 1$ of the GdL3 and GdL4 solutions showed a strong dependency on the calcium concentration resulting in the decrease to $66 \%$ and $61 \%$ of the initial $\mathrm{r} 1$ values, respectively. This is favorable for novel fMRI techniques as it could allow transforming the decrease in extracellular $\mathrm{Ca} 2+$ concentration into a positive MR contrast during the neuronal activity. Financial support of the Max-Planck Society, the Hertie Foundation, the Louis-Jeantet Foundation and the Centre National de la Recherche Scientifique (CNRS, France) is gratefully acknowledged. J.H. thanks the Fonds der Chemischen Industrie for a PhD scholarship. This work has been performed within the frame of the European COST Action D38 "MetalBased Systems for Molecular Imaging Applications".

\section{J078}

\section{PLURONIC F127 FERROGELS FOR MAGNETICALLY CONTROLLED DRUG RELEASE}

Jian Qin ${ }^{2}$, Andrea Fornara ${ }^{2}$, Sophie Laurent ${ }^{1}$, Robert N. Muller ${ }^{1}$, Mamoun Muhammed ${ }^{2}$

${ }^{1}$ NMR and Molecular Imaging Laboratory, University of Mons, Mons, Belgium, ${ }^{2}$ Functional Materials Division, Royal Institute of Technology (KTH), Stockholm, Sweden.

Pluronics are a class of ABA-type triblock copolymers. In particular, Pluronic F127 (PF127) consists two dissimilar moieties, which are 200 hydrophilic ethylene oxide units (A) and 65 hydrophobic propylene oxide units (B). This copolymer shows unique amphiphilic property, self-assembling into micelles to generate a lyotropic liquid crystalline gel phase. Hydrogels of PF127 exhibit low toxicity and are good candidates for drug delivery systems (DDS). Interestingly, aqueous solution of PF127 with concentration above 15-20\% displays reversible thermal gelation. This unique property of being liquid at room temperature and a solid gel at body temperature is ideal for injectable DDS which in principle avoids surgery during implantation. However, the release rate of a compound from PF127 hydrogels is not yet controllable. In order to control the release behavior, we introduce magnetic nanocrystals to PF127 hydrogels and study swelling behaviors, permeability and elasticity depending upon magnetic stimuli. Here, we prepared PF127 ferrogels via incorporating magnetic Fe3O4 nanocrystals into PF127 hydrogels. Two different particle sizes of $\mathrm{Fe} 3 \mathrm{O} 4$ nanocrystals, 10 and $25 \mathrm{~nm}$, were loaded in the ferrogel with the same concentration. The $\mathrm{Fe} 3 \mathrm{O} 4$ nanocrystals were synthesized through the method of high temperature decomposition of iron oleate complex in the presence of oleic acid as a capping agent. The obtained particles exhibit narrow size distribution and high crystallinity. In addition, these nanoparticles display superparamagnetic property that does not retain magnetization when the magnetic field is removed. The hydrophobic cavities of the micellar structures in PF127 hydrogels can accommodate hydrophobic magnetite nanoparticles as well as hydrophobic drugs. As a result, the microscopic morphology of the ferrogel could be altered when subjected to the magnetic field. Indomethacin (IMC) 
is used as a model drug and the release behaviors have been monitored in vitro. Results show that variation of magnetic field ends with distinct release rate and larger particles enhance release rate more than the smaller counterparts. To conclude, an injectable PF127 ferrogel with tunable release rate has been developed for loading and delivering hydrophobic drugs. Stability, biodistribution and toxicity of the ferrogel under physiological environment are currently under investigation.

\section{J079}

\section{NEW SUITABLE BIMODAL CONTRAST AGENTS FOR MRI AND OPTICAL IMAGING}

Sophie Laurent ${ }^{1}$, Luce Vander Elst ${ }^{1}$, Chantal Galaup ${ }^{2}$, Claude Picard ${ }^{2}$, Robert N. Muller ${ }^{1}$

${ }^{1} \mathrm{NMR}$ and Molecular Imaging Laboratory, University of Mons, Mons, Belgium, ${ }^{2}$ Laboratoire SPCMIB, Univeristé Paul Sabatier, Toulouse, France.

Three Gd- and Tb-PMN-tetracetate derivatives $1-3(\mathrm{PMN}=[2,6-$ pyridinediylbis (methylenenitrilo)] tetraacetic acid, respectively $\mathrm{R}=\mathrm{H}$ (1), COO- (2), $\mathrm{CONHCH} 2 \mathrm{CH} 2 \mathrm{OCH} 2 \mathrm{CH} 2 \mathrm{OCH} 2 \mathrm{COpeptide(3))are} \mathrm{syn-}$ thesized and evaluated for a potential use as bimodal contrast agents for MRI and optical imaging (figure 1). The peptide complexes 3 (TLVSSL) was selected to target apoptotic cells. Gd-complex: O-17 chemical shift analysis shows the presence of 2 coordinated water molecules. The proton relaxivity is increased as compared to Magnevist or Omniscan as a result of the presence of 2 water molecules in the first coordination sphere (figure 1). The water residence time is short (32$42 \mathrm{~ns}$ at $310 \mathrm{~K}$ ) and close to the optimal value. The possible interaction of the complex with serum albumin was tested. No binding of the Gdcomplex to HSA could be detected. The stability was first tested in a phosphate buffer $(\mathrm{pH}=7)$. No significant change of the relaxivity was observed. The possible transmetallation process was then assessed by the measurement of the evolution of the proton longitudinal paramagnetic relaxation rate (R1P) of a phosphate buffer containing an equimolar amount of Gd-complex and $\mathrm{Zn}$ ions $(2.5 \mathrm{mM})$. The stability versus $\mathrm{Zn}$ (II) ions is comparable (complex 1) or higher (complexes 2 and 3 ) to that of Gd-DTPA-BMA. Tb-complex: The comparison of the luminescence data in heavy and hydrogenated water confirms the presence of two water molecules in the first coordination sphere of the complexes. Experiments in phosphate buffer and in presence of bidentate coordinating anions (citrate, carbonate, bicarbonate) showed the kinetic inertness of the Tb-complexes. The values of the quantum yield obtained upon ligand excitation were determined to be 8.5, 10 and $4 \%$ for complexes 1, 2 and 3 respectively. The $\Phi$ tot value is within the actual range of commercially available di-aquo $\mathrm{Tb}$ complexes. The $\mathrm{Gd}$ and $\mathrm{Tb}$ complexes show interesting properties either for MRI or for optical imaging: for the Gd-complex, a high proton relaxivity and an acceptable stability in physiological medium; for the Tb-complex, energy transfer from pyridine to terbium and a long luminescence lifetime to avoid the overlap with biological background. The first tests on apoptotic cells with Gd-PMN peptide are very promising.
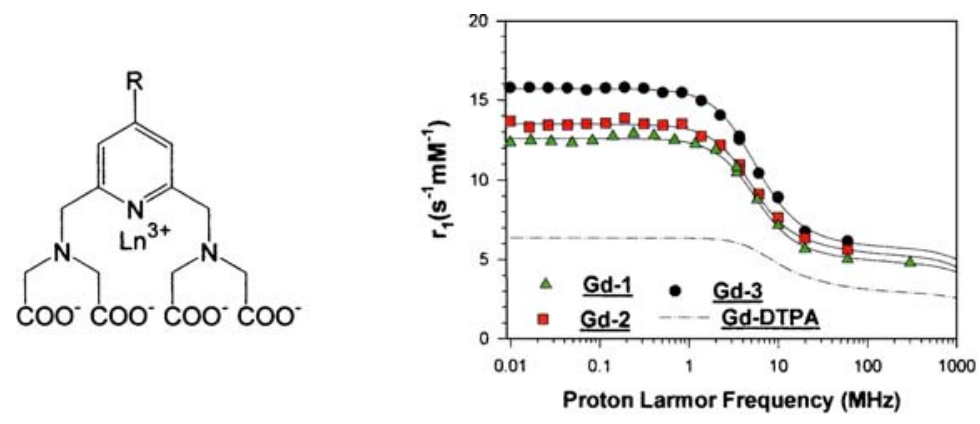

\section{Figure 1 : Structures of Ln-PMN derivatives and NMRD profiles of their Gd-complexes}

\section{J080}

\section{COMPARISON OF GADOLINIUM LOADED AND EMPTY CARBON NANOTUBES AS HIGH PERFORMANCE MRI CONTRAST AGENT}

Annie M. Tang ${ }^{1,3}$, Kelvin Wong ${ }^{1,2}$, Jeyarama S. Ananta ${ }^{4}$, Edmund Y. Lam $^{3}$, Lon J. Wilson ${ }^{4}$, Stephen T. Wong ${ }^{1,2}$

${ }^{1}$ Center for Biotechnology and Informatics, The Methodist Hospital Research Institute, Houston, TX, USA, ${ }^{2}$ Department of Radiology, The Methodist Hospital Research Institute and Weill Cornell Medical College, New York, NY, USA, ${ }^{3}$ Department of Electrical and Electronic Engineering, The University of Hong Kong, Hong Kong, China, ${ }^{4}$ Department of Chemistry, Rice University, Houston, TX, USA.

Introduction: Gadolinium loaded ultra-short single walled carbon nanotubes (US-tubes) has been proposed as an effective high $r_{2}$ relaxivity contrast agent for use in MR molecular imaging. However, the true active ingredients for inheriting its high performance $r_{2}$ characteristics, is remain unknown. In this study, we compare the $\mathrm{R}_{2}$ and $\mathrm{R}_{2}$ * relaxation rate of the US-tubes with/without Gadolinium ions encapsulation. Methods: Two types of US-tubes MRI phantoms were prepared: (i) Gadonanotubes with $41 \mu \mathrm{M}$ Gd and (ii) empty US-tubes. Each type of nanotubes was dispersed in pluronic solution with carbon concentration of $275 \mathrm{mg} / \mathrm{L}$ and transferred to a 1 cc syringe for MRI imaging. MRI imaging was performed in a $3 \mathrm{~T}$ MRI scanner using a $35 \mathrm{~mm}$ volume coil with $2 \mathrm{D}$ spin echo $(\mathrm{TR}=1500 \mathrm{~ms}, \mathrm{TE}=20-80 \mathrm{~ms}$ with $10 \mathrm{~ms}$ increment $)$ and $2 \mathrm{D}$ gradient echo $(\mathrm{alpha}=90, \mathrm{TR}=1500 \mathrm{~ms}, \mathrm{TE}=20-80 \mathrm{~ms}$ with $5 \mathrm{~ms}$ increment) sequences to evaluate the $\mathrm{R}_{2}$ and $\mathrm{R}_{2}{ }^{*}$ respectively. A $1 \mathrm{~mm}$ thick single slice was obtained at each TE cutting in the middle of the $3.5 \mathrm{~cm}$ long filled syringe placed parallel to the main magnetic field. $\mathrm{T}_{2}$ and $\mathrm{T}_{2} *$ were determined by linear fitting of the measured image intensities at each TE at each phantom into $\log (\mathrm{S})=\log \left(\mathrm{S}_{0}\right)-\mathrm{TE} / \mathrm{T}_{2}$ and $\log (\mathrm{S})=\log \left(\mathrm{S}_{0}\right)-\mathrm{TE} / \mathrm{T}_{2}{ }^{*}$. Results and Discussions: Table 1 shows the estimated $R_{2}$ and $R_{2} *$ relaxation rates of the phantoms. $R^{2}$ represents the coefficient of determinations of the linear fitting. Both Gadonanotubes and empty US-tubes show high $\mathrm{R}_{2}$ and $\mathrm{R}_{2}$ * relaxation rates with the $\mathrm{R}_{2}$ * being $6-11 \%$ stronger than $R_{2}$. The slight increase in $\mathrm{R}_{2} *$ may attribute to bulk magnetic susceptibility effects due to the presence of metal ions inside the US-tubes. Nethertheless, the $\mathrm{R}_{2}{ }^{*}$ observed is small when compared with that of other superparamagnetic contrast agents which usually show a few times increase in $\mathrm{R}_{2}{ }^{*}$. This may be due to the low concentration (in $\mu \mathrm{M}$ ) and lose density packing of the metal ions inside the tubes. For empty US-tubes, it is observed that its relaxivities are as good as Gadolinium loaded US-tubes. Additional $\mathrm{Gd}^{3+}$ loading does not 
significantly increase the relaxivities of the US-tubes. Therefore, it is reasonable to consider using empty US-tubes as a high performance MRI contrast agent as well as drug/gene delivery carrier, with the delivery items being encapsulating inside its hollow interior space.

\begin{tabular}{|c|c|c|}
\hline & Gadonanotubes & Empty US-tubes \\
\hline $\mathrm{R}_{2}\left(\mathrm{~s}^{-1}\right)$ & $26.76\left[\mathrm{R}^{2}=0.99\right]$ & $23.67\left[\mathrm{R}^{2}=0.99\right]$ \\
\hline $\mathrm{R}_{2} *\left(\mathrm{~s}^{-1}\right)$ & $29.72\left[\mathrm{R}^{2}=0.99\right]$ & $25.23\left[\mathrm{R}^{2}=0.99\right]$ \\
\hline
\end{tabular}

Table 1

J081

DOTA-LIKE COMPLEXES BEARING

A BISPHOSPHONATE MOIETY-BONE-SEEKING AND ION-SENSING AGENTS

Vojtech Kubicek, Ivan Lukeš

Depatment of Inorganic Chemistry, Charles University in Prague, Faculty of Science, Prague 2, Czech Republic.

Three bone-targeting metal carriers for applications in molecular imaging have been prepared. DOTA-like core was used as a metal chelating group. The chelator was attached to a bisphosphonate moiety that is known to adsorb efficiently on bone tissue. The compounds form stable DOTA-like complexes with $\mathrm{Ln}$ (III) ions. The bisphosphonate group is not coordinated. An in vitro study was performed using $159 \mathrm{~Tb}$ complexes as reporters and an aqueous suspension of hydroxyapatite as a model of bone tissue. The results have shown a strong and swift adsorption of the complexes on hydroxyapatite. In vivo SPECT/CT experiments using $177 \mathrm{Lu}$ have shown a significant uptake and a long retention of the complexes in joints and on bone surface of Lewis rat. Studied Gd(III) complexes exhibit MRI properties that are common for low-molecular compounds with one water molecule coordinated to the metal ion. Relaxivity (MRI efficiency) of the complexes is increased by presence of the rich second hydration sphere. Further, upon adsorption on the surface of hydroxyapatite, relaxivity was significantly improved. In addition, the complexes interact with divalent metal ions. The interaction of Gd(III) complexes results in a formation of polymeric bisphosphonate complexes and consequently in an increase (300-500\%) of relaxivity. In the field of MRI contrast agents, this is the highest response on presence of metal ions ever reported. On basis of the results, it could be concluded, that the studied compounds are promising bone-targeting (MRI, PET, SPECT) and metal-ion-responsive (MRI) contrast agents.

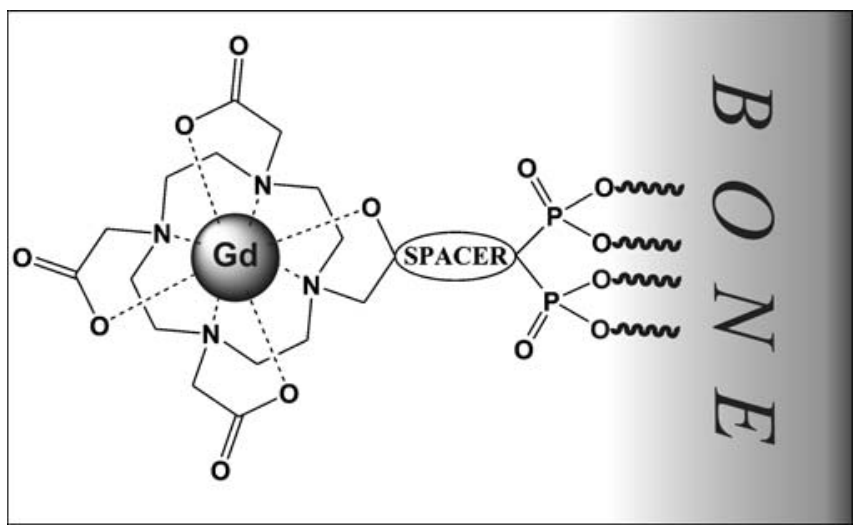

$\mathbf{J 0 8 2}$

\section{SPECIFIC DELIVERY AND RELEASE OF CYTOTOXIC GD3+ IONS TO TUMOR CELLS THROUGH VITAMIN B12 BIOCONJUGATES}

Eliana Gianolio $^{1}$, Patrizia Siega ${ }^{2}$, Jochen Wuerges ${ }^{2}$, Francesca Arena ${ }^{1}$, Silvano Geremia $^{2}$, Silvio Aime ${ }^{1}$, Lucio Randaccio ${ }^{2}$

${ }^{1}$ Chemistry IFM, University of Torino, Torino, Italy, ${ }^{2}$ Chemical Science, University of Trieste, Trieste, Italy.

Mammalian cells require Vitamin B12 as enzyme cofactor. Mammals have developed a specific internalization pathway for this essential micronutrient, and fast proliferating cell types require even higher amounts of it. This observation has given considerable potential to the use of vitamin B12 analogues as imaging and therapeutic agents. In this poster we describe the synthesis and applications of a VitB12-Gd-DTPA derivative to specifically deliver and release cytotoxic Gd3+ ions to tumor cells. Two new bioconjugates of vitamin $\mathrm{B} 12$ resulting from the esterification of the ribose 5'-hydroxyl of VitB12 with the metal-chelating agents DTPA or TTHA have been synthesized and characterized. The major difference between the two systems is the denticity of the ligands, that results in significant differences in the ability to release $\mathrm{Gd} 3+$ ions to cells and in different hydrolysis rates of the ester linkage between VitB12 and the ligand. Hydrolysis rate constants of $4.1 \times 10^{-}-3 \min ^{-1}$ and $4.9 \times 10^{-4} \mathrm{~min}-1$ and $\mathrm{t} 1 / 2$ values of $175 \mathrm{~min}$ and $1414 \mathrm{~min}$ have been determined for 1 and 2, respectively. As far cell labelling concerns, whereas 1 showed an increased uptake in K562 cells, internalization of 2 was negligible at all the investigated concentrations. It has been demonstrated that, in the case of 1 , the internalization process involves free $\mathrm{Gd} 3+$ ions and that the presence of the VitB12 moiety is crucial in the cell recognition process. Viability tests showed that in the presence of 1 the cell viability is markedly reduced while the effect of 2 is negligible. One can surmize that the involvement of the ester oxygen atom in Gd-coordination in 1 is responsible for its lower stability towards hydrolysis and in its higher ability to release $\mathrm{Gd} 3+$ ions at the binding sites on the cellular membrane. The high toxicity observed for K562 cells treated with 1 appears as a further indication of the massive uptake of $\mathrm{Gd} 3+$ ions.

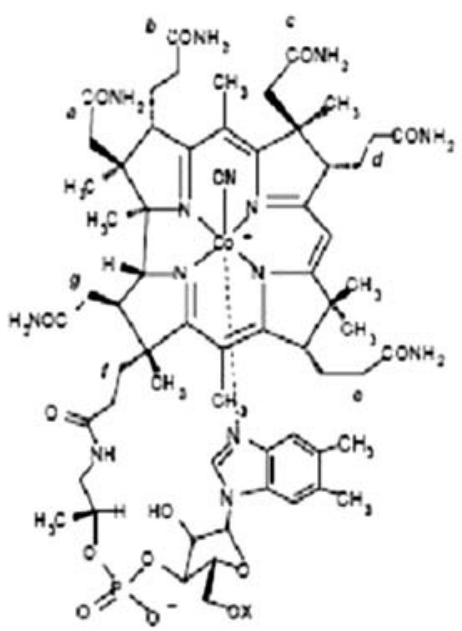


$\mathbf{J 0 8 3}$

\section{REDUCTION/DISSOLUTION OF THE INORGANIC BETA-MNOOH NANOPHASE IN THE FERRITIN CAVITY TO YIELD A HIGH SENSITIVY, BIOLOGICALLY COMPATIBLE MRI AGENT}

Ferenc K. Kalman ${ }^{1}$, Simonetta Geninatti- Crich $^{1}$, Ibolya Szabó ${ }^{1}$, Stefania Lanzardo $^{2}$, Silvio Aime ${ }^{1}$

${ }^{1}$ Department of Chemistry, Center for Molecular Imaging, University of Torino, Torino, Italy, ${ }^{2}$ Department of Clinical and Biological Sciences, University of Torino, Torino, Italy.

An innovative approach to the design of MR Contrast Agents has been pursued through the entrapment of Mn(II) aqua ions inside the inner cavity of Apoferritin. This task has been addressed by partial dissolution of the previously formed beta-MnOOH inorganic phase. The dissolution of beta$\mathrm{MnOOH}$ occurs via the reduction of $\mathrm{Mn}(\mathrm{III})$ to $\mathrm{Mn}$ (II) operated with aminopolycarboxilic acids that also act as coordination ligands for sequester- ing weakly coordinated manganese ions on the outer surface of the protein. The reductive treatment has allowed to generate an apoferritin-based nanocarrier (Mn-Apo) containing up to $300-400 \mathrm{Mn}$ (II) aqua ions encapsulated in the inner cavity. This yielded to the remarkable relaxivity value (per apoferritin) of ca. $7000 \mathrm{mM}-1 \mathrm{~s}-1$. Mn-Apo being formed by endogeneously occuring molecules and ions, displays a high biocompatibility. It targets hepatocytes with high efficiency and is able to discriminate healthy cells from hepatocarcinoma ones, on the basis of the different expression of ferritin receptors. Competition studies with native ferritin confirmed that cellular uptake involves these receptors and the lower ferritin uptake by hepatoma is a consequence of their lower expression on liver tumor cells. Mn-apo was injected in C57BL/6 mice at a dose of $0.01 \mathrm{mmol} / \mathrm{Kg}$ of $\mathrm{Mn}$ and a very high Signal Intensity (SI) enhancement of the liver in the MRI images was detected 30 ' and $1 \mathrm{~h}$ after the administration. $24 \mathrm{~h}$ after the decreased liver SI indicated the complete elimination of the probe. In conclusion, Mn-loaded apoferritin is a very efficient probe specific for liver imaging. In particular, it could be useful in the diagnosis of a variety of liver diseases involving an alteration in the hepatic iron storing capabilities (e.g. fibrosis, cirrhosis, cancer).
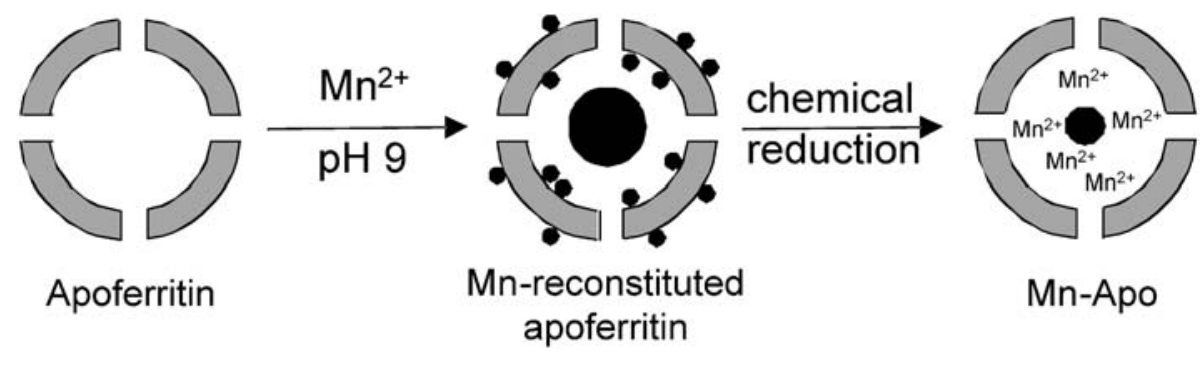

J084

DEVELOPMENT OF NOVEL LACZ RESPONSIVE ENHANCED GD-BASED 1H MRI AGENTS

Jian-Xin Yu, Ralph Mason; Radiology, The University of Texas Southwestern Medical Center at Dallas, Dallas, TX, USA.

Gene therapy holds great promise for treating cancer and has been successfully exploited in several clinical trials. A major current obstacle to implementation is to establish a method of assessment of therapeutic gene expression in terms of heterogeneity and longevity in tissues. The lac $Z$ gene encoding $\beta$-galactosidase ( $\beta$-gal) has been well characterized and extensively used as reporter gene in immunosorbent assays, in situ hybridizations, classification of mycobacteria and gene expression studies. A variety of lacZ gene reporters has been developed, such as colorimetric, fluorescent, chemiluminescent, radiotracers for PET or SPECT, 19F MRS/MRI, and MRI probes, though most have only been utilized in vitro, with a very few successful applications in vivo so far. We now report the exploration of a supramolecular approach to lacZ responsive Gd-based 1H MRI agents, generating high relaxivity by forming higher molecular weight and rigidity of the complexes. We have designed a novel class of enzyme-activated Gd3+based MRI contrast agents for in vivo detection of $\beta$-gal activity (see Figure). We anticipate that, following cleavage by $\beta$-gal, the released and activated aglycone, which is an Fe3+-ligand will spontaneously trap endogenous Fe3+ at the site of enzyme activity forming a highly stable complex, exhibiting restricted motion of the $\mathrm{Gd} 3+$ chelates enhancing relaxivity and providing contrast based on gene (viz. enzyme) stimulated local accumulation. We will present the design, synthesis and evaluation of 2-[( $\beta$-D-galactopyranosyl) oxy]-5-\{N,N-bis[2-[N',N'-bis(carboxymethyl)amino] ethyl]benzene $\}$ benzaldehyde nicotinoyl and isonicotinoyl hydrazones, and the characterization of these target molecules with $\beta$-gal reaction, the Fe-complex formation, and the resulting relaxation changes. Acknowledgements Supported by the DOD Prostate New Investigator Award PC050808 W81XWH-05-1-0593 (JXY).

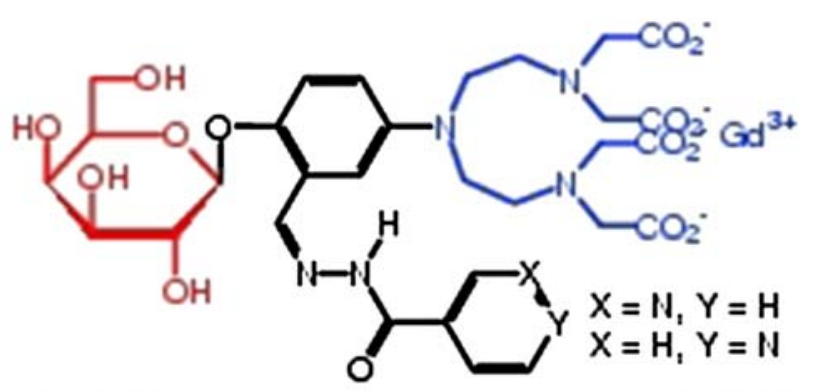

Glycosyl Part Blocked Fe(III) Ligand Gd(III) Signal Part

\section{J085}

COMPARISON OF DIFFERENT FLUORINATED ASPGLYMEDOTA- AND GLUGLYME-DOTA-GD-COMPLEXES

Markus Plaumann, Mireille Bipoun, Dieter Leibfritz; Institute of Organic Chemistry, University of Bremen, Bremen, Germany.

$\mathrm{Gd}^{3+}$-DOTA-complexes are important contrast agents for MRI. The syntheses of fluorinated Gd-contrast agents permit recording ${ }^{19} \mathrm{~F}$-images without any background signals and classical ${ }^{1} \mathrm{H}-\mathrm{MR}$ images at the same time. 3,5-Bis(trifluoromethyl)benzylamine and 3,5-Bis(trifluoromethyl)phenylhydrazine were selected as model compounds to synthesized six AspGlyMe-DOTA- and six GluGlyMe-DOTA-Gd-complexes. All complexes were characterized concerning to their lipophilic properties and relaxation times $\left(\mathrm{T}_{1}\right) . \mathrm{T}_{1}$-measurements were performed at $8.4 \mathrm{~T}$ and $293 \mathrm{~K}$ sample temperature in $1 \mathrm{mmol} / \mathrm{L}$ solutions. It could be shown that a change from L-aspartic acid to L-glutamic acid has no real influence of the ${ }^{1} \mathrm{H}$ - and ${ }^{19} \mathrm{~F}_{-} \mathrm{T}_{1}$-values. The lipophilicity of these complexes (log k'w) was determined by reversed phase chromatography by adapting methanol content in different runs with respect to the hydrophobic interaction of the analytes with the RP packing and plotting the logarithm of retention against the methanol content. 
Extrapolation to $0 \% \mathrm{MeOH}$ gives log k'w. A comparison shows a high lipophilicity for the bisaromatic GluGlyMe-DOTA-Gd-complexes (log k'w> 6.3) and a lower log k'w value of 6.04 for all bisaromatic AspGlyMe-DOTAcomplexes. The monoaromatic complexes with one free carbon acid have lower log k'w-values. A log k'w of 1.6 could be measured for the 3,5-Bis (trifluoromethyl)benzylamine-AspGlyMe-DOTA-complex which is of 1.1 lower than the appropriate GluGlyMe-DOTA-complex. Similar behavior can be observed for the mono 3,5-Bis(trifluoromethyl)phenylhydrazine complexes $\left(\log \mathrm{k}^{\prime} \mathrm{w}=2.00\right.$ and 2.8). The AspGlyMe-DOTA-complex is the more lipophilic molecule. These results show the influence of an additional $-\mathrm{CH}_{2}-$ group in the chain length of a spacer molecule and the effect of a second aromatic system to the lipophilicity of the molecules. An exchange of an amino group (-NH-) against a methylene group $\left(-\mathrm{CH}_{2}-\right)$ has just a small contribution for changing the lipophilicity. Additionally, no real influence to the relaxation times $\left({ }^{1} \mathrm{H}-\mathrm{T}_{1}\right.$ and $\left.{ }^{19} \mathrm{~F}-\mathrm{T}_{1}\right)$ was observed.

\section{J086}

\section{DEVELOPMENT OF BIO-RESPONSIVE CONTRAST AGENTS FOR MAGNETIC RESONANCE IMAGING: POTENTIAL APPLICATIONS OF A NOVEL PRECURSOR}

Kirti Dhingra $^{1}$, Anurag Mishra ${ }^{1}$, Joern Engelmann ${ }^{2}$, Almut Schüz ${ }^{1}$, Santiago Canals ${ }^{3}$, Rolf Pohmann ${ }^{2}$, Michael Beyerlein ${ }^{1}$, Martin E. Maier $^{4}$, Nikos K. Logothetis ${ }^{1,5}$

${ }^{1}$ Dept. of Physiology of cognitive Processes, Max Planck Institute for Biological cybernetics, tuebingen, Germany, ${ }^{2}$ Dept. of High field MR centre, Max Planck Institute for Biological cybernetics, tuebingen, Germany, ${ }^{3}$ Campus de San Juan, Instituto de Neurociencias CSICUMH, San Juan de Alicanta, Spain, ${ }^{4}$ Institute for Organic Chemistry, Tuebingen, Germany, ${ }^{5}$ Imaging Science and Biomedical engineering, Manchester, United Kingdom.
Magnetic resonance imaging (MRI) is one of the powerful imaging modality. To circumvent its low sensitivity, there has been a substantial interest on the development of the contrast agents. In the present scenario, there is a need to develop contrast agents which are target specific and can report the changes in the physiological environment around them. On the similar lines we are reporting here a novel precursor (tris-tert-Bu-(Z)-Ser-DO3A (Figure 1)). This precursor contains an amine and a carboxylate groups in an orthogonally protected condition, which allows their selective deprotection and coupling to different moieties. Out of the various possibilities, we explored two strategies of coupling that lead to a potential targeted $\mathrm{CA}$ and another $\mathrm{CA}$ with potential of tracing neuroanatomy in the brain. The special design of these agents not only provides the stability against their enzymatic degradation which is important for their in vivo applicability but also has the possibility to amplify its signal once recognized by the target site. This could be done by exploiting the biotin/strept(avidin) high affinity and the pretargeting strategy, which is well established in nuclear medicine. The CA when bound to avidin showed an enhancement in the relaxivity ( $\mathrm{r} 1$ and $\mathrm{r} 2$ ) at $1.5 \mathrm{~T}$. A substantial increase of $\geq 1000 \%$ in $\mathrm{r} 2$ was observed at all magnetic fields studied $(1.5 \mathrm{~T}, 3 \mathrm{~T}, 7 \mathrm{~T}, 9.4 \mathrm{~T})$ while $\mathrm{r} 1$ showed an increase of $260 \%$ at $1.5 \mathrm{~T}$ and an expected decrease with further increase of field strength. The relaxivity changes at $1.5 \mathrm{~T}$ suggest the structural requirement of a $\mathrm{CA}$ to fit in to avidin and optimize the parameters determining relaxivity of the complex matches well with our synthesized agent. Using the same precursor, we have also synthesized a CA which can potentially be used for tracing the neuronal tracks in the brain. Biocytin was used as the basic tracer. Coupling an MR detectable moiety to a well known neuroanatomical tracer would open up new possibilities to noninvasively study the neuronal networks by MRI.

\section{Figure 1: Structure of precursor}

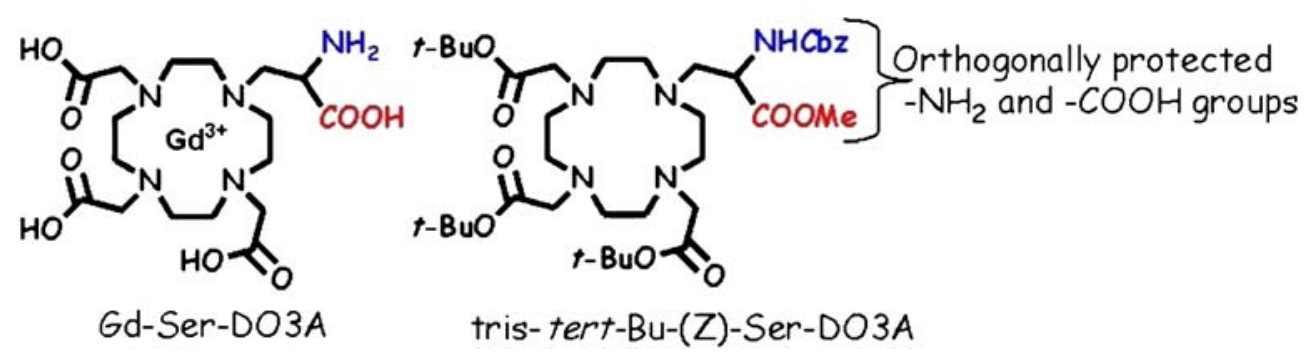

J087

\section{REACTION: RELEASE ACTIVATION OF IRON OXIDE NANOPARTICLES A NOVEL ENVIRONMENTALLY SENSITIVE MRI PARADIGM \\ Dorit Granot $^{1}$, Erik Shapiro ${ }^{1,2}$ \\ ${ }^{\mathrm{D}}$ Department of Diagnostic Radiology, Yale University School of Medicine, New Haven, CT, USA, ${ }^{2}$ Department of Biomedical Engineering, Yale University, New Haven, CT, USA.}

Here we introduce a new paradigm in MRI contrast agents with the potential to report on molecular events. Cells are labeled with biopolymer-coated particles which are cleavable by a specific enzyme. This coating restricts the approach of water, preventing the magnetic core from relaxing protons. A reactive enzyme, which can be engineered as a reporter protein, cleaves the particle coating, releasing the magnetic center, increasing the relaxivity of the agent. We demonstrate the principal of enzyme-mediated changes in nanoparticle relaxivity in cell free assays, in-cellulo, and in-vivo. Dextranase is an enzyme which cleaves dextran at $\mathrm{pH}<6.0$. Feridex is an FDA-approved dextran coated iron oxide nanoparticle. Incubation of Feridex with dextranase in acetate $\mathrm{pH} 6$ or citrate $\mathrm{pH} 5.5$ buffers resulted in $\mathrm{R} 2$ increases of $110 \%$ and $56 \%$ versus Feridex without enzyme, respectively. Next, cells were co-labeled with Feridex and dextranase and incubated $2 \mathrm{~h}$. R2 increased 56\% versus cells labeled only with Feridex. Confocal microscopy confirmed the presence of dextranase and Feridex in the same cells. Lastly, two sets of MCF7 cells were prepared to determine the extent of the effect of the cleavage of dextran coat in vivo. One set was labeled only with Feridex, the other set labeled with both Feridex and dextranase as above. Cells 
were injected into the hind limb of mice. 3D T2* GRE and multi-slice T2 weighted SE MRI of hind limbs at 1 day and 4 days post injection demonstrate that the susceptibility effect generated from the labeled cells is enhanced in the side injected with cells co-labeled with Feridex and dextranase compared to the leg injected with Feridex labeled cells only.
This study illustrates that enzyme-triggered removal of the thick polymer coat indeed reveals itself as shorter T2 relaxation times and larger susceptibility weighted signal voids. These experiments form the backbone for new classes of potential in vivo MRI reporter genes/agents that can report on cellular changes such as stem cell differentiation.
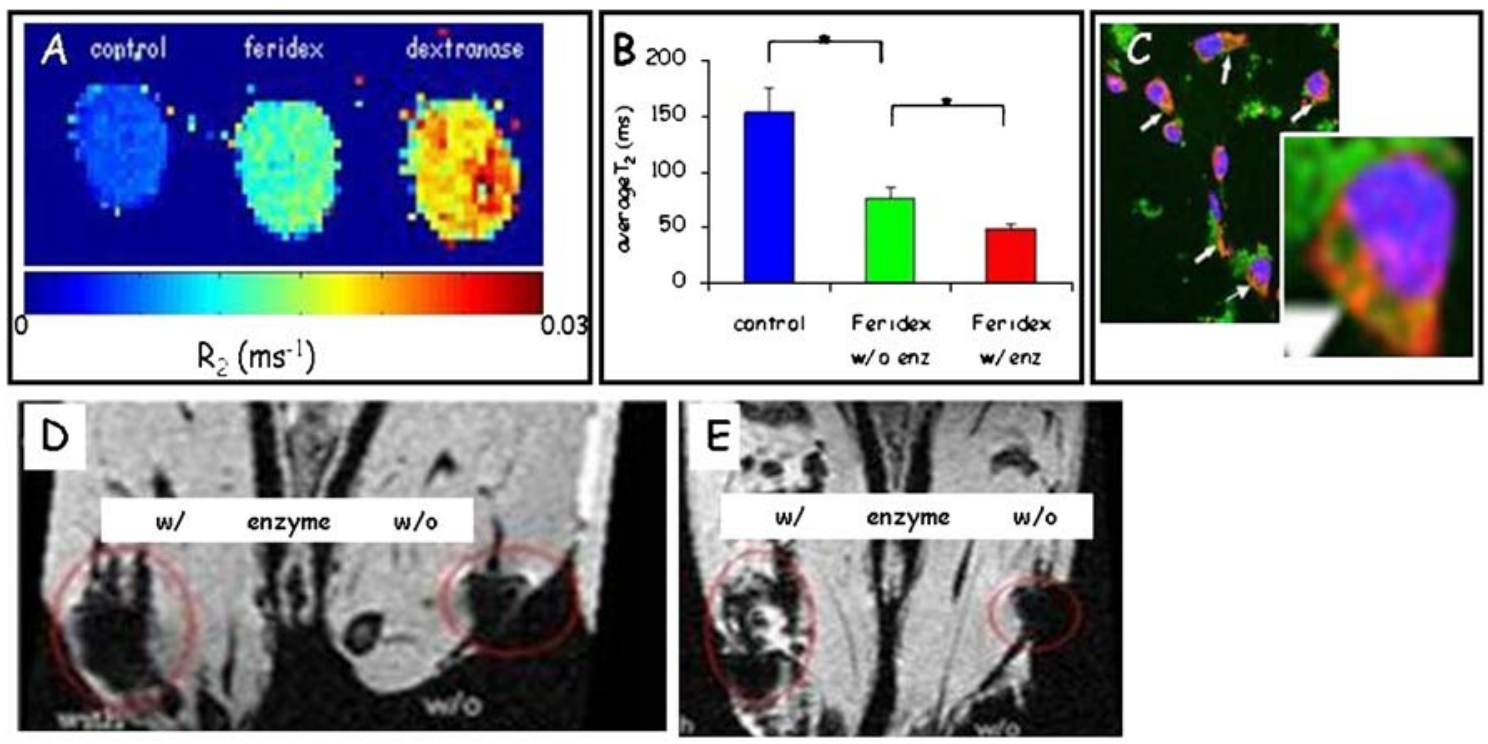

Reduction of $T 2$ following enzymatic cleavage of Feridex coating. A) T2 maps of cells in agar after 24 hours incubation. B) Graphical presentation of T2 values of cell samples. C) Confocal fluorescence microscopy of cells co-labeled with green fluorescent feridex and red fluorescent dextranase. D, E) 3D GRE images from the same animal showing both sites of labeled cell injections (circled) at 1 day (D) and 4 days (E) post injection. Left side is cells colabeled with Feridex and dextranase; right side is Feridex labeled cells only

\section{J088}

\section{DETECTION OF A DENDRITIC PARACEST AGENT IN AN EXPERIMENTAL HUMAN MALIGNANT GLIOMA RAT MODEL}

M. M. Ali ${ }^{1}$, Asm Iskander ${ }^{1}$, Nadimpalli Ravi S Varma ${ }^{1}$, Branislava Janic $^{1}$, Robert A. Knight ${ }^{1}$, Marty Pagel ${ }^{2}$, Ali S. Arbab ${ }^{1}$

${ }^{1}$ Radiology, Henry Ford Health System, Detrit, MI, USA, ${ }^{2}$ Biomedical Engineering and Chemistry, University of Arizona, Tucson, AZ, USA.

PARACEST (PARAmagnetic Chemical Exchange Saturation Transfer) MRI contrast agents have advantages relative to T1- or T2relaxivity MRI contrast agents. However, a major problem for in vivo PARACEST applications is the temporal resolution and sensitivity of the agent. We have recently reported that 1.62 and $1.43 \mathrm{mM} \mathrm{Eu-}$ DOTA-Gly and Yb-DOTA-Gly are required to achieve a 3\% CEST effect in solution which may limit in vivo applications. To overcome this problem, an average of 41 small molecule Eu-DOTA-Gly PARACEST agents were conjugated to a Generation-5 PAMAM dendrimer through an EDC/NHS coupling method. This G5-Eu PARACEST agent showed a tremendous improvement in sensitivity on a per-molecule basis. The solution study showed that $45 \mu \mathrm{M}$ G5$\mathrm{Eu}$ was required to generate $3 \%$ of CEST effect 1 . Besides, dendrimerbased MRI contrast agents passively target tumor tissues via the Enhanced Permeability and Retention (EPR) effect, which may further improve the detection of dendritic PARACEST agents within tumors. Here, we report the detection of a dendritic PARACEST agent within malignant glioma. Intracranial implanted U251 glioma was produced in nude rats and MRI was performed on day 20-22 following the implantation of the tumor. A series of RARE MRI experiments were conducted with presaturation applied at $+55 \mathrm{ppm}, 20 \mu \mathrm{T}$ and $3.67 \mathrm{sec}$ to generate the PARACEST effect ( $5 \mathrm{sec}$ TR, $80 \mathrm{sec} / \mathrm{image})$. After acquiring five pre-injection images, $0.04 \mathrm{mmol} / \mathrm{kg}$ of $\mathrm{G} 5$-Eu in $800 \mu \mathrm{L}$ was injected via the tail vein catheter, and a series of images were acquired to monitor the dynamic pharmacokinetics of the agent for $50 \mathrm{~min}$. Post-injection images were subtracted from pre-injection images to measure the PARACEST effect. Unfortunately, the pharmacokinetics of G5-Eu was not clearly visualized in the rat glioma tumor but the agent was clearly visualized in the internal carotid artery. Administration of $800 \mu \mathrm{L}$ of G5-Eu to a rat model showed a dynamic change in the PARACEST effect, culminating in a $30 \%$ CEST effect 30 min after administration in the internal carotid artery. The change in image contrast of the internal carotid artery was assigned to the accumulation of the PARACEST agents in the internal carotid artery. We are exploring to detect dendritic PARACEST agent in the rat glioma by modifying smaller size dendritic PRARCEST agents. Ref: 1. MM Ali, B Yoo and MD Pagel, Molecular Pharmaceutics, 2009 (in press)

J089

SYNTHESIS OF AN ISOTOPIC LABELLED GD-COMPLEX Markus Plaumann, Dieter Leibfritz

Institute of Organic Chemistry, University of Bremen, Bremen, Germany. 
Different techniques in diagnostic clinical imaging and biomedical research need different contrast agents. Therefore, many synthesis strategies were developed. A new method allows an isotopic labelling of a contrast agent, which can be used for MRI or PET, with deuterium or tritium. Reaction of 1,4,7,10-tetraazacyclododecane (cyclene) with dimethylformamidedimethyl acetal (DMF-DMA) results in 1,4,7,10-tetraazacyclo[5.5.1.0] tridecane, ${ }^{[1]}$ which was hydrolyzed with $\mathrm{H}_{2} \mathrm{O}$ or $\mathrm{D}_{2} \mathrm{O}$ in methanol to the appropriate aldehyde. After reaction with $\mathrm{HCN}$, the cyanhydrine was synthesized and following hydrolyzed with diluted acid to the carbon acid. Reaction with tert-butylbromoacetate gave the new ligand system. The t- butyl esters were cleaved of with trifluoroacetic acid. For MRI measurement the $\mathrm{Gd}^{3+}$-complexes were obtained by adding $\mathrm{GdCl}_{3}$ in an aqueous/ethanol solution of the ligand and following neutralisation with $1 \mathrm{M} \mathrm{NaOH}$. The complex were purified by HPLC and characterized by ESI-MS and NMR (8.4 T). The same reaction should be possible for using $\mathrm{T}_{2} \mathrm{O}$ instead of $\mathrm{H}_{2} \mathrm{O}$ or $\mathrm{D}_{2} \mathrm{O}$. This allows the synthesis of tritium labelled tracer molecules, which can also be used, depending of the metal ion, for MRI or PET. The new ligand system (figure 1) allows additionally varied reactions, like amidation or esterification, at the unprotected carbon acid. [1] Ferrand, A.-C. et al., Chem. Eur. J. 2007, 13, 8678-8687.
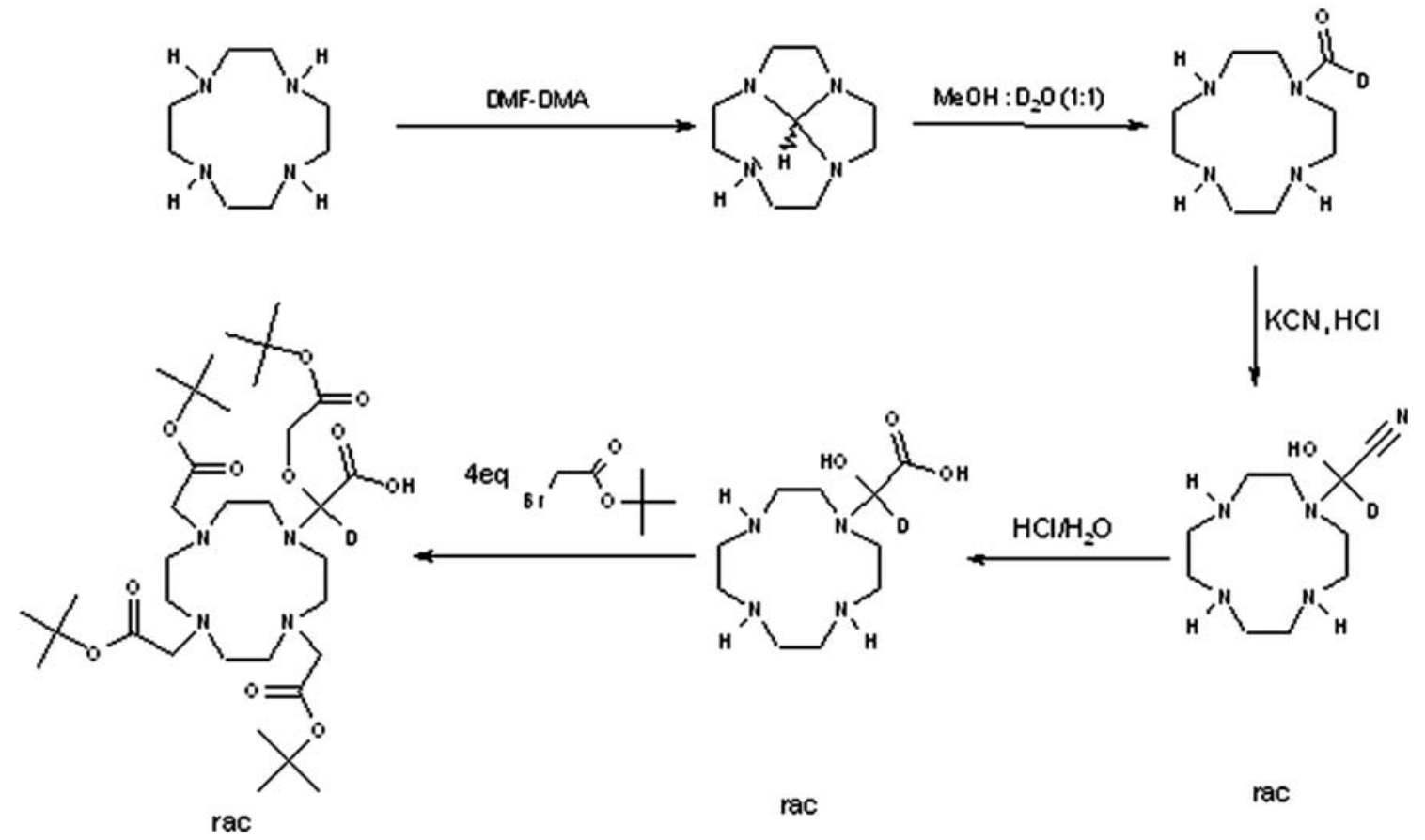

Figure 1: Synthesis scheme for the new ligand system.

\section{J090}

INTEIN-MEDIATED PROTEIN LIGATION

OF HER2-AFFIBODIES TO SUPERPARAMAGNETIC IRON OXIDE NANOPARTICLES FOR DISCRIMINATE TARGETING OF HER2-POSITIVE BREAST CANCERS

Andrew Elias, Andrew Tsourkas

Bioengineering, University of Pennsylvania, Philadelphia, PA, USA.

Over the past several decades, it has been revealed that the onset and progression of cancer can be characterized by a myriad of molecular and cellular processes. Often, malignant cells can be identified by the up-regulation of specific cell surface receptors. These unique expression profiles have recently been exploited as targets for molecular imaging contrast agents. The ability to non-invasively acquire information about the molecular make-up of malignant cells will allow for the early identification of disease. The HER2 affibody, a stable three-helix bundle molecule composed of 58 amino acids, presents a particularly promising targeting agent for imaging breast cancer because of its small size and high affinity for the HER2/neu receptor, which is commonly overexpressed in breast cancer. HER2 affibodies were conjugated to superparamagnetic iron oxide nanoparticle (SPIO) through an intein mediated protein ligation. This unique conjugation approach allowed for efficient and site-specific conjugation. Further, flow cytometric analysis and MR imaging confirmed that HER2 affibody-directed SPIO specifically labeled HER-2 positive cancer cells. It is envisioned that HER2 affibody-labeled SPIO may serve as a diagnostic tool for the efficient and discriminate detection of HER2-positive breast cancers.

\section{J091}

\section{MOLECULAR MR IMAGING OF CATHETER-INDUCED INJURY DURING ENDOVASCULAR INTERVENTIONS IN A MODEL OF SACCULAR ANEURYSM}

Michael Deleo, Matthew Gounis, Alexei A. Bogdanov; Radiology, University of Massachusetts Medical School, Worcester, MA, USA.

Catheter-induced vascular injury is not visible using standard digital biplane fluoroscopy. The molecular environment in the damaged vessel results in inflammatory changes including activation of the key inflammatory mediator NF-kappa-B. The enzyme myeloperoxidase (MPO) is known to play significant roles in the progression in various vascular pathologies. We performed feasibility experiments in an attempt to detect areas of vascular injury using the paramagnetic imaging probe (di-5-HT-GdDTPA) with proven sensitivity and specificity for MPO. We used elastase-mediated degradation of vascular wall in situ for generating saccular aneurysms at the base of right carotid artery in NZ white rabbits $(n=5)$. After 21 days, a $4 \mathrm{~F}$ guide catheter 
was secured under fluoroscopic guidance at the neck of the aneurysm. Under 3-D roadmap, a hypotube was advanced through a microcatheter and a fixed volume of $0.2 \mathrm{ml}$ saline into the vascular wall. Forty-eight hours after the injection, animals were subjected to $3 \mathrm{~T}$ MRI using 3D T1W-FFE sequence. Animals then received an intra-arterial injection of di-5-HT-GdDTPA $(0.1 \mathrm{mmol} / \mathrm{kg})$; $3 \mathrm{~h}$ after the administration, animals were subjected to MRI using the same pulse sequence. Post-contrast MRI revealed focal areas of enhancement at the dome of the aneurysm consistent with the area of vascular perforation with the hypotube. MPO activity in the vessels was determined and correlated with the enhancement area. We confirmed that aneurysms demonstrated a higher enhancement ratio compared to the left carotid artery in the same animals. In conclusion, we showed that: 1) focal vascular injury can be imaged using the MPO-specific paramagnetic probe; 2) MPO likely plays a role in the acute response to catheter-induced endothelial disruption; 3) the MPO-specific imaging probe enables MR imaging of very small areas of vessel injury; 4) MRI ER measurements can be used for predicting the extent of inflammation in aneurysms.
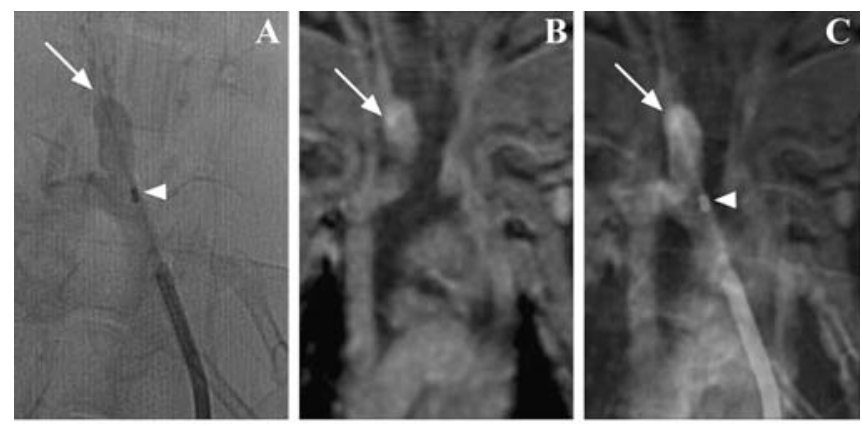

Saline injection under fluorscopic guidance (A), MPO probe enhanced MRI $(B)$, superimposed image verifying focal MR enhancement a result of injury (C).

\section{J092}

\section{DETECTION OF UROKINASE PLASMINOGEN ACTIVATOR ENZYME ACTIVITY WITH PARACEST MRI}

Byunghee Yoo', Vipul R. Sheth ${ }^{2}$, Aswin V. Sundaram ${ }^{2}$, M. M. Ali ${ }^{3}$, Marty Pagel ${ }^{1,4}$

${ }^{\top}$ Biomedical Engineering, University of Arizona, Tucson, AZ, USA, ${ }^{2}$ Biomedical Engineering, Case Western Reserve University, Cleveland, OH, USA, ${ }^{3}$ Radiology, Henry Ford Hospital, Detroit, MI, USA, ${ }^{4}$ Chemistry, University of Arizona, Tucson, AZ, USA.

Urokinase Plasminogen Activator (uPA) is an enzyme biomarker of metastatic breast cancer and pancreatic cancer. PARACEST (PARAmagnetic Chemical Exchange Saturation Transfer) MRI contrast agents have been developed that can detect enzyme activity [1,2]. We have exploited this methodology to create a PARACEST agent that can detect the activity of urokinase Plasminogen Activator. The PARACEST agent consisted of a Tm-DOTA chelate with a peptidyl ligand, CBZ-Gly-Gly-Arg. A solid phase peptide synthesis method that was modified to use Fmoc chemistry was used to create the PARACEST agent [3]. The Z-spectrum of $50 \mathrm{mM}$ of ZGGR-(Tm-DOTA) showed a CEST effect at $-52 \mathrm{ppm}$, which disappeared after incubation of the sample with $33 \mathrm{nM}$ of uPA. A PARACEST agent that is unresponsive to uPA enzyme activity, Yb-DOTA-Gly4, was included in the sample as a control, and showed a CEST effect at -16 ppm that did not change after incubation with uPA. These results demonstrate that ZGGR-(Tm-DOTA) can detect the presence of uPA enzyme by monitoring the difference in CEST effects between this uPA-responsive agent and a control agent. To prepare for in vivo studies of uPA with ZGGR-(Tm-DOTA), we have developed a simple linear calibration that translates the PARACEST effect to the concentration of the agent [4]. This calibration is based on a two-pool model of exchangeable protons on the agent and water, and assumes complete saturation of the agent pool with no direct saturation of the water pool. However, multiple pools are anticipated within in vivo tissues, and partial saturations of the agent and water may to occur in vivo. To address these issues, we have used our linear calibration method to develop a ratiometric approach that compares the PARACEST effects of a responsive and an unresponsive agent, which can cancel the effects of multiple pools and partial saturations. This ratiometric approach emphasizes how one of the primary advantages of PARACEST MRI - the selective detection of two MRI contrast agents during a single study — can be used to perform quantitative molecular imaging studies. References: 1 . Yoo B, Pagel MD. JACS, 2006, 128(43):14032-14033. 2. Yoo B, Raam M, Rosenblum R, Pagel MD. Contrast Media Molec Imag, 2007, 2:189-198. 3. Yoo B, Pagel MD. Bioconj Chem, 2007,18:903-911. 4. Ali MM, Yoo B, Pagel MD. Molec Pharm, 2009, published on-line, hardcopy in press.

\section{J093}

VALIDATION OF PH MEASUREMENTS DETERMINED FROM A SINGLE PARACEST MRI CONTRAST AGENT Vipul R. Sheth ${ }^{1}$, Yuguo $\mathrm{Li}^{2}$, Marty Pagel ${ }^{2,3}$

${ }^{1}$ Biomedical Engineering, Case Western Reserve University, Cleveland, $\mathrm{OH}, \mathrm{USA},{ }^{2}$ Biomedical Engineering, University of Arizona, Tucson, AZ, USA, ${ }^{3}$ Chemistry, University of Arizona, Tucson, AZ, USA.

The in vivo measurement of tumor extracellular $\mathrm{pH}$ can be used to study the effects of $\mathrm{pH}$-altering therapy and to predict the efficacy of $\mathrm{pH}$ dependent therapy[1]. We have previously developed a single PARACEST MRI contrast agent, Yb-DO3AoAA, that can accurately measure $\mathrm{pH}$ without the need to account for the concentration of the agent[2]. We have shown that the chemical exchange rates between the amide and water and between the amine and water have different dependencies on $\mathrm{pH}$, and that the ratio of these PARACEST effects is correlated with $\mathrm{pH}$. Importantly, this correlation with $\mathrm{pH}$ is independent of the concentration of the agent, so that this single agent can accurately measure $\mathrm{pH}$ without requiring a second MRI contrast agent to account for concentration. In this study, we have developed a theoretical correlation that directly determines $\mathrm{pH}$ from the PARACEST effects of Yb-DO3AoAA. We have also measured $\mathrm{pH}$ with $\mathrm{Yb}-\mathrm{DO} 3 \mathrm{AoAA}$ and validated our $\mathrm{pH}$ measurements with MR spectroscopy of IEPA. To accomplish this study, 1,4,7,10-tetraazacyclododecane-1.4.7.10-tetraacetic acid o-aminoanilide (DO3A-oAA) was synthesized and used to chelate $\mathrm{Yb}(\mathrm{III})$ using standard methods. A calibrated microelectrode was used to measure the $\mathrm{pH}$ of buffered solutions of Yb-DO3AoAA and IEPA, which ranged in $\mathrm{pH}$ from 5.7 to 7.9 in $\sim 0.2 \mathrm{pH}$ increments. The chemical shift of IEPA was measured with MR spectroscopy relative to a TSP chemical shift reference, which was used to measure $\mathrm{pH}[3]$. The CEST spectrum of $\mathrm{Yb}-\mathrm{DO} 3 \mathrm{AoAA}$ was acquired with selective saturation applied at +8 and $-11 \mathrm{ppm}$ at $10 \mu \mathrm{T}$ for $2.5 \mathrm{sec}$. These spectra were fit to a three-lorentzian lineshape model to determine the CEST effects of Yb-DO3AoAA and measure $\mathrm{pH}$. The QUEST method was used to measure the exchange rates[4]. The estimated exchange rates of the amide and amine were fit to the acid-base catalyzed exchange model to reveal the underlying catalysis mechanisms[5]. This validated method will be used to study the effects of $\mathrm{pH}$-altering therapy and to predict the efficacy of a $\mathrm{pH}$-dependent therapy on mouse models of breast cancer. References 1. Raghunand N, Gillies RJ. Drug Resist Updates, 2002, 3, 39. 2. Liu G, Li Y, Pagel MD, Magn Resn Med, 2007, 58, 1249. 3. van Sluis, R, Bhujwalla, Z. M., Raghunand, N., Ballesteros, P., Alvarez, J., Cerdan, S., Galons, J. P., Gillies, R. J. 1999, 41, 743. 4. M. T. McMahon, A. A. Gilad, J. Zhou, P. 
Z. Sun, J. W. Bulte, P. C. van Zijl, Magn Reson Med 2006, 55, 836. 5. E. Grunwald, E. K. Ralph, Acc. Chem. Res. 1971, 4, 107.

J094

SYNTHESIS AND MAGNETIC RELAXATION PROPERTIES OF PARAMAGNETIC GD-COMPLEXES OF NEW DTPA-BIS (AMIDE)DERIVATIVES

D. Sinha $^{1,2}$, Anjani K. Tiwari ${ }^{1}$, Gauri Shukla ${ }^{1,2}$, Shubhra Chaturvedi ${ }^{1}$, Harish Chandra $^{2}$, Anil K. Mishra ${ }^{1}$

${ }^{1}$ dcrs, inmas, Delhi, India, ${ }^{2}$ chemistry, delhi university, Delhi, India.

Introduction:-Magnetic resonance imaging (MRI) is at present well established as a safe and efficient imaging technique for the human body in clinical diagnosis. Gd(DTPA)2- is most commonly used wellestablished contrast agent in clinical use for MRI due to its high relaxivity, high chemical stability and low toxicity. However, its passive and nonspecific distribution in vivo limits its utility. Attempts to decrease the side effects and improve the tissue-and/or organ-specificity, has led to the preparation and study of a wide range of complexes. Among them, Gd-[DTPA-bis(amide)] derivatives have been successfully used in clinical MRI due to their potential target specificities after certain modification. Materials and Methods:- Herein, we report the synthesis and characterization of $\mathrm{Gd}(\mathrm{III})$ complexes incorporating in a new type of DTPA-bis(amides) functionalized by heterocyclic moiety as a precursor for various neurotransmitters to achieve enhanced specificity. The compounds were synthesized by the condensation reaction of DTPAbis (anhydride) with different optically active L-amino acids (Trp and His) and were characterised on the basis of different spectroscopy techniques. Results and Discussion:-Their potential application as brainspecific and low-osmolarity MRI contrast agents has been evaluated by in vitro and in vivo experiments. The gadolinium (III) complexes of the ligands were prepared and then studied in vitro by $17 \mathrm{O}$ NMR spectroscopy and by nuclear magnetic relaxation dispersion (NMRD) measurements. The residence time of the coordinated water in gadolinium (III) complexes was obtained from $17 \mathrm{O}$ NMR relaxometric T2 measurements. At $310 \mathrm{~K}$, the $\tau \mathrm{M}$ values were obtained in the range of $560-640$ ns. As shown by the analysis of the proton NMRD profiles, the larger proton relaxivities of the gadolinium (III) complexes at $310 \mathrm{~K}$ relative to that of the parent Gd-DTPA complex are mainly because of the increase in the rotational correlation time. Conclusion:- the in vivo and in vitro studies have proven that novel Gd [DTPA-bis(amide)] derivatives could be promising brain-specific MRI contrast agents and provides the stable imaging contrast for several hours with a lower toxicity.

J095

\section{"SMART" MRI CONTRAST AGENTS: ENZYME RESPONSIVE NANOSYSTEMS}

$\underline{\text { Sara Figueiredo }}^{2,1}$, Evelina Cittadino ${ }^{1}$, Enzo Terreno ${ }^{1}$, João Nuno Moreira $^{3}$, Carlos F. Geraldes ${ }^{2}$, Silvio Aime

${ }^{1}$ Department of Chemistry IFM and Molecular Imaging Center, University of Turin, Turin, Italy, ${ }^{2}$ Department of Biochemistry, Faculty of Sciences and Technology and Center for Neurosciences, University of Coimbra, Coimbra, Portugal, ${ }^{3}$ Lab. of Pharmaceutical Technology, Faculty of Pharmacy and Center for Neuroscience and Cell Biology, University of Coimbra, Coimbra, Portugal.

Assessment of a given enzymatic activity is an important task in Molecular Imaging investigations. When Magnetic Resonance is the chosen imaging modality, it is necessary to design highly sensitive systems in order to overcome the relatively low sensitivity of the technique. Therefore, we have envisaged an approach to enzymeresponsive agents based on the use of liposomes loaded with a high number of paramagnetic metal complexes as highly-sensitive MRI probes. Liposomes are self-assembled vesicles formed by saturated and/ or unsaturated phospholipids widely used in drug delivery procedures. The MR response of a liposome encapsulating a Gd(III)-based agent depends upon the permeability of the liposome membrane to water molecules and the integrity of the nanovesicles. The main objective of this work is the design of paramagnetic liposomes whose stability, and consequently their MRI response, is modulated by the activity a given enzyme. This task has been accomplished by incorporating in the liposome membrane an amphiphilic peptide acting as substrate for Matrix Metallo Proteinases (MMPs). The peptide, obtained from solid phase synthesis, was conjugated to a stearic acid chain (See Figure 1). The clinically approved MR contrast agent Gd-HPDO3A was loaded in the inner aqueous cavity of the vesicle. The in vitro experiment reported in Figure 1 shows that the presence of collagenase (namely MMP-1) triggers the MRI contrast generated by the probe. It was demonstrated that this occurs as a consequence of the release of the paramagnetic payload from the liposomal vesicle. The performance of the probe was also tested in vivo on mice bearing melanoma tumor xenografts. Finally, the herein proposed nanoprobe could act as an efficient theragnostic agent by exploiting the well known ability of liposomes to act as drug carriers.
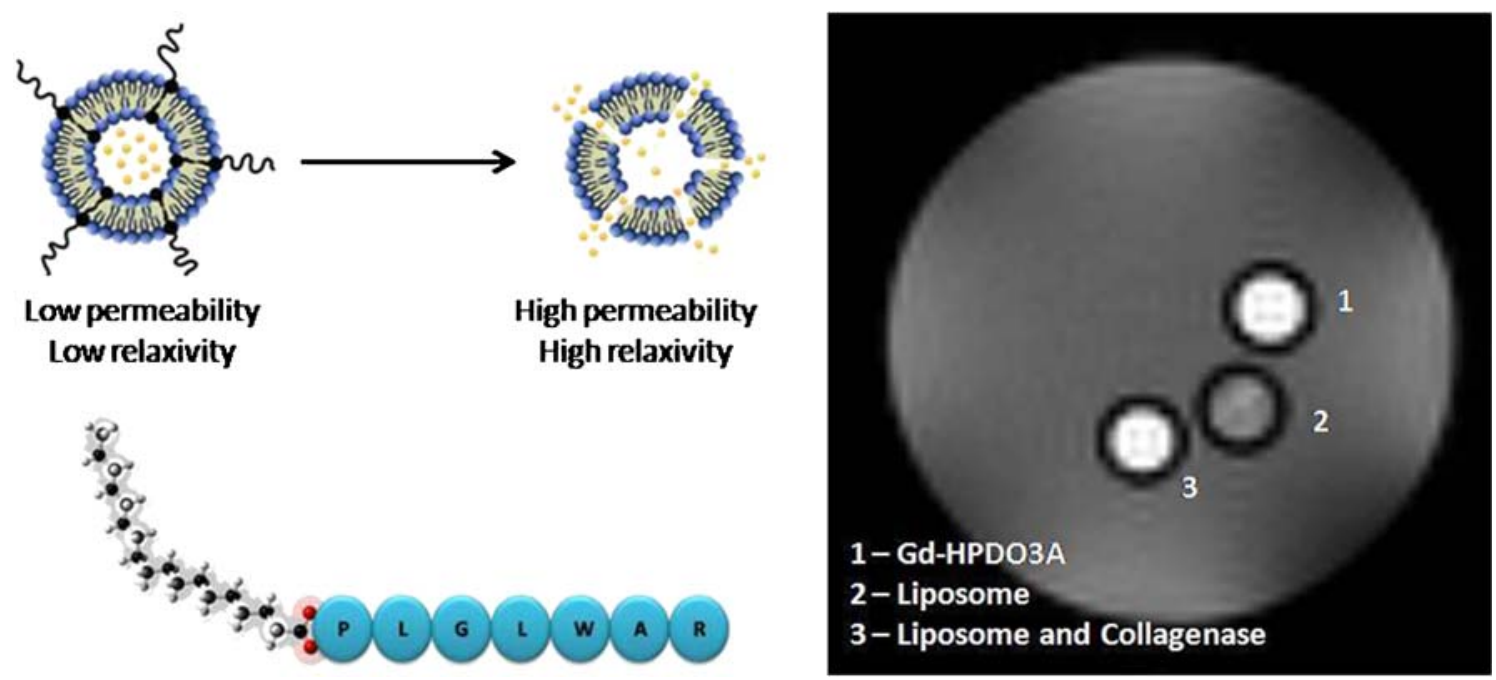

1-Gd-HPDO3A

2-Liposome

- Liposome and Collagenase 
J096

\section{MULTI-COLORED EU(III)-BASED PARACEST AGENTS}

Subha Viswanathan ${ }^{1}$, S James Ratnakar ${ }^{1}$, Kayla N. Green ${ }^{1}$, Zoltan Kovacs ${ }^{1}$, Dean Sherry ${ }^{1,2}$

${ }^{1}$ Advanced Imaging Research Center, UT Southwestern Medical Center, Dallas, TX, USA, ${ }^{2}$ Department of Chemistry, University of Texas, Dallas, Dallas, TX, USA.

Magnetic Resonance Imaging (MRI) is one of the most versatile and powerful diagnostic tools in modern medicine. Current diagnostic contrast agents for MRI are chiefly based on paramagnetic Gd(III) complexes that function by shortening the T1 (longitudinal relaxation) of bulk water. Another way to generate image contrast is to alter the total water signal by a process called Chemical Exchange Saturation Transfer or CEST, which depends on the transfer of spin magnetization between two pools of protons. To observe CEST, the two exchanging pools of protons must be in slow-to-intermediate exchange relative to the frequency difference $(\Delta \omega)$ between the two pools (kex $\leq \Delta \omega$ ), where kex is the proton exchange rate between the two pools. One of the primary advantages of CEST as a contrast mechanism is that the effect is detectable only when a selective RF irradiation pulse is applied at the specific frequency of the exchangeable protons. Consequently, if the resonance frequencies of the exchangeable protons are well separated, multiple agents can be used in one imaging experiment and selectively turned on and off by applying the appropriate frequency-selective RF pulse. PARACEST agents based on $\operatorname{Ln}(\mathrm{III})$ ions are particularly attractive in this respect because lanthanide ions across the series can be used to generate markedly diverse complexes with exchangeable protons covering a wide range of frequencies. "Multi-colored" MRI using PARACEST agents would be extremely useful to image multiple biomarkers simultaneously, since any parameter that alters the water exchange kinetics will in turn alter image contrast. To date, however, it has been largely the $\mathrm{Eu}(\mathrm{III})$ complexes that have displayed sufficiently slow water exchange kinetics to satisfy the kex $\leq \Delta \omega$ requirement. Complexes of other lanthanide ions that induce large paramagnetic shifts [Tm(III), Tb(III) and $\mathrm{Yb}(\mathrm{III})]$ have faster than optimal water-exchange kinetics and consequently their CEST properties are limited. In this work, we report the synthesis and characterization of three novel Eu(III) complexes (1-3) that show excellent promise as "multi-colored" PARACEST agents.

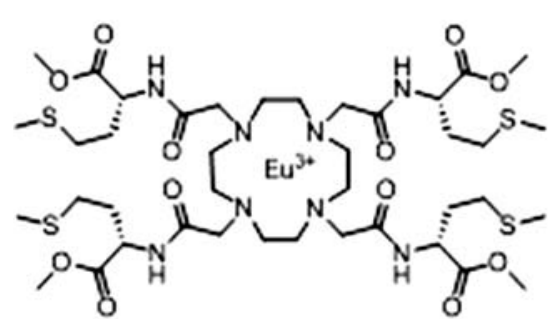

IOTA-Met-().Me

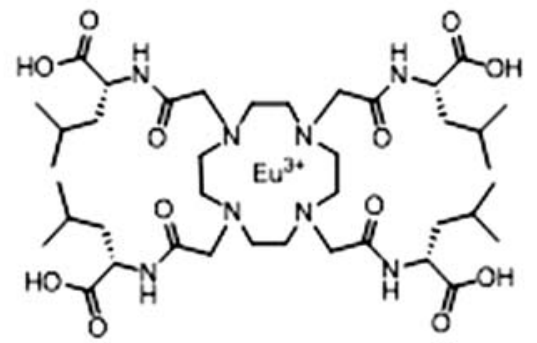

DOTA-Leu-OH

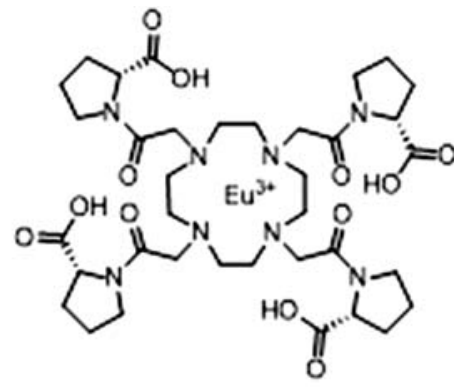

DOTA-Pro-OH
FERRITIN COORDINATES WITH USPIO IN TRACKING C6 RAT GLIOMA CELLS BY MRI

Jiandong Wang ${ }^{2,1}$, Guangming $\mathrm{Lu}^{2,1}$

${ }^{1}$ Nanjing Jinling Hospital, Nanjing, China, ${ }^{2}$ Radiology, Nanjing University, Nanjing, China.

Abstract Superparamagnetic iron oxide (SPIO) is used extensively as a MRI contrast agent in tracking cells. However, the concentration of SPIO is diluted with cell division and SPIO degradation. Ferritin is involved in iron storage in microbial, plant and animal species. $\mathrm{H}$ and $\mathrm{L}$ subunits of ferritin assemble into a hollow spherical apoferritin, which can accommodate up to 4,000 iron atoms as ferrihydrite. More recently, ferritin has been used as an endogenous reporter for MRI. The shortage of ferritin as a MRI reporter is the low sensitivity of detection. We hypothesized that overexpressed ferritin could sequester extra iron atoms degraded from SPIO, and would coordinate with SPIO in tracking cells. In this study, parental C6 rat glioma cells and C6 cells overexpressing ferritin were labeled with SPIO and bilaterally subcutaneously inoculated to SpragueDawley rats. The rats were scanned 2 weeks after tumor inoculation for T2 relaxation time with a SIMENS $3 \mathrm{~T}$ in vivo. Our data showed that $\mathrm{T} 2$ relaxation time in ferritin overexpressed C6 cells was significantly shorter than that in the parental C6 cells. Prussian blue stain results confirmed that C6 cells transfected with ferritin gene accumulated significantly more iron than the wild-type C6 cells. In conclusion, introduction of ferrtin gene could prolong the monitoring time for SPIO in tracking cells, and the sensitivity of detection for ferritin as an endogenous MR reporter was
2

3

increased by accommodation of iron atoms degraded from SPIO. Key words: ferritin; USPIO; MRI; cell tracking

J098

\section{ENHANCING THE SIGNAL-POLARIZATION OF PHOSPHOROUS-31 NUCLEI TO GENERATE AN IN VIVO PH IMAGING}

Natalie M. Barkey $^{1}$, Gary Martinez ${ }^{3}$, Robert Gillies ${ }^{2}$

${ }^{1}$ Imaging, Moffitt Cancer Center, Tampa, FL, USA, ${ }^{2}$ Imaging, Moffitt Cancer Center, Tampa, FL, USA, ${ }^{3}$ Imaging, Moffitt Cancer Center, Tampa, FL, USA.

One of the most powerful tools available to chemists and clinicians is the ability to obtain images on a molecular (eg: molecules in solution) as well as a macromolecular (eg: tissues in a living organism) scale. Nuclear Magnetic Resonance (NMR) and Magnetic Resonance Imaging (MRI) are two of the most commonly employed Magnetic Resonance (MR) techniques in the laboratory and clinical settings. Dynamic Nuclear Polarization (DNP) has gained much attention over the last several years as a means to apply the use of less sensitive nuclei (eg: 13C, 31P, 15N) that are present in much lower concentrations (endogeneous and injected) to in vivo studies. A major limitation of DNP is a tremendous dependence on a compound's longitudinal relaxation time (T1). In order for use of DNP on a compound in vivo to be feasible, a sufficiently long T1 (>11s) is required. To date, DNP has been used both in solution and in vivo to study the polarization of $13 \mathrm{C}$ enriched compounds almost exclusively. However, it is also possible to generate hyperpolarized 31P nuclei. Cancer cells are known to possess a 
lower extra-cellular $\mathrm{pH}$ than the surrounding, healthy tissue; thus, the ability to obtain in vivo images of tissues based on $\mathrm{pH}$ level is a potentially invaluable diagnostic tool. We plan to utilize 3-aminopropylphosphonate (APP) to probe the effectiveness of DNP on 31P nuclei. 3-APP has previously proven to be an effective $\mathrm{pH}$ indicator in vitro and is known to reside in the extracellular compartment exclusively. However, a weak signalto-noise ratio (SNR) has precluded its study in vivo. It is our belief that the signal amplification obtained through the use of 31P DNP will substantially increase the SNR of 3-APP, thereby rendering it a reliable probe for in vivo $\mathrm{pH}$ determinations. Initial experiments were conducted on both natural 3 APP and perdeuterated 3-APP in both $\mathrm{D} 2 \mathrm{O}$ and $\mathrm{H} 2 \mathrm{O}$ at three field strengths $(300 \mathrm{mHz}, 500 \mathrm{mHz}$ and $600 \mathrm{mHz})$, two temperatures $\left(22^{\circ} \mathrm{C}\right.$ and $\left.37^{\circ} \mathrm{C}\right)$ and $\mathrm{pH}$ values both above and below the $\mathrm{pKa}$. Initial results indicate that the $\mathrm{T} 1$ value of both compounds is inversely proportional to field strength and directly proportional to temperature. As expected, the longest relaxation times are observed with d7-APP in D2O, with the longest recorded time being $15 \mathrm{~s}\left(\mathrm{~d} 7-\mathrm{APP}\right.$ in $\mathrm{D} 2 \mathrm{O}$ at $37^{\circ} \mathrm{C}$ and $\left.300 \mathrm{mHz}\right)$. These results indicate that the relaxation of 3-APP is controlled mainly by chemical shift anisotropy (CSA) at high field and joints controlled by dipolar relaxation at low fields. Polarization studies of 3-APP are currently ongoing.

\section{Novel Multi-Modality Probes}

\section{J099}

\section{MONITORING THE MIGRATION AND EXPRESSION OF REPORTER GENE IN MAGNETICALLY LABELED TRANSGENIC AC133+ PROGENITOR CELLS CARRYING HNIS IN GLIOMA}

Ali S. Arbab, Nadimpalli Ravi S Varma, Asm Iskander, M. M. Ali, Branislava Janic

Cellular and Molecular Imaging Laboratory, Henry Ford Hospital, Detroit, MI, USA.

Purpose: Recently we have successfully tracked the migration and homing of $\mathrm{AC} 133+$ progenitor cells (AC133PCs) at the site of neovascularization in subcutaneous as well as in intracranial tumors by in vivo and ex vivo MRI. The tracking was possible due to magnetic labeling of cells using ferumoxides and protamine sulfate (FePro). Accessibility, easy harvesting and established techniques for genetic manipulation renders AC133PCs as attractive cellular vehicles when systemic or local gene delivery is required. The purpose of the study was to investigate the ability of AC133PCs to carry a gene to the tumor sites and use these transgenic cells as cellular probes to track the migration of the cells by magnetic resonance imaging (MRI). Material and Methods: Lentiviral vectors carrying human sodium iodide symporter (hNIS) with different promoters (such as CMV and EF1) were used to optimize the transfection efficiency of AC133PCs. Transgenic cells were also subjected to long term culture to determine the peak transfection time and the stability of transfection. For animal studies, transgenic cells were magnetically labeled using FePro on day four following transfection and the viability of the cells was determined. Mixtures of transgenic and transgenic plus magnetically labeled or nontransgenic magnetically labeled AC133PCs were administered intravenously in rats bearing U251 glioma on day 11 following intracranial implantation of $5 \times 105$ U251 cells. MR images were obtained pre and 7 days post administration of the cells to determine the migration of transgenic cells in the tumors. Following MRI, SPECT images were obtained to determine the expression of hNIS in the tumors using Tc- $99 \mathrm{~m}$ pertechnetate (Tc-99m). Collected brains were analyzed histochemically to determine the iron labeled cells and cells expressing hNIS. Results: Both
CMV and EF1 promoters equivocally expressed hNIS in AC133PCs, which was determined by Tc-99m uptake study. MRI images showed low signal intensity areas in tumor that received magnetically labeled cells, which was significantly different from the MRI that was obtained before the administration of cells in the same animal. SPECT images showed increased Tc-99m activity in tumor that received transgenic cells compared to the tumor that received only magnetically labeled cells. Histochemically, both iron and hNIS positive cells were determined in the tumors. Conclusion: Transgenic AC133PCs can be used both as gene carrier/ delivery system and probes for cellular MRI.

\section{$\mathbf{J 1 0 0}$}

\section{PEPTIDE MODIFIED HIGH DENSITY LIPOPROTEIN NANO-} PARTICLES FOR MULTIMODAL MOLECULAR IMAGING OF THE TUMOR VASCULATURE

Wei Chen $^{2}$, Peter A. Jarzyna ${ }^{2,1}$, Geralda A. van Tilborg ${ }^{5}$, Van A. Nguyen ${ }^{3}$, David P. Cormode ${ }^{2}$, Ahmed Klink ${ }^{2,6}$, Arjan Griffioen ${ }^{7}$, Gwendalyn J. Randolph $^{3}$, Edward A. Fisher ${ }^{8}$, Willem J. Mulder ${ }^{1,3}$, Zahi Fayad ${ }^{2,4}$

${ }^{1}$ Radiology, Mt. Sinai School of Medicine, New York, NY, USA,

${ }^{2}$ Translational and Molecular Imaging Institute, Mt. Sinai School of Medicine, New York, NY, USA, ${ }^{3}$ Department of Gene and Cell Medicine, Mt. Sinai School of Medicine, New York, NY, USA, ${ }^{4}$ The Zena and Michael A. Wiener Cardiovascular Institute and Marie-Josee and Henry R. Kravis Center for Cardiovascular Health, Mt. Sinai School of Medicine, New York, NY, USA, ${ }^{5}$ Image Sciences Institute, Utrecht University, Utrecht, Netherlands, ${ }^{6}$ Paris Cardiovascular Research Center, INSERM Assistance Publique-Hopitaux de Paris, Hopital Europeen Georges Pompidou, Paris, France, ${ }^{7}$ Angiogenesis Laboratory, Department of Pathology, Research Institute Growth and Development, Maastricht University and Hospital, Maastricht, Netherlands, ${ }^{8}$ Leon H. Charney Division of Cardiology, NYU Center for the Prevention of Cardiovascular Disease, New York University School of Medicine, New York, NY, USA.

Introduction: We previously modified high density lipoprotein (HDL), an endogenous nanoparticle, to function as a versatile contrast agent platform for magnetic resonance (MR) molecular imaging of atherosclerosis. In the present study, we utilize HDL as a multimodality nanoparticle platform for cancer imaging by either exploiting their nonspecific accumulation in tumors or by specifically targeting angiogenically activated tumor blood vessels via a rerouting strategy. Methods and Results: We reconstituted discoidal HDL (rHDL) and included amphiphilic gadolinium chelates for magnetic resonance imaging (MRI) and near infrared (NIR) fluorescent dyes for optical imaging. To reroute rHDL nanoparticles to angiogenic endothelial cells, rHDL nanoparticles were functionalized with $\alpha v \beta 3$ integrin-specific cyclic 5-mer RGD peptides, termed as rHDL-RGD (A). The uptake of rHDL and rHDL-RGD was examined in vitro on cultured macrophages and endothelial cells using optical techniques and MRI. It was observed that rHDL was phagocytosed by macrophages, while $\alpha v \beta 3$ integrin-specific rHDL-RGD nanoparticles were preferentially taken up by endothelial cells. The uptake of both particles in mouse tumors was evaluated in vivo using MRI (B) and NIR (C) imaging. We found both rHDL and rHDL-RGD to accumulate in tumors, but with very different accumulation/binding kinetics as observed by NIR imaging. Moreover, confocal microscopy revealed rHDL-RGD to be associated with tumor endothelial cells (D), while rHDL nanoparticles were mainly found in the interstitial space of the tumor. Conclusion: This study demonstrated the ability to reroute the rHDL nanoparticles from its natural target to angiogenic tumor blood vessels. The versatility of this platform and its potential for multimodality imaging of tumor-associated processes was demonstrated. 
A

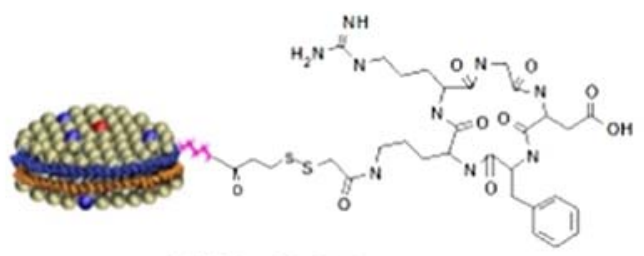

rHDL-RGD
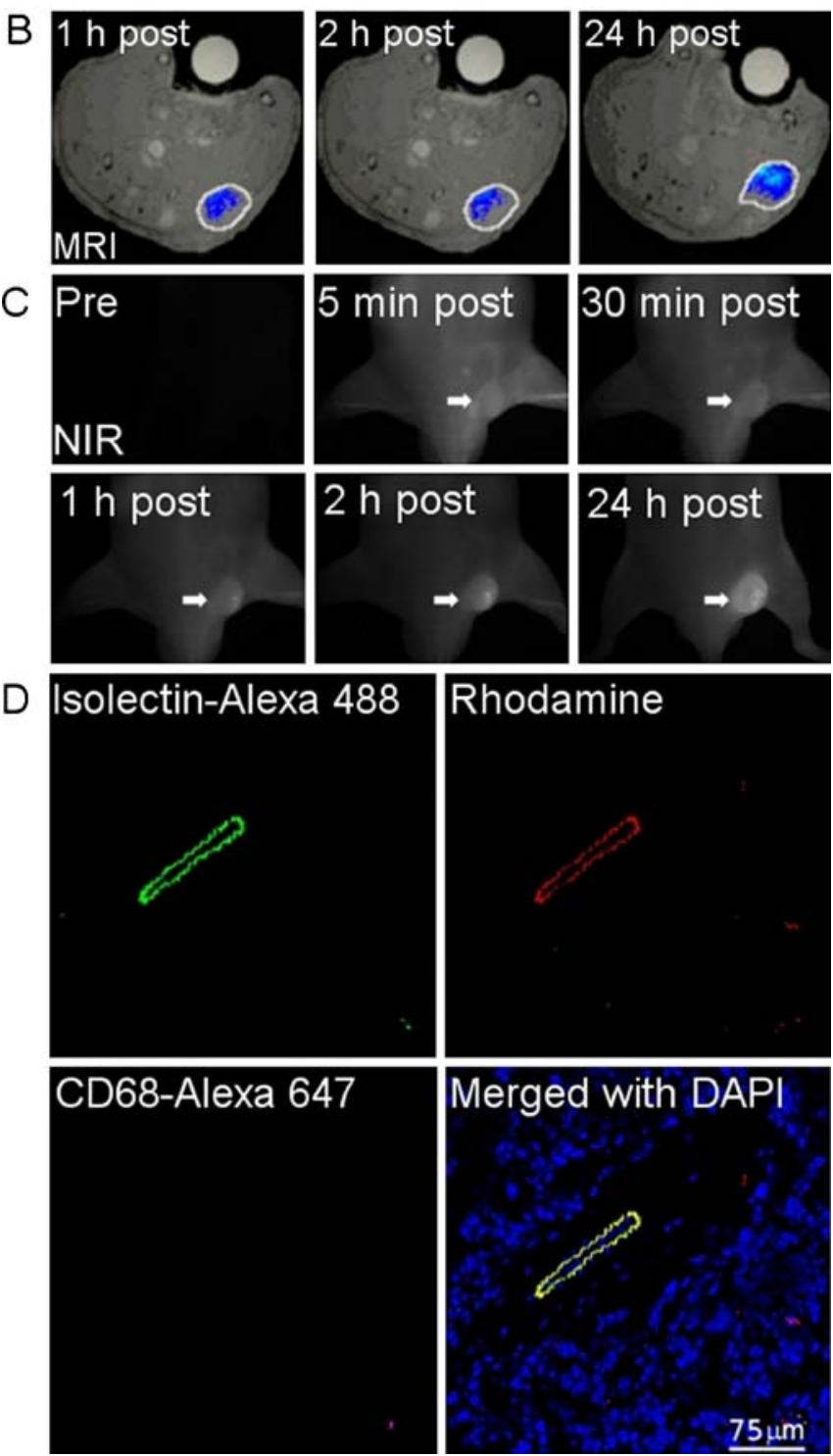

(A) Schematic of rHDL-RGD structure. (B) MR imaging of tumor postinjection of rHDL-RGD. The enhanced pixels are colored-coded. (C) Near infrared fluorescent imaging of tumor pre- and post-injection of rHDL-RGD. (D) Confocal images of a tumor tissue section. Angiogenic endothelial cells (Isolectin-Alexa 488, green), rHDL-RGD (Rhod-amine, red), Macrophages (CD68-Alexa 647, magenta), nuclei (DAPI, blue).
$\mathbf{J 1 0 1}$

\section{DEVELOPMENT OF NEW IN VIVO GD-BASED NEUROANATOMICAL TRACERS FOR MAGNETIC RESONANCE IMAGING}

Anurag Mishra $^{1}$, Kirti Dhingra ${ }^{1}$, Ritu Mishra ${ }^{2}$, Almut Schüz ${ }^{1}$, Joern Engelmann $^{2}$, Nikos K. Logothetis ${ }^{1,3}$, Santiago Canals ${ }^{1,4}$

${ }^{1}$ Department for Physiology of Cognitive Processes, Max PLanck Institute for Biological Cybernetics, Tuebingen, Germany, ${ }^{2}$ HighField MR Center, Max PLanck Institute for Biological Cybernetics, Tuebingen, Germany, ${ }^{3}$ Imaging Science and Biomedical Engineering, University of Manchester, Manchester, United Kingdom, ${ }^{4}$ Instituto de Neurociencias CSIC-UMH, Campus de San Juan, San Juan de Alicante, Spain.

Gaining insights into brain function by identifying neuronal circuits connecting different regions of the brain is of prime interest in cognitive neuroscience. For visualization of anatomical connections, the tract-tracing technique has been proved to be an extremely useful tool which provides valuable information on afferent and efferent connectivity of the brain. A variety of neuroanatomical tracers exists, and numerous investigations using classical tracers have contributed valuable descriptions of connectivity in the mammalian brain (1). These studies, however, require fixed, histologically processed tissue for data analysis and therefore cannot be applied for noninvasive/ longitudinal investigations. Manganese-enhanced Magnetic Resonance Imaging is a recently introduced non-invasive technique that represents the first effort in the direction of studying neuronal connectivity in vivo by means of MRI (2). It is based on the paramagnetic properties of $\mathrm{Mn} 2+$ ion and on the fact that, once taken up by neurons, $\mathrm{Mn} 2+$ is transported anterogradely along the axon. However, the technique presents several drawbacks that can challenge its applicability, the most important being the potential toxicity of the ion in the tissue. In this study, we have developed an entirely new tracing technique where we have synthesized stable and bifunctional neuronal tracers that allow both, in vivo brain connectivity studies by means of MRI and postmortem microscopic investigation in fixed tissue, in the same experimental animal. Existing tracer molecules with excellent tracing properties could be covalently connected to organic macrocyclic moiety caging gadolinium as MR reporter. We started with biocytin and biotinylated-dextranamine (BDA) as model molecules which are well known anterograde and also retrograde (as opposed to $\mathrm{Mn} 2+$ ) neuronal tracer. Due to high interaction of biotin-avidin, both tracers can be visualized in postmortem tissue by using a host of avidin conjugated markers at the light- and electron microscope level. We have performed in vitro cell studies in neuroblastoma cell lines to prove their uptake competence and in vivo MR/ histological visualization to prove their in vivo tracing efficiency as potential neuroanatomical tract-tracers. Our results reveal that these new tracers allow for a new strategy of neuronal tracttracing, combining the powerful spatial resolution of the conventional microscopic techniques and in vivo visualization by MRI. References. (1) Prog Neurobiol (2000), 62, 327 and (2) Neuroimage (2008), 40, 458. 
$\mathbf{J 1 0 2}$

\section{A DUAL-LABELED CYSTINE KNOT PEPTIDE FOR PET AND NEAR-INFRARED FLUORESCENCE IMAGING OF INTEGRIN EXPRESSION IN LIVING SUBJECTS}

Richard Kimura $^{1,2}$, Zheng Miao ${ }^{2}$, Zhen Cheng ${ }^{2}$, Jennifer R. Cochran ${ }^{1}$, Sanjiv S. Gambhir ${ }^{1,2}$

${ }^{1}$ Bioengineering, Stanford University, Stanford, CA, USA, ${ }^{2}$ Molecular Imaging Program, Stanford University, Stanford, CA, USA.

Knottins are small $(\sim 3 \mathrm{kDa})$ polypeptides stabilized by a central cystine knot motif which endows them with exceptional in vivo stability. Using yeast surface display and high-throughput screening methods, we evolved knottins to bind integrin receptors with high affinity and unique specificities. Previously, we tested the ability of these engineered peptides to target integrins in small animal tumor models using near-infrared fluorescence (NIRF) imaging and positron emission tomography (PET). Here, we report the in vivo performance of a knottin peptide conjugated to both PET and NIRF imaging agents. We created an orthogonallyprotected peptide-based linker for stoichiometric coupling of 64Cu-DOTA and Cy5.5 onto the $\mathrm{N}$-terminus of an integrinbinding knottin peptide. We show that Cy5.5 profoundly affects the pharmacokinetics of the tracer and significantly increases its tumor retention compared to the knottin peptide conjugated to 64Cu-DOTA alone. At 1 and $24 \mathrm{hr}$ post injection, the $64 \mathrm{Cu}-$ DOTA-labeled knottin peptide showed tumor uptake values of $4.47 \pm 1.21$ and $1.48 \pm 0.51 \% \mathrm{ID} / \mathrm{g}$, respectably, compared to 4.27 \pm 0.46 and $5.05 \pm 0.05 \% \mathrm{ID} / \mathrm{g}$ for the dual-labeled $64 \mathrm{Cu}-\mathrm{DOTA}-$ Cy5.5 knottin peptide $(P<0.05, n=3)$. Furthermore, this duallabeled PET/NIRF tracer demonstrates excellent tumor-to-normal tissue contrast within minutes after injection, and the tumor signal that results persists well beyond $24 \mathrm{~h}$. The robust imaging signal generated over this broad imaging window makes this dual-labeled PET/NIRF agent potentially useful for both preoperative nuclear imaging as well as real-time optical imaging during minimally invasive procedures, such as accurately determining tumor margins during surgical resection.

\section{$\mathbf{J 1 0 3}$}

\section{PRETARGETED IMAGING USING ULTRAFAST "CLICK" CHEMISTRY}

Neal K. Devaraj, Rabi Upadhyay, Scott Hilderbrand, Ralph Weissleder Systems Biology, Harvard Medical School / Massachusetts General Hospital, Boston, MA, USA.

We have used click chemistry to label receptors on live lung cancer cells pretargeted by modified monoclonal antibodies. The reaction rate of the click reaction is several orders of magnitude higher than coupling reactions that have been previously used to label live cells. The signal intensity can be systematically amplified by increasing the loading of click reagent on the antibody. The high rate of reaction coupled with the ability to amplify signal allows for fast detection ( $<30 \mathrm{~min}$ ) of pretargeted antibody on live cells even at low concentrations of labeling agent. The speed, sensitivity, and ability to perform the reaction in whole serum at $37^{\circ}$ suggests that this technology will be readily translatable to in vivo applications. We have begun to explore this in mouse models bearing cancer xenografts.

$\mathbf{J 1 0 4}$

GOLD NANOPARTICLES COATED WITH GADOLINIUM CHELATES AS A MULTIMODAL CONTRAST AGENT

Ji-Ae Park ${ }^{1}$, Kyeong Min Kim ${ }^{1}$, Jong Guk Kim ${ }^{1}$, Jin Su Kim ${ }^{1}$, Sang$\overline{\mathrm{Keun}} \mathrm{Woo}^{1}$, Gi Jeong Cheon ${ }^{2}$, Sang Moo Lim² ${ }^{2}$, Hee Kyung Kim ${ }^{3}$, Joo Hyun $\mathrm{Kim}^{3}$, Yongmin Chang ${ }^{3}$

${ }^{1}$ Laboratory of Nuclear Medicine Research, Korea Institute of Radiological \& Medical Sciences, Seoul, Korea, South, ${ }^{2}$ Department of nuclear Medicine, Korea Institute of Radiological \& Medical Sciences, Seoul, Korea, South, ${ }^{3}$ Department of Medical \& Biological Engineering, Kyungpook National University, Daegu, Korea, South.

The development of contrast agents (CAs) for use across multiple imaging modalities such as PET/CT, PET/MRI, SPECT/CT, and $\mathrm{MRI} /$ optical is a versatile research area gaining a lot of interest in recent years. In contrast, MRI/CT multi-modality has rarely been explored although the two techniques are separately applied to the same patient to improve the accuracy of diagnosis or assess the efficacy of treatment routines. Hence, development of new multi-modal probes integrating the advantages of MR and CT techniques offers many beneficial clinical applications. Current CAs for CT are based on iodinated small molecules effect rapid renal clearance and vascular permeation, necessitating short imaging time. The fact that gold has a higher X-ray absorption coefficient than iodine $(5.16$ and $1.94 \mathrm{~cm} 2 / \mathrm{g}$, respectively, at $100 \mathrm{keV}$ ) encouraged researchers in this field to study the feasibility of gold nanoparticles (AuNPs) as a potential CT CAs. Imaging gold at $80-100 \mathrm{keV}$ reduces interference from bone absorption and takes advantage of lower soft tissue absorption which would reduce patient radiation dose. Furthermore, the AuNPs are bio compatible and non toxic in vivo. Combining MRI CAs to AuNPs surface can make an extremely attractive multi-modal contrast agent. With the ability to carry large payloads of active magnetic centers, nanoparticle-based MR CAs can work at much lower concentrations and also be made target-specific. In this study, we described the systhesis and characterization of gold nanoparticles functionalized by gadolinium complex of DTPA-bis(amide) conjugate of cysteine, $\mathrm{Au} @ \mathrm{GdL}$, as both an MRI and a CT contrast agents. The modified synthetic procedure has been successfully applied to achieve a high number of GdL up to 2900 per gold nanoparticle. $\mathrm{Au} @ \mathrm{GdL}$ exhibits not only high and relaxivities in the order of $10^{\wedge} 5 \mathrm{mM}-1 \mathrm{~s}-1$ but excellent $\mathrm{CT}$ contrast properties. In vivo MR and CT studies reveal the liver and brain tumor enhancement of Au@GdL. This demonstrates the potential clinical uses of the Au@GdL as a potential multi-modal contrast agent. 


\section{$\mathbf{J 1 0 5}$}

\section{DEVELOPMENT OF A LIVER-SPECIFIC PROBE FOR MR AND NIRF DUAL IMAGING} Takehiro Yamane $^{1,2}$, Kenjiro Hanaoka ${ }^{1,2}$, Yasuaki Muramatsu ${ }^{1,2}$, Keita Tamura $^{3}$, Yusuke Adachi ${ }^{3}$, Yasushi Miyashita ${ }^{3}$, Tetsuo Nagano ${ }^{1,2}$ ${ }^{1}$ Graduate School of Pharmaceutical Sciences, The University of Tokyo, Tokyo, Japan, ${ }^{2}$ CREST, JST, Tokyo, Japan, ${ }^{3}$ Graduate School of Medicine, The University of Tokyo, Tokyo, Japan.

Magnetic resonance imaging (MRI) can provide the three-dimensional images inside the body noninvasively. Therefore, MRI is useful for the diagnoses of various diseases. On the other hands, gadolinium $\left(\mathrm{Gd}^{3+}\right)$ complexes are commonly used as MRI contrast agents, which make MR images clearer. Recently, many researchers have challenged to image specific diseases or biomolecules by functionalizing $\mathrm{Gd}^{3+}$ complexes. However, this attempt has some problems. For example, because of the low sensitivity of MRI, it is difficult to obtain the detailed information of the $\mathrm{Gd}^{3+}$ complexes about its localization inside the body, which may be useful for the rational design of MRI contrast agents. Therefore, we tried to devise the novel design to functionalize $\mathrm{Gd}^{3+}$ complexes utilizing the fluorescent dyes, because we think that the biodistribution of $\mathrm{Gd}^{3+}$ complexes can be controlled by the chemical properties of fluorescent dyes. In addition, the localization of $\mathrm{Gd}^{3+}$ complexes with fluorescent dyes also can be observed at cellular levels by the fluorescence microscopy. i.e., fluorescent dyes were used as both a functional moiety and a fluorescent tag. Among various fluorescent dyes, we chose indocyanine green (ICG) as a scaffold of fluorescent dye. It is well-known that ICG has near-infrared fluorescence (NIRF) and also accumulates to the liver specifically, so it is used as a diagnostic agent for hepatic functions. On the basis of the above concept, we have designed and synthesized the liver-specific MRI-NIRF dual imaging probe, Cy7-PEG-Gd, combining $\mathrm{Gd}^{3+}$ complexes with cyanine dyes. Firstly, we examined the $R_{1}$ of probes at $20 \mathrm{MHz}$. The $R_{1}$ of Cy7-PEG-Gd was $18 \mathrm{mM}^{-1} \mathrm{sec}^{-1}$ in PBS buffer and increased to $20 \mathrm{mM}^{-1} \mathrm{sec}^{-1}$ in the presence of $4.5 \%$ human serum albumin. These values were about 5 times higher than that of Gd-DTPA. Secondly, when Cy7-PEG-Gd was injected into the tail vein of nude mice, the large enhancement of the liver contrast was observed in $T_{1}$-weighted MR image. The specific accumulation and the retention of Cy7-PEG-Gd in the liver were also observed in fluorescence images. Moreover, the different biodistribution of $\mathrm{Gd}^{3+}$ complexes was observed by modifying the structure of fluorescent dyes. Thus, Cy7-PEG-Gd has a potential for the diagnoses of liver diseases such as liver cancer, etc.

\section{$\mathbf{J 1 0 6}$}

\section{PEPTIDE-TARGETED MULTIMODAL NANOAGENTS FOR THE DETECTION OF THROMBI}

Jason R. McCarthy ${ }^{1,3}$, Purvish Patel $^{2}$, Ion Botnaru ${ }^{2}$, Farouc A. Jaffer ${ }^{1,2}$, Ralph Weissleder ${ }^{1}, 3$

${ }^{1}$ Center for Molecular Imaging Research, Massachusetts General Hospital and Harvard Medical School, Boston, MA, USA, ${ }^{2}$ Cardiovascular Research Center, Massachusetts General Hospital, Boston, MA, USA, Center for Systems Biology, Massachusetts General Hospital and Harvard Medical School, Boston, MA, USA.
As one of the leading causes of death in the developed world, thrombosis underlies several life-threatening cardiovascular syndromes including stroke, myocardial infarction, and pulmonary embolism. It is thus important to develop imaging strategies to improve the diagnosis, risk stratification, and treatment of thrombosis syndromes. To this end, novel multimodal nanoagents have been developed in order to detect and monitor thrombogenesis and fibrinolysis in vivo. These agents, based upon magnetofluorescent nanoparticles, are targeted to different components of thrombi, namely fibrin and activated coagulation factor XIII (FXIIIa), via peptide affinity ligands. Importantly, fibrin is constitutively present in thrombi, whereas FXIIIa activity is only transient and is a hallmark of acute thrombi. Thus, the labeling of the nanoagents with spectrally distinct fluorophores and coinjection may allow for the determination of thrombus age and lysability via fluorescence imaging in two separate channels. As demonstrated by in vitro and in vivo studies, both nanoagents possess high affinities for thrombi, and enable mutimodal fluorescence and magnetic resonance imaging.

\section{$\mathbf{J 1 0 7}$}

\section{MULTI-MODALITY IMAGING ENDOTHELIAL PROGENITOR CELLS WITH "POSITIVE" MR CONTRAST AND NEAR- INFRARED FLUORESCENCE}

Shenghong Ju ${ }^{1}$, Yiru Qiu ${ }^{1}$, Cong $\mathrm{Li}^{2}$, Gaojun Teng ${ }^{1}$

${ }^{1}$ Radiology, Zhongda Hospital, Southeast University, Nanjing, China, ${ }^{2}$ Radiology and Molecular Imaging Center, School of Pharmacy, Fudan Unversity, Shanghai, China.

The ability to tracking the endothelial progenitor cells (EPCs) that actively involve in the atherosclerosis, ischemia and tumor associated angiogenesis using multimodal imaging technologies provides unprecedented opportunities to develop potential EPCdominated therapeutic applications. Non-invasively and dynamically monitoring the homing, migration and proliferation of EPCs would be of significant advantage in this quest. The multimodal strategy incorporating T1-weighted MR and optical imaging can complement their strengths to provide images with high sensitivity and spatial-resolution as well as the reduced artificial background. Here we developed a bCD-PLL-Cy5.5 conjugate 1 , in which the bacterial cytosine deaminase (bCD) protein was modified with poly-L-lysine that was labeled with T1-weighted MR contrast agent and near-infrared (NIR) fluorophore. Conjugate 1 showed low cytotoxicity (up to $3 \mu \mathrm{M})$ in EPCs that were isolated from the rabbit peripheral blood, and the normalized cell viabilities maintained above $90 \%$ after incubation for 1-5 days. Fluorescence microscopy of live cells indicated the rapid cellular uptake of 1 into EPCs even in $15 \mathrm{~min}$, and flow cytometry studies demonstrated the time-dependent internalization of conjugate 1 with the maximum uptake in $24 \mathrm{~h}$ after the treatment. In vitro MR phantom studies demonstrated the significant reduction of the T1 value of the EPC pellet $(340 \mathrm{~ms})$ that was pre-treated with $2 \mu \mathrm{M}$ conjugate 1 for $24 \mathrm{~h}$ compared with the control cell pellet $(1080 \mathrm{~ms})$ treated with PBS lonely. Our preliminary data show that conjugate 1 as a multimodal imaging media is promising in tracking the delivery and therapeutic response of the EPCs in vivo. 

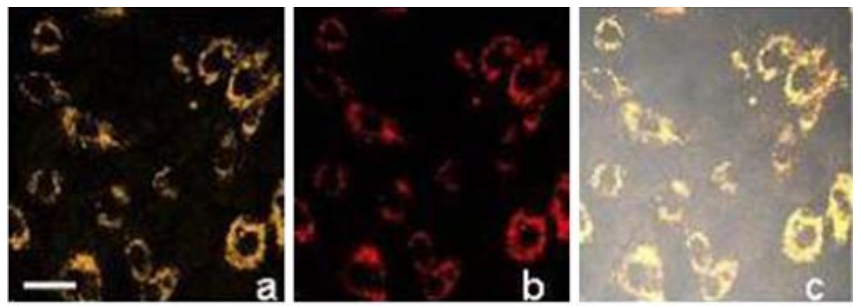

Rhodamine

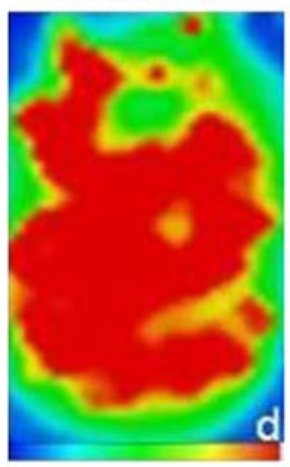

Cy5.5

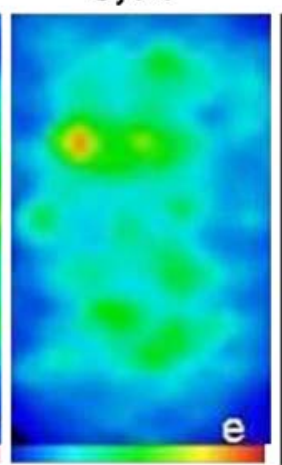

ECV 304
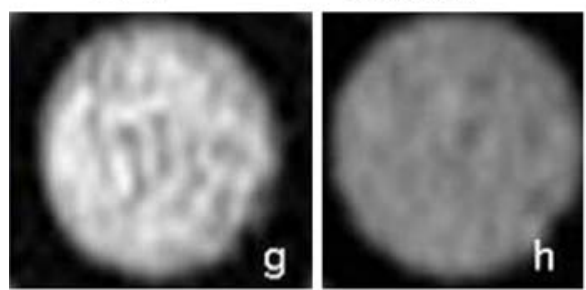

$\mathrm{T}_{1}$-weighted images
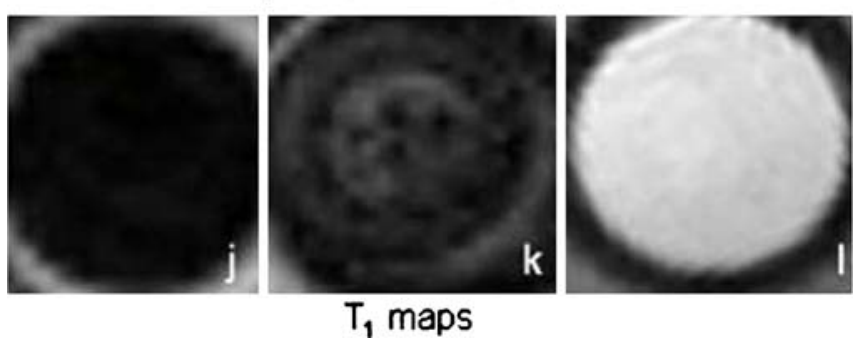

Figure 1. Panels a-c demonstrate the rhodamine (a), Cy5.5 (b) and merged confocal fluorescence image (c) of live EPCs treated with $2 \mu \mathrm{M}$ bCD-PLLCy5.5, 1 for $24 \mathrm{~h}$. The scale bar presents $15 \mu \mathrm{m}$. Panels d-f present the NIR optical imaging of the cell pellets of EPC (d), ECV304 (e) treated with $2 \mu \mathrm{M}$ conjugate 1 for $24 \mathrm{~h}$. Panel $\mathrm{f}$ shows the EPC pellet without the treatment. Panels g-i present the T1-weighted images of EPC pellets treated with $2 \mu \mathrm{M}$ conjugate 1 for $24 \mathrm{~h}(\mathrm{~g})$, without any treatment $(\mathrm{h})$ and double distilled water (i). Panels $\mathrm{j}-1$ present the $\mathrm{T} 1$ map of the cell panels shown in $\mathrm{g}-\mathrm{i}$.
J108

A ROBUST MURINE MODEL OF HUMAN OSTEOSARCOMA: USE OF MULTIMODALITY NONINVASIVE IMAGING FOR ORTHOTOPIC XENOGRAFT INDUCTION, MONITORING, AND MOLECULAR CHARACTERIZATION

Matt Steensma ${ }^{1,3}, \underline{\text { Kim Hardy }}{ }^{1}$, Milton Gross ${ }^{2}$, Rick Hay ${ }^{1}$, George Vande Woude ${ }^{1}$

${ }^{1}$ Hay Lab, Van Andel Research Institute, Grand Rapids, MI, USA, ${ }^{2}$ Nuclear Medicine Service, Department of Veterans' Affairs Healthcare System, Ann Arbor, MI, USA, ${ }^{3}$ Department of Surgery, Memorial Sloan-Kettering, Manhattan, NY, USA.

Study goals: to establish a robust murine model for human osteosarcoma (OS) and characterize the model by multimodality noninvasive imaging. There's compelling need to develop targeted therapies for human OS, aggressive bone malignancies that predominantly affect children. Current preclinical OS models pose limited availability (canine OS) or challenging logistics (high morbidity/mortality in mice). We've optimized a strategy for raising human OS orthotopic xenografts in immunocompromised mice to ensure high xenograft induction rate, low peri-induction morbidity/mortality, and a reliable caricature of clinical OS behavior. Mice were injected with cultured human OS cell suspensions into the tibia under ultrasound guidance. This ensured accurate intramedullary delivery and precluded inadvertent contralateral cortical bone perforation with cell spillage outside bone. Lower peri-procedural distress, morbidity, and mortality occurred with slower small volume injectates. Longitudinal X-Ray monitoring showed tumors grew rapidly, progressively destroying the proximal tibial and distal femur and expanding into peri tibial soft tissue. As most primary human OS tumors overexpress the Met receptor tyrosine kinase (www.vai.org/met/) product, we evaluated OS xenografts by Met-directed radioimmunoscintigraphy. Serial low-resolution gamma-camera images were acquired. Tumor-bearing hindlimbs were harvested, preserved, and re-interrogated by SPECT/CT. Region-of-interest analysis determined the tumors exhibited rapid uptake and prolonged retention of I-125 activity; with substantial parenchymal inhomogeneity. We conclude induction of human OS orthotopic tibial xenografts under ultrasound guidance results in low host morbidity/ mortality, efficient tumor implantation, rapid tumor growth and bone erosion, and sustained Met oncogene expression, as confirmed by multimodality noninvasive imaging. Such characteristics make this an attractive preclinical model for evaluating new diagnostic and therapeutic strategies against human OS. 

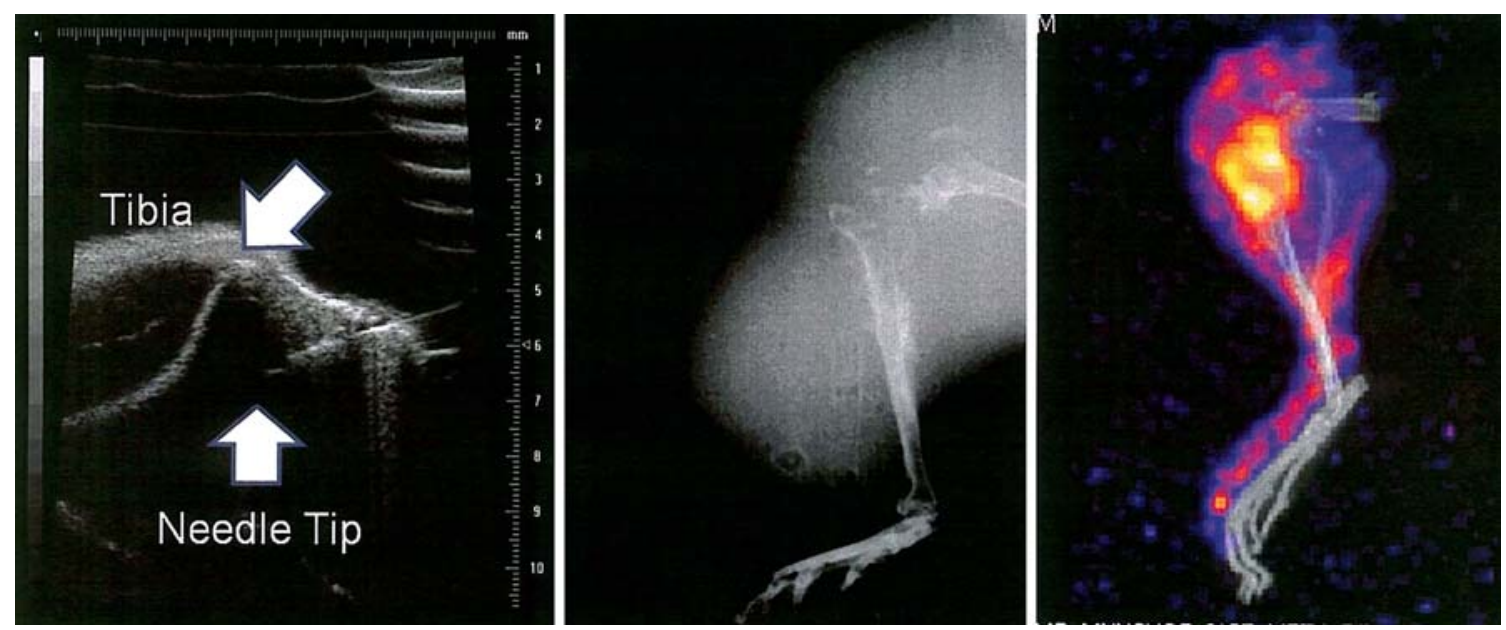

Left: Ultrasound Guidance of Anterior Medical Tibial Injection with a $27 \mathrm{~g}$ needle Mid: Day 21 Post Injection—x-ray Demonstrates extensive lysis, fibular involvement Right: Met4 Radiolabel SPECT/CT

$\mathbf{J 1 0 9}$

MULTIFUNCTIONAL, POLYMER-COATED IRON OXIDE NANOPARTICLES FOR IMAGING OF GENE DELIVERY

Gitte O. Knudsen ${ }^{1}$, David P. Cormode ${ }^{1}$, Nicole Parker ${ }^{2}$, Peter A. Jarzyna $^{1}$, Torjus Skajaa ${ }^{1}$, Karen C. Briley-Saebo ${ }^{1}$, Zahi Fayad ${ }^{1}$, Savio L. Woo ${ }^{2}$, Willem J. Mulder ${ }^{1}$

${ }^{1}$ Translational and Molecular Imaging Institute, Mount Sinai School of Medicine, New York, NY, USA, ${ }^{2}$ Department of Gene and Cell Medicine, Mount Sinai School of Medicine, New York, NY, USA.

Introduction: Gene therapy could improve outcomes in many diseases, but toxicity issues with virus based delivery vectors have delayed the clinical application of this technology. In the current study we developed a versatile nanoparticle platform and investigated its suitability for multimodal imaging of gene delivery. Methods: Iron oxide nanocrystals were coated with the DNA binding polymer, PMAL, and varying quantities of polyethylene glycol (PEG) functionalized lipids (A). The resulting nanoparticles (NPs) were characterized with respect to diameter, stability in serum, toxicity, DNA loading capacity and zeta potential. In vitro transfection experiments were performed using a variety of cancer cell lines. Confocal microscopy and fluorescence imaging were used to determine transfection efficiency of the cells. Preliminary in vivo experiments with the NP-DNA complexes were carried out in mouse models of cancer of which the tumors and liver were visualized with MRI and fluorescence imaging in vivo and with TEM and confocal microscopy ex vivo. Results: The mean diameters of the NPs were 19, 19 and $25 \mathrm{~nm}$ for respectively $0 \%, 5 \%$ and $10 \%$ PEG functionalized lipids in the coating. Importantly, we determined the effect of PEG-lipid on NP stability in serum and complexation efficacy (B). After $24 \mathrm{~h}$ of incubation in $10 \%$ FBS the size of the $0 \%$ PEG-lipid particle doubled while the size of the particle with $10 \%$ PEG did not significantly change. We found $5 \%$ of PEGlipid in the coating to be optimal, since these NPs only marginally aggregate in serum and adequately bound DNA. Cell viability assays showed low toxicity compared to other transfection agents. NP uptake by cells and subsequent gene expression was established as determined by confocal microscopy imaging (C and D). MRI, TEM and fluorescence imaging of injected mice showed that our particles accumulated in both the liver and tumors. Conclusion: We have developed a flexible, multifunctional, polymer-coated iron oxide nanoparticle platform that can be employed for intracellular delivery of DNA with low toxicity and can be visualized with MRI and optical techniques. 

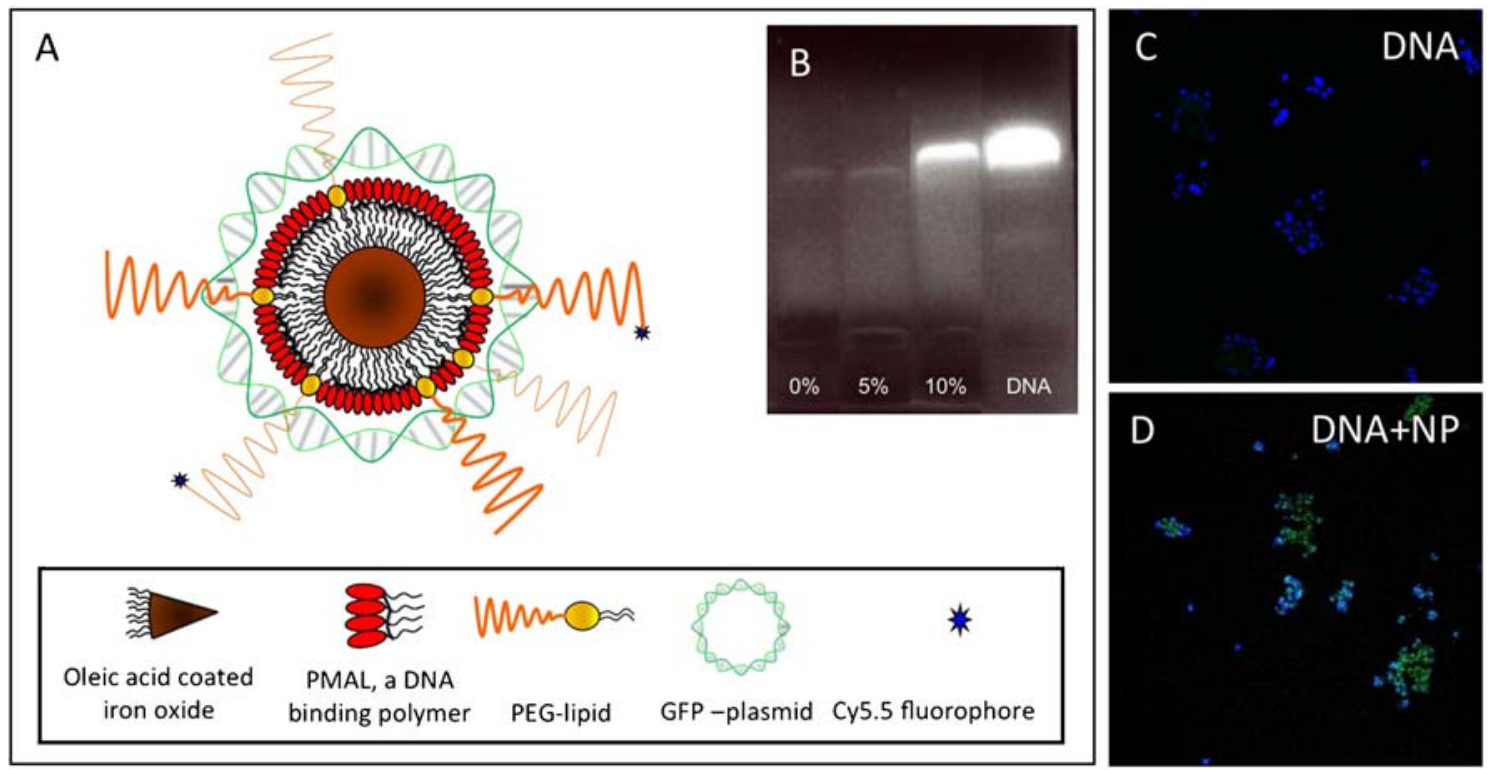

A) Fluorescent iron oxide nanoparticle (NP) coated with PEG lipids and the DNA binding polymer PMAL for MRI and fluorescence visualization of gene delivery. B) DNA bound to these NP when the proportion of PEG in the coating was $<10 \%$. Confocal microscopy showing HepG2 cells incubated with GFP encoding DNA only C) or with DNA complexed with NPs D). The images show the blue nucleus channel merged with the green GFP channel.

$\mathbf{J 1 1 0}$

\section{MANGANESE DOPED SILICON QUANTUM DOTS TARGETED TO MACROPHAGE FOR MRI AND OPTICAL IMAGING OF ATHEROSCLEROSIS}

Chuqiao $\mathrm{Tu}^{1}$, Xuchu $\mathrm{Ma}^{1,2}$, Susan Kauzlarich ${ }^{2}$, Angelique Louie ${ }^{1}$ ${ }^{1}$ Department of Biomedical Engineering, University of California, Davis, Davis, CA, USA, ${ }^{2}$ Department of Chemistry, University of California, Davis, Davis, CA, USA.

Of the 780,000 cardiac deaths reported per year in the US, more than half are "sudden death" without any advance warning of pathology. Increasing evidence indicates that it is vulnerable atherosclerotic plaque rupture that leads to acute infarction or sudden death. Therefore considerable interest exists in early detection of these vulnerable plaques that could allow for significant decrease of morbidity and mortality in affected patients. However, sensitive detection and differentiation of vulnerable versus stable atherosclerotic plaques in vessels with mild-severity stenoses remains limited with routine angiography or ultrasound. We are developing contrast agents for targeted multimodality molecular imaging that will allow assessment of the molecular and cellular composition of plaques with the goal of diagnosing plaque stability. Semiconductor silicon quantum dots (QDs) have experienced intense interest for biomedical imaging in the last decade due to their biocompatibility and desirable property of bright, environmentally stable luminescence. However, to date the reported silicon QDs are single functionality and solely used for optical imaging. Herein, we report the synthesis of manganese doped silicon QDs coated with dextran sulfate to target for uptake by macrophages, one of the earliest cellular components of atherosclerosis. Transmission Electron Microscopy (TEM) images show that isolated QDs have an average core diameter of $4.3 \pm$ $1.0 \mathrm{~nm}$ and the overall sizes range from 8.3 to $43 \mathrm{~nm}$ measured by Dynamic Light Scattering (DLS). The dextran sulfate coated nanoparticles have an R1 relaxivity of $25.50 \pm 1.44 \mathrm{mM}-1 \mathrm{~s}-1$ and an R2 relaxivity of $89.01 \pm 3.26 \mathrm{mM}-1 \mathrm{~s}-1\left(37^{\circ} \mathrm{C}, 1.4 \mathrm{~T}\right)$ and emit strong fluorescence at $441 \mathrm{~nm}$ with a quantum yield of $8.1 \%$ in water. Cell studies show that the QDs specifically accumulate in macrophages and are nontoxic to mammal cells, and produce distinct contrast in both $\mathrm{T} 1$ weighted magnetic resonance imaging and optical imaging. The uptake specificity of the QDs by macrophages is verified by nontargeted QDs uptake and competitive inhibition experiments. Macrophages are associated with plaques vulnerable to rupture, therefore the QDs are promising for detection of macrophage-rich plaques in atherosclerosis.

\section{$\mathbf{J 1 1 1}$}

EVALUATION OF [18F]FBAU AS A PET PROBE IN F98-TK/ LUC GLIOMA-BEARING RAT MODEL

Yi-Chun Chien ${ }^{1,2}$, Jeng-Jong Hwang ${ }^{1}$

${ }^{1}$ Department of biomedical image and radiological sciences, National yang-ming university, Taiwan, ROC., Taipei, Taiwan, ${ }^{2}$ Department of medical image and radiological sciences, I-Shou university, Taiwan, ROC., Kaohsiung, Taiwan.

The 1-(2-deoxy-2-[18F]fluoro- $\beta$-D-arabinofuranosyl)-5-bromouracil ([18F]FBAU) is a substrate for thymidine kinase, DNAincorporable thymidine analogues, and the blood clearance of [18F]FBAU in vivo study is very slow. It have been reported that $[76 \mathrm{Br}] \mathrm{FBAU}$ was used as PET reporter probe using mouse models 
of human glioma. But it has been noted that the use of FBAU labeled with $76 \mathrm{Br}(\mathrm{t} 1 / 2=16.2 \mathrm{~h})$ has disadvantages on the potentially high radiation dose to the patient. On the other hand $18 \mathrm{~F}$-fluoride production is shorter half-life time, $109 \mathrm{~min}$, and relatively readily available. To date taking advantage of $[18 \mathrm{~F}]$ FBAU, we will investigate the drug as PET probe in gliomabearing rat model. F98, rat glioma cell line, was transfected with dual reporter gene herpes simplex virus type 1 thymidine kinase (HSV1-tk) and luciferase (luc), renamed as F98-tk/luc. Then 106 F98-tk/luc cells in Hanks' buffer without $\mathrm{Mg} 2+$ and $\mathrm{Ca} 2+$ were inject into the left brain region of male Fischer 344 rates (1214 wk old). The HSV1-tk gene is transcribed and then translated an enzyme, HSV1-TK. After administration of a radiolabeled probe, like [18F]FBAU, and transport into the cell, the probe is phosphorylated by HSV1-TK. The phosphorylated radiolabeled probe does not readily cross the cell membrane and is trapped within the cell. And the radioactivity of $[18 \mathrm{~F}]$ is detected by positron emission tomography (PET). Luciferase could react with the substrates "luciferin", resulting in the formation of a luciferase bound peroxy-luciferin intermediate, which releases photons of visible light detected by IVIS system, bioluminescent imaging (BLI). Finally combining with ex vivo autoradiography, we can trace the brain tumor growth. The in vitro data show that the plating efficiency (PE) of F98 and F98-tk/luc are similar, the tumor doubling time of F98 and F98-tk/luc are 27.53 hours and 28.8 hours, the half maximal inhibitory concentration (IC50) of gancyclovir (GCV) for these cells is about $9 \mu \mathrm{M}$, and the F98-tk/ luc cells express 3 photons/second/cell. The multimodalities of molecular images show that the $[18 \mathrm{~F}] \mathrm{FBAU}$ is superior to the traditional tracer, [18F]FDG, using F98-tk/luc glioma-bearing rat model. In conclusion, [18F]FBAU could be a potential PET probe for brain tumor image. In future, we will attempt to estimate [18F]FBPA-Fr and [123I] FIAU as probes compare with [18F]FBAU in F98-tk/luc glioma-bearing rat model for brain cancer therapies and diagnosis.
$\mathbf{J 1 1 2}$

\section{ACIDIC PH-RESPONSIVE POLYMERIC MICELLES FOR DUAL IMAGING: OPTICAL AND MR}

GuangHui Gao ${ }^{1}$, Geun Ho $\mathrm{Im}^{2,3}$, Jung Hee Lee ${ }^{2,3}$, Doo Sung Lee ${ }^{1}$ "Polymer Engineering and Science, Sungkyunkwan University, "Suwon, Korea", Korea, South, ${ }^{2}$ Department of Radiology, Samsung Medical Center, Seoul, Korea, South, ${ }^{3}$ School of Medicine, Sungkyunkwan University, Seoul, Korea, South.

Molecular imaging, as a very efficient tool, is becoming extensive in biomedicine, such as diagnosis of the pathologic tissue and cell, discovery and development of drugs, and real-time monitoring disease treatment. Specially, functional polymeric micelles are employed as carriers to conjugate and/or encapsulate imaging markers for targeting the pathologic area by stimuli-response mechanism, including $\mathrm{pH}$, temperature and so on. In this research, we developed a $\mathrm{pH}$-responsive block copolymer, methoxy poly(ethylene glycol)-b-poly( $\beta$-amino ester) (mPEG-PAE) to target an acidic $\mathrm{pH}$ environment for dual imaging. In our system, the polymeric micelle, formed by a diblock copolymer in the aqueous media, contains a hydrophilic MPEG component and a $\mathrm{pH}-$ responsive degradable PAE component. The $\mathrm{pH}$-responsive PAE segment with ionizable tertiary amino groups on its backbone, can accept protons at low $\mathrm{pH}$ to be soluble or release protons and at basic $\mathrm{pH}$ environment to be transformed into a hydrophobic segment, which conjugated with a fluorescein dye (MPEG-PAE-F). Furthermore, we considered that if we encapsulate $\mathrm{Fe} 3 \mathrm{O} 4$ nanoparticles into the $\mathrm{pH}$ responsive polymeric micelle MPEG-PAE-F, the resulting Fe3O4loaded $\mathrm{pH}$-responsive MPEG-PAE-F could be used as a contrast agent for optical and MR imaging to target and, simultaneously, image the $\mathrm{pH}$ stimuli tissue. The more acidic $\mathrm{pH}$ of most tumors or pathologic tissues is a significant character, lower than the normal tissue and blood ( $\mathrm{pH} 7.2 \sim 7.4$ ). Therefore, this material would be a potential targeting agent for diagnosis of tumors or pathologic tissues, especially monitoring disease therapy.

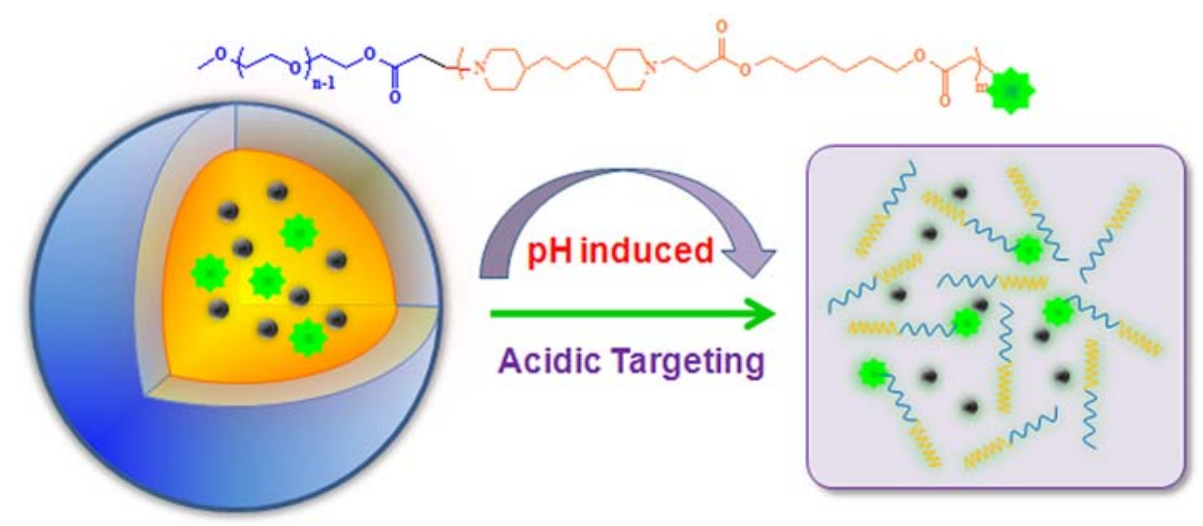

Figure. the schemic diagram of acidic $\mathrm{pH}$ induced demicellization and release of the dual imaging probes

\section{$\mathbf{J 1 1 3}$}

\section{QUANTITATIVE IMAGING OF A CELL-INTERNALIZED CONTRAST AGENT}

Anke de Vries ${ }^{1}$, Maarten Kok $^{1}$, Holger Gruell ${ }^{1,2}$, Gustav J. Strijkers ${ }^{1}$, Klaas Nicolay

${ }^{1}$ Biomedical Engineering, Eindhoven University of Technology, Eindhoven, Netherlands, ${ }^{2}$ Biomolecular Engineering, Philips Research Europe, Eindhoven, Netherlands.
In recent years, MRI has become the imaging modality of choice for many molecular imaging applications. For this, targeted Gd-based MRI contrast agents are under development, which can be employed in the detection and characterization of diseases such as cancer. Uptake of targeted Gd-based contrast agents generally leads to an increase in signal intensity on $\mathrm{T}_{1}$-weighted images. However, it is difficult to quantify contrast agent concentration using MRI, because several mechanisms influence the relaxivity of the contrast agent such as cellular internalization, compartmentalization, and processing of the 
contrast agent by cells after binding of the targeting ligand. Quantification, however, is essential for applications in monitored drug delivery and molecular imaging. For quantification, nuclear techniques such as SPECT are more suitable. Therefore, a combined MRI/SPECT contrast agent marries the best of both worlds; high-resolution localization of MRI with quantification potential of SPECT. In this study we demonstrated the quantification potential of a combined MRI/SPECT contrast agent upon labeling activated endothelial cells. The cells were incubated with RGDconjugated paramagnetic Gd-containing liposomes that were additionally radiolabeled with ${ }^{111}$ Indium. $\mathrm{R}_{1}$ and $\mathrm{R}_{2}$ relaxation rates were determined as a function of incubation time. Quantification of ${ }^{111}$ Indium was performed by SPECT and a $\gamma$-counting Wizard, which could be directly related to the Gd content. Incubation times were varied, resulting in an uptake (as percentage of the total incubated amount) from $0.7 \%$ of the liposomes at $1 \mathrm{~h}$ up to $4.6 \%$ at $24 \mathrm{~h}$ of incubation time. SPECT-determined uptake scaled with $\mathrm{Gd}$ content, while changes in $\mathrm{R}_{1}$ as function of incubation time did not scale with Gd content (Table 1). The latter was previously attributed to internalization of the contrast agent in poorly water-accessible intracellular compartments (1). This study showed that combining SPECT with MRI adds quantitative information to the MR contrast, which aids in understanding the contrast agent cellular labeling and internalization mechanisms as well as hold promise for future in vivo quantitative molecular and cellular imaging studies. Ref 1) Kok et al., Magnetic Resonance in Medicine 2009

Table 1: The uptake of ${ }^{111}$ Indium, $\mathrm{R}_{1}, \mathrm{R}_{2}$ relaxation rates and Gadolinium content vs incubation time.

\begin{tabular}{|c|c|c|c|c|c|c|}
\hline & $\begin{array}{c}\text { \% Uptake of } \\
111 \text { Indium }\end{array}$ & $\left.\mathrm{R} 1 \mathrm{~s}^{-1}\right)$ & $\mathrm{R} 2\left(\mathrm{~s}^{-1}\right)$ & $\begin{array}{c}\text { Total amount of } \\
\text { Gadolinium (nmol) }\end{array}$ & $\begin{array}{c}\text { Gadolinium } \\
(\mathrm{mM})\end{array}$ & $\begin{array}{c}\text { Gadolinium (mM) } \\
\text { from ref }(1)\end{array}$ \\
\hline $\begin{array}{c}\text { Incubation Time: } \\
0 \mathrm{~h}\end{array}$ & $0.0 \pm 0.0$ & $0.5 \pm 0.0$ & $28.4 \pm 1.3$ & $0.0 \pm 0.0$ & $0.0 \pm 0.0$ & $0.0 \pm 0.0$ \\
\hline $\begin{array}{c}\text { Incubation Time: } \\
1 \mathrm{~h}\end{array}$ & $0.7 \pm 0.1$ & $1.3 \pm 0.2$ & $56.4 \pm 4.9$ & $6.0 \pm 1.0$ & $0.6 \pm 0.02$ & $0.3 \pm 0.01$ \\
\hline $\begin{array}{c}\text { Incubation Time: } \\
3 \mathrm{~h}\end{array}$ & $3.6 \pm 0.9$ & $1.8 \pm 0.2$ & $83.6 \pm 11.5$ & $10.2 \pm 2.4$ & $1.3 \pm 0.2$ & $1.0 \pm 0.02$ \\
\hline $\begin{array}{c}\text { Incubation Time: } \\
24 \mathrm{~h}\end{array}$ & $4.6 \pm 0.9$ & not & $\begin{array}{c}\text { not } \\
\text { measurable }\end{array}$ & $37.4 \pm 4.0$ & $3.7 \pm 0.4$ & $3.3 \pm 0.29$ \\
\hline
\end{tabular}

\section{$\mathbf{J 1 1 4}$}

\section{BIOIMAGING OF NEURON-SPECIFIC MICRORNA124A USING SMART MAGNETIC FLUORESCENCE NANOPARTICLE PROBES}

Do Won Hwang ${ }^{1}$, Hae young $\mathrm{Ko}^{1}$, In Chan Song ${ }^{2}$, Dong Soo Lee ${ }^{1}$, Soonhag Kim ${ }^{1}$

${ }^{1}$ Department of Nuclear Medicine, Seoul National University, Seoul, Korea, South, ${ }^{2}$ Radiology, Seoul National University, Seoul, Korea, South.

Purpose: The investigation of neuronal microRNAs (miRNAs) expression pattern non-invasively to reveal their modulating effect in cellular processes will play an important role in the understanding of neuronal development in living organism. Here, we report on the development of a novel imaging platform for monitoring the miRNA124a during neurogenesis using rhodamine-coated cobalt ferrite (MF) nanoparticles linked to a quenching molecule containing a miR124a binding sequence (MF-miR124a beacon). Methods: A cobalt-ferrite nanoparticle surrounded by fluorescent rhodamine (designated MF) within a silica shell matrix was conjugated with miR124a beacon which is composed of fluorescence-quencher molecules and amine-linked oligonucleotides containing the miR124a binding sequences (MFmiR124a beacon). The expression level of miR124a on neuronal differentiation was examined in vitro by treating them onto P19 cells which were differentiated into neuron by retinoic acid. Results: Gel electrophoresis revealed the size difference pattern between MF particle and MF-miR124a beacon, confirming a successful conjugation. The gradual decrease of the magnetic signal intensity was observed as the concentration of MF-miR124a beacon was increased. The fluorescence of the above beacon-transfected cells increased gradually according to the progress of neuronal differentiation of P19 cells days after retinoic acid treatment. The bright fluorescence of the MF miR124a beacon in P19 cells of 6 days after neuronal differentiation disappeared almost to the original quenched states by anti-miR124a. In vivo fluorescence images showed the enhanced fluorescence signals in mice bearing P19 cells within a PLLA scaffold after induction of neuronal differentiation, while MR images showed in vivo cellular tracking containing MFmiR124a beacon. Conclusion: We developed a fluorescence signaltunable molecular beacon system based on multimodal nanoparticle to detect neuronal miRNA expression level during neuronal differentiation. These studies represent the first step toward the nanotechnology advancement of imaging miRNAs biogenesis and suggest a wide range of clinical application in monitoring miRNA related to cellular proliferation and differentiation.

\section{$\mathbf{J 1 1 5}$}

\section{MULTIMODALITY INVESTIGATION OF HIGH DENSITY} LIPOPROTEIN ACTION IN ATHEROSCLEROSIS

Torjus Skajaa ${ }^{1,2}$, David P. Cormode ${ }^{1}$, Peter A. Jarzyna ${ }^{1}$, Edward A. Fisher $^{3}$, Ronald E. Gordon ${ }^{4}$, Zahi Fayad ${ }^{1}$, Willem J. Mulder ${ }^{1}$

${ }^{1}$ Translational and Molecular Imaging Institute, Mount Sinai School of Medicine, New York, NY, USA, ${ }^{2}$ Clinical Institute, Aarhus University Hospital, Aarhus, Denmark, ${ }^{3}$ Departments of Medicine (Cardiology) and Cell Biology, NYU School of Medicine, New York, NY, USA, ${ }^{4}$ Department of Pathology, Mount Sinai School of Medicine, New York, NY, USA. 
Introduction Lipoproteins are endogenous nanoparticles and the primary vehicles of lipid transportation within the body. It is well recognized that high density lipoprotein (HDL) has an important role in atherosclerosis and is pivotal for reverse cholesterol transport. In the current study we exploit fluorescent and superparamagnetic iron oxide HDL (FeO-HDL, A) to investigate the uptake mechanism and metabolism of HDL in atherosclerosis. FeO-HDL is a multimodal nanoparticle platform detectable by MRI, optical imaging and TEM, thereby enabling its visualization at the anatomical, cellular and subcellular level. Methods The nanoparticle was synthesized and extensively characterized. The uptake of FeO-HDL in various cell lines in vitro was analyzed with imaging techniques, while uptake mechanisms and metabolism were investigated at sub-cellular detail using TEM. In vivo experiments included high resolution MRI of the abdominal aorta and liver of apoE-KO and wild type mice. Liver biopsies as well as aortic sections of sacrificed animals were further studied with fluorescence imaging and TEM. In addition cholesterol efflux was measured and the dynamics of lipid exchange between HDL particles were investigated. Results Characterization of FeO-HDL revealed close resembling to native HDL, in terms of physical properties and cholesterol efflux capacity. In vitro experiments showed that the particles were avidly taken up by macrophages (B). In vivo experiments on apoE-KO mice revealed a substantial signal decrease in the liver and lesioned vessel wall on T2*- weighted MR images (C). Ex vivo results confirmed the presence of $\mathrm{FeO}-\mathrm{HDL}$ in atherosclerotic plaques using optical imaging (D,E), while TEM on liver tissue revealed trafficking of the HDL within the hepatocyte and macrophage cells and localization of the HDL particles in cell compartments. Conclusion HDL mimicking iron oxide nanoparticles enable the multimodality investigation of receptor mediated interactions and metabolism of high density lipoproteins in cardiovascular disease.

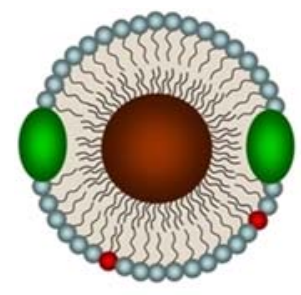

FeO-HDL $T 2^{(\star)}$-weighted MRI, fluorescence

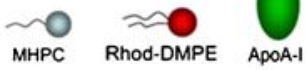

A
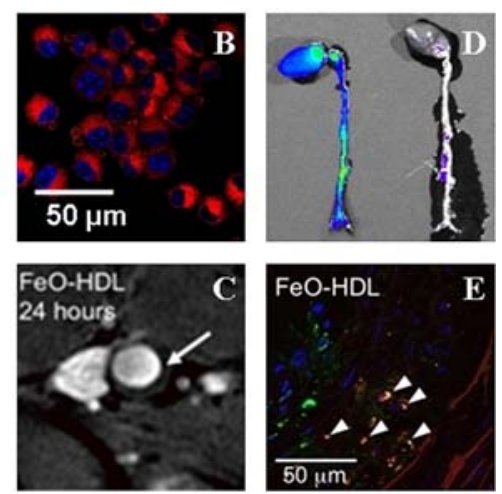

A, schematic depiction of $\mathrm{FeO}-\mathrm{HDL}$. B, confocal image of macrophages incubated with $\mathrm{FeO}-\mathrm{HDL}$. $\mathbf{C}$, in vivo $\mathrm{MR}$ image of apoE KO mouse aorta, showing signal decrease in the atherosclerotic vessel wall. $\mathbf{D}$, optical imaging of whole excised aorta $24 \mathrm{~h}$ after injection of $\mathrm{FeO}-\mathrm{HDL}$, as well as control aorta. E, optical imaging of aorta section.

\section{J116}

TUMOR-TARGETED IMAGING AND DELIVERY OF SIRNA TO ADENOCARCINOMA CELLS

Zdravka Medarova, Mohanraja Kumar, Anna Moore

Molecular Imaging Laboratory, Department of Radiology, Massachusetts

General Hospital, Charlestown, MA, USA.
The fast developing field of RNA interference requires monitoring of siRNA delivery to targeted organs by non-invasive imaging. Here, we have synthesized and tested a tumor-targeted nanoparticle imaging probe (MN-EPPT-siSurvivin) to specifically shuttle siRNA to tumors with subsequent detection by imaging. MN-EPPT-siSurvivin consists of superparamagnetic iron oxide nanoparticles (for magnetic resonance imaging), labeled with Cy5.5 dye (for near-infrared in vivo optical imaging), conjugated to peptides (EPPT) specifically targeting the tumor antigen uMUC-1, and to DY547-labeled synthetic siRNA targeting the tumor-specific anti-apoptotic gene birc5, which encodes Survivin. The siRNA was conjugated through a disulfide linker, which is degraded in the reducing intracellular environment, followed by dissociation of the siRNA from the nanoparticles, as shown by confocal microscopy. Probe uptake by human breast, colorectal, and pancreatic adenocarcinoma cells was demonstrated by flow cytometry. The feasibility of MRI and optical imaging of probe uptake by these cells was demonstrated in vitro in cell phantom studies. Silencing efficacy was assessed by quantitative RT-PCR. Gel electrophoresis demonstrated dissociation of the siRNA from the nanoparticles under reducing conditions at a ratio of $\sim 3$ siRNAs per nanoparticle. Following a $48-\mathrm{hr}$ incubation, $57.8+/-5.3,97.9+/-0.8$ and $80.7+/-2.5 \%$ of pancreatic, breast, and colorectal cells, respectively took up the probe. This resulted in a significant reduction in birc5mRNA, as measured by qRTPCR. MRI and optical imaging permitted visualization of all three cell lines following incubation with the probe, due to the T2 effect of the iron oxide core and the fluorescence of the Cy5.5 dye on MN and the DY547 dye on the siRNA. There is a lack of clinically-relevant noninvasive imaging strategies for the direct monitoring of siRNA delivery. Our approach is highly novel in that it utilizes a single nanoparticle as the backbone for a combined imaging/therapy probe. This strategy permits the simultaneous tumor-specific delivery of siRNA to tumors and the imaging of the delivery process. The significance of this research agenda is defined by the potential to apply it to many human cancer studies, including basic tumor biology and therapy. Ultimately, the developed technology can be applicable in a clinical setting as related iron oxides are already in clinical use.

\section{$\mathbf{J 1 1 7}$}

\section{SYNTHESIS OF MULTILAYER NANOPARTICLE COMPOSITE WITH MULTIMODAL IMAGING PROPERTIES}

Mehmet V. Yigit, Anna Moore, Zdravka Medarova

Athinoula A. Martinos Center for Biomedical Imaging, Massachusetts General Hospital, Charlestown, MA, USA.

Contrast agents that can be detected using multiple imaging modalities hold clear advantages over single-modality ones. Examples include extending the specificity and sensitivity of detection and combining functional and anatomic information in a single acquisition. Herein, we report a multilayered nanoparticle composite (AuMN), whose synthesis does not involve the use of toxic chemicals. AuMN can be used as a contrast agent for MRI and CT due to its iron oxide and gold content and is clinically applicable due to its biocompatible and biodegradable properties. AuMN can also be used as a Raman probe by introducing Raman active dyes on the gold surface. AuMN consists of a 3-5 nm core of iron oxide covered with dextran and a gold shell. The final PEG coating stabilizes the AuMN from agglomeration and increases the blood circulation for potential in vivo applications (Fig. A). The size of AuMN was determined by DLS and TEM. UV-vis spectroscopy was performed to obtain an indication of gold coating. Further confirmation was obtained through ICP elemental analysis. The relaxivity of the particles was determined by NMR Spectroscopy. Attenuation coefficient values were obtained from micro-CT. The nanoparticle has a 
median diameter of $77 \mathrm{~nm}$. The intense reddish color and distinctive absorbance at $525 \mathrm{~nm}$ is a strong indication of gold in the colloid. The presence of superparamagnetic iron oxide was confirmed by relaxivity studies. MRI revealed that AuMN and MN nanoparticles are comparably suitable for detection using T2-weighed MR sequences (Fig. B). CT scans confirmed that the AuMN has a similar attenuation coefficient value as commercially available pure gold nanoparticles (AuNP), significantly higher than parental MN (either diluted or concentrated) (Fig. C). The described AuMN is valuable because it represents one of the first examples of a clinically acceptable innately multimodal agent and could potentially be used in pre-clinical and clinical diagnostics, disease staging, and monitoring of therapy.

\section{A AuMN Synthesis}

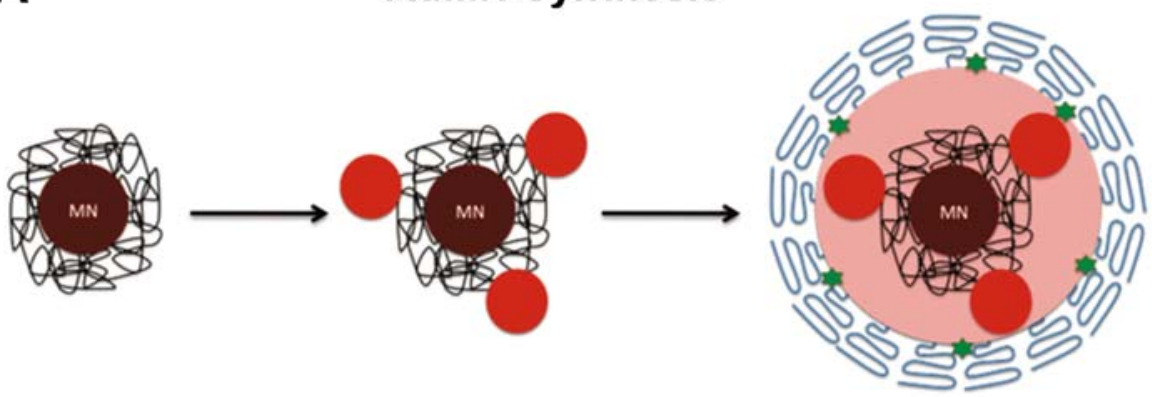

B MRI T2 map

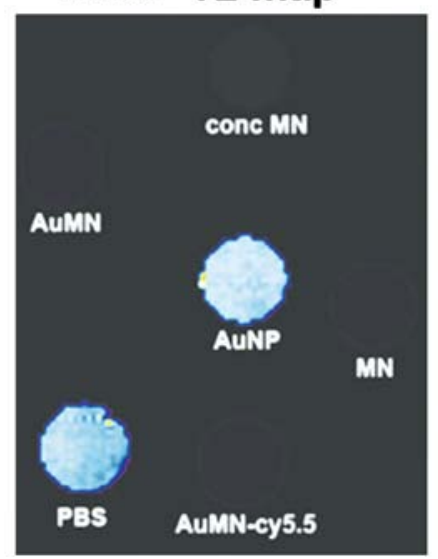

$\mathbf{J 1 1 8}$

MULTIFUNCTIONAL NANOPARTICLES FOR THERANOSTIC APPLICATIONS IN COLORECTAL LIVER METASTASES

Ravi Shukla ${ }^{1}$, Ajit Zambre ${ }^{1}$, Nripen Chanda ${ }^{1}$, Kavita K. Katti ${ }^{1}$, Klaus Thaler $^{2}$, Evan J. Boote ${ }^{1}$, Kattesh V. Katti ${ }^{1}$, Raghuraman Kannan ${ }^{1}$ ${ }^{1}$ Radiology, University of Missouri-Columbia, $\overline{\text { Columbia, MO, USA }}$, ${ }^{2}$ Surgery, University of Missouri, Columbia, MO, USA.

According to the American Cancer Society, colorectal cancer (CRC) ranks second in occurrence in the U.S. Over $50 \%$ of CRC patients develop liver metastases (LM). The 5-year survival rate of untreated CRC and LM patients is $<3 \%$. Treatment modalities for LM include chemotherapy, surgical resection and/or ablation of liver metastases. Successful surgical resection of LM improves 5-year survival up to $40 \%$ in best case scenarios. Unfortunately, only $15-20 \%$ of patients are
C
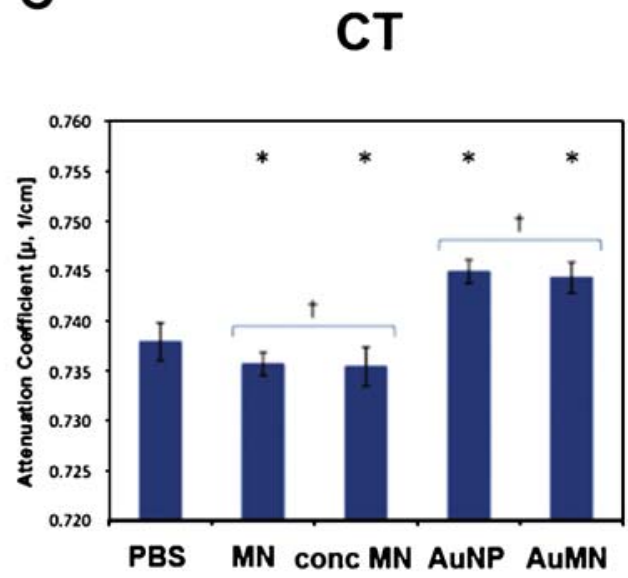

suitable for surgical resection. Overall, the average survival time of patients with LM with all therapeutic modalities (including surgery and modern chemotherapy) is not more than 20 months. In addition, existing diagnostic and therapeutic modalities for LM suffer serious limitations-cancer recurrence is observed in large group of patients within one year of surgery, attributed to undetected micro-metastases at the time of surgery. We have developed new theranostic agents for the accurate detection, treatment, and monitoring of the therapy response of liver metastases of colorectal cancer, using nanotechnology principles. Our research involved design of new multifunctional nanoconstructs comprising a gold nanoparticle (AuNP), Anti-Epidermal Growth Factor Receptor (Anti-EGFR), and Bevacizumab (Anti-VEGF therapy agent) as a $\mathrm{CT}$ theranostic with which to detect, treat, and monitor therapeutic response in liver metastases (LM) arising from Colorectal cancer (CRC) (Figure 1). We will present the following results in our research poster: (i) Synthesis, characterization and in vitro stability studies of 
theranostic agents, Anti-EGFR-AuNP (20 nm and 3.1 AR gold nanorod) and Anti-EGFR-AuNP-Bevacizumab (20 nm and $3.1 \mathrm{AR}$ gold nanorod); (ii) Binding affinity and VEGF inhibition activity (anti angiogenesis assay) of theranostic agents toward metastatic colorectal cancer; and (iii) results from the tumor targeting characteristics and imaging utility of targeted gold nanoconjugates as contrast enhancers in computer tomographic (CT) imaging of LM tumors in mice model (Figure 2).
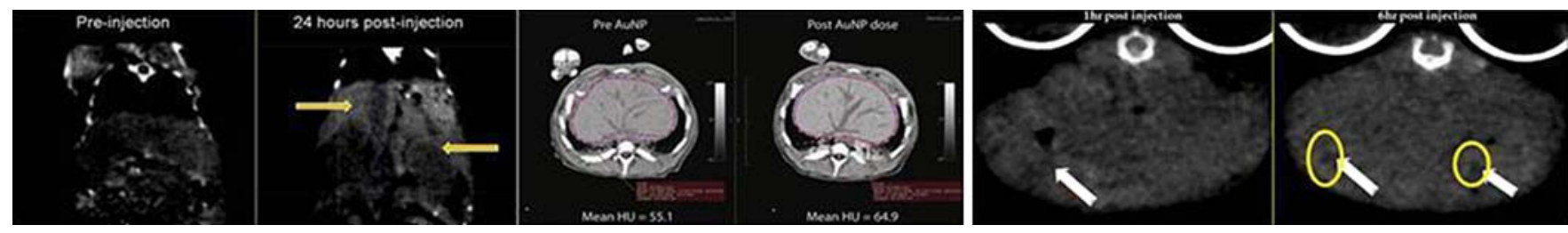

Figure 1: (A) CT contrast observed in liver after injecting with AuNPs (Pre injection and post injection); (B) CT contrast in swine liver after injecting with AuNPs; (C) Delineation of LM from rest of liver tissues using AuNP as contrast agent in mice model with liver C26 metastatic colorectal cancer.

$\mathbf{J 1 1 9}$

\section{SIGMA 2 RECEPTOR AS POTENTIAL INDICATOR OF STEM CELL DIFFERENTIATION}

Jodi Haller ${ }^{1}$, Joseph A. Frank ${ }^{1}$, Irina Panyutin ${ }^{1}$, Robert Mach ${ }^{2}$, Chenbo Zeng ${ }^{2}$ ${ }^{1}$ Radiology and Imaging Sciences, National Institute of Health, Bethesda, MD, USA, ${ }^{2}$ Radiological Sciences, Washington University School of Medicine, St. Louis, MO, USA.

The sigma-2 receptor (SR2), initially reported as a subtype of the opiate receptor (1), has since been distinguished functionally and pharmacologically as a separate receptor system (2). S2r is a potential biomarker of proliferative status of solid tumors with 10 fold higher expression in proliferating than in quiescent tumor cells (3). While the natural ligands remain to be determined, a number of specific synthetic probes using $\mathrm{N}$-substituted-9-azabicyclo[3.3.1]nonan-3 $\alpha$-yl carbamate analogs have been designed and implemented for cancer diagnosis and therapy (4). The purpose of this study was to investigate S2r expression in stem cells (SC) including embryonic (ESC), neural progenitor (NPC), amniotic fluid (AFSC, MTA Wake Forrest) and mesenchymal (BMSC) and differentiated cells astrocytes and fibroblasts. We incubated $2 \times 105$ live cells with fluorescently-labeled sigma 2 probe, SW120 (ex: $465 \mathrm{~nm}$, emiss: $520 \mathrm{~nm}$ ) (2) for $10 \mathrm{~min}$ and washed for elimination of all non-specific binding with immediate assay of mean fluorescence via flow cytometry. Below is an example of the normalized mean fluorescence SW120 for SC, clearly demonstrating the differences between multipotent SC versus differentiated astrocytes and fibroblasts. ESC have highest overall fluorescence, exceeding all other SC and the MCF-7 cell line (data not shown). One of the major challenges of stem cell therapy is the inability to monitor the differentiation of cells using non-invasive imaging. These promising results suggest that the sigma 2 receptors may be used to monitor the changes from multipotent SC to differentiated cells. The addition of MR or nuclear medicine contrast agents to SW120 probe may enable multimodal imaging to monitor stem cell differentiation in experimental studies. 1) Martin WR J Pharamacol Exp Ther 1976;197:517, 2) Vangveravong S Bioorganic and Medic Chem 2006;14:6988, 3) Mach RH Cancer Res 1997; 57:156, 4) Kashiwagi, H Molecular Cancer 2007,6:48

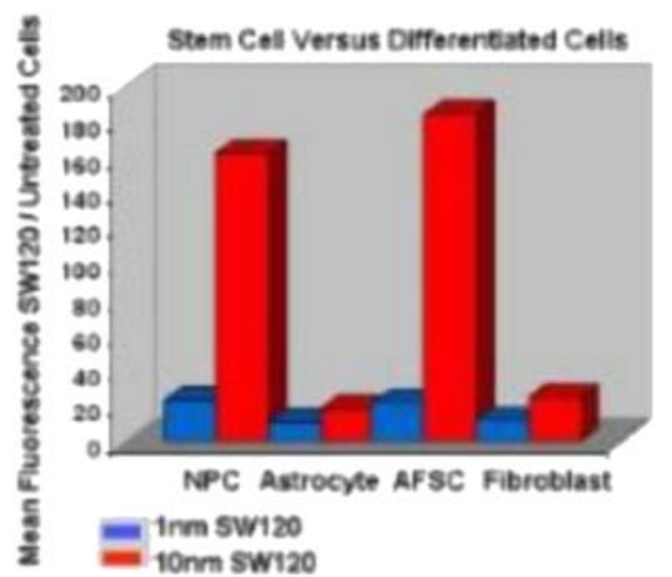

$\mathbf{J 1 2 0}$

\section{INTEGRATION OF OPTICAL LABELS FOR RAPID AND QUANTITATIVE EVALUATION OF PRECLINICAL NANOSCALE CT CONTRAST AGENTS}

Naomi Matsuura $^{1}$, Melissa L. Hill ${ }^{2}$, Ivan Gorelikov ${ }^{1}$, Farnaz Niroui ${ }^{1}$, Joydeep Chaudhuri ${ }^{1}$, James G. Mainprize ${ }^{1}$, Martin J. Yaffe ${ }^{1}$, John Rowlands ${ }^{1}$

${ }^{1}$ Imaging Research, Sunnybrook Health Sciences Centre, Toronto, ON, Canada, ${ }^{2}$ Medical Biophysics, University of Toronto, Toronto, ON, Canada.

INTRODUCTION Computed tomography (CT) enables high resolution, whole-body imaging with excellent depth penetration. The development of new targeted radiopaque CT contrast agents can provide the required sensitivity and localization for the successful detection and diagnosis of smaller lesions representing earlier disease. Nanoscale, perfluorooctylbromide (PFOB) droplets have been used as untargeted contrast agents in X-ray imaging, and form the basis of a promising new group of agents that can be developed for targeted CT imaging. For successful targeting to disease sites, new PFOB droplet formulations tailored for ideal in vivo performance (e.g., 
biodistribution, toxicity, and pharmacokinetics) must be developed. The integration of an optical marker within the PFOB agent can complement CT imaging by allowing the high-resolution, direct assessment of single droplet interactions with target cells for the rapid and quantitative evaluation of new types of PFOB formulations early in their preclinical development. METHOD In this work, we demonstrate a method to synthesize PFOB droplets labeled with fluorescent quantum dot (QD) nanoparticles, and experimentally determine the effectiveness of PFOB-QD droplets as in vitro, high-resolution, optical imaging probes to complement in vivo $\mathrm{CT}$ contrast imaging. Differentially-loaded suspended and pelleted macrophage cells were used to assess the optical and CT contrast, and anionic and nonionic fluorosurfactants were used to test the interactions of different PFOB-QD droplet formulations with cells. Fluorescence and electron microscopy were used to verify cell loading, optical contrast was determined using flow cytometry and fluorometry, and CT image contrast was quantified using a $49 \mathrm{kV}$ x-ray spectrum. RESULTS The PFOB-QD droplets loaded into cells resulted in linear dependence between agent loading, fluorescence and CT contrast. The presence of QDs in the PFOB droplets did not affect the CT contrast of PFOB droplets alone, but significantly increased the CT contrast compared with unloaded tissue-mimicking cell pellets $(\sim 350 \mathrm{HU}$ enhancement). PFOB-QD droplets stabilized using nonionic surfactants demonstrated less loading compared to anionic droplets. CONCLUSIONS QD-labeling of PFOB droplets was successfully demonstrated as an effective in vitro tool for the rapid and high-resolution assessment of new PFOB-based CT agents with target cells. The QD label can also be used to trace the biodistribution and pharmacokinetics of PFOB agents on a cellular level and to validate in vivo CT imaging data via histopathology.

$\mathbf{J 1 2 1}$

\section{SENTINEL NODE DETECTION WITH PET/MR/FLUORESCENT MULTI-MODAL IMAGING USING 68GA-SCN-NOTA SILICA NANOPARTICLE}

Young-Hwa Kim $^{1,2}$, Hyewon Youn ${ }^{1,4}$, Keon Wook Kang ${ }^{1,3}$, Jae Min Jeong $^{1,3}$, Dong Soo Lee ${ }^{1,4}$, June-Key Chung ${ }^{1,2}$

${ }^{1}$ Nuclear Medicine, Seoul National University College of Medicine, Seoul, Korea, South, ${ }^{2}$ Biomedical Sciences, Seoul National University College of Medicine, Seoul, Korea, South, ${ }^{3}$ Laboratory of Molecular Imaging and Therapy, Cancer Research Institute, Seoul National University College of Medicine, Seoul, Korea, South, ${ }^{4}$ Institute of Radiation Medicine, Medical Research Center, Seoul National University College of Medicine, Seoul, Korea, South.

Purpose: Recently, nanoparticles have shown great promise and powerful tools for bio-imaging. We examined the feasibility of PET/ $\mathrm{MR} /$ fluorescent multi-modal imaging system for sentinel node detection by using functionalized silica-nanoparticle. Materials and Methods: We developed $50 \mathrm{~nm}$ sized amino PEGylated silica nanoparticles containing near infrared fluorescent dye, NIR-797 and Co ferrite core (MNP-SiO2). MNP-SiO2 were conjugated with a chelating agent to amine, labeled with the $68 \mathrm{Ga}$, and injected subcutaneously into foot-pad of mice. Whole-body images were obtained using an animal PET/CT scanner, microMR imaging system, and Maestro optical imaging system. After acquiring PET and MR images, fusion of PET and MR images was performed using four fiducial markers in the Functional Image Registration (FIRE) program. Results: PET, MR and fluorescent signals were observed from the lymph nodes draining foot-pad in living mice. $\mathrm{PET} /$ fluorescent signals were also observed ex vivo after dissecting the lymph node. And we also observed respectively images of PET/ MR are correlated with fusion images. Conclusion: We demonstrated that our novel probes, 68 Ga-SCN-NOTA- MNP-SiO2 nanoparticles can define sentinel nodes in mice following footpad injection. Multi-modal silica nanoparticles may have a potential for visualizing sentinel nodes using the PET, MR, and fluorescent scanner during the operation and peri-operation.

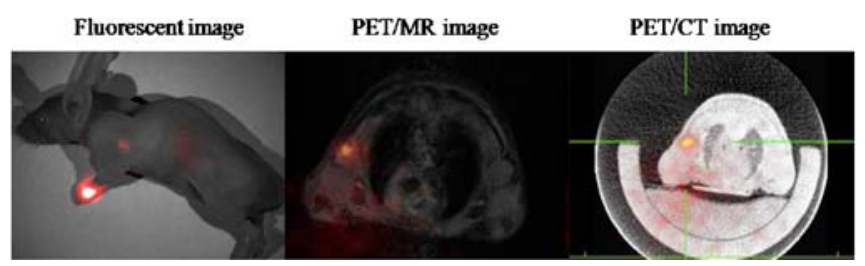

$\mathbf{J 1 2 2}$

\section{A QUAD MODE NANOPARTICLE SYSTEM FOR MOLECULARLY TARGETED THERANOSTICS} OF BREAST CANCER

Wenxue Chen ${ }^{1}$, Rizia Bardhan ${ }^{2}$, Carlos J. Perez-Torres ${ }^{5,3}$, Marc Bartels $^{1}$, Robia G. Pautler ${ }^{3}$, Naomi J. Halas ${ }^{4,2}$, Amit Joshi ${ }^{1}$

${ }^{1}$ Radiology, Baylor College of Medicine, Houston, TX, USA, ${ }^{2}$ Chemistry, Rice University, Houston, TX, USA, ${ }^{3}$ Molecular Physiology and Biophysics, Baylor College of Medicine, Houston, TX, USA, ${ }^{4}$ Electrical and Computer Engineering, Rice University, Houston, TX, USA, ${ }^{5}$ Translational Biology and Molecular Medicine, Baylor College of Medicine, Houston, TX, USA.

We report on the efficacy of a novel theranostic approach, which exploits the tunable plasmonics of silica core gold nanoshells (GNS) to dramatically enhance NIR fluorescence, and provide targeted photothermal therapy. Additionally, super paramagnetic iron oxide (SPIO) is incorporated within the nanostructure to significantly enhance the T2 relaxivity of the hybrid GNS, and provide an additional therapeutic option via magnetically induced hyperthermia. Methods: The core-shell diameter ratio of GNS was tuned so that the plasmon overlapped with emission spectra of ICG dye. $(\sim 810 \mathrm{~nm})$. A $5 \mathrm{~nm}$ SPIO layer was grown over the Au surface. The outermost layer comprised of silica doped with ICG. Hybrid GNS were functionalized with HER-2 targeting antibody. Magnetic resonance imaging (MRI) and Fluorescence optical imaging (FOI) were used to validate the molecular specificity of antiHER2-GNS towards breast cancer cell lines SKBR3 (HER2-positive) and MDA-MB-231 (HER2-negative) as control. For therapy cells were exposed to NIR irradiation $(808 \mathrm{~nm})$ and assessed by a cell viability assay. Results: Magnetic and ICG doped GNS had 45X the quantum yield relative to free ICG, and a T2 relaxivity of $390 \mathrm{mM}-1 \mathrm{~s}-1$ on a 9.4 T MR scanner. In vitro FOI revealed that GNS binding occurred predominantly around the cell membrane (Fig 1A (red)). GNS preferentially bound to cells overexpressing HER2 (SKBR3 in Fig1A vs. MDA-MB-231 in Fig 1B). MRI showed that SKBR3 cells labeled with GNS-anti-HER2 (Fig 1C) had 4 times the number of hypointense pixels as MDA-MB-231 cells labeled with the same agent(Fig1D). After photothermal therapy, live cells were stained green with Calcein and dead cells were stained red with Propidium Iodide. Cell death was significantly observed in SKBR3 cells labeled with GNS-anti-HER2.(Fig1E) This effect was not observed in HER2 negative cells MDA-MB231(Fig.1F). Conclusion: Enhanced Multimodal molecular theranostics by immune targeting of cancer cells can be effectively achieved by nanoscale redesign of existing FDA cleared agents. 

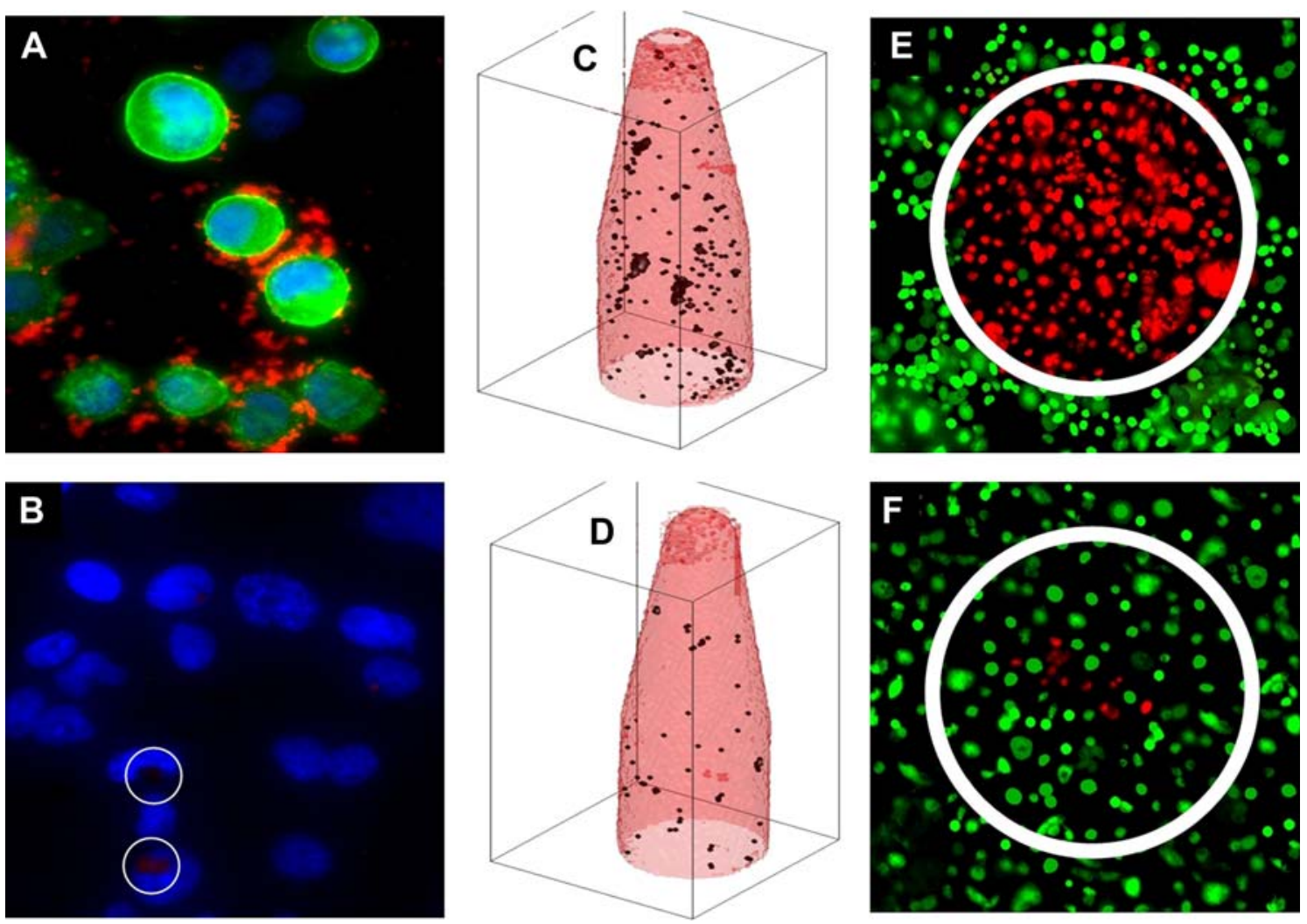

\section{GOLD NANOPARTICLES FOR MULTIMODAL IMAGING AND THERAPY}

Christophe Alric ${ }^{1}$, Jacqueline Taleb ${ }^{2}$, Imen Miladi ${ }^{2}$, Géraldine Le Duc ${ }^{3}$, Céline A. Mandon ${ }^{2}$, David Kryza ${ }^{4}$, Claire Billotey ${ }^{2}$, Pierre Mowat ${ }^{1}$, Marie Dutreix ${ }^{6}$, Marc F. Janier ${ }^{2}$, Pascal Perriat ${ }^{5}$, Olivier Tillement ${ }^{1}$, Stephane Roux ${ }^{1}$

${ }^{1}$ Laboratoire de Physico-Chimie des Matériaux Luminescents UMR 5620 CNRS, Université Claude Bernard Lyon 1, Villeurbanne, France, ${ }^{2}$ Université Claude Bernard Lyon 1, Laboratoire CREATIS-Animage, Villeurbanne, France, ${ }^{3}$ Biomedical Beamline ID 17, European Synchrotron Radiation Facility, Grenoble, France, ${ }^{4}$ Institut des Sciences Pharmaceutiques et Biologiques-Faculté de Pharmacie, Université Claude Bernard Lyon 1, Lyon, France, ${ }^{5}$ Matériaux Ingénierie et Science, INSA de Lyon, Villeurbanne, France, ${ }^{6}$ Translational Department, Institut Curie, Orsay, France.

The fight against the cancer requires the development of new multifunctional contrast agents combining imaging and therapy. Contrary to commercial contrast agents that are rapidly eliminated from the organism and are unable to combine several imaging techniques, nanoparticulate contrast agents can realize multimodal imaging and a longer half life inside the body. We recently developed the synthesis of gold nanoparticles functionalized by gadolinum chelates (Au@DTDTPA-Gd50). They are composed of a gold core (mean diameter of $\sim 2.5 \mathrm{~nm}$ ) encapsulated within a multilayered organic shell of $\sim 150$ ligands (DTDTPA) which entraps $\sim 50$ gadolinium ions. The contrast enhancement in MRI comes from the presence of gadolinium ions inside the organic shell, whereas the gold core provides a strong X-ray absorption. After intravenous injection to healthy mice and rats, the biodistribution of these nanoparticles was monitored by MRI (at $7 \mathrm{~T}$ ) and X-ray imaging, using the Synchrotron Radiation Computed Tomography (SRCT) technique at the ESRF. MRI and X-ray images showed a contrast enhancement that appears first in kidneys and then in the bladder due to the presence of paramagnetic gold nanoparticles. Thus, the Au@DTDTPA-Gd50 nanoparticles freely circulate in the blood pool without undesirable accumulation in liver and in spleen and are efficiently cleared by renal elimination. This was confirmed by ICP analysis and by SPECT (using gamma-emitting $99 \mathrm{mTc}$ instead of Gd ions): gold nanoparticles were essentially present in urine and kidneys but the intravenous injection of Au@DTDTPA-Gd50 to rats bearing a tumor in brain ( $9 \mathrm{~L}$ gliosarcoma) induces a contrast enhancement in the tumor zone. In addition to imaging, $\mathrm{Au} @$ DTDTPA-Gd50 nanoparticles can be applied for radiotherapy. Recently we showed that the high energy photons irradiation of cancerous cells after incubation in presence of Au@DTDTPA generates a larger number of DNA strand breaks. The radiosensitizing effect of gold nanoparticles was exploited for the destruction of tumors after IT or IV injection. The intratumoral injection in osteosarcoma followed by a X-ray irradiation improves the survival of tumor bearing rats. A longer survival time was also observed when gliosarcoma bearing rats were treated by X-ray beam after IV 
injection of Au@DTDTPA. Finally, this study confirms that $\mathrm{Au} @$ DTDTPA-Gd50 nanoparticles freely circulate in the blood pool and that they can be used in vivo as bimodal contrast agent for medical imaging. These nanoparticles could therefore be a promising tool for preclinical studies in oncology.

\section{$\mathbf{J 1 2 4}$}

\section{HIGH-THROUGHPUT MALDI FOR IDENTIFICATION OF CANCER-SPECIFIC SMALL MOLECULE TARGETING LIGANDS}

Jeong Heon Lee ${ }^{1}$, Miyoung $\mathrm{Ha}^{3}$, Hak Soo $\mathrm{Choi}^{1}$, Rafiou Oketokoun ${ }^{1}$, Khaled A. Nasr ${ }^{1}$, Gabriel A. Tobon ${ }^{1}$, Yangsun $\mathrm{Kim}^{3}$, John V. Frangioni ${ }^{1,2}$

${ }^{1}$ Medicine, Beth Israel Deaconess Medical Center, Boston, MA, USA, ${ }^{2}$ Radiology, Beth Israel Deaconess Medical Center, Boston, MA, USA, ${ }^{3}$ Hudson Surface Technology, Inc., Newark, NJ, USA.

Background: The field of molecular imaging is in need of highthroughput techniques for discovering cancer cell-specific, small molecule targeting ligands. These ligands will lay the foundation for the next generation of contrast agents in image-guided surgery and PET. The goal of this study was to develop a fully automated, high- accuracy, and large-scale MALDI system for encoder-less identification of small molecules created from combinatorial libraries of 5,000-10,000 molecules each. Materials and Methods: A highdensity, 5,076 spot small molecule array (HDMA) plate was developed for simultaneous matrix/sample spotting using a microarraying robot and ink-jet printer. A test library of 47 small molecules spanning a wide range of mass $(147$ to $1,592 \mathrm{~m} / \mathrm{z})$ and chemical structure were prepared and analyzed by imaged-based MALDI-TOF. Data mining and annotation were performed using a Bruker UltraFlex III, along with FlexImaging and JChem API based enumeration software. Results: The inkjet printer produced highly precise and uniform DHB pre-spotting onto the HDMA plate. The microarraying robot was capable of spotting 5,076 300-micron diameter spots on each HDMA plate. The specially patterned surface of the plate provided sample focusing and eliminated crosscontamination. Highly accurate and reproducible spectra were achieved from all 5,076 spots having different ionization yields. The pattern recognition by color-coding was obtained according to specified molecular weight. Conclusions: The multiplexed platform we describe automates all key functions needed for small molecule identification and removes the need for molecule encoding during combinatorial library preparation. It should now be possible to identify novel targeting ligands for molecular imaging applications that are created using combinatorial libraries.

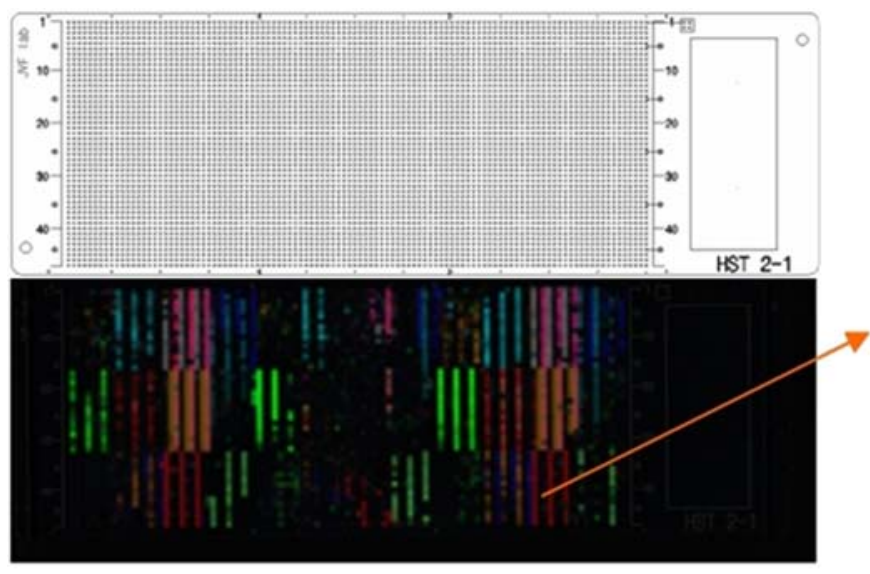

$\mathbf{J 1 2 5}$

\section{MULTIMODAL IMAGING PROBES FOR TARGETED IMAGING OF VASCULAR INFLAMMATION}

Benjamin R. Jarrett, Angelique Louie

Biomedical Engineering, University of California, Davis, CA, USA.

Challenges in diagnostic imaging have lead to an explosion of interest in combining modalities to more accurately interpret abnormalities in vivo. Two modalities that have raised expectations for highly sensitive and high resolution imaging are Positron Emission Tomography and Magnetic Resonance Imaging

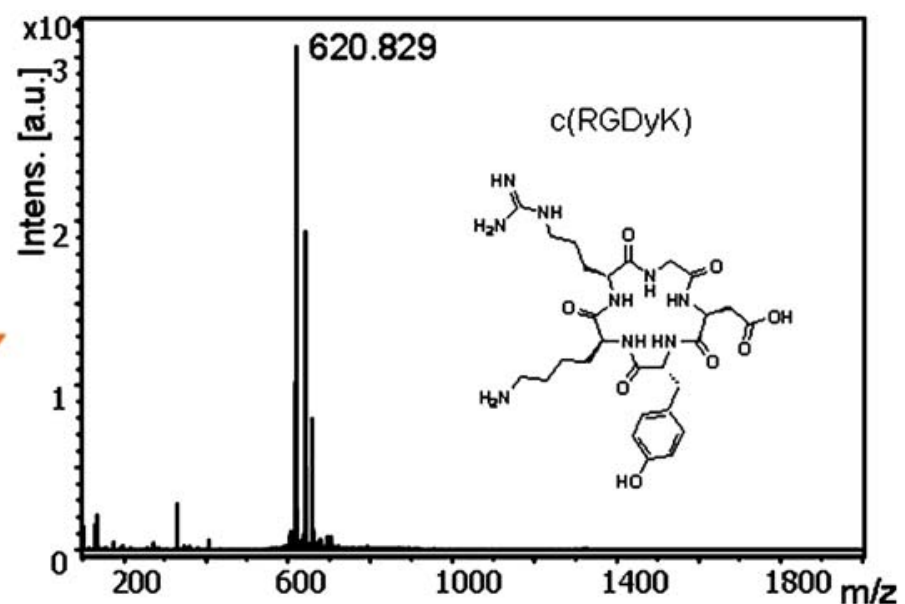

(PET/MRI). PET and MRI are complementary to each other given the high anatomical spatial resolution of MRI and the unparalleled sensitivity and functional imaging of PET. We have developed combined MRI/PET agents comprised of either protein-gadolinium complexes or iron oxide nanoparticles that are both coupled to chelated positron emitting ions; these are targeted towards inflammatory events, such as atherosclerotic plaques. Dual mode imaging of animal models of atherosclerotic plaque was performed and demonstrate signal enhancement in experimentally generated plaques. The image below shows overlay of PET and MR showing correlation of PET signal with the injured vessel of carotid arteries in a coronal view. The results here serve as a foundation for developing methods to image markers of vascular inflammation in 
vivo. The ultimate goal is to assess plaque composition in vivo using the sensitivity of PET to identify putative lesions, and then using the high resolution of MRI for determination of plaque content.

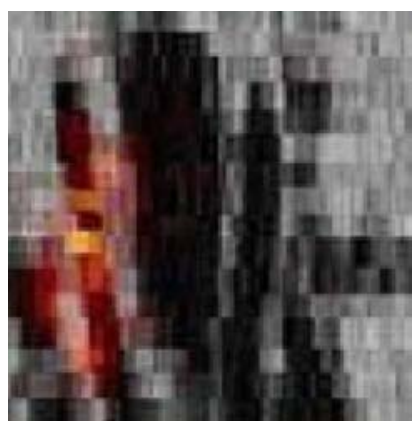

$\mathbf{J 1 2 6}$

\section{ALGINATE MICROCAPSULES WITH INTRINSIC RADIOPACITY FOR X-RAY IMAGING AND IMMUNOPROTECTION OF TRANSPLANTED CELLS \\ Dian R. Arifin ${ }^{1,2}$, Sameer Manek ${ }^{1,2}$, Emma Call ${ }^{2}$, Aravind Arepally ${ }^{1}$, Jeff W. Bulte ${ }^{1,2}$ \\ ${ }^{1}$ Radiology and Radiological Sciences, Johns Hopkins University, Baltimore, MD, USA, ${ }^{2}$ Institute for Cell Engineering, Johns Hopkins University, Baltimore, MD, USA.}

Cell transplantation is a powerful strategy to treat a wide range of diseases. Microencapsulation of engrafted cells provides immunoprotection for the cells and host, eliminating the use of immunosuppression. Our goal is to explore the potential of various microcapsule compositions for cell immunoprotection and $\mathrm{X}$-ray visualization. The following microcapsules were prepared: Alginate beads crosslinked with $10 \mathrm{mM} \mathrm{Ba}^{2+}$ (A), $20 \mathrm{mM}$ $\mathrm{Ba}^{2+}(\mathrm{B}), 50 \mathrm{mM} \mathrm{Ba}^{2+}(\mathrm{C})$ or $100 \mathrm{mM} \mathrm{Ca}^{2+}(\mathrm{D})$, crosslinked with clinical grade protamine sulfate and further crosslinked with a final layer of alginate. Conventional alginate/poly-L-lysine/alginate beads gelled with $100 \mathrm{mM} \mathrm{Ca}{ }^{2+}$ (E) were included. in vitro mechanical strength of microcapsules (Figure 1) was measured by agitating the capsules with glass beads (black bars) and subjecting the capsules to osmotic pressure gradients (white bars). Intact capsules were counted after the treatment. in vitro time stability was determined by continuously monitoring the capsule size changes in physiological condition for 30 days. The data are presented as the ratio of transient capsule size to initial size (Figure 2, capsules A: $\mathbf{\square}$, $\mathrm{B}$ : triangle, C: empty box, D: $\bigcirc$ and E: $\bullet$ ). The viability of mouse insulinoma $\beta$ TC6 cells encapsulated in each capsule composition was determined by fluorescein diacetate/propidium iodide staining. Microcapsule phantoms in saline were imaged using a micro-CT scanner: $75.16 \mathrm{kV}$ and $240.3 \mu \mathrm{A}$ tube settings, $64 \mathrm{~mm}$ detector setting and number of projections $=512.20 \mathrm{mM} \mathrm{Ba}{ }^{2+}$-gelled alginate/protamine sulfate/ alginate capsules (B) were the most promising capsules for immunoprotection of engrafted cells based on the mechanical strength (Figure 1), time stability (Figure 2) and cell viability (Figure 3 ). A cluster of $100 \mathrm{~B}$ capsules appeared as bright signals in micro-CT while D capsules did not (Figure 4). To our knowledge, they are the first inherently radiopaque microcapsules, which can be visualized by CT without the need of coencapsulating contrast agents.
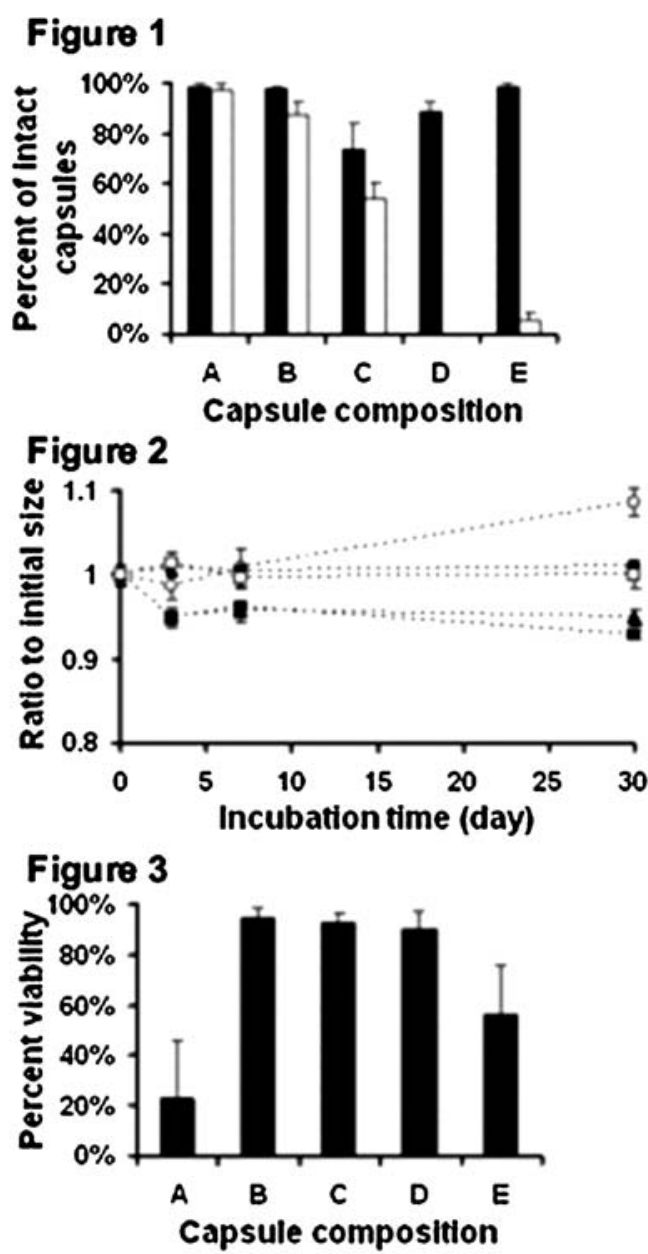

Figure 4
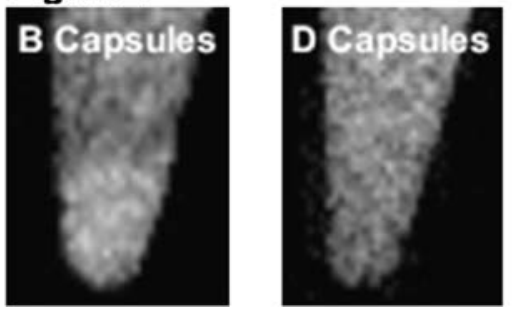

$\mathbf{J 1 2 7}$

SIMULTANEOUS PET-MR IN A RAT ISCHEMIC STROKE MODEL USING A FIBRIN TARGETED DUAL PET-MR PROBE

Ritika Uppal, Ilknur Ay, Ciprian Catana, Thomas Benner, $\underline{\text { Peter }}$ Caravan

Martinos Center for Biomedical Imaging, Massachusetts General Hospital, Charlestown, MA, USA.

Molecular MR probes provide sub-millimeter characterization in deep tissue. However high resolution MRI is time consuming and impractical over a large field of view, while faster low resolution imaging suffers partial volume effects. PET probes display high sensitivity of detection, but poor spatial resolution. A dual PET-MR 
probe may enable rapid identification of lesions by PET, followed by detailed lesion characterization by MR in the PET-defined region of interest. To test this hypothesis, we developed a dual PET-MR probe comprising a short fibrin-specific peptide, 3 GdDOTA moieties for MR enhancement and a [Cu-64]DOTA moiety for PET detection. An aged thrombus was delivered by catheter through the internal carotid to the middle cerebral artery bifurcation to induce ischemic stroke in rats. Rats were imaged $2 \mathrm{hrs}$ post stroke induction on a clinical $3 \mathrm{~T}$ MRI with a home-built surface coil and a MR-compatible human PET scanner that allows simultaneous acquisition of both PET and MR data. Following baseline MRI, the probe $(400 \mu \mathrm{Ci} / \mathrm{rat})$ was injected via the femoral vein and PET data were collected while the MR imaging was repeated. After $30 \mathrm{~min}$, a bolus of $30 \mu \mathrm{mol} / \mathrm{kg}$ Gd cold probe was administered i.v. and perfusion MR was performed followed by high resolution MRI. After 2 hrs imaging, the animals were euthanized and the clot and organs removed, weighed, and counted. Thrombus presence resulted in a right side flow deficit in the MR angiogram. The PET activity concentrated in the thrombus and in the neck around the area of the surgical intervention (arrows). Besides clot, most activity was found in the kidneys. First pass of the probe demonstrated a perfusion deficit by MR. High resolution MR showed thrombus enhancement in the internal carotid at the position showing enhancement on the PET image. Ex vivo analysis confirmed high $(>20)$ clot:blood and clot:brain probe levels. The dual PET-MR probe allows rapid lesion detection using PET and enables high resolution thrombus characterization with MR

Figure. Simultaneous PET-MRI of rat with thromboembolic stroke

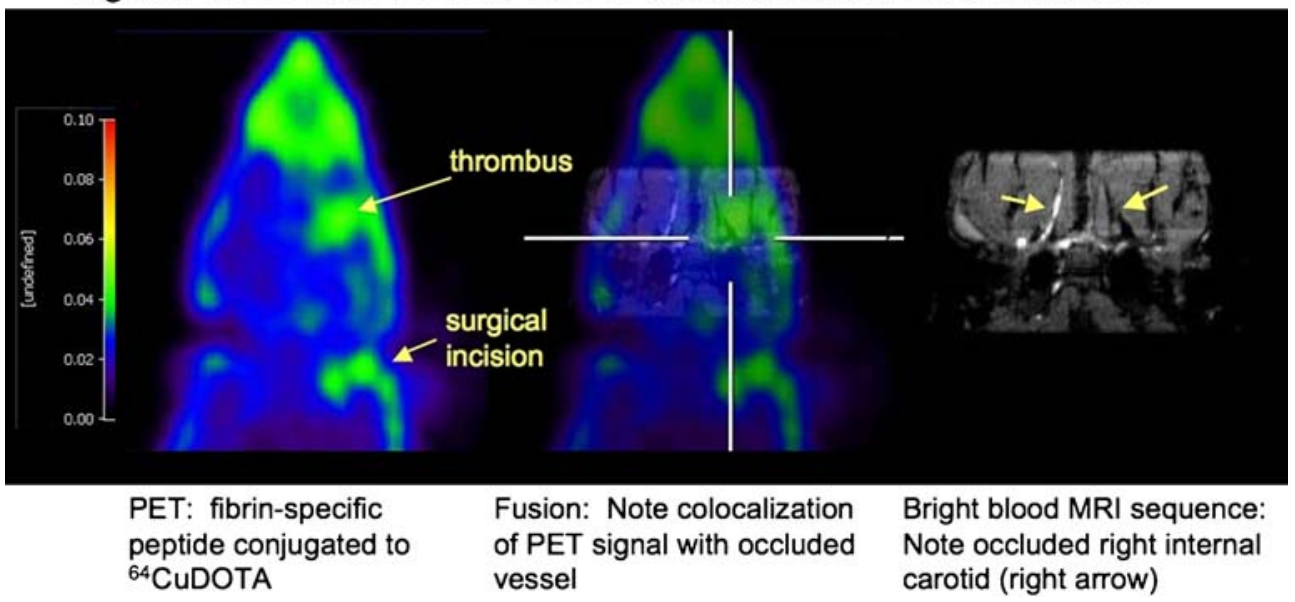

\section{$\mathbf{J 1 2 8}$}

\section{COMPARISON OF A LIPID- VS. PEPTIDE-BASED DELIVERY SYSTEM FOR INTRACELLULAR TARGETED MR IMAGING PROBES}

Rajendra Joshi ${ }^{1}, \mathrm{Wu} \mathrm{Su}^{2}$, Ritu Mishra ${ }^{1}$, Joern Engelmann ${ }^{1}$

${ }^{1}$ High Field MR Center, Max Planck Institute for Biological Cybernetics, Tuebingen, Germany, ${ }^{2}$ Department of Chemistry, University of Leicester, Leicester, United Kingdom.

Magnetic Resonance Imaging (MRI) is meanwhile one of the most important medical diagnostic tools. Its specificity and sensitivity can be further extended by contrast agents (CAs). As many clinically valuable targets like DNA, mRNA or protein/enzymes reside inside the cell membrane, development of efficient intracellular targeted MR CA is required. However, prerequisite for intracellular targeting is not only the efficient delivery inside the cell but also the colocalization with the target. Recently, cell penetrating peptides (CPP) are used to achieve an efficient uptake of cargo molecules. However, it has been shown that these conjugates were predominantly taken up by an endosomal mechanism preventing a proper interaction with targets located in the cytosol. Cholesterol coupling has been reported to facilitate cellular import of siRNAs for effective silencing of protein expression [1]. We developed a contrast agent based on a lipid mediated delivery system by using cholesterol. Uptake and MR contrast enhancement ability was compared with a CPP based CA previously reported by our group [2]. To image the presence of specific mRNAs the probes composed of Gd-DOTA, FITC, a sequence to bind to target mRNA (DsRed), and CPP (D-Tat) or cholesterol for cellular delivery. Fmoc continuous solid phase chemistry was used for synthesis. Fluorescence and MR studies were performed using a mouse fibrosarcoma cell line expressing DsRed protein and its parent cell line deficient of the target sequence. Fluorescence spectroscopy showed that the CPP based CA (CPP-CA) could enter efficiently in both cell types without observable toxicity up to a concentration of $5 \mu \mathrm{M}$. The cholesterol based CA (Chol-CA) was even more efficient. However, this conjugate was not soluble in aqueous solution at concentrations $>3 \mu \mathrm{M}$. Both probes were able to enhance MRI contrast in labeled target containing as well as non-targeted parent cells. Intracellular relaxation rate increased already at a labeling concentration of $1 \mu \mathrm{M}$ for CPP-CA and $0.5 \mu \mathrm{M}$ for Chol-CA. However, fluorescence microscopy demonstrated that Chol-CA was also predominantly localized inside endosomes. Coupling of cholesterol further improved uptake and contrast enhancement. However, the reduced solubility in physiological aqueous media is restricting the applicability for MR imaging purposes. In addition, endosomal entrapment poses a still unsolved problem. Modifications to circumvent both drawbacks have to be implemented to achieve a sufficient cytosolic distribution of CA. [1] Nature Biotechnol. 2007;25,1149; [2] CMMI 2007;2,42. 


\section{FUNCTIONALIZATION OF POLYAMIDOAMINE DENDRIMERS FOR MULTI-MODALITY MOLECULAR IMAGING}

Pasan Fernando ${ }^{1}$, Lihui $\mathrm{Wei}^{1}$, Corinne Bensimon ${ }^{1}$, Adam Smith ${ }^{1}$, Yin Duan $^{1}$, Shaan Jamshaid ${ }^{2}$, Terrence Ruddy ${ }^{2}$

${ }^{1}$ MDS Nordion, University of Ottawa Heart Institute, Ottawa, ON, Canada, ${ }^{2}$ University of Ottawa Heart Institute, Ottawa, ON, Canada.

Polyamidoamine (PAMAM) dendrimers are small molecules that have tremendous potential as targeting entities for drug delivery and hold great promise for applications in molecular imaging. In order to exploit their use in medical imaging, dendrimers must not only have favorable biological properties but also be tailored to suit an imaging modality of choice. Here we used PAMAM dendrimers with poly(ethylene) diamine core structures to study the effects of surface functionality on cellular properties. Generation-four (G4) dendrimers were conjugated with fluorescein isothiocyanate (FITC) at a variety of molar ratios. Preliminary results revealed that conjugation of G4:FITC at molar ratios of 1:10 and 1:20 yielded approximately 3 and 5 molecules of FITC per G4 respectively whereas lower yields of FITC molecules were found at higher conjugation ratios. Initial time-course experiments indicated that the G4:FITC conjugate had crossed the plasma membrane by $1 \mathrm{~h}$ following incubation with $\mathrm{HeLa}$ cells. Cytotoxicity after $24 \mathrm{~h}$ in HeLa cells was not observed when low molar ratios of G4: FITC were used. G4:FITC conjugates at low ratios demonstrated efficient uptake and remained extra-nuclear with diffuse localization throughout the cytoplasm. To further functionalize the G4 dendrimer, we conjugated the bi-functional chelate S-2-(4-Isothiocyanatobenzyl)1,4,7,10-tetraazacylododecane-tetraacetic acid (p-SCN-Bn-DOTA) as a means of generating a PAMAM tractable for nuclear imaging. Preliminary analyses revealed that at molar ratios of G4:DOTA of 1:10 and 1:20, approximately 4 and 5 molecules of DOTA were found per G4. We are currently investigating the effects In-111-DOTA-G4 conjugates on cell viability and toxicity on a panel of cultured cells. In an attempt to develop versatile multi-modal vehicles for optical and nuclear imaging, we are designing bi-functionalized G4 conjugates (fluorophore + novel bi-functional chelates) for labeling a variety of stem cells for both in vitro analyses and in vivo tracking in small animals.

\section{MULTIMODAL FLUORINE CONTAINING NANOEMULSIONS WITH BIOSENSING PROPERTIES}

Jelena M. Janjic $^{1,2}$, Michael J. Patrick ${ }^{3}$, Brigitte F. Schmidt ${ }^{3}$, Robbie Mailliard $^{4}$, Alan S. Waggoner ${ }^{3}$, Eric Ahrens ${ }^{1,2}$

${ }^{1}$ Biological Sciences, Carnegie Mellon University, Pittsburgh, PA, USA, ${ }^{2}$ Pittsburgh NMR Center for Biomedical Research, Carnegie Mellon University, Pittsburgh, PA, USA, ${ }^{3}$ Molecular Biosensor and Imaging Center, Carnegie Mellon University, Pittsburgh, PA, USA, ${ }^{4}$ Celsense Inc., Pittsburgh, PA, USA, ${ }^{5}$ Department of Cell Biology and Physiology, School of Medicine, University of Pittsburgh, Pittsburgh, PA, USA.

Currently, more than 700 clinical trials involving the transplantation of cells are underway. For emerging cellular therapeutics, a non-invasive method of imaging the behavior and movement of cells following injection is widely needed. 19F cell tracking methodologies rely on ex vivo cell labeling with perfluorocarbons, followed by injection or transplantation into a subject. The cell migration is then monitored in vivo using spindensity weighted 19F MRI. 19F MRI selectively detects the perfluorocarbon (e.g. perfluoropoylether, PFPE) labeled therapeutic cells with no background, and 1H MRI of the subject provides an anatomical context. Recently, our laboratory added a fluorescence detection modality to $19 \mathrm{~F}$ containing nanoemulsions (Janjic et al, JACS 2008, 130 (9): 2832-2841). These nanoemulsions were designed and synthesized as self-delivering into a wide range of cell types, including primary and non-phagocytic cells, using fluorescently conjugated PFPEs. Key results showed a linear correlation between 19F NMR and fluorescence signals, both in vitro and in labeled cells. The exact mechanism of the PFPE nanoparticle entry to the labeled cells and their fate upon long term culturing remains unknown. In order to study the mechanism of entry and the behavior of PFPE nanoparticles in labeled cells, new nanoemulsions containing biosensing modalities covalently conjugated to PFPE oils were developed. Synthetic strategy and design of novel PFPE nanoemulsions with biosensing properties will be presented. Demonstrative fluorescent microscopy studies in labeled cells will be shown. The utility of incorporating biosensors (e.g. $\mathrm{pH}$, protease or esterase sensors) into MRI reagents will be discussed in detail and supporting results shown. Figure 1 shows preliminary results for the first generation of $19 \mathrm{~F}$ nanoemulsions with biosensing properties incorporating $\mathrm{pH}$ sensing ability.
A

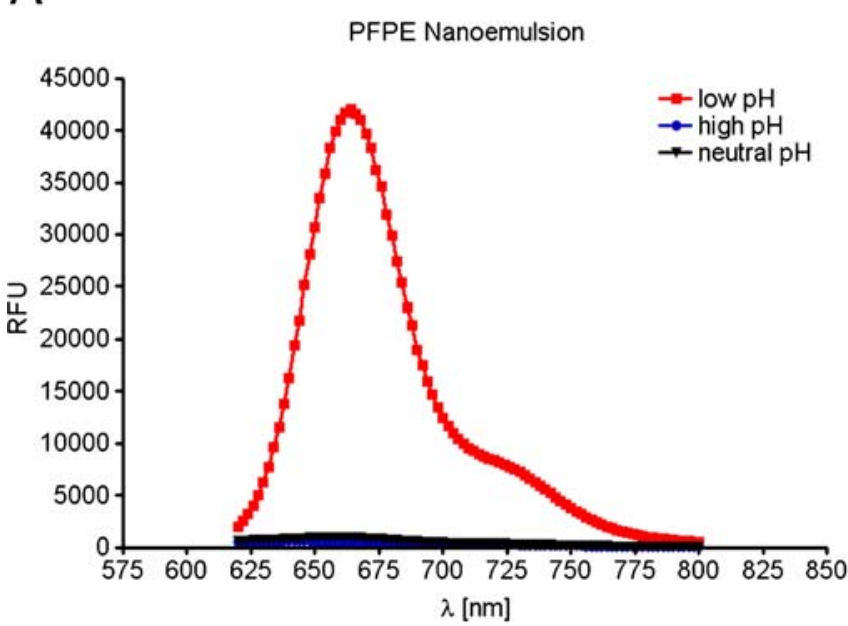

B

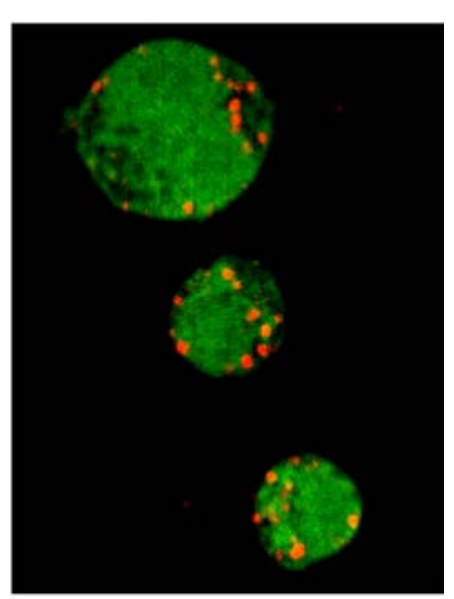

19F MRI nanoparticle with biosensing properties. (A) In vitro response of the novel PFPE nanoemulsion to changes in $\mathrm{pH}$; (B) Confocal microscopy of GFP expressing Murine derived dendritic cells (FSDC) cells (green) labeled with fluorescent biosensing PFPE nanoemulsion (red). 
J131

\section{INFLUENCE OF NEAR-INFRARED DYE LABELING} ON THE BIODISTRIBUTION OF 111IN-D2TIM

Xuejuan Wang, Melissa B. Aldrich, Lakshmi Sampath, Milton Marshall, Eva Sevick

Center for Molecular Imaging, The Brown Foundation Institute of Molecular Medicine, The University of Texas Health Science Center at Houston, Houston, TX, USA.

Dual labeled imaging probes with nuclear and optical reporters meet the unique need for noninvasive diagnostic imaging with radionuclides and for subsequent near-infrared (NIR) fluorescence imaging. Trastuzumab, a humanized monoclonal antibody against the human epidermal growth factor receptor 2 (HER2), is currently used for the treatment of patients with metastatic breast cancer overexpressesed HER2. Methods: Dual labeled trastuzumab-based conjugates, containing DTPA and IRDye $800 \mathrm{CW}$, were synthesized and optimized according to the radiolabeling yield and the affinity to HER2. The free DTPA and IRDye800CW were quantified via a HPLC system and a Odysse system for evaluation of conjugate purity. The metabolic stabilities of conjugutes were evaluated in the human serum via ITLC, and their immunoreactivities were determined in vitro using both the Lindmo assay and flow cytometry to monitor by their ability to bind to SK-BR3 human breast cancer cells, expressing high level of HER2. Biodistributions of $111 \mathrm{In}-\mathrm{D} 2 \mathrm{TI} 3$ and $111 \mathrm{In}-\mathrm{D} 2 \mathrm{TI} 5$ were compared with 111In-D2T in athymic mice bearing SK-BR-3 xenografts, and the influence of dye-to-protein ratio on the affinity was also evaluated in vivo. Results: 111In-D2TI3 and 111In-D2TI5(labeling yield above $90 \%$, radiochemical purity above $99 \%$ ) were found to be stable at 4 degree over $14 \mathrm{~d}$. The serum degradations of dual labeled agents at 37 degree were approximately $8 \% / \mathrm{d}$ and $15 \% / \mathrm{d}$, respectively, comparing to $5 \% / \mathrm{d}$ of $111 \mathrm{In}-\mathrm{D} 2 \mathrm{~T}$. Lindmo assays did not show any appreciable differences in immunoreactivity of the conjugates. In contrast, flow cytometry results showed that an increase dye-toprotein ratio decreased the binding affinity of dual labeled conjugates to $\mathrm{SKBr} 3$ cells which was reflected in reduction in tumor accumulation of $111 \mathrm{In}-\mathrm{D} 2 \mathrm{TI} 3$ and $111 \mathrm{In}-\mathrm{D} 2 \mathrm{TI} 5$ in the biodistribution study. The accumulation of dual labeled conjugates in the tumor reached their maximum at $24 \mathrm{~h}$, while the tumor uptake of $111 \mathrm{In}-\mathrm{D} 2 \mathrm{~T}$ peaked at $48 \mathrm{~h}$ post-injection. Increased dye-to-protein ratio resulted in decreasing the tumor uptakes. Renal uptake also increased significantly with increasing dye-to-protein ratio.

Biodistribution in SKB-r3 tumor-bearing nude mice after injection of[|\#11\#|] 111In-labeled trastuzumab conjugates $(\% \mathrm{ID} / \mathrm{g}, \mathrm{m}=$ dye-toprotein ratio)

\begin{tabular}{|c|c|c|c|}
\hline $48 \mathrm{~h}$ & $\mathrm{~m}=0$ & $\mathrm{~m}=3$ & $\mathrm{~m}=5$ \\
\hline blood & $0.93 \pm 0.33$ & $0.92 \pm 0.28$ & $8.00 \pm 0.18$ \\
\hline liver & $8.33 \pm 4.09$ & $8.09 \pm 1.39$ & $28.37 \pm 4.55$ \\
\hline kidney & $5.43 \pm 2.42$ & $25.99 \pm 1.52$ & $1.27 \pm 0.35$ \\
\hline muscle & $0.98 \pm 0.54$ & $1.18 \pm 0.14$ & $6.02 \pm 1.26$ \\
\hline bone & $2.92 \pm 0.39$ & $6.54 \pm 2.52$ & $6.77 \pm 1.73$ \\
\hline tumor & $15.76 \pm 2.61$ & $8.84 \pm 1.85$ & \\
\hline
\end{tabular}

$\mathbf{J 1 3 2}$

\section{DETECTION OF INTRACELLULAR CANCER MARKERS USING CLIO-VT680 NANOPARTICLES AND A CHIP-BASED NMR BIOSENSOR}

Jered B. Haun, Hakho Lee, Ralph Weissleder

Center for Systems Biology, Massachusetts General Hospital, Boston, MA, USA

The ability to detect and molecularly analyze small and unique cancer cell populations would allow rapid detection of rare cells in clinical specimen, enable marker profiling, and usher in a new era of medical diagnostics where rational treatment decisions could be based on quantitative molecular phenotypes. Recently we developed a miniaturized diagnostic magnetic resonance (DMR) sensor system that consists of an array of microcoils, microfluidic networks, on-board spectrometer, and portable magnets that is capable of rapidly detecting biological targets in microliter sample volumes and in multiplexed fashion. The DMR technique employs magnetic nanoparticles that act as proximity sensors that modulate the bulk spin-spin relaxation time of surrounding water molecules, leading to amplification of the molecular signature. While highly sensitive and specific, to date this system has has been limited to detection of extracellular tumor markers such as Her2/neu and EGFR. Therefore, we investigated techniques that would enable rapid interrogation of intracellular markers in clinical tumor samples ex vivo. Using numerous cancer cell lines as a model and CLIO magnetic nanoparticles that were modified with a cytokeratin 18 specific antibody and VT680 fluorescent dye (CK18-CLIO-VT680), we systematically optimized detection sensitivity and specificity with regards to the cell permeabilization strategy and type of magnetic nanoparticle used, magnetic nanoparticle incubation conditions, and clinical sample preservation method. To increase sample throughput, these studies were performed using flow cytometry by making use of the VT680 fluorescent signal. Promising conditions were then validated using the DMR sensor. Additionally, the specificity of cytokeratin tagging was demonstrated by confocal microscopy. Based on these optimization studies, we have successfully detected cytokeratins 18 and 19 as well as activated molecules related to the EGFR signaling pathway (phosho-EGFR, phospho-S6 ribosomal protein) in fewer than 100 cells using the miniaturized DMR system. These techniques will be tested in future validation studies using clinical tumor biopsy specimen. 


\section{$\mathbf{J 1 3 3}$}

\section{IMAGING BREAST CANCER METASTASIS TO LYMPHATICS AND BONE USING AN ANTIBODY-BASED IMAGING AGENT}

Lakshmi Sampath ${ }^{1,2}$, Rashieda Hatcher ${ }^{1}$, Sunkuk Kwon ${ }^{2}$, Pumin

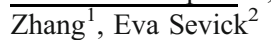

${ }^{1}$ Molecular Physiology and Biophysics, Baylor College of Medicine, Houston, TX, USA, ${ }^{2}$ Center for Molecular Imaging, University of Texas Health Science Center - Houston, Houston, TX, USA.

Breast cancer is the second leading cause of cancer death in women. Staging of breast cancer is based on size of primary tumor, extent of lymph node involvement, and metastatic spread. Seventy percent of all patients with metastatic disease die due to bone involvement wherein they suffer debilitating pain from bone loss, fractures and hypocalcaemia. Clinical diagnostic methods to detect metastasis include bone scan, Xray and MRI. There is a need for development of diagnostic imaging agents to detect metastases early with improved sensitivity. About $30 \%$ of breast cancer patients are affected by gene amplification or over expression of the Human Epidermal Growth Factor Receptor-2 (HER2), known to correlate with aggressive tumor growth and poor prognosis. Our lab has recently synthesized a dual-labeled antibodybased imaging agent that can detect HER2 overexpressing tumors in a subcutaneous xenograft mouse model. We conjugated trastuzumab (anti-HER2 Ab) with IRDye800 and DTPA for chelation to In-111 in order to assess HER2 levels using near-infrared fluorescence and SPECT imaging respectively. In this study, we used the transgenic MMTV-neu mouse model to determine if the antibody-based imaging agent can be used as a diagnostic agent to detect metastatic spread. This mouse model develops HER2 overexpressing mammary gland tumors around 6 months of age with metastasis to the lung and bone after 8 months. We injected approximately 100 ug of (InDTPA)n-trastuzumab-(IRDye800)m via tail vein and imaged uptake at 0,24 and $48 \mathrm{~h}$. In addition to the primary tumor, we were able to visualize agent uptake in bone (Fig. 1A,B) and lymph nodes (Fig. 1C). Imaging agent accumulation in the knee region of the bone corresponds with reports from the literature as the site of osteolytic bone lesions. This animal model allows us to study metastasis in an immune-competent mouse, mimicking functional lymphatics in the spread of cancer. We believe that the dual-labeled trastuzumab-based imaging agent can be used as an effective diagnostic biomarker for early detection of breast cancer related lymph and bone metastasis.

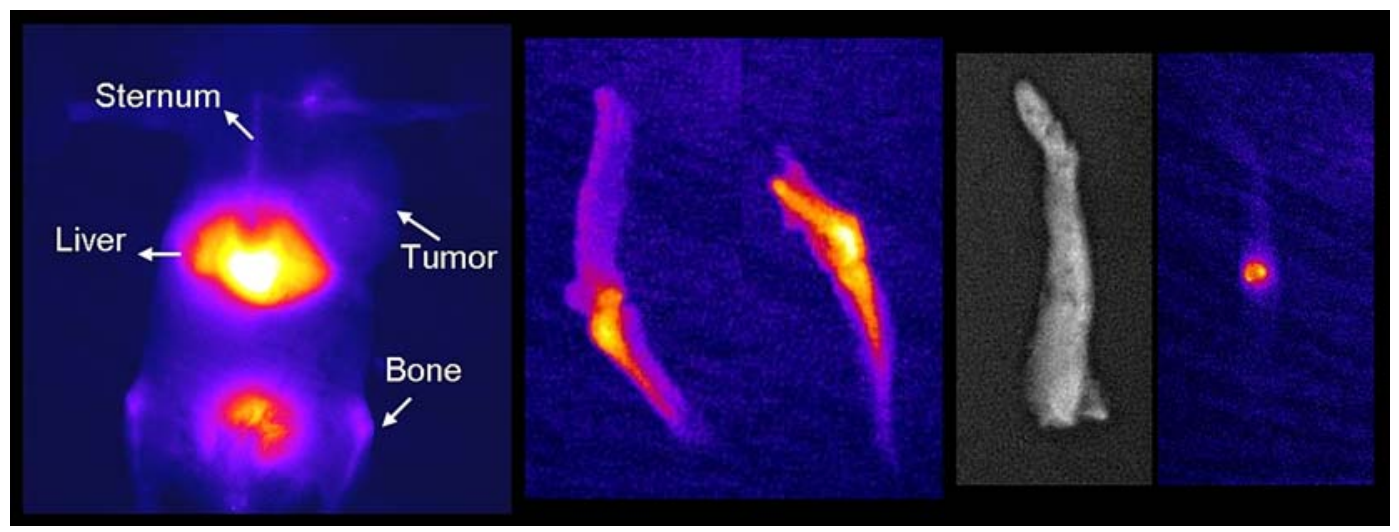

Fig. 1: (A) Whole-body near infrared fluorescence imaging of MMTV-neu mice. (B) Ex-vivo imaging of agent accumulation in bone. (C) White light (left) and fluorescence image (right) of ex-vivo mammary gland with lymph node

\section{J134}

\section{MULTIFUNCTIONAL CONTRAST AGENTS FOR MOLECULAR IMAGING OF PROSTATE CANCER}

Huifang Zhai ${ }^{1,2}$, Prasanta K. K. Nanda ${ }^{1}$, Charles J. Smith ${ }^{1}$, Ashley N. Brown ${ }^{5}$, Haibo $\mathrm{Lin}^{3}$, Ping $\mathrm{Yu}^{3}$, Gary L. Sieckman ${ }^{5}$, Timothy J. Hoffman ${ }^{4,5}$, Yash Sethi ${ }^{1}$, Lixin Ma ${ }^{1,5}$

${ }^{1}$ Radiology, University of Missouri-Columbia, Columbia, MO, USA,

${ }^{2}$ Nuclear Science and Engineering Institute, University of MissouriColumbia, Columbia, MO, USA, ${ }^{3}$ Physics, University of MissouriColumbia, Columbia, MO, USA, ${ }^{4}$ Internal Medicine, University of Missouri-Columbia, Columbia, MO, USA, ${ }^{5}$ Biomolecular Imaging Center, Harry S. Truman Memorial Veteran's Hospital, Columbia, MO, USA.

Development of efficacious receptor-targeting contrast agents is particularly important for early detection of prostate cancers. When using non-invasive magnetic resonance imaging (MRI) for molecular imaging, sensitivity and specificity are critical issues. A multimodal approach such as MRI/optical and MRI/positron emission tomography can solve the problems associated with individual imaging modality. In our previous study, a near-infrared fluorescent bombesin (BBN) conjugate, AlexaFluro680-BBN[7-14], has shown high efficacy for gastrin releasing peptide receptor (GRPR) targeted imaging in human cancer mouse models. The GRPR is over-expressed on human cancer cells including prostate, breast and small cell lung cancers, which makes it an attractive molecular target for early imaging and therapy of cancer patients. In this work, we report results on the synthesis and characterization of hybrid nanoparticle contrast agents, AlexaFluro750BBN[7-14]-SPIO and Gd-DOTA-BBN[7-14]-SPIO for dual-modality molecular imaging of prostate cancers. The nanoparticles consist of a biocompatible super-paramagnetic iron oxide (SPIO) core, and AlexaFluro750-BBN or Gd-DOTA-BBN conjugates on the surface of SPIO. Atomic force microscope (AFM) was used to measure the size of the nanoparticle conjugates. The characterizations were performed by IC50 (the half maximal inhibitory concentration) binding affinity, Prussianblue staining reaction, and fluorescence microscopic imaging in PC-3 prostate cancer cells. MRI relaxivity enhancements were determined using spin-echo sequences on $7 \mathrm{~T}$ MRI. In vitro and in vivo imaging efficacy was evaluated and compared to non-targeted contrast agents, i.e. Gd-DTPA and SPIO, using MRI/optical imaging in PC-3 cells and 
SCID tumor xenografts. Our results show that the BBN-based multifunctional SPIO nanoparticles demonstrated high binding affinity and specificity and a subsequent retention of the ligands in GRPR overexpressing prostate cancer cells via agonist initiated receptor-mediated endocytosis. This agonist initiated receptor-mediated endocytosis approach may offer a dynamic mechanism on nanoparticle accumulation and signal amplifications in cancer cells. The work is supported by DOD Prostate Cancer Research Program New Investigator Award PC081264 and NIH P50-CA-103130.

\section{$\mathbf{J 1 3 5}$}

\section{SMART PROBES FOR THERANOSTIC APPLICATIONS USING DUAL PURPOSE IMAGING AND THERAPEUTIC RADIONUCLIDES}

Daniel Y. Lee, King Li, Ching-Hsuan Tung

Radiology, The Methodist Hospital Research Institute, Houston, TX, USA.

The treatment of cancer remains a challenging problem that poses significant obstacles in the development of new effective drugs. The approach we have taken combines the power of molecular imaging with the utility of certain radionuclides that exhibit selective cytotoxic profiles due to its mode of decay and the physical characteristics of emitted particles. Here we describe the adaptation of peptide-based imaging probes designed to be activated by the action of proteases that are almost universally overexpressed in solid malignancies. These peptides are designed to have discrete functional domains that confer cell penetrating activity upon release of a control domain that masks its presentation via electrostatic interactions. Site-specific catalysis by a protease such as cathepsin B known to be overexpressed by malignant tumors is achieved with the proper configuration of naturally occurring and $\mathrm{D}$-isomer amino acid residues positioned adjacent to a linker that forms a stable beta turn. Radionuclide attachment is achieved with a macrocyclic chelator conjugated to the cell penetrating peptide (CPP) domain. The complete "smart" probe is equipped with the gamma and Auger electron-emitting radionuclide, indium-111, and the alphaemitter, actinium-225, that permit tomographic imaging and antitumor applications. The action of these highly reactive emission particles is strictly distance dependent due to their short range of travel within tissue. This characteristic limits the kill zone to the intracellular compartment, i.e., the nucleus (for Auger electrons), or up to one or two cell diameters (alpha particles). By exploiting the intracellular targeting activity of the CPP the delivery of such short-range radionuclides may yield excellent tumor cell specificity and potent cytotoxicity. In contrast to external radiation therapy, the mechanism of radionuclide-induced double-strand DNA breaks is independent of molecular oxygen; thus this mode of therapy is predicted to be less prone to variability as a result of tumor hypoxia. Moreover, the mechanism of cell membrane translocation by CPPs appears to circumvent the cell's efflux system that often gives rise to multidrug resistance in cancers. We present preliminary data on the optimization of smart probe design and in vitro characterization.

\section{$\mathbf{J 1 3 6}$}

FURTHER DEVELOPMENTS OF THE LABPET II, A 64-CHANNEL APD-BASED DETECTOR MODULE WITH PET AND COUNTING CT IMAGING CAPABILITIES Christian Thibaudeau $^{1}$, Melanie Bergeron ${ }^{2}$, Catherine M. Pepin ${ }^{2}$, Henri Dautet $^{3}$, Murray Davies ${ }^{3}$, Réjean Fontaine ${ }^{1}$, Roger Lecomte ${ }^{2}$

${ }^{1}$ Electrical engineering, Universite de Sherbrooke, Sherbrooke, QC, Canada, ${ }^{2}$ Nuclear medicine and radiobiology, Universite de Sherbrooke, Sherbrooke, QC, Canada, ${ }^{3}$ PerkinElmer Optoelectronics, VaudreuilDorion, QC, Canada.
CT imaging currently represents the standard approach to provide anatomical reference in PET molecular imaging. Since both PET and CT rely on detecting radiation to generate images, it would only appear logical to use the same detection system for data acquisition. Merging PET and CT hardware imposes extremely stringent requirements on detectors, including wide dynamic range with high signal-to-noise ratio, high pixelization and very high count rate capabilities. The APD-based LabPET II module is proposed as the building block for the first truly combined PET/CT scanner. The module is made of two $4 \times 8$ APD pixel arrays mounted side by side in a custom ceramic holder with each element having an active area of $1.1 \times 1.1 \mathrm{~mm}^{2}$ at a $1.2 \mathrm{~mm}$ pitch and coupled to a 12-mm high LYSO scintillator block array. While the initial version of the module was made of pyramidal shaped crystals $\left(1.35 \times 1.35 / 1.2 \times 1.2 \mathrm{~mm}^{2}\right.$, top/bottom $)$, a newer version was designed with a simpler rectangular geometry $\left(1.2 \times 1.2 \mathrm{~mm}^{2}\right)$, a better reflective material optimizing the shift of refractive index between crystals and reflector, and an enhanced APD quantum efficiency to improve intrinsic detector performance. Mean energy resolution was improved to $20 \pm 2 \%$ (formerly $24 \pm 1 \%$ ) at $511 \mathrm{keV}$ and to $46 \pm 5 \%$ (formerly $48 \pm$ $3 \%)$ at $60 \mathrm{keV}$. The valley-to-peak ratio in the latter spectra, which provides a measure of the SNR in CT mode, was improved to $0.2 \pm 0.15$ (formerly $0.7 \pm 0.1$ ). A noise threshold of $29 \pm 5 \mathrm{keV}$ (formerly $37 \pm$ $3 \mathrm{keV}$ ) was also estimated in $\mathrm{CT}$ mode. These intrinsic detector performance characteristics make the LabPET II module suitable for counting CT imaging with efficient energy discrimination.
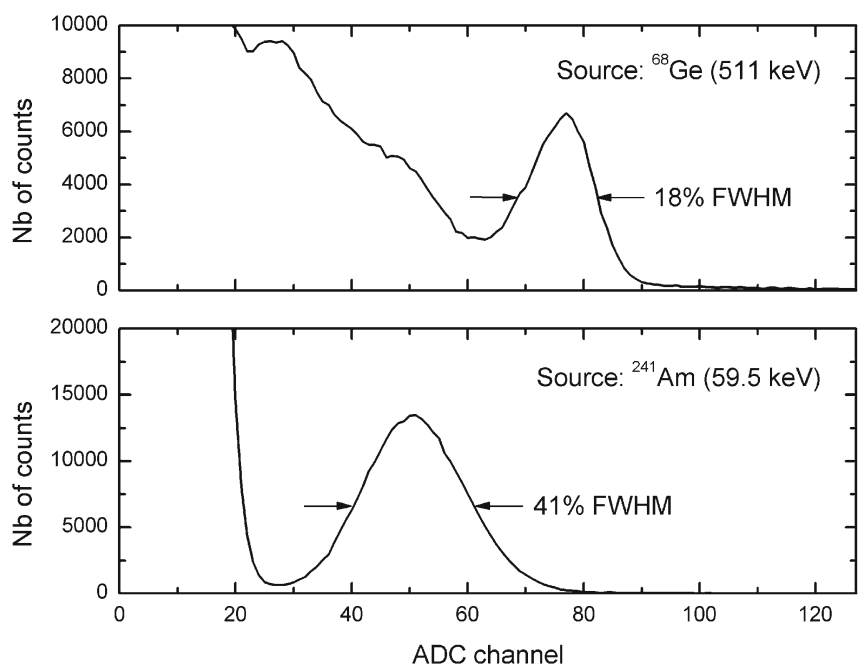

$\mathbf{J 1 3 7}$

NOVEL GENERATION OF NANOPARTICLE-INCORPORATED MICROBUBBLES AS MULTIMODAL CONTRAST AGENTS

Minseok Seo $^{1,2}$, Ivan Gorelikov ${ }^{2}$, Naomi Matsuura ${ }^{1,2}$

${ }^{\mathrm{D}}$ Department of Medical Biophysics, University of Toronto, Toronto, ON, Canada, ${ }^{2}$ Imaging Research, Sunnybrook Health Sciences Centre, Toronto, ON, Canada.

There is great interest in designing new medical imaging contrast agents that can permit multiple imaging applications using a single agent. This would provide concurrent contrast imaging for multiple modalities (e.g., magnetic resonance, x-ray computed tomography, ultrasound, and optical imaging) with complementary spatial, temporal and depth resolution for the more accurate diagnosis and local treatment of diseased tissue. Solid nanoparticles (NPs) are promising multimodal imaging and therapy agents. Microbubbles have long been 
used as effective contrast agents for ultrasound imaging in patients. Here, we present a new strategy for the simple and robust incorporation of various medical nanoparticles into monodisperse microbubbles based upon the controlled $\mathrm{pH}$-based regulation of the electrostatic attraction between NPs and the microbubble shell. Using this simple approach, microfluidic-generated, protein-coated, perfluorobutane microbubbles $(3-8 \mu \mathrm{m})$ were incorporated with various NPs, including quantum dots, iron oxide NPs, and gold nanorods. This work reveals the potential of modular synthesis of a variety of NP-microbubble constructs to represent flexible, user-defined multimodal imaging agents tailored for specific applications and disease types.

\section{Novel Optical Probes}

\section{$\mathbf{J 1 3 8}$}

\section{IMAGING OXIDATIVE STRESS WITH A NOVEL BIOLUMINESCENT SENSOR}

Julie Czupryna, Andrew Tsourkas

Bioengineering, University of Pennsylvania, Philadelphia, PA, USA.

Oxidative stress can occur when intracellular concentrations of reactive oxygen species (ROS) significantly increase and/or antioxidant mechanisms are compromised. Severe oxidative stress can damage various vital cellular components, namely DNA, RNA, proteins and lipids. Pathologic conditions that may result from accumulation of this damage include atherosclerosis, cystic fibrosis, type-2 diabetes and neurodegenerative disorders such as Parkinson's or Alzheimer's disease. ROS are also involved in cellular signaling and have been garnering attention for their role in cell death pathways. The ability to investigate oxidative stress/ROS via in vivo imaging could evidently provide unique insight into the aforementioned maladies and cellular processes. Recently, our group has developed a bioluminescent sensor that is responsive to conditions of cellular oxidative stress. We have evaluated the ability of this sensor to detect oxidative stress in multiple cell lines (HeLa, MCF7 , and $293 \mathrm{~T}$ cells) with several oxidative stress induction methods (e.g. Antimycin A, $\mathrm{H} 2 \mathrm{O} 2$, and paraquat) and achieved consistent, significant results. It is envisioned that the real-time, non-invasive imaging capability of our sensor will be useful in research pursuits involving the development/evaluation of new therapeutics for oxidative stress-related disorders as well as the identification effective antioxidants. Additionally, since tumorigenesis and anti- cancer drug resistance have been attributed to the ability of cells to evade caspase-dependent death, our sensor may be valuable in the examination of alternative cell death pathways involving ROS.

\section{J139}

\section{CASPASE-ACTIVATED CELL-PENETRATING PROBES FOR IN VIVO OPTICAL IMAGING OF RETINAL NEURODEGENERATION}

$\underline{\text { James R. Johnson }}{ }^{1}$, Brandon Kocher ${ }^{1}$, Dustin J. Maxwell ${ }^{1}$, Qing $\overline{\text { Chang }}^{2}$, Edward M. Barnett ${ }^{2}$, David Piwnica-Worms ${ }^{1}$

${ }^{1}$ Department of Radiology, Washingon University School of Medicine, St. Louis, MO, USA, ${ }^{2}$ Department of Ophthalmology and Visual Sciences, Washingon University School of Medicine, St. Louis, MO, USA.

Glaucoma is an optic neuropathy characterized by the death of retinal ganglia cells (RGCs), axon loss, and an excavated appearance to the optic nerve head. RGC death mechanisms in animal models of glaucoma and human glaucoma involve apoptosis. Regardless of trigger, the apoptotic cascade produces cell suicide by invoking caspase activation, a mechanism conserved in RGCs. To monitor cellular commitment to apoptosis, a series of activatable, cell-penetrating peptide-probes (CPP)s were developed to measure cytosolic activity of caspase-3. Our quenched probe (KcapQ647) incorporates a CPP sequence, and a fluorophorequencher pair linked by a caspase labile site. This structural motif allows for efficient cell uptake and renders the probe optically silent until cleavage by caspase-3. To monitor cellular uptake and further study internalization mechanisms, a nonquenched probe (Kcap647) was prepared. Herein we applied these probes to a retinal ganglial cell (RGC-5) culture model to further characterize biological events behind probe activation. RGCs readily differentiate into neurites upon treatment with $2.5 \mu \mathrm{M}$ staurosporine (SS) for $1 \mathrm{~h}$, allowing more accurate modeling of the differentiated state on neurodegeneration. Both native and differentiated RGCs show a concentration-dependent uptake of Kcap647. In both RGC types, $10 \mu \mathrm{M}$ Doxorubicin (Dox) treatment for $3 \mathrm{hrs}$ followed by KcapQ647 exposure produced distinct probe activation as observed by fluorescence microscopy. Based on these RGC results, KcapQ647 was applied to an in vivo rat glaucoma model. Intravitreal (eye) administration of KcapQ647 showed activation in RGCs with a dose-dependent response to N-methyl D-aspartate neurotoxicity with $>3.5$-fold signal increases when compared to vehicle controls. This RGC cell-type-specific property of our probes provides a noninvasive tool for in vivo imaging of retinal pathology.
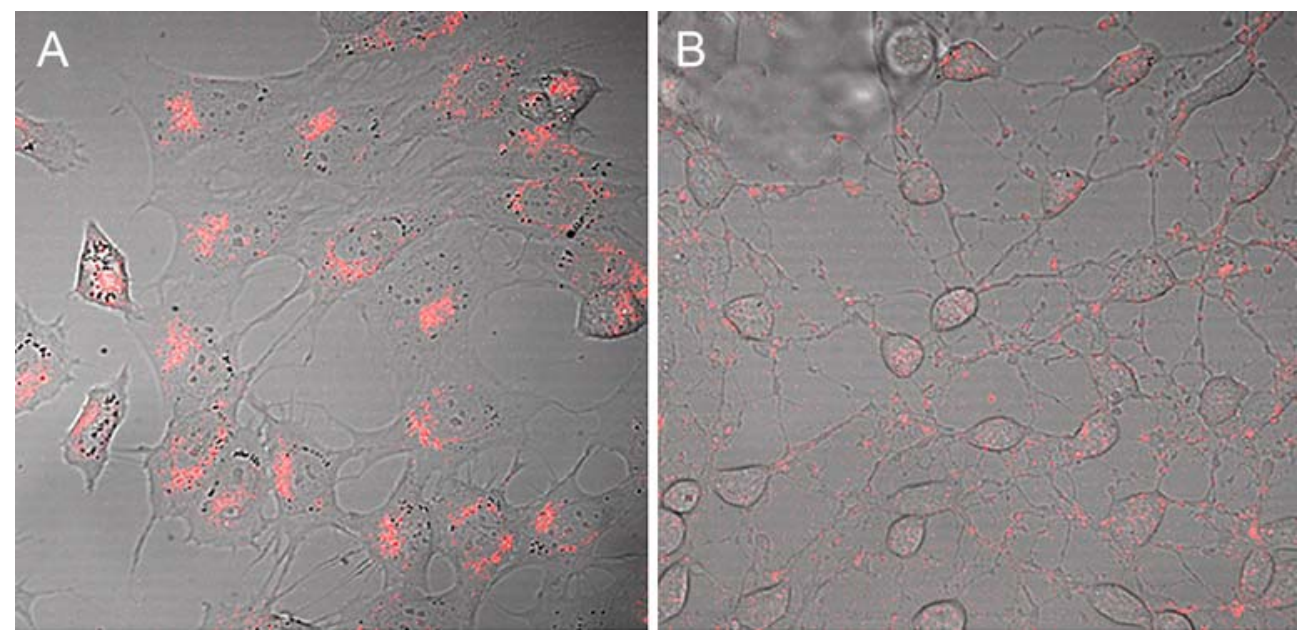

Fluorescence microscopy images of native (A) and SS-treated (B) RGC-5 cells after 3 hrs exposure to $10 \mu \mathrm{M}$ Kcap647. 
$\mathbf{J 1 4 0}$

\section{IN VIVO MULTICOLOR TUMOR-SPECIFIC PAINTING USING DEHALOGENASE-BASED PROTEIN-TAG (HALO-TAG) FLUORESCENT LIGANDS}

Hisataka Kobayashi ${ }^{1}$, Nobuyuki Kosaka ${ }^{1}$, Mikako Ogawa ${ }^{1}$, Natasha Karassina $^{2}$, Cesear Corona ${ }^{2}$, Mark G. McDougall ${ }^{2}$, Clifford Hoyt ${ }^{3}$, Richard Levenson ${ }^{3}$, Georgyi V. Los ${ }^{3}$, Peter Choyke ${ }^{1}$

${ }^{1}$ Molecular Imaging Program, National Cancer Institute/NIH, Bethesda, MD, USA, ${ }^{2}$ Promega Corporation, Madison, WI, USA, ${ }^{3}$ Cambridge Research Inc., Woburn, MA, USA.

Background: in vivo fluorescence imaging can be performed either with endogenous fluorescent proteins or exogenously introduced fluorescent probes bound to targeting molecules. However, endogenous fluorescent proteins, such as GFP, cannot be altered after transfection, and therefore if new colors are desired, the cells must be rederived. Exogenously targeted fluorescence probes are limited by the heterogeneous expression of naturally occurring cellular targets. Transfectable fusion protein tags produce specific receptor expression, which can be imaged in vivo with fluorescently labeled ligands of varying colors, thus overcoming the limitations of current methods. Methods: We adapted the dehalogenasebased protein-tag (HaloTag) system to in vivo cancer imaging by introducing highly expressed HaloTag receptors (HaloTagR) in SHIN3 cancer cells followed by externally injected fluorophore-conjugated dehalogenase-reactive ligands (tag). Tumor nodules arising from a single transfected cell line were stably labeled with fluorescent tag using fluorophores, which varied in emission from green to near infrared. in vivo spectral fluorescence imaging of the cell line (HaloTagR-SHIN3) was performed in live animals with peritoneal tumor dissemination. Results: The tumor nodules arising from HaloTagR-SHIN3 were successfully labeled with 4 different fluorophore-conjugated HaloTag-ligands (HaloTagL-Alexa488, HaloTagL-TMR, HaloTagL-Dy633, and HaloTagLIR800) each emitting light at different wavelengths ranging from visible to near infrared in vivo and ex vivo. When these fluorescent ligands were alternated on serial in vivo imaging it was possible to assess interval growth by the different color it produced. Fluorescence was retained in histological specimens after fixation. Conclusion: This tagging system is useful both for in vivo and in vitro imaging without requiring modification of the underlying cell line. This strategy can overcome some of the limitations associated with the current use of endogenous fluorescent proteins and exogenous targeted optical agents.
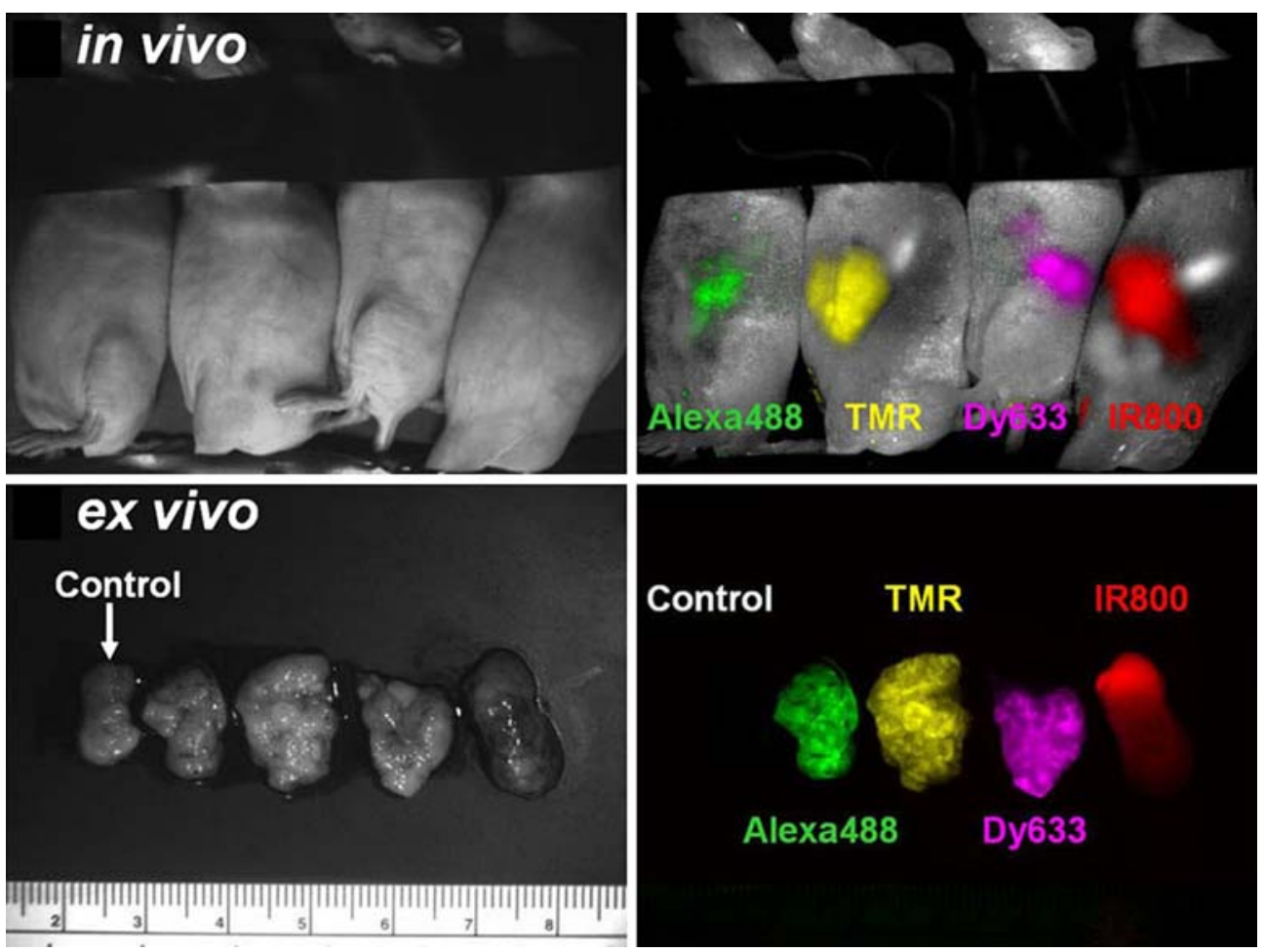

VALIDATION OF ITGA9 AS A TARGET FOR THE OPTICAL IMAGING OF LYMPHANGIOGENESIS

Pier-Anne Lachance $^{1,2}$, Eva Sevick ${ }^{1}$

${ }^{1}$ Institute of Molecular Medicine, University of Texas Health Science Center, Houston, TX, USA, ${ }^{2}$ Molecular Physiology and Biophysics, Baylor College of Medicine, Houston, TX, USA.

The lymphatic system is crucial for the maintenance of tissue fluid balance, for transport of lipids and large proteins, and for providing a compartment for the immune response. Lymphangiogenesis, the process of forming new lymphatic vessels, is thought to play a crucial

role in an array of diseases including cancer metastasis and chronic inflammation. In our program, we are validating integrin subunit $\alpha 9$ (ITGA9) as the first molecular target for the process of lymphangiogenesis and developing an optical imaging agent specific for ITGA9. ITGA9 is necessary for the proper development of the lymphatic vasculature and ITGA9 expression level is increased on lymphatic endothelial cells (LECs) in vitro when cells are treated with prolymphangiogenic factors (vascular endothelial growth factor (VEGF) family and the hepatocyte growth factor (HGF)). We hypothesize that ITGA9 modulates LEC proliferation and motility during lymphangiogenesis and therefore could be used as a marker for this process. We have selected two peptide motifs, with amino acid sequences MLDG and EIDGIEL, that specifically recognize the ligand binding site of 
ITGA9. Four peptides were synthesized and conjugated to a near infrared dye, IRDye800CW: IRDye800-PLAEIDGIELTY, IRDye800GGGPLAEIDGIELA, IRDye800-ARTMLDGLNDY, and IRDye800ARAMLDGLNDY. We have measured the IC50 value for all the peptides to be in the nanomolar range. Fluorescence microscopy was used to determine binding and specificity of all peptides on SW-480 human colon cancer cells transfected with human ITGA9 and cultured human LECs. Finally, we have assessed the expression on ITGA9 in two proliferating lymphatic mouse models: (1) a conditional knockout mouse model which shows uncontrolled LECs proliferation; and (2) a Matrigel plug mouse model, in which Matrigel enriched with growth factors (VEGF-C and HGF) is injected subcutaneously to promote lymphangiogenesis. Immunohistochemistry showed that ITGA9 is expressed on proliferating lymphatics in both models and immunoblot analysis showed an increase in expression in tissue undergoing lymphangiogenesis compared to the resting lymphatics. These animal models will provide means to validate and optimize the candidate imaging conjugate in vivo for the imaging of lymphangiogenesis. Supported in part by R01 HL 092923

\section{$\mathbf{J 1 4 2}$}

\section{THE ROLE OF H-TYPE DIMER FORMATION IN DESIGNING IN VIVO ACTIVATABLE OPTICAL MOLECULAR IMAGING PROBES}

Mikako Ogawa, Nobuyuki Kosaka, Peter Choyke, Hisataka Kobayashi NCI / NIH, Molecular Imaging Program, Bethesda, MD, USA.

Background: in vivo molecular imaging with target-specific activatable "smart" probes enables improved cancer detection. Dimerization and fluorescence quenching have been shown to occur in concentrated aqueous solutions of various fluorophores. We hypothesized that fluorophore dimerization and quenching after conjugation to targeting proteins can occur at low concentrations because protein molecules stabilize the fluorophore dimers based on physico-chemical interactions. Methods: Eight rhodamine derivatives were conjugated to the cancer targeting molecules, avidin and trastuzumab, which target Dgalactose receptor and HER2/neu antigen, respectively. The H-dimer formation was investigated by measuring the absorption spectrum. To dissociate the dimerized fluorophores, the conjugates were treated with SDS. The quenching efficiency was also determined. In vitro fluorescence microscopy experiments and in vivo, fluorescence endoscopic imaging in mice bearing peritoneal tumors were performed with a highly quenched probe (avidin-TAMRA) or a minimally quenched probe (avidin-Alexa488) to demonstrate the fluorescence activation effects. Results: A large proportion of R6G and TAMRA formed Htype dimers in both trastuzumab and avidin conjugates at low fluorophore concentrations. The effect was least marked with Alexa488 and most marked with TAMRA. There was a strong positive relationship between the extent of H-dimer formation and fluorescence quenching efficiency in both avidin and trastuzumab conjugates. The fluorescent signal on the surface of cells was brighter in trastuzumabAlexa488 than trastuzumab-TAMRA. Fluorescence was observed inside the cell after $8 \mathrm{hrs}$ of incubation with both conjugates, and it was brighter with trastuzumab-TAMRA. The tumors were clearly visualized with avidin-TAMRA, with low background fluorescence in vivo; in contrast, the background fluorescence was high for avidinAlexa488. Discussion: Here, we demonstrate that H-dimer formationderived fluorescence quenching occurs when fluorophores are conjugated to proteins, including antibodies and receptor ligands for target-specific imaging. After internalization and dissociation of the H-dimer in the target cell, dequenching occurs permitting high target to background imaging. Thus, H-dimer formation is a promising mechanism for activation of target-specific macromolecular optical probes for molecular imaging.

\section{$\mathbf{J 1 4 3}$}

\section{ULTRA-LOW BACKGROUND NEAR-INFRARED FLUOROPHORES FOR TUMOR TARGETING} Hak Soo Choi ${ }^{1}$, Maged Henary ${ }^{2}$, Khaled A. Nasr ${ }^{1}$, Jeong Heon Lee ${ }^{1}$, Fangbing Liu $^{1}$, Lucjan Strekowski ${ }^{2}$, Gabor Patonay ${ }^{2}$, John V. Frangioni ${ }^{1,3}$ ${ }^{1}$ Medicine, Beth Israel Deaconess Medical Center, Boston, MA, USA, ${ }^{2}$ Chemistry, University of Georgia, Atlanta, GA, USA, ${ }^{3}$ Radiology, Beth Israel Deaconess Medical Center, Boston, MA, USA.

Background: Near-infrared (NIR) fluorescence imaging has the potential to revolutionize image-guided surgery. However, the ideal fluorophores needed for intraoperative imaging have yet to be described. Over the last decade, our laboratory has been developing the FLARE ${ }^{\mathrm{TM}}$ (Fluorescence-Assisted Resection and Exploration) intraoperative imaging system, which is capable of simultaneous, realtime acquisition of two independent NIR fluorescent images along with color video images. Nevertheless, the fundamental limitation to the future success of this technology is the development of imaging probes that perform optimally in the body. The goal of this study was to develop such optical probes. Materials and Methods: A heptamethine indocyanine backbone was systematically derivatized to produce $\mathrm{N}$ hydroxysuccinimide (NHS) ester-conjugatable NIR fluorophores having net charges of $-4,-2,0,+2$, and +4 . Full-spectrum absorbance and fluorescence spectrophotometers were used to characterize these dyes in terms of their extinction coefficient, quantum yield, and stability in serum. NIR fluorophores were injected intravenously into mice, rats, and pigs, and their biodistribution and clearance were measured over a period of $4 \mathrm{~h}$. $5 \times 10^{6}$ of integrin $\alpha_{\mathrm{v}} \beta_{3}$-targeted M21 and M21-L cells were re-suspended in 50\% Matrigel and injected subcutaneously in the flank of athymic nu/nu mice. Cyclic RGDyK (cRGD)-conjugated NIR dyes were administered intravenously at a dose of $40 \mathrm{pmol} / \mathrm{g}$ for human melanoma targeting. Results: All fluorophores had high extinction coefficient $(121,000$ $\left.309,000 \mathrm{M}^{-1} \mathrm{~cm}^{-1}\right)$ and quantum yield $(9.3 \%-16.9 \%)$ in $100 \%$ serum at $37^{\circ} \mathrm{C}$, and excitation and emission wavelengths near $800 \mathrm{~nm}$. Dye ZW-1 had remarkably low uptake in normal tissue, and rapid elimination from the body within $4 \mathrm{~h}$. Using animal models of human melanoma, receptor-specific imaging with high tumor-to-background ratio (TBR) could be obtained with the optimal dye conjugated to cRGD, representing a 3.4-fold improvement over commercially available fluorophores (TBR of $17.2 \mathrm{vs.} 5.0$ ) at $4 \mathrm{~h}$ postinjection. Conclusion: We demonstrate that properly designed NIR fluorophores have improved optical and in vivo properties, including 800$\mathrm{nm}$ fluorescence, high extinction coefficient and quantum yield, no serum protein binding, rapid renal clearance, and ultra-low non-specific tissue uptake. This new class of NIR fluorophores lays the foundation for designing agents having optimal optical properties, biodistribution, elimination from the body, and receptor-based targeting for image-guided surgery.

\section{J144}

\section{NEW MATERIALS FOR IMAGING REACTIVE OXYGEN SPECIES IN VIVO}

Niren Murthy

Biomedical Engineering, Georgia Tech, Atlanta, GA, USA.

Inflammatory diseases, such as acute liver failure and congestive heart failure, cause millions of deaths each year and new strategies for treating and diagnosing inflammatory diseases are greatly needed. In this presentation, I will present 2 new materials that are designed to image reactive oxygen species in vivo and diagnosis inflammatory diseases. The first contrast agent, termed the peroxalate nanoparticles images hydrogen peroxide. The overproduction of hydrogen peroxide is implicated in the development of numerous diseases and there is currently great interest in developing contrast agents that can image hydrogen peroxide, in vivo. In this presentation, we 
demonstrate that nanoparticles formulated from peroxalate esters and fluorescent dyes can image hydrogen peroxide in vivo with high specificity and sensitivity. The peroxalate nanoparticles image hydrogen peroxide by performing a three component chemiluminescent reaction between hydrogen peroxide, peroxalate esters and fluorescent dyes. The peroxalate nanoparticles have several attractive properties for in vivo imaging, such as tunable wavelength emission $(460-630 \mathrm{~nm})$, nanomolar sensitivity for hydrogen peroxide and excellent specificity for hydrogen peroxide over other reactive oxygen species. The peroxalate nanoparticles were capable of imaging hydrogen peroxide in the peritoneal cavity of mice, during an LPS-induced inflammatory response. We anticipate numerous applications of peroxalate nanoparticles for in vivo imaging of hydrogen peroxide, given their high specificity, sensitivity, and deep tissue imaging capability. The second imaging agent I will discuss is a new family of fluorescent dyes, termed the hydrocyanines, which can image radical oxidants, such as superoxide in vivo (in mice) by fluorescent imaging.

\section{$\mathbf{J 1 4 5}$}

\section{IDENTIFICATION AND APPLICATION OF A FLUORESCENT GENERAL ANESTHETIC}

Ivan Dmochowski

Chemistry, UPenn, Philadelphia, PA, USA.

Despite surgical use for more than 160 years, we still know very little about how anesthetics achieve their clinical function. Our lab, in collaboration with others, has recently discovered the first fluorescent general anesthetic molecule, 1-aminoanthracene (1-AMA). This compound provides unprecedented opportunities for molecular and target discovery. We have shown that 1-AMA reversibly immobilizes a variety of model organisms, including tadpoles, zebrafish, and nematodes. Remarkably, this compound can be visualized in the brain, and throughout the whole body of living, immobilized animals, using a variety of different fluorescence imaging techniques (e.g., confocal laser scanning microscopy, fluorescence lifetime imaging, spectral deconvolution imaging). This fluorophore provides images of a working anesthetic at unprecedented spatial resolution, and we are now using 1-AMA to identify new anesthetic molecules in highthroughput screens. We are also using 1-AMA for mechanistic studies in vivo, with the goal of identifying the protein targets of general anesthetics.

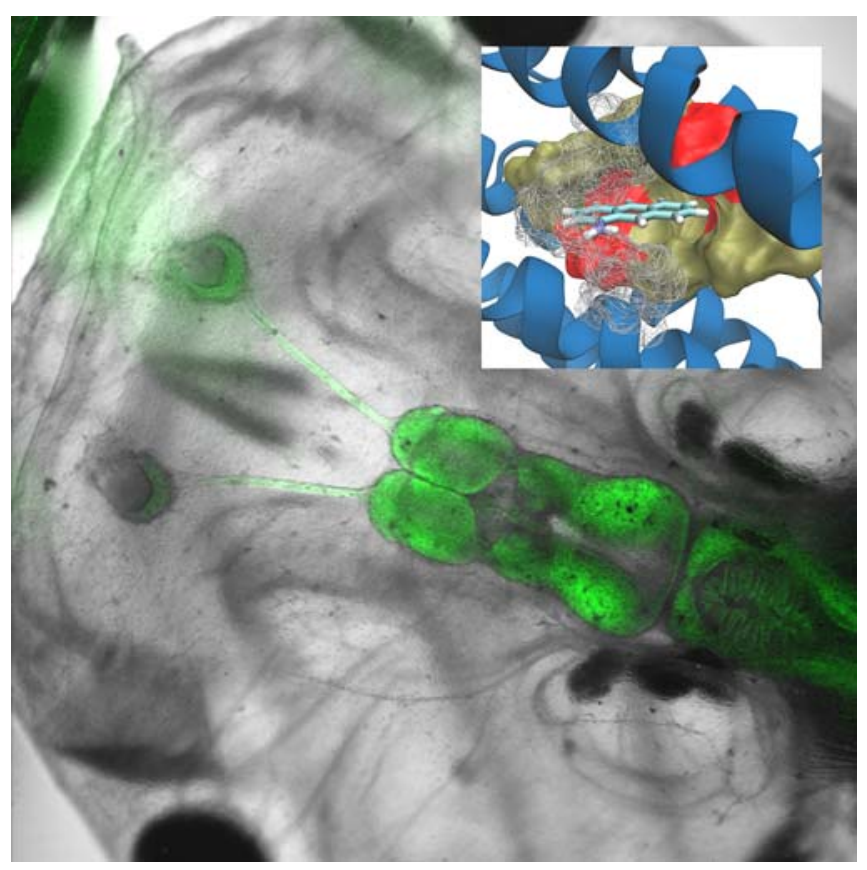

\section{$\mathbf{J 1 4 6}$}

VISUALIZING SPATIOTEMPORAL DYNAMICS OF CELLCYCLE PROGRESSION OF CANCER CELLS IN VIVO

AKi HANYU $^{1}$, Hirofumi Inoue ${ }^{1,2}$, Yasumichi Inoue ${ }^{1}$, Asako SakaueSawano $^{3,4}$, Atsushi Miyawaki ${ }^{3,4}$, Takeshi Imamura ${ }^{1}$

${ }^{1}$ Cancer Institute Division of Biochemistry, JAPANESE FOUNDATION FOR CANCER RESEARCH(JFCR), koto-ku, Japan, ${ }^{2}$ Division of Biochemistry and Molecular Genetics Department of System Biology, Ehime University Graduate School of Medicine, Toon, Japan, ${ }^{3}$ Laboratory for Cell Function and Dynamics, Advanced Technology Development Group, Brain Science Institute, RIKEN, Wakocity, Japan, ${ }^{4}$ Life Function and Dynamics, ERATO, JST, , Wako-city, Japan.

The cell-cycle transition from G1 to S phase has been difficult to visualize. We have established Fluorescent Ubiquitination-based Cell Cycle Indicator (Fucci) System that effectively labels individual G1 phase nucleired and those in S/G2/M phases green (Sakaue-Sawano et al., Cell, 2008). In this study, we generated several cancer cells constitutively expressing the Fucci probes, in which every cell nucleus exhibits either red or green fluorescence. We then demonstrated time-lapse imaging to explore the spatiotemporal patterns of cell-cycle dynamics of human cervical cancer HeLa cells during the development of tumors across blood vessels in live mice. Moreover, we investigated the cell-cycle patterns of human breast cancer MDA-MD-231 cells during the development of tumors at bone metastasis sites in live mice. These Fucci-expressing cancer cell lines will serve as powerful model systems for studying molecular mechanisms of cancer development and metastasis coordinated with cell cycle.

\section{$\mathbf{J 1 4 7}$}

\section{MOLECULAR IMAGING AGENTS SPECIFIC FOR THE ANNULUS FIBROSUS OF THE INTERVERTEBRAL DISC}

Summer Gibbs-Strauss ${ }^{1}$, Carrie Vooght ${ }^{1}$, Kenneth M. Fish ${ }^{3}$, Tiberiu M. Siclovan $^{3}$, Nicole E. Barnhardt ${ }^{3}$, Cristina A. Tan Hehir ${ }^{3}$, John V. Frangioni ${ }^{1,2}$ ${ }^{1}$ Hematology/Oncology, Beth Israel Deaconess Medical Center, Boston, MA, USA, ${ }^{2}$ Radiology, Beth Israel Deaconess Medical Center, Boston, MA, USA, ${ }^{3}$ Molecular Imaging and Diagnostic Advanced Technology Program, GE Global Research, Niskayuna, NY, USA.

Background: Low back pain is a prevalent medical condition that is difficult to diagnosis and treat. Current imaging methods are unable to correlate pain reliably with spinal structures, and surgical removal of painful damaged or degenerating discs is technically challenging. The intervertebral disc consists of the nucleus pulposus, annulus fibrosus and cartilaginous end-planes. Its composition changes significantly with age, creating difficulty in differentiating structural changes related to age versus those associated with degeneration and back pain. A contrast agent specific for the intervertebral disc could assist in the detection, diagnosis, and surgical treatment of low back pain. Materials and Methods: Physicochemical and optical properties of FM-type fluorophores were measured. Structure-activity relationships between chemical structure and in vivo uptake in nerves and other spinal structures were established. Two novel FM fluorophores, with improved optical properties for imaging the annulus fibrosus of intervertebral discs, were synthesized and evaluated in mice, rats, and pig. Results: After a single systemic injection, 8 of 8 FM fluorophores provided high contrast imaging of the trigeminal ganglia, while 6 of 8 provided high contrast imaging of the dorsal root ganglia. Unexpectedly, 3 of 8 FM fluorophores also provided high contrast imaging of annulus fibrosus tissue of the intervertebral discs in mice, rats, and pig. The intervertebral disc-specific fluorescence of the $3 \mathrm{FM}$ fluorophores was confirmed through dual channel fluorescence imaging with a co- 
injected near-infrared fluorophore specific for hydroxyapatite of bone. These findings were also confirmed histologically in mice, rats and pig. The chemical structural motif corresponding to annulus fibrosus uptake was determined. Conclusions: We present the first known contrast agent specific for the annulus fibrosus of intervertebral discs, and identify the chemical structural motif that mediates uptake. The FM fluorophores could be used for image-guided surgery to assist in the removal of the intervertebral disc. The FM fluorophores also lay the foundation for nonoptical diagnostic agents since the chemical structural motif that corresponds to annulus fibrosus uptake is amenable to derivatization for magnetic resonance imaging and positron emission tomography.

\section{$\mathbf{J 1 4 8}$}

\section{OPTICAL IMAGING OF TUMORS USING A NEAR-INFRARED SYNTHETIC PROBE FOR CELL DEATH}

Bryan A. Smith ${ }^{1}$, Walter J. Akers ${ }^{2}$, W. Matthew Leevy ${ }^{1}$, Andrew J. Lampkins $^{1}$, Shuzhang Xiao ${ }^{1}$, William R. Wolter ${ }^{3}$, Mark A. Suckow ${ }^{3}$, Samuel Achilefu ${ }^{2}$, Bradley D. Smith ${ }^{1}$
${ }^{1}$ Department of Chemistry and Biochemistry, University of Notre Dame, Notre Dame, IN, USA, ${ }^{2}$ Department of Radiology, Washington University School of Medicine, St. Louis, MO, USA, ${ }^{3}$ Freimann Life Science Center, University of Notre Dame, Notre Dame, IN, USA.

The ability to non-invasively detect cell death in vivo could potentially revolutionize the diagnosis and treatment of tumors. Annexin V, a protein that binds exposed phosphatidylserine (PS) during cell death, is commonly used to determine drug efficacy and cell viability in vitro; however, its poor clearance has limited its use in vivo. We have developed a near-infrared probe containing a carbocyanine dye conjugated to a zinc (II)-2,2-dipicolylamine ( $\mathrm{Zn}$ (II)-DPA) coordination complex, which binds PS on apoptotic and necrotic cells. Using tumor-bearing mouse and rat models, the probe (PSS794) was visualized at the site of the tumor. Ex vivo analysis revealed heterogeneity of probe localization in the tumor, with most of the probe concentrated in the necrotic core. The ability to detect cell death in the tumor could have important implications for imaging tumor drug delivery and efficacy.
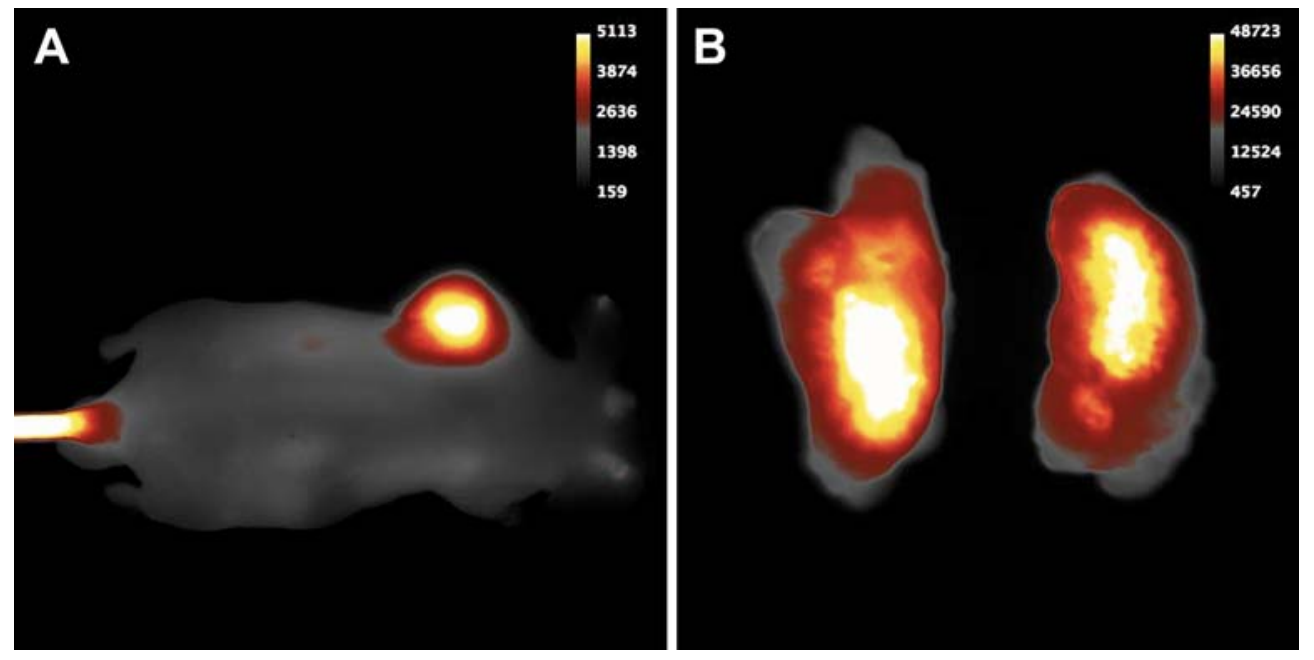

Figure 1. Image of an athymic mouse bearing a subcutaneous EMT-6 mammary tumor 24 hrs post-administration of PSS794 (A). Ex vivo image of a PAIII prostate tumor $24 \mathrm{hrs}$ post-injection of PSS794 (B). The tumor was sliced in half and placed so that the central core was facing the camera. Images were acquired using a Kodak image station.

\section{$\mathbf{J 1 4 9}$}

\section{NEAR-INFRARED FLUORESCENCE IMAGING OF MELANOCORTIN 1 RECEPTOR EXPRESSION WITH A CY5.5-LABELED A-MELANOCYTE-STIMULATING HORMONE ANALOG}

Hongguang Liu ${ }^{1,2}$, Gang Ren ${ }^{1}$, Zheng Miao ${ }^{1}$, Peizhen $\mathrm{Han}^{2}$, Sanjiv S. ${\text { Gambhir }{ }^{1} \text {, Zhen Cheng }}^{1}$

${ }^{1}$ Molecular Imaging Program at Stanford (MIPS), Department of Radiology and Bio-X Program, Stanford University, Palo Alto, CA, USA, ${ }^{2}$ Institute of Radiation Medicine, Chinese Academy of Medical Sciences, Peking Union Medical College, Tianjin, China.

The $\alpha$-melanocyte-stimulating hormone $(\alpha-\mathrm{MSH})$ receptor (melanocortin type 1 receptor, or MC1R) is known to be overexpressed in most of murine and human melanoma, making it a promising molecular target for melanoma imaging and therapy. The purpose of this study was to evaluate a near-infrared fluorophore, Cy5.5 conjugated $\alpha$-MSH analog (CyMSH,
Fig.a), as a contrast agent to visualize tumor MC1R expression in vivo. Methods: $\alpha$-MSH analog containing $\alpha$-MSH core sequences, His-D-PheArg-Trp, was designed and synthesized using Fmoc/HBTU chemistry on a solid-phase peptide synthesizer and conjugated with Cy5.5 through the Nterminal cysteine. The binding affinity was determined using a competitive receptor binding assay. Melanoma B16F10, TXM13 and A375M, which has high, medium and low MC1R expression, respectively, were chosen to evaluate the MC1R imaging profiles of CyMSH in vitro and in vivo. Results: CyMSH was successfully synthesized and displayed high MC1R binding affinity $(0.6 \mathrm{nmol} / \mathrm{L})$. In vitro cell fluorescence imaging study revealed that the probe showed high, medium and low cell staining in B16F10, TXM13 and A375M cell lines, respectively, which was in consistence with their receptor expression levels. Co-incubation the probe with a large excess of the $\alpha$-MSH peptide NDP specifically inhibited the probe uptakes in B16F10 and TXM13 cells. In vivo optical imaging detected little fluorescence in B16F10 tumor (Fig.b), which was mainly caused by the high melanin content in B16F10 tumor that absorbed nearly all excitation and emission NIR lights. To the contrary, melanoma TXM13 with much 
less melanin content and medium level of MC1R expression could be clearly visualized with CyMSH (Fig.c). Finally, amelanotic A375M with the lowest MC1R expression showed poor tumor/normal contrast (Fig.d).
Conclusion: This study suggests that the combination of the specificity of $\alpha$ MSH peptide with NIR fluorescence detection may be applied to molecular imaging of MC1R expression in melanoma with low melanin content.

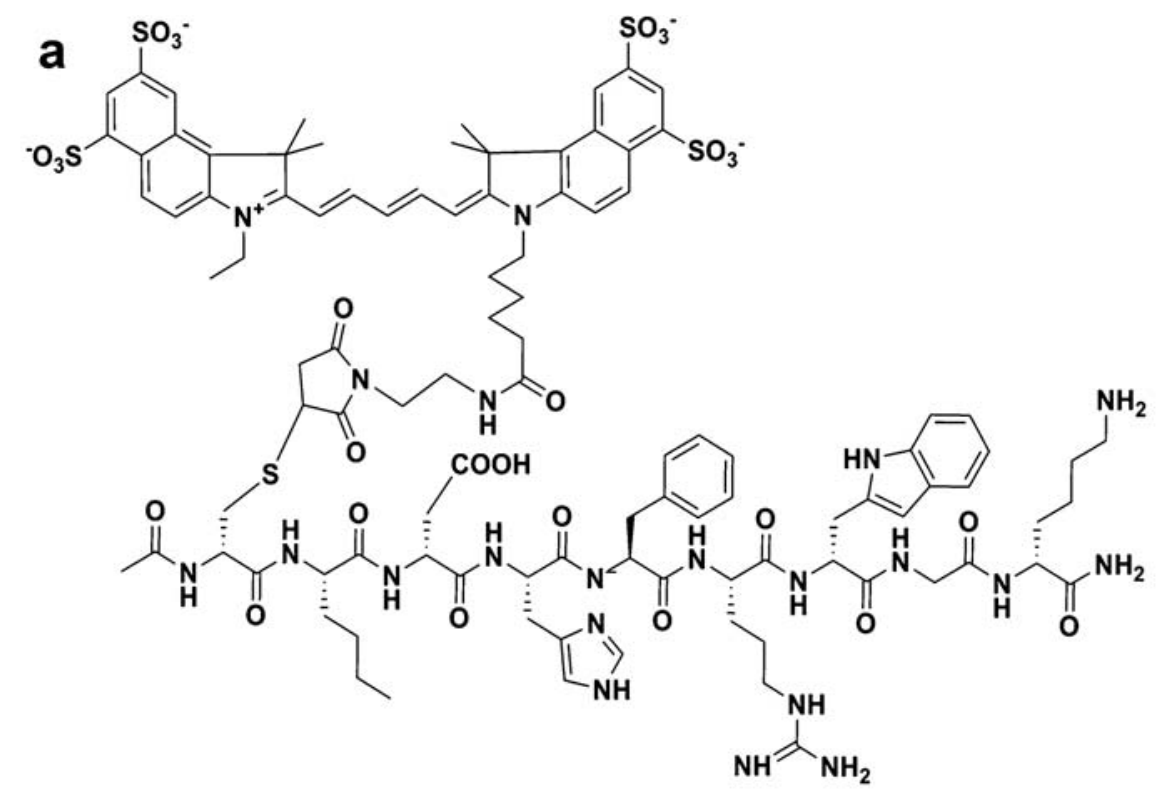

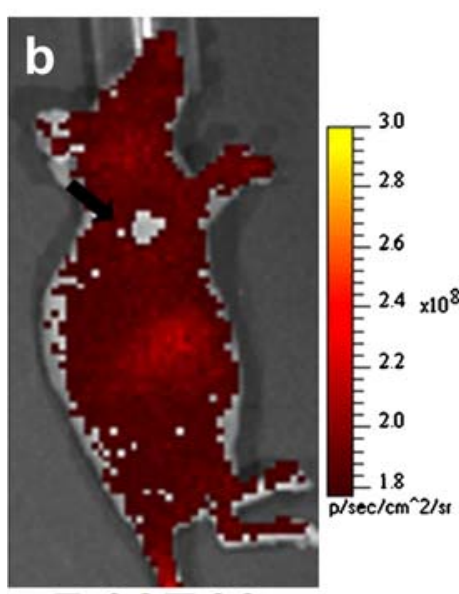

B16F10

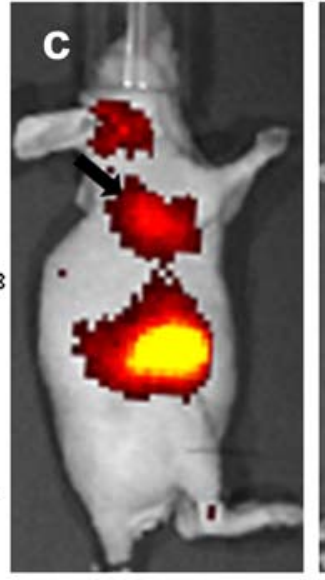

TXM13

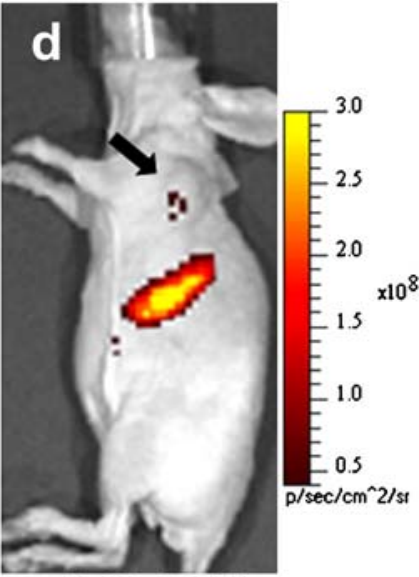

A375M
$\mathbf{J 1 5 0}$

BLACK HOLE QUENCHER 3 (BHQ-3) QUENCHES FLUORESCENCE FROM IRDYE800 BUT IS UNSTABLE IN VIVO-A TALE OF TWO COMPOUNDS

Karen E. Linder, Edmund Metcalfe, Palaniappa Nanjappan, Thangavel Arunachalam, Tina Marie Skedzielewski, Kimberly Ramos, Adrian Nunn, Michael Tweedle, Rolf E. Swenson

Ernst Felder Laboratories, Bracco Research USA Inc., Princeton, NJ, USA.

Introduction: Fluor/quencher-containing protease-cleavable peptides, if properly designed, are optically quenched until cleaved by their target protease. Enzymatic cleavage causes an increase in fluorescence due to separation of the fluor $(\mathrm{Fl})$ and quencher $(\mathrm{Q})$. This strategy has been successfully used with many Fl/Q pairs, but little has been reported with IRDye800, a near IR fluor that has shown utility for in vivo optical imaging. We explored the use of the highly conjugated azo-containing compound BHQ-3 as a contact quencher for IRDye800 and find unexpectedly that IRDye800/BHQ-3 is a suitable Fl/Q pair, despite the lack of proper spectral overlap for fluorescence resonance energy transfer (FRET) applications. However, in vivo instability was observed. Methods: IRDye800-PLGLK(BHQ-3)AR-NH $\mathrm{N}_{2}$ (1) and a "non-cleavable" control IRDye800-(1,13-diamino-4,7,10-trioxatridecane)-BHQ-3 (2) were prepared and characterized by LC/MS and UV/Vis/Fl spectroscopy. For in vitro cleavage studies, compounds $(0.5 \mathrm{nmol}, 5 \mu \mathrm{M})$ were incubated at RT in a 96-well plate for $60 \mathrm{~min}$ with $30-40 \mathrm{ng}$ of various activated matrix metalloproteases in MMP buffer. Cleavage as evident from increase of $\mathrm{Fl}$ signal at $780 \mathrm{~nm}$ was monitored in a plate reader. For in vivo metabolism studies, $40 \mathrm{nmol}$ of 
$\mathbf{1}$ or $\mathbf{2}$ was injected via tail vein into normal male Balb/c mice $(n=4)$. Serial plasma samples were collected from 3-60 min post injection. Plasma and urine extracts were analyzed by LC/MS. Stability and recovery from mouse plasma was also tested. Results: Both $\mathbf{1}$ and $\mathbf{2}$ showed expected mw and spectral peaks for both IRDye800 and BHQ3. Treatment of $\mathbf{1}$ with MMP-13 caused $\mathrm{Fl}$ to rise from $30 \pm 4 \mathrm{RFU}$ at $\mathrm{t}=$ 0 to $1723 \pm 492$ after $60 \mathrm{~min}(\mathrm{n}=3)$, a 60 -fold increase in Fl with enzyme treatment. Slower cleavage was seen with other MMPs. Under similar conditions, 2 had values of 119 and 123 at $\mathrm{t}=0$ and $60 \mathrm{~min}$, revealing incomplete quenching but no metabolism by MMPs. In vivo studies with $1(n=3)$ showed peptide cleavage in normal mice. Most metabolites were fluorescent and did not contain BHQ-3. Significant metabolism of 2 in normal mice $(n=4)$ was evident by 3 min P.I. By $20 \mathrm{~min}$, almost no unmetabolized 2 remained. LC/MS of plasma extracts showed the metabolite had a mw of 1394, consistent with azo bond cleavage in the BHQ-3 moiety. Conclusion: Although IRDye800/ BHQ-3 construct 1 showed good contact quenching prior to enzymatic cleavage, the compound was unstable in normal mice. Metabolism studies with 2 revealed in vivo instability of the azo bond in BHQ-3 quencher. Thus, BHQ-3 should be used with caution in vivo.

\section{$\mathbf{J 1 5 1}$}

\section{IMAGING LYMPHATICS WITH A VARIETY OF NEAR INFRARED-LABELED OPTICAL AGENTS \\ Joy L. Kovar ${ }^{1}$, Melanie A. Simpson ${ }^{2}$, D. M. Olive ${ }^{1}$}

${ }^{1}$ Molecular Biology, LI-COR Biosciences, Lincoln, NE, USA, ${ }^{2}$ Biochemistry, University of Nebraska-Lincoln, Lincoln, NE, USA.

The lymphatic system is a specialized network of the circulatory system responsible for fluid balance in the body, absorption and transport of fats and fatty acids, and supporting the immune response. Enhanced permeability and retention (EPR) is a phrase that describes phenomena of the tumor microenvironment, in which vasculature is relatively discontinuous, allowing molecules to diffuse from the bloodstream into the surrounding tumor tissue. In addition, the lymphatic drainage for these EPR regions is poor, allowing molecules to accumulate. Near infrared (NIR) imaging technology can be used noninvasively to visualize accumulation of labeled fluorophores within tumor tissue, providing sensitive and high resolution detection of tumors in mice. Imaging of fluorophores such as IRDye $800 \mathrm{CW}$ (em: $789 \mathrm{~nm}$ ) or IRDye 700DX (em: $700 \mathrm{~nm}$ ) spectrally minimizes interfering autofluorescent signals that generate background noise, which improves image resolution and quality. Images presented here demonstrate the EPR effect in the tumor microenvironment, tracking of lymph flow, and identification of lymph nodes of interest with a variety of NIR-labeled optical agents.

\section{$\mathbf{J 1 5 2}$}

PHOTONIC CRYSTAL PROBE FOR SPECIFIC PROTEIN DETECTION USING IN EXTREMELY LOW SAMPLE VOLUME Murukeshan V. Matham ${ }^{1}$, Saraswathi Padmanabhan ${ }^{1}$, Shinoj vengalathundakal $\mathrm{K}^{1}$, Padmanabhan Parasuraman ${ }^{2}$

${ }^{1}$ Mechanical and Aerospace Engineering, Nanyang Technological University, SINGAPORE, Singapore, ${ }^{2}$ SBIC, Singapore Bioimaging Consortium, Singapore, Singapore.

The recognition of specific protein based on immuno binding in $50 \mathrm{~nL}$ sample volume was achieved. MCF-7 breast carcinoma cells were lysed and immobilized inside a hollow core photonic crystal fiber. After immobilization the fiber was first processed with Primary antibody (Estrogen Receptor alpha), later followed by Alexa ${ }^{\mathrm{TM}}$ Fluor 488 (Green fluorescent dye) and 555 (Red Fluorescent dye) labeled Goat anti-rabbit IgG (secondary antibody). The fluorescence fingerprints of the Estro- gen Receptor alpha protein were observed under fluorescence microscope and its optical characteristics were also analyzed using the spectrophotometer. The research is expected to find potential biosensoing and therapeutics applications.

\section{$\mathbf{J 1 5 3}$}

THE STUDY OF LYMPHATIC VASCULATURE USING NEAR INFRARED IMAGING AGENTS

Cynthia Davies-Venn ${ }^{1,2}$, Sunkuk Kwon ${ }^{1}$, Melissa B. Aldrich ${ }^{1}$, Joy L. Kovar $^{3}$, D. M. Olive ${ }^{3}$, Eva Sevick ${ }^{1}$

${ }^{1}$ Center for Molecular Imaging, University of Texas Health Science Center-Houston, Houston, TX, USA, ${ }^{2}$ Translational Biology and Molecular Medicine, Baylor College of Medicine, Houston, TX, USA, ${ }^{3}$ Biotechnology, LI-COR Biosciences, Lincoln, NE, USA.

Lymphedema and lipedema are disorders in which blockage of the lymphatics prevents the return of lymph to the blood, and leads to fluid accumulation in the extremities of the affected individual. The high sensitivity of near infrared (NIR) imaging provides a unique opportunity for the non-invasive observation of lymphatic channels and architecture, which can in turn provide information about the pathophysiology of lymphatic disease. Indocyanine Green (ICG), a widely available, FDA approved fluorophore has been used clinically to image the lymphatics in normal subjects and lymphedema patients (Rasmussen et al. Curr Opin Biotechnol. 2009). However, it is limited by its dim signal, low quantum efficiency and consequent requirement for high concentrations of dye to obtain an adequate signal in mice using non-intensified cameras. We have utilized several new imaging probes, which are conjugates of IRDye $800 \mathrm{CW}$, a stable NIR dye that shows a thirty- fold higher quantum efficiency than ICG, and has shown high sensitivity imaging in a variety of fluorescence applications. The conjugates have the added advantage of possessing characteristics which improve lymphatic entry and retention as compared to IRDye 800 alone. Probes include conjugates of an abundant serum protein, a peptide, and an ECM polysaccharide polymer which binds the LYVE-1 receptor in lymphatic vasculature. Initial results showed that unlike ICG, the conjugates did not show quenching at high concentrations, and had a brighter fluorescent intensity but comparable propulsive frequency to ICG. In vivo imaging in mice showed that the IRDye 800 conjugates were taken up via the lymphatic capillary plexus, and displayed propulsion through the conducting lymphatic vessels from the inguinal to the axillary lymph node. These results indicate that the conjugates have significant potential to provide high quality lymphatic imaging in microdose quantities. This work is supported in parts by R01 CA112679 and R01 HL092923

\section{$\mathbf{J 1 5 4}$}

\section{SYNTHESIS AND EVALUATION OF IFENPRODIL CONJUGATES AS FLUORESCENT PROBES FOR NMDA NR2B RECEPTOR CONFOCAL MICROSCOPY IMAGING} Cédric Bouteiller ${ }^{1}$, Javier Becerril-Ortega ${ }^{2}$, Laetitia Mony ${ }^{3}$, Olivier Nicole $^{2}$, Barre Louisa ${ }^{1}$, Alain Buisson ${ }^{2}$, Cecile Perrio ${ }^{1}$

${ }^{1}$ Laboratoire de Développements Méthodologiques en TEP, CI-NAPS, UMR 6232, CYCERON, Caen, France, ${ }^{2}$ Equipe Physiopathologie de la Synapse, CI-NAPS, UMR 6232, CYCERON, Caen, France, ${ }^{3}$ Laboratoire de Neurobiologie, Ecole Normale Supérieure, Paris, France.

The N-Methyl-D-aspartate receptor (NMDAR) is a ligand-gated cationic channel that plays a critical role in the regulation of neuronal communication and synaptic function in the central nervous system, including neurotoxicity associated with stroke and neurodegenerative (Alzheimer's and Parkinson's) diseases. Most native NMDARs appear 
to function as heterotetrameric channel assemblies composed of two glycine-binding NR1 and two glutamate-binding NR2 subunits. The subunit composition (NR2A versus NR2B) and compartmental localization (synaptic or extrasynaptic sites) of NMDARs in neurons affect channel activity supporting the hypothesis that the spatial distribution of scaffolding complexes critically determines the full spectrum of NMDARs signaling. With the aim of relating the function of distinct NMDARs populations to physiological and pathological processes for the understanding of cellular/synaptic mechanisms involved in learning, memory and certain central disorders, we undertook the development of fluorescent probes for NR2B receptor imaging. These latters were elaborated as covalent conjugates of the potent and selective noncompetitive, voltage-independent NR2B-selective antagonist ifenprodil \{4-[2-(4-benzylpiperidin-1-yl)-1-hydroxy-propyl]-phenol\}, with a fluorescent moiety. The benzylic hydroxy group in ifenprodil was replaced by an amine function for dye conjugaison via a spacer. The influence of the spacer and of the fluorophore (dansyl, coumarin or cyanine) on the activity at NRB-containing receptors was examined in a functional test by monitoring intracellular calcium concentration with videomicroscopy in HEK-293 cell transfected with NR1-1a and NR2B subunits and by electrophysiology. Results including chemistry, in vitro evaluation and imaging studies will be presented.<smiles>CC([C@H](O)c1ccc(O)cc1)N1CCC(Cc2ccccc2)CC1</smiles>

( \pm )-ifenprodil

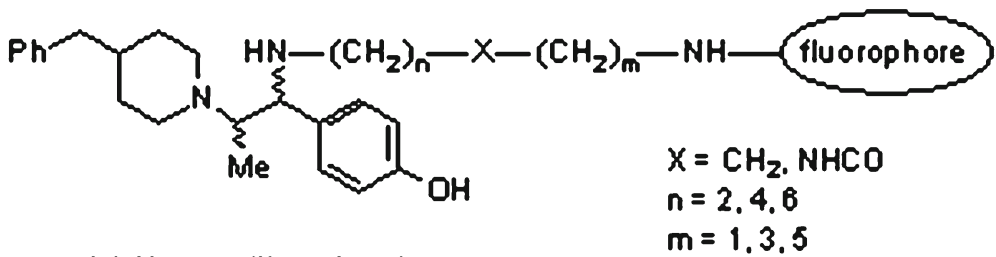

(t) ifenprodil conjugates
$\mathbf{J 1 5 5}$

DEVELOPMENT OF NOVEL "FUNCTIONAL" FLUORESCENT LABELING REAGENTS FOR VARIOUS LIGAND MOLECULES Tomoya Hirano $^{1}$, Jun Akiyama ${ }^{2}$, Hiroyuki Kagechika ${ }^{2,1}$

${ }^{1}$ Institute fo Biomaterials and Bioengineering, Tokyo Medical and Dental University, Tokyo, Japan, ${ }^{2}$ School of Biomedical Sciences, Tokyo Medical and Dental University, Tokyo, Japan.

Fluorescent ligand, which consists of fluorophore and ligand molecule, can visualize the localization of its target receptor protein in living cells, tissues and organisms by fluorescent microscopy, and it can also provide pharmacological information such as biding affinity. A large change of fluorescent properties of the fluorescent ligand by binding with its receptor would be ideal for highly sensitive detection or high contrast imaging. So we developed a novel "functional" fluorescent labeling reagents for various ligands, whose fluorescent intensity can be increased after binging with receptor. Our labeling reagent consists of four parts, fluorophore, flexible liker, quencher and amino reactive group, which forms covalent bond with ligand molecule. Before binding with the receptor, a fluorophore and quencher would intramolecularly interact with each other in a hydrophobic manner, resulting in quenching its fluorescence, and after binding, this interaction would be inhibited by some amino acid side chains around ligand binding domain, which can change local environment around fluorophore and quencher aggregate, resulting in restoring fluorescence. As a fluorophore, we chose tricarbocyanine dye, which has near-IR fluorescence, and tested four linkers and seven quencher candidates. We synthesized these conjugates and tested their fluorescent properties, and dabcyl group, as a quencher, containing conjugate showed the largest quenching ability in aqueous solution, and restoring its fluorescence completely by inhibiting the hydrophobic interaction between tricarbocyanine group and dabcyl group by adding cyclodextrin, which can include dabcyl group, or the change of the solvent polarity. Our labeling reagents can be utilized with various ligands, such as nuclear hormone receptor ligands.
$\mathbf{J 1 5 6}$

NEAR-INFRARED QUANTITATIVE FLUORESCENCE
IMAGING OF RENIN ACTIVITY IN KIDNEY TISSUE

Dorin V. Preda $^{1}$, Jun Zhang ${ }^{1}$, Kristine Vasquez ${ }^{1}$, Jeff Morin ${ }^{1}$, M. David Percival $^{2}$, Daigen $\mathrm{Xu}^{2}$, Dan J. McKay ${ }^{2}$, Michael Klimas ${ }^{3}$, Bohumil Bednar $^{3}$, Cyrille Sur ${ }^{3}$, Karen Madden ${ }^{1}$, Wael Yared ${ }^{1}$, Milind Rajopadhye ${ }^{1}$, Jeffrey D. Peterson ${ }^{1}$

${ }^{1}$ VisEn Medical, Inc., Bedford, MA, USA, ${ }^{2}$ Merck Frosst Centre For Therapeutic Research, Kirkland, QC, Canada, ${ }^{3}$ Merck Research Laboratories, West Point, PA, USA.

The Renin-Angiotensin System (RAS) is well studied for its regulation of blood pressure and fluid homeostasis. Renin, the rate-limiting enzyme of RAS, cleaves angiotensinogen to form angiotensin I (AngI). Ang-I is further cleaved by angiotensin converting enzyme, to produce the main effector of RAS, angiotensin II (Ang-II). Ang-II induces increases in blood pressure and fluid retention in the kidneys and stimulates cardiac hypertrophy. Abnormal RAS function has been linked to hypertension and cardiovascular disease. Our objective was to synthesize low molecular weight peptide substrates containing two internally quenched near infrared (NIR) fluorochromes, which are selectively activated by renin cleavage, providing an agent to noninvasively image and quantify the signal of renin activity in the kidneys by Fluorescence Molecular Tomography (FMT). To address selectivity of activation, the agents were tested for activation by a protease panel containing renin from various species, cathepsins (D and $G$ ) and human neutrophil elastase. Based on its activation profile, a rodent renin specific construct (VM3102) was selected for further in vivo studies. The pharmacokinetic profile of this agent was assessed in mice by measuring plasma fluorescence at different time points after intravenous injection with the agent. Renin activity in renal tissue was induced in mice by short-term low salt diet combined with diuretic treatment. This treatment resulted in upregulation of renin activity which was quantified by measuring the increase in the fluorescence signal in the kidneys as compared to the controls using FMT. These 
studies illustrate the potential of NIR fluorescence imaging to noninvasively quantify renin activity in tissues in vivo. This approach could provide new opportunities to assess disorders linked to altered renin/RAS activity and to closely monitor the efficacy of therapeutic treatments.
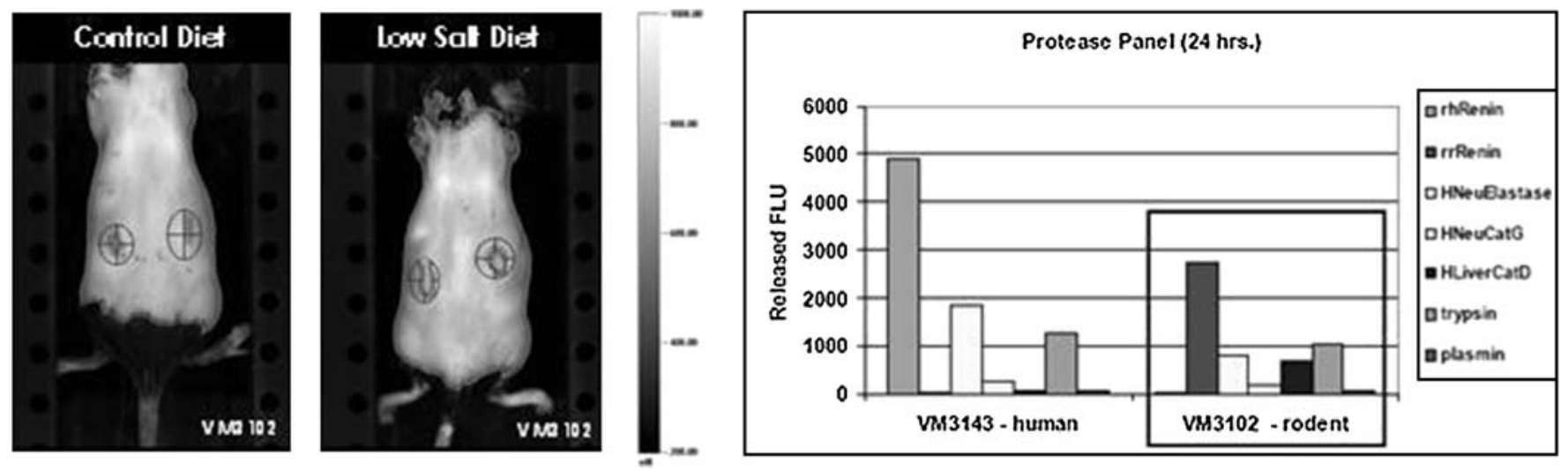

\section{NEAR-INFRARED DUAL-CHANNEL PROBES FOR IMAGING HYPOCHLOROUS ACID}

Fangwei Shao ${ }^{1,2}$, Jose-Luiz Figueiredo ${ }^{1,2}$, Cory Siegel ${ }^{1,2}$, Ralph Weissleder ${ }^{1,2}$, Scott Hilderbrand ${ }^{1,2}$

${ }^{1}$ Center for Molecular Imaging Research, Massachusetts General Hospital/Harvard Medical School, Boston, MA, USA, ${ }^{2}$ Center for Systems Biology, Massachusetts General Hospital/Harvard Medical School, Boston, MA, USA.

Hypochlorous acid, one of the most powerful natural oxidants is produced by myeloperoxidase (MPO), a heme enzyme secreted by neutrophils and macrophages that converts hydrogen peroxide to $\mathrm{HOCl}$ in the presence of chloride ions. Reactive oxygen species (ROS) like $\mathrm{HOCl}$ play numerous roles in many pathologic process, including cancer, atherosclerosis and arthritis. Fluorescent probes for hypochlorous acid, therefore, would be highly valuable for improved disease diagnosis. However, the lack of fluorescent, near-infrared (NIR) probes for selective detection of $\mathrm{HOCl}$ presents a signficiant obstacle to fluorescence-based imaging of MPO associated $\mathrm{HOCl}$. Two cyanine-based dyes (CyClO-1 and $\mathrm{CyClO}-2$ ) have been developed as fluorgenic probes for hypochlorous acid. The $\mathrm{Cy} 7$ analogs are prepared via a simple one-pot procedure in good yield. Both probes have excellent water solubility and NIR optical properties. In their reduced non-activated forms, $\mathrm{CyClO}-1$ and CyClO-2 absorb and emit above $800 \mathrm{~nm}$. After $\mathrm{HOCl}$ dependent oxidation, the absorption and fluorescence emission spectra of the probes blue-shift more than $120 \mathrm{~nm}$. This shift in fluorescence emission upon oxidation allows for independent monitoring of the probe distribution and activation via excitation of the probes at 640 and $750 \mathrm{~nm}$, respectively. A greater than 40-fold increase in fluorescence emission monitored at $720 \mathrm{~nm}$ is observed after oxidation of CyClO-2. Activation is also observed for both probes in the presence of MPO and $\mathrm{H} 2 \mathrm{O} 2$. The sensors are not activated by the MPO/H2O2 system when co-incubated with a MPO inhibitor, confirming that MPO-derived $\mathrm{HOCl}$ is the cause of oxidative activation. ROS screening demonstrates selective response to hypochlorous acid with little or no activation in the presence of common biological oxidants such as hydrogen peroxide, superoxide, hydroxyl radical, alkyoxyl radical, peroxynitrite, and nitric oxide. The selective activation by $\mathrm{HOCl}$ and NIR fluorescence makes these agents promising candidates for application to in vivo imaging of MPO activity.

\section{$\mathbf{J 1 5 8}$}

\section{NOVEL LONG-WAVELENGTH FLUORESCENT DYE SETA-633 FOR FLUORESCENCE LIFETIME IMAGING OF ANTIGEN-ANTIBODY REACTIONS}

Yevgen A. Povrozin ${ }^{1}$, Olga S. Kolosova ${ }^{1}$, Olena M. Obukhova ${ }^{1}$, Anatoliy L. Tatarets ${ }^{1}$, Vadim I. Sidorov ${ }^{1}$, Ewald Terpetschnig ${ }^{2}$, Leonid D. Patsenker ${ }^{1}$

${ }^{1}$ Organic Luminophores and Dyes, SSI "Institute for Single Crystals", Kharkov, Ukraine, ${ }^{2}$ SETA BioMedicals, Urbana, IL, USA.

We have developed a new long wavelength fluorescent label Seta633, which is available as free carboxylic acid, amino-reactive NHS ester and thiol-reactive maleimide. This dye exhibits a long-wavelength absorption maximum at $633 \mathrm{~nm}$, emission maximum at $644 \mathrm{~nm}$, very high extinction coefficient of 250,000 M-1 cm-1, quantum yield of $7 \%$, and a fluorescence lifetime of $0.25 \mathrm{~ns}$ measured in phosphate buffer $\mathrm{pH} 7.4$ in its free form. After covalent attachment to an antibody (human immunoglobulin, IgG) the quantum yield and fluorescence lifetime change significantly: quantum yields up to $26 \%$, and fluorescence lifetimes up to 1.49 ns were measured for these conjugates. These large changes make it a perfectly suitable label for the fluorescence lifetime based visualization of interaction of an antigen with an antibody. Tracing of such interaction is shown in the Figure: Seta-633-mono-NHS was labelled to biotin and used as a reporter molecule for the interaction with a specific antibody (anti-biotin antibody, affinity isolated). As a comparison the label was also treated with a non-specific antibody (IgG). The obtained results clearly reveal the potential of Seta-633 as a unique lifetime label: when bound to biotin (molecular mass, $\sim 244 \mathrm{Da}$ ) Seta-633 exhibits a short lifetime of $0.66 \mathrm{~ns}$ and upon 
interaction with the specific antibody (anti-biotin antibody, molecular mass, $\sim 160 \mathrm{kDa}$ ) the fluorescence lifetime gradually increases to $1.82 \mathrm{~ns}$ (a 2.8-fold increase compared to the initial value). Importantly, the sensitivity and selectivity of the specific interaction was not affected by the presence of bovine serum albumin (BSA), a known model transport protein with affinity for many types of ligands. The high selectivity and sensitivity of Seta-633 make it a perfect tracer and analytical tool in immunoreaction-based imaging or other binding assays of small molecular analytes using fluorescence lifetime as a read-out parameter.

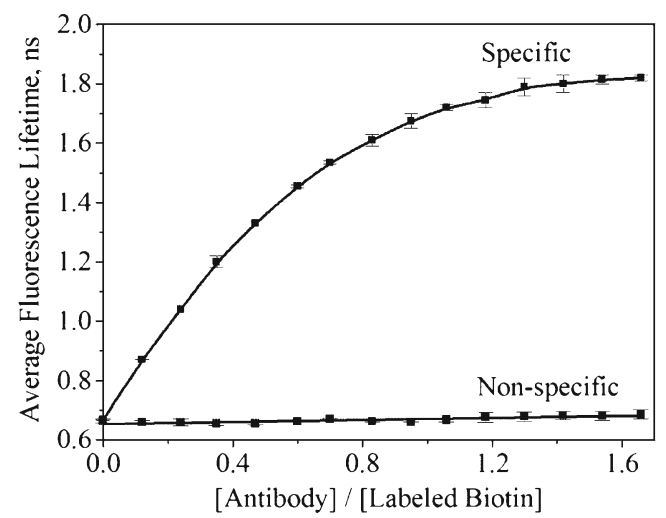

Changes in average fluorescence lifetime of Seta-633-biotin upon titration with specific (monoclonal) and non-specific antibody to biotin.

J159

LONG-WAVELENGTH DICYANOMETHYLENE SQUARAINE DYES FOR BIOLOGICAL IMAGING APPLICATIONS

Anatoliy L. Tatarets $^{1}$, Tatyana S. Dyubko ${ }^{1,2}$, Leonid D. Patsenker ${ }^{1,3}$, Ewald Terpetschnig ${ }^{3}$

${ }^{1}$ State Scientific Institution "Institute for Single Crystals" National Academy of Sciences of Ukraine, Kharkov, Ukraine, ${ }^{2}$ Institute for Problems of Cryobiology and Cryomedicine, National Academy of Sciences of Ukraine, Kharkov, Ukraine, ${ }^{3}$ SETA BioMedicals, LLC, Urbana, IL, USA.

We have synthesized a series of symmetrical and unsymmetrical dicyanomethylene squaraine dyes and investigated their spectral properties free in solutions and after interaction with Bovine Serum Albumine (BSA) and cells such as human fibroblasts and Saccharomyces Cerevisiae yeast. Depending on the nature of the terminal heterocyclic moiety these dyes absorb and emit in a wide spectral range. They have long-wavelength absorption and emission maxima in chloroform between 647 and $757 \mathrm{~nm}$, extinction coefficients between 104,000 and $208,000 \mathrm{M}^{-1} \mathrm{~cm}^{-1}$ and quantum yields as high as $80 \%$. All dicyanomethylene squaraine dyes exhibit additional absorption bands in the 378-396 nm range with extinction coefficients of about $29,000-44,000 \mathrm{M}^{-1} \mathrm{~cm}^{-1}$. Dye containing 5-nitroindolenine moiety absorbs not only in the red and UV but also in blue spectral region $(468 \mathrm{~nm})$ with extinction of $32,000 \mathrm{M}^{-1} \mathrm{~cm}^{-1}$. This makes dicyanomethylene squaraines also suitable for use with the blue (380, 405 and 470-nm) diode lasers excitation. Absorption and emission spectra in methanol are blue-shifted by 10-30 nm compared to chloroform and the quantum yield are somewhat lower. Squaraines form non-fluorescent aggregates in aqueous media. As a result the long-wavelength absorption band becomes broader and a new band appears. The fluorescence intensity of dyes substantially increases in presence of BSA with quantum yields as high as $95 \%$. These dyes were found to readily stain cells of different nature. The figure shows a human fibroblast cell stained with non-covalent squaraine probe. The obtained results evidence that the developed squaraine probes are perfectly suited for in-situ biological imaging and fluorescence-based quantification of proteins.
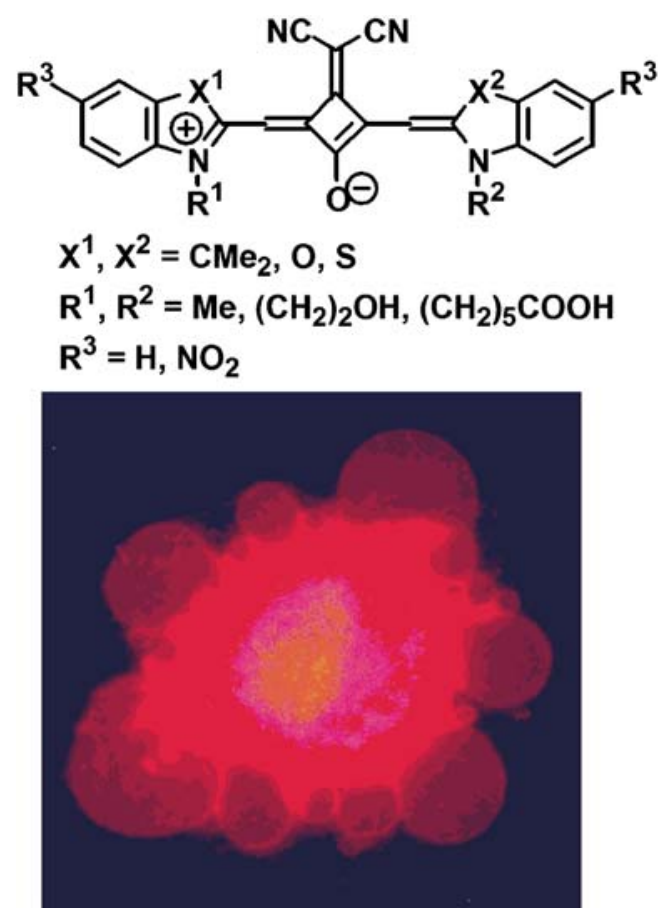

$\mathbf{J 1 6 0}$

\section{STABILIZED FRET PROBES FOR DETECTING NF-KAPPAB P50 PROTEIN}

Alexei A. Bogdanov, Surong Zhang, Valeriy G. Metelev

Radiology, University of Massachusetts Medical School, Worcester, MA, USA.

We previously synthesized fluorescence resonance energy transfer (FRET) duplex oligonucleotide (ODN) probes encoding transcription factor NF-kappaB p50 binding site by conjugating a pair of near-infrared fluorochromes (Cy5.5, donor, and IRDye800CW, acceptor) to novel internucleoside linkers. These ODN probes were successfully used for optical sensing of NF-kappaB p50 protein-ODN duplex interactions in vitro. We further modified the structure of ODN probes by using 3'- and 5'-hybrid tetraphosphorothioate (PS)/ phosphodiester (PO) hybrid ODNs (Figure) for stabilizing the probes against the degradation by exonucleases, which are responsible for the instability of ODN probes in living cells. The properties of hybrid PO/PS ODNs' were investigated to determine the efficiency of modification with fluorochromes, melting temperatures, and the rate of degradation by Exo III and DNAse I. We further investigated the specificity of transcpription 
factor NF-kappaB p50 protein binding to the obtained probes, as well as nonspecific protein binding. The FRET efficiency was 75$80 \%$. The hybrid PO/PS ODNs had approximately 10-degree lower melting temperatures than PO ODNs duplex probes. The efficiency of FRET in PO/PS probes did not change in the presence of Exo III, whereas the PO FRET probe showed rapid increase of donor fluorescence indicating duplex degradation. Randomly cleaving DNAse I resulted in rapid loss of FRET in both $\mathrm{PO}$ and $\mathrm{PO} / \mathrm{PS}$ probes. The binding of recombinant purified NF-kappaB p50 protein dimers was verified by using electrophoretic mobility shift assay. We determined that at the ratio of p50:ODN (12:1, molar) $92 \%$ of PO duplex was bound to NF-kappa B p50. Non-specific (scrambled) PO/PS ODN probe binding was at the level of $5-15 \%$. Therefore, hybrid $\mathrm{PO} / \mathrm{PS} \mathrm{ODN}$ reporter probes retain the specificity of PO reporters but had a higher stability in the presence of a model exonuclease.
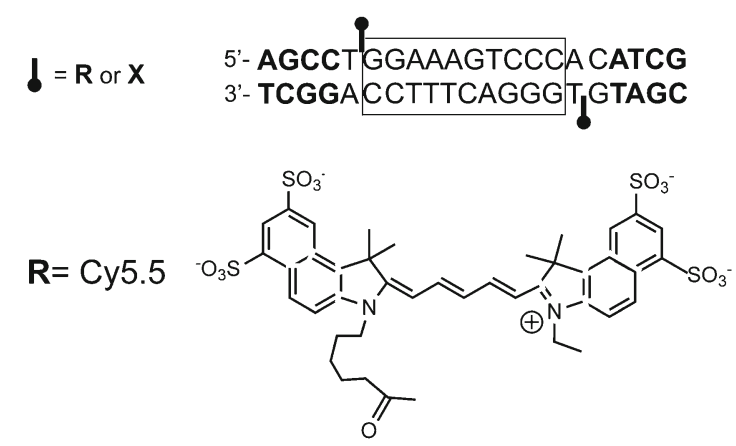

Donor

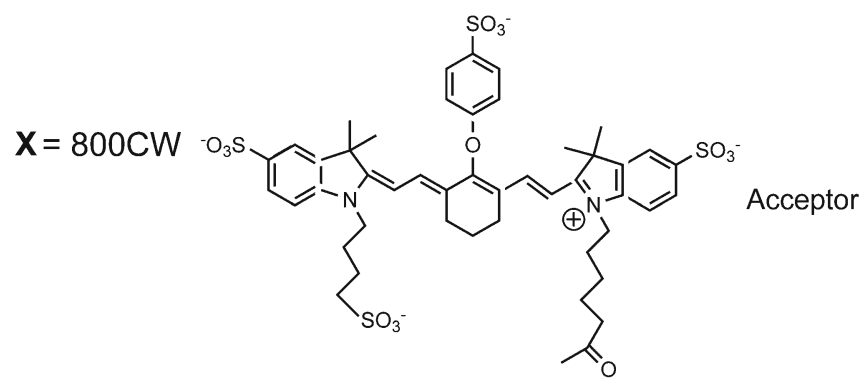

J161

EVOLUTION OF GROUP 14 RHODAMINES AS A PLATFORM FOR FAR-RED TO NIR EMITTING FLUORESCENCE PROBES IN COMBINATION WITH PET STRATEGY

Yuichiro Koide $^{1,2}$, Yasuteru Urano ${ }^{1}$, Tetsuo Nagano ${ }^{1,2}$

${ }^{1}$ Pharmaceutical Science, The University of Tokyo, Tokyo, Japan, ${ }^{2}$ CREST, JST, Tokyo, Japan.

Far-red to NIR emitting fluorescent dyes enjoy great deal of interest because of their possible application for in vivo imaging and several attractive features for biological applications, for example, the background biological autofluorescence from serum, proteins, and other macromolecules, problems arising from scattered light, and the greatly reducible risk of cell damage by excitation light in this region. The wavelengths of absorption and emission of group 14 xanthenes and rhodamines, which was introduced silicon, germanium, or tin atom at 10 position of the xanthene chromophore, showed large bathochromic shift compared to original rhodamines, which is derived from the stabilization of LUMO energy levels by the $\sigma^{*}-\pi^{*}$ conjugation between group 14 atom-C (methyl) $\sigma^{*}$ orbitals and a $\pi^{*}$ orbital of fluorophore. Those group 14 xanthenes and rhodamiens possess virtue of original rhodamines, including high quantum efficiency in aqueous media $\left(\Phi_{\mathrm{fl}}=\right.$ $0.3 \sim 0.45$ ), tolerance to photobleaching, high water solubility, and so on. We found that, unlike other NIR emitting fluorescence dyes, group 14 rhodamines have high values of reduction potentials, and, therefore, we speculated that they could be controlled their NIR fluorescence through PeT process. We carefully investigated whether group 14 rhodamines could be highly activated by PeT strategy, using newly synthesized SiR-derivatives bearing various electron density of the benzene moiety which was tuned in a fine manner by introducing methyl, methoxy, and dimethylamine groups into benzene moiety, and, as results, we found that $\mathrm{Si}-\mathrm{Rhodamine}(\mathrm{SiR})$ and Ge-Rhodamine (GeR) can be reached to nearly equal to 0 in $\Phi_{\mathrm{fl}}$ through PeT process, and that the threshold level between fluorescence on and off lay around 1.3-1.5 V for the SiRs, which is about $0.1 \mathrm{~V}$ lower than that of TokyoGreens. That is to say, SiR and GeR are able to be drastically activated greater than 100-fold in its fluorescence through PeT strategy. As application of this strategy for developing NIR fluorescence probes, we actually designed and developed two kinds of novel fluorescence probes in far-red to NIR region, $\mathrm{pH}$-sensor for acidic environment and $\mathrm{Zn}^{2+}$ sensor.

\section{$\mathbf{J 1 6 2}$}

MULTI-COLOR LUMINESCENCE IMAGING OF DUAL INTERACTION OF SMADS IN LIVING XENOPUS EMBRYO $\underline{\text { Naoki Hida }}^{1}$, Masaki Takeuchi ${ }^{1}$, Mayuri Tashiro ${ }^{1}$, Chiyo Takagi ${ }^{2}$, $\overline{\text { Naoto Ueno }}^{2}$, Takeaki Ozawa ${ }^{1}$

${ }^{1}$ University of Tokyo, Tokyo, Japan, ${ }^{2}$ National Institute for Basic Biology, Okazaki, Japan.

Describing networks of protein-protein interactions is very important because the networks play key roles in numerous crucial biological processes. Protein-fragment complement assay (PCA) is an effective methodology to assess protein-protein interactions in living subjects of interest. Particularly the assays using fluorescent proteins are powerful techniques, but they do not directly track interactions or enable quantitative analyses. The chromophore formation of fluorescent proteins and the irreversible reaction of the fragments' complementation limit temporal analysis of proteinprotein interactions. By contrast, PCAs using bioluminescent proteins can overcome those drawbacks. We herein describe an imaging method for real-time analysis of Smad1-Smad4 and Smad2-Smad4 interactions using multicolor luciferases with different spectral characteristics. We develop a carboxy-terminal fragment (McLuc) engineered from a click beetle luciferase, which has the ability to complement multiple $\mathrm{N}$-terminal luciferases. The McLuc were connected directly with Smad4 and N-terminal fragments of Click Beetle Red luciferase $(605 \mathrm{~nm})$ and Emerald luciferase $(535 \mathrm{~nm})$ were connected with Smad1 and Smad2 respectively. We succeeded in imaging of Smad1-Smad4 and Smad2-Smad4 interactions by using band-pass filters $(536 \pm 10 \mathrm{~nm}$ and $624 \pm 25 \mathrm{~nm}$ ) in early developing stages of a single living Xenopus laevis embryo. The specific patterns of the bioluminescence were identical to those obtained from the independent experiments of imaging of each Smads interaction. We demonstrated the value of this method by application of dual protein-protein interactions with shared protein using PCA based on multicolor luciferases. This technique supports quantitative analyses and imaging of versatile protein-protein interactions with a selective luminescence wavelength in opaque or strongly auto-fluorescent living subjects like Xenopus embryo. 


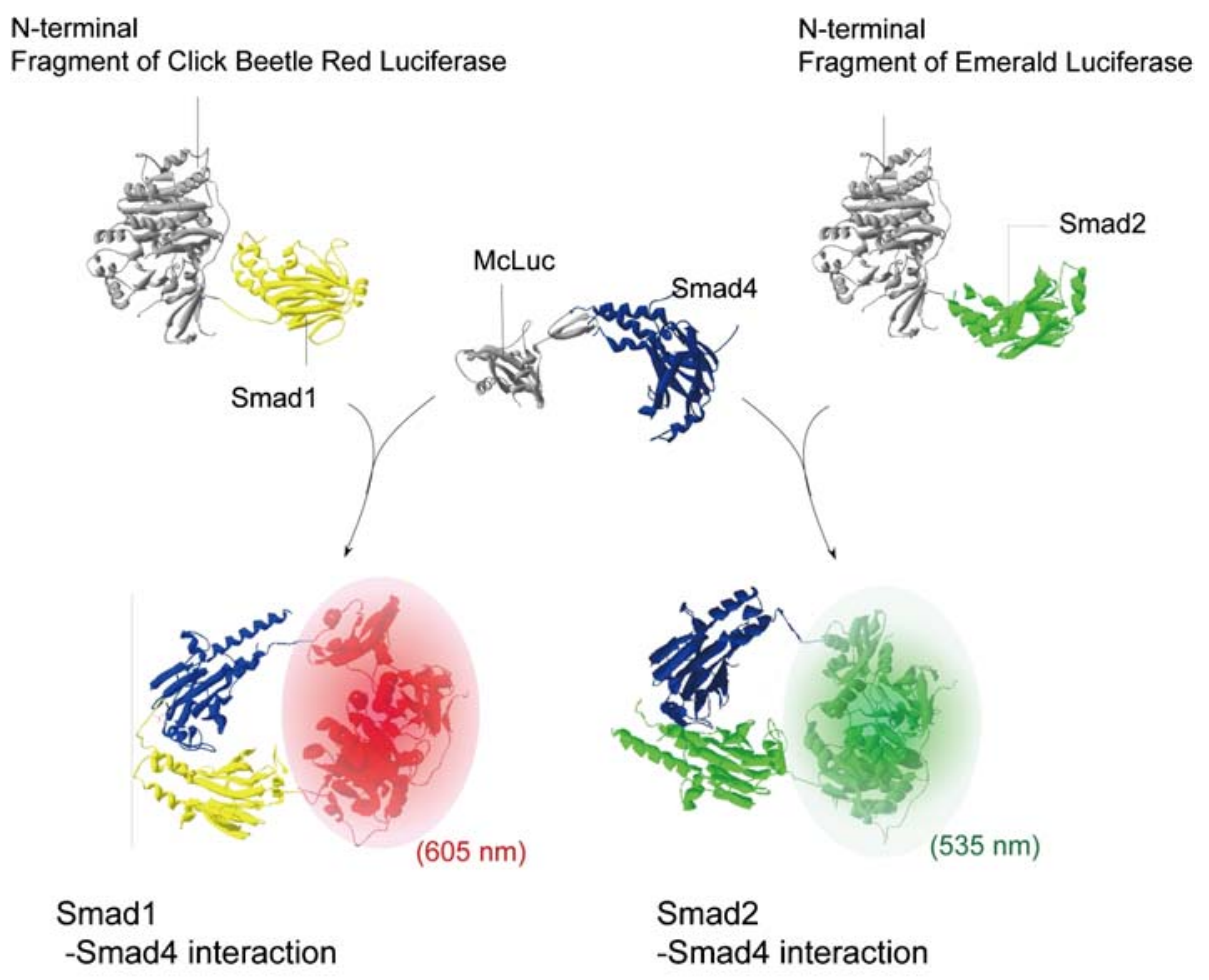

Schematic design of Smads probes

$\mathbf{J 1 6 3}$

\section{LONG-WAVELENGTH, NEXT GENERATION FLUORESCENT PROBES AND LABELS FOR BIOLOGICAL IMAGING AND SINGLE MOLECULE SPECTROSCOPY WITH EXTREME CHEMICAL AND PHOTOCHEMICAL STABILITY}

Leonid D. Patsenker ${ }^{1,2}$, Oleksii P. Klochko ${ }^{1}$, Yuliia O. Kudriavtseva ${ }^{1}$, Yevgen A. Povrozin ${ }^{1}$, Ewald Terpetschnig ${ }^{2}$

${ }^{1}$ SSI "Institute for Single Crystals", NAS Ukraine, Kharkiv, Ukraine,

${ }^{2}$ SETA BioMedicals, LLC, Urbana, IL, USA.

We have developed novel, long-wavelength absorbing and emitting hydrophobic probes and water-soluble reactive labels of the SeTau series. These extremely bright dyes have certain advantages as compared to Alexa 647 and Alexa 660, currently the brightest standards available on the market: The Figure below shows that SeTau dyes exhibit extremely high photo- and chemical stability in presence of oxidizing agents such as ozone and hydrogen peroxide. The spectral and photophysical characteristics of selected SeTau and Alexa dyes and their IgG conjugates measured in phosphate buffer $\mathrm{pH} 7.4$ are provided in the Table. SeTau dyes have extinction coefficients comparable to the Alexa dyes. SeTau-640 and SeTau-665 are the dyes with extremely high quantum yields (Q.Y.s) and their mono-exponential fluorescence lifetimes $(\tau)$ of $2.4 \mathrm{~ns}$ and $3.5 \mathrm{~ns}$ are several times longer compared to Alexa 647, which is an advantage for their use in the FLT-based assays and FLIM applications. SeTau-665 has an additional absorption band in the Vis / Near UV and therefore is excitable not only with 635 and 670-nm diode lasers but also with the 370-nm, and 405-nm lasers. SeTau-665 also exhibits an extremely large Stokes' shift of $54 \mathrm{~nm}$, which is about 3-times larger than that of Alexa $647(19 \mathrm{~nm})$ and about twice that of Alexa 660 $(27 \mathrm{~nm})$. The data show that SeTau dyes are truly advanced probes and labels for biological species (proteins, immunoglobulins, oligonucleotides, peptides), cells and drugs and are expected to have widespread use in biological imaging, single molecule spectroscopy, microarrays, proteomics, immunology, cytology, genomics, drug screening, cellular and molecular biology. Spectral characteristics in phosphate buffer $\mathrm{pH} 7.4$

\begin{tabular}{|c|c|c|c|c|}
\hline Dye & $\lambda_{\max }(\mathrm{Abs})[\mathrm{nm}]$ & $\lambda_{\max }(\mathrm{Em})[\mathrm{nm}]$ & Q.Y. [\%] & $\tau[\mathrm{ns}]$ \\
\hline SeTau-640 & 640 & 656 & 40 & 2.4 \\
\hline SeTau-655 & 655 & 673 & 25 & 0.9 \\
\hline SeTau-655 - IgG conjugate $(\mathrm{D} / \mathrm{P}=1)$ & 657 & 675 & 31 & 1.8 \\
\hline SeTau-665 & 665 & 719 & 56 & 3.5 \\
\hline Alexa 647 & 649 & 668 & 32 & 1.0 \\
\hline Alexa 647 - IgG conjugate $(\mathrm{D} / \mathrm{P}=1)$ & 652 & 670 & 42 & 1.5 \\
\hline Alexa 660 & 663 & 690 & 37 & 1.2 \\
\hline
\end{tabular}




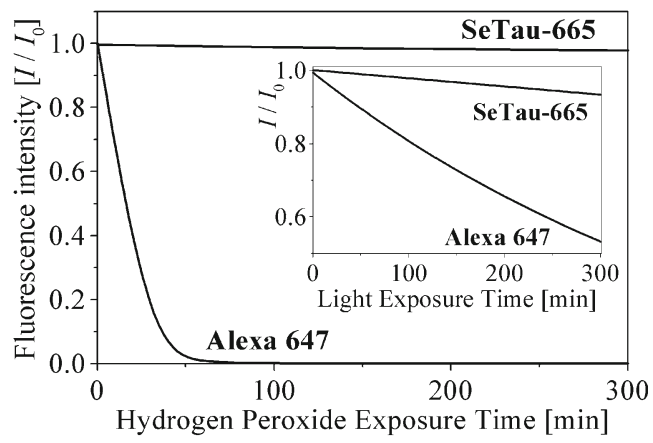

$\mathbf{J 1 6 4}$

\section{NOVEL HYPOXIA-SENSITIVE PROBES WITH NEAR- INFRARED FLUORESCENT EMISSION}

Kazuki Kiyose $^{1,2}$, Tetsuo Nagano ${ }^{1,2}$

${ }^{1}$ Department of Pharmaceutical Sciences, The University of Tokyo, Tokyo, Japan, ${ }^{2}$ CREST, JST, Tokyo, Japan.

Hypoxia, which is caused by an inadequate oxygen supply, is a feature of several disease state, including cancer, cardiopathy, and vascular diseases. Among them, solid tumor hypoxia is especially important, because it has been closely related to the resistance to cancer therapies, such as chemotherapy or radiotherapy. Therefore, hypoxia-specific molecular probes as diagnostic agents have been needed. Indeed, many groups reported some hypoxia-selective probes, representatively for positron emission tomography (PET), and received good results. However, there are few fluorescent probes for detecting hypoxia. Because fluorescence imaging has fine temporal and spatial resolution, and can be safely performed with simple devices and facilities, hypoxia-selective fluorescent probe can be a superior diagnostic agent for solid tumor. Then, we focused on hypoxia-selective bioreduction, which was demonstrated by various reductases, and designed and synthesized some fluorescent probes which become fluorescent when they reduced by reductases under hypoxic conditions. We previously developed some probes whose fluorescence intensity drastically increased after bioreduction by various reductases. However, all probes have visible absorption and emission, so they are not suitable for in vivo imaging. Then, we focused on near-infrared region (NIR), which is relatively poorly absorbed by biomolecules, hemoglobin as the principal absorber of visible light, water and lipids as the principal absorber of infrared light, and so it can penetrate deeply into tissues. This time, we developed two series of NIR fluorescent probes for detecting hypoxia based on two different molecular designs. In both design, carbocyanines were used as fluorophore, and FRET mechanism was used as fluorescence control method. In one series, fluorescence intensity increased by hypoxia-selective bioreduction, and in the other series, fluorescence ratio changed. We applied these probes to living cells and showed the possibility of live-cell hypoxia-selective fluorescence imaging using them. Now we are applying these probes to xenograft model mice, and trying to carry out in vivo tumor-hypoxia imaging.

\section{$\mathbf{J 1 6 5}$}

CyLoP-1: A NOVEL CELL PENETRATING PEPTIDE WITH REMARKABLE CELLULAR UPTAKE FOR CYTOSOLIC TARGETING

Ritu Mishra, Deepti Jha, Sven Gottschalk, Kamil Ugurbil, Joern Engelmann

High Field MR Center, Max Planck Institute for Biological Cybernetics, Tuebingen, Germany.
Endocytosis is a fundamental process through which essential biomolecules otherwise unable to permeate the plasma membrane are taken up by cells. This process is mainly used to fulfill the nutritional requirements of cells. The idea of exploiting this uptake mechanism to transfer exogenous macromolecules into cells is widely used. After crossing the cellular membrane via endocytosis most macromolecules enter the so-called endo-Iysosomal degradation pathway. This still is a severe hindrance for the development of therapeutics and diagnostic tools based on intracellular targets like. DNA or oligonucleotides. Cell penetrating peptides (CPPs) seemed to be promising tools for improving intracellular delivery of cargos. However, recent studies questioned the maintenance of biological activity of specific molecules following CPPassisted uptake. For most CPPs it has now been established that the mechanism of internalization mainly involves endocytosis/macropinocytosis, by this limiting access to targets located in the cytosol. Inspired by Crotamine, a toxin in snake venom, we developed the novel CPP CyLoP-1 that was able to pass through the cellular membrane barrier. Recently, we have shown its capability of delivering a MRI contrast agent into the cytosol. Herein we present the characterization of CyLoP-1 in comparison to other well-studied CPPs. The fluorescently labeled CPPs were assessed for cellular and cytosolic localization in 3T3 fibroblasts by fluorescence spectroscopy and microscopy. We also studied the distribution in vivo by injecting CyLoP-1 into mice followed by ex vivo observation of fluorescence in organs. CyLoP-1 required the presence of serum for solubility in medium and for cytosolic delivery. Without serum CyLoP-1 stuck to the outer membrane. In contrast, arginine-rich CPPs showed diffuse labeling in serum-free medium. Cytosolic delivery of CyLoP-1 was maintained at $4{ }^{\circ} \mathrm{C}$ indicating the uptake to be independent or complementary to endocytosis. In vivo studies showed that the peptide was readily dispersed into the blood stream after ip injection and it excretion via the kidneys after $4 \mathrm{~h}$. Apart from the kidneys and the bladder, many other organs (liver, spleen, lungs) were fluorescent. Interestingly, CyLoP-1 showed faint labeling in the brain as well. Our study reveals that CyLoP-1, a cysteine and arginine-rich peptide, is remarkably different in comparison to other available CPPs. Its peculiar cytosolic localization makes it a likely candidate for targeted delivery of drugs and agents for molecular imaging.

\section{$\mathbf{J 1 6 6}$}

\section{DESIGN AND SYNTHESIS OF A HIGHLY SENSITIVE FLUORESCENT CHEMOSENSOR FOR ZINC ION BASED ON AN ICT-BASED APPROACH}

Kenjiro Hanaoka $^{1,2}$, Tetsuo Nagano ${ }^{1,2}$

${ }^{1}$ Graduate School of Pharmaceutical Sciences, The University of Tokyo, Tokyo, Japan, ${ }^{2}$ JST CREST, Tokyo, Japan.

Fluorescence imaging is a powerful tool for the visualization of biological molecules in living cells or slice tissues, and is important for elucidating biological phenomena. On the other hand, zinc ( $\mathrm{Zn} 2+)$ is the second most abundant heavy metal ion after iron in the human body, and chelatable $\mathrm{Zn} 2+$ has many important roles in biological systems. However, its mechanisms of action are little known in comparison with those of other cations, such as $\mathrm{Ca} 2+, \mathrm{Na}+$, and $\mathrm{K}+$. Therefore, there is considerable interest in detecting chelatable $\mathrm{Zn} 2+$ in biological systems. Many Zn2+-selective fluorescent sensor molecules have been developed for measuring $\mathrm{Zn} 2+$ in living cells. Quinolinebased fluorescent sensor molecules (6-methoxy-8-(p-toluenesulfonamide)quinoline (TSQ) and its derivatives) are the most widely used fluorescent $\mathrm{Zn} 2+$ chemosensors. Recently, several fluorescein-based sensors have also been reported. These chemosensors employ the photoinduced electron transfer (PeT) mechanism, and have been successfully used for imaging intracellular $\mathrm{Zn} 2+$. On the other hand, if the fluorescence could be quenched completely in the absence of $\mathrm{Zn} 2+$, chemosensors would offer a better signal-to-noise ratio. So, our new 
approach to highly sensitive off-on fluorescence detection of $\mathrm{Zn} 2+$ is based on controlling internal charge transfer (ICT) within a dye platform to promote a change in the absorption wavelength upon $\mathrm{Zn} 2+$ binding. i. e., even if the sensor shows a weak fluorescence in the absence of $\mathrm{Zn} 2+$, the fluorescence of the sensor molecule can be further suppressed by selecting an appropriate excitation wavelength, which is hardly absorbed by the sensor molecule without $\mathrm{Zn} 2+$. On the basis of the above concept, we have designed and synthesized a novel, highly sensitive off-on fluorescent sensor molecule for $\mathrm{Zn} 2+$. This compound showed pronounced fluorescence enhancement with a blue shift in the absorption spectrum upon $\mathrm{Zn} 2+$ addition, and this also showed high selectivity for $\mathrm{Zn} 2+$ over other cations. Further, we explored the utility of the blue shift of this compound in the absorbance spectrum when $\mathrm{Zn} 2+$ was added. Our design strategy should be applicable to a range of off-on fluorescent sensor molecules for $\mathrm{Zn} 2+$ and other molecules of interest in biological applications.

\section{J167}

\section{LUMINESCENT PROBES USING A SELF-IMMOLATIVE LINKER STRATEGY: APPLICATION TO THE DETECTION OF PEPTIDASES}

Virgile Grandclaude $^{1,2}$, Jean-Alexandre Richard ${ }^{1,2}$, Yves Meyer ${ }^{1,2}$, Pierre-Yves Renard $^{2}$, Anthony Romieu ${ }^{2}$, Marc Massonneau ${ }^{1}$, Pauline Noack-Fraissignes $^{1}$

${ }^{1}$ Chemistry, Quidd, Saint Etienne du Rouvray, France, ${ }^{2}$ COBRA, UMR 6014 CNRS, Mont Saint Aignan, France.

Molecular imaging is of particular interest to detect biological disorders, provide accurate diagnosis and speed up drug development. Proteases play a fundamental role in most biological processes and diseases and thus constitute an attractive target. Luminescence spectroscopy happens to be a simple, cheap and very sensitive technique. Thus, we devised a general strategy relying on flexible and water soluble phenol based luminescent species (pro-fluorescent or chemiluminescent) for the synthesis of activable probes to detect peptidases. Our probes are composed of three parts: 1) a peptide sequence recognized by the targeted enzyme; 2) a luminophore which allows the emission of light; 3) a self-immolative linker [1] connecting the two moieties. We describe here our investigations concerning the synthesis of water soluble pro-fluorescent coumarin derivatives displaying emission wavelengths in the near infrared range (between $640 \mathrm{~nm}$ and $720 \mathrm{~nm}$ ) [2]. Our efforts now focus on the rigidification of the fluorophore structures to improve spectroscopic properties and thus use them for in-vivo applications. References: (1). Meyer, Y.; Richard, J.A.; Massonneau, M.; Renard, P.-Y.; Romieu, A., Org. Lett. 2008, 10, 1517-1520 (2). Richard, J.-A.; Massonneau, M.; Renard, P.-Y.; Romieu, A., Org. Lett. 2008, 10, 4175-4178

\section{$\mathbf{J 1 6 8}$}

\section{IN VITRO ZINC(II) SENSING USING TETRAMETHYL-M-} BENZIPORPHODIMETHENE AS A FLUORESCENCE PROBE Gao Fong Chang, Pei-Chuen Chiang, Chen-Hsiung Hung chemistry, academia sinica, Taipei, Taiwan.

Zinc is, after iron, the second most abundant transition metal in mammals, where it plays important roles in various biological processes such as neurotransmission, signal transduction and gene expression. In order to have better understanding for these zinc related biological processes or diseases, the quantitative and real time monitoring of zinc ions is important. A series of tetramethyl-m-benziporphodimethene compounds have been developed in our lab as novel $\mathrm{Zn}^{2+}$ specific chemosensors. These sensors show fluorescence switch-on upon $\mathrm{Zn} 2+$ binding with no background fluorescence. The long absorption and emission wavelength at nearly $600 \mathrm{~nm}$ and $672 \mathrm{~nm}$, respectively, make it an ideal sensing molecule with better penetration and less scattering in living cell. Competition studies on physiologically important metal ions demonstrate that only $\mathrm{Zn}^{2+}, \mathrm{Cd}^{2+}$ and $\mathrm{Hg}^{2+}$ turn on the fluorescence emission, and $\mathrm{Zn}^{2+}$ can readily replace $\mathrm{Cd}^{2+}$, $\mathrm{Hg}^{2+}$ or other metal ions in subject. It is found that different functional groups on the para position of meso phenyl rings showed a similar stability constant $\left(1.46 \times 10^{5} \sim 2.05 \times 10^{5}\right)$ and revealed that the modification on meso phenyl rings does not significantly affect the binding ability of the central core. The strong binding abilities prove that these compounds have low detection limit toward $\mathrm{Zn}^{2+}$ and are suitable for being a $\mathrm{Zn}^{2+}$ sensor. Results from the cell line experiments using zinc complex of benziporphodimethene confirmed that the fluorophore can penetrate into cells. Strong fluorescence also detected when the probes are treated into $\mathrm{Zn}^{2+}$ enriched Hela cell or treated into etoposide induced apoptotic cells. Currently, the imaging of living animal using zebrafish as a model is undergoing. In summary, the newly developed tetramethyl-m- benziporphodimethenes are fluorescent compounds with good potential for imaging physiological zinc ions.

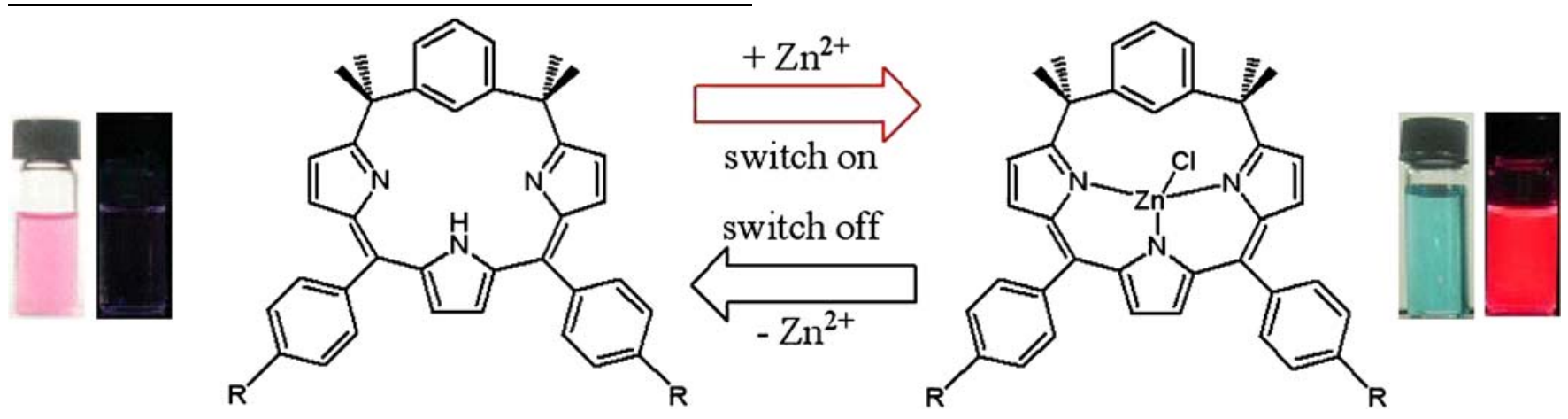

J169

OPTICAL IMAGING OF BACTERIAL INFECTION IN MURINE MODELS USING A NOVEL SYNTHETIC NEAR-INFRARED FLUORESCENT PROBE

Alexander White $^{1,2}$, Bradley D. Smith ${ }^{1,2}$

${ }^{1}$ Chemistry and Biochemistry, University of Notre Dame, Notre Dame, IN, USA, ${ }^{2}$ Notre Dame Integrated Imaging Facility, University of Notre Dame, Notre Dame, IN, USA.
Recent advances in optical imaging of bacterial infection have been propelled by the invention of genetic methods that produce fluorescent and bioluminescent bacteria, and also the discovery of synthetic fluorescent probes that selectively target bacterial cell surfaces. Optical imaging is an effective method for conducting longitudinal studies of bacterial infection in small animals. It can be used to address questions in medical microbiology concerning migration and colonization and it is an attractive method for determining the efficacy of antibiotic therapies. We have discovered that synthetic zinc coordination 
complexes can target the anionic molecular structures on the bacterial surface. Furthermore, fluorescent conjugates of these complexes can very selectively target bacterial cells in the presence of healthy animal cells, and we have successfully imaged localized bacterial infection in living animals (Bioconj. Chem., 2008, 19, 686-692). Here we report a second-generation bacterial imaging probe that exhibits superior performance. We can detect localized murine infections of Grampositive Staphylococcus aureus at levels of $10^{6} \mathrm{CFU}$. The probe clearance time is only a few hours and it can be used to conduct longitudinal imaging studies that evaluate the efficacy of antibiotic drugs in living animals.

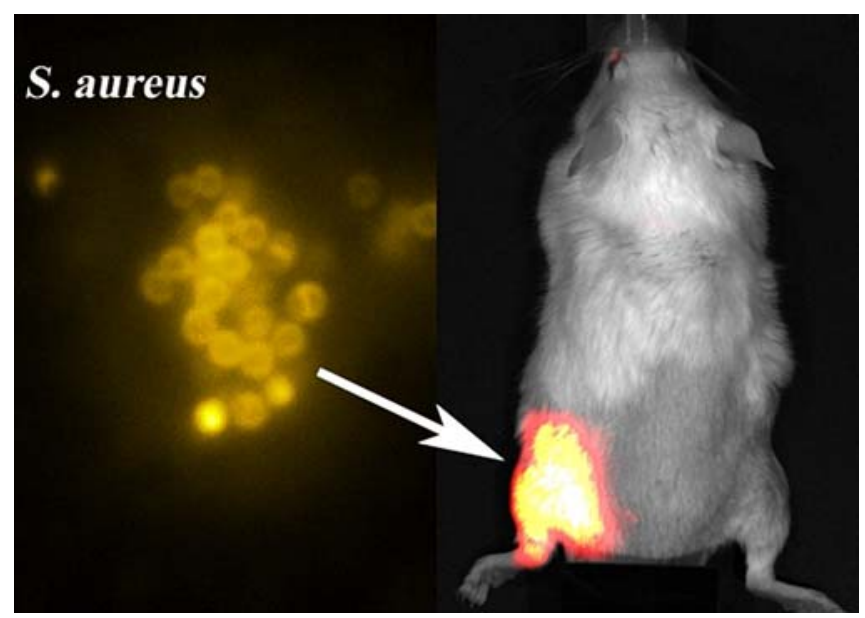

J170

IN VIVO IMAGING OF XENOGRAFT TUMORS USING AN EPIDERMAL GROWTH FACTOR RECEPTOR-SPECIFIC AFFIBODY MOLECULE LABELED WITH A NEAR-INFRARED FLUOROPHORE

Haibiao Gong, Joy L. Kovar, Garrick M. Little, Huaxian Chen, D. M. Olive

LI-COR Biosciences, Lincoln, NE, USA.

Due to its long wavelength, both the light absorption and scattering of near-infrared (NIR, 700-900 nm) light are reduced in animal tissues. This advantageous property, together with the low tissue autofluorescence in the NIR region, makes fluorescence labels with NIR emission highly desirable for optical animal imaging. In this study, we labeled an epidermal growth factor (EGF) receptor (EGFR)-specific Affibody molecule (Eaff) with the IRDye800CW-maleimide dye (Ex/ Em: 774/789 nm), and tested the binding of the labeled molecule (Eaff800) in cell culture and xenograft mouse tumor models. Unlike EGF, Eaff did not activate the EGFR signaling pathway. Both platebased assay and microscopic examination showed that Eaff 800 was bound and taken up specifically by EGFR-overexpressing A431 cells. High concentrations of unlabeled Eaff or EGF competed the cellular binding and uptake of Eaff800. When Eaff 800 was i.v. injected into nude mice bearing A431 xenograft tumors, the accumulation of probe in the tumor could be identified $1 \mathrm{hr}$ post-injection, and became most prominent after $1 \mathrm{~d}$. Images of dissected tissue sections demonstrated that the accumulation of Eaff 800 was highest in the liver, followed by the tumor and kidney. Moreover, in combination with an HER2specific probe Haff682, Eaff800 could be used to distinguish between A431 (EGFR-overexpressing) and SKOV3 (HER2-overexpressing) tumors. Interestingly, the organ distribution pattern and clearance rate of Eaff800 were different from those of Haff682. While Haff682 accumulated predominantly in the kidney, more Eaff 800 was found in the liver. Because of their favorable specificity, pharmacodynamic and pharmacokinetic characteristics, Affibody-based optical imaging agents should find broad usage in preclinical studies of human disease.

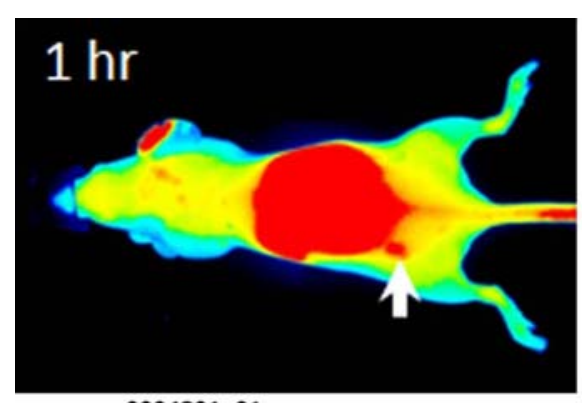

0004801_01

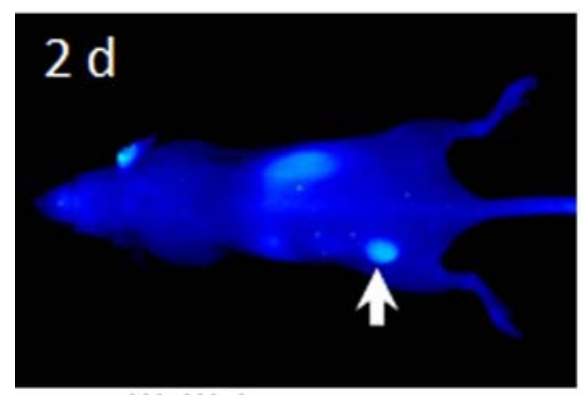

0004893_01

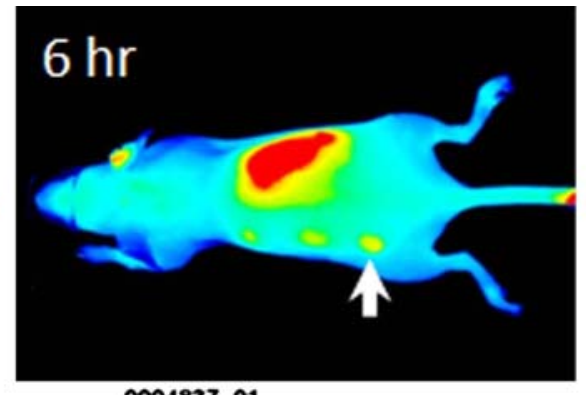

0004837_01

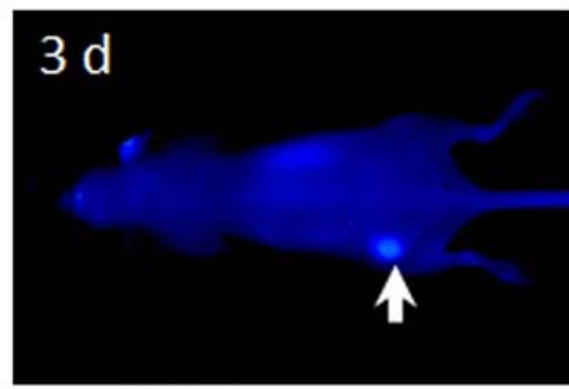

0004921_01

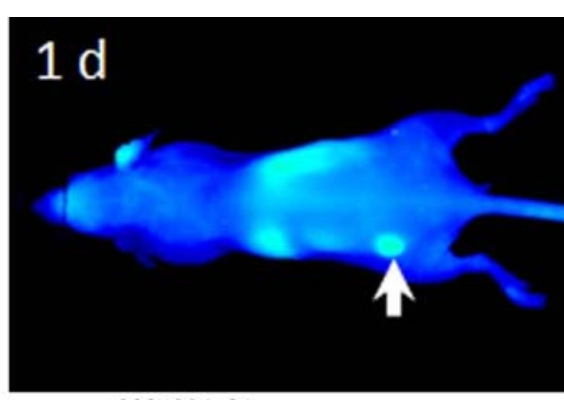

0004864_01

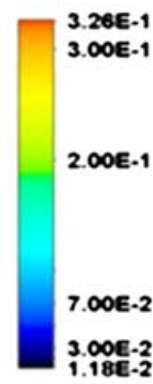

$1.00 E-2$ 
J171

\section{MEASUREMENTS OF SPECTRALLY-RESOLVED FLUORESCENCE LIFETIME OF INDOCYANINE GREEN}

Anna Gerega, Norbert Zolek, Tomasz Soltysinski, Daniel Milej, Piotr Sawosz, Adam Liebert

Polish Academy of Sciences, Institute of Biocybernetics and Biomedical Engineering, Warsaw, Poland.

Indocyanine Green (ICG) is a fluorescent dye which is widely used in biomedical studies due to its low toxicity and high absorption in the near infrared region. In particular, it is used as a contrast agent in blood flow and blood volume studies of the human brain. Kinetics of the inflow and washout of the dye may be potentially used for the estimation of the brain tissue perfusion. It has been reported earlier that fluorescence of the exogenous dye circulating inside the cortex of the brain can be excited and detected noninvasively at the surface of the human adult head using multichannel timeresolved monitoring device. Fluorescence lifetime $\tau$ is an important parameter which influence the signal detected in in-vivo measurements and potentially provides the capability of sensing of environment of the dye in the tissue. We constructed a time-resolved multichannel spectral system and applied it to determination of the fluorescence lifetime of the dye in different solvents. Fluorescence of ICG of different concentrations was excited by femtosecond pulses generated with the use of MaiTai laser. Excitation wavelength was tuned within the range of 736-791 nm. The fluorescence photons were acquired using polychromator PMLSpec, equipped with 16channel photomultiplier tube detector connected to time correlated single photon counting card SCP 830 (Becker \& Hickl, Germany). For the collected fluorescence spectra of ICG, the analysis of the distribution of times of arrival (DTA) of fluorescence photons was carried out. In order to determine the fluorescence lifetime, for the acquired DTAs, fitting procedure was applied. For this purpose single- and double-exponential decay models were used depending on the solvent in which ICG was dissolved. For this fitting procedure the measured instrumental response function of the setup was taken into account. For all solutions of ICG, fluorescence lifetime $\tau$ was determined. $\tau$ is equal to $0.16 \mathrm{~ns}$ in water, $0.15 \mathrm{~ns}$ in physiological salt (using single-exponent decay profile); for ICG in milk the value of $\tau$ strongly depends on concentration of ICG and varies from 0.07 to $0.37 \mathrm{~ns}$ for $\tau_{1}$ and from 0.61 to $0.82 \mathrm{~ns}$ for $\tau_{2}$ (using double-exponent decay profile) for the concentration of ICG 160 and $1.7 \mu \mathrm{M}$, respectively. We applied the spectrally-resolved method for estimation of dynamic of changes of fluorescence spectrum just after dissolving the ICG in the solvent. This attempt was made in the human blood and it will help to understand in-vivo data obtained during intravenous injection of ICG.

\section{$\mathbf{J 1 7 2}$}

\section{NOVEL NIR FLUOROPHORES FOR VISUALIZATION OF ENDOCRINE GLANDS AND THEIR TUMORS}

Hak Soo Choi ${ }^{1}$, Maged Henary ${ }^{2}$, Eiichi Tanaka ${ }^{3}$, Yoshitomo Ashitate ${ }^{1,3}$, Merlijn Hutteman ${ }^{1,5}$, Lucjan Strekowski ${ }^{2}$, Gabor Patonay ${ }^{2}$, John V. Frangioni ${ }^{1,4}$

${ }^{1}$ Medicine, Beth Israel Deaconess Medical Center, Boston, MA, USA, ${ }^{2}$ Chemistry, University of Georgia, Atlanta, GA, USA, ${ }^{3}$ Surgery, Hokkaido University Graduate School of Medicine, Hokkaido, Japan, ${ }^{4}$ Radiology, Beth Israel Deaconess Medical Center, Boston, MA, USA, ${ }^{5}$ Surgery, Leiden University Medical Center, Leiden, Netherlands.

Background: Highly sensitive and specific visualization is the key to image-guided surgery using near-infrared (NIR) fluorescent light. For example, identification of normal endocrine glands and their tumors are of critical importance to preserve normal tissue and remove diseased tissue, respectively. We hypothesized that it should be possible to design NIR fluorescent indocyanine derivatives that are extracted from the blood by certain endocrine tissues, leading to improved contrast for image-guided surgery. Materials and Methods: A series of indocyanine derivatives were developed, which exhibited high cellular uptake and high quantum yield in the presence of bodily fluids. A gel-filtration chromatography system capable of simultaneous, full-spectrum (300$900 \mathrm{~nm}$ ) absorbance and fluorescence was used to characterize the fluorophores in PBS and serum in vitro, and in bodily fluids after intravenous injection into insulinoma-bearing mice (Jackson Laboratories, Bar Harbor, ME). Biodistribution and pharmacokinetics in endocrine gland systems were demonstrated via intravenous injection of $20 \mathrm{pmol} / \mathrm{g}$ of NIR fluorophore into $300 \mathrm{~g}$ Sprague-Dawley rats or $30 \mathrm{~kg}$ Yorkshire pigs. Realtime intraoperative imaging of various glands and tumors in mice, rats, and pigs was performed for $1 \mathrm{~h}$ post-injection using the FLARETM imaging system equipped with $667 \mathrm{~nm}$ and $707 \mathrm{~nm}$ emission filters. Results: MHI84, a lipophilic NIR fluorophore was successfully developed for uptake in endocrine tissues. This fluorophore has an extinction coefficient of $148,000 \mathrm{M}^{-1} \mathrm{~cm}^{-1}$ and a quantum yield of $6.8 \%$ in serum. After $1 \mathrm{~h}$ of systemic circulation, intravenously injected MHI-84 accumulated mainly in adrenal glands and thyroid glands in rats and pigs, which were visualized using a real-time, intraoperative imaging system. Furthermore, rapid tumor targeting in insulinoma was seen within $10 \mathrm{~min}$ after intravenous injection of $40 \mathrm{pmol} / \mathrm{g}$ of MHI- 84 into mice, which permitted image-guided resection of even sub-millimeter tumors. Conclusion: In this study, we developed a lipophilic NIR fluorophore, MHI-84, and demonstrated its uptake into endocrine glands and early-stage endocrine tumors. When used with the FLARE $^{\mathrm{TM}}$ imaging system, MHI- 84 enables real-time avoidance of normal endocrine tissue and real-time resection of malignant tissue.

\section{$\mathbf{J 1 7 3}$}

HER2- AND EGFR-SPECIFIC AFFIPROBES-A NOVEL RECOMBINANT OPTICAL PROBES FOR CELL IMAGING Ilya Lyakhov ${ }^{1,2}$, Rafal Zielinski ${ }^{2}$, Gabriela Kramer-Marek ${ }^{2}$, Robert Fisher $^{1}$, Oleg Chertov $^{1}$, Lakshman Bindu ${ }^{1}$, Jacek Capala ${ }^{2}$

${ }^{1}$ PCL, SAIC-Frederick, Frederick, MD, USA, ${ }^{2} \mathrm{ROB}, \mathrm{NCI} / \mathrm{NIH}$, Bethesda, MD, USA.

The human epidermal growth factor receptors EGFR (Her1, ErbB1) and HER2 (HER2/neu, ErbB2, or c-erb-b2) are members of EGFR (ErbB) family of cell-surface receptors/tyrosine kinases: EGFR, HER2, HER3, and HER4. There are several identified ligands for these receptors (except HER2), including EGF and TGF-alpha, that induce receptor dimerization followed by tyrosine autophosphorylation, which leads to cell proliferation. EGFR and HER2 are found overexpressed in a number of cancer types, such as breast, ovarian, salivary gland, stomach, kidney, colon, prostate, and non-small cell lung cancer. Research has shown that EGFR- or HER2-positive cancers represent a more aggressive disease with greater likelihood of recurrence, poorer prognosis, and decreased survival rate compared to EGFR- or HER2negative cancers. The details of HER2 proto-oncogenic function, its internalization mechanism, and subcellular trafficking are not fully understood, partially because the availability of imaging reagents and tools for detection of EGFR and HER2 is still restricted to medium/low affinity antibody conjugated to small-molecule dyes. We have created photo-stable and relatively simple-to-prepare imaging probes for in vitro staining of EGFR and HER2. These new reagents, called Affiprobes, consist of the targeting moiety, a HER2- or EGFR-specific Affibody ${ }^{\circledR}$ molecule, and a fluorescent detection moiety mCherry (Red), or EGFP (Green). These fluorescent proteins were chosen due to their high stability and solubility and low toxicity for eukaryotic cells. Furthermore, HER2 and EGFR receptors can be detected simultaneously using corresponding optical filter sets. Our flow cytometry and confocal microscopy experiments demonstrated high specificity and signal/background ratio of Affiprobes. This type of optical probe can be 
easily extended for targeting other cell-surface antigens/receptors using different Affibody ${ }^{\circledR}$ molecules and fluorescent proteins. Funded by NCI Contract No. HHSN261200800001E.

\section{J174}

RAPID AND EFFICIENT LABELLING OF CELLS FOR OPTICAL IMAGING USING NOVEL CELL ENTRY PEPTOIDS IN MODELS OF ACUTE INFLAMMATION

Kevin Dhaliwal $^{1}$, Asier Unciti-Broceta ${ }^{2}$, Geraldine Escher ${ }^{2}$, Nicolaos Avlonitis $^{1,2}$, Neil A. McDonald ${ }^{1}$, John Simpson ${ }^{1}$, Haslett Chris ${ }^{1}$, Mark Bradley ${ }^{2}$

${ }^{1}$ Centre for Inflammation Research, University of Edinburgh, Edinburgh, United Kingdom, ${ }^{2}$ Department of Chemistry, University of Edinburgh, Edinburgh, United Kingdom.

Cell therapy is increasingly being recognised as a therapeutic intervention in acute and chronic conditions. The role played by innate immune cell ingress is also recognised as a key pathological determinant of disease progression. Neutrophil mediated tissue damage is central to the pathogenesis of both acute and chronic inflammatory conditions. Alongside the neutrophil, monocytes are recruited at early stages of acute inflammation. The temporal ingress of these monocytes may play a pivotal role in the ongoing neutrophil infiltration and subsequent pathology. Currently there are significant difficulties in labelling these cells ex vivo prior to adoptive transfer in preclinical small animal models because of concerns of length of labelling procedure and potential toxic effects. We report on the development and application of novel cell entry peptoids that allowed the rapid labelling and simultaneous imaging of two primary cells in vivo in models of lung inflammation. Chemistries were developed to link our recently described cell entry peptoids to the cyanine dyes, Cy5.5 and Cy7, which were assessed for cytotoxicity and cytokine liberation from labelled cells alongside labelling speed and efficiency and cellular retention of the label. We also evaluated the possibility of using both tags in the same animal to monitor the tracking of two innate immune cell types simultaneously after adoptive transfer in acute inflammatory models. In particular we assessed the utility of detecting monocyte infiltration to sites of ongoing neutrophil ingress. We report on the bio-distribution of these labelled cells using multispectral reflectance and tomographic systems and ex vivo by flow cytometry. CONCLUSIONS: The ability to monitor the neutrophil and the monocyte ingress in real-time using non invasive imaging may instruct the application of intervention at specific time points.

\section{$\mathbf{J 1 7 5}$}

\section{NERVE-SPECIFIC FLUORESCENT CONTRAST AGENTS FOR IMAGE-GUIDED SURGERY}

Summer Gibbs-Strauss $^{1}$, Khaled A. Nasr ${ }^{1}$, Tiberiu M. Siclovan ${ }^{3}$, Kenneth M. Fish ${ }^{3}$, Nicole E. Barnhardt ${ }^{3}$, Cristina A. Tan Hehir ${ }^{3}$, John V. Frangioni ${ }^{1,2}$

${ }^{1}$ Medicine, Beth Israel Deaconess Medical Center, Boston, MA, USA, ${ }^{2}$ Radiology, Beth Israel Deaconess Medical Center, Boston, MA, USA, ${ }^{3}$ Molecular Imaging and Diagnostic Advanced Technology Program, GE Global Research, Niskayuna, NY, USA.

Background: Nerve damage is the major morbidity associated with numerous surgical procedures. Although human nerve anatomy is well known, performing surgery that spares nervous structures is still a difficult task due to patient-to patient variability and the difficulty of nerve visualization. A nerve-specific fluorescent contrast agent, especially one with near-infrared (NIR) absorption and emission wavelengths, would be of immediate benefit to patients and surgeons for image-guidance during various surgical procedures. Fluorescent contrast agents can now be visualized during surgery using the
FLARE $^{\text {TM }}$ (Fluorescence-Assisted Resection and Exploration) system, which is currently in clinical trials. Materials and Methods: Two nervespecific contrast agents, one a derivative of the other, were assessed for physiochemical and optical properties in solvents with varied proton content. Both nerve-specific dyes were excited with ultraviolet light and emitted at green wavelengths. The agents were evaluated in mice, rats and pigs for accumulation in nervous, adipose and muscle tissues. Results: Due to the lipophilic nature of these agents, a formulation (10\% DMSO, 5\% Cremophor, 65\% serum and 20\% Hepes) was developed to enable intravenous (IV) administration. The quantum yield of the agents was similar in the IV formulation to that of their preferred solvent. Both agents were found to accumulate in nervous tissues including the brachial plexus, trigeminal ganglia, optic nerve, sciatic nerve, vagus nerve, phrenic nerve, facial nerves, spine, and femoral nerves in mice, rats and pigs. Kinetic rodent studies demonstrated peak nerve to muscle contrast $4 \mathrm{~h}$ after intravenous administration of either nerve-specific agent. Both contrast agents showed significant accumulation in adipose tissue as well as nervous tissue, and thus ratiometric imaging was employed to increase nervous contrast. Using the FLARETM imaging system, nervous tissue and blood vessels could be imaged simultaneously during surgery. Conclusions: Two contrast agents specific for nervous tissue have been characterized in vitro and in vivo. Although the current compounds are not ideal for clinical use, their structural similarity enabled recognition of a pharmacophore that provides nervous tissue contrast. This pharmacophore could be further derivatized to improve in vivo performance with the goals of increased nerve tissue specificity, decreased adipose tissue accumulation, and NIR absorption and emission wavelengths.

\section{$\mathbf{J 1 7 6}$}

\section{INHERENT CO-REGISTRATION OF NEAR-INFRARED FLUORESCENCE AND HEMATOXYLIN EOSIN ON THE SAME HISTOLOGICAL SLIDE}

Summer Gibbs-Strauss $^{1}$, Vida Kianzad ${ }^{1}$, Fangbing Liu ${ }^{1}$, Hak Soo Choi $^{1}$, John V. Frangioni ${ }^{1,2}$

${ }^{1}$ Medicine, Beth Israel Deaconess Medical Center, Boston, MA, USA, ${ }^{2}$ Radiology, Beth Israel Deaconess Medical Center, Boston, MA, USA.

Background: During the development of novel near-infrared (NIR) fluorescent contrast agents, it is always necessary to co-localize the agent with normal or diseased tissues on histological slides. Hematoxylin and eosin (H\&E) staining is the gold standard used by pathologists for tissue staining. We hypothesized that because of the fluorescent spectra of H\&E-stained tissue, it should be possible to perform simultaneous imaging using NIR fluorophores, which would eliminate the co-registration problems that plague conventional analysis. Such a technology would also enable automated tissue analysis using NIR fluorescent antibodies. Materials and Methods: Three examples of simultaneous NIR fluorescence and H\&E microscopy were performed using mouse, pig and human tissues. Human prostate cancer was used in this study as an example of a diseased tissue where diagnostic accuracy would be enhanced by immunofluorescence staining. The primary antibody $\alpha$-methyl-CoA racemase (AMACR) was used with an 800-nm secondary antibody for microscopy. Pig sentinel lymph node (SLN) mapping was performed using HSA800 under guidance from the FLARE (Fluorescence-Assisted Resection and Exploration) imageguided surgery system. The resected SLN was fixed, frozen and cryosectioned for H\&E staining. Metastatic melanoma was modeled through intravenous administration of IR786 stained B16 cells. The cells were tracked in the lung tissue using IR786 fluorescence. Fluorescent signal was confirmed to originate from melanoma cells using antibody co-staining. Results: In both the human prostate and mouse lung examples the NIR secondary antibody was readily visible on the H\&E stained slide without cross-interference from the H\&E 
stain. Similarly, HSA800-based SLN mapping and IR786 stained cells were readily visible on the $H \& E$ stained slides. The $H \& E$ stained slide retained its original information, and under white light looked identical to standard H\&E stain. The fluorescence was perfectly co-registered with the H\&E staining as the same slide was used for both microscopy images. Conclusions: The fluorescence emission spectra of H\&E stain does not interfere with NIR fluorescence counterstaining whether the fluorophore is administered in vivo or as an antibody stain ex vivo. The fluorescent co-staining of microscopy slides with standard H\&E stain enables perfect co-registration of gold standard pathology with functional fluorescence information.

\section{$\mathbf{J 1 7 7}$}

\section{ZIPPER MOLECULAR BEACONS: A GENERALIZED} METHODOLOGY FOR ACTIVATABLE PROTEASE PROBES Tracy W. Liu, Juan Chen, Pui-Chi Lo, Brian Wilson, Gang Zheng Medical Biophysics, University of Toronto/ Ontario Cancer Institute, Toronto, ON, Canada.

Optical molecular imaging has become a powerful tool for biochemical and cellular studies. With the continual development of peptide-based "smart" probes, the in vivo optical imaging of specific molecular targets, biological pathways and disease progression is gaining momentum. Although activatable molecular beacons (MB) have shown great promise, several challenges have arisen. The total dependence of the fluorescence and PDT quenching upon the random folding of the petide linker before protease cleavage limits the sequences to ones with natural conformations that bring the quencher and active moiety in close proximity in order for effective silencing to occur. The non-specific and passive nature of the PS's delivery to target cells after protease cleavage is suboptimal. Lastly, MB are activated extracellularly, so that the activated probes may diffuse to non-target cells/tissues before target cell uptake occurs. To address these concerns, we have developed a general strategy, zipper molecular beacons (ZMB). The objective is to balance maximal quenching efficiency and optimal two-step activation (protease cleavage with zipper dissociation), while enhancing target cell uptake. This strategy also eliminates the peptide sequence-dependence of conventional protease beacons. This ZMB concept is a generalizable approach to improve the functionality of a wide range of diagnostic/therapeutic probes through a simple switching of substrate sequences.
$\mathbf{J 1 7 8}$

\section{POLYMERIC NANOSENSOR FOR IMAGING MATRIX METALLOPROTEINASE EXPRESSION}

Seulki Lee ${ }^{1}$, Xiaoyuan Chen ${ }^{1}$, Ick Chan Kwon ${ }^{2}$, Kwangmeyung Kim ${ }^{2}$ ${ }^{\mathrm{T}}$ Radiology, Stanford University School of Medicine, Stanford, CA, USA, ${ }^{2}$ Biomedical Research Center, Korea Institute of Science and Technology, Seoul, Korea, South.

Several strategies of development of activatable probes have been developed, however, most of which have relatively low quenching efficiency and thus suboptimal target-to-background contrast. We report here a new protease activatable strategy based on a polymeric nanoparticle platform. It was hypothesized that chemically labeled activatable probes on the surface of nanoparticles induce an higher specificity and sensitivity of the probe than the simple fluorogenic peptide in vivo, since i) the nanoparticles can deliver the probe effectively to the disease sites, i.e. tumors region, by the enhanced permeation retention effect, and ii) the fluorescence labeled on the nanoparticles via peptide substrate can be dual-quenched via both the dye-dark quencher and dye-dye self-quenching mechanisms. When the nanosensor is exposed to the specific MMPs of interest, cleavage of the NIR dye-substrate occurs owing to specific substrate recognition by the MMPs and this is manifested in the form of pronounced NIR fluorescence signal recovery due to dequenching of the dye. We thus prepared nanosensors that were labeled with MMP activatable fluorogenic peptide, Cy5.5-GPLGVRGK(BHQ-3)GG, on the surface of hydrophobic 5beta-cholanic acid conjugated glycol chitosan nanoparticles. The resulting particles were spherical, approximately $250 \mathrm{~nm}$ in diameter, and were well-dispersed in aqueous buffer. The spectrofluorometry clearly demonstrated that significant time-dependent recovery of the fluorescence signals occurred against MMP-2, 9, and 13 ( $>30$-folds vs. without MMP) and the signals was proportionally $(\mathrm{r} 2=0.99)$ increased depending on the MMP-2 concentrations $(0.75$ to $15 \mathrm{nmol} / \mathrm{L})$. Intravenous administration of the nanosensor in MMP-2/9positive SCC-7 xenograft model verified the enzyme specificity of the nanosensor in vivo. The design platform of nanosensor is flexible and fine-tunable for a wide array of applications, such as detection of biomolecules, early diagnosis of disease, and monitoring therapeutic efficacy in vivo.
A B

\section{Multi-quenched State}

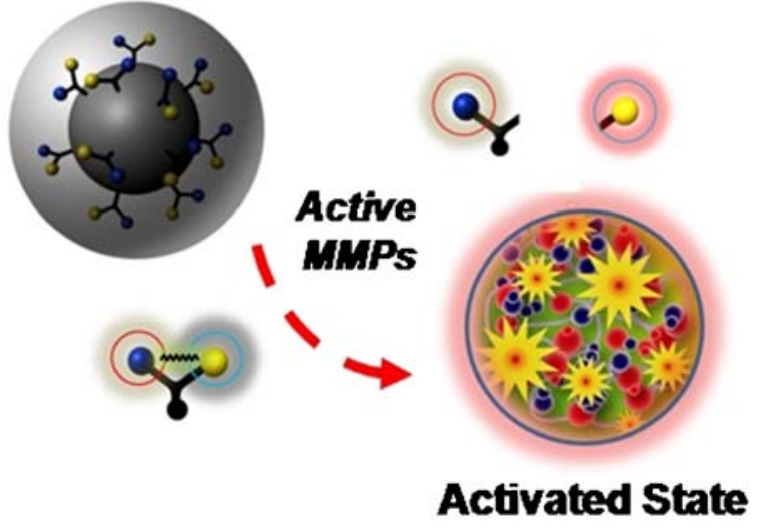

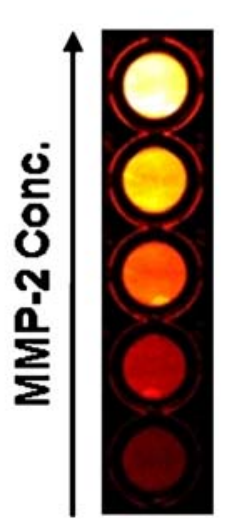

C
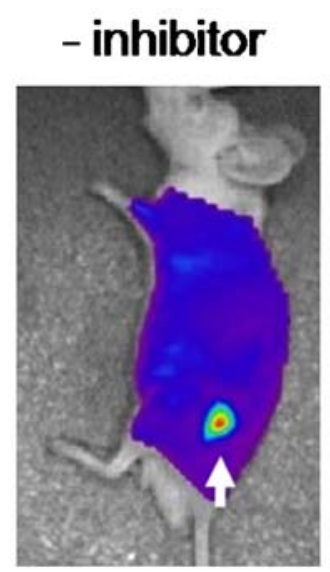

+ inhibitor

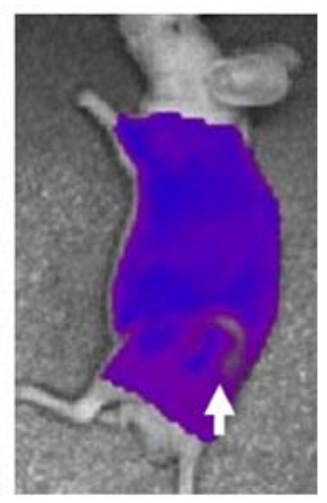

Figure. (A) A schematic diagram of the nanosensor. (B) Fluorescence image sections of a microplate containing the nanosensor and various concentrations of MMP-2 $(0.75,1.9,3.8,7.5,15 \mathrm{nmol} / \mathrm{L})$. (C) NIR images of SCC7 tumor bearing mice after intravenous injection of the nanosensor with or without MMP inhibitor. 
$\mathbf{J 1 7 9}$

ACTIVATABLE PHOTOSENSITIZER DESIGN USING FRET QUENCHING: FROM SMART PROBES TO THERAPEUTICS Jonathan F. Lovell ${ }^{1}$, Juan $\mathrm{Chen}^{2}$, Brian Wilson ${ }^{2}$, Gang Zheng ${ }^{2}$ ${ }^{1}$ Biomedical Engineering, University of Toronto, Toronto, ON, Canada, ${ }^{2}$ Department of Medical Biophysics, University of Toronto, Toronto, ON, Canada.

Activatable photosensitizers hold potential as therapeutic agents that are specifically activated and are toxic to diseased cells. Improved methods to rationally design and analyze activatable photosensitizers can facilitate their production and implementation. Forster resonance energy transfer (FRET) quenchers represent an attractive building block for activatable photosensitizers, however little is known quantitatively about FRET quenching of photosensitizers. To examine quenching of singlet oxygen generation, we linked various quenchers to a photosensitizer using a short spacer. Quenchers sharing moderate spectral overlap with the fluorescence emission of the photosensitizer quenched most of the singlet oxygen, while quenchers with weaker spectral overlap displayed minimal quenching. In agreement with FRET deactivation, extension of the linker to a polyproline peptide resulted in only the quenchers with much greater spectral overlap maintaining good quenching efficiency. There was strong correlation between fluorescence quenching and singlet oxygen quenching, demonstrating that fluorescence intensity can be an indicator for the singlet oxygen production status of activatable photosensitizers. Existing fluorescent smart probes could be converted to therapeutic activatable photosensitizers with the guidelines governing FRET quenching. The presented findings show that FRET based quenchers can effectively decrease singlet oxygen production and therefore be used to facilitate the rational design and analysis of activatable photosensitizers.

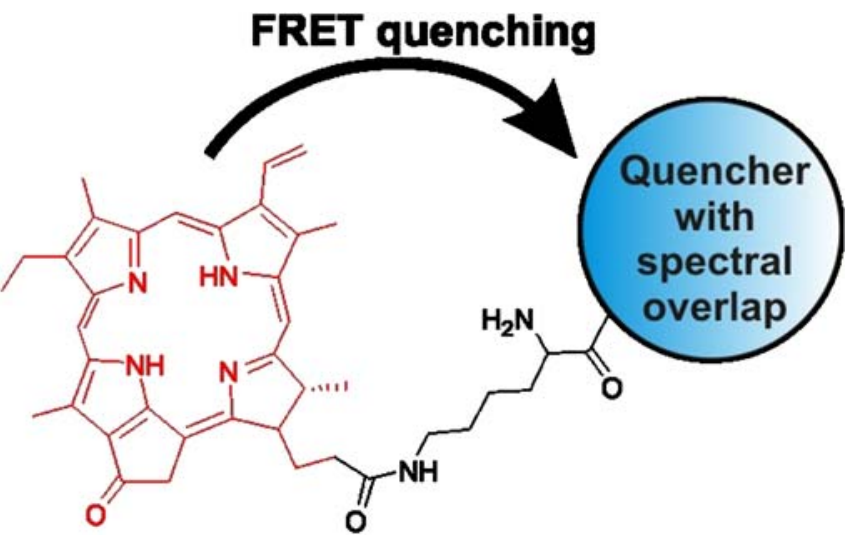

Schematic representation of FRET quenching of a photosensitizer, pyropheophorbide-a.

\section{$\mathbf{J 1 8 0}$}

\section{FLUORESCENT REAGENTS FOR MULTIPLEXED LIFETIME-BASED OPTICAL IMAGING}

Ivana Miletto $^{1,2}$, Paolo Soligo ${ }^{3}$, Salvatore Coluccia ${ }^{1,2}$, Giuseppe Caputo ${ }^{1,2}$ ${ }^{1}$ Nanostructured Interfaces and Surfaces Centre of Excellence, University of Turin, Turin, Italy, ${ }^{2}$ Department of Inorganic, Physical and Materials Chemistry, University of Turin, Turin, Italy, ${ }^{3}$ Cyanine Technologies s.r.l., Turin, Italy.

Fluorescence Lifetime Imaging is an Optical Imaging methodology that is gaining more and more importance due to its great potential. Each fluorescent dye has its own lifetime; by detecting differences in lifetimes it is possible to distinguish even dyes having the same fluorescent color as well as to identify autofluorescence. In this contribution we present the development of a series of Cy5.5® analogues cyanine dyes with the same fluorescent emission but different lifetimes. Methods. Cyanine dyes were synthesized and purified following standard methodologies and characterized via 1H-NMR spectroscopy, UV-Vis absorption and photoemission spectroscopy. Fluorescence lifetimes were measured using a timecorrelated single photon counting (TCSPC) technique with excitation source NanoLed at $636 \mathrm{~nm}$ and impulse repetition rate of $1 \mathrm{MHz}$ at $90^{\circ}$ to a TBX-4 detector. The detector was set to $700 \mathrm{~nm}$ with a $5 \mathrm{~nm}$ band pass. The instrument was set in the Reverse TAC mode, where the first detected photon represented the start signal by the time-to-amplitude converter (TAC), and the excitation pulse triggered the stop signal. DAS6 decay analysis software was used for lifetime calculation. Experiments were repeated using a commercial pre-clinical fluorescence lifetime optical imaging system with $665 \mathrm{~nm}$ laser excitation and $700 \mathrm{~nm}$ emission collection longpass filter. Results and Conclusion. A series of Cy5.5 $\mathbb{R}$ analogues characterized by significantly different fluorescent lifetimes has been prepared by inserting structural modifications that does not interfere neither with the absorption/emission wavelength and with the bioconjugation properties. Dyes show a trend in fluorescence lifetime consistent with the structural modifications on each dye. Experimental data have been collected by testing these cyanine dyes for antibody conjugation and by using the bioconjugates in optical imaging applications on a commercial fluorescence lifetime optical imager. Results show the possibility of multiplexing analysis in pre-clinical settings using three different dyes excited by a single laser source at $665 \mathrm{~nm}$.

\section{$\mathbf{J 1 8 1}$}

\section{DEVELOPMENT OF CATHEPSIN-B SPECIFIC DENDRITIC NEAR-INFRARED FLUORESCENT PROBE FOR THE DETECTION OF METASTATIC BREAST CANCER IN THE LYMPH NODES}

David M. Pham ${ }^{1}$, Bo Chen ${ }^{2}$, Francis C. Szoka ${ }^{2}$, Ella F. Jones ${ }^{1}$ ${ }^{D}$ Department of Radiology and Biomedical Imagaing, UCSF, San Francisco, CA, USA, ${ }^{2}$ Department of Biopharmaceutical Sciences, UCSF, San Francisco, CA, USA.

Background: Metastatic breast cancer primary route of proliferation is through the lymphatic system. Accurate assessment of lymph nodes is critical for staging the disease progression and determination of therapeutic options for patients. Lymphadenectomy is the current clinical method for lymph nodes assessment, which involves microscopical examination of surgically removed lymph nodes. This painful and invasive procedure causes nodal destruction and swelling and is not always analytically reliable. Molecular imaging affords non-invasive options for visualizing lymph nodes. However, available imaging techniques cannot accurately ascertain the malignancy of the infected lymph nodes, because they show only anatomical structures without revealing the bioactivities associated with disease progression. Cathepsin-B (Cat-B) is an established metastatic breast cancer biomarker and implicated in the invasion of cancerous cells into the lymph nodes by destruction of the basement membranes. Method: We have developed a near infrared optical imaging probe with potentials in highlighting diseased lymph nodes by selective activation with Cat-B. The developed molecular probe is consisted of three components: 1) a dendritic polyester platform, 2) Cat-B specific peptidic periphery reporters, 3) molecular weight modifier $\mathrm{mPEG}$ surface groups. Natively, the peptidic reporter is non-emissive, because the fluorescence of the conjugated cyanine dye is extinguish by the quencher moiety due to fluorescence resonance energy transfer (FRET) effect. Fluorescence is activated when Cat-B proteolytically removes the fluorescence quenching moieties from the probe. In vivo, this 'light 
switch' design will highlight the bioactivity of Cat-B, affording a viable method for non-invasive assessment of lymph nodes. Results: The synthesized peptidic reporter NH2-GK(5-FAM)QVSGFRFGC (DACM)G-CONH2 have been assayed with Cat-B, and shown to have comparable specificity with known Cat-B substrates (Table 1).
Conclusion: The demonstrated Cat-B specificity of the peptidic reporter warrants further in vitro and in vivo investigations of this probe. The in vitro results will highlight the promising utility of this probe in non-invasive assessment of metastatic breast cancer in the lymph nodes.

Table 1: Proteolytic Kinetics of Cathepsin-B Substrates

\begin{tabular}{|c|c|c|c|}
\hline Cathepsin B Substrates & $\mathrm{Km}(\mathrm{mM})$ & $\mathrm{kcat}(1 / \mathrm{s})$ & $\mathrm{kcat} / \mathrm{Km}(1 / \mathrm{M} * 1 / \mathrm{s})$ \\
\hline Cbz-FR-AMC (@ $\mathrm{pH} 6.0)$ & 0.6 & 33.8 & 54.6 \\
\hline NH2-GK(5-FAM)QVSGFRFGC(DACM)G-CONH2 (@ $\mathrm{pH} 5.5)$ & 1.0 & 45.5 & 47.8 \\
\hline NH2-GK(5-FAM)QVSGFRFGC(DACM)G-CONH2 (@ $\mathrm{pH} 7.4)$ & 2.8 & 40.8 & 14.6 \\
\hline
\end{tabular}

\section{$\mathbf{J 1 8 2}$}

\section{IN VIVO IMAGING OF TUBERCULOSIS WITH NEAR INFRARED FLUOROGENIC PROBES}

Jianghong Rao ${ }^{1}$, Ying Kong ${ }^{2}$, Hequan Yao ${ }^{1}$, Hongjun Ren ${ }^{1}$, Selvakumar Subbian $^{2}$, Jeffrey D. Cirillo ${ }^{2}$

${ }^{1}$ Stanford University School of Medicine, Palo Alto, CA, USA, ${ }^{2}$ Texas A\&M University System Health Science Center, College Station, TX, USA.

Tuberculosis (TB) is a major cause of morbidity and mortality worldwide. The long generation time and difficulty of manipulating Mycobacterium tuberculosis has encumbered progress toward understanding pathogenesis and developing improved antimicrobials and vaccines. Small animal models, including mice and guinea pigs, are widely used as human disease surrogates for tuberculosis. We hypothesize that the ability to monitor tuberculosis viability and disease processes through real-time live animal imaging will greatly facilitate progress in combating tuberculosis by allowing rapid evaluation of therapeutics and vaccines without the need for cumbersome colonybased quantification. By targeting the beta-lactamase expressed in $\mathrm{M}$. $\mathrm{Tb}$, we developed a series of near infrared fluorogenic substrates and demonstrated that non-invasive real-time imaging can be used to monitor acute tuberculosis infections in mice. After subcutaneous and aerosol infection, fluorogenic substrates can be injected i.v. and produce a strong fluorescent signal when cleaved by M. TB BlaC. This fluorescence can be detected and quantified, allowing direct observation of bacterial pulmonary infections in living mice. We compared four different substrates that differ in charge and carrier molecules for live animal imaging properties. We found that one of the probes, due to its ability to traverse mammalian membranes and be retained within eukaryotic cells, performs optimally in live animal imaging studies. These substrates have the advantage that they can also be used directly for fluorescent confocal microscopy and FACS to directly visualize infected cells. Non-invasive imaging of TB in vivo will facilitate progress in tuberculosis research, providing a rapid method for quantification of bacterial numbers during infections in animals and in vitro.

\section{$\mathbf{J 1 8 3}$}

CELL UPTAKE STUDIES OF FLUORESCENT LABELED PHOSHOPEPTIDE-CELL-PENETRATING PEPTIDE HYBRIDS Susan Richter ${ }^{1}$, Ralf K. Bergmann ${ }^{1}$, Ines Neundorf ${ }^{2}$, Anette G. BeckSickinger $^{2}$, Jens Pietzsch ${ }^{1}$, Jörg Steinbach ${ }^{1}$, Frank Wuest ${ }^{3,1}$

${ }^{1}$ Radiopharmacy, Forschungszentrum Dresden-Rossendorf, Dresden, Germany, ${ }^{2}$ Biochemistry, University of Leipzig, Leipzig, Germany, ${ }^{3}$ Oncology, University of Alberta, Edmonton, AB, Canada.
Introduction and Aim: Phosphopeptides are very useful reagents to study signal transduction pathways related with cellular protein phosphorylation/dephosphorylation processes. Phosphopeptides also have been identified as important drug candidates to modulate intracellular signal transduction pathways. The present study describes the use of cell-penetrating peptides (CPPs) sC18 and hCT(18-32)-k7 coupled to Polo-like kinase-1 (Plk-1)-binding hexaphosphopeptide HMet-Gln-Ser-pThr-Pro-Leu-OH to investigate cell uptake of the corresponding phosphopeptide-CPP hybrids. Materials and Methods: Peptide syntheses were accomplished using a combination of automated and manual solid-phase peptide syntheses following standard Fmoc chemistry. Fluorescence labeling was carried out via coupling 5 (6)-carboxyfluoresceine (CF) to the N-terminal end of the phosphopeptide using HATU as the activation agent, DIPEA as the base, and DMF as the solvent. Cell uptake studies of CF-Met-Gln-Ser-pThr-Pro-LeusC18 (CF-I) and CF-Met-Gln-Ser-pThr-Pro-Leu-hCT(18-32)-k7 (CFII) were performed using the human breast cancer cell line MCF-7 and human cervical carcinoma epithelial (HeLa) cells on a Zeiss Axio fluorescence confocal microscope. Results: Peptide hybrids CF-I and CF-II showed cellular uptake in both cell lines after incubation at $37^{\circ} \mathrm{C}$ for $60 \mathrm{~min}$. Cell uptake was more pronounced in HeLa cells compared to MCF-7 cells. Phosphopeptide conjugated to $\mathrm{sC} 18$ showed higher cell uptake compared to phosphopeptide-hCT(18-32)-k7 conjugate. As expected, CF-labeled phosphopeptide without being conjugated to CPPs as molecular shuttles was not internalized into both cell lines. Quantification of cellular uptake using flow cytometry analysis and fluorine-18 labeled phosphopeptide-CPP hybrids are currently in progress. Conclusion: Cell-penetrating peptides sC18 and hCT(1832)-k7 are useful drug delivery systems enabling sufficient membrane transport of phosphopeptides to further promote studies on intracellular metabolic pathways involving phosphopeptides.

\section{$\mathbf{J 1 8 4}$}

\section{MOLECULAR IMAGING OF NEUTROPILIN-1 RECEPTOR USING PHOTOACOUSTIC SPECTROSCOPY IN BREAST TUMORS}

Keith Stantz ${ }^{1,4}$, Minsong $\mathrm{Cao}^{3}$, Bo Liu ${ }^{1}$, Kathy D. Miller ${ }^{2}$, Robert Kruger $^{7}$, Lili Guo ${ }^{5,6}$

${ }^{1}$ School of Health Sciences, Purdue University, West Lafayette, IN, USA, ${ }^{2}$ Hematology/Oncology, Indiana University School of Medicine, Indianapolis, IN, USA, ${ }^{3}$ Radiation Oncology, Indiana University School of Medicine, Indianapolis, IN, USA, ${ }^{4}$ Radiology, Indiana University School of Medicine, Indianapolis, IN, USA, ${ }^{5}$ Biochemistry/ Molecular Biology, Indiana University School of Medicine, Indianapolis, IN, USA, ${ }^{6}$ Eli Lilly and Company, Indianapolis, IN, USA, ${ }^{7}$ OptonSonics, Inc., Oriental, NC, USA. 
Our purpose is to develop and test a molecular probe that can detect the expression of neutropilin-1 receptor in vivo using fluorescence imaging and photoacoustic spectroscopy. Introduction. Neutropilin-1 receptor (NPR-1) is expressed on endothelial cells and some breast cancer cells, and binds to vascular endothelial growth factor VEGF165, a growth factor associated with pathological tumor angiogenesis. This receptors is co-expressed with VEGFR and shown to enhance the binding of VEGF165 to VEGFR2; therefore, it has the potential to be used as a marker of angiogenic activity and targeted for therapy. Material and Methods. A peptide specific to NPR-1 receptor was synthesized and conjugated to a near infrared fluorochrome (IRDye800CW). This peptide (40 microg) was intravenously injected into three xenograft mouse models of breast cancer, VEGF transcripted MCF-7 tumors. Probe kinetics was monitored in vivo via near infrared fluorescence (NIRF) within an optical imager for up to 72 hours within the tumor and compared to other organs (liver, muscle) for binding specificity. After approximately 4-6 hours preliminary spectroscopic data was acquired within a small animal photoacoustic CT scanner (PCT-S). A multivariate fitting algorithm was used to spectrally deconvolve the IRDye $800 \mathrm{CW}$ from endogenous hemoglobin signature (hemoglobin concentration, $\mathrm{CtHb}$, and oxygen saturation, $\mathrm{SaO} 2$ ). Calibration phantoms were used to provide a tumor average measure of the NPR-1 concentration in NIRF imager and voxel concentrations in PCT-S. Results. Dynamics of the NIR fluorescence signal within the first hour after injection indicates specific binding compared to muscle. After $4-6 \mathrm{~h}$, a clear signal within the tumor is observed, and an average tumor-tomuscle ratio of $2.00(+/-0.27)$ measured. Measured photoacoustic spectra clearly indicated the presence of the NPR-1 probe was also identified within the tumor vasculature using photoacoustic spectroscopy, and a volumetric image was created to determine its distribution within the tumor. From calibration phantoms, the average tumor concentration from both NIRF and PCT-S was measured to be in the 200-250 nano-molar range. Conclusion. These preliminary results show the ability to image in vivo the expression of the neutropilin-1 receptor in breast tumors using NIR fluorescence imaging, and photoacoustic spectroscopy to measure NPR-1 expression and the hemoglobin state $(\mathrm{CtHb}$, and $\mathrm{SaO} 2)$ of the tumor.

\section{$\mathbf{J 1 8 5}$}

\section{A PHOTODYNAMIC MOLECULAR BEACON BASED ON A LINEAR SUPERQUENCHER}

Jonathan F. Lovell ${ }^{1}$, Juan Chen ${ }^{2}$, Gang Zheng ${ }^{2}$

${ }^{\mathrm{T}}$ Biomedical Engineering, University of Toronto, Toronto, ON, Canada, ${ }^{2}$ Department of Medical Biophysics, University of Toronto, Toronto, ON, Canada.

Photodynamic molecular beacons hold potential to target unwanted cells expressing mRNAs through Photodynamic Therapy (PDT). To reduce background effects, it is imperative that the beacons produce little singlet oxygen production in the closed state. One method to improve inactive state quenching of photodynamic molecular beacons is to use a group of multiple quenching subunits, known as a superquencher. This approach has previously used with a branched quencher design. Here we describe the first linear superquencher for quenching singlet oxygen, synthesized with a completely solid phase synthesis approach. A porphyrin conjugated solid support was developed and the molecular beacon quencher was composed of multiple Blackberry quencher subunits. This solid phase synthesis eliminated multiple conjugation and purification steps. Multiple quenching units altered the HPLC retention time, greatly facilitating purification of the full length product. Most importantly, multiple quenchers were more effective than a single quencher in terms of quenching background beacon singlet oxygen production. We examined the effects of 1,2 or 3 multiple quenchers on beacon performance with respect to stability (using thermal denaturation), kinetic activation, fluorescence and singlet oxygen production and describe the optimal superquencher design.

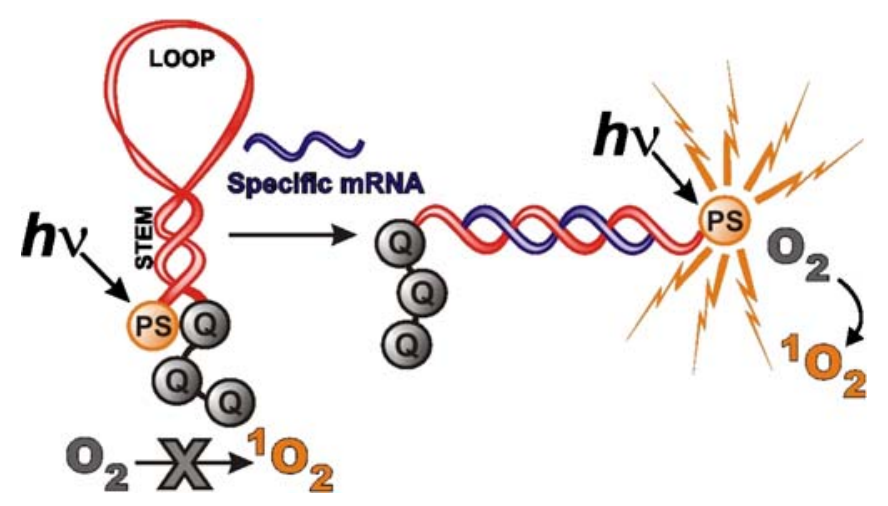

Schematic representation of the photodynamic beacon with with a linear superquencher. In the closed conformation (left) the beacon does not produce singlet oxygen. As the beacon hybridizes to a specific mRNA (right), it opens and the superquencher no longer can deactivate singlet oxygen generation.

\section{J186}

\section{IN VIVO BIOLUMINESCENCE IMAGING OF FURIN ACTIVITY IN BREAST CANCER CELLS USING LUMINOGENIC SUBSTRATES}

Anca Dragulescu-Andrasi, Gaolin Liang, Jianghong Rao Stanford University School of Medicine, Palo Alto, CA, USA.

Furin, a proprotein convertases family endoprotease, processes numerous physiological substrates and is overexpressed in cancer and inflammatory conditions. Non-invasive imaging of furin activity will offer a valuable tool to probe the furin function over the course of tumor growth and migration in the same animals in real time and directly assess the inhibition efficacy of drugs in vivo. Currently available fluorescence-based furin activity assays are limited to cultured cells. Here, we report successful bioluminescence imaging of furin activity in xenografted MBA-MB-468 breast cancer tumors in mice with luminogenic probes. The probes are conjugates of furin substrate, a consensus amino acid motif R-X-K/R-R (X, any amino acid), with the firefly luciferase substrate $\mathrm{D}$-aminoluciferin. In the presence of luciferase reporter, the probes are unable to produce bioluminescent emission without furin activation. Blocking experiments with a furin inhibitor and control experiments with a scrambled probe showed that the bioluminescence emission in the presence of firefly luciferase is furin-dependent and specific. After the furin activation, a 100-fold increase in the bioluminescent emission was observed in vitro and 50fold contrast between the probe and control was seen in the same tumor xenografts in mice. In summary, we have successfully developed first luminogenic probes for specific imaging of furin activity in vivo. Its availability could facilitate the study of furin function in tumorigenicity and discovery of new drugs for furintargeted cancer therapy. 
$\mathbf{J 1 8 7}$

\section{A QUANTITATIVE CONTINUOUS ENZYME ASSAY FOR INTRAMOLECULARLY-QUENCHED FLUOROGENIC PHOSPHOLIPASE SUBSTRATES}

Theresa M. Mawn, Anatoliy V. Popov, Jim Delikatny

Radiology, University of Pennsylvania, Philadelphia, PA, USA.

There has been recent growth in the development of activatable fluorescent molecular imaging probes, generally designed by placing fluorochromes on a cleavable substrate in close proximity to one another, such that they self-quench, but fluoresce upon separation via enzymatic cleavage of the substrate.

Although these probes offer excellent contrast, detection of enzyme activity is largely qualitative. In order to assess the effectiveness of a probe, it is useful to have a quantitative measure, such as the enzymesubstrate kinetic parameters. We have developed an assay to determine the kinetic parameters for Pyro-PL-BHQ, a fluorescent probe specific to phosphatidylcholine-specific phospholipase C. This assay is useful for comparing other fluorescent activatable phospholipase probes. The development of this assay includes corrections for intermolecular quenching, calibration, optimization of reaction mixtures, and determination of kinetic and inhibition parameters. Surface dilution kinetics was used to determine kinetic parameters: 1) the fluorescent substrate was varied while at a fixed mole fraction (Figure 1A);2) mole fraction was varied while holding the substrate constant (Figure 1B). Kinetic parameters (Vmax, $\mathrm{Km}$ and $\mathrm{Ks}$ ) were obtained from fitted data. We found that a mole fraction less than 0.005 will avoid aggregate quenching, yet we designed a method to correct for data at mole fractions greater than 0.005 . Fluorescence was calibrated to moles of product released. Egg-PtdCho lipid dispersions were found to be the optimal environment for enzyme reactions. The kinetic parameters were determined: $V \max =160.7( \pm 29.2) \mathrm{nmol} / \mathrm{min} / \mathrm{mg}, \mathrm{Km}=0.003 \pm 0.00007$ (mole fraction, unitless), and $\mathrm{Ks}=0.35( \pm 0.03) \mu \mathrm{M}$. From the inhibition study, a $\mathrm{Ki}=59 \mu \mathrm{M}$ was found.
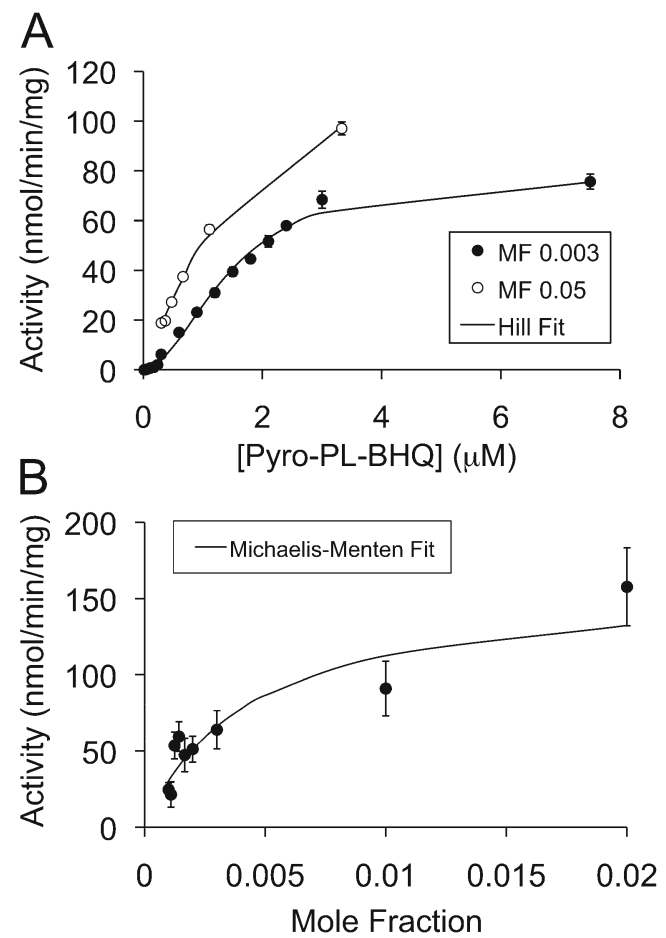

Figure 1 Surface dilution kinetics. (A) Total lipid ([PtdCho] + [PyroPL-BHQ]) was varied while holding mole fraction constant at 0.003 and 0.05 . (B) Mole fraction was varied by increasing [PtdCho] and holding substrate ([Pyro-PL-BHQ]) constant.

\section{$\mathbf{J 1 8 8}$}

\section{A PHOTOACOUSTIC TECHNIQUE TO ESTIMATE SUBSURFACE LASER FLUENCE FOR QUANTITATIVE MOLECULAR IMAGING}

Janaka C. Ranasinghesagara, Yan Jiang, Roger J. Zemp

Department of Electrical and Computer Engineering, University of Alberta, Edmonton, AB, Canada.

Photoacoustic imaging has recently shown great promise as a molecular imaging modality due to its ability to noninvasively visualize optical contrast of tissues with ultrasonic spatial resolution. It is being used to estimate blood oxygen saturation in blood vessels and image the distribution of nano-scale targeted contrast agents used in molecular imaging. When laser pulses are fired into a tissue, chromophores absorb the optical energy which is converted to thermal and acoustic energy. The photoacoustic signal amplitude is proportional to the local laser fluence, the local chromophore concentration and the respective molar extinction coefficients. To estimate chromophore bio-distribution quantitatively, the effective laser fluence at each subcutaneous location must be known. We developed a unique technique which will potentially calculate the local laser fluence at the subcutaneous locations from photoacousticbased estimates of tissue optical properties. In this technique, we focus a small laser spot on to the surface of a tissue and capture the photoacoustic signal by a focused ultrasound transducer. While translating the laser spot, the photoacoustic signal was measured by the stationary ultrasound transducer. The photoacoustic amplitude versus light spot translation distance provides an estimate of the normalized Green's function for light transport, which can then be used for estimating the desired laser fluence. We present experimental measurements for an Intralipid phantom with reduced scattering coefficients of 3,4 , and $5 \mathrm{~cm}-1$, and show that Monte Carlo simulations of light transport accurately reproduce experimental curves.

\section{$\mathbf{J 1 8 9}$}

IMMOBILIZED ACTIVATABLE BIOLUMINESCENT PROBES FOR IN VIVO IMAGING OF PROTEASE ACTIVITY IN TUMORS

Jianghong Rao, Zuyong Xia

Stanford University School of Medicine, Palo Alto, CA, USA.

We report here a novel strategy for in vivo imaging of enzyme activity at tumor, which provides a powerful tool for in vivo study the roles of enzymes in tumor biology and dynamic imaging of tumor response to drug treatment. In previous examples, fluorescent activatable probes are designed to image enzymes particularly proteases. One of the concerns with these examples is whether the enhanced fluorescence signal observed in tumors all came from the enzyme activation at the tumors or some came from enzymes at other locations but accumulated at the tumors due to the enhanced permeation effect (EPR). In addition, in spite of the amplification feature of the design, the observed contrast in vivo is generally small in these examples. In this presentation, we describe an immobilized activatable bioluminescent probe that we believe is able to truly 
image the extracellular protease activity in vivo. First, we took advantage of bioluminescence resonance energy transfer and generated a chemically quenched bioluminescent protein the conjugation with small chemical dyes. Protease cleavage led to the release of the quencher from the protein and thus the activation of the emission from the bioluminescent protein. This bioluminescence based detection offers a much greater sensitivity than the fluorescence based imaging. Furthermore, to ensure that the activated protein does not travel within the body and get accumulated into the tumors, we further fused the probe with the collagen binding protein. The binding of the probe to the abundant collagens restrained the probe mobility so the enzyme activated signals precisely reflect the local enzyme activity at tumor (Figure 1). With the designed probe, we were able to image the MMP activity exclusively in tumors with high sensitivity. Our approach opens a new avenue for study enzyme functions in vivo, which may have a significant impact on cancer research and tumor treatment.

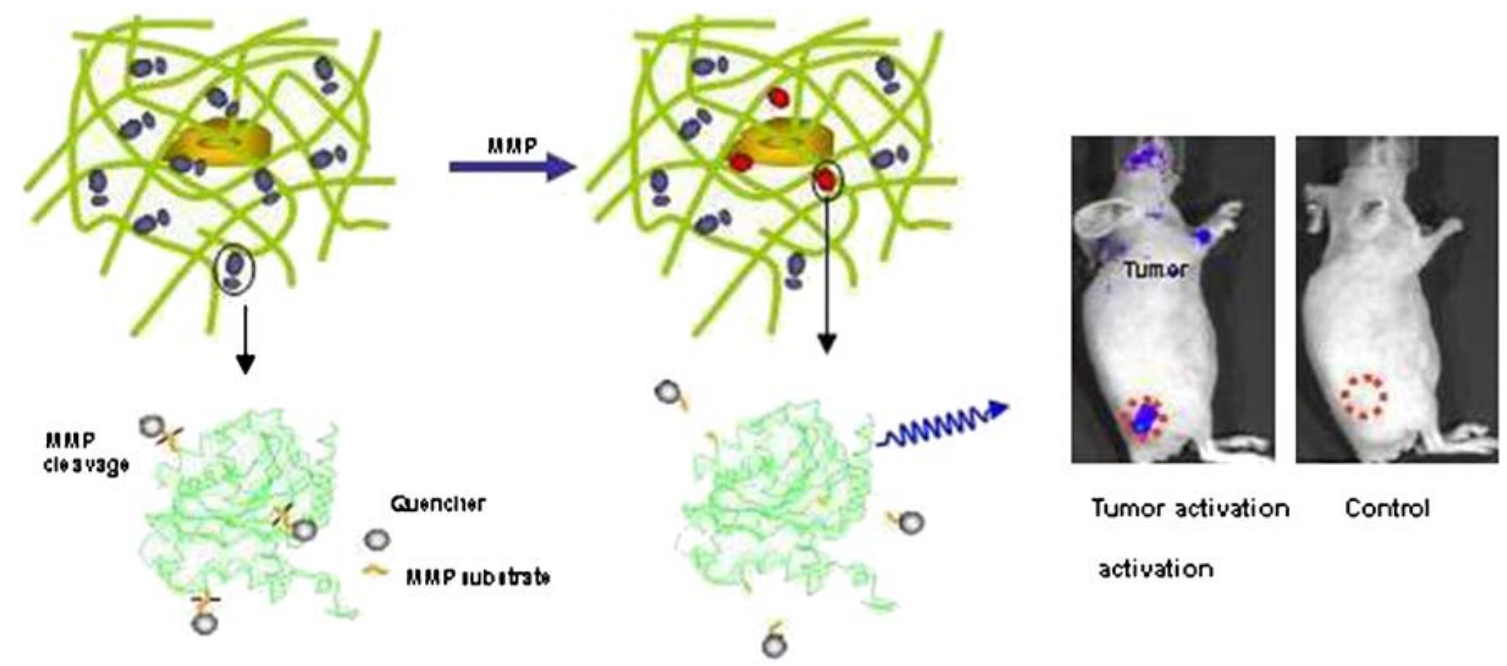

Figure 1. Immobilized activatable bioluminescent probe for in vivo imaging of enzyme activity. Bioluminescent proteins are chemically quenched with small dyes but can be activated by prote ase cleavage to restore the emission. Furthermore, the probe is fused to a collagen binding protein so it is immobilized at the extracellular matrix. MMP activity can thus be locally mapped and imaged.

J190

\section{FLUORESCENT ALKYL PHOSPHOLIPID ANALOGS FOR FLUORESCENCE IMAGING OF TUMORS IN VITRO AND IN VIVO}

Anatoly N. Pinchuk, Irawati K. Kandela, Marc A. Longino, Jamey P. Weichert, William R. Clarke

Cellectar, Madison, WI, USA.

CLR1401 (18-p-(iodophenyl)octadecyl phosphocholine, NM404), a radioiodinated alkyl phosphocholine analog, undergoes prolonged ( $>80$ days in mouse models) and selective retention in a wide variety of malignant tumors (Pinchuk AN et al. J Med Chem, 2006, $49,2155)$. On the basis of imaging and tissue distribution studies in several rodent tumor models, CLR1401 was chosen for follow-up evaluation in human cancer patients. We have synthesized a number of fluorescent alkyl phospholipid analogs which bear structural resemblance to CLR1401. The fluorophores in these probes are incorporated into hydrophobic alkyl chain of CLR1401. The selective tumor uptake of fluorescent alkyl phospholipid analogs was exemplified using BODIPY-modified phenyloctadecyl phospho- choline (CLR1501). In preliminary in vitro studies, A375 (melanoma) and 704sk (normal skin) cells were incubated with $7.5 \mu \mathrm{M}$ of CLR1501 for $24 \mathrm{~h}$. Results are shown in Figs 1 and 2. This procedure was repeated with 5 additional tumor cell lines: HCT-116 (colorectal adenocarcinoma), MES SA/Dx-5 (uterine sarcoma), Mia Paca-2 (pancreatic carcinoma), Ovcar-3 (ovarian adenocarcinoma) and U87-MG (glioblastoma). All 5 tumor cell lines demonstrated significant uptake of CLR1501 similar to CLR1401. Intracellular localization of CLR1501 was investigated in U87-MG (glioblastoma cells) using co-staining of intracellular organelles. Accumulation of CLR1501 was observed primarily in cytoplasm (green signal, Fig. 3). Yellow signals show co-localization of CLR1501 in mitochondria and cyan (blue-green) signals demonstrate co-localization in endoplasmic reticulum (ER). The cyan signals are observed in proximity to the nuclear membrane. This experiment has also demonstrated that CLR1501 does not enter the nucleus. In a subsequent study, athymic nude mice inoculated with Panc-1 (pancreatic carcinoma) were injected with CLR1501 24 and $96 \mathrm{~h}$ prior to imaging. In vivo fluorescence imaging has demonstrated pronounced accumulation of CLR1501 in tumors versus non-target organs and tissues. 


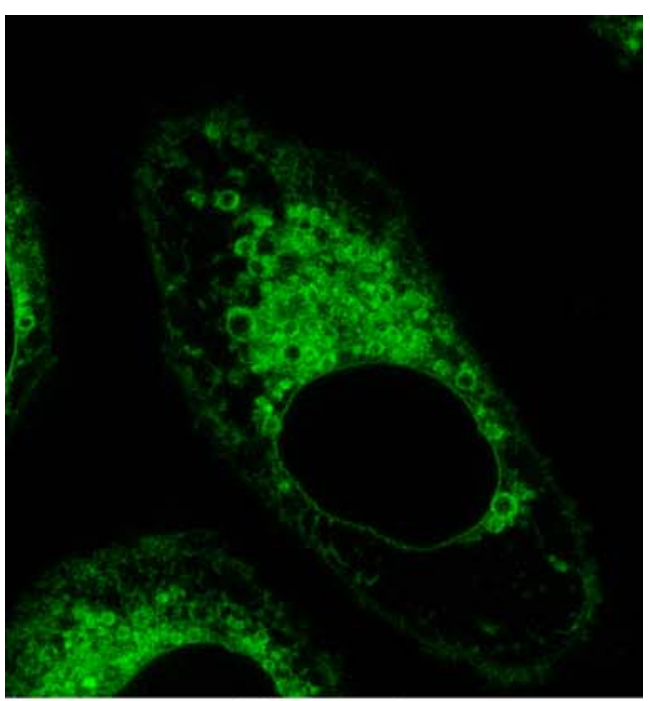

Figure 1

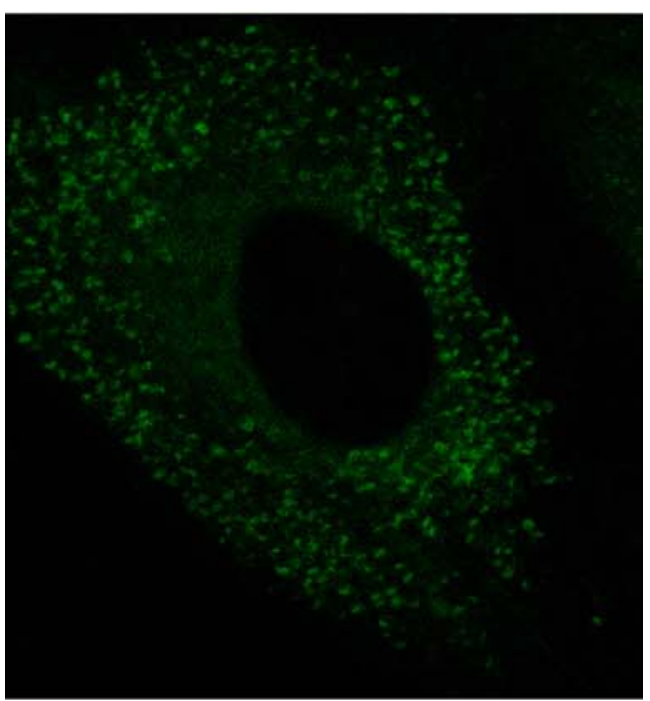

Figure 2

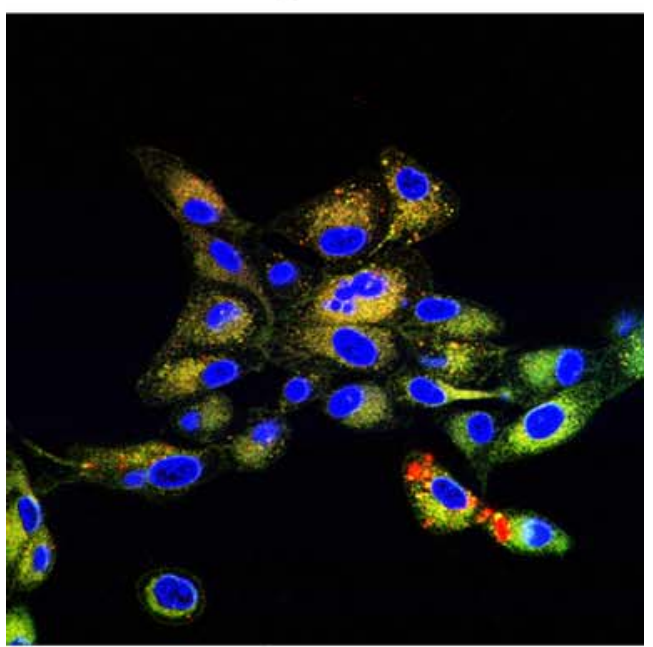

Figure 3
Figures 1 and 2: Selective incorporation of CLR1501 into A375 melanoma cells (Fig 1) versus 704sk normal skin cells (Fig 2). A375 cells showed internalization of CLR1501 into numerous fluorescent vesicles scattered throughout the cytoplasm. In 704sk cells, the CLR 1501 appeared to be transported to the lysosomes. Figure 3: The U87MG cells incubated with CLR1501 and co-stained with: Hoechst 33342 (blue) for nucleus; Blue-White DPX (blue) for endoplasmic reticulum; Mitotracker (red) for mitochondria. CLR1501: green.

\section{J191}

\section{MOLECULAR IMAGING AGENTS FOR INTRAOPERATIVE DIAGNOSIS AND TUMOR RESECTION}

Greg M. Thurber, Jose-Luiz Figueiredo, Ralph Weissleder

Center for Systems Biology, Mass General Hospital/Harvard Medical School, Boston, MA, USA.

Molecular imaging agents have paved the way for better diagnosis, staging, and treatment of cancer. So far, these agents have not been extensively used in the operating room, and surgeons rely primarily on visual (white light) images for tumor resection. Fluorescent molecular imaging probes have the ability to give real time feedback on what tissue to remove and provide images from the whole tumor down to the cellular level. The ability of the surgeon to visualize molecular expression intra-operatively will allow for more complete resections and potentially less radical surgery, resulting in improved patient morbidity and mortality. Using near infrared fluorescent antibodies and activatable fluorescent probes, we visualized cancer cells and tumor stromal tissue in subcutaneous and orthotopic models of several cancers. Localized fluorescence was sufficient to clearly delineate tumors even in highly autofluorescent organs such as the colon, and images could be sampled at a video frame rate $(30 \mathrm{fps})$ for real time feedback. A tumor to background ratio of 9 to 1 was achieved in one model system. An analysis of the transport and uptake rates for both types of probes is presented, which can provide guidance in the use of these probes and improve the current agents.

\section{$\mathbf{J 1 9 2}$}

\section{MULTI-FERROIC ACTIVATABLE PROBES FOR PROTEASE IMAGING}

Seulki Lee $^{1}$, Jin Xie ${ }^{1}$, Ki Young $\mathrm{Choi}^{2}$, Kwangmeyung Kim ${ }^{2}$, Ick Chan Kwon $^{2}$, Xiaoyuan Chen ${ }^{1}$

${ }^{1}$ Radiology, Stanford University School of Medicine, Stanford, CA, USA, ${ }^{2}$ Biomedical Research Center, Korea Institute of Science and Technology, Seoul, Korea, South.

Various strategies that amplify the fluorescent signal or boost specific target recognition properties drive the development of highly-sensitive activatable imaging probes. With their unique characteristics, such as strong optical fluorescence quenching efficacy, intense luminosity, and high photostability, certain types of metal-based imaging probes are suitable candidates for molecular optical imaging applications. We describe herein the potential of matrix metalloproteinase (MMP) activatable multi-ferroic imaging probes. We designed and prepared $20 \mathrm{~nm}$ dumbbell-shaped Au-Fe3O4 nanoparticles which were stabilized by NIR dye labeled MMP-2/9 substrate and biopolymer. Dopamine (Da)-based MMP substrate, Cy5.5-GPLGVRGDaKDaKDa, was anchored on the $\mathrm{Fe} 3 \mathrm{O} 4$ side and the PEG5k was linked to the Au side. The probes were lyophilizable and easily dispersed in aqueous buffer after reconstitution. The probe showed multi-quenching efficiency due to a combination of static quenching, fluorescence resonance energy transfer (FRET) and surface plasmon resonance (SPR) quenching mecha- 
nisms. The spectrofluorometry plots showed significant recovery of the fluorescent signals only in the presence of MMP-2, which was again inhibited by MMP inhibitors. Furthermore, the probe showed linear relationship between the recovered fluorescence signal and MMP-2 concentration in the range of sub-picomolar to nanomolar range. High florescent signal intensity in MMP-2/9-positive SCC-7 tumor xenograft model was also detected after intratumoral injection of the multiferroic probe. This platform technology can be widely applicable to any target proteases by changing the specific peptide motif between fluorophore and the multi-ferroic nanoparticle.

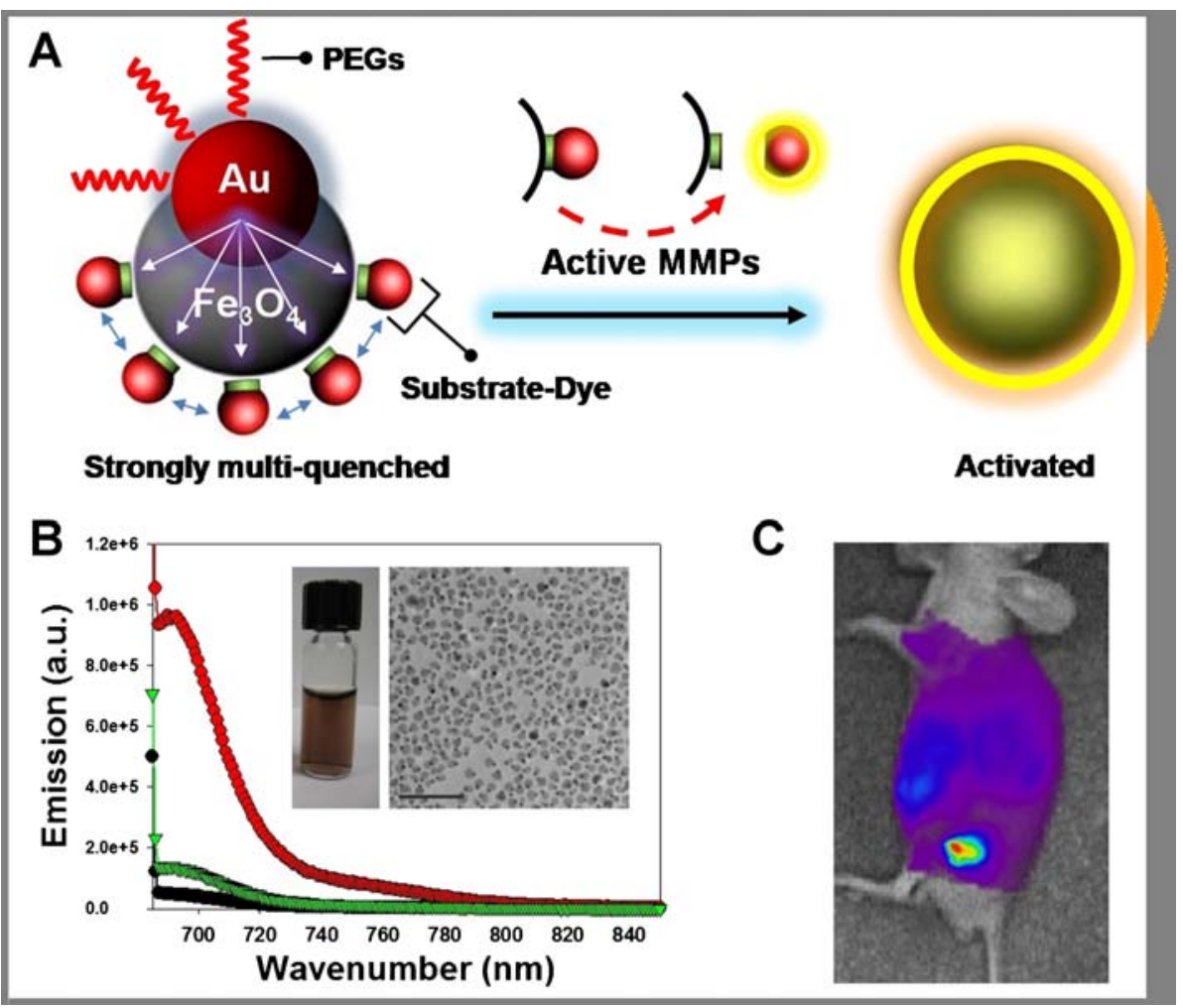

Figure. (A) Schematic diagram of the probe. (B) Fluorescence emission spectra of the probe in the presence of 15 nM MMP-2 (red), MMP-2 with inhibitor (1,10-phenanthrolone, $1 \mathrm{mM}$ ) (green) and buffer (black). Inset; photograph of the probe dispersed in water and TEM image showing the hybrid nanoparticle. (C) In vivo image of MMPs-positive SCC7 tumor bearing mouse.

\section{J193}

\section{QUANTIFYING GENE EXPRESSION IN LIVING BREAST CANCER CELLS USING MOLECULAR} BEACON-CONJUGATES

Antony K. Chen, Andrew Tsourkas

Bioengineering, University of Pennsylvania, Philadelphia, PA, USA.

Variations in gene expression are a major underlying determinant for dictating cell behavior. Accordingly, methods to measure gene expression, such as RT-PCR and DNA microarrays, have proven to be invaluable in understanding cell regulatory processes and disease mechanisms. However, these methods generally provide only the relative change in gene expression for a population of cells. Under many circumstances it is the stochasticity of RNA expression within single cells that drives phenotypic diversity and dictates cell fate. We have developed a molecular imaging probe, Quantitative Molecular Beacon (QMB), that allows for an absolute measure of gene expression in single living cells. Analogous to conventional molecular beacons, QMBs consist of a hairpin-forming antisense oligonucleotide labeled with a reporter fluorophore and a quencher. However, QMBs are also labeled with a reference dye, which remains unquenched regardless of the probe configuration. The reference signal allows us to determine the distribution and number of QMBs within each cell, while the ratio of reporter-to-reference signal provides us with a measure of probe hybridization. By comparing the QMB fluorescent signals to externally constructed standardization curves, we were able to quantitatively measure the stochasticity in the expression of the proto-oncogene cmyc within single living cells.

\section{J194}

\section{IMAGING AVB3 INTEGRIN EXPRESSION IN TRANSGENIC MOUSE GLIOMA MODEL USING IRDYE $800 \mathrm{CW}$ RGD PROBE \\ Jelena Vider, Ruimin Huang, Ronald G. Blasberg \\ Neurology, MSKCC, New York, NY, USA.}

Background and objective: Integrin $\alpha \mathrm{V} \beta 3$ has been demonstrated to be necessary for tumor-induced angiogenesis in gliomas. Previously it was shown that integrin $\alpha \mathrm{V} \beta 3$ is expressed in activated endothelial cells and glioma cells and its expression is associated with the grade of glioma malignancy. RGD (Arg-Gly-Asp) is a specific binding sequence for integrin receptors including $\alpha \mathrm{V} \beta 3$. It 
has been shown that near-infrared (NIRF) fluorescence imaging is feasible in xenograft models. Our objective is to image the expression of $\alpha \mathrm{V} \beta 3$ during gliomagenesis, since $\alpha \mathrm{V} \beta 3$ is expressed at a low level in normal brain. Methods: The RCAS/TVA transgenic mouse model was used to study glioma. Mice with intracranial injection of RCAS-PDGF (tumor group) and mice without injection (control group) were compared. Both groups were injected with 1 nmole (100 ul) of IRDye 800CW RGD (ex/em 778/789 nm) (LiCOR Biosciences, NE) in PBS. Mice were imaged immediately and 48 hours after injection with CRi Maestro imaging system using deep red filter set (ex $661 \mathrm{~nm} / \mathrm{em} 700 \mathrm{~nm}$ longpass). Mice were sacrificed after the last imaging session. Brain tissue was collected, imaged and fix-frozen for histological studies. Results: Non-specific signal from the IRDye $800 \mathrm{CW}$ RGD probe was detected immediately after injection. $48 \mathrm{~h}$ later, the tumor area could be visualized by NIR fluorescence imaging in the living animal and confirmed by ex vivo imaging of the sectioned brain. No fluorescence signal was observed in non-tumor brain areas or in the brain of control animals. Conclusions: Our study demonstrates the ability to image integrin $\alpha \mathrm{V} \beta 3$ expression in a well established transgenic mouse glioma model using a NIRF IRDye $800 \mathrm{CW}$ RGD probe. It suggests that this RGD probe might penetrate the blood-brain-barrier and the NIRF dye allows the probe detectable in the deep tissue like brain. The potential for screening drugs targeting integrin expression in gliomas will be assessed in the future.

\section{In Vivo}

\section{Glioma -}
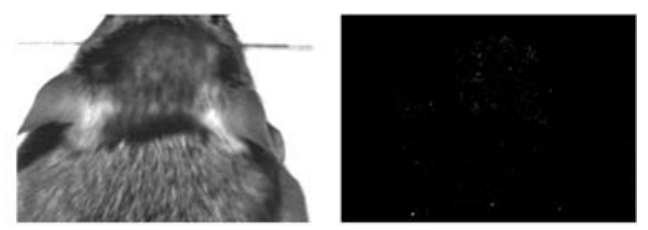

\section{Glioma +}

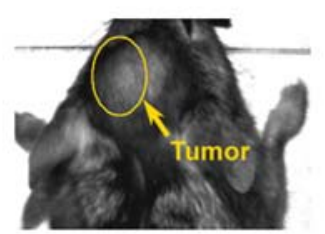

Photograph

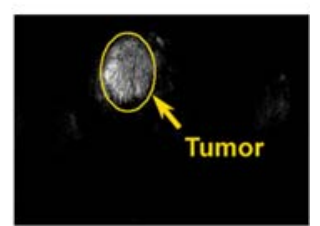

Probe-specific spectrum

Ex Vivo
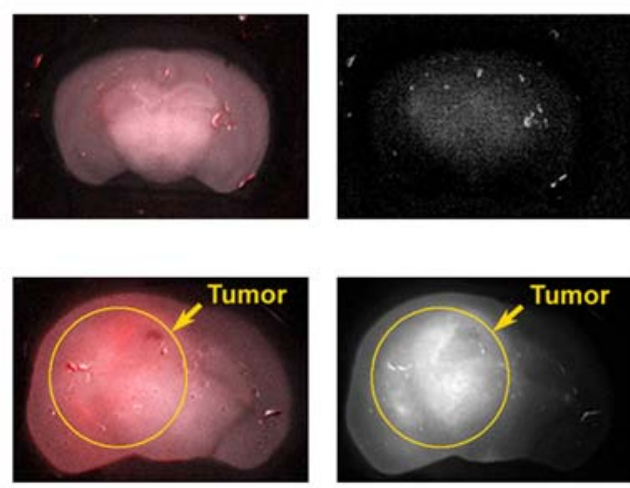

Pseudo-color overlay

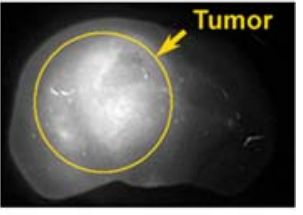

Probe-specific spectrum
$\mathbf{J 1 9 5}$

SYNTHESIS AND APPLICATION OF A HIGHLY SPECIFIC SUBSTRATE OF NEUTROPHIL ELASTASE FOR IN VIVO OPTICAL IMAGING OF LUNG INJURY

Nicolaos Avlonitis $^{1,2}$, Kevin Dhaliwal ${ }^{1}$, Geraldine Escher $^{2}$, Neil A McDonald $^{1}$, John Simpson ${ }^{1}$, Haslett Chris ${ }^{1}$, Mark Bradley ${ }^{2}$

${ }^{1}$ Centre for Inflammation Research, University of Edinburgh, Edinburgh, United Kingdom, ${ }^{2}$ Department of Chemistry, University of Edinburgh, Edinburgh, United Kingdom.

Neutrophil elastase (NE) has been postulated to be a key mediator of pulmonary tissue injury in vivo. $\mathrm{NE}$ is expressed both at the surface of neutrophils as part of neutrophil extracellular traps and free in the tissue milieu. Despite numerous experimental systems and models centrally implicating $\mathrm{NE}$, there remain no descriptions of imaging active NE in vivo in situ. Gauthier described a highly specific peptide as a substrate for NE. Herein, we report the synthesis of highly specific substrates of neutrophil elastase using a reporting FRET NIR fluorochrome pair using Cy5.5 and Cy7 (the dyes were synthesized following the methodology published in our group Org. Biomol. Chem., 2009, 7, 856). Using freshly isolated human neutrophils, we demonstrate active elastase imaging by confocal and flow cytometry. Extending to in vivo experimentation in models of lung inflammation, we show that this peptide allows real-time in vivo imaging of elastase activity measurable by reflectance and tomographic optical imaging. Inhibition of free elastase by antiproteinases is also demonstrated.

J196

TARGETING FLUORESCENT EGF IN PANCREAS TUMORS LEADS TO HIGH CONTRAST BUT LOW ACTUAL BINDING TO TUMOR

Kimberley Samkoe ${ }^{1}$, Omar Pardesi ${ }^{1}$, Julia A. O'Hara ${ }^{1,2}$, Brian W. Pogue ${ }^{1,3}$

${ }^{1}$ Thayer School of Engineering, Dartmouth College, Hanover, NH, USA, ${ }^{2}$ Department of Radiology, Dartmouth Medical School, Hanover, NH, USA, ${ }^{3}$ Department of Surgery, Dartmouth Medical School, Hanover, NH, USA.

The ability to image tumor contrast with molecular specificity is thought to be enhanced with the use of small molecular tracers with high affinity to their target and fast metabolic clearance rates. The fluorescently tagged EGF would be an ideal candidate for imaging aggressive tumors that undergo antibody therapy, such as in the pancreas. The AsPC-1 pancreas tumor was grown orthotopically in mice and used for fluorescence contrast analysis. Uptake of the EGF contrast agent was high, with near $3: 1$ contrast relative to the normal pancreas at long times after injection. The ability to image this molecular specific uptake with fluorescence tomography is very good, but the challenge is to determine the level of specific binding. To analyze this, slices of dissected tumors were used ex vivo, with imaging the fluorescence immediately after removal and then after a saline wash process, designed to remove the interstitially distributed contrast agent. Analysis of molecular specific binding in this manner indicated that approxiately $10 \%$ of the EGF tagged agent was bound, whereas $90 \%$ was sufficiently mobile to be washed away. This study indicates that while uptake of the contrast agent was good, and imaging was quite possible, there appears to be an endemic problem in achieving specific bound contrast levels. The 
use of this in MRI-guided fluorescence tomography is discussed, and examples of images shown.

\section{J197}

\section{SYNTHESIS AND IN VIVO EVALUATION OF SELF- QUENCHING REPORTERS FOR NEUTROPHIL ELASTASE} Nicolaos Avlonitis ${ }^{1,2}$, Kevin Dhaliwal ${ }^{1}$, Neil A. McDonald ${ }^{1}$, John Simpson $^{1}$, Haslett Chris ${ }^{1}$, Mark Bradley ${ }^{2}$

${ }^{1}$ Centre for Inflammation Research, University of Edinburgh, Edinburgh, United Kingdom, ${ }^{2}$ Department of Chemistry, University of Edinburgh, Edinburgh, United Kingdom.

The approach of using internally quenched fluorescence via the use of mono-dispersed dendrimers offers a very simple method of "internal FRET" and was initially described in our group as a method of assaying proteases. This approach relies on multiple copies of a single type of fluorophore, giving rise to internally quenched fluorescence and occurs for small Stoke's shift dyes, when the emission and excitation wavelengths of the fluorophore are close together. In this situation the dendrimer generates a high-local concentration of fluorophore, which permits efficient quenching while hydrolysis releases multiple copies of the dyes causing a large increase in emission. Herein, we describe the synthesis of a selfquenching substrate, which is highly specific for neutrophil elastase (NE) and it's use in preclinical applications in models of lung inflammation.

\section{J198}

\section{TARGETED PEPTIDES FOR MEDULLOBLASTOMA IDENTIFIED WITH PHAGE DISPLAY}

Henry Haeberle

Radiology, Stanford, Stanford, CA, USA.

Medulloblastoma is an undifferentiated, neuroepithelial tumor of the cerebellum and the most common malignant brain tumor in children. While surgical resection has significantly improved survival rates for medulloblastoma patients, approximately onethird die and $20 \%$ of survivors sustain severe neurological damage. This neurological damage is caused mostly from inexact tumor resection, and this presents a treatment dilemma for the neurosurgeon since larger resections correlate with improved patient outcome, but such resections are more likely to damage surrounding healthy brain tissue. Molecular probes with specificity for medulloblastoma would serve to guide surgeons and help identify tumor margins for improved resection. To visualize tumor cellsurface markers, we have screened a phage display library of peptides against malignant tissue derived from a mouse model of medulloblastoma (ptc +/-; p53 -/-). We have identified 49 unique phage clones that bind medulloblastoma tissue, and several of these were isolated repeatedly suggesting significance as strong candidate probes. These clones bear sequences with similarity to several mammalian genes for whom binding partners have been identified, including the alpha-subunit of the $\mathrm{K}+$ channel delayed-rectifier Kv9.1. Repeated identification of phage encoding peptides from different DNA sequences suggests that key molecular targets have been identified and that these screens will contribute to our understanding of disease mechanisms. These data suggest that phage display strategies can identify peptide sequences for development a molecular probes, but will also identify surface key genes markers for a greater understanding of basic mechanisms of disease.
J199

SIMULTANEOUS IMAGING OF CANCER BIOMARKERS WITH A NOVEL MOLECULAR IMAGING PLATFORM Ann-Marie Broome ${ }^{1,4}$, Gopal Ramamurthy ${ }^{2}$, Nihir R. Bhavsar ${ }^{1}$, Daniel Glazer $^{2}$, Gail Newton ${ }^{3}$, James Basilion ${ }^{2,4}$

${ }^{1}$ Biomedical Engineering, Case Western Reserve University, Cleveland, OH, USA, ${ }^{2}$ Radiology, University Hospital, Cleveland, OH, USA, ${ }^{3}$ Pathology, Brigham and Women's Hospital, Boston, MA, USA, ${ }^{4}$ National Foundation for Cancer Research, Case Western Reserve University, Cleveland, $\mathrm{OH}$, USA.

The genomics revolution has enabled investigators to discern changes in gene expression that are indicative of the biological states of cancer. These so called cancer signatures are manifested by changes in the expression of more than one gene product and can be utilized to interrogate intricately choreographed spatio-temporal molecular events during cancer progression. Given that disease is recognized both by its complexity and progression, methods that rely on a single biomarker are self-delimiting. Molecular imaging of cells or cellular processes can be obtained by targeting multiple cell surface receptors implicated in tumorigenesis, such as the epidermal growth factor receptor (EGFR) and transferrin receptor (TfR), and by imaging using a robust, noninvasive reporter complex. Historically, $\beta$-galactosidase ( $\beta$ gal) has been widely used and remains exceedingly popular as a reporter enzyme. By undertaking a totally novel strategy, we exploit transcomplementation of $\beta$ gal to reconstitute enzymatic activity at the site of multiple marker overexpression. We expand an intuitive molecular approach to identify enzyme fragments capable of proper orientation to reconstitute $\beta$ gal activity. In essence, we have engineered completely unique $\beta$ gal fragments from those previously identified by truncation mutants. These studies show that the $\beta$ gal can be completely renatured from its fragmented monomers. Individually, the monomer fragments are small, stable, and enzymatically inactive. When the correct combination of $\beta$ gal fragments are incorporated in bacteria or in mammalian cells, transcomplementation occurs and full enzymatic activity is restored. Further, we demonstrate the utility of these fragments for targeted-complementation in live cell assays and demonstrate the robustness of the system with an eye on utilizing these fragments to investigate molecular signatures associated with disease. By expanding the number of biomarkers available to diagnostic and therapeutic purview, we envision a novel and innovative platformbased approach to disease identification, staging, and treatment.

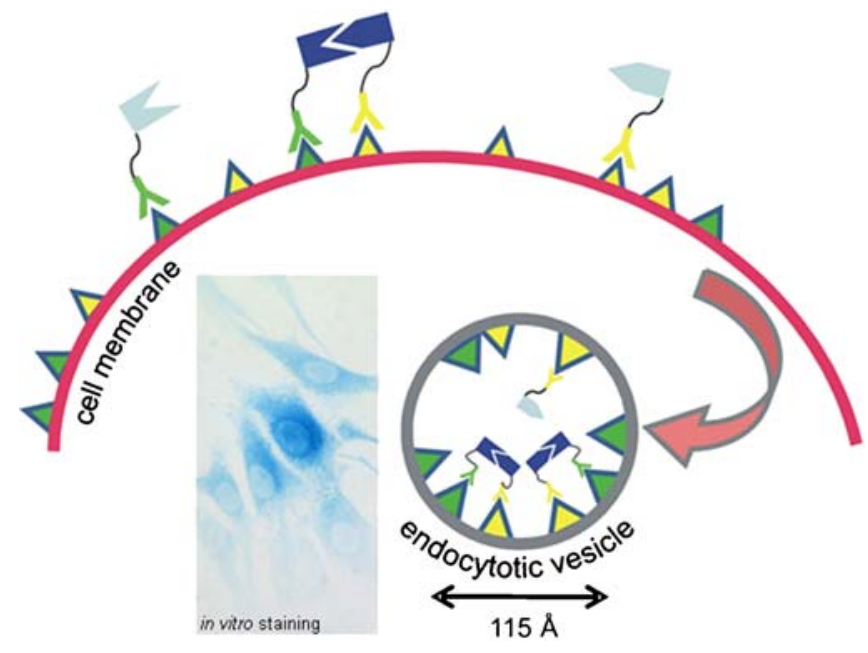

Multi-valent imaging complex 
$\mathbf{J 2 0 0}$

\section{SMALL MOLECULE PROBES FOR LIVE CELL IMAGING OF MEMBRANE PROTEINS WITH AN N-TERMINAL CYSTEINE}

Hongjun Ren ${ }^{1}$, Ke Zhan ${ }^{2}$, Zuyong $\mathrm{Xia}^{2}$, Jianghong Rao ${ }^{2}$

${ }^{1}$ Chemistry, Zhejiang University, Hangzhou, China, ${ }^{2}$ Radiology, Stanford University School of Medicine, Stanford, CA, USA.

Genetic fusion of green fluorescent protein (GFP) and its color variants to proteins of interest enables direct visualization of protein dynamics, localization, and interactions in single living cells. The relatively large size ( $\sim 238$ amino acids) of GFPs however may potentially alter structure and/or function of the host proteins, such as mislocalization or misexpression. Additionally, GFP based fluorescent imaging requires certain copies of transcripts and time of fluorophore maturation, with the stability of such fluorescent tags usually causing target protein dynamic study difficult since the accumulation of fluorescent-tagged protein builds up substantial level of background. Chemical labeling methods have been developed as an alternative to address these drawbacks, however the size of the labeled complex remains large in these systems. In order to develop a smaller fluorescent tag that could enable us to directly visualize interested protein in living cells, we have taken advantage of the cyclization reaction between the cyano precursor (CBT) and cysteine residue. Our in vivo study indicates that synthesized Rhodamine-CBT with a tetra-ethyl linker labels overexpressed membrane protein specifically in living cells. With the TEV protease substrate sequence inserted between the murine Ig $\kappa$-chain leader sequence and cyan fluorescent protein (CFP), we have TEV protease treatment in the expressed HeLa cell culture followed by Rhodamine-CBT labeling. The TEV protease removed $\mathrm{N}$-terminal murine Ig $\kappa$-chain leader sequence efficiently for $30 \mathrm{~min}$ at $37^{\circ} \mathrm{C}$ and Rhodamine-CBT ligated to the newly generated $\mathrm{N}$-terminal cysteine of expressed CFP at the cell surfaces within $5 \mathrm{~min}$ at $4^{\circ} \mathrm{C}$. When 15 to $60 \mathrm{~min}$ incubations were followed at $37^{\circ} \mathrm{C}$, Rhodamine-CBT showed an endocytozed pattern into the cytoplasm along with the labeled CFP. Our results demonstrate that the CBT based ligation reaction can specifically label membrane proteins terminated with $\mathrm{N}$-terminal cysteine on living cells. In comparison to other chemical labeling approaches, this system offers a single amino acid tag in a simple one-step labeling procedure, with minimal perturbations to the cells. This simple ligation reaction is compatible with mild physiological conditions and proceeds with a high degree of specificity. It offers an important alternative to currently available protein labeling methods, and opens new horizon of studying the protein dynamics in living cells.

MOLECULAR IMAGING OF C-MET KINASE ACTIVITY

Craig J. Galban ${ }^{1}$, Limin Zhang ${ }^{2}$, Shama Virani ${ }^{2}$, Yu Zhang ${ }^{2}$, Mahaveer S. Bhojani ${ }^{2}$, Brian D. Ross ${ }^{1}$, Alnawaz Rehemtulla ${ }^{2}$

${ }^{1}$ Radiology, University of Michigan, Ann Arbor, MI, USA, ${ }^{2}$ Radiation Oncology, University of Michigan, Ann Arbor, MI, USA.

The receptor tyrosine kinase c-Met and its ligand hepatocyte growth factor/scatter factor $(\mathrm{HGF} / \mathrm{SF})$ modulate signaling cascades implicated in cellular migration, invasion, proliferation, survival, and angiogenesis. Consequently, dysregulation of the c-Met/HGF pathway can compromise the cellular capacity to moderate these activities, and lead to cellular proliferation, therapeutic resistance and metastasis in various human malignancies. The therapeutic abrogation of HGF/Met receptor coupling or c-Met receptor-mediated signaling events may provide promising clinical insights into more effective strategies for preventing of tumor growth and spread. In our present study, we constructed a reporter molecule whose bioluminescent activity can be used as surrogate for c-Met tyrosine kinase activity. C-Met activity in cultured cells and tumor xenografts was monitored quantitatively and dynamically in response to the activation or inhibition of the HGF/c-Met signaling pathway via a c-Met kinase inhibitor and an HGF neutralizing antibody. Treatment with the c-Met inhibitor and the HGF neutralizing antibody stimulated the reporter's bioluminescence activity in a dose dependent manner as well as caused rapid regression of U-87 MG tumor xenografts. Results obtained from these studies provide unique insights into the pharmacokinetics and pharmacodynamics of agents that modulate cMet activity, validating c-Met as a target for human glioblastoma therapy and reveal the utility of this reporter for developing optimal dosing and schedule parameters for maximal tumor control in cancer treatment.

$\mathbf{J 2 0 2}$

\section{FLUORESCENT CYANINE LABELING REAGENTS FOR MOLECULAR IMAGING}

YoungSoo Kim ${ }^{1}$, Jinwoo Park ${ }^{1,2}$, Jong Joo Na ${ }^{2}$, Dong Jin Kim ${ }^{1}$

${ }^{1}$ Life Sciences Research Division, Korea Institute of Science and Technology, Seoul, Korea, South, ${ }^{2}$ Department of Biotechnology, BioActs, Incheon, Korea, South.

Molecular imaging has become one of the most powerful detection tools in life sciences and it has been stimulated by development of various fluorescent labeling reagents. Diverse biomolecules such as proteins, antibodies, nucleic acids, lipids, and polymers are common labeling targets for in vitro/vivo study and drug discovery. A collection of twenty one novel cyanine dyes, Flamma Fluor P, was synthesized for staining diverse biomolecules, and the photophysical characteristics relevant to applications in bio imaging were analyzed in detail. Their emission spectrum range covers from $550 \mathrm{~nm}$ to $800 \mathrm{~nm}$ and they are highly pure, water soluble, $\mathrm{pH}$ insensitive, temperature stable, photostable, and reactive in both aqueous and organic conditions. These labeling reagents retain a unique binding site and can be readily conjugated to amino, hydroxyl, and thiol groups. Therefore, they enable facile staining of biomaterials missing amine or thiol groups such as carbohydrates. Moreover, via michael addition reaction, they do not leave by-products like N-hydroxysuccimide during conjugation.

$\mathbf{J 2 0 3}$

\section{MOLECULAR IMAGING OF LIPID PEROXYL RADICALS IN LIVING CELLS WITH A BODIPY-ALPHA-TOCOPHEROL ADDUCT \\ Armen Khatchadourian $^{1}$, Katerina G. Krumova ${ }^{2}$, Sebastien Boridy ${ }^{1}$, An Thien $\mathrm{Ngo}^{2}$, Dusica Maysinger ${ }^{1}$, Gonzalo $\mathrm{Cosa}^{2}$ \\ ${ }^{1}$ Pharmacology, McGill University, Montreal, QC, Canada, ${ }^{2}$ Chemis- try, McGill University, Montreal, QC, Canada.}

An increasing number of reports discuss the role reactive oxygen species (ROS) have in cellular pathologies and cellular signaling 
processes. Critical to elucidating the underlying chemical mechanism behind these biological processes is the development of novel sensors and reporters with chemical sensitivity and, more importantly, molecular specificity, enabling the spatial and temporal monitoring of a specific ROS concentration in live cells. Here we report for the first time on the application of BODIPY-alpha-Tocopherol adduct (B-TOH), a novel lipophilic fluorescent antioxidant indicator, toward detection of peroxyl radicals in model lipid membranes and their imaging in the lipid membrane of live cells. Studies conducted in model lipid membranes show a 5-fold fluorescence enhancement upon reaction of liposome-embedded B-TOH with peroxyl radicals. The enhancement is independent of the solution $\mathrm{pH}$ and membrane composition. In studies in live cells performed under states of growth factor withdrawal and increased oxidative stress, a significant increase in B-TOH emission was also observed. Exogenous sources of free radicals were utilized herein, namely,
N,N'-dimethyl-4,4'-bipyridinium dichloride (also known as methyl viologen or paraquat) and uncoated nonemissive CdTe nanoparticles, a source of $\mathrm{Cd}(2+)$. The recorded fluorescence intensity of $\mathrm{B}-\mathrm{TOH}$ was proportional to the concentration of the dye and to the level of cellular oxidative stress. By employing fluorescent dyes such as Lysotracker and Nile Red, we demonstrate the formation of peroxyl radicals in subcellular locations in rat pheochromocytoma (PC12 cells) and in primary mouse hippocampal neural cells under oxidative stress conditions. Specifically, we observed peroxyl radicals in lysosomes. The assessment of the subcellular distribution of B-TOH in living cells deprived from growth factors and/or under oxidative stress may be useful in the future in determining subcellular sites of lipid peroxidation. In summary, results from this study underscore the potential of $\mathrm{B}-\mathrm{TOH}$ as a sensitive and specific probe enabling the molecular imaging of peroxyl radicals in the lipid membranes of live cells.

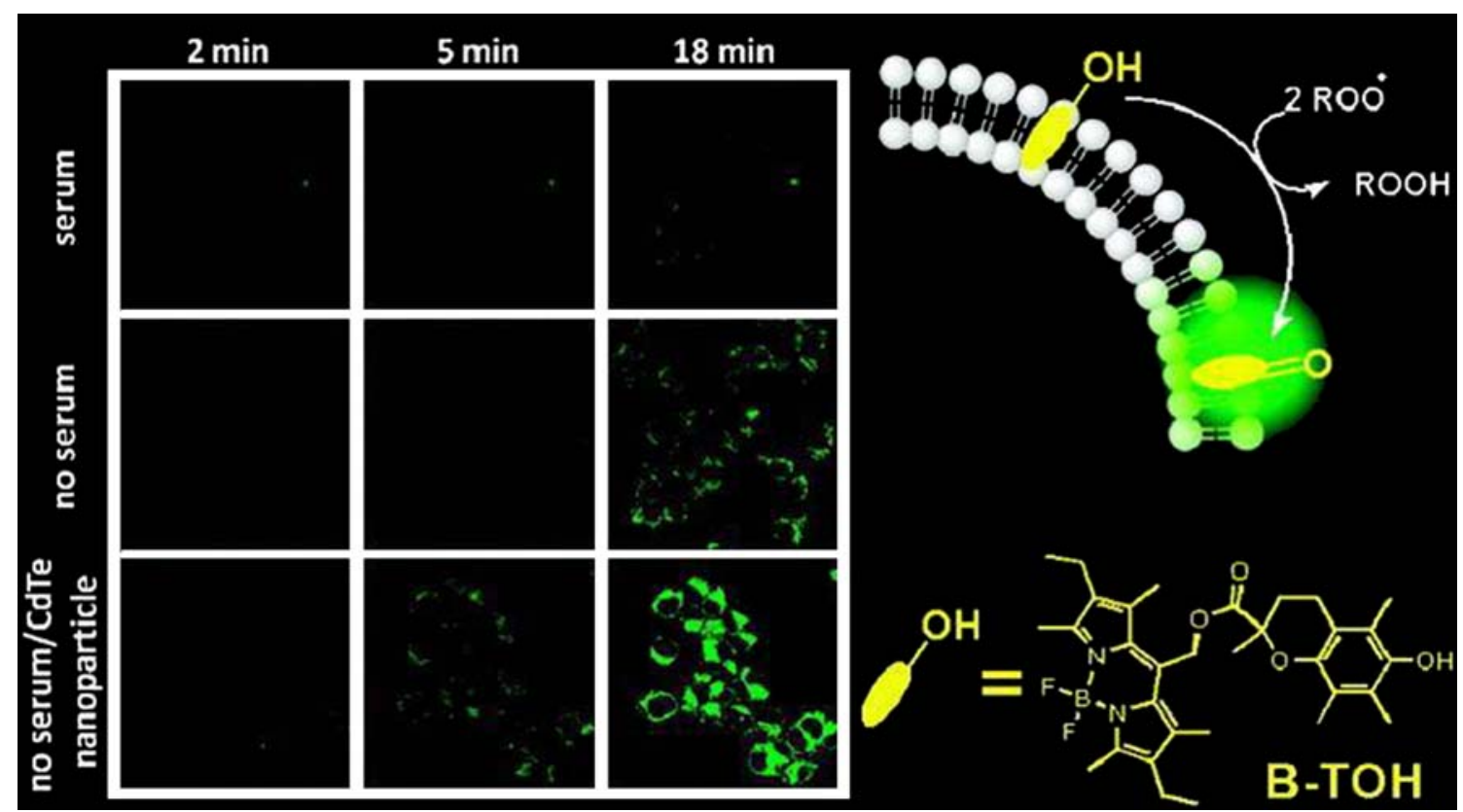

$\mathbf{J 2 0 4}$

\section{MOLECULAR IMAGING OF RETROGRADE NEURAL TRANSPORT USING AN OPTICAL AGENT}

Dawid Schellingerhout, Lucia LeRoux, Sebastian Bredow, Juri G. Gelovani Unit 370, University of Texas M D Anderson Cancer Center, Houston, TX, USA.

Goal: To develop an agent for imaging retrograde axonal transport in nerve tissue, based on optical labeling of a fragment of tetanus toxin protein. Methods: A His-tagged recombinant tetanus toxin fragment (TTF) was produced in E.coli, purified, and labeled with a variety of Alexa fluorophors for optical imaging studies. Gel electrophoresis (SDS-PAGE) and quantitative immunodetection studies were performed. Cell uptake studies were performed to assess in vitro efficacy. Labeled TTF was injected into the soleus muscle of $\mathrm{C} 57 \mathrm{bl}$ and $\mathrm{Balb} / \mathrm{C}$ mice, and Wistar rats, and imaging performed with the IVIS 200 (Xenogen). In situ validation was performed with laser scanning confocal microscope FV 1000 (Olympus) utilizing intact glycerol mounted samples, and cryosections. Results: Gel electrophoresis and quantitative immunodetection indicated that the integrity and immune reactivity of the protein was preserved after labeling. Cell uptake assays indicated robust uptake in differentiated $\mathrm{PC} 12$ cells. In vivo optical imaging 
demonstrated the uptake of TTF-Alexa in the sciatic nerve. Confocal microscopy studies on intact excised nerve segments and cryosections confirmed the compound uptake in nerve fascicles of the sciatic nerve. Axonal nerve uptake and superficial lymphatic uptake were clearly distinguishable, and transport was shown to be nerve-specific. Immunohistochemistry on cord sections demonstrated the presence of the agent in spinal cord neurons. Conclusion: Fluorescently labeled TTF is taken up into motor nerve endings after intramuscular injection, and is retrogradely transported in axons.
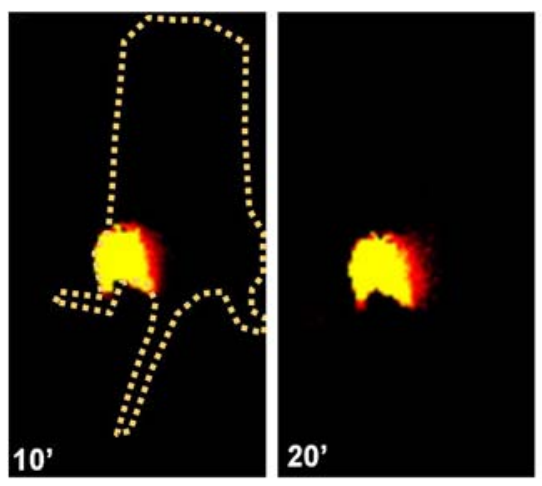
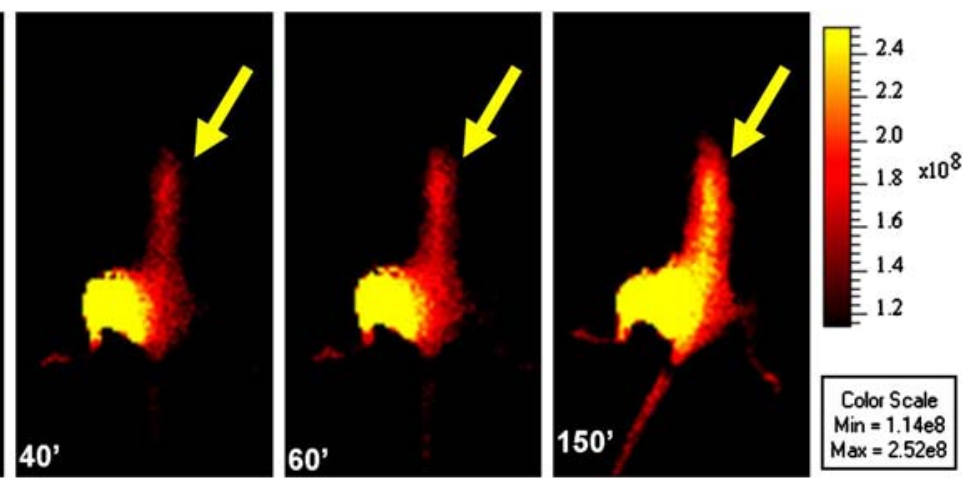

\section{NEAR-INFRARED FLUORESCENT NON-PEPTIDE BIVALENT INTERGRIN AVB3 ANTAGONIST FOR TUMOR IMAGING}

Zheng $\mathrm{Li}^{1}$, Feng $\mathrm{Li}^{1}$, Jiacheng Liu ${ }^{1}$, Daniel Y. Lee ${ }^{1}$, Gouri S. Jas ${ }^{2}$, Guoting Qin ${ }^{1}$, Jiawei Zhang ${ }^{1}$, Zheng-Zheng Shi ${ }^{1}$, King Li ${ }^{1}$

${ }^{1}$ Radiology, TMHRI, Houston, TX, USA, ${ }^{2}$ Department of Chemistry, Biochemistry, Baylor University, Waco, TX, USA.

Integrin $\alpha v \beta 3$ has been intensively studied due to its important role in tumor angiogenesis and tumor metastasis. It is highly expressed on activated tumor endothelial cells and fast-growing tumor cells but not resting endothelial cells and normal tissues, making it an attractive molecular target for tumor therapy and imaging. Although a series of monomeric and multivalent arginine-glycine-aspartic acid (RGD) peptides have been reported as potential ligands for targeting $\alpha v \beta 3$ in molecular imaging, non-peptide antagonists were rarely used and even less have used rational design for development. In this study, a bivalent non-peptide small molecule intergrin $\alpha \mathrm{v} \beta 3$ antagonist (IA) has been synthesized based on the in silico rational design approach. A near-infrared (NIR) fluorescent imaging probe has been developed based on this bivalent compound. In vitro binding assays have shown that the dimeric IA (IC50 $=0.40 \pm$ $0.11 \mathrm{nM}$ ) complex exhibited significantly improved integrin $\alpha \mathrm{v} \beta 3$ affinity when compared to the IA monomer (IC50=22.33 \pm $4.51 \mathrm{nM}$ ), resulting in an over 50 fold improvement in receptor affinity. Fluorescence microscopy studies demonstrated integrinmediated endocytosis of bivalent IA-Cy5.5 in U87 cells which was effectively blocked by unlabeled bivalent IA. We also demonstrated promising tumor accumulation of this NIR imaging probe in U87 mouse xenografts.

\section{DEVELOPMENT OF A FAST ACTIVATING NEW NEAR INFRARED-LABELED AGENT FOR DETECTING CATHEPSIN K ACTIVITY}

Sylvie Kossodo ${ }^{1}$, Kevin Groves ${ }^{1}$, Jun Zhang ${ }^{1}$, Emma Handy ${ }^{1}$, Garry J. Cuneo $^{1}$, Chiara Buono ${ }^{1}$, Jeffrey D. Peterson ${ }^{1}$, Milind Rajopadhye ${ }^{1}$, Karen Madden ${ }^{1}$, Michael Klimas ${ }^{2}$, Cyrille Sur ${ }^{2}$, Patrick Leung ${ }^{2}$, Maureen Pickarski ${ }^{2}$, Le Duong ${ }^{2}$, Bohumil Bednar ${ }^{2}$

${ }^{1}$ VisEn Medical, Bedford, MA, USA, ${ }^{2}$ Merck Research Laboratories, West Point, PA, USA.

Cathepsin K (CatK), a lysosomal cysteine protease with strong collagenolytic activity, is expressed primarily in osteoclasts, chondrocytes and synovial fibroblasts. Since CatK is critically involved in bone resorption and collagen degradation, CatK inhibitors are being evaluated in clinical trials for osteoporosis and the treatment of women with breast cancer and bone metastases. Clearly, a specific imaging agent allowing the detection, quantification and monitoring of CatK activity in vivo would prove valuable in pre-clinical and clinical settings. Herein, we report the design, synthesis and evaluation of a fast-activating nearinfrared fluorescence (NIRF) CatK imaging agent. The agent, VM269, was developed based on a human CatK-cleavable sequence, a pair of self-quenching NIRF fluorochromes and a pharmacokinetic modifier to confer increased blood half-life. The agent is optically quenched in its native form, but upon cleavage by CatK, becomes highly fluorescent with ex/em at 674/692 nm. In vitro selectivity was assessed by cleaving VM269 with a panel of enzymes and monitoring released fluorescence over time. The agent was preferentially cleaved by CatK (Figure 1). Cell uptake assays showed that VM269 was activated by human synovial 
fibroblasts from rheumatoid arthritic patients and rabbit synoviocytes. In human osteoclasts on bone, VM269 localized to lysosomes and the resorption lacunae (Figure 2a). The activation was significantly decreased in synovial fibroblasts treated with specific CatK inhibitors (Fig.2b). VM269 exhibited blood half-life of 25 minutes providing good distribution in target tissue, thus enabling a suitable window for in vivo imaging. These results highlight the potential of VM269 for detecting and monitoring CatK activity in osteoporosis and arthritis, as well as in atherosclerosis, lung remodeling, obesity and cancer.

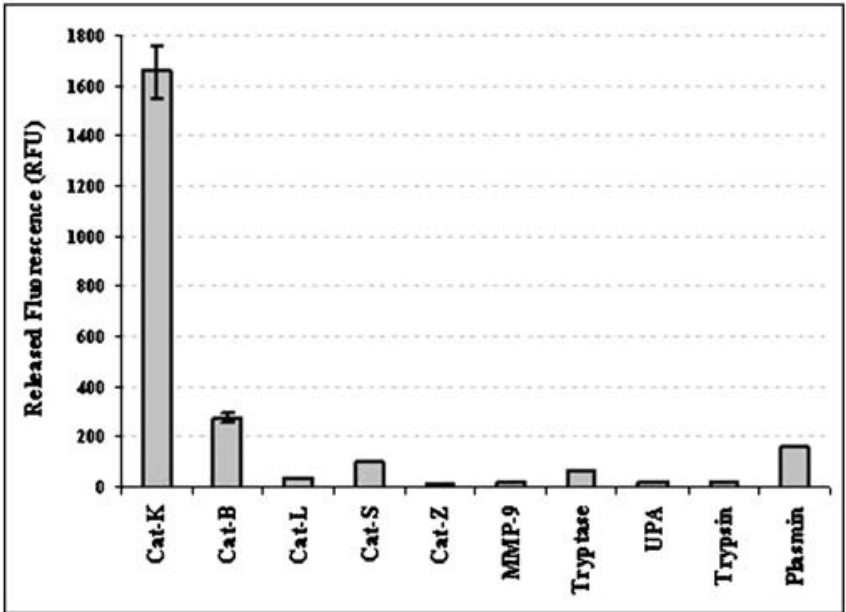

Figure 1. a
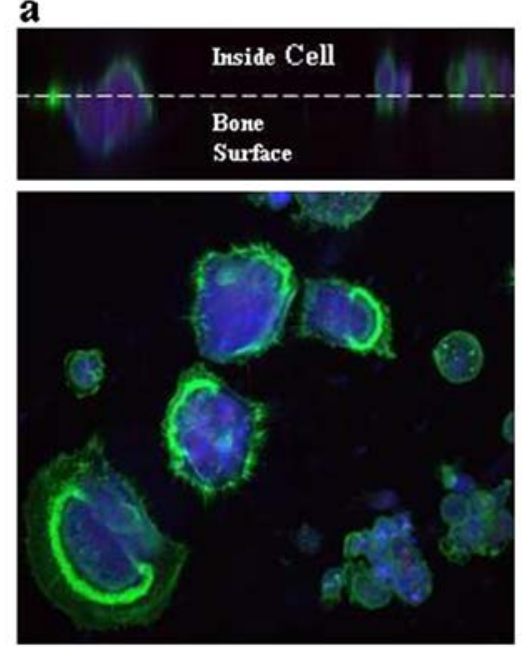

b

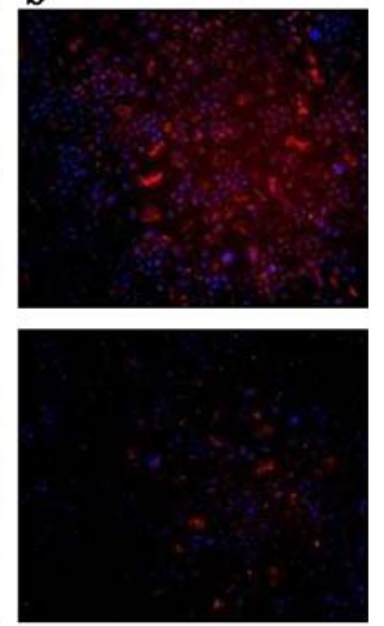

Figure 2.

Fig.1: VM269 activation by a panel of enzymes (at 5 h). Fig.2: (a) Activation of VM269 by osteoclasts. Green: Phalloidin, Blue: VM269, Red: Lysotracker. (b) Activation of VM269 hy human synovial fibroblasts in the absence (top) or presence (bottom) of a specific CatK inhibitor. Blue: DAPI, Red: VM269.

\section{Novel PET/SPECT Probes}

$\mathbf{J 2 0 7}$

TARGETING GLP-1R FOR PET IMAGING OF PANCREATIC $\beta$ CELL MASS USING ${ }^{68}$ GA-LABELED GLP-1 PEPTIDE ANALOGS $\underline{\mathrm{Su}-T a n g} \mathrm{Lo}^{1}$, Mai Lin ${ }^{1}$, Michael Long ${ }^{1}$, Eunice Murage ${ }^{2}$, Dean Sherry $^{3,1}$, Jung-Mo Ahn ${ }^{2}$, Xiankai Sun ${ }^{1,3}$

${ }^{1}$ Department of Radiology, UT Southwestern Medical Center at Dallas, Dallas, TX, USA, ${ }^{2}$ Department of Chemistry, University of Texas at Dallas, Dallas, TX, USA, ${ }^{3}$ Advanced Imaging Research Center, UT Southwestern Medical Center at Dallas, Dallas, TX, USA.

The sparsely distributed $\beta$ cell population in the pancreas along with the physical location of the pancreas imposes a great challenge to the development of an imaging technique to non-invasively determine $\beta$ cell mass $(\mathrm{BCM})$ change in the progression of diabetes. It is known that glucagon-like peptide-1 receptor (GLP-1R) is mainly expressed by pancreatic $\beta$ cell islets. As such, GLP-1R has become an attractive biomarker for the monitoring of $\mathrm{BCM}$ change. However, its natural ligand, GLP-1 peptide, is of no use in this regard due to its extremely short biological half life $\left(\mathrm{t}_{1 / 2} \sim 2 \mathrm{~min}\right)$. To overcome this limitation, we have designed and synthesized several analogs of GLP-1 peptide to improve the in vivo stability while maintaining their binding affinity to GLP-1R and agonist activity. Among them, a bicyclic peptide analog
(EM2198) showed the best results. In this study, we further evaluated the potential of this peptide when labeled with ${ }^{68} \mathrm{Ga}$ for PET imaging of BCM. For ${ }^{68} \mathrm{Ga}$ labeling, a bifunctional chelator (NOTA-maleimide: maleimidobenzyl-1,4,7-triazacyclononane-1,4,7-triacetic acid-maleimidoethylacetamide) was coupled to the cysteine residue at the C-terminus of the peptide, EM2198. The peptide conjugate was characterized by HPLC and MALDI-MS. After labeled with ${ }^{68} \mathrm{Ga}$ under mild conditions $(\mathrm{RCY}=70 \%$ ), the peptide was evaluated in normal healthy and streptozocin (STZ)-induced diabetic C57BL/6 mice by a dynamic scan $(0-30 \mathrm{~min})$ and a static imaging procedure at $2 \mathrm{~h}$ post-injection (p.i.), which were performed on a Siemens Inveon PET/CT multimodality system. Post-PET biodistribution was conducted to verify the imaging findings. As revealed by the results of both PET imaging and post-PET biodistribution, the peptide showed rapid and preferential uptake $(<10 \mathrm{~min})$ and retention $(>2 \mathrm{~h})$ in the pancreas area of the normal mice. Encouragingly, the uptake ratio of pancreas-to-liver in normal mice was significantly higher than that in the STZ-induced diabetic mice at $2 \mathrm{~h} \mathrm{p}$.i. ( 1.8 vs $1.0 ; p<0.05)$. This indicates that the BCM change could be reflected by the PET signal intensity decreases in the pancreas during the progression of diabetes. However, high renal accumulation was also observed in both animal models throughout the imaging study. In summary, a ${ }^{68} \mathrm{Ga}$ labeled bicyclic GLP-1 peptide analog has shown potential to enable non-invasive assessment of BCM by PET. Acknowledgements: The authors acknowledge the financial support of the work by National Institute of Health (P01 DK058398), Juvenile Diabetes Research Foundation (37-2009-13), and Welch Foundation (AT-1595). 
$\mathbf{J 2 0 8}$

\section{${ }^{64}$ CU-ATSM IMAGING FOR CANCER STEM CELL-RICH REGIONS: IN VIVO AND IN VITRO CHARACTERIZATION}

Yukie Yoshii $^{1}$, Yasushi Kiyono ${ }^{1}$, Atsuo Waki ${ }^{1}$, Ryo Watanabe ${ }^{2}$,

Yuya Suzuki $^{2}$, Tetsuya Mori ${ }^{1,4}$, Hiroshi Yoshii ${ }^{3}$, Takako Furukawa ${ }^{5}$, Tatsuya Asai ${ }^{2,1}$, Takashi Kudo ${ }^{1}$, Hidehiko Okazawa ${ }^{1}$, Michael J. Welch ${ }^{4}$, Yasuhisa Fujibayashi ${ }^{1,5}$

${ }^{1}$ BIRC, University of Fukui, Eiheiji, Fukui, Japan, ${ }^{2}$ Faculty of Engineering, University of Fukui, Fukui, Japan, ${ }^{3}$ Faculty of Medical Sciences, University of Fukui, Eiheiji, Fukui, Japan, ${ }^{4} \mathrm{MIR}$, Washington University School of Medicine, St. Louis, MO, USA, ${ }^{5}$ MIC, National Institute of Radiological Sciences, Chiba, Japan.

Introduction: Cancer stem cells are recently noticed to contribute to tumor malignant behaviors, such as resistance to therapy and metastasis ability in tumors. On the other hand, it has been also known that tumor hypoxia is associated with such tumor malignancy. This indicates that tumor hypoxia might be a specific environment to keep up cancer stem cells within tumors. In this study, we examined relationships between existence of cancer stem cells and accumulation of hypoxia imaging agent ${ }^{64} \mathrm{Cu}$-diacetyl-bis $\left(N^{4}\right.$-methylthiosemicarbazone) $\left({ }^{64} \mathrm{Cu}-\mathrm{ATSM}\right)$ in vivo and conducted in vitro characterization. Methods: Double-tracer autoradiography and immunohistchemistry was performed with mouse colon carcinoma (Colon-26) tumor-bearing mice. In autoradiography, mixture of $74 \mathrm{MBq}$ of ${ }^{18} \mathrm{FDG}$ and $0.37 \mathrm{MBq}$ of ${ }^{64} \mathrm{Cu}-\mathrm{ATSM}$ was intravenously injected. The distribution of radio-labeled tracers was compared with the immunohistochemical staining for CD133 expression, which reflected the characteristic of cancer stem cells in Colon-26 cells. Additionally, ${ }^{64} \mathrm{Cu}$-ATSM uptake and survival of $\mathrm{CD} 133^{+}$cells under hypoxia was also examined with Colon-26 cells in vitro. Results: In Colon-26 tumors, ${ }^{64} \mathrm{Cu}$-ATSM localizes preferentially in regions with a high density of $\mathrm{CD}_{133^{+}}$cells. Density of $\mathrm{CD}_{133^{+}}$cells was highest in regions of high ${ }^{64} \mathrm{Cu}$-ATSM uptake and lowest in regions of high uptake of ${ }^{18} \mathrm{FDG}$. In addition, we found that in vitro culturing of Colon-26 cells under hypoxia increased both ${ }^{64} \mathrm{Cu}-\mathrm{ATSM}$ uptake and the proportion of $\mathrm{CD}_{133^{+}}$cells present. Conclusion: Our findings show that, in Colon-26 tumors, ${ }^{64} \mathrm{Cu}-\mathrm{ATSM}$ accumulates in $\mathrm{CD} 133^{+}$ cell-rich regions and that these cells would be resistant to hypoxic environment. Therefore, ${ }^{64} \mathrm{Cu}-\mathrm{ATSM}$ could be a potential imaging agent for cancer stem cell-rich regions within tumors.
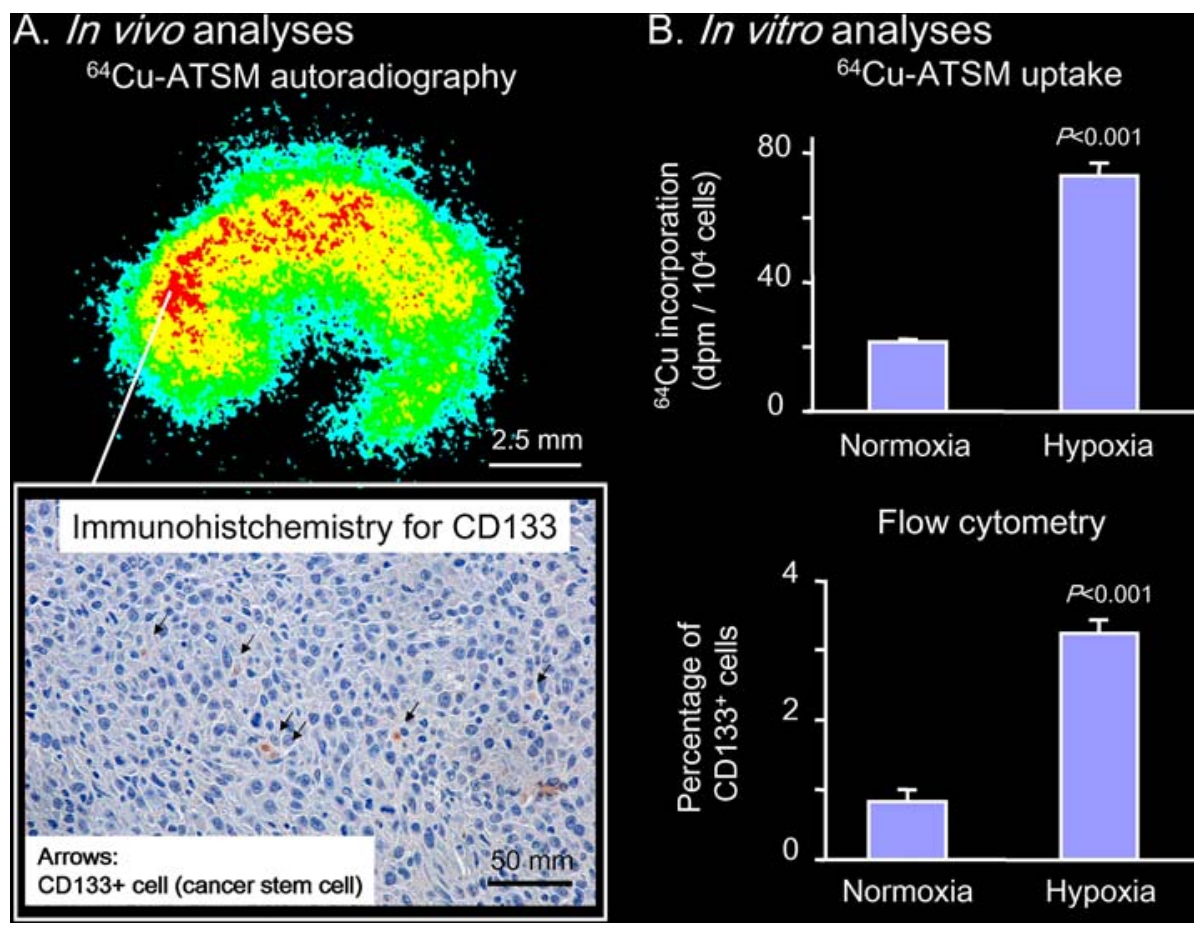

J209

\section{APTAMER IMAGING WITH CU-64 LABELED AS1411: EFFECT OF CHELATORS ON LABELING YIELD AND UPTAKE IN LUNG CANCER CELL}

Junling Li, Huaiyu Zheng, John O. Trent, Paula J. Bates, Chin Ng University of Louisville, Louisville, KY, USA.

Introduction: Radiolabeled aptamers hold promises as diagnostic imaging agents targeting specific human cancer. AS1411 is a 26-mer G-rich aptamer that has unmodified phosphodiester linkage and forms a
G-quadruplex structure which is highly resistant to degradation by serum enzymes. AS1411 is bound to nucleolin, a multifunctional protein over-expressed on various cancer cells. The choice of a chelator used for labeling AS1411 with Cu-64 can potentially affect the labeling yield and the uptake in lung cancer cell, so we performed the initial evaluation with these four chelators: 1. DOTA-NHS, 2. SCN-BnDOTA, 3. SCN-Bn-NOTA, and 4. CB-TE2A. Method: AS1411 was conjugated with four chelators in molar ratios of 1:150 in potassium carbonate buffer of $\mathrm{pH} 9-10$. CB-TE2A was activated by HBTU, EDC, $\mathrm{HOBt}$ and pyridine in DMSO prior to conjugation. $\mathrm{Cu}-64$ was mixed with the conjugates for $1 \mathrm{hr}$ at different $\mathrm{pH}$ and temperature: potassium 
citrate of $\mathrm{pH} 7.0$ at $60^{\circ} \mathrm{C}$ for chelators 1,2 and 3; and ammonium acetate of $\mathrm{pH} 8.0$ at $95^{\circ} \mathrm{C}$ for chelator 4 . The in vitro cellular uptake was studied in HTB177 non-small lung cancer (NSLC) cells. Five $\mu \mathrm{Ci}$ of $64 \mathrm{Cu}-\mathrm{AS} 1411$ was added to $1 \times 10^{\wedge} 6$ cells in each well and incubated at $37^{\circ} \mathrm{C}$ for $1,3,6,12,24$, and $48 \mathrm{~h}$. Percent uptake normalized to cell number was calculated by $100 \mathrm{x}$ (counts in cells/ total counts). Results: The labeling yield was $65 \pm 15 \%(n=3), 15 \pm 6 \%(n=2), 75 \pm 24 \%(n=2)$ and $36 \pm 19 \%(n=3)$, and the specific activity was 2.0-74 $(n=3)$, 1.0-2.2 $(n=2), 6.0-14.2(n=2)$, and 7.6-29.2 $\mathrm{mCi} / \mu \mathrm{mol}(n=3)$ for 64Cu-DOTA-NHS-AS1411，64Cu-SCN-Bn-DOTA-AS1411，64Cu-
SCN-Bn-NOTA-AS1411, and 64Cu-CB-TE2A-AS1411 respectively. Radiochemical purity for all four compounds was greater than $99 \%$ based on HPLC analysis. Percent uptake in NSLC cells for four labeled compounds is summarized in Table 1. Conclusion: AS1411 labeled with $\mathrm{Cu}-64$ and four different chelators demonstrated different labeling yield. The order of percent uptake in lung cancer cells was: DOTANHS $>$ CB-TE2A $>$ SCN-Bn-DOTA $>$ SCN-Bn-NOTA. These preliminary results suggest that either DOTA-NHS or CB-TE2A can be used to label AS1411 with $\mathrm{Cu}-64$ in order to explore the potential of aptamer imaging for in vivo lung cancer detection.

Table 1. Percent Uptake of Different Labeled Compounds ( $n=15$ for each time point for chelators 1 and 4 and $n=10$ for chelators 2 and 3 ).

\begin{tabular}{|c|c|c|c|c|c|c|}
\hline Labeled Compound & $1 \mathrm{~h}$ & $3 \mathrm{~h}$ & $6 \mathrm{~h}$ & $12 \mathrm{~h}$ & $24 \mathrm{~h}$ & $48 \mathrm{~h}$ \\
\hline $64 \mathrm{Cu}-$ DOTA-NHS-AS1411 & $2.65 \pm 0.39$ & $10.11 \pm 0.38$ & $20.30 \pm 1.19$ & $24.60 \pm 1.65$ & $20.41 \pm 0.54$ & $14.99 \pm 0.97$ \\
\hline $64 \mathrm{Cu}-\mathrm{SCN}-\mathrm{Bn}-$ DOTA-AS1411 & N/A & N/A & $0.95 \pm 0.06$ & $2.07 \pm 0.13$ & $4.06 \pm 0.29$ & $0.58 \pm 0.12$ \\
\hline $64 \mathrm{Cu}-\mathrm{SCN}-B$-NOTA-AS1411 & $0.17 \pm 0.03$ & $0.29 \pm 0.07$ & $0.33 \pm 0.08$ & $0.48 \pm 0.03$ & $0.78 \pm 0.09$ & $0.71 \pm 0.04$ \\
\hline $64 \mathrm{Cu}-\mathrm{CB}-\mathrm{TE} 2 \mathrm{~A}-\mathrm{AS} 1411$ & $2.30 \pm 0.28$ & $4.51 \pm 0.82$ & $5.35 \pm 0.61$ & $6.20 \pm 0.14$ & $12.55 \pm 0.94$ & $13.7 \pm 0.35$ \\
\hline
\end{tabular}

$\mathbf{J} 210$

\section{DEVELOPMENT OF IN VIVO IMAGING AGENTS TARGETING GLUCAGON-LIKE PEPTIDE-1 RECEPTOR (GLP-1R) IN PANCREATIC ISLETS} Hiroyuki Kimura $^{1}$, Yu Ogawa ${ }^{1}$, Hidekazu Kawashima ${ }^{2}$, Eri Mukai ${ }^{3,4}$, Kentaro Toyoda $^{3}$, Hiroyuki Fujimoto ${ }^{3,4}$, Konomu Hirao ${ }^{5}$, Kenji Nagakawa ${ }^{5}$, Masahiro Ono ${ }^{1}$, Nobuya Inagaki ${ }^{3}$, Hideo Saji ${ }^{1}$ ${ }^{1}$ Department of Patho-functional Bioanalysis, Kyoto University, Kyoto, Japan, ${ }^{2}$ Department of Diagnostic Imaging and Nuclear Medicine, Kyoto University, Kyoto, Japan, ${ }^{3}$ Department of Diabetes and Clinical Nutrition, Kyoto University, Kyoto, Japan, ${ }^{4}$ Japan Association for the Advancement of Medical Equipment, Tokyo, Japan, ${ }^{5}$ Research \& Development Division, Arkray, Inc, Kyoto, Japan.

Objectives: The beta cell mass is known to decrease as diabetes progresses. Although a technique for measurement of beta cell mass in vivo would be useful for the early diagnosis of diabetes, a non-invasive method to detect pancreatic beta cell has not been developed. GLP-1 plays an important role in glucose metabolism and homeostasis. High densities of GLP-1R expression in pancreatic islets would offer an attractive target for imaging. In this study, based on the GLP-1 antagonist exendin-(9-39), we developed in vivo imaging agents specifically targeting GLP-1R. Methods: The derivatives of both N-succinimidyl 4-[18F]fluorobenzoate ([18F]SFB) and [19F]SFB-labeled [exendin-(9-39)] were synthesized and evaluated. [18F]SFB was synthesized by our Onepot procedures. To evaluate the distribution of [18F]FB-[exendin(9-39)] derivatives in vivo, a biodistribution study was carried out in 6-week-old male ddY mice. Animal PET imaging studies of [18F]FB-[exendin-(9-39)] derivatives were performed. Results: The decay-corrected radiochemical yield of [18F]FB-[exendin- $(9-$ 39)] derivatives was about $7 \sim 18 \%$ and the radiochemical purity was greater than $99 \%$. In vitro binding study showed that the affinity of FB-[exendin-(9-39)] derivatives to GLP-1R was similar to non-labeled exendin-(9-39) (IC50: 1.4 2.4 nM). The in vivo biodistribution of [18F]FB-CompA[exendin-(9-39)] and [18F]FB-
CompB[exendin-(9-39)] in mice showed the highest uptake in the pancreas $(5.7 \% \mathrm{ID} / \mathrm{g}$ at $15 \mathrm{~min}$ and $7.3 \% \mathrm{ID} / \mathrm{g}$ at $30 \mathrm{~min}$ postinjection, respectively) and a significant reduction of pancreas and lung uptake was confirmed in GLP-1R knockout mice. Preadministration of excess nonradioactive exendin(9-39) significantly blocked the radio activity of pancreas and lung after [18F]FB-exendin(9-39) derivatives injection. Moreover, we succeeded in visualizing the pancreas by animal PET. Conclusion: For non-invasive imaging of pancreatic beta cell, exendin-(9-39) and GLP-1R can serve as a useful probe and target molecule, respectively.

\section{$\mathbf{J} 211$}

\section{SMALL-ANIMAL PET OF AT1R EXPRESSION IN HUMAN PANCREAS AND BREAST TUMOR XENOGRAFTS}

Jinsong Xia, William B. Mathews, Nedim C. Gulaldi, Zsolt Szabo Johns Hopkins Hospital, Baltimore, MD, USA.

INTRODUCTION: Inhibition of the angiotensin subtype 1 receptor (AT1R) has been reported to suppress tumor growth, metastasis and angiogenesis in experimental breast and pancreas tumor models. Translation of this novel methodology could benefit greatly from noninvasive imaging technology that can assess molecular heterogeneity and predict response to treatment. The objective of this study was to evaluate [11C]KR-31173 as a potential first positron emission tomography (PET) probe to quantify the AT1R in subcutaneous xenograft mouse models. METHODS: Human cancer cells (CRL-2119, MDA-MB-231) were implanted subcutaneously in the shoulder region 14-21 days before initiation of the study. [11C]KR-31173 (200 $\mu \mathrm{Ci}$ in $0.2 \mathrm{cc}$ ) was injected through a tail vein into female SCID mice bearing MDA-MB-231 subcutaneous xenografts and male SCID mice bearing CRL-2119 xenografts and tumors were imaged by small-animal PET/CT in vivo. A receptor blocking experiment with the potent AT1R antagonist SK 1080 administered intra- 
peritoneally was performed in parallel to evaluate specific binding. RESULTS: Greater than $99 \%$ radiochemical purity and specific activities of at least $200 \mathrm{GBq} / \mu \mathrm{mol}$ were routinely achieved. Small-animal PET showed rapid and clear tumor localization in both CRL-2119 and MDA-MB-231 xenograft models. This tracer exhibited predominantly liver clearance and fast clearance from the circulation, specific uptake in heart and lungs, and only background level activity in the muscles. Small animal CT allowed localization of lesions for quantification. Quantitative analysis of PET images resulted in a tumor-to-muscle ratio of $2.3 \pm 0.48$ at $60 \mathrm{~min}$ after tail vein injection. This value was reversed to 0.84 in animals pretreated with SK-1080, which confirmed the receptor specificity of $[11 \mathrm{C}] \mathrm{KR}-31173$ in vivo. CONCLUSIONS: [11C]KR-31173 specifically accumulated at the target site, for the first time enabling detailed in vivo visualization of AT1R in tumor models. The results support continual investigation of [11C]KR-31173 PET for non-invasive prediction and assessment of tumor response to AT1R molecular/gene manipulation therapy.

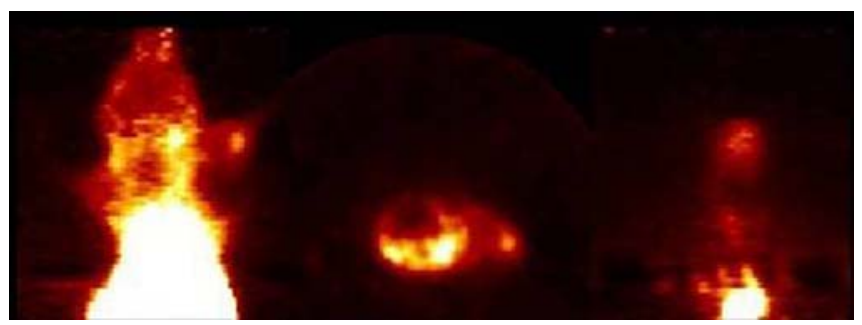

$\mathbf{J} 212$

\section{CHARACTERIZATION OF INDIUM-LABELED GLP-1 DERIVATIVES FOR BETA CELL MASS ESTIMATION} Vanessa A. Rota ${ }^{1,3}$, Babak Behnam Azad ${ }^{2}$, Leonard G. Luyt ${ }^{2}$, Savita Dhanvantari ${ }^{1,3}$

${ }^{1}$ Department of Medical Biophysics, The University of Western Ontario, London, ON, Canada, ${ }^{2}$ Department of Chemistry, University of Western Ontario, London, ON, Canada, ${ }^{3}$ Lawson Health Research Institute, London, ON, Canada.

Type 1 diabetes mellitus (T1DM) is a disease that results in an absolute decline in pancreatic beta cell mass. The ability to accurately measure changes in beta cell mass non-invasively in vivo would be useful for the detection of changes in beta cell mass that precede diabetes, as well as for the assessment of islet transplantation therapy. One possible suitable target for imaging is the glucagon-like peptide-1 receptor (GLP-1R), which is expressed on the surface of the beta cell. In our previous work, we generated eight 115/113In -labeled GLP-1-derived peptides and screened them for binding affinity for GLP-1R. Three peptides were identified that had an IC50 similar to that of GLP-1 (10nM): 37Lys-AEEA-In-DOTA-GLP-1 (LCE0028), 37Lys-In-DOTA-GLP-1 (LCE0031) and 22Lys-AEEA-In-DOTA-GLP-1 (LCE0039). We have subsequently characterized these potential SPECT probes as follows: 1) activation of the GLP-1R; 2) imaging of beta cells in vitro; and 3 ) in vivo biodistribution. To confirm GLP-1R activation, Chinese hamster ovary $(\mathrm{CHO})$ cells that over-express the GLP-1R were incubated for 30 min with $1 \mu \mathrm{M}$ IBMX and
$10 \mathrm{nM}-1 \mathrm{pM}$ peptide, and intracellular cAMP levels were determined by radioimmunoassay. LCEs 0028 and 0031 increased intracellular cAMP levels by 15 -fold on average, similar to the increase induced by native GLP-1, thus confirming receptor activation. In vitro imaging experiments were performed using INS-1 cells, a beta cell line that expresses the GLP-1R. Specific binding of LCEs 0028 and 0031 to GLP-1R was determined in the presence of unlabeled exendin-4, a GLP-1R analogue. After $30 \mathrm{~min}$ incubation with 111In-labeled peptides in the presence or absence of exendin-4, gamma camera imaging showed that both probes specifically targeted the GLP-1R on INS-1 cells. Finally, biodistribution studies were conducted in C57/BL6 mice following tail vein injection of 0.3-0.6 MBq of [111In]LCE0039. Significant uptake of the 111 Inlabelled probe in the pancreas was detected at $4 \mathrm{~h}(4 \% \mathrm{ID} / \mathrm{g} ; n=2)$ and $24 \mathrm{hrs}(2 \% \mathrm{ID} / \mathrm{g} ; n=2)$ post-injection. In conclusion, we have generated three GLP-1 derivatives that specifically bind to the GLP-1R on beta cells. We will use these probes for SPECT imaging of changes in beta cell mass changes during the progression of diabetes.

$\mathbf{J} 213$

\section{CU-LABELED MULTIVALENT $\alpha$-MELANOCYTE} STIMULATING HORMONE ANALOGS FOR MICROPET IMAGING OF MELANOCORTIN 1 RECEPTOR EXPRESSION Hongguang Liu ${ }^{1,3}$, Zheng Miao ${ }^{1}$, Gang Ren ${ }^{1}$, Lei Jiang ${ }^{1}$, $\overline{\text { Richard Kimura }}^{2}$, Jennifer R. Cochran ${ }^{2}$, Peizhen $\mathrm{Han}^{3}$, Zhen Cheng ${ }^{1}$ ${ }^{1}$ Molecular Imaging Program at Stanford (MIPS), Department of Radiology and Bio-X Program, Stanford University, Palo Alto, CA, USA, ${ }^{2}$ BioEngineering, Stanford University, Palo Alto, CA, USA, ${ }^{3}$ Institute of Radiation Medicine, Chinese Academy of Medical Sciences, Peking Union Medical College, Tianjin, China.

Multivalent peptides have been explored as a useful strategy to construct molecular imaging probes and drug delivery carriers. It is generally accepted that multivalency has advantages over monovalency for improving binding affinities and even activity. Herein by using multivalent $\alpha$-melanocyte stimulating hormone ( $\alpha$-MSH) analogs, B16F10 melanoma-bearing mice and microPET imaging technology, we systematically investigated the influence of multivalent effect on $\alpha$-MSH analogs' binding affinity and in vivo melanoma targeting profiles. Methods: Three $\alpha-\mathrm{MSH}$ analogs named as MSH1, MSH2 and MSH4 were designed and synthesized, which contained one, two or four valency of an $\alpha$-MSH core sequence, His-d, Phe-Arg-Trp, respectively (Fig. a). $\alpha-\mathrm{MSH}$ analogs tetramer was constructed using the multiple antigenic peptide (MAP) scaffold. 1,4,7,10-tetraazacyclododecane-1,4,7,10tetraacetic acid (DOTA) was conjugated to the lysine residue of peptides for radiolabeling with a $\mathrm{PET}$ radioisotope, $64 \mathrm{Cu}$. In vitro binding affinity assays were performed with B16F10 melanoma cell line. After radiolabeling with $64 \mathrm{Cu}$, the in vivo performances of the peptides were evaluated in subcutaneous B16F10 melanoma xenografted mice by microPET imaging followed by biodistribution studies. Results: In the receptor competition binding assays, DOTA-MSH4 showed highest binding affinity (IC50=1.00 nM) which is consistent with its highest ligand density. However, in vivo study demonstrated poor performance of MSH4 as an imaging agent due to its lowest tumor uptake and highest kidney accumulation. In comparison, DOTA-MSH2 displayed medium affinity (IC50=2.06 nM), yet highest tumor uptake and lowest kidney accumulation (Fig. b). Further blocking study of DOTA$\mathrm{MSH} 2$ confirmed its tumor targeting specificity in vivo. Conclu- 
sion: Multivalency effects have complex impact to peptides' in vivo behaviors. Eventhough MSH tetramer shows the higher binding affinities in vitro, the better in vivo tumor targeting ability is achieved by MSH dimer. Cu-64 labeled dimeric DOTA-MSH2 has been identified as an ideal melanoma PET imaging probe.

a

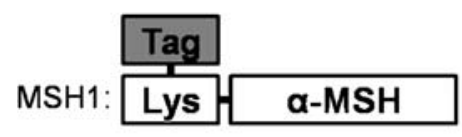

MSH4 b

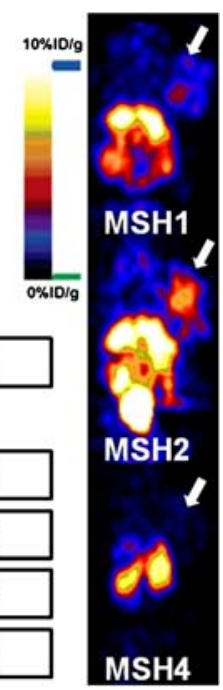

$\mathbf{J 2 1 4}$

\section{NOVEL 67/68GALLIUM-RADIOPHARMACEUTICALS FOR MONITORING MDR1 P-GLYCOPROTEIN-MEDIATED TRANSPORT AT THE BLOOD-BRAIN BARRIER (BBB) \\ Jothilingam Sivapackiam, Scott Harpstrite, Julie Prior, David Piwnica-Worms, Vijay Sharma \\ Washington University School of Medicine, St. Louis, MO, USA.}

Emerging models of transporter-mediated efflux pathways at the blood-brain barrier suggest a role for multidrug resistance (MDR1) P-glycoprotein (Pgp) in development of neurodegenerative diseases. Additionally, Pgp is also a well-characterized target for chemotherapeutic resistance in cancer. Thus, we have synthesized, characterized, and evaluated two novel 67/68gallium-radiopharmaceuticals for their potential to serve as imaging agents for probing Pgp-mediated functional transport in vitro and in vivo. The crystal structure data demonstrated the central gallium(III) involved in an octahedral geometry coordinated simultaneously and symmetrically with four nitrogen atoms in the equatorial plane and two axial phenolate oxygen atoms. Employing unlabeled gallium(III) complexes, the cytotoxicity profiles (using MTS-based 3-day screening protocol) were found to be modified by the expression of Pgp in human epidermal carcinoma cells suggesting their recognition by Pgp as its transport substrates. To confirm these results unambiguously, 67/68gallium-radiopharmaceuticals were obtained by treatment of hexadentate ligands with $67 / 68 \mathrm{Ga}$ (acetylacetonate) 3 in ethanol using either microwave irradiation $(60 \mathrm{~W} ; 15 \mathrm{~min})$ or heated at $100^{\circ} \mathrm{C}$ for $30 \mathrm{~min}$, purified on a $\mathrm{C}-18$ column employing an eluent mixture of ethanol and saline in a ratio of 9:1 using the HPLC system, equipped with a radiodetector. Purified gallium-67/ 68 complexes demonstrated accumulation in human epidermal carcinoma cells, and uptake profiles were found to be inversely proportional to Pgp expression. Additionally, LY335979 (1 $\mu \mathrm{M})$, a
Pgp specific inhibitor, reversed efflux of radiotracers in Pgp expressing $\mathrm{KB}$ 8-5 cells, equivalent to levels found in Pgp negative KB 3-1 cells, thus demonstrating the specificity of these agents to probe the transporter-mediated activity in cellulo. Furthermore, 67/68 Ga-complexes showed brain uptake and retention in mdrla/1b(-/-) gene-deleted (dKO) mice compared with facile excretion patterns in their wild-type (WT) counterparts, with insignificant differences in blood pharmacokinetics, thus consistent with their recognition by Pgp, expressed at the capillary bloodbrain barrier in vivo. Finally, 67/68 Ga-complexes also displayed retention and delayed clearance in livers of dKO mice compared with efficient clearance profiles in WT mice, conferring their Pgp recognition characteristics. These agents could offer potential leads for enabling noninvasive SPECT/PET monitoring of the functional transport activity of MDR1 Pgp in vivo.

\section{$\mathbf{J} 215$}

DISCOVERY AND IN VIVO EVALUATION OF TWO-HELIX SMALL PROTEINS FOR HER2 MOLECULAR IMAGING Gang Ren ${ }^{1}$, Jack M. Webster ${ }^{2}$, Rong Zhang ${ }^{2}$, Zheng Miao ${ }^{1}$, Sanjiv S. Gambhir ${ }^{1}$, Faisal A. Syud ${ }^{2}$, Zhen Cheng ${ }^{1}$

${ }^{1}$ Stanford University, Mountain View, CA, USA, ${ }^{2}$ General Electric Company, Global Research Center, Niskayuna, NY, USA.

Affibody molecules, nonimmunogenic 3-helix scaffold-based proteins $(\sim 7 \mathrm{kDa})$, have drawn lots of attentions as rapidly developing imaging probes for a variety of molecular targets. We've recently shown that monomeric Affibody constructs substantially perform better in vivo relative to their dimeric construct despite the latter's better affinity. Additional advantages expected for smaller protein constructs include the possibility of facile synthetic generation, easier library generation for screening, economic viability, and potentially lower immunogenic potential. In this report, we further develop strategies for discovery smaller Affibody analogs, 2-helix proteins, for in vivo cancer molecular imaging. Methods: The anti-human epidermal growth factor receptor type 2 (HER2) Affibody ZHER2:342 was used as a model protein. $\alpha$-Helix 3, not believed to be in direct contact with the target, was truncated, preserving helices 1 and 2 that contain the binding domain. A number of both sequence mutations and synthetic strategies to optimize the affinity of anti-HER2 2-helix proteins have been developed. A library of 2helix proteins $(>30)$ was synthesized. Peptides were then site specifically conjugated with 1,4,7,10-tetraazacyclododecane-1,4,7,10tetraacetic acid (DOTA) and labeled with PET radionuclide, $68 \mathrm{Ga}$ or $64 \mathrm{Cu}$. The resulting radiolabeled peptides were further evaluated for HER2 microPET imaging in SKOV3 tumor mice. Results: Several constrained 2-helix constructs with high HER2 affinity were successfully identified. Conjugation with DOTA still preserved high HER2 binding affinity (DOTA-MUT-DS, KD=4.76 nM). Radiolabeled $(68 \mathrm{Ga}$ or $64 \mathrm{Cu})$ probe displayed high stability in mouse serum and specificity towards HER2 in cell culture. Biodistribution and microPET imaging studies further showed that DOTA-MUT-DS had rapid and high SKOV3 tumor accumulation with lower nonspecific accumulation in normal organs (Figure). The specificity of radiometal labeled MUT-DS for SKOV3 tumors was confirmed by monitoring of modulation of HER2 protein upon treatment of 17DMAG or unlabeled Affibody in vivo. Conclusions: 2-helix proteins with high affinity to biomarkers can be discovered and used for in vivo applications. $68 \mathrm{Ga}$ or $64 \mathrm{Cu}-\mathrm{DOTA}-\mathrm{MUT}-\mathrm{DS}$ is a promising PET probe for imaging HER2 expression in vivo. 


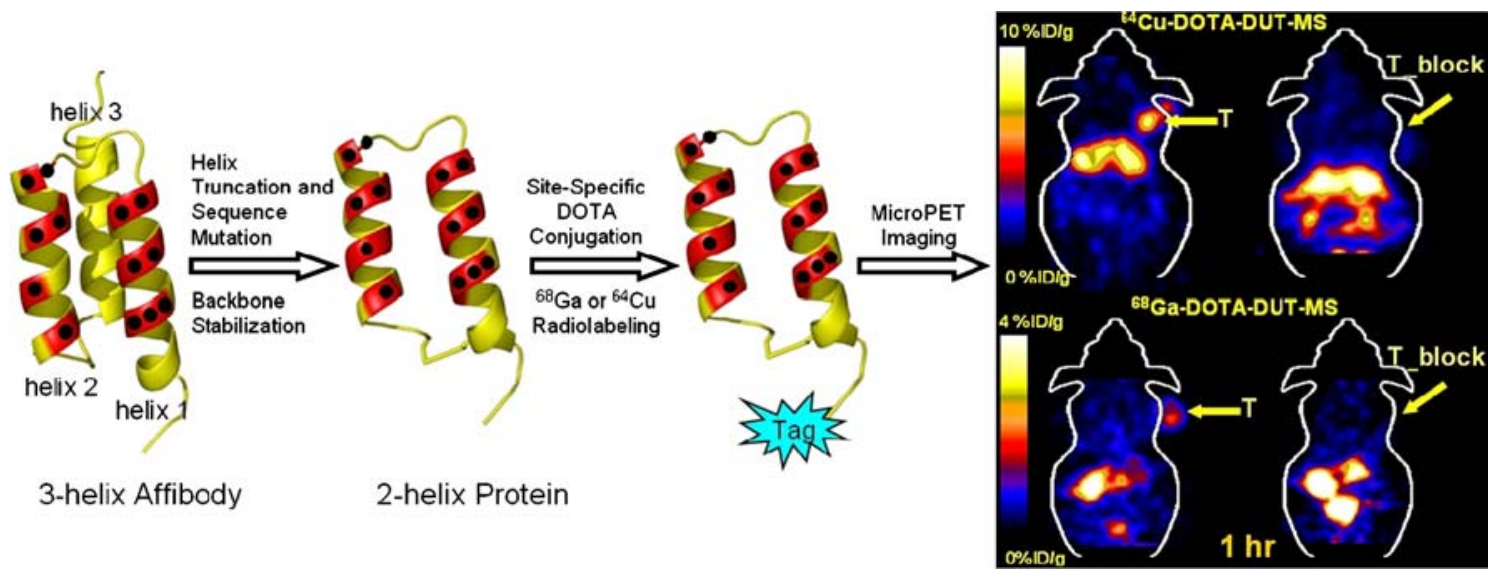

$\mathbf{J} 216$

\section{AFFIBODY BASED MOLECULAR PROBES FOR EGFR PET AND OPTICAL IMAGING \\ $\underline{\text { Zheng Miao }}^{1,2}$, Gang Ren ${ }^{1,2}$, Lei Jiang ${ }^{1,2}$, Hongguang Liu ${ }^{3,2}$, Yihong Wang ${ }^{1,2}$, Zhen Cheng ${ }^{1,2}$, Sanjiv S. Gambhir ${ }^{2,1}$ \\ ${ }^{1}$ Radiology, Stanford University, Palo Alto, CA, USA, ${ }^{2}$ Molecular Imaging Program at Stanford, Stanford University, Palo Alto, CA, USA, ${ }^{3}$ Institute of Radiation Medicine, Peking Union Medical College, Beijing, China.}

The purpose of this study is to site-specifically label an Affibody protein (Ac-Cys-ZEGFR:1907) binding to epidermal growth factor receptor (EGFR) with Cy5.5 and 64Cu-DOTA, investigate its in vitro cell uptake and binding specificity, study Cy5.5-ZEGFR:1907 and 64Cu-DOTA-ZEGFR:1907 in vivo tumor imaging ability, and evaluate the potential of EGFR PET imaging with 64Cu-DOTAZEGFR:1907 for clinical translation. Methods: Molecular probe Cy5.5-ZEGFR:1907 and 64Cu-DOTA-ZEGFR:1907 were made through solid peptide phase synthesis of Ac-Cys-ZEGFR:1907 followed by site-specific conjugation with Cy5.5-mono-maleimide and DOTA-mono-maleimide respectively. DOTA-ZEGFR:1907 was then labeled with $64 \mathrm{Cu}$ in sodium acetate buffer $(\mathrm{pH}=5.0)$ at $40^{\circ} \mathrm{C}$. Cell uptake assay was performed using EGFR expression A431 cell line (cervical cancer cell line) with/without pre-incubation of unlabeled Ac-Cys-ZEGFR:1907 for blocking. Cy5.5-ZEGFR:1907 (500 pmol) or 64Cu-DOTA-ZEGFR1907 $(40-70 \mu \mathrm{Ci})$ was injected into A431 tumor bearing nude mice through tail-vein for optical imaging and microPET imaging respectively. Bio-distribution studies were performed at 1, 4 and $24 \mathrm{~h}$ post-injection (p.i.). Results: in vitro microscopy study showed that majority of Cy5.5-ZEGFR:1907 specifically bound to A431 cell surface in $1 \mathrm{~h}$. Optical imaging of Cy5.5-ZEGFR:1907 successfully demonstrated EGFR receptor mediated targeting to A431 tumor. For radiolabeled probe, it displayed high $(27 \%$ at $1 \mathrm{~h})$ and specific in vitro cell uptake. In vivo microPET imaging showed high tumor accumulation $(>10 \% \mathrm{ID} / \mathrm{g}$ at $4 \mathrm{~h}$ p.i. $)$ and good contrast of 64Cu-DOTA-ZEGFR:1907. Bio-distribution studies demonstrated that 64Cu-DOTA-ZEGFR:1907 had high tumor uptakes, and also high blood, liver and kidney uptakes, while kidney and blood uptake dropped significantly $24 \mathrm{~h}$ p.i.. Conclusions: Cy5.5 and 64Cu labeled ZEGFR:1907 can provide high sensitivity, receptor specific optical and PET imaging of EGFR positive tumors. $64 \mathrm{Cu}$ DOTA-ZEGFR:1907 is mainly clearly through kidney-urinary system. Three-helix Affibodies are excellent protein scaffolds for the development of PET probes for clinical translation.
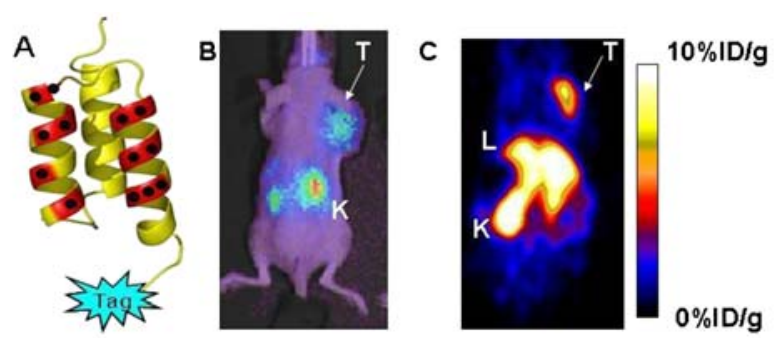

Figure: Three-helix Affibody based probe ${ }^{64} \mathrm{Cu}-\mathrm{DOTA}-\mathrm{Z}_{\text {EGFR-1907 }}$ and Cy5.5- $Z_{\text {EGFR:1907 }}$ for EGFR PET and optical imaging. (A) Cartoon of Affibody based probes, the black dots and red regions indicate the amino acid residues responsible for receptor binding. Optical (B) and microPET imaging (C) of a mouse bearing

A431 tumor xenograft at $2 \mathrm{~h} \mathrm{p.i..} \mathrm{Arrows} \mathrm{were} \mathrm{pointed} \mathrm{at} \mathrm{tumors.} \mathrm{T,}$ tumor; L, liver; K, kidney.

\section{$\mathbf{J} 217$}

DETECTION OF MYCOBACTERIAL GRANULOMA IN MICE WITH 99MTECHNETIUM LABELED CEFTIOFUR USING MULTIPLEXED MULTIPINHOLE SPECT

Pradip Chaudhari ${ }^{1}$, Domokos Mathe ${ }^{2}$, Lajos Balogh ${ }^{2}$, Andras Polyak ${ }^{2}$, Madhumanjiri M. Gatne ${ }^{4}$, Gyozo A. Janoki ${ }^{3}$

${ }^{1}$ Comparative Oncology, Advanced Centre for Treatment, Research and Education in Cancer, Tata Memorial Centre, Navi Mumbai, India, ${ }^{2}$ Experimental Nuclear Medicine, National "Frederic Joliot Curie" Research Institute for Radiobiology and Radiohygiene, Budapest, Hungary, ${ }^{3}$ Radiopharmacy, Medi-Radiopharma Ltd, Erd, Hungary, ${ }^{4}$ Pharmacology, Bombay Veterinary College, Mumbai, India.

Cephalosporines are broad spectrum antibiotics, active against both Gram-positive and -negative bacteria. Ceftiofur, a third generation cephalosporine was labeled with $99 \mathrm{mTc}$ to evaluate its potential for mycobacterial infection imaging. Aim of the study: To evaluate 99mTechenetium labeled Ceftiofur for imaging tubercular infection in mice. Materials and Methods: Labeling of Ceftiofur with $99 \mathrm{mTc}$ was carried out by using stannous chloride as reducing agent. Radiochemical purity of $99 \mathrm{mTc}$-Ceftiofur was determined by thin layer chromatography using saline and methanol: water (70:30) as mobile phases. Pulmonary and hepatic granulomae were formed by $\mathrm{M}$. bovis BCG strain injected into the mediastinum and liver of $\mathrm{Nu} / \mathrm{Nu}$ mice. Two weeks later $99 \mathrm{mTc}$-Ceftiofur was injected into the mice through the tail vein for multiplexed multipinhole SPECT/CT imaging studies. 
Animals were anesthetized while imaging. Images were acquired at $30 \mathrm{~min}, 1 \mathrm{~h}$, and $3 \mathrm{~h}$ post-injection. Results: The radiochemical purity (RCP) of $99 \mathrm{mTc}$-Ceftiofur was $>90 \%$. The in vitro stability of the complex was confirmed by carrying out RCP check over a period of 4 hours. The 3 hour post-injection images revealed that the labeled compound is excreted via renal system and GI-route. Renal clearance was seen maximum at $3 \mathrm{~h}$. The activity in the large-intestine was observed maximum at $3 \mathrm{~h}$. Intense radiopharmaceutical uptake was seen at the liver lesion at $3 \mathrm{~h}$. Mediastinal lesions were also visualized at the reconstructed SPECT images. Gross pathology, histology and Ziehl-Neelsen staining confirmed the presence of M. bovis in all of the detected pulmonary and liver lesions with a maximal and minimal diameter of $3 \mathrm{~mm}$ and $0.8 \mathrm{~mm}$. Conclusion: $99 \mathrm{mTc}-$ Ceftiofur was labeled with $>90 \%$ radiochemical purity of the complex. The clearance of the radiopharmaceuical appears to be through the renal and hepatic route. $99 \mathrm{mTc}$-Ceftiofur localizes in granulomae caused by $\mathrm{M}$. bovis BCG. The avidity of the labeled complex for BCG injected lesion warrants a further detailed evaluation to confirm the utility for labelled Ceftiofur as a M. tuberculosis imaging agent.

\section{$\mathbf{J} 218$}

\section{CB1 IMAGING IN OBESE RHESUS MONKEY FAT USING THE PET TRACER $\left[{ }^{18}\right.$ F]MK-9470}

Sandra Sanabria $^{1}$, Terence G. Hamill ${ }^{1}$, Stephen Krause ${ }^{1}$, WaiSi Eng ${ }^{1}$, Tung M. Fong ${ }^{2}$, Jacquelynn J. Cook ${ }^{1}$

${ }^{1}$ Imaging, Merck, West Point, PA, USA, ${ }^{2}$ Metabolic Disorders Department, Merck, Rahway, NJ, USA.
Various in vivo experimental studies have demonstrated that cannabinoid-1 receptor (CB1R) inverse agonists can lead to weight loss and improved metabolic profile in both animals and humans. It has been suggested that the favorable effects may result from blockade of CB1R in the brain and fat tissue. The development of $\left[{ }^{18} \mathrm{~F}\right] \mathrm{MK}-9470$ as a CB1R selective PET tracer has advanced the understanding of the relationship between central target engagement and pharmacological effects in both animal and human studies. We investigated the possibility of estimating the CB1R availability in rhesus monkey adipose tissue. Three obese rhesus monkeys with $58 \pm 3 \%$ body fat (by DEXA) were included in the study. Animals underwent a $3 \mathrm{~h}$ dynamic PET scan (head to hip) starting with $\left[{ }^{18} \mathrm{~F}\right] \mathrm{MK}-9470$ bolus injection. The CB1R inverse agonist taranabant was administered $\sim 60$ min into the scan as a bolus $(0.8 \mathrm{mg} / \mathrm{kg})$ plus infusion $(0.64 \mathrm{mg} / \mathrm{kg} / \mathrm{h})$ until termination of the scan. Tracer input function was measured in 2 animals. For each scan, regions of interest were defined in brain, arm muscle, liver and abdominal fat. Radioactivity was observed in interclavicular and abdominal fat following $\left[{ }^{18} \mathrm{~F}\right] \mathrm{MK}-9470$ administration. The lack of homogeneity in abdominal fat uptake compromised accurate comparison between animals. Radioactivity has also been observed in interclavicular fat in lean rhesus during dosimetry studies. Taranabant displaced $\left[{ }^{18} \mathrm{~F}\right] \mathrm{MK}-9470$ uptake in the brain resulting in $\sim 80 \%$ CB1R occupancy by the end of the scan. In contrast, in fat, liver and muscle, no activity displacement was observed suggesting uptake was non-specific in these regions. $\left[{ }^{18} \mathrm{~F}\right] \mathrm{MK}-9470$ metabolism and brain uptake in the obese animals were similar to measured values by our group in lean rhesus. These findings suggest that specific binding to $\mathrm{CB} 1 \mathrm{R}$ in rhesus monkey fat tissue cannot be observed using $\left[{ }^{18} \mathrm{~F}\right] \mathrm{MK}$ 9470. The activity uptake in adipose tissue could be attributed, at least in part, to the highly lipophilic nature of the PET tracer.
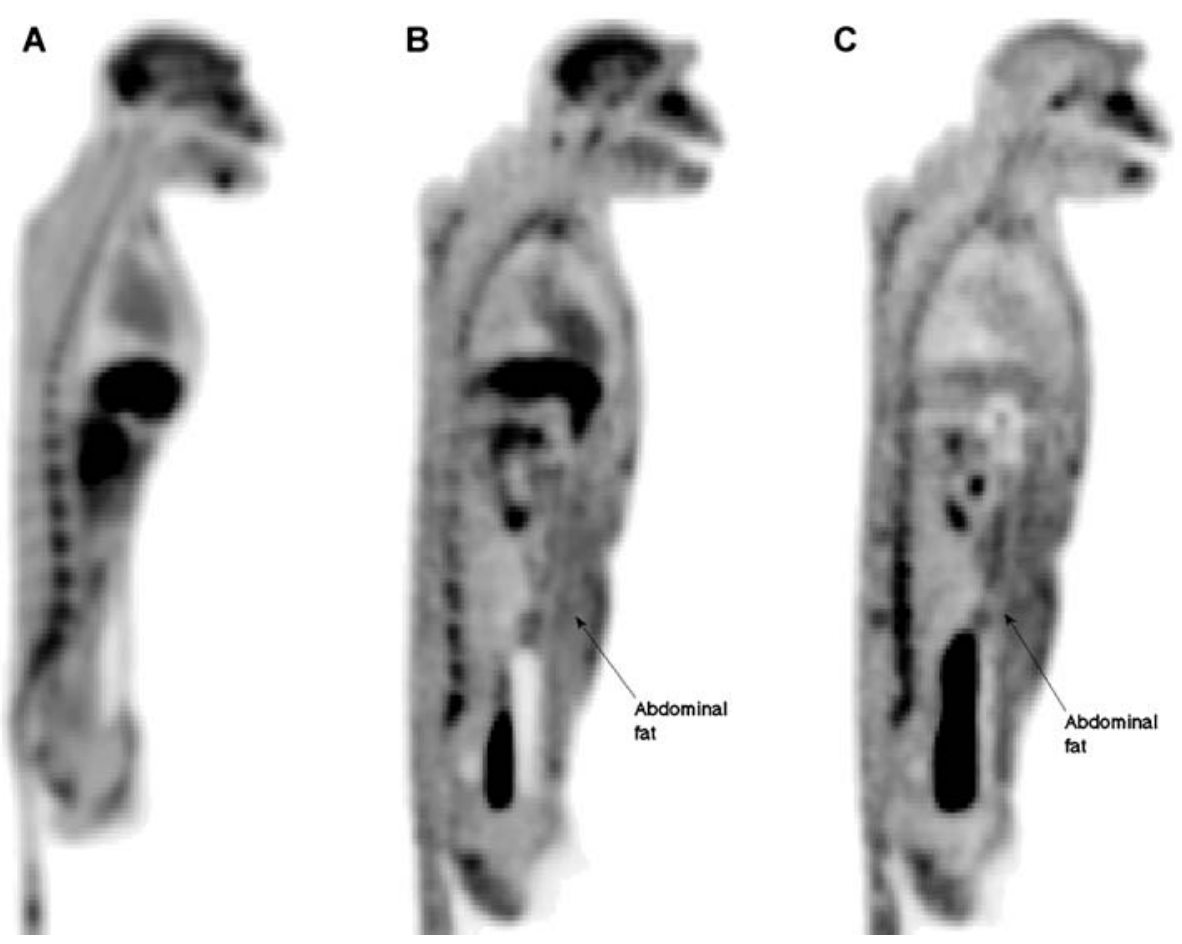

$\left[{ }^{18} \mathrm{~F}\right] \mathrm{MK}-9470$ uptake at baseline in lean (A) and obese (B) monkeys. Taranabant displaced radioactivity in the brain but not in abdominal fat in obese rhesus (C) 
$\mathbf{J 2 1 9}$

ESTIMATION OF THE $\left[{ }^{18}\right.$ F]FAc CONTRIBUTION TO BRAIN ACTIVITY DURING $\left[{ }^{18}\right.$ F]FAHA PET IMAGING IN RHESUS MONKEY

Sandra Sanabria ${ }^{1}$, Hong Fan ${ }^{1}$, Kerry Riffel ${ }^{1}$, Mangay Williams ${ }^{1}$, Juri G. Gelovani $^{2}$, Eric Hostetler ${ }^{1}$

${ }^{1}$ Imaging, Merck, West Point, PA, USA, ${ }^{2}$ Department of Experimental Diagnostic Imaging, The University of Texas M. D. Anderson Cancer Center, Houston, TX, USA.

Central HDAC (histone deacetylases) inhibitors have been proposed as potential treatments for a variety of neurodegenerative and cognitive disorders. In vivo HDAC imaging is possible using the HDAC substrate PET tracer $\left[{ }^{18} \mathrm{~F}\right]$ FAHA. $\left[{ }^{18} \mathrm{~F}\right]$ Fluoroacetate $(\mathrm{FAc})$ is the product of the FAHA-HDAC interaction and rapid hydrolysis and trapping of FAc is expected to be the mechanism providing the image. FAc is rapidly formed in the periphery and although low BBB permeability has been reported it may contribute to the PET signal during brain studies. We previously reported central HDAC imaging in rhesus monkey using $\left[{ }^{18} \mathrm{~F}\right] \mathrm{FAHA}$ but did not consider the contribution from circulating FAc in our analysis. We present here the $\left[{ }^{18} \mathrm{~F}\right]$ FAHA-FAc brain kinetics analysis during FAHA studies and re-evaluate the suitability of the tracer for central HDAC imaging. $\left[{ }^{18} \mathrm{~F}\right] \mathrm{FAc}$ and $\left[{ }^{18} \mathrm{~F}\right] \mathrm{FAHA}$ brain imaging was performed in 2 rhesus monkeys under baseline conditions. $\left[{ }^{18} \mathrm{~F}\right] \mathrm{FAHA}$ scans were also performed during dosing of the HDAC inhibitor F-SAHA. The $\left[{ }^{18} \mathrm{~F}\right] \mathrm{FAc}$ impulse response function $\operatorname{IRF}(\mathrm{FAc})$ was estimated using data driven methods: $\mathrm{TAC}(\mathrm{FAc})=$ Plasma(FAc)*IRF(FAc). When $\left[{ }^{18} \mathrm{~F}\right] \mathrm{FAHA}$ is injected, both $\left[{ }^{18} \mathrm{~F}\right] \mathrm{FAHA}$ and $\left[{ }^{18} \mathrm{~F}\right] \mathrm{FAc}$ in plasma contribute to brain activity. The contribution from FAHA alone was estimated as TAC(FAHA) $=\mathrm{TAC}(\mathrm{FAHA}+\mathrm{FAc})$-Plasma(FAc)*IRF(FAc). The corrected FAHA $\mathrm{V}_{\mathrm{T}}$ was used as HDAC availability index. $\left[{ }^{18} \mathrm{~F}\right] \mathrm{FAc}$ displayed slow brain kinetics with negligible clearance. After $\left[{ }^{18} \mathrm{~F}\right] \mathrm{FAHA}$ injection, the brain activity due to FAHA alone (after correction for FAc contribution) cleared with no evidence of retention from $\left[{ }^{18} \mathrm{~F}\right] \mathrm{FAc}$ formed in the brain. The test retest reproducibility of $\left[{ }^{18} \mathrm{~F}\right] \mathrm{FAHA} \mathrm{V}_{\mathrm{T}}$ was bad $(50 \%)$ and although a reduction in $\mathrm{V}_{\mathrm{T}}$ (FAHA) was observed during F-SAHA dosing, the changes were not dose dependent. In plasma, the only labeled metabolite was $\left[{ }^{18} \mathrm{~F}\right]$ FAc and the $\left[{ }^{18}\right.$ F]FAHA fraction was dependent on F-SAHA dose. At baseline and $0.001 \mathrm{mg} / \mathrm{kg}$ F-SAHA infusion, $\left[{ }^{18} \mathrm{~F}\right] \mathrm{FAHA}$ fraction was negligible after $15 \mathrm{~min}$. For F-SAHA doses higher than $0.01 \mathrm{mg} / \mathrm{kg},\left[{ }^{18} \mathrm{~F}\right]$ FAHA fraction was between $20 \%$ and $45 \%$ at $15 \mathrm{~min}$ and $~ 10 \%$ at $2 \mathrm{~h}$. If the $\left[{ }^{18} \mathrm{~F}\right] \mathrm{FAc}$ contribution to the brain is ignored, the changes in $\left[{ }^{18} \mathrm{~F}\right] \mathrm{FAHA}$ binding reflect changes in tracer metabolism and not HDAC inhibition. Although it is possible to correct for $\left[{ }^{18} \mathrm{~F}\right] \mathrm{FAc}$ contribution from plasma to the measured brain activity during $\left[{ }^{18} \mathrm{~F}\right] \mathrm{FAHA}$ studies our results indicate that $\left[{ }^{18} \mathrm{~F}\right] \mathrm{FAHA}$ is an unsuitable tracer for central HDAC imaging and cannot be used for target engagement of an HDAC inhibitor.

$\mathbf{J 2 2 0}$

WHOLE-BODY BIODISTRIBUTION

OF [11C]GLYCYLSARCOSINE AS A NOVEL CANCER PROBE TARGETING PEPTIDE TRANSPORTERS: NON-HUMAN PRIMATE STUDY

Sosuke Miyoshi $^{1,2}$, Akihiro Noda ${ }^{1,2}$, Yoshihiro Murakami ${ }^{1,2}$, Keisuke Mitsuoka ${ }^{1,2}$, Akihiko Fujikawa ${ }^{1,2}$, Akinori Iwashita, ${ }^{1,2}$, Satoshi Minoshima ${ }^{3}$, Ichiro Matsunari ${ }^{1}$, Shintaro Nishimura ${ }^{1,2}$

${ }^{1}$ The medical and pharmacological research center foundation, Hakuicity, Japan, ${ }^{2}$ Bioimaging, Astellas Pharma Inc., Tsukuba-city, Japan, ${ }^{3}$ Radiology, University of Washington, Seattle, WA, USA.
$\mathrm{H}+$ /peptide transporters are expressed functionally in many types of human cancer cells and promising molecular targets for cancer detection using PET. Recently, we have developed a peptide PET tracer [11C]glycylsarcosine (Gly-Sar) and demonstrated better distinction between tumors and inflammatory tissues than [18F]FDG in rodents (JNM April cover \& 49; 615-22: 2008). As a part of efforts to translate these findings to humans, we investigated biodistribution of [11C]Gly-Sar in non-human primates as compared to other oncologic PET tracers. Whole-body [11C]Gly-Sar PET imaging was performed in rhesus monkeys. The same animals underwent PET imaging with $[18 \mathrm{~F}]$ FDG, [18F]FLT, [11C]choline, [11C]acetate, and [18F]FMISO. In addition, biodistribution of $[11 \mathrm{C}] \mathrm{Gly}-\mathrm{Sar}$ was evaluated in mice implanted with human cancer xenograft (human pancreatic tumor cells, AsPC-1) using microPET system. The results showed the most significant advantage of [11C]Gly-Sar PET tracers was related to the markedly lower background activity except for those derived from urinary system, allowing more distinct detection of tumors. Its biodistribution in non-human primate was smilar to that observed in mice by microPET imaging in which clear delineation of implanted tumor was attained. This preclinical evaluation supports that $[11 \mathrm{C}] \mathrm{Gly}-\mathrm{Sar}$ is a promising candidate tracer for peptide transporter PET imaging in humans.
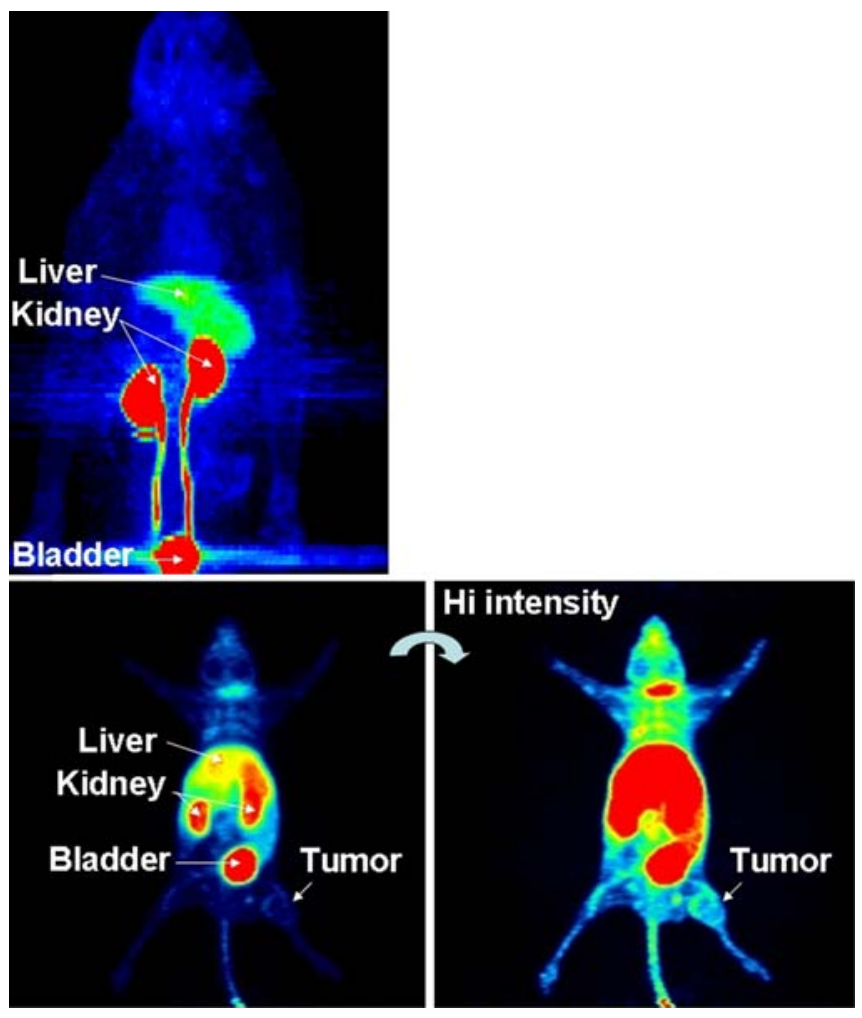

PET imaging of [11C]Gly-Sar in monkey and mouse with tumor

$\mathbf{J} 221$

NOVEL RADIOSYNTHESIS OF THE KAPPA RECEPTOR LIGAND $\left[{ }^{11} \mathrm{C}\right]$-GR103545 BY DIRECT FIXATION OF $\left[{ }^{11} \mathrm{C}\right]-\mathrm{CO}_{2}$ : SYNTHESIS OF $\left[{ }^{11} \mathrm{C}\right]$-CARBAMATES

Alan A. Wilson, Armando G. Garcia, Sylvain Houle, Neil Vasdev; PET Centre, Centre for Addiction and Mental Health, Toronto, ON, Canada. 
$\left[{ }^{11} \mathrm{C}\right]$-carbon dioxide is the most readily available source of cyclotron-produced carbon-11. Nevertheless, direct fixation of $\left[{ }^{11} \mathrm{C}\right]-\mathrm{CO}_{2}$ into complex biologically interesting molecules has been severely limited. Recent advances in "green" chemistry have produced some interesting carbamate producing reactions using carbon dioxide which can be applicable to carbon-11 chemistry (1). Here we describe the efficient radiosynthesis of the potent kappa opioid agonist $\left[{ }^{11} \mathrm{C}\right]-\mathrm{GR} 103545$ (2) by fixation of $\left[{ }^{11} \mathrm{C}\right] \mathrm{CO}_{2}$ into the corresponding amine precursor followed by alkylation of the intermediate carbonic acid. Methods: A solution of norcarbamoyl amine $(0.1 \mathrm{mg})$ in DMF $(40 \mu \mathrm{L})$ and the phosphazene base BEMP (3 $\mu \mathrm{L}$ ) was treated with cyclotron-produced $\left[{ }^{11} \mathrm{C}\right]-\mathrm{CO}_{2}$ in $\mathrm{N}_{2}$ $(20 \mathrm{~mL} / \mathrm{min})$ at ambient temperature. When radioactivity had peaked, a solution of dimethyl sulphate $(10 \mu \mathrm{L})$ in DMF $(400 \mu \mathrm{L})$ was added then immediately quenched with HPLC buffer. The resulting $\left[{ }^{11} \mathrm{C}\right]-\mathrm{GR} 103545$ (2) was purified by HPLC and formulated for imaging studies. Results: Trapping of $\left[{ }^{11} \mathrm{C}\right]-\mathrm{CO}_{2}$ in the amine solution was $>95 \%$. Incorporation of $\left[{ }^{11} \mathrm{C}\right]-\mathrm{CO}_{2}$ into the product was between 50 and $60 \%$. Isolated radiochemical yields (uncorrected for decay) of formulated, sterile, radiochemically pure $\left[{ }^{11} \mathrm{C}\right]-$ GR103545 were 10-14\% with specific activities greater than $50 \mathrm{GBq} / \mu \mathrm{mole}$ at end-of-synthesis. Chiral HPLC confirmed conservation of optical purity. Other primary and secondary aliphatic amines gave similar results while aromatic amines were more problematic. Conclusions: $\left[{ }^{11} \mathrm{C}\right]$-methylcarbamates, such as $\left[{ }^{11} \mathrm{C}\right]-$ GR103545, can be readily produced by direct fixation of amines in the presence of agents such as BEMP. Only $0.1 \mathrm{mg}$ of precursor was employed which is also advantageous when supplies are limited. The quality and quantity of product is suitable for PET imaging studies. References: (1)-Hooker, JT et al Angew. Chemie 2009, 121, 3534-3537 (2) - Ravert, HT et al Nucl. Med. Biol. 2002, $29,47-53$

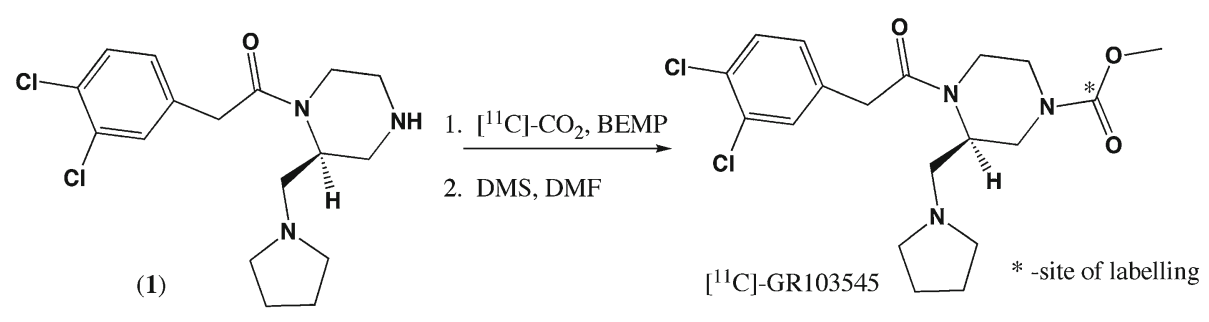

Radiosynthesis of $\left[{ }^{11} \mathrm{C}\right]-\mathrm{GR} 103545$ by direct fixation of $\left[{ }^{11} \mathrm{C}\right]-\mathrm{CO}_{2}$.

$\mathbf{J} 222$

QUANTIFICATION OF METABOTROPIC GLUTAMATE SUBTYPE 5 RECEPTOR WITH BOLUS PLUS CONSTANT INFUSION ADMINISTRATION OF $^{18}$ F-SP203 IN HEALTHY HUMANS

Yasuyuki Kimura $^{1}$, Sami S. Zoghbi ${ }^{1}$, Fabrice G. Siméon ${ }^{1}$, Jun Hatazawa $^{2}$, Victor W. Pike ${ }^{1}$, Robert B. Innis ${ }^{1}$, Masahiro Fujita ${ }^{1}$

${ }^{1}$ Molecular Imaging Branch, National Institute of Mental Health, Bethesda, MD, USA, ${ }^{2}$ Department of Nuclear Medicine and Tracer Kinetics, Osaka University Graduate School of Medicine, Suita, Japan.

We developed a new PET ligand, ${ }^{18} \mathrm{~F}-\mathrm{SP} 203$, to image metabotropic glutamate subtype 5 receptors (mGluR5) ${ }^{1}$. The binding was quantifiable by a bolus injection followed by serial acquisition of brain and arterial plasma data $^{2}$. Bolus plus constant infusion (B/I) of a radioligand may allow us less invasive studies than a bolus injection because total distribution volume $\left(V_{\mathrm{T}}\right)$ can be calculated from a ratio of brain to ${ }^{18} \mathrm{~F}-\mathrm{SP} 203$ in plasma at a single time point. The purpose of this study was to evaluate $\mathrm{B} / \mathrm{I}$ of ${ }^{18} \mathrm{~F}-\mathrm{SP} 203$ to quantify $V_{\mathrm{T}}$ of mGluR5 by comparing the 2 methods, the ratios of brain to plasma at a single time point and the two-compartmental analysis of $2 \mathrm{~h}$ data in the same scans.Methods: Five healthy subjects were injected with about $220 \mathrm{MBq}$ of ${ }^{18} \mathrm{~F}-\mathrm{SP} 203$ and scanned for $5 \mathrm{~h}$. The same ratio of bolus-toconstant infusion $\left(\mathrm{K}_{\text {bolus }}=219 \mathrm{~min}\right)$ was applied in all scans to achieve equilibrium in $2 \mathrm{~h}$. The concentrations of ${ }^{18} \mathrm{~F}$-SP203 were measured in the arterial plasma using radio-HPLC. We calculated $V_{\mathrm{T}}$ by two methods: 1) Ratios of radioactivity in the brain regions to unmetabolized ${ }^{18} \mathrm{~F}-\mathrm{SP} 203$ in the arterial plasma at one time point of $2 \mathrm{~h}$ and 2) Nonlinear least squares analysis with an unconstrained two-tissue compartment model using $2 \mathrm{~h}$ data. Results: The concentration of radioactivity was stable from $100 \mathrm{~min}$ to $300 \mathrm{~min}$ in most brain regions and arterial plasma indicating that equilibrium was achieved (Figure). $V_{\mathrm{T}}$ calculated from the ratio at $2 \mathrm{~h}$ was similar to that calculated by the two-compartment model with differences $10 \pm 5 \%$ (Table). Conclusion: B/I administration of ${ }^{18} \mathrm{~F}-\mathrm{SP} 203$ is an alternative method to measure the distribution volume of mGluR5 with less invasive procedures. References 1. Siméon, F. et al. J. Med. Chem. 50, 3256. 2. Brown, A.K. et al. J Nucl Med 49, 2042.

Distribution volume of ${ }^{18} \mathrm{~F}-\mathrm{SP} 203$ in brain regions calculated from two methods

\begin{tabular}{|c|c|c|}
\hline & \multicolumn{2}{|c|}{ Distribution volume $\left(\mathrm{mL} \& \# 9679 ; \mathrm{cm}^{-3}\right)$} \\
\hline Region & $2 \mathrm{TCM}$ & Brain/plasma ratio \\
\hline Temporal cortex & $28.6 \pm 3.0$ & $28.9 \pm 1.8$ \\
\hline Frontal cortex & $25.8 \pm 3.2$ & $24.9 \pm 1.8$ \\
\hline Thalamus & $19.7 \pm 2.9$ & $17.4 \pm 2.1$ \\
\hline Cerebellum & $17.5 \pm 1.1$ & $16.3 \pm 1.7$ \\
\hline
\end{tabular}




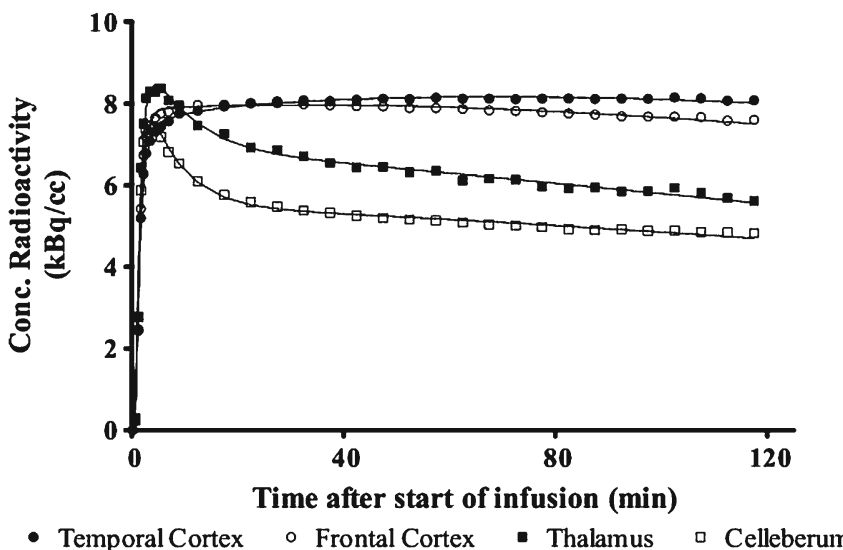

Time course of radioactivity in brain of a representative subject.

$\mathbf{J 2 2 3}$

PET STUDIES USING $\left[{ }^{11} \mathrm{C}\right]$ TELMISARTAN

FOR THE EVALUATION OF ORGANIC ANION

TRANSPORTING POLYPEPTIDE FUNCTION

AND INVESTIGATION OF DETERMINATION FACTOR FOR ITS NONLINEAR PHARMACOKINETICS

Tadayuki Takashima ${ }^{1}$, Yoshinobu Hashizume ${ }^{2}$, Machiko Murai ${ }^{1}$, Kazuya Maeda $^{3}$, Yasuhiro Wada ${ }^{1}$, Hisashi Doi ${ }^{2}$, Hiroyuki Kusuhara ${ }^{3}$, Yuichi Sugiyama $^{3}$, Yasuyoshi Watanabe ${ }^{1}$

${ }^{1}$ Molecular Probe Dynamics Laboratory, RIKEN, Center for Molecular Imaging Science, Kobe, Hyogo, Japan, ${ }^{2}$ Molecular Imaging Labeling Chemistry Laboratory, RIKEN, Center for Molecular Imaging Science, Kobe, Hyogo, Japan, ${ }^{3}$ Department of Molecular Pharmacokinetics, Graduate School of Pharmaceutical Sciences, the University of Tokyo, Tokyo, Japan.

Telmisartan, a nonpeptide angiotensin II subtype 1 receptor antagonist, is the substrate for organic anion transporting polypeptide 1B3 (OATP1B3) and excreted by hepatobiliary transport. Telmisartan showed nonlinear pharmacokinetics and large interindividual variability at therapeutic dose. However, the determination factor for nonlinear pharmacokinetics of Telmisartan was not directly demonstrated by in vivo plasma pharmacokinetic data. Positron Emission Tomography (PET) is a useful tool for functional analysis of drug transporters in vivo since it has higher sensitivity and spacio-temporal resolution to evaluate the tissue uptake and subsequent efflux, separately. We performed a quantitative PET study in normal Sprague-Dawley (SD) rats using $\left[{ }^{11} \mathrm{C}\right]$ Telmisartan as a PET tracer, and compared to the results using Oatp inhibitor rifampicin-treated rats to evaluate Oatp function on hepatic uptake. In addition, $\left[{ }^{11} \mathrm{C}\right]$ Telmisartan PET study with unlabeled Telmisartan $(1-10 \mathrm{mg} / \mathrm{kg})$ was conducted to evaluate the determination factor for its nonlinear pharmacokinetics. After a bolus injection of $\left[{ }^{11} \mathrm{C}\right]$ Telmisartan, a 90-minute PET scan was conducted, and tissue distribution of its radioactivity was analyzed. Carbon-11 labeled Telmisartan is rapidly taken up into the liver in normal rats with hepatic blood flow-dependent level, and then the radioactivity derived from its acylglucuronide was excreted into bile. When Oatp inhibitor Rifampicin was infused, hepatic uptake clearance of $\left[{ }^{11} \mathrm{C}\right]$ Telmisartan was decreased with increasing rifampicin infusion rate. Co-administration with unlabeled Telmisartan at $10 \mathrm{mg} / \mathrm{kg}$ by bolus injection decreased hepatic uptake clearance of $\left[{ }^{11} \mathrm{C}\right]$ Telmisartan, whereas biliary excretion of the radioactivity was increased with increasing unlabeled Telmisartan dose. These results show that $\left[{ }^{11} \mathrm{C}\right]$ Telmisartan is taken up into liver via Oatps in rats, and one of determination factors for its nonlinear pharmacokinetics is saturation of hepatic uptake process, which Oatp mediated transport is considered to be predominantly related. We suggest that PET study using $\left[{ }^{11} \mathrm{C}\right]$ Telmisartan could be useful for the analysis of rat Oatp function in hepatic uptake, and these results show that $\left[{ }^{11} \mathrm{C}\right]$ Telmisartan is expected to be the functional PET probes for OATP1B3 function in human. Also PET study is a powerful tool for evaluating hepatobiliary transport and elucidating the transporter related factor for its nonlinear pharmacokinetics.

PEPTOID-BASED PET IMAGING PROBES: A PILOT STUDY WITH A VEGFR2 SPECIFIC LIGAND

Guiyang Hao $^{1}$, Luis M. De Leon-Rodriguez ${ }^{2}$, Michael Long ${ }^{1}$, Rolf Brekken $^{3}$, Dean Sherry ${ }^{2}$, Xiankai Sun ${ }^{1,2}$

${ }^{1}$ Department of Radiology, UT Southwestern Medical Center at Dallas, Dallas, TX, USA, ${ }^{2}$ Advanced Imaging Research Center, UT Southwestern Medical Center at Dallas, Dallas, TX, USA, ${ }^{3}$ Department of Surgery, UT Southwestern Medical Center at Dallas, Dallas, TX, USA.

Peptoids, oligomers of N-substituted glycines, are a specific subclass of peptidomimetics. They are closely related to their natural peptide counterparts, but differ chemically in that their side chains are appended to nitrogen atoms along the molecule's backbone, rather than to the $\alpha$-carbons as in peptides. Peptoids are not sensitive to peptidases or proteases and they are even easier and more economical to be synthesized than peptides. Recently a dimeric peptoid, GU40C4 (J. Am. Chem. Soc. 2008, 130, 5744-5752), was reported as a highly potent antagonist of Vascular Endothelial Growth Factor Receptor 2 (VEGFR2) activation inhibiting angiogenesis and tumor growth in vivo. The purpose of this work was to evaluate the potential of this dimeric peptoid as PET imaging agent of VEGFR2. GU40C4 was firstly modified by attaching a cysteine residue to the C-terminus, by which a bifunctional chelator (DOTA: 1,4,7,10-tetraazacyclododecane-1,4,7,10tetraacetic acid) was then conjugated with the peptoid via the maleimide-thiol chemistry. The DOTA-GU40C4 conjugate was characterized by HPLC and MALDI-MS, and labeled with ${ }^{64} \mathrm{Cu}$ (Purity $>98 \%$, Specific activity $>10 \mathrm{GBq} / \mu \mathrm{mol})$. After receiving $4.4 \mathrm{MBq}$ of ${ }^{64} \mathrm{Cu}$-DOTA-GU40C4 via the tail-vein, the SCID mice bearing an A673 tumor in the right flank or U87MG tumors in the right and left shoulders were scanned by dynamic $(0 \sim 60 \mathrm{~min})$ and static imaging procedures at $4 \mathrm{~h}$ and $24 \mathrm{~h}$ post-injection (p.i.) on a Siemens Inveon PET-CT Multimodality system. In both A673 and U87MG tumor models, ${ }^{64} \mathrm{Cu}$-DOTA-GU40C4 showed significant tumor accumulation, which was increasing with time out to $24 \mathrm{~h}$ p.i. (A673 tumor: $1.4 \pm 0.4$, $2.0 \pm 0.3$, and $3.3 \pm 0.6 \% \mathrm{ID} / \mathrm{g}$ at 1,4 and $24 \mathrm{~h}$, respectively; U87MG tumor: $1.6 \pm 0.1$ and $1.9 \pm 0.3$ at 4 and $24 \mathrm{~h}$, respectively). However, the liver uptake of ${ }^{64} \mathrm{Cu}$-DOTA-GU40C4 was rather high in both tumor models (A673: $37.6 \pm 1.9,33.2 \pm 3.8$, and $23.8 \pm 2.5 \% \mathrm{ID} / \mathrm{g}$ at 1,4 and $24 \mathrm{~h}$, respectively; U87MG: $31.3 \pm 1.5$ and $22.5 \pm 1.8 \% \mathrm{ID} / \mathrm{g}$ at 4 and $24 \mathrm{~h}$, respectively). This is likely due to the expression of VEGFR2 in the liver and the positive charge of ${ }^{64} \mathrm{Cu}-\mathrm{DOTA}-\mathrm{GU} 40 \mathrm{C} 4$. In conclusion, we have shown the potential of using peptoids to develop stable PET imaging probes. GU40C4, a VEGFR2 targeting peptoid ligand, may find application in PET imaging of tumor angiogenesis. Acknowledgements: The authors acknowledge the financial support of this work by National Institute of Health (P01 DK058398). 
$\mathbf{J 2 2 5}$

\section{AGOUTI-RELATED PROTEIN: AN EXCELLENT PEPTIDE SCAFFOLD FOR IN VIVO TUMOR MOLECULAR IMAGING}

$\underline{\text { Lei Jiang }}^{1,2}$, Richard Kimura ${ }^{1}$, Zheng Miao ${ }^{1}$, Adam P. Silverman ${ }^{1}$, $\overline{\text { Gang Ren }}^{1}$, Hongguang Liu ${ }^{1}$, Peiyong Li ${ }^{2}$, Sanjiv S. Gambhir ${ }^{1}$, Zhen Cheng ${ }^{1}$

${ }^{1}$ Department of Radiology, Molecular Imaging Program at Stanford and Bio-X Program, Stanford, CA, USA, ${ }^{2}$ Department of Nuclear Medicine, Ruijin Hospital, Shanghai, China.

Agouti-related protein ( $\mathrm{AgRP})$ is a $4-\mathrm{kDa}$ cystine-knot peptide of human origin with four disulfide bonds and four solvent exposed loops that are amenable to directed evolution. An AgRP-based combinatorial library has recently been screened to identify mutants that bind $\alpha v \beta 3$ integrin with high affinity and specificity. In this study, for the first time we evaluated the feasibility of using AgRP peptides for in vivo cancer molecular imaging. Methods: AgRP peptides were prepared using solid-phase peptide synthesis and an oxidative folding reaction. Receptor competition binding assays were performed to measure their affinities to $\alpha v \beta 3$ integrinexpressing tumor cells. Mutant 7C-AgRP, which showed high av $\beta 3$ integrin binding affinity, was site-specifically conjugated to 1, 4, 7, 10-tetra-azacyclododecane-N, N', N', N'"'-tetraacetic acid (DOTA), and the resulting bioconjugate DOTA-7C-AgRP was then labeled with $64 \mathrm{Cu}$ for biodistribution and microPET imaging studies in mice bearing U87MG xenografts. The mouse serum stability and metabolite analysis of $64 \mathrm{Cu}-\mathrm{DOTA}-7 \mathrm{C}-\mathrm{AgRP}$ were performed to evaluate the in vitro and in vivo stability of the probe. Results: DOTA-7C-AgRP (IC50=22.6 $\pm 3.9 \mathrm{nM}$ ) was successfully radiolabeled with $64 \mathrm{Cu}$ in good yield and high radiochemical purity (>99\%). Radiolabeled 7C-AgRP showed fast blood clearance, good tumor uptake and retention $(2.70 \pm 0.93$ and $2.37 \pm 1.04 \% \mathrm{ID} / \mathrm{g}$ at 2 and $24 \mathrm{~h}$, respectively) and excellent tumor imaging ability (20.87 and 11.32 at $4 \mathrm{~h}$ for tumor-to-blood and tumor-to-muscle, respectively). Integrin $\alpha v \beta 3$ binding specificity of the probe was confirmed in vitro and in vivo by using large excess of unlabeled c (RGDyK). Stability and Metabolite assays further demonstrated that the AgRP peptide was very stable in vitro and in vivo. Conclusion: 64Cu-DOTA-7C-AgRP shows great potential as a molecular probe for $\alpha v \beta 3$ integrin-positive tumor PET imaging. AgRP is an excellent peptide scaffold for further development of peptides for tumor imaging and therapy.
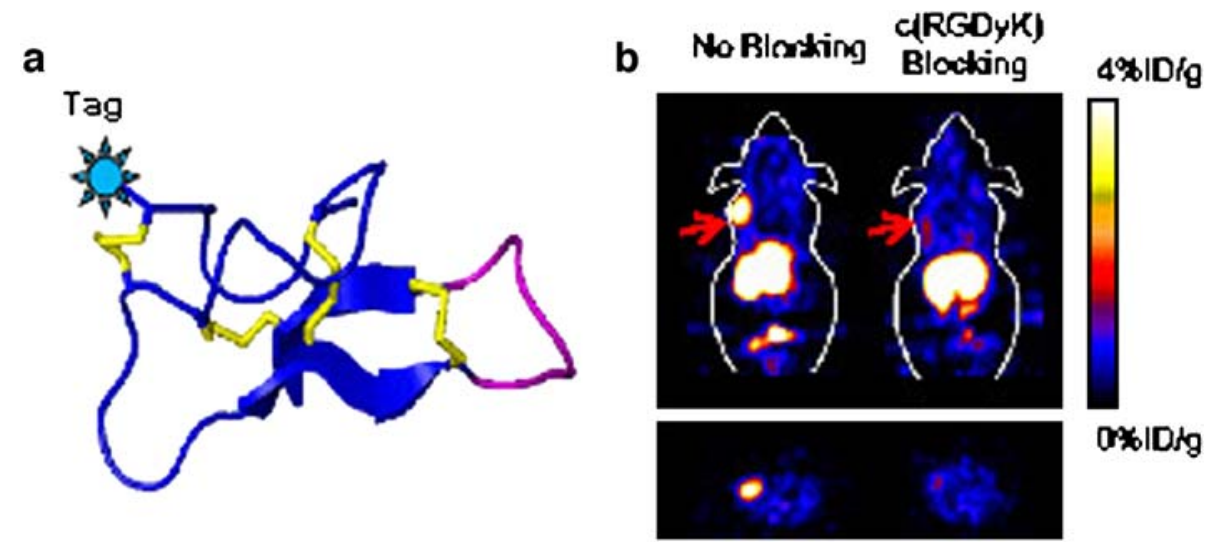

(A) AgRP based peptide scaffold for molecular probe development (the loop that was mutated for tumor targets recognition in our studies is indicated in purple). (B)The coronal and transaxial microPET images of U87MG tumor-bearing mice at $1 \mathrm{~h}$ after injection of $2.22 \mathrm{MBq}$ of $64 \mathrm{Cu}-$ DOTA-7C-AgRP with or without c(RGDyK).

J226

DEVELOPMENT OF A F3 PEPTIDE RADIOLIGAND FOR SPECT IMAGING OF TUMOR ANGIOGENESIS $\underline{\text { Marcian Van Dort }}{ }^{1}$, Brian D. Ross ${ }^{1}$, Mahaveer S. Bhojani ${ }^{2}$, Alnawaz Rehemtulla ${ }^{2}$, Rajesh Ranga ${ }^{2}$

${ }^{1}$ Radiology, University of Michigan, Ann Arbor, MI, USA, ${ }^{2}$ Radiation Oncology, University of Michigan, Ann Arbor, MI, USA.

Angiogenesis plays a key role in the growth and metastasis of solid tumors since new blood vessel formation bring in oxygen and nutrients catalyzing the dispersion of cancer cells to distant sites. Although recent advances in cellular and molecular biology have led to the identification of several novel angiogenic biomarkers and their signaling pathways, their translation to clinical oncology has been slow. This is partly due to a lack of clinically useful imaging ligands to noninvasively monitor the activity of these biomarkers. F3 peptide, a 33 amino acid fragment of HMGN2 protein, initially identified by the Ruoslahti group using phage display techniques, has been shown to target the vasculature of a variety of tumors by binding to endothelial cells. We focused on F3 peptide as a template for development of a radioiodinated ligand for SPECT imaging of angiogenesis. For this purpose, we synthesized the radioiodinated, thiol-specific, prosthetic labeling reagent: $N$-[2-(3-[ $\left[{ }^{125} \mathrm{I}\right]$ iodobenzoyl $)$ aminoethyl $]$ maleimide using a radioiododestannylation technique. Site-specific coupling of this reagent to the $\mathrm{C}$-terminal cysteine residue of $\mathrm{F} 3$ peptide provided the $\left[{ }^{125} \mathrm{I}\right]$-labeled F3 peptide radioligand $\left(\left[{ }^{125} \mathrm{I}\right] \mathrm{F} 3\right)$ in $72 \%$ overall radiochemical yield and $>99 \%$ radiochemical purity after HPLC 
purification. In preliminary cellular uptake studies, $\left[{ }^{125} \mathrm{I}\right] \mathrm{F} 3$ incubated with F3 peptide receptor overexpressing MDA-435 cells displayed a 13 -fold increase in uptake of radioactivity as compared to controls. Cell uptake studies conducted in the presence of a blocking dose of unlabeled $\mathrm{F} 3$ peptide $(10 \mu \mathrm{M})$ showed a $50 \%$ decrease in radioactivity uptake. These promising initial results have prompted in vivo SPECT imaging studies with $\left[{ }^{125}\right.$ I]IBDG in a nude mouse model having MDA435 xenografts. Disclosure of author financial interests or relationships: M. Van Dort, None; Mahaveer Bhojani, None; Rajesh Ranga, None; A. Rehemtulla, None; B. Ross, None.

J227

\section{SYNTHESIS OF NEW QUINOLINE DERIVATIVES AS CHELATING AGENTS: RADIOSYNTHESIS AND PRELIMINARY EVALUATION OF $\left[{ }^{18} \mathrm{~F}\right] 2$ - FLUOROQUINOLIN-8-OL FOR IMAGING AMYLOID \\ PLAQUES}

Neil Vasdev ${ }^{1,2}$, Pengpeng Cao ${ }^{1,2}$, Erik M. van Oosten ${ }^{1,2}$, Alan A. Wilson ${ }^{1,2}$, Sylvain Houle ${ }^{1,2}$, Peter P. Antich ${ }^{3}$, Frederick J. Bonte ${ }^{3}$, Padmakar V. Kulkarni ${ }^{3}$

${ }^{1}$ PET Centre, Centre for Addiction and Mental Health, Toronto, ON, Canada, ${ }^{2}$ University of Toronto, Toronto, ON, Canada, ${ }^{3}$ Radiology, University of Texas - Southwestern Medical Center, Dallas, TX, USA.

Purpose: Elevated levels of metal ions in amyloid plaques provide a promising target for imaging with chelating agents, as demonstrated by radioiodinated Clioquinol. The present work aims to synthesize several quinoline derivatives for PET. A fluorinated analog was developed and labelled with ${ }^{18} \mathrm{~F}$ for imaging amyloid plaques in rodent models with PET. Procedures: Over 10 new quinolines were synthesized and characterized. Furthermore, benzyloxy-2-chloroquinoline was prepared and reacted with $\left[\mathrm{K}_{222}\right]\left[{ }^{18} \mathrm{~F}\right]$ in DMSO at $135^{\circ} \mathrm{C}$, followed by catalytic hydrogenation. The product, $\left[{ }^{18} \mathrm{~F}\right] 2$-fluoroquinolin-8-ol, was purified by HPLC and formulated in $70 \mathrm{~min}$. Preliminary imaging studies were carried out in male Swiss Webster mice $(25 \mathrm{~g} ; n=4)$, following a 6 week preinjection of synthetic Abeta (1-42) peptide aggregates (10 $\mu \mathrm{g}$ in $25 \mu \mathrm{L}$ PBS) into the hippocampus (brain sections showed a positive Congo Red stain). Control mice received saline injections. Image acquisition (Siemens Inveon PET-CT) was started after i.v. injection of the tracer $(50-80 \mu \mathrm{Ci})$. Time-activity curves for brain activity were generated, taking the initial value obtained at $0.5 \mathrm{~min}$ as $100 \%$. Results: 4-Fluoro-quinoline derivatives were unstable in our hands and a rearrangement product with $\mathrm{MeOH}$ was identified by X-ray crystallography. However, the 2-fluoro-analog was synthesized and $\left[{ }^{18} \mathrm{~F}\right] 2$-fluoroquinolin-8-ol was subsequently prepared in $30 \pm 4 \%(n=5)$ radiochemical yield (uncorrected), with $>99 \%$ radiochemical purity. Specific activity was $1248 \mathrm{mCi} / \mu \mathrm{mol}$ and $\log \mathrm{P}(\mathrm{pH} 7.4)$ was $2.07 \pm 0.03$. PET images showed fast uptake and rapid washout of the tracer from the control mouse brain. For control animals, brain activity at $6.5 \mathrm{~min}$ was $13 \pm 4 \%$ and reached a plateau. Experimental animals had much slower washout $(24 \pm 5 \%$ at $6.5 \mathrm{~min})$ and reached a plateau, $21 \pm 5 \%$ at 10 min. Experimental animal brain activity was $180 \%$ of the control animals at $6.5 \mathrm{~min}$ and remained at $140 \%$. Initial brain uptake was $6-8 \% \mathrm{ID} / \mathrm{g}$, comparable to that of $\left[{ }^{11} \mathrm{C}\right] \mathrm{PIB}$. Preliminary images in a double transgenic mouse model (APP/PS1) showed specific uptake. Further imaging studies and the syntheses of new quinoline derivatives are concurrently under development. Conclusions: We have prepared a new $\left[{ }^{18} \mathrm{~F}\right]$-labelled radiotracer based on a hydroxyquinoline derivative for imaging amyloid plaques. These studies warrant further evaluation of this class of molecules as amyloid imaging agents.

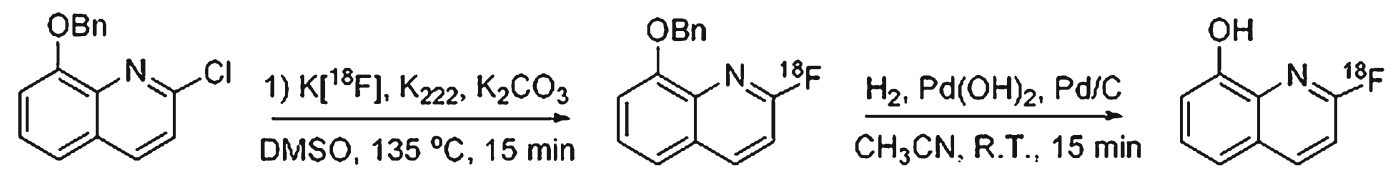

$\mathbf{J} 228$

\section{QUANTITATIVE MEASUREMENTS OF RADIOACTIVITY IN A MICROFLUIDIC CHIP DURING THE RADIOCHEMICAL SYNTHESIS OF PET IMAGING PROBES}

Jennifer Cho, Sebastian Olma, Kan Liu, Yi-Chun Chen, Clifton K. Shen, R Michael van Dam, Arion Chatziioannou; Crump Institute for Molecular Imaging, UCLA School of Medicine, Los Angeles, CA, USA.

One of the main research interests at our institute is the development of a versatile, modular and automated microfluidic platform capable of synthesizing 18F-labeled small molecules for PET imaging on demand. Although there is active research in the development of microfluidic devices for this application, not many tools are yet available for monitoring and analyzing the chip development process. Applying microfluidic systems to radiochemical synthesis is still in its nascent stage and one of the obstacles hindering the development of microfluidic chips is the lack of dedicated imaging systems. Therefore, an imaging system that can pinpoint the exact location of failure in a microfluidic chip during radiochemical synthesis and quantify the $2 \mathrm{D}$ distribution of radiolabeled molecules will provide researchers with a valuable tool to help design and develop many types of microfluidic chips. A new imaging system was developed for the detection of radiolabeled molecules in a microfluidic chip. The detector consisted of a charge coupled device (CCD) optically coupled to a lens. Proof of principle was demonstrated with two approaches: scintillation approach and Cerenkov radiation approach. In the scintillation approach, phosphor powder ( $\mathrm{ZnS}: \mathrm{Ag})$ was integrated into the microfluidic chip. The phosphor converts the high energy of beta particles emitted from PET probes into visible light photons that can be, in turn, detected by the system. In the Cerenkov radiation approach, we have developed a novel method for quantitative imaging of beta particles in a microfluidic chip by utilizing visible light emissions from Cerenkov radiation. We have theoretically proved and empirically demonstrated 
that beta particles emitted from PET imaging probe can produce visible light in a microfluidic chip from Cerenkov radiation. This technology has not been previously tried or reported to the best of our knowledge. The imaging system was used to evaluate the feasibility of these two approaches as quantitative imaging methods. The system spatial resolution, minimum detectable activity and dynamic range were evaluated. This technology is already being used at our institute to pinpoint the location of failures or abnormality in a microfluidic chip. We anticipate that quantitative activity measurements during the PET imaging probe synthesis will be presented at the time of the conference. These novel methods of quantitative imaging of beta particles in a microfluidic chip in-situ will provide researchers with a simple yet robust quantitative imaging tool for microfluidic applications.

\section{J229}

AN 18F-LABELED KNOTTIN PEPTIDE FOR TUMOR AVB3 INTEGRIN PET IMAGING

$\underline{\text { Zheng Miao }}^{2,1}$, Gang Ren ${ }^{2,1}$, Lei Jiang ${ }^{2,1}$, Richard Kimura ${ }^{4,3}$, Jennifer R. Cochran ${ }^{4,3}$, Zhen Cheng ${ }^{2,1}$, Sanjiv S. Gambhir ${ }^{2,1}$

${ }^{1}$ Radiology, Stanford University, Palo Alto, CA, USA, ${ }^{2}$ Molecular Imaging Program at Stanford, Stanford University, Palo Alto, CA, USA, ${ }^{3}$ BioX program, Stanford University, Palo Alto, CA, USA, ${ }^{4}$ Bioengineering, Stanford University, Palo Alto, CA, USA.

Knottins are small constrained polypeptides that share a common disulfide-bonded framework and a triple-stranded beta-sheet fold. Our objectives were to: 1) site-specifically label an integrin $\alpha v \beta 3$ binding knottin peptide, $2.5 \mathrm{D}$, with $18 / 19 \mathrm{~F}$, 2) characterize $18 \mathrm{~F}$ labeled 2.5D (18F-FB-2.5D) in living subjects with microPET imaging methods, and 3) evaluate the translational potential of 18F-FB-2.5D. Methods: 2.5D was prepared by solid phase synthesis and oxidized using glutathione. $2.5 \mathrm{D}$ was reacted with $\mathrm{N}$ succinimidyl-4-18/19F-fluorobenzoate (18/19F-SFB) in DMSO at $60^{\circ} \mathrm{C}$. Competition binding assays were performed with human glioblastoma U87MG cells using 125I-labeled echistatin. Approximately $100 \mathrm{uCi} 18 \mathrm{~F}-\mathrm{FB}-2.5 \mathrm{D}$, with and without a molar excess of $\mathrm{c}(\mathrm{RGDyK})$, was injected via the tail vein of U87MG tumor bearing mice for microPET imaging performed at $0.5 \mathrm{~h}, 1 \mathrm{~h}$ post-injection (p.i.) In addition, biodistribution studies were conducted at $0.5 \mathrm{~h} \mathrm{p}$. i. Finally, dynamic microPET scanning for a $35 \mathrm{~min}$ p.i. duration was also performed. Results and Conclusions: 19F-FB-2.5D competed with 125I-echistatin for binding to cell surface integrins with an IC50 of $162 \pm 21 \mathrm{nM}$. Radiofluorinated $2.5 \mathrm{D}$ displayed a high specific activity $(75-100 \mathrm{GBq} / \mu \mathrm{mol})$ throughout the course of our studies. In vivo microPET imaging showed that the radiotracer rapidly accumulated at the tumor $(2.7 \% \mathrm{ID} / \mathrm{g}, n=3)$, and also rapidly cleared through the kidneys. These pharmacokinetics leads to promising tumor-to-normal tissue contrast (Figure1). Bio-distribution studies demonstrated that $18 \mathrm{~F}-\mathrm{FB}-2.5 \mathrm{D}$ had moderate tumor uptakes at $0.5 \mathrm{~h} \mathrm{p}$.i. and that co-injection of $\mathrm{c}(\mathrm{RGDyK})$ significantly reduced tumor uptake $(1.90 \pm 1.15 \% \mathrm{ID} / \mathrm{g}$ vs $0.57 \pm$ $0.14 \% \mathrm{ID} / \mathrm{g}, 70 \%$ inhibition, $P<0.05)$. Dynamic scanning showed high kidney uptake at early time points $(51.8 \% \mathrm{ID} / \mathrm{g}$ at $4 \mathrm{~min}$ p.i. $)$, followed by rapid clearance within a half an hour $(6.2 \% \mathrm{ID} / \mathrm{g}$ at 35 min p.i.), Finally, the probe displayed relatively low uptake in the liver $(1.5 \% \mathrm{ID} / \mathrm{g}$ at $35 \mathrm{~min}$ p.i.). Collectively, knottins are excellent peptide scaffolds for development of PET probes for clinical translations.
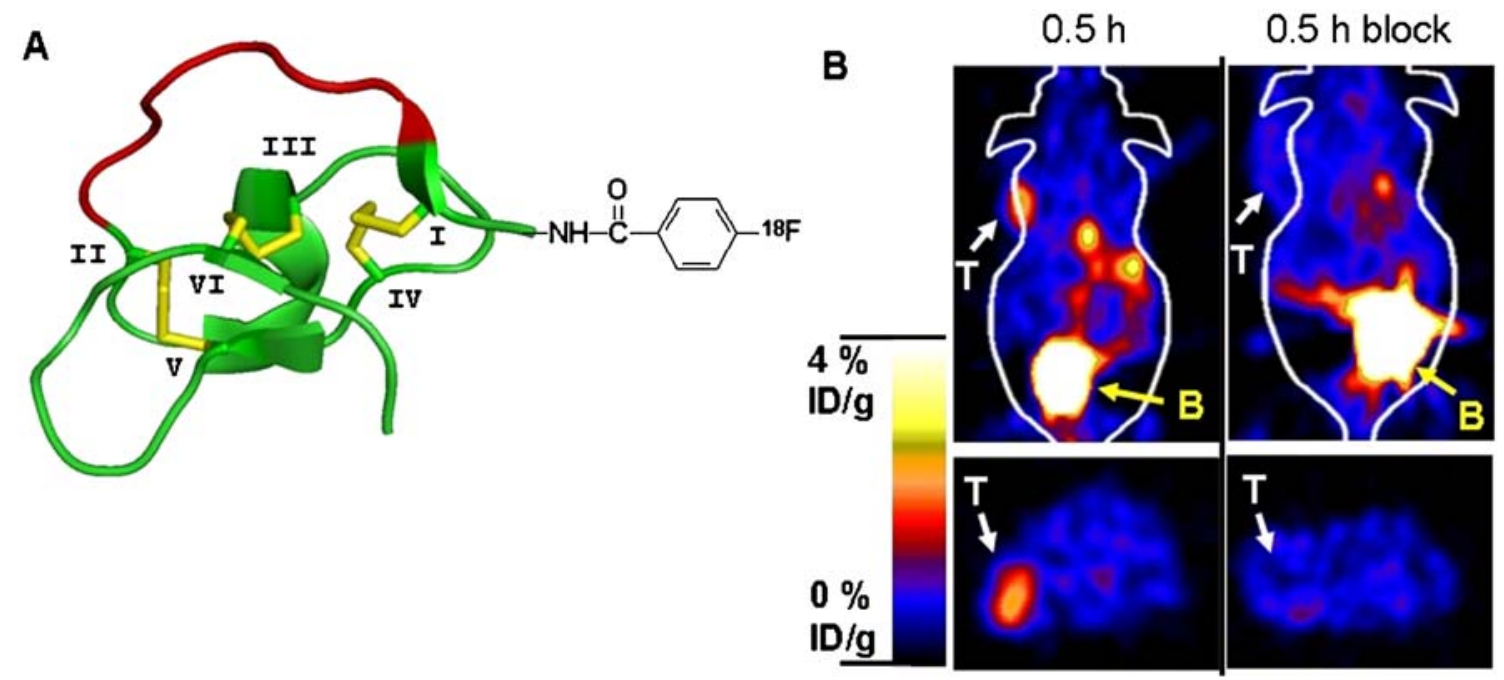

Figure: (A) Cartoon representation of a knottin scaffold showing the location of the conjugated radiolabel. The loop that was mutated for integrin binding is indicated in red. (B) The coronal and transaxial microPET images of U87MG tumor-bearing mice at $0.5 \mathrm{~h}$ after injection of ${ }^{18} \mathrm{~F}-\mathrm{FB}-2.5 \mathrm{D}$ with or without $\mathrm{C}(\mathrm{RGDyK}) \mathrm{co}$-injection. Tumor(T) and Bladder(B) are indicated by arrows. 


\section{IMAGING THE TYROSINE KINASE DOMAIN OF EPIDERMAL GROWTH FACTOR RECEPTOR} Glenn Cartwright $^{1}$, Uwe Ackermann ${ }^{2}$, Graeme O'Keefe ${ }^{2}$, $\overline{\text { Angela Rigopoulos }}^{1}$, Diana $\mathrm{Cao}^{1}$, Henri Tochon-Danguy ${ }^{2}$, Andrew M. Scott ${ }^{1,2}$ ${ }^{1}$ Ludwig Institute for Cancer Research, Heidelberg, VIC, Australia, ${ }^{2}$ Centre For PET, Austin Health, Heidelberg, VIC, Australia.

Background: Epidermal Growth Factor Receptor (EGFR) is involved in stimulating the growth of many human tumours including glioma. Central to EGFR activation is the intracellular tyrosine kinase domain. Therapeutic strategies targeting EGFR include antibodies such as Cetuximab and Tyrosine kinase Inhibitors (TKI), such as gefitinib and erlotinib. AG1478 is a TKI that has been shown to be a potent inhibitor of EGFR-positive tumours. This study aimed to assess the utility of the 11C labeled AG1478 as an imaging agent using the EGFR-positive A431 tumour xenograft model in nude mice. Methods: 11C-AG1478 was synthesised from 7-desmethyl AG1478 dissolved in DMF. Following the addition of $\mathrm{NaCO} 3, \mathrm{C}-11$ methyl iodide was distilled into the reactor vial and heated to $80^{\circ} \mathrm{C}$ for $5 \mathrm{~min}$. The radiotracer was purified by semipreparative HPLC and reformulated in $10 \%$ ethanol/saline for injection into tumour bearing mice. A431 xenografts were established in the right shoulder of BALB/c nude mice following the injection of 6x106 A431 cells. When tumours were approximately $600 \mathrm{~mm} 3$. Following injection of 11C-AG1478, mice were scanned using a Phillips Allegretto small animal PET camera for $60 \mathrm{~min}(12 \times 5 \mathrm{~min}$ frames $)$. Results: The pharmacokinetics determined a rapid 11C-AG1478 clearance with $\sim 7 \%$ injected dose/gram [ID/g] detectable 5 minutes post-injection in blood. Biodistribution analysis following PET imaging studies revealed similar biodistribution patterns in major organs including liver, kidney and bowel: $>10 \% \mathrm{ID} / \mathrm{g}$ at $5 \mathrm{~min}$ decreasing rapidly to $2 \% \mathrm{ID} / \mathrm{g}$ by $45 \mathrm{~min}$ post injection. A low level of $\sim 5 \% \mathrm{ID} / \mathrm{g}$ 11C-AG1478 was evident in A431 tumours from $5 \mathrm{~min}$ through $45 \mathrm{~min}$ post injection. Tumour imaging values were below the limit of quantitation. Conclusions: The results indicate the need to improve the stoichiometry of TKI binding to the ATP pocket of the wild-type EGFR kinase domain to permit imaging of tumour kinase activation status in vivo.

\section{9mTc-LABELLED FUCOIDAN \\ AS P-SELECTIN-TARGETED IMAGING AGENT \\ FOR THE IN VIVO SCINTIGRAPHIC \\ DETECTION OF PLATELET ACTIVATION \\ AND ACCUMULATION \\ Laure Bachelet, Francois Rouzet, Alain Meulemans,}

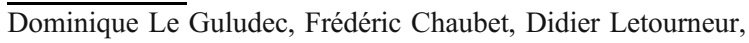
Jean-Baptiste Michel

INSERM U698, Paris, France.

Introduction: P-selectin, a cell adhesion molecule expressed on activated platelets and endothelial cells, plays an important role by mediating leucocytes/platelets or endothelium interactions. P-selectin is a potential marker of platelet or endothelium activation. Thereby, Pselectin-targeted imaging agents could help evaluation of cardio/ neurovascular diseases. P-selectin is also known to strongly interact with polysaccharides like fucoidan, a natural sulfated poly-L-fucose. Fucoidan exhibits a high affinity (KD $1 \mathrm{nM}$ ) for P-selectin. The intensity of fucoidan binding to activated platelets is dependent on the level of platelet activation. The present work relates to the synthesis and use of $99 \mathrm{mTc}$-labelled fucoidan for the in vivo scintigraphic detection of platelet activation and accumulation. Methods: Fucoidan was labelled with technetium-99 $\mathrm{m}(99 \mathrm{mTc})$ using a classical stannous reaction. $99 \mathrm{mTc}$-labelled fucoidan was injected in rats with either endocarditic vegetations, aneurysmal or atrial trombi, models associated with platelet activation. Ex vivo autoradiography was performed on histological sections. Results: 99mTc-labelled fucoidan allowed the in vivo visualization of platelet-rich endocarditic vegetations, atrial and aneurismal thrombi. These data were confirmed by ex vivo autoradiography showing the histological co-localization of the $99 \mathrm{mTc}$ signal in activated platelets-rich valves vegetations or thrombi with a high signal to background ratio of 8-10. Conclusion: $99 \mathrm{mTc}$-labelled fucoidan is a new P-selectin-targeted imaging agent for the evaluation of cardiovascular diseases associated with platelet or endothelium activation.
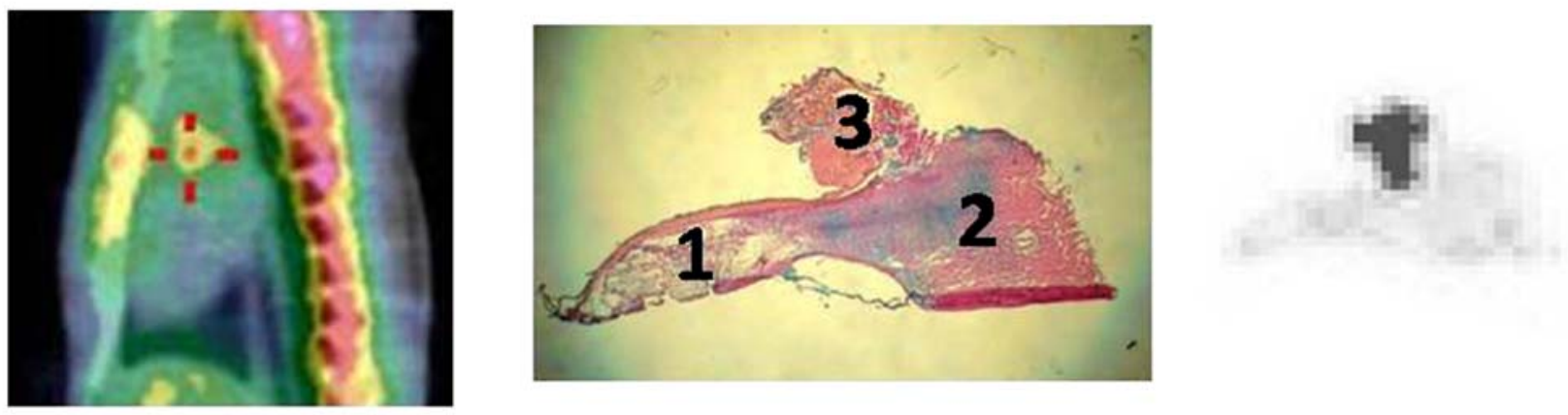

Figure shows in vivo SPECT imaging (left), histology (middle) and autoradiography (right) of heart in rat model of endocarditis. SPECT shows $99 \mathrm{mTc}-$ labelled fucoidan in the left ventricle. One histologic section vegetation is restricted to the valve (3) whereas the aorta (1) and the subvalvular myocardium (2) were normal. On the autoradiography, the signal from 99mTc-labelled fucoidan is exactly co-localized with the valvular vegetation. 
$\mathrm{J} 232$

111IN-LABELED AGOUTI RELATED PROTEINS FOR SPECT/CT IMAGING OF TUMOR $\alpha$ VB3 INTEGRIN $\underline{\text { Lei Jiang }}^{1,2}$, Zheng Miao ${ }^{1}$, Richard Kimura ${ }^{1}$, Gang Ren ${ }^{1}$, Hongguang Liu ${ }^{1}$, Adam P. Silverman ${ }^{1}$, Peiyong $\mathrm{Li}^{2}$, Sanjiv S. Gambhir ${ }^{1}$, Jennifer R. Cochran ${ }^{1}$, Zhen Cheng ${ }^{1}$

${ }^{1}$ Department of Radiology, Molecular Imaging Program at Stanford, Stanford, CA, USA, ${ }^{2}$ Department of Nuclear Medicine, Ruijin Hospital, Shanghai, China.

Agouti-related protein (AgRP) is a 4-kDa cystine-knot peptide of human origin with four disulfide bonds and four solvent exposed loops that are amenable to directed evolution. By replacement of a six amino acid loop in AgRP with a nine amino acid loop containing an RGD motif, engineered AgRP mutants that bind to $\alpha v \beta 3$ integrin with high affinity and high specificity have been discovered. In this study, the AgRP mutant 7C was labeled with $111 \mathrm{In}$ and used for SPECT/CT imaging of av $\beta 3$ integrin expression in living subjects. Methods: AgRP 7C was synthesized using solid-phase peptide synthesis and an oxidative folding reaction. 1, 4, 7, 10-tetra-azacyclododecane-N, N', N", N"' -tetraacetic acid (DOTA) was site specifically coupled to the N-terminus of the peptides. Receptor competition binding assay was then performed to measure the $\alpha v \beta 3$ integrin binding affinity of the resulting bioconjugate. Radiolabeling of DOTA-7C was achieved by incubating the peptide with $111 \mathrm{InCl} 3$ in $\mathrm{NaOAc}$ buffer $(\mathrm{pH} 5.5)$ at $80^{\circ} \mathrm{C}$ for 45 min. Biodistribution and microSPECT/CT imaging studies were then performed to evaluate the in vivo performance of the probes using mice bearing U87MG xenografts. Results: DOTA-conjugated AgRP 7C displayed high $\alpha v \beta 3$ integrin binding affinity (nM range), and was easily labeled with $111 \mathrm{In}$ in high yield and purity $(>99 \%)$. Moreover, this SPECT probe exhibited excellent stability in PBS buffer ( $\mathrm{pH} 7.4)$ and mouse serum. 111In-

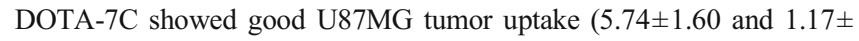
$0.21 \% \mathrm{ID} / \mathrm{g}$ at 0.5 and $24 \mathrm{~h}$, respectively). High tumor-to-normal organ ratios were also obtained for this probe ( 25.8 and 39.8 at $2 \mathrm{~h}$ for tumor-toblood and tumor-to-muscle, respectively). The tumor uptake of $111 \mathrm{In}$ DOTA-7C could also be specifically inhibited by co-injection with large excess of the $\alpha v \beta 3$ integrin-binding peptide $c($ RGDyK). Finally, excellent and specific tumor imaging of $111 \mathrm{In}$-DOTA-7C was also demonstrated by small-animal SPECT/CT imaging at $1 \mathrm{~h}$ after injection. Conclusion: AgRP peptide 7C labeled with $111 \mathrm{In}$ exhibited high tumor uptake, demonstrating great potential for SPECT/CT imaging of $\alpha v \beta 3$ integrin expression in living subjects.

\section{$\mathbf{J} 233$}

\section{C-KIT-SPECIFIC PET/SPECT IMAGING IN A MOUSE MODEL OF SMALL CELL LUNG CANCER}

Chisato Yoshida ${ }^{1,2}$, Atsushi B. Tsuji ${ }^{1}$, Hitomi Sudo ${ }^{1}$, Aya Sugyo ${ }^{1}$, Chizuru Sogawa ${ }^{1}$ Chika Murai ${ }^{1}$, Masayuki Inubushi ${ }^{1}$, Yasushi Arano ${ }^{2}$, Misturu Koizumi ${ }^{1}$, Tsuneo Saga ${ }^{1}$

${ }^{1}$ Diagnostic Imaging Group, Molecular Imaging Center, National Institute of Radiological Sciences, Chiba, Japan, ${ }^{2}$ Graduate School of Pharmaceutical Sciences, Chiba University, Chiba, Japan.

C-kit is a $145 \mathrm{kDa}$ transmembrane glycoprotein and a tyrosine kinase type receptor for the stem cell factor. C-kit is well known to be a protooncogene, and mutations of this gene induce ligand-independent tyrosine kinase activation and autophosphorylation, which leads to uncontrolled cell proliferation and carcinogenesis. C-kit is highly expressed in gastrointestinal stromal tumors (GISTs), small cell lung cancer (SCLC), etc.
SCLC, representing about $20 \%$ of all lung cancer types, is an aggressive form of lung cancers, and tends to develop metastases to lymph nodes and other organs at an early stage. The overall prognosis remains poor, though SCLC is sensitive to chemotherapy and radiation. Imatinib, a specific inhibitor of c-kit-tyrosine kinase, has been found to be highly effective against the chemotherapy-resistant GISTs and may be applicable for SCLC. Because not all SCLC express c-kit, we need a c-kit-specific imaging method to select SCLC patients who will respond to imatinib treatment and to broaden treatment options. In this study, we labeled anti-c-kit antibodies (IgG and Fab) with 125I, 111In and 64Cu, and assessed the in vitro and in vivo characteristics. Methods; We labeled murine anti-c-kit antibodies with 125I using a chloramine-T, 111In using a CHX-A"-DTPA and 64Cu using a p-Bn-DOTA, and performed cell binding, competitive inhibition and internalization assays using a human SCLC cell line, SY, expressing c-kit. We also performed biodistribution, PET and SPECT imaging using mice bearing SY tumors. Results: Cell binding and competitive inhibition assays showed that radiolabeled antibodies retained immunoreactivity to c-kit and specifically bound to SY cells. Internalization assay showed that IgG was quickly internalized into cells, while Fab was slowly internalized. Biodistribution experiments showed that tumor uptake of $111 \mathrm{In}$-IgG were $15.5 \pm 1.9,23.3 \pm 2.3$ and $20.3 \pm 1.6 \% \mathrm{ID} / \mathrm{g}$ after 24,48 and $96 \mathrm{~h}$, respectively, and those of $64 \mathrm{Cu}$-Fab were $2.6 \pm 0.7,6.1 \pm 0.2$ and $6.2 \pm 2.0 \% \mathrm{ID} / \mathrm{g}$ after 1,6 and $12 \mathrm{~h}$ respectively. In contrast, tumor uptake of 125I-labeled IgG and Fab remained low and decreased with time, probably because of the internalization and dehalogenation of 125I-labeled antibodies, suggesting that labeling with metal radionucleotide is suitable for imaging. In fact, we got clear planar and SPECT images of xenografted tumors at day 4 after injection of 111In-IgG, and PET images at $6 \mathrm{~h}$ after injection of 64Cu-Fab. Conclusion: $111 \mathrm{In}-\mathrm{IgG}$ and $64 \mathrm{Cu}-\mathrm{Fab}$ used in this study would be useful for SPECT or PET imaging of c-kit expression in SCLC.

\section{$\mathbf{J 2 3 4}$}

\section{AUTOMATIC SYNTHESIS OF $16 \beta-\left[{ }^{18}\right.$ F]FLUORO-5 $\alpha-$ DIHYDROTESTOSTERONE USING CASSETTE-TYPE $\left[{ }^{18} \mathrm{~F}\right]$ FDG SYNTHESIZER}

Yasushi Kiyono $^{1}$, Tetsuya Mori ${ }^{1,3}$, Tatsuya Asai $^{2}$, Yukie Yoshii ${ }^{1}$, Hidehiko Okazawa $^{1}$, Carmen S. Dence ${ }^{3}$, Michael J. Welch ${ }^{3}$, Yasuhisa Fujibayashi ${ }^{1}$

${ }^{1}$ Biomedical Imaging Research Center, University of Fukui, Fukui, Japan, ${ }^{2}$ Department of Nuclear Power and Energy Safety Engineering, Graduate School of Engineering, University of Fukui, Fukui, Japan, ${ }^{3}$ Department of Radiology, Washington University School of Medicine, St. Louis, MO, USA.

Objectives: The androgen receptor (AR) expression in prostate cancer is an important factor to predict tumor responsiveness to hormonal therapy. $16 \beta-\left[{ }^{18} \mathrm{~F}\right]$ fluoro-5 $\alpha$-dihydrotestosterone $\left(\left[{ }^{18} \mathrm{~F}\right] \mathrm{FDHT}\right)$ has been developed as one of the radiopharmaceuticals for AR PET imaging, however, the institutions which can use $\left[{ }^{18} \mathrm{~F}\right] \mathrm{FDHT}$ are limited because the reported synthesis method includes complex reaction conditions and prevents to use automatic modules. Automatic $\left[{ }^{18} \mathrm{~F}\right] \mathrm{FDG}$ synthesizers which can produce high-quality product with the operation easy enough for routine work, are widely used in clinical PET centers for diagnosis of cancer. Therefore, in this study we developed a new labeling method of $\left[{ }^{18} \mathrm{~F}\right]$ FDHT to adjust a cassette-type $\left[{ }^{18} \mathrm{~F}\right] \mathrm{FDG}$ synthesizer. Methods \& Results: A TRACERlab $\mathrm{MX}_{\mathrm{FDG}}$ was chosen as the module for $\left[{ }^{18} \mathrm{~F}\right] \mathrm{FDHT}$ because it is widely used as a simple automatic FDG synthesizer and it has the flexibility in programming and accepts modification to the cassette. The cassette was prepared from the FDG synthesis cassette with a little modification keeping aseptic condition. Optimum labeling condition was investigated by changing several conditions (amount of precursor and $\mathrm{NaBH}_{4}$, 
temperature and time of labeling, reduction and hydrolysis etc.)and the optimized condition is as follows: four milligrams of $16 \alpha$-[[(trifluoromethyl)sulfonyl]oxy]-3,3-(ethylenedioxy)androstan-17-one in acetonitrile was heated at $125^{\circ} \mathrm{C}$ for $10 \mathrm{~min}$ with dried $\left[{ }^{18} \mathrm{~F}\right]$ fluoride. The resultant solution was evaporated, and then reduced with $\mathrm{NaBH}_{4}$ in ethanol at room temperature. The hydrolysis with $0.1 \mathrm{~N} \mathrm{HCl}$ was carried out at $85^{\circ} \mathrm{C}$ for $15 \mathrm{~min}$. We performed 10 trials to synthesize $\left[{ }^{18} \mathrm{~F}\right] \mathrm{FDHT}$. The radiochemical yield estimated by analytical HPLC was $52.4 \pm 8.6 \%$. Investigation of purification condition is going on. Conclusion: $\left[{ }^{18} \mathrm{~F}\right] \mathrm{FDHT}$ was synthesized with a cassette-type FDG synthesizer. This system would enable us to produce $\left[{ }^{18} \mathrm{~F}\right] \mathrm{FDHT}$ safely for clinical use.

\section{$\mathbf{J} 235$}

\section{PURIFICATION AND IN-PROCESS ANALYSIS OF $\left[{ }^{18}\right.$ F]FDG} PRODUCED WITH THE PET BIOMARKER-GENERATOR

Anthony M. Giamis, David Patton, Ronald Nutt

Advanced Biomarker Technologies, Knoxville, TN, USA.

Introduction: There continues to be only one widely used biomarker, $\left[{ }^{18} \mathrm{~F}\right] \mathrm{FDG}$, in clinical PET and only a few biomarkers for drug development. One of the primary reasons for this lack of development is due to the nature of the production and distribution network for the single $\left[{ }^{18} \mathrm{~F}\right] \mathrm{FDG}$ biomarker. Advanced Biomarker Technologies (ABT) has created a manufacturing platform for the medical and biotechnology industries. This Biomarker-Generator allows you to produce $\left[{ }^{18} \mathrm{~F}\right]$ FDG "on-demand" for human injection and/or research subjects. Experimental: The Biomarker-Generator consists of a lower-energy positive ion accelerator which operates at $<20$ watts on target, producing $\left[{ }^{18} \mathrm{~F}\right]$ fluoride at $1-2 \mathrm{mCi}$ per minute The BiomarkerGenerator then uses microfluidic technology to manage the synthesis and control the volume. The crude $\left[{ }^{18} \mathrm{~F}\right] \mathrm{FDG}$ is purified by a single use microfluidic cartridge which neutralizes the hydrolysis conditions, removes unreacted $\left[{ }^{18} \mathrm{~F}\right]$ fluoride, and strips kryptofix from the mixture. The resulting flow is passed through a sterile filter into the final product vial. A sample from the dose vial is removed using microfluidic automation for quality assurance. An early generation micro Total Analytical System ( $\mu \mathrm{TAS}$ ) tests for $\mathrm{pH}$, kryptofix, organic solvents, radiochemical purity, and color/turbidity. Concurrent with the analysis, the $\left[{ }^{18} \mathrm{~F}\right] \mathrm{FDG}$ dose is compounded with saline and prepared for release. Results and Discussion: The purification, dilution, and compounding of the $\left[{ }^{18} \mathrm{~F}\right] \mathrm{FDG}$ dose was completed in 2.5 minutes. The analysis was timed for $15 \mathrm{~min}$. A full batch record was produced with the final release of $\left[{ }^{18} \mathrm{~F}\right] \mathrm{FDG}$ dose for each of 10 individual runs. Conclusion: We have demonstrated the capability to produce, purify, and qualify a single dose of $\left[{ }^{18} \mathrm{~F}\right] \mathrm{FDG}$ in an economic, efficient, and timely manner using microfluidic methods. Future improvements remain in terms of user interface and further streamlining the process.

\section{$\mathbf{J} 236$}

\section{THE C-TERMINAL CYS-(HIS) 6 SEQUENCE ON C2A ALLOWS FOR EFFICIENT SITE SPECIFIC RADIOLABELING WITH TECHNETIUM TRICARBONYL}

Richard Tavaré, Rafael Torres Martin de Rosales, Phil Blower, Greg Mullen; Division of Imaging Sciences, King's College London, London, United Kingdom.

Site specific radiolabeling of protein based imaging agents has led to a reduction of inactive protein and hence improved target affinity and biodistribution, as shown in development of AnnexinV imaging studies. The C2A domain of Synaptotagmin I, similar to AnnexinV, has been used previously as a cell death imaging agent (apoptosis/ necrosis) due to its ability to bind phosphatidylserine (PS) in the presence of calcium. Here we present an engineered $\mathrm{C} 2 \mathrm{~A}$ that contains a C-terminal cysteine followed by a HexaHistidine tag $(\mathrm{C} 2 \mathrm{AcH}$, $16.5 \mathrm{kDa})$ to be efficiently site specifically radiolabeled using technetium tricarbonyl $\left[\left(\mathrm{H}_{2} \mathrm{O}\right)_{3}{ }^{99 \mathrm{~m}} \mathrm{Tc}(\mathrm{CO})_{3}\right]^{+}$. The single cysteine also has the added benefit to allow for site-specific labeling of maleimideconjugated fluorophores and chelators for preclinical imaging. These proteins were characterized by electrospray mass spectrometry, SDS/ PAGE and size exclusion chromatography. When the thiol on the cysteine is blocked via fluorosceine-5-maleimide, the radiolabeling efficiency decreases from $90 \%$ to $50 \%$ when using the IsoLink kit for $30 \mathrm{~min}$ at $37^{\circ} \mathrm{C}$, indicating cysteine involvement of metal coordination. Protein derivatives without the HexaHistadine tag do not radiolabel above $15 \%$. Cysteine involvement in the mechanism of $\left[\left(\mathrm{H}_{2} \mathrm{O}\right)_{3}{ }^{99 \mathrm{~m}} \mathrm{Tc}\right.$ $\left.(\mathrm{CO})_{3}\right]^{+}$binding was further analyzed with an experiment showing that rhenium tricarbonyl labeled $\mathrm{C} 2 \mathrm{AcH}$ inhibits the formation of a dimer via thiols as compared to the non-rhenium tricarbonyl labeled control. The radiolabeled proteins were tested for binding to red blood cells (RBC) with exposed PS in a calcium dependant manner. The $\mathrm{Ec}_{50}$ of $\mathrm{C} 2 \mathrm{AcH}$ is $0.76 \mathrm{mM} \mathrm{Ca}^{2+}$, while the fluorescein conjugate, $\mathrm{C} 2 \mathrm{AcH}-$ FITC, has an $\mathrm{Ec}_{50} 1.03 \mathrm{mM} \mathrm{Ca}{ }^{2+}$. Furthermore, $\mathrm{C} 2 \mathrm{AcH}$ that was conjugated with an amine-directed NHS derivative, N-(benzyloxycarbonyloxy) succinimide that nonspecifically modifies Lys residues, the resultant conjugate did not bind to the red blood cells. We conclude that (i) the combination of Cys and a His-tag greatly enhances the speed and efficiency of labeling with $\left[\left(\mathrm{H}_{2} \mathrm{O}\right)_{3}{ }^{99 \mathrm{~m}} \mathrm{Tc}(\mathrm{CO})_{3}\right]^{+}$compared to either the His-tag or the Cys alone and this sequence deserves further evaluation as a radiolabeling tag; (ii) non-site-specific modification of $\mathrm{C} 2 \mathrm{~A}$ via lysine residues impairs target binding affinity; (iii) ${ }^{99 \mathrm{~m}} \mathrm{Tc}-\mathrm{C} 2 \mathrm{AcH}$ has excellent radiolabeling, stability and PS binding characteristics and warrants in vivo evaluation as a cell-death imaging agent.

$\mathbf{J} 237$

\section{HUMAN SERUM ALBUMIN DOMAIN III-PROTEIN SCAFFOLD WITH CONTROLLED SERUM PHARMACOKINETICS}

Vania Kenanova $^{1}$, Tove Olafsen ${ }^{1}$, Felix Bergara ${ }^{1}$,

Lawrence E. Williams ${ }^{2}$, Anna M. Wu ${ }^{1}$

${ }^{1}$ Molecular and Medical Pharmacology, University of California Los Angeles, Los Angeles, CA, USA, ${ }^{2}$ Radiology Division, City of Hope National Medical Center, Duarte, CA, USA.

Our objective is to generate a small, non-immunogenic protein scaffold with controlled serum kinetics and protein architecture suitable for modification. When attached to low molecular weight tumor targeting agents (peptides, aptamers, small chemicals) with transient serum persistence and low target uptake, the scaffold would provide tailored pharmacokinetics, improved target accumulation, and residues for functional group conjugation. We have engineered several versions of a small $(23,12$ or $11 \mathrm{kDa})$, non-immunogenic scaffold based on human serum albumin domain III (HSA DIII; $23 \mathrm{kDa}$ ). HSA DIII binds the neonatal $\mathrm{Fc}$ receptor $(\mathrm{FcRn})$, thereby extending the serum half-life of albumin. In order to modulate the serum persistence of the scaffold we perturbed the DIII-FcRn interaction by individually mutating conserved histidine residues H535, H510 and H464 to alanine, and also expressed the DIIIa $(12 \mathrm{kDa})$ and DIIIb $(11 \mathrm{kDa})$ half domains. To evaluate the effect of each mutation, fusion proteins consisting of the anti-CEA T84.66 diabody (Db; dimer of two scFv; $55 \mathrm{kDa})$ and either the HSA 
DIII wild type (WT) or one of the H535A, H510A and H464A variants were generated, labeled with I-124 and injected in xenografted mice that were imaged serially by microPET/CT. Image analysis of the DbDIIIs and $\mathrm{Db}$ at $4,20,28,44$, and $51 \mathrm{~h}$ showed segregation of kinetics, beginning at $28 \mathrm{~h}$. At $4 \mathrm{~h}$, the tumor-to-soft tissue ratios were 2.4 (WT and $\mathrm{H} 535 \mathrm{~A})$ and $2.5(\mathrm{H} 510 \mathrm{~A}, \mathrm{H} 464 \mathrm{~A}$ and Db), reaching $4.5(\mathrm{WT})<4.9$ $(\mathrm{H} 535 \mathrm{~A})<5.2(\mathrm{H} 510 \mathrm{~A})<7.6(\mathrm{H} 464 \mathrm{~A})<25.1(\mathrm{Db})$ at $51 \mathrm{~h}$. MicroPET image quantification of relative blood pool activity (ROI over the heart) at each time point was used to calculate the mean residence time of the proteins in the serum as follows: Db-DIII WT(56.7 h) $>\mathrm{H} 535 \mathrm{~A}(25 \mathrm{~h})>$ $\mathrm{H} 510 \mathrm{~A}(20 \mathrm{~h})>\mathrm{H} 464 \mathrm{~A}(17 \mathrm{~h})$, compared to $2.9 \mathrm{~h}$ for the $\mathrm{Db}$ alone. Biodistribution at $51 \mathrm{~h}$ confirmed the order of clearance from slowest to fastest: Db-DIII WT $>$ H535A $>$ H510A $>$ H464A $>$ Db with 4.0, 2.0, $1.8,1.6$ and $0.08 \% \mathrm{ID} / \mathrm{g}$ of remaining radioactivity in blood, respectively. The microPET/CT imaging studies demonstrate that attenuating the DIII-FcRn interaction provides a way of controlling the serum persistence of the entire Db-DIII fusion protein without compromising tumor targeting. Residue H464 appears to be most crucial for FcRn binding, followed by $\mathrm{H} 510$ and H535, with both DIIIa and DIIIb participating in the FcRn interaction. Future conjugation of tumor targeting peptides to the DIII scaffolds will enable identification of optimal targeting moiety-scaffold pharmacokinetics combinations for either imaging or therapeutic applications.

\section{$\mathbf{J} 238$}

\section{[C-11]MK-4232: THE FIRST PET TRACER FOR THE CALCITONIN GENE-RELATED PEPTIDE (CGRP) RECEPTOR AND ITS EVALUATION IN RHESUS MONKEY}

Sandra Sanabria, Hong Fan, Stacey O'Malley, Patti Miller, Zhizhen Zeng, Christine Ryan, Kerry Riffel, Mangay Williams, WaiSi Eng, Steve N. Gallicchio, Ian Bell, Harold Selnick, Chris Salvatore, Richard Hargreaves, Stefanie Kane, Sam Graham, Cyrille Sur, Jacquelynn J. Cook, Eric Hostetler

Merck Research Laboratories, West Point, PA, USA.
CGRP is a potent neuropeptide whose agonist interaction with the CGRP receptor (CGRP-R) results in vasodilation. This process is implicated in the cause of migraine headaches, and CGRP-R antagonists in clinical development have proven effective in treating migraine-related pain in human. CGRP-R is expressed in blood vessels throughout the periphery as well as in the CNS. However, it is not clear what role the inhibition of central CGRP$\mathrm{R}$ plays in migraine pain relief. Therefore, we desired to discover a CGRP-R PET tracer, which may be useful in helping understand the contribution of central CGRP-R antagonism to successful migraine treatment. These efforts have resulted in the identification of [C-11]MK-4232, a PET tracer for quantification of CGRP-R availability in the CNS. [C-11]MK-4232 $(\mathrm{Kd}=$ $0.27 \mathrm{nM}, \log P=3.38$ ) was synthesized via alkylation of the corresponding des-N-methyl precursor with [C-11]methyl triflate at room temperature. PET imaging studies were carried out in propofol anesthetized rhesus monkeys. Administration of [C-11] MK-4232 as an IV bolus provided high uptake in the cerebellum and brain stem, consistent with CGRP-R distribution in both monkey and human. Lower tracer uptake was observed in all other brain regions; lowest uptake was observed in white matter. Pre-administration of the potent CGRP antagonist MK-3207 resulted in plasma concentration-dependent blockade of tracer uptake in all brain regions. Tracer kinetic modeling was used to determine [C-11]MK-4232 total distribution volume, and receptor occupancy was calculated assuming the non-displaceable distribution volume was the same in all regions under baseline and blockade conditions. A sigmoidal relationship was observed when plotting MK-3207 plasma concentration vs. CGRP-R occupancy. In vitro studies in rhesus monkey and human cerebellum homogenate indicate a similarly high binding potential (Bmax/ $\mathrm{Kd}$ ) in both species, suggesting a signal of useful magnitude in human. Additionally, Scatchard plots are consistent with binding to a single site. In conclusion, [C-11]MK-4232 is a selective, high affinity, moderately lipophilic PET tracer that is useful for quantifying CGRP-R occupancy in monkey and is promising for use in human studies.
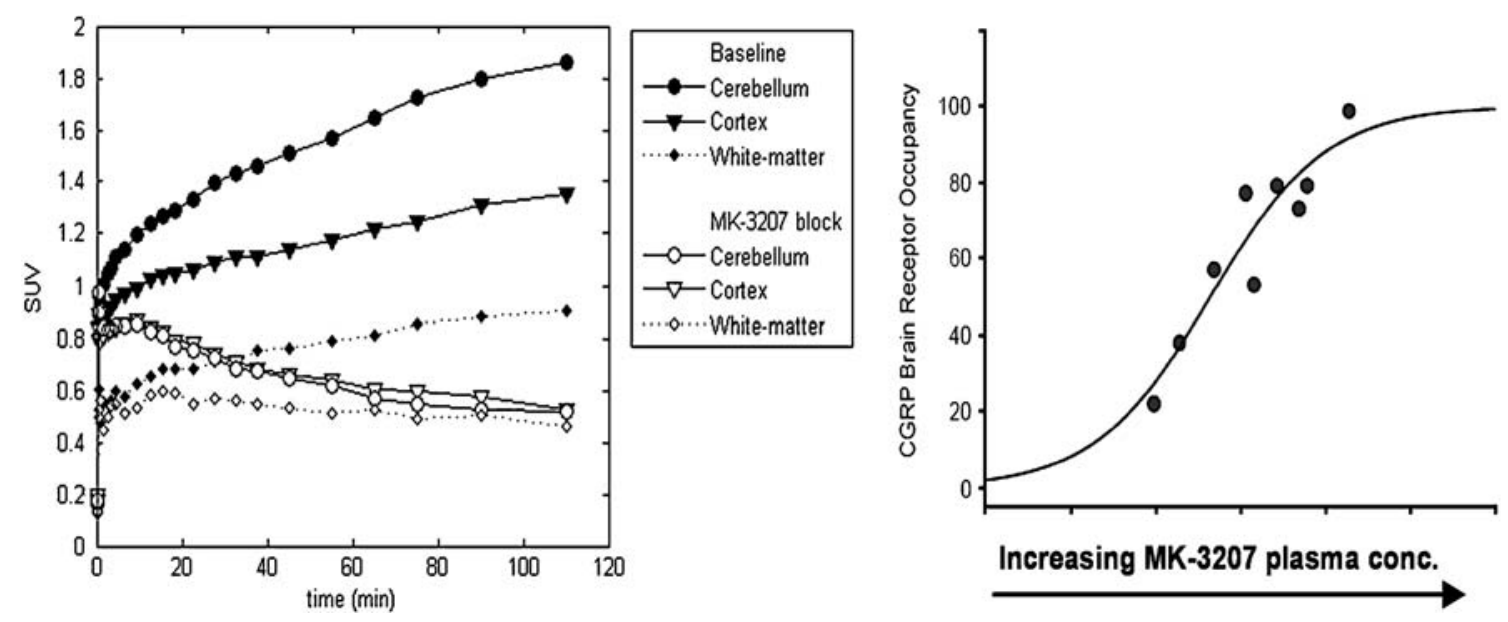
J239

PET IMAGING OF PROTEOLYTIC TGF-p1 ACTIVATION REACTION IN MICE

Yousuke Kanayama $^{1}$, Chinuyo Sumita ${ }^{1}$, Koki Hasegawa ${ }^{1}$, Yasuhiro Wada ${ }^{1}$, Emi Hayashinaka ${ }^{1}$, Shuichi Enomoto ${ }^{1,2}$, Soichi Kojima ${ }^{3}$, Yasuyoshi Watanabe ${ }^{1}$

${ }^{1}$ RIKEN Center for Molecular Imaging Science, Kobe, Japan, ${ }^{2}$ Graduate School of Medicine, Dentistry and Pharmaceutical Sciences, Okayama University, Okayama, Japan, ${ }^{3}$ Molecular Ligand Biology Research Team, RIKEN, Wako, Japan.

Transforming growth factor beta (TGF- $\beta$ ) is a multifunctional cytokine that regulates cellular proliferation, differentiation, apoptosis, and other function in many cell types. It is involved in pathogenesis of various kinds of diseases including hepatic cirrhosis, atherosclerosis, lung fibrosis, myelofibrosis and rheumatoid arthritis via stimulating the excessive production of extracellular matrices from mesenchymal cells and suppressing the growth of epithelial cells. There is growing requirement for the evaluation method of in vivo distribution and activities of TGF- $\beta$. On the other hand, TGF- $\beta$ is biosynthesized as an inactive latent form, which consists of latency-associated protein (LAP) and active TGF- $\beta$, and converted to its active form by proteolytic processing of the propeptide. Kojima et al. have found that during the proteolytic TGF- $\beta$ activation reaction, plasma kallikrein cleaved between Arg58 and Leu59 within the propeptide sequence, and also developed antibodies that specifically recognize each cleaved site (International Publication WO2005/023870). In this study, we investigated the PET imaging of the in vivo distribution and activities of TGF- $\beta$ activation reaction using ${ }^{64} \mathrm{Cu}$-labeled anti-Leu59 cleavage siterecognizing monoclonal antibody (anti-L59-mAb) as a PET probe. The anti-L59-mAb was conjugated with 1,4,7,10-tetraazacyclododecane-N, $\mathrm{N}^{\prime}, \mathrm{N}$ ",N"'-tetraacetic acid and subsequently labeled with ${ }^{64} \mathrm{Cu}$. A group of mice (BALB/c nu/nu, male, 10-week-old) were prepared as acute inflammation models induced by intramuscular injection of $30 \mu \mathrm{l}$ turpentine oil into the right foot. Two, four, and seven days after turpentine oil injection, the mice were subjected to the PET imaging. Twenty-four hours prior to the PET imaging, $30 \mathrm{MBq} / 0.1 \mathrm{nmol}$ of 64Cu-labeled anti-L59-mAb was intravenously injected to each mouse. Another group of inflammation model mice were subjected to tissue sampling for the immunohistochemical analysis and Western blotting for the LAP fragment. As a result, the PET images clearly showed high accumulation in the inflammatory region of the right foot of mice, and the distributions altered with the elapsed time after turpentine oil injection. The biochemical analyses also showed that the inflammatory region included the LAP fragments with the Leu59 cleavage site. The PET imaging of antiL59-mAb was thus successfully visualized in vivo distribution of LAP fragments where the TGF- $\beta 1$ activation reaction occurred.

\section{$\mathbf{J} 240$}

\section{RE AND TC TRICARBONYL COMPLEXES WITH ESTABLISHED AFFINITY FOR 5-HT1A RECEPTORS: A 3D QSAR STUDY}

${ }_{\text {Zélia Barbosa }}{ }^{1}$, Antero Abrunhosa ${ }^{2}$, Isabel R. Santos ${ }^{3}$

${ }^{1}$ Farmácia, Escola Superior de Tecnologia da saúde de Coimbra, S. Martinho do Bispo, Portugal, ${ }^{2}$ Instituto de Ciências Nucleares Aplicadas à Saúde, Universidade de Coimbra, Coimbra, Portugal, ${ }^{3}$ Instituto Tecnológico e Nuclear, Sacavém, Portugal.
Introduction: Amongst all 5-HT receptors, the 5-HT1A subtype is by far the best studied and appears to be involved in important psychiatric disorders such as anxiety and depression. The availability of a fully aqueous-based kit preparation of the precursor $[99 \mathrm{mTc}$ $(\mathrm{H} 2 \mathrm{O}) 3(\mathrm{CO}) 3]+$ prompted the research on organometallic complexes for in vivo SPECT imaging of 5-HT1A receptors. In our study we aimed at establishing models to relate the 3D molecular properties of such organometallic compounds with their in vitro affinity for the 5HT1A receptor. Methods: $20 \mathrm{Re}$ and Tc tricarbonyl compounds with documented affinity for the 5HT1A receptor were selected. 3D structures, when available, were obtained from crystallographic data. In the absence of such data the closest crystallographic match was used for the skeleton of the molecule, minimized using MM3 or AM1 algorithms. Using dedicated molecular modeling software based of semi-empirical calculations (CNDO), a database of about 150 molecular descriptors was created. Models were obtained by Multiple Linear Regression and PRESS analysis was used to validate them. Results and discussion: Several models were obtained with high degree of correlation $(\mathrm{r} 2>0.8)$ and good validation results. Critical parameters for the affinity of these compounds for the 5HT1A receptor include linker size, lipophilicity (cLogP), hydrogen bonding capacity and the presence of a bifunctional ligand. It was expected that these models could be helpful on elucidating the mechanisms involved in the 5-HT1A binding process, and on improving our ability to design compounds with better affinity for this receptor.

\section{J241}

\section{c-MET POSITIVE TUMOR IMAGING USING I-125 cMBP-CLICK-RGD}

Eun-Mi Kim $^{1,2}$, Dong Wook Kim ${ }^{1,2}$, Su-Jin Cheong ${ }^{1,2}$,

Chang-Moon Lee ${ }^{1,2}$, Sun-Hee Kim ${ }^{1,2}$, Hwan-Jeong Jeong ${ }^{1,2}$, Seok Tae Lim ${ }^{1,2}$, Myung-Hee Sohn ${ }^{1,2}$

${ }^{1}$ Nuclear Medicine, Chonbuk National University Hospital, Jeonju, Korea, South, ${ }^{2}$ Cyclotron research center, Chonbuk National University Medical School and Hospital, Jeonju, Korea, South.

Purpose: We recently identified that GGG- or AOC- containing KSLSRHDHIHHH peptide (cMBP) specifically targets c-Met receptor tyrosine kinase in vivo and in vitro. Although U87MG tumor xenografts could be visualized by SPECT/microCT using the various 125I labeled cMBP, image contrast and overall quality were unremarkable. We hypothesize that a peptide ligand recognizing both c-Met and integrin will be advantageous because of its dual-receptortargeting ability. Methods: A cMBP-click-RGD heterodimer was synthesized from azide linked cMBP-GGG- $\beta$ AK and alkyne terminal $\mathrm{c}(\mathrm{RGDyK})$ through a click reaction then labeled with I-125. Receptor binding affinity was tested in vitro and in vivo. Biodistribution was studied in U87MG bearing athymic mice. Results: cMBP-click-RGD had comparable integrin $\alpha v \beta 3$-binding affinity with $c($ RGDyK) and comparable c-Met-binding affinity with cMBP. In the previous study, I-125 cMBP-GGG showed tumor to blood ratio and tumor to muscle 2.94, 1.52 at $4 \mathrm{hr}$, respectively. I-125 cMBP-click-RGD had higher tumor to blood ratio and tumor to muscle ratio compared with monomeric I-125 cMBP-GGG-SC $(\mathrm{T} / \mathrm{B}=5.92, \mathrm{~T} / \mathrm{M}=6.45$ at $4 \mathrm{hr})$. And I-125 cMBP-RGD showed significantly fast blood clearance, but kidney uptake was more increased. Conclusion: Dual integrin $\alpha v \beta 3$ and c-Met recognition showed significantly improved tumor-targeting efficacy and pharmacokinetics compared with I-125 cMBP-GGG-SC monomeric analogs. 
Figure 3. Pinhole planar and reconstructed coregistered transverse SPECT/CT images of ${ }^{125}$-CMBP.click-RGDyK at $1 \mathrm{hr}$ in U87MG xenograft mice (14.8 MBq, acquisition time $=5 \mathrm{~min})$. Reconstructed coregistered coronal slice image $(B)$ and maximum-intensity-projection SPECT/CT image (C) of 1251-CMBP-clickRGDyK (32 projections, 60 sec/projection).

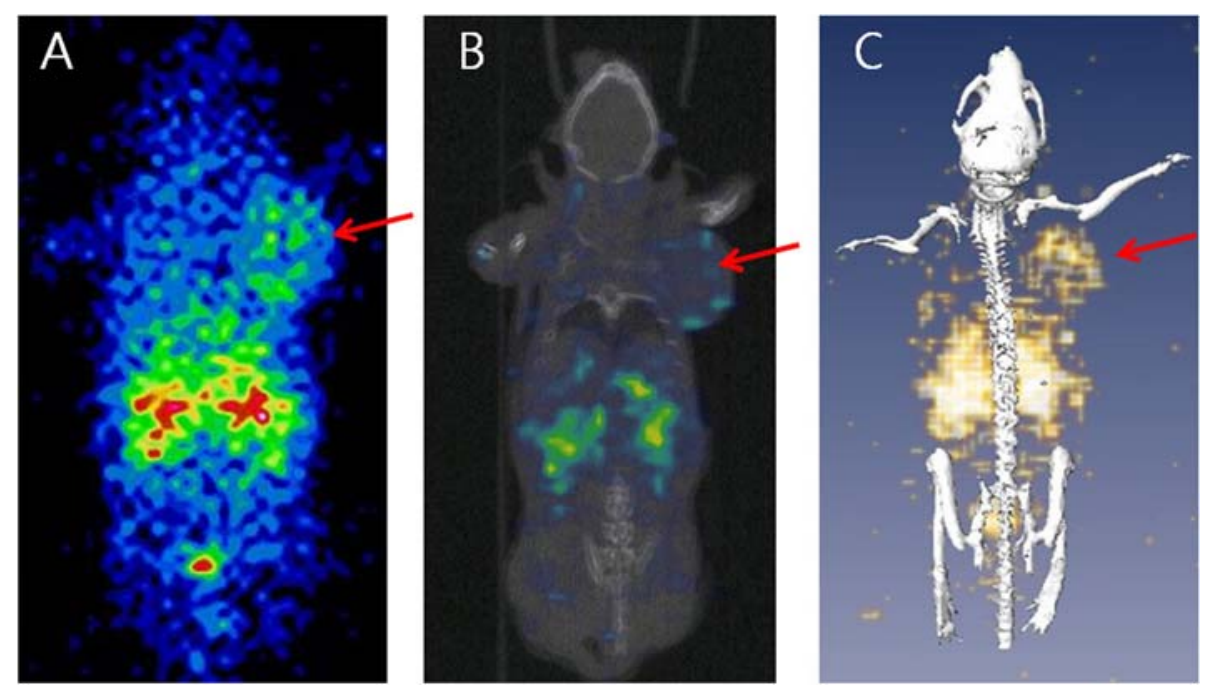

SYNTHESIS AND BIOLOGICAL EVALUATION

OF RADIOIODINE LABELED BENZIMIDAZOLE DERIVATIVE FOR SPECT IMAGING OF NR2B SUBTYPE OF THE NMDA RECEPTOR

Fuchigami Takeshi ${ }^{1}$, Yamaguchi Hiroshi ${ }^{1,2}$, Kosugi Mutsumi ${ }^{1}$, Biao Le ${ }^{1}$, Mikako Ogawa $^{1}$, Yasuhiro Magata ${ }^{1,2}$

${ }^{1}$ Photon Medical Research Center, Hamamatsu University School of Medicine, Hamamatsu, Japan, ${ }^{2}$ Molecular Imaging Frontier Research Center, Hamamatsu University School of Medicine, Hamamatsu, Japan.

Objectives: NMDA receptor NR2B subtype has been implicated in modulating functions such as learning and memory processing, as well as related disorders. However, there is no appropriate ligand for in vivo imaging. On these bases, we developed several novel benzimidazole derivatives for imaging of NMDA receptor NR2B subtype using SPECT. Method: We designed and synthesized new iodinated compounds, IBZIM, ISFIM, and IPHIM to maintain binding affinity for the NMDA receptor NR2B subtype. Compared with methanesulfonamide group, hydroxyl group is lower molecular weight and $\mathrm{pKa}$ value, which is expected to make significant difference in binding character and BBB penetration each other. The binding affinities of these compounds for the NR2B were measured by displacement of $[3 \mathrm{H}]$ Ifenprodil to rat cortical membranes. In vitro regional distributions of $125 \mathrm{I}$ labeled ligands on the rat brain slices were evaluated by an autoradiography method. Biodistribution studies in normal mice of 125I labeled ligands were performed. Results: IBZIM and ISFIM showed high affinity for the NR2B ( $\mathrm{Ki}=$ 1.4 and $1.0 \mathrm{nM}$, respectively). On the contrary, IPHIIM was approximately 20 -fold less potent than IBZIM $(\mathrm{Ki}=30.1 \mathrm{nM})$. In in vitro autoradiography experiments, [125I]ISFIM showed a high accumulation in the cerebrum and very low in the cerebellum. This distribution pattern was quite similar to mRNA distribution of NR2B subunit. Moreover the bindings of [125I]ISFIM in the cerebrum were selectively inhibited by NR2B antagonist. The biodistribution studies in normal mice demonstrated that brain to blood ratios of both [125I]IBZIM and [125I]ISFIM increased with time and showed 1.04 and 1.11 at $180 \mathrm{~min}$ post-injection, respectively. The highest brain uptake was $0.79 \%$ dose/g at $180 \mathrm{~min}$ post-injection of [125I] ISFIM, which is significantly higher than that of [125I]IBZIM $(0.52 \%$ dose $/ \mathrm{g})$. However the uptake of [125I]ISFIM in the cerebrum were also observed. Conclusion: Very high affinity for the NR2B subtype of the NMDA receptor radioiodine labeled ligand, [125I] ISFIM, has been developed. [125I]ISFIM displayed specific binding to the NR2B subunit of NMDA receptors in in vitro experiments, but not in vivo studies. Since it is unclear the reasons why the binding characters were quite different between in vitro and in vivo studies, further investigations are now undertaken.

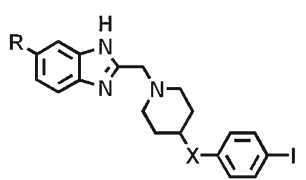

IBZIM; $\mathrm{R}=\mathrm{OH}, \mathrm{X}=\mathrm{CH}_{2}$ ISFIM; R= $\mathrm{NHSO}_{2} \mathrm{Me}, \mathrm{X}=\mathrm{CH}_{2}$ IPHIM; R=OH, $X=0$

$\mathbf{J} 243$
IDENTIFICATION AND CHARACTERIZATION OF A NEW PEPTIDE (CAIX-P1) FOR TARGETING CARBONIC
ANHYDRASE IX
Vasileios Askoxylakis $^{1,2}$, Walter Mier ${ }^{1,3}$, Annette Altmann ${ }^{1}$, Jurgen Debus ${ }^{2}$, Uwe Haberkorn ${ }^{1,3}$
${ }^{1}$ Clinical Cooperation Unit Nuclear Medicine, German Cancer Research Center, Heidelberg, Germany, ${ }^{2}$ Radiooncology and Radiation Therapy, University of Heidelberg, Heidelberg, Germany, ${ }^{3}$ Nuclear Medicine, University of Heidelberg, Heidelberg, Germany. 
Carbonic anhydrase IX (CaIX) is a transmembrane enzyme found to be overexpressed in a variety of tumours and associated with tumour hypoxia. We identified CaIX-P1, a linear dodecapeptide binding carbonic anhydrase IX, using recombinant CAIX and phage display technology and evaluated the binding and metabolic properties of it. Phage display was performed with a 12 amino acid phage display library by panning against a recombinant extracellular domain of CaIX. The identified peptide CaIXP1 was produced by Fmoc solid phase peptide synthesis and labeled with [125]I or [131]I. Binding, kinetic and competition studies were performed on the colorectal carcinoma cell line HCT 116 and on human umbilical vein endothelial cells (HUVEC). FACS analysis was done with the FITC-labeled CaIX-P1 peptide and rhodamine-labeled anti-human CaIX $\mathrm{mAb}$. Organ distribution studies were carried out in Balb/c nu/nu mice, carrying HCT 116 tumors transplanted subcutaneously into the trunk. RNA expression of CaIX in HCT 116 and HUVEC cells was investigated by RT-PCR. RT-PCR demonstrated a higher expression of CaIX in HCT 116 cells, compared to HUVEC. In vitro binding experiments of [125]I-labeled-CaIX-P1 revealed an increased uptake of the radioligand in HCT 116 cells. Binding of the radioligand was inhibited up to $90 \%$ by the unlabelled peptide, but not by other negative control peptides at the same concentration. FACS analysis showed a correlation between CaIX-P1 binding and rhodamine-labeled anti-human CaIX mAb uptake in HCT 116 cells. Organ distribution experiments revealed a higher accumulation of CaIX-P1 in HCT 116 tumours than in most of the organs. These data indicate that CaIX-P1 is a candidate for the development of a new ligand targeting carbonic anhydrase IX.

\section{J244}

\section{DEVELOPMENT OF ${ }^{76} \mathrm{Br}$-META- BROMOBENZYLGUANIDINE $\left({ }^{76} \mathrm{Br}-\mathrm{MBBG}\right)$ FOR IMAGING NEUROENDOCRINE TUMOR}

Hirofumi Hanaoka $^{1}$, Shigeki Watanabe ${ }^{2}$, Satoshi Watanabe ${ }^{2}$, Yasuhiko Iida $^{1}$, Noriko S. Ishioka ${ }^{2}$, Keigo Endo ${ }^{1}$

${ }^{1}$ Gunma University Graduate School of Medicine, Maebashi, Japan, ${ }^{2}$ Quantum Beam Science Directorate, Japan Atomic Energy Agency (JAEA), Takasaki, Japan.

Objectives: ${ }^{123}$ I-meta-Iodobenzylguanidine $\left({ }^{123} \mathrm{I}-\mathrm{MIBG}\right)$ has been used for the diagnosis of norepinephrine transporter (NET)-expressing neuroendocrine tumors, such as pheochromocytoma and paraganglioma, and ${ }^{131}$ I-MIBG has been used for the systemic radionuclide therapy. ${ }^{123}$ I-MIBG has high specificity and detectability not only for primary tumors but also metastatic lesions. However, ${ }^{123}$ I-MIBG is not enough for imaging NET expression of tumors because of less in resolution and quantification compared to positron emission tomography (PET). In this study, we synthesized ${ }^{76} \mathrm{Br}$ (positron emitter, half-life: $16.2 \mathrm{~h}$ ) labeled MIBG analog, ${ }^{76} \mathrm{Br}$ meta-bromobenzylguanidine $\left({ }^{76} \mathrm{Br}-\mathrm{MBBG}\right)$, and evaluated as a PET tracer for imaging NET expression of neuroendocrine tumors, which enable to stratify patients for potential response to ${ }^{131} \mathrm{I}-\mathrm{MIBG}$ therapy. Methods: We have produced no-carrier-added ${ }^{76} \mathrm{Br}$ via the ${ }^{76} \mathrm{Se}(\mathrm{p}, \mathrm{n}){ }^{76} \mathrm{Br}$ reaction using $\mathrm{Cu}_{2}{ }^{76} \mathrm{Se}$ target. ${ }^{76} \mathrm{Br}-\mathrm{MBBG}$ was prepared by halogen exchange reaction between the ${ }^{76} \mathrm{Br}$ and the iodine of non-radioactive MIBG. Biodistribution studies were performed in normal mice or NET-positive PC-12 tumor-bearing mice by administration of mixed solution of ${ }^{76} \mathrm{Br}-\mathrm{MBBG}$ and ${ }^{125} \mathrm{I}$ MIBG. The tumor was imaged using ${ }^{76} \mathrm{Br}-\mathrm{MBBG}$ and FDG with an animal PET scanner. Results: After labeling experiment, $20 \mathrm{MBq}$ of ${ }^{76} \mathrm{Br}-\mathrm{MBBG}$ was obtained and the labeling yield was $44 \%$. In biodistribution studies, ${ }^{76} \mathrm{Br}-\mathrm{MBBG}$ showed high accumulation in the NET positive organs (adrenal and heart) in normal mice, and showed high accumulation in the tumor $(32.0 \pm 18.6 \% \mathrm{ID} / \mathrm{g}$ and $23.7 \pm 5.9 \% \mathrm{ID} / \mathrm{g}$ at $3 \mathrm{~h}$ and $6 \mathrm{~h}$ after administration, respectively) in tumor-bearing mice. The biodistribution of ${ }^{76} \mathrm{Br}-\mathrm{MBBG}$ is similar to that of ${ }^{125} \mathrm{I}-\mathrm{MIBG}$ in all mice. ${ }^{76} \mathrm{Br}$-MBBG PET showed very clear image of the tumor, which was comparable to ${ }^{18} \mathrm{~F}-\mathrm{FDG}$ PET. Furthermore, ${ }^{76} \mathrm{Br}-\mathrm{MBBG}$ detected very small tumor weighting about $10 \mathrm{mg}$. Conclusions: ${ }^{76} \mathrm{Br}-\mathrm{MBBG}$ showed high tumor accumulation and provided clear PET image, indicating that ${ }^{76} \mathrm{Br}-\mathrm{MBBG}$ would be a potential tracer for imaging NET expressing neuroendocrine tumors.

\section{$\mathbf{J} 245$}

\section{COMPARISON OF $\left[{ }^{18}\right.$ F]FDG and $\left[{ }^{18}\right.$ F]FOLATE IN A RABBIT MODEL OF ATHEROSCLEROSIS BY PET AND AUTORADIOGRAPHIC IMAGING}

Dinko Gonzalez Trotter ${ }^{1}$, Maria S. Michener ${ }^{1}$, Daniel J. Rubins ${ }^{1}$, Hyking D. Haley $^{1}$, Amy Vanko ${ }^{1}$, Donna L. Suresch ${ }^{1}$, Brett Connolly ${ }^{1}$, Paul McQuade ${ }^{1}$, Sumith A. Kularatne ${ }^{2}$, Philip S. Low ${ }^{2}$,

Michael Klimas ${ }^{1}$, Jacquelynn J. Cook ${ }^{1}$

${ }^{1}$ Imaging Departmnet, Merck \& Co., Inc., West Point, PA, USA, ${ }^{2}$ Department of Chemistry, Purdue University, West Lafayette, IN, USA.

The folate receptor is highly expressed in the kidney cortex, several tumor cell types and activated macrophages, making it an attractive target for imaging in oncology and inflammatory diseases. Activated macrophages are present in the plaque of atherosclerotic animal models and patients, and it has been reported that $\left[{ }^{18} \mathrm{~F}\right] \mathrm{FDG}$ PET can detect these lesions non-invasively. The folate tracer $\left[{ }^{18} \mathrm{~F}\right]$ Folate was compared to $\left[{ }^{18} \mathrm{~F}\right] \mathrm{FDG}$ by in vivo $\mathrm{PET}$ imaging, ex vivo autoradiography and histology in a rabbit model of atherosclerosis. Six rabbits were started on a highcholesterol diet and balloon-injured in the aorta. Approximately twenty weeks after initiation of the diet, rabbits were dynamically imaged for $3 \mathrm{~h}$ with $\left[{ }^{18} \mathrm{~F}\right] \mathrm{FDG}$ using a commercially-available small-animal PET camera. Three of the rabbits were later scanned for $2 \mathrm{~h}$ with $\left[{ }^{18} \mathrm{~F}\right]$ Folate (Folate group) while the rest were rescanned with $\left[{ }^{18} \mathrm{~F}\right] \mathrm{FDG}$ (FDG group). After the final PET scans, rabbits were euthanized, the aorta extracted for autoradiographic imaging, H\&E and macrophage RAM-11 staining. PET and autoradiographic signals were quantified for inter-group comparison. The $\left[{ }^{18} \mathrm{~F}\right]$ Folate specificity for the folate receptor was confirmed in vivo with $\mathrm{SUV}=3.4 \pm 0.66$ (mean \pm S.E.M.) uptake localized in the kidney cortex. Final PET scans' in vivo signal in the aorta (\%ID) was $0.049 \pm 0.005$ for the FDG group, and $0.011 \pm 0.004$ for Folate group (last $30 \mathrm{~min}$. frame). H\&E and RAM-11 staining showed substantial plaque and macrophages in both groups, with autoradiography showing that $\left[{ }^{18} \mathrm{~F}\right] \mathrm{FDG}$ co-localized with the RAM-11 staining better than $\left[{ }^{18} \mathrm{~F}\right]$ Folate (see Figure). The autoradiographic signal in plaque normalized to injected dose $\left(\mathrm{PSL} / \mathrm{mm}^{\wedge} 2\right)$ was $290 \pm$ 83 for $\left[{ }^{18} \mathrm{~F}\right] \mathrm{FDG}$ and $92 \pm 22$ for $[18 \mathrm{~F}]$ Folate. From these results it was concluded that $\left[{ }^{18} \mathrm{~F}\right] \mathrm{FDG}$ provided a better signal in plaque and macrophage-targeting specificity than $\left[{ }^{18} \mathrm{~F}\right]$ Folate in this rabbit model of atherosclerosis. 


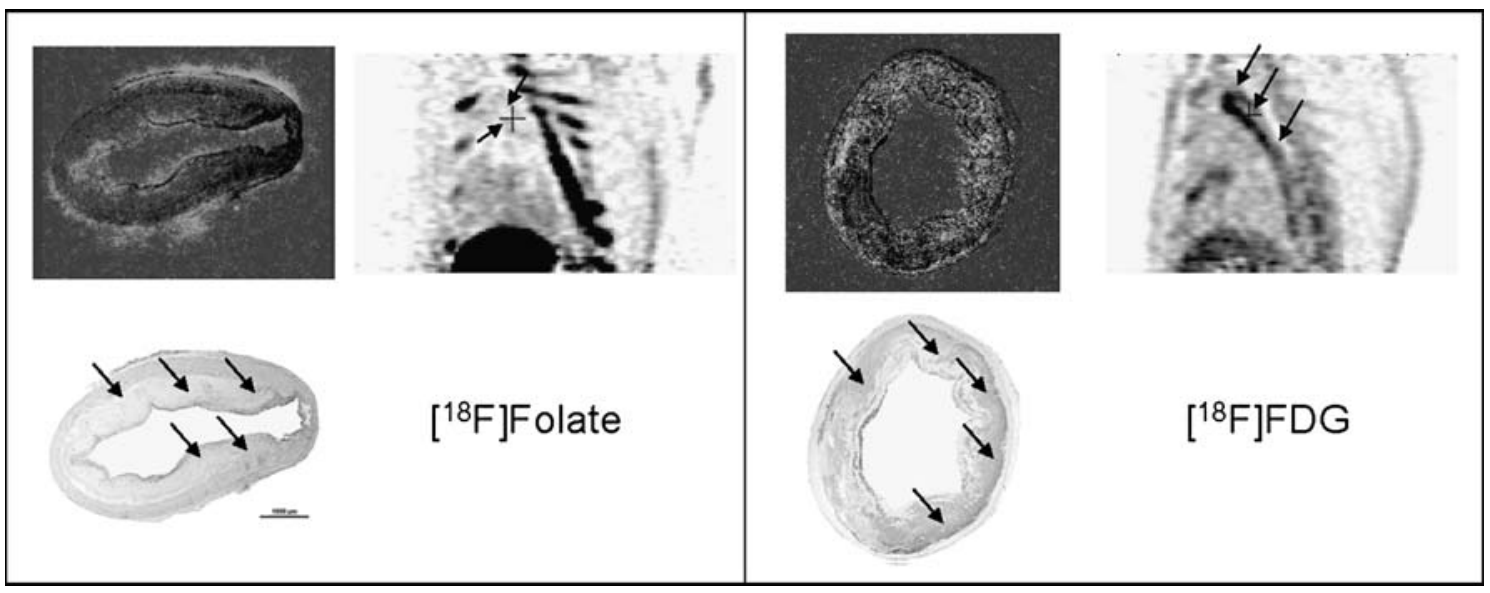

$\left[{ }^{18} \mathrm{~F}\right]$ Folate and $\left[{ }^{18} \mathrm{~F}\right] \mathrm{FDG}$ autoradiographic overlay with H\&E (top left corner of panels) and dark grey RAM-11 macrophage staining (bottom left corner of panels). Sagittal $\left[{ }^{18} \mathrm{~F}\right]$ Folate and $\left[{ }^{18} \mathrm{~F}\right] \mathrm{FDG}$ PET (right side of panels) images of the same rabbit showing differences in aortic uptake (SUV scale $0-1$, no attenuation correction)

\section{J246}

\section{PET IMAGING OF THE PANCREAS WITH $\left[{ }^{68} \mathrm{Ga}\right]$ DOTA-OCTREOTIDE; A POTENTIAL BIOMARKER FOR BETA CELL MASS MEASUREMENT}

Takeo Sako ${ }^{1,3}$, Yousuke Kanayama ${ }^{1}$, Koki Hasegawa ${ }^{1}$, Mie Nishimura ${ }^{1}$, $\overline{\text { Yilong Cui }}^{1}$, Yosky Kataoka ${ }^{1}$, Yasuhiro Wada ${ }^{1}$, Michio Senda ${ }^{2,3}$, Yasuyoshi Watanabe ${ }^{1}$

${ }^{1}$ Center for Molecular Imaging Science, RIKEN, Kobe, Japan, ${ }^{2}$ Imagebased Medicine Center, Institute of Biomedical Research and Innovation, Kobe, Japan, ${ }^{3}$ Graduate School of Medicine, Kobe university, Kobe, Japan.

Introduction: Recently, several papers have reported that not only in type 1 diabetes mellitus (DM) but in type $2 \mathrm{DM}$ the pancreatic beta cell mass (BCM) decreased even before appearance of abnormal blood sugar level and clinical symptoms. However, BCM is currently determined precisely at biopsy, though the pancreas is not an ideal organ for this method. Non-invasive method to quantify BCM could be useful for realizing detection and treatment of DM at earlier stage. Now we focus on Somatostatin receptors highly expressed in the beta cells and have developed a PET probe from Octreotide, a Somatostatin analog, for beta cell PET imaging. Method: We injected $\left[{ }^{68} \mathrm{Ga}\right] \mathrm{DOTA}-$ Octreotide into the tail vein of male rats under isoflurane anesthesia and performed emission scans in the abdomen for $90 \mathrm{~min}$ after injection. To investigate the specificity of the probe, we conducted emission scans in the rats treated by un-labeled Octreotide administered at $1 \mathrm{~min}$ before the injection of radio-labeled Octreotide. Also, we did the PET studies in the diabetic rats made by pharmacological treatment of Streptozotocin (STZ). After the PET scan, we took tissues and organs from the rats and measured their radioactivity with gamma counter. Results: The PET studies show that $\left[{ }^{68} \mathrm{Ga}\right]$ DOTA-Octreotide was highly accumulated in the pancreas of normoglycemic rats but significantly decreased in the rats treated by un-labeled Octreotide or by STZ. These results were coherent to the biodistribution data. Conclusion: The accumulation of $\left[{ }^{68} \mathrm{Ga}\right]$ DOTA-Octreotide in the pancreas is specific and reflects $\mathrm{BCM}$. Therefore, this $\mathrm{PET}$ probe can be a potential biomarker for $\mathrm{BCM}$ measurement.

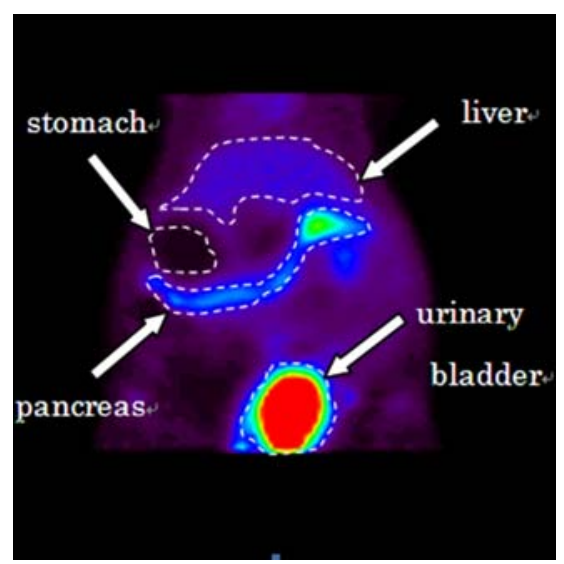

10-90 min static image of normoglycemic rat abdomen (MAP reconstruction)

$\mathbf{J} 247$

\section{APPLICATION OF THE CU-FREE CLICK REACTION} IN PRETARGETED RADIOIMMUNOIMAGING AND -THERAPY Pascal Renart Verkerk ${ }^{1}$, Sandra M. van den Bosch ${ }^{1}$, Raffaella Rossin ${ }^{1}$, Wolter ten Hoeve ${ }^{2}$, Laurens H. Pellikaan ${ }^{1}$, Lobke Maas ${ }^{1}$, Johan Lub ${ }^{1}$, Marc S. Robillard ${ }^{1}$

${ }^{1}$ Biomolecular Engineering, Philips Research, Eindhoven, Netherlands, ${ }^{2}$ Syncom, Groningen, Netherlands.

Pre-targeting combines the excellent tumor-seeking properties of MAbs with the fast clearance of low MW probes and is a highly sought-after approach for RIT. To address the drawbacks of the currently used biological antibody-probe binding systems we designed a novel approach based on the $\mathrm{Cu}$-free click reaction, employing azide-conjugated MAbs and radiolabeled cyclooctyne derivatives. These functionalities are small, abiotic, and bioorthogonal and have recently been applied in bio-conjugations in 
mice [1]. However, the quantities $(160 \mu \mathrm{mol} / \mathrm{Kg})$ were orders of magnitude higher than the limit for a typical RIT application. Up to now the usefulness of this reaction for pretargeted RIT has remained unexplored. To this end, we developed $177 \mathrm{Lu}-\mathrm{DOTA}$ conjugates with three promising cyclooctyne moieties (1-3) and evaluated their stability, reactivity and in vivo behavior at low concentrations. The labeling of $1-3$ with c.a. $177 \mathrm{Lu}$ was complete after $10 \mathrm{~min}$ at $45^{\circ} \mathrm{C}$ in acetate buffer $(\mathrm{pH} 8)$. After $4 \mathrm{~h}, 1$ showed complete stability in PBS and $10 \%$ decomposition in $50 \%$ mouse serum, while 2 and 3 were stable in both media for that time. The blood kinetics and biodistribution of 1-3 was evaluated in tumorbearing mice $(n=4)$. Probes $1-3$ cleared from the blood with halflives ranging from $3 \mathrm{~min}$ (1) to $200 \mathrm{~min} \mathrm{(3).} \mathrm{None} \mathrm{of} \mathrm{the}$ compounds showed marked accumulation in any non-target organs, including liver and kidney. The reactivity of 1-3 towards N3-MAb (3.44 $\mu \mathrm{M} \mathrm{MAb}$ and 1 eq probe per N3) was studied in PBS, $50 \%$ mouse serum and rat blood using SDS-PAGE. After $2 \mathrm{~h}$ incubation in $\mathrm{PBS}$ at $37^{\circ} \mathrm{C}$, the $\%$ antibody-bound probe (corrected for nonspecific binding) was 34,61 , and 71 for $1-3$, respectively. In blood, the reactivity of 1 towards N3-MAb increased to $45 \%$. On the contrary, the reactivity of 2 and 3 showed a 2 -fold decrease in serum and blood, possibly due to non-covalent interactions with plasma proteins, which is in agreement with the long blood halflives. In conclusion, the $\mathrm{Cu}$-free click reaction has proven to occur efficiently in vitro for the coupling of small probes to MAbs at the low concentrations relevant for RIT $(\sim 1 \mu \mathrm{mol} / \mathrm{Kg})$. The validation of tumor pre-targeting with the $\mathrm{Cu}$-free click reaction in mice is underway. [1] Bertozzi cs PNAS 2007, 104, 16793

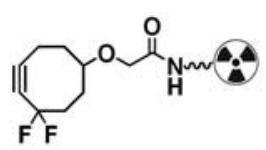

1

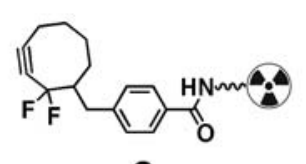

2
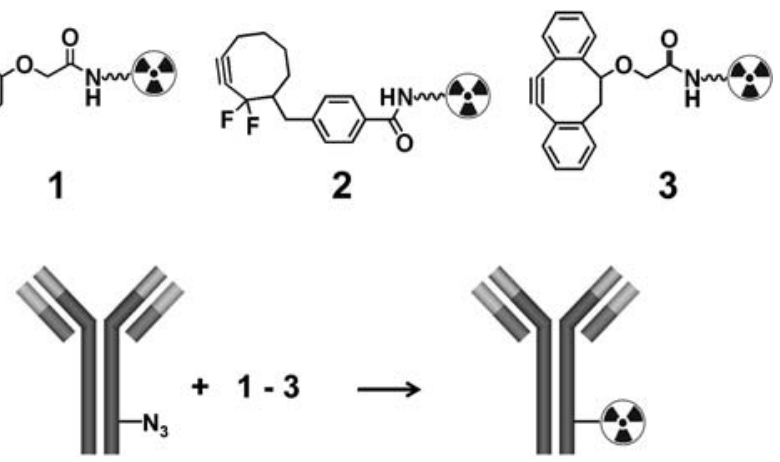

$1-3$

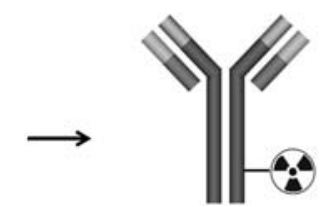

dysfunction may precipitate several neurological and psychological disorders. The aim of this project was to develop an effective SPECT imaging tracer for Histamine H3 receptors. Candidate SPECT molecules were chosen from a library of compounds within Wyeth's H3 drug development program. Methods: Radiolabelling of WYE230949 was carried out by iododestannylation and produced a high yield, purity and specific activity. in vitro radioligand binding assays were performed using rat brain homogenates to determine the dissociation constant $\left(\mathrm{K}_{\mathrm{d}}\right)$ and binding capacity $\left(\mathrm{B}_{\max }\right)$. Biodistribution, total brain uptake and regional brain binding were determined following i.v. injection of $\left[{ }^{125} \mathrm{I}\right]-$ WYE230949 to anaesthesia exposed and nonanaesthesia exposed rats. Blood and plasma clearance were determined by sampling from the femoral artery in anesthetised animals. Rats were killed and tissues harvested at either $30 \mathrm{~min}$ or $2 \mathrm{~h}$ post injection. Time activity curves were constructed from midbrain slices $(4 \mathrm{~mm})$ following i.v. injection of $\left[{ }^{125} \mathrm{I}\right]-$ WYE230949 using in vivo microSPECT. Results: Binding of $\left[{ }^{125} \mathrm{I}\right]-$ WYE230949 to rat brain homogenates obtained $K_{d}$ and $B_{\max }$ values of $6.9 \pm 1.3 \mathrm{nM}$ and $509 \pm 35 \mathrm{fmol} / \mathrm{mg}$ protein respectively. Brain uptake was $0.111 \pm 0.008 \% \mathrm{ID} / \mathrm{g}$ tissue and $0.063 \pm 0.007 \% \mathrm{ID} / \mathrm{g}$ tissue in anaesthetised rats at $30 \mathrm{~min}$ and $2 \mathrm{~h}$ respectively. Relative ROI to cerebellar ratios were calculated and the whole body and brain biodistribution determined in both anaesthesia exposed and non-anaesthesia exposed rats. Conclusion: $\left.{ }^{125} \mathrm{I}\right]-$ WYE230949 displayed good in vitro binding characteristics. Results from further in vivo characterisation experiments will be presented in order to determine the potential of $\left[{ }^{125} \mathrm{I}\right]-$ WYE230949 as a SPECT tracer for histamine H3 receptors. Research Support: This work was supported by an award (Ref: NS_AU_084) from the Translational Medicine Research Collaboration-a consortium made up of the Universities of Aberdeen, Dundee, Edinburgh and Glasgow, the four associated HNS Health Boards (Grampian, Tayside, Lothian and Greater Glasgow \& Clyde), Scottish Enterprise and Wyeth Pharmaceutical. Dr S Champion is funded by the Scottish Imaging Network: A Platform for Scientific Excellence (SINAPSE).

\section{$\mathbf{J} 249$}

\section{SYNTHESIS AND BIODISTRIBUTION OF ${ }^{18}$ F-PROPYL- RHODAMINE B AND ${ }^{18}$ F-DIETHYLENEGLYCOL- RHODAMINE B: PROMISING MYOCARDIAL PERFUSION IMAGING AGENTS}

Vijay Gottumukkala $^{1,2}$, Tobias K. Heinrich ${ }^{1,2}$, Amanda F. Baker ${ }^{1}$, Patricia Dunning $^{1}$, Frederic H. Fahey ${ }^{1,2}$, S. T. Treves ${ }^{1,2}$,

Alan B. Packard ${ }^{1,2}$

${ }^{1}$ Division of Nuclear Medicine, Children's Hospital Boston, Boston, MA, USA, ${ }^{2}$ Harvard Medical School, Boston, MA, USA.

\section{CHARACTERISATION OF NOVEL HISTAMINE H3 RECEPTOR TRACERS FOR SPECT}

$\underline{\text { David Y. Lewis }}{ }^{1}$, Sue Champion ${ }^{1}$, Deborah Dewar ${ }^{1}$, Garry D. Honey ${ }^{3}$, Jonathan Gross ${ }^{4}$, Albert J. Robichaud ${ }^{4}$, Andrew Welch ${ }^{5}$, David Wyper ${ }^{2}$, Sally Pimlott ${ }^{2}$

${ }^{1}$ Division of Clinical Neuroscience, University of Glasgow, Glasgow, United Kingdom, ${ }^{2}$ Department of Clinical Physics, University of Glasgow, Glasgow, United Kingdom, ${ }^{3}$ Translational Medicine Research Centre, Wyeth Research, Dundee, United Kingdom, ${ }^{4}$ Wyeth Research Chemical Sciences, Princeton, NJ, USA, ${ }^{5}$ The John Mallard PET Centre, University of Aberdeen, Aberdeen, United Kingdom.

Introduction: There is clear need for a greater understanding of the relationship between drug dose versus receptor occupancy and efficacy in both the pre-clinical and clinical trials. Histamine receptor H3
Rhodamine dyes are lipophilic cationic compounds that are known to accumulate in the myocardium and also to be substrates for Pgp, the protein implicated in MDR1 multidrug resistance. We therefore hypothesized that an ${ }^{18} \mathrm{~F}$-labeled rhodamine dye might be developed as a PET myocardial perfusion agent. However, microPET imaging showed that, in mice, ${ }^{18} \mathrm{~F}$-ethyl-rhodamine $\mathrm{B}$ was rapidly excreted through the gall bladder with minimal accumulation in the heart, suggesting in vivo hydrolysis of the ester bond of the ${ }^{18} \mathrm{~F}$-ethyl group and loss of the ${ }^{18} \mathrm{~F}$ label. In vitro serum stability studies of the ethyl ester revealed rapid decomposition in mouse serum, but much slower decomposition in rat and human sera. A biodistribution study of this compound in rats confirmed the higher stability and 
showed the expected uptake in the myocardium. To further explore this family of compounds, ${ }^{18} \mathrm{~F}$-propyl-rhodamine $\mathrm{B}$ and ${ }^{18} \mathrm{~F}$ diethyleneglycol-rhodamine $\mathrm{B}$ were synthesized by ring opening reaction of rhodamine lactone with ${ }^{18} \mathrm{~F}$-tosylate derivative. Male Fischer rats were injected with $100-150 \mu \mathrm{Ci}$ of ${ }^{18} \mathrm{~F}$-propyl-rhodamine $\mathrm{B}$ or ${ }^{18} \mathrm{~F}$-diethyleneglycol-rhodamine $\mathrm{B}$ and imaged beginning immediately after injection for 1 hour. The results (Figure 1) clearly show that both the ${ }^{18} \mathrm{~F}$-propyl-rhodamine $\mathrm{B}$ and the ${ }^{18} \mathrm{~F}$ diethyleneglycol-rhodamineB have high myocardial uptake and very low uptake in the liver. The time activity curve of ${ }^{18} \mathrm{~F}$-diethyleneglycol-rhodamine $\mathrm{B}$ showed a faster clearance from the liver compared to ${ }^{18} \mathrm{~F}$-propyl-rhodamine $\mathrm{B}$ at the end of 1 hour. There was no clearance from the myocardium during this period for either compound. In summary, both the propyl and the diethyleneglycol esters show higher myocardial uptake and lower liver uptake than the ethyl ester, presumably because of the increased stability of the ester linkage. ${ }^{18} \mathrm{~F}$-diethyleneglycol-rhodamine $\mathrm{B}$ shows higher uptake in the myocardium and faster clearance from the liver than ${ }^{18} \mathrm{~F}$ propyl-rhodamine B suggesting it is of potential interest for PET imaging of myocardial infraction.

\section{${ }^{18} \mathrm{~F}$-propyl-rhodamine B in Fischer rat}
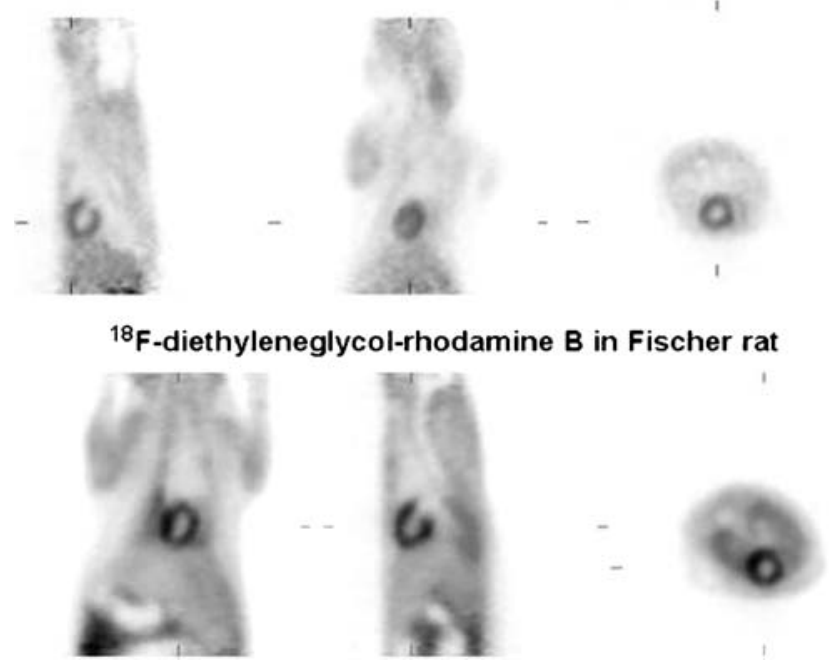

Figure 1

\section{$\mathbf{J} 250$}

\section{IN VITRO CHARACTERIZATION OF A NOVEL PEPTIDE HETERODIMER FOR SPECIFIC BREAST CANCER PET IMAGING}

Véronique Dumulon-Perreault $^{1}$, François Bénard ${ }^{2}$, Céléna Dubuc ${ }^{1}$, Patrick Fournier $^{1}$, Simon Authier ${ }^{2}$, Marie-Claude Tremblay ${ }^{1}$, Réjean Langlois ${ }^{1}$, Samia Ait-Mohand ${ }^{1}$, Brigitte Guerin ${ }^{1}$

${ }^{1}$ Médecine Nucléaire et Radiobiologie, Université de Sherbrooke, Sherbrooke, QC, Canada, ${ }^{2}$ British Columbia Cancer Research Center, Vancouver, BC, Canada.

Objectives: In the past few years, many molecules have been synthesized to bind specifically to peptide receptors. Breast cancer cells express a lot of peptide receptors. Some of them, gastrin releasing peptide receptor (GRPR) and neuropeptide Y subtype 1 receptor (NPY Y1R) are overexpressed and could be used as potential targets for specific PET imaging of breast cancer. The objective of this study is to evaluate a peptide heterodimer targeting both receptors at the same time. The (64Cu/DOTA)-Heterodimer is tested as a novel agent for breast cancer PET imaging. Methods: in vitro experiments were performed to characterize the (64Cu/DOTA)Heterodimer. Competition assays were done on T47D and MCF-7 human breast cancer cells to determine the inhibition constant of the tracer. The expression of each receptor was measured on the cell culture with $125 \mathrm{I}-\mathrm{BBN}$ or $125 \mathrm{I}-\mathrm{NPY}$. Plasmatic stability in mouse plasma was performed to validate the stability of the compound and also the stability of the $\mathrm{Cu} / \mathrm{DOTA}$ complex. Finally, the cellular uptake and the internalization of the (64Cu/DOTA)-Heterodimer were verified in vitro with T47D cells. Results: The expression of NPY Y1R has been proven on MCF-7 and T47D cells. However, T47D cells seemed to have a lower expression level of NPY Y1R than the MCF-7 cells. The GRPR is present only on T47D cell line. The NPY peptide segment of the DOTA-Heterodimer that binds to the NPY Y1R has an inhibition constant (Ki) of $271 \pm 179 \mathrm{nM}$ and $2093 \pm 922 \mathrm{nM}$ on MCF-7 and T47D cells respectively. The BBN peptide segment, which binds to GRPR, has a $\mathrm{Ki}$ of $0.91 \pm 0.32 \mathrm{nM}$ on T47D cells. The plasmatic stability showed that the DOTA has a high efficacy keeping the $64 \mathrm{Cu}$ with a percentage of linked copper-64 to the peptide of $95 \%$ after $1 \mathrm{~h}$. The cell uptake experiments on the T47D line showed rapid internalization with progressive uptake up to two hours after incubation. Conclusions: We have already successfully achieved binding of a DOTAHeterodimer to two families of receptors. The new tracer $(64 \mathrm{Cu} /$ DOTA)-Heterodimer is very stable in mouse plasma and has a good affinity for both receptors. Effective internalization of the peptide occurred in T47D cells.

\section{$\mathbf{J} 251$}

64CU TE3A-EA FOR IMMUNOTHERAPY: RADIOCHEMICAL SYNTHESIS AND THEIR PRE-CLINICAL EVALUATION Sweta Singh ${ }^{1,2}$, Nisha Saini ${ }^{1}$, B. Singh ${ }^{2}$, Anil K. Mishra ${ }^{1}$ ${ }^{\mathrm{DCRS}}$, INMAS, Delhi, India, ${ }^{2}$ Chemistry, BHU, Varansi, India.

Sweta Singh, Nisha, B.Singh, A. K. Mishra* Department of Chemistry, Banaras Hindu University, Varanasi-221005,INDIA Division of Cyclotron and Radiopharmaceutical Sciences, Institute of nuclear medicine and Allied Sciences, Brig. S. K. Mazumdar Road, Delhi-110054, India. Aim PET radiotracers, which incorporate longer-lived radionuclides, enable biological processes to be studied over many hours, at centres remote from a cyclotron. This work examines the radioisotope characteristics, synthesis of ligands, imaging performance, radiation dissymmetry and pre-clinical studies of $62 \mathrm{Cu}$ and $64 \mathrm{Cu}$, to assess their merits for PET imaging applications. Material and Methods: All used chemicals were purchased from commercial sources, while TE3A-EA was synthesised by starting from Cyclam in three step reaction.. Analytical $1 \mathrm{H}$ and $13 \mathrm{C}$ NMR spectra were recorded on a Bruker $400 \mathrm{MHz}$ spectrometer. Mass Unit mass determinations were carried out by using a electro spray ionization mass spectrometry (ESI-MS) on an SL 1200 system (Agilent) with ion trap detection in the positive and negative ion modes. After incubation at $90^{\circ} \mathrm{C}$ for $30 \mathrm{~min}$, ITLC and HPLC analyses were carried out to check the 
radiochemical purity of complex. Biological experiments were performed on male Wistar rats. Results: It was found that the complex formation is dependent on temperature. The optimal temperature range for the Complexation was $70-80^{\circ} \mathrm{C}$. The characteristics checked were buffer $\mathrm{pH}$ and excess of ligand. The optimal $\mathrm{pH}$ interval for rapid complex formation was 5.5-6.5 and excess of ligand was necessary. Finally, 1-aminoethyl-5, 8, 12-tri (carboxymethyl)-Cyclam can be easily labelled by $\mathrm{Cu}-64$ with high radiochemical purity. Biodistribution studies in rats confirmed that radiolabelled complexes under study were rapidly cleared from the blood, other organs and tissues. The main elimination pathway of radioactivity was urinary excretion. No specific accumulation of radioactivity in any organ and tissue was detected. Conclusion: It turned out that Complexation and biodistribution results of radiolabelled 1-aminoethyl-5, 8, 12-tri (carboxymethyl)-Cyclam are comparable to those of known ligand. So it can go for next phase of clinical trials.

$\mathbf{J} 252$

\section{BIODISTRIBUTION AND RADIATION DOSIMETRY OF CA-IX PET IMAGING AGENT 18F-VM4-037 MEASURED IN HEALTHY VOLUNTEERS*}

Mohan Doss ${ }^{1}$, R. Katherine Alpaugh ${ }^{2}$, Jian Q. Yu ${ }^{1}$

${ }^{1}$ Diagnostic Imaging, Fox Chase Cancer Center, Philadelphia, PA, USA, ${ }^{2}$ Protocol Support Lab, Fox Chase Cancer Center, Philadelphia, PA, USA.

Objectives: Human safety, biodistribution, dosimetry, and imaging characteristics of the CA-IX PET imaging agent 18F-VM4-037 or (S)3-(4-(2-fluoroethoxy)phenyl)-2-(3-methyl-2-(4-((2-sulfamoylbenzo[d] thiazol-6-yloxy)methyl) -1H-1,2,3-triazol-1-yl) butanamido) propanoic acid were evaluated in a Phase 0 clinical trial (ClinicalTrials. gov Identifier: NCT00884520). 18F-VM4-037 is a novel PET imaging that targets hypoxia by focusing on CA-IX, a carbonic anhydrase enzyme that, when elevated in tumors, is indicative of poor prognosis for the patient. Identifying such patients through PET imaging can help to optimize the treatments. Methods: Four healthy volunteers aged 30-65 were administered $550-730 \mathrm{MBq}$ of $18 \mathrm{~F}-\mathrm{VM} 4-$ 037 intravenously and imaged with a PET/CT scanner. Five sequential whole body PET/CT scans with ultra-low dose CT (CT parameters: $140 \mathrm{kV}, 10 \mathrm{~mA}, 0.8 \mathrm{sec}$ rotation, Helical) from top of skull to mid thigh were obtained over 2.2 hours following the injection. In addition, a PET/CT scan covering mid thigh to toes was obtained after the first whole body PET scan. Blood samples were obtained at multiple time points and analyzed. 99\% of VM4-037 was found to be intact at 90 minutes post injection. Vital signs and $E K G$ were monitored prior to, periodically during, and right after imaging. The residence times were determined from multi-exponential regression of organ ROI data normalized by injected dose. Radiation dose estimates were calculated using the MIRD schema with OLINDA/ EXM, using voiding bladder model with 4.8 hour voiding interval. All subjects had 24 hour follow-up visit. Results: No adverse events were noted during the 24 hour post injection monitoring period. Kidneys and liver showed significantly enhanced uptake in comparison to other organs, and there was little renal clearance of the activity. The top four highest-dose organs and their respective mean

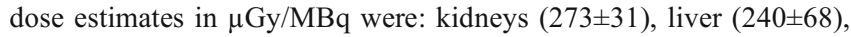
gallbladder $(63 \pm 17)$, and. urinary bladder $(41 \pm 6)$. The effective dose was $28 \pm 1 \mu \mathrm{Sv} / \mathrm{MBq}$. Conclusions: The high uptake in liver and kidneys may reduce lesion visibility in these organs, due to intense background activity. The doses to kidney, liver and gallbladder are higher than those for $18 \mathrm{~F}-\mathrm{FDG}$, while the doses to the other organs are lower. Further study with patients is in progress. *This work was supported by Siemens Molecular Imaging Inc.

\section{$\mathbf{J} 253$}

\section{POSITRON EMITTING TOMOGRAPHY (PET) RADIOTRACERS SUITABLE FOR DETERMINING IN-VIVO OCCUPANCIES OF SEVERAL ALLOSTERIC MODULATORS OF METABOTROPIC GLUTAMATE RECEPTOR SUBTYPE 1 (mGLuR1)}

Waisi Eng ${ }^{1}$, Hiroshi Kawamoto ${ }^{2}$, Stacey O'Malley ${ }^{1}$, Stephen Krause ${ }^{1}$, Christine Ryan ${ }^{1}$, Zhizhen Zeng ${ }^{1}$, Kerry Riffel ${ }^{1}$, Toshifumi Kimura ${ }^{2}$, Saturo Ito ${ }^{2}$, Gentaroh Suzuki ${ }^{2}$, Jacquelynn J. Cook ${ }^{1}$, Satoshi Ozaki ${ }^{2}$, Hisashi Ohta ${ }^{2}$, Cyrille Sur ${ }^{1}$, Richard Hargreaves ${ }^{1}$, Donald Burns ${ }^{1}$, Eric Hostetler ${ }^{1}$

${ }^{1}$ Imaging, Merck Research Labs, West Point, PA, USA, ${ }^{2}$ Tsukuba Research Institute, Merck Research Labs, Tsukuba, Japan.

Demonstration of in vivo target engagement is important both for preclinical validation of therapeutic target(s), and for ensuring that pharmacological and therapeutic hypotheses are adequately tested in a clinical proof of concept study. PET imaging with specifically developed novel radiotracers have been widely used to visualize CNS receptor systems, to monitor receptor engagement / occupancy by drug candidates, and to establish the exposure-occupancy relationships of these drug candidates to help select appropriate doses for clinical studies. We describe here the challenges encountered in our development of three radiotracers $([18 \mathrm{~F}] \mathrm{MK}$ 1312, [18F]MK-4908 and [11C]MG1-0567) belonging to three structural classes that are suitable for determining the in vivo mGluR1 occupancy by three allosteric modulators (MK-5435, MK-7643 and MG1-0567). In-vitro binding and autoradiography studies failed to reveal significant binding differences among these tracers and allosteric modulators. However, results of PET studies in anesthetized rhesus monkeys uncovered a required close-match between the structure of a tracer and that of the corresponding allosteric modulator in order for the former to correctly reveal the in vivo mGluR1 occupancy by the latter. This was evident in light of the failure of a given mGluR1 allosteric modulator to achieve complete blockade of a "mismatched" tracer (thus leading to erroneous occupancies being reported) at plasma levels, at which it was independently shown to be able to achieve complete blockade of the "matched" tracer. Therefore, a unique, structurally matched tracer had to be designed for each of the three unique structural class of modulators in our studies. Taken together, these data suggest that the allosteric modulator and PET tracer pairs may each have a unique (but somewhat overlapping) binding site, and that great care must be taken in selecting an appropriate PET tracer for quantifying target engagement by the modulator. 


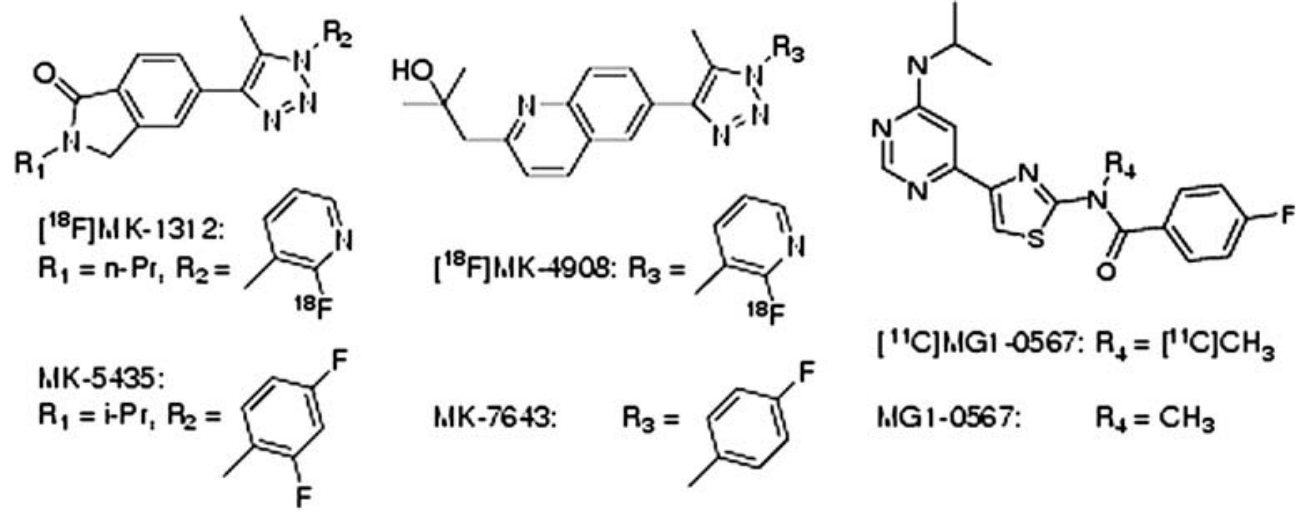

$\mathbf{J} 254$

\section{DETERMINATION OF OCULAR RESIDENCE TIMES OF ${ }^{124}$ I-LABELLED EYE DROP FORMULATIONS IN RABBITS WITH SMALL ANIMAL PET}

Gloria Stundner ${ }^{1}$, Thomas Wanek ${ }^{1}$, Daniel Dangl ${ }^{2}$, Margit Hornof ${ }^{2}$, Herbert Kvaternik $^{3}$, Oliver Langer ${ }^{1}$, Claudia Kuntner ${ }^{1}$

${ }^{1}$ Health \& Environment, Austrian Research Centers GmbH - ARC, Seibersdorf, Austria, ${ }^{2}$ Research Department, Croma-Pharma G.m.b.H, Leobendorf, Austria, ${ }^{3}$ Seibersdorf Laboratories, Austrian Research Centers GmbH - ARC, Seibersdorf, Austria.

AIM: Eye drop formulations containing a chitosan-N-acetylcysteine conjugate (chitosan-NAC) are supposed to show longer ocular residence times as compared to formulations containing unmodified chitosan, due to interaction between thiol groups of chitosan-NAC with cysteine-rich mucin (MUC5AC). The aim of this study was to measure ocular residence times of new eye drop formulations, labelled with iodine-124, using small animal positron emission tomography (PET) imaging in rabbits. METHODS: Two different eye drop formulations, containing $0.3 \%$ and $0.5 \%$ of chitosan-NAC, spiked with ${ }^{124}$ I-labelled chitosan-NAC, were compared with an aqueous solution of sodium $\left.{ }^{[24} I\right]$ iodide and a formulation containing unmodified chitosan $(0.5 \%)$, spiked with ${ }^{124}$ I-labelled chitosan, in groups of 4 rabbits per formulation. Each rabbit received one drop $(35 \mu \mathrm{L})$ of the test formulation in the conjunctival sac of the right eye. Radioactivity distribution in the head region was measured at 5 different time points between 0 and $22 \mathrm{~h}$ after topical eye drop application. Regions of interest were manually defined in the reconstructed PET images and activity concentration was quantified as percent applied dose per gram tissue $(\% \mathrm{ID} / \mathrm{g})$. RESULTS: Clear differences were observed in the ocular residence times of unmodified and thiolated chitosan formulations. At $3 \mathrm{~h}$ after application, ocular activity uptake values were $0.5 \pm 0.0 \% \mathrm{ID} / \mathrm{g}$ for sodium $\left[{ }^{124} \mathrm{I}\right]$ iodide, $4.7 \pm 5.3 \% \mathrm{ID} / \mathrm{g}$ for the formulation containing $0.5 \%$ unmodified chitosan, and $8.8 \pm 7.9$ and $12.1 \pm 10.8 \% \mathrm{ID} / \mathrm{g}$ for the formulations containing $0.3 \%$ and $0.5 \%$ thiolated chitosan, respectively. For the thiolated chitosan formulations, radioactivity was still detectable on the ocular surface at $22 \mathrm{~h}$ after application, which was not the case for the two control formulations. Ocular surface distribution of the ${ }^{124}$ I-labelled $0.3 \%$ chitosan-NAC formulation was homogeneous during the entire observation period, whereas the highest activity levels were detected in the inner canthus for the $0.5 \%$ chitosanNAC formulation. CONCLUSION: Our data demonstrate that ocular residence times of ${ }^{124}$ I-labeled eye drop formulations can be measured with small animal PET. We were able to show that chitosan-NAC formulations have increased ocular residence times as compared to an unmodified chitosan formulation.

\section{$\mathbf{J} 255$}

\section{DESIGNING ZN(II) AND CU(II) THIOSEMICARBAZONE DERIVATIVES AS PROBES FOR IN VITRO FLUORESCENCE IMAGING}

Anjani K. Tiwari, Himanshu Ojha, Nitin Kumar, Anil K. Mishra Department of cyclotron \& radiopharmaceuticals, Institute of nuclear medicine \& Allied sciences, Delhi, India.

Anjani K Tiwari1, Himanshu Ojha1, Nitin Kumar1, A. K. Mishra1*1. Division of Cyclotron and Radiopharmaceutical Sciences, Institute of nuclear medicine and Allied Sciences, Brig. S. K. Mazumdar Road, Delhi-110054, India. Aim There is a wide interest in designing novel imaging probes for biological targets, which can be employed in vivo with a range of molecular imaging techniques to attain research and clinical objectives. Non-invasive techniques such as PET (positron emission tomography) and SPECT (single photon emission computerised tomography), can be used to follow the in vivo distribution of radiolabelled metal complexes of interest in terms of therapeutic and imaging applications. Fluorescence microscopy has been recently used to follow the uptake of such molecules in living cells. Material and Methods: All used chemicals were purchased from commercial sources, and final compounds were synthesised by using a common procedure, Analytical $1 \mathrm{H}$ and $13 \mathrm{C}$ NMR spectra were recorded on a Bruker $400 \mathrm{MHz}$ spectrometer. Mass Unit mass determinations were carried out by using a electro spray ionization mass spectrometry (ESI-MS) on an SL 1200 system (Agilent) with ion trap detection in the positive and negative ion modes. Results: We aimed to design and deliver novel, small, metal-based probes that can be radiolabelled and are fluorescent for simultaneous optical and radiochemical (dual mode) imaging. We report here our results towards a series of potentially biologically active metal complexes for use as imaging probes and/or as therapeutic agents, and a qualitative observation of their uptake in cancer cells. We sought to enhance the rather weak fluorescence of the known $\mathrm{Zn}(\mathrm{ATSM})$ systems by 
incorporation of an aromatic ligand backbone, which could also improve the cell uptake. Finally have synthesised a new family semicarbazonato metal complexes featuring flat, aromatic backbones and allyl groups at the exocyclic nitrogens. These were characterised in solution by spectroscopic methods The $\mathrm{Zn}(\mathrm{II})$ complex was found to be intrinsically fluorescent so its uptake in MCF-7 cancer cells was monitored by confocal fluorescence imaging. The uptake in HeLa cells was tested by epifluores-cence imaging. Fast high purity radiolabelling with $64 \mathrm{Cu}(\mathrm{II})$ was performed by transmetallation from the corresponding $\mathrm{Zn}$ (II) complex using $64 \mathrm{Cu}(\mathrm{OAc}) 2$. These intrinsically fluorescent probes are highly cytotoxic in MCF-7 cancer cell lines.

\section{$\mathbf{J} 256$}

\section{ADVANCED SINGLE AMINO ACID CHELATES (SAAC) THAT REDUCE HEPATOBILIARY CLEARANCE OF ${ }^{99 m}$ Tc-Tyr3-OCTREOTIDE}

Kevin P. Maresca, John C. Marquis, Genliang Lu, Shawn M. Hillier, Frank J. Femia, Craig N. Zimmerman, William C. Eckelman, John L. Joyal, John W. Babich

Molecular Insight Pharmaceuticals Inc, Cambridge, MA, USA.

Single Amino Acid Chelators (SAAC) for the incorporation of the $\{\mathrm{M}$ $(\mathrm{CO}) 3\}+$ core $(\mathrm{M}=\mathrm{Tc} / \mathrm{Re})$ have been evaluated in a variety of biological systems. However, the lipophilicity of these compounds, including our prototypic (S)-(2-amino-6-(bis(pyridin-2-ylmethyl)amino)hexanoic acid) SAAC, (1) afford highly stable but hydrophobic ${ }^{99 \mathrm{~m}} \mathrm{Tc}$ chelate conjugates with increased liver/gut clearance, diminishing their utility for abdominal imaging. The goal of this study was to design more hydrophilic chelators with the potential to favor renal clearance. Methods: A series of novel ${ }^{99 \mathrm{~m}} \mathrm{Tc}$-SAAC complexes were synthesized and evaluated for stability, lipophilicity, tissue distribution, and clearance in normal rats. New chelators were incorporated into Tyr3-octreotide by solid phase peptide synthesis, labeled with ${ }^{99 \mathrm{~m}} \mathrm{Tc}$, and evaluated for in vitro binding to AR42J (SSTR2 positive) cells, as well as tissue distribution, tumor uptake, and clearance in AR42J xenograft mice. Results: A series of lysine SAAC chelators incorporating polar functionalities were prepared to afford (S)-2amino-6-((carboxymethyl)(pyridin-2-ylmethyl)amino)hexanoic acid (2), (S)-2-amino-6-(bis(thiazol-2-ylmethyl)amino)hexanoic acid (3), (S)-2amino-6-((carboxymethyl)(thiazol-2-ylmethyl)amino)hexanoic acid (4), (2-amino-6-(bis((1-X-1H-imidazol-2-yl)methyl)amino)hexanoic acid) where $\mathrm{X}=$ methyl (5), 2,2-dimethoxyethyl (6), 2-ethoxyethyl (7), and carboxymethyl (8). Radiolabeling with ${ }^{99 \mathrm{~m}} \mathrm{Tc}$ was achieved at low ligand concentrations $(10-6 \mathrm{M})$ and complexes were stable for at least $24 \mathrm{~h}(>90 \% \mathrm{RCP})$. Log $\mathrm{P}$ values ranged from -1.1 to -2.3 . The novel chelators showed some improvement over 1 with respect to hepatobiliary clearance in rats. The lysine SAAC chelators were subsequently incorporated into Tyr3-octreotide and radiolabeled with ${ }^{99 \mathrm{~m}} \mathrm{Tc}$ for comparison in AR42J xenograft mice. The results demonstrated significant differences in liver and/or gut uptake of the peptides, with conjugates $\mathbf{4}$ and $\mathbf{8}$ showing a substantial decrease in hepatobiliary clearance, implicating increased renal clearance. The profile obtained was similar to that of ${ }^{111} \mathrm{In}$ DOTA-Tyr3-octreotide. Furthermore, the ${ }^{99 \mathrm{~m}}$ Tc-SAAC-Tyr3-octreotide analog 8 possessed good tumor uptake of $4.5 \% \mathrm{ID} / \mathrm{g}$ at $4 \mathrm{hrs}$. Conclusions: SAAC chelators containing imidazole donating groups were functionalized with polar substituents to dramatically reduce hepatobiliary clearance. These novel chelators applied to Tyr3octreotide have led to the development of a ${ }^{99} \mathrm{~m}$ Tc-radiopharmaceutical with a desirable pharmacokinetic profile.

\section{$\mathbf{J} 257$}

A NOVEL SYNTHESIS OF 2'-DEOXY-2'-[ ${ }^{18}$ F]FLUORO-9- $\beta$-DARABINO-FLURANOSYLGUANINE $\left(\left[{ }^{18}\right.\right.$ F $]$ F-AraG),

FOR IMAGING T CELL ACTIVATION WITH PET

Mohammad Namavari ${ }^{1}$, Ya-Fang Chang ${ }^{1}$, Brenda Kusler ${ }^{1,2}$, Shahriar Yaghoubi $^{1}$, Beverly S. Mitchell ${ }^{1,2}$, Sanjiv S. Gambhir ${ }^{1}$

${ }^{1}$ Radiology, Stanford University, Stanford, CA, USA, ${ }^{2}$ Medicine/ Oncology, Stanford University, Stanford, CA, USA.

2'-Deoxy-9- $\beta$-D-arabino-furanosylguanine (AraG) is a guanosine analog that has proven efficacy in the treatment of $\mathrm{T}$ cell lymphoblastic disease. It is metaboloized in a unique fassion by deoxyguanosine kinase and incorporated into mitochondrial. Also, 2'-deoxy-2'-fluoro-9- $\beta$-D-arabinofuranosylguanine (F-AraG) is known to exhibit selective T-cell toxicity. We were therefore intersted in the synthesis of 2'-deoxy-2'-[ $\left.{ }^{18} \mathrm{~F}\right]$ fluoro-9$\beta$-D-arabino-furanosylguanine $\left(\left[{ }^{18} \mathrm{~F}\right] \mathrm{F}\right.$-AraG) as a novel PET imaging agent. We report for the first time the synthesis of $\left(\left[{ }^{18} \mathrm{~F}\right] \mathrm{F}-\mathrm{AraG}\right)$ as an imaging agent. The partially protected 2-N-acetyl-6-O-((4-nitrophenyl) ethyl)guanosine derivative 1 was converted to 3',5'-di-O-trityl (2) and 2',5'-di-O-trityl (3) derivatives. Trityl derivatives 2 and 3 were separated on a silica gel column. Treatment of trityl derivative 2 with $\mathrm{CF}_{3} \mathrm{SO}_{2} \mathrm{Cl} /$ DMAP afforded 2-N-acetyl-6-O-((4-nitrophenyl)ethyl)-9-( 3',5'-di-O-trityl-2'-O-trifyl- $\beta$-D-ribofuranosyl)guanine $(4,65 \%)$ as a precursor for $\left(\left[{ }^{18} \mathrm{~F}\right]\right.$ F-AraG. $\left[{ }^{18} \mathrm{~F}\right] \mathrm{F}$-AraG was prepared from precursor 4 in $8-12 \%$ radiochemical (decay corrected). The specific activity ranged $0.6-1.3 \mathrm{Ci} / \mu \mathrm{mol}$. The synthesis utilizes the reaction between the leaving group triflate in 4 and anhydrous $\left[{ }^{18} \mathrm{~F}\right] \mathrm{KF} / \mathrm{Kryptofix} 2.2 .2$ at $85^{\circ} \mathrm{C}$ for $40 \mathrm{~min}$ to produce 2-N-acetyl-6-O-((4-nitrophenyl)ethyl)-9-(3',5'-di-O-trityl- $\left[{ }^{18} \mathrm{~F}\right]-2^{\prime}$ fluoro- $\beta$-D-arabinofuranosyl)guanine (5). Finally, acid hydrolysis of 5 produces the final $\left[{ }^{18} \mathrm{~F}\right] \mathrm{F}-\mathrm{AraG}$. Preliminary cell uptake experiments indicate that the CCRF-CEM cell line and activated primary thymocytes take up the $\left[{ }^{18} \mathrm{~F}\right] \mathrm{F}-\mathrm{AraG}$. Competition assays show that the cold derivative F-AraG competes with the uptake of $8-\left[{ }^{3} \mathrm{H}\right]-$ AraG, indicating similar uptake pathways. In conclusion, for the first time to our knowledge, $\left[{ }^{18} \mathrm{~F}\right] \mathrm{F}-\mathrm{AraG}$ has been successfully synthesized by direct fluorination of an appropriate precursor of a guanosine nucleoside. This approach also maybe useful for the synthesis of other important PET probes such as $\left[{ }^{18} \mathrm{~F}\right] \mathrm{FEAU},\left[{ }^{18} \mathrm{~F}\right]$ FMAU and $\left[{ }^{18} \mathrm{~F}\right] \mathrm{FBAU}$ which currently are synthesized by multiple steps and involved lengthy purification processes.

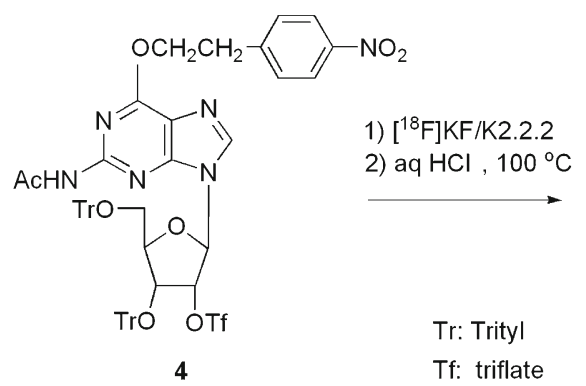

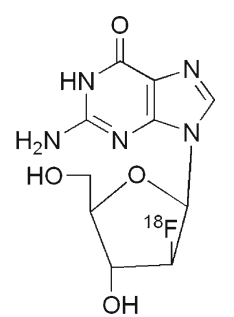

$\left[{ }^{18} \mathrm{~F}\right] \mathrm{F}-\mathrm{AraG}$ 
$\mathbf{J} 258$

\section{NOVEL MOLECULAR PET PROBES FOR DIAGNOSIS AND TREATMENT STRATIFICATION OF LYMPHOPROLIFERATIVE DISORDERS IN VIVO}

$\underline{\text { Dean O. Campbell }}^{1,2}$, Chengyi J. Shu ${ }^{3}$, Jason T. Lee ${ }^{1,2}$, Jordan C. Wengrod ${ }^{4}$, Gerald Toy ${ }^{1,2}$, Matthias R. Benz ${ }^{5}$, N. Satyamurthy ${ }^{1}$, Johannes Czernin ${ }^{5}$, Caius G. Radu ${ }^{1,2}$

${ }^{1}$ Medical and Molecular Pharmacology, UCLA, Los Angeles, CA, USA, ${ }^{2}$ Crump Institute for Molecular Imaging, UCLA, Los Angeles, CA, USA, ${ }^{3}$ Department of Immunology, Netherlands Cancer Institute, Amsterdam, Netherlands, ${ }^{4}$ New York University School of Medicine, New York, NY, USA, ${ }^{5}$ Ahmanson Biological Imaging Division, UCLA, Los Angeles, CA, USA.

Deoxycytidine kinase (DCK) is a key enzyme in the nucleoside salvage pathway, the fundamental biochemical pathway which controls nucleotide pools for DNA replication and repair. DCK is predominantly expressed in hematopoietic cells and other rapidly proliferating tissues. The enzyme is typically upregulated in cancers and other cellular proliferative disorders that arise from these tissues. DCK is also responsible for activating a widely-used class of cancer therapeutic prodrugs. These nucleoside-analog prodrugs include gemcitabine, decitabine, fludarabine and araC. Since loss of DCK activity is a major path of resistance to therapy with these drugs, the ability to use PET to non-invasively assay DCK activity is very vital for treatment selection. We recently developed 1-D-(2'-deoxy-2'-[18F]fluoroarabinofuranosyl) cytosine $([18 \mathrm{~F}] \mathrm{FAC})$ as a novel DCK-specific PET probe able to assay DCK activity in vivo. However, [18F]FAC is also a substrate for cytidine deaminase (CDA), which deaminates, thus deactivating, the probe in vivo. We set out with the objective to synthesize novel DCK-specific PET probes based on [18F]D-FAC that retain specificity for DCK while being resistant to CDA. Five novel FAC-analog probes were synthesized taking advantage of the differential selectivity of DCK and CDA. All five probes were shown to be resistant to CDA and phosphorylated by DCK in vitro with varying kinetics. Two of these probes, showed the most favorable biodistribution and sensitivity in mice. The probes were taken up preferentially by lymphoid organs. Preliminary data in murine models of cancer and autoimmune disorders suggest that these probes could enable a wide range of immune monitoring applications and could guide treatment selection in lymphoid malignancies as well as potentially other cancers.

\section{$\mathbf{J} 259$}

\section{INVESTIGATION OF C-TERMINAL MODIFICATIONS} FOR THE OPTIMIZATION OF A BOMBESIN ANTAGONIST FOR MOLECULAR IMAGING OF PROSTATE AND BREAST CANCER

$\underline{\text { Brienne Bottenus }}^{1,4}$, Gary L. Sieckman ${ }^{4}$, Tammy L. Rold ${ }^{2,4}$, $\overline{\text { Samantha Sublett }}^{3,4}$, Said Diabes Figueroa ${ }^{4}$, Silvia Jurisson ${ }^{1}$, Timothy J. Hoffman ${ }^{4,2}$

${ }^{1}$ Chemistry, University of Missouri, Columbia, MO, USA, ${ }^{2}$ Internal Medicine, University of Missouri, Columbia, MO, USA, ${ }^{3}$ Nuclear Science and Engineering, University of Missouri, Columbia, MO, USA, ${ }^{4}$ Harry S Truman VA Hospital, Columbia, MO, USA.

Objective: The purpose of this study was to determine what effect alterations to the $\mathrm{C}$-terminus of the truncated bombesin antagonist sequence, coupled to DOTA, would have on binding affinity to the bombesin receptor subtype II (BB2r) positive tumors in vitro as well as in vivo. The compounds are labeled with In-111 and Ga-67 for SPECT imaging purposes. Methods: All peptides of the sequence, DOTA-8Aoc(D)F -Q-W-A-V-G-H-L-(X) (X = amide, methyl amide, ethyl amide, propyl amide, butyl amide, hydrazine, or methyl ester) were synthesized using standard Fmoc protected solid phase peptide synthesis on chemically modified resins and labeled with Ga-nat/67 and In-nat/111. IC50 values were determined for each metal labeled peptide in the $\mathrm{BB} 2 \mathrm{r}$ expressing cell lines, PC-3 and T-47D. In vivo biodistributions will be performed in SCID mice bearing PC-3 tumor xenografts and tissues evaluated to determine $\% \mathrm{ID}$ and $\% \mathrm{ID} / \mathrm{g}$ in each organ/tissue for peptides with IC50 values $<10 \mathrm{nM}$. Micro-SPECT imaging is currently being carried out in SCID mice bearing PC-3 tumor xenografts at $4 \mathrm{~h}$ p.i. Results: IC50 results suggest that the more electron donating groups on the $\mathrm{C}$-terminus have an increased binding affinity to BB2r positive tumors. The amidated C-terminus peptide labeled with indium has an IC50 value of $\sim 200 \mathrm{nM}$ while the butyl amide has an IC50 value of 3.14 \pm $0.42 \mathrm{nM}$. Preliminary biodistributions with the Indium-111 conjugates, however, exhibit the propyl amide having slower clearance from nontarget tissues when compared to the ethyl amide, as well as lower tumor uptake and thus lower target to non-target ratios in all tissues and organs studied Conclusions: Current results suggest that factors such as hydrophobicity and receptor affinity may play an equal role in determining the optimal C-terminal modification for radiolabelled bombesin antagonists for GRPr targeting peptides. Biodistributions and Micro-SPECT imaging are currently underway in order to ascertain which $\mathrm{C}$-terminal modification creates the optimal BB2r molecular diagnostic agent. Work was supported by the US Department of Veteran's Affairs Merit Review

\section{$\mathbf{J} 260$}

\section{RADIOFLUORINATED HISTAMINE $\mathrm{H}_{3}$ RECEPTOR ANTAGONIST AS A POTENTIAL PROBE FOR IN VIVO BRAIN IMAGING WITH PET}

Svetlana V. Selivanova $^{1}$, Michael Honer ${ }^{1}$, Claudia Keller ${ }^{1}$, Kathleen Isensee $^{2}$, Holger Stark ${ }^{2}$, Pius A. Schubiger ${ }^{1}$, Simon M. Ametamey ${ }^{1}$ ${ }^{1}$ Swiss Federal Institute of Technology, Zurich, Switzerland, ${ }^{2}$ Johann Wolfgang Goethe University, Frankfurt am Main, Germany.

The histamine $\mathrm{H}_{3}$ receptor $\left(\mathrm{H}_{3} \mathrm{R}\right)$ is one of the four known subtypes of Gprotein coupled histamine receptors. $\mathrm{H}_{3} \mathrm{R}$ is identified as a regulator of the synthesis and release of histamine and other important neurotransmitters, such as acetylcholine, noradrenalin, dopamine, and serotonin. The $\mathrm{H}_{3} \mathrm{R}$ plays a role in cognition and memory processes and is implicated in different neurological disorders, including Alzheimer's disease, schizophrenia, and narcolepsy. in vivo studies of the receptor occupancy using radiolabeled tracers would be very useful for CNS drug discovery. We report here the synthesis and in vitro characterization of a novel F-18 labeled high-affinity $\mathrm{H}_{3} \mathrm{R}$ antagonist, $\left[{ }^{18} \mathrm{~F}\right]-\mathrm{ST} 889\left(\mathrm{~K}_{\mathrm{i}}\left(h \mathrm{H}_{3} \mathrm{R}\right)=0.094 \mathrm{nM}\right.$ for the cold reference compound $\left.\left[{ }^{19} \mathrm{~F}\right]-\mathrm{ST} 889\right)$. Radiofluorination was performed in one step from a mesylate precursor followed by semi-preparative HPLC purification within $2 \mathrm{~h}$ from the end of bombardment. The radiolabeled probe for biological applications was obtained after reformulation using solid phase exchange technique. Decay-corrected radiochemical yields were in the range of $8-16 \%$. Typically, $3.3-7.0 \mathrm{GBq}$ of the product was isolated with effective specific activities reaching 60-110 GBq/ $\mu \mathrm{mol}$. The purity was always higher than $99 \%$. In addition, the compound showed high plasma stability with a decomposition of $<3 \%$ after the incubation at $37 \mathrm{C}$ for $120 \mathrm{~min}$. in vitro autoradiography with $\left[{ }^{18} \mathrm{~F}\right]-\mathrm{ST} 889$ was carried out on horizontal rat brain slices with the radiotracer alone or in the presence of $100 \mu \mathrm{M}$ histamine for blocking experiments. Tracer binding showed heterogeneous distribution with the highest concentration in the striatum and cerebral cortex (Figure 1). The binding to these brain regions was found to be specific by blocking with histamine. We conclude that $\left[{ }^{18} \mathrm{~F}\right]-\mathrm{ST} 889$ is a promising tracer for $\mathrm{H}_{3} \mathrm{R}$ in vivo imaging and quantification using PET. Further in vitro characterization and in vivo PET imaging studies are presently underway in our laboratory. 

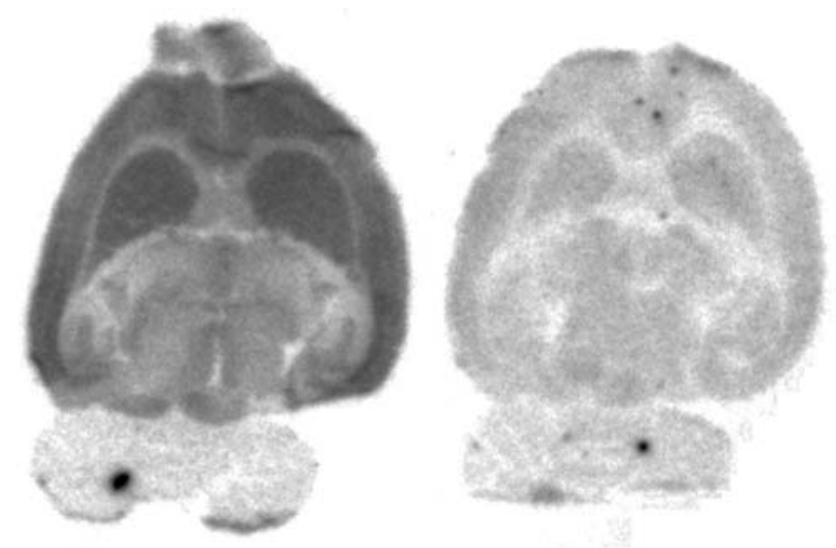

Figure 1. in vitro autoradiography in rat brain slices. Blocking (on the right) confirms the specificity of the tracer binding.

AUTOMATED RADIOCHEMISTRY PLATFORM (ARC-P): PLUG-AND-PLAY RADIOCHEMISTRY MODULES FOR RECONFIGURABLE RADIOSYNTHESIS Mark A. Eddings $^{1,2}$, Sebastian Olma ${ }^{1,2}$, Mingwei Wang ${ }^{1,2}$, Yuliang Deng $^{1,2}$, Huijiang Ding ${ }^{1,2}$, N. Satyamurthy ${ }^{1,2}$, Clifton K. Shen ${ }^{1,2}$, R Michael van Dam ${ }^{1,2}$

${ }^{1}$ Molecular and Medical Pharmacology, UCLA, Los Angeles, CA, USA, ${ }^{2}$ Crump Institute for Molecular Imaging, UCLA, Los Angeles, CA, USA.

To address the demand for increasing diversity of PET probes, flexible platforms are needed that can easily be configured to perform radiosyntheses for new probe development as well as subsequent routine use. We have developed such a platform based on a plug-andplay, modular approach where standalone modules perform welldefined chemical unit operations. The system is configured by connecting modules in the sequence of desired chemical unit operations that constitute the radiosynthesis. Replumbing and reprogramming are avoided, simplifying the setup of new syntheses without engineering expertise, despite differences in number of pots, number of purification steps, and reaction conditions. We have designed, constructed, and validated the following modules: reagent delivery module (RDM), pressurized-reaction module (PRM), cartridge purification module (CPM), and microwave reaction module (MRM). Each module has a touchscreen interface and has one input line to accept fluid delivery from the previous module and one output line to transfer to the next module. Modules were independently tested with non-radioactive and radioactive solutions to verify their basic liquid-handling functionality. Solutions are rapidly delivered by the RDM and through the CPM with minimal loss and carryover in the manifold and connections. The PRM incorporates robotics and a gasket sealing mechanism to broaden the range of accessible reaction conditions by enabling high temperature $\left(200^{\circ} \mathrm{C}\right)$ and pressure reactions $(100+\mathrm{psi})$. The MRM comprises a miniature microwave cavity for performing reactions and evaporations. The modules have been combined to perform several different radiosyntheses: [18F]FNB, [18F]FDG, [18F]FLT, and [18F]SFB, with comparable radiochemical yield and purity as compared with manual syntheses in our lab and literature values. Future efforts will focus on miniaturization, development of measurement/analysis modules to monitor reactions, and implementation of more complex (2- and 3pot) F-18 radiosyntheses.

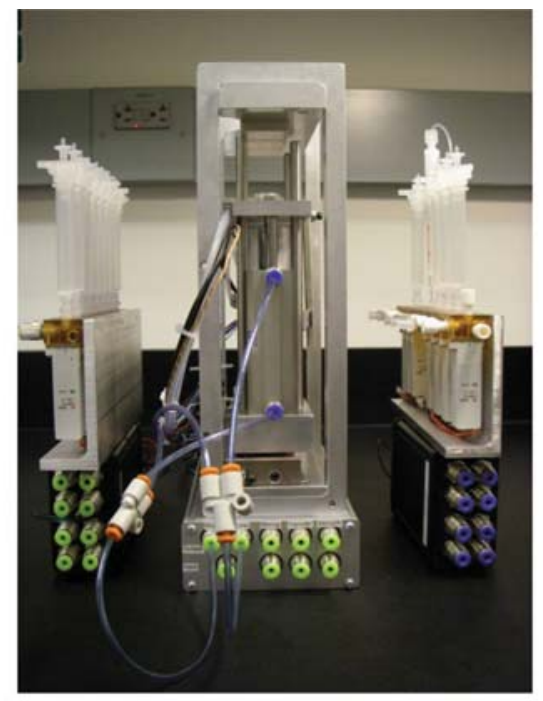

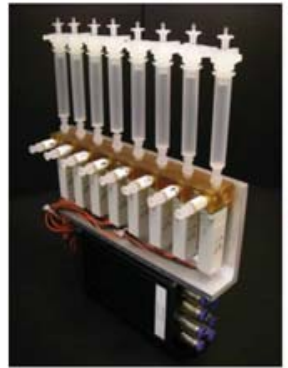

RDM

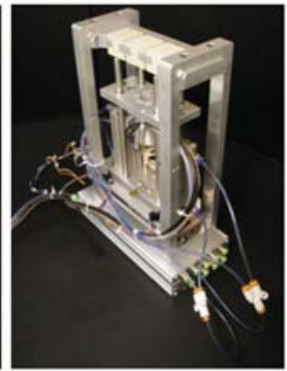

PRM

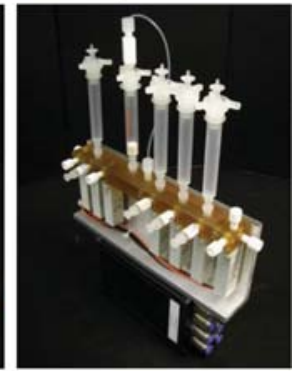

CPM
J262

\section{RADIOSYNTHESIS OF N-[C-11]METHYL-HYDROXYFASUDIL FOR IMAGING RHO KINASES (ROCKS)}

Ana C. Valdivia, Samantha Mason, Jeffrey Collins, Rob S. Beanlands, Jean N. DaSilva

National Cardiac PET Centre, University of Ottawa Heart Institute, Ottawa, ON, Canada.

Introduction: The small GTP-binding protein Rho kinases (ROCKs) play a central role in diverse cellular functions including smooth muscle contraction, actin cytoskeleton organization, cell adhesion, cell motility, and proliferation. Two ROCKs are expressed in murine tissues including the aorta, brain, heart and urinary bladder. Increased ROCK activity is observed in cerebral ischemia, coronary vasospasm, hypertension, vascular inflammation, arteriosclerosis and atherosclerosis. Strategies that specifically target and inhibit ROCKs in the vascular wall and inflammatory cells may have clinical benefits for theraphy. This study was aimed at developing N-[C-11]Methyl-hydroxyfasudil as a radiotracer for imaging ROCKs using PET. Experimental: The N-[C-11]Methyl-hydroxyfasudil (3) was synthesized from fasudil (1) in 6 steps: 1) protection of the amine in the diazepane ring; 2) formation of the isoquinolinone $\mathrm{N}$-oxime; 3) rearrangement of the N-oxime; 4) preparation of the sodium salt/benzo-15crown-5 complex (2); 5) methylation and 6) deprotection, (Scheme 1). The final unlabeled product (3) was characterized by NMR and HRMS spectroscopy. Results: Radiolabeling of N-Boc hydroxyfasudil was achieved by methylating the pre-formed sodium salt complex (2) using [C-11]methyl iodide, and then deprotected under acidic conditions to afford N-[C-11]Methyl-hydroxyfasudil (3). The use of a crown ether to trap sodium allows a rapid and clean reaction without byproducts and facilitate methylation. N-[C-11]Methyl-hydroxyfasudil was obtained in a radiochemical yield of $88 \%$ and radiochemical purity 
$>97 \%$. Coinjection with the standard in analytical HPLC confirmed the identity of $\mathrm{N}-[\mathrm{C}-11]$ Methyl-hydroxyfasudil. The radiosynthesis time was approx. $20 \mathrm{~min}$ from end-of-bombardment (EOB).
Conclusion: N-[C-11] Methyl-hydroxyfasudil was synthetized and radiolabeled in high yield and purity. Animal studies using microPET are currently in progress.

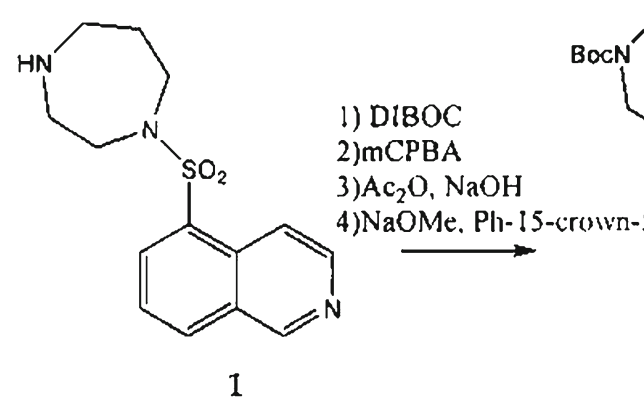

Scheme 1. Radiosynthesis of N-[C-11]Methyl-hydroxyfasudil.

\section{EVALUATION OF ENOTA AND PNOTA LIGANDS} AS POTENTIAL BIFUNCTIONAL CHELATES FOR GA-68 Hendrik P. Engelbrecht ${ }^{1}$, Leanne Forbis ${ }^{1}$, Stacy Wilder ${ }^{1}$, Melchor V. Cantorias ${ }^{1}$, John R. Lever ${ }^{2}$, Lisa Watkinson ${ }^{3}$, Shuang Liu ${ }^{4}$, Cathy S. Cutler ${ }^{1}$

${ }^{1}$ Missouri University Research Reactor (MURR), Missouri University, Columbia, MO, USA, ${ }^{2}$ Department of Radiology, University of Missouri, Columbia, MO, USA, ${ }^{3}$ Harry S. Truman Memorial Veterans Hospital, VA, Columbia, MO, USA, ${ }^{4}$ School of Health Sciences, Purdue University, West Lafayette, IN, USA.

Objective: The aim of the study was to evaluate potential bifunctional chelates for binding high specific activity Ga-68 at room temperature. The use of high specific activity radiotracer enables the use of less bioconjugated targeting molecules during the labeling procedure and which is important if a potent biologically active targeting moiety is being used. The use of less bioconjugated targeting molecules necessitates a chelating system with a high affinity and stability constant for the chosen isotope. For these reasons the in vitro and in vivo stability of ENOTA and PNOTA (4,7,4',7'-tetraacetic acid-bis$1,1 ', 1,4,7$-triazacyclononylethane and propane) complexes of Ga-68 $\left(t^{1} / 2=68 \mathrm{~min}\right)$ were tested. Methods: The radiolabeling was performed at ambient temperature for $15 \mathrm{~min}$ and the labeling yield for the Ga-68ENOTA and Ga-68-PNOTA complexes were confirmed by radio-TLC and HPLC. The in vitro stability was investigated by adding the radiocomplexes to hydroxyapatite (HA) and rat serum at $37^{\circ} \mathrm{C}$ and monitoring the percentage of intact radiolabeled complex over time by radio-TLC. The in vivo stability of both complexes was tested in CF-1 normal mice. Results: Both the complexes had $<90 \%$ labeling after $15 \mathrm{~min}$ at room temperature and exhibited excellent in vitro stability with only $<3.1 \%$ decomposition at $60 \mathrm{~min}$ in both the HA and rat serum at $37^{\circ} \mathrm{C}$. The in vivo studies showed both complexes to have fast blood clearance through the urine with only 15.3 and $6.3 \%$ ID remaining in the blood at 2 min post injection for the Ga-68-ENOTA and Ga-68PNOTA respectively. The urine contained $89 \%$ of the injected dose of Ga-68-ENOTA and 70\% of Ga-68-PNOTA at $60 \mathrm{~min}$ after injection. Due to the excretion through the urine, the kidneys contained $7.6 \%$ ID and $8.7 \%$ ID of the ENOTA and PNOTA complexes respectively after $2 \mathrm{~min}$ that decreased to $<0.7 \%$ ID at $60 \mathrm{~min}$. The highest liver uptake for both complexes was at 2 min with $3.2 \%$ ID that decreased to less than $0.3 \%$ ID at 60 min. Conclusion: Both Ga-68 complexes exhibited excellent in vitro as well as in vivo stability and these chelating agents are currently being pursued as bifunctional chelating agents with various targeting molecules.

\section{$\mathbf{J 2 6 4}$}

RADIOPHARMACOLOGICAL INVESTIGATION OF 3-[4'-[18F] FLUOROBENZYLIDENYL]-INDOLIN-2-ONE

$\underline{\text { Ralf K. Bergmann }^{1}}{ }^{\text {, Torsten Kniess }}{ }^{1}$, Manuela Kuchar ${ }^{1}$, Jörg Steinbach ${ }^{1}$, Frank Wuest ${ }^{2}$

${ }^{1}$ Radiopharmaceutical Biology, Forschungszentrum Dresden, Dresden, Germany, ${ }^{2}$ Cross Cancer Institute, University of Alberta, Edmonton, $\mathrm{AB}$, Canada.

Introduction and Aim: The radiopharmacological evaluation of the new compound 3-[4'-[18F]fluorobenzylidenyl]-indolin-2-one is described. The compound is a radiolabeled derivative of an inhibitor of the VEGFmediated signaling through the Flk-1/KDR (VEGFR-2) tyrosine kinase receptor pathway (SU5416 (NSC 696819) [3-(3,5-dimethyl-1H-pyrrol2-ylmethylene)-1,3-dihydro-indol-2-one]). Materials and Methods: The radiosynthesis was accomplished by Knoevenagel condensation of 4$[18 \mathrm{~F}]$ fluorobenzaldehyde with oxindole in a remotely controlled synthesis module. The radiotracer was obtained after a two-step labeling procedure in $4 \%$ decay-corrected radiochemical yield at a specific activity of $48-61 \mathrm{GBq} / \mu \mathrm{mol}$ within $90 \mathrm{~min}$. The radiochemical purity after semi-preparative HPLC purification exceeded 98\%. The biodistribution, metabolism and excretion were studied in Wistar rats. Small animal PET studies in rats and $\mathrm{FaDu}$ tumor bearing nude mice were carried out. Results: After distribution the radiotracer was rapidly accumulated in the adrenals, liver and kidneys, however, it was cleared from these and the most other organs. Only the adipose tissue retained the activity over $60 \mathrm{~min}$. Unexpected high transient uptake was observed in the brain, pancreas, heart and lung. The fast blood clearance of 3-[4'-[18F]fluorobenzylidenyl]-indolin-2-one was caused by excretion, approximately one half each was renal and biliary excreted and other metabolic processes. Only the original compound was entering the brain. In arterial blood plasma were two more polar radiometabolites detected. The blood clearance was fast and could be described by a two phase elimination with half-lifes of 8 and $267 \mathrm{~s}$. Consequently, in small animal PET studies with FaDu tumor bearing mice - no specific uptake in the tumors could be detected. Conclusion: 
The investigation of the 3-[4'-[18F]fluorobenzylidenyl]-indolin-2one revealed that the uptake in high perfused organs was transiently, and only in the adipose tissue the compound was accumulated. However, it seems to be it is not applicable for angiogenesis imaging.

\section{J265}

\section{PET IMAGING OF RENAL AT ${ }_{1}$ RECEPTORS WITH $\left[{ }^{11} \mathrm{C}\right]$} METHYL-LOSARTAN IN RATS

Rawad E. Antoun ${ }^{1,2}$, Tayebeh Hadizad ${ }^{1}$, Sheryn Kirkpatrick $^{1,2}$, Mireille Lortie $^{1}$, Robert A. deKemp ${ }^{1}$, Rob S. Beanlands ${ }^{1,2}$, Jean N. DaSilva ${ }^{1,2}$

${ }^{1}$ National Cardiac PET Center, University of Ottawa Heart Institute, Ottawa, ON, Canada, ${ }^{2}$ Cellular and Molecular Medicine, University of Ottawa, Ottawa, ON, Canada.

Background: The Renin-Angiotensin System is involved in the regulation of blood pressure via activation of the Angiotensin II Type 1 Receptor $\left(A T_{1} R\right)$. The expression pattern of $A T_{1} R s$ is altered in cardiac and renal diseases, such as hypertension and heart failure. Structure-activity relationship studies reported similar binding affinities for $O$-methyl-Losartan and the clinically used Losartan for $\mathrm{AT}_{1} \mathrm{Rs}\left(\mathrm{IC}_{50}=32 \mathrm{nM} \& \mathrm{IC}_{50}=19 \mathrm{nM}\right.$, respectively $)$. This work presents the initial assessment of $O-\left[{ }^{11} \mathrm{C}\right]$ methyl-Losartan in rats in small animal PET for non-invasive imaging studies of $\mathrm{AT}_{1} \mathrm{Rs}$. Methods: Dynamic PET studies (60 min) with $1.5-2.1 \mathrm{mCi}(2.1-$ $3.7 \mu \mathrm{g})$ i.v. were performed on two male Sprague-Dawley rats $(n=6$ scans) using $\left[{ }^{11} \mathrm{C}\right]$ Methyl-Losartan and the Siemens Inveon camera. Data was reconstructed into a dynamic histogram with scatter and attenuation correction. Regions of interest were drawn around the left atrial (LA) cavity and both kidneys. Time activity curves (TAC) were normalized to peak blood activity. Distribution volume (DV) values were generated using a Logan graphical analysis for the kidneys using the LA cavity as the input function without metabolite correction. Results: $\left[{ }^{11} \mathrm{C}\right]$ Methyl-Losartan displays high uptake in $\mathrm{AT}_{1} \mathrm{R}$-rich kidneys, good signal contrast between 5 and $30 \mathrm{~min}$, and slow renal washout relative to blood activity. Logan DVs showed low variability, with right and left kidney values of $1.60 \pm 0.18 \mathrm{ml} / \mathrm{g}$ and $1.58 \pm 0.18 \mathrm{ml} / \mathrm{g}$ respectively. Summary: Biodistribution results at $10 \mathrm{~min}$ post-injections displayed highest tracer uptake in the kidney cortex and outer medulla; 10.6 and 3.7 ratio-to-blood, respectively, and $75-90 \%$ specific binding of $\left[{ }^{11} \mathrm{C}\right]$ Methyl-Losartan to $\mathrm{AT}_{1} \mathrm{Rs}$. These results suggest that $\left[{ }^{11} \mathrm{C}\right]$ MethylLosartan has potential for PET imaging of renal $\mathrm{AT}_{1} \mathrm{Rs}$ in vivo. Further studies are in progress to asses binding selectivity with PET, and the presence of labeled metabolites in regions of interest. We thank the Canadian Institutes of Health Research MOP-80203 for funding this work.

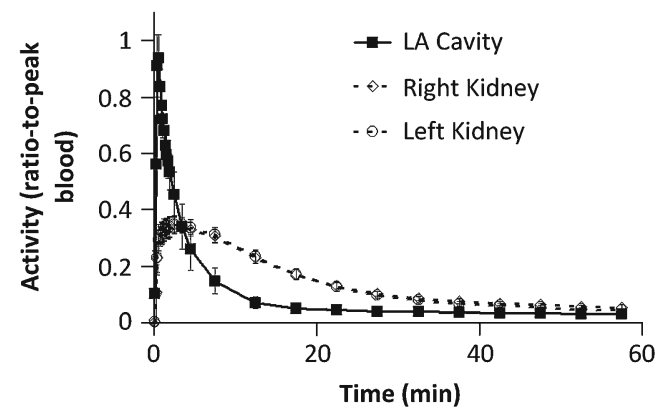

Figure 1-Renal TACs of [C-11]Methyl-Losartan (mean $\pm \mathrm{SD}, n=6$ ).
J266

SYNTHESIS AND EVALUATION OF 99MTC-DO3A-AMINO ACID CONJUGATES AS A POTENTIAL PROBE FOR TUMOR IMAGING WITH SPECT

D. Sinha $^{1,2}$, Gauri Shukla ${ }^{1,2}$, Anjani K. Tiwari $^{1}$, Anil K. Mishra ${ }^{1}$, Harish Chandra ${ }^{2}$

${ }^{1}$ radiopharmaceuticals, Institute of nuclear medicine \& allied sciences, Delhi, India, ${ }^{2}$ Chemistry, Delhi University, Delhi, India.

Introduction:-In recent years several amino acids have been labeled with either gamma or positron-emitting radio nuclides and are widely used for the diagnosis of tumor. It has been observed that most tumor cells show an increased uptake of amino acids as compared to normal cells and malignant transformation increases the use of amino acids for energy, protein synthesis, and cell division. Keeping these considerations, Novel 99mTc-DO3AAmino acid conjugates have been synthesized and evaluated as potential radiopharmaceuticals for tumor imaging. Methods: The compounds were obtained by following two step synthesis procedures. optically active heterocyclic L-amino acids [Histidine and Tryptophan,] reacts with chloroacetyl chloride in the presence of triehylamine in dichloromethane to give the $\mathrm{N}$ alkylated derivative of the corresponding amino acids, which further react with $\mathrm{DO} 3 \mathrm{~A}$ at $70^{\circ} \mathrm{C}$ for $5 \mathrm{~h}$ in DMF to give the desired products. The compounds were characterized by different spectroscopic techniques Results: The compounds were labeled with $99 \mathrm{mTc}$. The radiolabeling efficiency was more than $95 \%$ and the complexes were stable for about $24 \mathrm{~h}$ under physiological conditions. Blood kinetics study of the conjugates showed a bi-exponential patter as well as rapid clearance of radioactivity from the blood circulation. The biological $t 1 / 2(F)$ and $t 1 / 2(S)$ were found to be $50 \mathrm{~min}$ for DO3A-(His) and 22 min for DO3A-(Trp) and t1/2 (slow) $6 \mathrm{~h}$ $30 \mathrm{~min}$ and $5 \mathrm{~h} 30 \mathrm{~min}$ for DO3A-(His), and DO3A-(Trp) respectively. Tumor imaging was performed in mice bearing EAT tumor and both DO3AAmino acid conjugates showed significant accumulation at the tumor site. The mice depicted the beginning of the accumulation of activity in tumor at $30 \mathrm{~min}$, which reached to maximum at $90 \mathrm{~min}$. Biodistribution study of DO3A-(His) 2 and DO3A-(Trp)2 in normal rabbit and mice bearing EAT tumor showed showed significant accumulation in the brain and at tumor site respectively. The receptor binding study of the compounds on established human tumor cell line ( $\mathrm{U} 87-\mathrm{MG}$ ) showed $\mathrm{KD}=1.10 \mathrm{nM}$ and $1.65 \mathrm{nM}$ for DO3A-(His) and DO3A-(Trp) respectively by Scatchard plot analysis. Conclusions: Both radiotracers can be produced in high radiochemical yield and high radiochemical purity from stable precursors and are potentially valuable agent for imaging brain and other tumors with SPECT. Biodistribution studies of the compounds showed rapid and persistent accumulation of radioactivity in tumors with an excellent signal to background ratio, which warrants their further in vivo evaluation for clinical applications.

\section{$\mathbf{J} 267$}

SYNTHESIS AND EVALUATION OF ${ }^{99} \mathrm{~m}_{\text {TC-INTRODUCED }}$ BIOTIN AS A LABELING AGENT FOR ANTIBODIES Hidekazu Kawashima $^{1,2}$, Makoto Hirasawa ${ }^{2}$, Hiroyuki Kimura ${ }^{2}$, Masahiro $\mathrm{Ono}^{2}$, Hideo Saji ${ }^{2}$

${ }^{1}$ Nuclear Medicine and Diadnostic Imaging, Kyoto Univ., Kyoto, Japan, ${ }^{2}$ Patho-Functioal Bioanalysis, Kyoto Univ., Kyoto, Japan.

Objectives: Considering in vivo labeling of antibodies with ${ }^{99 \mathrm{~m}} \mathrm{Tc}$ for the diagnosis of tumor by SPECT, it is very available to utilize specific binding between streptavidin and biotin from the point of view of the high affinity and the convenience. In this study, for the purpose of introduction of ${ }^{99 \mathrm{~m}} \mathrm{Tc}$ into streptavidin-conjugated antibody efficiently, biotin derivative labeled with compact ${ }^{99 \mathrm{~m}}$ Tc-tricarbonyl complex was developed. Methods: D-biotin was converted into norbiotinamine through Curtius rearrangement, followed by a condensation reaction with histidine under water soluble carbodiimide as a catalyst to generate the histidinyl biotin (His-biotin), which possesses a ligand 
field to ${ }^{99 \mathrm{~m}}$ Tc-tricarbonyl complex. The tricarbonyl complex was synthesized from ${ }^{99}{ }^{9} \mathrm{TcO}_{4}{ }^{-}$using IsoLink kit. React with His-biotin for $50 \mathrm{~min}$ at $75^{\circ} \mathrm{C}$, then ${ }^{99 \mathrm{~m}} \mathrm{Tc}$-labeled biotin ${ }^{99 \mathrm{~m}} \mathrm{Tc}$-His-biotin) was obtained by HPLC purification. The affinity of ${ }^{99 \mathrm{~m}} \mathrm{Tc}$-His-biotin for streptavidin was measured by ultrafiltration with centrifugal filter (10,000 Mw cut-off). Results: ${ }^{99 \mathrm{~m}} \mathrm{Tc}-$ His-biotin was prepared from the reaction mixture of IsoLink kit in a $70 \%$ radiochemical yield, with a radiochemical purity higher than $98 \%$. When incubated with $160 \mu \mathrm{g} / \mathrm{ml}$ of streptavidin for $60 \mathrm{~min}$ at $37^{\circ} \mathrm{C}, 99 \%$ of ${ }^{99 \mathrm{~m}} \mathrm{Tc}$ His-biotin was bound. Moreover, this binding was inhibited almost completely in the presence of $10 \mu \mathrm{M}$ of biotin. Conclusion: ${ }^{99 \mathrm{~m}} \mathrm{Tc}-\mathrm{His}-$ biotin, a ${ }^{99 \mathrm{~m}} \mathrm{Tc}$-labeled biotin employing histidine as a ligand field was successfully synthesized. It is suggested that this compound could have a potential as an in vivo ${ }^{99 \mathrm{~m}} \mathrm{Tc}$-labeling agent for anti-tumor antibody.

$J 268$

\section{DETECTION OF GOLD NANOPARTICLES IN SMALL NUMBERS OR SINGLE CELLS}

Evan J. Boote $^{1}$, John A. Viator ${ }^{2}$, Ravi Shukla ${ }^{1}$, Ajit Zambre ${ }^{1}$,

Kavita K. Katti $^{1}$, Nripen Chanda ${ }^{1}$, Raghuraman Kannan ${ }^{1}$, Kattesh V. Katti ${ }^{1}$ ${ }^{1}$ Radiology, University of Missouri-Columbia, Columbia, MO, USA,

${ }^{2}$ Department of Biological Engineering, University of MissouriColumbia, Columbia, MO, USA.

The ability to detect and track small numbers of gold nanoparticle (AuNP) labeled cells will be a critical contribution to the ongoing work to identify effective targeting vectors. In addition, the increased signal-to-noise ratio that makes such detection possible will also be greatly beneficial to imaging methods applied. Lastly, single AuNP bearing cell detection against a background (e.g. blood) opens the possibility for sensitive tests for the spread of malignant cells. In this presentation, we will demonstrate the ability to detect small concentrations of gold nanoparticles in clusters of cells. This has been done using an ultra-high resolution computed tomography system (Xradia). As can be seen in the image, the computed tomography system is capable of resolving uptake of AuNP into single cells. At higher resolutions, there are high intensity (representing high uptake of AuNP) regions in some single cells and lesser uptake of AuNP (resulting in lower attenuation) in others. These are PC-3 (prostate cancer) cells irrigated and washe with the EGCG-stabilized AuNP. In another experiment, a small number of PC-3 cells $(10$ per $\mathrm{cm} 3)$ were detected in a photoacoustic experiment with the cells circulated through a chamber. This result indicates the potential of these labeled AuNP to provide detect cells circulating through the blood stream.

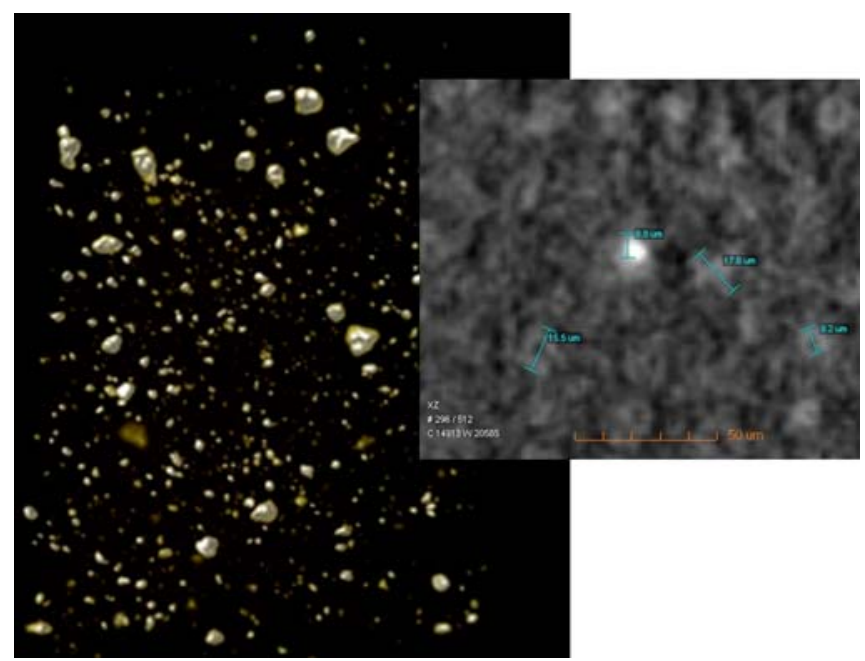

Left - a 3D rendering of ultra high resolution CT data Right - a cross sectional image shown at smaller scale
J269

DISCOVERY OF PEPTIDE LIGANDS BY PHAGE DISPLAY FOR PET/SPECT/OPTICAL IMAGING OF PSMA

Duanwen Shen ${ }^{1}$, Marie T. Dickerson ${ }^{2}$, Susan L. Deutscher ${ }^{2}$, Wilson B. Edwards $^{1}$

${ }^{1}$ Radiology, Washington University, St. Louis, MO, USA, ${ }^{2}$ Biochemistry, University of Missouri, Columbia, MO, USA.

Prostate specific membrane antigen (PSMA) is a transmembrane glycoprotein expressed on the surface of human prostate cancer (PC) cells as a non-covalently bound homodimer. PSMA is target for imaging or therapy of PC because it has limited expression in normal prostate and other tissues but over-expression in PC during all stages of tumor development. Our objective is to develop peptides for PSMA imaging by screening a library of phage displayed peptides against PSMA. In an initial step, the library was pre-cleared by i.v. administration in a mouse to remove phage that bind to vascular proteins thus clearing the phage pool of nonspecific binders. The resulting library was then screened against the ecto-domain of PSMA under low stringency conditions. After two rounds of screening, six clones were isolated and tested for specific binding to PSMA on human PC cells (LnCAP) by confocal immuno-fluorescence microscopy to determine whether the library was biased toward PSMA binding. LnCAP cells (PSMA positive) were grown on chamber slides and incubated with clonal phagebearing peptides. Cell-associated phage clones were visualized with anti-phage antibody after rinsing, fixing, and permeabilization. To determine whether the clones would specifically bind PSMA expressed on LnCAP cells, they were incubated with and without soluble PSMA $\left(37^{\circ} \mathrm{C}, 100 \mathrm{nM}\right)$. Inhibition of internalization of all six clones indicated it was mediated by PSMA. Internalized phage were visualized in the optical plane through the middle of the cells which is consistent with cytosolic uptake. Furthermore, a reduction in temperature $\left(4^{\circ} \mathrm{C}\right)$ resulted in very little internalization which is further indicative of receptor mediated endocytosis. These results show that the phage library is indeed biased toward PSMA binding and not randomly distributed. The observed binding and subsequent internalization was mediated by PSMA, and we will be moving forward with this library for additional screening.

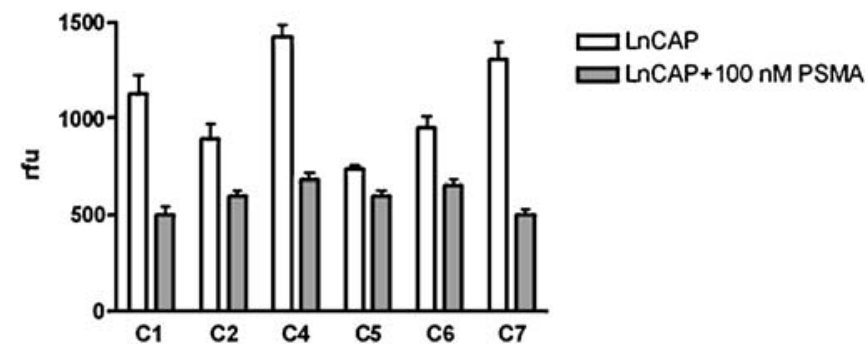

$\mathbf{J} 270$

ONE-STEP PREPARATION FOR CANNABINOID-1

RECEPTOR LIGAND [18F]MK-9470

Hsiaoju Lee, Herve Da Costa, Andrei O. Koren, David Alagille, David M. Ocame, Gilles Tamagnan Institute of Neurodegenerative Diseases, New Haven, CT, USA. 
Objectives: The cannabinoid receptor (CB1R), mainly found in the brain, is one of the most abundant $G$ protein-coupled receptors. It has been identified to play a role in various psychiatric disorders and its ligands have been shown to exhibit therapeutic effects in conditions such as addiction and obesity. This receptor is also a target for imaging agent development to noninvasively study the role this receptor plays as well as longitudinally monitor treatment progress. Several potential positron emitting tomography ligands, radiolabled with $\mathrm{C}-11$ or $\mathrm{F}-18$, have been developed by various groups. Of those, [18F]MK9470 was shown to be promising due to its high selectivity and high affinity toward CB1R, and the results from initial in vivo imaging studies. Previous attempts to radiolabel this ligand involved a two-step reaction, synthesis of $[18 \mathrm{~F}]$ $\mathrm{FCH} 2 \mathrm{CH} 2 \mathrm{Br}$ and radiolabeling of its hydroxyl precursor with $[18 \mathrm{~F}] \mathrm{FCH} 2 \mathrm{CH} 2 \mathrm{Br}$. This reaction suffers several disadvantages such as more complex reaction setup, longer reaction period and lower reaction yield. Here we illustrated a one-step radiolabeling condition to produce [18F]MK9470 with high radiochemical purity and specific activity. Methods: A new tosylated precursor was dissolved in acetonitrile and introduced to azeotropic distilled [18F]F- in a GE TRACERlab FX synthesis module. The reaction was carried out at $100^{\circ} \mathrm{C}$ for $10 \mathrm{~min}$. The reaction mixture was purified with an acidic reverse phase HPLC system. Collected fractions were concentrated with a tC18 Sep-Pak column and formulated in $50 \mathrm{mM}$ ascorbic acid solution for subsequent MicroPET studies. Results: The total synthetic time was less than $60 \mathrm{~min}$ with radiochemical yields of greater than $25 \%$. The reaction gave good specific activity and radiochemical purity. Animal studies are in progress. Conclusions: A novel one-step method was developed for routine production of [18F]MK9470 in a commercially available GE synthesis module. The shorter synthetic time, reduced complexity and higher yield make this radiopharmaceutical a good tool to assess in vivo CB1R activities in various neurological diseases.

$\mathbf{J 2 7 1}$

\section{EVALUATION OF ${ }^{18}$ F-CLOFARABINE AS A PET PROBE IN A MOUSE MODEL BEARING HUMAN GLIOBLASTOMA}

Chun-Yi Wu ${ }^{1}$, Pei-Chia Chan ${ }^{2}$, Hsin-Ell Wang ${ }^{2}$, Ren-Shyan Liu ${ }^{1,3}$ ${ }^{1}$ MAGIC, Dept NM, Faculty of Medicine, National Yang-Ming University, Taipei, Taiwan, ${ }^{2}$ Biomedical Imaging and Radiological Sciences, National Yang-Ming University, Taipei, Taiwan, ${ }^{3}$ NPCC, Dept NM, Taipei Veterans General Hospital, Taipei, Taiwan.

Objectives: Clofarabine is now a next-generation tumor treatment drug. This study aimed to evaluate the fluorinated clofarabine $\left({ }^{18} \mathrm{~F}\right.$ clo) as a PET probe for monitoring glioblastoma in a mouse model. Methods: Starting from benzoate-protected arabinose triflate, ${ }^{18} \mathrm{~F}$ clofarabine was prepared in $3.5 \mathrm{~h}$ via a two-step synthesis with high radiochemical purity $(\geqq 98 \%)$ and acceptable radiochemical yield (10-15\%, decay corrected). NOD-SCID mice were inoculated with GBM glioblastoma cells in the left thigh or in the brain. F344 rats were inoculated with F98 glioma cells in the brain. The microPET imaging of ${ }^{18} \mathrm{~F}$-clo was performed on day 12 after tumor inoculation in the animals bearing tumor. Results: For ${ }^{18} \mathrm{~F}$-clo, GBM cells exhibited the moderate cell-to-medium $(\mathrm{C} / \mathrm{M})$ ratio during $2 \mathrm{~h}$ incubation when comparing with other cells (21.61). The microPET imaging showed different tumor contrasts depending on tumor-bearing sites and species. The tumor-tomuscle ratio was only 1.51 in the left thigh tumor-bearing mice. However, the radioactivity retained in the tumor bear in the brain was higher than normal brain to achieve 12.4 of tumor-to-brain ratio due to the low dCK activity in the brain. In addition, ${ }^{18} \mathrm{~F}$-clo seems not be allowed to pass through blood-brain-barrier in F344 rat. The bearing GBM tumors in the mice or rats were confirmed by biopsy and H\&E stain. Conclusions: In this study, the fluorine-18 labeled clofarabine is successfully prepared via a two-step synthesis. This study demonstrated that ${ }^{18} \mathrm{~F}$-clo is a promising PET probe for detection of brain tumors in the mice model.

\section{$\mathbf{J} 272$}

\section{HUMAN RADIATION DOSIMETRY AND WHOLE-BODY BIODISTRIBUTION FOR A NOVEL AMYLOID IMAGING AGENT-18F-AV-45}

Kun-Ju Lin ${ }^{1,2}$, Mei-Ping Kung ${ }^{3}$, Wen-Chuin $\mathrm{Hsu}^{4}$, Ing-Tsung $\mathrm{Hsiao}^{2}$, Shiaw-Pyng Wey ${ }^{2}$, Daniel M. Skovronsky ${ }^{5}$, Yau-Yau Wai ${ }^{6}$,

Hsiu-Ping Chang ${ }^{1}$, Chuan-Wei Lo ${ }^{1}$, Cheng-Hsiang Yao ${ }^{1}$, Tzu-Chen Yen ${ }^{1}$ ${ }^{1}$ Nuclear Medicine, Chang Gung Memorial Hospital, Taoyuan, Taiwan, ${ }^{2}$ Medical Imaging and Radiological Sciences, Chang Gung University, Taoyuan, Taiwan, ${ }^{3}$ Radiology, University of Pennsylvania, Philadelphia, PA, USA, ${ }^{4}$ Neurology, Chang Gung Memorial Hospital, Taoyuan, Taiwan, ${ }^{5}$ Avid Radiopharmaceuticals, Philadelphia, PA, USA, ${ }^{6}$ Radiology, Chang Gung Memorial Hospital, Taoyuan, Taiwan.

18F-AV-45 ( (E)-4-(2-(6-(2-(2-(2-F-18 fluoroethoxy) ethoxy) ethoxy) pyridin-3-yl) vinyl) -N-methylbenzenamine, also known as florpiramine F-18) is a novel radiopharmaceuticals that selectively binds to amyloid plaques. The present study sought to measure whole body biodistribution and dosimetry in Alzheimer disease (AD) patients and healthy elderly human subjects. The optimal imaging time with $18 \mathrm{~F}-\mathrm{AV}-45$ was determined. Method: Three AD and three healthy elderly control subjects underwent dynamic brain PET/CT study, and another 3 healthy elderly subjects underwent whole body scan up to $3 \mathrm{hr}$ after intravenous injection of $370 \mathrm{mCi}$ of $18 \mathrm{~F}-\mathrm{AV}-45$. The source organs were delineated on PET/CT. Brain magnetic resonance imaging was conducted for three $\mathrm{AD}$ subject to provide structural reference. Results: The high uptakes of radioactivity were found in the gallbladder wall (critical organ receiving $58.7 \mathrm{~Gy} / \mathrm{MBq}$ ), liver, and upper large intestine wall. High uptake was observed in the hepatobiliary system included the gallbladder (ranging 116.0 29.9 Gy/MBq) and liver (ranging 71.5 41.7 Gy/MBq). The effective dose equivalent and effective dose for $18 \mathrm{~F}-\mathrm{AV}-45$ were $23.37 \pm 2.54 \mathrm{~Sv} / \mathrm{MBq}$ and $16.87 \pm 0.60 \mathrm{~Sv} / \mathrm{MBq}$, respectively. These values are comparable to that of other F-18 labeled radiopharmaceuticals. PET brain imaging demonstrated that the radiopharmaceutical localized in the frontal, parietal and precuneus cortex in the $\mathrm{AD}$ brains consistent with the pattern of amyloid distribution. The optimum imaging time of $18 \mathrm{~F}-\mathrm{AV}-45$ for plaques in the brain was determined to be a 5 to 10 minute scan taken anytime between 30 and $60 \mathrm{~min}$ after injection of the radiopharmaceutical. Conclusion: $18 \mathrm{~F}-\mathrm{AV}-45$ is safe with appropriate biodistribution for imaging amyloid plaques in human. The optimal scanning time for brain can be reached quickly after injection. 


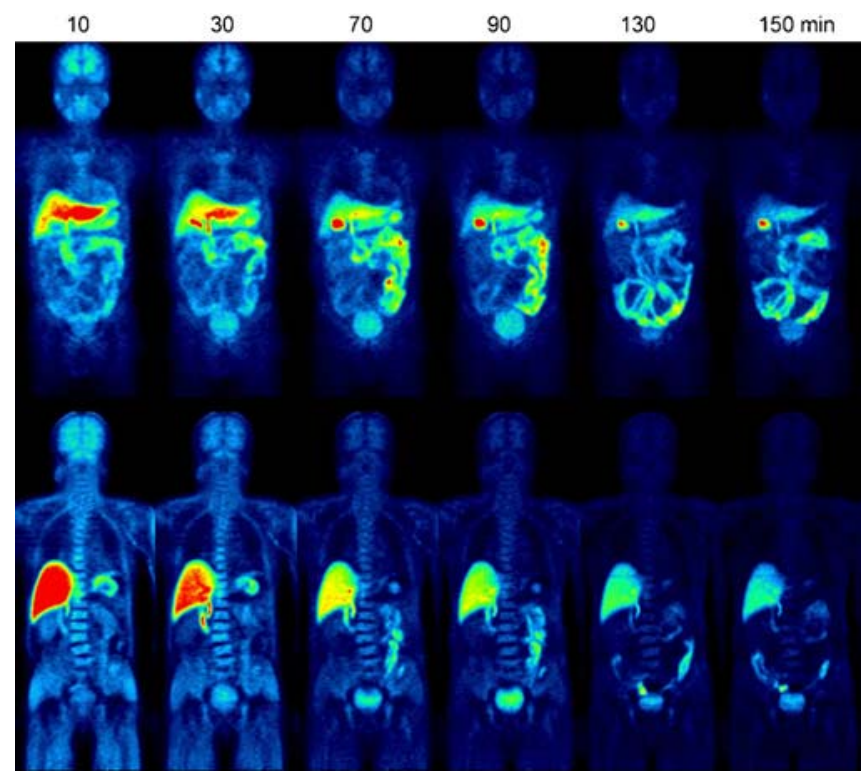

Coronal images of sequential whole body PET images.

\section{$\mathbf{J} 273$}

\section{BINDING CHARACTERISTICS OF F-18-AV-133,} A TETRABENAZINE DERIVATIVE, IN RATS

Hsin-Hsin Tsao $^{1}$, Shiaw-Pyng Wey ${ }^{1}$, Kun-Ju Lin ${ }^{1,2}$, Jyuhn-Huarng Juang ${ }^{3}$, Daniel M. Skovronsky ${ }^{4,5}$, Tzu-Chen Yen ${ }^{2}$, Mei-Ping Kung ${ }^{1,5}$

${ }^{1}$ Department of Medical Imaging and Radiological Sciences, Chang Gung University, Taoyuan, Taiwan, ${ }^{2}$ Department of Nuclear Medicine, Chang Gung University and Memorial Hospital, Taoyuan, Taiwan, ${ }^{3}$ Division of Endocrinology and Metabolism, Chung Gung University and Memorial Hospital, Taoyuan, Taiwan, ${ }^{4}$ Avid Radiopharmaceuticals, Philadelphia, PA, USA, ${ }^{5}$ Department of Radiology, University of Pennsylvania, Philadelphia, PA, USA.

Objectives: The C-11 or F-18 labeled tetrabenazine derivatives targeting vesicular monoamine transporters (VMAT2), a potential biomarker for beta cell mass (BCM), have shown some promising results. In the present study we examined the binding characteristics of F-18-AV-133, a potential PET tracer for BCM imaging, in rat pancreas and rat brain, respectively. Methods: Exocrine and islet cells were isolated from Sprague-Dawley rats. Membrane homogenates prepared from pancreatic exocrine and islet cells as well as from brain striatum and hypothalamus regions were used for in vitro binding studies. In vitro and ex vivo autoradiography studies with F-18-AV-133 were performed on brain and pancreas sections. Islet beta cells were confirmed via immunohistochemistry with anti-insulin antibody. Results: Excellent binding affinities of F-18-AV-133 were observed in rat striatum and hypothalamus homogenates with $\mathrm{Kd}$ values of 0.19 and $0.25 \mathrm{nM}$, respectively. Islet cell homogenates, however, showed two saturable binding sites (site $\mathrm{A}: \mathrm{Kd}=$ $6.76 \mathrm{nM}, \mathrm{Bmax}=60 \mathrm{fmol} / \mathrm{mg}$ protein; site $\mathrm{B}: \mathrm{Kd}=241 \mathrm{nM}, \mathrm{Bmax}=$ $1500 \mathrm{fmol} / \mathrm{mg}$ protein). Similar B sites were also observed in exocrine cells $(\mathrm{Kd}=209 \mathrm{nM})$. In vitro autoradiography of F-18-AV-133 using frozen sections of rat pancreas in optimum conditions showed labeling of islets, as evidenced by co-localization with anti-insulin antibody. Ex vivo VMAT2 pancreatic autoradiography in the rat however was not successful, in contrast to the excellent ex vivo autoradiography of VMAT2 binding sites in the brain. Conclusions: F-18-AV-133 is an excellent imaging agent for mapping VMAT2 sites in rat brain and specifically binds islet cells in vitro and postmortem. Additional optimization may be required to achieve ex vivo islet beta cell labeling in rats.
$\mathbf{J 2 7 4}$

\section{${ }^{11}$ C-LABELED CETROZOLE: A BETTER PET TRACER FOR AROMATASE}

Kayo Takahashi ${ }^{1}$, Takamitsu Hosoya ${ }^{2}$, Kayo Onoe ${ }^{1}$, Hisashi Doi ${ }^{1}$, Hiroko Nagata' $^{1}$, Toshiyuki Hiramatsu ${ }^{3}$, Xiao-Le Li ${ }^{3}$, Yasuhiro Wada ${ }^{1}$, Tadayuki Takashima ${ }^{1}$, Yumiko Katayama ${ }^{1}$, Hajime Yamanaka ${ }^{1}$, Masaaki Suzuki ${ }^{1}$, Hirotaka Onoe ${ }^{1}$, Yasuyoshi Watanabe ${ }^{1}$

${ }^{1}$ Center for Molecular Imaging Science, RIKEN, Kobe, Japan, ${ }^{2}$ Graduate School of Biomedical Science, Institute of Biomaterials and Bioengineering, Tokyo Medical and Dental University, Tokyo, Japan, ${ }^{3}$ Department of Blological Information, Graduate School of Bioscience and Biotechnology, Tokyo Institute of Technology, Yokohama, Japan.

Aromatase is an enzyme that converts androgens to estrogens. The enzyme in the brain is involved not only in the regulation of the sexual behavior but also in synaptic plasticity, neuroprotection and emotional behavior. To investigate the functional role of the enzyme in living body, we previously developed $\left[{ }^{11} \mathrm{C}\right]$ vorozole and ${ }^{18} \mathrm{~F}$-labeled analogs as PET tracers for aromatase. However, these radiolabeled tracers showed relatively high nonspecific binding. To overcome this disadvantage, we developed a novel PET tracer for aromatase, $\left[{ }^{11} \mathrm{C}\right]$ cetrozole. $\left[{ }^{11} \mathrm{C}\right]$ Cetrozole showed less nonspecific binding in the cortices as compared with $\left[{ }^{11} \mathrm{C}\right]$ vorozole. The time-activity curves of $\left[{ }^{11} \mathrm{C}\right]$ cetrozole in the monkey brain delineated that the standardized uptake value of $\left[{ }^{11} \mathrm{C}\right]$ cetrozole peaked at around $5 \mathrm{~min}$ and declined with time. On the other hand, reuptake of radioactivity was observed after $70 \mathrm{~min}$ of the time course in PET scan with $\left[{ }^{11} \mathrm{C}\right]$ vorozole. This phenomenon suggests that the radiolabeled metabolites of $\left[{ }^{11} \mathrm{C}\right]$ vorozole were incorporated into the brain, and it is not suitable for quantitative analyses. Taken together, $\left[{ }^{11} \mathrm{C}\right]$ cetrozole is a better PET tracer for aromatase to assess the pathophysiology of diseases or disorders related to sex steroids in living body.

\section{$\mathbf{J} 275$}

\section{NUMERICAL SIMULATION OF HEAT TRANSFER IN CONVENTIONAL VS. MICROFLUIDIC REACTORS AND EXPERIMENTAL BENEFITS FOR [18F]PET-TRACER SYNTHESIS}

Victor Samper ${ }^{1}$, Stefan Riese ${ }^{2}$, Christian Rensch ${ }^{1}$, Christoph Boeld ${ }^{1}$, Gerald Reischl ${ }^{3}$, Nicole Heumesser ${ }^{3}$, Marko Baller ${ }^{1}$

${ }^{1}$ GE Global Research, Garching b. München, Germany, ${ }^{2}$ GEHC, GE, Münster, Germany, ${ }^{3}$ Radiopharmacy, University Hospital Tübingen, Tübingen, Germany.

Objectives The increased surface to volume ratio offered by microfluidic synthesisers compared to conventional PET synthesizers (ca. 1 cc volume), enhances heat transfer to and from reagents. The objective of this work was to simulate the thermal performance of a microfluidic system and to demonstrate feasibility by performing $18 \mathrm{~F}$ labelling of NITTP to give [18F]FMISO in a microfluidic reactor. Methods A numerical model of a planar microfluidic system was defined. It consisted of a $200 \mu \mathrm{l}$ microchannel reactor in Topas 6015 cyclic olefin copolymer (COC). The channel cross-section was $500 \mu \mathrm{m}$ $\mathrm{X} 500 \mu \mathrm{m}$ and the channel-to-heating-surface distance was $1.75 \mathrm{~mm}$. A second model was constructed for a 1 cc cylindrical reactor, also from COC, with a wall thickness of $1.5 \mathrm{~mm}$. The reactor external surfaces were heated to $120^{\circ} \mathrm{C}$ by a contact heater. The temperatures and heating rates of the reactors and reagents were modeled, with and without preheating of the reactors. A similar microfluidic reactor was fabricated in COC and used to label NITTP. Results The wall and centre temperatures of the fluid are within $5^{\circ} \mathrm{C}$ of each other and $5^{\circ} \mathrm{C}$ of the final target temperature $\left(120^{\circ} \mathrm{C}\right)$ after $15 \mathrm{~s}$ for the pre-heated micro- 
fluidic reactor. With no pre-heating, the system was $\sim 30$ s slower, without any increase in the wall to centre fluid temperatures. By comparison, the $1 \mathrm{cc}$ reactor requires $\sim 280 \mathrm{~s}$ to reach the same point without pre-heating, and shows a peak wall to centre fluid temperature difference of $\sim 60^{\circ} \mathrm{C}$. The macro setup becomes $20-30$ s faster with preheating, but this results in a $\sim 70^{\circ} \mathrm{C}$ peak discrepancy between the centre and wall temperatures of the fluid. Experimental results showed that microfluidic and conventional syntheses approach similar yields on the time frame of $300-600 \mathrm{~s}$ at $100^{\circ} \mathrm{C}^{*}$. The advantage of the rapid heating and tight temperature control for the microfluidic reactor was demonstrated by reducing the reaction time to $60 \mathrm{~s}$ with a resulting increase in yield from $60 \%$ to $80 \%$. Conclusions Microfluidic reactors with large surface to volume ratios for the fluid, result in improved temperature uniformity and transient response when compared to conventional reactors. In the case described, the maximum benefits are realised by pre-heating the microreactor. Experimental results demonstrate that the thermal response of the microreactor enables labelling of NITTP in $<60 \mathrm{~s}$. * Patt $\mathrm{M}$ et al. Preparation of [18F]fluoromisonidazole by nucleophilic substitution on... J Radioanal Nucl Chem 240, 925-7, 1999 Work funded in part by Bavarian State (Germany)

\section{$\mathbf{J} 276$}

\section{IMAGING AND BIOCHEMICAL MANIPULATION} OF COBALAMIN METABOLISM IN BREAST TUMORS Douglas A. Collins ${ }^{1}$, Joseph A. Bauer ${ }^{2}$, Michael K. O'Connor ${ }^{1}$ ${ }^{1}$ Radiology, Mayo Clinic, Rochester, MN, USA, ${ }^{2}$ Biochemistry, Cleveland Clinic, Cleveland, OH, USA.

Malignant tumors have been shown to accumulate cobalamin $(\mathrm{Cbl})$ to a greater extent than normal tissue. We have utilized an Indium-111 (In-111) radiolabeled 5'deoxyadenosylcobalamin (AC) analog (In-111AC) to image $\mathrm{Cbl}$ metabolism in human patients with known suspicious masses. Retrospectively, Cbl metabolism was clinically imaged in $87.8 \%(43 / 49)$ of a variety of human tumors. The highest average tumor to background ( $\mathrm{T}$ : B) ratio was 5.8 (range 2.0-22.5) in 16/17 ductal and lobular breast carcinomas. We subsequently investigated our observation, that the administration of non-labeled Cbl or dexamethasone (DEX) $\sim 24 \mathrm{hrs}$ prior to the injection of In-111AC appeared to increase the T:B ratios in several of the human breast tumors compared to other tumors. In 20 gm nude mice burdened with MDA-MB-231 human breast tumor xenografts, gamma well counting of tumor and tissue samples demonstrated that the $24 \mathrm{hr}$ pulse chase method, with IP pre-administration of $2.0 \mathrm{ug}$ of AC or DEX $24 \mathrm{hrs}$ prior to IP injection of $0.2 \mathrm{ug}$ of In-111 AC, respectively increased activity in tumors by $90.6 \%$ and $44.8 \%$ compared to controls. Quantitative immunohistologic staining of breast tumors for the transcobalamin receptor (TC-R) excluded acute upregulation in the number of TC-R as a possible mechanism for the increased tumor concentration of the tracer. Except for the liver and kidneys, the remainder of normal tissue sampled demonstrated partially saturable, tissue specific suppression of In-111AC uptake. We hypothesize that there is an innate number and recycling of TC-R in tissue, which is enhanced in tumors. These findings could optimize targeted delivery of $\mathrm{Cbl}$ analogs to image or treat a variety of breast tumors.

\section{$\mathbf{J} 277$}

\section{PRELIMINARY RESULTS OF RADIOIODINATED ATP} SYNTHASE MONOCLONAL AB AS A THERAGNOSTIC TARGET FOR ANGIOGENESIS IMAGING Bok-Nam Park $^{1}$, Joon-Kee Yoon ${ }^{1}$, Kyung-Han Lee ${ }^{2}$

${ }^{1}$ Nuclear Medicine and Molecular Imaging, Ajou University, Suwon, Korea, South, ${ }^{2}$ Nuclear Medicine, Samsung Medical Center, Sungkyunkwan University, Seoul, Korea, South.
Objectives: ATP synthase which has been known as an angiostatin receptor, presents on the extracellular surface of a variety of tumor cells, therefore, it could be a potent theragnostic imaging target for tumors. In this study, we synthesized ${ }^{125}$ I-labeled ATP synthase monoclonal $\mathrm{Ab}\left({ }^{125} \mathrm{I}\right.$-ATP $\left.\mathrm{mAb}\right)$ and evaluated its in vitro cellular binding and in vivo distribution in normal mice. Methods: ${ }^{125} \mathrm{I}-\mathrm{mAb}$ was prepared using Iodogen tube method. In vitro labeling efficiencies and retention rates were measured. Cellular binding was tested in a variety of cancer cells at $30 \mathrm{~min}, 1,2,4$ and $24 \mathrm{hr}$ after adding ${ }^{125} \mathrm{I}$ ATP mAb. Pin hole gamma camera images were acquired at $2 \mathrm{hr}$ after intravenously injection of ${ }^{125} \mathrm{I}$-ATP mAb into normal mice $(n=3)$. And then, organs were removed and counted using a gamma counter for biodistribution. Results: Average labeling efficiency of ${ }^{125} \mathrm{I}-\mathrm{ATP} \mathrm{mAb}$ was gradually increased by the increment of $\mathrm{mAb}$ dose; 47.5, 69.0, 74.4, 75.9 and $91.5 \%$ at 5, 10, 20, 40 and $80 \mu \mathrm{g} \mathrm{Ab}$. Retention rates of ${ }^{125}$ I-ATP mAb were $87.3 \%, 67.6 \%, 43.6 \%$, and $27.2 \%$ at $1,2,4$, and $6 \mathrm{hrs}$, respectively. Among 9 cancer cells tested, MKN-45 (human gastric adenocarinoma) showed the highest cellular binding at $24 \mathrm{hr}$ $(2.0 \pm 0.0 \%)$. Gamma camera images demonstrated no significant thyroid uptake at $2 \mathrm{hr}$ after injeciton. The radioactivity measured on a gamma couter revealed high uptake $(\% \mathrm{ID} / \mathrm{g})$ in spleen, kidney, lung, heart and thyroid, but, poor uptake in liver (Fig. 1). Conclusion: ATP synthase mAb was easily labeled to radioiodine with a sufficient labeling efficiency, and its binding ability was confirmed in cancer cells. Therefore, although it needs further evaluation in tumor models, radioiodinated ATP synthase $\mathrm{mAb}$ could be valuable as a target for theragnostic angiogenesis imaging.

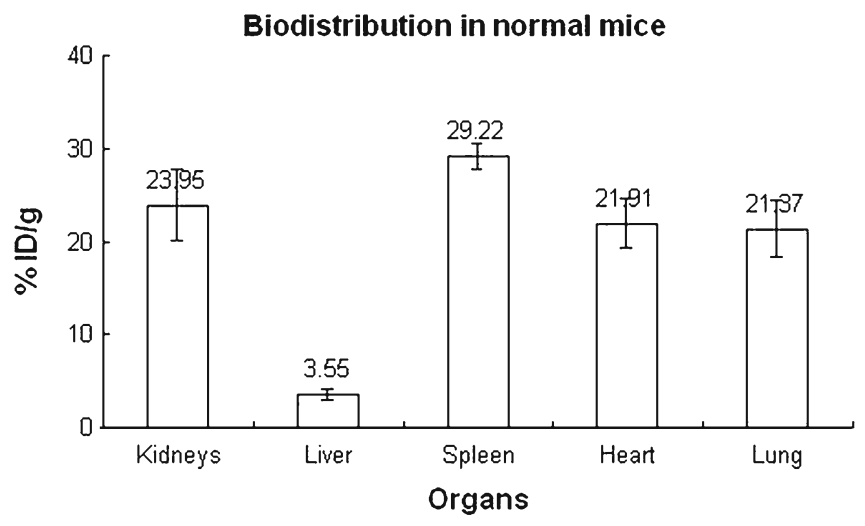

$\mathbf{J} 278$

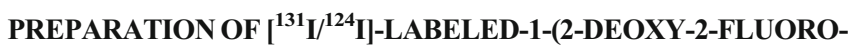
B-D-ARABINOFURANOSYL)-5-IODOCYTOSINE (FIAC) FOR MONITORING HERPES SIMPLEX VIRUS TYPE 1 THYMIDINE KINASE GENE EXPRESSION

${ }_{\text {Wen-Yi Chang }}{ }^{1}$, Wei-Ti Kuo ${ }^{1}$, Chuan-Lin Chen ${ }^{1}$, Ren-Shyan Liu ${ }^{2}$, $\overline{\text { Hsin-Ell Wang }}^{1}$

${ }^{1}$ Department of Biomedical Imaging and Radiological Sciences, National Yang-Ming University, Taipei, Taiwan, ${ }^{2}$ Department of Nuclear Medicine, Veterans General Hospital, Taipei, Taiwan.

Objective: The aim of this study was to synthesize and evaluate a novel iodinated nucleoside as a herpes simplex virus type 1 thymidine kinase gene expression probe for SPECT or PET. Methods: The labeling precursor 5-tributylstannyl 1-(2-deoxy-2fluoro- $\beta$-D-arabino- furanosyl)cytosine (FTAC) was prepared via a five-step syntheses starting from the $1,3,5$ tribenzoyl- $\alpha$-D-ribofur- 
anose, the total yield was about $30 \%$. The radiolabeling of FIAC with ${ }^{131} \mathrm{I}$ and ${ }^{124} \mathrm{I}$ was accomplished by the iododestannylation reaction of the FTAC under the mild condition. In vitro cellular uptake study was processed by adding $6 \mu \mathrm{Ci}{ }^{131}$ I-FIAC to a well containing $1 \times 10^{6}$ HSV1-tk retroviral vector transduced murine sarcoma cells (NG4TL4-STK, tk+) or wild type NG4TL4 (tk-) cells. The radioactivity accumulated in cells was assayed and recorded in cell-to-medium ratio at different time points. The animal model was achieved by subcutaneously inoculating about put $1 \times 10^{6}$ $\mathrm{tk}(+)$ and $\mathrm{tk}(-)$ cells in the right and left flanks of syngeneic FVB/N mice to grow tumors. In-vivo biodistribution and imaging studies were performed 8 to 10 days after tumor transduction for sufficient growth to facilitate experiments. Results: Radiolabeling of ${ }^{131} \mathrm{I} /{ }^{124} \mathrm{I}-$ FIAC was obtained with high radiochemical yield (in average $\geqq$ $90 \%$ ) and radiochemical purity ( $\geqq 95 \%)$. In vitro cellular uptake study of ${ }^{131}$ I-FIAC was effectively phosphorylated by cytosolic nucleoside kinases and specifically incorporated into a thymidine kinase-expressing NG4TL4-STK $(\mathrm{tk}+)$ cell rather than a thymidine kinase-deficient NG4TL4 (tk-) cell. The radioactivity accumulation in $\mathrm{tk}(+)$ cells was 247.3 folds higher than that of $\mathrm{tk}(-)$ cells at $8 \mathrm{~h}$ incubation. Furthermore, biodistribution studies showed significantly higher radioactivity retention in $\mathrm{tk}(+)$ tumors than those in $\mathrm{tk}(-)$ tumors and normal tissue in the whole experimental periods (2 days).The results of scintigraphic planar gamma imaging and PET imaging were consistent with those in-vivo biodistribution results. Conclusion: This study clearly demonstrates that radioiodine-labeled cytosine derivative ${ }^{131}$ I-FIAC is a novel and promising probe for non-invasive monitoring of HSV1-tk transgene expression.

\section{J279}

\section{SYNTHESIS OF 5- ${ }^{123 / 131}$ I-IODO-2'-DEOXYCYTIDINE AS A POTENTIAL PROLIFERATION PROBE FOR TUMOR DIAGNOSIS}

Wei-Ti Kuo ${ }^{1}$, Wen-Yi Chang ${ }^{1}$, Ren-Shyan Liu $^{2}$, Hsin-Ell Wang ${ }^{1}$, Chuan-Lin Chen ${ }^{1}$

${ }^{1}$ Biomedical Imaging and Radiological Sciences, National Yang-Ming University, Taipei, Taiwan, ${ }^{2}$ Department of Nuclear Medicine and National PET/Cyclotron Center, Veterans General Hospital, Taipei, Taiwan.

Objective: The purpose of this investigation was to optimize the 5-iodo2 -deoxycytidine labeling with radioiodinated nuclides and evaluate this iodinated nucleoside as a proliferation imaging probe for tumor diagnosis. Materials and methods: The radiolabeling of 5- iodo-2'deoxycytidine (ICdR) with $\mathrm{Na}^{123} \mathrm{I}$ and $\mathrm{Na}^{131}$ I was accomplished via the iododestannylation reaction of the 5- tributylstannyl-2'-deoxycytidine at the mild oxidative condition. In-vitro cellular uptake study was evaluated by adding $6 \mu \mathrm{Ci}{ }^{131} \mathrm{I}-\mathrm{ICdR}$ to a well containing $7 \times 10^{6}$ murine sarcoma cells NG4TL4 cells at various incubation time points $(0.5,1,2,4,8 \mathrm{~h})$ and radioactivity accumulated in cells was expressed with cell-to-medium ratio at different time points. The animal model was achieved by subcutaneously inoculating about $1 \times 10^{6}$ NG4TL4 cells in the right flank of syngeneic $\mathrm{FVB} / \mathrm{N}$ mice to grow tumors and scintigraphic planar gamma imaging study with ${ }^{123} \mathrm{I}-\mathrm{ICdR}$ was performed 10 days after tumor transduction for sufficient growth to facilitate imaging. Results: Radiolabeling of ${ }^{123 / 131} \mathrm{I}-\mathrm{ICdR}$ was achieved with high radiochemical yield (in average $\geqq 85 \%$ ) and radiochemical purity ( $\geqq 95 \%$ ). In vitro cellular uptake study of ${ }^{131} \mathrm{I}-\mathrm{ICdR}$ was reveal a linear uptake and the cell to medium ratio $(\mathrm{C} / \mathrm{M})$ that reach to $72.64 \pm$ 7.94 after $8 \mathrm{~h}$ incubation. Furthermore, the planar gamma imaging of sarcoma-bearing mice revealed high tumor-to-muscle ratio that reach to 19.88 at $8 \mathrm{~h}$ post administration of ${ }^{123} \mathrm{I}-\mathrm{ICdR}$ via the tail vein. Conclusion: ${ }^{123 / 131} \mathrm{I}-\mathrm{ICdR}$ could be prepared by using a simple synthetic method with high radiochemical yield and purity, and further studies should clarify the usefulness of this compound as a tumor proliferation probe.

\section{$\mathbf{J 2 8 0}$}

\section{A NON-INVASIVE, TRANSLATIONAL BIOMARKER OF KETAMINE-INDUCED NEURONAL DEATH IN RATS: MICROPET IMAGING USING [18F]-ANNEXIN-V}

Xuan Zhang ${ }^{1}$, Merle G. Paule ${ }^{1}$, Glenn Newport ${ }^{1}$, Xiaoju Zou ${ }^{1}$, Natalya V. Sadovova ${ }^{2}$, Marc Berridge ${ }^{3,4}$, Scott Apana ${ }^{3}$, Joseph P. Hanig ${ }^{5}$, William Slikker ${ }^{1}$, Cheng Wang ${ }^{1}$

${ }^{1}$ National Center for Toxicological Research, US Food and Drug Administration, Jefferson, AR, USA, ${ }^{2}$ Toxicologic Pathology Associates, Jefferson, AR, USA, ${ }^{3}$ 3D Imaging, LLC, Little Rock, AR, USA, ${ }^{4}$ Radiology, University of Arkansas for Medical Sciences, Little Rock, AR, USA, ${ }^{5}$ Center for Drug Evaluation and Research, US Food and Drug Administration, Silver Spring, MD, USA.

Ketamine is a dissociative anesthetic that is primarily used for the induction and maintenance of general anesthesia. It has been reported that suppression of NMDA receptor function by ketamine may trigger apoptosis of neurons and cause neurotoxicity in neonatal rats when given repeatedly during the brain growth spurt period. Noninvasive and quantitative imaging of neuronal activity in the brains of rodents may, allow for detection of toxic alterations induced by ketamine. In all apoptotic neurons, phosphatidylserine (PS) is rapidly redistributed from the inner to the outer surface of the plasma membrane and can be selectively recognized and bound by annexin V. Because the small animal high-resolution positron emission tomography microPET scanner can provide in vivo molecular imaging at sufficient resolution to resolve neuronal activity in the rat brain, it has been proposed as a minimally invasive method for detecting apoptosis using the tracer [18F]-labeled annexin V. In this study, the effect of ketamine on the metabolism and integrity of the rat brain were evaluated by investigating the uptake and retention of [18F] fluorodeoxyglucose (FDG) and $[18 \mathrm{~F}]$-annexin $\mathrm{V}$ using microPET imaging. On PND 7, rat pups in the experimental group were exposed to 6 injections of ketamine $(20 \mathrm{mg} / \mathrm{kg}$ at $2 \mathrm{~h}$ intervals) and control rat pups received 6 injections of saline. On PND 35, 1 mci of [18F] fluorodeoxyglucose (FDG) or [18F]-annexin V was injected into the tail vein of treated and control rats and static microPET images were obtained over $1 \mathrm{~h}$ (FDG) and $2 \mathrm{~h}$ (Annexin V) following the injection. Radiolabeled tracer accumulation in the region of interest (ROI) which include of the frontal cortex and lateral ventricle areas was converted into Standard Uptake Values (SUVs). After the injection, radiotracer quickly distributed into the brains of both ketamine- and saline-treated rats. Compared with the control group, no significant difference was found in [18F] FDG uptake in the ROI in the brains of ketamine-treated rats compared with saline-treated controls. The uptake of $[18 \mathrm{~F}]$-annexin $\mathrm{V}$, however, was significantly increased in the ROI of ketamine-treated rats. Additionally, the duration of annexin $\mathrm{V}$ tracer wash-out was prolonged in the ketamine-treated animals. These results demonstrate that microPET imaging is capable of distinguishing differences in retention of [18F]-annexin $\mathrm{V}$ in different brain regions and suggests that this approach may provide a minimally invasive biomarker of neuronal apoptosis in rats. 
J281

CLINICAL-GRADE $\left[{ }^{18}\right.$ F $_{\text {FPPRGD }}$ : AN AUTOMATED MULTI-STEP RADIOSYNTHESIS FOR HUMAN PET STUDIES Frederick T. Chin, Bin Shen, Shuanglong S. Liu, Rhona A. Berganos, Edwin Chang, Erik Mittra, Xiaoyuan Chen, Sanjiv S. Gambhir Department of Radiology, Stanford University, Stanford, CA, USA.

Introduction. In positron emission tomography (PET) imaging, radiolabeled RGD-peptides are one of the most promising radiotracers for noninvasive imaging of $\alpha_{\mathrm{v}} \beta_{3}$ expression for potential applications in tumor staging, monitoring response to therapy, and characterizing metastatic potential. Here we report a reliable, routine and automated radiosynthesis of a new clinical-grade ${ }^{18} \mathrm{~F}$-RGD-peptide tracer $\left(\left[{ }^{18} \mathrm{~F}\right] \mathrm{FPPRGD} \mathrm{D}_{2}\right)$ which is formed by conjugating 4-nitrophenyl-2-[ $\left[{ }^{18} \mathrm{~F}\right]$ fluoropropionate $\left(\left[{ }^{18} \mathrm{~F}\right] \mathrm{NPE}\right)$ as a prosthetic group with the $\mathrm{PEG}_{3}-\mathrm{E}[\mathrm{c}(\mathrm{RGDyK})]_{2}$ peptide and was evaluated both in mouse and human. Experimental. Optimized radiochemistry was performed in a GE TRACERLab $\mathrm{FX}_{\mathrm{FN}}$ synthesis module with a customized module $(\mathrm{CM})$. $\left[{ }^{18} \mathrm{~F}\right] \mathrm{NPE}$ was prepared [1] and purified by prep-HPLC. $\left[{ }^{18} \mathrm{~F}\right] \mathrm{NPE}$ fraction was transferred into the $\mathrm{CM}$ and processed with a $\mathrm{C}-18$ SPE cartridge. Trapped $\left[{ }^{18} \mathrm{~F}\right] \mathrm{NPE}$ was eluted with $\mathrm{Et}_{2} \mathrm{O}$ and passed through a $\mathrm{Na}_{2} \mathrm{SO}_{4}$ drying cartridge. $\mathrm{Et}_{2} \mathrm{O}$ was removed by He stream and then a peptide solution $(2 \mathrm{mg} / 200 \mu \mathrm{L}$ $\mathrm{DMSO} / 40 \mu \mathrm{L}$ DIPEA) was added to the vial containing dried $\left[{ }^{18} \mathrm{~F}\right]$ NPE. Peptide mixture was heated at $60^{\circ} \mathrm{C}$ for $20 \mathrm{~min}$, acidified, and then purified by prep-HPLC. Formulated $\left[{ }^{18} \mathrm{~F}\right] \mathrm{FPPRGD} \mathrm{P}_{2}$ was sterilefiltered and then analyzed for quality control. Imaging with clinicalgrade $\left[{ }^{18} \mathrm{~F}\right] \mathrm{FPPRGD} \mathrm{D}_{2}$ in mouse and human were completed by microPET and human PET scanners. Results and Discussion. All radiochemical yields $(\mathrm{RCY} \pm \mathrm{SD})$ are decay-corrected to end-ofbombardment. After $170 \mathrm{~min}$ radiosynthesis, $\left[{ }^{18} \mathrm{~F}\right] \mathrm{FPPRGD}_{2}$ afforded consistent RCYs $(14.4 \pm 2.8 \% ; n=5)$ with specific radioactivity of 900 $\pm 257 \mathrm{mCi} / \mu \mathrm{mol}(33.3 \pm 9.5 \mathrm{GBq} / \mu \mathrm{mol})$. High radiochemical and chemical purities exceeded $99 \%$ via HPLC analysis. As a better alternative to traditional rota-evaporation alone $(9.9 \pm 2.8 \%, n=3)$, more $\left[{ }^{18} \mathrm{~F}\right] \mathrm{NPE}$ is available for peptide coupling when the SPE process uses $\mathrm{Et}_{2} \mathrm{O}$ and a $\mathrm{Na}_{2} \mathrm{SO}_{4}$ cartridge $(91.8 \pm 2.6 \%, n=9)$ followed by gentle evaporation of $\mathrm{Et}_{2} \mathrm{O}$ with a $\mathrm{He}$ stream in the CM. The $\mathrm{Na}_{2} \mathrm{SO}_{4}$ cartridge gave higher coupling yields $(79.3 \pm 8.5 \%, n=9$ vs. without cartridge $30 \pm 35 \%, n=3$ ) since residual moisture was removed after SPE processing of $\left[{ }^{18} \mathrm{~F}\right]$ NPE. PET imaging in mouse (Normal/Tumorxenografted*: bladder $>>$ kidneys $>$ gut/liver $>>$ tumor*/brain/feces) and initial biodistribution studies in human have been done. Conclusion. A multi-step radiosynthesis of clinical-grade $\left[{ }^{18} \mathrm{~F}\right] \mathrm{FPPRGD}_{2}$ was implemented in a commercial automated synthesis module with a $\mathrm{CM}$ to provide the tracer routinely for animal/clinical PET studies. Reference. [1] Guhlke, S. et. al. (1994) Appl. Radiat. Isot. 45, 715-727.

\section{$\mathbf{J} 282$}

\section{SSR180575, A CARBON-11 LABELED RADIOLIGAND FOR PET-IMAGING THE PERIPHERAL BENZODIAZEPINE RECEPTORS}

Frederic Dolle $^{1}$, Raphael Boisgard ${ }^{1,2}$, Fabien Chauveau ${ }^{1,2}$, Herv Boutin ${ }^{1,2}$, Sabine Boisnard ${ }^{3}$, Thomas Rooney ${ }^{3}$, Jesus Benavides ${ }^{3}$, Philippe Hantraye ${ }^{4}$, Bertrand Tavitian ${ }^{1,2}$

${ }^{1}$ DSV, I2BM, SHFJ, CEA, Orsay, France, ${ }^{2}$ INSERM U803, CEA, Orsay, France, ${ }^{3} \mathrm{CNS}$ Department, Sanofi-Aventis, Vitry-sur-Seine, France, ${ }^{4}$ I2BM, MIRCen, CEA, Fontenay aux Roses, France.
Introduction: Microglia activation is considered as the predominant cellular response to inflammation within the CNS, a process characterized by a drastic change in the morphology of these cells and by the notable overexpression of the peripheral benzodiazepine receptor (PBR or TSPO $18 \mathrm{kDa}$ ). Since over two decades, these binding sites are clearly recognised as early markers of neuroinflammation, supporting extensive efforts into the design of radiolabeled ligands for PET imaging. Today, [11C]PK11195 is still considered as the compound of reference, but several new structures, all belonging to other chemical classes (e.g. [18F]FEDAA1106, [11C]PBR$28,[18 \mathrm{~F}] \mathrm{DPA}-714$ and [11C]DPA-713) are already proposed as promising alternative ligands. Another attractive chemical class of structures, truly underinvestigated to date, are the indoleacetamides. Within this class, we have labeled SSR180575 with carbon-11, and pharmacologically evaluated it in a rat model of neuroinflammation (unilaterally, AMPA-induced, striatum-lesioned rats) with PET. Methods: Carbon-11 labeling of SSR180575 comprises : (1) trapping at $-10^{\circ} \mathrm{C}$ of $[11 \mathrm{C}] \mathrm{MeOTf}$ in DMF $(0.3 \mathrm{~mL})$ containing $0.2-0.3 \mathrm{mg}$ of the indole precursor for labeling and $4 \mathrm{mg}$ of $\mathrm{K} 2 \mathrm{CO} 3$ (excess) ; (2) heating at $120^{\circ} \mathrm{C}$ for $3 \mathrm{~min}$; (3) dilution of the residue with $0.5 \mathrm{~mL}$ of the HPLC mobile phase and (4) purification using semipreparative reversed-phase HPLC (Zorbax ${ }^{\circledR}$ SB-C-18). PET-imaging (Focus 220 Concorde) includes control kinetics and displacement experiments with PK11195 and SSR180575 (1 mg/kg). Results: Starting from a $55 \mathrm{GBq}$ cyclotron-produced [11C]carbon dioxide batch, 4.5-5.0 GBq of [11C]SSR 180575, >99\% radiochemically pure and ready-to-inject, were obtained within $25 \mathrm{~min}$. Specific radioactivities ranged from 50 to $90 \mathrm{GBq} / \mu \mathrm{mol}$. In PET experiments, [11C]SSR180575 showed a higher contrast between the lesioned area and the corresponding area in the intact contralateral hemisphere when compared to [11C]PK11195 (ratio ipsi/contra at $20 \mathrm{~min}$ post-injection: [11C]SSR180575:2.7, $n=4$; [11C]PK11195, 1.7, $n=5$ ). Furthermore, [11C]SSR180575 was displaced by non labeled PK11195 or SSR180575. Immunohistochemical analyses correlate with PET-imaging and showed strong activation of microglia in and around the lesion. Conclusions: The decay-corrected overall yields for the preparation of [11C] SSR180575 were 19.1-21.2\% $(n=10)$. Dynamic $\mu$ PET studies in rats demonstrate the potential of [11C]SSR180575 to image neuroinflammation.

\section{Ultrasound Probes}

\section{$\mathbf{J 2 8 3}$}

\section{DESIGN AND TESTING OF A NOVEL ULTRASOUND CONTRAST AGENT FOR MOLECULAR ULTRASOUND IMAGING OF TUMOR ANGIOGENESIS \\ Juergen K. Willmann $^{1}$, Richard Kimura ${ }^{1,2}$, Nirupama Deshpande ${ }^{1}$, Amelie M. Lutz ${ }^{1}$, Jennifer R. Cochran ${ }^{2}$, Sanjiv S. Gambhir ${ }^{1,2}$ \\ ${ }^{1}$ Radiology, Stanford University, Stanford, CA, USA, ${ }^{2}$ Bioengineering, Stanford University, Stanford, CA, USA.}

Molecular ultrasound imaging is increasingly being recognized as a promising and powerful molecular imaging tool. In this proof-ofprinciple study we hypothesized that a new class of molecular ultrasound contrast agents could be developed using small ( $\sim 3 \mathrm{kDa})$, conformationally constrained integrin-binding peptides that are coupled onto the surface of contrast microbubbles. Directed evolution was used to engineer a small, disulfide-constrained cystine knot peptide (knottin) to bind to $\alpha v \beta 3$-integrins with nanomolar affinity (knottinIntegrin). A molecular ultrasound imaging contrast agent was designed by attaching 
the knottinIntegrin onto the shell of perfluorocarbon-filled microbubbles (MB-Knottinintegrin). A knottin peptide with a scrambled sequence was used to create control microbubbles (MB-KnottinScrambled). Binding of MB-Knottinintegrin and MB-KnottinScrambled to $\alpha v \beta 3$-integrin-positive cells and control cells was assessed and compared to microbubbles labeled with anti- $\alpha v \beta 3$-integrin monoclonal antibodies $(\mathrm{MB} \alpha \mathrm{v} \beta 3)$ and microbubbles labeled with $\mathrm{c}$ (RGDfK) (MBcRGD). In vivo imaging signal of contrast-enhanced ultrasound using the different types of microbubbles was quantified in 49 mice bearing human ovarian adenocarcinoma xenograft tumors. Tumor tissue was stained for $\alpha v \beta 3$ integrin and CD31, a vascular endothelial cell marker. MB-KnottinIntegrin attached significantly more $(P<.0001)$ to $\alpha v \beta 3$-integrin-positive cells $(0.47 \pm 0.21$ microbubbles per cell) than to control cells $(0.02 \pm 0.02)$. Control MBKnottinScrambled adhered less to $\alpha v \beta 3$-integrin-positive cells $(0.17 \pm$ 0.12) than MB-KnottinIntegrin. After blocking of integrins, attachment of MB-KnottinIntegrin to $\alpha v \beta 3$-integrin-positive cells significantly decreased $(P<.04)$. In vivo ultrasound imaging signal was higher following administration of MB-KnottinIntegrin compared to MB $\alpha \mathrm{v} \beta 3$, MBcRGD, and control MB-KnottinScrambled $(P<.018)$. Following in vivo blocking of integrin receptors, the imaging signal after administration of MB-KnottinIntegrin significantly $(P<.018)$ dropped by $64 \%$. Ex vivo immunofluorescence confirmed integrin expression on tumor endothelial cells of human ovarian adenocarcinoma xenograft tumors. In summary, our study shows that knottin peptides are a promising platform for designing novel contrast agents for molecular ultrasound imaging of tumor angiogenesis. The design of small, evolvable peptide ligands that can be coupled with the surface of microbubbles may facilitate the translation of molecular ultrasound from preclinical animal models to clinical applications in patients in the near future.

\section{A NOVEL SMART AGENT FOR PHOTOACOUSTIC MOLECULAR IMAGING}

Keith B. Hartman, Anca Dragulescu-Andrasi, Jelena Levi, Adam de la Zerda, Sanjiv S. Gambhir

Molecular Imaging Program (MIPS), Stanford University, Stanford, CA, USA.

Photoacoustic imaging is an exciting new modality that offers higher spatial resolution and deeper penetration than traditional optical imaging. We have designed an activatable (smart) photoacoustic molecular imaging agent which can be activated by matrix metalloprotease-2 (MMP-2), a secreted enzyme commonly present in many cancers. The smart probe developed couples a fluorescent dye (cy5.5) to a photoacoustic dye via an enzyme-recognized peptide sequence (RVGLP). In close spatial proximity, the fluorescent dye silences the photoacoustic dye because of its significantly greater coefficient of absorption (approximately 3-6-fold greater). A scrambled control peptide sequence (PGLRV) probe was also synthesized. Upon cleavage of the enzyme-specific peptide sequence, diffusion of the photoacoustic dye away from the fluorescent dye allows it to produce a greater photoacoustic signal. The activated and control probes were each incubated with either the activated enzyme (recombinant human MMP$\left.2,37^{\circ} \mathrm{C}\right)$ or the non-activated enzyme $\left(0^{\circ} \mathrm{C}\right)$ for 1 hour prior to suspension in an agar phantom. The samples were at a concentration of approximately $100 \mu \mathrm{M}$ and imaged in triplicate. The measured photoacoustic signals for the agar alone, unactivated probe, and activated smart probe were 232 relative units (RU) +/- 35 RU, $2751 \mathrm{RU}+/-306 \mathrm{RU}$, and $3529 \mathrm{RU}+/-255 \mathrm{RU}$, respectively. All combinations of data comparisons are statistically significant $(p<0.05)$. The scrambled control probe did not lead to any detectable cleavage.Mouse studies with the activatable probe show a statistically significant $(p<0.05)$ signal enhancement of $256,260 \mathrm{RU}+/-105,802 \mathrm{RU}$ as compared to normal regions of the mouse where the signal is $802 \mathrm{RU}+/-101 \mathrm{RU}$. These experiments validate the first photoacoustic smart probe and the strategies developed can be used to synthesize other smart probes after further validation in various mouse models. Figure: Shown is a sagittal mouse photoacoustic image (color scale) with three subcutaneous sites (arrows) of activated smart probe superimposed on the standard ultrasound image (greyscale). The color scale bar is in relative units. Note the very high $(\sim 300$-fold $)$ signal to background.

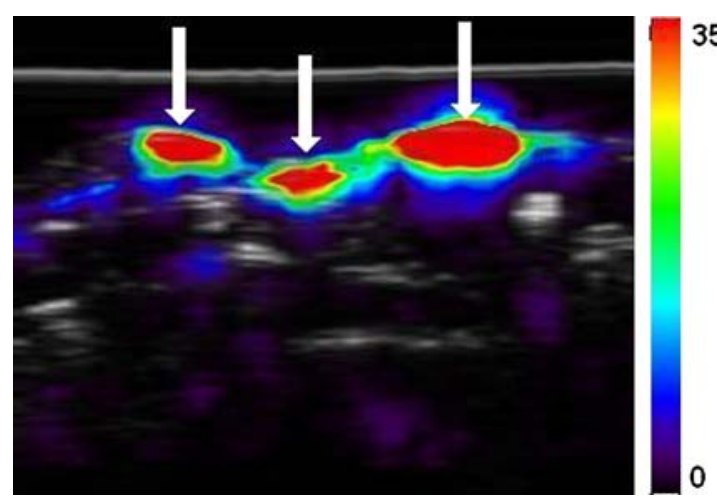

$350,000 \mathrm{RU}$

\section{$\mathbf{J} 285$}

\section{HIGH-FREQUENCY IN VIVO ULTRASOUND IMAGING OF SIZE-ISOLATED MICROBUBBLES}

Shashank R. Sirsi ${ }^{1}$, Jameel Feshitan ${ }^{1}$, Shunichi Homma ${ }^{2}$, Mark A. Borden ${ }^{1}$

${ }^{1}$ Chemical Engineering, Columbia University, New York City, NY, USA, ${ }^{2}$ Cardiology, Columbia University, New York City, NY, USA.

High-frequency ultrasound imaging using microbubble contrast agents is becoming increasingly popular for small animal research. Previous in vivo studies have relied on highly polydisperse microbubble suspensions, which may provide a complex and varied acoustic response. In order to investigate the effect of size on contrast enhancement, lipid-coated microbubbles of 1-2, 4-5, and 6-8 $\mu \mathrm{m}$ diameter were isolated using a differential centrifugation technique previously developed in our lab [Feshitan et al., J. Colloid Interface Sci., 2009]. Size-isolated and polydisperse microbubbles were imaged in mice over a range of concentrations using a Visualsonics Vevo 770 scanner with a $40-\mathrm{MHz}$ probe. Contrast 
enhancement was monitored continuously in the kidney of the mouse following a bolus injection of the microbubble suspension. Time-intensity data was fit to a single-compartment pharmacokinetic model, and contrast enhancement was measured as the area under the curve (AUC) and contrast persistence ( $\left.t^{*}\right)$. Our results demonstrate that contrast enhancement is a strong function of microbubble size and concentration. For $0.1-\mathrm{mL}$ bolus injections of $\sim 10^{\wedge} 8 \# / \mathrm{mL}$, the AUC increased over 4-fold for $6-8$ vs. 4-5 $\mu \mathrm{m}$ diameter microbubbles. Surprisingly, we found that 1-2 $\mu \mathrm{m}$ diameter microbubbles did not enhance the signal at any concentration, and in fact attenuated the signal at higher concentrations $\left(\sim 10^{\wedge} 9 \# / \mathrm{ml}\right)$. At lower microbubble concentrations, $\left(\sim 10^{\wedge} 7 \# / \mathrm{ml}\right)$ only 6-8 $\mu \mathrm{m}$ diameter microbubbles showed contrast enhancement, whereas $4-5 \mu \mathrm{m}$ and $1-2 \mu \mathrm{m}$ diameter microbubbles produced no increase in grayscale signal intensity. Contrast persistence was also found to increase with microbubble size and concentration. For example, $t *$ increased from $120 \pm 26 \mathrm{sec}$ to $442 \pm 102 \mathrm{sec}$ for $4-5 \mu \mathrm{m}$ vs. $6-8 \mu \mathrm{m}$ microbubbles $\left(10^{\wedge} 8 \# / \mathrm{mL}\right)$. For high-frequency imaging in mice, we conclude that large microbubbles strongly enhance contrast, while small microbubbles mainly attenuate. The results suggest that larger microbubbles can enhance ultrasound images at much lower concentrations without sacrificing persistence, which may be important for applications in molecular imaging and drug delivery.

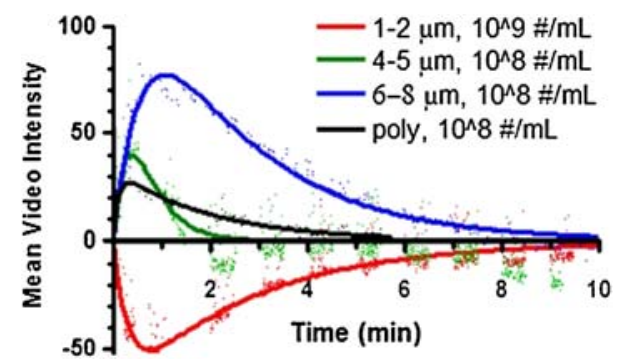

$\mathbf{J} 286$

\section{ULTRASOUND MOLECULAR IMAGING OF TUMOR ANGIOGENESIS WITH A NOVEL $\alpha$ VB3-INTEGRIN TARGETED MICROBUBBLE CONTRAST AGENT} Joshua Rychak ${ }^{1}$, Alexander L. Klibanov ${ }^{2}$, Jose L. Tlaxca ${ }^{3}$, Michael B. Lawrence ${ }^{3}$, Christopher R. Anderson ${ }^{1}$

${ }^{1}$ Targeson LLC, Charlottesville, VA, USA, ${ }^{2}$ Cardiology, University of Virginia, Charlottesville, VA, USA, ${ }^{3}$ Biomedical Engineering, University of Virginia, Charlottesville, VA, USA.

Ultrasound molecular imaging is an emerging technique for sensitive detection of intravascular targets. Molecular imaging of angiogenesis has strong potential for both clinical use and as a research tool in tumor biology and the development of antiangiogenic therapies. We have developed a robust microbubble (MB) ultrasound contrast agent platform to which targeting ligands can be conjugated. Microbubbles are synthesized with PDP reactive groups on their surface, which enables covalent conjugation of thiol-bearing targeting ligands by disulfide exchange. Here, we utilized a cyclic RGD (cRGD) pentapeptide containing a cysteine group for targeting $\alpha \mathrm{V} \beta 3$ integrins, which are overexpressed on the angiogenic endothelium. To enable detection of peptides bound to MB surfaces, the cRGD peptide was labeled with FITC and reacted with PDP-MB. The fluorescence of covalently bound dye was detected by flow cytometry and fluorescence microscopy. Additionally, the FITC-cRGD load on MB surfaces was quantified by plate fluorometry $(\sim 500,000$ molecules/MB). Targeted adhesion of cRGD-MB was demonstrated in an in vitro flow adhesion assay with recombinant $\alpha \mathrm{V} \beta 3$ integrin substrates and $\alpha \mathrm{V} \beta 3$ integrin expressing endothelial cells (bEnd.3). At 1 dynes/cm2, cRGD-MB exhibited 5-fold higher adhesion to immobilized recombinant $\alpha \mathrm{V} \beta 3$ integrin than nontargeted control MB. Similarly, cRGD-MB showed significantly greater adhesion to bEnd.3 EC compared to non-targeted control MB. The specificity of cRGD-MB for $\alpha \mathrm{V} \beta 3$ integrin was shown by treating bEnd.3 EC with an anti- $\alpha \mathrm{V}$ monoclonal antibody, which reduced adhesion of cRGD-MB by $80 \%$. A murine model of prostate cancer was used to assess targeted adhesion in vivo, and cRGD-MB, but not control MB, showed a strong signal in tumors by contrast ultrasound imaging. These results demonstrate the functionality of a novel microbubble contrast agent covalently coupled to an RGD peptide for ultrasound molecular imaging of $\alpha \mathrm{V} \beta 3$ integrins. This contrast agent is expected to be a useful tool in basic science and drug discovery research. Supported by NIH 1R43CA137913.

$\mathbf{J} 287$

\section{ULTRASOUND RADIATION FORCE APPLICATION TO KDR-TARGETED CONTRAST AGENT IN TUMOR ANGIOGENESIS MODEL}

Isabelle Tardy, Peter Frinking, Martine Theraulaz, Xavier Fouillet, Sibylle Pochon, Marcel Arditi, Francois Tranquart, Michel Schneider Bracco Research SA, Geneva, Switzerland.

Ultrasound molecular imaging using targeted microbubbles is an active and rapidly expanding area of research that provides a noninvasive means for visualising events occurring at a molecular level. For example, it allows highlighting the in vivo expression of angiogenic markers such as vascular endothelial growth factor receptor 2 (VEGFR2), one of the main regulators of angiogenesis. To improve binding of targeted microbubbles, radiation force has been suggested to push targeted microbubbles from the luminal space towards the endothelium of the vessel wall. In this work, the effect of the radiation force on targeted microbubble binding was investigated in a rat tumor model. Prostate adenocarcinoma was grown in Copenhagen rats by orthotopic injection of the $G$ Dunning R-3327 tumor cells in one lobe of the ventral prostate. BR55 microbubbles, a phospholipid-based contrast agent functionalized with a heterodimeric peptide specific for VEGFR2 (KDR/ Flk-1) was evaluated in the tumor and healthy tissue of the prostate. The expression of VEGFR2 on the neovessels was confirmed by immunohistology on tumor cryosections. Radiation 
force pulses (3.5 MHz, $38 \mathrm{kPa}, 98 \%$ duty cycle) were applied to the microbubbles in the prostate (approx. $2 \mathrm{~cm}$ in width) during 2 min following microbubble injection using a linear array probe (L9-3) connected to a Philips iU22 ultrasound scanner. Microbubble binding was determined by imaging targeted microbubbles using Power Modulation (L9-3 probe, 4.0 MHz, $180 \mathrm{kPa}$ ) from 2 to $10 \mathrm{~min}$ after agent injection. Different agent concentrations $(0.2-$ $0.8 \mu \mathrm{L}$ gas $/ \mathrm{kg}$ ) were used. As a reference, microbubble binding without radiation force at the highest concentration $(0.8 \mu \mathrm{L}$ gas $/ \mathrm{kg})$ was determined. Compared to the reference, radiation force improved bubble binding efficiency in tumor tissue by a factor of 3-10, depending on agent concentration. At the highest concentration, increased binding by a factor of 3 was also obtained in healthy tissue. However, this increase in nonspecific binding was not observed at lower concentrations. For an optimal concentration $(0.4 \mu \mathrm{L}$ gas $/ \mathrm{kg})$, the net effect of radiation force resulted in a 3-4 fold improvement in binding specificity compared to the reference. The absence of any endothelium injury induced by radiation force in the tumor and adjacent tissue was confirmed by histopathology. Radiation force was shown to improve the binding efficiency and specificity of targeted microbubbles in pathological tissue and to allow the use of a reduced concentration of contrast agent.

$\mathbf{J} 288$

\section{DEVELOPING SMART CONTRAST AGENTS FOR US IMAGING USING ENZYME-SPECIFIC AGGREGATION OF TARGETED MICROBUBBLES}

Mohammad Eghtedari, Robert F. Mattrey

Department of Radiology, University of California in San Diego, San Diego, CA, USA.

Background: A major goal of molecular imaging is the detection of enzymes in vivo. Our project aims to develop smart ultrasound (US) contrast agents for this purpose. US is a promising molecular imaging tool that has high sensitivity to microbubbles as well as high spatial and temporal resolution. Since backscatter is related to the radius of particles raised to the sixth power, we hypothesized that if enzymes can induce aggregations, the aggregate will be detectable. The proposed system consists of two populations of particles each carrying one pair of an adhesion molecule pair, such as avidin/biotin, one of which is blocked by an enzyme specific cleavable molecule. Once the blocking molecule is cleaved, aggregation will occur. Methods: We chose perfluoroctyl-bromide (PFOB) emulsion since it is invisible until aggregation occurs, and avidin/biotion as well as polyacrylic acid (PAA) as aggregating agents as proof of concept. We produced a positively charged and biotin-labeled emulsion of PFOB $(\sim 150 \mathrm{~nm})$. We assessed particle size distribution (Malvern Mastersizer), degree of particle precipitation (observation), backscatter (Panametrics pulser/receiver 5300, V306 transducer at $2.25 \mathrm{MHz}$ ), and echogenicity (US imaging) of suspensions containing PFOB emulsion alone, avidin alone, PAA alone, PFOB emulsion + free biotin + avidin, PFOB emulsion + graded concentrations of avidin, and PFOB emulsion + graded concentrations of PAA. Results: Particle size increased in the presence of avidin or PAA, while PFOB alone generated minimal backscatter and was not visible on imaging, backscatter increased $>20 \mathrm{~dB}$ and the suspension became echogenic in the presence of avidin or PAA. No change in echogenicity was observed when free biotin was added. Conclusion: This experiment confirms that controlled aggregation induces signal. Our research is now focused on producing an enzyme controlled process. Study supported by the ICMIC P50 CA128346 and the Roadmap R21 EB005360.

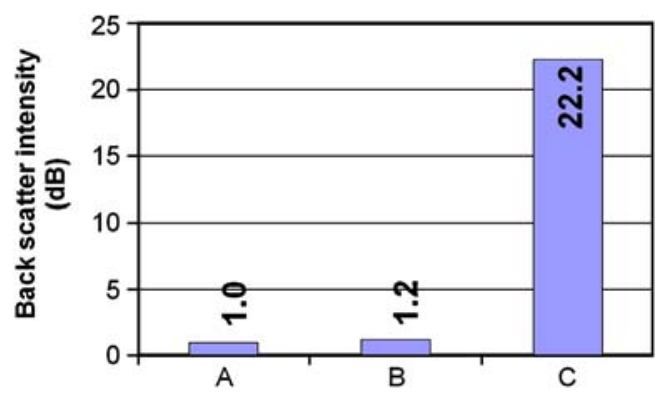

Ultrasound backscatter intensity of water (A), PFOB micelles before aggregation (B), and PFOB micelles after adding PAA that causes aggregation (C). Aggregation enhanced backscatter signal $>20 \mathrm{~dB}$.

J289

\section{MAXIMUM INTENSITY PERSISTENCE ANALYSIS IS A SENSITIVE AND RELIABLE TOOL TO QUANTITATE TUMOR ANGIOGENESIS WITH ULTRASOUND IMAGING}

Marybeth A. Pysz, Cedric Panje, David S. Wang, Nirupama Deshpande, Juergen K. Willmann

MIPS, Radiology, Stanford University, Stanford, CA, USA.

The purpose of our study was to compare two post-processing techniques, maximum intensity persistence (MIP) technique versus a traditional time-intensity curve analysis, for quantitation of high-resolution (40 MHz) ultrasound (US) imaging intensities of microbubbles (MBs) in a subcutaneous angiosarcoma tumor xenografts in mice $(n=6)$. Contrast MBs were injected $(100 \mu \mathrm{l})$ intravenously using an infusion syringe pump, and varied by concentration and infusion rate as follows: $5 \mathrm{E} 5 \mathrm{MBs}$ (injected at $1.2 \mathrm{ml} / \mathrm{min}$ ), 5E6 (at $1.2 \mathrm{ml} / \mathrm{min}$ ), 5E7 (at $1.2 \mathrm{ml} / \mathrm{min}$, repeated twice), 5E8 (at $1.2 \mathrm{ml} / \mathrm{min}$ ), 5E7 (at $0.6 \mathrm{ml} / \mathrm{min}$ ), and $5 \mathrm{E} 7 \mathrm{MBs}$ (injected at $2.4 \mathrm{ml} / \mathrm{min}$ ). Regions of interest (ROIs) were drawn over the tumors, and images were processed with MIP persistence (expressed as \% vascular tumor area), or no persistence (traditional time-intensity curve analyses, expressed as upslope, timeto-peak, and peak enhancement). After imaging, tumors were excised, immunostained for CD31 expression (a marker for tumor endothelial cells), and analyzed for microvessel density (MVD). Both MIP and peak enhancement values increased with increasing MB concentrations $(P<0.004)$. MIP analysis was more sensitive 
in detecting tumor angiogenesis at low MB concentrations (5E5 and 5E6) than all three parameters from the time-intensity analysis (Figure). At two repeated injections of $5 \mathrm{E} 7 \mathrm{MBs}$, absolute values of both MIP and peak enhancement were not significantly different $(P>.05)$. MIP $(P=0.78)$ and peak enhancement $(P=0.55)$ were not affected by variation of infusion rates $(0.6 \mathrm{ml} / \mathrm{min}, 1.2 \mathrm{ml} / \mathrm{min}$, and $2.4 \mathrm{ml} / \mathrm{min})$, but different flow rates significantly $(P<0.001)$ affected both time to peak and the upslope values of the traditional time-intensity curve analysis. Ex vivo MVD highly correlated $\left(\mathrm{R}^{\wedge} 2>0.9\right)$ with both in vivo MIP and peak enhancement values. Our results suggest that MIP is a straight forward post-processing approach that allows a reliable and accurate assessment of tumor angiogenesis in vivo. Since MIP can be applied during real-time ultrasound imaging, it may be used as a promising bedside tool for tumor angiogenesis quantification in future clinical trials in cancer patients.

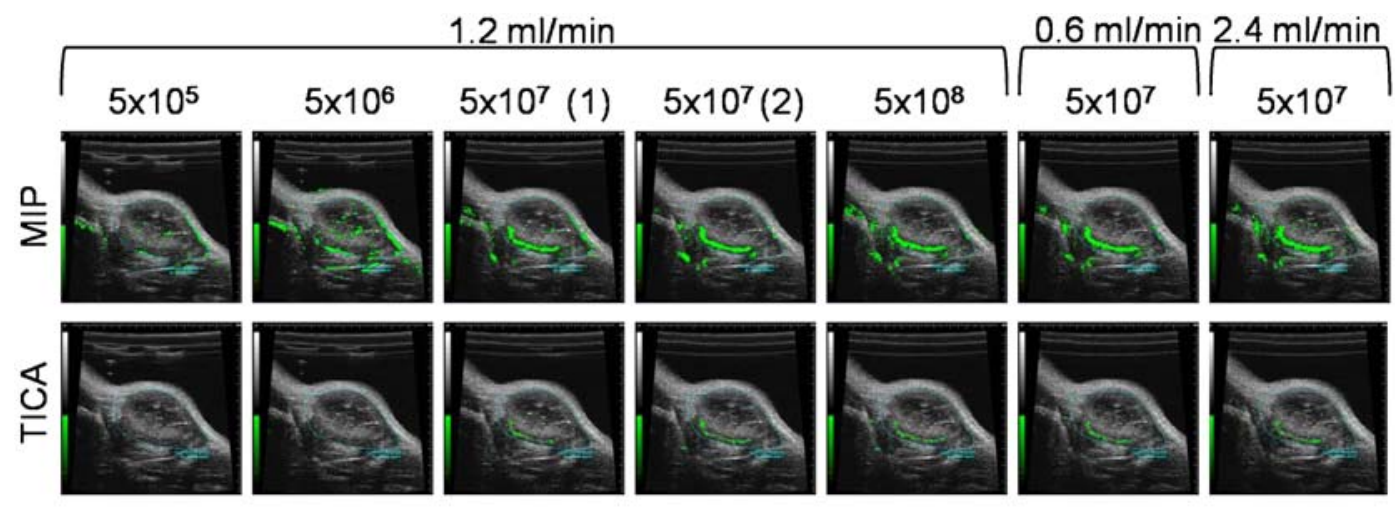

Figure: US image intensities (shown by green signal overlay) increase with increasing MB concentration and are not changed by infusion rate when images are processed using MIP persistence, or time-intensity curve analysis (TICA). Notably, MIP-processed signals are more sensitive than TICA-processed signals at lower concentrations of contrast agent.

$\mathbf{J 2 9 0}$

PRE-CLINICAL EVALUATION OF NOVEL CLINICALLYTRANSLATABLE KDR-TARGETED MICROBUBBLES FOR MOLECULAR ULTRASOUND IMAGING OF ANGIOGENESIS IN CANCER

Marybeth A. Pysz ${ }^{1}$, Cedric Panje ${ }^{1}$, Sanjiv S. Gambhir ${ }^{1}$,

Michel Schneider $^{2}$, Juergen K. Willmann ${ }^{1}$

${ }^{1}$ MIPS, Radiology, Stanford University, Stanford, CA, USA, ${ }^{2}$ Bracco Research, Geneva, Switzerland.

The purpose of this study was to develop and test novel human vascular endothelial growth factor receptor type 2 (KDR)-targeted contrast microbubbles (KDR-MBs) using binding chemistry that allows a future use in patients. Previous studies have shown that molecular ultrasound using biotinylated anti-VEGFR2 antibodies conjugated to streptavidin-contrast MBs allows VEGFR2/KDR imaging at the molecular level in murine tumor xenografts. To develop clinically-translatable KDR-MBs, a peptide that binds to KDR with high affinity was isolated with phage display and coupled onto the surface of perfluorobutane-filled, lipid-shelled contrast MBs. First, binding specificity of KDR-MB compared to control MB was shown on KDR-expressing (PAE-KDR) and negative (PAE) cells under flow stress conditions $\left(100 \mathrm{~s}^{\wedge}(-1)\right)$ in a parallel plate flow chamber $(P<0.05)$. In vivo binding specificity of KDR-MB was then tested in human LS174T colon carcinoma tumor xenografts in mice $(n=6)$ using a high-resolution $(40 \mathrm{MHz})$ ultrasound system. In vivo imaging signal was significantly higher $(P<0.001)$ with KDR-MB compared with control MB, and significantly $(P<0.007)$ dropped by $60 \%$ following receptor blocking with an anti-KDR antibody, thus, confirming in vivo binding specificity of KDR-MBs for detection of angiogenic vessels (Figure). To test the use of KDR-MBs for monitoring anti-angiogenic therapy, mice with $(n=4)$ and without $(n=4)$ antiangiogenic treatment were imaged every $24 \mathrm{~h}$ for a total of 3 days. In vivo imaging signal was significantly $(P<0.03)$ reduced in anti-angiogenic treated tumor-bearing animals compared with nontreated animals. Ex vivo analysis confirmed both reduced microvessel density and KDR expression levels in treated versus non-treated tumors. Our results suggest that human KDR-targeted microbubbles can be developed that allow detection, quantification, and monitoring of KDR expression at the molecular level in vivo. Our study is a further step towards a future clinical translation of molecular ultrasound imaging in cancer patients. 

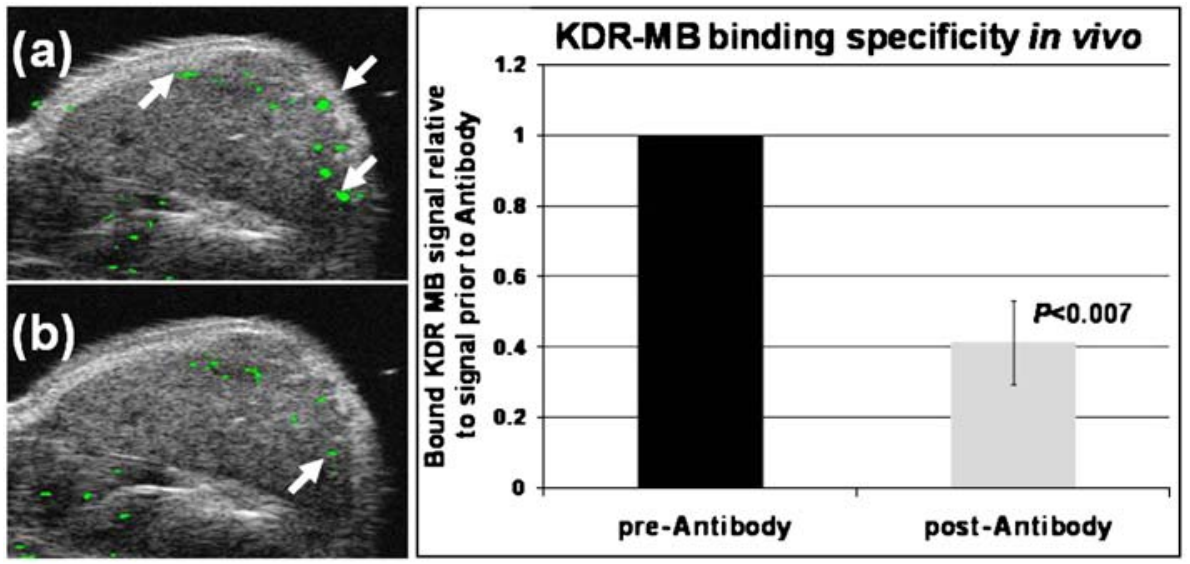

Figure: A) Clinically translatable human KDR-targeted MBs specifically bind to angiogenic vessels in tumorbearing mice (arrows point to green signal from bound microbubbles, overlaid on B-mode ultrasound image). B) Image signal intensity dropped significantly after in vivo blocking of receptors with antibodies. C) Bar chart summarizes in vivo blocking experiments for 3 tumorbearing mice.

\section{J291}

ENHANCED ULTRASOUND-MEDIATED GENE DELIVERY WITH CATIONIC MICROBUBBLES IN A MOUSE MODEL OF TUMOR ANGIOGENESIS

David S. Wang ${ }^{1}$, Marybeth A. Pysz ${ }^{1}$, Ramasamy Paulmurugan ${ }^{1}$,

Cedric Panje ${ }^{1}$, Sanjiv S. Gambhir ${ }^{1}$, Michel Schneider ${ }^{2}$,

Juergen K. Willmann ${ }^{1}$

${ }^{1}$ Department of Radiology, MIPS, Stanford University School of Medicine, Stanford, CA, USA, ${ }^{2}$ Bracco Research SA, Geneva, Switzerland.

$<\mathrm{p}>$ Sonoporation with ultrasound (US) and microbubbles (MBs) is a promising strategy for efficient gene therapy. While maintaining US contrast properties, MBs may be modified into vehicles for therapeutic delivery. This study sought to determine whether novel cationic MBs, which directly bind DNA through charge interactions, would enhance USmediated gene delivery (UMGD) in a mouse model of tumor angiogenesis. $<\mathrm{p}>$ By varying shell composition, cationic (zeta potential $+28 \mathrm{mV}$ ) and control neutral MBs were produced. Plasmids encoding for red shifted click beetle luciferase (CBluc) driven by a CMV promoter were charge coupled with cationic MBs and used for in vitro UMGD in SVR cells, an angiosarcoma-like cell line. Luciferase activity was evaluated in $24 \mathrm{hrs}$. To establish mouse models of tumor angiogenesis, SVR cells were implanted subcutaneously in nude mice hindlimbs. Regional in vivo UMGD was performed by insonation $\left(2 \mathrm{~W} / \mathrm{cm}^{2}, 50 \%\right.$ duty cycle, $\left.5 \mathrm{~min}\right)$ of tumor xenografts after tail vein administration of plasmid-bearing cationic MBs. CBluc expression was assessed 12, 24, 36, and $48 \mathrm{hrs}$ following UMGD. $<$ p $>$ SVR cells transfected by UMGD with cationic MBs expressed luciferase in vitro. As shown in the table and figure, in vivo CBluc activity of tumor-bearing hindlimbs was significantly increased at $12,24,36$, and $48 \mathrm{hrs}(P<0.05$, all time points) in mice who received US and cationic MBs $(N=7)$ relative to those who received US and neutral MBs $(N=4)$, US but no MBs $(N=4)$, and cationic MBs but no US $(N=4)$. In vivo expression was validated ex vivo. $<p>$ Cationic MBs enhance UMGD in a mouse model of tumor angiogenesis. These MBs may be further modified for molecular targeting, providing a promising theranostic approach for targeted gene delivery and imaging of tumor neovasculature.

\begin{tabular}{|c|c|c|c|c|}
\hline \multicolumn{5}{|c|}{ In vivo click beetle luciferase activity (mean max radiance \pm SE photons/sec/cm $2 / \mathrm{sr}$ ) } \\
\hline & 12 hours & 24 hours & 36 hours & 48 hours \\
\hline$<$ P ALIGN=LEFT $>$ A) Cationic MBs, with US $+</$ P $>$ & $8.1 \pm 1.5 \times 10^{4}$ & $7.4 \pm 1.3 \times 10^{4}$ & $9.3 \pm 2.0 \times 10^{4}$ & $5.9 \pm 0.8 \times 10^{4}$ \\
\hline$<$ P ALIGN=LEFT $>$ B) Neutral MBs, with US $</$ P $>$ & $2.5 \pm 0.5 \times 10^{4}$ & $3.0 \pm 1.1 \times 10^{4}$ & $2.8 \pm 0.6 \times 10^{4}$ & $2.5 \pm 0.5 \times 10^{4}$ \\
\hline$<$ P ALIGN=LEFT $>$ C) No MBs, with US $</$ P $>$ & $1.2 \pm 0.7 \times 10^{4}$ & $6.4 \pm 3.6 \times 10^{3}$ & $3.7 \pm 1.8 \times 10^{3}$ & $3.1 \pm 1.1 \times 10^{3}$ \\
\hline$<$ P ALIGN=LEFT>D) Cationic MBs, no US $</ P>$ & $9.8 \pm 1.6 \times 10^{2}$ & $6.5 \pm 1.5 \times 10^{2}$ & $1.1 \pm 0.5 \times 10^{3}$ & $1.3 \pm 0.4 \times 10^{3}$ \\
\hline
\end{tabular}



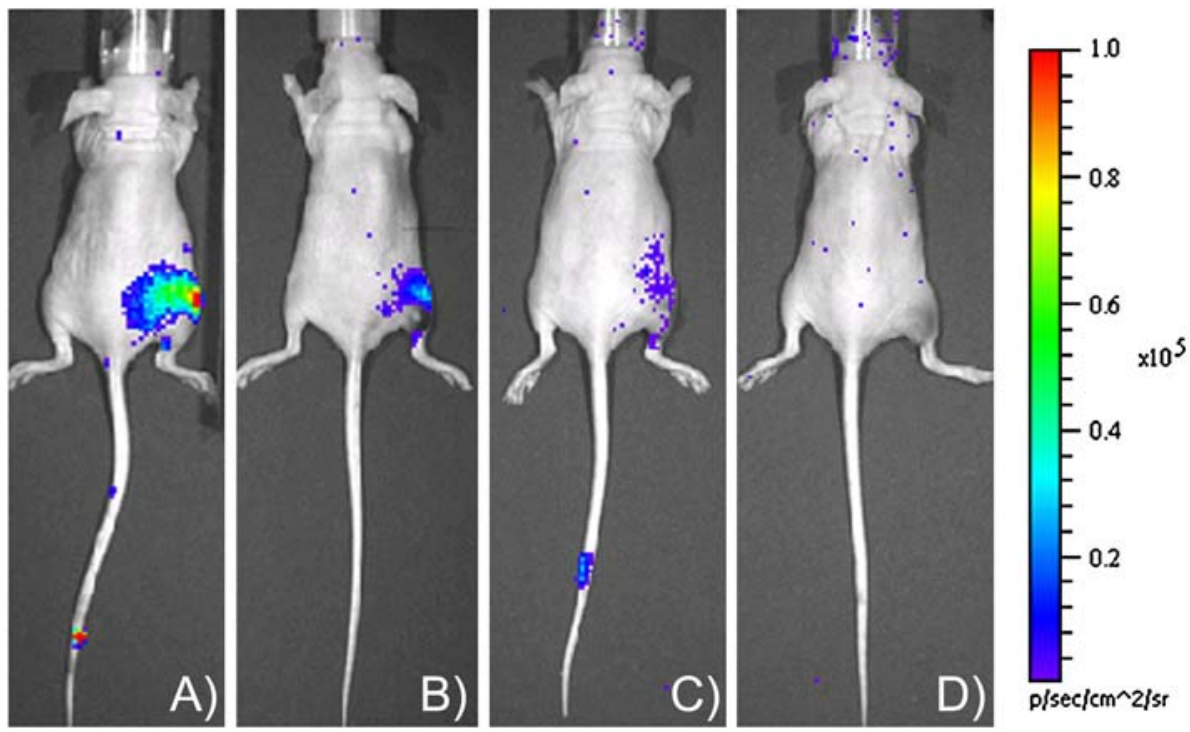

Representative images of CBluc expression in hindlimb tumor xenografts 36 hrs after UMGD using A) cationic, B) neutral, and C) no MBs. For negative control D), mice received cationic MBs but no US.

\section{$\mathbf{J 2 9 2}$}

\section{STIMULUS-RESPONSIVE CONTRAST AGENTS}

FOR SITE-SPECIFIC ULTRASOUND IMAGING

Andrew P. Goodwin ${ }^{1}$, Mark J. Hsu ${ }^{2}$, Mohammad Eghtedari ${ }^{3}$, Sadik C. Esener ${ }^{1,4}$, Robert F. Mattrey ${ }^{3,4}$

${ }^{1}$ Nanoengineering, University of California San Diego, La Jolla, CA, USA, ${ }^{2}$ Electrical Engineering, University of California San Diego, La Jolla, CA, USA, ${ }^{3}$ Radiology, University of California San Diego, La Jolla, CA, USA, ${ }^{4}$ Moores Cancer Center, La Jolla, CA, USA.

Background: Ultrasound is a safe, portable imaging method that can reach tissues as deep as $20 \mathrm{~cm}$ and can be operated with inexpensive, ubiquitous equipment. While microbubbles are effective ultrasound contrast agents, there is currently no mechanism of activation in vivo to obtain site-specific contrast enhancement. We report here the design of a novel perfluorocarbon gas-filled microbubble whose shell stiffness can be altered by specific local conditions or enzymes. When the shell is rigid, oscillation is dampened, but when made elastic backscatter and harmonic signals increase dramatically, allowing their detection. Methods: For proof of principle we produced $2.5 \mu \mathrm{m}$ perfluorobutane-filled microbubbles that were stabilized by combinations of non-toxic, biocompatible polymers and phospholipids, where the polymer is crosslinked by a reversible linker to rigidify the microbubble shell. We made two such formulations where the polymer was crosslinked by disulfide bonds coupled to poly(acrylic acid) (PAA). Microbubble suspensions were then placed in a phantom and the backscatter and harmonic signals measured using a Panametrics pulser/receiver 5300 equipped with a V306 transducer at 2.25 MHz. These measurements were repeated several times over 60 minutes following the addition of a disulfide bond reducer (TCEP). Results: When the crosslinks were cleaved chemically, backscatter increased by approximately $10 \mathrm{~dB}$ (see Figure) and harmonic signal was enhanced by more than $15 \mathrm{~dB}$. Note that
TCEP had no effect on the microbubble backscatter when the polymer contained no crosslinkable groups (Figure). Conclusion: Using this technique, site-specific ultrasound imaging can be achieved in response to $\mathrm{pH}$, oxidizing or reducing environments, or proteolytic enzymes. We aim in our future work to replace the disulfide bond linker with a peptide cleavable by thrombin, an enzyme that is active in acute thrombosis to detect acute thrombus formation in veins and tumors.

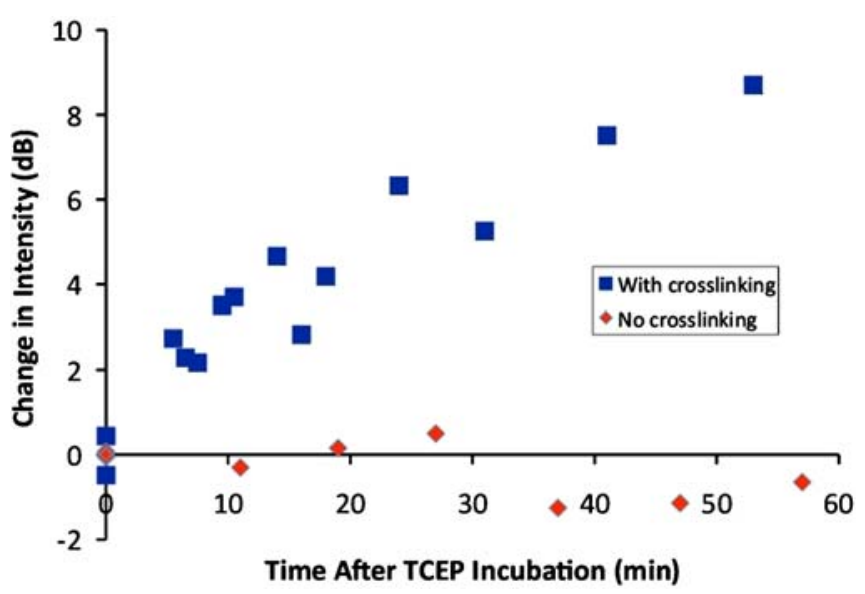

Figure: Addition of TCEP cleaves disulfide crosslinks, increasing microbubble backscatter signal. Microbubbles with no disulfide bonds are unaffected.

A NOVEL METHOD OF MEASURING SPLENIC INFLAMMATION IN RESPONSE TO CONTRAST ENHANCED ULTRASOUND USING AN AD5-COX2-LUC ANIMAL MODEL Jason M. Warram ${ }^{1}$, Kenneth Hoyt ${ }^{2}$, Karri Folks ${ }^{2}$, Kurt R. Zinn ${ }^{1,2}$

${ }^{1}$ Molecular and Cellular Pathology, University of Alabama at Birmingham, Birmingham, AL, USA, ${ }^{2}$ Radiology, University of Alabama at Birmingham, Birmingham, AL, USA. 
The demand for high-contrast ultrasonography has driven the development of novel ultrasound contrast agents (UCA, i.e. microbubbles) for improved diagnostic imaging, allowing for contrast-enhanced ultrasound (CEUS). CEUS applications in echocardiography are now approved by the FDA. One potential problem with CEUS is that at high pressure amplitudes (termed mechanical index; MI) above the resonance frequency threshold commonly used in high MI ultrasound (US), microbubbles exhibit acoustic cavitations causing the outer shell to collapse producing mechanical perturbations and the potential for nearby tissue damage. Intravascular exposure of these cavitations and subsequent shear forces may lead to endothelial and blood cell destruction. The goal of the current research was to develop a method to detect inflammatory change associated with CEUS exposure. To this end, an inflammation sensing model was applied. One week prior to US imaging, 25 mice were injected IV (6x108 pfu) with an adenoviral (Ad) vector encoding firefly luciferase under the control of a Cox 2 promoter. Mice then received a titrated IV bolus of UCA (Definity, Lantheus Medical Imaging) with 4 dose groups $(n=5)$ spanning between $1 \mathrm{ul}$ and 60ul. US imaging of the mouse liver was performed continuously using a SONIX RP scanner (Ultrasonix Medical Corp) and a linear array probe (L14-5). Scanning was externally triggered 10 frames/second and performed at a MI value of 1.4 corresponding to a peak negative pressure of 3.6 MPa. Bioluminescent imaging was performed both prior to US exposure and intermittently out to 18 days to detect luciferase expression. There was a UCA dose-dependent inflammatory response in the spleen which collectively peaked at day 3 post CEUS and returned to baseline 4 days thereafter. In addition, control groups that included US alone $(n=5)$ and UCA alone $(n=5)$ displayed only baseline signal in the spleen. The imaging studies demonstrate the potential for bioluminescence to monitor cox2-induced luciferase expression in the spleen following high MI CEUS. It is hypothesized that observed splenic inflammation from CEUS exposure may be related to RBC destruction and subsequent splenic perfusion of Heme, a known inducer of tissue inflammation.

\section{J294}

\section{INCREASING ULTRASOUND SENSITIVITY} TO PERFLUORCARBON EMULSION DROPLETS Saleh Amirriazi ${ }^{1}$, Mohammad Eghtedari ${ }^{3}$, Seunghan $\mathrm{Oh}^{2}$, Seong Deok Kong ${ }^{2}$, Sungho $\mathrm{Jin}^{2}$, Robert F. Mattrey ${ }^{3}$

${ }^{1}$ Bioengineering, University of California, San Diego, San Diego, CA, USA, ${ }^{2}$ Material Science, University of California, San Diego, San Diego, CA, USA, ${ }^{3}$ Radiology, University of California, San Diego, San Diego, CA, USA.

Background: Phase transition of perfluorocarbon (PFC) droplets to microbubbles (MBs) to improve ultrasound (US) sensitivity to emulsions (10 orders of magnitude) has been shown possible by several laboratories. Increasing sensitivity to targeted droplets is critical because their longer circulating time and larger particle count is superior to targeted MBs. The major limitation to use low boiling point PFCs, which causes side effects by spontaneously vaporizing in vivo, and the need for high US energy to cavitate the PFCs. While small particles are preferred, they require more US energy to induce phase transition. We hypothesized that PFCs with a higher boiling point can be used to prevent spontaneous vaporization in vivo, and the US energy needed to vaporize the droplet can be lowered if nucleation sites are provided within the droplets. In this study we aimed to define the differences in the US energy required to vaporize a PFC before and after iron oxide nanoparticles (IONP) are added. Methods: We manufactured PFCcoated $50 \mathrm{mg}$ IONP, followed by suspension in $20 \mathrm{ml}$ of PFC. We then produced a lipid emulsion that generated a $60 \% \mathrm{w} / \mathrm{v}$ iron oxide-loaded droplet with a mean diameter of $200 \mathrm{~nm}$. We built a PDMS phantom that allows the delivery of liquids to, and the recovery of generated MBs from, a small chamber; the generated MBs were guided to an imaging phantom where they will be detected and counted using a harmonic imaging system (Figure). The chamber was positioned at the focal zone of a high intensity focused ultrasound system as water, pure PFC, and emulsions of PFC with or without encapsulated IONP were circulated through the chamber at different ambient temperatures. Result \& Conclusion: While water required high US pressure to produce bubbles, non-emulsified PFP vaporized at room temperature with minimal US pressure but required higher pressure to vaporize when emulsified. The presence of IONP within the PFC decreased the US pressure needed to induce vaporization.

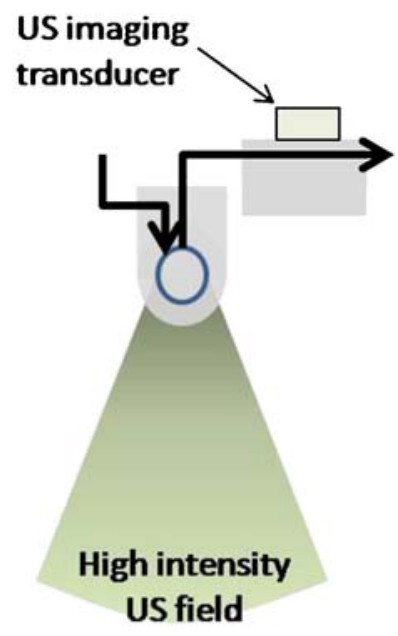

$\mathrm{PFC}$ vaporization/MB detection setup

\section{J295}

\section{SIZE-TAILORING CONTRAST AGENT DIAMETER: A MECHANISM TO IMPROVE THE SENSITIVITY OF ULTRASOUND MOLECULAR IMAGING}

Jason Streeter, Ryan Gessner, Iman Miles, Paul A. Dayton

Joint Dept of Biomedical Engineering, University of North CarolinaNorth Carolina State University, Chapel Hill, NC, USA.

Ultrasonic molecular imaging relies on microbubble contrast agents (MCAs) bearing ligands to target specific molecular markers of disease. After intravenous administration, targeted agents bind to endothelial cells in the vasculature expressing characteristic epitopes and enhance the backscatter from diseased tissue. Prior studies have shown that only a small percentage of injected MCAs are retained at the target site; thus 
enhancing US sensitivity to small populations of targeted microbubbles is essential to improve the efficacy of this technique. We hypothesized that tailoring larger microbubble size distributions would enhance the backscatter intensity per bound agent. To test this, we performed acoustic studies of MCAs with different mean diameters in-vitro and invivo. We used size-sorting methods optimized by Feshitan et al (J Colloid Interface Sci. 2009) to selectively isolate size distributions of biotinylated lipid-encapsulated microbubbles. Our in-vitro tests consisted of conjugating size-sorted distributions to Thermanox coverslips coated with avidin. Adherent bubbles were acoustically examined with a $15 \mathrm{MHz}$ pulse-echo system, which raster scanned in two dimensions over the plate. Adherent MCA concentrations were determined optically, by raster scanned microscope image captures across the same coverslips, and data were correlated offline. In-vivo experiments were conducted with non-targeted agents administered in a rodent model to determine the relationships between MCA diameter, injected dose, and echogenicity. Results illustrate that the backscatter intensity strongly depends on mean diameter of the targeted MCA population and concentration. In-vitro data of targeted agents illustrate an intensity increase of $\sim 12 \mathrm{~dB}$ between a 1.1 micron mean diameter and a 3.2 micron mean population. In-vivo, data show a $\sim 15 \mathrm{~dB}$ intensity increase between 1.2 micron distribution and a 3.3 micron distribution for the same concentration. Data illustrate that the same signal intensity can be achieved with 20 fold fewer contrast agent by optimizing the bubble population. In conclusion, scattered US signal intensity from MCAs is significantly affected by population diameter, and substantial enhancement of sensitivity to small numbers of MCAs can be achieved by tailoring microbubble size distributions for targeting applications.

\section{J296}

\section{NANOPARTICLE-LOADED PERFLUOROCARBON DROPLETS FOR ULTRASOUND IMAGING AND THERAPY OF CANCER}

Naomi Matsuura $^{1}$, Ross Williams ${ }^{1}$, Nikita Reznik ${ }^{2}$, Ivan Gorelikov ${ }^{1}$, Joydeep Chaudhuri ${ }^{1}$, John Rowlands ${ }^{1,2}$, Kullervo Hynynen ${ }^{1,2}$, F. Stuart Foster ${ }^{1,2}$, Peter N. Burns ${ }^{1,2}$

${ }^{1}$ Imaging Research, Sunnybrook Health Sciences Centre, Toronto, ON, Canada, ${ }^{2}$ Medical Biophysics, University of Toronto, Toronto, ON, Canada.

Introduction. Nanoparticle-loaded perfluorocarbon (PFC) droplets can be used for targeted imaging and therapy of cancer with ultrasound. Because of the enhanced permeability of tumour vascular endothelium, droplets with diameters from 100-300 nm can extravasate into tumours. When exposed to ultrasound, droplets can be converted to gas, which can be used for the detection and treatment of solid tumours. At low frequency, inertial cavitation of droplets can be used to enhance ultrasound ablation and to locally release encapsulated nanoparticles; at high frequency, droplet vapourization can be exploited for the detection of tumours using established ultrasound imaging techniques. Methods. In this work, we report on the in vitro and in vivo interaction of ultrasound with droplet emulsions composed of various PFC's (PFP, PFH, PFOB), with and without nanoparticle incorporation. The droplet diameters ranged from 100 $300 \mathrm{~nm}$ as measured by dynamic light scattering. Ultrasound experiments to measure droplet cavitation at low frequency were performed at $1 \mathrm{MHz}$ using a single-element transducer with passive detection at $500 \mathrm{kHz}$; high frequency experiments to detect droplet vapourization were performed with a high-power flash mode in conjunction with pulse-echo detection at $18 \mathrm{MHz}$ using a linear array transducer. Results. In vitro experiments demonstrated that the cavitation and vapourization pressure thresholds could be manipulated by varying droplet composition so that phase conversion is obtained at clinically relevant pressures. The incorporation of nanoparticles lowered the inertial cavitation threshold at $1 \mathrm{MHz}$ by $20 \%$, permitting controlled nanoparticle release from perfluorohexane droplets in macrophage cells in vitro. In vivo experiments demonstrated that perfluoropentane droplets can be converted to gas and imaged at high frequency in hepatomas in mice, using brief high pressure flashes to achieve the conversion. Finally, fluorescent quantum dot nanoparticles were used to trace the biodistribution of droplets within rabbits and mice using fluorescence histology. Conclusions. This work shows that PFC droplets are a promising agent for both therapeutic and diagnostic applications of ultrasound to cancer. Nanoparticles can be encapsulated within the droplets, providing a novel way to extend the functionality of PFC droplets to other imaging and therapy modalities, while constraining the biophysical interactions to those determined by the properties of the encapsulating droplet. Practical issues and requirements for further droplet development will be discussed.

\section{$\mathbf{J} 297$}

\section{MICROBUBBLE LABELING AND IN-VIVO ULTRASOUND} IMAGING OF NEURAL PROGENITOR CELLS

Wenjin Cui ${ }^{1}$, Marika Decyk ${ }^{1}$, Malak Itani ${ }^{1}$, Yuko Kono ${ }^{1,2}$, Robert F. Mattrey ${ }^{1}$

${ }^{1}$ Radiology, University of California, San Diego, San Diego, CA, USA, ${ }^{2}$ Medicine, University of California, San Diego, San Diego, CA, USA.

Background: It is known that neural progenitor cells (NPCs) accumulate in tumors, providing an opportunity for tumor detection if NPCs are labeled with a reporter. Because microbubbles cannot target extravascular sites, we hypothesized that microbubble-labeled NPCs could serve to target and detect tumors by ultrasound (US). This study aimed to optimize NPC microbubble uptake and determine if these labeled cells could be detected in-vivo. Methods: Since we showed that positively charged microbubbles are more efficient at labeling phagocytic cells, we prepared positively charged microbubbles by sonicating a lipid mixture that contained $10 \mathrm{~mol} \%$ of DSTAP in the presence of PFC vapor. C17.2 NPCs (acquired from Evan Y. Snyder, MD, Burnham Institute) were grown in a $24-w e l l$ plate to $50-70 \%$ confluence (approximately $2^{*} 10^{\wedge} 6$ cells per well) and $30-50 \mu \mathrm{l}$ of microbubbles $\left(10^{\wedge} 8 / \mathrm{ml}\right)$ added, along with $3 \mathrm{ml}$ of cell culture medium. The culture dish was covered with parafilm and inverted to float the microbubbles against the adherent NPCs. To study uptake, cultures were observed for 30 minutes with confocal microscopy $(0.7 \mu \mathrm{m}$ slice thickness). For imaging, NPCs were cultured for 24 hours and the cells were washed with PBS to remove all free microbubbles and resuspended to produce $\sim 1.6 \times 10^{\wedge} 6 \mathrm{NPCs} / \mathrm{ml}$. Cell viability was assessed by trypan blue exclusion. The right jugular vein of a mouse was exposed and $0.25 \mathrm{ml}$ of NPCs was injected. The mouse heart was imaged using harmonic imaging with a Seimens Antares equipped with a VFX 13-5 transducer transmitting at $5 \mathrm{MHz}$ center 
frequency at low mechanical index $(<0.2)$. Results \& Conclusion: Trypan blue staining revealed $>90 \%$ viability. Confocal microscopy showed microbubble internalizations, and single bubble movement within the cell was traced. In-vivo imaging of the mouse heart showed echogenic filling of the left ventricular (arrow in Figure) indicating that microbubble-labeled NPCs are not only visible on US, but also cross the lung to enter the systemic circulation, enabling the study of tumor targeting.

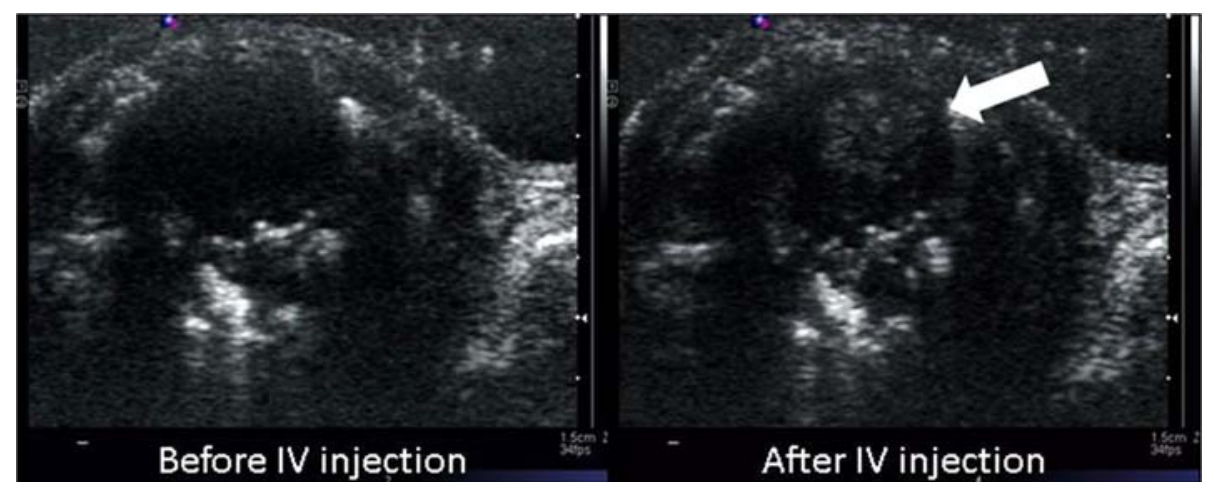

\section{Imaging Disease/Organ Processes}

\section{Cancer}

\section{$\mathbf{J} 298$}

\section{GA68-DOTA-NOC PET/CT IN NEUROENDOCRINE}

TUMORS-COMPARISON WITH IN111-DTPA-OCTREOTIDE Yodphat Krausz $^{1}$, Nanette Freedman ${ }^{1}$, Rina Rubinstein ${ }^{1}$, Eyal Mishani ${ }^{1,2}$, Efi Lavie $^{3}$, Avihai Y. Bross ${ }^{3}$, Dani Vaknin ${ }^{3}$, Kobi Ben-Meir ${ }^{4}$, Roni Michael ${ }^{4}$, Marina Orevi ${ }^{1}$, Sagi Tshori ${ }^{1}$, Asher Salmon ${ }^{5}$, Benjamin Glaser ${ }^{6}$, Roland Chisin ${ }^{1}$, David J. Gross ${ }^{6}$

${ }^{1}$ Nuclear Medicine, Hadassah-Hebrew University Medical Center, Jerusalem, Israel, ${ }^{2}$ Cyclotron Unit, Hadassah-Hebrew University Medical Center, Jerusalem, Israel, ${ }^{3}$ Division of Radiopharmaceuticals, Soreq, Nuclear Research Center, Yavne, Israel, ${ }^{4}$ Cyclotron Unit, Soreq, Nuclear Research Center, Yavne, Israel, ${ }^{5}$ Oncology, Hadassah-Hebrew University Medical Center, Jerusalem, Israel, ${ }^{6}$ Endocrinology and Metabolism Service, Hadassah-Hebrew University Medical Center, Jerusalem, Israel.

Ga68-DOTA-1-NaI3-octreotide (Ga68-DOTA-NOC) is a novel agent for PET/CT imaging of neuroendocrine tumors (NETs) that has theoretical advantages of improved spatial resolution and broader somatostatin receptor specificity in comparison to the "current standard" SPECT/CT with In111-DTPA-octreotide (OCT). The aim of our prospective study was to compare Ga68-DOTA-NOC with OCT in evaluation of the extent of tumor involvement in NET patients. Patients and Methods: Nineteen patients [7F, age range 25-81 yrs] with pathology-proven carcinoid [ $n=8]$, pancreatic NETs [ $n=9]$ and NE carcinoma of unknown origin [ $n=2]$, were referred for staging or for follow-up after biopsy $[n=9]$ or surgery $[n=10]$. Whole body Ga68-DOTA-NOC PET/CT and OCT, including planar, SPECT and SPECT/CT images were acquired within the time frame of 10 65 days [median 24]. Ga68-DOTA-NOC scans were evaluated qualitatively and semi-quantitatively, using standardized uptake values (SUVs). Findings with both tracers were compared by a region-by-region analysis. Results: Focal Ga68-DOTA-NOC uptake compatible with high density of somatostatin receptors in tumor sites was seen on all scans, often in multiple sites, with mean SUV ranging from 1 to 155 . Physiological uptake was documented in the pituitary, adrenals and spleen. In 37 regions of 18 patients, findings on Ga68-DOTA-NOC and OCT were comparable, although the Ga68-DOTA-NOC image quality was superior to that of OCT in all 19 studies, having better resolution and target-tobackground contrast. In 6 regions of 5 patients, while findings were seen with both techniques, Ga68-DOTA-NOC yielded additional foci. In 18 regions of 12 patients, foci were detected on Ga68-DOTA-NOC scans only, with CT verification for 10 of these regions, and impact on staging in 4 patients. In 23 regions of 13 patients, very faint foci were seen on OCT only; however, most had no anatomical verification, suggesting false-positive findings. Conclusions: Data of Ga68-DOTA-NOC and OCT were comparable in 18/19 patients. However, Ga68-DOTA-NOC images were clearer, facilitating rapid and unequivocal identification of the presence, location and intensity of tumor foci. Ga68-DOTA-NOC detected additional tumor sites in 12 patients, with impact on staging in $4 / 19$ patients, though further work is required for complete anatomical validation. Ga68-DOTA-NOC PET/CT was better tolerated by patients, with shorter scan duration and no need for repeated scan or bowel preparation. This prospective comparative study suggests that PET imaging with Ga68-DOTA-NOC may replace OCT for evaluation of NETs.

\section{J299}

TUMOR ANGIOGENESIS DETECTION WITH BR55, A LIPOPEPTIDE-BASED CONTRAST AGENT FOR MOLECULAR ULTRASOUND IMAGING

Sibylle Pochon $^{1}$, Isabelle Tardy ${ }^{1}$, Philippe Bussat ${ }^{1}$, Thierry Bettinger ${ }^{1}$, Martine Theraulaz ${ }^{1}$, Patricia Emmel ${ }^{1}$, Radhakrishna Pillai ${ }^{2}$, Mathew von Wronski ${ }^{2}$, Michel Schneider ${ }^{1}$

${ }^{1}$ Bracco Research SA, Geneva, Switzerland, ${ }^{2}$ Bracco Research USA, Princeton, NJ, USA. 
The recruitment of new blood vessels is a prerequisite of tumor development and among the specific molecular markers of angiogenesis, the vascular endothelial growth factor receptor 2 (VEGFR2, also called KDR in man and Flk-1 in rodents) is recognized as a major player. VEGFR2 expression has been linked to the progression and aggressiveness of many types of tumors. Numerous angiogenesis inhibitors are in clinical trials, several of which target the VEGF/VEGFR2 pathway. Molecular imaging of this receptor is obviously an important issue to better detect tumors. Targeted-microbubble-enhanced ultrasonography is a very sensitive and convenient modality for the real-time and noninvasive imaging of intravascular markers with good spatial resolution. We designed BR55, a KDR-specific contrast agent for the early detection of tumoral angiogenesis. In contrast with previously published studies using VEGFR2-targeted microbubbles, BR55 doesn't require an antibody for binding nor use a biotin/streptavidin coupling strategy. BR55 is a phospholipidbased contrast-agent functionalized with a heterodimeric peptide that binds to VEGFR2 (KDR/Flk-1) with high affinity. BR55 microbubbles were evaluated on recombinant proteins and on cells expressing VEGFR2 to demonstrate their binding efficiency and specificity. BR55 microbubble interaction with the mouse (Flk-1) and human (KDR) receptor was shown and could be inhibited with an excess of free VEGF or a VEGFR2-specific antibody. Then BR55 was evaluated in a rat prostate tumor model and compared to streptavidin microbubbles coupled to a biotinylated VEGFR2-specific antibody. Tumor perfusion was assessed during the vascular phase of BR55, and then the malignant lesion was highlighted by specific accumulation of the targeted microbubbles on KDR-expressing endothelium. A tumor to prostate ratio of 20 was observed. The expression of VEGFR2 in tumor cryosections was confirmed by immunohistochemistry. BR55 was shown to specifically recognize the VEGFR2 marker of angiogenesis in vitro and in vivo with an efficacy comparable to that of antibodyconjugated microbubbles. The potential use in man of targeted ultrasound contrast agents such as BR55 opens a new era in medical imaging. The information provided by molecular imaging combined with the assessment of tumor perfusion will likely prove useful in characterizing a tumor's phenotype, detecting metastases, monitoring tumor response to therapy, and for finding the optimal time window for treatments and dose ranging of anti-cancer drugs.

$\mathbf{J 3 0 0}$

IMAGING THE TUMOR ANGIOGENESIS: MAGNETIC RESONANCE IMAGING (MRI), SYNCHROTRON RADIATION-BASED MICRO COMPUTED TOMOGRAPHY (SRMCT), POSITRON EMISSION TOMOGRAPHY (PET) AND HISTOLOGICAL EXAMINATION TO FOLLOW THE VESSEL FORMATION

Marco Dominietto $^{1}$, Sabrina Lang ${ }^{2}$, Steffi Lehmann ${ }^{1}$, Ruth Keist ${ }^{1}$, Bert Müller ${ }^{2}$, Markus Rudin ${ }^{1}$

${ }^{1}$ Institute for Biomedical Engineering, ETH Zurich, Zurich, Switzerland, ${ }^{2}$ Biomaterials Science Center, University of Basel, Switzerland, Basel, Switzerland.

Several techniques are available to visualize and evaluate the functionality of the neovasculature during tumor angiogenesis. By means of MRI is it possible to get anatomy, tumor blood volume (TBV) and flux (TBF), and vessel size index (VSI). With PET is it possible to visualize the hypoxic regions. Nevertheless to visualize the $3 \mathrm{D}$ capillary architecture $(5-10 \mu \mathrm{m})$ ex-vivo $\mathrm{SR} \mu \mathrm{CT}$ is needed. These imaging modalities are complemented by histology and immunohistochemistry. The aim of this work is to characterize vessel formation using these techniques in order to elucidate multiple aspects of the angiogenic process. Eighteen $\mathrm{balb} / \mathrm{c}$ nude mice were injected subcutaneously with $10^{\wedge} 6 \mathrm{C} 51$ cells (colon carcinoma). For a first group of mice $(N=6)$ anatomical images, TBV, TBF, VSI maps were recorded using MR. Thereafter, mice were perfused with different contrast agents and the tumors explanted for SR $\mu \mathrm{CT}$ measurements. For the second group $(N=6)$ MRI-PET longitudinal studies have been carried out. Measurements were performed every 3 days. The MR protocol was the same as for the first group, PET protocol consists of the injection of the radiotracer [18F]-FMISO to visualize the hypoxic regions. The experiment with the third group $(N=6)$ comprized MRI and immuno-staining techniques. MRI acquisition (same protocol as for first experiment) and histological examination using the endothelial marker CD31 were performed every 3 days. The results show us the formation of different regions during tumor growth. These regions differ from hysiological behavior and hypoxic level. Images from $\mathrm{SR} \mu \mathrm{CT}$ show a chaotic structure of the vessel architecture at the capillary level. The use of complementary imaging systems is possible to analyze multiple aspects of the vessel network formation in tumor tissue.
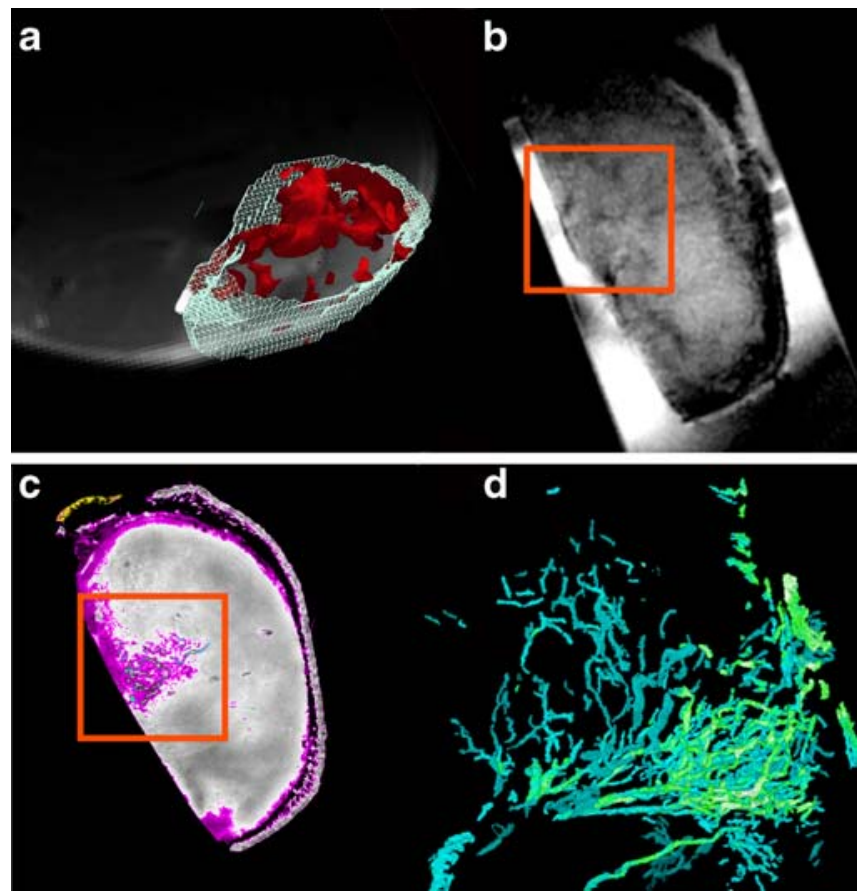

a) $3 \mathrm{D}$ tumor reconstruction from in-vivo MRI. In red are visible the perfused regions. b) ex-vivo MRI of the tumor sample. c) co-registration of the ex-vivo MRI with $\mathrm{SR} \mu \mathrm{CT}$ data. d) 3D reconstruction of $\mathrm{SR} \mu \mathrm{CT}$ in the red rectangular. 


\section{$\mathbf{J 3 0 1}$}

\section{IN VIVO IMAGING OF COLON MUCOSA TARGETED SOLID LIPOSOME NANO-PARTICLE VECTORS IN MICE WITH MR IMAGING AND FLUORESCENT IMAGING}

Zheng Weiliang

Radiology, Zhejiang University, Hangzhou City, China.

Purpose To probe the possibility of monitoring adhesion of mucosa targeted solid liposome nano-particle vectors loaded with MR contrast GdDTPA and fluorescence FITC with in vivo MR imaging and fluorescent imaging. Method A novel vector of loaded stearic acid solid liposome nano-particles (SLNs) loaded with Gd-DTPA and ODA-FITC (Fluorescein isothiocyanate labeled otcadecylamine) was synthesized with solvent diffusion method in aqueous system. MR scans of axial fat suppression FLAIRT1WI in 7 mice before, during and 40 minutes after infusion of suspensions of Gd-ODA-FITC and ODA-FITC SLNs were untertaken in 5 mice. Samples of the colon correlated to the enhanced area were obtained for microscopy examination. Signal intensity (SI) of ROIs in the wall of rectum or colon, muscles of the pelvis near the rectum and background were measured and corresponding relative signal intensity(SIr) values were subsequently calculated. SIr values between the two groups and preand post-infusion were compared with t pared analysis. Results Signal intensity of the colon wall was greatly enhanced as segmental or annular patterns after infusion of Gd-ODA-FITC SLNs. Values of SIr of the rectum at pre- and post- infusion in the Gd-ODA-FITC group were 0.86 \pm .051 and1.47 \pm .191 (mean \pm SD) respectively with $P<0.001$ while those in ODA-FITC group were $0.84 \pm .041$ and $0.87 \pm .147$ with $P>0.05$. Signal increase rate was $0.74 \pm .198$ which was significantly higher than that in ODA-FITC group with $P<0.01$. Under the fluorescent microscopy green fluorescent material was found in the whole wall of the rectum or colon which was comparable to the MR findings. Linear green fluorescent material was found along the serosa and large amount of fluorescent material was observed in the intersticial space.Conclusion In-vivo MR imaging can reveal the phenomenon of adhesion and absorption of mucosa targeted SLNs carriers. With additional FITC labeling bimodal MR imaging and fluorescent imaging may hold promise in the study colon mucosa mediated drug delivery with SLNs.

\section{$\mathbf{J 3 0 2}$}

\section{LONGITUDINAL AND MULTI-MODAL IN VIVO IMAGING OF TUMOR HYPOXIA AND ITS DOWNSTREAM MOLECULAR EVENTS}

$\underline{\text { Steffi Lehmann }}^{1}$, Daniel P. Stiehl ${ }^{2}$, Michael Honer ${ }^{3}$, Marco Dominietto ${ }^{1}$, Ruth Keist ${ }^{1}$, Simon M. Ametamey ${ }^{3}$, Roland H. Wenger ${ }^{2}$, Markus Rudin ${ }^{1,4}$ ${ }^{1}$ Institute for Biomedical Engineering, University / ETH Zurich, Zuerich, Switzerland, ${ }^{2}$ Institute of Physiology, University of Zurich, Zurich, Switzerland, ${ }^{3}$ Institute for Pharmaceutical Sciences, ETH Zurich, Zurich, Switzerland, ${ }^{4}$ Institute of Pharmacology and Toxicology, University of Zurich, Zurich, Switzerland.

The importance of tumor hypoxia is receiving increased attention in the field of cancer research. Not only are hypoxic tumor cells more resistant to radio- and chemotherapy, but also, tumor hypoxia triggers the stabilization and subsequent activation of Hypoxia Inducible Factor (HIF), an oxygen sensitive transcription factor, which has been shown to promote tumor cell survival, malignancy and metastasis. To study the relationship between tumor growth, tumor hypoxia, stabilization and activation of HIF we have generated in vivo imaging tools which allow monitoring these processes in a mouse allograft tumor model in a longitudinal and non-invasive manner. We used Positron Emission Tomography (PET) with the hypoxia sensitive tracer $\left[{ }^{18} \mathrm{~F}\right]$-FMISO to quantitatively assess hypoxia in C51 tumor allografts, s.c. implanted into the neck of Balb/c nude mice. In the same tumor model, we followed the stabilization and activity of HIF-1 $\alpha$ using bioluminescence imaging of C51 cells stably expressing either a HIF-1 $\alpha$ - luciferase fusion construct or luciferase driven from a HIF sensitive promoter. Interestingly, we did not observe a major change in tumor hypoxia throughout the observation period of 14 days. However, HIF-1 $\alpha$ levels and HIF activity showed marked temporal variations. When comparing the respective readouts as a function of time, we found a good correlation between HIF- $1 \alpha$ levels and HIF activity. In contrast, there was no significant correlation between the $\left[{ }^{18} \mathrm{~F}\right]$-FMISO PET and the HIF readouts (Fig. 1). In vivo results were confirmed in vitro by immunohistochemical analysis of tumor sections. The tools developed in this work will be of value when monitoring the efficacy of therapeutic interventions targeting the HIF pathway. Moreover, our observations are of high interest with regard to $\left[{ }^{18} \mathrm{~F}\right]$-FMISO PET hypoxia imaging in clinical cancer diagnostics.

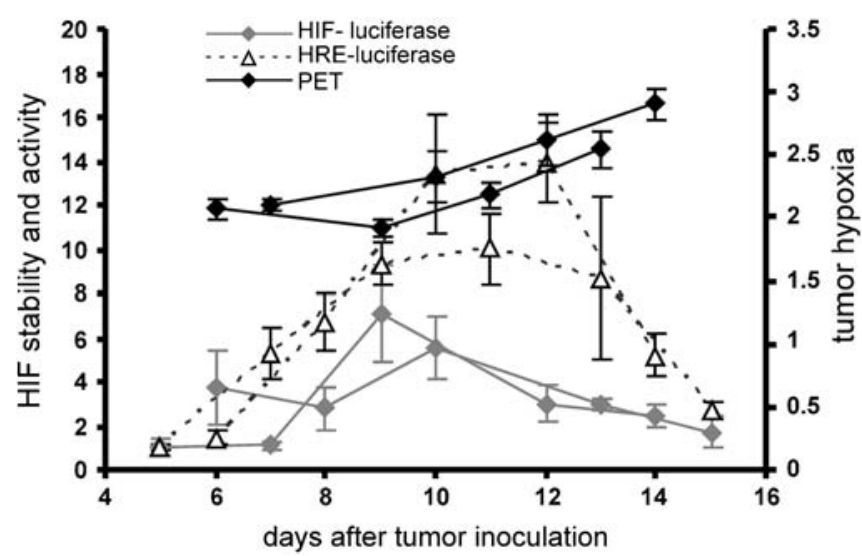

Fig. 1. Luciferase activity was measured using the Xenogen IVIS 100. Tumor hypoxia was determined by quantifying the uptake of $\left[{ }^{18} \mathrm{~F}\right]$ FMISO. Values (mean \pm sem) are shown for two groups of animals for each readout, relative to those measured on day 5 for the luciferase- and day 6 for the PET experiments, respectively.

\section{$\mathbf{J 3 0 3}$}

\section{IMAGING OF TUMOR NEOVASCULARIZATION IN TRANSGENIC MOUSE MODELS USING A NOVEL PET ${ }^{64} \mathrm{Cu}$-DOTA-KNOTTIN PEPTIDE}

Carsten H. Nielsen ${ }^{1,2}$, Nadia Withofs ${ }^{1}$, Richard Kimura ${ }^{1}$, Phuoc T. Tran ${ }^{3,4}$, Zheng Miao ${ }^{1}$, Jennifer R. Cochran ${ }^{5}$, Zhen Cheng ${ }^{1}$, Dean W. Felsher ${ }^{3}$, Juergen K. Willmann ${ }^{1}$, Sanjiv S. Gambhir ${ }^{1,5}$

${ }^{1}$ MIPS, Radiology, Stanford University, Stanford, CA, USA, ${ }^{2}$ Cluster for Molecular Imaging, Clinical Physiology, University of Copenhagen, Copenhagen, Denmark, ${ }^{3}$ Division of Oncology, Medicine \& Pathology, Stanford University, Stanford, CA, USA, ${ }^{4}$ Radiation Oncology, Stanford University, Stanford, CA, USA, ${ }^{5}$ Bioengineering, Stanford University, Stanford, CA, USA.

Despite advances in cancer management the mortality of lung cancer is still strikingly high. A major problem is that lung cancer patients typically are diagnosed when they have advanced stage disease thus eliminating the option of curative surgery. Once a tumor reaches $1-3 \mathrm{~mm}$ in diameter it is dependent on angiogenesis to support further growth. In this study we demonstrate the potential of using a novel 64Cu-DOTA-Peptide (Knottin $2.5 \mathrm{~F}$ ) to image angiogenesis (through $\alpha_{\mathrm{v}} \beta_{3}, \alpha_{\mathrm{v}} \beta_{5}$, and $\alpha_{5} \beta_{1}$ targeting) associated with malignancy in a transgenic mouse model mimicking the development of lung cancer. Conditional transgenic mice with the oncogenes cMyc and K-ras induced by doxycyline were screened for lung lesions using respiratory gated microCT. Mice $(N=7)$ with positive $\mathrm{CT}$ findings underwent microPET/microCT imaging with Knottin $2.5 \mathrm{~F}$ $(\sim 100 \mu \mathrm{Ci})$. In addition mice were imaged with FDG-microPET/microCT. The PET-tracers uptake in the lesions was estimated by volumetric ROIs drawn based on the microCT images and tumor to normal lung ratios 
calculated. Ex vivo gamma counting was also performed on tumor- and normal lung sections and tumor tissue was stained for integrins $\left(\alpha_{\mathrm{v}^{-}}\right.$ subunit) and CD31. Lung lesions, as small as $3^{3} \mathrm{~mm}^{3}$ in volume, were successfully imaged with Knottin $2.5 \mathrm{~F}$ and FDG. Overall, the $\% \mathrm{ID} / \mathrm{g}$ in the tumors was lower for Knottin $2.5 \mathrm{~F}$ compared to FDG, but the tumor to normal lung ratio $(6.01 \pm 1.92$ vs. $4.36 \pm 2.15)$ was significantly higher $(P \leq$ 0.04 ) for Knottin $2.5 \mathrm{~F}$. Ex vivo gamma counting supported the PET findings showing higher uptake in tumor sections than in normal lung.
Immunofluorescence confirmed expression of integrins ( $\alpha_{\mathrm{v}}$-subunit) on tumor vasculature. In conclusion, we demonstrate the feasibility of using Knottin $2.5 \mathrm{~F}$ as an imaging agent to image angiogenesis occurring during tumorigenesis in a transgenic mouse model. Knottin $2.5 \mathrm{~F}$ yields a higher tumor to background ratio as compared to FDG and shows minimal uptake in the thorax making it a promising imaging agent to characterize lung lesions. The improved visualization may facilitate better characterization of malignant lesions in patients undergoing PET/CT.
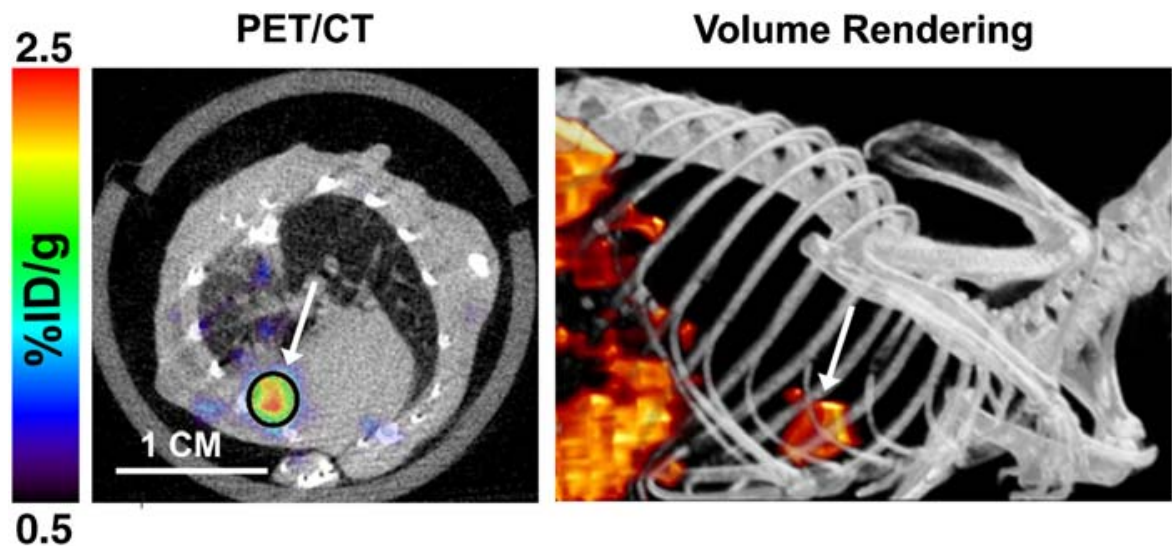

Figure 1. Left: MicroPET/microCT cross section of a transgenic mouse imaged with Knottin 2.5F. A nodule $\sim 3^{3} \mathrm{~mm}^{3}$ immediate adjacent to the heart is clearly visible (arrow). Right: Volume rendering of the same mouse. The Knottin 2.5F uptake is shown in color. Uptake is seen in the liver (left). The low background in the lungs makes it easy to delineate the nodule (arrow).

J304

NON-INVASIVE MONITORING OF CANCER THERAPEUTIC EFFICACY IN VIVO USING A NOVEL NEAR INFRAREDLABELED AGENT AND FLUORESCENCE MOLECULAR TOMOGRAPHY (FMT)

Kevin Groves, Sylvie Kossodo, Jun Zhang, Emma Handy, Garry J. Cuneo, Jeffrey D. Peterson, Milind Rajopadhye

VisEn Medical, Bedford, MA, USA.

Matrix metalloproteinases (MMPs) are key mediators in tumor growth, neo-vascularization and metastasis. MMPs are components of the cancer degradome and as such, clinically-relevant biomarkers of cancer progression. The aim of this investigation was to develop a fast-acting and rapidly clearing near-infrared fluorescence (NIRF) imaging agent which could be used to detect tumor-associated MMP activity in vivo noninvasively and in real time. A novel activatable NIRF agent was developed based on the MMP-cleavable sequence PLGVR. This agent is optically quenched in its native form but upon cleavage by selected enzymes, becomes highly fluorescent with an excitation at $750 \mathrm{~nm}$ and emission at $770 \mathrm{~nm}$. In vitro selectivity was assessed by cleaving the imaging agent with a panel of enzymes and monitoring released fluorescence over time. The agent was preferentially cleaved by MMP-7, $-9-12$, and -13 . In vivo selectivity was determined in two tumor models known to express MMPs: human colorectal HT-29 and mouse breast carcinoma 4T-1 tumors implanted in nude mice. Using quantitative fluorescence molecular tomography (FMT), tumor fluorescence due to in vivo activation of the agent was clearly detectable as early as $4 \mathrm{~h}$ after injection of the imaging agent, peaking at $6 \mathrm{~h}$ and mostly washed out by $96 \mathrm{~h}$. Importantly, this imaging agent was used as a mechanistic biomarker for therapeutic efficacy: mice with established tumors were treated with either the broadspectrum antibiotic doxycyline or a combination of the non-protein thiol
$\mathrm{N}$-acetyl-cysteine and a synthetic pan-MMP inhibitor. One day only after beginning the treatment, at a time when there was no effect on tumor size, both regimes inhibited MMP signal in tumors by $70 \%$ and $88 \%$ respectively ( $p=0.026$ and 0.0042 as compared to vehicle-injected control mice). These results underscore the value of NIRF optical imaging agents and FMT in improving the early detection and monitoring of cancer treatment.

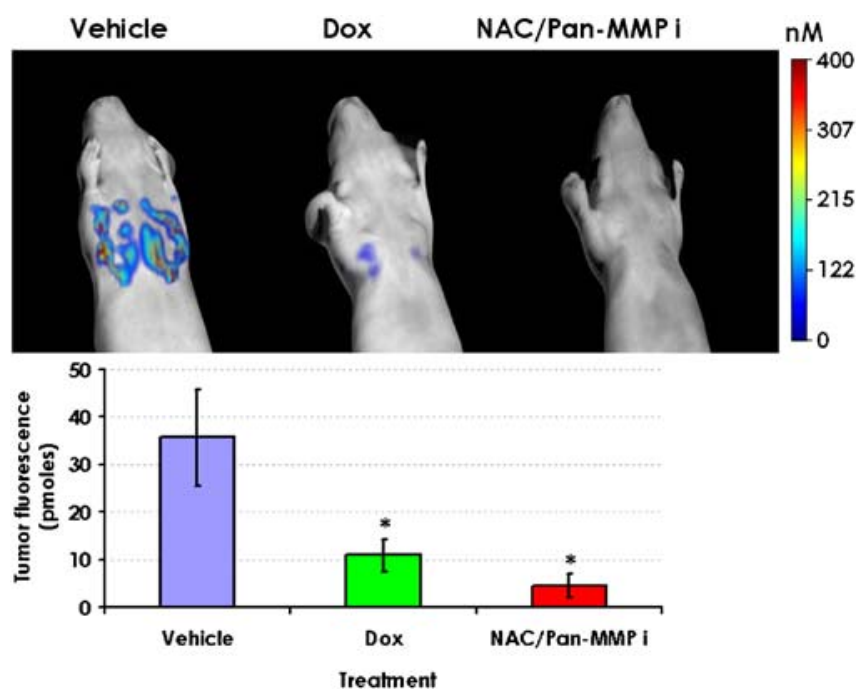

Representative volume renderings of mice treated with vehicle, doxycycline or a combination of $\mathrm{N}$-acetyl-cysteine with a pan-MMP inhibitor, showing the significant inhibition of MMP fluorescent signal in tumors following chemotherapy. 
$\mathbf{J 3 0 5}$

IN VIVO MR EVALUATION OF TARGETED CELL MIGRATION TO CANCER BY THE OVER-EXPRESSED CCR2

Bo-Kyeong Kang ${ }^{1}$, Jin Young Sohn ${ }^{3,2}$, Jewon Ryu ${ }^{3,2}$, Hyeujin Chang ${ }^{3,2}$, Kyung Won Lee ${ }^{3,2}$, Jin Seong Lee ${ }^{1,3}$

${ }^{1}$ Radiology, Asan Medical Center, Seoul, Korea, South, ${ }^{2}$ Asan Institute for Life Sciences, Asan Medical Center, Seoul, Korea, South, ${ }^{3}$ Institute for Innovative Cancer Research, Asan Medical Center, Seoul, Korea, South.

Cell therapy is the emerging technology for cancer treatment. For effective cell therapy, targeted cell migration to cancer is very important. Chemokines and chemokine receptors is essential to the targeted cell migration. So, we investigated whether over-expressed chemokine receptor enhances the migration of monocytes toward cancer cells. MCP-1/CCR2 system is known to be the most potent in the migration of monocyte and macrophages. The cloned CCR2 plasmids were transfected to THP-1 cell lines of monocytes. For transmigration tests, THP-1 cells were added to upper chamber and A549 cells (human lung carcinoma) were in the lower chamber of Transwell plates. After 3, 24, or 48 hours of incubation, the number of migrated THP-1 cells in lower chamber was counted. For in vivo evaluation, A549 (human lung carcinoma) cells were inoculated in the thigh muscle of the nude mice, then SPIO (superparamagnetic iron oxide)-labeled CCR2-overexpressed THP-1 cells and contolTHP-1 cells were intravenously administrated. In vivo magnetic resonance $(\mathrm{MR})$ image with $\mathrm{T} 2 *$-weighted gradient echo sequence was performed to detect the migrated THP-1 cells in the cancer, and was correlated with microscopic pathologic examination. CCR2overexpressed THP-1 expressed higher level of CD11b (integrin alpha $\mathrm{M}$ ) and more rapidly migrated to the lower chamber in timedependent manner in the transwell migration test. On in vivo MR examination, more areas of signal drop by migrated cells were demonstrated in cancer masses of CCR2-overexpressed THP-1 administered group, and confirmed by histopathologic examination. Over expressed CCR2 on THP-1 increases the expression of CCR2 and $\mathrm{CD} 11 \mathrm{~b}$ by $\mathrm{MCP}-1$ application and promotes the targeted migration of cells toward the A549 cancer. Over-expressed chemokine receptor could be useful to enhance the targeted migration of immune cells toward cancer mass.

\section{J306}

COMPARISON OF WHOLE-TORSO DIFFUSION WEIGHTED MRI AND 11C-CHOLINE PET/CT IN RECURRENT PROSTATE CANCER PATIENTS

Matthias Eiber $^{2}$, Bernd J. Krause ${ }^{1}$, Hubert Kubler ${ }^{3}$, Markus Schwaiger ${ }^{1}$, Ambros J. Beer ${ }^{1}$

${ }^{1}$ Department of Nuclear Medicine, Technische Universität München, Klinikum rechts der Isar, Munich, Germany, ${ }^{2}$ Department of Radiology, Technische Universität München, Munich, Germany, ${ }^{3}$ Department of Urology, Technische Universität München, Munich, Germany.

Aim: To assess to potential of whole torso MRI (from base of the skull to proximal thigh) including diffusion weighted imaging (DWI) in recurrent prostate cancer patients in relation to $11 \mathrm{C}$-Choline PET/CT. Material/Methods: 22 patients underwent whole torso MRI at $1.5 \mathrm{~T}$ using axial DWI (b-values of 50 and $600 \mathrm{~s} / \mathrm{mm} 2$ ), T1w and STIR (each section thickness $5 \mathrm{~mm}$ ) and 11C-Choline PET/CT. Diagnostic performance for MRI and 11C-Choline PET/CT was assessed using the ADC and SUV, respectively, combined with a 5-point scale of diagnostic certainty for calculation of ROC-curves. ADC-values and SUVs were compared by using linear-regression analysis. All clinical findings (incl. bone scintigraphy, follow-up imaging) and consensus of $11 \mathrm{C}-$ Choline PET/CT and MRI served as standard of reference. Results: In total 83 lesions were considered as malignant: 10 local recurrences, 45 lymph node, 35 bone and 5 lung metastases. Overall sensitivity, specificity and accuracy for 11C-Cho-PET/CT were $94.3 \%, 76.3 \%$ and $93.1 \%$, for whole-torso MRI $77.9 \%, 94.1 \%$ and $81.0 \%$, respectively. AUC was significantly higher for $11 \mathrm{C}-$ Choline PET/CT than for MRI ( 0.865 and 0.802 , respectively). While $8 / 10$ local recurrences were only detected in 11C-Choline PET/CT, 6 bone metastases were only found by MRI. 2 lymph nodes and 3 bone metastases were retrospectively detected only by consensus reading of both modalities. A highly significant difference $(p<0.01)$ could be found for the mean ADCs and SUVs for benign and malignant retroperitoneal and pelvic lymph nodes with a moderate and highly significant inverse correlation $(r=-0.54, p<$ 0.0001). Conclusion: Whole-body MRI including DWI is a promising tool for assessment of lesions in recurrent prostate cancer patients. Our preliminary data suggest that MRI is inferior to $11 \mathrm{C}$-Choline PET/CT concerning local recurrence, but seems to have complementary value in assessment of lymph node and bone metastases.

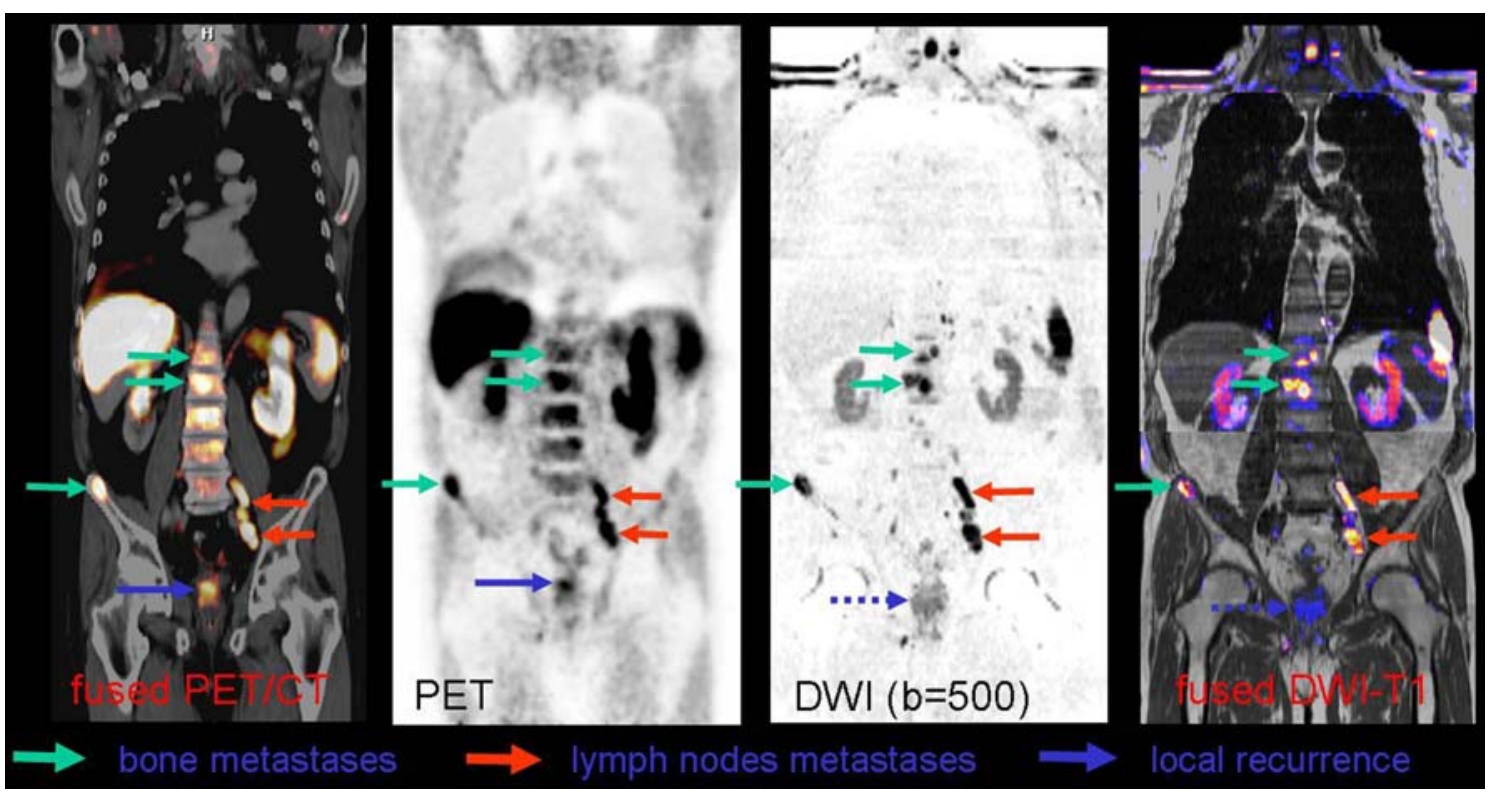


$\mathbf{J 3 0 7}$

\section{MOLECULAR IMAGING ASSESSMENT OF THE EFFECT OF ACUTE AND CHRONIC HYPOXIA IN GLIOBLASTOMA MULTIFORME PROGRESSION AND RESISTANCE TO RADIATION THERAPY}

Cheng- Hung Lee ${ }^{1}$, Ren-Shyan Liu ${ }^{2}$, Chia-Hung Hsieh ${ }^{1}$

${ }^{T}$ Graduate Institute of Basic Medical Science, China Medical University, Taichung, Taiwan, ${ }^{2}$ MAGIC, NRPGM, Dept. of NM, Faculty of Medicine, National Yang-Ming University, Taipei, Taiwan.

Tumor hypoxia or a reduction of the tissue oxygen tension is a key microenvironmental factor for tumor development, angiogenesis, growth and therapy resistance in many different human cancers. There is ample evidence to suggest that hypoxia plays a role in many aspects of cancer progression. Tumor hypoxia exists both chronically, due to the poorly organized vasculature of solid tumors, and acutely, due to fluctuations in blood flow. However, which hypoxia, either chronic or acute, played the main role in promotion of cancer progression or therapy resistance has not been addressed. The purpose of the present study was to determine whether experimentally imposed hypoxic stress in vivo, either chronic or acute, affects the process of tumor progression and the efficiency of radiotherapy in tumor-bearing mice. The human glioblastoma multiforme (GBM) cell line was stably transfected with a retroviral vector bearing a HIF-1-inducible "sensor" reporter gene (NESHSV1-tk/GFP:dMODC fusion) and a constitutively expressed "beacon" reporter gene (DsRed2/ XPRT) which allowed dynamic monitoring of HIF-1 signal transduction activity and tumor growth by nuclear imaging and optical imaging. We exposed tumor cells or mice bearing human glioblastoma multiforme (GBM) to low oxygen conditions (5-7\% $\mathrm{O} 2$ breathing) daily during tumor growth in an attempt to induce additional chronic $(2 \mathrm{~h} /$ day $)$ and acute $(12 \mathrm{x}$ $10 \mathrm{~min} /$ day) hypoxia in cells or tumors with or without radiotherapy. The results demonstrated that the acute but not the chronic hypoxia treatment significantly increased the tumor growth, invasion, angiogenesis and radioresistance in the mice. Furthermore, acute hypoxia resulted in a significant enhancement in HIF-1 signal transduction activity as compared with chronic hypoxia and control mice. Our data suggest that acute hypoxia is more efficiency than chronic hypoxia in promoting tumor progression and inefficiency of radiation therapy in GBM and its mechanism may be due to enhancement of the HIF-1 signal transduction activity.

\section{$\mathbf{J 3 0 8}$}

QUANTITATIVE OPTICAL IMAGING DEMONSTRATES ENHANCED LYMPHATIC DRAINAGE FROM VEGF-C OVEREXPRESSING TUMORS

Steven Proulx, Stefanie J. Derzsi, Matthias Rinderknecht,

Michael Detmar

Swiss Federal Institute of Technology (ETH) Zurich, Zurich, Switzerland.

Research on lymphatic metastases has been hindered by a lack of imaging methods to measure lymphatic flow from tumors to draining lymph nodes (DLN). The goal of the current study was to develop optical imaging biomarkers of DLN function in tumor models and test the effect of VEGF-C overexpression on lymphatic flow in vivo. A mouse melanoma cell line (B16-F1) was stably transfected with a pcDNA3.1 vector harboring human full length VEGF-C or empty vector and overexpression of VEGF-C was validated with real time RTPCR and ELISA. B16-VEGFC and B16-pcDNA tumors $\left(2 \times 10^{\wedge} 5\right.$ cells $)$ were induced in the footpads of C57/BL6 mice ( $n=6$ each group) and allowed to grow for 21 days. Dynamic near infrared fluorescence imaging (NIRF) of the DLN was performed for 1 hour after intradermal injection of indocyanine green (ICG) dye at the tumor site. Immunofluorescence staining for lymphatic and blood vessels using LYVE-1 and MECA-32 antibodies, respectively, was performed on frozen sections of both primary tumors and DLNs. Stable transfection of full length human VEGF-C into B16-F1 cells led to a strong increase in VEGF-C expression, as detected by real-time RT-PCR and ELISA of the highest expressing clone versus empty vector control. In vivo NIRF imaging of B16-pcDNA tumor DLNs versus non-tumor DLNs demonstrated enhanced flow in the tumor bearing animals. The time to peak enhancement was reduced from $42.8+/-11.5$ to $9.9+/-7.2$ min $(p=0.02)$ in the tumor-bearing animals. The clearance rate of ICG from the DLN increased from $0.76+/-0.28 \%$ per minute to $3.43+/-1.74 \%$ per minute $(p=0.01)$ in mice bearing tumors. VEGFC expression significantly decreased the time to peak enhancement $(2.7+/-0.5$ minutes, $p=$ $0.05)$ and increased the clearance rate of ICG dye $(7.25+/-0.81 \%$ per minute, $p=0.002$ ) versus B16-pcDNA tumor DLNs. Quantification of LYVE-1 staining revealed an increased lymphatic density in DLNs from B16-VEGFC tumors versus B16-pcDNA tumors (15.5\% versus $9.9 \%, p=0.01$ ). Tumor draining lymph nodes demonstrated enhanced lymphatic drainage that was further accelerated from tumors overexpressing human VEGF-C. The developed quantitative imaging technique could be useful to test lymphatic function during novel therapeutic interventions in preclinical studies and may have potential translational value as a predictive biomarker of lymphatic metastasis.

\section{J309}

CHARACTERIZATION OF A CELL-PENETRATING, OXYGENDEPENDENT DEGRADABLE STREPTAVIDIN AS A HYPOXIAINDUCIBLE FACTOR-1-ACTIVE TUMOR IMAGING AGENT Masashi Ueda ${ }^{1,2}$, Takashi Kudo ${ }^{2}$, Shinae Kizaka-Kondoh ${ }^{3}$, $\overline{\text { Azusa Miyano }}^{2}$, Kei Ogawa ${ }^{2}$, Masahiro Ono ${ }^{2}$, Takahiro Mukai ${ }^{4}$, Yuji Kuge ${ }^{5}$, Masahiro Hiraoka ${ }^{3}$, Hideo Saji ${ }^{2}$

${ }^{1}$ Radioisotopes Research Laboratory, Kyoto University Hospital, Kyoto, Japan, ${ }^{2}$ Grad. Sch. of Pharmaceutical Sciences, Kyoto University, Kyoto, Japan, ${ }^{3} \mathrm{Grad}$. Sch. of Medicine, Kyoto University, Kyoto, Japan, ${ }^{4} \mathrm{Grad}$. Sch. of Pharmaceutical Sciences, Kyushu University, Fukuoka, Japan, ${ }^{5}$ Central Institute of Isotope Science, Hokkaido University, Sapporo, Japan.

Introduction Hypoxia-inducible factor-1 (HIF-1) is a master transcriptional activator of various genes related to malignant phenotypes, and thus, the imaging of HIF-1-active tumors is important for targeting cancer therapy and predicting prognosis. We have developed a protein containing a protein transduction domain (PTD), an oxygen-dependent degradation domain (ODD) of HIF-1 $\alpha$, and a monomeric streptavidin (SAV) (PTDODD-SAV; POS), and succeeded in tumor hypoxia imaging using ${ }^{123} \mathrm{I}-$ labeled POS ( $\left({ }^{123}\right.$ I-IPOS). In the present study, we aimed to demonstrate that the accumulation of radioiodinated POS reflects the expression of HIF-1. Methods POS was overexpressed in Escherichia coli and ${ }^{125} \mathrm{I}-$ IPOS was obtained by the incubation of POS and ${ }^{125} \mathrm{I}$-labeled biotin derivative. The tumoral accumulation of ${ }^{125}$ I-IPOS was compared with HIF-1 activity using mice carrying tumors with the HIF-1-dependent luciferase reporter gene. The radioactivity in the tumors was analyzed by a size-exclusion chromatography. The intratumoral distribution of ${ }^{125}$ I-IPOS was examined by autoradiography, and then the same sections were subjected to double-fluorescent immunostaining for pimonidazole (PIMO), which is a hypoxic marker, and HIF-1. Results ${ }^{125}$ I-IPOS was obtained with radiochemical yield of $65 \%$ and radiochemical purity of greater than $95 \%$. More than $77 \%$ of radioactivity was eluted in the highmolecular-weight fraction, indicating the radioactivity in the tumors was mainly derived from ${ }^{125}$ I-IPOS. The tumoral accumulation of ${ }^{125}$ I-IPOS was positively and significantly correlated with HIF-1 activity $(R=0.71$, $P<0.05$ ). On immunohistochemistry, HIF-1-positive areas were not identical to the PIMO-positive areas but mainly existed both in the PIMO-positive areas and in the areas surrounding them. The intratumoral distribution of ${ }^{125}$ I-IPOS was heterogeneous and coincided with the HIF1-positive area. Conclusion The accumulation of radioiodinated POS reflects the expression of HIF-1. Thus, ${ }^{123}$ I-IPOS would be a useful imaging probe for HIF-1-active tumors. 


\section{J310}

\section{MOLECULAR CHARACTERIZATION OF THE RELATIONSHIP BETWEEN HYPOXIA, TOTAL CHOLINE AND BREAST CANCER STEM CELL MARKERS}

Balaji Krishnamachary, Marie-France Penet, Sridhar Nimmagadda, Yelena Mironchik, Meiyappan Solaiyappan, Dmitri Artemov, Kristine Glunde, Arvind P. Pathak, Paul T. Winnard, Venu Raman, Martin Pomper, Zaver M. Bhujwalla

JHU ICMIC Program, The Russell H. Morgan Department of Radiology and Radiological Science, The Johns Hopkins University School of Medicine, Baltimore, MD, USA.

The discovery of cells with stem-like characteristics in several cancers, that are the most likely to be resistant to therapy and to lead to recurrence and metastasis, is offering new paradigms for understanding and treating tumor recurrence and metastasis. Some of the molecular and functional markers that can be used to identify populations enriched with cells with stem-like characteristics are (i) CD44+/ CD24- or low phenotype for breast cancer, (ii) exclusion of Hoechst 33342 (ABCG2 transporter/BCRP) and rhodamine-123 (MDR1), (iii) increased aldehyde dehydrogenase (ALDH1) activity, and (iv) tumor growth from low cell inoculums. Recent studies suggest that hypoxia provides a suitable niche for stem cells to maintain their precursor status. In tumors, hypoxia is also a major cause of radiation and chemoresistance. We previously observed an association between hypoxia, total choline, and increased expression of CD44, a marker associated with stem-like breast cancer cells. Here we have performed molecular characterization of the role of hypoxia in increasing stem-like characteristics using MDA-MB-231 breast cancer cells stably expressing short hairpin RNA (shRNA) against HIF-1 $\alpha$. Additional molecular imaging validation was performed using tumors derived from MDA-MB-231 cells stably transfected with red-fluorescent tdTomato protein (RFP) expressed under control of the VEGF hypoxia response element (HRE). We confirmed with imaging, that hypoxia, increased CD44 expression, and increased total choline are co-localized in MDA-MB-231 breast cancer xenografts (Figure 1a, b). Molecular regulation of some stem-like breast cancer cell markers by hypoxia was also confirmed (Figure 1c, d). These data suggest that noninvasive imaging of hypoxia or metabolism can identify regions most likely to contain stem-like breast cancer cells, and that hypoxic environments and choline metabolism may be targeted to reduce stem-like cell burden and minimize tumor recurrence.
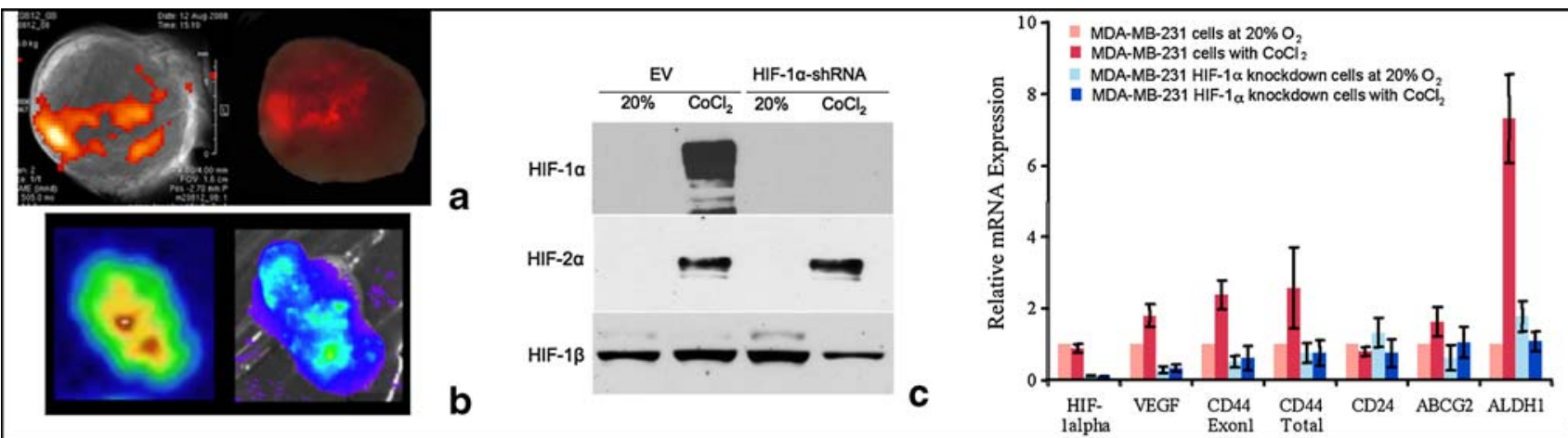

Figure 1a. Distribution of total choline (left) in an MDA-MB-231 HRE-RFP tumor $\left(\sim 250 \mathrm{~mm}^{3}\right)$. Distribution of RFP in $2 \mathrm{~mm}$ slice obtained from the $4 \mathrm{~mm}$ MRSI slice (right); the RFP image has not been warped to the MRS image but the association between total choline and hypoxia is evident. Figure 1b. SPECT image (left) from $2 \mathrm{~mm}$ fresh tissue slice of MDA-MB-231 tumor showing the distribution of CD44 expression. RFP image of this tissue section (right) obtained with Xenogen IVIS showing co-localization of fluorescence with high CD44 antibody expression. Figure 1c. MDA-MB-231 empty vector (EV) cells or a transduced population of MDA-MB-231 cells expressing HIF-1 $\alpha$ shRNA were maintained in an incubator at $37^{\circ} \mathrm{C}$ with or without $200 \mu \mathrm{M}$ of the hypoxia mimetic $\mathrm{CoCl}_{2}$. Resolved proteins were transferred to nitrocellulose membrane and probed with monoclonal antibody against HIF-1 $\alpha$ and re-probed with monoclonal antibody recognizing HIF-1 $\beta$. Figure 1d. MDA-MB-231 cells and a transduced population of MDA-MB-231 cells stably expressing HIF-1 $\alpha$ shRNA were maintained in an incubator at $37^{\circ} \mathrm{C}$ with or without $200 \mu \mathrm{M}$ of the hypoxia mimetic $\mathrm{CoCl}_{2}$. Twenty four hours later, total mRNA was isolated and cDNA prepared. Gene expression for VEGF and CD 44 was assessed by q-RT-PCR using specific primers. Two different CD44 primers spanning Exon 1 or designed from published data (CD44 total) were designed to recognize all isoforms of CD44. Relative mRNA expression is displayed normalized to values obtained for MDA-MB-231 cells maintained at $20 \% \mathrm{O}_{2}$. Values are Mean + S.E. obtained from three separate experiments.

\section{J311}

\section{PET IMAGING OF HEPATOCELLULAR CARCINOMA WITH L-[METHYL- ${ }^{11}$ C]METHIONINE}

Yu Kuang ${ }^{1}$, Nicolas Salem ${ }^{1}$, Fangjing Wang ${ }^{2}$, David J. Corn ${ }^{1}$, Jeffrey A. Kolthammer ${ }^{2}$, Bernadette Erowku ${ }^{1}$, Haibin Tian ${ }^{1}$, Ann-Marie Broome ${ }^{1,2}$, Zhenghong Lee ${ }^{1,2}$

${ }^{1}$ Radiology, University Hospitals Case Medical Center, Cleveland, $\mathrm{OH}$, USA, ${ }^{2}$ Biomedical Engineering, Case Western Reserve University, Cleveland, OH, USA.

L-[methyl- $\left.{ }^{11} \mathrm{C}\right]$ methionine (Met) was developed for imaging the amino acid (AA) transport, which is believed to be generally increased in tumor. Met mainly incorporates into protein synthesis as well as phosphatidylcholine (PC) synthesis via phosphatidylethanolamine (PE) methylation pathway. However, its metabolic routes in hepatocellular carcinoma (HCC) are not clearly identified. Addressing this issue will help to better understand how Met's uptake impacts the interpretation of the PET imaging data. Furthermore, the temporal information from Met metabolism will help to optimize imaging timing for a better signal and background ratio in clinical studies. In this study, (1) Dynamic scans on woodchuck models of HCC with Met were acquired for $60 \mathrm{~min}$. The images showed that HCC region had a higher uptake than surrounding hepatic tissue. The tumor uptake of Met stabilized at 20 min post-injection. The average signal to background ratio was 1.96; (2) A woodchuck HCC cell lines WCH17 was used to characterize the transport mechanism of Met during PET imaging. There was a $\mathrm{Na}$ dependency of Met uptake, which indicated an energy-dependent transport of Met in HCC. Uptake was dominated by BCH-sensitive transport, i.e. transport through system L. However, inhibition by MeAIB was insignificant for Met uptake, which rules out a relevant transport through system A; (3) To investigate the metabolic fate of Met uptake in $\mathrm{HCC},{ }^{3} \mathrm{H}-$ Met was pulsed on $\mathrm{WCH} 17$ cells for $5 \mathrm{~min}$ and then chased with cold media to simulate the rapid blood clearance of ${ }^{11} \mathrm{C}$-Met. Metabolite analysis found that the major intracellular ${ }^{3} \mathrm{H}$ activity was located mostly in protein phase, which is fairly stabilized within $55 \mathrm{~min}$ chase. In contrast, the uptake of 
Met in primary hepatocytes mainly reflects PC synthesis through PE methylation pathway; (4) In addition, cycloheximide was used to inhibit the protein synthesis pathway in $\mathrm{WCH} 17$, the conversion from water soluble phase to PC occurs slowly. The result suggested that the pathway from S-adenosylmethionine to lipid class is impaired in HCC which lacks PE N-methyltransferase 2 (PEMT2) activity. WCH17 cells were transfected with PEMT2 expression plasmid fused with CMV promoter to confirm that lower PEMT2 activity in WCH17 diverted the metabolic pathway of Met into protein synthesis. The relationship between PEMT2 and cell proliferation in HCC was also determined. Taken together, the study demonstrated that the different metabolisms between HCC and surrounding hepatic tissue contributed to the enhanced uptake of Met in HCC for PET imaging, which is not FDG-avid.

\section{J312}

\section{CHARACTERIZING POROSITIES IN THE FIBRILLAR COLLAGEN MESH OF THE EXTRACELLULAR MATRIX (ECM) OF SOLID TUMORS}

Samata M. Kakkad ${ }^{1}$, Meiyappan Solaiyappan ${ }^{1}$, Kristine Glunde ${ }^{1}$, Brian O'Rourke $^{2}$, Arvind P. Pathak ${ }^{1}$, Venu Raman ${ }^{1}$, Marie-France Penet ${ }^{1}$, Zaver M. Bhujwalla ${ }^{1}$

${ }^{1}$ JHU ICMIC Program, Department of Radiology and Radiological Science, The Johns Hopkins University School of Medicine, Baltimore, MD, USA, ${ }^{2}$ Institute of Molecular Cardiobiology, The Johns Hopkins University School of Medicine, Baltimore, MD, USA.
Quantitative analyses of fibrillar collagen distribution in the extracellular matrix $(E C M)$ from images obtained with second harmonic generation (SHG) microscopy (Figure 1a) is important to understand the role of these fibers in altering the transport characteristics of ECM in tumors. Such analyses are especially important to understand how hypoxic microenvironments typically found in tumors influence fiber distribution and transport. A major obstacle in quantitative characterization of fibrillar collagen distribution arises from the complex intersecting patterns of the fibers. Here we present a practical approach to characterize the distribution of collagen fibers by analyzing the interstices in the fibrillar mesh. Because of its robustness, our method requires minimal preprocessing steps and can be adapted to images of varying quality. We applied this approach to process previously acquired images from tumor xenografts derived from MDA-MB-231 human breast cancer cells genetically engineered to fluoresce under hypoxia (Figure 1b). Second harmonic images were obtained using the $880 \mathrm{~nm}$ laser line of a two-photon microscopy system. Images were filtered to exclude noise and non-fibrillar shapes in the image (Figures 1c-e). A 3D volume of the collagen fiber mesh, where the distances from each empty voxel representing the interstices within the mesh to the nearest collagen fiber voxel was computed (Figure 1f), providing a cumulative distance distribution as a measure of mesh porosity (Figure $1 \mathrm{~g}$ ). The histogram plots demonstrated a significant difference (Kolmogorov-Smirnov (K-S) test, $p<0.01$ ) in the distribution of fibers between hypoxic and non-hypoxic regions, despite the underlying geometric complexity of the mesh. Such methods could help track changes in fibrillar collagen that influence diffusive transport through the ECM of solid tumors. (a)
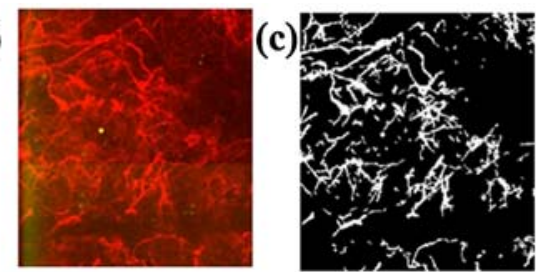

(b)

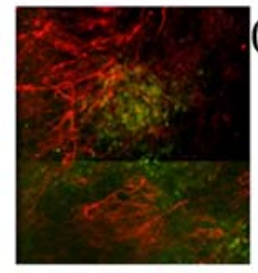

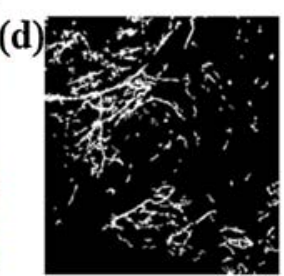

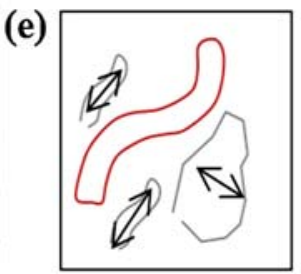

(f)

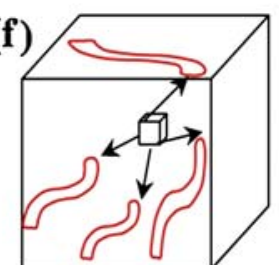

(g)

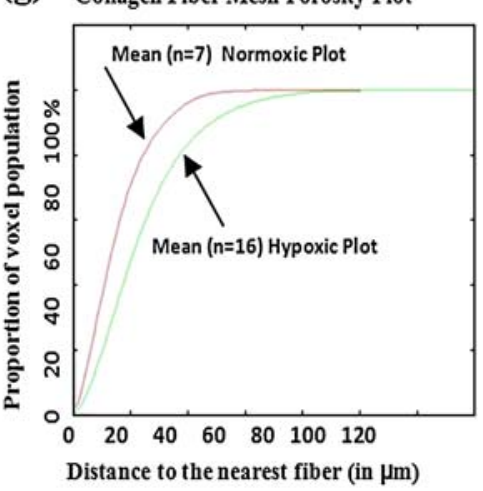

Figure 1. Construction of collagen fiber mesh porosity plot : (a),(b) composite images of the collagen fibers (red) in (a) normoxic and (b) hypoxic (green) regions; (c),(d) corresponding images after preprocessing using the filter (e) to identify fiber-like objects (red) among various non-fiber like objects in the image; (f) computing the distances in $3 \mathrm{D}$ to the surrounding fibers from each voxel to determine the nearest distance to a fiber; $(\mathrm{g})$ cumulative distance distributions demonstrating the differences in the mean distance to the nearest fiber from 7 normoxic regions (red) and 16 hypoxic regions (green).

J313

NEAR INFRARED FLUORESCENT IMAGING OF METASTATIC OVARIAN CANCER USING FOLATE RECEPTOR TARGETED HDL NANOCARRIERS

$\underline{\text { Ian Corbin }}{ }^{1}$, Kenneth K. $\mathrm{Ng}^{1}$, Han $\mathrm{Li}^{2}$, Lili Ding ${ }^{1}$, Andrea Jurisicova ${ }^{2}$, Gang Zheng ${ }^{1}$

${ }^{1}$ Medical BIophysics, Ontario Cancer Institute, University of Toronto,

Toronto, ON, Canada, ${ }^{2}$ Department of Obstetrics and Gynecology, Samuel Lunenfeld Research Institute, Mount Sinai Hospital, University of Toronto, Toronto, ON, Canada.
Ovarian cancer is the leading cause of death from gynecologic malignancies and is ranked as the 4th most common cancer mortality among women. This disease often follows a clinically silent course, thus at the time of diagnosis the majority of patients present with advanced disease that has spread beyond the ovaries. Given that metastatic disease from ovarian cancer is confined to the peritoneal cavity, intraperitoneal routes of drug delivery have been explored and show increased patient disease free periods. Unfortunately, the majority patients still succumb to their disease due to relapse or incomplete therapy due to dose limiting toxicities. These grim results have lead to active investigation of 
molecular targeted therapies which aim to address this major clinical need. Our group has recently engineered a folate receptor (FR) targeted High Density Lipoprotein (HDL) based nanocarrier that displays exceptional drug delivery properties. FR is constitutively over-expressed in the majority of ovarian cancers, hence FR is an appropriate target for ovarian cancer therapy. In this study we compared the targeting efficacy of intravenous versus intraperitoneal administered FR targeted fluorescent labeled HDL nanocarriers in a syngenetic orthotopic mouse model of ovarian cancer. Intravenous delivery resulted in high uptake of the fluorescent nanoparticle in healthy organs such liver and spleen, with relatively little uptake of the nanocarrier in solid metastatic lesions or free circulating cancer cells. Conversely, near infrared fluorescent images following the intraperitoneal administration of FR targeted HDL showed a disposition favoring selective tumor uptake. Fluorescence levels of the FR targeted nanoparticle within the solid tumor lesions equaled and even surpassed that seen in the host liver. Moreover, the uptake of FR targeted HDL within the free circulating cancer cells in the peritoneal cavity showed fluorescence intensities 1000 fold greater via intraperitoneal administration compared to the intravenous method of delivery. In summary, our findings demonstrate that local region intraperitoneal administration of FR targeted HDL nanocarriers selectively accumulates in both solid metastatic tumors and free circulating cancer cells. This is an attractive drug delivery system as it may provide an efficacious means of selectively delivering chemotherapeutics at a high dose to ovarian cancer cells while sparing healthy tissue from toxic adverse effects, thus improving our management and treatment of this highly aggressive gynecological malignancy.

\section{$\mathbf{J 3 1 4}$}

\section{PREDICTION OF THE NEOPLASTIC REGION IN ORAL CANCER USING MULTIPLE MOLECULAR-SPECIFIC CONTRAST AGENTS DETECTED WITH WIDEFIELD FLUORESCENCE IMAGING}

Kelsey J. Rosbach ${ }^{1}$, Darren Roblyer ${ }^{1}$, Michelle D. Williams ${ }^{3}$, Ann M. Gillenwater ${ }^{2}$, Rebecca Richards-Kortum ${ }^{1}$

${ }^{1}$ Bioengineering, Rice University, Houston, TX, USA, ${ }^{2} \mathrm{Head}$ and Neck Surgical Oncology, M. D. Anderson Cancer Center, Houston, TX, USA, ${ }^{3}$ Pathology, M. D. Anderson Cancer Center, Houston, TX, USA.

Background: Early detection of pre-cancerous lesions and cancer in the oral cavity dramatically improves patient survival, however, dysplasia can be difficult to detect under white light inspection alone. Non-invasive, molecular-specific optical imaging has the potential to rapidly assess expression of biomarkers that indicate neoplastic changes, which may aid in detection of neoplasia at the point of care. Information about biomarker expression may also aid in determination of an appropriate surgical margin, ensuring that all neoplastic tissue is removed without removing excess normal tissue. Methods: In this study, we used two targeted fluorescence contrast agents to gain information about two different biomarkers commonly overexpressed in oral cancer. The first agent was 2-NBDG (Invitrogen, CA), a fluorescently tagged glucose molecule to target overexpression and activity of GLUT transporters. The second was a fluorescently tagged epidermal growth factor (EGF) peptide to target overexpression of the EGF receptor. Both contrast agents were topically applied to freshly resected oral lesions $(n=20)$ and imaged with a widefield fluorescence microscope (16 $\mu \mathrm{m}$ resolution, $5 \times 7 \mathrm{~cm} \mathrm{FOV)}$ after a 20 minute incubation period. Using Matlab software (MathWorks, MA), the fluorescence images were converted to binary using a threshold value set above the average intensity of a histologically normal region from the same patient. The binary image was smoothed to identify a contiguous region. The outline of this region (a potential "margin") was overlaid onto a pathology map to assess agreement to the gold standard. Results: The predicted margin from both contrast agents matches the pathology well. Not only can invasive cancer be detected, but difficult to identify regions of mild dysplasia are also recognized as abnormal. Varying the threshold adjusts the sensitivity and specificity of this margin, which can be calculated based on the area of the region correctly diagnosed or not. IHC confirms expression level of EGF receptors and GLUT transporters. Conclusions: We have demonstrated that non-invasive optical imaging of biomarker expression may help identify oral neoplasia. This may assist a clinician in choosing a region to biopsy for initial diagnosis or in determining an appropriate surgical margin during treatment. The technique can also be applied to other epithelial cancers, thus impacting a large range of clinical cases.

\section{J315}

\section{INTRAVITAL MOLECULAR IMAGING OF THE BIRTH OF A TUMOR FROM CANCER STEM CELLS USING INTRAVITAL MICROSCOPY}

Natesh Parashurama ${ }^{1}$, Neethan Lobo ${ }^{2}$, Michael F. Clarke ${ }^{2}$, Sanjiv S. Gambhir ${ }^{1}$

${ }^{1}$ Department of Radiology, Molecular Imaging Program at Stanford, Stanford University, Stanford, CA, USA, ${ }^{2}$ Institute for Stem Cells and Regenerative Medicine, Stanford University, Stanford, CA, USA.

Despite phenotypic enrichment of breast cancer stem cells (CSC), the early detection of breast cancer by targeting CSC remains elusive. We hypothesized that incorporating imaging into CSC assays could provide novel insights into the early mechanisms of tumor initiation from CSC. Furthermore, we reasoned that imaging CSC can be done in the adult, postnatally developing mammary gland, and regenerating mammary gland after transplantation. To image the 4th adult mammary gland, a surgical flap was created and stabilized in an anesthetized, transgenic, virgin, BL6 eGFP (and DsRed) female mouse in which mammary ducts express high levels of fluorescent protein. Mammary ducts and lobules could be visualized with 4,10 , and $20 \mathrm{x}$ objectives in both adult $(N=10)$ and developing $($ age $=3-4.5$ weeks, $N=4)$ eGFP + mice. This technique, which utilizes a multichannel intravital microscope, has a spatial resolution of approximately $1 \mu \mathrm{m}$, and depth penetration of approximately $150 \mu \mathrm{m}$. Furthermore, vascular structures, ranging from $1-500 \mu \mathrm{m}$, were imaged using a fluorescent $(750 \mathrm{~nm})$ intravascular dye $(N=5$ mice $)$. To image regenerating mammary ducts, 50,000 cells of an eGFP+, Linpopulation, which contains rare mammary stem cells, were transplanted into the cleared fat pads of wild type hosts. Regenerating glands were repetitively imaged at 8 and 12 weeks after transplantation $(N=5)$. Importantly, cellular structures in living subjects could be resolved for the first time in all systems. Furthermore, we imaged 5000 Lineage-, CD44+, human breast CSC that had been lentivirally transduced with reporter gene expressing tomato fluorescent protein, and transplanted into the mammary fat pad of NOD-SCID mice for 10 days. Having 
established these systems, we will image single CSC, in both mouse and humans, and corresponding changes in microvasculature in tandem. These approaches should markedly change our understanding of breast tumor development in living subjects, and advance the early detection of, or early therapeutic targeting of CSC.
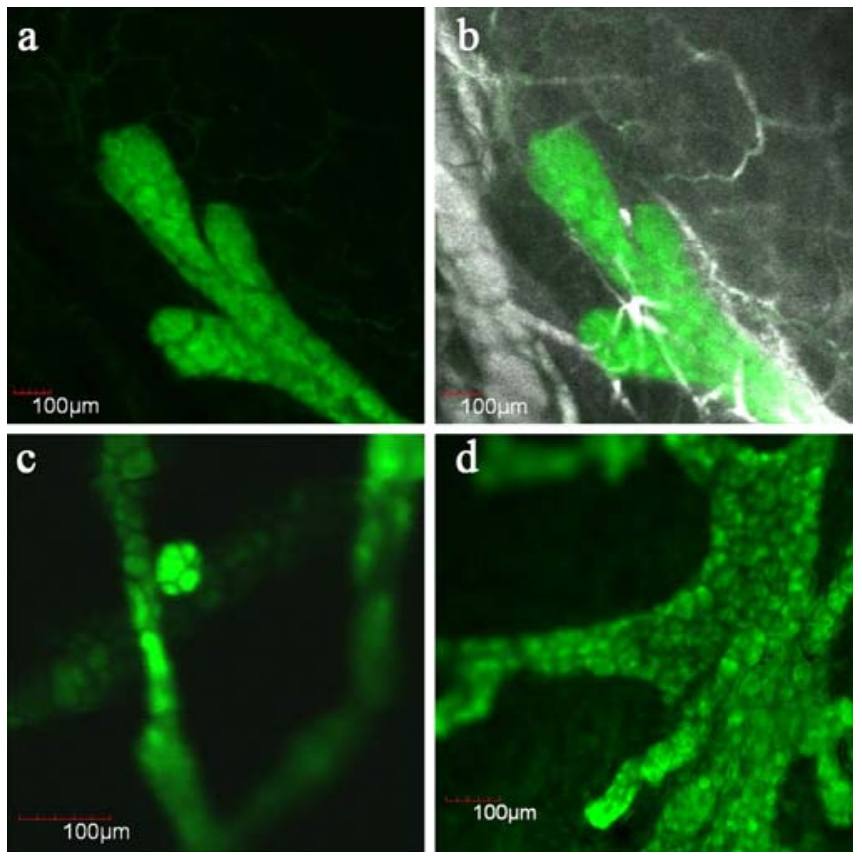

Figure 1. Repetitve, non destructive intravital imaging of the mammary gland in living mice. All images were captured with a live mouse with surgically exposed mammary gland in eGFP + transgenic mouse, $488 \mathrm{~nm}$ laser, as part of a depth stack of several images a) Intravital image of a portion of the eGFP + mammary gland in live transgenic mouse $4 \mathrm{x}$ objective and $\mathrm{zoom}=2 \mathrm{x}$. Demonstrates cellular detail in the normal duct. b) Same as (a) except a second channel added, at 750nm, which demonstrates the normal duct surrounded by normal microvasculature (near Infra-red emission), 1 hour after fluorescent dye injection c) Image of regenerated gland obtained, 8 weeks after transplantation, 10X objective zoom $=4 \mathrm{x}$, demonstrating duct and budding area near duct, and cell-like detail in walls of duct d) Image of primary duct and branches of 4.5 week old eGFP + mouse, $4 x$ objective, zoom $=3 x$ Demonstrates cellular level detail, with numerous subcellular features present.

\section{J316}

THE ROLE OF FDG PET/CT IN PERIAMPULLARY CARCINOMA MANAGEMENT

Jyotsna Rao, Kavitha Nallapareddy, Alka A. Chengapa,

Sikandar M. Shaikh

PET/CT, Apollo Gleneagles PET/CT Ctr, Hyderabad, India.

Periampullary carcinoma is difficult to diagnose and has a poor prognosis with very low five year survival rates. However, FDG
PET/CT may help in staging and further management and we present retrospective data analysis from our center. Material and method: Data analysis of patients referred between May 2005 and December 2008 was done retrospectively. Patients were scanned per standard whole body protocol after intravenous injection of $10-15 \mathrm{mCi}$ of F18 FDG after 4 hours of fasting. Oral and intravenous contrast was given to patients. Reporting was done jointly by a radiologist and PET physician. Follow up was obtained where possible. Results: 19 patients with the diagnosis or suspected diagnosis of periampullary carcinoma were referred to the center.Of the 19 patients, $1(5 \%)$ was referred for diagnosis, 6/19(32\%) for staging, 10/19(50\%) for restaging and $2 / 19(13 \%)$ for monitoring response to therapy. The 1 patient referred for diagnosis showed metastases but the primary was negative on PET. Of the 6 patients scanned for staging, in $1 / 6$ $(16.5 \%)$ the primary was negative on PET with metastases showing FDG uptake. The other $4 / 6(67 \%)$ patients showed both the primary and metastases on PET/CT. 1/6(16.55\%) staged patient showed only the primary tumor on PET/CT. Of the 10 patients scanned for restaging, $3 / 10(30 \%)$ were negative, $2 / 10$ $(20 \%)$ showed tumour recurrence and metastases, the rest $5 / 10$ $(50 \%)$ showed metastases only with $1 / 5(20 \%)$ of them showing a liver lesion only on PET. The two patients scanned to monitor response to therapy showed residual viable primary tumour with metastases. Metastatic sites in the positive patients included nodes, liver, spleen, peritoneum and skeleton. Conclusion: PET/CT may be helpful in the management of periampullary carcinoma and should be considered in the work up although,it is not a common malignancy.

\section{$\mathbf{J 3 1 7}$}

\section{THE ROLE OF FDG PET/CT IN THE MANAGEMENT OF GALL} BLADDER CARCINOMA

Jyotsna Rao, Kavitha Nallapareddy, Alka A. Chengapa,

Sikandar M. Shaikh

Apollo Gleneagles PET CT Ctr, Hyderabad, India.

India has the highest incidence of gall bladder carcinoma in the world. It is surgically curable in stage I and II although, the overall prognosis is poor. We present our experience in FDG PET/ $\mathrm{CT}$ in the management of gall bladder carcinoma. Aim: To evaluate the role of FDG PET/CT in the management of gall bladder carcinoma. Material and method: A retrospective data analysis of patients for FDG PET/CT for gall bladder carcinoma evaluation between May 2005 and December 2008 was done. All patients were scanned after intravenous injection of $10-15 \mathrm{mCi}$ of F18 FDG after 4 hours of fasting. Oral and intravenous contrast was given to patients who were scanned per standard whole body protocol. Retrospective data analysis of 24 patients with gall bladder carcinoma referred to our center was done. Patients were injected with $10-15 \mathrm{mCi}$ of F18 FDG following 4 hours of fasting. Oral and intravenous contrast was given and patients scanned per standard whole body protocol. Reporting was done jointly by a radiologist and PET physician. Follow up data were obtained where possible. Results: $1 / 24(5 \%)$ of the patients was referred for diagnosis of malignancy. 9/24(37\%) patients were referred for staging where PET/CT identified the primary in all patients. CT showed positive nodal disease in $1 / 9(11 \%)$ patient. Of the $12 / 24$ $(50 \%)$ restaged patients, $2 / 12(16 \%)$ were negative, $1 / 12(8 \%)$ was positive only on PET in the liver, 3/12(25\%) showed local recurrence with metastases and 6/12(50\%) showed metastases only. In $2 / 24(8 \%)$ of the patients referred to monitor response to therapy, 
viable metastases were identified. Metastatic sites in the positive patients included the liver, spleen, peritoneum, adrenals and nodes. Conclusion: Our experience indicates that in gall bladder carcinoma, although uncommon, FDG PET/CT may have a role to play in patient management from the time of diagnosis to monitoring response to therapy.

\section{J318}

\section{THE ROLE OF FDG PET/CT IN OCULAR MALIGNANCY}

Jyotsna Rao, Kavitha Nallapareddy, Alka A. Chengapa,

Sikandar M. Shaikh

Apollo Gleneagles PET CT Ctr, Hyderabad, India.

Ocular malignancies, primary and metastatic are not very common and are difficult to manage needing a multidisciplinary approach. We present our experience in FDG PET/CT at our center in patients referred for various intra and extra ocular malignancies. Aim: To assess the utility of FDG PET/CT in the management of ocular malignancies. Material and method: A retrospective data analysis of patients referred for intra and extra ocular malignancy from May 2005 to December 2008 was done. Patients were injected with $10-15 \mathrm{mCi}$ of intravenous F18 FDG after 4 hours of fasting. Oral and intravenous contrast was also given and patients were imaged per standard whole body protocol. The scans were reported jointly by a radiologist and PET physician. Follow up was obtained where possible. Results: Retrospective analysis of reports of 14 patients referred with ocular malignancy was done. $4 / 14(29 \%)$ patients were referred for staging, 9/14(64\%) for restaging and $1 / 14(5 \%)$ to monitor response to therapy. Tumor types included $7 / 14(50 \%)$ patients with lymphoma, 4/14(28\%) with melanoma, 2(/14(14\%) with mucoepidermoid tumor and 1/14 $(8 \%)$ with lacrimal gland tumor. $2 / 4(50 \%)$ staged patients showed the primary orbital tumor on PET/CT and liver and lung lesions on CT. $1 / 4(25 \%)$ in post operative staging showed extensive nodal disease. $1 / 4(25 \%)$ staged patient showed primary tumor and nodal disease. $4 / 9(44 \%)$ of the restaged patients were negative, $2 / 9(22 \%)$ showed lung lesions on CT only and $2 / 9(22 \%)$ patients showed recurrence at the primary site and nodal disease, $1 / 2(50 \%)$ of them showing more nodal abnormalities on CT. 1/9(12\%) restaged patient showed a lung lesion only on CT. 1 patient who showed extensive nodal tumor recurrence on the baseline restaging scan showed residual disease on a follow up post therapy scan suggestive of partial response.Metastatic sites in the melanoma patients included nodes, liver, bones and lungs. Extra nodal sites of involvement in the lymphoma patients included the spleen, liver, pancreas and stomach. Conclusion: Our initial experience shows that FDG PET/CT may be helpful in the management of ocular malignancy of various types although, only a small group of patients was analyzed at our center.

\section{J319}

THE ROLE OF FDG PET/CT IN CUTANEOUS LYMPHOMA Jyotsna Rao, Kavitha Nallapareddy, Alka A. Chengapa, Sikandar M. Shaikh

Apollo Gleneagles PET CT Ctr, Hyderabad, India.

Cutaneous lymphomas are relatively rare but F18 FDG PET/CT has been reported to be useful in their management. We present our experience in the imaging of this malignancy which has a broad spectrum of presentation. Aim: To evaluate the role of FDG $\mathrm{PET} / \mathrm{CT}$ in the management of cutaneous lymphoma. Material and method: A retrospective analysis of 12 reports of patients with cutaneous lymphoma who were referred to our center between May 2005 and December 2008 was performed. Patients were injected intravenously with $10-15 \mathrm{mCi}$ of F18 FDG after $4 \mathrm{~h}$ of fasting and scanned per standard whole body protocol after giving oral and intravenous contrast. The scan were reported jointly by a radiologist and PET physician. Follow up was obtained where possible. Results: $2 / 12(17 \%)$ were referred for staging of which $1 / 2$ $(50 \%)$ was negative with the other showing gluteal region tumor, nodal disease and additional bone CT lesions. Of the 7/12(58\%) referred for restaging, $3 / 7(43 \%)$ of the scans were reported negative and $2 / 7(29 \%)$ of the restaged patients showed extensive cutaneous and nodal recurrence. $1 / 7$ of the restaged patient (14\%) showed a subcentimeter lung nodule with no abnormality on PET. 1/7(14\%) showed a subcentimeter nodule only on CT and cutaneous abnormality only on PET at the primary site. Of the $3 / 12(25 \%)$ patients who were referred to monitor response to chemotherapy, 2/ $3(66 \%)$ showed residual viable cutaneous and subcutaneous tumour and $1 / 3(34 \%)$ showed sclerotic bony lesions on CT alone. 1/12 (8\%) patient was referred 5 times for indications of staging, restaging and monitoring response to therapy. 1/12(8\%) was referred twice for restaging and monitoring response to therapy. Conclusion: In the small group of patients with cutaneous lymphoma referred to our center, FDG PET/CT proved useful in their management.

\section{$\mathbf{J 3 2 0}$}

\section{THE ROLE OF FDG PET/CT IN THYROID CARCINOMA}

Jyotsna Rao, Kavitha Nallapareddy, Alka A. Chengapa, Sikandar M. Shaikh

Apollo Gleneagles PET CT Ctr, Hyderabad, India.

Thyroid cancer represents $1 \%$ of all new cancers appearing every year and has a more favorable prognosis compared to other cancers. Nuclear scanning with I131 plays an important role in its management and will continue to do so particularly in well differentiated tumors. We present our experience with F18 FDG PET/CT with thyroid carcinoma. Aim: To assess the role of FDG $\mathrm{PET} / \mathrm{CT}$ in the management of thyroid cancer. Material and method: Retrospective data analysis of patients referred to our centre from May 2005 to December 2008 was done. The patients were injected intravenously with $10-15 \mathrm{mCi}$ of F18 FDG after 4 hours of fasting after giving oral and intravenous contrast. Scanning was done per standard whole body protocol. The scan was reviewed jointly by a radiologist and PET physician. Follow up was obtained where possible. Results: 42 patients were referred to our center for evaluation for various indications of thyroid malignancy. 1/42(2\%) referred for diagnosis showed the primary with associated metastases. $4 / 42(9 \%)$ referred for staging showed the primary tumor and metastases. 36/42(86\%) were referred for restaging of which $12 / 36(33 \%)$ were negative on PET/CT. 6/36 (17\%) showed positive findings on CT alone-4/6(66\%) showed subcentimeter lung nodules, $1 / 6(17 \%)$ showed nodes, subcentimeter lung nodule and a liver lesion and $1 / 6(17 \%)$ showed a nodule in the ampullary region. $1 / 36(2 \%)$ was positive only on PET for metastatic nodes. $10 / 17(49 \%)$ restaged patients who were positive on PET/CT showed only metastases, 5/17(29\%) showed local tumour recurrence and $2 / 17(9 \%)$ showed local tumor recurrence and metastases. $1 / 17(6 \%)$ showed diffuse activity in the thyroid bed suggestive of post treatment change secondary to external radiation. 
$10 / 36(28 \%)$ of the restaged patients had documented rising thyroglobulin of which $1 / 10(10 \%)$ was negative on PET/CT and $1 / 10(10 \%)$ showed a subcentimeter lung nodule only on CT. 1/42 (2\%) patient who was initially staged with PET/CT was referred to monitor response to radio iodine ablation and showed reduced activity and size in the primary and metastases. Metastatic sites in the positive patients included nodes, lungs, liver, bones, brain and adrenals. Conclusion: FDG PET/CT proved useful in the management of patients with thyroid carcinoma, particularly while restaging them.

\section{J321}

\section{FDG PET/CT AND CARCINOMA CERVIX}

Jyotsna Rao, Kavitha Nallapareddy, Alka A. Chengapa, Sikandar M. Shaikh

Apollo Gleneagles PET CT Ctr, Hyderabad, India.

Carcinoma cervix is the most common female malignancy in India and early diagnosis and treatment lead to significant five year survival rates. We present our experience in PET/CT imaging in carcinoma cervix. Aim: To evaluate the role of FDG PET/CT in the management of carcinoma cervix. Material and method: Retrospective data analysis of patients referred to our centre from January 2006 to December 2008 was done. Patients were injected intravenously with $10-15 \mathrm{mCi}$ of F18 FDG after 4 hours of fasting and scanned per standard whole body protocol. Oral and intravenous contrast was given to patients. The scans were reported by a radiologist and PET physician jointly. Follow up was obtained where possible. Results: Data of 74 patients with cervical malignancy were analyzed.7/74(9\%) were referred for staging and 66/74 (89\%) for restaging. $2 / 7(28 \%)$ of the staged patients showed only the primary tumor. $5 / 7(72 \%)$ of the staged patients showed the primary and metastases. $17 / 66(26 \%)$ of the restaged patients were negative on PET/CT. 23/66(34\%) showed only metastases. 9/66 (14\%) showed local tumor recurrence and metastases.4/66(7\%) showed only local tumor recurrence. $8 / 66(12 \%)$ of the restaged patients showed metastases on CT alone and 1/66(1\%) showed a bulky cervix with no FDG uptake. 1/66(1\%) showed focal FDG uptake in the cervix with no obvious corresponding CT abnormality. $1 / 66(1 \%)$ showed a mildly hypermetabolic right lung opacity suggestive of a benign etiology . $2 / 66(2 \%)$ of the restaged patients showed breast and axillary nodal uptake. 1/74(1\%) patient was referred to monitor response to therapy who showed resolution of a previous node and decrease in metabolism of an abdominal wall metastasis. Conclusion: FDG PET/CT proved useful in the management of carcinoma cervix patients at our center and is to be considered part of the treatment protocol, particularly for restaging patients.

\section{$\mathbf{J 3 2 2}$}

\section{FDG PET/CT IN PEDIATRIC ONCOLOGY}

Jyotsna Rao, Kavitha Nallapareddy, Alka A. Chengapa, Sikandar M. Shaikh

Apollo Gleneagles PET CT Ctr, Hyderabad, India.

Treatment and outcome for pediatric malignancy have improved over the years and at least $70 \%$ are now curable. Keeping this in view, early diagnosis, staging, restaging and monitoring response to therapy become important in management. We present our experience with FDG PET/CT in pediatric malignancies. Aim: To assess the role of FDG PET/CT in the management of pediatric oncology. Material and method: A retrospective analysis of data of pediatric patients ranging from infancy to age 16 referred for various oncologic indications from May 2005 to December 2008 to our center was done. Patients were injected intravenously with F18 FDG 4 hours after fasting. Oral and intravenous contrast was given to patients. Sedation was given when required. Scans were reported jointly by a radiologist and PET physician. Follow up was obtained where possible. Material and method: Retrospective data analysis of 69 pediatric patients was performed. 62/69(89\%) were referred for whole body PET/CT scans for the following indications lymphoma $37 / 62(60 \%)$, soft tissue sarcoma 13/62(21\%), ovarian tumor (granulosa cell tumor) 4/62(6\%), neuroblastoma 3/62(5\%), Wilm's tumor $1 / 62(2 \%)$, granulosa cell tumor of the brain stem $1 /$ $62(1 \%)$ and pinealoblastoma $3 / 62(5 \%)$. $7 / 69(12 \%)$ were referred for brain scans for glioma $4 / 7(57 \%)$, medulloblastoma $2 / 7(29 \%)$ and chordoma $1 / 7(14 \%) .1 / 62(1 \%)$ patients who had whole body scans was referred for diagnosis, $1 / 62(1 \%)$ was referred for staging, 54/ $62(88 \%)$ patients were referred for restaging and $6 / 62(10 \%)$ were referred to monitor response to therapy. Of the brain scans performed, $1 / 7(14 \%)$ was referred for diagnosis and the remaining $6 / 7(86 \%)$ were scanned for evaluation after therapy, radiation and/ or chemotherapy. $24 / 54(44 \%)$ of the restaged patients were negative on PET/CT. The remaining $30 / 54(56 \%)$ patients referred for diagnosis, staging and restaging were positive for tumor, primary and/or metastases. Of the 7 brain PET/CT scans performed, the $1 / 7(14 \%)$ performed for diagnosis was positive, $2 / 7(29 \%)$ of the patients referred after treatment of brain tumor were negative and the remaining $4 / 7(57 \%)$ were positive for tumor. Conclusion: FDG PET/CT in pediatric oncology proved useful at our center and should be considered more often during management.

\section{$\mathbf{J 3 2 3}$}

\section{DETECTING RESPONSE TO TREATMENT IN HUMAN BREAST ADENOCARCINOMA USING A CO-ADMINISTRATION OF HYPERPOLARIZED $\left[1{ }^{13} \mathrm{C}\right]$ PYRUVATE AND $\left[1,4-{ }^{13} \mathrm{C}_{2}\right]$} FUMARATE

Timothy Witney $^{1,2}$, Mikko I. Kettunen ${ }^{1,2}$, De-En $\mathrm{Hu}^{1,2}$, Ferdia Gallagher ${ }^{3,1}$, Kevin Brindle ${ }^{1,2}$

${ }^{1}$ Dept. of Biochemistry, University of Cambridge, Cambridge, United Kingdom, ${ }^{2}$ Cancer Research UK Cambridge Research Institute, Cambridge, United Kingdom, ${ }^{3}$ Dept. of Radiology, Addenbrooke's Hospital, University of Cambridge, Cambridge, United Kingdom.

Tumor response to therapy is assessed primarily in the clinic by monitoring reductions in tumor size. However, this approach lacks sensitivity since in many cases several weeks may elapse before there is evidence of tumor shrinkage. The aim of this study was to determine whether hyperpolarized ${ }^{13} \mathrm{C}$ MRS could be used to detect response to therapy in a model of human breast adenocarcinoma. Addition of $75 \mathrm{mM}$ hyperpolarized $\left[1-{ }^{13} \mathrm{C}\right]$ pyruvate to a suspension of untreated MDA-MB-231 cells resulted in detectable exchange of label with added lactate, in the reaction catalyzed by lactate dehydrogenase. Co-administration of $20 \mathrm{mM}$ hyperpolarized $\left[1,4-{ }^{13} \mathrm{C}_{2}\right]$ fumarate to untreated cells resulted in no detectable appearance of signal corresponding to hyperpolarized $\left[1,4-{ }^{13} \mathrm{C}_{2}\right]$ malate. Treatment of cells with the topoisomerase type I 
inhibitor, doxorubicin, for $72 \mathrm{~h}$ resulted in a 7.5 -fold increase in annexin $\mathrm{V}$ staining, indicating the induction of apoptosis, as measured by flow cytometry. Low levels of necrosis were also observed by SYTOX Red staining. At this time point a $48 \%$ drop in pyruvate-lactate exchange $(p<0.05)$, coinciding with a $91 \%$ reduction of intracellular $\mathrm{NAD}(\mathrm{H})$, was observed (Fig. $1 \mathrm{~A}+\mathrm{B}$ ). Peaks corresponding to hyperpolarized $\left[1,4-{ }^{13} \mathrm{C}_{2}\right]$ malate were also observed $72 \mathrm{~h}$ post treatment (Fig.1C $+\mathrm{D}$ ). The largest decrease in pyruvate-lactate exchange was evident at $96 \mathrm{~h}$ when the majority of cells were necrotic. A further $220 \%$ increase in fumarate-malate flux $(p<0.001)$ was also observed $96 \mathrm{~h}$ post treatment. These data show that the decrease in lactate-pyruvate exchange coincides with both the induction of cell death and the depletion of the intracellular $\operatorname{NAD}(\mathrm{H})$ pool in breast cancer cells. Fumarate-malate flux also appears to indicate the onset of necrosis. Future studies will focus on determining whether hyperpolarized pyruvate and fumarate can be used as markers of cell death in implanted breast cancer tumors following treatment.

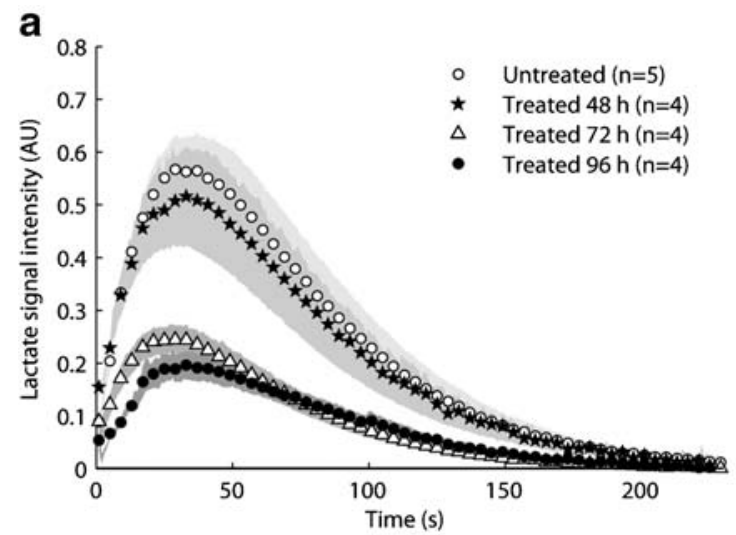

b
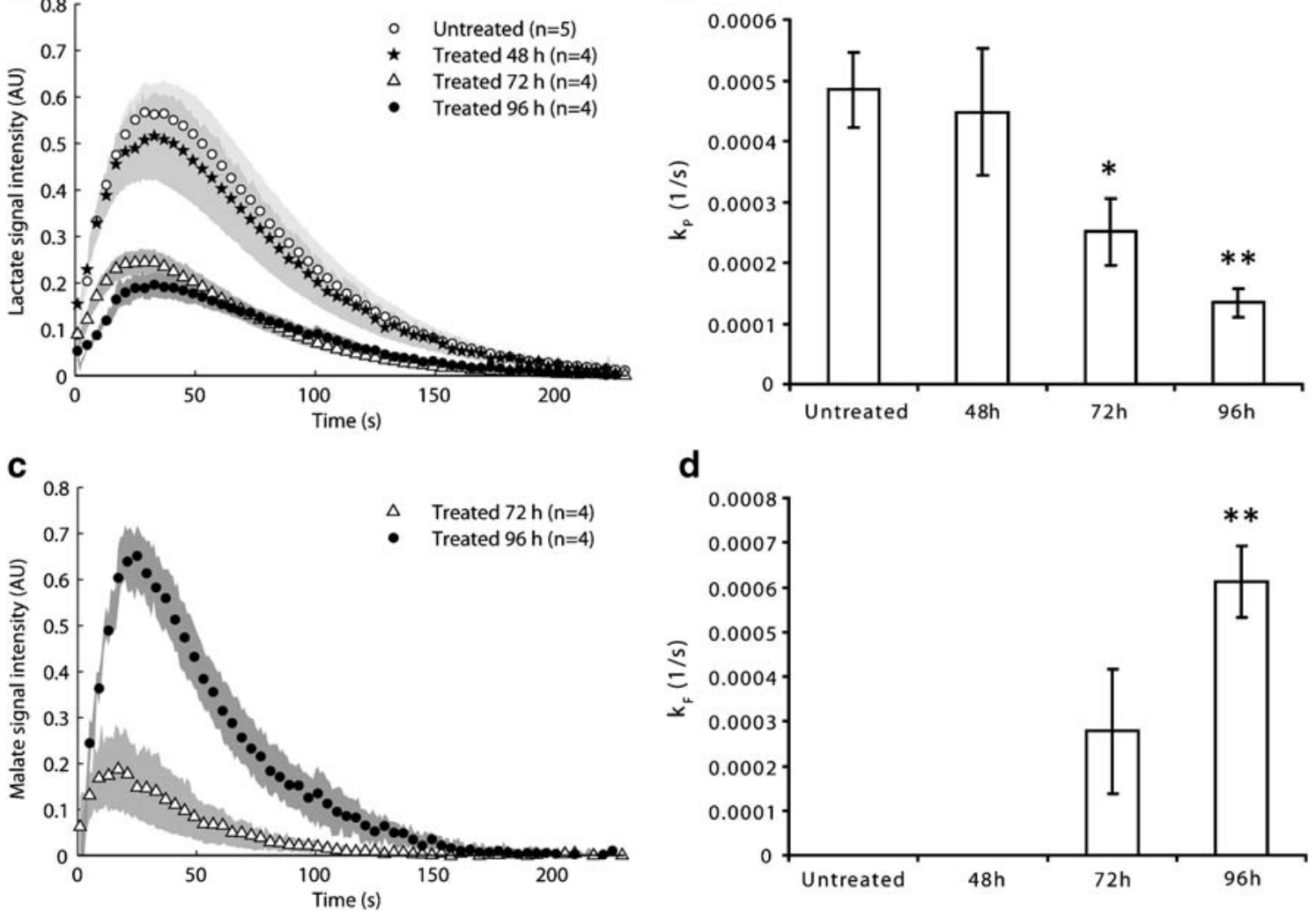

Fig.1- Effect of doxorubicin treatment on hyperpolarized pyruvate-lactate exchange $(A+B)$ and hyperpolarized fumarate-malate exchange $(C+D)$ in $M D A-M B-231$ cells.

\section{$\mathbf{J 3 2 4}$}

MOLECULAR MRI ASSESSMENT OF VASCULAR ENDOTHELIAL GROWTH FACTOR RECEPTOR-2 IN RAT C6 GLIOMAS

Ting $\mathrm{He}^{1,3}$, Nataliya $\mathrm{Smith}^{1}$, Debra Saunders ${ }^{1}$, Sabrina Doblas ${ }^{1}$, Jessica Hoyle $^{1}$, Robert Silasi-Mansat ${ }^{2}$, Florea Lupu ${ }^{2}$, Megan Lerner ${ }^{4}$, Daniel J. Brackett ${ }^{4}$, Rheal Towner ${ }^{1,3}$

${ }^{1}$ Advanced Magnetic Resonance Center, Oklahoma Medical Research Foundation, Oklahoma City, OK, USA, ${ }^{2}$ Cardiovascular Biology, Oklahoma Medical Research Foundation, Oklahoma City, OK, USA, ${ }^{3}$ Oklahoma Center for Neurosciences, University of Oklahoma Health Sciences Center, Oklahoma City, OK, USA, ${ }^{4} \mathrm{O}^{\prime}$ Donoghue Research Institute, University of Oklahoma Health Sciences Center, Oklahoma City, OK, USA.
Evaluation of angiogenesis is important for tumor early diagnosis and treatment. The quantitative and dynamic in vivo assessment of tumor angiogenesis can be achieved by molecular magnetic resonance imaging (mMRI). Vascular endothelial growth factor (VEGF) and VEGF receptors (VEGFRs) are the main regulatory systems for angiogenesis. Limited research has been done using mMRI to target VEGF/VEGFRs. In our study, we aimed to assess the expression of VEGFR2 at late-stage development of tumor in a C6 glioma model by using specific molecular probes (anti-VEGFR2-albumin-Gd-DTPA-biotin) with mMRI. The differential uptake of probes that are conjugated by a monoclonal antibody $(\mathrm{mAb})$ to VEGFR2, shown by varied increases in MR signal intensity (see Fig. 1) and corresponding decreases in T1 values during a 2 hour period, demonstrated the heterogeneous expression of VEGFR2 in different tumor regions compared to normal brain tissue. These 
results correlated with VEGFR2 expression by immunohistochemistry. Microscopic fluorescence imaging for the biotin group with streptavidin-Cy3 confirmed a sustained retention of the VEGFR2 probe in glioma tissue. The angiogenesis process was also assessed using MR angiography (MRA), which indicated a significant increase in tumor blood volume and a change in vasculature morphology induced by the tumor. Together these results suggest mMRI can be very useful in assessing and characterizing the expression of specific angiogenic markers in vivo and help establish angiogenesis associated with tumor progression.

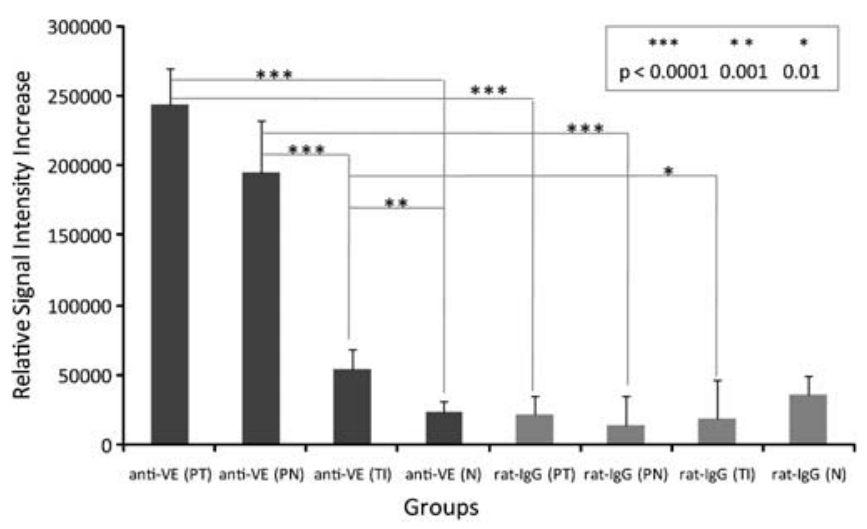

Figure 1: Differences in signal intensities in different brain regions (PT is peripheral tumor, $\mathrm{PN}$ is peripheral necrosis, TI is tumor interior, and $\mathrm{N}$ is normal brain tissue; anti-VE is for the anti-VEGF-R2 probe; rat-IgG is non-specific normal rat $\mathrm{IgG}$ coupled to albumin-Gd-DTPA-biotin) measured between $2 \mathrm{hrs}$ post- and pre- administration of control-IgG or VEGFR2-mAb linked mMRI agents.

\section{$\mathbf{J 3 2 5}$}

\section{EVALUATION OF POSITRON EMISSION MAMMOGRAPHY} (PEM) IN BREAST CANCER

Sunyoung Jang ${ }^{1,2}$, Lee P. Adler ${ }^{3}$, Dennis Nelson ${ }^{4}$, Jianqiao Luo ${ }^{5}$, Edward N. Sternick $^{1,2}$, David E. Wazer ${ }^{1,2}$

${ }^{1}$ Radiation Oncology, Brown University Medical School, Providence, RI, USA, ${ }^{2}$ Rhode Island Hospital, Providence, RI, USA, ${ }^{3}$ Adler Institute for Advanced Imaging, Jenkintown, PA, USA, ${ }^{4}$ MIMvista, Inc., Cleveland, OH, USA, ${ }^{5}$ Radiology, Virginia Commonwealth University, Richmond, VA, USA.

Objective: Positron emission mammography (PEM) as a dedicated breast imaging system has demonstrated the improved spatial resolution (FWHM: $1.5 \mathrm{~mm}$ ) and higher sensitivity over conventional whole body PET and PET/CT scanners (FWHM: 4 to $6 \mathrm{~mm}$ ) and higher specificity over the dedicated breast-MR system. Previously authors presented the metabolic tumor volume (MTV) comparison of PEM to PET (WMIC 2008). The purpose of this study is to obtain the tumor imaging characteristics using PEM for the potential use in surgical or radiation treatment planning. Methods: Nine female patients $(51.9$ years \pm 6.2$)$ underwent PEM and PET/CT imaging for breast cancer evalua- tion. PEM images of patients' both breasts were acquired in the cranio-caudal (CC) and medio-lateral oblique (MLO) views under mild compression. Three dimensional tumor sizes, standard uptake value (SUV), and tumor-to-normal tissue contrast were measured. Tumor sizes were measured using the $40 \%$ threshold and SUV threshold methods. The gradient-based edge detection was used for contrast measurements. For the SUV threshold method an SUV of 2.5 was used for the tumors with max SUVs greater than 4.0, and an SUV of 2.0 was used for the tumors with the max SUV equal to or less than 4.0. Tumor sizes were measured in axial, sagittal, and coronal lengths for CC and MLO images and compared with the corresponding lengths in PET images. Results: Averages of the maximum SUVs were 5.2 and 10.3 for PEM and PET, respectively. PEM produced mean distinctive tumor-tonormal tissue contrast value of 0.75 and PET yielded that of 0.76. The average ratios of PEM tumors to those of PET for three dimensional sizes were ranged between 0.8 and 1.0 for the $40 \%$ threshold method, and between 0.62 and 0.72 for the SUV threshold method. Conclusions: PEM images produced smaller max SUVs than PET while it demonstrated distinctive tumor-tonormal tissue contrast values similar to PET. In general, three dimensional lengths of PEM tumors were agreed better with the $40 \%$ threshold than the SUV threshold method. PEM has shown important contributions as a complimentary means for the screening and biopsy, and potentials for surgical or radiation treatment planning in breast cancer. The generation of the combined information of tumor extent or tumor bed, and its metabolism are under investigation for radiation treatment planning, since PEM offers not only complimentary functional information to mammography, but also more accurate delineation of tumor margins than PET in breast cancer.

\section{J326}

USING TARGET-SPECIFIC AGENTS TO IMAGE OSTEOSARCOMA Wei Wang ${ }^{1}$, Xiuchun Qiu ${ }^{2}$, Jin Sun ${ }^{1,3}$, Fujun Zhang ${ }^{1,4}$,

Arlin G. Cameron ${ }^{1}$, Lawrence A. Donehower ${ }^{1}$, Jason Yustein ${ }^{1}$, Alan Davis ${ }^{1}$, Elizabeth Olmsted-Davis ${ }^{1}$, Adrienne McNees ${ }^{1}$, Juliet Wendt ${ }^{1}$, Michel Mawad ${ }^{1}$, Shi Ke ${ }^{1}$

${ }^{1}$ Baylor College of Medicine, Houston, TX, USA, ${ }^{2}$ The Fourth Military Medical University, Xi'an, China, ${ }^{3}$ Southeast University, Nanjing, China, ${ }^{4}$ Sun Yat-sen University, Guangzhou, China.

Chemokine receptors play crucial role at all stages of neoplastic transformation and progression. They are targets of growing interest for new therapeutic drugs. The most important chemokine ligand-receptor interaction is CXCL12 (stromal cell-derived factor1, SDF-1) ligand with its exclusive receptor CXCR4. Chemokine receptor CXCR4 is involved in many malignant tumor development, especially in cancer bone metastasis and osteosarcoma. The conjectural role of CXCR4 at the tumor development may become a critical therapeutic target. Several CXCR4 antagonists have been proposed as potential drugs. Noninvasive evaluation of CXCR4 expression in tumors and its changes during treatments is critical need for developing target-specific therapeutic agents, selecting target-positive diseases, and following-up the biological effects of the treatment. We have synthesized peptide based CXCR4 imaging agents and labeled with near-infrared dye for in vitro and in vivo studies. Cell binding studies demonstrated those agents bound to the human osteosarcoma cells. Near infrared optical imaging demonstrated those agents can be used to detect 
osteosarcoma at early and late stages of the disease. Whole body images showed the high signal intensity in the tumor, liver, and thymus region. Positron emission tomography (PET) showed the tumors have high metabolic status of $18 \mathrm{~F}-\mathrm{FDG}$. A vasculature contrast agent and computed tomography (CT) demonstrated hyper-vasculature in the tumor region. In conclusion, we developed a molecular imaging agent that specific target to CXCR4 chemokine receptor. We can image osteosarcoma formation in both early and late disease stages using this agent. WW, XQ: These authors contributed equally to this work. Grant support: DoD W81XWH-08-1-0489, Department of Defense, Department of Radiology research fund, Curtis Hankamer Basic Research Fund and the L.E. and Josephine S. Gordy Memorial Cancer Research Fund, China State Scholarship Fund, Dan L. Duncan Cancer Center Scholar, American Society for Clinical Oncology and Hope Street Kids Foundation, and National Science Foundation of China grants (30471988 and 30873027).

\section{J327}

\section{MOLECULAR IMAGING OF PANCREAS CANCER} WITH A HIGHLY SELECTIVE ANTIBODY CONJUGATE Jan Grimm $^{1}$, Holger Kalthoff ${ }^{2}$, Peter M. Smith-Jones ${ }^{1}$, Hedvig Hricak ${ }^{1}$ ${ }^{\mathrm{T}}$ Radiology, Memorial Sloan Kettering Cancer Center, New York, NY, USA, ${ }^{2}$ Molecular Oncology, University Hospital Schleswig Holstein, Campus Kiel, Kiel, Germany.

Introduction: Pancreas adenocarcinoma is one of the deadliest cancers with a very poor prognosis, mainly because of the late diagnosis. The diagnosis can be challenging given the often subtle imaging findings of small tumors. To date no specific imaging agent is available to target pancreatic cancer in vivo and to aid in the diagnosis. An important aspect in improving survival is thus the development of new tools for a more specific diagnosis. We propose to utilize a unique monoclonal antibody targeting a novel mucinous antigen found in pancreas adenocarcinoma (RA-96) that will be labeled primarily for PET imaging to facilitate clinical translation but we also aim to explore conjugates for MR and optical imaging as well. Materials and Methods: The antibody was coupled covalently to iron oxide nanoparticles. Spheroids of CAPAN-2 cells were incubated with the nanoparticle-mabRA96 conjugate and imaged with a T2-weighted sequence. Additionally the antibody was radiolabeled with $\mathrm{I}-124$ and injected into nude mice, bearing pancreatic tumors (CAPAN-2) and prostatic (PC3) tumors on the contralateral flank as controls. Dynamic imaging over 72 hours revealed uptake only in the pancreatic and not in the control tumor. Results: Targeting of mabRA-96 conjugated to iron oxide particles in vitro to CAPAN-2 spheroids was demonstrated with MR imaging, confirming, that the conjugate retained the affinity and that MR imaging with nanoparticles specific for pancreatic cancer is feasible as well. Staining of histologic slides confirmed the specificity of the conjugate for the RA96 antigen as well. We also demonstrated in vivo targeting with radioiodinated mabRA-96 to implanted pancreatic tumors. Discussion: We demonstrate the feasibility to image pancreatic adenocarcinoma with mabRA-96. The study is fulfilling an urgent need to provide an imaging agent to specifically image pancreatic adenocarcinoma. Furthermore, our approach of utilizing a unique monoclonal antibody (mabRA-96) is readily clinical translatable for PET imaging.

\section{J328}

\section{IMAGING NASAL TUMOR XENOGRAFTS USING RETINOID ACID DERIVATIVES}

Wei Wang ${ }^{1}$, Fujun Zhang ${ }^{1,2}$, Jin Sun ${ }^{1,3}$, Arlin G. Cameron ${ }^{1}$, Juliet Wendt ${ }^{\text {, Michel Mawad }}{ }^{1}$, Shi Ke ${ }^{1}$

${ }^{1}$ Baylor College of Medicine, Houston, TX, USA, ${ }^{2}$ Sun Yat-sen University, Guangzhou, China, ${ }^{3}$ Southeast University, Nanjing, China.

Retinoids are natural and synthetic vitamin A derivatives that regulate a multitude of biological processes in mammalian cells. Those include metabolism, development, cell proliferation, differentiation, and carcinogenesis. Retinoids have been used to prevent diseases and tumor formation, include the head and neck, lung, esophagus, and bladder cancers. It also shows the effects of reverse oral premalignancy and significantly decreases second primary tumors. Retinoids treatments benefit those smoking-related second primary tumors in the small size, short-time, well selected patient population. However, the anti-cancer effects of retinoids are poorly understood. The purpose of our study was to develop imaginable retinoid agents and help understanding retinoids actions in the in vitro and in vivo studies. We synthesized retinoid acid agents and labeled with reporters. Optical whole images showed the high signal intensity from tumor and liver region. The distributions of those retinoids were determined by molecular imaging modalities. PET imaging showed the nasal tumor has high metabolic status of $18 \mathrm{~F}-\mathrm{FDG}$. Vasculature contrast agent and CT image demonstrated the increasing blood vessels in the tumor region. In conclusion, we demonstrate our synthetic imaginable retinoids can be used for imaging studies. These labeled retinoids could also be used to evaluate the retinoid receptor status in pre-clinical researches. We can quantify the uptakes and correlate imaging agent with the disease-response. The imaginable retinoids will allow us to further develop imageguided therapy that using biological dose rather than maximum tolerant doses. We can use imaging method to select target-positive diseases, define the dosage and frequency of administration; as well as following the agents' traffic in the body. Those agents have potential to detect retinoid receptor positive tumors and/or diseases. WW, FZ: These authors contributed equally to this work.

\section{J329}

\section{MRI MONITORING OF TUMOR RESPONSE FOLLOWING ANGIOGENESIS INHIBITION IN AN EXPERIMENTAL HUMAN GLIOMA MODEL}

M. M. Ali, Branislava Janic, Abbas Babajani-Feremi, Nadimpalli Ravi S Varma, Asm Iskander, John Anagli, Ali S. Arbab Radiology, Henry Ford Health System, Detrit, MI, USA.

Anti-angiogenic treatments of malignant tumors targeting vascular endothelial growth factor receptors (VEGFR) tyrosine kinase are being used in different early stages of clinical trial. Recently, VEGFR tyrosine kinase inhibitor (Vetanalib, PTK787) has been used in glioma patient in conjunction with chemotherapy and radiotherapy but results are yet to be published. However, changes in the tumor size, tumor vascular permeability, vascular density, expression of VEGFR2, and changes other angiogenic factors are not well documented following the treatment with PTK787. The 
purposes of this study were to determine the tumor size, vascular permeability, distribution volume, and expression of VEGFR2 in treated and non-treated implanted glioma (U-251) by in vivo MRI and single photon emission computed tomography (SPECT). The findings were compared and correlated with histochemical and western blotting studies. Groups of rats carrying intracranial glioma (U-251) were subjected to treatment with either PTK787 or vehicles-only for two weeks. The treatment was started 7 days after the implantation of tumor cells. Following the treatment; tumor size, tumor vascular permeability transfer constant (Ktrans), distribution volume (FV), and expression of VEGFR2 (KDR) were assessed by in vivo MRI and SPECT studies (Tc-99-HYNICVEGF-c). Following imaging studies, animals were sacrificed and tumors as well as contralateral brain were collected for histochemistry and western blotting studies to evaluate therapy response by monitoring multiple angiogenesis biomarkers. Dynamic contrast-enhanced MRI (DCE-MRI) using a macromolecular contrast agent (albumin-(GdDTPA)20 showed significantly increased Ktrans at rim of the treated tumor compared to that of the central part of the treated as well as vehicle treated tumors. Size of the tumors was also increased in treated group. Expression of KDR detected by Tc-99m-HYNIC-VEGFc SPECT also showed significantly increased activity at the treated tumors. In PTK-treated tumors, histological staining revealed increase in VEGF, KDR, and VEGFR3 expression and microvessel density in the close proximity to the tumor border. Western blotting study indicated increased KDR, VEGFR3 and EGFR expressions at the peripheral part of the treated tumors compared to that of vehicletreated and central part of the treated tumors. These findings indicate that PTK treatment induced over expression of VEGF as well as the Flk-1/KDR receptor tyrosine kinase especially at the rim of the tumor as indicated by DCE-MRI, SPECT imaging and immunohistochemistry.

\section{$\mathbf{J 3 3 0}$}

\section{MESENCHYMAL STROMA CELLS FROM PATIENTS} WITH MYELODYSPLASTIC SYNDROME AND ACUTE MYELOID LEUKEMIA SHOW DISTINCT CYTOGENETIC AND DNA-MUTATION DATA AS COMPARED WITH BONE MARROW LEUKEMIC BLASTS

Olga Blau ${ }^{1}$, Wolf-Karsten Hofmann ${ }^{1}$, Claudia Dorothea Baldus ${ }^{1}$, Gundula Thiel $^{2}$, Florian Nolte ${ }^{1}$, Elke Schuemann ${ }^{2}$, Ouidad Benlasfer ${ }^{1}$, Annette Sindram ${ }^{1}$, Eckhard Thiel ${ }^{1}$, Igor Wolfgang Blau ${ }^{1}$

${ }^{1} \mathrm{CBF}$, Hematology and Oncology, Charité University Berlin, Berlin, Germany, ${ }^{2}$ Genetics Laboratory and Out-Patients' Clinic, Berlin, Germany.

Bone marrow mesenchymal stroma cells (BMSC) are key components of the hematopoietic microenvironment. BMSC from patients with acute myeloid leukemia (AML) and myelodisplasic syndrome (MDS) display functional and quantitative alterations. To gain insight into these questions, we carried out cytogenetic investigation, FISH analyses, and mutation examinations of FLT3 and NPM1 genes of both hematopoietic (HC) and BMSC derived from 53 AML and 54 MDS patients and 35 healthy donors after in vitro culture expansion. Clonal chromosomal aberrations were detectable in BMSC from $12 \%$ of patients. Using FISH we have assume that cytogenetic markers in BMSC were always distinct as the aberrations in $\mathrm{HC}$ from the same individual. $17 \%$ and $12 \%$ of AML patients showed FLT3 and NPM1 mutations in HC, respectively. In BMSC, we could not detect mutations of NPM1 and FLT3, independent from the mutation status of HC. For control analysis, BMSC cultures from 35 healthy donors were prepared under the same conditions. BMSC from healthy donors showed normal diploid karyotypes and absence of DNA-mutations of NPM1 and FLT3 genes. Our data indicate that BMSC from MDS and AML patients are characterized by genetic instability. Lack of aberrations as detected in $\mathrm{HC}$ and appearance of novel clonal rearrangements in BMSC may suggest enhanced genetic susceptibility and potential involvement of BMSC in the pathogenesis of MDS and AML.

\section{J331}

LONGITUDINAL ASSESSMENT OF INTRACRANIAL TUMOR PROGRESSION IN AN ORTHOTOPIC MURINE GLIOBLASTOMA MODEL UTILIZING CT AND ULTRASOUND IMAGING

Kim Hardy, David Monsma, Dawna Dylewski, Stephanie Scott, Qian Xie, Craig P. Webb

Animal Imaging Services, Van Andel Research Institute, Grand Rapids, MI, USA.

The objective of this study was to assess the potential utility of Computer Axial Tomography (CT) and Ultrasound (Sonography) imaging modalities in the early detection and assessment of disease progression in an orthotopic glioblastoma (GBM) model. Ten 7 week old nude male immune-compromised mice were randomly assigned into two study arms; 5 mice were implanted intracranially with $1.5 \times$ $10 \Lambda 6$ U251 human glioblastoma cells/3ul, and 5 mice were implanted in a similar fashion with 3 ul Phosphate Buffered Saline (PBS). At Day 10 post implantation, a dual-modality imaging approach using both CT and Ultrasound was initiated and continued 3 times weekly until animals became moribund. All personnel were blinded to treatment arm assignments and data analysis until study completion. Both CT and Ultrasound technologies accurately classified between the U251 cell and PBS implanted mice from Day 14 post cranial injection. These findings were consistent with disease progression (as assessed by time from injection) and preceded the classical terminal endpoint of weight loss by Day 32 . It is significant to note that both imaging modalities demonstrated evidence of disease onset earlier than indicated by loss of body weight. Our data suggests that CT and Ultrasound technologies may be useful in the early detection and assessment of tumor progress in preclinical models of glioblastoma, thereby facilitating longitudinal studies where each mouse serves as its own internal control and reducing number of animals required.

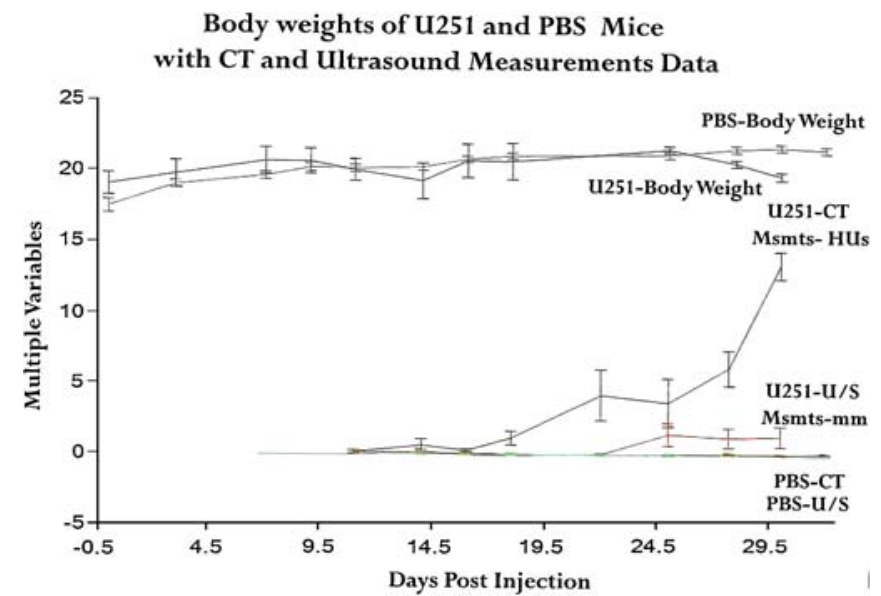




\section{$\mathbf{J 3 3 2}$}

\section{SYNTHESIS AND CHARACTERIZATION OF A 68GA-LABELED LINEAR PEPTIDE FOR NON-INVASIVE DETECTION OF UROKINASE-TYPE PLASMINOGEN ACTIVATOR RECEPTOR (UPAR) EXPRESSION IN HUMAN CANCER Morten Persson ${ }^{1,2}$, Jacob Madsen ${ }^{2}$, Michael Ploug ${ }^{3}$, Andreas Kjaer ${ }^{1,2}$ ${ }^{\mathrm{T}}$ Cluster for Molecular Imaging, University of Copenhagen, Copenhagen, Denmark, ${ }^{2}$ Department of Clinical Physiology, Nuclear Medicine and PET, Rigshospitalet, Copenhagen, Denmark, ${ }^{3}$ Finsen Laboratory, Rig- shospitalet, Copenhagen, Denmark.}

Background \& Aim: The urokinase-type plasminogen activator receptor (UPAR) has been shown to facilitate cancer cell invasion and metastasis development. UPAR is over-expressed in various cancers, including human breast, prostate and colorectal cancer, and over-expression has been shown to correlate with poor prognosis due to increased rates of metastatic relapse. The proven correlation between uPAR expression and metastatic potential provides an opportunity to develop an in vivo imaging agent, which could identify cancer patients at increased risk for metastatic disease. Methods \& Results: We have developed a new DOTA-conjugated 68Ga-labeled linear peptid tracer for non-invasive detection of uPAR expressing cancers, named 68Ga-DOTA-AE105. The tracer was synthesized using standard solid-phase peptide chemistry and subsequently N-terminal conjugated with DOTA. The DOTAconjugated peptide was radiolabeled with $68 \mathrm{Ga}$ using a $68 \mathrm{Ge} /$ $68 \mathrm{Ga}$ generator system, which render the production of the tracer independent of a onsite cyclotron. The new PET tracer was characterized in vitro in four different human cancer cell lines (H727: neuroendocrine, HT-29: colorectal, A2780: ovarian and U87MG: glioblastome) with an increasing expression of uPAR. The tracers were incubated with each cell line for $30 \mathrm{~min}$ and subsequently washed with PBS to remove any unbound tracer. The bound radioactivity was measured in a gamma counter and the results were compared to UPAR expression data determined by ELISA on cell extracts. A good correlation between uPAR expression level determined by ELISA and binding of the tracer was observed. U87MG showed to have the highest expression of UPAR and as expected, also the highest binding capacity of the new tracer. In addition, A2780 xenotransplanted mice were injected intravenously with 68Ga-DOTA-AE105; killed 1.5 hrs post injection and the radioactivity concentration in the tumor and different organs was measured in a gamma counter. The xenotransplanted tumor showed higher uptake pr. gram tissue of the tracer compared to all other organs, except liver and kidney, and with a tumor-tomuscle-ratio of 5.7. Conclusion: Our data demonstrate that the new PET tracer 68 Ga-DOTA-AE105 is a promising radiotracer for noninvasive detection of UPAR in different human cancers. Good in vitro binding properties and a high tumor-to-muscle ratio in vivo have been found. The use of generator produced $68 \mathrm{Ga}$ could further support translation into the clinic.

$\mathbf{J 3 3 3}$

\section{A COMPARTMENT MODELING FOR ESTIMATION OF TUMOR HYPOXIA BASED ON 64CU-ATSM PET SCAN} Fan $\mathrm{Li}^{1}$, Jesper T. Joergensen ${ }^{1}$, Jacob Madsen ${ }^{2}$, Andreas Kjaer ${ }^{1,2}$ ${ }^{T}$ Faculty of Health Sciences, University of Copenhagen, Cluster for Molecular Imaging, Copenhagen N, Denmark, ${ }^{2}$ Department of Clinical Physiology, Nuclear Medicine and PET, Rigshospitalet, Copenhagen Oe, Denmark.
Introduction/Aim: Tumor hypoxia is an important parameter for planning of cancer treatment and the extent of hypoxia has influence on the outcome of radiation- and chemotherapy. $64 \mathrm{Cu}-$ diacetyl-bis(N4-Methylthiosemicarbazone) (64Cu-ATSM) is a positron-emitting hypoxia tracer, which is trapped in hypoxic cells, and it has been used to visualize tumor oxygenation by imaging with PET. We present a mathematical model to estimate hypoxic specific binding based on 64Cu-ATSM PET imaging. Methods: Nude NMRI mice, implanted with cells from a tumor cell line derived from human colorectal cancer (HT29) were used as animal model. A dose of approximately $20 \mathrm{MBq} 64 \mathrm{Cu}-\mathrm{ATSM}$ was injected through the tail vein while the mice were positioned in the PET scanner and $1.5 \mathrm{~h}$ dynamic PET acquisition was performed to obtain time-activity curves(TACs). In addition, a 7 minutes small animal CT with intravascular contrast was performed directly after the PET scan. SUV values were calculated based on obtained PET scanning data. In order to obtain TACs for kinetic analysis, an image-based input function derived from left ventricle (LV) was used by drawing ROIs placed in the left ventricular cavity on PET/CT images. Tumor TACs were obtained from tumor-bearing regions in a similar way. To evaluate the time-activity data with the presented model, a non-linear least squares fit was performed to estimate model parameters. Tracer retention kinetic was analyzed using an irreversible 3-compartment model. For this, a secant version of Levenberg-Marquardt algorithm was used. Results: Both input function TAC obtained from $\mathrm{LV}$ and $\mathrm{TAC}$ from tumor bearing regions were fitted satisfactorily with the weight parameters, which reflected the signal contributions from the respective compartments. Hypoxic parameters as $\mathrm{k}^{\prime}{ }^{\prime}=\mathrm{k} 2+\mathrm{k} 3$ and the contribution caused of hypoxia signal $\mathrm{wA}^{\prime}=\mathrm{wAk} 1 \mathrm{k} 3 /(\mathrm{k} 2+\mathrm{k} 3)$ were estimated to be $0.0013-0.0024$ and $1 \mathrm{e}-5-9 \mathrm{e}-4$, respectively. Conclussion: The present study showed that a non-invasive input function is feasible for hypoxia modeling studies of 64Cu-ATSM in mice. The model output in shape of TAC was in agreement with $1.5 \mathrm{~h}$ dynamic PET data. The compartment model described seems to be physiologically realistic and general enough to visualize tracer transport and uptake mechanism. Moreover, the model is capable of predicting late tracer accumulation based on early dynamic PET data. Currently, comparison with molecular markers of hypoxia is being undertaken as further validation of the model.

\section{$\mathbf{J 3 3 4}$}

\section{MAGNETIC RESONANCE APPROACH IN THE IMAGING OF THE TUMOR ANGIOGENESIS: SIMULTANEOUS ACQUISITIONS OF VESSEL SIZE INDEX, TUMOR BLOOD VOLUME AND TUMOR BLOOD FLOW}

Marco Dominietto, Ruth Keist, Steffi Lehmann, Markus Rudin Institute for Biomedical Engineering, ETH Zurich, Zurich, Switzerland.

A crucial step in tumor progression is angiogenesis, i.e. the formation of neovasculature to recruit nutrients from the hosting tissue. The aim of the current work is to study the physiological behavior of the novel vessels in the course of tumor development addressing aspects such as distribution of vessel diameters (vessel size index, VSI), tumor blood flow (TBF) and volume (TBV) by means of magnetic resonance imaging (MRI). Six balb/c nude mice were injected subcutaneously with $10^{\wedge} 6$ C51 cells (colon carcinoma). Mice were measured every three 
days following implantation using a 4.7T Pharmascan MRI Scanner (Bruker Biospin, Ettlingen, Germany). Iron oxide nanoparticles (Sinerem ${ }^{\circledR}$, Guerbet SA, Paris, France) was used as intravascular contrast agent (CA). High-resolution MR images were recorded prior and after $\mathrm{CA}$ administration yielding information on VSI and TBV. CA administration during dynamic MRI data acquisition yielded relative TBF values Mice were sacrificed 12 days following tumor inoculation and the tumors were explanted for histological examination using the endothelial marker CD31. All images were co-registered enabling the study of spatial correlations. Tumors growth was found to be heterogeneous with distinct differences with regard to the tumor vasculatures. Within individual regions we observed a correlation between VSI, TBF and TBV during tumor development. Measurement of VSI, TBF and TBV allows the quantitative characterization of the angiogenic vessels both at a anatomical (vascular network structure) and functional (blood supply) level. In vivo MRI results have been validated by comparing with the data from histology. This study demonstrates the feasibility to follow the evolution of the angiogenesis non-invasively by means of these macroscopic physiological parameters.
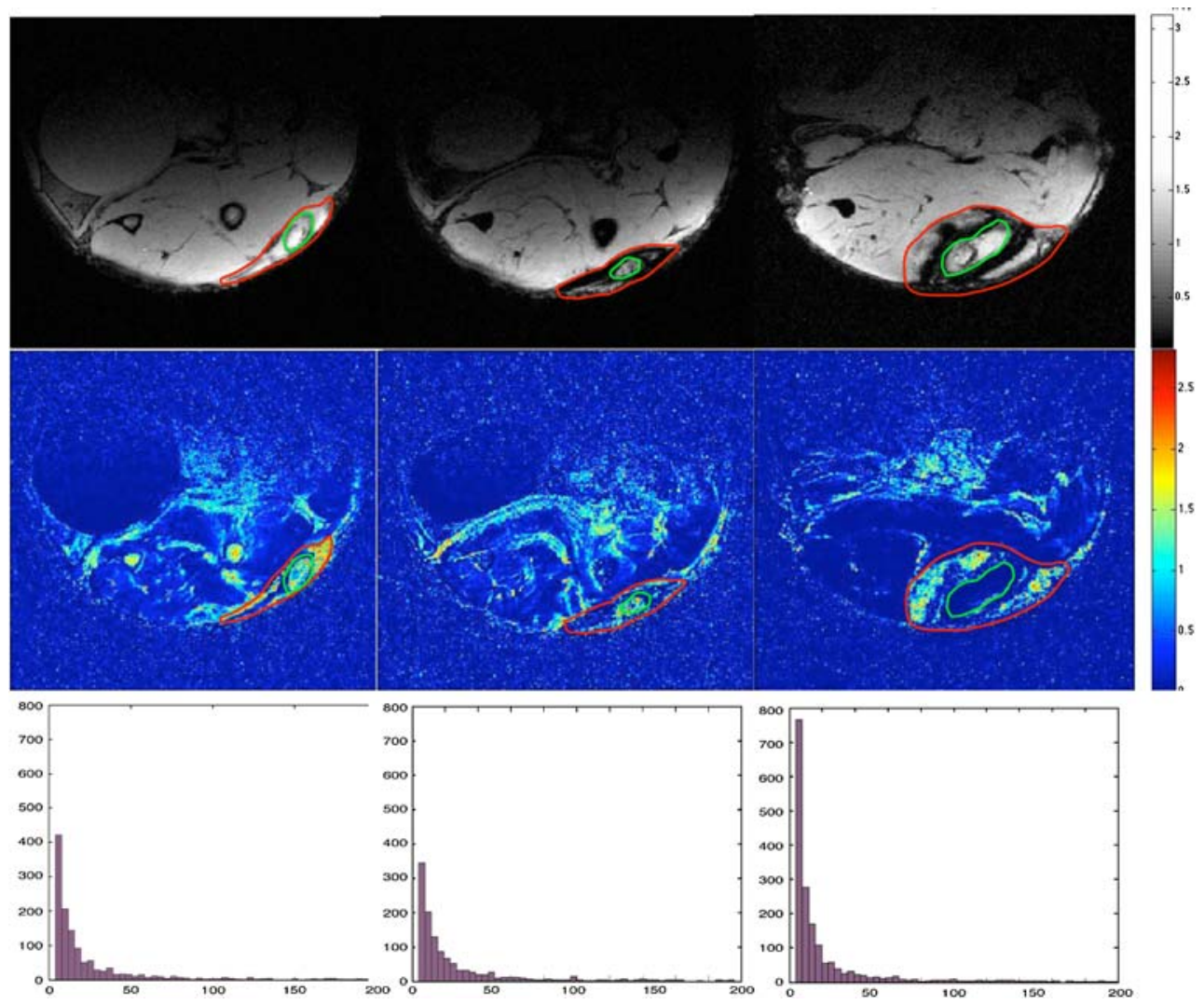

Tumor growth at day 7, 10 and 13 from the cells inoculation: anatomical images (1st row), tumor blood volume (2nd row) and vessel size index (3rd row). Vessel size index is represented as the number of the pixel (y-axis) comprises the average vessel diameters (x-axis)

\section{$\mathbf{J 3 3 5}$}

\section{BASIC EVALUATION OF ZN-EDDA FOR CANCER IMAGING} Takako Furukawa $^{1,2}$, Yuriko Saito ${ }^{1,3}$, Sumitaka Hasegawa ${ }^{1}$, Tsuneo Saga ${ }^{1}$, Yasuhisa Fujibayashi ${ }^{2,1}$

${ }^{1}$ Molecular Imaging Center, National Institute of Radiological Sciences, Chiba, Japan, ${ }^{2}$ Biomedical Imaging Research Center, University of Fukui, Yoshida, Japan, ${ }^{3}$ Department of Molecular Imaging and Radiotherapy, Graduate School of Pharmaceutical Sciences, Chiba University, Chiba, Japan.
Zinc-63 is a positron emitter with half life of $30 \mathrm{~min}$. It can be produced using a small clinical cyclotron targeting $\mathrm{Cu}-63$, the most abundant $\mathrm{Cu}$ isotope in nature, which makes $\mathrm{Zn}-63$ a potentially attractive radionuclide for clinical imaging. Zinc, one of the essential trace elements, is contained in many enzymes and functional proteins such as transcription factors as a critical component. The usefulness of radiolabeled Zn-EDDA in imaging of pancreatic function was reported earlier: the uptake of $\mathrm{Zn}$ EDDA into pancreas was shown to reflect the active production of $\mathrm{Zn}$-containing enzymes. As $\mathrm{Zn}$ is essential for cell proliferation, it 
would be reasonable to expect that rapidly growing tissues, such as cancer, need larger amount of $\mathrm{Zn}$ than the others. Accordingly, we were prompted to explore the possibility to use radiolabeled $\mathrm{Zn}$ EDDA for cancer imaging. We examined cellular uptake of Zn-65 labeled $\mathrm{Zn}$-EDDA, $\mathrm{Zn}$ content, and expression of $\mathrm{Zn}$ transporters, comparing several cancer cell lines, together with the biodistribution of Zn-65 labeled Zn-EDDA in tumor-bearing mice. The cellular uptake was found to be correlated with $\mathrm{Zn}$ content, but not with the expression of any particular transporter tested, suggesting that the uptake reflects the physiological needs of the cells. The accumulation of $\mathrm{Zn}-65$ in the tumor xenografts ranged 2.3-4.2 \% ID/g, tumor/blood ratio 3.5-8.1, and tumor/muscle ratio 5.5-12. Bone, liver, kidney and upper intestine, other than pancreas, accumulated high $(5<\% \mathrm{ID} / \mathrm{g})$ amount of the radioactivity. Zn-EDDA would have potential as a cancer imaging agent, though the use would be restricted in limited tissues.

\section{$\mathbf{J 3 3 6}$}

\section{MONITORING OF POTENTIAL GROWTH IN MICE OF HUMAN NEUROBLASTOMA TUMOR-INITIATING CELLS BY MRI AT 1.5T}

Gabriella Baio $^{1}$, Maria V. Corrias ${ }^{2}$, Claudio Gambini ${ }^{3}$, Michele Cilli ${ }^{4}$, Giuseppe Taverniti ${ }^{4}$, Carlo E. Neumaier ${ }^{1}$

${ }^{1}$ Department of Diagnostic Imaging, National Cancer Institute, IST, Genoa, Italy, ${ }^{2}$ Laboratory of Oncology, Gaslini Institute, Genoa, Italy, ${ }^{3}$ Service of Pathology, Gaslini Institute, Genoa, Italy, ${ }^{4}$ Animal Facility, National Cancer Institute, IST, Genoa, Italy.

Tumor-initiating cells(TIC) have been described in different solid tumors, including neuroblastoma(NB). Since metastatic cells are responsible for the majority of cancer-related death,it is conceivable that metastatic cells may be enriched in TICs.We illustrated the in vivo MR experiments with metastatic NB cells present in the UCB of a child that developed NB at 3 months.Given the limited amount of GD2(+)NB cells recovered from the patient's $\mathrm{UCB}$ and the intent of avoiding any in vitro manipulation,we orthotopically injected them in the adrenal gland of a single NOD/SCID.As a control,we orthotopically injected the GD2(-) hematopoietic cells.Every 2 weeks,MR at $1.5 \mathrm{~T}$ was performed. Hystopathological analysis was performed blind to the MRI data. MRI demonstrated the absence of tumor growth in the adrenal gland(Fig1 AB), then confirmed by hystopatology(Fig1C). Mice showed a progressive enlargement of the spleen that became 3 times greater than normal(Fig2).We also observed a change in signal intensity of the kidneys cortex and medulla,better appreciated on T2-FS weighted images,as compared with normal mice (Fig3).Hystopatholgical analysis showed an intense spleen metaplasia involving the myeloid component(FigAF); the kidneys appeared slightly hydropic and they showed sign of focal glomerulonephritis,involving the tube and the glomerulo(FigAG). These alterations can be ascribed to cytokines/growth factors and albumin present in the cell suspensions. To limit loss of precious cells, in fact, extensive washing was avoided. In conditions where potential TICs are in limited number and in vitro manipulation or expansion is not recommended monitoring their fate in vivo is challenging.MRI demonstrated that NB cells present in the UCB were not tumorigenic in our animal model.Probably,GD2-positive cells from the patient's UCB did not have tumor-initiating capacity because stage $4 \mathrm{~s}$ often undergo spontaneous regression. However,MRI allowed a safe,well tolerated,longitudinal monitoring and the observed organ alterations were fully confirmed by blind histopatology.This is the first report of MRI of small animals injected with human tumor cells infiltrating UCB.
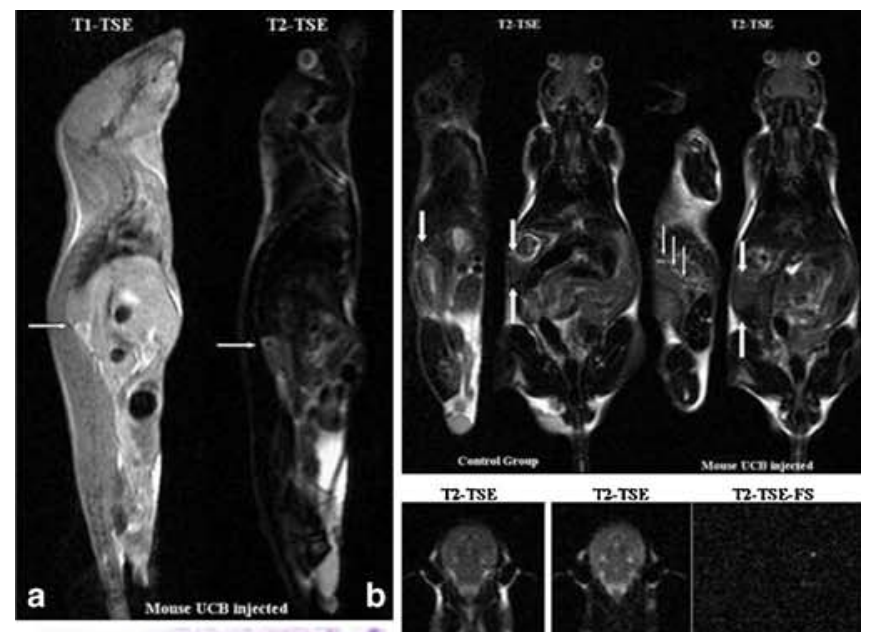

c
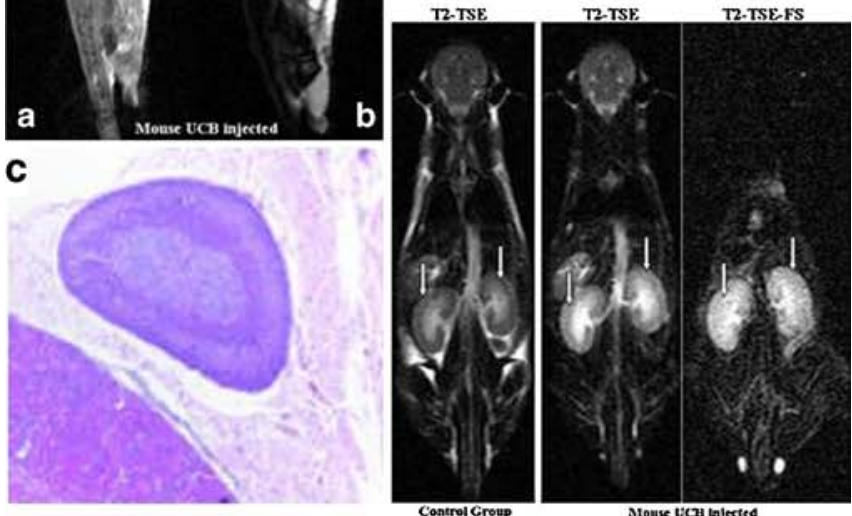

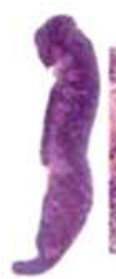

a $\mathrm{mm}:$

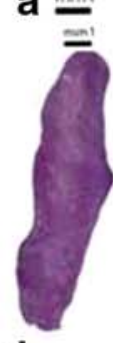

d

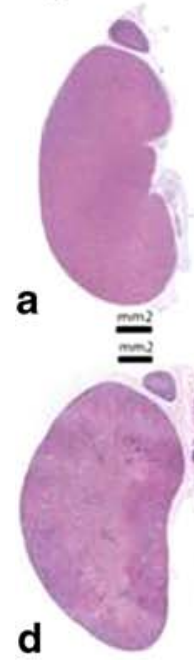

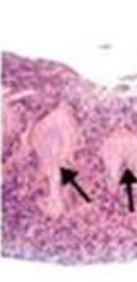
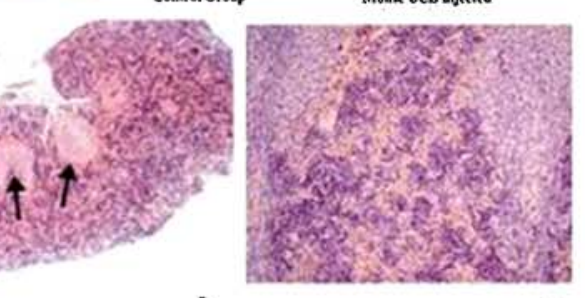

b

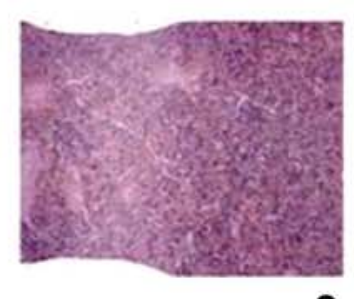

e

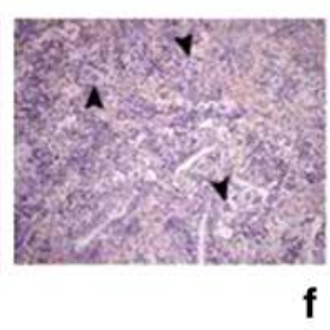

f

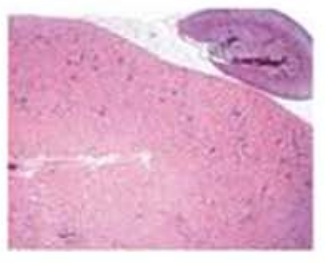

b

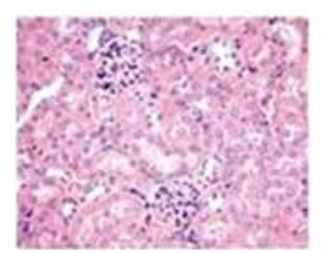

c

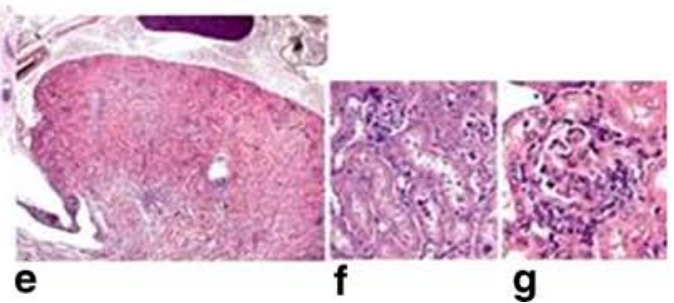


$\mathbf{J 3 3 7}$

QUANTITATIVE ASSESSMENT OF OSTEOLYSIS

IN A PRECLINICAL DISEASE MODEL OF MULTIPLE MYELOMA USING MICRO-COMPUTED TOMOGRAPHY

Neill Gingles ${ }^{1}$, Sally-Ann Ricketts ${ }^{1}$, Juliana Bales ${ }^{1}$, Arvind Parmar ${ }^{1}$, Sangeetha Palakurthi ${ }^{2}$, Anne-Marie Mazzola ${ }^{2}$

${ }^{1}$ Imaging, Translational Sciences, AstraZeneca, Macclesfield, United Kingdom, ${ }^{2}$ Cancer Bioscience, AstraZeneca R\&D Boston, Waltham, MA, USA.

Tumour induced bone loss or osteolysis is an important clinical manifestation of multiple myeloma (MM) resulting in pathologic fractures and severe bone pain in affected patients. A pre-clinical model of multiple myeloma involving the intravenous injection of human multiple myeloma (MM1.S) cells into immunocompromised mice has been shown to recapitulate the clinical phenotype of osteolysis. The aim of this study was to assess the utility of micro-CT to characterize osteolysis in the preclinical MM model, by quantification of bone volume and architectural changes. The overall objective of this study was to establish the use of micro-CT to assess/characterize the disease state in this model for further drug development studies. High-resolution $\mathrm{CT}$ images $(18 \mu \mathrm{m})$ were acquired using a pre-clinical CT scanner from eight severe combined immunodeficiency (SCID) mice leg samples. Seven samples were derived from SCID mice 45 days post intravenous implantation of MM.1S and one from a naïve mouse. Quantitative analysis of bone morphometry parameters from reconstructed images was carried out using Inveon Research Workplace software. Analysis of reconstructed CT images showed that osteolytic lesions induced in the MM model were present largely at the proximal tibial metaphysis near the femorotibial joint, with differing severity within the samples tested. Visible lesions were observed from 3D rendered CT images with a quantifiable reduction in bone volume and disruption of trabecular bone microarchecture (Table 1) These data correlated well with histological scoring assessment of lesions in terms of the rank severity of samples. This study has demonstrated that micro-CT is a capable modality for the assessment of osteolysis from the preclinical MM model. Moreover, micro-CT protocols developed in this study will provide a way of highlighting qualitative and quantitative changes in bone within the model, and could be used as a tool for drug target validation or quantifying the effects of novel therapeutics on bone disease progression.

Table 1. Morphometry parameters calculated from reconstructed micro-CT murine tibial bone samples

\begin{tabular}{|c|c|c|c|c|c|c|c|c|}
\hline Parameter/ Sample ID \# & $8^{*}$ & 16 & 17 & 21 & 22 & 23 & 25 & 29 \\
\hline Total bone volume / Total volume ratio (\%) & 63.52 & 38.37 & 56.07 & 63.17 & 39.34 & 47.38 & 32.49 & 24.63 \\
\hline Total bone surface Area / Total Bone Volume & 15.80 & 8.03 & 15.06 & 14.68 & 20.79 & 16.57 & 20.55 & 22.35 \\
\hline Trabecular Bone Volume / Total Volume (\%) & 31.99 & 14.27 & 23.86 & 29.04 & 16.19 & 16.36 & 8.47 & 8.06 \\
\hline Trabecular Bone Surface Area / Trabecular Bone Volume & 42.82 & 57.67 & 45.39 & 40.03 & 50.44 & 53.35 & 66.95 & 63.91 \\
\hline Trabecular Thickness (mm) & 0.05 & 0.03 & 0.04 & 0.05 & 0.04 & 0.04 & 0.03 & 0.03 \\
\hline Trabecular Number (mm-1) & 6.85 & 4.12 & 5.42 & 5.81 & 4.08 & 4.36 & 2.84 & 2.58 \\
\hline Trabecular Spacing (mm) & 0.10 & 0.21 & 0.14 & 0.12 & 0.21 & 0.19 & 0.32 & 0.36 \\
\hline
\end{tabular}

* naïve mouse control sample

$\mathbf{J 3 3 8}$

\section{IMAGING MELANOMAS WITH NONLINEAR INFRARED TRANSIENT ABSORPTION MICROSCOPY}

Ivan Piletic $^{1}$, Thomas Matthews ${ }^{1}$, Kelly Nelson ${ }^{2}$, Maria A. Selim³ ${ }^{3}$, Warren Warren $^{1}$

${ }^{1}$ Chemistry, Duke University, Durham, NC, USA, ${ }^{2}$ Dermatology, Duke University, Durham, NC, USA, ${ }^{3}$ Pathology, Duke University, Durham, NC, USA.

Early detection of melanomas in order to limit metastases, is unquestionably an important strategy to reduce its mortality rate. Diagnosis of melanomas in the clinic typically involves a two stage process: lesions are visually inspected by eye or dermoscope, and those deemed 'suspicious' are excised, stained and viewed in a microscope by a pathologist. Unfortunately, both stages suffer from drawbacks. Visual/dermoscopic inspection is fairly subjective as many skin lesions (including melanomas) share similar characteristics such as irregular shape and pigmentation. Histopathological examination improves diagnostic accuracy, but it is not feasible for patients possessing numerous dysplastic nevi since they all cannot be evaluated in such an invasive manner. Even histopathological assessment is hampered by false negatives that increase mortality and false positives that drive up the cost of healthcare with unnecessary, expensive, and invasive procedures. Thus, development of better ways to detect and grade (stage) skin cancers, both before and after excision, could have an enormous impact on public health and associated health care costs. Nonlinear optical microscopy has the potential to address both challenges since it may be used to noninvasively probe lesions with sub-cellular resolution and novel contrast that is not available in histopathology. Optically imaging pigmented skin lesions such as melanoma for diagnostic purposes is challenging because the melanin pigments contained within absorb light effectively and possess a low fluorescence quantum yield. We address this challenge by directly detecting the absorptive properties of melanins in order to provide molecular contrast at high resolution in skin lesions. Specifically, we exploit the process of transient absorption to target melanins whereby a pump pulse is used to create an excited state population that is subsequently monitored by a probe pulse. The two main types of melanin (eumelanin and pheomelanin) exhibit unique spectroscopic signatures that may be used to identify them in tissue as well as report on their structural characteristics which has long been debated in the literature. In fact, we have been able to microscopically resolve eumelanin and pheomelanin in human melanoma tissue sections. This has clinical importance since recent studies suggest altered melano- 
genesis in melanoma lesions, but this kind of information cannot be obtained by conventional histopathology or dermoscopy.

\section{J339}

CHARACTERISATION OF THE TUMOUR GROWTH DYNAMICS OF RAT MODELS OF HUMAN GLIOBLASTOMA DISPLAYING AN INVASIVE OR AN ANGIOGENIC PHENOTYPE BY MULTI-TRACER PET AND MRI

Thomas Viel ${ }^{1}$, Jan Jikeli ${ }^{1}$, Roland Ullrich ${ }^{1}$, Parisa Monfared ${ }^{1}$, $\overline{\text { Daniel Rudan }}^{1}$, Philipp Euskirchen ${ }^{1}$, Gabriele Schneider ${ }^{1}$, Bernd Neumaier ${ }^{1}$, Frits Thorsen ${ }^{2}$, Mathias Hoehn ${ }^{1}$, Rolf Bjerkvig ${ }^{2}$, Hrvoje Miletic $^{2}$, Andreas H. Jacobs ${ }^{1}$

${ }^{1}$ Laboratoire for Gene therapy and Molecular Imaging, MPI for Neurological Research, Cologne, Germany, ${ }^{2}$ Department of Biomedicine, University of Bergen, Bergen, Norway.

Despite aggressive multimodal treatment strategies median survival of patients with glioblastoma is still limited to 1-2 years. For the development of more efficient treatments, it is crucial to better understand the molecular processes of the disease progression such as deregulation of the cell cycle, neovascularisation, tumour cell migration and invasion. Molecular imaging technology will help to determine the dynamics of some of these important molecular alterations in vivo. Tumour tissues from two human gliomas serially passaged in nude rats, were maintained in culture as spheroids. Tumour A displayed an invasive while tumour B showed an angiogenic phenotype. Spheroids were stereotactically transplanted in the right brain hemisphere of nude rats, and the development of the brain tumours was followed by [18F]FDG-, [11C]MET-, and [18F]FLT-PET imaging together with magnetic resonance imaging (T1-weighted, T2- and $\mathrm{T} 2 *$-weighted sequences before and after contrast agent injection; ADC maps). Non-invasive observations were correlated with immunohistochemical analysis. Implantation of spheroids allows the maintenance of the original human tumour characteristic. The first group of rats, injected with spheroids derived from glioblastoma A, displayed a highly invasive tumour, with neither signs of angiogenesis nor disturbed vasculature. Tumour development was revealed by a decrease of FDG uptake in the right brain hemisphere. Only a very small increase of MET uptake could be observed in the region correlating with the low FDG uptake area, whereas no increase of FLT uptake could be detected. The extent of the infiltrative tumours can be observed in T2 and T1 MR images, but contrast is very low and no contrast enhancement could be observed after Gadolinium injection. The second group of rats, injected with spheroids derived from glioblastoma $\mathrm{B}$, presented circumscribed tumours with extensive angiogenesis and some necrotic areas. PET showed low uptake of FDG in the right brain hemisphere correlating with high uptake of MET and very high uptake in FLT. The tumour was discernible already on the pre-contrast agent MR scans. Here, contrast was strongly enhanced after Gadolinium injection. Multi-modal molecular imaging is the basis for the determination of the dynamic behaviour of tumour characteristics in vivo. These glioblastoma models will be useful for the development of improved imaging-based treatment strategies, such as anti-proliferative or antiangiogenic regimens which can be followed by serial MET-PET, FLTPET and MR imaging.

\section{J340}

DOES DIFFERENT DISTRIBUTION OF ${ }^{62}$ CU-ATSM AND ${ }^{18}$ F-FDG REFLECT RESISTANT TISSUE OF CANCERS? Myungmi $\mathrm{Oh}^{1}$, Takashi $\mathrm{Kudo}^{1}$, Yasushi Kiyono ${ }^{1}$, Yasuhisa Fujibayashi ${ }^{1}$, Hidehiko Okazawa ${ }^{1,2}$

${ }^{\mathrm{T}}$ Biomedical Imaging Research Center, University of Fukui, Eiheiji-cho, Japan, ${ }^{2}$ Research and Education Program for Life Science, University of Fukui, Eiheiji-cho, Japan.

Introduction: Since hypoxic tissue in malignant tumor is reported to be resistant to treatment such as radiation and chemotherapy, determination of hypoxic region of tumors is useful to choose appropriate therapeutic methods. In the present study, $\left[{ }^{62} \mathrm{Cu}\right]-$ diacetyl-bis(N4-methlythiosemicarbazone) $\left({ }^{62} \mathrm{Cu}-\mathrm{ATSM}\right)$ was used to delineate the hypoxic tissue in the head and neck cancer, and compared its distribution with that for $\left[{ }^{18} \mathrm{~F}\right]$-fluorodeoxy glucose $\left({ }^{18} \mathrm{~F}-\mathrm{FDG}\right)$. Methods: Thirty patients with lung cancer underwent ${ }^{62} \mathrm{Cu}$-ATSM and ${ }^{18} \mathrm{~F}$-FDG PET within a few days interval. Before ${ }^{62} \mathrm{Cu}-A T S M$ PET scans, ATSM was labeled with $600-800 \mathrm{MBq}$ of ${ }^{62} \mathrm{Cu}$ eluted from a ${ }^{62} \mathrm{Zn} /{ }^{62} \mathrm{Cu}$ generator, and 20 min-dynamic PET data were acquired after the tracer injection. Accumulation of tracer for each PET image was converted to standardized uptake values (SUV). After co-registeration of ${ }^{18} \mathrm{~F}-\mathrm{FDG}$ and ${ }^{62} \mathrm{Cu}-\mathrm{ATSM}$ images with anatomical information of individual $\mathrm{CT}$ or MR image, multiple small regions of interest (ROIs) were drawn on tumor mass and applied to both PET images. SUV values were obtained for all ROIs and the slopes of regression line between SUVs for ${ }^{18}$ F-FDG and ${ }^{62} \mathrm{Cu}-\mathrm{ATSM}$ of each tumor were determined. Results: In the peripheral region of tumor mass, relative uptake of ${ }^{62} \mathrm{Cu}$ ATSM was higher than that of ${ }^{18} \mathrm{~F}-\mathrm{FDG}$, and the center of the tumor showed the other tendency (spatial mismatching). Thus, the relationship of the SUVs for ${ }^{18} \mathrm{~F}-\mathrm{FDG}$ and ${ }^{62} \mathrm{Cu}-\mathrm{ATSM}$ showed negative correlation in most of squamous cell carcinomas. However, a few cases of adenocarcinoma showed similar and spatially homogenous accumulation in the tumor mass, and SUVs for the two tracers showed positive correlation (matched distribution). The mean of regression slopes for all tumor lesions was $-0.26 \pm 0.28$ (mean $r=0.45$, p-value $<0.001$ ). Advanced cancers were treated by chemoradiation and regions of high accumulation of ${ }^{62} \mathrm{Cu}-\mathrm{ATSM}$ tended to show residual tissue after the treatment. Conclusion: In patients with head and neck cancer, intratumoral distribution of ${ }^{18} \mathrm{~F}$ FDG and ${ }^{62} \mathrm{Cu}$-ATSM showed negative correlation although adenocarcinoma showed positive regression. Our results indicate that intratumoral regions of high glucose metabolism and high accumulation of ${ }^{62} \mathrm{Cu}$-ATSM tend to show different distribution especially in squamous cell carcinomas. The region of high ${ }^{62} \mathrm{Cu}-$ ATSM accumulation may represent resistant tissue.

\section{J341}

IS THERE A SPECIES SPECIFIC UPTAKE OF [11C]CHOLINE AND [18F]FECH IN XENOGRAFT-MODELS IN PROSTATE CANCER?

Damaris Kukuk $^{1}$, Gerald Reischl ${ }^{2}$, Olivier Raguin ${ }^{3}$, Nadine Kemmler ${ }^{1}$, Andreas Schmid $^{1}$, Stefan Wiehr ${ }^{1}$, Julia G. Mannheim ${ }^{1}$, Daniel Bukala ${ }^{1}$, Olivier Duchamp ${ }^{3}$, Walter Ehrlichmann ${ }^{2}$, Martin S. Judenhofer ${ }^{1}$,

Bernd J. Pichler ${ }^{1}$

${ }^{1}$ University of Tuebingen, Laboratory for Preclinical Imaging and Imaging Technology of the Werner Siemens-Foundation, Tuebingen, Germany, ${ }^{2}$ Department of Radiology, University Hospital of Tuebingen, Radiopharmacy, Tuebingen, Germany, ${ }^{3}$ Oncodesign, Dijon Cedex, France.

Prostate cancer is the second leading cause of death in men in the industrial nations. Although PET-[18F]FDG has proven to be the gold standard in cancer detection, it has not been reliably used to detect prostate cancer. For this reason new tracers like [11C]Choline and $[18 \mathrm{~F}] \mathrm{FECh}$ have been developed. However, in clinical use there is controversy about their benefit especially in early diagnosis of prostate cancer. We found in our studies in xenograft-models in BALB/c-nude male mice with the two human hormone-independent prostate cancer cell lines PC-3 and DU145 a very low uptake of [18F]FECh and [11C] Choline with a tumor-to-muscle ratio (T/M) of $1.17 \pm 0.41$ in PC-3 $(n=$ 7), $1.60 \pm 0.34$ in DU145 $(n=7)$ and with $[18 \mathrm{~F}] \mathrm{FECh}$ a T/M ratio of $1.24 \pm 0.25$ in PC-3 $(n=10)$ and $1.42 \pm 0.04$ in DU145 $(n=11)$. In continuative studies we investigated the uptake characteristics of the two hormone-dependent CWR22 and PAC120 xenograft tumor models in mice before and after surgical castration. Here we found an uptake 
for [18F]FECh in CWR22 with a T/M of $2.03 \pm 0.41$ before castration $(n=5)$ and $0.98 \pm 0.13$ three weeks after castration $(n=5)$ but no $[18 \mathrm{~F}]$ FECh uptake was detected in PAC120 tumors. No [11C]Choline uptake was found in the hormone-dependent tumor models. In our latest study both the subcutaneous CWR22 and PAC120 tumor model in RH-rnurnu male rats show a good [11C]Choline uptake. A T/M ratio of 2.46 in PAC120 $(n=2)$ and $2.51(n=2)$ in CWR22 tumors was measured. With $[18 \mathrm{~F}] \mathrm{FECh}$ a T/M ratio of $1.91 \pm 0.66(n=6)$ in PAC120 was measured $30 \mathrm{~min}$ after tracer injection. Based on these results, we conclude that [11C]Choline uptake in hormone-dependent tumors is species dependent. The next steps will focus on uptake studies in the hormone-dependent tumor models in rats after surgical castration. Additionally, we will perform a cross-correlation of the PET data with MR-spectroscopy and immunohistochemistry.

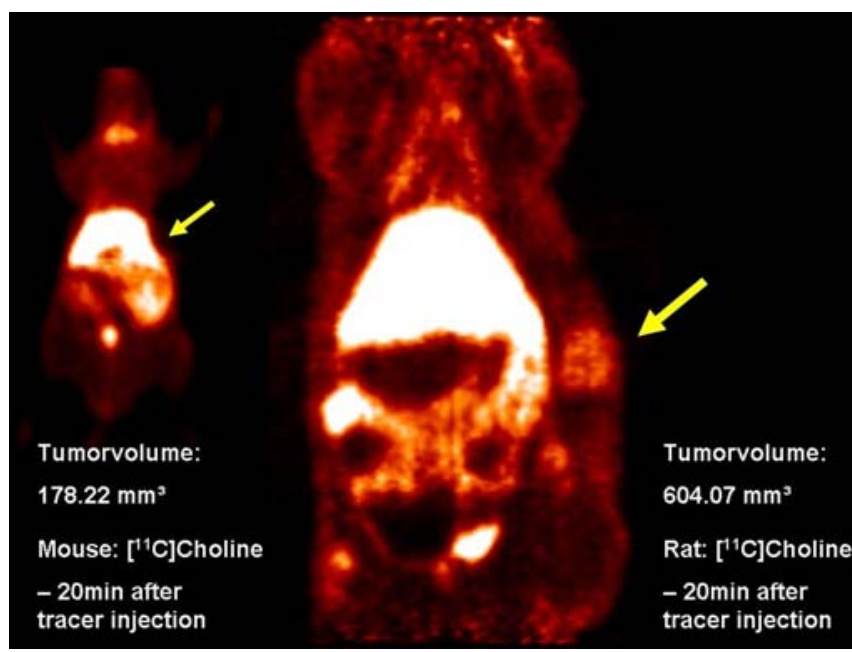

Figure 1: Shown are [11C]Choline PET images of a mouse and a rat with a human hormone-dependent PAC120 prostate cancer xenograft tumor.

$\mathbf{J 3 4 2}$

[18F]-FLT PET OF MOUSE XENOGRAFT TUMOR MODELS FOR ASSESSING TREATMENT RESPONSE: MAKING A CASE TO RANDOMIZE TREATMENT GROUPS BASED ON BASELINE TUMOR FLT UPTAKE AND NOT TUMOR VOLUME

Kenneth Zasadny, Kyle Kuszpit, Michael J. Callahan, Aijun Zhu, Anne M. Burkholder, Richard L. Harris, Laigao Chen Bioimaging, Pfizer, Inc., Groton, CT, USA.

[18F]-FLT PET is used to assess the effect of cancer therapy on tumor cell proliferation. The assumption that tumors of approximately the same size should be at approximately the same phase of growth/proliferation has led to the convention in pre-clinical cancer research of randomizing mouse xenograft tumor models into treatment groups based on caliper-measured tumor volume. Randomizing treatment groups by baseline tumor FLT uptake may improve detection of treatment effects. Recently we investigated the effects of Docetaxel on tumor proliferation using [18F]-FLT PET as a biomarker. At days 1, 7 and 14 post initial treatment, vehicle control mice showed significant increases in tumor FLT uptake relative to baseline regardless of the magnitude of their baseline FLT measurement. In the Docetaxel treatment group at the same time points, the magnitude of change in tumor FLT uptake relative to baseline was correlated with the magnitude of each subjects baseline measurement, where lower proliferators $(<1.6$ max tumor $\mathrm{SUV} /$ mean liver SUV) at baseline showed net increases in tumor FLT uptake, and higher proliferators $(>1.6$ max tumor SUV/mean liver SUV) showed net decreases in tumor FLT uptake. Our results suggest that the magnitude of treatment response in tumor as measured by [18F]-FLT PET is correlated with baseline FLT uptake. Treatment groups dominated by either low or high proliferators at baseline may affect the ability to differentiate treatment response. Based on these results, we advocate randomizing treatment groups based on baseline tumor FLT uptake together with consideration of tumor volume.

\section{$\mathbf{J 3 4 3}$}

\section{REAL TIME IMAGING OF CANCER-CELL DYNAMICS}

OF COLONY FORMATION ON THE LUNG IN THE LIVE MOUSE IDENTIFIES POTENTIAL METASTATIC STEM CELLS Hiroyuki Kimura $^{1,3}$, Katsuhiro Hayashi ${ }^{3}$, Kensuke Yamauchi ${ }^{3}$, Norio Yamamoto $^{3}$, Hiroyuki Tsuchiya ${ }^{3}$, Katsuro Tomita ${ }^{3}$,

Michael Bouvet $^{2}$, Robert M. Hoffman ${ }^{1,2}$

${ }^{1}$ AntiCancer, Inc., San Diego, CA, USA, ${ }^{2}$ Department of Surgery, University of California San Diego, San Diego, CA, USA, ${ }^{3}$ Department of Orthopaedic Surgery, Kanazawa University School of Medicine, Kanazawa, Japan.

Although many experimental animal models have been developed to obtain lung metastasis, the lung is the most challenging organ to observe in live animals because of its anatomical location. We have developed a new in vivo mouse model to image cancer cell dynamics in the lung in real-time. We were able to observe single cancer cells seeding the lungs of live mice and follow them at any time in the same mouse. Endotracheal intubation on the nude mouse was performed under anesthesia for ventilation. Controlling the flow volume enables lung expansion adequate for imaging. The right chest wall was then opened in order to place a flat transparent plate on the lung. Green fluorescent protein (GFP) or red fluorescent protein (RFP) or both, were expressed in the cancer cells. The labeled cancer cells were injected in the tail vein of the mouse. The lung was directly imaged using the Olympus OV-100 Small Animal Imaging System with variable magnification. After each observation, the chest wall was sutured and the air was suctioned in order to re-inflate the lung. In this way, the mouse could be kept alive, and we could observe the same developing metastatic colony over time by repeatedly re-opening the chest wall using the procedure described above. Fluorescent cancer cells were video imaged arresting and colonizing in the lung. About $5 \mathrm{~s}$ after the tail vein injection of cancer cells, they reached the lung and embolized in the capillaries. The embolized cancer cells gradually disappeared from the lung over time. After $24 \mathrm{~h}$, only a few cells were still arrested in the lung. These arrested cells started proliferating and by one week after the tail injection, they grew up from single cells to tumor colonies. The ability of these rare cells to form colonies suggests they are stem-cell like. Observations have been carried out for up to $8 \mathrm{~h}$ per session and repeated up to 3 times per mice thus far. This model makes it possible to observe real time monitoring of cancer-cell dynamics of colony formation in the lung and to identify potential metastatic stem cells. These stem-like cells will be isolated and further characterized.

\section{J344}

\section{MOLECULAR IMAGING OF CANCER CACHEXIA}

Marie-France Penet, Balaji Krishnamachary, Samata M. Kakkad, Paul T. Winnard, Dmitri Artemov, Zaver M. Bhujwalla JHU ICMIC Program, The Russell H. Morgan Department of Radiology and Radiological Science, The Johns Hopkins University School of Medicine, Baltimore, MD, United States, Baltimore, MD, USA.

One of the main causes of mortality from cancer is cachexia, a poorly understood syndrome, characterized by progressive weight loss due to 
metabolic alterations, depletion of lipid stores and severe loss of skeletal muscle protein. The ability to arrest or reverse this condition would have a tremendous impact on improving quality of life, treatment outcome, and increasing life expectancy. Here we have used magnetic resonance spectroscopic imaging (MRSI) to understand the metabolism of cachectic tumors and its consequences on normal tissue. Cachectic (MAC16) and non-cachectic (MAC13) murine colon adenocarcinoma cell lines were inoculated in male mice. Cachectic MAC16 tumors induced extensive weight loss unlike MAC13 tumors, which although histologically similar, do not alter body weight. Cachectic MAC16 tumors had higher total choline levels compared to non-cachectic MAC13 tumors, identified in high-resolution spectra of extracts, as due to increased choline and phosphocholine. Thus, enzymes in choline phospholipid metabolism present novel molecular targets to arrest cancer-induced cachexia. Lipid maps indicated a profound depletion of the lipid signal in normal tissue but not tumor tissue in MAC16 tumor bearing mice (Figure 1). These studies are initial steps towards a systemic understanding of the effect of cancer on host organs and tissues - 'the tumor macroenvironment'. The ability to non-invasively image the onset of cachexia early on, preferably before weight loss occurs is critically important to treat the condition, design and optimize therapeutic strategies, and detect response to such treatments.

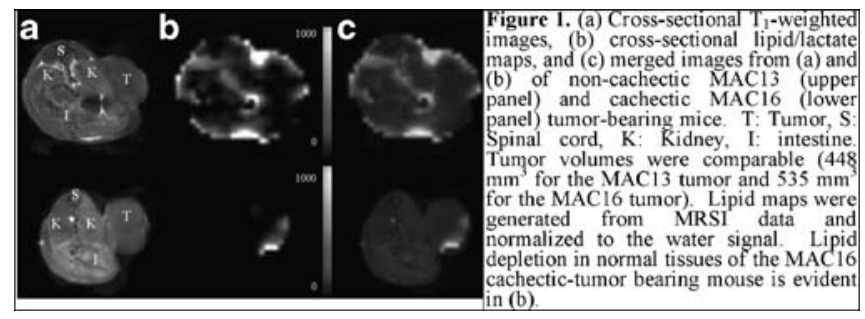

J345

PDGF-BB NORMALIZES MICROMORPHOLOGY AND VESSEL FUNCTION IN VEGF-INDUCED SQUAMOUS CELL CARCINOMAS Wiltrud Lederle $^{1,2}$, Julia Heusel ${ }^{2}$, Eva C. Woenne ${ }^{3}$, Stefan Zwick ${ }^{3}$, Jessica Bzyl ${ }^{1}$, Mihaela Skobe ${ }^{4}$, Fabian Kiessling ${ }^{1}$, Norbert Fusenig ${ }^{5}$, Margareta M. Mueller ${ }^{2}$

${ }^{1}$ Department of Experimental Molecular Imaging, RWTH Aachen University, Aachen, Germany, ${ }^{2}$ Tumor and Microenvironment (A101), German Cancer Research Center, Heidelberg, Germany, ${ }^{3}$ Department of Medical Physics in Radiology, German Cancer Research Center, Heidelberg, Germany, ${ }^{4}$ Department of Oncological Sciences, Mount Sinai School of Medicine, New York, NY, USA, ${ }^{5}$ Division of Differentiation and Carcinogenesis (A080), German Cancer Research Center, Heidelberg, Germany.

Introduction: VEGF often induces formation of immature vessels with increased permeability, resulting in hemorrhages and reduced vessel functionality. Vessel maturation and stabilization is mainly mediated by PDGF-BB, leading to resistance towards anti-angiogenic therapy. Thus, the assessment of tumor vascularization including vessel permeability and function by non-invasive imaging techniques will ultimately improve early diagnosis and characterization of the tumor vascularization with respect to anti-angiogenic treatment. Methods: s.c. tumors and surface transplants of HaCaT-VEGFtransfectants and a 1:1 mixture of VEGF- and HaCaT-PDGF-B transfectants were characterized by $H \& E$ staining and indirect immunofluorescence for epithelial structure, vessel density and vessel maturation. The analysis of tumor vascularization and vessel function was complemented by functional vessel imaging (DCE-MRI) followed by post-processing according to the two compartment model of Brix. Results: De novo expression of murine VEGF-164 in before non tumorigenic $\mathrm{HaCaT}$ cells induced malignant and invasive tumors. However, histological analyses demonstrated an ulcerated, disorganized tumor epithelium infiltrated by immature blood vessels. The combined expression of hPDGF-BB and mVEGF led to similar tumor growth, but epithelial morphology and vessel maturation were markedly improved together with a higher microvessel density. Functional vessel imaging with DCE-MRI revealed an increased amplitude $\mathrm{A}$ in the VEGF- plus PDGF-xenografts, demonstrating an increased mean blood volume. In contrast, the rate constant kep, a parameter influenced by vessel permeability, was decreased compared to the VEGF-xenografts. Conclusions: These data clearly demonstrate that solid tumors with a functional vasculature arise from the concerted action of different cytokines and emphasize the need for a multi-factorial therapy. On the other hand, DCE-MRI data coincide with histological data, thus highlighting functional vessel imaging as a powerful non-invasive tool for the characterization of tumor vascularization with respect to vessel density, status and function. Acknowledgement: This work was supported by the European Union FP6 Cancer Degradome and the Deutsche Forschungsgemeinschaft (SFB-TR23 (project Z1) and SPP1190 Project MU1830/3-1)

\section{J346}

\section{AN AUTOMATED HISTOGRAM BASED SELF-NORMALIZATION METHOD FOR COMPARISON OF INTRA-STUDY CBV MAPS} Ravi T. Seethamraju ${ }^{1}$, Geoffrey Young ${ }^{2}$

${ }^{1}$ MR R and D, Siemens Medical Solutions, USA Inc., Malden, MA, USA, ${ }^{2}$ NeuroRadiology, Brigham and Women's Hospital, Boston, MA, USA.

Introduction: Dynamic susceptibility contrast perfusion weighted MRI (DSCPWI) is a technically robust method of assessing vascularity in brain tumor. Cerebral blood volume (CBV) measurements made with DSCPWI are quantitative in a relative rather than an absolute sense. Relative CBV maps (relCBV) from the same patient produced at different time points are often difficult to compare due to variation in the sequences and due to limitations of the DICOM format. To mitigate this relCBV measurements in the tumor are typically normalized to the those in the contra-lateral normal appearing white matter (NAWM), to yield a dimensionless ratio called normalized $\mathrm{CBV}$ (nCBV). In the appropriate population, $\mathrm{nCBV}$ thresholds between 2.0 and 4.0 have been shown to have a high specificity for presence of high grade glioma (HGG), and thus are potentially valuable for monitoring progression and therapeutic response during therapy, but the reliability of the nCBV values is operator dependent. In order to increase the robustness of the nCBV ratios and allow reliable longitudinal comparison, the method of selecting NAWM must be automated. Here we introduce a method to automate this procedure based on a histogram. Method: Based on a validation cohort consisting of 12 patients enrolled after resection of pathologically confirmed HGG for treatment with whole brain radiation (RT) we observed that on a image pixel intensity range of $0-4095$, the pixels for NAWM appear as the highest peak between the pixel intensities of 5113583. By normalizing the pixel intensities of the relCBV map with the pixel intensity of this peak we get an automated normalized CBV (anCBV) for each of the pixels. Results: Fig 1. shows that in an anCBV map while all intensities below NAWM are suppressed, pixels with anCBV $=2-4$ are highlighted. After segmentation for removal of normal gray matter and blood vessels the anCBV maps should be reliable for longitudinal comparison during tumor burden during follow up because the anCBV normalization is automated. 

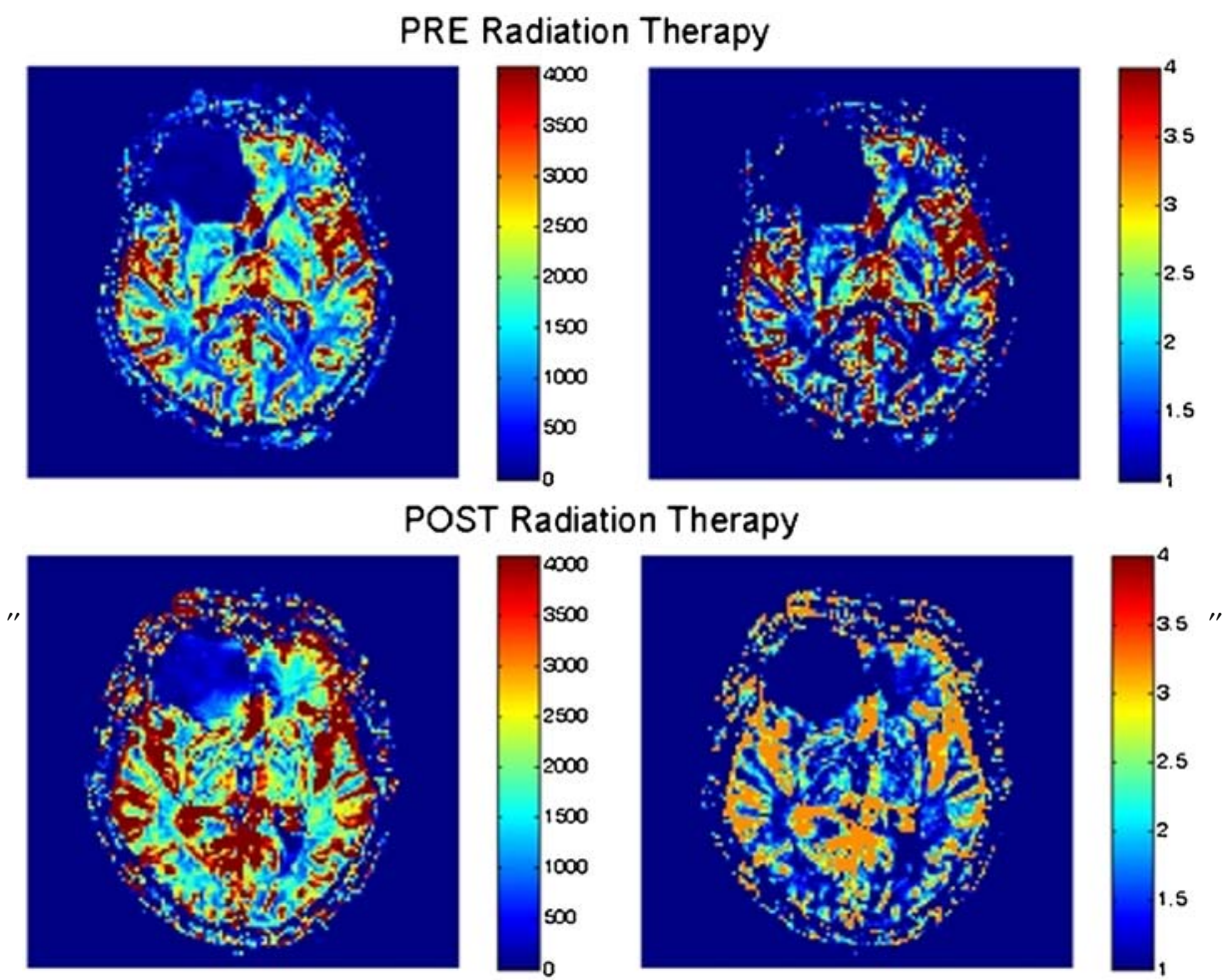

Fig 1. RelCBV maps are shown on the left and anCBV maps on the right. The map on the right now enhances the regions that have CBV greater than NAWM while suppressing regions of NAWM. See difference in maps of anCBV post RT while they appear similar with relCBV maps.

J347

DUAL BLOOD MONITORING OF NF-KAPPAB ACTIVATION AS CORRELATED TO TUMOR FORMATION IN VIVO

Christian Badr ${ }^{1,2}$, Johanna M. Niers ${ }^{1,2}$, Lee-Ann Tjon-Kon-Fat ${ }^{1}$, David Noske $^{2}$, Thomas Wurdinger ${ }^{2,1}$, Bakhos A. Tannous ${ }^{1}$

${ }^{1}$ Neurology, Massachusetts General Hospital and Harvard Medical School, Boston, MA, USA, ${ }^{2}$ Neuro-oncology Research Group, VU Medical Center, Cancer Center, Amsterdam, Netherlands.

Nuclear factor kappa B (NFkB) is a transcription factor that plays a major role in many human disorders, including immune diseases and cancer. Monitoring of expression of this transcription factor should facilitate a better understanding of NFkB activation in pathological processes. We designed a reporter system based on NFkB responsive promoter elements driving expression of the secreted Gaussia luciferase (Gluc). We show that this bioluminescent reporter is a highly sensitive tool for non-invasive monitoring of the kinetics of NFkB activation and inhibition over time, both in conditioned medium of cultured cells, as well as in blood and urine of animals. NFkB activation was successfully monitored in real time in endothelial cells in response to tumor angiogenic signaling, as well as in monocytes in response to inflammation. Further, we demonstrated dual blood monitoring of both NFkB activation during tumor development as correlated to tumor formation using the NFkB Gluc reporter, as well as the secreted alkaline phosphatase reporter. These reporter systems provide useful tools for monitoring NFkB activity in real time in vitro and in vivo and could be applied for dual blood monitoring of any two different in vivo processes simultaneously.

\section{J348}

MOLECULAR IMAGING ASSESSMENT OF THE IMPACT OF CXCR7/RDC1 IN HUMAN LUNG CANCER PROGRESSION Pei-shan Hung, Chih-Chao V. Liu, Chia-Hung Hsieh

Graduate Institute of Basic Medical Science,China Medical University, Taichung, Taiwan.

CXCR7, formerly called RDC1 is a recently deorphanized Gprotein coupled receptor which binds with high affinity the inflammatory and homing chemokines CXCL11/ITAC and CXCL12/SDF-1. Several reports have recently documented that CXCR7 functions as a chemokine receptor for SDF-1/CXCL12, which regulates a spectrum of normal and pathological processes. In this study, the role of CXCR7/RDC1 in lung cancer was explored. Real-time PCR and western blotting assays demonstrate that the levels of CXCR7 expression decrease as the tumors become more aggressive. The human lung adenocarcinoma cell line 
with high invasiveness and metastasis, CL1-5, has lower expression of CXCR7 than the CL1-1 cells, which is the low invasive and metastatic subpopulation. In vitro and in vitro molecular imaging assay together with CXCR7 loss of function and CXCR7 gain of function techniques display that alterations in CXCR7 expression are associated with enhanced angiogenesis, invasive and metastatic activities. These results differ from those in a previous report on other carcinoma cells. Our data suggest that the alternation of CXCR7 expression plays a critical role in tumor angiogenesis, invasion and metastasis in the lung cancer progression.

\section{J349}

CLINICAL VALUE OF 18F FLUORODEOXYGLUCOSE POSITRON EMISSION TOMOGRAPHY IN POSTOPERATIVE RECURRENT LUNG CANCER PATIENTS WITH STAGE I DISEASE

Masahiro Endo $^{1}$, Kyoichi Kaira ${ }^{2}$, Nobuyuki Yamamoto ${ }^{2}$, Takehiko Okumura ${ }^{3}$, Yasuhisa Ohde ${ }^{3}$, Kazuo Nakagawa $^{3}$, Haruhiko Kondo ${ }^{3}$ ${ }^{1}$ Diagnostic Radiology, Shizuoka Cancer Center, Shizuoka, Japan,

${ }^{2}$ Thoracic Oncology, Shizuoka Cancer Center, Shizuoka, Japan,

${ }^{3}$ Thoracic Surgery, Shizuoka Cancer Center, Shizuoka, Japan.

Background and Purpose; The 18F fluorodeoxyglucose positron emission tomography (FDG PET) is useful imaging modality for diagnosing, and evaluating response to treatment in patients with non-small cell lung cancer (NSCLC). Primary tumor maximum standardized uptake value (SUVmax) of FDG PET has been studied as a potential factor for survival or recurrence. However, few studies were reported the significance of FDG PET for the diagnosis of recurrent tumor and/or the relationship between the preoperative SUVmax of primary tumor and early recurrence rate as compared with conventional radiological examinations in patients with stage I lung cancer. The purpose of this study was to assess the clinical value of FDG PET for diagnosing the recurrence of postoperative stage I lung cancer patients. Materials and Methods; A total of 73 consecutive recurrent lung cancer patients (45 males, 28 females; mean age, 67 years) who underwent pathologically proven complete resection with stage I disease between September 2002 and June 2007 were enrolled in this study approved by the institutional review board. All patients underwent preoperative FDG PET. The relation of SUVmax value between preoperative primary tumor and recurrent lesion was analyzed retrospectively, and the SUVmax of preoperative primary tumor was compared with the diagnosis of recurrence using conventional radiological examinations. Results; At diagnosis 41 patients had stage IA disease and 32 stage IB disease. The cases comprised 52 adenocarcinoma, 12 squamous cell carcinoma and 11 others. Out of 73 patients, 52 patients performed FDG PET at the time of the tumor recurrence. Median SUVmax of preoperative primary tumor was 9.3 (range 1.6-24.2) and that of the recurrent lesion was 6.2 (range 1.0-17.2). The correlation coefficient of the SUVmax between preoperative primary and recurrent tumor was 0.57 (95\%C.I.: $0.35-0.73)$ at Pearson's test, demonstrating significance $(p<0.0001)$. FDG PET was more useful tool than conventional radiological examination for diagnosing the tumor recurrence in proportion with the SUVmax of preoperative primary lesion, and the correlation coefficient was 0.43 (95\%C.I.: 0.18 $0.64)$ at Spearman's test $(p=0.0013)$. However, FDG PET was not compared with conventional radiological examination for diagnosing brain and intrapulmonary metastases. Conclusion: It is considered that the tumor characteristics of FDG uptake on the recurrent tumor has been carried, and so FDG PET can be used for assessment for diagnosing the recurrence of postoperative stage I lung cancer patients.

\section{J350}

\section{LONGITUDINAL AND REAL-TIME IMAGING OF THE PHARMACOLOGICAL STIMULATION OF HIF ACTIVITY IN TUMOR ALLOGRAFTS} Steffi Lehmann ${ }^{1}$, Daniel P. Stiehl ${ }^{2}$, Roland H. Wenger ${ }^{2}$, Markus Rudin ${ }^{1}, 3$

${ }^{1}$ Institute for Biomedical Engineering, ETH/University of Zurich, Zurich, Switzerland, ${ }^{2}$ Institute of Physiology, University of Zurich, Zurich, Switzerland, ${ }^{3}$ Institute of Pharmacology and Toxicology, University of Zurich, Zuerich, Switzerland.

The oxygen-sensitive transcription factor Hypoxia Inducible Factor (HIF) plays a key role in regulating adaptation of cells to decreased oxygen levels. Under normoxic conditions, HIF is hydroxylated by prolyl hydroxylase domain proteins (PHD) and thereby marked for proteasomal degradation. Depending on molecular oxygen as a co-factor, PHD activity is inhibited during hypoxia which leads to the stabilisation of HIF. In cancer, HIF is activated in response to tumor hypoxia and promotes the survival and proliferation of tumor cells by inducing genes mainly involved in angiogenesis and anaerobic glycolysis. Moreover, HIF activity has been implied to reduce the effectiveness of chemo- and radiation therapy. To further investigate the impact of increased HIF levels on tumor progression we s.c. implanted murine C51 cancer cells, stably expressing luciferase from a HIF responsive promoter, into the neck of $\mathrm{Balb} / \mathrm{c}$ nude mice. Mice were treated with the PHD inhibitor dimethyloxalyglycine (DMOG) every 48 hours for 10 days to stimulate HIF activity in the tumors. As expected, DMOG treatment resulted in increased tumor growth rates. However, using bioluminescence imaging we observed a decreased HIF activity in tumors of DMOG treated animals (Fig. 1). Recent data (Mazzone et al., Cell, 2009) suggest increased HIF levels in endothelial cells to promote the formation of more mature tumor blood vessels leading to better oxygenated tumors. We are currently investigating if the changes in HIF activity in our model are dependent on the pharmacological inhibition of PHDs in host endothelial cells. Alternatively, a negative feedback mechanism within the tumor cells themselves may account for a decrease in HIF activity as it has been observed previously upon exposing cells to chronic hypoxia conditions in vitro. In any case, decreased HIF activity in tumors may enhance the success of radiation and chemotherapy.

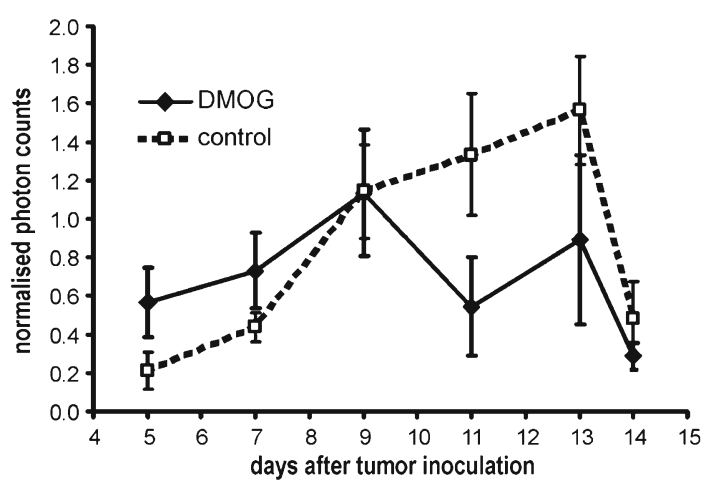

Fig. 1 Monitoring the effect of DMOG treatment on HIF activity in C51 tumors. HIF induced luciferase photon counts were normalised to counts from control tumors of the same size, constitutively expressing luciferase (CMV-luciferase). Values are shown as means \pm sem relative to day 5 . 


\section{J351}

PET-IMAGING OF VEGF RECEPTORS IN TUMOR ANGIOGENIC VASCULATURE WITH SCVEGF-PEG-HBED$\mathrm{CC} /{ }^{68}$ GA AND SCVEGF-PEG-NOTA $/{ }^{68}$ GA TRACERS

Matthias Eder ${ }^{2}$, Arcadius Krivoshein ${ }^{1}$, Marina Backer ${ }^{1}$, Joseph M. Backer ${ }^{1}$, Uwe Haberkorn ${ }^{3}$, Michael Eisenhut ${ }^{2}$

${ }^{\mathrm{T}}$ SibTech, Inc., Brookfield, CT, USA, ${ }^{2}$ German Cancer Research Center (DKFZ), Heidelberg, Germany, ${ }^{3}$ Department of Nuclear Medicine, University of Heidelberg, Heidelberg, Germany.

Receptors for vascular endothelial growth factor (VEGF) play the key role in angiogenesis and are important targets for therapy of malignant tumors. Imaging of VEGF receptors during anti-angiogenic therapy would provide important information, which can influence patient management. The aim of this study was to develop an easy-to-label positron-emitting targeted tracer for imaging VEGF receptors. As targeting protein we used engineered single-chain (sc) VEGF expressed with cysteine-containing tag (Cys-tag) for site-specific conjugation of various payloads. scVEGF was site-specifically derivatized with PEGylated chelating agents PEG-HBED-CC or PEG-NOTA, which both allow fast labeling with ${ }^{68} \mathrm{Ga}$ at room temperature. scVEGF-PEG-HBED-CC was synthesized by activating a single carboxyl group of the $[\mathrm{Fe}$ (HBED-CC)] $]^{-}$complex with N-hydroxysuccinimide. Reaction of the activated complex with $5 \mathrm{kDa}$ NH2-PEG-maleimide was followed by site-specific conjugation thiol group in Cys-tag of scVEGF. The scVEGF-PEG-NOTA conjugate was synthesized using $5 \mathrm{kDa}$ NHSPEG-Maleimide and p- $\mathrm{NH}_{2}$-Bn-NOTA. ${ }^{68} \mathrm{Ga}$ complexation was performed at $\mathrm{pH} 4.2$ at room temperature. The functional activity of the radiolabeled tracers was tested using cell binding assays on 293/KDR cells, a derivative of HEK-293 cells engineered to express $\sim 10^{6}$ VEGFR-2/cell. Biodistribution and PET imaging studies were performed on HT29 tumor-bearing mice after 1, 2, 3 and $4 \mathrm{~h}$. The radiolabeling of scVEGF-PEG-HBED-CC proved more efficient than scVEGF-PEG-NOTA allowing to stop the reaction after 4 minutes ( $>97 \% \mathrm{RCY})$. Radioligand cell binding assays performed on $293 / \mathrm{KDR}$ cells revealed no change in the binding properties of ${ }^{68} \mathrm{Ga}$ labeled scVEGF relative to the parental protein. The tracers were stable in $50 \%$ human serum for at least $72 \mathrm{~h}$. Both tracers showed comparable biodistribution like low liver uptake or similar tumor accumulation. The fast and easy-to-label with ${ }^{68} \mathrm{Ga}$ conjugates scVEGF-PEG-HBED-CC and scVEGF-PEGNOTA revealed comparable in vivo characteristics allowing $\left[{ }^{68} \mathrm{Ga}\right]$ PET imaging of VEGF receptors in angiogenic vasculature after $1.5 \mathrm{~h}$. Our results indicate that both scVEGF-PEG-HBED-CC $/{ }^{68} \mathrm{Ga}$ and scVEGF-PEG-NOTA $/{ }^{68} \mathrm{Ga}$ are highly promising tracers for PET imaging of VEGF receptors for diagnostic and treatment management purposes.

\section{$\mathbf{J 3 5 2}$}

\section{${ }^{18}$ F-FLT KINETIC CHANGES AT 2 AND 6 WEEKS DURING TREATMENT OF HIGH GRADE RECURRENT GLIOMA Christiaan Schiepers ${ }^{1}$, Magnus Dahlbom ${ }^{1}$, Wei Chen ${ }^{1}$, Timothy Cloughesy $^{2}$, Johannes Czernin ${ }^{1}$, (Henry) Sung-Cheng Huang ${ }^{1}$ ${ }^{1}$ Molecular \& Medical Pharmacology, David Geffen School of Medicine at UCLA, Los Angeles, CA, USA, ${ }^{2}$ Neurology, David Geffen School of Medicine at UCLA, Los Angeles, CA, USA.}

High grade gliomas have a poor prognosis. ${ }^{18} \mathrm{~F}$-deoxy-fluorothymidine (FLT) is an in-vivo marker of cellular proliferation that provides prognostic information by identifying responders. Changes in FLT kinetics during treatment with bevacizumab and irinotecan were investigated with compartmental modeling. Methods: 15 patients (6 M,9F), 26-77 yr, with high-grade recurrent glioma (2 WHO grade III, 13 WHO grade IV) were investigated. Each had 3 PET studies, at baseline (study 1), after one course (2 weeks, study $2)$, and at the end of therapy (6 weeks, study 3$)$. FLT (1.5 MBq/kg) was administered and dynamic PET acquired for $1 \mathrm{hr}$. Curves of blood clearance and tumor uptake were derived from factor images or summed frames. A VOI (volume-of-interest) was delineated with thresholding techniques, or defined as a fixed cube of $10 \times 10 \times$ $12 \mathrm{~mm}^{3}$ on tumor. A 3-compartment, 2-tissue model was applied to estimate the rate constants. An input function was derived from the transverse sinus and corrected for metabolites and partial volume. No corrections were applied to the output function. FLT-uptake in SUV was also measured. Results were stratified to actual clinical survival. Results: A linear relationship was found between transport rate k1 and influx rate $\mathrm{Ki}(r=0.86)$, and between $\mathrm{Ki}$ and SUV $(r=0.91)$. The regression slopes were the same for all 3 studies. Absolute parameter values did not differ significantly between studies, but the change between baseline and study 2 or 3 showed significant differences for $\mathrm{k} 1, \mathrm{Ki}$ and SUV $(p<0.05)$. Comparing factor vs summed images, similar results were obtained for a threshold of $70 \%$ vs $75 \%$, respectively. Viable tumor volume (voxels inside the VOI) was 255 vs $244 \mathrm{~mL}$, respectively; linear regression provided 1.05 as slope and 0.08 as intercept. A fixed cube VOI of $1.2 \mathrm{~mL}$ produced the best goodness-of-fit and lowest variations among patients in the parameter estimates. Three groups were distinguished based on survival, $<6$ months (G1,n=4), 6-12 months (G2,n=6), or $>1$ yr $(\mathrm{G} 3, n=5)$. None of the rate constants was significantly different between groups. Long survivors (G3) showed significantly different SUV-changes between study 1 and 3, whereas short survivors (G1, G2) did not. Conclusions: FLT kinetics during treatment of recurrent glioma show a linear relation between $\mathrm{Ki}$ and SUV. A significant change of $\mathrm{Ki}$ or SUV between study 1 and 3 was predictive of a favorable clinical outcome. A summed image 45$60 \mathrm{~min}$ after FLT injection and cubic VOI were sufficient to measure SUV-changes and predict therapy response.

\section{$\mathbf{J 3 5 3}$}

\section{NON-INVASIVE IMAGING OF RADIATION INDUCED TUMOR-INFILTRATING MACROPHAGES}

Rao V. Papineni ${ }^{1}$, Parmeswaran Diagaradjane ${ }^{2}$, William McLaughlin ${ }^{1}$, Sunil Krishnan $^{2}$

${ }^{1}$ Carestream Molecular Imaging, Carestream Health, Inc., New Haven, CT, USA, ${ }^{2}$ Radiation Oncology, MD Anderson Cancer Center, Houston, TX, USA.

Macrophage migration to sites of tissue injury has been described in many models of infection, sepsis, inflammation, cancer, ischemiareperfusion, and trauma. Using a thermal injury mouse model, we have recently demonstrated that both the endogenous peritoneal macrophages and the exogenous murine cells that were transplanted into the peritoneal cavity of mice migrated to the site of inflammation induced by thermal injury to the hindlimb. Using a similar experimental approach, we explored the possibility that endogenous macrophages (with or without an accompanying payload) might migrate to a tumor, subjected to focused radiation therapy as the primary inflammatory trigger. We evaluated this possibility in a subcutaneous colorectal cancer xenograft model in nude mice using localized $\gamma$-radiation (8 Gy, a dose used clinically for palliation of pain due to due tumor encroachment of surrounding normal structures). A commercially available Multimodal in vivo imaging system was used to obtain $\mathrm{X}$ ray and fluorescence (NIRF) images at different time intervals. We demonstrate that peritoneal macrophages migrate to the irradiated region of the animal (figure below). Trafficking of peritoneal macrophages to the irradiated tumor has immense potential in the develop- 
ment of platforms for targeted delivery of drugs, vectors, nanoparticles, and other payloads directly and in high concentrations to the tumor using a radiation beam physically collimated to conform to the edges of the tumor, minimizing unnecessary exposure of other parts of the body to these agents. The role of clinically relevant radiation doses, in the infiltration of compartmentalized macrophages into the tumor environment, and the prospects of developing novel therapeutic strategies utilizing cell trafficking will be discussed.

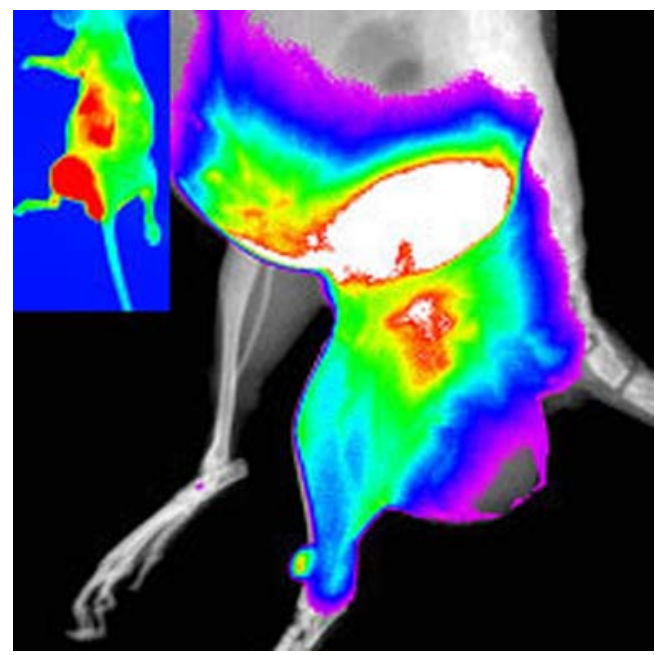

Radiation induced tumor infiltration of NIR fluorescence signal.

\section{J354}

\section{RODENT GLIOMA THERAPY AT 21T. THE FUNDAMENTAL DIFFERENCE BETWEEN SODIUM AND PROTON DIFFUSION MRI}

Victor D. Schepkin, Cathy W. Levenson, Fabian Calixto Bejarano, William W. Brey, Peter L. Gor'kov

CIMAR, NHMFL/FSU, Tallahassee, FL, USA.

The fundamental difference between responses in diffusion and sodium was revealed during the first two days following rodent glioma BCNU chemotherapy (Fig.1). All in vivo MRI experiments were performed using a unique wide bore $21 \mathrm{~T}$ magnet. On the third day after therapy and, later, when apoptotic tumor cell destruction occurs, a strong correlation between tumor sodium and diffusion was observed. Findings of tumor cell destruction and apoptosis were confirmed by histology. After the initiation of chemotherapy, diffusion in the tumor remained unchanged during $\sim 2$ days. No change in tumor diffusion is evidence that cell destruction has not yet occurred. There were no delays, however, in the tumor sodium increases during the first days after chemotherapy. It is important to note that at this time there were also no alterations in the tumor volume. Thus, the observed total sodium increase is due to an increase of intracellular sodium and, from our estimation, it was up to $\sim 65 \mathrm{mM}$. Such a level of intracellular $\mathrm{Na}$ is usually required to initiate irreversible cell damage. There is mounting evidence in vitro that increased intracellular $\mathrm{Na}$ is capable of initiating apoptosis. In our experiments, an increase of intracellular sodium occurs prior to the structural apoptotic changes and, most importantly, it is happening in vivo. Thus, if chemotherapeutic response in vivo is mediated by an increase of intracellular sodium, then a more direct way of affecting intracellular sodium, beyond chemotherapy, is needed. Consequently, this could lead to a change of target and strategy for future drug development. Sodium and ADC function as independent windows for tumor therapy, correlating with the intensity of therapeutic tumor cell destruction. Dual sodium and diffusion MRI is a valuable in vivo approach for tumor therapy efficacy and anticancer drug development. The study was supported by NIH/NCI grant R21 CA119177.

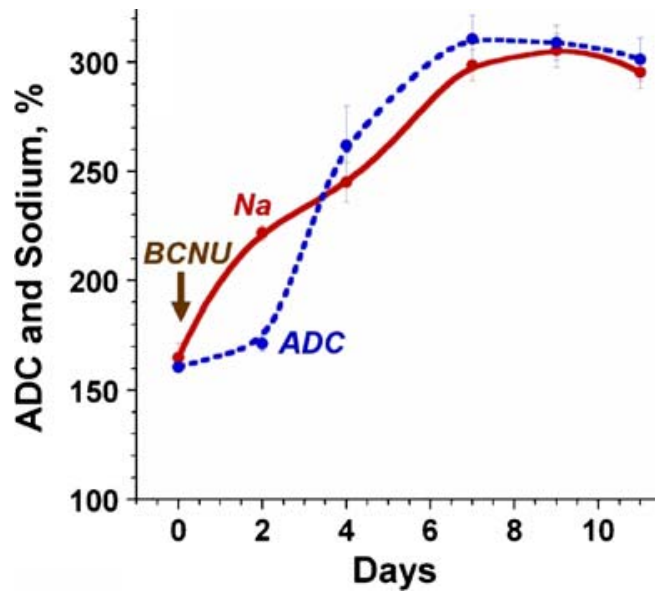

Fig. 1. Sodium and diffusion responses of rat glioma during BCNU chemotherapy $(26.6 \mathrm{mg} / \mathrm{kg})$. The results are presented in percent relative to a normal rat brain.

\section{$\mathbf{J 3 5 5}$}

MULTIPLEX IMAGING OF SINGLE TUMOR CELLS USING QUANTUM DOT-BASED APTAMERS

Ju Ri Chae ${ }^{1}$, Won Jun Kang ${ }^{1}$, Ye Lim Cho ${ }^{1}$, Jong Doo Lee ${ }^{1}$, Soonhag Kim ${ }^{2}$

${ }^{1}$ Department of Radiology, Division of Nuclear Medicine, Yonsei University College of Medicine, Seoul, Korea, South, ${ }^{2}$ Department of Nuclear Medicine, Seoul National University College of Medicine, Seoul, Korea, South.

Background: Aptamer is a small oligonucleotide that binds to specific targets similarly to antibodies, but has advantages such as a lack of immunogenicity, small size, and ease of synthesis. QDs are promising imaging probes because of broad absorption with a narrow emission spectra, high quantum yields, low photobleaching, and chemical stability. Surface-modified QDs can easily be conjugated with ligands such as aptamer or peptides that specifically target bio-molecules. We report the development of a multiplex cellular imaging system that simultaneously targets three different molecular markers in a single cancer cell. Methods: Three different QDs with distinct emission spectrum ranges as follows: $605 \mathrm{~nm}, 655 \mathrm{~nm}$, and $705 \mathrm{~nm}$ were chosen. AS1411 that binds to the nucleolin, TTA1, and MUC-1 were selected to target cancer tissue. Four cancer cell lines, Hela, NPA, C6, PC-3, and a normal cell line, $\mathrm{CHO}$ were individually evaluated. Results: In $\mathrm{CHO}$ cells, the normal healthy cells, QD-conjugated aptamers showed undetectable fluorescence intensity. There was a significant difference in fluorescence activity according to the 3 different types of aptamer. QD-AS1411 showed high levels of fluorescent activities in all 4 different cancer cell lines while QD-TTA1 and QD-MUC-1 displayed significant fluorescent signals in the C6 and HeLa cell lines, respectively. These results demonstrated that nucleolin, tenascin $\mathrm{C}$, and mucin proteins had different cellular expression in different cancers. Triple cellular imaging using 3 different QDconjugated aptamers was performed in a single cell simultaneously by confocal microscopy. Three QD-aptamers showed relatively good fluorescence signals on cellular surfaces in C6 and HeLa cell lines. However, fluorescent activity of QD-TTA1 was very weak in 
PC3 and NPA cell lines. The fluorescent signals of QD-AS1411, QD-TTA1, and QD-MUC-1 were overlapped in a localized area of C6 and HeLa cells. Conclusion: In this study, we successfully visualized a multiplex imaging by QD-conjugated aptamers that targeted highly expressed membrane proteins in cancers. Simultaneous multiplex imaging demonstrated the co-localization and co-expression of nucleolin, tenascin $\mathrm{C}$, and mucin proteins in the cellular membrane of a single cancer cell.

\section{$\mathbf{J 3 5 6}$}

A NOVEL PEPTIDE FOR FIBROSARCOMA TUMOR TARGETING AND NONINVASIVE IMAGING IN VIVO Erh-Hsuan Lin ${ }^{1}$, Alexander C. Wu ${ }^{1,2}$, Wei-Hsiang Wang ${ }^{1}$, Win-Ping Deng ${ }^{1}$

${ }^{1}$ Biomedical materials and Engineering, Taiepi Medical University, Taipei City, Taiwan, ${ }^{2}$ Cancer Center, Taipei Medical University Hospital, Taipei, Taiwan.

A novel peptide specifically targeting fibrosarcoma cells was identified from a phage display library. The phage clone displaying a 12-mer amino acid peptide SATTHYRLQAAN, denominated TK4 in this report, was isolated after four rounds of biopanning. In vitro analysis using ELISA, immunofluorescence, and flow cytometry confirmed the binding affinity of TK4-displaying phage to fibrosarcoma cells. In vivo distribution of TK4-displaying phage exhibited a highly specific homing to tumor xenograft in mouse after systemic administration through tail vein. The phage titers recovered from normal tissues including heart, lung, liver and kidney showed a reduction of $>75 \%$ to that from tumor. Competition assay using a synthetic TK4 peptide substantially reduced the homing of TK4-displaying phage to tumor, confirming that the binding affinity of TK4 phage to tumor cells strictly depends on its displayed peptide. The synthetic TK4 peptide conjugated with 131I was intravenously administrated to mice bearing fibrosarcoma tumors. Four hours after injection, the accumulation of TK4 signal in tumor can be imaged with a digital $\Gamma$-camera. The biodistribution of TK4 peptide showed an increase of radioactivity in tumor $>5$-fold in comparison with surrounding muscle $4 \mathrm{hr}$ after injection. In summary, the peptide TK4 discovered in this report represents a novel peptide with specific binding affinity to fibrosarcoma cells in vitro and in vivo, and is sufficient for non-invasive molecular imaging in small animal. The TK4 peptide holds promise as a lead peptide for future applications in peptide-based tumor diagnosis and therapy.

\section{$\mathbf{J 3 5 7}$}

IN VIVO EVALUATION OF GENE THERAPY BY LOCKED NUCLEIC ACID (LNA) AND PEPTIDE NUCLEIC ACID (PNA)-MICRORNA-21 INHIBITOR

$\underline{\text { Hyunhee Kim }}^{1,2}$, Hyewon Youn ${ }^{1,3}$, Keon Wook Kang ${ }^{1,2}$, $\overline{\text { Dong Soo Lee }}^{1,3}$, June-Key Chung ${ }^{1,2}$

${ }^{1}$ Nuclear Medicine, Seoul National University College of Medicine, Seoul, Korea, South, ${ }^{2}$ cancer research institute, Seoul National University College of Medicine, Seoul, Korea, South, ${ }^{3}$ Institute of Radiation Medicine in Medical Research Center, Seoul National University College of Medicine, Seoul, Korea, South.

Introduction: Although microRNA-21 (miR-21) could be used to mediate gene silencing in mouse models, challenges still exist about stability and therapeutic efficacy. We compared the efficacy of cytotoxic tumor therapy by using locked nucleic acid (LNA) and peptide nucleic acid (PNA)-anti-miR-21 with unmodified miR-21 inhibitor in xenograft model. Methods: Unmodified, LNA and PNAmodified anti-miR-21 were incubated at $37^{\circ} \mathrm{C}$ for $2.5,24,48,72$ and 96 in mouse serum and visualized their stability by denaturing poly- acrylamide gel electrophoresis. DU145 cells were transfected with retroviral vector EF1 $\alpha$-Fluc, which express firefly luciferase under EF1 $\alpha$ promoter (EF1 $\alpha$-Fluc), and the stable cells were selected by Geneticin for two weeks. DU145-Fluc cells were then implanted subcutaneously into nude mice, and anti-miR-21s were injected intratumorally. The IVIS100 imaging system was used for the bioluminescent data acquisition and analysis. Results: Unmodified anti- miR-21 was degraded within $24 \mathrm{~h}$ of incubation. In contrast, LNA and PNA-anti-miR-21 were more stable and did not show signs of degradation after $96 \mathrm{~h}$ of incubation. The DU145-Fluc cells demonstrated an excellent correlation between luciferase activity and cell number. Reduction of tumor size in xenografted DU145-Fluc cells was observed in PNA-anti-miR-21 groups, compared with unmodified and LNA- anti-miR-21 group. Conclusion: Our imaging system could help to verify that PNA-anti-microRNA-21 may be beneficial for clinical application for efficacy and stabilization of anti microRNA.

\section{J358}

\section{COMPARISON OF ACCUMULATION OF 64CU-ATSM} IN DIFFERENT TYPES OF XENOGRAFTS

Jesper T. Joergensen $^{1,2}$, Jacob Madsen ${ }^{2}$, Andreas Kjaer ${ }^{1,2}$

${ }^{T}$ Cluster for Molecular Imaging, Department of Biomedical Sciences, University of Copenhagen, Copenhagen, Denmark, ${ }^{2}$ Department of Clinical Physiology, Nuclear Medicine and PET, Rigshospitalet, Copenhagen Oe, Denmark.

Aim: To compare uptake of 64Cu-diacetyl-bis(N4-methyl-thiosemicarbazone) (64Cu-ATSM) in different tumor xenografts and investigate whether it would be beneficial to PET scan nine hours after injection (late), instead of one (early). Methods: Twelve Nude NMRI mice were divided into groups of 3 and had 107 tumor cells of either MCF-7 (breast cancer), HT-29 (colorectal cancer), A2780 (ovarian cancer), or H727 (lung carcinoid), implanted in each flank. After 2-4 weeks, a dose of approximately $10 \mathrm{MBq} 18 \mathrm{~F}-\mathrm{FDG}$ was administered i.v. and the mice were PET scanned for 20 minutes 1 hour after injection using a small animal PET scanner, followed by a 7 minutes small animal CT scan. The following day, each mice had between $10-20 \mathrm{MBq} 64 \mathrm{Cu}-$ ATSM administered i.v. and was PET scanned for $20 \mathrm{~min}$, 1- and 9 hours after injection, again in each case followed by a 7 minutes CT scan. PET data were reconstructed using MAP algorithm, ROIs were drawn on fused PET/CT images, and SUV values and T/M-ratio were calculated. Finally, the correlations between SUV values from the $18 \mathrm{~F}-\mathrm{FDG}$ and the $64 \mathrm{Cu}-\mathrm{ATSM}$ scan were analyzed by linear regression. Results: All tumors had SUVmean values between 0.25 and 0.5 with regard to the FDG scan, and the SUVmean values for the 1 hour $64 \mathrm{Cu}-\mathrm{ATSM}$ scan were at a similar level. While no increase in 64Cu-ATSM uptake were seen in the MCF-7 and A2780 xenografts after 9 hours, SUVmean values for the HT29 and H727 increased by 1.83 and 1.46 folds respectively. T/M-ratios were in average between 1.5 and 2.5 for the all xenograft types 1 hour after injection. Nine hours after injection this ratio was increased to 3.2 and 3.8 for the HT29 and H727 xenografts respectively. No correlation between SUV for FDG and $\mathrm{Cu}-$ ATSM were seen. Conclusion: Differences in SUV between of xenograft derived from different cell lines was observed with HT29 and H727 having the highest uptake. Moreover, for these xenografts it would be beneficial to PET scan late after injection to achieve increase in SUV.

\section{J359}

\section{PEPTIDE-GUIDED THERANOSTIC NANOPARTICLE FOR IMAGING AND THERAPY OF IL-4 RECEPTOR POSITIVE TUMOR}

Moon Hee Na, Byung-Heon Lee

Cell \& Matrix Biology Research Institute, Kyungpook National University, Daegu, Korea, South. 
Byung-Heon Lee1, Moon-Hee Na1, Hai-Yan Hong1, Xiaofeng He1, Kai Wang1, Ick-Chan Kwon2, Maggie Lu3, and In-San Kim1 1Department of Biochemistry and Cell Biology, School of Medicine, Kyungpook National University, Daegu, Korea, 2Korea Institute of Science and Technology, Seoul, Korea, 3Industrial Technology Research Institute Targeted imaging and therapy of tumor can enhance the efficiency of tumor detection and treatment. IL-4 receptor (IL-4R) has been shown to be abundantly present on various tumor cells, such as lung, ovary, and prostate tumor, and suggested as a novel targeting molecule. We have previously identified a peptide, named AP-1, which has homologous sequences with IL-4 and binds to IL-4R. In order to target tumor through the IL-4R, we employed AP-1 as a targeting ligand to deliver imaging agents and therapeutic drugs to tumor. In vitro binding assays showed that AP-1 preferentially bound H226 human lung cancer cell line that highly express IL-4R, as compared to H460 human lung cancer cell line that little express IL-4R. To examine the in vivo homing of AP-1 to tumor, it was conjugated with the Cy7.5 near-infrared fluorescence (NIRF) dye that has long wavelength and strong tissue penetration ability. The tumor homing of the Cy7.5-AP-1 that was injected into the tail vein of nude mice bearing H226 tumor xenografts was demonstrated by in vivo imaging of NIRF signal. Moreover, chitosan nanoparticles labeled with AP-1 and Cy7.5 bind preferentially to $\mathrm{H} 226$ cells and are uptaked by tumor cells. Accordingly, they home to tumor in vivo at higher levels than unlabeled control nanoparticles. To examine the targeted therapy, liposomes containing doxorubicin were labeled with AP-1. When intravenously injected into mice bearing $\mathrm{H} 226$ tumor, the AP-1-labeled liposomes inhibited the tumor growth more significantly than unlabeled control liposomes and free doxorubicin given at the same concentration. These results suggest that AP-1 could be a useful ligand for targeted imaging and therapy of tumor through the IL-4R.

\section{$\mathbf{J 3 6 0}$}

\section{MOLECULAR IMAGING OF MET-INDUCED-MOTILITY BRINGS NEW INSIGHTS INTO UNDERSTANDING BREAST CANCER METASTASIS}

Galia Tsarfaty ${ }^{2}$, Tammy Diamant ${ }^{1}$, Dganit Shkedy ${ }^{1}$,

Adi Laser Azogui ${ }^{1}$, Shira Israeli ${ }^{1}$, Judith Horev ${ }^{1}$, Sari Natan ${ }^{1}$, Dvora Ronen ${ }^{1}$, Miriam Shaharabany ${ }^{1}$, Ilan Tsarfaty ${ }^{1}$

${ }^{1}$ Tel Aviv University, Tel Aviv, Israel, ${ }^{2}$ Department of Diagnostic Imaging, Sheba Medical Center, Ramat Gan, Israel.

Met tyrosine kinase receptor signaling induces diverse biological responses including cell motogenesis, invasion and metastasis. Met induced cell motility is mediated by the formation of membrane structures such as ruffles, pseudopodia and blebs. Membrane blebbing, a common phenomenon often associated with unfavorable conditions, is also involved in physiological processes like mitosis and developmental cell migration as well as in motility during invasion and metastasis. In this work we demonstrate that overexpression of GFPMet results in its constitutive activation, cell rounding, detachment and dynamic non-apoptotic membrane blebbing. Bleb retraction results in numerous membrane microspikes where CFP-Met, YFP-actin and membrane markers accumulate. Expression of Dominant-Negative (DN) YFP-Met alone does not induce any membrane blebbing. Moreover, co-expression of wt CFP-Met and DN YFP-Met significantly reduces membrane blebbing. Co-expression of YFP-Grb2 and CFP-Met altered bleb morphology to a podium structure in a dosedependent manner. Co-expression of Met and Grb2 induced long podia structures with high local concentration of Met and Grb2 that were colocalized at the tip of the podia. The co-localized Met and Grb2 were shown to be phosphorylated. Co-expression of Met and Gab1 revealed highly dynamic podium. Co-expression of Met and K-Ras dramatically increased bleb size and dynamics. Actin seems to be organized in fibers, probably contributing to the stability of these structures. We hypothesize that Grb2 overexpression alters and "normalizes" Met phenotype by stabilizing the podia structures as compared to blebs. Using fiber confocal microscopy, we were able to demonstrate that Met-Grb2 induced podia structures in the living mouse, suggesting that these podia have a role in vivo. Moreover, we demonstrate that bleb formation in vivo is an initiating step of cell motility tumor dissemination and local metastasis. This model may shed a light on the novel mechanisms of cancer cell motility toward metastasis.

\section{J361}

\section{IMAGING OF HEPATOCELLULAR CARCINOMA WITH [18F] FLUORO-2-DEOXY-GALACTOSE PET/CT HAS A HIGH SENSITIVITY}

Michael Sørensen $^{1,2}$, Dirk Bender ${ }^{1}$, Susanne Keiding ${ }^{1,2}$

${ }^{1}$ PET Center, Aarhus University Hospital, Aarhus, Denmark, ${ }^{2}$ Dept. of Medicine V (Gastroenterology/Hepatology), Aarhus University Hospital, Aarhus, Denmark.

Background and aims. Hepatocellular carcinoma, HCC, is a common malignant complication to liver cirrhosis. Early diagnosis is important, but difficult because of difficulties in distinguishing benign cirrhotic liver tissue from malignant tissue using common diagnostic imaging modalities (e.g. MRI, CT, and ultrasound). The aim of this project was to study the feasibility of imaging HCC by PET after injection of the hepatocyte-specific tracer [18F]fluoro-2-deoxy-galactose, FDGal. Galactose is very specifically taken up by hepatocytes and an increased uptake is expected in malignant cells. PET using the sugar analogue $18 \mathrm{~F}$-deoxy-glucose (FDG) is widely used in oncology to detect malignant tissue but for HCC it only detects about $2 / 3$ of the nodules. Methods. Ten patients with liver cirrhosis and newly diagnosed HCC were included. Diagnosis was based on contrast enhanced dual-phase CT (ceCT), MRI, ultrasound, and/or biopsies according to existing internationally approved guidelines. After overnight fasting the patients underwent a static PET scan from base of the skull to mid-thighs one hour after injection of $200 \mathrm{MBq}$ FDGal. All patients had a ceCT performed; six patients also had a FDG PET scan. Results. All patients had several HCC nodules in the liver and two patients had extrahepatic disease. All nodules were detected by FDGal, except in one case where there was a small hyper dense lesion on ceCT. FDG PET was only positive in $2 / 3$ of the cases in accordance with previous findings. All FDG negative cases were detected by FDGal PET. In one case both FDG and FDGal revealed a metastasis in the axilla that was not found by ceCT. In two cases FDGal PET found HCC not otherwise detected by conventional ceCT nor by FDG PET. Conclusions. FDGal PET seems promising as a novel imaging modality in detecting HCC with better results than FDG PET/CT. It seems, that FDGal PET in conjunction increases sensitivity and specificity, but this needs to be further evaluated in a larger clinical trial.

\section{J362}

CORRELATION OF GENE EXPRESSION OF GLUT1, HK1 AND HK2 WITH F-18-FDG-PET UPTAKE AND CELLULAR PROLIFERATION IN GASTROENTEROPANCREATIC NEUROENDOCRINE TUMORS

Tina Binderup $^{1,2}$, Ulrich Knigge ${ }^{1,3}$, Annika Loft ${ }^{2}$, Andreas Kjaer ${ }^{1,2}$

${ }^{1}$ Cluster for Molecular Imaging, University of Copenhagen, Copenhagen, Denmark, ${ }^{2}$ Dept. Clinical Physiology, Nuclear Medicine and PET, Rigshospitalet, Copenhagen, Denmark, ${ }^{3}$ Dept. Surgical Gastroenterology, Rigshospitalet, Copenhagen, Denmark.

Introduction: Neoplastic tissue exhibits high glucose utilization, reflected in an over-expression of glucose transporters and hexokinases. F-18-Fluo- 
rodeoxyglucose positron emission tomography (FDG-PET) is widely used for imaging of this increased metabolic activity characteristic of malignant cells. In comparison to other neoplasms, the majority of gastroenteropancreatic neuroendocrine tumors (GEP-NETs) are slow growing with low proliferation index. Accordingly, FDG-PET seems less suitable for imaging of these neoplasms. The aim of this study was to see whether this generally low-malignant characteristic was reflected in the expression profile of glycolytic associated genes and how the metabolic activity correlated with proliferation index and FDG-PET imaging. Materials \& Methods: Using real-time PCR, gene expression of facilitative glucose transporter 1 (GLUT1), hexokinase 1 (HK1) and 2 (HK2) were studied in 34 GEP-NETs with 14 colorectal adenocarcinomas (CRA) as controls. When available, Ki67 index as determined by immunohistochemistry and 18F-FDG-PET imaging was compared with gene expression. Maximal Standardized Uptake Value (SUVmax) was used for quantification of tracer accumulation. Results: FDG-PET identified malignant foci in significantly fewer GEPNETs ( 4 of $11,36 \%$ ) than CRAs ( 6 of $7,86 \%), \chi 2, p=0.04$ and significantly fewer GEP-NETs showed upregulation of GLUT1 compared to CRAs. Thus, GLUT1 was upregulated in $41 \%$ of GEP-NETs compared to $86 \%$ of CRA tumors $(\chi 2, p=0.005)$. HK1 was upregulated in $41 \%$ of GEP-NETs and $71 \%$ of the CRA tumors $(\chi 2, p=0.057)$ whereas HK2 was upregulated in $53 \%$ of the GEP-NETs and $64 \%$ of the CRA tumors $(\chi 2, p=0.47)$. There was a significant correlation between the Ki67 proliferation index of the GEP-NETs and the GLUT1 gene expression $(R=0.38, p=0.036)$ whereas no significant correlation between Ki67 index and HK1 or HK2 gene expression was found. By multivariate analysis with GLUT1, HK1 and HK2 in the model, GLUT1 independently predicted the SUVmax of the positive FDG-PET scans ( $2=0.51, p=0.030)$. Conclusion: The gene expression results, with GLUT1 being less frequently upregulated in GEPNETs, confirmed the lower metabolic activity of GEP-NETs compared to the more aggressive CRAs. GLUT1 seemed to predict the aggressiveness of the GEP-NETs, as determined by the Ki67 index, whereas HK1 and HK2 did not, and GLUT1 was the only independent predictor of FDG accumulation as determined by SUVmax.

\section{$\mathbf{J 3 6 3}$}

ANGIOGENESIS AND CELL TRACKING WITH IRON OXIDELABELED TUMOR CELLS: CORRELATION BETWEEN CELL GROWTH AND THE FORMATION OF THE TUMOR VASCULAR BED USING HIGH RESOLUTION MAGNETIC RESONANCE (MR) ANGIOGRAPHY, T1 AND T2* MAPPING AND HISTOLOGY

Piotr A. Wielopolski, Gyula Kotek, Sandra van Tiel,

Gabriela N. Doeswijk, Lejla Alic, Gabriel P. Krestin, Monique R. Bernsen Department of Radiology, Erasmus MC, University Medical Center, Rotterdam, Netherlands.

Purpose: We correlate super paramagnetic iron oxide (SPIO) labeled tumor cells growth and distribution with high resolution magnetic resonance (MR) angiography, $\mathrm{T} 1$ and $\mathrm{T} 2 *$ parametric mapping and histology. Material and Methods: Imaging was performed repeatedly on Brown Norway rats using BN175, a soft tissue sarcoma cell line. Tumor cells were labeled with SPIO particles with pre-defined protocols for the cell line and injected subcutaneously in the hind limbs. Using a 3.0T scanner with small $1-2 \mathrm{~cm}$ internal diameter loops for signal reception, high resolution $\mathrm{MR}$ imaging and angiography (MRA) were performed. Blood circulating Gd-loaded liposomes were used to delineate the vascular bed. At $50-72 \times 50$ $72 \times 100 \mu \mathrm{m} 3$ voxels, 3D T1 - T2*- and proton-density-weighted scans were performed and data volume rendered to provide reconstructions illustrating spatial-temporal relationships of vascular growth/angiogenesis, tumor size and signal distribution of labeled cells. Parametric T1, T2 and T2* maps were collected on all scanning days (minimum 5) for quantitative data analysis. Histology was used to correlate the end-stage MR data to recognize viable and non-viable tumor regions. Results and discussion: Tumor growth was visualized with increased recruitment of vascular components around the tumor mass for a period of three weeks. Dark SPIO labeled cells distributed non-uniformly during tumor growth. The vascular bed was denser in places where the contrast of labeled cells faded more rapidly. Time-resolved volume rendered reconstructions provided a cue to interpretation, showing vascular redistribution and enhancement patterns within the tumor volume in combined data sets with the different imaging contrasts acquired. Parametric maps showed shorter $\mathrm{T}^{*} *$ in certain tumor regions in comparison to controls using non-labeled tumor cell injections. Conclusion: Multicontrast datasets using SPIO labeled tumor cells in combination with time-resolved volume rendering provides an insight to the process of tumor growth and a novel tool to facilitate the interpretation of vascular bed formation, vascular recruitment and cell proliferation.

\section{J364}

\section{GLUCOSE BASED DETECTION OF ENDOGENOUS} PROSTATE CANCERS IN PTEN MUTANT MOUSE MODELS Muhan Chen, Jernej Murn, Hyejin Cho, Danielle Grace, Christopher Pratt, Lloyd C. Trotman

Cancer Center, Cold Spring Harbor Laboratory, Cold Spring Harbor, NY, USA.

PTEN is a tumor suppressor that is among the most frequently lost or mutated genes of human cancer. More than half of the 220,000 men diagnosed with prostate cancer in the United States each year are expected to exhibit alterations of PTEN at the gene or protein level. By genetically mutating Pten in mice we have established a variety of highly faithful models for human prostate cancer. Malignant neoplasms exhibit an elevated rate of glucose uptake and glycolysis over normal cells. This characteristic forms the basis for positron emission tomography (PET) using glucose analogues such as 18F-2-deoxy-D-glucose (18FDG) to visualize primary tumors and metastases. Therefore, radiolabeled 2-DG is generally used in small animal studies. However, FDG-PET fails to visualize prostate tumors in our Pten mutant mice, which is in part caused by the unspecific uptake of FDG-PET by muscles and bladder. Here we demonstrate that a fluorophore-labeled variant of 2-DG (IRDye 800CW 2DG) allows for sensitive visualization of endogenous prostate tumors in Pten mutant mouse models. Dose-dependent and specific accumulation in these tumors was established and correlated to tumor progression using Magnetic Resonance Imaging (MRI) and post-mortem pathology. The use of this infrared-based detection system thus allows for simple non-invasive monitoring of prostate tumorigenesis and its therapy in genetically engineered mouse cohorts. Advantages of this system over the PET and MRI modalities will be discussed.

\section{$\mathbf{J 3 6 5}$}

\section{IN VIVO UPTAKE OF $\left[{ }^{18}\right.$ F]FDG AND $\left[{ }^{18}\right.$ F]FAZA IN EMT6} MURINE CANCER MODEL

Thomas Wanek $^{1}$, Gloria Stundner ${ }^{1}$, Oliver Langer ${ }^{1,2}$,

Claudia Kuntner ${ }^{1}$

${ }^{1}$ Molecular Medicine, Health and Environment, Austrian Research Centers GmbH-ARC, Seibersdorf, Austria, ${ }^{2}$ Department of Clinical Pharmacology, Medical University of Vienna, Vienna, Austria.

Positron emission tomography with $\left[{ }^{18} \mathrm{~F}\right]$-fluoroazomycin arabinoside (FAZA) delivers valuable information about tumor oxygenation. A number of studies investigated the role of $\left[{ }^{18} \mathrm{~F}\right] \mathrm{FAZA}-\mathrm{PET}$ in murine cancer models. Even though these studies concluded in promising results direct comparison of studies remained challenging as tumor size and time points after injection varied. Aim of this study was to assess 
the influence of tumor size on $\left[{ }^{18} \mathrm{~F}\right] \mathrm{FDG}$ and $\left[{ }^{18} \mathrm{~F}\right] \mathrm{FAZA}$ uptake in subcutaneously implanted EMT6 tumors. Furthermore $\left[{ }^{18} \mathrm{~F}\right] \mathrm{FAZA}$ uptake under different breathing protocols (either pure oxygen or room air) was investigated at different time points after injection. METHODS: Twelve EMT6 tumor bearing NRMI nu/nu mice were repeatedly monitored over a period of 21 days. Ten and 17 days after subcutaneous injection of $2.5 \times 10^{6}$ EMT6 cells in the right flank, subjects were investigated with $\left[{ }^{18} \mathrm{~F}\right] \mathrm{FDG}$ on a small animal PET system. On the day following $\left[{ }^{18} \mathrm{~F}\right] \mathrm{FDG}$ scan, consecutive scans with $\left[{ }^{18} \mathrm{~F}\right] \mathrm{FAZA}$ were recorded, either under oxygen breathing $\left(100 \% \mathrm{O}_{2}\right)$ or room air breathing conditions $\left(21 \% \mathrm{O}_{2}\right)$. Dynamic imaging was performed for both breathing conditions. Additional static recordings were made 2 and $5 \mathrm{~h}$ past injection of $\left[{ }^{18} \mathrm{~F}\right] \mathrm{FAZA}$. Anaesthesia was performed using 2\% isofluran. PET images were normalized and regions of interest were manually drawn over the whole tumor. For comparison, standardized uptake values of voxels $\geq 75 \%$ of maximum SUV and tumor-to-background (T/B) ratios were calculated. RESULTS: No significant difference in $\left[{ }^{18} \mathrm{~F}\right] \mathrm{FDG}$ and $\left[{ }^{18} \mathrm{~F}\right]$ FAZA uptake was found between the small $(0.38 \pm 0.11 \mathrm{cc})$ and large $(1.56 \pm 0.46 \mathrm{cc})$ tumor group. Dynamic imaging showed a linear increase in tumor to background ratio of $\left[{ }^{18} \mathrm{~F}\right] \mathrm{FAZA}$ in tumor region $(R=0.99)$ for mice breathing room air while $\mathrm{T} / \mathrm{B}$ ratio of oxygen breathing subjects stabilizes at a lower level. An elevated, but not significant increase in T/B ratio was found between mice breathing different atmospheres $\left(2.62 \pm 1.07\right.$ for $100 \% \mathrm{O}_{2}, 3.66 \pm 1.26$ for room air) $2 \mathrm{~h}$ past injection. Five hours past injection T/B ratios increased to $4.73 \pm 1.70$ for $100 \% \mathrm{O}_{2}$ and $6.65 \pm 1.03$ for room air breathing subjects. Statistically significant differences $(P>0.01)$ were found between the different time points $(2$ and $5 \mathrm{~h})$ after injection under the same atmospheric conditions. CONCLUSION: This study found no dependence of $\left[{ }^{18} \mathrm{~F}\right] \mathrm{FDG}$ and $\left[{ }^{18} \mathrm{~F}\right] \mathrm{FAZA}$ uptake from tumor size within the period of study but underlines the importance of standardized imaging time points. Further studies on different tumors and other hypoxia-sensitive radiotracers are in preparation.

\section{J366}

\section{COMPARISONS BETWEEN DIFFERENT PET TRACER UPTAKES, MR CONTRAST AGENT ENHANCEMENT} AND MR SPECTROSCOPY USING SIMULTANEOUS PET/MR IN A MOUSE BRAIN TUMOR MODEL

Hans F. Wehrl ${ }^{1}$, Martin S. Judenhofer ${ }^{1}$, Carsten Calaminus ${ }^{1}$, Kathy Hasenbach ${ }^{2}$, Nadine Kemmler ${ }^{1}$, Andreas Schmid ${ }^{1}$, Alexander Sauter ${ }^{1}$, Gerald Reischl ${ }^{3}$, Ghazaleh Tabatabai ${ }^{2}$, Bernd J. Pichler ${ }^{1}$ ${ }^{1}$ Laboratory for Preclinical Imaging and Imaging Technology of the Werner Siemens-Foundation, University of Tuebingen, Tuebingen, Germany, ${ }^{2}$ Department of Neurology, University Hospital Zurich, Zurich, Switzerland, ${ }^{3}$ Radiopharmacy, University of Tuebingen, Tuebingen, Germany.

One novelty offered by simultaneous PET/MR is the synchronic acquisition of multiple diagnostic parameters, such as PET tracer uptake and various MR based imaging techniques. This new advantage is realized in our study, where we focused on the comparison of different PET tracer uptakes with MR contrast agent uptake and MR spectroscopy in SMA560 brain tumor bearing mice. As an initial approach, the influence of a PET tracer on MR spectra was evaluated. Therefore, three $\left[{ }^{1} \mathrm{H}\right]-\mathrm{MR}$ spectra of the tumor side were obtained. Subsequently, $\left[{ }^{11} \mathrm{C}\right]$ choline PET tracer (a marker for membrane activity) was i.v. injected into the tail vain and the PET acquisition was started. Shortly after the PET tracer injection another set of MR spectra were obtained, exactly colocalized to the three pre PET tracer spectra. The integrals of the choline signals, an indirect measurement of the choline concentration, in the MR spectra were compared between the pre and post PET tracer application. In addition, we performed further PET/MR measurements using $\left[{ }^{11} \mathrm{C}\right]$ choline, $\left[{ }^{18} \mathrm{~F}\right]$ fluoro-Lthymidine (FLT) (cell proliferation) and $\left[{ }^{11} \mathrm{C}\right]$ methionine (MET) (amino acid detection) as PET-tracers in the same brain tumor model. The data was acquired in combination with MR chemical shift imaging spectroscopy $\left(\right.$ CSI, TR $=1800 \mathrm{~ms}, \mathrm{TE}=135 \mathrm{~ms}$, voxel size $\left.=2.35 \mathrm{~mm}^{3}\right)$. Gd contrast agent enhanced MR with enhancement. Choline/creatine MR-CSI maps were computed and compared with the PET choline uptake. The MR choline spectra integral was [9.84 \pm 0.73$]$ a.u. before $\left[{ }^{11} \mathrm{C}\right]$ choline PET tracer injection and $[9.68 \pm 0.94]$ a.u. after injection, which indicates the PET tracer having no significant influence on the MR spectra. This reflects a much lower sensitivity (micromolar range) of MR compared to PET (picomolar range). The CSI choline/creatine maps showed only a partial match with the choline PET data. We speculate MR spectroscopy and choline PET measuring not exactly the same metabolic processes (deposited choline content vs. elevated choline turnover). This mismatch was also seen between PET tracer uptake and MR contrast agent enhancement, which might be important for clinical practice, like evaluation of biopsy navigation and therapy decision making (e.g. tumor resection vs. irradiation). Our current studies concentrate on the investigation of this mismatch effect in more detail by correlating the PET/MR data with histology of the mice brains, determining necrotic and proliferating tumor areas.

\section{$\mathbf{J 3 6 7}$}

\section{REAL-TIME IMAGING OF HIF-1A STABILIZATION AND DEGRADATION: SPATIAL DISTRIBUTION IN NORMAL AND CANCER CELLS}

Ekaterina Moroz ${ }^{1}$, Sean Carlin ${ }^{2}$, Katerina Dyomina ${ }^{1}$, Sean Burke ${ }^{2}$, Gol Golshani ${ }^{1}$, Howard T. Thaler ${ }^{3}$, Ronald G. Blasberg ${ }^{1}$, Inna S. Serganova ${ }^{1}$

${ }^{1}$ Neurology, Memorial Sloan-Kettering Cancer Center, New York, NY, USA, ${ }^{2}$ Medical Physics, Memorial Sloan-Kettering Cancer Center, New York, NY, USA, ${ }^{3}$ Epidemiology and Biostatistics, Memorial Sloan-Kettering Cancer Center, New York, NY, USA.

HIF-1 $\alpha$ is overexpressed in many human cancers compared to normal tissues due to the interaction of a multiplicity of factors and pathways that reflect specific genetic alterations and extracellular stimuli. Subcellular distribution of HIF-1 $\alpha$ was found to be cell specific and the nuclear localization of HIF-1 $\alpha$ was shown to be associated with shorter survival in patients with cancers. The goal of the study was to analyze the degradation process and the subcellular localization of HIF-1 $\alpha$ in normal and cancer cells. HIF-1 $\alpha /$ FLuc and HIF-1 $\alpha(\Delta$ ODDD $) / F l u c$ reporter systems were developed and used to quantify the half-life of these fusion proteins in reporter-transduced NIH3T3, HEK293 and tumorigenic PTEN-negative U87 cells under normoxia, hypoxia and treatment with $\mathrm{CoCl} 2$ (a hypoxia mimetic). Using immunofluorescence microscopy, we observed different patterns of subcellular localization of HIF- $1 \alpha /$ FLuc fusion protein between normal cells and cancer cells; similar differences were observed for HIF-1 $\alpha$ in both reporter-transduced and wild-type cells. A dynamic cytoplasmicnuclear exchange of the fusion protein and HIF-1 $\alpha$ was observed in NIH3T3 and HEK293 cells under different conditions (normoxia hypoxia and $\mathrm{CoCl} 2$ treatment). In contrast, U87 cells showed a more persistent nuclear localization pattern that was less affected by different growing conditions. Employing a kinetic model for protein degradation, we were able to distinguish two components of HIF-1 $\alpha /$ FLuc protein degradation and quantify the half-life of HIF-1 $\alpha$ fusion proteins. The rapid clearance component (t1/2 4-6 min) was abolished by the hypoxia-mimetic $\mathrm{CoCl} 2$, MG132 treatment and deletion of the ODD domain, and reflects the oxygen/VHL-dependent degradation pathway. The slow clearance component (t1/2 200 $\mathrm{min})$ is consistent with other unidentified non-oxygen/ VHL-dependent degradation pathways. Overall, the continuous bioluminescence readout of HIF- $1 \alpha /$ FLuc stabilization in vitro and in vivo will facilitate the development and validation of therapeutics that affect the stability and accumulation of HIF-1 $\alpha$. 
J368

\section{IDENTIFYING MICROENVIRONMENTS OF METASTASIZING CELLS}

Paul T. Winnard, Venu Raman, Marie-France Penet, Dmitri Artemov, Zaver M. Bhujwalla, Russell H. Morgan

Department of Radiology and Radiological Science, The Johns Hopkins University Medical School, Baltimore, MD, USA.

We frequently observe cancer cells expressing hypoxia induced, i.e., hypoxia response element (HRE) promoter controlled, enhanced green or red fluorescence protein (EGFP/RFP) in lymph nodes and ascites fluid from primary orthotopic breast or prostate tumors derived from these cells (Figure 1A-B). One explanation is that metastatic cells are from hypoxic microenvironments within the primary tumor. The other possibility is that cells metastasize to the lymph node or peritoneal cavity where environments are unable to sustain oxygenation, resulting in hypoxia. By deriving tumors from cells stably transfected with optical reporters with different half-lives under transcriptional control by the hypoxia inducible factor (HIF) -1, we can determine if cancer cells disseminate from hypoxic regions, or become hypoxic in lymph nodes or ascites fluid. Hypoxia is a major cause of resistance to treatment, which may explain in part, the refractory characteristics of metastatic disease. MCF-7 cells were transiently co-transfected with $0.5 \mu \mathrm{g}$ of p5HRE-RFP plus p5HRE-d2EGFP. Fluorescent protein stability was longitudinally evaluated by microscopy, following withdrawal of a $24 \mathrm{~h}$ exposure of $200 \mu \mathrm{M}$ of the hypoxia mimetic $\mathrm{CoCl}$. As shown in Figure 1C-D, green fluorescence from d2EGFP was lost within $24 \mathrm{~h}$. The d2EGFP is engineered with a PEST motif at the carboxy-terminus, targeting the protein to proteosomal degradation. RFP has no PEST motif and is not subject to degradation. These studies demonstrate the feasibility of using such constructs to understand the role of hypoxic environments in metastasis. The presence of the long-lived RFP in the absence of EGFP in metastatic sites will indicate that the cells originated from hypoxic tumor regions but that the metastatic sites are not hypoxic, i.e., the short-lived EGFP will be degraded following synthesis at the primary tumor site and no new translation product of EGFP will be observed in the normoxic metastatic site. Additional control of reporter half-lives can be obtained in the future by using degron sequences.

\section{$\mathbf{J 3 6 9}$}

\section{MULTIMODAL MOLECULAR IMAGING OF BRAIN CANCER USING NANOPARTICLES FUNCTIONALIZED WITH IGFBP7 SINGLE DOMAIN ANTIBODY}

Homam Albaghdadi $^{1}$, Umar Iqbal ${ }^{1}$, Roger Mackenzie ${ }^{1}$, Desvaux Celine ${ }^{2}$, Teodor Veres ${ }^{2}$, Danica Stanimirovic ${ }^{1}$, Barbara Blasiak ${ }^{4}$,

Boguslaw Tomanek ${ }^{3,4}$, Garnette Sutherland ${ }^{3,4}$, Abedelnasser Abulrob ${ }^{1}$

${ }^{1}$ Institute for Biological Sciences, NRC, Ottawa, ON, Canada, ${ }^{2}$ Institute for Industrial Materials, NRC, Montreal, QC, Canada, ${ }^{3}$ Institute for Biodiagnostics, NRC, Calgary, AB, Canada, ${ }^{4}$ Dept of Clinical Neurosciences, University of Calgary, Calgary, $\mathrm{AB}$, Canada.

Glioblastoma multiforme are highly malignant and invasive forms of brain tumors. We have previously reported that the insulin-like growth factor binding protein-7 (IGFBP7) is a unique molecular marker of glioblastoma vessels distinctly over-expressed in tumor compared to non-tumor vasculature, making it an excellent candidate for therapeutic and diagnostic targeting. Single-domain antibodies ( $\mathrm{sdAb}$ ) consist of the $\mathrm{VH}$ domain of the heavy-chain antibodies found in camelid species. These antibodies are small $(\sim 13 \mathrm{kDa})$, stable, and have high affinities for their antigens. Here, we evaluated the ability of nanoparticles (NPs) targeted with the $\mathrm{sdAb}$ against IGFBP7 to deliver imaging payload to the tumor. The near-infrared dye, cy5.5, and IGFBP7 $\mathrm{sdAb}$ were conjugated to a PEGylated superparamagnetic iron oxide NPs to create a dual-modality contrast agent for near-infrared fluorescence imaging and magnetic resonance imaging (MRI). NPs were injected intravenously into CD1 nude mice harboring 10-day old intracranial U87MG tumors. Mice were then imaged using an eXplore Optix pre-clinical optical imager. At $48 \mathrm{~h}$, mice were sacrificed, saline perfused and the brains were harvested, fixed in formalin and sectioned for immunohistochemistry. In a separate study, imaging of tumor-bearing mice was assessed using MRI to determine tumor contrast enhancement using IGFBP7 functionalized NPs. The data indicates enhanced tumor targeting with IGFBP7-functionalized NPs compared to non-targeted NPs and support the application of $\operatorname{sdAb}$ targeted NPs in diagnostic imaging of brain tumors.

\section{$\mathbf{J 3 7 0}$}

\section{MOLECULAR IMAGING OF BRAIN TUMOR VESSELS USING} ANTI-IGFBP7 SINGLE DOMAIN ANTIBODY CONSTRUCTS

Umar Iqbal $^{1}$, Homam Albaghdadi ${ }^{1}$, Roger Mackenzie ${ }^{1}$,

Boguslaw Tomanek ${ }^{2,3}$, Garnette Sutherland ${ }^{2,3}$, Danica Stanimirovic ${ }^{1}$, Abedelnasser Abulrob ${ }^{1}$

${ }^{1}$ Cerebrovascular Research, National Research Council of Canada Institute for Biological Sciences, Ottawa, ON, Canada, ${ }^{2}$ National Research Council of Canada - Institute for Biodiagnostics, Calgary, ON, Canada, ${ }^{3}$ Department of Clinical Neurosciences, University of Calgary, Calgary, ON, Canada.

Glioblastoma multiforme are highly malignant and invasive forms of brain tumors. We have previously reported that insulin-like growth factor binding protein-7 (IGFBP7) is a unique molecular marker of human glioblastoma vessels that can be exploited in molecular imaging of tumor angiogenesis. Single-domain antibodies $(\mathrm{sdAb})$ consist of the $\mathrm{VH}$ domain of the unique heavy-chain antibodies found in camelid species. These antibodies are small $(\sim 13 \mathrm{kDa})$, stable and have high affinities for their antigens. Here we evaluated the ability of various anti-IGFBP7 $\mathrm{sdAb}$ constructs (monomer and pentamer) to target brain tumor vessels in an orthotopic brain tumor model in mice. sdAbs were labeled with the near-infrared dye, cy5.5, injected intravenously into CD1 nude mice harboring 10-day old intracranial U87MG tumors and imaged using the eXplore Optix preclinical optical imager at various time points up to 72 hours. At the end of each experiment, mice were sacrificed, saline perfused and the brains were harvested, fixed in formalin and sectioned for fluorescence microscopy. The optical imaging data indicated that the anti-IGFBP7 $\mathrm{sdAb}$ constructs can localize and retain in the brain tumor region but not in normal brain. Ex-vivo sectioning of brains from brain tumor bearing mice injected with labeled sdAbs, confirmed the specific binding of the $\mathrm{sdAb}$ to the brain tumor vessels. Furthermore, sdAbs were able to bind selectively to tumor vessels in human glioma patient samples, comparable to commercially available antibodies. These data support the use of antiIGFBP7 $\mathrm{sdAb}$ for molecular imaging of brain tumors.

\section{$\mathbf{J 3 7 1}$}

\section{BRAIN-TUMOR TARGETING AND IMAGING BY SAPOSIN C-ASSOCIATED FLUORESCENT NANOVESICLES}

Xiaoyang $\mathrm{Qi}^{1}$, Zhengtao $\mathrm{Chu}^{1}$, Keith Stringer ${ }^{2}$, Balveen $\mathrm{Kaur}^{3}$, Antonio Chiocca $^{3}$, Ray Takigiku ${ }^{4}$, Paul Correa ${ }^{4}$, Brian Gray ${ }^{5}$,

Koon Y. Pak ${ }^{5}$

${ }^{1}$ Human Genetics, Cincinnati Children's Hospital Research Foundation, Cincinnati, OH, USA, ${ }^{2}$ Pathology, Cincinnati Children's Hospital Research Foundation, Cincinnati, OH, USA, ${ }^{3}$ Neurological Surgery, The Ohio State University, Columbus, OH, USA, ${ }^{4}$ Bexion Pharmaceuticals, Covington, KY, USA, ${ }^{5}$ Molecular Targeting Technologies, Inc, West Chester, PA, USA.

Saposin C (SapC) is a small lysosomal protein (80 aa) which can induce acidic phospholipid membrane fusion. Nanovesicles composed 
of SapC and the phospholipid dioleoylphosphatidylserine (DOPS) are stable, $200 \mathrm{~nm}$ in size and have high affinity for phosphatidylserine (PS)-enriched membrane surfaces which are common in many types of tumor cells. We observed that SapC- DOPS nanovesicles targeted and accumulated in glioblastoma multiforme (GBM) tumors. Our preliminary experiments indicated that a far-red emitting membrane intercalating dye, CellVue Maroon (CVM, ex. max. $647 \mathrm{~nm}$; em. max. $667 \mathrm{~nm}$ ) carried by SapC-DOPS nanovesicles specifically stained the GBM tumors as imaged in live animals using an IVIS imaging system. SapC is essential for this GBM-targeting. Functional organization studies map fusogenic domains to the N-terminal half of SapC. These domains are required for GBM-selected targeting of the nanovesicles. To investigate molecular targets, we developed a rapid screen method by subcutaneously implanting luciferase transfected GBM cells in animals for in vivo image analysis. After treating the GBM cells with PSspecific binding proteins or peptides, SapC-DOPS targeting was blocked without significant detectable CVM signal in GBM implanted animals. This suggests that PS may be the molecular target of the nanovesicles. In chronic and acute toxicity studies, SapC-DOPS nanovesicles showed no gross toxicity signs in mice. These intriguing observations support the use of SapC-DOPS as a potentially safe vehicle for delivery of imaging agents to improve diagnosis of brain tumors and also to assist in their surgical ablation.

\section{J372}

\section{IN VIVO MONITORING OF FLUORESCENTLY LABELED CANCER CELLS}

Eva-YiWen Barton, Angel Ang, Khanh Urban, Chaincy Kuo,

Kevin P. Francis, Jae-Beom Kim

Oncology, Caliper Life Sciences, Alameda, CA, USA.

Whole body in vivo imaging has contributed significantly to the detection of disease progression and drug efficacy for past several years. With the constant introduction of sensitive imaging instruments, applications in fluorescent imaging have expanded to monitoring tumor cell growth and gene expression. In addition, there has been explosion in development of variety of fluorescent proteins from existing red fluorescent proteins. The new fluorescent proteins have improved brightness, photostability and better tissue penetration of signals with far red wavelength. Previously, we developed brighter bioluminescent cancer cell lines using enhanced firefly luciferase 2 (luc2). Using one of them, 4T1-luc2 cells, we were able to detect a single bioluminescent cell in vivo. To expand the applications of genetically labeled fluorescent tumor cells, we have developed single and dual labeled 4T1 and PC3M cell lines using tdTomato fluorescent protein and luc2. First, we engineered a vector encoding tdTomato protein in which expression is under the control of human ubiquitin $\mathrm{C}$ promoter. Parental 4T1 and PC3M cells were stably transfected with tdTomato vector and antibiotic resistant cells were selected to generated 4T1-tdT and PC3MtdT respectively. In parallel, single luciferase 2 labeled cells were transfected with tdTomato vector to develop dual labeled cell lines (4T1-luc2-tdT and PC3M-luc2-tdT). Our initial analyses showed that the expression level of tdTomato protein was unstable over time in both single and dual labeled cells. Therefore, we attempted single cell cloning and isolated individual clones of both single and dual labeled cells. Positive clones were subjected to fluorescent activated cell sorting (FACS). Once cells were isolated, luciferase expressions were confirmed in dual label cell lines. Cultures were maintained to test the stability of tdTomato and luc2 expression levels over 4 weeks. The results indicate that cloned populations have stable expression of both reporters. We confirmed that the growth rates of both single and dual labeled cells were comparable to those of the parental cells. To monitor tumor growth in vivo, cells were implanted subcutaneously and orthotopically into $\mathrm{nu} / \mathrm{nu}$ mice. Tumor progressions were monitored using a cooled CCD camera non-invasively in real time. Our data demonstrate that we could follow primary tumor growth and metastases in vivo using fluorescent (and bioluminescent) imaging. These dual reporter systems can be applied to monitor pathway specific signaling pathways in the animals.

\section{J373}

\section{INITIAL DATA FROM A FIRST PET/MRI-SYSTEM AND ITS APPLICATIONS IN CLINICAL STUDIES USING MRI BASED ATTENUATION CORRECTION}

$\underline{\text { Armin Kolb }}^{1}$, Matthias Hofmann ${ }^{1,3}$, Vesna Sossi ${ }^{1,4}$, Hans F. Wehrl ${ }^{1}$, Alexander Sauter $^{1,2}$, Andreas Schmid ${ }^{1}$, Martin S. Judenhofer ${ }^{1}$, Heinz-Peter W. Schlemmer ${ }^{2}$, Claus Claussen ${ }^{2}$, Bernd J. Pichler ${ }^{1}$ ${ }^{1}$ Laboratory for Preclinical Imaging and Imaging Technologies of the Werner Siemens Foundation, University of Tuebingen, Tuebingen, Germany, ${ }^{2}$ Department of Radiology, University of Tuebingen, Tuebingen, Germany, ${ }^{3}$ Max Planck Institute for Biological Cybernetics, Tuebingen, Germany, ${ }^{4}$ University of British Columbia, Vancouver, BC, Canada.

First prototype clinical PET/MRI scanners are readily available to be tested in patient studies. In addition to provide a unique possibility for multifunctional imaging with temporally and spatially matched data, PET/MRI also provides anatomical information that can be used for attenuation correction with no radiation exposure to the subjects. When both, PET and MRI, are necessary for a clinical diagnosis, the ability to perform simultaneous PET and MRI in one machine, results in a reduced scan time. Three PET/MRI systems have already been delivered to clinical centers and they are showing promising results in first patient studies. For quantitative PET imaging attenuation correction is obligatory. In current PET/MRI setups, there is no possibility to perform a transmission scan. Thus, the attenuation map needs to be derived from the MRI image instead. Based on the MRI image, we computed the attenuation map of the patient using an adaptation of our correction method which uses a database of MRI-CT template images that are registered to the patient image. The MR-based attenuation corrected PET images were quantitatively compared to the PET/CT images. Here we describe three studies in the field of neuro-oncology and a patient with cerebral stroke. The main aim of the PET/MRI examinations was the exclusion of metastases for the determination of further therapy strategies. Furthermore, the exact coregistration of PET and MRI data as well as the good soft tissue contrast from the MR images supports the radiotherapy planning. One patient suffered from pharyngeal carcinoma received a [18F]FDG-PET/CT examination for tumor staging. Incidentally, PET/CT revealed a hypodense area of the temporal right lobe, obviously revealing an old cerebral infraction. In addition to a better soft tissue contrast, MRI offers advanced diagnostic options like MRI spectroscopy and MRI angiography. These two imaging options showed a reduced choline area and peripheral stenosis of the right arteria cerebri media, adding additional data for the diagnosis along to the reduced FDG uptake in this area. Finally, we performed a full set of phantom scans according to the NEMA protocol to investigate the PET imaging performance. Detailed studies assessing potential mutual interferences between PET and MRI showed only minor, for in vivo imaging, not relevant effects.

\section{J374}

\section{MOLECULAR IMAGING OF FIBRIN IN A MOUSE TUMOR MODEL}

Ritika Uppal, Zdravka Medarova, Christian T. Farrar, Guangping Dai, Anna Moore, Peter Caravan

Martinos Center for Biomedical Imaging, Massachusetts General Hospital, Charlestown, MA, USA.

Introduction: Since Trousseau suggested a correlation between the hemostatic system and cancer biology in 1865 , several clinical studies 
have shown that the expression of procoagulants and fibrinolytic factors by tumor cells and/or stromal cells correlates with advanced disease and poor outcome for cancer. Cellularly, both tumor and wound are characterized by the presence of fibrin clots. The success of fibrin-specific MR probes in identifying thrombi in vivo suggests their potential use in identifying and characterizing primary and metastatic tumors. The aim of this work is to compare the established fibrin-specific probe EP-2104R and untargeted GdDTPA using steady-state and dynamic contrast enhanced imaging in a breast cancer xenograft mouse model. Methods: Tumors were grown in the flank of 5-6 wk old nude mice $(N=10)$ by subcutaneous injection of human breast adenocarcinoma cells. Imaging was performed at $9.4 \mathrm{~T}$ prior to and following i.v. injection of either $200 \mu \mathrm{mol} / \mathrm{kg}$ GdDTPA or $20 \mu \mathrm{mol} /$ $\mathrm{kg}$ EP-2104R. The imaging sequences included multislice FLASH at low resolution $(0.3 \times 0.3 \mathrm{~mm} 2, \mathrm{AT}=13 \mathrm{~s})$ and higher resolution $(0.15 \times$ $0.15 \mathrm{~mm} 2,16$ averages, $\mathrm{AT}=7 \mathrm{~min}$ ) and inversion recovery $\mathrm{T} 1$ maps. Frozen tumor sections were stained with a fibrin-specific antibody (Mouse anti-human fibrin II beta-chain antibody (clone NYBT2G1). ROIs were drawn in the tumor core, tumor periphery, adjacent muscle, and air outside the animal and contrast to noise ratios for tumor core or periphery relative to muscle were calculated as $\mathrm{CNR}=\{\mathrm{SI}($ tumor)-SI(muscle) $\} / \mathrm{SD}$ (air) Results and Conclusions: The kinetics of contrast enhancement and washout from the tumor core were similar for both GdDTPA and EP2104R. However, the clearance rate for EP-2104R was significantly slower than GdDTPA $(2.5 \mathrm{X}, p<0.05)$ from the tumor periphery (figure). T1 values measured at 40 min post EP-2104R were reduced significantly in both the tumor core $(p<0.0001)$ and the periphery $(p<0.0001)$ compared to pre-contrast agent. The CNR in the periphery was $80 \%$ higher for EP-2104R compared to GdDTPA $(p<0.05)$. The persistent enhanced CNR and shortened T1 in the periphery following EP-2104R correlates with rich staining of fibrin in the periphery. This peripheral tumor enhancement with EP-2104R may be a general steady state marker for solid tumors.
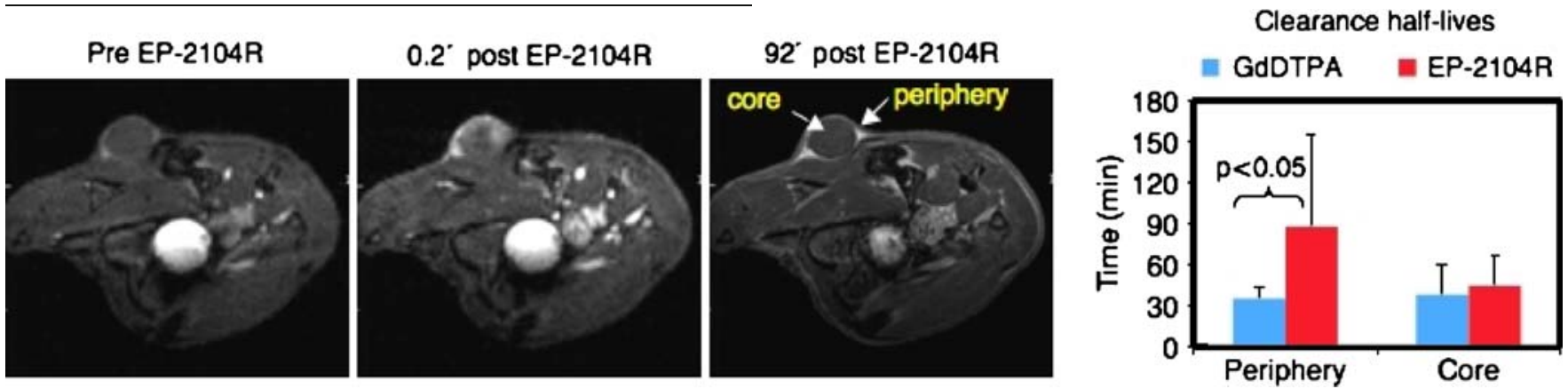

J375

MONITORING OF MYELOMA CELLS HOMING IN VIVO THROUGH MULTIFUNCTIONAL IMAGING REPORTERS Zheng-Zheng Shi ${ }^{1}$, Yongdong J. Feng ${ }^{2}$, Jianguo Wen ${ }^{2}$, Daniel Y. Lee ${ }^{1}$, King Li ${ }^{1}$, Chung-Che (Jeff) Chang ${ }^{2}$

${ }^{1}$ Radiology, The Methodist Hospital Research Institute, Houston, TX, USA, ${ }^{2}$ Hematology, The Methodist Hospital Research Institute, Houston, TX, USA.

Multiple myeloma is an incurable hematological malignancy of plasma cell origin. In an effort to better understand this disorder, animal models of myelomas have been developed to define mechanisms of myeloma cell homing to bone marrow and factors within bone marrow that promote myeloma cell growth, invasion, and drug resistance. The purpose of this study is to noninvasively image and monitor myeloma cells in live animals to characterize their trafficking and growth patterns. We have constructed a lentiviral vector (pLenti7.3/V5-DEST) that expresses a fusion gene cassette consisting of luciferase (luc), hygromycin phosphotransferase (Hy), and HSV1-tk under the control of CMV promoter, as well as an Emerald GFP gene (EGFP) under the control of the SV40 promoter. This lentivector was used to transduce human myeloma cell line RPMI 8226. A subline of RPMI 8226 cells stably expressing the lentivector has been established by hygromycin selection and flow cytometry sorting on EGFP. The expression of luciferase was confirmed and the HSV1-tk activity was evaluated by in vitro uptake of FHBG (44 times higher than the non-transduced parental line). We also confirmed that the transduction did not change cellular functionality: the subline had similar colony forming ability as the parental cell line, and contained a similar side population (SP, i.e., stem cells) in flow cytometry assay. One million of the multi-reporter expressing myeloma cells mixed with 100 thousand bone marrow stromal cells were injected intravenously to immunocompromised NOD/SCID Il2rg (-/-) mouse to induce the myeloma disease. The mouse was imaged weekly after the injection with a Xenogen IVIS 200 system for in vivo bioluminescence. Of 8 mice tested, 6 demonstrated a bioluminescence signal at either of their femoral regions between 2-5 weeks after the injection. To confirm the lesion containing engineered RPMI 8226 cells, bone marrow cells were harvested from the femoral bone and examined with fluorescence microscopy and flow cytometry. The majority of the harvested marrow cells expressed GPF, and in flow cytometry the virtual SP cells were all positive for GFP, suggesting that the putative myeloma stem cells remained in the xenograft lesion. Additionally, the presence of HSV1-tk in these cells was verified by ganciclovir sensitivity. These results suggest that the engineered myeloma cells maintain long-term stable expression of multifunctional imaging reporters amenable to longitudinal in vivo assessment of myeloma development in a murine model.

\section{J376}

USING HDL-LIKE PEPTIDE-PHOSPHOLIPID SCAFFOLD FOR THE CYTOSOLIC DELIVERY

Zhihong Zhang ${ }^{1,3}$, Mi Yang ${ }^{1,2}$, Juan Chen ${ }^{1}$, Lili Ding ${ }^{1}$, Honglin Jin ${ }^{1,3}$, Ian Corbin ${ }^{1}$, Weiguo Cao ${ }^{1,4}$, Gang Zheng ${ }^{1}$

${ }^{1}$ medical biophysics, Ontario Cancer Insititute, University of Toronto, Toronto, ON, Canada, ${ }^{2}$ Oncology, Drum Tower Hospital, Nanjing, China, ${ }^{3}$ Britton Chance Center for Biomedical Photonics, Wuhan National Laboratory for Optoelectronics-Huazhong University of Science and Technology, Wuhan, China, ${ }^{4}$ Department of Chemistry, Shanghai University, Shanghai, China.

The HDL receptor, scavenger receptor class B type I (SR-BI), is overexpressed in several types of cancer cells. Neutral lipid cargo of HDL is selectively taken up from HDL by the cancer cells through a unique lipid transfer mechanism which allows for cytosolic deliver of the lipids to cells. The HDL cytosolic delivery is an attractive release mechanism as it overcomes limitations of endosomal sequestration following endocytosis uptake. To exploit such non-endocytic mechanisms, we created a HDL-like peptide-phospholipid scaffold (HPPS) with a precisely controlled sub-30 $\mathrm{nm}$ core-shell structure for the efficient and 
non-toxic cytosolic transport of functional active cargos to cancer cells. Our studies showed that stable HPPS particles could be formulated with phospholipids, cholesteryl oleate, optical probes or therapy agents and apoA-I mimetic peptide. The particle displayed similar binding activity to SR-BI as native HDL. Confocal images proved core loaded dyes could be delivered to cancer cells through cytosolic delivery without lysosomal colocalization. Furthermore, the uptake of the fluorescent dye, DIRBOA HPPS by MT1 cells (SRBI+) was quantified by flow cytometry at different time points. Our results confirmed that the intracellular uptake of DIRBOA occured very quickly $(<10 \mathrm{~min})$. A lipid derivative of paclitaxel, paclitaxel oleate (PTX-OL), was synthesized and incorporated into HPPS. PTX-OL HPPS was shown, by MTT assay, to be selectively toxic to cancer cells expressing SR-BI. In summary, our initial findings suggest that the HPPS nanocarrier is particularly useful cytosolic delivery strategy for cancer diagnostic and therapeutic agents.

\section{J377}

INTRAOPERATIVE FDG PET/CT OF COLORECTAL CANCER SURGICAL SPECIMENS FOR VERIFICATION OF STAGING OF DISEASE AND ASSISTANCE WITH PATHOLOGY SAMPLING Nathan C. Hall ${ }^{1}$, Douglas Murrey ${ }^{1}$, Edward Martin ${ }^{2}$, Jun Zhang ${ }^{1}$, Michael V. Knopp ${ }^{1}$

${ }^{1}$ Radiology, The Ohio State University, Columbus, OH, USA, ${ }^{2}$ Surgery, The Ohio State University, Columbus, OH, USA.

Introduction: Accurate staging is critical to guiding appropriate therapy for a variety of cancer types including colorectal cancer. Given that a single positive lymph node advances a patient to stage III and necessitates chemotherapy, it would follow that an accurate evaluation all the nodes in the surgical specimen is warranted, however, the current method of lymph node evaluation requires identification and evaluation of only 15 nodes. Routinely, only a small fraction of total surgical specimens undergo microscopic pathologic evaluation. We present methodology which will assist pathologists in sampling of surgical specimens and demonstrates potential for real time intraoperative staging and verification of resection of disease. Methods:On the day of surgery, patients were injected with $18 \mathrm{~F}$ FDG intravenously approximately 100 minutes before surgery. Intraoperatively, a handheld gamma probe was used to help locate and identify primary and metastatic sites of tumor. Standard surgical resection of disease occurred with additional information from the handheld gamma probe. A microPET/CT scan of the resected specimen was performed to identify specimen margins and potential sites of metastatic disease. All surgical specimens were sent for standard pathologic evaluation. Results: Micro PET/CT images of surgical specimens were obtained for accurate localization of hypermetabolic malignancy. The example case images demonstrate a primary lesion in the sigmoid colon and five hypermetabolic lymph nodes within the mesenteric fat. These nodes were later confirmed to contain tumor. Image based staging of was accurate based on final pathologic confirmation and was available before the patient awoke from anesthesia. Conclusions: Intraoperative specimen imaging following preoperative 18F-FDG administration demonstrates great potential for accurate real time staging of disease and assistance for guiding pathologic sampling for verification of accurate staging of disease. Intraoperative imaging based verification of staging could not only guide pathologic staging but direct medical management of patients with less time investment and resources.

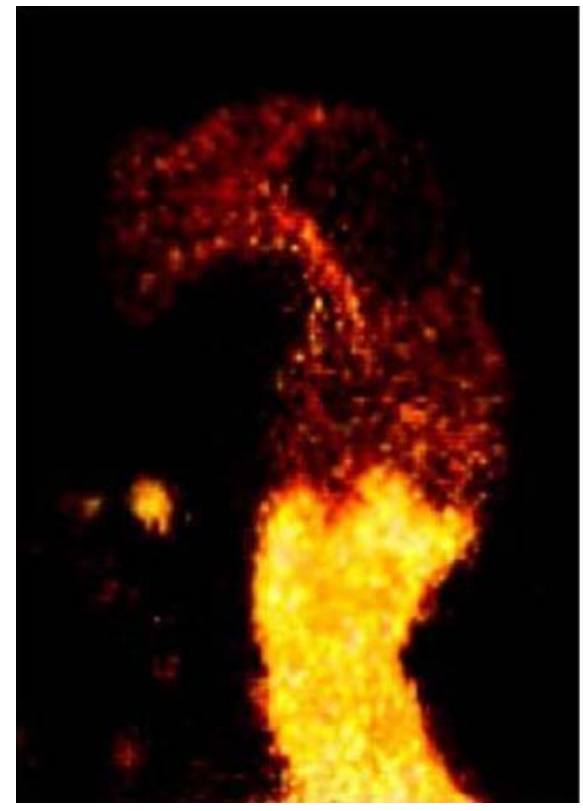

$\mathbf{J 3 7 8}$

IN VIVO COMPARISON OF [18-F]FLUOROCHOLINE AND [11-C]CHOLINE FOR PET IMAGING OF HEPATOCELLULAR CARCINOMA

Jeffrey A. Kolthammer ${ }^{1}$, Haibin Tian ${ }^{2}$, Chunying $\mathrm{Wu}^{2}$, Yu Kuang ${ }^{1,2}$, Nicolas Salem ${ }^{1,2}$, David J. Corn ${ }^{2}$, Zhenghong Lee ${ }^{2,1}$

${ }^{1}$ Biomedical Engineering, Case Western Reserve University, Cleveland, $\mathrm{OH}$, USA, ${ }^{2}$ Radiology, University Hospitals Case Medical Center, Cleveland, OH, USA.

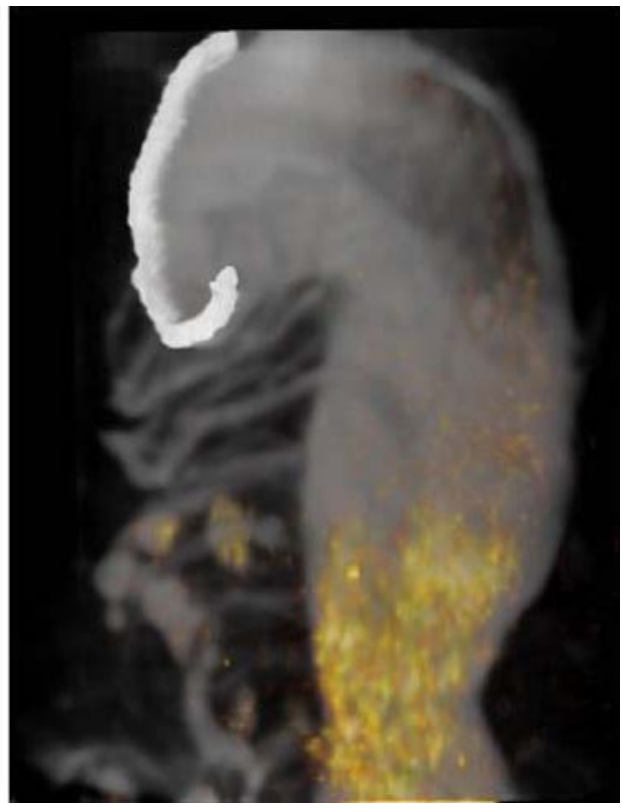

[18F]Fluorocholine was developed as a PET tracer with a longer halflife, but analog to [11C]-labeled choline, which is involved in cell membrane synthesis with high uptake in some tumor cells. These two agents' performance in PET imaging of hepatocellular carcinoma (HCC) is compared using an Eastern woodchuck (Marmota monax) model in this study. PET data were acquired for $1 \mathrm{~h}$ on two animals each with IV administration of [N-methyl(-11C)]-Choline (CHOL) and [18F]-Fluoroethylcholine (FCH) on separate occasions. List-mode PET data were reconstructed as a dynamic sequence; suspected tumor regions in the liver were identified using contrast-enhanced $\mathrm{CT}$ and 
perfusion-phase PET images. Time-activity curves for the regions of increased uptake $(N=12)$ and surrounding hepatic regions were calculated. Results: The two tracers demonstrate similar blood-pool dynamics; FCH shows higher transient kidney signal up to $10 \mathrm{~min}$ post-injection. The concentration of tracer in the tumor regions stabilized 30-45 min post-injection; background liver uptake increased initially more gradually, but continued increasing through $1 \mathrm{~h}$ post-injection. Both tracers accumulated at a similar timeconstant in the hepatic tissues surrounding the liver tumor, with higher overall uptake for FCH: $1.56 \pm 0.26$ times that of CHOL-SUV at $1 \mathrm{~h}$. Tumor uptake at 30-60 min post-injection were similar between the two tracers $(1.02 \pm 0.16$, ratio $\mathrm{FCH}-\mathrm{SUV}$ to $\mathrm{CHOL}-$ SUV) and visual contrast to local liver background at late time points was positive in all cases; however, the increased background uptake with $\mathrm{FCH}$ led to lower contrast. Conclusions: Increased initial uptake in liver tumors, relative the surrounding hepatic tissues, is observed with both tracers. At later time points, the tumors accumulated $\mathrm{CHOL}$ and $\mathrm{FCH}$ at similar levels, with FCH's contrast degraded by higher concentration in surrounding liver. The cause of the agents' different relative uptake in HCC and the surrounding hepatic tissues is a topic for further study.

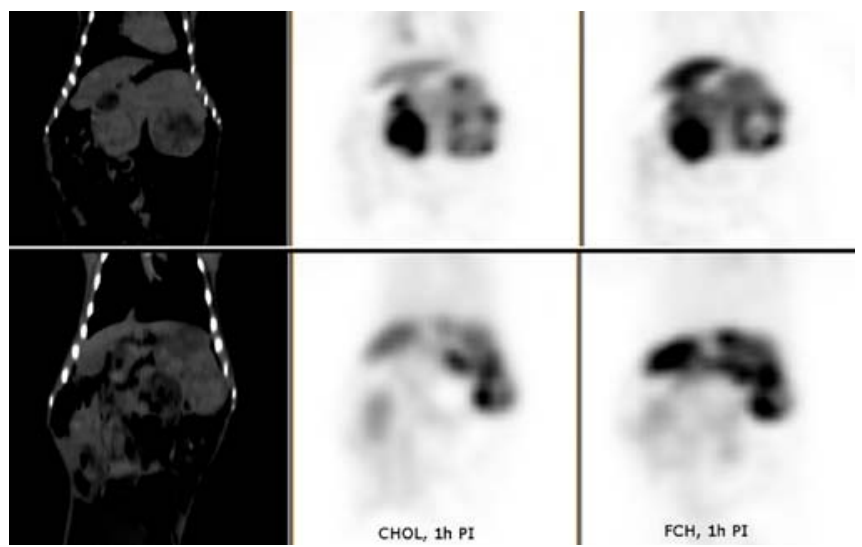

Representative coronal slice at $1 \mathrm{~h}$ post injection for two animals; left to right: CT, CHOL-PET, FCH-PET

J379

\section{A SMART THERANOSTIC NANOPARTICLE FOR TUMOR THERAPY AND IMAGING, WHICH CAN AMPLIFY ITS OWN HOMING SIGNAL IN SITU}

Byung-Heon Lee

Cell \& Matrix Biology Research Institute, Kyungpook National University, Daegu, Korea, South.

Author block: Byung-Heon Lee1, Kai Wang1, Ji-Young Lee1, Xiao-Feng He1, Rang-Woon Park1, Yu-Kyoung Oh2, and In-San Kim1 1Department of Biochemistry and Cell Biology and Cell \& Matrix Research Institute, School of Medicine, Kyungpook National University, Daegu, South Korea; 2Department of Life Science, Korea University, Seoul, South Korea The induction of apoptosis plays a pivotal role in cancer treatment such as irradiation and chemotherapy. In this regard, ligands that can target apoptosis may be useful for selectively delivery of therapeutics and imaging agents to tumor. Using phage display technology, we have previously identified a 6 -mer peptide ligand that targets apoptotic cell and named it ApoPep-1 (Apoptosis-targeting Peptide-1). When injected intravenously into nude mice bearing a tumor xenograft, fluorescein-conjugated ApoPep-1 selectively homed to apoptotic cells at tumor tissues, which was demonstrated by TUNEL staining. To examine whether such an apoptosis-recognizing peptide can selectively deliver therapeutics to tumor, liposomes containing doxorubicin (Lipo-DOX) was labeled with ApoPep-1. Treatment of H460 tumor bearing mice with either Lipo-DOX or ApoPep-1Lipo-DOX (at the concentration of $1 \mathrm{mg}$ DOX/ $\mathrm{kg}$ body weight; injected every other day for total 7 times) significantly inhibited tumor growth as compared to HEPES- or DOX-treated groups. More importantly, ApoPep-1-Lipo-DOX more efficiently inhibited tumor growth than Lipo-DOX. When the apoptosis of tumor is induced by the treatment, this may recruit more ApoPep-1-LipoDOX to tumor tissue and in turn amplify apoptosis leading to efficient inhibition of tumor growth. This hypothesis was confirmed by imaging with Apopep-1-Lipo-DOX-Cy7.5 or Lipo-DOXCy7.5. After $2 \mathrm{~h}$ circulation, the optical imaging was taken and then tumors were isolated to take ex vivo optical imaging. The imaging signal was much higher in Apopep-1-Lipo-DOX-Cy7.5treated tumors than Lipo-DOX-Cy7.5-treated tumors, nevertheless, tumor sizes were significantly smaller in Apopep-1-Lipo-DOXCy7.5-treated mice than Apopep-1-Lipo-DOX-Cy7.5-treated mice. These results suggest that ApoPep-1-conjugated nanoparticle can amplify its own homing signal in situ and therefore can be developed into a smart theranostic nanoparticle for tumor therapy and imaging.

\section{$\mathbf{J 3 8 0}$}

IMAGING OF MYOCARDIAL MUSCLE APOPTOSIS AFTER MYOCARDIAL ISCHEMIA AND REPERFUSION WITH AN APOPTOSIS TARGETING PEPTIDE

\section{Byung-Heon Lee}

Cell \& Matrix Biology Research Institute, Kyungpook National University, Daegu, Korea, South.

Author block: Byung-Heon Lee1, Kai Wang1, Rang-Woon Park1, Chan-Il moon2, In-San Kim1 1Department of Biochemistry and Cell Biology and Cell \& Matrix Research Institute, School of Medicine, Kyungpook National University, Daegu, South Korea; 2Department of Cardiology, Gil hospital of Gacheon Medical School, In-Chon, South Korea Myocardial apoptosis and necrosis play an important role in the pathogenesis of various myocardial diseases. Its noninvasive identification and localization therefore may help in the diagnosis of these disorders, as well as in prognosis and assessment of treatment response. Using phage display technology, we have previously identified a 6-mer peptide ligand that targets apoptotic cell and named it ApoPep-1 (Apoptosis-targeting Peptide-1). When injected intravenously into ischemia and reperfusion (I/R) rat, fluorescein-conjugated ApoPep-1 selectively targeted to apoptotic cells at myocardial tissue, which was demonstrated by H\&E and TUNEL staining. To image apoptosis in I/R model, Cy7.5 labeled ApoPep-1 was injected intravenously after $\mathrm{I} / \mathrm{R}(30 \mathrm{~min} / 2 \mathrm{~h})$. Peptides were allowed to circulate and optical imaging was taken at $1 \mathrm{~h}, 2 \mathrm{~h}, 4 \mathrm{~h}$, and $6 \mathrm{~h}$. In contract to sham and control peptide groups, Cy7.5-ApoPep-1 group showed significant signals from $\mathrm{I} / \mathrm{R}$ heart, interestingly, the signal was increasing from $1 \mathrm{~h}$ to $6 \mathrm{~h}$. And ex vivo imaging showed the peptide targeting area was co-localized with damaged area. These results suggest that ApoPep-1 is a useful tool for the apoptosistargeting ligand-based diagnosis of myocardial diseases, as well as prognosis and assessment of treatment response. 


\section{J381}

STUDY THE DIFFERENCE IN THE CYTOSKELETON

OF CERVICAL CANCER AND NORMAL EPITHELIAL CELLS USING ATOMIC FORCE MICROSCOPE (AFM) AND CONFOCAL SCANNING LASER MICROSCOPE (CSLM) Ravi Gaikwad $^{1}$, Igor Sokolov ${ }^{1,2}$

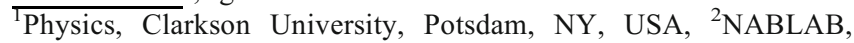
Clarkson University, Potsdam, NY, USA.

Cervical cancer is one of the most leading causes of cancer in the US, a lot of research has been done in the early detection of this cancer. The existing techniques are traditional and based on the observation of growth of malignant cells in the cervix. Malignant cells differ from normal cells in terms of cell growth, organization of cytoskeleton and interaction with extracellular matrix. The cytoskeleton plays an important role in its motility and other structural properties. Our study is focused on understanding the organization of the cytoskeleton of cancer and normal cells using microscopy techniques like Atomic force microscope (AFM) and Confocal scanning laser microscope (CSLM). Our hypothesis is that the cytoskeleton fibers beneath the plasma membrane have a specific distribution, density and organization for normal and malignant cells. The cytoskeleton is exposed by using nonionic detergent Triton X-100 in low concentration to remove the membrane, soluble proteins and organelles from the cell. The molecules of the cytoskeleton fibers is then stained with fluorescent dye Rodamine R6G (10-3M) for imaging with CSLM and consequently with AFM. CSLM and AFM have an advantage over other existing methods like optical and electron microscope because of its spatial resolution and ease of sample preparation. A 3-D structure of the cell containing information of the cytoskeletal filaments can be constructed using CSLM while high resolution 3-D images are consequently obtained using AFM. This allows determining the spatial parameters of interest, prominent among them are the surface fiber density, texture aspect ratio and the density of summits. Hence we can quantify the difference in the observed cytoskeleton by its volume and density for cancer as well as normal cells.

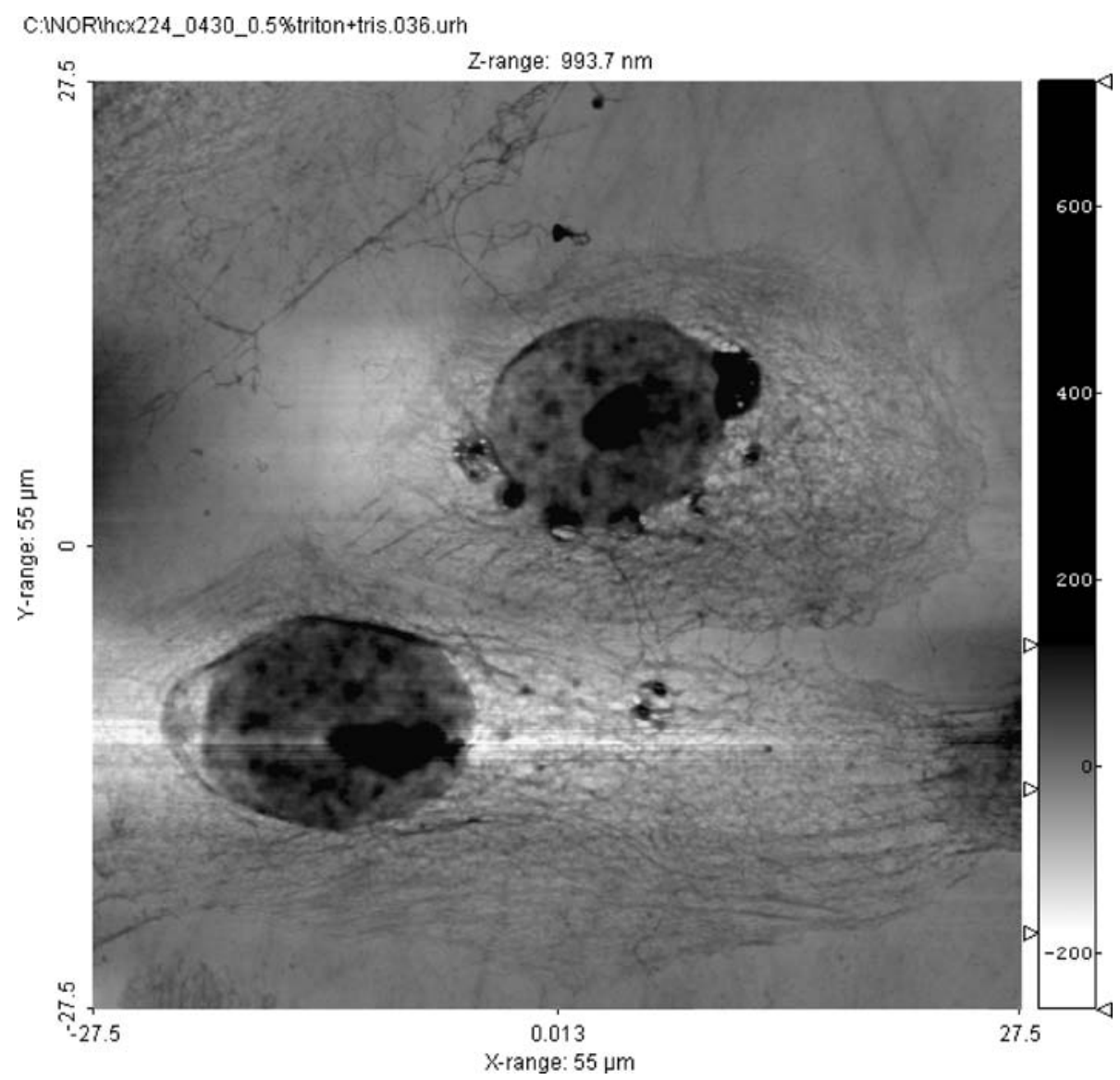

$\mathbf{J 3 8 2}$

OPTIMAL CRITERIA FOR CHARACTERIZING BORDERLINE ADRENAL FDG UPTAKE

Sunhee Kim

UPMC, Pittsburgh, PA, USA.
PURPOSE There have been several previous studies on the role of F-18 FDG PET in characterizing adrenal lesion. It appeared that each study used different criteria to call positive adrenal lesions such as background activity, SUVmean of the liver, SUVmax of the liver and adrenal/liver SUVmax $>1.5$. It is critical to get consensus of these parameter to characterize adrenal lesions accurately and consistently. The goal of our study is to compare the 


\begin{tabular}{|c|c|c|c|c|c|}
\hline & SN $\%$ & SP $\%$ & PPV $\%$ & NPV \% & ACC $\%$ \\
\hline Visual assessment using background activity & 100 & 0 & 7 & 0 & 7 \\
\hline Visual assessment using liver activity & 100 & 30 & 10 & 100 & 34 \\
\hline Quantitative assessment using liver SUV mean & 100 & 7 & 7 & 100 & 14 \\
\hline Quantitative assessment using liver SUV max & 100 & 93 & 50 & 100 & 93 \\
\hline $\begin{array}{c}\text { Quantitative assessment using adrenal } \\
\text { SUV max/liver SUVmax }>1.5\end{array}$ & 0 & 100 & 0 & 93 & 93 \\
\hline
\end{tabular}

each parameter with histopathologic and clinical correlation and to find the best PET parameter. METHOD AND MATERIALS Total of 29 adrenal lesions, 25 patients (mean age 68, M:F=17:12) with F18 FDG PET/CT without IV contrast are identified. Inclusion criteria is any adrenal lesions with mid FDG activity and the exclusion criteria is any lesion without activity and/or adrenal lesions with moderate to severe FDG activity. 5 types of readings using 5 different parameters were obtained and compared with clinical and histopathologic correlation. RESULTS Among 29 lesions with borderline FDG activity, only 2 lesions were malignant. The sensitivity (SN), specificity (SP), PPV, NPV and accuracy (ACC) are as following Table. CONCLUSION Among 5 parameters, the overall accuracy is the highest using parameter of SUVmax of the liver and adrenal SUVmax/liver SUVmax $>1.5$ in characterizing borderline adrenal FDG activity. The SUVmax of the liver consistently demonstrate high SN and SP and adrenal SUVmax/liver SUVmax $>1.5$ has lowest sensitivity. Comparison of several criterias for characterizing adrenal uptake of F-18 FDG

\section{$\mathbf{J 3 8 3}$}

\section{MR IMAGING OF HEPATIC TUMOR IN H-RAS 12V TRANSGENIC MICE}

Seong Hoon Park $^{1}$, Bang Dong-Ho ${ }^{1}$, Moon Hyung-Bae ${ }^{2}, \mathrm{Yu}$ Dae-Yeul ${ }^{3}$, Byun Seung Jae $^{4}$, Pyoung Suk Lim ${ }^{5}$, Kwon-Ha Yoon ${ }^{1}$, Dae Won Kim ${ }^{6}$ ${ }^{1}$ radiology, Radiological Imaging Science, Wonkwang University School of Medicine, Iksan, Korea, South, ${ }^{2}$ pathology, Wonkwang University School of Medicine, Iksan, Korea, South, ${ }^{3}$ Functional Genomics Research Center, Bioscience and Biotechnology, Daejeon, Korea, South, ${ }^{4}$ vascular and Transplantation surgery, Radiological Imaging Science, Wonkwang University School of Medicine, Iksan, Korea, South, ${ }^{5}$ Internal medicine, Gunsan Medical Center, Gunsan, Korea, South, ${ }^{6}$ Neurosurgery, Wonkwang University School of Medicine, Iksan, Korea, South.

PURPOSE: To evaluate characteristics of MR imaging of hepatic tumor in H-ras $12 \mathrm{~V}$ transgenic mice using 4.7T micro MR system. MATERIALS \& METHODS: Hepatocellular carcinoma lines were established to create an insertion of the H-ras $12 \mathrm{~V}$ transgene under the control of the albumin enhancer/promoter. Six H-ras $12 \mathrm{~V}$ transgenic mice underwent $\mathrm{T} 1$ weighted image without fat saturation and with fat saturation, T2 weighted image without fat saturation, and Gd-EOB-DTPA enhanced T1 weighted image with fat saturation when these mice were $4,6,8$ and 9 months old age. MR imaging was performed with a 4.7-T scanner (Phamascan; Bruker, Germany) using a mouse body coil. The diagnosis of hepatic tumor was confirmed by means of pathologic examination. For imaging analysis, we recorded MR characteristics including size, number and signal intensity of the tumor in each sequence. The MR images were correlated with pathologic examinations. RESULTS: Four of six transgenic mice had six tumors when these were 4 months old age. When all transgenic mice were six months old age, all mice had tumor in their liver. The smallest tumor detected on micro-MR was $400 \mu \mathrm{m}$. In MR imaging, almost hepatic tumors had high signal intensities on T1WI without fat saturation and these high signal intensities dropped on T1WI with fat saturation. These tumors had very low signal intensity on hepatobiliary phase of $\mathrm{T} 1$ weighted image with fat saturation. Pathologi-cally, these tumors had well defined margin, prominent fat component and trabecular pattern without capsule, and were confirmed as hepatocellular adenoma or well-differentiated hepatocellular carcinoma. CONCLUSIONS: MiroMR imaging reflect characteristics of hepatic tumor in live murine model, very well. The Gd-EOB-DTPA enhanced T1 weighted image is helpful to detect hepatic tumor in $\mathrm{H}$-ras $12 \mathrm{~V}$ transgenic mice.

\section{$\mathbf{J 3 8 4}$}

\section{INCREMENTAL VALUE OF 18F FDG PET-CT OVER CECT IN THE SURVEILLANCE OF LARGE BOWEL MALIGNANCIES}

Swagat Dash

Nuclear Medicine \& PET-CT, Sir Ganga Ram Hospital, New Delhi, India.

Aim: To determine the impact of whole body $18 \mathrm{~F}$ FDG PET-CT in decision making in the management of large bowel malignancy patients.Material and Methods: A double blind prospective study of 31 patients of suspected or biopsy proven large bowel malignancy with normal or raised serum CEA level was carried out using whole body PET scan $60 \mathrm{~min}$ after the injection of $8-10 \mathrm{mCi}$ of 18 F-FDG along with CECT. Oral contrast was used in all the patients to better delineate the abdominal tumors. Intravenous and rectal contrast were also used in selected cases. Both the scans were interpreted by two independent observers.Result: All 31 patients were grouped in two categories, one with normal CEA level and the other with elevated CEA level. Out of these 31 patients, 3 patients had CEA level within normal range and 28 patients had raised CEA level. In the patients with normal CEA level, PET CT was able to detect local disease and the results were discordant with CECT abdomen and pelvis. In the other group of 28 patients with unexplained rise in serum CEA levels, in 17 patients PET CT and CECT results were concordant in detecting locoregional and distant metastatic disease. In 2 patients PET CT and CECT results were discordant.In these patients CECT findings suggestive of post radiation fibrosis were detected to have recurrence on the PET CT scan, which were later proven histopathologically as well.PET CT demonstrated an absence of any abnormal FDG avid focus in the whole body survey in 9 patients. Out of these 9 patients, the PET CT results were concordant with CECT scan in 6 patients and there was no evidence of active disease on the CECT scan as well. However PET CT results were discordant with CECT in 3 patients.In these 3 patients, CECT suggested lesions suspicious of local recurrence or metastases. Of these 3 CECT positive patients, 2 were negative histopathologically whereas 1 had positive biopsy for mucinous adenocarcinoma of rectum which could be the possible explanation for non FDG avid lesions on PET CT. Conclusion:18F FDG PET CT as an isotropic metabolic imaging 
modality has an incremental role over CECT in the oncological work up of colorectal and other large bowel malignancies. The ability to detect metabolic activity helps in detecting occult malignancies and in lesion characterization when used in surveillance for local residual / recurrent disease or in unsuspected distant metastasis.

\section{$\mathbf{J 3 8 5}$}

RADIOMICS OF LUNG CANCER: QUANTITATIVE IMAGE FEATURES AS "IMAGING BIOMARKERS"

Virendra Kumar ${ }^{1}$, Robert A. Gatenby ${ }^{2,3}$, Edward A. Eikman ${ }^{3}$, $\overline{\text { Claudia Berman }}^{3}$, Dmitry Goldgof ${ }^{4}$, Lawrence Hall ${ }^{4}$, Robert Gillies ${ }^{1,3}$

${ }^{1}$ Molecular and Functional Imaging, H. Lee Moffitt Cancer Center and Research Institute, Tampa, FL, USA, ${ }^{2}$ Integrated Mathematical Oncology, H. Lee Moffitt Cancer Center and Research Institute, Tampa, FL, USA, ${ }^{3}$ Radiology, H. Lee Moffitt Cancer Center and Research Institute, Tampa, FL, USA, ${ }^{4}$ Computer Engineering, University of South Florida, Tampa, FL, USA.

Conventional radiology has largely utilized subjective or qualitative terminology to characterize the appearance of pathological features on radiographic images. For diagnosis or evaluation of treatment, tumors are characterized as having, e.g. "irregular borders, central necrosis, etc." Currently available positron emission tomography (PET) and computed tomography (CT) can provide objective, quantitative anatomical and functional information about lung tumors. These image features result from cellular, physiologic and genetic alterations that are related to the underlying cancer. Automated or semi-automated methods can be developed to efficiently extract quantifiable information from radiologic images, i.e. "radiomics." Currently, the only two commonly used quantitative measures in tumor radiology are tumor size (RECIST or bi-dimensional measures) and standardized uptake value in PET scanning. We have extracted and quantified $\sim 30$ features from CT images of lung lesions. The current work is semi-heuristic in that all features have associated specific hypotheses relating to what is measured and how the features will perform as diagnostic indicators (Table 1). Features are extracted using a multidimensional seed growing approach to automatically segment the tumor. Once segmented, tumor region and boundary features are quantified. Table 1 lists representative values from a single patient in a retrospective study of CT images from 50 non small cell lung cancer patients whose clinical outcome and tumor gene expression data are known. From this study, features will be analyzed in terms of dynamic range, variance, information content and predictive value through correlation with gene expression and clinical outcome. It is our view that a large proportion of information contained in currently available imaging data are underutilized and foresee that extraction and quantification of these data, in combination with gene expression information, will result in improved diagnosis, personalized cancer therapy and, hence, improved outcomes.

Table 1: Representative list of features and example values obtained for one patient.

\begin{tabular}{|c|c|c|c|}
\hline Feature & Calculation & Hypothesis & Examples of Values \\
\hline $\begin{array}{l}\text { 1. Area } \\
\text { 2. Perimeter }\end{array}$ & $\begin{array}{l}\text { 1. Area in } \mathrm{mm}^{2} \\
\text { 2. Perimeter in } \mathrm{mm} \text { with snake }\end{array}$ & $\begin{array}{l}\text { Larger tumors are later stage and area is good } \\
\text { measure of size }\end{array}$ & $\begin{array}{l}\text { Area }=1201 \mathrm{~mm}^{2}(875 \text { voxels }) \\
\text { Perimeter }=152.4 \mathrm{~mm}\end{array}$ \\
\hline $\begin{array}{l}\text { 3. Volume }(\mathrm{V}) \\
\text { 4. Surface area } \\
\text { (SA) } \\
\text { 5. SA/V }\end{array}$ & $\begin{array}{l}\text { 3. Volume in } \mathrm{mm}^{3} \text { by } 3 \mathrm{D} \\
\text { reconstruction } \\
\text { 4. Surface are in } \mathrm{mm}^{2} \text { with 3D snake } \\
\text { 5. } \mathrm{In} \mathrm{mm}^{-1}\end{array}$ & $\begin{array}{c}\text { Volume is a better measure of size. Lesions } \\
\text { with higher SA/V are more lobulated and } \\
\text { perhaps more invasive, less responsive/worse } \\
\text { outcome }\end{array}$ & $\begin{array}{c}\text { Volume }=2615 \mathrm{~mm}^{3} \\
\mathrm{SA}=1099.3 \mathrm{~mm}^{2} \\
\mathrm{SA} / \mathrm{V}=0.42 \mathrm{~mm}^{-1}\end{array}$ \\
\hline $\begin{array}{l}\text { 6. Tumor margin } \\
\text { gradients }\end{array}$ & $\begin{array}{l}\text { 6. Attenuation gradient across } 5 \text { pixels } \\
\text { at the edge of the tumor, measured } \\
\text { every } 5 \text { degree from centroid. } \\
\text { Expressed as HU per mm (mean } \pm \mathrm{SD} \text { ). }\end{array}$ & $\begin{array}{l}\text { Lesions with diffuse margins (low gradient) } \\
\text { have increased partial volumes at edge and } \\
\text { should also be a characteristic of having } \\
\text { small spiculations and more invasive. }\end{array}$ & Slope $($ mean \pm SD $)=133.5 \mathrm{HU} / \mathrm{mm}$ \\
\hline 7. Heterogeneity & $\begin{array}{l}\text { 7. Mean, SD and range of } \mathrm{HU} \text { values } \\
\text { over entire volume of lesion and } \\
\text { histogram analysis. }\end{array}$ & $\begin{array}{c}\text { More heterogenous lesions may contain 'hot } \\
\text { spots' of resistant volumes }\end{array}$ & $\begin{array}{c}\text { Mean } \pm \text { SD }=19.33 \pm 76.59 \mathrm{HU} \\
\text { Min. }=-249 H U, \text { Max. }=118 \mathrm{HU} \\
\text { Skewness=-1.06, Kurtosis }=7.15\end{array}$ \\
\hline $\begin{array}{l}\text { 8. Circularity } \\
\text { 9. Mean radius }\end{array}$ & $\begin{array}{l}\text { 8. Circularity } \\
\text { 9.Mean } \pm \text { SD of distance from center of } \\
\text { mass to edge }\end{array}$ & $\begin{array}{l}\text { Oblong tumors are growing along existing } \\
\text { vasculature, thus more likely to have } \\
\text { mediastinal or distant metastasis }\end{array}$ & $\begin{array}{l}\text { Circularity }=0.83 \\
\text { Mean radius }=19.97 \pm 3.24 \mathrm{~mm}\end{array}$ \\
\hline $\begin{array}{l}\text { 10. Low density } \\
\text { areas with in } \\
\text { tumor }\end{array}$ & $\begin{array}{l}\text { 10. Total fractional volume and size } \\
\text { distribution (mean } \pm \mathrm{SD})\end{array}$ & $\begin{array}{l}\text { Low density areas represent necrosis which } \\
\text { would be a negative predictor for outcome }\end{array}$ & $\begin{array}{l}\text { Relative volume of low density } \\
\text { area }=0.21 \\
\text { Mean } \pm \mathrm{SD}=4.89 \pm 8.35 \mathrm{~mm}^{3}\end{array}$ \\
\hline
\end{tabular}

\section{$\mathbf{J 3 8 6}$}

QUANTITATIVE MRI ASSAYS OF ANGIOGENESIS WITH MICROSCOPIC CORRELATION IN A BEVACIZUMABTREATED XENOGRAFT CANCER MODEL

Hans-Jürgen Raatschen

$\overline{\text { Radiology and Nuclear }}$ Medicine, Charité Berlin, Berlin, Germany.
Objective: To compare two different techniques for measuring tumor vascularity, in vivo contrast-enhanced MRI and perfusion-dependent fluorescent microscopy, to determine if the techniques are in agreement and to validate both for use in monitoring tumor microvessel response to angiogenesis inhibition. Materials and Methods: MDA-MB 435 cancer xenografts were produced subcutaneously in thirteen 4-week-old female nude rats. When tumors reached a diameter of $1 \mathrm{~cm}$, treatment 
with saline (control group, $n=5$ ) or the monoclonal anti-VEGF antibody bevacizumab was initiated at two different dose levels $(0.1 \mathrm{mg}, n=5$ and $1.0 \mathrm{mg}, n=3)$ and continued over 10 days with a total of four administrations given every third day. One day after the final injection, dynamic MRI enhanced with macromolecular albumin$(\text { Gd-DTPA })_{30}$ was performed and followed immediately by intravascular vital staining of those microvessels with functional flow using a fluorescently-labeled lycopersicon esculentum lectin-containing perfusate that can be measured with fluorescent microscopy. Quantitative MRI assays of tumor vascularity expressed as fractional plasma volume (fPV, \%) were calculated based on an established two-compartment pharmacokinetic model and were compared to the microscopic assays of vascularity, expressed as the percent area density of lectin-stained vessels. Results: MRI-assayed tumor vascularity, the fractional plasma volume (fPV), was significantly higher $(p<0.05)$ in the control saline-treated tumors $(5 \pm 1 \%)$ than in either of the two angiogenically-inhibited tumor groups $(3 \pm 0.5 \%$ and $2 \pm 0.8 \%$, respectively). Microscopic measurements of lectin area densities were significantly higher $(p<0.05)$ in the control group $(14.6 \pm 2.9 \%)$ compared to the reduced microscopic assays of vascular richness in the two Avastintreated tumor groups $(10.9 \pm 0.7 \%$ and $5.7 \pm 0.5 \%)$. MRI-assayed fPV showed a strongly positive and significant correlation with lectin-areadensity $\left(\mathrm{r}^{2}=0.74, p<0.001\right)$. Conclusion: An MRI-based method to measure richness of vascularity in tumors, fractional plasma volume, and a quantitative microscopic method for estimating perfused microvessels are in good agreement both for control and angiogenicallyinhibited tumors. These results comparing two methods that are fundamentally different in approach serve to validate each.

Tumor vascularity in bevacizumab-treated MDA-MB 435 xenografts

\begin{tabular}{|c|c|c|}
\hline Bevacizumab dose $(\mathrm{mg})$ & MRI-assayed fractional plasma volume (fPV, \%) & microscopic lectin area density $(\%)$ \\
\hline control & $5 \pm 1$ & $14.6 \pm 2.9$ \\
\hline 0.1 & $3 \pm 0.5^{*}$ & $10.9 \pm 0.7^{*}$ \\
\hline 1.0 & $2 \pm 0.8^{*}$ & $5.7 \pm 0.5 \dagger$ \\
\hline
\end{tabular}

${ }^{*} p<0.05$ vs. control; $\uparrow p<0.05$ vs. $0.1 \mathrm{mg}$

\section{$\mathbf{J 3 8 7}$}

\section{MULTIPARAMETRIC IMAGING FOR TUMOR CHARACTERIZATION AND THERAPY RESPONSE MONITORING IN AN ENDOGENOUS MOUSE MODEL OF PANCREATIC DUCTAL ADENOCARCINOMA (PDAC)} Manuela R. Gretzinger ${ }^{1}$, Jens Siveke ${ }^{3}$, Andreas Steingötter ${ }^{1,2}$, Ken Herrmann $^{2}$, Irina Heid $^{3}$, Yvonne Kosanke ${ }^{1,2}$, Roland M. Schmid ${ }^{3}$, Markus Schwaiger ${ }^{1}$, Ernst J. Rummeny ${ }^{1}$, Rickmer Braren ${ }^{1}$

${ }^{1}$ Insitut für Röntgendiagnostik, Technische Universität München, München, Germany, ${ }^{2}$ Klinik und Poliklinik für Nuklearmedizin, Technische Universität München, München, Germany, ${ }^{3}$ Klinik und Poliklinik für Innere Medizin 2, Technische Universität München, München, Germany.

Introduction: Pancreatic Adenocarcinoma (PDAC) is highly resistant to chemo- and radiation-therapy. Conditional KrasG12D-based mouse models develop PDAC from preneoplastic PanIN and IPMN lesions, closely resembling the human condition. A detailed knowledge of the spontaneous course of tumor progression is necessary for the correct interpretation of spontaneous and drug induced changes in tumor physiology and biology. We therefore conducted this multimodal imaging study, using a clinical $1.5 \mathrm{~T}$ MRI and a small animal PET system to monitor spontaneous tumor progression and therapy response. Results: On T2w MRI PDAC were highly heterogeneous in appearance. Animals with advanced stage disease often exhibited additional pathologies, such as ascites, pleural effusion and metastases in liver and lung. Premalignant PanIN and IPMN lesions were not distinguishable from PDAC based on T2w MRI. In contrast, transplanted tumors grew faster and were more homogenous in appearance. Murine PDAC showed specific [18F]FDG uptake (100\%). [18F]FLT uptake was seen in only one tumor, which was histologically characterized by an anaplastic sarcomatoid phenotype. [18F]FDG and [18F]FLT uptake were $3,39 \pm 1,99$ and $1,21 \pm 0,52 \% \mathrm{ID} / \mathrm{g}$ respectively for endogenous and $2,15 \pm 0,96$ and $1,33 \pm 0,43 \% \mathrm{ID} / \mathrm{g}$ respectively for transplanted tumors. Initial [18F]FDG uptake was not predictive with regard to tumor growth kinetics. ADC values $(0.99 \mathrm{~s} / \mathrm{mm} 2 \pm 0.23)$ and histological grading of tumors correlated well. ADC values and $[18 \mathrm{~F}]$ FDG uptake correlated moderately $\left(\mathrm{R}^{\wedge} 20.53\right)$ in fast growing KrasG12D;Tgfa;p53+/fl and transplanted tumors only. Ktrans values of untreated solid tumors were ranging from $0.14 \mathrm{~min}-1$ to $0.69 \mathrm{~min}-1$, indicating a highly variable degree of tumor perfusion. 6 animals were subjected to a standard Gemcitabine ${ }^{\circledR}$ therapy regimen. Only one animal with a fast growing PDAC, exhibiting a high initial ktrans value and low ADC value showed good treatment response, reflected in a dramatic decrease in ktrans and ADC value on day 1 after treatment onset. Conclusion: Multiparametric imaging of endogenous and transplanted PDAC in mice is feasible and reveals high individual heterogeneity in tumor morphology and tumor progression. A combination of DCE-MRI, DWI and [18F]FDG PET is most useful for the evaluation of tumor perfusion, composition and metabolic activity. Our study emphasizes the necessity for an individualized tumor assessment and response monitoring in complex preclinical tumor models as well as the need for novel therapy regimens for the treatment of PDAC.

\section{$\mathbf{J 3 8 8}$}

IMAGING OF HYPOXIC PHENOTYPE IN HEAD AND NECK SQUAMOUS CELL CARCINOMA

Jonna Sinkkonen $^{1}$, Antti Silvoniemi ${ }^{1,2}$, Sarita Forsback ${ }^{1}$, Nina Savisto ${ }^{1}$, Olof Solin $^{1}$, Reidar Grénman ${ }^{2}$, Heikki Minn ${ }^{1,3}$, Tove J. Grönroos ${ }^{1}$

${ }^{1}$ Turku PET Centre, University of Turku, Turku, Finland, ${ }^{2}$ Department of Otorhinolaryngology, Turku University Hospital, Turku, Finland, ${ }^{3}$ Department of Oncology and Radiotherapy, Turku University Hospital, Turku, Finland.

Aims: Radiotherapy continues to play an essential role in cancer treatment but may fail due to the presence of tumor hypoxia. PET/ 
CT is being increasingly used in oncology for treatment planning. HNSCC are a group of cancers that in particular confers resistance to treatment due to hypoxia. Hypoxia is also known to affect the glycolytic activity of tumors. The aim of this study was to study whether the glycolytic activity and the proportion of hypoxia vary in cell lines and experimental tumors originating from different HNSCC patients. [18F]FDG and [18F]EF5 PET tracers were used to study this phenomena. Materials and methods: Four cell lines established from patients with HNSCC were cultured in chamber slides or subcutaneously induced into nude mice. Experimental hypoxia was induced by culturing cells for $1-12 \mathrm{~h}$ in the presence of $1 \%$ oxygen in a special oxygen incubator. The uptake of $[18 \mathrm{~F}]$ FDG and $[18 \mathrm{~F}] \mathrm{EF} 5$ was determined in vitro utilizing a digital autoradiography method and by using cells under normal culturing circumstances as controls. Tracer uptake intensity per cell growth area was determined and changes in the uptake induced by hypoxia compared between the different cell lines. Experimental tumors were induced subcutaneously on the back of male nude mice and the growth rate of the tumors determined. Dynamic whole body $\mathrm{PET} / \mathrm{CT}$ scans were performed with both tracers within an average interval of two-days, at least once per animal. Results: A clear difference in the growth rate and pattern was seen between the different cell lines. Furthermore, a clear distinction in the uptake of $[18 \mathrm{~F}] \mathrm{FDG}$ and $[18 \mathrm{~F}] \mathrm{EF} 5$ was detected between cell lines. An increased uptake of $[18 \mathrm{~F}] \mathrm{EF} 5$ was seen in some cell lines, especially $1-3 \mathrm{~h}$ after induction of hypoxia, whereas some cell lines did not respond to the decreased oxygen level at all. Differences in the [18F]FDG uptake were not as distinct as for $[18 \mathrm{~F}] \mathrm{EF} 5$, but in average those cell lines showing an increased [18F]EF5 uptake also showed an increased uptake rate of [18F] FDG, which however was more obvious in outer, growing periphery of tumors. These results were confirmed by in vivo PET/CT scans, clearly showing an inverse uptake pattern for the two tracers. Conclusion: Our preliminary results show a great variety in the glycolytic and hypoxic properties of patients diagnosed with HNSCC, which furthermore most probably will play an important role in the context of treatment planning. Hence, we suggest that the relationship between different phenotypes and the uptake of PET tracers should be validated in more details.

\section{J389}

THERMALLY CROSS-LINKED SUPERPARAMAGNETIC IRON OXIDE NANOPARTICLES ENHANCED MR IMAGING FOR DETECTION OF LYMPH NODE METASTASES IN MURINE MELANOMA MODELS; IN VIVO

\section{AND EX VIVO STUDY}

Seong Hoon Park ${ }^{1}$, Se Woong Lim ${ }^{1}$, Pyoung Suk Lim ${ }^{4}$, Byun Seung Jae ${ }^{3}$, Hye-Won Kim ${ }^{1}$, Dae Won Kim${ }^{6}$, Sangyong Jon ${ }^{2}$, Hun Soo Kim ${ }^{5}$, Kwon-Ha Yoon ${ }^{1}$

${ }^{1}$ Radiology, Radiological Imaging Science, Wonkwang University School of Medicine, Iksan, Korea, South, ${ }^{2}$ Life science, Gwangju Institute of Science and Technology, Kwangju, Korea, South, ${ }^{3}$ Vascular and transplantation surgery, Wonkwang university hospital, Iksan, Korea, South, ${ }^{4}$ Internal medicine, Kunsan medical center, Kunsan, Korea, South, ${ }^{5}$ Pathology, Wonkwang University School of Medicine, Iksan, Korea, South, ${ }^{6}$ Neurosurgery, Radiological Imaging Science, Wonkwang University School of Medicine, Iksan, Korea, South.

Purpose : To assess the feasibility of Thermally Cross-Linked Superparamagnetic Iron Oxide Nanoparticles (TCL-SPION) on MR imaging for lymph node metastases in murine melanoma models. Materials and Methods : B16F1 human melanoma cells were injected into thigh of C57BL/6 mice subcutaneously. $(N=10)$ MR imaging were performed 3 weeks after tumor injection using $4.7 \mathrm{~T}$ MR scanner. In vivo and $E x$ vivo MR images for both inguinal lymph nodes with $\mathrm{T} 2$ weighted fast spin echo and gradient echo sequence were obtained before and after intravenous injection of TCL-SPION. MR signal intensities and sizes were measured in each inguinal lymph nodes and the relative contrast enhancement ratio (CER) were calculated. All lymph nodes were evaluated histopathologically. Results : Histopathology confirmed 9 metastatic lymph nodes in 20 inguinal lymph nodes. The CER of metastatic lymph nodes were significantly higher than that of non metastatic lymph nodes in both In vivo and Ex vivo MR images. But there was no significant difference in size between metastatic and non metastatic lymph nodes. Conclusion ; TCL-SPION enhanced MR imaging could detect early lymph node metastasis non invasively both In vivo and Ex vivo studies.

\section{J390}

\section{NONINVASIVE EVALUATION OF INTRATUMORAL DISTRIBUTION OF 111IN-LABELED BLOCK COPOLYMER MICELLES IN MICE BY HIGH RESOLUTION AND HIGH SENSITIVITY MICROSPECT/CT IMAGING}

Bryan Hoang $^{1}$, Helen Lee ${ }^{1}$, Christine Allen ${ }^{1,2}$, Raymond M. Reilly ${ }^{1,3}$ ${ }^{1}$ Pharmaceutical Sciences, University of Toronto, Toronto, ON, Canada, ${ }^{2}$ Chemistry, University of Toronto, Toronto, ON, Canada, ${ }^{3}$ Medical Imaging, University Health Network, Toronto, ON, Canada.

To date, information on the tumor penetration and intratumoral distribution of block copolymer micelles (BCMs) has been quite limited. With the advent of numerous formulations relying on $\mathrm{BCMs}$ in clinical evaluation (NK911, SP1049C, Genexol-PM), there is an impetus to develop a radiolabeled formulation that can be used to gain invaluable insight into the intratumoral distribution of the micelles. This information could then be used to direct formulation strategies as a means to optimize treatment outcomes. As such, 111In-labeled BCMs were prepared and the utility of these nanosystems for noninvasive evaluation of tumor accumulation, penetration, and intratumoral distribution by MicroSPECT imaging was investigated. 111 In-was chelated to purified DTPAPEG-b-PCL and incorporated into pre-formed MePEG-b-PCL BCMs. Subsequently, mice bearing MDA-MB-231 tumor xenografts were administered $1 \mathrm{mCi}$ of $111 \mathrm{In}-\mathrm{BCMs}$ via intravenous tail vein injection and imaged at various timepoints post injection using a NanoSPECT/CT animal imager. Tumor accumulation was quantified using non-invasive region of interest (ROI) analysis of the acquired image reconstructions and correlated with values obtained by tumor excision and counting with a gamma counter. Appreciable tumor $(9.48 \%$ i.d/g) uptake was observed for $111 \mathrm{In}$ BCMs at 48 h.p.i. $111 \mathrm{In}-\mathrm{BCMs}$ were preferentially localized in perivascular regions of the tumors, implying their non-specific extravasation to tumors through the hyperpermeable tumor vasculature due to the enhanced circulation profile of the micelles and the exploitation of the EPR effect. Analysis of the transversal slices revealed heterogeneous intratumoral distribution, with predominant uptake around the tumor periphery and incomplete tumor penetration. This critical information, that is otherwise unavailable through traditional assessment of biodistribution, could have profound implications in terms of the ability of such a micelle formulation to eradicate all or most tumor cells in a lesion. Lastly, a correlation between MicroSPECT/CT ROI analysis and the biodistribution data obtained by $\gamma$-counting of excised tissues was found ( $r=0.91, P=0.03$ ), implying that imaging can be used as a non-invasive tool to accurately quantify relative tissue concentrations of $111 \mathrm{In}-\mathrm{BCM}$ s. In conclusion, microSPECT/CT imaging enabled the accurate quantification of the tumor uptake of the micelles and visualization of their degree of tumor penetration. In this way, the image-based assessment provided unique information that is not obtainable using conventional methodologies. 
J391

IDENTIFICATION OF CELL SURFACE TARGET COMBINATIONS FOR HETERO-MULTIVALENT LIGAND DEVELOPMENT FOR IMAGING AND TREATMENT OF METASTATIC MELANOMA

$\underline{\text { David L. Morse }^{1,2}}$, Yoganand Balagurunathan ${ }^{3}$, Galen Hostetter ${ }^{3}$, Haiyong $\operatorname{Han}^{3}$, Robert Gillies ${ }^{1,2}$

${ }^{1}$ Imaging, H. Lee Moffitt Cancer Center \& Research Institute, Tampa, FL, USA, ${ }^{2}$ Oncologic Sciences, College of Medicine, University of South Florida, Tampa, FL, USA, ${ }^{3}$ The Translational Genomics Research Institute, Phoenix, AZ, USA.

Ligand-based targeting of imaging or therapeutic agents to the surface of tumor cells holds considerable promise as a strategy for improving the detection and treatment of cancers. Monomeric ligands such as monoclonal antibodies have demonstrated clinical potential as tumor targeting agents. A major limitation of the monomeric ligand approach is that a single target must be highly overexpressed on the surface of tumor cells and minimally expressed in normal tissues, which rarely occurs in solid tumors. Through non-covalent crosslinking of combinations of heterologous cell surface receptors, multivalent ligands containing multiple binding domains may overcome this limitation. Due to cooperative binding, multimeric ligands have a high affinity for cells expressing all targeting proteins and only minimal binding to cells that express none or some of the targets. However, identifying combinations of targets that are expressed concurrently in tumor cells, but not in normal cells, is a challenging task. We have used gene expression profiling to identify such combinations in melanoma. We generated DNA microarray expression profiles for 129 normal tissue samples representing 34 different organ sites and 19 melanoma samples. Expression data of cell surface genes were analyzed using a multivariate rule-based computational approach to identify combinations expressed in melanoma, but not in normal tissues. Protein expression was validated for six of the three-gene combinations using immunohistochemistry on melanoma and normal tissue microarrays (TMA). The melanoma TMA contained 20 controls (including normal skin), 34 benign nevi, 61 dysplastic nevi, 22 melanoma in situ, 130 malignant melanoma and 20 melanoma metastases representing 164 independent cases. The normal TMA contained 282 tissue cores representing 66 normal tissue types. One combination, CCBP2, GPR34 and LRP12 was co-expressed in $8 \%$ of malignant melanomas and $30 \%$ of metastatic melanomas by a pathology score of $\geq 2+$, and were not co-expressed in any of the benign nevi, dysplastic nevi or melanoma in situ samples at this level. These targets individually scored $\geq 2+$ in only three normal tissues and were not coexpressed in any normal tissue at this level. Hence, this target combination can be used to develop hetero-multivalent ligands for the imaging and/or treatment of disseminated melanoma. Metastatic melanoma is currently untreatable and targeted delivery of imaging and therapies could improve patient survival.

\section{$\mathbf{J 3 9 2}$}

UNKNOWN PRIMARY TUMORS: TRACING THE ORIGIN USING DUAL MODALITY PET/CT

Mir Abrar T. Ali ${ }^{1}$, Jyotsna Rao ${ }^{2}$, Sandeep Kumar Ganji ${ }^{3}$,

Venkateswara Rao M. Meka1, Atul Gada ${ }^{2}$, Manigandan Saikumar ${ }^{1}$, Manohar M. Babu ${ }^{4}$

${ }^{1}$ Department of Biomedical, University College of Engineering (Autonomous), Osmania University, Hyderabad, Hyderabad, India, ${ }^{2}$ PET/CT Center, Apollo Gleneagles, Apollo Hospitals, Hyderabad, India, ${ }^{3}$ Department of Radiology, University of Texas Southwestern Medical Center-Dallas, Dallas, TX, USA, ${ }^{4}$ Cylotron, Apollo Gleneagles PET/CT Centre, Hyderabad, India.
The unknown primary tumors UPT's are defined as Metastases in which the cancerous tissue origin (primary source) is not known. These tumors are hard to detect as there source(s) is unknown and hence treatment plan most often concentrate on the metastatic cancer growth. The most frequent sites for metastatic disease from cancer of an unknown primary tumor are the lymph nodes of the supraclavicular and cervical regions. Even though fluorine $18 \mathrm{~F}$ fluorodeoxyglucose (FDG) positron emission tomography (PET) has been demonstrated as an efficient method to localize unknown primary tumors there still remains case in which a separate anatomical scan (like CT or MRI) is needed. With the advent of the PET along with CT scan, the field of unknown primary tumors detection is revolutionized. In this abstract we would like to share our experience of detecting and in treatment planning of unknown primary tumors. Our case study consists of 12 subjects (average of 57.8 years with a standard deviation of 12.3 years). Most of the subjects have a history of metastases or have been sent for further evaluation for possible metastases. Most of the subjects have been found to have a marked increased in the nodular regions (like right cervical, paratracheal, abdominal, peritoneal etc). We first separated the CT and PET (after complete reports have been handed the subject physician) reports and analyzed the subject data individually and noted the findings and course of actions for each report (i.e. CT and PET separately). Later we looked at the PET findings in the light of CT reports and noted the course of actions. The systemic analysis of these notes cleared showed that FDG PET findings in the light of CT findings greatly changed the future course of action for each subject and in some cases helped identify the primary origin of the malignancy. This study (and our encounter so far with unknown primary tumors with dual modality $\mathrm{PET} / \mathrm{CT}$ ) greatly support and provide further evidence to advantages of FDG-PET/CT over FDG-PET, which is currently known to be an empirical assumption without sufficient cases to support it.

\section{Imaging Disease/Organ Processes}

\section{Cardiovascular and MSK}

\section{J393}

\section{FDG PET IMAGING IN A RABBIT MODEL \\ OF ATHEROSCLEROSIS: COMPARISON OF UPTAKE IN TWO MODELS AIMED AT PRODUCING CHARACTERISTICS OF UNSTABLE VERSUS STABLE AORTIC PLAQUES}

Kenneth Zasadny, Michael J. Callahan, Laigao Chen, Serguei Liachenko

Bioimaging, Pfizer, Inc., Groton, CT, USA.

FDG uptake in preclinical rabbit models of atherosclerosis has been shown to be correlated to the density of macrophages as determined by histology (Tawakol,A.,J Nucl Cardiol, 2005. 12(3):p.294). Macrophage density is expected to be elevated in unstable atherosclerotic plaque relative to stable plaque. Rabbits fed high cholesterol diets spontaneously produce atherosclerotic plaques in ascending and descending aorta. Furthermore, diets made up of coconut oil based fats $(\mathrm{CNO})$ versus peanut oil fats $(\mathrm{PNO})$ are expected to produce more unstable plaque with possibly higher macrophage content. The purpose of this study was to investigate the ability of FDG PET imaging to differentiate the two models along with correlation to high resolution MRI conducted on ex vivo aortic samples. Twelve rabbits were used in the study, 2 controls fed regular chow diets and 10 animals fed high cholesterol diets: $5 \mathrm{CNO}$ and $5 \mathrm{PNO}$, for 15 weeks, 2 controls and $3 \mathrm{CNO}$ and 3 PNO were included in imaging. All animal 
study procedures conducted were approved by an institutional review committee(IACUC). After overnight fasting, rabbits were injected with $3.5 \mathrm{mCi}$ FDG via ear vein under isofluorane anesthesia. After FDG injection, animals were returned to home cage and allowed to recover for a 3.5 hour uptake period. After the uptake phase, rabbits were injected with ketamine/xylazine and PET scanned for $20 \mathrm{~min}$ over the level of the heart with a Siemens F220 microPET scanner. Following the 20 minute emission scan, $1 \mathrm{mCi}$ FDG was administered via ear vein followed by $5 \mathrm{~min}$ emission scanning for anatomical localization of blood vessels by blood flow. PET scans were analyzed for FDG uptake by SUV and volume of elevated FDG uptake over normal control levels. After necropsy, ascending aorta sections were scanned by high resolution MRI for quantitative plaque volume determination. Both high fat diets produced elevated aortic FDG uptake over control animals with visualization of ascending and descending aorta. In a standard ascending aorta segment analyzed, mean SUV was $0.72,0.67$ and 0.34 for $\mathrm{CNO}, \mathrm{PNO}$ and controls and were not significantly different between $\mathrm{CNO}$ and PNO diets. Percentage of high FDG uptake voxels correlated with ex vivo MRI scans. High cholesterol diets in rabbits produced FDG avid atherosclerotic plaques. Diets aimed at producing characteristics of a more unstable plaque (increased macrophage content) did produce a significantly higher FDG uptake versus chow diet. These results also confirm that FDGPET can non-invasively image aortic plaque models in rabbits quantitatively.

\section{J394}

\section{VCAM-1 TARGETED PET-CT DETECTS INFLAMMATORY ATHEROSCLEROTIC PLAQUES}

Matthias Nahrendorf ${ }^{1}$, Edmund J. Keliher ${ }^{1}$, Peter Panizzi ${ }^{1}$,

Hanwen Zhang ${ }^{1}$, Jose-Luiz Figueiredo ${ }^{1}$, Elena Aikawa ${ }^{1}$, Peter Libby ${ }^{2}$, Ralph Weissleder ${ }^{1}$

${ }^{1}$ Center for Systems Biology, Massachusetts General Hospital, Boston, MA, USA, ${ }^{2}$ Cardiovascular Division, Brigham \& Women's Hospital, Boston, MA, USA.

Background: Hybrid PET-CT imaging of VCAM-1 expression and vascular anatomy may facilitate simultaneous assessment of atherosclerotic lesion biology and morphology, and enhance risk assessment in individual patients. We used combined in vitro/in vivo screening of candidate affinity ligands and developed a PET reporter for imaging VCAM-1 expression with high sensitivity, specificity and translational potential. Methods and Results: Three different phage display-derived VCAM-1 affinity peptides were tested using immobilized VCAM-1, VCAM-1 expressing cells and apoE-/- mice. A compound with a linear peptide and arborising tetrameric design showed high affinity $(86.6 \mathrm{nM})$ and specificity for VCAM-1 (97\% inhibition with soluble VCAM-1). This lead compound was derivatized with 18 Fluorine to synthesize the clinically viable $\mathrm{PET}$ agent $18 \mathrm{~F}-4 \mathrm{~V}$. In vivo PET-CT imaging showed robust uptake of $18 \mathrm{~F}-4 \mathrm{~V}$ in plaque laden arterial sections from 8 apoE-/- mice, significantly higher than in 4 wild type mice and attenuated by atorvastatin treatment $(p<0.05)$. 18F-4V uptake was confirmed in excised aortas, colocalized with atherosclerotic plaques delineated by Oil Red $\mathrm{O}$ staining and correlated with mRNA levels of VCAM-1 measured by quantitative RT-PCR (R2= $0.62, p=0.03$ ). Conclusion: $18 \mathrm{~F}-4 \mathrm{~V}$ allows noninvasive PET-CT imaging of VCAM-1 in atheromata, has sufficient dynamic range to quantify treatment effects, and correlates with inflammatory gene expression. This approach lends itself to seamless translation to human PET-CT imaging.

\section{J395}

2-[18F]-FLUORO-2-DEOXY-D-GLUCOSE (18FDG)-UPTAKE REFLECTS COLLAGENOLYTIC ACTIVITY OF CATHEPSIN K AS MEASURED BY QPCR

$\underline{\text { Sune F} . ~ P e d e r s e n ~}^{1,2}$, Martin Graebe ${ }^{3,1}$, Anne Mette F. Hag ${ }^{1,2}$, Liselotte Hoejgaard $^{2,1}$, Henrik Sillesen ${ }^{3,1}$, Andreas Kjaer ${ }^{1,2}$

${ }^{1}$ Cluster for Molecular Imaging, University of Copenhagen, Copenhagen, Denmark, ${ }^{2}$ Department of Clinical Physiology, Nuclear Medicine \& PET, Rigshospitalet, Copenhagen, Denmark, ${ }^{3}$ Department of Vascular Surgery, Rigshospitalet, Copenhagen, Denmark.

Elevated inflammatory and collagenolytic activity is a hallmark of the advanced atherosclerotic lesion. To uncover a correlation between molecular markers of atherosclerotic plaque vulnerability and the imaging modality positron emission tomography (PET), we utilized the PET tracer and sugar analog 2-[18F]-fluoro-2-deoxy-D-glucose (18FDG) and real-time quantitative PCR (qPCR). The gene expression of several molecular markers of atherosclerotic plaque vulnerability: Cluster of differentiation-68 (CD68), interleukin-18 (IL-18), matrix metalloproteinase-9 (MMP-9) and cathepsin K was measured in carotid plaques from 17 patients with confirmed advanced atherosclerotic disease. Additionally the gene expression of the glycolytic enzymes; glucose transporter-1 (GLUT-1) and hexokinase 2 (HK2) was measured. Finally the gene expression data was compared with 18FDG accumulation calculated as SUVmean and SUVmax in the atherosclerotic lesions. Univariate analyses revealed that gene expression of the selected molecular markers of plaque vulnerability and glycolysis correlated with 18FDG-uptake. This suggest that $18 \mathrm{FDG}$ is an indicator of plaque vulnerability as well as it confirms the involvement of the glycolytic enzymes in 18 FDG uptake and metabolism. The gene expression of the macrophage marker CD68 did not correlate with GLUT-1 gene expression raising the question if $18 \mathrm{FDG}$ accumulation is primarily down to macrophage infiltration of the vulnerable plaque. Multivariate linear regression demonstrated that the gene expression of GLUT-1, HK2, CD68 and cathepsin $\mathrm{K}$ each offered independent information about 18FDG-uptake in the final models (SUVmean: $\mathrm{R} 2=0.26 ; p<0.0001$, SUVmax: $\mathrm{R} 2=0.30 ; p<0.0001)$. It therefore seems likely that 18 FDG-uptake in atherosclerotic plaques is a composite indicator of macrophage load, inflammatory activity and collagenolytic plaque destabilization driven by cathepsin $\mathrm{K}$ gene expression.

\section{J396}

\section{PROTEASE-SENSING MOLECULAR OPTICAL IMAGING OF HUMAN CAROTID ATHEROMATA TO COMPLEMENT ANATOMY-BASED IMAGING}

Dong-Eog Kim ${ }^{1}$, Jeong-Yeon Kim ${ }^{1}$, Dawid Schellingerhout ${ }^{2}$, Soo-Min Shon ${ }^{1}$, Seulki $\mathrm{Lee}^{3}, \mathrm{Kas} \mathrm{\textrm {Kim } ^ { 3 }}$, Eric $\mathrm{Cha}^{3}$

${ }^{1}$ Molecular Imaging and Neurovascular Research Lab, Department of Neurology, Dongguk University Ilsan Hospital, Goyang, Korea, South, ${ }^{2}$ Departments of Radiology and Experimental Diagnostic Imaging, University of Texas M.D. Anderson Cancer Center, Houston, TX, USA, ${ }^{3}$ Biomedical Research Center, Korea Institute of Science and Technology, Goyang, Korea, South.

Background \& Objective: Protease-sensing molecular optical imaging has a potential to identify vulnerable atherosclerotic plaques in vivo before infarcts occur. The identification of high-risk patients prior to the development of the clinical events will allow interventions to prevent infarcts and stroke before they happen. For human trials and clinical applications of the technique, several translational issues need to be resolved. We studied if the 
molecular optical imaging complement current structural atherosclerosis imaging methods and provide more comprehensive plaque characterization. Methods: We carefully compared molecular imaging data with angiography, ultrasonography, pathology, and other clinical data, all of which were prospectively collected from the 50 consecutive patients who underwent carotid endarterectomy or carotid angioplasty with stenting. We also studied if the molecular imaging reflected therapeutic effects of statin. Results: Firstly, we showed that CatB or MMP-sensing molecular optical imaging precisely reflected pathophysiologic alteration of atherosclerosis and stain-mediated therapeutic effect on it. Secondly, degree of carotid stenosis or ultrasonographic echodensity, key information acquired from anatomy-based conventional imaging to guide therapeutic planning, was only weakly correlated with the inflammatory proteolytic enzyme activities on the molecular imaging. Thirdly, strong protease activity signals were observed mainly in the carotid bifurcation area and around the ulcero-hemorrhagic lesions. Lastly, we revealed that distribution patterns of the protease imaging signals had clear difference between symptomatic plaques and asymptomatic ones. Conclusion: Our study shows that detection of protease activity in atherosclerotic plaques in vivo could provide a powerful tool for evaluation of vascular inflammation, for determination of individualized therapeutic strategies, and for monitoring the effects of therapeutic interventions. In addition, our study could justify clinical trials to evaluate the use of protease-sensing molecular optical imaging in human atherosclerosis patients.

\section{J397}

\section{NEAR-INFRARED FLUORESCENT IMAGING OF MICE AORTAS REVEALS THAT EXERCISE REDUCES MMP ACTIVITY AS WELL AS ATHEROSCLEROTIC PLAQUE SIZE}

Soo-Min Shon ${ }^{1}$, Jeong-Yeon Kim ${ }^{1}$, Sang-Wuk Jeong ${ }^{1}$, Seulki Lee ${ }^{2}$, Kas Kim $^{2}$, Eric $\mathrm{Cha}^{2}$, Dong-Eog Kim ${ }^{1}$

${ }^{1}$ Neurology, Dongguk Univ. International Hospital, Goyang-si, Korea, South, ${ }^{2}$ Biomedical Research Center, Korea Institute of Science and Technology, Seoul, Korea, South.

Background \& Objective: Protease-sensing molecular optical imaging has a potential to identify vulnerable atherosclerotic plaques. We investigated if matrix metalloproteinase(MMP-2/9) nearinfrared fluorescent(NIRF) imaging of mice aortas could reflect potential anti-atherosclerotic effect of exercise to attenuate the MMP activity as well as plaque growth, when the exercise was performed after the plaque formation. Methods: We used 8 weekold ApoE knock-out mice fed on a normal $\operatorname{diet}(n=9)$ or western $\operatorname{diet}(n=18)$ for 18 weeks. For the last 10 weeks, half of the animals on a western diet were trained( $30 \mathrm{~min} / \mathrm{day}, 5$ days/week) to run on a treadmill with a rubber belt driven at a controlled speed $(17 \mathrm{~m} / \mathrm{min})$. During the last week, serum cholesterol levels were measured. Then, the animals' aortas were imaged ex vivo using a NIRF imaging system 24 hours after the intra-venous injection of activatable Cy5.5 MMP probe. The levels of superoxide dismutase (SOD) and lipid peroxide(LPO) of homogenized aortic tissues were also measured. From the light image, surface lesion areas were quantified using an image analyzer and expressed as a percentage of the total surface area. After normalization of the MMP images, the median NIRF signal intensities of the entire tissue were calculated. Results: MMP-related signal intensities were significantly higher in the western diet group(73.9 \pm 78.1 arbitrary units, A.U.) than in the normal chow diet group $(6.2 \pm 9.4$ A.U.) or western diet + exercise group(13.5 \pm 8.4 A.U., $p<0.05$, ANOVA). On the light image, plaque area percentages were also higher in the western diet group $(38.9 \pm 11.6 \%)$ than in the normal diet group
$(8.8 \pm 8.3 \%)$ or western diet + exercise group $20.7 \pm 5.8 \%, p<0.05$, ANOVA). LPO levels were higher in the western diet group $(42.9 \pm$ $3.1 \mathrm{nmol} / \mathrm{ml})$ than in the normal diet group $(13.0 \pm 10.25 \mathrm{nmol} / \mathrm{ml})$ or western diet + exercise group $(21.2 \pm 0.9 \mathrm{nmol} / \mathrm{ml}, p<0.05$, ANOVA). In contrast, SOD levels were lower in the western diet $\operatorname{group}(0.02 \pm 0.02 \mathrm{unit} / \mathrm{ml})$ than in the normal diet $\operatorname{group}(0.08 \pm 0.07$ unit $/ \mathrm{ml})$ or western diet + exercise $\operatorname{group}(0.05 \pm 0.03$ unit $/ \mathrm{ml})$, which however did not reach a statistical significance $(p=0.19$, ANOVA). There were no inter-group differences in the cholesterol levels. Conclusion: Using the NIRF MMP imaging, we demonstrated that exercise performed after atheromata formation could attenuate the MMP activity as well as plaque size in ApoE knock-out mice. Despite the worry about the exercise-induced oxidative stress, we observed that exercise could reduce lipid peroxidation of the aortic tissue.

\section{J398}

ENGINEERING ATTENUATED SALMONELLA TYPHIMURIUM TO SELECTIVELY TARGET AND DELIVER PROTEIN IN INFARCTED MYOCARDIUM

Uyenchi N. Le ${ }^{1}$, Jin-Sook Kwon ${ }^{2}$, Vu Hong Nguyen ${ }^{1}$, Hyeong Seok Kim ${ }^{3}$, Sheng Nan Jiang ${ }^{1}$, Yeongjin Hong ${ }^{4}$, Myung Geun Shin ${ }^{5}$, Joon Haeng Rhee, Hee-Seung Bom ${ }^{1}$, Sanjiv S. Gambhir ${ }^{6}$, Youngkeun Ahn ${ }^{2}$, Hyon El Choy ${ }^{4}$, Jung-Joon Min ${ }^{1,7}$

${ }^{1}$ Nuclear Medicine, Chonnam National University Medical School, Gwangju, Korea, South, ${ }^{2}$ Cardiovascular Medicine, Chonnam National University Medical School, Gwangju, Korea, South, ${ }^{3}$ Forensic Medicine, Chonnam National University Medical School, Gwangju, Korea, South, ${ }^{4}$ Microbiology, Chonnam National University Medical School, Gwangju, Korea, South, ${ }^{5}$ Laboratory Medicine, Chonnam National University Medical School, Gwangju, Korea, South, ${ }^{6}$ Molecular Imaging Program at Stanford, Department of Radiology \& Bioengineering, Bio-X Program, Stanford University, Stanford, CA, USA, ${ }^{7}$ Bioimaging Research Center, Gwangju Institute of Science and Technology, Gwangju, Korea, South.

Gene-based therapeutic approaches offer a potential strategy to redress myocardial dysfunction for the patients with refractory angina despite conventional medical or surgical treatment. Optimizing the specific affinity of the cardiac vectors would improve the efficiency of gene/ protein delivery, and reduces unwanted transfection of non-cardiac tissues. However, none of the cardiac vectors so far have specificity for the infarcted myocardium. In this study, we explored bacterial tropism for infarcted myocardium. We constructed a Salmonella typhimurium defective in ppGpp synthesis to express and secrete the reporter gene (RLuc 8$)$ by the regulation of an inducible $\mathrm{P}_{\mathrm{BAD}}$ promoter. A bacterial expression plasmid encoding a variant of Renilla luciferase (RLuc8) was constructed, in which the pelB leader sequence and a histidine tag (6x His) were fused to the amino- and carboxy-terminus, respectively, of RLuc8. An occlusion at the left coronary artery generated the myocardial infarction (MI) in the left ventricle of Sprague-Dawley rats. $2 \times 10^{8}$ cfu of the Salmonellae were intravenously injected and bacterial tropism for MI was observed by cooled CCD camera. To assess the systemic or local toxicity after bacterial injection, we measured Creactive protein and procalcitonin in the rats' serum and measured infarct size by TTC staining before and after bacterial injection. The Salmonella were found to accumulate in infarcted myocardium. RLuc8 gene delivered by the engineered Salmonellae was expressed and its translated product was secreted specifically in the infarcted myocardium under the stringent control of the $\mathrm{P}_{\mathrm{BAD}}$ promoter after $\mathrm{L}$ arabinose administration. No sign of serious local or systemic inflammatory reactions was noted following intravenous administration of attenuated Salmonella. Thus, MI-targeting bacteria can potentially deliver therapeutic molecules to salvageable myocardium. Taken together, the development of MI-targeting bacteria opens many new avenues for molecular imaging and therapy, including tissue- 
specific targeting with signal amplification based on bacterial proliferation, in vivo tissue-specific drug delivery, and the design of imageable therapeutic probes.

\section{J399}

\section{TOWARD A PET TRACER FOR QUANTITATION OF PANCREATIC $\beta$-CELL MASS}

Brett Connolly, Amy Vanko, Paul McQuade, Ilonka Guenther, Karen Schlingmann, Xiangjun Meng, Rikki N. Waterhouse, Donald Burns, Richard Hargreaves, Eric Hostetler Imaging Research, Merck Research Laboratories, West Point, PA, USA.

Diabetes mellitus is a chronic disorder with the characteristic feature of hyperglycemia due to a deficient insulin secretory response and destruction of pancreatic islet $\beta$-cells. A major goal of diabetic treatments is to stabilize or increase the number of $\beta$-cells. Therefore, a non-invasive imaging agent for determining $\beta$-cell mass is desirable. However, $\beta$ cells account for only around $1 \%$ of the pancreas, making detection via imaging difficult. In an effort to discover a PET tracer for imaging $\beta$-cell mass, immunohistochemistry studies focusing on cell surface receptors expressed on $\beta$-cells were conducted using normal and diabetic human, monkey or rodent pancreas. Of the results obtained glucagon-like peptide 1 receptor (GLP-1R) showed promise due to its apparent lack of expression in the exocrine pancreas. Exendin-4, a 39 amino acid peptide mimetic of the incretin hormone GLP-1, binds to GLP-1R with pmol affinity (Curr Drug Metab, 2006, 7; 367) and is claimed as a therapeutic agent for type 2 diabetes. Several publications (e.g. Regul Pept. 2006; 137:162) report that an In-111 labeled Exendin4 analog localizes in mouse pancreas and may be useful for assessing GLP-1R density in pancreas. Exendin- 4 was therefore chosen as our proof of concept molecule for detection of islet $\beta$-cells via GLP-1R. Similar to work previously reported; the C-terminus was modified with lysine and functionalized with the bi-functional chelator DOTA. Subsequently, labeling with Copper-64 and purification yielded [64Cu]DOTA-Exendin in high radiochemical purity and with a specific activity $>200 \mathrm{Ci} / \mathrm{mmol}$. Ex vivo autoradiographic studies were carried out on Sprague Dawley rats in which $[64 \mathrm{Cu}]$ DOTA-Exendin was administered alone or co-injected with the GLP-1R antagonist Exendin(9-39) (2.94 mg/kg). Animals were euthanized, pancreas extracted and immediately frozen and sectioned. The sections were dried and apposed to a phosphor image plate, scanned and afterwards immunostained for insulin for coregistration of islets with the autoradiographic data. Results of the combined images revealed that [64Cu]DOTA-Exendin-4 bound specifically to the islet cells and that this uptake could be blocked by the co-administration of Exendin (9-39). Tracer uptake in the exocrine pancreas was extremely low. Additionally, insulin immunostaining coincided with [64Cu]-DOTA-Exendin-4 uptake, demonstrating that tracer uptake is islet-specific. These results indicate that further in vivo evaluation of radiolabeled Exendin-4 as a potential PET tracer for $\beta$-cell mass is warranted.

\section{$\mathbf{J 4 0 0}$}

LUMINAL ENDOTHELIAL PHOSPHATIDYLETHANOLAMINEA CRITICAL ANTICOAGULANT AND NOVEL BIOMARKER FOR ENDOTHELIAL HEALTH AND DYSFUNCTION

Zhixin Li, Ming Zhao

medical college of wisconsin, Milwaukee, WI, USA.

OBJECTIVE: It has long been speculated that phosphatidylethanolamine (PE) is physically present at the luminal endothelial surface, where it functions as a critical anticoagulant. But neither the presence nor the distribution profile of vascular PE has ever been characterized due to a lack of investigate probes on a tissue level. The objective of this work was to address this knowledge gap and to develop a novel biomarker for vascular health and diseases. METHOD: Duramycin-biotin and Gd-Duramycin were synthesized as PE-specific probes. The rat aortic arch and branches were selected as major conduit vessels under significant hemodynamic burden. Duramycin binding in the vessels was characterized by histology and high-resolution MRI. In addition, the vessels from normotensive and hypertensive salt-sensitive rats were investigated. RESULTS: There is an extraordinarily high level of PE at the luminal endothelial surface of aortic flow dividers (Fig 1B, C). Significant PE was also identified at the ascending aorta and the inner curvature of the aortic arch (Fig 1D, E). The PE distribution profile was captured in high-resolution MRI using Gd-Duramycin (Fig 1F). In hypertension, which is tenaciously associated with endothelial dysfunction, there is a markedly diminished PE level at the aforementioned vascular regions. Furthermore, a reversal of hypertension is accompanied with a re-population of the vascular surface by PE. CONCLUSION: Novel findings from this study include: 1) For the first time the physical distribution of vascular PE is described using PEspecific molecular probes. 2) There is a high likelihood that the distribution pattern of PE is correlated with hemodynamics. 3) PE may serve as an important biomarker for endothelial health and dysfunction, which can be imaged in high resolution on a tissue level. The characterization of vascular PE will have fundamental significance to the understanding of hemostasis and vascular physiology, and provide a definitive biomarker for therapeutics.

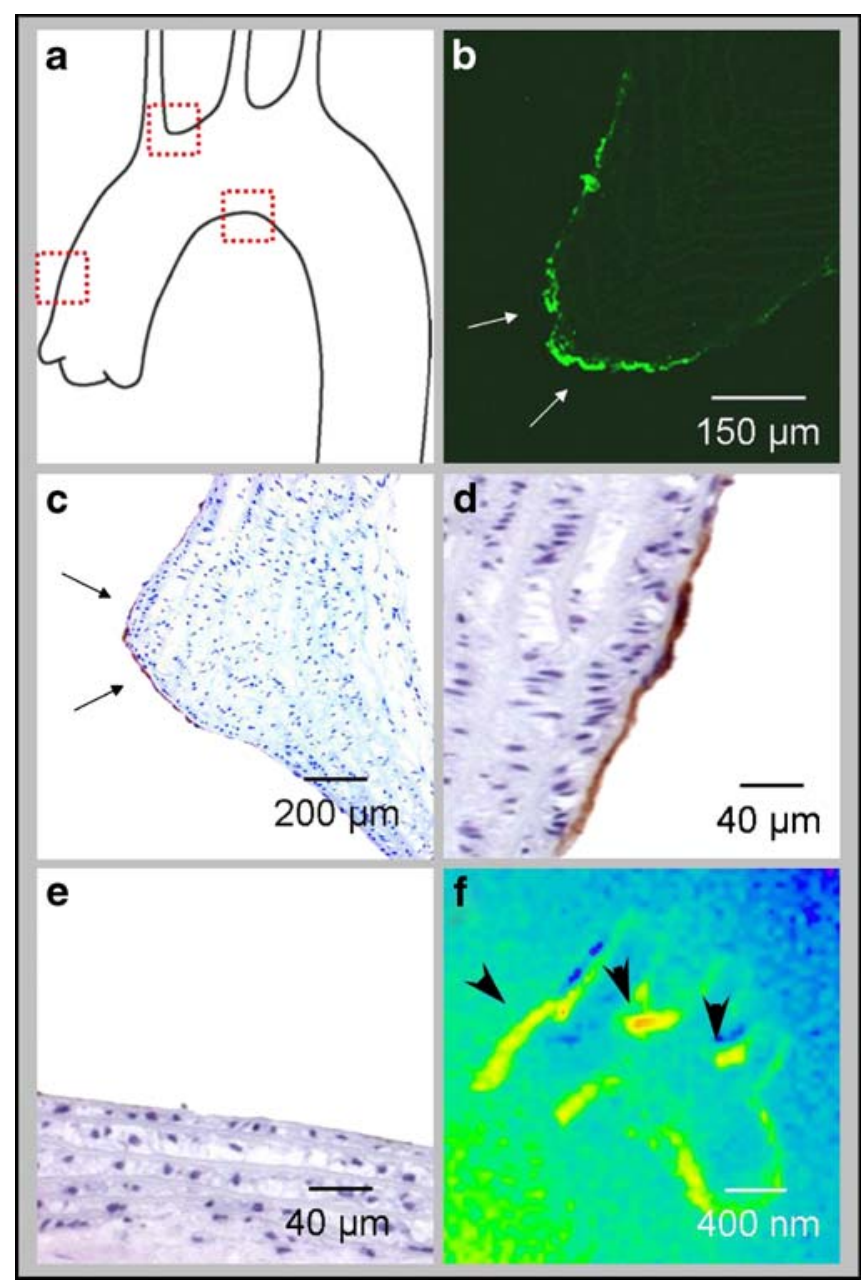


$\mathbf{J} 401$

\section{A TRANSGENIC MOUSE MODEL FOR PET IMAGING OF PANCREATIC ISLETS}

Rebecca M. McGirr ${ }^{1}$, Siu-Pok Yee ${ }^{1,2}$, Michael S. Kovacs ${ }^{1,3}$ Ting-Yim Lee ${ }^{1,4}$, Savita Dhanvantari ${ }^{1,4}$

${ }^{1}$ Lawson Health Research Institute, London, ON, Canada, ${ }^{2}$ Oncology, University of Western Ontario, London, ON, Canada, ${ }^{3}$ Chemistry, University of Western Ontario, London, ON, Canada, ${ }^{4}$ Medical Biophysics, University of Western Ontario, London, ON, Canada.

Positron emission tomography (PET) is an attractive modality with which to detect molecular function in vivo, since it provides quantitative information on the tissue distribution of a radiolabelled tracer. We are using the PET reporter gene HSV1-sr39tk and [18F] FHBG (fluorohydroxymethyl-butyl-guanine) as the reporter probe to image mechanisms of beta cell regeneration in endogenous pancreatic islets. To this end, we have made a transgenic mouse expressing sr39tk, under the control of the mouse insulin promoter (MIP-sr39tk). Western blot analysis and fluorescence immunocytochemistry showed robust sr39tk expression that is restricted to pancreatic islets. Islet architecture was normal, and beta cell mass could be determined using either insulin or sr39tk immunoreactivity. Glucose tolerance, as determined by area under the curve intraperitoneal glucose tolerance tests (AUC IPGTT), was also normal in transgenic mice compared with non-transgenic littermates, indicating that expression of sr39tk does not affect beta cell function in vivo. For PET scans, mice were injected i.v. with $200 \mu \mathrm{Ci}$ [18F]FHBG. Dynamic PET scans were acquired for $1 \mathrm{~h}$ and static images were subsequently acquired for $30 \mathrm{~min}$. Dynamic scans showed washout of the probe over time in the kidney, and accumulation of the probe in the pancreas. Static PET scans of female transgenics showed $10 \%$ higher probe uptake $(p<0.01, n=3)$ when compared with nontransgenic littermates, as measured by standardized uptake values (SUVs) in a region of interest that encompassed the gut. Biodistribution of [18F]FHBG showed a 7-fold ( $p<0.01, n=3)$ elevation of $18 \mathrm{~F}$ activity in the pancreas of transgenic mice compared with non-transgenic littermates, confirming that the increase in SUV was due to increased probe uptake in pancreatic beta cells. In mice treated with streptozotocin to induce diabetes, [18F]FHBG uptake decreased to levels of nontransgenic mice. There was also a negative correlation between SUV and AUC IPGTT, indicating that molecular PET reflects beta cell function. We conclude that our MIP-sr39tk mouse can be used as a model for imaging changes in beta cell mass during the progression of diabetes as well as regeneration of the endogenous islet. We will use this mouse for longitudinal studies of changes in beta cell mass over time after induction of diabetes and in response to therapies that increase functional beta cell mass. Acknowledgements: This work is funded by an operating grant and scholarship from the Canadian Diabetes Association to S. Dhanvantari.

\section{$\mathbf{J 4 0 2}$}

\section{TARGETED IMAGING OF HEARTS IN HEALTHY} AND DISEASED MODELS BY HEART-HOMING LIPOSOMES Hua Zhang ${ }^{1}$, Ning $\mathrm{Li}^{2}$, Jai Woong $\mathrm{Seo}^{1}$, Katherine D. Watson ${ }^{1}$, Lisa M. Mahakian $^{1}$, Nipavan Chiamvimonvat ${ }^{2}$, Katherine Ferrara ${ }^{1}$ ${ }^{1}$ Biomedical Engineering, University of California, Davis, Davis, CA, USA, ${ }^{2}$ Internal Medicine, University of Califnornia, Davis, Davis, CA, USA.

Background and aim: Previously, we reported on accumulation of ${ }^{18} \mathrm{~F}$ labeled peptide (CRPPR)-targeted liposomes in the normal mouse heart ${ }^{1}$. Within $100 \mathrm{~s}, 44 \pm 9 \%$ injected dose/gram accumulated in the heart and remained bound over $90 \mathrm{~min}$. We now evaluate the potential for delivery of targeted liposomes to diseased hearts over time, and explore the use of PET to visualize and quantify accumulation. Methods: Two disease models were studied, each resulting in damage to the left ventricle. First, an ischemia- reperfusion (IR) model was evaluated, in which the left anterior descending artery (LAD) was ligated for 1 hour and released; the animals were intravenously injected with ${ }^{18} \mathrm{~F}$-CRPPR-liposomes $24 \mathrm{hrs}$ later and imaged over $60 \mathrm{~min}$. Second, a myocardial infarction (MI) model was evaluated, where the LAD was ligated one week before injection and imaging. The in vivo biodistribution and accumulation kinetics of the ${ }^{18} \mathrm{~F}$-liposomes were assessed by microPET. Wild type (WT) mice were imaged in parallel with IR or MI mice. The left wall thickness and volume are quantified using ultrasound and compared with PET and histopathology. Results: Compared with WT mice, reduced ${ }^{18} \mathrm{~F}$ accumulation was observed in the IR $(80 \%)$ and MI $(82 \%)$ hearts. Accumulation in the left wall of the left ventricle decreased as compared with the right wall. The accumulation ratios (left wall vs. right wall) are $1.02 \pm 0.09,0.75 \pm 0.03$, and $0.84 \pm 0.17$ for WT, IR, and MI, respectively, however substantial activity remains within the affected wall. Regions of decreased accumulation are visualized with PET in both the IR and MI models. The kinetics of particle accumulation are unchanged between WT, IR, and MI mice. $1 \mathrm{H}$. Zhang, J. Kusunose, A. Kheirolomoom et al., Biomaterials 29 (12), 1976 (2008).

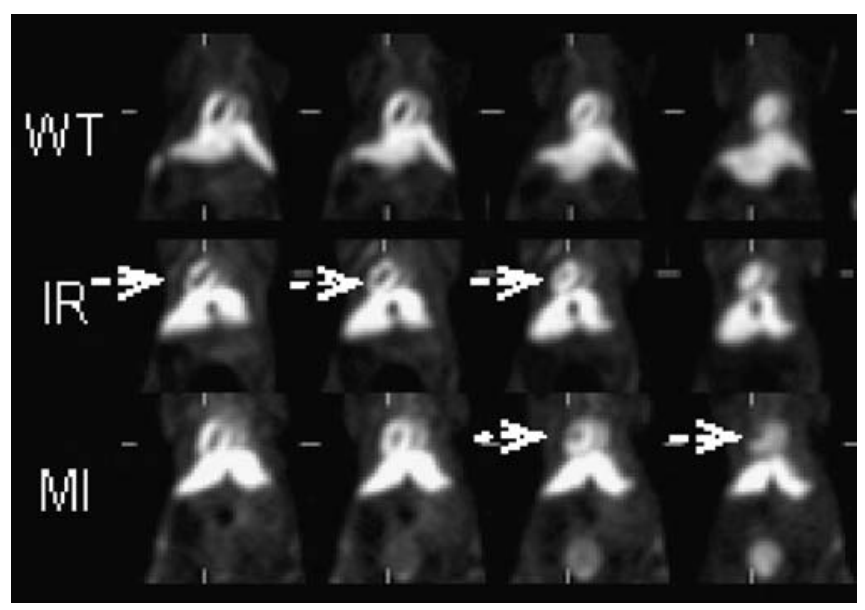

The microPET coronal images from WT (upper), IR (middle) and MI (lower) mice over the first 60 minutes after intravenously injection of F-18 CRPPR-liposome. Max gray scale (white) $=$ (maximum value in heart), and Min gray scale $($ black $)=0$. Such gray scale is chosen for better visualization of damaged parts of the hearts, which are highlighted by arrows.

\section{$\mathbf{J 4 0 3}$}

\section{IN VIVO MONITORING OF AUTOLOGOUS FAT GRAFTING AND ADIPOSE-DERIVED REGENERATIVE CELLS THROUGH BIOLUMINESCENCE IMAGING}

Dan Ansaldi ${ }^{1}$, Min $\mathrm{Zhu}^{2}$, Kai Pinkernell ${ }^{2}$, Mark Roskey ${ }^{1}$, Kevin P. Francis ${ }^{1}$, Ning Zhang ${ }^{1}$

${ }^{1}$ Caliper Life Sciences, Alameda, CA, USA, ${ }^{2}$ Cytori Therapeutics, Inc., San Diego, CA, USA.

Background: Adipose-derived regenerative cells (ADRCs) have shown considerable promise in cell therapy research and are currently being investigated clinically in ADRC enhanced autologous fat grafting. The cells share some characteristics with bone marrow derived cells but can be harvested in large numbers with less morbidity from adipose tissue than from marrow. Here we demonstrate the ability to non-invasively monitor fat grafts and ADRCs in vivo by utilizing IVIS ${ }^{\circledR}$ bioluminescent imaging technology. Methods and Results: Inguinal adipose depots were harvested from beta-actin-luc, GAPDH-luc, and Ubc-luc transgenic mice implanted (without ADRC enhancement) into the dorsal subcutaneous space of non-transgenic recipients $(n=3$ per group). Comparison of the different strains confirmed in vitro 
data showing beta-actin-luc adipose tissue to have the highest luciferase signal among the donor types. Luciferase activity demonstrated survival of donor fat out to at least 5 months. An increase in signal intensity could be seen over the first 11 days, most likely due to establishment of perfusion and consequent improvement in metabolic activity and luciferin availability within the grafted tissue. In another study subset, ADRCs were harvested from beta-actin-luc inguinal fat pads by enzymatic digestion and centrifugation and implanted subcutaneously into non-transgenic recipient mice $(1 \times$ $10^{\wedge} 6$ ADRCs in $100 \mu \mathrm{l}$ Lactated Ringer). Utilizing bioluminescent imaging, we were able to monitor the engraftment of these cells in vivo, with an increase in signal intensity up to 11 days, most likely reflecting the same increases in metabolic activity and luciferin availability seen with intact adipose tissue. Conclusion: These findings demonstrate the utility of bioluminescent imaging for tracking adipose tissue and ADRCs longitudinally in vivo. Such an approach may allow further investigation of both intrinsic and external factors that regulate fat tissue and ADRC survival and homing in animal tissues.

\section{J404}

\section{QUANTITATIVE DYNAMIC SPECT/CT IMAGING}

\section{OF ATHEROSCLEROTIC PLAQUES IN ApoE-/- MICE}

Benjamin M. Tsui ${ }^{1}$, Jianhua $\mathrm{Yu}^{1}$, Yuchuan Wang ${ }^{2}$, Greta S. Mok ${ }^{3}$, Sridhar Nimmagadda ${ }^{1}$, Djahida Bedja ${ }^{4}$, Kathleen L. Gabrielson ${ }^{4}$, Frank Bengel ${ }^{1}$, Martin Pomper ${ }^{1}$

${ }^{1}$ Department of Radiology, Johns Hopkins University, Baltimore, MD, USA, ${ }^{2}$ Department of Radiology, Harvard University, Boston, MA, USA, ${ }^{3}$ Department of Radiology, Chinese University of Hong Kong, Hong Kong, China, ${ }^{4}$ Department of Comparative Medicine, Johns Hopkins University, Baltimore, MD, USA.
The goal of the study is to develop quantitative Tc-99m Annexin-V pinhole SPECT/CT method to investigate the characterization and progression of atherosclerotic plaques in ApoE-/- mice over time. Fourteen batches of mice (41 ApoE-/- and 21 controls) were imaged at $2-3$ weeks intervals from $\sim 18$ to $\sim 35$-weeks old. The ApoE-/- mice were fed with a high fat diet that accelerated the development of atherosclerotic plaques. At each time point The animals were imaged with Tc-99m Annexin-V (A5) pinhole SPECT with $\sim 1.25 \mathrm{~mm}$ resolution for apoptosis and co-registered microCT using Fenestra VG for contrast enhancement (CE) to provide anatomic correlation with SPECT. Autoradiography and histological data of the excised aortas were obtained after the last imaging session to confirm Tc-99m A5 uptake in plaques. An equivalent SPECT standardized uptake value (SSUV) value, defined as the mean activity value over a focal Tc-99m A5 uptake region normalized by the injected Tc-99 activity and body weight, was determined from the SPECT images. The quantitative SSUV values over time were used to characterize the progression of the plaques. From the 41 ApoE-/- mice, we found 23 with suspicious to significant focal Tc-99m A5 uptakes in the aortic arch area consistently for 3 or more imaging time points. Among them, 13 were found with monotonic increase in SSUV values over time, 4 with initial increase followed by a decrease, 4 with initial decrease followed by an increase, and 2 with monotonic decrease. Calcium deposits were observed in the aortic arch area in the CE CT images on 23 of the 41 animals, correlating with low uptake of A5. The SSUV values provide a quantitative means to analyze the dynamic Tc-99 m A5 SPECT images of atherosclerotic plaques. The analysis demonstrates that the development of plaque can be a dynamic process that follows one of several possible progression patterns. Further correlation with histological analysis will provide deeper mechanistic understanding of the development of plaque in this animal model.

\section{DYNAMIC QUANTITATIVE UPTAKE OF ANNEXIN V IN ATHEROSCLEROTIC PLAQUES IN APOE-/- MICE*} SPECT standardized uptake values (SSUV) of ${ }^{99 \mathrm{~m} T C-A n n e x i n ~} \mathrm{~V}$ uptake in plaques.

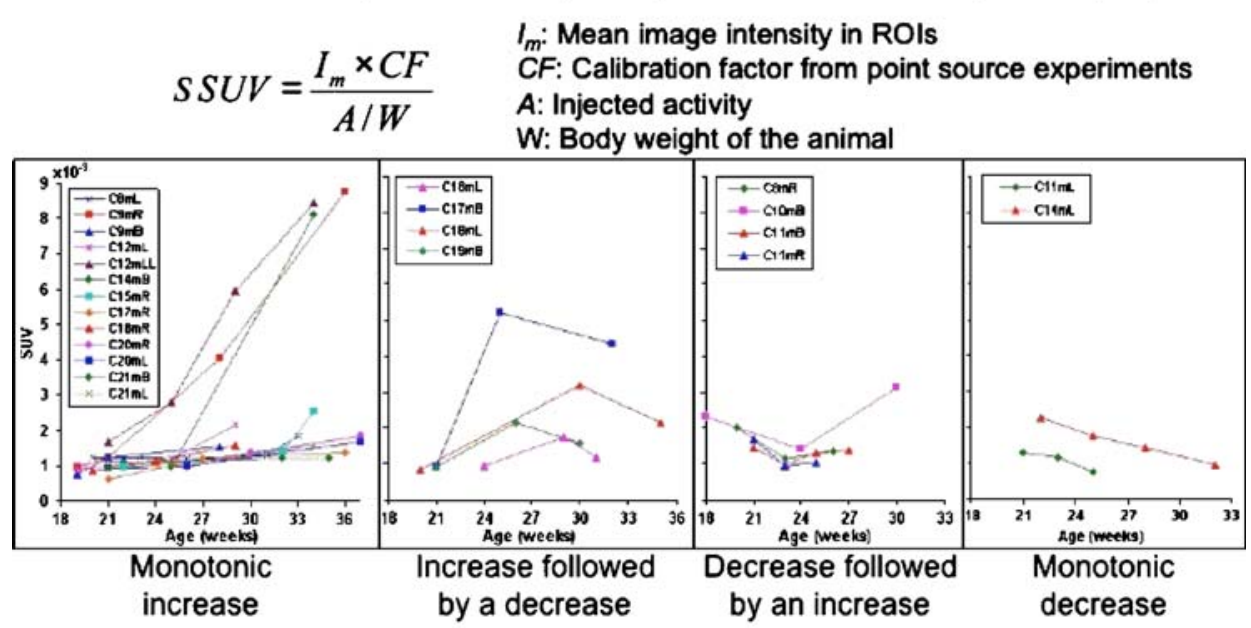

The formation and progression of atherosclerotic plaques is a dynamic process.

- From 23 ApoE-\% mice 
$\mathbf{J} 405$

68 Ga-DOTA-RGD-peptide-BIODISTRIBUTION AND BINDING INTO ATHEROSCLEROTIC PLAQUES IN MICE

Johanna Haukkala $^{1}$, Iina Laitinen ${ }^{1}$, Pauliina Virsu ${ }^{1}$, Peter Iveson $^{2}$, Ian Wilson ${ }^{2}$, Hege Karlsen ${ }^{3}$, Alan Cuthbertson ${ }^{3}$, Jukka Laine ${ }^{4}$, Pia Leppänen ${ }^{5}$, Seppo Ylä-Herttuala ${ }^{5}$, Juhani Knuuti ${ }^{1}$, Anne Roivainen ${ }^{1,6}$ ${ }^{1}$ Turku PET Centre, Turku University, Turku, Finland, ${ }^{2}$ GE Healthcare Biosciences, Medical Diagnostics, London, United Kingdom, ${ }^{3} \mathrm{GE}$ Healthcare MDx Research, Nycovein 2, Oslo, Norway, ${ }^{4}$ Department of Pathology, Turku University Hospital, Turku, Finland, ${ }^{5}$ A.I. Virtanen Institute, University of Kuopio, Kuopio, Finland, ${ }^{6}$ Turku Centre for Disease Modelling, University of Turku, Turku, Finland.

Rupture of unstable atherosclerotic plaque is main cause of acute coronary syndrome and myocardial infarction. Identification of plaques vulnerable to rupture, might guide the local therapy and prevent clinical complications. Increased density of microvessels is associated with intraplaque haemorrhage and plaque rupture and increased expression of $\alpha v \beta 3 / \alpha v \beta 5$ integrin is involved in angiogenesis and inflammatory process in the atherosclerotic plaques. The 68Ga-DOTA-RGD peptide binds with high affinity to $\alpha v \beta 3 / \alpha v \beta 5$ integrin. The aim of this study was to investigate the uptake of $68 \mathrm{Ga}-\mathrm{DOTA}-\mathrm{RGD}$ peptide in atherosclerotic plaques. Uptake of intravenously administered $68 \mathrm{Ga}-$ DOTA-RGD peptide was studied ex vivo in excised tissue samples and aortic sections of six LDLR-/-ApoB100/100 atherosclerotic mice and six control mice. The uptake of the tracer in aortic cryosections was examined by using digital autoradiography. Subsequently, the autoradiographs were combined with histological and immunohistological analysis of the sections. DOTA-RGD peptide was successfully labelled with the generator-produced $68 \mathrm{Ga}$. The tracer had reasonable good specific radioactivity $(8.7 \pm 1.1 \mathrm{GBq} / \mu \mathrm{mol})$ and was quite stable in vivo. According to ex vivo biodistribution results 68Ga-DOTARGD was cleared rapidly from the blood circulation and excreted through the kidneys to the urine with high radioactivity in the intestine, lungs, spleen and liver. Autoradiography results showed significantly higher uptake of 68 Ga-DOTA-RGD peptide in the atherosclerotic plaques compared with healthy vessel wall (mean ratio $\pm \mathrm{SD} 1.4 \pm 0.1, p=0.0004)$. We observed that 68Ga-DOTA-RGD is accumulated into the plaques of atherosclerotic mice. Further studies are warranted to assess the uptake of this tracer into human atherosclerotic plaques.

\section{J406}

HYBRID IN VIVO FMT-CT IMAGING OF CATHEPSIN ACTIVITY IN ATHEROSCLEROSIS WITH CUSTOMIZED PROTEASE NANOSENSORS

Matthias Nahrendorf ${ }^{1}$, Peter Waterman ${ }^{1}$, Greg M. Thurber ${ }^{1}$, Kevin Groves $^{2}$, Milind Rajopadhye ${ }^{2}$, Peter Panizzi ${ }^{1}$, Brett Marinelli ${ }^{1}$, Mikael Pittet ${ }^{1}$, Filip K. Swirski ${ }^{1}$, Ralph Weissleder ${ }^{1}$

${ }^{1}$ Center for Systems Biology, Massachusetts General Hospital, Boston, MA, USA, ${ }^{2}$ Visen Medical, Bedford, MA, USA.

Objective: Cathepsins are emerging biomarkers of inflammatory diseases. In atherosclerosis, these proteases are secreted by inflammatory macrophages and digest the extracellular matrix of the fibrous cap and destabilize atheromata. Their function can be monitored with fluorescence molecular tomography (FMT) and activatable probes. To address two major constraints currently associated with imaging of murine atherosclerosis (lack of highly sensitive probes and absence of anatomical information), we compared protease sensors (PS) of variable size and pharmacokinetics and fused FMT datasets to computed tomography (FMT-CT). Methods and results: Fusion of FMT and CT was achieved with a multimodal imaging cartridge containing fiducial markers detectable by both modalities. A high-resolution $\mathrm{CT}$ angiography protocol accurately localized fluorescence to the aortic root of atherosclerotic apoE-/- mice. To identify suitable sensors, we first modeled signal kinetics in-silico and then compared three probes with identical oligo-L-lysine cleavage sequences targeted by cathepsins: PS-5, $5 \mathrm{~nm}$ in diameter containing 2 fluorochromes, PS-25, a $25 \mathrm{~nm}$ version with an elongated lysine chain and PS-40, a polymeric nanoparticle. Serial FMT-CT showed fastest kinetics for PS-5 but surprisingly, highest fluorescence in lesions of the aortic root for PS40 (FMT@24 hrs, PS-5: 10.6 \pm 2.2 pmol, PS-25: 26.8 \pm 4.4 pmol, PS40: $50.5 \pm 5.8, p<0.05)$. PS-40 robustly reported therapeutic effects of atorvastatin, corroborated by ex vivo imaging and qPCR for cathepsin B expression $(p<0.05)$. Conclusions: FMT-CT is a robust and observer-independent tool for non-invasive assessment of inflammatory murine atherosclerosis. Reporter containing nanomaterials may have unique advantages over small molecule agents for in vivo imaging.

\section{$\mathbf{J 4 0 7}$}

\section{TRIPLE ISOTOPE DIGITAL AUTORADIOGRAPHY TO CONTRAST OXIDIZED LDL ANTIBODIES WITH ${ }^{18}$ FDG AND CONTROL ANTIBODIES IN ATHEROSCLEROTIC PLAQUE IMAGING}

Anders Orbom $^{1}$, Bo Jansson ${ }^{2}$, Jan Nilsson ${ }^{3}$, Sven-Erik Strand ${ }^{1}$

${ }^{\mathrm{T}}$ Medical Radiation Physics, Lund University, Lund, Sweden, ${ }^{2}$ BioInvent International $\mathrm{AB}$, Lund, Sweden, Slinical Science, Lund University, Lund, Sweden.

Introduction: Clinically, FDG is routinely used to diagnose and stage Atherosclerosis. However, it is a marker for metabolism and inflammation and not specific to the acute form of instable "vulnerable" atherosclerotic plaques. It has been shown that antibodies specific for a specific epitope on oxidized low-density lipoprotein (oxLDL) causes rapid regression of plaques in a mice model (Schiopu et al. 2007, J Am Coll Card). We propose that similar anti oxLDL antibodies can be used for imaging to improve specificity in diagnosis and staging. Digital autoradiography offers sufficient resolution to evaluate targeting accuracy to plaques and simultaneous imaging of several isotopes enables comparison of the contrast between the aorta wall and plaques for both antibodies and ${ }^{18}$ FDG in the same animal. Methods: Apobec LDLR-/- mice with atherosclerotic plaques received ${ }^{131}$ I-labelled anti oxLDL and ${ }^{125} \mathrm{I}-$ labelled control antibodies i.v. Food was removed at $67 \mathrm{~h}$ PI and between 68 and $75 \mathrm{~h} \mathrm{PI}{ }^{18}$ FDG was given i.v. The mice were sacrificed $1 \mathrm{~h}$ after FDG injection and the aorta opened and mounted on a glass slide after fixation. Each slide was imaged immediately after mounting for approx $1 \mathrm{~h}$ using a double-sided silicon-strip detector (Biomolex 700 Imager, Bimolex AS, Oslo) and then again at least two days later for at least $8 \mathrm{~h}$. The aortas were subsequently stained with Oil Red O. Contributions from different isotopes were separated using both energy spectra $\left({ }^{125} \mathrm{I} /{ }^{131} \mathrm{I}\right)$ and half-life $\left({ }^{18} \mathrm{~F}\right)$. Results: The animal study and data analysis is currently ongoing but preliminary data show that isotope separation is successful. Results will be presented on contrast for the different radiolabels and digital images of activity uptake correlated to histology. Pictures from individual animals of ${ }^{18} \mathrm{~F},{ }^{125} \mathrm{I}$ and ${ }^{131} \mathrm{I}$ isotope separated images will be presented and compared with the standard oil red lipid stain of each individual targeted aorta. Conclusions: Our results show the feasibility of simultaneous imaging of ${ }^{125} \mathrm{I} /{ }^{131} \mathrm{I}$-labelled antibodies and ${ }^{18} \mathrm{FDG}$. We hope to expand the method to include in vivo evaluation of two different antibodies and the relative metabolic activity in tissue using $\mu$ SPECT/PET. 
$\mathbf{J 4 0 8}$

EXPLORING NOVEL OPTICAL IMAGING METHODS FOR QUANTIFYING MUSCLE DAMAGE AND INFLAMMATION IN MOUSE MODELS OF MUSCULAR DYSTROPHY

Andreas R. Baudy $^{1,2}$, Eric Hoffman ${ }^{1,2}$, Kanneboyina Nagaraju ${ }^{1,2}$ 'Genetic Medicine, Children's National Medical Center, Washington, DC, USA, ${ }^{2}$ George Washington University, Washington, DC, USA.

Live-Imaging is revolutionizing the way in which disease progression can be monitored and how therapeutic intervention can be assessed. Currently, the three most prominent technologies being used for small animal imaging are: MRI, Optical Imaging, and PET. While each has its pros and cons, a major advantage of Optical Imaging is that it does not require an extensive infrastructure to support its operation. Advancement in Optical Imaging will better enable investigators to conduct experiments that provide rich, whole-body information about specific biological events occurring in the given specimen. In skeletal muscle diseases, such as Limb Girdle and Duchenne Muscular Dystrophy, large amounts of degeneration and inflammation occur within the myofiber environment. Herein, we investigate the ability of two commercially available near-IR dyes to detect these features in the ART Explore Optix Optical Imaging system within the context of the mdx and SJL mouse models. The first dye we tested was a Cy5.5-10 kDa dextran conjugate, and was chosen because it's previously been shown that $10 \mathrm{kDa}$ dextrans are effective at entering leaky muscle. The second dye we tested, Visen ProSense 680, is currently used for detecting Cathepsin-related inflammation; this was chosen since Cathepsins have been shown to be upregulated in Muscular Dystrophy. Interestingly, the Cy5.5-10 kDa dextran was found at significantly higher levels in the $\mathrm{mdx}$ and SJL models compared to BL6, indicating its entry into damaged muscle, and potential as use of a marker of myofiber damage. We are currently investigating the activity of ProSense 680 in these models, as well as validating our results by assessing the degree to which the dyes co-localize with inflammatory foci and degenerating fibers in frozen cross-sections of multiple skeletal muscles from each model.

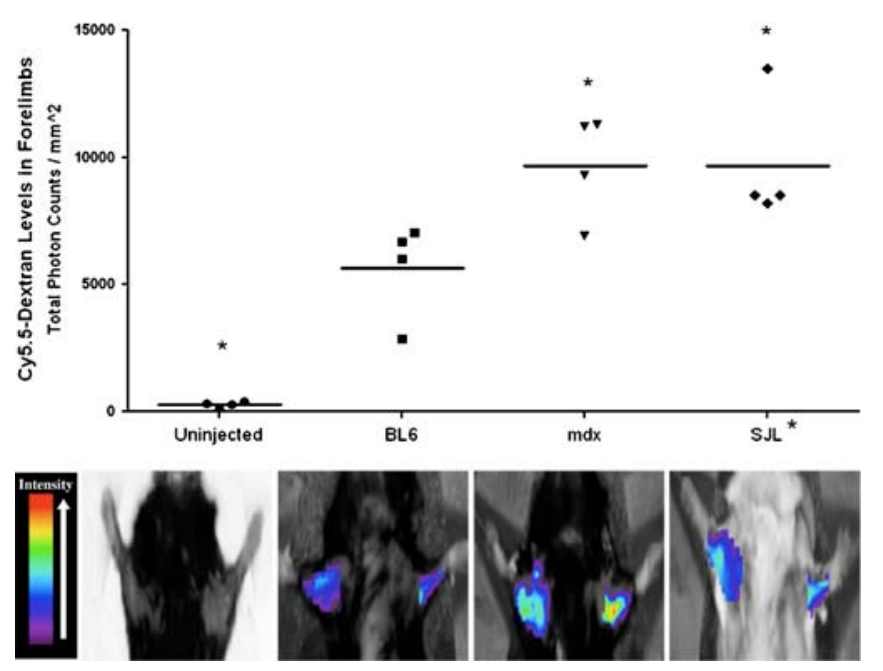

Mice were I.P. injected with $150 \mu 1$ of Cy5.5-10 kDa Dextran dye 24 hours prior to scanning of forelimbs with the Explore Optix Optical Imager. A significant increase in signal intensity was detected in both $\mathrm{mdx}$ and SJL models, suggesting preferential localization of dye to damaged myofibers. ( ${ }^{*}$ p-value $=<0.05$ compared to BL6)

\section{J409}

COMBINED DYNAMIC AND ELECTROCARDIOGRAM-GATED 82-RB-PET WITHOUT LIST MODE DATA ACQUISITION: QUANTIFICATION OF UNCERTAINTY ASSOCIATED WITH George Sayre $^{1}$, Stephen Bacharach ${ }^{1}$, Michael Dae ${ }^{1,2}$, Youngho Seo ${ }^{1}$ ${ }^{R}$ Radiology and Biomedical Imaging, University of California - San Francisco, San Francisco, CA, USA, ${ }^{2}$ Medicine, University of California - San Francisco, San Francisco, CA, USA.

Background. Rb-82 Positron Emission Tomography (Rb-82-PET) is being increasingly used to evaluate patients with suspected or known ischemic heart disease. For scanners incapable of list-mode data acquisition or for clinical practices where list mode is impractical, separate dynamic and electrocardiogram-gated (ECG-gated) acquisitions are needed when both left ventricular ejection fraction (LVEF) and myocardial flow reserve (MFR) evaluations are desired. We investigated the feasibility of combining these two acquisitions into one protocol without relying on list-mode data acquisition. We applied a mathematical data-fitting method that can estimate clinical uncertainty associated with incomplete dynamic data sets that necessarily result from the combined dynamic/gated acquisitions on detectors not operating in list-mode. Methods. Eight complete, separate TACs were compiled from Rb-82-PET dynamic acquisitions of four patients. In order to simulate incomplete TACs typical of sequential dynamic multiframe and ECG-gated acquisitions on detectors not operating in listmode, TAC time points following a two-minute dynamic acquisition were replaced with a single, averaged point that would be provided by an ECG-gated study. Time-integrated TACs (ITACs), which are needed for myocardial blood flow (MBF) and MFR calculations, were computed from the incomplete TACs with several functional fits of the data gap regions. Results. The ITACs calculated from the incomplete TACs were compared to the gold-standard (complete-data) ITACs. A free-parameter exponential function (FPE), $\exp \left(-\mathrm{a}^{*} \mathrm{x}^{\wedge} \mathrm{p}\right)$, resulted in incomplete ITACs that best approximated the gold-standard ITACs: approximately $6 \%$ average percent difference, and approximately $6 \%$ standard deviation. Conclusions. The average percent difference and standard deviation between the gold-standard and FPEincomplete ITACs were deemed acceptable by an experienced cardiologist and an expert data analyst of dynamic 82Rb-PET studies for myocardial blood flow calculations. Therefore, our results suggest that a combined dynamic/ECG-gated acquisition protocol for $82 \mathrm{Rb}$ PET, even with a substantial time difference between the final dynamic acquisition and the average ECG-gated point, produce acceptable accuracies in both MFR and LVEF measurements. However, additional ITAC comparisons are needed to strengthen the argument for this simplified clinical protocol.

\section{$\mathbf{J 4 1 0}$}

QUANTITATIVE ASSESSMENT OF GLUCOSE METABOLISM IN THE VESSEL WALL OF AAA WITH FDG-PET/CT INCLUDING CT BASED PARTIAL VOLUME CORRECTION

$\underline{\text { Ralph A. Bundschuh }}{ }^{1}$, Markus Essler ${ }^{1}$, Christian Reeps ${ }^{2}$, Jaroslav Pelisek ${ }^{2}$, Michael Herz ${ }^{1}$, Sibylle Ziegler ${ }^{1}$, Markus Schwaiger ${ }^{1}$, Stephan G. Nekolla ${ }^{1}$

${ }^{1}$ Department of Nuclear Medicine, Klinikum rechts der Isar der TU Muenchen, Munich, Germany, ${ }^{2}$ Department of Vascular Surgery, Klinikum rechts der Isar der TU Muenchen, Munich, Germany.

Objectives: In aneurysm of the abdominal aorta (AAA) macrophage infiltration and MMP activation are correlated with progression. Enhanced glucose metabolism and hence increased FDG uptake in PET/CT examinations may show such infiltration. For quantitative analysis of tracer uptake in the aortic wall, we built a software tool including a CT-based partial volume correction (PVC). Methods: To validate the analysis software a model of the aorta was built, the aortic wall (thickness 5, 10, and $20 \mathrm{~mm}$ ) 
was made of radioactive (F-18) wax, while the blood pool and background activity was simulated with N-13 and F-18 in watery solution. 19 patients with AAA underwent FDG-PET/CT including CT angiography of the abdomen. The three transaxial slices with the highest FDG uptake in the aorta were analyzed for mean SUV in the vessel wall with (SUVcorr) and without PVC (SUVunc). SUVcorr values were compared according to whether the patients were symptomatic or not and correlated to macrophage infiltration and MMP 2, and 9 expression found in histopathological sections after surgery. Results: In the phantom study the recovery of the wall activity was in mean $65 \%$ of the real activity in the modeled walls and the partial volume correction improved it significantly to $92 \%$ especially in the $5 \mathrm{~mm}$ wall. In patient studies corrected SUV values were significantly $(p<0.0001)$ higher compared to uncorrected ones (up to a factor of 3.2, mean 2.3). Significant $(p<0.05)$ differences were found for SUV values in the 14 non-symptomatic patients (mean SUV 2.6) compared to the 5 symptomatic patients (mean SUV 4.5). For 17 patients (two patients did not receive surgery) best correlation with SUVcor was found for macrophage infiltration ( $22=0.62)$, while MMP9 and MMP2 showed no correlation $(\mathrm{r} 2=0.15$ and $\mathrm{r} 2=0.28)$. Conclusions: Using CT derived PVC, tracer uptake in the vessel walls was improved significantly. Patient studies showed significant different uptake patterns in sympromatic patients than in asymptomatic patients. A correlation between macrophage infiltration and FDG uptake was found. Hence FDG-PET/CT might give additional information for the therapy decision of AAAs. Except for AAAs this analysis tool may be usefule for other disease like aortitis, disections of the aorta.

\section{J411}

EVALUATION OF THE THERAPEUTIC EFFECTS OF BEE VENOM IN ACUTE MONO-ARTHRITIC RATS BY BONE SCINTIGRAPHY WITH TC-99M DPD

Soon-Ah Park ${ }^{1}$, Chung Yong Yang ${ }^{2}$, Gyung Jae $\mathrm{Oh}^{3}$, Dae-Weung Kim ${ }^{1}$ ${ }^{\mathrm{T}}$ Nuclear Medicine, Wonkwang university College of Medicine, Iksan, Korea, South, ${ }^{2}$ Physical Medicine and Rehabilitation, Wonkwang university College of Medicine, Iksan, Korea, South, ${ }^{3}$ Preventive Medicine, Wonkwang university College of Medicine, Iksan, Korea, South.

Objectives: Several recent studies have shown that bee venom (BV) has an anti-inflmmatory effect on arthritis. The present study was aimed to evaluate the therapeutic effect of BV by bone scintigraphy with Tc-99m DPD (3,3diphosphono-1,2-propan-dicarbonacid) . Methods: Mono-arthritis was induced by intra-articular injection of carrageenan in the Sprague-Dawley rats. Administration of BV $(0.8 \mathrm{mg} / \mathrm{kg})$ was conducted at 30 minute before and at $4 \mathrm{~h}$ after the induction of mono-arthritis. We assigned rats to BVbefore, BV-after, control groups and compare the results of each group with the weight loading test, diameter measurement of the inflamed swelling and bone scintigraphy. Rats received an intravenous injection of $37 \mathrm{MBq}$ of Tc$99 \mathrm{~m}$ DPD by tail vein and then scanning was performed at 2 hours and 24 hours, respectively. Visual assessment and quantitative analysis were conducted in both knee. Results: BV-before and BV-after groups was more improved than control groups in weight load test $(p<0.05)$. Diameter of arthritic knee in BV-after group was smaller than that of BV-before group at 2 time points of $8 \quad(p<0.05)$. Bone scintigraphy showed lower activity in BV-before group than control-before group at 2 hours image $(p<0.05)$. However, significant difference between BV-before and BV-after was not observed at 2 hours and 24 hours images. Conclusion: BV had some therapeutic effect in carrageenan induced mono-arthritis. Bone scintigraphy with Tc-99m DPD had provided visual and quantitative information as an objective tool for the evaluation of response of BV in acute arthritis model and it had suggested that bee venom may have preventive effect against development of acute monoarthritic disease.

\section{J412}

\section{EVALUATION OF CARDIAC PET HEART STUDIES WITH THE PRESENCE OF TC99M ISOTOPE}

Laszlo Papp, Jasmin Sengupta, Maaz Zuhayra

Department of Nuclear Medicine, UK-SH Campus Kiel, Kiel, Germany.

Aim: In everyday nuclear medicine practice cardiac studies may include more functional modalities for the same diagnose. This situation may happen if a stress/rest cardiac SPECT is performed followed by a cardiac PET study. While SPECT images may detect dark areas in the heart referring to closed vessels after a heart attack, PET images can prove if the dark area is alive or not. In practice all of these acquisitions can be performed on the same day with a strict order. Our goal was to evaluate the possible effects of the Tc99m isotopes on the PET acquisitions by the means of quantitative measurements. Materials and methods: A Jaszczak heart phantom installed into a 6 liters cylinder phantom was used for our evaluations. Two different experiments were performed. In the first experiment $5 \mathrm{Mbq}$ F18 was administered to the wall of the heart phantom. The surrounding area was filled up with water. In the second experiment next to the $5 \mathrm{Mbq}$ F18, $24 \mathrm{Mbq} \mathrm{Tc} 99 \mathrm{~m}$ was administered to the wall as well. For both experiments 15 PET acquisitions were performed after one another to detect possible different SUV changes over time. The trues and randoms were collected. An isocount VOI was determined around the heart wall with the same isocount parameters on all reconstructed images. SUV based statistical values were gained from the VOIs for both experiments. In order to avoid specific results, both experiments were repeated two more times. Results: There were no significant differences in the trues or randoms, although 3D VOI statistics represented significant differences. In those cases when Tc99m was present as well, $6 \%+-1 \%$ of SUV value decrease occurred. This loss was increasing over time which accorded to the higher half-life of Tc99m comparing to the F18. Conclusions: Although visually there were no detectable differences between the experiment images, quantitatively SUV loss occurred when Tc99m isotope was present as well. Since this loss was increasing over time, a logical explanation is the increasing scatter ratio due to the presence of $\mathrm{Tc} 99 \mathrm{~m}$. As the next step of our research we will determine the relation between SUV loss ratio and the dose ratio between F18 and Tc99m. Additionally different scatter windows will be set for the acquisitions.

\section{$\mathbf{J 4 1 3}$}

STUDYING INDIRECT $\mathrm{CA}^{2+}$ ALTERATIONS FOLLOWING MYOCARDIAL INFARCTION IN A MURINE MODEL

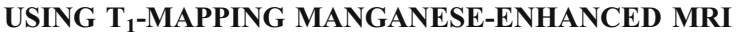
Ben Waghorn ${ }^{1,2}$, Jimei Liu ${ }^{1}$, Nathan Yanasak ${ }^{1}$, Tom C. $\mathrm{Hu}^{1,2}$

${ }^{1}$ Small Animal Imaging, Department of Radiology, Medical College of Georgia, Augusta, GA, USA, ${ }^{2}$ NRE and Medical Physics Programs, Georgia Institute of Technology, Atlanta, GA, USA.

Intracellular $\mathrm{Ca}^{2+}$ overload in the myocardium following ischemia is known to exacerbate myocardial injuries. Manganese-enhanced MRI (MEMRI) has been used to monitor $\mathrm{Ca}^{2+}$ in vivo, where $\mathrm{Mn}^{2+}$ acts as a $\mathrm{Ca}^{2+}$ analogue. Ex vivo studies observe an increase in intracellular $\mathrm{Ca}^{2+}$ following myocardial infarction, but to the best of our knowledge this increase has not been detected using MEMRI. The goal of this study is to focus on the use of MEMRI to quantify $\mathrm{Mn}^{2+}$ efflux in a mouse myocardial infarction model. $\mathrm{T}_{1}$-mapping of myocardial infarcted mice were performed on a 7.0-T 20-cm horizontal bore Bruker BioSpec MRI spectrometer (Bruker Instruments, Billerica, MA). Mice $(n=94)$ were divided into Control, Sham (open chest surgery) and myocardial 
infarction (MI, left anterior descending coronary artery ligation) groups. Each group was infused with 190 nmoles/g BW $\mathrm{MnCl}_{2}$ and $\mathrm{T}_{1}$-maps were acquired pre- and at multiple post-infusion time points between 0 $24 \mathrm{hrs}$. In-house software was used to isolate and segment the myocardium from the $T_{1}$-maps in order to provide an unbiased analysis of the regional $\Delta \mathrm{R}_{1}$ values $\left(\Delta \mathrm{R}_{1}=1 / \mathrm{T}_{1}\right.$ post $-1 / \mathrm{T}_{1}$ pre$\mathrm{MnCl}_{2}$ infusion). First-order exponential fits were applied to the in vivo efflux data. $\mathrm{Mn}^{2+}$ efflux rates in the remote, viable regions of the MI myocardium were not significantly different than the Control and Sham groups (Figure 1a). However, the infarcted site had a significantly reduced efflux half-life $(p<0.05)$. Studying the regional variations in half-life within the MI model (Figure 1b), demonstrated that there were regional variations in efflux half-life across both the infarcted and adjacent zones. $\mathrm{T}_{1}$-mapping MEMRI has been shown to be sensitive to alterations in myocardial $\mathrm{Mn}^{2+}$ efflux rates in the diseased heart. Results from this study suggest that increased $\mathrm{Mn}^{2+}$ efflux in the diseased zones may accompany an increase in $\left[\mathrm{Ca}^{2+}\right]_{\mathrm{i}}$, consistent with ex vivo studies. This technique could potentially be developed to provide in vivo assessment of $\mathrm{Ca}^{2+}$ handling alterations.
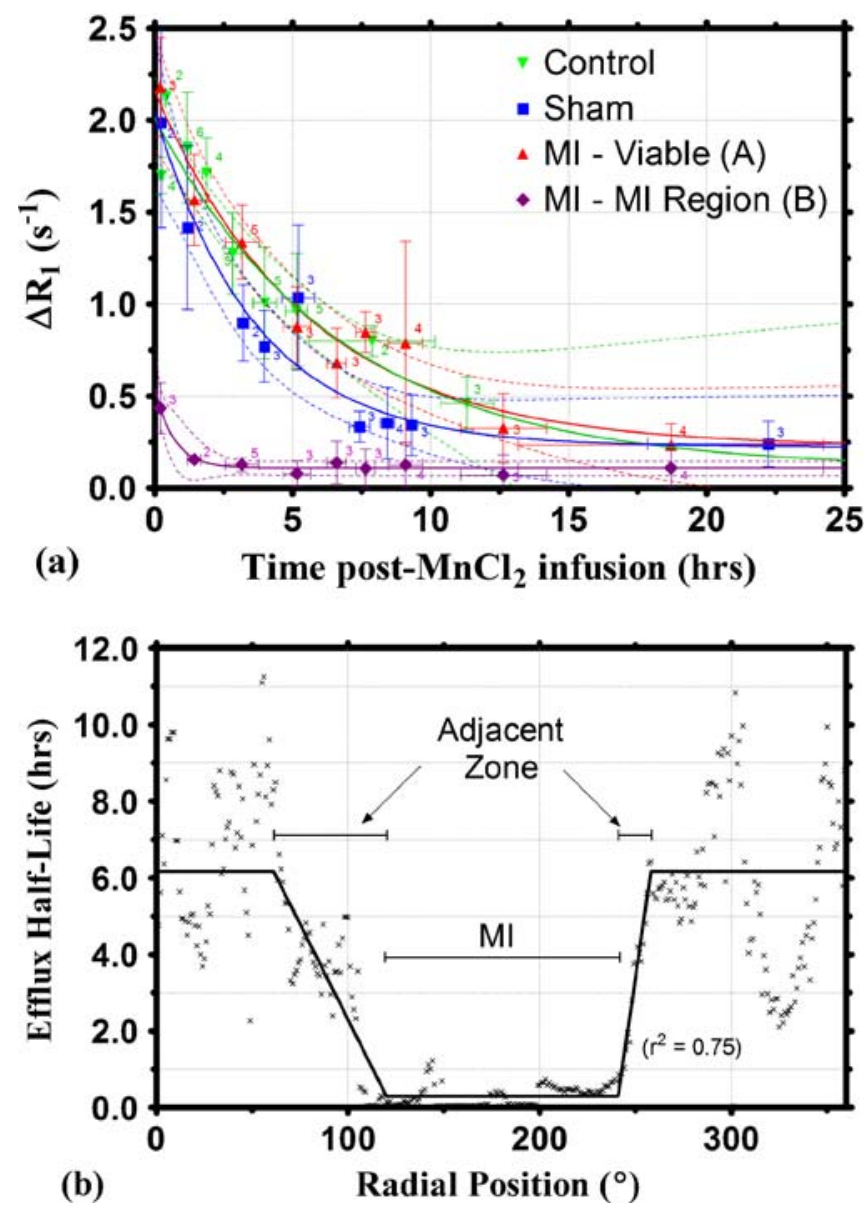

Figure 1

J414

IN VIVO IMAGING OF INFLAMMATION IN ATHEROSCLEROTIC LESIONS OF MOUSE MODELS OF ATHEROSCLEROSIS USING $\alpha_{v} \beta_{3}$ INTEGRIN BIOMARKER AND FLUORESCENCE MOLECULAR TOMOGRAPHY

Bohumil Bednar, Shu-An Lin, Brett Connolly, Michael Klimas, Cyrille Sur

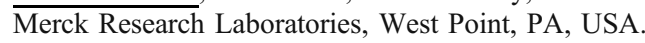

The atherosclerosis is an inflammatory disease leading to plaque formation associated with macrophage accumulation in the arterial walls and their transformation into foam cells regulated by $\alpha_{v} \beta_{3}$ integrin. Conventional imaging techniques largely assess luminal narrowing, calcification, or morphological abnormalities of atherosclerotic plaque, which have been shown to be poor indicators of vulnerable plaque and thus poor predictors of heart attack and stroke. Detection of the vulnerable plaque can be achieved by molecular imaging of $\alpha_{\mathrm{v}} \beta_{3}$ integrins expressed by macrophages. In this study, we describe noninvasive detection of inflammation associated with atherosclerotic lesions of apolipoprotein E-deficient (Apoe-/-) and human cholesteryl ester transfer protein (CETP) knockin/LDL receptor-deficient (C57BL/ 6-Tg(CETP)-Ldlrtm1) mice using newly developed optical molecular probes of $\alpha_{v} \beta_{3}$ integrin and fluorescence molecular tomography (FMT). Transgenic as well as control $\mathrm{C} 57 \mathrm{Bl} / 6$ mice were fed a cholesterol enriched diet ( $9 \%$ fat, $0.15 \%$ cholesterol) to induce atherosclerosis. The animals were injected iv with optical probes and fluorescence was measured in vivo 24 and 48 hours after the probe injection. The aortas of some animals were then excised and fluorescence of the probes measured on intact arteries ex vivo. Selected parts of the arteries were sectioned for further histology analysis. In vivo measurements detected longitudinal increase in the fluorescence in the thorax area of athero mice, which correlated well with the development of atherosclerotic lesions in the aortic arch, and carotid, and subclavian arteries detected in similar studies by anatomic imaging methods. The fluorescence of $\alpha_{v} \beta_{3}$ integrin probes associated with atherosclerotic lesions of athero mice was three to five fold higher when compared with control animals. Ex vivo imaging of the dissected vessels and histopathology confirmed association of the fluorescence with the atherosclerotic lesions. This study demonstrated that the $\alpha_{v} \beta_{3}$ integrin optical reporter can be used as a molecular imaging biomarker for detecting longitudinally inflammation in atherosclerosis. Such a biomarker can potentially serve in the preclinical testing of therapeutics and early diagnosis of atherosclerosis in patients.

\section{J415}

IMAGING OF MICROVASCULAR CHANGES IN DIABETIC RATS USING BLOOD POOL AGENT

Zdravka Medarova ${ }^{1}$, Zeynep Onder $^{1}$, Marytheresa Ifediba $^{1}$, Dale L. Greiner ${ }^{2}$, Alexei A. Bogdanov ${ }^{3}$, Anna Moore ${ }^{1}$

${ }^{1}$ Molecular Imaging Laboratory, Department of Radiology, Massachusetts General Hospital, Charlestown, MA, USA, ${ }^{2}$ Department of Medicine, University of Massachusetts Medical School, Worcester, MA, USA, ${ }^{3}$ Department of Radiology, University of Massachusetts Medical School, Worcester, MA, USA.

Type 1 diabetes develops as a result of autoimmune lymphocytic infiltration of the pancreas which progresses over many years and culminates in the destruction of insulin-producing beta-cells. This process is associated with islet microvascular dysfunction. Therefore it is important to monitor the dynamics of pancreatic microvasculature non-invasively. Here, we describe the application of the long-circulating, nanosized paramagnetic T1 contrast agent, PGC-GdDTPA-F (Protected graft copolymer bearing covalently linked gadolinium diethylenetriaminepentaacetic acid residues and labeled with fluorescein) for the non-invasive semi-quantitative evaluation of vascular changes in RT6-depleted BB-DR rats as a model of type 1 diabetes. Diabetic animals and non-diabetic controls were monitored using magnetic resonance imaging (MRI) following the I.V. injection of PGC-GdDTPA-F. MR imaging was performed on a $9.4 \mathrm{~T}$ horizontal bore scanner equipped with an RF transmit/ receive volume coil. $3 \mathrm{D}$-angiograms were obtained using a gradient echo pulse sequence with first-order flow compensation (GEFC). The following imaging parameters were utilized: $\mathrm{TE} / \mathrm{TR}=2.43$ / $40 \mathrm{~ms}, \mathrm{FOV}=38.4 \times 38.4 \mathrm{~mm}$, Spatial resolution $=0.3 \times 0.312 \times$ 
$0.3 \mathrm{~mm} /$ pixel, matrix size $=128 \times 160 \times 128$, and a total imaging time of $13 \mathrm{~min} 32 \mathrm{sec}$. The pancreas images were manually segmented and subjected to region-of-interest (ROI) analysis determination of pancreas-associated $\mathrm{T} 1$ relaxation times. We observed a significantly greater accumulation of PGC-GdDTPA-F in the pancreata of diabetic animals, as compared to non-diabetic controls at $1 \mathrm{hr}$ post-injection (Figure). No differences were seen in the blood pool, kidney, or muscle or in any of the organs at 24-hrs after injection. These results suggest that there were measurable differences in regional blood volume/regional permeability in the pancreas of diabetic vs. healthy control animals. This study was supported by 1R24DK080264 from NIH.
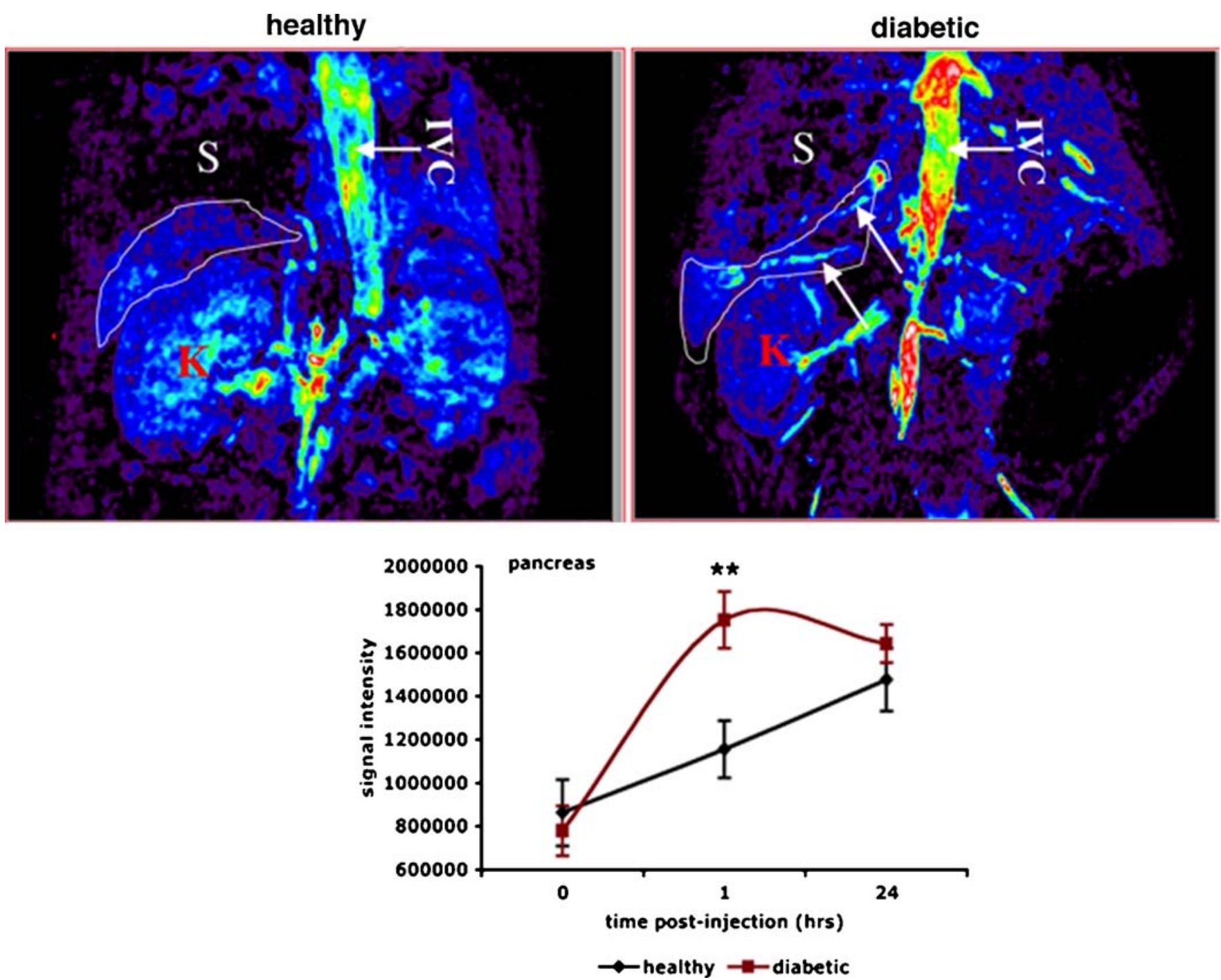

MR images at $1 \mathrm{hr}$ post PGC-Gd injection. Pancreas is outlined. S, stomach; IVC, inferior vena cava, K, kidney. Arrows point to blood vessels.

$\mathbf{J 4 1 6}$

\section{STANDARDIZED MULTIPARAMETRIC VISUAL ANALYSIS OF VESSEL PATHOLOGIES IN MOUSE MODELS OF ATHEROSCLEROSIS}

Sven Hermann ${ }^{1}$, Timo Ropinski ${ }^{2}$, Stefan Wagner $^{3}$, Klaus Kopka ${ }^{3}$, Klaus Hinrichs $^{2}$, Michael Schafers ${ }^{1}$

${ }^{1}$ European Institute of Molecular Imaging, University of Münster, Münster, Germany, ${ }^{2}$ Visualization and Computer Graphics Research Group, University of Münster, Münster, Germany, ${ }^{3}$ Department of Nuclear Medicine, University Hospital Münster, Münster, Germany.
Cardiovascular diseases, mainly caused by atherosclerosis, are the main cause of death worldwide. Major questions remain with respect to the underlying molecular pathomechanisms but also regarding the assessment of the individual risk to suffer from a cardiovascular event. Molecular imaging of the vascular system using specific biomarkers has the potential to answer these questions. To reach this goal emerging novel biomarkers and imaging technologies have to be evaluated in mouse models of human atherosclerosis in comparison to wild type mice. This is significantly hampered by the complex anatomy of the arterial tree (aorta, carotids, bracheocephalic artery etc.) and the size of the structures, even more aggravated by variations 
between subjects. Aim of this project is the multiparametric analysis of atherosclerotic lesions using a standardized and robust algorithm including normalization of the vessel architecture and comparison to a control group. Aortic arches of ApoE (high cholesterol diet) and wildtype mice were investigated using small animal-CT and -PET with F-18-FDG (inflammation), F-18-Fluoride (calcification) and F18-CGS (matrix metallo proteinases 2, 9). Before preparation of the aortic arch animals were perfused with the intravascular contrast agent Microfil ${ }^{\circledR}$. Then the samples were embedded in paraffin and measured by PET and CT. Accurate image fusion is based on three radiolabeled marker spheres (diameter: $2-3 \mathrm{~mm}$ ) which were also embedded in the paraffin block. The new analysis algorithm comprises a visual analysis of the complex vessel architecture, by considering the vessel lumen, wall thickness and intensity of radiotracer uptake in an integrated way. Because of the normalization of the vessel architecture the derived parameters in the aortic arch of each individual can be compared to a control group. Current data show pathological findings in typical segments of the normalized aortic arch in ApoE deficient mice with high cholesterol diet. The exploited techniques enable to analyze the complex arterial tree of mice multiparametrically in a standardized way, and have proven to be reliable and robust. The comparison to the control group is work in progress and will be presented. In conclusion the new integrated visual analysis approach shows promising results in first measurements. Next steps are evaluation and adaption of this analysis algorithm for in vivo studies, not only in the preclinical setup but also in the clinical scenario.

\section{$\mathbf{J 4 1 7}$}

\section{ATHEROSCLEROSIS IN APOE-DEFICIENT MICE STUDIED WITH ${ }^{18}$ F-FDG SMALL ANIMAL PET: REGIONAL DIFFERENCES AND EFFECT OF DIET}

Anne Mette F. Hag ${ }^{1,2}$, Sune F. Pedersen ${ }^{1,2}$, Rebecca Myschetzky ${ }^{1,2}$, Michelle Kaijer ${ }^{1,2}$, Andreas Kjaer ${ }^{1,2}$

${ }^{1}$ Cluster for Molecular Imaging, Faculty of Health Sciences, University of Copenhagen, Copenhagen, Denmark, ${ }^{2}$ Department of Clinical Physiology, Nuclear Medicine \& PET, Rigshospitalet, Copenhagen, Denmark.

\footnotetext{
Aim: To validate the use of ${ }^{18} \mathrm{~F}-\mathrm{FDG}$ as a tracer for imaging regional atherosclerosis in mice. Methods: Two groups of 16-weeks old ApoE-deficient $\left(\mathrm{ApoE}^{-/}\right)$mice were investigated. One group $(n=12)$ received a high-fat Western type diet (diet-group) for 8 weeks, while the other group $(n=12)$ received normal chow (control-group). Approximately $15 \mathrm{MBq}$ of ${ }^{18} \mathrm{~F}-\mathrm{FDG}$ was administered to the mice as well as $0.2 \mathrm{~mL}$ of a contrast-agent. Three hours later a PET/CT scan was performed. SU-values for the aortic arch, the thoracic aorta, and the abdominal aorta were calculated, as well as SUV for the entire aorta. Unpaired or paired t-tests were used to compare groups. Results: Comparing the aorta of the two groups, $\mathrm{SUV}_{\text {mean }}$ for the diet-group was significantly higher than in the control-group $(0.76 \pm$ 0.09 vs. $0.52 \pm 0.05, p<0.05)$. Regionally, a significant increase was found in both the aortic arch $(0.75 \pm 0.07$ vs. $0.53 \pm 0.03, p<0.05)$ and the thoracic aorta $(0.69 \pm 0.10$ vs. $0.39 \pm 0.04, p<0.05)$, while the increase in the abdominal aorta was non-significant. Within the control-group, the $\mathrm{SUV}_{\text {mean }}$ for the abdominal aorta was highest $(0.67$ $\pm 0.05)$, while the value for the thoracic aorta $(0.39 \pm 0.04)$ was lowest. The SUV-values were different (aortic arch vs. thoracic aorta, $p<0.01$, aortic arch vs. abdominal aorta, $p<0.05$, thoracic vs. abdominal aorta, $p<0.001)$. In the diet-group, there were no differences. Conclusion: ${ }^{18}$ F-FDG PET can be used to non-invasively study the regional development of atherosclerosis in mice. The pattern observed, with highest $\mathrm{SUV}_{\text {mean }}$ in the abdominal aorta, corresponds well to observations in humans, indicating that $\mathrm{ApoE}^{-/-}$mice may be a valid model for the study of atherosclerosis.
}

\section{J418}

THE SCAVENGER RECEPTOR CLASS B TYPE I-DEFICIENT,
HYPOMORPHIC APOLIPOPROTEIN E-R61 MOUSE-A NEW
TRANSGENIC MOUSE MODEL EXHIBITING DIET-INDUCED
OCCLUSIVE CORONARY ATHEROSCLEROSIS
AND MYOCARDIAL INFARCTION Michael T. Kuhlmann ${ }^{1}$, Sven Hermann ${ }^{1}$, Klaus Tiemann ${ }^{2}$, Michael Schafers ${ }^{1,3}$

${ }^{1}$ European Institute of Molecular Imaging, University of Münster, Münster, Germany, ${ }^{2}$ Department of Cardiology and Angiology, University Hospital Münster, Münster, Germany, ${ }^{3}$ Department of Nuclear Medicine, University Hospital Münster, Münster, Germany.

Common transgenic mouse models of atherosclerosis are characterized by plaque development in the aorta and its branches; but in none of these models this leads to complete coronary occlusion and myocardial infarction (MI). Thus, at present there is no suitable mouse model mimicking the clinical pathogenesis of MI. Recently, a new transgenic mouse was described, lacking the Scavenger Receptor class B type I (SR-BI K.O.) and expressing reduced levels of an ApoE4-like murine ApoE isoform (ApoE-R61(hypo)), that develops occlusive coronary atherosclerosis, MI and cardiac dysfunction under a high-fat /highcholesterol- (HFC-)diet. Aim of the present study was to further characterize this mouse strain with special focus on molecular imaging techniques. All mice were fed a normal chow diet before starting the experiments. After onset of the HFC-diet SR-BI K.O./ApoE-R61(hypo) mice died within 4 weeks. Histological analysis of the explanted hearts and the aortic arch revealed massive plaque development in the aorta and the coronary arteries, coronary occlusion, as well as tissue breakup and extensive fibrosis in the myocardium. TUNEL staining and immunohistochemistry demonstrated cell death, apoptosis (caspase-3), inflammation (MRP-14; activated macrophages) and myocardial remodelling (MMP-9). The massive plaque development in the coronary artery system was also visualized by 3D-CTimaging of explanted hearts perfused with microfil ${ }^{\circledR}$. Serially performed echocardiography documented a dramatic drop in myocardial pump function, but in F-18-FDG-PET scans only in some mice this was accompanied by focal defects in F-18-FDG-uptake. In most animals myocardial F-18-FDG-uptake was rather inhomogeneous, maybe indicating multiple micro-infarction events. In contrast, the hearts of HFC-diet fed SRBI wildtype/ApoE-R61(hypo) mice appeared histological quite normal and functional inconspicuous. Interestingly, extensive coronary plaque deposition was found also in some homozygous double-transgenic mice which continued with normal chow diet. But in those animals this led not to complete occlusion and MI. In conclusion, the present results indicate that the SR-BI K.O./ApoE-R61(hypo) mouse-strain seems to be a valuable animal model for the development of new sophisticated molecular probes for all steps in the course of atherosclerosis, from early plaque development until coronary occlusion and MI. In addition, the model has the advantage that the progression of atherosclerosis can be altered (and therefore adopted to the experimental needs) by modifying the lipid content in the diet.

\section{J419}

\section{ANALYSIS OF LIPID-BASED MR CONTRAST AGENTS TO DETECT ATHEROSCLEROTIC PLAQUES IN THE AORTIC ARCH}

Brigit den Adel $^{1,2}$, Linda M. van der Graaf ${ }^{1,2}$, Bianca Hogers ${ }^{1}$, Glenda S. van Bochove ${ }^{4}$, Marco C. DeRuiter ${ }^{1}$, Klaas Nicolay ${ }^{4}$, Robert E. Poelmann ${ }^{1,2}$, Louise van der Weerd ${ }^{1,3}$,

${ }^{1}$ Department of Anatomy and Embryology, Leiden University Medical Center, Leiden, Netherlands, ${ }^{2}$ Molecular Imaging Laboratories Leiden, Leiden University Medical Center, Leiden, Netherlands, ${ }^{3}$ Department of Radiology, Leiden University Medical Center, Leiden, Netherlands, ${ }^{4}$ Department of Biomedical Engineering, Eindhoven University, Eindhoven, Netherlands. 
Introduction: Atherosclerosis is the main cause of morbidity and mortality in Western societies; the disease is usually not identified until a clinical event occurs. Several MRI studies have shown that atherosclerosis can be successfully detected with contrast agents (CA). It remains challenging to discriminate intimal thickening and plaque burden in areas with moving structures like the aortic arch, and little is known about the time course of enhancement. In the present study we aim to determine the optimal time curve for in vivo visualization of atherosclerosis in the aortic arch of ApolipoproteinE deficient (ApoE-/-) mice, using lipid-based MR CA compared to a conventional Gd-containing CA using retrospectively gated cine MRI. Methods: Meglumine gadoterate (Dotarem $\left.{ }^{\circledR}\right)$, Gd-containing NIR664-conjugated micelles and liposomes were applied in 3 groups ( $n=5$ per group) of $10-14$ months old male ApoE-/- mice. Mice were imaged using a 9.4T vertical Bruker MRI system. The aortic arch of mice was imaged at baseline and 6-12 hour intervals for 6 days following i.v. injection of $\mathrm{CA}$, using equivalent doses of $\mathrm{Gd}(50 \mu \mathrm{mol} / \mathrm{kg})$. Using IntraGate software, retrospectively gated cine FLASH images with 10 cardiac frames were obtained from 6 slices (TR $31 \mathrm{~ms} / \mathrm{TE} 3 \mathrm{~ms}$, NA 400 , MTX $128 * 128$, FOV $18 * 18$, hermite FA $15^{\circ}$, res. $141 \mu \mathrm{m})$. Immunohistochemistry and scanning confocal microscopy were performed to correlate CA with atherosclerotis. Results: Contrast enhancement (CE) in the aortic wall was observed within $6 \mathrm{hrs}$ after Dotarem injection. Both micelle- and liposome-injected mice showed a bi-phasic $\mathrm{CE}$, with a 1 st peak in contrast-to-noise-ratio (CNR) about $12 \mathrm{hrs}$ after injection. A 2nd wave of more focal CE was observed with peak CNR around 60 72 hrs. Plasma R1 measurement showed a pattern inversely related to aortic $\mathrm{CE}$, suggestive for organ retention of micelles and liposomes, and release in the blood $\sim 1.5$ days post injection. Histology demonstrated a correlation between site of MRI CE and the presence of atherosclerotic plaques. The lipid-based CAs were found both extra- and intracellular in the plaques. Conclusions: Retrospectively gated MRI of the aortic arch allows visualization of atherosclerotic plaques in mice. Kinetics of CE indicates lipid- based CA have a complex biodistribution involving multiple organ systems. Passive uptake of $\mathrm{CA}$ in atherosclerotic plaques shows retention in several plaque components. We conclude that the optimal imaging moment may vary for different animal models and $\mathrm{CA}$, as the standard $24 \mathrm{~h}$ interval was not nearly optimal in our animal model.

\section{$\mathbf{J 4 2 0}$}

\section{DETECTION OF THROMBUS COMPOSITION AND PROPAGATION FROM SITES OF DISRUPTED ATHEROSCLEROTIC PLAQUES BY MRI}

Alkystis Phinikaridou, Kevin J. Hallock, Ye Qiao, James A. Hamilton Biophysics, Boston University School of Medicine, Boston, MA, USA.

Acute coronary syndromes (ACS) such as myocardial infarction and unstable angina are a major cause of mortality in industrialized natios. Histological studies have demonstrated that ACS are triggered by disruption of vulnerable atherosclerotic plaques which result in luminal thrombosis. However, little is known about the factors that determine thrombus composition, propagation, and size. We studied plaque disruption in a rabbit model of controlled atherothrombosis using in vivo,ex vivo MRI and histology. Examination of disrupted aortic plaques $(n=20)$ revealed that thrombi propagated both parallel and anti-parallel to blood flow. An example of a ruptured plaque with thrombus propagation antiparallel to the direction of blood flow is shown in Figure 1. We found that thrombi attached at the site of plaque rupture are platelet-rich whereas the thrombi at the propagated site become enriched in fibrin. The changes in thrombus composition were reflected in the \% magnetization transfer rate and apparent diffusion coefficient which were: $9.5 \pm 2 \%$ and $0.52 \pm 0.05 \mathrm{~mm} 2 / \mathrm{s}$ at the site of rupture and $43 \pm 1$ and $0.4 \pm 0.05 \mathrm{~mm} 2 / \mathrm{s}$ at the propagated site. Our histological studies suggest that thrombus propagation occurs over inflamed and lipid-rich plaques.

Figure 1

In vivo after triggering

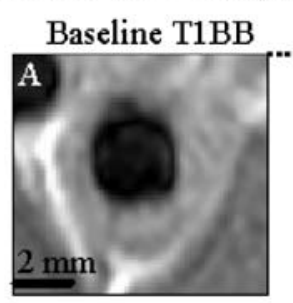

T1BB \& Gd-DTPA
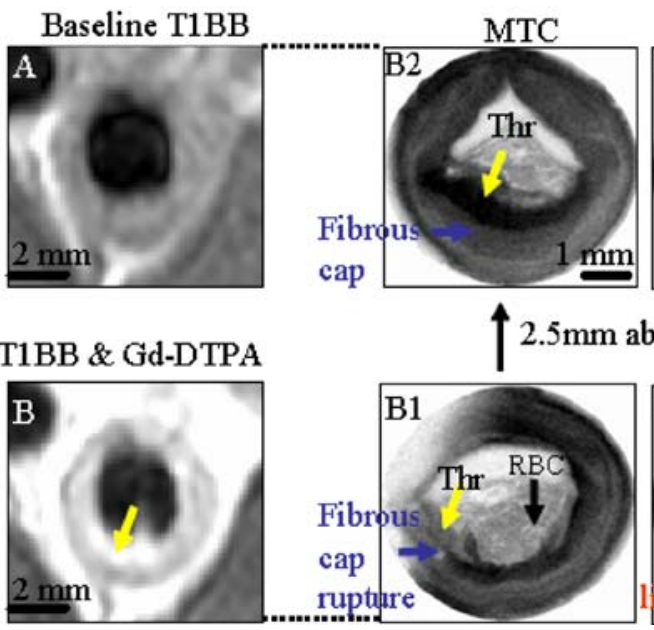

Ex vivo

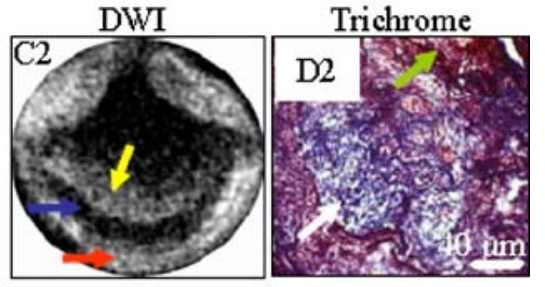

$2.5 \mathrm{~mm}$ above

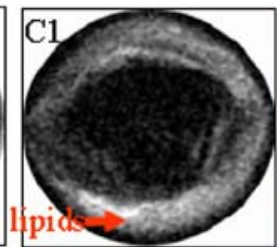




\section{J421}

THE KINETICS OF GD-DTPA UPTAKE MAY DISCRIMINATE STABLE FROM VULNERABLE ATHEROSCLEROTIC PLAQUE Alkystis Phinikaridou, Christopher Sucato, James A. Hamilton Biophysics, Boston University School of Medicine, Boston, MA, USA.

In vivo MRI has the capacity to identify atherosclerotic plaque components and features associated with symptomatic plaques. Contrast enhanced MRI (CE-MRI) using gadolinium has improved the discrimination between the fibrous cap and the lipid core and the visualization of coronary atherosclerosis. Furthermore, dynamic CE-MRI has shown that uptake of Gd-DTPA is correlated with neovascularization and inflammation, both of which are increased in vulnerable plaques. To study the uptake of Gd-DTPA in plaques at different stages, we employed in vivo MRI (3 Tesla) in a rabbit model that develops plaques that resemble most of the histological plaque classifications categorized by the American Heart Association for human plaques. We used dynamic CE-MRI to study the amount and temporal uptake of GdDTPA in a quantitative manner, which could help to optimize the timing of the contrast-enhanced imaging and differentiate stable from vulnerable atherosclerotic plaques. An example of gadolinium uptake into the vessel wall and part of the atherosclerotic plaque as well as comparison of gadolinium uptake in four different plaques are shown in Figure 1.
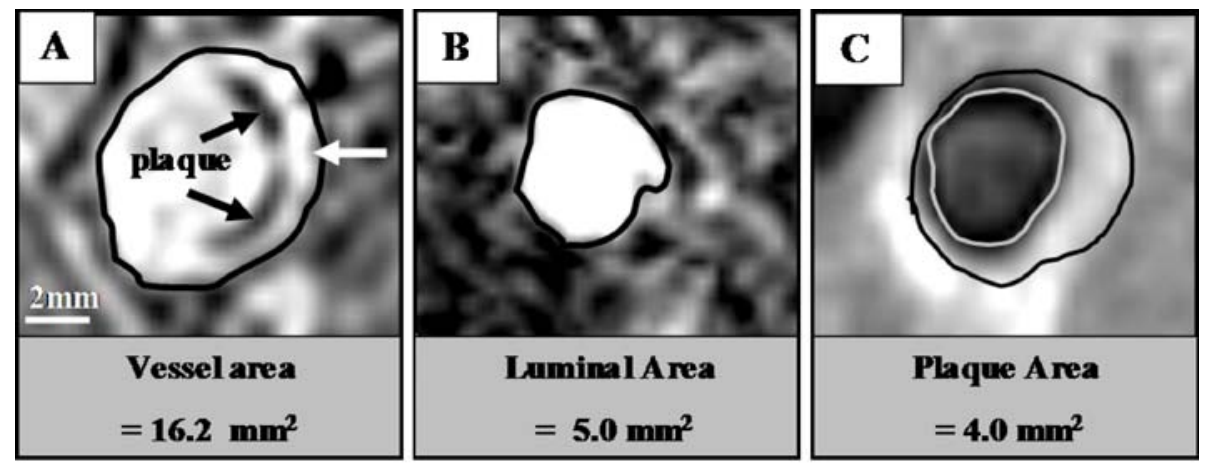

\section{Changes in the signal intensity vs. time}

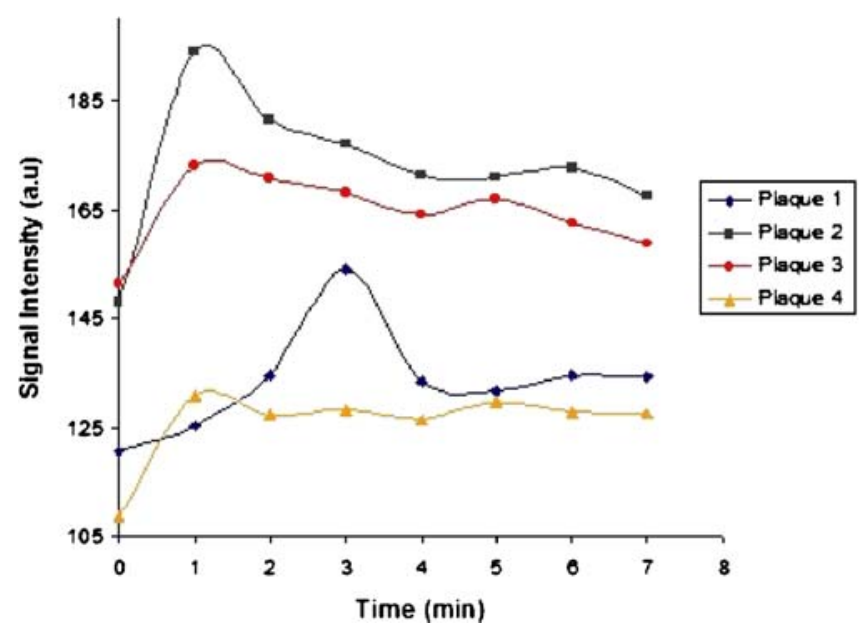

Figure 1: Contrast-enhanced images with gadolinium show leakage of the gadolinium in the adventitial and part of the plaque. (A) Contrastenhanced flow-compensated image identified the outer (adventitial) contour of the vessel wall (white arrow). Part of the plaque was also highlighted by the contrast agent whereas the other part appeared as a signal void (black arrows). (B) Flow-encoding phase-contrast image showed the unobstructed part of the lumen. (C) T1-weighted, black-blood image revealed the entire plaque area (circumscribed by the lines). (D) Examples of differential uptake of gadolinium in four different atherosclerotic plaques. 
$\mathbf{J 4 2 2}$

IMPROVED MYOCARDIAL INFARCT ASSESSMENT WITH ${ }^{13} \mathrm{~N}$-AMMONIA FLOW QUANTIFICATION IN RATS Miran Kenk, Stephanie Thorn, Jennifer M. Renaud, Ran Klein, Myra A. Kordos, Julia Lockwood, Samantha Mason, Jeffrey Collins, Marc Lamoureux, Rob S. Beanlands, Jean N. DaSilva, Robert A. deKemp Cardiac PET Centre, University of Ottawa Heart Institute, Ottawa, ON, Canada.

Objectives: ${ }^{18} \mathrm{~F}$-FDG PET is commonly used in conjunction with relative blood flow imaging to define regions with reduced flow and impaired metabolic activity, indicating myocardium that has undergone infarction (defect). We sought to evaluate defect contrast following left coronary artery (LCA) ligation in rats using small animal PET imaging of relative uptake with ${ }^{13} \mathrm{~N}$-ammonia and ${ }^{18} \mathrm{~F}$ FDG compared to absolute myocardial blood flow (MBF) with ${ }^{13} \mathrm{~N}$-ammonia. We hypothesized that absolute MBF quantification may provide a more accurate indication of defect severity. Methods: Six male Sprague-Dawley rats (200-250 g) underwent LCA ligation surgery. Following recovery, 8-11 days post-surgery, rats were injected $1.8-3.1 \mathrm{MBq}$ of ${ }^{13} \mathrm{~N}$-ammonia and scanned for $30 \mathrm{~min}$ with the Siemens InveonTM camera. After $30 \mathrm{~min}$ washout, 51.8-96.2 MBq of ${ }^{18} \mathrm{~F}-\mathrm{FDG}$ was injected and rats scanned for $60 \mathrm{~min}$. Dynamic images were reconstructed for ${ }^{13} \mathrm{~N}$-ammonia and ${ }^{18}$ F-FDG using OSEM3D/MAP $(\beta=0)$. FlowQuant $(C$ automated analysis software was used to create polar maps of the left ventricular myocardium tracer uptake at $t=2-30 \mathrm{~min}$ for ${ }^{13} \mathrm{~N}$ ammonia, $t=35-60 \mathrm{~min}$ for ${ }^{18} \mathrm{~F}-\mathrm{FDG}$, and $\mathrm{MBF}(\mathrm{mL} / \mathrm{min} / \mathrm{g})$ using a one-compartment model and the first 4 min of ${ }^{13} \mathrm{~N}$-ammonia data. A 17-segment model was applied to the polar maps and defect contrast was computed as the segment with the lowest mean compared with a normal region contra-lateral to the area of infarction. Results: Similar defect to normal zone contrast was observed for both ${ }^{13} \mathrm{~N}$-ammonia and ${ }^{18} \mathrm{~F}$-FDG relative uptake images in the rats $(0.70$ and 0.77 defect/normal ratio, respectively; $p=\mathrm{NS}$ ). Defect contrast with ${ }^{13} \mathrm{~N}$-ammonia MBF imaging (0.30 ratio) was found to be significantly higher compared to both ${ }^{13} \mathrm{~N}$-ammonia and ${ }^{18} \mathrm{~F}$-FDG uptake imaging $(p<0.01)$. In addition, ${ }^{13} \mathrm{~N}$-ammonia uptake contrast was observed to be less variable across the rat population in comparison to ${ }^{18} \mathrm{~F}-\mathrm{FDG}$ uptake contrast $(p=0.03)$. Conclusions: These results suggest that MBF quantification in rats with ${ }^{13} \mathrm{~N}$-ammonia and small animal PET imaging may be more sensitive for the assessment of infarct severity compared to relative uptake imaging.

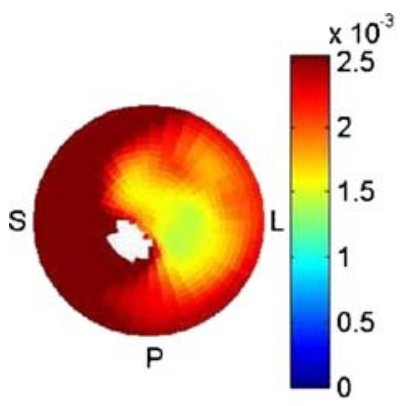

${ }^{13} \mathrm{~N}$-ammonia Uptake

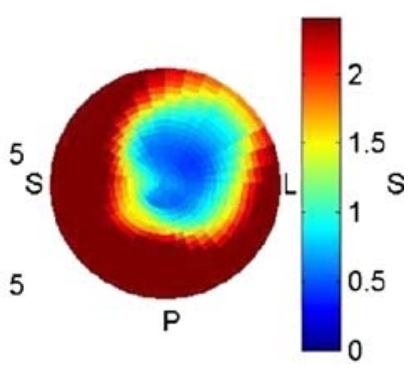

${ }^{13} \mathrm{~N}$-ammonia Flow

Representative left ventricle polar maps of ${ }^{13} \mathrm{~N}$-ammonia uptake and flow.

\section{$\mathbf{J 4 2 3}$}

\section{${ }^{11}$ C-ACETATE FOR MYOCARDIAL FLOW QUANTIFICATION IN MICE WITH SMALL ANIMAL PET IMAGING}

Jennifer M. Renaud, Marc Lamoureux, Myra A. Kordos, Samantha Mason, Jean N. DaSilva, Rob S. Beanlands, Robert A. deKemp Cardiac PET Centre, University of Ottawa Heart Institute, Ottawa, ON, Canada.

Objectives: Measurements of absolute myocardial blood flow (MBF) and relative flow homogeneity are important surrogate outcomes in cardiovascular research. Clinically, ${ }^{13} \mathrm{~N}$-ammonia is the accepted PET tracer for both relative uptake imaging and absolute MBF quantification, however ${ }^{11} \mathrm{C}$-acetate has also been proposed as a potential alternative. Neither tracer has been validated for small animal PET imaging. The purpose of this study was to compare relative tracer homogeneity and uptake rates (K1) using ${ }^{13} \mathrm{~N}$ ammonia and ${ }^{11} \mathrm{C}$-acetate, as estimates of $\mathrm{MBF}$ in mice. Methods: Five normal FVB mice were IV-injected with $30-60 \mathrm{MBq}$ of ${ }^{13} \mathrm{~N}$ ammonia and imaged for $30 \mathrm{~min}$, then scanned 15 min later with ${ }^{11} \mathrm{C}$-acetate using the same protocol. Dynamic images $(12 \times 10 \mathrm{~s}, 3 \times$ $60 \mathrm{~s}$ and $5 \times 300 \mathrm{~s}$ frames) were reconstructed using OSEM3D/MAP $(\beta=0)$. FlowQuant $\odot$ automated analysis software was used to create polar maps of the left ventricle myocardium tracer uptake at $T=$ 1.5-3 $\mathrm{min}$ and $\mathrm{K} 1(\mathrm{~mL} / \mathrm{min} / \mathrm{g})$ using a one-compartment model and the first $4 \mathrm{~min}$ of data. Results: Similar myocardium to blood contrast ratios (1.68 vs. 1.66) were observed in ${ }^{13} \mathrm{~N}$-ammonia and ${ }^{11} \mathrm{C}$-acetate images $(p=\mathrm{NS}) .{ }^{13} \mathrm{~N}$-ammonia showed better relative uptake homogeneity compared to ${ }^{11} \mathrm{C}$-acetate $(\mathrm{COV}=8.9 \%$ vs. $13 \% ; p=0.004)$, however ${ }^{11} \mathrm{C}$-acetate had almost threefold improvement in $\mathrm{K} 1$ homogeneity $(\mathrm{COV}=23 \%$ vs. $65 \% ; p=0.001)$. The ${ }^{11} \mathrm{C}$ acetate $\mathrm{K} 1$ values were approximately $50 \%$ higher than the standard ${ }^{13} \mathrm{~N}$-ammonia $\mathrm{K} 1$ values $(\mathrm{K} 1=4.09$ vs. $6.21 ; p=0.005)$. Conclusion: This pilot study demonstrates a highly uniform myocardial blood flow distribution with ${ }^{11} \mathrm{C}$-acetate, suggesting that this tracer may be more precise than ${ }^{13} \mathrm{~N}$-ammonia for mouse imaging studies of MBF. 

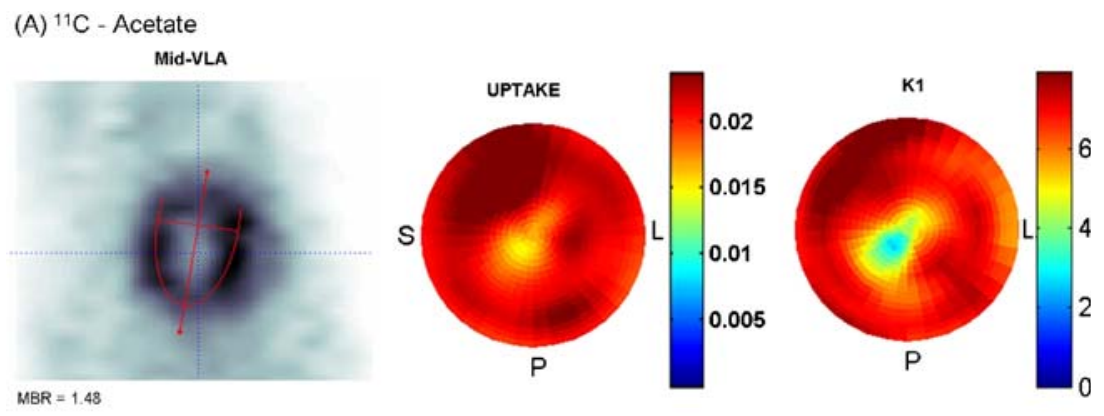

(B) ${ }^{13} \mathrm{~N}$ - Ammonia
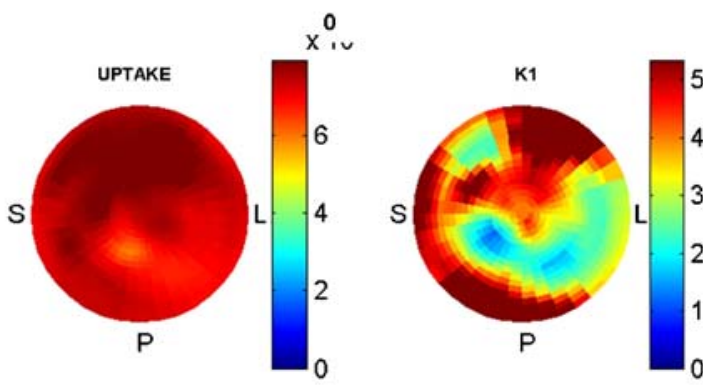

Figure 1. Comparison of the contrast in the mid-vertical long axis (mid-VLA) of the myocardium, and the uptake and $\mathrm{K} 1$ polar maps for (A) ${ }^{11} \mathrm{C}$ acetate and (B) ${ }^{13} \mathrm{~N}$-ammonia.

\section{J424}

\section{INCREASED FDG UPTAKE IN ACUTE PERI-INFARCT AREA IN POST-MYOCARDIAL INFARCTION RATS}

Stephanie Thorn, Miran Kenk, Jennifer M. Renaud, Ran Klein, Myra A. Kordos, Julia Lockwood, Samantha Mason, Jeffrey Collins, Marc Lamoureux, Rob S. Beanlands, Jean N. DaSilva, Robert A. deKemp Cardiac PET Centre, University of Ottawa Heart Institute, Ottawa, ON, Canada.

Background: Myocardial viability assessment with FDG PET provides a means to evaluate cardiac energy utilization following ischemic insult. Alterations in oxidative and anaerobic metabolism occur during the acute post-infarct phase in the myocardium. These metabolic changes are being investigated in a short-term rat myocardial infarction model in the infarct, peri-infarct and contra-lateral normal zones. Methods: Five male Sprague Dawley rats (200-250 g) underwent left coronary artery ligation. At 811 days post-surgery, rats were anaesthetized with isoflurane and scanned with FDG (51.8-81.4 MBq) for $60 \mathrm{~min}$ in a Siemens InveonTM scanner.
Dynamic images were reconstructed with $3 \times 60 \mathrm{~s}$ and $11 \times 300 \mathrm{~s}$ frames with OSEM3D/MAP $(\beta=0)$. FlowQuant $\odot$ automated analysis software was used to create polar maps in the LV myocardium of relative uptake at $t$ $=35-60 \mathrm{~min}$ for $18 \mathrm{~F}$-FDG. A 17 -segment model was applied to the polar maps, and the segment with the lowest mean FDG uptake was designated as the infarct segment. The segment value (above $75 \%$ threshold) surrounding the defect (peri-infarct) was compared with a normal region contra-lateral to the area of infarction. Results: Left coronary artery ligation resulted in a defect size encompassing more than $43 \%$ of the left ventricle. Segment mean values $(\mathrm{MBq} / \mathrm{cc})$ in the infarct zone $(0.0016 \pm$ $0.0007)$ were significantly reduced compared to the peri-infarct $(0.0054 \pm$ $0.0014)$ and normal contra-lateral zone $(0.0044 \pm 0.0013)(p<0.011$, paired t-test). Additionally, there was a significant increase in FDG uptake in the peri-infarct zone compared to the normal contra-lateral area $(p<0.003$, paired t-test). Conclusions: These results suggest that FDG uptake may be increased in the peri-infarct zone after acute myocardial infarction in rats. An increase in FDG uptake in the peri-infarct area may reflect a shift towards increased myocardial glucose metabolism or glucose uptake by inflammatory cells.

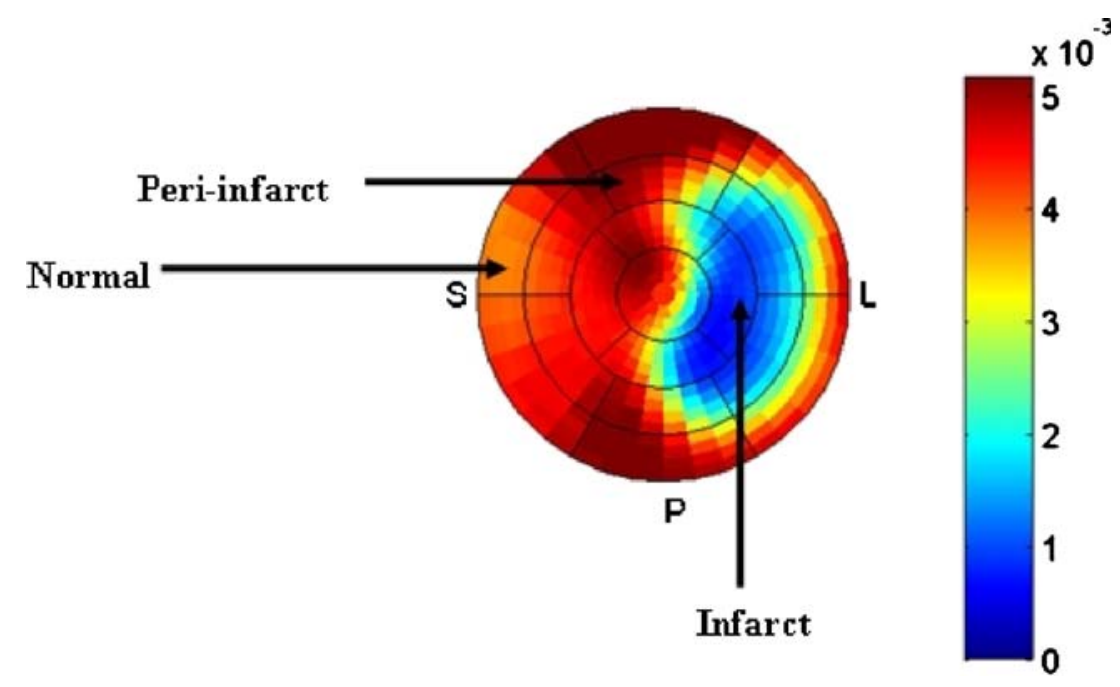


$\mathbf{J 4 2 5}$

LONGITUDINAL EVALUATION OF METABOLIC RATES FOR GLUCOSE AND FATTY ACID IN NORMAL AND SPONTANEOUSLY HYPERTENSIVE RAT HEARTS WITH DYNAMIC MICROPET AND MICROSPECT IMAGING Bryan W. Reutter, Ronald H. Huesman, Kathleen M. Brennan, Rostyslav Boutchko, Stephen M. Hanrahan, Grant T. Gullberg Radiotracer Development \& Imaging Technology, Lawrence Berkeley National Laboratory, Berkeley, CA, USA.

In the case of hypertrophy caused by pressure overload (hypertension) there is an increase in cardiac mass and modification of cardiac metabolism. This study was designed to study the changes in glucose and fatty acid metabolism in the left ventricle with the progression of hypertrophy in spontaneously hypertensive rats (SHR). Dynamic F-18-FDG PET data were acquired with the microPET II. Dynamic I-123-BMIPP pinhole SPECT data were acquired with a dual-head GE Millennium VG Hawkeye SPECT/ CT scanner equipped with custom pinhole collimators. Slow rotation of the SPECT camera necessitated modeling the radiotracer time variation in the dynamic image reconstruction algorithm, which yielded time-activity curve estimates directly from the projection data. Previous studies have shown that under normal fasting conditions, exogenous fatty acids are the preferred metabolic substrate of the heart, accounting for $70-80 \%$ of the energy production. Our results show that in the case of hypertension in the SHR model the heart relies on glycolysis as the primary pathway for energy production, whereas the normotensive (WKY) rat relies primarily on fatty acid metabolism and oxidative phosphorylation as its main source of energy. The results indicate that the metabolic rate of the glucose analog F-18-FDG decreases with age in the SHR and WKY rat but that it remains considerably higher in the hypertensive SHR model than in the normotensive WKY rat. Whereas for the metabolism of fatty acids in the heart the reverse is true: the metabolic rate of the fatty acid analog I-123-BMIPP decreases with age in both the WKY rat and the SHR model but it remains considerably higher in the WKY rat than in the SHR model. Not only does hypertension have an effect on rate-limiting enzymes for glucose and fatty acid oxidation but in the case of the SHR model the transport of long chain fatty acids across cell membranes is limited.
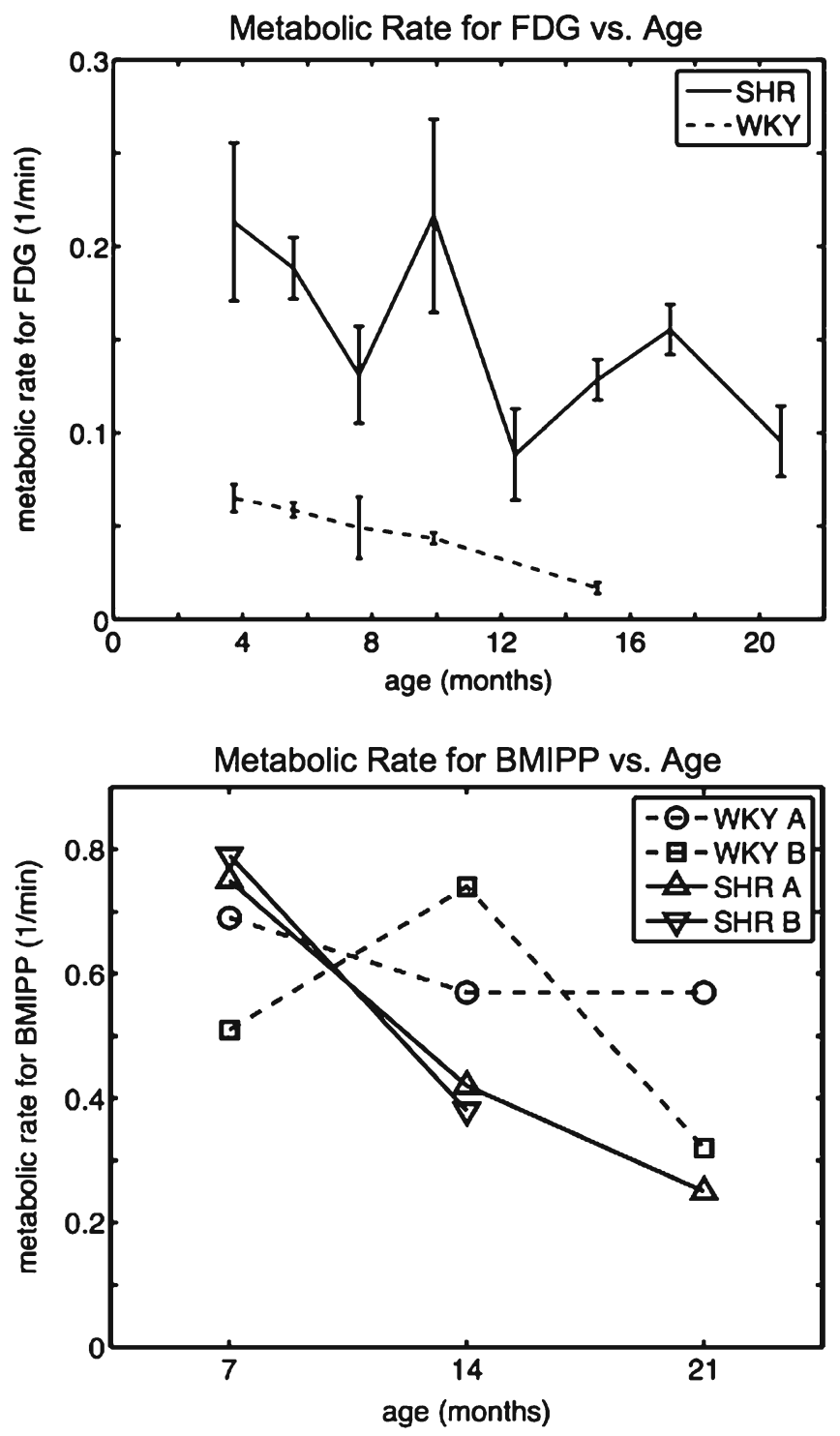

Metabolic rates for FDG vs. age (upper) and for BMIPP vs. age (lower).

$\mathbf{J 4 2 6}$

NON-INVASIVE MULTIMODALITY ASSESSMENT OF ANTIINFLAMMATORY EFFECTS OF THE PPAR-GAMMA AGONIST-PIOGLITAZONE WITH DYNAMIC CONTRAST ENHANCED MRI

Claudia Calcagno $^{1}$, Esad Vucic ${ }^{1,2}$, Steve Dickson ${ }^{1}$, James H. Rudd ${ }^{1}$, John Whitbread $^{3}$, Ronald Law ${ }^{3}$, Zahi Fayad ${ }^{1,2}$

${ }^{1}$ Radiology, Mount Sinai School of Medicine, New York, NY, USA,

${ }^{2}$ Medicine, Mount Sinai School fo Medicine, New York, NY, USA,

${ }^{3}$ Takeda Pharmaceuticals North America, Takeda Pharmaceuticals

North America, Deerfield, IL, USA. 
Introduction. Inflammation is believed to be a major contributor to plaque instability and rupture. Pioglitazone, a PPAR- $\gamma$-agonist, has been shown to delay the progression of atherosclerosis in mouse models and exhibits positive effects on survival in high risk populations for cardiovascular disease in humans. It is also known to have anti-inflammatory action, promote reverse cholesterol transport and have positive effects on lipoprotein profiles. Aim. In this study we aimed to develop sensitive non-invasive imaging techniques to assess the efficacy of anti-inflammatory therapy in atherosclerosis. We used dynamic contrast enhanced (DCE) MRI to monitor changes in inflammation in aortic plaques of atherosclerotic rabbits after pioglitazone treatment. Anatomical changes were investigated using multi-contrast MRI. Methods. 11 atherosclerotic New Zealand White rabbits underwent DCE and multicontrast (T1, T2 and PD) MRI. After baseline imaging, rabbits were divided into a control $(n=6)$ and treatment group $(n=5)$. The control group maintained a high cholesterol diet while the treatment group received $10 \mathrm{mg} / \mathrm{kg}$ pioglitazone admixed to it. Both groups were scanned again after 1 and 3 months. DCE-MRI images were analyzed by calculating the area under the signal intensity versus time curve (AUC) of the contrast agent uptake in the atherosclerotic plaque. Results. Compared to baseline, at 1 month, the treatment group showed a trend towards decreasing AUC values whereas controls showed no change. Imaging at 3 months after treatment showed a significant reduction of AUC in the treatment group compared to baseline $(p=0.045)$ and no difference in the control group $(p=0.83)$. No significant change in vessel wall area was detected by using multi-contrast MRI over the entire period in both groups. Conclusion: In this study we showed that DCE-MRI successfully detected changes in vessel wall inflammation after 3 months of treatment with pioglitazone. On the contrary vessel wall area measured with multi-contrast anatomical MRI scans showed no change upon treatment.

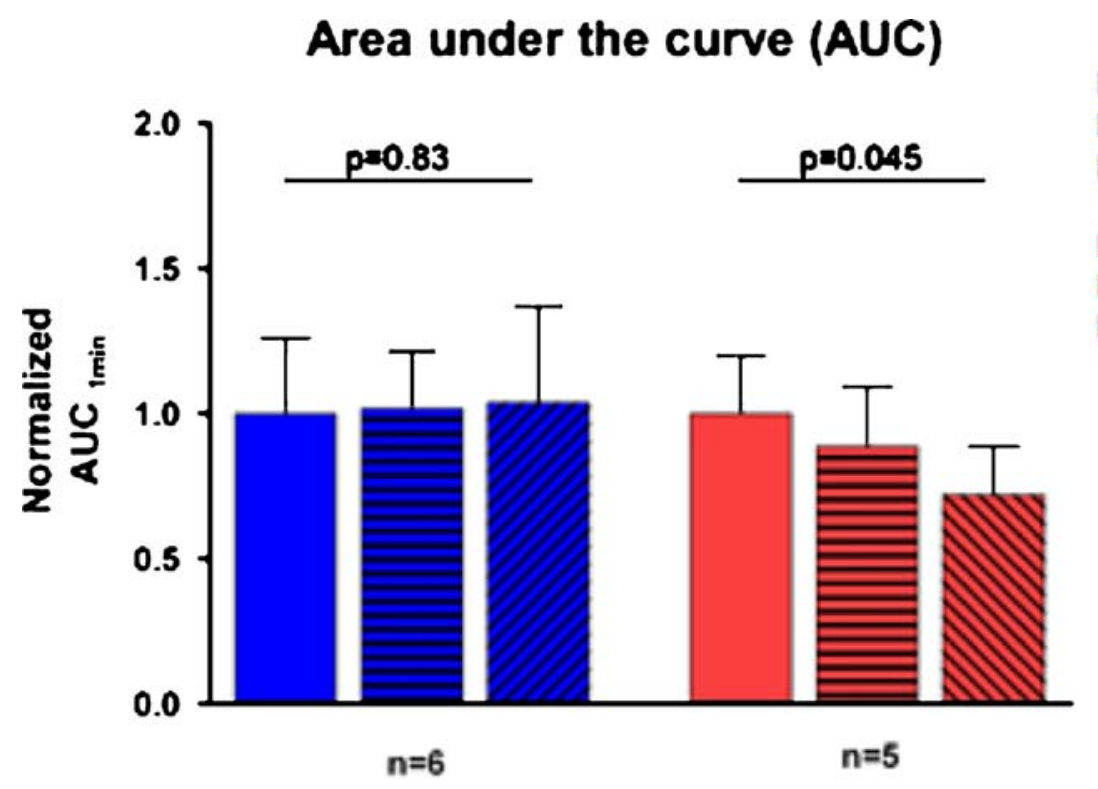

\section{Central Nervous System}

$\mathbf{J 4 2 7}$

EFFECTS OF AMYLOID PLAQUE MODULATING ANTIBODYTREATMENT IN ARCAß MICE USING NON-INVASIVE NEAR INFRARED FLUORESCENCE (NIRF)

Felicitas L. Kranz ${ }^{1}$, Bertram L. Koelsch ${ }^{1}$, Thomas Mueggler ${ }^{1}$, $\overline{\text { Karl-Heinz Altmann }}^{2}$, Roger M. Nitsch ${ }^{3}$, Markus Rudin ${ }^{1,4}$

${ }^{1}$ Institute for Biomedical Engineering, University and ETH Zurich, Zurich, Switzerland, ${ }^{2}$ Institute of Pharmaceutical Sciences, ETH Zurich, Zurich, Switzerland, ${ }^{3}$ Division of Psychiatry Research, University of Zurich, Zurich, Switzerland, ${ }^{4}$ Institute of Pharmacology and Toxicology, University of Zurich, Zurich, Switzerland.
Non-invasive assessment of amyloid plaques in brains of Alzheimer's disease (AD) patients would be of high value for diagnosis and evaluation of plaque modulating therapy. This can be achieved by specific labeling of deposits using PET tracers or fluorescent dyes. In this study we used the fluorescent dye AOI987 (Hintersteiner, 2005), which emits in the NIRF spectral domain and binds specifically to amyloid plaques in brains of different AD mouse models, to monitor the effects of amyloid reducing therapy in $\operatorname{arcA} \beta$ (tg) and wildtype (wt) mice in a longitudinal manner. Baseline images were recorded in 16 months old mice prior to treatment onset. Thereafter, the animals were divided into 4 groups, receiving weekly either antibody treatment $(\mathrm{Ab})$ or PBS: $\mathrm{A}=\operatorname{tg}, \mathrm{PBS}$; $\mathrm{B}=$ $\operatorname{tg}, 4$ weeks $\mathrm{Ab} ; \mathrm{C}=\operatorname{tg}, 8$ weeks $\mathrm{Ab} ; \mathrm{D}=$ wt,PBS. NIRF imaging was performed 4 and 8 weeks after treatment begin. Retention of AOI987 in mouse brains (180 min after dye injection) was increased 
in $\mathrm{B}$ and $\mathrm{C}$ after 4 weeks of treatment compared to A. Maintenance of treatment $(\mathrm{C})$ led to reduced fluorescent intensity as compared to the 4 week value. In contrast, high signal intensity was preserved in B, for which treatment was discontinued after 4 weeks. This observation is indicative of plaque fragmentation corresponding to increased surface area of $A \beta$ aggregates available for dye binding, prior to amyloid removal from brains of Ab-treated mice. The study demonstrates that NIRF imaging in combination with amyloid specific dyes is an attractive preclinical tool for non-invasive assessment and efficacy of therapy modulating cerebral amyloid plaque load.

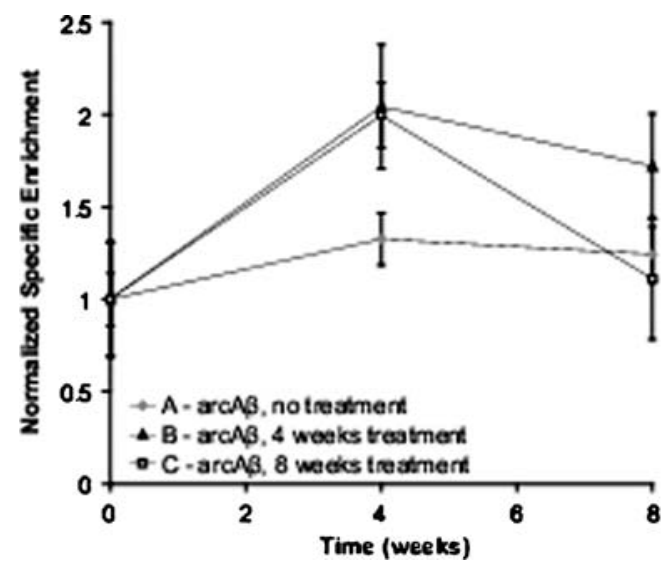

Normalized specific enrichment in tg mice relative to wt. Increase of fluorescence signal in 4 weeks Ab-treated tg $(B \& C)$ compared to nontreated tg (A). After 8 weeks decrease of signal in continuously Abtreated animals (C) opposed to constant fluorescent signal in 4 weeks Ab-treated animals (B).

\section{$\mathbf{J 4 2 8}$}

\section{NON-INVASIVE DETECTION OF AMYLOID PLAQUES} BY COMBINED MULTI-FUNCTIONAL AND MORPHOLOGICAL IMAGING IN TRANSGENIC MOUSE MODELS OF ALZHEIMERS DISEASE

Florian C. Maier $^{1}$, Detlef Stiller ${ }^{2}$, Hans F. Wehrl ${ }^{1}$, Rebecca Radde ${ }^{3}$, Gerald Reischl $^{4}$, Anke Stahlschmidt ${ }^{4}$, Daniel Bukala $^{1}$, Julia G. Mannheim ${ }^{1}$, Stefan Wiehr ${ }^{1}$, Mathias Jucker ${ }^{3}$, Claus Claussen ${ }^{5}$, Bernd J. Pichler ${ }^{1}$

${ }^{1}$ University of Tuebingen, Laboratory for Preclinical Imaging and Imaging Technology of the Werner Siemens-Foundation, Tuebingen, Germany, ${ }^{2}$ Drug Discovery Support, Boehringer Ingelheim Pharma $\mathrm{GmbH} \& \mathrm{Co}$. KG, Ingelheim, Germany, ${ }^{3}$ University of Tuebingen, Hertie Institute for Clinical Brain Research, Tuebingen, Germany, ${ }^{4}$ University of Tuebingen, Radiopharmacy, Tuebingen, Germany, ${ }^{5}$ University Hospital Tuebingen, Department of Radiology, Tuebingen, Germany.

Neuroimaging technology and the development of new animal models of neurodegenerative diseases provide powerful tools to study disease characteristics and progression. Longitudinal noninvasive detection and quantification of amyloid plaques in transgenic mouse models of Alzheimers Disease is of major interest for the evaluation of new treatment strategies directed against the formation of $A \beta$ deposits. Our aim was the characterisation of two different transgenic mouse models using [11C]PIB and Arterial Spin Labeling (ASL) concerning amyloid burden and cerebral blood flow. Transgenic APP/PS21 mice / Tg2576 mice and littermate controls were injected intravenously with $7.6 \pm 2.8 \mathrm{MBq}[11 \mathrm{C}] \mathrm{PIB}$ (specific activity $50 \mathrm{GBq} / \mu \mathrm{mol})$. Dynamic small animal PET scans were performed for $1 \mathrm{hp} . \mathrm{i}$. The mice were anesthetised with $1.5 \%$ isofluran in $100 \%$ oxygen. In addition, 3D MR images and ASL were acquired for each mouse. The PET images were analysed using cortical regions as target and the cerebellum as internal reference. The obtained time activity curves were processed with the noninvasive Logan Plot and the simplified reference tissue model (SRTM) by Lammertsma. Furthermore, the animals were analysed histopathologically. Both analysis methods revealed significant differences in the binding potential (BP) of [11C]PIB in cortical regions of transgenic APP/PS21 mice (Logan 0.28 \pm 0.10 ; SRTM $0.32 \pm 0.18$ ) in comparison to littermate controls (Logan $0.13 \pm 0.07$; SRTM $0.05 \pm 0.07, n=8, p<0.05)$. In sharp contrast, transgenic Tg2576 mice (Logan $0.05 \pm 0.01$; SRTM $0.02 \pm 0.02$ ) could not be differentiated from age matched controls (Logan $0.06 \pm 0.02$; SRTM $0.01 \pm 0.01, n=4)$ with $[11 \mathrm{C}] \mathrm{PIB}$. These results were correlated with the plaque distribution pattern assessed by histological analysis, thus confirming a specific binding of [11C]PIB. Furthermore, transgenic APP/PS21 mice showed higher rCBF-values, while transgenic Tg2576 mice displayed a decreased $\mathrm{rCBF}$ when compared to control animals. These results clearly elucidate the potential of [11C]PIB for imaging amyloid plaques in APP/PS21 mice in sharp contrast to Tg2576 mice, even in young animals (confirmed by histology). The differences obtained by ASL regarding $\mathrm{rCBF}$ furthermore show that both mouse models might be characterised by a totally different pathologic state. As both the SRTM and the Logan-Plot are known to be dependent upon differences in local perfusion of target and reference region, the assessment of rCBF with both small animal PET and MRI technology are used to confirm the obtained data and to cross validate both measurement approaches.

\section{J429}

\section{FUNCTIONAL IMAGING OF THE RAT BRAIN WITH MICRO-ULTRASOUND}

John Sun, Liis Lindvere, Adrienne Dorr, Bojana Stefanovic, F. Stuart Foster

Sunnybrook Health Sciences Center, Toronto, ON, Canada.

Linear array based Micro-Ultrasound provides 40-150 um resolution over a significant depth of field at frames rates as high as $1000 \mathrm{fps}$. Current imaging modalities for investigating in vivo brain function are challenged to provide this combination of imaging parameters. Therefore, the present experiment was carried out to investigate the potential of Micro-Ultrasound in neuroimaging of rodents. Adult male Sprague-Dawley rats were anesthetized with isoflurane. To enable high frequency ultrasound imaging of the brain, stereotaxic surgery was done to prepare a small cranial window. Non-linear Ultrasound imaging (pulse inversion and amplitude modulation) was performed using high frequency linear array (Vevo2100, VisualSonics), equipped with a $20-\mathrm{MHz}$ center frequency probe. The probe was positioned appropriately to reveal 2 regions of interest, the forelimb representation in the primary somatosensory cortex (S1FL) and primary motor cortex (M1). Ultrasound contrast agent (Micromarker, VisualSonics) at $40 \mathrm{uL} / \mathrm{min}$ and $2 \times 10^{\wedge} 9$ microbubbles $/ \mathrm{mL}$ was infused through the tail vein. When signal intensity reached a steady state, a contrast disruption pulse was delivered to assess brain reperfusion during electrical stimulation to the forelimb and $10 \% \mathrm{CO} 2$ inhalation. Average signal intensity from S1FL and M1 regions were fit to a monoexponential model to estimate the slope and plateau values of the reperfusion curves. Slope and plateau are indices of rate of flow and total blood volume 
respectively. An increase in both the initial slope and plateau of the reperfusion curve were observed in the S1FL (but not in M1) during the electrical stimulation to the forepaw $(P<0.01)$ and in both the S1FL and M1 during the CO2 inhalation $(P<0.05)$. As expected, the electrical stimulation and $\mathrm{CO} 2$ inhalation was thus found to result in a transient increase in cerebral blood flow and volume. These data demonstrate the great potential of the use for Micro-Ultrasound for functional brain imaging in animal models. The imaging parameters tradeoff afforded by nonlinear contrast Micro-Ultrasound with depth penetration sufficient to enclose the entire cerebrum-provides a unique window into the study of cerebral hemodynamics, both in the cortex and in the deep grey matter.

\section{Somatosensory Cortex Reperfusion}
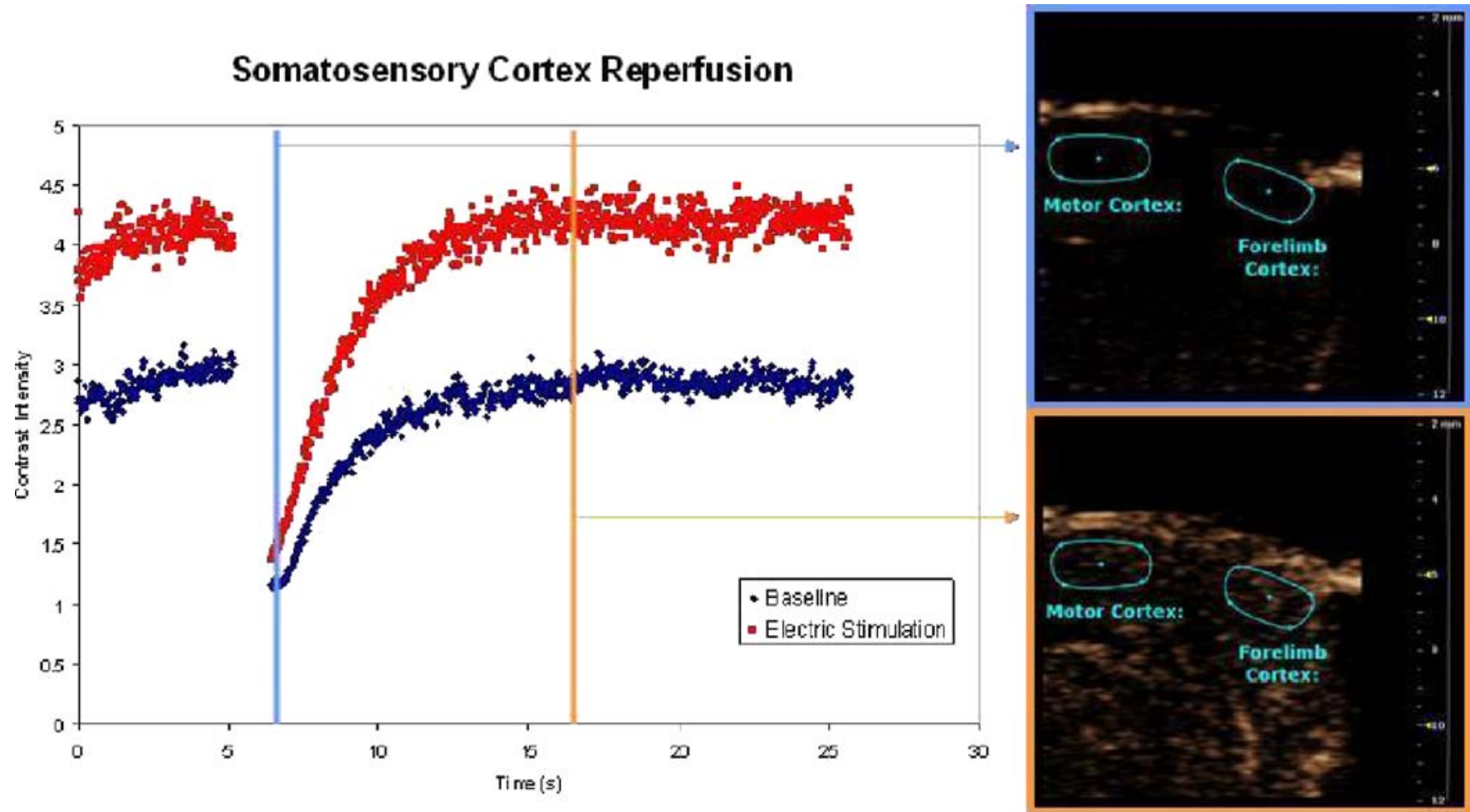

$\mathbf{J 4 3 0}$

\section{IN VIVO IMAGING OF IMMUNE CELLS INFILTRATING THE CNS OF THE mSOD1 MOUSE MODEL OF ALS} Zheng-Zheng Shi ${ }^{1}$, David R. Beers ${ }^{2}$, Shixiang Wen ${ }^{2}$, Jenny S. Henkel ${ }^{2}$ Radiology, The Methodist Hospital Research Institute, Houston, TX, USA, ${ }^{2}$ Neurology, The Methodist Hospital Research Institute, Houston, TX, USA.

Amyotrophic lateral sclerosis (ALS) is a devastating adult-onset, rapidly progressing, neurodegenerative disease that selectively destroys upper and lower motoneurons, resulting in paralysis and death typically within $3-5$ years. The ubiquitous overexpression of mSOD1 in transgenic mice induces a neurodegenerative disease similar to that seen in ALS patients. Current evidence suggests that motoneuron injury in ALS and transgenic mSOD1 mice is non-cell autonomous and involves microglia and infiltrating immune cells. Our recent data indicate that microglia play a pivotal role in disease progression in $\mathrm{mSOD} 1 \mathrm{mice}$, and CD4+ T cells have a critical function modulating microglial activation and slowing motoneuron degeneration. Importantly, we have established that transplanted GFP-expressing immune cells, including T-lymphocytes and monocytes, enter the CNS at sites of injury in mSOD1 mice. In the present study we tracked the infiltrating immune cells in vivo as they entered the spinal cord by transplanting mSOD1 mice at 80 days of age with bone marrow cells expressing luciferase under control of a promoter regulated by tetracycline. Since tetracycline does not cross the bloodbrain-barrier, this allowed us to shut off luciferase expression in the periphery, thus eliminating any inference from peripheral luciferase bioluminescence. The donor-derived luciferase-expressing immune cells were followed in mSOD1 mice using the Xenogen IVIS 200 system for in vivo bioluminescence imaging. Without tetracycline, the luminescence is widely observable (Figure 1A). With tetracycline, the luminescence is not detectable 20 days after transplant (B) but is easily observed near endstage, 65 days after transplant $(\mathrm{C})$. By identifying the infiltrating immune cells and observing CNS entry as it occurs, we will improve our understanding about CNS-immune interactions in a chronic neurodegenerative disease. In addition, this model can be used to evaluate a drug's effectiveness, as the luciferase immune cells will infiltrate the CNS only at sites of injury. 


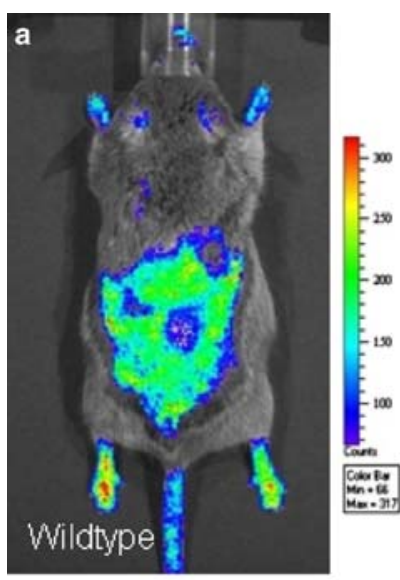

wio Tet

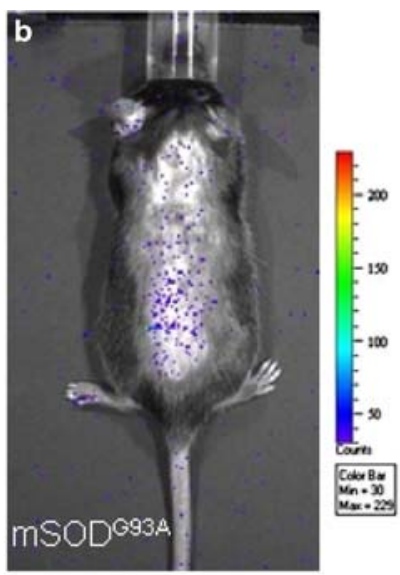

100 days $w /$ Tet

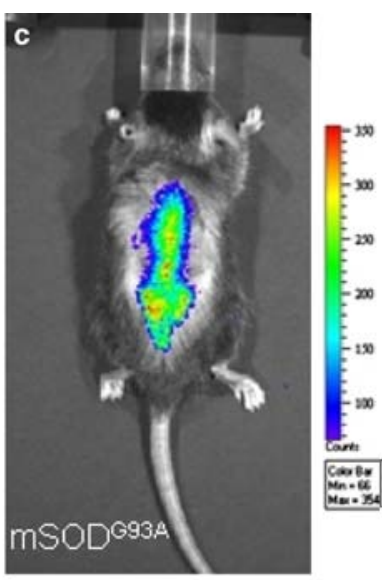

Endstage w/ Tet
$\mathbf{J} 431$

\section{MAPPING NEURO-INFLAMMATION IN EARLY STAGES OF EPILEPTOGENESIS WITH PET AND [18F]PBR111 IN THE RAT BRAIN}

Paul Callaghan, Stefanie Dedeurwaerdere, Tien Pham, Christian Loch, Andrew Katsifis, Marie-Claude Gregoire

RRI, ANSTO, Menai, NSW, Australia.

Objective: Assess the mapping of microglia activation with in vivo and in vitro techniques on the same animal in an early phase of an epilepsy Kainic Acid (KA) rat model. Methods: Two groups of 4 rats were investigated. Rats of the first group (KA) were injected with repeated low dose KA treatment (total dose $10-15 \mathrm{mg} / \mathrm{kg}$ ), with status epilepticus reversed after 4 hours with diazepam $(4 \mathrm{mg} / \mathrm{kg})$, while rats of the second group (control) were injected with saline. PET/CT was performed after 7 days. Rats were injected with a low mass $(0.1 \mathrm{~nm}+/-$ $0.08)$ of $[18 \mathrm{~F}] \mathrm{PBR} 111$ and imaged for 50 minutes. Arterial blood samples were collected during the scan, and parent compound arterial concentration was derived in order to assess the total volume of distribution (Vt) in 20 Regions-Of-Interest. After the scan, the rats were sacrificed. 125I-CLINDE autoradiography and OX42 immunohistochemistry were performed on consecutive sections in vitro to determine relative PBR density and assess microglial activation. Results: KA group showed an average increase in $\mathrm{Vt}(216 \%)$ compared to control group. Amygdala, hippocampus and frontal cortex average increases were respectively 488, 264 and $263 \%$. Significant increases in PBR density were observed in vitro in regions including hippocampus (Dentate gyrus 264\%, CA1 mid 710\%; CA2 mid 630\%, CA3 Mid $1011 \%$, caudal $602 \%$; ventral subiculum $767 \%$ ), piriform cortex $(695 \%)$ and basolateral/medial amygdala $(622 \%)$, in each case strongly associated with OX42 signal. In vivo mild increases in hippocampal and cortical ROIs correlate with anatomical heterogeneity in PBR density and OX42 labelling increases seen in vitro within these structures, and was confirmed by inspection of Vt parametric volumes. Conclusion: This study confirms that brain regions important in the generation of seizures in the KA model of status epilepticus display significant microglial activation and relative PBR density increases. Moreover, in vivo imaging with [18F]PBR111 showed a very good correlation with in vitro data. These results show that it will be possible to follow-up the patterns of activated microglia during the whole epileptogenesis phase with this radioligand.

\section{$\mathbf{J 4 3 2}$}

\section{EFFECTS OF LONG-TERM ADDICTION OF COCAINE} ON RAT DOPAMINERGIC AND SEROTONERGIC SYSTEMS ASSAYS BY MULTIMODALITIES OF MOLECULAR IMAGING Tong-Hsien Chow ${ }^{1}$, Wei-Chan $\mathrm{Lin}^{1,2}$, Chia-Hui Chu ${ }^{1}$, Chi-Wei Chang ${ }^{1,3}$, Ren-Shyan Liu ${ }^{3}$, Shyh-Jen Wang ${ }^{3}$, Victor F. Pang ${ }^{4}$, Jyh-Fei Liao, Chen Chang ${ }^{6}$, Jeng-Jong Hwang ${ }^{1}$

${ }^{1}$ Department of Biomedical Imaging and Radiological Sciences, National Yang-Ming University, Taipei, Taiwan, ${ }^{2}$ Department of Radiology, Taipei Veterans General Hospital, Taipei, Taiwan, ${ }^{3}$ Department of Nuclear Medicine, Taipei Veterans General Hospital, Taipei, Taiwan, ${ }^{4}$ Department of Veterinary Medicine, National Taiwan University, Taipei, Taiwan, ${ }^{5}$ Department of Pharmacology, National Yang-Ming University, Taipei, Taiwan, ${ }^{6}$ Institute of Biomedical Sciences, Academia Sinica, Taipei, Taiwan.

Dopaminergic and serotonergic systems are associated with high susceptibility to cocaine abuse. We aimed to investigate the influence of both systems of rat brain by long-term (three doses per week for four months) addiction of cocaine via simulating human chronic psychostimulant abusers. Animal locomotion was observed to estimate the external behavior expression. The neuronal imaging of [11C]raclopride microPET and [123I]ADAM (2-((2-((dimethylamino)methyl)phenyl) thio)-5-iodophenylamine) gamma scintigraphy were applied for specific binding assays (striatum/midbrain) in dopamine D2 receptor and serotonin transporter after cocaine treatment, respectively. MicroMRI and immunohistochemistry (IHC) were carried out to assess the functional and anatomical changes of rat brain. Animal behavioral assay showed that the maximal locomotion was observed in the first 10 weeks and gradually recovered to the baseline at the 11th week after 33 doses of cocaine injections. MicroPET brain images displayed the decrease of $[11 \mathrm{C}]$ raclopride uptake at $\mathrm{D} 2$ receptors in the striatum and midbrain during and after withdrawal of cocaine injection. [123I] ADAM gamma images showed the similar results up to the 8th week 
and followed by recovery during the 12 th to 24 th weeks. Though microMRI indicated that no imaging evidence of brain hemorrhage or edema were found even two months after cessation of cocaine treatment, however, IHC examination revealed serious neuronal damage presented by the decrease in tyrosine hydroxylase $(\mathrm{TH})$-like
IHC positive expression and the increase in glial cell level in critical brain regions, which were the sequela of the cocaine addiction. Thus, cocaine produces a predominance of dopamine over serotonin, which is associated with hyper-locomotion, D2 receptor impairment and neuron deficit.
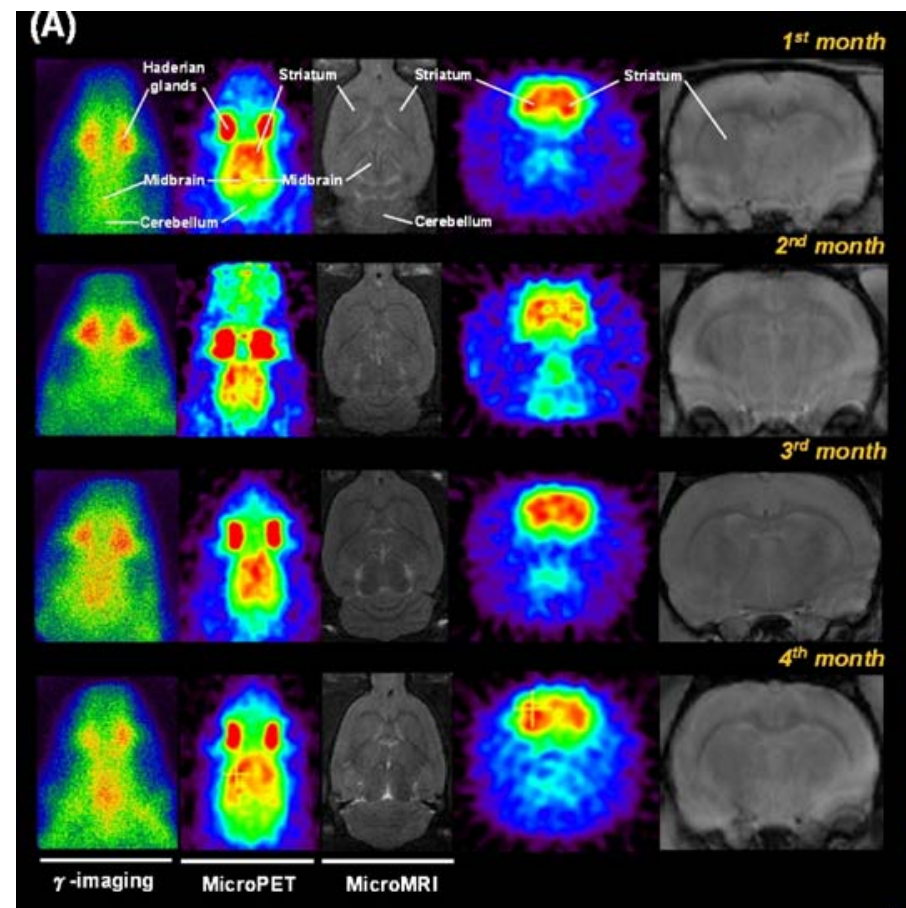

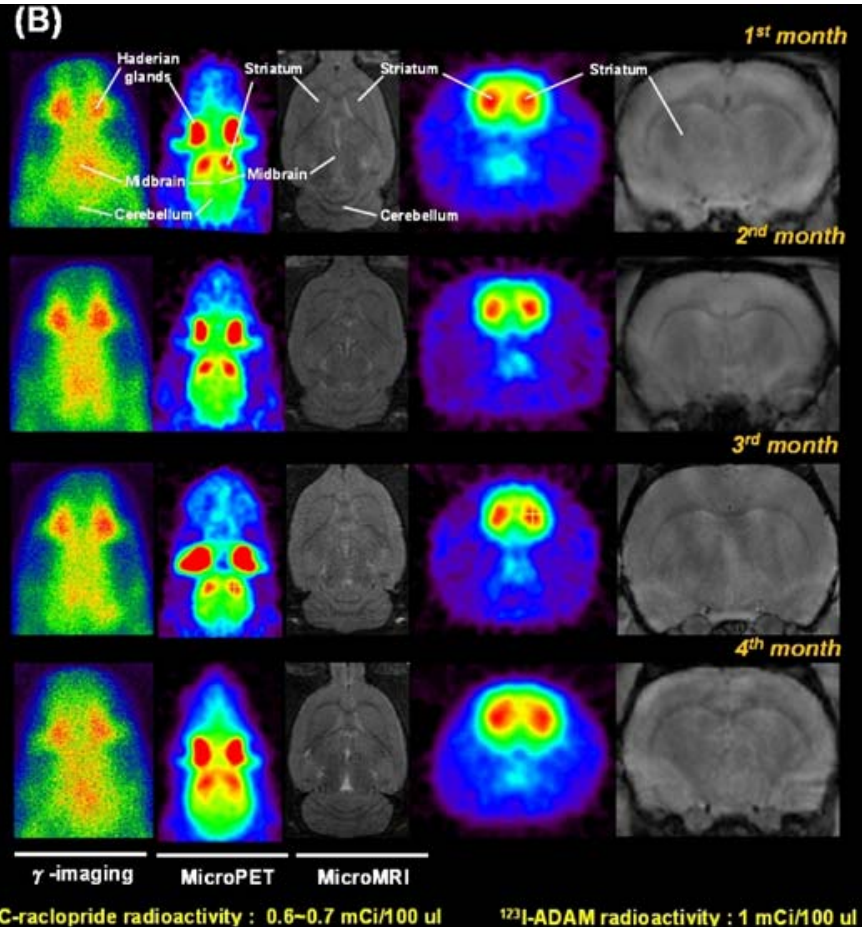

Figure 1. Multimodalities of neuronal imaging: gamma scintigraphy (column 1); microPET (columns 2 and 4) and microMRI (columns 3 and 5) of rat brains were shown after (A) cocaine (20 mg/kg) and (B) normal saline administration for four months. Images were obtained from the same rat in (A) and (B), respectively.

$\mathbf{J 4 3 3}$

VOLUMETRIC MRI AND MRS PROVIDE SENSITIVE MEASURES OF ALZHEIMER'S DISEASE NEUROPATHOLOGY IN INDUCIBLE TAU TRANSGENIC MICE (RTG4510)

Dewen Yang ${ }^{1}$, Zhiyong Xie ${ }^{1}$, Diane Stephenson ${ }^{2}$, Carol Hicks ${ }^{2}$, Daniel Morton $^{3}$, Tracy Brown ${ }^{2}$, Renuka Sriram ${ }^{1}$, Sharon $\mathrm{O}^{\prime} \mathrm{Neill}{ }^{2}$, Thomas Bocan ${ }^{1}$

${ }^{1}$ BioImaging Center of Emphasis, Global Reaserch \& Development, Pfizer Inc, Groton, CT, USA, ${ }^{2}$ CNS Research Unit, Global Reaserch \& Development, Pfizer Inc, Groton, CT, USA, ${ }^{3}$ Toxicologic Pathology, Global Reaserch \& Development, Pfizer Inc, Groton, CT, USA.

Objective: Determine if in vivo high resolution 3D MRI and localized $1 \mathrm{H}$ MR spectroscopy (MRS) can detect CNS findings resembling Alzheimer's disease in rTg4510 mice that overexpress human phosphorylated tau protein. Methods: Seven double transgenic rTg4510 female mice and 7 wild type (wt) female mice $(22-31 \mathrm{~g})$ were evaluated at 5 months of age. After an initial $5-10 \mathrm{~min} 2.5 \%$ isoflurane induction period, all mice were anesthetized and maintained with 1.6-2.0\% isoflurane in oxygen air delivered via a mouth piece. MRI/S were performed using a
4.7T magnet (Bruker Biospec 47/40). A 72-mm volume coil was used as the RF transmitter; a mouse brain quadrature surface coil was used as the receiver. T2-weighted 3D RARE (field of view $=16 \times 16 \times 19.2 \mathrm{~mm} 3$, spatial resolution $=125 \times 125 \times 300 \mu \mathrm{m} 3, \mathrm{TR}=2600 \mathrm{~ms}, \mathrm{TE}=23 \mathrm{~ms}$, RARE factor $=$ 16 , total imaging time $=44 \mathrm{~m} 22 \mathrm{~s}$ ) scans were performed. Following morphological imaging, a rectangular volume of interest $(7.8 \times 2.6 \times$ $2.6 \mathrm{~mm} 3$ ) was positioned in the hippocampal and upper thalamus areas for a localized 1H MRS. To acquire the spectrum, a PRESS sequence with the following parameters was used: $\mathrm{TR}=2500 \mathrm{~ms}, \mathrm{TE}=11.4 \mathrm{~ms}$, VAPOR water suppression and 256 averages, with $10 \mathrm{~m} 40$ s acquisition time. Resultant spectra were analyzed using LCModel (Provencher, 2001) and students t-test was used for statistical evaluation of the differnecs in the animal groups. Results: Mean hippocampal and whole brain volumes in transgenic mice were $27 \%$ and $15 \%$ smaller than wt controls $(p<0.0001)$, respectively. The mean volumes of the cerebellums were not statistically different between the two groups $(p=0.21)$. Ventricular dilation was visible in 4 of $7 \mathrm{rTg} 4510$ mice. The myo-inositol to total creatine ratio $(\mathrm{Ins} / \mathrm{Cr})$ was significantly higher in hippocampus of $\mathrm{rTg} 4510$ mice relative to wt mice $(p=0.0345)$. The $\mathrm{N}$-acetylaspartate to total creatine ratio (NAA/Cr) and glutamate to total creatine ratio $(\mathrm{Glu} / \mathrm{Cr})$ were less in $\mathrm{rTg} 4510$ mice; however, the differences were not statistically significant. All MRI/S findings were confirmed by 
histology and immunohistochemistry which demonstrated ventricular dilation, hippocampal neuronal loss, hyperphosphorylated tau deposition and glial activation. Conclusion: In vivo MRI/S is a sensitive, non-invasive tool to detect brain atrophy and related biochemical changes in $\mathrm{rTg} 4510$ mice and provides a translational tool to model Alzheimer's disease progression and assess drug efficacy.

\section{$\mathbf{J 4 3 4}$}

\section{SEX DIFFERENCES IN OFF MEDICATION SCHIZOPHRENIC SUBJECTS USING PET AND [F-18]FALLYPRIDE}

Patrizia Riccardi

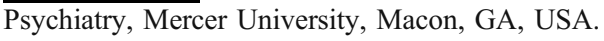

Post mortem and imaging studies of the brain in schizophrenic subjects have reported abnormal dopaminergic neurotransmission in striatum, extrastriatal and cortical regions which has been related to positive symptoms and cognitive impairments. Previous imaging studies of extrastriatal Dopamine D2/3 receptors in schizophrenic subjects have reported lower thalamic bp than healthy controls. Despite a considerable literature concerning the role of dopamine in schizophrenia few studies have evaluated sex differences in D2/ 3r. [18F]fallypride PET studies were performed in off medication schizophrenic patients and healthy controls to examine sex differences in striatal and extrastriatal DA D2r. Method: Schizophrenic patients $(n=11$, $6 \mathrm{M}$, age $30.5 \pm 8.0$ years) and age and sex matched healthy controls underwent a medical history, physical and neurological examination, and brain MRI and PET scans. Regions of interest (ROI) were identified on thin section T1 weighted MRI images, and automatically transferred to the coregistered PET images. ROI included the caudate, putamen, ventral striatum, medial thalamus, posterior thalamus, substantia nigra, amygdala, temporal cortex, anterior cingulate, and hippocampus. Exploratory voxelwise analysis also was performed using SPM2. Result: ROI analysis found sex differences in the left thalamus with males having a higher bp $(4.49 \pm$ $0.45)$ than females $(3.79 \pm 0.41 ; \mathrm{p}=0.03)$. The difference for the mean of the right + left medial thalamus also was higher in males $(4.49 \pm 0.42)$ than in females $(3.83 \pm 0.48 ; p=0.04)$. When subjects were examined as a whole, we found a decreased $\mathrm{bp}$ in the left medial thalamus in patients compared to controls but the finding was due to the females. The mean in male patients (4.49) was not different from the mean in male controls (4.55), but the mean for female patients (3.79) is significantly lower and was responsible for the difference between the group as a whole. Parametric analysis showed two significant clusters of female $>$ male bp which are significant at a cluster level at a $p<0.0001$. The clusters involve the globus pallidus extending into the ventral pallidum and slightly into the lateral thalamus. Discussion: This is the first time that a sex difference in thalamic D2/3r is reported in schizophrenic subjects. Several studies have reported a decrease bp in the left medial thalamus between schizophrenic and healthy subjects. Our results show that the decreased D2/3r in schizophrenic subjects is determined by significant differences in female subjects.

\section{$\mathbf{J 4 3 5}$}

\section{AUTORADIOGRAPHIC DETECTION} OF NEUROINFLAMMATION ASSOCIATED WITH DIASCHISIS AFTER FOCAL CEREBRAL ISCHEMIA IN RATS

Nicolas Arlicot ${ }^{1}$, Simon Roussel ${ }^{2}$, Edwige Petit ${ }^{2}$, Andrew Katsifis ${ }^{3}$, Jerome Toutain ${ }^{2}$, Denis Guilloteau ${ }^{1}$, Myriam Bernaudin ${ }^{2}$, Sylvie Chalon ${ }^{1}$

${ }^{1}$ Equipe 3 "Neurotransmission: de l'imagerie moléculaire à la clinique", UMR Inserm U930 - CNRS ERL3106 - University of Tours - CHRU, Tours, France, ${ }^{2}$ CERVOxy group "Hypoxia and cerebrovascular physiopathology", CI-NAPS, UMR 6232, University of Caen, University of Paris Descartes, CNRS, CEA, CYCERON, Caen, France, ${ }^{3}$ Radiopharmaceuticals Research Institute, ANSTO, Sydney, NSW, Australia.
Ischemic stroke is a leading cause of death and disability in the increasingly ageing population of developed countries. Neuroinflammatory response is involved in the pathophysiology of stroke and affects brain areas not directly concerned by the blood supply decrease. We aimed to evaluate and quantify, in these regions, microglial activation, a major feature of neuroinflammation, regarding the intensity of induced ischemia in a rat model of stroke using CLINDE, a promising radioligand for the Translocator Protein (TSPO). Transient focal ischemia was produced in 17 adult male Sprague-Dawley rats by intraluminal Middle Cerebral Artery Occlusion (MCAO) during $30(n=$ $8)$ or $90 \min (n=9)$ followed by reperfusion. Cerebral T2 MRI was performed at 20 days post-occlusion (dpo) for measurement of infarct volume. Ex vivo autoradiographic studies were undertaken on ischemic rats by i.v. injection of $\left[{ }^{125} \mathrm{I}\right]$ CLINDE (3 MBq in saline) at $21 \mathrm{dpo}$. Brains were removed $30 \mathrm{~min}$ after tracer injection, frozen, and coronal sections (20 $\mu \mathrm{m}$-thickness) were exposed. Autoradiograms were scanned and $\left[{ }^{125} \mathrm{I}\right]$ CLINDE uptake was quantified with an objective and relevant method based on volume and intensity of gray levels using $\beta$ vision + software. Near-adjacent slices were also used for immunohistochemical staining of activated microglia using OX42 as primary antibody. Infarcted volumes evaluated by MRI varied from 17 to $345 \mathrm{~mm}^{3}$ (30 min MCAO group: $95 \pm 22 \mathrm{~mm}^{3}$; 90 min MCAO group: $\left.158 \pm 43 \mathrm{~mm}^{3}\right)$. We obtained a good spatial correspondence between ischemic areas and significant increase of $\left[{ }^{125} \mathrm{I}\right]$ CLINDE binding $(p<$ 0.01 ), which also overlapped with OX42+ cells. $\left[{ }^{125}\right.$ I]CLINDE uptake in both ipsilateral thalamus and substantia nigra pars reticulata $(\mathrm{SNr})$ was significantly higher $(p<0.01)$ than that in intact contralateral tissue and colocalised with activated microglia. The intensity of the $\left[{ }^{125} \mathrm{I}\right]$ CLINDE binding in thalamus was positively correlated $\left(\mathrm{r}^{2}=0.88\right)$ to the ischemic volume, whereas no correlation was found in $\mathrm{SNr}$. We evidenced neuroinflammation associated with ipsilateral diaschisis produced by focal ischemia in both thalamus, reflecting retrograde neurodegeneration correlated with the severity of cortical lesion, and, for the first time using molecular imaging modalities, in $\mathrm{SNr}$, assuming an anterograde process due to the lesion in the striatum. Functional in vivo imaging using potent TSPO radioligands like CLINDE is expected to expand the understanding of neuroinflammation in stroke and guide therapeutic innovation. This work is supported in part by the EC-FP6project DiMI, LSHB-CT-2005-512146.

\section{$\mathbf{J 4 3 6}$}

\section{COMPARISON OF PIB DISTRIBUTION ON PET WITH BETA-AMYLOID DEPOSITS AT AUTOPSY}

Val Lowe ${ }^{1}$, Joseph E. Parisi ${ }^{2}$, Clifford R. Jack ${ }^{1}$, Kejal Kantarci ${ }^{1}$, Matthew L. Senjem ${ }^{4}$, Stephen Weigand ${ }^{4}$, Brad Kemp $^{1}$, Ronald C. Petersen ${ }^{3}$ ${ }^{1}$ Radiology, Mayo Clinic, Rochester, MN, USA, ${ }^{2}$ Pathology, Mayo Clinic, Rochester, MN, USA, ${ }^{3}$ Neurology, Mayo Clinic, Rochester, MN, USA, ${ }^{4}$ Biostatistics, Mayo Clinic, Rochester, MN, USA.

Background: A comparison of the distribution of PiB accumulation as seen on PET with the distribution of amyloid seen on autopsy is of great interest in understanding $\mathrm{PiB}$ binding in vivo. Very limited autopsy/PiB correlative data exists in the literature. In this report we describe the findings of PiB scans as compared to autopsy in two male subjects with the clinical diagnosis of non-amnestic mild cognitive impairment (naMCI) and Alzheimer's disease (AD). Methods: Two male subjects who underwent antemortem PiB PET scans subsequently came to autopsy. One patient had the diagnosis of $\mathrm{AD}(\mathrm{CDR}$ lobal $=2)$ and the other of non-amnestic, multi-domain MCI (naMCI) (CDRglobal=0.5). They expired 11 and 17 months after their PiB scans respectively. The quantitative distribution of PIB on PET imaging was compared in the frontal (Fr), parietal (Par), temporal (Tem), occipital (Occ) and hippocampal (Hip) regions to the distribution diffuse plaques, cored plaques and vascular amyloid, evaluated with beta- 
amyloid immunohistochemistry (Novacastra, NCL-B-amyloid; clone 6F/3D) at autopsy. Results: PiB scans showed increased PiB binding in the patient with $\mathrm{AD}$ (Global $\mathrm{PiB}$ cerebellar ratio=2.8) but not in the patient with naMCI (global $\mathrm{PiB}$ cerebellar ratio=1.4). Regional $\mathrm{PiB}$ cerebellar ratios were 2.8 and $1.4 ; 2.9$ and $1.4 ; 2.4$ and $1.2 ; 2.1$ and 1.3; and 1.6 and 1.2 in Fr, Par, Tem, Occ, and Hip regions in the AD and naMCI subjects respectively. At autopsy, the AD patient showed frequent numbers of diffuse and cored amyloid plaques in neocortical areas (Fr, Par, Tem and Occ (calcarine)), moderate numbers in medial temporal (Hip) area and absent deposits in cerebellum. The degree of amyloid angiopathy varied from absent (Tem and Par) to patchy mild leptomeningeal $>$ cortical (calcarine) to moderate (Fr and cerebellum), with mild to moderate severity. In the patient with naMCI, there was a conspicuous absence of amyloid deposits (diffuse and compact plaques) and amyloid angiopathy in all areas sampled. Conclusions: These results are consistent with the expected finding that $\mathrm{PiB}$ accumulation occurs in patients with $\mathrm{AD}$. The findings are also consistent with the idea that patients with naMCI may not have $\mathrm{AD}$ as the pathological substrate, but rather have prodromal, non- $\mathrm{AD}$ dementia. $\mathrm{PiB}$ distribution on PET is most closely associated with microscopic amyloid deposits at autopsy. Grant support: National Institute on Aging [P50 AG16574, U01 AG06786, and R01 AG11378]; the Robert H. and Clarice Smith and Abigail Van Buren Alzheimer s Disease Research Program.

\section{$\mathbf{J 4 3 7}$}

\section{RELATIVE INTEREST OF CT SCANNER IMAGING OVER OPTICAL FLUORESCENCE MICROSCOPY FOR PRECLINICAL RESEARCH ON GLIOBLASTOMA}

$\underline{\text { Franck Debarbieux }}^{1,2}$, Fabio Stanchi $^{2}$, Alain Bonissent ${ }^{1}$, Charles Hemmer $^{1}$, Christophe Meessen ${ }^{1}$, Geneviève Rougon ${ }^{2}$, Pierre Delpierre ${ }^{1}$, Christian Morel $^{1}$

${ }^{1}$ CPPM, CNRS-in2p3-Université de la méditerranée, Marseille Cedex 09, France, ${ }^{2}$ IBDML, CNRS-Université de la méditerranée, Marseille Cedex 09, France.

We have previously shown that PIXSCAN, our X-ray micro-CT prototype based on the XPAD2 photon counting detector was useful to monitor the development of tumors at low irradiation dose in a murine model of lung cancer (WMIC2008). In order to challenge PIXSCAN in conditions where contrast was less favorable for tumor detection, we have investigated the possibility to monitor brain tumor development in the context of an orthotopic glioblastoma xenograft model. Although endogenous X-ray contrast between pathological and normal tissue was too low to detect millimetric glioblastoma, more than $75 \%$ of tumors $(n=9)$ could be successfully revealed by intravenous injection of iodinated contrast agent $(200 \mu$ IOMERON 350). Patterns and properties of tumor vessels obviously favored focal accumulation of iodine and resulted in $\sim 60 \mathrm{HU}$ extra absorption inside the tumor versus healthy brain. This imaging protocol was then used to test whether an anti-angiogenic treatment already used for colorectal and lung cancers (Avastin) would also reduce the tumor burden in glioblastoma. Avastin was delivered to two groups of mice at two different stages of the disease, namely 2 or 4 weeks after tumor implantation: during 3 weeks mice received twice a week intravenous injection of Avastin diluted in PBS $(10 \mathrm{mg} / \mathrm{kg})$ or the same volume of PBS alone. Tomographic reconstructions of their brain were performed at the end of the treatment protocol in the presence of iodinated contrast agent. In control groups, tumors could be detected in most of the mice $(80 \%, n=5$ and $75 \%, n=4)$ whereas these percentages significantly decreased to $40 \%(n=5)$ and $25 \%(n=8)$ for the groups receiving late and early Avastin treatment, respectively. These results could either support a significant tumor size reduction by Avastin or an Avastininduced change in vessel permeability preventing iodine accumulation hence imaging sensitivity. To decide between these two alternatives we used additional imaging modalities. As implanted glioblastoma cells had been transfected with a GFP expressing vector, we performed on the same animals optical epifluorescence imaging and in vivo two-photon microscopy in addition to the CT scan. Similar millimetric green tumors were observed in both treated and non treated mice suggesting that Avastin mainly modulates vessels' permeability. The sum of information given by these 3 complementary techniques are instrumental for a better knowledge of glioblastoma physiopathology and their treatment.

\section{$\mathbf{J 4 3 8}$}

\section{MR DETECTION OF MACROPHAGE USING SPIO AFTER TRANSIENT CERVICAL NERVE ROOT COMPRESSION IN THE RAT}

Daniel L. Thorek, Christine L. Weisshaar, Beth A. Winkelstein, Andrew Tsourkas

Bioengineering, University of Pennsylvania, Philadelphia, PA, USA.

Transient compression of the cervical spinal nerve roots, as can occur during motor vehicle and sports injuries, can produce cervical radicular pain. This potentially disabling neuropathological condition presents with pain radiating down the arm and can severely impact the quality of life. The economic and societal costs associated with this syndrome are staggering. Diagnosis and management of patients with back pain is often hindered because it is not possible to correlate any radiological findings with the presence or extent of pain symptoms. Transient loading of the $\mathrm{C} 7$ nerve root in a rat model has been shown to lead to inflammation and macrophage infiltration into the affected tissue associated with persistent pain in rat models. Long-circulating superparamagnetic iron oxide (SPIO) nanoparticles are known to localize to sites of inflammation, through accumulation in activated macrophage. We have shown that systemically delivered dextran-coated SPIO nanoparticles ( $30 \mathrm{~nm}$ diameter) can be used to identify these sites of neuroinflammation. Male Holtzmann rats underwent a $\mathrm{C} 6 / \mathrm{C} 7$ hemilaminectomy and facetectomy on the right side to expose the dorsal roots at $\mathrm{C} 7$. Rats in the injured group $(n=6)$ underwent a 15 minute transient compression of the $\mathrm{C} 7$ dorsal nerve root by application of a 10 gmf microvascular clip. Animals in the sham group $(n=4)$ received no compression. After a 6 day postoperative period, the C6-C7 spinal levels of all rats were $\mathrm{T} 2 *-\mathrm{w}$ imaged with a surface coil and $9.4 \mathrm{~T}$ magnet. Hypointense signal regions corresponding to the level of the injured nerve root were detected 1 day following the tail vein injection of $10 \mathrm{mg} / \mathrm{kg}$ of iron; 7 days after the surgery. Specifically, the normalized signal in the ipsilateral nerve roots was $59.29 \%$ lower than the normalized signal in the uninjured contralateral nerve roots $(p<$ $0.05)$. No significant difference in normalized signal was observed between sides in pre-contrast images. Further, no significant difference in normalized signal was observed post-contrast between the ipsalateral and contralatal nerve roots in the sham animals not undergoing transient compression. The presence of accumulated SPIO localized to activated macrophage was confirmed by histology. This work may provide the basis for the use of SPIO in a clinical setting to enable radiological detection of nerve root injury.

\section{J439}

MR IMAGING STUDY OF MULTIPLE SCLEROSIS MODEL USING ULTRASMALL SUPERPARAMAGNETIC NANOPARTICLES OF IRON OXIDE COMBINED WITH MAGNETIC TRANSFER IMAGING Jingjing Lu ${ }^{1}$, Zhengyu Jin ${ }^{1}$, Fang Wang ${ }^{1}$, Yan $\mathrm{Xu}^{2}$

${ }^{\mathrm{R}}$ Radiology, Peking Union Medical College Hospital, Beijing, China, ${ }^{2}$ Neurology, Peking Union Medical College Hospital, Beijing, China. 
Experimental allergic encephalomyelitis(EAE) is an ideal model of human multiple sclerosis. In the study, EAE models were induced in female Lewis rats. Local institutional review committee on animal care approved the experiments, which were performed according to the guidelines of the committee on animal research at our institution. Before and after onset of symptoms, plain MRI, Gd-DTPA enhanced imaging, USPIO enhanced imaging, and magnetic transfer imaging were performed in the experimental $\operatorname{rats}(n=11)$ and control $\operatorname{rats}(n=10)$. The sensitivity of USPIO and Gd-DTPA enhancement in detecting the lesions in EAE rats was calculated. Magnetic transfer rate(MTR) of USPIO enhanced area before and after symptoms' onset in EAE rats were respectively calculated. HE, myelin, and Prussian blue staining of the rats' brain section were performed after imaging. There were no abnormally enhanced lesions shown in Gd-DTPA enhanced scan, while abnormally USPIO enhanced lesions were shown in EAE rats' brain. The MTR values of USPIO enhanced area before symptom onset were significantly different from these at the same areas after symptom onset $(27.5 \pm 1.6$ vs $25.3 \pm 2.2, P<0.05)$. Inflammation cells and demyelinating lesions were found in USPIO enhanced area histopathologically. There were no positive findings in control rats. In conclusion, the sensitivity of USPIO enhanced scan in detecting EAE lesions was much higher than Gd-DTPA enhanced imaging. MT imaging combined with USPIO enhanced scan, was helpful to determine the pathological base of the EAE lesions.

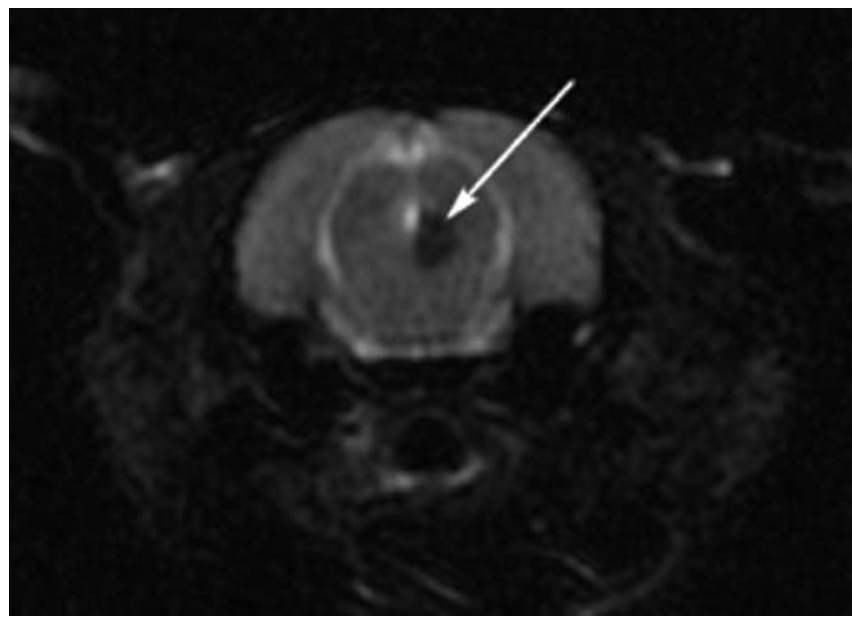

Iron oxide nanoparticles- enhanced MR T2 weighted image of the EAE rat.

\section{J440}

MRI OF ACUTE AND RELAPSING EXPERIMENTAL ALLERGIC ENCEPHALOMYELITIS: CORRELATION WITH PATHOLOGY Jingjing Lu ${ }^{1}$, Zhengyu Jin ${ }^{1}$, Fang Wang ${ }^{1}$, Yan $\mathrm{Xu}^{2}$

${ }^{\mathrm{T}}$ Radiology, Peking Union Medical College Hospital, Beijing, China, ${ }^{2}$ Neurology, Peking Union Medical College Hospital, Beijing, China.

Experimental allergic encephalomyelitis(EAE) is an ideal model of human multiple sclerosis. In the study, acute and relapsing EAE model was induced by intradermal inoculation with guinea pig CNS homogenate in 12 female Lewis rats. Another 12 rats served as control. The clinical presentation and body weight of the animals were recorded daily. Routine MRI, Gd- enhanced MRI were performed when EAE animals showed the initial symptoms. Ultrasmall superparamagnetic iron oxide(USPIO) colloid solution was also administrated intravenously and MRI was performed again after $24 \mathrm{~h}$. The brain was removed instantly after USPIO-enhanced imaging. The pathological exams including HE staining, myelin sheath staining and Prussian staining were performed. The imaging findings were observed in correlation with pathological results. The acute EAE rats showed decrease of body weight on the 6th to 7 th day after inoculation, and the clinical symptoms appeared on the 10th to 11 th day after inoculation. While the relapsing EAE rats showed second decrease of body weight and symptoms on the 25th to 27th days. Routine MRI did not show any definite abnormalities. The Gd- enhanced MRI found the diffuse thickening and enhancement of brain meninges. In acute EAE, the USPIO- enhanced MRI showed areas of low signal intensity at white matter of medulla oblongata on T2WI,gradient $\mathrm{T} 2 * \mathrm{WI}$ found more foci of low signal intensity in cerebellar white matter besides the lesions in the brain stem. In relapsing EAE, the USPIOs were mainly deposited in basal ganglia together with in brain stem. The range of abnormal signal intensity was larger in animal with higher clinical scores than that with lower score. There were no abnormal findings in control animal. The pathological exam found "perivasular cuff" in the brain white matter in EAE animals, some accompanied with adjacent demyelination. The Prussian staining found blue particles within the cytoplasm of the macrophages around the lesion, which corresponded to the area of low signal intensity on T2WI. In conclusion USPIO- enhanced MRI could reveal acute and relapsing EAE lesions which were not capable of being shown on routine MRI and Gd- enhanced MRI. It can image the macrophages around the lesions in vivo. USPIO is important for future research and application in MS patients.

J441

NO EVIDENCE OF LEVODOPA OR DOPAMINE AGONIST TREATMENT INFLUENCE ON THE PROBABILITY OF DEVELOPING DYSKINESIA IN A 6-OHDA MODEL OF PD Vesna Sossi, Katie Dinelle, Siobhan E. McCormick, Rick Kornelsen, Raul de la Fuente-Fernandez

UBC, Vancouver, BC, Canada.

Background. There is ongoing controversy on the effect of levodopa and dopamine (DA) agonist treatment on the risk of developing motor complications in the management of Parkinson's disease (PD). Previous studies in human and animal models of PD have identified disease severity and abnormal dopamine release patterns as two important contributors to the development of dyskinesia. In particular abnormal dopamine release patterns have been shown to precede development of motor fluctuations. In the present study we have used Positron Emission Tomography imaging and the unilateral 6-hydroxydopamine (6-OHDA) rat model of PD to investigate (i) if pramipexole, a D2/D3 agonist and levodopa differentially alter central DA kinetic patterns (ii) if the two treatments affect behavioral outcomes measured with the Assesment of Involuntary Movement(AIM) scores differently and (iii) if lesion-induced changes in central DA kinetics contribute to the propensity of developing motor complications independently of dopaminergic denervation (DD) severity. Methods: 27 unilaterally 6OHDA lesioned rats underwent a 11C-dihydrotetrabenazine (DTBZ, a VMAT2 marker), and two 11C-raclopride (RAC, D2-like receptor antagonist) scans (baseline and 45 after I.P. administration of levodopa/ benserazide $(50 \mathrm{mg} / \mathrm{kg}, 15 \mathrm{mg} / \mathrm{kg}$ prepared) to investigate levodopaderived changes in synaptic DA levels $(\Delta(\mathrm{DA}))$. After being assigned to three treatment groups (saline $N=7)$, pramipexole $(N=11)$ and levodopa $(N=9)$ and undergoing treatment for approximately 4 weeks, they underwent an identical scanning protocol to compare imaging outcomes, followed by AIM assessment. DD as estimated from the pretreatment DTBZ scan ranged between $17 \%$ and $98 \%$. Results. ANOVA analysis showed no treatment effect for $\Delta(\mathrm{DA})(p=0.83)$, and no significant change after treatment $(p=0.7)$. ANCOVA analysis likewise showed no treatment effect on the severity of AIM assessment ( $p=$ 0.31 ), identifying however a significant dependence of AIM on the 
pretreatment value of $\Delta(\mathrm{DA})$ and $\mathrm{DD}(p=0.003$ and $p=0.007$ respectively). Conclusion. These data show that (i) neither treatment has an effect on levodopa-derived changes in synaptic DA levels or AIM scored, thus neither treatment is likely to change the risk to develop motor complications and (ii) both lesion severity and lesion induced changes in $\Delta(\mathrm{DA})$ contribute to the probability of developing motor complications independently of disease severity thus confirming the important role of lesion (disease) induced alteration of central DA kinetics in motor complications.

\section{J442}

\section{IMAGING PSYCHOSTIMULANT-INDUCED ALTERED BRAIN GENE ACTIVITIES IN VIVO}

Christina Liu, Jia-Qian Ren, Philip K. Liu

Radiology, Massachusetts General Hospital, Charlestown, MA, USA.

Introduction: Altered gene activities are underlying causes of many neurological disorders including drug addiction. Most techniques in detecting gene expression at gene transcription level require in vitro processing of postmortem or biopsy samples, with limited longitudinal applicability. Over the past years, we have reported a novel magnetic resonance imaging (MRI) platform for live brain imaging of gene activities and its utility in the neuroscience research (Liu et al, J Neurosci 2007, 2009, Liu et al. Molecular Imaging 2007, and Liu et al. FASEB 2007, 2008). The goal of this study was to apply this technique to detect psychostimulant-induced gene activation associated with drug exposure in the living mouse brains. Methods: MRI probes in which an iron-based T2 contrast agent (SPION) was conjugated to phosphorothioate-modified oligodeoxynucleotides (sODN) with sequence complementary to intracellular targets of interest were used. The targets were mRNA of the immediate early genes (IEGs) for the transcription factors (Fos) or their product activator protein (AP-1) whose inductions are accepted markers of neural activation. MRI R2* maps were obtained from live mice to assess localized enhanced probe retention due to elevated mRNA or AP-1 level after amphetamine (AMPH) stimulation. Results: We observed regional enhancement in the R2* maps of live mouse brain images encompassing the dopaminergic pathway and its projections after AMPH in animals that received probes targeting Fos mRNA or AP-1, not of randomized sequence. Such enhancement was ameliorated by pretreatment of D1/D5 receptor antagonist SCH23390. Conclusion: The ability to detect, visualize, and report endogenous gene transcription using advanced imaging technology in live subjects holds great promise in facilitating the discovery and evaluation of potential therapies or interventions for drug addiction. (NS045845 \& NS057556 to PKL, DA024235 \& DA026108 to CHL, P41RR14075)

\section{J443}

\section{LOWER TEMPORAL AND FRONTAL METABOLISM IN POSTMENOPAUSAL WOMEN TAKING PROGESTERONE- PLUS-ESTROGEN COMPARED TO WOMEN TAKING UNOPPOSED ESTROGEN}

Cheri L. Geist ${ }^{1}$, Daniel H. Silverman ${ }^{1}$, Heather A. Kenna ${ }^{2}$, Natalie L. Rasgon ${ }^{2}$

${ }^{1}$ Department of Molecular and Medical Pharmacology, UCLA School of Medicine, Los Angeles, CA, USA, ${ }^{2}$ Department of Psychiatry and Behavioral Sciences, Stanford University School of Medicine, Stanford, CA, USA.

Background: Previous studies have offered mixed support with respect to a potential neuroprotective role for estrogen; even less has been established concerning differential cerebral metabolic effects of unopposed estrogen versus progesterone-plus-estrogen regimens. Methods: 53 postmenopausal women at risk for Alzheimer's Disease and receiving estrogen therapy opposed $(n=$ $35)$ or unopposed $(n=18)$ by progesterone were recruited. All subjects underwent FDG-PET; data were independently analyzed by statistical parametric mapping (SPM) and standardized volume of interest (sVOI) methods of analysis. The latter measured activity values within 240 standardized regions of interest that were clustered into 47 volumes. Results: Unopposed estrogen users were similar to estrogen-plus-progesterone users in years of education ( $\mathrm{av} \pm \mathrm{SD} ; 16 \pm 1.5$ vs $16 \pm 2.2)$, age at menarche $(13 \pm 1.8$, $13 \pm 1.5)$, age at recruitment $(58 \pm 5.6,58 \pm 4.4)$, and length of HT use $(10 \pm 5.7,9 \pm 5.7)$ respectively. They differed in age at menopause $(42 \pm 8.0,49 \pm 3.7)$-with the younger age of the unopposed estrogen group reflecting the prevalence of surgically induced menopause among some of those women- and therefore years of endogenous estrogen exposure. Analyses with SPM showed that postmenopausal women taking progesterone-plusestrogen had lower metabolism than postmenopausal women taking unopposed estrogen within right mesial temporal and left inferior lateral temporal regions $(t=4.51$ and $t=4.64$, respectively, at voxels of peak significance, $p<0.0005)$. The sVOI analyses corroborated SPM analyses, demonstrating that progesterone-plus-estrogen users had lower mean metabolism in corresponding temporal regions than unopposed estrogen users; $\mathrm{rMT} \mathrm{F}=0.001, p=0.004$, and liLT $\mathrm{F}=$ $4.660, p=0.036$ after correction for years of endogenous estrogen exposure. SPM analyses also demonstrated that women taking progesterone-plus-estrogen had lower metabolism than women taking unopposed estrogen in the right inferior frontal cortex $(t=$ $3.65, p<0.0005)$. The sVOI analyses corroborated SPM analyses, with progesterone-plus-estrogen users having lower mean metabolism than unopposed estrogen users in the right posterior inferior frontal gyrus $(\mathrm{av} \pm \mathrm{SD} 1.14 \pm 0.03$ vs $1.16 \pm 0.03, t=2.29, p=0.026)$. In the other direction, unopposed estrogen users did not have any areas demonstrating significantly lower metabolism after correction, than progesterone-plus-estrogen users. Conclusion: Postmenopausal women whose hormone therapy regimens included progesterone had lower metabolism in frontal and temporal brain regions than those taking unopposed estrogen.

\section{J444}

\section{REGIONAL RED BLOOD CELL MASS DETERMINATION IN THE RAT BRAIN BY QUANTITATIVE MMP-SPECT TECHNOLOGY}

$\underline{\text { Domokos Mathe }}^{1}$, István Portöro ${ }^{2}$, Gyozo A. Janoki ${ }^{4}$, László G. Puskás ${ }^{5}$,

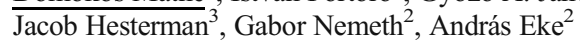

${ }^{1}$ Nat.Inst.Radbiol.Radhyg., Budapest, Hungary, ${ }^{2}$ Human Physiology and Clinical Experimental Medicine, Fact. Medicine Semmelweis University, Budapest, Hungary, ${ }^{3}$ InviCRO, LLC, Boston, MA, USA, ${ }^{4}$ Medi-Radiopharma Ltd, Érd, Hungary, ${ }^{5}$ Avidin Ltd, Szeged, Hungary.

Objectives: Regional oxygen supply by red blood cells (RBCs) within the regional cerebral blood volume (rCBV) has a major impact on tissue functions in the brain. It could be used as a biomarker for tumor and peritumoral tissue oxygenation and inflammatory reactions. Our aim was - by using a small animal imaging system with its proprietary Multiplexed-Multipinhole Collimation for in vivo quantitative SPECT- to develop a method for imaging RBC mass in the brain. As a test, $\mathrm{rCBV}$ was decreased by increasing the vascular tone via the $\mathrm{NO}$ / cGMP pathway by L-NAME. Methods: Male Wistar rats $(n=4)$ were anesthetized by a 1:1 mixture of Ketamine-Xylasine solution given i.p. in a dose of $2.5 \mathrm{~mL} / \mathrm{kg}$ bdw for induction, followed by an hourly maintaining dose of $1.5 \mathrm{~mL} / \mathrm{kg}$ bdw. Catheters were inserted into the femoral artery and vein. RBCs were labeled with $99 \mathrm{mTc}$ 
using stannous pyrophosphate as reducing agent (20 $\mu \mathrm{g} \mathrm{Sn}$ (II)/ $/ \mathrm{kg}$ bdw, i.v.). Thirty minutes later, $1 \mathrm{~mL}$ of pre-treated arterial blood was withdrawn and gently mixed with $1 \mathrm{~mL}$ of $99 \mathrm{mTc}$-pertechnetate solution of $\sim 200 \mathrm{MBq}$ activity, and allowed to stand for 10 minutes prior to re-injection. Labeled RBCs were re-injected (in $0.7 \mathrm{~mL}$ with approximate activity of $70 \mathrm{MBq}$ ) for mapping RBCs 5 minutes postinjection. Two animals were treated by L-NAME $(100 \mathrm{mg} / \mathrm{kg}$ bdw, i.v. $)$. Scans were acquired for control and at 44 minutes following the L-NAME injection. Animals were sacrificed by saline infusion (a total of $100 \mathrm{~mL}$ ) given via the arterial line with concomitant drainage via the venous line in order to remove blood from the brain's parenchyma. Cerebral RBC mass (CRBCM) was characterized by activities normalized by the brain's volume. Results: No activities were found in the thyroids and in the stomach, the sites where free $99 \mathrm{mTcpertechnetate}$ in blood accumulates if present; an evidence of a larger then $99 \%$ purity of radiolabeling. Hot spots in the brain were detected at sites of venous sinuses and the circus of Willis. CRBCM decreased in the L-NAME treated animals, as anticipated, while $99 \mathrm{mTc}$-activity became a magnitude smaller $(0.03 \mathrm{mBq} / \mathrm{cm} 3)$ after saline infusion demonstrating the specificity of the $99 \mathrm{mTc}$-radiolabeling for RBCs. Conclusions: Quantitative NanoSPECT technology is efficient to assess regional $\mathrm{RBC}$ mass in the rodent brain for tumor biomarker monitoring. Acknowledgemnets: EC-FP6 EMIL,HU NKTH grants 2008ALAP1-01569/2008 and GLINOLID

\section{J445}

\section{IMAGING IMPAIRMENT OF MITOCHONDRIAL FUNCTION IN ISCHEMIC BRAIN: A PET STUDY IN MONKEYS WITH AN ANIMAL PET}

Hideo Tsukada

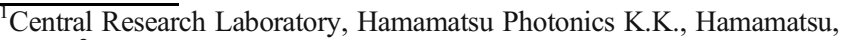
Japan, ${ }^{2}$ CREST, JST, Kawaguchi, Japan.

Preclinical evaluation of novel neuronprotectant candidates with nonhuman primate model of ischemic brain using PET is useful to bridge the efficacy determined in the in vivo evaluation with rodent model and the neuroprotective effects in patients with ischemic insult. In order to evaluate the possibility to apply the mitochondrial complex-1 (MC-1) activity as an index of the neuronal function, we used monkey model of transient occlusion of the right middle cerebral artery (MCA) with clipping for $3 \mathrm{hrs}$, followed by the reperfusion by removing the clip. PET scans were conducted before (normal condition) and 1, 3, and 7 days after the ischemia/reperfusion with a high-resolution animal PET (SHR-7700, Hamamatsu Photonics, Hamamatsu, JAPAN) under isoflurane anesthesia. Five young adult male monkeys (Macaca fascicularis) were imaged with $\left[{ }^{11} \mathrm{C}\right]$ Flumazenil for central benzodiazepine receptor (CBR) binding to determined neuronal damage, and also with $\left[{ }^{18} \mathrm{~F}\right] \mathrm{BMS}-747,158-02$ to assess mitochondrial MC-1 activity. In the control condition, $\left[{ }^{18} \mathrm{~F}\right] \mathrm{BMS}-747,158-02$ provided the sufficient uptake into the monkey brain, and showed the constant levels $15 \mathrm{~min}$ after injection and thereafter. Metabolic analysis indicated that $\left[{ }^{18} \mathrm{~F}\right]$ BMS-747,158-02 was relatively stable in the monkey plasma. Neuronal damaged area determined with $\left[{ }^{11} \mathrm{C}\right]$ Flumazenil gradually expanded with time up to 7 days after ischemia/reperfusion, showing ca. 25 and $40 \%$ reduction of CBR binding at 1 and 7 days post, respectively in ischemic-induced damaged cortical area compared with normal condition. In contrast, MC-1 activity measured with $\left[{ }^{18} \mathrm{~F}\right] \mathrm{BMS}-747,158-02$ revealed the marked reduction of ca. $70 \%$ compared with normal condition from 1 day after ischemia/reperfusion, and remained the reduction constant up to 7 days post. These results suggested that $\left[{ }^{18} \mathrm{~F}\right] \mathrm{BMS}-747,158-02$, developed as PET ligand for myocardial imaging, might be useful to assess the mitochondrial MC-1 activity in the brain, and also that $\left[{ }^{18} \mathrm{~F}\right]$ BMS-747,158-02 could be proper ligand for imaging neuronal damages induced by ischemic insult. Supported by CREST, JST, JAPAN.

\section{J446}

\section{WHITE MATTER ABNORMALITIES AND VARIANTS OF GENES ENGAGED IN THE NEURONAL MIGRATION AND MYELINATION PROCESSES IN PEOPLE WITH EARLY ONSET SCHIZOPHRENIA (EOS). DIFFUSION TENSOR MAGNETIC RESONANCE IMAGING (DTI) AND GENETICS STUDY}

Piotr Gebski ${ }^{1}$, Marta Gawlowska ${ }^{2}$

${ }^{\mathrm{D}}$ Department of Radiology, Medical University of Lodz, Lodz, Poland, ${ }^{2}$ Department of Affective and Psychotic Disorders, Medical University of Lodz, Lodz, Poland.

Neuroimaging studies (MRI) as well as post mortem examinations have confirmed early speculations that the brain structure is disordered in schizophrenia. Growing body of evidence suggests that reduced organization in white matter tracts, connecting different brain regions, rather than abnormalities within the gray matter of particular locations themselves, are responsible for the clinical symptoms observed in this disorder and therefore supports the theory of schizophrenia being a neurodevelopmental disorder. The structural and functional brain abnormalities reported in patients with schizophrenia, might be due to aberrant neuronal migration or myelination aberrations, since the final condition and position of neurons affects neuronal function, morphology, and eventually organization of white matter. The goal of this study was to investigate brain white matter abnormalities by using diffusion tensor imaging (DTI) in patients with schizophrenia. The main aim was to find association between alternations in white matter structure and gene variants engaged in the neuronal migration and myelination processes in people with early onset schizophrenia. Four genes that are proved to be engaged in the neurodevelopmental processes were selected to this study: MAG (myelin-associated glycoprotein), QKI (quaking gene), CNP (2',3'cyclic nucleotide-3'-phosphodiesterase) and HAR1F(human accelerated region $1 \mathrm{~F}$ ). Each gene were genotyped for SNPs (single nucleotide polymorphisms), searching for those that might be associated with clinical symptoms of schizophrenia. Some of selected gene variants were choosen on the basis of former positive result of other genetic studies. Our preliminary DTI results indicate that reduced organization of white matter, as inferred from anisotropy, in the schizophrenia group compared to controls, was found in the number of locations including: the right inferior occipito-frontal fasciculus, the left arcuate fasciculus, anterior and middle portions of the cingulum bundle bilaterally, bilaterally in the internal capsule, the fornix and the corpus callosum.

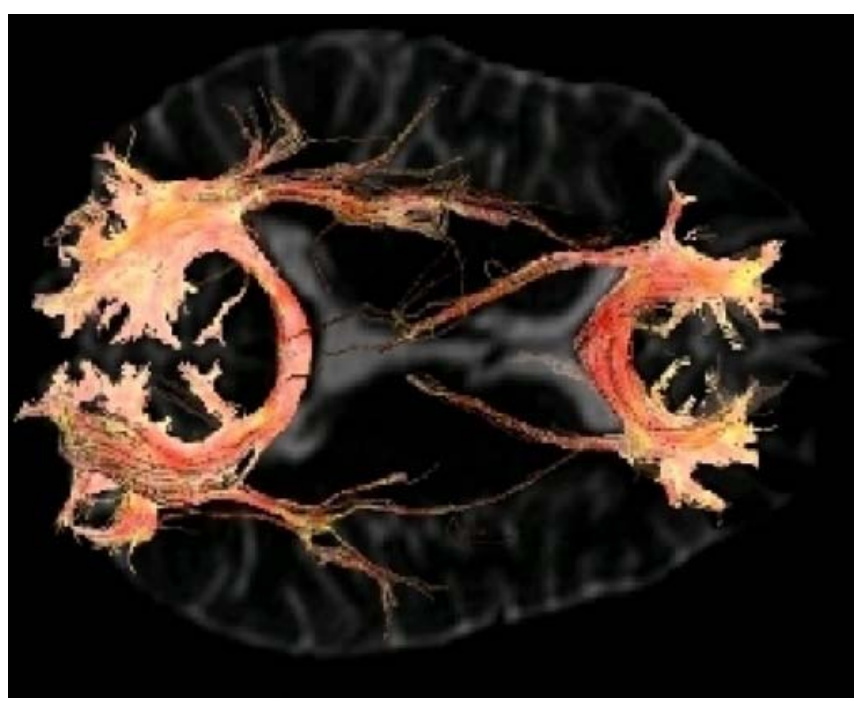




\section{J447}

\section{PATLAK GRAPHICAL ANALYSIS OF ${ }^{18}$ F-FDOPA WITH A STANDARD INPUT FUNCTION TO DIAGNOSE PARKINSON'S DISEASE}

Christiaan Schiepers, Magnus Dahlbom, Johannes Czernin, (Henry) Sung-Cheng Huang

Molecular \& Medical Pharmacology, David Geffen School of Medicine at UCLA, Los Angeles, CA, USA.

Objective: Quantitative imaging with FDOPA is a reliable method to diagnose Parkinson's disease (PD). The need for an input function (IF) limits the application for routine clinical studies. We used a pump for standardization of the tracer administration. Influx rate was calculated with Patlak graphical analysis. Results were compared to full dynamic imaging and kinetic modeling. Methods: Six patients with a clinical diagnosis of PD and 2 controls were analyzed. ${ }^{18} \mathrm{~F}-\mathrm{FDOPA}(3-11 \mathrm{mCi})$ was delivered over $2 \mathrm{~min}$; the infusion setup took 3-5 min preparation. The dynamic PET acquisition time was $125 \mathrm{~min}$. The cerebellar uptake curve was used as IF. Each patient's IF was normalized to the peak, and the average of these 6 provided a "standard" IF. An individual IF was derived by scaling the standard IF by the tail (last 5 points of the cerebellar curve). Regions of interest (ROI) of the left and right putamen and caudate, were manually drawn on parametric images, or delineated by thresholding parametric or summed images. Results of 3 image planes were averaged. No corrections were applied for partial volume effects or metabolites of the IF. Patlak analysis was applied to 6 frames of 5 min (half an hour). The start of the plot was varied from 60-95 min, and influx rate and distribution volume were estimated for the caudate and putamen. Results: For the manual method and full dynamic imaging of PD patients, influx rate was $0.0086 \pm 0.0020 \mathrm{~min}^{-1}$ for the caudate and $0.0058 \pm 0.0020 \mathrm{~min}^{-1}$ for the putamen. The Patlak plot using the individual IF yielded $0.0080 \pm$ $0.0032 \mathrm{~min}^{-1}$ for the caudate and $0.059 \pm 0.0019 \mathrm{~min}^{-1}$ for the putamen. The standard IF yielded $0.0079 \pm 0.0034 \mathrm{~min}^{-1}$ for the caudate and $0.0057 \pm$ $0.0021 \mathrm{~min}^{-1}$ for the putamen. Correlations between individual and standard IF were highest for a Patlak plot from 65-95 min: caudate ( $r=$ $0.99)$ and putamen $(r=0.97)$. The two controls had values that were on average $1.7 \mathrm{x}$ higher for the caudate and $2.2 \mathrm{x}$ higher for the putamen, compared to PD. Conclusion: Utilizing a standardized i.v. FDOPA administration, a standard IF scaled by actual cerebellar uptake, and dynamic imaging from 60-90 min, accurate estimates of FDOPA influx rate were obtained with Patlak graphical analysis. PD patients had values less than $50 \%$ of controls for the putamen. The $2 \mathrm{~min}$ infusion and half hour acquisition can easily be implemented in daily routine.

\section{$\mathbf{J 4 4 8}$}

\section{ANALYSIS OF EX VIVO PRESERVATION POSSIBILITIES} OF MANGANESE INDUCED CONTRAST IN MOUSE BRAIN Brigit den Adel ${ }^{1,2}$, Anastasia Diamantopoulou ${ }^{4}$, Robert E. Poelmann ${ }^{1,2}$, Melly S. Oitzl ${ }^{4}$, Louise van der Weerd ${ }^{1,3}$

${ }^{1}$ Anatomy and Embryology, Leiden University Medical Center, Leiden, Netherlands, ${ }^{2}$ Molecular Imaging Laboratories Leiden, Leiden University Medical Center, Leiden, Netherlands, ${ }^{3}$ Department of Radiology, Leiden University Medical Center, Leiden, Netherlands, ${ }^{4}$ Leiden Amsterdam Center for Drug Research, Leiden University, Leiden, Netherlands.

Introduction: Manganese enhanced MRI (MEMRI) exploits the paramagnetic Mn2+ ion's ability to act as a calcium analogue and locally shorten T1 relaxivity. Mn2+ enters active cells through voltage-gated $\mathrm{Ca} 2+$ channels. Memri has been successfully employed to image brain activity and cardiac viability, yet ex vivo high-resolution scans would allow a far easier assignment and localization of contrast enhancement. Standard PFA fixation results in rapid decline of Mn-enhanced contrast.
Therefore, we assessed the potential to detect Mn induced contrast in vivo and ex vivo using different fixation methods. Methods: A group $(n=8)$ of $8-10$ weeks old male C57B16Jico mice were imaged with a vertical 9.4T Bruker MRI system. Both before and 24 hours after intraperitoneal injection of $80 \mathrm{mg} / \mathrm{kg}$ of a Tris buffered (pH7.4) $105 \mathrm{mM} \mathrm{MnCl} 2$ solution, mouse brains were scanned with 3 separate MRI protocols: T1W 3D RARE (TE $8.1 \mathrm{~ms} /$ TR $300 \mathrm{~ms}$, rare factor 4, 2 averages, BW $75 \mathrm{kHz}$, matrix $256 * 160 * 160$, resolution $(100) 3 \mu \mathrm{m})$ rare inversion recovery (TE $8.7 \mathrm{~ms} / \mathrm{TR} 3500 \mathrm{~ms}$, TI $1000 \mathrm{~ms}, 8$ averages, BW $80 \mathrm{kHz}$, matrix $256 * 128$, resolution $(100) 2 \mu \mathrm{m}, 19 * 0.55 \mathrm{~mm}$ slices) and a multislice T1 measurement (TE $10 \mathrm{~ms} / \mathrm{TR}$ sequence: 8000-55003000-1500-1250-1000-750-500 ms, BW $50 \mathrm{kHz}$, matrix 128*128, resolution (195) $2 \mu \mathrm{m}, 10 * 0.55 \mathrm{~mm}$ slices). After the contrast-enhanced MRI scan, mice were sacrificed and perfused with saline followed by perfusion with 1) saline, 2) $4 \%$ buffered paraformaldehyde (PFA), 3) 4\% PFA with $2 \%$ glutaraldehyde or 4) 4\% PFA with $3.5 \%$ glutaraldehyde. One hour after dissection, the ex vivo imaging protocol was started, and repeated 5 times over a period of at least 12 hours. Results: Mn injection induced a $20-30 \%$ decrease in T1 relaxation times in the olfactory bulb, hippocampi, prefrontal cortex and cerebellum, whereas contrast did not significantly alter in the musculature surrounding the head. Without fixation, contrast gradually blurred within a few hours, PFA fixation allowed for longer retention of contrast, yet diffusion and decrease of contrast of $>20 \%$ was apparent after 4-5 hours. A more "rigid" tissue fixation was obtained using PFA with glutaraldehyde. Highest concentrations of $4 \%$ PFA with $3.5 \%$ glutaraldehyde showed a mean decline of $5 \%$ in contrast after 12 hours. Conclusions Aldehyde fixation in increasing concentrations decreases the effusion speed of $\mathrm{Mn} 2+$ ions out of the distinct brain regions, allowing for a larger time frame to perform high resolution ex vivo scans. Using this protocol, high-resolution corroboration of activated brain areas becomes a realistic option.

\section{J449}

\section{LATERALIZATION OF SEX-SPECIFIC REGIONAL METABOLIC DECLINE IN AD SUBJECTS OF THE ALZHEIMER'S DISEASE NEUROIMAGING INITIATIVE}

Thalia Wong, Cheri L. Geist, Magnus Dahlbom, Daniel H. Silverman University of California, Los Angeles, Los Angeles, CA, USA.

Objectives: To assess the effect of sex on Alzheimer's-related regional cerebral metabolic decline in longitudinally followed patients. Methods: Analyses of 88 brain FDG PET scans from the first 44 subjects who were prospectively recruited for the Alzheimer's Disease Neuroimaging Initiative (ADNI) and completed follow-up of their neuropsychologic and neuroimaging evaluations to at least two years after the time of their baseline evaluations are reported here. These subjects include $18 \mathrm{Nl}$ controls, 16 with $\mathrm{MCI}$, and 10 with $\mathrm{AD}$. Regional cerebral activity was analyzed by standardized VOI (sVOI) analyses and statistical parametric mapping methods (SPM). For the former, activities in 47 sVOIs were quantified for each scan, and normalized to pontine activity in each scan; for the latter, voxel-by-voxel normalization was to mean whole brain voxel activity. Results: The most significantly declining regions in male and female $\mathrm{AD}$ subjects were found in temporal regions. The sVOI analysis demonstrated that females declined most significantly in left temporal cortex $(t=5.35, p=$ 0.005 and $t=4.31, p=0.01$, for lateral and medial regions, respecctively), by 2.4-4.1 percent per year. The SPM analysis corroborated the left temporal metabolic decline identified in sVOI analyses $(z=4.09$ at voxel of peak significance, $p<0.0005$ ). The sVOI analyses further demonstrated that males declined most significantly in right temporal cortex, in lateral regions $(t=5.15, p=0.005)$, by $2.4-2.7$ percent per year. The SPM analysis corroborated the right temporal metabolic decline $(z=4.11, p<0.0005)$. Among males, the next most significantly declining region identified by $\mathrm{sVOI}$ analyses was the posterior cingulate cortex $(t=4.48, p=0.01)$, again 
corroborated by SPM analysis. Interestingly, comparably significant decline in the posterior cingulate cortex of either hemisphere over the same period of time was not found among the female subjects, by either of the methods of analysis. No regions differed significantly in their rates of metabolic decline between males and females from either the MCI or N1 subject groups. Conclusion: Both male and female AD patients demonstrated significant temporal metabolic decline over a two year period, but the side of the brain most affected differed consistently with sex (greater on left for females, and on right for males).

\section{$\mathbf{J 4 5 0}$}

\section{DIRECT INJECTION OF A UBIQUITIN PROTEASOME} INHIBITOR INTO THE SUBSTANTIA NIGRA OF THE RAT RESULTS IN REDUCED + DTBZ BINDING

Scott Mackey $^{1,2}$, Jing $\mathrm{Yu}^{1,2}$, Rick Kornelsen ${ }^{1,2}$, Chenoa L. Mah ${ }^{1,2}$, Doris J. Doudet ${ }^{1,2}$

${ }^{1}$ Medecine/Neurology, University of British Columbia, Vancouver, BC, Canada, ${ }^{2}$ Pacific Parkinson's Reserch Center, University of British Columbia, Vancouver, BC, Canada.

Several converging lines of evidence suggest that dysfunction of the ubiquitin proteasome system, the principal cytoplasmic mechanism for the removal of damaged or misfolded proteins, may be involved in the etiology of Parkinson's disease. Recent efforts to study this possibility in an animal model exposed to a ubiquitin proteasome inhibitor, such as MG-132, epoxomycin, or lactacystin have been hampered by technical issues surrounding the administration of the toxin. While systemic administrations have met with mixed success, all direct injections of proteasome inhibitor into either the substantia nigra or the striatum have reproduced key behavioral and pathological elements of Parkinson's disease. In this study, we injected 2, 10, or $20 \mu \mathrm{g}$ of lactacystin directly into the right substantia nigra of 15 rats (5 rats in each dose group). Both before and after surgery, the animals' behavior was assessed with the ledge beam and elevated body swing tasks. Two animals from each group were scanned for $(+)-[11 \mathrm{C}]$ dihydrotetrabenazine (+DTBZ) binding at 2 weeks post-surgery and two more at 8 weeks. The binding potential of + DTBZ in the left and right striata was calculated by the Logan method. The animals were sacrificed shortly after scanning for postmortem processing with TH immunohistochemistry. Following surgery, the animals in the larger dose groups $(10 \mu \mathrm{g}$ and $20 \mu \mathrm{g})$ displayed an exaggerated leftward head-tilt (i.e contralateral to the lesion) in their home cages and were more impaired than the animals in the $2 \mu \mathrm{g}$ dose group on the ledge beam and elevated body swing tasks compared to preoperative performance. Interestingly, all dose groups, despite differences in the severity of post-surgical behavioral deficits, displayed an almost total abolition of +DTBZ binding in the striatum ipsilateral to the injection site compared to both the contralateral striatum and the pre-surgical values. Analysis of the postmortem TH immunohistochemistry is underway. These data suggest that the intracerebral administration of lactacystin may be a viable method to induce in animal models reproducible behavioral and physiological aspects of Parkinson's disease.

\section{J451}

\section{RESEARCH AND FUNDING OPPORTUNITIES IN GENETIC STUDIES AND MOLECULAR IMAGING AT NIDA}

Da-Yu Wu, Jonathan D. Pollock

Division of Basic Research, National Institute on Drug Abuse, Bethesda, MD, USA.

Recent advancement of molecular imaging technology, such as MRI, PET, SPET and optical imaging technologies has enabled exciting progress in non-invasive and minimally invasive in vivo gene expression detection in the central nervous system (CNS), brain development and functional neural circuitry research. Non-invasive brain imaging offers a unique opportunity to examine the inner workings of the brain and to monitor normal and abnormal changes in structure and function, and to study neurological disorders, such as substance abuse and addiction. The National Institute on Drug Abuse (NIDA), part of the National Institute of Health, is strongly interested in supporting and fostering innovative research on the genetics and epigenetics of brain development, neural plasticity and brain function relevant to drug addiction. Molecular imaging on how specific gene expression in the brain dictates neuronal activity and neural function and connectivity, and the consequences of substance abuse or diseases for gene regulation are important areas of NIDA supported research. Imaging of brain can encompass multiple levels of analysis, e.g. from molecules to cell activity to neural connections, using multi-modality approaches and animal models, with the ultimate goal of non-invasive imaging of human brain structure and function. The goal of NIDA supported research is to develop innovative brain imaging capabilities that will eventually significantly advance our understanding of human brain structure and function. In this presentation, we will present NIDA's scientific interest, research initiatives, and funding opportunities for genetic and epigenetic research, and molecular imaging.

\section{$\mathbf{J 4 5 2}$}

\section{RELATIONSHIP OF EDUCATIONAL STATUS TO METABOLISM OF BRAIN REGIONS AFFECTED BY EARLY ALZHEIMER'S DISEASE}

Kate Longstaffe, Magnus Dahlbom, Cheri L. Geist, Daniel H. Silverman UCLA, Los Angeles, CA, USA.

Objective: Development of Alzheimer's disease (AD) is associated with declining glucose metabolism of non-motor/non-sensory areas of cerebral cortex, which has been sensitively detected through neuroimaging with PET. Apart from aging, lower educational level is the strongest environmental risk factor yet to be identified for being eventually diagnosed with $\mathrm{AD}$. The nature of the relationship between educational status and metabolism of involved brain regions, however, has not been extensively explored. Methods: Fifty subjects who had completed at least two years of neuropsychologic and neuroimaging follow-up after time of recruitment were identified from the Alzheimer's Disease Neuroimaging Initiative database. Half were clinically and neuropsychologically characterized as being normal, with Clinical Dementia Rating (CDR) scores of 0, and half were considered to be mildly cognitively impaired, with CDR scores of 0.5 ; all were right-handed, and they were selected for study without initial attention to educational status. Resting brain PET data, acquired $30 \mathrm{~min}$ after administration of FDG, were analyzed by statistical parametric mapping and standardized volume of interest (sVOI) methods of analysis, in both cases normalizing to whole brain activity. Of 47 total sVOI's quantitatively assessed, 10 cortical regions were selected a priori for this analysis, based upon previously established involvement by hypometabolism in early $\mathrm{AD}$, including the left and right parietotemporal, posterior cingulate, Broca's (and contralateral), medial posterior temporal, and lateral posterior temporal areas. Results: Examination of the distribution of educational levels among the subjects, revealed that $20(40 \%)$ had less than 14 years of education (i.e., no college or university degree), $20(40 \%)$ had $\geq 18$ years (at least Masters-level training), and $20 \%$ had $14-17$ years. The number of years of education was most significantly positively correlated with posterior temporal cortex on the right, particularly in the lowest educational stratum $(r=0.62, p=0.004$; with 1 anomalous point censored, $r=0.73, p<0.0005$ ). A substratified analysis by cognitive subgroups revealed that this relationship was strongest for the cognitively normal subgroup. Conclusion: Years of education is strongly positively correlated with metabolism of posterior temporal cortex, an area 
affected by early Alzheimer's disease, particularly among subjects with relatively low levels of education (less than college degree).

\section{J453}

\section{BRAIN SPECT FOR QUANTITATIVE EVALUATION OF ENZYME REPLACEMENT THERAPY IN TYPE VI MUCOPOLYSACCHARIDOSIS}

Chin-Ho Tsao ${ }^{1}$, Bing-Fu Shih ${ }^{1}$, Ren-Shyan Liu ${ }^{2,3}$

${ }^{\mathrm{T}}$ Dept NM \& Dept Pediatrics, Mackay Memorial Hospital, Taipei, Taiwan, ${ }^{2}$ MAGIC, Dept NM, Faculty of Medicine, National YangMing University, Taipei, Taiwan, ${ }^{3}$ NPCC, Dept NM, Taipei Veterans General Hospital, Taipei, Taiwan.

Objective: Type VI mucopolysaccharidosis (MPS) is a lysosomal storage disease caused by a decreased amount or function of $\mathrm{N}$ acetylgalactosamine-4-sulfatase (arylsulfatase B). Enzyme replacement therapy (ERT) has been accepted as the safer option than stem cell transplantation. Nevertheless, identification of its efficiency with clinical markers has been a challenge. We assessed cerebral perfusion to provide quantitative measures of MPS VI-affected patients (pts) with ERT. Materials and Methods: Five pts with MPS type VI, age 7, 8, 8, 14 and 21 years (males: 3; females: 2) were enrolled in this study. All pts were assessed for urine glycosaminoglycan (GAG) excretion, amplitude of shoulder joint mobility (SJM), 3 min stair climbing, 6 min \& 12 min walking test, brain MRI and Tc-99m ECD brain SPECT before and after ERT. The brain perfusion scan was taken $30 \mathrm{~min}$ after injection of $740 \mathrm{MBq}$ Tc-99m ECD. Neurogam software (GE) was applied to quantitate perfusion changes. Results: After ERT in 3 months, results of walking test and SJM did not correlate with GAG levels. White matter lesions and ventricular enlargement were found in MRI but looked no apparent change. Three pts revealed improvement of cerebral perfusion. Tc-99m ECD activity was increased to $2.8 \%, 4.6 \%$ and $62.6 \%$, respectively and was correlated with GAG levels. Two patients without apparent change of cerebral perfusion revealed no decrease in GAG levels. Conclusions: Cerebral perfusion correlates well with GAG levels for monitoring the effect of ERT on MPS. Tc-99 m ECD brain SPECT provides a new reference value to quantitatively evaluate MPS VI pts with ERT.

\section{J454}

\section{MONITORING OF TISSUE VIABILITY AND LESION GROWTH IN FOCAL CEREBRAL ISCHEMIA IN RATS USING 201TLDDC SMALL-ANIMAL SPECT}

Jürgen Goldschmidt ${ }^{1}$, Oliver S. Grosser ${ }^{2}$, Holger Amthauer ${ }^{2}$, Klaus G. Reymann ${ }^{1}$, Henning Scheich ${ }^{1}$, Ulrich H. Schröder ${ }^{1}$

${ }^{1}$ Leibniz Institute for Neurobiology, Magdeburg, Germany, ${ }^{2}$ Klinik für Radiologie und Nuklearmedizin, Universitaetsklinikum Magdeburg, Magdeburg, Germany.

In vivo monitoring of lesion size and lesion growth in focal cerebral ischemia in rodents is usually done using magnetic resonance imaging. But the diffusion- and perfusion-weighted images obtained with MRI cannot provide direct information on tissue viability in acute cerebral ischemia. In particular, it has remained highly controversial whether or to what degree irreversible damage in the ischemic core can be detected using diffusion weighted imaging. We here introduce a novel method for in vivo monitoring of tissue viability and lesion growth in focal cerebral ischemia in rodents. The method is based on high-resolution SPECT-imaging of the spatiotemporal patterns of the $\mathrm{K}+$-probe $201 \mathrm{Tl}+$ in the brain after intravenous injections of the lipophilic chelate complex 201thallium diethyldithiocarbamate (201TIDDC). 201TIDDC is an FDA-approved radiopharmaceutical that had been introduced in 1987 for SPECT-imaging of cerebral blood flow in humans (van Royen et al., J Nucl Med 28:178). It is known that considerable fractions of $201 \mathrm{Tl}$ are retained in the CNS after intravenous 201TIDDC injections, but the mechanism of retention had not been clarified. We analyzed the spatial and temporal patterns of $\mathrm{Tl}+$-uptake and distribution in rat brain at the cellular level using a histochemical method for heavy metal detection and in vivo using high-resolution multi-pinhole SPECTimaging. We provide evidence that, after crossing the blood-brain barrier, $\mathrm{Tl}+$ is released from TIDDC into the CNS extracellular space, from which neurons and glial cells take up the ion. This indicates that 201TIDDC can be used for imaging CNS K+-metabolism and tissue viability in essentially the same manner as $201 \mathrm{Tl}+$ is used for imaging myocardial $\mathrm{K}+$-metabolism and viability. Accordingly we find, upon induction of focal cerebral ischemia by endothelin-mediated reversible occlusion of the middle cerebral artery, with SPECT-imaging as well as with thallium histochemistry, a core region in the affected brain area, in which $\mathrm{Tl}+$-uptake is markedly reduced. This core region expands over time, obviously reflecting breakdown of $\mathrm{K}+$-gradients and lesion growth. Due to the long half-life of $201 \mathrm{Tl}(73 \mathrm{~h})$ the spatiotemporal patterns of tissue viability and lesion growth in focal cerebral ischemia can be monitored, depending on the dose injected, over a period of about three days after a single injection of 201TIDDC. 201TIDDC small-animal SPECT is a convenient metabolic imaging technique that offers advantages over both PET and MRI in assessing tissue viability in rodent models of cerebral ischemia in vivo.

\section{$\mathbf{J} 455$}

\section{IN VIVO TOMOGRAPHIC OPTICAL MOLECULAR IMAGING OF BRAIN TUMORS IN MICE} Eric M. Soehngen ${ }^{1,2}$, Ralf B. Schulz ${ }^{1}$, Angelique B. Ale ${ }^{1}$, Marta P. Zientkowska ${ }^{1}$, Annette Foerschler ${ }^{3}$, Claus Zimmer ${ }^{3}$, Juergen Schlegel ${ }^{2}$, Vasilis Ntziachristos ${ }^{1}$

${ }^{1}$ Institute for Biological and Medical Imaging, Helmholtz Zentrum Munich, Munich, Germany, ${ }^{2}$ Department of Neuropathology, Technical University Munich, Klinikum rechts d. Isar, Munich, Germany, ${ }^{3}$ Department of Neuroradiology, Klinikum Rechts d. Isar, Technische Universität München, Munich, Germany.

INTRODUCTION Fluorescent molecular tomographic (FMT) imaging can noninvasively monitor molecular function in living animals using specific fluorescent probes. However, macroscopic imaging methods such as FMT exhibit low anatomical details. To overcome this, we report a quantitative technique to image both structure and function by combining FMT and computed tomography (CT) imaging. We show that FMT-CT imaging can produce three-dimensional, multimodal images of living mouse brains. METHODS The approach, applied to in-vivo protease imaging of brain tumorogenesis in mouse models, is based on a free-space hybrid optical tomography and X-ray CT system. The novel hybrid system registers optical data onto X-ray CT data acquired in the same geometry . LN18 human glioblastoma cells were stereotactically implanted in the right brain lobe of 6-8 weeks old nude mice at a depth of $2,8 \mathrm{~mm}$. Tumor growth was assessed by MR Imaging. 4 weeks after implantation, mice were injected with an optical reporter agent, activated by Cathepsine B expression on tumor cells $24 \mathrm{~h}$ before in-vivo imaging was performed. After imaging, mice were euthanized and brains were investigated histologically. RESULTS and DISCUSSION The results of the reconstructed volume using a Tikhonov reconstruction method are showing a pronounced signal from the tumor site, which was not detectable in the control group. Based on the comparison, we conclude that combined FMT-CT imaging of fluorescent molecular probes could be valuable for brain tumor drug development and other neurological applications. 


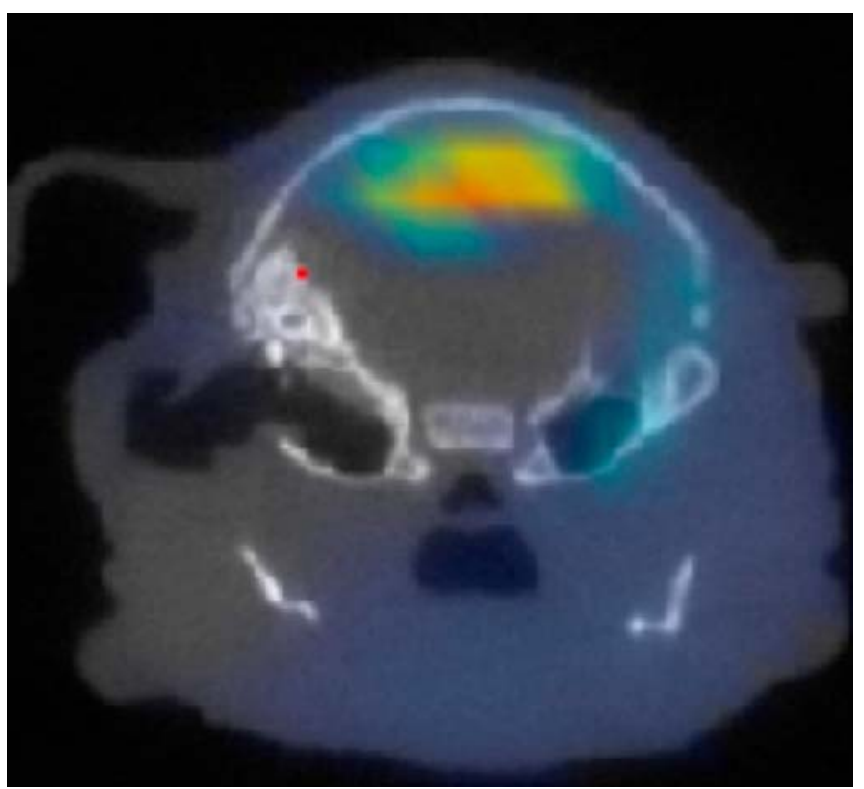

FMT-XCT imaging of LN18 human glioblastoma in nude mouse brain

\section{$\mathbf{J 4 5 6}$}

\section{EXPERIENCES WITH I-123- VASCULAR ENDOTHELIAL GROWTH FACTOR SCINTIGRAPHY FOR VISUALISATION OF BRAIN TUMORS}

Shuren $\mathrm{Li}^{1}$, Elke Dimou ${ }^{1}$, Georg Widhalm ${ }^{3}$, Christine Marosi ${ }^{2}$, Peter Angelberger ${ }^{4}$, Robert Dudczak ${ }^{1}$

${ }^{1}$ Department of Nuclear Medicine, Medical University of Vienna, Vienna, Austria, ${ }^{2}$ Internal Medicine, Medical University of Vienna, Vienna, Austria, ${ }^{3}$ Neurosurgery, Medical University of Vienna, Vienna, Austria, ${ }^{4}$ Austrian Research Center Seibersdorf, Seibersdorf, Austria.

Aim: Some brain tumors such as glioblastoma is characterized by hypervascularity and invasive potential. The high level expression of VEGF receptors on vascular endothelial cells of tumors provides the molecular basis for successful use of radiolabeled VEGF as tumor angiogenesis tracers. We report our experience with 123I-VEGF for imaging in patients with brain tumor. Materials and Methods: 19 patients (Glioblastoma before the treatment, $n=8$; glioblastoma after the treatment, $n=5$; Glioma grade I or II, $n=3$; Brain lymphoma or other tumors, $n=3$;) were injected with 123I-VEGF165 (mean dose $189 \pm 15 \mathrm{MBq}$ ) for imaging purposes. Dynamic acquisition for the first $20 \mathrm{~min}$ and singlephoton emission tomography (SPET) imaging at $30 \mathrm{~min}$ and 18 hours post-injection were undertaken. Results: Glioblastomas could be visualized in 7 of 8 patients $(88 \%)$ shortly after application of 123I-VEGF165 and were still demonstrated clearly 18 hours post injection. Negative scan results were obtained in one patient with small size of glioblastoma (diameter $<2.5 \mathrm{~cm}$ ) and 5 patients with glioblastoma after receiving radiotherapy and/or chemotherapy and in 3 patients with benign glioma. Weak positive results were obtained in 3 patients with brain lymphoma or other tumors. No side effects were observed in patients after i.v. administration of 123I-VEGF. Conclusion: These results indicate that scanning with 123 I-VEGF165 can visualize various tumors and their metastases expressing receptors for VEGF165. 123I-VEGF165 scintigraphy may be useful for visualization of tumor angiogenesis. *This study was partly supported by the Jubiläumsfonds of Austrian Nationalbank (Project No: 11707)
$\mathbf{J 4 5 7}$

\section{6-[18F]FLUORO-PBR28, A NOVEL TSPO 18 KDA} RADIOLIGAND FOR PET-IMAGING NEUROINFLAMMATION $\underline{R a p h a e l ~ B o i s g a r d ~}^{1,2}$, Annelaure Damont ${ }^{1}$, Agnes Blossier ${ }^{1,2}$, Benoit Jego ${ }^{1,2}$, Karine Siquier ${ }^{1,2}$, Michael Kassiou ${ }^{3,4}$, Frederic Dolle ${ }^{1}$, Bertrand Tavitian ${ }^{1,2}$

${ }^{1}$ I2BM, Service Hospitalier Frederic Joliot, CEA, Orsay, France, ${ }^{2}$ INSERM U803, Laboratoire Imagerie Moleculaire Experimentale, CEA, Orsay, France, ${ }^{3}$ Brain and Mind Research Institute, Sydney, NSW, Australia, ${ }^{4}$ School of Chemistry, University of Sydney, Sydney, NSW, Australia.

The peripheral benzodiazepine receptor (PBR or TSPO $18 \mathrm{kDa}$ ) is expressed by microglial cells in many neuropathologies involving neuroinflammation. [11C]PK11195 is today the most widely used radioligand for the in vivo imaging of PBR using PET, and this in spite of its low brain uptake and its high level of non-specific binding. Numerous PK11195 challengers are currently under investigation [1], and of particular interest are the N-benzyl-N-(2-phenoxyaryl)-acetamides, a series which includes [11C]PBR28 [2]. A fluorine-containing analogue, namely 6-fluoro-PBR28 (N-(2-methoxybenzyl)-N-(6-fluoro-4-phenoxypyridinyl-3-yl)acetamide), has been labeled with the longer half-life positron-emitter fluorine-18 and pharmacologically evaluated in a rat model of neuroinflammation (unilaterally, AMPA-induced, striatumlesioned rats) with PET. Methods. 6-Fluoro-PBR28, as well as the corresponding precursors for labeling, were synthesized from 4-chloro-3nitropyridine. 6-Fluoro-PBR28 was labeled with fluorine-1 8 by nucleophilic heteroaromatic substitution using K[18F]F-Kryptofix ${ }^{\circledR 222}$, purified by HPLC (Waters Symmetry ${ }^{\circledR}$ C-18) and formulated for i.v. injection. The AMPA rat model was used to study in vitro and in vivo specific and non-specific binding using autoradiography and $\mu$ PET imaging on a Concorde Focus P220 PET camera, including displacement with PK11195 and non-labeled 6-fluoro-PBR28 (1 mg/kg). Results. Starting from a $37 \mathrm{GBq}$ cyclotron-produced [18F]fluoride batch, 3.3$3.7 \mathrm{GBq}$ of $6-[18 \mathrm{~F}]$ fluoro-PBR $28,>99 \%$ radiochemically pure and readyto-inject, were obtained within 90 minutes. In PET experiments, 6-[18F] fluoro-PBR28 showed a higher contrast between the lesioned area and the corresponding area in the intact contralateral hemisphere (ratio ipsi/contra at 60 min post-injection: 6-[18F]fluoro-PBR28:2.2). Furthermore, 6[18F]fluoro-PBR28 was displaced by PK11195 or 6-fluoro-PBR28. Finally, modelisation of the PET data using the "simplified-referencetissue-model" showed increased binding potential (BP) in comparison to the BP of [11C]PK11195 measured in the same model (2.0 00.6 versus $1.1 \pm 0.2)$. Immunohistochemical analyses correlate with PET-imaging and showed strong activation of microglia in and around the lesion. Conclusion. Dynamic $\mu$ PET studies in rats demonstrate the potential of 6 [18F]fluoro-PBR28 to image neuroinflammation. [1] Chauveau et al. Eur. J. Nucl. Med. Mol. Imag. (2008), 35, 2304-2319. [2] Briard E et al. J. Med. Chem. (2008), 51, 17-30.

\section{$\mathbf{J 4 5 8}$}

INFLUENCE OF MILD, EXPERIMENTAL KETONEMIA

ON CEREBRAL METABOLISM IN RATS:

A PET STUDY WITH [11C]ACETOACETATE

\section{AND [18F]FLUORODEOXYGLUCOSE}

Sebastien Tremblay ${ }^{1,2}$, Fabien Pifferi ${ }^{1}$, Mélanie Fortier ${ }^{1}$ Jennifer Tremblay Mercier ${ }^{1}$, Etienne Croteau ${ }^{2}$, Jean-François Beaudoin ${ }^{2}$, M'hamed Bentourkia $^{2}$, Roger Lecomte ${ }^{2}$, Stephen Cunnane ${ }^{1}$

${ }^{1}$ Research Center on Aging CSSS- Sherbrooke University Geriatric Institute., Sherbrooke University, Sherbrooke, QC, Canada, ${ }^{2}$ Sherbrooke Molecular Imaging Center, Department of Nuclear Medicine and Radiobiology, Sherbrooke University, Sherbrooke, QC, Canada. 
Neurodegenerative disease severely impairs the quality of life in the elderly and drains precious resources from the health care system. Using positron emission tomography (PET) and [18F] fluorodeoxyglucose (18FDG), lower brain uptake of glucose in elderly people, and more significantly in patients with Alzheimer's disease (AD) has been reported on several occasions. Ketones (acetoacetate, $\beta$-hydroxybutyrate) are the preferred alternative source of energy for brain when glucose is low and can supply up to $70 \%$ of its energy requirements. Recent studies demonstrate that raising blood ketones induce short-term improvement in cognitive function in $\mathrm{AD}$ and experimental hypoglycaemia induced in type 1 diabetics. Alternative sources of energy substrates for the aging brain need to be explored including the mechanism underlying the brain's ability to utilize ketones. In the present study, brain energy substrate availability was assessed in Fisher rats by PET imaging. Mild experimental ketonemia was induced by a ketogenic diet (KD) or by $48 \mathrm{~h}$ fasting (FS). Two tracers were used, one for ketone kinetics, $[11 \mathrm{C}]$ acetoacetate $([11 \mathrm{C}] \mathrm{AcAc})$, and $18 \mathrm{FDG}$ as analog of glucose. Each animal was its own control, starting first on the normal diet, then undergoing $48 \mathrm{~h}$ fasting, followed finally by 2 weeks on ketogenic diet. In each diet group, expression of the transporters of glucose and ketones (GLUT1 and MCT1) was measured in the brain and relevant metabolites were measured in blood. Compared to the controls, MCT1 increased 2 fold in the KD group but did not change in FS. No significant difference was observed for GLUT1. Significant differences across the three groups were observed for plasma levels of $\beta$-hydroxybutyrate, acetoacetate (AcAc), glucose, triglycerides, glycerol and cholesterol. No significant differences were observed for free fatty acids, insuline and lactate. Compared to controls, the brain uptake of $[11 \mathrm{C}] \mathrm{AcAc}$ was 2.1 and 2.3 fold higher in the $\mathrm{KD}$ and $\mathrm{FS}$ animals, respectively. Similar trends were observed for 18FDG uptake (1.9 to 2.6 times increase for KD and FS, respectively). These results suggest that energy substrate availability to the brain was increased in the $\mathrm{KD}$ and FS groups. Since fasting is not a practical long term way to improve brain energy substrate availability, we are now testing whether mild, experimental ketonemia alters brain energy substrate availability and cognitive function in elderly humans. This study was supported by CFI and a Canada Research Chair (SCC).

\section{J459}

\section{IMAGING NEUROINFLAMMATION IN SPINAL CORDS OF EAE RATS USING THE TSPO PET LIGAND, [18F]DPA-714} Raphael Boisgard ${ }^{1,2}$, Galith Abourbeh ${ }^{1,2}$, Renaud Maroy ${ }^{1}$, Annelaure Damont ${ }^{1}$, Michael Kassiou ${ }^{3,4}$, Frederic Dolle ${ }^{1}$, Bertrand Tavitian ${ }^{1,2}$

${ }^{1}$ I2BM, LIME, SHFJ, CEA, Orsay, France, ${ }^{2}$ INSERM U803, CEA, Orsay, France, ${ }^{3}$ Brain and Mind Research Institute, , Sydney, NSW, Australia, ${ }^{4}$ School of Chemistry, University of Sydney, Sydney, NSW, Australia.

Multiple sclerosis (MS) is a chronic inflammatory demyelinating disease of the CNS. The animal models of MS, collectively known as experimental autoimmune encephalomyelitis (EAE), can be elicited by immunizing with CNS tissue or with components of CNS myelin. Activation of microglia/ peripheral macrophages has been illustrated in acute and chronic MS/ EAE lesions, and importantly also in remote projection areas and histologically normal appearing white matter. Since microglia activation occurs at early disease stages, and is associated with elevated expression of the peripheral benzodiazepine receptor (PBR), PET imaging of microglia activation using PBRspecific radioligands such as [18F]DPA-714 [1,2] could offer a valuable tool for disease monitoring. EAE was induced in Lewis rats by injecting a fragment of myelin basic protein (MBP). MBP68-86 in saline $(0.5 \mathrm{mg} / \mathrm{ml})$ was emulsified with complete Freund's adjuvant (CFA), followed by injection of the emulsion $(0.1 \mathrm{ml})$ into the hind footpads. Control rats were injected with a saline/CFA emulsion. MicroPET and/or biodistribution studies of control and EAE rats were carried out 11-12 days post immunization. Rats were injected with [18F]DPA-714, followed by a 60 min scan. At the end of scan, rats were sacrificed, and the radioactivity content of selected organs was measured. EAE induction using the aforementioned protocol results in an acute disease with extensive neuroinflammation in the spinal cord. Biodistribution and PET studies of EAE and control rats revealed no statistically significant differences in radioactivity uptake values in peripheral organs. Conversely, a 4-fold higher uptake of [18F]DPA-714 was measured in spinal cords of EAE rats compared to those of controls. Importantly, the particular architecture of the spinal cord and the uptake of [18F]DPA-714 in the vertebrae exerted significant partial volume effect, which misrepresented the differential radioactivity uptake in the spinal cords of EAE vs. control rats using conventional 2D OSEM reconstruction algorithm. Nevertheless, this difference was revealed using 3D OSEM-MAP reconstruction. Using [18F]DPA-714, microglia activation in spinal cords of EAE-induced rats could be monitored by PET. To the best of our knowledge, this is the first illustration of spinal cord lesions by PET molecular imaging using PBR ligands. [1] Kassiou M et al. J. Nucl. Med. 49: 814-824 (2008). [2] Damont A et al. J. Label. Compds Radiopharm. 51:286-292 (2008).

\section{Immune System}

\section{J460}

\section{MANGANESE ENHANCED MRI (MEMRI) OF THE OLFACTORY TRACT OF MICE WITH INDUCED NEUROPSYCHIATRIC LUPUS DEPRESSION}

Tammar Kushnir ${ }^{1}$, Shaye Kivity ${ }^{2}$, Galia Tsarfaty ${ }^{1}$, Nancy Agmon-Levin ${ }^{2}$, David Manor ${ }^{1}$, Miri Blank ${ }^{2}$, Eli Konen ${ }^{1}$, Joab Chapman ${ }^{3}$, Yehuda Shoenfeld ${ }^{2}$

${ }^{1}$ Dept. of Diagnostic Imaging, The Chaim Sheba Medical Center, Tel Hashomer, Israel, ${ }^{2}$ Center for Autoimmune Diseases, The Chaim Sheba Medical Center, Tel Hashomer, Israel, ${ }^{3}$ Dept. of neurology, Sagol Neuroscience Center, The Chaim Sheba Medical Center, Tel Hashomer, Israel.

Background:MEMRI allows in vivo mapping of functional neuronal connections in the brain. The $\mathrm{Mn}^{+2}$ ion enters neurons through voltagegated calcium channels, transfer along axons and cross synapses, e.g., along the olfactory tract of a rodent following $\mathrm{Mn}^{+2}$ administration to the nasal cavity. The method was used here to investigate the olfactory function in an experimental model of mice with Neuropsychiatric systemic lupus erythematosus (NPSLE). This autoimmune illness, involving the central nervous system, is manifested by cognitive impairment, depression and psychosis. Elevated levels of Antiribosomal phosphoproteins antibodies (anti-Rib PAbs) are found in $30-40 \%$ of NPSLE patients. NPSLE-induced mice demonstrated depression-like behavior, expressed as increased immobility during forced swimming test and diminished sense of smell. The symptoms were significantly alleviated by specific anti-anti-RibPAbs treatment, and by long-term antidepressant therapy. The anti-RibPAbs specifically stained neurons in the limbic system, including the olfactory cortex. Aims:to find out if MnEMRI of the limbic/olfactory cortex can characterize the differences between NPSLE-induced mice and healthy control group. Methods: 4 groups of 10 mice were checked. All were Intracerebraventricular (ICV) injected to the right hemisphere: 20 with anti-RibPAbs from a patient with NPSLE and 20 with non specific Human IgG antibodies (control). To half of each group's mice $4 \mu$ $0.5 \mathrm{M} \mathrm{MnCl}_{2}$ was administered to the left nostril 40 hours before the 
MRI scan. All the mice had depression, smell (using Menthol) and MRI tests. MRI measurements were performed using 3T HDx GE system, mice volume coil and 3D IR SPGR $\mathrm{T}_{1} \mathrm{~W}$ sequence. $\mathrm{Mn}^{+2}$ enhancements were measured in a series of ROIs across the olfactory tract including the amygdala. Results:Significant increased immobility was detected for the NPSLE group compared to the control group in the forced swimming test, indicating depression-like behavior, concomitant with significant smell threshold impairment in the diseased mice. MnEMRI of NPSLE-mice demonstrated significant reduction in normalized (to bulb value) $\mathrm{Mn}^{+2}$ enhancement ratios, compared to healthy mice. It should be noted that there was also (less significant) difference in MRI intensities between the groups without $\mathrm{Mn}$, indicating a general damage to the tissue following the anti-RibPAbs injection. Conclusions:MnEMRI may serve as a tool to monitor olfactory/limbic abnormalities in NPSLE induced mice and may indicate that olfactory abnormalities are associated with these autoimmune conditions.

\section{J461}

MODULATION OF PHENOTYPE OF CANCER CELLS GENERATED BY CANCER GENE THERAPY USING MANT2 (ADENINE NUCLEOTIDE TRANSLOCATOR) SHRNA VECTOR AND IN VIVO BIOLUMINESCENT IMAGING OF ITS ANTI-TUMOR EFFECTS

Yong Hyun Jeon ${ }^{1,3}$, Yun $\mathrm{Choi}^{2}$, Lee You La ${ }^{1}$, Hwang Mi Hye ${ }^{1}$, Lee Sang Woo ${ }^{1}$, Ahn Byeong-Cheol ${ }^{1}$, Chul Woo Kim ${ }^{2}$, June-Key Chung ${ }^{3}$, Lee Jaetae ${ }^{1}$

${ }^{1}$ Nuclear Medicine, School of Medicine, Kyungpook National University, Daegu, Korea, South, ${ }^{2}$ Pathology, Seoul National University Hospital, Seoul, Korea, South, ${ }^{3}$ Nuclear Meicine, Seoul National University Hospital, Seoul, Korea, South.

Objective: Various strategies for the modulation of phenotype of cancer cells to increase anti-tumor immune response are being investigated. We have previously reported on the anti-tumor effects of mANT (adenine nucleotide translocator) 2 shRNA therapy in human breast cancer model. Here, we investigated whether vectorbased mANT2 shRNA gene therapy could modulate the phenotype of cancer cells and generate the anti-tumor effects using bioluminescent imaging (BLI) in immunocompetent mice. Materials and Methods: Fluc expressing TC-1 cells (TC-1/Fluc) were provided from T.-C Wu. TC-1/Fluc cells were treated with PBS, scramble shRNA DNA, and mANT2 shRNA DNA using Lipofectamine 2000. Apoptosis rate (\%) was measured using FACS analysis (Annexin V and PI staining). Two days later, the level of gene expression of MHC class I $(\mathrm{H} 2 \mathrm{~Kb})$ and Fas was analyzed using FACS analysis. At 10 days after tumor challenge to three different sites of mice, tumor bearing mice were administered with PBS, scramble DNA, mANT2 shRNA DNA once per 2 days for 6 days, respectively. Anti-tumor effects were visualized with bioluminescent imaging for 24 days. Results: The apoptosis rates was highest in mANT2 shRNA treated cells among three groups (PBS, scramble shRNA, and mANT2 shRNA, 12, 14, and 47\%, respectively). The mean fluorescent intensity (MFI) of MHC class I was 18,349 $\pm 125,21,212 \pm$ 110 , and 30,018 \pm 210 , in PBS, scramble, mANT2 shRNA vector treated cell, respectively. The MFI of Fas was 4,043 $\pm 225,4,684 \pm 210$, and $7,638 \pm 115$ in PBS, scramble, mANT2 shRNA DNA treated cells. In vivo tumor imaging revealed the significant tumor growth inhibition in mANT2 shRNA treated tumor but not PBS or scramble vector treated tumor $(P<0.01)$. Conclusion: Our data suggest that mANT2 shRNA gene therapy could modulate the phenotype of cancer cells in vitro and generate strong anti-tumor effects in immunocompetent mice. Cancer gene therapy using mANT shRNA vector could be useful tool to enhance anti-tumor immunity in immunosuppressive cancer subjects being treated with immunotherapy.

\section{$\mathbf{J 4 6 2}$}

\section{CLINICAL VALIDATION OF EXPERIMENTAL RADIOIMMUNOIMAGING OF HUMAN AL AMYLOID DEPOSITS}

Jonathan Wall $^{1}$, Stephen J. Kennel ${ }^{1}$, Tina A. Richey ${ }^{1}$, Alan Stuckey ${ }^{1}$, Sallie D. Macy $^{1}$, David W. Townsend ${ }^{1}$, Bjoern Jakoby ${ }^{3,4}$, Karen Wells ${ }^{2}$, Alan Solomon ${ }^{1}$

${ }^{1}$ Department of Medicine, University of Tennessee Graduate School of Medicine, Knoxville, TN, USA, ${ }^{2}$ Department of Radiology, University of Tennessee Graduate School of Medicine, Knoxville, TN, USA, ${ }^{3}$ Siemens Medical Solutions, Knoxville, TN, USA, ${ }^{4}$ The University of Surrey, Guildford, United Kingdom.

Amyloidosis is a protein-folding disorder characterized by the aggregation and deposition of proteinaceous fibrils in vital organs and tissues leading to organ dysfunction and ultimately death. Amyloid can affect any organ or tissue however, the kidneys, pancreas, liver, spleen, nervous tissue and heart constitute the major sites of deposition in patients with familial or sporadic forms of peripheral amyloid disease. The most common form of sporadic, peripheral amyloid is immunoglobulin light chain (LC) amyloidosis (AL), a monoclonal plasma cell dyscrasia that has an incidence of 5 per 100,000 persons per year in the USA. It is one fifth as common as multiple myeloma but more devastating with a median survival of only 13.2 months due partly to the rapidly progressive nature of the organ destruction, the lack of effective anti-amyloid therapeutics and the inability to successfully diagnose the disease before organ failure occurs. We have previously described a monoclonal antibody (mAb), designated 11-1F4 that binds to a conformational epitope present on LC fibrils but not the monomeric LC precursor proteins. When administered to mice bearing either $\mathrm{AL \kappa}$ or $\mathrm{AL} \lambda$ amyloidomas the $\mathrm{mAb} 11-1 \mathrm{~F} 4$ expedited dissolution of the amyloid material by opsonizing the fibrils and inducing cell-mediated removal. Furthermore, when radioiodinated and injected into mice with human AL $\kappa$ and AL $\lambda$ amyloidomas the $\mathrm{mAb} 11-1 \mathrm{~F} 4$ co-localized with the material in an isotype-independent manner, as evidenced using microSPECT and now microPET imaging. Under the auspices of an Investigational New Drug application we now have studied the distribution of ${ }^{124}$ I-labeled 11$1 \mathrm{~F} 4 \mathrm{mAb}$ in patients with $\mathrm{AL}$ amyloidosis. Each of the 11 patients received $<2 \mathrm{mCi}$ and $<1 \mathrm{mg}$ of ${ }^{124} \mathrm{I}-11-1 \mathrm{~F} 4$ and the distribution of the reagent was visualized using PET/CT imaging. In $\sim 40 \%$ of the patients the $\mathrm{mAb}$ co-localized with biopsy proven sites of $\mathrm{AL}$ amyloidosis, as evidenced in the PET data. As predicted by the preclinical murine imaging studies, the ${ }^{124} \mathrm{I}-11-1 \mathrm{~F} 4 \mathrm{mAb}$ bound

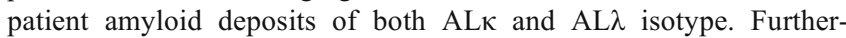
more, there was no evidence of $\mathrm{mAb}$ binding to tissues that did not contain amyloid, which was further predicted by our murine model of AL amyloidoma. These studies provide the first clinical validation of the fibril-specific immunoreactivity of the mAb 111F4. The data correlate well with our preclinical imaging studies, and further support the use of this reagent as an immunotherapeutic for patients with AL amyloidosis.

$\mathbf{J} 463$

\section{ALTERED DISTRIBUTION OF AA AMYLOID PATHOLOGY IN IMMUNOCOMPROMIZED MICE: A QUANTITATIVE SPECT IMAGING STUDY}

Jonathan Wall, Tina A. Richey, Alan Stuckey, Emily Paulus, Alan Solomon, Stephen J. Kennel

Department of Medicine, University of Tennessee Graduate School of Medicine, Knoxville, TN, USA. 
Amyloidosis is a protein-folding disorder characterized by the aggregation and deposition of proteinaceous fibrils in vital organs and tissues. The most common form of non-cerebral amyloidosis Worldwide and the second most prevalent form of peripheral amyloidosis in the USA is reactive (AA) amyloidosis which is associated with chronic inflammatory disorders such as arthritis and tuberculosis. The incidence of AA in US patients with rheumatoid or psoriatic arthritis can be as high as $26 \%$. Effective translation of novel therapeutic and diagnostic agents for AA requires a tractable animal model that reliably recapitulates human pathology. In this regard, the experimental murine model of systemic AA amyloidosis provides reproducible, controllable amyloid deposition involving multiple organs. We have used these mice to study novel immunotherapeutic approaches for the treatment of AA amyloidosis. In particular, we have shown that AA amyloidosis induced in transgenic H2-Ld-huIL-6 Tg Balb/c (C) mice could be alleviated by multiple infusions of a fibril-reactive murine monoclonal antibody $(\mathrm{mAb})$, designated 11-1F4. Furthermore, AA deposition was completely prevented by prophylactic mAb administration. Through the RAID Program at the National Cancer Institute we now have a chimeric (c) form of mAb 11-1F4 for study. This necessitates the use immunocompromized mice with AA amyloidosis. Therefore, $\mathrm{AA}$ has been induced in scid [CB17/Icr.Cg-Prkdc ${ }^{\text {scid }}$ Lyst $\left.{ }^{\text {bg }} \mathrm{Crl}\right]$ and Rag1-/- [C.129S7(B6)-Rag $1^{\text {tmlMom} / J] ~ m i c e ~ b y ~ i n j e c t i n g ~ p r o-i n f l a m-~}$ matory silver nitrate solution and amyloid enhancing factor. AA deposits were detected in both strains of mice as evidenced histologically and in SPECT images using 125I-labeled serum amyloid P component as a tracer. Remarkably, the distribution of the amyloid differed in the immunocompromized strains as compared to similarly treated Balb/c mice. Hepatic amyloid was greater in the scid and Rag1-/- mice, retaining $\sim 40-50 \%$ ID/g of ${ }^{125} \mathrm{I}-\mathrm{SAP}$ at $24 \mathrm{~h}$ post-injection as compared to $\sim 20 \%$ in Balb/c mice. In contrast, scant splenic AA was seen in the scid and Rag1-/- mice $(<10 \% \mathrm{ID} / \mathrm{g})$ as compared to $\sim 50 \% \mathrm{ID} / \mathrm{g}$ in the $\mathrm{Balb} / \mathrm{c}$. These biodistribution data correlated with the mean voxel intensity values obtained from SPECT images. The studies demonstrate that AA amyloidosis can be induced in immunocompromized mice but the distribution of the AA deposits induced in scid and Rag1-/- mice differs from that in Balb/c mice, possibly due to the to the lack of functional lymphocytes or follicular integrity in the spleen.

\section{J464}

\section{NON-INVASIVE IMAGING OF SCHISTOSOMIASIS TO ASSESS DISEASE BURDEN}

Jason D. Balkman ${ }^{1,2}$, Nicolas Salem ${ }^{1,3}$, Zhenghong Lee $^{3}$, Christopher L. King ${ }^{2}$, James Basilion ${ }^{1,4}$

${ }^{1}$ Department of Biomedical Engineering, Case Western Reserve University, Cleveland, OH, USA, ${ }^{2}$ Center for Global Health and Disease, Case Western Reserve University, Cleveland, OH, USA, ${ }^{3}$ Department of Radiology, University Hospitals Case Medical Center, Cleveland, OH, USA, ${ }^{4}$ NFCR Center for Molecular Imaging, Case Western Reserve University, Cleveland, OH, USA.

Schistosomes are chronic intravascular helminth parasites of humans causing a heavy burden of disease worldwide. Diagnosis of schistosomiasis requires the detection of schistosome eggs in the feces and urine of infected individuals. This method unreliably measures disease burden due to poor sensitivity and wide variances in egg shedding. In vivo imaging of schistosome parasites could potentially better assess disease burden, improve management of schistosomiasis, facilitate vaccine development, and enhance study of the parasite's biology. The following work uses Positron Emission Tomography (PET) to image schistosomes in vivo. This technique is based on the high metabolic demand for glucose of Schistosoma mansoni, and the hypothesis that 2-deoxy2[18F]fluoro-D-glucose (FDG), a glucose analog and PET tracer, can be avidly taken up by the parasite. Both larvae and young adult worms were FDG avid in vitro. Adults possessed 50-100 times greater uptake per worm compared to larval stages. PET imaging of Schistosoma mansoni infected mice showed preferential FDG uptake in the mesenteric region. The number of worms perfused from infected animals correlated with the mean FDG uptake density $(\mathrm{nCi} / \mathrm{ml})$ derived from imaging analyses $(\mathrm{R}$-squared $=0.97$, $P<0.01)$. Treatment of infected animals with praziquantel completely eliminated adventitious FDG uptake in one animal, and diminished tracer uptake in all other cases. These results demonstrate the potential utility of Positron Emission Tomography to quantitatively image schistosomiasis in vivo. This methodology can be rapidly applied to humans for translational research toward novel therapies, diagnosis, and vaccine development.
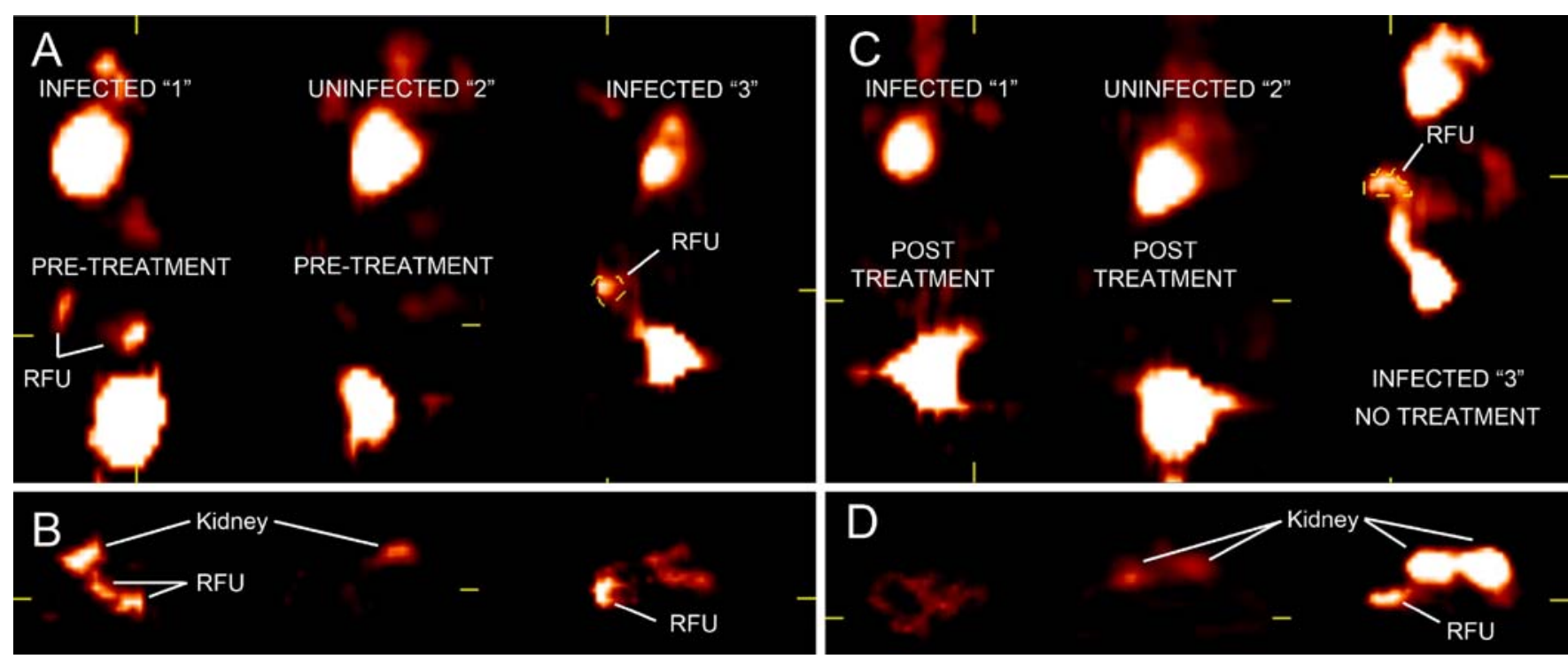

In vivo imaging of Schistomiasis. (a) Coronal and transverse (b) slices of two infected nude mice ("1" and "3"), and an uninfected control mouse ("2"). Mean liver uptake taken from each mouse was used to eliminate all lesser signals, revealing two distinct regions of focal (mesenteric) uptake (RFU) in the gut of mouse " 1 ", and a single RFU in mouse " 3 ". (c, d) Reimaging of the same mice three days following treatment of mouse "1" with praziquantel. These images are representative of 5 similar studies. 


\section{$\mathbf{J} 465$}

COMPARISON OF 18F-FOLATE, 68GA-DOTA-FOLATE AND 18F-FDG IN A RODENT INFLAMMATION MODEL: IMPLICATIONS FOR INFLAMMATION MONITORING

Marie-Jose Belanger ${ }^{1}$, Xiangjun Meng ${ }^{1}$, Paul McQuade ${ }^{1}$, Sumith A. Kularatne ${ }^{2}$, Philip S. Low ${ }^{2}$, Denise C. Welsh ${ }^{1}$, Amy Vanko ${ }^{1}$, Donna L. Suresch ${ }^{1}$, Ilonka Guenther ${ }^{1}$, Dinko Gonzalez Trotter ${ }^{1}$

${ }^{1}$ Imaging, Merck and Co, West Point, PA, USA, ${ }^{2}$ Chemistry, Purdue University, West Lafayette, IN, USA.

Objective: In this work, we directly compare the uptake of a $18 \mathrm{~F}$ or $68 \mathrm{Ga}$ labeled Folate analogue (18F-Folate or 68Ga-DOTA-Folate) with $18 \mathrm{~F}-\mathrm{FDG}$ in a rodent hind paw inflammation model within the same animal and imaging session. Folate receptors are expressed on activated macrophages, making them an attractive target for monitoring and treating inflammatory diseases. Method: Seven SD rats had one hind paw injected with $0.1 \mathrm{ml}$ of Complete Freund's Adjuvant (CFA-1 mg/ $\mathrm{ml})$. After $72 \mathrm{~h}$, the rats were injected with $0.25 \mathrm{mCi} 18 \mathrm{~F}$-Folate $(n=3)$ or $68 \mathrm{Ga}$-DOTA-Folate $(n=4)$. These animals were then imaged for 60 mins using a small-animal PET system. Subsequently, rats were injected with $0.75 \mathrm{mCi} 18 \mathrm{~F}-\mathrm{FDG}$ and imaged for 90 mins. The initial 10 mins were acquired to correct for Folate contamination in the FDG scan. Each hind paw ROI was drawn on the summed 18F-FDG image and the standardized uptake value (SUV) was tallied at the end of each scan. A signal-to-noise ratio $(\mathrm{SNR})$ was defined as: $\mathrm{SNR}=\left(\mathrm{SUV}_{\mathrm{CFA}^{-}}\right.$ $\left.\mathrm{SUV}_{\text {normal }}\right) /\left(\sigma_{\mathrm{CFA}}{ }^{2}+\sigma_{\text {normal }}\right)^{1 / 2}$ and measures the ability of the tracer to detect changes. Results: The SUVs for all three tracers were larger in the inflamed paw than in the normal paw for all rats. The uptake of $68 \mathrm{Ga}-\mathrm{DOTA}$-Folate was not significantly different than $18 \mathrm{~F}$-Folate in either the inflamed paws or the normal paws, and were subsequently grouped together as Folate tracers. In the inflamed paw of all seven rats, the ratio of $18 \mathrm{~F}-\mathrm{FDG}$ to Folate is $2.9 \pm 0.6$ while in the normal paw, the $18 \mathrm{~F}-\mathrm{FDG} /$ Folate is $0.9 \pm 0.2$. SNR of both $18 \mathrm{~F}-\mathrm{FDG}$ and Folate was very similar ( 3.7 for $18 \mathrm{~F}-\mathrm{FDG}$ and 4.4 for Folate). Conclusion: This direct SNR comparison mitigates the uptake advantage of $18 \mathrm{~F}-\mathrm{FDG}$ over folate tracers in the inflamed paw. It suggests that Folate tracers might be equivalent to $18 \mathrm{~F}-\mathrm{FDG}$ to monitor inflammation.

\begin{tabular}{|c|c|c|}
\hline Tracer & Inflammed paw & Normal Paw \\
\hline 18F-Folate $(\mathrm{n}=3)$ & $0.78 \pm 0.08$ & $0.37 \pm 0.06$ \\
\hline 68Ga-DOTA-Folate $(\mathrm{n}=4)$ & $0.88 \pm 0.06$ & $0.41 \pm 0.05$ \\
\hline 18 F-FDG $(\mathrm{n}=7)$ & $2.52 \pm 0.58$ & $0.33 \pm 0.07$ \\
\hline
\end{tabular}

SUV mean \pm standard deviation $(\sigma)$ at the end of the scan

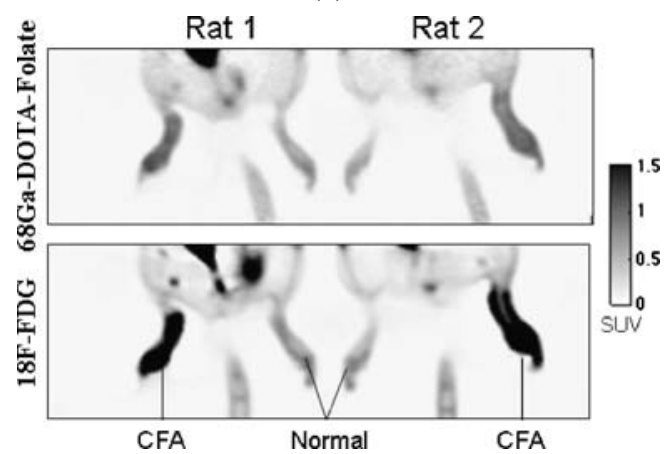

Summed 68Ga-DOTA-Folate and summed 18F-FDG images for the same two rats.

\section{J466}

\section{LONGITUDINAL OPTICAL IMAGING IN MOUSE DSS INDUCED COLITIS, AN ANIMAL MODEL OF INFLAMMATORY BOWEL DISEASE}

Kent T. Miner, Helen McBride

Inflammation Research, Amgen, Inc., Thousand Oaks, CA, USA.

Mouse models of inflammatory bowel disease (IBD) are an important tool in the preclinical development of compounds to treat human IBD. Currently our methods of measuring the severity of disease are indirect (weight loss) and labor intensive (histopathology). Non-invasive optical imaging offers the promise of reducing the amount of time to a decision regarding efficacy as well as providing objective measures of disease severity. Optical imaging using functional probes can also give new insights into the mechanisms driving inflammation in these models and the timing of particular events during the course of the disease such as reactive oxygen species (ROS) formation and glucose utilization. Such information is important when deciding whether and when intervention in a specific pathway might be efficacious in disease. Imaging also has the potential to give new insights in the mechanism of action of molecules and signaling pathways during drug discovery. We show here longitudinal imaging data of a mouse model of IBD, DSS induced colitis, with two optical imaging probes: L-012, a bioluminescent probe for ROS and IRDye800CW 2-DG, a fluorescent alternative to $18 \mathrm{FDG}$.

\section{$\mathbf{J} 467$}

\section{VISUALIZATION OF POLYCYTHEMIA VERA DISEASE DEVELOPMENT IN MICE WITH BIOLUMINESCENCE IMAGING \\ Weisheng Zhang, Joe Zhu, Yanhong Ma, Shuxia Zhao, Kimberly A. Bettano, Xianlu Qu, Gary Marshall, Jonathan R. Young, Martin L. Scott Merck, Boston, MA, USA.}

Polycythemia vera (PV) is a myeloproliferative disorder involving hematopoietic stem cells. A recurrent somatic missense mutation in JAK2 (JAK2V617F) is thought to play a causal role in PV. 
Therefore, targeting Jak2 will likely provide a molecular mechanism based therapy for PV. To facilitate the development of such new and specific therapeutics, we created a bone marrow transplant mouse model that co-expresses both mutant Jak2 and Luciferase 2 (Luc2) genes. Jak2V617F-Luc2 mice developed disease with clinical features closely resembling PV and similar to previously described Jak2V617F-GFP mice. Importantly, mutant Jak2V617F cells that also expressed Luc2 could be readily visualized by bioluminescent imaging (BLI), which was utilized to study the development of PV in vivo. We also generated mice carrying a kinase inactive mutant Jak2 (Jak2K882E) that did not activate the downstream signaling pathway and failed to develop disease, demonstrating that the PV disease was dependent on constitutive activation of the Jak2 kinase activity. We further showed that the Jak2V617F mutation caused increased stem cell renewal activity and skewed cell differentiation which was at least in part due to deregulated transcriptional programming in the hematopoietic compartment. Thus, the Jak2V617F-Luc2 PV mice serve as a valuable model enabling further insight into the mechanism and development of PV. These mice will also be a useful preclinical model to characterize novel JAK2 inhibitors for the treatment of PV.

\section{J468}

\section{COMPARING THE MR APPEARANCE OF THE MOUSE LYMPHATIC SYSTEM IN DIFFERENT STRAINS USED FOR CANCER RESEARCH}

Vasiliki Economopoulos $^{1,2}$, Jennifer C. Noad ${ }^{1,2}$, Jonatan A. Snir ${ }^{1,2}$, Paula Foster ${ }^{1,2}$

${ }^{1}$ Imaging Research Laboratories, Robarts Research Institute, London, ON, Canada, ${ }^{2}$ Medical Biophysics, University of Western Ontario, London, ON, Canada.

Immune compromised mice are routinely used in experimental cancer models to allow the transplantation and survival of human cancer cell lines. Nude mice are one such example. Nude mice lack a thymus (and as a result $\mathrm{T}$ cells) and have an under-developed lymphatic system. Despite this, nude mice are often used to investigate cancer metastasis in lymph nodes. In our lab we are interested in tracking cancer cells in the lymphatic system using MRI. The goal of this experiment was to investigate the normal MR appearance of the lymph nodes in 3D images of the whole mouse body. Methods: Three strains of mice (Nude, NOD/SCID, C57/ BL6) were imaged weekly for one month after arrival in the housing barrier. Nude and NOD/SCID mice were returned to ventilated racks within an external barrier between scanning sessions. Imaging was performed at 1.5 Tesla using a high performance gradient coil insert and a custom-built mouse body RF coil. Steady state free precession (SSFP) images of the whole mouse were acquired in approximately 35 minutes with a $200 \mu \mathrm{m}$ isotropic spatial resolution. Results: 3DSSFP images of the whole mouse body allowed for visualization of all lymph nodes. Lymph nodes were not detected in the NOD/ SCID mice, although lymphatic vessels were present. Lymph nodes in the nude mice were variable in appearance. The axillary nodes sometimes appeared with a hyperintense center (see Figure). All nodes in the C57/B16 mice appeared with similar signal intensity and with high contrast due to the surrounding fat. These differences were obvious at the first scan and did not change over the 4 weeks of imaging. All mice remained healthy. The volume of the inguinal nodes was not significantly different, the popliteal nodes were smaller in nude mice and the axillary nodes were larger in the nude mice. In nude mice all nodes increased slightly in size between week 1 and 2. Discussion: The normal lymph nodes in different strains of mice used for cancer research can appear quite different; even the same type of node within one mouse. It is important to understand the baseline MR appearance of nodes, especially when monitoring potential changes in node size or when looking for abnormal nodes due to cancer.

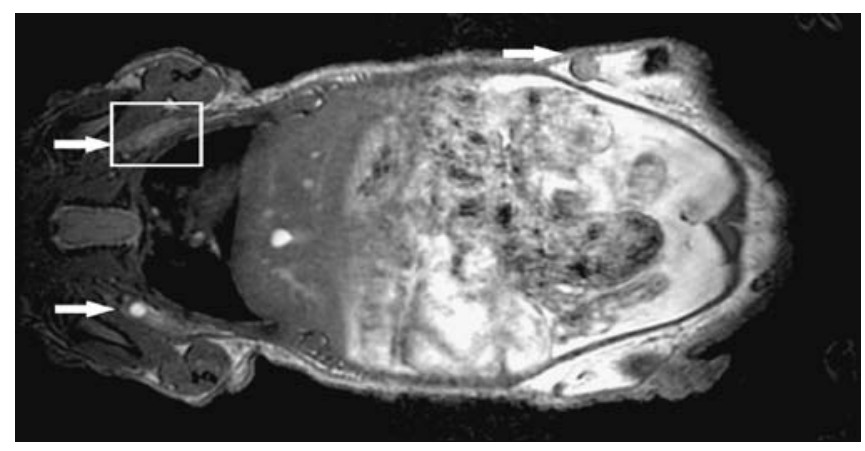

Image of nude mouse. Lymph nodes (arrows) have a varying appearance.

\section{J469}

\section{NONINVASIVE IMAGING OF BETA-CELL MASS USING AN INSULIN-SPECIFIC SOLUBLE T CELL RECEPTOR} Zdravka Medarova $^{1}$, Zeynep Onder ${ }^{1}$, Jessie Gavarret ${ }^{2}$, Daniel D. Williams ${ }^{1}$, Nikolai Lissin ${ }^{2}$, Rebecca Ashfiled ${ }^{2}$, Anna Moore ${ }^{1}$

${ }^{1}$ Molecular Imaging Laboratory, Department of Radiology, Massachusetts General Hospital, Charlestown, MA, USA, ${ }^{2}$ ImmunoCore Ltd, Oxon, United Kingdom.

The reduction of beta-cell mass (BCM) appears to be a key element in the pathology of both type 1 and type 2 diabetes. Since estimation of BCM is limited to autopsy, there is a growing unmet need to assess the level of beta-cell destruction non-invasively. This would allow not only for early diabetes detection, but also for assessment of the efficiency of therapeutic interventions. Here, we describe the application of a novel beta-cell specific contrast agent (Cy5.5-TCR) for the tracking of beta-cell mass and its reduction in the streptozotocin (STZ)-induced mouse model of type 1 diabetes. The contrast agent consists of a soluble mouse T-cell receptor, recognizing Insulin B chain15-23 peptide presented by $\mathrm{H}-2 \mathrm{Kd}$ on the surface of betacells, which has been identified as beta-cell autoantigen (1). For in vivo near-infrared optical imaging it was labeled with Cy5.5 dye. Cy5.5-TCR was injected intravenously into healthy and STZ-induced diabetic mice. NIRF imaging was performed before, as well as 1, 4, 21,26 , and $45 \mathrm{~h}$ after injection. At 21 -hrs after injection, a subgroup of mice were sacrificed, pancreata excised, and imaged ex vivo. Fluorescence microscopy of frozen pancreatic tissue sections was performed to correlate Cy5.5-TCR accumulation and insulin. Our results demonstrated a significantly lower pancreatic accumulation of Cy5.5-TCR in STZ-induced animals relative to healthy controls at 21hrs after injection ( $p=0.006$, Fig A). This finding was confirmed by ex vivo imaging of excised pancreas at $21 \mathrm{~h}$ (Fig. B). Fluorescence intensity also allowed us to reliably separate animals based on blood glucose values. In conclusion, this study holds promise for noninvasive estimation of beta-cell mass based on specific recognition between surface-expressed beta-cell autoantigen and TCR, which recognizes this autoantigen in the context of MHC class I protein. 1. Wong et al J Exp Med 183:67-76, 1996 
A
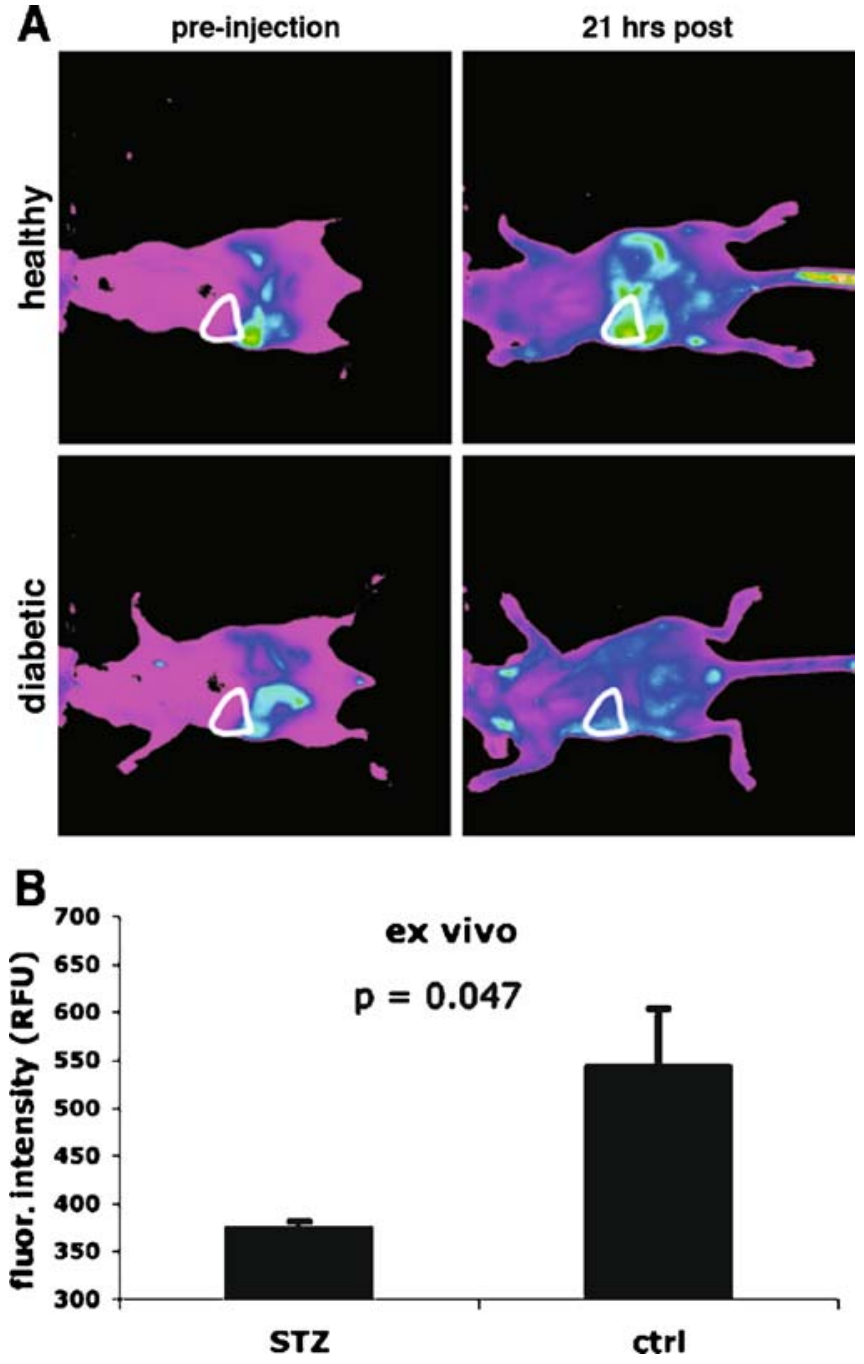

J470

SYNERGISTIC ANTITUMOR EFFECTS OF COMBINED HMUC1 DNA VACCINATION AND MANT2 SHRNA

Yong Hyun Jeon ${ }^{1,3}$, Yun $\mathrm{Choi}^{2}$, Lee You La ${ }^{1}$, Hwang Mi Hye ${ }^{1}$, Lee Sang Woo $^{1}$, Ahn Byeong-Cheol ${ }^{1}$, Chul Woo Kim ${ }^{2}$, June-Key Chung ${ }^{3}$, Lee Jaetae ${ }^{1}$

${ }^{1}$ Nuclear Medicine, School of Medicine, Kyungpook National University, Daegu, Korea, South, ${ }^{2}$ Pathology, Seoul National University College of Medicine, Seoul, Korea, South, ${ }^{3}$ Nuclear medicine, Seoul National University College of Medicine, Seoul, Korea, South.

Objective: Previously, we demonstrated that adenine nucleotide translocator 2 (ANT2) silencing by DNA vector-based siRNA (ANT2 shRNA-1) effectively induces tumor growth inhibition in nude mice cancer models. Here, we developed a novel combination therapy to enhance the efficiency of hMUC1 DNA vaccination in conjunction with mANT2 shRNA-1 treatment in a C57BL/6 mice cancer model (immunocompetent mice). Materials and Methods: We established a stable cell line (B16F1/hMUC1-Fluc: BMF) expressing the hMUC1 and Fluc genes using a lentivirus system. Cell proliferation assay (MTT assay) and apoptosis analysis (Annexin $\mathrm{V}$ and PI staining) was performed in PBS, scramble shRNA DNA, and mANT2 shRNA transfected BMF cells. At 14 days after tumor challenge, mice were dived into five groups for in vivo therapy (PBS, scramble shRNA, mANT2 shRNA, phMUC1, mANT2 shRNA + pMUC1 group). mANT shRNA or scramble shRNA DNA was administered to tumor bearing mice once per 2 days for 6 days. And, mice were immunized with pcDNA or phMUC1 DNA at 19 and 26 days post tumor challenge. Tumor growth was monitored using bioluminescence and tumor volume measurement. The killing activity of cytotoxic T cell (CTLs) was measured using $\mathrm{LDH}$ assay and the number of $\mathrm{CD} 8+\mathrm{IFN} \gamma+\mathrm{T}$ cells was determined in splenocytes and tumor infiltrating cells using FACS analysis. Results: MTT assay and apoptosis analysis showed the reduction of cell proliferation and increased apoptotic cell death in BMF cells treated with mANT2 shRNA, compared with those treated with PBS or scramble DNA. Slight tumor growth inhibition was observed in mANT2 shRNA or phMUC1 therapy group alone but not in PBS and scramble group, and significant tumor growth inhibition was monitored in mANT2 shRNA plus phMUC1 group for 40 days compared with single therapy group $(P<0.01)$ by using bioluminescent imaging and tumor measurement. The number of hMUC1-assoicated CD8 + IFNr + T cells was the most increased in combination group among five groups. Furthermore, combination therapy generated more hMUC1-associated tumor infiltrating CD8 + IFN- $\gamma+\mathrm{T}$ cells than other single therapy. The killing activities of hMUC1-associated CTLs against BMF cells were the most enhanced in combination group (phMUC1 and mANT2 shRNA) among five groups. Conclusion: Our finding suggest that combined hMUC1 DNA vaccination and mANT2 shRNA therapy could generate more enhanced anti-tumor immune response than single therapy and concurrently induce synergistic antitumor effects. This combination strategy would be helpful for treatment of immune suppressed patients with cancer.

J471

SPECT IMAGING OF AL AMYLOID IN MICE USING AN ANTIBODY TO A FIBRIL-SPECIFIC, CRYPTIC EPITOPE Jonathan Wall $^{1}$, Stephen J. Kennel ${ }^{1}$, Tina A. Richey ${ }^{1}$, Alan Stuckey ${ }^{1}$, Sallie D. Macy ${ }^{1}$, Robin Barbour ${ }^{2}$, Peter Seubert ${ }^{2}$, Alan Solomon ${ }^{1}$, Dale Schenk $^{2}$

${ }^{1}$ University of Tennessee Graduate School of Medicine, Knoxville, TN, USA, ${ }^{2}$ Elan Pharmaceuticals, South San Francisco, CA, USA.

Amyloidosis is a protein misfolding disorder, associated with a diverse group of often fatal hereditary and sporadic diseases characterized by the deposition of protein fibrils in vital organs and tissues. The incidence of peripheral amyloidosis in the USA, is estimated to be $\sim 5300$ per yr. Treatment options for peripheral amyloidosis currently are limited and focus on reducing synthesis of the amyloidogenic protein e.g., with high dose chemotherapy but there are currently 31 new trials for treating light chain (AL), reactive (AA), and senile cardiac (ATTR) amyloidosis underway. Unfortunately, there are no methods available in the USA to monitor changes in amyloid burden during disease progression or response to therapy either clinically or during clinical trials. In a routine screen of monoclonal antibodies (mAbs) that bind AA amyloid deposits we identified a reagent, designated 7D8 that exhibited cross-reactivity with AL amyloid, composed of immunoglobulin light chains. The mAb 7D8 bound relatively specifically to $\mathrm{V} \lambda 6$ light chain fibrils with an apparent midpoint concentration $\left(\mathrm{EC}_{50}\right)$ of $\sim 0.1 \mu \mathrm{M}$; however the interaction with monomeric $\mathrm{V} \lambda 6$ was weaker $\left(\mathrm{EC}_{50}>0.5 \mu \mathrm{M}\right)$. Furthermore, the presence of 160 -fold excess of 
monomeric V $\lambda 6$ did not inhibit the binding of mAb 7D8 to fibrils. Immunostaining of $\mathrm{AL}$ amyloid in tissue samples was also achieved using mAb 7D8 at $3 \mu \mathrm{g} / \mathrm{mL}$. These data support the cross-reactivity of this $\mathrm{mAb}$ to an as yet unidentified cryptic epitope present on $\mathrm{AL}$ amyloid fibrils but not the monomeric precursor proteins. To evaluate amyloid-specific binding of this reagent in vivo we injected $\sim 200 \mu \mathrm{Ci}$ of ${ }^{125}$ I-labeled mAb 7D8 $(\sim 20 \mu \mathrm{g})$ into mice bearing sub cutaneous human ALk or AL $\lambda$ amyloidomas. After $48 \mathrm{~h}$ the distribution of the ${ }^{125}$ I-7D 8 was visualized by microSPECT imaging. The mean $\%$ injected dose per gram in the amyloid mass was $9.9 \%$ and $17.4 \%$ for AL $\lambda$ and $\kappa$ amyloid, respectively, which was significantly greater than the $1.4 \%$ and $2.4 \%$ injected dose observed using an isotype-matched control mAb. The amyloid-toliver ratio was 2.5:1 and 3.6:1 rendering the amyloid masses readily visible above background in the microSPECT images. These data indicate that mAb 7D8 selectively binds amyloid fibrils, even in the presence of significant concentrations of monomeric precursor protein and that when radiolabeled this reagent can be used to image AL amyloid deposits in vivo.

J472 FLUORESCENCE MOLECULAR TOMOGRAPHIC IMAGING
OF PROTEASE ACTIVITIES AND INTEGRINS

IN THE INFLAMED GASTROINTESTINAL TRACT OF MICE Hilla Wahnishe $^{1}$, David Miller ${ }^{1}$, Victoria Lyo ${ }^{2}$, Nigel W. Bunnett ${ }^{2}$, $\overline{\text { Eileen F. Grady }}^{2}$, Ella F. Jones ${ }^{1}$

${ }^{1}$ Department of Radiology \& Biomedical Imaging, UCSF, San Francisco, CA, USA, ${ }^{2}$ Department of Surgery, UCSF Center for the Neurobiology of Digestive Diseases, San Francisco, CA, USA.

BACKGROUND: Inflammatory bowel disease (IBD), including Crohn's disease and ulcerative colitis, and irritable bowel syndrome (IBS) are diseases that present with increasing frequency in industrialized countries and predispose patients for cancer. Direct in vivo identification of protease activity and integrins during intestinal inflammation could yield novel targets for diagnosis and treatment. METHODS: We determined the feasibility of in vivo quantitative imaging of intestinal inflammation using commercial probes in a mouse model of inflammatory bowel disease. To characterize cathepsins, matrix metalloproteinases (MMP) and integrins in colitis, we injected $2 \mathrm{nmol}$ ProSense, MMPSense and IntegriSense probes intravenously in IL10-/- mice treated with piroxicam (a non-steroidal drug that accelerates colitis). Mice were imaged at $0-24 \mathrm{~h}$ after injection and the images were quantified by Fluorescence Molecular Tomography (FMT). RESULTS: With all probes used, control mice had minimal signal. ProSense signal, indicating cathepsin activity, was significantly higher in the mice with colitis $24 \mathrm{~h}$ after injection (Fig. 1A, 149 pmol ProSense) vs. the negative control (Fig. 1B, 6.4 pmol ProSense). IntegriSense signal, indicating integrin $\alpha v \beta 3$, was significantly increased in mice with colitis $4 \mathrm{~h}$ after injection (117-296 pmol IntegriSense in mice with colitis vs. 21 pmol IntegriSense in controls), thus neovasculature can be a marker in inflamed tissues. No alteration of MMPs was detected at $24 \mathrm{~h}$ after injection in this model. CONCLUSION: Cathepsins and integrins may serve as feasible markers to non-invasively quantify disease intensity in deep tissues by use of fluorescence molecular tomography. This technology could assess new drug efficacy and monitor treatment for colitis. Supported by DK43207, DK52388, CCFA \#1730, NIH 1 R01 CA 135626-0.
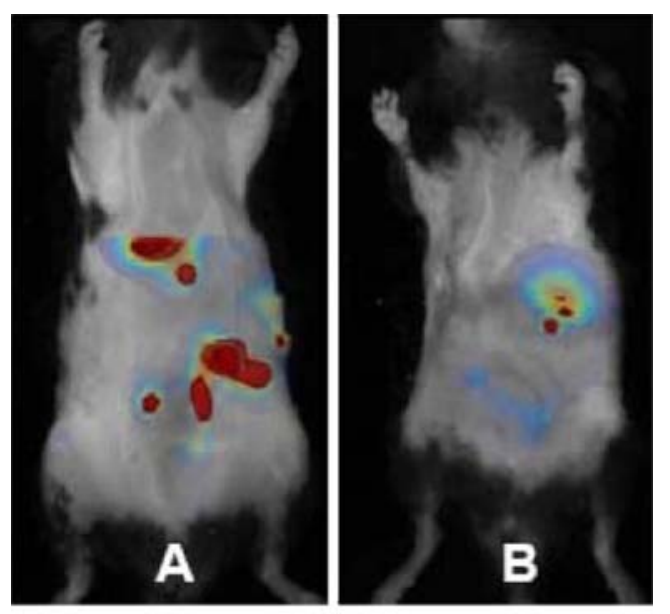

Figure 1. IL-10-\% mice with piroxicam-induced intestinal inflammation (A) and control (B). Mice were injected with ProSense 680 and imaged after $24 \mathrm{~h}$.

Imaging Methodology

CT

J473

FULLY-AUTOMATED DOUBLE SELF-GATING ALGORITHM FOR SMALL ANIMAL CT

Jan Kuntz $^{1}$, Julien Dinkel ${ }^{2}$, Tobias Baeuerle ${ }^{1}$, Michael Grasruck ${ }^{3}$, Wolfhard Semmler $^{1}$, Soenke Bartling ${ }^{1}$

${ }^{1}$ Medical Physics in Radiology, DKFZ, Heidelberg, Germany, ${ }^{2}$ Radiology, DKFZ, Heidelberg, Germany, ${ }^{3}$ Healthcare, Siemens, Forchheim, Germany.

In small animal $\mathrm{CT}$, physiologic respiratory and cardiac movements cause motion artifacts in reconstructed slice images. Therefore we developed and evaluated a fully-automated intrinsic gating algorithm for both, respiratory and cardiac motion. Mice $(n=5)$ and rats $(n=5)$ were examined using a flat-panel cone-beam CT scanner. The diaphragm was automatically detected in projection images using a variation analysis of projections acquired at different time points but at same projection angles. Within a ROI, automatically placed over the diaphragm and extended over the heart, the center of mass in longitudinal direction was calculated as motion parameter. The resulting motion signal provided large peaks that were caused by respiratory activities. These peaks were converted into a binary respiratory gating signal using upper limit detections. To extract cardiac gating signals, local maxima detection was applied after band pass-filtering. Projection data was resorted with respect to the binary gating signals, resulting in a phase-sensitive gated dataset that was reconstructed using a filtered backprojection. To evaluate this method, the volume determinability was investigated in phantom studies using a custom-made motion phantom with a moving acrylic glass ball. The real volume of the acrylic glass ball was compared to the segmented volume of the moving phantom. Intrinsically gated images were compared to simultaneously acquired extrinsic gating (gold standard). Image quality for mice and rat scans was analyzed by rating, cardiac ejection volumes of mice were compared as functional parameters. 
In all cases reliable results were obtained using fully-automated intrinsic gating. Volume measurements of the phantom showed an error of less than $6 \%$. Improvement of image quality over nongated scans was highly significant $(p<0.01)$ for automatic intrinsic as well as extrinsic cardiac gating. Image quality as determined by rating was ascertained to be equivalent for both gating techniques. Mean cardiac ejection volumes of mice correlated well with $31.0 \mu \mathrm{l} \pm 4.6 \mu \mathrm{l}$ for extrinsic gating and $31.2 \mu \mathrm{l} \pm 3.6 \mu \mathrm{l}$ for automatic intrinsic gating. The fully-automated gating algorithm evaluated in this study can be applied reliably on cone-beam CT measurements of mice and rats. It is assumed that the algorithm can be used for wide range of species and different cone-beam CT types.

\section{J474}

\section{REPLACING VASCULAR CORROSION CASTING BY IN-VIVO MCT IMAGING FOR BUILDING 3D CARDIOVASCULAR MODELS IN MICE}

Bert Vandeghinste $^{1}$, Bram Trachet ${ }^{1}$, Marjolijn Renard ${ }^{2}$, Christophe Casteleyn $^{3}$, Steven Deleye ${ }^{1}$, Philippe Joye ${ }^{1}$, Stefaan Vandenberghe ${ }^{1}$, Steven G. Staelens ${ }^{1}$, Patrick Segers ${ }^{1}$, Bart Loeys ${ }^{2}$

${ }^{1}$ Institute Biomedical Technology, Ghent University, Ghent, Belgium, ${ }^{2}$ Centre for Medical Genetics, Ghent University, Ghent, Belgium, ${ }^{3}$ Department of Morphology, Ghent University, Ghent, Belgium.

INTRODUCTION: Mice offer an accurate model to study cardiovascular disease. In this respect, Computational Fluid Dynamics (CFD) is often used to simulate the flow patterns inside the aorta. However, CFD requires reliable $3 \mathrm{D}$ computer models of the arterial system. These are usually created by vascular corrosion casting, which requires sacrificing the animals and thus excludes follow-up studies. In-vivo imaging with $\mu \mathrm{CT}$ could be an alternative, but traditional iodine-based contrast agents (needed to differentiate the aorta from surrounding tissues) cannot be used for mice since they are cleared too fast through the renal system. Fenestra VC (Advanced Research Technologies Inc., Saint Laurent, Canada) has a slow uptake in the liver, enabling 4 hours of contrastenhanced imaging. To investigate if reliable $3 \mathrm{D}$ geometrical models could be reconstructed using $\mu \mathrm{CT}$ and Fenestra VC, we compared these models with those obtained by casting the same animals. METHODS: Two 4-weeks-old and two 6-months-old wild type mice were placed on a diet of cooked yam and $1 \mathrm{ml}$ of physiological fluid was administered IP to prevent dehydration. The Ghent University ethical commission approved all experiments. First $0.015 \mathrm{ml} / \mathrm{gr}$ IV Fenestra VC-131 was administered through the tail vein, after which the animals were anesthetized using ketamine and xylazine. The mice were scanned 25 minutes after the injection of Fenestra in a FLEX Triumph $\mu$ CT scanner (Gamma Medica-Ideas, Northridge, USA) using $70 \mathrm{keV}, 170 \mu \mathrm{A}, 2 \times 2$ binning and $3.5 \times$ magnification, giving a spatial resolution of $46 \mu \mathrm{m}$. The mice were cast using Batson's No. 17 one week later. The casts were scanned using the same protocol and $\mu \mathrm{CT}$ system as the in-vivo acquisitions. Both image sets were manually segmented in Mimics (Materialise, Leuven, Belgium). RESULTS: The in-vivo images show adequate soft-tissue contrast adequately enabling 3D model generation. A comparison between diameters shows a 30\% difference in aortic arch diameter between in and ex-vivo experiments, including the aortic arch branches. This may be due to the stretching caused by the large injected contrast-agent volume on one hand and the shrinking by the cast material on the other hand. CONCLUSION: Our results show that in-vivo imaging using $\mu \mathrm{CT}$ and Fenestra $\mathrm{VC}$ can be used to build reliable 3D models.

Aortic arch diameters

\begin{tabular}{|c|c|c|}
\hline & Cast & In-vivo \\
\hline Ascending aorta & $1.08 \pm 0.10 \mathrm{~mm} \pm 0.08 \mathrm{~mm}$ \\
\hline Descending aorta & $0.87 \pm 0.11 \mathrm{~mm}$ & $1.13 \pm 0.10 \mathrm{~mm}$ \\
\hline Aortic arch & $0.93 \pm 0.08 \mathrm{~mm}$ & $1.24 \pm 0.06 \mathrm{~mm}$ \\
\hline Subclavian artery & $0.45 \pm 0.05 \mathrm{~mm}$ & $0.62 \pm 0.04 \mathrm{~mm}$ \\
\hline Left common carotid artery & $0.40 \pm 0.08 \mathrm{~mm}$ & $0.51 \pm 0.01 \mathrm{~mm}$ \\
\hline Brachiocephalic artery & $0.55 \pm 0.07 \mathrm{~mm}$ & $0.78 \pm 0.06 \mathrm{~mm}$ \\
\hline
\end{tabular}

$\mathbf{J 4 5}$

\section{NEGATIVE CONTRAST ENHANCEMENT ENABLES VISUALIZATION OF INTRAPERITONEAL TUMORS IN MICE USING CT}

Murtuza Rampurwala ${ }^{1}$, Murali K. Ravoori ${ }^{2}$, Wei $\mathrm{Wei}^{3}$, Valen Johnson ${ }^{3}$, Raghunandan Vikram $^{1}$, Vikas Kundra ${ }^{1,2}$

${ }^{1}$ Department of Diagnostic Radiology, University of Texas-M.D. Anderson Cancer Center, Houston, TX, USA, ${ }^{2}$ Department of Experimental Diagnostic Imaging, University of Texas-M.D. Anderson Cancer Center, Houston, TX, USA, ${ }^{3}$ Department of Biostatistics, University of Texas-M.D. Anderson Cancer Center, Houston, TX, USA.

Poor intrinsic soft tissue contrast of small animal CT limits evaluation of intra-abdominal structures. Long acquisition times can result in equilibration of standard intravascular-extracellular contrast (IE-IV), limiting tissue distinction. The purpose of this study was to determine whether a negative contrast strategy designed to enhancing normal tissue surrounding tumor, instead of the tumor itself, can assess intraperitoneal cancer burden in a mouse model. Animals were administered different types of contrast via various routes. Four groups of 3 mice each bearing intraperitoneal Hey A8 human ovarian cancer cells were administered serial dilutions of intravenous Fenestra LC (RES-IV), oral Gastroview and intraperitoneal Optiray 320. Other groups were administered intravenous Optiray 320 (IE-IV), oral Gastroview and intraperitoneal Optiray 320 in successive combinations. After CT, tumor and organ visualization, margin distinction, and Hounsefield Units (HU)were scored; and, in vivo CT-derived tumor weight was correlated with excised tumor weight. In general, the HU of organs increased with increasing amounts of contrast, whereas, that of tumor remained essentially stable. Both visualization and margin distinction of abdominal 
organs and tumor also generally increased with increasing contrast amount. The combination using RES-IV significantly improved both visualization and margin delineation of tumor adjacent to liver, spleen, or stomach compared to the IE-IV combination. Using the RES-IV combination, in vivo CT derived tumor weights correlated highly with ex vivo tumor weights $(r=0.96, p<0.0001$, coefficient of $\mathrm{x}$-variable $=1.18, n=15)$. Therefore, visualization and quantification of intraperitoneal tumors by $\mathrm{CT}$ is enabled by the negative contrast enhancement strategy; in addition, such a strategy makes possible anatomic localization of signals from functional imaging for combination/molecular imaging.

\section{J476}

\section{VOLUMETRIC QUANTIFICATION OF LUNG TUMORS USING MICRO-CT IN COMPLEX GENETICALLY ENGINEERED MOUSE MODELS}

Raquel Sevilla, Kimberly A. Bettano, Brian B. Haines, Melissa Chenard, Christopher Ware, Minilik H. Angagaw, Christopher T. Winkelmann, Christopher Tong, John F. Reilly, Cyrille Sur, Weisheng Zhang Merck \& Co., Boston, MA, USA.

Conditional expression of oncogenic K-rasLSL-G12D leads to spontaneous development of lung tumors in transgenic mice. Additional expression of p53LSL-R270H or/and deletion of the mouse endogenous p53 gene enhances tumor proliferation. These mouse models of human lung cancer have been invaluable to study in vivo lung cancer initiation and progression in a genetically and physiologically relevant context. However, it has been challenging to apply these models in oncology drug discovery due to the heterogeneity, multiplicity and complexity of tumor formation in these models. Here, we describe a novel analytical method to quantitatively measure total lung tumor burden in live animals using micro-CT imaging. Applying this methodology, we studied the kinetics of tumor development in K-ras, K-ras/p53 R270H and K-ras//p53 R270H/p53null models. Consistent with previous reports, lung tumors in these models developed in a timeand dose (Cre recombinase)-dependent manner. The double transgenic K-ras LSL-G12D/p53 LSL-R270H mice developed tumors faster and more robustly than mice harboring a single K-rasLSL-G12D oncogene. Tumors in triple transgenic mice were even more aggressive as expected. Erlotinib, a small molecule inhibitor of the epidermal growth factor receptor (EGFR), significantly inhibited tumor growth in K-ras LSL-G12D/p53 LSL-R270H mice. These results demonstrate that this novel imaging technique can be used to monitor both tumor progression and response to treatment, and therefore support a broader application of these genetically engineered mouse models in oncology drug discovery and development.

\section{J477}

\section{SPECTRAL COMPUTED TOMOGRAPHY AND TARGETED GOLD NANOPARTICLES FOR ANALYSIS OF ATHEROSCLEROTIC PLAQUE COMPOSITION}

David P. Cormode ${ }^{1}$, Ewald Roessl ${ }^{2}$, Axel Thran ${ }^{2}$, Torjus Skajaa ${ }^{1}$, Ronald E. Gordon ${ }^{3}$, Jens-Peter Schlomka ${ }^{2}$, Edward A. Fisher ${ }^{4}$, Willem J. Mulder ${ }^{1}$, Roland Proksa ${ }^{2}$, Zahi Fayad ${ }^{1}$

${ }^{1}$ Translational and Molecular Imaging Institute, Mount Sinai School of Medicine, New York, NY, USA, ${ }^{2}$ Sector Medical Imaging Systems, Philips Research Europe, Hamburg, Germany, ${ }^{3}$ Department of Pathology, Mount Sinai Hospital, New York, NY, USA, ${ }^{4}$ School of Medicine, New York University, New York, NY, USA.

Introduction Spectral computed tomography (CT) is a new imaging technique that can accurately distinguish and quantify different materials by analysis of their X-ray absorption at different wavelengths. In conventional CT imaging of atherosclerosis, iodine contrast agents are used to identify vessel wall narrowing, while calcifications can be identified as well. Lately, gold nanoparticles have been proposed as contrast agents for $\mathrm{CT}$ and exhibit excellent properties to differentiate with spectral CT targeted soft tissues in plaque and calcifications. Objective To investigate the potential of spectral CT to distinguish iodine and gold contrast agents from each other as well as muscle and bone tissue, for the purpose of imaging atherosclerotic plaque composition. Methods We synthesized a macrophage targeted, fluorescently labeled gold nanoparticle, while vascular phase iodine contrast agents were commercially sourced. Phantom imaging was performed using water or meat as a matrix, with calcium phosphate simulating bone and calcifications. The apoE knockout mouse was used as model of atherosclerosis. These mice were injected with gold nanoparticles, followed by iodine contrast agents at 24 hours and then imaged. Wild-type mice were used as controls. Aortic sections from these mice were imaged using confocal microscopy and transmission electron microscopy. Results Phantom imaging showed that the spectral CT system could distinguish gold, iodine, bone/calcifications and soft tissue simultaneously and quantitatively. Importantly, in apoE knockout mice spectral CT allowed the visualization of the mouse anatomy (A) as well as the areas of gold accumulation (B, C). We observed gold uptake by aortic plaques to extensively occur in the aorta. TEM imaging (D) and confocal microscopy of aortic sections confirmed that the gold particles localized in macrophages. Conclusions Spectral CT married with carefully chosen contrast agents can provide valuable and unique information on atherosclerotic plaque composition.
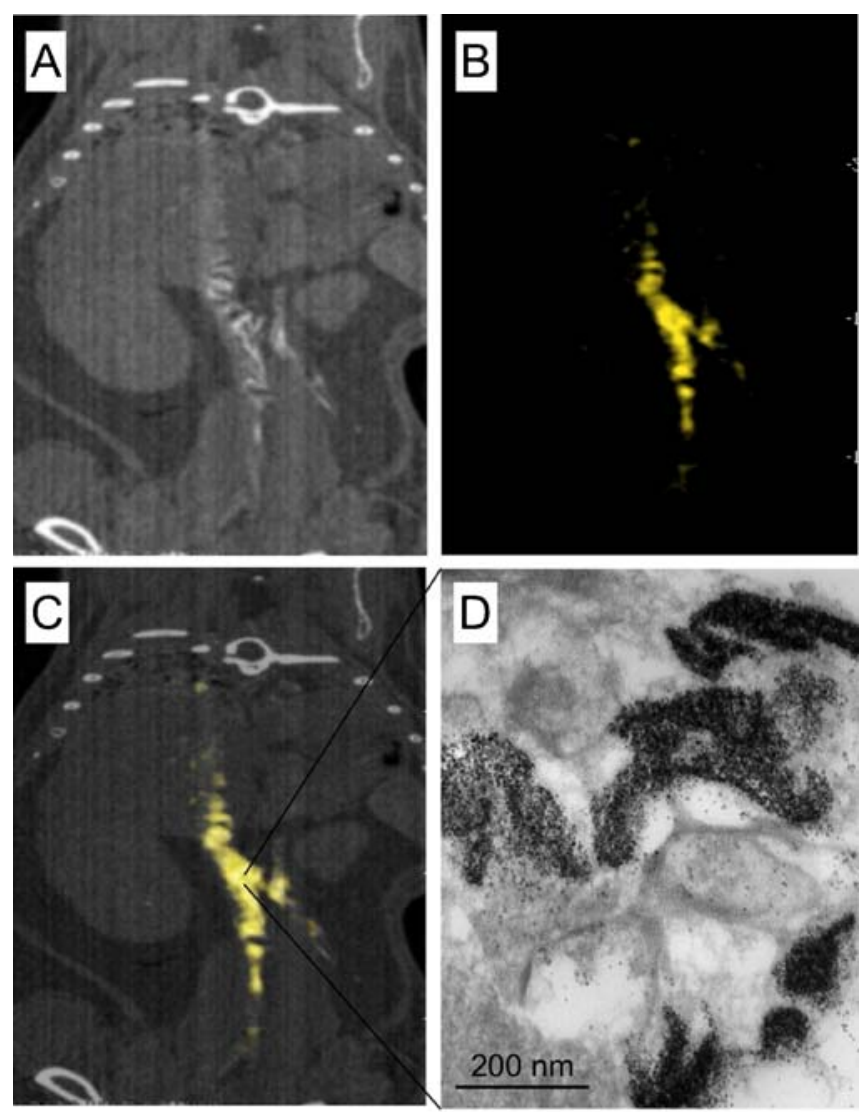

A) Conventional CT image of an apoE $\mathrm{KO}$ mouse injected 24 hours earlier with macrophage specific gold nanoparticles. B) Location of gold in A) as determined by spectral CT. C) Overlay of A) and B). D) TEM image of gold nanoparticle deposition in the aorta where spectral CT indicated high gold content. 


\section{J478}

\section{COMPUTED TOMOGRAPHY PATIENT'S EXAMINATION IN KENYA}

Anthony K. Shadrack

RADIATION PROTECTION BOARD, MINISTRY OF HEALTH, Kisumu, Kenya.

Abstract. Computed tomography (CT) is a very important tool in medical practice. The frequency on $\mathrm{CT}$ examinations is increasing all over the world, as per UNSCEAR 2000. CT contributes over $34 \%$ of collective dose from diagnostic X- Ray examinations in the world. Radiation doses received from CT exams are higher than those received in conventional radiology. The optimal use of CT equipment, considering optimized techniques, and the justification of examinations, are imperative in order to minimize the undesirable effects of radiation. The objective of the study was to establish the performance level of the Computed Topography (CT) scanners in Kenya in accordance with the International Commission on Radiation Protection (ICRP) and International Atomic Energy Agency (IAEA) standards, to estimate the patient dose and to develop effective performance tests criteria for quality assurance compliance of CT scanner. The dose measurements were measured using Axial slicing for two methods Dose in the air and in the phantoms. Scanning protocols were clinically used for brain, chest, abdomen and pelvis examinations. Head and Body phantoms model 76-414-4150 with the center and 4 holes in the peripheral were used with $10 \mathrm{~cm}$ dose length probe model 6000-100 and Victoreen model 4000M+. Computed Tomography Dose Index (CTDIw), Dose Length Product (DLP), Multiple Scan Average Dose (MSAD) and Effective Dose (E) for $10 \mathrm{CT}$ units from different companies have been investigated in different hospitals distributed all over Kenya. Mean CTDIw was found to be $83,98,94$ and 94 mGy for brain, chest, abdomen and pelvis respectively. Mean DLP was found to be 67,50 , 35 , and 31 for brain, chest, abdomen and pelvis respectively. Mean MSAD was found to be $67,0,47$ and 0 for brain, chest, abdomen and pelvis respectively. Mean E was found to be 67, 44, 29 and 50 for brain, chest, abdomen and pelvis respectively. The National Derived Reference Level (NDRL) in Kenya for radiological Computed tomography was found to be lower than the ICRP DRL. There was no clear documentation kept under a standard data form.

\section{J479}

DATA STITCHING OF CBCT BASED ON MATCHING TEMPLATE Jingjing Mao ${ }^{1}$, Jimin Liang ${ }^{1}$, Jie Tian ${ }^{1,2}$, Xiaochao $\mathrm{Qu}^{1}$, Xiangsi $\mathrm{Li}^{1}$, Jing $\mathrm{Xu}^{1}$

${ }^{1}$ Life Science Research Center, School of Electronics Engineering, Xidian University, Xi'an, China, ${ }^{2}$ Medical Image Processing Group, Institute of Automation,CAS, "Beijing,100080", China.

Cone beam CT system (CBCT) has advantages of high spatial resolution, efficient source utilization and short scan time. However, the size of the detector in most cone beam CT systems usually limits the size of the object to be detected. This paper presents a method for seamless image stitching using matching template by scanning the long object in subsections. Matching template is a widely used method for image stitching because of its high matching precision. Move the detector to scan the object in 360 degrees on several different heights. Then, match the data with the same scan angle but obtained from different heights using method of matching template based on OpenCV. Meanwhile, the data was selected by mean and variance to improve the matching accuracy and efficiency. Repeat this step in multiple degrees, and calculate the matching result according to the information acquired from all scan angles. Then complete the data stitching in 360 degrees and reduce the color difference using method of median filter. In this way, we can obtain the complete 2D projection data of the whole object from each angle which are necessary in 3D reconstruction, avoiding the distortion and mismatch caused by the incomplete data. Stitching algorithm for the projection data makes it possible to get the complete information of a big object using a detector of small size, which can significantly improve the performance-price ratio of the $\mathrm{CBCT}$ and has a good application prospect.
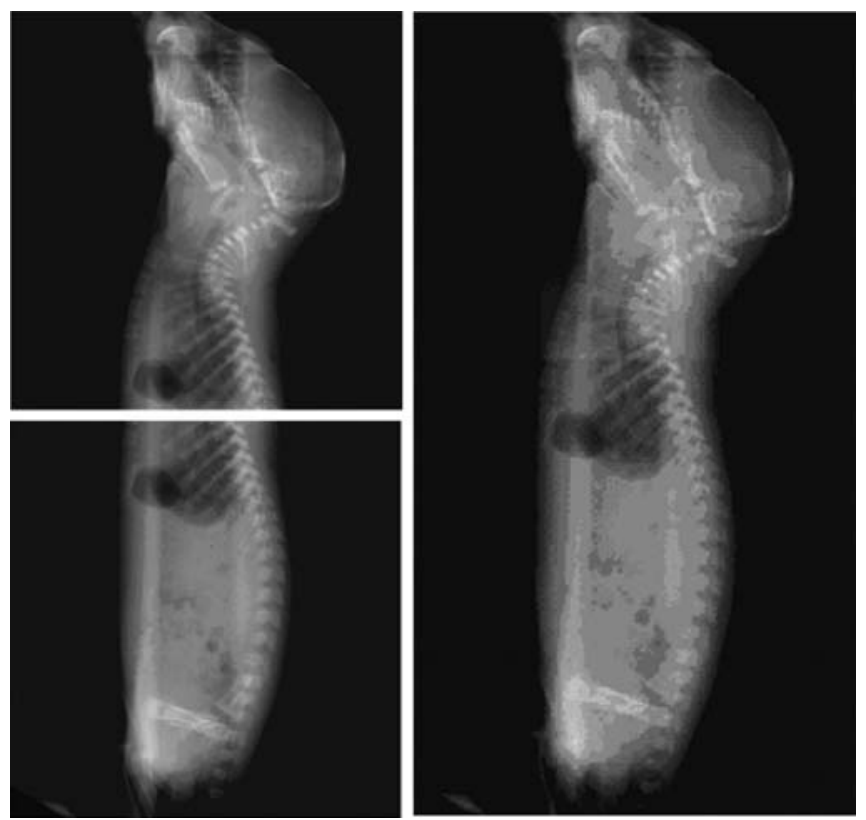

Fig.1 Result of CBCT data stitching

\section{$\mathbf{J 4 8 0}$}

\section{MICRO-CT SMALL ANIMAL IMAGING ENHANCED BY FENESTRA LC AND MEGLUMINE DIATRIZOATE}

Shouping Zhu, Jie Tian, Xibo Ma, Xing Zhang, Guorui Yan

Medical Image Processing Group, Institute of Automation,CAS, "Beijing, 100080", China.

Micro-computed tomography (Micro-CT) imaging, which could obtain high resolution anatomic information for small animals, has played a critical role in the development of molecular imaging. However, relative low soft tissue contrast resolution hinders the widespread of it. Fortunately, exogenous contrast agent can be adopted to overcome the problem in a certain extent. Fenestra LC, which is commercially available from ART Advanced Research Technologies (Montreal, Canada), is a liver contrast agent developed specifically for Micro-CT. Mouse imaging enhanced by Fenestra LC has been reported in many cases. In our experiment, besides Fenetra $\mathrm{LC}$, the meglumine diatrizoate is injected to the same mouse to enhance the kidney and bladder imaging simultaneously. We use a prototype Micro-CT system to scan a $15 \mathrm{~g}$ BALB/C mouse anesthetized by Urethane. The X-ray source is operated in a continuous mode with $50 \mathrm{KVp}$ and $1.0 \mathrm{~mA}$. 360 projections are acquired by a $360^{\circ}$ full scan. The FDK filtered backprojection method using GPU hardware is applied in 3D reconstruction. Fig.1 (a) show the image acquired at $2.5 \mathrm{~h}$ after injecting Fenestra LC $0.2 \mathrm{ml}$ through tail vein. It can be seen that the liver is enhanced by the agent clearly, but the kidney and bladder are difficult to distinguish from surrounding tissues. Then $0.2 \mathrm{ml}$ meglumine diatrizoate is injected through tail vein to the same mouse. And the mouse is scanned again 
by the Micro-CT system $3 \mathrm{~h}$ after Fenestra LC injection, namely 17 minutes after meglumine diatrizoate injection. Fig.1 (b) shows the enhanced results by both Fenestra LC and meglumine diatrizoate. Besides the liver, kidney and bladder are clearly shown in the image, which due to the effect of meglumine diatrizoate. The experiments results illuminate that liver, kidney and bladder can be enhanced simultaneously by Fenatra LC and meglumine diatrizoate.

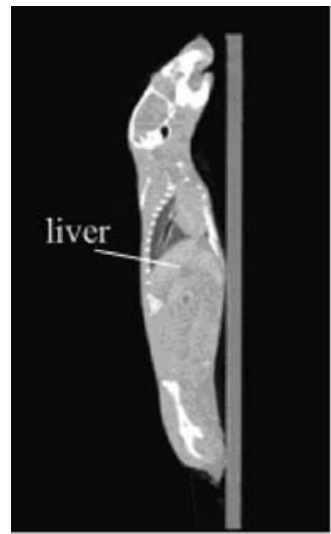

(a)

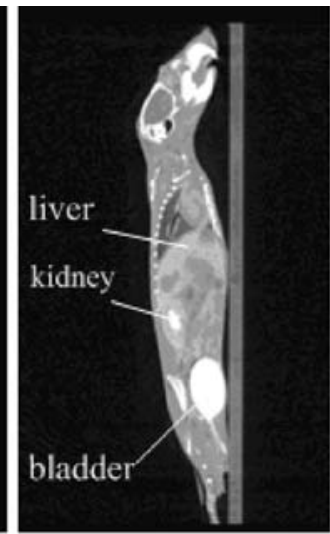

(b)
Fig.1 Mouse imaging enhanced by Fenestra LC (a) and enhanced by Fenestra LC and meglumine diatrizoate (b)

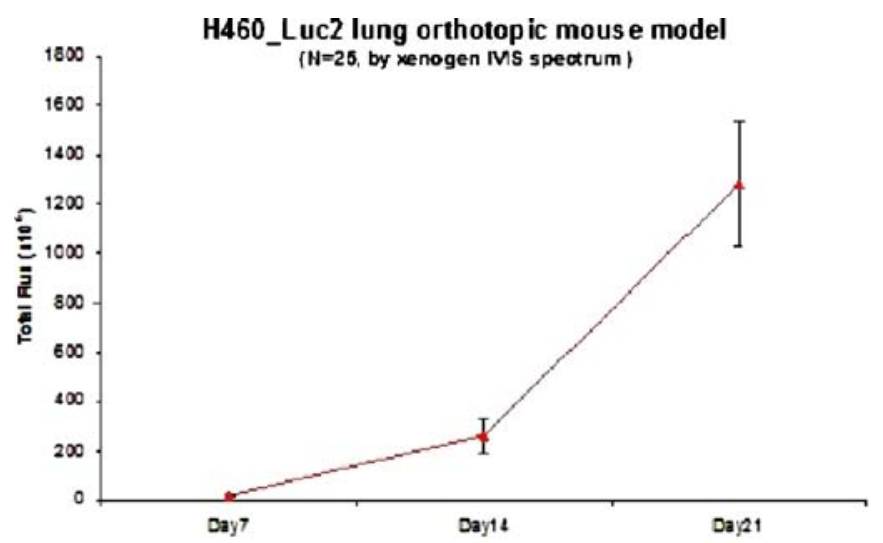

$\mathbf{J 4 8 2}$

\section{CONTRAST-ENHANCED IN VIVO MICRO-CT IMAGING}

Inneke Willekens $^{1,2}$, Tony Lahoutte ${ }^{1}$, Vicky Caveliers ${ }^{1}$, Nico Buls ${ }^{2}$, Rudi Deklerck $^{3}$, Axel Bossuyt ${ }^{1}$, Johan de Mey ${ }^{2}$

${ }^{1}$ In vivo Cellular and Molecular Imaging - ICMI, Vrije Universiteit Brussel, Jette, Belgium, ${ }^{2}$ Radiology, UZ Brussel, Jette, Belgium, ${ }^{3}$ Electronics and Informatics - ETRO - IRIS, Vrije Universiteit Brussel, Jette, Belgium.

Introduction: Micro-computed tomography offers high-resolution volumetric imaging of the inner anatomy of living small animals.

\section{J481}

\section{A NON-INVASIVE, NON-CONTRAST, UN-GATED, HIGH THROUGHPUT, LONGITUDINAL QUANTITATIVE COMPUTED TOMOGRAPHY TECHNIQUE FOR MULTI- FOCAL MURINE ORTHOTOPIC LUNG TUMOR IMAGING} Christopher M. Bull, Michael S. Westmore, Jeffrey A. Wolos, Hong Liu, Li C. Li

Molecular and Anatomical Imaging, Covance, Greenfield, IN, USA.

Current methods of lung tumor quantification by Computed Tomography $(\mathrm{CT})$ only allow for easy quantification of single tumors within the lung; not all cancer cell lines grow in this fashion. Currently we have developed a high throughput in-vivo assay that scans 12 mice in an 8 second scan. We have also developed an analysis technique that allows for the quantification of multi-focal tumor sites within the lung. Utilizing a standardized Region of Interest (ROI), we are able to use the free air space to correct tissue values within the lung field. In our method, athymic nude mice were injected with NCI-H460 luc into the left lung. CT tissue values were obtained with the start of the ROI at the level of the second thoracic vertebrae. At the same time points mice were also scanned in a Caliper IVIS system. The "raw" CT data shows an inverse quantity of tissue due to weight and tissue loss during the progression of the disease. The corrected CT tissue data correlates with the luminescence data obtained from the Caliper system. These results illustrate that we have developed a CT quantification method for multi-focal lung tumors. The method has potential benefits since it is fast, inexpensive and directly translatable to clinical studies.

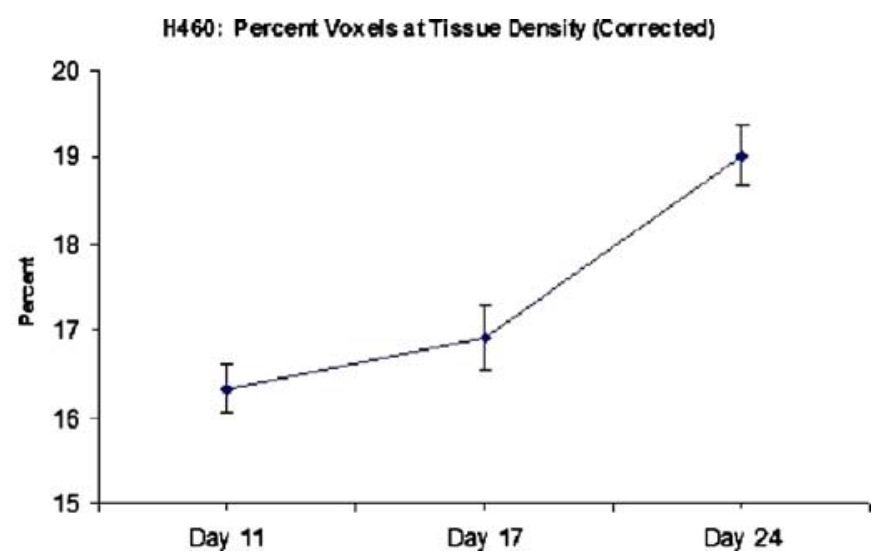

However, the contrast between the different soft tissues is inherently poor in micro-CT images. To improve the visualization of abdominal organs, attenuating exogenous contrast agents are used. Purpose: To evaluate the time-course of contrast-enhancement of spleen, liver, and blood using Exia 160XL in healthy mice. Methods: Healthy C57bl/6 mice $(n=12)$ were used. Anesthesia was induced with $5 \%$ isoflurane and maintained at $2 \%$ during the scan with spontaneous breathing via a mask. Exia 160XL (Binitio Biomedical, Inc., Ottawa, CA) was administered intravenously at a dose of $0.1 \mathrm{ml} / 20 \mathrm{~g}(n=6)$ or $0.2 \mathrm{ml} / 20 \mathrm{~g}(n=6)$. Exia 160 is an aqueous colloidal polydisperse contrast with prolonged blood-pool effect. Imaging was performed using 
micro-CT (SkyScan 1178 micro-CT system; SkyScan, Kontich, Belgium) at a resolution of $83 \mu \mathrm{m}$. The total acquisition time was 121 s. Each animal underwent a micro-CT scan before contrast injection, immediately after contrast injection and at $15 \mathrm{~min}$, $30 \mathrm{~min}, 45 \mathrm{~min}, 1 \mathrm{~h}, 2 \mathrm{~h}, 3 \mathrm{~h}, 4 \mathrm{~h}, 24 \mathrm{~h}$ and $48 \mathrm{~h}$ after contrast. Images were reconstructed using filtered backprojection (NRecon, SkyScan) and analysed using Amide (Loening et al). Regions of interest (ROIs) were drawn in spleen, liver, and left ventricle. The contrast enhancement was measured and expressed in function of time. Results: Our results show that the dose of $0.2 \mathrm{ml} / 20 \mathrm{~g}$ was lethal for the mice: they died $40 \mathrm{~h}$ after contrast injection. The Exia $160 \mathrm{XL}$ contrast enhancement of the spleen reaches a maximum at $30 \mathrm{~min}$ after the low dose and at $45 \mathrm{~min}$ after the high dose, while the maximum contrast in the liver occurs at $45 \mathrm{~min}$ after the low dose and at $4 \mathrm{~h}$ after the high dose. Conclusion: Administration of Exia $160 \mathrm{XL}$ results in longlasting blood-pool contrast with high contrast enhancement, however the administered dose is limited to $0.1 \mathrm{ml} / 20 \mathrm{~g}$. The high iodine content allows small injection volumes, which is preferred in serial small animal in vivo studies.

\section{Hybrid}

\section{$\mathbf{J 4 8 3}$}

\section{SIMULTANEOUS PERFUSION MEASUREMENTS USING COMBINED PET/MR}

Hans F. Wehrl ${ }^{1}$, Martin S. Judenhofer ${ }^{1}$, Florian C. Maier ${ }^{1}$, Stefan Wiehr ${ }^{1}$, Carsten Calaminus ${ }^{1}$, Julia G. Mannheim ${ }^{1}$, Petros Martirosian ${ }^{2}$, Kerstin Fuchs ${ }^{1}$, Gerald Reischl ${ }^{3}$, Vesna Sossi ${ }^{1}$, Fritz Schick $^{2}$, Bernd J. Pichler ${ }^{1}$

${ }^{1}$ Laboratory for Preclinical Imaging and Imaging Technology of the Werner Siemens-Foundation, University of Tuebingen, Tuebingen, Germany, ${ }^{2}$ Department of Radiology, Section on Experimental Radiology, University of Tuebingen, Tuebingen, Germany, ${ }^{3}$ Radiopharmacy, University of Tuebingen, Tuebingen, Germany.

Perfusion is a crucial factor in many diseases. However it is difficult to quantify perfusion, since it is dependent on a manifold of physiological parameters. Two modern imaging technologies that allow a measurement of perfusion are PET and MRI, although it is unknown if these measurements match exactly. Here we present initial results of simultaneous perfusion measurements using $\left[{ }^{15} \mathrm{O}\right]$ water PET and simultaneously arterial spin labeling (ASL) MRI techniques. A combined small animal PET/MR system, developed and built by our group was used in this study. The system allows simultaneous acquisition of PET and MRI data, therefore assuring that the animals under study are in the same physiological state during the perfusion measurements. This would be very difficult using sequential imaging methods, since e.g. changes in body temperature can have a profound effect on perfusion. Balb/c mice were placed inside the PET/MR scanner and a transversal brain slice was chosen as imaging region. The animals were breathing $100 \% \mathrm{O}_{2}(1.5 \mathrm{l} / \mathrm{min})$ with $1.5 \%$ isoflurane. An ASL-MR sequence (FAIR, TR=4.1 ms, TE=2.1 ms, TI=1800 ms), acquiring 40 label and control image pairs was started. During the run of the MR-ASL sequence an i.v. injection of $1.3 \mathrm{mCi}$ radioactive labeled $\left[{ }^{15} \mathrm{O}\right]$ water was given to the animal via the tail vain and a dynamic PET scan was started. The breathing gas was then switched to carbogen $(95 \%$ $\mathrm{O}_{2}, 5 \% \mathrm{CO}_{2}$ ). After a $15 \mathrm{~min}$ stabilization time, the above experiment (ASL and $\left[{ }^{15} \mathrm{O}\right]$ water PET) was repeated in the same animal in exactly the same position. To obtain an arterial input function for the $\left[{ }^{15} \mathrm{O}\right]$ water PET, the heart of the animal was subsequently positioned at the center of the FOV. The $\left[{ }^{15} \mathrm{O}\right]$ water injections were repeated for pure oxygen as well as carbogen breathing. Matlab was used to quantify the MR-ASL data, as well as PMOD for modeling the PET perfusion data with the heart slice for AIF generation. The ASL signal-time-course showed no influence by the PET tracer injection. Preliminary ASL-MR data showed a slight increase in cortex perfusion from $[69 \pm 23] \mathrm{ml} /$ $100 \mathrm{~g} / \mathrm{min}$ to $[101 \pm 27] \mathrm{ml} / 100 \mathrm{~g} / \mathrm{min}$ during carbogen breathing. Using the heart derived AIF the PET cortex perfusion for oxygen was $[94 \pm 25] \mathrm{ml} / 100 \mathrm{~g} / \mathrm{min}$ and $[148 \pm 20] \mathrm{ml} / 100 \mathrm{~g} / \mathrm{min}$ for carbogen. Besides differences in the absolute perfusion values between ASL-MR and PET, we observed also differences in the relative perfusion increase between oxygen and carbogen breathing $(46 \%$ for MR and $57 \%$ for PET). These differences could be caused by diverse modeling approaches and assumptions, which are currently investigated.

\section{J484}

\section{DUAL MODALITY IMAGING SYSTEM FOR SIMULTANEOUS MRI AND FMT MEASUREMENTS: TECHNICAL REALIZATION AND FEASIBILITY}

Florian Stuker $^{1,5}$, Katerina Dikaiou ${ }^{1,5}$, Christof Baltes ${ }^{1}$, Lucio Carrara ${ }^{3}$, Jorge Ripoll $^{2}$, Edoardo Charbon ${ }^{3}$, Markus Rudin ${ }^{1,4}$

${ }^{1}$ Institute for Biomedical Engineering, ETH and University Zurich, Zurich, Switzerland, ${ }^{2}$ IESL, FORTH, Heraklion, Greece, ${ }^{3}$ AQUA Group, EPFL, Lausanne, Switzerland, ${ }^{4}$ Institute of Pharmacology and Toxicology, University of Zurich, Zurich, Switzerland, ${ }^{5}$.., both authors contributed equally to this work, .., Switzerland.

The combination of complementary imaging methods providing either structural/functional or molecular information bear high potential for biomedical research. Here, we describe the combination of magnetic resonance imaging (MRI) with fluorescence molecular tomography (FMT) for simultaneous measurements. The technological challenge was the design of a very compact FMT system fitting in within the bore of a small animal MRI system. The setup uses free laser beam illumination for a non contact optical reflection measurement and fluorescence signal detection inside the bore close to the sample location using a single photon avalanche diode (SPAD) array. The illumination part is located outside the magnet bore (Fig. 1a). The illumination setup comprises a continuous wave laser source, a collimation lens with a pinhole, which is mapped by a $2 \mathrm{f}$ image to get a focal spot on the sample in the MRI bore and a scanning device to scan the point grid on the sample surface. This illumination geometry allows the flexibility to scan any kind of source pattern on the sample surface. Point scanning is necessary for collecting the set of images used for the tomographic data reconstruction. The incident light beam is deflected onto the sample by a small mirror mounted in the back plate of the sample platform (Fig. 1b). Fluorescence detection is achieved using a SPAD array detector, which was shown to operate at high magnetic fluxes $(9.4 \mathrm{~T})$. A low profile fixed focus objective lens was used to map the diffusive light pattern on the sample surface onto the $32 \times 32$ detector array. The appropriate fluorescence wavelengths were selected using band pass filters that were mounted on a filter wheel in front of the objective lens. The inner part of the rectangular radio frequency surface coil comprised a window for the sample illumination. First experiments with silicon phantoms demonstrated feasibility of simultaneous FMT/MRI data acquisition and allowed proper reconstruction of fluorescent dye concentration (Fig. 1c). 
a

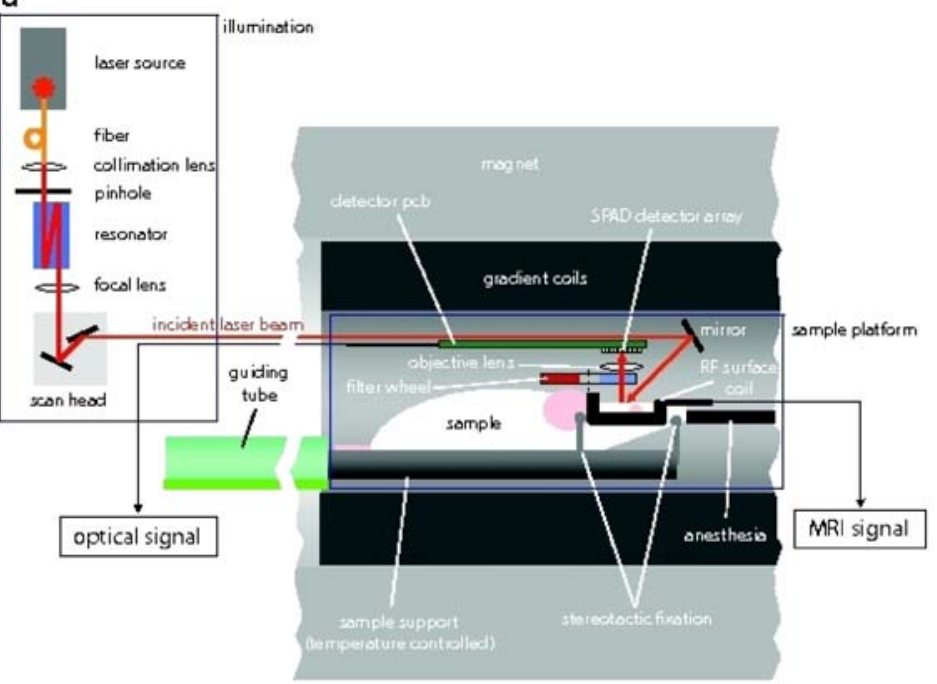

b

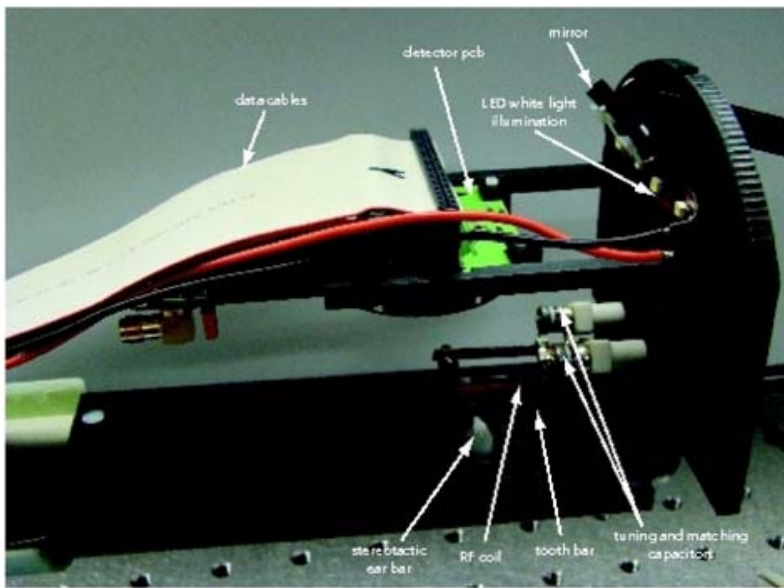

FMT

$c$
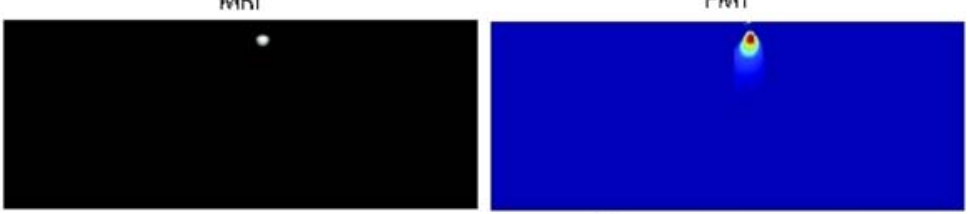

overlay

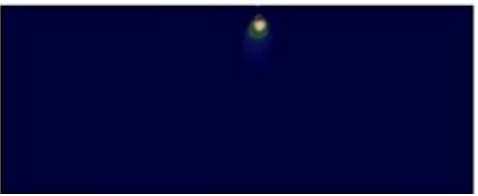

Fig 1: a) Schematic drawing of the FMT/MRI setup. b) Sample platform and its components c) Data set of a simultaneous acquired phantom measurement

$\mathbf{J} 485$

\section{HIGH-FREQUENCY ULTRASOUND GUIDED FLUORESCENCE IMAGING OF PROTOPORPHYRIN IX IN SKIN TUMORS}

Josiah Gruber $^{1}$, Brian W. Pogue ${ }^{1,2}$, Kimberley Samkoe $^{1}$, Edward Maytin ${ }^{4}$, Tayyaba Hasan

${ }^{1}$ Thayer School of Engineering, Dartmouth College, Hanover, NH, USA, ${ }^{2}$ Department of Surgery, Dartmouth College, Hanover, NH, USA, ${ }^{3}$ Wellman Center for Photomedicine, Harvard Medical School, Boston, MA, USA, ${ }^{4}$ Lerner Research Institute, Cleveland Clinic Foundation, Cleveland, OH, USA.

A novel instrument to image fluorescence in the layers of the skin has been developed to couple with high frequency ultrasound. The system uses small fiber optics that couple onto the side of the transducer, and couple to automated laser sources and detection spectrometers, to measure the transmitted and fluorescent light simultaneously. The ultrasound system is used to detect the layers of the skin, and then feed this into a segmentation and meshing algorithm, which preapares the image data space for estimation of the fluorescence yield in these layers. The fluorescence from protoporphyrin IX is present in skin tumors such as basal cell carcinoma, and is used for inducing photodynamic therapy. The mice studied are given aminolevulinic acid to stimulate production of the protoporphyrin IX, and imaging of maximal contrast is observed at 3-4 hours post administration. The recovery of fluorescence in the layers is achieved by acquiring mulitple overlapping transmission paths with the light signal, in a remittance mode, similar to the mode that ultrasound works in. The added information of the spatial layers makes the fluorescence recovery of PPIX signal highly linear and repeatible. This type of hybrid imaging instrument can be used for estimation of uptake of many types of fluorphores in vivo, while localizing the signal to the layers of origin. While simple point probe systems can achieve the same signal in homogeneous tissue, the combination of ultrasound with optics is required for accurate signal recovery in layered realistic tissues.

\section{J486}

\section{MRI DERIVED ARTERIAL INPUT FUNCTION FOR RADIONUCLIDES USING COMBINED AND SIMULTANEOUS PET-MRI INSERT}

Thomas S. $\mathrm{Ng}^{1}$, Daniele Procissi ${ }^{1}$, Yibao $\mathrm{Wu}^{2}$, Russell Jacobs ${ }^{1}$ ${ }^{\mathrm{T}}$ Biological Imaging Center, California Institute of Technology, Pasadena, CA, USA, ${ }^{2}$ Department of Biomedical Engineering, University of California, Davis, CA, USA. 
Quantitative molecular imaging requires real time measurement of the pharmacokinetics and pharmacodynamics of an injected agent in a living subject. A complete kinetic analysis of bloodstream delivered agent involves two major components. First, agent delivery by systemic circulation to areas of interest is determined by the arterial input function (AIF). This AIF can then be used to develop a pharmacokinetic model of agent uptake and clearance in specific regions of interest (ROIs). The gold standard for AIF determination remains arterial blood sampling. However, the procedure is invasive, and in small animals, can be difficult. Non invasive imaging methods have been demonstrated for image-derived input functions using PET alone. Yet, because the ROIs defined in these studies are determined primarily via transmission scans taken prior or after the emission scan, movement and spill-over artifacts remain a persistent concern. Simultaneous PET/ MR imaging is ideal to address these issues. High resolution MRI allows for a more robust ROI delineation, and the simultaneous nature of the image acquisition means that spill-over and motion artifacts can be reduced. We show here that MR angiography and dynamic contrast enhanced MR imaging techniques, using Gadolinium (Gd) based contrast agents, can provide a guide for accurate delineation of ROIs in PET images of radioactive 18F-FDG. We used MRI data to identify the ROIs corresponding to the arterial tree (Fig. $1 \mathrm{a} \& \mathrm{~b}$ ) with sufficient pixel signal for analysis in the PET images. We then obtain quantitative AIF and tumor uptake curves (Fig 1c). Complete spatial and temporal coregistration of the PET and MRI data was obtained for several examples demonstrating the versatility of a synergistic approach to AIF determination.
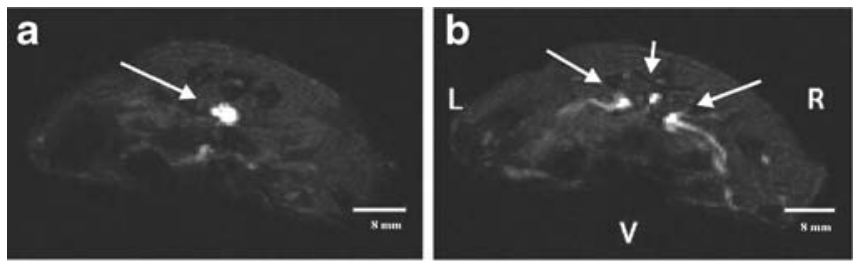

C

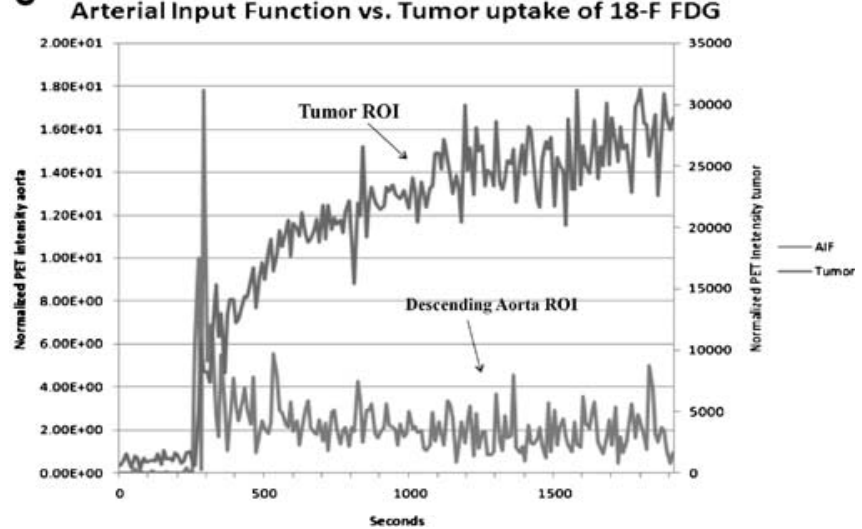

Axial time of flight MRI images : (a) at the descending aorta; (b) at the iliac arteries $(\mathrm{L}=$ left, $\mathrm{R}=$ right, $\mathrm{V}=$ ventral). (c) AIF and tumor uptake curves derived using coregistered MR and PET images

\section{$\mathbf{J 4 8 7}$}

\section{INVESTIGATION OF TUMOR RESPONSE AFTER PHOTODYNAMIC THERAPY USING SMALL-ANIMAL PET / MRI COREGISTRATION}

Kim Mongrain, Luc Tremblay, Jean-François Beaudoin, Johan E. van LIER, Roger Lecomte, Martin D. Lepage

Département de médecine nucléaire et de radiobiologie, Université de Sherbrooke, Sherbrooke, QC, Canada.
Introduction: Photodynamic therapy (PDT) is a promising cancer treatment that combines tumor-localizing photosensitizers (PS), light and oxygen to induce damage to the tumor tissue. A decrease in tumor perfusion and inflammation after treatment are typically observed after PDT. While ${ }^{18} \mathrm{~F}-\mathrm{FDG}$ is sensitive to both tumor metabolism and inflammation, MRI can provide complementary information on the tumor perfusion and inflammation following PDT. Methods: Fischer rats bearing two adenocarcinoma tumors were secured in a MRI-PET compatible cradle with a 3-point stereotactic reference frame. Data acquisition protocols for both modalities were adapted to allow normalization and coregistration of images using custom-developed software. Animals were injected i.v. with porfimer sodium (a clinically approved PS) at a therapeutic dose of $5 \mu \mathrm{mol} / \mathrm{kg}$ and treated with red light at fluence rates of $50 \mathrm{~mW} / \mathrm{cm}^{2}$ or $200 \mathrm{~mW} / \mathrm{cm}^{2}$. PET and MRI data were acquired at several time points before and after PDT ( $1 \mathrm{~h}, 3 \mathrm{~h}, 24 \mathrm{~h}$ and $48 \mathrm{~h})$. Tumor vasculature and inflammation were observed and characterized using dynamic contrast enhanced MRI at 7 T. PET images were acquired dynamically for 1 hour after a bolus injection of ${ }^{18} \mathrm{~F}$-FDG in an animal PET scanner. Results and Discussion: MR images obtained $1 \mathrm{~h}$ post PDT reveal regions of inflammation at both fluence rates, but, as expected, to a greater extent at $200 \mathrm{~mW} / \mathrm{cm}^{2}$. The size of the inflamed area increased with time. This was difficult to observe using PET as the diffuse weak signal in the inflamed area was contaminated by the large signal from neighboring tumor and heart. FDG uptake as well as MR perfusion of the contrast agent before and $1 \mathrm{~h}$ after treatment were similar for the control and treated tumor. However, at $24 \mathrm{~h}$ post PDT, even though FDG uptake of the treated tumor seems unchanged, the treated volume was clearly identified on the MR images as a symmetric spherical hypointense region. This is indicative of low perfusion suggesting a vascular effect of the PDT. Forty eight hours after treatment, both modalities reveal a regression in tumor volume as shown by the low intensity MR signal and an almost complete disappearance of FDG uptake. For both imaging modalities, comparison of the control and treated tumor over time clearly shows the progression of the control tumor and the regression of the treated tumor. Conclusion: Our results clearly demonstrate the synergy obtained by combining PET and MR image analysis to study tumor response to PDT.

\section{$\mathbf{J 4 8 8}$}

MOLECULAR ULTRASOUND IMAGING USING MAGNETIC NANOPROBES

Mohammad Mehrmohammadi ${ }^{1}$, Li L. Ma ${ }^{2}$, Min Qu ${ }^{1}$, Keith P. Johnston ${ }^{2}$, Konstantin Sokolov $^{1,3}$, Stanislav Y. Emelianov ${ }^{1}$

${ }^{1}$ Biomedical Engineering, The university of Texas at Austin, Austin, TX, USA, ${ }^{2}$ Chemical Engineering, The University of texas at Austin, Austin, TX, USA, ${ }^{3}$ Department of Imaging Physics, UT M.D. Anderson Cancer Center, Houston, TX, USA.

Understanding the molecular and cellular mechanism of diseases can lead to detecting the pathology at early stages of development. Therefore, efforts have been directed toward design and development of new imaging techniques, capable of visualizing biological events at molecular and cellular level. Currently, nuclear medicine imaging, magnetic resonance imaging (MRI), and optical imaging are the most widely used molecular imaging techniques. However, these methods have several deficiencies, such as the need for radioactive agents in radionuclide molecular imaging, lack of realtime in molecular MRI, and low penetration depth in molecular optical imaging. Ultrasound-a non-ionizing, real time, reliable, portable and cost effective technique, is an excellent imaging modality. Unfortunately, lack of sufficient contrast and spatial resolution limits the ability of ultrasound to image molecular events. Here, we introduce magneto-acoustic (MA) imaging, capable of visualizing the molecular content of tissue by utilizing magnetic nanoprobes which can be indirectly detected by ultrasound. 
Magneto-acoustic imaging utilizes a high strength magnetic field to induce motion in the magnetically labeled molecules or cells and ultrasound to detect the motion within the tissue. A set of experiments were performed on phantoms with living macrophage cells with internalized ultra-small magnetic nanoparticles. These labeled cells were mixed with gelatin and injected into normal tissue to form an inclusion. The tissue was scanned with a high-frequency, focused, single-element ultrasound transducer while a pulsed magnetic excitation field was applied to the sample. Several ultrasound pulse-echo signals were captured before, during and after the application of magnetic pulse. The displacement of tissue was computed using a block-matching cross-correlation approach. The maximum displacement at each position within the imaging plane was displayed in the MA image. The results show the contrast between the magnetically labeled cells inclusion and the tissue background. In conclusion, magneto-acoustic imaging was introduced as an ultrasound-based molecular imaging tool. Further, with the development of biocompatible nanoparticles that have the desired molecular targeting moiety, magneto-acoustic imaging can become a pathology-specific molecular imaging technique.

\section{J489}

\section{INTEGRATION AND CO-CALIBRATION OF AN OPTICAL TOMOGRAPHIC IMAGING SYSTEM INTO A SINGLE- GANTRY SPECT-CT SMALL ANIMAL IMAGER}

Liji Cao, Wolfhard Semmler, Joerg Peter

Div. Meidical Physics in Radiology, German Cancer Research Center, Heidelberg, Germany.

Objective: Simultaneous detection of optical and radio-pharmaceutical signals possibly using dual-labeled imaging probes in vivo with coregistered anatomical information not only provides complementary information which can improve diagnosis but also offers the opportunity to validate and quantify optical imaging by comparison with nuclear medicine modality. Intended for such application we integrated optical tomographic imaging components into a previously published co-calibrated SPECT-CT small animal imager [cao et al. NSSMIC08]. A geometric co-calibration method was applied to calculate the inherent mechanical displacements between the optical system and the SPECT-CT, yielding intrinsically co-registered tri-modal imaging without moving the subject across the modalities. Methods: The optical tomographic components consist of a displaceable light source, a CCD camera and VIS/NIR light reflectors. This design provides fully 360 degree detection of fluorescent or bioluminescent signals for any rotational gantry position. Geometric co-calibration was performed by imaging a cylindrical light source $(0.9 \mathrm{~mm}$ in diameter, $2.5 \mathrm{~mm}$ in length) firstly with $\mathrm{CT}$, and then with optical detection at 240 angular and 2 axial positions. Given the coordinates of the light source in the $\mathrm{CT}$ volume as well as the 2D locations of it at optical detector plane, the mechanical displacements (7 parameters for each view) were calculated with a hybrid method including both analytical and optimization steps. Results: The co-calibration process determines the intrinsic geometric parameters of the optical detection system as well as the positional relationship between SPECT-CT results and the optical images. In this way, the photon flux of each point on the object surface whose position is extracted from SPECT-CT data can be acquired from this optical system. Results show that the internal system errors are within sub-pixel range. Discussion: This integrated hardware with inherent cocalibration provides the possibility of simultaneous and intrinsically coregistered imaging with aforementioned three modalities. Such instrumentation offers not only an easy study management with different modalities but also the opportunity of direct comparison between nuclear and optical signals in vivo at the same time on the overlaid anatomical information, which may lead to the validation and quantification of optical imaging.

\section{J490}

\section{A NOVEL RECONSTRUCTION STRATEGY FOR FLUORESCENCE MEDIATED TOMOGRAPHY USING CO-REGISTERED CT-SPECT PRIORS}

Liji Cao, Josef K. Doll, Wolfhard Semmler, Joerg Peter

Div. Meidical Physics in Radiology, German Cancer Research Center, Heidelberg, Germany.

Objective: Fluorescence mediated tomography (FMT) reconstruction in the continuous wave (CW) mode constitutes an ill-posed inverse problem and artifacts are expected in the reconstruction results. Given an integrated SPECT-CT-OT small animal imaging system and duallabeling probes consisting of both NIR fluorophores and radionuclides the fluorescent tomography reconstruction may benefit from both the prior information of the subject's anatomy and the coupled radioactivity distribution acquired from simultaneous CT and SPECT scanning. Materials and Methods: All the measurements were taken from a selfdeveloped single-gantry SPECT-CT-OT small animal imaging system which yields intrinsically co-registered images of three modalities. There cylindrical resin phantoms, which approximate the optical properties of real tissues, were used in the experiments. Each phantom concludes a 2 mm-cylinder inclusion at different depth and was filled with a mixed solution of Tc99m and Cy5.5. Firstly, a 3D tetrahedral mesh representing the object was extracted from CT results by marching cubes followed with a constrained Delaunay tetrahedralization algorithm. The forward model of FMT based on Born normalization was calculated by Finite Element Method. The inverse problem was then solved by an MLEM procedure which includes SPECT prior information as a soft constraint to guide the solution to a reasonable convergence. Results: Results show that CT data provide an accurate surface representation of the imaging object with arbitrary geometries. Direct FMT reconstruction without including SPECT prior information is burdened by slow convergence, yields artifacts at boundary regions and represents different reconstructed quantities for same amount of fluorephore at different positions. Integrating the coupled SPECT prior as a soft constraint, however, guides the reconstruction results to a better solution, regardless of the position of the fluorephore inclusion, at a higher speed and with lower level of artifacts. Discussion: The possibility of improving FMT by means of coupled anatomic and nuclear imaging modalities was studied. Results demonstrate that the ill-posed FMT problem which is due to the high scattering probability of optical photons can be better solved with coupled prior information of high-energy photon distribution which yields low scatter events. This combined multimodality techniques are promising to facilitate quantitative analysis of fluorephore distributions for small animals without the limitation of penetration depth.

\section{J491}

PET, SPECT AND CT TRIMODALITY IMAGING OF A MOUSE INJECTED WITH MIXED MODALITY ISOTOPES

Kerrie H. Tainter ${ }^{1,6}$, Stephen Lokitz ${ }^{1}$, Chris Vascoe ${ }^{2}$, Joann Zhang ${ }^{3}$, Brent J. Coco $^{3}$, Koji Iwata ${ }^{3}$, Douglas J. Wagenaar ${ }^{4}$, J. M. Mathis ${ }^{5,6}$ ${ }^{1}$ PET Imaging Center, Biomedical Research Foundation, Shreveport, LA, USA, ${ }^{2}$ Radiochemistry, Biomedical Research Foundation, Shreveport, LA, USA, ${ }^{3}$ Preclinical Imaging, GE Healthcare, Waukesha, WI, USA, ${ }^{4}$ GammaMedica-Ideas, Northridge, CA, USA, ${ }^{5}$ Cellular Biology and Anatomy, LSU Health Sciences Center - Shreveport, Shreveport, LA, USA, 'Gene Therapy Program, LSU Health Sciences Center Shreveport, Shreveport, LA, USA.

Recent availability of commercial pre-clinical PET/SPECT/CT trimodality systems brings a unique opportunity to assess the imaging 
animals injected with mixed modality (PET/SPECT) isotopes. Our goal in this study was to characterize the image quality of PET and SPECT mouse images acquired in the presence both $\left({ }^{18} \mathrm{~F}-\mathrm{FDG}\right.$ and $\left.{ }_{99 \mathrm{~m}} \mathrm{Tc}-\mathrm{MDP}\right)$. Methods: Physiologically relevant tracer quantities (2$3 \mathrm{mCi}{ }^{99 \mathrm{~m}} \mathrm{Tc}-\mathrm{MDP}$ and $\left.200-300 \mathrm{uCi}{ }^{18} \mathrm{~F}-\mathrm{FDG}\right)$ were injected into a mouse. ${ }^{99 \mathrm{~m}} \mathrm{Tc}-\mathrm{MDP}$ was allowed 30 minutes uptake before SPECT acquisitions at small $(60 \mathrm{~mm})$ and larger $(100 \mathrm{~mm})$ Fields of View (FOVs). Following the SPECT series, ${ }^{18}$ F-FDG was injected and a second SPECT series was acquired. This allowed approximately 40 minutes for ${ }^{18} \mathrm{~F}$-FDG uptake before the PET acquisition. After the PET scan, a CT was acquired for anatomical reference. Utilizing the different half-lives of ${ }^{18} \mathrm{~F}$-FDG and ${ }^{99 \mathrm{~m}} \mathrm{Tc}$-MDP scans with different SPECT/PET isotope ratios were evaluated for signal and noise characteristics. Results: Pre- and Post- ${ }^{18}$ F-FDG SPECT series show that, while there is visible noise in the image, the pattern is largely circumferential and more prevalent at the outside of the FOV. Important bony features of the mouse are still readily identifiable in SPECT images. The PET image shows no signs of degradation with clearly defined uptake in expected organs such as the heart, brain, and bladder. Conclusions: Simultaneous SPECT and PET imaging is feasible using current imaging technologies. Consideration of the timing, amount, and type of injected SPECT and PET tracers is necessary to account for the presence and spatial distribution of scatter resulting from the PET tracer contaminating the SPECT image set.

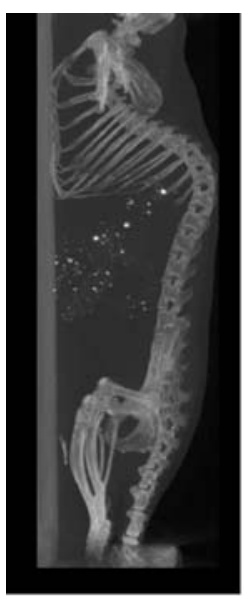

CT MIP

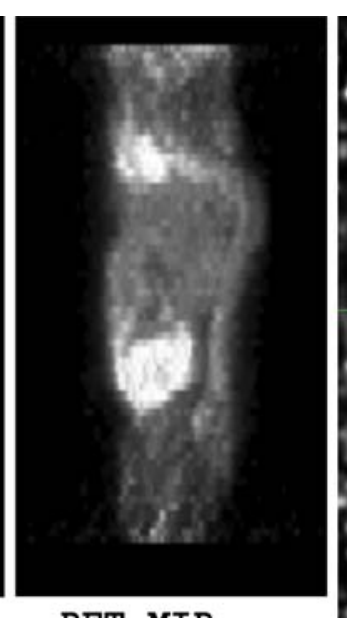

PET MIP

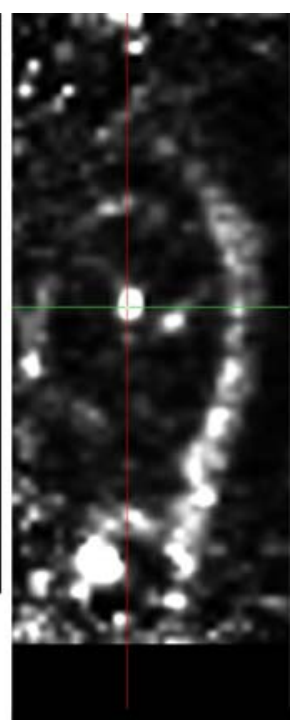

$\mathbf{J 4 9 2}$

\section{UTE-MR-BASED ATTENUATION MAPS FOR MICROPET/ MICROSPECT-MR}

Vincent Keereman $^{1}$, Steven Deleye ${ }^{1}$, Yves Fierens ${ }^{2}$, Steven H. Baete ${ }^{1,3}$, Tony Lahoutte ${ }^{4,5}$, Steven G. Staelens ${ }^{1}$, Yves De Deene ${ }^{1,3}$, Stefaan Vandenberghe ${ }^{1}$

${ }^{1}$ MEDISIP, Ghent University - IBBT - IBiTech, Ghent, Belgium, ${ }^{2}$ Radiology/BEFY, UZ Brussel, Brussels, Belgium, ${ }^{3}$ Laboratory for Quantitative Nuclear Magnetic Resonance in Medicine and Biology, ECNURAD, Ghent University, Ghent, Belgium, ${ }^{4} I n$-vivo Cellular and Molecular Imaging, UZ Brussel, Brussels, Belgium, ${ }^{5}$ Nuclear Medicine, UZ Brussel, Brussels, Belgium.

INTRODUCTION It is difficult to derive an attenuation map from MR images because of the low signal intensity in bone caused by the very short spin-spin relaxation time (T2). MR-based attenuation correction would however render a high-dose micro-CT obsolete in some cases.
For human applications the use of ultrashort echo time (UTE) sequences for MR-based attenuation correction has recently been proposed. We have investigated the possibility of applying the same technique to small animal imaging. METHODS A rat was euthanized and deep frozen overnight to assure no internal organs moved between $\mathrm{CT}$ and MR scans. A CT was acquired on a GE Triumph tri-modality system $(70 \mathrm{keV}, 200 \mathrm{mAs})$. MR images were acquired on a Philips Achieva $3.0 \mathrm{~T}$ system using a knee coil. A multi-echo UTE sequence with $3 \mathrm{D}$ radial sampling was used with $\mathrm{TE} 1=0.12 \mathrm{~ms}$, TE2 $=2.3 \mathrm{~ms}$ and $\mathrm{TR}=12 \mathrm{~ms}$. The reconstructed voxel dimensions for $\mathrm{CT}$ and $\mathrm{MR}$ were 0.3 and $0.5 \mathrm{~mm}$ respectively. The MR images at both echo times were used to derive the R2-map $(=1 / \mathrm{T} 2)$ of the imaging volume. A binary mask derived from the first echo image was applied to the R2-map to set all voxels containing air to zero. The R2-map then respectively shows high, medium and zero intensity in bone, soft tissue and air voxels and can easily be segmented into these tissue classes by tresholding. The CT image was also segmented by tresholding. A coronal slice of the corrected R2-map, segmented MRI and segmented CT containing the spine is shown in figure 1. RESULTS A voxel-by-voxel comparison of both segmented images was performed in the displayed coronal slice. $92 \%$ of voxels were assigned to the correct tissue class. Segmentation was difficult in the abdomen because the rat food also has a short $\mathrm{T} 2$ when frozen and is segmented as bone. Relaxometry measurements have shown that this problem does not arise at room temperature. CONCLUSION The feasibility of estimating an attenuation map from MR images acquired with a UTE sequence was shown. Comparison with segmented CT images shows that the accuracy of the segmentation should be sufficient for attenuation correction in small animal nuclear imaging.

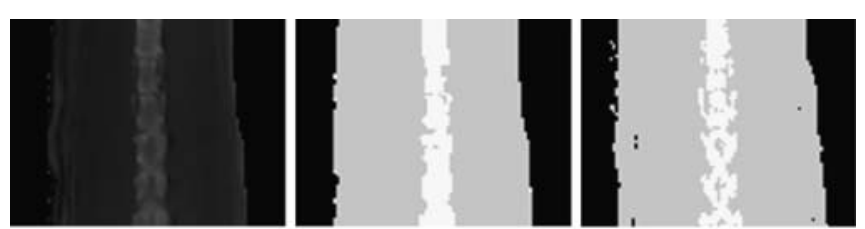

FIGURE 1: Coronal slice of the R2-map (left), segmented MR (middle) and segmented CT (right).

\section{J493}

\section{OPTIMIZING SENSITIVITY OF DETECTION OF SMALL POPULATIONS OF FLUORESCENT CELLS IN VIVO BY ANIMAL ROTATION}

Gilbert D. Feke, W. Matthew Leevy, Sean P. Orton, Benjamin F. Geldhof, William McLaughlin

Carestream Molecular Imaging, Carestream Health, Inc., New Haven, CT, USA.

To better optimize in vivo imaging for detection of fluorescent cells, a multimodal approach was used wherein X-ray images were precisely coregistered with fluorescence images of mice undergoing craniocaudal rotation (CR) - achieved by a commercially available multimodal imaging system fitted with a unique, automated animal rotation device. To test the efficacy of this approach, HeLa cells were labeled by incubation at $37^{\circ} \mathrm{C}$ for $4 \mathrm{hrs}$ with cationic latex nanospheres loaded with a near-infrared (NIR) fluorescent dye. $\mathrm{Nu} / \mathrm{Nu}$ mice each received dorsal punctile subcutaneous injections of a dilution series of these fluorescently labeled cells spanning population sizes from $1.5 \times 10^{4}$ to $1.5 \times 10^{6}$ cells, and were imaged $(750 \mathrm{~nm}$ excitation $/ 830 \mathrm{~nm}$ emission). The regions-of-interest (ROI) analysis defined fluorescent cell signals as the mean pixel value within ROIs thresholded at $50 \%$ of the maximum pixel value for each injection, and defined noise as the standard deviation of the pixel values within a ROI on the mouse which excluded the fluorescent signal entirely. The results show that detection of the smallest cell populations is most reliant upon optimization of the 
CR angle. For example, ROI identification of the $1.5 \times 10^{4}$ cell population in one of the mice was only possible within a $68^{\circ}$ range around the peak $\mathrm{CR}$ angle, which provided a signal-to-noise ratio (SNR) of 4.3. In contrast, ROI identification of the $1.5 \times 10^{6}$ cell population persisted through a $233^{\circ}$ range around the peak CR angle, which provided a SNR of 532. The analysis demonstrates a linear relationship between cell population size and SNR, which indicates that the fluorescence noise level can be equated to a cell-equivalent noise level by simple slope-intercept analysis. For example, for the mouse described above, the fitted noise level was 4415 cells. A decrease of the detected fluorescent signal due to nonoptimal CR angle has the effect of increasing the cell-equivalent noise level proportionately.
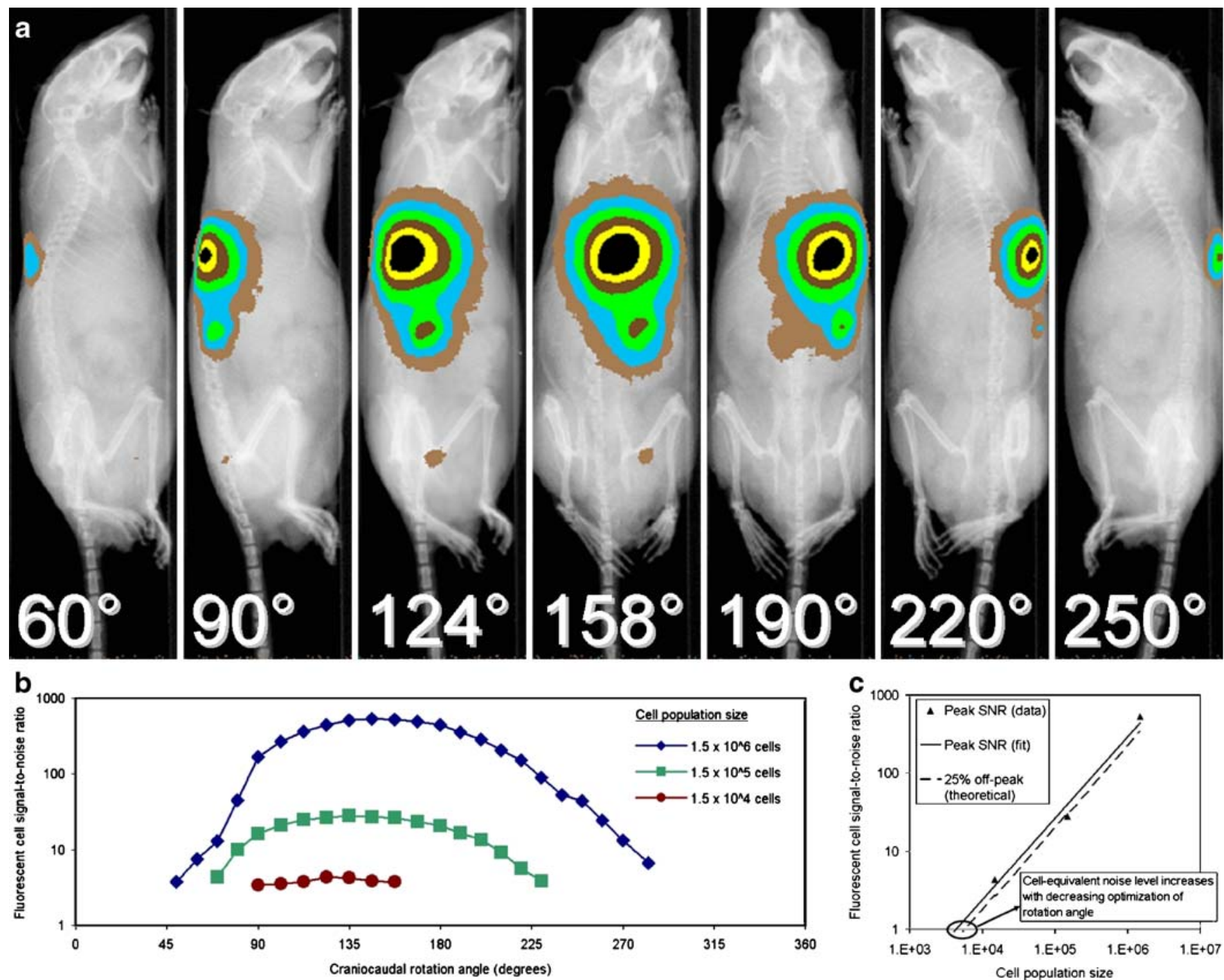

(A) X-ray images with fluorescent overlay for various CR angles. (B) SNR vs. CR angle. (C) SNR vs. cell population size.

J494

\section{AN INTEGRATED PET/MRI (IPET/MRI) SYSTEM FOR SMALL ANIMALS}

Seiichi Yamamoto ${ }^{1}$, Masao Imaizumi², Yoshikazu Kanai², Eiji Sugiyama $^{3}$, Masaaki Aoki ${ }^{3}$, Eku Shimosegawa $^{2}$, Jun Hatazawa ${ }^{2}$

${ }^{1}$ Kobe City College of Technology, Kobe, Japan, ${ }^{2}$ Nuclear Medicine, Osaka University Graduated School of Medicine, Osaka, Japan, ${ }^{3}$ Hitach Metal, Neomax Company, Saga, Japan.
An integrated PET/MRI (iPET/MRI) system for small animals was developed by combining a new MR-compatible PET and a permanent magnet open MRI. In the MRI, a tunnel is opened at the yoke of the magnet. The position sensitive photo-multiplier tube (PSPMTs) of the MR-compatible PET are positioned backside of the yoke where the magnetic field is low enough. Scintillators for the PET system is positioned at the center of the MRI magnets and the scintillation photons are changed its direction by slanted light guides and are fed to the PSPMTs by $75 \mathrm{~cm}$ long optical fiber bundles. The PET detectors 
employed two types of LGSO crystals $(1.9 \mathrm{~mm} \times 2.2 \mathrm{~mm} \times 6 \mathrm{~mm}$ and $7 \mathrm{~mm}$ ) with different decay times (33 ns and $43 \mathrm{~ns}$ ) for depth-ofinteraction (DOI) detection. Sixteen optical fiber based block detectors are arranged in $112 \mathrm{~mm}$ diameter ring. The transaxial field-of-view (FOV) of the PET system is $\sim 80 \mathrm{~mm}$ and axial FOV is $21 \mathrm{~mm}$. The axial FOV can be enlarged by a z-motion of the PET detector ring during MRI acquisition. The tranaxial and axial resolution at the center of the PET system was $2.9 \mathrm{mmFWHM}$ and $2.4 \mathrm{~mm} \mathrm{FWHM}$, respectively. Absolute sensitivity was $1.5 \%$ at the center of axial FOV. Phantom images showed that there was no artifact in either PET or MRI images. We could successfully obtain simultaneously measured small animal images using the iPET/MRI system. We confirmed that the iPET/MRI system has good performance and can be used for the simultaneous measurements of small animals.

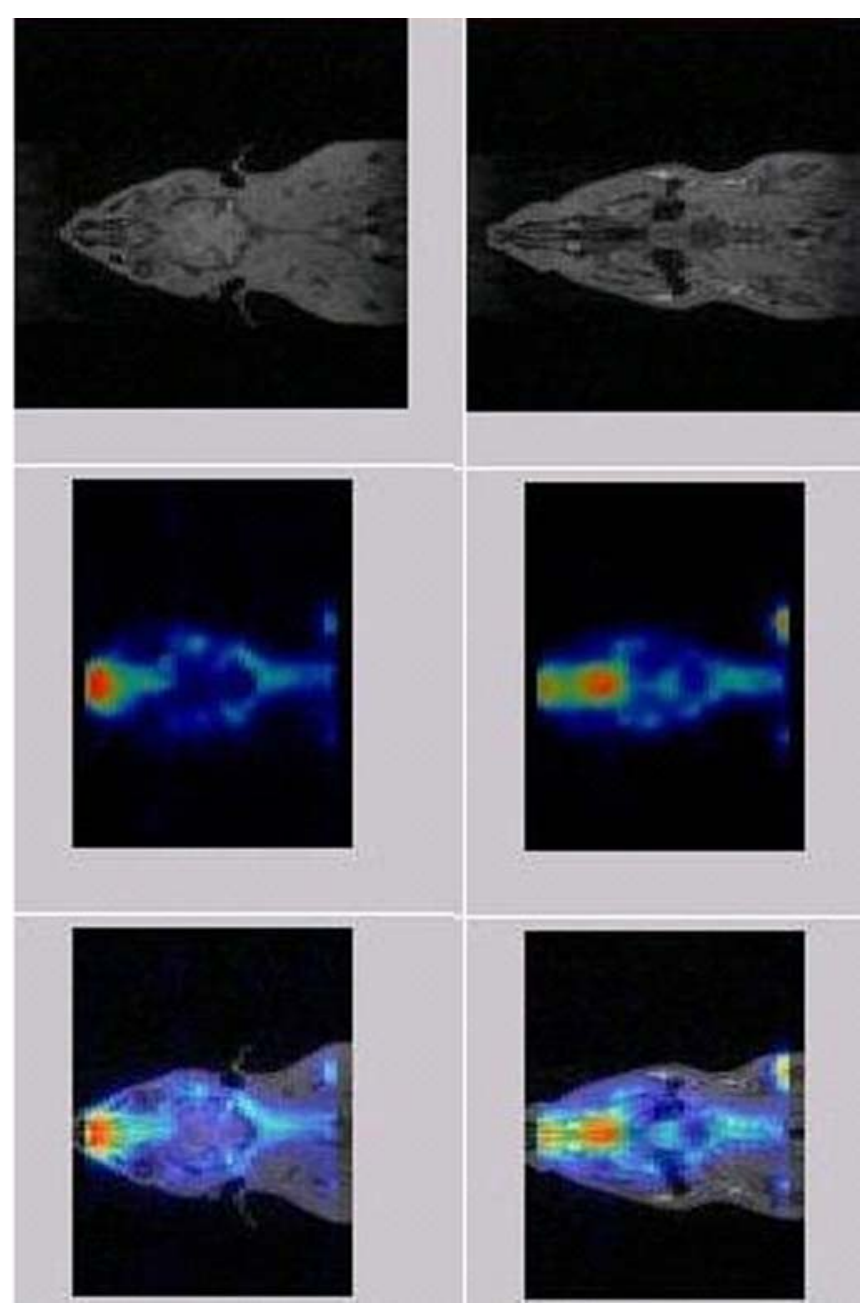

Simultaneously measured MRI (top), PET (middle) and fused (bottom) coronal images of rat brain (F-minus study) using the iPET/MRI system

\section{J495}

\section{QUANTITATIVE OPTOACOUSTIC SIGNAL EXTRACTION} USING SPARSE SIGNAL REPRESENTATION

Amir Rosenthal $^{1,2}$, Daniel Razansky ${ }^{1,2}$, Vasilis Ntziachristos ${ }^{1,2}$

${ }^{1}$ Helmholtz Zentrum München, Neuherberg, Germany, ${ }^{2}$ Technical University of Munich, Munich, Germany.
We report on a new quantification methodology of optoacoustic tomographic reconstructions under highly heterogeneous illumination conditions representative of realistic wholebody imaging scenarios. Our method relies on the different spatial characteristics of the absorption coefficient and the optical energy density within the medium. By using sparse-representation based decomposition [1], we exploit these different characteristics to extract both the absorption coefficient and the photon density within the imaged object from the optoacoustic image. In contrast to previous methods [2], this image normalization algorithm is not based on the solution of theoretical light transport equations and offers robust performance, as it does not require explicit knowledge of the illumination geometry, optical properties of the object and other unknown or loosely defined experimental parameters. It is therefore ideally suited for practical implementations in varying complexity tomographic schemes including multi-projection illumination systems and multi-spectral optoacoustic tomography studies of tissue biomarkers. The method was successfully tested on both numerically and experimentally generated data. References: [1] S.S.Chen, D.L. Donoho, and M.A. Saunders, "Atomic decomposition by basis pursuit," SIAM Rev. Vol. 43, pp. 129-159, 2001. [2] B. T. Cox, S. R. Arridge, K. P. Kostli, and P. C. Beard, "2D quantitative photoacoustic image reconstruction of absorption distributions in scattering media using a simple iterative method," Applied Optics Vol. 45, pp. 1866-1875, 2006.
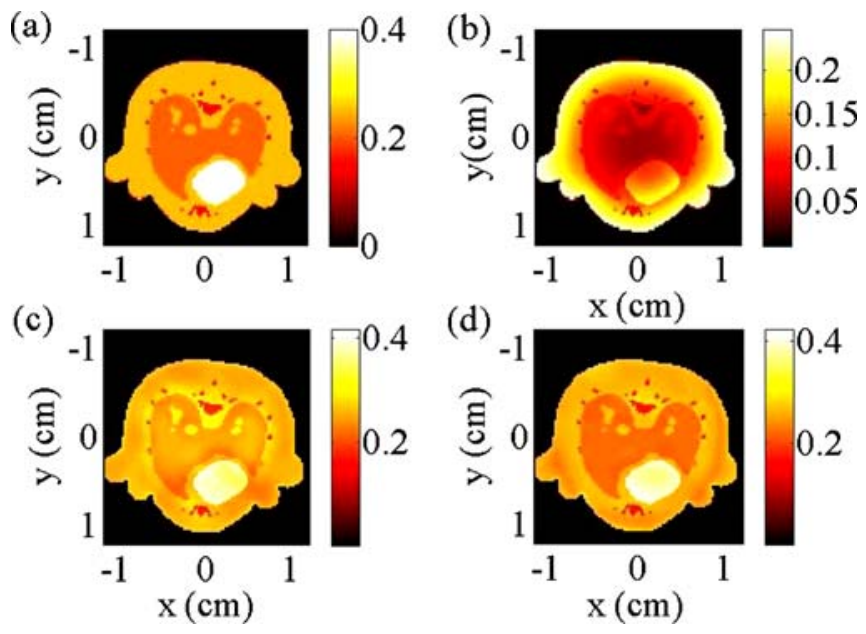

(a) An absorption coefficient map with the geometry of a mouse's torso and (b) its corresponding optoacoustic image. (c) The reconstruction of the absorption coefficient of the object obtained by the decomposition algorithm with the Haar and Fourier bases, and (d) with expanded libraries which included a user-defined function and an analytical solution to the light diffusion equation

\section{J496}

\section{FACTORS AFFECTING PARTIAL VOLUME CORRECTION OF PET-CT IMAGES BASED ON THE METHOD OF RECOVERY COEFFICIENTS}

David Yerushalmi $^{1,3}$, Frezghi Habte ${ }^{2,3}$, Sanjiv S. Gambhir ${ }^{2,3}$

${ }^{\mathrm{T}}$ Bioengineering, Stanford University, Stanford, CA, USA, ${ }^{2}$ Radiology, Stanford University, Stanford, CA, USA, ${ }^{3}$ Molecular Imaging Program at Stanford (MIPS), Stanford University, Stanford, CA, USA. 
Accurate quantitation of focal tracer uptake in PET-CT images is relevant to a number of applications including treatment monitoring and earlier disease detection. Blurring due to the partial volume effect limits accurate measurement of activity concentration in small tumors. Partial volume correction using recovery coefficients (RC) is an established method for more accurately estimating focal uptake in PET images however its application for quantitation of relatively small lesions has been unreliable. Here we demonstrate the use of phantom studies and computer simulations to investigate the relative effect of different factors contributing to the observed variability including noise, slice thickness, and object placement. A small hollow sphere (radius $=3.95 \mathrm{~mm}$ ) was filled with a calibrated concentration of activity $(\sim 1 \mu \mathrm{Ci} / \mathrm{mL})$. A series of PET-CT scans were performed by varying scan time and object location in order to independently measure the effect of these experimental parameters. In a related study, the Point spread function (PSF) for the PET-CT scanner was measured by imaging a point source positioned about the center of the FOV. The imaging process was then modeled as the convolution of a discretized spherical object with a Gaussian kernel based on prior experimental measurements. Model data was reformatted to account for changing PET slice thickness and object position to better understand the sensitivity of quantitation accuracy as a function of these parameters and for comparison with experimental data. Simulation results suggest that the error due to object offset relative to detector center may be as large as $12 \pm 7 \%$. This is much larger than the contribution of noise $(\sim 2-3 \%)$, and also depends on the accuracy of the PSF measurement at any given position. These results better characterize the factors that limit application of the recovery coefficients and suggest the need for more accurate methods to estimate the scanner PSF.
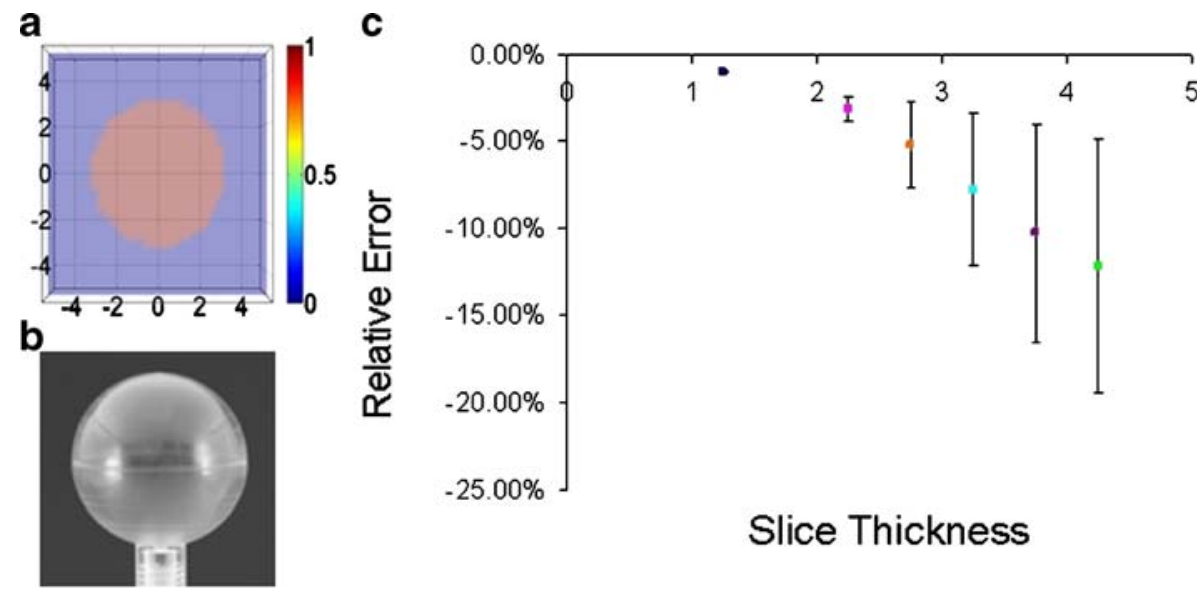

Slice Thickness

Figure: Cross-section through discretized object used for simulation (A), Image of physical phantom used for data acquisition experiments (B), Plot showing predicted Percent Relative Error estimated based on a simulation and showing increasing average error and variability with increasing slice thickness $(\mathrm{C})$.

J497

\section{A MULTIMODAL POST PROCESSING FRAMEWORK FOR ACTIVATION STUDIES OF THE RAT BRAIN}

Steven G. Staelens ${ }^{1}$, Tine Wyckhuys ${ }^{2}$, Steven Deleye ${ }^{1}$, Hans Hallez ${ }^{1}$, Stefaan Vandenberghe ${ }^{1}$, Bregt Van Nieuwenhuyse ${ }^{2}$, Kristl Vonck ${ }^{2}$

${ }^{1}$ Medical Signal and Image Processing group, Ghent University IBBT, Gent, Belgium, ${ }^{2}$ Laboratory for Clinical and Experimental Neurophysiology, Ghent University Hospital, Gent, Belgium.

INTRODUCTION: A stimulus-on versus stimulus-off imaging study is often used to evaluate the brain's response to a presented visual, electrical or chemical trigger. Clinical software and human templates already exist but given the recent advent of ultrahigh resolution $\mu$ SPECT and $\mu$ PET, a larger need rises for a post processing platform to perform these subtraction molecular imaging studies also in small animals. We have designed such a multimodal framework to perform $\mu$ SPECT activation studies in rats thereby making use of $\mu \mathrm{CT}$ and MRI for anatomical land marking. METHODS: Our software solution is a combination of Amide, MRIcroN and a custom made Matlab implementation. We have studied the performance of different deep brain stimulations for which 6 rats were implanted with a multi-polar stimulation electrode in the right hippocampus. Each animal underwent a 99mTc-HMPAO $\mu$ SPECT with the Milabs U-SPECT-II and a $\mu \mathrm{CT}$ scan with the GMI X-O CT before and after stimulation. Two line markers in oblique positions filled with low activity of $125 \mathrm{I}$ are used to register the $\mu$ SPECT and $\mu \mathrm{CT}$ images. Afterwards the animals were sacrificed, their electrode was removed and a MRI scan was performed using the wrist coil of a Siemens Trio 3T. RESULTS: A semi-automated five step procedure delivers the activation map: (i) first the stimulus-on $\mu \mathrm{CT}$ and the MRI are registered to the stimulus-off $\mu \mathrm{CT}$ followed by (ii) the fusion of the off/on $\mu$ SPECT scans with their off/on $\mu \mathrm{CT}$ counterparts. From the MRI, (iii) the rat brain is extracted, which is used as a mask for the calculation. Afterwards, (iv) both off/on $\mu$ SPECT scans are normalized and subtracted within this MRI brain mask. Finally, (v) the $\mathrm{Z}$-score, representing the activation map, is achieved by dividing the result with the standard deviation of the masked 
stimulus-off $\mu$ SPECT. CONCLUSION: This semi-automated approach allows the experimental neuroscientist to draw conclusions on the location, spatial extent and intensity of the small animal brain's response to the stimulus.
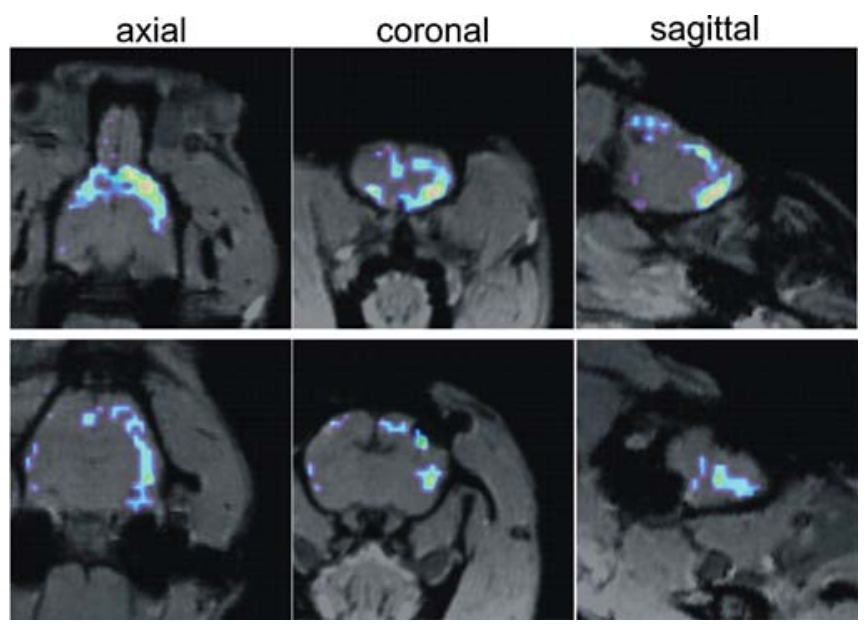

Illustrative outcome of the multimodal post processing framework: activation map (Z-score) as the reponse to a stimulation train.

J498

\section{SEMI-ANALYTICAL MODEL-BASED ACOUSTIC INVERSION ALGORITHM FOR QUANTITATIVE OPTOACOUSTIC TOMOGRAPHY}

Amir Rosenthal $^{1,2}$, Daniel Razansky ${ }^{1,2}$, Vasilis Ntziachristos ${ }^{1,2}$ ${ }^{1}$ Helmholtz Zentrum München, Neuherberg, Germany, ${ }^{2}$ Technical University of Munich, Munich, Germany.

We present a fast model-based inversion algorithm for two- and three-dimensional optoacoustic tomography. The algorithm provides modeling flexibility essential for image quantification and is shown to overcome several substantial reconstruction-related artifacts of commonly used backprojection inversion schemes [1]. In contrast to previous model-based approaches, which are based on numerical solutions to the acoustic propagation problem [2], the semi-analytical solution presented here is exact for piecewise planar acoustic-source functions, which significantly improves the accuracy and computational speed. In addition, our method requires solving the forward acoustic problem only once, thus reducing the computational complexity. The solution is saved in a matrix, which is inverted in order to reconstruct the image. The inverted matrix does not depend on the data. Thus, the same inverse matrix may be used for different experimental data obtained by the same system. If the inverse matrix of the system has been calculated beforehand, the reconstruction may be performed in real time. The algorithmic performance is demonstrated using numerical simulation studies and experimentally on tissue-mimicking optically heterogeneous phantoms. References: [1] M. Xu and L. V. Wang, "Universal back-projection algorithm for photoacoustic-computed tomography," Phys. Rev. E 71 (1): 016706 (Part 2, Jan. 2005). [2] Huabei Jiang, Zhen Yuan, and Xuejun Gu, "Spatially varying optical and acoustic property reconstruction using finite-element-based photoacoustic tomography,” J. Opt. Soc. Am. A Vol. 23, pp. 878-888, 2006.

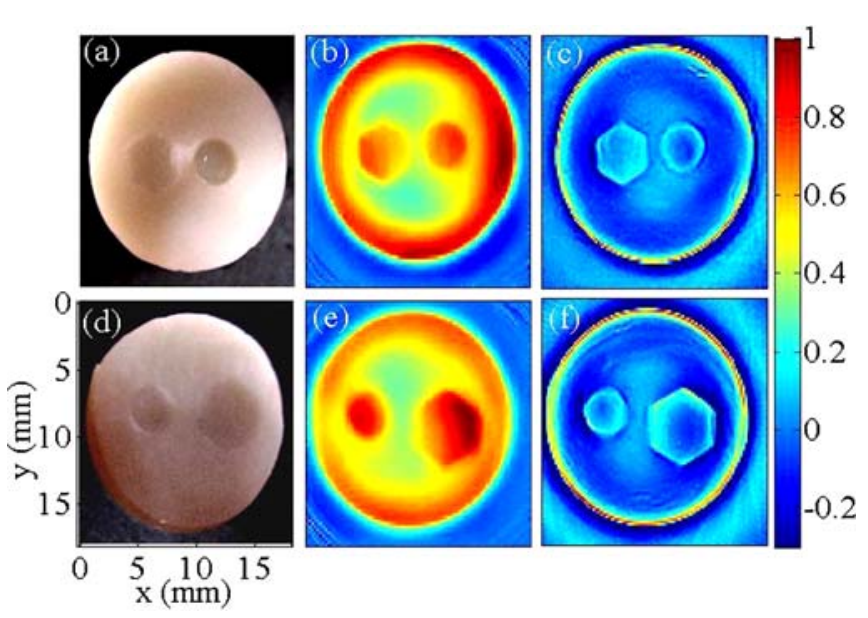

Experimental reconstruction of two phantoms with insertions. The phantoms were uniformly illuminated from their sides. The phantoms are shown in the first column; the model-based reconstruction is shown in the second column; and the back-projection reconstruction is shown in the third column. The figure clearly shows that the back-projection reconstruction suffers from negativevalue artifacts and does not depict the light attenuation along the phantom. In contrast, the model-based reconstructions do not suffer from these deficiencies.

\section{STATIONARY SPECT CAMERA FOR SIMULTANEOUS SPECT-MRI PRE-CLINICAL IMAGING}

Dirk Meier ${ }^{1}$, Douglas J. Wagenaar ${ }^{1}$, Bjorn M. Sundal ${ }^{1}$, Gunnar E. Maehlum ${ }^{1}$, Bradley E. Patt ${ }^{1}$, Si Chen ${ }^{2}$, Jingyan $\mathrm{Xu}^{2}$, Benjamin M. Tsui ${ }^{2}$, Seung Hoon $\mathrm{Ha}^{3}$, Mark Hamamura ${ }^{3}$, Werner W. Roeck ${ }^{3}$, Orhan Nalcioglu ${ }^{3}$

${ }^{1}$ Gamma Medica - Ideas, Fornebu, Norway, ${ }^{2}$ Johns Hopkins University, Baltimore, MD, USA, ${ }^{3}$ University of California, Irvine, CA, USA.

The objective is to develop a SPECT camera for simultaneous SPECT-MRI in pre-clinical imaging. SPECT provides extreme molar sensitivity and the ability to image multiple biological processes through energy discrimination. MRI provides information on anatomy and a-priori data for SPECT attenuation correction, motion correction and blood flow. Our goal is to operate the SPECT inside the MRI and to acquire in vivo images using both modalities simultaneously with the same FOV. Until today it was impossible to operate conventional SPECT inside the MRI because of mutual interference. The new SPECT technology is based on pixellated radiation sensors (CZT, ASICs) and enables SPECT inside MRI. The SPECT camera fits into conventional high field MRI systems with a min. 12-cm bore size. The SPECT uses an MR-compatible multi-pinhole collimator (32 apertures of $\varnothing 2-\mathrm{mm}$ ) for mice with $\varnothing 30-\mathrm{mm}$ FOV. The radiation detector has $1.6-\mathrm{mm}$ intrinsic spatial resolution. We operated the SPECT inside the MRI and acquired SPECT and MRI data. We reconstructed SPECT images from capillary tubes and measured $2.5-\mathrm{mm}$ FWHM at the peak of the LSF. We measured 5.4-keV FWHM energy resolution at $140 \mathrm{keV}$ inside a 3-T MRI system. The SPECT affected the noise in the spin-echo MR image, but did not affect the gradient-echo MR images. New 
detector technology (CZT, ASICs) enables simultaneous SPECT/ MRI. The new SPECT camera operates inside an MRI system and delivers images with minimum interference effects from the two imaging modalities.

Characteristics of the SPECT camera

\begin{tabular}{|c|c|}
\hline modalities & simultaneous SPECT and MRI \\
\hline & dynamic SPECT without camera motion \\
\hline SPECT camera specifications & multi-isotope SPECT \\
\hline & $\varnothing 30 \mathrm{~mm} \times 120 \mathrm{~mm}$ FOV (mice) \\
\hline & $2.5 \%$ geometric collimator sensitivity \\
\hline & 32 x $\varnothing 2$ mm apertures \\
\hline sensor technology & 1.6 mm intrinsic spatial resolution \\
\hline & pixellated CZT, direct radiation sensor \\
\hline
\end{tabular}

$\mathrm{CZT}=$ cadmium zinc telluride, $\mathrm{ASIC}=$ application specific integrated circuits, $\mathrm{FOV}=$ field of view

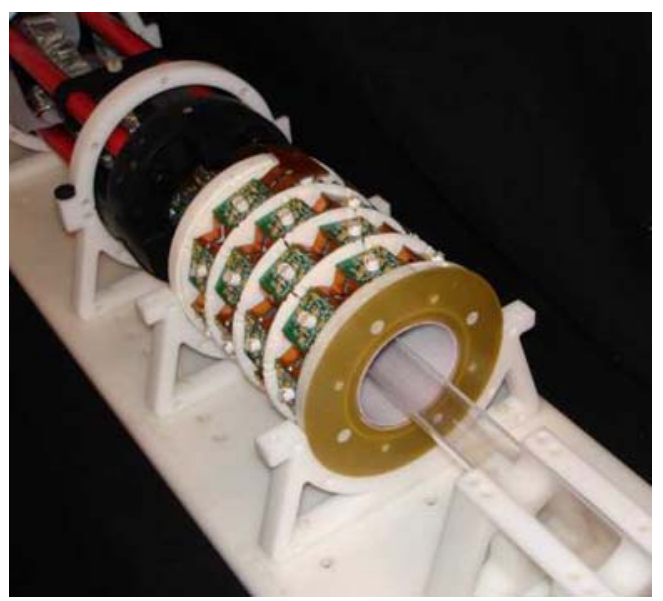

The MR-compatible SPECT camera.

\section{$\mathbf{J 5 0 0}$}

\section{BRIDGE CAPSULE: HOLDING FIXTURE TO IMPROVE REPRODUCIBILITY AND REGISTRATION DURING LONGITUDINAL MULTIMODAL IMAGING}

Daisuke Kokuryo, Yuichi Kimura, Takayuki Obata, Taiga Yamaya, Hiroo Ikehira, Ichio Aoki, Iwao Kanno

Biophysics Group, Molecular Imaging Center, National Institute of Radiological Sciences, Chiba, Japan.

Introduction: The purpose of this study was to develop a holding fixture, termed the 'Bridge Capsule', for longitudinal multimodal observation of rodents with postural reproducibility. Previously, several holding fixtures have been proposed for rodent multimodal molecular imaging (Chow PL: Phys. Med. Biol., 2006, Jan ML: IEEE Trans. Med. Imaging, 2005, Christian N: Radiother. Oncol, 2008, Rommel D: Nucl. Med. Biol., 2008), but postural immobilization has not been considered in depth. If the posture is altered while switching between imaging modalities during longitudinal observation, misregistration can easily occur. Therefore, we present here a holding fixture that can reliably reproduce the posture of the animal between imaging experiments. Specifics of the Bridge Capsule design are presented and details of the reproducibility between fixing and re-fixing are reported. Methods: With the Bridge Capsule, both the head and lower body of the rodent are fixed. The head is immobilized by placing it into the stereotaxic component (A), which is made from polyacetal resin. A second component, made from the vinyl-silicone utilized in dental implants, is then molded to cover the lower body (B). Both of these components sit on a stage (C) made with stereo-lithography. The vinyl-silicon hardens in about five minutes. Image registration between different modalities is improved by using position markers consisting of silicon tubes filled with a contrast agent or radioisotope inserted in the lower body fixture (B). Results and Discussion: The Bridge Capsule had no effect on the quality of MR and PET images, and the positional accuracy of the spinal column between fixing and refixing was within $1.0 \mathrm{~mm}$ in-plane. We conclude that the Bridge Capsule is a useful tool for enhancing reproducibility and registration in multimodal imaging experiments.

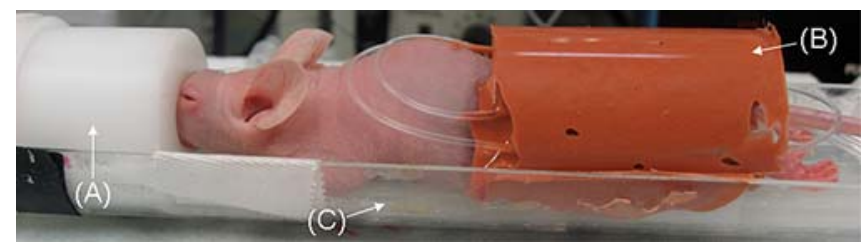


J501

PERFORMANCE ASSESSMENT OF A DUAL-MODALITY FMT/MRI SYSTEM FOR SIMULTANEOUS MEASUREMENTS $\underline{\text { Katerina Dikaiou }}^{1}$, Florian Stuker ${ }^{1}$, Christof Baltes ${ }^{1}$, Lucio Carrara ${ }^{3}$, Jorge Ripoll $^{2}$, Edoardo Charbon ${ }^{3}$, Markus Rudin ${ }^{1,4}$

${ }^{1}$ Institute for Biomedical Engineering, ETH and University of Zurich, Zurich, Switzerland, ${ }^{2}$ Institute of Electronic Structure and Laser, FORTH, Heraklion, Greece, ${ }^{3}$ Aqua Group, EPFL, Lausanne, Switzerland, ${ }^{4}$ Institute of Pharmacology and Toxicology, University of Zurich, Zurich, Switzerland.

A compact system for simultaneous fluorescence molecular tomography (FMT) and magnetic resonance imaging (MRI) of the murine brain has been developed. The goal is to combine structural/functional and molecular information from the two modalities and improve the FMT reconstruction by use of prior information. A $4 \times 4 \mathrm{~cm}^{2} \mathrm{FOV}$ is detected in a $9.4 \mathrm{~T}$ small animal magnet using a surface coil. The system's optical counterpart has a non-contact geometry for increased flexibility and operates in reflectance mode. The signal from a $0.8 \times 0.8 \mathrm{~cm}^{2} \mathrm{FOV}$ on the sample is collected with a $32 \times 32$ single photon avalanche diode (SPAD) detector array with a mounted microlens and corresponding bandpass filters. Due to non-uniform sensitivity of the array, a filtering step is applied prior to reconstruction. For performance evaluation, glass tubes of $1.5 \mathrm{~mm}$ diameter filled with $20 \mu \mathrm{l}$ of AOI987 (Hintersteiner et al.,2005) in various concentrations were embedded along the y-axis at $z=1.5 \mathrm{~mm}$ depth in a silicon-based phantom with tissue-similar optical properties and measured with a $660 \mathrm{~nm}$ cw laser at $670 \mathrm{~nm}$ (intrinsic) and at $720 \mathrm{~nm}$ (fluorescence). A tube with stock solution of $0.24375 \mathrm{mM}$ concentration is properly reconstructed (Fig.1a,1b). The geometrical accuracy of the reconstruction was not affected by gradient switching, yet the intensity of the reconstructed signal amplitude was slightly reduced. Two $1.5 \mathrm{~mm}$ tubes at a $2 \mathrm{~mm}$ center-tocenter distance could be clearly resolved (Fig.1c). The reconstructed fluorescence intensity for a dilution series made from the stock solution depended linearly on the respective concentration (Fig.1d). These data demonstrate that FMT using a $32 \times 32$ SPAD detector array is feasible inside a small animal MR system. The hybrid FMT/MRI will enable simultaneous structural/functional and molecular imaging of the murine brain. ${ }^{*} \mathrm{~K}$. Dikaiou and F.Stuker contributed equally to this work.

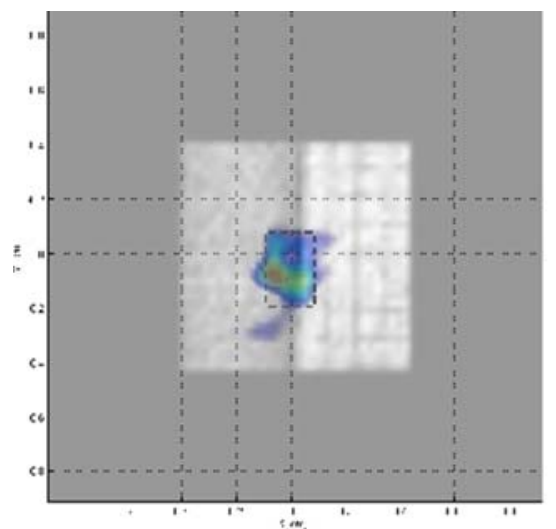

$1 \mathbf{a}$

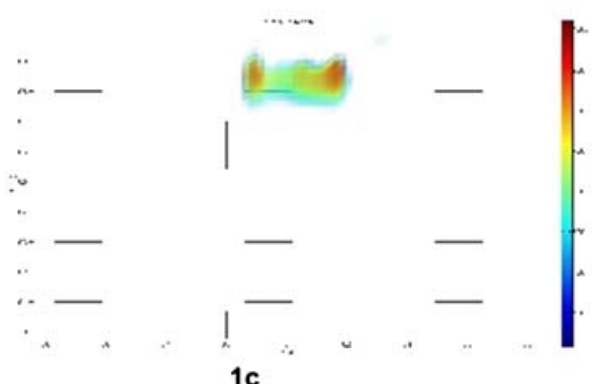

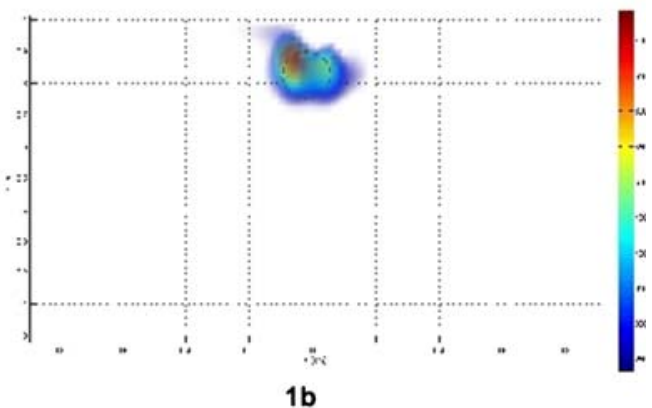

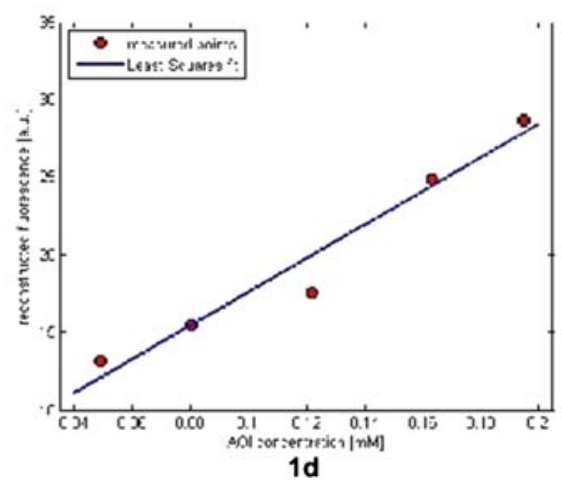

1a,1b: xy- and xz-views of reconstructed fluorescence of AOI987-filled tube. Tube outline in dashed gray.1c: xy-view of FMT reconstruction for identical tubes at $2 \mathrm{~mm}$ center-to-center distance. 1d: Reconstructed fluorescence values for a dilution series and least-squares fit.

$\mathbf{J 5 0 2}$

\section{PREDICTING TUMOUR LOCATION WHEN IMAGING EARLY GROWTH PHASE WITH PET/CT}

Catriona J. Wimberley ${ }^{1}$, Di Xiao ${ }^{2}$, David Zahra ${ }^{1}$, Rachael Shepherd ${ }^{1}$, Paula Berghofer $^{1}$, Emma Millard $^{1}$, Olivier Salvado ${ }^{2}$, Marie-Claude Gregoire ${ }^{1}$ ${ }^{1}$ Radiopharmaceutical Research Institute, ANSTO, Lucas Heights, NSW, Australia, ${ }^{2}$ Biomedical Imaging, CSIRO ICTC - The Australian e-Health Research Centre (AEHRC) Royal Brisbane and Women's Hospital, Brisbane, QLD, Australia.

Assessing tumour metabolism during very early growth phase with PET/ CT is hampered by the difficulties in outlining it on the CT data. This study aims at using a single CT scan of a mouse performed on the day of inoculation, to locate the tumour site from any CT acquired subsequently. The method relies on two assumptions that are validated in this study: 1) The tumour grows at the site of tumour cell inoculation; 2) An accurate mouse skeleton registration method allows prediction of the tumour location. Validation of 1) Two cohorts of mice (total $n=47$ ) were inoculated in the flank with human tumour cells. After inoculation, a cross was drawn on the skin over the site and each subsequent day the site examined for signs of tumour growth. The first tumour measurement by palpation occurred on day 3-4 after inoculation at a mean distance of $0.3 \pm$ $1.05 \mathrm{~mm}$ of the cross, with an estimated tumour volume of $22.83 \pm$ $14.52 \mathrm{~mm}^{3}$ and a length:width ratio of $1.3 \pm 0.33$. Validation of 2) An improved 3D shape context based non-rigid registration method was developed for implementing mouse hind limb skeleton registration and ROI mapping. To validate, on the day of inoculation a CT scan was 
performed on one mouse from cohort 1 , and two mice from cohort 2 (total $n=3$ ), with an external marker (diameter $2.2 \mathrm{~mm}$ ) strapped to the inoculation site. The co-registration software extracted the mouse hind limb skeleton and marker from the CT data. Every 3-4 days another CT scan was performed on each mouse. The animal was installed and restrained on a dedicated bed and scanned with and without the external marker. The software registered the mouse hind limb skeleton from the initial scan onto each current scan and mapped the original marker. Mean absolute distance between registered skeleton surfaces (MAD) and marker position distance (MPD) from the predicted marker to the actual marker were used to assess the accuracy of the method. MAD for 3 mice was $0.16 \pm 0.02 \mathrm{~mm}$. And MPD for 3 mice was $1.98 \pm 0.75 \mathrm{~mm}$ (mean MPD, mouse 1: $2.32 \mathrm{~mm}$; mouse 2: $2.22 \mathrm{~mm}$; and mouse 3: $1.40 \mathrm{~mm}$ ). The decrease in error could be due to improved accuracy of manual placement of the external marker over the cross. This study shows that it is possible to estimate the tumour location during early growth phase using a CT on the day of inoculation. This tool will be helpful for the follow-up of tumour biochemistry with $\mathrm{PET} / \mathrm{CT}$.

\section{J503}

\section{A FREELY AVAILABLE TOOLBOX FOR THE CO- REGISTRATION AND THE CORRELATION OF FLUORESCENCE DIFFUSE OPTICAL TOMOGRAPHY AND SMALL-ANIMAL PET IMAGING} Albertine Dubois $^{1,2}$, Anikitos Garofalakis ${ }^{1,2}$, Daniel M. Dupont ${ }^{1,2}$, Renaud Maroy $^{1}$, Bertrand Kuhnast ${ }^{1}$, Frederic Dolle ${ }^{1}$, Frederic Duconge ${ }^{1,2}$, Bertrand Tavitian ${ }^{1,2}$

${ }^{1}$ DSV-I2BM-SHFJ-LIME, CEA, Orsay, France, ${ }^{2}$ U803, INSERM, Orsay, France.

We recently developed methods to experimentally evaluate the fluorescence Diffuse Optical Tomography (fDOT) limits by using PET as a calibration reference. Characteristics of fDOT performance such as the sensitivity, the linearity of the reconstructed values and the accuracy in predicting the actual concentration of optical probes inside mice have been determined. PET is a highly quantitative imaging modality that can act as a golden standard and hence can be used to calibrate fDOT in a more realistic manner by using dual purpose probes. In this study, we developed an automated toolbox for the coregistration of small-animal PET with fDOT technology in order to obtain fusion images with information from both modalities. Oligonucleotides tagged with fluorescent probes and the radioactive isotope fluorine-18 were co-injected in mice. The fluorescence signal was measured with an optical tomography prototype TomoFluo3D (CEALETI, France). PET and fDOT imaging were performed subsequently using an animal supporting bed containing multimodal fiducial markers that enables the co-registration of independently acquired images. For the selection of the volumes of interest (VOIs) an automatic segmentation tool was used that takes into account the pharmacokinetics of the fluorine-18 probes as provided from dynamic PET scans. The reconstructed fDOT signal was correlated against the corresponding PET signal by performing multimodality measurements from specific VOIs, including kidneys and liver. All the computerized treatments were integrated with in-house image processing software BrainVISA, available for free download on the Internet. We successfully managed to coregister and correlate the TomoFluo3D results with PET reconstructions and hence to evaluate multimodality measurements. The automation of the co-registration and the selection of VOIs as well as their integration into a graphical interface allowed fast and easy processing and guaranteed the objectivity of the results. In this study, we have developed a new toolbox for the co-registration and the correlation of independently acquired $\mathrm{fDOT}$ and PET images. With the aid of this toolbox a prototype fDOT system has been tested and evaluated for in-vivo imaging by using the PET technique as a golden standard. The reconstructed fDOT signal was well correlated against the corresponding PET signal from kidneys and liver. Several performance aspects of the optical prototype like the linearity, the quantification limit and the accuracy of reconstructing multiple sources of fluorescence have also been determined.

\section{$\mathbf{J 5 0 4}$}

\section{HARDWARE AND SOFTWARE TOOLS FOR BRIDGING MACROSCOPIC AND MICROSCOPIC MOLECULAR IMAGING OF CANCER}

Rafiou Oketokoun $^{1}$, Summer Gibbs-Strauss ${ }^{1}$, John V. Frangioni ${ }^{1,2}$

${ }^{1}$ Medicine, Beth Israel Deaconess Medical Center, Boston, MA, USA,

${ }^{2}$ Radiology, Beth Israel Deaconess Medical Center, Boston, MA, USA.

Background: Clinical molecular imaging, i.e., positron-emission tomography (PET), single photon emission computed tomography (SPECT), and magnetic resonance imaging (MRI) result in 3-D images of relatively low resolution. On the contrary, histological analysis, utilizing tumors removed during surgery, results in high-resolution 2-D images that are sparsely sampled in the third dimension. We hypothesized that innovations in hardware and software could lay the foundation for fusing macroscopic, clinical imaging data sets with microscopic, histological data sets so that the molecular basis for contrast seen during clinical imaging could be validated. Materials and Methods: A Nikon TE2000-E microscope was fused with a 10-micron step size Velmex bislider stage platform to create a system capable of automatically scanning up to 561 " $\times 3$ " glass slides or 232 " $\times 3$ ". Custom software was written that acquires simultaneous color H\&E images and near-infrared (NIR) fluorescence images created using a new staining technique (see abstract by Gibbs et al.). An Oracle database and WebLogic server were used to store and distribute digital data. Human prostate cancer specimens were co-stained with an AMACR primary antibody, NIR fluorescent secondary antibody and H\&E. Results: After an automated calibration sequence, the microscopy system was capable of scanning a large number of histological slides at micron resolution, and stitching them together into a single high-resolution image. Being acquired from the same slide, H\&E and NIR fluorescence images were inherently co-registered, and using the H\&E image, multi-spectral NIR fluorescence imaging could be performed. Oracle database functions permitted rapid movement of the field-of-view to regions of interest, storage of intermediate zoomed images for rapid zoom-in and zoom-out, as assembly of high-resolution 2-D images into 3-D data sets. Using the WebLogic server, these data sets could be shared over the Internet with all investigators working on the project. Conclusions: The hardware and software tools we describe create inherently co-registered 3-D data sets from large numbers of high-resolution 2-D data sets and permit fusion of H\&E data with NIR fluorescence data. Future work will focus on co-registering the initial macroscopic images with the final data sets.

\section{J505}

\section{SIMULTANEOUS PET AND THREE-DIMENSIONAL FLUORESCENCE OPTICAL TOMOGRAPHY IMAGING: SYSTEM CHARACTERIZATIONS AND APPLICATIONS} Changqing Li ${ }^{1}$, Yongfeng Yang ${ }^{1}$, Guobao Wang ${ }^{1}$, Gregory S. Mitchell ${ }^{1}$, Richard M. Leahy ${ }^{2}$, Jinyi Qi ${ }^{1}$, Simon R. Cherry ${ }^{1}$

${ }^{1}$ Biomedical Engineering, University of California Davis, Davis, CA, USA, ${ }^{2}$ Electrical Engineering, University of Southern California, Los Angeles, CA, USA.

We have developed an innovative simultaneous PET and multi-spectral three-dimensional (3D) fluorescence optical tomography (FOT) imaging system based on a truncated conical mirror inserted into the gantry of a microPET II scanner. We also have integrated PET and optical reconstruction. Using a sparsity regularization, the target prior from reconstructed PET images is incorporated into the reconstruction algorithm for 3D FOT imaging. We have demonstrated that, with the use of the PET data, 3D FOT images have a spatial resolution close to that of microPET II and quantitation 
of fluorophore concentration has also been improved substantially. The fluorescence measurements from 3D FOT system have been calibrated and the 3D FOT system noise evaluated. Mouse-shaped phantoms with realistic absorption and scattering properties are used for spectral optimization of 3D FOT studies. With phantom experiments, the dependence of the spatial resolution and measurement sensitivity of 3D FOT system are evaluated with and without the PET data. This combined system will be particularly useful for activatable fluorescent probes, and probes whose fluorescent yield depends on their environment, as PET will provide information on the total probe concentration at a given location, while FOT will provide information on the local environment or activation of the probe.

\section{J506}

\section{DEVELOPMENT OF A PHOTONIC METHOD FOR PROTEIN PATTERNING}

Jonathan M. Bélisle $^{1,2}$, Darío Kunik ${ }^{1,2}$, Santiago Costantino ${ }^{1,3}$

${ }^{1}$ Hopital Maisonneuve-Rosemont, Universite de Montreal, Montreal, QC, Canada, ${ }^{2}$ Institut de Génie Biomédical, Université de Montreal, Montreal, QC, Canada, ${ }^{3}$ Depertment of Ophthalmology, Université de Montreal, Montreal, QC, Canada.
Spatial distributions of proteins play fundamental roles in the development and life of living organism. Morphogen distributions control differentiation and chemoattractants help cells to sense direction telling them where to migrate and extend. To study in a controlled manner the precise details of chemotaxis, it is necessary to elaborate reliable methods to mimic spatial distributions of proteins found in vivo. We developed a direct and simple method to create protein patterns with a micron size resolution which is called LAPAP (Laser-assisted protein adsorption by photobleaching). The method's key principle is photobleaching fluorophores with a laser to adsorb them onto a glass substrate in order to produce a protein pattern. The dynamic range of LAPAP was characterized in terms of laser power, dwell time and fluorophore concentration. We found that the amount of substrate bound protein can be modulated by three orders of magnitude. As proof of principle for the method's functionality, gradients of a peptide from laminin were patterned in vitro using LAPAP. Axons from dorsal root ganglion cells showed a great guidance response to the peptide's gradients. In conclusion, LAPAP is a method to create functional substrate-bound protein patterns of subcellular resolution with a high dynamic range.
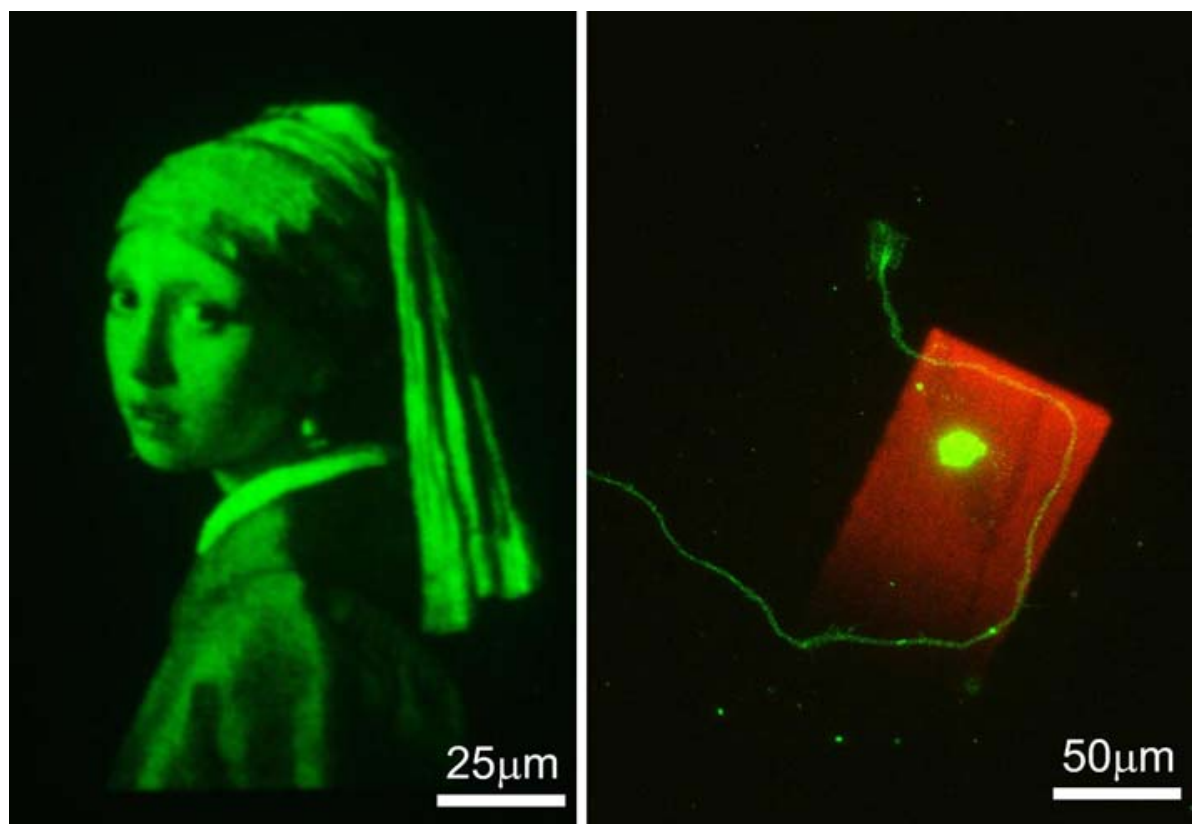

Streptavidin-Cy5 miniature reproduction of Vermeer's Girl With a Pearl Earring that demonstrates the flexibility and dynamic range of the technique, and an example of axonal guidance by a gradient of IKVAV peptide. DRG neuron stained with Alexa 546 in green and laminin peptide gradient visualized by streptavidin-Cy5 in red.

\section{$\mathbf{J 5 0 7}$}

EARLY DETECTION OF METASTASIS TUMORS IN MOUSE LIVER MODEL USING GATED SMALL ANIMAL FDG PET AND CONTRAST CT IMAGES

Sang-Keun Woo, Gi Jeong Cheon, Kyeong Min Kim, Taesup Lee, Jaeho Jung, Yong Jin Lee, Joo Hyun Kang, Ji-Ae Park, Jin Su Kim, Jong Guk Kim, Sang Moo Lim

Molecular Imaging Research Center, KIRAMS, Seoul, Korea, South.

Small animal PET studies allow non-invasively molecular imaging of physiological events in small animal models of disease. The combination of PET and CT image provides quantitative in vivo. The aim of this study was to identify the precise location of the liver metastasis tumor by using gating PET/CT method. LS174T-FL cells harvested after trypsinization, suspended in PBS, and stored on ice. In the liver metastasis model, $5 \times 10(6)$ cells were injected into the spleen of nude mice. One to two weeks later, the liver with metastatic tumors was placed on a special stage. Respiratory gating was realized with the help of an external trigger device (BioVET) synchronized with the listmode acquisition. PET imaging studies was performed with a dedicated small animal R4 PET scanner. PET imaging was started $60 \mathrm{~min}$ after the administration of $18 \mathrm{~F}-\mathrm{FDG}$ via tail vein injection. Mice were placed on an acryl plate for fusion between PET and CT images. Contrast enhanced CT images were obtained at three hours after injection of contrast agent (Fenestra LC). Dosage was based on body weight of each mouse at $10 \mathrm{uL} / \mathrm{g}$. The $\mathrm{CT}$ images obtained with a Inveon $\mathrm{CT}$ scanner (Siemens) can be used to improve the anatomical localization of uptake in the small animal PET images. Fused image in liver metastasis tumor model showed a good correlation of images from both 
PET and CT. The gated PET/CT images show the better image quality, compared to that with no gating, in terms of contrast and size of interested region, particularly with high uptake of FDG and contrast. In the gated CT image, the SNR of 0 bin, 2 bin, 4 bin and 8 bin number on the CT image were 5.4, 5.5, 4.9 and 5.3, respectively. The contrast values of each bin number the CT image were 2.77, 3.93, 4.99 and 3.8, respectively. The small size of liver metastasis tumor $(1 \mathrm{~mm})$ was clearly visualized by gated PET/CT image. The gated contrast CT images showed sufficient contrast for identification of small animal anatomical structures. We performed to monitor and to quantify microcirculatory parameters in the tumor grown in the liver. The use of gated PET and gated contrast CT allowed a precise and improved detection of tumors in liver models.

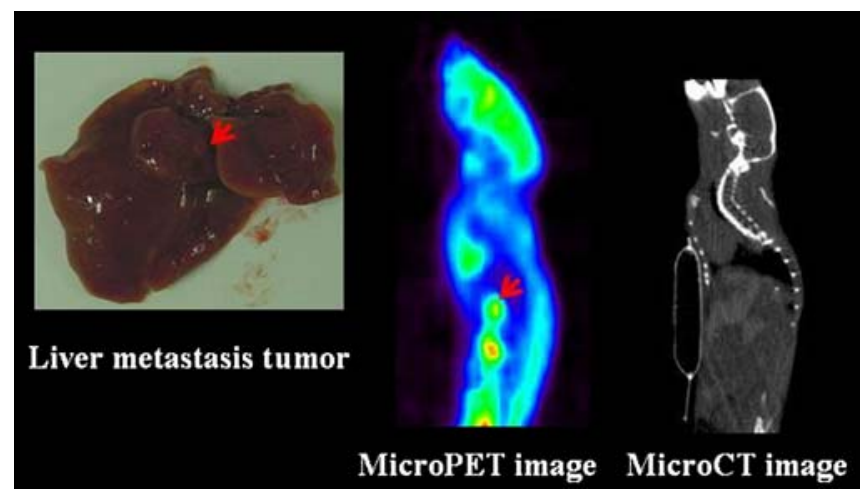

J508

\section{SMALL ANIMAL PET/MRI: AN IN VIVO STUDY IN ONCOLOGY USING A U87MG MOUSE GLIOMA MODEL}

Martin S. Judenhofer ${ }^{1}$, Hans F. Wehrl ${ }^{1}$, Julia G. Mannheim ${ }^{1}$, Olivier Raguin ${ }^{2}$, Virginie Bari ${ }^{2}$, Olivier Duchamp ${ }^{2}$, Gerald Reischl ${ }^{3}$, Bernd J. Pichler ${ }^{1}$

${ }^{1}$ Laboratory for Preclinical Imaging and Imaging Technologies of the Werner Siemens-Foundation, University of Tuebingen, Tuebingen, Germany, ${ }^{2}$ Oncodesign, Dijon, France, ${ }^{3}$ Department of Radiopharmacy, University of Tuebingen, Tuebingen, Germany.

First PET/MRI systems have been developed and are at a stage to be used for in vivo animal imaging experiments. The combination of PET and MRI in one device allows the use dedicated PET/MRI imaging protocols which take advantage of simultaneous acquired PET and MRI data. A PET insert based on LSO-APD detectors was built to be operated in a $7 \mathrm{~T}$ small animal MRI system. This work shows results in the field of tumour imaging in mice using a orthotropic model of glioma (U87MG). The tumour model was investigated for its suitability in an initial experiment where tumour growth and the therapeutic effect of the cell cytotoxic drug Carmustin were assessed by MRI volumetric measurements. In a second experiment two groups of Carmustin and vehicle treated mice were scanned using the PET/MRI system. Treatment was initiated after the first PET/MRI measurements at day 10 after tumour inoculation. Treatment was performed using $20 \mathrm{mg} / \mathrm{kgBW}$ Carmustin every 4 days for 3 times. For MR imaging t2 and t1 weighted imaging as well as post gadolinium-based-contrast-agent imaging was used to visualize brain tumours. PET imaging was performed using [C-11]choline injected intravenously and measured for $20 \mathrm{~min}$ after a $40 \mathrm{~min}$ uptake period. PET and MR imaging was performed simultaneously. The initial MRI experiments showed a reproducible tumour growth after inoculation for approximately 4 weeks up to a size of $50 \mu \mathrm{L}$. Treatment effects were detectable due to reduced tumour growth and significantly increased life span of the mice. The measurements with the PET/MRI system showed that co-registration of the data is very accurate. PET data were evaluated based on regions drawn in the post contrast agent MR images. Measurements at day 8 after treatment showed increased tumour growth $(1.4+-0.3$ fold $)$ in vehicle groups vs. treatment groups $(1.1+-0.3$ fold $)$ in the volumetric measurements from the MRI. These data correlated well with the uptake of [C-11]choline being higher in the vehicle group (1.3 +-0.1 tumour/BG ratio) compared to the treatment group $(1.1+-0.1$ tumour/BG ratio). These first results showed clearly one benefit of using combined PET/MRI as scan times were halved due to simultaneous measurements. Furthermore, a more accurate analysis of the PET data can be achieved by using MRI guided ROIs. Further experiments will investigate a larger cohort of animals to evaluate the potential of correlating consecutive PET and MRI measurements to better estimated early therapeutic effects. In addition, in current studies we are perform MR spectroscopy along with [C-11]Methionine PET scans.

\section{J509}

MOLECULAR IMAGING USING COMBINED FLUORESCENCE TOMOGRAPHY, DIFFUSE OPTICAL TOMOGRAPHY, AND X-RAY COMPUTED TOMOGRAPHY

William C. Barber $^{1}$, Yuting Lin ${ }^{2}$, Jan S. Iwanczyk ${ }^{1}$, Einar Nygard ${ }^{1,3}$, Neal E. Hartsough $^{1}$, Nail Malakhov ${ }^{1,3}$, Orhan Nalcioglu ${ }^{2}$, Gultekin Gulsen ${ }^{2}$ ${ }^{1}$ DxRay Inc., Northridge, CA, USA, ${ }^{2}$ University of California Irvine, Irvine, CA, USA, ${ }^{3}$ Interon ASA, Asker, Norway.

We have developed and fabricated a novel multi-modality preclinical imager by combining non-contact fluorescence tomography (FT) and high-efficiency high-resolution x-ray computed tomography $(\mathrm{CT})$. The anatomical data from CT provides a-priori morphological information to the FT reconstruction to create functional images with accurate localization of fluorophore distribution. The construction of a combined FT/CT system will create a new molecular imaging tool for stem cell and cancer research. The system offers improved cell tracking methods which are important tools in the development of cellular therapies for disease treatment. We use a high-efficiency high-resolution flat panel detector to perform CT imaging. We use a prototype fully non-contact charge coupled device (CCD) based FT system that can acquire FT as well as diffuse optical tomography (DOT) measurements. The CT and FT systems are combined onto a single gantry with a common patient table. Accurate localization of the fluorophore concentration is achieved by using a-priori information about the morphology of the phantom during the reconstruction of the FT image. We constructed multi-modality phantoms with multiple compartments to mimic background optical heterogeneity. The absorption maps obtained from DOT at $785 \mathrm{~nm}$ and $830 \mathrm{~nm}$ were used as functional a priori information. Indocyanine-Green (ICG) was used as the fluorophore. Furthermore, iodine was added to the different compartments at different concentrations for $\mathrm{CT}$ contrast. The structure of the phantom was obtained by the CT system and was used as morphological a priori information. The experimental study confirmed that the fluorophore concentration of an inclusion deeply embedded inside a heterogeneous background can only be recovered accurately when both functional and structural a priori information is available. This unique multi-modality imaging methodology that combines functional and anatomical a priori information to FT reconstruction provides a new approach to molecular imaging. We have demonstrated that a combined FT/DOT/CT system has the potential for quantitative molecular imaging. The fluorophore concentration can only be accurately recovered by using a priori information about the optical properties of the background from DOT and the morphology of the phantom from CT during the reconstruction of the FT image. 


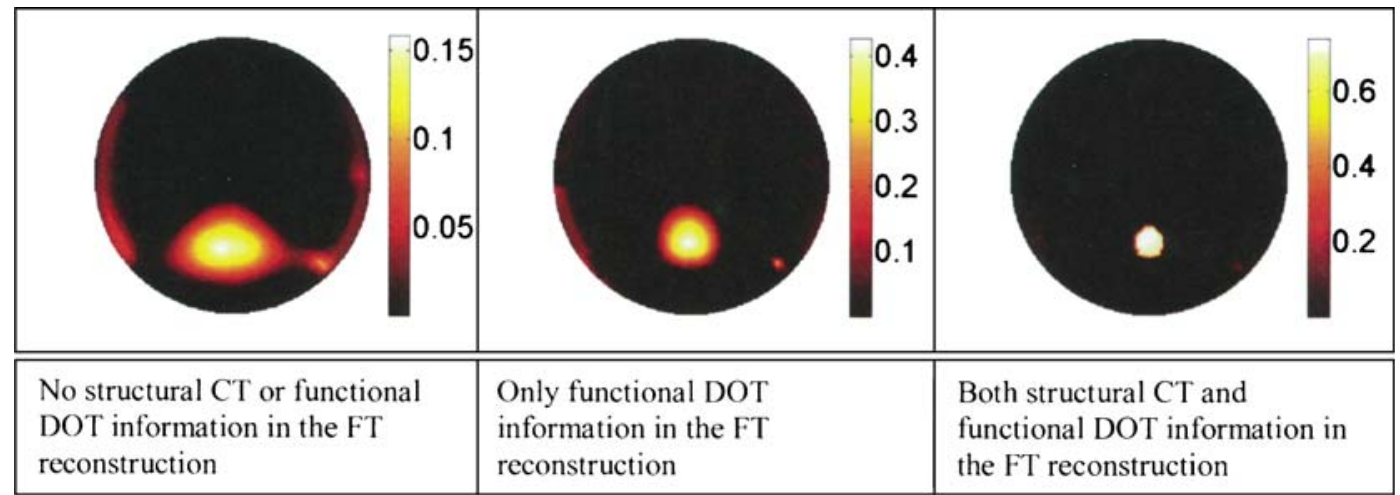

$\mathbf{J 5 1 0}$

\section{QUALITATIVE COMPARISON OF 15O-H2O PET AND FAIR ARTERIAL SPIN LABELING REGIONAL CEREBRAL BLOOD FLOW IMAGING METHODS IN RATS}

Jeffrey M. Moirano ${ }^{1}$, Samuel A. Hurley ${ }^{1}$, Luke A. Lopas ${ }^{2}$,

$\overline{\text { Krishna N. Kurpad }}^{1}$, Alexander K. Converse ${ }^{2}$

${ }^{1}$ Department of Medical Physics, University of Wisconsin, Madison, WI, USA, ${ }^{2}$ Waisman Center for Brain Imaging and Behavior, University of Wisconsin, Madison, WI, USA.

Objective: Regional cerebral blood flow (rCBF) imaging plays an important role in investigations of the pathology and physiology of the brain, and such methods are widely employed in both clinical and preclinical imaging. The gold standard for rCBF measurement is $15 \mathrm{O}-$ $\mathrm{H} 2 \mathrm{O}$ PET, but as MRI scanners have become more prevalent, arterial spin labeling (ASL) methods have been increasingly relied on. This work aims to qualitatively evaluate and compare these measures separately, with the overall goal of quantitative comparison of perfusion measures using simultaneous PET/MR in rats. Methods: A small rat radiofrequency head coil was developed for the Varian 4.7T small animal MR system in order to meet the space constraints of a multimodality PET/MR system. Rat brain $\mathrm{rCBF}$ images were created using this coil and a flow-sensitive alternating inversion-recovery (FAIR) MRI sequence. $(128 \times 128$ matrix $)$ PET images were collected using a Concorde Microsystems P4 microPET scanner. Between 0.4 $1 \mathrm{mCi} 15 \mathrm{O}-\mathrm{H} 2 \mathrm{O}$ were injected intravenously into the tail, and data was collected for $120 \mathrm{~min}$. Processed images were manually aligned to a rat atlas image using the Spamalize software package. Five equal sized regions of interest were drawn on the atlas image and used to collect the average pixel intensity of the region of interest for both the PET and MRI images. Analysis was done by arranging the regions in rank order for comparison. Results: The rank order of the five regions of interest was very similar for both the 15O-H2O PET and ASL MRI images. The calculated Spearman rank order correlation coefficient (rs) was 0.9, which is significant at the .05 level for $n=5$. Conclusion: Our methodology for $15 \mathrm{O}-\mathrm{H} 2 \mathrm{O}$ PET and FAIR ASL seem to be in qualitative agreement based on the rank order comparison of regions of interest, indicating that we are able to assess $\mathrm{rCBF}$ in the rat brain. Using a MRI compatible PET insert, we hope to directly compare and validate these methods by taking simultaneous data sets using these methods.

\section{J511}

DUAL MR AND OPTICAL IMAGING OF TUMORS TO SUPPORT MOLECULAR IMAGING

Olivier M. Girard ${ }^{1,2}$, Ahmet Erten ${ }^{2,3}$, Mark Bydder ${ }^{1}$, Milan T. Makale ${ }^{2}$, David A. Cheresh $^{2}$, Sadik C. Esener ${ }^{2,3}$, Robert F. Mattrey ${ }^{1,2}$

${ }^{1}$ Radiology, University of California, San Diego, San Diego, CA, USA, ${ }^{2}$ Moores Cancer Center, University of California, San Diego, San Diego, CA, USA, ${ }^{3}$ Electrical Engineering, University of California, San Diego, San Diego, CA, USA.
Magnetic Resonance Imaging (MRI) holds great promise to become an efficient Molecular Imaging (MI) modality. MRI's ability to probe deep tissue properties in vivo, while benefiting from highly resolved anatomical detail is one of the main motivations for advancing MRI for MI. MRI however suffers from lack of sensitivity to T2 and more so T1 agents as compared to nuclear or optical imaging. Although the lack of sensitivity can in part be overcome by increased contrast agent (CA) dose, this approach leads to loss in specificity. More important the large dose of a targeted agent leads to receptor saturation and poor target to background ratio. We aimed to develop an in vivo mammalian imaging model that allows for high MRI sensitivity as well as optical imaging to assess and validate MI paradigms for MR detection particularly when agents are targeted to tumors. In this study we report on the design and implementation of a highly sensitive MRI coil coupled to an MR compatible dorsal skinfold window chamber that allows for intravital microscopy of the same tumor. This system is then best suited to study dually labeled MR and Optical contrast agents. We built a detachable inductively-coupled MR coil tuned for 3T to allow MR imaging of many mice implanted with window chambers using the same coil or to generate reproducible MR images of the same mouse over time. The inductive coupling was necessary to minimize the coil space requirement and to increase the distance of the connector from the preparation to avoid shorting. Dually labeled targeted iron oxide particles can then be imaged by MR and intravital optical microscopy to validate their accumulation. The high resolution images of a normal window chamber and the position of the coil are shown (Figure).
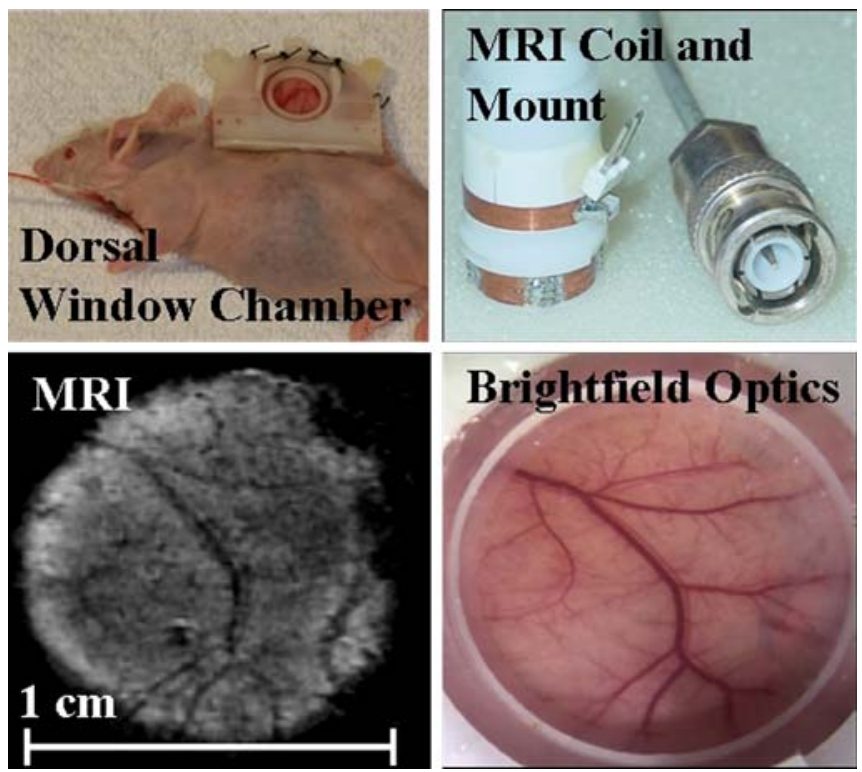


\section{J512}

\section{CONSTRUCTION AND USE OF A SIMPLE MULTI-MODALITY, MULTI-ANIMAL MOUSE HOLDER}

Timothy Doyle

Pediatrics, Stanford University, Stanford, CA, USA.

Small animal multi-modality molecular imaging often requires the use of multiple instruments and a large number of animals to generate statistically valid data. The ability to place the specimen animals in a manifold that allows simple translation between instruments, and hold several animals simultaneously lead to the creation of a simple holder that can be made from items found within the imaging lab, which is cheap enough to be, if necessary, disposable. The manifold can hold up to 4 mice with isoflurane gas delivery to each animal, and is compatible with MRI, MicroPET, MicroCT and MicroSPECT. Single animal holders can also be simply adapted for gated studies with the inclusion of appropriate third party gating hardware. The device is made from a $60 \mathrm{ml}$ syringe, opened to allow placement of the specimen mouse, with a second smaller syringe providing isoflurane delivery to the nose of the animal. This manifold can be made by users in under $30 \mathrm{~min}$. Individual holders can be attached to each other with plastic screws and nuts, and up to four have been successfully used in MicroCT, MicroPET and MicroSPECT allowing more efficient use of scan time by the end users. These devices are now routinely used at the Stanford Small Animal Imaging Facility for both gated MicroPET/CT scans and high-volume animal scanning.

\section{J513}

\section{COMPARISON OF LEFT VENTRICULAR EJECTION FRACTION OBTAINED WITH CORONARY COMPUTED TOMOGRAPHY ANGIOGRAPHY AND 13N-AMMONIA PET IN PATIENTS WITH CORONARY ARTERY DISEASE $\underline{\text { Erick Alexánderson }}^{1,2}$, Juan M. Ochoa ${ }^{1}$, Gabriela Meléndez ${ }^{2}$, Rodrigo Jácome ${ }^{1}$, Aloha Meave $e^{1,2}$ \\ ${ }^{1}$ PET/CT Cyclotron Unit, Universidad Nacional Autónoma de México, México D.F, Mexico, ${ }^{2}$ Nuclear Cardiology and Cardiac CT, Instituto Nacional de Cardiología "Ignacio Chávez”, Mexico D.F, Mexico.}

INTRODUCTION: Left ventricular ejection fraction (LVEF)reflects left ventricular function and is a parameter used to stratify patient with coronary artery disease (CAD). In normal subjects, LVEF increases during stress while in patients with $\mathrm{CAD}$ there is an inverse relationship between the degree of ischemia and the change seen in stress LVEF. LVEF at rest and during peak pharmacologic stress can be obtained using $13 \mathrm{~N}$-ammonia gated PET. LVEF can also be obtained with Coronary Computed Tomography Angiography (CCTA). By Hybrid PET/CT systems we can be able to evaluated LVEF in both technologies. The aim of this study is compared the LVEF obtained from $13 \mathrm{~N}$-ammonia rest-stress PET and by CCTA in patient with suspected CAD. METHODS: We studied 26 patients, 20 men $(76.9 \%)$ and 6 females $(23 \%)$ with suspected CAD whom underwent a rest and pharmacologic (adenosine) stress $13 \mathrm{~N}$-ammonia gated PET and, CCTA scan by a PET/CT Biograph 64 scanner (Siemens). LVEF in PET was obtained by the Cedars Cardiac Quantification (QGS) and, Circulation software for coronary CCTA. Statistical analysis was made with SPSS V.16. RESULTS: Mean LVEF measured during rest PET was similar to those obtained by CT $(59.2 \pm 9 \%$ and $58.8 \pm 12.3 \%$ respectively), with an ICC for single measures of 0.728 (IC $0.48-0.868, p<0.001$ ). In 18 of $26(69 \%)$ patients, ischemia was detected. Rest PET LVEF was statistically different in non ischemic and ischemic patients $(64.87 \pm 7 \%$ and $56.7 \pm 10 \%$, respectively; $p=0.027)$, as in CT $(67 \pm 8.9 \%$ and $55.3 \pm 12 \%$; $p=0.013$ ). Delta of LVEF obtained by PET (stress LVEF - rest LVEF) was statistically different in non ischemic (median 2 ( -2 to 15$)$ ) and ischemic patients (median -6.5 ( -20 to 9 )). CONCLUSION: PET and CT allows the measurement of LVEF. There is a good correlation between rest PET and CT LVEF. New hybrid systems open the possibility to obtain more information about ventricular function.

\section{J514}

\section{CVP: A TOOL FOR THE FUSION, REGISTRATION, ANNOTATION, AND VISUALIZATION OF MULTI- MODALITY DATASETS}

Peter Kok ${ }^{1,4}$, Sven Mieog ${ }^{3}$, Alexander Vahrmeijer ${ }^{3}$, Clemens Lowik ${ }^{2}$, Boudewijn Lelieveldt ${ }^{1,4}$, Jouke Dijkstra ${ }^{1}$

${ }^{1}$ Radiology, LUMC, Leiden, Netherlands, ${ }^{2}$ Endocrinilogy, LUMC, Leiden, Netherlands, ${ }^{3}$ Surgery, LUMC, Leiden, Netherlands, ${ }^{4}$ Quantitative Imaging Group, Delft University of Technology, Delft, Netherlands.

Background. In order to understand molecular processes in living subjects, we would like to have a technology which can zoom in from the organ level down to the atomic detail. This can be not be accomplished by current available technology. Aim. To be able to understand and visualize images from different sources at different scales, a system is needed that can handle different resolutions, data types and time points and allows to relate and visualize these images by image fusion. Material \& Methods. The Cyttron Visualization Platform (CVP) is a system developed by the Cyttron consortium and is aimed at visualizing multimodal datasets at various resolutions. Multiple datasets can be loaded and visualized at the same time, augmenting the amount of information the user can extract from them. The platform is highly extensible and allows for various custom visualization methods, filters, readers and step-by-step user tasks to be added. Results. As an example we show the fusion of high resolution HE stained microscopic images with Fluorescent images. The microscopic images have first been stitched, creating a new high resolution image which can be imported into the CVP together with multiple other images of the same sample. Since the nature of the images can be very different, the most flexible approach is the manual registration by indicating multiple corresponding landmarks in the different images. For the visualization, different blending techniques have been implemented which can be adjusted by the user during review.

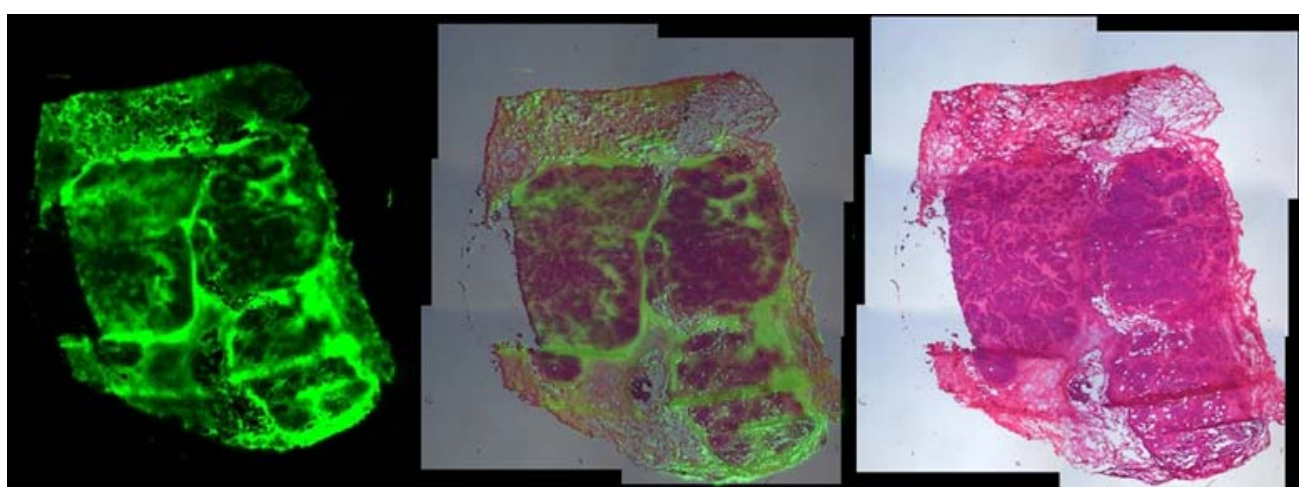

A: Original Fluorescent image B: Registered and blended $50 \%$ C: Original HE stained image 
Imaging Methodology

\section{MRI}

J515

IN VIVO INJECTION OF SINGLE-WALLED CARBON NANOTUBES (SWCNT): NONINVASIVE DETECTION AND BIOLOGICAL IMPACTS USING MR TECHNIQUES Achraf Al Faraj $^{1}$, Florence Fauvelle ${ }^{2}$, Ghislaine Lacroix ${ }^{3}$, Florence Lagarde $^{4}$, Cyrille Richard ${ }^{5}$, Yannick Cremillieux ${ }^{1}$, Emannuelle Canet-Soulas ${ }^{1}$

${ }^{1}$ Creatis-LRMN, CNRS 5220, INSERM U630, University Lyon 1, Lyon, France, ${ }^{2}$ Biophysique Cellulaire et Moléculaire, Laboratoire de RMN, CRSSA, La Tronche, France, ${ }^{3}$ Institut National de l'Environnement et des Risques Industriels, Verneuil-en-Halatte, France, ${ }^{4}$ Laboratoire des Sciences Analytiques, CNRS UMR 5180, University Lyon 1, Villeurbanne, France, ${ }^{5}$ Faculté de pharmacie, CNRS UMR 8151, INSERM U 640, Université Paris Descartes, Paris, France.

SWCNT hold promise for applications in nanomedicine field as contrast agents or target delivery carriers. When administered in vivo, their biodistribution and pharmacological profile have to be fully characterized.
MRI was applied for noninvasive SWCNT monitoring as standard radio- or fluorescently-labeled pharmaceuticals are not well suited for in vivo long term follow-up. The biodistribution of albumin well-dispersed raw (R) and purified (P) pristine SWCNT and carboxyl functionalized (f-) SWCNT were assessed in blood and target tissues after their intravenous injection in a 2week study by longitudinal in vivo MRI and their potential effect on liver metabolism by HR-MAS spectroscopy after liver biopsy. Ex vivo Raman spectroscopy, iron assay and histology were performed to correlate with the MRI readouts. An iron oxide contrast agent Sinerem $(c$ was also included as a positive control for contrast MRI. SWCNT physicochemical properties were characterized by Raman spectroscopy and Inductively Coupled PlasmaOptical emission spectrometry (ICP-OES) for iron impurities dosage. Iron impurities allowed raw SWCNT in vivo detection by MRI using gradient echo sequence. A transitional accumulation in spleen and liver were observed by MRI. Ex vivo Raman spectroscopy, iron assays and histology confirmed MRI readouts. Moreover, no presence of acute toxicological effect on liver metabolism was observed after injection of both welldispersed and functionalized SWCNT assessed using ex vivo High Resolution Magic Angle Spinning (HR-MAS) ${ }^{1} \mathrm{H}$ NMR. HR-MAS combined to pattern recognition methods (Principal Component and Partial Least Squares-Discriminant Analysis), is a well-established technique for studying the endogenous metabolic changes caused by drug toxicity and disease processes. No clustering of NMR spectra was found using PCA (specific biomarkers of toxicity). PLS-DA allowed the sole clustering of spectra according to age-related organ maturation.
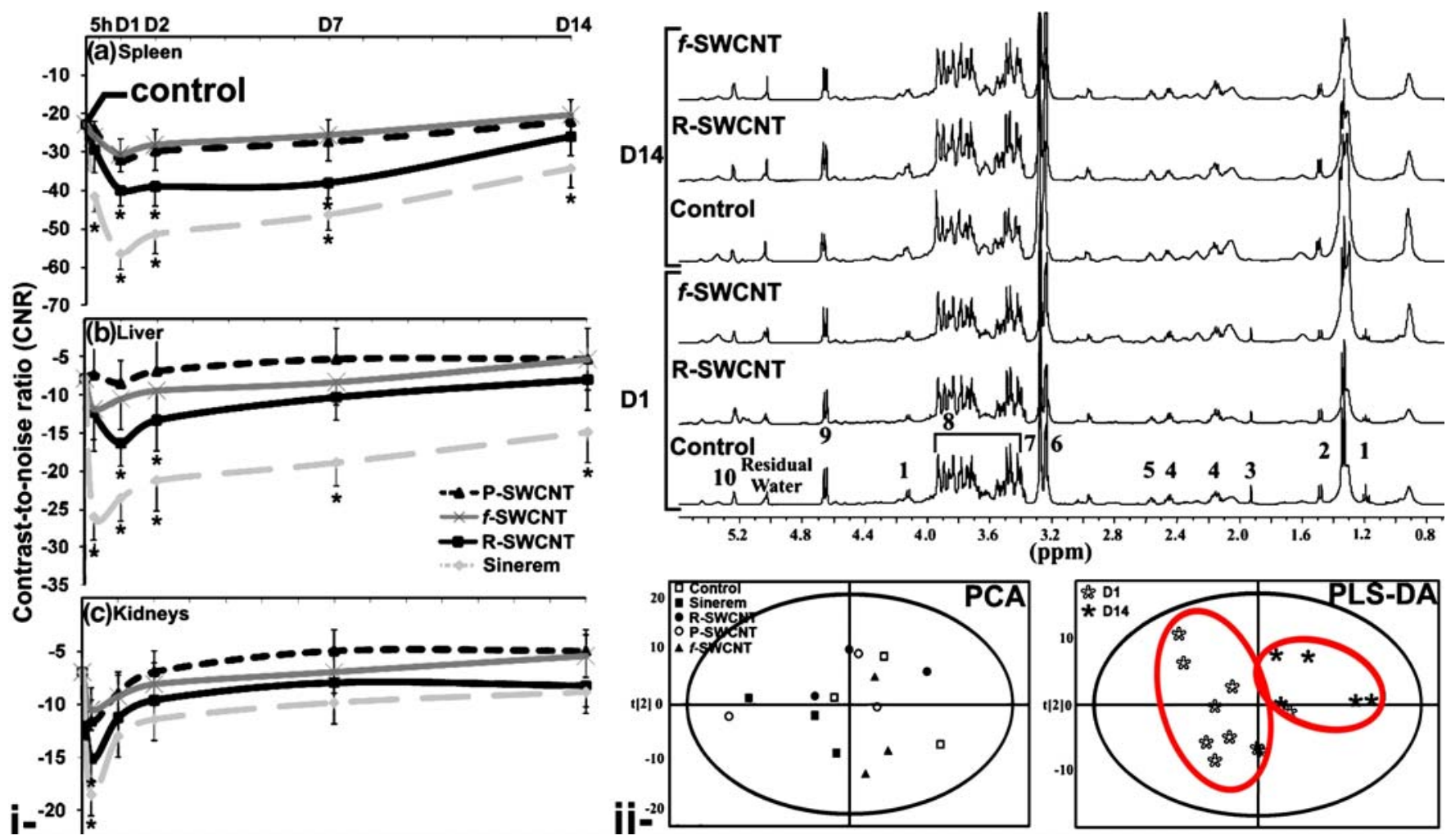

Figure1: i- MRI contrast-to-noise variations of Sinerem, R-, P- and f-SWCNT injected groups compared to control values. ii- HR-MAS ${ }^{1} \mathrm{H}$ NMR spectra of liver sample with the applied PCA and PLS-DA score plot.

J516

\section{MONITORING OF ANTI-ANGIOGENIC THERAPY BY USING} MULTI-PARAMETRIC MR IMAGING TECHNIQUE

Janine Ring $^{1}$, Thorsten Persigehl ${ }^{1}$, Walter Heindel ${ }^{1}$, Stefanie Remmele ${ }^{2}$, Christoph Bremer ${ }^{1}$

${ }^{1}$ Department of Clinical Radiology, University of Muenster, Muenster, Germany, ${ }^{2}$ Philips Medical Systems, Philips Hamburg, Hamburg, Germany.
Introduction Early monitoring of anti-angiogenic tumor treatment and the analysis of its microvasculature is of high interest in oncology research. Steady-state MRI, using long-circulating USPIOs, allows sensitive $\Delta \mathrm{R} 2$ and $\Delta \mathrm{R} 2 *$ relaxometry, which gives insight into physiologic parameters like the vascular volume fraction (VVF) and the mean vessel size of a tumor (VSI). Apparent diffusion coefficient (ADC) derived from diffusion weighted imaging (DWI) allows for the non-invasive assessment of the tissue cellularity. The purpose of our 
study was to evaluate the usage of multi-parametric "steady-state" MR imaging and DWI for the early assessment of anti-angiogenic treatment effects using multi-targeted tyrosine kinase inhibitor (SU11248). Material and Methods MDA-MB 435 bearing nude mice $(n=10)$ were treated over one week with SU11248 $(60 \mathrm{mg} / \mathrm{kg})$ or saline as control respectively. MR imaging by using DWI, multi-spin-echo and multigradient-echo sequence was applied before and after USPIO injection on baseline and follow up. The VSI map was calculated using ADC and $\triangle \mathrm{R} 2 / \mathrm{R} 2 *$ maps. Furthermore, the VVF, as a known surrogate marker of the micro vessel density (MVD), was calculated by calibrating $\Delta \mathrm{R} 2 *$ of the tumor by $\Delta \mathrm{R} 2 *$ of muscle. MR results were compared with the change of the tumor size and morphometric analysis of the tumor tissue on CD31 stained tumor sections measuring the MVD and the vessel diameter. For statistical analysis a t-test was performed. Results SU11248 treatment revealed a significant reduction of $\Delta \mathrm{R} 2 *$ (baseline: $18.75 \pm 1.51$ /follow-up: $10.41 \pm 0.21$ ), VVF (baseline: $4.00 \pm$ 0.52/follow-up:1.67 \pm 0.08 ) and vessel size (baseline:36.83 \pm 2.90 /follow-up $21.47 \pm 0.12$ ) compared to control. ADC-maps showed a clear water diffusion increase in terms of a reduced tumor tissue cellularity. In line with $\triangle \mathrm{R} 2 *$ and VVF, the histological counted MVD decreased significantly after treatment compared to the control group (SU11248:27.25 $\pm 2.52 /$ control:43.20 \pm 0.09$)$. Immunohistologically measured mean vessel diameters confirmed the MR vessel size distribution (SU11248:16.30 $\pm 1.09 /$ control:22.75 \pm 1.00 ) although the absolute vessel diameters were overestimated with MRI. Moreover, SU11248 therapy over one week resulted in a tumor size regression, whereas the untreated control group showed a tumor size progression. Conclusion Multi-parametric MR imaging allows anti-angiogenic tumor treatment monitoring by non-invasive visualization of tumor microvascular and cellularity changes. This could support further understanding of anti-angiogenic action in early treatment monitoring in patients.

\section{J517}

\section{NON-INVASIVE, HIGH-RESOLUTION MONITORING OF TUMOR ANGIOGENESIS BY CONTRAST ENHANCED MR IMAGING}

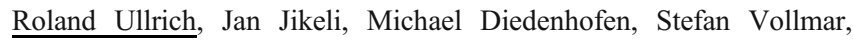
Mathias Hoehn

Max-Planck-Institute for Neurological Research, Cologne, Germany.

In this study we present a protocol for tumor vessel imaging providing vessel maps for microvascular density, microvascular and macrovascular blood volume in a subcutaneous NSCLC model in nude mice. The experiments were performed on a 7T BioSpec Bruker scanner using the sequences MGE and MSME acquired before and after i.v. injection of the iron oxide contrast agent Endorem (30 mg Fe/kg). Microvascular density was derived from the $\mathrm{N}$-map as determined by $\mathrm{N} \sim \mathrm{Q} 3 / \mathrm{ADC}$ with $\mathrm{Q}=\Delta \mathrm{R} 2 /(\Delta \mathrm{R} * 2) 2 /$,3 . The ADC-map was calculated from the diffusionweighted images with a mono-exponential fit. We determined microvascular vessel volume represented by the ratio of $\Delta R * 2 /(\Delta R 2)$, where $\Delta \mathrm{R} * 2=\ln ($ GEpre/GEpost $) / \mathrm{TE} \sim$ total Tumor Blood Volume $(\mathrm{TBV})$ and $\Delta \mathrm{R} 2=\ln ($ SEpre/SEpost $) / \mathrm{TE} \sim$ micro TBV. Moreover, we compared the MR data to immunohistological endothelial cell staining. The sprouting and growth of angiogenic tumor vessels were monitored 1 day, 4, 8 and 14 days after tumor cell injection. Simultaneously, BLI was measured for monitoring tumor growth. The spreading of tumor microvessels $(3-8 \mu \mathrm{m})$ as well as tumor macrovessels (size $10-30 \mu \mathrm{m}$ ) into the tumor was clearly visible beginning at 4 days after subcutaneous tumor injection. This process of formation of tumor micro- and macrovessel continued during the next 10 days and was paralleled by an increase in tumor growth as assessed by BLI. Most importantly, coregistration of the MR images with immunohistochemical tissue sections of the tumor, stained with CD31 for positive endothelial cells, resulted in a good colocalization between the tumor vessels detected by MRI and CD-31 positive cells (see Figure 1). The presented method provides a useful tool to non-invasively monitor tumor angiogenesis. Due to its high resolution it will allow noninvasive monitoring of the evolution of microvascular as well as macrovascular tumor vessel density. This now opens new possibilities to follow inhibition of this process as important end-point for the in vivo evaluation of anti-angiogenic treatment targets.

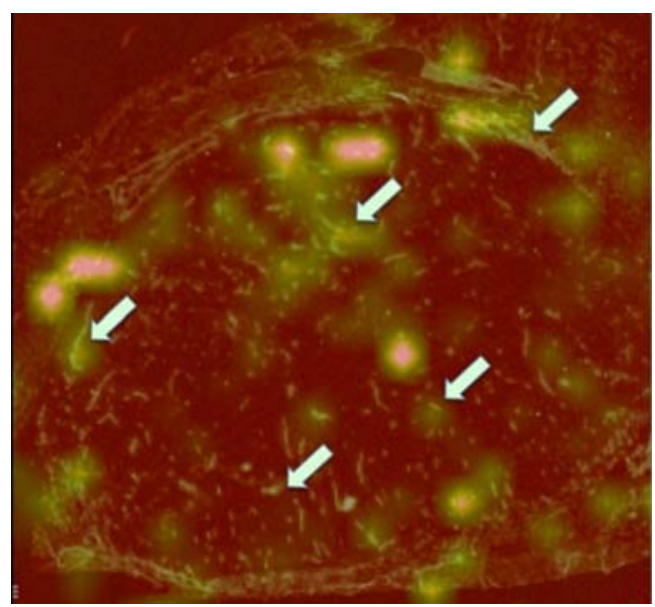

\section{J518}

MRI OF A SINGLE HISTOLOGICAL SLICE USING INDUCTIVELY COUPLED SELF-RESONANT MICROCOILS Rob Nabuurs, Andrew Webb, Louise van der Weerd Radiology, C2S, LUMC, Leiden, Netherlands.

Introduction.Histology remains the gold standard to validate changes in MR image contrast, either to examine a specific pathological substrate or to validate targeted MR contrast agents. However, precisely registration between histology sections and MR data remains difficult. Potential solution is the direct MR imaging of a histology slide, a concept shown by Meadowcroft et al.[MRM 57:835-841], who developed a highly specialized coil for similar purposes. Here we demonstrate a very simple, broadly applicable approach using inductively coupled coils. Using these easily produced and replaceable coils, high resolution images of histological samples can be acquired rapidly. Methods.A self-resonant square loop coil $(15 \times 15 \mathrm{~mm})$ was placed directly on top of a microscope slide (Fig 1a). The slide was positioned to assure strong coupling with the standard $25 \mathrm{~mm}$ Bruker birdcage coil. The variable capacitors of the birdcage were used to impedance match the low frequency resonance of the coupled resonators at $400 \mathrm{MHz}$. $60 \mu \mathrm{m}$ fixed human brain sections were imaged with and without histology coil on a vertical bore 9.4T Bruker AV400WB with $1 \mathrm{Tm}-1$ actively shielded gradients. Results.MR images (Fig.1) showed $4.3 \times$ SNR increase, corresponding to $\sim 20$ fold reduction in imaging time, while image quality improved by showing features not being distinguishable using only the volume resonator (Fig.1d\&e). Fig.1f, with $60 \times 60 \mu \mathrm{m}$ in-plane resolution, shows that SNR allows even higher spatial resolution to be attained. Discussion.We showed that inductivelycoupled coils can produce high quality images from single histology slides. The approach is simple and robust, and reduces losses from multiple components and finite lead lengths, particularly important at high field. SNR improvement compared to the volume coil in our experimental design compares favorably to more sophisticated approaches involving, for example, cryogenic coils. Therefore, this design could serve as a useful tool, for example, in the development of MR contrast agents. 

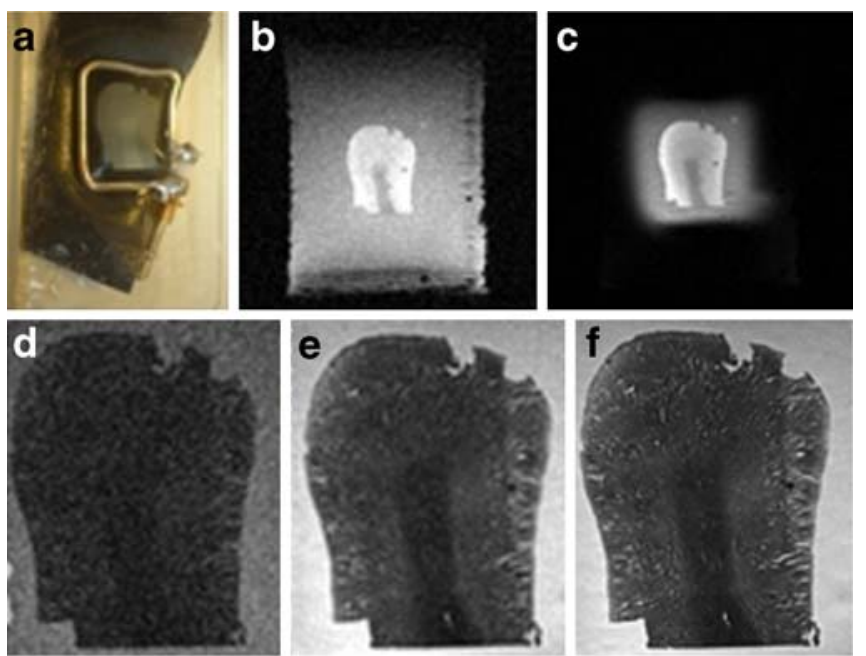

Figure 1.(a) Self-resonant loop placed on top of a $60 \mathrm{~mm}$ thick brain slice. (b) \&(c) Low-resolution scout images of the histology slice from the volume coil and "histology" coil, respectively;

GE: TR $T E=75 / 3 \mathrm{~ms}, \mathrm{FOV}=50 \times 50 \mathrm{~mm},[128128], \mathrm{NEX} 10, \mathrm{EST}=1.36 \mathrm{~min}$;

Comparison of image quality from volume (d) and histology coil (e)

using SE:TR/TE=2500/20 ms,FOV=16 ×16 mm,[128 128],

NEX $=4, E S T=20 \mathrm{~min}$; ( $f$ ) High resolution image acquired with the histology coil, like (e) with [256 256], NEX $=24, E S T=4$ hours.
J519

DIRECT IMAGING OF SPIOS IN MICE USING MAGNETIC PARTICLE IMAGING: INSTRUMENT CONSTRUCTION AND 3D IMAGING

Patrick Goodwill $^{1}$, Steven Conolly ${ }^{2}$

${ }^{1}$ Joint Graduate Group in Bioengineering, University of California Berkeley / UCSF, Berkeley, CA, USA, ${ }^{2}$ Bioengineering, University of California, Berkeley, Berkeley, CA, USA.

Magnetic Particle Imaging (MPI) is a new imaging modality that promises long-term detection and tracking of nano-mol/L concentrations of super-paramagnetic iron oxide (SPIO) particles commonly used as MRI contrast agents. The MPI method directly detects the magnetization from an SPIO whose saturation magnetization approaches $0.6 \mathrm{~T}$, or $10^{\wedge} 6$ times larger than the magnetization detected by MRI at 7 Tesla. The estimated $20 \mathrm{nmol} / \mathrm{L}$ detection limit [1], corresponding to a 200x SNR boost over MRI at detecting SPIOs, still needs to be experimentally verified. We have developed a MPI scanner capable of imaging a whole mouse. The system directly detects the magnetization of iron with remarkable sensitivity and resolution over a $3 \mathrm{~cm}$ field of view. Our system is a variant of MPI [1] that enables small receive bandwidths at high frequencies, with a clear path towards body noise dominance [2]. The system is capable of full XYZ imaging without moving the sample. To achieve this, water cooled electromagnets move the field free point up to $1.5 \mathrm{~cm}$ in any direction. The electromagnets are powered using standard MRI gradient amplifiers, requiring up to 450 Amp pulses to image the entire FOV. Continuous RF power of 10mT@240 kHz is well below the SAR limit for a mouse, and elicits a strong signal from the $50 \mathrm{~nm}$ SPIO particles used in this study. The system has a permanent $\mathrm{NdFeB}$ magnet gradient $(\mathrm{dB} / \mathrm{dz}=6500 \mathrm{mT} / \mathrm{m}, \mathrm{dB} / \mathrm{dx}, \mathrm{y}=4500 \mathrm{mT} / \mathrm{m})$, and is controlled by a custom built MRI console capable of working at low frequencies. We anticipate uses for MPI in angiography and cell tracking, and so we have imaged an angiography phantom that shows high contrast and resolution (see Figure). We have also used the system to image preserved mice injected with tracer (not shown). Conclusion: We successfully built a mouse sized MPI system that directly detects the magnetization of iron with remarkable sensitivity and resolution. Significant gains in SNR and resolution are possible as we continue to develop pulse sequences, reduce vibration, improve electronics, and develop new hardware.
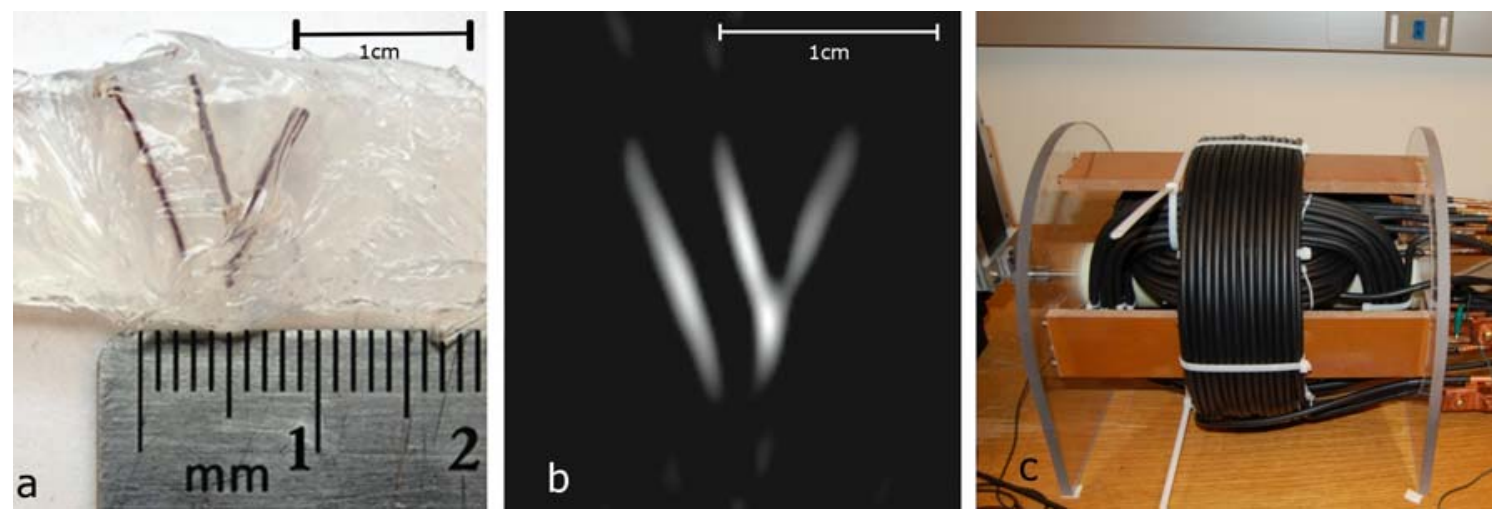

[a] Vascular phantom constructed of plastic tubing with $0.38 \mathrm{~mm}$ inner diameter. [b] MPI image of vascular phantom with FOV $=2.5 \mathrm{~cm} \times 3 \mathrm{~cm}$. Note: millimeter resolution. [c] Magnetic Particle Imager capable of fitting a mouse. 


\section{J520}

\section{ULTRASHORT TE IMAGING OF IRON-OXIDE NANOPARTICLES PRODUCES POSITIVE CONTRAST WITH LOW NANOMOLAR SENSITIVITY AT CLINICAL FIELD STRENGTHS}

Ravi T. Seethamraju $^{1}$, Sonia Nielles-Vallespin ${ }^{4}$, Ralph Weissleder ${ }^{3}$, David E. Sosnovik ${ }^{2,3}$

${ }^{1}$ MR R and D, Siemens Medical Solutions, USA Inc., Malden, MA, USA, ${ }^{2}$ Radiology, Martinos Center for Biomedical Imaging, Charlestown, MA, USA, ${ }^{3}$ Center for Molecular Imaging, Boston, MA, USA, ${ }^{4}$ MR PLM AW, Siemens AG, Erlangen, Germany.

Introduction: There is currently intense interest in the use of positive contrast methods to image iron-oxide based contrast agents. Although $\mathrm{T} 2 *$ based methods are sensitive to low nanomolar concentrations of iron-oxides, concerns have been raised regarding the negative contrast produced with this approach. Offresonance and other positive contrast methods based on the $\mathrm{R} 2 *$ properties of iron-oxide have been developed. These methods, however, can suffer from reduced sensitivity and nonlinearity, particular at high fields. Iron-oxide nanoparticles also exert significant R1 effects, particularly at low fields. We thus aimed to exploit the R1 effects of iron-oxide with an ultra-short TE (UTE) imaging sequence, and characterize the sensitivity of this approach as a function of field strength. Material and methods: The iron-oxide nanoparticle CLIO (cross linked iron-oxide: size $30 \mathrm{~nm}, \mathrm{R} 1$ at $0.47 \mathrm{~T}=26.4$ and $\mathrm{R} 274.9$ ) was studied. Imaging was performed on clinical $3 \mathrm{~T}$ and $7 \mathrm{~T}$ systems with a 3D UTE sequence with TE set to $70 \mu \mathrm{s}$. Other parameters included $\mathrm{FA}=90, \mathrm{TR}=2.9 \mathrm{~ms}(3 \mathrm{~T})$ and $8.54 \mathrm{~ms}(7 \mathrm{~T})$ and $1 \mathrm{~mm}$ Isotropic resolution. A phantom was created with 6 tubes containing CLIO concentrations of 0,10,50,100,200 and $400 \mathrm{nM}$ surrounded by water. Scanning was performed with a 12 channel head coil at 3T and an 8 channel phased array and detunable TEM volume coil at 7T. Results: Fig 1 shows the CNR generated by the UTE sequence as a function of field strength and CLIO concentration. At 3T a CLIO concentration of $10 \mathrm{nM}$ could be resolved, producing a CNR of 7.25. As can be seen in Fig 1, CNR increased linearly with CLIO concentration. No contamination from R2* effects was seen due to the ultrashort TE of 70 us. A CLIO concentration of $10 \mathrm{nM}$, however, could not be resolved at 7T due to the reduction in the $\mathrm{R} 1$ of CLIO with increasing field. A concentration of $50 \mathrm{nM}$, however, produced a CNR at $7 \mathrm{~T}$ of $>15$. Discussion: Iron-oxide nanoparticles have high R1 values at 1.5-3 Tesla. The use of a UTE sequence allowed low NM amounts of iron-oxide to be detected with a positive contrast approach without a significant loss of sensitivity. Moreover, the images were free of R2* effects and had a linear response to concentration at $3 \mathrm{~T}$. UTE sequences thus provide an attractive tool with which to image ironoxide Nanoparticles at standard clinical field strengths.

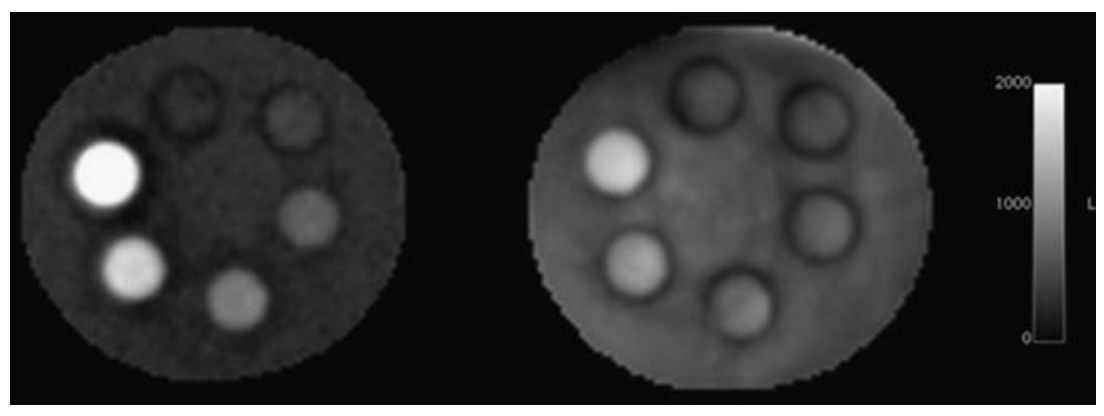

J521

\section{HAND-HELD CONCEPT OF A MAGNETIC PARTICLE IMAGING DEVICE}

Timo F. Sattel ${ }^{1}$, Sven Biederer ${ }^{1}$, Tobias Knopp ${ }^{1}$, Kerstin Luedtke-Buzug ${ }^{1}$, Bernhard Gleich $^{2}$, Jörn Borgert ${ }^{2}$, Thorsten M. Buzug ${ }^{1}$

${ }^{1}$ University of Luebeck, Institute of Medical Engineering, Luebeck, Germany, ${ }^{2}$ Tomographic Imaging Group, Philips Technologie GmbH Forschungslaboratorien, Hamburg, Germany.

Recently, B. Gleich and J. Weizenecker published a method for the quantitative measurement of the spatial distribution of superparamagnetic nanoparticles (Nature 435, 2005, 1214-7). Magnetic particle imaging (MPI) uses the nonlinear magnetization characteristics of the particles. A sinusoidally oscillating magnetic field that is overlayed to the nanoparticles stimulates a response that can be described by Langevin's theory of paramagnetism. The temporal change of the particle magnetization can be measured via appropriate receive coils. Due to magnetization saturation of the nanoparticles this leads to harmonics in the acquired signal spectrum. After separation of the higher harmonics from the fundamental driving frequency, the energy of the measured signal can be exploited to estimate the concentration of the nanoparticles. However, for spatial coding, two types of magnetic fields must be superimposed that allows for the measurement of the spatial distribution of the particles, i.e. a static selection field and an oscillating drive field. In this contribution we propose a novel asymmetric single-side coil design, comprising all field generating and signal receiving coils on one side. Using two transmit coils where one fits concentrically into the other transmit coil. That way, 
a field is produced by superposition that annihilates at two points at the axis of the coils arrangement, one on the front side (the patient side) and the other on the back side (the device side). We will present simulations and first experimental results of a single-side MPI device. The performance with respect to resolution and contrast is lower than that of a symmetric design. However, the results of this new concept are promising and the optimization process of the set-up is in the very beginning. With this new design, the patient does no longer need to fit into a tube-like coil assembly. Even when considering the limitations in penetration depth and resolution of this concept, it is reasonable that future single-sided MPI scanners will have improved imaging characteristics and single-sided 3D imaging become feasible.

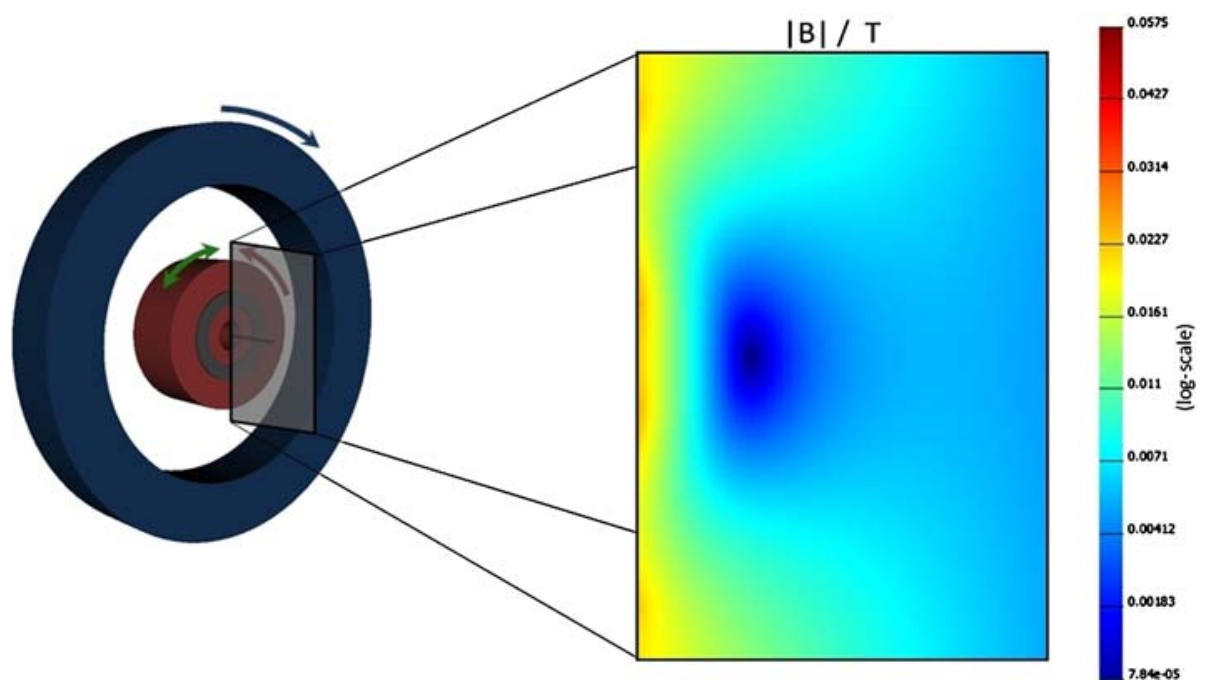

Single-side MPI line scanner. A flat coil arrangement produces a field-free point at either side of the assembly.

J522

\section{EFFECT OF STANDARD WATER PHANTOM ADC VALUE CORRECTION ON AN INTER-CENTER STANDARIZATION STUDY}

Hua dan Xue, Shuo Li, Zhengyu Jin

Department of Radiology, Peking Union Medical College Hospital, Beijing, China.

Purpose: To evaluate the effect of ADC value correction using standard water phantom in a multi-center standarization study for ADC value. Methods: 10 healthy volunteers aged 20-30 yr were included in each of the four medical centers. All centers were equipped with a $1.5 \mathrm{~T}$ MRI scanner. ADC value of a standard water phantom (pADC) was measured using the same scanning protocol. ADC values were also obtained in 24 organs over the whole body for each volunteer using whole body DWI sequence. Correlations between the pADC and variation of each organs' ADC value were evaluated using Spearman's coeffients. Coherence of ADC value between different hospitals (one way ANOVA) were also evaluated in each organ befor and after correction with the phantom $\mathrm{ADC}$ value $(\mathrm{cADC}=\mathrm{ADC} / \mathrm{pADC}$, $\mathrm{cADC}=$ corrected $\mathrm{ADC})$. Results: $\mathrm{ADC}$ value of 10 organs had moderate to obvious relation with $\mathrm{pADC}(r>0.4), 5$ organs showed mild relation $(0.4>\mathrm{r}>0.3)$, and 9 showed no significant relation. There were significant $\mathrm{ADC}$ value difference between four centers in all organs except central zone of the prostate gland and the junctional zone of the uterus $(p<0.01)$. While after correction with the phantom, cADC values of thalamus, gray matter, parotid gland, submandibular gland, gastric wall, spleen, pancreas, vertebral bodies and uterus wall showed no more significant differences(see table, $p<0.01$ ). Conclusion: With correction of the standard water phantom, part of the "systemic" ADC value variation between different medical centers can be overcome. Yet there is still reasons for these variation which needs further study to promote inter-center ADC value standarization. 
Inter-center cADC value comparison ( mean cADC value and One Way ANOVA results)

\begin{tabular}{|c|c|c|}
\hline organs (mean cADC value) & $\mathrm{F}$ & Sig. \\
\hline $\begin{array}{l}\text { gray matter } \\
(0.474)\end{array}$ & 0.861 & 0.468 \\
\hline $\begin{array}{l}\text { white matter } \\
\qquad(0.396)\end{array}$ & 16.182 & 0.000 \\
\hline $\begin{array}{l}\text { pons } \\
(0.442)\end{array}$ & 9.917 & 0.000 \\
\hline $\begin{array}{c}\text { caudate nucleus } \\
(0.365)\end{array}$ & 37.788 & 0.000 \\
\hline $\begin{array}{l}\text { putamen } \\
(0.356)\end{array}$ & 29.140 & 0.000 \\
\hline $\begin{array}{l}\text { thalamus } \\
(0.409)\end{array}$ & 0.600 & 0.618 \\
\hline $\begin{array}{l}\text { mammal glands } \\
(0.907)\end{array}$ & 5.059 & 0.004 \\
\hline $\begin{array}{l}\text { left ventricle wall } \\
\qquad(1.021)\end{array}$ & 18.679 & 0.000 \\
\hline $\begin{array}{l}\text { gastric wall } \\
(1.104)\end{array}$ & 0.961 & 0.392 \\
\hline $\begin{array}{l}\text { left hepatic lobe } \\
\qquad(0.711)\end{array}$ & 13.703 & 0.000 \\
\hline $\begin{array}{l}\text { right hepatic lobe } \\
\qquad(0.668)\end{array}$ & 7.865 & 0.000 \\
\hline $\begin{array}{l}\text { spleen } \\
(0.547)\end{array}$ & 1.886 & 0.145 \\
\hline $\begin{array}{c}\text { pancreas } \\
(0.991)\end{array}$ & 0.418 & 0.741 \\
\hline $\begin{array}{l}\text { renal medulla } \\
\quad(1.082)\end{array}$ & 15.640 & 0.000 \\
\hline $\begin{array}{l}\mathrm{T} 8 \text { vertebral body } \\
\qquad(0.265)\end{array}$ & 0.088 & 0.966 \\
\hline $\begin{array}{l}\text { T8-9 intervertebral disc } \\
(0.721)\end{array}$ & 9.113 & 0.000 \\
\hline $\begin{array}{l}\text { L2 vertebral body } \\
\qquad(0.260)\end{array}$ & 0.832 & 0.483 \\
\hline $\begin{array}{l}\text { L2-3 intervertebral disc } \\
(0.865)\end{array}$ & 28.573 & 0.000 \\
\hline $\begin{array}{l}\text { central zone of prostate gland } \\
(0.772)\end{array}$ & 1.157 & 0.350 \\
\hline $\begin{array}{l}\text { peripheral zone of prostate gland } \\
\qquad(0.887)\end{array}$ & 32.673 & 0.000 \\
\hline $\begin{array}{l}\text { uterus wall } \\
\quad(0.703)\end{array}$ & 1.585 & 0.222 \\
\hline $\begin{array}{l}\text { junctional zone of the uterus } \\
\qquad(0.933)\end{array}$ & 0.771 & 0.524 \\
\hline $\begin{array}{l}\text { parotid gland } \\
\quad(0.686)\end{array}$ & 3.895 & 0.014 \\
\hline $\begin{array}{l}\text { submandibular glands } \\
(0.668)\end{array}$ & 2.942 & 0.042 \\
\hline
\end{tabular}

OPTIMIZATION AND VALIDATION STUDY ON FePro METHOD TO MAGNETICALLY LABEL CELLS FOR MRI Branislava Janic $^{1}$, Ali M. Rad ${ }^{1}$, E. Kay Jordan ${ }^{2}$, Asm Iskander ${ }^{1}$, M. M. Ali ${ }^{1}$, Nadimpalli Ravi S Varma ${ }^{1}$, Joseph A. Frank ${ }^{2,3}$, Ali S. Arbab ${ }^{1}$;

${ }^{1}$ Cellular and Molecular Imaging Laboratory, Henry Ford Hospital, Detroit, MI, USA, ${ }^{2}$ Raiology and Imaging Sciences, Clinical Center, The National Institutes of Health, Bethesda, MD, USA, ${ }^{3}$ Intramural Research Program, National Institute of Biomedical Imaging and Bioengineering, Bethesda, MD, USA.

Background and Objective: Current method to magnetically label cells using ferumoxides (Fe)-protamine (Pro) sulfate (FePro) is based on generating FePro complexes in a serum free media that are then incubated overnight with cells for the efficient labeling. However, this labeling technique requires long $(>12-16 \mathrm{~h})$ incubation time and uses relatively high dose of Pro $(5-6 \mu \mathrm{g} / \mathrm{ml})$ that makes large extracellular FePro complexes. These complexes can be difficult to clean with simple cell washes and may create low signal intensity on $\mathrm{T} 2 *$ weighted MRI that is not desirable. The purpose of this study was to revise the current labeling method by using low dose of Pro and adding $\mathrm{Fe}$ and Pro directly to the cells before generating any FePro complexes. Experimental Approaches and Results: Human tumor glioma (U251) and human monocytic leukemia cell (THP-1) lines were used as model systems for attached and suspension cell types, respectively and dose dependent (Fe 25 to $100 \mu \mathrm{g} / \mathrm{ml}$ and Pro 0.75 to $3 \mu \mathrm{g} / \mathrm{ml}$ ) and time dependent $(2$ to $48 \mathrm{~h})$ labeling experiments were performed. Labeling efficiency and cell viability of these cells were assessed. Prussian blue staining revealed that more than $95 \%$ of cells were labeled. Intracellular iron concentration in U251 cells reached $\sim 35 \mathrm{pg}$-iron/ cell at $24 \mathrm{~h}$ when labeled with $100 \mu \mathrm{g} / \mathrm{ml}$ of Fe and $3 \mu \mathrm{g} / \mathrm{ml}$ of Pro. However, comparable labeling was observed after $4 \mathrm{~h}$ across the described FePro concentrations. Similarly, THP-1 cells achieved $\sim 10 \mathrm{pg}$-iron/cell at $48 \mathrm{~h}$ when labeled with $100 \mu \mathrm{g} / \mathrm{ml}$ of $\mathrm{Fe}$ and $3 \mu \mathrm{g} / \mathrm{ml}$ of Pro. Again, comparable labeling was observed after $4 \mathrm{~h}$ for the described FePro concentrations. FePro labeling did not significantly affect cell viability. There was almost no extracellular FePro complexes observed after simple cell washes. To validate and to determine the effectiveness of the revised technique we applied the revised method to label various types of stem as well as mature differentiated cells. Human T-cells, human hematopoietic stem cells (hHSC), human bone marrow stromal cells (hMSC) and mouse neuronal stem cells (mNSC C17.2) were labeled. Labeling for 4 hours using $100 \mu \mathrm{g} / \mathrm{ml}$ of $\mathrm{Fe}$ and $3 \mu \mathrm{g} / \mathrm{ml}$ of Pro resulted in very efficient labeling of these cells, without impairing their viability and functional capability. Conclusions: The new technique with short incubation time using $100 \mu \mathrm{g} / \mathrm{ml}$ of $\mathrm{Fe}$ and $3 \mu \mathrm{g} / \mathrm{ml}$ of Pro is effective in labeling different cell types for cellular MRI.

\section{J524}

DYNAMIC CONTRAST-ENHANCED FOLATE RECEPTOR TARGETED MR IMAGING USING A GD-LOADED PEGDENDRIMER-FOLATE CONJUGATE IN A MOUSE

XENOGRAFT TUMOR MODEL

Wei-Tsung Chen ${ }^{1,2}$, Dhakshanamurthy Thirumalai ${ }^{3}$, Tiffany Ting-Fang Shih $^{2,4}$, Ran-Chou Chen ${ }^{1,5}$, Hsing-Yang Tu ${ }^{1}$, Wen-Yuan Hsieh ${ }^{3}$, PangChyr Yang ${ }^{6}$, Jim Chin-I Lin ${ }^{3}$

${ }^{1}$ Radiology, Taipei City Hospital, Taipei, Taiwan, ${ }^{2}$ Radiology, Medical School, National Taiwan University, Taipei, Taiwan, ${ }^{3}$ Union Chemical Laboratory, Industrial Technology Research Institute, Hsingchu, Taiwan, ${ }^{4}$ Medical Imaging, National Taiwan University Hospital, Taipei, Taiwan, ${ }^{5}$ Biomedical Imaging and Radiological Sciences, National Yang Ming University, Taipei, Taiwan, ${ }^{6}$ Medicine, National Taiwan University, Taipei, Taiwan. 
Purpose: To validate a folate receptor (FR) targeted dendrimer, PEGG3-(Gd-DTPA)11-(folate)5, for its ability to detect FR positive tumors, by using dynamic contrast enhance MRI. Procedures: KB cells, FR siRNA knockdown KB cells, and FR negative HT-1080 cells, were incubated with fluorescein-labeled dendrimer and their cellular uptake was observed. Folate competition study was also performed. Dynamic contrast enhanced MRI was performed on mice bearing KB and HT1080 tumors and the enhancement patterns and parameters were analyzed. The Type A time intensity curve represents an rapidly rising slope followed by a second slow rising phase. Type B represents a rapidly rising slope, followed by a plateau. Type $\mathrm{C}$ represents a rapidly rising slope, followed by a wash-out phase in the latter portion. Results: Green fluorescence was found in the KB cells in the cellular uptake experiment, but was less seen in FR siRNA knockdown KB cells and FR negative HT-1080 cells. Decreased green fluorescence was also seen in 500X free folate competition group. In the dynamic contrast enhanced MRI, the 30 minute washout percentage was $-4 \pm 18 \%$ in the KB tumors and $39 \pm 23 \%$ in the HT- 1080 tumors. A $17 \%$ cut-off point gave a sensitivity of $94.4 \%$, and a specificity of $93.8 \%$. All 5 type A curves were found in the KB tumors. Twelve of 13 type $\mathrm{B}$ curves were revealed in the KB tumors. Seventeen of 18 type C curves were seen in the HT-1080 tumors $(p<0.001)$. Conclusions: We have demonstrated the targeting ability of PEG-G3-(Gd-DTPA)11-(folate) 5 in vitro and in vivo. A $17 \%$ cut-off point for a 30 minute washout percentage can be used as a useful parameter for the diagnosis of FR positive tumors.

Distribution of the enhancement patterns of the KB and HT-1080 tumors after PEG-G3-(Gd-DTPA)11-(folate)5 injection.

\begin{tabular}{|c|c|c|c|c|}
\hline & type A curve & type B curve & type C curve & total \\
\hline KB tumor & 5 & 12 & 1 & 18 \\
\hline HT-1080 tumor & & 1 & 17 & 18 \\
\hline & 5 & 13 & 18 & 36 \\
\hline
\end{tabular}

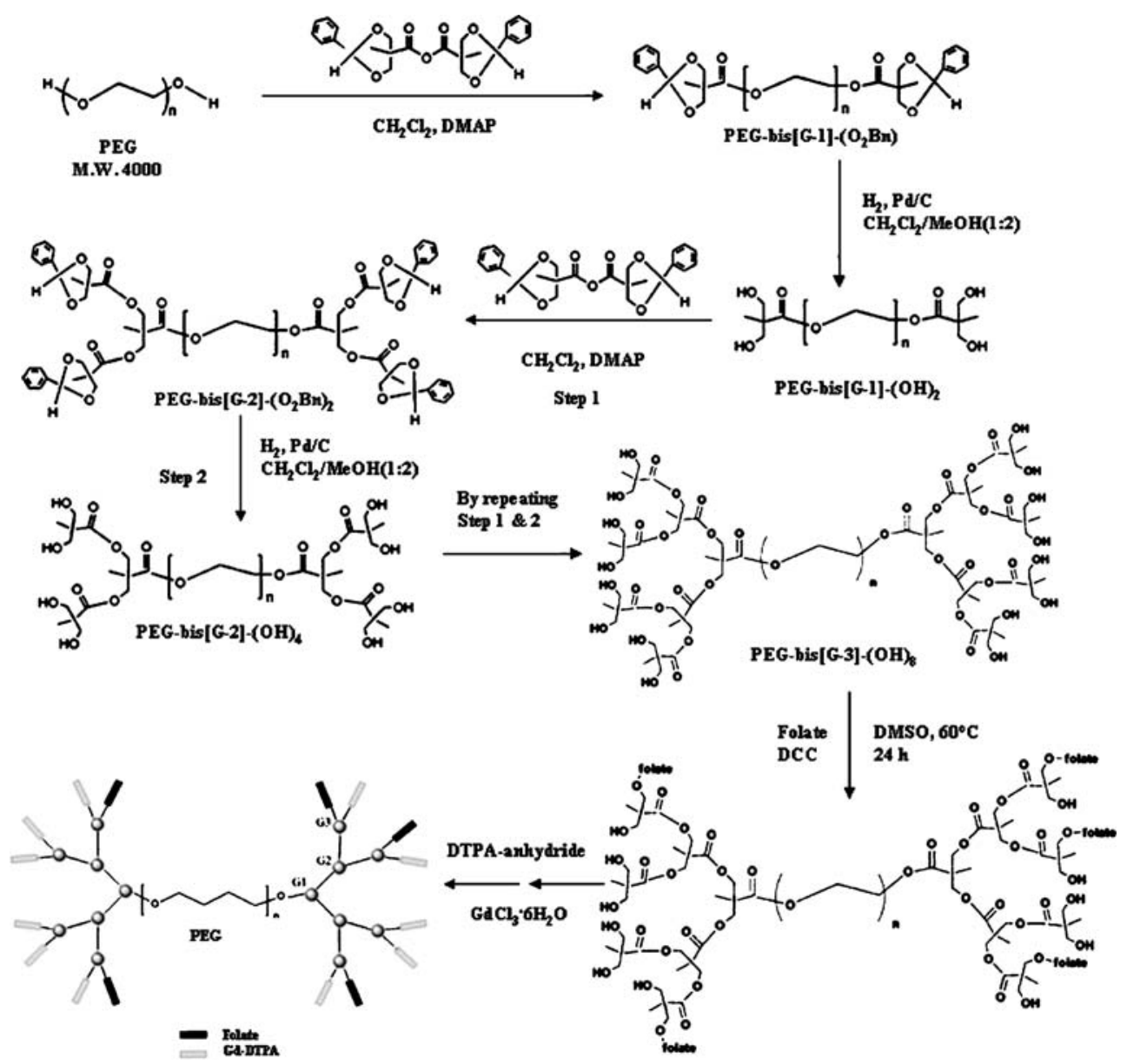

The synthesis scheme of the PEG-G3-(Gd-DTPA)11-(folate)5 dendrimer. 
TOWARDS NOVEL MRI ${ }^{13}$ C-HYPERPOLARIZED PROBES: PARA-HYDROGENATION STUDIES ON UNSATURATED GLUCOSE CONTAINING SUBSTRATES

Francesca Reineri ${ }^{1}$, Daniela Santelia ${ }^{1}$, Alessandra Viale ${ }^{1}$, Tomas Trtek $^{2}$, Erika Cerutti $^{1}$, Silvio Aime ${ }^{1}$, Stephen S. Premkumar ${ }^{1}$, Roberto Gobetto ${ }^{1}$, ${ }^{1}$ Dept. of Chemistry IFM, University of Torino, Torino, Italy, ${ }^{2}$ Institute of Organic Chemistry and Biochemistry, Academy of Sciences of the Czech Republic, Prague, Czech Republic.

Introduction. Much attention has been devoted in recent years to the development of hyperpolarized ${ }^{13} \mathrm{C}$ contrast agents for ${ }^{13} \mathrm{C}$-magnetic resonance imaging, which can be produced either by Dynamic Nuclear Polarization (DNP) or by Para Hydrogen Induced Polarization (PHIP). The most challenging application of ${ }^{13} \mathrm{C}$-hyperpolarized molecules deals with their use as metabolic imaging reporters. Up to now, in the clinical practice, the most important "in vivo" metabolic imaging assay is represented by the PET measure of glucose uptake. We wonder whether a ${ }^{13} \mathrm{C}$-hyperpolarized glucose derivative could be considered as a viable MRI alternative to the complex and expensive PET procedure. As an hydrogenation route to glucose can not be easily envisaged, we have synthesized and tested for para-hydrogenation a set of molecules in which the sugar moiety is bound to an hydrogenable synthon (butynoic acid), in order to select suitable candidates for an "in vivo" MRI method for the assessment of glucose cellular uptake. Methods. Para-enriched hydrogen $(52 \%)$ was prepared storing $\mathrm{H}_{2}$ over $\mathrm{Fe}_{2} \mathrm{O}_{3}$ at $77 \mathrm{~K}$ for one hour. Para-hydrogenation reactions were carried out in ALTADENA conditions in a $5 \mathrm{~mm}$ NMR tube equipped with a Young valve, using [Bis(diphenylphosphino)butane](1,5-cyclooctadiene)rho$\operatorname{dium}(\mathrm{I})$ tetrafluoroborate as the catalyst, in an acetone- $\mathrm{d}^{6} / \mathrm{CD}_{3} \mathrm{OD} 9: 1$ mixture. A magnetic field cycle, consisting in quickly inserting the tube into a $\mu$-metal shield (field strength $0.1 \mu \mathrm{T}$ ), and then slowly removing the shield, was applied to the samples in order to convert the antiphase ${ }^{13} \mathrm{C}$ signal to an in-phase one. NMR spectra were recorded on a Bruker Avance 600 spectrometer, operating at $600 \mathrm{MHz}$ for the proton and $150 \mathrm{MHz}$ for ${ }^{13} \mathrm{C}$. Single scan spectra were acquired. Results. Butynoic acid ester derivatives of glucose are hydrogenated in high yield and afford enhanced ${ }^{1} \mathrm{H}$ and ${ }^{13} \mathrm{C}$ NMR spectra after para-hydrogenation. The obtained PHIP patterns can be explained by calculating the spin level populations in the parahydrogenated products. A short spacer between the unsaturated moiety and the glucose unit is efficient in maintaining the ${ }^{13} \mathrm{C}$ relaxation time of the carbonyl group in the butenoic moiety long enough for manipulations and NMR/MRI experiments. These molecules may find interesting applications in ${ }^{13} \mathrm{C}$-MRI as hyperpolarized probes for assessing the activity of glucose transporters in cells.

\section{ACCELERATED 3D HIGH RESOLUTION MAGNETIC RESONANCE ANGIOGRAPHY OF CAROTID ARTERY STENOSES IN MICE}

David Ratering ${ }^{1}$, Christof Baltes ${ }^{1}$, Christine Lohmann ${ }^{2}$, Christian M. Matter ${ }^{2}$, Markus Rudin ${ }^{1,3}$

${ }^{1}$ Institute for Biomedical Engineering, University and ETH Zurich, Zurich, Switzerland, ${ }^{2}$ Institute of Physiology, University Zurich and Cardiology University Hospital Zurich, University Zurich, Zurich, Switzerland, ${ }^{3}$ Institute of Pharmacology \& Toxicology, University Zurich, Zurich, Switzerland.

Three dimensional high-resolution MR angiography (3D-MRA) has been proven to be a useful tool for the visualization of carotid artery stenoses. However, 3D-MRA in small rodents is hampered by long acquisition times due to sensitivity issues associated with small voxel dimensions, putting high demands on the physiological stability of the animal preparation. The combination of 3D-MRA with the parallel imaging technique SENSitivity Encoding (SENSE) has been shown to considerably reduce acquisition times in human 3D-MRA, while still preserving good image quality. In this work the question was addressed whether accelerating high resolution 3D-MRA affects the reliability and accuracy of detecting vascular lesions in male Apolipoprotein E (ApoE) knockout mice, an accepted model for atherosclerosis. Experiments have been carried out on a Bruker Pharmascan 47/16 (Bruker BioSpin MRI, Ettlingen, Germany) animal MR system operating at $200 \mathrm{MHz}$ using a linear polarized volume resonator for excitation and a four element $(2 \times 2)$ phased array surface coil for signal reception. Data acquisition was performed using a $3 \mathrm{D}$-gradient echo sequence with acquisition parameters: $\mathrm{FOV}=17 \times 19 \times 20 \mathrm{~mm}^{3}$, spatial resolution $=80 \times$ $80 \times 80 \mu \mathrm{m}^{3}$, pulse angle $=80^{\circ}, \mathrm{TE} / \mathrm{TR}=3.7 / 70 \mathrm{~ms}$. Data were acquired using full sampling and with acceleration factors (R) ranging from 2.0 to 3.3. The effect of accelerated data collection on the angiograms was evaluated by comparing maximum intensity projections (MIPs) (Fig.1a) as well as morphometric analyses (Fig.1b) obtained from accelerated and fully sampled data sets. MIPs obtained from accelerated data showed minimal differences in vessel representation compared to the reference MIP: stenoses could be depicted reliably even for $\mathrm{R}$ values of 3.3. Morphometric analysis at various levels with regard to the stenoses revealed that accelerating data acquisition did not reduce the accuracy for acceleration factors up to 3.3 .<smiles>CC#CC(=O)OC[Pb]C(=O)OC=CC(=O)OC(C)=O</smiles>

Schematic representation of the para-hydrogenation of butynoic acidglucose derivatives 

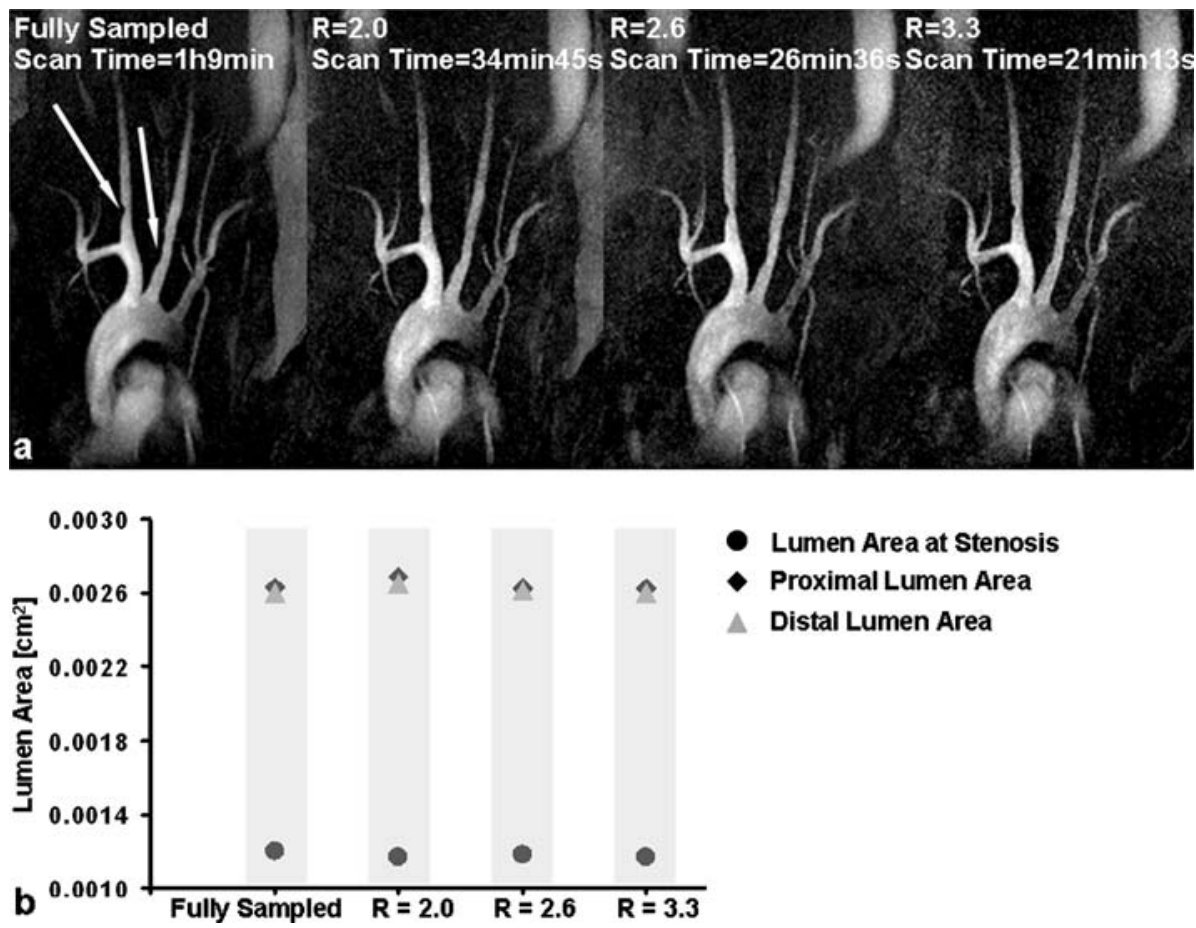

- Lumen Area at Stenosis

- Proximal Lumen Area

$\Delta$ Distal Lumen Area

Fig.1: a: Horizontal MIPs of aortic arch, carotid and subclavian arteries for different R-values. Stenoses are indicated by arrows. b: Lumen areas calculated proximal, distal and at the site of stenosis for different acceleration factors.

J527

\section{IN VIVO DETECTION OF BOTH SINGLE CELLS}

AND DEVELOPING METASTASES USING SSFP AT 1.5T

Emeline J. Ribot ${ }^{1}$, Francisco Martinez ${ }^{1}$, Brian K. Rutt ${ }^{2}$,

Paula Foster $^{1}$

${ }^{1}$ Robarts Research Institute, London, ON, Canada, ${ }^{2}$ Dept of Radiology, Stanford, CA, USA.

A complete analysis of experimental metastasis by MRI requires imaging protocols with the sensitivity and tissue contrast needed to detect both single cancer cells and metastases. Large mouse brain tumors have been imaged in vivo using balanced steady state free precession (SSFP) at $4.7 \mathrm{~T}$. However, SSFP contrast at $9.4 \mathrm{~T}$ prevented the same tumors from being detected (1). This is because short repetition times (TR) need to be used with SSFP at high field to minimize banding artifacts and because SSFP contrast, related to $\mathrm{T} 2 / \mathrm{T} 1$, is affected by the increasing $\mathrm{T} 1$ values with high fields. A short TR SSFP sequence has also been used to detect single iron labeled cells in vivo in the mouse brain at $1.5 \mathrm{~T}$ (2), however visibility of small brain metastases was poor. In this study we show that a modified SSFP sequence, with long TR, permits the detection of both iron labeled cancer cells and the developing metastases, in one scan at 1.5T. Methods: Brain metastatic breast cancer cells were labeled with iron and injected into the heart in nude mice. SNR, CNR and the number of voids were measured in SSFP images acquired at $1.5 \mathrm{~T}$ with a range of parameters varied, including flip angle $(\alpha)$, receiver bandwidth (BW) and TR/TE. Results: With our optimal SSFP parameter set $\left(\mathrm{BW}=21 \mathrm{kHz}, \mathrm{TR} / \mathrm{TE}\right.$ of $26 / 13 \mathrm{~ms}$ and $\alpha=35^{\circ}$ ) both single ironlabeled cancer cells and metastases could be visualized in vivo.
With RF phase cycling implemented for b-SSFP at $1.5 \mathrm{~T}$ banding artifacts are diminished and long TRs can be employed to enhance detection of iron labeled cells; the longer the TR, the greater the dephasing and the darker the void. Tumor-brain contrast was strongly influenced by the use of small $\alpha$ and was reduced when the same parameters were used ex vivo. The ability to monitor the fate of both single iron labeled cells and developing metastases in vivo in the same image set will allow for important spatial and temporal relationships between the arresting cancer cells and the developing metastases, to be carefully analyzed. (1) Miraux et al. JMRI 2008 (2) Heyn et al. MRM 2006

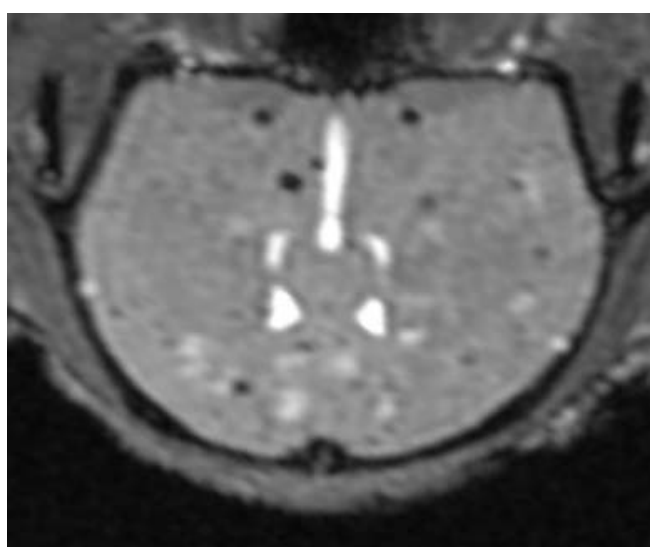


$\mathbf{J 5 2 8}$

\section{FEASIBILITY OF KEYHOLE ACQUISITION FOR IMPROVED TEMPORAL RESOLUTION DYNAMIC CONTRAST ENHANCED MR IMAGING OF ATHEROSCLEROTIC PLAQUE AT 3T \\ Venkatesh Mani, Claudia Calcagno, Zahi Fayad}

Department of Radiology, Mount Sinai School of Medicine, New York, NY, USA.

Introduction: Plaque neovascularization is a hallmark of vulnerable atherosclerotic lesions. Parameters reflecting micro-vessel perfusion, permeability and extracellular leakage space are determined from T1weighted dynamic contrast enhanced (DCE) MR images acquired at several time points after contrast agent injection. DCE imaging in atherosclerosis however places stringent requirements on spatial resolution for accurate vessel wall delineation ( $0.5 \mathrm{~mm}$ in-plane), temporal resolution for accurate estimation of arterial input function (under $10 \mathrm{~s} /$ frame) and volumetric coverage for imaging the whole lesion $(>10 \mathrm{~mm})$. We hypothesize that high spatial and temporal resolution 3D bright blood DCE MR imaging of atherosclerosis is feasible at $3 \mathrm{~T}$ using keyhole acquisitions. Methods: Abdominal aortas of atherosclerotic rabbits were imaged with a 3D GRE sequence on a $3 \mathrm{~T}$ MR scanner $(5$ slices; TR/TE= $19 / 4.9 \mathrm{~ms}$; voxel size $0.5 \times 0.5 \times 3 \mathrm{~mm}$; SPIR fat suppression; flip angle= $50^{\circ}$; 40 measurements) following injection of $0.1 \mathrm{mmol} / \mathrm{Kg}$ of Gd-DTPA after the 2nd image without (temporal resolution $12.9 \mathrm{~s}$ ) and with keyhole acquisitions (25\% acquisitions, $8.1 \mathrm{~s}$ temporal resolution, $32 \mathrm{~s}$ reference scan). The area under the signal intensity vs. time curve (AUC-5 minutes), transfer constants from plasma to tissue compartment and vice versa (Ktrans and Kep) were calculated for each pixel on the image. Results: The image shows AUC, Ktrans and Kep maps of atherosclerotic lesions obtained using the keyhole acquisitions. Keyhole imaging enabled acquisition of $37 \%$ higher temporal resolution with similar spatial resolution and coverage as non keyhole imaging. Kinetic parameters obtained showed correlation between keyhole and non keyhole acquisitions. Conclusions: This study shows feasibility of high spatial and temporal resolution 3D bright blood DCE MR imaging of atherosclerotic lesions in rabbits at $3 \mathrm{~T}$ using keyhole acquisitions with adequate SNR to accurately evaluate kinetic parameters.

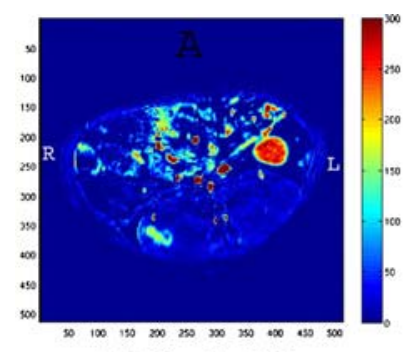

AUC 5 Min a.u.

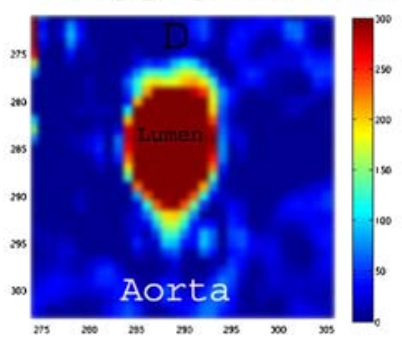

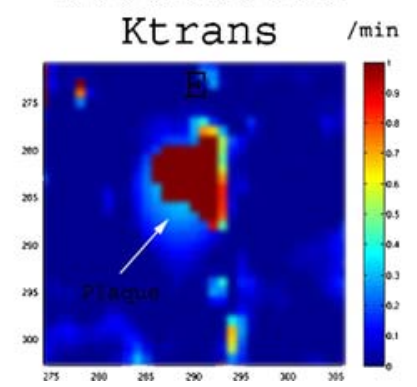
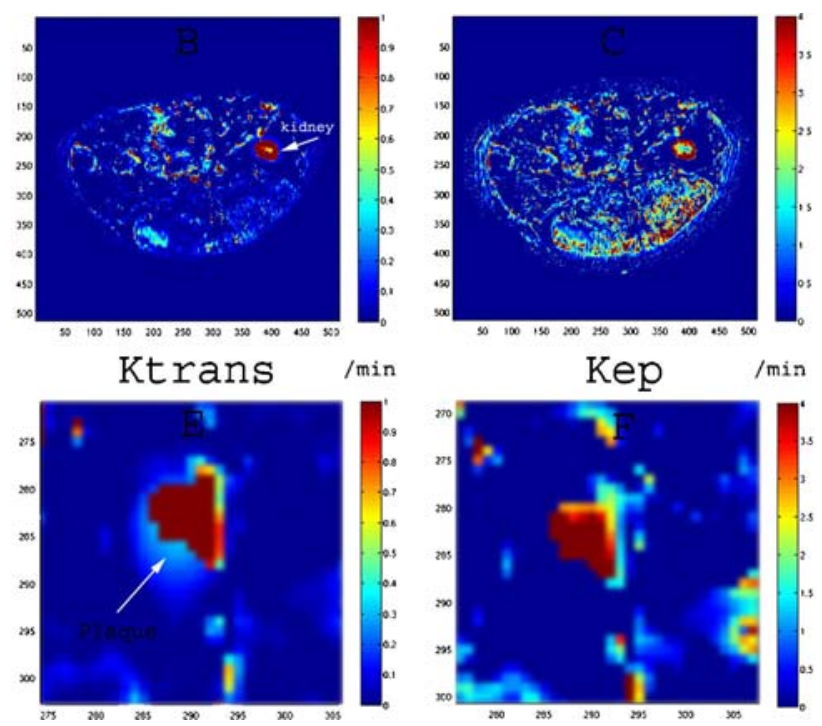

Figure 1: AUC (A, D), Ktrans (B, E)and Kep (C, F) maps of atherosclerotic lesions obtained using the DCE MRI keyhole acquisitions. Top panels show the whole rabbit and the bottom panels show the zoomed in view of the abdominal aorta.

J529

\section{IN VIVO CEREBROMICROVASCULATURAL VISUALIZATION USING USPIO AND 3D TRUEFISP MRI AT 9.4T. APPLICATION TO A GLIOMA BEARING MOUSE MODEL \\ Wadie Ben Hassen ${ }^{1}$, Veronique Bouchaud ${ }^{1}$, Emeline J. Ribot ${ }^{2}$, Jean-Michel Franconi ${ }^{1}$, Pierre Voisin ${ }^{1}$, Eric Thiaudiere ${ }^{1}$, Sylvain Miraux ${ }^{1}$ ${ }^{1} \mathrm{CNRS} /$ Univ Bordeaux2, RMSB, Bordeaux, France, ${ }^{2}$ Imaging Research Laboratories, Robarts Research Institute, London, ON, Canada.}

Imaging methods for assessing the cerebromicrovasculature is therefore important in a wide range of neuroscience-research fields, particularly to understand tumor models and to visualize the efficiency of a therapeutic treatment. To visualize the cerebromicrovasculatre on mice models, we proposed a novel method based on 3D TrueFISP imaging at high magnetic field and systemic injection of Ultra Small Particles of Iron Oxide. It has been recently showed that artifact-free TrueFISP imaging can be obtained on mice models at high field thanks to alternating radiofrequency phase method and a SOS reconstruction [1]. This method was employed to generate high resolution 3D images (9.4T Bruker MRI system, (Ettlingen, Germany), TE/TR : 3.5/ $7 \mathrm{~ms}, 150 \mu \mathrm{m}$ isotropic resolution, $12 \mathrm{~min}$ of acquisition time) of the entire mouse head before and after USPIO injection $(150 \mu \mathrm{mol}$ $\mathrm{Fe} / \mathrm{kg}$ ) in the tail vein. Same imaging sequence was repeated during one hour after the contrast agent injection. Brain vasculature 
and microvasculature map was obtained according to equation : 1/ $\mathrm{TE}^{*} \ln ($ Spre contrast/Spost contrast). Experiments on normal mouse brain show the entire vasculature of the brain. Major veins and major arteries were visible. Once more, smaller vessels such as arterioles and venules (not visible with classical Magnetic Resonance Angiography technique) can also be visualize. On the glioma bearing mice models, the technique clearly shows neo-vascularisation of the tumor. The advantage of TrueFISP method is to generated imaging with high signal-to-noise ratio and high spatial resolution and to be very sensitive to decrease of signal due to USPIO. The method appears free of motion and susceptibility artifact compared to classical $\mathrm{T} 2 *$ gradient echo. Compared to DeltaR2 imaging [2] with fast spin echo method, total acquisition time is largely reduced and consequently allows to generate dynamic images. These preliminary results show the potential of the proposed method to visualize mouse brain microvascularization. It could be used to assess efficiency of a therapeutic treatment against glioma or other brain diseases. Due to its high sensitivity, the method can also be applied in molecular imaging based on functionalized-USPIO. [1] Miraux S et al. 3D TrueFISP imaging of mouse brain at 4.7T and 9.4T. J Magn Reson Imaging. 2008 Aug;28(2):497-503. [2] Lin CY et al. In vivo cerebromicrovasculatural visualization using 3D DeltaR2-based microscopy of magnetic resonance angiography (3DDeltaR2-mMRA). Neuroimage. 2009 Apr 15;45(3):824-31.

$\mathbf{J 5 3 0}$

\section{STRUCTURAL SPECTROSCOPYTM_A TRANSLATIONAL, MAGNETIC RESONANCE BASED, STRUCTURE BIOMARKER FOR CHARACTERIZING FINE ANATOMICAL STRUCTURES}

Timothy W. James ${ }^{1}$, John Heinrich ${ }^{1}$, Pawel F. Tokarczuk ${ }^{2}$,

Stephen J. Paisey ${ }^{2}$, Andrew J. Stewart ${ }^{2}$

${ }^{1}$ OsteoTronix, Ltd., Swansea, United Kingdom, ${ }^{2}$ The Experimental MRI Center, Department of Biosciences, Cardiff University, Cardiff, United Kingdom.

Purpose: Structural Spectroscopy ${ }^{\mathrm{TM}}$ is a new technique for characterizing anatomical structures using magnetic resonance data. Data is acquired from rectangular prisms located in the anatomical region and orientation of interest using a novel pulse sequence. The resulting one-dimensional signal profiles are then analyzed using customized signal processing algorithms to determine the spacing and/or size distribution of the anatomical elements of interest. Structural Spectroscopy is, like MRI, noninvasive and fully translational from preclinical to clinical applications. However, it offers the further advantages of very short acquisition time $(<1 \mathrm{~min}$.)) and the ability to identify structural features as small as 50 microns Methods: Structural Spectroscopy was implemented on a $9.4 \mathrm{~T}$ Bruker $^{\mathrm{TM}}$ preclinical imaging system running standard Paravision ${ }^{\mathrm{TM}} 4.0$ software. The acquisition protocol uses the geometry editor and OsteoTronix ${ }^{\mathrm{TM}}$ application interface to locate a rectangular prism within the anatomy (for example, a rat spine), run the pulse sequence, and collect the profile data. All image and profile data is stored and exported in standard DICOM format. Results: The acquisition protocol produces a one-dimensional profile consisting of magnetic resonance signal strength as a function of position along the prism and structural spectra are generated from segments of interest along the anatomical region defined by the prism. As an example, a Structural Spectrum from a region of porous bone shows a principal peak at a structural frequency of approximately $2 / \mathrm{mm}$, indicating typical structural spacings of about $0.5 \mathrm{~mm}$. A spectrum from a denser region exhibits a peak at $4 / \mathrm{mm}$ representative of the finer structure in that region and higher frequency peaks corresponding to trabecular web thickness. The spacings derived from the structural spectra have been correlated with the spacings observed in microCT from the corresponding regions. Conclusions: Structural Spectroscopy appears to be a very promising technique for the evaluation of anatomical structures, with potential applications in preclinical and clinical research and diagnosis and monitoring of therapy in a variety of clinical settings. Refinement of acquisition and analysis techniques, implementation on other platforms and investigation of applications including osteoporosis, angiogenesis, organ fibrosis and mammary ductal structures is continuing and will also be presented.

\section{J531}

\section{A PHASE-CORRELATION METHOD FOR REGISTRATION OF DYNAMIC CONTRAST-ENHANCED MAGNETIC RESONANCE IMAGES OF THE HUMAN CAROTID ARTERY \\ Sarayu Ramachandran, Claudia Calcagno, Zahi Fayad}

Translational and Molecular Imaging Institute, Mount Sinai School of Medicine, New York, NY, USA.

Introduction: Dynamic contrast enhanced magnetic resonance (DCEMR) has recently been used to investigate the composition of atherosclerotic plaques. Patient motion and poor signal-to-noise ratio can degrade the images, necessitating registration during postprocessing. In this study we propose a phase-correlation method for registration, and compare it to a squared-difference registration method [Kerwin, Magn Reson Med. 2002] in DCE-MRI studies. Methods: Both algorithms were tested on DCE-MRI of the common carotid arteries of 10 patients. DCE-MR images were acquired using a black-blood double inversion recovery turbo spin-echo sequence. To ensure the presence of motion between frames, random translational shifts were introduced for each frame (maximum shift of 3 pixels). Registration was performed on a small region of interest $(80 \times 80$ pixels) that was centered on the lumen of the carotid. Both algorithms were tested with and without Kalman filtering on this dataset. Performance was evaluated by computing the correlation coefficients (CC) between consecutive frames and a mean CC for each patient. Paired t-test was used to compare the $\mathrm{CC}$ for each method to unregistered data. Results: Figure 1 shows a plot of mean $\mathrm{CC}$ values for each patient for unregistered data and after registration by each method with and without filtering. Both methods show a significant improvement in $\mathrm{CC}$ values over unregistered values (phasecorrelation, $p<0.01$; squared-difference, $p<0.001$; both methods after Kalman filtering, $p<0.001)$. Performance of phase-correlationbased registration was validated against the squared-difference method. The results were promising for the phase-correlation method considering the faster computation time of this method, as well as its flexibility to be extended to correct for rotational motion in the future. A simple registration method is presented for improved DCE-MRI of carotid arteries for atherosclerotic plaque assessment. 


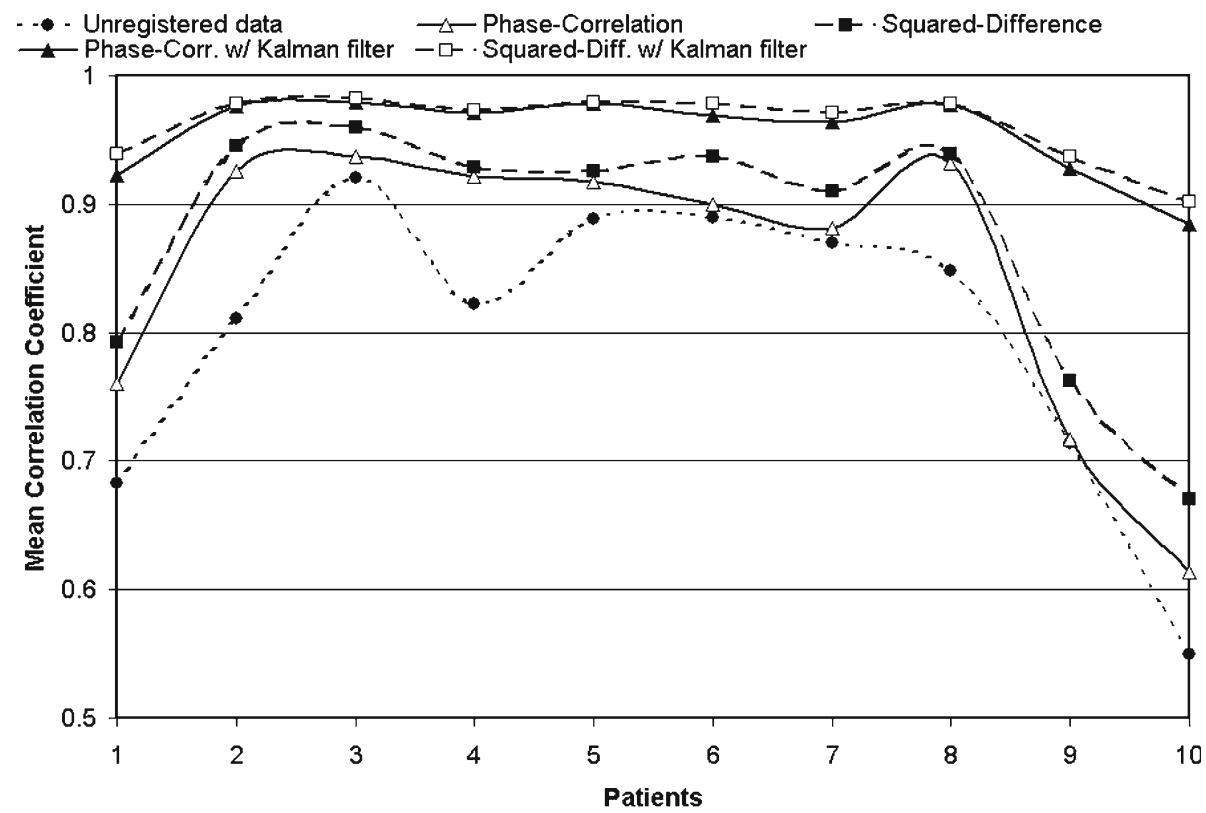

Fig 1. Plot of mean CC for each patient before and after registration

J532

\section{CONTRAST BEHAVIORS OF IDQCS MR IMAGING WITH VARIOUS METAL SALTS}

Jee H. Cho ${ }^{1}$, Sangdoo Ahn ${ }^{2}$, sung Lan Jeon ${ }^{1}$, Chulhyun Lee ${ }^{1}$

${ }^{1}$ MRI team, Korea Basic Science Institute, Chungbuk, Korea, South,

${ }^{2}$ Chemistry, Chung-Ang, Seoul, Korea, South.

Recently, a new method for contrast enhancement in magnetic resonance imaging based on the detection of relatively strong signals from intermolecular multiple quantum coherences (iMQCs) both in vitro and in vivo has been reported. These signals are strongly depending on the local magnetic properties of samples which can be modified by appropriate contrasting agents. In this study, several metal salts have been tested as contrast agents affecting the iMQCs signals at $14.1 \mathrm{~T}$ $\mu \mathrm{MRI}$ on the purpose of looking for a good contrasting condition fitting to the new method. The results show that the iMQCs microscopic images are readily observable and have unique contrast depending on the micro-structures of samples. The detailed contrast characteristics of iMQCs MR images with these metal salts for several phantoms and model cell systems will be discussed.

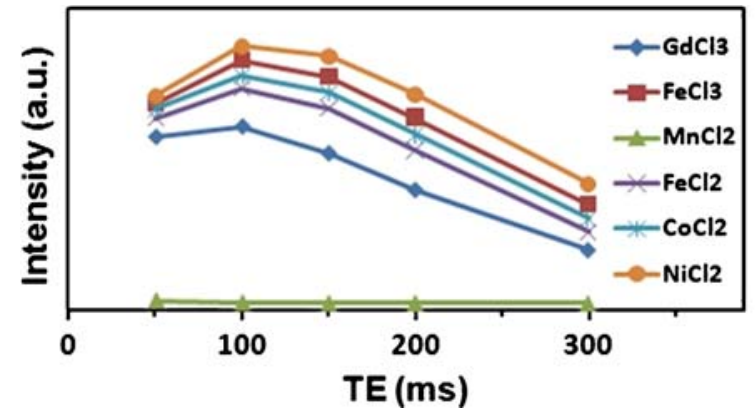

Contrast behaviors of iDQC MR images with various metal salts

\section{J533}

\section{DEVELOPMENT OF 14.1T MR MICROSCOPE}

Tomohiro Ueno ${ }^{1}$, Munehiro Inukai ${ }^{2}$, Shin-ichi Urayama ${ }^{1}$, Kazuyuki Takeda ${ }^{2}$, Shouichirou Kanayama ${ }^{1}$, Naozo Sugimoto ${ }^{1}$, Hidenao Fukuyama ${ }^{1}$

${ }^{1}$ Graduate School of Medicine, Kyoto University, Kyoto, Japan,

${ }^{2}$ Graduate School of Science, Kyoto University, Kyoto, Japan.

We have been developing an MRI with very high spatial resolution, "MR Microscope", by using a 14.1T NMR magnet. The MR Microscope is designed for visualizing small tissue in vitro and will be applicable for pathological tests without using cryo-sectioning and for molecular

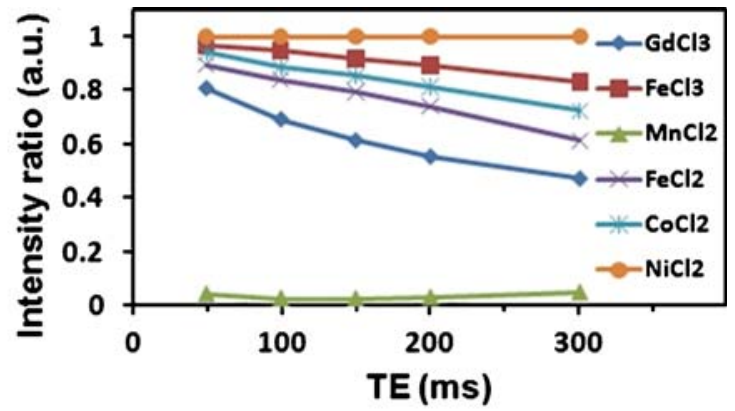

imaging. The target voxel size of the MR Microscope is $10 \mu \mathrm{m} \times$ $10 \mu \mathrm{m} \times 10 \mu \mathrm{m}$ in a $128 \times 128 \times 128$ matrix with moderate temporal resolution. In order to attain the goal, we installed the 14.1T NMR magnet with $48 \mathrm{~mm}$ horizontal bore (Japan Superconductor Technology, Inc., Japan) and a $600 \mathrm{MHz}$ NMR spectrometer with an $1 \mathrm{~kW}$ RF power amplifier (Thamway, Inc., Japan). As for gradient control system, we chose three 400W high speed amplifiers (KEPCO, Inc., USA) which were driven by an FPGA module (National Instruments, Inc., USA). All other components such as an MRI probe (solenoid type Rx/Tx coil of ID $5 \mathrm{~mm}$ and $5 \mathrm{~mm}$ long), gradient coils (triaxial coils on parallel plane bobbins), were built by ourselves. With the above system, we could get less than $1 \mathrm{ppm}$ magnetic field homogeneity in the sample space, and $0.62 \mathrm{~T} / \mathrm{m}$, 
$0.56 \mathrm{~T} / \mathrm{m}, 1.3 \mathrm{~T} / \mathrm{m}$ gradient field strength for 3 orthogonal axes. The difference in the gradient fields was caused by the output voltage limitation of the gradient amplifiers. Using the ordinary spin echo sequence, we accomplished $50 \mu \mathrm{m}$ resolution with 3D image of a dried young sardine, which was soaked in water beforehand and fixed in agar gel. We are trying to obtain higher spatial resolution by improving the MRI probe and the gradient coils. We will present our current status of the deveplopment.

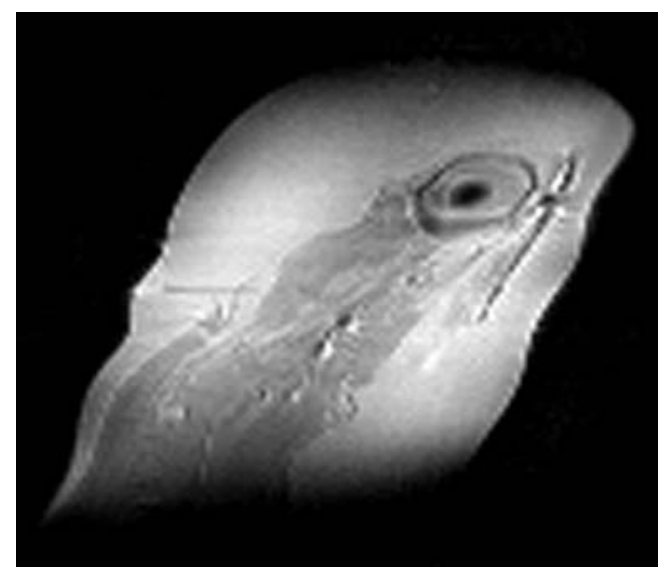

$\mathbf{J 5 3 4}$

\section{MEMRI OBSERVATION OF PAIN-RELATED NERVOUS \\ SYSTEMS OF RODENTS}

Jee H. Cho, Chulhyun Lee

MRI team, Korea Basic Science Institute, Chungbuk, Korea, South.

2D slice extracted from 3D image of a dried young sardine in agar gel. The structure of the head, such as the eyeball, is clearly seen.

\begin{abstract}
Manganese-enhanced MRI (MEMRI) techniques can be useful to understand functions of pain-related nervous systems though systematic studies have not been conducted well. To observe responses of electrical stimulated pain for rodents, MR imaging were taken for brain and spinal cord with $\mathrm{MnCl}_{2}$ injection at $4.7 \mathrm{~T}$ animal MRI scanner. The MEMRI images responding to pain in the brain cortex showed remarkable signal enhancement in the primary sensory area, which corresponded to sensory tactile electric stimulation at hindlimb region. The signal enhancement could be suggested to functional increases in calcium influx and brain activation by electric stimulation. Also, injection of $\mathrm{MnCl}_{2}$ into the lumbar of rat and dissection of its SC made it possible to observe manganese enhanced patterns of the SC in the serial $\mathrm{T}_{1}$ weighted images at $6,12,24$, and 48 hours after $\mathrm{MnCl}_{2}$ administration. This study would be useful for the decision of grade and clinical effect of spinal cord injury.
\end{abstract}
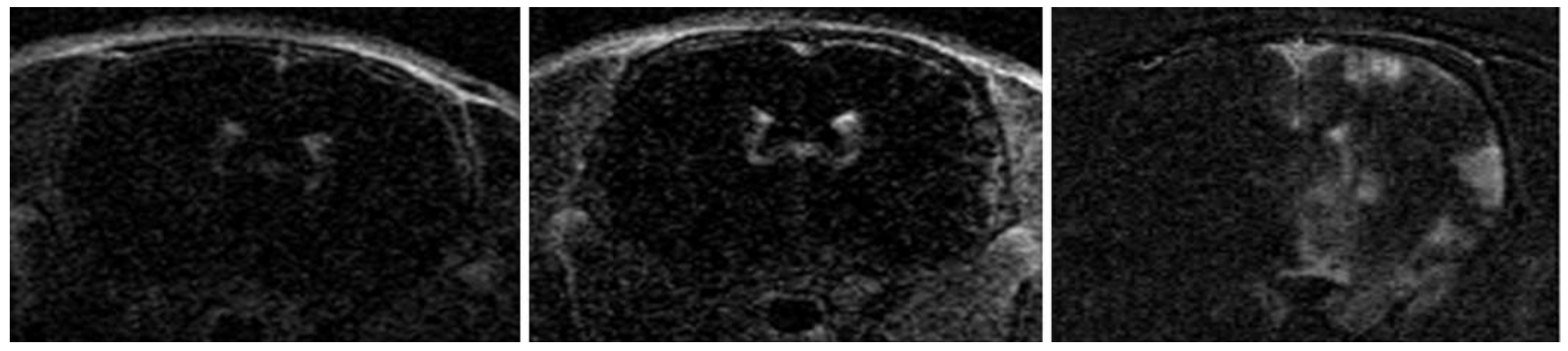

MEMRI for a naïve(left), sham stimulation(middle) and electric stimulation(right) rat

\section{$\mathbf{J 5 3 5}$}

\section{USE OF ULTRASHORT TE (UTE) SEQUENCES}

TO INCREASE MR SIGNAL SPECIFICITY TO IRON OXIDE NANOPARTICLES (IONP)

Olivier M. Girard $^{1}$, Lilach Agemy ${ }^{2}$, Kazuki N. Sugahara ${ }^{2}$,

Erkki Ruoslahti $^{2}$, Graeme M. Bydder ${ }^{1}$, Robert F. Mattrey ${ }^{1}$

${ }^{1}$ Radiology, University of California, San Diego, San Diego, CA, USA,

${ }^{2}$ Burnham Institute for Medical Research, University of California, Santa Barbara, Santa Barbara, CA, USA.

There is great interest in IONP for molecular imaging with MRI because of their 2 to 3 orders of magnitude greater MR sensitivity than Gd chelates. IONP causes T2* shortening effects that induce signal loss because IONP strongly dephase protons located in or adjacent to their hydration sphere. A major challenge is recognition that detected low signal is due to IONP and not normally occuring low signal tissues, particular when SNR is low. To aid in this recognition, a pre-image is typically acquired; however, because of the slow accumulation of IONP in tissues, two different sessions are needed making co-localization challenging. Since IONP shortens both $\mathrm{T} 1$ and $\mathrm{T} 2 *$, and since $\mathrm{T} 1$ shortening cannot be detected with standard MR imaging if there is no detectable signal, we optimized a UTE sequence (TE $\sim \mu \mathrm{s}$ ) from which we subtract the signal acquired from a 2 nd echo acquired at $\mathrm{TE} \sim 6 \mathrm{~ms}$ to reduce the long $\mathrm{T} 2$ components. Resulting contrast combines both $\mathrm{T} 1$ and $\mathrm{T} 2 *$ effects, and thus increases MRI specificity to IONP. For this study we used an IONP particle coated with the peptide CREKA that specifically targets tumor vessels that contain clotted plasma proteins. This causes additional clotting and further amplifies tumor accumulation. We showed that this approach successfully occludes the majority of tumor vessels without causing clotting in normal vessels. We scanned mice using a $2.5 \mathrm{~cm}$ cylindrical coil at $3 \mathrm{~T}$. They had an orthotopically implanted prostate cancer. They were imaged before and after the administration of either non-targeted IONP or IONP targeted using the CREKA peptide. Targeted IONP were easily detected as voxels with both short $\mathrm{T} 1$ and $\mathrm{T} 2 *$. The subtraction image had the greatest contrast since signal from tissues with relatively long $\mathrm{T} 2 \mathrm{~s}$ was reduced. While typically a pre-image is 
needed to recognize that the signal loss is due to IONP, our proposed MR IONP detection scheme could allow post IONP imaging only because of its greater specificity.

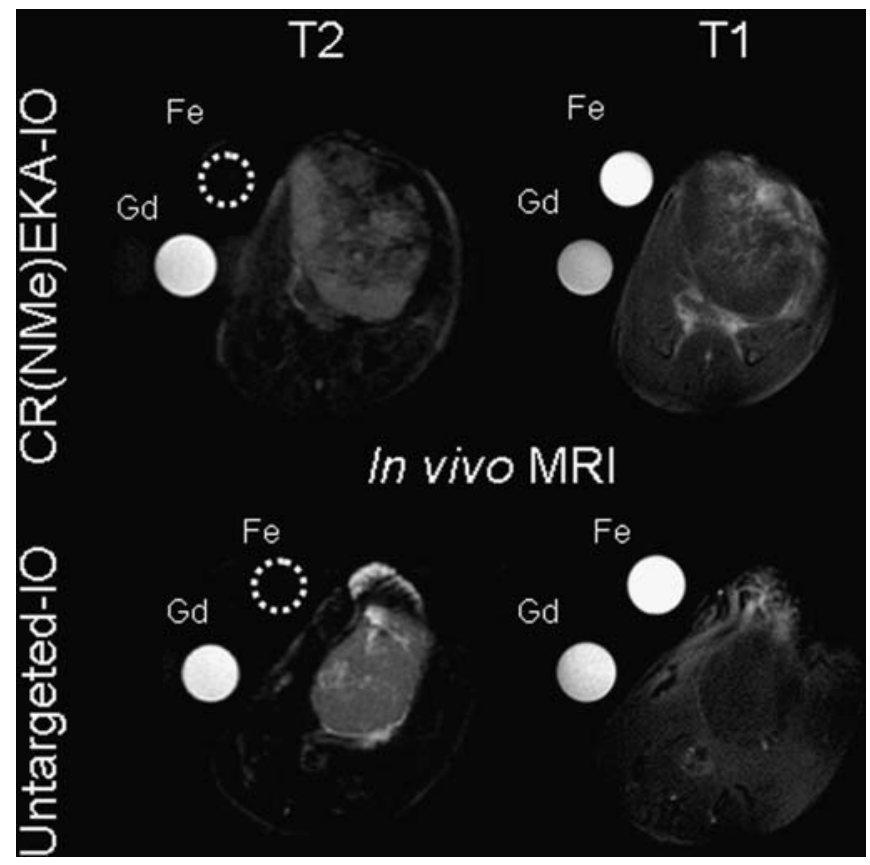

Gd and IONP tubes were added as reference standards.

$\mathbf{J 5 3 6}$

\section{MRI-COUPLED VIDEO RATE OPTICAL OXIMETRY TOMOGRAPHY}

Zhiqiu $\mathrm{Li}^{1}$, Venkataramanan Krishnaswamy ${ }^{1}$, Kimberley Samkoe ${ }^{1}$, Brian W. Pogue ${ }^{1,2}$

${ }^{1}$ Thayer School of Engineering, Dartmouth College, Hanover, NH, USA, ${ }^{2}$ Department of Surgery, Dartmouth Medical School, Hanover, $\mathrm{NH}$, USA.

Optical tomography of deep tissue allows spectroscopy and fluorescence sampling of deep tissue, and unfortunately while it could be done quickly, essentially all optical scanners require significant amounts of time to complete whole body scans. Spectral encoding methods can be used for parallel delivery of multiple sources of light to tissue, assuming that the detection method can decode the source spectrum in the transmitted signal. The advantage of this encoding approach is that the signal is separated prior to detection, avoiding inherent dynamic range limitations or temporal sequencing of other encoding approaches. A parallel version of this spectral encoding approach is demonstrated with two wavelength bands, 16 sources, and 16 parallel spectrometer detectors. The instrument can record transmission data through an animal at 50 frames per second. Tissue phantoms have been used to verify the abilty to image pulsatile function, exogeneous uptake and blood oximetry imaging. In vivo measurements and tissue phantom data have been used to show how fast uptake of exogenous agents can be imaged through bulk tissue, and how those signals can be localized to the compartment of interest in the MR image volume. The system allows tissue oximetry because the two wavelength bands straddle the isobestic point of oxy- and deoxy-hemoglobin, similar to the way fingar pulse oximetry works. This system allows fast drug kinetic imaging and pulse oximetry imaging of the organs of rodents all within the MR scanner.
$\mathbf{J 5 3 7}$

\section{RELAXATION ENHANCEMENT BY LONGITUDINAL MULTISPIN ORDERS-A NOVEL APPROACH FOR HYPERPOLARIZED IMAGING EXPERIMENTS} Prasanta Dutta $^{1}$, Gary Martinez ${ }^{1}$, S. Sendhil Velan ${ }^{2}$, Robert Gillies ${ }^{1}$ ${ }^{1}$ Imaging, Moffitt Cancer Center, Tampa, FL, USA, ${ }^{2}$ Laboratory of Molecular Imaging, Singapore Bioimaging Consortium, Singapore, Singapore.

There is a great interest in increasing the longitudinal relaxation of nuclear spins for hyperpolarized imaging experiments. In this work we propose that the longitudinal multispin orders (LOMO) might enhance the longitudinal relaxation. LOMO correspond to the non-equilibrium population distribution and can be created in spin systems that exhibit $\mathrm{J}$ couplings, dipolar couplings or quadrupolar couplings (1). It can also be created via crosscorrelated relaxation between different relaxation pathways present for the given spin system (2). The rate at which these populations return to equilibrium following a perturbation is determined by the corresponding transition probability W. Considering a two-spin-1/2 spin system AX, the observed intensities of $\mathrm{A}$ and $\mathrm{X}$ transitions are proportional to $\mathrm{Az}$ and $\mathrm{Xz}$ i.e., the macroscopic longitudinal magnetization. These vectors $\mathrm{Az}$ and $\mathrm{Xz}$ are proportional to population differences between these states. The rate at which populations corresponding to each state returns to equilibrium following a perturbation is governed by the relevant set of transition probabilities W. The W1's are single quantum relaxation probabilities, W0 is the zero quantum relaxation probability, while $\mathrm{W} 2$ is the double quantum relaxation probability (1). Within the dipolar relaxation framework, we can readily estimate that: $1 / \mathrm{T} 1(\mathrm{~A}): 1 / \mathrm{T} 1(\mathrm{X}): 1 / \mathrm{T} 1(\mathrm{AzXz})=5: 5: 3$. Thus it is very clear that the relaxation rates differ for the various LOMO's in any given spin system. We will choose some 13C-labelled organic molecules for creating LOMO states and their T1's will be measured by $7 \mathrm{~T}$ Varian Scanner. Under the framework of dipolar mechanism the LOMO appears to have longer T1's and might be suitable for storing the hyperpolarized state of different hyperpolarized compounds and useful for bio-imaging. (1) Velan SS, J Magn Reson, 171, 345, 2004. (2) Kumar A., Grace RCR, Madhu PK. Prog Nucl Magn Reson Spectrosc., 37, 191, 2000.

\section{$\mathbf{J 5 3 8}$}

\section{A COMBINATION OF EDGE-CORRECTION}

AND CORRELATION DETECTOR FUNCTIONS FOR MR SPECTROSCOPIC AND MR SPECTROSCOPIC IMAGING DATA CORRECTION

Sandeep Kumar Ganji ${ }^{1}$, Mir Abrar T. Ali ${ }^{2}$, Venkateswara Rao M. Meka ${ }^{2}$ ${ }^{1}$ Radiological Sciences Graduate Program, The University of Texas Southwestern Medical Center, Dallas, TX, USA, ${ }^{2}$ Department of Biomedical Engineering, University College of Engineering (Autonomous), Hyderabad, India.

The spectral data of both Magnetic Resonance Spectroscopy (MRS) and Magnetic Resonance Spectroscopic Imaging (MRSI) depend on the assumption that the subject is not moving and that the reconstruction algorithm accounts for any motion which might occur during the scan. Many a times the data is corrupted by artifact errors which degrades overall data quality, submerging any clinically significant data, which is especially true in whole brain imaging studies where in both MRS and MRSI used. The correction algorithm is based on applying the smoothness of gradient detectors for edge motion detection in combination with a motion-energy velocity function for spatio-temporal edge detection. Similarities between the neighboring displacement vectors (pixel values) may be used in order to estimate the actual displacement from the image motion/displacement. Though here in our poster only a special case will be discussed (which is any extension of our previous work), which assumes that images (w.r.to their spectral data) have a distribution of displacements vectors along an edge (shifts). We have used a MRS/MRSI raw data for our analysis, and then corrected the spectral data using the method after introduction of significant motional noise into the data sets. In this poster, we would like to present the preliminary insights we obtained by using the above methodology. 


\section{IDENTIFICATION OF PANCREATIC CANCER STEM CELLS AND EARLY DETECTION OF PANCREATIC CANCER BY OPTICAL AND ENHANCED MAGNETIC RESONANCE MOLECULAR IMAGING \\ Yang Cao ${ }^{1}$, Raymond $\mathrm{Ngo}^{1}$, Ryan Quiroz ${ }^{1}$, David Ang ${ }^{1}$, Hongxiang Hui ${ }^{2}$, Yongming G. Tang ${ }^{3}$, Yung-Ya Lin ${ }^{1}$ \\ ${ }^{1}$ Chemistry and Biochemistry, UCLA, Los Angeles, CA, USA, ${ }^{2}$ Medicine, UCLA, Los Angeles, CA, USA, ${ }^{3}$ Medical Genetics, Cedars-Sinai Medical Center, Los Angeles, CA, USA.}

Pancreatic cancer is the fourth leading cause of cancer-related death in both men and women in US. Due to difficulties in diagnosis and resistance to chemotherapy as well as radiotherapy, pancreatic cancer has the worst survival of any solid tumor-five-year survival rate $<4 \%$ in US. Since emerging evidence suggests there are putative pancreatic cancer stem cells (PCSC), we developed new approaches based on optical and magnetic resonance molecular imaging to identify and image pancreatic cancer stem cells in vivo. First, in vitro identification of the PCSC is done by fluorescence microscopy of YAG-AB-quantum dots incubated with PCSC in cell media, where antibody $\mathrm{AB}=\mathrm{CD} 24$, CD44, and ESA (Figs. A, B). After the binding was confirmed and the PCSC was identified, we then imaged PCSC via magnetic resonance microimaging with new superparamagnetic nanoparticles that exhibit unique T1/T2 values and new radio-frequency pulse sequences that generated T1/T2-weighted imaging for enhanced imaging contrast and sensitivity of the diluted PCSC (only $0.5 \%$ of cell population) (Figs. C, D). Consequently, the intellectual merits of this cross-disciplinary project are: (1) Bioconjugation chemistry - conjugated quantum dots and magnetic nanoparticles with specific PCSC biomarker antibody. (2) Material chemistry-synthesized iron-oxide magnetic nanoparticles that exhibit unusual T1/T2 relaxation effects to surrounding water protons; (3) Spin engineering-developed new radio-frequency pulse sequences to enhance the contrast and detection sensitivity for the magnetic nanoparticles and therefore the PCSC biomarker and PCSC. It was demonstrated that the proposed new approaches based on optical and magnetic resonance molecular imaging may help make early detection of pancreatic cancers and targeted therapy possible. Its implication to the new tumor model-mutation only at the stem cell or progenitor cell level-will be discussed.
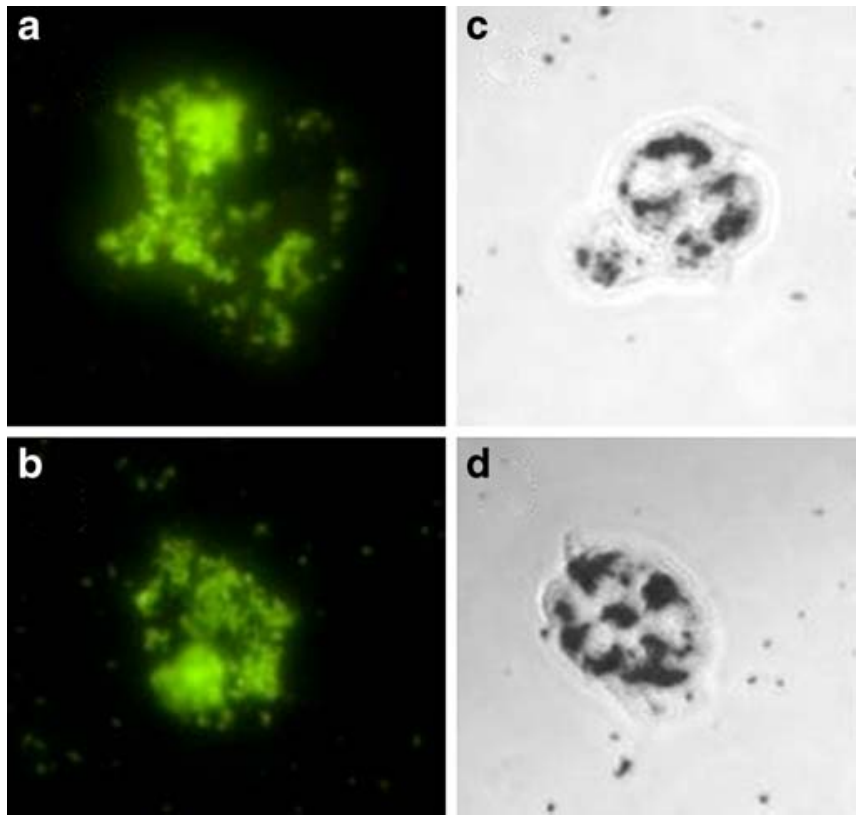

IMAGING CHARACTERIZATION OF HYPERSECRETING OR NON-HYPERSECRETING ADRENAL ADENOMAS: COMPARISON BETWEEN IODINE-131 NOR-CHOLESTEROL UPTAKE AND MAGNETIC RESONANCE SIGNAL INTENSITY

Simone Maurea $^{1}$, Mario Fusari ${ }^{1}$, Carmine Mollica ${ }^{2,3}$,

Massimo Imbriaco ${ }^{1}$, Leonardo Pace ${ }^{1}$, Marco Salvatore ${ }^{1}$

${ }^{1}$ Radiologia e Medicina Nucleare, Dipartimento di Scienze Biomorfologiche e Funzionali (DSBMF), Università degli Studi di Napoli Federico II (UNINA), Napoli, Italy, ${ }^{2}$ Istituto di Biostrutture e Bioimmagini (IBB), Consiglio Nazionale delle Ricerche (CNR), Napoli, Italy, ${ }^{3}$ Diagnostica per immagini, Fondazione SDN (IRCCS), Napoli, Italy.

The aim of this study was to compare the results of nor-cholesterol scintigraphy and those of magnetic resonance imaging (MRI) in characterizing adrenal adenomas and differentiating between hypersecreting and non-hypersecreting lesions. We studied 41 patients $(18 \mathrm{M}$ and $23 \mathrm{~F}$, mean age $47 \pm 15$ years) with hypersecreting ( $n=19)$ or nonhypersecreting $(n=22)$ unilateral adrenal tumors; all patients underwent iodine-131 nor-cholesterol adrenal scintigraphy and MRI studies. Pathology examinations $(n=26)$ or follow-up data $(n=15)$ were obtained showing 34 adenomas, 2 cysts, 1 myelolipoma, 1 pheochromocytoma, 1 carcinoma and 2 metastases. Imaging studies were qualitatively evaluated and the corresponding results were classified as true-positive, true-negative, false-positive and false-negative calculating diagnostic accuracy of each test; furthermore, nor-cholesterol uptake was semi-quantitatively measured using a four-point score from 0 to 3 , while MRI signal intensity ratios (SIRs) were quantitatively assessed using region of interest (ROI) analysis. The diagnostic accuracies of nor-cholesterol scintigraphy (95\%) and MRI (93\%) to identify adrenal adenomas were comparable; however, while a significantly $(p=0.01)$ higher nor-cholesterol uptake was observed in hypersecreting $(n=19)$ adenomas compared to non-hypersecreting $(n=$ 15) lesions, no significant differences in MRI SIRs were found in this comparative analysis; furthermore, no significant difference in tumor size occurred between hypersecreting and non-hypersecreting adenomas. In conclusion, adrenal scintigraphy using nor-cholesterol and MRI are both able to accurately identify cortical adenomas; in particular, while quantitative analysis of nor-cholesterol uptake is effective to differentiate between hypersecreting and non-hypersecreting adenomas, MRI SIRs evaluation is not useful for this purpose. These findings have relevant clinical implications particularly in patients with non-hypersecreting adenomas; in such patients, laboratory data are not helpful for lesion diagnosis, while imaging findings allow tumor characterization; in particular, the lower nor-cholesterol uptake observed in non-hypersecreting adenomas might reflect the normal hormone synthesis status of these lesions and, thus, regular secretion.

\section{J541}

IMPROVEMENT OF WHOLE BODY DIFFUSION-WEIGHTED IMAGE QUALITY USING 3D-GAUSSIAN FILTER AND TRILINEAR INTERPOLATION

WonHo Lee, Sang-Keun Woo, Kyeong Min Kim, Ji-Ae Park, Sang Moo Lim, Jong Guk Kim, Jin Su Kim, Gi Jeong Cheon Molecular Imaging Research Center, Kirams, Seoul, Korea, South.

Diffusion-weighted image (DWI) provides functional information of water molecules in the body and can be used detection of tumor. Recent advances in MR technology, fast imaging sequence like echo planar imaging (EPI), high gradient amplitudes, multichannel coils and parallel imaging, enable the acquisition whole body DWI. However, whole body (WB) DWI has disadvantage of magnetic susceptibility and respiration artifacts. To eliminate these artifacts, optimized scan protocol and breathhold or respiratory triggered scanning are used. However, noise in 
the DWI image still present. In this study, we used image processing (3D Gaussian filtering and trilinear interpolation) method to enhance WB-DWI image quality. The WB-DWI and T1 images obtained with a 3T Siemens (Tim trio) scanner. WB-DWI images were acquired using EPI sequence (TR : $4700 \mathrm{~ms}$, TE: $67 \mathrm{~ms}, \mathrm{FOV}=350 \mathrm{~mm}, 108 \times 128 \times 30$ matrix with $2.7 \times 2.7 \times 3.0 \mathrm{~mm}$ spatial resolution, 30 axial slices and slice thickness $=$ $3.0 \mathrm{~mm}$, ) WB-T1 images were acquired using VIBE sequence (TR : $2.72 \mathrm{~ms}$, TE: $1.04 \mathrm{~ms}, \mathrm{FOV}=500 \mathrm{~mm}, 234 \times 384 \times 128$ matrix with $2.0 \times$ $1.3 \times 2.0 \mathrm{~mm}$ spatial resolution, 128 coronal slices and slice thickness= $2.0 \mathrm{~mm}$, ). The WB-DWI and $\mathrm{T} 1$ images have to be processed on workstation. The 3D data sets from the different axial series are combined one series. Then, DWI images ( $b$-value $=0)$ are processed 2 image filters (3D Gaussian, 3D median) and trilinear interpolation using Matlab. To evaluate processed image quality used CNR and SNR (Table. 1). Filtering (3D gaussian, 3D median) methods improved uniformity and SNR compared with original DWI image (original image uniformity: 0.2 , filtering method uniformity: 0.18). The 3D Gaussian among filtering methods was more effective than 3D median. Trilinear interpolation method reduced Mean Square Error (MSE) about $27.8 \%$ compared with T1. The use of 3D Gaussian filtering with trilinear interpolation method improved DWI image quality. This method would be useful for reducing noise and improving image visualization in WB-DWI study.

\begin{tabular}{|c|c|c|c|c|c|c|}
\hline & Ori & MF & GF & Ori+TI & MF+TI & GF+TI \\
\hline CNR & 2.57 & 2.50 & 2.55 & 2.58 & 2.51 & 2.56 \\
\hline SNR & 5.35 & 11.47 & 11.06 & 5.35 & 11.46 & 11.66 \\
\hline
\end{tabular}

Ori: Original DWI image (b-value=0), MF: 3D Median filtering $(3 \times 3 \times 3)$, GF: $3 \mathrm{D}$ Gaussian filtering $(3 \times 3 \times 3)$, TI: Trilinear Interpolation, CNR: $(\mathrm{C}-\mathrm{N}) / \mathrm{N}$ (C: same as SNR ROI, N: right inferior lung, $30 \times 30 \times 30 \mathrm{~mm}^{\wedge} 3$ sphere), SNR: mean/SD (ROI: liver center, $30 \times 30 \times 30 \mathrm{~mm}^{\wedge} 3 \mathrm{sphere)}$

\section{Imaging Methodology}

\section{Optics}

J542

\section{D OPTOACOUSTIC TOMOGRAPHY SYSTEM FOR MOLECULAR AND FUNCTIONAL WHOLE BODY IMAGING IN SMALL ANIMALS}

Alexander A. Oraevsky ${ }^{1,2}$, Hans-Peter F. Brecht ${ }^{1}$, Sergey A. Ermilov ${ }^{1}$, ${\text { Richard } \mathrm{Su}^{1} \text {, Andre Conjusteau }}^{1}$, Ketan $\mathrm{Mehta}^{1}$, Matthew P. Fronheiser ${ }^{2}$ ${ }^{1}$ R\&D, Fairway Medical Technologies, Houston, TX, USA, ${ }^{2}$ Seno Medical Instruments, San Antonio, TX, USA.

3D optoacoustic tomography system was developed combining advantages of pulsed optical spectroscopy and high-resolution ultrasonic detection, characterized and shown to produce high-contrast 3D maps of optical absorbance through the whole body of an animal with resolution better than $0.5 \mathrm{~mm}$. Much smaller tissue structures and microvessels can be visualized in case of sufficient contrast. An ultrawide-band of ultrasonic frequencies present in optoacoustic signals contains wealth of information, which can be revealed through proper filtering and post-processing. We demonstrated that either larger anatomy, such as organs or major vessels, or the smaller structures (kidney medullas, ovarian arteries) and even microvasculature can be visualized depending on methods of signal and image processing. Figure below shows an exemplary image. The endogenous image contrast is based on the optical absorption of hemoglobin and oxy-hemoglobin, the main chromophores of blood in the near-infrared spectral range. Visualization of these chromophores at different optical illumination wavelengths provides not only anatomical information on tissue and vasculature, but also can be used to generate functional maps of blood concentration and its oxygen saturation. This system was also used to generate molecular images of cancer related protein receptors of BT474 cells. Acquisition of the latter images was facilitated by the use of gold nanorods covalently conjugated to an antibody raised against HER2/neu antigens. An exceptionally strong and tunable optical absorption in gold nanorods followed by effective energy conversion into heat makes these nanoparticles a superior optoacoustic contrast agent. Administration of these conjugates into mice resulted in an enhanced contrast of adenocarcinoma tumors relative normal tissues.
Optoacoustic tomography enhanced with contrast agents has the potential to become a useful molecular imaging modality for preclinical research.

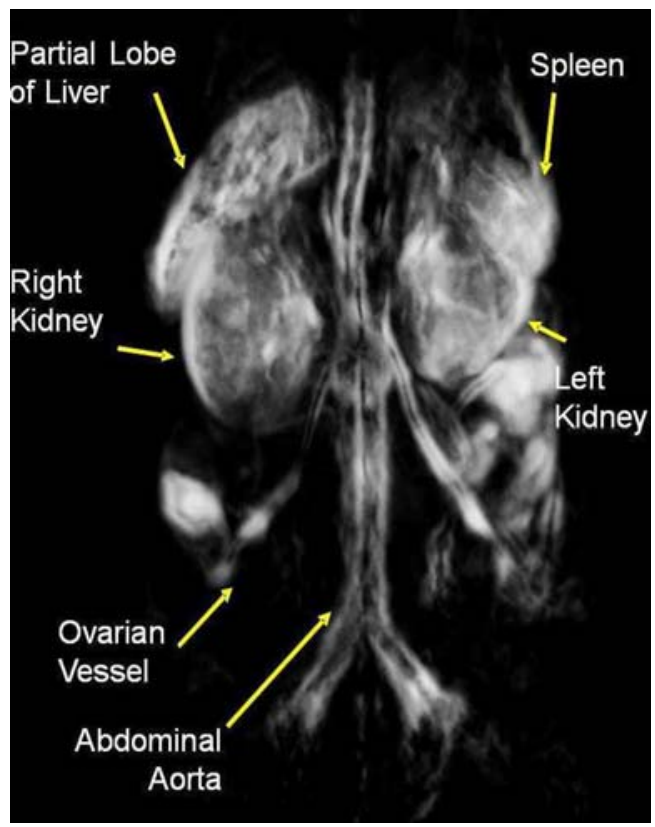

3D image of a live mouse showing internal organs and vessels.

\section{J543}

CLINICAL AND PRECLINICAL METHODS FOR IMAGING ENDOGENOUS FLUORESCENCE FROM GLIOMAS

Frederic Leblond $^{1}$, Pablo Valdes ${ }^{1}$, Kathryn Fontaine ${ }^{1}$, Dax S. Kepshire ${ }^{1}$, Brent T. Harris ${ }^{2}$, Songbai Ji ${ }^{1}$, Alex Hartov ${ }^{1,3}$, David W. Roberts ${ }^{3,4}$,

Brian W. Pogue ${ }^{1}$, Keith D. Paulsen ${ }^{1,3}$

${ }^{1}$ Thayer School of Engineering, Dartmouth College, Hanover, NH, USA, ${ }^{2}$ Department of Pathology, Dartmouth Medical School, Hanover, NH, USA, ${ }^{3}$ Norris Cotton Cancer Center, Dartmouth-Hitchcock Medical Center, Lebanon, NH, USA, ${ }^{4}$ Department of Neurosurgery, Dartmouth-Hitchcock Medical Center, Lebanon, NH, USA. 
Metabolic pathways of some high-grade gliomas, which account for approximately $40 \%$ of all intracranial tumors, lead to preferential accumulation of the fluorescent molecule Protoporphyrin IX (PpIX). We have developed clinical and preclinical fluorescence imaging methods to image gliomas in vivo. Clinically, we are using PpIX imaging during surgery to guide tumor resection. This procedure has become routine practice based on evidence suggesting a correlation between extent of tumor resection and patient prognosis. We have completed a 24-patient clinical study showing the effectiveness of this approach. A surgical microscope was equipped with a blue light source and filters optimized for surface fluorescence imaging. For example, Figs. 1(a) and (b) show, respectively, white-light and fluorescence images acquired one hour after a patient was given a $20 \mathrm{mg} / \mathrm{kg}$ oral dose of ALA. ALA is a prodrug known to enhance endogenous fluorescence in gliomas due to PpIX. Pathological analysis of corresponding histological sections (Fig. 1(c)) reveal that fluorescent areas had cellular features consistent with a high-grade glioma, i.e., glioblastoma multiforme (GBM). In parallel to the human studies, we have developed a near-infrared preclinical tomography fluorescence system able to non-invasively detect orthotopic gliomas. The instrument is based on a non-contact, single-photon counting fan-beam acquisition system interfaced with a microCT system. Animal modeling consists in injection of U251 glioma cells into the mouse brain. Tumors were incubated for 14 days and imaged in vivo with magnetic resonance to assess size and location (Fig. 1(e)). Fig. 1(d) shows the reconstructed fluorescence image following administration of ALA superimposed on microCT image to create a hybrid structural-functional image of the fluorescence contrast. Recovery of the spatial distribution of fluorescence yield is robust and indicates an effective PpIX concentration near $5 \mu \mathrm{g} / \mathrm{ml}$ based on phantom calibration experiments. Fig. 1(f) shows a histological section of the animal cerebrum with positive fluorescence showing infiltration by tumor cells in a pattern similar to that seen in a GBM, such as is shown in Fig. 1(c).
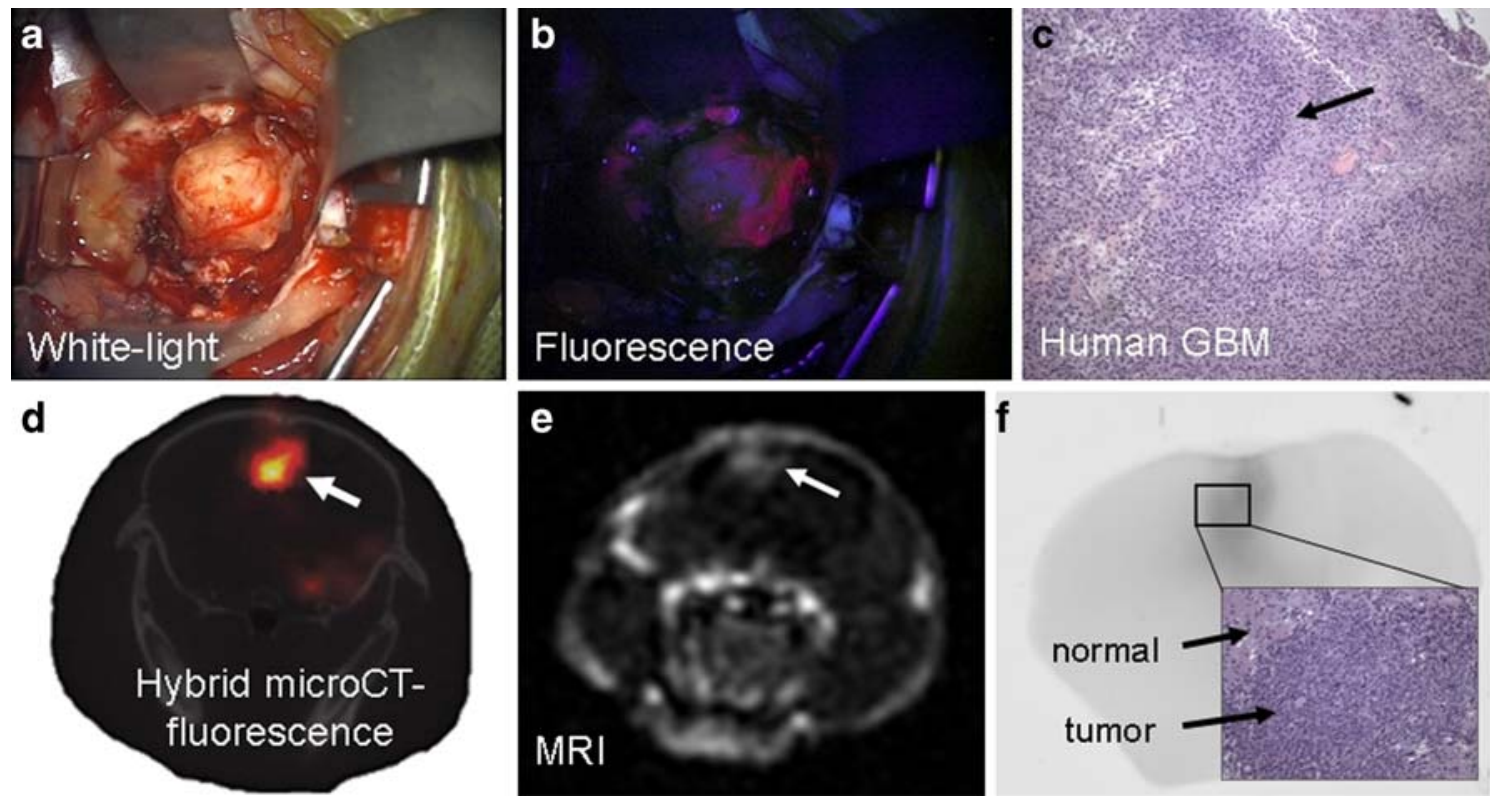

J544

\section{PHOTOACOUSTIC IMAGING OF THE EYE FOR IMPROVED DISEASE DETECTION} $\frac{\text { Adam de la Zerda }}{\text { Sanjiv S. Gambhir }}^{1,4}$, Yannis M. Paulus ${ }^{3}$, Darius M. Moshfeghi ${ }^{3}$,

${ }^{1}$ Electrical Engineering, Stanford University, Stanford, CA, USA, ${ }^{2}$ Radiology, Stanford University, Stanford, CA, USA, ${ }^{3}$ Ophthalmology, Stanford University, Stanford, CA, USA, ${ }^{4}$ Bioengineering, Stanford University, Stanford, CA, USA.

Ophthalmologists lack good functional and molecular imaging tools for the evaluation of angiogenesis in patients. Solving this need would allow earlier detection and improved intervention to multiple ophthalmic diseases including macular degeneration and ocular malignancies. We report on the first demonstration of photoacoustic retinal imaging in living animals. The photoacoustic device can not only be used with photoacoustic contrast agents for molecular imaging but can be used without contrast agents, in which case the eye blood vessels are visualized. The device has high spatial resolution $(250 \mu \mathrm{m})$ and high depth of penetration allowing it to visualize the retina, choroid, sclera and optic nerve. This is particularly important as most eye diseases originate deep in the eye where they cannot be visualized with existing imaging tools. The device illuminates the eye with a wide laser beam at a safe power $\left(800 \mathrm{~nm}\right.$ wavelength at $\left.0.5 \mathrm{~mJ} / \mathrm{cm}^{2}\right)$. The light is absorbed by hemoglobin producing ultrasound waves that emerge from the eye and detected by an ultrasound transducer. The transducer scans the eye from multiple locations creating a threedimensional map of blood distribution in the eye. We scanned living rats and rabbits eyes ( $n=3$ of each animal) and enucleated pigs eyes $(n=3)$. Along with the photoacoustic scan, we acquired an ultrasound scan to visualize the eye's anatomy. The field of view included most of the eyeball ( $\sim 2 \mathrm{~cm}$ in diameter) and time of acquisition was 20 minutes using a $25 \mathrm{MHz}$ transducer (Fig. 1). This is the first demonstration of photoacoustic ocular imaging in living animals. Such system has uses not only in clinical diagnosis but also in the drug development process where ophthalmic 
diseased animal models can be scanned for the first time with high depth of penetration.

\section{Rabbit's Eye}

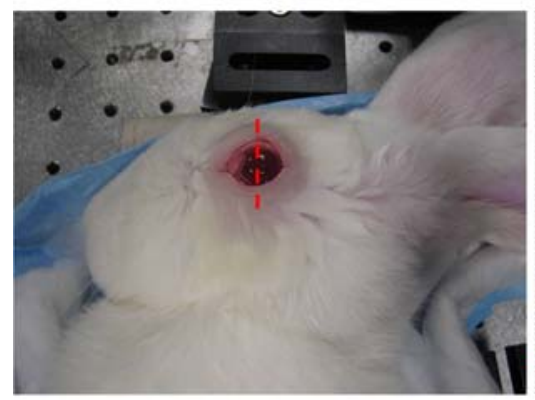

Ultrasound (anatomy)

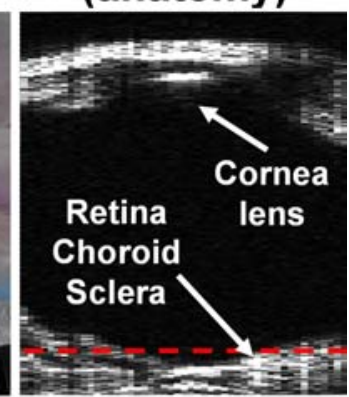

Photoacoustic Photoacoustic (blood distribution) transverse

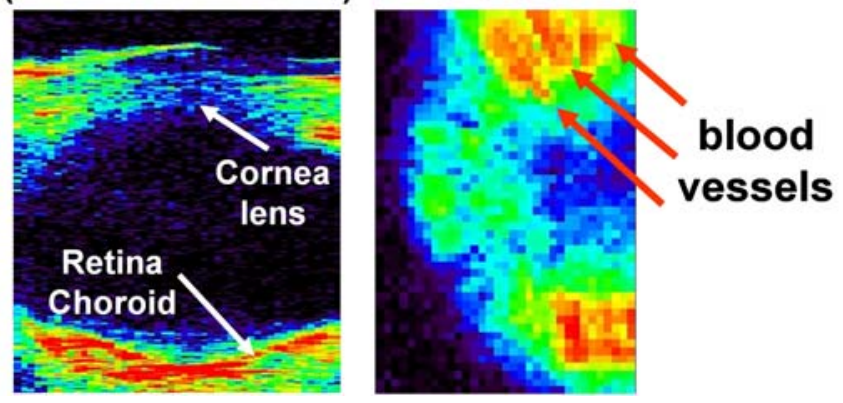

Photoacoustic and ultrasound images of a live rabbit's eye. Using safe laser exposure, photoacoustic signals were measured to image both the retina and choroid visualizing individual blood vessels.

\section{J545}

\section{TIME RESOLVED FLUORESCENCE DIFFUSE OPTICAL TOMOGRAPHY APPLIED TO PROSTATE CANCER DIAGNOSIS: FEASIBILITY OF FLUORESCENT INCLUSION LOCALIZATION}

Lionel Herve $^{1}$, Mathieu Debourdeau ${ }^{1}$, Laurent Guyon ${ }^{1}$,

Jerome Boutet $^{1}$, Philippe Peltie ${ }^{1}$, Didier Vray ${ }^{2}$, François Duboeuf ${ }^{2}$, Jean-Marc Dinten ${ }^{1}$

${ }^{1}$ CEA, LETI-MINATEC, Grenoble, France, ${ }^{2}$ Creatis LRMN, Lyon, France.

Prostate cancer diagnosis currently relies on biopsies undertaken on suspicious patients. As their sampling pattern is either systematic or random, the procedure may miss small and medium size tumors. To increase the diagnosis specificity, we propose a tool combining fluorescence and ultrasound modalities to guide biopsies: the ultrasound modality provides morphological information and the optical modality detects and localizes fluorophore marked tumors. This feasibility experiment is performed on a phantom composed by Polyvinyl Alcohol (PVA), titanium dioxide, ink and silica so as to mimic prostate tissues both on their optical (absorption $\mu$ a and diffusion $\mu$ 's) and ultrasound properties. $2 \mathrm{~cm}$ deep $5 \mu$ fluorescent (IndoCyanine Green) ICG inclusions with concentrations ranging from $0.1 \mu \mathrm{M}$ to $5 \mu \mathrm{M}$ were inserted to simulate a marked tumor. Optical measurements performed on prostate are challenging because of the high absorption coefficient $(\mu \mathrm{a}=0.3 \mathrm{~cm}-1)$, depth of potential fluorescent inclusion $(3 \mathrm{~cm})$ and reflection geometry leading to high levels of background signal. To exploit optical signal at best, a time-resolved acquisition chain is used. It is composed by a Titanium-sapphire (Spectra-Physics) laser source delivering femtosecond pulses at $770 \mathrm{~nm}$ and $80 \mathrm{MHz}$ and a gated CCD camera (KENTECH) to analyze the response of the medium. Light is guided from the laser source to a commercially available probe (Vermon) and from the probe to the CCD camera by optics fibers. Six excitation $(25 \mathrm{~mW})$ and four detection points are located on two rows located on the head of the ultrasonic probe. The fluorescence yield is reconstructed by processing the acquired timeresolved signal (time delay ranging from 0 to $4 \mathrm{~ns}$ with $25 \mathrm{ps}$ steps) of each source-detector combination. These computations are based on the use of the intensity and the mean time of flight of each signal. We proved that this choice of features are optimal since these variables are uncorrelated as opposed to zeroth order and first order moments which are often used for analyzing time responses but are highly correlated $(\mathrm{R} 2>0.95)$. Our choice of features generally leads to non linear computation but this difficulty vanished when localized inclusions are considered. Reconstructions performed on experimental data acquired on the prostate mimicking phantom show that a resolution (in all directions) better than $2 \mathrm{~mm}$ is achievable. At the WMIC conference we will disclose the reconstruction algorithm, the results of the linearity measurements and the depth limits of the system.

\section{J546}

\section{CONTINUOUS ACQUISITION SCANNER FOR WHOLE-BODY} MULTISPECTRAL OPTOACOUSTIC TOMOGRAPHY

Rui Ma, Vasilis Ntziachristos, Daniel Razansky

Technical University of Munich and Helmholtz Center Munich, Institute for Biological and Medical Imaging (IBMI), Neuherberg, Germany.

By combining optical contrast with ultrasonic diffraction-limited resolution optoacoustics holds a great promise for many biological and medical imaging applications. It has been already proven capable of high-resolution visualization of vascular anatomy, tumor angiogenesis as well as other functional contrast in living tissues of small animals and humans. More recently, by applying illumination at several optical wavelengths, multispectral optoacoustic tomography (MSOT) was able to resolve distribution of smart molecular agents and fluorescent proteins with both high sensitivity and spatial resolution in optically opaque organisms and tissues. In this work, we considered the complete three-dimensional tomographic problem with size and geometry of realistic whole-body small animal imaging applications. The main difficulty arising from three-dimensional optoacoustic imaging is the long acquisition times associated with recording signals from multiple spatial projections. The acquired signals are also generally weak and the signal-to-noise ratio is low, problems that are usually solved by multiple averaging, which only further complicates matters and makes imaging challenging for most applications, especially those dealing with living subjects. Finally, when considering multispectral data acquisition, in which the same tomographic data is recorded at several different wavelengths, the imaging times become unrealistic. The scanner, presented herein, uses instead a fast continuous data acquisition approach that greatly shortens recording times over multiple projection angles. For tomographic data acquisition the samples are continuously rotated 360 degrees and the data is recorded without averaging. In this way, two dimensional image acquisition having 300 angular projections only takes about 10 seconds, while full multispectral three-dimensional image can normally take about 15 minutes to acquire with a single ultrasonic detector. We have tested various performance characteristics of the newly introduced system on tissue-mimicking phantoms containing known concentrations of fluorescent molecular 
agent as well as small animals. In-plane spatial resolution on the order of $50 \mu \mathrm{m}$ and vertical resolution of about $150 \mu \mathrm{m}$ were demonstrated in both phantom experiments and imaging of optically opaque organisms having typical cross-section dimensions between $1-20 \mathrm{~mm}$. These initial results confirmed availability of the system for high resolution whole-body visualization of molecular probes and other biomarkers.

\section{J547}

\section{FULL OPTICAL RING TOMOGRAPHY IMAGING SYSTEM} Joerg Peter;

Medical Physics, German Cancer Research Center, Heidelberg, Germany.

An optical tomographic imaging instrument is presented, applicable for non-contact bioluminescence imaging and fluorescence mediated imaging/tomography. The imager consists of a hexagonal assembly of six $25 \mathrm{~mm} \times 100 \mathrm{~mm}$ microlens array (MLA) based photon sensor detectors that completely enclose the imaged object circumferentially, forming an inner bore opening of $60 \mathrm{~mm}$. The individual detector fields-of-view merge at a radius of $25 \mathrm{~mm}$ yielding complete, untruncated coverage of cylindrical objects equal or less than $50 \mathrm{~mm}$ in diameter (mice). Each detector consists of CMOS photon sensors (48 microns feed size) placed at the resultant focal planes of the MLA's (480 microns lens diameter) as determined by the uniform lens focal length of $2.2 \mathrm{~mm}$. The gap between MLA and CMOS is filled out by a bore hole septum mask (400 microns hole diameter) to suppress light cross-talk between detector entities. In total, the detector has an effective thickness of $6 \mathrm{~mm}$, including housing. All detectors are mounted on a rotatable gantry within a cylindrical light-tight enclosure measuring $125 \mathrm{~mm}$ in outer diameter. Adjacent to each detector, six laser diode generated light beams can be projected at any axial position towards the imaged object for fluorochrome excitation and rotate with the gantry for arbitrary object surface positioning. Mechanically attached to the light beam assembly are retractable diffuser light fibers used for large area illumination. These sources are also deployed for extracting a set of reflectance images to be used for object surface reconstruction. Finally, retractable band-pass filters can be moved in front of the detectors when fluorescence meditated imaging is performed. The imaged object is restrained inside a self-contained glass cylinder compartment of $45 \mathrm{~mm}$ in diameter that also provides mountings for ventilation and for miscellaneous monitoring equipment. The overall size of the whole assembly, particularly the cylindrical imaging enclosure is small enough for integration with secondary imaging modalities such as PET and MRI with which the optical ring tomographic imaging system is compatible. This allows for concurrent multi-modal data acquisition at identical fields-of-view. Besides, the imager carries a number of advantages such as complete object coverage with improved dynamic coverage as compared to mirrorbased, single camera setups. A fully operational prototype instrument was build and is evaluated experimentally. Imaging capabilities of this novel approach are presented using phantom and mouse objects.

\section{J548}

FAST DIGITAL MICROMIRROR BASED DIFFUSE OPTICAL TOMOGRAPHY

$\underline{\text { Samuel Bélanger }}^{1,2}{ }^{\text {, Maxime Abran }}{ }^{1}$, Christian Casanova ${ }^{2}$, Frédéric Lesage ${ }^{1}$

${ }^{1}$ Génie Biomédical, Polytechnique de Montréal, Montréal, QC, Canada, ${ }^{2}$ Laboratoire des neurosciences de la vision, Ecole d'optométrie, Montreal, QC, Canada.

In this work a new acquisition scheme based on a pair of Digital Micromirror Devices (DMD) is developed. By using a single detector and pairs of illumination-detection patterns, the system is used to record relevant measures to recover a $3 \mathrm{D}$ absorption images. A reconstruction algorithm is implemented on a GPU providing video rate optical tomography. Optical imaging in turbid media has seen rapid development over the last few years. Applications in cancer detection, brain activation monitoring, small animal fluorescence imaging have been demonstrated. With the emergence of optical tomography, systems with an increasing number of sources and detectors have been built to enhance image quality by taking a larger number of measures. In particular, it has been recognized that having dense sets of measurements enable the imaging of complex structures. The use of exogenous molecular compounds has led to a multitude of applications with new avenues and questions. For example, the use of dynamical information, e.g. pharmaco-kinetics, is gaining interest associated with the ability of optical techniques to use many tracers simultaneously. However the technology to perform fast and complete measurements of this dynamic information is lacking. In this work, spatially modulated imaging is used to achieve a high acquisition rate for tomography. In distinction with previous work, DMD boards are used to illuminate but also measure light patterns on the boundary of an object at frequencies as high as $10 \mathrm{kHz}$. An acquisition card is connected to the DMDs to control the pattern changes. Light is detected with a single photomultiplier tube, aligned with the reception mirrors. The single detector system leads to lower cost, easy integration of detection technologies (e.g. time-domain) and high throughput. By pre-computing the regularized reconstruction matrices, it is possible to perform live 3D imaging. Validation experiments were performed with a $10 \mathrm{~cm} \mathrm{X}$ $6.5 \mathrm{~cm} \mathrm{X} 2 \mathrm{~cm}$ liquid phantom made of a mixture of intralipid (Liposin III) and india ink. The optical properties were adjusted to match that of biological tissue (scattering coefficient of $10 \mathrm{~cm}-1$, absorption coefficient of $0.01 \mathrm{~cm}-1$ ). Inclusions were added to the phantom to create a change in optical properties and results confirm the ability of the system to perform tomography. A fluorescence application of the same system was also explored.

\section{J549}

\section{IN VIVO IMAGING UTILIZING CERENKOV LUMINESCENCE} Matthew Silva ${ }^{1}$, Robbie Robertson ${ }^{1}$, Melissa Germanos ${ }^{1}$,

Simon R. Cherry ${ }^{2}$

${ }^{1}$ Imaging Sciences, Millennium Pharmaceuticals, Inc., Cambridge, MA, USA, ${ }^{2}$ Biomedical Engineering and Center for Molecular and Genomic Imaging, University of California Davis, Davis, MA, USA.

We have discovered a novel method of optical imaging that utilizes the Cerenkov radiation emitted by high-energy positron-emitting isotopes and high-sensitivity optical (CCD) detection, which we have termed Cerenkov Luminescence Imaging (CLI). For decay energies exceeding $260 \mathrm{keV}$, the positron velocity transiently exceeds the speed of light in tissue, which is slower than the speed of light in a vacuum due to interaction with the medium and is proportional to the refractive index of the medium. The emitted light is a continuous spectrum weighted heavily in the UV-blue bands. In several experiments, we have confirmed the discovery of this imaging method and have demonstrated that it is suitable for in vivo imaging with commercial luminescence instruments (e.g., Xenogen IVIS, Caliper Life Science, Alameda, CA). In the included figure, light output from a well of FDG (left) as well as from a human xenograft models (CWR22-RV1) are shown (right). The signal of the xenograft is $12.3 \times$ over background. This phenomenon, not previously described for these imaging tracers has several potential applications, especially for in vivo molecular imaging studies. We anticipate that this finding could have a substantial 
impact in the areas of optical, radio-imaging and molecular imaging, especially for researchers who are combining the modalities, and may provide a cost-effective solution for laboratories currently limited to optical imaging techniques. In addition, we have utilized the Cerenkov radiation as an excitation source for fluorophores enabling the in vivo use of UV-excitable probes. Figure 1. Left: A well-plate containing several wells of saline (rows A and B) and a single well containing $200 \mathrm{uCi}$ of [18F]FDG (Row D, Column 6). Note the luminescence detected from the well containing the FDG. Right: A SCID mouse bearing a flank CWR22-RV1 prostate tumor xenograft and injected with $\sim 300 \mathrm{uCi}$ of FDG. After 5 minutes of distribution, there is increased luminescence in the xenograft as well as in the snout and eyes. (Note that the red circles in both images are ROIs used for quantification.)
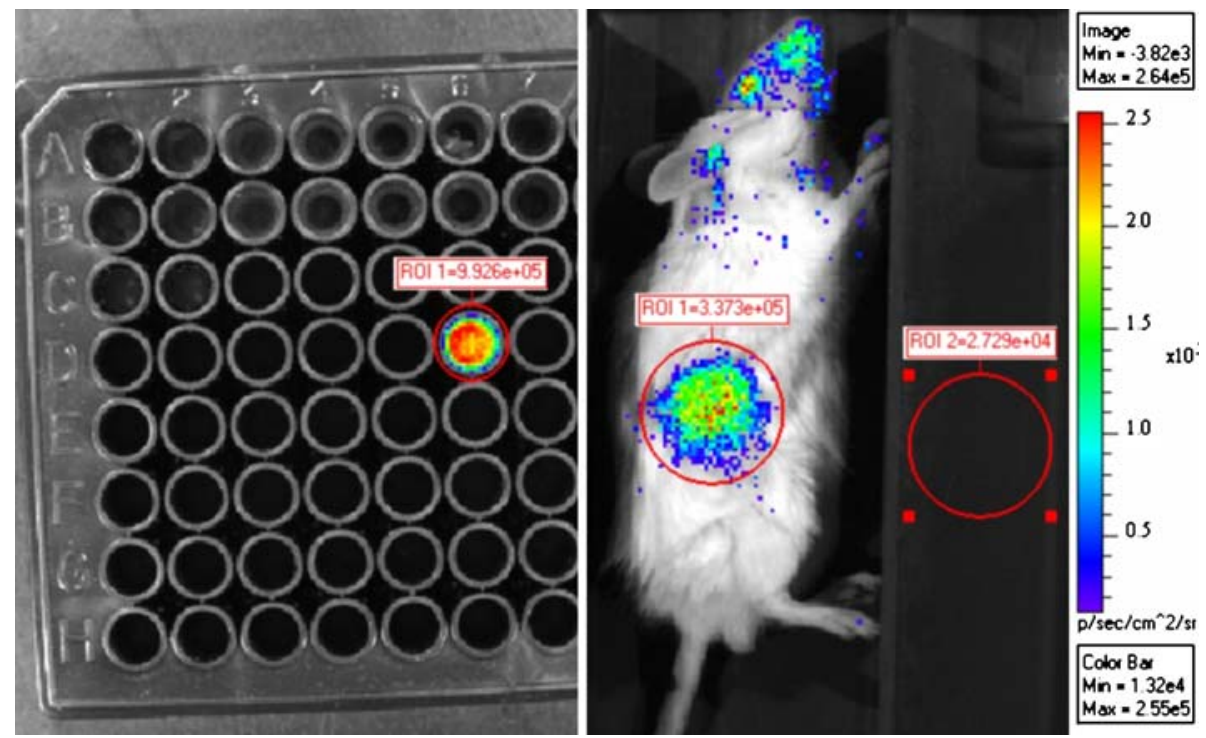

Figure 1.

\section{DUAL-MODE FAST SCANNING ULTRASOUND- PHOTOACOUSTIC SYSTEM FOR PRECLINICAL MOLECULAR IMAGING}

Janaka C. Ranasinghesagara, Huihong Lu, Kory W. Mathewson, Tyler Harrison, Roger J. Zemp;

Dept. of Electrical and Computer Engineering, University of Alberta, Edmonton, AB, Canada.

In recent years, photoacoustic imaging has shown the ability to noninvasively visualize optically-absorbing structures with high ultrasonic spatial resolution. It is being used for molecular imaging to map the distribution of chromophores, targeted contrast agents, and optically-absorbing products of gene expression. However, photoacoustic imaging lacks structural information. High-frequency ultrasound imaging has become an important tool for pre-clinical structural and molecular imaging. We developed a fast scanning dual mode ultrasound-photoacoustic imaging system. This system uses a voice-coil stage to scan an ultrasound-photoacoustic probe (with a $25-\mathrm{MHz}$ focused single element transducer and a unique light-delivery system) at rapid rates, producing up to 30 ultrasound frames per second with a focal resolution of $\sim 150 \mathrm{um}$. Since the pulse repetition rate of the laser determines the photoacoustic-mode scanning speed, current frame rate in the photoacoustic mode is limited by our $20 \mathrm{~Hz}$ laser. Ultrasound and photoacoustic interlaced pulse sequences permit spatial registration of optical and acoustic images. We use this system to image a human finger, showing clearly visible microvasculature of the finger in context with the surrounding tissue. We envision that this technique will be valuable for pre-clinical molecular and functional imaging.

\section{IN VIVO FLUORESCENCE KINETIC IMAGING FOR IMPROVED CONTRAST AND STUDIES OF TEMPORAL BIODISTRIBUTION} James Mansfield $^{1}$, L. Ashley Aronow ${ }^{2}$, Allison Curtis ${ }^{2}$, Elizabeth M. Hillman ${ }^{3}$, Thomas Krucker ${ }^{2}$, Richard Levenson ${ }^{1}$; ${ }^{1} \mathrm{CRi}$, Woburn, MA, USA, ${ }^{2}$ Novartis Institutes of BioMedical Research, Cambridge, MA, USA, ${ }^{3}$ Biomedical Engineering, Columbia University, New York, NY, USA.

In vivo fluorescence imaging has added an easy and economical modality to the rapidly growing field of molecular imaging. It offers the possibility to perform experiments analogous to bioluminescence imaging but at longer and more efficient wavelengths. A further distinction is the translational and research aspects of the imaging of fluorophore-labelled antibodies and activatable reagents. One unique aspect limiting the sensitivity of in vivo fluorescence methodologies is the confounding effect of tissue autofluorescence, which is addressable through the proper use of spectral imaging. However, like many other molecular imaging modalities, reagent-based in vivo fluorescence imaging also has to contend with sensitivity and contrast problems due to non-specific signals and long wash-out times, both limiting the detection of specifically bound reagent and preventing accurate determination of uptake rates. To address this issue, we have developed a kinetic imaging modality that combines rapid imaging (up to 15 frames/sec monochrome or $10 \mathrm{sec} /$ frame multispectrally) with an advanced data processing methodology which combined allow determination of (1) the rates of change of the intensity of the fluorophore in each pixel of the image and (2) the rate of uptake and wash-out in the animal. By utilizing the uptake and wash-out rate information, a much higher contrast image of the accumulating fluorophore can be obtained in a much shorter period of time (minutes and hours vs. days). This 
broadly applicable temporal-biodistribution methodology can be used, for example, to differentiate between the rate of liver uptake vs. the rate of tumor uptake and quantitate each signal relative to general body distribution, and since it can be combined with multispectral imaging, it can be used to quantitate the biodistribution of multiple fluorophores simultaneously.
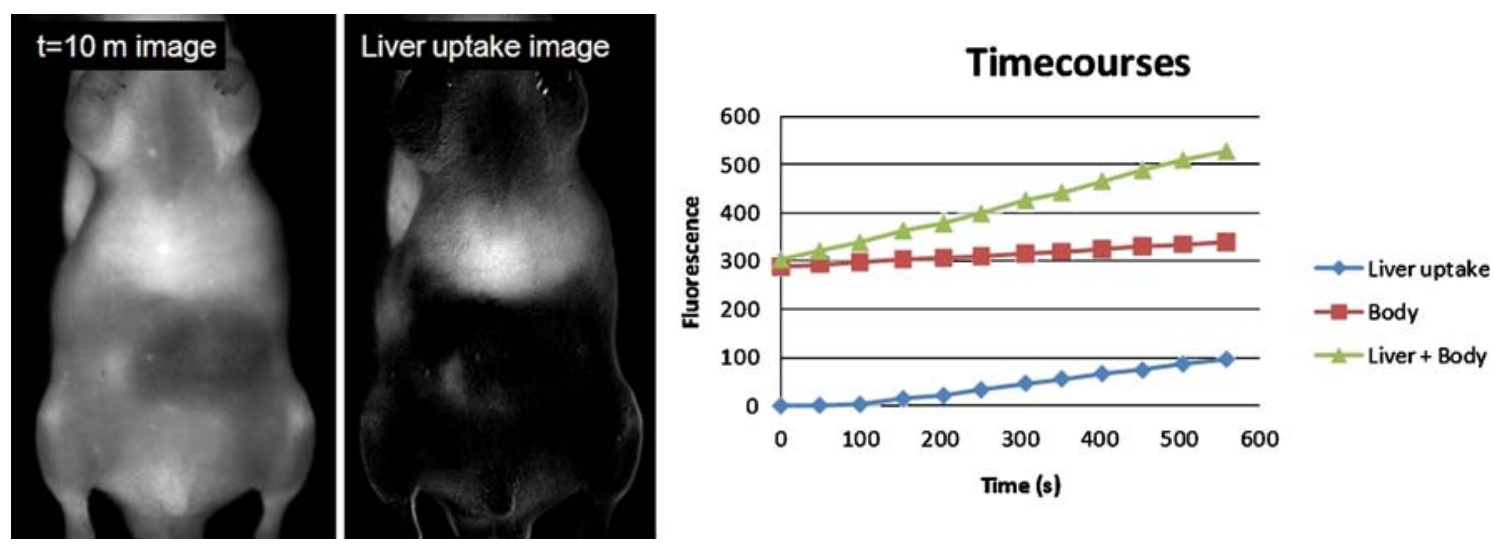

Figure 1: Mouse with tail vein injection of fluorescently labeled liver antibody. Left image is $10 \mathrm{~min}$ post injection. Right image shows specific liver uptake. Graph shows relative accumulation rates.

\section{$\mathbf{J 5 5 2}$}

\section{TIME DOMAIN FLUORESCENCE LIFETIME TOMOGRAPHY FOR DETECTING IN-VIVO FLUORESCENT PROTEIN EXPRESSION}

Anand T. Kumar $^{1}$, Euiheon Chung ${ }^{4}$, Scott B. Raymond ${ }^{2}$, ${ }_{\text {Dai Fukumura }}^{4}$, Rakesh K. Jain ${ }^{4}$, Brian J. Bacskai ${ }^{3}$, David A. Boas ${ }^{1}$

${ }^{1}$ Radiology, Massachusetts General Hospital, Charlestown, MA, USA, ${ }^{2}$ Health Sciences technology, Massachusetts Institute of Technology, Boston, MA, USA, ${ }^{3}$ Neurology, Massachusetts General Hospital, Boston, MA, USA, ${ }^{4}$ Radiation Oncology, Massachusetts General Hospital, Boston, MA, USA.

Intrinsically fluorescent proteins, such as the green fluorescent protein (GFP) and other spectral variants, have served as powerful tools to visualize disease mechanisms at the cellular level. FPs have been extensively employed in fluorescence lifetime imaging (FLIM) techniques to visualize excited-state reactions such as fluorescence resonance energy transfer and in steady state intra-vital microscopy as markers of gene expression and for studying tumor patho physiology. Whole body small animal imaging applications with FPs are however limited, partly owing to a large background tissue auto-fluorescence that is significant in the visible region, where most currently available FPs absorb. Previous attempts at whole animal imaging have been primarily restricted to continuous wave $(\mathrm{CW})$ detection which is insensitive to lifetime. In addition, the FP signal in a $\mathrm{CW}$ measurement is indistinguishable from the tissue auto-fluorescence (AF) background. Multi-spectral detection alleviates this problem to some extent, using un-mixing techniques that use a priori spectral profiles of the autofluorescence and the FP fluorescence. The use of time domain (TD) detection allows the visualization of FPs in-vivo using an additional dimension, namely lifetime. We have performed preliminary time domain studies with mouse tumor models that indicate that the autofluorescence signal has a non-exponential decay that is distinct from the single exponential decay of many FPs. This suggests the potential for temporal un-mixing, analogous to spectral un-mixing, using basis functions that are experimentally characterized from nude mice. We illustrate this approach by presenting lifetime resolved images of FP expression within intact mice models of breast adenocarcinoma using
TD detection. We discuss numerical simulations to estimate the threshold limits for the separation of the AF and FP components using the TD approach. We also present lifetimes of multiple red FPs and discuss their suitability for in-vivo lifetime multiplexing applications. These results suggest the potential for exploiting fluorescence lifetime for imaging FPs for a variety of whole body small animal imaging applications.

\section{$\mathbf{J 5 5 3}$}

QUANTITATIVE OPTICAL WHOLE ORGAN ENDPOINT IMAGING IN CARDIOVASCULAR DRUG DISCOVERY RESEARCH

Gordon M. Turner $^{1}$, Zhiping Chen ${ }^{1}$, Peter E. O'Donnell ${ }^{1}$, Sara Knight ${ }^{1}$, Qing Nie $^{1}$, Galina Deyneko ${ }^{1}$, Jean-Rene Galarneau ${ }^{2}$, Judit Markovits ${ }^{2}$, Michael Hansen ${ }^{3}$, Jeffrey Tsao ${ }^{3}$, Jennifer R. Allport ${ }^{1}$

${ }^{1}$ Cardiovascular and Metabolism Disease Area, Novartis Institutes for Biomedical Research, Cambridge, MA, USA, ${ }^{2}$ Preclincal Safety, Novartis Institutes for Biomedical Research, Cambridge, MA, USA, ${ }^{3}$ Global Imaging Group, Novartis Institutes for Biomedical Research, Cambridge, MA, USA.

Quantitative whole organ imaging is a frequent requirement in cardiovascular drug discovery research, but until recently few tools have been available to enable quantitative metrics to be obtained easily from the large number of samples generated in preclinical drug discovery. Histological serial sectioning can provide quantitative readouts on thin tissue sections, but full organ coverage is extremely labor intensive and impractical for more than an occasional example specimen. Macroscopic whole organ imaging is also possible, but fails to provide quantitative volumetric data. We report here on the development of two complementary technologies that have been deployed by our laboratories to enable whole organ imaging with volume throughput capabilities suitable for preclinical drug discovery research: optical projection tomography (OPT) and whole slide digital imaging (WSI) and analysis. OPT provides volumetric organ data at 15 micron resolution and when paired with the sub-cellular resolution of WSI, provides for high resolution images of the entire organ pathology without sacrifice of the cellular and molecular level detail provided by histological thin sections. Here we present the application of these technologies to the volumetric measurement of atherosclerosis in both mouse and rabbit disease models, and discuss the application of this technology to other organs. Furthermore, the details of the OPT device 
design, sample throughput and processing workflow, and computational infrastructure for image reconstruction using graphics processors (GPUs) are described.

\section{$\mathbf{J 5 5 4}$}

\section{CHARACTERIZATION OF HUMAN LYMPHATIC ARCHITECTURE AND (DSY)FUNCTION WITH NEAR- INFRARED FLUORESCENCE IMAGING} John C. Rasmussen $^{1}$, I-Chih Tan ${ }^{1}$, Milton Marshall ${ }^{1}$, Kristen E. Adams ${ }^{1}$, Caroline E. Fife ${ }^{2,3}$, Erik A. Maus ${ }^{2,3}$, Latisha A. Smith ${ }^{2,3}$, Eva Sevick ${ }^{1}$

${ }^{1}$ The Brown Foundation Institute of Molecular Medicine, University of Texas Health Science Center Houston, Houston, TX, USA, ${ }^{2}$ Department of Internal Medicine, Division of Cardiology and Hyperbaric Medicine, University of Texas Health Science Center Houston, Houston, TX, USA, ${ }^{3}$ Center for Lymphedema Management, Memorial Hermann Hospital, Houston, TX, USA.

Lymphatic failure manifests in a condition known as lymphedema that regardless of the etiology of onset (whether hereditary or acquired) is demarked by chronic swelling and fibrosis of the affected tissue. While the role of the lymphatic system is recognized, its function in disease is little understood due primarily to the lack of real time imaging techniques. We recently completed a feasibility trial in which lymphatic architecture and function were imaged in real time in 24 control subjects and 20 subjects diagnosed with unilateral lymphedema of arms or legs. After informed consent, multiple $100 \mathrm{ml}$ intradermal injections containing $25 \mu \mathrm{g}$ indocyanine green were administered bilaterally in either arms or legs. The limbs were illuminated with $785 \mathrm{~nm}$ near-infrared (NIR) light and the resultant $830 \mathrm{~nm}$ fluorescent signal was imaged using a custom, 16 bit, intensified $\mathrm{CCD}$ camera. The movement of the dye was tracked from injection sites to the nodal basin, and the apparent lymph velocity was computed for each observed packet of lymph. The time period between sequential lymph propulsion events was also quantified. Marked differences were observed between the lymphatic architectures of control and lymphedema subjects. Statistical analyses of control subjects found subtle but significant differences in the left and right velocities in both arms $(0.84 \mathrm{~cm} / \mathrm{s}$ and $0.76 \mathrm{~cm} / \mathrm{s}$, $p=8.1 \mathrm{e}-5)$ and legs $(0.99 \mathrm{~cm} / \mathrm{s}$ and $0.87 \mathrm{~cm} / \mathrm{s}, p=0.032)$ but not in the periods of contralateral limbs. The difference in velocity in control arms $(0.79 \mathrm{~cm} / \mathrm{s})$ and legs $(0.94 \mathrm{~cm} / \mathrm{s})$ is small but significant $(p=1.22 \mathrm{e}-7)$ while the difference in period is not. Significant differences in period exist between control (arms: $48.2 \mathrm{~s}$; legs: $52.2 \mathrm{~s}$ ) and both asymptomatic (arms: $39.6 \mathrm{~s}, p=2.68 \mathrm{e}-6$; legs: $65.3 \mathrm{~s}, p=0.0022$ ) and symptomatic (arms: $33.2 \mathrm{~s}$, $p=4.14 \mathrm{e}-5$; legs: $72.1 \mathrm{~s}, p=0.00014)$ limbs. Significant differences were also found between the velocities in the control $(0.94 \mathrm{~cm} / \mathrm{s})$ and both asymptomatic $(0.83 \mathrm{~cm} / \mathrm{s}, p=0.0084)$ and symptomatic $(0.78 \mathrm{~cm} / \mathrm{s}, p=$ $0.0036)$ legs but not in the arms of control and lymphedema subjects. No significant differences were found between the velocities or periods in the asymptomatic and symptomatic limbs indicating that lymphedema may be a systemic disease regardless of onset etiology. These results show the utility of NIR fluorescence imaging following microdose administration of imaging agent for evaluation of lymphatic function. Supported by the American Cancer Society and the Longaberger Foundation through Research Scholar Grant RSG-06-213-01-LR.

\section{$\mathbf{J 5 5 5}$}

QUANTITATIVE UNMIXING OF SPECTRA FOR RAMAN MOLECULAR IMAGING OF LIVING SUBJECTS Kranthi K. Kode $^{1}$, Cristina Zavaleta ${ }^{2}$, Sanjiv S. Gambhir ${ }^{3,2}$, David Paik ${ }^{2}$ ${ }^{1}$ Computational \& Mathematical Engineering, Stanford University, Stanford, CA, USA, ${ }^{2}$ Radiology, Stanford University, Stanford, CA, USA, ${ }^{3}$ Bioengineering, Stanford University, Stanford, CA, USA.

PURPOSE: In recent years, surface enhanced Raman spectroscopy has gained interest as a tool for noninvasive molecular imaging of living subjects because of its potential for greater multiplexing as compared to fluorophores. Various strategies have been developed for small-animal optical imaging based on Raman spectrocopy and Raman nanoparticles. However, quantitative unmixing of the Raman spectra poses a significant challenge due to significant autofluorescence in vivo which causes low SNR. We have developed a reliable algorithm to quantitatively unmix the spectra of multiplexed Raman nanoparticles in living subjects. METHOD AND MATERIALS: Surface-enhanced Raman scattering nanoparticles were used to demonstrate whole-body Raman imaging, nanoparticle pharmacokinetics, multiplexing, and in vivo tumor targeting, using an optical microscope adapted for small-animal Raman imaging. Three female 8-week-old nude mice were used for all Raman spectroscope studies. The unmixing process was carried out on Raman spectra of 4 nanoparticles injected simultaneously 1) using a 'polyfit' background subtraction followed by linear least squares on the Raman spectra followed by 2) our unmixing algorithm that parameterizes individual Raman peaks and uses Levenberg Marquardt optimization, accounting for changes in peak position and width. The results from least squares were used as initial estimate for the optimization process. RESULTS: We modeled individual Raman peaks to accurately represent the Raman spectra of nanoparticles and developed an unmixing method that avoids carrying out dubious preprocessing techniques such as blind baseline correction for background subtraction. The signal to noise ratio was estimated to be 0.0010 for the combined spectra. Five Raman peaks having the greatest area under the curve were modeled for each nanoparticle. Percent error in the estimate of concentrations of each nanoparticle for method 1) were $26 \%, 27 \%, 45 \%$, $65 \%$ and corresponding errors for methods $1+2$ ) were $23 \%, 16 \%, 6.7 \%$, $16 \%$. CONCLUSION: While the narrow peaks of the Raman spectra provide specificity of each nanoparticle's spectrum, their steep slopes can lead to large errors in the presence of very minor spectral shifts or changes in width. Our results demonstrate that accounting for this effect can lead to better and more relaiable performance in quantitative unmixing thereby enabling Raman spectroscopic imaging in living subjects.

\section{J556}

\section{STEM CELLS TRACKING WITH OPTICAL IMAGING: IN VITRO AND IN VIVO STUDIES FOR NEW THERAPEUTICAL APPROACHES} Chiara Garrovo $^{1}$, Natascha Bergamin ${ }^{2}$, Serena Zacchigna ${ }^{3}$, Daniela Cesselli ${ }^{2}$, Federica d'Aurizio ${ }^{2}$, Patrizia Marcon ${ }^{2}$, Stefania Biffi ${ }^{1}$, Mauro Giacca ${ }^{3}$, Carlo Alberto Beltrami ${ }^{2}$, Vito Lorusso ${ }^{1,4}$ ${ }^{1}$ 1. Optical Imaging, CBM, Area Science Park, Trieste, Italy, ${ }^{2} 2$. Centro Interdipartimentale di Medicina Rigenerativa, University of Udine, Udine, Italy, ${ }^{3}$ 3. Molecular Medicine, International Centre for Genetic Engineering and Biotechnology (ICGEB), Trieste, Italy, ${ }^{4} 4$. Ephoran Multi Imaging Solutions, Colleretto Giocosa, Torino, Italy.

Stem cells are characterized by the ability to renew themselves into specialized cell types and stem cell therapy is believed to have potential to highly improve the treatment of very different types of human diseases. In our study, multipotent adult stem cells (MASC) were obtained from human adipose tissue and labelled with $\mathrm{DiD}$, a carbocyanine dyes with spectral characteristics of absorbance at $644 \mathrm{~nm}$ and emission at $665 \mathrm{~nm}$. Herein, we reported the investigation on labelled MASC (MASC-DiD) both in vitro and in vivo using NIR time domain (TD) optical imaging and confocal microscopy into two well defined and different diseases: the collagen VI-related muscular dystrophies and acute myocardial infarction (AMI). Collagen VI-related muscular dystrophies, as Bethlem myopathy (BM) and Ullrich congenital muscular dystrophy (UCMD), are characterized by skeletal muscle diseases whereas AMI determines the loss of contractile tissue. In order to study collagen VI-related muscular dystrophies, healthy mice have been treated intramuscularly (im) in the left tibialis anterior muscle with $2 \times 10^{6}$ MASC-DiD and monitored for 48 hours. We analyzed MASC-DiD biodistribution from the site of injection specifically to the controlateral leg (not treated), evaluating the capability of stem cells to migrate to different regions after im injection. At 
the end of the experiments, mice were sacrificed for the ex vivo analysis of the single organs. In the second experiments, 30 minutes after ligation of the descending coronary artery, mice were injected into the border zone of the infarction with $1 \times 10^{6}$ MASC-DiD in order to assess the ability of injected cells to home to the damaged area. Cells' migration was evaluated 96 hours after the treatment performing ex vivo analysis of the organs from both pathological and control mice. In vivo results were confirmed both by ex vivo NIR time domain (TD) optical imaging of the tissues collected after mice sacrifice and by the confocal analysis of formalin-fixed specimens. While in the dystrophy model, labelled cells were detected in several districts, in the AMI model cells were mainly localized in the heart. In summary, the studies indicate that MASC-DiD, previously proved to be effective on stem cells based therapy, can be injected and monitored both in healthy mice and in the pathologic models and that the applicability of NIR time domain (TD) optical imaging allows to easily comprehend how different diseases and different routes of administration can influence their biodistribution, availability and capability to regenerate in vivo.

\section{J557}

\section{A NEW AVENUE FOR IMAGE ANALYSIS BASED ON THE FOURIER DECOMPOSITION OF ACQUIRED SIGNALS: APPLICATION ON IN VIVO OPTICAL IMAGING OF THE VISUAL CORTEX}

Matthieu Vanni $^{1}$, Jean Provost ${ }^{2,3}$, Samuel Bélanger ${ }^{1,2}$, Frédéric Lesage $^{2}$, Christian Casanova ${ }^{1}$

${ }^{1}$ Universite de Montreal, Ecole d'Optometrie, Montreal, QC, Canada, ${ }^{2}$ Ecole Polytechnique, Departement de Genie Electrique, Montreal, QC, Canada, ${ }^{3}$ Columbia University, Biomedical Engineering Department, New York, NY, USA.

Continuous stimulation is a new paradigm that proved to provide high signal to noise ratios and reduce acquisition times in optical imaging and fMRI. The use of periodically changing stimuli results in a response at a specific frequency that can be quantified away from physiological noise. However, this method is currently limited to the information contained in a single harmonic and cannot be used to fully characterize the neural response profile. In this study, it is proposed that by using multiple harmonics, additional information can be gathered such as, in visual neurosciences, motion sensitivity, visual acuity and binocular integration. Those last parameters are fundamental in understanding the mechanisms subtending visual perception and in evaluating the impact of diseases such as stroke, retinopathies and neuronal diseases. Optical imaging of intrinsic signal was performed on the visual cortex of mice, rats rabbits, and cats. The cortical representation of the visual field (visuotopy) was generated using periodically bars moving at different frequencies. The optical responses were modeled as a convolution between hemodynamic and neuronal responses. Then, by modeling the affinity of the neuron by a Gaussian response, the ratio of the second to first harmonic was related to the shape of the neuronal response and used to estimate the portion of the visual field integrated (i.e. the receptive field). The paradigm was finally adapted to estimate the selectivity to motion (i.e. response to one direction but not to its opposite) and binocularity (i.e. level of the contribution of each eye to the response). Using the continuous paradigm, visuotopic maps were obtained ten times faster than those obtained with episodic paradigms. Moreover, responses of up to four simultaneous stimulations were extracted in one recording session. The hemodynamic response profile was estimated and shown to be homogeneous throughout the cortex. This result is of importance since it confirms that relative harmonic quantifications can be used to quantify neuronal responses. The measures of receptive field size (related to visual acuity), motion sensitivity and binocular integration in different cortical areas showed good agreement with those obtained with standard episodic paradigms. In conclusion, Fourier decomposition can be used to evaluate a large number of neural properties in a limited recording time. This paradigm could be easily translated to other modalities such as fluorescence imaging, in vivo microscopy and fMRI. Supp: NSERC and CIHR to CC and NSERC to FL

\section{J558}

\section{OPTICAL PROJECTION TOMOGRAPHY: A NEW OPTION FOR IMAGING MAMMALIAN CARDIOVASCULAR DEVELOPMENT}

Gregory A. Anderson ${ }^{1,2}$, Jian Yang ${ }^{2}$, Mark Henkelman ${ }^{1,2}$

${ }^{1}$ Medical Biophysics, University of Toronto, Toronto, ON, Canada, ${ }^{2}$ Mouse Imaging Centre, The Hospital For Sick Children, Toronto, ON, Canada.

A multitude of molecular mechanisms control the differentiation and assembly of the cell lineages involved in cardiovascular development, and our understanding of this process has grown significantly through the characterization of signaling modulators such as those in the transforming growth factor-beta (TGF- $\beta$ ) superfamily. Endoglin is a transmembrane receptor for the TGF- $\beta$ superfamily that is expressed in endothelial cells (ECs) from early gestation to the adult stage. In humans, the critical role of endoglin in vascular development is shown in patients with Hereditary Hemorrhagic Telangiectasia type 1 (HHT1). In these patients, mutations in the endoglin gene lead to arteriovenous malformations in the pulmonary, cerebral, and hepatic circulations, which can evoke complications later in life such as stroke and internal hemorrhaging. Mice bearing a homozygous null mutation in endoglin (Eng-/-) die at mid-gestation due to circulatory failure and hemorrhage. These mutant embryos show numerous lesions in their cardiovascular systems: their ECs lack integrity, angiogenesis in the yolk sac is abrogated, and their hearts fail to undergo the normal morphogenetic looping that generates the four-chambered heart. Moreover, we have shown that endoglin is necessary for endocardial cushion formation during heart development, and that the other developmental defects are secondary lesions. To characterize the heart development defect in Eng-/- mice we need to create a visual atlas of the vascular anatomy of these embryonic mice so as to elucidate the role endoglin plays in both arteriovenous specification and heart valve development. Optical Projection Tomography (OPT) is a new and powerful imaging modality that can fill this role. Essentially an optical version of X-ray computed tomography, OPT is ideally suited to visualize the complex 3-dimensional structure of the mouse embryo because it provides greater molecular specificity, cellular resolution, and larger specimen coverage than other comparable imaging tools. OPT allows us to map and register the mutant heart phenotype of our endoglin null mice to phenotypically normal wild-type mice in 3D, thus allowing us to further characterize the functional role of endoglin.

\section{$\mathbf{J 5 5 9}$}

\section{A PRACTICAL SCANNER FOR SMALL ANIMAL} PHOTOACOUSTIC COMPUTED TOMOGRAPHY IMAGING Timothy Morgan $^{1}$, Robert Kruger ${ }^{2}$, Paul Picot ${ }^{1}$, Michael M. Thornton ${ }^{1}$ ${ }^{1}$ Endra, Inc., Boston, MA, USA, ${ }^{2}$ OptoSonics, Inc., Oriental, NC, USA.

Interest in photoacoustic imaging of small animals has increased in the last few years due to its capability of achieving nano-molar sensitivity at depths greater than $20 \mathrm{~mm}$ in turbid tissue, without the degraded resolution associated with other optical techniques. Early designs of photoacoustic instruments were developed as proof of principle devices that often focused on the technology and paid little attention to features that would allow non-imaging experts to use the system routinely. Animal handling and preparation were often difficult and time consuming, requiring animals to be placed in tubes and submerged underwater. Detector sizes and geometries often required the animal to be rotated and involved long scan times. Here, we present a practical photoacoustic computed tomography scanner, designed for simple animal handling, system setup, and workflow to facilitate routine use in small animal studies. The device uses a $10 \mathrm{~cm}$ radius hemispherical detector array with 128 piezoelectric transducers arranged to provide uniform sampling when rotated axially, resulting in a field of view greater than $20 \mathrm{~mm}$ in diameter. The detector is filled with acoustic coupling fluid, and an animal tray provides convenient placement of the animal in a horizontal position. The animal tray and animal remain 
stationary during the scan. Acoustic coupling fluid is placed between the animal tray and the area of the animal being scanned, rather than completely submerging the animal. In this position, the head and tail of the animal are stationary and freely accessible, providing simple management and routing of tubes for gas anesthesia and tail catheterizations. A special purpose acquisition system has been developed and samples the 128 transducers at up to $40 \mathrm{MHz}$, without multiplexing. Photoacoustic excitation is by $7 \mathrm{~ns}$ pulses from a Nd:YAG laser-pumped optical parametric oscillator, permitting multispectral imaging between $690 \mathrm{~nm}$ and $980 \mathrm{~nm}$ at $25 \mathrm{~mJ}$ per pulse. A 5-wavelength scan using 60 views and 8 pulses per view can be performed in less than 10 minutes, including animal preparation and positioning. A protocol-driven user interface allows free choice of scan parameters and requires minimal user interaction during procedures. Studies showed contrast sensitivity of $350 \mathrm{nM}$ equivalent ICG within a $1.1 \mathrm{~mm}$ vessel in a $25-\mathrm{mm}$ tissueequivalent phantom. Images of point-like objects showed a spatial resolution of $280 \mu \mathrm{m}$ FWHM. The ease of animal handling, scan speed, and image quality of this scanner make it a practical, viable laboratory tool that is currently in routine use.

\section{J560}

NON-NEGATIVE MATRIX FACTORIZATION APPLIED TO IN VIVO OPTICAL FLUORESCENCE SPECTROSCOPY: A BLIND SOURCES SEPARATION METHOD TO UNMIX INTRINSIC AND SPECIFIC FLUORESCENCE

Anne-Sophie Montcuquet ${ }^{1,2}$, Lionel Herve ${ }^{1}$, Laurent Guyon ${ }^{1}$, Jérôme I. Mars ${ }^{2}$, Jean-Marc Dinten ${ }^{1}$

${ }^{1}$ DTBS/LISA, CEA LETI Minatec, Grenoble, France, ${ }^{2}$ Department Images-Signal, GIPSA-Lab, Grenoble, France.

Fluorescent imaging in diffusive media is an emerging imaging modality for medical applications: injected fluorescent markers bind specifically to targeted compounds, like carcinoma. The region of interest is illuminated with near infrared light and the emitted back fluorescence is analyzed to localize the fluorescence sources. To investigate thick media for medical diagnostic application, as the fluorescence signal gets exponentially weak with the light travel distance, any disturbing signal, such as biological tissues intrinsic fluorescence (IF), may be a limiting factor. To remove these unwanted contributions, a spectroscopic approach is explored. A feasibility experiment is performed on a mouse: a capillary tube filled with $5 \mu$ Indocyanine Green (ICG) at $10 \mu \mathrm{Mol} / 1$ is inserted subcutaneous to simulate a marked tumor (See Figure 1, left). The animal is illuminated along a line with a planar laser at $690 \mathrm{~nm}$. The fluorescence signal emitted back from the mouse is collected by an imaging spectrometer coupled with a charge-coupled device camera (Andor Technologies) above the animal. The fluorescence acquisition was processed with Nonnegative matrix factorization (NMF) algorithm which approximates the acquisition image as the product of two non negative matrices i.e. the spectra and the weighting factors of the fluorescence sources (IF and ICG). Spectroscopic measurements being the sum of nonnegative components, the method is suitable for spectroscopic measurements. We processed the NMF algorithm on the fluorescence acquisition obtained along the laser line on the back of the mouse. On Figure1 (right), the original signal and the IF and ICG contributions obtained by the algorithm are represented. We see that they are correctly separated despite their fluorescence spectra are overlapping. We found that in vivo spectrally resolved acquisition combined to NMF processing successfully filters fluorescence contributions of interest from measurements impaired by unwanted signals.
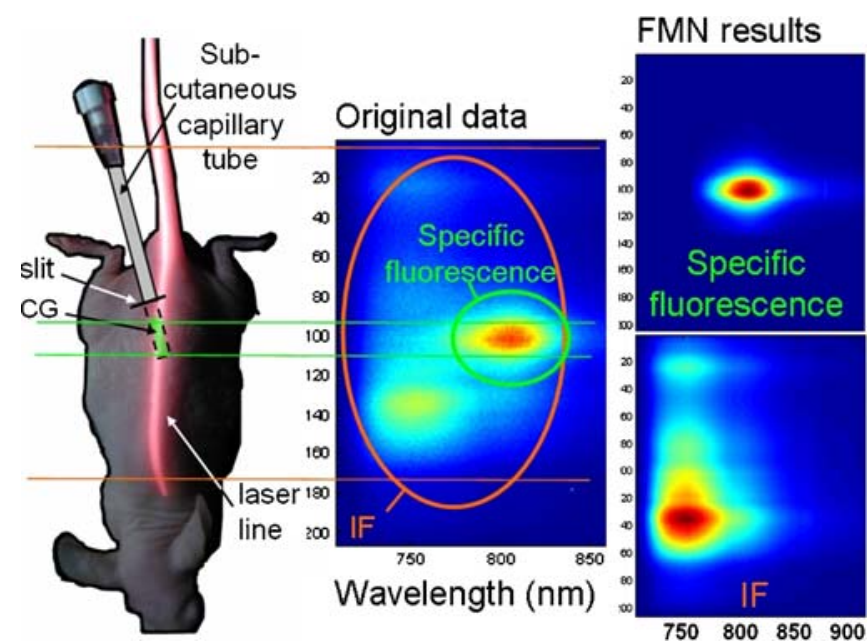

J561

ISOFLURANE ANESTHESIA INHIBITS FIREFLY LUCIFERASE IN A DOSE-DEPENDENT WAY: A PITFALL FOR IN VIVO BIOLUMINESCENCE IMAGING Marleen Keyaerts $^{1,2}$, Isabel Remory ${ }^{1,3}$, Vicky Caveliers ${ }^{1,2}$, Lea O. Tchouate Gainkam ${ }^{1}$, Jan I. Poelaert ${ }^{3}$, Axel Bossuyt ${ }^{1,2}$, Tony Lahoutte ${ }^{1,2}$

${ }^{1}$ In Vivo Cellular and Molecular Imaging Laboratory, Vrije Universiteit Brussel, Brussel, Belgium, ${ }^{2}$ Department of Nuclear Medicine, Universitair Ziekenhuis Brussel, Brussel, Belgium, ${ }^{3}$ Department of Anaesthesiology, Universitair Ziekenhuis Brussel, Brussel, Belgium.

Bioluminescence imaging (BLI) offers unique possibilities to study biological processes in intact organisms in a quantitative, non invasive and repeated approach. Rodents are anesthetized during imaging and BLI cameras are routinely provided with an isoflurane anesthesia unit. Isoflurane is animal- and user-friendly because of its fast induction and recovery. However a direct inhibition of the luciferase enzyme by anesthetics has been described and might influence in vivo quantification. Aim: To assess the impact of isoflurane anesthesia on the bioluminescent signal intensity in vitro and in vivo. Methods: For in vitro assessment, $10^{6}$ Fluc + R1M cells were plated in $25 \mathrm{~cm}^{2}$ flasks. Cells were exposed to $\mathrm{O}_{2}$ /isoflurane mixtures starting $10 \mathrm{~min}$ before and continuously throughout BLI. D-luciferin was added to reach a $0.15 \mathrm{mg} / \mathrm{ml}$ concentration. For in vivo analysis, a mouse bearing a sc Fluc + R1M tumor was injected ip with $30 \mathrm{mg} / \mathrm{kg}$ of D-luciferin under 
$2.5 \%$ isoflurane anesthesia. Kinetic BLI data were acquired using list mode acquisition while the amount of isoflurane was varied between 2.5 and $5 \%$. Results: Cells incubated with $1.5 \%$ isoflurane showed a decrease in signal of $38.5 \pm 2.2 \%(p<.001)$ compared to control samples $\left(100 \% 0_{2}\right)$. Isoflurane at $3 \%$ shows a dose-dependent decrease of $29.8 \pm$ $0.8 \%$ compared to the intensity at $1.5 \%$ isoflurane. No statistical difference was detected between different $0_{2}$ levels $(100-50-25 \%$ in $\mathrm{N}_{2}$ ), confirming that the inhibition is not due to a decrease in oxygen but the increase in isoflurane. In vivo imaging also shows the presence of isoflurane dose-dependency of the BLI signal (see fig). Preliminary results with desflurane and sevoflurane show similar in vitro effects. Conclusion: Isoflurane anesthesia significantly reduces the intensity of the BLI signal both in vitro and in vivo, indicating the presence of a direct inhibitory effect. Researchers should be aware of this impact on BLI quantification and should standardize the use of gas anesthetics thoroughly.

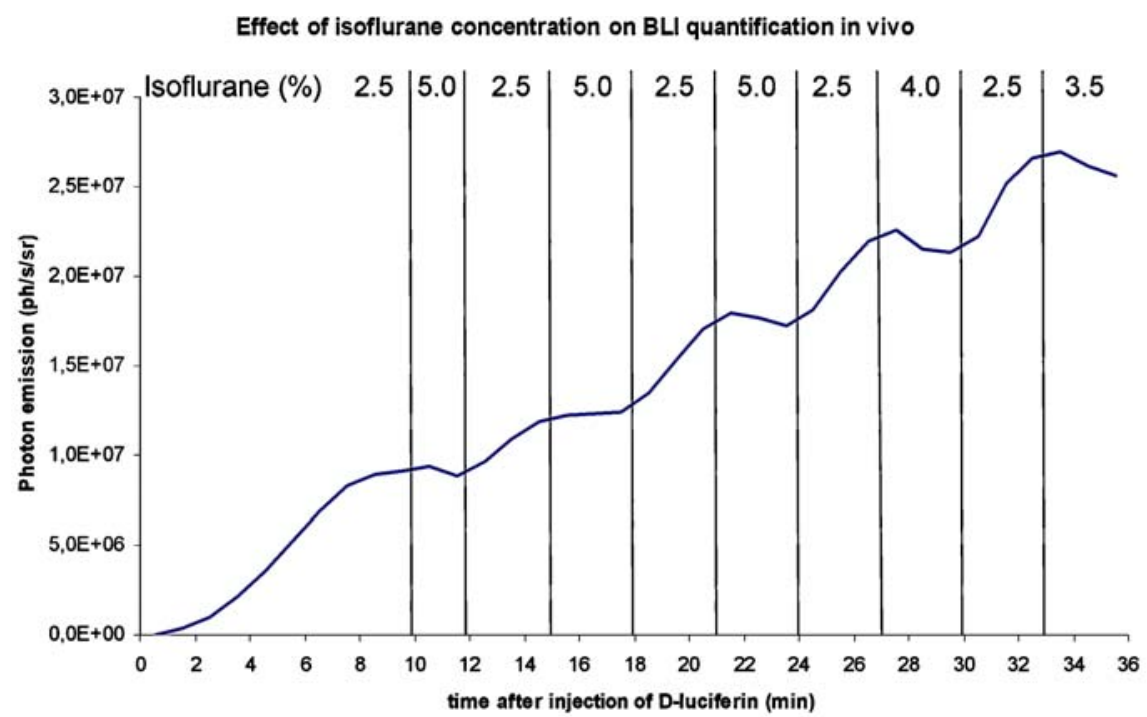

\section{Evolution of the bioluminescent signal over time from a R1M-Fluc+ subcutaneous tumor in a mouse anesthetized with isoflurane. Isoflurane was vaporized in oxygen and the concentration of isoflurane was changed over time as indicated above the curve. The figure shows the negative and concentration-dependent effect of isoflurane on the in vivo BLI quantification.}

NIR FLUORESCENCE IMAGING PROVIDES FIRST GLIMPSE OF LYMPHATIC (DYS)FUNCTION

Sunkuk Kwon ${ }^{1}$, Philip King ${ }^{2}$, Philip E. Lapinski ${ }^{2}$, Guillermo Oliver ${ }^{4}$, Thomas W. Glover ${ }^{3}$, Eva Sevick ${ }^{1}$

${ }^{1}$ IMM, UTHSC-H, Housron, TX, USA, ${ }^{2}$ Microbiology and Immunology, University of Michigan, Ann Arbor, MI, USA, ${ }^{3}$ Human Genetics, University of Michigan, Ann Arbor, MI, USA, ${ }^{4}$ Genetics / Tumor Cell Biology, St. Jude Children's Research Hospital, Memphis, TN, USA.

The lymphatics play vital roles in edema prevention, immune surveillance, lymphocyte circulation, as well as fluid/protein homeo- stasis. All of these functions may require a regulated lymph flow generated from intrinsic lymph pumping (due to contractions of lymphatic smooth muscle) and extrinsic lymph pumping (caused by skeletal muscle movement and respiration). The alteration of these functions causes edema and may be associated with obesity, diabetes, as well as other diseases. Despite its importance, little is known about the lymphatic function, due mainly to the lack of diagnostic imaging approaches. Recently, we have developed in vivo dynamic near-infrared (NIR) fluorescence imaging methods for quantifying propulsive lymph flow in humans as well as in murine models of human lymphatic and vascular disease. Specifically, we show abnormal lymphatic function and architecture in genetically engineered mice, with mutations in forkhead transcription factor-2 (FOXC2+/- mice), in homeobox gene Prox1 (Prox1+/- mice), and inducible knockout of p120RasGTPaseactivating protein (p120RasGAP; RASA1 mice). FOXC2+/- mice provide a murine model of lymphedema distichiasis; Prox $1+/-$ mice 
may mimic the human condition of lipidemia; and mutations in RASA1 are associated with capillary malformation - arteriovenous malformations (CM-AVM) in humans. Our NIR imaging results on FOXC2+/mice show normal trafficking of an imaging agent along the collecting lymphatic vessels within the dermis. However, a fluorescent intensity profile as a function of time shows non-coordinated propagation of lymphatic contraction. In addition, we found abnormal lymph drainage along the retroperitoneal lymphatic vessels. In Prox1+/- mice, we found diffused dye patterns as well as tortuous and mispatterned lymphatic vessels, which may impact adipose cell recruitment. Our imaging results on RASA1 mice show extensive lymphatic hyperplasia but we could not find any vascular abnormalities. Based upon our ability to non-invasively and quantitatively image lymph propulsion and lymphatic architecture in normal and diseased mice, our results demonstrate that non-invasive NIR fluorescence imaging can be used to image functional lymph flow and remodeling in transgenic mice. The transgenic models and imaging together may potentially provide a method to investigate new lymphovascular therapeutics for the human conditions of lymphedema distichiasis, lipidemia, as well as CM-AVM.

\section{J563}

\section{CO-REGISTRATION ON MULTI-VIEW IMAGING SYSTEM} FOR MOTION ISSUES IN OPTICAL IMAGING

Mickael Savinaud $^{1,2}$, Raphael Boisgard ${ }^{4}$, Serge Maitrejean ${ }^{3}$, Nikos Paragios ${ }^{1,2}$ ${ }^{1}$ Laboratoire MAS, Ecole Centrale Paris, Paris, France, ${ }^{2}$ Equipe GALEN, INRIA Saclay-Ile de France, Paris, France, ${ }^{3}$ Biospace Lab, Paris, France, ${ }^{4}$ LIME - U803, CEA - Inserm, Orsay, France.
Advances in optical imaging instrumentation have opened new research perspectives for freely moving animals. Several studies highlighted the impact of anesthetics agent and animal handling for in vivo studies [1] and thus raised interest in non anesthetized animal experiments, especially in optical imaging. Dealing with freely moving animals reinforces the need of simultaneous acquisition from different part of the mouse. Moreover significant displacements of the animal during monitoring period may also blur of the luminescent data. Therefore to address these issues, we proposed to expand our previous work about cinematic acquisition to a novel framework for multi-views recording. For that purpose, we used new device (Photon Imager, Biospace Lab) which enable high time resolution acquisitions [2]. We added a multiviews system composed of two mirrors and an animal stage which position could be easily adjusted. This setup was applied to in vivo imaging of freely moving mouse (NMRI) bearing a PC12 tumor injected ten days before experiments in the striatum (10000 cells in $0.5 \mu \mathrm{L}$ ). To enhance signals localization throughout the entire video, we filter these biological data by a non rigid deformation field which measure animal movement [3]. In addition, the development of automatic ROI tracking provided several statistical and visual criteria for our motion correction approach. Combining these methods, we were able to significantly improve the localization of the signal and the discrimination of emitting surfaces with a spatial resolution compatible with optical imaging experiments. Experimental results (Fig. 1) demonstrate the interest of the proposed framework for signal localization analysis and its ability to recover all the biological data. References: [1] Hildebrandt I, Anaesthesia and other considerations for in vivo imaging of small animals, ILAR Journal, 2008. [2] Roncali, E., A new device for real time bioluminescence imaging in moving rodents, JBO, (September 2008). [3] Savinaud, M., Motion based enhancement of optical imaging, Proc. IEEE ISBI 09, (June 2009).
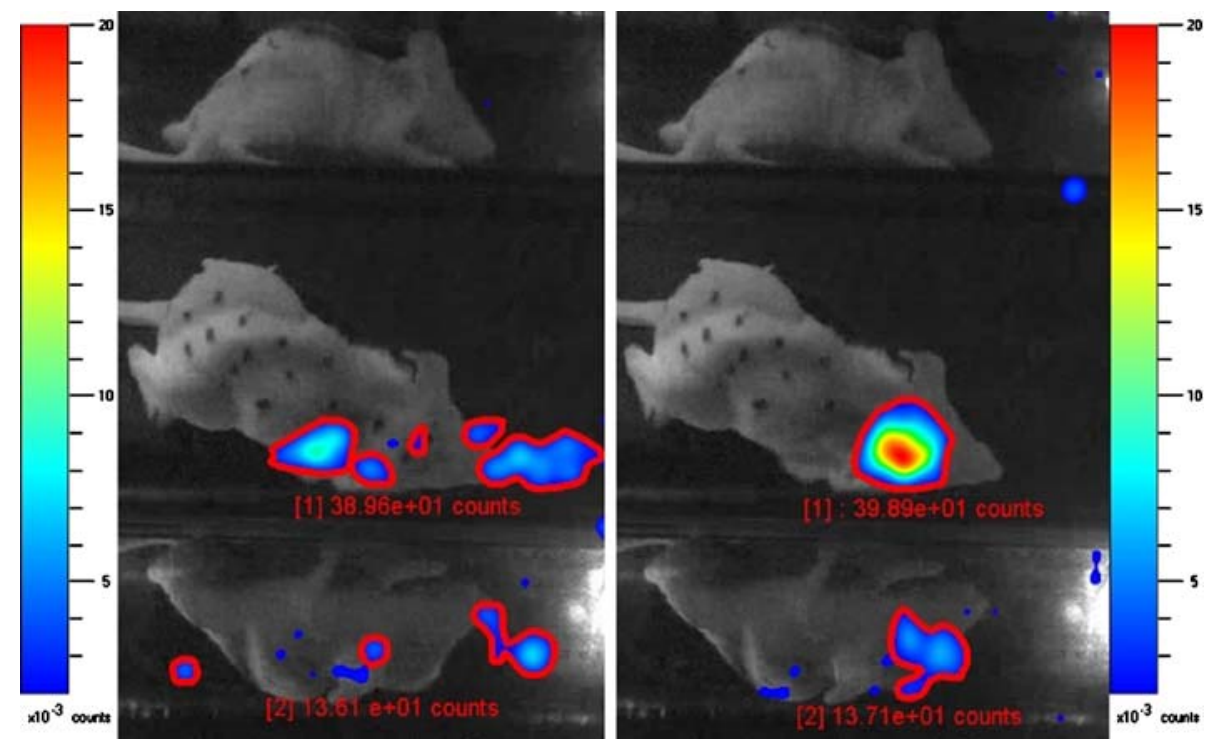

Figure 1: Scene image and bioluminescence data (exposure time $=576 \mathrm{~ms}$ ) overlay without and with registration (respectively left and right). 
J564

IN VIVO IMAGING OF CANCER METASTASIS IN MICE USING A NEWLY DEVELOPED NEAR-INFRARED FLUORESCENCE IMAGING SYSTEM

Kentaro Hizume $^{1}$, Tatsuya Ikehara ${ }^{1}$, Atsushi Yajima ${ }^{1}$, Yoshio Tsunazawa $^{1}$, Ichiro Oda ${ }^{1}$, Hideyuki Saya ${ }^{2}$

${ }^{1}$ Technology Research Laboratory, SHIMADZU CORPORATION, Kyoto, Japan, ${ }^{2}$ Institute for Advanced Medical Research, Keio University School of Medicine, Tokyo, Japan.

Introduction: Optical imaging for small animals is a powerful technique to visualize biological dynamics easily and non-invasively. Especially the light in the near-infrared (NIR) spectral region (700-900 nm), often referred to as "the near-infrared window" can penetrate deeper in the tissue due to the low light absorption by oxy- or deoxy -hemoglobin. In addition the autofluorescence, which determines detection limits in many cases, is remarkably lower in the NIR range than in other wavelength range including visible light. These two facts make the NIR fluorescence imaging a proper method to detect biological changes in the deep tissue. Recent development of imaging probes that emits the NIR fluorescence is also accelerating the progress. Fluorescence imaging apparatus: The NIR fluorescence imaging system that we are developing aims at multidirectional view of a small animal such as mice. Conventional imaging systems irradiate mice to excite fluorophores in one direction and observe fluorescence from mice in one or three direction. On such system, however, it is difficult to detect slight fluorescence in the hidden side of the view unless the animal is rotated. Our imaging system can detect fluorescence signals simultaneously in five directions. The images of the top, side and bottom view is projected on the plane of a CCD by 4 mirrors. The excitation system is also multi directional. NIR laser diodes are arranged around the animal so as to irradiate the uneven surface such as mouse efficiently from various sides. Thus the system can detect the fluorescence of higher intensity from the whole body with a better signal to noise ratio. Detection of cancer metastasis: We observed lung metastases in $\mathrm{Balb} / \mathrm{c}$ mice by injecting the NIR fluorescence imaging agents IntegriSense ${ }^{\circledR}$ which targets integrin $\alpha v \beta 3$. To induce cancer metastasis, Balb/c mice were orthotopically transplanted with $1 \times 10^{\wedge} 54 \mathrm{~T} 1$ mouse breast adenocarcinoma cells into mammary fat pad, yielding lung metastases in the majority of mice within 4 weeks. At 4 weeks after the lung metastatic lesions were detected non-invasively in living mice using our imaging system in 24 hours after injecting intravenously IntegriSense ${ }^{\circledR}$.

$\mathbf{J 5 6 5}$

\section{A MULTI-FREQUENCY ANALYSIS OF FLUORESCENCE LIFETIME DIFFUSE OPTICAL TOMOGRAPHY}

Ralph Nothdurft, Joseph P. Culver, Samuel Achilefu

Radiology, Washington University School of Medicine, St. Louis, MO, USA.

The ability to distinguish fluorophores is often critical with in vivo molecular imaging. The distinction may need to be made between either multiple reporters or different states of a single reporter due to conformational or other environmental interactions. Fluorophore lifetime (FLT) is an exquisitely sensitive contrast with which to differentiate fluorphores in vivo. Lifetime is largely independent of the amount of dye present so that FLT provides a mechanism independent of the in vivo delivery kinetics. We have recently demonstrated in vivo imaging of single FLT's with diffuse optical tomography. An approach for separating multiple lifetimes in vitro using multiple frequency domain measurements was previously developed (Lakowicz \&
Gryczynski, 1991). The theory uses models of the amplitude and phase response versus frequency for both single and multiple lifetime solutions. In this work we adapt the multi-frequency approach to diffuse optical tomography with time-domain illumination and detection. A pulsed laser illuminates the subject while a gated image intensifier provides a measure of the emitted light as a function of time. Illumination from multiple points provides datasets for the tomographic reconstruction. Applying a Fourier transform to our time-domain data yields equivalent synthetic, multi-frequency data. Subsequently a tomographic reconstruction is performed via the normalized Born method using data from each particular frequency. The amplitude modulation and phase that emerge for each frequency from our experimental reconstructions (to date in phantoms) closely mirror the theoretical response for dyes in isolation. The implications for in vivo imaging will be discussed.

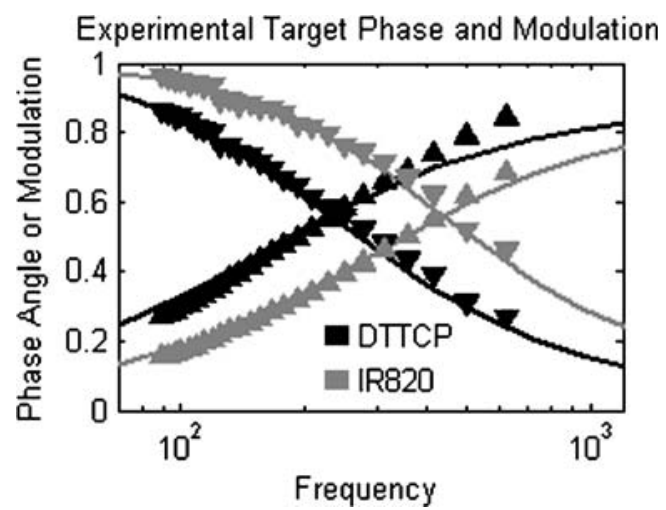

Frequency dependence of the Phase and Amplitude modulation from DOT images of a fluorescent tube inclusion in a slab phantom. DTTCP $(\tau=1.57 \mathrm{~ns})$ black, IR820 ( $\tau=0.54 \mathrm{~ns})$ grey. Experimental results (triangles) closely match the best-fit single lifetime theoretical curves.

\section{$\mathbf{J 5 6 6}$}

THE EFFECT OF ANESTHESIA ON BIOLUMINESCENCE LIGHT PROPAGATION IN THE MOUSE

Bradley J. Beattie ${ }^{1}$, Marleen Keyaerts ${ }^{2}$, Valerie A. Longo ${ }^{3}$, Ronald G. Blasberg ${ }^{1,3}$

${ }^{1}$ Neurology, MSKCC, New York, NY, USA, ${ }^{2}$ In Vivo Cellular and Molecular Imaging Laboratory, Vrije Universiteit Brussel, Brussel, Belgium, ${ }^{3}$ Radiology, MSKCC, New York, NY, USA.

The wavelength dependent difference in photon absorption between oxy- and deoxy-hemoglobin is well known, with large increases in absorption for deoxyhemoglobin occurring in the 600-800 $\mathrm{nm}$ range. Similarly, it is well known that anesthetic agents used in small animal bioluminescence studies, such as isoflurane, can depress breathing rates resulting in a reduction in blood oxygen tension. In spite of this confluence of knowledge, little attention has been given to the potential that the anesthesia, routinely used in bioluminescence studies, might modulate the measured bioluminescence signal thereby mimicking or obscuring changes in the biological parameter of interest. Because different wavelengths are affected unevenly, these changes in photon absorption can distort the depth information used in spectrally resolved bioluminescence tomography efforts and lead to errors in the 
reconstructed source distribution. Our goal in this study is to measure the magnitude of the changes in the bioluminescence signal as it varies with differing depths of anesthesia. We accomplish this using a constant light source (specifically a tritium-powered phosphorescent bead - Mb-Microtec, $\mathrm{CH}$ ) placed within fasted nude (nu/nu) mice using a rectal catheter. Given no movement of the bead or surrounding tissues, changes in the light signal reaching the skin surface can be attributed to changes in the propagation of the light. Mice were imaged using an IVIS 200 optical imager. Serial images were acquired using $20 \mathrm{~nm}$ bandpass filters covering the range from 560 to $660 \mathrm{~nm}$. This sequence was repeated continuously while the mice were given isoflurane at levels ranging between 1 and $5 \%$ with intervening photographs to monitor for movement. Regions of interest were drawn on the luminescence images (corrected for background) and the measured light intensity plotted as a function of time for each wavelength. For these beads having approximately $5 \mathrm{~mm}$ of overlying tissue, the measured light intensity was seen to drop by nearly a factor of two for wavelengths above $600 \mathrm{~nm}$. Little to no change in the signal was seen for the $560 \mathrm{~nm}$ and $580 \mathrm{~nm}$ wavelengths as was expected given the small difference between oxy- and deoxy-hemoglobin at these wavelengths. These results suggest that blood oxygenation levels may be a significant confound requiring consideration and/or correction in bioluminescence comparisons and in spectrally resolved bioluminescence tomography studies.

$\mathbf{J 5 6 7}$

\section{MOLECULAR IMAGING USING NANOTUBES AND A CLINICAL} OPTICAL BREAST IMAGING SYSTEM

$\underline{\text { Stephanie M. Van de Ven }}{ }^{1}$, Niculae Mincu ${ }^{2}$, Jean Brunette ${ }^{2}$, Guobin $\mathrm{Ma}^{2}$, Mario Khayat ${ }^{2}$, Debra M. Ikeda ${ }^{3}$, Sanjiv S. Gambhir ${ }^{1,3}$

${ }^{1}$ Radiology, MIPS, Stanford University, Stanford, CA, USA, ${ }^{2}$ Clinical Systems, ART Advanced Research Technologies Inc., Montreal, QC, Canada, ${ }^{3}$ Radiology, Stanford University Medical Center, Stanford, CA, USA.

Aim Optical imaging studies have shown that differences in intrinsic optical absorption often are not pronounced enough to detect breast masses against normal tissue background. A possible solution is to enhance lesions with agents that change light transmission. Singlewalled carbon nanotubes (SWNT) could potentially be used as lightabsorbing contrast agents for optical breast imaging. We studied phantoms containing varying concentrations of SWNT to determine if SWNT produced detectable optical transmission changes in a clinical optical breast scanner. Methods Six concentrations of SWNTs $(0.8,1.6$, $2.4,4,6.4,20 \mathrm{nM})$ were measured in specifically designed phantoms of 3 sizes $(200 \mathrm{~mm} 3,780 \mathrm{~mm} 3,1570 \mathrm{~mm} 3)$ on a clinical optical breast scanner using 4 wavelengths $(684,732,781,827 \mathrm{~nm})$. Each phantom was placed in the scanner tank where a patient's breast normally would be positioned, and the tank was filled with optical matching medium (OMM), mimicking the average absorption and scattering of a normal breast. We first performed scans using only OMM to acquire the background signal. Then, SWNT absorption scans were done and compared to background absorption. Measurements were repeated on 2 days, 4 weeks apart, to assess reproducibility. Results All phantoms of different sizes and SWNT concentrations were detected by our system at all 4 wavelengths, with best results obtained at $684 \mathrm{~nm}$. SWNT absorption was between $10 \%$ and $80 \%$ higher than background absorption (Fig. 1). Optical absorption signal (y) was dependent on phantom size and SWNT concentration (x), e.g. for $200 \mathrm{~mm} 3$ at
$684 \mathrm{~nm}: \mathrm{y}=0.0003 \ln (\mathrm{x})+0.0038, \mathrm{R} 2=0.93$. Reproducibility was excellent with an Intraclass Correlation Coefficient of 0.95 (95\% CI 0.92-0.97). Conclusion Nanomolar concentrations of SWNT in phantoms were reproducibly detected. This shows the potential of using highly light-absorbing contrast, with appropriate targeting ligands, as molecular imaging agents for breast disease using a commercially available clinical optical breast scanner.

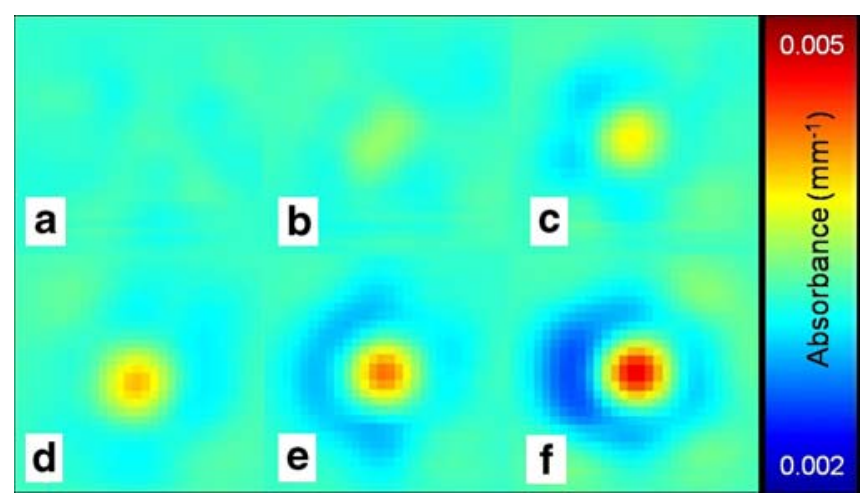

Figure 1. Optical absorption measurements at $684 \mathrm{~nm}$ : (a) optical matching medium only; (b-f) $200 \mathrm{~mm} 3$ phantom with different SWNT concentrations, i.e. 0.8 (b), 1.6 (c), 2.4 (d), 6.4 (e), and $20 \mathrm{nM}$ (f).

\section{J568}

OPTICAL MICROTOMOGRAPHY OF EARLY MOUSE EMBRYO WITH LASER SCANNING MICROSCOPY Maria A. Pogorelova ${ }^{1,2}$, Alexander G. Pogorelov ${ }^{2}$

${ }^{1}$ Biological faculty,Embryology, Moscow State University, Moscow, Russia, ${ }^{2}$ Institute of Theoretical and Experimental Biophysics RAS, Pushchino, Russia.

The early embryo membrane is highly permeable for water, which makes the cell very sensitive to osmotic shock. The cell volume alteration plays an important role in regulation of key cellular functions, including metabolism, protein synthesis, cell death and proliferation. Qualitative relationships between osmotic characteristics of the incubation medium and the volume of the mammalian early embryo have been shown experimentally. However, it is difficult to find the quantitative proportion between these characteristics, because it is hard to measure the volume of objects with such small sizes. This problem may be solved by three-dimensional reconstruction (3-DR). In our study the keeping of the intact volume (shape) of the embryo compartments was based on freeze-drying technique. Different variants of osmotic shock were modeled by changes of $\mathrm{NaCl}$ contents in the incubation medium. After cryfixation in liquid propane and subsequent low-temperature dehydration, the embryo was immersed in the Epon medium. A Zstack of optical slices at a step of $2 \mu \mathrm{m}$ between the layers was obtained in a confocal microscope (Zeiss 510, Germany). 3-DR was performed in the $3 \mathrm{ds} \max$ medium. Figure 1 illustrates the comparative results of the volume reconstruction of the embryo after hyperosmotic shock. Our data indicate that a long-term hypoosmotic $(70 \mathrm{mM} \mathrm{NaCl})$ shock results in the embryo volume recovery. A hyperosmotic $(280 \mathrm{mM} \mathrm{NaCl})$ medium induced 
irreversible shrinkage of embryonic cells. Anisotonic conditions induced both changes in the volume parameters and qualitative transformation of the shape. We observed the formation of outgrowths ("blebs") in the medium with a high $\mathrm{NaCl}$ content (Figure 1B). The developed technology of LSM microtomography of early mouse embryo allows us to measure the cell volume corresponding to the like life state. The obtained quantitative data correspond to the qualitative effects observed in experiments in vitro.

a

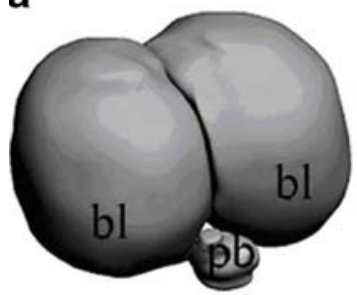

b

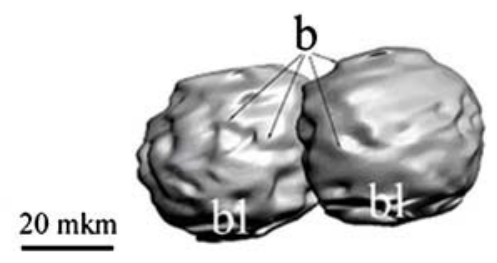

regions and then repeating the training. Typically, only a few rounds of this are required. Depending on the set of images used for training, the entire training process can take a few seconds to a few minutes to complete. The resulting classifiers can used to differentiate relevant tissue regions (e.g., malignant and normal epithelia, stroma, necrosis, etc.) and to segment cellular compartments (nuclei, cytoplasm, and membrane) to allow for detailed, spatially resolved cell-by-cell multiparameter quantitation. The accuracy (vs. manual visual examination by expert pathologists) and practicality of the system has been demonstrated across hundreds of human clinical breast cancer specimens. Its generalizable software can be used for many different tasks as well, since it is easy for non-technical users to train on specimens of their choice. Once the classifiers and downstream quantitative analytical methods are created and validated, having such tools in place will be particularly valuable to developers of in-vivo imaging methodologies, since the histological correlates of in-vivo images can be evaluated automatically, and without ongoing recourse to potentially scarce pathologist input.

J570

Figure 1. Two-cell mouse embryos images obtained with 3-D reconstruction. (A) Control embryo cryofixed immediately after extraction from the oviduct; (B) $30 \mathrm{~min}$ incubation in hypertonic conditions; b- blebs, bl- blastomer, pb-polar body.

J569

\section{MACHINE-LEARNING IMAGE ANALYSIS FOR AUTOMATED MORPHOLOGY-DRIVEN QUANTITATIVE TISSUE AND MARKER ASSESSMENT: A COMPLEMENT TO IN-VIVO IMAGING \\ $\underline{\text { Richard Levenson, Tyna A. Hope, Clifford Hoyt }}$ \\ CRI, Woburn, MA, USA.}

Development of in-vivo imaging methods often depends upon a correlative step involving analysis of tissue sections.This is especially true when molecular imaging approaches are involved. Preclinical and clinical drug trials often also have a similar dependence on histological correlation. However, obtaining accurate, quantitative, and potentially multiplexed analysis of tissue sections remains a challenge. CRI has developed multispectral hardware and powerful machine-learning imaging software to create an automated microscopy platform that can perform the necessay steps without need for hands-on expertise. A Nuance ${ }^{\mathrm{TM}}$ multispectral imaging system was integrated with an automated microscope platform including a 200 -slide robotic slide handler to create an intelligent slide imaging system $\left(\operatorname{Vectra}^{\mathrm{TM}}\right)$. The multispectral capability permits quantitative detection of multiple molecular signals in either brightfield or fluorescence mode. The system also includes a powerful automated image segmentation software (inForm ${ }^{\mathrm{TM}}$ ) that uses "learn-by-example" methods to create multi-class classifiers. While the feature selection and neural-net-based learning procedure are sophisticated and effective, the technical components are hidden from the user. Errors in classification can be readily corrected by amending the training
TRUNCATED TOTAL LEAST SQUARES METHOD WITH A MODIFIED GENERALIZED CROSS VALIDATION FOR THE RECONSTRUCTION OF BIOLUMINESCENCE TOMOGRAPHY

Xiaowei He ${ }^{1,2}, \underline{\text { Jie Tian }}^{1,3}$, Jimin Liang ${ }^{1}$, Xiaochao $\mathrm{Qu}^{1}$

${ }^{1}$ Life Science Research Center, School of Electronics Engineering, Xidian University, xi'an, China, ${ }^{2}$ School of Information Science and Technology, Northwest University, xi'an, China, ${ }^{3}$ Medical Image Processing Group, Institute of Automation,CAS, "Beijing,100080", China.

Reconstruction of the bioluminescent source distribution inside a biological tissue from the optical signals measured on the body surface is the key problem of bioluminescence tomography (BLT), which is ill-posed in nature. Geometric errors are inescapable as well as measurement noise in the BLT, which make the solution of such problem more difficult. Especially geometric errors that directly affect the calculation of coefficient matrix in the linear system equation have not been fully investigated in the literatures. Regularization technique such as Tikhonov's method has played an important role in solving the BLT inverse problem but the choosing of appropriate regularization parameter is very crucial. In this work, with the finite element method (FEM) solving the diffusion approximation model of the photon propagation, the truncated total least squares (TTLS) method is applied to deal with the illposedness of BLT inverse problem and a modified generalized cross validation (GCV) criterion is proposed to determine the proper regularization parameter. In this algorithm, both errors/noise are taken into account and the geometric errors are treated in a new fashion. In order to enhance numerical stability and efficiency, permissible source region set as Fig.1 (b) is incorporated as a priori information during the reconstruction process. Simulations with a heterogeneous mouse chest phantom as shown in Fig.1 (a) reveal the feasibility of the proposed algorithm and evaluate its performance in terms of source location, density, and robustness against noise. The reconstructed results demonstrate that the proposed algorithm performs better than the directly Tikhonov's method, especially when both sides of the system equation are contaminated by errors/noise. 


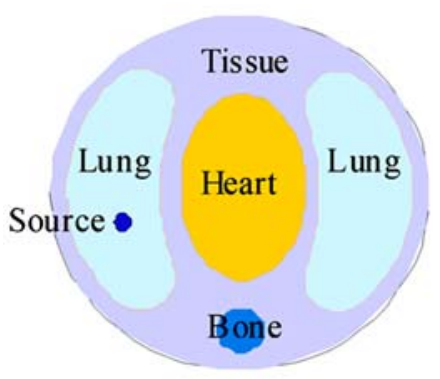

d

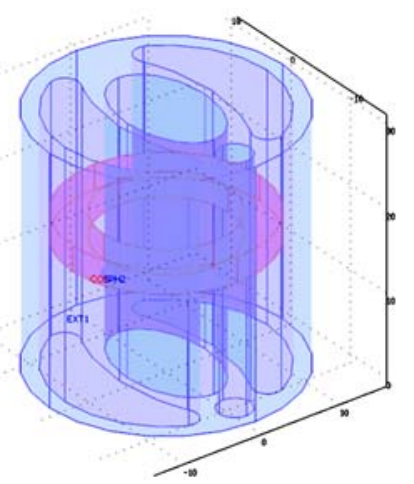

b

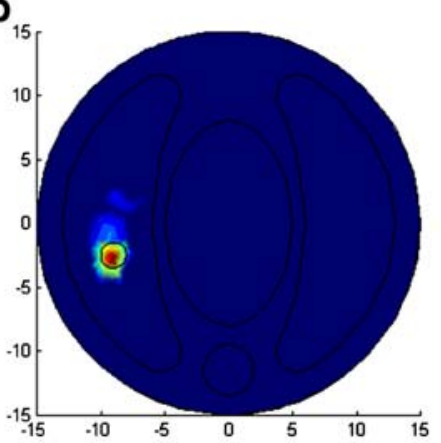

e

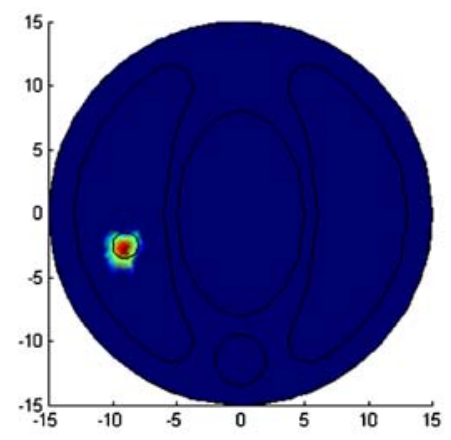

C
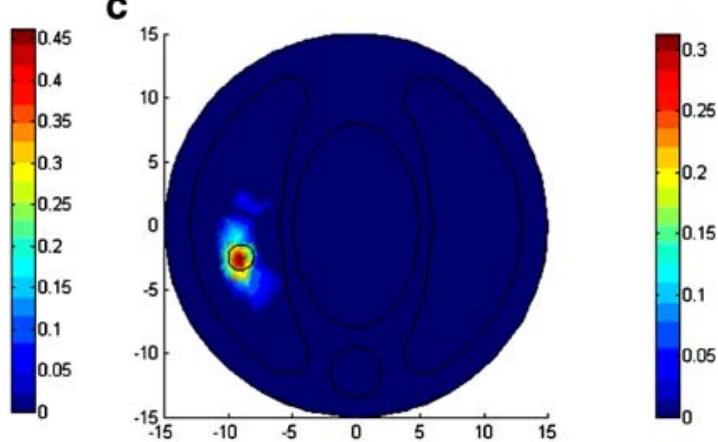

f

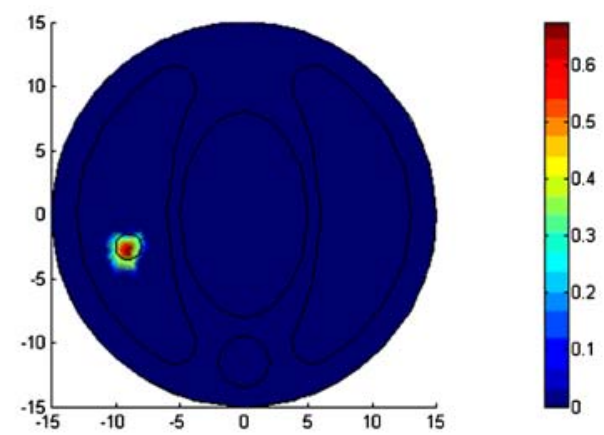

Fig.1 Simulation setups and results (a)XY view,(b)3D view,(c) (d) Results under noise-free with Tikhonov method and the proposed method,(e) (f)Corresponding results under $10 \%$ noise and 0.02 error,respectively.

J571

\section{HYBRID OPTIMIZATION METHOD FOR BLT BASED ON ADAPTIVE FEM}

Qitan Zhang ${ }^{1}$, Shuping Gao ${ }^{1}$, Jimin Liang ${ }^{1}$, Jie Tian ${ }^{1,2}$, Xiaochao $\mathrm{Qu}^{1}$, Runqiang Han ${ }^{1}$, Heyu Huang ${ }^{1}$

${ }^{1}$ Life Science Research Center, School of Electronics Engineering, Xidian University, Xi'an, China, ${ }^{2}$ Medical Image Processing Group, Institute of Automation,CAS, "Beijing,100080", China.

Molecular imaging is a rapidly expanding new technique which has been attracting more and more attention for real-time and non-invasive observation of physiological and pathological changes in-vivo. As a promising molecular imaging technique, bioluminescence tomography (BLT) system has been used to address the needs for 3D localization and quantification of bioluminescent source distribution in small animals. For highly scattered tissue studied in BLT, photon propagation can be well described by the steady-state diffusion equation (DE). Therefore the reconstruction of source position and energy density is converted to an inverse problem of DE. As an efficient numerical method, adaptive finite element method (AFEM) has shown its potential in BLT light source reconstruction. By auto-adapted reduction of mesh size and increment of the basis function order, both convergence speed and accuracy of the reconstruction can be improved in BLT. Priori knowledge as permissible source region is also incorporated to reduce the ill-possedness, where the region is adjusted according to the reconstructed result in AFEM. However, the refinement of mesh and the introduction of higher order basis function complicate the following optimized solution process. So, we employ hybrid optimization method(HOM) according to the characteristics of the system equation in different levels. For the first level of uniform spatial grid and large permissible source region, conjugate gradient
method(CGM) with high convergence speed is adopted to find a approximate solution. But in the reconstruction of subsequent finer grid, where the unknown source is restricted in a smaller permissible source region, according to the previous results, Landweber iteration method (LIM) is selected to achieve a better estimate of the true solution. Numerical simulation results show that our method is feasible to obtain a better reconstruction of both location and energy density with reduced time cost.

\section{J572}

\section{A LEVEL SET BASED BIOLUMINESCENCE TOMOGRAPHY}

Kai Liu, Jie Tian, Dan Liu, Junting Liu, Xin Yang, Min Xu

Medical Image Processing Group, Institute of Automation,CAS, "Beijing,100080", China.

Bioluminescence imaging (BLI) makes it possible to elucidate molecular and cellular signatures to better understand the effects of human disease in small animal models in vivo. Using the BLI data on the surface of object, bioluminescence tomography (BLT) can facilitate to further localize and quantify the signatures in biological tissues. In the contribution, a novel level set based method is presented. The numerical experiments have shown that the proposed method is reliable and fairly robust with respect to high noise levels with a bigger domain of interest. In order to evaluate the performance of the proposed method, experiments using measurements corrupted by different Gaussian noise are carried out, and the Powell method is also compared with them. In figure 1, (a) and (b) are based on the proposed method, and can work well with high noise level. Otherwise, (c) based on the Powell method can not recover the location of sources. It is only when domain of interest is diminished and noise is reduced that 
one may get reliable results. (d) represents the evolution of the source strength, and the relative error is within 20\%. Furthermore, the relative error of the strength correlates well with the signal to noise ratio levels, and the variance for the linear regression is 0.433 , as shown in (e). It clearly suggests that this method is nonsensitive to noise. Hence, even if the experimental conditions are relatively poor, the proposed method may still get reliable reconstruction results. What's more, the runtime for all cases is about $1000 \mathrm{~s}$ with little variation. By contrast, the process based on the Powell method requires about 2400s.

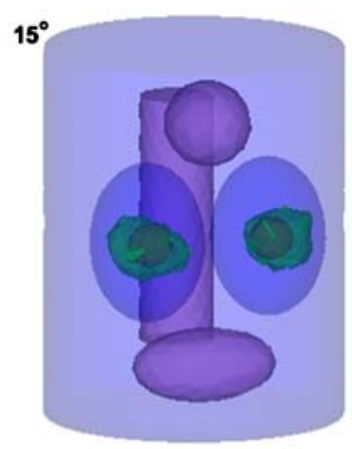

(a)

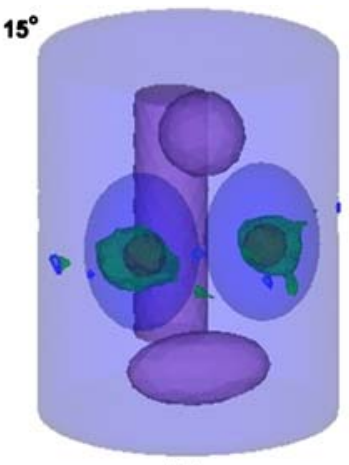

(b)

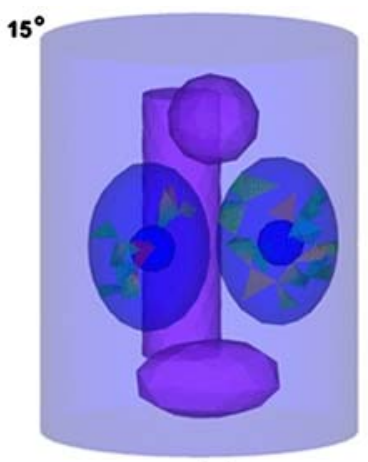

(c)

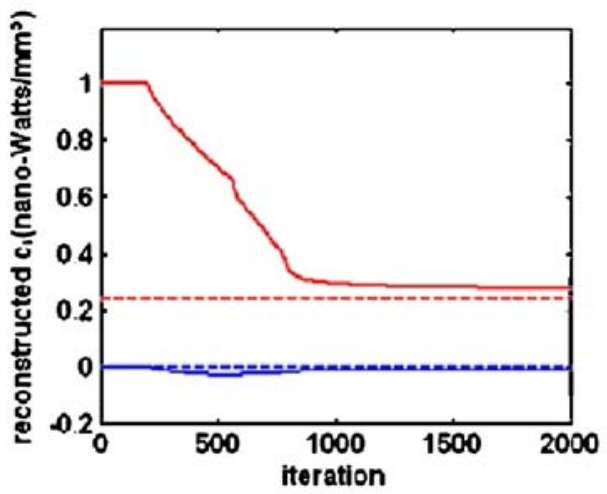

(d)

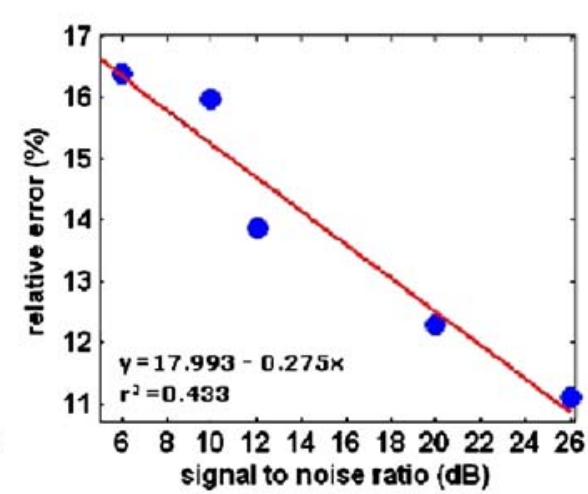

(e)

Figture 1: BLT reconstructions of bioluminescent sources in the heterogeneous experiments from BLI measurements corrupted by $1 \%$ (a), $25 \%$ Gaussian noise levels. (c) The reconstruction results based on the Powell method with $25 \%$ Gaussian noise. (d) Source stength ci (i=1,2) evolution during reconstructions as a function of iteration associated (b). (e) Correlation between the SNR and corrsponding relative error.

J573

\section{MEASURING SUBCELLULAR PROTEIN CONCENTRATIONS FROM IMMUNOFLUORESCENCE IMAGES}

Mark Migueis $^{2}$, Jeffrey J. Carson ${ }^{1,2}$, Savita Dhanvantari ${ }^{1,2}$

${ }^{1}$ Imaging Program, Lawson Health Research Institute, London, ON, Canada, ${ }^{2}$ Department of Medical Biophysics, The University of Western Ontario, London, ON, Canada.

Fluorescence microscopy has been used traditionally in cell and molecular biology for qualitatively evaluating many molecular mechanisms, including intracellular prohormone sorting. In order to extract quantitative information from microscope images, we have developed a method for determining concentration values from fluorescently-labeled proteins within cells using immunofluorescence deconvolution microscopy. First, protein concentration values were derived by removing spectral crosstalk between fluorophores using linear unmixing algorithms developed in Matlab. Microscopy images of phantom slides containing Alexa Fluor 488 (green) and Alexa Fluor 594 (red) fluorophores were used to determine spectral correction algorithms in Matlab that determined fluorophore concentration at individual pixels. Second, ratios of protein concentrations within selected regions of interest (ROIs) of cells were used to derive a measure of co-localization. We then applied our method to examine hormone sorting into secretory granules using Neuro2a (N2a) cells stably expressing proglucagon $(\mathrm{N} 2 \mathrm{a} \mathrm{wt})$ and prohormone processing enzyme carboxypeptidase E (N2a-CPE), and stained for proglucagon and a Golgi marker, p115. Concentration maps for proglucagon and p115 were produced using our customized algorithms, and ratio maps were computed by determining the concentration ratio of glucagon to $\mathrm{p} 115$. Co-localization measures were determined by calculating the average protein concentration ratio found within ROIs selected based on the reference protein in ImageJ. Concentration maps of $\mathrm{N} 2 \mathrm{a}$ cells showed that the concentration of p115 was $1 \mu \mathrm{M}$ and that of proglucagon was $0.09 \mu \mathrm{M}$. Ratio maps and co-localization measures showed that, within the Golgi, the ratio of proglucagon to p115 concentration 
was lower in N2a-CPE cells compared with N2a wt cells. These results indicate that the presence of CPE had an effect on the sorting of proglucagon. Therefore, we successfully applied our method to answer questions regarding molecular mechanisms of intracellular proglucagon sorting. We conclude that our method can extract values for protein concentration from immunofluorescence images, thus allowing for wide application to questions in cell and molecular biology, including the quantitative determination of intracellular protein localization and trafficking.

\section{$\mathbf{J 5 7 4}$}

\section{A STUDY OF PHOTON PROPAGATION IN FREE-SPACE FOR NONCONTACT OPTICAL IMAGING}

Lin Wang ${ }^{1}$, Jimin Liang ${ }^{1}$, Jie Tian ${ }^{1,2}$, Xiaochao Qu ${ }^{1}$, Xueli Chen ${ }^{1}$, Nunu Ren ${ }^{1}$

${ }^{1}$ Life Science Research Center, School of Electronics Engineering, Xidian University, Xi'an, China, ${ }^{2}$ Medical Image Processing Group, Institute of Automation, CAS, "Beijing, 100080", China.

With the development of the optical marker technique and optical imaging technology, noncontact optical imaging has attracted more and more attention as an emerging technology in the field of molecular imaging. Compared with the traditional fiber-based optical imaging, noncontact optical imaging has many advantages on sensitivity, spatial resolution, image quality and system simplicity. However, it is still a challenging topic to model the photon propagation in free-space for this noncontact measurement. Thus, to develop a general and effective model for free-space photon propagation, which simulates the noncontact detection scheme in such an optical imaging area, is becoming an urgent problem to be solved. In this contribution, we present an effective simulation model to depict the photon propagation in free-space, which can handle the transport process of photons escaping from phantom surface and captured by the CCD camera. This model is based on the Lambertian source theory and combining with the imaging law of lens. Firstly, the imaging spot of the escaped photon must be calculated according to the imaging law of lens. In particular, the effects of aperture stop and depth-of-field have been taken into account, which can more accurately and effectively simulate the CCD camera adopted in real optical experiments. Secondly, a discretizing method for lens is adopted in our processing to avoid the vignetting phenomenon of the optical system. Finally, the energy distribution on the detector plane can be calculated through the Lambert's cosine law This model has been implemented with $\mathrm{C}++$ language for the platform of Molecular Optical Simulation Environment which has been developed recently to simulate photon propagation both in turbid media and in free-space. Some interesting results have also been obtained in simulations, and further improvement will go on in future.

J575

\section{THE STUDY OF BIOLUMINESCENCE THREE-DIMENSIONAL IMAGING DEVICE}

Qiujuan Gao, Jie Tian, Xin Yang, Min Xu, Xibo Ma, Dong Han

Medical Image Processing Group, Institute of Automation,CAS, "Beijing, 100080", China.

As a novel molecular imaging technique, bioluminescence tomography (BLT) is attracting more and more attention. Bioluminescence signals coming from the animal can be captured by nitrogen-cooled CCD camera. BLT is to reconstruct the bioluminescent source distribution inside a living animal from optical signals measured on the surface of the animal. Because bioluminescence signal is dimmer than fluorescence signal, and the signal decays over time, so there is an increasing interest in multiple view bioluminescence tomography, since multiple view imaging device can capture more images simultaneously, thus improving the reconstruction accuracy. Our proposed device is mainly for parallel acquisition of multiple view bioluminescent data. The three-dimensional bioluminescence imaging device refers to a unique mirror module, which includes four mirrors with stages. These mirror stages are right triangular blocks making $45^{\circ}$ to the animal around the animal in the up, down, left and right orientation, see figure 1(a). We can acquire four images using one CCD through twice photograph using the device. The orientation of the small animal is perpendicular to the paper plane. When CCD is located at the top of the left mirror, we can acquire the dorsal, abdominal and left side images simultaneously, figure (b) is the image we captured. When CCD is located at the top of the right mirror, we can acquire the right side image of animal. Some experiments have been performed to demonstrate the feasibility of this system. It is shown that bioluminescent signals collected using our system can produce a better reconstruction quality while reducing the data acquisition time, as compared to the sequential data acquisition mode.
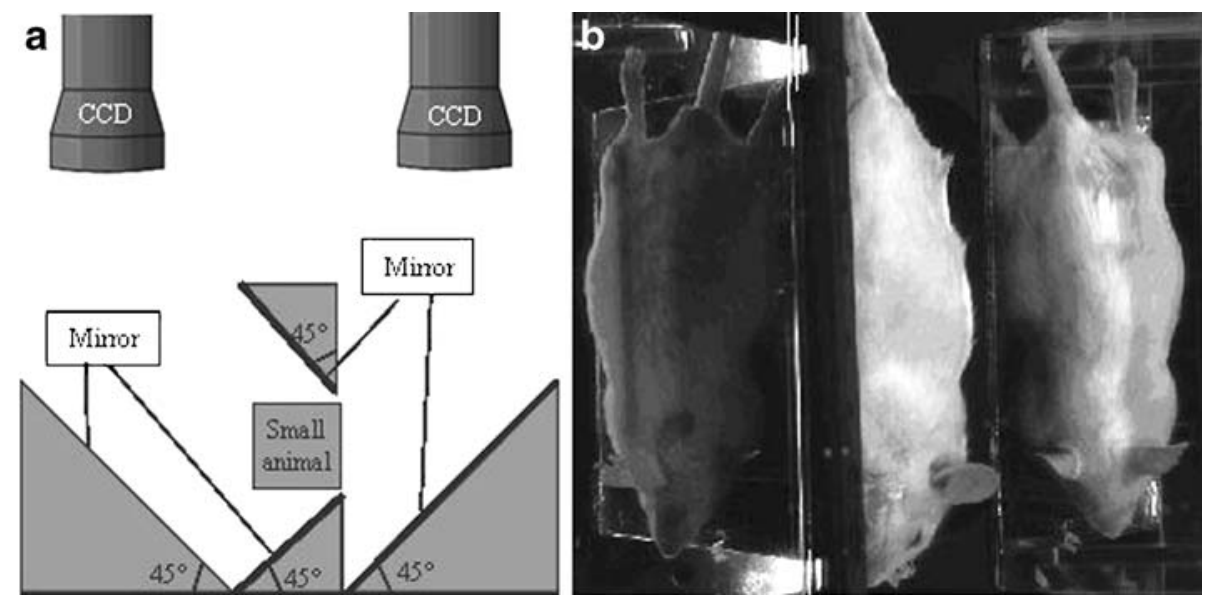

Figure 1 Structure and captured image of three-dimensional bioluminescence imaging device (a) Structure of three-dimensional bioluminescence imaging device; (b) Picture of three images through once photograph 
J576

FLUORESCENCE ENDOMICROSCOPY VERSUS CONFOCAL MICROSCOPY FOR IMAGING AND CHARACTERIZING HUMAN HEAD AND NECK SQUAMOUS CELL CARCINOMA Muriel Abbaci ${ }^{1}$, Stephane Temam ${ }^{2}$, Odile Casiraghi ${ }^{3}$, Philippe Vielh ${ }^{3}$, Jacques Bosq $^{3}$, Corinne Laplace-Builhé ${ }^{1}$

${ }^{1}$ Platform of Cellular Imagery and Cytometry, UPRES EA 4040, IRCIV, Institut Gustave Roussy, Villejuif, France, ${ }^{2}$ Department of Otorhinolaryngology and Head and Neck Surgery, Institut Gustave Roussy, Villejuif, France, ${ }^{3}$ Department of Pathology, Institut Gustave Roussy, Villejuif, France.

Purpose: Fibered confocal endomicroscopy (CEM) is a non invasive imaging tool enabling "optical biopsies" of tissues at cell and molecular level. Clinical studies have successfully reported the accuracy of CEM for the characterization of gastrointestinal, dermatologic and ocular diseases. In this study, we assess the potential use of endomicroscopy in combination with fluorophores clinically approved or undergoing early clinical trials, to characterize human head and neck premalignant and malignant lesions. Method: 70 fresh surgical specimens of head and neck squamous cell carcinoma (HNSCC) were obtained. Normal mucosa and tumoral tissues were analysed. Five different dyes able to stain cell structures or extracellular matrix were evaluated alone or combined. En face images were achieved using both fibered confocal microscopy (CellVizio ${ }^{\circledR}$, Mauna kea Tech.) and conventional confocal microscopy (Leica SPE). Cellular and subcellular morphology and distribution were examined on the fluorescence images obtained with both systems. CEM and confocal images were then compared to the corresponding histological H\&E sections to diagnose and grade lesions. Results: In normal mucosa samples, an homogeneous and regular nuclear distribution was showed in squamous epithelium with both CEM and confocal imaging. Expected changes in cell density were also seen in basal, intermediate and superficial layers. Fluorescence imaging of different subtypes of squamous cell carcinoma, from poorly to well differentiated carcinoma, verrucous or sarcomatoid, presented distinct features when compared to normal squamous epithelium. Images provided clear information on the heterogeneous distribution of cancerous cells surrounded by stroma. Nuclei size and shape irregularities were well defined and independent of the fluorophore tested. Changes in nuclear-cytoplasmic ratio and higher nuclear density could also be noticed. Disorders of keratinisation like dyskeratosis and keratin pearls were clearly discerned by CEM and the images corroborated well with histological data. Conclusion: Fluorescence confocal endomicroscopy allows to make the diagnosis of HNSCC on surgical specimens. Suitable combinations of stains, such as acriflavin-fluorescein improve significantly the contrast of CEM images that are closely comparable to conventional confocal imaging.

\section{J577}

HIGH END ROBOTIC AND COMPUTER TECHNOLOGIES APPLIED TO SMALL ANIMAL OPTICAL MOLECULAR IMAGING

Jean Beaudry $^{1}$, Heidi Ligeret ${ }^{1}$, Bin Zhang $^{1}$, Matthieu Boffety ${ }^{2}$, Marc Massonneau ${ }^{1}$

${ }^{1}$ Laboratoires Quidd Inc, Sherbrooke, QC, Canada, ${ }^{2}$ Institut Langevin / Laboratoire d'Optique Physique, ESPCI Paris Tech, Paris, France.

In vivo molecular imaging of small animals represents the path forward towards new and exciting breakthroughs in preclinical and clinical studies. In order to be able to achieve higher standards in term of sensitivity, precision and reproducibility, a solid integration of different highly advanced technologies is needed. This integration allows the device to be ready for new modules and therefore be a platform to a multimodal approach. To be able to open up the technological boundaries, we have developed a new generation of small animal optical imager using the latest advances in optical, computer and robotic technologies. This new device includes various interactive procedures that takes into account the contours of the animal and automatically positions the camera ideally with regards to optical and experimental constraints. More than this, the system allows the same experimental conditions to be reproduced with an advanced repositioning algorithm. An animal can therefore be analyzed over multiple days in the "same" test conditions. The system includes state of the art detection technology and will remain up to date thanks to its modular design. It also includes high end linear motor allowing fast movement and high repeatability all with easy control. The sensitivity, precision and reproducibility of this device were evaluated in both in vitro conditions (using calibrated luminescent sources in tissue phantoms) and in in vivo models (using optical probes specifically targeting molecular events generated in various pathological models). The results show significant progress in optical molecular imaging quantification achieving an unmatched level of reproducibility for small animal longitudinal studies. Finally, the device is equipped with software allowing a high degree of control over the data and giving the user powerful data analysis features. The software furthermore offers a technology that lets the user build a 3D reconstruction of the animal and the luminescence source from 2D images. These technological advances are promising and open the door to a new universe of possibilities regarding multimodal analysis while giving the small animal optical molecular imaging a high sensitivity, precision and reproducibility platform.

\section{$\mathbf{J 5 7 8}$}

\section{A GENERAL STUDY OF OPTIMAL GEOMETRIC PARAMETERS FOR TIME RESOLVED DIFFUSE OPTICAL TOMOGRAPHY}

Jerome Boutet, Ludovic Lecordier, Laurent Guyon, Lionel Herve, Mathieu Debourdeau, Jean-Marc Dinten

CEA-LETI-MINATEC, Grenoble, France.

Fluorescence Diffuse Optical Tomography is a promising technique for cancer diagnostic. It provides a way to characterize and localize tumors with a good accuracy and without ionisation of tissues. This technique can be performed by continuous or time resolved measurements. Time-resolved imaging is based on the use of subnanosecond laser pulses for excitation combined to fast response detection, providing photon time of flight. Some applications, such as breast cancer diagnostics, allow us to choose between two possible geometries: transmission or reflection. Others, like prostate cancer require working in reflection. First, we will present a comparison between geometries on a time resolved acquisition chain. It is composed by a Titanium-sapphire laser source delivering femtosecond pulses at $775 \mathrm{~nm}$ and a gated CCD camera to analyze the response of the medium. A complete acquisition and reconstruction study has been carried out in comparable conditions for both geometries. The fluorescence yield is reconstructed by processing the acquired time-resolved signal of each source-detector combination. These computations are based on the use of the integral (intensity) and the mean time (mean time of flight) of each signal. The influence of number, step and relative position of the sources and detectors will be demonstrated by simulations and experiments. We show how the temporal step can be optimized to accelerate time of flight measurements and increase signal without degrading spatial resolution. These optimal parameters are evaluated thorough a set of experiments performed on a breast phantom reproducing both its optical and geometrical properties. Finally we will show how time resolved approach is particularly pertinent for reflection geometry. 


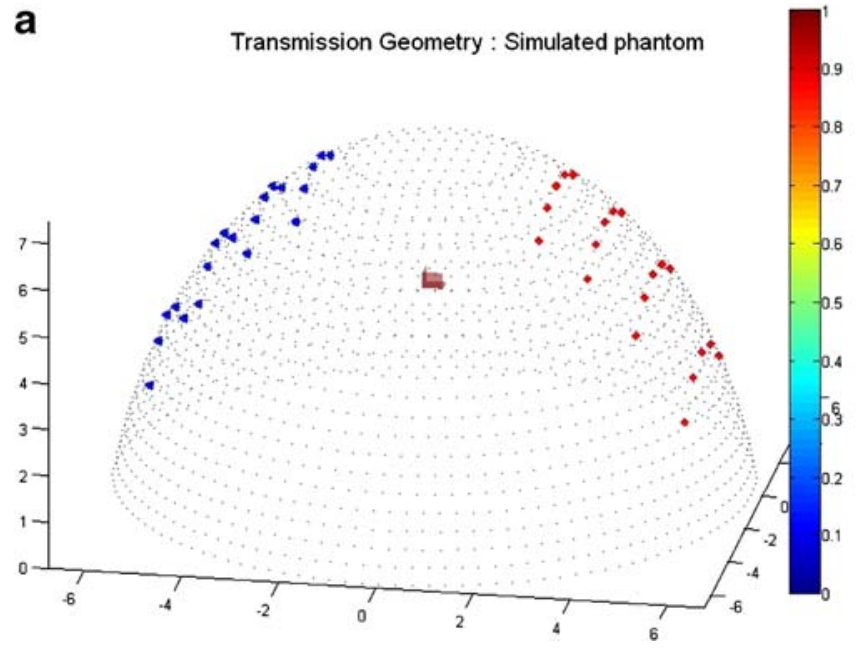

J579

\section{COMPARISON OF ROI ANALYSIS METHODS} FOR THE MEASUREMENT OF BIO-LUMINESCENCE SIGNAL Yoon Oh Tak ${ }^{1,3}$, Hyeon Sik Kim ${ }^{1}$, Hyeong Ju Park ${ }^{1}$, Eun Seo Choi ${ }^{2}$, $\overline{\text { Byeong-il Lee }}^{1}$

${ }^{1}$ nuclear medicine, Chonnam National University Hwasun Hospital, Hwasun, Jeonnam, Korea, South, ${ }^{2}$ Physics, Chosun Univeristy, Gwangju, Korea, South, ${ }^{3}$ Medical Image Science, Inje University, Kimhae, Korea, South.

Introduction: Image analysis using ROI(region of interest) is widely used technique. The difference of selected area and noise among acquired images is directly affected to the calculated number of photons. This study deals that the development of photon counting method and the comparison among results measured by proposed methods. Objective and method: The equipment of ALIS(Animal Lighting Imaging System), which is lab-made optical image equipment, is used for the acquisition of bioluminescence images. As a source of bio-luminescence, tubes containing light emitting bacteria of $\triangle$ ppGpp_lux are used. Each tube has different CFU of $4.0 \times 10^{9} \mathrm{CFU}$ $\left(\mathrm{L}_{1}\right), 5.0 \times 10^{9} \mathrm{CFU}\left(\mathrm{L}_{2}\right)$ and $1.0 \times 10^{1 \circ} \mathrm{CFU}\left(\mathrm{L}_{3}\right)$. Exposure time is controlled as 10, 20, 30, 60, 90, and 120 seconds. Identical ellipticalshaped ROI, which enclose a tube, is applied to all the acquired images. Four different photon counting methods are applied to each registered ROI. The each method as follows: photon counting within $\operatorname{ROI}\left(\mathrm{S}_{1}\right)$, photon counting within ROI after automatic threshold process $\left(\mathrm{S}_{2}\right)$, photon counting within ROI after subtraction of background noise in an additional larger ROI than firstly selected $\mathrm{ROI}\left(\mathrm{S}_{3}\right)$, and photon counting after performing noise rejection in $S_{2}$ as in $S_{3}\left(S_{4}\right)$. Total counts(T) is obtained by the summation of photons at each pixel and average count $(\mathrm{A})$ is calculated by the total counts divided by the total number of pixels. The $\mathrm{T}$ and $\mathrm{A}$ are calculated by the proposed four methods and compared with each other. Results: In $\mathrm{S}_{1}$ and $\mathrm{S}_{2}, \mathrm{~T}$ 's are slowly reduced after 30 seconds elapsed but T's are linear increased along the exposure times in the case of $\mathrm{S}_{3}$ and $\mathrm{S}_{4}$. Average values also show similar response. When $\mathrm{L}_{1}$ tube is used as a source, the differences of $\mathrm{T}$ between $\mathrm{S}_{1}$ and $\mathrm{S}_{2}$ represented that it contains high noise signal. However linearity of photon counts at $S_{3}$ and $S_{4}$ was improved. Conclusion: The quantification results obtained by different ROI measurement methods provide same patterns along exposure times although different brightness is employed in bio-luminescence imaging.

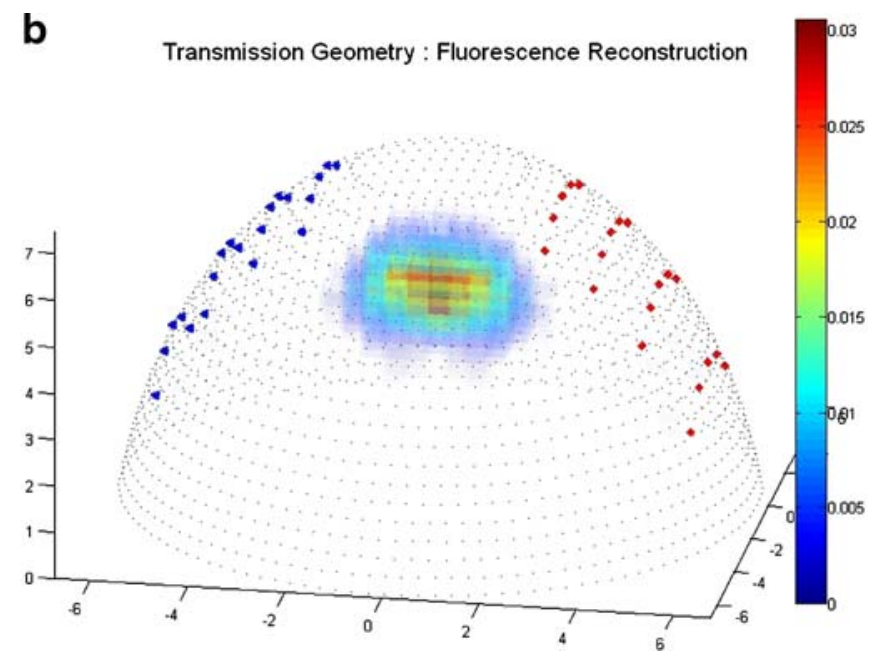

Applied noise rejection methods for the external noise and the background noise at the selected areas realize well-established linear response of photon counts to exposure time. The quantification of bioluminescence image could be accurately improved by employing proper noise rejection method.

\section{J580}

\section{PERFORMANCE OF THE RED-SHIFTED FLUORESCENT PROTEINS IN MULTISPECTRAL OPTOACOUSTIC} TOMOGRAPHY (MSOT)

Nikolaos C. Deliolanis, Adrian Taruttis, Amir Rosenthal, Daniel Razansky, Vasilis Ntziachristos

Institute for Biological and Medical Imaging, Technical University of Munich and Helmholtz Zentrum Munich, Munich, Germany.

Fluorescent proteins (FPs) have emerged as a very powerful imaging tool in biomedical research. So far FPs have been used in traditional fluorescence imaging modalities, such as fluorescence microscopy or fluorescence epillumination imaging, but with limited penetration depth. Optoacoustic tomography (OT) is an emerging imaging technology that combines the advantage of the high contrast optical imaging with the higher resolution of ultrasonic imaging in deep-tissue that can resolve fluorophore concentration in small animals by multispectral data acquisition and processing. The development of a new generation of fluorescent proteins operating in the near red part of the spectrum combined with the use of appropriate excitation wavelengths can open exciting possibilities in deep-tissue imaging. In this work we explore the feasibility to use fluorescent proteins as reporter genes in multispectral optoacoustic tomography (MSOT) in deep-tissue applications both with analytical study and experiments. In order to predict the performance of the fluorescent proteins in deep tissue imaging, we employ a simple photon propagation model for transillumination imaging, that takes into account the spectral tissue attenuation and the molar extinction coefficient of the proteins. Comparative analysis of among the currently available fluorescent proteins shows that mRaspberry is the optimum choice, not only because it can produce 3 times stronger signal compared to any other available fluorescent protein at the moment, but also has a much steeper absorption drop above $615 \mathrm{~nm}$, which can be easily separated from the hemoglobin absorption in MSOT. The results show the great potential of this method to image FPs in murine models. 


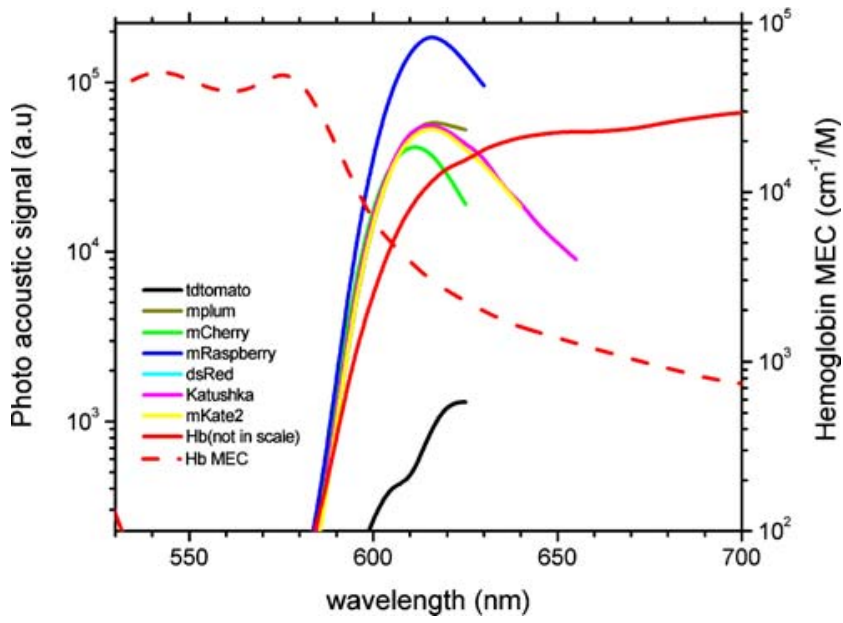

J581

\section{ENGINEERING BACTERIA FOR TARGETED TUMOR SPECIFIC GENE EXPRESSION}

Nha Ca T. Nguyen, Hee Jung Lee, Vu Hong Nguyen, Jung-Joon Min; Nuclear Medicine, Chonnam National University Medical School, Hwasun, Korea, South.

Attenuated Salmonella typhimurium defective in ppGpp synthesis have a propensity to naturally accumulate and replicate in a wide variety of solid tumors. Bacterial tropism for solid tumor leads us to engineer the Salmonellae as a drug delivery vehicle. Thus, we explored to engineer bacteria to express target protein specifically in the tumor region using promoters that are preferentially activated in tumors. We exploited some promoters which are regulated by hypoxic condition with different manner: promoters of pepT, ansB and pflE genes. These promoters' sequences were amplified directly from bacteria by PCR and cloned to pGEM-Teasy vector. To evaluate the expression of these promoters, reporter gene (Firefly luciferase or Renilla luciferase) was inserted in the downstream of these promoters with the same direction. The reporter genes controlled by Lac promoter was used as a positive control and a promoterless vector as a negative control. To evaluate the hypoxia-specific expression, the regulation of reporter genes under the control of such promoters was studied in vitro under anaerobic and aerobic condition as well. FNR is a global transcription regulator that can control gene expression in hypoxic condition. In this case, pepT and pflE promoters have FNR-binding site for this regulator to control gene expression. Because there was no exact consensus, we optimized nucleotide sequence in the binding site. During cell culture, reporter gene expression was significantly increased in hypoxic condition compared to that in normoxic condition, which was shown in Luciferase assay or Western blot analysis. We administered the $S$. typhimurium ( $\triangle \mathrm{ppGpp}$ ) strain carrying pPepT-Fluc, pAnsB-RLuc8, pPflE-RLuc8 or pLac-RLuc8 $\left(5 \times 10^{7} \mathrm{CFU}\right)$ intravenously in BALB/c mice bearing CT-26 colon cancer. Whole body bioluminescence imaging, necropsy imaging and imaging of isolated organs showed that the bacterial signal was significantly higher in tumor and was negligible in liver, spleen or lung with Salmonellae carrying pPepTFluc, pAnsB-RLuc8, or pPflE-RLuc8 as compared to the signal by the positive control. In conclusion, the tumor-specific gene expression by tumor-targeting bacteria would be a good strategy to enhance therapeutic gene expression in tumor as well as to reduce un-wanted expression in normal organs.

\section{$\mathbf{J 5 8 2}$}

\section{TIME-RESOLVED DETECTION OF FLUORESCENCE OF INDOCYANINE GREEN CIRCULATING IN THE HUMAN HEAD}

Adam Liebert $^{1}$, Michal Kacprzak ${ }^{1}$, Tomasz Soltysinski ${ }^{1}$, Anna Gerega ${ }^{1}$, $\overline{\text { Piotr Sawosz }}^{1}$, Daniel Milej ${ }^{1}$, Joanna Maczewska ${ }^{2}$, Wojciech Weigl ${ }^{3}$, Katarzyna Fronczewska ${ }^{2}$, Leszek Krolicki ${ }^{2}$, Ewa Mayzner-Zawadzka ${ }^{3}$, Roman Maniewski ${ }^{1}$

${ }^{1}$ Department of Biophysical Measurements and Imaging, Institute of Biocybernetics and Biomedical Engineering, Warsaw, Poland, ${ }^{2}$ Department of Nuclear Medicine, Medical University of Warsaw, Warsaw, Poland, ${ }^{3}$ Department of Anesthesiology and Intensive Care, Medical University of Warsaw, Warsaw, Poland.

Fluorescence techniques based on inflow of exogeneous optical markers are commonly used in small animal studies. Detection of fluorescence light originating from inclusions located deeply in the tissue structures is very difficult because of strong light scattering. Recently, it was reported that the fluorescence light can be excited in the human brain and detected non-invasively on the surface of the head with the use of time-resolved technique. Potentially, this method can be useful in measurement of brain tissue perfusion and detection of bloodbrain-barrier disruption. In present study we analyzed inflow pattern of indocyanine green (ICG) at different sites on the head, at different source-detector separations and at multiple emission wavelengths. For ICG excitation a MaiTai femtosecond laser source operating at wavelength of $760 \mathrm{~nm}$ was used. We used three different light detection strategies: (i) simultaneous detection of reflectance and fluorescence at 8 source-detector pairs distributed on both hemispheres of the brain (ii) detection of fluorescence at 4 source-detector separations, (iii) detection of fluorescence in 16 spectral channels with the use of multichannel PMT and polychromator. The time-resolved detection in all three strategies was possible with the use of timecorrelated single photon counting. For blocking of the excitation light a high-pass filter was utilized. A volunteer was examined in the sitting position. ICG was injected rapidly into forearm vein. Time-resolved fluorescence was detected at different sites on the head and showed similar pattern as reported previously. Increase of mean time of flight of distributions of times of arrival of fluorescence photons was observed, however, in some of the channels this effect was not clearly visible. The multidistance measurement revealed strong dependence of the pattern of fluorescence signal on the source-detector separation. In multi-wavelength measurement, the fluorescence signals were detected in 6 channels, covering range of about $80 \mathrm{~nm}$. Its maximum has been found at about $820 \mathrm{~nm}$. Our study shows that the fluorescence light originating from ICG circulating in the human head can be successfully detected on the surface of the tissue at different sites, at different source-detector separations and with evaluation of the spectrum of fluorescence emission. Experiments carried out allow for optimization of the fluorescence detection setup and for better understanding of the pattern of changes of time-resolved signal detected on the head of adult after ICG bolus injection.

\section{$\mathbf{J 5 8 3}$}

\section{THE GEOMETRIC TRANSFER FUNCTION FOR A NOVEL MLA BASED OPTICAL DETECTOR}

Joerg Peter

Medical Physics, German Cancer Research Center, Heidelberg, Germany.

A theoretical approach has been developed and is presented for the geometric transfer function (GTF) of microlens array (MLA) based light detectors intended for in vivo optical molecular imaging. It not only is used to analyze the imaging properties of such detector layout but also to support a mathematical model for inverse 
mapping of the three-dimensional object space onto arbitrary allocation of detectors in space. The detector's response characteristics are described in terms of geometry factor and the derived measures of geometric efficiency, spatial resolution, and depth of field. We apply a mathematical concept that shows similarity to the physics of parallel beam collimators as used in scintillation gamma cameras. The depth-dependent, though shift invariant geometry factor is described as the superposition of all physical entities comprising a single detector element: lens - septum hole-sensor lattice field. The field of view (FOV) of MLA detectors as employed here is essentially constant, forming a cuboid imaging volume. As all detector entities are identical the geometric efficiency is proportional to the fraction of the overall detector FOV with respect to the projected object volume. Even though a single detector does not fully cover a mouse transaxially (detector size: $25 \mathrm{~mm} \times 100 \mathrm{~mm}$ ) full coverage of the object volume is achieved by hexagonal allocation of six detectors which form a superimposed untruncated cylindrical imaging volume inside a maximum diameter of $50 \mathrm{~mm}$. Analysis of the system response has been accomplished in terms of an effective point spread function (PSF) derived by measurements of luminescent point sources. Because the PSF of an MLA detector depends on the position of the point source relative to the entity pattern, we have derived an effective GTF by means of the Fourier transform. The MLA based light detector does not form a depth of focus plane. Rather, the detector's depth of field is associated with an intrinsic spatial resolution that is best in front of the detector and degrades with distance. For the detector at hand, sub-millimeter spatial resolution is attained up to about $75 \mathrm{~mm}$ depth of field. By employing a newly developed inverse mapping algorithm that takes into account the effective GTF, resolution could be significantly improved (up the $300 \%$ as compared to intrinsic resolution) inside the adequate object space ( $60 \mathrm{~mm}$ diameter). It is interesting to note that the $1 \mathrm{~mm}$ depth of field limit could not be enlarged because of increasing interferences of entity FOV's which scale with detector distance.

\section{J584}

TRACKING IN VIVO BIODISTRIBUTION OF BIOLOGICS USING FLUORESCENCE LIFETIME GATING

Allison Curtis $^{1}$, L. Ashley Aronow ${ }^{1}$, Abhiruchi Agarwal ${ }^{1}$, Brian Granda $^{2}$, Rainer Kneuer ${ }^{3}$, Thomas Krucker ${ }^{1}$

${ }^{1}$ Molecular Imaging, Novartis Institutes for BioMedical Research, Cambridge, MA, USA, ${ }^{2}$ Analytical Sciences, Novartis Institutes for BioMedical Research, Cambridge, MA, USA, ${ }^{3}$ Global Imaging Group, Novartis Pharma AG, Basel, Switzerland.

Optical molecular imaging of fluorescently labeled biologics (antibodies, proteins), in living animals, can be used to non-invasively measure biodistribution and pharmacokinetics. As the use of biologics for therapeutic and diagnostic applications becomes more and more prevalent, determining an accurate time course of specific distribution (vs., non-specific accumulation in tissue, cellular uptake, and clearance) is essential to avoid misinterpretation of measured signals. For instance, the unspecific accumulation of a labeled antibody in tissue can interfere with the therapeutic/ diagnostic utility of the antibody. The majority of in vivo optical imaging systems available measure amount of fluorescence in tissue, without considering whether this is the conjugated antibody itself or an accumulation of the free fluorophore. One way to address this problem is by using fluorescence lifetime. Fluorescence lifetime gives additional quantitation to an image and will vary given the tissue environment or size of the fluorophore. Using a commercially available time domain optical imaging device, differentiation was made between free and conjugated fluorophore in subcutaneous injections in nude mice. Through the use of exponential fits to temporal point-spread functions, lifetime values can be used to gate intensity images thereby making the measurement more accurate and specific. A study was designed to systematically address the ability of the time domain optical imaging system to track the condition of a Cy5.5-labeled antibody in vivo, determining how the in vivo fluorescence lifetime values change in tissue over time. Three groups of nude mice were imaged for one week, focusing on the distribution of a Cy5.5-labeled mouse IgG, Cy5.5 only and trypsinated Cy5.5-IgG in the liver and muscle. By measuring the different lifetime values of Cy5.5-IgG and Cy5.5, we were able to determine how much of the measured signal was free versus conjugated fluorophore. This study was repeated with a fluorescently labeled targeted antibody to compare how well lifetime gating can account for specific tissue distribution. In vivo data was compared to standard ex vivo and in vitro assays for protein detection. The use of this methodology has the potential to not only aid in more accurate detection of labeled biologics, but also reveal significant details about their specific activity and metabolism in vivo.

\section{$\mathbf{J 5 8 5}$}

\section{INFERRING THE STRENGTH OF PROTEIN INTERACTIONS FROM FRET DATA}

Catherine Lichten $^{2,1}$, Peter Swain ${ }^{1}$

${ }^{\mathrm{C}}$ Centre for Systems Biology, University of Edinburgh, Edinburgh, United Kingdom, ${ }^{2}$ Physiology, McGill University, Montreal, QC, Canada.

Advances in technology allow researchers to collect increasingly sophisticated data, but another important step is developing analysis techniques to extract information from this data. One experimental tool with potential to yield further useful information is fluorescence resonance energy transfer (FRET), which can reveal interactions between proteins and possibly also be used to quantify the strength of those interactions in terms of the proteins' dissociation constant (Kd). Several analytical techniques exist to correct FRET data for the effects of spectral overlap between fluorophores and normalize it for varying fluorophore concentrations, but a remaining challenge is to quantify FRET results in terms that are independent of experimental setup. Moreover, existing analysis techniques incorporate experimentally measured parameters in calculations but neglect to address how the error of these measurements affects the resulting quantities and the data's interpretation. The goal of this work is to develop a method for extracting the Kd from FRET data while taking into account experimental measurement errors, and to explore how measurement errors and experimental design impact the quality of this Kd estimate. Using a Bayesian framework, we developed an algorithm for inferring the values of the two unknown parameters: $\mathrm{Kd}$ and $\mathrm{E}$, the efficiency of energy transfer from the donors to the acceptors. We use Monte Carlo optimization to minimize differences between experimental observations and predictions from a mathematical model of FRET. Applying our method to simulated data, we measured the error in the estimate of $\mathrm{Kd}$ and $\mathrm{E}$ under varying experimental conditions. Finally, we validated our method and theoretical results with experiments on solutions of fluorescently tagged, interacting proteins. Analyzing FRET data from a single cell or vesicle yielded infinite solutions for the values of $\mathrm{E}$ and $\mathrm{Kd}$, but analyzing an ensemble of data from samples containing varying concentrations of donors and acceptors yielded a unique pair of values. The accuracy of this estimate in turn depended on both the noise present in the data and the extent of the variation in the fluorophore concentrations. Our work has applications for extracting additional quantitative information from FRET data and for evaluating the feasibility of FRET as a tool for measuring protein interaction strength under various experimental conditions. 
J586

MINI-FLARE: A COMPACT AND ERGONOMIC DUAL-CHANNEL NEAR-INFRARED FLUORESCENCE IMAGE-GUIDED SURGERY SYSTEM

Alan Stockdale $^{1}$, Rafiou Oketokoun ${ }^{1}$, Sylvain Gioux ${ }^{1,3}$, John V. Frangioni ${ }^{1,2}$

${ }^{1}$ Medicine, Beth Israel Deaconess Medical Center, Boston, MA, USA,

${ }^{2}$ Radiology, Beth Israel Deaconess Medical Center, Boston, MA, USA,

${ }^{3}$ Engineering, Boston University, Boston, MA, USA.

Background: We have previously developed and translated to the clinic a cart-based, mobile, image-guided surgery system that provides simultaneous imaging of color video and two independent channel of near-infrared (NIR) fluorescence. In this study, we designed, constructed, and validated a more compact and mobile version of the

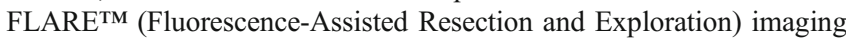
system with many of the same capabilities. Materials and Methods: The device was enabled by the construction of compact, high-power LEDbased light modules with integrated linear drivers and heat dissipation technology. Custom optics were used to mount a dual band (700 nm and $800 \mathrm{~nm}$ ) emission filter in front of a single IEEE-1394 Firewire NIR camera, while a second Firewire camera was used to simultaneously capture color video. A custom articulated arm and sterile drape permit translation to the clinic. Results: The final light source/image capture head has a radius of 7.5", is 9" high, and weighs less than $9 \mathrm{lbs}$. An umbilical cord consisting of two Firewire cables, a power cable, a control line cable, and cooling lines, connect the device to a portable cart that holds the computer, monitors, circulating chiller, and power sources. A total of 18 LED modules produce 20,9001x of white light, $1.7 \mathrm{~mW} / \mathrm{cm}^{2}$ of $670 \mathrm{~nm}$ light, and $8.6 \mathrm{~mW} / \mathrm{cm}^{2}$ of $760 \mathrm{~nm}$ light, at a 12 " working distance and with a $10-\mathrm{cm}$ field-of-view. The dual-band NIR fluorescence emission filter permits sequential acquisition of $700 \mathrm{~nm}$ and $800 \mathrm{~nm}$ fluorescence emission images from a single NIR camera, both of which are simultaneous with color video images. The imaging head is supported by a flexible arm that allows below horizontal through past vertical positioning of the head. The entire imaging head/support arm system is mounted on a movable stand that allows maximum horizontal and vertical adjustments for positioning the imaging system. The modular design of the LED light source and filters permits any set of NIR wavelengths to be used with the system. Conclusion: We have developed a lightweight, maneuverable, and inexpensive dual-channel NIR fluorescence system for image-guided surgery. This system should lower the barrier for adoption of the technology in outpatient clinics and developing countries.

\section{$\mathbf{J 5 8 7}$}

\section{THEORETICAL AND EXPERIMENTAL PREDICTION OF DETECTION SENSITIVITY IN OPTOACOUSTIC IMAGING OF MOLECULAR BIOMARKERS}

Daniel Razansky, John Baeten, Vasilis Ntziachristos

Technical University of Munich and Helmholtz Center Munich, Institute for Biological and Medical Imaging (IBMI), Neuherberg, Germany.

In the recent years optoacoustic tomography has evolved into a powerful imaging method with the capacity to visualize structural, functional and molecular information in intact diffuse tissues. Due to its scattering-free ultrasonic resolution, the method mitigates the common drawbacks of the optical imaging methods, which are plagued by the multiple scattering of light in tissue reducing imaging resolution and penetration depths. However, while the feasibility of different optoacoustic imaging implementations has been showcased, little is known on the sensitivity performance from a theoretical and systematic standpoint. Determination of the absolute detection limit, as it relates to the sensitivity of optoacoustic tomography in imaging small volumes of markers and lesions is not straightforward. This is due to the fact that experimental determination of the sensitivity remains difficult since no established technique exists so far to reproducibly create small lesions containing well-defined concentrations of marker. Here we use instead a theoretical analysis of optoacoustic signal generation and propagation in order to predict detection limits by imaging larger amounts of the marker and placing the measured value on the appropriate parameterdependent signal intensity curve. A performance estimate of the particular experimental system and the expected signal-to-noise-ratio for smaller amounts of markers can then be made. A major finding of this study is that while optoacoustic signals depend linearly on concentration, they exhibit a non-linear dependence not only as a function of target depth but also as a function of its volume. The steep attenuation of light causes a drop in the energy absorbed by targets as a function of depth. On the other hand, the resulting light energy deposition is decreasing with target volume, simultaneously reducing the detected optoacoustic signal as a function of square root of the deposited energy. It was further found that for small targets (in practice, less than $0.2-0.5 \mathrm{~mm}$ in size), effects of ultrasonic dispersion start playing increasingly dominant role in the reduction of the detected optoacoustic signal intensity, owing to increased attenuation of high frequency sound components. This important finding demonstrates that it would be inaccurate to linearly extrapolate the detection limits of optoacoustics from data obtained on larger lesions. All these effects create a complex estimation model on detection limits, which is defined also in the context of lesion size and depth, not only lesion concentration.

\section{$\mathbf{J 5 8 8}$}

DYNAMIC GAIN COMPENSATION ALLOWS QUANTITATIVE RECOVERY OF FLUORESCENCE TOMOGRAPHY INDEPENDENT OF TUMOR POSITION WITHIN THE ANIMAL Dax S. Kepshire ${ }^{1}$, Brian W. Pogue ${ }^{1,2}$, Frederic Leblond ${ }^{1}$

${ }^{1}$ Thayer School of Engineering, Dartmouth College, Hanover, NH, USA, ${ }^{2}$ Department of Surgery, Dartmouth Medical School, Hanover, $\mathrm{NH}$, USA.

Using fluorescence tomography to compare uptake of agents in orthotopic versus subcutaneous tumors requires an imaging approach which is sensitive to the large change in dynamic range of the signal, and an image recovery process which is verified to provide a flat field response. In many instances these two features are considered separate issues, but in this paper, we present results showing how the former can impact the latter issue. Data is used from a new fluorescence tomography system which couples to MicroCT imaging for use in rodent tumor studies. The system uses single photon counting PMT detectors, with an algorithm to pre-sample the signal and provide dynamic gain compensation. This signal sampling approach dramatically increases the effective dynamic range from the original level of three orders of magnitude, to allow up to six orders of magnitude in sensitivity. This greater signal to noise level then provides the reconstruction algorithm with superior ability to produce reconstructed images which have a flat field response, i.e. the ability to recover the same signal intensity independent of changes in position. This is a highly desirable feature in diffuse tomography, and challenging to achieve because of the highly ill-posed nature of the inversion problem. Dynamic gain compensation, produces a higher signal to noise level, which thereby results in a flatter field because the regularization parameter has more effect upon the recovered image. This approach is demonstrated in simulations and phantoms, as well as in animal data with orthotopic glioma models. The orthotopic models require this added feature, because the pathophysiological growth features of the tumor can be drastically different in orthotopic versus subcutaneous growth, because of the difference in the blood brain barrier properties. The system is used to analyze delivery of small molecular contrast EGF 
tagged with fluorescence, and if it is able to penetrate the blood brain barrier to gain localization in regions of low grade disease.

J589

\section{EXPERIMENTAL EVALUATION OF A MICROLENS ARRAY} BASED OPTICAL DETECTOR

Tobias Senkbeil, Daniel Unholtz, Mathies Breithaupt, Wolfhard Semmler, Joerg Peter

German Cancer Research Center (DKFZ Heidelberg), Heidelberg, Germany.

Objective: We have developed an optical detector intended for optical tomographic and multi-modal (MRI, PET) small animal molecular imaging applications. This detector is experimentally investigated regarding its optical imaging properties. Methods: A detector unit consists of two CMOS photodiode array image sensors, two microlens arrays and a septum mask. The sensors, each containing $512 \times 1000$ pixels, are arranged to form a total sensitive area of $25 \mathrm{~mm} \times 100 \mathrm{~mm}$. They are mounted in an aluminum housing and aligned to an integrated septum mask of $51 \times 200$ parallel boreholes. This mask prevents optical cross-talk and is important when used in dual-modal MRI operation as it shields, in combination with the aluminum housing, RF emissions interfering with the MRI system. The microlens arrays are mounted on top of the septum mask with the microlenses aligned to the subjacent boreholes. We determined the imaging performance in terms of spatial resolution and sensitivity, whereby two different data processing modes were investigated: center focal point extraction data, and the full set of sensor data undergoing a mathematical inverse mapping algorithm developed recently. Applying the slanted edge method (ISO 12233) the spatial frequency response was assessed for different object-detector distances, from which the spatial resolution was derived, respectively. Additionally, a sector star target was imaged for visual confirmation. In order to quantify the sensitivity of the detector at various distances, a calibrated light source was used. Results: The spatial resolution for center focal point extraction images corresponds to the microlens pitch $(480 \mu \mathrm{m})$. As expected, the mapping model yields higher spatial resolution for distances of up to $45 \mathrm{~mm}$. Furthermore, the model also improves sensitivity. Compared to a conventional optical camera (Hamamatsu Orca II-BT-512G) though, sensitivity and spatial resolution are inferior. All acquired images are impaired by light reflections in the boreholes of the septum mask. While this degrades spatial resolution to a certain degree, it primarily lowers image contrast. Discussion: Significant improvements in sensitivity and spatial resolution resulting from the employment of the mapping model justify its computational burden. The most important issue as a consequence of this study concerns the improvement of the comparatively low sensitivity. We are now incorporating a cooling system for the imaging sensors to improve the SNR. Spatial resolution, on the other hand, is sufficient for the intended use.

\section{$\mathbf{J 5 9 0}$}

CERENKOV LUMINESCENCE TOMOGRAPHY: A NEW APPROACH FOR IMAGING $\beta$-EMITTING RADIONUCLIDES IN SMALL ANIMALS

Changqing Li ${ }^{1}$, Robbie Robertson ${ }^{2}$, Gregory S. Mitchell ${ }^{1}$, Matthew Silva ${ }^{2}$, Simon R. Cherry ${ }^{1}$

${ }^{1}$ Biomedical Engineering department, University of California Davis, Davis, CA, USA, ${ }^{2}$ Imaging Sciences, Millennium Pharmaceuticals, Inc., Cambridge, MA, USA.

Cerenkov radiation is a well-known phenomenon in which optical photons are emitted by moving charged particles when they exceed the speed of light in a medium. We have observed optical photons emitted from $18 \mathrm{~F}$ and $11 \mathrm{C}$ solutions with a CCD camera and the optical photon intensity is proportional to the radionuclide activity. As predicted, the Cerenkov signal is also increased as the refractive index of the medium is increased. We have performed phantom and in vivo mouse imaging studies to demonstrate that measurements of these emitted optical photons on the mouse surface can be used to reconstruct the radiation activity distribution inside mice using Cerenkov luminescence tomography (CLT), a method similar to bioluminescence optical tomography, in which optical photon propagation in the tissues is modeled with the diffusion equation and the optical emission source is reconstructed iteratively with a preconditioned conjugate gradient method (PCG). Cerenkov imaging will allow a method to noninvasively image the location of both $\beta+$ and $\beta$ - emitting radionuclides, which may have important applications in monitoring radiotherapeutic compounds and also permits imaging of PET radiotracers with conventional optical imaging systems.

\section{J591}

ACCURATE DETERMINATION OF OPTICAL PROPERTIES OF SOLID PHANTOMS WITH TIME DOMAIN, FREQUENCY DOMAIN AND CONTINUOUS WAVE TECHNIQUES

Jean-Pierre Bouchard, Isabelle Noiseux, Jean-François Cormier, Michel Fortin, Pascal Gallant, Sébastien Leclair, Jocelyne Osouf, Ozzy Mermut

Biophotonics, INO, Quebec, QC, Canada.

Tissue phantoms of known optical properties are necessary for the standardization, testing and calibration of optical molecular imaging instrumentation. Solid tissue simulating phantoms offer the benefits of ease of use and long term stability of their optical characteristics. They are therefore the preferred tool for intensity and time/frequency response standardization. Reference phantoms should be accurately characterized in a manner that is laboratory and technique independent in order to provide a strong basis for collaborative work and instrumentation development. Recent inter-laboratory comparison of phantom characterization results have shown discrepancies between retrieved optical properties as high as $30 \%$. Absolute accuracy of the metrology of scattering and absorption coefficient of turbid media is therefore still an open subject. In order to address and assess the accuracy, we present a comparative study of three characterization methods: time-domain transmittance, frequency-domain transmittance and a continuous wave system based on total reflection and total transmission. Systematic error sources of each characterization method have been theoretically studied and corrected for in the data analysis when possible. A homogeneous tissue simulating phantoms set was fabricated and characterized using the three different measurement approaches. Error propagation for each measurement technique has been performed to quantify the level of agreement between the results. This level of intersystem agreement provides an estimation of the true absolute accuracy of the characterization.

\section{J592}

\section{DEVELOPMENT OF CALIBRATED FLUORESCENT PHANTOM FOR FLUORESCENT IMAGING}

Isabelle Noiseux, Jean-Pierre Bouchard, Jean-François Cormier, Michel Fortin, Pascal Gallant, Sébastien Leclair, Jocelyne Osouf, Ozzy Mermut

Biophotonics, INO, Quebec, QC, Canada.

In the field of fluorescence-based molecular imaging, development of optical standards and imaging phantoms with stable fluorescence properties and accurately characterized optical properties is necessary. Standards are largely used in scientific instrumentation; they are used in system calibration, inter-system comparisons, instrument development as well as for methodology implementation, to name a few. The goal of the phantom is to mimic the optical parameters of tissues, specifically their absorbance, luminescence and scattering properties. For fluores- 
cence imaging of tissues, determining the optical properties is critical for retrieving the true fluorophore concentration of embedded structures. Currently, there are no standard fluorescent phantoms with controlled scattering properties available. We fabricated solid fluorescent phantoms based on polyurethane. A variety of fluorophores were used to create fluorescent phantoms including CyDye Fluor, bright Alexa Fluor and Quantum Dots. The fluorophores were uniformly embedded inside the solid phantom matrix. It is observed that incorporation of fluorophore in the polyurethane matrix causes a decrease in the fluorescence emission. Hence, we define a method to obtain the fluorescence emission characteristics of the fluorophore at a precise tuned concentration. Lastly, we assess the optical stability of the fluorescence phantom by tracking the intensity over a period of more than 3 months. Fluorescent inclusions are incorporated into a matrix for which scattering and absorbance properties are well characterized and mimic the optical properties and luminescence of tissues.

\section{J593}

COMPUTATIONAL MODELS OF OPTICAL EXTINCTION MEASURE THE DEPTH OF FLUORESCENT MOLECULES EMBEDDED IN TISSUE AND IMPROVE QUANTIFICATION

Douglas O. Wood, Douglas Vizard, Sean P. Orton

Carestream Molecular Imaging, New Haven, CT, USA.

We have investigated the wavelength dependent extinction (absorption and scattering) of optical and near-infrared light in various tissue types using both real tissues and biophysically realistic phantoms. From these measurements we created parametric models of the extinction and incorporated the extinction model into our existing model for spectral unmixing. With the combined model, we can estimate the depth of the embedded fluorescent emission while at the same time separating the emission produced by different fluorescent molecules and by unwanted tissue auto-fluorescence. Our data was collected using a commercially available multi-modal, multi-spectral imaging system that takes an exciter-side approach to fluorescent imaging (i.e. varying the wavelength of the excitation while keeping the emission filter constant). Because normal tissues tend to have much greater extinction in the blue than in the red, we find that an exciter-side approach is better able to exploit the spectral effects of extinction than an emission-based imaging system. If a good model for the extinction properties of the tissue is known and it is a reasonably good assumption that the tissue is homogeneous, we find that including extinction in our unmixing model improves the quantification and the overall quality of our results.

\section{$\mathbf{J 5 9 4}$}

\section{QUANTITATIVE IN VIVO 3D FLUORESCENCE LIFETIME} IMAGING OF A LIVER-SPECIFIC AGENT

Guobin Ma, Dao Chao Huang, Marilyse Piché, Muriel Jean-Jacques, Simon Fortier, Mario Khayat;

ART Advanced Research Technologies, Inc., Montreal, QC, Canada.

The interest in small animal fluorescence imaging has increased considerably in the last decade thanks to increasing applications in the study of gene expression, metabolism, disease models, drug delivery, and treatment efficacy. One common issue encountered when performing quantitative in vivo imaging is the elimination of tissue autofluorescence. The other is the proper separation of free and bound fluorescent tags of biomarker that target specific organs or disease tissue. Using a time-resolved platform (Optix), we present a method to differentiate signal due to tissue autofluorescence by lifetime. We demonstrate the ability to distinguish fluorophore that is bound or detached from a biomarker. With time-of-flight information, we quantitatively recover the $3 \mathrm{D}$ distribution of the biomarker. The imaging agent used in the study is a liver-targeted biomarker. In the experiment, Cy5.5 tagged agent was administered to several mice through tail vein injection. The mice were then imaged using Optix at different time (range from 10 to $90 \mathrm{~min}$ ) after injection. Results show that the agent starts to accumulate in the liver region as early as $10 \mathrm{~min}$ post injection. 20 min later, there is some signal from the bladder region. However, based on fluorescence lifetime, this signal is different. The signal in the liver is from the Cy5.5 bound to the agent, while the signal in the bladder is from the unbound Cy5.5. Using our reconstruction tool, we recovered the 3D distribution of Cy5.5 in mice (Figure 1). Using lifetime information, it is found that at 60 minutes post injection, the overall percentage of Cy5.5 bound to the agent, free Cy5.5, and tissue autofluorescence are respectively 52\% (liver), 39\% (bladder), and $9 \%$ (other region) in mouse body. In summary, these results demonstrate that, through the use of lifetime and 3D analysis, time-domain in-vivo fluorescence imaging can provide a quantitative tool to assess metabolism and accurately track the distribution of therapeutic agents in time.

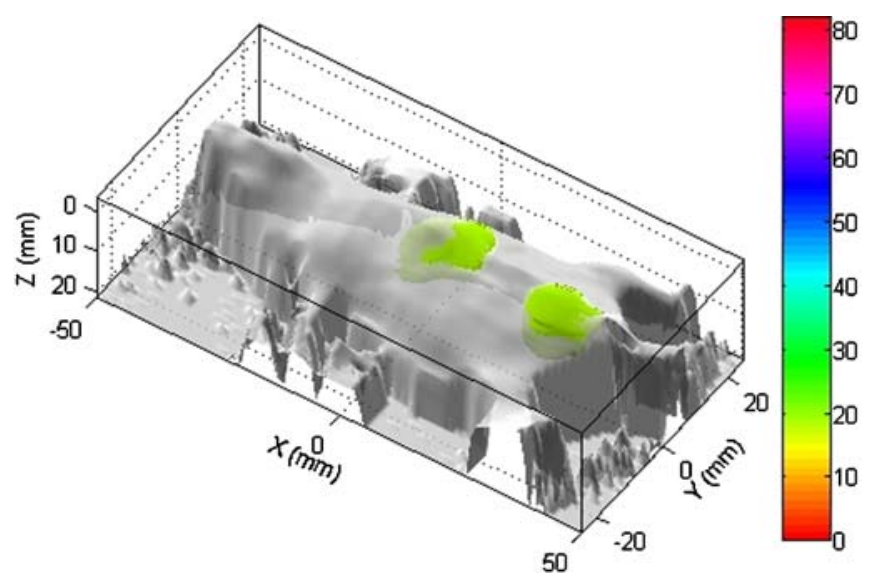

FIg. 1. Recovered 3D distribution of Cy5.5 in mouse 4 based on measurement $60 \mathrm{~min}$ after injection of liver-targeted agent tagged with Cy5.5.

\section{J595}

SURFACE PROFILING FROM SHAPE, SHADOW, AND SHADING FOR SMALL ANIMAL OPTICAL TOMOGRAPHY

Gregory S. Mitchell ${ }^{1}$, Changqing $\mathrm{Li}^{1}$, Peter Harvey ${ }^{2}$, Richard Levenson ${ }^{2}$, Simon R. Cherry ${ }^{1}$

${ }^{1}$ Biomedical Engineering, University of California, Davis, Davis, CA, USA, ${ }^{2}$ Cambridge Research \& Instrumentation, Inc., Woburn, MA, USA.

In small animal optical tomography, surface profiling is necessary in order to obtain a finite-element representation of the animal. This representation is used to create a subject-specific forward model of light propagation, and thus to relate the observed surface distribution of emission light to the distribution of internal sources. We present a simple and fast method of obtaining a mm-precision surface profile of a mouse in our tomographic optical imaging system. The system has four mirrors in a pyramidal geometry around the transparent and horizontal animal bed, which allows the camera, located on the axis of the pyramid, to obtain four simultaneous views of the animal. Two projections of the animal visual hull are given by white light images with broad illumination. Light-emitting diodes at a variety of angles allow surface points to be extracted from the two views which observe the transition from light to shadow at each axial location. Additional surface points are obtained by interpolation using shape from shading techniques. In our system the mouse is stationary and there are no moving parts. Calibrated surface profiles are obtained for phantom 
objects and both nude and fur-covered mice. The surface profile can eventually register to and warp a segmented atlas of a representative animal; the warped atlas can then be used to introduce non-uniform optical properties into the forward model.

J596

CONTINUOUS, QUANTITATIVE, MOLECULAR MONITORING OF A NEAR INFRARED FLUOROPHORE USING A NOVEL, MICROFABRICATED, IMPLANTABLE BIOSENSOR

Natesh Parashurama ${ }^{1}$, Thomas D. O'Sullivan ${ }^{2}$, Adam de la Zerda ${ }^{1}$, Ofer Levi ${ }^{3}$, James S. Harris ${ }^{2}$, Sanjiv S. Gambhir ${ }^{1}$

${ }^{1}$ Department of Radiology, Molecular Imaging Program at Stanford, Stanford University, Stanford, CA, USA, ${ }^{2}$ Electrical Engineering, Stanford University, Stanford, CA, USA, ${ }^{3}$ Institute of Biomaterials and Biomedical Engineering and Department of Electrical and Computer Engineering, University of Toronto, Toronto, ON, Canada.

Current approaches to detect fluorescence in anesthetized subjects utilize CCD cameras and generally take snapshots of a particular molecular process. Alternatively, continuous molecular monitoring in a freely moving subject would be useful in preclinical disease models for monitoring drug delivery, stem cell growth, and metastases. With these objectives in mind, we fabricated a novel cylindrical device $(8 \mathrm{~mm}$ diameter) containing a $1.5 \mathrm{~mW}, 670 \mathrm{~nm}$ vertical-cavity surface-emitting laser (VCSEL), un-cooled Gallium Arsenide photodiode, an integrated collimation lens, and a commercially available fluorescence emission filter. The field of view was $(3 \times 3 \times 3 \mathrm{~mm})$, and for Cy5.5 dye, the in vitro sensitivity was $100 \mathrm{nM}(\mathrm{R} 2=0.891)$, with signal saturation at $30 \mu \mathrm{M}$. The in vivo sensitivity curve was linear $(\mathrm{R} 2=0.856)$ and correlated well with $\mathrm{CCD}$ camera data $(\mathrm{R} 2=0.99)$, and the in vivo sensitivity was $1 \mu \mathrm{M}$. We then utilized this device in a glioblastoma (U87 cell line) tumor xenograft model in nude mice in which Cy5.5RGD specifically binds to integrin receptors on tumor cells. After tailvein injection of Cy5.5-RGD $(50 \mu \mathrm{M}$ in $50 \mu \mathrm{l})$, the signal was background corrected, and signal to noise ratio was $23.69 \pm 2.73$ in tumors and $7.59 \pm 1.39$ in non tumor tissue. This data was statistically significant $(P<0.018, N=2$ mice $)$. This agreed with the specificity of RGD-Cy5.5 uptake in tumors versus control tissue. Furthermore, we were able to continuously monitor probe uptake, and compare kinetics between mice. We found that the $90 \%$ of the max signal occurs at $13.27 \pm 4.11 \mathrm{~min}(N=3$ mice $)$. Lastly, we demonstrated intraperitoneal (IP) injection of the Cy5.5-RGD (50 $\mu \mathrm{M}$ in $50 \mu \mathrm{l})$ resulted in a delayed $90 \%$ of the max signal (approximately 55 minutes) when compared to tail vein injection (approximately $13 \mathrm{~min}$ ). This is the first demonstration of using a VCSEL for the detection of a molecular probe and is a proof-of-principle that should allow expansion of this technology for implanting sensors in freely moving subjects. a
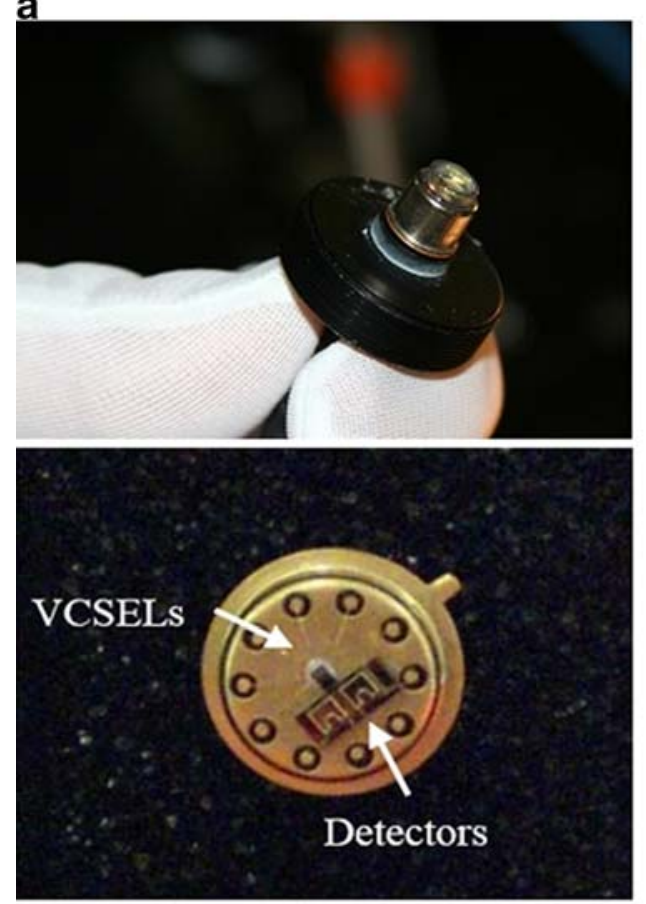

b

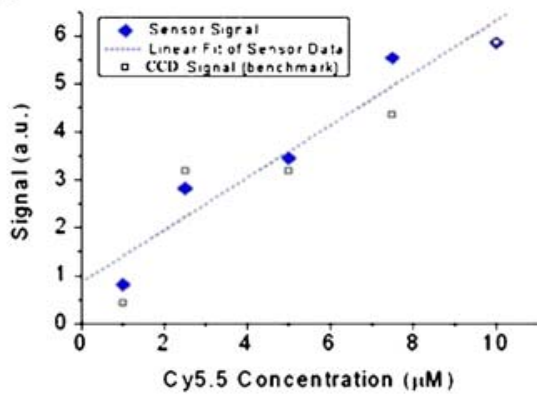

C

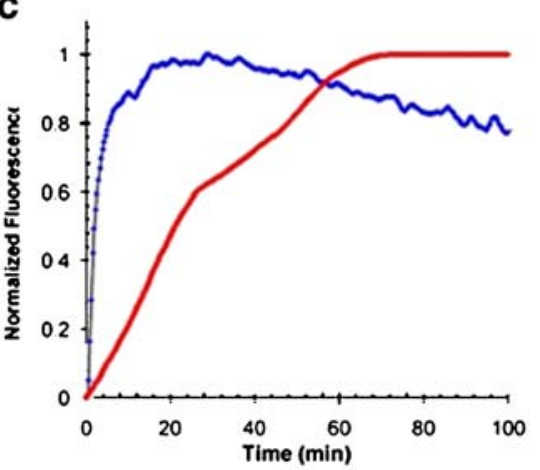

Figure 1. Integrated biosensor for continuous sensing a) Top Panel- The VCSEL (vertical cavity surface emitting laser) and detector integrated sensor device Bottom Panel-The microfabricated in integrated sensor design with laser and detector on same chip c) The sensor detecting Cy5.5 dye in a live mouse d) Kinetic sensing data demonstrating increased signal after IV injection of RGD-Cy5.5 for two different sensors within one tumor, and control site away from tumor. 
J597

\section{MEASURING MOLECULAR MOBILITY WITH MACRO FLUORESCENCE IMAGING}

Claudia Y. Lee ${ }^{1}$, Andrew Zepfel ${ }^{2}$, Sean R. Gallagher ${ }^{1}$

${ }^{1}$ UVP LLC, Upland, CA, USA, ${ }^{2}$ The Vaccine Development Institute, Pitzer College, Claremont, CA, USA.

We demonstrate that molecular mobility can be measured by macro imaging with steady state fluorescence anisotropy. A standard macro imaging system with overhead UV lamp, emission filter and a cooled CCD camera with additional linear polarizers can perform fluorescence anisotropy macro imaging (FAMI). The FAMI system produces results comparable to other anisotropy instruments on top of the capability to perform macro imaging which had been frequently applied to quantify molecular expression in vivo. We applied this technique to examine GFP in live plants and observed systematic differences in different areas non-invasively.

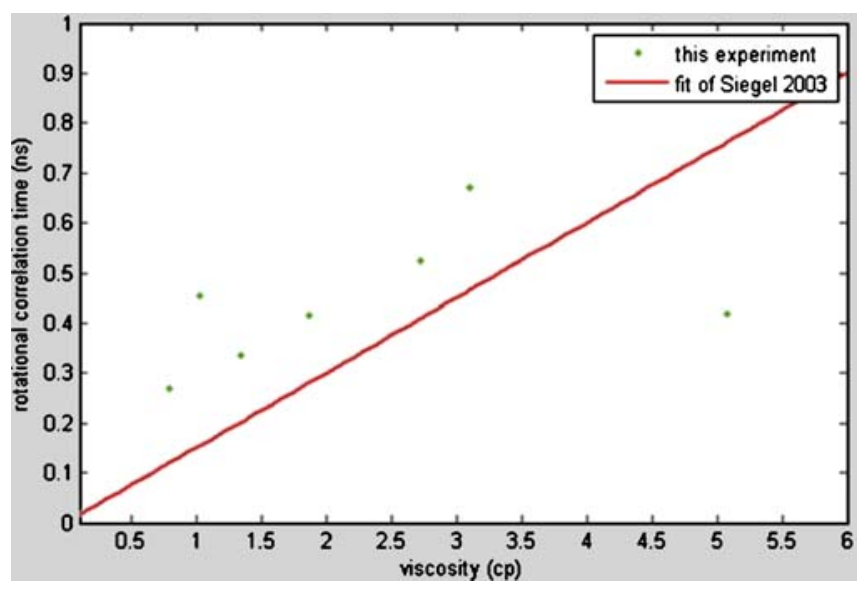

Mobility of fluorescein molecules in 7 drops of various glycerol concentration a $10 \times 8 \mathrm{~cm}$ plane
J598

\section{IN VIVO IMAGING OF A UV-EXCITABLE FLUORESCENT AGENT IN A HUMAN TUMOR MODEL UTILIZING CERENKOV LUMINESCENCE IMAGING}

Matthew Silva ${ }^{1}$, Robbie Robertson ${ }^{1}$, Simon R. Cherry ${ }^{2}$,

Melissa Germanos

${ }^{1}$ Imaging Sciences, Millennium Pharmaceuticals, Inc., Cambridge, MA, USA, ${ }^{2}$ Biomedical Engineering and Center for Molecular and Genomic Imaging, University of California Davis, Davis, CA, USA.

The in vivo use of UV-excitable fluorophores, such as those used for time-resolved fluorescence (TRF), is limited by the complications for probe excitation. Specifically, the tissue penetration limitations of UV lead to poor excitation of agents, such as lanthanides. These probes are of interest, however, due to the large Stokes shift and the minimization of background autofluorescence. Our laboratory has discovered an alternative method of excitation by utilizing the discovery of Cerenkov Luminescence Imaging (CLI). Briefly, high energy positron emitters, including $18 \mathrm{~F}$, have a velocity transiently exceeding the speed of light in a medium, resulting in the emission of Cerenkov radiation, a continuous spectrum of radiation weighted toward to the UV-blue bands. We utilized the Cerenkov radiation to excite a Europium III [Eu(III)] quantum dot (RadiantDOTTM, BioPal, Inc., Worcester, MA) in vivo after intratumoral injection of the probe. This technique by-passes the need for external excitation while allowing for real-time imaging of UV-excitable or TRF targeted probes. First, an experiment was conducted comparing wells of $425 \mu \mathrm{Ci}$ [18F]FDG with and without $10 \mu \mathrm{L}$ RadiantDOT $(\sim 50 \mu \mathrm{g}$ $\mathrm{Eu}(\mathrm{III}))$. Using $20 \mathrm{~nm}$ filters, images were acquired at 580, 600, 620 , and $640 \mathrm{~nm}$. The signal from the $620 \mathrm{~nm}$ band (610$630 \mathrm{~nm})$ had a 15 -fold increase in signal in the [18F]FDG + RadiantDOT sample; whereas the other bands had a $1.6 \pm 0.6$-fold increase. These results are consistent with the fact that the $\mathrm{Eu}(\mathrm{III})$ probe is excited at $310 \mathrm{~nm}$ by the Cerenkov radiation and emits at $615 \mathrm{~nm}$. Below are the data from a proof-of-concept experiment demonstrating in vivo imaging of the RadiantDOT in a SCID mouse bearing a lymphoma xenograft. $100 \mu \mathrm{L}$ of RadiantDOT was injected $\sim 5 \mathrm{~mm}$ intratumorally, and the animal was injected $\mathrm{i}$. v. with $300 \mu \mathrm{Ci}[18 \mathrm{~F}]$ FDG. After 20 minutes of distribution, the animal was scanned (IVIS 200, Caliper Life Science, Alameda, CA). Due to abstract limitations, we are only showing the bioluminescence image that was acquired (left). Note that the cell line is not luciferase expressing and no external fluorescence excitation is used. On the right, the excised tumor is shown; note the focal luminescence emanating from a region measure at $5.6 \mathrm{~mm}$ from the surface.
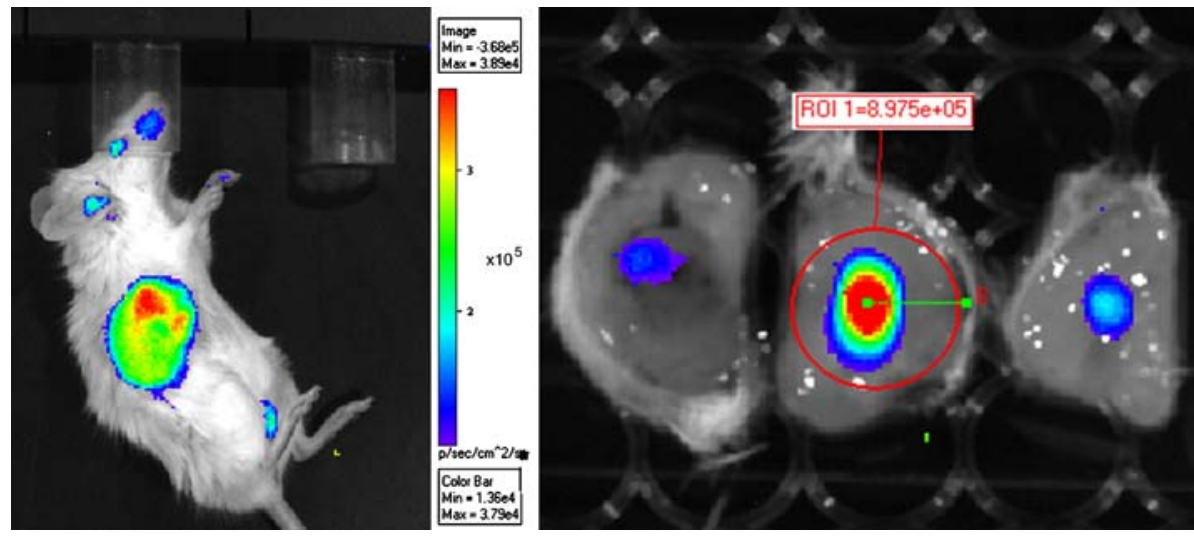


\section{J599}

\section{TRI-MODALITY IMAGING: BIOLUMINESCENCE, FLUORESCENCE, AND PLANAR X-RAY}

Jay Whalen, David Nilson, Victor Ninov, Heng Xu, Brad Rice, Ed Lim, Ning Zhang

Caliper Life Sciences, Alameda, CA, USA.

Imaging of bioluminescent reporters, fluorescent proteins, and nearinfrared (NIR) probes in pre-clinical animal models has become a powerful tool for the study of disease. While optical imaging provides exquisite sensitivity and specificity to molecular targets, it typically lacks anatomical information due to the scattering of light in tissue. Combining optical molecular imaging with anatomical planar x-ray helps overcome this limitation. We have developed a high sensitivity instrument that is optimized for in vivo detection of both fluorescent and bioluminescent probes. The system includes a cooled (-90C) CCD camera, light-tight imaging chamber, extensive filter capabilities, and complete computer automation. A moveable CsI scintillator plate has been added to provide planar x-ray images. The same high-sensitivity CCD camera is used to image both the scintillator plate and the optical signals emitted from the subject, so co-registration of the images is straightforward. The x-ray imaging time is rapid $(<10 \mathrm{~s})$ resulting in a low animal dose of $\sim 1 \mathrm{mGy}$ per image. Several animal models have been evaluated to test the system. In an orthotropic mammary fat pad 4T1-luc tumor model, we injected an Avastin/CF750 infrared probe to demonstrate coregistration of bioluminescence, fluorescence, and x-ray in the same system. Further, several contrast agents have been studied including, Aurovist, where vasculature in the body and tumor is visible. Finally, overlaying the $\mathrm{x}$-ray component with fluorescent and bioluminescent data will elucidate the source location by coupling the radiance emitted from the surface to the skeletal and soft tissue of a small animal.

\section{$\mathrm{J} 600$}

TAKING A CLOSER LOOK: VALIDATING MACROSCOPIC OPTICAL IMAGING RESULTS WITH MICROSCOPY

James Mansfield $^{1}$, Richard Levenson ${ }^{1}$ ${ }^{1}$ CRi, Woburn, MA, USA, ${ }^{2}$ Bioengineering, Rice University, Houston, TX, USA.

In vivo optical imaging is a valuable technique for monitoring the distribution of proteins and other biomarkers in living animals quickly and effectively. However, a critical and sometimes overlooked component of the method development of these experiments is the validation of the results obtained in vivo from intact animals through some other, complementary, technique. Of the two major small animal optical imaging modalities, bioluminescence and fluorescence, only the latter offers the capability to easily validate in vivo results through ex vivo or in situ microscopic imaging. Near-IR-labeled antibodies are widely imaged in vivo to determine the biodistribution of a protein or as a means of quantitating tumor size non-invasively. However, as these probes can sometimes bind to necrotic regions in tumors or get trapped in interstitial spaces, it is important to ascertain their biodistribution with appropriately high resolution in order to qualify the in vivo results. In vivo fluorescence data from whole animals and from whole organs, both in situ and ex vivo, were compared to the corresponding microscopic images from the same animals. In one model, AF647labeled anti-EGFR antibodies were injected into mice with subcutaneous tumors and imaged. In another model, fluorescent images of H\&E tissue sections from excised tumors from mice that had been injected with Cy5-labeled anti-Her2 were acquired using with multispectral microscopy to generate simulated brightfield images, which resemble standard DAB-stained immunohistochemistry samples that can be readily interpreted by pathologists. Microscopic imaging indicated that the labeling pattern was often consistent with specific binding to the corresponding antigen. However, in some instances, binding of the label to necrotic regions was demonstrated, affecting the validity of whole-tumor intensity estimations: strong labeling in some tumors could be shown to be due to prominent binding to necrotic regions rather than to viable tumor. In vivo tumor intensity signals, however, agreed closely with ex vivo whole-organ results. In vivo imaging, while useful and practical, requires careful validation of the model system being imaged to ensure correct interpretation of results. Two good methods of validation are the imaging of tissues, either in situ or as whole, excised organs, and the high-resolution imaging of tissue sections on a microscope. Of the popular optical imaging modalities, only fluorescence imaging is readily amenable to such validation.

\section{J601}

\section{OPTIMIZATION OF SIGNAL-TO-NOISE RATIO} AND STABILITY OF A CELL-FREE LUCIFERASE ASSAY Carola Heneweer $^{1,2}$, Marleen Keyaerts ${ }^{1,3}$, Ronald G. Blasberg ${ }^{1}$ ${ }^{1}$ Neurology and Radiology, Memorial Sloan Kettering Cancer Center, New York, NY, USA, ${ }^{2}$ Clinic for Diagnostic Radiology, University Hospital Schleswig-Holstein, Campus Kiel, Kiel, Germany, ${ }^{3}$ In Vivo Cellular and Molecular Imaging Laboratory, Vrije Universiteit, Brussel, Belgium.

Bioluminescence imaging (BLI) provides immense sensitivity. This requires special attention to avoid high background noise, especially in cell-free systems. Aim: To optimize signal-to-noise ratios (SNR) and stability of a cell-free BLI assay. Methods: The buffer used contained HEPES, $\mathrm{CaCl}_{2}$ and $\mathrm{MgSO}_{4}$, ATP and Firefly luciferase (Luc). In positive samples D-luciferin (lcn) was added. Negative controls were measured in the absence of $1 \mathrm{cn}$. The effect of stabilizing agents such as tergitol, prionex and DTT on the SNR was tested. The assay was compared when performed under sterile (SC) and non-sterile (NSC) conditions. BLI signals were measured using a CCD camera. Results: High BLI signals $\left(110^{6} \mathrm{ph} / \mathrm{s} / \mathrm{sr}\right)$ were found in negative controls under NSC. They were dependent on ATP, $\mathrm{MgSO}_{4}$ and Luc and significantly reduced by $95 \%$ under SC. Camera background was $610^{3} \mathrm{ph} / \mathrm{s} / \mathrm{sr}$. BLI signals under NSC yielded $29 \%$ of SC signal intensities for positive controls. This resulted in a 20 -fold higher SNR for SC compared to NSC. Signals of negative controls decreased more under NSC than SC (fig A). Positive controls decreased to $0.04 \%$ (NSC) and $8 \%$ (SC) resulting in a 500-fold higher SNR for SC compared to NSC after $4 \mathrm{~h}$ of incubation (fig A). Addition of stabilizing agents entailed higher signals for positive controls staying stabile for at least $10 \mathrm{~h}$ for both SC and NSC (fig B). After $21 \mathrm{~h}$, BLI signals decreased by $86 \%$ for SC and $99.94 \%$ for NSC. Negative controls diminished within the first $4 \mathrm{~h}$ to $1 \%$ (SC) and $8 \%$ (NSC) compared to initial signals. The highest SNR for SC was reached between $4 \mathrm{~h}$ and $10 \mathrm{~h}$ which was up to 1000 -fold higher than SNR for NSC (fig B). With stabilizing agents, SNR increased 9-fold under SC and 4-fold under NSC compared to buffers without stabilizing agents. Conclusion: Working under SC dramatically increases SNR, mainly by reducing background signals due to non-specific luciferase activity. Addition of stabilizing agents results in further increase of SNR, and provides stabile assay conditions for at least $10 \mathrm{~h}$. 
a Time profile for buffers without stabilizing agents

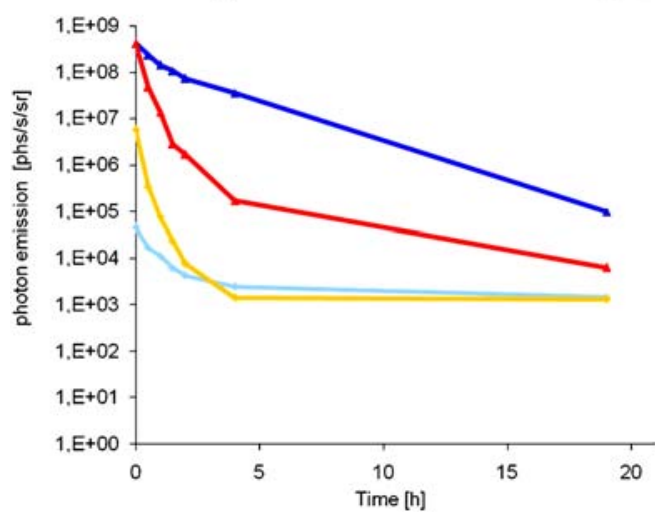

$\mathbf{J 6 0 2}$

\section{DEVELOPMENT OF A PHYSIOLOGICALLY-BASED PHARMACOKINETIC MODEL FOR INTRAMOLECULARLY-QUENCHED FLUOROGENIC MOLECULAR BEACONS \\ Theresa M. Mawn, Anatoliy V. Popov, Jim Delikatny}

Radiology, University of Pennsylvania, Philadelphia, PA, USA.

One major factor limiting the development of activatable molecular beacons is quantification, i.e. accurately determining the degree of probe activation against a background of absorption and excretion. As the activated fluorescent probe is re-circulated, the overall increase in background signal leads to inaccurate measurements of activated probe. In order to overcome some of these limitations and extract more quantitative results, we developed a physiologically-based pharmacokinetic (PBPK) model to describe how Pyro-PL-BHQ, a phospholipase probe designed and synthesized by our group, is absorbed, distributed, metabolized and b

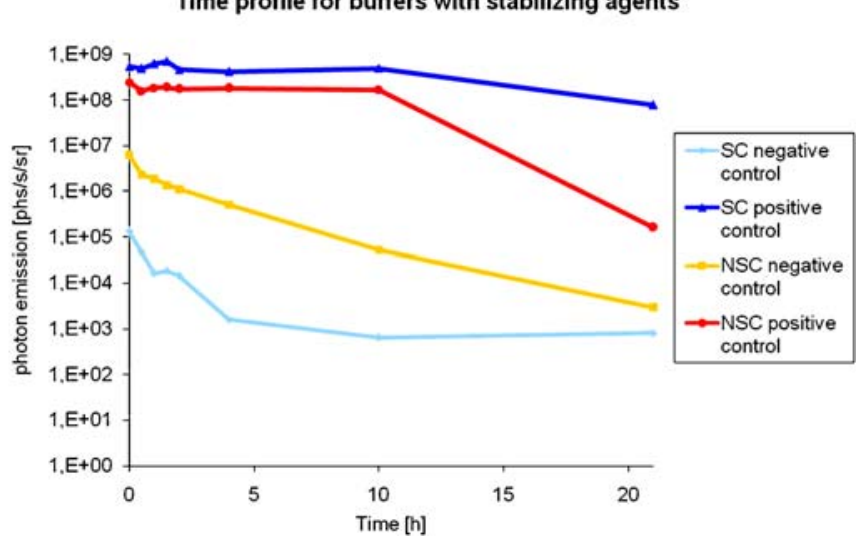

excreted in a tumor xenograft mouse model. This model is designed to allow future synthesized probes to be quantitatively compared for their efficiency as in vivo molecular beacons. The PBPK model consists of 4 compartments: tumor, periphery, liver/gastrointestinal (GI), and blood. The periphery and tumor compartments are considered diffusion-limited. Elimination occurs via the liver/GI as a saturable process. The derived set of mass balance equations was solved by fitting simulated data to actual in vivo fluorescent metabolite measurements in order to determine the rate processes involved and the amount of substrate (parent and metabolite) in each tissue compartment over time. The simulations show that Pyro-PL-BHQ is rapidly absorbed and metabolized in the tumor to fluorescent product and quickly returned to the system. However, only a minimal amount of fluorescent metabolite is reabsorbed by the tumor; the majority is absorbed by the periphery. Thus, the fluorescence observed in the tumor, according to these simulations, is largely due to the concentration of the metabolite in the tumor blood compartment. By $24 \mathrm{~h}$, the majority of the fluorescent metabolite was being excreted by the liver/GI compartment.
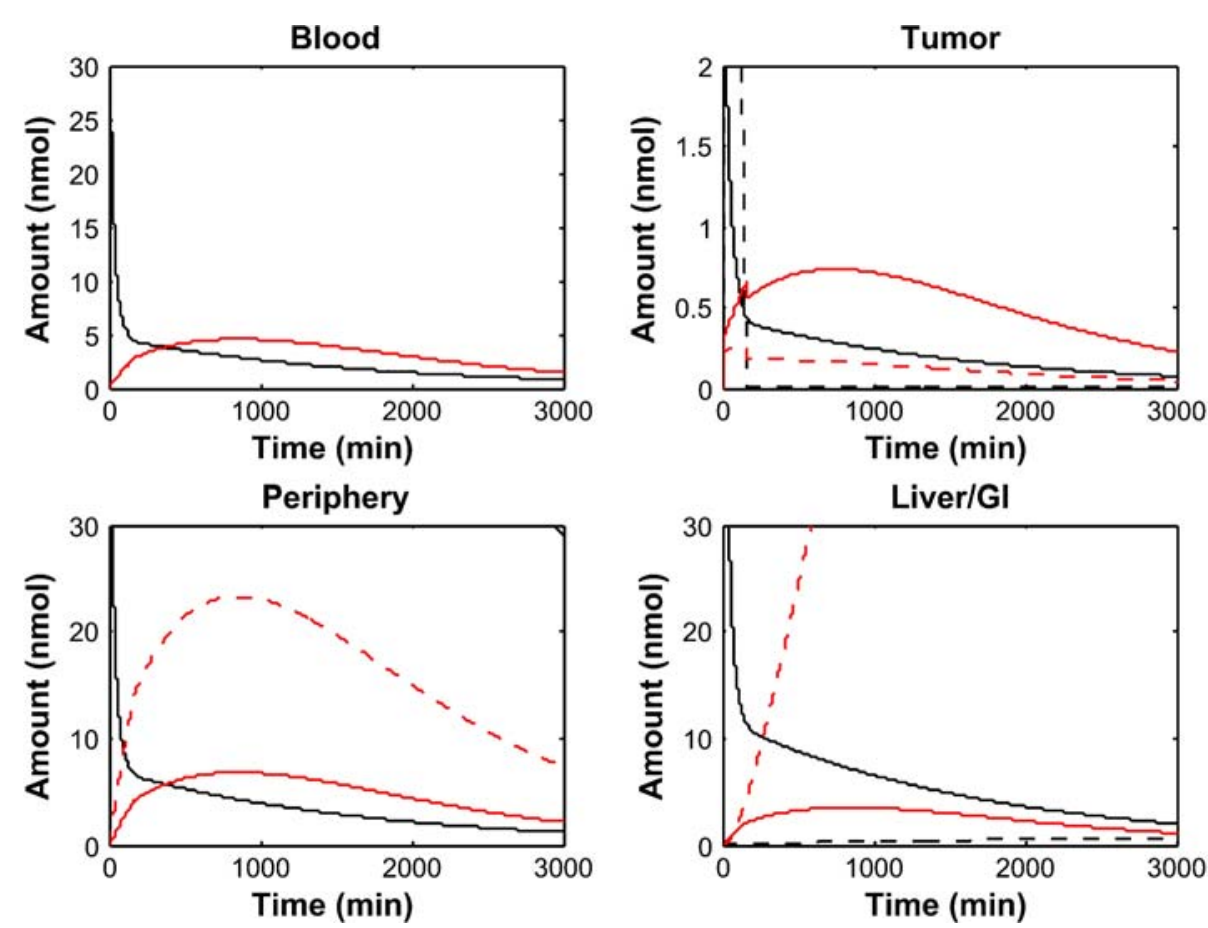

Figure 1 Model simulations of Pyro-PL-BHQ parent (black) and metabolite (red) distribution in tissue blood (solid) and cellular matrix (dashed) compartments. 


\section{$\mathrm{J} 603$}

\section{A MODEL OF IMAGE PROCESSING FOR BIOLUMINESCENCE IMAGING TECHNOLOGY}

Zhijun Chang, Jie Tian, Min Xu, Chenghu Qin, Dan Liu, Kai Liu Medical Image Processing Group, Institute of Automation,CAS, "Beijing,100190", China.

Imaging of small animals has become an important tool for biomedical research at the anatomical, functional, cellular and molecular levels. To study a small animal using molecular imaging techniques, the animal organ/tissue is typically transfected with a reporter gene in a viral promoter, and the image processing plays an important role in emerging bioluminescence imaging (BLI) technology. The bioluminescent image has four key features: Weak signal, Fuzzy edges, Low SNR and Poor visualization. We developed an effective image processing model of the bioluminescent image according to its key features. The model starts with initializing a bioluminescent image and a background image, and they must be coincident, and in this section, We designed a special data structure to store the image information and to meet the necessary of repeating operation. Then the initial data will be eliminated noise, enhanced, segmented, added pseudo-color. At last, the model overlays the background image and the bioluminescent image. Eliminating noise which mainly comes from system error can effectively improve the SNR. It is necessary to enhance image in the model because of weak signal character of the bioluminescent image. The goal of segmenting is to recognize the region of interesting (ROI) in the bioluminescent image. To overlay two images makes us can obtain the accurate position of ROI, thus we can ensure the focus in the body of the small animal. According to the calibrated parameter which we got from the calibration experiment used the integrating sphere (Labsphere company), we can quickly convert the gray value to the photon density. The library of algorithms of different section of the model of image processing can be easily extended by the all-purpose data structure. The proposed mode efficiently achieves the target and lays the foundation for the further analysis(e.g Fig.1). We believe that the bioluminescence imaging technology will be applied in more fields.
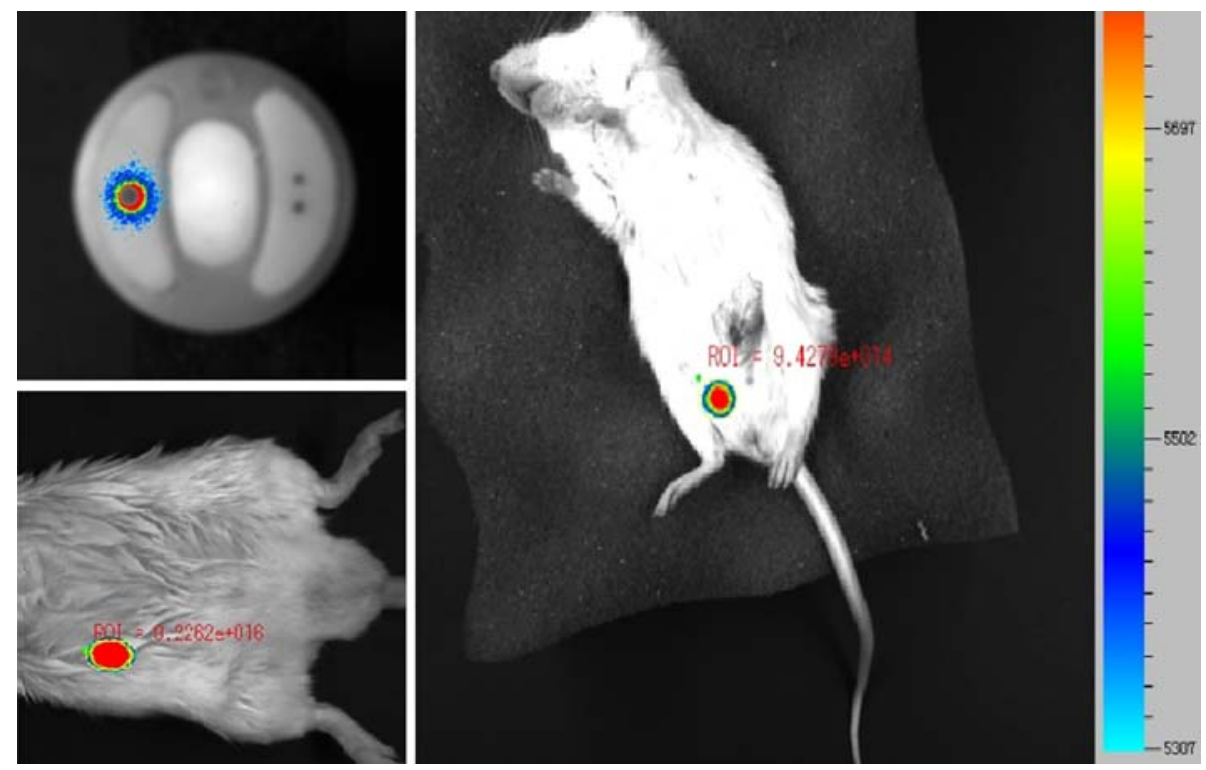

Fig.1 Test rusults of proposed mode

$\mathbf{J 6 0 4}$

\section{IMPROVED SENSITIVITY OF DETECTION WITH TRANSMISSION NORMALIZED FLUORESCENCE IMAGING}

Heng Xu, Ning Zhang, Dan Ansaldi, Ed Lim, Chaincy Kuo, Brad Rice Caliper Life Sciences, Alameda, CA, USA.

In vivo fluorescence imaging constantly faces the challenge of autofluorescence background when detecting a fluorescence moiety in animal tissue. This problem is further exacerbated when detecting deeptissue probes with an EPI-illumination geometry where the tissue autofluorescence background can be overwhelming. Transillumination (TRANS) fluorescence imaging is generally a superior approach for detecting NIR fluorophores in deep tissue. The improved sensitivity of detection is largely due to decrease in autofluorescence background when emitted fluorescence signal from the target is captured from the opposite side of the illumination light source. However, TRANS fluorescence imaging still poses challenges for target detection, as illumination in the TRANS mode may generate a background signal pattern similar to that of the fluorescence moiety target, which obscures the delineation of the target from the background. On the other hand, the reverse correlation between the distance of excitation light source and the brightness of fluorescence emission makes optimal fluorescence detection dependent on fine positioning adjustment of the light source. Furthermore, tissue heterogeneity as well as animal geometry regulate photon migration and pose additional challenges to both interpretation and quantification of the data. In this study, we introduce a new imaging and data processing algorithm to circumvent the limitations of both EPI and conventional TRANS fluorescence imaging. This involves a raster scan of a subject with transillumination light at the emission wavelength (no excitation filter) to establish a reference image in the scanned area of interest. A subsequent raster scan with an excitation light that is optimal for the fluorescence target generates a second image. The fluorescence image is then divided by the transmission image to produce a transmission normalized fluores- 
cence (TNF) image with improved sensitivity of target detection. We applied this algorithm to a mouse cadaver phantom model and showed improved detection of several fluorescence dyes implanted into the thoracic cavity, achieving $\sim 10$ picomole sensitivity for infrared dyes and sub picomole sensitivity for quantum dots. We further tested this algorithm in a lung tumor model and demonstrated the detection of lesions targeted by a fluorescence probe. Thus, this innovative TNF algorithm provides the means to observe deep tissue lesions beyond superficial detection. Moreover, TNF algorithm can be incorporated to the fluorescent tomography to provide improved $3 \mathrm{D}$ reconstruction.

\section{$\mathbf{J 6 0 5}$}

\section{A DEPTH-RESOLVED FLUORESCENCE LIFETIME IMAGING TECHNIQUE FOR SMALL ANIMALS}

Kenneth M. Tichauer ${ }^{1}$, Mamadou Diop ${ }^{1,2}$, Keith St. Lawrence ${ }^{1,2}$, Ting-Yim Lee L,3 $^{1,3}$

${ }^{1}$ Imaging Division, Lawson Health Research Institute, London, ON, Canada, ${ }^{2}$ Medical Biophysics, University of Western Ontario, London, ON, Canada, ${ }^{3}$ Imaging Laboratories, Robarts Research Institute, London, ON, Canada.

The development of fluorescent molecular imaging probes that absorb and emit light in the near-infrared - where tissue absorption is lowhas made it possible to do whole-body molecular imaging of small animals. This abstract presents a novel technique for imaging depth and lifetime (decay) of fluorophores in-vivo with a fluorescence lifetime imaging (FLI) machine (eXplore Optics, GE Healthcare). Such information can localize fluorescent inclusions, resolve contributions from multiple probes based on differences in lifetime, and provide information about the surrounding tissue, since lifetime of some fluorophores change with microenvironmental factors such as $\mathrm{pH}$. The shape of the time-resolved signal arising from a fluorophore inclusion is dependent on the width of the excitation pulse, the absorption and scattering properties of the tissue, the depth of the fluorophore, and the lifetime of the fluorophore. The algorithm we developed attempts to decompose time-domain data from multiple fluorophores at multiple depths into a weighted sum of terms each pertaining to fluorescence with a specific lifetime from a fluorophore at a specific depth. The purpose of this abstract is to demonstrate that if the absorption and scattering properties of the tissue are known then multiple lifetimes from fluorophores at multiple depths can be resolved both theoretically and in a phantom. Simulated data was produced using the solution of the time-domain diffusion equation for light propagation from a fluorescent inclusion in tissue. Two fluorophores (one with a lifetime of $0.5 \mathrm{~ns}$ and another with a lifetime of $1.0 \mathrm{~ns}$ ) were modeled at various depths. When the simulated data was analyzed it was found that as long as the signal to noise ratio was greater than $100: 1$, more than $95 \%$ of the signal could be attributed to terms with the correct lifetime and depth. The fitting becomes highly unpredictable under conditions of low signal to noise, which is directly proportional to the intensity of the laser and the duration of the scan. In addition, a phantom with known optical properties was imaged with the eXplore Optics machine. There were three inclusion of the fluorescent dye, Cy5.5 (Amersham, GE Healthcare): two at $2 \mathrm{~mm}$ depth and one at $5 \mathrm{~mm}$ depth. The lateral diameter of the inclusions was $5 \mathrm{~mm}$. Greater than $90 \%$ of the signal was found in the expected voxels. The majority of signal outside of the expected voxels formed predictable cones about the inclusions, probably owing to the diffusion of signal into adjacent voxels suggesting that filtering may be used as a correction.
J606

\section{REGULARIZATION TECHNIQUES FOR LUMINESCENT SOURCE SHAPE RESOLUTION IN OPTICAL TOMOGRAPHY Chaincy Kuo, Brad Rice, Qing Feng}

Biophotonic Imaging R\&D, Caliper Life Sciences, Alameda, CA, USA.

3D localization and quantification of luminescent sources in living tissue are sought for small-animal imaging, in which 2D planar data images of surface radiance are acquired in a single viewing angle. The photon propagation is approximated as a diffuse process for relevant wavelengths in a homogeneous medium. Closed-form analytical solutions for source intensity and location using this model are not available, therefore the parameters of interest are determined using inverse techniques for the ill-posed problem comprised of a Green's function kernel. Incorporating image data analogous to multi-angle projection data improves resolution for source localization and intensity by reducing non-uniqueness. In the bioluminescent case, image data acquired through a number of narrow-bandwidth filters spanning the luciferase emission spectrum offers the discriminating information analogous to projection data. For fluorescence tomography involving transillumination data, the excitation photon injection position in tissue is varied, while emission photon data collection position at the opposing tissue boundary remains the same. In this work, we demonstrate that luminescent sources with extended shape can be resolved by addressing the ill-posedness of the inverse problem using regularization techniques and by improving the tissue boundary delineation (Figure 1). Regularization is imposed here by biasing parameters with low kernel sensitivity towards zero intensity. We will also show that absolute tomographic quantification of source intensities in units of cell numbers and picomoles for luciferase/fluorescent protein cell lines and fluorophore compounds, respectively, is possible with calibration in the imaging instrument. Experiments are first performed on mouse-shaped tissue phantoms and in-vivo studies with calibrated sources for algorithm validation.
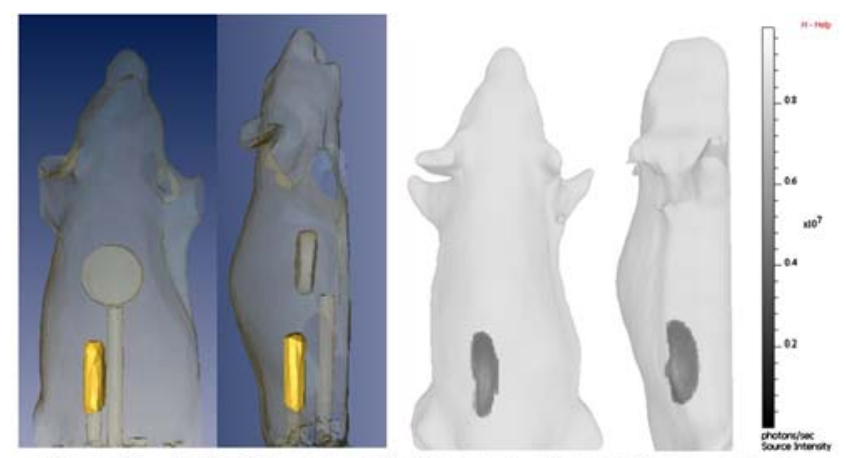

Figure 1. Left: CT segmented mouse-shaped tissue phantom with luminescent material filling hollow rod bore, rendered as bright surface. Disk and secondary rod hollows are visualized as well. Right: Phantom surface determined by structured light analysis in white-black shading, and reconstruction of luminescent material in black-white fade.

\section{$\mathbf{J 6 0 7}$}

\section{DEPTH DETERMINATION OF BIOLUMINESCENT} REPORTER SYSTEMS IN SMALL ANIMALS

Christian Klose ${ }^{3}$, Alexander D. Klose ${ }^{1}$, Bradley J. Beattie ${ }^{2}$

${ }^{1}$ Radiology, Columbia University, New York, NY, USA, ${ }^{2}$ Memorial Sloan Kettering Cancer Center, New York, NY, USA, ${ }^{3}$ Fluorotom LLC, Roseville, CA, USA. 
An image analysis tool is presented for calculating the actual location and light emission strength of bioluminescent luciferase/luciferin reporter systems in small animal tissue. A computationally fast algorithm uses spectral information of bioluminescence light in twodimensional (2D) images generated with planar surface imaging technology that is currently available for small animals. This approach has a significant advantage for practitioners: accurate temporal reporter probe alterations can be analyzed in the same small animal in longitudinal in vivo studies. Overall, bioluminescence light is scattered in animal tissue leading to diffuse light distributions at the tissue surface. Planar images of the measured bioluminescence light, however, do not reveal the depth location and emission strength of the reporter probes. Therefore, a computational tool is devised to predict depth information from multi-spectral planar images that are taken at different wavelengths $(560 \mathrm{~nm}-660 \mathrm{~nm})$ of the bioluminescence spectrum. This technology is based on a numerical light propagation model and a machine intelligence algorithm to predict reporter probe depth and emission strength. The interpretation system uses depthdependent spectral information of bioluminescence light for estimating the depth of light source. The system estimates reliable values between $1 \mathrm{~mm}$ and $20 \mathrm{~mm}$ with an uncertainly of up to $\pm 10 \%$. Experimental data from small animals with luciferase-expressing tumors located in lung, kidney, and liver are shown. Magnetic resonance images (MRI) confirm resulting data of depth estimations.

\section{$\mathrm{J} 608$}

\section{DETECTING SINGLE STEM CELLS ANYWHERE IN A MOUSE WITH CRYO-IMAGING}

David L. Wilson $^{1,2}$, Grant Steyer ${ }^{1}$, Debashish Roy ${ }^{1}$, Madhusudhana Gargesha ${ }^{1}$, Yuan Lin ${ }^{5}$, Marc S. Penn ${ }^{4,3}$

${ }^{1}$ Biomedical Engineering, Case Western Reserve University, Cleveland, OH, USA, ${ }^{2}$ Radiology, Case Western Reserve University, Cleveland, OH, USA, ${ }^{3}$ Skirball laboratory for Cardiovascular Cellular Therapeutics, Cleveland Clinic Foundation, Cleveland, OH, USA, ${ }^{4}$ Cardiology, Cleveland Clinic Foundation, Cleveland, OH, USA, ${ }^{5}$ Case Comprehensive Cancer Center, Case Western Reserve University, Cleveland, OH, USA.

We created and used a cryo-imaging system which provides single-cell detection of fluorescently labeled stem cells throughout a mouse. The Case cryo-imaging system consists of a fluorescence microscope, robotic positioner, customized whole mouse cryostat, controller, and multi-scale visualization/analysis software. It alternates between sectioning (10-40 microns) and imaging, collecting tiled color and fluorescent block face images, creating $>60 \mathrm{~GB}$ at microscopic resolution. Software detects fluorescent cell clusters and estimates the number of cells per cluster. In validation experiments, auto-fluorescence was readily rejected and software analysis of images at nominal resolution compared favorably to manual micro-sphere counting in super high resolution gold standard images. In bone marrow transplantation studies, HSCs were labeled with a dual reporter (fluc/EGFP), allowing us to follow homing/engraftment in vivo using bioluminescence imaging with centimeter resolution followed by cryo-imaging with single cell sensitivity to exactly determine cell location. In several experiments, we used cryo-imaging to assess MSC treatment of myocardial infarction following tail vein injection. With 100,000 injected cells, we detected about $650 / 11000$ cells in the heart/ lungs at $24 \mathrm{hr}$, giving a $6 \%$ delivery ratio. Days later, numbers of cells reduced in both organs. Unlike some previous reports, we identified cells in the heart with our thorough methods, giving credence to intravenous injection as a viable method of therapeutic intervention (FASEB J. $21: 3197-3207 ; 2007)$, possibly via a paracrine mechanism. In another experiment, we labeled HSCs with EGFP under the tie promoter and applied them in a tail vein injection to a mouse with xenograft tumor. In $>300$ sections, we found just three instances of fluorescent micro-vessel sections, indicating that the exogenous cells had participated in angiogenesis. Patency was determined by the presence of a dye within the vasculature. We believe that the resolution, sensitivity, and analysis/ visualization software of cryo-imaging provides an excellent complement to existing in vivo imaging and unique opportunities for quantifying stem cell experiments in homing/engraftment, delivery, homing factors, cell enhancement factors, differentiation, etc.

\section{J609}

MULTIPHOTON IMAGING OF MELANOMA XENOGRAFT TUMOR USING ULTRASHORT LASER PULSES

Yair Andegeko, Dmitry Pestov, Vadim V. Lozovoy, Marcos Dantus Department of Chemistry, Michigan State University, East Lansing, MI, USA.

Multiphoton imaging has become a standard in the biomedical imaging field due to its high peak power intensities, narrow focal plane excitation, and highly focused signal with the absence of out-of-focus photobleaching, photodamage and fluorescence scattering. The low absorption coefficient of near- infrared laser intensities enables imaging of cells and tissue without thermal damage, a feature that is especially important when imaging highly pigmented cells like melanocytes or tissues like kidney. In nonlinear optical microscopy, the control over the laser pulse characteristics at the sample and the acquisition of conclusive, reproducible data depend on the ability to deliver transform limited pulses, i.e. without spectral phase distortions, at the focal plane of the microscope objective. Efforts to compensate chromatic dispersion, such as the use of prism pairs, are able to correct second-order dispersion, also known as group delay dispersion, but not higher-order dispersion. When left uncompensated, these distortions prevent the efficient use of pulses shorter than $30 \mathrm{fs}$. Here we introduce newly developed method called multiphoton interference intrapulse phase scan (MIIPS) that allows delivering transform limited pulses with spectral bandwidth of $100 \mathrm{~nm}$ (full width at half maximum) or more. In this study we are using a laser system capable of delivering pulses as short as $12 \mathrm{fs}$ (at least ten times shorter than typical two-photon microscopes), outfitted with a pulse shaper. We demonstrate the dependence of two-photon excitation fluorescence on pulse duration, by imaging a fluorescently labeled mouse kidney with $12 \mathrm{fs}$ and 100 fs laser pulses, keeping the energy and other experimental parameters unchanged. Our results show the expected gain of 8-fold in fluorescence signal. We then further demonstrate the advantage of ultra-short pulses when imaging unstained xenografts of melanoma tumors, getting a 20 -fold increase in the Second Harmonic Generation (SHG) and auotofluorescence signal. This improvement in signal to noise ratio enables us to identify various biological effects of treatment on the melanoma xenograft specimens, and therefore demonstrates the importance of dispersion-free imaging system. The greater efficiency in signal allows imaging with lower laser intensities, thereby reducing photobleaching and photodamage.

\section{$\mathrm{J} 610$}

NUMERICAL AND EXPERIMENTAL STUDIES OF GFP EXPRESSING SMALL ANIMAL MODELS WITH THE EQUATION OF RADIATIVE TRANSFER

Ludguier D. Montejo ${ }^{1}$, Alexander D. Klose ${ }^{2}$

${ }^{1}$ Department of Biomedical Engineering, Columbia University, New York, NY, USA, ${ }^{2}$ Department of Radiology, Columbia University, New York, NY, USA. 
We present results from numerical and experimental studies in imaging of green fluorescence protein (GFP) in small animals. Fluorescence imaging has proven to be a valuable tool in exploring the effectiveness of pharmacological intervention for a wide variety of diseases and disorders. It has a unique ability to visualize disease-related processes at the molecular and cellular level prior to macroscopic tissue changes. However, to obtain accurate quantification and localization of fluorescing target proteins or cells it is necessary to develop an algorithm that accurately models light-tissue interactions in small animals. Beside optical image reconstruction that provides three-dimensional tomographic maps of fluorescent reporter probes, fluorescence imaging algorithms also provides a means for determining the optimal experimental conditions for imaging. Therefore, we implemented a numerical model for light propagation in animal tissue that is based on the frequency domain (FD) equation of radiative transfer (ERT). The FD-ERT is solved with discrete-ordinates and the first order step-method on multilevel block-structured grids. The accuracy of our method is verified by comparing the predicted camera images of a small animal containing fluorescence sources to experimental data of the same setup. Moreover, we used a GFP expressing small animal cancer model to perform numerical and initial experimental studies. For example, Fig. 1 represents the expected fluence at the surface of a mouse torso from GFPexpressing cells $0.5 \mathrm{~cm}$ interior to the surface. Furthermore, the effects of modulation frequency, excitation wavelength, and fluorophore location on the strength of the signal at the animal surface due to the fluorophore emission are presented. This work was supported in part by the National Cancer Institute, NCI grant NCI-4R33CA118666, which is part of the U.S. National Institutes of Health.

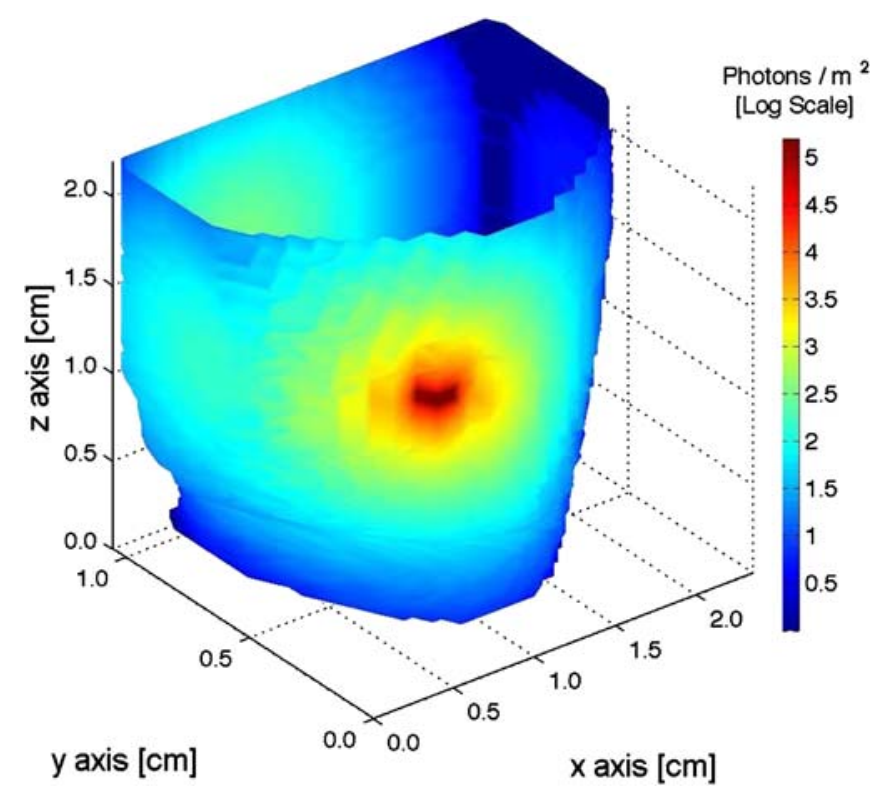

Figure 1: Predicted fluence at the surface of a mouse torso from an internal GFP source using the FD-ERT algorithm.

\section{Imaging Methodology}

\section{PET}

J611

\section{ACCURATE ESTIMATION OF TOTAL VOLUME OF DISTRIBUTION IN PET WITH LIMITED BLOOD SAMPLING}

Aniket D. Joshi, Terence G. Hamill, Kerry Riffel, Sandra Sanabria Imaging, Merck and Co., Inc., West Point, PA, USA.

We propose a modified Logan plot method for estimating total volume of distribution $\left(V_{\mathrm{T}}\right)$ of a PET tracer using input function measured from $\sim 15 \mathrm{~min}$ after tracer injection. Furthermore, since tracer concentration measured in arterial and venous samples is similar after $\sim 15 \mathrm{~min}$, venous samples may suffice for implementing this method. Logan plot implementation (Eq. 1, Logan et al. 2000) requires the knowledge of input function, $C_{\mathrm{p}}(\mathrm{t})$ starting from tracer injection. The linear regression slope in Eq. 1 corresponds to $V_{\mathrm{T}}, C(\mathrm{t})$ is the time activity curve for a regionof-interest (ROI) and $T_{i}$ is midpoint time for frame $i$. Eq. 1 was modified by expressing the integral term on the right hand side as the sum of two integrals: a constant term $\int C_{\mathrm{p}}(\mathrm{t})$ from 0 to $T^{\prime}$ (represented as $\mu$ ) and $\int C_{\mathrm{p}}(\mathrm{t})$ from $T^{\prime}$ to $T_{i}$ (Eqs. 2,3). $T^{\prime}$ is the earliest time from which the tracer concentration in arterial and venous plasma coincides as well as the Logan plot becomes linear. First, the parameter $\mu$ (constant for all ROIs) was estimated by fitting kinetically distinct curves obtained from cluster analysis to Eq. 3. Subsequently, $V_{\mathrm{T}}$ and $I N T$ values were obtained for several anatomically defined ROIs using Eq. 3 and the estimated $\mu$ value. Baseline studies with the mGluR5 tracer $\left[{ }^{18} \mathrm{~F}\right] \mathrm{F}$ PEB were performed in 4 rhesus monkeys (120 min duration scans). The tracer arterial input curve was measured from start of the scan. Both $V_{\mathrm{T}}$ and $I N T$ were estimated using the Logan analysis (Eq. 1) and the proposed method (Eq. 3 with $T^{\prime}=30 \mathrm{~min}$ ). The correlation between parameters estimated using both methods and the estimation bias using the modified approach were calculated. $V_{\mathrm{T}}$ and INT values estimated using both methods showed high correlation $\left(R^{2}>0.92\right.$ and $\mathrm{R}^{2}>0.9$, respectively). Using the modified approach, bias for $V_{\mathrm{T}}$ was between $92 \%$ and $108 \%$ and for INT was between $74 \%$ and $101 \%$ (100\% implies no bias). The results show that the quantification of reversible tracers using reduced number of arterial samples and potentially venous samples alone is possible. The technique is general enough to be applied to other reversible tracers where avoiding arterial sampling is highly desirable.

$$
\begin{aligned}
\frac{\int_{0}^{T_{i}} C(t) d t}{C\left(T_{i}\right)} & =V_{T} \frac{\int_{0}^{T_{i}} C_{p}(t) d t}{C\left(T_{i}\right)}+I N T \\
& =V_{T} \frac{\left\{\int_{0}^{T^{\prime}} C_{p}(t) d t+\int_{T^{\prime}}^{T_{i}} C_{p}(t) d t\right\}}{C\left(T_{i}\right)}+I N T \\
& =V_{T} \frac{\left\{\mu+\int_{T^{\prime}} C_{p}(d t)\right\}}{C\left(T_{i}\right)}+I N T
\end{aligned}
$$


$\mathrm{J} 612$

\section{SYSTEM DESCRIPTION AND PERFORMANCE CHARACTERIZATION OF NANOPETTM/CT, A NEW ULTRA-HIGH RESOLUTION, HIGH SENSITIVITY PRE-CLINICAL PET-CT SYSTEM FOR RODENTS} York Haemisch ${ }^{1}$, Gabor Nemeth ${ }^{2}$, Peter Major ${ }^{2}$, Tamas Bükki ${ }^{2}$, Paul Marsden $^{3}$, Greg Mullen ${ }^{3}$, Istvan Szanda ${ }^{3}$, Laszlo Nagy ${ }^{2}$

${ }^{1}$ Bioscan Inc., Washington, DC, USA, ${ }^{2}$ Mediso Inc., Budapest, Hungary, ${ }^{3}$ Guy's, King's and St Thomas' School of Medicine, King's College, London, United Kingdom.

Introduction: The usability of imaging methods for pre-clinical research largely depends on the combination of spatial resolution and sensitivity provided by an imaging system. One of the biggest challenges is achieving those in a large enough volume at reasonable costs. Simultaneously, e.g. for neurological research on the brain of mice, sub-mm resolution is desired in a small volume at the centre. The NanoPETTM/CT design aims to meet these requirements using the most advanced, commercially available components forming an $18 \mathrm{~cm}$ diameter PET-detector polygon from 12 detector modules of $81 \times 39$ LYSO crystals $(1.12 \times 1.12 \times 13 \mathrm{~mm} 3)$, tightly packed (pitch $1.17 \mathrm{~mm}$, PF 94\%) and coupled to two 256-channel PSPMT's (Hamamatsu H9500) each via a thin light guide. The modular design was favoured over the block design in order to avoid inhomogeneities in the centre of the FOV. A total of 37,908 detector elements and 24 PS-PMT's provide an AFOV of $95 \mathrm{~mm}$ and TFOV between 45 and $123 \mathrm{~mm}$. The diameter of the gantry opening is $160 \mathrm{~mm}$ and allows imaging of larger rodents (e.g. rabbits). The system employs advanced crystal recognition algorithms; algorithms for correction of PSF and other physical effects are under development. The PET system is complemented by an x-ray CT system with variable magnification, consisting of an $8 \mathrm{~W}$ micro-focus source and a $5 \times 17.5 \mathrm{~cm} 2$ flat panel detector, operated in helical mode, providing voxel sizes down to $9 \mu \mathrm{m}$. Both systems are aligned on the same axis with minimal axial separation. Methods: PET system characterization follows the new NEMA-NU4-2008 standard for small animal PET systems. Spatial resolution, system sensitivity, energy and timing resolution will be measured. Initially standard FORE/2D FBP and FORE/MLEM/OSEM reconstruction will be used; advanced 3D algorithms are under development. The CT subsystem will be evaluated using standard micro-CT resolution and contrast phantoms. Results: Measurements of spatial resolution and sensitivity will be presented. Characterization of energy- and timing resolution will be discussed as well as initial examples of images of micro-phantoms and animals will be shown. Conclusions: The NanoPETTM/CT represents a new generation of pre-clinical PET-CT scanners aiming at highest possible performance achieved under industrial standards of manufacturing quality (ISO 9001), compliance with industry standards (CE Medical, CFR21, ch. 11) and by using the highest quality components offering a high quality imaging instrument to pre-clinical researchers in an economically viable way.

\section{J613}

EVALUATION OF [18F]FLT UPTAKE WITH VARIOUS ANAESTHETICS AND DIFFERENT BREATHING PROTOCOLS IN JOINT-INFLAMMATION AND CT26 MOUSE TUMORS

Kerstin Fuchs ${ }^{1,2}$, Damaris Kukuk ${ }^{2}$, Daniel Bukala², Julia G. Mannheim², $\overline{\text { Stefan Wiehr }}{ }^{2}$, Martin S. Judenhofer ${ }^{2}$, Gerald Reischl $^{3}$, Martin Röcken ${ }^{1}$, Manfred Kneilling ${ }^{1}$, Bernd J. Pichler ${ }^{2}$

${ }^{1}$ Department of Dermatology, University of Tuebingen, Tuebingen, Germany, ${ }^{2}$ Laboratory for Preclinical Imaging and Imaging Technology of the Werner Siemens-Foundation, University of Tuebingen, Tuebingen, Germany, ${ }^{3}$ Department of Radiopharmacy, University of Tuebingen, Tuebingen, Germany.
While studying [18F]FLT uptake in an experimental model of arthritis and an exogenous CT26 colon cancer model we surprisingly detected significant differences in arthritic joints and subcutaneous CT26 tumors between dynamic and static PET scans. Thus, we suspected that different anaesthetics, anaesthetic times and breathing protocols using air and $\mathrm{O} 2$ were responsible for changes in [18F]FLT uptake in these animal models. We induced arthritis by injecting intra peritoneal auto-antibodies against Glucose-6-phosphate-isomerase (GPI) and CT26 carcinomas by subcutaneous inoculation of CT26 cancer cells into the flank of Balb/c mice. At days 5-7 after arthritis induction and at days 8-12 after tumor inoculation, mice were injected intravenously with $11 \mathrm{MBq}$ [18F]FLT and dynamic or static micro PET scans were performed. Three different anaesthesia protocols (1,5\% Isoflurane; Midazolam/Medetomidin; Ketamin/Rompun) and two different breathing protocols $(\mathrm{O} 2$; air) were used to investigate potential differences in $[18 \mathrm{~F}] \mathrm{FLT}$ uptake. Mice were kept under anaesthesia or alternatively left awake after [18F]FLT injection for static PET scans which were performed 30 min (arthritis model) or $2 \mathrm{~h}$ (tumor model) after injection. [18F]FLT uptake in arthritic joints and CT26 tumors were also analyzed by ex vivo biodistribution and histology. In dynamic scans we found the highest tracer uptake in arthritic joints when mice underwent Ketamin/Rompun or Midazolam/Medetomidin anaesthesia. Interestingly, using Ketamin/Rompun anaesthesia with oxygen we found significant differences ( $3.6 \mathrm{vs} .2 .3 \% \mathrm{ID} / \mathrm{cc}$ ) when mice were kept awake or under anaesthesia during PET tracer uptake. In contrast we observed the highest [18F]FLT uptake in CT26 tumors using Isoflurane/air (6.44\%ID/cc-sleeping protocol) and Isoflurane/O2 (6.38\% $\mathrm{ID} / \mathrm{cc}$-sleeping protocol) anaesthesia. Lowest [18F]FLT uptake in CT26 tumors was measured using Ketamin/Rompun anaesthesia with air $(5.41 \% \mathrm{ID} / \mathrm{cc}-$ sleeping protocol). All anaesthesia protocols with air breathing measurements showed no differences between dynamic and static scans and denote the highest [18F]FLT uptake in both mouse models. Therefore, the best choice to compare dynamic with static scans and to exclude anaesthesia alterations is to use an Isoflurane/air anaesthesia protocol. The various protocols indicate that the main factor influencing [18F]FLT uptake is the breathing (air versus O2) during PET tracer uptake. Thus, investigators should plan very carefully their imaging studies, especially when a combination of dynamic and static scans is required for comparison.

\section{J614}

EFFECT OF POSITRON RANGE ON PARTIAL VOLUME EFFECT AND ACCURACY OF PARTIAL VOLUME CORRECTION IN SMALL ANIMAL BRAIN POSITRON EMISSION TOMOGRAPHY: A SIMULATION STUDY Wencke Lehnert $^{1,2}$, Marie-Claude Gregoire ${ }^{3}$, Steven Meikle ${ }^{1,2}$

${ }^{1}$ Ramaciotti Imaging Centre, Brain \& Mind Research Institute, The University of Sydney, Sydney, NSW, Australia, ${ }^{2}$ Discipline of Medical Radiation Sciences, Faculty of Health Sciences, The University of Sydney, Sydney, NSW, Australia, ${ }^{3}$ Radiopharmaceutical Research Institute, ANSTO, Sydney, NSW, Australia.

Objectives: To investigate 1. the effect of positron range on partial volume effect (PVE) and accuracy of correction using the geometric transfer matrix (GTM) method, 2. whether a spatially variant point spread function (PSF) is necessary for PVC of a rat brain at or close to the centre of the field of view (CFOV), Methods: Monte Carlo simulations of microPET Focus 220 studies were performed using PET SORTEO. To evaluate the PSF, acquisitions of $11 \mathrm{C}$ and $18 \mathrm{~F}$ point sources, as well as a hypothetical isotope with zero positron range (zero-PR source), were simulated. The point sources were positioned at regular intervals from the CFOV to a radius of $2 \mathrm{~cm}$ in the transverse direction and $2 \mathrm{~cm}$ offset in the axial direction. Binary images of anatomical rat brain regions extracted from a stereotaxic 
atlas were used as approximations of the rat brain and convolved with the PSF to calculate activity retention in each region and spillover between regions. Dynamic rat brain PET studies of $11 \mathrm{C}$ Raclopride with a BPND=3 in the striatum were simulated with the brain at the CFOV and shifted $\pm 1 \mathrm{~cm}$ in the $\mathrm{x}, \mathrm{y}$ and $\mathrm{z}$ directions. An additional rat brain simulation was performed with the zero-PR source. Results: There was no significant deterioration in spatial resolution as a function of distance with $7 \%$ increase in FWHM from $0.2 \mathrm{~cm}$ to $2 \mathrm{~cm}$. Thus a spatially invariant PSF measured at $8 \mathrm{~mm}$ offset was used for PVC. The $11 \mathrm{C}$ activity retention in striatum, cerebellum and remaining brain was $43.2 \%, 52.4 \%$ and $73.7 \%$ respectively. This represents an increase in PVE of $11.1 \%$, $7.4 \%$ and $3.7 \%$, respectively, compared to $18 \mathrm{~F}$ and $17.2 \%, 11.8 \%$ and $5.8 \%$ compared to the zero-PR source. As a comparison, a Gaussian fit to the measured PSF would underestimate PVE in the striatum by $18.9 \%$ for $11 \mathrm{C}(12.9 \%$ for $18 \mathrm{~F})$ which is close to the effect of zero positron range. Comparing the dynamic rat brain PET simulations of $11 \mathrm{C}$ and the zero-PR source, it was found that $5 \%$ in the bias of BPND is due to positron range of $11 \mathrm{C}$. The $11 \mathrm{C}$ BPND bias for the brain at the CFOV was $-35.34 \%$ without PVC and $1.30 \%$ with PVC. Correction with a Gaussian function leaves a bias of BPND of $-15.92 \%$. The accuracy of BPND after PVC for the mispositioned brain was within $6 \%$. Conclusion: For microPET brain studies, positron range has a significant impact on PVE and has to be taken into account for accurate PVC even for low energy positron emitters such as $18 \mathrm{~F}$ or $11 \mathrm{C}$. PVC using a spatially invariant PSF measured at a small $(<1 \mathrm{~cm})$ offset from the CFOV is accurate if the brain is positioned within a radius of $1 \mathrm{~cm}$ from the CFOV.

\section{$\mathbf{J} 615$}

\section{IN VIVO SCATCHARD STUDIES OF THE VESICULAR} MONOAMINE TRANSPORTER IN MICE

Katie Dinelle ${ }^{1}$, James E. Holden ${ }^{3}$, Vesna Sossi ${ }^{2}$, Doris J. Doudet ${ }^{4}$

${ }^{1}$ Pacific Parkinson's Research Centre, University of British Columbia, Vancouver, BC, Canada, ${ }^{2}$ Physics and Astronomy, University of British Columbia, Vancouver, BC, Canada, ${ }^{3}$ Medical Physics, University of Wisconsin, Madison, WI, USA, ${ }^{4}$ Medicine, University of British Columbia, Vancouver, BC, Canada.

The last decades have seen large growth in the use of mice in medical and biological research, mostly due to the increase in genetically engineered animals that can be produced in a relatively short period of time. The ability to characterize normal or abnormal aspects of specific brain function in these animals, longitudinally and in vivo is thus of great interest. We have previously demonstrated the feasibility of performing evaluation of receptor/transporter density $\left(\mathrm{B}_{\max }\right)$ and apparent affinity $\left(\mathrm{K}_{\mathrm{d}}{ }^{\mathrm{app}}\right)$ in both monkeys and rats using tracers of the dopamine D1 and D2 receptor and of the vesicular monoamine transporter (VMAT2). Such studies not only allow investigation of the biology of systems of interest at baseline and/or under challenge conditions, they also allow evaluation of the effect of injected mass on the binding parameters, an effect of the utmost importance in small animal imaging. In this study, we used our in vivo Scatchard method [1], to evaluate the density and affinity of the VMAT2 in mice using $\left[{ }^{11} \mathrm{C}\right]-(+)-\alpha$-dihydrotetrabenazine positron emission tomography (PET). Six C57B16 mice were scanned on a microPET Focus 120. Each animal underwent 3 scans under isofluorane anaesthesia at decreasing specific activity (SA) as described in [1]. The amount of activity injected was 174 $\pm 30 \mu \mathrm{Ci} / 100 \mathrm{~g}$ of body weight. The $\mathrm{SA}$ ranged from $5000 \mathrm{Ci} /$ $\mathrm{mmol}$ to $16 \mathrm{Ci} / \mathrm{mmol}$, corresponding to a range of injected masses of $0.009 \mu \mathrm{g}$ to $3.7 \mu \mathrm{g} / 100 \mathrm{~g}$ body weight. A mouse atlas was used to guide the placement of ROIs on the striatum and cerebellum in each image. The binding potential at each SA was calculated using the Logan method and linear fits were used to calculate $\mathrm{B}_{\max }$ and $\mathrm{K}_{\mathrm{d}}{ }^{\text {app}}$. The average VMAT2 $\mathrm{B}_{\max }$ was $54 \pm$ $16 \mathrm{pmol} / \mathrm{mL}$ and the apparent $\mathrm{K}_{\mathrm{d}}{ }^{\text {app }}$ was $50 \pm 14 \mathrm{pmol} / \mathrm{mL}$. The apparent affinity in these mice was remarkably similar to that of the VMAT2 $\mathrm{K}_{\mathrm{d}}$ app in the rat striatum [1]. The transporter occupancy curve showed negligible occupancy $(<1 \%)$ at specific activities $\geq 2000 \mathrm{Ci} / \mathrm{mmol}$, and $10 \%$ occupancy at $185 \mathrm{Ci} / \mathrm{mmol}$. These values compare well with similar data in rats. In conclusion, these preliminary data demonstrate the feasibility of performing long, complex and meaningful imaging studies in subjects as small as mice. Further studies in lesioned mice are ongoing. These data also demonstrate the importance of controlling for the mass of injected ligand in in-vivo studies especially for small animals considering that a relatively large amount of activity has to be administered to a mouse to obtain adequate images and kinetic curves. [1] Sossi et al (2007) JCBFM 27.

\section{J616}

\section{IMAGING PERFORMANCE OF A LARGE AXIAL FOV} SMALL ANIMAL PET SCANNER

Melanie Bergeron $^{1}$, Jules Cadorette ${ }^{5,4}$, Chloé Bureau-Oxton ${ }^{2}$, Jean-François Beaudoin ${ }^{5}$, Marc-André Tétrault ${ }^{3}$, Martin D. Lepage ${ }^{4}$, Ghislain Robert ${ }^{4}$, Réjean Fontaine ${ }^{3}$, Roger Lecomte ${ }^{1}$

${ }^{1}$ Nuclear Medicine and Radiobiology, Université de Sherbrooke, Sherbrooke, QC, Canada, ${ }^{2}$ Physics, Université de Sherbrooke, Sherbrooke, QC, Canada, ${ }^{3}$ Electrical and Computer Engineering, Université de Sherbrooke, Sherbrooke, QC, Canada, ${ }^{4}$ Gamma Medica-Ideas, Sherbrooke, QC, Canada, ${ }^{5}$ CIMS, Université de Sherbrooke, Sherbrooke, QC, Canada.

Small animal research drives the need for high resolution and high sensitivity PET imaging. High resolution and good imaging performance were reached with LabPET4 and LabPET8 scanners, based on LGSO/LYSO phoswich detectors $\left(2 \times 2 \times 12 / 14 \mathrm{~mm}^{3}\right.$ crystals) read out by APDs and parallel digital electronics, despite their relatively small axial FOV $(3.75 / 7.5 \mathrm{~cm})$. However, the need for better sensitivity and whole-body dynamic imaging capabilities has motivated the development of a new version, LabPET12, having an $11.4 \mathrm{~cm}$ axial FOV and 9216 pixel detectors. Performance evaluation was conducted with different energy windows in order to investigate high-resolution and high-sensitivity modes. Reducing the energy threshold from $350 \mathrm{keV}$ to $100 \mathrm{keV}$ increased sensitivity from $2.8 \%$ to $8 \%$. Maximum peak NEC count rates were achieved with a $200 \mathrm{keV}$ threshold at $449 \mathrm{kcps}$ with $66 \mathrm{MBq}$ for the mouse and $179 \mathrm{kcps}$ with $81 \mathrm{MBq}$ for the rat NEMA phantoms. Scatter fraction varied from 14 to $34 \%$ with the mouse phantom and from 27 to $41 \%$ for the rat phantom when lowering the energy threshold from 350 to $100 \mathrm{keV}$. Phantom and animal images were obtained with three different energy windows (200-, 250- and 350-650 keV). The NEMA image quality phantom was filled with 2.8/4.2/4.6 MBq of ${ }^{18} \mathrm{~F}$ and imaged during 20 minutes. Recovery coefficients were measured for hot spots from 1 to $5 \mathrm{~mm}$ and are reported below. Images of the Ultra Micro Hot Spot Phantom acquired during 30 minutes using 9.7/15.6/15.4 MBq of ${ }^{18} \mathrm{~F}$, made it possible to clearly distinguish $1.0 \mathrm{~mm}$ hot spots. $\mathrm{Na}^{18} \mathrm{~F}$ imaging of mice was also performed using 8.7/7.9/8.0 MBq. The LabPET12 was shown to be capable of fast imaging using low radioactivity amounts, with emphasis on high resolution or high sensitivity, at the user need. 


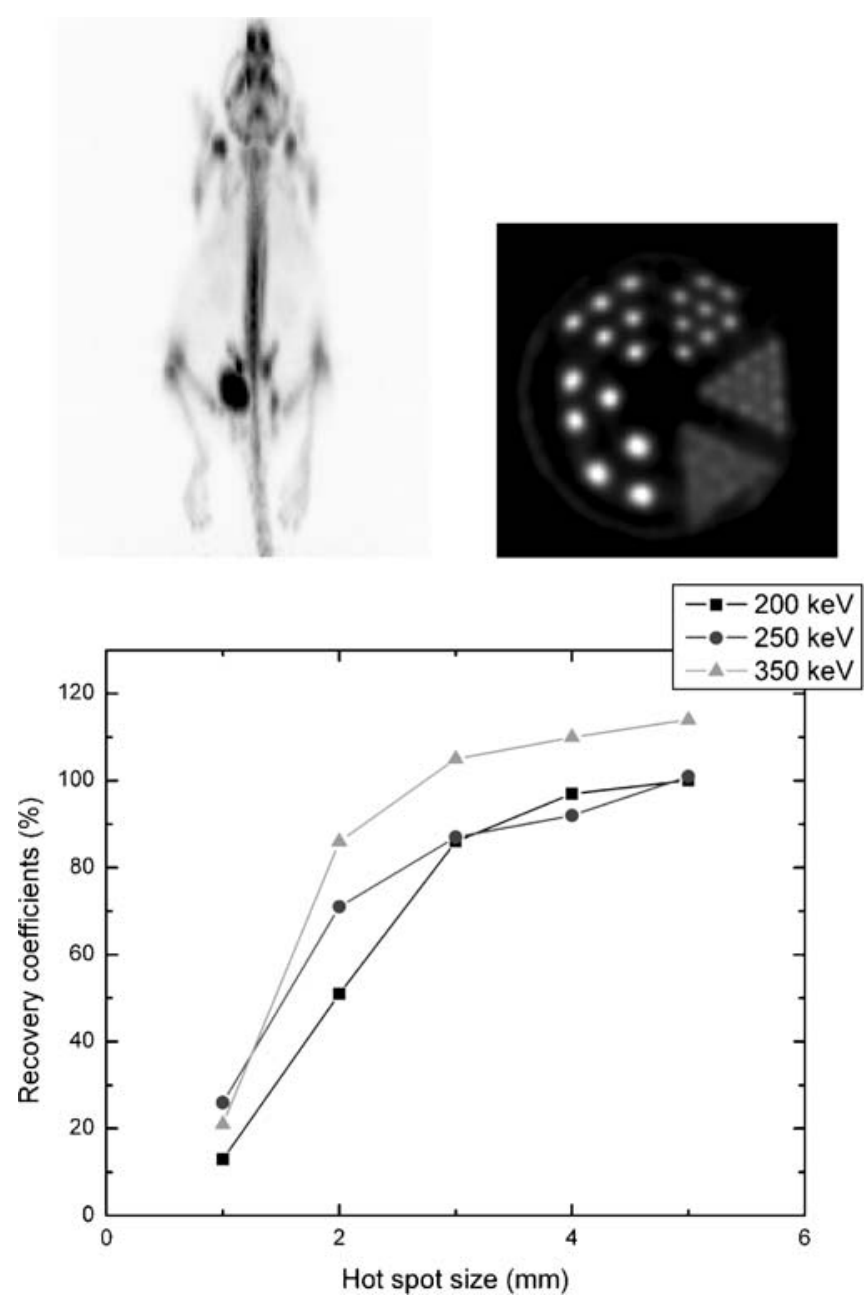

First row: NaF mouse image and ultra micro hot spot phantom with 350-650 keV energy window Second row: Recovery coefficients with different energy windows for Ultra Micro Hot Spot phantom

$\mathbf{J 6 1 7}$

\section{EVALUATION OF PET/AUTORADIOGRAPHY COMPARISON FOR BRAIN IMAGING IN MICE: FEASIBILITY OF DAT QUANTIFICATION}

Kristina Fischer $^{1}$, Daniel Bukala ${ }^{1}$, Andreas Schmid ${ }^{1}$, Carina Stegmayr ${ }^{3}$, Ulrich Ruess $^{3}$, Julia G. Mannheim ${ }^{1}$, Andreas von Ameln-Mayerhofer ${ }^{3}$, Anke Stahlschmidt ${ }^{2}$, Vesna Sossi ${ }^{1}$, Bernd J. Pichler ${ }^{1}$

${ }^{1}$ Laboratory for Preclinical Imaging and Imaging Technologies, University of Tuebingen, Tuebingen, Germany, ${ }^{2}$ Radiopharmacy, University of Tuebingen, Tuebingen, Germany, ${ }^{3}$ Neuropharmacology, University of Tuebingen, Tuebingen, Germany.

Quantitative assessment of [11C]Methylphenidate (MP) binding using PET is often used to evaluate the integrity of the dopaminergic neurons in the striatal regions. Considering the small size of the mouse brain, and striata in particular, it is important to validate the in-vivo PET measurements (resolution of $\sim 1.5 \mathrm{~mm} 3$ ) with a spatially more accurate imaging technique. Autoradioagraphy (AR) is often used for this purpose. The aim of this work is to first provide an evaluation of the accuracy of DAT binding quantification with PET and then to examine the impact of different methodological approaches on the comparison outcome. For this purpose mice were lesioned with 6-OHDA $(2 \mu \mathrm{g} / \mu \mathrm{l})$ into the right striatum (str) using stereotactic coordinates. Two weeks after lesioning [11C]MP $(12 \pm 5 \mathrm{MBq})$ dynamic PET scans were acquired. Mice were positioned with stereotactic holders in the center of FOV under temperature controlled conditions. Brains were removed immediately after completion of PET experiments and cut into $20 \mu \mathrm{m}$ slices for a total of $80-100$ slices. Slices were readout with a pixel size of $50 \mu \mathrm{m}$ using a phosphor imager. For each slice AR determined binding potential (BPND) was expressed as (conc(str)-conc(cer))/conc (cer) and denervation severity (DS) as 1-BPND_L/BPND_C. For each striatum, BPND was averaged over (i) central 50 slices to match PET ROI location and alternatively (ii) first, middle and last 15 slices to determine DS uniformity. DAT BPND from PET measurements was evaluated by using SRTM and by using the last $30 \mathrm{~min}$ of data acquisition to obtain approximate measure of BPND consistent with AR. DS severity was defined as above. A large gradient in DS across AR slices was observed for two animals leading to a very variable DS assessment according to slice selection from $75 \%$ to $0 \%$ while a more uniform distribution was observed for another 2 animals. A related variability in the correlation between AR and PET determined DS was observed (from $\mathrm{r} 2=0.3$ to $\mathrm{r} 2=0.53$, using RTM) with best correlation obtained when the central 50 AR slices were used. With the ratio method this correlation increased to 0.66 . These data show there can be variability in the DS gradient itself which can introduce variability in the AR to PET comparison. In addition, PET analysis methods can influence the comparison between PET and AR BP determination. These preliminary data show good agreement between DAT BP as determined by PET and AR and showed the need of a systematic comparison protocol. Further studies including bolus infusion protocols, will be conducted to confirm these results.

\section{J618}

\section{CARDIAC PERFUSION AS A FUNCTION OF CARDIAC HYPERTROPHY USING MICROPET/CT IMAGING OF F-18-FLUORODIHYDROROTENOL} Grant T. Gullberg ${ }^{1,2}$, James P. O'Neil ${ }^{1}$, Mustafa Janabi ${ }^{1}$, Kathleen M. Brennan ${ }^{1}$, Hilla Wahnishe $^{2}$, Youngho Seo ${ }^{2}$, Henry VanBrocklin ${ }^{2,1}$

${ }^{1}$ Radiotracer Development \& Imaging Technology, Lawrence Berkeley National Laboratory, Berkeley, CA, USA, ${ }^{2}$ Radiology, University of California San Francisco, San Francisco, CA, USA.

The goal of this project is to develop radionuclide molecular imaging technologies for the study of heart failure. The work involves molecular imaging of the spontaneous hypertensive rat (SHR) as a model of hypertensive related pathophysiology, utilizing a microPET/CT scanner to quantify the changes in perfusion as a function of hypertrophy and age. Methods: Normotensive Wistar Kyoto rats (WKY) and SHR rats were imaged using the microPET/ CT Inveon scanner (Siemens). Dynamic gated list mode data of approximately $1,500,000,000$ counts were acquired over $60 \mathrm{~min}$ immediately after injecting a dose of $1-2 \mathrm{mCi}$ of $\mathrm{F}$-18-fluorodihydrorotenol (FDHROL). The data were reconstructed both as static images and as a dynamic sequence of 3D images for obtaining time activity curves for the blood input sampled from the left ventricular blood pool and for the left ventricular myocardial for the purpose of measuring the washin and washout of FDHROL from the myocardium. Results: F-18-fluorodihydrorotenol demonstrated outstanding flow versus extraction characteristics. It also provided high contrast ratios of heart to lung, which allowed for improved visualization of the right ventricle. Conclusion: F-18-fluorodihydrorotenol provides an outstanding tracer for analyzing perfusion in small animal models. It has characteristics similar to that of a chemical microsphere. Its 
excellent extraction versus flow provides an excellent marker of flow with better statistics for improved precision and bias of estimated perfusion parameters. In addition, its improved contrast allows for the differentiation of subtle differences within the left ventricle as well as the right ventricle which enables the potential for modeling pulmonary hypertension.

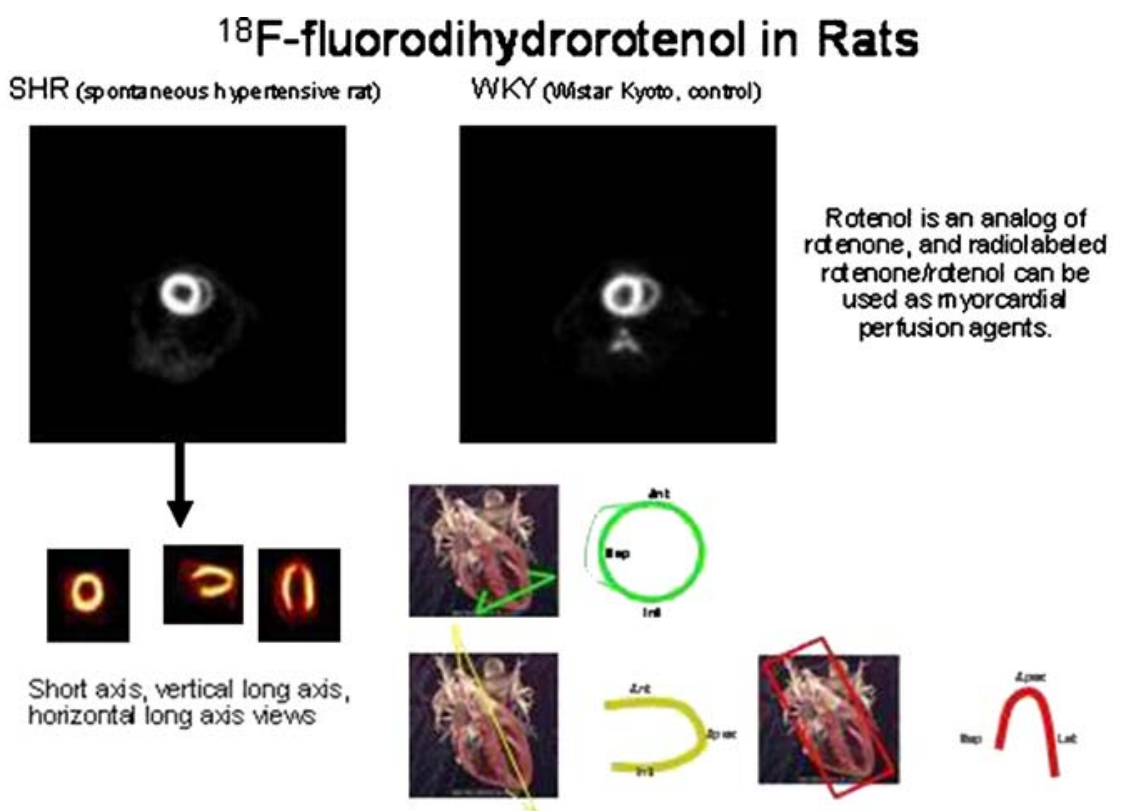

Figure 1. Cardiac microPET study in rats using ${ }^{18} \mathrm{~F}$-fluorodihydrorote nol $\left({ }^{18} \mathrm{FDHROL}\right)$. The image on the left is a transaxial slice through the heart of a spontaneous hypertensive rat model. The image on the right is a transaxial slice through the heart of a WKY control rat.

J619

\section{PRECLINICAL AND CLINICAL PET DETECTOR DESIGN CONSIDERATIONS USING SILICON PHOTOMULTIPLIERS (SPM)}

Hao Peng, Peter D. Olcott, Craig S. Levin

School of Medicine, Stanford University, Palo Alto, CA, USA.

We are developing a new high-resolution PET block detector using the silicon photomultiplier (SPM) for both clinical ( $\sim 3 \mathrm{~mm}$ resolution) and preclinical $(\sim 1 \mathrm{~mm}$ resolution) PET/MRI applications. This detector exhibits the advantages of compact size, high gain and the ability to operate in a strong magnetic field. In this work, we investigated coupling arrays of $3 \mathrm{~mm}$ and $1 \mathrm{~mm}$ scintillation crystals to a $4 \times 4$ array of $3 \mathrm{~mm}$ SPM pixels. In the first case the $3 \mathrm{~mm}$ crystals were coupled one-to-one to the matching SPM pixels. In the case of coupling the array of $1 \mathrm{~mm}$ crystals each element is 1/9th smaller in area than a single SPM pixel; thus, for uniform photon positioning a light diffuser is required to share light from each $1 \mathrm{~mm}$ element into the $3 \mathrm{~mm} \mathrm{SPM}$ pixels. To reduce the number of SPM readout channels from 16 to 4 while maintaining the detector's performance, a novel charge multiplexing circuit was built. For the $3 \mathrm{~mm}$ crystal pitch array, $3 \times 3$ crystals were coupled one-to-one to a $3 \times 3$ portion of the $4 \times 4$ SPM array; the individual crystals can be clearly resolved with average peak-to-valley ratio of $23.1+/-0.8$; the $511 \mathrm{keV}$ photopeak energy resolution of the global energy spectrum (after normalization per $3 \mathrm{~mm}$ crystal) is 15.9 $+/-0.4 \%$ FWHM. No significant degradation was found when compared to the same configuration except without any multiplexing (energy resolution $15.3+/-0.2 \%$ ). For the case of a $6 \times 6$ array of $1 \mathrm{~mm}$ crystals directly coupled to $2 \times 2$ pixels of the SPM array without a light diffuser, due to highly non-uniform light sharing, individual $1 \mathrm{~mm}$ crystals were not well resolved. There was also no distinguishable $511 \mathrm{keV}$ photopeak. When we coupled an $8 \times 8$ array of $1 \mathrm{~mm}$ crystals through a $1.5 \mathrm{~mm}$ thick light guide to a $3 \times 3$ portion of the $4 \times 4$ SPM array all 64 crystals are resolved with an average peak-to-valley ratio of $4.48+1$ -2.06. The $511 \mathrm{keV}$ photopeak energy resolution of the global energy spectrum (after the normalization per $1 \mathrm{~mm}$ crystal) is $21.2+/-0.4 \%$ FWHM. 


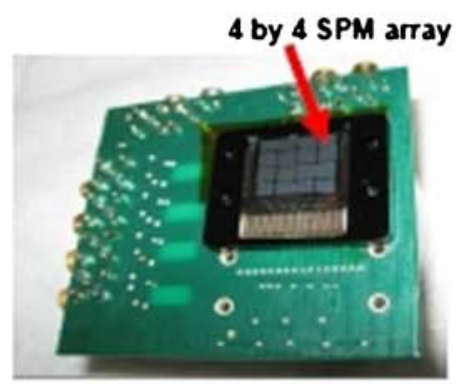

$3 \mathrm{~mm}$ (4x4 array)

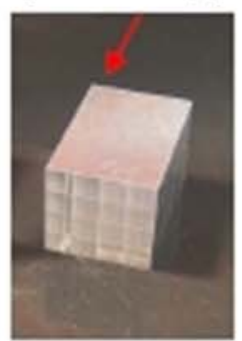

$1 \mathrm{~mm}$ (8x8 array)

Picture of the $4 \times 4$ array of $3 \mathrm{~mm}$ SPM pixels (top left): $4 \times 4$ array of $3 \mathrm{~mm}$ pixels and $8 \times 8$ array of $1 \mathrm{~mm}$ pixels are shown at the bottom left, the test board (top left) consist of the circuits necessary to test the SPM amay and the charge multiplexed readout scheme. The different crystal arrays (bottom left) were coupled to the SPM array as described. The capacitive multiplexing scheme is shown on the right. Along each row. the cathodes are connected together and then into two splitting capacitors. The splitting capacitors are weighted by the row. and the capacitance is made to sum to a constant. Along each column, the anodes are connected together and into the same network as for the anodes.
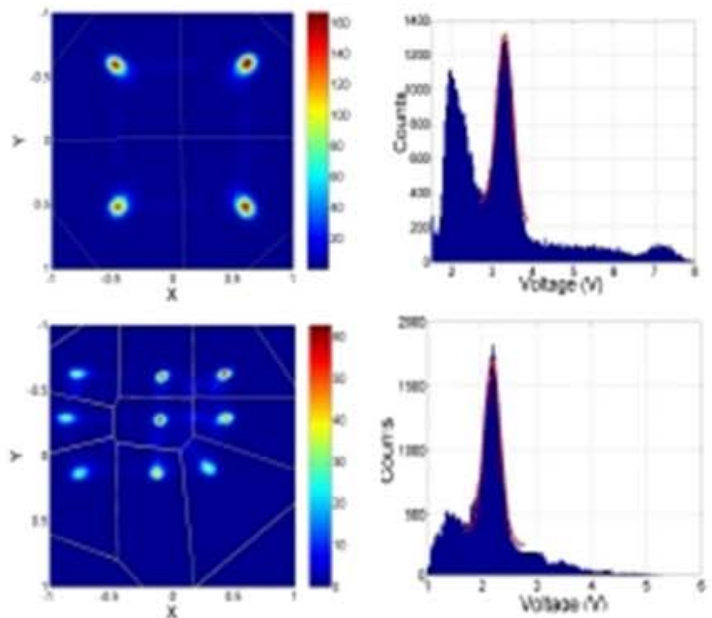

Results from $3 \mathrm{~mm}$ scintilation crystals coupled without and with mulkiplexing of the SPM array signa's. (Top) For the one-to-one coupling case, a $2 \times 2$ portion of the $4 \times 4$ SPM array (that has 3 $\mathrm{mm}$ pixels) was used which was covered by a $2 \times 2$ portion of the $4 \times 4$ scintilation crystal array ( $3 \mathrm{~mm}$ pixels). The average energy resolution of the four non-multiplexed SPM chamels is $15.3+$ $0.2 \%$ FWHM for the $511 \mathrm{keV}$ photopeak. (Bottom) For the mukiplexed case, a $3 \times 3$ portion of the SPM array was tested with a $3 \times 3$ portion of the LYSO crystal array. The $511 \mathrm{keV}$ photopeak energy resolution of the glcbal energy spectrum (after normalization per crystal) is $15.9+1-0.4 \%$. The dfference between the voltage here and that of the non-multiplexing case is due to the different amplifier settings.
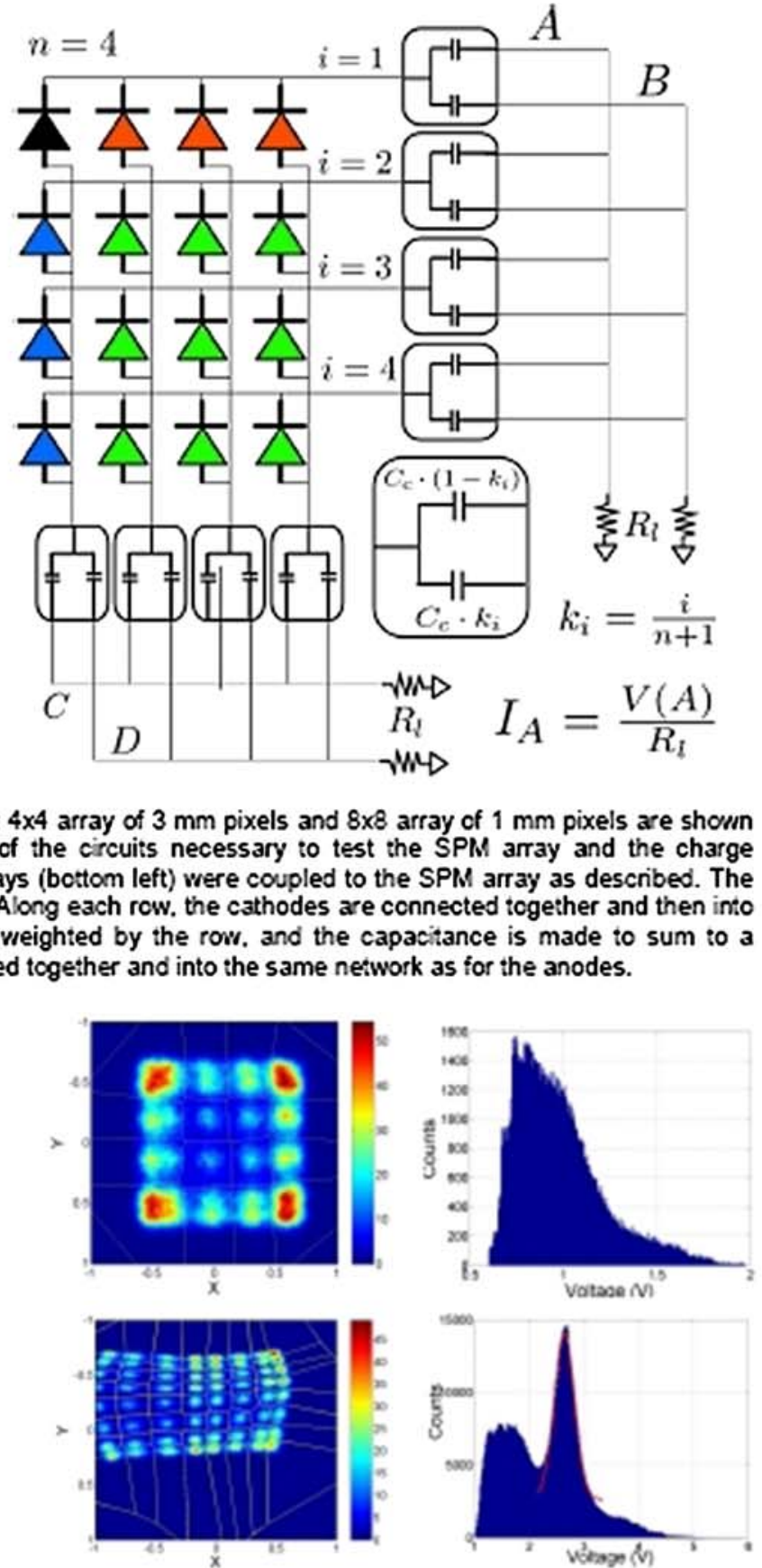

Results from $1 \mathrm{~mm}$ scintilation crystals coupled without and with scintillation light sharing over the SPM array. Both cases used multiplexing of the SPM array signals. (Top) For the directly coupled case, a $2 \times 2$ portion of the $4 \times 4$ SPM array was used which was covered by a $6 \times 6$ portion of the $8 \times 8$ scint illation crystal array $(1 \mathrm{~mm}$ pixels). Without a light diffuser present. the $1 \mathrm{~mm}$ crystal array could not be clearly resolved at the edge $\propto$ corners and the energy spectrum is poor as the size of the SPM pixels $(3 \mathrm{~mm})$ is too coarse to resolve the $1 \mathrm{~mm}$ scintillation crystals and there is significant light lost between SPM pixels. (Bottom) When the $8 \times 8$ crystal amay was coupled to the $3 \times 3$ porticn of the $4 \times 4$ SPM with a $1.5 \mathrm{~mm}$ thick light diffuser, all 64 crystals in the array are clearly resolved and only slight distortions are observed at the edge and corner of the flood histogram. The $511 \mathrm{keV}$ photopeak energy resolution of the global energy spectrum (after normalization per crystal) is $21.2+1-0.4 \%$. 
$\mathbf{J 6 2 0}$

\section{MONITORING SMALL ANIMAL ANESTHESIA WITH A VIDEO CAMERA}

Richard Taschereau, Brittany Berry-Puzey, David Stout, Arion Chatziioannou Molecular \& Medical Pharmacology, UCLA, Los Angeles, CA, USA.

Small animals used in preclinical PET studies are anesthetized during imaging procedures to prevent motion artifacts. Gas anesthetics provide excellent flexibility, enabling real time control of the depth of anesthesia. This approach becomes particularly important for long data acquisition times like those involved in dynamic studies. In these situations, the animal can undergo significant changes in its rate of respiration and anesthesia needs from the beginning to the end of the study. An automated method of anesthesia monitor and control will enable investigators to concentrate on other study aspects and can provide a more consistent level of anesthesia. We report on a system integrated to the PETBox $^{\mathrm{TM}}$ (a new bench top PET imaging device) that uses a video camera to monitor animal breathing as an indicator of the degree of anesthesia. A color video camera is placed on one side of the gantry looking at the animal with a short focal length lens. Video frames are acquired at $30 \mathrm{fps}$. To facilitate image segmentation, a colored translucent acrylic sheet is placed behind the mouse as a backdrop. The color image is segmented into two regions (mouse and background), and cropped to a smaller region centered on the mouse back. The difference between each successive frame is calculated continuously creating a time series. The Fourier Transform of this time series enables the separation of the base respiratory motion. In Figure 1 we show an example of the Fourier Transform obtained with an anesthetized mouse over a $20 \mathrm{~s}$. time period. The highest peaks are located at 2.1 (the fundamental), 4.1, 6.2 and 8.3 (second, third and fourth harmonics) cycles per second corresponding to a respiratory rate of 125 breaths per min. In contrast, awaken mice have a breathing frequency in the range of 200-350 breaths per minutes. In future development, the breathing frequency will be used as input to a bio-feedback system controlling isoflurane flow

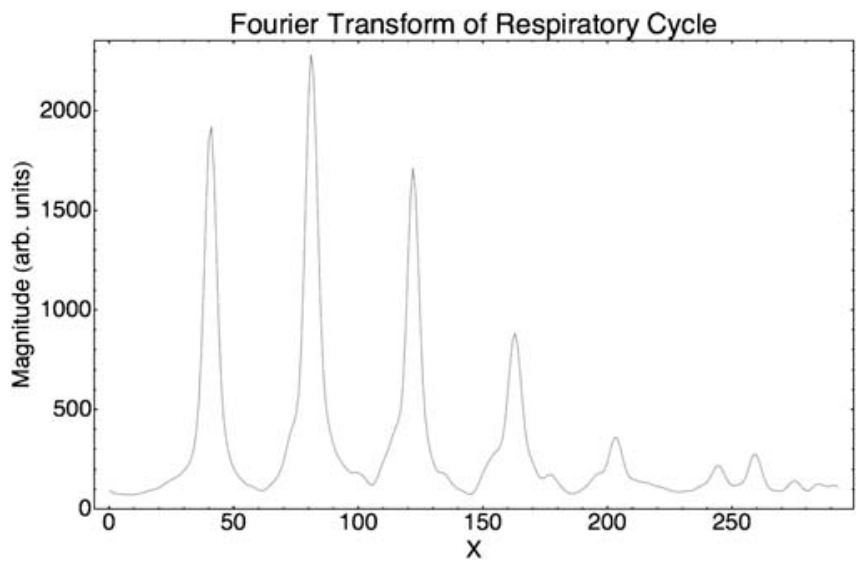

Fig. 1. Fourier Transform of the time series of the difference of successive frames. The fundamental breathing frequency and higher harmonics can be identified.

$J 621$

$\left[{ }^{18}\right.$ F]-TAGGED ION-EXCHANGE BEADS MAKE EXCELLENT POINT SOURCES FOR HIGH RESOLUTION PET CAMERAS Alan A. Wilson ${ }^{1}$, Katie Dinelle ${ }^{2}$, Sylvain Houle ${ }^{1}$, Peter Bloomfiled ${ }^{1}$ ${ }^{1}$ PET Centre, Centre for Addiction and Mental Health, Toronto, ON, Canada, ${ }^{2}$ Brain Research Centre, Vancouver Hospital and Health Science Centre, Vancouver, BC, Canada.
Point sources of radioactivity are used in tomographic imaging to evaluate functions of the imaging system such as image resolution and spatial variation of the point spread function. High resolution cameras demand point sources which combine the features of high radioactivity, small unit volume, ease of handling, and reproducible preparation. We describe here the preparation and application of $\left[{ }^{18} \mathrm{~F}\right]$-radiolabelled ion-exchange resin beads as suitable point sources for evaluation of PET cameras. Methods: Resin beads, (AG 1-X8, 20-50 mesh) were incubated with stirred, aqueous solutions of $\left[{ }^{18} \mathrm{~F}\right]$-fluoride and the rate of uptake of radioactivity measured. Variables included time, temperature, bead concentration, specific activity, and counterion. Uptake of $\left[{ }^{18} \mathrm{~F}\right]$-fluoride onto the beads were determined two methods: a) filtration:incubation was terminated by aspirating out the beads onto a filter and the filter was then washed with water. The filter was then measured for trapped radioactivity. b) sampling: uptake of the radioactivity was monitored by removing and counting small aliquots of solution from the vials into tared tubes at the appropriate times. For PET imaging individual beads were mounted in the tip of a spinal needle which, while increasing the effective size of the source, maintained the source extent to be smaller than the sampling distance of the tomograph. Results: Kinetic measurements demonstrated that uptake of $\left[{ }^{18} \mathrm{~F}\right]$-fluoride by the resin beads followed fast first order kinetics and was dependent upon bead conc., temp., and counter ion (fluoride being optimal). Added $\left[{ }^{19} \mathrm{~F}\right]$ fluoride inhibited the uptake of radioactivity. The kinetics under no-carrieradded conditions could adequately be described by the first order equation: $\%$ uptake/bead $=100 *\left(\left(1-\exp \left(-\mathrm{k}^{*}[\mathrm{~B}] * \mathrm{t}\right)\right)\right) / \mathrm{B}$ where: $\mathrm{k}=$ rate constant $/$ bead $/$ $\mathrm{ml} ;[\mathrm{B}]=\#$ beads $/ \mathrm{ml} ; \mathrm{B}=\#$ beads; and $\mathrm{t}=$ incubation time. The process was adapted for the practical production of $\left[{ }^{18} \mathrm{~F}\right]$-tagged beads as point souces using a commercial radiotracer production box. Mounted beads were used a point sources on an HRRT PET camera to measure the resolution of the tomograph following the NEMA NU 2-2001 specifications, enabling the image resolution, full width half-maximum, to be measured as $3.1 \mathrm{~mm}$ radial and tangential, and $3.4 \mathrm{~mm}$. axially at a distance $10 \mathrm{~mm}$ from the centre of the transaxial FOV. Conclusions: Ion-exchange beads can rapidly and practically be tagged with $\left[{ }^{18} \mathrm{~F}\right]$. The tagged beads have the desirable properties of a point source including small size, suitable isotope, ease of handling, and high activity.

\section{$\mathbf{J} 622$}

\section{MICRO PET IMAGING OF RAT BRAIN TUMOR WITH INFUSION OF I-124-GD LABELED METALLOFULLERENES FOLLOWED BY INTRAPERITONEAL INJECTION OF F-18 FDG}

Jianqiao Luo, John D. Wilson, Jerry I. Hirsch, Panos P. Fatouros Radiology, Virginia Commonwealth University Medical Center, Richmond, VA, USA.

OBJECTIVE: This study was designed to investigate a dual isotope technique to provide anatomical localization of I-124 infusion site and FDG uptake of a brain tumor using microPET imaging. METHOD: Two tumor-bearing rats were infused intratumorally with $2.1 \mathrm{uCi}$ I-124 Gd Tagged Metallofullerenes. The Gd MR label will permit MRI detection as well. A 2-hour post I-124 infusion PET scan was performed on each animal to identify the infusion site and possible free I-124 distribution. Following I-124 imaging, $32 \mathrm{uCi}$ and $182 \mathrm{uCi}$ of F-18 FDG were administered respectively by IP injection to highlight brain anatomy at different image contrasts. Each animal was imaged for 5 minutes using a micro PET scanner (Rodent R4, Concorde Microsystems), 350-550 keV energy window, without transmission scanning. F-18 imaging started 5 and 45 minutes subsequently after the IP injection. Sinograms were generated by histogramming (3-D, span 3 and 31 ring difference) of list mode data. Image reconstruction algorithms (FBP, 3D OSEM, 3D ReProjection with or without Hanning Filter) were used for comparison. Image processing and analysis were conducted on ASIPro VM platform. 
The infusion site and tumor were measured by FWHM from Gaussian fit of count profiles. RESULT: The post infusion PET scan showed low activity concentration of free I-124 in thyroid. The F-18 FDG distribution demonstrated better anatomy and tumor uptake when $182 \mathrm{uCi}$ was administered and waiting for $45 \mathrm{~min}$ compared with $32 \mathrm{uCi}$ and $5 \mathrm{~min}$ waiting. The 3D OSEM reconstruction improved spatial resolution and contrast compared with the FBP and 3DRP algorithms. The infusion site was $5.21 \mathrm{~mm}$ (FWHM). CONCLUSION: Dual isotope imaging of I-124 and F-18 labeled compounds demonstrated both tumor uptake and anatomical structure. Image quantification is dependent upon the reconstruction algorithm selected and the acquisition or processing protocol.

\section{Micro PET Images of A Rat Brain with intratumoral Infusion of I-124-Gd Metallofullerenes followed by Intraperitoneal injection of F-18 FDG}
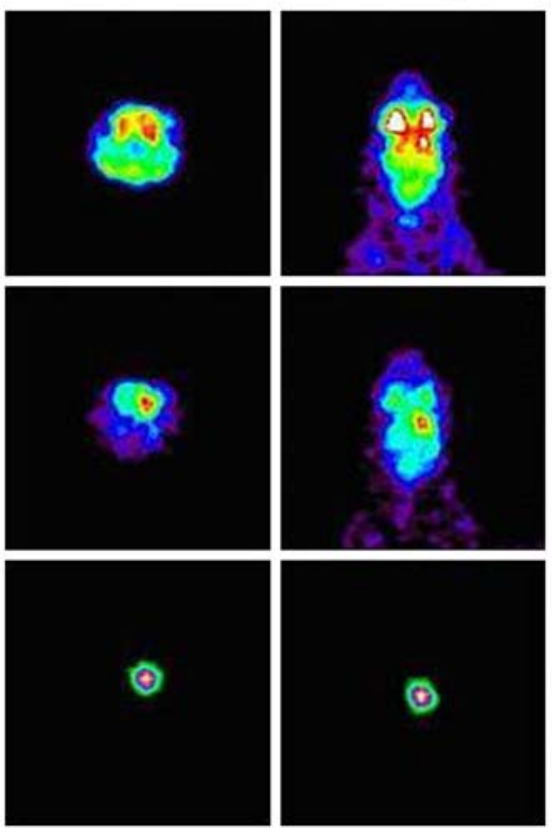

$\mathbf{J} 623$

A METHOD FOR IMPROVING THE QUANTITATIVE ACCURACY OF IMAGE RECONSTRUCTION

IN SMALL-ANIMAL PET CANCER STUDIES

Nikolai V. Slavine, Peter P. Antich

Radiology, UT Southwestern Medical Center, Dallas, TX, USA.

Modern small-animal PET systems require fast image reconstruction procedure that can operate on list-mode data and take into account most of the effects that could affect quantitation. Our image reconstruction technique is based on the one-pass list-mode high resolution iterative algorithm with system matrix modeling and was improved by image deblurring procedure and incorporating into the system model more realistic resolution kernel for describing the double Compton scattering [1]. Corrections for the ratios of geometrical efficiencies, radioisotope decay in time and photon attenuation are included in the algorithm. In our new reconstruction approach, we consider conditions that current resolution kernel could changed during the image iteration procedure, but average value will give us the same point spread function value in the median plane between PET detectors. We obtain images with higher resolution to see more important detail moreover decreased imaging processing time and noise level on reconstructed images. Data acquisition was performed on the small-animal PET system [2] which was tested with different radioactive sources, phantoms and animals to achieve high sensitivity and spatial resolution. The quantitative accuracy in PET reconstruction is particularly poor for small tumors because of partial volume errors. This effect is highly

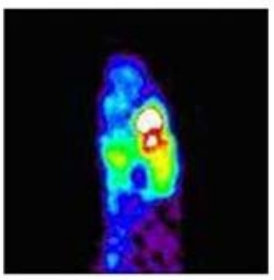

\author{
-124 Gd \& F-18 FDG \\ imaging: \\ 45 min post F-18 FDG \\ injection
}

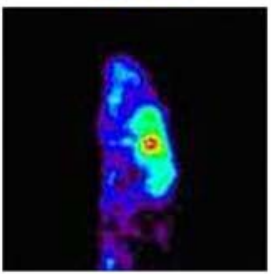

I-124 Gd \& F-18 FDG

imaging:

5 min post F-18 FDG

injection

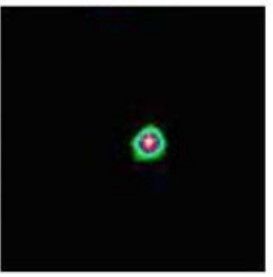

\author{
I-124 Gd imaging: \\ 2 hours post $1-124 \mathrm{Gd}$ \\ infusion. \\ prior to F-18 FDG \\ Injection
}

relevant to the advancement of molecular medicine, particularly in regard to early cancer diagnosis and prognosis. Recently [3] we described a new practical method for radioactivity distribution analysis in volume of interest from reconstructed PET images. We are going to combine both our methods with partial volume correction techniques. Our study provides further evidence validating the use of this reconstruction approach to enhance the assessment of characteristics of small-animal tumor growth, identifying metastases and potentially determining the effectiveness of cancer treatment. [1] Antich P et al Application of Expectation Maximization Algorithms for Image Resolution Improvement in a Small-Animal PET System. IEEE Trans Nucl Sci, 53: 684-690, 2005. [2] Tsyganov E et al UT SW Small Animal Positron Emission Imager. IEEE Trans Nucl Sci, 53:2591-2600, 2006. [3] Slavine N, Antich P. Practical Method for Radioactivity Distribution Analysis in Small-animal PET Cancer Studies, Appl Radiat and Isot, 66:1861-1869, 2008.

$\mathbf{J 6 2 4}$

\section{REPRODUCIBILITY OF INTERNATIONAL HARMONIZATION PROJECT (IHP)-BASED INTERPRETATIONS OF POSTTHERAPY PET/CT IN PATIENTS WITH HODGKIN'S AND AGGRESSIVE NON-HODGKIN'S LYMPHOMA \\ Damita Thomas $^{1}$, Kevin Berbaum ${ }^{1}$, David Bushnell ${ }^{2,1}$, Michael Graham ${ }^{1}$, $\overline{\text { Malik E. Juweid }}^{1}$ \\ ${ }^{1}$ Radiology/Div of Nuclear Medicine, University of Iowa Hospitals and Clinics, Iowa City, IA, USA, ${ }^{2}$ Radiology/Division of Nuclear Medicine, Veterans' Administration Medical Center, Iowa City, IA, USA.}


Background: IHP-based interpretation of PET/CT post-tx of lymphoma is now widely used but there is little data regarding its reproducibility and accuracy. We determined interobserver variability using IHP criteria and compared its accuracy with semiquantitative approaches using the residual mass (RM) absolute standardized uptake value (SUVmax) alone and the ratio of the RM SUVmax to mediastinal blood pool (MBP) SUVmean. Methods: Three expert nuclear medicine physicians blinded to patient $(\mathrm{pt})$ outcome independently visually interpreted post-tx PET/CT scans based on the IHP-criteria. RMs were scored on a scale of $0-4$, where 3-4 was positive for $R M s \geq 2 \mathrm{~cm}$ whereas $1-4$ was positive for $\mathrm{RMs}<2 \mathrm{~cm}$; scans without RMs were similarly scored, depending on whether there were PET positive or negative. RM SUVmax, MBP SUVmean and the RM SUVmax to MBP SUVmean ratio were determined. Since outcome data were available on all 50 pts (median follow-up of pts without progression=49 months), the positive predictive value (PPV), negative predictive value (NPV) and accuracy for prediction of residual disease/progression were compared between the IHPcriteria and the semiquantitative approaches. Results: A total of 50 post-tx scans in 50 pts with aggressive NHL $(n=24)$ and HL $(n=26)$ were interpreted by the 3 readers. On a patient-by-patient basis, the 3 readers had were in complete agreement with respect to final scan interpretation (i.e., pos/neg) $90 \%$ of the time using the IHPcriteria. Percentage of PET + scans ranged from $20 \%-30 \%$ and PET + RMs from $45 \%-55 \%$ for the 3 readers. On a per-patient basis, visual criteria resulted in PPVs of $47 \%-70 \%$; NPVs of $83 \%-85 \%$ and accuracies of $72 \%-82 \%$ for the 3 readers. In contrast, cut-off RM SUVmax of 2.5 or cut-off RM SUVmax to MBP SUVmean ratio of 1.75 resulted in PPV, NPV and accuracy of $75 \%, 83 \%$ and $82 \%$. Conclusions: Visual IHP-criteria provide fairly reproducible interpretations among nuclear medicine experts with fair to moderate diagnostic/ prognostic accuracy. Compared with the IHP criteria, the semiquantitative approaches provide, at least for some readers, substantial improvement in PPV and overall accuracy. These approaches should, therefore be considered for a more consistent scan interpretations after standardization of the timing of imaging after FDG injection.

\section{J625}

RESOLUTION RECOVERY BY ITERATIVE DECONVOLUTION FOR IMAGING MONKEY BRAIN WITH A HUMAN PET CAMERA Nicolas Costes $^{1}$, Vincent Leviel ${ }^{2}$, Franck Lavenne ${ }^{1}$, Gérard Gimenez ${ }^{1}$, Anthonin Reilhac ${ }^{3}$

${ }^{1}$ PET department, CERMEP - imagerie du vivant, LYON, France, ${ }^{2} \mathrm{U} 486$, INSERM, LYON, France, ${ }^{3}$ Biospective, MONTREAL, QC, Canada.

Imaging primate brain with whole body human PET camera is relevant since transaxial field of view and accessibility of microPETs are reduced. But monkey's cerebral brains structures are small and close to the PET resolution, leading to a partial volume effect (PVE), affecting time activity curves (TAC) and binding quantification. Most efficient PVE correction methods rely on having delineated anatomical brain structures spatially registered with the PET images. Recently, recovery methods based on iterative deconvolution have been proposed and their performances compared with GTM standard method (Tohka, J 2008). These promising recovery methods are here applied to dynamic PET acquisitions of dopamine transporter imaging with [11C] PE2I of a MPTP-treated Parkinson's monkey model. Optimization, performance and accuracy is assessed with actual data compared to simulations. For each monkey, five (one-month spaced) scans of [11C]PE2I were recorded on a Siemens HR + PET camera. Regional TACs extracted from delineated striatal regions were processed with an SRTM model to quantify binding potential (BP), $\mathrm{k} 2$ efflux rate, and delivery ratio between target and reference region (R1). Bias, standard error and intraclass coefficient were computed.
Moreover, thanks to the generation of voxel based corrected dynamic images, parametric images of binding potential were computed with the SRTM model. Statistical parametric mapping revealed the improvement obtained with the method in term of sensitivity thanks to the recovery of true $\mathrm{BP}$, especially with the low level of binding reached because of the treatment. Complete quantitative evaluation of the performance will be provided for the conference. Preliminary results show that iterative deconvolution of the PET image is an efficient alternative method for resolution recovery and partial volume correction, without anatomical information, allowing the computation of quantitative parametric image of binding potential. Preliminary results show that iterative deconvolution of the PET image is an efficient alternative method for resolution recovery and partial volume correction, without anatomical information, allowing the computation of quantitative parametric image of binding potential.

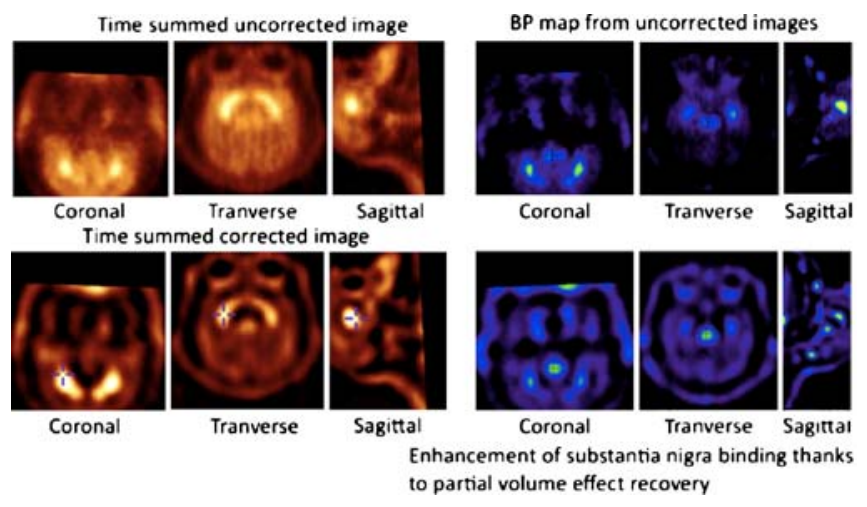

J626

\section{QUANTITATIVE FDG PET IMAGING IN MICE FOR EVALUATION OF INTRAVENOUS INJECTION CATHETER SIZES}

Douglass C. Vines $^{1,2}$, David E. Green ${ }^{1}$, Shawn Stapleton ${ }^{3}$, Justin Grant ${ }^{1}$, Stephen L. Breen ${ }^{1,2}$

${ }^{1}$ Radiation Medicine Program, Princess Margaret Hospital, Toronto, ON, Canada, ${ }^{2}$ Radiation Oncology, University of Toronto, Toronto, ON, Canada, ${ }^{3}$ Medical Biophysics, University of Toronto, Toronto, ON, Canada.

Purpose: Many imaging studies of mice often require access to the venous system for delivery of an imaging agent. In PET 30 gauge (Ga) needles are commonly used for mouse tail vein injections, a larger $26 \mathrm{Ga}$ angiocatheter may be preferred for subsequent delivery of CT or MR agents to achieve higher flow rates. The purpose of this study was to evaluate both qualitatively and quantitatively the use of a $26 \mathrm{Ga}$ angiocath and $30 \mathrm{Ga}$ needle catheter in mice using PET. Methods: Mice were fasted $4 \mathrm{~h}$ then injected with 3.1-14.5 MBq FDG via lateral tail vein using either a $26 \mathrm{Ga}$ angiocath $(n=10)$ or $30 \mathrm{Ga}$ needle $(n=10)$ attached to polyethylene tubing with a blunt $30 \mathrm{Ga}$ luer lock end. During injection, qualitative assessment of the injection was performed and recorded as good (G) [no resistance, blood return after injection, and no tail blanching], intermediate (I) [slight resistance, no blood return, and slight blanching of tail], or poor (P) [significant resistance and tail blanching, no blood return]. After 60 min uptake, a 10 min PET scan of the body followed by a 2 min tail scan were acquired using a Siemens Focus 220 scanner. Images of tail injection sites were quantitatively assessed by determining a mean percent injected dose (\%ID) from a 3D region-of-interest. To assess baseline amount of FDG in the tail, 2 additional mice were injected by intra- 
peritoneal (IP) method and scanned after 60 min uptake. High frequency ultrasound (US) imaging was performed on 5 mice tails with catheters remaining at the FDG injection site to evaluate catheter location with respect to the tail vein. Results: Mean \%ID of the tail for IP injections was 0.7. Qualitative (QL) and quantitative (\%ID) data for both 26 and $30 \mathrm{Ga}$ are listed in the table. Of the 5 mice tails imaged by US, one had a $30 \mathrm{Ga}(\mathrm{G})$ injection and 4 had $26 \mathrm{Ga}$ (2G and $2 \mathrm{I})$ injections. US confirmed placement of $26 \mathrm{Ga}(n=2)$ and $30 \mathrm{Ga}(n=1)$ inside the tail vein. US also revealed venous constriction at the tip of the $26 \mathrm{Ga}(n=2)$ and that the angiocath was larger than the diameter of the vein for all $26 \mathrm{Ga}$ mice. Conclusions: The success rate for FDG injection with $26 \mathrm{Ga}$ angiocath and $30 \mathrm{Ga}$ needle catheter was 50 and $90 \%$, respectively. The use of a $26 \mathrm{Ga}$ angiocath may permit venous access and higher flow rates for various imaging agents. However, the success rate of $26 \mathrm{Ga}$ injection is low, which may be related to the size of the catheter.

Qualitative and Quantitative data

\begin{tabular}{|c|c|c|c|c|c|c|}
\hline \multicolumn{9}{|c|}{ QL } & \multicolumn{3}{c|}{ \%ID } \\
\hline & G & I & P & G & I & P \\
\hline $26 \mathrm{Ga}$ & 5 & 4 & 1 & 0.9 & 10.0 & 16.3 \\
\hline $30 \mathrm{Ga}$ & 9 & 1 & 0 & 0.8 & 5.2 & 0 \\
\hline
\end{tabular}

\section{$\mathrm{J} 627$}

\section{DYNAMIC PET TO ASSESS EARLY DIABETIC CARDIOMYOPATHY AND DIASTOLIC DYSFUNCTION IN A PRECLINICAL MODEL}

Said Daibes Figueroa ${ }^{1,3}$, Jenna M. Rehmer ${ }^{1,2}$, Nathan Rehmer ${ }^{1,2}$, Samantha Sublett $^{1,3}$, Tammy L. Rold ${ }^{1,2}$, Lixin Ma ${ }^{1,3}$, Rebecca I. Schneider ${ }^{1,2}$, Adam T. Whaley-Connell ${ }^{1,2}$, Jim R. Sowers ${ }^{1,2}$, Timothy J. Hoffman ${ }^{1,2}$

${ }^{1}$ Research Services, Harry S Truman VA Hospital, Columbia, MO, USA, ${ }^{2}$ Department of Internal Medicine, University of MissouriColumbia, Columbia, MO, USA, ${ }^{3}$ Department of Radiology, University of Missouri-Columbia, Columbia, MO, USA.

Diastolic dysfunction (DD) is often present in pre-diabetic patients that manifest insulin resistance but have not developed clinical hyperglycemia. Thereby, recognition of diabetic associated DD represents a vital moment by which clinicians can intercede to potentially arrest the development of diabetic related cardiovascular disease (CVD) at an early stage. PET allows scientists the capability to noninvasively monitor disease progression and response to therapy interventions. Objective: To explore the utility of dynamic PET imaging in early detection and monitoring of DD as a secondary effect from diabetic cardiomyopathy in a transgenic diabetic model. Methods: Two groups [obese diabetic (ZFM) and lean controls (ZLM)] $(N=6)$ of the transgenic Zucker male rat model were employed. They were imaged longitudinally via microPET at 5, 7 and 12 weeks (wks). Fasted subjects were administered $18.5 \mathrm{MBq}$ of 18F-FDG under anesthesia gas. Dynamic list-mode PET imaging data was collected for 60 minutes and sorted into a multi-frame 3D sinogram, and later reconstructed employing a 3D-RAMLA algorithm. Left ventricle (LV) and myocardium uptake, with a 2.5 zoom, were investigated using the Imalytics Workspace via SUV, ROI, time activity curves and T/NT ratio analysis comparing both groups. Imaging findings were correlated to blood metabolite sampling data. Results: PET imaging demonstrated an average LV SUV of $2.1 \pm 0.8$ for both groups at 5 and $7 \mathrm{wks}$ at $60 \mathrm{~min}$ PI, however at $12 \mathrm{wks}$ the ZFM exhibited an SUV of $1.9 \pm 0.3$ vs $5.8 \pm 1.4$ for the ZLM. At 12 wks the ZFM exhibited hyperglycemia and peak insulin resistance with diminished $\mathrm{LV}$ uptake. ROI analysis at 5 and 7 wks for the ZLM group demonstrated favorable uptake and LV differentiation in the short axis and horizontal long axis views. Early indications of LV hypertrophy and dysfunction was noted in four of the subjects analyzed (ZFM group) when assessing the vertical long axis views at 12 wks. Time activity curves and T/NT ratios were very comparable among both groups at 5 and 7 wks, however the opposite was seen at 12 wks. Conclusions: A preclinical model with early indicators for diabetic LV cardiomyopathy was characterized via dynamic PET molecular imaging. There was an apparent correlation between the 18F-FDG uptake time course imaging results and tissue functional metabolite findings. Dynamic PET imaging offers researchers a promising approach for noninvasive evaluation of the tissue inflammatory pathways leading to CVD as a secondary effect from the progression of diabetes.

\section{J628}

\section{MEASURING THE CEREBRAL METABOLIC RATES OF GLUCOSE IN RATS USING FDG-PET AND WHOLE BLOOD SAMPLES}

Hsiao-Ming C. $\mathrm{Wu}^{1,3}$, Neil G. Harris ${ }^{2}$, Sima Ghavim² ${ }^{2}$, Waldemar Ladno ${ }^{1}$, Richard L. Sutton ${ }^{2}$

${ }^{1}$ Crump Institute for Molecular Imaging, University of California, Los Angeles, Los Angeles, CA, USA, ${ }^{2}$ UCLA Brain Injury Research Center, University of California, Los Angeles, Los Angeles, CA, USA, ${ }^{3}$ Department of Molecular and Medical Pharmacology, University of California, Los Angeles, Los Angeles, CA, USA.

Measuring the cerebral metabolic rates of glucose (CMRG) using FDGPET in rats requires the FDG concentrations in plasma (i.e. input function; IF). Traditionally these values have been obtained from plasma after serial arterial blood samples have been hand-drawn and centrifuged. Several alternative methods, including the image-derived blood curve, have been used to avoid the technical challenges of blood sampling. Most of these methods use whole-blood (WB) FDG concentrations in the CMRG calculations. The purpose of this study was to evaluate the validity of using WB samples in calculation of the CMRG. Methods: Eight male, SD rats $(\mathrm{wt}=316 \pm 50 \mathrm{~g}$ ) with a wide range $(8.1-19.8 \mathrm{mM})$ of plasma glucose concentrations were studied. Each rat had surgery to catheterize both femoral arteries. A $60 \mathrm{~min}$ dynamic FDG-PET scan and a 10 min CT scan were taken immediately after the surgery. During the scan, 18 blood samples $\left(\sim 100 \mu \mathrm{L}\right.$ each; $\left.\mathrm{IF}_{\text {manual-wB }}\right)$ were hand-drawn from the right artery and 18 blood samples $(\sim 0.56 \mu \mathrm{L}$ each; $\left.\mathrm{IF}_{\text {auto-WB}}\right)$ were machine-drawn from the left artery using an automated microfluidic device. Aliquots of the hand-drawn blood samples were centrifuged immediately to obtain plasma (pl) FDG curve ( $\left.\mathrm{IF}_{\text {manual-pl }}\right)$. A population FDG plasma:WB ratio curve was derived from the hand-drawn 
blood samples and used to calculate the plasma FDG concentrations $\left(\mathrm{IF}_{\text {auto-pl }}\right)$ in the machine-drawn blood samples. The CMRG of the frontal cortex of each rat were calculated using the conventional FDG compartment $(3 k)$ model, Patlak analysis, and the Operational equation. Using the CMRG obtained from the $\mathrm{IF}_{\text {manual-pl }}$ as the gold standards, those obtained from other blood curves were evaluated. Results: Table 1 lists the CMRG ( $\mu \mathrm{mol} / 100 \mathrm{~g} /$ min) estimated from the different methods using the four different blood curves as the IF. The CMRG measurement obtained using the whole blood samples were significantly ( $p<0.01$; labeled with '*') larger than those measured using the traditional $\mathrm{IF}_{\text {manual-pl }}$ method. No significant $(p>0.3)$ difference was found for the CMRG obtained by using the $\mathrm{IF}_{\text {manual-pl }}$ versus the $\mathrm{IF}_{\text {auto-pl. }}$. Conclusions: Quantitative FDG-PET study in rats using whole blood FDG concentrations significantly overestimated regional CMRG. Our study indicates that the CMRG can be measured reliably using dynamic FDG-PET and whole blood samples if a population FDG plasma:WB ratio curve is established for use in calculations.

\begin{tabular}{|c|c|c|c|c|}
\hline & IF manual-pl & IF manual-WB & IF auto-pl & IF auto-WB \\
\hline Compartment model & $\mathbf{6 0 . 9} \pm \mathbf{5 . 6}$ & $68.8 \pm 7.6^{*}$ & $59.9 \pm 6.9$ & $68.3 \pm 8.4^{*}$ \\
\hline Patlak analysis & $\mathbf{5 9 . 5} \pm \mathbf{7 . 3}$ & $66.6 \pm 9.5^{*}$ & $58.7 \pm 7.2$ & $66.6 \pm 9.5^{*}$ \\
\hline Operational equation & $\mathbf{6 5 . 9} \pm \mathbf{7 . 7}$ & $87.8 \pm 9.2^{*}$ & $66.0 \pm 7.9$ & $89.9 \pm 10.2^{*}$ \\
\hline
\end{tabular}

J629

\section{IMPROVEMENTS OF DEPTH OF INTERACTION ENCODING DETECTORS WITH PHOSPHOR-COATED CRYSTALS}

Emilie Roncali, Huini Du, Yongfeng Yang, Simon R. Cherry Biomedical Engineering, UC Davis, Davis, CA, USA.

Incorporating depth of interaction (DOI) information in the design of positron emission tomography (PET) detectors enables the reduction of parallax errors in small ring diameter PET scanners and the enhancement of the sensitivity, as longer crystals may be used. Several depthencoding detector designs have been proposed and there has been significant progress in this important area in recent years. An innovative solution combining the advantages of continuous DOI information and single-ended readout of the scintillator has been proposed by $\mathrm{Du}$ et al [1]. The surface of lutetium oxyorthosilicate (LSO) crystals has been partially coated with a yttrium-aluminiumgallium oxide (YGG) phosphor and coupled to a photodetector, which detects both the mixture of the scintillation light from the LSO and the light converted by the phosphor. This converted light is wavelength shifted and presents a longer decay time due to the phosphor. The decay time of the recorded light pulse depends on the fraction of light converted by the YGG coating, which is related to the depth of interaction. Therefore, measuring the decay time variations provides information regarding the DOI. A resolution of $8 \mathrm{~mm}$ has been achieved using photomultiplier tube readout of the phosphor-coated scintillator array [1]. To improve upon these results, several concepts are being explored. First, the use of silicon photomultipliers is expected to enhance the depth of interaction resolution, since the photodetection efficiency they exhibit at the phosphor emission wavelengths is higher than the quantum efficiency of photomultiplier tubes. Initial experiments conducted with Multi-Pixel Photon Counter devices (MPPC, Hamamatsu Photonics) produced promising results in decay time measurements. Additional testing will be performed on devices from other manufacturers. Secondly, optimization of the phosphor material and coating processes is being investigated. Finally, two methods are being implemented to provide additional information to the pulse shape analysis to gain in robustness and accuracy of DOI determination. The first option consists of using spectral information, although currently used photodetectors are not wavelength sensitive. A second alternative involves exploring variations in rise times along the crystal length. Preliminary results using MPPCs showed a difference of 4 ns with $20 \mathrm{~mm}$ LSO crystals coated with phosphor and warrant further investigation. [1] H. Du et al., "Continuous depth-ofinteraction encoding using phosphor-coated scintillators" Phys. Med. Biol. 54 (2009) 1757-1771

\section{$\mathbf{J 6 3 0}$}

\section{ASPECTS OF HIGH-RESOLUTION PET FOR PLANT STUDIES}

Simone Beer ${ }^{1}$, Jonas Buehler ${ }^{2}$, Thomas Hombach ${ }^{2}$, Siegfried Jahnke ${ }^{2}$, Horst Larue $^{1}$, Christoph Parl ${ }^{1}$, Gerd Roeb ${ }^{2}$, Ulrich Schurr ${ }^{2}$, Matthias Streun ${ }^{1}$, Karl Ziemons ${ }^{1}$

${ }^{1}$ Central Institute for Electronics, Research Center Juelich, Juelich, Germany, ${ }^{2}$ ICG-3: Phytosphere, Research Center Juelich, Juelich, Germany.

Positron emitters like ${ }^{11} \mathrm{C},{ }^{13} \mathrm{~N}$ and ${ }^{18} \mathrm{~F}$ are widely used in clinical diagnosis and animal studies, but can as well be applied to study noninvasively metabolic and physiological functions in plants. ${ }^{11} \mathrm{CO}_{2}$, for example, can be applied to leaves to trace the movement of photosynthetic products in plants. The monitoring of the labeled compounds within the intact plant has been realized so far by a set of collimated detectors, by detectors in coincidence mode [1] or by a planar camera [2]. These studies are limited to plants or part of plants growing in a planar fashion. A new Plant Tomographic Imaging System (PlanTIS), based on ClearPET technology [3], is used in our center to study the dynamics of carbon supply from source leaves to different plant organs including roots in soil and bulky organs such as beets or fruits. The scanner consists of LSO and LuYAP scintillator crystals in phoswich configuration, which are coupled directly to position sensitive PMT's. Data are acquired in list mode and are reconstructed iteratively. We will demonstrate the initial performance of the scanner and first applications, like a 5 minute scan of barley roots in soil after ${ }^{11} \mathrm{C}$ uptake by the leaves (fig.1). We will discuss the main challenges of this new application in comparison with small-animal PET, e.g. the requirements of deadtime correction, influence of activity from outside the field of view due to the gas supply and questions of attenuation in soil. [1] Minchin P E H, Thorpe M R 2003 Using the short-lived isotope ${ }^{11} \mathrm{C}$ in mechanistic studies of photosynthate transport Funct. Plant Biol. 30 83141 [2] Kawachi N, et al. 2006 Kinetic analysis of carbon-11-labeled carbon dioxide for studying photosynthesis in a leaf using Positron Emitting Tracer Imaging System IEEE Trans. Nucl. Sci. 53 2991-7 [3] Ziemons K, 
et al 2005 The ClearPET project: development of a 2nd genertion highperformance small animal PET scanner Nucl. Instr. Meth. A 537 307-11

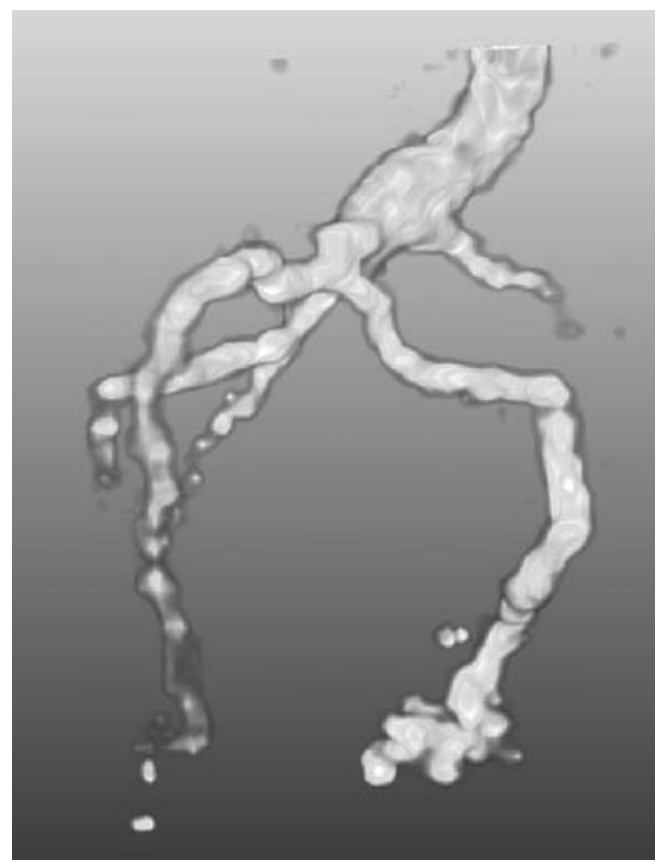

PET scan of a barley root in soil after ${ }^{11} \mathrm{C}$ application to a leaf.

\section{$\mathbf{J 6 3 1}$}

\section{QUANTIFICATION OF F-18 MICRO PET IMAGING OF ECT PHANTOM WITH HOT SPHERES IN ADJUSTED BACKGROUND ACTIVITY}

Jianqiao Luo $^{1}$, Sunyoung Jang ${ }^{2}$

${ }^{1}$ Radiology, Virginia Commonwealth University Medical Center, Richmond, VA, USA, ${ }^{2}$ Radiation Oncology, Brown University Medical Center, Providence, RI, USA.

Purpose: To investigate the image quantification and corrections in micro PET Imaging the ECT phantom data with radioactive hollow spheres at different background activities were acquired and reconstructed using four reconstruction algorithms. Methods: Six hollow spheres (Data Spectrum Micro Spheres: dia. of 12.43, 9.89, 7.86, 6.23, 4.95 and $3.95 \mathrm{~mm}$ ) in a cylinder(net volume: $110 \mathrm{~mL}$ with hot rods insert) were injected with 6.29 MBq of F-18 FDG and the cylinder was filled sequentially with $175.38 \mathrm{MBq}$ of F-18 FDG in 7 doses to adjust target to background ratios (sphere over cylinder) from $14.7,7.4,5.6,4.5,3.8,3.3$ to 2.9 , respectively. The phantom was imaged using a micro PET scanner (Rodent R4, Concorde Microsystems) with 1-bed imaging for $5 \mathrm{~min}$., $350-550 \mathrm{keV}$ energy window, no transmission scan. First imaging started with no background activity, followed by 7 scans of increased activity in the cylinder. List mode data were rebined by histogramming (3D, span 3 and 31 ring difference) to produce sinograms for mage reconstruction. Four reconstruction algorithms (FBP, 3D OSEM, and 3D Re-Projection) were used for comparison. Image processing and analysis were conducted on ASIPro-VM platform. Results: Image quality improved using OSEM reconstruction especially when background activity increased. Measured ratios of activity concentration agreed with dose calibrator reading for two large spheres but much lower when diameter of the spheres was below $7.86 \mathrm{~mm}$. Sphere activities were under estimated for large spheres but over counted for spheres less than $6.23 \mathrm{~mm}$. Higher sensitivity (by ROI counts) was observed for spheres near center of rotation particularly for lower background activities. Sphere sizes measured by FWHM of Gaussian fit of profiles were under estimated and changed with background activities. Conclusions: Image quantification for micro PET depends on size of the object, background activity and reconstruction algorithm. Correction factors should be used whenever necessary. And quality control in acquisition and processing is key to obtain reliable images in micro PET imaging.

Measured Ratios (sphere/cylinder) of Activity Concentration

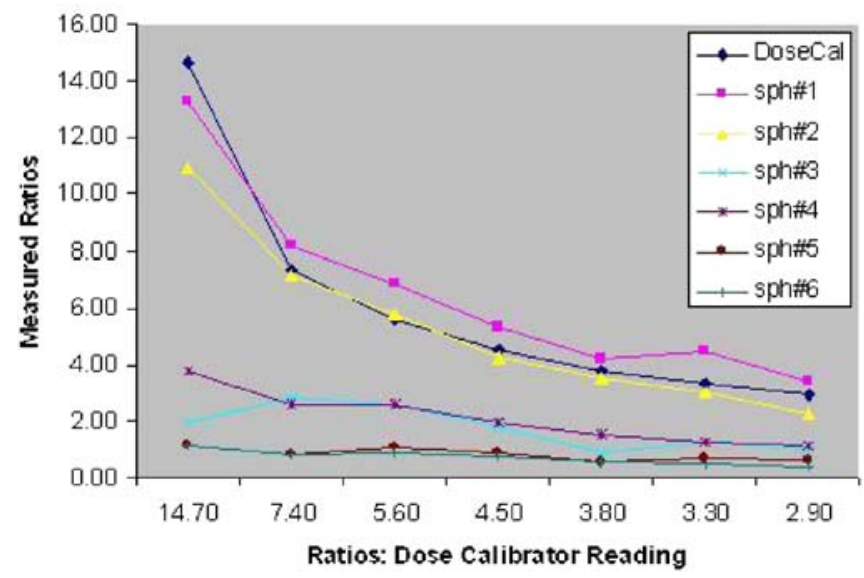

J632

\section{MEASUREMENT OF THE SIGNAL-TO-NOISE RATIO IN RECONSTRUCTED FDG PET BRAIN IMAGES}

Brad Kemp, Val Lowe, Brian Mullan

Department of Radiology, Mayo Clinic, Rochester, MN, USA.

The signal-to-noise ratio (SNR) characteristics of three 3D reconstruction algorithms were evaluated for brain PET studies on the GE Discovery RX PET/CT system. The three 3D algorithms were: Fourier-rebinning weighted least-squares ordered subset expectation maximization (FORE-WLS-OSEM), fully 3D OSEM (3D-OSEM) and reprojection (RP). Point source experiments were conducted to select the parameters that match the resolution of the algorithms. To investigate how SNR varies with noise equivalent counts (NEC), a phantom filled with $\mathrm{C}-11$ was scanned for 8 halflives. A pulse generator operating at 60 beats $/ \mathrm{min}$ was connected to the scanner and at $10 \mathrm{~min}$ intervals a list mode acquisition was acquired. The list data was 'unlisted' into a gated study consisting of 20 time bins (replicates), which were reconstructed with the three algorithms. A SNR image at each time point was generated as the ratio of mean and standard deviation (noise) images, and the regression of SNR as a function of NEC was calculated. This gated replicate method was applied to eight FDG patient scans. For a linear reconstruction the SNR is expected to vary as $\mathrm{NEC}^{\wedge} 0.5$, and the SNR for FORE-WLS-OSEM, 3D-OSEM and RP varied as $\mathrm{NEC}^{\wedge} 0.26, \mathrm{NEC}^{\wedge} 0.45$ and $\mathrm{NEC}^{\wedge} 0.50$, resp, with FORE-WLSOSEM images having the highest and RP images the lowest SNR. The SNR of the patient scans have the same ranking as for the phantom study, with the mean images for each reconstruction method very similar. The differences in SNR are due to the noise (see Figure). The RP noise images contain the highest magnitude of noise which extends throughout the image. The 3D-OSEM noise 
images contain localized noise that resemble the mean image; the 3D-OSEM images have the lowest noise in low uptake regions. Knowledge of the SNR of a reconstruction algorithm is important in understanding its performance.

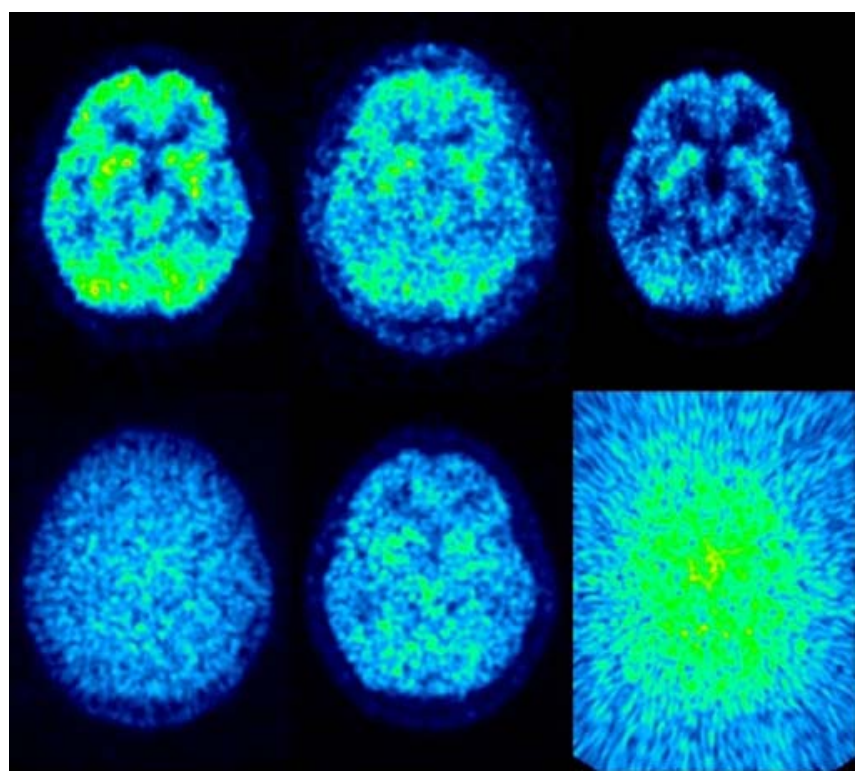

SNR (top row) and noise (bottom row) images for FORE-WLS-OSEM, 3D-OSEM and RP reconstruction algorithms (left to right). Each row of images is set to the same intensity range.

J633

\section{ALGORITHMS FOR THE DETECTION AND LOCALIZATION OF STEM AND PROGENITOR CELLS IN LOW SIGNAL-TO-NOISE RATIO PET STUDIES}

Melissa Freedenberg ${ }^{1}$, Simon R. Cherry ${ }^{1}$, Alice F. Tarantal ${ }^{2,3}$

${ }^{\mathrm{D}}$ Department of Biomedical Engineering, University of California, Davis, CA, USA, ${ }^{2}$ Center of Excellence in Translational Human Stem Cell Research, California National Primate Research Center, University of California, Davis, CA, USA, ${ }^{3}$ Departments of Pediatrics and Cell Biology and Human Anatomy, University of California, Davis, CA, USA.

We present a simple, probabilistic two-dimensional method for reconstructing and localizing very low signal-to-noise ratio (SNR) PET data from radiolabeled cells. Dynamic quantification of stem cell transport in vivo is a major challenge which must be addressed to determine the efficacy of targeted stem cell therapies. PET radionuclides with long half-lives are ideal for translational dynamic stem cell imaging over several days since PET can measure very small concentrations of radiotracer deep inside tissue. However, traditional PET scanners and reconstruction software have not been optimized for stem cell imaging, which inherently has a low SNR. Traditional PET applications, such as imaging accumulation of ${ }^{18} \mathrm{~F}$-fluorodeoxyglucose in tumors, are characterized by high injected activities of several $\mathrm{mCi}$, and therefore high tissue concentrations and relatively high SNR. Images of radio- labeled stem cells have a much lower SNR because the amount of radioactivity that a stem cell can be labeled with is limited by cell toxicity; a typical stem cell injection of several million cells has only a total of a few $\mu \mathrm{Ci}$ of activity. Further complicating matters, the LSO or LYSO crystals on which many PET scanners are based produce their own background radiation. Preliminary studies have shown that these PET scanners and associated image reconstruction methods are ineffective for imaging low radionuclide activities relevant to stem cell trafficking studies. Our current aim is to increase the detection limits for stem cell imaging by designing a probability-weighted backprojection image reconstruction method to identify stem cell foci in vivo. $\langle\mathrm{p}\rangle$ Experiments were performed using an young monkey-sized, water-filled phantom containing a single $1 \mathrm{~mL}$ positron-emitting source to simulate a low SNR signal in tissue. Long-duration scans were acquired using the microPET P4 scanner as the source decayed over many orders of magnitude of activity levels. Projection data were reorganized into 2-D datasets using single-slice rebinning (SSRB), and each SSRB plane was reconstructed using a modified voxel-driven backprojection algorithm. The probability that each backprojected voxel originated from either phantom or background was calculated based on the number of counts in each voxel, and probability maps of the source distribution were created to determine the location of the activity. This work has facilitated interpretation of PET data in very low SNR situations where only a small fraction of the lines of response contain events. $<$ P $>$

\section{$\mathbf{J} 634$}

DEVELOPMENT OF MICROFLUIDIC PLASMA COUNTING SYSTEM FOR SMALL ANIMAL MOLECULAR IMAGING USING PET —MEASUREMENT OF SMALL RADIOACTIVITY CONCENTRATION-

Yuichi Kimura $^{1}$, Chie Seki ${ }^{1}$, Nobuya Hashizume ${ }^{2,1}$,

Takahiro Nishimoto $^{2}$, Keishi Kitamura ${ }^{2,1}$, Iwao Kanno ${ }^{1}$

${ }^{1}$ Molecular Imaging Center, National Radiological Sciences, Chiba, Japan,

${ }^{2}$ Technology Research Laboratory, Shimadzu Corporation, Kyoto, Japan.

[Introduction] The system for $\mu$ L-ordered blood sampling and plasma radioactive concentration measurement, Microfluidic Mouse Plasma Counting System ( $\mu \mathrm{FmPC}$, patent pending), is developing. $\mu \mathrm{FmPC}$ should measure very small radioactivity concentration, from several to hundreds $\mathrm{Bq} / \mu \mathrm{L}$. This study aimed to investigate the accuracy on such small radioactivity concentration. [Method] In $\mu \mathrm{FmPC}$, blood was sampled using microfluidic technique, and it was stocked in channels on a plastic disc (CD-Well). The blood was then separated by rotating the disc. Because the channels had exact cross-sectional area of $0.067 \mathrm{~mm} 2$, the volumes of blood and separated plasma could be derived by measurement of the length using a flatbed scanner. The amount of radioactivity was acquired by a storage phosphor screen. In the experiments, one and three $\mu \mathrm{L}$ of FDG solutions containing the radioactivity ranging from 4 to $128 \mathrm{~Bq} / \mu \mathrm{L}$ were introduced in the channels. The radioactivity was measured using Imaging Plate (IP) and BAS-5000 (Fujifilm Corp., Japan) with 120 -minute exposure. CD-Well was also scanned by GT-X970 scanner (Seiko EPSON, Japan) in $2400 \mathrm{dpi}$ in order to measure the volume of solution. The activity concentration of given FDG was compared with a photo-stimulated luminescence value (PSL) derived from $\mu \mathrm{FmPC}$. [Results and Discussion] Measured images were shown in Fig. 1: a scanned image (A) and an image from IP 
(B). The position of introduced FDG was detected as shown in (C) with black thick curves, and it was applied to the IP image to read out an amount of radioactivity. (D) was a superimposed image. From 120-minute exposure, the PSL was correlated with the given concentration well $(y=1.8 \times+4.4$ for $1 \mu \mathrm{L}$ case and $\mathrm{y}=$ $2.1 \times+6.6$ for $3 \mu \mathrm{L}$ case. $\mathrm{r} 2=1.00$ for both cases). We can conclude that the concept of $\mu \mathrm{FmPC}$ had potential to measure small radioactivity concentration.

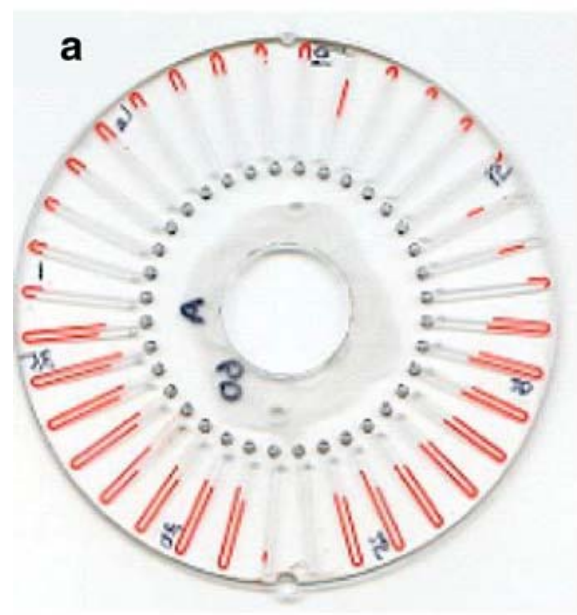

b

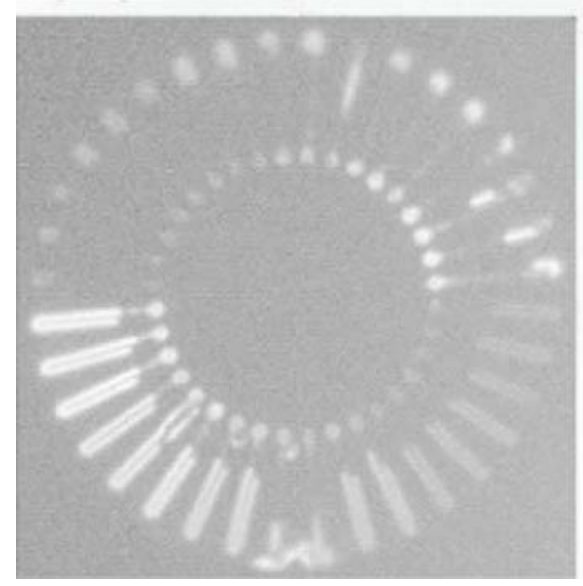

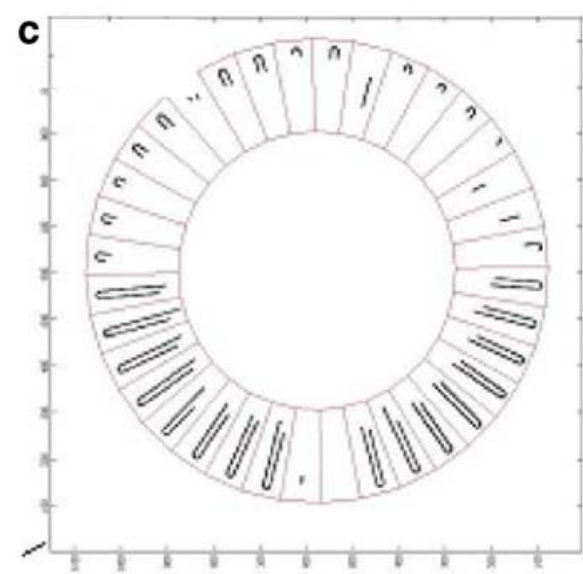

d

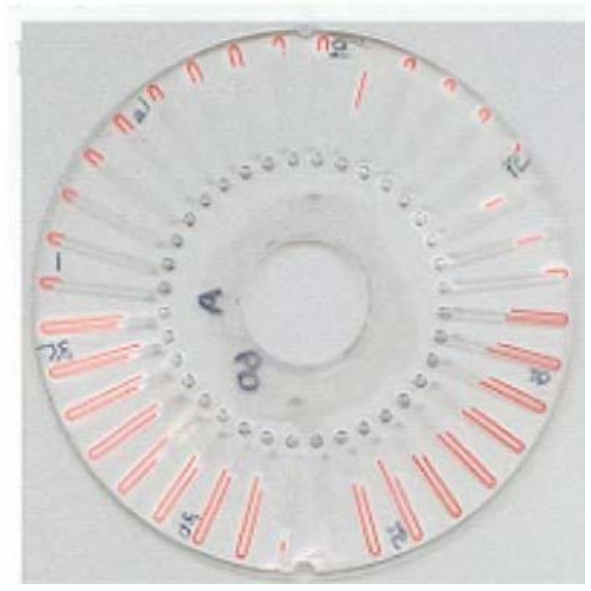

Fig.1 Measured images

\section{PET-HAT: AN WEARABLE PET SYSTEM FOR BRAIN RESEARCH}

Seiichi Yamamoto ${ }^{1}$, Manabu Honda ${ }^{2}$, Keiiji Shimizu ${ }^{3}$, Michio Senda ${ }^{3}$ ${ }^{1}$ Kobe City College of Technology, Kobe, Japan, ${ }^{2}$ National Center of Neurology and Psychiatry, Tokyo, Japan, ${ }^{3}$ Institute of Biomedical Research and Innovation, Kobe, Japan.

We developed a low cost, small size, wearable brain PET system for brain research named PET-Hat. The PET-Hat employed double counter-balanced systems for the mechanical supports. The detector ring can freely move with the counter balanced systems. These motions enable subject to move relatively freely with the PET during acquisition. The detector consists of a GSO block, a tapered light guide, and a flat panel photomultiplier tube (FP-PMT). Two types of GSOs are used to form depth-of-interaction (DOI) detector to minimize the ring diameter. The tapered light guide is used to increase the size of the GSO blocks and reduce the number of FPPMTs. Sixteen detector blocks are arranged in a $280 \mathrm{~mm}$ diameter ring. Transaxial and axial field-of-view (FOV) are $22 \mathrm{~cm}$ and $5 \mathrm{~cm}$, respectively. Energy resolution of the block detectors was $\sim 15 \%$ FWHM and timing resolution was $\sim 4.6$ nsFWHM. Transaxial resolution and axial resolution at the center of the FOV were $\sim 4.5 \mathrm{mmFWHM}$ and $\sim 3.5 \mathrm{mmFWHM}$, respectively. Sensitivity was $\sim 1 \%$ at the center of the axial FOV. Hoffman brain phantom images are successfully obtained. We conclude that the PET-Hat is 
a prom-ising, low cost, small size, wearable brain PET system for brain functional studies.

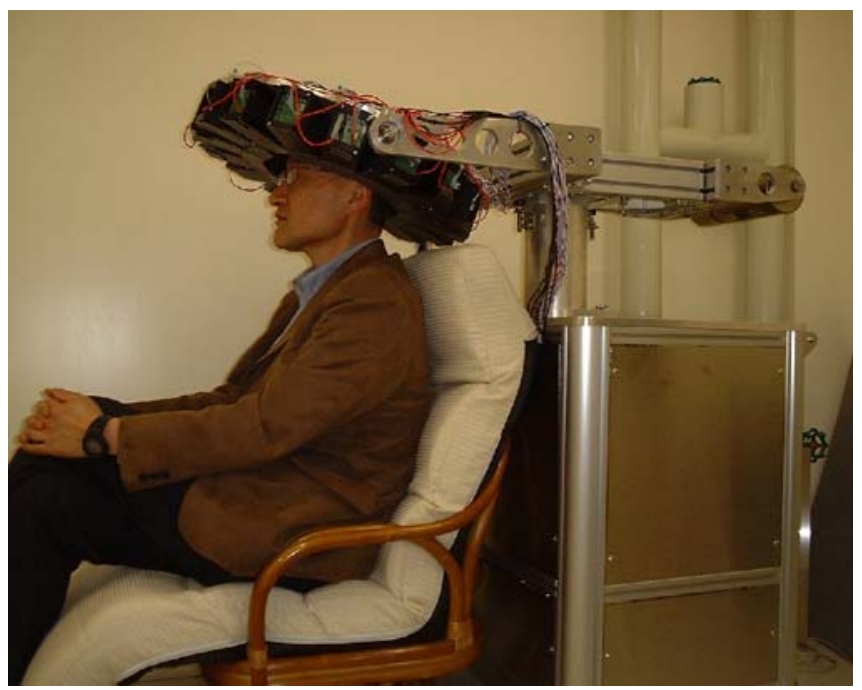

Photograph of the developed PET Hat with a subject

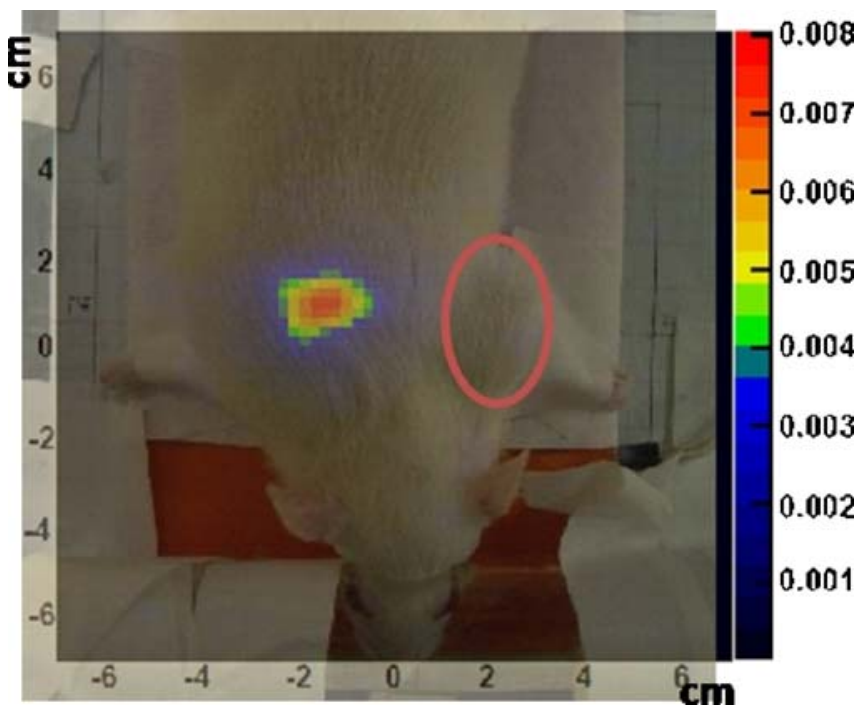

Fig 1. Image of Au-198-nano-particle (412 keV). Au-198 was accumulated in the liver (color contour). Ring area shows the rat MRMT1.

\section{$\mathbf{J} 637$}

\section{MOTION-CORRECTED MICROPET BRAIN IMAGING OF CONSCIOUS RATS}

Roger Fulton ${ }^{1,2}$, Steven Meikle ${ }^{1}$, Andre Z. Kyme ${ }^{1}$, Victor Zhou ${ }^{1}$, $\overline{\text { Kata Popovic }}^{1}$, Michel Kassiou ${ }^{1}$, Mahmood Akhtar ${ }^{1}$

${ }^{1}$ University of Sydney, Sydney, NSW, Australia, ${ }^{2}$ Department of Medical Physics, Westmead Hospital, Sydney, NSW, Australia.

Introduction Small laboratory animals are usually anaesthetised during PET imaging to avoid motion artifacts. However anaesthesia can confound observations of brain function by altering the brain's physiological state. In this paper we report the development and successful application of a motion correction technique that enables microPET brain imaging to be performed in conscious rats using a conventional preclinical PET scanner. Methods Rats were implanted with telemetric temperature probes to enable continuous stress monitoring. Training was conducted over 4 days for habituation to the microPET environment including an open-ended tubular burrow. On day 5 , rats were injected with $60 \mathrm{MBq}$ of [18F]FDG and, after a 20 min uptake period, placed in the burrow with their head protruding. During the $10 \mathrm{~min}$ emission scan and $20 \mathrm{~min}$ transmission scan, head pose was measured at $30 \mathrm{~Hz}$ using an optical motion tracking system that tracked a lightweight marker glued to the fur. The motion data were synchronized to the PET list mode data using an external trigger, 
and used to correct both emission and transmission data for motion by applying transformations to coincidence lines of response. Reconstruction was performed with Fourier rebinning and OSEM, including corrections for attenuation and scatter. Results Rats generally tolerated the imaging procedure without undue stress. None exceeded a core body temperature of $39^{\circ} \mathrm{C}$ which was a predetermined criterion for aborting the study. Rotations and translations spanned $100^{\circ}$ and $50 \mathrm{~mm}$, respectively. Without motion correction images were severely blurred, but were markedly improved after motion correction (Figure 1). Conclusion Imaging the brains of awake rats appears to be feasible. This development permits avoidance of anaesthetic effects, and may facilitate the development of entirely new experimental paradigms that require animals to be in a conscious state during imaging.

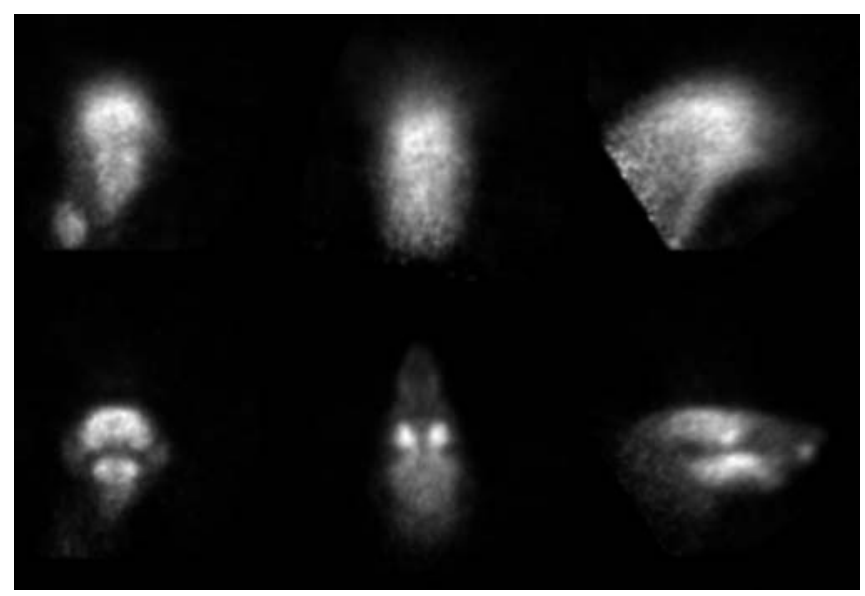

Figure 1. Reconstructed slices of a rat brain in 3 orthogonal planes without motion correction (upper row) and with motion correction (lower row).

\section{J638}

\section{ADVANCED ANALYSIS OF REGIONAL MYOCARDIAL PERFUSION USING DYNAMIC $\left[{ }^{15} \mathrm{O}\right]-\mathrm{H}_{2} \mathrm{O}$ PET: AN AUTOMATED AND ROBUST APPROACH USING STATISTICAL DATA ANALYSIS AND IMAGE SEGMENTATION}

Klaus P. Schäfers ${ }^{1}$, Kambiz Rahbar $^{2}$, Lars Stegger ${ }^{2}$, Otmar Schober ${ }^{2}$, Michael Schafers, 2

${ }^{1}$ European Institute of Molecular Imaging, University of Münster, Münster, Germany, ${ }^{2}$ Department of Nuclear Medicine, University Hospital Münster, Münster, Germany.

Introduction: The analysis of myocardial perfusion with $\left[{ }^{15} \mathrm{O}\right]-\mathrm{H}_{2} \mathrm{O}-$ PET is most challenging due to short radioactive half life (2 min) and fast water transit time. Methods based on tracer kinetic modelling have been developed relying on either manually defined regions-of-interest (ROI) or calculating pixel-by-pixel perfusion images. Both methods are usually limited in accuracy since they are either user dependent and need additional (e.g. blood pool) images accurately registered to the dynamic water study, or they are prone to image noise. We demonstrate an advanced and automated analysis method for calculating regional myocardial blood flow (MBF) based on statistical image processing and semi- automated region definition. Methods: Dynamic $\left[{ }^{15} \mathrm{O}\right]-\mathrm{H}_{2} \mathrm{O}$ data were acquired on a Siemens Ecat Exact 47 PET scanner in 31 normal subjects $(15 / 16 \mathrm{~m} / \mathrm{w}$, age $46+/-15)$ under rest, adenosine stress, and "cold-pressure-test" conditions. From the coincidence sinogram data, tissue and blood component images were generated using cluster and factor analysis techniques. The component images were manually oriented and myocardial contours were automatically defined using a previously developed approach. The left ventricle was segmented into 17 myocardial and one whole-heart (WH) 3D-ROIs. An input time activity curve (TAC) was generated from the inner myocardial contour. Tissue TACs were calculated and tracer kinetic modelling was performed to determine regional myocardial perfusion, tissue fraction and spill-over fractions. The results of the advanced algorithm were compared to a validated reference method. Additionally, the inter-observer variability was tested with two observers for the advanced and reference algorithm. Results: The comparison between the WH-MBF values of the reference and advanced algorithm show good correlation (correlation coefficient $r^{2}=0.73, p<0.001$ ). The automated MBF calculation demonstrated far less inter-observer variability $\left(\mathrm{r}^{2}=\right.$ $0.92)$ compared to the reference method $\left(r^{2}=0.75\right)$. The time for complete analysis could substantially be reduced from $\sim 2 \mathrm{~h}$ to $\sim 10$ min by minimising user interaction. Conclusions: An advanced method for myocardial perfusion analysis with $\left[{ }^{15} \mathrm{O}\right]-\mathrm{H}_{2} \mathrm{O}-\mathrm{PET}$ could be developed allowing fast and reliable regional data analysis mostly independent on user variability.

\section{J639}

DEVELOPMENT OF MICROFLUIDIC PLASMA COUNTING SYSTEM FOR SMALL ANIMAL MOLECULAR IMAGING USING PET - PRINCIPLE VALIDATION FOR BLOOD TRANSPORTATION SYSTEM-

Nobuya Hashizume $^{1}$, Yuichi Kimura ${ }^{2}$, Chie Seki $^{2}$, Takahiro Nishimoto ${ }^{1}$, Keishi Kitamura', Iwao Kanno $^{2}$

${ }^{1}$ Technology Research Laboratory, Shimadzu Corp., Kyoto, Japan, ${ }^{2}$ Molecular Imaging Center, National Institute of Radiological Sciences, Chiba, Japan.

Introduction Fully quantitative functional imaging using PET requires frequent measurement of radioactivity concentrations in arterial plasma. However, a small size of rodents limits an amount of blood sample to a few $\mu \mathrm{L}$ and it makes the quantitative rodent imaging difficult. This study aimed to develop $\mu \mathrm{L}$-ordered blood sampling and radioactivity measurement system, Microfludic Mouse Plasma Counting System ( $\mu \mathrm{FmPC}$, patent pending). System $\mu \mathrm{FmPC}$ was designed to have the following functions: a precise $\mu \mathrm{L}$-ordered blood sampling, maximally 36 blood samplings without coagulation provision, centrifuge, and radioactivity measurement in both of whole blood and plasma. Fig. 1 shows the overview of $\mu \mathrm{FmPC}$. The blood sampling was archived by a microfluidic polydimethylsiloxane (PDMS) chip (A), consisting of a micro channel with precise cross-section area $\left(0.062\left[\mathrm{~mm}^{2}\right]\right)$. The amount of sampled blood was determined by a photo-detector equipped above (A). The blood was sectioned to the predetermined volume with air bubbles, and was transferred into a CD-Well (B) by flushing air. The CD-well engraved 36 U-shaped channels with accurate crosssection area that had hydrophilic inside walls and specially designed inlets to ensure the entire sampled blood proceeding into a channel. After the final blood sampling, the system centrifuged blood samples. The radioactivity and the volume of whole blood and plasma in each channel were measured by a storage phosphor screen and a flatbed 
scanner to derive radioactivity concentration $[\mathrm{Bq} / \mu \mathrm{L}]$, respectively. During the later period of PET scan when the intervals of blood samplings were 5 to $10 \mathrm{~min}$, the channels on (A) were filled with heparinized saline to prevent coagulation during the intervals. Performance Test The $\mu \mathrm{L}$-ordered blood sampling with $\mu \mathrm{FmPC}$ was evaluated using $40 \%$ sucrose solution under $75 \mathrm{mmHg}$ pressure that had the equivalent viscosity and pressure of arterial blood. Then it was tested using rat blood as well. The $\mu \mathrm{L}$-ordered blood sampling was realized for the both sucrose solution and blood. Conclusion We concluded that $\mu \mathrm{FmPC}$ had potential to realize $\mu \mathrm{L}$-ordered blood sampling.

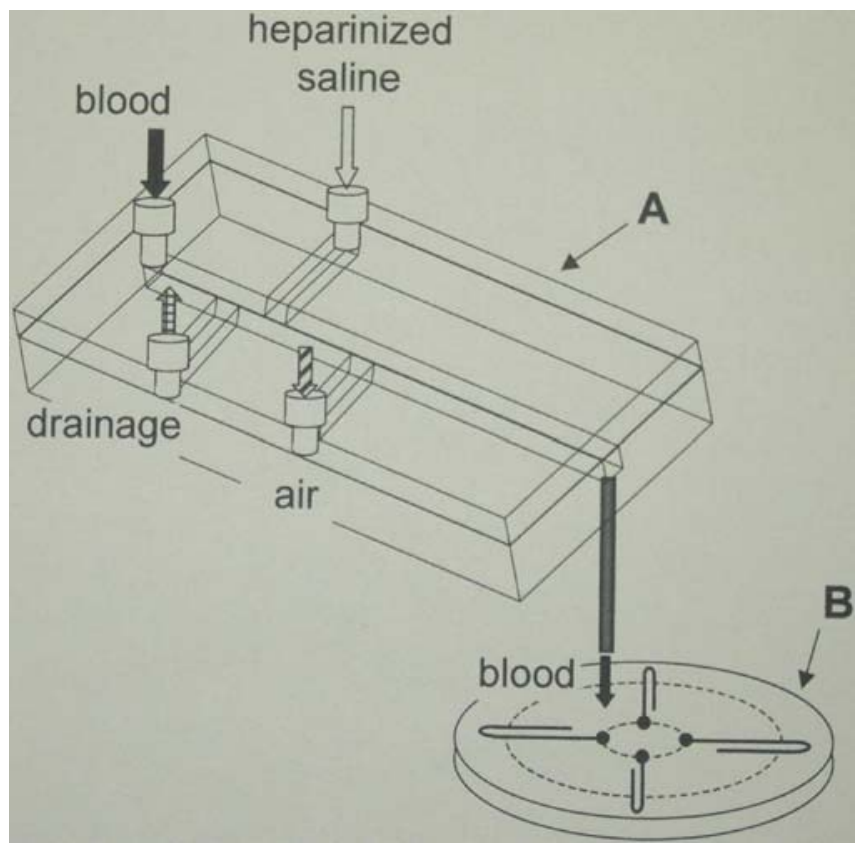

Fig. 1 Schematic of $\mu \mathrm{FmPC}$

\section{J640}

\section{HIGH QUALITY AND HIGH ACCURACY SMALL ANIMAL PET IMAGING}

Yasuhiro Wada $^{1,2}$, Keiichi Matsumoto ${ }^{3,1}$, Emi Hayashinaka ${ }^{1}$, Yasuyoshi Watanabe ${ }^{1,2}$

${ }^{1}$ Center for Molecular Imaging Science, RIKEN, Kobe, Japan, ${ }^{2}$ Physiology, Osaka City Univ., Osaka, Japan, ${ }^{3}$ Kyoto College of Medical Science, Kyoto, Japan.

Positron Emission Tomography (PET) is a useful tool for molecular imaging. Because it has a capability to obtain quantitative images, however, proper parameter settings for data acquisition, histogramming, various corrections and image reconstruction are required. For example, energy window and coincidence timing window for data acquisition, span, ring difference and dead time correction for histogramming, and choice of algorithm for image reconstruction. Especially, for attenuation correction, segmentation procedure is essential for quantitative images. This procedure can cause size error of attenuation objects if threshold settings are not proper. Consequently, this brings low accurate PET images, even for a small animal.
To reduce statistical noise on PET images, it has to be considered about not only emission scan's Noise Equivalent Counts (NEC), also counts for normalization data. In addition, number of counts of transmission and blank scan are must be considered when transmission based attenuation correction is applied. We have been using microPET P4 (Siemens, Knoxville TN USA) installed in Osaka City Univ. and microPET Focus220 installed in Kobe RIKEN for more than 7 years. Scan objects size and weight is wide-ranging, its from a mouse which weight is approximately $20 \mathrm{~g}$ to a monkey or a rabbit which weight is approximately $3,000-6,000 \mathrm{~g}$. And animal folder is used sometimes, but animal folders create complicated attenuation object structure and less accurate images due to complicated attenuation and scatter corrections. The optimization of parameter settings for acquisition, histogramming and reconstruction during the more than 7 years experience will be reported in this study. In RIKEN, these parameter settings are used for daily small animal PET study and obtain high quality and high accuracy PET images routinely.

\section{J641}

\section{EVALUATION OF MYOCARDIUM TO BLOOD POOL SPILLOVER CORRECTION IN QUANTIFICATION MYOCARDIAL BLOOD FLOW WITH ${ }^{82}$ Rb PET}

Ran Klein $^{1,2}$, Chietsugu Katoh ${ }^{3}$, Keiichiro Yoshinaga ${ }^{4}$, Nagara Tamaki ${ }^{4}$, Andy Adler ${ }^{5}$, Rob S. Beanlands ${ }^{1}$, Robert A. deKemp ${ }^{1,2}$

${ }^{1}$ Cardiac PET Centre, University of Ottawa Heart Institute, Ottawa, ON, Canada, ${ }^{2}$ School of Information Technology and Engineering, University of Ottawa, Ottawa, ON, Canada, ${ }^{3}$ Health Sciences, Hokkaido University School of Medicine, Sapporo, Japan, ${ }^{4}$ Department of Nuclear Medicine, Hokkaido University School of Medicine, Sapporo, Japan, ${ }^{5}$ Systems and Computer Engineering, Carleton University, Ottawa, ON, Canada.

Background: Quantification of Myocardial Blood Flow (MBF) with PET can be achieved by compartmental modelling between an input (blood) function and an output (myocardium) function. Input functions are commonly derived directly from the image (IDIF) using a region of interest (ROI) overlapping the left ventricle cavity. However, due to the limited resolution of PET, IDIF suffer of contamination from myocardium signal spillover. In this work we evaluate the benefit of spillover correction of the input function (SCIF) in quantification of $\mathrm{MBF}$ using ${ }^{82} \mathrm{Rb}$ dynamic PET. Method: A total of 60 images (20 patients each at rest, vasodilator induced stress, and cold pressor test) were processed using automatically derived ROIs and a 2 compartment kinetic model to quantify ID-MBF. The same TACs were then corrected for spillover and MBF quantification was repeated to obtain SC-MBF. The extraction function was adjusted to calibrate SC-MBF to ID-MBF. Results: Without calibrating the extraction function, the MBF values obtained with SCIF correlated well with MBF values obtained with IDIF $\left(r^{2}=0.93\right)$, but SC-MBF underestimated MBF (linear regression: $\mathrm{SC}-\mathrm{MBF}=0.57 \mathrm{X}$ ID-MBF + 0.069 ), confirming the need for a new extraction function. With the new extraction fraction SC-MBF with ID-MBF correlated similarly well $\left(r^{2}=0.92\right)$ and linear regression $\mathrm{SC}-\mathrm{MBF}=0.92 \mathrm{X}$ ID-MBF +0.11 (left plot). The stress SC-MBF values varied less $(\mathrm{CV}=20 \%)$ than ID-MBF $(\mathrm{CV}=25 \%)$, resulting in better ability to delineate between rest and stress (right plot). The variances of ID$\mathrm{MBF}$ and SC-MBF were similar for rest and CPT scans. Conclusion: Spillover correction benefits MBF quantification using ${ }^{82} \mathrm{Rb}$ PET, which may lead to more sensitive imaging tools for tracking disease progression and evaluating therapies. 
Flow Values - Method Comparison

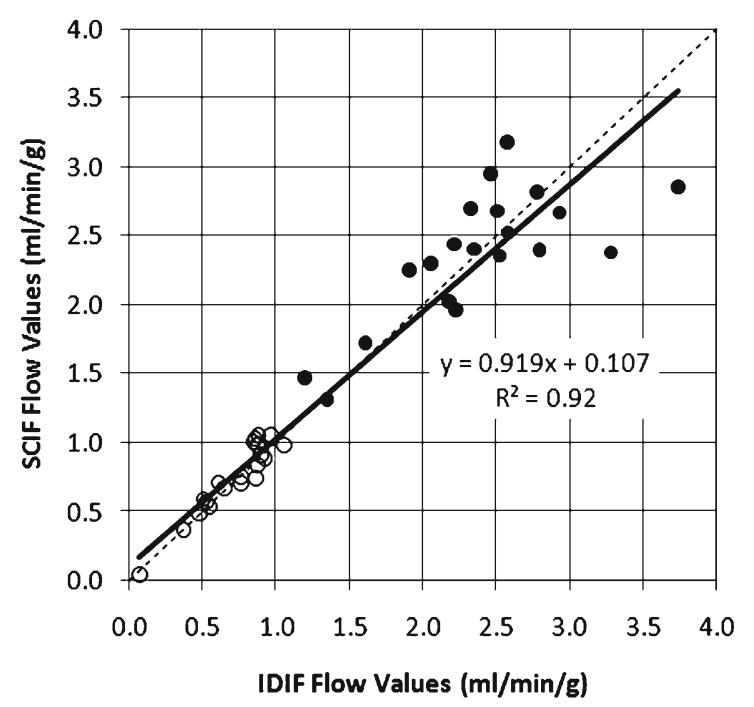

Flow Values - Group Deliniation

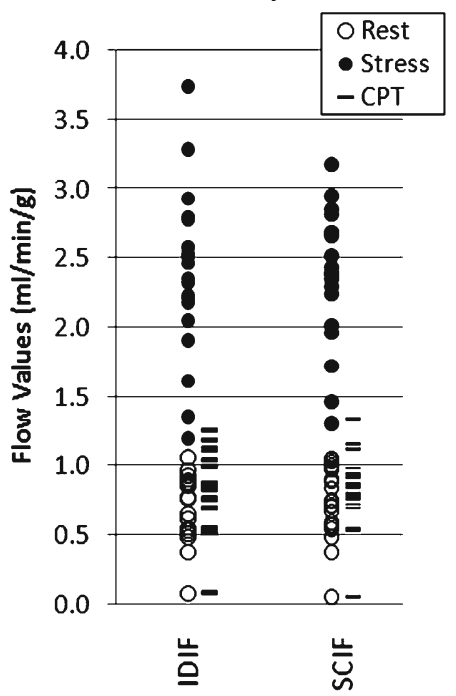

$\mathrm{J} 642$

\section{VERIFICATION OF AN MCNP BASED PET SIMULATION TOOL ON NANOPET/CT SYSTEM}

Judit Lantos $^{2}$, Szabolcs Czifrus ${ }^{2}$, Gergely Patay ${ }^{1}$, Tamas Bükki ${ }^{1}$ ${ }^{\mathrm{T}} \mathrm{R} \& \mathrm{D}$, Mediso Ltd, Budapest, Hungary, ${ }^{2}$ Institute of Nuclear Techniques, Budapest University of Technology and Economics, Budapest, Hungary.

Introduction: The general purpose MCNP/MCNPX code serves in many cases as reference, but it is not immediately capable for simulating all processes of a Positron Emission Tomograph, such as the selection of coincidence events, accounting for detector dead time etc. Therefore a claim arouses to supplement/modify MCNP so that it is able to model PET systems. Simulation results can be compared to the results of GATE, a GEANT4 based Monte Carlo code, which is directly suited to study the physical behavior of medical nuclear imaging systems. Furthermore, the developed MCNP-based simulation tool is verified using measured performance parameters of the NanoPETTM/CT system. Methods: In order to make MCNP capable for the PET simulations, a computer program has been developed. As input, the program uses the ptrac file created as a standard option when running MCNP. This file contains all the information necessary for modelling the behavior of a PET. When processing the ptrac information, the filter program follows the energy transfer steps, computes the detector- and energy-dependent Gaussian energy broadening, identifies the scintillating detector pixels, simulates the paralyzable dead-time of the detector modules accounting for the activity of the source, puts the detected signals into time windows and finally selects the coincidence events. Taking into account the parameters of the detector system, this program produces also the data of the LORs (lines of response), which can be further processed by reconstruction programs. Results: With the aid of the above outlined program, the NanoPET ${ }^{\mathrm{TM}} / \mathrm{CT}$, a new ultra-high resolution, high sensitivity pre-clinical PET-CT system is modelled and a series of performance simulations has been carried out, such like spatial resolution, system sensitivity and noise equivalent count rate according to the NEMA-NU4-2008 standard. We have implemented the same PET model in GATE as well, and compared the results of the performance simulations. Finally, we have compared the results of the Monte Carlo evaluations to some real measurements carried out on the NanoPET TM/CT system. Reasonable agreement of simulated and measured results is found and will be presented.

\section{J643}

\section{IN-VIVO BONE DENSITY AND MASS ESTIMATES}

Douglas Vizard $^{1}$, Douglas O. Wood ${ }^{1}$, William McLaughlin ${ }^{1}$, Rao V. Papineni ${ }^{1}$, Gilbert D. Feke $^{1}$, W. Matthew Leevy ${ }^{2,1}$, Sean P. Orton ${ }^{1}$

${ }^{1}$ Carestream Molecular Imaging, New Haven, CT, USA, ${ }^{2}$ University of Notre Dame, South Bend, IN, USA.

An analytical suite of software has been assembled for estimating bone density and bone mass for small animal in-vivo/ex-vivo measures using a multi-modal digital imaging system. The in-vivo value of animal experimentation, the quantitative and ergonomic benefits of digital imaging, and the multimodal optical system all contribute to new opportunities for experimental design. Achieving the important goal of the in-vivo estimate focuses upon validating/correlating in-vivo and ex-vivo measures. The essential task of calibrating X-ray the attenuation coefficient of bone in varying tissue depths for "soft" X-rays is challenging due to the heterogeneity of the X-ray energy spectrum, but is surmountable and has been implemented in the system software. Combining the high-resolution enabled by soft X-ray imaging with analytical measurement creates unparalleled opportunities for structural studies (inset image of Metatarsal). The simple reporting of actual density (e.g., CaPO4-equivalence, g/cm3) in bones having cylindrical symmetry is now in practice and being applied to significant animal disease models. Additionally, bone mass estimates applicable to clearly visualized bones with intractable symmetries (e.g., lumbar or fibula segments) are possible, and very sensitive estimates of calcifications in biopsy samples are being achieved. 


\section{Density Measures along the Metatarsals of Mouse}

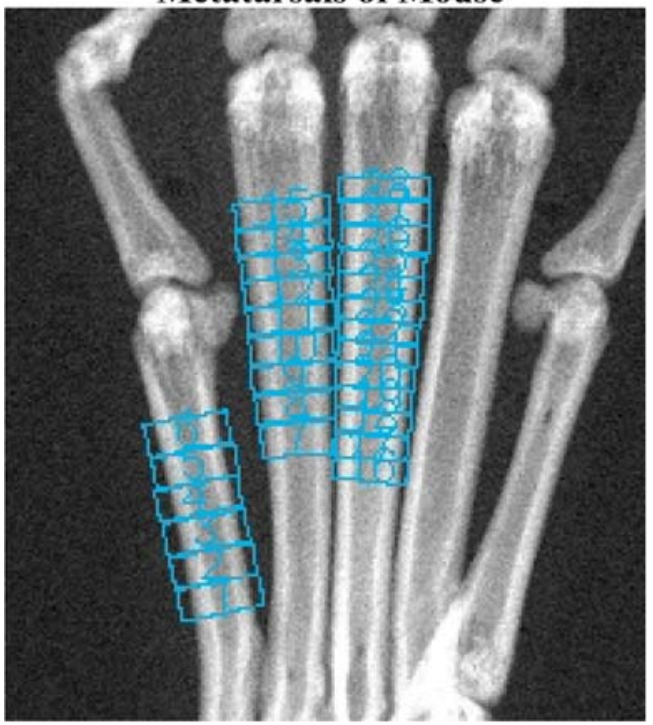

scatter corrected. This work will optimize the transmission acquisition parameters for a Siemens dedicated PET system. The goal is to find an energy window with optimum SNR within the minimum scan time while maintaining accurate mu-values. The default energy window for transmission imaging on this system is $120 \mathrm{keV}-125 \mathrm{keV}$ which minimizes contamination from "outside" influences but results in the rejection of usable data. Methods Thirteen energy windows were selected and used to acquire data. Each new dataset was acquired with a different window beginning at $120 \mathrm{keV}-125 \mathrm{keV}$ adjusted in $5 \mathrm{keV}$ increments on the upper and lower bound to $110 \mathrm{keV}-145 \mathrm{keV}$. A water bottle was imaged using these protocols so that measurements of the attenuation coefficients for water could be assessed with the changing energy windows. ROIs were drawn on the phantom at 9 locations in the volume. Results The resulting measurements show that the transmission acquisition protocol for this system can be further optimized from the default parameters. The table provided is sorted by $\%$ difference from water mu-value @ $511 \mathrm{keV}$ and then by StDev. This indicates an optimum window of $120 \mathrm{keV}-145 \mathrm{keV}$. Conclusions This project shows that transmission measurements should be performed with the above suggested acquisition parameters. These parameters result in high quality quantitative data that yields excellent results for attenuation correction and better anatomical localization through improved contrast. The attached images show the result of the optimized protocol compared to the default settings. The tissue contrast is much greater as indicated by the visible plastic of the bottle.

\begin{tabular}{|c|c|c|c|}
\hline Energy Window $(\mathrm{keV})$ & Water mu-value & StDev & \% Difference from water @ 511 keV \\
\hline $120-145$ & 0.0965 & 0.015 & 0.574 \\
\hline $120-140$ & 0.0947 & 0.016 & 1.269 \\
\hline $115-145$ & 0.0929 & 0.018 & 3.253 \\
\hline $120-135$ & 0.0928 & 0.018 & 4.314 \\
\hline $115-140$ & 0.0921 & 0.015 & 5.280 \\
\hline $110-145$ & 0.0909 & 0.012 & 6.305 \\
\hline $120-130$ & 0.0899 & 0.020 & 6.563 \\
\hline $115-135$ & 0.0897 & 0.015 & 7.308 \\
\hline $120-125$ & 0.0890 & 0.028 & 8.143 \\
\hline $115-130$ & 0.0881 & 0.017 & 9.046 \\
\hline $110-135$ & 0.0873 & 0.014 & 11.495 \\
\hline $110-130$ & 0.0850 & 0.015 & 11.893 \\
\hline $115-125$ & 0.0846 & 0.019 & \\
\hline & & & \\
\hline
\end{tabular}

J644

\section{OPTIMIZATION OF TRANSMISSION IMAGING FOR A SMALL ANIMAL PET SCANNER}

Dustin Osborne

Siemens, Knoxville, TN, USA.

Objectives Transmission imaging is used on small animal dedicated PET systems. This allows PET only acquisitions to be attenuation and
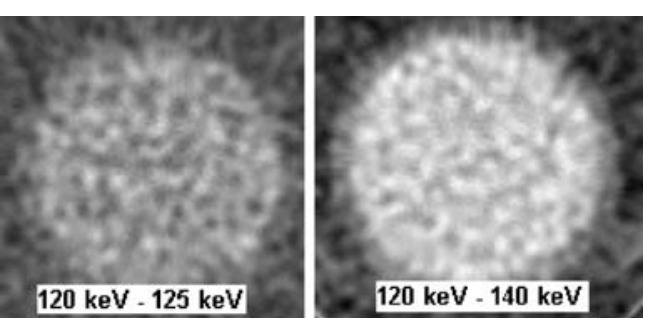


\section{PROGRESS IN SIPM BASED $\mathrm{LaBr}_{3}$ :Ce SCINTILLATION DETECTORS FOR TIME-OF-FLIGHT PET}

${ }_{\text {Dennis R. Schaart }}^{1}$, Stefan Seifert ${ }^{1}$, Herman van Dam ${ }^{1}$, Ruud Vinke ${ }^{2}$, Peter Dendooven $^{2}$, Herbert Löhner ${ }^{2}$, Freek J. Beekman ${ }^{1,3}$

${ }^{1}$ Applied Sciences, Delft University of Technology, Delft, Netherlands, ${ }^{2}$ KVI, University of Groningen, Groningen, Netherlands, ${ }^{3}$ Image Sciences Institute, University Medical Centre Utrecht, Utrecht, Netherlands.

Scintillation detectors based on $\mathrm{LaBr}_{3}: \mathrm{Ce}$ crystals and silicon photomultipliers (SiPMs) are promising for time-of-flight (TOF) positron emission tomography (PET). $\mathrm{LaBr}_{3}: \mathrm{Ce}$ is a fast $(\sim 16 \mathrm{~ns}$ decay time) and bright ( 70.000 photons/MeV) scintillator, while SiPMs offer low time jitter and high gain. Coincidence resolving times (CRTs) better then 160 ps FWHM have been reported in the peer-reviewed literature for single $\mathrm{LaBr}_{3}: \mathrm{Ce}(5 \%)$ crystals on photomultiplier tubes (PMTs). This work is aimed at TOF-PET detectors based on SiPMs, which have the advantage of MRI compatibility. TOF studies were performed with two small $(3 \mathrm{~mm} \times 3 \mathrm{~mm} \times 5 \mathrm{~mm})$, bare $\mathrm{LaBr}_{3}: \mathrm{Ce}(5 \%)$ crystals optically coupled to $3 \mathrm{~mm} \times 3 \mathrm{~mm}$ SiPMs (Hamamatsu MPPC S10362-33-050C). Detector pulses were digitized at $8 \mathrm{GS} / \mathrm{s}$ and 10 bit resolution using two synchronized Acqiris DC282 digitizers. Cubic spline interpolation was performed on the sampled pulses and a leading edge trigger was applied to the rising edge of the interpolated pulses. CRTs close to $\sim 100$ ps FWHM, corresponding to $\sim 1.5 \mathrm{~cm}$ FWHM TOF position resolution, were obtained with a $0.5 \mathrm{~mm}$ diameter $\mathrm{Na}-22$ point source of annihilation photons placed in between the two detectors. Figure 1 shows the coincidence timing spectra measured with the Na-22 source placed at 3 different positions spaced $2 \mathrm{~cm}$ apart. All source positions are clearly resolved. Measurements on detectors consisting of large, monolithic $\mathrm{LaBr}_{3}: \mathrm{Ce}(5 \%)$ crystals (e.g. $18 \mathrm{~mm} \times$ $16 \mathrm{~mm} \times 10 \mathrm{~mm}$ ), coupled to an array of $4 \times 4 \mathrm{SiPMs}$ (Hamamatsu S11064-050P(X)), are underway. In these detectors, photon traveling times within the crystal will affect the timing resolution. Therefore, a correction for the resulting time walk is applied, making use of the independently measured position and depth of interaction (DOI). Initial results of this work will be presented at the conference.

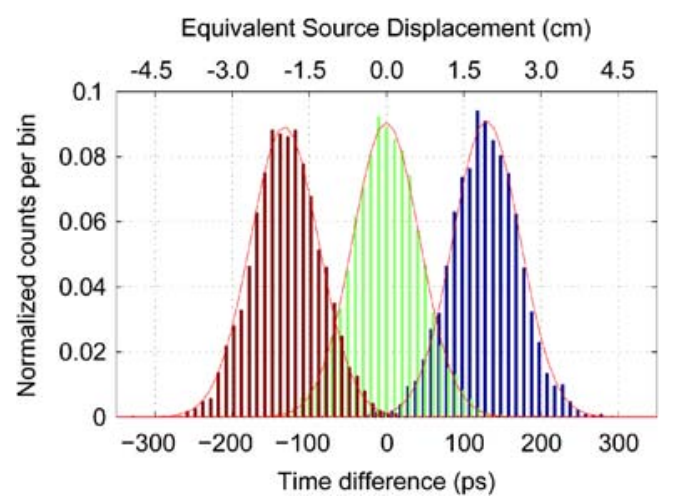

Timing spectra for Na-22 source locations $\mathrm{x}_{1}=2 \mathrm{~cm}$ (green), $\mathrm{x}_{2}=0 \mathrm{~cm}$ (blue), and $\mathrm{x}_{3}=-2 \mathrm{~cm}$ (red), obtained with two $\mathrm{LaBr}_{3}: \mathrm{Ce} / \mathrm{SiPM}$ detectors. FWHM CRTs are 103.4 ps, $99.5 \mathrm{ps}$, and 101.8 ps for $\mathrm{x}_{1}, \mathrm{x}_{2}$, and $\mathrm{x}_{3}$, respectively.
IN VIVO IMAGING OF TUMORS WITH ANTIBODY PROBES USING SEMICONDUCTOR COMPTON CAMERA

$\underline{\text { Shuichi Enomoto }}^{1,2}$, Yousuke Kanayama ${ }^{1}$, Shinji Motomura ${ }^{1}$, Tomonori Fukuchi ${ }^{1}$, Chinuyo Sumita ${ }^{1}$, Yasuko Matsumoto ${ }^{1}$, Emi Hayashinaka ${ }^{1}$, Yasuhiro Wada ${ }^{1}$, Yasuyoshi Watanabe ${ }^{1}$

${ }^{1}$ RIKEN Center for Molecular Imaging Science, Kobe, Japan, ${ }^{2}$ Graduate School of Medicine, Dentistry and Pharmaceutical Sciences, Okayama University, Okayama, Japan.

Multiple molecular imaging, which allows simultaneous visualization of the molecular dynamics in various biological processes, is required to achieve more advanced and precise diagnosis. To realize the multiple molecular imaging, we have developed a Compton camera composed of two Ge semiconductor detectors, which we call "GREI (Gamma-Ray Emission Imaging)". Owing to the collimator-less measurement principle and the excellent energy resolution of Ge detector, GREI enables to distinguish various radionuclides by emitted $\gamma$-ray energy in a wide range from $200 \mathrm{keV}$ to $2 \mathrm{MeV}$. Furthermore, multidirectional projection of the $\gamma$-ray source distribution can be obtained even by a fixed-angle imaging. Using GREI, if each molecule is labeled with different radionuclides, the in vivo distributions of the multiple molecules are able to be visualized simultaneously. In parallel with the system development, exploration of imaging probes which have suitable characteristics for GREI is also required. To explore such probes, PET imaging is of assistance to evaluate the availability of each probe because of its high quantifiability and spatial resolution. In this study, we studied in vivo imaging of tumor-bearing mice with antibody probes using small animal PET and GREI. Cetuximab, an antiepidermal growth factor receptor (EGFR) antibody, was conjugated with 1,4,7,10-tetraazacyclododecane-1,4,7,10-tetraacetic acid (DOTA) and subsequently labeled with ${ }^{64} \mathrm{Cu}$. Tumor-bearing mice were prepared by subcutaneous injection of A431, BT474, SKOV3, and C6 cells separately to $\mathrm{BALB} / \mathrm{c}$ nu/nu mice 10 to 14 days before imaging. The tumor-bearing mouse was injected the ${ }^{64} \mathrm{Cu}$-DOTAcetuximab and after 23 hours imaged by a small animal PET and subsequently the GREI. After 15 hours of imaging, the subject was immediately sacrificed for sampling of tissues, and then those radioactivities were measured. As a result, the similar accumulation in the tumor region was observed in both PET and GREI images. On the basis of the single molecular PET imaging, exploration of multiple molecular probes for the GREI is able to be advanced effectively.

J647

\section{IN VIVO TRACKING OF 18F-FDG-LABELED INDUCED PLURIPOTENT STEM CELLS TO TUMOR-BEARING MICE} Takahito Nakajima ${ }^{1,2}$, Hirofumi Hanaoka ${ }^{2}$, Keigo Endo $^{1}$

${ }^{\mathrm{D} D i a g n o s t i c ~ r a d i o l o g y}$ \& Nuclear medicine, Gunma university, Maebashi, Japan, ${ }^{2}$ Bioimaging analysis informatics, Gunma university, Maebashi, Japan.

We assessed the feasibility of microPET tracking of 18F-FDGlabeled induced pluripotent stem cells (iPS cells) in tumor-bearing mice. METHODS: Immature iPS cells were labeled with $18 \mathrm{~F}-\mathrm{FDG}$ after $30 \mathrm{~min}$ fasting in glucose free medium. 18F-FDG-labeled iPS cells were intravenously injected into mice with implanted HT-29 and LL2 tumors. The images of $18 \mathrm{~F}-\mathrm{FDG}$ as free tracer also were evaluated for comparison. After injection of 18F-FDG-labeled iPS cells or only $18 \mathrm{~F}-\mathrm{FDG}$ into tumor-bearing mice, PET images were acquired at $10 \mathrm{~min}, 60 \mathrm{~min}, 3 \mathrm{~h}$ and $6 \mathrm{~h}$. RESULTS: At $10 \mathrm{~min}$, 
microPET images of living mice indicate that tail-vein-injected labeled iPS cells traffic to the lungs. Continuously, the images at the point of $60 \mathrm{~min}$ after iPS cells injection showed the accumulation at the livers and the subcutaneous tumors. At $6 \mathrm{~h}$, these accumulation were disappeared and redistributed with myocardium and muscles. The images were not similar to that of free $18 \mathrm{~F}-\mathrm{FDG}$. CONCLUSION: MicroPET tracking of $18 \mathrm{~F}-\mathrm{FDG}-\mathrm{labeled}$ iPS cells is feasible and iPS cells have potentially accumulation to tumors.

\section{J648}

STUDY OF GEOMETRIC AND ACCEPTANCE-ANGLE EFFECTS IN DUAL-PLANE POSITRON IMAGING SYSTEM

Yu-Ching Ni, Meei-Ling Jan, Hsuan-Yu Lai, Tien-Hsiu Tsai, Fan-Pin Tseng, Zhi-Kun Lin

Radiation Application Technology Center Physics Department, Institute of Nuclear Energy Research, Taoyuan, Taiwan.

Dual-plane positron imaging has applied on breast, small animal, and plant imaging recently. This kind of imaging system, compared to conventional ring-type PET, doesn't have data with complete angle. In this research, a simple histogram and indexes to find the best geometry with sufficient sampling are introduced. The trade-off between sensitivity and resolution of dual-plane positron system affected by geometry and acceptance angle is also discussed. The geometries of dual-plane positron system were built with various ratios of detector area to detector-to-detector distance(DD) $(4 \times 2: 4,4 \times 2: 3,4 \times 2: 2,4 \times 2: 1)$ using GATE. 3D images were implemented by the planar tomography reconstruction. Since DDs influence the maximum acceptance angle, the LORs (lines of response) within acceptance angles at different DDs were calculated. The skewness and kurtosis of the LOR-numberacceptance-angle histograms were analyzed. To estimate the effects on spatial sensitivity and resolution by acceptance angle, a $0.4 \mathrm{~mm}$ diameter point source was scanned with various DDs. The percentages of accepted counts with various DDs and acceptance angles were analyzed for sensitivity study. And the images of point source were presented for resolution evaluation. Furthermore, the percentages of parallax-error-count at different DDs were verified by simulation using two-layer crystal design. The skewness values of LOR-numberacceptance-angle histograms are $0.69,0.33,0.02$ and 0.51 ; the kurtosis values are $-1.14,-1.55,-1.62$ and -1.22 at above-mentioned DDs respectively. The optimum system geometry is not only covered more angles but also has more uniform histogram. It reveals that the geometric setup with $4 \times 2: 2$ has the smallest skewness and kurtosis, although $4 \times 2: 1$ has the widest angle range due to the largest ratio of detector area to DD. Enlarging the maximum acceptance angle by decreasing DD, the spatial resolution along direction perpendicular to detector plane is improved about 15\%; however, the other directional resolutions are degraded $8.8 \%$ due to parallax error. To reject large angle LORs by choosing a small acceptance angle can eliminate unwanted data to reduce parallax error and scatter, however, it will cause counts lose. The trade-off between sensitivity and resolution of dual-plane positron system by choosing proper acceptance angle will be discussed in the conference.

\section{J649}

\section{QUANTIFICATION ACCURACY PARTIAL VOLUME} EFFECT OF THE INVEON SMALL ANIMAL PET SCANNER Julia G. Mannheim, Martin S. Judenhofer, Vesna Sossi, Bernd J. Pichler Laboratory for Preclinical Imaging and Imaging Technology of the Werner Siemens-Foundation, University of Tuebingen, Tuebingen, Germany.
Small animal PET scanners are used increasingly in biomedical research. Previous studies already evaluated the performance parameters of the Inveon small animal PET scanner, such as energy \& spatial resolution, sensitivity, image quality and noise equivalent count rate (NECR). This study concentrates on quantification accuracy and partial volume effects (PVE). The dynamic range and quantification accuracy in a homogeneous phantom was determined over an activity range from $37 \mathrm{MBq}-2 \mathrm{MBq}$. To determine the influence of the emission activity contaminating the transmission data three attenuation measurements were acquired before, during and after the emission scan (at 37, 15.8 \& $0 \mathrm{MBq}$ ). Including the high emission activity of $37 \mathrm{MBq}$ for attenuation correction the scanner shows small deviations from the true activity. However when using a transmission scan with a lower emission activity for attenuation correction the deviation increases. The influence of the object size on the quantification accuracy was determined using three different sized phantoms mimicking the imaging conditions of mice and rats. FBP with a segmented attenuation correction was used as reference and deviations were calculated. The deviation between the three applied reconstruction algorithms goes up to $85 \%$ for large sized objects. The PVE was investigated in dependence of the object size and location within the PET FOV. The NEMA micro hollow sphere phantom including four different sphere sizes was measured with a sphere to background ratio of $8: 1$. The recovery of the biggest sphere with a diameter of $7.86 \mathrm{~mm}$ is about $60 \%$. The smallest sphere (Ø $3.95 \mathrm{~mm}$ ) has a recovery of $30 \%$. To determine the variation in PVE in dependence to the FOV position a single sphere with an inner diameter of $3.2 \mathrm{~mm}$ located in a cylindrical phantom was used and moved axial and transaxial through the FOV. The percentage changes of the partial volume effect along the FOV goes up to $30 \%$. Concluding, the Inveon PET scanner is well suitable for small animal imaging although the imaging and data correction protocols should be selected and adapted to the individual study situation if accurate quantification is required. This is especially the case for calibration of the system which should be performed at activity levels similar to the ones used in the imaging study. A major impact on quantification was observed by the object size, which could be problematic in mouse studies in general and in rodent brain studies in specific. Thus, recovery algorithms and advanced reconstructions need to be implemented.

\section{$\mathbf{J} 650$}

\section{IN VIVO PET QUANTIFICATION OF DA-RECEPTOR AND -TRANSPORTER BINDING POTENTIAL IN MICE USING CONSTANT INFUSION PROTOCOLS}

Kristina Fischer ${ }^{1}$, Kerstin Fuchs ${ }^{1}$, Julia G. Mannheim ${ }^{1}$, Anke Stahlschmidt ${ }^{3}$, Andreas von Ameln-Mayerhofer ${ }^{2}$, Vesna Sossi ${ }^{1}$, Bernd J. Pichler ${ }^{1}$

${ }^{1}$ Laboratory for Preclinical Imaging and Imaging Technologies of the Werner Siemens Foundation, University of Tuebingen, Tuebingen, Germany, ${ }^{2}$ Department of Neuropharmacology, University of Tuebingen, Tuebingen, Germany, ${ }^{3}$ Department of Radiopharmacy, University of Tuebingen, Tuebingen, Germany.

Positron emission tomography has been widely used for quantification of DA-transporters and -receptors in humans, non human-primates and recently with advanced scanner technology in rodents. To calculate parameters of receptor- and transporter binding tracer injection as a bolus is the conventional approach, but there are many advantages of bolus plus constant infusion administration. The aim of the current study was the development of a bolus/infusion (B/I) protocol of [11C] Methylphenidate (MP) and [11C]Raclopride (RAC) in mice as a basis for future transporter and receptor binding studies. We further investigated the changes in specific binding of tracer after a pharmacological challenge. In the $\mathrm{B} / \mathrm{I}$ experiments, performed in mice, the tracer was applied as a fast bolus $(1 \mathrm{~min})$ followed by a constant 
infusion for $120 \mathrm{~min}$ with different bolus to infusion rates (Kbol) between 100 and 150 min using a computer controlled infusion pump. Sixteen consecutive frames were acquired for $120 \mathrm{~min}$ after tracer injection start via tail vein catheter under temperature controlled conditions. Perturbations of the equilibrium state were introduced by injecting unlabelled tracer in both experiments. In addition, cold MP and D-Amphetamine were injected as an indirect stimulus in experiments with [11C]RAC. To calculate binding potential, regions of interest were placed over bilateral striata and cerebellum; the ratio (str/ cer) was determined. In B/I studies carried out with [11C]MP, steadystate equilibrium was reached with a Kbol of 150 min 2000 seconds after start of infusion, with constant str/cer ratios around 2.3 during equilibrium period. Cold MP (3 mg/kg) administration induced a decrease in binding ratio of $39 \%$. In B/I experiments with [11C]RAC steady state equilibrium was also reached with a Kbol of $150 \mathrm{~min}$ within 2000 to 3000 seconds, with constant str/cer ratios around 2.7. Almost complete displacement of [11C]RAC from striatal binding sites was caused by injecting unlabelled RAC $(0.3 \mathrm{mg} / \mathrm{kg})$; striatum and cerebellum time activity curves became very similiar with str/cer ratios around 1.5. Indirect displacement with unlabeled MP $(5 \mathrm{mg} / \mathrm{kg})$ and DAmphetamine $(2.5 \mathrm{mg} / \mathrm{kg})$ resulted also in a decrease of [11C]RAC binding. Str/cer ratio was 2.3 after challenge with unlabeled MP and 1.8 after challenge with D-Amphetamine. The results clearly indicate the applicability of $\mathrm{B} / \mathrm{I}$ experiments in mice. We also showed that $\mathrm{B} / \mathrm{I}$ protocols are a useful and sensitive approach to detect changes in synaptic neurotransmitter concentrations in mouse brain.

\section{$\mathbf{J 6 5 1}$}

\section{IN VIVO IMAGING OF TUMOR-INDUCED LYMPH NODE LYMPHANGIOGENESIS BY IMMUNO-POSITRON EMISSION TOMOGRAPHY}

Viviane Mumprecht $^{1}$, Michael Honer ${ }^{1}$, Benjamin Vigl ${ }^{1}$, Steven Proulx ${ }^{1}$, Eveline Trachsel $^{2}$, Manuela Kaspar ${ }^{2}$, Nadja Banziger-Tobler ${ }^{1}$, Roger Schibli ${ }^{1}$, Dario $\mathrm{Neri}^{1}$, Michael Detmar ${ }^{1}$

${ }^{1}$ Institute of Pharmaceutical Sciences, Swiss Federal Institute of Technology, Zurich, Switzerland, ${ }^{2}$ Philochem AG, Swiss Federal Institute of Technology, Zurich, Switzerland.

Metastasis to regional lymph nodes is a prognostic indicator for cancer progression. There is a great demand for sensitive and noninvasive methods to detect metastasis to the lymph nodes. While conventional in vivo imaging approaches have focused on the detection of cancer cells, lymphangiogenesis within tumor draining lymph nodes might be the earliest sign of metastasis. In mouse models of lymph node lymphangiogenesis, we found that systemically injected antibodies to lymphatic epitopes accumulated in the lymphatic vasculature in tissues and lymph nodes. Using a radiolabeled antibody against a lymphatic specific epitope, we imaged, for the first time, inflammation-and tumor-draining lymph nodes with expanded lymphatic networks in vivo by positron emission tomography. This method may open up new avenues for the early detection of metastases and for radioimmunotherapy and the images obtained might be used as biomarkers for the progression of diseases associated with lymphangiogenesis.

\section{$\mathbf{J} 652$}

\section{STRIATAL DAT BINDING AND REVERSAL TASK PERFORMANCE IN RHESUS}

Alexander K. Converse, Jeffrey M. Moirano, Elizabeth O. Ahlers, Julie A. Larson, Dhanabalan Murali, Bradley T. Christian, Onofre T. DeJesus, Robert J. Nickles, James E. Holden, Colleen F. Moore, Mary L. Schneider University of Wisconsin, Madison, WI, USA.
GOALS: As part of a larger experiment to determine the effects in offspring of maternal exposure to alcohol and/or stress during pregnancy in rhesus, we imaged striatal DAT binding by [F-18]FECNT with PET in control subjects. In a separate session the same subjects performed a reversal task to measure executive function. Expecting that reduced dopaminergic transmission would impair executive function, we hypothesized that across individuals, task performance would negatively correlate with striatal DAT binding. METHODS: Ten rhesus macaques were scanned under isoflurane anesthesia in a microPET P4 (Concorde). Data were acquired following iv injection of a bolus of FECNT. After corrections including attenuation and scatter, images were aligned to template regions of interest and time activity curves were determined. Striatal to cerebellar distribution volume ratios were calculated using the Logan graphical reference tissue method. Reversal task performance was measured by total correct trials in a 30 minute session. The Pearson product moment coefficient, r, was calculated for correct trials vs. FECNT DVR. RESULTS: Across all ten subjects, the correlation coefficient for task performance vs. DAT binding was $r=-0.448(p=0.26,2$-tailed, $n=10)$. Two subjects attempted the task in only 3 and 7 trials compared to a range of 18-67 for the remaining eight subjects. Post hoc analysis of the correlation without these two subjects yielded $r=-0.638$ ( $p=0.089$, 2-tailed, $n=8)$. CONCLUSION: This result suggests that across the participating subjects ( 8 of 10), executive function may be impaired by increased DAT density perhaps through reduced dopaminergic transmission. This work was supported by NIH R01 AA12277.

\section{$\mathbf{J} 653$}

\section{ASSESSMENT OF PET QUANTITATIVE ACCURACY WHEN USING A STEROTACTIC HEAD HOLDER FOR THE RHESUS MONKEY}

Elizabeth O. Ahlers, Jeffrey M. Moirano, Bradley T. Christian, James E. Holden, Colleen F. Moore, Mary L. Schneider, Alexander K. Converse University of Wisconsin - Madison, Madison, WI, USA.

OBJECTIVES: Scatter correction is commonly applied to PET data and typically decreases the observed radioactivity. However, limitations and assumptions present in the algorithm can lead to streaking, the appearance of activity outside the subject, or abnormally low counts. Therefore, the scatter correction algorithms need to be periodically evaluated in order to improve them [1]. In animal studies, a stereotactic head holder is often used to hold the head in place to improve image quality by preventing motion. To determine the implications for [18F] fallypride binding measures in rhesus macaque brain, especially the cerebellum, we investigated the effect of the head holder on scatter correction in a phantom study. METHODS: The phantom was a $8 \mathrm{~cm}$ long, $6.5 \mathrm{~cm}$ inside diameter cylinder filled with F-18 in water with three $1.5 \mathrm{~cm}$ diameter tubes evenly spaced at radius $2 \mathrm{~cm}$ containing either air, plastic, or C-11 in saline. The phantom was placed in a head holder made with a plastic U-shaped frame. Data were acquired over 20 min when the scatter corrected C-11 activity approximated the [18F] fallypride activity seen in rhesus cerebellum, $26 \mathrm{nCi} / \mathrm{cc}(\mathrm{LOW})$, and the surrounding F-18 was approximately $166 \mathrm{nCi} / \mathrm{cc}(\mathrm{HI})$. Two different VOIs for each radio-isotope were used, one in planes that contained the head holder frame and one in planes that did not. Scans were conducted on a microPET P4 scanner. Events were histogrammed into 3D sinograms (span 3, ring-difference 31). Reconstructions were done using $1 \mathrm{x}$ zoom FBP no filter, attenuated corrected, and normalized using MicroPET Manager 2.4 with 4-byte histograms. RESULTS: Analysis of the phantom data showed that scatter correction is not adversely affected by the presence of the head holder frame. For both LOW and HI, the difference between the planes with the frame and without the frame is $4 \%$. CONCLUSIONS: A rhesus cerebellum, often 
used as a reference region, would most likely be in the planes with the head holder frame.. If scatter correction was adversely affected by the head holder frame, this could compromise the data gathered from the cerebellum, thus compromising its use as a reference region. However, the phantom scan shows that the head holder frame does not adversely affects scatter correction. REFERENCES: [1] Zaidi, H., Montandon, M. 2007 Scatter Compensation Techniques in PET. PET Clinics 2: 219-234

\section{$\mathbf{J} 654$}

\section{DENOISING OF DYNAMIC PET DATA USING THE EMPIRICAL MODE DECOMPOSITION} Andrew McLennan, Sir Michael Brady

Engineering Science, University of Oxford, Oxford, United Kingdom.

Objectives: The reconstruction of dynamic Positron Emission Tomography (dPET) data can result in extremely noisy images if noise inherent in PET is not accounted for. The benefits of preprocessing of projection data and/or post-processing of reconstructed dPET images using the Empirical Mode Decomposition (EMD), with the aim of improving overall image quality, are examined. We investigate the ability of the transform to ensure temporally smooth Time Activity Curves (TACs) without the need to specify a prior set of temporal basis functions with which any given TAC can be approximated. Methods: The EMD algorithm decomposes signals into empirically determined frequency modes and residual functions. Each Intrinsic Mode Function (IMF) contains a range of frequencies with varying amplitudes. As noise in PET is high frequency and non-stationary, we compare various IMF thresholding methods with the aim of removing noise whilst retaining true pharmacokinetics. Taking projection data, we denoise each temporal Line of Response using the EMD algorithm and use these results in the image reconstruction process. We also analyse when EMD is only applied to unprocessed dynamic image sequence, and when a combination of pre/post processing is performed. Results: A range of pharmacokinetic activity curves are tested. The pre-processing of dPET data, by ensuring consistency between neighbouring temporal frames, enables less noisy reconstructions to be formed even though the data may no longer strictly follow a Poisson distribution. Image post-processing also leads to improved signal to noise ratios and reduced mean square error when compared to Gaussian smoothing. Conclusions: The Empirical Mode Decomposition, when used to temporally regularise dPET reconstructions, enables less noisy images to be formed and does not require a temporal basis set to be specified a priori.

\section{$\mathbf{J 6 5 5}$}

\section{QUANTITATION ERROR ASSESSMENT FOR SMALL ANIMAL} PET

Frezghi Habte $^{1,3}$, Carsten H. Nielsen ${ }^{1,3}$, Gang Ren ${ }^{1,3}$, Shahriar Yaghoubi ${ }^{1,3}$, $\overline{\text { Nadia Withofs }}^{1,3}$, Timothy Doyle ${ }^{2,3}$, Laura J. Pisani ${ }^{1,3}$, Craig S. Levin ${ }^{1,3}$, Sanjiv S. Gambhir ${ }^{1,3}$, David Paik ${ }^{1,3}$

${ }^{1}$ Radiology, Stanford University, Stanford, CA, USA, ${ }^{2}$ Pediatrics, Stanford University, Palo Alto, CA, USA, ${ }^{3}$ Molecular Imaging Program, Stanford University, Palo Alto, CA, USA.

Image quantitation in PET depends on many factors including pre-scan animal preparation, accuracy of calibrations, understanding error correction mechanisms and reliability of the entire data analysis procedure. Hence, the goal of this study is to identify the major sources of errors and optimize data analysis procedures to improve accuracy. We monitored the accuracy of calibration parameters on a monthly basis and compared the biodistribution of various organs/tumors using both microPET and a gammacounter. We also conducted a phantom study with F-18 filled spheres to demonstrate the consistent underestimation of organ activity observed by microPET in various studies for organs expressing high tracer uptake. The biodistribution comparison showed close agreement $(<10 \%$ difference) for organs/tumors with lower uptake $(>10 \mathrm{uCi} / \mathrm{mL})$ while significantly larger difference $(>30 \%)$ for organs with higher uptake. This observation was in close agreement with previous studies. We further verified the significant underestimation of PET concentration for regions with high tracer uptake using phantom studies. In our study, we have applied the vendor recommended attenuation and scatter corrections despite their minimum effect on mouse imaging. On the other hand, the vendor recommended global deadtime correction has limitations showing consistent count loss in high activity $(>10 \mathrm{uCi} / \mathrm{mL})$ regions of interest. This suggests that further evaluation of the microPET deadtime correction methods may be needed or additional post-reconstruction deadtime correction should be applied for more accurate quantitation.
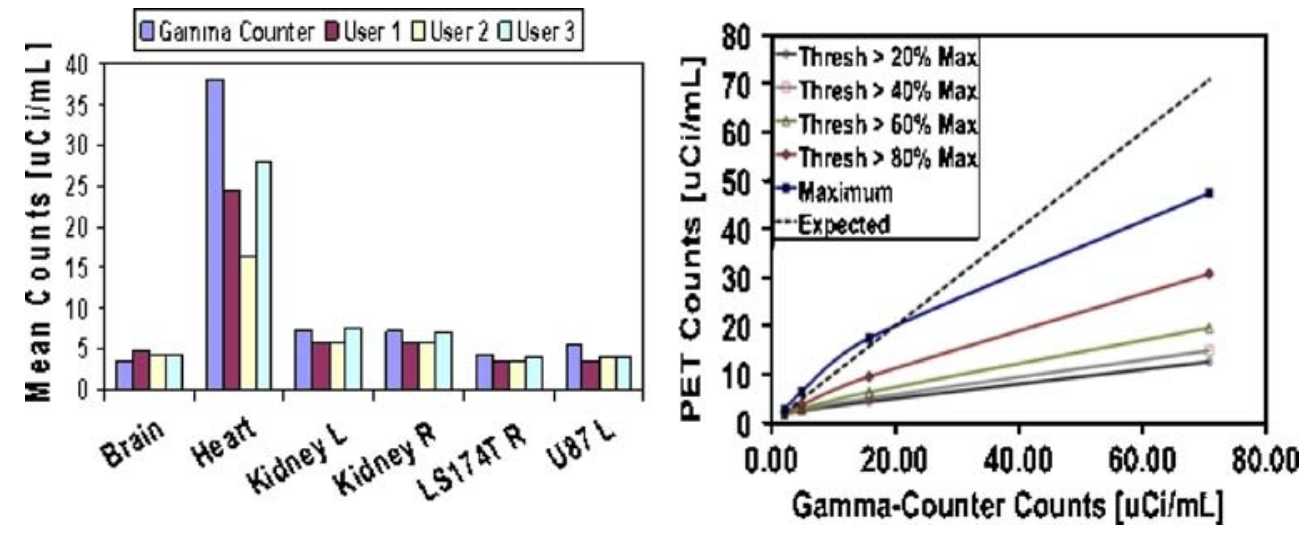
J656

EFFECT OF INTER- AND INTRA- USER VARIABILITY IN QUANTIFICATION OF MOLECULAR IMAGING DATA Frezghi Habte $^{1,2}$, Shradha Budhiraja ${ }^{1,2}$, Timothy Doyle ${ }^{2,3}$, Craig S. Levin ${ }^{1,2}$, David Paik ${ }^{1,2}$

${ }^{1}$ Radiology, Stanford University, Stanford, CA, USA, ${ }^{2}$ Molecular Imaging Program, Stanford University, Palo Alto, CA, USA, ${ }^{3}$ Pediatric, Stanford University, Palo Alto, CA, USA.

In molecular imaging, absolute image quantitation is generally considered to be critical since it reduces subjectivity and provides important additional information that adds confidence in the data analysis process. Quantitation, however, heavily depends on the region of interest (ROI) analysis method, which normally is performed by manually defining an ROI and querying its statistics. Depending on how the ROI is drawn, the geometry and size of the ROI and the specific software tool used introduce variability and limit the accuracy. To characterize variability, nine experienced users with no restriction on data analysis tools were selected to read five different data sets three times for each data set. The images were acquired using microPET. In addition, we conducted a survey to study the users' confidence and subjectivity involved in the analysis. Our result indicates that high inter-user variability (s.d. $>30 \%$ of the inter-user mean) occurred while the intra-user variability is relatively low (s.d. $<10 \%$ of the inter-user mean). We also observed a pattern where each user consistently either under- or over-estimates the mean quantitative value, demonstrating the high degree of subjectivity. There was no direct correlation observed between the inter-user variability and confidence of the users on drawing the specific ROIs. Thus, software tools capable of defining ROIs semiautomatically may be required to improve quantitation accuracy and reduce variability in analysis of molecular imaging data.

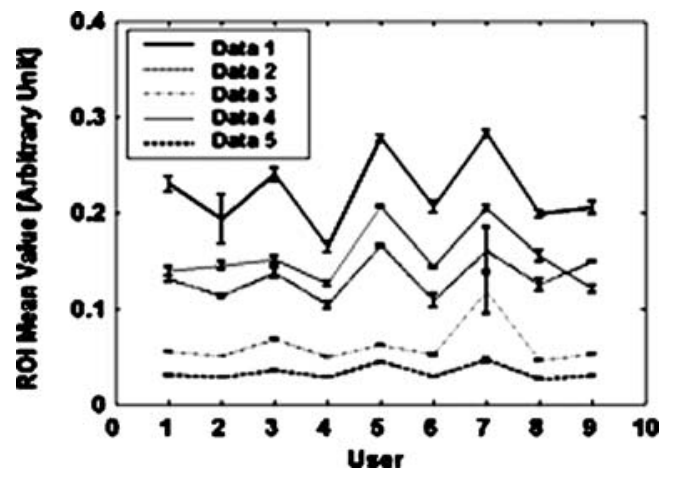

$\mathbf{J} 657$

\section{IMPLEMENTATION OF PET-SORTEO ADAPTED TO THE INVEON PET SCANNER FOR DUAL MOUSE IMAGING \\ Joel Bertinshaw, David Zahra, Marie-Claude Gregoire ANSTO, Menai, NSW, Australia.}

Medical imaging research involving large mice cohorts can be significantly streamlined by performing PET scans with two mice in the field of view (FOV). However, positioning within the FOV and signal cross-contamination from two mice can affect the quality of reconstructed scans. To assess the loss in signal integrity when imaging two mice, PET-SORTEO ${ }^{1}$ Monte Carlo simulator has been implemented to isolate the potential factors of information loss and to validate new correction methods. As the first step, this study aims to validate the implementation of SORTEO for the Siemens ${ }^{\mathrm{TM}}$ Inveon
PET system with a series of phantom studies. The spatial resolution was evaluated using a single line source mouse phantom. A teflon capillary with a $0.28 \mathrm{~mm}$ inner diameter was filled with F-18 and set in a $28 \mathrm{~mm}$ diameter cylinder filled with gelatine. For comparison three scans of the same phantom were performed: (i) alone and centred (ii) $15 \mathrm{~mm}$ off-centre (iii) off-centre with with an identical cold phantom. Cross-contamination was assessed by imaging the same cylinder filled with uniform activity in the same method as above. Scan lengths were set to record 5 billion counts. The Inveon scanner was defined in SORTEO using a series of parameters to set scanner geometry and other operational characteristics such as processing dead time. To validate CT and PET scans of the physical phantoms were segmented to create the emission and attenuation volumes. Scan conditions were replicated as close as possible to the physical studies within SORTEO. Scans were reconstructed using 3D-OSEM-MAP (Fastmap, $\beta=0.5$ ). Initial results from the physical scans indicate a spread of spatial resolution with a FWHM of $1.77 \mathrm{~mm}, 2.10 \mathrm{~mm}$ and $2.14 \mathrm{~mm}$ and a FWTM of $3.26 \mathrm{~mm}, 3.65 \mathrm{~mm}$ and $3.9 \mathrm{~mm}$ for the centre, off-centre and dual phantoms respectively. The dual uniform phantom showed a marked signal decrease of $9.4 \%$ at the centre edge as compared with the off-centre phantom. These results will be compared with the reconstructed images from the SORTEO simulation and used to validate and fine tune the scanner parameters within the software. 1. A. Reilhac et al., "PET-SORTEO: a Monte Carlo-based Simulator with high count rate capabilities," IEEE Transactions on Nuclear Science 51, 1 (2004): 46-52.

\section{$\mathbf{J} 658$}

EXTRACTING PURELY IMAGE-DERIVED ARTERIAL INPUT FUNCTIONS FROM THE CAROTID ARTERY USING A GENERALIZED EXPECTATION-MAXIMIZATION ALGORITHM WITH PARTIAL VOLUME CORRECTION FOR PET-CT STUDIES OF HEAD AND NECK CANCER George Sayre, Youngho Seo

Radiology and Biomedical Imaging, University of California - San Francisco, San Francisco, CA, USA.

Background: Correlations between tumor characteristics and radionuclide kinetics with PET-CT data may improve optimization of radiation treatments. PET activity maps require arterial input functions (AIFs) to be determined for calculation of kinetic-rate constants. The goal of this project was to extract AIFs from the carotid artery without intrusive blood sampling. We propose an algorithm that: 1) delineates the carotid artery in head-and-neck PET-CT images (using a generalized expectation-maximization approach); and 2) decreases associated partial-volume errors. Methods and Materials: Using several kineticrate constants derived from blood-brain-barrier studies, $\{\mathrm{K} 1=$ $(2.5,2.5,3.98), \mathrm{k} 2=(3.98,2.5,2.5) \mathrm{mL}-\mathrm{g}-1 \mathrm{~s}-1 \times 1000\}$, to approximate a range of interactions between the carotid and its surrounding tissue, and a realistic AIF profile obtained from the aortic arch of a human patient, we constructed three unblurred computer phantoms of the carotid and its immediate surrounding tissue. The size and shape of these phantoms were based upon manual delineation of a real carotid in a CT image of a human patient. The phantoms were blurred with an isotropic full width at half $\max$ (FWHM) of $6.5 \mathrm{~mm}$ to simulate more realistic PET images. First-order kinetics was assumed, and thus, the logical comparison metric was the integrated AIF (I-AIF). I-AIFs were extracted from the blurred phantom data sets using our algorithm and were compared with the unblurred phantom I-AIFs. Results: Applying our algorithm to the blurred phantoms resulted in I-AIFs that differed from their gold-standard counterparts by $7.5 \%, 5.98 \%$, and $5.0 \%$. The validation process for the phantom corresponding to $5 \%$ error is visually illustrated in Figure 1. Discussions and Conclusions: We 
developed an algorithm to extract arterial input functions from the carotid artery for PET/CT studies of head and neck cancer without need for intrusive blood samples. Future investigations into region-based, partial-volume correction schemes may lead to improved accuracy.

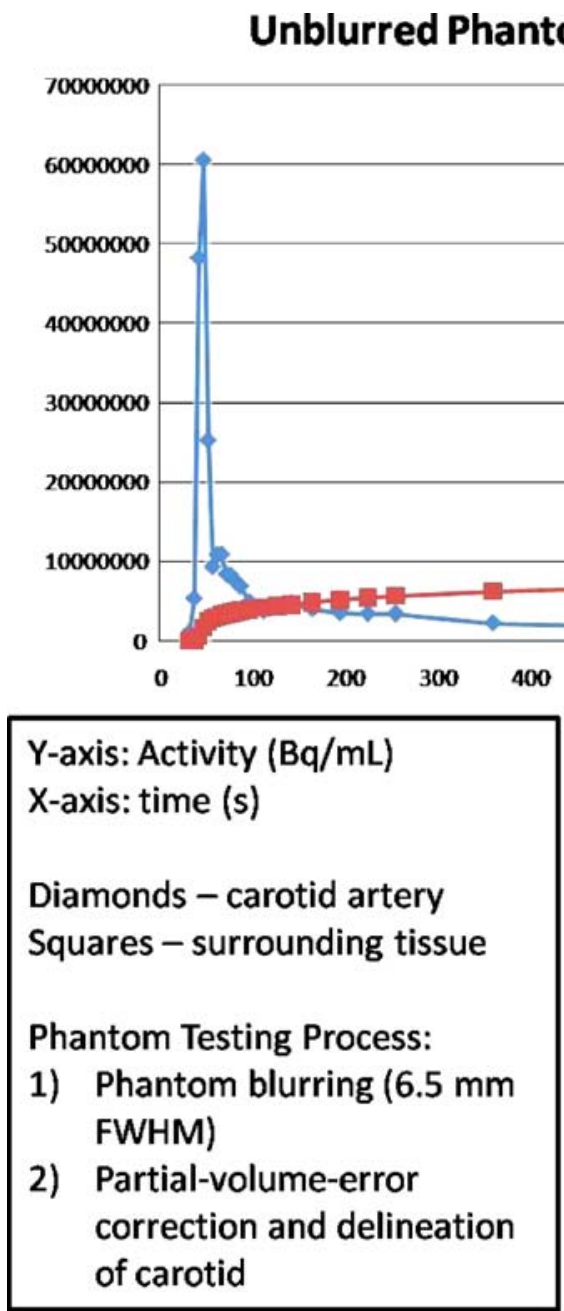

Figure 1. Phantom validation process.
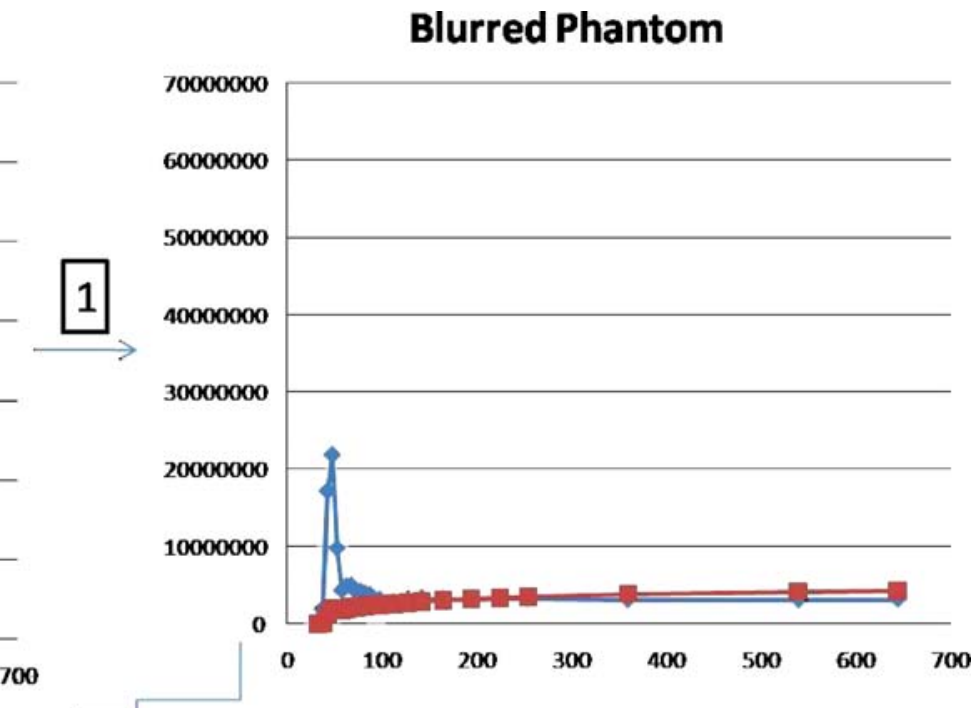

2 Extracted Time Profiles

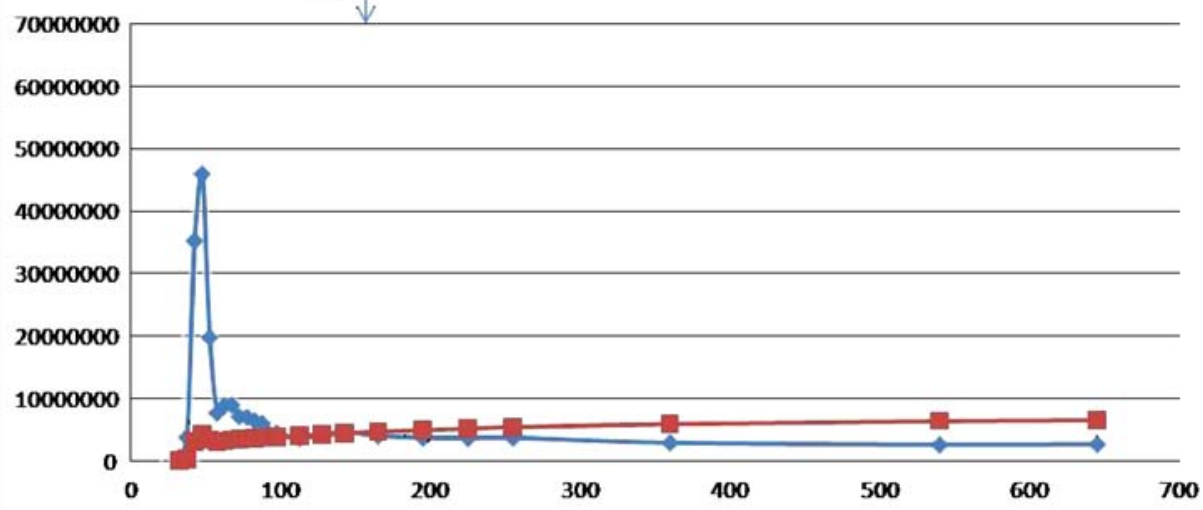

$\mathbf{J} 659$

HEPATIC BLOOD PERFUSION CAN BE ESTIMATED

NON-INVASIVELY BY DYNAMIC PET USING GLUCOSE ANALOGS: VALIDATION OF A PORTAL VENOUS MODEL IN PIG STUDIES

Michael Winterdahl ${ }^{1}$, Susanne Keiding ${ }^{1,2}$, Michael Sørensen ${ }^{1}$, Frank V. Mortensen $^{3}$, Ole L. Munk ${ }^{1}$

${ }^{1}$ PET Center, Aarhus University Hospital, Aarhus, Denmark, ${ }^{2}$ Medical Department V, Aarhus University Hospital, Aarhus, Denmark, ${ }^{3}$ Department of Surgery, Aarhus University Hospital, Aarhus, Denmark.

Hepatic blood perfusion can be quantified by kinetic analysis of the timeactivity curves (TACs) in liver tissue measured by dynamic PET in relation to the tracer input from the hepatic artery (HA) and the portal vein (PV) measured by blood sampling. In humans, blood sampling from the portal vein is not possible. The purpose of the study was to examine if a portal venous model can replace portal venous blood sampling for the estimation of hepatic blood perfusion. We used three radio-labeled glucose analogs: $\left[{ }^{11} \mathrm{C}\right]$ methyl-glucose, MG [1], $\left[{ }^{18} \mathrm{~F}\right]$ fluoro-2-deoxy-galactose, FDGal [2], and $\left[{ }^{18} \mathrm{~F}\right]$ fluoro-2-deoxy-glucose, FDG [1]. In pigs studies time-activity curves from the portal vein and the femoral artery were measured by blood sampling, following iv injections of MG, FDGal or FDG. Flows in HA and PV were measured by surgically placed ultrasound transit-time flowmeters. The study was performed according to guidelines of the Danish Animal Experimentation Act. Dispersion and delay from arterial TAC to portal venous TAC was described by a portal venous model with a single parameter, $\beta$ [3]. Average values of $\beta$ were estimated for each of the three glucose analogs (Table 1). For each injection: 1) a simulated tissue TAC was calculated using the measured dual-input TAC (arterial samples + PV samples, measured flow-ratio) and a one-tissue compartment model with a 'true' perfusion, F true; 2) a non-invasive dual-input TAC (arterial samples + PV model, average flow-ratio) was calculated; 3 ) hepatic blood perfusion was estimated using the simulated tissue TAC and the non-invasive PVmodel dual input, Fest. The relative deviation $\left(\mathrm{F}_{\text {est }}-\mathrm{F}_{\text {true }}\right) / \mathrm{F}_{\text {true }}$ was not significantly different from zero for any of the three glucose analogs (Table 1). In conclusion, hepatic perfusion can be quantified by dynamic PET with radio-labeled glucose analogs using glucose analog specific average values of $\beta$ for the non-invasive portal venous model. For clinical use, the method needs validation in humans. Arterial blood sampling may 
be replaced by scan data from the abdominal aorta [4], which will make the method completely non-invasive. 1) Munk et al, J Nucl Med 2001;42:795;
2) Sørensen et al, Am J Phys 2008;295:G27; 3) Munk et al, Am J Phys 2003;4:G671; 4) Keiding et al, Eur J Nucl Med 2000;42:1673.

\begin{tabular}{|c|c|c|c|}
\hline Table 1 & MG & FDGal & 10 \\
\hline Number of pig studies & 6 & 6 & $0.50 \pm 0.05$ \\
\hline$\beta(\mathrm{min})$ & $0.57 \pm 0.09$ & $0.82 \pm 0.08$ & $23.00 \pm 22.94$ \\
\hline Relative deviation of perfusionestimates (\%) & $-4.28 \pm 9.30$ & $-8.98 \pm 12.60$ & 2 \\
\hline
\end{tabular}

Data are given as mean \pm standard error of the mean.

\section{J660}

QUANTITATIVE ACCURACY OF MAP RECONSTRUCTION FOR DYNAMIC IMAGING IN SMALL ANIMAL PET

Ju-Chieh (Kevin) Cheng, Xiaodong Liu, Richard Laforest

Radiology, Washington University in St. Louis, St. Louis, MO, USA.

The 3D maximum a posteriori (MAP) image reconstruction algorithm has been developed to improve the spatial resolution by an accurate modeling of the PET system response to the detection of gamma rays. The objective of this study is to evaluate the quantitative accuracy of the MAP reconstruction algorithm and compare with the 2D filtered-backprojection (2D-FBP), a standard image reconstruction algorithm, in the microPET-FOCUS small animal PET cameras. A special attention was given to that whether the quantitative accuracy is preserved for various level of count density encountered in typical dynamic scanning as well as the imaging of cardiac activity concentration in small animal studies. Specially designed phantoms were used for evaluation of the spatial resolution, image quality, and quantitative accuracy. Normal mice were employed to evaluate the accuracy of the blood time activity concentration extracted from left ventricle ROIs to actual blood activity concentration measured from arterial blood sampling. Images were reconstructed with FORE rebinning followed by 2D-FBP with a ramp filter operated at Nyquist frequency. MAP images were reconstructed from 3D sinograms for 20 iterations. The spatial resolution obtained with MAP reaches values of $1.24 \mathrm{~mm}$ while the 2D-FBP has value of $1.7 \mathrm{~mm}$; this result is consistent with what has been reported before. Importantly, the spatial resolutions achieved by MAP are all obtained with lesser noise than those by 2D-FBP. For MAP the spatial resolution improves with the number of iterations but converges to a constant value at 18 iterations. The noise in the images increases rapidly until that point and then slowly increases. For all the MAP images, the measured activity concentration in the largest sphere has the expected concentration relative to the background area. The recovery coefficients have been also shown to be independent of the count density, and the value is the lowest for the smallest sphere. The 2D-FBP does not perform as well, yielding recovery coefficients lower than those observed with MAP. However, a small positive bias was observed in MAP reconstructed images for frames of short duration. This bias is present in the uniform area for count density of less than 0.05 million counts $/ \mathrm{mL}$. For the dynamic mouse study, it was observed that MAP (even gated at diastole) cannot predict accurately the blood activity concentration due to some small remaining spill-over activity from the Myocardium into the left-ventricle (i.e. partial volume effect), but it still performs better than 2D-FBP.

\section{J661}

EVALUATION OF THE SIEMENS TRUEX RECONSTRUCTION ALGORITHM FOR QUANTITATIVE PET STUDIES

David M. Dommett, Nicholas Keat, William A. Hallett

Clinical Imaging Centre, GlaxoSmithKline, London, United Kingdom.
The Siemens TrueX reconstruction algorithm incorporates a direct measurement of the scanner system model, and offers improved spatial resolution compared with conventional techniques. The aim of this work was to investigate the suitability of this algorithm for research applications where quantitative accuracy of PET images is paramount. Hot and cold sphere emission data from a NEMA image quality phantom was acquired on a Siemens TruePoint PET-CT scanner with a range of total detected events. This data was reconstructed using the TrueX algorithm using different combinations of subsets, iterations and smoothing. The same data was also reconstructed using conventional filtered back projection (FBP). Contrast recovery, background variability and linearity were investigated. For hot sphere contrast recovery TrueX performance was superior to FBP provided sufficient iterations and subsets were used. For cold sphere contrast recovery FBP outperformed TrueX. The convergence of results was slower for cold spheres. The background variability was generally lower for TrueX. Linearity was determined by comparing hot and cold sphere contrast recovery and was inferior for TrueX compared to FBP. TrueX offers improved spatial resolution and noise suppression compared with FBP, but should be used with care if quantification is of primary importance. The TrueX reconstruction parameters need to be optimised as there is a trade off between contrast recovery and background variability.

\section{$\mathrm{J} 662$}

\section{CONTINUOUS MINIATURE CRYSTAL ELEMENT DETECTOR DESIGNS WITH DEPTH OF INTERACTION POSITIONING CAPABILITY}

Robert Miyaoka ${ }^{1}$, Xiaoli Li ${ }^{2}$, Tom K. Lewellen ${ }^{1}$

Radiology, University of Washington, Seattle, WA, USA, ${ }^{2}$ Physics, University of Washington, Seattle, WA, USA.

Objectives: We have developed a series of continuous miniature crystal element (cMiCE) positron emission tomography (PET) detectors with depth of interaction (DOI) positioning capability as a lower cost design alternative to finely pixilated discrete crystal detectors. To achieve high intrinsic spatial resolution in three dimensions (3D) a statistics based positioning algorithm is used. Methods: In this work we report on two detector designs. The first consists of a $50 \mathrm{~mm}$ by $50 \mathrm{~mm}$ by $15 \mathrm{~mm}$ monolithic crystal of LYSO coupled to an 8 by 8 multi-anode photomultiplier tube (MA-PMT). Experimental data were collected for this implementation. The second design consists of a $49.6 \mathrm{~mm}$ by $49.6 \mathrm{~mm}$ by $15 \mathrm{~mm}$ crystal readout by a 16 by 16 array of solid-state photosensors (e.g., silicon photomultipliers, SiPM) placed on the entrance surface of the scintillator crystal. Our hypothesis is that this sensor on the entrance surface (SES) design will lead to better positioning performance than conventional placement of the sensors on the back surface of the scintillator. Simulations were conducted to evaluate the SES design. Both designs use a statistics based positioning 
method that requires characterizing the light response function of the detector in 3D. To support real time processing of detected events, we separate the 3D positioning method into a $2 \mathrm{D}(\mathrm{X}, \mathrm{Y}), 1 \mathrm{D}(\mathrm{Z}), 2 \mathrm{D}(\mathrm{X}, \mathrm{Y})$ series of positioning steps. The proposed methodology is being implemented in a field programmable gate array and will support real time positioning at the detector module level. Results: The average X, $\mathrm{Y}$ intrinsic spatial resolution for the $\mathrm{cMiCE}$ detector using conventional readout and an 8 by 8 MA-PMT was $1.74+/-0.35 \mathrm{~mm}$ full width at half maximum (FWHM) for a useful imaging area of $44.6 \mathrm{~mm}$ by $44.6 \mathrm{~mm}$. The average DOI positioning resolution was 5.1+/-0.7 mm FWHM using our simplified 2D, 1D, 2D positioning methodology. The average $\mathrm{X}, \mathrm{Y}$ intrinsic spatial resolution for the cMiCE detector using SES readout and a 16 by 16 sensor array was $0.87+/-0.1 \mathrm{~mm}$ FWHM for a useful imaging area of $46.5 \mathrm{~mm}$ by $46.5 \mathrm{~mm}$. The average DOI positioning resolution was $2.35+/-0.55 \mathrm{~mm}$ FWHM. Conclusion: cMiCE detectors using a $15 \mathrm{~mm}$ thick LYSO crystal have been evaluated. While the design using a PMT provided very good intrinsic $\mathrm{X}, \mathrm{Y}$ spatial resolution and DOI positioning estimation to support high resolution imaging, simulation results indicate that positioning performance will be significantly improved through the use of newly available SiPM arrays and the SES readout configuration.

\section{Imaging Methodology}

\section{SPECT}

J663

\section{DUAL ISOTOPE NANOSPECT/CT FOR QUANTIFICATION OF NET-TARGET SPECIFICITY OF IMAGING AGENTS}

Nicole Hijnen ${ }^{1}$, Anke de Vries ${ }^{1}$, Holger Gruell ${ }^{1,2}$

${ }^{1}$ Biomedical NMR, Eindhoven University of Technology, Eindhoven, Netherlands, ${ }^{2}$ Biomolecular Engineering, Philips Research, Eindhoven, Netherlands.

Molecular Imaging allows the in vivo visualization of a diseased tissue with the help of specific agents which home in on disease-related markers. Concentrations of disease-related markers are in the range of $\mu \mathrm{mol} / \mathrm{L}$ to $\mathrm{nmol} / \mathrm{L}$, requiring specific ligands with high affinity as well as a sensitive imaging technique. SPECT can be employed to image radiolabeled ligands with nanomolar sensitivity. The energy-resolved detectors used in SPECT cameras offer the possibility of simultaneous imaging of two isotopes with different $\gamma$-emission characteristics. Dual isotope SPECT can be exploited to image the biodistribution of two different radiotracers labeled with two different isotopes in the same animal, thereby excluding any inter-animal variations. We developed dual isotope SPECT imaging protocols to assess the specificity of a ligand (cRGD-peptide) in binding to an angiogenesisrelated marker $(\alpha v \beta 3)$ in comparison to a potential non-specific control (cRAD-peptide) simultaneously in the same animal. The isotope couple $111 \mathrm{In} / 177 \mathrm{Lu}$ was chosen based on their $\gamma$-emission and radiochemical properties. SPECT protocols for quantitative imaging were developed to correct for spectral overlap of the two isotopes. Dual isotope imaging was tested in vivo using $111 \mathrm{In}$ - and $177 \mathrm{Lu}$-labeled peptide tracers, cRGDDOTA and cRAD-DOTA, in tumor-bearing mice (glioblastoma-astrocytoma, U87MG). In dual isotope studies, specific tumor uptake of $3.2 \pm 0.4$ $\% \mathrm{ID} / \mathrm{g}$ was found for $\mathrm{cRGD}$ compared to $0.2 \pm 0.1 \% \mathrm{ID} / \mathrm{g}$ for $\mathrm{cRAD}(n=9)$. Interchanging isotopes did not influence the biodistribution of the peptides. The results of the dual isotope studies were confirmed by single isotope scans and biodistribution using $\gamma$-counting. In conclusion, SPECT single and dual isotope imaging showed tumor uptake of cRGD, while cRAD showed no specific uptake. Dual isotope SPECT imaging using combined $111 \mathrm{In} / 177 \mathrm{Lu}$ was shown to be a unique, successful method for sensitive in vivo quantification of the net target specificity of ligands in molecular imaging research, excluding inter-animal variations, physiological changes as well as reducing the amount of animals required.

J664

\section{CLINICAL IMAGING OF THALLIUM TRANSPORT IN THE HUMAN OLFACTORY NERVE}

Hideaki Shiga $^{1,2}$, Junichi Taki ${ }^{3}$, Kohshin Washiyama ${ }^{4}$, Sayaka Yagi ${ }^{2}$, Ryohei Amano $^{4}$, Mitsuru Furukawa ${ }^{2}$, Seigo Kinuya ${ }^{3}$, Takaki Miwa ${ }^{1,2}$; ${ }^{1}$ Otorhinolaryngology-Head and Neck Surgery, Kanazawa Medical University, Kanazawa, Japan, ${ }^{2}$ Otorhinolaryngology-Head and Neck Surgery, Graduate School of Medical Science, Kanazawa University, Kanazawa, Japan, ${ }^{3}$ Biotracer Medicine, Graduate School of Medical Science, Kanazawa University, Kanazawa, Japan, ${ }^{4}$ Forefront Medical Technology, Graduate School of Medical Science, Kanazawa University, Kanazawa, Japan.
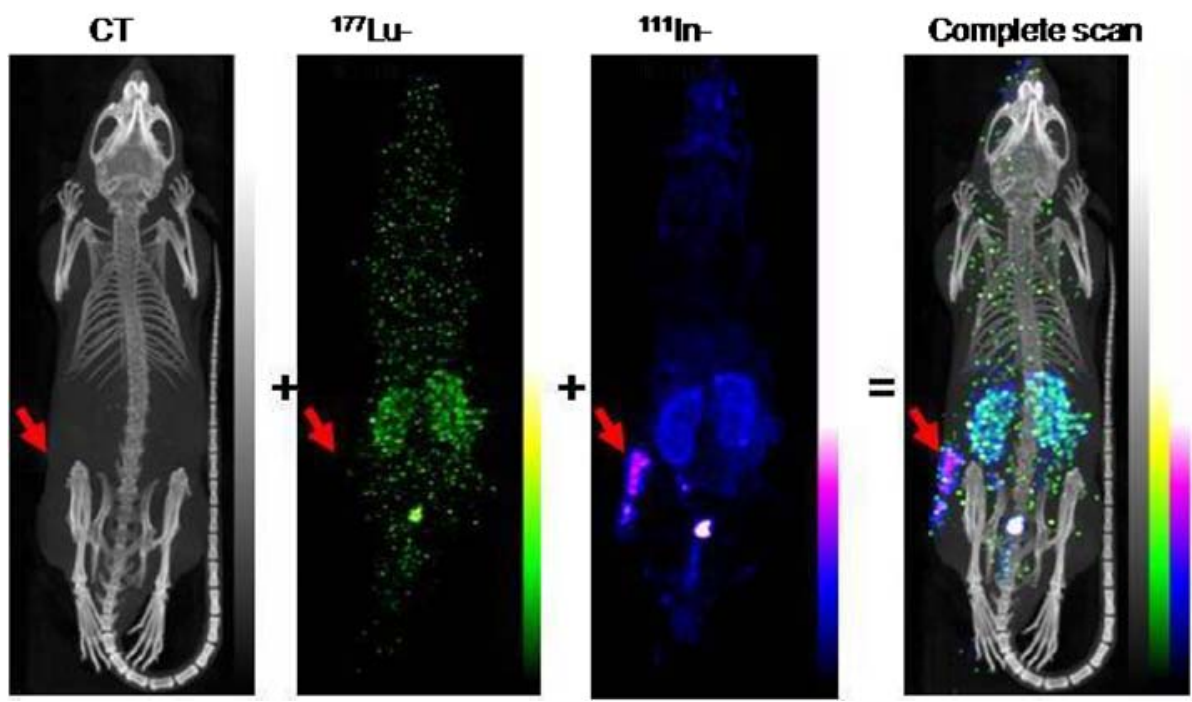
Background: Viruses can invade the central nervous system via the nose and olfactory nerve. However, this route has not been confirmed, because it is difficult to directly view olfactory transport to the human brain. Here, we show olfactory transport to the human brain using an imaging method with nasal thallium-201 $\left({ }^{201} \mathrm{Tl}\right)$ administration. Methods: Healthy volunteers gave informed consent $(n=5)$. They were first assessed for their olfactory threshold, and then received ${ }^{201} \mathrm{TlCl}$ $(0.3 \mathrm{ml}, 74 \mathrm{MBq} / \mathrm{ml})$ onto one side of the olfactory clefts. At $30 \mathrm{~min}$ and $24 \mathrm{~h}$ after ${ }^{201} \mathrm{Tl}$ administration, they were scanned by single photon emission computed tomography (SPECT)/CT. Separate MRI images were merged with the SPECT images by using the mutual information method. The higher uptake of the ${ }^{201} \mathrm{Tl}$ (yellow to red colors) was regarded as the peak of ${ }^{201} \mathrm{Tl}$ uptake each time. Results: ${ }^{201} \mathrm{Tl}$ was taken up mainly in the upper nasal turbinate (olfactory epithelium area) under the cribriform lamina $30 \mathrm{~min}$ after administration. The bulk of the ${ }^{201} \mathrm{Tl}$ entered the olfactory bulb in the anterior skull base through the cribriform lamina $24 \mathrm{~h}$ after administration. ${ }^{201} \mathrm{Tl}$ uptake in the olfactory bulb area was confirmed on the fusion image. No participants experienced any adverse events, including olfactory disturbances. Conclusions: Nasal ${ }^{201} \mathrm{Tl}$ administration could safely mark the direct pathway to the brain via the nose. This study is the first to show olfactory transport to the anterior skull base in humans. Our imaging method may help assess the olfactory neurons as a route of infection in patients with central nervous system disorders.

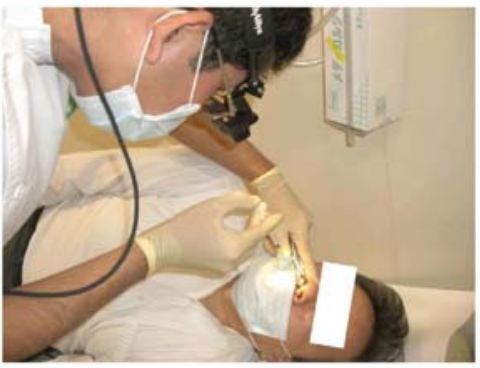

${ }^{201} \mathrm{TICl}$ nasal administration

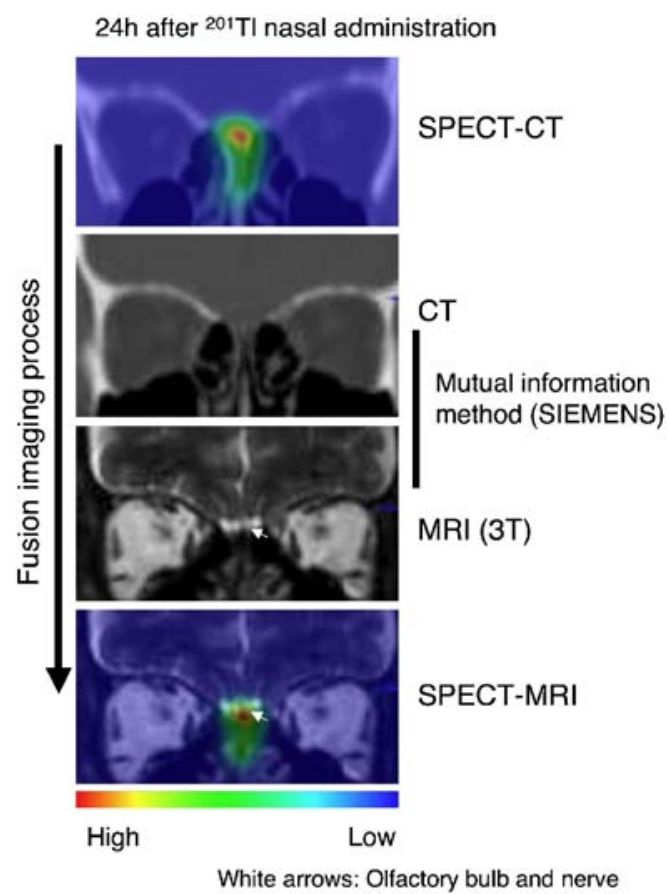

attenuation, scatter, and geometric blurring caused by radionuclide collimators. The conversion factors between image values and tracer concentrations (in $\mathrm{Bq} / \mathrm{ml}$ ) were calculated from uniform phantom filled with aqueous solution of $111 \mathrm{InCl} 3$ using the same acquisition protocol and reconstruction parameters as for patient studies. In addition, the spatial resolution of the reconstructed images was measured from a point source phantom. The measured spatial resolution was modeled into a point spread function (PSF), and the PSF was implemented in a deconvolultion-based partial volume error (PVE) correction algorithm. The recovery capability to correctly estimate true tracer concentration values was tested using prostate-like and bladder-like lesion phantoms fitted in the modified NEMA/IEC body phantom. Patients with biopsy-proven prostate cancer $(n=10)$ who were undergoing prostatectomy were prospectively enrolled in the preoperative SPECT/CT studies at the San Francisco VA Medical Center. The CT portion of SPECT/CT was used for CT-based attenuation map generation as well as an anatomical localization tool for clinical interpretation. Pathologic Gleason grades were compared with in vivo "antibody uptake value" (AUV) normalized by injected dose, effective half-life, and injection-scan time difference. AUVs
Objectives: We have developed an in vivo antibody uptake quantification method using $111 \mathrm{In}$-capromab pendetide SPECT/CT technology. Our goal is to evaluate this noninvasive antibody quantification method for potential prostate tumor grading. Methods: In vivo tracer quantification needs a quantitative SPECT reconstruction so that tracer concentration values could be extracted from the image space. Our phantom experiments focused on the robustness of an advanced iterative reconstruction algorithm that involves corrections for photon 
were calculated in each lobe of prostate gland with cylindrical volumes of interest (VOIs) having dimensions of $1.5 \mathrm{~cm}$ both in diameter and height. Results: Reconstructed SPECT images further corrected by the deconvolution-based PVE correction could recover true tracer concentrations in volumes as small as $7.77 \mathrm{ml}$ up to $90 \%$ in phantom measurements. From patient studies, there was a statistically significant correlation ( $\rho=0.7107, P=0.03301$ ) between higher AUVs (from either left or right lobe) and higher components of pathologic Gleason scores. Conclusion: Our results strongly indicate noninvasive prostate tumor grading potential using quantitative $111 \mathrm{In}$ capromab pendetide SPECT/CT for prostate cancer evaluation.

\section{J666}

\section{CHARACTERIZATION OF THE INVEON MULTI-MODAL SPECT SYSTEM}

Douglas J. Rowland, Sara St. James, Simon R. Cherry

Biomedical Engineering, University of California Davis, Davis, CA, USA.

The UC Davis Center for Molecular and Genomic Imaging recently installed a Siemens Inveon Multi-Modal SPECT/CT system and we will present our characterization of the system. The Siemens SPECT system is composed of two NaI(Tl)-PMT modules oriented at 180 degrees. The modules have a large active area of $15 \mathrm{~cm} \times 15 \mathrm{~cm}$. Individual crystals $(2 \mathrm{~mm} \times 2 \mathrm{~mm} \times 10 \mathrm{~mm}$ with a $2.2 \mathrm{~mm} \times 2.2 \mathrm{~mm}$ pitch) make up the $68 \times 68$ array. Projection formation is based on pinhole technology in which tungsten collimators can be interchanged to provide different resolution and sensitivity combinations (4 single pinhole collimators at $0.5,1.0,2.0$ or $3.0 \mathrm{~mm}$; two 5 pinhole collimators at 0.5 or $1.0 \mathrm{~mm}$ ). System stability is being assessed in order to develop periodic tests to ensure the long-term integrity of the system. These measurements include energy resolution, integral and differential uniformity and quantitative accuracy using a Co-57 source. Other measurements to be presented will include resolution, sensitivity and energy resolution for different nuclides (Tc-99 m, In-111, I-123 and I-125) and collimators. Axial uniformity will be evaluated for single bed position and helical scans using a micro-Defrise phantom. The integral and differential uniformity has been evaluated daily in order to look at the day-to-day variation. The results over a four-day span show a maximum of 5 and $10 \%$ change in uniformity for heads 1 and 2 , respectively. This test is being continued on a weekly basis and results will be presented. Initial tests of the axial uniformity have begun using the $1 \mathrm{~mm}$ pinhole collimators. The helical scanning of the micro-Defrise phantom has shown that this collimator has a good axial uniformity. Our initial studies show that the system is operational and the long-term study of system stability will be presented at this meeting. The characterization of a new system such as the Inveon MM SPECT is an important step to optimizing scan protocols for animal imaging and producing quality animal images.

\section{$\mathbf{J} 667$}

EFFECT OF COLLIMATOR PINHOLE SIZE IN LOW-DOSE, FAST-SCAN IMAGING APPLICATIONS USING A PRECLINICAL SPECT/CT SYSTEM

Ciara Finucane $^{1,2}$, Jacob Hesterman ${ }^{2}$, Christian Lackas ${ }^{2,3}$, Jack Hoppin ${ }^{3,2}$, Stephen Mather $^{1}$

${ }^{1}$ Molecular Oncology and Imaging, Queen Mary University, London, United Kingdom, ${ }^{2}$ Imaging, Bioscan, Inc, Washington DC, DC, USA, ${ }^{3}$ Imaging, InviCRO, Boston, MA, USA.

An inherent trade-off exists in SPECT imaging between resolution, sensitivity, and field of view. In preclinical SPECT, different pinhole geometries may be used to vary these parameters on an application-specific basis. This study examines the trade-off between resolution and sensitivity at low activity concentrations and short scan times (as found in dynamic or gated imaging) for three different pinhole diameters. SPECT acquisitions were performed using a commercial small-animal SPECT/CT system with four detectors and inter-changeable multiplexing nine-pinhole collimators. A bone imaging agent (Tc99m-MDP) was injected into four mice with two mice each receiving doses of only $5 \mathrm{MBq}$ and $50 \mathrm{MBq}$. Whole-body scans were acquired with durations of 5,10 , and $15 \mathrm{~min}$. All scans were repeated with sets of nine-pinhole collimators having pinhole diameters of $1.0 \mathrm{~mm}$, $1.4 \mathrm{~mm}$, and $2.0 \mathrm{~mm}$. Identical reconstruction parameters were used for all scans. The experiment was repeated using a hot-rod phantom using the same parameters. At the $50 \mathrm{MBq}$ activity, the higher achievable resolution of the smaller-diameter pinholes is evident in mouse and phantom images regardless of scan duration. At the $5 \mathrm{MBq}$ activity, sensitivity becomes the critical factor in image quality; a qualitative and quantitative difference is observed as a function of pinhole diameter with the larger-pinhole collimators producing higher quality images. The attached image shows results obtained with 15 -minute whole-body scans at $5 \mathrm{MBq}$ and $50 \mathrm{MBq}$ for each of the three pinhole diameters. This study demonstrates the tradeoff between sensitivity and resolution and illustrates the importance of collimator selection when planning imaging studies. The choice of collimator depends on dose, target, and time restrictions. Low-count or rapid, high-throughput studies benefit from a more sensitive collimator, while applications needing high-resolution images require smaller-diameter pinhole collimators and adequate consideration of dose and acquisition time.

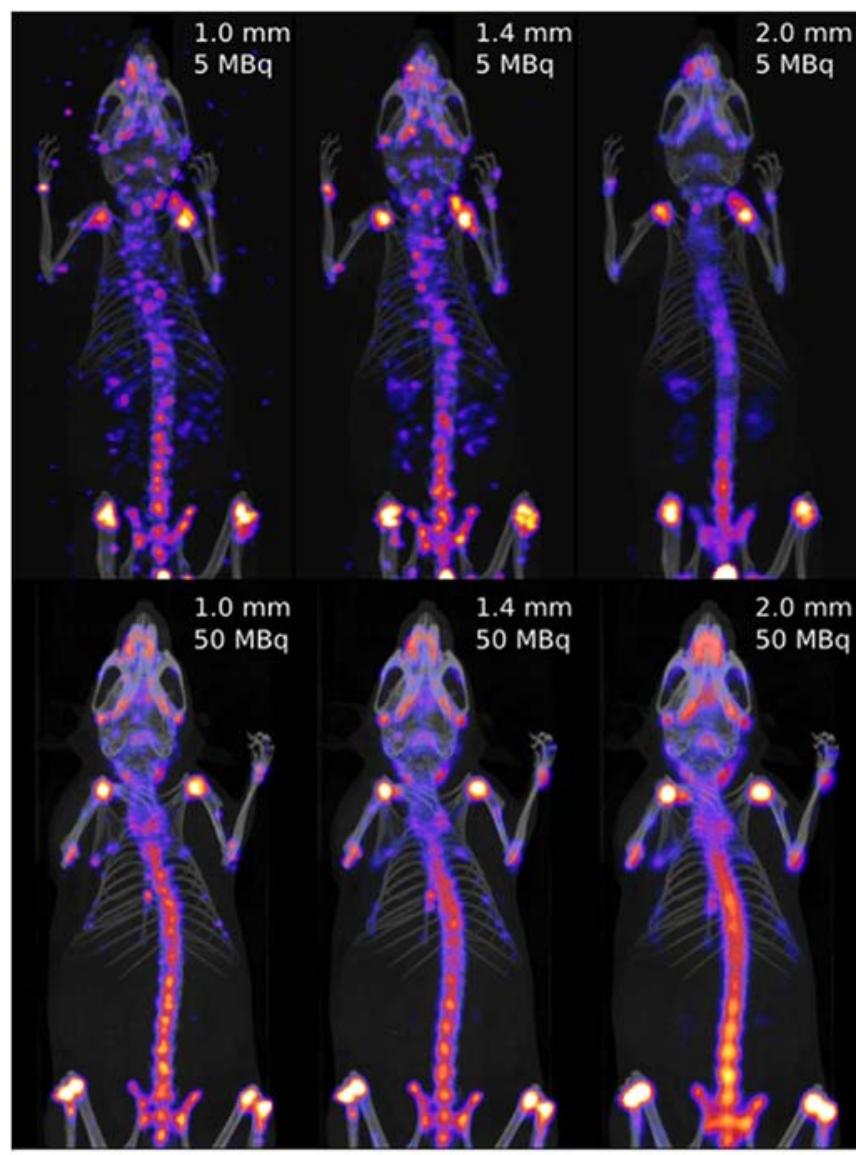

15-minute scan time for all SPECT images shown 


\section{J668}

ANALYSIS OF AEROSOL DEPOSITION PATTERNS IN MICE AND RATS USING MANUAL AND AUTOMATED QUANTIFICATION METHODS

Benjamin Gershman ${ }^{1,5}$, Jacob McDonald ${ }^{3}$, Phil Kuehl ${ }^{3}$, Jack Hoppin ${ }^{4}$, Daniel J. Irwin ${ }^{2}$, Jacob Hesterman ${ }^{5}$, Ky Harlin ${ }^{4}$, Christian Lackas ${ }^{4}$, Tamara Anderson ${ }^{2}$, Jeffrey P. Norenberg ${ }^{2}$

${ }^{1}$ Northeastern University, Boston, MA, USA, ${ }^{2}$ College of Pharmacy, University of New Mexico, Albuquerque, NM, USA, ${ }^{3}$ Chemistry and Inhalation Exposure, Lovelace Respiratory Institute, Albuquerque, NM, USA, ${ }^{4}$ InviCRO, LLC, Boston, MA, USA, ${ }^{5}$ Bioscan, Inc., Washington, DC, USA.

Objectives: The evaluation of the deposition pattern of inhaled radiolabeled sulfur-colloid particles in mice and rats using manual and automated quantification techniques. Methods: A novel smallanimal inhalation system was used to deliver nose-only exposure of radiolabeled sulfur colloid particles to mice and rats. Aerosols were generated with a series of compressed air jet nebulizers with and without active aerosol drying to achieve a series of particle sizes $(0.5$, $1.0,3.0$ and 5.0 mass median micron diameter). Subjects were euthanized immediately post-exposure and sequentially imaged using a dedicated small-animal SPECT/CT system. Quantification of deposited radioactivity was performed on the following regions: exterior of the head, oral and nasal passages, trachea and bronchus, bifurcation between the lungs, each lung individually and the stomach. In addition to whole organ quantification, an algorithm was developed for segmenting the lungs via topographical thinning or "onion peeling". Results: Quantitative and qualitative analysis presented evaluating whole-organ uptake as well as $3 \mathrm{D}$ lung deposition as a function of particle size. The study demonstrated that an increase in particle size increases gastrointestinal uptake and modifies the deposition of the labeled particles in the lung. Conclusions: We have developed and presented a method for imaging and analyzing radiolabeled sulfurcolloid particles in the lungs of mice and rats.

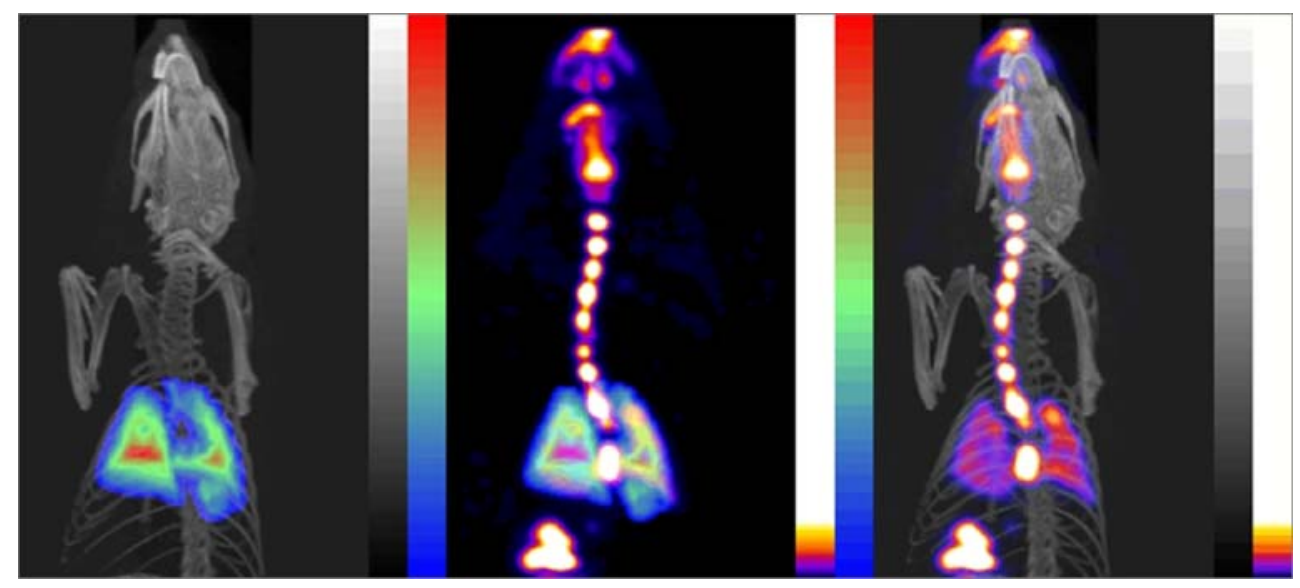

Tc99m-labeled sulfur colloid uptake in lungs of rat: X-ray CT and mathematical 'onion' (left), SPECT and onion (center), SPECT/CT (right). Assessing radiopharmaceutical concentration across onion layers enables calculation of aggregate profile 3D deposition.

J669

\section{FEASIBILITY AND PERFORMANCE OF SIMULTANEOUS SPECT-CT IMAGING}

Derek W. Austin, Dustin Osborne, Mu Chen, JunJun Deng, Shikui Yan Preclinical Solutions, Siemens Healthcare, Knoxville, TN, USA.

Acquiring SPECT and X-ray CT data simultaneously may improve correlation and registration of SPECT-CT images, increase throughput, and benefit the scanned subject by minimizing time under anesthetic. We have acquired SPECT and CT data simultaneously on a preclinical imaging system (Siemens Inveon Multimodality) using two SPECT detectors that are mounted coplanar and orthogonal to an X-ray micro-CT system. Figure 1a shows a SPECT projection of a Co-57 point source through a 5-pinhole tungsten collimator in a $30-300 \mathrm{keV}$ energy window with the X-ray tube at $70 \mathrm{kVp}$ and $\mathrm{X}$ ray shutter fixed open. Although the number of X-ray events (less than $20,000 /$ second) collected by the SPECT detector was significant when the shutter was open, these events could be discriminated by setting an appropriate energy window. An energy spectrum is shown below in Figure $1 \mathrm{~b}$ with the X-ray tube at $70 \mathrm{kVp}$ and shutter open. After applying a $20 \%$ energy window for Co-57 (110-134 keV), the improved image in Figure 1c was obtained. Yet, during a simultaneous SPECT-CT scan with typical scan parameters, the X-ray shutter was closed $\sim 90 \%$ of the total scan time. That is, during 10 minutes of SPECT-CT acquisition, the multiple X-ray exposures summed to only $\sim 1 \mathrm{~min}$. When the X-ray shutter was closed, a high signal-to-noise ratio was maintained on the SPECT system (data not shown here). Similarly, the effects of the Co-57 gamma emission had little impact on the quality of the CT images due to the high X-ray flux, as illustrated by the CT projection image in Figure 1d. However, gammas emitted from the subject may add some noise to CT images. In this paper, we evaluate the performance of simultaneous SPECT-CT tomographic scans and present data demonstrating the impact on SPECT image quality in the presence of X-rays and the impact on X-ray CT image quality in the presence of low-to-medium energy gamma emission. 


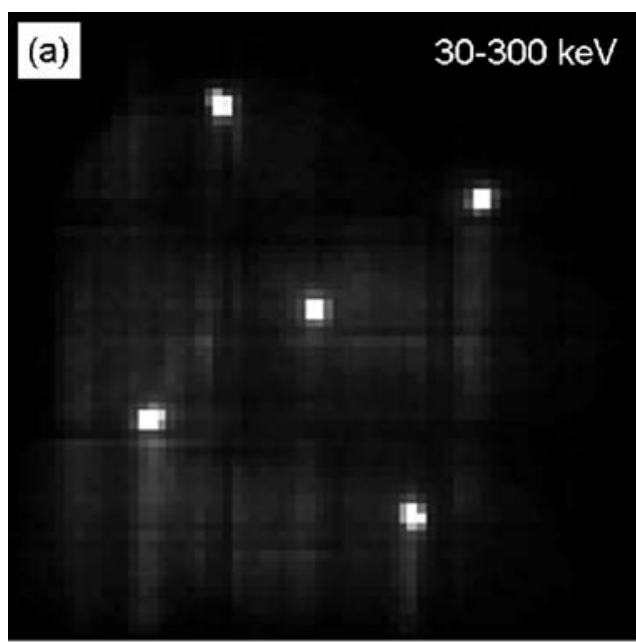

\section{(b)}

\section{SPECT Detector Spectrum}
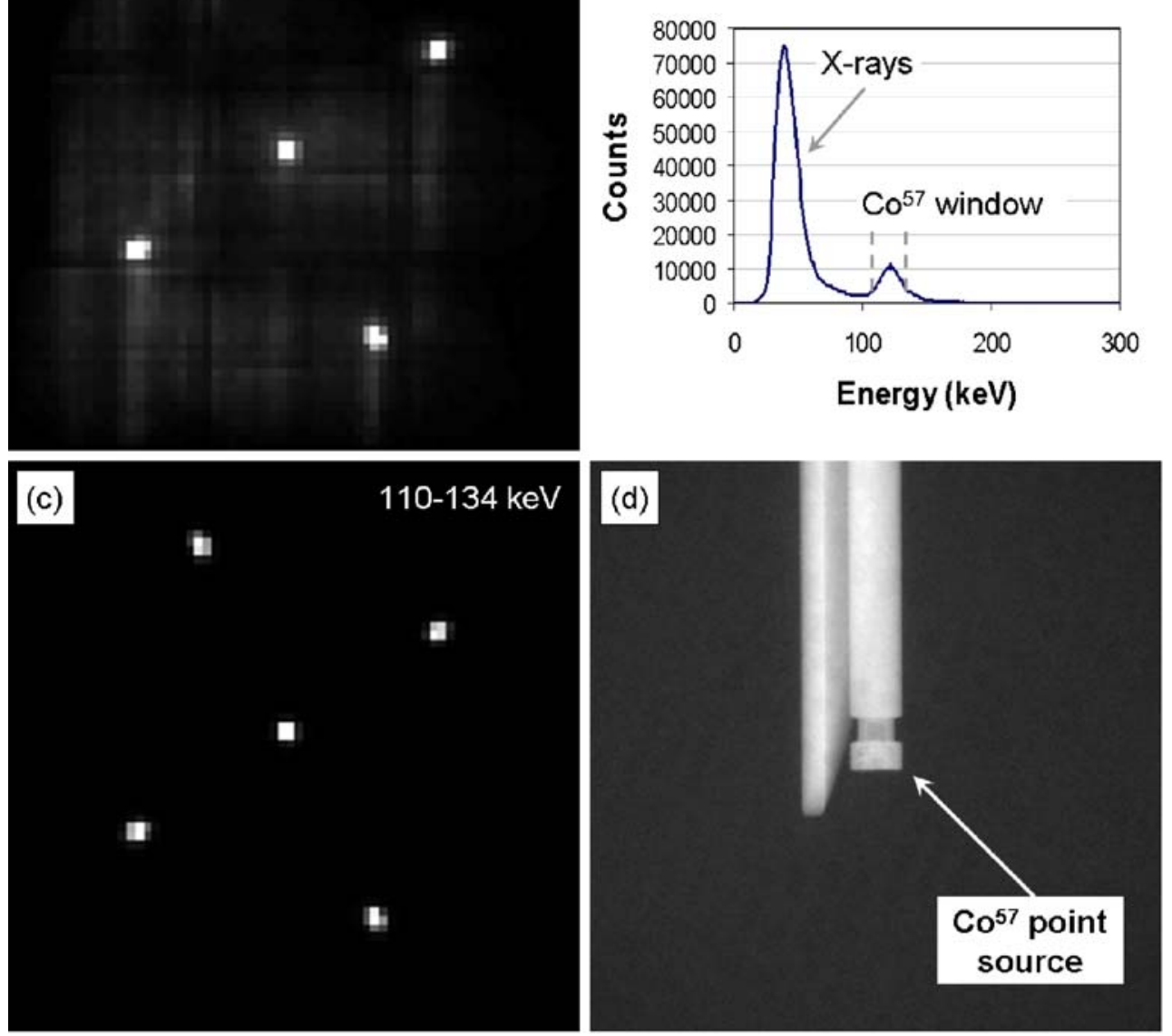

configurable CZT detector heads. Furthermore, dramatic improvement in energy resolution (from $9.5 \%$ to $4 \%$ at $140 \mathrm{keV}$ ) results in improved conspicuity for low contrast lesions. A three-site clinical trial for women at high risk or with radiographically dense breasts is planned to confirm the

MOLECULAR BREAST IMAGING WITH HIGHPERFORMANCE CZT DETECTORS

$\underline{\text { Doug Wagenaar }^{1} \text {, Rex Moats }}{ }^{1,2}$, Dirk Meier ${ }^{1}$, Gunnar E. Maehlum ${ }^{1}$, $\overline{\text { Bjorn M. Sundal }}^{1}$, Kevin Parnham ${ }^{1}$, Bradley E. Patt ${ }^{1}$

${ }^{1}$ Gamma Medica-Ideas, Northridge, CA, USA, ${ }^{2}$ Radiology, CHLA/ USC, Los Angeles, CA, USA.

Many molecular imaging agents are under development in both SPECT and PET for the diagnosis and treatment of breast cancer. These include molecules targeted to hormone receptors and biological processes associated with breast cancer. Research on radiolabeled nanoparticles and reporter gene techniques has been reported. Concerning single photon (SPECT) imaging hardware, improvements in spatial resolution and detection sensitivity result in lower patient dose, improved quantitative accuracy, and better patient management decisions. We describe a new dual-head, room-temperature semiconductor CZT imaging system that can be used for single- and dual-tracer molecular imaging of the breast. The agent 99mTc-sestamibi (MIBI, formerly marketed as Miraluma) has proven in scintimammography studies with Anger cameras over 15 years to be an effective breast cancer imaging tracer. The CZT system with dual-head images MIBI uptake from opposing MLO and CC views for 5-10 minutes per view per breast, totaling less than 40 minutes per patient. Detection sensitivity for lesions less than $1 \mathrm{~cm}$ is dramatically improved over Anger camera-based scintimammography studies in preliminary clinical trials involving more than 1500 patients. This is due to the improved spatial resolution derived from the close proximity of the smaller, more easily promising results of the initial clinical studies. Funding from the NIH National Cancer Institute grant \# R42CA128407-03 is gratefully acknowledged.

\section{J671}

NUCLEAR MICROSCOPY: IN VIVO CELLULAR IMAGING AT ULTRA-HIGH RESOLUTION

Doug Wagenaar ${ }^{1}$, Dirk Meier ${ }^{1}$, Rex Moats ${ }^{1,2}$, Gadi Pelled ${ }^{3}$, Dan Gazit $^{3}$, Bradley E. Patt ${ }^{1}$

${ }^{1}$ Gamma Medica-Ideas, Northridge, CA, USA, ${ }^{2}$ Radiology, CHLA/ USC, Los Angeles, CA, USA, ${ }^{3}$ Cedars Sinai, Los Angeles, CA, USA.

Autoradiography is nuclear imaging with fine-grain film to detect beta-particle emissions from the nuclides $3 \mathrm{H}$ (tritium), $14 \mathrm{C}$, and $32 \mathrm{P}$, and it has been the workhorse of the in vitro biomedical research lab since the $1960 \mathrm{~s}$. The spatial resolution of film autoradiography is generally sub-cellular-i.e., $\sim 1$ micron, and has not yet been equaled with digital detection techniques. Optical imaging allows the visualization of single cells within a living system, but currently fields-ofview and depths are limited to just a few hundred microns due to scattering of light. Nuclear microscopy is the new field of in vivo cellular imaging in small animals such as mice. Cells are radioactively labeled - either ex vivo (e.g., oxime) or in vivo using 
reporter gene methods. Radionuclides are single-photon emitters with gamma-ray and x-ray energy in the range 15-40 keV-sufficiently high to penetrate tissues of the specimen but low enough to collimate (and shield) to high resolution. Resolution is targeted to be $\sim 15$ microns with 3D FOV of $1 \mathrm{~cm} 3$. High resolution image formation is achieved with a gold foil of 75 micron thickness through which a coded aperture pattern has been etched. Magnification of five allows the $1 \mathrm{~cm} 2 \mathrm{FOV}$ to project onto a $6 \mathrm{~cm} \times 6 \mathrm{~cm}$ double-sided silicon detector with 59 micron pixel pitch. This megapixel $(1028 \times 1028)$ detector has a $1.0 \mathrm{~mm}$ silicon thickness, sufficient for detecting the low energy photons with high efficiency. Progress in the fabrication, assembly, and operation of the first prototype will be reported. Nuclear Microscopy is a new method for achieving ultra-high resolution in vivo in small laboratory animals, and may find use in monitoring stem cell and genetic engineering therapy research. Support from the California Institute for Regenerative Medicine is gratefully acknowledged.

\section{Imaging Methodology}

\section{Ultrasound}

\section{J672}

\section{NONLINEAR MOLECULAR IMAGING WITH A LINEAR} ARRAY BASED MICRO-ULTRASOUND SYSTEM

Andrew Needles ${ }^{1}$, James Mehi $^{1}$, Corina Bilan ${ }^{1}$, Marcel Arditi ${ }^{2}$, Emmanuel Gaud ${ }^{2}$, Peter Frinking ${ }^{2}$, Desmond Hirson ${ }^{1}$, F. Stuart Foster ${ }^{3,1}$ ${ }^{1}$ VisualSonics, Toronto, ON, Canada, ${ }^{2}$ Bracco Research, Geneva, Switzerland, ${ }^{3}$ Sunnybrook Health Sciences Centre, Toronto, ON, Canada.

Recently, a linear array based micro-ultrasound system with a 64 channel beamformer has been developed. This talk will focus on the implementation of a real-time nonlinear contrast mode on this array based system, capable of performing in vivo molecular imaging with targeted microbubble contrast agents. A modified micro-ultrasound scanner (Vevo 2100, VisualSonics) was operated at 18-24 MHz with a 256 element linear array transducer $(\mathrm{MS}-250, \mathrm{fc}=21 \mathrm{MHz}$ ). in vitro experiments were conducted with MicroMarker (VisualSonics) contrast agent flowing at $30 \mathrm{~mm} / \mathrm{s}$ through a $1 \mathrm{~mm}$ wide vessel within a polyvinyl alcohol (PVA) cryogel phantom. Low amplitude $(350 \mathrm{kPa})$ narrowband pulses (6 cycles) were used with the following multipulse sequences: pulse-inversion (PI), amplitude-modulation (AM) and their combination (PIAM). These sequences allow isolation of nonlinear bubble echo signals at the subharmonic ( $\mathrm{SH})$ and also fundamental (FUND - only for AM and PIAM) frequencies. The signals were digitally bandpass filtered (BPF) around the SH, FUND or SH + FUND frequency bands. Mean echo power values were calculated for bubbles and PVA, and contrast-to-tissue-ratios (CTR) were determined. From the in vitro results, the improvement in CTR at $18 \mathrm{MHz}$ was maximized $(13 \mathrm{~dB})$ with either AM or PIAM, compared to raw non-processed echo signals, whereas at $24 \mathrm{MHz}$ the highest improvement in CTR $(15 \mathrm{~dB})$ was obtained with PI $+\mathrm{SH}$. Guided by these results, in vivo images (Fig. 1) were obtained using $\mathrm{AM}$ in the hind limb of mice with induced inflammation, with a targeted agent specific to P-selectin as well as an isotype control agent. The detected signal, from the targeted agent, was 2-3 times higher than from the control agent. This ratio is substantial, compared to using frame subtraction with B-mode imaging, which showed no significant difference between the targeted and control agents. Nonlinear molecular imaging with micro-ultrasound is now possible with an array based system in the 18-24 MHz range. Advantages of nonlinear FUND or SH detection have been demonstrated, which allow substantial improvements in CTR over traditional frame subtraction-based techniques.
Control

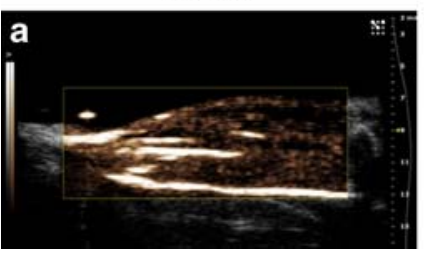

Figure 1
Targeted

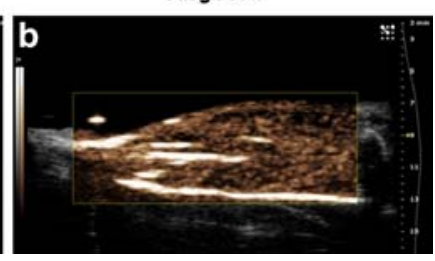

\section{$\mathrm{J} 673$}

NOVEL ULTRASOUND AND DCEMRI ANALYSES AFTER
ANTIANGIOGENIC TREATMENT WITH A SELECTIVE
VEGF RECEPTOR INHIBITOR Katherine D. Watson ${ }^{1,2}$, Xiaowen $\mathrm{Hu}^{1}$, Heather A. Lindfors ${ }^{1}$, Chun-Yen Lai ${ }^{1}$, Dana D. Hu-Lowe ${ }^{3}$, Theresa A. Tuthill ${ }^{5}$, David R. Shalinsky ${ }^{4}$, Katherine Ferrara ${ }^{1}$

${ }^{1}$ Biomedical Engineering, University of California, Davis, CA, USA, ${ }^{2}$ Molecular and Cellular Inegrative Physiology, University of California, Davis, CA, USA, ${ }^{3}$ Cancer Biology, Pfizer La Jolla, San Diego, CA, USA, ${ }^{4}$ Translational Medicine, Pfizer La Jolla, San Diego, CA, USA, ${ }^{5}$ Molecular Imaging, Pfizer New London, New London, CT, USA.

MRI is frequently used to assess the effects of anti-angiogenic treatments. However, ultrasound is more cost-effective and enables a simpler analysis than MRI due to the purely intravascular nature of the tracer. We report a novel imaging protocol to quantify tumor vascular perfusion using ultrasound with results comparable to those obtained using MRI. AG-028262 (Pfizer Oncology), a potent inhibitor of VEGFR (VEGF-R1, -R2, and -R3) autophosphorylation, was employed in a rat tumor model to decrease tumor growth and enable a direct comparison of the effects of this agent on tumor vascular perfusion by each imaging modality. Methods: $1 \times 106 \mathrm{R} 3230 \mathrm{AC}$ rat adenocarcinoma cells were injected SC into the hind flank of Fischer344 rats. Two 12-h interval doses of AG-028262 were administered $\mathrm{PO}$ at $30 \mathrm{mg} / \mathrm{kg}$ when tumors reached $5 \mathrm{~mm}$ in diameter. Dosing was repeated at $7 \& 14$ days. Ultrasound and MR images were acquired before and after each treatment period. Seven $\mathrm{MHz}$ contrastassisted ultrasound was recorded over a 20 -second acquisition interval using a high mechanical index (1.9) destructive pulse and low mechanical index (0.1) imaging sequence. Spoiled gradient $1.5 \mathrm{~T}$ magnetic resonance sequences with gadolinium contrast were acquired over 2 min with 2 -sec time resolution and with both the tumor and arterial input recorded. For ultrasound, a time to $80 \%$ contrast replenishment for each tumor voxel was calculated; for MR imaging, a measure of local flow rate was estimated from a linear fit of minimum to maximum intensities. Results and conclusion: AG028262 significantly decreased tumor growth after each dose administered. The mean ultrasound time to $80 \%$ contrast replenishment increased from $3.3 \mathrm{~s}$ in controls to $5.7 \mathrm{~s}$ at $24 \mathrm{~h}$ after treatment, where both the absolute time and percent change were significantly different than control animals $(p<0.05)$. The MR-based measure of $80 \%$ contrast inflow ranged from 20 to $122.5 \mathrm{~s}$ while ultrasoundbased time to contrast replenishment spanned 1.3 to $6.8 \mathrm{~s}$ with a correlation observed between ultrasound and MRI measures $(\mathrm{r} 2=$ 0.74). The results demonstrate similar trends in ultrasound and MRbased measures in response to VEGFR antagonism by AG-028262 and the utility of ultrasound as a tool to quantify tumor perfusion. 
J674

\section{MOLECULAR IMAGING DRUG DELIVERY WITH INTRAVASCULAR ULTRASOUND USING TARGETED ULTRASONIC CONTRAST AGENTS}

Linsey C. Phillips ${ }^{1}$, Alexander L. Klibanov², Brian R. Wamhoff ${ }^{2}$, John A. Hossack ${ }^{1}$

${ }^{1}$ Biomedical Engineering, University of Virginia, Charlottesville, VA, USA, ${ }^{2}$ Dept. of Medicine, Cardiovascular Division, University of Virginia, Charlottesville, VA, USA.

Complications due to atherosclerosis lead to millions of deaths worldwide each year. Detection of atherosclerotic lesions and treatment with localized drug delivery may be aided by intravascular ultrasound (IVUS) imaging of targeted ultrasonic contrast agents (microbubbles) to inflammatory cell surface markers such as vascular cell adhesion molecule-1 (VCAM-1). Although IVUS imaging is clinically used for visualizing arterial lumens our group has recently used IVUS to achieve gene delivery under non-clinical ultrasound conditions. The goals of these studies were to detect targeted microbubbles with IVUS and enhance drug/gene delivery through molecular targeting. VCAM-1 expression was induced in confluent mouse vascular smooth muscle by treatment with IL-1 $\beta$ $(0.1-10 \mathrm{ng} / \mathrm{ml})$ for $48 \mathrm{~h}$. Molecular targeted fluorescent (DiI) microbubbles $(\mathrm{d}=2.4+/-1.5 \mu \mathrm{m})$ composed of a phosphatidylcholine lipid shell with biotinylated polyethylene glycol spacers were conjugated via streptavidin to biotinylated VCAM-1 antibodies, isotype control $\mathrm{IgG}$ antibodies, or no antibodies for control. Cells were exposed to the bubbles for a total of 5 minutes and then washed twice with saline before fluorescent imaging at $512 \mathrm{~nm}$ at $10 \times$ magnification. Cylindrical flow chambers $2.5 \mathrm{~mm}$ wide with thin polystyrene membranes coated with VCAM-1 or casein allowed for in vitro optical and acoustic inspection under flow. A commercially available intravascular ultrasound catheter transducer was used for imaging at $40 \mathrm{MHz}$ (Boston Scientific Atlantis). Delivery with control or targeted bubbles $\left(1.5 \times 10^{\wedge} 7 / \mathrm{ml}\right)$ with CMVGFP plasmid $(3 \mu \mathrm{g} / \mathrm{ml})$ was performed with a single element transducer at $1 \mathrm{MHz}$, with a 50 cycle pulse at a PRF of $100 \mathrm{~Hz}$ for 25 seconds per cell. 5.3 times as many VCAM-1 targeted bubbles were adherent to cells treated with $1 \mathrm{ng} / \mathrm{ml}$ IL- $1 \beta$. Molecularly targeted microbubbles resulted in 65 fold and 100 fold increases in bubble adherence to the inflamed cells over biotin and IgG targeted bubbles respectively. At an equivalent density of $3 \times$ $10^{\wedge} 4$ bubbles $/ \mathrm{mm} 2$, IVUS image intensity increased 4.1 fold over that of non bubble coated surfaces. Rupture of microbubbles from the single element transducer resulted in a $53 \%$ reduction in image intensity. Targeted microbubbles resulted in a $50 \%$ increase in gene transfection over non targeted bubbles. Our results demonstrate the use of IVUS to detect targeted microbubbles to VCAM-1 expression on inflamed smooth muscle in vitro and subsequently deliver a gene. These targeted bubbles have clinical potential for early atherosclerosis detection.

\section{$\mathbf{J} 675$}

\section{A DUAL-FREQUENCY TRANSDUCER DESIGNED TO IMPROVE HIGH-RESOLUTION ULTRASONIC TARGETED MOLECULAR IMAGING}

Ryan Gessner ${ }^{1}$, Mike Lee ${ }^{2}$, Marc Lukacs ${ }^{2}$, F. Stuart Foster ${ }^{2}$, Paul A. Dayton ${ }^{1}$ ${ }^{1}$ Joint Department of Biomedical Engineering, University of North Carolina and North Carolina State University, Chapel Hill, NC, USA, ${ }^{2}$ Biomedical Engineering, University of Toronto, Toronto, ON, Canada.
With recent advances in genetic engineering and developments of animal models of human disease, there is an increased need for highresolution molecular imaging techniques. Ultrasound (US) is a popular small animal imaging modality for this purpose because of its portability, cost, and real-time imaging capability. One of the challenges in US molecular imaging is that only a very small dose of total injected contrast is retained at the target site. We hypothesized two improvements to counter this limitation. The first improves sensitivity to small numbers of microbubble contrast agents (MCAs) by utilizing ultraharmonic detection. The second improves the percentage of injected MCAs retained at the target site by utilizing radiation force to enhance binding. While these ideas have previously been examined, they have not yet been implemented together within either preclinical or clinical high-resolution US imaging systems. The primary reason these two methods of increasing MCA sensitivity have not been implemented in high-resolution molecular imaging to date is that both require a transducer that can excite them near their resonant frequencies - typically $2-5 \mathrm{MHz}$ - which is too low for high-resolution imaging. Hence, we have developed a dual-frequency confocal transducer, consisting of a $2.5 \mathrm{MHz}$ outer element and a $30 \mathrm{MHz}$ inner element. The low frequency element can be utilized to enhance adhesion of molecular targeted MCAs by producing radiation force, and it can facilitate ultraharmonic detection by maximizing nonlinear oscillations near the MCAs' resonant frequencies which are then detected at $30 \mathrm{MHz}$ without signal from tissue. Preliminary studies have shown this probe to be a versatile new means to improve molecular targeted imaging in both of the proposed ways. We can both destructively and nondestructively image MCAs in-vivo. These techniques have boosted our contrast sensitivity, allowing us to detect MCAs with contrast-to-tissue ratios $15 \mathrm{~dB}$ greater than standard b-mode imaging. Additionally, ultraharmonic imaging is not susceptible to tissue motion, allowing high frame-rate image acquisitions without respiratory gating. The probe has proven adept at producing sufficient radiation force to enhance binding of targeted agents as well by repeatedly pushing a polydisperse distribution of MCAs moving with a linear flow velocity of $44 \mathrm{~mm} / \mathrm{s}$ a distance of $200 \mu \mathrm{m}$ perpendicular to their direction of motion and against buoyancy in $10 \mathrm{~ms}$. This displacement increases the retention of targeted MCAs and concurrently, the scattered signal intensity.

\section{J676}

\section{INVESTIGATIONS INTO THE MECHANISMS} OF PULSED-HIFU MEDIATED DELIVERY

Victor Frenkel $^{1}$, Hilary Hancock ${ }^{1}$, Shutao Wang ${ }^{2}$, Vesna Zderic ${ }^{2}$, Mark Palmeri $^{3}$, Eitan Kimmel ${ }^{4}$

${ }^{1}$ Radiology and Imaging Sciences, Clinical Center, Bethesda, MD, USA, ${ }^{2}$ Dept. of Biomedical Engineering, George Washington University, Washington, DC, USA, ${ }^{3}$ Dept. of Biomedical Engineering, Duke University, Durham, NC, USA, ${ }^{4}$ Dept. of Biomedical Engineering, Technion, Haifa, Israel.

HIGH intensity focused ultrasound exposures are presently being used to thermally ablate tissue. Continuous exposures enable the build-up of heat to destroy the tissue by coagulative necrosis. Pulsed exposures reduce temporal average energy deposition so that temperature elevations are substantially reduced, making the exposures nondestructive. As a result, less robust non-thermal mechanisms may occur, which can generate effects such as rendering the tissue more permeable for the uptake of therapeutic agents in a variety of formulations. We recently provided preliminary evidence for a novel ultrasound mechanism for enhancing delivery, where the effects are 
non-thermal and cannot be explained by the occurrence of acoustic cavitation alone. The mechanism is based on the generation of radiation forces, and the localized displacements they produce, which can be on the order of hundreds of microns. A key characteristic is the high shear stress occurring off axis of the focal zone, where gradients in displacement are the highest. It is suggested that the resulting strain is large enough to open cell to cell-to-cell junctions in both the vasculature and the parenchyma for improving extravasation and interstitial transport, respectively, as observed in a variety of tissue models. In order to support this model we carried out systematic investigations using a range of methodologies for studying the potential contributions of the different ultrasound mechanisms. This includes simulations and direct measurements for thermal effects, and monitoring for acoustic emissions and broadband noise for acoustic cavitation. Both simulations and radiation force impulse imaging were used to characterize the displacements, as well as the resulting strain. The results of these studies were correlated with in vivo imaging of fluorescently labeled agents, as well as electron and brightfield microscopy. Time course studies on the reversibility of the effects were also performed. Additional investigations into the irregular nature of the tumor microenvironment employed a number of techniques, where observed effects included increases in hydraulic conductivity, as well as a reduction in interstitial fluid pressure. Experiments in whole blood clots showed similar beneficial effects, such as improved binding and penetration of thrombolytics. A comprehensive understanding of the ultrasound mechanisms for producing these effects will be essential for optimization of the exposures, and help expedite their implementation into drug/device applications for clinical purposes.

\section{J677}

\section{MAXIMUM INTENSITY OVER TIME FOR POST PROCESSING OF DYNAMIC CONTRAST-ENHANCED ULTRASOUND: A RELIABLE METHOD TO ASSESS ANTIANGIOGENIC THERAPY EFFECTS IN TUMOR XENOGRAFTS}

Jessica Bzyl $^{1}$, Wiltrud Lederle ${ }^{1}$, Jessica Gätjens ${ }^{1}$, Michaela Socher ${ }^{2}$, Peter Hauff $^{3}$, Wolfhard Semmler ${ }^{2}$, Rolf W. Günther ${ }^{4}$, Fabian Kiessling ${ }^{1}$, Moritz Palmowski ${ }^{1,4}$

${ }^{1}$ Experimental Molecular Imaging, RWTH Aachen University, Aachen, Germany, ${ }^{2}$ Medical Physics in Radiology, German Cancer Research Center, Heidelberg, Germany, ${ }^{3}$ Global Drug Discovery, Bayer-Schering Pharma AG, Berlin, Germany, ${ }^{4}$ Department of Diagnostic Radiology, RWTH Aachen University, Aachen, Germany.

Objectives: Our aim was to prospectively compare two postprocessing techniques for dynamic contrast enhanced ultrasound and to evaluate their capability to monitor antiangiogenic therapy. Materials and Methods: Mice with s.c. epidermoid carcinoma xenografts were examined during administration of polybutylcyanoacrylate-microbubbles using high frequency ultrasound $(40 \mathrm{MHz})$. Cine loops were acquired and analyzed using conventional timeintensity (TI) and maximum intensity over time (MIOT) curves. Influences of fast $(50 \mu 1 / 2$ s) vs. slow $(50 \mu 1 / 10$ s) injection of microbubbles on both types of curves were investigated. The sensitivity of both techniques to monitor effects of antiangiogenic treatment (SU11248) was examined. Correlative histological analysis was performed for vessel density. Mann-Whitney test was used for statistical analysis. Results: Injection rates significantly influenced upslope, time-to-peak and peak enhancement of conventional TI curves $(p<0.05)$ while maximum enhancement of MIOT curves (representing relative blood volume) was alsmost not affected. Additionally, antiangiogenic therapy effects could be assessed more reliably and earlier (already after 1 day of therapy; $p<0.05$ ) with maximum enhancement of MIOT curves compared to peak enhancement of TI curves. Immunohistochemistry validated the significantly $(p<0.01)$ lower vessel densities in treated tumors and high correlation $(\mathrm{R} 2=0.95)$ between vessel density and maximum enhancement of MIOT curves was observed. Conclusions: MIOT is less susceptible to variations of the injections' speed and cardiac output. It is capable of indicating the effects of antiangiogenic tumor therapies earlier, more reliably and with lower standard deviations than conventional TI curves. It can easily be translated into clinical practice and thus may provide a promising tool for cancer therapy monitoring.

\section{J678}

\section{SONOPORATION INDUCED DRUG UPTAKE BY ENDOTHELIAL CELLS WITH ULTRASOUND AND CD31-TARGETED MICROBUBBLES}

Klazina Kooiman $^{1}$, Miranda Harteveld ${ }^{1}$, Nico de Jong ${ }^{1,2}$

Biomedical Engineering, Erasmus MC, Rotterdam, Netherlands, ${ }^{2}$ Interuniversity Cardiology Institute of the Netherlands, Utrecht, Netherlands.

Molecular imaging using ultrasound makes use of targeted ultrasound contrast agents that consist of encapsulated gas microbubbles. Until now, targeted microbubbles have only been used for imaging, not drug delivery. This study focuses on inducing sonoporation with targeted microbubbles in endothelial cells. Sonoporation is a method that induces transient cell membrane pores by oscillating or jetting microbubbles so that therapeutics can enter the cell. So far, sonoporation has only been induced using non-targeted microbubbles [1,2]. In an in vivo situation, this may be challenging since there may not be sufficient microbubble-cell contact due to the blood flow. A microbubble targeted to the cell membrane may overcome this, but it is not known whether sonoporation can still be induced. CD31 was chosen as target since this adhesion molecule is expressed constitutively by endothelial cells [3]. Biotinylated lipid coated microbubbles with a C4F10 gas core were made by sonication [4]. CD31 antibody was conjugated via avidinbiotin bridging [5]. Microbubbles were added to human umbilical vein endothelial cells and allowed to adhere. Microbubble-cell behavior upon insonification at $1 \mathrm{MHz}$ (10 cycle sine-wave bursts) was studied with the Brandaris 128 high-speed camera. Microbubble diameter (D) - time curves were extracted from the recordings and Dmax-Dmin $(\Delta \mathrm{D})$ normalized to the resting diameter $\mathrm{D} 0$ were determined. The cellimpermeable propidium iodide (PI; $25 \mu \mathrm{g} / \mathrm{ml}$ ) was used as indicator for increased cell membrane permeability due to sonoporation. On average, $\sim 1$ microbubble bound to each cell. At $80 \mathrm{kPa}$ peak negative acoustic pressure $\left(\mathrm{P}_{-}\right)$, the targeted bubbles $(\mathrm{D} 0=3-4 \mu \mathrm{m})$ oscillated spherically, shrank by $\sim 20 \%$ but were not destroyed. $\Delta \mathrm{D} / \mathrm{D} 0$ was 0.1 . Although cell deformation was not detectable, PI was taken up locally in the area surrounding the bubble (area $\sim 4 \times$ the bubble D0). Sonoporation was also studied when targeted bubbles were insonified at a higher $\mathrm{P}_{-}$of $200 \mathrm{kPa}$. At this $\mathrm{P}_{\text {, }}$, bubble oscillation was violent, non-spherical, and jets were observed. $\Delta \mathrm{D} / \mathrm{D} 0$ was 1.4. Cell deformation of $1 \mu \mathrm{m}$ was detected and PI was taken up and was found to be distributed throughout the entire cell. This study reveals that targeted microbubbles can induce sonoporation. This feature may now be used in molecular imaging using ultrasound, thereby combining imaging and drug delivery. [1] van Wamel et al, J Control Rel 2006 [2] Schneider, J Endourol 2008 [3] DeLisser et al, Immunol Today 1994 [4] Klibanov et al, Invest Radiol 2004 [5] Lindner et al, Circulation 2001 
J679

BINDING FORCE OF TARGETED MICROBUBBLES MEASURED BY ULTRASOUND RADIATION FORCE

Toshihiko Sugiura $^{1}$, Klazina Kooiman ${ }^{1}$, Marcia Emmer ${ }^{1}$, Hendrik J Vos ${ }^{1}$, Nico de Jong ${ }^{1,2}$, Annemieke van Wamel ${ }^{1}$

${ }^{1}$ Biomedical Engineering, Erasmus MC, Rotterdam, Netherlands, ${ }^{2}$ Interuniversity Cardiology Institute of the Netherlands, Utrecht, Netherlands.

Targeted microbubbles are used for molecular imaging using ultrasound. For such applications, the strength of adhesion between a microbubble and a cell surface is important. This research focuses on the evaluation of the binding force between biotinylated lipid coated microbubbles and a streptavidin-coated surface, which mimics a cell surface. Controlled ultrasound radiation force is used to determine the binding force by detaching microbubbles bound to the surface. Biotinylated lipid coated microbubbles with a C4F10 gas core were made by sonication [1]. The back of an Opticell membrane was coated with streptavidin $(5 \mu \mathrm{g} / \mathrm{ml})$, followed by washing, blocking and washing. Microbubbles were allowed to adhere to the coated membrane by flotation. After washing, the Opticell was mounted in the set-up such that the bound microbubbles were on top of the membrane. Microbubbles were insonified at $15 \mathrm{MHz}$. This frequency was higher than the resonant frequency of the microbubbles $(5 \mathrm{MHz})$, allowing to insonify microbubbles without causing large oscillations that can lead to shrinkage or breakage of the microbubbles. The experiment was performed at $37^{\circ} \mathrm{C}$. A linearized equation for radiation force was used for calculating the strength of the radiation force for detaching microbubbles. Detachment of microbubbles (radius $1.23 \mu \mathrm{m}$ ) from the surface was observed if the peak negative acoustic pressure was 1.6 $\mathrm{MPa}$. The radiation force required for detaching one microbubble in this experiment was $\sim 50 \mathrm{nN}$. The contact area of 1 microbubble with the streptavidin-coated surface can be estimated as $0.08 \mu \mathrm{m} 2$, assuming that the contact area is defined as the area where the distance between the microbubble and the surface is less than $10 \mathrm{~nm}$. According to [2] and taking our microbubble composition into consideration, the amount of biotin-streptavidin bonds in this area is $\sim 160$. From these assumptions, the binding force per bond in our study can be evaluated as $\sim 300 \mathrm{pN}$. This is comparable to the reported unbinding force of one bond, $257 \pm 25 \mathrm{pN}$ [3]. In conclusion, detachment of biotinylated microbubbles from a streptavidin-coated surface was successfully achieved by transmitting ultrasound with a frequency higher than the resonant frequency. From calculating the radiation force, the binding force was evaluated as $\sim 50 \mathrm{nN} /$ bubble or $\sim 300 \mathrm{pN} /$ bond. This method will be useful to assess binding forces of newly developed targeted ultrasound contrast agents. [1] Klibanov et al, Invest Radiol 2004 [2] Takalkar et al, J Control Rel 2004 [3] Moy et al, Science 1994

\section{$\mathbf{J} 680$}

\section{EVALUATION OF DETECTION SCHEMES FOR CYANOACRYLATE MICROBUBBLE QUANTIFICATION}

Monica Siepmann, Martin P. Mienkina, Georg Schmitz

Ruhr-University, Bochum, Germany.

Cyanoacrylate microbubbles (CMB) have shown promising results for ultrasound molecular imaging in small animal experiments. Doppler imaging of their destruction is an established method for the quantification of targeted microbubbles. Doppler methods rely on the decorrelation between echoes due to the destruction of MB. Their sensitivity depends on the pulse repetition frequence (PRF) and on the dissolution time of the released gas bubble. In this work we evaluate a detection scheme consisting of low intensity detection pulses with an intermittent destruction pulse as an alternative to conventional Doppler sequences of pulses with constant amplitude. Comparing the scattering of an intact $\mathrm{MB}$ with the echo of a released gas bubble after destruction is expected to result in stronger decorrelation than the decorrelation during dissolution, which is employed in Doppler processing. Experimental CMB were embedded in gelatine, mimicking targeted MB. Rfsignals were acquired with a Sonix RP (Ultrasonics, Toronto) commercial ultrasound scanner using a L14-5 linear array transducer. For amplitude modulation the number of transmitting elements was varied from 128 to 2 . It was verified that the low intensity pulses are non-destructive. Then, ultrasound echoes were acquired of a sequence consisting of 10 low intensity pulses, one destruction pulse and further non-destructive pulses. The sequence was acquired with different pulse repetition frequencies of 5 and $10 \mathrm{kHz}$. Doppler sequences consisting of 8 high amplitude pulses were acquired at $5 \mathrm{kHz}$ PRF. Power Doppler processing was applied using a wallfilter of $140 \mathrm{~Hz}$ which corresponds to the standard system settings. Baseline signals without contrast agents were acquired for all sequences and the signal gain was calculated. The experiment with the proposed sequence was repeated 8 times at the same position to ensure the same amount of destroyed MB. Power Doppler processing yields a signal gain of $9.1 \mathrm{~dB}$. Employing the proposed sequences for both PRFs an increase of signal intensity after the destruction pulse was detected, suggesting the release of a free gas bubble. The gain was $10.0 \mathrm{~dB}$ for $10 \mathrm{kHz}$ and $3.4 \mathrm{~dB}$ for $5 \mathrm{kHz}$ PRF respectively. Thus, at sufficiently high $\mathrm{PRF}$, this method provides an increased sensitivity than Doppler processing for targeted MB. The performance with respect to Doppler imaging is potentially further increased in the presence of blood flow.

\section{$\mathbf{J} 681$}

\section{POWER MODULATION HARMONIC IMAGING} OF ULTRASOUND CONTRAST AGENTS CAN SENSITIVELY MONITOR THE THERAPEUTIC RESPONSE OF BREAST CANCER TO BEVACIZUMAB

Kenneth Hoyt ${ }^{2}$, Jason M. Warram ${ }^{1}$, Heidi R. Umphrey ${ }^{2}$, Lin E. Belt ${ }^{2}$, Sharon Samuel ${ }^{2}$, Karri Folks ${ }^{2}$, Cecil Stockard ${ }^{1}$, Mark E. Lockhart ${ }^{2}$, William Grizzle ${ }^{1}$, Michelle Robbin ${ }^{2}$, Kurt R. Zinn ${ }^{1,2}$

${ }^{1}$ Pathology, University of Alabama at Birmingham, Birmingham, AL, USA, ${ }^{2}$ Radiology, University of Alabama at Birmingham, Birmingham, AL, USA.

A current dilemma in cancer therapy is ascertaining if and when an individual patient responds to a therapeutic agent. With the advent of low mechanical index (MI) power modulated harmonic imaging (PMHI) ultrasonography, ultrasound (US) contrast agents (UCA) flowing through both microcirculation and large vessels can be visualized. Using an UCAspecific PMHI technique, the objective of this study was to assess changes in breast cancer vasculature in response to bevacizumab therapy. Twenty nude athymic mice were implanted with 2LMP breast cancer cells in left and right inguinal regions of the mammary fat pad (10 controls and 10 in therapy group). All mice were imaged daily using a bioluminescence imaging system (IVIS system, Xenogen Inc.) to detect firefly luciferase expression and monitor tumor growth. Three weeks post cell implantation, baseline US images were acquired following a $30 \mathrm{uL}$ i.v. bolus injection of UCA (Definity, Lantheus Medical Imaging). Subsequently, the therapy mice group received a $0.1 \mathrm{mg}$ i.p. injection of bevacizumab (Genentech). Six days after baseline imaging, all mice were reimaged. All scans were performed using an iU22 system (Philips Medical Systems) equipped with an L12-5 transducer. Prior to and after UCA destruction via high MI "flash" frame sequences, microvascular flow (reperfusion) was monitored for 20 s using 
low MI PMHI. DICOM data was processed using custom software developed using Matlab (Mathworks). For a given tumor ROI, the following metrics: Area under the curve (AUC), peak intensity (Ipk), and time to peak intensity (Tpk), was derived from an average time-intensity curve. Subsequently, mice were sacrificed and tumors surgically excised. Tumor response to therapy was assessed histologically via CD31 staining and quantification of tumor microvessel density (MVD). For each mouse, the tumor exhibiting greatest intratumoral blood flow on day 6 was selected for reference to baseline data. Of the 20 mice, 3 controls were excluded from analysis due to inadequate US baseline images. A comparison between control and therapy group PMHI-based tumor perfusion metrics revealed larger increases in AUC $(p<0.01)$, Ipk $(p<0.01)$, and Tpk $(p=0.20)$ for the therapy group whereas increases in tumor area were more pronounced in the control group $(p=0.18)$. MVD counts were significantly higher in the control versus therapy group $(p<0.01)$. This study demonstrates that PMHI of UCA is a sensitive and promising modality for assessing changes in breast cancer vasculature in response to bevacizumab therapy.

\section{Imaging Molecular and/or Cellular Processes}

\section{Antibody}

\section{$\mathrm{J} 682$}

\section{F-LABELED DIABODIES FOR MICROPET IMAGING OF PROSTATE STEM CELL ANTIGEN (PSCA)-EXPRESSING XENOGRAFTS}

$\underline{\text { Eric J. Lepin }}{ }^{1}$, Sebastian Olma ${ }^{1}$, Mingwei Wang ${ }^{1}$, Robert E. Reiter ${ }^{2}$, Clifton K. Shen ${ }^{1}$, Anna M. Wu ${ }^{1}$

${ }^{1}$ Mol. \& Med Pharmacology, Crump Institute -UCLA, Los Angeles, CA, USA, ${ }^{2}$ Department of Urology, UCLA, Los Angeles, CA, USA.

Purpose: PSCA is a cell surface glycoprotein expressed in normal human prostate and bladder over-expressed in most prostate cancers and pancreatic carcinoma. We have previously evaluated 124I-labeled anti-PSCA antibody fragments ranging from 50 to $150 \mathrm{kDa}$ using microPET imaging. Among these fragments the $2 \mathrm{~B} 3$ diabody ( $\mathrm{scFv}$ dimer, $50 \mathrm{kDa}$ ) which shows the fastest blood clearance was able to detect PSCA expressing xenografts 4 hours post injection (Leyton et al., Prot. Eng. Des. Sel. 22:209, 2009). Since in this time frame, $18 \mathrm{~F}$ is a superior radiolabel for diabody PET imaging compared to 124I (Cai et al., J. Nucl. Med. 48:304, 2007), we are developing rapid methods to radiolabel diabodies with $18 \mathrm{~F}$. Here, we report radiolabeling results, in vitro and microPET evaluation of anti-PSCA diabodies labeled using N-succinimidyl-4-[18F]fluorobenzoate(18F-SFB). Methods: We developed a one-pot microwave reactor-based method for synthesis of $18 \mathrm{~F}-\mathrm{SFB}$. Three affinity variants of $2 \mathrm{~B} 3$ diabody (P, A2, and A11) were conjugated to $18 \mathrm{~F}-\mathrm{SFB}$ and radiochemical yield (RCY) determined using ITLC. Immunoreactivity of HPLC purified 18F-FB-diabodies was determined using SKW 6.4 cells transfected with PSCA. 18F-FB-A2 diabody was purified using spin columns and injected intravenously into male SCID mice bearing LAPC-9 human prostate cancer xenografts. Two hour microPET dynamic scans were started at injection, followed by static microPET/CT scans and biodistribution studies performed $4 \mathrm{~h}$ post injection. Regions of interests were drawn and microPET images quantified using AMIDE. Results: We successfully labeled P, A2 and A11 anti-PSCA diabodies using $18 \mathrm{~F}-\mathrm{SFB}$ with a RCY of $6-11 \%$ after 45 min of reaction. Following size exclusion chromatography the radiochemical purity of $18 \mathrm{~F}$ FB-A2 diabody was greater than $98 \%$ and immunoreactivity $14 \pm 1.7 \%$. For in vivo studies, mice $(n=3)$ bearing tumors were injected with 1.5 to 2.1 MBq of 18F-FB-A2 diabody. Biodistribution studies showed a tumor uptake of $2 \pm 0.2 \% \mathrm{ID} / \mathrm{g}$ and tumors were readily visualized by microPET with a tumor-to-soft tissue ratio of $6 \pm 1.5,4 \mathrm{~h}$ post injection. Conclusion: Rapid labeling of small biomolecules such as anti-PSCA diabodies with $18 \mathrm{~F}$ enable PET imaging of antigen expressing tumors within a few hours.

\section{$\mathbf{J} 683$}

FLUORESCENCE LIFETIME IMAGING OF ACTIVATABLE TARGET SPECIFIC MOLECULAR PROBES

Raphael Alford ${ }^{1}$, Mikako Ogawa ${ }^{1}$, Moinuddin Hassan ${ }^{2}$, Amir Gandjbakhche ${ }^{2}$, Peter Choyke $^{1}$, Hisataka Kobayashi ${ }^{1}$

${ }^{1}$ Molecular Imaging Program, National Cancer Institute, National Institutes of Health, Bethesda, MD, USA, ${ }^{2}$ Laboratory of Integrative and Medical Biophysics, National Institute of Child Health and Human Development, National Institutes of Health, Bethesda, MD, USA.

In vivo optical imaging using fluorescently labeled self quenched monoclonal antibodies, activated through binding and internalization within target cells, results in excellent target-to-background ratios. We hypothesized that fluorescence lifetime imaging (FLI) could be used to detect the internalization of antibodies targeting the HER2/neu receptor, and might provide an alternative means of confirming internalization. Two imaging probes were synthesized, consisting of the antibody, trastuzumab (targeting HER2/neu), and conjugated to Alexa Fluor 750 in ratios of either 1:8 or 1:1. Fluorescence intensity and lifetime of each conjugate were initially determined in PBS at $\mathrm{pH} 6$ and 7.2. Since the $1: 8$ conjugate is self quenching, fluorescence lifetime of each probe was also determined after exposure to the known dequencher SDS. in vitro imaging experiments were performed using 3T3/HER2+ and BALB/3T3 (HER2-) cell lines. Following 8 hours of incubation with HER2+ cells, the Trastuzumab-Alexa Fluor 750 1:8 conjugate was internalized resulting in increased fluorescence intensity. Changes in fluorescence lifetime correlated with temperature- and time-dependent cellular internalization. in vivo imaging studies in mice with dual flank tumors (3T3/HER2+ and BALB/3T3 (HER2-)) did not detect a difference in fluorescence lifetime. In conclusion, fluorescence lifetime imaging monitors the internalization of target-specific activatable antibodyfluorophore conjugates in vitro. Challenges remain in adapting this methodology to in vivo imaging.

\section{J684}

\section{AN ENGINEERED ANTIBODY FRAGMENT FOR PET IMAGING OF ALCAM-POSITIVE TUMORS}

Katelyn E. McCabe ${ }^{1}$, Bin Liu ${ }^{2}$, James D. Marks ${ }^{2}$, Hong $\mathrm{Wu}^{3}$, Anna M. Wu ${ }^{1}$ ${ }^{1}$ Crump Institute for Molecular Imaging, Molecular and Medical Pharmacology, UCLA, Los Angeles, CA, USA, ${ }^{2}$ Anesthesia, UCSF, San Francisco, CA, USA, ${ }^{3}$ Molecular and Medical Pharmacology, UCLA, Los Angeles, CA, USA.

Activated leukocyte cell adhesion molecule (ALCAM/CD166) is a transmembrane protein that has recently been identified as a prospective pancancer biomarker. While ALCAM has shown initial promise as a therapeutic target, its potential as an imaging target has not yet been explored. To investigate this possibility, a human anti-ALCAM scFv was reformatted to generate a bivalent diabody that retains the variable domains of the scFv. Diabodies undergo higher uptake in tumors than scFvs as a result of their higher avidity, but still exhibit rapid blood clearance in vivo. Due to these favorable pharmacokinetic properties, diabodies have been found to be better molecular imaging probes than both smaller scFvs and larger intact antibodies. The high-affinity $55 \mathrm{kDa}$ anti-ALCAM diabody produced showed specific binding to ALCAM-positive human pancreatic cancer cell lines as determined by flow cytometry. To create a PET imaging probe, the 
diabody was conjugated to 1,4,7,10-tetraazacyclododecane-1,4,7,10-tetraacetic acid (DOTA) and subsequently labeled with ${ }^{64} \mathrm{Cu}$. Radiometal labeling efficiency was $77 \pm 6 \%(n=3)$. Mice bearing BxPC3 (ALCAMpositive) and C6 (rat glioma; ALCAM-negative) tumors were imaged at 4 and 21 hours post-injection of ${ }^{64} \mathrm{Cu}$-DOTA-diabody, and biodistribution studies were carried out after the second scan. MicroPET images at 4 and 21 hours showed high uptake in the ALCAM-positive tumor compared to the ALCAM-negative tumor (see Figure). Biodistribution studies at 21 hours confirmed targeting of the probe, with $2.50 \pm 0.52 \% \mathrm{ID} \mathrm{g}^{-1}$ in the ALCAMpositive tumor compared to $1.03 \pm 0.13 \% \mathrm{ID} \mathrm{g}^{-1}$ in the ALCAM-negative tumor $(n=4)$. These findings show promise for ALCAM as an imaging target, and indicate that the ${ }^{64} \mathrm{Cu}$-DOTA-diabody is a suitable agent for PET imaging of ALCAM-positive tumors.

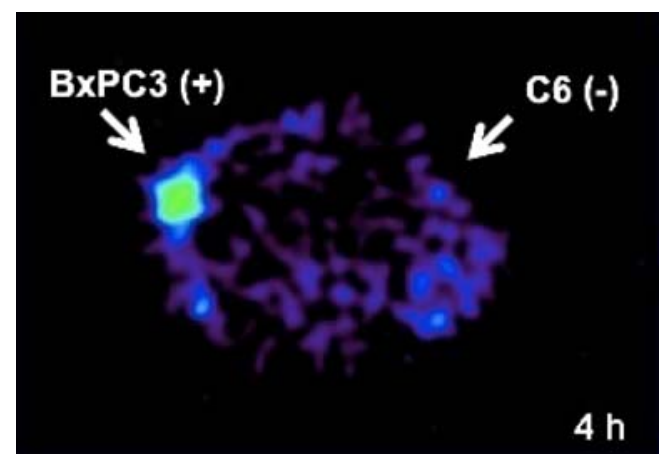

\section{$\mathbf{J} 685$}

MOLECULAR IMAGING OF PANCREATIC BETA-CELL MASS: AN ANTIBODY MEDIATED OPTICAL IMAGING APPROACH

Divya Vats ${ }^{1}$, Florian Stuker ${ }^{1}$, Katerina Dikaiou ${ }^{1}$, Haiyan Wang ${ }^{2}$, Hugues Matile $^{2}$, Cristiano Migliorini ${ }^{2}$, Markus Rudin ${ }^{1}$

${ }^{1}$ Animal Imaging Center, Institute for Biomedical Engineering, ETH honggerberg, Zurich, Switzerland, ${ }^{2}$ Pharma research and Metabolic diseases Basel, F.Hoffmann-La Roche Ltd, Basel, Switzerland.

Loss of beta-cell ( $\beta$-cell) mass and function in type 2 diabetes leads to metabolic dysregulation and inability to maintain normoglycemia. Imaging of pancreatic $\beta$-cell mass would therefore provide a valuable research and diagnostic tool for quantifying progression to diabetes and response to therapeutic intervention. This work aims at developing an imaging strategy for $\beta$-cell mass targeting highly $\beta$-cell specific surface proteins using fluorescently labeled antibodies. Recent gene expression studies of pancreatic $\beta$ cells mass showed that the protein TMEM27 is selectively expressed on $\beta$-cells and in the proximal tubules of the kidneys. Targeting in vivo TMEM27 with fluorescently labeled antibodies may provide a highly specific tool for in vivo imaging of the pancreatic $\beta$-cells mass using fluorescence imaging. Experimental: The imaging strategy was evaluated in two animal models: subcutaneous insulinomas and transgenic mice expressing human TMEM27 (hTMEM27. in vivo fluorescence imaging was carried out in nude mice with subcutaneously insulinomas comprising hTMEM27 over-expressing $\beta$-cells, to evaluate for the efficiency and specificity of TMEM27 antibody labeling. Tomographic reconstruction of the tumor was performed as a proof of concept of targeting in vivo $\beta$-cells. Fluorescence-reflectance imaging was then applied to visualize in vivo antibody distribution in RIPhTMEM27 transgenic mice (mice over expressing hTMEM27 under rat insulin promoter in $\beta$-cells) and to quantitatively assess signal-to-background ratios. Three optical imaging systems have been used-eXplore Optix (ART, Montreal, $670 \mathrm{~nm}$ ), Maestro 5000 (CRI, Woburn MA, broadband) and a home-built fluorescence molecular tomography (FMT, $680 \mathrm{~nm}$ ) system. in vivo findings were validated using histological analysis. Results and conclusion: Our data shows that the fluorescently labeled antibody is specific to TMEM27 on $\beta$-cells and can be detected by optical imaging of hTMEM27 over-expressing tumors as well and in pancreatic islets (ex vivo analysis). This suggests TMEM27 to be a potential $\beta$-cell marker for targeted imaging, thus offering possibilities for monitoring the normal and diseased pancreas (and in turn $\beta$-cells mass) in vivo. First in vivo studies, however, indicated that targeted imaging of pancreatic $\beta$-cells using fluorescently labeled antibodies suffer from the long antibody circulation time and from antibody accumulation in adjacent organs (kidney, liver), which degrades the signal-to-background ratio. Further analysis is ongoing.

\section{J686}

\section{IMMUNO-PET IMAGING OF LUNG METASTASIS OF COLON CANCER WITH GA-68-NOTA FAB FRAGMENT OF ANTI-TAG-72 HUMANIZED ANTIBODY}

Jaeho Jung ${ }^{1}$, Taesup Lee ${ }^{1}$, Joo Hyun Kang ${ }^{1}$, Sang-Keun Woo ${ }^{1}$, Kwang Sun Woo ${ }^{1}$, Weesup Chung ${ }^{1}$, HyoJeong Hong ${ }^{2}$, Sang Moo Lim ${ }^{1}$, Gi Jeong Cheon ${ }^{1}$

${ }^{1}$ Molecular Imaging Research Center, KIRAMS, Seoul, Korea, South, ${ }^{2}$ Therapeutic Antibody Research Center, KIRBB, Daejeon, Korea, South.

Objectives: The tumor associated glycoprotein-72 (TAG-72) is expressed in the majority of human adenocarcinomas of colon, ovary, pancreas, and breast. $3 \mathrm{E} 8$ is anti-TAG-72 humanized antibody. Antibody fragments have some advantages such as improved pharmacokinetics and reduced immunogenicity compared to whole IgG. We investigated the feasibility of Ga-68NOTA-3E8 Fab for imaging lung metastasis of colon cancer in small animal PET. Methods: SCN-Bn-NOTA and 3E8 Fab was allowed to react for overnight. purified by dialysis, purity was check by SDS-PAGE. Ga-68 labeling performed at room temperature for $30 \mathrm{~min}$ and labeling yield check by ITLC-SG and citrate buffer. in vitro TAG-72 specific binding of NOTA-3E8 Fab was assessed by cell binding assay. Biodistribution of Ga-68-NOTA3E8 Fab was obtained in normal Balb/c mice. For lung metastasis model, LS174T-L cells, expressing firefly luciferase gene, were injected into tail vain of Balb/c nude mouse. Metastasis tumor in lung was confirmed by bioluminescent image. Small animal PET image obtained in colon cancer lung metastasis model with Ga-68NOTA 3E8 Fab. Results: Molecular weight of 3E8 Fab fragment was $25 \mathrm{Kd}$ by SDS-PAGE. Ga-68-NOTA-3E8 Fab showed radiolabeling yield with $>99 \%$ and stable in serum condition during $2 \mathrm{~h}$. Cell binding activity of Ga-68-NOTA-3E8-Fab was $1.95 \%$ ID in TAG-72 expressed tumor cells. In the biodistribution study, Ga-68-NOTA-3E8 Fab showed fast blood and lung clearance, but high uptake in liver. Lung metastasis of colon cancer was confirmed by bioluminescent image at 5 to 7 days. Small animal PET images showed that Ga-68-NOTA-3E8 Fab have high tumor uptake in lung metastasis of colon cancer at $1 \mathrm{~h}$ post injection. Conclusions: Ga-68-NOTA-3E8 Fab was easily prepared and showed high stability and favorable pharmacokinetics for tumor imaging. Ga-68-NOTA-3E8 Fab could be useful for a radioimmunoconjugate for immuno PET imaging in TAG-72 expressing tumor. 
Immuno PET of Ga-68-NOTA-3E8 Fab in Lung Metastasis model

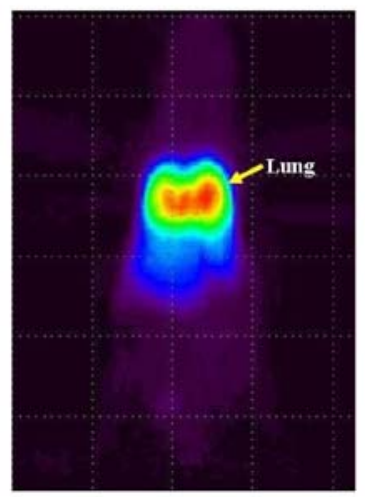

: Lung metastasis of colon cancer at $1 \mathrm{~h}$ post injection with Ga-68-NOTA 3E8 Fab. Apoptosis, Autophagy, Necrosis

$\mathbf{J} 687$

DIRECT INJECTION CHEMOTHERAPY OF A SYNTHETIC HYDRAPHILE CHANNEL IN LIVING MICE MONITORED BY OPTICAL IMAGING WITH A NEAR INFRARED FLUORESCENT PROBE

$\underline{\text { Seth T. Gammon }}^{1,4}$, Bryan R. Smith ${ }^{3}$, James R. Johnson ${ }^{4,3}$, Wei Wang ${ }^{2}$, David Piwnica-Worms ${ }^{4}$, George W. Gokel ${ }^{2}$, W. Matthew Leevy ${ }^{3,1}$

${ }^{1}$ Carestream Molecular Imaging, Carestream Health, Inc., Saint Louis, MO, USA, ${ }^{2}$ Dept. of Chemistry \& Biochemistry, University of Missouri - St. Louis, St. Louis, MO, USA, ${ }^{3}$ Department of Chemistry and Biochemistry, Notre Dame, Sound Bend, IN, USA, ${ }^{4}$ Molecular Imaging Center, Department of Radiology, and Department of Developmental Biology, Washington University School of Medicine, St. Louis, MO, USA.
Imaging of phosphatidylserine serine (PS) exposure by fluorescence methods readily allows for the detection of cell death in vitro via FACS assays, but strategies for detecting cell death in vivo are still under development. Zinc dipicolylamine (DPA-Zn2+) conjugates have been shown to bind PS on dying cells in vitro, but have yet to be applied to analysis of tissues in living mice. Herein, we test the ability of DPA-Zn2+ to measure cell death in vivo. The DPA-Zn2+ complex (PSS-794), 1, is conjugated to a near infrared (NIR)

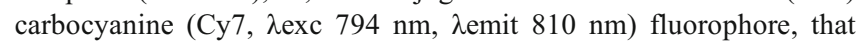
is nearly optimal for balancing photon penetration through tissue and CCD sensitivity. $50 \mu \mathrm{L}$ of a $10 \mathrm{mM}$ solution in EtOH of synthetic hydraphile, 2 , which is known to be toxic to a variety of cells by disrupting ion gradients, was then locally injected into the leg muscle tissue of nude mice. $75 \mu \mathrm{L}$ of $1 \mathrm{mM}$ PSS-794 was then injected i.v., and mice were imaged at multiple time points on a Kodak in vivo FX to determine if exposed PS could be effectively documented. At $12 \mathrm{~h}$ post injection of PSS-794, cell death was readily discernable at the hydraphile injection site. Ethanol, the carrier for 2, was utilized as the non-target control injected in the opposite leg. Target to non-target ratios at 12 hours were $1.73 \pm 0.03$ (SEM), $n=3$ and $p<0.01$. An untargeted $\mathrm{Cy} 7$ fluorophore was identically administered i.v. as a negative control for non-specific accumulation of probe at the site of injury. Critically, the fluorophore did not selectively accumulate at the site of hydraphile injection in a separate cohort of 3 mice. The site of cell death was well localized to the injection site, as expected, because hydraphiles readily insert directly into membranes. This leads us to the hypothesis that hydraphiles may be utilized as a direct injection chemotherapeutic. Finally, PSS-794, a small molecule fluorescent probe, could be effectively utilized in vivo for monitoring cell death. Future approaches may utilize multispectral imaging to further discern apoptotic cells from necrotic cells.

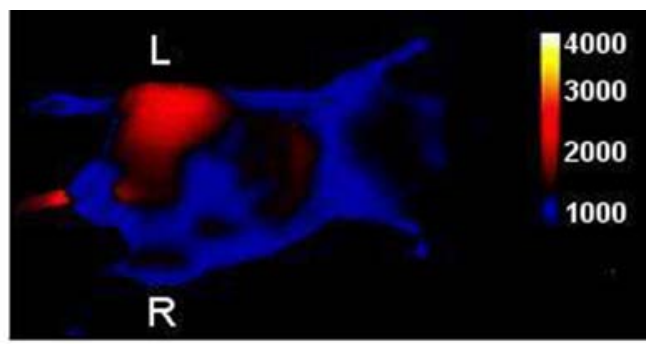

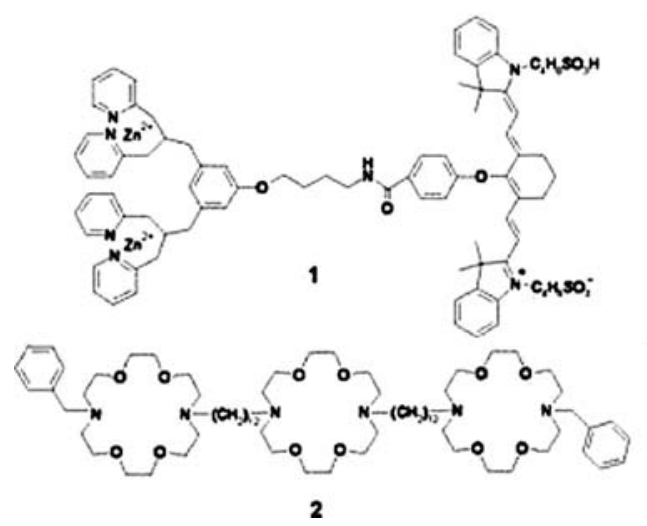

Left leg muscle (L) was injected with 2 to induce cell death, and the contra lateral leg muscle was injected with vehicle. 1 was then administered IV, and the mouse was then subsequently imaged. The site of cell death is readily visualized.

$\mathbf{J 6 8 8}$

DISTINCT PATTERNS OF CARDIOMYOCYTE APOPTOSIS IN ACUTE ISCHEMIC INJURY AND HEART FAILURE DETECTED IN-VIVO WITH MOLECULAR MRI

David E. Sosnovik ${ }^{1,2}$, Elena Aikawa ${ }^{1}$, Matthias Nahrendorf ${ }^{1}$, Guangping Dai ${ }^{2}$, Jose-Luiz Figueiredo ${ }^{1}$, Peter Panizzi ${ }^{1}$, Takashi Matsui ${ }^{3}$, Anthony Rosenzweig ${ }^{3}$, Ralph Weissleder ${ }^{1}$, Lee Josephson ${ }^{1}$

${ }^{1}$ Center for Molecular Imaging Research, Massachusetts General Hospital, Harvard Medical School, Charlestown, MA, USA, ${ }^{2}$ Martinos Center for Biomedical Imaging, Massachusetts General Hospital, Harvard Medical School, Charlestown, MA, USA, ${ }^{3}$ Beth Israel Deaconess Medical Center, Harvard Medical School, Boston, MA, USA.
BACKGROUND: Cardiomyocyte (CM) apoptosis plays an important role in acute ischemia and chronic heart failure. However, the spatial pattern of $\mathrm{CM}$ apoptosis in both conditions has not been well characterized. We thus aimed to use the apoptosis sensing nanoparticle, AnxCLIO-Cy5.5, to perform high-resolution in-vivo MRI of CM apoptosis in mouse models of ischemia-reperfusion and heart failure. METHODS: 13 mice were exposed to transient coronary ligation and injected with AnxCLIO-Cy5.5 $(n=7)$ or a control nanoparticle $(n=6)$. Cine and T2* weighted MRI was performed at 9.4 Tesla within 4-6 hours of reperfusion. CM apoptosis in heart failure was studied in mice with over-expression of Gaq and postpartum cardiomyopathy. MRI at $9.4 \mathrm{~T}$ was performed in 5 postpartum mice injected with AnxCLIO-Cy5.5 and 5 with the control probe. RESULTS: The uptake of AnxCLIO-Cy5.5 in ischemic injury was most frequently seen in the 
midmyocardium (Fig 1A). In severe ischemic injury, however, the uptake of the probe extended transmurally (Fig 1B,C). The contrast-to-noise ratio (CNR) between the injured and uninjured myocardium was significantly greater in the mice injected with AnxCLIO-Cy5.5 than the control agent (CNR 8.82 vs 3.78, $p<0.05$ ). In the Gaq mice the uptake of AnxCLIO-Cy5.5 occurred in isolated clusters, most frequently in the subendocardium and anterolateral wall (Fig 1D, E). Myocardial T2* was significantly lower (7.6 vs $16.8 \mathrm{~ms}, p<0.05$ ) in the mice injected with AnxCLIO-Cy5.5 than the control probe. FIGURE: In-vivo MRI of AnxCLIO-Cy5.5 in a mouse with (A) moderate and (B) severe ischemic injury. (C) Fluorescence microscopy $(100 \mathrm{x})$ of the injured myocardium shown in panel B. (D) In-vivo and (E) exvivo MRI of a Gaq mouse with heart failure. (F) Fluorescence microscopy (400x) of a Gaq mouse showing an isolated apoptotic $\mathrm{CM}$ with probe uptake. CONCLUSION: CM apoptosis in acute ischemia occurs in a dense pattern, most frequently in the midmyocardium. In contrast, in heart failure $\mathrm{CM}$ apoptosis occurs in isolated clusters, most frequently in the subendocardium. These distinct patterns may represent important differences in the pathophysiology of CM apoptosis and may have important implications for the development and monitoring of anti-apoptotic therapies in acute ischemia and heart failure.
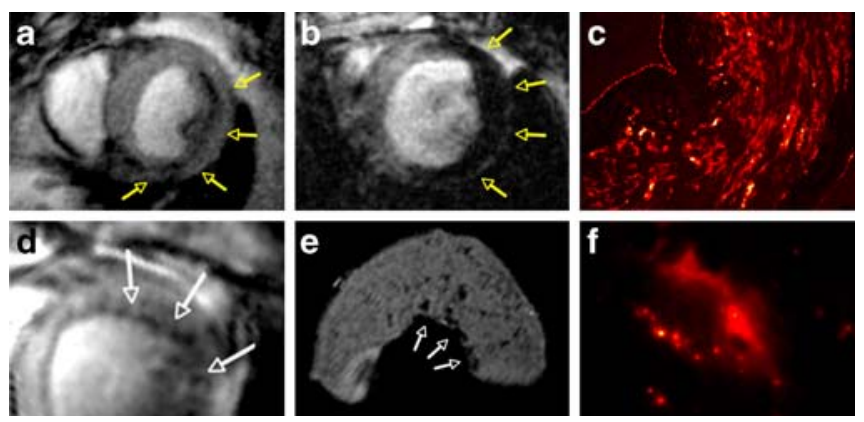

$\mathbf{J 6 8 9}$

\section{BLOOD MONITORING OF APOPTOSIS IN VIVO}

Johanna M. Niers, Lee-Ann Tjon-Kon-Fat, Grant Lewandrowski, $\overline{\text { Bakhos A. Tannous }}$

Neurology, massachusetts General Hospital and Harvard Medical School, Boston, MA, USA.

We have developed two reporters for real-time monitoring of apoptosis in the blood of living animals. In the first reporter, we fused a green fluorescent protein (GFP) to the $\mathrm{N}$-terminus of the secreted Gaussia luciferase (Gluc; including its signal sequence, ss) separated by a DEVD sequence, a caspase 3 cleavage site (GFP-DEVD-ssGluc). This fusion protein resides in the cytoplasm where the Gluc is not active. Upon caspase 3 activation during apoptosis, the DEVD sequence is cleaved, freeing ssGluc which can then enter the endoplasmic reticulum where it is folded properly (active) and is secreted outside of the cell. We showed that when human glioma cells are transduced with a lentivirus vector expressing this reporter and treated with doxorubicin, a chemotherapeutic agent which kills cells by apoptosis, an increase in Gluc signal was observed over time in the conditioned medium reaching $>20$-fold higher at 12 hrs post-treatment. Further, when mice bearing glioma tumors expressing GFPDEVD-ssGluc were treated with the same agent, a similar increase in Gluc activity was observed over time in blood of mice which is correlated at the tumor site by in vivo bioluminescence imaging after injection of coelenterazine and acquiring photon counts using a CCD camera. We have also designed another reporter in which the DEVD sequence was cloned in the middle of ssGLuc sequence. In this case, upon caspase activation, a decrease in Gluc activity was observed over time reaching $>80 \%$ less in conditioned medium of cells or in blood of mice bearing tumors in vivo. These reporters provide great tools for monitoring of apoptosis in culture and in vivo and are well suited for high-throughput screening applications.

\section{GCD camea}
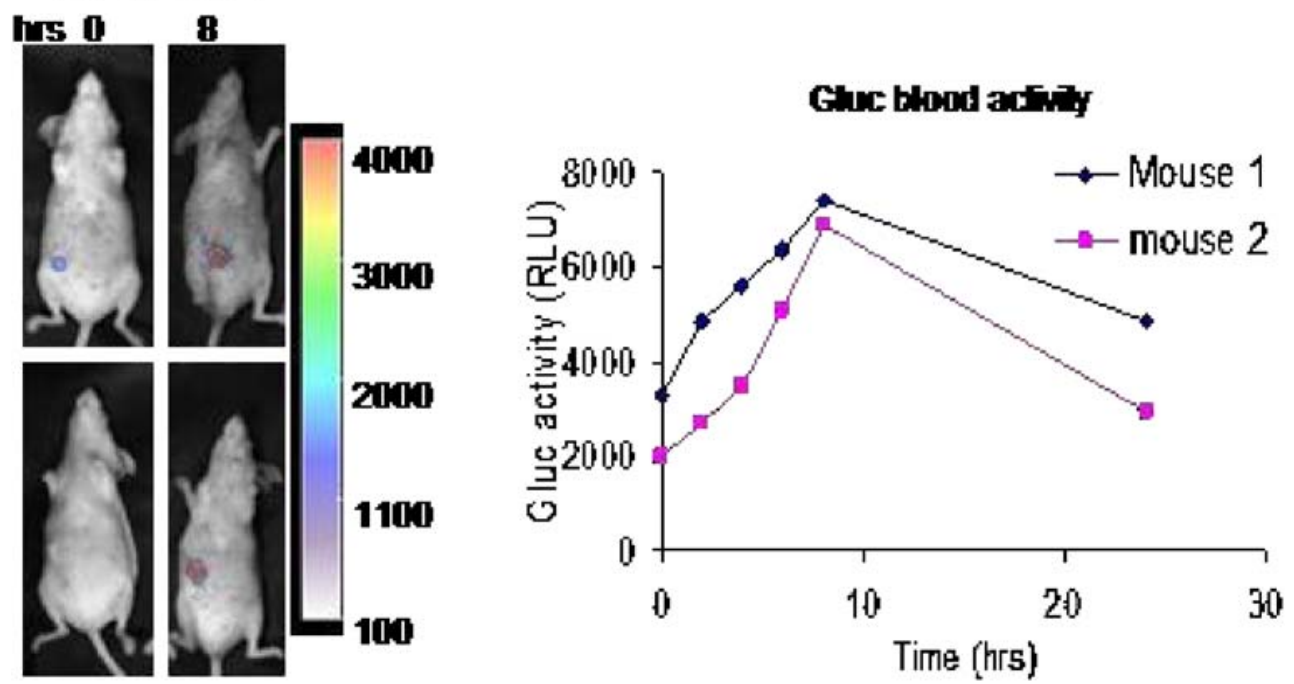

Figure. Blood monitoring of apoptosis. Gli36 human glioma cells expressing GFP-DEVDssGluc were implanted subcutaneously. One week later, tumors were injected with doxorubicin and Gluc activity was assayed in $5 \mu \mathrm{l}$ blood at different time points after addition of coelenterazine (100 $\mu \mathrm{M})$ using a luminometer. Before and $8 \mathrm{hrs}$ post-treatment, mice were i.v. injected with coelenterazine and Gluc activity in tumors were imaged using a CCD camera. 
$\mathbf{J 6 9 0}$

DEVELOPMENT AND EVALUATION OF XENOGRAFT MOUSE TUMOR MODEL FOR DETECTION OF CELL DEATH USING $\left[\mathrm{Cy}_{5,5}\right]$ ANNEXIN $V$ AND FLOURESCENCE MOLECULAR TOMOGRAPHY AND $\left[{ }^{64} \mathrm{Cu}\right]$ ANNEXIN V AND PET

Alexa Gleason, Brett Connolly, Amy Vanko, Shailendra Patel, Raymond E. Gibson, Caroline Houde, Huseyin Mehmet, Cyrille Sur, Bohumil Bednar

Merck Research Laboratories, West Point, PA, USA.

Development of cancer therapeutics as well as the evaluation of efficacy in patients benefits from early detection of tumor cell death using noninvasive imaging, which is enabled by specific imaging biomarkers. Development of molecular imaging biomarkers of cell death requires a well defined animal model. Recent findings indicate that the vast majority of cell death mechanisms can be detected and visualized in vivo with Annexin $\mathrm{V}$, which senses and binds to lipid alterations on the membrane surface of dying cells, irrespective of their dying mechanism. Here we report the development of a xenograft mouse tumor model of cell death after treatment with the commercial therapeutic Taxotere, based on subcutaneously implanted cancer cells with a genetically encoded optical reporter luciferase. From two evaluated human colon cancer cell lines (HCT116 and HT29) and human breast cancer cell line MDA-MB231 only the HT29 cells developed reproducible tumors after subcutaneous implantation of 1.5$2 \times 10^{6}$ cells into flanks of athymic mice. Nine out of ten injected animals developed tumors in the range of 100-150 mg within four weeks, with minimal detected cell death and skin scaring. Animals in the treatment cohort were treated with three daily iv doses of $12 \mathrm{mg} / \mathrm{kg}$ Taxotere. The cell death model based on HT29 cells has been evaluated in two independent studies with 15 animals in both treatment and control cohorts. The cell death in the tumors of the treatment cohort when compared with control animals, detected by the increase in the fluorescence of $\left[\mathrm{Cy}_{5.5}\right]$ Annexin $\mathrm{V}$ reporter using fluorescence molecular tomography (FMT), has been found in both studies statistically significant ( $p=0.04$ and $p=0.02$ ). The results obtained using optical imaging have been confirmed in one study by parallel PET measurements using $\left[{ }^{64} \mathrm{Cu}\right]$ Annexin $\mathrm{V}$ reporter $(p<0.05)$. Histology of dissected tumors in the parallel optical and PET measurement provided a good correlation between cell death measured in tumor sections by TUNEL and caspase 3 activation assays with in vivo measurement of cell death by the binding of optical reporter $\left[\mathrm{Cy}_{5.5}\right]$ Annexin $\mathrm{V}$. Thus, we believe the xenograft mouse tumor model based on human colon cancer cells HT29 with the described treatment by Taxotere can serve well in the development of molecular imaging biomarkers of cell death.

\section{J691}

\section{LIVE IMAGING OF APOPTOSIS}

Michal Eifer, Natalie Yivgi ohana, Michal Neeman, Atan Gross Biological regulation, Weizmann Institute, Rehovot, Israel.

Apoptosis, the process of programmed cell death, is fundamental to many biological events. A precise balance between cell proliferation and cell death is required for tissue homeostasis, in which old or damaged cells are deleted and replaced by new cells. Defects in apoptosis contribute to many diseases such as cancer, stroke, and neurodegenerative conditions. Furthermore, many cytotoxic cancer therapies are designed to induce cancer cell apoptosis. Our aim was to establish an imaging technique that would enable us to monitor the activation of apoptosis in cells and living animals. Our experimental strategy is based on the protein complementation assay (PCA). One such PCA is the split-YFP complementation, which tests potential protein-protein interactions by assaying for reassembly of amino- and carboxy-terminal fragments of YFP fluorescent protein appended to the candidate interacting proteins. In the present study we used proteins known to interact during apoptosis: mitochondrial Second mitochondria-derived activator of caspase (Smac)/DIABLO cloned upstream to the C-terminus of YFP, and cytosolic X-linked inhibitor of apoptosis protein (XIAP) cloned upstream to the N-terminus of YFP. For in-vitro imaging, MCF-7 cells expressing the two proteins were treated with etoposide (DNA damage reagent) and followed by the DeltaVision system. Six hours after etoposide treatment YFP (green) fluorescence was detected at the mitochondria. The increase in YFP signal was highly correlated with a decrease in Tetramethyl Rhodamine Methyl Ester (TMRM) fluorescence (red), indicating a decrease in mitochondrial membrane potential known to occur during apoptosis. We conclude that this protein-complementation technique enable the imaging of intra-cellular protein interactions known to occur during apoptosis. Such a method would be valuable for identifying novel proteins involved in the apoptotic process as well as for in vivo evaluation of apoptosis during physiological and pathological conditions.

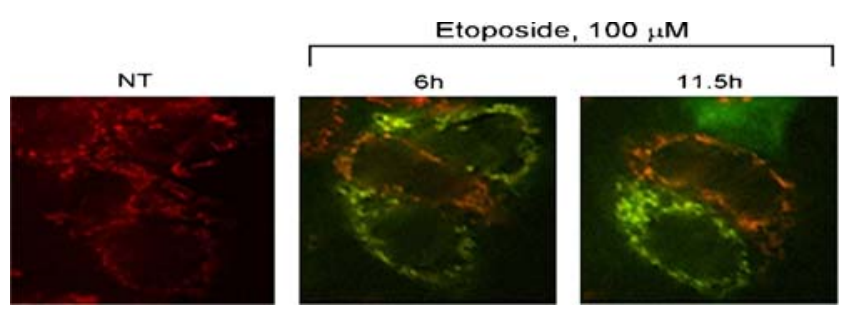

J692

\section{MOLECULAR NMR AND EPR IN VIVO DETECTION OF APOPTOSIS USING SPECIFIC PHOSPHATIDYLSERINE- TARGETED IRON OXIDE PARTICLES \\ Kim A. Radermacher ${ }^{1}$, Sebastien Boutry ${ }^{2}$, Isabelle Mahieu ${ }^{2}$, Sophie Laurent ${ }^{2}$, Luce Vander Elst ${ }^{2}$, Caroline Bouzin ${ }^{3}$, Olivier Feron ${ }^{3}$, Robert N. Muller ${ }^{2}$, Benedicte F. Jordan ${ }^{1}$, Bernard Gallez ${ }^{1}$ \\ ${ }^{1}$ Biomedical Magnetic Resonance Unit, Catholic University of Louvain, Bruxelles, Belgium, ${ }^{2} \mathrm{MR}$ and Molecular Imaging Laboratory, University of Mons, Mons, Belgium, ${ }^{3}$ Unit of Pharmacology and Therapeutics, Catholic University of Louvain, Bruxelles, Belgium.}

The aim of the study was to develop a new molecular marker for non invasive diagnosis and monitoring of cell death in order to evaluate the efficacy of anti-cancer treatments. A phosphatidylserine-targeted peptide, isolated by phage-display, was coupled to pegylated ultrasmall particles of iron oxides (USPIO). USPIO particles are used as negative contrast agent for magnetic resonance imaging (MRI) due to strong $\mathrm{T} 2$ and $\mathrm{T} 2 *$ effects and besides this method they can also be quantitatively detected by electron paramagnetic resonance 
(EPR). Apoptosis has been induced in TLT (transplantable liver tumor) cells by incubation with staurosporine. This apoptosis model has been previously proven by flow cytometry. Cell fixation of USPIO particles grafted with our peptide, with a scrambled peptide or the ungrafted particles was measured by X-band EPR After intravenous injection of the grafted or control particles into tumorbearing mice, their concentration was evaluated in irradiated and non-treated TLT tumors ex vivo by X-band EPR and in vivo by Lband EPR, as well as by MRI. In irradiated tumors was greater accumulation of the targeted USPIO particles compared to control particles or compared to the targeted particles in untreated tissues. The major finding of the present investigation is that functionalization of the surface of iron oxide particles with our hexapeptide allows the sensitive detection and mapping of tumor cell death after cytotoxic treatment. This molecular targeted system should be evaluated further as a potential biomarker of tumor response to treatment.
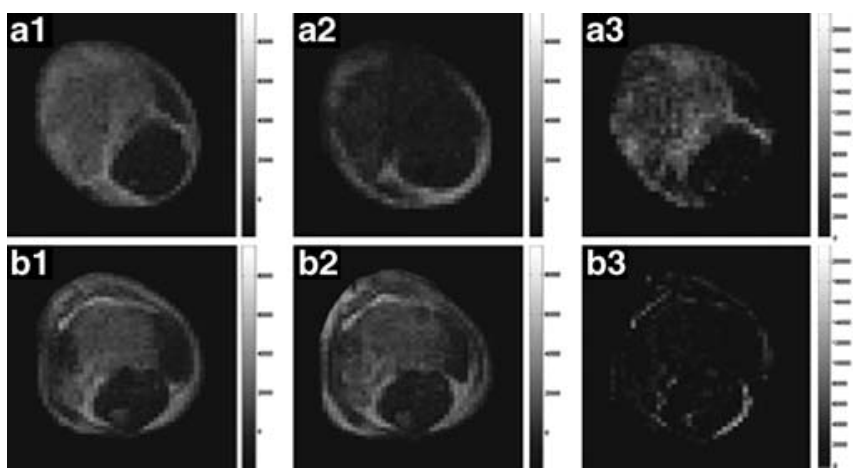

Axial slices of irradiated murine tumors obtained by T2-weighted MRI. The images of the first row come from a mouse that was injected with phosphatidylserine-targeted particles (A) and the second row from a mouse where control particles were injected (B). On the left side are the pre-contrast images (A1, B1) and than the 3 hours post-contrast images (A2, B2). Subtraction images (A3, B3) were obtained by subtracting the corresponding post-contrast images from the pre-contrast images.
J693

\section{COMPARISON OF THE SPECIFICITY AND SENSITIVITY OF A NOVEL TARGETED AGENT FOR DETECTING TUMOR CELL DEATH WITH ANNEXIN V}

$\underline{\text { Israt S} \text {. Alam }}^{1,2}$, Andre Neves ${ }^{1,2}$, Timothy Witney ${ }^{2}$, Joan Boren ${ }^{1,2}$, Kevin Brindle $^{1,2}$

${ }^{1}$ Cancer Research UK Cambridge Research Institute, Cambridge, United Kingdom, ${ }^{2}$ Department of Biochemistry, University of Cambridge, Cambridge, United Kingdom.

The exposure of phosphatidylserine (PS) on the surface of dying cells has been used as a marker of cell death. The phospholipid is externalized during apoptosis and becomes accessible intracellularly, during subsequent necrosis, due to the disruption of plasma membrane integrity. Annexin V (AnxV), a 35-kDa, PS-binding protein, commonly used for the detection of dying cells, has been extensively validated in vitro and also undergone advanced clinical trials, for detecting tumor cell death post-treatment. However, biodistribution issues, in particular high renal, liver and gut accumulation, have prevented $\mathrm{AnxV}$ from being used routinely in the clinic. The $\mathrm{C} 2 \mathrm{~A}$ domain of Synaptotagmin $\mathrm{I}$ is a smaller $(14.7 \mathrm{kDa})$ protein, which like $\mathrm{AnxV}$, binds to PS in a $\mathrm{Ca} 2+$ dependent manner and with high affinity. We have produced a novel site-directed mutant of $\mathrm{C} 2 \mathrm{~A}(\mathrm{C} 2 \mathrm{Am})$, in which a serine residue has been replaced by a single cysteine residue (S78C), providing a unique site for the modification of the protein with fluorescent, radionuclide or MRI-detectable labels. We assess here the potential of a fluorescent derivative of $\mathrm{C} 2 \mathrm{Am}$ to detect cell death in murine lymphoma and human breast carcinoma cell lines using flow cytometry. We compared the sensitivity and specificity of the new agent for apoptotic and necrotic cells with that of a commercially available fluorescent derivative of AnxV. We show that the novel C2Am agent is capable of detecting similar levels of cell death, as AnxV, throughout the time course of treatment with conventional chemotherapies. More importantly, the C2Am probe showed up to 3-fold and 4-fold more specific binding to apoptotic and/or necrotic cells, versus viable cells, in the lymphoma and breast carcinoma cell models, respectively. This new probe opens a route for the development of a new generation of more specific molecular imaging probes for the clinical detection of cell death.
A

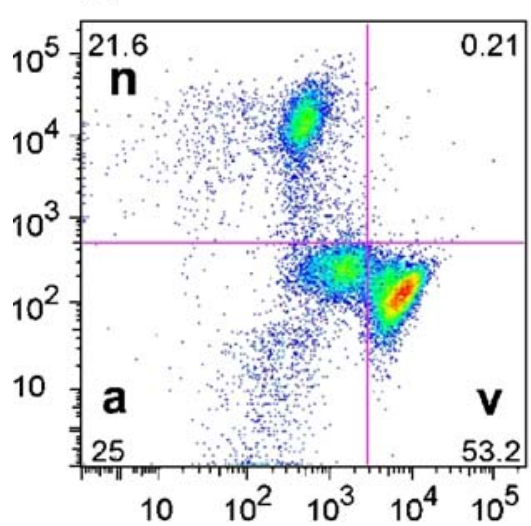

B

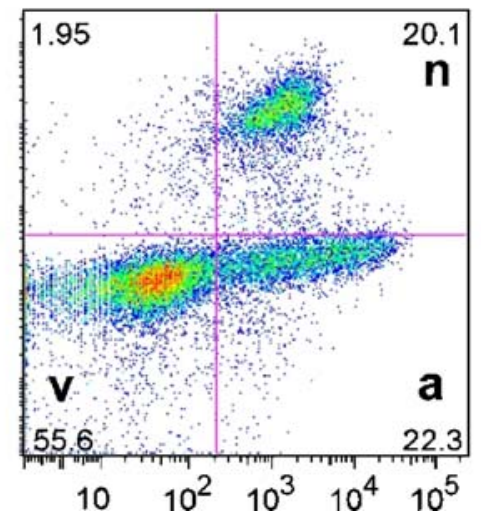

C

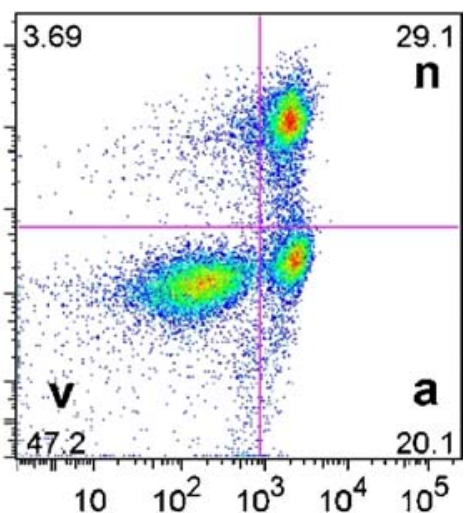

Figure 1-Labeling of dying murine lymphoma cells with C2Am or AnxV. Dual scatter plots of cellular viability, assessed using NADH autofluorescence (A), $\mathrm{C} 2 \mathrm{Am}(\mathrm{B})$ or AnxV (C) labeling (x-axis) versus Sytox Green dead cell staining (y-axis). C2Am (B) is more specific for apoptotic (a) and necrotic cells (n), than viable cells (v), compared with $\mathrm{AnxV}(\mathrm{C})$. The values in each quadrant represent the $\%$ of each cell fraction. 


\section{J694}

\section{DEATH RECEPTOR 5 (DR5) AGGREGATION BY TRA-8 AGONIST ANTIBODY IS ENHANCED BY CHEMOTHERAPY IN TRIPLE NEGATIVE (TN) BREAST CANCER CELLS}

Edward Ivey $^{1}$, Sharon Samuel ${ }^{1}$, Hyunki Kim ${ }^{1,2}$, Kurt R. Zinn ${ }^{1,3}$

${ }^{1}$ Department of Radiology, University of Alabama at Birmingham, Birmingham, AL, USA, ${ }^{2}$ Department of Biomedical Engineering, University of Alabama at Birmingham, Birmingham, AL, USA, ${ }^{3}$ Comprehensive Cancer Center, University of Alabama at Birmingham, Birmingham, AL, USA.

Introduction. The purpose of this study was to image DR5 aggregation induced by TRA8, and determine if chemotherapy would enhance aggregation. Aggregation is part of the signaling pathway and precedes cell death. Mesenchymal TN cancer cells are sensitive to TRAIL apoptosis (Breast Cancer Res Treat 113(2): 217-30, 2008). An important aspect of effective therapy with TRAIL and antibody agonists is DR5 aggregation. Methods. Cy5.5 was conjugated to anti-DR5 antibody (TRA-8) at a 3:1 molar ratio. Cy5.5-TRA8 (+/- carboplatin@ @ $100 \mu \mathrm{M})$ was incubated with SUM149, SUM159, MB-MDA-231, and 435 cells at a concentration of $2.5 \mu \mathrm{M}$ for $12 \mathrm{~h}$, then imaged with a Leica DMIRE2 inverted microscope (Leica) equipped with a Nuance camera (CRI). Results. TRA-8-induced DR5 aggregation and capping in TN breast cancer cell lines, which with significantly enhancement by carboplatin. This aggregation preceded the death of the TN breast cancer cells. Figure 1 presents representative images showing this enhancement in the SUM159 TN breast cancer cell line. After $12 \mathrm{hrs,} \mathrm{DR5-aggregates}$ (capped cells) averaged $45+5 \%$ for TRA- $8+$ carboplatin, significantly higher $(p<0.05)$ than $28+1 \%$ for TRA- 8 alone. Importantly, a similar effect was not found in the control, non-TN 435-breast tumor cell line (data not shown). Conclusions. These initial observations are important, and lay the foundation for evaluating additional TN cell lines, and additional modulators in combination with TRA-8. The technique may also be applicable for biopsy specimens in neoadjuvant clinical trials, and enable potential responders to be identified. An approach to image TRA-8 induced DR5 aggregation in mice is being developed.
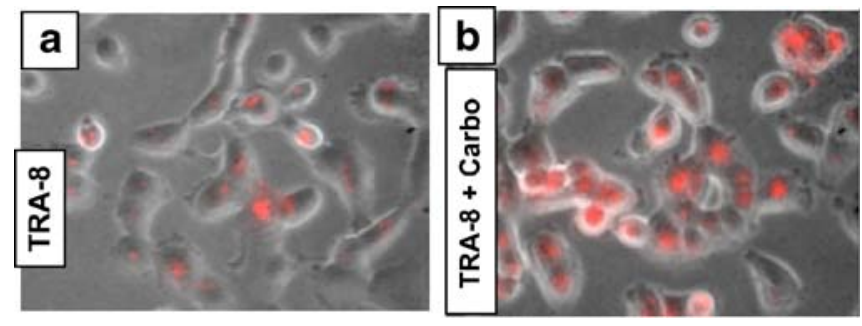

\section{$\mathbf{J 6 9 5}$}

\section{BIOLUMINESCENCE IMAGING OF APOPTOSIS UTILIZING} A CASPASE-3/7 SPECIFIC PROBE

Matthew Silva, Robbie Robertson, Donna Cvet

Millennium Pharmaceuticals, Inc., Cambridge, MA, USA.

Apoptosis, or programmed cell death, is induced during cancer drug therapy. It is of great interest to monitor this process to determine the efficacy and characteristics of experimental therapeutics in vivo. We have developed an optical probe to monitor caspase-3/7 activity in living cells using a modified circular luciferase/DEVD molecular probe (optimized from Akira Kanno, et al. Angewandte Chemie Volume 119, Issue 40). The probe utilizes dual, split luciferase and $\mathrm{Npu}$ DnaE-intein cassettes, along with strong binding domains to enhance circularization of the split luciferase. Circularization attenuates the activity of luciferase when there is no active caspase-3/7 present in the cell. Upon induction of apoptosis the circular probe is cleaved at the DEVD caspase-3/7 recognition sequence allowing the luciferase to linearize and become active. Preliminary results have been obtained utilizing this probe in vivo using transfected Hela cells implanted into nude mice and treated with $200 \mu \mathrm{g} / \mathrm{kg}$ staurosporine. The data acquired every hour for $3 \mathrm{~h}$ post treatment and is normalized to wild-type luciferase controls. One hour post treatment, the signal in the staurosporine group was $3 \mathrm{x}$ higher than the untreated group, consistent with the induction of caspase-3/7. By $2 \mathrm{~h}$, the signal in the treatment group was nearly returned to baseline however remained higher than the untreated group. Additional studies are in process to provide additional confirmation of the probe specificity as well as test the effect of various therapeutics and treatment regimes. Ultimately, the ability to image apoptosis and other cellular events non-invasively, in real-time and in vivo is a powerful tool for analyzing acute drug activity, studying the effects of scheduling, and will aid in pre-clinical drug combination studies.

\section{Energy Metabolism}

\section{J696}

\section{MEASUREMENT OF FDG TRANSPORT AND UPTAKE RATE CONSTANTS IN CELL CULTURE: ASSAY WITH A MICROFLUIDIC CELL CULTURE ARRAY COUPLED WITH A PSAPD CAMERA}

Wei Sha, Zeta Yu, Nam Vu, Arion Chatziioannou, Hsian Rong Tseng, (Henry) Sung-Cheng Huang

Molecular and Medical Pharmacology, UCLA, Los Angeles, CA, USA.

Objective: Values of FDG transport and phosphorylation rate constants (k1, $\mathrm{k} 2, \mathrm{k} 3$ and $\mathrm{k} 4$ ) in cells can be assayed with a microfluidic cell culture chip coupled with a PSAPD (MF-PSAPD). In this study, we use this assay platform to examine the effects of medium glucose level on FDG transport and phosphorylation rate constants in two cell lines. Methods: For the MFPSAPD platform, a switching strategy that consists of multiple mediuminfusion cycles, each of which has a tracer incubation (TI) period followed by a background-removal (BR) period was employed. P8 and CaP8 cell lines were cultured in separate wells in the platform. The wells (containing about 200-300 cells each) were perfused with culture medium containing $0.5,1.0$, or $1.5 \mathrm{~g} / \mathrm{L}$ glucose. An equally-spaced switching strategy with 12 cycles of TI and BR periods (5 min each) was used to provide the FDG kinetics in cells, and a regular 3-compartment FDG model was used to fit the measured kinetics to give estimates of the transport and phosphorylation rate constants. Results: The measured kinetics were well fitted by the compartmental model (R-Square $=0.9999$ on average). The coefficients of variation of the estimated rate constants were $10 \%, 26 \%$ and $44 \%$ for $\mathrm{k} 1, \mathrm{k} 2$ and k3, respectively. For both cell lines, estimates of $\mathrm{k} 2$ and $\mathrm{k} 3$ did not correlate with medium glucose level ( $p>0.05$, F-test). The value of $\mathrm{k} 1$ though was inversely proportional to glucose level (R-Square $=0.892$ for CaP8 and R-Square $=0.928$ for P8), and the resulting cell glycolytic rate $(\mathrm{GR}=\mathrm{k} 1 * \mathrm{k} 3 /(\mathrm{k} 2+\mathrm{k} 3) *[\mathrm{glc}])$ was not affected by medium glucose level ( $p=0.418$ for CaP8 and $p=0.053$ for P8), for both cell lines. The results indicate that both cell lines regulated well the transport of glucose into cells, adapting to changes in medium glucose levels and maintaining stability in the cellular glycolytic process. Conclusion: The MF-PSAPD platform with a kinetic switching strategy was shown to provide reliable estimates of cellular FDG transport and uptake constants. Both $\mathrm{P} 8$ and $\mathrm{CaP} 8$ cell lines can regulate well the glucose transport into cells to maintain their glycolytic metabolic rate adapting to environmental glucose level changes. 


\section{MONITORING 3D HIGH CELL DENSITY CULTURES BY NON-INVASIVE AND NON-DESTRUCTIVE MEDICAL IMAGING TECHNIQUES}

Julie A. Chouinard $^{1,2}$, Jacques A. Rousseau ${ }^{1}$, Pierre Proulx ${ }^{2}$, Patrick Vermette $^{2}$, Roger Lecomte ${ }^{1}$

${ }^{1}$ Médecine Nucléaire et Radiobiologie, Université de Sherbrooke, Sherbrooke, QC, Canada, ${ }^{2}$ Génie chimique et génie biotechnologique, Université de Sherbrooke, Sherbrooke, QC, Canada.

Bioreactors are used to engineer 3D tissue substitutes. These systems can recreate and modulate the cell culture's environmental hydrodynamics and the mechanical stimuli, known to influence intracellular signaling and phenotype. One of the main challenges facing tissue engineering is the lack of adequate vascularization in cell constructs. Mammalian cells have important nutrient needs and are very sensitive to metabolic wastes. Hence, micro-vessel networks must be developed to support functional and viable tissues. A better understanding of the hydrodynamic, biochemical and mechanical factors modulating cell behaviors within the bioreactor 3D environment is essential in order to achieve tissue constructs of clinical use. Imaging techniques borrowed from the medical field can be of tremendous interest for tissue engineers to assess tissues grown under bioreactor conditions in a non-invasive and non-destructive fashion. Positron Emission Tomography (PET), for instance, can be used to monitor cell metabolism and proliferation, hypoxia, apoptosis, angiogenesis and perfusion without interfering with normal tissue growth. Moreover, the same sample can be used as its own reference for day-to-day follow-up. In this study, PET imaging with $18 \mathrm{~F}-\mathrm{FDG}$ was used to obtain information on flow and cell metabolism in $3 \mathrm{D}$ high cell density cultures grown in a perfusion bioreactor system. Mathematical modelling was used to extract information on the flow patterns within the bioreactor immediately following FDG injection. Data on nutrient diffusion within growing construct could also be isolated. After radioactivity washout from the fluid, FDG uptake in the tissue was measured as a marker of cell metabolism, hence, of tissue growth. PET imaging is a promising mean to provide real-time follow-up information on $3 \mathrm{D}$ tissue cultures in bioreactors nondestructively.

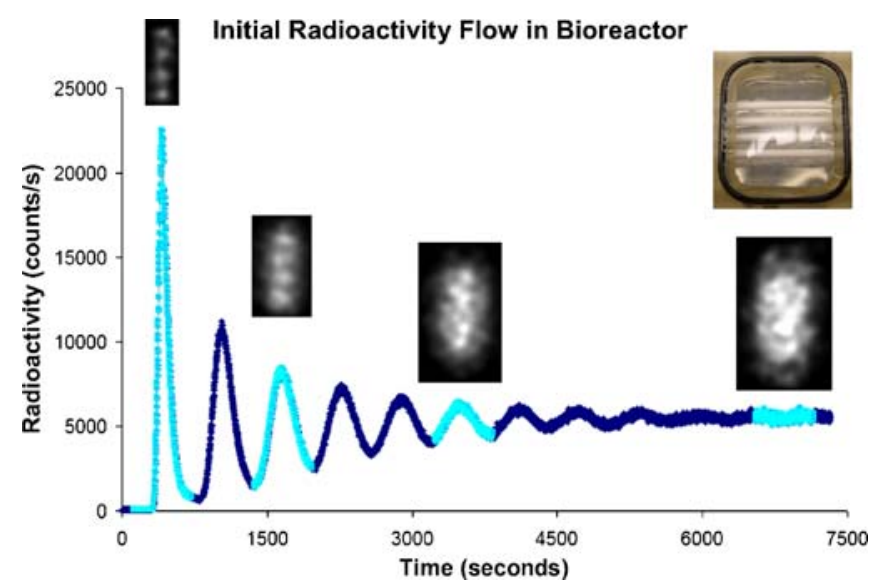

Total 18FDG radioactivity (cps) in bioreactor following introduction into the constant flowing fluid. Highlighted parts of the curve represent the time interval used for the reconstructed images shown above. Top right corner: Top view picture of the culture chamber with its 4 hollow fibres and surrounding gel containing the cells.

\section{DYNAMIC IMAGING OF RADIOLABELED PROBES USING A PSAPD BETA CAMERA SYSTEM}

Nam Vu, Zeta Yu, Wei Sha, (Henry) Sung-Cheng Huang, Hsian Rong Tseng, Arion Chatziioannou

Molecular and Medical Pharmacology, UCLA, Los Angeles, CA, USA.

A novel camera system has recently been developed for imaging and quantification of charged particle emitting probes within a microfluidic platform. The imaging system employs a prototype position sensitive avalanche photodiode (PSAPD) in close contact with the substrate layer of a microfluidic chip. Using a pneumatically operated valve system in the control layer enables control of the flow of solutions inside a microfluidic chip on the order of seconds. An integrated PSAPD beta camera can then be used to monitor the flow of radiotracers through the microfluidic system. The dynamic imaging capability of the system was tested using a microfluidic chip. A dynamic sequence was used to cycle $[18 \mathrm{~F}]$ FDG solution in and out of chambers with a frequency of 6 cycles/ hr. After loading the $[18 \mathrm{~F}] \mathrm{FDG}$ solution, it is held inside the chambers for 5 mins during the tracer incubation (TI) period. This is followed by a background removal (BR) period where all of the $[18 \mathrm{~F}]$ FDG solution is washed out from the chambers. Transition between the TI and BR periods takes less than $1 \mathrm{sec}$. for each chamber. ROI analysis of the count rate showed that cycling of [18F]FDG solution was constant after correction for $18 \mathrm{~F}$ decay. Analysis of the standard deviation in the measured counts also showed that the noise in the dynamic acquisition was limited by Poisson statistics. For cells cultured in microfluidic chips, the dynamic imaging capability of the PSAPD camera and switching flow control can be used to collect useful information on the transport kinetics of [18F]FDG. For these experiments cancer cell lines were cultured inside an array of $4 \times 4$ chambers. Cells were cultured in chambers using cell medium with 2 different glucose concentrations $2.8 \mathrm{nM}$ (low) and $8.4 \mathrm{nM}$ (high). As the cycling sequence progressed, the $[18 \mathrm{~F}] \mathrm{FDG}$ activity increased inside the cell. This was observed as a rise in the count rate during the BR periods. For cells cultured in the lower $2.8 \mathrm{nM}$ glucose medium we observed a steeper slope in the $[18 \mathrm{~F}] \mathrm{FDG}$ uptake compared to the cells cultured the higher $8.4 \mathrm{nM}$ glucose concentration. This indicates that cells cultured in a low glucose environment may compensate by increasing the rate of $[18 \mathrm{~F}] \mathrm{FDG}$ transport into the cytoplasm. These dynamic data sets can also be incorporated into tracer kinetic models in order to calculate the transport rate constants for the each of the cell cultures.

\section{J699}

\section{OBSERVATIONS ON THE EFFECT OF RAPAMYCIN TREATMENT ON $\left[1{ }^{11}\right.$ C $]$ ACETATE PET IMAGING OF HCC}

Nicolas Salem $^{1,2}$, Yu Kuang ${ }^{1,2}$, Jeffrey A. Kolthammer ${ }^{1}$, David J. Corn ${ }^{2}$, Bernadette Erowku ${ }^{2}$, Haibin Tian ${ }^{2}$, Fangjing Wang ${ }^{1}$, Zhenghong Lee ${ }^{2,1}$ ${ }^{1}$ Biomedical engineering, Case Western Reserve University, Cleveland, OH, USA, ${ }^{2}$ Radiology, University Hospitals Case Medical Center, Cleveland, OH, USA.

Inhibition of the mammalian target of rapamycin (mTOR) with the use of chemotherapeutic agents has demonstrated synergistic effects in treating hepatocellular carcinoma (HCC). However, the need for imaging agents capable of evaluating the response to such treatment remains as FDG is not predictive of clinical 
outcome during mTOR inhibition therapy. $\left[1-{ }^{11} \mathrm{C}\right]$ acetate $\left({ }^{11} \mathrm{C}\right.$-Act $)$ uptake in HCC was shown to be associated with increased lipid synthesis and a study implicated the mTOR complex 1 in the regulation of lipogenesis in epithelial cells. In this study, we investigated the effects of mTOR inhibition with rapamycin on PET imaging of $\mathrm{HCC}$ with ${ }^{11} \mathrm{C}$-Act in a woodchuck model. Pulsechase experiments with $\left[1-{ }^{14} \mathrm{C}\right]$ acetate $\left({ }^{14} \mathrm{C}\right.$-Act $)$ conducted on cells derived from an adult woodchuck hepatoma and on freshlyisolated rat hepatocytes after rapamycin treatment showed a decrease in the incorporation of the radiolabel into lipid-soluble compounds. PET imaging with ${ }^{11} \mathrm{C}$-Act on a woodchuck with multiple tumors which was treated 1 week with rapamycin showed a decreased SUV in three tumors, and increased SUV in one tumor compared to the baseline scan. The SUV in a tumor of a sham-treated woodchuck was increased compared to the baseline scan. The clearance of ${ }^{11} \mathrm{C}$-Act from tumors with decreased SUV after rapamycin treatment was greater. The same woodchucks were injected with ${ }^{14} \mathrm{C}$-Act and the radioactivity content in the lipid-soluble fraction was inconsistent with the PET imaging results. ${ }^{11} \mathrm{C}$-Act uptake is affected by rapamycin treatment in some tumors. The exact mechanisms of action are currently under investigation.

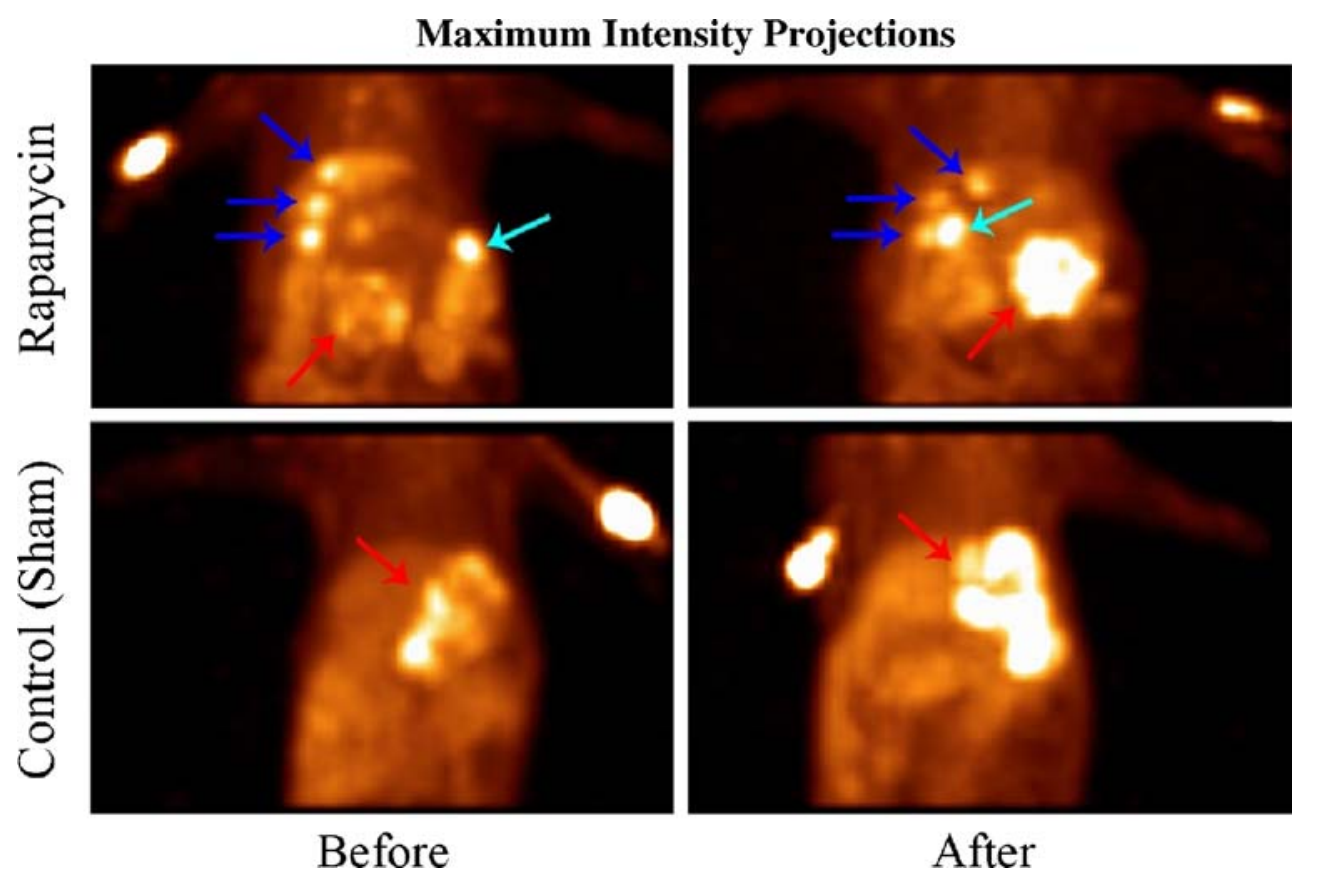

$\mathbf{J 7 0 0}$

DOUBLE TROUBLE: A 2-HIT HYPOTHESIS OF NEURONAL AND GLIAL DYSFUNCTION IN METHAMPHETAMINE ABUSE

Napapon Sailasuta ${ }^{1}$, Osama Abulseoud ${ }^{2}$, Kent Harris ${ }^{1}$, Brian D. Ross ${ }^{1}$ ${ }^{1}$ Clinical Spectroscopy, Huntington Medical Research Institutes, Pasadena, CA, USA, ${ }^{2}$ Psychiatry, University of Southern California, Los Angeles, CA, USA.

Background: Persistent neurochemical abnormalities could predispose to drug addiction. We used novel carbon-13 magnetic resonance spectroscopy to quantify neuronal glutamate and glial metabolic flux rate in frontal brain of abstinent methamphetamine abusers. Methods: Five methamphetamine addicted women, abstinent 30-190 days, were compared with five age-matched women with no history of illicit drug use. MRI localized MRS assays were performed on a conventional MR scanner, measuring steady-state glutamate and $\mathrm{N}$-acetyl aspartate concentrations and glial tricarboxylic acid cycle rate following intravenous infusion of [1-13C] acetate. Results: Abstinent methamphetamine abusers demonstrated significant reduction of glial TCA cycle rate (HC03 0.025 vs $0.063 \mu \mathrm{mol} / \mathrm{gm} / \mathrm{min}, P=0.001)$. In the same brain region, glutamate concentration was elevated while $\mathrm{N}$-acetylaspartate, a neuronal marker was reduced. Conclusions: These MRS results are the first to define glial injury in frontal brain, a region relevant to drug abuse. An increase in cerebral glutamate concentration with reduction in NAA, a neuronal marker, indicates that effect of methamphetamine abuse is not limited to glial dysfunction. At the time of this Report, 2 of 5 subjects have relapsed into methamphetamine abuse. This leads us to propose a 2-hit mechanism for addiction: First, glial energy failure limits clearance of glutamate, second, neuronal injury results from glutamate excitotoxicity. If addiction is the expression of the necessity for methamphetamine abusers to maintain a high ambient cerebral glutamate concentration to overcome the reduced glial TCA rate, treatments directed at restoring glial metabolic rate could be effective. 
J701

\section{EVOLVING ROLE OF PET/CT IN FOLLOW UP OF PATIENTS WITH OVARIAN MALIGNENCY \\ Madhuri A. Shimpi, Balkundi A. Krishna \\ Nuclear Medicine, P.D.Hinduja National Hospital, Mumbai, India.}

Introduction: This is a retrospective study of 38 patients of ovarian carcinoma to asses the utility of PET-CT in detection of occult disease during post treatment follow-up. Results: The 38 patients were grouped into two categories. The Group I consisted of 27 patients with positive CT scan of which 19 patients showed evidence of disease (70\%) while the remaining 8 patients did not show evidence of disease in this patients 7 patients remained disease free while 1 patient showed disease recurrence., suggesting CT scan FALSE POSITIVE in 26\% of patients and PET scan FALSE NEGATIVE in 3\% of patients. In group II, patients with raised CA125 were compared with PET-CT findings. There were 22 patients in this group of which PET-CT scan revealed disease in 20 patients (91\%) suggesting good correlation with tumor marker. Conclusion: Our study demonstrates specificity of $91 \%$ for PET -CT scan in detection of recurrence while the CT scan shows FALSE POSITIVE rate on $26 \%$. The PET-CT scan also upstaged disease in $15 \%$ of patients thereby altering the treatment approach.

\section{$\mathbf{J 7 0 2}$}

\section{${ }^{18}$ F-FDG MicroPET/CT DIFFERENTIATES TRASTUZUMAB (HERCEPTIN)-RESPONSIVE FROM UNRESPONSIVE HUMAN BREAST CANCER XENOGRAFTS IN ATHYMIC MICE}

Kristin McLarty ${ }^{1}$, Aisha Fasih ${ }^{1}$, Deborah A. Scollard ${ }^{1}$, Susan J. Done $^{2,3}$, Douglass C. Vines ${ }^{4,5}$, David E. Green ${ }^{4}$, Danny L. Costantini ${ }^{1}$, Raymond M. Reilly ${ }^{1,6}$

${ }^{1}$ Department of Pharmaceutical Sciences, University of Toronto, Toronto, ON, Canada, ${ }^{2}$ Department of Medical Biophysics and Department of Laboratory Medicine and Pathobiology, University of Toronto, Toronto, ON, Canada, ${ }^{3}$ Ontario Cancer Institute and Department of Pathology, University Health Network, Toronto, ON, Canada, ${ }^{4}$ STTARR Innovation Centre, Radiation Medicine Program, Princess Margaret Hospital, Toronto, ON, Canada, ${ }^{5}$ Department of Radiation Oncology, University of Toronto, Toronto, ON, Canada, ${ }^{6}$ Department of Medical Imaging, University of Toronto, Toronto, ON, Canada.

Breast cancers (BC) with high HER2 expression are most likely to respond to trastuzumab (Herceptin $\AA$ ), however, the mechanisms of action of trastuzumab are complex and there are no established biomarkers to accurately monitor treatment outcome in individual patients. Therefore, our aim was to determine if there were any changes in ${ }^{18}$ F-FDG uptake in human $\mathrm{BC}$ xenografts in athymic mice treated with trastuzumab that were associated with response to the drug and which could have utility in monitoring response in patients. Methods: Baseline tumor uptake of ${ }^{18} \mathrm{~F}$-FDG was measured in mice with MDAMB-361 HER2-overexpressing xenografts and MDA-MB-231 xenografts with very low HER2 expression by microPET imaging on Day 0. Mice were treated with PBS or trastuzumab $(4 \mathrm{mg} / \mathrm{kg}$ ) and microPET imaging was repeated 2 days post-treatment. Maintenance doses of trastuzumab $(2 \mathrm{mg} / \mathrm{kg})$ or PBS were administered on Days 7 and 14 and mice were imaged again on Days 9 and 16. Tumor uptake was measured as \% ID/g by volume-of-interest analysis on Days 2, 9 and 16, followed by biodistribution studies on Day 16. Tumor growth was measured and a tumor growth index (TGI) was calculated. Results:
Trastuzumab treatment resulted in a significant decrease in tumor uptake of ${ }^{18} \mathrm{~F}$-FDG in HER2-overexpressing MDA-MB-361 xenografts compared to PBS-treated control mice after 16 days of treatment $(2.6 \pm$ 0.8 vs. $4.6 \pm 1.8 \% \mathrm{ID} / \mathrm{g}$, respectively; $P<0.03$ ), but not after 2 or 9 days of treatment $(P=0.28-0.32)$. In contrast, there was no significant change in the tumor uptake of MDA-MB-231 xenografts with low HER2 expression during the entire course of therapy ( $4.4 \pm 1.7$ vs. $3.6 \pm$ $1.1 \% \mathrm{ID} / \mathrm{g}$, respectively; $P=0.31$ ). Trastuzumab resulted in significant growth inhibition of MDA-MB-361 xenografts compared to PBStreated controls as early as 10 days from initiation of treatment (TGI $0.7 \pm 0.2$ vs. $1.7 \pm 0.3$, respectively; $P<0.0005$ ), whereas no tumor growth inhibition was observed for MDA-MB-231 xenografts $(5.3 \pm 2.7$ and 5.2 $\pm 3.0 ; P=0.95)$. Conclusion: Changes in the tumor uptake of ${ }^{18}$ F-FDG accurately identified responding and non-responding human breast cancer xenografts in athymic mice treated with trastuzumab (Herceptin ${ }^{\circledR}$ ), however, diminished glucose utilization did not precede changes in tumor volume.

\section{Imaging Molecular and/or Cellular Processes}

\section{Gene Expression}

\section{J703}

\section{GOING DEEPER THAN MICROSCOPY WITH MULTISPECTRAL OPTOACOUSTIC TOMOGRAPHY (MSOT) OF FLUORESCENT PROTEINS IN-VIVO} $\underline{\text { Daniel Razansky }}{ }^{1}$, Martin Distel ${ }^{3}$, Claudio Vinegoni ${ }^{2}$, Reinhard Koester $^{3}$, Vasilis Ntziachristos ${ }^{1}$

${ }^{1}$ Technical University of Munich and Helmholtz Center Munich, Institute for Biological and Medical Imaging (IBMI), Neuherberg, Germany, ${ }^{2}$ Center for Systems Biology, Harvard Medical School, Boston, MA, USA, ${ }^{3}$ Institute for Developmental Genetics, Helmholtz Center Munich, Neuherberg, Germany.

Fluorescence proteins (FP) have become essential reporter molecules for studying life at the cellular level, from interrogating molecular pathways and protein function to cell migration and organism development, re-defining the ways we look into biology. Despite significant technological progress, high-resolution imaging is limited by photon scattering, therefore state of the art optical microscopy typically operates at depths between a few hundred microns to one millimeter. Correspondingly, a significant part of in-vivo biological research revolves around optically transparent stages of development or postmortem studies of chemically treated specimen. This work reports on the development of multi-spectral optoacoustic tomography (MSOT) method that utilizes illumination of the sample at different wavelengths for effective detection of distinct spectral signatures from FPs, dyes and other chromophores. We describe previously undocumented capacity of visualizing optical reporter molecules deep inside optically diffuse tissues with spatial resolution of about 38 microns, while simultaneously providing the reference anatomical images. Fig. 1a shows three-dimensional optoacoustic images of a head of a living adult zebrafish having cross-section of about $6 \mathrm{~mm}$. The multispectral (MSOT) reconstruction is shown in Fig. 1b revealing the precise location of mCherry FP expression in the brain in high congruence with the corresponding histology. Moreover, as demonstrated in phantom experiments, several centimeters of penetration with nearly same resolution can be achieved. Indeed, size of many important model organisms, e.g. worms, developing and adult insects and vertebrates including small mammals and their extremities, lie in this range and 
could be visualized. As such, MSOT can find very diverse applications in studying signaling pathways and gene expression, morphogenesis, decease progression and many other targeted mechanisms through whole bodies of opaque living organisms and animals.

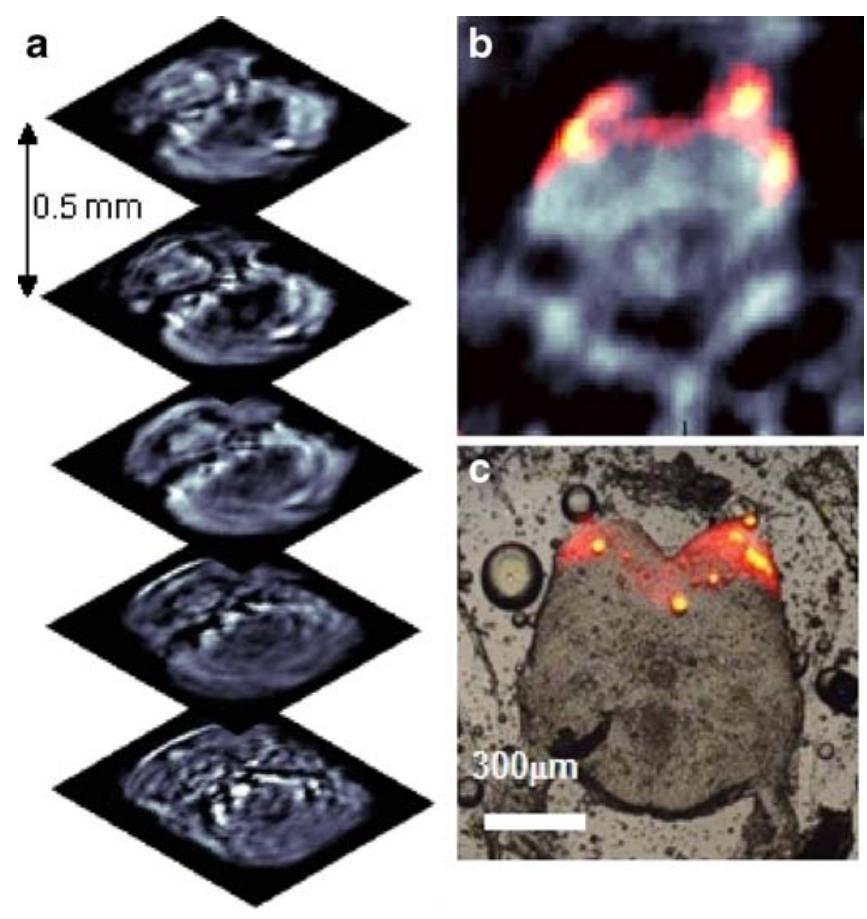

$\mathbf{J 7 0 4}$

\section{TRANSGENIC MICE FOR MULTIMODAL IMAGING OF ANGIOGENESIS}

Benjamin B. Bartelle, Cesar Berrios-Otero, Anne E. Friedland, Daniel H. Turnbull

Skirball Institute of Biomolecular Molecular Medicine, New York University Sackler Institute of Graduate Biomedical Sciences, New York, NY, USA.

The formation of new blood vessels via the process of angiogenesis is critical for normal development in every organ system, and underlies a host of pathologies including most cancers. We have developed transgenic mice in which vascular endothelial cells are biotinylated during angiogenesis. These mice are highly useful for targeted in vivo imaging of angiogenesis using avidinated agents for any imaging modality. We developed a transgene, consisting of an engineered Biotin Ligase (BirA) and a cluster of BirA substrate sequences (Biotags) fused to a transmembrane domain. Transgenic mice were generated, expressing the Biotag-BirA cassette from a minimal element of the endothelial promoter Tie2. Transgene expression and in vivo biotinylation was confirmed and characterized in E12 embryos using Western blot and IHC. To test efficacy of in vivo labeling, matrigel was implanted subcutaneously into adult wild type and transgenic animals to induce angiogenesis. After 1-2 weeks of vascularization, avidinated fluorophores were injected intravenously and matrigel plugs were imaged in vivo with fluorescence microscopy $(\mathrm{A}, \mathrm{B})$, and ex vivo at higher resolution with confocal microscopy $(\mathrm{C})$. Fluorescence microscopy showed specific labeling of developing vasculature in the transgenic mice(A) compared to wildtype(B). Confocal microscopy showed labeling was limited to newly forming vasculature within the matrigel of Tie2-Biotag mice(C). Addition of the angiogenic factor VEGF to the matrigel induced more vasculature with broader labeling within a shorter time period. Ongoing studies are focused on testing avidinated MRI and ultrasound contrast agents in adult and embryonic Tie2Biotag mice. In the future, these approaches can be expanded to in vivo analyses of Tie2 expression during angiogenesis in a wide variety of disease and injury mouse models, including tumor progression, wound healing, and response to ischemia.
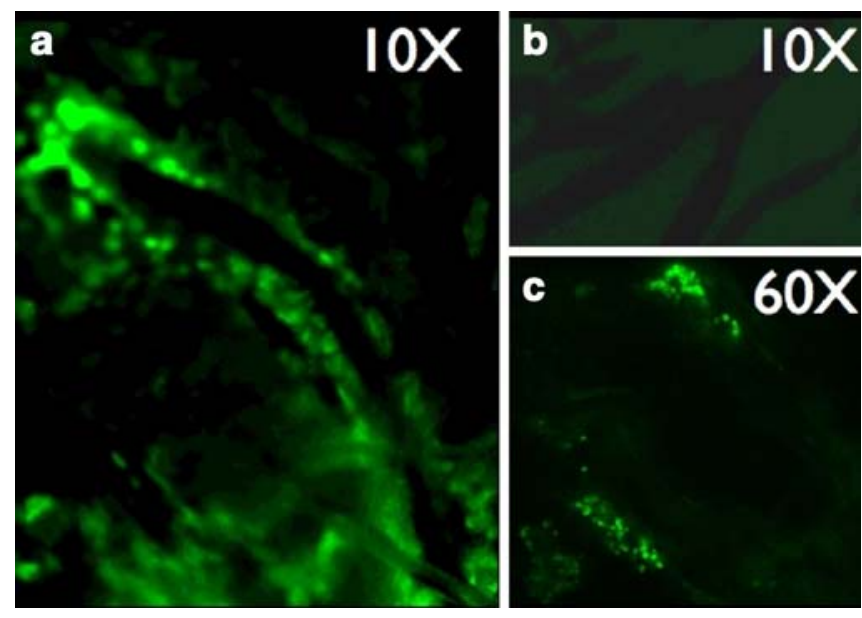

A: Tie2-Biotag mouse blood vessel injected with Avidin-FITC. B: Wild type mouse injected with Avidin-FITC shows no vascular labeling C: Branching vessel in Avidin-FITC labeled Tie2-Biotag mouse

$\mathbf{J 7 0 5}$

\section{A NEW PYRIMIDINE-SPECIFIC HUMAN-DERIVED REPORTER GENE FOR PET IMAGING IN HUMANS: TRUNCATED MUTANT DEOXYCYTIDINE KINASE} Yury Likar $^{1}$, Juan Zurita ${ }^{1}$, Konstantin Dobrenkov ${ }^{1}$, Larissa Shenker ${ }^{1}$, Anton Neschadim ${ }^{2}$, Jeffrey Medin ${ }^{2}$, Laurence James ${ }^{1}$, Shangde Cai ${ }^{1}$, Hedvig Hricak $^{1}$, Vladimir Ponomarev ${ }^{1}$

${ }^{1}$ MSKCC, New York, NY, USA, ${ }^{2}$ Ontario Cancer Institute, Toronto, ON, Canada.

Background: PET imaging of HSV1-tk reporter gene expression can be compromised in patients receiving nucleoside-based anti-viral treatment. Purpose: To create a new human-derived reporter gene for successful PET imaging in patients treated with antiviral drugs. Methods: Using U87 human glioma cells we compared novel human derived reporter genes fused with green fluorescent protein (GFP): human deoxycytidine kinase (dCK), its mutants containing R104M and D133A with (hdCKTM) or without (hdCKDM) S74E substitutions and the truncated variant of hdCKDM lacking nuclear localization signal at the N-terminus (h $\triangle \mathrm{dCKDM}$ ). U87 cells transduced with HSV1-tk were used as reference cell line. The in vitro 2-hour radiotracer uptake assay 
was performed with $3 \mathrm{H}-\mathrm{PCV}, 3 \mathrm{H}-\mathrm{FEAU}$ and $3 \mathrm{H}-\mathrm{FAC}$. In vitro sensitivity to Gemzar, Ara-C and GCV was measured using WST-test. $18 \mathrm{~F}-\mathrm{FHBG}$ and 18F-FEAU accumulation were assessed in subcutaneous xenografts using PET followed by tissue sampling. Results: Nuclear (for hdCK/GFP, hdCKDM/GFP, hdCKTM/GFP) or pancellular (for $\mathrm{h} \triangle \mathrm{dCKDM} / \mathrm{GFP}$ ) localization were observed in transduced cells. In vitro $3 \mathrm{H}-\mathrm{FEAU}$ accumulation was observed in hdCKDM, hdCKTM, h $\triangle \mathrm{dCKDM}$ and HSV1-tk transduced cells. There was no accumulation of $3 \mathrm{H}-\mathrm{FEAU}$ in hdCK+ and non-transdcued cells. $3 \mathrm{H}-$ PCV accumulation was observed only in HSV1-tk transduced cells. No $3 \mathrm{H}-\mathrm{PCV}$ accumulation was observed in hdCK and its mutants hdCKDM, hdCKTM, h $\triangle \mathrm{dCKDM}$ and non-transduced cells. Accumulation of 3H-FAC was observed in non-transduced and all transduced cells. Non-transduced cells and cells transduced with hdCK and its mutants have showed similar sensitivity to GCV. In contrast, transduced cells have shown significantly lower sensitivity to pyrimidine based drugs (Ara-C, Gemzar) compared with non-transduced cells. In vivo accumulation of $18 \mathrm{~F}-\mathrm{FEAU}$ was observed in $\mathrm{h} \triangle \mathrm{dCKDM}+$ and HSV1-tk+ tumors. Only HSV1-tk expressing tumors have shown 18F-FHBG accumulation. No 18F-FHBG accumulation was observed in non-transduced, hdCK and $\mathrm{h} \triangle \mathrm{dCKDM}+$ tumors. Conclusion: We developed and tested a series of new human-derived reporter genes based on hdCK that showed specificity and high phosphorylation activity with pyrimidine nucleoside analogs. $\mathrm{h} \triangle \mathrm{dCKDM}$ reporter gene can be used for PET imaging with $18 \mathrm{~F}-\mathrm{FEAU}$ in patients without interference from acycloguanosine-based antiviral analogs. The suicidal activity towards clinically used pyrimidine-based drugs will allow safe elimination of $\mathrm{h} \triangle \mathrm{dCKDM}$-transduced cells.

J706

\section{TO ESTABLISH TSA-INDUCIBLE GENE EXPRESSION} FOR DRUGS SCREENING AND SUICIDE GENE THERAPY Ren-Shyan Liu ${ }^{1,2}$, Luen Hwu ${ }^{1}$, Yc Chen $^{1}$, Kuan-Hung Lin ${ }^{1}$, Wd Kuo ${ }^{3}$, Hsin-Ell Wang ${ }^{3}$

${ }^{1}$ MAGIC, Dept NM, Faculty of Medicine, National Yang-Ming University, Taipei, Taiwan, ${ }^{2}$ NPCC, Dept NM, Taipei Veterans General Hospital, Taipei, Taiwan, ${ }^{3}$ Institute of Biomedical Imaging and Radiological Sciences, National Yang-Ming University, Taipei, Taiwan.

Objectives: Histone deacetylase inhibitors (HDACi) such as trichostatin A (TSA) has been shown to inhibit cancer cell proliferation, induce apoptosis and regulate the expression of genes involved in cell cycle. The cell cycle regulated genes, p21 and Gadd45, could be upregulated and induced by TSA. This study aimed to establish a TSA-inducible reporter gene system for HDACi drug screen and gene therapy. Methods: A p21-driven reporter composed of p21 promoter, luciferase (Fluc), DsRed monomer (DsRedm) and truncated HSV-1 thymidine kinase SR39 mutant (ttkSR39) gene, was constructed and transfected into the NSCLC cell line H1299 after DNA sequencing verification. Several putative stably p21-driven reporter expressed cells were obtained after G418 drug selection and the fluorescent microscopic examination. TSA was applied to these stable cell lines, and its effect was determined by luciferase assay and 3H-FEAU uptake. The stably p21-driven reporter expressed cell clones were inoculated in SCID mice, and optical imaging and microPET imaging with ${ }^{124}$ I-FEAU were performed. Results: The p21-driven reporter expressed H1299 cells grew well in G418-containing culture medium. In vitro study of treatment with TSA showed remarkable increase in luciferase and TK activity. In vivo study confirmed the H1299-transfected p21-driven reporter tumor xenograft has obvious luciferase activity and ${ }^{124}$ I-FEAU uptake. Conclusions: The p21-driven reporter stably expressed clones are
TSA inducible. These clones could be used for HDACi drugs screening and could be combined with GCV for suicide gene therapy.

\section{IVIS: H1299-p21-3H 2-2}
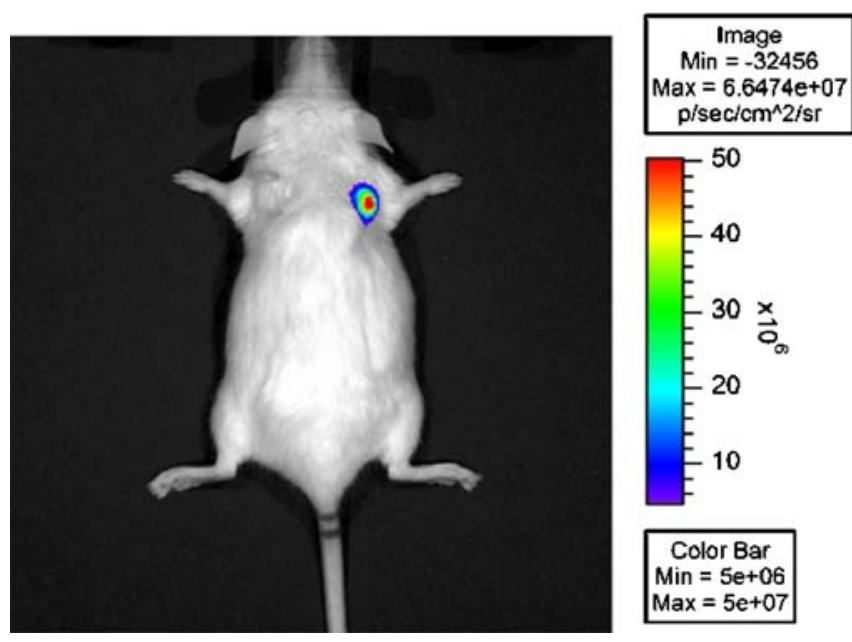

J707

MOLECULAR IMAGING OF DUAL OPTICAL AND NUCLEAR REPORTER SYSTEMS FOR MONITORING THE CHARACTERISTICS AND PROGRESSION OF TUMOR IN CT-26/TK-LUC XENOGRAFTS

Tong-Hsien Chow ${ }^{1}$, Hsin-Ell Wang ${ }^{1}$, Ren-Shyan Liu ${ }^{1,2}$, Victor F. Pang ${ }^{3}$, ${ }_{\text {Wei-Chan Lin }}^{1,4}$, Yu-Cheng Kuo ${ }^{1,5}$, Chia-Hui Chu ${ }^{1}$, Jeng-Jong Hwang ${ }^{1}$ ${ }^{1}$ Department of Biomedical Imaging and Radiological Sciences, National Yang-Ming University, Taipei, Taiwan, ${ }^{2}$ Nuclear Medicine, Taipei Veterans General Hospital, Taipei, Taiwan, ${ }^{3}$ Veterinary Medicine, National Taiwan University, Taipei, Taiwan, ${ }^{4}$ Radiology, National YangMing University Hospital, Taipei, Taiwan, ${ }^{5}$ Radiation Oncology, China Medical University Hospital, Taipei, Taiwan.

Noninvasive molecular genetic imaging combined with optical and nuclear reporter systems are becoming beneficial for diagnostic and therapeutic purposes. The quantified result from reporter gene imaging is able to provide real-time biological information in biomedical researches. Calibration of tumor size in animal models is considered to be a method of choice in most studies involved in tumor monitoring, while the exact volume of tumor underneath is still hardly to be explored. The goal of this study was to investigate the correlation among various modalities offering tumor volume information, and growth characteristics in tumor-bearing mice. Murine colorectal adenocarcinoma (CT-26/tk-luc) xenografted BALB/c mice were used. Imaging modalities including bioluminescence imaging (BLI), 131IFIAU gamma scintigraphy, 18F-FEAU microPET, 18F-FDG microPET, and ultrasonic imaging were applied. Histopathology was also performed. The in vitro and in vivo doubling time of tumor growth were similar between reporter genes transfected and parental cells. The quantified results obtained from BLI, 131I-FIAU gamma imaging and 18F-FEAU microPET all showed similar patterns of tumor growth kinetic when before tumor size less than $1000 \mathrm{~mm} 3$ and gradually decline afterwards. 18F-FDG metabolism, on the contrary, within ROIs of 
the same late stage tumor was increased. Ultrasonic imaging and histopathology showed some anatomical change, which was closely correlated in the time sequence to reporter gene imaging during tumor progression. The optical and nuclear reporter systems combined with multi-imaging modalities are useful to depict tumor characteristic and quantification. In addition, the system has the potential to enhance in inter-translational studies for diagnostic and therapeutic effectiveness.

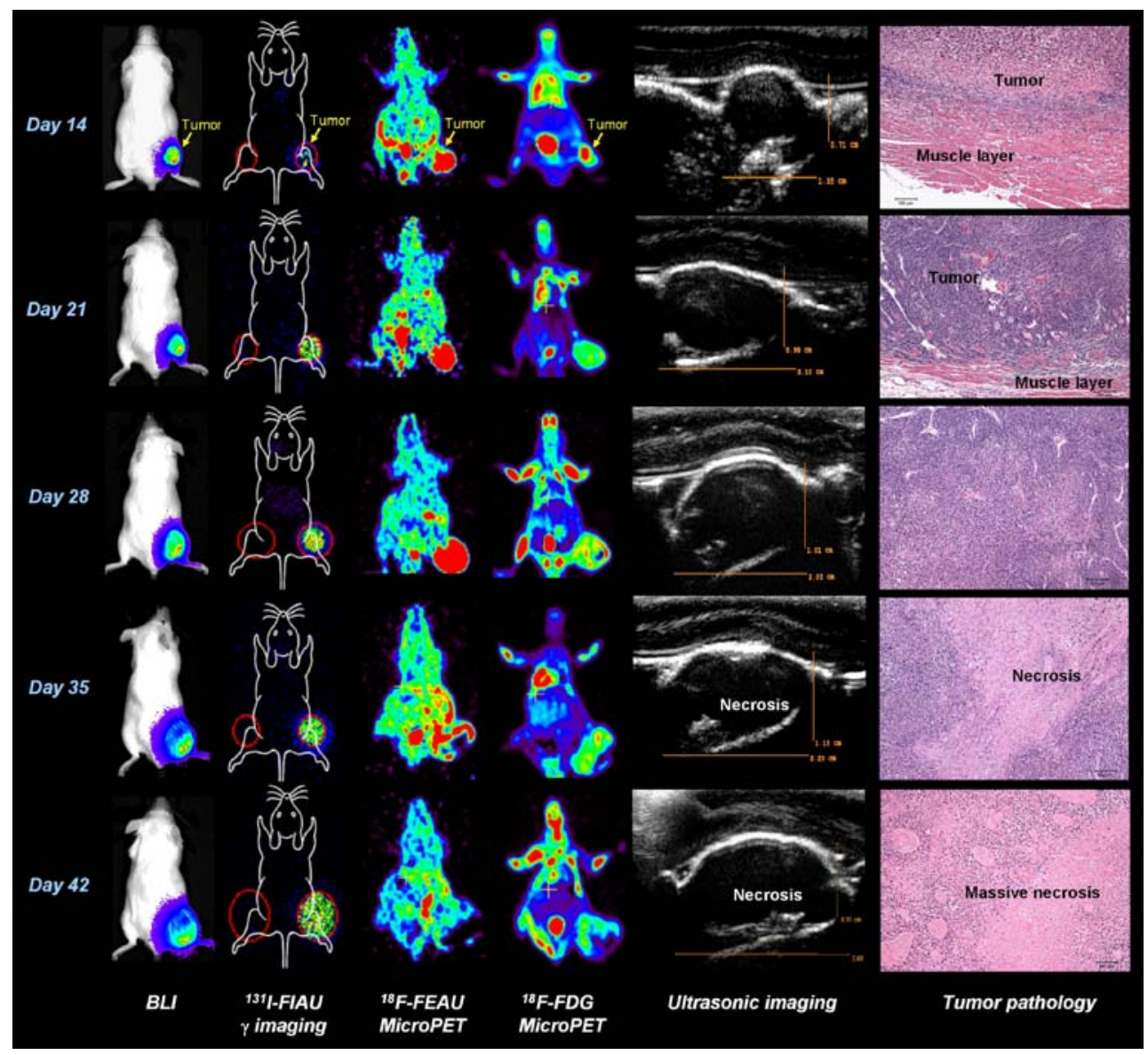

Figure Multi-imaging modalities of BLI, ${ }^{131}$ I-FIAU gamma scintigraphy, ${ }^{18} \mathrm{~F}$-FEAU microPET, ${ }^{18} \mathrm{~F}-\mathrm{FDG}$ microPET as well as ultrasonic imaging in CT-26/tk-luc tumor xenografted mice. The tumor images from different imaging modalities were performed at corresponding time point. Ultrasonic imaging and tumor pathology were carried to examine the anatomical and pathological changes in tumor progression.

$\mathbf{J 7 0 8}$

PROMOTER FUNCTIONALITY STUDIES IN LENTIVIRAL TRANSDUCED HUMAN CARD BLOOD DERIVED STEM CELLS Nadimpalli Ravi S Varma, Branislava Janic, M. M. Ali, Asm Iskander, Ali S. Arbab

Radiology, Henry ford Hospital, Detroit, MI, USA.

Human cord blood derived hematopoietic stem cell can renew it-self and can differentiate to a variety of specialized cells. Transplantation of umbilical cord blood in children with Fanconi anemia and in other therapeutic use is growing rapidly. There is growing interest to use stem cells as gene carrier or delivery systems. Investigators have used different promoters systems for lentivector based transfection studies and reported varying degree of transfection efficiency in different cells lines. However, there is limited data available on relative strengths of different promoters in transfecting cord blood stem cells using lentivectors, thus it is important to analyze the promoter strength in the cord blood stem cells before using them as gene delivery vehicles or in therapeutic use. Four different promoter systems were used in this study; 1) Human cytomegalovirus (CMV) promoter, 2) Simian virus 40 (SV40) promoter, 3) mammalian UbiquitinC (UBC) promoter and 4) cellular polypeptide chain elongation factor 1 alpha (EF1) promoter systems in umbilical cord blood derived stem cells. Vectors containing any of the four promoters were able to transfect the stem cells, however both EF1 and CMV promoters robustly drove the expression of reporter gene (GFP), while SV40 and UBC promoters showed very low level of expression of reporter gene (GFP). Methods : Lentivirus was produced by transient transfection of $293 \mathrm{TN}$ cells (SBI, USA) Lentiviral titers were determined by analysis of GFP expression in HeLa cells. HSCs were transfected with all four different lentivirus. The GFP expression by transduced cells was analyzed using fluorescence microscope and 
flow cytometer. Results: The GFP expression in stem cells was analyzed at days 6,28 and 52 after transduction. Both fluorsence microscopic and flowcytometric analysis reveled that $\mathrm{EF} 1$ and $\mathrm{CMV}$ promoter drove high level expression of GFP, while SV40 and UBC promoters drove low level expression of GFP. Conclusions: The four different promoters were analyzed for their transcriptional activates in freshly isolated cord blood stem cells. A high level expression of GFP reporter gene was achieved from the EF1 and CMV promoters, while little expression of GFP was detected from the SV40 and UBC promoters. This study indicates lentivectors containing EF1 and CMV promoters can be used for the high-level stable gene expression in cord blood derived stem cells.

J709

\section{NONINVASIVE IMAGING OF CANCER GENE THERAPY IN ORTHOTOPIC MOUSE MODELS USING A NOVEL SYSTEMICALLY DELIVERED BI-DIRECTIONAL TRANSCRIPTIONAL TARGETING VECTOR}

Byeong Cheol Ahn ${ }^{1,4}$, Young Il Kim ${ }^{2}$, Sunetra Ray ${ }^{1}$, Regina Katzenberg ${ }^{2}$, Lawrence V. Hofmann ${ }^{2}$, Sanjiv S. Gambhir ${ }^{1,3}$

${ }^{1}$ Molecular Imaging Program at Stanford, Radiology, Stanford University, Stanford, CA, USA, ${ }^{2}$ Interventional Radiology, Stanford University, Stanford, CA, USA, ${ }^{3}$ Bioengineering, Stanford University, Stanford, CA, USA, ${ }^{4}$ Nuclear Medicine, Kyungpook National University, Deagu, Korea, South.

Weak cancer specific promoters, such as Survivin promoter(pSurv), need an amplification strategy to be used for cancer gene therapy. Gene therapy ideally requires non invasive tools to image the delivery of therapeutic genes. We report the development of a novel adenoviral vector carrying a bidirectional transcriptional amplification GAL4-VP2 system which can amplify pSurv activity leading to correlated expression of a reporter gene (firefly luciferase; FL) and a therapeutic gene (TRAIL) [Ad-pSurv-TRAIL-G8-FL]. Methods; MCA-RH7777 (Morris hepatoma) and BRL 3A (Buffalo rat liver) cells were transfected with 100 MOI Ad-pSurv-TRAIL-G8-FL and bioluminescence imaging (BLI) was performed at $72 \mathrm{hrs}$. Comparison of FL and TRAIL activities was also performed with HCT116 cells. For animal studies, MCA-RH7777 cells $\left(1 \times 10^{6}\right)$ were surgically implanted in the liver of Buffalo rats. Tumor formation was confirmed with FDG-PET at 14 days after implantation. $10^{9}$ PFU of Ad-pSurv-TRAIL-G8-FL was administered via tail-vein. BLI was performed to evaluate FL expression in tumor and non-tumor regions at $2 \mathrm{~d}$ after the virus delivery. Results; MCA-RH7777 cells show 3 fold higher FL activity than BRL 3A cells $\left(4.2 \times 10^{7} \pm 4.2 \times 10^{6}\right.$ vs. $\left.1.4 \times 10^{7} \pm 1.9 \times 10^{6} \mathrm{p} / \mathrm{s} / \mathrm{cm}^{2} / \mathrm{sr}, p=0.002\right)$. Expression levels of FL and TRAIL were well correlated $\left(\mathrm{R}^{2}=0.93\right)$. PET imaging 2 wks after tumor implantation showed FDG uptake in tumor implanted area $(0.62 \pm 0.13 \% \mathrm{ID} / \mathrm{gm})$. Tumor region showed 29 fold higher light output than normal liver $\left(9.2 \times 10^{3}\right.$ vs. $\left.3.1 \times 10^{2} \mathrm{p} / \mathrm{s} / \mathrm{cm}^{2} / \mathrm{sr}, p=0.02\right)$ in BLI. Conclusions; An adenoviral vector carrying Survivin promoter driven both the TRAIL therapeutic gene and the FL reporter gene is more active in hepatoma cell lines compared to normal liver cell lines. Most importantly, we report for the first time the iv injection of such a vector to achieve highly specific liver tumor targeting in living rats. The bidirectional transcriptional amplification system using GAL4-VP2 preserves promoter specificity and has the capability of showing therapeutic gene expression non-invasively in a rat orthotopic hepatic tumor model.

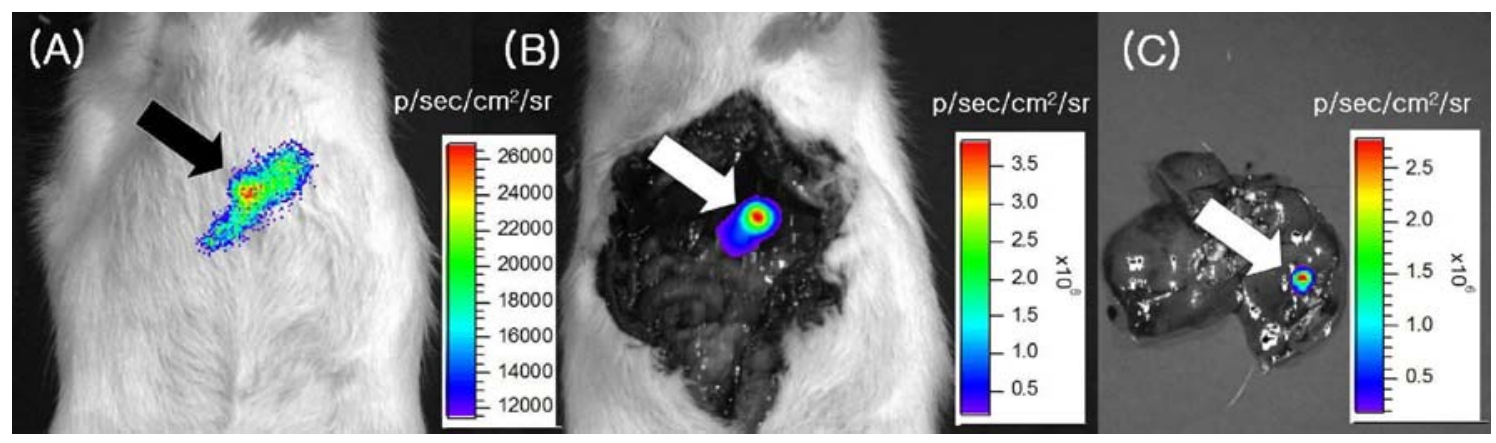

(A) Intact Rat, (B) open abdomen, (C) ex vivo bioluminescence imaging after tail-vein injection of gene delivery vehicle followed by D-Luciferin intraperitoneal delivery 48 hours later. Luciferase activity is only observed from the area of implanted tumor showing highly specific targeting (Arrow).

$\mathbf{J} 710$

\section{BIOINFORMATICS APPROACH FOR IN VIVO IMAGING OF ENDOGENOUS MICRORNA TARGETS DURING NEUROGENESIS}

Yeona Jin, Young Ha Kim, Do Won Hwang, Dong Soo Lee, Soonhag Kim nuclear medicine, Seoul national university college of medicine, Seoul, Korea, South.

MicroRNAs (miRNAs), a class of small non-coding RNAs, have been reported to be functionally involved with cellular metabolism and a variety of diseases. The importance of miRNA expression and functional targeting has recently become a focus of intense research. However, their endogenous molecular targets have not been clearly identified despite multiple attempts in prior studies using bioinformatics. Methods: In silico predicted targets of miR124a and miR9* from the PicTar database (DB) and heterogeneous microarray data from the NCBI GEO DB were investigated by scoring each of the targets according to the values of the PicTar scores and gene expression for neuronal differentiation among brain tissues. Genes with more than 50 points were considered to be endogenous targets of miR124a or miR9* and further validated by RT-PCR during neuronal differentiation of P19 cells and in response to miR124a or miR9*. Among them, SOX2 was selected for imaging of miR ${ }^{*}$-mediated repression during neurogenesis both in vitro and in vivo. Results: Our bioinformatics strategy and in vitro validation by the PCR, identified 16 out of 337 miR124apredicted targets and 5 out of 299 miR9*-predicted targets were significantly and directly down-regulated by each of the miRNAs during neurogenesis. In vitro and in vivo bioluminescent imaging system was successfully monitored the miR $9 *$-mediated repression of SOX2 during neuronal differentiation of the P19 cells. Conclusion: We demonstrate that our bioinformatics approach offers a powerful and precise method for the identification of novel miR124a and miR9* endogenous targets during neuronal differentiation. This bioinformatics approach, using microarray data available from public DBs, provides a practical means for identifying the endogenous targets of other miRNAs. 


\section{J711}

\section{EVALUATION OF HUMAN HEMATOPOIETIC STEM CELL AS GENE DELIVERY VEHICLES IN MOUSE BREAST CANCER MODEL}

Nadimpalli Ravi S Varma, Branislava Janic, M. M. Ali, Asm Iskander, Ali S. Arbab

Radiology, Henry ford Hospital, Detroit, MI, USA.

Enormous potential exists for gene therapy as alternative treatment for the breast cancer. However, lack of an efficient carrier or delivery vehicle hampers the therapeutic potential of vectors containing therapeutic gene after local of systemic administration. Stem cells are being used to carry therapeutic genes to the sites of interest. The purpose of this study was to determine the potential of cord blood derived CD34+/CD133+ hematopoietic stem cells (cbHSCs) as gene delivery vehicles and to determine their ability to cross transfect the surrounding tumor cells, when administered locally in a mouse model of human breast cancer. Two million MDA-MB-231 cells were subcutaneously implanted in the right flank of nude mice to develop the breast cancer. The cbHSCs were transfected with replication competent adenoviral vector carrying herpes simplex virus thymidine kinase (HSV-tk) and human sodium iodide symporter (hNIS) gene. Viral transfected cbHSCs (5X105 transgenic cbHSCs in $5 \mu \mathrm{l}$ ) were administered locally into the tumor ( 5 points, $2 \mu \mathrm{l} /$ point) when the largest tumor diameter reached $0.5 \mathrm{~cm}$. For control mice non-transgenic cbHSCs $(5 \mathrm{X} 105 \mathrm{cbHSCs}$ in $5 \mu \mathrm{l})$ were administered locally. To compare the cross transfection efficiency with naked viral vectors, set of mice carrying breast cancer also received $5 \mu$ of adenoviral particles (6X107 viral particles, equivalent to viral load of the transfected cells). Single photon emission computed tomography (SPECT) images were obtained on days 3,7 and 14 after the local injection. Optimal transfection: After intra-tumor injection of transfected cbHSCs, animals were scanned on days 3, 7 and 14. Significantly higher amount of Tc$99 \mathrm{~m}$ activity was observed on day 14 after the administration of transfected cbHSCs. Based on the percent whole body activity, it is indicated that 14 days after the administration of cells or viral vectors, optimal transfection could be achieved for in vivo SPECT image acquisition. All mice that received transfected cells showed significantly higher activity of Tc-99m in the tumors compared to that in mice receiving non-transfected cells or viral vectors only. These finding were further verified with immunohistochemistry. The results further agreed with the SPECT studies. The higher amount of Tc-99m in the tumors administered with transfected cbHSCs indicates the cross transfection efficiency of the transfected cbHSCs. Our study indicates that transfected HSCs carrying replication competent viral vector can be used for local gene delivery vehicles for gene therapy in breast cancer.

\section{J712}

CONDITIONAL VIRAL VECTORS FOR SPECIFIC LABELING AND NONINVASIVE IMAGING OF NEURONAL CELL POPULATIONS IN MOUSE BRAIN

Veerle Reumers, Rik Gijsbers, Abdelilah Ibrahimi, Greetje Vande Velde, Jaan Toelen, Sarah-Ann Aelvoet, Sylvie De Swaef, Irina Thiry, Chris Van den Haute, Zeger Debyser, Veerle Baekelandt

Molecular Medicine, Katholieke Universiteit Leuven, Leuven, Belgium.

In vivo bioluminescence imaging (BLI) would be a very valuable tool to monitor neuroregeneration and neurodegeneration in intact animals. We have previously shown that in vivo BLI accurately reports on viral vector-mediated expression of a transgene of interest in rodent brain.
Viral vectors can locally transduce both dividing and postmitotic cells by a single stereotactic injection in the adult brain. We now aimed to develop viral vectors that specifically target certain neuronal cell populations in order to noninvasively follow transgene expression in these cells. We designed conditional lentiviral vectors (LV) and recombinant adeno-associated viral vectors (rAAV) based on Cremediated recombination. In the construct the cDNA of enhanced green fluorescent protein (eGFP) and of firefly luciferase (Fluc) are present in antisense orientation and flanked by two mutually exclusive lox sites. Upon Cre recombination the cDNA between 2 lox sites will be inverted and thereby induce expression. Mice were imaged using an optical CCD-camera (IVIS 100). First, we validated the conditional viral vectors in cell culture. Efficient expression of both transgenes was demonstrated only in the presence of the Cre recombinase. Next, conditional LV were stereotactically injected in the subventricular zone (SVZ) of Nestin-Cre transgenic mice, that express Cre in the endogenous neural stem cells (eNSC). We have previously shown that BLI can be used for noninvasive detection and quantification of the migration of neuroblasts by LV marking of the eNSC. However, the BLI signal originating from different cell types at the site of injection hampered accurate quantification of the signal originating from the eNSC. By using conditional LV, the BLI signal originated specifically from the eNSC and their migration could be precisely monitored. In another approach, the conditional rAAV were injected in the substantia nigra (SN) of DAT-Cre transgenic mice, that express Cre driven by the dopamine transporter promoter (DAT), ensuring specific expression in the dopaminergic neurons. A BLI signal could be detected at 2 weeks post injection. Specific labeling of the dopaminergic cells was confirmed with confocal histological analysis. Conclusion: We developed a new conditional viral vector-based system for specific labeling of neuronal cell populations in vivo. We applied these viral vectors to specifically label the eNSC in the SVZ and the dopaminergic cells in the SN. This approach holds promise to noninvasively follow up endogenous neurogenesis and neurodegeneration over time using BLI.

\section{$\mathbf{J} 713$}

\section{IN VIVO MONITORING OF ELECTROPORATION-BASED NON-UNION FRACTURE REPAIR USING BIOLUMINESCENCE AND MICRO CT IMAGING}

$\underline{\text { Gadi Pelled }}^{1,2}$, Nadav Kimelman Bleich ${ }^{2}$, Olga Mizrahi ${ }^{2}$, Yoram Zilberman $^{2}$, Dan Gazit ${ }^{1,2}$

${ }^{1}$ Surgery, Cedars Sinai Medical Center, Los Angeles, CA, USA, ${ }^{2}$ Skeletal Biotech Laboratory, Hebrew University of Jerusalem, Jerusalem, Israel.

Non-union fractures are a major challenge with no optimal solution in orthopedics up to date. Recent findings indicate that in vivo DNA electroporation (use of short high-voltage pulses to overcome the barrier of the cell membrane) is a safe, simple, and effective technique of gene delivery. We hypothesized that direct in vivo electroporation of an osteogenic gene (BMP-9) into a non-union radius bone defect site would induce complete bone regeneration and fracture repair. A $1.5 \mathrm{~mm}$ segmental defect was created in the radius bone of $\mathrm{C} 3 \mathrm{H} / \mathrm{HeN}$ mice. A $1.5 \mathrm{~mm}$ long collagen sponge was implanted in the defect site. After 10 days, needle electrodes were placed under fluoroscopic guidance in the defect site, such as that the implanted sponge was located between the electrodes (Fig. 1A). Plasmids encoding either for Luciferase or BMP-9 genes were injected into the collagen sponge. Eight pulses at $100 \mathrm{~V}$ were generated using an ECM 830 electroporator. Each pulse was of $20 \mathrm{~ms}$ with $100 \mathrm{~ms}$ interval. In vivo gene expression was monitored longitudinally using bioluminescence imaging. Five weeks post electroporation the limbs were subjected to highresolution $\mu \mathrm{CT}$ scanning which enabled quantitative analysis of bone 
formation at the site of the defect. Our results showed that Luciferase expression was evident till day 24 post electroporation (Fig. 1B). Prominent bone formation was seen in the defect site electroporated with BMP9, based on the $\mu \mathrm{CT}$ analysis (Fig. 1C). The new bone was sufficient to bridge the defect gap. No bone formation was noted in the limbs electroporated with Luciferase, 5 weeks post electroporation
(Fig. 1C). Our results indicate that bone regeneration in a non-healing fracture model can be attained using in vivo electroporation of an osteogenic gene. This bone regeneration strategy could revolutionize orthopedic treatment in patients suffering from major bone loss. Moreover, our method may pave the way for the regeneration of other skeletal tissues such as cartilage and tendon.
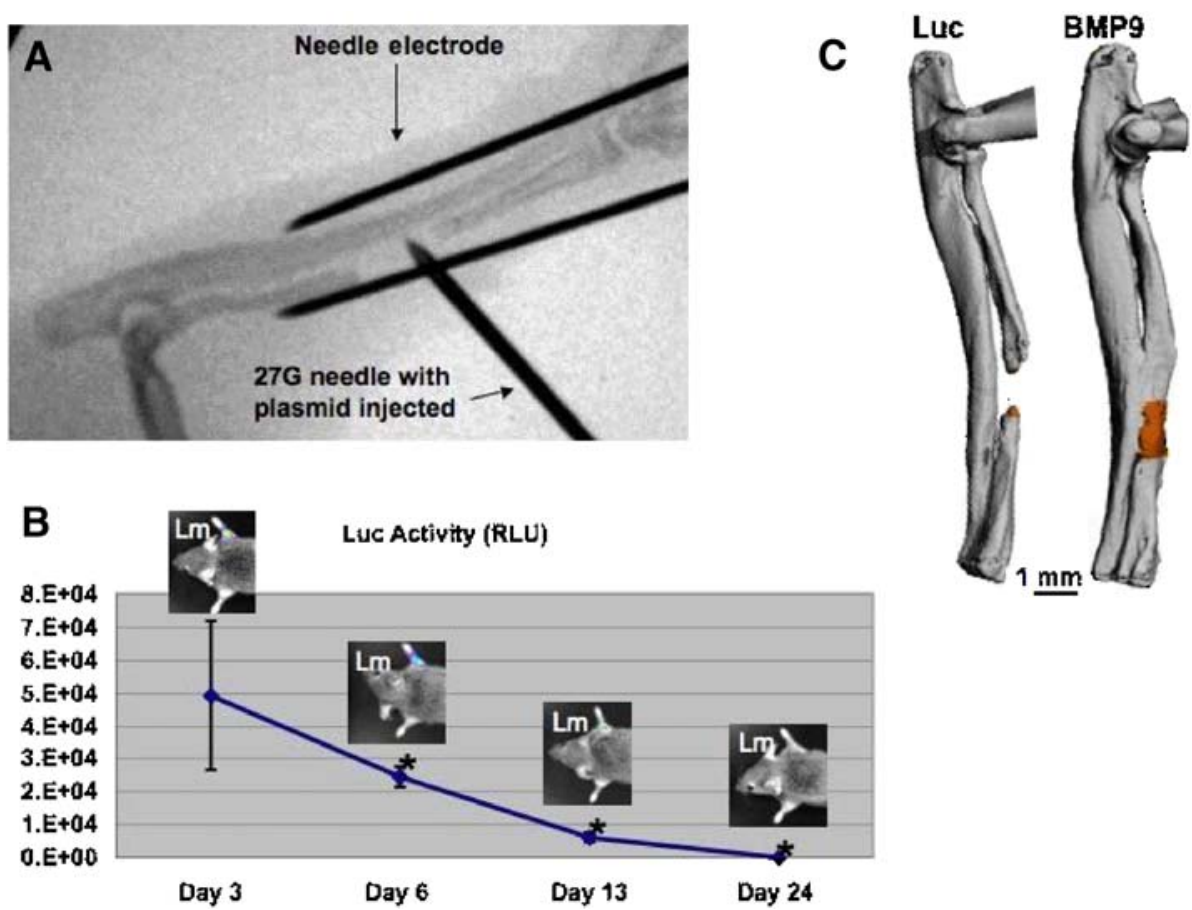

$\mathbf{J} 714$

\section{IMAGING SYSTEM FOR MICRO RNA EXPRESSION ASSOCIATED WITH MYOCYTE DIFFERENTIATION USING MOLECULAR BEACON}

Ye Lim Cho ${ }^{1}$, Won Jun Kang, Ju Ri Chae ${ }^{1}$, Jong Doo Lee ${ }^{1}$, Soonhag Kim ${ }^{2}$ ${ }^{1}$ Department of Radiology, Division of Nuclear Medicine, Yonsei University College of Medicine, Seoul, Korea, South, ${ }^{2}$ Department of Nuclear Medicine, Seoul National University College of Medicine, Seoul, Korea, South.

Background: Differentiation of progenitor cell after administration is crucial for the therapeutic effect. Molecular beacon is a specifically designed DNA structure that can be applied to monitor gene expression. Of several miRNAs, miRNA-206 and miRNA-26a are known to be highly expressed during myocyte differentiation. We designed in vitro and in vivo imaging system that can simultaneously demonstrate separate miRNA expression during myocyte differentiation using molecular beacon. Material and method: Mouse miRNA-26a (5'TTCAAGTAATCCAGGATAGGCT 3') and miRNA-206 (5'TGGAATGTAAGGAAGTGTGTGG3') were synthesized. Molecular beacons of miRNA-26a (miRNA-26a beacon 6FMQ1: 6FAM-5' AAGTTCATTAGGTCCTATCCGAAACTT 3'-BHQ1) and miRNA 206 (miRNA-206 beacon TRdQ2: Texas Red-5' ACCTTA CATTCCTTCACACACCAAGGT 3'-BHQ2) were synthesized. Mouse miRNA-133a (5'TTTGGTCCCCTTCAACCAGCTG 3') was used as control miRNA. C2C12 cell (skeletal myoblast) was adopted as miRNA expressing cells and Hela cell (cervix cancer cell) was adopted as control cells with no natural miRNA expression. miRNA26a-beacon and miRNA206-beacon were co-transfected to Hela cell simultaneously, and miRNA26a and miRNA206 were applied with different concen- tration $(0,0.25,0.5,0.75,1 \mathrm{~nm}) . \mathrm{C} 2 \mathrm{C} 12$ cells were co-transfected with miRNA26a-beacon and miRNA206-beacon, and differentiation was induced. Confocal images were obtained at D0, D2, D4, D6 after differentiation induction. Results: Fluorescence intensity was increased according to miRNA level administered in Hela cell by both miRNA26a and miRNA206. miRNA133a, negative control, did not show increased fluorescence intensity after administration. On confocal images, similar incremental pattern of fluorescence was observed on Hela cell. C2C12 cells showed increased fluorescence in propotion to differentiation progression without target miRNA administration. Conclusion:We demonstrated that miRNA expression after cellular differentiation could be visualized using molecular beacon in skeletal myoblast cells.

\section{$\mathbf{J} 715$}

\section{DICER SUBSTRATE SIRNA-MEDIATED SILENCING OF HUMAN SODIUM IODIDE SYMPORTER (hNIS) GENE IN VITRO AND IN VIVO}

Won Woo Lee ${ }^{1}$, So Yeon Park ${ }^{1}$, Young So ${ }^{2}$

${ }^{1}$ Nuclear Medicine, Seoul National University College of Medicine, Seoul, Korea, South, ${ }^{2}$ Nuclear Medicine, Konkuk University School of Medicine, Chungju, Korea, South.

Purpose: We designed a human sodium iodide symporter (hNIS)targeted dicer substrate small interfering RNA (dsiRNA) and evaluated the silencing effects of the dsiRNA in vitro and in vivo. Methods: The dsiRNA (25/27R form) has a 2-nt 3' overhang at the antisense and 2-nt DNA bases at the 3' end of the sense strand. Three stably hNISexpressing cell lines ( 2 cancer cell lines, C6-hNIS and CT26-hNIS; 1 stem cell line, rMSC-hNIS) were transfected by the dsiRNA at 0.001 , 
$0.01,0.1,1$, and $30 \mathrm{nM}$ using lipofectamine 2000. $48 \mathrm{~h}$ after the transfection, I- 125 uptake tests were performed and compared to the effects of specific hNIS inhibitor, NaClO4 $(300 \mu \mathrm{M})$. CT26-hNIS tumor cells were transfected using the dsiRNA $(30 \mathrm{nM})$ and then implanted to the male Balb/C nude mouse (7.0X10-5 cells). Control tumor (CT26-hNIS cells transfected using negative siRNA) model was also established in the same nude mouse (8.8X10-5 cells). NanoSPECT-CT images were obtained $1 \mathrm{hr}$ after tail vein injection of $0.1 \mathrm{mCi}$ of I-125. Result: The dsiRNA transfection $(30 \mathrm{nM})$ to C6-hNIS cells completely inhibited I-125 uptake as $74.5 \pm 18.9$ picomole/106 cells. The $1 \mathrm{nM}$ concentration of dsiRNA inhibited $50 \%$ of I-125 uptake, but lower concentrations $(0.001 \sim 0.1 \mathrm{nM})$ had no effect on I-125 uptake. The dsiRNA transfection ( $30 \mathrm{nM})$ to CT26-hNIS cells near completely inhibited I-125 uptake as $136.5 \pm 32.7$ picomole/106 cells. The $1 \mathrm{nM}$ concentration of dsiRNA inhibited $81 \%$ of I-125 uptake, and even the lowest concentration tested $(0.001 \mathrm{nM})$ inhibited $17 \%$ of I- 125 uptake. The dsiRNA transfection $(30 \mathrm{nM})$ to rMSC-hNIS cells completely inhibited I125 uptake as $143.5 \pm 29.0$ picomole/ 106 cells. The concentration dependent inhibition of I-125 uptake was also observed in rMSC-hNIS cells. The dsiRNA-transfected tumor in the nude mouse showed lower I-125 uptake on the nano-SPECT-CT images than the negative control tumor up to 2 weeks after implantation. The I- 125 uptakes in both dsiRNA-treated and negative tumors were completely suppressed after $\mathrm{NaClO} 4$ i.p. injection $(10 \mathrm{mg} / \mathrm{kg})$. Conclusion: The hNIS-targeted dsiRNA silenced the hNIS function in vitro and in vivo. The delivery systems for dsiRNA can be effectively developed using the combination of hNIS-targeted dsiRNA/its modifications and hNIS expression monitor systems.

\section{$\mathbf{J} 716$}

\section{MONITORING PROMOTER ACTIVITY ENHANCEMENT OF 5MMTERT-NIS SYSTEM BY INCREASE OF C-MYC FOLLOWING NF-kB INDUCTION}

$\underline{\text { Seung Hoo Kim }}^{1,2}$, Hyewon Youn ${ }^{1,3}$, Hye Kyung Chung ${ }^{1,4}$, Keon Wook Kang $^{1,3}$, Jae Min Jeong ${ }^{1,3}$, Dong Soo Lee ${ }^{1,3}$, June-Key Chung ${ }^{1,3}$ ${ }^{1}$ Department of Nuclear Medicine, Seoul National University College of Medicine, Seoul, Korea, South, ${ }^{2}$ Department of Tumor Biology, Seoul National University College of Medicine, Seoul, Korea, South, ${ }^{3}$ Institute of Radiation Medicine, Medical Research Center, Seoul National University College of Medicine, Seoul, Korea, South, ${ }^{4}$ Laboratory of In Vivo Molecular Imaging, Department of Nuclear Medicine, Chonnam National University Medical School, Gwangju, Korea, South.

Objectives: The transcription factor c-myc is known to be activated by NF$\mathrm{kB}$, and c-myc is one of activators known to transactivate hTERT promoter. Hence, activated NF-kB could participate in the transactivation of hTERT promoter indirectly. Our purpose is to visualize the enhanced promoter activity of 5 mmTERT-NIS by NF-kB activation. Materials and methods: Human hepatocelluar carcinoma cell line having TERT promoter/NIS gene (Hep3B-5mmTERT-NIS) was produced using a retrovirus. After transfection, TNF- $\alpha(0,1,3$ and $5 \mathrm{ng} / \mathrm{ml})$ were treated for $1 \mathrm{hr}$ with or without $10 \mu \mathrm{M}$ Bay 11-7082, an inhibitor of NF-kB for $4 \mathrm{hr}$. After that, the expression of c-myc and hNIS mRNA were evaluated by RT-PCR. Luciferase assay using a plasmid vector harboring firefly luciferase gene under NF-kB promoter (pcDNA3.1-NF-kB-Luci) were also performed for evaluation of NF-kB activation. Changes of NIS expression by enhancing $5 \mathrm{mmTERT}$ promoter activity were monitored and Iodide uptake assay was also performed. Results: The incensement of c-myc and hNIS mRNA by TNF- $\alpha$ treatment was observed in RT-PCR. TNF- $\alpha$ induced NF-kB activation were observed by enhanced luciferase gene expression. Iodide uptake by TNF- $\alpha$ was also significantly increased. The effect of TNF- $\alpha$ treatment was blocked by inhibitors of NFkB, Bay 117082. Conclusion: TNF- $\alpha$-induced NF-kB activation induced increase of NIS gene expression under 5mmTERT promoter. TNF- $\alpha$ pretreatment before radioiodide gene therapy using 5 mmTERT-NIS system may be useful for combination tumor therapy.
$\mathbf{J} 717$

\section{HIGH MICROPET BACKGROUND RADIOACTIVITY IN NUCLEOSIDE ANALOG HSV1-TK IMAGING: NO ROLE OF BACTERIA BUT SIMPLE STRATEGIES REDUCE IT} Alessandro Ruggiero $^{1}$, Peter Brader ${ }^{1}$, Inna S. Serganova ${ }^{2}$, Valerie A. Longo $^{3}$, Hedvig Hricak ${ }^{1}$, Ronald G. Blasberg ${ }^{2,1}$

${ }^{1}$ Department of Radiology, Memorial Sloan-Kettering Cancer Center, New York, NY, USA, ${ }^{2}$ Department of Neurology, Memorial SloanKettering Cancer Center, New York, NY, USA, ${ }^{3}$ Small Animal Imaging Core Facility, Memorial Sloan-Kettering Cancer Center, New York, NY, USA.

Background: Monitoring adoptive cell-based therapies and viral vector tracking in gene therapy are the most challenging and clinically relevant applications of reporter gene imaging. Herpes simplex virus type 1 thymidine kinase gene (HSV1-tk), either wild type or the sr39tk mutant, currently are the most commonly used radiotracer-based reporter genes. However, the main limitation of using radionucleoside analogs is the high intestinal background radioactivity. We hypothesized that endogenous expression of thymidine kinase in bacterial flora could phosphorylate and trap such radiotracers, contributing to high intestinal radioactivity levels. We therefore compared the background activity of those three different radionucleosides analogs and explored different strategies to accelerate the fecal elimination of the radiotracer. Methods: Intestinal radioactivity was assessed by microPET imaging and ex vivo tissue counting following injection of 18F-FEAU, 124I-FIAU or 18F-FHBG in bacteria-free mice raised in a sterile environment and in the same strain of animals kept in a normal environment. We explored the use of an osmotic laxative (Nulytely ${ }^{\circledR}$ ) and/or a 100\% enzymatically hydrolyzed liquid diet (Peptamen(). Results: No significant difference in PET imaging and in ex vivo radioactivity assays were observed between germ free and normal mice. $18 \mathrm{~F}-\mathrm{FHBG}$ intestinal activity was substantially higher than that of $18 \mathrm{~F}$ FEAU and 124I-FIAU; intestine-to-blood activity ratio was 20-fold higher for 18 F-FHBG. The combination of Peptamen ${ }^{\circledR}$ and Nulytely ${ }^{\circledR}$ was most effective; it reduced the dehydration caused by Nulytely ${ }^{\circledR}$ alone, lowered intestinal radioactivity values and increased (2.2-fold) the HSV1-tk transduced xenograft-to-intestine ratio for 18F-FEAU. Conclusions: Intestinal bacteria in germ-free mice have little or no role in contributing to high intestinal radioactivity. The combination of Peptamen ${ }^{\circledR}$ and Nulytely ${ }^{\circledR}$ increases radiotracer elimination by increasing bowel motility without dehydration.

\section{J718}

EVALUATION OF THE THERAPEUTIC EFFICACY

OF VEGFR2-BLOCKING ANTIBODY USING SODIUMIODIDE SYMPORTER MOLECULAR IMAGING IN TUMOR XENOGRAFT MODELS

Su-Jin Cheong, Chang-Moon Lee, Eun-Mi Kim, Hwan-Jeong Jeong, Dong Wook Kim, Seok Tae Lim, Myung-Hee Sohn

Chonbuk National University Medical School, "Geumam-dong, Dukjin-gu, Jeonju, Jeonbuk", Korea, South.

Human specific vascular endothelial growth factor receptor 2 (VEGFR2)-blocking antibody (TTAC-0001) has inhibitory effects in tumor growth and angiogenesis in vivo. The human sodium/iodide symporter (hNIS) gene is very useful as a molecular imaging reporter gene in tumor imaging and radiotherapy. Here, we investigated the evaluation possibility of therapeutic efficacy in hNIS gene transfected tumor xenograft mice by a gamma imaging system during TTAC0001 treatment. hNIS gene transfected human breast cancer cells (MDA-MB-231-hNIS) were injected subcutaneously into the right flank of BALB/c nude mice. When tumor volume reached approximately 200-250 $\mathrm{mm} 3$, we randomly assigned five animals to each of 
four groups. The animals were intravenously injected with the following every three day for 16 days: control-PBS (group 1); TTAC-0001 $50 \mu \mathrm{g} /$ mouse (group 2); $100 \mu \mathrm{g} /$ mouse (group 3); $150 \mu \mathrm{g} /$ mouse (group 4). At one week and two weeks after the first injection of TTAC-0001, gamma imaging was performed to monitor the effects of tumor therapy. We used a CD31 stain to verify therapeutic effects of TTAC-0001. All mice treated with TTAC-0001 demonstrated markedly reduced tumor growth rates compared to the control group. In vivo gamma imaging results showed that at one week after the first injection of TTAC-0001, the 125I uptake in tumor was maximum 1.8 fold lower than PBS-treated tumor. After two weeks of treatment, 125I uptake of PBS-treated tumor was increase 2.1 fold than before one week. 125I uptake of TTAC-0001 treated tumor was maximum 2.3 fold lower than PBS-treated tumor. CD31 staining for angiogenesis showed that vessel formation was significantly decreased in TTAC-0001 treated tumors. This study demonstrates the power of molecular imaging using a gamma imaging system for evaluating the therapeutic efficacy of TTAC-0001 treatment in hNIS gene transfected tumor xenograft mice. Molecular imaging systems can be useful for the evaluation and development of effective diagnostic and/or therapeutic antibodies for specific target molecules.

\section{J719}

\section{ANALYSIS OF THE VEGFR2-luc-KI MOUSE: A REPORTER MOUSE WITH BIOLUMINESCENT VASCULATURE} Scott K. Lyons ${ }^{1}$, Kevin Brindle ${ }^{1,2}$

${ }^{1}$ Dept of Molecular Imaging (room 140), CRUK Cambridge Research Institute, Cambridge, United Kingdom, ${ }^{2}$ Department of Biochemistry, University of Cambridge, Cambridge, United Kingdom.

A reporter mouse has been developed with bioluminescent blood vessels as a consequence of the firefly luciferase gene (luc) being knocked-into the endogenous VEGFR2 locus. Certain non-labelled tumor xenografts can be implanted in these mice subcutaneously and the resultant tumors produce bioluminescence, presumably as a result of the in-growth of host-derived bioluminescent vasculature. Curiously however, not all tumour types appear to behave in the same way when implanted into this mouse line, despite the fact that they grow normally and to an equivalent size. One tumor line, LL2 (Lewis Lung Carcinoma), appears to show a robust induction of bioluminescence over the course of tumor development, whereas other cell lines (e.g HT29 (colorectal adenocarcinoma)) bioluminesce to a lesser extent. We describe here what is currently understood about the extent of induced bioluminescence over the course of tumor development in vivo in relation to underlying tumor biology. In addition to immunohistochemical analysis of the tumors, we present the effects of VEGF overexpression and the administration of an anti-vascular drug on in vivo bioluminescent read-out. Taken together these data should provide a deeper insight into the underlying biological nature of induced bioluminescence in these reporter mice.

$\mathbf{J 7 2 0}$

\section{MicroPET OF MUSCULAR GENE DELIVERY USING HUMAN COPPER TRANSPORTER 1 AS A REPORTER GENE} $\underline{\text { Fangyu Peng }}^{1,2}$, Otto Muzik ${ }^{3,4}$

${ }^{1}$ Radiology, UT Southwestern Med Center, Dallas, TX, USA, ${ }^{2}$ Advanced Imaging Research Center, UT Southwestern Medical Center, Dallas, TX, USA, ${ }^{3}$ Pediatrics, Wayne State University, Detroit, MI, USA, ${ }^{4}$ Radiology, Wayne State University, Detroit, MI, USA.

OBJECTIVES: To study human copper transporter 1 (hCtr1) as a reporter gene for imaging muscular gene delivery in gene therapy. METHODS: To study its potential as a reporter gene for imaging gene delivery, the hCtr1 cDNA was subcloned to construct a plasmid vector and an adenoviral vector encoding hCtr1 as a reporter gene. The hCtr1 plasmid DNA was delivered to the skeletal muscle of right leg of a mouse by intramuscular injection using PolyTAT peptide as a delivery vehicle, with intramuscular injection of control plasmid to the skeletal muscle of left leg. The DNA carrier based on TAT-peptide, amino acid residues $47-57$ of the transactivating transcriptional activator protein from HIV-1, was prepared by oxidation of CGRKKRRQRRRGC peptide. TAT peptide has been ascribed with ability to translocate cargos across cell membranes. Similarly, the adenovirus encoding hCtrl was also injected to the skeletal muscles of right leg for muscular gene delivery, with intramuscular injection of control adenovirus to the left leg. The mice were subjected to MicroPET at 48 hours post injection of the hCtr1 plasmid DNA or the hCtr1 adenovirus, using copper-64 chlorides as a reporter probe. Upon completion of MicroPET, postmortem tissues were harvested, weighted, and counted for radioactivity with a gamma counter. RESULTS: On the MicroPET images, focal increased uptake of copper-64 chlorides was visualized in the muscles of the right leg injected with the hCtr1 plasmid conjugated with PolyTAT peptide, compared with that in the muscles of left leg injected with control plasmid. Slight increase of the copper-64 chlorides radioactivity was detected in the muscles injected with the adenovirus encoding hCtr1, compared with that detected in the muscles of left leg. However, the radiotracer activity detected in the muscles injected with the hCtr1 plasmid DNA conjugated with PolyTAT peptide is significantly higher than that in the muscles injected with the hCtr1 adenovirus, which may be related to lower expression or functional activity of hCtr1 in the muscles transduced by the hCtrl adenovirus. CONCLUSIONS: The hCtrl holds potential as a reporter gene for imaging muscular gene delivery with positron emission tomography using copper-64 chlorides as a reporter probe. It needs further investigation to test other gene delivery vehicles or methods to optimize delivery of the hCtr1 reporter gene to muscular tissues.

\section{$\mathbf{J} 721$}

IMAGING TUMOR GROWTH USING MAGA, MODIFIED FERRITIN SUBUNITS AND MRI

Donna E. Goldhawk ${ }^{1}$, Rene Figueredo ${ }^{2}$, Claude Lemaire ${ }^{3}$, Paula Foster $^{4}$, Savita Dhanvantari ${ }^{1,5}$, James Koropatnick ${ }^{2,6}$, R. Terry Thompson ${ }^{1,7}$, Frank Prato ${ }^{1,7}$

${ }^{1}$ Imaging, Lawson Health Research Institute, London, ON, Canada, ${ }^{2}$ London Regional Cancer Program, London, ON, Canada, ${ }^{3}$ Physics \& Astronomy, University of Waterloo, Waterloo, ON, Canada, ${ }^{4}$ Imaging, Robarts Research Institute, London, ON, Canada, ${ }^{5}$ Diabetes \& Metabolism, Lawson Health Research Institute, London, ON, Canada, ${ }^{6}$ Oncology, University of Western Ontario, London, ON, Canada, ${ }^{7}$ Medical Biophysics, University of Western Ontario, London, ON, Canada.

Overexpression of the iron binding proteins, MagA and ferritin, in cancer cell lines provides a model for examining gene-based contrast for molecular imaging using MRI. A number of challenges exist in the development of reporter gene expression for MRI, including an understanding of how iron is effectively converted by cells into a contrast agent We have analyzed mouse tumors formed from engineered cell lines to correlate MRI signal void with tumour morphology and iron uptake. Human MDA-MB-435 cells, were transfected with either MagA or HF + LF (heavy and light ferritin chains, lacking iron response elements). Clones expressing high levels of the target gene were selected and expanded in culture in the presence or absence of iron supplementation. Subcutaneous injections of 10 million cells into the hind limb of nude mice were monitored for tumor formation over 9 weeks. MRI at $3 \mathrm{~T}$, using a custom built radio frequency coil and gradient insert, was performed on formalinfixed mice: 3D steady state free precession; TR/TE $16 / 8 \mathrm{~ms}$; band width $62 \mathrm{kHz}$; scan time $21 \mathrm{~min}$. The ratio of tumor void volume to total tumor 
volume was compared among samples and correlated to histological analyses (Figure) for iron, apoptosis, necrosis and angiogenesis. Untransfected as well as engineered tumors exhibited a poorly differentiated phenotype, characteristic of aggressive growth from cells that have lost their parent cell properties. The large nuclei with mitotic figures and irregular cell shape are hallmarks of MDA-MB-435. In addition, all tumors showed varying degrees of cell death and blood vessel infiltration. However, tumors which formed subsequent to iron supplementation displayed the greatest Prussian Blue staining. These data indicate that gene-based iron contrast has potential for tumor imaging by MRI.

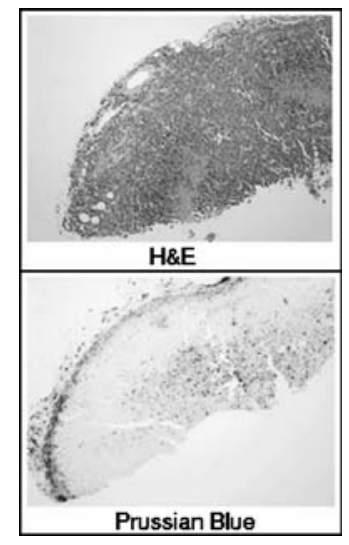

Correlating MagA activity with tumor histology. Tumor sections 3 weeks post-injection show a poorly differentiated phenotype (H\&E) with iron staining (Prussian Blue) along the outer margin and inner core.

\section{J722}

\section{DEVELOPMENT OF DUAL MODALITY FUSION REPORTERS USING HUMAN SODIUM/IODIDE SYMPORTER AND MONOMERIC RED FLUORESCENT PROTEIN}

Kwang Il Kim, Jae Jun Park, Joo Hyun Kang, Yong Jin Lee, Taesup Lee, Inho Song, Gi Jeong Cheon, Chang Woon Choi, Sang Moo Lim Molecular Imaging Research Center, Korea Institute of Radiological and Medical Sciences, Seoul, Korea, South.

Purposes: To develop a noninvasive dual-reporter gene system for nuclear and optical imaging, we constructed fusion reporter vector concurrently expressing human sodium/iodide symporter (hNIS) and monomeric red fluorescent protein (mCherry), and evaluated the function of this fusion reporter system in cancer cells. Methods: Human NIS and mCherry genes were fused by the insertion of hNIS in pmCherry-N1 (Clontech), and transfected into SK-Hep-1 cells to generate SK-Hep-1-hNIS/mCherry cells. After selection with geneticin, the stable transfectant was sorted for positive mCherry expression using dissecting pipette under the fluorescent microscopic view. I-125 uptake assays, fluorescent microscopy, and fluorescent imaging were performed on SK-Hep-1-hNIS/mCherry cells. These cells were inoculated subcutaneously into the flanks of nude mice. After incubation, scintigraphic and fluorescence images were acquired and quantitated. Results: The results of radioiodide uptake and fluorescence intensity correlated well with viable cell numbers. SK-Hep-1-hNIS/ mCherry cells accumulated I-125 up 260 times higher than that of parental cells. Also these cells showed cytoplasmic red fluorescence, while parental cells were not. However, fluorescence intensity was about 10 fold lower than that of SK-Hep-1-mCherry cells. The radioactivity from in vivo scintigraphic images and the intensity from fluorescent images were also found to be proportional to the tumor size. Conclusions: The developed dual-reporter imaging method using hNIS and mCherry genes reflected viable cell numbers and correlated well. This suggests that hNIS/mCherry dual-reporter protein can be a useful tool for noninvasive dual imaging.

\section{J723}

\section{OPTICAL BIOLUMINESCENCE AND NANOSPECT IMAGING OF NIS-FLUC DUAL REPORTER FUSION IN BREAST CARCINOMA CELLS}

Padmanabhan Parasuraman ${ }^{1}$, Ramasamy Paulmurugan ${ }^{2}$, Loyola DSilva $^{1}$, George K. Radda ${ }^{1}$, Kishore Bhakoo ${ }^{1}$

${ }^{1}$ Laboratory of Molecular Imaging, Singapore BioImaging Consortium (SBIC), Singapore, Singapore, ${ }^{2}$ MIPS, Radiology, Stanford University, Palo Alto, CA, USA.

Background: The sodium/iodide symporter (NIS) is over expressed in epithelial cells of thyroid and lactating breast. The basal expression of NIS gene has been detected in $80 \%$ of breast carcinoma specimens. Endogenous NIS expression in thyroid tumor has been used for radioiodine ablation. But it is least explored in breast carcinomas. In this study, we evaluated endogenous NIS expression in a number of breast carcinoma cell lines of estrogen-receptor (ER) positive (MCF7; T47D; BT-549), ER-negative (MB-231; MB-435) and a non-breast control cell-line (293T). Methods: All cell-lines were transfected with either FLUC-NIS fusion (CMV promoter-Fluc-NIS) or NIS transgene (CMV promoter-NIS), and dual imaged by optical bioluminescence (optical CCD camera imaging and luminometry) and nanoSPECT imaging. For nanoSPECT imaging and uptake studies (gamma-counter), we used Tc99m, which is also taken up by via the NIS channel1. Results: The uptake of Tc99m in either the ER positive cells (MCF7, T47D, BT-549) or ER negative cells (MB-231, MB-435) was minimal,, ranging from 5-15\% compared to control cells $(293 \mathrm{~T})$. In the ER positive cells there little variation in the uptake of Tc99m. All cell-lines transfected FLUC-NIS fusion showed significant expression of FLUC activity as measured by luminometry and optical imaging. Moreover, cells transfected with either the bi-fusion construct FlucNIS or NIS alone, in both ER positive and negative lines, showed increased uptake (3-4 fold increase), both by the gamma-counter and by SPECT imaging. However, there was some variation (not significant) between the levels of Tc99m uptake as determined by gamma-counter vs SPECT imaging. Comparisons between NIS-FLUC fusion vs FLUC alone, using optical imaging and luminometry, showed some variation in their respective signals, with Fusion being $40 \%$ less then Fluc alone. However, interestingly the NIS activity in cells expressing FLUC-NIS fusion is almost similar to those with NIS alone. Conclusion: We report the use of NIS reporter constructs that will be useful for both multimodality molecular imaging applications and for examining the potential of $131 \mathrm{I}$ to ablate breast cancer in animals models. The FLUC/optical imaging system can be used monitor the ablation response. Such studies will aid in the translation of these ablation approaches to the clinic. 1-Ryo UY et al. Thyroid Imaging agents: a comparison of I-123 and Tc-99m pertechnetate. Radiology, 148; 819-822, 1983

\section{J724}

\section{NEGATIVE CONTROL OF 5' UNTRANSLATED REGION OF SODIUM IODIDE SYMPORTER GENE TO ENHANCE GENE EXPRESSION}

Chien-Chih Ke ${ }^{1}$, Luen Hwu ${ }^{1}$, Yi-Jie Chen ${ }^{1}$, Wei-Ying Kuo ${ }^{1}$, Ren-Shyan Liu ${ }^{1,2}$

${ }^{1}$ MAGIC, Dept NM, Faculty of Medicine, National Yang-Ming University, Taipei, Taiwan, ${ }^{2} \mathrm{NPCC}$, Dept NM, Taipei Veterans General Hospital, Taipei, Taiwan.

Objective: The rapid radioiodide efflux weakens the efficacy of sodium iodide symporter (NIS) in cancer gene imaging and radioiodide therapy. Untranslated regions (UTR) of specific genes are important regulator of mRNA stability, localization and translation. However, UTRs possess positive regulation in some genes but negative regulation in others. The strength of regulation by UTRs also differs in various genes. This study aim to up-regulate NIS expression by control of UTR. Material and Method: Anaplastic thyroid cancer cell line ARO and non-small cell lung cancer cell line H1299 were transfected with constructs which contains coding region of 
NIS gene (NIS-CDS) equipped with or without 5'UTR of NIS gene. $48 \mathrm{hr}$ after transfection, radioiodide uptake was assayed. Total RNA were isolated from cells and realtime PCR was carried out to evaluate the NIS gene expression. Cells were also transfected with construct containing firefly luciferase equipped with or without 5'UTR of NIS gene. Results: 5'UTR of NIS gene has a negative control in NIS protein translation in both thyroid cancer and lung cancer cells. In the presence of 5'UTR, the luciferase activity had $30 \%$ reduction, compared to that without 5'UTR. In cellular uptake, ARO cells transfected with 5'UTR-containing NIS construct accumulated $90 \%$ of I-125 compared with NIS-CDS construct. H1299 cells transfected with NIS-CDS construct had 3 times of I-125 accumulation compared with 5'UTR-containing NIS construct. Conclusion: To enhance the expression of NIS gene, NISCDS serves as a better expressing construct than that contains 5'UTR

\section{Imaging Molecular and/or Cellular Processes}

\section{Immune Cells}

J725

\section{MONITORING ADOPTIVE CELLULAR IMMUNOTHERAPY} IN GLIOMA PATIENTS USING PET REPORTER GENE IMAGING Shahriar Yaghoubi ${ }^{1}$, Michael Jensen ${ }^{2}$, N. Satyamurthy ${ }^{3}$, Frezghi Habte ${ }^{1}$, David Paik $^{1}$, Johannes Czernin ${ }^{3}$, Sanjiv S. Gambhir ${ }^{1}$

${ }^{1}$ Radiology, Stanford University, Stanford, CA, USA, ${ }^{2}$ Cancer Immunotherapeutics and Tumor Immunology, Beckman Research Institute, Duarte, CA, USA, ${ }^{3}$ Molecular and Medical Pharmacology, UCLA, Los Angeles, CA, USA.
Noninvasive imaging of cell trafficking should allow early assessment of efficacy and potential adverse effects of adoptive cellular immunotherapy in cancer patients. Under a FDA authorized IND $(61,880)$, we have imaged the biodistribution of the positron emission tomography (PET) reporter probe, 9[4-[18F]fluoro-3-(hydroxymethyl)butyl $]$ guanine $\left(\left[{ }^{18} \mathrm{~F}\right] \mathrm{FHBG}\right)$ in glioma patients (two hours after injecting $<7 \mathrm{mCi}$ intravenously). These patients had recurrent glioblastoma multiforme. They were infused autologous cytolytic T lymphocyte (CTL) clones (12 sessions, $10^{7}$ to $10^{8}$ cells each session) into the site of surgically removed recurrent tumor. The CTLs expressed Herpes Simplex virus type 1 thymidine kinase (HSV1-tk) PET reporter gene (confirmed by Ganciclovir sensitivity and tritiated Penciclovir uptake assays) and IL-13 zetakine. We previously reported the case of a glioma patient imaged with $\left[{ }^{18} \mathrm{~F}\right] \mathrm{FHBG}$ only after cell infusions (Yaghoubi et. al. Nature Clinical Practice Oncology 6(1): 53-58). In this patient we observed tumor signals that were about 2.6 fold higher, when compared to tumor signals in control glioma patients who had not been infused CTLs. Biopsy had confirmed presence of CTLs at tumor resection site as well as in a newly formed tumor in the corpus callosum. Here, we report imaging another patient who was imaged both before and after CTL infusions with $\left[{ }^{18} \mathrm{~F}\right] \mathrm{FHBG}$. The Figure illustrates brain $\left[{ }^{18} \mathrm{~F}\right] \mathrm{FHBG}$ PET images pre and post CTL infusions. Comparing Tumor/brain intensity ratios, the ratio is 1.6 fold higher after cell infusions. Comparing tumor/meninges ratios, the ratio is 4.2 fold higher after cell infusions. Another observed difference is that in pre-cell infusions the signal is observed on the walls of the tumor resection site; whereas after infusions, the signal emanates from the whole resection site. Additional studies are planned for imaging patients pre and post CTL infusions that should allow statistical analysis. Meanwhile, these recent data, using the prescan of the same patient as a control image provide further demonstration of PET reporter gene based imaging of therapeutic cells in cancer patients.

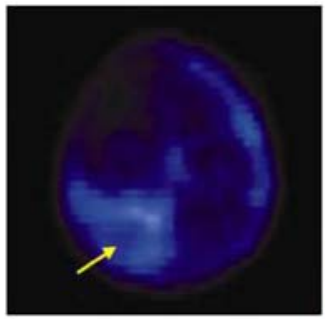

Transverse

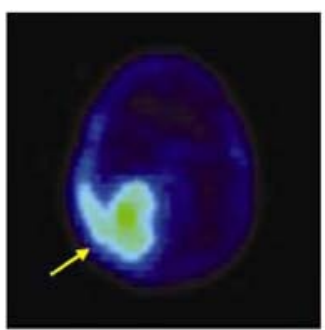

Arrows indicate the tumor resection site where CTLs had been Injected.

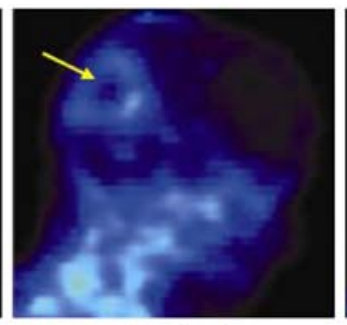

Sagital
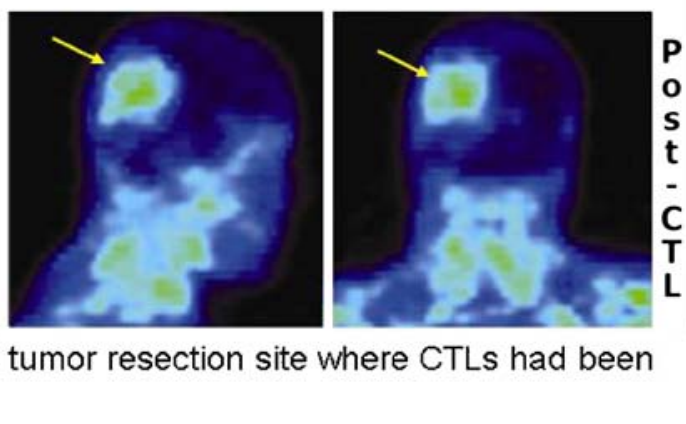

SUV

0.3

0.2

0.1

0.05
J726

NON INVASIVE SMALL ANIMAL PET IMAGING OF TH1 CELL TRAFFICKING IN MOUSE MODELS FOR INFLAMMATION USING INTRACELLULAR [64Cu]PTSM LABELLING

Christoph M. Griessinger ${ }^{1}$, Stefan Wiehr ${ }^{1}$, Daniel Bukala ${ }^{1}$, Martin Röcken $^{2}$, Walter Ehrlichmann ${ }^{3}$, Gerald Reischl ${ }^{3}$, Manfred Kneilling ${ }^{2}$, Bernd J. Pichler ${ }^{1}$

${ }^{1}$ Deparmtent of Radiology; Laboratory for Preclinical Imaging and Imaging Technology of the Werner Siemens-Foundation, University of Tübingen, Tübingen, Germany, ${ }^{2}$ Department for Dermatology, University of Tübingen, Tübingen, Germany, ${ }^{3}$ Radiopharmacy, University of Tübingen, Tübingen, Germany.
T-helper cells play an important role in the mediation of autoimmune diseases. To get a better understanding about the migration properties of $\mathrm{T}$ cells, high sensitive multimodality imaging is an important tool for preclinical studies. $T$ cell migration kinetics, homing or proliferation sites in inflammation models are still unknown. In previous work we focused on labelling strategies for Th1 cells using $64 \mathrm{Cu}$ and investigated potential effects to cell viability of functionality. The goal of the continuative studies was to visualize the migration of ovalbumin-T cell-receptor transgenic Thelper cells (OVA-Th1) in an animal model for delayed-type hypersensitivity reaction (DTHR) and lung inflammation. We used the lipophilic radioactive marker [64Cu]PTSM as intracellular label of the Th1 cells for PET. Previous results considering the effects of this labelling strategy on viability and functionality of the target cells revealed that in vivo imaging of 
Th1 cells is possible for up to 48 hours. BALB/c mice were sensitized at the abdomen with the hapten TNCB and one week later, cutaneous DTHR was elicited at the right ear. To induce OVA-specific lung inflammation the mice were sensitized i.p. with OVA and were challenged intranasal twice four weeks later to induce lung inflammation. OVA-Th1 cells were isolated from the spleen and lymph nodes of DO.11.10 mice, activated with OVA and cultured for $12-14$ days. $2 \mathrm{E}+06$ cells $/ \mathrm{ml}$ Th1 cells were labelled with $0.7 \mathrm{MBq}[64 \mathrm{Cu}]$ PTSM for 3 hours and injected (i.p., $1 \mathrm{E}+07[64 \mathrm{Cu}] \mathrm{Th} 1$ cells/animal) in diseased and control mice. Static PET-scans in combination with CT, autoradiography, and biodistribution-analysis were performed 24 and 48 hours after Th1 cell injection. Analysing T cell trafficking in DTHRmice we detected a 2 -fold enhanced $[64 \mathrm{Cu}] \mathrm{Th} 1$ cell accumulation in the cervical draining lymph node of the TNCB-treated right ear compared to the lymph node of the untreated left ear. Ex vivo FACS-analysis confirmed accumulation of OVA-Th1 cells in the draining right cervical lymph node. Analysing OVA-specific Th1 cell migration in lung inflammation we detected accumulating [64Cu]OVA-Th1 cells in lung tissue already $24 \mathrm{~h}$ after the final challenge. Data were further confirmed ex vivo by biodistribution and autoradiography, $24 \mathrm{~h}$ and $48 \mathrm{~h}$ after OVA-challenge. As a new approach to investigate Th1 cell migration for longer time periods, we labelled Th1 cells with an [111In]DOTA-coupled CD3-monoclonal antibody for SPECT-imaging. Using this method, we were able to visualize subcutaneously injected OVA-Th1 cells in vivo for up to 216 hours.

\section{J727}

\section{LABELLING DENDRITIC CELLS WITH FERIDEX HAS IMPLICATIONS FOR IN VIVO MIGRATION AS ASSESSED WITH CELLULAR MAGNETIC RESONANCE IMAGING}

Sonali N. de Chickera $^{1,2}$, John Barrett ${ }^{2}$, Sakina Thawer, ${ }^{2,5}$, Jonatan A. $\mathrm{Snir}^{3,4}$, Roja Rohani ${ }^{3,4}$, Paula Foster ${ }^{3,4}$, Gregory A. Dekaban ${ }^{2,5}$ ${ }^{1}$ Anatomy and Cell Biology, University of Western Ontario, London, ON, Canada, ${ }^{2}$ Biotherapeutics, Robarts Research Institute, London, ON, Canada, ${ }^{3}$ Biomedical Physics, University of Western Ontario, London, ON, Canada, ${ }^{4}$ Imaging, Robarts Research Institute, London, ON, Canada, ${ }^{5}$ Microbiology \& Immunology, University of Western Ontario, London, ON, Canada.

Dendritic cells (DCs) serve as important sentinels in the immune system and have the ability to stimulate potent adaptive immune responses. For this reason, the DC-based cancer vaccine shows much promise in the field of cell-based cancer immunotherapies. In this context, DCs serve as a natural adjuvant to deliver antigen to naïve $\mathrm{T}$ cells in vivo. As a result, migration of injected DCs to regional lymph nodes (LNs) is a critical step for $\mathrm{T}$ cell priming. Accordingly, immunotherapeutic applications of in vitro-generated DCs require reliable, safe, and non-invasive methods experimentally in mice and in a clinical setting to confirm therapeutic numbers of cells are reaching a targeted LN. This study employs cellular magnetic resonance imaging (MRI) to track DC migration. In order to distinguish cells of interest from surrounding tissues, Feridex, a superparamagnetic iron oxide (SPIO) particle, was used to label murine bone marrow-derived DCs (BMDCs) in vitro. Our data show that the presence and intensity of Feridex-induced signal voids in MR images correlates with the presence of BMDCs in the popliteal LNs over time. While our previous data indicate that Feridex has no significant effect on BMDC phenotype or maturation, it appears to decrease the efficiency of in vivo BMDC migration to popliteal LNs by $\sim 30 \%$. Time course and competition experiments involving Feridex-labelled and unlabelled BMDCs indicate Feridex likely physically impedes migration. Using a magnet-based method that doubles the amount of Feridex taken up by BMDCs, however, did not lead to increased sensitivity with respect to detecting smaller numbers of DC in target LNs. In contrast, magnetic separation of Feridex- labelled BMDCs from unlabelled BMDCs leads to a greater signal void intensity, indicating that more Feridex-labelled BMDCs reached the LNs. By maturing BMDCs in the presence of a TLR agonist, PGE-2, and cytokine cocktail, Feridex-labelled BMDC migration to LNs is restored to unlabelled control levels. We have also optimized the conditions required to transduce DC with different viral vectors (recombinant adenovirus, myxoma virus and herpes simplex virus type 1) in the presence and absence of Feridex. The optimized viral transduction protocol involves loading immature BMDCs with viral vectors prior to labelling with Feridex, which allows for improved recombinant gene expression. These findings could have implications regarding therapeutic numbers of Feridex-labelled DCs required for effective anti-tumour responses in clinical patients.

\section{J728}

\section{NEAR-INFRARED OPTICAL IMAGING OF IMMUNOTHERAPEUTIC CELLS -BASED CANCER THERAPY USING NATURAL KILLER CELLS LABELED WITH FLUORESCENT NANOCRYSTALS Yong Taik Lim, Young-Woock Noh, Bong Hyun Chung} Bionanotechnology research center, KRIBB, Daejeon, Korea, South.

We described the development of near-infrared optical imaging technology for the monitoring of immunotherapeutic cells-based cancer therapy using natural killer (NK) cells labeled with fluorescent nanocrystals. Although NK cell-based immunotherapeutic strategies have drawn interest as potent preclinical or clinical methods of cancer therapy, there are few reports documenting the molecular imaging of NK cell-based cancer therapy, primarily due to the difficulty of labeling of NK cells with imaging probes. Human natural killer cells (NK92MI) were labeled with anti-human CD56 antibody-coated quantum dots (QD705) for fluorescence imaging. The FACS analysis showed that the labeled NK92MI cells with anti-human CD56 antibody-coated QD705 have no effect on the cell viability. The effect of anti-human CD56 antibody-coated QD705 labeling on NK92MI cell function was investigated by measuring interferon gamma (IFN-gamma)production and cytolytic activity. Finally, the NK92MI cells labeled with antihuman CD56 antibody-coated QD705 showed similar therapeutic effect as that of naïve NK92MI cells. The image of intratumorally injected NK92MI cells labeled with anti-human CD56 antibody-coated could be acquired using near-infrared optical imaging both in vivo and in vitro. This result demonstrates that the immunotherapeutic cells labeled with fluorescent nanocrystals can be a versatile platform for the effective tracking of injected therapeutic cells using optical imaging technology, which is very important in the cell-based cancer therapy.

\section{J729}

\section{SUPERPARAMAGNETIC OXIDES DO NOT AFFECT DENDRITIC CELL FUNCTION IN AN IN VIVO} PROLIFERATION ASSAY AND AS A TUMOR VACCINE Richard Tavaré $^{1}$, Pervinder Sagoo ${ }^{2}$, Gopal Varma ${ }^{1}$, Tobias Schaeffter ${ }^{1}$,

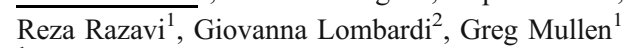

${ }^{1}$ Division of Imaging Sciences, King's College London, London, United Kingdom, ${ }^{2}$ Department of Nephrology and Transplantation, King's College London, London, United Kingdom.

Magnetic Resonance Imaging (MRI) provides insightful information that can be combined to give anatomical, temporal and quantitative data for cell tracking studies. When used for cellular therapy, the migration 
of dendritic cells (DCs) from the periphery to lymph nodes (LNs) is critical in that DCs must interact with T cells in lymph nodes to induce their immunological effect. In this study, murine dendritic cells are labeled with the superparamagnetic iron oxide (SPIO) Endorem ${ }^{\circledR}$ and this label has the potential of being harmful to cellular function and viability. To date most analysis of DC function post labeling has been in vitro while in vivo analysis has yielded varying results [1,2]. Thorough in vitro characterization of labeled cells was performed, including trypan blue, MTT, phenotype analysis by flow cytometry, and in vitro proliferation assays. In order to analyzed the effect of SPIO labeling in vivo, murine bone marrow derived DCs we used in an in vivo functional assay to monitor the ability of SPIO labeled and unlabeled, ovalbumin pulsed DCs to both migrate from the heel after a subcutaneous injection to the popliteal LN and initiate the proliferation of CFSE-labeled OT-II CD4+ T cells. We also look at the ability of SPIO labeled and unlabeled DCs to be used as a vaccine for ovarian cancer. In these experiments, ovalbumin pulsed DCs are injected four days before the intravenous injection of an ovalbumin expressing B16 melanoma tumor cell line. Two weeks after tumor cell injections, the lungs are isolated and tumor nodules are counted to show vaccine efficacy. Using serial MRI (3T Philips Achieva) on days 0, 1, 2 and 4, we show that SPIO labeled cells do migrate to the popliteal $\mathrm{LN}$ within 24 hours by monitoring the increased hypointensity due to the injected SPIO labeled cells. Also, the growth in LN over the 4 days is the same it the DCs are SPIO labeled or unlabeled. The SPIO has no effect on the ability of the DCs to initiate T cell specific proliferation as shown by the calculated proliferation index of CFSE dividing T cells. As a cancer vaccine, SPIO labeled DCs did not show a difference when compared to unlabeled DCs to eradicate the growth of tumor nodules. This shows that SPIO labeled DCs are efficient at initiating a strong, antigen specific autologous response for an ovalbumin expressing tumor cell line. 1. Baumjohann D, et al. Eur. J. Immunol. 2006;36(9):2544-55. 2. Dekaban GA, et al. J. Immunother. 2009;32(3):240-51.

\section{$\mathbf{J 7 3 0}$}

FEASIBILITY OF HUMAN SODIUM IODIDE SYMPOTER GENE AS A REPORTER GENE TO MONITOR THE DC CELL VACCINE MIGRATION IN IMMUNOCOMPRISED MICE

Lee You La, Yong Hyun Jeon, Ahn Byeong-Cheol, Lee Sang Woo, Lee Jaetae

Nuclear Medicine, School of Medicine, Kyungpook National University, Daegu, Korea, South.

Objective: In this study, we investigated the feasibility of sodium iodide symporter gene as a reporter gene to monitor the DC cell vaccine migration in immunocompetent mice. Methods: 0, 20, 40, 50, 100, $200 \mathrm{MOI}$ of recombinant adenovirus that expresses hNIS and EGFP under the control of a CMV promoter (Ad-hNIS-EGFP, ANE) was transduced to DC cell vaccine. Functional gene expression of hNIS and EGFP was confirmed using I-125 uptake and flow cytometry. ANE (100 MOI) infected DC cells and non-infected DC cells were intradermally administered into thigh of right hind leg. Infected DC cell vaccine was serially monitored for 1, 7, 24,
$96 \mathrm{hr}$ after cell injection using micro-PET and gamma camera. After imaging acquisition, biodistribution study was performed following Tc$99 \mathrm{~m}(10 \mathrm{uCi})$ injection to mice immunized with DC cell vaccine. Results: I-125 uptake was increased in ANE infected cells in a virus dosedependent manner. Flow cytometry showed the enhanced EGFP gene expression in ANE infected cells in a virus dose-dependent manner. ANE infected DC cell vaccine was monitored at injection site by both microPET imaging and scintigrapy for 4 days post- injection of DC cell vaccine. Biodistribution results showed higher uptake of Tc-99 $\mathrm{m}$ in secondary immune organs (draining lymph nodes and spleen) and injection site of mice immunized with ANE infected DC cell vaccine than that of mice immunized with non-infected DC cell vaccine. Conclusions: Our finding suggests that hNIS could be used as a beneficial reporter gene to monitor the migration of DC cell vaccine, and subsequently hNIS expressing DC cell vaccine may be a helpful bio-material for determination of optimal injection site of DC cell vaccine administration.

\section{J731}

\section{CHARACTERIZATION OF MPIO LABELED BONE MARROW} DERIVED MURINE MACROPHAGES

\section{Kevin Tang}

Yale University, New Haven, NJ, USA.

Cellular MRI is a newly emerging field of imaging research that combines the ability to generate high resolution in vivo MRI data with contrast agents for labeling cells. The use of micron sized iron-oxide particles (MPIOs) for cell-specific imaging by MRI has become increasingly popular for its increased iron content per labeled cell over smaller particles, and has been demonstrated in a number of different disease models. Early detection of the immune response is essential for identification and early treatment of diseases such as HIV, cancer, and stroke. However, the early immune response has not been wellcharacterized. Macrophages are key players in the innate immune response and important markers of local inflammation. Being highly phagocytic, macrophages are highly suitable for use with MPIOs for single cell detection by MRI. Here, we evaluated the effects of MPIO labeling on bone marrow derived murine macrophages ability to secrete cytokines, maintain phagocytotic ability and migratory capacity. MPIOs used were polystyrene/divinyl benzene coated and $1.63 \mu \mathrm{m}$ in diameter. Labeling with MPIOs on its own did not induce production of TNF- $\alpha$ or IL-12. Furthermore, MPIO labeling did not inhibit the macrophages ability to produce TNF- $\alpha$ and IL-12 in the presence of LPS, an endotoxin known to induce cytokine production by macrophages (Figure 1a). Labeled macrophages were also tested for the ability to continue phagocytosis. Green fluorescent MPIOs were incubated with macrophages for $4 \mathrm{~h}$ followed by incubation with red fluorescent MPIOs for an additional 4 hours. Figure 1b shows macrophages with two different MPIOs internalized within them, indicating the ability to continue phagocytosis after labeling. To test for migration, transwell migration assays were performed on labeled macrophages using MCP-1 as the chemoattractant. Labeled macrophages were found to maintain their migratory capacity across a $5 \mu \mathrm{m}$ pore size 24 -well transwell insert.

\begin{tabular}{|c|c|c|c|}
\hline Samples & $\begin{array}{c}\text { TNF- } \boldsymbol{\alpha} \\
(\mathbf{n g} / \mathbf{m l})\end{array}$ & $\begin{array}{c}\text { IL-12 } \\
(\mathbf{n g} / \mathbf{m l})\end{array}$ & $\begin{array}{c}\text { Transwell } \\
\text { migration }\end{array}$ \\
\hline 2ug/m1LPS & $2.061+/-.034$ & 0.169 & - \\
\hline 2ug/m1LPS + MPIOs & $1.566+/-.136$ & 0.142 & - \\
\hline MPIOs & $-0.03+/-0.031$ & $.045+/-.048$ & $\gamma$ \\
\hline Control & $-.010+/-.001$ & $.023+/-.012$ & $\gamma$ \\
\hline
\end{tabular}

Figure 1: Left) Table showing cytokine release data for labeled and unlabeled cells, both with and without LPS treatment Also shown are the results from transwell migration assays. Right) Three color fluorescence image showing blue -DAPI stained nucleus, green - first labeling MPIOs, and red - second wave of MPIOs. 


\section{J732}

\section{A SOPHISTICATED FLOW SYSTEM TO INVESTIGATE EARLY ATHEROSCLEROSIS USING MOLECULAR CONTRAST AGENT}

$\underline{\text { Anne-Lise Aulanier }}^{1,6}{ }^{\text {, Linda B. Andersen }}{ }^{3,6}$, Kristina D. Rinker ${ }^{2,4}$, Robert Shepherd $^{2,4}$, Richard Frayne ${ }^{1,5}$

${ }^{1}$ Electrical and Computer Engineering, University of Calgary, Calgary, $\mathrm{AB}$, Canada, ${ }^{2}$ Centre for Bioengineering Research and Education, University of Calgary, Calgary, AB, Canada, ${ }^{3}$ Radiology, University of Calgary, Calgary, AB, Canada, ${ }^{4} \mathrm{Chemical}$ and Petroleum Engineering, University of Calgary, Calgary, AB, Canada, ${ }^{5}$ Clinical Neurosciences, University of Calgary, Calgary, AB, Canada, ${ }^{6}$ Seaman Family MR Research Centre, Foothills Medical Centre, Calgary, AB, Canada.

Introduction: Atherosclerosis is a condition in which plaques build up in the innermost layer of the arterial wall resulting in thickening and hardening of arteries. Poorly understood and defined predisposing stimuli that increase the endothelium permeability are believed to be the initiating step for atherosclerotic lesion development, followed by the adhesion of circulating monocytes to the surface of the endothelial cells. Monocyte adhesion to the endothelium and accumulation/differ- entiation into macrophages within the intima are the events that inspired development of our in vitro model system, and our interest in early lesion detection. Methods: The experimental setup included human endothelial cells cultured on a glass slide and placed within a flow chamber. The cells were activated by a 4-h incubation with the cytokine TNF- $\alpha$ prior to initiating flow. A pump generated a steady flow of MM6 monocytes $(0.25 \mathrm{ml} / \mathrm{min}, 3 \mathrm{e} 5$ cells $/ \mathrm{mL})$ over the endothelial cells for $1 \mathrm{hr}$. Separately, unlabeled MM6 were diluted in a 96 well plate and imaged on a 3 T MR scanner. Results: Circulating MM6 were observed to adhere to the endothelial surface and accumulate on a glass slide (Fig). Unlabeled MM6 were also detected via MR (8e6 cells, Fig). Different imaging contrast agents are under investigation to evaluate the potential of directly labeling monocytes and/or macrophages with molecular contrast agents in vitro. Further investigation will determine whether labeled monocytes can be visualized localizing to atherotic arterial endothelium. Conclusion: We have demonstrated cell adhesion between activated endothelial cells and circulating MM6. Additionally, we have shown MR visualization of unlabeled MM6 in a 96 well plate. These results suggest the feasibility of using a MR compatible in vitro flow system to characterize monocyte recruitment to activated endothelium. This approach has the advantage of easily and inexpensively enabling experimental determination of the complex etiology of early atherosclerotic lesions.
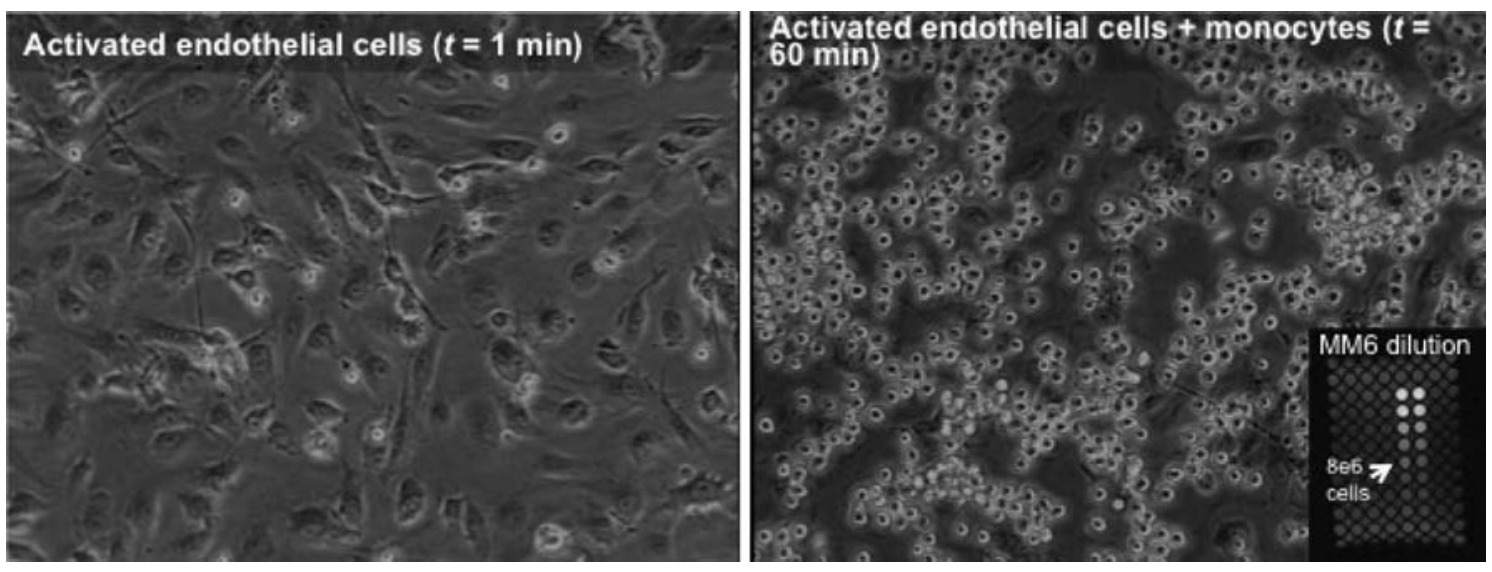

\section{UPTAKE AND TRAFFICKING OF FUNCTIONALIZED CDSE NANOPARTICLES IN LIVING IMMUNE CELLS}

$\underline{\text { Jerilyn A. Timlin }}^{1}$, Jesse Aaron ${ }^{1}$, Adrienne Greene ${ }^{2}$, George D. Bachand $^{2}$

${ }^{1}$ Bioenergy and Defense Technologies, Sandia National Labs, Albuquerque, NM, USA, ${ }^{2}$ Center for Integrated Nanotechnology, Sandia National Labs, Albuquerque, NM, USA.

Nanomaterials, including semiconductor quantum dots (QDs), have gained widespread interest for use as optical biological tracers with demonstrated potential for a variety of applications including cancer research. Nanomaterials can elicit unique and potentially deleterious physiological responses that are not observed with bulk materials of the same type. However, systematic studies of QD toxicology have lagged behind their development and application. As part of a larger effort to assess the toxicology of engineered nanoparticles, we have employed multispectral and hyperspectral fluorescence microscopy and imaging techniques to study surface functionalized (both $-\mathrm{COOH}$ and -NH2) CdSe QD interactions with living immune cells. Using high-speed, dual-color total internal reflectance fluorescence (TIRF) microscopy we interrogate nanoparticles contained in the cell membrane and study their membrane diffusion characteristics and internalization rates. Single QD tracking on the cell surface reveals a range spanning $>10$ orders of magnitude in effective $2 \mathrm{D}$ diffusion constants, indicating a highly complex, heterogeneous environment. Further, QD internalization rates were found to be on the order of $10 \mathrm{~min}$ in cultured Rat basophilic leukemia mast cells (RBL-2H3) cells. We also employ hyperspectral confocal microscopy and multivariate image analysis to study the kinetics and distribution of QDs after internalization. QDs of different sizes can be discriminated on the basis of their spectral emission and thus this method enables localization of several different quantum dot sizes simultaneously with subcellular spatial resolution. We will present our imaging results as a function of QD size, surface properties, and long term cytotoxicity to give a greater mechanistic understanding of nanomaterial interactions in biological systems. 


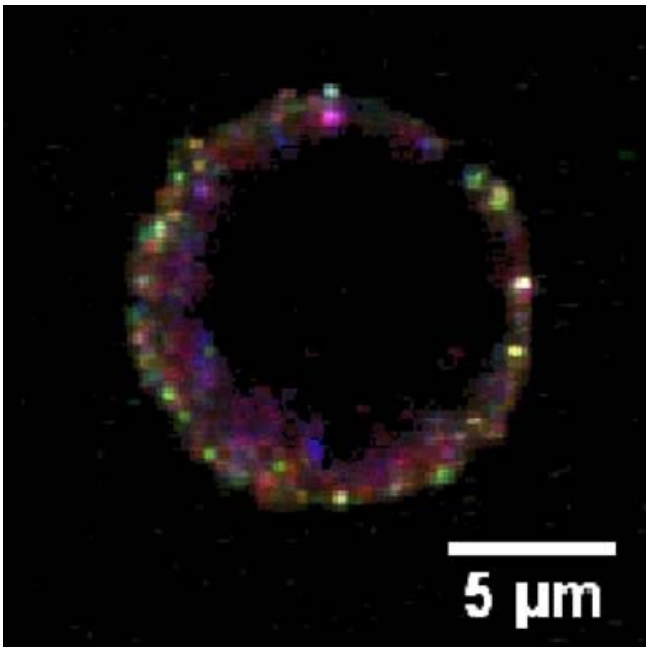

Hyperspectral confocal microscopy shows the distribution of three COOH-functionalized QD populations (QD545 in blue, QD605 in red, and QD655 in green) in a live cell

\section{Imaging Molecular and/or Cellular Processes}

\section{Infection}

\section{J734}

\section{TECHNITIUM-99M LABELED HYDROXYPROPYL B-CYCLODEXTRIN (TC-99M HPBCD) AS A LIGAND FOR BACTERIAL MEMBRANE PROTEINS AND AN IMAGING TOOL FOR VASCULAR PROSTHESIS INFECTION Geetanjali Arora $^{1}$, Guru P. Bandopadhyaya ${ }^{1}$, Jaya Shukla ${ }^{1}$, P. P. Kotwal ${ }^{2}$}

${ }^{1}$ Department of Nuclear Medicine, All India Institute Of Medical Sciences, Delhi, India, ${ }^{2}$ Dept of Orthopaedics, All India Institute of Medical sciences, Delhi, India.

Infection remains a major cause of patient morbidity and mortality inspite of our increased understanding of microorganisms and increased availability of antimicrobial therapy. The role of radionuclide imaging in the evaluation of suspected infection patient varies with the situation. In painful joint replacement, radionuclide studies are the primary diagnostic imaging modality for differentiating infection from other causes of prosthetic failure. The vascular prosthesis infection is most commonly caused by staphylococcus. HP $\beta C D$ is a chemically modified $\beta$-cyclodextrin (oligosaccharides) and is suitable for parenteral use. HP $\beta C D$ tightly binds to maltose binding protein of bacterial membrane without being metabolized or producing any chemotactic response. These properties of HP $\beta C D$ can be exploited for imaging septic infection after labeling with an imagable radionuclide. In the present study, HP $\beta C D$ was labeled with Tc-99m by Stannous Chloride reduction method at $\mathrm{pH} 6.5$ and 7.4. The labeled compound was characterized and labeling yield was determined by ITLC method using methyl ethyl ketone as a solvent. Normal biodistribution of the radiopharmaceutical was observed in Wistar albino rats $(n=3 ; 250+25 \mathrm{~g}$ each). The efficacy has been tested in patients of known infection as well as in patients without infection. Results indicated that a maximum labeling yield $(>95 \%)$ was achieved at $\mathrm{pH}$ 6.5. Rf value of Tc-99m HPBCD, as determined by ITLC method, was in the range of $0.18-0.19$. The radiolabelled compound was stable for 6 hrs. The in vivo tracer distribution studies, on rats, revealed blood pool activity in heart and kidneys in the first 5 min. Thyroid was not seen indicating absence of any free pertechnetate. On delayed images activity was seen only in kidneys and bladder indicating renal route of excretion. The tracer uptake was seen in areas of infection when injected intravenously to patients of known prosthesis infection. No uptake was seen in patients without infection, thus establishing the efficacy of the radiopharmaceutical in detection of infections. To conclude, HP $\beta C D$ could be successfully labeled and characterized. The initial results were suggestive of the potential application of Tc- $99 \mathrm{~m}$ HP $\beta C D$ as an infection imaging agent because of its binding to the bacterial periplasmic protein. It has the advantage of being costeffective; easy availability and easy method of preparation as compared to other infection imaging agents such as Ga-67 citrate; radiolabelled leukocytes and F-18 FDG that are presently being used in nuclear medicine.

\section{$\mathbf{J} 735$}

BIOIMAGING AND THERAPEUTIC SCREENING OF NOVEL ANTIBIOTICS IN MURINE MODELS OF PSEUDOMONAS LUNG INFECTION

$\underline{\text { Neil A. McDonald }}^{1}$, Geraldine Escher ${ }^{2}$, Nicolaos Avlonitis ${ }^{1,2}$, Tom S. Wilkinson $^{3}$, John R. Govan ${ }^{1}$, Haslett Chris ${ }^{1}$, Mark Bradley ${ }^{2}$, John Simpson $^{1}$, Kevin Dhaliwal ${ }^{1}$

${ }^{1}$ Centre for Inflammation Research, University of Edinburgh, Edinburgh, United Kingdom, ${ }^{2}$ Department of Chemistry, University of Edinburgh, Edinburgh, United Kingdom, ${ }^{3}$ Department of Microbiology, Swansea University, Swansea, United Kingdom.

Nosocomial infection is a major cause of morbidity and mortality, confounded by the significant emergence of multi drug resistant pathogens. In order to enable the screening and validation of alternative antibiotic regimes, we developed a reproducible model of murine pneumonia using a laboratory strain of Pseudomonas aeuriginosa genetically engineered to express luciferase. Optical imaging of relative bacterial load in vivo in situ was multiplexed with fluorescent probes to detect inflammatory enzymatic activity and pulmonary vascular leak. Initiation of pneumonia by direct intratracheal intubation of mice was followed serially in cohorts of animals in which antibiotic regimes were administered. Initially, the model was validated using intramuscular administration of ceftazidime. Screening was performed with reflectance and tomographic optical imaging in the setting of established infection. Pathogen specific phage was isolated by selective culture, purified and tagged with near infra red flurophore. In vitro and in vivo studies demonstrated the utility of using phage in pneumonia without any adverse toxic effects. The early bactericidal effects of phage were accompanied by attenuated inflammatory events as evidenced by optical imaging. We also tested small peptide antibiotics (secretory leucocyte protease inhibitor; SLPI and elafin) which similarly showed bactericidal effects in vivo and in vitro but were accompanied by increased neutrophil ingress and inflammatory activity in the lung. Pre instillation of adenovirus encoding elafin/SLPI into the murine lung was used as a technique to augment their pulmonary specific expression. Similar to exogenous peptide administration, transgene expression was associated with increased neutrophil ingress. This highlighted the pleiotropic nature of these small peptides as antibiotics and chemoattractants for innate immune cells. This supports studies we have conducted in the past. Conclusions: We report the development of murine models of lung infection that permit the rapid screening of novel antibiotics using optical imaging platforms.

\section{J736}

DOSIMETRY OF TC99M ANTIGRANULOCYTE MOLECULAR IMAGING IN LOWER EXTREMITIES INFECTION Maria E. Lyra ${ }^{1,2}$, George S. Limouris ${ }^{2}$

${ }^{1}$ Radiology, Radiation Physics Unit, University of Athens, Athens, Greece, ${ }^{2}$ Nuclear Medicine, University of Athens, Athens, Greece. 
Aim: The localization and delineation of sites of focal inflammation are crucial in the management of patients with infectious processes. The scintigraphic detection of infection and inflammation is in last years successfully covered by Tc99m -antigranulocyte Fab' fragment molecular imaging in gamma camera. Tc99m-labeled Fab' fragment of IMMU-MN3, is an immunoglobulin GI murine monoclonal antibody. It reacts strongly with nonspecific cross-reacting antigen present on human granulocytes. In this study, patient specific dosimetric calculations of radiopharmaceutical Tc99m- Anti-Granulocyte Fab' fragment imaging of lower limbs are evaluated. Methods: The practical dosimetric individualized study was based on 22 patients that were referred for a radionuclide scan on the suspicion of an infectious lesion in the extremities. The Fab' fragment is provided in a ready to label lyophilized kit. Tc99m -antigranulocyte Fab' has a biologic half-life of $10.5 \mathrm{~h}$. Planar imaging was completed at $3 \mathrm{~h}, 6$ $8 \mathrm{~h}$ and $24 \mathrm{~h}$ post injection by an El Scint SPX4 SPECT gamma camera. The distribution and dosimetry is evaluated using the information from simultaneous anterior and posterior whole body scintigrams, as well as from tomographic SPECT 360 degrees views. The geometric means of conjugate views and ROI analysis are used to determine target organ and pathologic area uptakes, mean residence times and absorbed radiation dose estimates. The effective half-life of the radiopharmaceutical was calculated from the obtained attenuation curves of the imaging data. 3 regions of interest (ROI) were drawn on each one of the images: One at the pathologic area, a ROI for background counts and a ROI close to the lesion area for the evaluation of the projection of the infectious area. An index Infection Projection Ratio (IRP) of the lesion was calculated.Results: Based on MIRD schema, the residence time of the radiopharmaceutical to the source organ as well as its cumulative activity was calculated for each administration of the cases. The absorbed dose to the inflammation site and most critical organs were calculated for each case. The indices IRP represent the ratio of the counts in the pathologic area to the counts of a normal area adjacent to the infected tissue. Conclusions: Our results demonstrate of Tc-99m Anti-Granulocyte Fab' a difference in absorbed dose and mean residence time in normal and pathologic areas. The calculated indices IPR contribute to the conclusion that Tc99m -antigranulocyte Fab' demonstrates the full extent of a lesion specifically for each patient

\section{$\mathbf{J 7 3 7}$}

\section{SINGLE PARTICLE TRACKING THE HEPATITIS C VIRUS RECEPTOR COMPLEX}

$\underline{\text { Helen J. Harris }}{ }^{1}$, Caroline Clerte ${ }^{2}$, Christopher J. Mee ${ }^{1}$, Michelle J. Farquhar $^{1}$, Peter Balfe ${ }^{1}$, Pierre-Emmanuel Milhiet ${ }^{2}$, Jane McKeating ${ }^{1}$ ${ }^{1}$ Institute of Biomedical Reseach, University of Birmingham, Birmingham, United Kingdom, ${ }^{2}$ Centre de Biochimie Structurale, Université de Montpellier, Montpellier, France.

Hepatitis $\mathrm{C}$ virus (HCV) entry is dependent on host cell expression of scavenger receptor BI (SR-BI), tetraspanin CD81 and tight junction (TJ) proteins Claudin-1 (CLDN1) and Occludin (OCLDN). SR-BI and CD81 interact with $\mathrm{HCV}$ particles, however the exact role(s) played by each of the 'entry' factors in the multi-step entry process is unclear. Hepatocytes are the primary reservoir supporting HCV replication in the liver and are highly polarized, with TJs separating their basolateral (BL) and apical domains. TJ proteins form homo- and hetero-dimeric complexes both within and between adjacent cells, raising questions as to their accessibility to $\mathrm{HCV}$ particles and their role in the virus entry process. We have exploited novel techniques such as FRET, TIRF and single particle tracking (SPT) with the human hepatocyte derived HepG2 cell line to characterise HCV receptor complex association and motility in polarized cells. Transduction of HepG2 cells with monomeric fluorescent tagged CD81, CLDN1 and OCLDN enabled us to study protein-protein interaction(s) at the $\mathrm{BL}$ and $\mathrm{TJ}$ membranes. CLDN1 and OCLDN were enriched at TJs and associated with a defined stoichiometry at both the TJ and BL membranes. In contrast, CD81 expression was reduced at the TJs and was only found to associate with CLDN1 and OCLDN at the BL membrane. We hypothesize an important role for BL expressed forms of CD81-CLDN1 in the lateral movement of particles leading to virus internalization. We recently demonstrated that HepG2 polarization restricts $\mathrm{HCV}$ entry and were interested to study the dynamic movement(s) of CD81 in polarized and non-polarized HepG2 cells. We utilised SPT on a TIRF microscope with fluorescent labelled antiCD81 FAb fragments. HepG2 polarization restricted the localized movement(s) of CD81 to the periphery of the cell. In summary, FRET and SPT data support a model where CD81-CD81 and CD81-CLDN1 motility at the BL membrane may be critical for the lateral movement and entry of $\mathrm{HCV}$ particles.

\section{$\mathbf{J 7 3 8}$}

\section{HIGH-THROUGHPUT SCREENING OF SMALL MOLECULE LIGANDS SPECIFIC FOR PATHOGENIC BACTERIA}

Jeong Heon Lee ${ }^{1}$, Hak Soo $\mathrm{Choi}^{1}$, Rafiou Oketokoun ${ }^{1}$, Sunny Park ${ }^{3}$, John V. Frangioni ${ }^{1,2}$

${ }^{1}$ Medicine, Beth Israel Deaconess Medical Center, Boston, MA, USA, ${ }^{2}$ Radiology, Beth Israel Deaconess Medical Center, Boston, MA, USA, ${ }^{3}$ Medicine, Brigham and Women's Hospital, Boston, MA, USA.

Background: Molecular imaging of pathogenic bacteria requires the development of small molecule targeting ligands with high affinity and specificity to their cell surface. We hypothesized that a glass slidebased screening system could be developed for such a purpose by combining robotic microarraying, advances in glass slide derivatization, and near-infrared (NIR) fluorescence detection. Materials and Methods: Several live pathogenic bacteria, such as $\mathrm{S}$. aureus and P. aeruginosa, E. coli, and B. subtilis were labeled by simple mixing with SYBR $(\mathrm{Em}=$ $500 \mathrm{~nm}$ ), BIODIPY $(\mathrm{Em}=600 \mathrm{~nm}$ ), MHI-84 (a custom NIR fluorophore; $700 \mathrm{~nm})$ and IR-786 $(800 \mathrm{~nm})$. A model library of small molecules and positively charged polymers (polyarylamine and polyL-lysine) specific for bacteria was prepared and micro-arrayed onto PEG-NHS functionalized glass slides. A Nikon TE2000E computercontrolled, 6-channel microscope was used for automated sample imaging and deconvolution of images. This microscope permitted quantitative scoring of each small molecule "spot" as a function of the number of a particular bacteria binding to the spot. Results: Using a 10X objective, real-time deconvolution, and multi-spectral imaging, each chemical spot could be scored for bacterial binding affinity in less than $500 \mathrm{msec}$. Glass slides having 5,076 conjugated small molecule test compounds could be screened in less than $3 \mathrm{~h}$ using this system. In particular, the highest affinity was obtained from vancomycin spots for S. aureus showing specific binding of more than 10,000 cells in a 300micron spot, while almost no binding for P. aeruginosa. Gramicidin S also showed relatively high affinities to E. coli and S. aureus $(8,000$ and 7,000 cells per spot) compared to B. subtilis $(<200$ cells per spot). Other small molecules, such as tryptamine, 2-amino-5,6-dimethylbenzimidazole, tryptophanamide, and tyrosine, showed low binding affinity and no specificity to all species. Conclusions: In this study, the compounds vancomycin and gramicidin showed strong binding to susceptible bacteria, and resulted in a high signal-to-background ratio during quantitative scoring. The key to successful diagnosis, and ultimately treatment, of pathogenic bacteria is the development of high affinity small molecule targeting ligands. By combining multispectral fluorescence imaging with robotic microarraying, we have developed a system that permits rapid, quantitative screening of targeting ligands and one-step conjugation to contrast agents and radiotracers. 


\section{Imaging Molecular and/or Cellular Processes}

\section{Inflammation}

J739

IN VIVO MULTI-COLOR IMAGING REVEALED ABNORMAL MULTI-CELLULAR KINETICS IN METABOLIC SYNDROME AND THROMBOSIS

Satoshi Nisimura ${ }^{1,2}$, Mika Nagasaki ${ }^{1}$, Ichiro Manabe ${ }^{1,2}$, Koji Eto ${ }^{3}$, Takashi Kadowaki ${ }^{4}$, Ryozo Nagai ${ }^{1}$

${ }^{1}$ Department of Cardiovascular Medicine, The University of Tokyo, Tokyo, Japan, ${ }^{2}$ PRESTO, Japan Science and Technology Agency, Tokyo, Japan, ${ }^{3}$ Division of Stem Cell Therapy, Center for Stem Cell Biology and Regenerative Medicine, Institute of Medical Science, The University of Tokyo, Tokyo, Japan, ${ }^{4}$ Department of Metabolic Diseases, The University of Tokyo, Tokyo, Japan.

To elucidate the molecular mechanisms of pathological conditions consisted by the complicated and multi-cellular abnormal interactions in common diseases, such as metabolic syndrome, arteriosclerosis, and thrombosis, an intravital visualization technique was needed to clarify the cellular mechanisms. We developed a novel "in vivo multi-color imaging method" by combining single-photon spinning disk confocal, and multi-photon resonance scanning system. It enabled us to precisely evaluate the threedimensional structures in living tissue (b), and the multi-cellular dynamics in vivo with high time and spatial resolutions (d-h). Using this technique, we focused on the metabolic syndrome and how obese adipose tissue plays a bad role in obesity. Our imaging specifically visualized adipocytes, macrophages, lymphocytes, endothelium, and blood cells in adipose in vivo (b-e). First, we elucidated the close spatial and temporal relationships between angiogenesis and adipogenesis, and both were augmented in obesity, (c, 2007 Diabetes). In microcirculation of obese adipose, tissue, inflammatory cellular dynamics based on chronic inflammation, such as adhesion and rolling of leukocyte and platelet were observed (f,g, $2008 \mathrm{JCI})$. Recently, we also clarified that the $\mathrm{CD} 8+\mathrm{T}$ cells and macrophage interactions were important for this inflammatory processes (2009 Nat Med). Surprisingly, our novel technique clearly visualized single platelets in vivo for the first time (h). We elucidated the complicated cellular mechanisms of developing thrombus consisting by multiple cell types including endothelium, leukocyte, and platelets. This imaging method enables us to analyze single platelet function, effect of anti-platelet drugs, and gene function in vivo in single platelet levels.

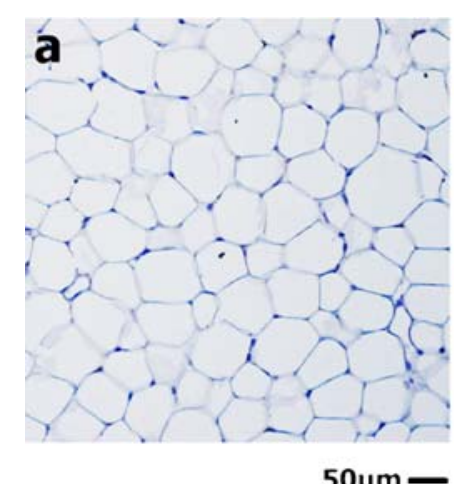

Conventional Imaging using fixed adipose tissue

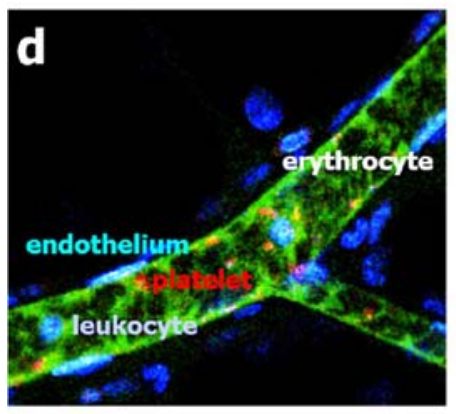

multi-color in vivo imaging revealed cell dynamics

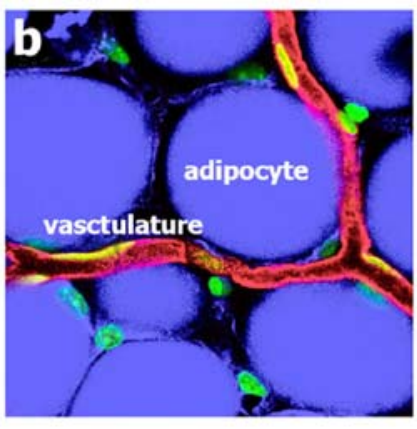

50 um (lean adipose)

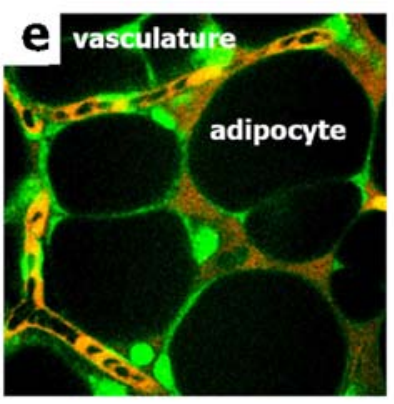

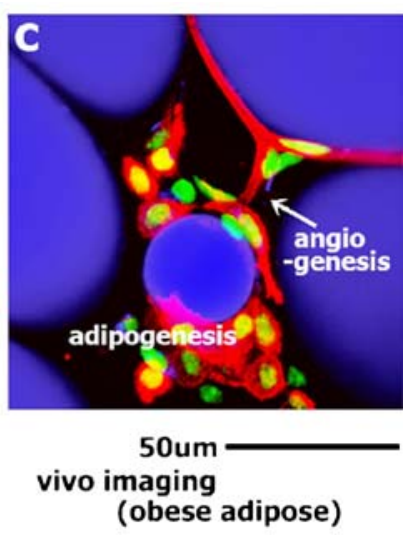

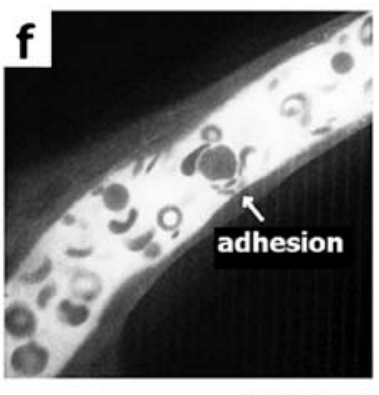

$10 \mathrm{um}-$

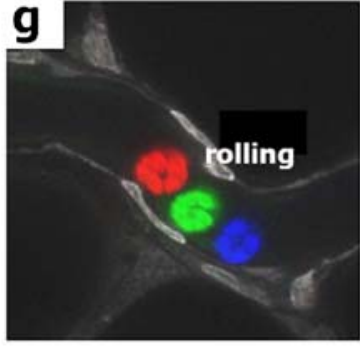

10um

Inflammation in obese adipose h

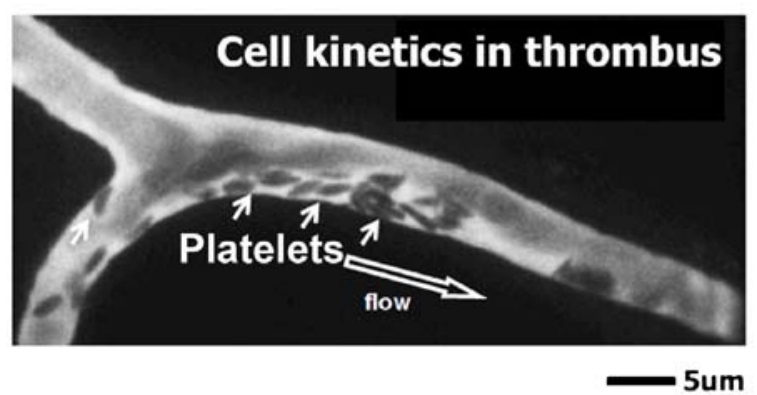

Single platelet kinetics in developing thrombus 
Obese adipose tissue in metabolic syndrome and thrombosis visualized by "in vivo multi-color molecular imaging" method

\section{$\mathbf{J 7 4 0}$}

\section{VALUE OF P-SELECTIN ULTRASOUND MOLECULAR IMAGING TO PREDICT LOCAL RESPONSE TO TNF ANTAGONIST TREATMENT}

Thierry Bettinger, Isabelle Tardy, Philippe Bussat, Francois Tranquart, Patricia Emmel, Michel Schneider

Novel Agents Formulation, Bracco Research Geneva, Plan-Les-Ouates / Geneva, Switzerland.

Introduction: New strategies have recently emerged for the treatment of inflammatory diseases, such as rheumatoid arthritis. They rely on the use of TNF $\alpha$ antagonists, and despite accepted effectiveness, up to $40 \%$ of the patients fail to respond to the treatment. Thus, diagnostic tools are needed to predict responsiveness and to improve patient care. Molecular ultrasound imaging using targeted microbubbles allows to monitor non-invasively the expression of vascular biomarkers in real time. Pre-treatment with TNF $\alpha$ antagonist has been shown to reduce the expression of various vascular markers, such as P-selectin, ICAM-1 and VCAM-1 (Esposito, 2007, J Leuk Biol). The objective of the present study was to assess the potential of targeted ultrasound contrast agents (TUCA) to monitor the expression of P-selectin (CD62P) following injection of the TNF $\alpha$ antagonist etanercept. Materials and Methods: Twenty four hours before the onset of inflammation, rats were pretreated either with a s.c. injection of etanercept $(0.45 \mathrm{mg} / \mathrm{kg})$ or saline in control animals. Inflammation was induced in the hind limb by i.m. injection of LPS in the tibialis cranialis $(1.1 \mathrm{mg} / \mathrm{kg})$. Binding of streptavidin-bearing microbubbles (Target-Ready Contrast Agent, Visualsonics, Toronto, Canada) functionalized with a biotinylated Pselectin antibody (LYP20 clone, Biocytex) was evaluated in the inflamed and control hind limb of the rat, $24 \mathrm{~h}$ after LPS injection. The overexpression of P-Selectin was confirmed by immunohistochemistry in absence of etanercept injection. Blood concentration of TNF $\alpha$ was monitored during the course of the experiment. Results: In controls animal, strong binding was observed in the inflamed hind limb, $10 \mathrm{~min}$ after microbubble injection (echo signal: 20土12) as opposed to the contralateral muscle $(0.7 \pm 0.3)$. A two-fold decrease in

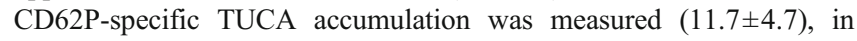
animals pre-treated with etanercept in comparison to control animals. The effect of the etanercept dose on the TNF $\alpha$ blood concentration and on TUCA accumulation was also followed over time. Conclusion: This study shows the ability of TUCA to monitor P-selectin expression in the course of an anti inflammatory treatment with etanercept in the rats. Therefore, ultrasound molecular imaging has the potential to become a powerful diagnostic tool to monitor anti inflammatory treatment in man.

\section{$\mathbf{J 7 4 1}$}

\section{ULTRASOUND MOLECULAR IMAGING OF CORONARY ENDOTHELIAL P-SELECTIN IN A MYOCARDIAL ISCHEMIA-REPERFUSION PIG MODEL}

Anne Broillet $^{1}$, Robert Rieben ${ }^{2}$, Alexandre Helbert ${ }^{1}$, Thierry Bettinger ${ }^{1}$, Philippe Bussat $^{1}$, Pranitha Kamat ${ }^{2}$, Francois Tranquart ${ }^{1}$, Michel Schneider ${ }^{1}$ ${ }^{1}$ Bracco Research SA, Plan-les-Ouates, Switzerland, ${ }^{2}$ Department of Clinical Research, University of Bern, Bern, Switzerland.

In the United States alone, 5-6 million patients annually visit emergency departments due to chest pain. A large majority of them does not have an acute coronary syndrome (ACS) but for those with ACS, standard testing procedures such as ECG and serum troponin are frequently non-diagnostic. Contrast-enhanced echocardiography (CEE) can often provide useful diagnostic information but in some cases wall motion and perfusion have returned to normal at the time of examination. During ACS, the ischemia/reperfusion phenomenon induces over-expression of various inflammatory markers such as PSelectin (CD62P). The purpose of this study was to investigate the use of a P-selectin-binding ultrasound contrast agent for CD62P imaging in a pig myocardial ischemia/reperfusion model. The left anterior descending artery (LAD) was occluded in five pigs $(30 \mathrm{~kg}$ ) with a coronary intervention catheter. The balloon was inflated for $20 \mathrm{~min}$ under angiographic control to completely occlude the vessel. The $20 \mathrm{~min}$ ischemia period was followed by $6 \mathrm{~h}$ of reperfusion. CEE was performed using a Siemens Sequoia 512 equipment with a 3V2c (shortaxis view) and a $4 \mathrm{C} 1$ probe (subxyphoid view). Occlusion and reperfusion were confirmed by CEE after injection of control microbubbles. Binding of streptavidin-bearing microbubbles (Target-Ready Contrast Agent, Visualsonics, Toronto, Canada) functionalized with a biotinylated P-selectin antibody (AK4 clone, BD Bioscience) was evaluated in the myocardium, at baseline, and between $30 \mathrm{~min}$ and $6 \mathrm{~h}$ after the onset of reperfusion. P-selectin expression in the myocardium was also assessed by immunohistochemistry. After inflation of the balloon, CEE with control bubbles showed the absence of signal in the LAD territory indicating complete occlusion of the artery. No perfusion defect was detected by CEE after reopening and during the entire reperfusion period $(6 \mathrm{~h})$. P-selectin microbubbles generated stronger echo enhancement in the ischemic area compared to the non-ischemic area. Signal enhancement in the ischemic area was detected from 1 to $6 \mathrm{~h}$ with peak intensity between 2 and $4 \mathrm{~h}$ after reperfusion. The signal measured in the ischemic area at peak enhancement was 2-4 times higher than in the normal area. These results were correlated with immunohistochemical observations. The ability to non-invasively detect P-selectin signal several hours after an ischemic event by ultrasound molecular imaging with targeted agents could markedly improve the efficiency of the triage and management of patients presenting with chest pain.

\section{$\mathbf{J} 742$}

\section{ANTI-MRP14-CY5.5 - A NOVEL TRACER FOR IN VIVO MONITORING OF INFLAMMATORY BOWEL DISEASE WITH OPTICAL IMAGING METHODS}

Michel Eisenblaetter ${ }^{1}$, Dominik Bettenworth ${ }^{2}$, Andreas Lügering ${ }^{2}$, Thomas Vogl $^{3}$, Christoph Bremer ${ }^{1}$

${ }^{1}$ Department of Clinical Radiology, University Hospital of Münster, Münster, Germany, ${ }^{2}$ Department of Medicine B (Gastroenterology), University Hospital Münster, Münster, Germany, ${ }^{3}$ Department of Immunology, University Hospital Münster, Münster, Germany.

Purpose: Murine models of inflammation are powerful tools in preclinical research, for example in evaluation of new drugs. Generally, monitoring of disease activity by e.g. histological changes or proinflammatory cytokines requires an ex vivo preparation and therefore precludes ongoing, continuous surveillance. Thus, specific in vivo imaging with the opportunity of in vivo follow-up examinations would be desirable. Activated macrophages play a crucial role during initialisation and maintenance of inflammatory process. Their expression of Myeloid Related Protein (MRP) 14 during activation has been shown to be strictly correlated with disease activity. In previous studies, feasibility of specific MRP-Targeting using a fluorescence-labelled MRP14-Antibody could be shown. This study should confirm the feasibility of MRP-Targeting for monitoring of disease activity in experimental inflammatory bowel disease (IBD) in vivo, using Fluorescence Mediated Tomography (FMT). Materials and Methods: Cy5.5 was either coupled to an MRP14-antibody or to Immunoglobulin $\mathrm{G}$ serving as control for non-specific label distribution. IBD was 
induced in C57BL/6 WT mice by addition of 2\% Dextran-Sodiumsulfate (DSS) to drinking water. The course of IBD was monitored by weight loss. FMT was performed either at definite times before and after DSS-application or weight-loss-dependant. Tracers were applicated in amounts of $2 \mathrm{nmol}$ per animal, $24 \mathrm{~h}$ prior to FMT. For correlation of imaging findings, immunohistochemistry of sections of inflamed tissue was performed and colonic changes were scored histologically. For statistical analysis, a student-t-test was performed. Results: In mice with severe inflammation - strong weight loss and histologic evidence of colitis, injection of Anti-MRP14-Cy5.5 resulted in fluorescence intensity which was about two-fold higher compared to IGG-Cy5.5 injected animals (585.3 vs. 205.6; $p<0.05$ ). Rapid weightloss $(18 \% / 7 \mathrm{~d})$ correlated with high anti-MRP14-Cy5.5 fluorescence in FMT (1263.5) and was histologically proven to correspond to strong colitis, while mice presenting with slower weight-loss $(10 \% / 10 \mathrm{~d})$ and moderately elevated MRP-fluorescence (363.5), showed only rare signs of colonic inflammation. In healthy animals, no significant traceruptake in the abdomen could be observed. Conclusion: Anti-MRPCy5.5 combined with FMT allows sensitive and specific detection of phagocyte activity represented by MRP14 expression in vivo and can therefore be regarded a suitable probe for in vivo-examination of inflammation, such as IBD.

\section{$\mathbf{J 7 4 3}$}

DYNAMIC M1-ACTIVATED MACROPHAGES MIGRATION INSIDE AORTIC ATHEROSCLEROTIC PLAQUES: IN VIVO PRECLINICAL MRI STUDY IN APOE2 KNOCK IN MICE Amine Bessaad

Creatis-Lrmn, Villeurbanne, France.
The inflammatory process is a crucial event in atherogenesis[1,2]. The chemotaxic response through gamma interferon (INF $\gamma$ ) provides macrophage accumulation into plaques[3] and high resolution MRI permits the detection of their dynamic recruitement [4,5]. Our purpose is the MRI characterization of plaque inflammation and macrophages trafficking to aortic arch in ApoE2 (K.I) mice using iron oxide Anionic Magnetic Nano Particules (AMNP) labelling. Method: Bone Marrow cells were cultured in presence of M-CSF and Flt3-Ligand then activated by INF $\gamma$. they were labelled for 6 hours in AMNP solution [6]. 4 million cell /mouse were injected after baseline MRI and followed during 3 days. A high resolution multi-contrast MRI protocol was performed on a $4.7 \mathrm{~T}$ : a proton density and a $\mathrm{T} 2$ weighted spin echo (SE) sequences, two multi TE gradient echo (GE) sequences. Mice were euthanizedand ascendant aortic arch was taken for histological Prussian blue, for iron and IHC analysis using anti(CD11b, F4/80, and MHC-II) molecules for macrophages (figure 1). Results: more than 108 macrophages were obtained. This number reduced to 4.107 to after labelling. MRI shows a local signal loss inside the vessel wall, (mean signal over the 3 days is $-27 \%$ to $-37 \% n=6$ ), wich is confirmed by co-localization with prussian blue positive cells and activated macrophages (MHCII). Moreover a dynamic intraplaque trafficking was observed during the 3 days with intimal and/or adventitial infiltration, confirmed also by confocal imaging.Conclusion: MRI macrophage labelling allows to assess specifically atherosclerotic plaque inflammation status in-vivo. As ApoE2 Ki mice is an excelent model for plaque inflammation this method can be used to follow-up in-vivo treatment accuracy and may provide a new index of the plaque evolution (i.e, migration and intimal/adventitial distribution of macrophages). [1] F.K.Swirsky 2007, [2] D.B.Weinreb 2007, [3]A. Mantovani 2002, [4] B.Qiu 2007,[5] M.F.Kircher, [6] C.Wilhelm 2008 a

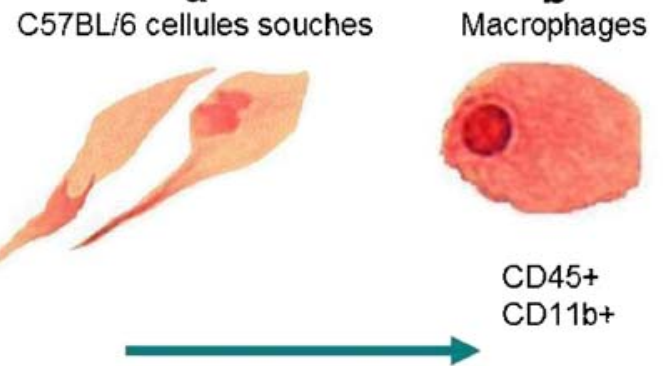

Culture cellulaire + différenciation

( 8 jours)

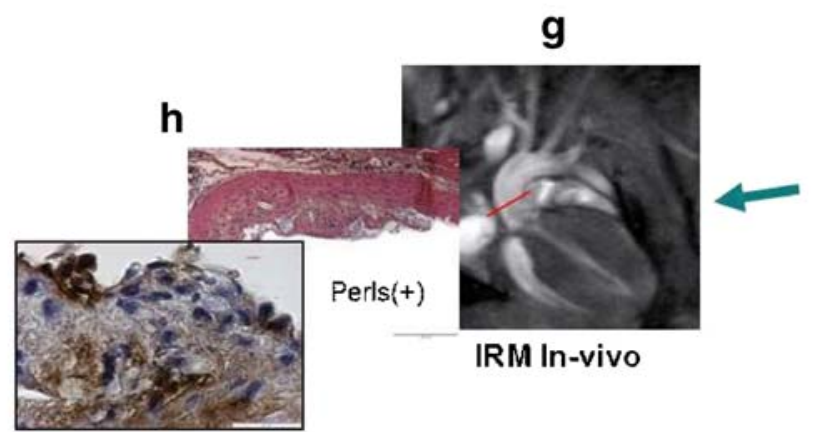

MHC-II $\{+\}$

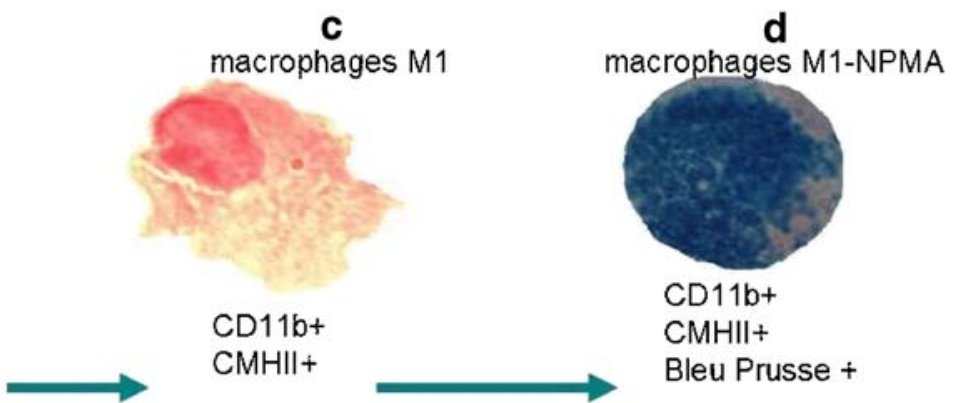

Activation M1 ( 3 jours)

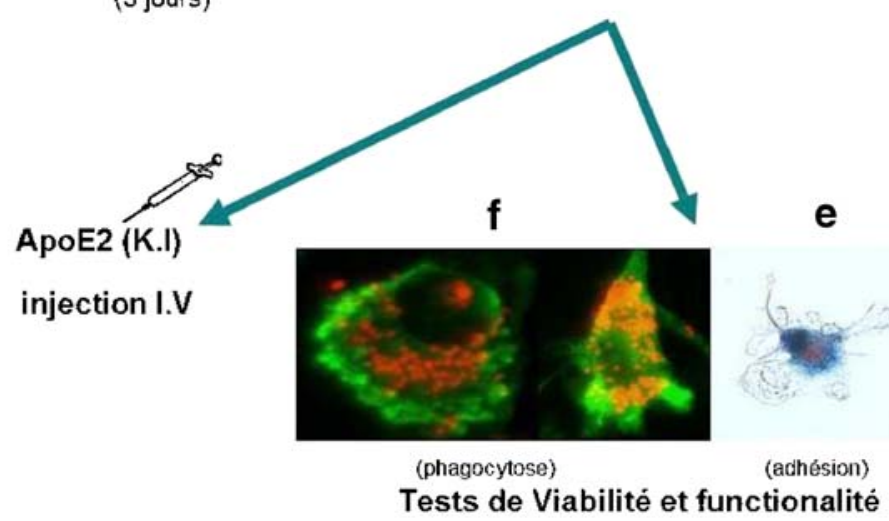




\section{J744}

\section{ATHEROSCLEROSIS-ASSOCIATED LY-6CHI BLOOD MONOCYTOSIS IMPAIRS WOUND HEALING IN MICE WITH MYOCARDIAL INFARCTION}

Peter Panizzi ${ }^{1}$, Filip K. Swirski ${ }^{1}$, Jose-Luiz Figueiredo ${ }^{1}$, Peter Waterman ${ }^{1}$, David E. Sosnovik $^{1}$, Elena Aikawa ${ }^{1}$, Peter Libby ${ }^{2}$, Mikael Pittet ${ }^{1}$, Ralph Weissleder ${ }^{1}$, Matthias Nahrendorf ${ }^{1}$

${ }^{1}$ Center for Systems Biology, Massachusetts General Hospital, Boston, MA, USA, ${ }^{2}$ Cardiovascular Division, Brigham \& Women's Hospital, Boston, MA, USA.

Background: Monocytes are key cellular protagonists of tissue repair and their specific subtypes regulate a carefully balanced healing program after myocardial infarction (MI). In MI tissue, one subset (Ly-6Chi in the mouse) dominates on days 1-4 after ischemic injury and orchestrates inflammation while another subset (Ly-6Clo) accumulates on days 5-7 and promotes angiogenesis and scar formation. Here we tested the hypothesis that chronically elevated Ly-6Chi blood levels induced by hypercholesterolemia in apoE-/- mice negatively affect infarct healing. Methods and Results: Flow cytometric enumeration of cells isolated from 5 day old murine infarcts showed $>10$-fold higher numbers of Ly-6Chi monocytes in apoE-/- compared to wild type mice $(14.4 \pm 5.8 \times 104$ vs $1.1 \pm 0.1 \times 104, p<0.05)$, accompanied by a pro-inflammatory pattern of gene expression (e.g. increased TNF- $\alpha$ and MPO and decreased TGF- $\beta$ mRNA in apoE-/- infarct extracts, $p<$ $0.001)$. Immunostaining showed high abundance of the protease cathepsin B and F4/80+ phagocytes ( $p<0.05$ apoE-/- vs wild type). To relate inflammatory activity to left ventricular remodeling, we used a combination of noninvasive molecular and physiologic imaging. Hybrid Fluorescence Molecular Tomography/Computed Tomography (FMT-CT) on day 5 post MI showed 2.9-fold higher protease activity and 2.6-fold higher phagocytic rates in infarcts of atherosclerotic mice $(p<0.05)$. Serial MRI volumetry showed that the prolonged inflammation in apoE-/- infarcts resulted in accelerated deterioration of ejection fraction between day 1 and 21 after MI $(17 \pm 4$ vs $5 \pm 3 \%$ decline in wild type, $p<0.05)$. Conclusion: Atherosclerosis-associated blood monocytosis disturbs resolution of inflammation in healing murine infarcts and consequently enhances left ventricular remodeling. This finding identifies monocyte subsets as potential therapeutic targets to augment tissue repair after infarction and prevent post-MI heart failure.

\section{$\mathbf{J 7 4 5}$}

\section{OXAZINE CONJUGATED NANOPARTICLE DETECTS} IN VIVO REACTIVE OXYGEN SPECIES GENERATION Peter Panizzi $^{1,2}$, Matthias Nahrendorf ${ }^{1,2}$, Moritz Wildgruber ${ }^{1,2}$, Peter Waterman $^{1,2}$, Jose-Luiz Figueiredo ${ }^{1,2}$, Elena Aikawa ${ }^{2}$, Jason R. McCarthy $^{1,2}$, Ralph Weissleder ${ }^{1,2}$, Scott Hilderbrand ${ }^{1,2}$

${ }^{1}$ Center for Systems Biology, Massachusetts General Hospital, Boston, MA, USA, ${ }^{2}$ Center for Molecular Imaging Research, Massachusetts General Hospital, Charlestown, MA, USA.

Inflammation is an ubiquitous response to acute or chronic tissue injury. Reactive oxygen and nitrogen species (ROS/RNS) generated during inflammation are causal to or can exacerbate pathogenesis of Alzheimer disease, atherosclerosis, cancer, ischemia-reperfusion injury in stroke, myocardial infarction and organ transplantation. The current lack of suitable probes has limited the in vivo imaging of reactive oxygen species (ROS). ROS are often generated by ischemia-induced inflammation; defining the extent of tissue involvement or ROS-related damage would have a significant clinical impact. We present the preparation and demonstration of a fluorogenic sensor for monitoring peroxynitrite (ONOO-) and myeloperoxidase (MPO) mediated hypo- chlorous acid ( $\mathrm{HOCl} / \mathrm{OCl}-)$ production. The sensor consists of a long circulating biocompatible nanoparticle that targets phagocytic cells in vivo and is coated with $\sim 400$ quenched oxazine fluorophores that are released by reaction with $\mathrm{HOCl}$ or ONOO-, but stable towards oxidants such as hydroxyl radical, hydrogen peroxide, and superoxide. MPOdependent probe activation is chloride ion dependent and is negated in MPO inhibitor treated neutrophils. Flow cytometric profiling and microscopic fluorescence imaging in mouse hearts after myocardial infarction showed probe activation in neutrophil-rich ischemic areas, making the MPO/ROS sensor a novel prognostic indicator. In summary, we present a novel MPO/ROS sensor that enables imaging of ROS production resulting from ischemia-induced inflammation in living cells and in a mouse model of cardiovascular disease. Attachment of a fluorogenic oxazine probe to a dextran-coated iron oxide nanoparticle favorably alters the washout kinetics of small fluorescence sensors and results in high detection sensitivity, likely enabling ROS studies by whole animal fluorescence imaging. Importantly, this advance would allow for high throughput screening of anti-inflammatory molecules in vivo. Finally, clinical monitoring of ROS production by macroscopic and endoscopic fluorescence imaging may enable detection of inflammation in atherosclerosis, cancer, metastasis and organ rejection.

\section{J746}

\section{MRI STUDY OF THE EFFECTS OF MATERNAL} INFLAMMATION ON THE OFFSPRING

Sylvie Girard $^{1}$, Luc Tremblay ${ }^{2}$, Melanie Archambault ${ }^{2}$, Guillaume Sebire $^{1}$, Martin D. Lepage ${ }^{2}$

${ }^{1}$ Pediatric, Universite de Sherbrooke, Sherbrooke, QC, Canada, ${ }^{2}$ Nuclear Medicine and Radiobiology, Universite de Sherbrooke, Sherbrooke, QC, Canada.

Objectives: Maternal infection/inflammation affects newborns, leading to a heavy burden of both neurodevelopmental diseases and enhanced susceptibility for later brain damage. Currently, no therapeutic strategy exists to protect the newborn brain against such systemic inflammatory aggressions. Indeed, the pathophysiological sequence linking maternal inflammation and fetal brain damage remains obscure. We tested the hypothesis that systemic maternal infection impacts the placenta through neurotoxic cytokines - especially interleukin-1 (IL-1) - that would subsequently affect the neurodevelopment. We used MRI to investigate the link between maternal inflammation and perinatal brain damage. Model: Studies were performed on an established rat model of prenatal inflammation that mediates the development of brain damage. Anesthetized animals were imaged using a small-animal $7 \mathrm{~T}$ MRI system at gestational day 17 (G17). Placentas and fetuses were visualized with a fast spin-echo pulse sequence. Gd-DTPA was injected i.v. and its distribution monitored by T1-weighted images. The rat was then injected every 12 hours either with lipopolysaccharide (LPS) to induce inflammation or vehicle until G20 when the MRI procedure was repeated. In a separated set of experiments, radiolabeled IL-1 $\beta$ was injected at G20 and its distribution in the fetal organs was studied. MRI results were correlated with histology and immunohistochemistry. Results: We observed that Gd-DTPA was rapidly distributed in the placenta of control dams at both mid and late gestation. In LPS-treated dams, the placental perfusion was decreased or even completely absent. This abnormal placental perfusion was correlated to the presence of apoptosis, infiltration of macrophages and overexpression of IL-1 $\beta$. Radiolabeled IL-1 $\beta$ injected at G20 revealed that inflammation increased placental permeability to the cytokine, which was detected within the fetuses brain. Placental defects triggered by systemic LPS exposure were also associated with significant perinatal death. Conclusion: In summary, we showed that maternal inflammation had a major impact on the placenta and on the pups, which might be 
mediated via cytokines, especially IL-1. MRI could be a useful tool to detect in utero inflammation and to test the efficiency of antiinflammatory treatments.

J747

\section{NON-INVASIVE QUANTITATIVE FLUORESCENCE \\ MOLECULAR IMAGING OF INFLAMMATION AND TREATMENT RESPONSE OF LIVER GROWTH FACTOR IN TOBACCO-INDUCED MURINE EMPHYSEMA} Sandra Perez-Rial ${ }^{1}$, Laura del Puerto-Nevado ${ }^{1}$, Angelos Kyriazis ${ }^{2}$, Juan José Díaz-Gil ${ }^{3}$, Nicolás González-Mangado ${ }^{1}$, Jesús Ruiz-Cabello ${ }^{2}$, Germán Peces-Barba ${ }^{1}$

${ }^{1}$ Experimental Pulmonary Laboratory, Fundación Jiménez DíazCAPIO-CIBERES, Madrid, Spain, ${ }^{2}$ Instituto de Estudios Biofuncionales, Universidad Complutense de Madrid-CIBERES, Madrid, Spain, ${ }^{3}$ Experimental Biochemistry, Hospital Puerta de Hierro, Madrid, Spain.

We report the utility of near infrared imaging agents, in combination with fluorescence molecular imaging (FMI), for the non-invasive quantitative imaging of mouse lung inflammation in tobacco-induced emphysema model. We used Liver Growth Factor (LGF) in a model of chronic tobacco exposure in mice in order to test its regenerative capacity in this system and visualize inflammation. Methods. 15 male AKR/J mice were estudied after 6 months of cigarette smoke exposure.
After 6 months, one tobacco exposed group was treated with LGF $(1.8 \mu \mathrm{g} /$ mouse, i.p.). We visualized the molecular process through FMI by injecting via tail vein with 2 nmoles of a matrix metalloproteases activable fluorescent probe (MMPSense680, VisenMedical). We adquired images 3 hours after probe injection with IVIS Lumina (Xenogen) and quantifications were made with Living Image software. At the end of exposition period mice were canullated and Compliance were measured with a small animals ventilator. Finally, mice were sacrified, and Mean Linear Intersection (LM) was calculated with standard image analysis software (Leica Qwin). Results. Quantification by FMI revealed in vivo metalloproteases activity within the lung associated with the inflammatory response which decreased in response to LGF treatment. Results were correlated with pulmonary function and morphometric analysis and revealed good correlation between these measures and quantification of MMPSense680 activation. We have demonstrated the ability of FMI to non-invasively visualize and quantify inflammation in the lung and monitor therapeutic efficacy in vivo. Tobacco group presents an increased of Compliance and LM values respect to Control group, and increased values of metalloproteases expresion in lung due to tobacco exposition. In the other hand, LGF treatment produced a reduction in the Compliance and LM after tobacco exposition as well as metalloproteases expresion observed with FMI images. All the variables measured are represented in the Table 1. Conclusion. Tobacco exposure is characterized by increased levels of metalloproteases, Compliance and LM. LGF was able to ameliorate previously established tobacco lesion in lung, including improvement of lung function, lung morphometry and inflammation status in AKR/J mice.

\begin{tabular}{|c|c|c|c|}
\hline & $\begin{array}{c}\text { FMI EFFICIENCY } \\
\text { (a.u. }\end{array}$ & $\begin{array}{c}\text { COMPLIANCE } \\
(\mathrm{ml} / \mathrm{cmH} 2 \mathrm{O})\end{array}$ & LM $(\mu \mathrm{m})$ \\
\hline CONTROL GROUP & $3.6 \times 10-4 \pm 2.45 \times 10-5$ & $0.137 \pm 0.0015$ & $32.78 \pm 0.7$ \\
\hline TOBACCO GROUP & $6.06 \times 10-4 \pm 3.84 \times 10-5^{*}$ & $0.186 \pm 0.006^{*}$ & $38 \pm 1.32^{*}$ \\
\hline LGF GROUP & $3.22 \times 10-4 \pm 1.68 \times 10-5^{\wedge}$ & $0.116 \pm 0.008^{\wedge}$ & $32.88 \pm 1.24^{\wedge}$ \\
\hline
\end{tabular}

Values are presented as mean \pm SEM. $\left({ }^{*}\right) p<0.05$ versus Control group; $\left(^{\wedge}\right) p<0.05$ versus Tobacco group.

\section{$\mathbf{J} 748$}

\section{PEGYLATION OF VAP-1 TARGETING 68GA-DOTAVAP-P1 PEPTIDE IMPROVES PET IMAGING OF INFLAMMATION} Anu K. Autio $^{1}$, Tiina Henttinen ${ }^{2}$, Henri Sipilä ${ }^{1}$, Sirpa Jalkanen ${ }^{3}$, Anne Roivainen ${ }^{1,4}$

${ }^{1}$ Turku PET centre, Turku, Finland, ${ }^{2}$ Department of Biology,Division of Genetics and Physiology, University of Turku, Turku, Finland, ${ }^{3}$ MediCity Research Laboratory, University of Turku, Turku, Finland, ${ }^{4}$ Turku Centre for Disease Modelling, University of Turku, Turku, Finland.

AIM: Vascular adhesion protein-1(VAP-1) is an adhesion molecule that has a key role in recruiting leukocytes into sites of inflammation. We have previously shown that 68 Gallium labeled VAP-1 targeting peptide (68Ga-DOTAVAP-P1) is a potential PET imaging agent (Lankinen P et al. 2008; Ujula $T$ et al. 2009). Although capable of assessing rat osteomyelitis, the circulatory half-life of 68Ga-DOTAVAP-P1 is quite poor $(26 \mathrm{~min})$. In this study, we preliminary evaluated a new PEGylated 68Ga-DOTAVAP-P1 for in vivo imaging of inflammation. METHODS: Linear 9-aa 68Ga-DOTAVAP-P1 peptide and its PEGy- lated analog having 8-amino-3,6-diooxaoctanoyl linker (polyethylene glycol, PEG derivative) between DOTA and peptide were studied. A sterile skin/muscle inflammation was induced by s.c. injection of turpentine oil in Spraque-Dawley rats. Whole-body distribution kinetics and assessment of inflammation by 68Ga-DOTA-peptides were evaluated in vivo by dynamic PET imaging. Rat plasma and urine obtained at different time points after intravenous injection of $68 \mathrm{Ga}-$ DOTA-peptides were analyzed by reversed-phase radio-HPLC. In addition, plasma protein binding and water-octanol partition coefficient $(\log \mathrm{D})$ were assessed. RESULTS: The circulatory half-life of PEGylated 68Ga-DOTAVAP-P1 was $120 \pm 6$ min (mean $\pm \mathrm{SD}, n=3$ per each time point, Fig. A) and $99 \pm 2 \%$ of urine excreted radioactivity originated from unchanged $68 \mathrm{Ga}$-DOTA-peptide. Inflammation-tomuscle ratios by PET were $6.3 \pm 1.6$ and $7.3 \pm 2.1$ for 68Ga-DOTAVAP-P1 $(n=3)$ and its PEGylated analog $(n=3)$, respectively. The PEGylated peptide showed slower renal excretion (Fig B, C) but similar liver uptake as the original peptide. Both plasma protein binging and hydrophilicity were slightly lower for PEGylated $68 \mathrm{Ga}-$ DOTAVAP-P1 compared to the original peptide (14\% vs. $16 \%$; $\log \mathrm{D}-4.3$ vs. -4.5). CONCLUSION: PEGylation prolonged circulatory half-life of the 68Ga-DOTAVAP-P1 peptide, which led to higher target-to-backgroud ratio and improved in vivo PET imaging of inflammation. 


\section{口68Ga-DOTAVAP-P1 口PEGylated 68Ga-DOTAVAP-P1}

A

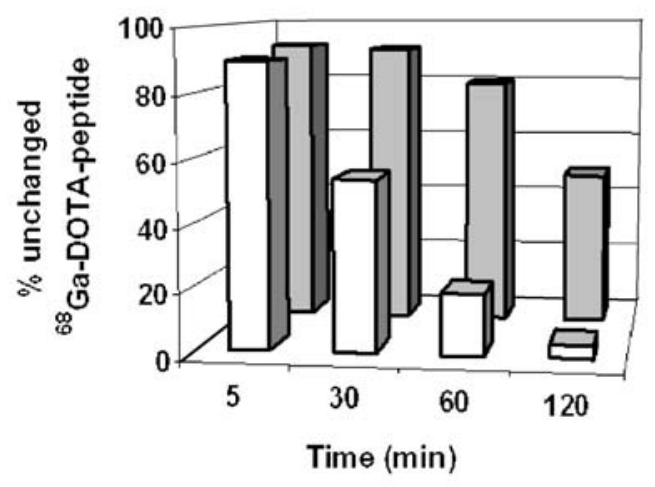

c

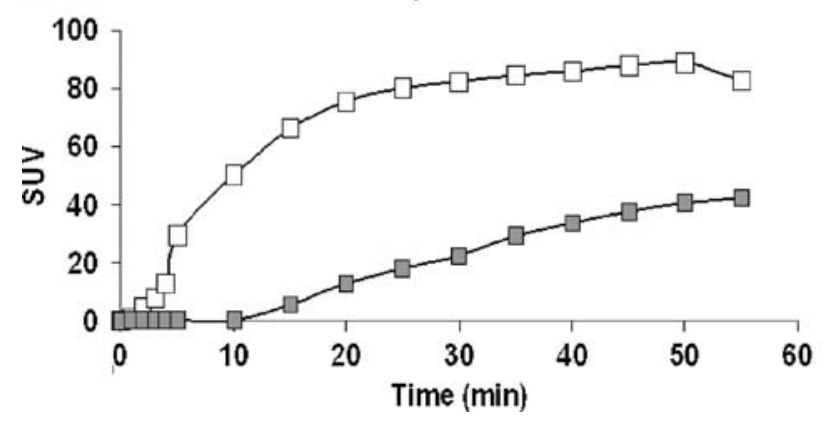

B

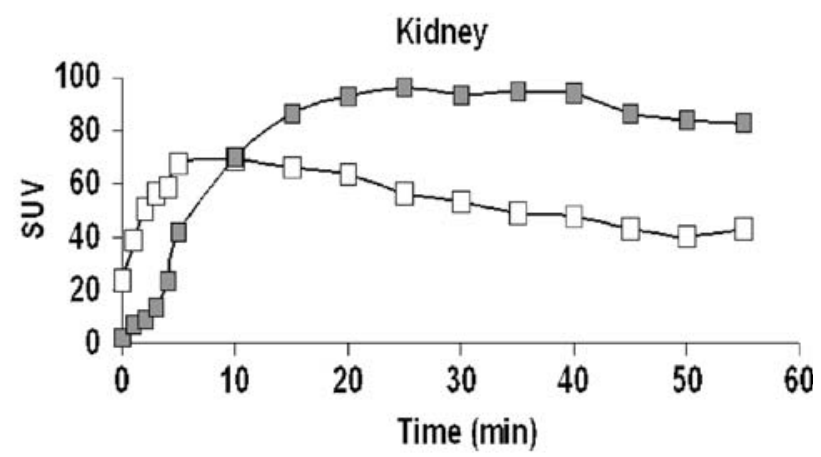

J749

\section{PRECLINICAL OPTICAL IMAGING OF MONOCYTE DEPLETION IN A MODEL OF LUNG INJURY- AS A PRELUDE TO A PHASE II CLINICAL STUDY}

Kevin Dhaliwal $^{1}$, Lois Alexander ${ }^{2}$, Mark Bradley ${ }^{2}$, Haslett Chris ${ }^{1}$, John Simpson ${ }^{1}$

${ }^{1}$ Centre for Inflammation Research, University of Edinburgh, Edinburgh, United Kingdom, ${ }^{2}$ Department of Chemistry, University of Edinburgh, Edinburgh, United Kingdom.

Acute lung injury (ALI) is a major cause of morbidity and mortality. Peripheral blood monocytes (PBMs) have been implicated in the pathogenesis of ALI but as yet there have been no targeted depletion studies in relevant murine models. We used a LPS model of lung injury and applied temporal monocyte depletion strategies. Primary endpoints of murine lung inflammation were total thoracic cathepsin and MMP activity measured with fluorescence molecular tomography alongside airway albumin leak. Temporal monocyte ablation attenuated lung injury as evidenced by optical imaging biomarkers. Furthermore, neutrophil ablation demonstrated the central role of the granulocytes as mediators of the PBM-induced vascular compromise in this model. Definitively, using a cd11b diptheria toxin receptor transgenic mouse, we adoptively transferred wild type PBMs to rescue the injury in the monocyte depleted mice. CONCLUSIONS: Mechanistic insights into the coordination of the acute inflammatory response to LPS have been elucidated. This murine modeling using optical imaging has led onto a phase II clinical study to deplete PBMs in healthy human volunteers with LPS injury.

\section{J750}

\section{DOSE OPTIMIZATION FOR IRON OXIDE PARTICLES ENHANCED MRI CELL TRACKING IN MURINE MYOCARDIAL INFARCTION MODEL}

Yidong Yang $^{1,2}$, Jimei Liu ${ }^{1}$, Yuhui Yang ${ }^{1}$, Nathan Yanasak ${ }^{1}$, Tom C. Hu ${ }^{1,2}$ ${ }^{1}$ Department of Radiology, Medical College of Georgia, Augusta, GA, USA, ${ }^{2}$ Radiological and Nuclear Engineering/Medical Physics Program, Georgia Institute of Technology, Atlanta, GA, USA.

Introduction: Inflammation plays a pivotal role in the cardiac remodeling process following myocardial infarction (MI). Our previous study showed the inflammatory cell infiltration could be monitored by iron oxide particles enhanced MRI. The inflammatory cells were labeled with micrometer-sized iron oxide particles (MPIO) via systemic injection. Signal attenuation at the MI site was observed in 
T2*-weighted MRI. However, the MPIO dose warrants further investigation. The purpose of this study is to optimize the dose for the MPIO-labeled inflammatory cell tracking. Methods: C57Bl/6 mice ( $n=17,6-11$ weeks) were used. One group (high dose group, $n=7$ ) received $0.04 \mathrm{mg} \mathrm{Fe} / \mathrm{g} \mathrm{MPIO}$, and another group (low dose group, $n=$ 4) received $0.01 \mathrm{mg} \mathrm{Fe} / \mathrm{g}$ MPIO. The animals underwent MI via permanent ligation of left anterior descending coronary artery 7 days post-MPIO injection. A control group $(n=6)$ underwent MI but without MPIO injection. MRI was performed at baseline, 3 days (D3), 7 days (D7) and 14 days (D14) post-MI in a 7-T spectrometer. Region-ofinterest (ROI) analysis was performed on $\mathrm{T} 2 *$-weighted images. A contrast-to-noise ratio (CNR) showing the signal intensity difference between the infarction site and the remote healthy myocardium was calculated. Results and Discussion: The T2*-weighted images were shown in Figure 1 and CNR data in Table 1. At $0.01 \mathrm{mg} \mathrm{Fe} / \mathrm{g}$ dose level, the CNR at the MI site was attenuated post-MI and significantly lower than the control group $(p<0.01)$. Comparing the two dose groups, the difference in CNR was not significant $(p>0.05)$. This indicates saturation may exist for inflammatory cells to in vivo take up the MPIO. The low dose may be sufficient for inflammatory cell labeling and tracking. However, the CNR is sensitive to the localization of ROI due to the inhomogeneous distribution of the signal intensity in the left ventricle wall. Therefore more non-biased analysis is warranted. Histology will be performed to correlate with the MRI findings.

Table 1: CNR measurements

\begin{tabular}{|c|c|c|c|c|}
\hline & Baseline & Day 3 & Day 7 & Day 14 \\
\hline MI-MPIO & $0.14 \pm 0.81$ & $-1.84 \pm 1.46$ & $-0.86 \pm 1.32$ & $-9.98 \pm 0.97$ \\
\hline $\begin{array}{c}\text { MI+MPIO } \\
(0.01 \mathrm{mg} \mathrm{Fe} / \mathrm{g})\end{array}$ & $0.40 \pm 1.04$ & $-6.80 \pm 1.38$ & $-8.80 \pm 1.14$ & $-10.79 \pm 1.25$ \\
\hline $\begin{array}{c}\text { MI+MPIO } \\
(0.04 \mathrm{mg} \mathrm{Fe} / \mathrm{g})\end{array}$ & $1.63 \pm 1.78$ & $-7.90 \pm 0.35$ & $-8.42 \pm 1.13$ & \\
\hline
\end{tabular}

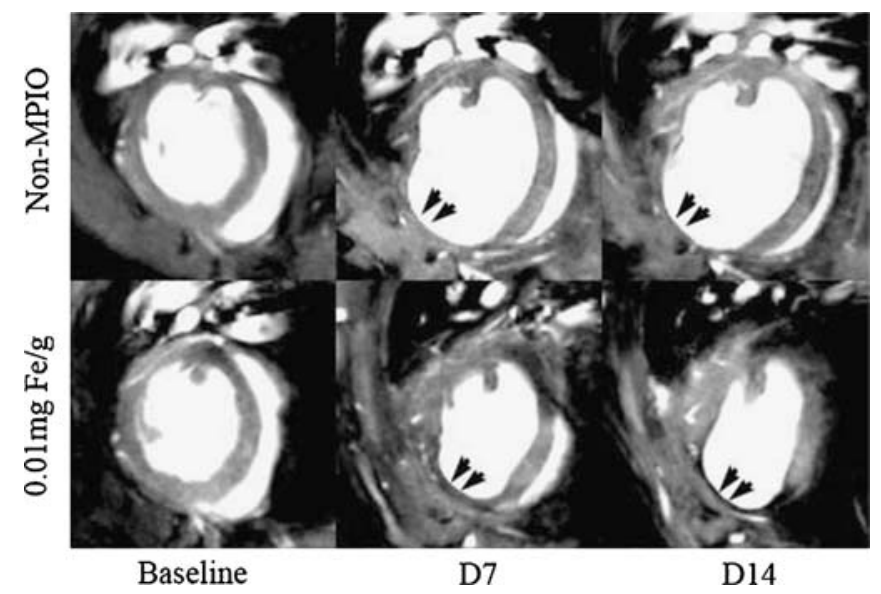

Figure 1

J751

\section{QUANTIFYING SUSCEPTIBILITY GRADIENT MAPPING IN A MODEL FOR ATHEROSCLEROSIS PROGRESSION}

Gopal Varma $^{1}$, Marcus R. Makowski ${ }^{1}$, Matthias Taupitz ${ }^{2}$, Stephen Keevil $^{3}$, Rene M. Botnar ${ }^{1}$, Tobias Schaeffter ${ }^{1}$

${ }^{1}$ Division of Imaging Sciences, King's College London, London, United Kingdom, ${ }^{2}$ Department of Radiology, Charité-Universitätsmedizin Berlin, Berlin, Germany, ${ }^{3}$ Medical Physics, Guy's \& St Thomas' NHS Foundation Trust, London, United Kingdom.
MRI using iron-oxide based contrast agents (CAs) was proposed for detection of atherosclerotic plaque. This results in a negative contrast. Positive contrast techniques have been developed for better detection ${ }_{[1]}$. Susceptibility gradient mapping (SGM) allows positive contrast by exploiting the local shift in $k$-space. This shift might be evaluated as a quantitative measure. In this work we want to quantify the progression of plaque using uptake of iron-oxide CA. The presence of CA perturbs the local field gradient, causing a shift of the echo-top during acquisition. SGM is based on calculation of the resulting shift in $k$ space $_{[2]}$. The shift is proportional to susceptibility gradient strength, and may thus be used to provide information regarding accumulation of CA. Phantom experiments were conducted using macrophage cells labeled by incubation with CA $(100 \mu \mathrm{g} / \mathrm{ml}$ for $2 \mathrm{hrs})$. These were serially diluted and suspended in gelatin. MRI was conducted on a $3 \mathrm{~T}$ Philips Achieva scanner. Images were acquired at multiple echo-times (TEs) to produce a $\mathrm{R}_{2}{ }^{*}$-map using a mono-exponential fit. The relaxation rate increased for increasing cellular concentrations (Fig.1a). The in-plane echo shift was also observed to increase with cell concentration (Fig.1b). A model for atherosclerosis was induced in ApoE-KO mice by feeding on a high-fat diet $(\mathrm{HFD})_{[3]}$. MRI was conducted on groups of at least 5 after $0,4 \& 8$ weeks of HFD. $200 \mu \mathrm{mol} /$ $\mathrm{kg}$ of VSOP CA was injected $24 \& 48 \mathrm{~h}$ prior to imaging. SGM was applied to acquisitions at $\mathrm{TE}=6.9 \mathrm{~ms}$. The in-plane shift was calculated for a region encompassing the entire vessel at the site of the plaque. The results show a quantifiable increase of the echo-shift for increasing duration on the HFD (Fig.1d). The echo-shift provided by SGM may thereby provide a quantifiable parameter to complement measuring the area of hypo-intensity from the negative contrast obtained by MRI. [1] Korosoglou et al.JACC(2008)52:483-490;[2]Dahnke et al.MRM(2008) 60:595-603;[3]Johnson et al.Circulation(2005)111:1422-1430 
a

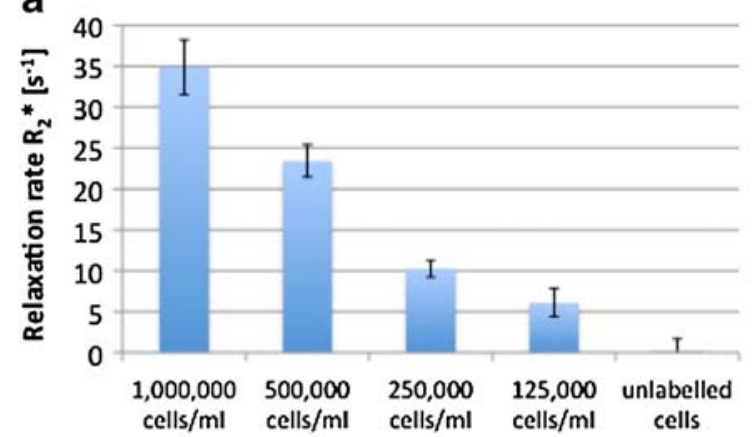

C

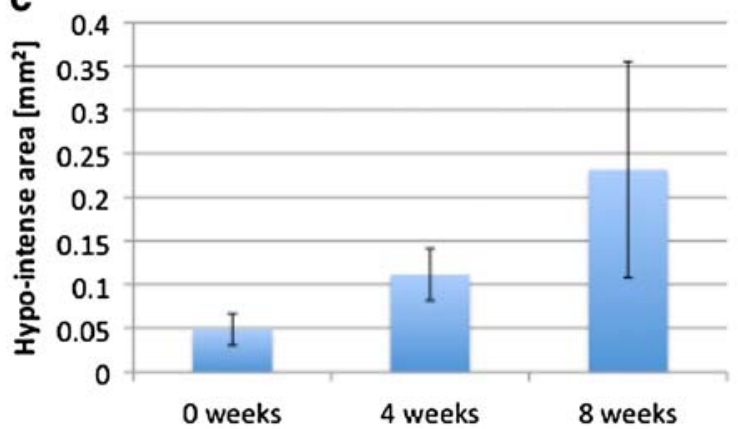

b

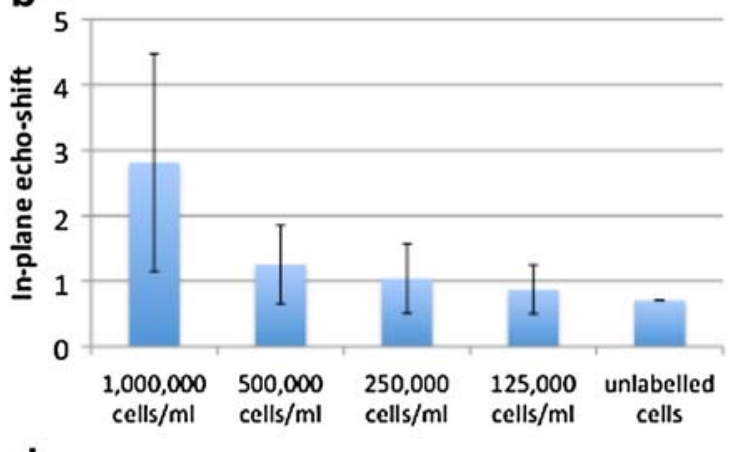

d

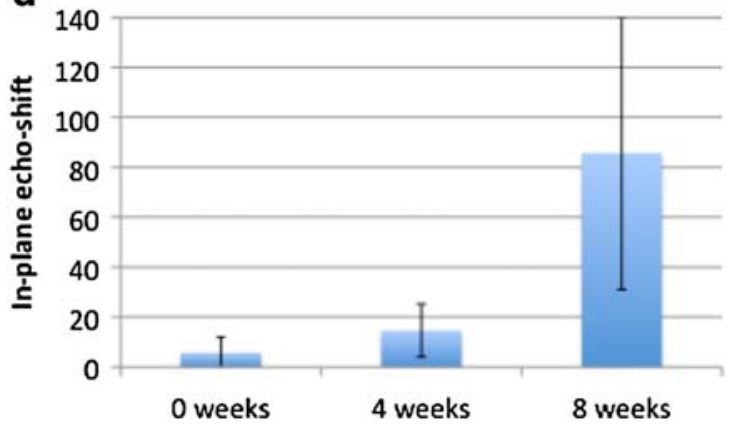

Figure 1. Charts of (a) $\mathrm{R}_{2} *$ \& (b) shift measured for the different labeled cell concentrations. Charts of (c) hypo-intense area \& (d) average shift from area of wall + lumen for different HFD duration.

IN VIVO DETECTION OF HYPOXIA DURING EARLY STAGES OF EXPERIMENTAL ARTHRITIS USING [18F] FMISO AND [18F]FAZA EVEN BEFORE HISTOLOGICAL VISIBLE JOINT INFLAMMATION

Kerstin Fuchs $^{1,2}$, Gerald Reischl ${ }^{3}$, Christoph M. Griessinger ${ }^{2}$, Daniel $\overline{\text { Bukala }^{2} \text {, Julia G. Mannheim }}{ }^{2}$, Denis Lamparter ${ }^{3}$, Stefan Wiehr ${ }^{2}$, Martin S. Judenhofer ${ }^{2}$, Martin Röcken ${ }^{1}$, Bernd J. Pichler ${ }^{2}$, Manfred Kneilling ${ }^{1}$ ${ }^{1}$ Department of Dermatology, University of Tuebingen, Tuebingen, Germany, ${ }^{2}$ Laboratory for Preclinical Imaging and Imaging Technology of the Werner Siemens-Foundation, University of Tuebingen, Tuebingen, Germany, ${ }^{3}$ Department of Radiopharmacy, University of Tuebingen, Tuebingen, Germany.

Detection of early stages of arthritis is essential to start early antiinflammatory treatment to prevent joint destruction. Hypoxia can induce angiogenesis via stabilization of the transcription factor hypoxia inducible factor (HIF)-1 $\alpha$ in resident and infiltrating cells and might be one of the early indicators of inflammation. Under hypoxic conditions HIF- $1 \alpha$ induces angiogenesis by upregulation of pro-angiogenic mediators such as bFGF and VEGF. We investigated hypoxia during early stages of experimental arthritis in vivo, using the established hypoxia PET-tracers 18F-Fluoromisonidazole ([18F]FMISO) and 18Ffluoroazomycin-arabinosid ([18F]FAZA), selectively accumulating in hypoxic tissue. The aim of this study was to investigate a new tool to examine initial phases of angiogenesis in autoimmune diseases such as rheumatoid arthritis by detection of hypoxia, before incidence of clinical symptoms and histological visible joint inflammation. We induced arthritis in Balb/c mice by intra peritoneal injection of auto- antibodies against Glucose-6 phosphate-isomerase (GPI). Mice underwent [18F]FMISO-, or [18F]FAZA in vivo PET investigations and magnetic resonance imaging ( 7 Tesla MRI) 6, 12, 48, and 52 hours after induction of arthritis. In addition, we performed H\&E-staining and real-time PCR analysis of gene expression patterns of joint tissue 6 and 12 hours after onset of arthritis. In vivo PET images confirmed accumulation of hypoxia tracer shortly after arthritis induction. Already $6 \mathrm{~h}$ after GPI serum injection [18F]FMISO and [18F]FAZA uptake in arthritic joints was 1,5 fold higher than in joints of healthy control mice. Importantly, at this time point nearly no signs of inflammation were visible in H\&E-stained joint sections. In line with the PET-data, RT-PCR analysis showed a 7,5 fold enhanced expression of HIF-2 $\alpha$ mRNA, and a up to 12 fold up regulated mRNA expression of proinflammatory mediators, such as, IL-1 $\beta$, IL- 6 even $6 \mathrm{~h}$ after a single GPI serum injection. Interestingly, pro-angiogenic mediators such as bFGF and VEGF were not elevated at the mRNA level at this early time point. Thus, non invasive in vivo examination of hypoxiainduced angiogenesis using [18F]FAZA and [18F]FMISO seems to be a new powerful tool to detect initial phases of angiogenesis in autoimmune diseases such as rheumatoid arthritis before joint inflammation is visible.

\section{$\mathbf{J 7 5 3}$}

\section{NEW PEPTIDE LIGANDS TO DETECT INFLAMMATORY BLOOD BRAIN BARRIER ALTERATIONS}

Klaus G. Petry, Antoine Vekris, Claudine Boiziau

Laboratoire de Neurobiologie des Affections de la Myéline, Université Bordeaux 2, Bordeaux, France. 
INTRODUCTION: In Multiple Sclerosis, the infiltration of the central nervous system (CNS) by immune cells (T cells, macrophages) depends on complex molecular alterations at microvascular endothelial cells at the blood brain barrier (BBB) that are still poorly understood. These molecular alterations provide the opportunity to identify and to target specifically the areas undergoing inflammation, potentially at a very early stage of acute inflammatory lesion formation. METHODOLOGY: To better characterize the early molecular inflammatory alteration events at the BBB, we have applied the approach of phage-peptide ligand screening ("phage display") in the early clinical phase of the experimental autoimmune encephalomyelitis (EAE) rat model of MS in comparison to healthy control rats. This strategy allowed the generation of two phage peptide ligand repertoires highly enriched for the respective physiological situation at the BBB. RESULTS: We have performed DNA subtraction of the two phage peptide ligand repertoires obtained with EAE rats versus healthy rats to further enrich and select peptide ligands that present specific binding with EAE rat CNS tissue. We have identified peptide ligands expressed by individual phages that bind to molecules expressed in various cells interacting at the BBB of CNS inflammation. The retained peptide ligands were analysed in silico and revealed that among the identified peptides 1 quintet, 15 trios and 38 duos of peptides are mimicking individual proteins in human, monkey, mouse and rat genomes. Some of the identified proteins are known to be expressed on $\mathrm{T}$ cells, macrophages and endothelial cells. Immunohistochemistry and PCR of specific binding of generated repertoires of selected phages to healthy or EAE spinal cord confirmed their discriminative reactivity. Labelling of phage peptide ligands with gadolinium allowed first in vivo MRI observation of inflammatory brain lesions. Supported by ANR-TecSan, CR Aquitaine, ARSEP.

\section{$\mathbf{J 7 5 4}$}

\section{MOLECULAR IMAGING OF INFLAMMATORY BOWEL DISEASE USING CONTRAST ULTRASOUND}

Joshua Rychak $^{1}$, Alexander L. Klibanov ${ }^{4}$, Christopher R. Anderson ${ }^{1}$, Klaus Ley ${ }^{3}$, Theresa T. Pizarro ${ }^{2}$

${ }^{1}$ Targeson, Inc, Charlottesville, VA, USA, ${ }^{2}$ Pathology, Case Western Reserve University, Cleveland, OH, USA, ${ }^{3} \mathrm{La}$ Jolla Institute for Allergy and Immunology, La Jolla, CA, USA, ${ }^{4}$ Internal Medicine, University of Virginia, Charlottesville, VA, USA.

Crohn's disease (CD) is a chronic, relapsing inflammatory condition of the GI tract with no known cure. It follows a cyclical progression, with alternating periods of flare ups with disease remission. No conventional imaging technique is currently able to directly identify the location and extent of inflammation, which may be crucial for diagnosing and managing the disease. A safe, cost-effective molecular imaging technique able to monitor inflammation could be of significant utility in pre-clinical and clinical trials of CD therapeutics, in addition to assisting in routine patient care. We have investigated contrast ultrasound as a method for molecular imaging of inflammation in the context of $\mathrm{CD}$. In the current study, we utilized the SAMP/YitFc (SAMP) mouse model of CD to examine the feasibility of this technique. SAMP mice develop progressive, chronic intestinal inflammation with similar appearance to the human disease. Lipid encapsulated perfluorocarbon microbubbles bearing antibody or glyococonjugate ligands against a panel of pro-inflammatory endothelial adhesion molecules were used as ultrasound contrast agents. Microbubble preparations were administered intravenously in random order, and microbubble accumulation and wash out was measured by non-destructive contrast ultrasound imaging. The contrast signal was quantified in three regions of interest corresponding approximately to the terminal ileum, mesenteric lymph node, and mid-gut. We observed a significant contrast signal from microbubbles targeted to MAdCAM-1 and VCAM-1, moderate signal from microbubbles targeted to ICAM-1 and P-/E-selectin, and negligible signal from microbubbles targeted to Eselectin only or from control (isotype-targeted) microbubbles. Additionally, we investigated the use of targeted microbubbles to monitor MAdCAM-1 expression over the course of the disease in this model (940 weeks). We observed significant contrast enhancement at all time periods for MAdCAM-1 targeted microbubbles, with minimal signal from control microbubbles or in healthy control mice. Histological evaluation by blinded GI pathologist revealed that disease severity (as assessed by a metric encompassing villus distortion, active inflammation, and chronic inflammation) steadily increased over 9-40 weeks; however, our molecular imaging data suggests that MAdCAM-1 upregulation within the affected bowel precedes histopathologically detectable signs of inflammation. This suggests that molecular imaging of MAdCAM-1 may enable detection of disease prior to the appearance of clinical symptoms.

\section{$\mathbf{J 7 5 5}$}

\section{BIOLUMINESCENCE IMAGING OF MYELOPEROXIDASE ACTIVITY IN VIVO}

Shimon Gross, Seth T. Gammon, Britney L. Moss, David Piwnica-Worms Molecular Imaging Center, Mallinckrodt Institute of Radiology and Department of Developmental Biology, Washington University Medical School, St. Louis, MO, USA.

The myeloperoxidase (MPO) system of activated phagocytes contributes to the pathogenesis of inflammatory disease states ranging from atherosclerosis to cancer. We recently showed that upon systemic administration, the small molecule luminol enables noninvasive bioluminescence imaging (BLI) of MPO activity in vivo. In a focal acute arthritis model, we now further characterized the time-course of MPO activity induced by intra-articular injection of lipopolysaccharide (LPS) in wild type $\left(\mathrm{Mpo}^{+/+}\right)$and gene-deleted $\left(\mathrm{Mpo}^{-/}\right)$mice. LPS $(20 \mu \mathrm{g})$ was injected into the left ankle joint of $\mathrm{Mpo}^{+/+}$mice or $\mathrm{Mpo}^{-/-}$mice $(n=5$ each). Vehicle (saline) was injected into the right ankle joints. Before and up to $120 \mathrm{~h}$ after LPS injection, luminol was administered by i.p. injection $(200 \mathrm{mg} / \mathrm{kg})$ and mice were imaged 10 min later with a cooled CCD imaging system. Luminol-BLI produced a peak signal of 30-fold over background at $48 \mathrm{hrs}$ in $\mathrm{Mpo}^{+/+}$mice; there was no significant signal detected from the joints of similarly-treated $\mathrm{Mpo}^{-/-}$mice (Figure 1). Histological analysis showed matched infiltration of neutrophils in both $\mathrm{Mpo}^{+/+}$and $\mathrm{Mpo}^{-/-}$mice. In an acute dermatitis model, we applied phorbol 12-myristate 13-acetate (PMA; $100 \mu \mathrm{M})$ on the ear lobes of $\mathrm{Mpo}^{+/+}$and $\mathrm{Mpo}^{-/-}$mice $(n=5$ each). Three hrs post PMA stimulation, luminol-BLI revealed a detectable area of signal confined to the PMA application site, which by $24 \mathrm{~h}$ reached $13.2 \pm 0.2$ - fold over background. However, by $27 \mathrm{~h}$ post PMA, a second proximal structure could be detected, suggesting the involvement of regional lymph nodes. Again, bioluminescence co-localized with histological sites of inflammation and was totally abolished in $\mathrm{Mpo}^{-/-}$mice, despite massive infiltration of neutrophils in vivo, confirming the specificity for MPO activity. Luminol-BLI provides a noninvasive, specific, and highly sensitive optical readout of phagocytic-mediated MPO activity in vivo and may enable novel diagnostic applications in a wide range of acute inflammatory conditions. 


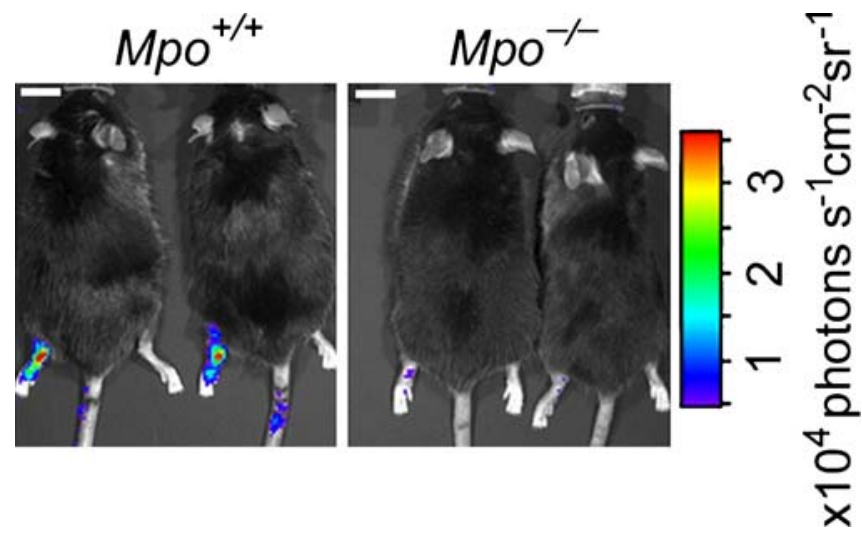

Figure 1. Bioluminescence imaging of MPO activity in vivo with luminol in an acute focal arthritis model.

\section{Imaging Molecular and/or Cellular Processes}

\author{
None of the Above
}

$\mathbf{J} 756$

\section{PET IMAGING OF THE PRE-METASTATIC NICHE WITH ${ }^{64}$ CU-CB-TE2A-LLP2A, A HIGH AFFINITY LIGAND FOR INTEGRIN $\alpha_{4} \beta_{1}$}

Monica Shokeen $^{1}$, Alexander Zheleznyak ${ }^{1}$, Jessica M. Wilson ${ }^{1}$, Kim Nguyen $^{1}$, Ruiwu Liu ${ }^{2}$, Lynne Collins ${ }^{3}$, Riccardo Ferdani ${ }^{1}$, David Piwnica-Worms ${ }^{3}$, Kit S. Lam ${ }^{2}$, Carolyn Anderson ${ }^{1}$

${ }^{1}$ Radiological Sciences, Washington University School of Medicine, Saint Louis, MO, USA, ${ }^{2}$ Division of Hematology \& Oncology, University of California-Davis Cancer Center, Davis, CA, USA, ${ }^{3}$ Molecular Imaging Center, Washington University School of Medicine, Saint Louis, MO, USA.

Introduction: Despite the advances made in cancer diagnosis and treatment, metastasis remains the primary cause of morbidity and mortality in cancer patients. Recent studies have established a role for bone marrowderived hematopoietic progenitor cells (HPCs) in the metastatic process. HPCs that express vascular endothelial factor receptor 1 (VEGFR1) and integrin $\alpha_{4} \beta_{1}$ (also known as very late antigen 4 (VLA-4)) arrive at sites of metastasis and form a pre-metastatic niche/microenvironment for initiating metastasis. We aim to address an imaging strategy for in vivo imaging of HPCs at pre-metastatic sites before a secondary tumor is established. Methods: The mouse model of tumor metastasis was $\alpha_{4} \beta_{1}$ negative MDAMB-231/luc human breast tumor cells. Implanted through arterial circulation (left ventricle (l.v.)), the tumor cells spontaneously metastasized to distant sites including bone. The mice were monitored over a period of 30 days post-implantation by a combined bioluminescence (BLI)/microPET/microCT imaging study to determine whether increases in PET signal with ${ }^{64} \mathrm{Cu}-\mathrm{CB}-\mathrm{TE} 2 \mathrm{~A}-\mathrm{LLP} 2 \mathrm{~A}$, which has pM affinity for $\alpha_{4} \beta_{1}$, was observed prior to tumors appearing in the bone. For microPET studies, images were acquired at 4 and $24 \mathrm{~h}$ after the administration of $180-200 \mu \mathrm{Ci}$ ( $2 \mu \mathrm{g} / \mathrm{mouse})$ of ${ }^{64} \mathrm{Cu}-\mathrm{CB}-\mathrm{TE} 2 \mathrm{~A}-\mathrm{LLP} 2 \mathrm{~A}$. Standard uptake values (SUVs) from microPET images were calculated using Amide and ASIPro. Simultaneous BLI imaging was done to track the luciferase transfected tumor cells. Results: Thus far two independent studies $(n=7$ and 8 mice respectively) have suggested an influx of $\alpha_{4} \beta_{1}$-positive HPCs prior to tumor cells homing to the site of metastasis. Cumulative SUV data from an experiment with 8 mice imaged on days -3 (control), 4, 10, 16 and 30 post l.v. tumor cell injection is presented in Figure 1. These data show an increased uptake of ${ }^{64} \mathrm{Cu}-\mathrm{CB}-\mathrm{TE} 2 \mathrm{~A}-\mathrm{LLP} 2 \mathrm{~A}$ over 30 days $(p<0.0001)$ at the potentially metastatic bone sites (right and left legs). Data validation using histology and FSCS analysis is underway. Conclusions: The presented data suggest feasibility of the pre-metastatic niche imaging by using the multi-modality approach described herein.

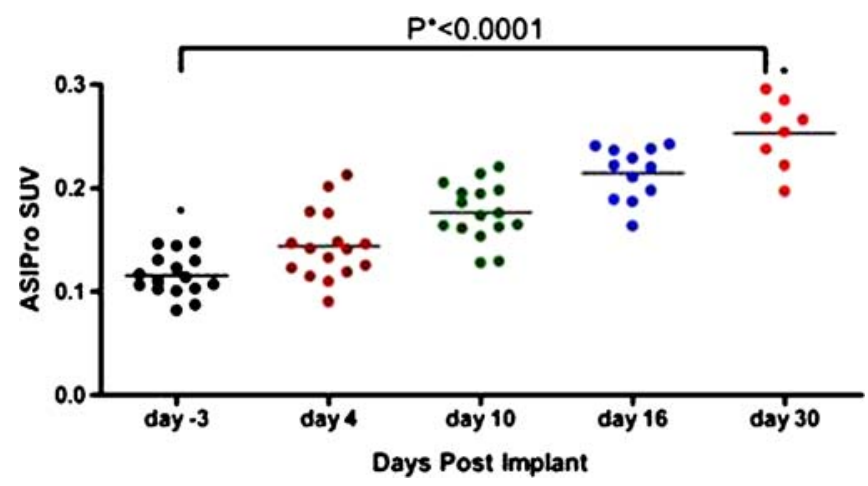

Figure 1.

$\mathbf{J 7 5 7}$

B-CELL SUB-CELLULAR LOCALIZATION OF GLUCOSE STIMULATED MN UPTAKE BY X-RAY FLUORESCENCE : IMPLICATIONS FOR PANCREATIC MRI

Lara Leoni $^{1}$, Anita Dhyani ${ }^{1}$, Patrick J. La Riviere ${ }^{1}$, Brian B. Roman ${ }^{1,2}$ ${ }^{1}$ Radiology, University of Chicago, Chicago, IL, USA, ${ }^{2}$ Committee on Medical Physics, University of Chicago, Chicago, IL, USA.

Manganese enhanced MRI (MEMRI) main applications have been traditionally in brain and cardiac functional studies. Recently there has been a renewed interest in $\mathrm{Mn}$ and novel applications are being investigated. We have shown MEMRI can be successfully employed to assess glucose activation and therefore $\beta$-cell function in isolated human islets as well as in the endogenous pancreas in rodents. Mn has been shown to enter $\beta$-cells through voltage-gated calcium channels but how it is metabolized and stored intracellularly and how it contributes to MR enhancement has not been investigated before. In this study isolated human islets perfused with $50 \mu \mathrm{M} \mathrm{Mn}$ and $2 \mathrm{mM}$ or $16 \mathrm{mM}$ glucose were found to have about $11 \%$ and $38 \%$ drop in T1 relaxation time respectively. Similarly, their MR signal had a $15 \%$ and $45 \%$ enhancement over untreated islets indicating a passive uptake of $\mathrm{Mn}$ in baseline conditions and a prevalent active one in a stimulating environment. To evaluate how Mn affects MR signal and how it is metabolized in $\beta$-cells, X-Ray Fluorescence Spectroscopy was used to both quantify and localize Mn within MIN-6 mouse insulinoma cells. Cells were exposed to $50 \mu \mathrm{M} \mathrm{Mn}$ and $2 \mathrm{mM}, 16 \mathrm{mM}$ glucose and $30 \mathrm{mM} \mathrm{KCl}$, fixed and imaged at the Advance Photon Source at Argonne National Laboratory. As expected only trace amounts of Mn were detected in untreated cells, $0.114 \mathrm{ug} / \mathrm{cm} 2$ with no difference in distribution across the cell. Analogous to MRI contrast data, an increase in $\mathrm{Mn}$ was measured upon exposure to $2 \mathrm{mM}$ glucose. The overall cellular concentration was about $0.86 \mathrm{ug} / \mathrm{cm} 2$, with the average nuclear concentration being slightly lower at $0.81 \mathrm{ug} / \mathrm{cm} 2$ and the cytoplasm showing diffuse $\mathrm{Mn}$ at an overall concentration about $1.0 \mathrm{ug} / \mathrm{cm} 2$. $16 \mathrm{mM}$ glucose stimulation caused a further increase in the cytoplasmic $\mathrm{Mn}$, especially the perinuclear area to $2.7 \mathrm{ug} / \mathrm{cm} 2$ whereas nuclear levels increased to $0.9 \mathrm{ug} / \mathrm{cm} 2$. The most dramatic increase was due to fast depolarization caused by $\mathrm{KCl}$ with a total cell concentration of $8.0 \mathrm{ug} / \mathrm{cm} 2$. Mn preferentially accumulated in high density areas within the cytoplasm at $16.5 \mathrm{ug} / \mathrm{cm} 2 \mathrm{Mn}$ versus an average $5.2 \mathrm{ug} / \mathrm{cm} 2$ measured in the nuclei. To the best of our knowledge this is the first report of Mn elemental analysis on Mn treated $\beta$-cells and the data well support its use as a MRI reporter of $\beta$-cell function. 


\section{Imaging Molecular and/or Cellular Processes}

\author{
Signal Transduction
}

$\mathbf{J 7 5 8}$

\section{EFFECTS OF CAMP DEPENDENT PROTEIN KINASE ACTIVATOR AND INHIBITOR ON IN VIVO ROLIPRAM BINDING TO PHOSPHODIESTERASE 4 \\ IN CONSCIOUS RATS}

Tetsuji Itoh ${ }^{1,2}$, Kohji Abe ${ }^{2,3}$, Jinsoo Hong ${ }^{1}$, Osamu Inoue ${ }^{3}$, Victor W. Pike $^{1}$, Robert B. Innis ${ }^{1}$, Masahiro Fujita ${ }^{1}$

${ }^{1}$ Molecular Imaging Branch, National Institute of Mental Health, Bethesda, MD, USA, ${ }^{2}$ Developmental Research Laboratories, Shionogi \& Co., Ltd., Toyonaka, Japan, ${ }^{3}$ Division of Health Sciences, Osaka University Graduate School of Medicine, Suita, Japan.

Background and aims: Phosphodiesterase-4 (PDE4), a major phosphodiesterase isozyme hydrolyzing the second messenger cAMP, may play a pivotal role in brain diseases and the effects of centrally acting drugs. Rolipram is a selective inhibitor of PDE4 and $\left[{ }^{11} \mathrm{C}\right]$ rolipram PET scans should allow the function of the cAMP second messenger cascade to be explored. cAMP dependent protein kinase (PKA) phosphorylates PDE4 and increases both enzyme activity and affinity of rolipram binding. In the present PET study, we examined effects of PKA modulators in conscious rats on the binding of $\left[{ }^{11} \mathrm{C}\right](R)$-rolipram in comparison to the much less active enantiomer $\left[{ }^{11} \mathrm{C}\right](S)$-rolipram. Methods: Dibutyryl cyclic-AMP (10 nmol; db-cAMP; cAMP analogue and PKA activator; $n=12,6$ for $\left[{ }^{11} \mathrm{C}\right](R)$-rolipram and 6 for $\left[{ }^{11} \mathrm{C}\right](S)$-rolipram) or Rp-adenosine-3',5' cyclic monophosphorothioate (100 nmol; Rp-cAMPS; PKA inhibitor; $n=$ 14,7 for $\left[{ }^{11} \mathrm{C}\right](R)$-rolipram and 7 for $\left[{ }^{11} \mathrm{C}\right](S)$-rolipram) was injected into the left striatum at $2.5,1.5$, and $0.5 \mathrm{~h}$ before the scan. At the same time, saline was administered into the right striatum. The animals were given an intravenous bolus injection of $\left[{ }^{11} \mathrm{C}\right](R)$-rolipram $(61 \pm 19 \mathrm{MBq})$ or $\left[{ }^{11} \mathrm{C}\right]$ $(S)$-rolipram $(46 \pm 9 \mathrm{MBq})$ and were imaged for $100 \mathrm{~min}$. The binding activity of $\left[{ }^{11} \mathrm{C}\right](R)$ - and $\left[{ }^{11} \mathrm{C}\right](S)$-rolipram in striatum was measured and expressed as standardized uptake value (SUV). The area under the curve (AUC) was compared between left and right-side striatum for each of $(R)$ and $(S)$-rolipram. Results: db-cAMP significantly increased AUC of $\left[{ }^{11} \mathrm{C}\right]$ $(R)$-rolipram compared to the saline injection side by $11 \%$ (Table. $p<0.01$ ). In contrast, Rp-cAMPS significantly decreased AUC of $\left[{ }^{11} \mathrm{C}\right](R)$-rolipram by $11 \%(p<0.01)$. On the other hand, the effects of neither of these PKA modulators were not observed in $\left[{ }^{11} \mathrm{C}\right](S)$-rolipram scans. Conclusion: Significant increase and decrease of $\left[{ }^{11} \mathrm{C}\right](R)$-rolipram binding were observed by PKA activator and inhibitor, respectively. These effects were not caused by changes in brain regional blood flow, since there were no effects when $\left[{ }^{11} \mathrm{C}\right](S)$-rolipram was used. These results support the value of measuring $\left[{ }^{11} \mathrm{C}\right](R)$-rolipram binding in brain for assessing responses to physiological or pharmacological challenges to the cAMP second messenger system.

Uptake $(\mathrm{SUV} \times \min )$ of $\left[{ }^{11} \mathrm{C}\right]$ rolipram in rat striatum treated with PKA modulators

\begin{tabular}{|c|c|c|c|c|}
\hline & {$\left[{ }^{11} \mathrm{C}\right](R)$-rolipram } & & {$\left[{ }^{11} \mathrm{C}\right](S)$-rolipram } & Drug \\
\hline db-cAMP $(\mathrm{N}=6)$ & Drug & Saline & $36 \pm 14$ & $36 \pm 14$ \\
\hline Rp-cAMPS $(\mathrm{N}=7)$ & $175 \pm 42^{*}$ & $158 \pm 38$ & $36 \pm 5$ & $37 \pm 6$ \\
\hline
\end{tabular}

$* P<0.01$ between drug and saline administration.

J759

\section{IMAGING LIGAND-DEPENDENT INTERACTION OF CXCR7 WITH $\beta$-ARRESTIN}

Kathryn E. Luker, Mudit Gupta, Gary Luker

Radiology, Microbiology and Immunology, University of Michigan, Ann Arbor, MI, USA.

CXCR7 recently was identified as a second receptor for chemokines CXCL11 and CXCL12. This seven-transmembrane receptor is reported to function both as a signaling molecule and a chemokine decoy receptor to regulate normal development and a wide range of disease processes. We hypothesized that binding to chemokine ligands promoted recruitment of the cytosolic adapter protein $\beta$-arrestin to CXCR7, providing the basis for an imaging assay in cell-based assays and living mice. We used firefly luciferase protein fragment complementation to quantify association of CXCR7 with $\beta$-arrestin in stably transduced cells and in an orthotopic mouse xenograft model of human breast cancer. In cellbased assays, we established that CXCR7 preferentially interacts with $\beta$-arrestin 2 as compared with $\beta$-arrestin 1 . Chemokine ligands significantly enhanced association of CXCR 7 with $\beta$-arrestin 2 in a dose-dependent manner. The magnitude of interactions between CXCR7 and b-arrestin 2 continued to increase over time in response to chemokine ligands, contrasting with the transient association of $\beta$-arrestin 2 with the related chemokine receptor CXCR4. We also determined that small molecule inhibitors of chemokine binding to CXCR7 promoted recruitment of $\beta$-arrestin 2 to CXCR7 in cell-based assays. In an orthotopic mouse xenograft model of human breast cancer, we used bioluminescence imaging to quantify association of CXCR7 and $\beta$-arrestin 2 following treatment with a CXCR7-targeted compound. These studies demonstrate ligand-dependent interactions of CXCR7 with $\beta$ arrestin 2 in intact cells and living mice, establishing an imaging reporter assay to investigate dynamic regulation of CXCR7 by chemokines and candidate therapeutic agents.

\section{$\mathbf{J} 760$}

\section{CT SCANS ROLE IN CRANIOFACIAL FEATURES OF MUTANT MICE WITH ALTERED LEVELS OF CELL CYCLE PROTEIN}

Paulina Wachowicz ${ }^{1}$, Guillermo de Carcer $^{1}$, Francisca Mulero ${ }^{2}$, Marcos Malumbres ${ }^{1}$

${ }^{1}$ Cell Division and Cancer Group, CNIO, Madrid, Spain, ${ }^{2}$ Molecular Imaging, CNIO, Madrid, Spain.

Purpose In our study, we developed the mouse model carrying the partial deletion of the gene implicated in the cell cycle. Due to CT technique we were able to compare the anatomic features in the heterozygous mice and their wild-type littermate in order to find out if 
the partial deletion of this protein can influence the anatomy of the animal. Methods We studied 30 mice, 15 heterozygous mice and 15 wild type littermate. The CT acquisition was performed with an eXplore Vista PET CT (GE Healthcare) apparatus using the following parameters: $200 \mu \mathrm{A}, 35 \mathrm{kV}, 160 \mu, 8$ shots and 360 projections. Recordings were subsequently analyzed with MMWKS software. Mice were anaesthetized with Ketamine $(75 \mathrm{mg} / \mathrm{Kg}$, Imalgene 500$)$ and Xilacine (1 mg/Kg, Ronpun) during acquisition. Results: The heterozygous mice displayed the significant craniofacial alterations as compared to wild-type mice of the same age and sex. Interestingly, none of other bones in the body was affected. The altered features include changes in the volume of the brain cavity and the slope of nasal bone. The heterozygous mouse showed augmented cranial volume and more pronounced nasal slope when compared to their wild type littermates. Although observed in both sexes, those variations were more pronounced in female mice. Conclusion: We show that the levels of our protein influence the anatomical changes of the cranium in mouse and the $\mathrm{CT}$ is an appropriate technique to show these alterations.
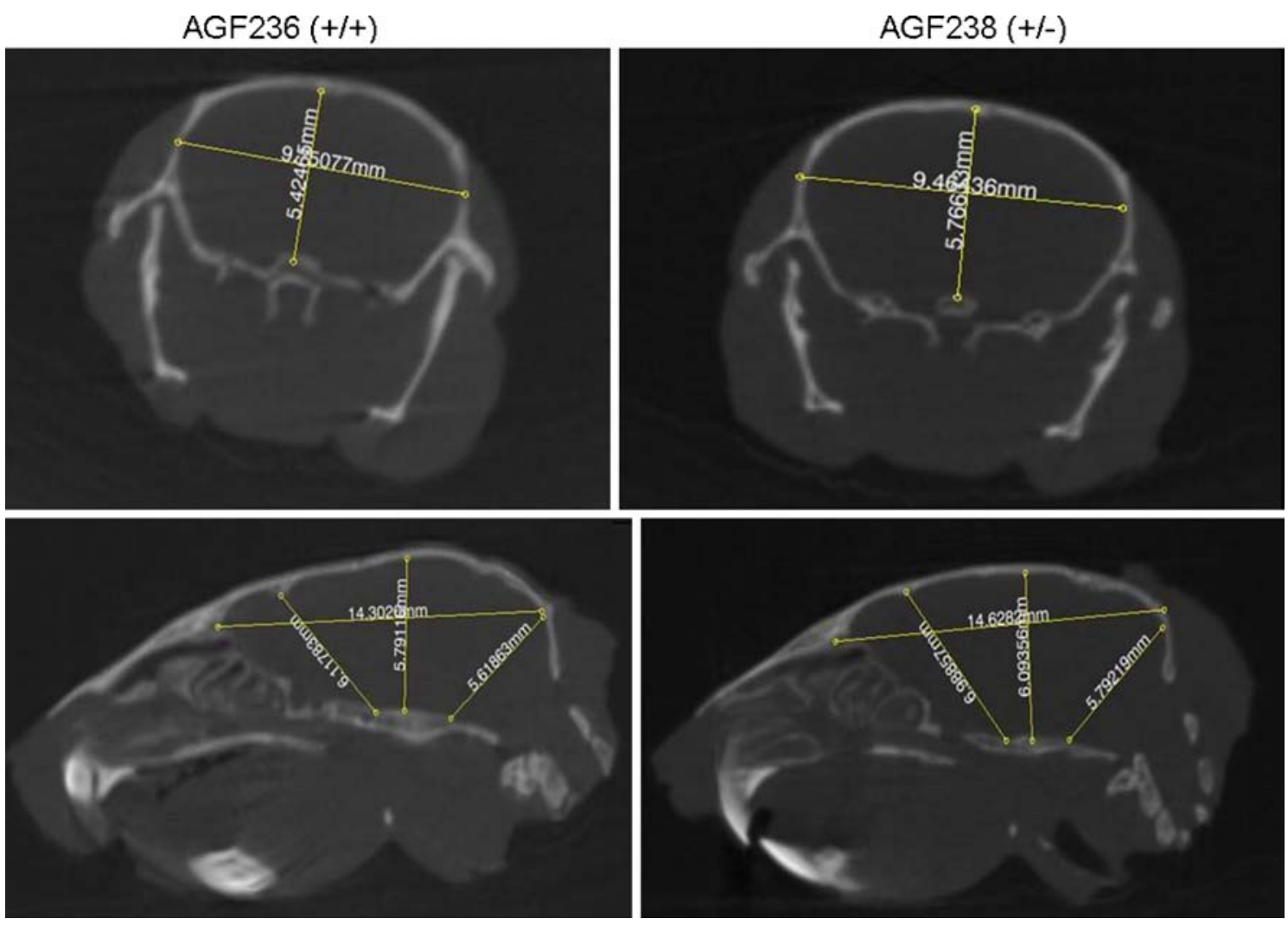

Cranium measures

\section{THE SRC-HOMOLOGY 2 (SH2) DOMAIN OF GROWTH FACTOR RECEPTOR BOUND PROTEIN 2 (GRB2) HAS A POTENTIAL FOR IN VIVO IMAGING, TARGETING ACTIVATED EPIDERMAL GROWTH FACTOR RECEPTOR (EGFR)}

Yuriko Saito $^{1,2}$, Takako Furukawa ${ }^{1}$, Yasushi Arano $^{2}$, Yasuhisa Fujibayashi ${ }^{3,1}$, Tsuneo Saga ${ }^{1}$

${ }^{1}$ Diagnostic Imaging Group, Molecular Imaging Center, National Institute of Radiological Sciences, Chiba, Japan, ${ }^{2}$ Department of Molecular Imaging and Radiotherapy, Graduate School of Pharmaceutical Sciences, Chiba University, Chiba, Japan, ${ }^{3}$ Biomedical Imaging Research Center, University of Fukui, Fukui, Japan.

Abnormal activation of EGFR is known to be involved in cancer pathogenesis and its malignancy. In order to generate an imaging probe that can capture EGFR activation, not the amount or structure of EGFR, we designed a new protein probe. We selected SH2 domain of Grb2, an adaptor protein of activated-EGFR, for the key component of the probe, and added transactivating transduction (TAT), flag and tyrosine residue, for protein transduction into cells, molecular analysis and radiolabeling, respectively, to produce a probe protein named TSF. Having confirmed that TSF can cross the cell membrane and bind to EGFR, we moved to examine the efficacy of TSF in vivo by labeling it with ${ }^{125} \mathrm{I}$. Labeling efficiency of ${ }^{125} \mathrm{I}$-TSF by chloramine-T method was about $40 \%$ and average of 0.8 molecule of ${ }^{125} \mathrm{I}$ was calculated to be on single TSF molecule. After column purification, the radiochemical purity was more than $99 \%$. The amount of ${ }^{125}$ I-TSF taken up into cells was significantly higher in EGFR positive A431 cells $(24.6 \pm 3.7 \%)$ than EGFR negative MDA-MB435 cells $(20.4 \pm 2.1 \%)$. When the biodistribution of ${ }^{125} \mathrm{I}$-TSF was examined in tumor-bearing mice at $0.5,1$, and $3 \mathrm{~h}$ after the administration from the tail vein, the radioactivity in A431 tumor was increased for $1 \mathrm{~h}$ after the administration, while that into the other tissues including MDAMB435 tumor was decreased over time. A431 tumor-to-tissue ratio was highest at $1 \mathrm{~h}$ after injection, and A431 tumor accumulated 1.6-fold more TSF than MDA-MB435 tumor. A431 tumor-to-blood and muscle ratios were 1.1 and 3.5, respectively. However, high accumulation of the radioactivity in stomach indicated rapid de-iodination of 
the labeled probe in vivo. In conclusion, $\mathrm{SH} 2$ domain of Grb2 has a potential as a key component of the probe to detect EGFR activation in vivo, though direct ${ }^{125}$ I-labeling would not be suitable.

$\mathbf{J} 762$

\author{
MOLECULAR IMAGING OF CHECKPOINT KINASE 1 \\ (CHK1) PHOSPHORYLATION BY DNA DAMAGE \\ USING FLUORESCENCE RESONANCE ENERGY TRANSFER \\ (FRET) SYSTEM YOU-RI LEE, JONG-HWA PARK, IN-SOO \\ YOON AND YE SUN HAN DEPARTMENT OF ADVANCED \\ TECHNOLOGY FUSION, KONKUK UNIVERSITY, \\ HWAYANG-DONG, GWANGJIN-GU, SEOUL, 143-701, KOREA \\ Ye Sun Han

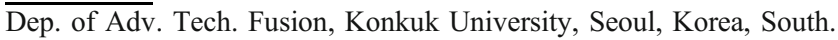

Replication stress and DNA damage cause the phosphorylation and activation of checkpoint kinase 1 (Chk1) through ataxia telangiectasia and Rad3-related protein (ATR). Activated Chk1 plays a critical role in cellular checkpoint responses by stabilizing stalled replication forks, and blocking the firing of late origins of replication forks, and arresting cells in G2/M. A major target for Chk1 in cell cycle checkpoints is a Cdc25 phsophatase, that dephosphatates and activates cyclin dependent kinases (Cdks), thereby promoting cell progression. Among the three isoforms of vertebrate Cdc25s, Cdc25A has shown to be essential for the Chk1-induced cell cycle arrest and the DNA replication checkpoint. In this work, we developed a fluorescence resonance energy transfer (FRET)-based molecular imaging system to monitor the phosphorylation of Chk1 by DNA damage. FRET is a process by which energy is transferred from one fluorescent molecule to another by way of dipole-dipole interactions during an excitation of the donor molecule, resulting to a excitation of acceptor molecule. It was successfully employed to monitor a distance between different fluorescent probes that are attached to macromolecules, binding interactions between different molecules, and conformational changes within the same molecule. To establish FRET-based Chk1 phosphorylation imaging system, gene fragments corresponding to the coding regions of Chk1 and Cdc25A were generated by PCR and separately fused with enhanced cyan fluorescent protein (ECFP) and enhanced yellow fluorescent protein (EYFP) coding regions. Human embryonic kidney 293 (HEK293) cells transfected with EGFP-Chk1 or/and EYFP-Cdc25A expressing vector were determined using confocal microscope with $440 \mathrm{~nm}$ excitation and $535 \mathrm{~nm}$ emission filter. Yellow fluorescences emitted by EGFP that was excited by ECFP were only observed in HEK293 cells co-transfected with EGFP-Chk1 and EYFP-Cdc25A expressing vectors, which were enhanced by DNA damage inducing agents. These results demonstrate that physical interactions between Chk1 and Cdc25A are increased by DNA damage inducing agents and FRET-based imaging system can be successfully used to monitor the phosphorylation of Chk1 by DNA damage.

\section{$\mathbf{J} 763$}

GENE DOSAGE EFFECT OF PKBALPHA/AKT1 IN VASCULARIZATION AND MINERALIZATION OF THE LONG BONES: MRI AND CT

Katrien Vandoorne ${ }^{1}$, Vicki Plaks ${ }^{1}$, Amnon Sharir ${ }^{3}$, Jeremy Magland ${ }^{4}$, Felix W. Wehrli ${ }^{4}$, Brian A. Hemmings ${ }^{5}$, Alon Harmelin ${ }^{2}$, Michal Neeman $^{1}$

${ }^{1}$ Biological Regulation, Weizmann Institute, Rehovot, Israel, ${ }^{2}$ Veterinary Resources, Weizmann Institute, Rehovot, Israel, ${ }^{3}$ Molecular Genetics, Weizmann Institute, Rehovot, Israel, ${ }^{4}$ Laboratory for Structural NMR Imaging, Department of Radiology, University of Pennsylvania, Philadelphia, PA, USA, ${ }^{5}$ Friedrich Miescher Institute for Biomedical Research, Basel, Switzerland.
PKBalpha/Akt1 is a major mediator of signaling of angiogenic growth factors, affecting endothelial cell survival, proliferation and differentiation as well as vascular permeability. In the study reported here, non invasive dynamic contrast enhanced MRI was applied demonstrating vascular defects at long bones associated with reduced size of PKBalpha/Akt1 deficient mice. Vascular deficiency was detected in the humeri of 40-day-old mice along with osteopenia revealed by $\mu \mathrm{CT}$ and $\mu$ MRI. These results implicated PKBalpha/Akt1 in regulation of overall size by affecting bone vasculature. Histological examination showed reduced blood vessel density, and mineralized bone density at the humerus, distal from the growth plate in PKBalpha/Aktl deficient mice. Remarkably, significant vascular and bone developmental defects were detected also for heterozygous mice lacking a single copy of PKBalpha/Akt1. Dynamic contrast enhanced MRI was performed using biotin-BSAGdDTPA as a macromolecular contrast media. Vascular defects in PKBalpha/Akt1 deficient mice were detected by MRI.

\section{$\mathbf{J} 764$}

\section{IMAGING CHEMOKINE RECEPTOR DIMERIZATION WITH FIREFLY LUCIFERASE COMPLEMENTATION Kathryn E. Luker, Mudit Gupta, Gary Luker} Radiology, Microbiology and Immunology, University of Michigan, Ann Arbor, MI, USA.

Cell culture models suggest that homodimerization and heterodimerization of seven-transmembrane receptors regulate processes including specificity of ligand binding and activation of downstream signaling pathways, making receptor dimerization a critical determinant of receptor biology and a promising new therapeutic target. Our objective was to develop an imaging system to detect and quantify receptor dimerization both in cell-based assays and animal models of disease. We used firefly luciferase protein fragment complementation to quantify homo- and hetero-dimerization of chemokine receptors CXCR4 and CXCR7, two seven-transmembrane receptors with central functions in normal development, cancer, and other diseases. Studies were performed in intact cells and an orthotopic mouse tumor xenograft model of breast cancer. Treatment with chemokine ligands and pharmacologic agents produced time- and dose-dependent changes in reporter signal. Changes in dimerization signals could be detected immediately after adding ligand, and the magnitude of change in bioluminescence increased over time. While chemokines regulated reporter bioluminescence for CXCR4 or CXCR7 homodimers, ligands did not alter reporter signals from receptor heterodimers. In a mouse tumor xenograft model of breast cancer, we used bioluminescence imaging to analyze dynamic changes in receptor homodimerization in response to CXCR4- and CXCR7-targeted agents. These studies show that ligands and targeted compounds modulate dynamics of CXCR4 and CXCR7 homodimers in a dose- and time-dependent manner. Firefly luciferase complementation enables function and therapeutic modulation of chemokine receptors to be translated readily from cell culture assays to mouse models of disease.

\section{$\mathbf{J} 765$}

REAL-TIME BIOLUMINESCENCE IMAGING OF LIGANDINDUCED IKB $\alpha$ SIGNAL TRANSDUCTION IN CELLULO AND IN VIVO

Britney L. Moss, David Piwnica-Worms

Mallinckrodt Institute of Radiology, Washington University, St. Louis, MO, USA. 
The transcription factor NF- $\mathrm{kB}$ regulates immunity, inflammation, and survival and is implicated in a variety of human pathologies. We have developed a bioluminescent imaging (BLI) reporter ( Fluc) for real-time in cellulo visualization of both degradation and NF$\kappa \mathrm{B}-$ dependent upregulation of a critical NF- $\mathrm{NB}$ inhibitor, I $\mathrm{I} \mathrm{B} \alpha$. Upon ligand stimulation, cytoplasmic I $\mathrm{I} \mathrm{B} \alpha$ complexed to $\mathrm{NF}-\mathrm{\kappa B}$ is targeted for proteasomal destruction by $\mathrm{I} \kappa \mathrm{B}$ Kinase (IKK)-mediated phosphorylation, which leads to translocation of NF- $\mathrm{KB}$ into the nucleus. A critical target of NF- $\kappa B$ transcriptional activity is the $\mathrm{I} \kappa \mathrm{B} \alpha$ gene, thus establishing a negative feedback loop. To image in real-time the dynamics of this feedback loop, we transiently expressed the pкB5$>\mathrm{I} \kappa \mathrm{B} \alpha$-Fluc reporter in HepG2 cells and used BLI to systematically evaluate the effects of ligand type, concentration, and duration upon degradation, re-synthesis, and stabilization of I $\mathrm{I} B \alpha$. Surprisingly, brief $5 \mathrm{~s}$ pulses of tumor necrosis factor $\alpha(\mathrm{TNF} \alpha)$ or interleukin $1 \beta$ (IL-1 $\beta$ ) produced robust degradation and re-synthesis of IкB $\alpha$ levels, suggesting that even instantaneous ligand exposure was capable of generating a substantial response in target cells. Varying ligand pulse duration from 5-900 s increased the degree of IкB $\alpha$ degradation and re-synthesis, but had less striking effects on the kinetics of these events, whereas modulation of ligand concentration between $0.06-570 \mathrm{pM}$ revealed strong impacts on both the amplitudes and kinetics of I $\mathrm{I} B \alpha$ processing. $\mathrm{TNF} \alpha$ and IL- $1 \beta$ elicited similar patterns in I $\mathrm{B} \alpha$ degradation, but had differing re-synthesis trends. While in cellulo experimentation can reveal much about the NF- $\mathrm{kB}$ circuitry within a cell or group of cells, of critical interest is how NF- $\mathrm{KB}$ signaling is regulated in vivo within tissues and under different types of physiological and pathological stimulation. To this end, we have employed somatic gene transfer by hydrodynamic injection, a technique that allows selective transfection of mouse liver with the $\mathrm{p \kappa B} 5->\mathrm{I} \kappa \mathrm{B} \alpha$-Fluc reporter gene. Remarkably, i.v. bolus TNF $\alpha$ stimulation ( $30 \mathrm{ng} /$ mouse) in these animals resulted in I $\mathrm{B} \alpha$ processing profiles strongly resembling the profiles seen in cellulo, but with even faster kinetics. Furthermore, increasing doses of $\mathrm{TNF} \alpha$ (from 1-30 ng/mouse) yielded increasing levels of degradation and re-synthesis, similar to what was seen in cellulo. These data demonstrate the utility of a novel real-time BLI tool to interrogate the dynamic and kinetic properties of a key regulatory node within the NF$\kappa \mathrm{B}$ pathway in cellulo and in vivo.

\section{J766}

\section{MDM2 SMALL MOLECULE INHIBITOR ACTIVATES P53 FUNCTION AND SENSITIZES GLIOMAS CELLS TO CHEMOTHERAPY AGENT}

Parisa Monfared, Daniel Rudan, Lars Franken, Thomas Viel, Gabriele Schneider, Eva Knoedgen, Yannic Waerzeggers, Philipp Euskirchen, Andreas H. Jacobs Laboratory for Gene Therapy and Molecular Imaging, Max-Planck Institute for Neurological Research with Klaus-JoachimZülch-Laboratories of the Max Planck Society, Koeln, Germany.

Introduction: Targeted therapies that inhibit the MDM2-p53 interaction and the downstream Rb-E2F signalling pathway have shown promising anticancer activity, but their efficacies in human gliomas have not been investigated. Recently, small-molecule antagonists of MDM2, the MDM2-inhibitiors, have been developed to inhibit the MDM2-p53 interaction and activate p53 signalling serving possible anti-cancer activity. Aim: To investigate the therapeutic potential of disrupting the MDM2-p53 interaction in human gliomas cells with various p53 status. We particularly followed whether MDM2- inhibition would sensitize gliomas to additional chemotherapy. Methods: Human glioma cell lines with various p53 status [U87Neo and U87dEFGR (p53wt/wt), U87-Neo-E6 (p53 inactivated), Gli36dEGFR (p53mt/mt), LN-18 (p53mt/wt), LN-308 (p53 null), CCF-STTG1 (p53WT-overexpress MDM2) and primary brain tumor patient's samples (glioblastoma, oligoastrocytomas grade III)] were treated with MDM2-inhibitor with and without BCNU. Detailed analysis of the expression of mdm2, p53, p21, and E2F1, as well as proliferative activity and apoptotic pathway was performed. Following treatment, luciferase activity was monitored in U87dEGFR-E2FLITG and U87dEGFR-p53-LITG in vitro and in vivo. Results: MDM2-inhibior alone and in combination with BCNU results in a dose- and time-dependent reduction in cell viability and proliferation. Western blot studies showed that MDM2-inhibition modifies expression of several cell cycle regulating genes and results in cell cycle arrest and induction of apoptosis. Moreover, MDM2-inhibition increases the effectiveness of sublethal BCNU doses causing significant growth inhibition. Notably, we found consistent and robust accumulation of $\mathrm{p} 53$ protein and downregulation of E2F-1 protein triggered by MDM2-inhibition alone and in combination with $\mathrm{BCNU}$ in primary glioma samples. Bioluminescence activity following treatment show to be dose and time dependent and correlated to $\mathrm{p} 53$ and E2F activity in vitro and in vivo. Conclusions: Our results demonstrate that MDM2 inhibition elicits a dose- and time-dependent antiproliferative effect of glioma growth and potentiates the effects of BCNU via $\mathrm{p} 53$-dependent and $\mathrm{p} 53$-independent mechanisms and that multiple cell cycle regulating genes are involved in the process. MDM2 inhibitors have a broad spectrum of antitumor activities in human cancers regardless of p53 status and may provide novel approaches for anti-glioma therapy.

\section{J767}

\section{IMAGING OF FCERI DYNAMICS AT THE MAST CELL} SYNAPSE

Jerilyn A. Timlin $^{1}$, Amanda Carroll-Portillo ${ }^{2,1}$, Kathrin Spendier ${ }^{3}$, Jesse Aaron', Janet Pfeiffer ${ }^{2}$, Keith Lidke ${ }^{3}$, Diane Lidke ${ }^{2}$, James L. Thomas ${ }^{3}$, Bridget Wilson ${ }^{2}$

${ }^{1}$ Bioenergy and Defense Technologies, Sandia National Labs, Albuquerque, NM, USA, ${ }^{2}$ Department of Pathology, University of New Mexico, Cancer Research and Treatment Center, Albuquerque, NM, USA, ${ }^{3}$ Department of Physics and Astronomy, University of New Mexico, Albuquerque, NM, USA.

High affinity IgE receptors (FceRI) on mast cells undergo spatial reorganization upon binding of a recognized antigen. We employed a multi-scale, multi-modal molecular imaging approach to visualize the dynamics of the FceRI receptor and related signaling components at early and late time points subsequent to antigen binding. Rat basophilic leukemia mast cells (RBL-2H3) primed with fluorescent anti-DNP IgE were engaged by surfaces presenting supported lipid bilayer-incorporated, monovalent DNP (mobile ligand) or chemically crosslinked, multivalent DNP (immobile ligand). Total internal reflection fluorescence (TIRF) microscopy, confocal fluorescence microscopy, and electron microscopy were used to visualize receptor reorganization within the contacting membranes. We have observed that dynamic receptor reorganization within the contacting membrane is markedly different upon binding of mobile and immobile ligands. We discovered that the 
"mast cell synapse" formed upon contact with a surface presenting mobile, monovalent ligand resulted in development of a large contact region localized to the central portion of the contacting membrane. Receptors remained mobile within this region and were capable of initiating a signaling cascade, leading to measurable degranulation. We measured bulk degranulation with a standard assay for $\beta$-hexosaminidase release. We also quantified single degranulation events using a mast cell line expressing a green fluorescent version of FasL protein, which is targeted to the secretory lysomes. This permits visualization of membrane fusion and granule release. Collectively, these data indicate mast cell signaling can occur in the absence of FceRI crosslinking, suggesting an alternative mechanism exists for mast cell signal transduction. The dynamics of other cellular components such as actin, cholesterol, secretory lysosomes, and downstream signaling molecules were also analyzed and will be presented.

\section{Imaging Molecular and/or Cellular Processes}

\section{Stem Cells and Tissue Regeneration}

\section{$\mathbf{J} 768$}

\section{OPTICAL IMAGING OF NEURAL PROGENITOR CELL} HOMING TO TUMORS OF NON-NEURONAL ORIGIN

Isabel G. Newton ${ }^{1}$, Steven Messina-Graham ${ }^{1}$, Annelie Abrahamsson ${ }^{2}$, Evan Y. Snyder ${ }^{3,4}$, Catriona Jamieson ${ }^{2}$, Robert F. Mattrey ${ }^{1}{ }^{1}$ Radiology, UCSD, San Diego, CA, USA, ${ }^{2}$ Medicine, UCSD, San Diego, CA, USA, ${ }^{3}$ Stem Cells and Regenerative Medicine, Burnham Institute, San Diego, CA, USA, ${ }^{4}$ Pediatrics, UCSD, San Diego, CA, USA.

PURPOSE: Murine neural progenitor cells (C17.2) demonstrate a tropism for gliomas, other neural diseases, and some non-neural cancers. If sufficiently robust, cellular homing to tumors could be exploited for targeted imaging and delivery of therapeutics. METHODS: We tested the hypothesis that IV-injected C17.2 cells home to solid non-neuronal tumors at a level that can be detected by optical imaging. Murine models of human chronic myeloid leukemia (CML), colon cancer (HT29) and lung cancer (LLC) were used. Neonatal rag2 mice underwent intrahepatic transplantation of human-derived blast crisis CML cells 8 weeks prior, allowing solid tumors to form. Adult nude mice received subcutaneous flank injections of LLC or HT29 cells and grown to $0.5 \mathrm{~cm}$ in diameter. C17.2 cells were stained with DiR and $10^{6}$ cells injected IV into mice bearing CML, LLC, HT29 and untransplanted controls $(N=10$ each). At $30 \mathrm{mins}, 24 \mathrm{hrs}$, and $48 \mathrm{hrs}$ post injection, fluorescence was imaged using a Caliper IVIS ${ }^{\circledR} 200$, cohorts were sacrificed and the viscera imaged in situ. The liver, spleen, kidney, marrow, brain, and tumors were harvested and, in a subset of CML and HT29 mice, imaged ex vivo. FACS analysis of human engraftment (CD45+) and DiR-positive cells was conducted on the tissues (except brain). RESULTS: From $30 \mathrm{~min}$ post injection of DiR-labeled C17.2, the lungs then liver and spleen demonstrated fluorescence. Signal appeared in the legs by $24 \mathrm{~h}$ and the head by $48 \mathrm{~h}$. No signal was detectable in vivo in the CML, LLC or HT29 tumors. Postmortem imaging of exposed viscera confirmed robust signal in the lungs, liver and spleen, with lower levels in the marrow and brain. Of the tumors imaged ex vivo, weak signal was seen in $60 \%$ of the CML tumors and none of the HT29 tumors. FACS confirmed the largest number of DiR-positive cells were in the liver and spleen followed by bone marrow. Whereas $30 \%$ of the CML tumors showed low levels of DiRpositive cells by FACS, none of the colon or lung tumors were positive. CONCLUSION: C17.2 cells weakly home to human CML tumors, detectable optically only when imaged ex vivo. C17.2 do not home to LLC lung cancer or HT29 colon cancer. The high proportion of $\mathrm{C} 17.2$ cells in the lungs, liver and spleen may constitute filtration, whereas homing likely accounts for C17.2 localization to the CML tumors, marrow and brain. Future studies will focus on optimizing the conditions under which C17.2 cells home to human CML tumors in order to exploit this property for the purpose of targeted imaging and drug delivery.

J769

\section{BIOLUMINESCENCE, TRANSGENIC REPORTER MICE, FIBERED CONFOCAL MICROSCOPY AND MICRO CT IMAGING DEMONSTRATE THE EFFECT OF SYNTHETIC OXYGEN CARRIERS ON STEM CELL-BASED BONE REGENERATION}

Gadi Pelled $^{1,2}$, Nadav Kimelman Bleich ${ }^{2}$, Yoram Zilberman ${ }^{2}$, Wafa Tawackoli $^{1}$, Zulma Gazit ${ }^{1,2}$, Dan Gazit ${ }^{1,2}$

${ }^{1}$ Surgery, Cedars Sinai Medical Center, Los Angeles, CA, USA, ${ }^{2}$ Skeletal Biotech Laboratory, Hebrew University of Jerusalem, Jerusalem, Israel.

Combining Mesenchymal Stem Cells (MSCs) and Bone Morphogenetic Proteins (BMPs) has been shown to promote fracture repair and rapid bone formation in vivo. It is also known that osteogenesis is highly dependent on vascularization and oxygen supply. In tissue engineering applications, cells are seeded within a biodegradable scaffold, which could reduce oxygen availability, thus limiting the osteogenic effect. We therefore hypothesized that oxygen-enriched scaffolds would enhance engineered MSC-based bone formation, in vivo. In order to test this hypothesis we have utilized perfluorocarbons synthetic oxygen carriers as a means to increase oxygen availability in hydrogels. Tet-off BMP2 MSCs (C3H10T1/2 MSC line that overexpress the BMP-2 gene under Tetracycline regulation) and Tet-off BMP2 Luc/GFP (Tet-off BMP2 MSCs that were genetically engineered to constantly express both luciferase and GFP reporter genes) were used. Aliquots of cells (one million Tet-off BMP2 cells or 3 million Tet-off BMP2 Luc/GFP cells) were resuspended in 50ul of fibrin gel (Tisseel, Baxter, Germany). Five or $10 \%$ (v/w) Heptacosafluorotributylamine (PFTBA, Sigma) was added to the hydrogels just before implantation. The cell-hydrogel mixture was injected subcutaneously in $\mathrm{C} 3 \mathrm{H} / \mathrm{HeN}$ mice or in Osteocalcin-Luciferase transgenic (Oc-Luc Tg) mice. Osteocalcin expression was noninvasively quantified using bioluminescence imaging (Roper Scientific, USA) of MSC implants in Oc-Luc Tg mice. In order to quantify bone formation, the ectopic implants were scanned in a high-resolution micro CT system (uCT 40, Scanco). Tet-off BMP2 Luc/GFP cell viability in the ectopic implants was quantified using bioluminescence and fibered confocal microscopy imaging (Cell Vizio, France). Mice were sacrificed after two weeks, and implants were harvested and fixed in $4 \%$ formalin. Bioluminescence imaging of Oc-Luc Tg mice indicated an increase in osteogenesis in PFTBAenriched grafts. Indeed, micro CT-based analysis of ectopic bone formation demonstrated significant 2.5 fold increase in bone volume 
in PFTBA-supplemented implants. Moreover, when Tet-off BMP2 Luc/GFP cells were implanted in PFTBA enriched fibrin gel, cell viability was enhanced significantly compared with implants that did not contain PFTBA, as demonstrated by bioluminescence and fluorescence imaging. We have shown, for the first time, that synthetic oxygen carriers supplementation of engineered MSCs implants enhances ectopic bone formation in vivo. This effect is correlated with the enhancement of post implantation cell viability.

$\mathbf{J 7 7 0}$

\section{MOLECULAR IMAGING OF CELL TRANSPLANTATION IN PORCINE MYOCARDIUM USING CLINICAL MRI AND PET-CT}

Natesh Parashurama ${ }^{1}$, Byeong Cheol $\mathrm{Ahn}^{1,4}$, David Yerushalmi ${ }^{1}$, Cristina Zavaleta $^{1}$, Jaehoon Chung ${ }^{2}$, Julia C. Swanson ${ }^{3}$, Fumiaki Ikeno ${ }^{2}$, Tomohiko Teramoto ${ }^{2}$, Jennifer Lyons ${ }^{2}$, Srabani Bhaumik ${ }^{5}$, Shahriar Yaghoubi $^{1}$, Phillip Yang ${ }^{2}$, Paul G. Yock ${ }^{2}$, Robert C. Robbins ${ }^{3}$, Sanjiv S. Gambhir $^{1}$

${ }^{1}$ Department of Radiology, Molecular Imaging Program at Stanford, Stanford University, Stanford, CA, USA, ${ }^{2}$ Cardiovascular Medicine, Stanford University, Stanford, CA, USA, ${ }^{3}$ Cardiothoracic Surgery, Stanford University, Stanford, CA, USA, ${ }^{4}$ Nuclear Medicine, Kyungpook National University, Daegu, Korea, South, ${ }^{5}$ GE Global Research Center, General Electric, Niskayuna, NY, USA.

Imaging cells after cardiac cellular therapy could be a promising approach to optimizing this new treatment strategy. Marrying the strengths of cardiovascular MRI (high spatial resolution) and PET (high sensitivity) enhances the ability to assess the cell location, viability, survival, and myocardial response to treatment. To build on our previous swine studies with adenovirally transduced cells, Rat Mesenchymal Stem Cells (rMSC) were stably transduced with a second generation, self-inactivating lentivirus carrying a ubiquitin-driven triple fusion (TF) reporter gene (RG), comprised of green fluorescent protein (GFP), firefly luciferase (Fluc) and a mutant truncated herpes simplex virus type 1 thymidine kinase (HSV1-tsr39tk). These TFRG expressing rMSC were loaded overnight with $12 \mu \mathrm{g} / \mathrm{ml}$ Iron bearing SPIO particles $(35 \mathrm{~nm})$ using a protamine transfection technique. To determine sensitivity, 3 injections of SPIO particles, at varying concentrations, were made into swine hearts, and a T2 weighted sequence, a short axis, Fiesta sequence, was applied to the $3 \mathrm{~T}$ MRI. Injection of $5 \mathrm{mg} / \mathrm{ml}$ of SPIO resulted in an SNR (signal to noise ratio) of 0.81 , injection of $12 \mu \mathrm{g} / \mathrm{ml}$ resulted in an SNR of 4.65 , and injection of $4 \mu \mathrm{g} / \mathrm{ml}$ resulted in an SNR of 5.73, where decreasing dose of SPIO leads to an increased signal intensity $(N=2$ swine). The MRI allowed simple detection of injection site after injection of 150-220 x106 cells. Next, clinical PET-CT was performed dynamically op to five hours after injection of F18-FHBG $(14 \mathrm{mCi})$, and nine hours after injection of the rMSC. Uptake of probe was measured as a SNR ratios 1.34 and 1.16 each injection $(N=2$ swine). Cell injection sites were localized to areas of heart by MRI T2 weighted images. Future work will aim to use swine TFRG-MSC in survival models of swine infarction. Studies of PET reporter genes in large animals should help translate stem cells to the clinic and establish the role for molecular imaging in monitoring stem cell therapies.
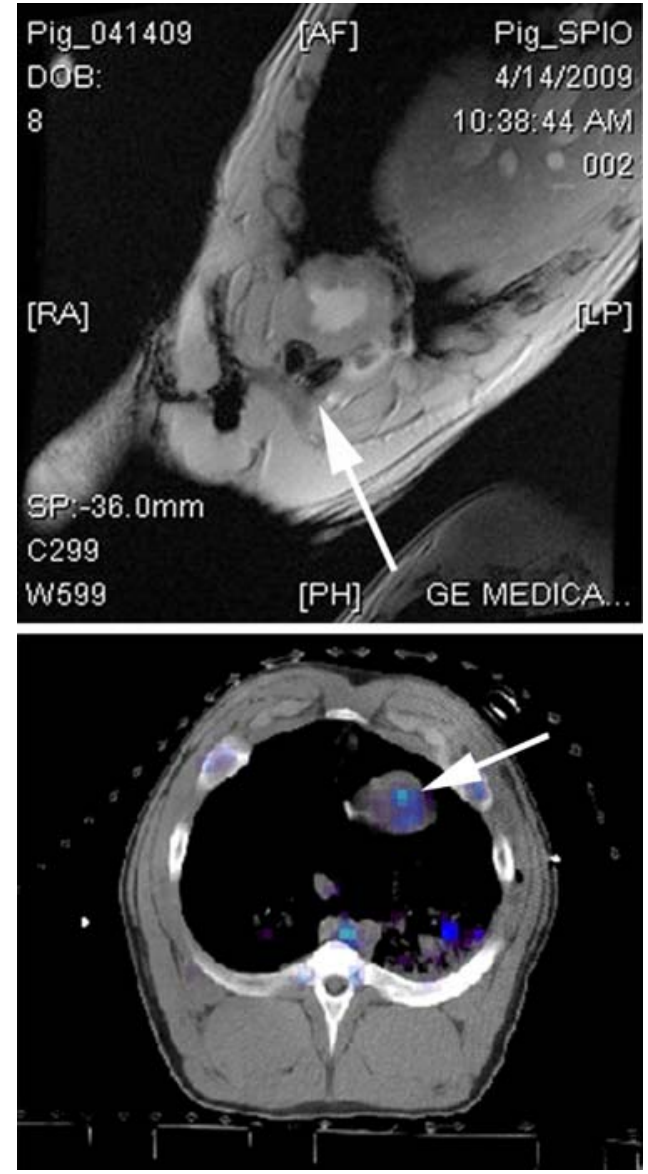

Figure 1. rMSC-TFRG implanted into Porcine swine and imaged with PET-CT and MRI. a) Fast gradient echo sequence sequence, short axis view, of the swine heart. $250 \times 10^{\wedge} 6 \mathrm{rMSC}-\mathrm{TFRG}$ cells labeled with $35 \mathrm{~nm}$ iron bearing SPIO and transplanted into swine hearts. Arrow demonstrates dephasing signal at apex of heart. b) PET-CT coregistered image $9 \mathrm{~h}$ after injection of rMSC and $5 \mathrm{~h}$ after injection of F18-FHBG. Transverse section demonstrate coregistered image of CT and PET of apex of heart. Arrow demonstrates signal at bottom of heart, consistent with cell injection and uptake.

\section{J771}

\section{IMAGING THE FATE OF GLIAL PRECURSORS TRANSPLANTED INTO THE BRAIN OF RAG2/SHIVERER DOUBLE KNOCKOUT MICE}

Nidhi Rumpal $^{1,2}$, Michael Gorelik ${ }^{1,2}$, Caroline Nelson ${ }^{2}$, Hwa Huang ${ }^{2}$, Sam L. Trumbo ${ }^{2}$, Jeff W. Bulte ${ }^{3,2}$, Douglas A. Kerr ${ }^{1,2}$, Piotr Walczak ${ }^{3,2}$ ${ }^{1}$ Neurology, Johns Hopkins University, Baltimore, MD, USA, ${ }^{2}$ Institute of Cell Engineering, Johns Hopkins University, Baltimore, MD, USA, ${ }^{3}$ Radiology, Johns Hopkins University, Baltimore, MD, USA.

Glial precursors (GRPs) have the potential to differentiate into astrocytes and/or oligodendrocytes and their downstream differentiation pathway is determined by numerous factors including the cell state at the time of transplantation, recipient's age, physiological and pathological environment. We are using Bioluminescence Imaging (BLI) as a noninvasive method of tracking the fate of these cells after transplantation. We have generated glial cell lines which express firefly luciferase gene under the control of promoters activated at various stages during differentiation of 
glial precursors. One such promoter is Opalin, which derives the expression of a transmembrane protein (Tmem10/opalin) expressed in myelinating oligodendrocytes. We have used cytomegalovirus (CMV) promoter driven luciferase signal as a marker for cell survival after transplantation. Rat GRP cells were stereotactically injected into the brain of immunodeficient (Rag2), myelin deficient (shi) neonatal (P1-P3) mice. This model eliminates graft rejection as a variable as well as allows for rigorous histological validation of new myelination with MBP antibody. Bioluminescence imaging at day 26 after transplantation demonstrated that 2/3 mice showed persistent CMV driven luciferase signal. Opalin driven luciferase signal was not detectable initially in any of the mice $(n=5)$, however, signal was clearly detectable at day 26 in $3 / 5$ mice (Fig 1). In conclusion, our data indicate that Opalin promoter is a good candidate for monitoring oligodendrocyte differentiation and in conjunction with bioluminescence imaging can be used to monitor and guide cell transplantation-based therapies for myelin disorders.

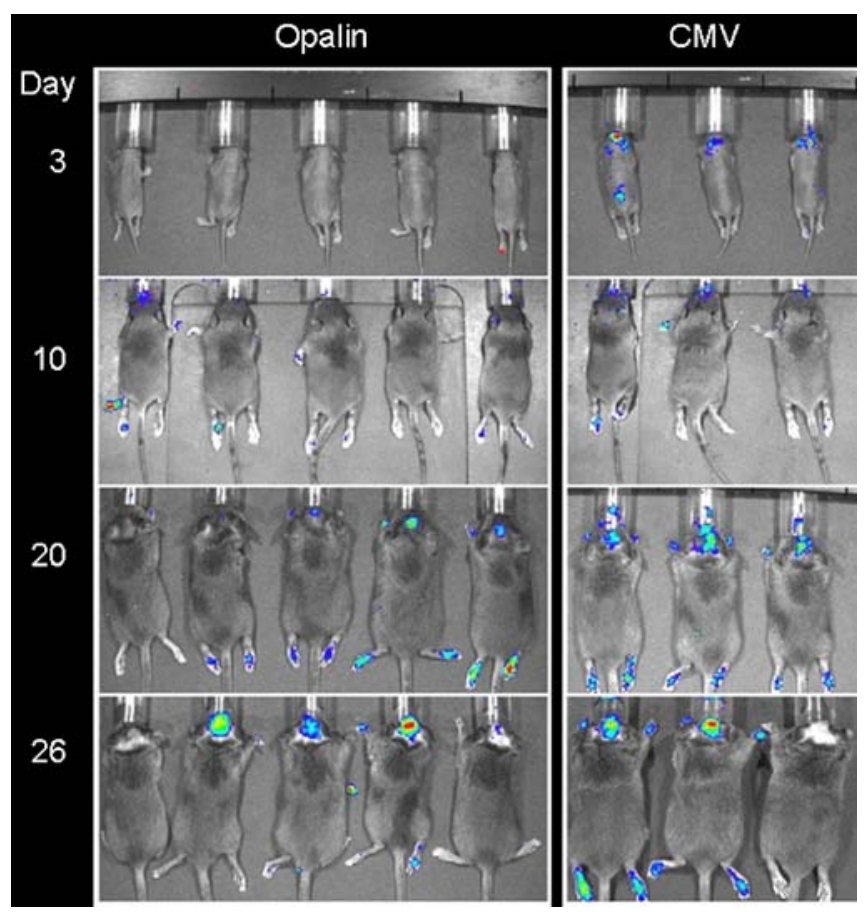

BLI signal from GRPs

\section{J772}

ENHANCED DETECTION OF SPIO-LABELED STEM CELLS WITH Z-SHIM ULTRA-SHORT ECHO TIME (ZUTE)

Philip Lee ${ }^{1}$, Xavier Golay ${ }^{2}$

${ }^{1}$ Lab of Molecular Imaging, Singapore Bioimaging Consortium, Singapore, Singapore, ${ }^{2}$ Brain Repair and Rehabilitation, UCL Institute of Neurology, London, United Kingdom.

Introduction In-vivo tracking of transplanted stem cells is paramount in regenerative studies. To increase detection sensitivity, cells are normally labeled with iron-oxide based nano-particles, which appear as dark voids in a typical $\mathrm{T} 2 *$-weighted MR image. Interpretation of such signal loss can be challenging due to partial volume and susceptibility artifacts. Here we present a technique that elucidates the presence of iron using a hybrid pulse sequence comprising Ultra-short Echo Time (UTE) 1 and local magnetic inhomogeneities rephasing with Z-gradient compensation. Method An excised rat heart injected with mononuclear cells, which have been labeled with SPIO, was scanned in the coronal plane. A 2D (thickness $=1 \mathrm{~mm}$ ) ZUTE radial pulse sequence was implemented for stem cell tracking at $9.4 \mathrm{~T}$, achieving a $\mathrm{TE}=192 \mathrm{us}$ for positive contrast $(\mathrm{TR}=150 \mathrm{~ms})$. Images were compared to a negative contrast MRI sequence (gradient-echo multi-slice: GEMS, TE $=3.40 \mathrm{~ms}, \mathrm{TR}=150 \mathrm{~ms}$ ), a positive contrast technique known as GRASP 2 and UTE. Contrast-to noise ratio (CNR) between labeled and unlabeled left ventricle is displayed. In-plane resolution was $156 \times 156 \mathrm{um}$. Results Figure 1 compares GEMS, GRASP, UTE and ZUTE images. All positive contrast techniques clearly illuminate iron-labeled cells in the left ventricle, with ZUTE having the highest CNR. This results from a combination of: (1) rephasing the regions surrounding the iron particles and (2) leveraging on T1-relaxation effect of iron (which can only be done at very short echo-time). Notice the heavier suppression of non-iron background signal with ZUTE. References 1) Philip Lee et al. WMIC 2008. Abstract 1048. 2) V. Mani et al. MRM 55(1); pp 126-135
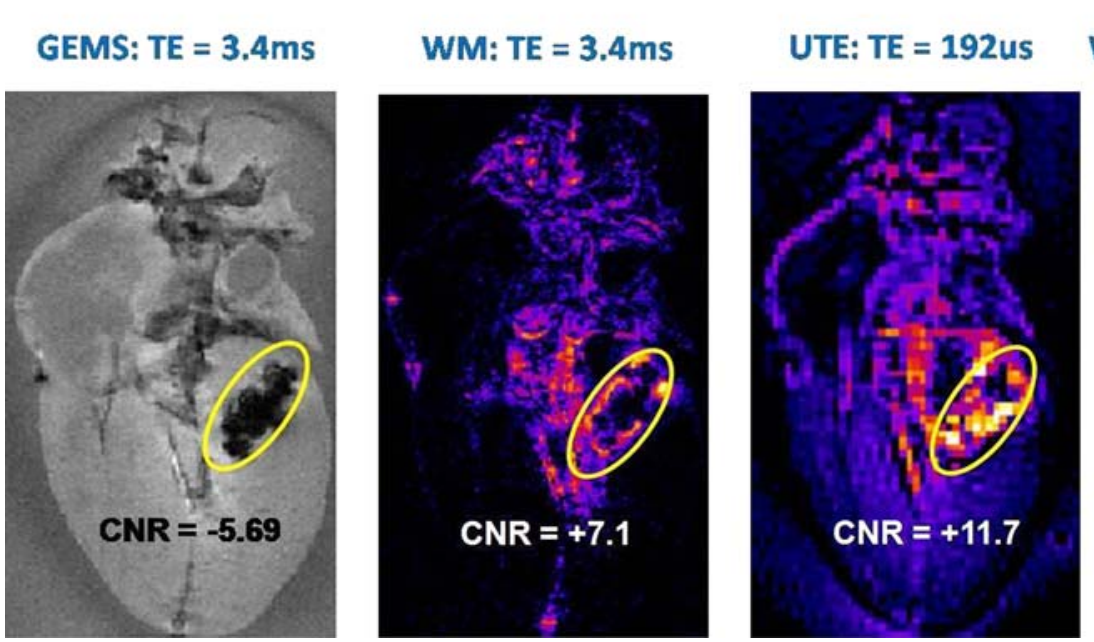

ZUTE: TE $=192$ us
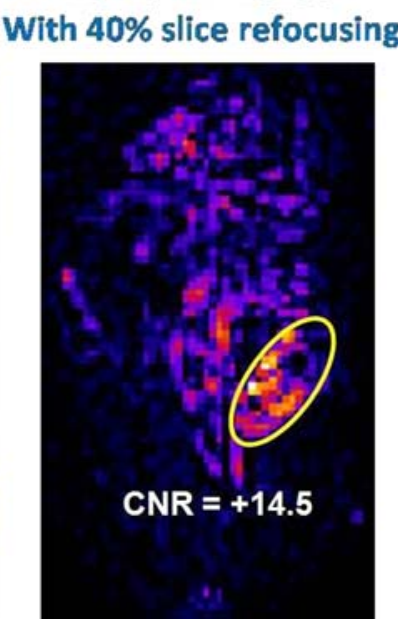

Figure 1: Stem-cell tracking comparison between Negative (GEMS) and Positive (GRASP, UTE, ZUTE) contrast techniques. 


\section{$\mathbf{J} 773$}

DYNAMICS OF INDUCTIVE DERMAL PAPILLA CELL MICROTISSUE FORMATION ON BIOMATERIAL SURFACES FOR HAIR FOLLICLE REGENERATION

HuiRu Tu ${ }^{1}$, Yi-Ching Huang ${ }^{1}$, Sung-Jan Lin ${ }^{1,2}$

${ }^{1}$ Institute of Biomedical Engineering, National Taiwan University, Taipei, Taiwan, ${ }^{2}$ Department of Dermatology, National Taiwan University Hospital and College of Medicine, Taipei, Taiwan.

Neogenesis of hair follicle (HF) in adult life has been demonstrated by transplanting cultured HF dermal papilla (DP) cells in dense aggregates or microtissues, an intercellular organization similar to the physiological condition of DP cells. We have proposed a 3-step approach for efficient HF neogenesis: expansion of DP cells, generation of inductive microtissues by cell self-assembly in a bioreactor, and transplantation of microtissues. We found poly (ethylene-co-vinyl alcohol) (EVAL) surface can facilitate DP microtissue formation and these DP microtissues are able to induce HF neogenesis in vivo. In this work, the dynamic cell behavior is investigated by time-lapse light microscopy and multiphoton imaging. We found that, seeded above a critical density, DP cells spontaneously grow into many dense spheroidal microtissues on EVAL surface. These microtissues have high cell viability, preserve DP markers and are able to induce new HFs in vivo. Dynamically, the formation of dermal papilla microtissues can be divided into 3 steps: active cell migration, intercellular collision and multicellular aggregation into microtissues. Interestingly, the formed microtissues are able to move collectively, a unique behavior similar to that of dermal papilla cells during hair follicle cycling in vivo. The selfaggregation process is associated with higher cell motility and declined cell-substrate adhesivity on EVAL. However, the declined adhesivity has the drawbacks of higher cell loss. We further found that specific extracellular matrix proteins can switch cell morphologies. Though fibronectin increases substratum adhesivity and reduces cell loss, it still keeps cells in a highly motile state. Fibronectin also increases cell growth rate. The higher motility and cell density increase the intercellular collision rate, thereby increasing the number of microtissues by $67 \%$. Other extracellular matrix proteins increase the adhesivity, but detrimentally compromises cell mobility, leading to a monolayered morphology. In conclusion, this system allows investigation of the self-assembling behavior of dermal papilla and can also help to produce dermal papilla microtissues on a large scale for HF regeneration.

\section{J774}

\section{PLASTICITY OF LONG TERM CULTURED CORD BLOOD} DERIVED AC133+ STEM CELLS

$\underline{\text { Branislava Janic }}{ }^{1}$, Nadimpalli Ravi S Varma ${ }^{1}$, Austin $\mathrm{Guo}^{2}$, Asm Iskander $^{1}$, M. M. Ali ${ }^{1}$, Ali S. Arbab ${ }^{1}$

${ }^{1}$ Cellular and Molecular Imaging Laboratory, Henry Ford Hospital, Detroit, MI, USA, ${ }^{2}$ Women Health Services, Gynecologic Oncology Research, Henry Ford Hospital, Detroit, MI, USA.

Stem/progenitor cells are central to the development of therapies for various diseases. Until recently, it has been considered that differentiation multipotency was the property exclusive for embryonic stem cells. However, numerous studies questioned the lineage commitment of different stem/progenitor cell populations. We hypothesize that under the adequate conditions, cord blood (CB) derived $\mathrm{AC} 133+$ progenitor cells can give rise to diverse lineages. However, the clinical application of this diverse potential is limited by low quantities of $\mathrm{AC} 133+$ cells that can be generated from patient CB. Hence, ability to in vitro amplify AC133+ cell numbers while preserving their plasticity is critically important. The objective of this study was to evaluate the capacity of $\mathrm{CB}$ AC133+ cells to differentiate, in vitro, towards mature endothelial cells (ECs) and neuronal cells (NC) and to give rise to cells of hematopoietic lineages after long term in vitro expansion. We hypothesized that differentiation multipotential of $\mathrm{CB}$ AC133+ will not be affected by long term in vitro culture. $\mathrm{CB} \mathrm{AC} 133+$ cells were cultured for 5 to 30 days. At days 15-20 and 25-30 cells were induced to differentiate for two weeks. Differentiation towards ECs was performed in the presence of FBS and $2 \mathrm{ng} / \mathrm{ml}$ of VEGF. Differentiation towards $\mathrm{NC}$ was performed in the presence of $\mathrm{FBS}, 1 \%$ $\mathrm{N} 2,20 \mathrm{ng} / \mathrm{ml}$ EGF, $20 \mathrm{ng} / \mathrm{ml} \mathrm{bFGF}$ and $50 \mathrm{ng} / \mathrm{ml}$ BDNF. Differentiated cells were analyzed for the expression of mature EC and NC markers. Functional ECs' characteristics were assessed by analyzing their chemotactic ability and DiI-Ac-LDL incorporation. Potential to give rise to cells of hematopoietic lineages was analyzed by Methocult ${ }^{\circledR}$ Colony-Forming Assay. After 2 weeks, cells differentiated towards ECs exhibited high expression levels of mature ECs markers (CD31, VEGFR-2 and vonWillebrand factor). Preserved ECs' functional capacity was demonstrated by high uptake of DiI-Ac-LDL, chemotactic ability and ECs' morphological characteristics. Cells differentiated towards NCs exhibited high levels of neuronal markers ( $\beta$ III tubulin, MAP-2, NeuN) and morphological changes typical for neuronal cells. Two weeks after being plated in Methocult ${ }^{\circledR}$ methylcellulose-based media CB $\mathrm{AC} 133+$ gave rise to colonies of different hematopoietic lineages. No difference in differentiation multipotential was observed between the cells differentiated at different times of primary culture. Therefore, long term in vitro culturing did not impede the $\mathrm{CB}$ $\mathrm{AC} 133+$ plasticity and potential to differentiate towards endothelial, neuronal and hematopoietic cells.

\section{$\mathbf{J 7 7 5}$}

\section{NOGGIN PROTEIN MEDIATED ANTI-VASCULOGENESIS} IMAGING IN A MOUSE MODEL SYSTEM

Alexei A. Bogdanov $^{1}$, Hye-Won Kang ${ }^{1}$, Ronn P. Walvick ${ }^{2}$

${ }^{1}$ Radiology, University of Massachusetts Medical School, Worcester, MA, USA, ${ }^{2}$ Worcester Polytechnic Institute, Worcester, MA, USA.

Noggin protein potently antagonizes bone morphogenetic proteins (BMP-4 and -7) and is capable of inhibiting vasculogenesis even in the presence of pro-vasculogenic VEGF and FGF-2. We found that human umbilical vein endothelial cells (HUVEC) do not express Noggin in culture in the absence of shear stress and used these cells for modeling of anti-vasculogenic effects of Noggin in vitro and in vivo. We hypothesized that high-efficiency transduction of human umbilical vein endothelial cells (HUVEC) with bicistronic lentiviral vector encoding Noggin and EGFP (enhanced green fluorescent protein) enables direct visualization of Noggin effects in homogenous primary cell populations in vitro and in vivo. By comparing HUVEC transduced with a control EGFP and EGFP/Noggin lentiviral vectors we showed that constitutive and orthotopic Noggin protein expression had no influence on cell proliferation, resulted in downregulation of BMP-4 transcript but showed no effect on BMP receptor transcripts. We demonstrated that in contrast to EGFP-only control, Noggin expression in endothelial cells abrogated endothelial migration in response to monolayer injury, blocked endothelial transmigration and caused abrogation of chord formation in vitro. Imaging experiments in vivo investigated vessel formation in model Matrigel implants in athymic mice using EGFP/anti-CD31-Cy5.5 imaging or magnetic resonance imaging of perfusion in the implants. The latter was facilitated by 
injecting a long-circulating paramagnetic nanopolymer (PGC-Gd). Both approaches demonstrated the lack of functional vessel formation after the adoptive transfer of EGFP/Noggin-expressing human endothelial cells in mice: the presence of EGFP fluorescence in EGFP/Noggin HUVEC implants correlated nor with Cy5.5 fluorescence, nor with PGC-Gd signal.

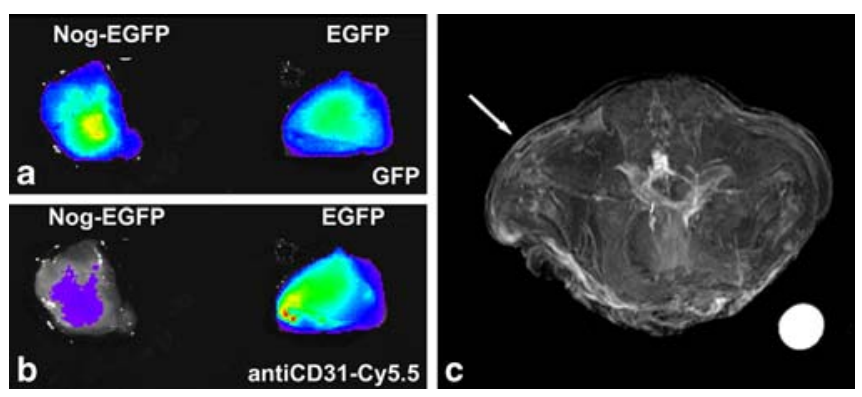

A- EGFP imaging; B- anti-human CD31 imaging ex vivo. C- In vivo 2T MRI (T1w SE 700/15 MIP image) after PGC-Gd I.V. injection (0.05 $\mathrm{mmol} / \mathrm{kg}$ ). Arrow :EGFP-HUVEC implant.

J776

\section{MONITORING STEM CELL TRANSPLANTATION}

\section{IN A MODEL OF VASCULAR INJURY USING MRI}

Jennifer C. Noad $^{1,2}$, Heather Broughton ${ }^{3}$, David Hess ${ }^{3}$, Paula Foster ${ }^{1,2}$

${ }^{1}$ Imaging, Robarts Research Institute, London, ON, Canada, ${ }^{2}$ Medical Biophysics, University of Western Ontario, London, ON, Canada,

${ }^{3}$ Krembil Centre For Stem Cell Biology, Robarts Research Institute, London, ON, Canada.
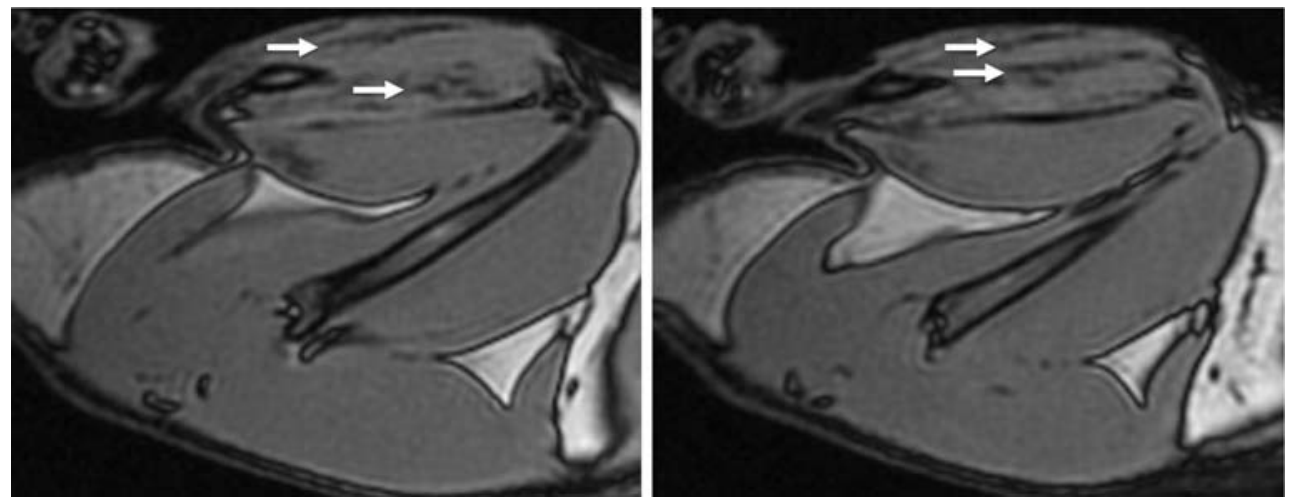

$\mathbf{J} 777$

THE INFLUENCE OF APOPTOSIS ON MR SIGNAL OF CONTRAST AGENT LABELED HUMAN MESENCHYMAL STEM CELL TRANSPLANTS

Alexander Nedopil, Christopher Klenk, Siyuan Liu, Daniel Golovko, Heike Daldrup-Link

Radiology, UCSF, San Francisco, CA, USA.
Stem cells are under investigation for their possible therapeutic effects in vascular injury. Many important questions persist, however, relating to the engraftment, migration, and survival of donor cells after transplantation. Cellular MRI allows for the in vivo tracking of cells after their labeling with magnetic nanoparticles. Here we show that cellular MRI can be used to monitor the homing of human bone marrow-derived stem cells after their transplantation in mice. Methods: Human multipotent mesenchymal stromal cells (MSCs) were isolated from bone marrow and labeled with magnetic nanoparticles (Feridex + protamine sulfate) to allow their detection by MRI. Cells were injected IV into immunodeficient mice (NOD/SCID IL-2R $\gamma$ null); either healthy controls, irradiated controls, or mice with a femoral artery ligation to create an ischemic vascular injury. Different cell concentrations were compared $(100,000,500,000)$. Injured mice were imaged before cell injection and 1 week after. Additionally irradiated control mice were injected with free iron. Imaging was performed at 1.5 Tesla with a custom-built, high performance gradient coil using a 3D steady-state free precession pulse sequence with a resolution of $100 \times 100 \times 200 \mu \mathrm{m}$. Results: Areas of signal loss were observed in the injured limb at 7 days after the IV administration of 500000 labeled human MSCs (Figure, arrows). This was in contrast to the contralateral limb and to injured mice with no MSCs injection. These results suggest the homing of MSCs at the site of injury. Iron labeled MSCs injected into control mice were detected as a gradual decrease in signal intensity in the spleen, but not liver or muscle, from 2 hrs to 4 days post injection. Free iron nanoparticles were detected within the liver and spleen, but not muscle, as areas of very low signal intensity. Discussion: Cellular MRI allows for monitoring of the fate of transplanted stem cells and will be a valuable tool for investigating therapeutic strategies in vascular injury. This report represents the first evidence that MRI can detect the homing of iron-labeled MSCs in a mouse model of hindlimb ischemia.
Purpose: To compare MR signal characteristics of contrast agent labeled apoptotic and viable human mesenchymal stem cells (hMSC) in matrix associated stem cell implants (MASI). Methods: hMSCs were labeled with Gd-DTPA or ferumoxides. One group (A) remained untreated while a second group (B) underwent Mitomycin C-induced apoptosis induction. Viability of group A and apoptosis of group B was confirmed by Caspase-assays, MTS bioassays and Trypan blue staining. Labeled and unlabeled viable and apoptotic hMSCs in agarose scaffold were implanted into cartilage defects of porcine patellae 
specimen and underwent MR imaging at $3 \mathrm{~T}$ and $7 \mathrm{~T}$, using T1-, T2and $\mathrm{T} 2 *$-weighted sequences. T1- and T2-relaxation times of the implants were calculated and compared between groups A and B using a t-test. Results: Gd-DTPA labeled hMSCs provided a strong T1-effect and ferumoxides labeled hMSCs provided a strong T2-effect. T1- and T2-relaxation times of labeled cells were significantly different from unlabeled controls. Apoptosis resulted in a significant shortening of T1 and T2-relaxation times of Gd-DTPA and ferumoxides labeled cell transplants. Conclusion: A thorough understanding of MR signal characteristics of Gd-DTPA and ferumoxides labeled hMSC transplants allows for conclusions about the viability of MASI in cartilage defects. The described MR signal characteristics of viable and apoptotic hMSCs, labeled with clinically applicable contrast agents, could be immediately applied as non-invasive outcome measures for the assessment of MASI therapies in clinical practice.

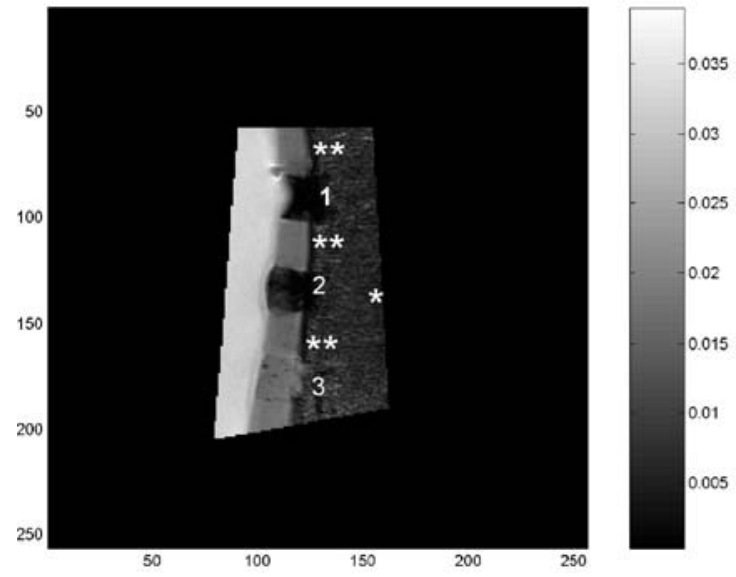

1. Fe - labeled apoptotic cells

2. $\mathrm{Fe}$ - labeled viable cells

3. Unlabeled apoptotic cells

* Bone

** Cartilage
$\mathbf{J 7 7 8}$

\section{MOLECULAR IMAGING ASSESSMENT OF THE ROLE OF SDF-1/CXCR7 IN MODULATING POST-ISCHEMIC ANGIOGENESIS DRIVEN BY ENDOTHELIAL PROGENITOR CELLS \\ Chia-Hung Hsieh, Chih-Chao V. Liu Graduate Institute of Basic Medical Science, China Medical University, Taichung, Taiwan.}

Recruitment and retention of circulating progenitor cells at the site of injured or ischemic tissues facilitates adult neo-vascularization. Endothelial progenitor cells (EPCs) have been introduced in cardiovascular medicine as cell therapy for promotion of angiogenesis. EPCs can repair damaged endothelium and attenuate the development and progression of atherosclerosis. Also, EPCs can form new vessels in ischemic areas and thus promote recovery after ischemic events. However, the molecular mechanism of EPCs involved in ischemiainduced angiogensis is still unclear. Recently, a new SDF-1 receptor named CXCR7 has been discovered and enhanced expression of CXCR7 was observed in the neurons and blood vessels after cerebral ischemia in the rodent brain. Based on this finding, CXCR7 may play a crucial role in ischemia-induced vasculogenesis. We used in vitro and in vitro molecular imaging approaches together with CXCR7 loss-offunction and CXCR7 gain-of-function techniques to test this hypothesis and further address its impact in the ischemia-induced vasculogenesis. We knocked down CXCR7 in a model of hind limb ischemia in mice and investigated its role in ischemia-induced angiogenesis. The results demonstrated that CXCR7 knockdown in the muscle of ischemic hind limbs inhibited angiogenesis and muscle regeneration in ischemic hind limbs. Furthermore, our results also demonstrated that knocked down CXCR7 in ischemic hind limbs also inhibited the proper migration of transplanted EPCs into ischemic tissues. These data indicate that CXCR7 plays an essential role in the EPCS migration and angiogenesis.

J779

MONITORING OF EARLY MIGRATION OF MESENCHYMAL STEM CELLS BY LABELING WITH ${ }^{{ }^{99} \mathrm{~m}}$ Tc-HMPAO

Joon-Kee Yoon ${ }^{1}$, Bok-Nam Park ${ }^{1}$, Wooyoung Shim ${ }^{2}$, Young Hwan Ahn ${ }^{3}$ ${ }^{T}$ Nuclear Medicine \& Molecular Imaging, Ajou University Medical School, Suwon, Korea, South, ${ }^{2}$ Molecular Science and Technology, Ajou University Medical School, Suwon, Korea, South, ${ }^{3}$ Neurosurgery, Ajou University Medical School, Suwon, Korea, South.

Purpose: ${ }^{99 \mathrm{~m}} \mathrm{Tc}$ is the most popular radioisotope in nuclear medicine imaging. We evaluate the usefulness of ${ }^{99 m} \mathrm{Tc}$ in the early monitoring of mesenchymal stem cells (MSCs) in acute cerebral trauma model. Methods: Rat bone marrow derived MSCs were isolated from femurs of male rats and labeled with ${ }^{99 \mathrm{~m}} \mathrm{Tc}-\mathrm{HMPAO}$. ${ }^{99 \mathrm{~m}} \mathrm{Tc}-\mathrm{MSCs}\left(20 \mathrm{MBq} / 10^{6}\right.$ cells for each rat) were injected via tail vein in acute trauma models $(n=14)$ and controls $(n=13)$. Labeling 
efficiency and retention rate were measured. Brains, lungs, livers, spleens, kidneys and hearts were removed at 4 hour after injection and $\%$ injected dose (\%ID) was calculated in gamma counter for biodistribution analysis. Confocal microscope with PKH26 was used to confirm the migration of MSCs to the traumatic brains. Results: Labeling efficiency of ${ }^{99 \mathrm{~m}} \mathrm{Tc}-\mathrm{MSCs}$ was $61 \pm 15 \%$, and retention rates were $72 \%, 45 \%, 31 \%$, and $16 \%$ at $1,3,6$, and 24 hours, respectively. ${ }^{99 \mathrm{~m}} \mathrm{Tc}-\mathrm{MSC}$ s were distributed mostly in the lungs, livers and spleens at 4 hour. ${ }^{99 \mathrm{~m}} \mathrm{Tc}-\mathrm{MSCs}$ uptake of brain was significantly higher in the traumatic rats than in controls (Fig. 1, $0.40 \%$ vs. $0.20 \%, p=0.0002$ ). In addition, in trauma models, ${ }^{99 \mathrm{~m}} \mathrm{Tc}$ MSCs uptake of traumatic hemispheres was significantly higher than that of contralateral ones $(0.27 \%$ vs. $0.13 \%, p=0.0001)$. Meanwhile, ${ }^{99 \mathrm{~m}} \mathrm{Tc}-\mathrm{MSC}$ uptake of the other organs was not significantly different between traumatic rats and controls (all $p>0.05$ ). As expected, in controls, radioactivity between 2 hemispheres was not significantly different $(0.10 \%$ vs. $0.10 \%, p=0.86)$. Confocal microscopic images also confirmed the presence of PKH26 and ${ }^{99 \mathrm{~m}}$ Tc-labeled MSCs in the traumatic hemispheres. On the contrary, non-traumatic hemispheres and control brains did not show MSCs. Conclusion: ${ }^{99 \mathrm{~m}} \mathrm{Tc}-\mathrm{HMPAO}$ was easily labeled to MSCs with sufficient labeling efficiency. ${ }^{99 \mathrm{~m}} \mathrm{Tc}$-labeled MSCs significantly migrated to the traumatic brains as early as 4 hour after injection. Therefore, ${ }^{99 \mathrm{~m}} \mathrm{Tc}$ could be used as an early monitoring method of MSCs transplantation.

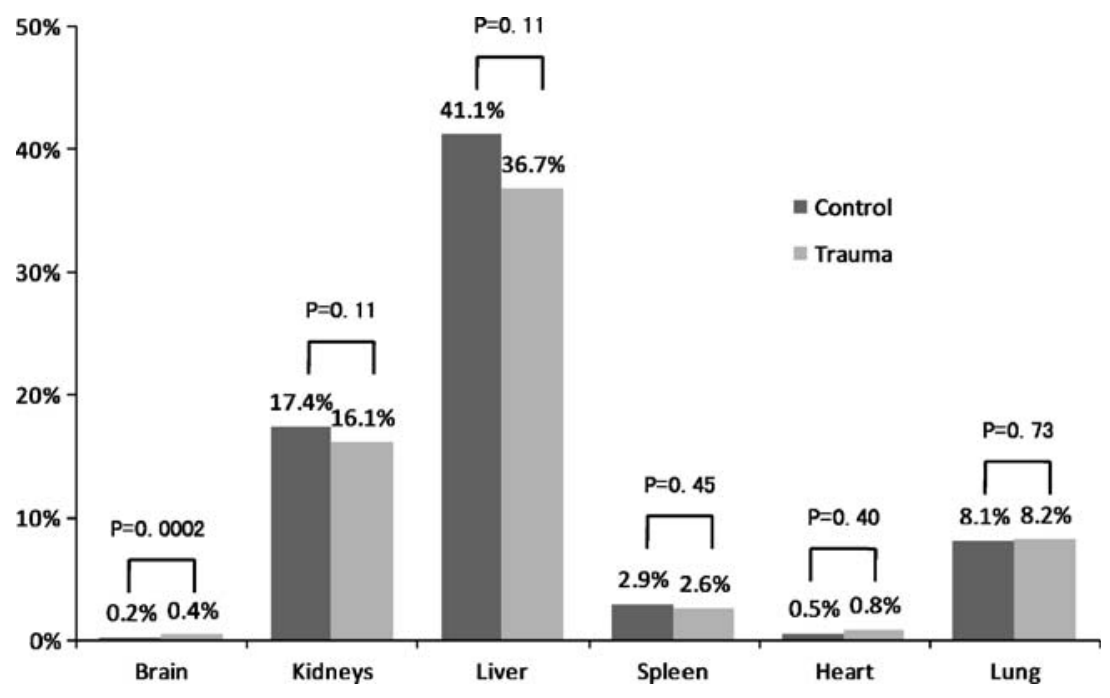

J780

\section{IN VIVO AND EX VIVO MICRO MRI ANALYSIS OF NATIVE AND STEM CELL-BASED ENGINEERED ANNULUS FIBROSUS}

Gadi Pelled $^{1,2}$, Yoram Zilberman ${ }^{2}$, Galit Saar ${ }^{3}$, Nadav Kimelman Bleich ${ }^{2}$, Hadassah Shinar ${ }^{3}$, Keren Keinan-Adamsky ${ }^{3}$, David Kaplan ${ }^{4}$, Carmen Preda $^{4}$, Dan Gazit ${ }^{1,2}$, Gil Navon ${ }^{3}$

${ }^{1}$ Surgery, Cedars Sinai Medical Center, Los Angeles, CA, USA, ${ }^{2}$ Skeletal Biotech Laboratory, Hebrew University of Jerusalem, Jerusalem, Israel, ${ }^{3}$ School of Chemistry, Tel Aviv University, Tel Aviv, Israel, ${ }^{4}$ Biomedical Engineering, Tufts University, Medford, MA, USA.

One of the major goals in tissue regeneration research is the establishment of quantitative imaging modalities for the evaluation of repair processes. In this study we hypothesized that advanced micro MRI methods could be used in order to quantitatively monitor the changes in the intervertebral disc (IVD) post-injury and provide an analytical tool for annulus fibrosus (AF) regeneration. Rat coccyegal nucleus pulposus
(NP) was removed under fluoroscopic guidance. Early changes in the IVD were analyzed on day 5 using T2 measurements; magnetization transfer contrast (MTC) and $1 \mathrm{H}$ or $2 \mathrm{H}$ double quantum filtered (DQF) microscopic MRI. Tissue-engineered AF composed of mesenchymal stem cells (MSCs), seeded on disc-shaped silk scaffold, were transplanted subcutaneously in $\mathrm{C} 3 \mathrm{H} / \mathrm{HeN}$ mice. Tissue formation in the engineered AF was analyzed longitudinally in vivo using $7 \mathrm{~T}$ MRI (Bruker, Germany) and histology. T2 and MTC map of the engineered AF were compared to the natural rat IVD. Results showed that within the intact IVD there was a clear distinction between the NP and the AF in the T2-weighted MTC and $1 \mathrm{H}$ and $2 \mathrm{H}$ DQF images, After NP removal the width of the disc was significantly decreased, and the distinction between the AF and the NP was lost in all the above MRI techniques, indicating an ingression of collagen from the AF into the NP, as was confirmed by histological analysis. T2 and MTC maps of the engineered AF displayed shortening of T2 and higher MTC values, mainly in the periphery of the scaffold, which was comparable to the intact $\mathrm{AF}$ (see figure). This observation was confirmed by histology. Our results demonstrate the possibility of utilizing quantitative, in vivo, micro MRI in order to monitor early IVD tissue damage and also AF tissue formation. 

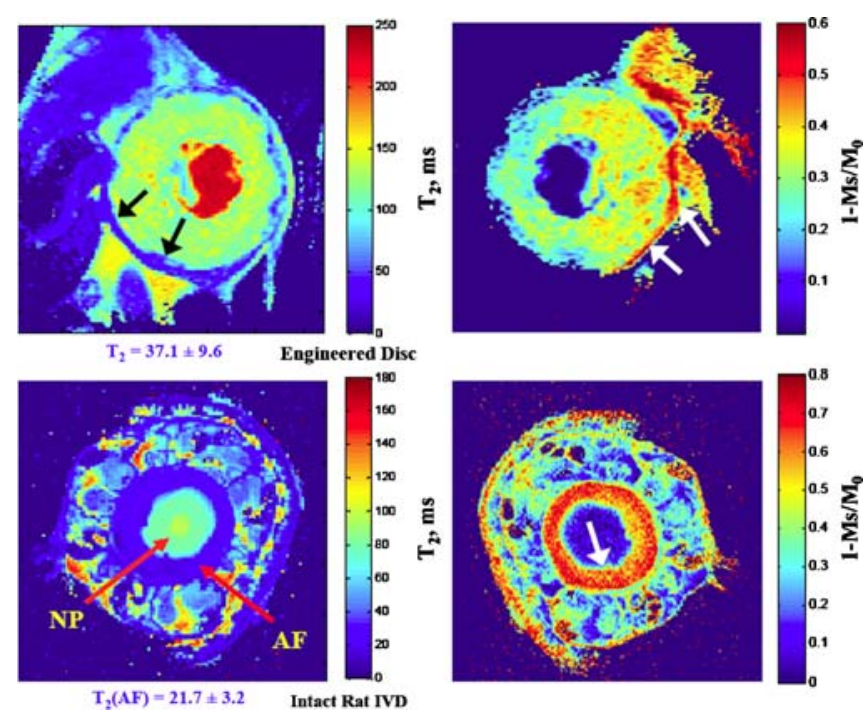

Intact and engineered $\mathrm{AF}$ comparison: discs that were made as described using silk scaffold for the AF portion were scanned using MRI at week 3 post implantation. The T2 and MTC maps display short T2 times and high MTC in the disc periphery (arrows) that was comparable with measurements in the native Rat IVD.

EVALUATION OF DOSE-DEPENDENT EFFECTS OF 111IN ON CANINE ENDOTHELIAL PROGENITOR CELLS

\section{FOR CELL TRACKING}

Kimberley Blackwood $^{1,2}$, Andrea J. Mitchell ${ }^{1,2}$, Wendy $\mathrm{Zhu}^{3}$, Qingping Feng ${ }^{1,5}$, Donna E. Goldhawk ${ }^{1}$, Savita Dhanvantari ${ }^{1}$, Gerald Wisenberg ${ }^{1,4}$, Frank Prato ${ }^{1,2}$

${ }^{1}$ Imaging Program, Lawson Health Research Institute, London, ON, Canada, ${ }^{2}$ Medical Biophysics, University of Western Ontario, London, ON, Canada, ${ }^{3}$ Physiology and Pharmacology, University of Western Ontario, London, ON, Canada, ${ }^{4}$ Division of Cardiology, London Health Science Centre, London, ON, Canada.

Introduction Transplantation of endothelial progenitor cells to assist angiogenesis has been evaluated in models of myocardial infarction. Cell tracking with the use of radiolabels like 111In have been instrumental in determining the retention of labeled cells at the infarction site. However, cellular safety limits of such radiolabels must be established to ensure that transplanted cells remain functional. We describe the dose dependent effects of $111 \mathrm{In}$ cytotoxicity on canine endothelial progenitor cells (cEPC) in vitro for autologous transplantation of cEPCs in a canine model of myocardial infarction. Methods Peripheral blood was taken from one female mongrel $\operatorname{dog}(30 \mathrm{ml})$ and mononuclear cells were isolated using density gradient centrifugation. Isolated cells were cultured in EBM-2 media supplemented with $10 \% \mathrm{FBS}$ and penicillin/streptomycin and adherent cEPCs were culture expanded up to passage 9 . On day 0 , cEPCs were collected and divided into 4 groups consisting of 3 radiolabeled groups and a control group $(5.79 \times 106$ cells/group). Radiolabeled groups were labeled with $111 \mathrm{In}$-tropolone for $30 \mathrm{~min}$, washed twice with HBSS, and cell pellet and supernatant activity was determined using a dose calibrator. Cell proliferation was assessed using the MTT assay at days $1,4,8$, and 10 and cell counting with a hemacytometer days 1,4 , and 9 . Viability was assessed using the trypan blue exclusion assay days 1, 4, and 9. Results Labeling efficiency for each radiolabeled group of cells was $94.3,92.8$, and $91.2 \%$ with cellular activities of $0.036,0.08$, and $0.198 \mathrm{~Bq} /$ cell respectively. MTT results demonstrated that cEPC proliferation was affected by $111 \mathrm{In}$ in a dose-dependent manner with the $0.198 \mathrm{~Bq} /$ cell group being significantly different from control at all time-points $(p<0.05)$. Lower cellular doses indicated a slight lag at early time-points after labeling (day 1 control vs. $0.08, p<0.01$; day 4 control vs. 0.032 and $0.08 p<0.05$ ), however were not significantly different from control at later time-points. Cell counting data also confirmed these results. Cell viability remained between $80-100 \%$ over the observation period. Conclusion Dose-dependent effects of 111In were seen on cell function such that cEPCs exhibited significantly slower proliferation rates at the highest dose. Viability data at late time-points suggest little direct effects from 111In at the levels tested over the period of observation. These data suggest the importance of safe cellular limits for angiogenic therapies using labeled transplanted cells.

$\mathbf{J 7 8 2}$

\section{TARGETING OF MAGNETICALLY LABELED GLIAL PROGENITORS TO CEREBRAL ENDOTHELIUM USING THE VLA-4/VCAM-1 ADHESION PATHWAY} Piotr Walczak $^{1,2}$, Michael Levy ${ }^{2,3}$, Michael Gorelik ${ }^{2,3}$, Douglas A. Kerr ${ }^{2,3}$, Jeff W. Bulte ${ }^{1,2}$

${ }^{1}$ Radiology, Johns Hopkins University, Baltimore, MD, USA, ${ }^{2}$ Institute for Cell Engineering, Johns Hopkins University, Baltimore, MD, USA, ${ }^{3}$ Neurology, Johns Hopkins University, Baltimore, MD, USA.

Introduction: One of the key bottlenecks in neural cell repair, in particularly for multifocal diseases, is the appropriate delivery and efficient targeting of cells to the affected areas. We hypothesized that intracerebral docking of intra-arterially injected glial restricted precursor cells (GRPs) can be modulated by the use of the VLA-4/VCAM-1 adhesion pathway. Following engineering of GRPs to induce VLA-4 expression and labeling with Feridex, we evaluated the global cerebral distribution of intra-arterially injected GRPs in an LPS-induced rat brain model of inflammatory disease. Methods: Adult Lewis rats were injected with lipopolysaccharide (LPS, to induce endothelial VCAM-1 expression, see schematic Figures C and D) given ip at $24 \mathrm{hrs}$ before cell injection. The right internal carotid artery (ICA) was cannulated with plastic tubing, and the animal was positioned inside of $40 \mathrm{~mm}$ diameter volume coil and placed in Bruker 9.4 Thorizontal bore magnet. The first $\mathrm{T} 2 *$-weighted image was acquired prior to cell injection. Immediately after, Feridex labeled VLA-4+ GRP cells were infused at $1 \mathrm{ml} / \mathrm{min}$. After the completion of cell injection a second T2*image was acquired. The control experiment consisted of transplantation of VLA-4- cells (C). At the end of imaging session animals were sacrificed for high resolution ex vivo MRI and histology. Results: GRP cells stably expressed VLA-4 as demonstrated by GFP fluorescence (A). The efficiency of Feridex labeling was high with nearly $100 \%$ cells labeled (B). MR imaging demonstrated that for VLA-4- cells there were no detectable hypointense signals in the brain (E). In contrast, for VLA-4+ cells, numerous hypointense regions were detected in the right hemisphere where the injection occurred $(\mathrm{F})$. Conclusions: We have demonstrated that through overexpression of VLA-4 integrin, transplanted GRPs can be effectively targeted to activated endothelium. We have shown that MRI cell tracking using Feridex-labeled cells is an excellent technique to monitor and evaluate this cerebral targeting and homing process. To the best of our knowledge, this is one of the first examples of a targeted cellular contrast agent for molecular imaging. Supported by: NMSS PP1491, NMSS RG3630, MSCRF 104062, TEDCO ESC-06-29-01. 

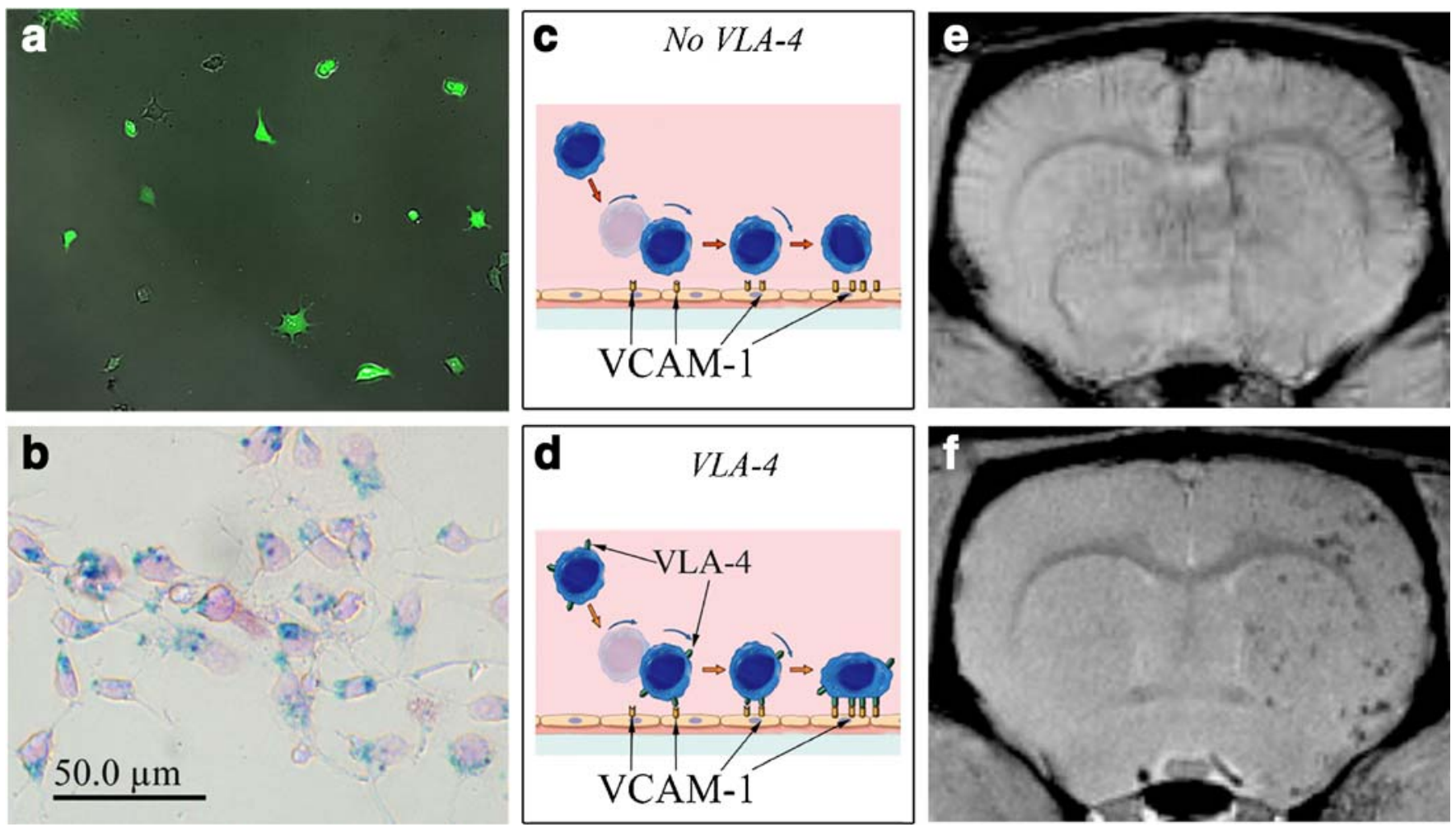

$\mathbf{J 7 8 3}$

\section{ESTABLISHMENT OF MESENCHYMAL STEM CELL-LIKE CELLS FROM PLACENTAL TISSUE}

Branislava Janic, Asm Iskander, Nadimpalli Ravi S Varma, Ali S. Arbab Cellular and Molecular Imaging Laboratory, Henry Ford Hospital, Detroit, MI, USA.

Mesenchymal stem cells (MSCs) are of great therapeutic potential due to their ability to self-renew and differentiate into different mesodermal cell lineages including osteocytes, adipocytes and chondrocytes. MSCs were found in bone marrow (BM) where they constitute only $0.01-$ $0.001 \%$ of cellular population. MSC were also found in cord and peripheral blood, however, at numbers even lower than in BM. Thus, the low frequency of this subpopulation of stem cells necessitate their in vitro amplification prior to therapeutic use, as well as further exploration of various tissues as the potential source of MSC. We established the new method for generating MSC-like cells from placental tissue, characterized their phenotype during long term in vitro culture and evaluated their capacity to differentiate, in vitro, towards osteocytes, adipocytes and chondrocytes. Inner placental membrane and Wharton's jelly were collected; tissues cut in small 1$2 \mathrm{~mm}$ size pieces and incubated in trypsin for $1 \mathrm{~h}$. After filtering through the $100 \mu \mathrm{m}$ filter, red blood cells were lysed and the isolated cells were plated on fibronectin coated dishes in low glucose DMEM media supplemented with $10 \%$ FBS and cultured until passage 17 th. Shortly after plating, cells exhibited morphological characteristics typical of MSC. Flow cytometry analysis of this MSC-like cells revealed that they expressed MSC markers such as CD105, CD90, CD166 and CD140B. These cells also exhibited high expression levels of HLA-ABC, and CD29 and CD44, molecules important for cell adhesion and homing, respectively. In parallel, commercially available Mesenchymal Stem Cell line (Cambrex) was used as a control for all the experimental conditions. Differentiation experiments were performed using commercially available Mesenchymal Stem Cells differentiation media (Gibco). Differentiation for two weeks demonstrated the capacity of MSC-like cells to give rise to adypocytes, osteocytes and chondrocytes. However, adipogenesis in these cells was of significantly less potency than in control MSC cells. Our study indicates that placental tissues besides cord blood may be utilized as a potential source of progenitor cells that exhibit MSC-like characteristics. Further studies are underway to determine whether these MSClike cells from placenta support hematopoiesis.

\section{J784}

\section{A LABELING TECHNIQUE FOR VISUALIZATION OF CARDIOMYOCYTES WITH OPTICAL IMAGING:} A PLATFORM FOR OPTIMIZING STEM CELL DELIVERY TECHNIQUES

Sophie E. Boddington ${ }^{1}$, Tobias D. Henning ${ }^{1}$, Priyanka Jha ${ }^{1}$, Christopher R. Schlieve $^{2}$, Lydia Mandrussow ${ }^{1}$, Heike Daldrup-Link ${ }^{1}$ ${ }^{1}$ Radiology, University of California, San Francisco, San Francisco, CA, USA, ${ }^{2}$ Gladstone Stem Cell Core, The J. David Gladstone Institutes, San Francisco, CA, USA.

Purpose: Human embryonic stem cell derived cardiomyocytes (hESCCM's) have demonstrated the ability to improve myocardial function following cardiac ischemia; however results from recent studies indicate that the functional benefits are transient because the majority of the transplanted cells undergo apoptosis and are washed away shortly after transplantation. A diagnostic technique that could directly visualize transplanted hESC-CM's in vivo, could help to direct and optimize stem cell delivery techniques and confirm a successful stem cell deposition in the myocardium. Thus, the purpose of this study was 
to develop a labeling technique for hESC's and hESC-CM's with the FDA approved contrast agent Indocyanine Green (ICG) for subsequent optical imaging (OI). Materials and methods: hESC were differentiated into hESC-CM's through the "hanging drop" method. Triplicate samples of hESC were labeled with $0.5,1.0,2.0$ and $2.5 \mathrm{mg} / \mathrm{ml}$ of ICG for 30,45 and $60 \mathrm{~min}$ at $37^{\circ} \mathrm{C}$. The viability of the cells was determined with the Trypan Blue Assay. Longitudinal OI studies were performed up to $120 \mathrm{~h}$ post labeling with both hESC and hESC-CM's. Variations of the labeling protocol, fluorescence signal and viability of labeled and unlabeled hESC's and hESC-CMs were compared for significant differences by an analysis of variance (ANOVA). Multiple comparison p-values were adjusted by Tukey-Kramer method or Bonferroni method. The expression of surface proteins was assessed by immunofluorescent staining. Results: hESC's labeled with $2 \mathrm{mg}$ ICG $/ \mathrm{ml}$ for $60 \mathrm{~min}$ achieved a maximum fluorescence with preserved viability. These parameters were therefore used for all subsequent experiments. Longitudinal studies of hESC's and hESC-CM's revealed that the fluorescent signal slowly decreased and was equivalent to controls at 120 hours post labeling. The fluorescence signal of the hESC's and hESC-CM at $1 \mathrm{~h}, 24 \mathrm{~h}$ and $48 \mathrm{~h}$ was significantly higher compared to pre-contrast data $(p<0.05)$. The signal at $120 \mathrm{~h}$ post labeling was not significantly different from baseline $(p>0.05)$. Immunohistochemistry revealed characteristic cell-specific surface markers post labeling for both hESC and CM's. Conclusion: hESC's and hESC-CM's labeled with ICG show a significant fluorescence up to $48 \mathrm{~h}$ and can be visualized non-invasively with OI. The labeling procedure does not impair the viability or functional integrity of the cells. The technique may be useful for assessing different delivery routes in order to improve cell retention, homing and engraftment of transplanted hESC-CM's.

\section{$\mathbf{J 7 8 5}$}

DILUTION AND CLEARANCE OF IRON OXIDE NANOPARTICLES FROM PROLIFERATING NEURAL STEM CELLS TRANSPLANTED INTO MOUSE BRAIN

Stacey M. Berman ${ }^{1,2}$, Martijn L. Chatrou ${ }^{3}$, Luc W. Starmans ${ }^{3}$, Assaf Gilad $^{1,2}$, Klaas Nicolay ${ }^{3}$, Jeff W. Bulte ${ }^{1,2}$, Piotr Walczak ${ }^{1,2}$

${ }^{1}$ Radiology, Johns Hopkins University, Baltimore, MD, USA, ${ }^{2}$ Institute of Cellular Engineering, Johns Hopkins University, Baltimore, MD, USA, ${ }^{3}$ Department of Biomedical Engineering, Eindhoven University of Technology, Eindhoven, Netherlands.

Introduction: Cell-based therapy for neurodegenerative diseases is actively pursued in preclinical models and has considerable potential for clinical translation. The purpose of this study was to determine the fate of superparamagnetic iron oxide nanoparticles at very late time points following the transplantation of labeled cells. Methods: Mouse neural stem cells (C17.2) were transduced with the lentiviral vector pLenti4-CMV-fLuc2 in order to express the bioluminescent reporter gene firefly luciferase. Cells $\left(3 \times 10^{5} / 3 \mu \mathrm{l}\right)$ labeled with iron oxide nanoparticles were stereotaxically implanted into corpus callosum of adult mice. BLI imaging was performed at various time points from 0 to 95 days after cell implantation. In vivo MRI was performed on a Bruker $9.4 \mathrm{~T}$ spectrometer at day 1 and day 95 after cell implantation with a T2-weighted spin echo sequence. Results: Implanted magnetically labeled, luciferase-expressing $\mathrm{C} 17.2$ cells generated detectable BLI signal (Figures A, B) on the day of transplantation. During a period of about 60 days, the BLI signal was gradually increasing to a plateau at the level of 1000-fold that of the initial signal (Graph A). Ninety-five days after transplantation, a strong BLI signal was detected originating from the entire brain area (Figure D), while the MRI hypointensities became undetectable (Figure E). Histology revealed that b-gal positive transplanted cells were detected in large numbers with quite limited migration, occupying a limited area in the proximity of the injection site (Figure F). Prussian Blue staining showed only few positive cells mostly within white matter tracks (Figure G). Conclusions: Transplantation of magnetically labeled, proliferating cells into mouse brain leads to dilution of iron oxide label with complete clearance of contrast agent over a period of 95 days. Lack of residual contrast indicates that a potential transfer of label to local tissue macrophages is followed by complete biodegradation of superparamagnetic iron oxide.
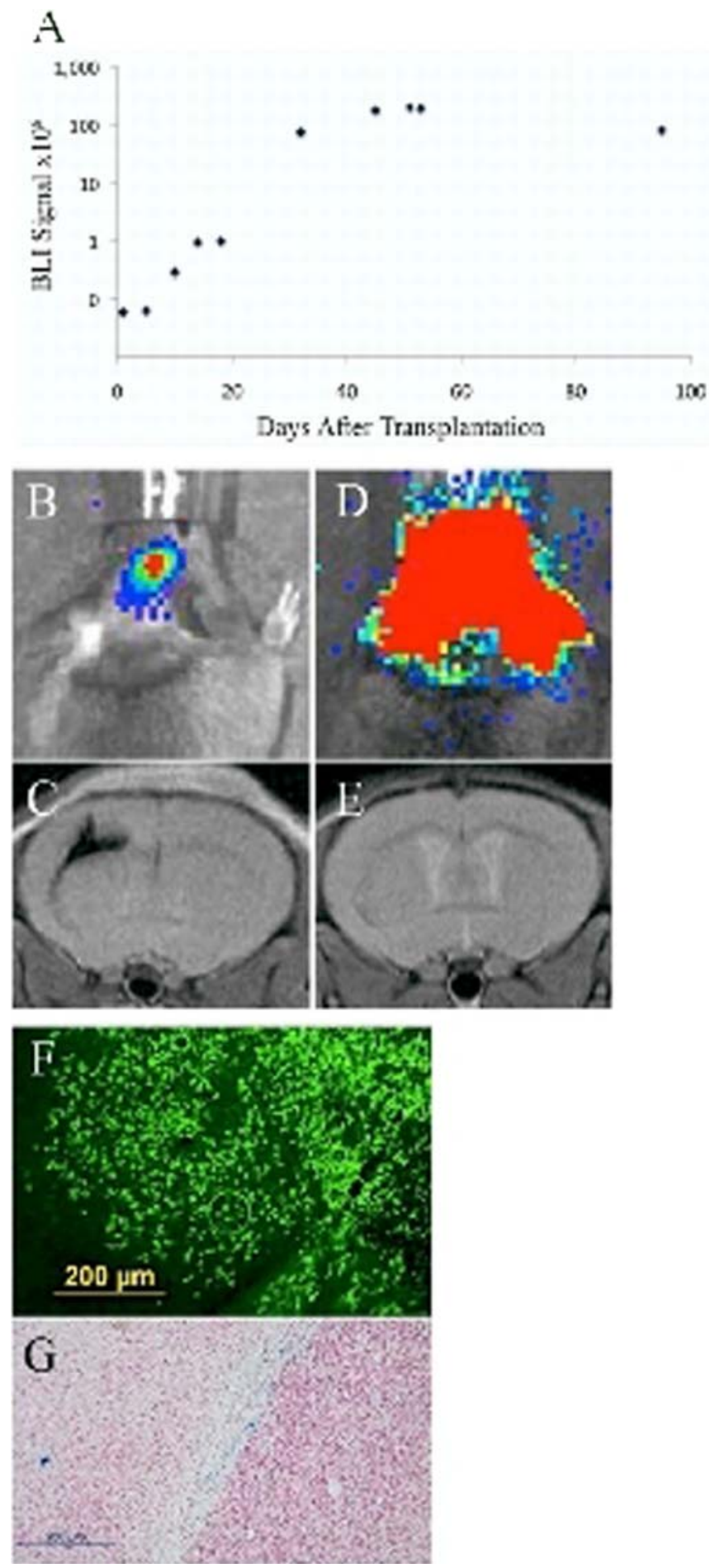
J786

SERIAL MONITORING AND QUANTIFICATION OF ENDOGENOUS NEUROBLAST MIGRATION RATES BY CELLULAR MRI

Dorit Granot $^{1}$, Dustin Scheinost ${ }^{1}$, Xenophon Papademetris ${ }^{1}$, Erik Shapiro $^{1,2}$

${ }^{1}$ Department of Diagnostic Radiology, Yale University School of Medicine, New Haven, CT, USA, ${ }^{2}$ Department of Biomedical Engineering, Yale University, New Haven, CT, USA.

In vivo labeling of endogenous neural progenitor cells in adult rat brains is an established method for tracking native cell migration in vivo. Briefly, micron sized iron oxide particles (MPIOs) are injected into the lateral ventricle proximal to the neural stem cell niche in the brain and are endocytosed by neural progenitor cell population, making them visible by susceptibility weighted gradient echo MRI. Here we extend this method to quantify cell migration rates and numbers over time. Using optimized
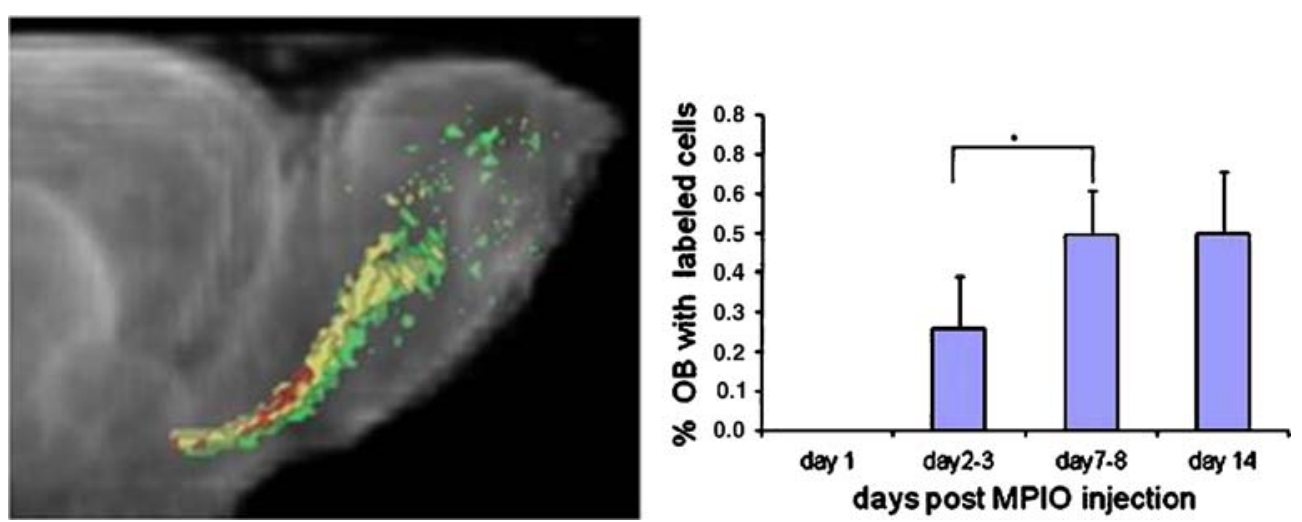

J787

IN VITRO AND IN VIVO ANGIOGENIC POTENTIAL OF LONG TERM CULTURE CORD BLOOD DERIVED AC133+ ENDOTHELIAL PROGENITOR CELLS (EPCS)

Branislava Janic, Nadimpalli Ravi S Varma, Asm Iskander, M. M. Ali, Ali S. Arbab

Cellular and Molecular Imaging Laboratory, Henry Ford Hospital, Detroit, MI, USA.

Endothelial progenitors cells (EPCs) are important for the development of cell therapies for vascular ischemic diseases. However, the successful clinical application of such therapy is limited by low quantities of EPCs that can be generated from patient and by the lack of adequate non-invasive imaging approach for in vivo monitoring of transplanted cells. Hence, ability to in vitro amplify and cryopreserve autologous EPCs while preserving their differentiation and angiogenic potential, as well as the ability to in vivo monitor biodistribution of transplanted EPCs are critically important. The objective of this project was to determine the ability of $\mathrm{CB} \mathrm{AC} 133+\mathrm{EPCs}$ to differentiate, in vitro and in vivo, towards mature ECs after long term in vitro expansion and cryopreservation and to use magnetic resonance imaging (MRI) to assess the in vivo angiogenic potential of ex vivo expanded CB AC133+ EPCs. CB derived AC133+ cells were cultured for 15-20 and 2530 days after which their angiogenic properties were assessed in vitro and in vivo. For the in vitro experiments cells were differentiated for two weeks in the presence of 5-10\% of FBS and $2 \mathrm{ng} / \mathrm{ml}$ of VEGF and analyzed for the expression of mature EC markers while their functional characteristics were assessed by analyzing their chemotactic ability and LDL lipoprotein uptake. In vivo differentiation was assessed cryopreservation.

\section{$\mathbf{J} 788$} AMNIOTIC MEMBRANE Erika Ruvalcaba $^{1}$, Clemente Ibarra ${ }^{1}$, Cristina Velasquillo ${ }^{1}$ tigation, Mexico, Mexico. labeling conditions, we conducted a longitudinal study to quantify the migration of labeled progenitors to the olfactory bulb (OB). Dose dependent studies revealed an optimal labeling dose of $20 \mu \mathrm{l}\left(6^{*} 107\right.$ MPIOs). Migration of labeled precursor cells in the RMS was detected as early as 1 day post-injection of MPIOs, and at 2-3 days signal voids were detected in the $\mathrm{OB}$ in the granule cell layer. Accumulation of labeled cells in the OB increased during the first week after injection, and spread to the outer edges of the $\mathrm{OB}$, the periglomerular layer. IHC confirmed the presence of MPIOs within doublecortin positive neural progenitor cells. By 3 days following injection, $0.26 \%$ of the volume of the OB contained labeled cells. By 8 days, this volume nearly doubled to $0.49 \%$. At 14 days post injection, no difference was measured in the occupancy in the OB. In this study we have tuned the in vivo cellular labeling protocol to enable visualization of early migration of cells into the OB. This facilitated quantification of this phenomenon. MRI detection of migration plateaus from week 1 to 2, indicating that the MPIO injection acts like a bolus, not a sustained slow release label. This migration rate matches nearly identically to classical neurogenesis studies employing immunohistochemical means.

in mouse matrigel angiogenic model. At days 15-20 and 25-30 of the primary culture, cells were magnetically (FePro) and fluorescently (DiI) labeled and intravenously injected into the matrigel bearing mice. Seven days after injection, mice were analyzed by MRI for the migration and neovascular incorporation of administered EPCs. The presence of labeled EPCs within the neovasculature was confirmed by immunohistochemistry. To determine the effect of cryopreservation on angiogenic properties of long term cultured $\mathrm{CB}$ AC133+ EPCs, at days 15-20 and 25-30 of the primary culture cells were labeled with FePro and DiI and cryopreserved for few weeks. Cryopreserved cells were then thawed, cultured for $30 \mathrm{~min}-2 \mathrm{~h}$ and IV administered to glioma bearing rats and MRI was obtained with a 7-Tesla system 7 days after. Control group of rats received long-term cultured magnetically labeled non-cryopreserved cells and no difference was observed between the groups. This study demonstrated that long term in vitro expanded cells preserved their angiogenic properties that were exhibited under in vitro and in vivo conditions and these properties were not affected by

CHARACTERIZATION AND ESEM IMAGING OF EPIDERMAL MURINE CELL PROLIFERATION OVER HUMAN

$\underline{\text { Ricardo Gómez }}{ }^{1}$, María Esther Martínez ${ }^{2}$, Carlos Landa ${ }^{1}$,

${ }^{1}$ Tissue Engineering, National Institute of Rehabilitation, Mexico City, Mexico, ${ }^{2}$ Membranes, National Institute of Nuclear Inves- 
Cells cultured in vitro conditions change in all metabolism ways this due to the Scaffold is an important component of tissue engineering; amniotic membrane is considered a potential matrix, providing support to growing cells. We isolated epidermal murine cells to evaluate his phenotype, viability and adhesion capacity over human amniotic membrane. The aim of this study was to investigate the possibility to use human amniotic membrane as scaffold for epidermal cells, thinking a future aplication in skin illness. Material and Methods: Epidermal cells were harvested from Balb/c skin samples free of subcutaneous tissue; the cells were plated until confluence and at the first passage were transferred to six well culture plates with amniotic membrane; cultures were maintained for two weeks, inmunohistochemistry assays were performed and environmental scanning electron microscope images were taken to assure cell attachment to the scaffold. Results: The cells attached in one day, almost $90 \%$ of the cells remain growing over the human amniotic membrane, the other $10 \%$ remain in the bottom of the culture plate; calcein incubation was used to assure cell viability and specific epidermal markers (cytokeratin 12, cytokeratyn 19 and vimentin) were use to perform inmunohistochemistry. Conclusion: Epidermal cells growth over human amniotic membrane and maintain their phenotype for two weeks; this yield the possibility to use this membrane in further investigations leading to epidermal regeneration.

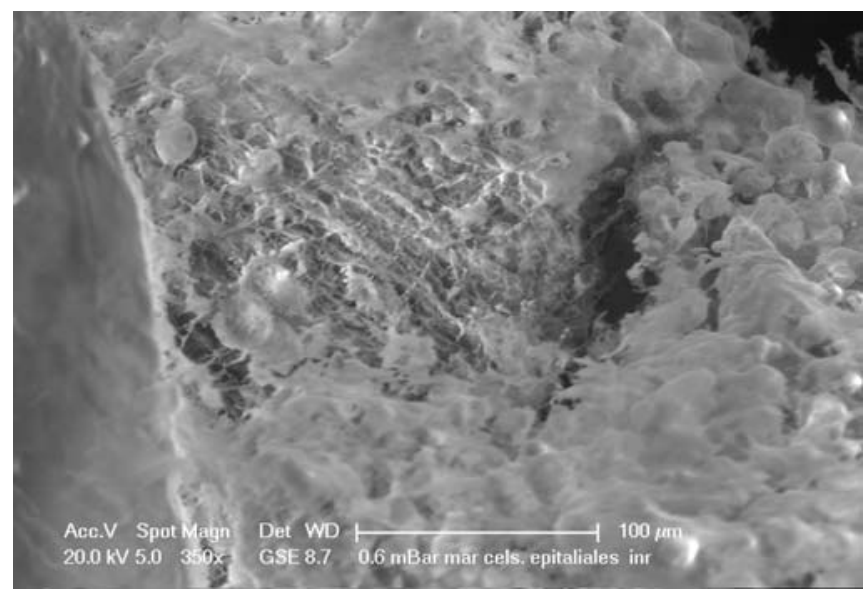

Epitelial murine cells in confluence over the amniotic membrane.

\section{J789}

\section{IMAGING IN BURN INJURY WITH MESENCHYMAL STEM} CELLS IN MICE MODEL

Ali A. Bonab $^{1,2}$, Ali M. Rad ${ }^{1,2}$, Shawn P. Fagan ${ }^{2}$, Biju Parekkadan ${ }^{2}$, Yong-Ming $\mathrm{Yu}^{2}$, Dionyssios Mintzopoulos ${ }^{2}$, Valeria Righi ${ }^{2}$, Alan J. Fischman ${ }^{2}$

${ }^{1}$ Radiology /Nuclear Medicine, MGH, Boston, MA, USA, ${ }^{2}$ Surgery, Shriners Children Hospital, Boston, MA, USA.

Despite significant work in stem cell treatment of thermal injuries the outcomes still are not convincing and new approaches are needed. Previously we have shown the trafficking(migration and accumulation) of systematically administered progenitor cells (PCs) to the sites of tumors using a specific technique. To date, no report has documented the use of our image trafficking technique as a potential therapeutic agents or molecular imaging probes.A mouse thermal injury model ( $25 \%$ total body surface area) was used in our study. PCs were isolated from mice bone marrow. Different markers (e.g. CD11b, CD106, CD90, SCA-1) were used to confirm our cell as progenitor cells (mesenchymal stem cells). Mesenchymal stem cells (MSCs) were magnetically labeled with ferumoxides-protamin sulfate (FePro) complexes before injection. Mean intracellular iron was determined by spectrophotometric methods using hydrochloric acid and potassium ferrocyanide. MSCs (3 million cells) were injected between 48 to $72 \mathrm{~h}$ after thermal injuries and mice were imaged $48 \mathrm{~h}$ after IV injection of MSCs in a $4.7 \mathrm{~T}$ horizontal bore magnet $(20 \mathrm{~cm}$ bore diameter, Magnex Scientific, using a Bruker Avance console). We acquired both positive and negative contrast MR images. Positive contrast was achieved with an off-resonance imaging (ORI) sequence employing fat suppression and narrowband water suppression pulses followed by wide-band spin-echo excitation and refocusing pulses. Further, a novel sequence combining the ORI scheme with a spin-locking train for T2 $\rho$ relaxation in the rotating frame was employed. Negative contrast was achieved with a series of gradient-echo $\mathrm{T} 2 *$-weighted images using FLASH (fast low-angle shot) with TE varying from $4 \mathrm{~ms}$ to $14 \mathrm{~ms}$. Mice were euthanized 2 weeks after imaging and histology were performed. Histochemical determination of iron labeled cells was also confirmed by Prussian blue staining. Our results show that MSCs were efficiently labeled with ferumoxides-protamine sulfate complexes. Labeling efficiency was more than $95 \%$, as determined by manual counting of prussian blue stained and unstained cells using a microscope. MR images clearly showed the trafficking of labeled MSCs to the thermal injury area and it was in agreement with histology sections stained for iron labeled cells. Our study shows the possible application of our imaging technique to aid in the development of stem cell technology as it applies to thermal injuries.

\section{$\mathbf{J 7 9 0}$}

\section{IN VIVO PRE-LABELING OF BONE MARROW CELLS WITH IRON PARTICLES FOR MRI}

Shruti Krishnamoorthy $^{2,1}$, Yuhua Chen ${ }^{2}$, Sonali N. de Chickera ${ }^{3}$, Emeline J. Ribot ${ }^{2}$, Paula Foster ${ }^{2,1}$

${ }^{1}$ Medical Biophysics, University of Western Ontario, London, ON, Canada, ${ }^{2}$ Imaging Research Laboratories, Robarts Research Institute, London, ON, Canada, ${ }^{3}$ Anatomy and Cell Biology, Robarts Research Institute, London, ON, Canada.

Many studies used the intravenous (IV) administration of iron oxide nanoparticles for labeling immune cells in vivo, allowing their detection by MRI in various experimental models involving inflammation or liver MRI. When this approach is taken cells of the reticuloendothelial system capture much of the iron nanoparticle dose. MR images show that the liver and spleen have very low signal intensity due to this iron uptake. The bone marrow also appears with low signal intensity. We hypothesize that bone marrow cells can be 'pre-labeled' invivo in healthy mice and that this approach will permit MR tracking of bone marrow cells involved in disease or tissue repair. The purpose of this experiment was to determine if iron uptake by cells results in low signal intensity in MR images of bone marrow and to optimize this method for pre-labeling. Methods: C57/BL6 mice were injected IV with superparamagnetic iron nanoparticles (SPIO, Feridex $4.5 \mathrm{mg} / \mathrm{kg}$ ). Bone marrow was harvested at either 1 or $24 \mathrm{hrs}$ post injection. Unlabeled cells were sorted from those containing iron using a magnetic column. Both groups of cells were stained with Perl's Prussian blue (PPB) to detect iron. Results: IV SPIO resulted in labeling bone marrow cells. Staining cells with PPB showed that more cells were iron-labeled in the bone marrow samples harvested at 24 hrs post injection; nearly all bone marrow cells in the iron-sorted population stained positive for PPB. A variety of cell types were ironlabeled. A representative PPB cell stain at 24 hours post iron administration is shown in the Figure below. To the best of our knowledge this is 
the first evidence of in vivo pre-labeling of bone marrow cells with iron particles. The bone marrow contains progenitors for various leukocytes and stem cells and the controlled mobilization of bone marrow has great potential for promoting recovery and repair. This new strategy for in vivo labeling of bone marrow has a large number of potential applications for cell tracking in regeneration.

\section{J791}

\section{A REAL-TIME MURINE MODEL OF REGENERATIVE THERAPY FOR MUSCULAR DISEASES}

Lisa M. Hoffman $^{1,2}$, Jennifer Hadway ${ }^{1,2}$, Siu-Pok Yee ${ }^{3}$,

Michael S. Kovacs $^{1,4}$, David Hill ${ }^{1}$, Savita Dhanvantari ${ }^{1,4}$, Ting-Yim Lee ${ }^{1,2}$

${ }^{1}$ Imaging, The Lawson Health Research Institute, London, ON, Canada,

${ }^{2}$ Imaging, Robarts Research Institute, London, ON, Canada, ${ }^{3}$ Genetics and Developmental Biology, University of Connecticut Health Center, Farmington, CT, USA, ${ }^{4}$ Medical Biophysics, University of Western Ontario, London, ON, Canada.

Duchenne muscular dystrophy (DMD) is a severe genetic neuromuscular disorder that affects 1 in 3500 boys, and is characterized by progressive muscle degeneration. In patients, the ability of resident muscle satellite cells (SCs) to repair damaged myofibers becomes increasingly inefficient. Therefore, transplantation of SCs from healthy subjects is a promising therapeutic approach to DMD. Assessing the efficacy of cell therapy for DMD is hampered by an inability of current methods (invasive muscle biopsies) to longitudinally assess cell survival, differentiation and function. Therefore, there is a critical need to develop a means to non-invasively assess $\mathrm{SC}$ function in vivo. As proof-of-principle that SC function and muscle regeneration can be assessed non-invasively, $\mathrm{C} 2 \mathrm{C} 12$ cells, an immortalized myoblast cell line, were transfected with a PET reporter gene ( $\mathrm{sr} 39 \mathrm{tk})$ construct under the control of a constitutive CMV promoter. One day post-transfection, cells were incubated for $30 \mathrm{~min}$ with $200-400 \mathrm{uCi} 18 \mathrm{~F}-\mathrm{FHBG}$, then imaged on a uPET scanner (GE Healthcare, eXplore Vista). Images were acquired for 10 minutes at an energy window of 250-700 kEv. Uptake and retention of 18F-FHBG occurs only in cells transfected with the sr39tk reporter construct. Using a PET reporter construct under the control of a muscle-specific Myogenin promoter, we can also target gene expression specifically to differentiating myoblasts. 1-5 million myoblasts transfected with either promoter construct were transplanted into the gastrocnemius muscles (GMs) of $\mathrm{C} 3 \mathrm{H}$ mice (shown below). The next day, 200-500 uCi F-18 FHBG was injected via a tail vein, and following a $2 \mathrm{~h}$ period, 18F-FHBG uptake in the GMs was demonstrated in images acquired for 30 minutes at an energy window of $250-700 \mathrm{kEv}$. We are now utilizing this technology to image myoblast differentiation and regeneration longitudinally in dystrophic mice, transplanting SCs harvested from transgenic mice that harbor the myogenin promoter-trifusion reporter.
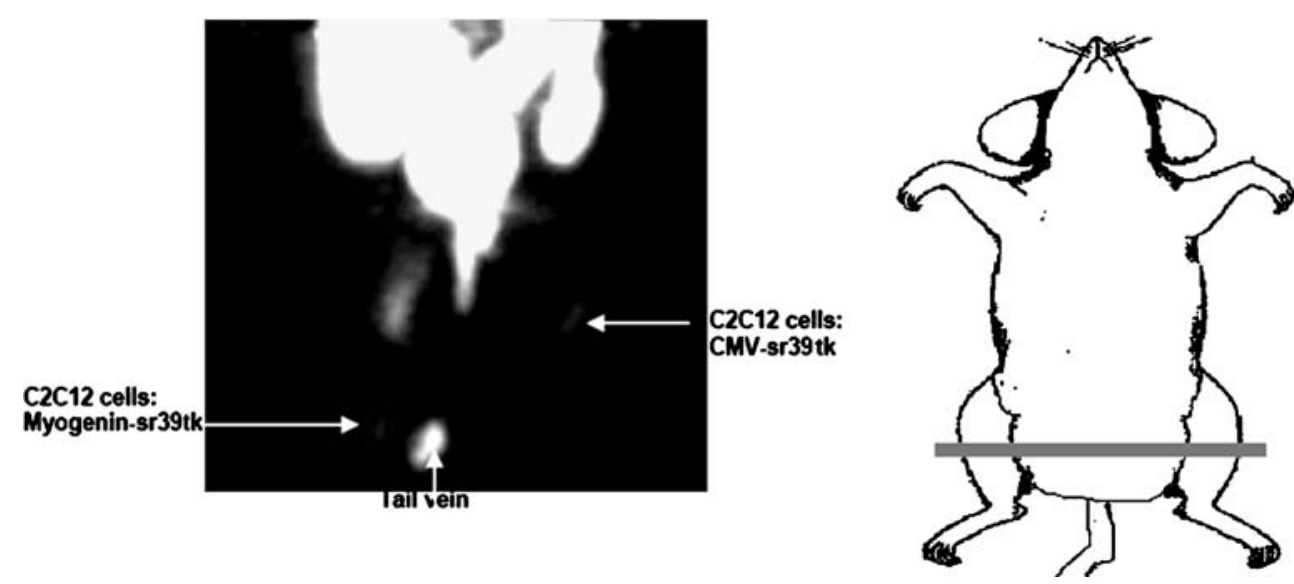


\section{LONG-TERM MONITORING OF PERSISTENCE, MIGRATION AND DIFFERENTIATE OF HSV1-TK EXPRESSED MESENCHYMAL STEM CELLS IN AN ISCHEMIA- REPERFUSION PORCINE MODEL WITH 18F-FEAU PET/CT AND MRI}

Mei Tian ${ }^{1}$, Guilherme Silva ${ }^{2}$, Frank C. Marini ${ }^{3}$, Yi Zheng ${ }^{2}$, Fred Baimbridge ${ }^{2}$, Xin Quan ${ }^{2}$, James Jackson ${ }^{4}$, Vincenzo Paolillo ${ }^{1}$, Uday Mukhopadhyay ${ }^{1}$, Mian M. Alauddin ${ }^{1}$, Agatha Borne ${ }^{5}$, Rajesh Uthamanthil $^{5}$, David Brammer ${ }^{5}$, Amer M. Najjar ${ }^{1}$, Suren Soghomonyan ${ }^{1}$, Hwan-Jeong Jeong ${ }^{1}$, Jesse M. Rios ${ }^{2}$, Osama Mawlawi ${ }^{6}$, Tinsu Pan ${ }^{6}$, Jason Stafford $^{6}$, Vikas Kundra ${ }^{1}$, Chun $\mathrm{Li}^{1}$, Elizabeth Shpall ${ }^{3}$, Emerson Perin ${ }^{2}$, Juri G. Gelovani ${ }^{1}$

${ }^{1}$ Experimental Diagnostic Imaging, MD Anderson Cancer Center, Houston, TX, USA, ${ }^{2}$ Stem Cell Center, Texas Heart Institute at St Lukes Episcopal Hospital, Houston, TX, USA, ${ }^{3}$ Stem Cell Transplantation and Cellular Therapy, MD Anderson Cancer Center, Houston, TX, USA, ${ }^{4}$ Nuclear Medicine, MD Anderson Cancer Center, Houston, TX, USA, ${ }^{5}$ Veterinary Surgery and Medicine, MD Anderson Cancer Center, Houston, TX, USA, ${ }^{6}$ Imaging Physics, MD Anderson Cancer Center, Houston, TX, USA.

Introduction: Intramyocardial transplantation of mesenchymal stem cell (MSCs) has shown promise for repair and restoration of myocardial function post infarction. However, the long-term fate and mechanisms remain unclear. The aims of this study were (1) to assess the feasibility of long-term monitoring of MSCs post transplantation using PET/CT/ MRI; (2) to investigate the role of myocardial lymphatic drainage in early distribution of MSCs and the contribution to restoration of myocardial lymphatic homeostasis. Methods: The animals were subjected to AMI and then divided into 4 study groups: Group I (with injections of non-transduced autologous MSCs), Group II (with injections of E86 retroviral vector producing cells of sr39HSV1-tk expression), Group III (with sr39HSV1-tk-MSC injections) and Group IV (with polyglutamic-Gd-DTPA-near infrared fluorescent agent (PGGd-NIRF) injections). [18F]FEAU-PET/CT images were performed and followed up for 35-148 days post implantation. Results: In Group I, no non-specific [18F]FEAU accumulation was observed in the myocardium. In Group II, very high levels of [18F]FEAU accumulation were observed in the injection site. In Group III, at $6 \mathrm{hrs}$ post implantation, the predominant localization of [18F]FEAU-derived radioactivity was observed in the sites of sr39HSV1-tk-MSC injection. Between 2 and 35 days post transplantation, [18F]FEAU signal gradually increased and peaked, both at the site of injection and in the anterior part of septum adjacent to infracted area, reflecting the sites of engraftment and proliferation of sr39HSV1-tk-MSCs. The sr39HSV1-tk-MSC-derived signals gradually decreased but were stably detectable in these regions during subsequent 4 months, as seen in the [18F]FEAU PET/CT images obtained at 106 and 148 days post sr39HSV1-tk-MSCs injection. In Group IV, the PET/CT, MRI and fluorescence images demonstrated the pattern of spatial-temporal dynamics of sr39-HSV1-tk-MSC distribution from the injection site to the infarct area through the myocardium and myocardial lymphatic system. Histopathological and IHC staining of heart tissues obtained post animal sacrifice confirmed the results of noninvasive imaging. Conclusions: Our studies demonstrated for the first time the feasibility and sufficient sensitivity of [18F]FEAU $\mathrm{PET} / \mathrm{CT}$ imaging for long-term in vivo monitoring ( $1 / 2$ year) of the fate of sr39HSV1-tk-MSCs after NOGA-assisted transendomyocardial delivery in a porcine AMI model and indicated that myocardial lymphatic system plays an important role in the mechanism of MSCmediated myocardial repair.
SODIUM IODIDE SYMPORTER (NIS) REPORTER GENE IMAGING FOR GUIDING ONCOLYTIC VIRAL THERAPY Alan R. Penheiter ${ }^{2}$, Stephen J. Russell ${ }^{2}$, Stephanie K. Carlson ${ }^{1,2}$; ${ }^{\mathrm{T}}$ Molecular Medicine, Mayo Clinic, Rochester, MN, USA, ${ }^{2}$ Radiology, Mayo Clinic, Rochester, MN, USA.

OBJECTIVE: To determine the ability of the NIS reporter gene and high-resolution pinhole micro-SPECT/CT to accurately and quantitatively image the intratumoral distribution of oncolytic viral infection in human pancreatic tumor xenografts. MATERIALS \& METHODS: Human BxPC-3 pancreatic xenografts were established in nude mice. Tumors were injected with a fusogenic, oncolytic measles virus engineered to express the sodium iodide symporter (MV-NIS). Mice were imaged in a dedicated small animal micro-SPECT/CT scanner (1 mm pinhole collimator) with 99TcO4 on days $2,3,4$, 7, and 14 postMV-NIS infection. PMOD imaging software was used to quantitate tumor dimensions and $99 \mathrm{TcO} 4$ localization. After imaging, tumors were immediately excised, weighed, counted in a dose calibrator, and either snap frozen or fixed in $10 \%$ formalin. Tumors were sectioned corresponding to coronal slices on microSPECT/CT images and stained with hematoxalin and eosin, or processed for autoradiography and subsequent immunohistochemistry (IHC) with an antibody against the measles $\mathrm{N}$ protein. Developed slides were scanned into a digital pathology workstation for quantitative analysis of infected and uninfected tumor cell volume, and tumor stromal components. Additional quantitative parameters were obtained from in vitro infection of BxPC-3 cells and from micro-SPECT/CT imaging of non-infected BxPC-3 tumors and BxPC-3 tumors stably- transduced with a NIS lentiviral construct. RESULTS: Pinhole micro-SPECT/CT imaging of MV-NIS infection strongly correlated with IHC and autoradiography. Comparison of IHC with autoradiography confirmed that all MV-NIS infected multinucleated syncytia within the tumor accumulated 99TcO4. Digital pathology and IHC sections were used to determined mean BxPC-3 cell volume, and the \% of tumor occupied by infected BxPC-3 cells, uninfected BxPC-3 cells, fibroblasts, extracellular matrix, and fluid. A nearly perfect correlation between the calculated $\%$ of infected BxPC-3 cells and the $\%$ injected dose $/ g$ (determined with micro-SPECT/CT) was observed. The sensitivity of micro-SPECT/CT for the detection of tumor infection was calculated to be $7.2 \%$ infected tumor cells (using a threshold of $1.5 \times$ control tumor uptake). It was also possible to resolve separate intratumoral zones of infection from the non-infected portions of the tumor. CONCLUSIONS: Molecular imaging using a NIS reporter and small animal pinhole 99TcO4 micro-SPECT/CT yields precise quantitation of the extent and localization of MV-NIS infection in human pancreatic xenografts.

\section{J794}

\section{A COMBINED FACTOR OF DEXAMETHASONE AND ASCORBIC ACID CAN ACTIVATE THE PROMOTER OF COLLAGEN TYPE 1 ALPHA 1}

Yunhui Kim ${ }^{1}$, James Dennis ${ }^{2}$, Stan Gerson ${ }^{3}$, Zhenghong Lee ${ }^{1,4}$ ${ }^{T}$ Radiology, Case werstern reserve university, Cleveland, OH, USA, ${ }^{2}$ Orthopedic, Case Western Reserve University, Cleveland, OH, USA, ${ }^{3}$ Hematology, Case Western Reserve University, Cleveland, OH, USA, ${ }^{4}$ Biomedical engineering, Case Western Reserve University, Cleveland, $\mathrm{OH}$, USA.

Human Mesenchymal Stem Cells (hMSCs) can differentiate into osteoblast in bone defect region. hMSCs have a great potential of the bone defect therapy. Collagen type 1 alpha 1 (Colla1) is one of the upregulated expressing gene during osteogenesis. To induce hMSCs to 
osteoblast in vitro, Osteogenic media containing 10-7 M Dexamethasone (Dexa), $0.05 \mathrm{mM}$ Ascorbic acid (AA), and $2 \mathrm{mM}$ betaglycerophosphate (BGP) are usually used. hMSCs were transduced with a lentivirus containing firefly luciferase (Luc) controlled by colla1 promoter. $1.5 \times 104 \mathrm{hMSCs}$ were seeded on 24 well tissue culture plate. Next day, we added 8 combinations of 3 factor; Dexa, AA, and BGP to Luc expressing hMSCs. At day 1, 4, 7, 11, 15, 20, 25, 28, and 35 , we measured luciferase activity to check the expression of the colla1 promoter. The luciferase activity by colla1 of normal media, Dexa, AA or BGP or AA/BGP treated hMSCs was less than Dexa/AA or Dexa/BGP or Dexa/AA/BGP treated one. Till day 25 , the luciferase activity by colla1 of Dexa/AA treated hMSCs was almost same Dexa/ AA/BGP treated one. But, at day 35, the luciferase activity by colla1 of Dexa and AA treated hMSCs was 1.6 fold higher than Dexa/AA/BGP treated one. In this study, we evaluate that Dexa/AA is the most effective combination to activate collal promoter.

\section{Imaging Molecular and/or Cellular Processes}

\section{Transport Systems}

\section{J795}

IMAGING P-GLYCOPROTEIN FUNCTION AT THE BLOOD BRAIN BARRIER WITH 11C-N-DESMETHYL-LOPERAMIDE Jeih-San Liow $^{1}$, Sami S. Zoghbi ${ }^{1}$, William Kreisl ${ }^{1}$, Neva Lazarova ${ }^{1}$, Nicholas Seneca $^{1}$, Robert Gladding ${ }^{1}$, Edward Tuan ${ }^{1}$, Andrew Taku ${ }^{2}$, Peter Herscovitch ${ }^{2}$, Victor W. Pike ${ }^{1}$, Robert B. Innis ${ }^{1}$

${ }^{1} \mathrm{MIB}$, NIMH/NIH, Bethesda, MD, USA, ${ }^{2} \mathrm{PET}$, CC/NIH, Bethesda, MD, USA.

Loperamide is an avid substrate for the efflux transporter, P-glycoprotein (P-gp). 11C-Loperamide has been proposed as a PET tracer for studying Pgp function at the blood-brain barrier. However, 11C-loperamide is rapidly metabolized to 11C-N-desmethyl-loperamide (11C-dLop), which is also a substrate for P-gp. Therefore, 11C-dLop is also a candidate PET tracer for P-gp function. We evaluated the utility of $11 \mathrm{C}$-dLop for quantifying P-gp function at the blood-brain barrier in mouse, monkey and human. After injection with $11 \mathrm{C}$-dLop, we measured brain radioactivity in P-gp knockout and wild type mice with PET (in vivo) and with radioactivity counting (ex vivo). For monkeys, dynamic PET scans were acquired at baseline and after P-gp blockade with a P-gp inhibitor, DCPQ (4, 8, or $16 \mathrm{mg} / \mathrm{kg}$ ). Unchanged radiotracer was measured in arterial blood samples. Some monkeys received PET scans with $15 \mathrm{O}-\mathrm{H} 2 \mathrm{O}$ for cerebral blood flow measurement. Eight healthy human subjects were also scanned at baseline with $15 \mathrm{O}-\mathrm{H} 2 \mathrm{O}$ followed by $11 \mathrm{C}$-dLop, both accompanied by arterial blood sampling. In the 11C-dLop PET scans, brain uptake was 2.5 fold greater in P-gp knockout mice than in wild type mice. Ex vivo measurements of brain radioactivity were 16-fold higher in knockout mice. For monkeys, brain uptake of 11C-dLop increased 5-fold after P-gp blockade. In a one-tissue compartment model, the increase in brain uptake was mainly due to increased $\mathrm{K} 1$, suggesting an increased rate of radiotracer entry rather than a decreased rate of removal. Regional radiotracer uptake correlated with cerebral blood flow, indicating a high single pass extraction of 11C-dLop. After correction for blood flow, the uptake of 11C-dLop was fairly uniform across brain regions, suggesting uniform P-gp function. A near linear dose-response curve between DCPQ and brain uptake of 11CdLop was observed. For human studies at baseline, brain uptake was low (standardized uptake value $\sim 15 \%$ ). The plasma concentration of 11C-dLop declined rapidly, but the composition of radioactivity in plasma was quite stable, with the parent constituting $85 \%$ of total radioactivity after $5 \mathrm{~min}$. In conclusion, brain uptake of 11C-dLop in mice and monkeys increased greatly after P-gp blockade. The single pass extraction was high for 11C$\mathrm{dLop}$ and required blood flow correction to measure P-gp function accurately. Human data at baseline confirmed that 11C-dLop is a substrate for P-gp. The low K1 is consistent with that seen in the monkey studies, suggesting that $\mathrm{P}$-gp rapidly ejects the radiotracer upon its entry into the lipid bilayer.

\section{J796}

REGIONAL CEREBRAL P-GLYCOPROTEIN FUNCTION STUDIED WITH (R)- $\left[{ }^{11}\right.$ CIVERAPAMIL AND SMALL ANIMAL POSITRON EMISSION TOMOGRAPHY IN RATS AFTER STATUS EPILEPTICUS

Claudia Kuntner $^{1}$, Jens P. Bankstahl ${ }^{3}$, Marion Bankstahl ${ }^{3}$, Thomas Wanek ${ }^{1}$, Johann Stanek ${ }^{2}$, Markus Mueller ${ }^{2}$, Wolfgang Loescher ${ }^{3}$, Oliver Langer ${ }^{1,2}$ ${ }^{1}$ Health \& Environment, Austrian Research Centers GmbH - ARC, Seibersdorf, Austria, ${ }^{2}$ Clinical Pharmacology, Medical University of Vienna, Vienna, Austria, ${ }^{3}$ Pharmacology, Toxicology \& Pharmacy, University of Veterinary Medicine, Hannover, Germany.

AIM: Multidrug efflux transporters like P-glycoprotein (P-gp) at the blood-brain barrier are believed to play an important role in resistance to antiepileptic drug treatment. (R)- $\left[{ }^{11} \mathrm{C}\right]$ verapamil (VPM) positron emission tomography (PET) can be used to measure cerebral P-gp function, but low brain uptake of VPM hampers the mapping of P-gp function in different brain regions. We investigated if this limitation can be overcome by P-gp modulation prior to PET imaging and if this method is suitable to quantify changes in cerebral P-gp function occurring in rats after status epilepticus (SE). METHODS: Two groups of naïve female Sprague-Dawley rats underwent single VPM PET scans at $2 \mathrm{~h}$ after intravenous administration of different doses of the third generation P-gp inhibitors elacridar (ELC) or tariquidar (TQD), and arterial blood sampling. Compartmental modelling was performed to estimate VPM brain distribution volumes (DV). A sigmoidal doseresponse curve was fitted to DV values measured after different inhibitor doses. To study regional differences in cerebral P-gp function, paired VPM PET scans, before and after administration of TQD (3 or $15 \mathrm{mg} / \mathrm{kg}$ ), were performed both in naïve rats and in rats at $48 \mathrm{~h}$ after pilocarpine-induced SE. RESULTS: Inhibitor administration resulted in up to 10-fold increased VPM brain DV values with half-maximum effect doses of $1.12 \pm 0.15$ and $3.02 \pm 0.19 \mathrm{mg} / \mathrm{kg}$ for ELC and TQD, respectively. In the paired PET scans, the $15 \mathrm{mg} / \mathrm{kg}$ TQD dose uniformly increased radioactivity distribution across different brain regions as compared to baseline scans, both in naive and post SE rats. In post SE but not in naive rats, the $3 \mathrm{mg} / \mathrm{kg}$ TQD dose resulted in obvious regional differentiation of brain radioactivity distribution, with lowest uptake observed in cerebellum. Immunohistochemical staining showed a 2-fold increased P-gp content in cerebellum of post SE compared to naive rats. CONCLUSION: Our data suggest that VPM PET in combination with administration of moderate doses of P-gp inhibitors can be used for the mapping of regional cerebral P-gp function. Moreover, our data point to regional changes in cerebral P-gp function occurring in post $\mathrm{SE}$ as compared to naïve rats.

\section{$\mathbf{J 7 9 7}$}

\section{FOAMY VIRUS AS A SAFE TOOL FOR GENE DELIVERY IN APPLICATION OF MOLECULAR IMAGING}

Liang-ting Lin, Yi-Jang Lee

Department of Biomedical Imaging and Radiological Sciences, National Yang Ming University, Taipei, Taiwan.

Foamy Virus is a member of retrovirus that was found in cats and chimpanzees with no pathogenicity or transmission in human and documented as a useful and safe vehicle for gene delivery. In this study, we have applied foamy viral-mediated transduction of reporter genes as a 
novel approach for non-invasive molecular imaging. A dual fluorescence reporter gene was constructed in the foamy-viral producing vectors and was named $\mathrm{p} \Delta \Phi$-DsRed-IRES-EGFP. Virus soup were prepared by cotransfection of vectors with three helper plasmids, including pCiGs $\Delta \Psi$, pCiPs, and pCiEs that took the responsibility for production of gag, pol, and env gene respectively. To increase the titers, collected virus soup was further subjected to ultracentrifugation. For optimizing the infection, we assayed the fluorescent on different time point from 2 to 14 days postinfection with continuous culturing. Human non-small lung cancer H1299 cells were infected with this constructed foamy virus, and the result showed that the frequency of infection was about $20 \%$. To investigate the difference between foamy virus and lentivirus, we prepared both virus soup in the same titering and reporter gene, EGFP. Intraperitoneal inoculations of both virus soups in mice were held for comparing the pathogenicity and immune response between each other. The established cells were sorted by FACS and subcutaneously implanted onto NOD/SCID mice for in vivo imaging observation. Distribution and kinetic were determined non-invasively by In Vivo Imaging System, IVIS50. Our preliminary data showed that foamy virus provided ability for gene transduction with no significant pathogenicity than lentiviral system in vivo. Thus, foamy virus is an effective and safe tool in gene delivery for molecular imaging.

\section{J798}

FEASIBILITY OF MEASURING MYOCARDIAL NORADRENALINE RE-UPTAKE IN MICE USING $\left[{ }^{11} \mathrm{C}\right] M$-HYDROXYEPHEDRINE AND SMALL ANIMAL PET Marilyn P. Law $^{1}$, Klaus P. Schäfers ${ }^{1,2}$, Stefan Wagner ${ }^{1}$, Otmar Schober ${ }^{1}$, Paulus Kirchhof ${ }^{3}$, Klaus Kopka ${ }^{1}$, Michael Schafers ${ }^{2}$

${ }^{1}$ Department of Nuclear Medicine, University Hospital, University of Münster, Münster, Germany, ${ }^{2}$ European Institute of Molecular Imaging, University of Münster, Münster, Germany, ${ }^{3}$ Department of Cardiology, University Hospital, University of Münster, Münster, Germany.
Introduction: Dysfunction of the sympathetic nervous system (SNS) contributes to many cardiac diseases. Small animal PET with appropriate radiotracers enables non-invasive quantitation of the SNS in mouse models of human disease. We are using $\left[{ }^{11} \mathrm{C}\right] m$-hydroxyephedrine $\left(\left[{ }^{11} \mathrm{C}\right] m \mathrm{HED}\right)$ to assess myocardial innervation (noradrenaline re-uptake) using the quadHIDAC small animal PET scanner (Oxford Positron Systems). Depending on the specific activity of the $\left[{ }^{11} \mathrm{C}\right] m$ HED preparation, a significant mass of stable mHED and precursor, the noradrenaline mimetic metaraminol, is injected and competes with $\left[{ }^{11} \mathrm{C}\right] m \mathrm{HED}$ for re-uptake sites. The present study investigates the effects of metaraminol on the myocardial kinetics of $\left[{ }^{11} \mathrm{C}\right]$ mHED. Methods: Anaesthetised mice (2 or 4) were placed on the scanner bed. $30 \mathrm{~s}$ after scan start, $\left[{ }^{11} \mathrm{C}\right] m \mathrm{HED}(<8 \mathrm{MBq})$ was injected via a tail vein into each animal simultaneously. 15 min after $\left[{ }^{11} \mathrm{C}\right]$ $m \mathrm{HED}$, one animal was injected with saline and the other(s) with metaraminol $(0.05-10 \mu \mathrm{mol} / \mathrm{kg})$. Listmode data were acquired for $60 \mathrm{~min}$. A second scan using $\left[{ }^{18} \mathrm{~F}\right] \mathrm{FDG}(<8 \mathrm{MBq})$ was carried out to visualise the heart. Time activity curves for myocardial regions of interest were computed using in-house software. Results: Myocardial uptake of radioactivity was visualised after injection of $\left[{ }^{11} \mathrm{C}\right] \mathrm{mHED}$ (a). Metaraminol displaced myocardial activity (b, mouse 1). $\left[{ }^{18} \mathrm{~F}\right] \mathrm{FDG}$ showed heart position (c). Injection of saline had no effect on the rate of loss of myocardial radioactivity after $\left[{ }^{11} \mathrm{C}\right] m \mathrm{HED}$ injection but metaraminol increased it (d, arrow). Metaraminol doses $<50 \mathrm{nmol} / \mathrm{kg}$ had no effect whereas doses $>1 \mu \mathrm{mol} / \mathrm{kg}$ caused complete loss of specifically bound radioactivity within $5 \mathrm{~min}$. Conclusions: These results show that $\left[{ }^{11} \mathrm{C}\right] m \mathrm{HED}$ can be used to study sympathetic innervation of the heart in mice using the quadHIDAC scanner if the total molar dose of metaraminol and $\left[{ }^{11} \mathrm{C}\right] \mathrm{mHED}$ injected is below $50 \mathrm{nmol} / \mathrm{kg}$. Our first studies of transgenic mouse models of human arrhythmogenic diseases using the methods to study the SNS reported here are in progress and will be presented. This work is supported in part by the Deutscheforschungsgemeinschaft SFB 656 project A5.
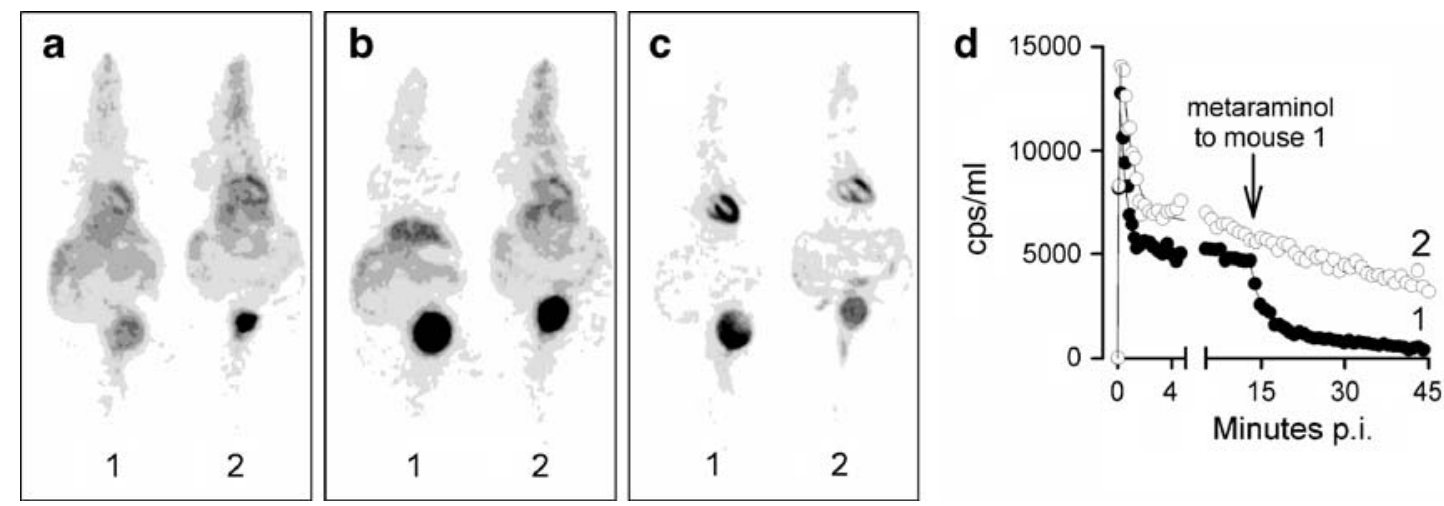

\section{DOWN-REGULATION OF RETINOIC ACID-INDUCED SODIUM IODIDE SYMPORTER EXPRESSION BY ESTROGEN RECEPTOR AGONIST IN BREAST CANCER CELLS}

Su-Jin Cheong, Chang-Moon Lee, Hwan-Jeong Jeong, Eun-Mi Kim, Seok Tae Lim, Myung-Hee Sohn, Dong Wook Kim

Nuclear Medicine, Chonbuk National University Medical School, Jeonju, Korea, South.
The sodium iodide symporter (NIS) mediates iodide uptake in the thyroid gland as well as in lactating breast, and is also expressed in the majority of breast cancers. Several hormones and factors including all-trans-retinoic acid (atRA) regulate the expression of NIS. Previous research reported a novel estrogen-responsive element sequence located in promoter region of NIS that specifically binds unliganded estrogen receptor- $\alpha(E R \alpha)$ mediates dependent activation of transcription in the RA-responsive mammary cancer cell model such as the MCF-7. In this study we examined the effect of estrogen receptor(ER) agonist (17- $\beta$-estradiol) and/or antagonist (tamoxifen or raloxifene) on atRA-induced NIS expression according to 
concentration of ER agonist NIS mRNA expression levels in MCF-7 cells were examined by RT-PCR and NIS functional activity was measured by iodide uptake assay after incubation with $17-\beta$-estradiol, tamoxifen or raloxifene in the presence of atRA. The effect of ER agonist or antagonist on iodide uptake was studied inMCF-7 cells after treatment with of without atRA. When the 17- $\beta$-estradiol or antagonist (tamoxifen or raloxifene) treated with alone, iodide uptake level was similar to background level. After treatment with 17- $\beta$-estradiol (10-15 to 10-10 M) in the presence of atRA, iodide uptake was stimulated up to 20 -fold as compared to background level. However, when treated with 17- $\beta$-estradiol of high concentration $(10-5 \mathrm{M})$ in the presence of atRA, iodide uptake was significantly decreased. No iodide uptake above background level was observed. When ER antagonist (tamoxifen or raloxifene) was combined with $17-\beta$-estradiol in the presence of atRA, the result of iodide uptake was also similar to previous result. Iodide uptake was significantly decreased according to increase of $17-\beta$-estradiol. The expression of NIS mRNA by RT-PCR also showed similar results. Estrogen receptor agonist (17- $\beta$-estradiol) down-regulates NIS gene expression and iodide uptake in ER-positive breast cancer cell line according to concentration of treatment.

\section{$\mathbf{J 8 0 0}$}

SYSTEM A AND SYSTEM L AMINO ACID TRANSPORT IMAGING WITH ${ }^{11} \mathrm{C}$ MEAIB AND ${ }^{11} \mathrm{C}$ MET PET STUDY

Ryuichi Nishii ${ }^{1}$, Tatsuya Higashi ${ }^{1}$, Shinya Kagawa ${ }^{1,2}$, Yoshihiko Kishibe $^{1}$, Masaaki Takahashi ${ }^{1}$, Tomoya Uehara ${ }^{3}$, Shigeki Nagamachi ${ }^{4}$, Yasushi Arano $^{3}$, Keiichi Kawai ${ }^{2,5}$

${ }^{1}$ Division of PET Imaging, Shiga Medical Center Research Institute, Shiga, Japan, ${ }^{2}$ Graduate School of Medical Science, Kanazawa University, Kanazwa, Japan, ${ }^{3}$ Graduate School of Pharmaceutical Sciences, Chiba University, Chiba, Japan, ${ }^{4}$ Department of Radiology, Faculty of Medicine, Miyazaki University, Miyazaki, Japan, ${ }^{5}$ Biomedical Imaging Research Center, University of Fukui, Fukui, Fukui, Japan.

Purpose: The aim of this study was to assess the uptake mechanism of $\alpha$-[N-methyl- $\left.{ }^{11} \mathrm{C}\right]$-methylaminoisobutyric acid $\left(\left[{ }^{11} \mathrm{C}\right]-\mathrm{MeAIB}\right)$ as specific substrate of the system A amino acid transport in human-derived carcinoma cells, compared with that of [S-methyl- $\left.{ }^{11} \mathrm{C}\right]-\mathrm{L}-$-methionine $\left(\left[{ }^{11} \mathrm{C}\right]-\mathrm{MET}\right)$, and to clarify the difference between system $\mathrm{A}$ and $\mathrm{L}$ amino acid transport imaging in clinical PET. Methods: Uptake study of amino acid radiotracers, $\alpha-\left[1-{ }^{14} \mathrm{C}\right]$-Methylaminoisobutyric acid $\left(\left[{ }^{14} \mathrm{C}\right]-\mathrm{MeAIB}\right)$ and $\left[\mathrm{S}-\mathrm{methyl}-{ }^{3} \mathrm{H}\right]-\mathrm{L}-$ methionine $\left(\left[{ }^{3} \mathrm{H}\right]-\mathrm{MET}\right)$ were performed in five types of human carcinoma cells (epidermal carcinoma: A431, colorectal carcinoma: LS180, lung carcinoma: PC14/GL, H441/GL, breast carcinoma: MDA-MB435). Inhibition studies of system A and L were also performed using MeAIB in $\mathrm{Na}^{+}$containing solution as a specific inhibitor of system $\mathrm{A}$, and $\mathrm{BCH}$ in $\mathrm{Na}^{+}$free solution as a specific inhibitor of system L. In clinical study, eight normal healthy subjects received intravenous injection of both $\left[{ }^{11} \mathrm{C}\right]-$ MeAIB and $\left[{ }^{11} \mathrm{C}\right]-\mathrm{MET}$, respectively in other day, and dynamic and static PET images were obtained. Plasma and pancreas time-activity curve were analyzed graphically using pharmacokinetic modeling with the 1 tissue-compartment modeling for $\left[{ }^{11} \mathrm{C}\right]$-MeAIB PET and the 2 tissue-compartment modeling for $\left[{ }^{11} \mathrm{C}\right]$-MET PET, followed by the voxel-based parametric image analysis. Results: In the cell uptake study, the uptake of $\left[{ }^{3} \mathrm{H}\right]$-MET is about $3-8$ times higher than $\left[{ }^{14} \mathrm{C}\right]-$ MeAIB in all carcinoma cells. Inhibition study of amino acid transport in carcinoma cells revealed that $\left[{ }^{14} \mathrm{C}\right]-\mathrm{MeAIB}$ was transported into these cells by the system $A$, while that of $\left[{ }^{3} \mathrm{H}\right]-$ Met was by the system L. In clinical PET imaging of $\left[{ }^{11} \mathrm{C}\right]-\mathrm{MeAIB}$ and $\left[{ }^{11} \mathrm{C}\right]-\mathrm{MET}$, peak plasma accumulation were observed in 2 min after injection and decreased tri-exponentially, while pancreas showed constant increased uptake because of system $\mathrm{A}$ and $\mathrm{L}$ amino acid transports. In pharmacokinetic modeling from the time-activity curves, parameters of $\mathrm{K} 1, \mathrm{~K} 2$ and Vt were $0.28 \pm 0.07,0.11 \pm 0.01,2.67 \pm 0.76$ for $\left[{ }^{11} \mathrm{C}\right]-$ $\mathrm{MeAIB}$ and $0.75 \pm 0.17,0.07 \pm 0.07,18.67 \pm 3.93$ for $\left[{ }^{11} \mathrm{C}\right]-\mathrm{MET}$, respectively $(\mathrm{P}<0.01)$. Conclusions: System $\mathrm{A}$ and $\mathrm{L}$ amino acid transport image analysis were obtained with $\left[{ }^{11} \mathrm{C}\right]$-MeAIB and $\left[{ }^{11} \mathrm{C}\right]-$ MET PET. These PET imaging would contribute to assess the activity of amino acid transports in various diseases such as neoplasm.

\section{IN VIVO WORK}

\section{Animals}

\section{J801}

IN VIVO HYPERPOLARIZED ${ }^{13} \mathrm{C}$ MR SPECTROSCOPIC IMAGING OF DISEASE PROGRESSION AND REGRESSION IN A TRANSGENIC MOUSE MODEL OF LIVER CANCER Simon $\mathrm{Hu}^{1,2}$, Asha Balakrishnan ${ }^{3}$, Robert Bok ${ }^{1}$, Peder E. Larson ${ }^{1}$, Sarah Nelson ${ }^{1,2}$, John Kurhanewicz ${ }^{1,2}$, Andrei Goga ${ }^{3}$, Daniel B. Vigneron $^{1,2}$

${ }^{1}$ Dept. of Radiology and Biomedical Imaging, UCSF, San Francisco, CA, USA, ${ }^{2}$ UCSF \& UCB Joint Graduate Group in Bioengineering, San Francisco and Berkeley, CA, USA, ${ }^{3}$ Dept. of Medicine, Division of Hematology/Oncology, UCSF, San Francisco, CA, USA.

Introduction: Recent development of hyperpolarized technology utilizing dynamic nuclear polarization has enabled the monitoring of ${ }^{13} \mathrm{C}$ metabolites in vivo at very high SNR [1]. The purpose of this preclinical study was to assess metabolism during disease progression and regression in a transgenic model of liver cancer using 3DMRSI with hyperpolarized ${ }^{13} \mathrm{C}_{1}$-labeled pyruvate. Methods: We used Tet-o-MYC/LAP-tTA double-transgenic mice in which the human MYC proto-oncogene is overexpressed only in the liver and can be switched off with doxycycline [2]. All studies were performed on a GE $3 \mathrm{~T}$ scanner with a custom ${ }^{1} \mathrm{H} /{ }^{13} \mathrm{C}$ mouse coil. ${ }^{13} \mathrm{C}$ 3D-MRSI data (TE/TR $=140 \mathrm{~ms} / 215 \mathrm{~ms}, 0.034 \mathrm{~cm}^{3}$ voxel size) were acquired with a double spin-echo compressed sensing pulse sequence [3] after injection of $0.35 \mathrm{~mL}$ of $80 \mathrm{mM}$ hyperpolarized ${ }^{13} \mathrm{C}_{1}$-pyruvate. Lactate area/total carbon (lac/tCar) and alanine area/ total carbon (ala/tCar) ratios were derived from the spectral arrays. Results: A total of 48 separate hyperpolarized experiments were performed on mice in various disease states (10 healthy, 9 before appearance of tumor on anatomic imaging, 17 with tumor confirmed with anatomic imaging, and 12 with tumor regression confirmed with anatomic imaging). Figure 1a shows a representative case of disease progression. Elevated ala/tCar was observed before a tumor was apparent on anatomic imaging, and elevated lac/tCar was observed afterward. Figure $1 \mathrm{~b}$ shows a representative case of disease regression (regression after MYC was switched off with doxycycline). Reductions in both tumor size and lac/tCar were observed. Discussion: This study demonstrated the ability of hyperpolarized ${ }^{13} \mathrm{C}$ to monitor cancer progression and treatment in a transgenic mouse model of liver cancer, showing the potential of this technique for future in vivo applications. References: [1] Ardenkjaer-Larsen et al. PNAS (2003) 100:10158 [2] Shachaf et al. Nature (2004) 431:1112 [3] Hu et al. JMR (2008) 192:258 


\section{A) DISEASE PROGRESSION}
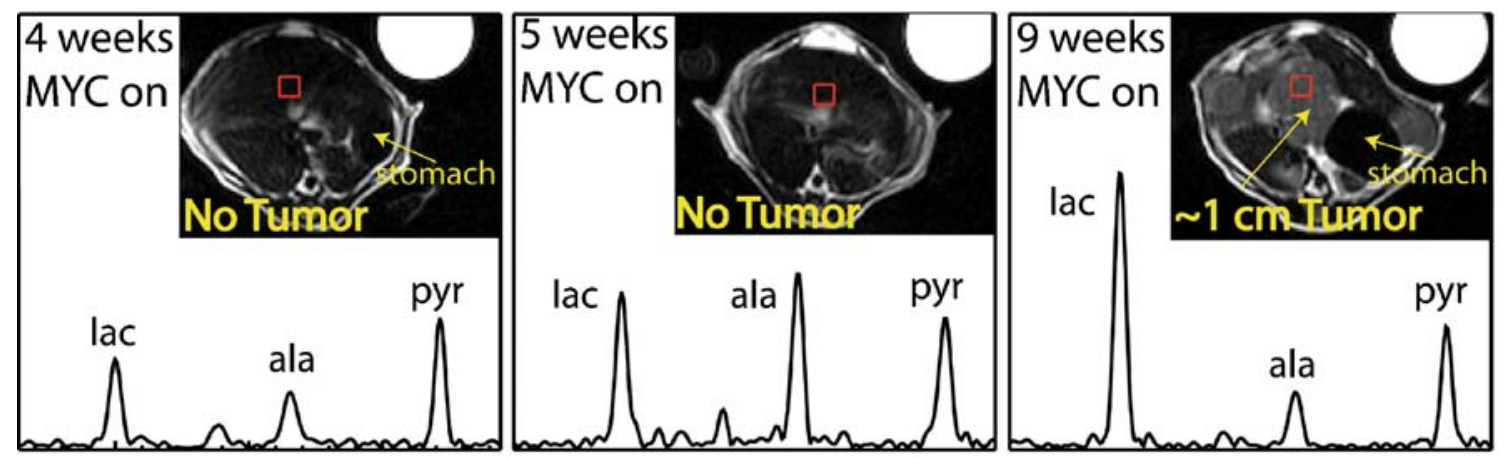

\section{B) DISEASE REGRESSION}
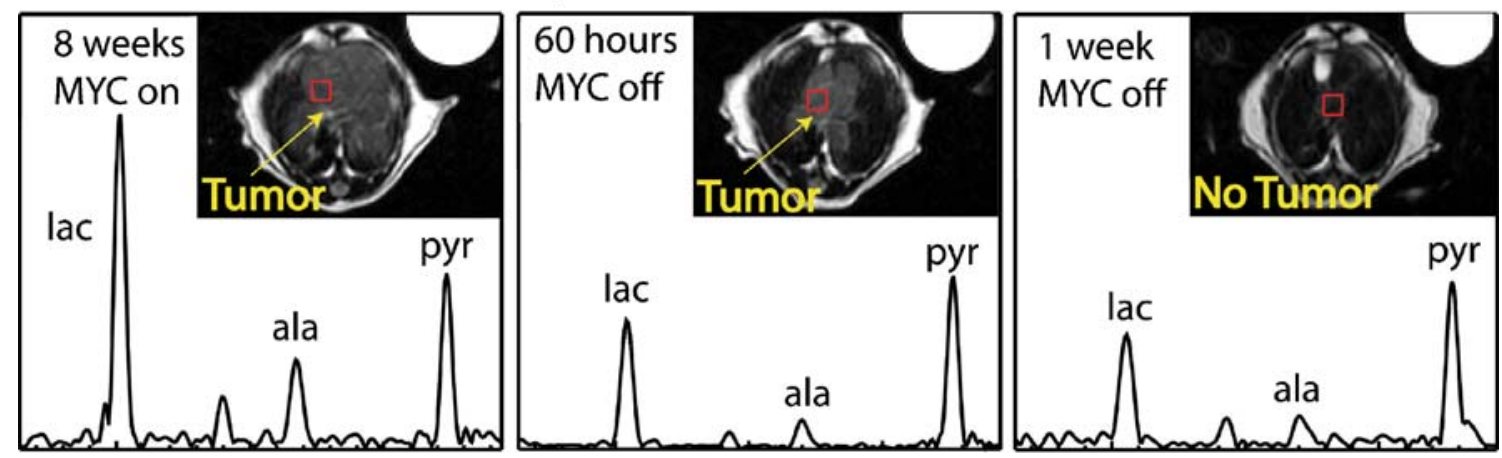

Figure 1: A) Note elevated alanine in middle spectrum and elevated lactate in right spectrum. B) Reduction of lactate was observed after MYC expression was switched off.

$\mathbf{J 8 0 2}$

COMPARISON OF LENTIVIRAL AND ADENO-ASSOCIATED VIRAL VECTORS FOR STABLE MRI REPORTER GENE EXPRESSION IN THE RODENT BRAIN

Greetje Vande Velde ${ }^{1,4}$, Janaki R. Rangarajan ${ }^{2,4}$, Tom Dresselaers ${ }^{3,4}$, Abdelilah Ibrahimi ${ }^{1,4}$, Zeger Debyser ${ }^{1,4}$, Veerle Baekelandt ${ }^{1,4}$, Uwe Himmelreich $^{3,4}$

${ }^{1}$ Molecular and Cellular Medicine, Katholieke Universiteit Leuven, Leuven, Belgium, ${ }^{2}$ Medical Imaging Research Center (Radiology ESAT/PSI), Katholieke Universiteit Leuven, Leuven, Belgium, ${ }^{3}$ Biomedical NMR unit, Katholieke Universiteit Leuven, Leuven, Belgium, ${ }^{4}$ Molecular Small Animal Imaging Centre (MoSAIC), Katholieke Universiteit Leuven, Leuven, Belgium.

AIMS: For delivery of MRI reporter genes (e.g. ferritin) to the rodent brain, lentiviral (LV) and adeno-associated viral (AAV) vectors are the preferred viral vector systems when aiming for stable, long-term labelling and in vivo visualization of cells. However, their potential limitations for MRI like the generation of unspecific contrast by the vector itself are often insufficiently addressed. Here, we evaluated the potential of LV and AAV to be used as MRI reporter gene expressing vectors to the rodent brain with regard to their background contrast, both in immunodeficient as in immunocompetent animals. METHODS: LV/AAV with and without MRI reporter, or PBS were stereotactically injected in the striatum of C57BL/6 and NOD-SCID mice. 3D T2*-weighted MR images and $\mathrm{T} 2 / \mathrm{T} 2 *$ maps were acquired at different time points post injection on a 9,4 $\mathrm{T}$ small animal scanner. After the last time point, animals were sacrificed, followed by ex vivo MRI of the excised brain and (immuno-)histochemical staining. MRI images were coregistered, intensity-normalized and the contrast was quantified using an in-house developed pipeline. RESULTS: Injection in rodent brain of LV/AAV without MRI reporter resulted in persisting hypointense contrast at the injection site on T2*-weighted MRI. This contrast correlated with the presence of $\mathrm{Fe} 3+$ and microglia/ macrophages and could not be explained by bleeding or damage inherent to the injection. When comparing a control LV (eGFP) with a LV encoding an MRI reporter (ferritin) in mouse brain, no significant differences in affected volume on MRI between LVeGFP and LV-FerrH could be observed. In immunodeficient mice, the background contrast of LV was significantly reduced. For AAV, the background contrast was reduced by about $50 \%$ as compared to LV. AAV-mediated ferritin overexpression resulted in more pronounced hypointense contrast as compared to AAV-control vector injection in immunocompetent mice. CONCLUSIONS: Viral vector injection itself results in hypointense contrast at the site of injection on T2*-weighted MRI that cannot be explained by mechanical damage and/or bleeding inherent to the injection alone, but correlates with the presence of $\mathrm{Fe} 3+$ and microglia at the site of injection. These results challenge the signal-to-noise properties of any putative MRI reporter gene to be detectable above this background signal in immunocompetent animals. AAV seems the most promising vector system for use with MRI reporter genes.

\section{$\mathrm{J803}$}

BIOLUMINESCENCE AND MICRO CT IMAGING FOR QUANTIFYING BONE TISSUE REGENERATION USING rAAV-BMP2-COATED CALVARIAL ALLOGRAFTS Gadi Pelled $^{1,2}$, Ayelet Ben Arav ${ }^{2}$, Wafa Tawackoli ${ }^{1}$, Zulma Gazit ${ }^{1,2}$, Edward M. Schwarz ${ }^{3}$, Dan Gazit ${ }^{1,2}$

${ }^{1}$ Surgery, Cedars Sinai Medical Center, Los Angeles, CA, USA, ${ }^{2}$ Skeletal Biotech Laboratory, Hebrew University of Jerusalem, Jerusalem, Israel, ${ }^{3}$ Center for Musculoskeletal Research, University of Rochester, Rochester, NY, USA. 
Bone tissue has regenerative abilities, however in vast bone loss complete regeneration will not occur. Recently, a novel approach, which revitalizes allograft bone by freeze-drying of recombinant adeno-associated virus (rAAV) encoding for an osteogenic gene on allograft bone has been developed. In this study, we aimed to investigate the kinetics of transgene expression induced by rAAV-coated calvaria allografts, its biodistribution (BD) and to quantify the osteogenic effect induced by rAAV-BMP2 coated allograft in a calvaria defect model. Hypothesis: rAAV-coated calvaria allografts would induce local and transient gene expression followed by critical-size bone defect regeneration. AAV-Luciferase (Luc) coated allografts were implanted in a calvaria defect induced in $\mathrm{FVB} / \mathrm{N}$ mice. In vivo Luc expression was monitored using bioluminescence imaging (BLI). On day 28 the internal organs were harvested and tested for Luc activity or for the existence of its DNA, using qPCR. Single strand (SS) or self complementary (SC) rAAV encoding for BMP2 were used to coat allografts, which were implanted in defects created in the calvaria of osteocalcin-Luc transgenic mice. Uncoated allografts and autografts served as controls. BLI of these mice provides an indication of osteocalcin activity. $\mu \mathrm{CT}$ scans were performed to evaluate bone formation. Results showed that BD of the Luc transgene was localized the calvaria up to Day 21 (Fig. 1A). No detectable levels of Luc were found in the mice internal organs. The osteogenic effect demonstrated by BLI in the transgenic mice showed a peak of expression on the first week, which was the highest with the SC-rAAV-BMP2 coated allografts (Fig. 1B). $\mu \mathrm{CT}$ analysis focused on a site of interest around the defect (Fig 1C) showed significantly higher BV values for SC-rAAV-BMP2 group compared to other groups (Fig 1D). Our results show feasibility of craniofacial regeneration using rAAV-BMP2-coated allografts and indicate its prominent local and transient osteogenic effect, leading to an increased BV over time. These results could prove to be an attractive therapeutic solution for the regeneration of severe bone defects.
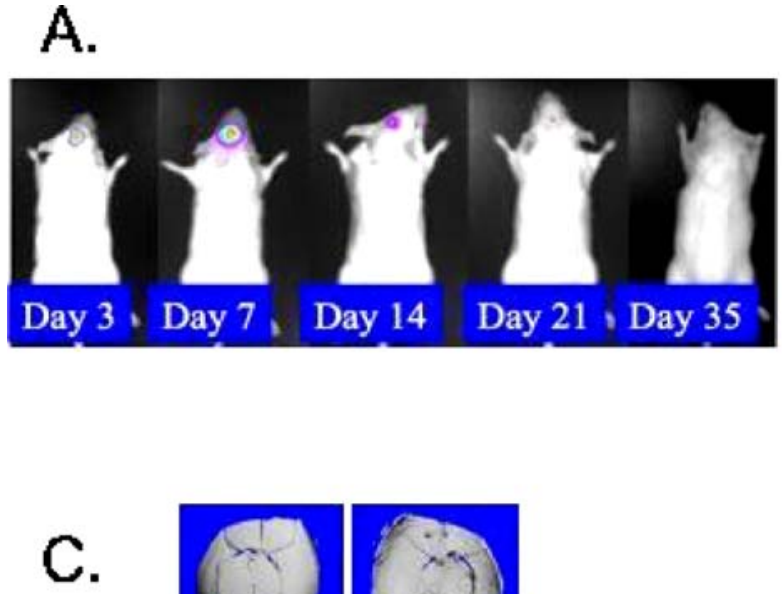

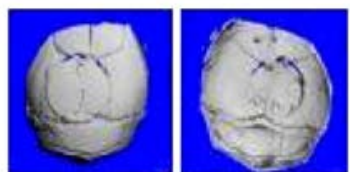

SS-FAAV-BMP2

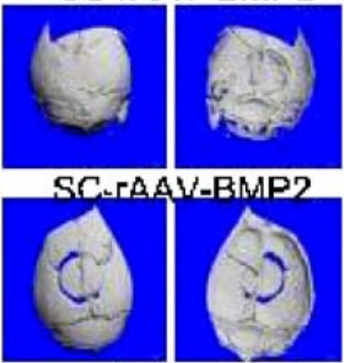

Autooraft

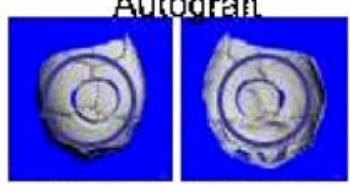

Uncoated Allograft

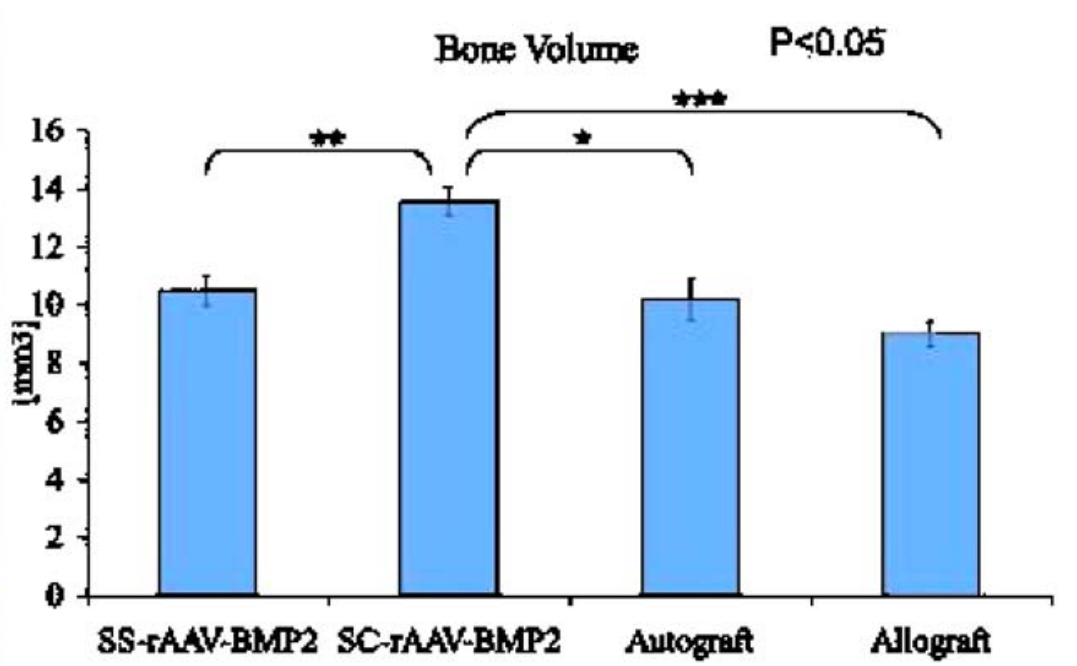

$\mathbf{J 8 0 4}$

MICROBUBBLE CONTRAST-ENHANCED ULTRASOUND AS A BIOMARKER IN MTOR INHIBITOR THERAPY: MOLECULAR IMAGING ASSESSMENT OF TUMOR GROWTH AND VASCULARIZATION IN A TRANSGENIC MOUSE MODEL OF MAMMARY CARCINOMA

$\underline{\text { Lei Zhao }}^{1}$, Xinkang Wang ${ }^{1}$, Yutian Zhan ${ }^{1}$, John Alvarez ${ }^{2}$, Robert $\overline{\text { Abraham }}^{3}$, Stephen A. Douglas ${ }^{1}$, Giora Z. Feuerstein ${ }^{1}$

${ }^{1}$ Discovery Translational Medicine, Wyeth, Collegeville, PA, USA,

${ }^{2}$ Clinical Translational Medicine, Wyeth, Collegeville, PA, USA,

${ }^{3}$ Discovery Oncology, Wyeth, Pearl River, NY, USA.

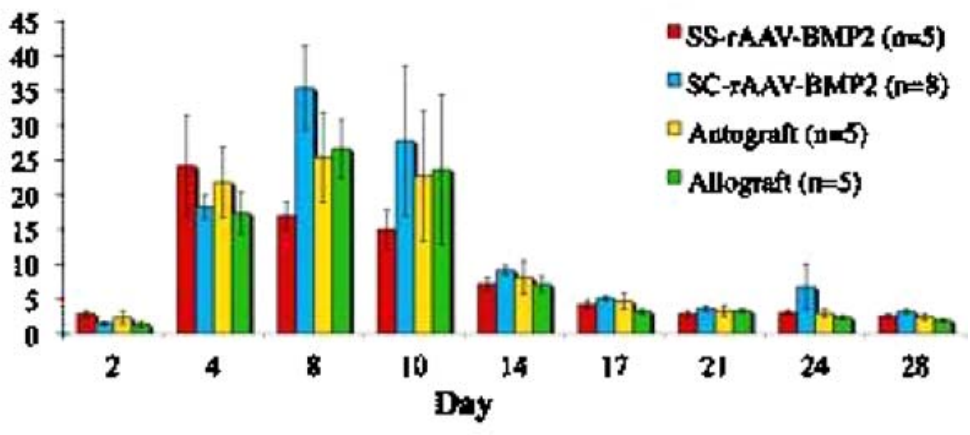

Introduction: Contrast-enhanced non-targeted and targeted microbubble-based ultrasonography (US) has emerged as novel technology for visualization and quantification of tumor microvasculature and angiogenesis. The current study examined the impact of temsirolimus (CCI779), an mTOR inhibitor, on tumor growth, microvascular density and neo-vasculature formation using three-dimensional (3D) volumetric assessment, non-targeted and VEGFR2-targeted microbubble contrastenhanced techniques in the polyomavirus middle T-antigen (PyMT) mouse model of mammary carcinoma. Methods: Heterozygous female PyMT mice at early ( 8 weeks) and advanced (11 weeks) phases of tumor development were subjected to baseline US (Vevo 770, Visualsonics) data acquisition (3D volumetric measurement, non-targeted 
contrast-enhanced microvascular density and VEGFR2-targeted neovasculature assessment) and subsequent administration of CCI-779 ( $25 \mathrm{mg} / \mathrm{kg}$, i.v., once weekly for 2 weeks). These tumor parameters were monitored longitudinally for 2 weeks and mice were sacrificed at 10 weeks (early phase) and 13 weeks (advanced phase) respectively. Texas red-dextran staining for tumor vasculature and VEGFR2 staining for neo-vasculature were assessed using histological approaches. Results: CCI-779 led to significant $85 \%$ reduction of tumor volume at 10 weeks $\left(2.5 \pm 0.3\right.$ in CCI-779 group v $17.1 \pm 1.8 \mathrm{~mm}^{3}$ in vehicle, $P<$ $0.01)$ and 13 weeks $\left(29.9 \pm 16.7\right.$ in CCI-779 group v $185.0 \pm 56.8 \mathrm{~mm}^{3}$ in vehicle, $P<0.05$ ). A marked $67 \%$ decrease of microvascular density was also observed in CCI-779-treated mice compared to the vehicle group at both early $(2.5 \pm 0.8 \%$ in CCI-779 group $\mathrm{v} 7.6 \pm 1.3 \%$ in vehicle, $P<0.05)$ and late tumor phases $(3.7 \pm 0.6 \%$ in CCI-779 group v $10.9 \pm 3.0 \%$ in vehicle, $P<0.05)$. The reduction in vascular density was verified by histological assessment using Texas red-dextran microvascular staining. VEGFR2 binding signal, an index reflecting absolute specific VEGFR2 binding to microvasculature, was diminished $70 \sim 76 \%$ upon CCI-779 administration (10 weeks, $2.5 \pm 0.3$ in CCI-779 group v $8.4 \pm 1.9$ a.u. in vehicle, $P<0.05 ; 13$ weeks, $2.4 \pm 1.0$ in CCI-779 group v $10.2 \pm 2.8$ a.u. in vehicle, $P<0.05$ ). Histological VEGFR2 staining further confirmed these findings. Conclusions: The current study demonstrated that CCI-779 plays an essential role in retarding tumor growth and neo-vasculature development in PyMT mice. These data also suggest that microbubble contrast-enhanced US can become a capable imaging modality for non-invasive and longitudinal compound screening in preclinical animal models of breast cancer.

\section{J805}

\section{MULTIMODAL IMAGING OF TRANSPLACENTAL INFECTION AND SUBSEQUENT BIRTH DEFECTS}

Jonathan Hardy ${ }^{1}$, Laura J. Pisani ${ }^{2}$, Christopher Contag ${ }^{1,2}$

${ }^{1}$ Department of Pediatrics, Stanford University School of Medicine, Stanford, CA, USA, ${ }^{2}$ Radiology, Stanford University, Stanford, CA, USA.

Approximately one fifth of all pregnancies result in miscarriage, the causes of which are largely unknown. Maternal factors such as age and substance abuse may increase the chances of miscarriage but these factors explain only a minority of cases. Few studies have addressed the underlying causes of spontaneous abortion or stillbirth, but it is estimated that many are due to infection. Perinatal bacterial infections can induce abortion, stillbirth, and neonatal fatalities. A variety of bacteria infect the fetus preferentially, often with only mild or no illness in the mother, which results in loss of the fetus for no readily apparent reason. Little is known of the pathogenesis of infections leading to abortions, because they occur spontaneously and not in a manner that facilitates useful sample collection. Therefore animal models of transplacental infection may yield important fundamental information that can be used to prevent bacteria from causing tragedy for pregnant women and their families. One of the most well characterized models of transplacental infection, and indeed infectious disease of any kind, is Listeria monocytogenes, a bacterium that is dangerous to pregnant women. The consumption of soft cheeses by pregnant women is discouraged because of $\mathrm{L}$. monocytogenes, which often kills in outbreaks associated with contaminated foods. We applied in vivo bioluminescence imaging (BLI) to this model in pregnant mice, using lux expressing L. monocytogenes, and have imaged the process of transplacental infection leading to abortion, stillbirth, and transmission of the bacteria to offspring. Here, we combine BLI with MRI to examine the process of infection and abortion of fetuses and reveal the actual defects in the infected fetuses in utero as they progress. We find that placental infection alone can cause spontaneous abortion and that stillborn pups are developmentally arrested, and display defects in skull and limb morphology.

\section{J806}

\section{DEVELOPMENT OF THE TRANSGENIC CYAN FLUORESCENT PROTEIN (CFP)-EXPRESSING NUDE MOUSE FOR “TECHNICOLOR" CANCER IMAGING}

Hop S. Tran $\mathrm{Cao}^{2}$, Jose Reynoso ${ }^{1}$, Meng Yang ${ }^{1}$, Hiroyuki Kimura ${ }^{2,1}$, Sharmeela Kaushal ${ }^{2}$, Cynthia S. Snyder ${ }^{2}$, Robert M. Hoffman ${ }^{1,2}$, Michael Bouvet ${ }^{2}$

${ }^{1}$ AntiCancer, Inc., San Diego, CA, USA, ${ }^{2}$ Dept. of Surgery, University of California San Diego, San Diego, CA, USA.

A major goal for in vivo biology is to develop models which can express multiple colors of fluorescent proteins in order to image multiple processes simultaneously in real time. Towards this goal, the cyanofluorescent protein (CFP) nude mouse was developed by crossing non-transgenic nude mice with the transgenic CK/ECFP mouse in which the $\beta$-actin promoter drives expression of CFP in almost all tissues. In crosses between nu/nu CFP male mice and nu/+ CFP female mice, approximately $50 \%$ of the embryos fluoresced blue. In the CFP nude mice, the pancreas and reproductive organs displayed the strongest fluorescent signals of all internal organs which vary in intensity. Orthotopic implantation of XPA-1 human pancreatic cancer cells expressing red fluorescent protein (RFP) or green fluorescent protein (GFP) in the nucleus and RFP in the cytoplasm was performed in female nude CFP mice. Color-coded fluorescence imaging of these human pancreatic cancer cells implanted into bright flue fluorescent pancreas of the CFP nude mouse afforded novel insight into the interaction of the pancreatic tumor and the normal pancreas, in particular the strong desmoplastic reaction of the tumor. The naturally enhanced blue fluorescence of the pancreas in the CFP mouse serves as an ideal background for color-coded imaging of the interaction of implanted cancer cells and the host. The CFP nude mouse will provide unique understanding of the critical interplay between the cancer cells and their microenvironment.

\section{$\mathbf{J 8 0 7}$}

\section{KINETICS OF UPTAKE OF RADIOIODINATED MAB TO A TARGET IN THE VASCULAR SPACE}

Stephen J. Kennel ${ }^{1}$, Saed Mirzadeh ${ }^{2}$, Wenbin Zeng ${ }^{1}$, Alan Stuckey ${ }^{1}$, David W. Townsend ${ }^{1}$, Jonathan Wall ${ }^{1}$

${ }^{1}$ Graduate School of Medicine, University of Tennessee, Knoxville, TN, USA, ${ }^{2}$ Oak Ridge National Laboratory, Oak Ridge, TN, USA.

Objectives: There is growing interest in delivery of drugs and radioisotopes with carriers designed to target molecular receptors in the vascular space. In contrast to targets outside of blood vessels vascular receptors are easily accessed and targeting is not significantly impacted by the size of the drug carrier. Although it is accepted that vascular targeting is very efficient, the kinetics of tracer-target interactions have not been evaluated over very short times. Methods: MAb 201B to murine thrombomodulin accumulates in lung, and has been used as a model for immunoimaging protocols and radioimmunotherapy. MAb 201B was radioiodinated with ${ }^{125} \mathrm{I}$ or ${ }^{124} \mathrm{I}$ using either chloramine $\mathrm{T}$ or a N-succinimidyl benzoate derivative to prevent dehalogenation. Accumulation and retention was monitored by standard biodistribution experiments or by dynamic microPET imaging after iv injection in mice. Results: Lung uptake and clearance $\left(\mathrm{t}_{1 / 2} \sim 40 \mathrm{hrs}\right)$ of ${ }^{125} \mathrm{I}$ MAb 201B was similar for both radioiodination methods. Lung uptake of ${ }^{124} \mathrm{I}$ MAb 201B occurred within seconds of injection as observed by dynamic microPET imaging with little ${ }^{124}$ I MAb detected in the peripheral 
circulation at any time. In contrast, distribution kinetics of control ${ }^{124} \mathrm{I}$ MAb 14 or ${ }^{124}$ I MAb 201B that had been diluted with excess cold MAb 201B equilibrated throughout the vascular space. Conclusion: Accumulation of iv injected, high-affinity MAbs that target epitopes in the vascular space occurs very rapidly and likely within the first blood pass. Research supported by University of TN Graduate school of Medicine and the DOE contract with ORNL ERKP 038.

\section{J808}

\section{MOTION TRACKING USING OPTICAL NAVIGATION DURING IN VIVO TWO-PHOTON MICROSCOPY}

James L. Schroeder ${ }^{1}$, Merav Luger-Hamer ${ }^{1}$, Randall Pursley ${ }^{2}$, Peter Kellman $^{1}$, Robert S. Balaban ${ }^{1}$

${ }^{1}$ Lab of Cardiac Energetics, NHLBI, Bethesda, MD, USA, ${ }^{2}$ Center for Information Technology, NIH, Bethesda, MD, USA.
We adapted a commercial multi-photon microscope to do real-time motion tracking of in-vivo tissue. Anesthetized animals were restrained on a hexapod micro-positioning stage. An upright microscope was equipped with an objective piezo stepper motor to rapidly adjust focal plane. Image acquisition was performed with a resonant scanning mirror at 24 frames per second while the piezo motor changed the focal plane of each frame. The resulting image sequence would oscillate focal plane up and down across a $20+$ micron depth at a period of up to $2 \mathrm{~Hz}$. The image stream was reassembled in real-time into a $3 \mathrm{D}$ volume, and a parallel processing algorithm compared each new volume to a previously acquired reference volume to calculate offsetting motion and make compensatory adjustment of the hexapod stage position. Using this system we tracked tissue during various physiological perturbations within several microns, and were able to observe dynamic physiological processes. Physiological perturbations included metabolic substrates, drug delivery, and anoxia. Image sequences were analyzed using principal component analysis to find significant trends and map them to spatial locations.
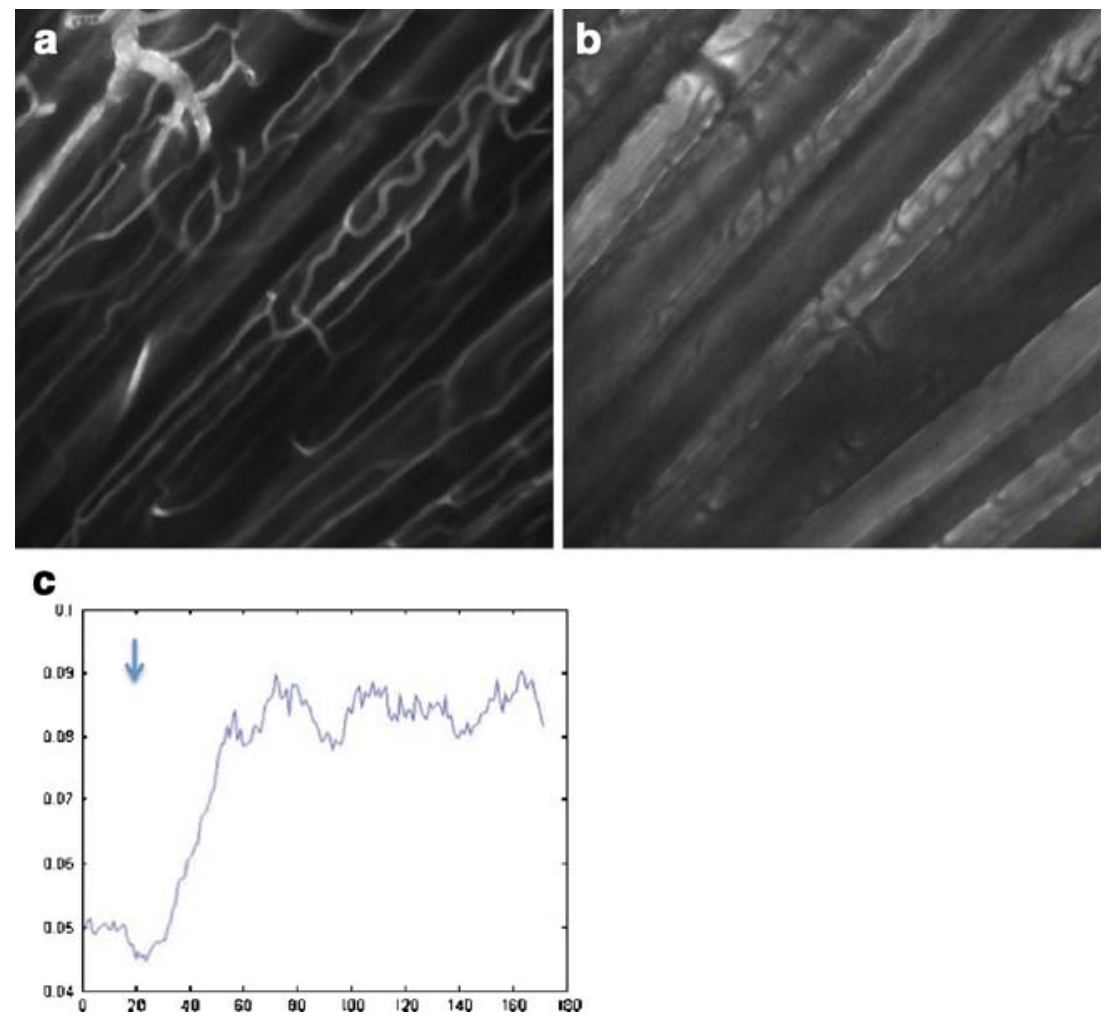

A: Principal component map for 515-650 nm (ANEPPS $d y e$ ) B: Principal component map for 430-500 nm (NADH autofl.) C: Channel B, graph of principal component fluorescence (arb. units) vs. time (total of $6 \mathrm{~min}$.) after systemic anoxia (arrow)

\section{J809}

OPTIMIZED TUMOR TARGETING OF NANOPARTICLEMEDIATED FLUORESCENT CONTRAST AGENTS

$\underline{\text { Steven D. Perrault }}^{1}$, Carl Walkey $^{1}$, Travis Jennings ${ }^{2}$, Hans C. Fischer ${ }^{1}$, Warren C W. Chan

${ }^{1}$ Institute of Biomaterials and Biomedical Engineering, University of Toronto, Toronto, ON, Canada, ${ }^{2}$ eBioscience, San Diego, CA, USA.

Nanoparticles are ideally suited to improving how diagnostic agents are delivered to tumors, because their size and surface chemistry properties can be engineered towards specific behaviours in vivo. Despite this, there has not been a systemic examination of how particle size and surface chemistry impacts tumor targeting behaviour for particles with diameters below $100 \mathrm{~nm}$. Here we mapped the blood half-life, tumor accumulation and permeation properties of gold nanoparticles varied in size $(15-100 \mathrm{~nm})$ and surface chemistry (poly-ethylene glycol). Half-life of the particles in circulation improved with larger molecular weight PEG and smaller core diameters, such that sub-100 $\mathrm{nm}$ particles can provide a broad range of blood pharmacokinetic behaviour ( $\mathrm{t} 1 / 2=0.4-$ $51.1 \mathrm{~h}$ ). Total tumor accumulation of $20,40,60,80$ and $100 \mathrm{~nm}$ particles was tested in a xenograft tumor model, finding that all 
particle sizes were able to access the tumor interstitial space via porous vasculature. Further, we found that tumor accumulation of specific particle designs was strongly dependent on their half-life in blood, and that smaller particles had relatively poor accumulation $(p<0.015)$. By measuring particle distribution within the tumor space using silver enhancement staining of histology samples, we found that large particles (i.e. $100 \mathrm{~nm}$ ) were highly localized around blood vessels and small (ie $20 \mathrm{~nm}$ ) particles had permeated far into the tumor mass at 8 hours post-injection. This fundamental characterization provided a basis for the rational design of nanoparticle vehicles to improve delivery of diagnostic agents to tumors. We engineered a novel detection strategy using particles with specific pharmacokinetic (t1/2 4 h), and tumor permeation $(70-90 \mathrm{~nm})$ properties to allow relatively efficient passive accumulation with perivascular localization. We tested this system using a fluorescent contrast agent and optical detection platform, finding an improved signal-to-noise and longer residency time within the tumor. These results provide a basis for the rationale design of tumor targeting nanoparticle vehicles, and a demonstration of how this can be applied to improve tumor detection.

\section{J810}

\section{IN VIVO INTRAVITAL FLUORESCENCE MICROSCOPY OF THE LUNG: TRAVELLING ABYSSAL ZONES \\ O. Lesur \\ $\overline{\text { Medicine, }}$ CHU Sherbrooke, CRC, MICU, FMSSS, Sherbrooke, QC, Canada.}

Clément Fournier, Paul Charette, Frédéric Chagnon, Bruno Hogue, Daniel M Payet, Steven Thomas, Peter Delaney, and Olivier Lesur. U de Sherbrooke QC Canada, Optiscan Pty Ltd, Victoria, Australia. There are no bedside clinical tools that have the acuteness/accuracy to assess in vivo a progression towards lung tissue remodeling/repair at the cellular level during ALI/ ARDS. Lung biopsies are invasive and current bedside clinical tools such as gas exchanges or chest X-Rays are neither sufficiently sensitive nor specific to assist physicians in detecting improvements in ARDS. A fluorescence confocal system (Optiscan FIVE 1) equipped with a laser "pen-like" probe allowed in vivo real-time distal airspaces -including very small airwaysimaging using systemic and topical/intrapulmonary staining with fluorophores. Optical resolution was $7 \mu \mathrm{m}$ with $4 \mu \mathrm{m} / \mathrm{sec}$ adjustments, and the imaging resolution was $1024 \times 1024$ pixels. For this goal, adult rats were anesthetized, ventilated, and the probe secured onto the visceral pleura through a transdiaphragmatic pathway. Nuclear staining of lung endothelial cells was secured by acridine orange $(0.05 \% \mathrm{I} / \mathrm{V})$, coupled with a plasmatic contrast using FITC-Dextran (70 kDa, I/V). Distal epithelial membrane staining was observed with FITC-R.Communis lectin and RTI40 Ab IT instillation (Dr L Dobbs). Cell apoptosis was screened by using YO-PRO-1® IT and IV labeling. Neutrophils were ex vivo stained with $\mathrm{PKH} 2$ and reinjected IV to visualize lung recruitment after PMA local or Bleomycin instillation. Preliminary assays offered a high-quality imaging. Data can be viewed as successive real-time captured images in area of interest, assembled and edited during static and dynamic ventilation with or without 3D reconstruction. Up to now, normal lungs and several models of airway and distal airspace injury have been studied, allowing assessment of lung microvascular circulation, epithelial injury, edema, and inflammatory cell recruitment. A new tool for realtime in vivo lung cellular imaging is proposed and presently in development. The ultimate objective is to provide physicians with additional knowledge on lung remodeling/repair, informative for diagnostic and prognostic translation. Supported by RSR-FRSQ and CRC CHUS and Fac Génie UDS

\section{J811} EVALUATION OF THE TSPO (18KDA)/PBR RADIOLIGAND
[18F] DPA-714 IN A RAT MODEL OF FOCAL CEREBRAL
ISCHEMIA

Abraham Martin ${ }^{1}$, Raphael Boisgard ${ }^{1}$, Benoit Theze ${ }^{1}$, Frederic Dolle ${ }^{2}$, Nadja Van Camp ${ }^{1}$, Michel Kassiou ${ }^{3}$, Bertrand Tavitian ${ }^{1}$

${ }^{1}$ Laboratoire Imagerie Moléculaire Expérimentale;

INSERM U803, CEA, DSV, I2BM, SHFJ, Orsay, France, ${ }^{2}$ Laboratoire Imagerie Moléculaire Expérimentale, CEA, DSV, I2BM, Orsay, France, ${ }^{3}$ Brain and Mind Research Institute, University of Sydney, Sydney, NSW, Australia.

Focal cerebral ischemia leads to an inflammatory reaction which involves an over-expression of the translocator protein, TSPO $(18 \mathrm{KDa})$, expressed in the monocytic lineage (microglia and monocyte) and astrocytes under such situation. The study of this receptor using radiolabeled ligands has revealed inflammatory processes with Positron emission tomography after cerebral ischemia. We performed [18F] DPA-714 PET and autoradiography to determine brain TSPO expression in rats at several days after a model of cerebral ischemia. In vivo PET imaging showed a significant increase at day 7 , day 11 , day $15(P<0.001)$ and day $21(P<0.05)$ on the injured side compared to the contralateral area. The highest binding value was reached at 11 days after ischemia relatively to 7 and 15 days $(P<0.05)$. [18F]DPA-714 uptake decreased at day 21 and day 30 with respect to the uptake at 11 days. In vitro binding using DPA-714 was performed at the same time points as in PET studies in order to confirm the results obtained in vivo. Displacement studies using in vivo PET imaging and in vitro binding with PK11195 and DPA-714 showed a faster and more complete displacement of [18F] DPA-714 binding from the lesion by DPA714 compared to PK11195. The immunohistochemistry studies showed an increase of TSPO expression of ameboid cells (monocytic lineage) in the ischemic area reaching the maximal number at day $11(P<0.001)$ in relation to the control. After this time point, microglia/macrophage started a progressive decrease in the core of the infarction coinciding with a centripetal migration of astrocytes towards the lesion, promoting the formation of the astrocytic scar. Minocycline was used as antiinflammatory strategy for decreasing TSPO expression but treated rats did not show a decrease of [18F] DPA-714 binding in the brain damage after a severe model of ischemia.[18F] DPA-714 appears as a good tracer for the study of inflammatory reaction presented after several days after stroke.

\section{J812}

\begin{abstract}
${ }^{18}$ F-FDG LABELING OF ADIPOSE DERIVED STEM CELLS FOR IN VIVO TRACKING WITH microPET IMAGING

Esmat Elhami ${ }^{1}$, Ganghong Tian $^{2}$, Bo Xiang ${ }^{2}$, Jixian Deng ${ }^{2}$, Rakesh C. Arora $^{3}$, Darren H. Freed ${ }^{3}$, Shadreck Mzengeza ${ }^{1}$, Andrew L. Goertzen $^{1}$

${ }^{1}$ Radiology, University of Manitoba, Winnipeg, MB, Canada, ${ }^{2}$ Cardiac Studies Group, Institute for Biodiagnostics, National Research Council Canada, Winnipeg, MB, Canada, ${ }^{3}$ Cardiac Science Program, St. Boniface General Hospital, Winnipeg, MB, Canada.
\end{abstract}

Adipose-derived stem cells (ADSCs) have several important advantages over other types of stem cells for cell therapy. Accurately monitoring the implanted stem cells is a key to understanding the mechanisms through which this novel therapy acts. Thus, in this work we characterize the in vitro uptake and retention of ${ }^{18}$ F-FDG (FDG) by ADSCs. Methods: Samples of rat ADSCs were prepared, each with $10^{5}>$ cells in $0.75 \mathrm{ml}$ glucose- 
free FBS culture medium. Cells were labeled with $205 \mathrm{kBq}$ FDG, and uptake periods of 5, 30, 60, 90 and 120 min were used. After an uptake period the medium was collected and the cells were washed twice with phosphate-buffered saline (PBS). The ADSCs were then lysed with $0.1 \%$ sodium dodecyl sulfate (SDS) and collected for subsequent protein assaying with Bradford reagent. The amount of FDG in the medium, PBS wash solution, and cell lysate was measured using a well counter and the ratio of FDG uptake in the cells calculated for each uptake period. In parallel experiments cells were labeled with $555 \mathrm{kBq}$ FDG for 90 minutes. After label removal cells were incubated in tracer-free medium for $0,0.5,2$ and $4 \mathrm{~h}$. After each efflux period, medium was removed and label uptake was measured as described above. Results: Our preliminary results show that the decay-corrected labeling efficiency is $10 \%, 19 \%, 25 \%, 26 \%$ and $34 \%$ for labeling periods of 5, 30, 60, 90 and $120 \mathrm{~min}$, respectively. Label retention show a rapid decrease indicating that the unbound FDG leave the cells rapidly. Conclusion: For in vivo cell tracking studies the parameter of interest is the amount of radiotracer that is present in the cells being labeled. Our data suggest that: 1) the ADSCs can be effectively labeled with FDG; 2) the maximum labeling efficiency is better than $20 \%$ of the starting activity; and 3) a labeling period of 60 minutes provides a reasonable balance between cell uptake, decay of the FDG and label efflux by the ADSCs.

\section{${ }^{18}$ F-FDG Uptake and Retention by the ADSCs}
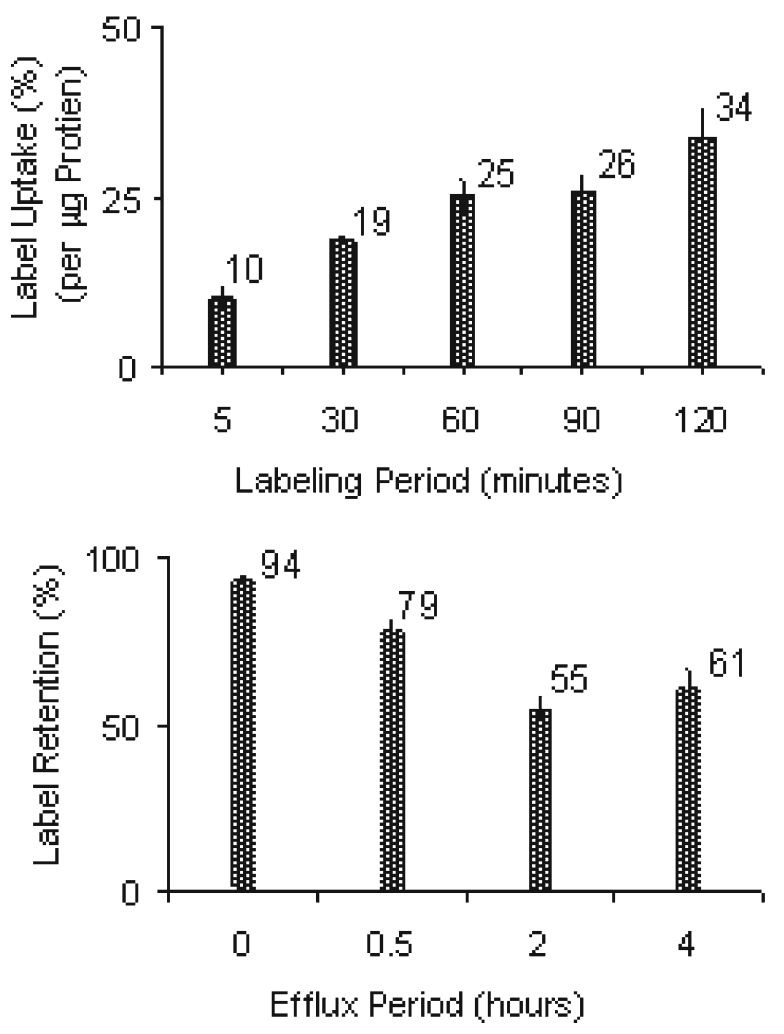

Each bar represents the mean value \pm SD for 6 replicate wells used in each group.
J813

IN VIVO TRACKING OF DUAL-LABELED MESENCHYMAL STEM CELLS HOMING INTO THE INJURED COMMON CAROTID ARTERY WITH MAGNETIC RASONANCE IMAGING Aihong Cao, Hongjian Shi, Gaojun Teng

southeast univercity, Nanjing, China.

Objective: To conduct in vivo, noninvasive magnetic resonance imaging of labeled rat bone mesenchymal stem cells (BMSCs) as they home to the site of injured common carotid artery following allograft transplantation. Methods: Our study was approved by the Institutional Committee on Animal Research. Purified rat BMSCs were dual-labeled with superparamagnetic iron oxide (SPIO) particle and fluorescent DiI dye, and subsequently transplanted into recipient rats injured in the left common carotid arteries. Immediately before and 3 hours, 3, 7 and 12 days after transplantation, the labeled cells were monitored in vivo using a 7T micro-magnetic resonance imaging (7T micro-MRI) scanner. The signal-to-noise ratios (SNR) at the injured sites were corroborated with histological examination using Prussian blue staining and fluorescent imaging. Results: Rat BMSCs were labeled with SPIO and DiI at $100 \%$ efficiency. Relative to the baseline level before transplantation, the SNR decreased significantly on $\mathrm{d} 3$ and $\mathrm{d} 7$ after injection in the experimental group (Dunnet t test, $P<0.05$ ), whereas insignificant differences were observed after $3 \mathrm{~h}$ and 12 days (Dunnet t test, $P>0.05$ ). In the control group, no significant differences in SNR were found among different time points (ANOVA, $P>0.05$ ). Histological analyses illustrated that red fluorescence and Prussian bluepositive cells were mainly distributed around the lesion areas of injured common carotid arteries. Conclusion: Rat BMSCs can be efficiently labeled with SPIO and DiI, and the directional homing of labeled cells to the site of injured common carotid arteries after intravascular transplantation could be tracked in vivo with 7T micro-MRI.

\section{J814}

AN INTEGRATED ENVIRONMENTAL CONTROL AND MOUSE HANDLING SYSTEM FOR PRECLINICAL IMAGING WITH PETbox

David Stout $^{1}$, Dennis Corey ${ }^{2}$, Richard Taschereau ${ }^{1}$, Brittany BerryPuzey $^{1}, \mathrm{Nam} \mathrm{Vu}^{1}$, Arion Chatziioannou ${ }^{1}$

${ }^{1}$ Molecular and Medical Pharmacology, UCLA Crump Institute, Los Angeles, CA, USA, ${ }^{2}$ VetEquip, , Pleasanton, CA, USA.

Preclinical imaging experiments using PET require anesthesia, positioning, heating and often pathogen control to acquire physiologically meaningful measurements. At present there are a few tools that can be used to control these factors, but they are not integrated with existing commercial PET systems. We describe an integrated mouse chamber for use with PETbox, a low cost, preclinical PET imaging system designed to provide dynamic and static imaging of PET probe biodistributions in vivo. Design criteria: Low cost, easy to use, with minimal connection requirements, easy to sterilize, providing a pathogen barrier, allowing for dynamic injections, enabling visualization of respiratory rate, providing optimal animal positioning within scanner for single and repeat studies, providing temperature controlled environment and gas anesthesia. The chamber must allow for the preparation work typically done inside a biosafety cabinet, such as vessel catheterizations, while still controlling temperature and anesthesia. The environmental system we designed is comprised of several 
components: a mouse chamber, a docking station providing heating power and anesthesia for use in a biosafety cabinet, a docking station within the PETbox gantry, a dual induction box for holding injected and non-injected animals and an anesthetic vaporizer providing isoflurane gas at a constant pressure. The chamber temperature is preset to $35.5 \mathrm{C}$, and the mouse temperature quickly equilibrates $(<1 \mathrm{~min})$ and remains constant for as long as needed. Anesthesia supply, exhaust and power for temperature control are automatically activated when the chamber is docked. The imaging process begins with mice being anesthetized using the induction box. If the scan is dynamic, starting from the time of injection, the mouse is first moved to the chamber in the docking station for catheter insertion. A small slot allows for the catheter to extend outside the chamber. The handle is designed to hold the syringe typically used for PET work using mice. Once the chamber is placed in the PETbox gantry, the scan can be started and the animal injected, leaving the catheter in place until after the scan. For static imaging, the mouse can be injected, returned to the second induction box for uptake and nonspecific probe clearance. When it is time to image, the mouse is positioned in the chamber, the cover put on, and the chamber moved to the PETbox gantry and inserted for imaging. All chamber parts are easy to sterilize using a spray bottle-based disinfectant.

\section{J815}

REAL-TIME VOLUMETRIC IN VIVO MAGNETIC PARTICLE IMAGING OF CEREBRAL PERFUSION

Jürgen Rahmer $^{1}$, Bernhard Gleich ${ }^{1}$, Jürgen Weizenecker ${ }^{2}$, Jörn Borgert ${ }^{1}$ ${ }^{1}$ Philips Research Europe - Hamburg, Hamburg, Germany, ${ }^{2}$ Fakultät für Elektro- und Informationstechnik, University of Applied Sciences, Karlsruhe, Germany.

We demonstrate real-time 3D imaging of a bolus of iron oxide nanoparticles passing through the brain of living mice using magnetic particle imaging (MPI) [1-3]. Particle concentrations were in the range between 27 and $103 \mu \mathrm{mol}(\mathrm{Fe}) / 1$. Methods Three anesthetized mice were imaged in an experimental MPI scanner [3]. The field of view of $16.8 \times 20.4 \times 12.0 \mathrm{~mm}^{\wedge} 3$ was positioned to cover the brain. Temporal resolution was 46 volumes/sec, scan duration was $40 \mathrm{~s}$. Iron particles (Resovist, Bayer Schering Pharma AG) were bolus injected into the tail vein of the mice. Concentrations were 56, 27, and $103 \mu \mathrm{mol}(\mathrm{Fe}) / 1$ for the three mice. Image resolution was about $1.5 \mathrm{~mm}$ in $\mathrm{AP}$ and $3 \mathrm{~mm}$ in SI and LR direction. MR scans of the sacrificed mice were acquired with a mouse coil in a medical $3.0 \mathrm{~T}$ MRI scanner using a TSE sequence. Results Fig. 1a shows a sagittal slice through the 3D MPI data of mouse \#1 when the bolus is passing the brain. Arteries are visible in the lower part (red arrow), while veins appear in the upper part of the image (blue and green arrow). Fig. 1b shows a manual fusion with the MRI data. Fig. 1c shows the temporal signal evolution in voxels within the regions indicated by the colored arrows. Signal from the arteries is modulated by the heart beat, while signal from the veins is rather smooth. The bolus passes the veins about a second later than the arteries, i.e., after perfusing the brain. Fourier analysis of the arterial signal yields a heart rate of $254 \mathrm{bpm}$. Fig. 1d shows the surface rendered MPI data at the same point in time as Figs. 1a and 1b. Conclusion MPI is a new imaging technique capable of $3 \mathrm{D}$ real-time imaging of iron oxide nanoparticles perfusing the brain in the blood stream. Applications could be perfusion imaging after stroke and functional imaging using particles with long blood retention time. References [1] Gleich B et al. Nature 435:1214-1217 (2005). [2] Conolly M et al. IEEE Trans. Med. Imag. (2009). [3] Weizenecker J et al. Phys. Med. Biol. 54:L1-L10 (2009).
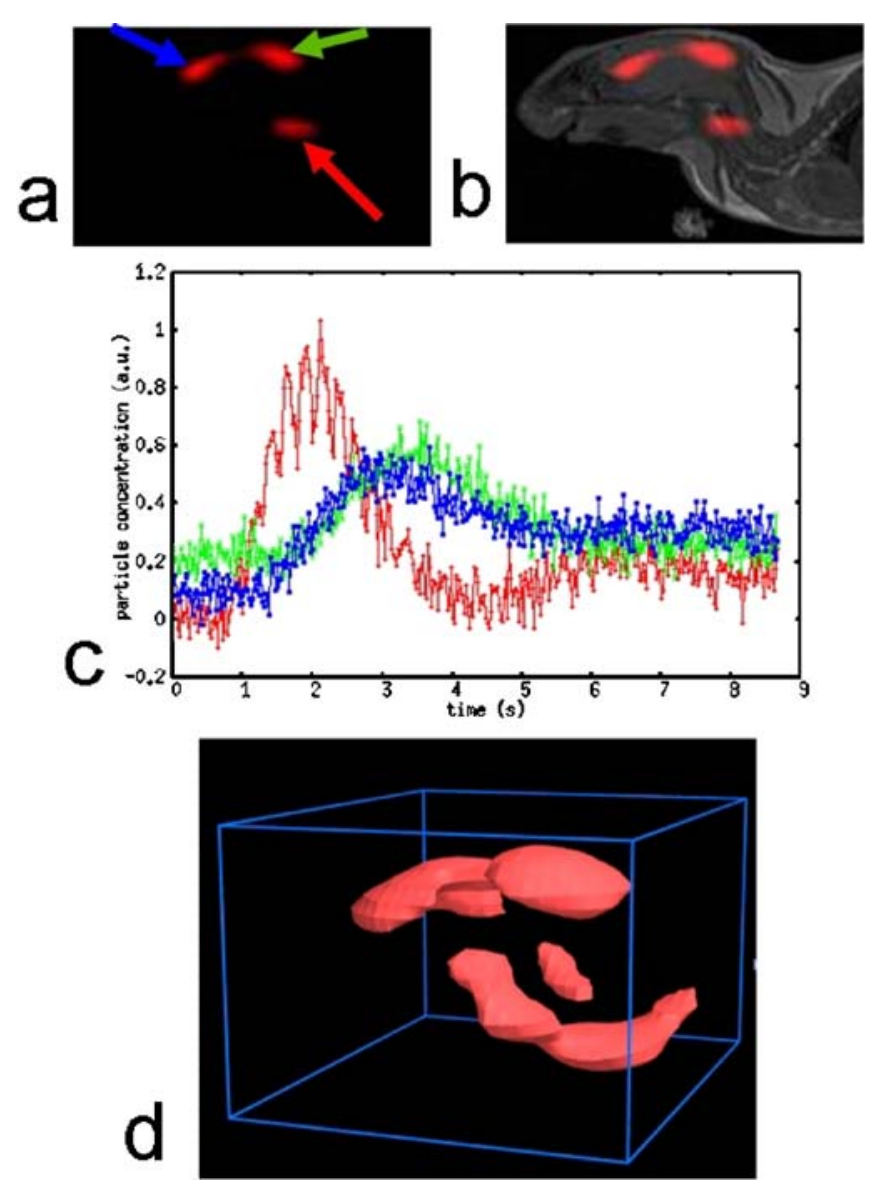

J816

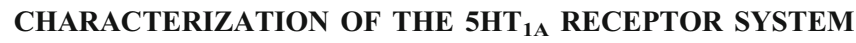 IN MICE USING PHARMACOLOGICAL FMRI}

$\underline{\text { Florence Razoux }}^{1}$, Thomas Mueggler ${ }^{1}$, Anna Buehler ${ }^{1}$, Holger Russig ${ }^{3}$, Christof Baltes $^{1}$, Isabelle Mansuy ${ }^{3}$, Markus Rudin ${ }^{1,2}$

${ }^{1}$ Institute for Biomedical Engineering, Zürich, Switzerland, ${ }^{2}$ Institute of Pharmacology \& Toxicology, Zürich, Switzerland, ${ }^{3}$ Brain Research Institute, Zürich, Switzerland.

To investigate the role of the $5 \mathrm{HT}_{1 \mathrm{~A}}$ receptor, known to be strongly implicated in the pathophysiology of depression and the action of antidepressant drugs, we established a pharmacological fMRI protocol for mice using the $5 \mathrm{HT}_{1 \mathrm{~A}}$ receptor agonist 8 -hydroxy-N(di-n-propyl)-aminotetralin (8-OH-DPAT) and the antagonist WAY100635 as pharmacological stimuli. The specificity of $5 \mathrm{HT}_{1 \mathrm{~A}}$ mediated fMRI response was investigated by phenotyping $5 \mathrm{HT}_{1 \mathrm{~A}}$ knock-out mice. Activation-related changes in regional cerebral blood volume (CBV) were measured using iron oxide nanoparticules (Endorem ${ }^{\circledR}$, Guerbert SA, Paris) as intravascular contrast agent (CA). Using R2 weighted sequences allowed estimating the amount of $\mathrm{CA}$ in tissue and, hence, the CBV. In wild-type C57B16 mice a dose-dependent decrease in relative $\mathrm{CBV}\left(\mathrm{CBV}_{\text {rel }}\right)$ has been observed 
following 8-OH-DPAT injection in a region specific manner, that reflects the distribution of the $5 \mathrm{HT}_{1 \mathrm{~A}}$ receptor in the murine brain (Figure). These effects could be antagonized by co-administration of 8-OH-DPAT and WAY-100635 indicative of the specificity of the interaction. Yet, in some brain areas administration of the antagonist alone prompted similar $\mathrm{CBV}_{\text {rel }}$ response than the agonist. As expected, the fMRI response induced by $0.1 \mathrm{mg} / \mathrm{ml} / \mathrm{kg}$ of $8-\mathrm{OH}-$ DPAT was reduced in the heterozygote and largely abolished in homozygous $5 \mathrm{HT}_{1 \mathrm{~A}}$ knock-out mice as compared to wild-type littermates. Finally, to improve the understanding of the interplay among brain regions, the fMRI responses in various brain regions-ofinterest were compared using cross-correlation analyses. The results demonstrate that functional investigation of the $5 \mathrm{HT}_{1 \mathrm{~A}}$ receptor by fMRI is possible in adult mice using the $5 \mathrm{HT}_{1 \mathrm{~A}}$ receptor agonist 8$\mathrm{OH}-\mathrm{DPAT}$. Functional responses can be analyzed with a time resolution $<1 \mathrm{~min}$. By correlating temporal profiles, functional interactions among brain regions might be identified: an attractive feature to elucidate networks.

\section{Anatomical references and corresponding activity maps $(0.1 \mathrm{mg} / \mathrm{kg}$ 8-OH-DPAT - integration period of $6 \mathrm{~min}$ )}
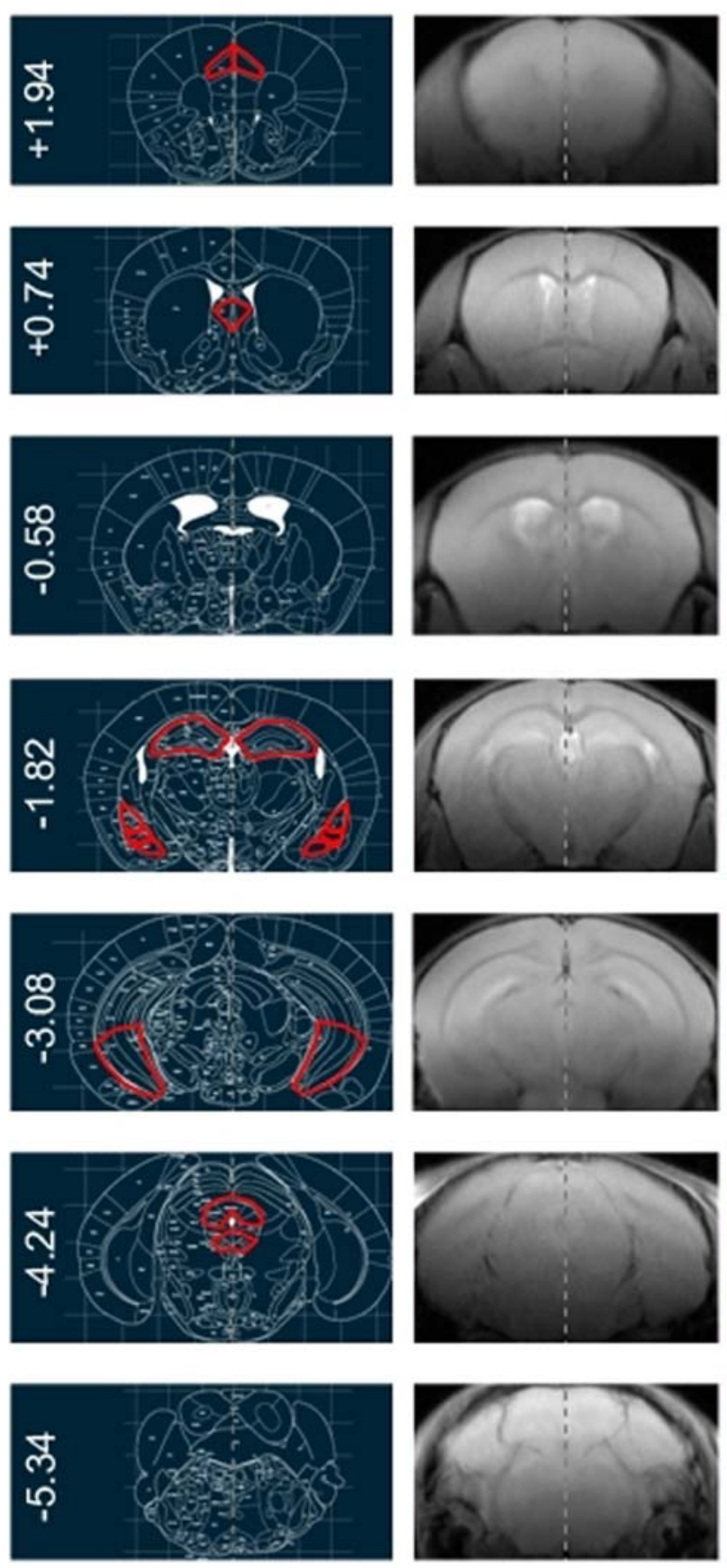

stereotaxic atlas *

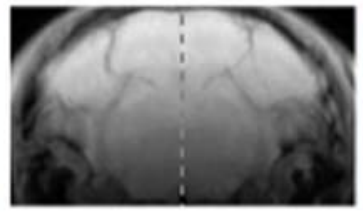

anatomy
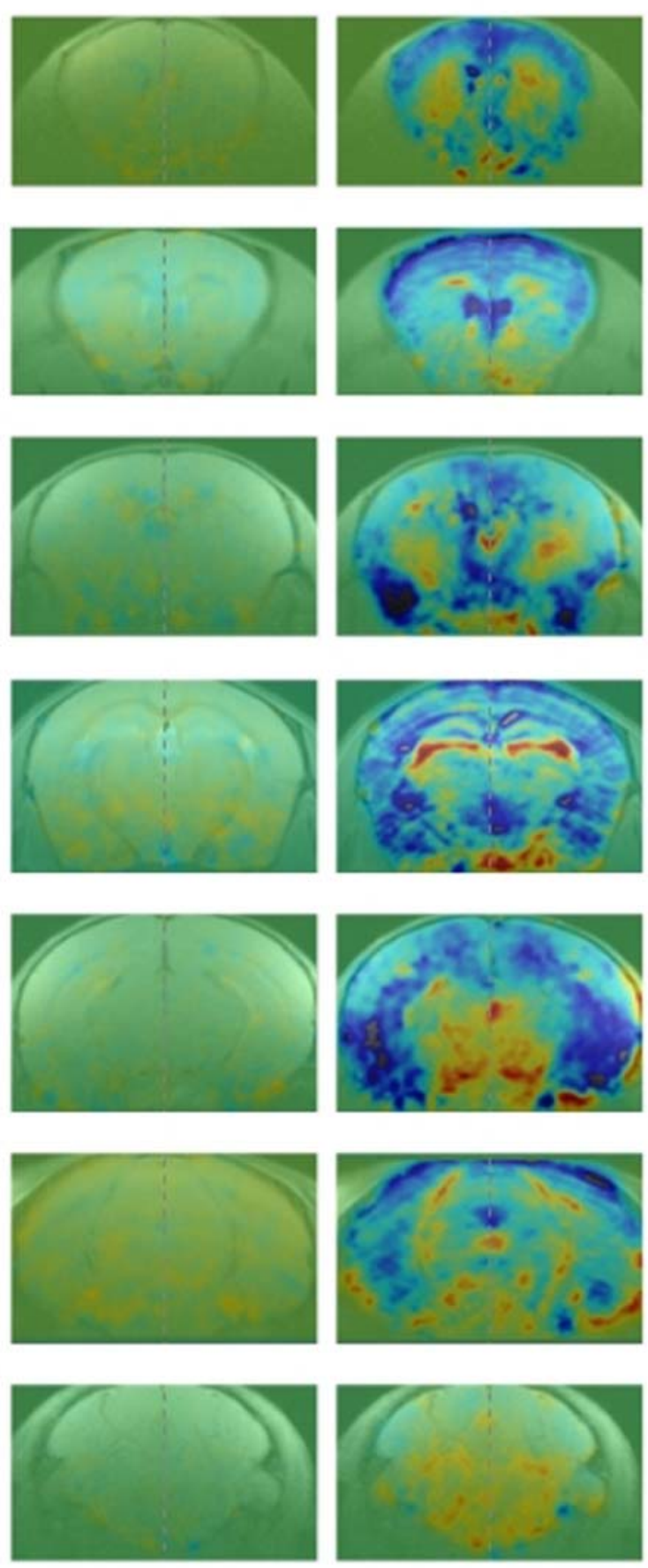

baseline

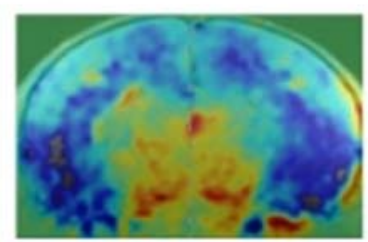

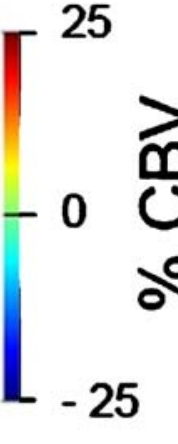




\section{J817}

PHARMACOKINETICS AND MR IMAGING OF RHESUS MONKEYS WITH PG-Gd, A BLOOD POOL IMAGING AGENT $\underline{\text { Xiaoxia Wen }}^{1}$, Edward F. Jackson ${ }^{2}$, Rajesh Uthamanthil ${ }^{3}$, Chaan S. Ng ${ }^{4}$, Juri G. Gelovani ${ }^{1}$, Mei Tian ${ }^{1}$, Chun $\mathrm{Li}^{1}$

${ }^{1}$ Exp. Diagnostic Imaging, U.T. M. D. Anderson Cancer Center, Houston, TX, USA, ${ }^{2}$ Imaging Physics, U.T. M. D. Anderson Cancer Center, Houston, TX, USA, ${ }^{3}$ Veterinary Medicine \& Surgery, U.T. M. D. Anderson Cancer Center, Houston, TX, USA, ${ }^{4}$ Diagnostic Radiology, U. T. M. D. Anderson Cancer Center, Houston, TX, USA.

Purpose: Blood-pool MRI contrast agents have enormous potential to aid cancer detection and diagnosis of diseases of the cardiovascular system. The purpose of this study was to evaluate PG-Gd [poly(Lglutamic acid)-benzyl-DTPA-Gd], a new macromolecular magnetic resonance imaging contrast agent, for its pharmacokinetics, biodistribution, and clearance in mice and nonhuman primates. Materials and Methods: Studies were performed in mice and rhesus monkeys at intravenous doses ranging from 0.01 to $0.08 \mathrm{mmol} \mathrm{Gd} / \mathrm{kg}$. Concentrations of PG-Gd in blood were determined by quantifying Gd content using inductively coupled plasma mass spectrometry. T1-weighted MR images were acquired at $1.5 \mathrm{~T}$ using a fast spoiled gradient echo imaging protocol. Small molecular weight contrast Magnevist was used as a control. Results: MRI imaging in monkeys showed prolonged circulation of PG-Gd in the blood pool. Enhancement of blood vessel and organs with high blood perfusion (heart, liver, and kidney) was cleared visualized at $2 \mathrm{hr}$ after contrast injection at doses ranging from 0.01 to $0.08 \mathrm{mmol} \mathrm{Gd} / \mathrm{kg}$. By 2 days after PG-Gd injection, most contrast agent was cleared from all major organs including kidney. By 8 days after contrast injection, no residual contrast could be detected by MRI. PG-Gd was cleared from both the hepatobiliary and renal routes. The mean resident time in monkey was $14 \mathrm{~h}$. In comparison, the behavior of PG-Gd in mice was different, exhibiting a shorter mean resident time of $4.2 \mathrm{~h}$. In comparison to PG-Gd, Magnevist was rapidly cleared from the blood pool of rhesus mnkey. Conclusion: The pharmacokinetics and elimination profile of PG-Gd, including vascular retention, renal excretion, and biodegradability of the polymer, are favorable for use in humans as an intravascular contrast agent for MRI. Biodegradable macromolecular contrast agents such as PG-Gd that can be cleared from the body after its use may be a valuable addition to clinically approved low-molecular-weight Gd(III) chelates.
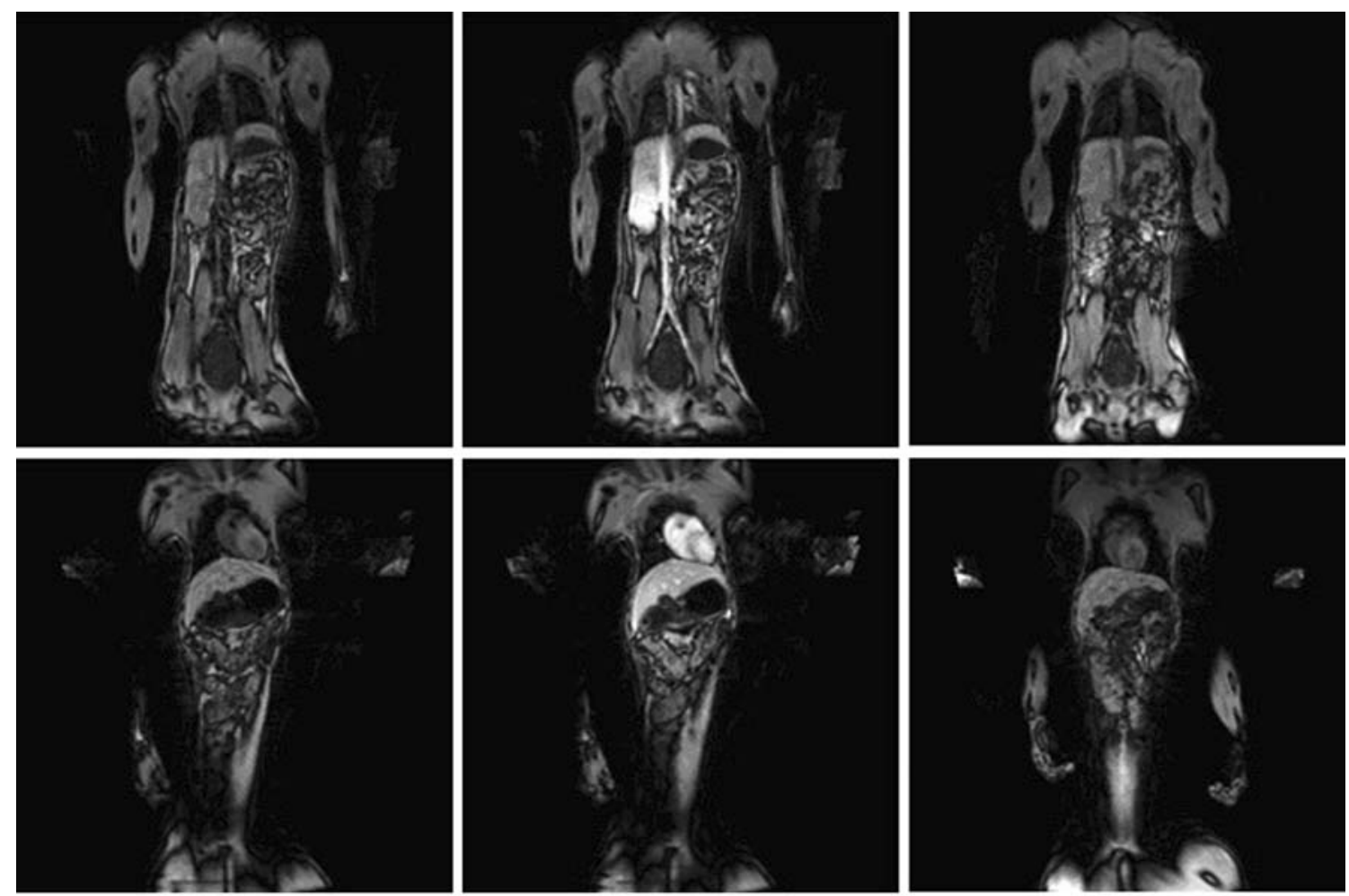

Pre-Contrast

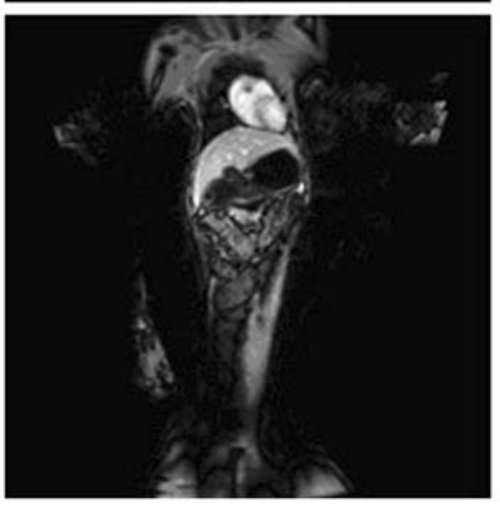

2 h Postcontrast

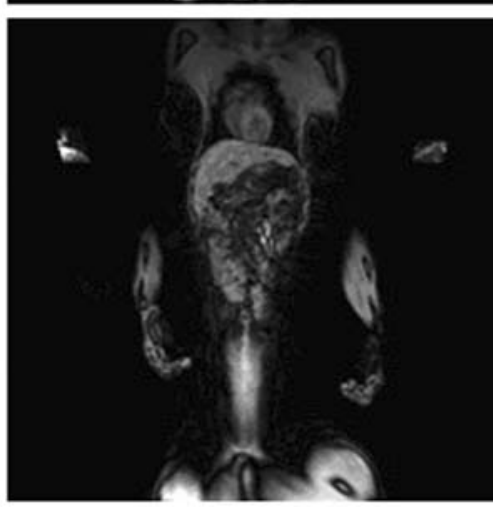

8 d Postcontrast

T1-weighted MR images of rhesus monkeys after i. v. injection of PG-Gd at a dose of $0.02 \mathrm{mmol} \mathrm{Gd} / \mathrm{kg}$.

\section{J818}

A MOUSE ANGIOGENESIS MODEL COMPRISING IMPLANTED HUMAN ENDOTHELIAL CELLS

Mary Ellen Maddalena ${ }^{1}$, Lisa Passantino ${ }^{1}$, Karen Saar ${ }^{1}$, Laura E. Lantry $^{1}$, Regi Thomas ${ }^{1}$, Jun Yan ${ }^{1}$, Edmund R. Marinelli ${ }^{2}$, Mathew von Wronski ${ }^{1}$, Adrian Nunn ${ }^{1}$

${ }^{1}$ Biology, Bracco Research USA, Princeton, NJ, USA, ${ }^{2}$ Chemistry, Bracco Research USA, Princeton, NJ, USA.
It is generally required that new imaging and/or therapeutic agents demonstrate efficacy and safety in animal models before testing in man. However, particularly for certain molecules such as antibodies and peptides, the targeting entities developed frequently display species specificity. Since the vasculature in rodent xenograft models is of rodent origin, testing imaging agents that selectively target human endothelial cell surface markers in such models can be problematic. We have optimized a previously published implant model combining human endothelial cells with Matrigel (and other support cells, growth factors, and matrix components) for both convenience and reliability, and characterized it to 
demonstrate an abundance of functional blood vessels lined with human endothelial cells within 7 days of implantation and lasting for at least 36 days post implant. High molecular weight dextran and Hoechst 33342 injected prior to removal of implants confirmed that a significant fraction of the "human" vessels were perfused. Immunohistochemistry of sectioned implants indicated that most of the human CD31-positive blood vessels in the implants also stained strongly with an anti-VEGFR-2 antibody. VEGFR-2 is a validated angiogenesis target and its high-level expression in the neovasculature of this model implies that these vessels are exhibiting an angiogenic phenotype and that this model will be useful for testing imaging and therapeutic agents directed against other human angiogenesis markers as well. Further characterization/ development of this model is ongoing to confirm its utility for testing human-selective endothelial targeting entities in vivo as well as to identify the optimal temporal window for testing a given agent using this model. It is expected that this model will be useful in longitudinal studies where the data from non-invasive imaging of human angiogenesis biomarkers over time will be correlated with the therapeutic response to angiogenesis inhibitors in the same animals. This will allow more definitive conclusions to be reached with a smaller number of test subjects than would be possible with a non-longitudinal study.

\section{J819}

EVALUATION OF FMISO UPTAKE IN RAT TUMOR TISSUE: COMPARISON WITH FDG UPTAKE

Toshiyuki Hatano $^{1}$, Songji Zhao ${ }^{1}$, Yuji Kuge ${ }^{1}$, Yan Zhao ${ }^{1}$, Ken-ichi Nishijima $^{1}$, Norihito Kuno ${ }^{2}$, Hiroko Hanzawa ${ }^{2}$, Takeshi Sakamoto ${ }^{2}$, Nagara Tamaki ${ }^{1}$

${ }^{1}$ Graduate School of Medicine, Hokkaido University, Sapporo, Japan,

${ }^{2}$ Central Research Laboratory, Hitachi, Ltd., Tokyo, Japan.

Purpose: Accurately imaging to identify hypoxic areas in tumors is useful for the radiation treatment planning. In hypoxic cells, genes involved in glucose metabolism are upregulated. On the other hand, fluorominosidazole (FMISO) and fluorodeoxyglucose (FDG) have been investigated as hypoxic and glucose metabolic markers, respectively. Thus, FMISO uptake may be relevant to FDG uptake, but the correlation remains unclear. In this study, we evaluated the intratumoral FMISO distribution, and compared it with FDG distribution and tumor histology. Methods: Rats were implanted with C6 glioma cells into the calf mustle. Nine days later, the rats were fasted overnight $(n=5)$, and injected with $\left[{ }^{18} \mathrm{~F}\right] \mathrm{FMISO}$ and $\left[{ }^{14} \mathrm{C}\right] \mathrm{FDG}$. Ninety minutes after the injection, the rats were injected with pimonidazole. Sixty minutes after the pimonidazole iv, the rats were sacrificed, and tumors tissues were sectioned into slices. The adjacent slices were frozen for autoradiography (ARG) and histochemical studies. $\left[{ }^{18} \mathrm{~F}\right] \mathrm{FMISO}$ ARG images were divided into the regions of $\left[{ }^{18} \mathrm{~F}\right] \mathrm{FMISO}$ high uptake (FMISO+) and low uptake (FMISO-). Seven regions of interest were randomly selected from FMISO+ and FMISO- regions. In these ROIs, $\left[{ }^{18} \mathrm{~F}\right]$ FMISO uptake, $\left[{ }^{14} \mathrm{C}\right] \mathrm{FDG}$ uptake, pimonidazole positive areas, Glut-1 positive areas, and Ki-67 positive cell ratios were evaluated. Results: FMISO+ regions and pimonidazole positive areas were similar (Fig. a; $\left[{ }^{18} \mathrm{~F}\right] \mathrm{FMISO}$ uptake in FMISO $+: 0.45 \pm 0.11 \% \mathrm{ID} / \mathrm{g} / \mathrm{kg}$, FMISO-: $0.18 \pm$ $0.04 \% \mathrm{ID} / \mathrm{g} / \mathrm{kg}, p<0.01$; Fig.b ; Pimonidazole positive areas in FMISO+ : $31 \pm 12 \%$, FMISO-: $13 \pm 9 \%, p<0.05$ ). Thus, $\left[{ }^{18} \mathrm{~F}\right] \mathrm{FMISO}$ uptake reflects tumors hypoxia. However, no significant difference in $\left[{ }^{14} \mathrm{C}\right] \mathrm{FDG}$ uptake was observed between FMISO+ and FMISO- regions (Fig. c; FMISO $+: 3.5 \pm 0.8 \% \mathrm{ID} / \mathrm{g} / \mathrm{kg}$, FMISO-: $3.2 \pm 0.7 \% \mathrm{ID} / \mathrm{g} / \mathrm{kg}, \mathrm{p}=$ ns). On the other hand, Glut-1 positive areas increased in FMISO+ regions. Ki-67 positive cell ratios were not significantly different between FMISO+ and FMISO- regions. Conclusions: $\left[{ }^{18} \mathrm{~F}\right] \mathrm{FMISO}$ uptake in tumors was correlated with pimonidazole uptake, but not with $\left[{ }^{14} \mathrm{C}\right]$ FDG uptake. Glut-1 expression increased in the regions of $\left[{ }^{18} \mathrm{~F}\right]$ FMISO high uptake. Ki-67 expression was not correlate with $\left[{ }^{18} \mathrm{~F}\right]$ FMISO uptake. These results are useful as fundamental data for radiation treatment planning using FMISO and FDG.

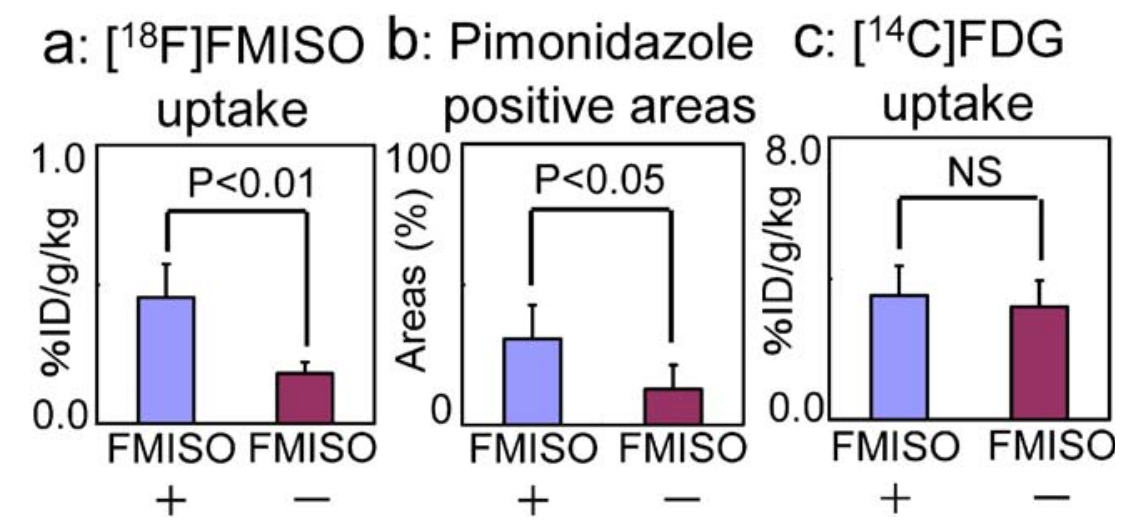

\section{J820}

USE OF CIS-[18F]FLUORO-PROLINE FOR ASSESSMENT OF COLLAGEN SYNTHESIS IN MUSCULOSKELETAL CONNECTIVE TISSUE: EFFECT OF EXERCISE

Dorthe Skovgaard $^{1,2}$, Michael Kjaer ${ }^{2}$, Malene Brandt-Larsen ${ }^{3}$, Jacob Madsen ${ }^{3}$, Andreas Kjaer ${ }^{1,3}$

${ }^{1}$ Cluster for Molecular Imaging, University of Copenhagen, Copenhagen, Denmark, ${ }^{2}$ Institute of Sports Medicine, University of Copenhagen, Copenhagen, Denmark, ${ }^{3}$ Dept. Clinical Physiology, Nuclear Medicine and PET, Rigshospitalet, Copenhagen, Denmark.

Introduction: Musculoskeletal connective tissues can adapt to acute and long term exercise regimes with an increase in collagen synthesis. However, in-vivo investigations of these exercise-induced modifications rely on invasive procedures, including biopsies obtained from the studied tissues. The aim of the present study was to investigate the possibility of applying the PET tracer, cis-[18F]fluoro-proline (Fpro), for non-invasive assessment of collagen synthesis in rat musculoskeletal tissues at rest and following short-term treadmill running (1 bout a day for 3 days). Methods: Musculoskeletal collagen synthesis was studied in rats with the use of FPro and PET/CT. An exercise group $(n=8)$ was investigated at rest and $24 \mathrm{~h}$ post-exercise. A control group $(n=10)$ sustained normal cage activity. At each session rats were PET scanned at two time points following injection of FPro: 60 min ("Fpro-transport") and 240 min p.i. ("Fpro-incorporation"). Standardized Uptake Values (SUV) were calculated for Achilles tendon, calf muscle and tibial bone (trabecular and cortical). The PETderived results were compared to mRNA expression of collagen type I and III using real-time PCR. Results: PET images revealed clearly, visible uptake of Fpro in musculoskeletal tissues. Trabecular and cortical bone had 
the highest SUV that increased significantly $(p<0.001)$ when comparing the early (60 min p.i.) and the late ( $240 \mathrm{~min}$ p.i.) PET scans. This was in contrast to a continuous decrease in SUV in tendon and muscle $(p<0.001)$. Exercise had no influence on SUV (both 60 and 240 min p.i.) in the investigated tissues. Compared to controls, tendon and muscle of the exercised rats exhibited enhanced gene expression of collagen type I and type III (range $\sim 2-5$ fold). Conclusion: The tissue-specific differences with the highest basal uptake in trabecular bone compared to that of other types of musculoskeletal tissues are in accordance with earlier studies relying on tissue incorporation of isotopic-labelled proline (e.g. $13 \mathrm{C}$ or $15 \mathrm{~N}$-proline). Fpro uptake was not changed following exercise, which was contradicted by an increased gene expression of collagen type I and III. This could indicate that the PET-based method is not sensitive enough to detect minor changes in collagen synthesis. Further studies with greater mechanical loading of the tissues and possible also applying a kinetic compartment model must be performed to establish whether cis-Fpro can be used for non invasive in-vivo assessment of exercise-induced changes in musculoskeletal collagen synthesis.

\section{J821}

\section{THE EFFECT OF INHALED GASES ON MICROBUBBLE HALF-LIFE}

Malak Itani, David J. Fisher, Robert F. Mattrey

Radiology, University of California, San Diego, San Diego, CA, USA.

Background: Contrast-enhanced ultrasound (CEUS) is gaining increasing importance in molecular imaging. Mixing isoflurane with a pressurized gas, typically oxygen $\left(\mathrm{O}_{2}\right)$, is a preferred method of anesthesia in small animals. However, the perfluorocarbon (PFC) gas within microbubbles (ultrasound contrast agents) is cleared by the lungs, so the type of gas filling the alveoli can impact microbubbles survival. Injectable anesthesia can be used to avoid this problem, but this can affect cardiac function, and may increase animal loss. The goal of our study was to investigate the effect of $\mathrm{O}_{2}$ and medical air (MA), as well as $\mathrm{O}_{2}$ mixed with $\mathrm{PFC}$ vapor, on microbubble survival. Methods: $12 \mathrm{NIH}$ Swiss mice received $0.1 \mathrm{ml}$ Definity diluted 1:20 with normal saline in the jugular vein. Timeintensity-curves (TIC) curves, representing the acoustic response of microbubbles over time, were acquired from the iliac vein using a $40 \mathrm{MHz}$ transducer of a Vevo770 (VisualSonics) ultrasound system at 5 frames per second. All animals were anesthetized with isoflurane mixed with MA and observed for $120 \mathrm{~s}$ after contrast injection. The gas was then changed for a period of $180 \mathrm{~s}$ in random order to: (a) MA (control); (b) $\mathrm{O}_{2}$; (c) $\mathrm{O}_{2}+$ perfluorohexane (PFH) (volume ratio 1:1.5); or (d) $\mathrm{O}_{2}+$ octafluoropropane (OFP) (volume ratio 1:1.5), OFP being the PFC used in Definity. After each experiment, the inhaled gas was reverted to MA (180 s). The slope of signal intensity decay was calculated for each gas and was analyzed using repeated measures analysis of covariance. Results: The best effect on microbubble survival was seen with MA (slope of signal decay in TIC: $-0.52 \pm .02$ units $/ \mathrm{sec})$. The fastest decay was seen with $\mathrm{O}_{2}(-$ 1.4 units/sec, $p<0.001$ relative to MA). The rate of signal loss was less with OFP (-1.1 units/sec) than PFH (-1.3units/sec), but both were greater than MA. Conclusions: The type of gas used with inhaled anesthesia affects microbubble half-life. Medical air has the least effect and should be used when imaging small animals. This study was supported in part by the ICMIC P50 CA128346 and the Roadmap R21 EB005360

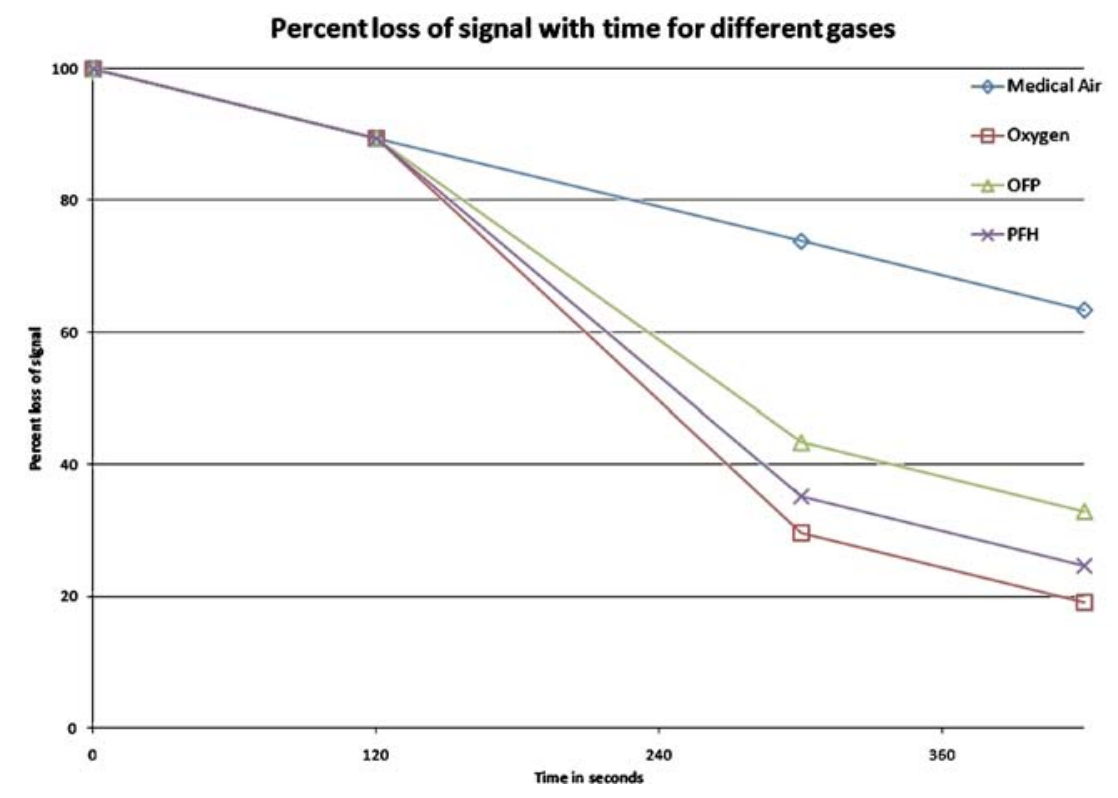

J822

SPECT/CT IMAGING OF 111In-LABELED ANTIBODIES TO VASCULAR AND TUMOR CELL TARGETS OF HUMAN OVARIAN CARCINOMA XENOGRAFTS

Tuulia Huhtala ${ }^{1}$, Pirjo Laakkonen ${ }^{1}$, Hanna Sallinen ${ }^{1}$, Seppo YläHerttuala $^{1,2}$, Kari Alitalo ${ }^{3,4}$, Ale Närvänen ${ }^{1,2}$

${ }^{1}$ Department of Biotechnology and Molecular Medicine, University of Kuopio, Kuopio, Finland, ${ }^{2}$ Biocenter Kuopio, University of Kuopio, Kuopio, Finland, ${ }^{3}$ Molecular/Cancer Biology Research Program, University of Helsinki, Helsinki, Finland, ${ }^{4}$ Ludwig Institute for Cancer Research, University of Helsinki, Helsinki, Finland.
Ovarian cancer is the fifth most common cancer in women. Due to the late diagnosis the overall 5-year survival rate is less than $40 \%$. The high death rate reflects the difficulty to detect small metastatic tumor nodules in the peritoneum undetectable by the surgeons. Epidermal growth factor receptor (EGFR) and vascular endothelial growth factor receptor 3 (VEGFR-3) are involved in tumor progression and monoclonal antibodies against these receptors have shown to interfere the growth of the tumors by inhibiting the function of the receptors. EGFR and VEGFR-3 receptors are expressed in the tumor area of ovarian carcinoma and corresponding monoclonal antibodies are potential diagnostic molecules for in vivo imaging. As an ovarian cancer model we used a SKOV-3 m cell line, which generates aggressive ovarian cancer with several clinical, histological and immuno- 
histological similarities to the human cancer. The long term biodistribution of commercially available antibody Cetuximab against EGFR and monoclonal antibody mF4-31C1 against VEGFR-3 was studied in nude mice with orthotopic SKOV-3 m xenografts. The biodistribution of intravenously injected 111-Indium labeled antibodies was followed up to 48 hours post injection using combined SPECT and CT imaging modality. After final imaging organ samples were collected and specific organ activity was measured. The accumulation of the i.v. injected antibodies to the tumor tissue and lymph nodes was verified using immunohistology. Imaging studies with SPECT/CT showed clear accumulation of both antibodies into tumor area. The tumor uptake was $8.78 \pm 0.74 \% \mathrm{ID} \mathrm{g}-1$ for Cetuximab and $5.77 \pm 0.62 \% \mathrm{ID} \mathrm{g}-1$ for $\mathrm{mF} 4-31 \mathrm{C} 1$ after $48 \mathrm{~h}$ post-injection. Cetuximab had lower liver tropism and faster tumor homing rate. In addition, after $48 \mathrm{~h}$ two out of five tumor-bearing mice showed a clear accumulation of the Inlabeled $\mathrm{mF} 4-31 \mathrm{C} 1$ at the left axillary area. Both i.v. administrated antibodies could also be detected from the tumor sections by immunohistological staining but only $\mathrm{mF} 4-31 \mathrm{C} 1$ from the lymph nodes. These results demonstrate the accumulation of the EGFR and VEGFR-3 specific antibodies to the orthotopic ovarian carcinoma tumors. Systemically administered they had slow pharmacokinetics which is typical for antibodies. Accumulation of $\mathrm{mF} 4-31 \mathrm{C} 1$ antibody to the lymph nodes suggests the remote activation of VEGFR-3 by the primary tumor.

\section{J823}

\section{CHARACTERIZING BREAST CANCER METASTASIS}

\section{TO THE BRAIN USING MRI}

Mevan C. Perera $^{1,2}$, Carmen Simedrea ${ }^{3,2}$, Jonatan A. Snir ${ }^{1,2}$, Catherine Ramsay ${ }^{1}$, Emeline J. Ribot ${ }^{1}$, Patricia S. Steeg ${ }^{4}$, Ann Chambers ${ }^{3,2}$, Paula Foster ${ }^{1,2}$

${ }^{1}$ Imaging Dept., Robarts Research Institute, London, ON, Canada, ${ }^{2}$ Medical Biophysics, University of Western Ontario, London, ON, Canada, ${ }^{3}$ London Regional Cancer Program, Victoria Hospital, London, ON, Canada, ${ }^{4}$ Women's Cancer Section, Laboratory of Molecular Pharmacology, Center for Cancer Research, National Cancer Institute, Baltimore, MD, USA.
Background: A wealth of information can be derived about the development of metastases from 3D cellular MRI, when compared to traditional histological analyses. To the best of our knowledge this is the first report of the in vivo spatial and temporal monitoring of the development of experimental brain metastasis in mice. Methods: Brain metastastic human breast cancer cells $(175,000$ MDA-MB-231BR, or MDA-MB-231BR/HER2) were injected into the left ventricle of the heart in nude mice. Mouse brains were imaged in vivo at days: 1,16,19,23,26 and 29 post injection, using a steady state free precession pulse sequence (3DSSFP) at 1.5 Tesla. 3D images were acquired at $100 \times 100 \times 200 \mu \mathrm{m}$ resolution in 1 hour. Brains were categorized into four regions for analysis: the cortex, central brain, olfactory and posterior brain. The tumor burden and distribution, individual tumor volumes and growth rates were measured for the whole brain over time for all mice. Results: Excellent tumor to brain contrast in optimized SSFP images allowed for the whole brain tumor burden to be measured at each timepoint. For mice injected with MDA-MB-231Br/HER2 cells, over $40 \%$ of the metastases that formed were detected in the cortex, $20-30 \%$ were in the central brain and $10-20 \%$ of the metastases were detected in both the olfactory and posterior brain regions (Figure). The tumor volumes ranged from $1 \mu \mathrm{m}^{3}$ at day 19 to $1 \mathrm{~mm}^{3}$ at day 29 . These were coarsely categorized into 'small' $\left(<30 \mu \mathrm{m}^{3}\right)$ or 'large' $\left(>30 \mu \mathrm{m}^{3}\right)$ metastases. At day $19,97 \%$ were classed as small metastases; at day $23,62 \%$ were small and $38 \%$ large; at day $26,48 \%$ were small and $52 \%$ were large, and at day $29,25 \%$ were small an d75\% were large. 'Small' metastases detected at the endpoint consisted of those detected for the first time, as well as metastases present in images at earlier time points, but which had slow growth. A comparison of the two cell lines showed a similar distribution. However, more metastases were detected in 231BR/HER2 injected mice. At day 19 there were on average $1.6 \mathrm{x}$ the number in the cortex, $1.9 \times$ more in the central region, $10 \times$ more in the posterior regions and $4 \times$ more in the olfactory region.

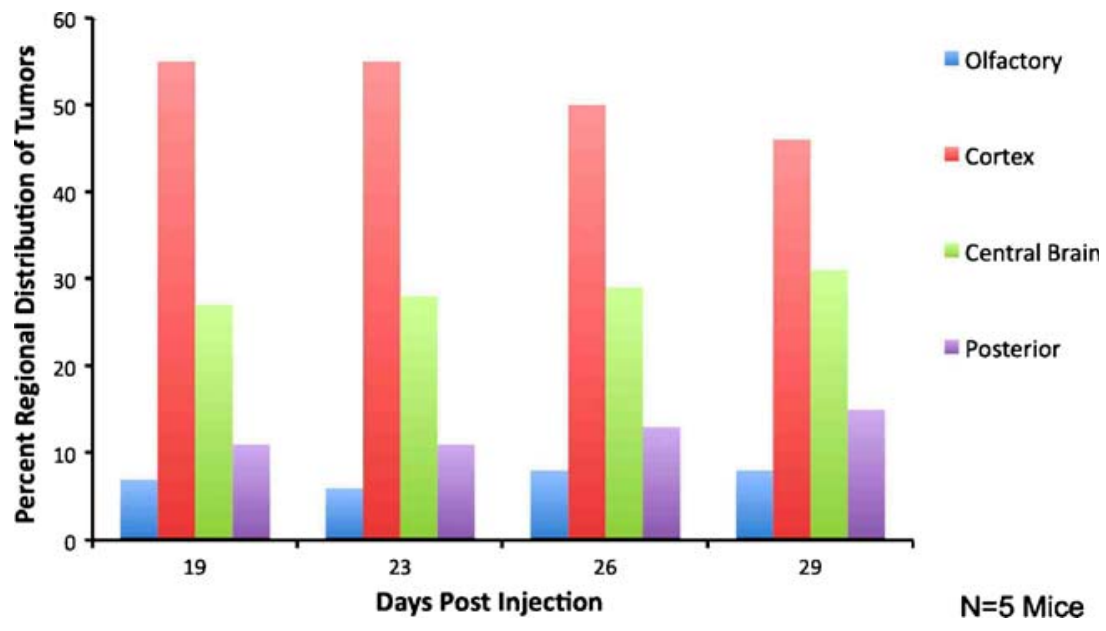

J824

DIRECT COMPARISON OF RETROSPECTIVELY GATED CARDIAC Micro-CT AND CONDUCTANCE CATHETER MEASUREMENTS IN MICE

Sarah A. Detombe $^{1,2}$, Fuli Xiang ${ }^{3}$, Joy Dunmore-Buyze ${ }^{1}$, Qingping Feng $^{3}$, Maria Drangova ${ }^{1,2}$

${ }^{1}$ Imaging Research Laboratories, Robarts Research Institute, London, ON, Canada, ${ }^{2}$ Dept. of Medical Biophysics, The University of Western Ontario, London, ON, Canada, ${ }^{3}$ Dept. of Physiology and Pharmacology, The University of Western Ontario, London, ON, Canada.
Background: The development of preclinical tools used to evaluate cardiac function in mouse models is a crucial area of research. Our group has developed a retrospectively gated micro-CT imaging technique capable of assessing heart function that can also be used to evaluate other preclinical tools. Conductance catheters are an established method of determining cardiac function in mice; however, a previous study showed that catheter-derived volumes are underestimated compared to MRI measurements, although the data were not acquired concurrently. In this study, we simultaneously acquire catheter and micro-CT data, in order to compare catheter-derived volumes with micro-CT derived volumes. Methods: 5 C57BL/6 mice were anesthe- 
tized and intubated, and a blood-pool contrast agent was injected to provide contrast between blood and tissue. A 1.4F Millar pressure conductance catheter was inserted through the carotid artery and into the left ventricle (LV). Animals were scanned at $80 \mathrm{kVp} / 50 \mathrm{~mA}$ for 10 rotations using the GE Locus Ultra; scan time was $50 \mathrm{~s}$. Cardiac waveforms were recorded during the scan, to enable retrospective gating; simultaneously, pressure-volume information was recorded with the calibrated catheter. 3D images were reconstructed at $12-\mathrm{ms}$ intervals throughout the cardiac cycle with an isotropic voxel spacing of $0.15 \mathrm{~mm}$. Results: The figure illustrates the mouse heart before (a) and during (b) catheterization. End diastolic volume (EDV) and end systolic volume (ESV) measured from the micro-CT scans were $60.5 \pm 9.6 \mu \mathrm{l}$ and $28.8 \pm 4.8 \mu \mathrm{l}$, respectively. EDV and ESV from the catheter measurements were $26.6 \pm 4.3 \mu \mathrm{l}$ and $9.0 \pm 5.5 \mu \mathrm{l}$, respectively. There was a significant difference between the micro-CT and catheter-derived volumes, for both $\operatorname{EDV}(p=0.0014)$ and $\operatorname{ESV}(p=$ 0.0024). Conclusion: One of the key advantages of this micro-CT technique is the ability to visualize the catheter in the LV. As shown in the figure, catheter placement is along the septum instead of running longitudinally through the chamber, as was previously thought to occur. This offers one explanation for the significantly lower volume measurements recorded by the catheter, and provides knowledge that will aid in the development of more accurate catheter tools in the future.
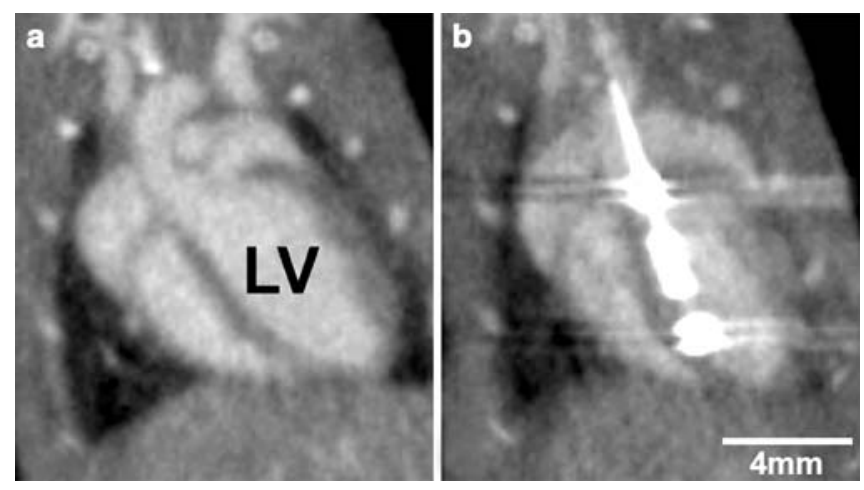

$\mathbf{J 8 2 5}$

HIGH 18F-fluoro-deoxyglucose (18F-FDG) UPTAKE IN MICROSCOPIC PERITONEAL TUMORS REQUIRES PHYSIOLOGICAL HYPOXIA

$\underline{\text { Xiao-Feng Li }}{ }^{1}$, Yuanyuan $\mathrm{Ma}^{2}$, Xiaorong Sun ${ }^{1}$, John L. Humm ${ }^{1}$, Clifton Ling $^{1}$, Joseph O'Donoghue ${ }^{1}$

${ }^{1}$ Medical Physics, Memorial Sloan-Kettering Cancer Center, New York, NY, USA, ${ }^{2}$ Pathology, Memorial Sloan-Kettering Cancer Center, New York, NY, USA.

The objective of this study was to examine 18F-fluoro-deoxy-glucose (18F-FDG) uptake in microscopic tumors grown intraperitoneally in nude mice and to relate this to physiological hypoxia and glucose transporter-1 (GLUT-1) expression. Methods: Human colon cancer HT29 and HCT8 cells were injected intraperitoneally into nude mice to generate disseminated tumors of varying sizes. Following overnight fasting, animals, either breathing air or carbogen $(95 \% \mathrm{O} 2 ; 5 \% \mathrm{CO} 2)$, were intravenously administered 18F-FDG together with the hypoxia marker pimonidazole (PIMO) and the cellular proliferation marker bromodeoxyuridine (BrdUrd) one hour before sacrifice. Hoechst
33342, a perfusion marker, was administered one minute before sacrifice. Following sacrifice, the intratumoral distribution of $18 \mathrm{~F}$ FDG was assessed by digital autoradiography of frozen tumor sections. This was compared with the distributions of PIMO, GLUT-1 expression, BrdUrd and Hoechst 33342 as visualized by immunofluorescent microscopy. Results: Small tumors $(<1 \mathrm{~mm}$ diameter) had high 18F-FDG accumulation and were uniformly hypoxic with high GLUT1 expression. Larger tumors (1-4 mm diameter) had low 18F-FDG accumulation and were not significantly hypoxic with low GLUT1 expression. Carbogen breathing significantly decreased 18F-FDG accumulation and tumor hypoxia in microscopic tumors but had little effect on GLUT1 expression. Conclusions: In microscopic tumors, high 18F-FDG uptake was spatially associated with physiological hypoxia and high GLUT-1 expression. However, in the absence of physiological hypoxia, high GLUT1 expression was insufficient to ensure high 18FFDG uptake.

\section{J826}

THE BIODISTRIBUTION OF $\left[{ }^{153} \mathrm{Gd}\right] \mathrm{Gd}$-labeled MR CONTRAST AGENTS DIFFERS GREATLY IN WILD-TYPE MICE AND A TRANSGENIC MOUSE MODEL OF RENAL FAILURE

Thaddeus J. Wadas ${ }^{1}$, Christopher D. Sherman ${ }^{1}$, Jeffrey H. Miner ${ }^{2}$, James R. Duncan $^{1}$, Carolyn Anderson ${ }^{1}$

${ }^{1}$ Mallinckrodt Institute of Radiology, Washington University School of Medicine, St. Louis, MO, USA, ${ }^{2}$ Department of Internal Medicine, Washington University School of Medicine, St. Louis, MO, USA.

Introduction: Nephrogenic Systemic Fibrosis (NSF), a severely debilitating, systemic fibrosing disorder has been observed in renally impaired patients that have been exposed to intravenously administered gadoliniumbased contrast agents. The goal of this work was to examine the biodistribution of gadolinium-153 labeled contrast agents having different chelate stabilities (Gd-DTPA-BMA vs. Gd-DTPA vs. Gd-DOTA) in the context of renal insufficiency using the transgenic Alport (AL) mouse, which is characterized by mutations in the Col4a3 gene, which encodes specialized chains of type IV collagen causing loss of kidney function. Methods: Biodistribution studies were performed in AL mice and aged matched wild-type (WT) control animals by injecting each animal with either $0.13 \mathrm{MBq}$ of carrier added ${ }^{153} \mathrm{Gd}$-DOTA, ${ }^{153} \mathrm{Gd}$-DTPA-BMA or ${ }^{153} \mathrm{Gd}$-DTPA, sacrificing each animal at selected time points post-injection and removing tissues of interest. The $\% \mathrm{ID} / \mathrm{g}$ and $\% \mathrm{ID} /$ organ for the blood and selected tissues were determined. Results: After 7 days, the effects of Gd-transchelation, which results from Gd being released from its chelating ligand, were still evident. For example, WT mice that received ${ }^{153 / \mathrm{Nat}} \mathrm{Gd}-$ DTPA-BMA had 25 fold and 5 fold more activity $(p<0.0001)$ in their liver tissue and 78 fold and 8 fold more activity $(p<0.0001)$ in their bone tissue than those animals that received ${ }^{153 / \mathrm{Nat}}$ Gd-DOTA and ${ }^{153 / \mathrm{Nat}}$ Gd-DTPA, respectively. Based upon these data the order of stability for these three complexes has been assigned as ${ }^{153 / \mathrm{Nat}}$ Gd-DOTA $>{ }^{153 / \mathrm{Nat}}$ Gd-DTPA $>$ ${ }^{153 / \mathrm{Nat}} \mathrm{Gd}-\mathrm{DTPA}-\mathrm{BMA}$. This same trend in stability was observed in AL mice, but the amount of transchelated gadolinium was greatly enhanced by renal impairment. For example, AL mice that received ${ }^{153 / \mathrm{Nat}}$ Gd-DTPABMA had 70 fold and 6 fold more activity $(p=0.0004)$, in their liver tissue and 469 fold and 17 fold more activity $(p=0.0156)$ in their bone tissue than did AL mice that received ${ }^{153 / \mathrm{Nat}}$ Gd-DOTA and ${ }^{153 / \mathrm{Nat}} \mathrm{Gd}-\mathrm{DTPA}$, respectively. Conclusion: The data clearly demonstrate that Gd-chelates from aliphatic chelators show greater accumulation in normal tissues compared to the macrocyclic chelator DOTA, and in the AL model, the enhanced retention of Gd is significantly more pronounced. These data suggest that linear chain Gd-based MR contrast agents are less stable than their macrocyclic counterparts and this illustrates how MR contrast agent instability combined with poor renal function contributes to the retention of residual gadolinium species that are believed to facilitate the development of NSF. 
J827

DIRECT MICROSCALE VISUALIZATION OF TARGETED QUANTUM DOT BINDING IN MULTIPLE TUMOR MODELS OF LIVING MICE USING INTRAVITAL MICROSCOPY

Bryan R. Smith ${ }^{1}$, Zhen Cheng ${ }^{1}$, Abhijit De ${ }^{1}$, Ai Leen Koh ${ }^{2}$, Robert Sinclair ${ }^{2}$, Sanjiv S. Gambhir ${ }^{1}$

${ }^{1}$ Radiology/Molecular Imaging, Stanford University, Stanford, CA, USA, ${ }^{2}$ Materials Science and Engineering, Stanford University, Stanford, CA, USA.

Targeted nanoparticles have begun to make major contributions in cancer diagnosis and monitoring of therapeutic response. However, comprehension of the mechanisms by which nanoparticles target tumors is critically lacking. Mechanistic insight is essential because it will lead to: 1 . Selection of the appropriate diagnostic entity for each disease/disease site 2. Optimization of nanoparticle parameters for superior targeting efficiency, and 3. Regulatory approval for nanoparticle formulations. We employed intravital microscopy to directly image the process of quantum dots (qdots) targeting tumor. In three different tumor models and two animal models, we surprisingly found that targeted qdots bound to tumor blood vessels only as aggregates, rather than as individual units. Further, we showed that in two of the three tumor models tested, qdots did not extravasate. We conjugated cyclic-RGD (targeting $\alpha v \beta 3$-integrins localized on tumor blood vessels and some tumor cells) to near-infrared emitting $(800 \mathrm{~nm})$ qdots of $~$ $20 \mathrm{~nm}$ hydrodynamic diameter. EGFP-SKOV-3, EGFP-LS174T, and EGFP-U87MG cells were implanted into the ears of mice or in surgically implanted dorsal window chambers (DSC) to form tumors. We injected AngioSense dye and $\sim 50$ pmol qdots into tail veins of mice to visualize the arrival and binding of qdots to tumor vessels. We observed RGD-conjugated qdot aggregates binding tumor blood vessels significantly $(P<0.001)$ more than controls (RAD, RGD-block, bare (no peptide) qdots, and RGDqdots in normal mice) in all tumor models. Interestingly, binding occurred more in the SKOV-3 DSC condition than in any other. We also showed that while we are capable of visualizing disperse qdot binding with our instrument, it did not occur in any of our models. Furthermore, while qdots did not extravasate in SKOV-3 nor LS174T models, we imaged their extravasation in our U87MG tumor model. We have thus demonstrated the utility of intravital microscopy to extract critical information that will aid in future nanoparticle design and approval in cancer diagnostics.
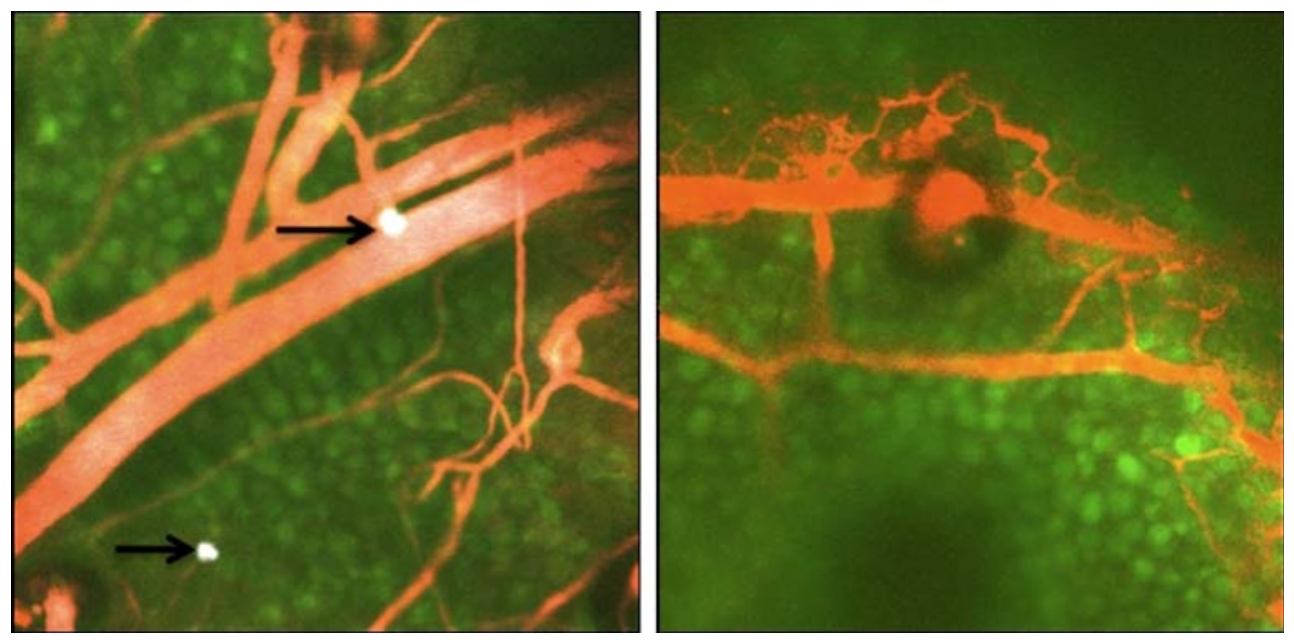

RGD-qdots were observed binding to tumor blood vessels significantly more $(P<0.001)$ than RAD-qdots. In typical images, RGD-qdot aggregates (left) bound to neovasculature, while RAD-qdots (right) did not as shown here in the SKOV-3 ear model.

J828

\section{THE MICROSCALE JOURNEY OF TARGETED CARBON} NANOTUBES IMAGED USING INTRAVITAL MICROSCOPY: FROM CIRCULATION TO TUMOR CELLS IN LIVING SUBJECTS

$\underline{\text { Bryan R. Smith }}^{1}$, Cristina Zavaleta ${ }^{1}$, Zhuang Liu ${ }^{2}$, Jennifer Prescher ${ }^{1}$, John Ramunas ${ }^{3}$, Ricky Tong ${ }^{4}$, Xiaoyuan Chen ${ }^{1}$, Hongjie Dai ${ }^{2}$, Sanjiv S. Gambhir ${ }^{1}$

${ }^{1}$ Radiology/Molecular Imaging, Stanford University, Stanford, CA, USA, ${ }^{2}$ Chemistry, Stanford University, Stanford, CA, USA, ${ }^{3}$ Neuroscience, Stanford University, Stanford, CA, USA, ${ }^{4}$ School of Medicine, Stanford University, Stanford, CA, USA.

Though nanoparticles have become invaluable in the molecular imaging toolkit, little is known about the mechanisms by which they target disease sites. To overcome this critical hurdle, we visualized targeted single-walled carbon nanotubes (SWNTs) entering tumor vasculature, specifically binding luminal targets, extravasating from vessels, and binding to tumor cells. To understand the fundamental mechanisms underlying SWNT tumor uptake, we confirmed and correlated our intravital microscopy (IVM) results with macroscopic Raman imaging, which quantitatively detects the SWNTs' intrinsic Raman signal. Targeted SWNTs were prepared by conjugating RGD peptides (targeting $\alpha \mathrm{v} \beta 3$-integrins expressed on tumor neovasculature and some tumor cells) and Cy5.5 dye. Dorsal chambers were surgically implanted into mice and EGFP-U87MG tumor cells (expressing $\alpha v \beta 3$-integrins) or EGFP-SKOV3 were inoculated. 25 mice (U87MG, SKOV-3, no tumor) were imaged with RGDSWNTs ( $\sim 60 \mathrm{pmol})$ and controls. Using IVM and Raman, mice were imaged during injection and frequently during the following month. Unlike controls, RGD-SWNTs were observed to bind tumor blood vessels. Within hours, SWNTs extravasated in U87MG tumor beds, but not in SKOV-3. While both RGD and 
control SWNTs were observed associated with some tumor cells in U87MG tumors, RGD-SWNTs not only bound more $(P<0.0001)$, but they differentially bound tumor cells over time compared with controls $(\mathrm{P}<0.007)$ and they persisted in tumor for over a month. Control SWNTs cleared within $\sim 1$ week. Also, we unexpectedly observed uptake of SWNTs by circulating white blood cells, which subsequently trafficked to tumor; FACS confirmed these results. In summary, IVM allowed detailed exploration of SWNT uptake in tumor, particularly unanticipated features. This work offers unprecedented understanding of the mechanisms/temporal framework of nanoparticle tumor uptake, which will translate into superior properties for clinical use.

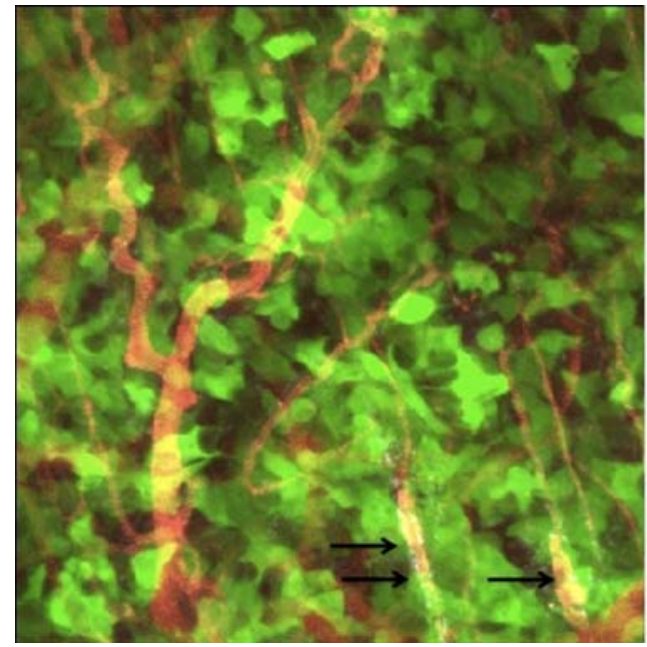

(Left) Image taken soon after RGD-SWNTs cleared from vessels ( $\sim 6$ hrs post-injection). Arrows indicate where RGD-SWNTs specifically bound vessels. (Right) Single frame capture chosen from a movie in which SWNT-laden WBCs are moving through tumor blood vessels at $\sim 4 \mathrm{~h}$ post-injection. Arrows designate circulating WBCs.

\section{J829}

\section{IN VIVO IMAGING OF SPINAL ANTERIOR HORN IN ALS MODEL MICE}

$\underline{\text { Kazunori Miyazaki }}{ }^{1}$, Masamoto Kazuto ${ }^{2,3}$, Makiko Nagai ${ }^{1}$, Nobutoshi Morimoto $^{1}$, Yasuyuki Ohta ${ }^{1}$, Yasushi Takehisa ${ }^{1}$, Yoshio Ikeda ${ }^{1}$, Tohru Matsuura $^{1}$, Takayuki Obata ${ }^{2}$, Iwao Kanno ${ }^{2}$, Koji Abe ${ }^{1}$

${ }^{1}$ Neurology, Graduate School of Medicine, Dentistry and Pharmaceutical Sciences, Okayama University, Okayama, Japan, ${ }^{2}$ Molecular Imaging Center, National Institute of Radiological Sciences, Chiba, Japan, ${ }^{3}$ The University of Electro-Communications, Chofu, Japan.

Introduction Amyotrophic lateral sclerosis (ALS) is a progressive and fatal disease that is caused by the selective death of motor neurons. The underlying mechanism of ALS has not yet been fully clarified. Recently, abnormality of the micro vessels in the anterior horn of the spinal cord has been reported in ALS model mice, but there is no previous report using in vivo imaging, which shows the capillary structures and the real-time blood flow of the anterior horn. In this report, we attempted to develop in vivo imaging in the anterior horn of the spinal cord in ALS model mice using multi-photon excitation microscopy. Methods We used ALS model transgenic (Tg) mice and non-Tg (WT) mice as controls. The 10-weekold (W) Tg mice were considered to be at the pre-symptomatic stage, the $16 \mathrm{~W}$ mice to be at the early-symptomatic stage, and the $19 \mathrm{~W}$ mice to be at the end stage of the disease. Physiological parameters of each mouse were monitored throughout all experiments. For in vivo imaging, laminectomy of the lumber vertebrae at the level of (L4-5) was performed in mice anesthetized with isoflurane. After intravenously injection of fluorescent dot, spinal column was stabilized and the image was captured. Results

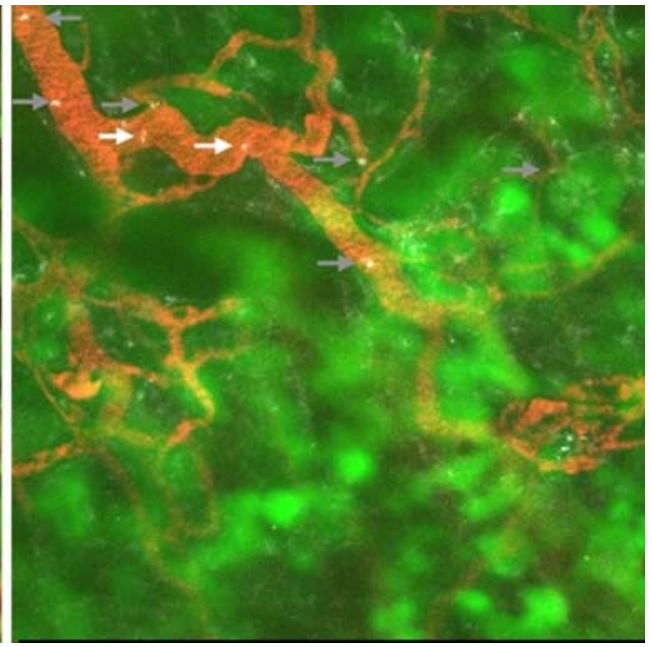

With multi-photon excitation fluorescence microscopy, we approached and monitored anterior horn of the spinal cord in ALS model Tg mice and WT controls. Capillary ultra structures were successfully visualized, and the diameter was measured. We also visualize red blood cell flow that appears as shadows against bright background and could measure average velocity. We could compare the fluxional microvascular condition between WT and Tg mice. Discussion Some reports showed the in vivo imaging of the dorsal horn of the spinal cord. In the present study, we focused on anterior horn and successfully visualized capillary ultra structure and red blood cell flow. In vivo imaging of anterior horn may useful to understand underlying mechanisms of the disorders that anterior horn is the main focus of disease such as ALS.

\section{J830}

\section{IN VIVO MONITORING OF MOUSE SKELETAL MUSCLE CA2+ EXCITATION DURING ACUPUNCTURE}

Jieming Liang, Paul Y.S. Cheung

Electrical and Electronic Engineering, The University of Hong Kong, Hong Kong, China.

$\sim$ INTRODUCTION $\sim$ During a typical acupuncture session, many practitioners will perform needle manipulations which consist of rapid bidirectional rotation and up-and-down pistoning. Previous study [1] showed that measurable tissue displacement occurred up to $4 \mathrm{~cm}$ away from the needle during acupuncture in human. A tight mechanical coupling between the needle and tissue allows movements of the needle to deliver a powerful mechanical signal to the tissue. This study aim to demonstrate how this mechanical signal will further induce cytosolic $\mathrm{Ca} 2+$ excitation in mouse skeletal muscle. $\sim$ MATERIAL AND METHODS $\sim$ In vivo experiments were carried out on 4-week-old C57BL/6 N mice. Mouse hind-limb muscle was transfected with GCaMP2 pcDNA3 by electroporation. In brief, Injection of $30 \mu \mathrm{g}$ expression plasmid followed by controlled electric pulses ( $30 \mathrm{~V} / 20 \mathrm{~ms}$ duration) delivered by needle 
electrodes inserted into the skeletal muscle. 1-2 weeks after transfection, the operated animal was anesthetized, shaved and mounted for observation. The acupuncture needle was inserted into the hind-limb muscle at acupoint ST36 (Fig. 1) and Ca2+ signal was monitored by confocal microscope during acupuncture. $\sim$ RESULTS $\sim$ Fluorescence microscopy reveals that $80 \%$ of all fibers exhibited a strong diffuse GCaMP2 signal. Fig. 2 shows a significant increase of fluorescence intensity in hind-limb muscle during acupuncture, which suggests a dependence of $\mathrm{Ca} 2+$ activation on acupuncture. $\sim$ CONCLUSIONS $\sim \mathrm{Ca} 2+$ signaling is a universal signaling mechanism of cellular communication. Cytosolic $\mathrm{Ca} 2+$ plays important physiological roles on diverse cell processes such as enzyme control, cell proliferation, gene regulation, contraction, exocytosis, and apoptosis. In this study, the mechanical signal of acupuncture needle manipulation was shown to result in $\mathrm{Ca} 2+$ excitation in mouse skeletal muscle. The finding may provide new insights on acupuncture mechanism. $\sim$ REFERENCES $\sim[1]$ Langevin HM et al. Ultrasound Med Biol 30:1173-83(2004).
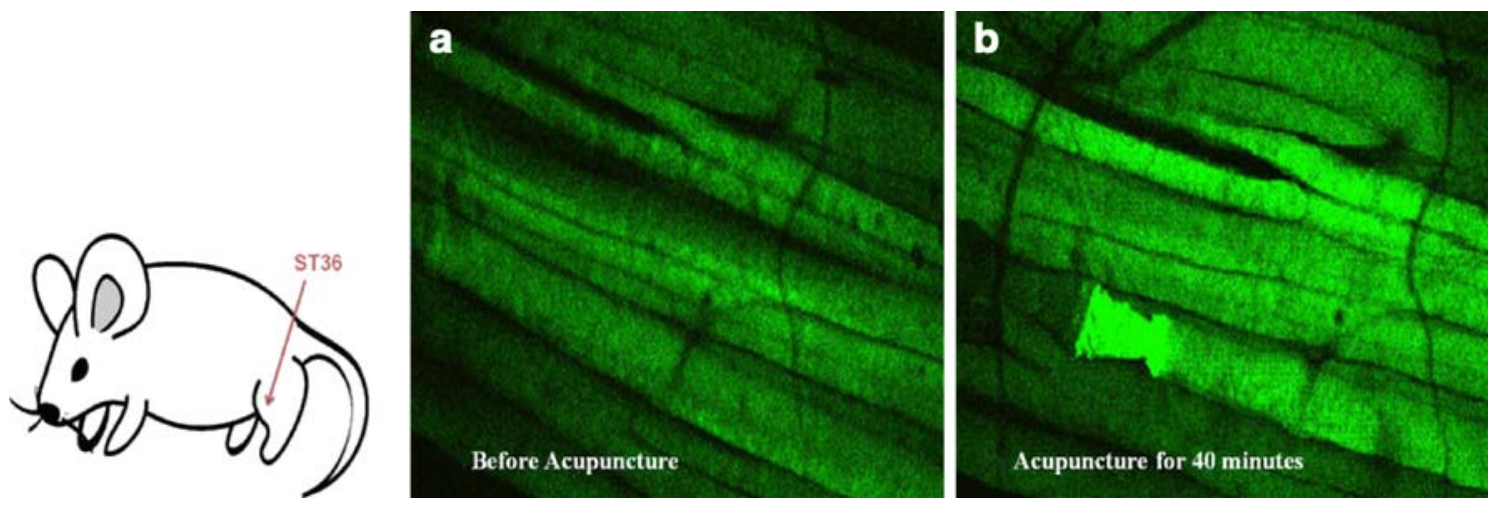

J831

\section{USE OF SPECT/CT TO ASSESS REGENERATION \\ OF AUTOTRANSPLANTED LYMPH NODE FRAGMENTS \\ AND ITS EFFECTS ON THE LYMPH DRAINAGE}

Katrin S. Blum $^{1,2}$, Catarina Hadamitzky ${ }^{2}$, Klaus F. Gratz ${ }^{3}$, Reinhard Pabst $^{2}$

${ }^{1}$ Institute of Diagnostic and Interventional Neuroradiology, Hannover Medical School, Hannover, Germany, ${ }^{2}$ Institute of Functional and Applied Anatomy, Hannover Medical School, Hannover, Germany, ${ }^{3}$ Clinic of Nuclear Medicine, Hannover Medical School, Hannover, Germany.

The combination of nuclear medicine and CT imaging is rapidly becoming a tool for precise functional and anatomic imaging. Lymphoscintigraphy with SPECT/CT is often used for presurgical localization of sentinel lymph nodes in patients with breast cancer and melanoma. The transplantation of lymph node fragments has positive effects on the regeneration and restoration of the lymph flow after lymphadenectomy in the pig model. The reintegration of the lymph node fragments was shown histologically but a major problem was the evaluation of lymph vessel regeneration and of the restoration of the lymph flow in vivo. Seventeen minipigs underwent autologous lymph node transplantation of the superficial lymph node in the left groin. The lymph node was excised, cut in 3-6 pieces and transplanted in 2 or 3 artificial subcutaneous pouches. The right superficial lymph node was removed as control. The lymph flow of both legs was investigated 5 and 8 months after surgery by using conventional lymphoscintigraphy and SPECT/CT with $10 \mathrm{MBq}$ Tc-99m-nanocolloid in combination with Berlin Blue injection in the draining area of both hind legs. The results were confirmed by dissection. The lymph flow from the injection site to the lymph node transplants in the groin was shown in 16 animals. Efferent lymph flow could be followed up to the lumbar trunks and to the retroperitoneal lymph vessels. Impairment of lymph flow was seen in 5 animals in the transplanted groin. One of the five developed a dermal backflow. In one animal no Tc-99m-nanocolloid enhancing spot was found in the left groin. Fourteen animals developed impaired lymph drainage in the right groin. Ten of these developed dermal backflow. Those results were more obvious in the groups 8 months after lymphadenectomy. SPECT/CT with injection of $10 \mathrm{MBq}$ Tc-99m-nanocolloid is an advantageous method to evaluate pathophysiologic changes of the lymph flow after lymphadenectomy. Impairment of lymph flow and dermal backflow development seems to be prevented by transplantation of lymph node fragments. SPECT/CT allows exact localization of the transplanted lymph node fragments in the threedimensional space.

\section{A NOVEL APPROACH FOR DYNAMIC ANALYSIS OF TRANSPLANTED HEMATOPOIETIC STEM/PROGENITOR CELLS IN VIVO BY NEAR-INFRARED FLUORESCENCE IMAGING}

Takashi Ushiki $^{1,2}$, Shinae Kizaka-Kondoh ${ }^{1}$, Eishi Ashihara ${ }^{3}$, Shotaro Tanaka ${ }^{4}$, Hideyo Hirai $^{3}$, Shinya Kimura ${ }^{3,5}$, Taira Maekawa ${ }^{3}$, Masahiro Hiraoka $^{1}$

${ }^{1}$ Department of Radiation Oncology \& Image-Applied Therapy, Kyoto University, Kyoto, Japan, ${ }^{2}$ Division of Hematology, Niigata University, Niigata, Japan, ${ }^{3}$ Department of Transfusion Medicine and Cell Therapy, Kyoto University Hospital, Kyoto, Japan, ${ }^{4}$ Department of Biochemistry, Tokyo Women's Medical University, Tokyo, Japan, ${ }^{5}$ Department of Internal Medicine, Saga University, Saga, Japan.

INTRODUCTION: Engraftment failure and graft-versus-host disease are major complications after bone marrow transplantation (BMT) 
along with the high mortality. The precise mechanisms underlying these complications remain unclear. The recent development of optical imaging may enable us to study the behavior of transplanted hematopoietic stem cells in vivo. Bioluminescence imaging is used to study the processes involved in BMT. However, since a large number of cells are required to detect bioluminescence, it is impossible to track the behavior of transplanted cells before engraftment. Herein, we present a novel method to track the circulating and homing donor cells before hematopoietic reconstitution by using near-infrared fluorescent (NIRF) dye. METHODS: We used BALB/c nu/nu (H2d) mice as recipients, and BALB/c nu/nu (for congenic studies) or C57BL/6 (H2b, for allogenic studies) mice as donors. The surface of bone marrow mononuclear cells (BMMNCs) was labeled with an NIRF dye such as Cy5.5 or Alexa Fluor ${ }^{\circledR 750}$ (AF750). The following BMT models were examined (1) congenic BMT, (2) allogenic T-cell-depleted (TCD) BMT, (3) allogenic TCD BMT + splenocyte transplantation, and (4) congenic intra-BMT. After BMT, the recipient mice were spatiotemporally observed for $24 \mathrm{hrs}$ by using the IVIS Spectrum system (Xenogen). Furthermore, BMMNCs from transgenic ICR mice expressing the enhanced green fluorescent protein (EGFP) (ICR/EGFP) and labeled with an NIRF dye were used for fluorescence-activated cell sorting (FACS) analysis. We estimated the percentages of EGFP + cells in the tibiae, peripheral blood (PB), and spleens of the recipients by using FACS at 24, 48, and $72 \mathrm{hrs}$ after BMT. RESULTS: In allogenic studies using ICR/EGFP mice as donors, the chimerism of EGFP + cells in the recipient's tibia reached about $50 \%$ at $72 \mathrm{~h}$, and FACS analysis revealed that these cells included all the fractions of leukocytes. Moreover, EGFP + granulocytes appeared in the PB. These results indicate that the hematopoietic reconstitution of the donor cells begins around $72 \mathrm{~h}$ after BMT. NIRF-labeled transplanted donor cells had a constant mean fluorescent intensity, which can be detected in vivo over $24 \mathrm{~h}$ by the IVIS Spectrum system. In the congenic BMT model, the homing of AF750-labeled BMMNCs was detected in the tibiae $1 \mathrm{~h}$ after BMT. CONCLUSIONS: This NIRF-labeling method is useful for analyzing the behavior of transplanted cells in murine BMT models. We can track the cells before hematopoietic reconstitution by using this novel method.

\section{J833}

ON THE KINETICS OF THE $\left[{ }^{18}\right.$ F]FAC SERIES OF PET PROBES FOR IMAGING LYMPHOID AND TUMOR TISSUES Jason T. Lee ${ }^{1,2}$, Koon-Pong Wong ${ }^{1,2}$, Dean O. Campbell ${ }^{1,2}$, Andrew Q. $\operatorname{Tran}^{1,2}$, N. Satyamurthy ${ }^{1,2}$, (Henry) Sung-Cheng Huang ${ }^{1,2}$, Caius G. $\operatorname{Radu}^{1,2}$

${ }^{1}$ Molecular \& Medical Pharmacology, University of California, Los Angeles, Los Angeles, CA, USA, ${ }^{2}$ Crump Institute for Molecular Imaging, Los Angeles, CA, USA.

PET imaging of the nucleoside salvage pathway for DNA synthesis and repair may allow monitoring of rapidly proliferating tissues in immune disorders and other diseases including cancer. We have recently reported the synthesis and evaluation of $1-\left(2^{\prime}-\right.$ deoxy- $-2-\left[{ }^{18} \mathrm{~F}\right]$ fluoroarabinofuranosyl) cytosine $\left(\left[{ }^{18} \mathrm{~F}\right] \mathrm{FAC}\right)$. FAC retention in tissues is proportional to the activity of deoxycytidine kinase (DCK), a rate-limiting enzyme of the salvage pathway. A potential limitation of FAC is its susceptibility to deamination by cytidine deaminase. To overcome this limitation we have developed five novel analogs of FAC which retain the affinity for DCK but are resistant to deamination. Current methods for analysis of their PET images based on percent injected dose per gram $(\% \mathrm{ID} / \mathrm{g})$ or standardized uptake value (SUV) with respect to reference tissues do not account for potential differences in transport and metabolism. To better understand the distinctive PET profiles of these probes and to identify the best FAC analog for measuring the salvage pathway, we sought to characterize their differential uptake and distributions in lymphoid and tumor tissues using compartmental tracer kinetic modeling. Dynamic microPET/CT imaging studies were performed after intravenous injection of probe in $\mathrm{C} 57 / \mathrm{B} 16$ mice. Probe retentions as measured by $\% \mathrm{ID} / \mathrm{g}$ of tissue were compared with corresponding DCK mRNA expressions. Kinetic analysis was performed with the Kinetic Imaging Software (KIS) model fitting module using Patlak plots for overall irreversible uptake and compartment models representing transport and retention due to DCK phosphorylation. We found that for three of the five new probes, PET signals in thymus, spleen, heart, lung, muscle, and brown fat had good correlations with DCK gene expression. Current analysis has been expanded to include imaging of severe combined immunodeficiency (SCID) mice for xenograft studies of tumor cell lines. The model parameter $\mathrm{k}_{3}$ of the $\mathrm{FAC}$ probes holds promise as a noninvasive measure of DCK gene expression in vivo and the lack of deamination of the new analogs indicates a significant improvement over $\left[{ }^{18} \mathrm{~F}\right] \mathrm{FAC}$ for measuring upregulated nucleotide salvage metabolism in immune and other diseases marked by rapid proliferation.

\section{J834}

\section{${ }^{11}$ C-Acetoacetate AS NEW HEART IMAGING RADIOPHARMACEUTICAL ${ }^{11} \mathrm{C}$-Acetate}

Etienne Croteau, Sebastien Tremblay, Véronique Dumulon-Perreault, Jonathan Audet, François Bénard, Roger Lecomte

Médecine Nucléaire et radiobiologie, Université de Sherbrooke, Sherbrooke, QC, Canada.

Aim: This study was designed to assess the potential of ${ }^{11} \mathrm{C}$ Acetoacetate as a cardiac imaging agent for the measurement of myocardial perfusion, oxidative metabolism, ventricular volume and the ejection fraction. Acetoacetate is a ketone body, that is first metabolized to acetoacetyl-CoA and then processed by the same oxidative metabolic pathways as Acetate, making it a potential agent for the measurement of myocardial oxygen consumption. Method: Four male Fischer rats (200 g) underwent longitudinal gated dynamic PET imaging following i.v. administration of either, ${ }^{11} \mathrm{C}$-Acetate or ${ }^{11} \mathrm{C}$-Acetoacetate. Both scans are done within $48 \mathrm{~h}$ and repeated 2 weeks later. A three compartment kinetic model was used to obtain the perfusion index $\left(\mathrm{K}_{1}\right)$ and the oxygen consumption $\left(\mathrm{K}_{2}\right)$. The ejection fraction and ventricular volume were obtained from a two minutes segment of the gated acquisition, taken ninety seconds after injection of the radiotracer. The amount of ${ }^{11} \mathrm{CO}_{2}$ released during expiration at 20 min post injection was also measured for $5 \mathrm{~min}$. Results: Overall results see table. According to our compartmental analysis, the cardiac perfusion and oxidative metabolism index values obtained with ${ }^{11} \mathrm{C}$-Acetoacetate are lower than those obtained with ${ }^{11} \mathrm{C}$-Acetate (Student-Tpaired $p<.05$ ). ${ }^{11} \mathrm{C}$-Acetoacetate data yield an increased ventricular volume compared to ${ }^{11} \mathrm{C}$-Acetate leading to a lower ejection fraction value. The residual overall ${ }^{11} \mathrm{C}$ metabolism was measured between 20 and 25 minutes following radiotracer administration, at that time, the amount of ${ }^{11} \mathrm{CO}_{2}$ released during expiration was higher for ${ }^{11} \mathrm{C}$-Acetoacetate than for ${ }^{11} \mathrm{C}$-Acetate. These observations are in agreement with a slower clearance rate of ${ }^{11} \mathrm{C}$-Acetoacetate as compared to ${ }^{11} \mathrm{C}$-Acetate. Conclusion: ${ }^{11} \mathrm{C}$-Acetoacetate proved to be a robust and reproducible radiotracer for the evaluation of myocardial metabolism of ketone bodies and the evaluation of the cardiac function. Furthermore the longer myocardial transit time of ${ }^{11} \mathrm{C}$-Acetoacetate leads to higher quality cardiac images. 
Myocardial PET Kinetic Analyses and Function

\begin{tabular}{|c|c|c|}
\hline & $11_{\text {C-acetoacetate }(\mathrm{n}=8)}$ & $3.97 \pm 0.96$ \\
\hline Myocardial Perfusion $\left(\mathrm{K}_{1} \mathrm{~min}^{-1}\right)$ & $3.02 \pm 1.24$ & $1.64 \pm 0.55$ \\
\hline Oxygen Consumption $\left(\mathrm{k} 2 \mathrm{~min}^{-1}\right)$ & $1.30 \pm 0.46$ & $60.6 \% \pm 3.4$ \\
\hline Ejection Fractions & $55.5 \% \pm 2.4 \%$ & $227 \pm 41$ \\
\hline Systolic Volume $(\mu \mathrm{L})$ & $248 \pm 47$ & $90 \pm 23$ \\
\hline Diastolic Volume $(\mu \mathrm{L})$ & $111 \pm 19$ & $3.4 \% \pm 1.2 \%$ \\
\hline $11 \mathrm{CO}_{2}$ exhaled $(20-25 \mathrm{~min})$ & $4.2 \% \pm 1.0 \%$ & \\
\hline
\end{tabular}

$\mathbf{J 8 3 5}$

\section{COMPARISON OF IN VITRO CHARACTERISTICS OF A MONOMERIC, DIMERIC AND TRIMERIC FIBRONECTIN-DERIVED LINEAR RGD-PEPTIDE}

Zhao-Hui Jin ${ }^{1}$, Takako Furukawa ${ }^{1,2}$, Atsuo $\mathrm{Waki}^{2}$, Kenichi Akaji ${ }^{3}$, Jean-Luc Coll $^{4}$, Tsuneo Saga ${ }^{1}$, Yasuhisa Fujibayashi ${ }^{1,2}$

${ }^{1}$ Diagnostic Imaging Group, Molecular Imaging Center, National Institute of Radiological Sciences, Chiba, Japan, ${ }^{2}$ Biomedical Imaging Research Center, University of Fukui, Fukui, Japan, ${ }^{3}$ Department of Organic and Medicinal Chemistry, Graduate School of Medical Science, Kyoto Prefectural University of Medicine, Kyoto, Japan, ${ }^{4}$ INSERM U823, Cibles Diagnostiques ou Thérapeutiques et Vectorisation de Drogues dans les Cellules Tumorales, Institut Albert Bonniot, Grenoble, France.

Multivalent interactions are frequently used to enhance ligand-receptor binding affinity. In this study a mono-, di- and trimeric AVTGRGDSY peptide derived from fibronectin were compared concerning the integrin receptor binding affinity and/or specificity. Methods: AVTGRGDSY monomer, dimer and trimer were synthesized, labeled with 125I or Cy5.5. Using human embryonic kidney cells HEK293 (naturally $\alpha \mathrm{V}$-positive and $\beta 3$-negative), HEK293( $\beta 1)$ ( $\beta 1$-transfected and $\alpha \mathrm{V} \beta 3$-negative), HEK293( $\beta 3$ ) ( $\beta 3$-transfected and strongly $\alpha \mathrm{V} \beta 3$ positive), and human glioblastoma cells U87MG (naturally $\alpha \mathrm{V} \beta 3$ positive), cell-binding assay, competitive inhibition assay, cell adhesion assay and confocal laser scanning microscopic study were performed to determine the bioactivities of these peptides. Results: The monomeric AVTGRGDSY showed specific binding to both HEK293( $\beta 1)$ and HEK293( $\beta 3)$ cells. Multimerization resulted in no change with HEK293 cells, diminished binding for HEK293( $\beta 1)$ cells, but substantially enhanced binding for $\alpha \mathrm{V} \beta 3$-positive HEK293 ( $\beta 3$ ) and U87MG cells in the presence of Mn2+. Moreover, the multimeric AVTGRGDSY peptides were found to be nearly comparable with the $\alpha \mathrm{V} \beta 3$-specific cyclo(RGDfV) peptide in specificity and affinity for targeting $\alpha \mathrm{V} \beta 3$ integrin. Conclusion: The multimeric AVTGRGDSY peptide might be an efficient $\alpha \mathrm{V} \beta 3$ - targeting molecule. The present study would be useful for better understanding of the molecular basis of the interaction between RGD ligands and integrin receptors.

J836

\section{D SURFACE CONSTRUCTION FOR BIOLUMINESCENCE} TOMOGRAPHY FROM ONE 2D PHOTOGRAPHIC IMAGE

Jianghong Zhong, Jie Tian, Xin Yang, Min Xu

Medical Image Processing Group, Institute of Automation,CAS, "Beijing, 100080", China.

The objective of our project is the study of Shape from Shading (SfS) problem for bioluminescence tomography. Our real time in vivo image system named WinMI can only collect the twodimensional bioluminescence image or photographic image through the CCD acquisition array. However, the image system needs the three-dimensional shape to localize and quantify bioluminescence sources inner the biological organization in vivo. In this study, SfS problem will be addressed as an energy minimization problem to obtain three-dimensional surface image, compared to the other shape recovery methods. A constraint which establishes a connection between intensity gradient space and solution surface is imposed into the corresponding energy functional in a direct energy minimization scheme. The reason of the usage of intensity gradient is its ability to force the solution surface as smooth as the actual surface and to establish an additional connection between image intensity and constructed surface gradient values. Since deterministic methods cannot avoid local minima, an alternative stochastic approach will be used. The above methods will be combined in a multiresolutional framework in order to couple the efficiency and accuracy properties. In a word, it is proved that the $3 \mathrm{D}$ surface reconstruction method is practical and will provide the original input information for the bioluminescence tomography reconstruction. 


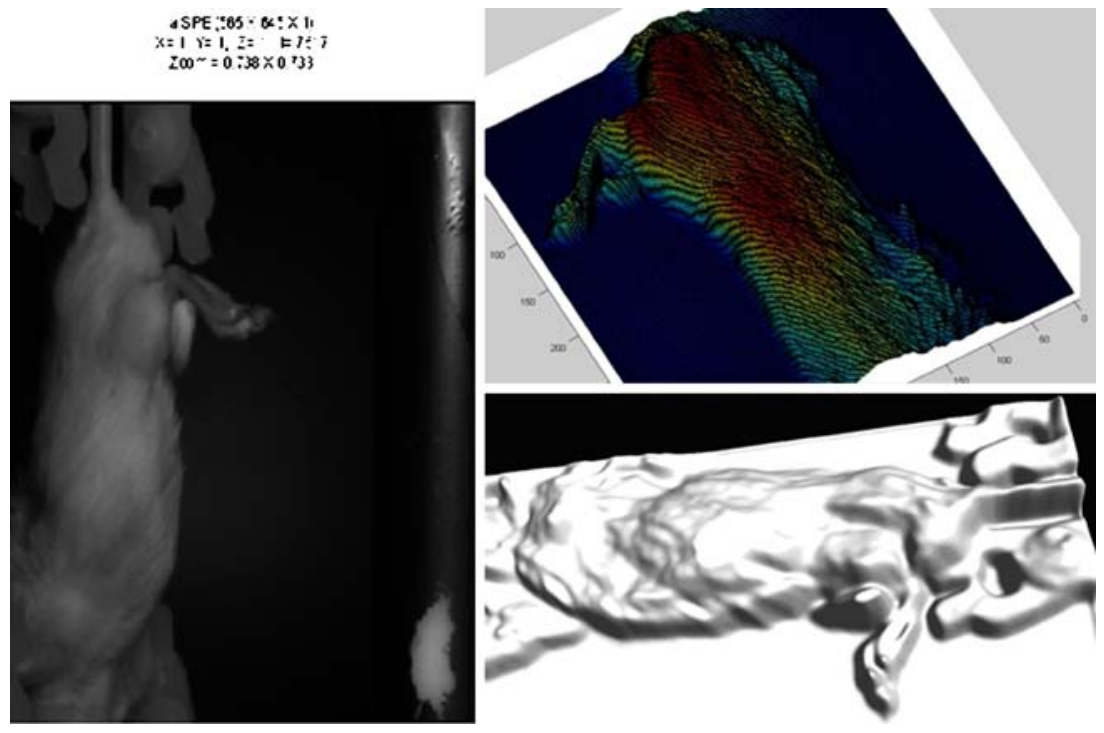

3D surface from a photographic image

$\mathbf{J 8 3 7}$

\section{MRI TRACKING OF ULTRASOUND GUIDED INTRAMYOCARDIALLY INJECTED STEM CELLS}

Jamal Guenoun, Miranda Harteveld, Annemieke van Wamel, Piotr A. Wielopolski, Gabriel P. Krestin, Monique R. Bernsen Erasmus MC, Rotterdam, Netherlands.

Purpose: To explore the feasibility and advantages of high resolution ultrasound guided stem cell delivery, a relatively under-utilized technique, in a rodent model of myocardial infarction. Material and methods: Cryoinfarction of the LV anterior wall was induced in 9 Wistar rats $(200-250$ g.) applying a $5 \mathrm{~mm}$. diameter cryoprobe (Brymill, UK) for $14 \mathrm{~s}$. Rat mesenchymal stem cells were isolated from bone marrow and magnetically labelled with SPIO, a T2 MRI contrast agent. A cell suspension containing 500.000 labeled cells $/ 20 \mu \mathrm{l}$ PBS was prepared one week after induction of a cryo-infarction. A $25 \mathrm{MHz}$ single element ultrasound transducer (Vevo 770, VisualSonics Inc., Canada) was used to image the anterior LV wall in high resolution B-mode. Under ultrasound guidance, cells in a $27 \mathrm{G}$ needle were injected intramyocardially, bordering the infarction. Infarct size was determined using a $2 \mathrm{D}$ cardiac gated inversion recovery sequence (delayed enhancement) at $3.0 \mathrm{~T}$. Stem cell deposition was monitored at 0,7 , 14 and 21 days post-injection using a 3D gradient echo scan. Results: SPIO-labeled stem cells were visualized near the infarct border as hypointense regions for at least 21 days. Cryoinfarction applied for $14 \mathrm{~s}$. produced transmural infarction of the left ventricle anterior wall, visualized on both short and long axis view delayed enhancement scans. MRI revealed a pneumothorax in one animal after the transthoracic injection, which spontaneously resolved. One animal died within 24 hours after cryoinfarction. Two animals died during follow-up. Conclusion: High resolution transthoracic echocardiography (TTE) can be used as an effective technique for targeted stem cell delivery in experimental cell tracking studies. The possibility of real-time anatomical information on infarct size and location poses an additional advantage for relatively accurate targeted delivery of the stem cells.

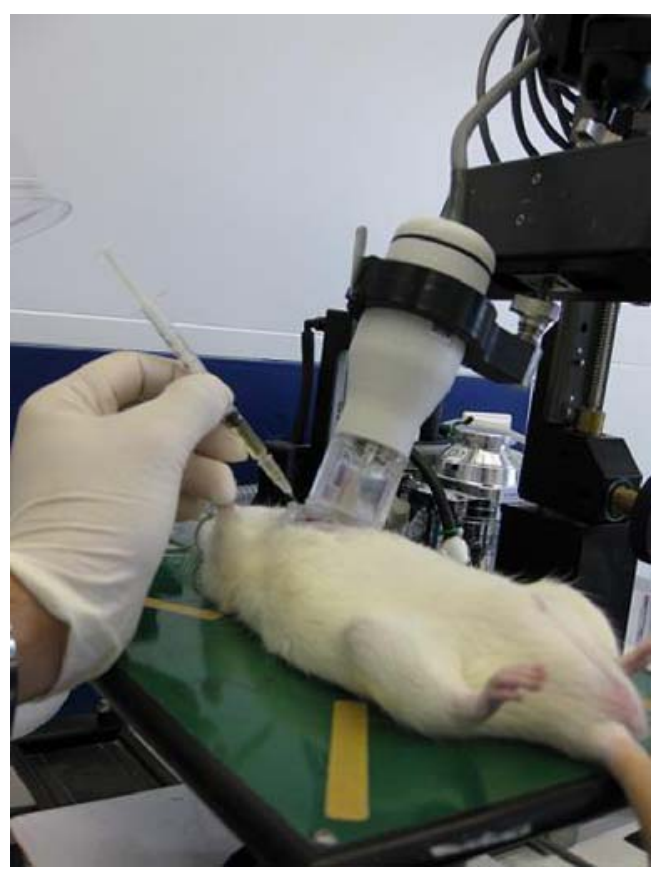

Ultrasound guided intramyocardial injection

$\mathbf{J 8 3 8}$

MAGNETIC RESONANCE IMAGING OF IRON OXIDE-LOADED HUMAN ENDOTHELIAL PROGENITOR CELLS REVEALS SHORT TERM DEATH OF INJECTED PROGENITORS

WITHOUT LOSS OF ANGIOGENIC ACTIVITY

Anita Gianella ${ }^{1}$, Uliano Guerrini ${ }^{1}$, Luigi Sironi ${ }^{1}$, Maurizio C. Capogrossi $^{3}$, Elena Tremoli ${ }^{1}$, Maurizio Pesce ${ }^{2}$

${ }^{1}$ Dipartimento di Scienze Farmacologiche, Universita' degli Studi di Milano, Milano, Italy, ${ }^{2}$ Laboratorio di Biologia Cellulare e Terapia Genica, Centro Cardiologico Monzino-IRCCS, Milano, Italy, ${ }^{3}$ Laboratorio di Patologia Vascolare, Istituto Dermopatico dell'Immacolata IDI-IRCCS, Roma, Italy. 
Background and Aims: The assessment of cell survival after transplantation is one of the major challenges in cell therapy. In preclinical models, genetic engineering by green fluorescent protein (GFP) or luciferase gene transfer, allows to follow cell fate in recipient tissues by immunohistochemistry on tissue sections and by bioluminescence in vivo detection. However, the translation of these techniques to clinical practice is difficult. A possible option has been offered by iron oxide particle loading of cells to be tracked by in vivo magnetic resonance imaging (MRI). Methods and Results: Human peripheral blood-derived "early" endothelial progenitor cells (hEPCs) were loaded with iron oxide particles (SPIOFeridex). These cells were studied in vitro and imaged by MRI following injection in a mouse model of hind limb ischemia (A). Our experiments showed that iron-loaded hEPCs maintained their phenotype and in vitro functional activity. Importantly, we were able to visualize the SPIO-loaded cells using in vivo MRI at 1 and 7 days after injection into the ischemic adductor muscle of pharmacologically immunosuppressed mice (B). Iron staining performed on hEPC-injected muscle histologic sections showed a positive correlation with MRI signal. Immunohistochemical staining using human HLA specific antibodies and quantitative PCR performed on DNA extracts from injected ischemic limbs revealed the absence of living human cells at 7 days after injection. Additionally, iron-positive cells were stained with mouse anti CD68 antibody (C), suggesting death of injected progenitor cells and uptake of the iron particles by host macrophages. In spite of their short survival in vivo hEPCs enhanced angiogenesis in ischemic adductor muscles (D). Conclusions: Our results confirm hEPCs pro-vascularization action and their limited life span after in vivo transplantation. Further, iron oxide particle uptake by host cells calls for caution about the use of iron-based contrast agents to detect stem cells engraftment and survival in vivo.

\section{J839}

\section{METABOLIC PET IMAGING IN EXPERIMENTAL MODEL OF ISCHEMIC STROKE}

Monica Sobrado ${ }^{1}$, Mercedes Delgado ${ }^{2}$, Encarnacion Fernandez-Valle ${ }^{3}$, Luis García $^{2}$, Jose Vivancos ${ }^{4}$, Igacio Larrañaga ${ }^{6}$, Jose Sanchez-Prieto ${ }^{5}$, Magdalena Torres ${ }^{5}$, Elena Gorospe ${ }^{6}$, M Angeles Moro ${ }^{1}$, Ignacio Lizasoain ${ }^{1}$, Miguel A. Pozo ${ }^{2,6}$

${ }^{1}$ Farmacologia, Universidad Complutense, Madrid, Spain, ${ }^{2}$ Cartografía Cerebral, Universidad Complutense, Madrid, Spain, ${ }^{3}$ CAI RM, Universidad Complutense, Madrid, Spain, ${ }^{4}$ Neurología, Hospital La Princesa, Madrid, Spain, ${ }^{5}$ Bioquímica y Biologia Molecular, Universidad Complutense, Madrid, Spain, ${ }^{6} \mathrm{I}+\mathrm{D}+\mathrm{i}$, Instituto Tecnológico PET, Madrid, Spain.

The aim of this study is to assess the progress of changes in cerebral metabolism in animal models of ischemic stroke by means of a highresolution positron emission tomography scanner used for small animal imaging. A time course study using RMI and PET imaging was carried out using adult male Fisher rats $(275 \pm 25 \mathrm{~g})$. Animals were distributed in three experimental groups: sham-operated $(n=3)$, permanent middle cerebral artery occlusion (pMCAO; $n=5)$ and transitory middle cerebral artery occlusion ( $\mathrm{tMCAO} ; n=5)$. The PET experiments were performed with 2-Deoxy-2-[F-18]fluoro-D-glucose (FDG) as a tracer with a specific activity of $1.500 \mathrm{Ci} / \mathrm{mmol}$ and produced by the Instituto Tecnológico PET (Madrid, Spain). Rats were anesthetized ( $2 \%$ isofluorane) and injected i.v. with $18.5 \mathrm{MBq}$ of FDG. After $75 \mathrm{~min}$ of uptake period, the animals were placed in a dedicated animal PET scanner (Albira PET, Oncovision, Spain) for $60 \mathrm{~min}$ of data acquisition. PET data were reconstructed using the OSEM algorithm and co-registered with the T2-weighted MRI of the same animal using the PMOD software (PMOD Technologies, Zurich, Switzerland). Regions of interest were drawn on the fused images. Longitudinal PET studies were performed at time $4 \mathrm{~h}, 24 \mathrm{~h}$ and $48 \mathrm{~h}$ after the onset of MCAO and compared with a control PET acquisition performed $24 \mathrm{~h}$ before. Our results show that: a) The neocortical infarct volume expressed as percentage of ipsilateral/contralateral hemisphere (\%I), measured on ADC maps, was significantly increased from 4 at $24 \mathrm{~h}$ after surgery in both models and then it remained stable until $48 \mathrm{~h}$. The \%I was significantly higher in pMCAO vs tMCAO model $(36 \pm 4 \%$ vs $5 \pm$ $6 \%, 78 \pm 4 \%$ vs $23 \pm 3 \%$ and $73 \pm 6 \%$ vs $27 \pm 8 \%$, at 4,24 and $48 \mathrm{~h}$ after surgery, respectively).b)The metabolic activity measured as FDG reuptake was not affected in sham-operated animals after surgery at any time studied. The FDG uptake was significantly reduced at 4, 24 and $48 \mathrm{~h}$ vs before surgery, in both models of ischemia. The FDG uptake was lower in pMCAO vs tMCAO model ( $70 \pm 8$ vs $83 \pm 3,54 \pm 7$ vs $69 \pm 16,55 \pm 8$ vs $75 \pm 22$, at 4,24 and $48 \mathrm{~h}$ after surgery, respectively). In conclusion, PET imaging with FDG is sensitive to metabolic changes associated to the experimental model of ischemic stroke.

\section{J840}

\section{QUANTIFYING DYNAMICS OF GASTRIC EMPTYING USING NON-INVASIVE IN VIVO FLUORESCENT REFLECTANCE IMAGING}

L. Ashley Aronow ${ }^{1}$, Allison Curtis $^{1}$, Renee Commerford ${ }^{2}$, Tara Mullarkey $^{2}$, Anne Basler ${ }^{3}$, Rainer Kneuer ${ }^{3}$, Thomas Krucker ${ }^{1}$

${ }^{1}$ Molecular Imaging, Novartis Institutes for BioMedical Research, Cambridge, MA, USA, ${ }^{2}$ Cardiovascular and Metabolism, Novartis Institutes for BioMedical Research, Cambridge, MA, USA, ${ }^{3}$ Global Imaging Group, Novartis Institutes for BioMedical Research, Basel, Switzerland.

Over the last decade, increasing incidence of obesity and diabetes has led to greater investment in research on metabolic disorders and their molecular pathways, focusing on characterizing healthy or pathological gastrointestinal function. Although a variety of methods have been applied to study gastric motility, many of them have technical limitations and are low throughput, e.g., imaging a single anesthetized animal per time point and requiring long acquisition times. In vivo optical imaging provides researchers with the ability to study a living system using simple, non-invasive techniques to investigate biological systems in action. The goal of this research was to adapt an in vivo method (Gremlich et al., Mol. Imaging 2004) using near-infrared (NIR) fluorescent reflectance imaging to allow for higher-throughput experimentation and dynamic quantitation of gastrointestinal transit. Using a two-dimensional timeresolved fluorescent reflectance system with dynamic imaging capabilities, we measured the percent and rate of gastric emptying of a semi-viscous nutrient meal either enriched or not with saturated fats and containing fluorescent labeled polystyrene beads in $\mathrm{nu} / \mathrm{nu}$, C57BL/6, and diet-induced obese (DIO) C57BL/6 mice. Three mice were imaged simultaneously at several time points as well as continuously to capture dynamics of motility and signal intensity. We tested the sensitivity of our assay with two known modulators of gastric emptying: a high fat meal and cholestykinin (CCK-8). Acquisition times were low (ms) limiting the time animals had to be anesthetized and allowing for multiple mice to be imaged simultaneously, providing a significant increase in efficiency over other imaging approaches. While continuous imaging was sensitive enough to detect the change in location of the bolus as it progressed through the gastrointestinal tract, motility was significantly slower. Discrete time points were chosen after observations that periods of wakefulness and ambulation restored normal digestion rates. As expected, CCK-8 significantly delayed gastric emptying under all experimental conditions tested. Meal fat type also significantly affected rates of gastric emptying in both control and CCK- 8 treated mice. This method provides a higher-throughput alternative for current gastric emptying methodologies. It allows for more animals 
to be imaged in less time and is sensitive enough to detect and measure changes in gastric emptying over the course of several hours, while providing a visual confirmation of food transit in a live animal.

\section{J841}

REAL-TIME INTRAOPERATIVE ASSESSMENT OF LEAKAGE AND STENOSIS OF GASTROINTESTINAL ANASTOMOSES USING A NATURAL NEAR-INFRARED FLUORESCENT ORAL AGENT

Yoshitomo Ashitate $^{1,4}$, Hak Soo $\mathrm{Choi}^{1}$, Merlijn Hutteman ${ }^{1,3}$, Rita Laurence $^{1}$, John V. Frangioni ${ }^{1,2}$

${ }^{1}$ Medicine, Beth Israel Deaconess Medical Center, Boston, MA, USA, ${ }^{2}$ Radiology, Beth Israel Deaconess Medical Center, Boston, MA, USA, ${ }^{3}$ Surgery, Leiden University Medical Center, Leiden, Netherlands, ${ }^{4}$ Surgical Oncology, Hokkaido University Graduate School of Medicine, Hokkaido, Japan.

Background: Much progress has been made in gastrointestinal surgery, but leakage and stenosis at the anastomosis site are still major complications. Although endoscopic evaluation, methylene blue administration, and barium as an oral contrast agent for x-ray fluoroscopy have been tried, no similar oral agent for near-infrared (NIR) fluorescence-guided surgery has been described. In this study, we demonstrate that a simple herbal supplement provides intense NIR fluorescence contrast that can be used for real-time intraoperative assessment of the anastomosis site. Materials and Methods: Chlorophyll is a natural, orally-ingestible agent found in typical human diets. Each $3 \mathrm{~g}$ of the herbal supplement chlorella powder (green microalgae) contains $60 \mathrm{mg}$ of chlorophyll. In initial experiments ( $n=3$ pigs), feed was withheld from swine $48 \mathrm{~h}$ before the study to exclude autofluorescence. After anesthesia, a midline abdominal incision was performed, the stomach and small intestine were exposed, and the nasogastric tube was inserted. For oral administration, chlorella was completely dissolved in water with $1 \%$ DMSO and $1 \%$ Cremophor. The FLARE ${ }^{\mathrm{TM}}$ intraoperative NIR fluorescence imaging system was used to simultaneously acquire color video, NIR fluorescence, andmerged images, as chlorella was infused via the nasogastric tube. Results: Chlorella exhibitedmultiple absorption peaks, with the longest at $668 \mathrm{~nm}$ in 1\% DMSO. Using $655 \mathrm{~nm}$ laser excitation, peak fluorescence emission was at $670 \mathrm{~nm}$. Stenosis, injury, gastrojejunostomy, and Roux-en Y models, which include both gastrojejunostomy and jejunostomy, were developed. When 10 to $60 \mathrm{ml}$ of chlorella was infused into the stomach, intense NIR fluorescence was observed, with an exposure time of from 60 to $250 \mathrm{msec}$. Contrast in the gastrointestinal lumen could be followed, and all models could be assessed. Conclusions: Simple administration of an herbal supplement provides sensitive, high-resolution, NIR fluorescent, image-guided assessment of gastrointestinal function during surgery. This technology holds the promise of real-time intraoperative assessment of anastomoses using invisible NIR fluorescent light.

\section{J842}

EVALUATION OF CELL TYPE AND INJECTION STRATEGY ON CELL RETENTION IN INFARCTED CANINE MYOCARDIUM

$\underline{\text { Kimberley Blackwood }}^{1,2}$, Andrea J. Mitchell ${ }^{1,2}$, Jane Sykes ${ }^{1}$, Lela $\overline{\text { Deans }}^{1}$, Gerald Wisenberg ${ }^{3}$, Frank Prato ${ }^{1,2}$

${ }^{1}$ Imaging Program, Lawson Health Research Institute, London, ON, Canada, ${ }^{2}$ Medical Biophysics, University of Western Ontario, London, ON, Canada, ${ }^{3}$ Division of Cardiology, London Health Sciences Centre, London, ON, Canada.

Introduction Given that cellular therapeutics have shown potential in augmenting various forms of cardiac disease, optimization of such therapies have been extensively investigated. Notably, parameters like cell type and route of delivery have been compared in animal models in an attempt to improve retention and engraftment of transplanted cells.
We present preliminary results comparing the effects of injection strategy and cell type on retention of radiolabeled cells in a reperfused canine model of myocardial infarction. Methods Four groups of canine cells were studied: bone marrow mononuclear cells (BMMNC), muscle satellite cells (MSC), bone marrow stromal cells (BMSC), and endothelial progenitor cells (EPC). Cells were labeled with $111 \mathrm{In}$ tropolone and autologously transplanted into infarcts $(2-3 \mathrm{~h} \mathrm{LAD}$ occlusion/ 2-3 h reperfusion) in the peri-infarct border region. Cells were transplanted in various ways: a) intracoronary (IC) injection using a balloon catheter or, b) intramyocardial (IM) injection directed i) epicardially following left thoracotomy or, ii) endocardially using an endocardial catheter system by x-ray angiography. BMMNCs were injected IC $(n=3)$, MSCs $(n=3)$ were injected IM epicardially, BMSCs $(n=2)$ were injected IM endocardially, while EPCs were injected IM epicardially $(n=2)$ and endocardially $(n=4)$. Immediately after injection, wholebody images of animals were acquired with a gamma camera. Cell retention following cell injection was based on heart activity regions of interest compared to total activity in the body after background correction to give heart:wholebody ratios. Ratios are expressed as a percentage and values are mean \pm SD. Results Data grouped by injection strategy revealed that it significantly affected cell retention demonstrating that the IC route was the least effective $(3.6 \pm 0.7 \%)$ compared to the endocardial $(26.3 \pm 13.9 ; p<0.05)$ and epicardial $(59.0 \pm 5.1 ; p<0.001)$ IM routes. Significant differences in cell retention were also found between the epicardial and endocardial injections with $p<0.001$, however larger variability in the latter group may account for these differences. No significance was found between EPC vs. MSC $(55.9 \pm 3.0 \%$ vs. $61.1 \pm 5.7 \%)$ epicardially injected and EPC vs. BMSCs $(32.3 \pm 13.3 \%$ vs. $14.3 \pm 13.7 \%)$ endocardially injected. Conclusion Intramyocardial injections through the epicardium lead to better cell retention compared to cells injected via the endocardium or intracoronary route. Data also suggests that cell type may not influence cell retention in a reperfused canine myocardial infarction model.

\section{J843}

\section{FEASIBILITY STUDY OF LOW DOSE DEXA IMAGING FOR BONE-DENSITY MEASUREMENT OF OSTEOPOROSIS MICE MODEL}

Sheng-Pin Tseng, Meei-Ling Jan, Yu-Ching Ni, Hsin-Chin Liang, Hong-Chih Lu, Tien-Hsiu Tsai

Institute of Nuclear Energy Research, Taoyuan County, Taiwan.

For small animal imaging, micro-CT is usually performed for osteoporosis examination. However, the micro-CT imaging provides high spatial resolution with the price of high radiation dose. Low dose dual energy Xray absorptiometry (DEXA) which is popular in clinical inspections for osteoporosis might be a good alternative. This work is to study the feasibility of applying DEXA method to bone-density measurement of mice. An x-ray tube was operated ranging from $20 \sim 50 \mathrm{kV}$, and two types of sensor, Gd2O2S/CCD-based and CsI/CMOS based detectors, were used to acquire the planar images with respective resolutions of $512 \times 512$ and $586 \times 560$ pixels for experiments. Phantoms with $0 \sim 2 \mathrm{M}$ consistencies of Potassium Iodide (KI) solution were scanned to evaluate the linearity of Gd2O2S/CCD-based and CsI/CMOS-based detectors and to find the suitable dual energy pairs of DEXA. C57/BL6 mice with osteoporosis after ovariectomy (OVX) surgery and the control group without OVX were scanned with x-ray dual energies of $25 \mathrm{kVp} / 50 \mathrm{kVp}$. Areal density images were calculated from the low/ high energy planar images using DEXA method. Results of KIconsistency experiment show the Gd2O2S/CCD-based detector has higher sensitivity and better linearity than CsI/CMOS-based detector. The areal density images of normal and osteoporosis mice are shown in the figure. The femur-head region and soft tissue were chosen as the regions of interest. The areal density of bone and bone-to-soft 
tissue ratio are 0.1058 and 3.6015 for normal mice and 0.0531 and 2.4122 for osteoporosis mice using Gd2O2S/CCD-based detector. In conclusion, the analysis of the experiment results reveals that the low dose DEXA imaging for osteoporosis inspection of mice is promising.
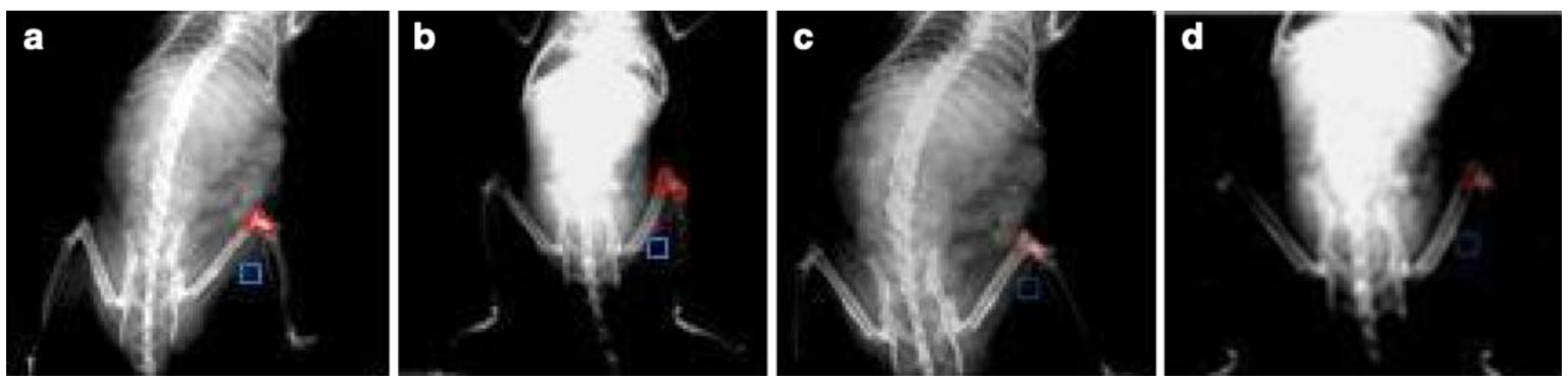

J844

PET IMAGING OF ANTI-PSMA ANTIBODIES AS NOVEL AGENTS FOR TARGETING PROSTATE CANCER

Stefan Wiehr ${ }^{1}$, Karen Alt ${ }^{3,4}$, Patrick Bühler ${ }^{4}$, Philipp Wolf ${ }^{4}$, Gerald Reischl $^{2}$, Walter Ehrlichmann ${ }^{2}$, Daniel Bukala ${ }^{1}$, Martin S. Judenhofer ${ }^{1}$, Hans F. Wehrl ${ }^{1}$, Andreas Schmid ${ }^{1}$, Bernd J. Pichler ${ }^{1}$

${ }^{1}$ Laboratory for Preclinical Imaging and Imaging Technology, Department of Radiology, University of Tuebingen, Tuebingen, Germany, ${ }^{2}$ Radiopharmacy, Department of Radiology, University of Tuebingen, Tuebingen, Germany, ${ }^{3}$ Department of Urology, University of Freiburg, Freiburg, Germany, ${ }^{4}$ Faculty of Biology, University of Freiburg, Freiburg, Germany.

The Prostate Specific Membrane Antigen (PSMA) has been shown to represent an excellent target for diagnosis and therapy of prostate cancer. In previous studies we generated monoclonal antibodies (mAbs) against native cell-adherent PSMA, radiolabled them with ${ }^{64} \mathrm{Cu}$ and successfully performed small animal PET imaging in SCID mice. The aim of this continuative study was to compare the original 3/ A12 mAb with two newly generated antibodies antibody fragments for imaging specific binding in human prostate tumors. Specific binding of the mAbs to distinct extracellular epitopes of cell-adherent PSMA was determined by flow cytometry. For PET imaging the mAbs and $\mathrm{F}(\mathrm{ab})_{2}-$ fragments were conjugated with the chelating agent DOTA and radiolabeled with ${ }^{64} \mathrm{Cu}$. The $\mathrm{mAb}$ were administered to SCID mice (20 $\mu \mathrm{g} \mathrm{mAb}$ corresponding to $7 \mathrm{MBq}$ ) bearing PSMA-positive or -negative human prostate carcinoma xenografts. To block the PSMAspecific receptors mice were injected once with 250 to $700 \mu \mathrm{g}$ non-radioactive $\mathrm{mAb} 3 \mathrm{~h}$ prior to injection of the corresponding radiolabeled $\mathrm{mAb}$. Mice were in vivo imaged using a small animal PET and MRI for exact localizing the tumors at 3, 24, and $48 \mathrm{~h}$ post injection. After the last scan the ex vivo biodistribution was performed by gamma-counting. The anti-PSMA mAbs called 3/A12 ( $\left.\operatorname{IgG}_{1}\right), 3 / \mathrm{F} 11$ $\left(\operatorname{IgG}_{2 \mathrm{a}}\right)$ and $3 / \mathrm{E} 7\left(\operatorname{IgG}_{2 \mathrm{~b}}\right)$ showed a high and specific binding to PSMAexpressing prostate tissue and were not influenced by the DOTAconjugation. Animal PET images revealed a percentage of the injected dose per cc $(\% \mathrm{ID} / \mathrm{cc})$ in $\mathrm{C} 4.2$ tumors of $12.8,12.7$ and 11.7 at $24 \mathrm{~h}$ and $14.1,15.7$ and 14.0 at $48 \mathrm{~h}$ after injection of $\left[{ }^{64} \mathrm{Cu}\right] \mathrm{DOTA}-3 / \mathrm{A} 12$, $\left[{ }^{64} \mathrm{Cu}\right]$ DOTA-3/F11 mAb or $\left[{ }^{64} \mathrm{Cu}\right]$ DOTA-3/E7. The binding to PSMA was blocked down to 3.0, 5.2 and $4.4 \% \mathrm{ID} / \mathrm{cc}$ already $3 \mathrm{~h}$ after injection of $700 \mu \mathrm{g}$ of non-radioactive antibodies. A reduced tumor uptake of 3.7 and $2.8 \% \mathrm{ID} / \mathrm{cc} 48 \mathrm{~h}$ after injection was obtained with the corresponding $\mathrm{F}(\mathrm{ab})_{2^{-}}$and $\mathrm{FAB}$-fragments, which showed a much faster blood clearance and extensive kidney uptake compared to the mAbs. Due to the high and specific binding to prostate cancer cells and their high

uptake in PSMA-positive tumors the mAbs 3/A12, 3/F11 and 3/E7 but not the tested antibody fragments represent excellent candidates for prostate cancer imaging and a high potential for radioimmunotherapy, where our future studies will focus on.

\section{J845}

QUANTITATION OF SODIUM IODIDE SYMPORTER (NIS) EXPRESSION IN XENOGRAFT TUMOR MODELS VIA IMMUNOBLOTTING AND SPECT/CT IMAGING

Fangbing Liu ${ }^{1}$, Elaine P. Lunsford ${ }^{1}$, Robert Lenkinski ${ }^{2}$, John V. Frangioni ${ }^{1,2}$

${ }^{1}$ Medicine, Beth Israel Deaconess Medical Center, Boston, MA, USA, ${ }^{2}$ Radiology, Beth Israel Deaconess Medical Center, Boston, MA, USA.

Background: Although the sodium iodide symporter (NIS) has been used for tracking tumor and stem cells, quantitation of NIS expression and sodium pertechnetate uptake in the xenograft tumors has not yet been documented. To validate the NIS model system, this study was aimed at quantifying Tc-99m uptake as a function of NIS expression in xenograft tumors. Materials and Methods: The full length cDNA of human NIS was stably transfected into the human prostate cancer cell line PC-3 using linearized plasmid or retroviral vector. Athymic nu/nu mice were subcutaneously inoculated with both stable NIS expressing cells (on one flank) and non-expressing control cells (on the other flank). Tc-99m pertechnetate was injected intravenously after tumors reached 0.2 to $0.5 \mathrm{~cm}$ in diameter, approximately 2 to 3 weeks postinoculation. Imaging was performed using SPECT/CT (Bioscan NanoSPECT/CT) using varying doses $(250 \mu \mathrm{Ci}, 500 \mu \mathrm{Ci}$ and $1000 \mu \mathrm{Ci})$ of Tc-99m and varying clearance time $(1 \mathrm{~h}, 2 \mathrm{~h}$ and $4 \mathrm{~h})$. Tc-99m uptake in the tumors was compared with the level of NIS expression in xenograft tumors by Western blot using a NIS-specific monoclonal antibody. Results: Stable NIS-expressing prostate cancer cell lines were obtained by both methods. Visible xenograft tumors derived from stable NIS expressing cells formed in the nude mice 2 to 3 weeks postinoculation. The level of NIS expression in xenograft tumors was measured by Western blot, and NIS activity in the tumor cells was quantified in vivo as well as with well counting of resected tissues. Parameters for SPECT/CT imaging of xenograft tumors were optimized. Exogenous NIS expressed in xenograft tumors retained the same specificity and sensitivity of the endogenous NIS expressed in thyroid and stomach. Conclusions: Ectopic NIS delivery permits noninvasive in vivo cancer cell tracking using SPECT/CT imaging. We have now defined the key parameters required for optimal Tc-99m uptake in xenograft tumors, as well as the expression level of NIS required for imaging other animal model systems. 


\section{J846}

\section{VISUALIZATION OF SPIO LABELED ANTIGEN-SPECIFIC HUMAN CYTOTOXIC NATURAL KILLER CELLS IN PROSTATE CANCER WITH MR IMAGING}

Reinhard Meier ${ }^{1,2}$, Daniel Golovko ${ }^{1}$, Sidhartha Tavri ${ }^{1}$, Christiane Knopp $^{4}$, Tobias D. Henning ${ }^{1,2}$, Guido Piontek ${ }^{3}$, Martina Rudelius ${ }^{3}$, Ernst J. Rummeny ${ }^{2}$, Winfried S. Wels ${ }^{4}$, Heike Daldrup-Link ${ }^{1}$

${ }^{1}$ Radiology, University of California San Francisco, San Francisco, CA, USA, ${ }^{2}$ Radiology, Technische Universität München, Munich, Germany, ${ }^{3}$ Pathology, Technische Universität München, Munich, Germany, ${ }^{4}$ Chemotherapeutisches Forschungsinstitut, Georg-SpeyerHaus, Frankfurt, Germany.

Purpose: to label the human natural killer (NK) cell line NK-92 and genetically engineered $\mathrm{NK}-92-\mathrm{scFv}(\mathrm{MOC} 31)-\zeta$ cells with superparamagnetic iron oxides (SPIO) for subsequent in vivo tracking to prostate cancer with magnetic resonance imaging (MRI). Methods: NK-92-scFv(MOC31)- $\zeta$ cells were genetically modified to express a chimeric antigen receptor specific to the tumor-associated EpCAM (epithelial cell adhesion molecule) antigen. NK-92 and NK-92-scFv (MOC31)- $\zeta$ cells were labeled with the FDA-approved SPIO Ferumoxides (Feridex, Berlex) using simple incubation and transfection (protamine and lipofectin) techniques. Labeling efficiencies were evaluated with MRI and spectrometry. Cell-surface expression of the chimeric antigen receptor $\mathrm{scFv}(\mathrm{MOC} 31)-\zeta$ and the specificity of NK cell killing was determined by fluorescence activated cell sorter (FACS) analysis and a FACS-based cell killing experiment. Subsequently, SPIO labeled NK-92 and NK-92-scFv(MOC31)- $\zeta$ cells were intravenously injected into twelve rats with subcutaneously implanted EpCAM positive DU145 prostate tumors. T2-weighted MR scans were obtained before, at $1 \mathrm{~h}$ and $24 \mathrm{~h}$ after cell injection (p.i.). The MR signal intensity of the tumors was quantified as $\Delta$ SI (\%) and correlated with immunohistochemistry. Results: NK-92 and NK-92-scFv(MOC31)- $\zeta$ cells could be efficiently labeled with Ferumoxides. The labeling efficiency was highest after transfection with lipofectin. Flow cytometry analysis of chimeric antigen receptor expression on the NK cells showed that unlabeled NK cells stained not significantly different than labeled NK cells. The antigen-specific cytolytic activity of the NK cells was preserved following labeling. After intravenous injection of $15 \times 106$ Ferumoxides-labeled NK-92$\operatorname{scFv}(\mathrm{MOC} 31)-\zeta$ cells into tumor-bearing rats, MR showed a significant signal decline of EPCAM-positive prostate cancers at $1 \mathrm{~h}$ and $24 \mathrm{~h}$ p.i.. The injection of $15 \times 106$ Ferumoxides-labeled parental NK-92 cells did not cause significant signal intensity changes of the tumors. Immunohistochemistry confirmed an accumulation of the genetically engineered, EPCAM targeted, but not the non-targeted NK cells in prostate cancers. Conclusion: NK-92-cells can be directed against EpCAM tumor antigens by genetic modification and can be efficiently labeled with SPIO without affecting the antigen-specific cytolytic activity. The accumulation of these labeled NK cells in EpCAM positive prostate tumors can be monitored with MRI.

\section{J847}

EVALUATION OF A NEW TARGETED FLUORESCENT PROBE IN HUMAN PRIMARY OVARIAN CANCER MODELS FOR MONITORING TUMOR GROWTH AND THERAPEUTIC RESPONSE

Tracy W. Liu, Jocelyn Stewart, Juan Chen, Lili Ding, Benjamin Neel, Gang Zheng

Medical Biophysics, University of Toronto/ Ontario Cancer Institute, Toronto, ON, Canada.
Ovarian cancer is the most frequent cause of death from gynecological cancer. It is characterized by few early symptoms, presents at advanced stages and has a poor overall survival. Thus, greater than $70 \%$ of patients are diagnosed with advanced stages (Stage III/IV) of this disease which has an associated 5-year survival rate of less than $30 \%$. Conversely, the 5-year survival rate of patients diagnosed with stage I ovarian cancer is greater than $90 \%$. The relationship between the stage at presentation and survival rate in ovarian cancer indicates a need to improve detection of early stage disease. Folate receptor (FR) targeted diagnostics and therapeutics have gained momentum in the past decade as FR is highly expressed in a variety of epithelial cancers particularly ovarian cancer $(\sim 90 \%, 30$ fold). Over $70 \%$ of newly diagnosed ovarian cancer overexpress FR. We have constructed a folate-peptide-fluorophore (PPF) that has shown preferential accumulation in tumour tissues with high folate receptor specificity and delivery efficiency. Many FR targeted therapeutics have had poor biodistribution due to clearance of the conjugates by the hepatobiliary system leading to accumulation in the gastrointestinal tract. However, PPF shows a favorable biodistribution profile despite this clearance pathway. Promising preliminary data indicates the selectivity capabilities, the sensitivity and therapeutic potential of PPF in detecting and treating primary human ovarian cancer in a SCID mouse xenograft model. With the targeting capabilities and sensitivity of PPF in detecting ovarian cancer, PPF is becoming a useful tool for in vivo detection of ovarian tumour growth and potentially early detection of this gynecological cancer may become clinically feasibly. In addition, PPF's capabilities may aid in surgical debulking of ovarian cancers by its fluorescenceimage guidance.

\section{J848}

\section{NOVEL NONLINEAR CONTRAST IMAGING TECHNIQUE USING HIGH FREQUENCY ULTRASOUND}

Tonya Coulthard, David Bates, Andrew Needles, Catherine Theodoropoulos, F. Stuart Foster

VisualSonics Inc., Toronto, ON, Canada.

MicroMarker contrast agents have been used with high frequency ultrasound to noninvasively enhance the visualization of the microvasculature in various tissues, organs and diseases. Previously contrast agent imaging had been completed using reference subtracted fundamental imaging using linear imaging. Recently nonlinear contrast agent imaging was implemented on the Vevo 2100, a linear array high frequency ultrasound system. This is a breakthrough in contrast agent imaging as amplitude modulation has been used to allow for nonlinear imaging at frequencies as high as $24 \mathrm{MHz}$ something not previously possible by a commercially available ultrasound system. Nonlinear contrast imaging is a substantial improvement over previous imaging techniques as it increases the signal to noise ratio by suppressing the tissue signal and increasing the sensitivity to MicroMarker contrast agents providing 10 to 30 fold times improvement in contrast imaging over linear techniques depending on the target tissue being imaged. Imaging the microvasculature is important for many different research areas; MicroMarker contrast agents can simply be used to visualize blood flow but can also be used to detect the expression of certain endothelial cell surface markers using Target-Ready MicroMarker contrast agents. This technique will be a valuable tool to assess flow in many organs \& tissues incl; the kidney, hind limb and tumors. E.g. an enhancement in contrast power was found to be $15 \mathrm{~dB}$ following contrast injection in the kidney while the time to peak following a bolus injection in the same organ was found to be approx. $2.5 \mathrm{~s}$. in the cortex and a $5 \mathrm{~s}$. delay in the medulla to about $5 \mathrm{~s}$. In addition the potential to examine the expression of 
biomarkers such as VEGFR2 and P-Selectin in vivo and in real-time suggests a significant breakthrough for understanding molecular processes in animal models. E.g. in a tumor model the VEGFR2 specific signal was found to be $300 \%$ higher than found for the negative control signal. Nonlinear contrast imaging can be completed in both $2 \mathrm{D} \& /$ or $3 \mathrm{D}$, for both the untargeted agent where blood flow is enhanced \&/or when using the targeted contrast agent. Quantification of nonlinear contrast imaging provides relative values of relative blood volume, relative blood velocity and in the case of targeted imaging relative expression of the specific molecular target. All imaging is done noninvasively while the contrast agent is injected intravenously so that the same animal can be used throughout the course of a study to enhance the power of the generated data.

\section{J849}

\section{DETECTION OF ABDOMINAL TUMOURS IN MOUSE MODELS} BY PET-MR IMAGING

Andreas Schmid $^{1}$, Heidi Braumüller ${ }^{2}$, Nadine Bauer ${ }^{2}$, Daniel Bukala ${ }^{1}$, Damaris Kukuk ${ }^{1}$, Hans F. Wehrl ${ }^{1}$, Walter Ehrlichmann ${ }^{3}$, Gerald Reischl $^{3}$, Roland Haubner ${ }^{4}$, Stefan Wiehr ${ }^{1}$, Bernd J. Pichler ${ }^{1}$

${ }^{1}$ Laboratory for Preclinical Imaging and Imaging Technologies of the Werner Siemens-Foundation, University of Tuebingen, Tuebingen, Germany, ${ }^{2}$ Department of Dermatology, Eberhard Karls University, Tuebingen, Germany, ${ }^{3}$ Department of Radiopharmacy, Eberhard Karls University, Tuebingen, Germany, ${ }^{4}$ Department of Nuclear Medicine, Universitiy of Innsbruck, Innsbruck, Austria.

Approximately every 5 th death of cancer in humans is caused by abdominal cancer and the 5-year relative survival rate of e.g. pancreatic cancer is only approx. 5\%. Therefore, abdominal tumour detection in mouse models in vivo is of major interest as it allows longitudinal studies to develop novel therapy strategies. Furthermore, imaging of the pancreas is of high interest in research on diabetes (beta-cell imaging). This work focuses on pancreatic cancer detection using PET-MR imaging in the Rip1-Tag2 mouse model, where tumour development starts at the age of 6 weeks. The mice die from invasive carcinomas leading to hypoglycaemia at an age of approximately 15 weeks. Aiming at therapy monitoring, we investigated several different approaches on visualizing tumour development in this mouse model using PET-MR imaging. We compared MRI protocols for best tumour delineation and used anatomical MR scans in combination with small animal PET to evaluate different tracers such as [F-18]FDG, [F-18]FLT, [Cu-64] RGD, [Ga-68]RGD and [Cu-64]labelled Th1-cells. Relaxationparameter (T1-, T2-) maps were measured and tumour burden was monitored using MRI. Respiratory gated MR protocols allow detection of the pancreatic tissue und segmentation from other organs in vivo. Evaluation of the T2- maps revealed significant higher values in spin-spin relaxation (T2) times in tumours (T2: $57 \pm 10 \mathrm{~ms}$ ) than in normal pancreatic tissue (T2: $37 \pm 7 \mathrm{~ms}$ ), while evaluation of T1-maps did not lead to successful tumour delineation. PET imaging with [F-18]FDG nor [F-18] FLT did not show specific tracer uptake in pancreatic tumour lesions. As RIP1-Tag2 mice are an ideal model for studying tumour angiogenesis, we used angiogenesis marker [Cu-64]RGD for activated integrin $\alpha v \beta 3$. However, the PET images show high non-specific uptake in gut and kidneys, so that the uptake in the tumours cannot be distinguished from the overall background. Higher uptake was seen with [Ga-68]RGD in tumours compared to healthy pancreatic tissue in PET and was verified by autoradiography. [Cu-64] labelled Th1-cells showed enrichment in tumour environment but cannot be used to identify and to localize tumours itself. Several approaches have been investigated to identify pancreatic tumours in vivo. In contrast to other tumours the gold standard in PET imaging ([F-18]FDG) fails. Ex vivo results using radiolabeled RGD peptides could be verified in vivo but are still under investigation. Further studies will include radiolabelled tumor antigen specific antibodies for PET imaging.

\section{$\mathbf{J 8 5 0}$}

\section{MicroPET IMAGING OF GENE TRANSFER IN A PULMONARY ANIMAL MODEL USING A ${ }^{64} \mathrm{Cu}$-Labeled SOMATOSTATIN ANALOG}

Jesse J. Parry $^{1}$, Thaddeus J. Wadas ${ }^{2}$, James Kozlowski ${ }^{2}$, Christopher D. Sherman $^{2}$, Steven Brody ${ }^{3}$, Carolyn Anderson ${ }^{2}$, Buck Rogers ${ }^{1}$

${ }^{1}$ Radiation Oncology, Washington University School of Medicine, St. Louis, MO, USA, ${ }^{2}$ Mallinckrodt Institute of Radiology, Washington University School of Medicine, St. Louis, MO, USA, ${ }^{3}$ Internal Medicine, Washington University School of Medicine, St. Louis, MO, USA.

Introduction. Gene therapy has been proposed for the treatment of pulmonary edema and acute lung injury by over expression of $\beta$ receptors and has shown to be effective for fluid clearance in both mice and rats. However, the extent of gene transfer can be a limiting factor in therapeutic outcomes. Therefore, a non-invasive method to assess the efficiency of gene transfer may increase the overall therapeutic efficacy. In this project, we evaluated human somatostatin receptor subtype 2 (SSTR2) as a reporter gene for non-invasively monitoring gene expression in an animal model such that it could be combined with a therapeutic gene in a single vector. $</ \mathrm{P}>$ Methods. AdSSTR2 was administered intratracheally either at $2 \times 10^{10}$ or $2 \times 10^{11}$ Viral particles (VP)/animal. This was accomplished by insertion of a 16 gauge $\times 2$ inch angiocatheter, which had been shortened $\sim 1 \mathrm{~cm}$, into the trachea of anesthetized $200 \mathrm{~g}$ Sprague Dawley rats. The virus was mixed 1:1 with surfactant in a total volume of $800 \mu \mathrm{l}$. The virus was instilled as four $200 \mu \mathrm{l}$ aliquots, 5 minutes apart, and rotating the animals 90 degrees after each. The animals recovered for three days, then $0.74 \mathrm{MBq}$ of ${ }^{64} \mathrm{Cu}-\mathrm{CB}-\mathrm{TE} 2 \mathrm{~A}-\mathrm{Y} 3-\mathrm{TATE}$ was intravenously injected into the rats. For biodistribution studies, tissues were harvested $4 \mathrm{~h}$ post-injection for radionuclide determination. For microPET imaging, images were acquired at both 1 and $4 \mathrm{~h}$ after the administration of $10 \mathrm{MBq}$ of ${ }^{64} \mathrm{Cu}-\mathrm{CB}-\mathrm{TE} 2 \mathrm{~A}-\mathrm{Y} 3-\mathrm{TATE}$. $</ \mathrm{P}>$ Results and Discussion. Lung uptake of radiotracer was found to be $0.81 \% \mathrm{ID} / \mathrm{g}$ and $0.83 \% \mathrm{ID} / \mathrm{g}$ for $2 \times 10^{10} \mathrm{VP} / \mathrm{animal}$ and $2 \times 10^{11} \mathrm{VP} /$ animal, respectively in biodistribution assays. This was significantly greater than lungs that received no virus $(0.13 \%$ $\mathrm{ID} / \mathrm{g}$ ). Blood and muscle uptake were $0.02 \% \mathrm{ID} / \mathrm{g}$ and $0.01 \% \mathrm{ID} / \mathrm{g}$, respectively. MicroPET imaging of the rat lungs showed high levels uptake and with uniform distribution of the radiotracer at 1 and $4 \mathrm{~h}$. Standard Uptake Value (SUV) analysis of the microPET images showed significantly greater uptake of tracer in AdSSTR2-administered rats compared to saline-treated control rats. SUV were $0.76 \%$ $\mathrm{ID} / \mathrm{cc}$ and $0.64 \% \mathrm{ID} / \mathrm{cc}$ for 1 and $4 \mathrm{~h}$, respectively, versus $0.05 \% \mathrm{ID} /$ $\mathrm{cc}$ and $0.04 \% \mathrm{ID} / \mathrm{cc}$ for controls. $</ \mathrm{P}>$ Conclusions. AdSSTR 2 delivery to the lungs of rats results in significant localized and sustained expression of SSTR2 as determined by biodistribution and microPET imaging studies. This suggests a strategy for the use of a SSTR2 reporter for monitoring markers of acute lung injury and protection of such injury using a combined reporter gene and gene therapy vector. 
J851

\section{SECRETORY GAUSSIA LUCIFERASE (SGLUC)-MONOMERIC RED FLUORESCENCE PROTEIN (mRFP)-TRUNCATED HERPES SIMPLEX VIRUS}

THYMIDINE KINASE (tTK) TRIPLE FUSION IMPROVES INTRACELLULAR LUCIFERASE ACTIVITY AND ENHANCES ITS IMAGING APPLICATIONS IN SMALL ANIMALS

Ramasamy Paulmurugan ${ }^{1,2}$, Ataya Sathirachinda ${ }^{2}$, Byeong Cheol

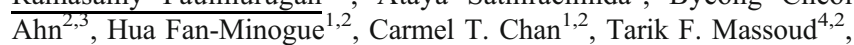
Sanjiv S. Gambhir ${ }^{1,2}$

${ }^{1}$ Radiology and Bioengineering, the Bio-X Program, Stanford University, Stanford, CA, USA, ${ }^{2}$ Molecular Imaging Program at Stanford (MIPS), Stanford University, Stanford, CA, USA, ${ }^{3}$ School of Medicine, Kyungpook National University, Daegu, Korea, South, ${ }^{4}$ Radiology, University of Cambridge, Cambridge, United Kingdom.

Gaussia luciferase, a bioluminescent photoprotein that uses coelenterazine as its substrate, has potential as a reporter gene for different biological applications. In this study we report that the 16-amino acids (1-16) at the $\mathrm{N}$-terminus of this protein are not only important for the secretory nature of this protein, but are also critical for its rapid maturation and proper functionality. We constructed a series of vectors expressing GLUC fusions and N-terminal truncation/substitution (see Figure) to prove the importance of the secretory peptide, and also to improve its intracellular retention for small animal imaging. The 293T, SKBr3 and CHO cells transfected with these constructs were assayed for GLUC-activity immediately after lysis and, at 2, 5, 12, 24, 48, 72 and $120 \mathrm{hrs}$ post-lysis. The results showed significant improvement in the intracellular activity only from the sGLUC fused to mRFP-tTK (10-fold). The cells transfected with tGLUC, tGLUCGFP and TK-Leader-tGLUC showed minimal intracellular activity when assayed immediately after lysis $(<1.0 \%$ of sGLUC), the signal increased over time and stabilized at $72 \mathrm{~h}$. The activity by tGLUC measured at $72 \mathrm{~h}$ was $120 \pm 10$-fold higher than the initial activity and was $\sim 80 \%$ of the sGLUC activity. Tumor xenografts of $293 \mathrm{~T}$ cells stably expressing tGLUC-GFP, sGLUC-GFP and sGLUC-mRFP-tTK in mice $(N=11)$ were imaged for GLUC, GFP and mRFP signals over time. The imaging results showed no GLUC-signals from the cells transfected with tGLUC-GFP, but showed significant level of fused GFP signal $(p<0.001)$. The sGLUC with C-terminal fusions showed a constant intracellular GLUC-signal over time, indicating the importance of the secretory peptide for GLUCmaturation. The enhanced fluorescent signal from GFP and RFP fused to the GLUC, than the corresponding fusions with other bioluminescent reporters, indicates its minimal steric hindrance. In conclusion, we have successfully developed a novel triple fusion that enhances imaging intracellular GLUC protein in small animals.

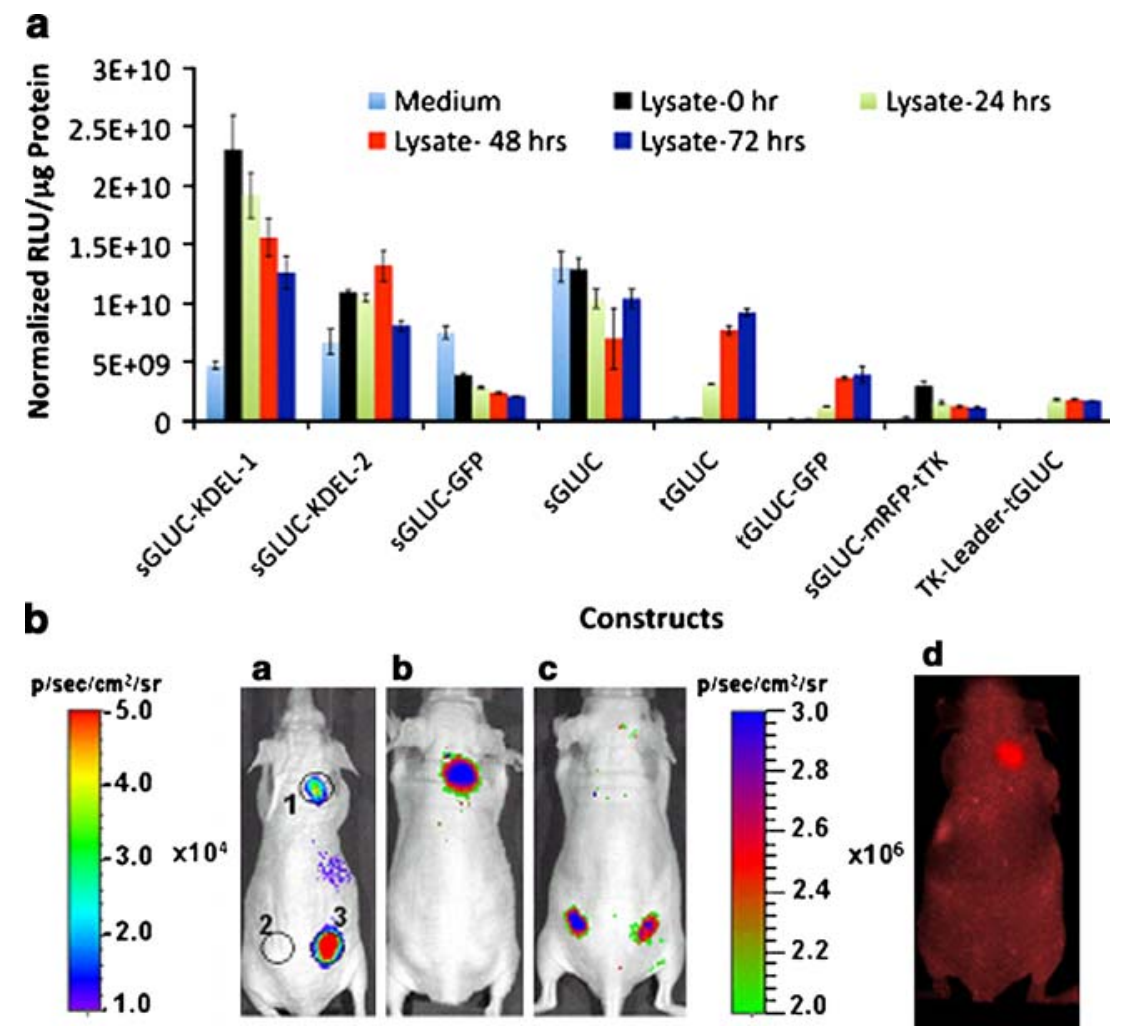

$\mathbf{J 8 5 2}$

EVALUATION OF A NEURO CT SCANNER FOR USE IN A PET/CT SYSTEM FOR PRIMATE IMAGING Charles M. Laymon ${ }^{1}$, Brian J. Lopresti ${ }^{1}$, M. T. Coleman ${ }^{1}$, Joanne L. Flynn ${ }^{2}$, Lonnie J. Frye ${ }^{3}$, James M. Mountz ${ }^{1}$, Darunee Whitt ${ }^{4}$, Jonathan Carney ${ }^{1}$

${ }^{1}$ Radiology, University of Pittsburgh, Pittsburgh, PA, USA, ${ }^{2}$ Molecular Genetics and Biochemistry, University of Pittsburgh, Pittsburgh, PA, USA, ${ }^{3}$ Center for Vaccine Research, University of Pittsburgh, Pittsburgh, PA, USA, ${ }^{4}$ Radiation Safety, University of Pittsburgh, Pittsburgh, PA, USA.
Preclinical large animal imaging, i.e. animals of size intermediate between rodents and humans, provides important information related to a variety of research projects, for example, oncologic and infectious disease studies. We report on the adaptation of a portable neuro CT unit, the CereTom, for this purpose. The scanner's light weight and mobility simplify its use in an animal imaging lab. Additionally, its lack of an integrated bed facilitates its incorporation into a multimodality PET/CT system. The scanner can operate in helical mode, enabling contrast enhanced scans, as well as in axial mode for high resolution studies. Imaging characteristics were measured in both modes using the American College of Radiology (ACR) CT phantom spanning the CT 
operating range (100-140 kV and 1-7 mA). Dosimetry measurements using a Perspex $16 \mathrm{~cm}$ (head) phantom and $10 \mathrm{~cm}$ ionization chamber were performed. In helical mode at $140 \mathrm{KV}, 7 \mathrm{~mA}$, and an axial scan rate of $1 \mathrm{~cm}$ per second, 6-mm cylinders differing from background by $6 \mathrm{HU}$ (ACR phantom module 2) were resolved in the resulting $1.8-\mathrm{mm}$ slices. Dosimetry measurements at $140 \mathrm{kV}$ yield a weighted CT dose index (CTDIw) of $4.07 \mathrm{mGy} / \mathrm{mAs}$. We thus expect a CTDIw value of 29 mGy for the helical scan parameters described above, a dose similar to that using full size scanners in a clinical setting. The scanner has been integrated into a nonhuman primate PET/CT system and used for several applications. Contrast enhanced images of macaque monkeys have been acquired in which good definition of the vascular system is observed. Studies of anesthetized, ventilated monkeys have also been performed in which a forced breath hold has been used to eliminate breathing-motion artifacts. We conclude that the imaging characteristics of the CereTom coupled with the advantages of small size, portability, and integration potential make the unit a valuable preclinical imaging tool.

\section{$\mathbf{J 8 5 3}$}

IN VIVO MOLECULAR IMAGING APPLICATIONS OF VOLUME PHOTOACOUSTIC TOMOGRAPHY FOR SMALL ANIMALS

Timothy Morgan ${ }^{1}$, Robert Kruger ${ }^{2}$, Paul Picot ${ }^{1}$, Keith Stantz ${ }^{3}$

${ }^{1}$ Endra, Inc., Boston, MA, USA, ${ }^{2}$ OptoSonics, Inc., Oriental, NC, USA,

${ }^{3}$ Purdue University, West Lafayette, IN, USA.

Photoacoustic tomography combines the sensitivity of optical imaging with the resolution of ultrasound, providing a three-dimensional map of molecular or physiological data. Recent technical advances in photoacoustics are enabling practical applications in molecular imaging. We have developed a second generation, volume photoacoustic tomography system, with nanomolar sensitivity and spatial resolution of $280 \mu \mathrm{m}$, at depths greater than $20 \mathrm{~mm}$, for quantitative molecular imaging in small animals. The spatially resolved concentrations of spectrally distinct chromophores can be determined through the use of multispectral imaging, enabling quantification of endogenous contrast (hemoglobin and oxygenation) and exogenous contrast (blood pool agents and molecularly tagged agents). Over the past five years we have routinely used photoacoustic imaging to study cancer and polycystic kidney-diseased animal models including tissue intraheterogeneity and labeled antibodies. In this study we will briefly describe the second generation scanner used in this study and the workflow involved in obtaining photoacoustic data. Minimal animal preparation is required and volumetric scan times are as short as one minute, or typically less than $25 \mathrm{~min}$ for full spectroscopy (14 wavelengths between $690-940 \mathrm{~nm}$ ). We demonstrate applications using endogenous contrast to measure the local concentrations of deoxygenated and oxygenated hemoglobin, providing a quantitative, non-invasive method to track hypoxia in tumors. We use the exogenous contrast agent ICG for blood flow measurements. In addition, we use HER2 antibodies labeled with Licor IRDye800CW to investigate tumor progression. This novel molecular imaging tool is routinely used in our laboratory for a variety of disease and physiology studies without the need for nuclide labeled molecules or specialized siting requirements.

\section{J854}

\section{DYNAMIC NEAR-INFRARED OPTICAL IMAGING OF 2-DEOXYGLUCOSE UPTAKE BY INTRACRANIAL TUMORS OF ATHYMIC MICE}

Heling Zhou, Li Liu, Kate Luby-Phelps, Debabrata Saha, Ralph Mason, Dawen Zhao

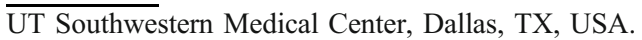

It is well recognized that cancer cells exhibit highly elevated glucose metabolism and up-regulated glucose transporters compared to nontumor cells. On this basis ${ }^{18} \mathrm{FDG}$, the glucose analogue, has been used as the most common PET radiotracer to visualize clinical tumors and their metastases. We have recently applied in vivo optical imaging to studying dynamic uptake of a near-infrared dye labeled 2-DG (IRDye800CW 2-DG, Li-Cor Bioscience) by brain tumors in orthotopic mouse xenografts. The orthotopic brain tumor model was established by surgically implanting human glioma U87-luc cells or breast cancer MDA-MB-231-luc cells directly into the right caudal nuclear region of a nude mouse. Intracranial tumor growth was monitored longitudinally by both bioluminescence imaging (BLI) and MRI. When tumor size reached $>5 \mathrm{~mm}$ diameter, in vivo fluorescence imaging of IRDye $800 \mathrm{CW} 2$-DG was performed. A series of real-time whole body images acquired immediately after i.v. infusion clearly visualized the near-infrared dye circulating into various internal organs sequentially, which validated a capability of the 2-DG dye as a probe to image deep-seated organs or tumors and also provides useful information on the first pass perfusion. Dynamic fluorescent imaging of mouse brain was then performed at different time points. Higher signal intensity in the brain region, but lack of contrast was found between the left (normal) and right (tumor) brain during the first $4 \mathrm{~h}$ after injection. However, significantly higher signal intensity was clearly seen in the tumor side of the brain than the contralateral normal side $24 \mathrm{~h}$ after injection (tumor/normal ratio $4.1 \pm 1.1$ ). In contrast, a control dye, IRDye800carboxyl, showed little difference (ratio $1.4 \pm 0.1$ ). These observations may suggest an optimal timing for imaging glucose uptake in clinical brain tumors, which are found to be sometimes indistinct from a high background signal during $18 \mathrm{FDG}$ PET, which is necessarily undertaken early due to the short half life of fluorine-18. After in vivo imaging at $24 \mathrm{~h}$, the mice brains were dissected and ex vivo fluorescence imaging performed on whole brain cryosections revealed distinct tumor margins. Moreover, microscopic fluorescence imaging identified cytoplasmic locations of the 2-DG dye in tumor cells. These results further suggest that the near-infrared dye labeled 2-DG may serve as a useful fluorescence imaging probe to assist gross resection of clinical brain tumors. Acknowledgements: Supported by DOD IDEA Award W81XWH-08-1-0583 and SAIRP U24 CA126608.

\section{$\mathbf{J 8 5 5}$}

\section{EARLY DETECTION OF HUMAN BREAST CANCER MCF-7} USING ANTI-EPCAM ANTIBODY CONJUGATED WITH CY5.5

Dao Chao Huang, Marilyse Piché, Muriel Jean-Jacques, Guobin Ma, Mario Khayat

ART Advanced Research Technologies, Inc., Montreal, QC, Canada.

Epithelial cell adhesion molecule (EpCAM), one of the first tumorassociated antigens identified, is overexpressed in a wide range of tumors including breast carcinomas, urothelial carcinomas, pancreatic carcinomas, and hepatocellular carcinomas. Mouse-derived mAbs directed to EpCAM have been successfully used in patients in small cell lung cancer diagnosis and also for adjuvant treatment of minimal residual disease of colon carcinoma. Thus EpCAM is a potential target for various cancer therapies as well as treatment monitoring. MCF-7 is a human breast cancer cell line being used in many pre-clinical assessment of drug and therapy development. In this study, we present our preliminary results of detecting MCF-7 cancer cells in mouse model by fluorescent imaging techniques using mouse-derived $\mathrm{mAb}$ conjugated with Cy5.5 directed to human EpCAM. During the experiment, MCF-7 breast cancer cells were labeled with anti-EpCAM PerCP-Cy5.5. Fluorescence-Activated Cell Sorting (FACS) analyses indicated that MCF-7 cells expressed EpCAM by over 90\%. To access 
the quality of the EpCAM expression, MCF-7 cells were stained with anti-EpCAM PerCP-Cy5.5, together with wheat germ agglutinin antibody-FITC to membrane, and Hoechst to nuclear staining. Confocal fluorescence microscope analyses showed that MCF-7 expressed EpCAM strongly in the cellular membrane and cytoplasm. These results confirmed the specificity of anti-EpCAM antibody to MCF-7 breast cancer cells. In our in vivo study, breast tumor growth in $\mathrm{nu} / \mathrm{nu}$ mice following subcutaneous injection of MCF-7 cells was imaged using Optix, a small animal fluorescent molecular imager. One week following tumor implantation, mice were tail vein injected $200 \mathrm{ng}$ of antiEpCAM PerCP-Cy5.5. Then the mice were imaged at many time points post injection. At $20 \mathrm{~h}$ post injection, strong fluorescence signal were detected in MCF-7 tumor region. Fluorescence lifetime analysis confirmed that the signal was from anti-EpCAM antibody-Cy5.5. It was also observed that the antibody was cleared out 7 days after the injection. These results demonstrated that using anti-EpCAM-Cy5.5 we can specifically and sensitively image MCF-7 tumor in the early stage by Optix. This opens the door for us to effectively monitor the treatment efficacy of breast tumor as well as other noninvasive in vivo tumor studies.

\section{J856}

\section{WHOLE-BODY FLUORESCENCE LIFETIME IMAGING OF HUMAN MELANOMA METASTASES IN MICE USING GREEN FLUORESCENCE PROTEIN (GFP)}

Dao Chao Huang, Marilyse Piché, Muriel Jean-Jacques, Guobin Ma, Mario Khayat

ART Advanced Research Technologies, Inc., Montreal, QC, Canada.

Although bioluminescence can be used to monitor tumor size, it is suitable mainly for cases where expression is limited to the tumor cells. Fluorescence optical imaging systems have the additional capacity to provide functional information about the biology of the tumor potentially providing more sensitivity for earlier detection. In this study, nude mice injected into the left cardiac ventricle with A375 human melanoma cells usually develop whole body metastases including liver, lung, osteolytic lesions within 2-3 weeks. We then used a time-domain in vivo small animal fluorescence molecular system (Optix) to study the cancer cell dissemination to different organs by GFP. A375 human melanoma cells expressing GFP were established following stable transfection of GFP driven by a CMV promoter. In order to access the detection limit of Optix, two calibration experiments were done before hand. First, different numbers of A375 cells expressing GFP were implanted subcutaneously in several mice and imaged using Optix. Preliminary quantitative analysis indicated that the minimal cell number of detectable GFP signal was 2000 cells in vitro and in vivo subcutaneous. Second, several small pieces of GFP expressing tumor tissue have been implanted in liver with different depth of live mice. Then the mice were imaged and the recovered volumes were examined. Optix eliminates non specific fluorescence signals by performing time resolved fluorescence measurements. As a result, the system can provide fluorescence lifetime, which is used to distinguish fluorophores with similar emission spectra according to their fluorescence lifetimes. In this experiment, fluorescence measurements by Optix were done at timed intervals following intracardiac administration of cancer cells. Our analysis indicated that GFP-expressed cancer cells could be detected at early time in bone, liver, lung of live animals. As predicted, the size of the lesions determined by the fluorescent signals increased progressively with time and correlated with histomorphometric analysis of tumor volume. These results indicate that, using time-domain fluorescence technology, the detection of tumor cells within bone, in liver and lung using GFP is both specific and sensitive and that the signals correlate well with tumor volumes. This provides a tool for longitudinal analysis of whole body metastasis noninvasive optical imaging in live animal, especially treatment monitoring which will be addressed as our next step.

\section{J857}

\section{AN INDUCIBLE NEUROGENESIS REPORTER MODEL FOR OPTICAL IMAGING}

Sebastien Couillard-Despres ${ }^{1,3}$, Jingzhong Zhang ${ }^{2}$, Katrin Stadler ${ }^{3}$, Wolfgang Wurst ${ }^{2}$, Ludwig Aigner ${ }^{1}$, Daniela Vogt-Weisenhorn ${ }^{2}$

${ }^{1}$ Institute for Molecular Regenerative Medicine, Paracelsus Medical University, Salzburg, Austria, ${ }^{2}$ Institute for Developmental Genetics, Helmholtz Zentrum Muenchen, National Research Center for Environmental Health, Munich, Germany, ${ }^{3}$ Clinic and Polyclinic for Neurology, University of Regensburg, Regensburg, Germany.

Doublecortin (DCX) is a microtubule-associated protein whose expression gets specifically induced upon the neuronal determination of multipotent neural progenitors. DCX expression remains in neuronal precursors and young neurons until cells acquire mature neuronal features, such as extensive synaptic contacts or expression of the marker NeuN. Hence, the expression profile of DCX is ideal to detect the generation of new neurons, i.e. a good marker of neurogenesis. We previously used the promoter sequences of DCX to drive expression of reporter genes in various transgenic models and demonstrated that it was possible to detect and monitor neurogenesis in vivo using optical imaging techniques (Couillard-Despres et al. Eur J Neurosci 2006; CouillardDespres et al. Mol Imaging 2008). In the current work, we developed a next generation reporter model allowing for an inducible and thus timeresolved optical imaging of neurogenesis. We placed the expression of the CreERT2 gene under the control of the DCX promoter (DCX-promoCreERT2). CreERT2 is a form of Cre recombinase requiring the administration of tamoxifen to be active and excise genomic fragments located between lox sequences. Mating this DCX-promo-CreERT2 mouse model with a second line of transgenic animal bearing lox-STOP-loxreporter genes enables for the specific and permanent activation of reporter expression in neuronal precursors/young neurons and their progeny. This new tool makes possible to pulse-label newly generated neurons in a timeresolved fashion and allows for the long-term analysis of their fate. Histological characterization of the CreERT2 activation pattern within newly generated neurons as been performed using a LacZ reporter and also using reporter for in vivo optical imaging. We believe that the DCX-promoCreERT2 reporter system will provide a more sensitive and powerful tool to monitor neurogenesis in vivo via optical imaging techniques.

\section{J858}

\section{A NOVEL PEPTIDE-PHOSPHOLIPID SCAFFOLD NANOCARRIER FOR TARGETED CANCER IMAGING}

Zhihong Zhang ${ }^{1,2}$, Juan Chen ${ }^{1}$, Lili Ding ${ }^{1}$, Honglin Jin ${ }^{1,2}$, Ian Corbin ${ }^{1}$, Weiguo Cao ${ }^{1}$, Mi Yang ${ }^{1}$, Qingming Luo ${ }^{2}$, Gang Zheng ${ }^{1}$

${ }^{1}$ Biophysics, University of Toronto, Toronto, ON, Canada, ${ }^{2}$ Biomedical photonics, Huazhong University of Science and Technology, Wuhan, China.

Naturally occurring lipoproteins are attractive as nanocarrier due to their high payload capacity, exquisite nano-size control and receptor recognition. However, the need to isolate apolipoproteins from fresh donor plasma has limited its application. Recently we created the HDLlike peptide-phospholipid scaffold (HPPS), a sub-30 nm core-shell lipid nanoparticle for targeted delivery of cancer imaging agents. HPPS was formulated with phospholipids, cholesterol oleate, cargo, and apopA-I mimic peptide. By conjugating with tumor-homing ligands, HPPS can selectively target cancer cells through specific surface receptors. For proof-of-concept, HPPS was core-loaded with near-infrared fluorescent probe DIR-BOA, and conjugated with epidermal growth factor (EGF) for targeting cancer cells overexpressing EGF receptor (EGFR). The receptor-specific uptake of the resulting nanoparticle, EGF(DIR-BOA) HPPS, was preformed on EGFR positive(e.g. KB) and EGFR negative (e.g. H520) cells using confocal imaging and flow cytometry. In vivo imaging was preformed on nude mice bearing dual tumor (KB and H520) using 
Maestro system. Confocal imaging showed high uptake of EGF(DIR-BOA) HPPS in EGFR positive cells and this uptake was significantly inhibited by adding excess EGF. In vivo optical imaging and tissue biodistribution further confirmed the targeting specificity of EGF(DIR-BOA)HPPS to EGFR positive tumors. In conclusion, EGF-HPPS particles are a novel class of lipid-based nanocarriers featuring precise size-control and offer excellent targeting efficacy for selective delivery of cancer diagnostics.

\section{J859}

\section{ACCUMULATION AND DISTRIBUTION OF IRON OXIDE LABELED STEM CELLS IN PROSTATE CANCER MODELS IN MICE}

Lyubov Ostrovskaya, Jiangyang Zhang, Dmitri Artemov

The Johns Hopkins University, Baltimore, MD, USA.

We compared, with in vivo and ex vivo MRI, accumulation of Iron Oxide labeled mesenchymal stem cells (MSCs) administered systemically in mouse tail vein in two prostate cancer models: fast growing rat MatLyLu and slow growing human PC3 tumors. With in vivo MRI and immunohistological staining, we demonstrated homing of intravenously injected stem cells to the vasculature of developing tumors. The rate of label accumulation in fast growing MatLyLu tumors was significantly higher than in slow growing PC3 tumors. Ex vivo MRI did not show difference in the amount of accumulated labeled cells, however, label distribution was different. In PC3 tumors, the label concentrated mostly on tumor periphery, while in MatLyLu tumors the labeled cells were located in the central regions of the tumor, presumably, in hypoxic areas. Different MSCs homing patterns between fast and slow growing tumors can be explained by recruitment of circulating stem cells to growing neovasculature. Dilution of the label during tumor growth can explain similar total accumulation of the label in both models determined quantitatively by ex vivo MR microscopic imaging.

\section{$\mathbf{J 8 6 0}$}

TUMOR GROWTH CHARACTERIZATION IN A RAT BONE CANCER PAIN MODEL USING MRI-PET COREGISTRATION Louis Dore-Savard $^{1}$, Luc Tremblay ${ }^{2,3}$, Karine Belleville ${ }^{1}$, Melanie Archambault $^{2,3}$, Jean-François Beaudoin ${ }^{2,3}$, Nicolas Beaudet ${ }^{1}$, Roger Lecomte $^{2,3}$, Philippe Sarret ${ }^{1}$, Martin D. Lepage ${ }^{2,3}$

${ }^{1}$ Physiology and biophysics, Universite de Sherbrooke, Sherbrooke, QC, Canada, ${ }^{2}$ Radiobiology and Nuclear Medicine, Universite de Sherbrooke, Sherbrooke, QC, Canada, ${ }^{3}$ Sherbrooke Molecular Imaging Center, universite de Sherbrooke, Sherbrooke, QC, Canada.

Half of the patients suffering from cancer cannot find relief with existing analgesic treatments. Severe cancer pain is typically induced by bone metastases involved in bone remodelling and nerve infiltration or compression. Despite many undesirable side effects, the use of analgesics relieving inflammation and neuropathy associated pain, such as nonsteroidal anti-inflammatory drugs or opiates, has not evolved significantly over the last decades. We have developed and characterized a bone cancer model to evaluate the analgesic potential of new pharmacological treatments. The primary aim of this study was to correlate pain development observed by behavioral tests during bone cancer growth with tumorinduced changes in bone morphology and molecular characteristics determined by MRI and PET imaging. Bone cancer was induced by the injection of syngenic malignant mammary carcinoma MRMT-1 cells in the medullar space of the femur of Sprague-Dawley rats. Behavioral indications of pain were evaluated over a 3-week period. Meanwhile, bone tumor development and structural damage to the femur were monitored by MRI ( $\mathrm{T}_{1}$-weighted images, Gd-DTPA $)$ and PET $\left(\mathrm{Na}^{18} \mathrm{~F}\right.$ and $\left.\left[{ }^{18} \mathrm{~F}\right] \mathrm{FDG}\right)$ at different times following cancer cells inoculation. PET scans were performed immediately following MRI acquisitions while the animal remained under anaesthesia in a home-built hind limbs positioning device. At 14 days following surgery, a significant reduction in the response threshold of hind paw withdrawal to von Frey stimulations and a decreased weight bearing on the affected limb were detected. Accordingly, MR images showed progressive damage to the bone environment from day 10 post-inoculation. Indeed, the tumor was readily detectable in the MR images by a relative increase in voxel intensities in the inoculated region. Evidence of tumor development was observed at later stage with PET and correlates exactly with MR in the coregistered images. Moreover, $\mathrm{Na}^{18} \mathrm{~F}$ distribution was highly modified compared to the contralateral hind limb in the tumor bearing bone indicating active remodelling in labelled zones and necrosis in unlabelled areas. In this study, MRI-PET coregistration was used to characterize tumor metabolism and growth in our model of bone cancer pain. The bimodal imaging procedure is expected to be useful in the complete evaluation of the analgesic potential of new pharmacological treatments, enabling early detection of cancer and close correlation between tumor growth, changes in bone morphology, metabolism and behavioral data.

\section{J861}

\section{LASER GUIDED GENOMIC ANALYSIS OF TISSUE} RESPONSE TO LASER INDUCED THERMAL STRESS

Mark A. Mackanos, Mike W. Helms, Christopher Contag

Pediatrics, Stanford University, Stanford, CA, USA.

Induction of heat shock protein (Hsp) expression correlates with a cytoprotective effect in cultured cells and with improved healing of damaged tissues in animal models and in humans. This family of proteins can also serve as indicators of thermal stress in cases of bun injury or surgical procedures that produce heat. Thus, a rapid in vivo readout for the induction of Hsp transcription would facilitate studies of Hsp genes and their encoded proteins as mediators of therapeutic effects and as reporters of thermal damage to tissues. A transgenic reporter mouse where the expression of luciferase is controlled by the regulatory region of the inducible $70 \mathrm{kDa} H s p$ (Hsp70A1) was produced in our lab, and was used to assess activation of Hsp70 transcription in live animals in response to rapid, high temperature stresses using in vivo bioluminescence imaging (BLI). This model noninvasively reveals levels of Hsp70 transcription in living tissues, and has utility in studies of the predictive and protective effects of Hsp70 expression, and of various responses to tissues. We determined the expression levels of Hsp70 after laser irradiation with a CO2 laser at 10.6 um in wavelength on the skin. Murine tissues were heated with pulse durations between 1 and $1000 \mathrm{~ms}$ with a $6 \mathrm{~mm}$ diameter flat-top beam. The thermal response was measured with BLI between 7 and 96 hours. The thermal response to $\mathrm{CO} 2$ laser stress was then used to perform microarray and RT-PCR analysis to identify the up-regulated genes involved in the thermal damaged laser region of the skin and was compared to the adjacent laser region. A fluence of $14.86 \mathrm{~J} / \mathrm{cm} 2$ provided a region of central damage, an adjacent region of upregulation, and a distant control region with no damage. Microarray analyses of these regions revealed that the laser treated region differed from the adjacent region in gene expression patterns with more genes decreased in the laser treated region. There were a number of gene increased after thermal stress and we selected 17 genes with a 10-fold increase of expression for comparison by RT-PCR analysis. Consistent with need to recruit cells to the damaged region, the chemokine CXCL3 was dramatically increased after thermal stress. The promoter of the CXCL3 gene may serve as an excellent indicator of cellular stress, survival, collateral damage, and wound healing.

\section{J862}

ULTRASMALL NEAR-INFRARED NON-CADMIUM QUANTUM DOTS FOR TUMOR IMAGING

Jinhao Gao ${ }^{1}$, Kai Chen ${ }^{1}$, Renguo Xie ${ }^{2}$, Xiaogang Peng ${ }^{2}$, Sanjiv S. Gambhir $^{1}$, Xiaoyuan Chen ${ }^{1}$

${ }^{1}$ Radiology, Stanford University, Stanford, CA, USA, ${ }^{2}$ Chemistry and Biochemistry, University of Arkansas, FayetteVille, AR, USA. 
Here we described the high tumor-uptake of ultrasmall near-infrared (NIR) quantum dots (QDs) because of the enhanced permeability and retention (EPR) effect. InAs/InP/ZnSe QDs coated by mercaptopropionic acid (MPA) exhibited the emission wavelength at about $800 \mathrm{~nm}$ (QD800-MPA) with very small hydrodynamic diameter $(\sim 10 \mathrm{~nm})$. Using 22B and LS174T tumors as the models, in vivo and ex vivo imaging showed that QD800-MPA was highly accumulated in the tumor area (Figure 1). The semi-quantitative analysis of region of interest (ROI) indicated that the tumor-uptake was about $13.5 \% \mathrm{ID} / \mathrm{g}$, which is very promising for the tumor detection in living mice. The ex vivo elemental analysis (Indium) using inductively coupled plasma (ICP) spectrometry confirmed the tumor-uptake of QDs. The ICP data are consistent with the in vivo and ex vivo fluorescence imaging. Human serum albumin (HSA) coated QD800MPA nanoparticles (QD800-MPA-HSA) showed lower localization in mononuclear phagocytic system (MPS)-related organs than QD800MPA plausibly due to the low uptake of QD800-MPA-HSA in macrophage cells. QD800-MPA-HSA may have great potential for in vivo fluorescence imaging.

a

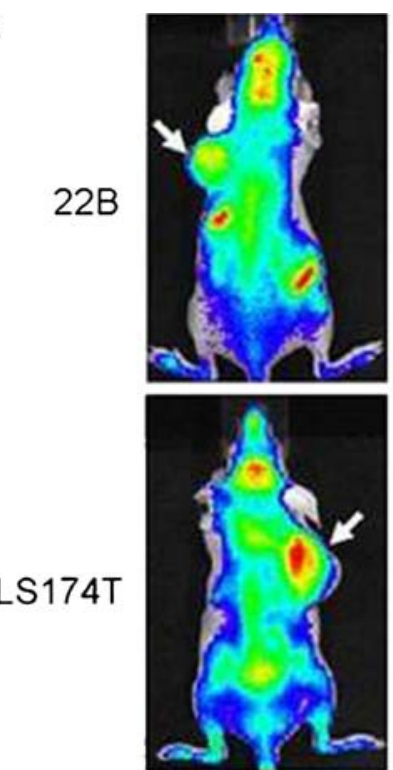

$1 \mathrm{~h}$

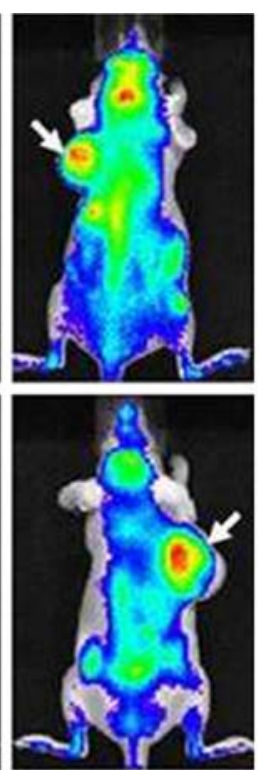

$4 \mathrm{~h}$ b

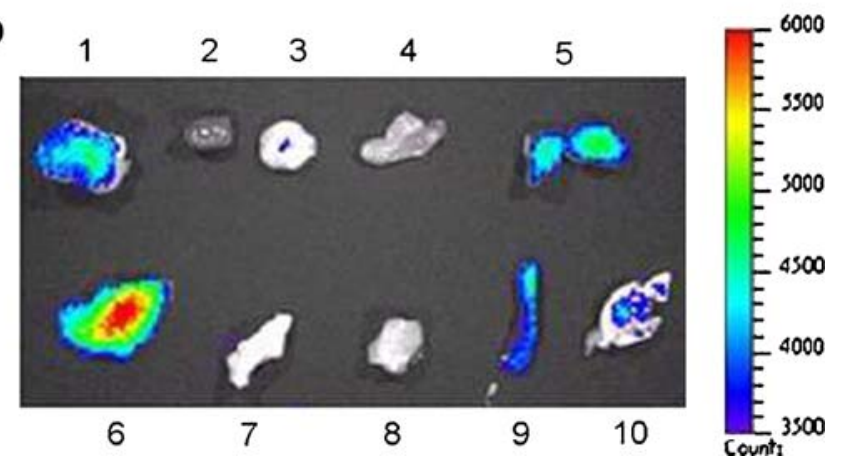

Figure 1. In vivo and ex vivo fluorescence imaging by the tail vein injection of QD800-MPA. (A) In vivo NIR fluorescence imaging of 22B tumor-bearing mice and LS174T tumor-bearing mice (arrows) at $1 \mathrm{~h}$ and $4 \mathrm{~h}$. (B) Representative ex vivo imaging at $4 \mathrm{~h}$ injection. 1, 22B tumor; 2 , heart; 3 , pancreas; 4 , intestine; 5 , kidney; 6 , liver; 7 , skin; 8 , muscle; 9, spleen; 10, lung.
J863

MICROPET STUDY OF GLOBAL CB1 CANNABINOID
RECEPTOR DISTRIBUTION IN MICE FED WITH DIETS
DIFFERING IN FAT CONTENT Olga Millan $^{1}$, Dolors de la Fuente ${ }^{1}$, Deborah Pareto ${ }^{1}$, Laura España ${ }^{1}$, Raul Herance ${ }^{1}$, Anna Berini ${ }^{1}$, Blanca Rubi ${ }^{2}$, Juan D. Gispert ${ }^{1}$, Rafael Maldonado ${ }^{2}$

${ }^{1}$ Institut d'Alta Tecnologia - CRC (IAT-PRBB), Barcelona, Spain, ${ }^{2}$ Dept. Ciències Experimentals i de la Salut, Universitat Pompeu Fabra, Barcelona, Spain.

The involvement of the endocannabinoid system in energy control has made the specific role of the $\mathrm{CB} 1$ cannabinoid receptor to be object of great interest in the pathophysiology of obesity. To further elucidate the mechanisms underlying this disease, we aimed to study changes in the distribution of CB1 cannabinoid receptors in mice exposed to three diets differing in fat content (normal, high-fat isocaloric or hypercaloric), and the consequences of returning to normal diet. We also aimed to correlate the changes on the distribution of $\mathrm{CB} 1$ receptors observed in vivo with the values obtained for the main metabolic parametres. To achieve these goals, microPET imaging with [11C]JHU75528 was used for the longitudinal noninvasive external detection of the distribution of CB1 receptors in vivo. Mice were imaged under basal conditions; after one, four and sixteen weeks of diet exposure, and after four weeks of re-exposure to normal diet. At the same time points, blood samples were taken and glucose, triglyceride, insulin, glucagon and amylin were measured in blood or plasma. As expected, a significant enhancement on body weight was only observed in mice exposed to hypercaloric diet in comparison to normal diet. MicroPET data showed a significant increase in tracer uptake associated to diet exposure in all the tissues analyzed. A correlation between weight and tracer uptake was also observed in these tissues. Interestingly, the tracer uptake pattern of brown fat was dependant on the diet. Thus, mice under normal and hypercaloric diets showed an increase in tracer uptake during the whole experimental sequence, whereas mice exposed to a high-fat isocaloric diet showed a decrease after diet withdrawal. A similar pattern of tracer uptake was revealed on the liver and the skeletal muscle of mice exposed to the different diets. This distribution pattern was not observed in subcutaneous fat, whose tracer uptake was also increased after withdrawal of the high-fat isocaloric diet. This study suggests the existence of compensatory effects on CB1 cannabinoid receptor distribution in those tissues involved in metabolic balance after withdrawal of a high-fat isocaloric diet.

\section{J864}

REAL-TIME FDG-PET IMAGING OF TUMOR RESPONSE TO DIFFERENT PHOTODYNAMIC THERAPY PROTOCOLS Nicole Cauchon, Roger Lecomte, Johan E. van LIER

Département de médecine nucléaire et radiobiologie, Université de Sherbrooke, Sherbrooke, QC, Canada.

Objectives: Photodynamic therapy (PDT) requires three basic elements, a photosensitizer, oxygen and light, to destroy tumor cells. Dynamic FDG-PET was used to monitor transient metabolic processes in realtime during tumor treatment by PDT. The effect of three photosensitizers and two different illumination protocols were investigated. Methods: Mice bearing two mammary tumors ( $\sim 7 \mathrm{~mm}$ diam) received 1 $\mu \mathrm{mole} / \mathrm{kg}$ of three different zinc sulfophthalocyanine-based photosensitizers: $\mathrm{ZnPcS} 2, \mathrm{ZnPcS} 4$ and $\mathrm{ZnPcS} 3 \mathrm{C} 6$. The following day, the animals were positioned in the Sherbrooke small-animal PET scanner and continuously infused with [18F]-FDG while dynamic images were acquired for $3 \mathrm{~h}$. Thirty min after starting FDG infusion, one tumor was exposed to red light $(680 \mathrm{~nm})$ from a laser diode using two light delivery protocols $(30 \mathrm{~min}$ continuous light or $40 \mathrm{~min}$ fractionated light $-5 \mathrm{~min}$ on, $2 \mathrm{~min}$ off). Images were reconstructed as $2 \mathrm{~min}$ frames 
and tumor, myocardium and blood time-activity curves (TACs) were extracted by ROI analysis. Results: FDG TACs during PDT showed distinct transient patterns. Variations in tumor uptake rate and response delay revealed tumor and systemic metabolic response processes that correlate with differences in mechanism of action between the drugs. For $\mathrm{ZnPcS} 2$ and $\mathrm{ZnPcS} 3 \mathrm{C} 6$, TACs were characterized by an initial drop and subsequent recovery of tumor FDG uptake rates. No drop in FDG uptake was detected with $\mathrm{ZnPcS4}$. At the end of treatment, continuous light delivery induced a reduction in FDG uptake by the treated tumor for all three drugs tested. Fractionated light treatment caused no change in FDG uptake pattern in the case of $\mathrm{ZnPcS} 2$. A higher drop in FDG tumor uptake was observed during and following ZnPcS3C6-PDT. However, surprisingly, FDG tumor uptake rates during and following ZnPcS4-PDT increased with the fractionated light protocol. Moreover, myocardial FDG TACs showed a reduction in cardiac activity immediately after continuous $\mathrm{ZnPcS} 2-$ or ZnPcS4-PDT, which correlated with elevated animal mortality. The fractionated light protocol stabilized the cardiac activity during these treatments and increased viability. In contrast, ZnPcS3C6-PDT did not induce any mortality or change in cardiac FDG uptake. Conclusion: Real-time tumor response to PDT in mice, as monitored by dynamic FDG-PET, demonstrates differences in tumor response mechanisms between different drugs and light delivery protocols. In addition, changes in cardiac FDG uptake rates could serve as an indicator of toxic sideeffects of the drug or cancer treatment protocol.

\section{$\mathbf{J 8 6 5}$}

\section{GOLD NANOTAGS FOR DUAL-MODALITY IMAGING OF CANCER}

Ming Xiao ${ }^{1,3}$, James O. Nyagilo ${ }^{4}$, Michael Long ${ }^{1}$, Daxing Xie ${ }^{5}$, JerTsong Hsieh ${ }^{5}$, Dongsheng $\mathrm{Xu}^{3}$, Digant P. Davé ${ }^{4}$, Xiankai Sun ${ }^{1,2}$ ${ }^{1}$ Department of Radiology, University of Texas, Southwestern Medical Center at Dallas, Dallas, TX, USA, ${ }^{2}$ Advanced Imaging Research Center, University of Texas, Southwestern Medical Center at Dallas, Dallas, TX, USA, ${ }^{3}$ College of Chemistry and Molecular Engineering, Peking University, Beijing, China, ${ }^{4}$ Department of Bioengineering, University of Texas at Arlington, Arlington, TX, USA, ${ }^{5}$ Department of Urology, University of Texas, Southwestern Medical Center at Dallas, Dallas, TX, USA.

Gold nanoparticles (AuNPs) have been well documented as surface enhanced Raman scattering (SERS) active substrates with potentials for various biomedical applications, and recently reported as X-ray based computed tomography (CT) contrast agents. Given the biocompatibility and tunable surface of AuNPs, we designed and synthesized AuNPbased Raman nanotags (AuNTs) as dual modality imaging agents, which would enable anatomical imaging of $\mathrm{CT}$ and cell surface receptor mapping via SERS on a single nanoplatform. The AuNTs were synthesized by attaching molecules of a Raman dye to the surface of AuNPs and then a monolayer of polyethylene glycol (PEG). The AuNPs and AuNTs were characterized by transmission electron microscope (TEM), dynamic light scattering (DLS), UV-Vis spectra, and SERS. The in vitro and in vivo evaluations of the AuNTs were carried out using monodispersed AuNPs of 40-nm diameter in rat serum and an orthotopic prostate cancer xenograft mouse model, respectively. TEM images before and after the AuNPs surface modification clearly showed the presence of a PEG layer, and the encapsulation of the Raman dye was confirmed by the characteristic Raman peaks on the SERS spectra. The AuNTs stayed intact up to 3 days in rat serum as determined by TEM. Comparing with Iohexol (Omnipaque $^{\mathrm{TM}}$, GE Healthcare), the AuNTs exhibited 30\% higher CT attenuation (Hounsfield units) at the same concentrations. Nude mice bearing PC-3 tumors were scanned before and after the intraveneous injection of $100 \mu \mathrm{L}$ of $12.5 \mathrm{mg} / \mathrm{mL}$ AuNTs, and FDG-PET/CT scans were performed to visualize the distal and localized prostate tumors.
Aided by AuNTs, tumors were revealed by CT at $24 \mathrm{~h}$ post-injection, which was in agreement with the FDG-PET/CT findings and ex vivo SERS. Ex vivo TEM images of tumor, normal liver, and normal spleen tissues demonstrated that most of the AuNTs were accumulated in endosomes and stayed intact. In summary, the AuNTs have shown potential as a nanoplatform to enable CT and SERS simultaneously for cancer detection. Acknowledgements: This work was partially supported by the National Science Foundation of China (20673008) and the USAMRMC Prostate Cancer Research Program (W81XWH-05-1-0592).

\section{J866}

\section{DETERMINATION OF NASAL RESIDENCE TIME USING A NOVEL LIVE FLUORESCENT IMAGING TECHNIQUE; THE EFFECT OF TRIMETHYL CHITOSAN ON NASAL \\ CLEARANCE RATE}

Bram Slütter $^{2}$, Ivo Que ${ }^{1}$, Niels Hagenaars ${ }^{3}$, Eric Kaijzel ${ }^{1}$, Clemens Lowik $^{1}$, Wim Jiskoot ${ }^{2}$

${ }^{1}$ Endocrinology, LUMC, Leiden, Netherlands, ${ }^{2}$ Division of Drug Delivery Technology, LACDR, Leiden, Netherlands, ${ }^{3}$ Department of Pharmaceutics, Utrecht Institute of Pharmaceutical Sciences, Utrecht University, Utrecht, Netherlands.

Introduction. Nasal administration of vaccines holds great promise, as it may ameliorate the use of needles and can increase the efficacy of the vaccine. The nasal epithelium is easily accessible, has relatively low enzymatic activity and is capable of eliciting systemic as well as local immune responses. Nonetheless, only one nasal vaccine is currently on

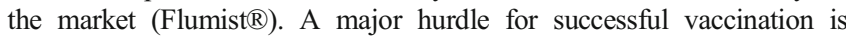
the relatively short residence time of the vaccine formulation in the nasal cavity, which hampers efficient uptake of the antigen through the nasal epithelium. Increasing the residence time of the antigen in the nasal cavity is therefore an interesting approach to improve nasal vaccine efficiency. Methods. Here we introduce a near-infrared fluorescent (NIRF) method to determine the nasal residence time of vaccines. A NIRF-probe (IRdye ${ }^{\mathrm{TM}} 800 \mathrm{CW}$, LI-Cor, USA) was covalently linked to a model antigen, ovalbumin (OVA). After nasal administration of the antigen to immune-deficient mice, the fluorescence intensity in the nasal cavity was assessed using an IVIS Spectrum ${ }^{\circledR}$ (CaliperLS, USA) and followed in time.In an attempt to increase the residence time, next to OVA alone, three different nanoparticulate vaccine formulations were included, to investigate the effect of the carrier on the nasal clearance rate. Results. We observed an exponential decay of fluorescence intensity allowing calculation of the nasal clearance rate. Trimethyl chitosan (TMC) nanoparticles significantly decrease nasal clearance rate. Interestingly, TMC nanoparticles also proved to be the most effective formulation in a vaccination study, using the same formulations. Conclusion. Using a live imaging technique we successfully studied the nasal residence time of vaccines. TMC nanoparticles are longer retained by nasal epithelium and may therefore be useful for nasal vaccination.

\section{$\mathbf{J 8 6 7}$}

USE OF FLUORESCENT LABELED EPIDERMAL GROWTH FACTOR TO IN VIVO IMAGE HEPATOCELLULAR CARCINOMA XENOGRAFTS

Kezheng Wang ${ }^{1}$, Baozhong Shen ${ }^{1}$, Tao Huang ${ }^{1}$, Xiaoyuan Chen ${ }^{2}$

${ }^{1}$ The medical imaging and nuclear medicine department, Harbin Medical University, Harbin, China, ${ }^{2}$ Department of Radiology, Bio-X \& Biophysics, Stanford University School of Medicine, Stanford, CA, USA.

Abstract Purpose The aim of this study was to investigate whether epidermal growth factor (EGF) labeled with Cy5.5 can specifically image EGF receptor (EGFR) in hepatocellular carcinoma (HCC) 
xenografts using near-infrared (NIR) imaging. Methods A fluorochrome probe was designed by coupling Cy5.5 to EGF peptide through acidylation. The probe's optical quality was determined by ultraviolet assay and flow cytometry was used to testify probe binding specificity. HepG2 cell line cellular membrane EGFR expression was qualified by immunofluorescence and the cells were transfected with lentivirus vectors expressing enhanced green fluorescence protein (GFP) gene. In vivo GFP fluorescent imaging of mice transplanted HepG2 tumors was executed to monitor tumor development. In vivo NIR imaging was performed after intravenous injection of $0.2 \mathrm{nmol}$ probes, with and without cetuximab preadministered for blocking. The fluorescence in tumor tissue frozen sections was examined under confocal microscopy and EGFR expression was analyzed by immunohistochemical assay. Results The excitation/emission wavelengths for the EGF-Cy5.5 conjugate and Cy5.5 were 674/697 nm and 674/712 nm, respectively. Flow cytometry confirmed the specificity of the probe to EGFR. EGFR expression in HepG2 cells was confirmed by immunofluorescence. Monitoring of the time-fluorescence intensity in mice and tissue frozen sections indicated that EGF-Cy5.5 accumulated in HepG2 tumors. Moreover, tumor uptake of EGF-Cy5.5 was blocked by cetuximab. Tissue immunohistochemistry staining indicated HepG2 had highly EGFR expression (3+). Conclusion Our data suggest EGF-Cy5.5 may be used as a specific NIR contrast agent for noninvasive imaging of EGFR expression in hepatocellular carcinoma xenograft mice models. Key Words: Near-infrared optical imaging; epidermal growth factor receptor (EGFR); Hepatocellular carcinoma;

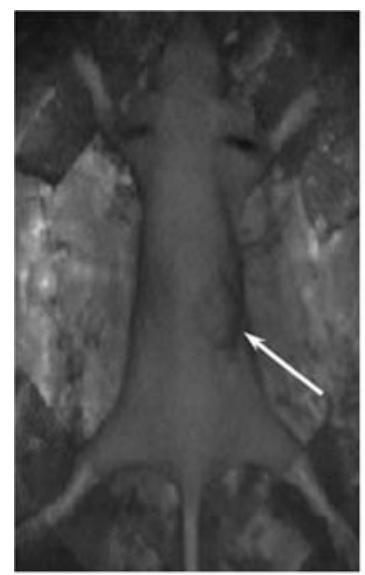

Before Injection

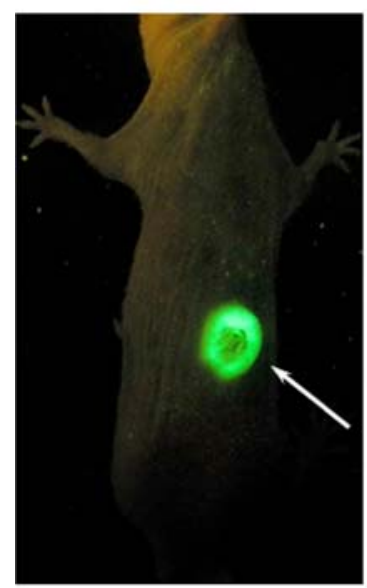

GFP Imaging

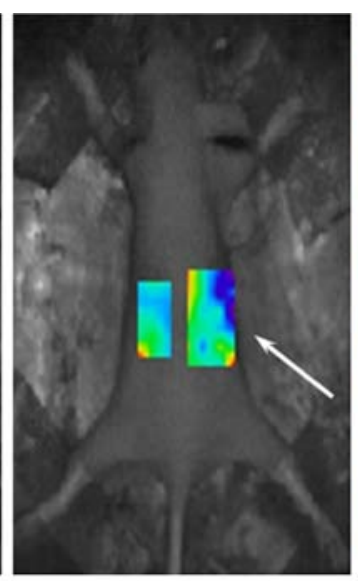

Localization Phase

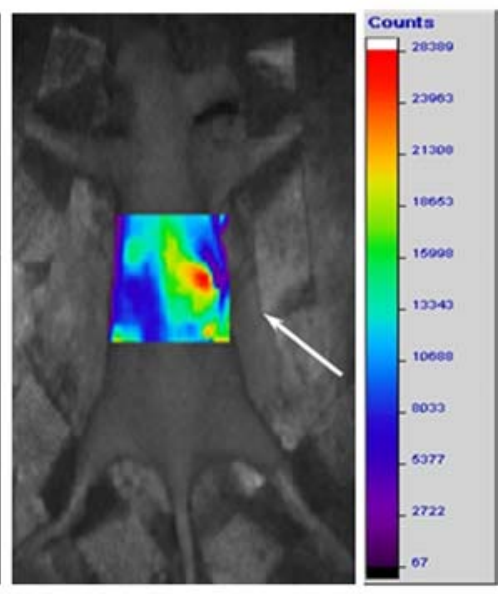

Postinjection 4h

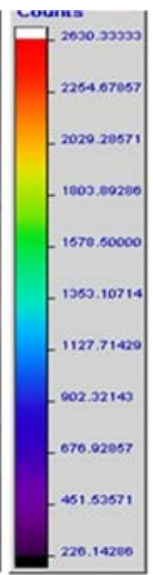

In vivo NIR and GFP fluorescence imaging of the mice bearing HepG2 hepatocellular carcinoma.

\section{J868}

EVALUATING MURINE INFLAMMATION IN AN ACUTE LUNG INJURY MODEL BY FLUORESCENCE TOMOGRAPHY AND MICRO-CT

Lynnette M. Gerhold ${ }^{1}$, Saad Sammani ${ }^{2}$, Biji Mathew ${ }^{2}$, Christian Wietholt $^{2}$, Lilana Moreno-Vinasco ${ }^{2}$, Patrick J. La Riviere ${ }^{3}$, Jeffrey S. Souris $^{3}$, Jeffrey Jacobson ${ }^{2}$, Charles Pelizzari ${ }^{4}$, Joe G. Garcia ${ }^{2,5}$, Chin-tu $\mathrm{Chen}^{3}$

${ }^{1}$ Optical Imaging Core Facility, The University of Chicago, Chicago, IL, USA, ${ }^{2}$ Medicine, The University of Chicago, Chicago, IL, USA, ${ }^{3}$ Radiology, The University of Chicago, Chicago, IL, USA, ${ }^{4}$ Radiation Oncology, The University of Chicago, Chicago, IL, USA, ${ }^{5}$ Prizker School of Medicine, The University of Chicago, Chicago, IL, USA.

Understanding, comparing, and validating a variety of molecular imaging modalities is critical for a core facility to effectively service their clientele. In the growing field of in vivo imaging, it is paramount not only to reliably reproduce data but that such data also confers with histology and other ex vivo methods. We compared two imaging modalities: the VisEn Fluorescence Molecular Tomography 1 system (FMT1) and the micro-CT option of the GE Triumph tri-modality imaging system in a lipopolysaccarride (LPS) -induced acute lung injury (ALI) model. The FMT1 has the ability to image in vivo targeted near-infrared fluorescent probes in the picomolar concentration range tomographically while the micro-CT provides high resolution $(60 \mu)$ anatomical detail of lung parenchyma and density changes that arise from vascular leakage. In these studies, we challenged C57BL/6 mice (8 wks old males) with intratracheal LPS $(2.5 \mathrm{mg} / \mathrm{kg}$ body wt.) or vehicle (control) administration. After LPS administration, the probes MMPsense680 and Prosense750, which label enzymatically activated MMPs and cathepsins, respectfully and are also associated with inflammation were administrated simultaneously by i.v. injections $(2 \mathrm{nM}) .18 \mathrm{~h}$ post LPS challenge and probes injection, mice were imaged first with the FMT1 followed by micro CT. Mice were then euthanized post imaging and evaluated for cellular and biochemical inflammatory indices by bronchoalveolar lavage (BAL) fluid and lung tissues proteins, leukocytes, BAL albumin, and histology. Preliminary results demonstrated that lung inflammation is detectable in the LPSinduced ALI as seen by increased concentrations of fluorescent probes in the lungs when compared to control mice that did not receive LPS administration. Similarly, in the micro-CT images, LPS challenged mice exhibited regions of denser tissue when compared to control mice. LPS-mediated alterations were seen in lung histology as well as in increased concentrations of BAL protein and albumin were measured, confirming the in vivo imaging results. In conclusion, we demonstrated that in vivo imaging can reproduce and confirm results obtained by time-consuming methods such as histology and other ex vivo/post mortem techniques. Our future studies will test the effectiveness of an alveolar and vascular barrier protective agonist (sphingosine 1phosphate (S1P) receptor agonist SEW-2871), which reduces the LPS-induced ALI. Furthermore, image registration methods for fluorescence imaging and micro-CT will be developed in an effort to correlate the results from both modalities.

\section{J869}

DETECTION OF AGE-RELATED CEREBRAL GLUCOSE METABOLISM ALTERATION WITH PET 18FDG IN MICROCEBUS MURINUS PRIMATE Olène Dorieux $^{1,2}{ }^{\text {, Martine Guillermier }}{ }^{1}$, Albertine Dubois ${ }^{3}$, Sebastien Jan $^{1}$, Fabienne Aujard ${ }^{2}$, Martine Perret ${ }^{2}$, Thierry Delzescaux ${ }^{1}$, Philippe Hantraye $^{1}$, Anne-Sophie Hérard ${ }^{1}$, Marc Dhenain ${ }^{1}$

${ }^{1}$ DSV, CEA, Fontenay aux Roses, France, ${ }^{2}$ UMR 7179, CNRS, Brunoy, France, ${ }^{3}$ DSV, CEA, Orsay, France. 
Alzheimer's disease (AD) is a neurodegenerative disease associated with aging. AD' brains display with amyloid plaques and altered tau proteins within neurofibrillary tangles. Magnetic Resonance Imaging (MRI) studies allow to observe cerebral atrophy in the brain, temporal lobe and hippocampus of AD patients. In addition the basal glucose metabolism decreases in frontal, temporal and parietal cortices detected by Positrons Emission Tomography examination. Microcebus murinus $(\mathrm{Mm})$ is small $(100 \mathrm{~g})$ lemurian Primate with a life span of 12 years. During aging some animals develop cognitive impairments and histological alterations such as amyloid plaques and accumulation of altered Tau proteins. Furthermore studies by MRI highlighted brain atrophy in a subpopulation of old $\mathrm{Mm}$. So the $\mathrm{Mm}$ was suggested to be a primate model of $\mathrm{AD}$. In our study we evaluated age-related cerebral glucose metabolism impairments in $\mathrm{Mm}$ by in vivo PET. Seven female (2-8 year) were evaluated. PET scans were recorded with a MicroPET ${ }^{\circledR}$ Focus 220 system after 2-[18]-fluoro-2-deoxy-D-glucose (FDG) injection. Mm were anesthetised during the whole procedure. FDG $(900 \mu \mathrm{Curie} / 100 \mathrm{~g})$ was injected through a venous catheter. MR images were recorded in the same animals (4.7 Tesla spectrometer). Three-D images were recorded with a fast spin echo inversion recovery sequence (IRRARE; isotropic resolution $=234 \mu \mathrm{m} ; \mathrm{TR} / \mathrm{TE} / \mathrm{TEw} / \mathrm{TI}=2500 / 6 / 45 /$ $200 \mathrm{msec}$, RARE factor=16). Anatomical images we registered on TEP images by rigid transformations. Activities were evaluated in the temporal lobe, the frontal cortex, the hippocampus, and in the whole brain by drawing out volumes of interest. Activity recorded in the temporal lobe normalized by the whole brain activity showed a lower glucose uptake in old M.m. than in young animals (ANOVA, $f=7,669$; $p=0,0394$, STATVIEW). This activity decrease in the old Microcebus brains evokes metabolism impairment described in Alzheimer patients. Our study has showed that Microcebus can be followed-up by PET scans and that this clinical examination allows evaluating age-related brain metabolism impairment.

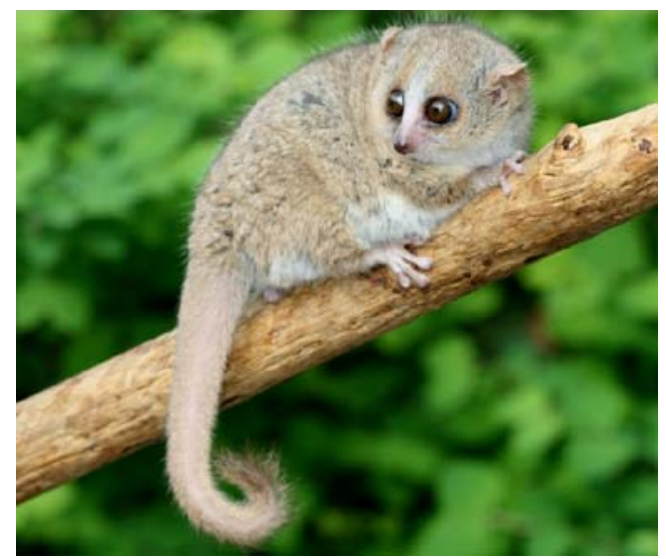

J870

\section{DYNAMIC TRACKING OF AML STEM CELLS IN VIVO}

Amanda Graham $^{1}$, Robert K. Stuart ${ }^{2}$, Amanda C. LaRue ${ }^{3}$, Michael Rosol $^{1}$

${ }^{1}$ Radiology and Radiological Science, Medical University of South Carolina, Charleston, SC, USA, ${ }^{2}$ Medicine, Medical University of South Carolina, Charleston, SC, USA, ${ }^{3}$ Pathology and Laboratory Medicine, Medical University of South Carolina, Charleston, SC, USA.

The aim of this study was to develop and use in vivo bioluminescent imaging to address key functional characteristics of acute myelog- enous leukemia (AML) stem cells from patients and normal hematopoeitic stem cells (HSC) from volunteers. We assessed AML stem cell engraftment and quantitatively compare it to normal HSC engraftment in a xenogeneic transplant model using in vivo bioluminescent imaging. Human hematopoietic stem cells (CD34 + CD38-) were isolated from both normal frozen cord blood and from peripheral blood of AML patients using modified Miltenyi Biotec (Auburn, CA) magnetic bead isolation kits. Isolated cells were transduced with a lentiviral vector (HIV-luc) pseudotyped with the VSV envelope able to express the firefly luciferase (fluc) gene in human HSC. Using a Caliper Life Sciences/Xenogen IVIS 200 optical imaging system, cells were assayed for fluc expression 48 hours after transduction. Transduced HSC and AML cells $(60,000$ each) were transplanted intravenously into sublethally irradiated adult NOD/SCID/IL2R $\gamma$ null mice and mice were imaged serially from day 1 to day 90 post transplantation. Luminescent signal from peripheral blood and marrow engraftment of cord blood HSC and AML stem cells was detectable within two weeks post-transplantation. Mice injected with AML cells showed peripheral blood enhancement, evidenced as whole body signal, while those injected with cord blood HSC demonstrated focal signal in the spine, sternum, and lower hindlimbs, as well as whole body signal representing blood. To our knowledge, we have demonstrated for the first time that AML stem cells can be efficiently labeled with a luciferase enzyme for dynamic tracking in vivo. This work has resulted in a useful animal model that will enable us to elucidate the engraftment patterns of AML cells in living animals, learn the dynamics of the interplay of different stem cell populations, and provide information on the efficacy of new treatments in the future.

\section{J871}

\section{IN VIVO PET STUDIES OF AT1R RECEPTOR EXPRESSION IN A PORCINE MODEL OF CHRONIC RENAL ARTERY STENOSIS TREATED BY STENT REVASCULARIZATION}

Nedim C. Gulaldi, Jinsong Xia, Kelvin Hong, William B. Mathews, Zsolt Szabo

Radiology, Johns Hopkins University, Baltimore, MD, USA.

Objectives: Chronic renal artery stenosis leads to hypertension and renal injury, core molecular components of its pathogenesis are the tissue hormone Angiotensin II (Ang II) and its subtype 1 receptor (AT1R). We investigated the AT1R with PET as a potential imaging biomarker of renal injury in renovascular hypertension and revascularization in a porcine model of renal artery stenosis. Methods: Six pigs underwent renal angiography followed placement of irritating coils into one of the renal arteries to induce chronic stenosis. Magnetic resonance angiography (MRAs) and PET were performed 17-21 days after coil insertion. PET imaging included a perfusion scan with O-15 water and an AT1R receptor scan with the selective radioligand C-11 KR31173. Three animals were treated by a stent placed in the stenosed artery $1-2$ days after PET imaging. These animals received a second MRA and PET scan to monitor the effect of treatment. To measure AT1R binding in vivo, cortical time activity curves were derived and analyzed with a parallel two tissue compartment model. Receptor binding was quantitatively expressed by the distribution volume of specific binding (DVsp) and by radioligand retention derived both from the time activity curves (Yret) and from the impulse response function (Fret). The hemodynamic effects of stenosis and revascularization were characterized by kidney size, degree of stenosis, contrast accumulation and the perfusion ratio obtained with O-15 water PET. Results: Radioligand retention was increased in kidneys with renal artery stenosis $(25.95 \pm 17.08 \%)$ compared to contralateral organs $(16.21 \pm 5.30 \%)$. Factor analysis revealed that $70 \%$ of the variance could be described by a single 
factor with the following rank contribution of the parameters: perfusion, contrast accumulation, kidney size, Yret, Fret, artery patency and DVsp. There was a strong negative correlation between the factor score and perfusion ratio $(R=0.908)$. Increased factor scores corresponding to AT1R upregulation were observed when revascularization treatment was complicated by thrombosis. Conclusion: Ischemia stimulates AT1R expression in the kidney triggering molecular events of chronic organ injury. The presented in vivo study suggests that treatment success or failure can be monitored at the molecular level using AT1R/PET which is therefore complimentary to anatomical imaging.

\section{Humans}

\section{J872}

\section{ADMINISTRATION OF SPIO-LABELLED HUMAN MONONUCLEAR CELLS INTO MAN: A PILOT STUDY} Jennifer M. Richards ${ }^{1}$, Catherine A. Shaw ${ }^{1}$, Ninian N. Lang ${ }^{1}$, Anne P. Atkinson $^{2}$, Kevin Dhaliwal ${ }^{3}$, Julie H. Crawford ${ }^{2,4}$, Anne Burdess ${ }^{1}$, Huw Roddie $^{2}$, Graham McKillop ${ }^{4}$, A. J. Simpson ${ }^{3}$, Thomas M. Connolly ${ }^{5}$, Giora Z. Feuerstein ${ }^{5}$, George R. Barclay ${ }^{2,4}$, Marc L. Turner ${ }^{2,4}$, David E. Newby ${ }^{1}$

${ }^{1}$ Centre of Cardiovascular Science, University of Edinburgh, Edinburgh, United Kingdom, ${ }^{2}$ Scottish National Blood Transfusion Service, SNBTS, Edinburgh, United Kingdom, ${ }^{3}$ Centre for Inflammation Research, University of Edinburgh, Edinburgh, United Kingdom, ${ }^{4}$ Department of Radiology, Royal Infirmary of Edinburgh, Edinburgh, United Kingdom, ${ }^{5}$ Translational Medicine Research Collaboration, TMRC, Dundee, United Kingdom.

Introduction: Labelling cells with super-paramagnetic iron nanoparticles (SPIO, Endorem) using protamine sulphate has been proposed. However, the translation of this method into a Good Manufacturing Practice (GMP)-compliant protocol presents many challenges that need to be overcome if cell-tracking studies are to be realised in man. Method: Up to 109 human peripheral blood mononuclear cells (HPBMCs) were isolated from $150 \mathrm{~mL}$ whole blood or $140 \mathrm{~mL}$ leucopheresis product and labelled with Endorem $(100 \mu \mathrm{g} / \mathrm{mL})$ and Protamine sulphate $(4 \mu \mathrm{g} / \mathrm{mL})$ for 3-4 hours in a GMP facility. Cell viability, determined using flow cytometry (propidium iodide/Annexin V), and the ability to migrate towards monocyte chemoattractant protein-1 (MCP-1) in a $5 \mu \mathrm{m}$ pore modified Boyden chamber were used to inform systematic modifications to the protocol. Six healthy human volunteers received a combination of intramuscular thigh injections of labelled cells (104109), unlabelled cells and Endorem alone, followed by imaging in a 1.5T MRI scanner. Two further volunteers received six increasing doses (104-109) of labelled cells intravenously over four visits, and safety was determined through clinical observation and haematological testing. Results: Cell clumping, marked cell loss and absent in vitro migratory capacity were observed when processing high cell numbers using the unmodified labelling method. Protocol modifications, including reduction of the incubation time to $2 \mathrm{~h}$, continuous agitation and avoidance of centrifugation, obviated these problems with good cell viability and in vitro MCP-1 dependent migration. Labelled cells administered intramuscularly into the thigh of a healthy volunteer were visualised in a clinical $1.5 \mathrm{~T}$ MRI scanner at a minimum threshold of 105 cells $/ \mathrm{mL}$. Intravenous administration of up to 109 labelled cells was well tolerated without evidence of clinical side-effects or derangements in coagulation. Conclusion: HPBMCs can be labelled with SPIOs under GMP compliant conditions and retain viability and migratory capacity. SPIO-labelled cells can be safely administered and imaged in vivo in man. This work was supported by an award from the Translational Medicine Research Collaboration.

\section{J873}

RETENTION INDEX FROM DUAL-TIME-POINT F-18 FDG PET/CT AS A PROGNOSTIC MARKER OF RECTAL CANCER Eun Kyung Park ${ }^{1}$, Min Ju Kim², Jae Gol Choe ${ }^{1}$

${ }^{1}$ Nuclear Medicine, Korea University Anam Hospital, Seoul, Korea, South, ${ }^{2}$ Radilogy, Korea University Anam Hospital, Seoul, Korea, South.

BACKGROUND: Dual-time-point FDG PET/CT has been shown to be useful in the differentiation between malignant and benign colorectal lesions. However, the in-depth value of FDG PET/CT in revealing biomolecular characteristics of tumor cells, other than diagnosis of malignant colorectal lesion is not well investigated. The aim of this study was to evaluate the usefulness of dual-time-point FDG PET/CT as a prognostic parameter in patients with rectal cancer. PATIENTS AND METHODS: Consecutive fifty-three patients (male/female: 33/ 20; mean age: 61.8 years; range: 34-79 years) with rectal cancer underwent FDG PET/CT for the initial staging work-up before treatment from August 2007 to February 2009. Histological confirmation was done in all patients with surgical resection. Twenty-four of these 53 patients also performed dual-time-point FDG PET/CT. On PET/CT, standardized uptake values of the rectal cancer at $1 \mathrm{hr}$ (SUV1) and $2 \mathrm{hr}$ (SUV2) following the injection of FDG were determined, and the retention index (RI) was calculated by dividing the difference between SUV2 and SUV1 by SUV1. The correlation of SUV1, SUV2, and RI was analyzed with histopathological and clinical results including pathologic $\mathrm{T}$ and $\mathrm{N}$ stages, tumor differentiation, distant metastasis and serum CEA level. RESULTS: All rectal cancer lesions demonstrated hypermetabolism both at $1 \mathrm{hr}$ and $2 \mathrm{hr}$ PET/CT images. SUV2 (mean \pm SD $14.6 \pm 5.5$ ) and SUV1 (12.1 \pm 5.7 ) was not significantly different $(p>0.05)$, however, no SUV2 was smaller than SUV1. RI was $28.7 \% \pm 13.4 \%$. RI demonstrated significant correlation with tumor differentiation and pathologic $\mathrm{T}$ staging. Well-differentiated or pathologic T1 rectal adenocarcinomas demonstrated significantly higher RI than that of moderately-differentiated or above T2 tumors $(p<0.05)$. However, RI was not significantly correlated with pathologic $\mathrm{N}$ staging and serum CEA level. SUV1 and SUV2 did not show any significant correlation with clinical and histopathological results. CONCLUSION: Retention Index demonstrated significant correlation with tumor differentiation and pathologic $\mathrm{T}$ staging, which suggests possibility of RI as an additional prognostic marker for rectal cancer.

\section{J874}

\section{"DOES THALLIUM SCAN INCREASE THE SPECIFICITY OF FDG PET-CT SCAN IN PRE AND POST TREATMENT ASSESSMENT OF MALIGNANT TUMORS?"}

Madhuri A. Shimpi, Balkundi A. Krishna

Nuclear Medicine, P.D.Hinduja National Hospital, Mumbai, India.

Aims \& Objectives: The development of imaging modalities can improve the accuracy of diagnosis, although findings based on a single modality cannot always help physicians to differentiate malignant tumors from other benign lesions. In such cases, the combined results of more than two imaging procedure are most reliable. We know that FDG positron emission tomography is more sensitive than other imaging methods \& 201 Tl SPECT imaging technique is more specific. The aim of this study was to increase 
the specificity of FDG PET-CT scan by using thallium tumor retention index- To differentiate benign from malignant lesions $\&$ tissue necrosis or fibrosis from tumor recurrence. Material and method: This is a prospective study of 27 patients (F: 10, M: 17). 22 of 27 patients had treatment for concerned malignancy in past (category I) whereas 7 were in pretreatment setting (category II). They initially underwent FDG-PET scan as per clinical indication followed by 201T1 SPECT-CT of suspicious lesions because of ambiguous findings on FDG-PET scan. For FDG-PET-CT scan; the method of semiquntitative analysis (SUV) by body weight method \& for 201Tl SPECT-CT scan early (EUR) and delay uptake ratio (DUR) were calculated. If the DUR of $201 \mathrm{Tl}$ in the lesion was increased or persistent as compared with EUR, the 201Tl SPECT was defined as positive for malignancy. Similarly we calculated EUR and DUR of known malignant sites as control in possible cases. Results: CATEGORY I: In decreasing ratio group benign lesions were found to be a fungus granuloma, reactive inflammatory changes $\&$ infectious lung nodules. In decreasing ratio but confirmed malignancy were found in scans done immediate after intervention. CATEGORY II: false positive was seen in 1 case which turned out to be a tuberculous lymphadenitis that was suspicious for lymphoma. While true negative lesions were due to tuberculous lymphadenitis and benign polyp. Interestingly, we found that 5 cases as control, all showed rising ratio. Conclusion: Our study has shown high accuracy of thallium ratios for differentiating benign from malignant lesions. Though FDG PET-CT scan is most sensitive modality at present, $201 \mathrm{Tl}$ scan as a complementary study can increase specificity by $85.7 \%$ with a sensitivity of $72 \%$, positive predictive value of $92.8 \%$ and with negative predictive value of $54.5 \%$., which we feel is a major impact of the technique in the management of patients.

Results

J875

\begin{tabular}{|c|c|c|c|c|}
\hline & \multicolumn{2}{|c|}{ category I } & \multicolumn{2}{|c|}{ categoryl } \\
\hline & 00 & 11 & 1 & 4 \\
\hline risinglconstant ratio & 4 & 5 & 2 & $\infty$ \\
\hline decreasing ratio & 4 & \multicolumn{2}{c}{} \\
\hline
\end{tabular}

\section{INCREMENTAL VALUE OF DUAL TRACER (SUBTRACTION) PROTOCOL OVER DUAL PHASE 99M TC SESTAMIBI OR 99M TC TETROFOSMIN PROTOCOL IN PARATHYROID SCINTIGRAPHY}

Parul Thakral

Dept. of Nuclear Medicine\& PET-CT, Sir Ganga Ram Hospital, New Delhi, India.

Aim: To determine whether performing a $99 \mathrm{~m}$ Tc-Pertechnetate $/ 99 \mathrm{~m} \mathrm{Tc}$ SestaMIBI or $99 \mathrm{~m}$ Tc Tetrofosmin subtraction imaging can obviate the need for performing dual phase imaging for localizing parathyroid adenoma Materials and Methods- 40 patients of clinical and biochemical findings of hyperparathyroidism had undergone parathyroid scintigraphy using 99mTc Tetrofosmin and 99m Tc Sestamibi dual phase and dual tracer subtraction protocols.Results- Out of 36 surgically diagnosed parathyroid adenomas, $99 \mathrm{mTc}$ Sestamibi/99mTc pertechnetate subtraction protocol could locate an adenoma in 34 patients while dual phase $99 \mathrm{mTc}$ sestamibi protocol was positive in only 32 patients. The sensitivity of dual tracer $99 \mathrm{~m}$ Tc Sestamibi protocol was $94.4 \%$, while that of dual phase 99mTc Sestamibi was $88.9 \%$. 99mTc Tetrofosmin/ 99m Tc pertechnetate subtraction protocol could locate adenoma in 33 patients with sensitivity of $91.7 \%$ while $99 \mathrm{mTc}$ Tetrofosmin dual phase could locate 28 adenomas with sensitivity of $77.8 \%$.Conclusion- $99 \mathrm{mTc}$-pertechnetate/ Sestamibi subtraction imaging appears be more reliable method of localizing parathyroid adenomas with the added advantage of thyroid delineation and reduction of the total time of investigation
J876

\section{OMEGA-3 FATTY ACID SUPPLEMENTATION DOES \\ NOT AFFECT BRAIN GLUCOSE UPTAKE DURING HEALTHY AGING: A PET STUDY}

Fabien Pifferi $^{1}$, Etienne Croteau ${ }^{2}$, Mélanie Fortier ${ }^{1}$, Eric Turcotte ${ }^{2}$, Stephen Cunnane ${ }^{1}$

${ }^{1}$ Research Center on Aging, Sherbrooke, QC, Canada, ${ }^{2}$ Clinical Research Center, University of Sherbrooke, Sherbrooke, QC, Canada.

Glucose is the brain's principle fuel, supplying 95\% of brain energy requirements under normal conditions. Regional changes in brain glucose uptake are usually studied using positron emission tomography (PET), with a glucose analogue-18fluorodeoxyglucose (18FDG). PET studies have shown for 20 years now that brain glucose uptake is impaired in Alzheimer's disease but also, to a lesser extent, during healthy aging. Impaired brain glucose uptake now appears to be a contributing factor to at least some types of declining brain function later in life. Omega-3 fatty acids, especially docosahexaenoic acid (DHA), are found in fish, especially marine cold water fish, but also in other edible marine organisms. DHA is a key fatty acid in mammalian brain structure and function. Animal studies on rodents and primates suggest DHA is linked to brain glucose uptake. Indeed, rats made deficient in omega-3 fatty acids have reduced brain DHA and reduced glucose uptake, which are associated with lower cognitive performance and decreased expression of brain glucose transporters (Pifferi et al 2005). In rhesus monkeys, short term DHA supplementation significantly increases regional cerebral blood flow, a parameter tightly linked to glucose uptake. Epidemiological studies in humans show that low intake of omega-3 fatty acids, mostly as fish, in the elderly is frequently, but not always, associated with an elevated risk of Alzheimer's disease (Cunnane et al 2009). Hypothesis: On the basis of these observations, we proposed the hypothesis that DHA supplementation could increase brain glucose uptake in healthy elderly patients. To assess this hypothesis, brain glucose uptake was measured by PET using $18 \mathrm{FDG}$ as tracer in young $(n=6$; mean age $=25.5 \pm$ 5.4 years old) and elderly ( $n=6$; mean age $=76.3 \pm 4.4$ years old) healthy subjects, before and after three weeks supplementation with a fish oil providing $680 \mathrm{mg} / \mathrm{d}$ DHA and $320 \mathrm{mg} / \mathrm{d}$ eicosapentaenoic acid. Oral glucose tolerance and DHA incorporation in plasma were also measured before and after supplementation. Results: Our elderly subjects have lower brain glucose uptake compared to young (-10 to $20 \%$ depending on brain region). However, DHA supplementation did not change this degree of hypometabolism, nor affect oral glucose tolerance in either group. Three weeks fish oil supplementation at about 4-5 times average intake in North America does not seem to change glucose uptake in healthy elderly compared to healthy young adults. Financial support: CIHR, CFI, Canada Research Chairs, the Department of Medicine of Sherbrooke University

\section{J877}

\section{ADDRESSING NATIONAL PATIENT SAFETY GOAL 9 IN A HOSPITAL BASED NUCLEAR MEDICINE PRACTICE} Deborah A. Hurley, Nathan C. Hall, Michael V. Knopp Radiology, The Ohio State University, Columbus, OH, USA.

Objectives: This study evaluated the ease of implementation of a pilot program for reduction of outpatient falls to provide continuity of care within our department and other clinical areas and determine the impact of technologist awareness of falls risks. Within our institution, a Falls Prevention Protocol is in place for inpatients in compliance with NPSG 9. The Nuclear Medicine Division developed a pilot program to extend this program to include outpatients. Methods: Using the Falls Prevention Protocol as a template, an Outpatient Falls Risk Worksheet was developed with several falls risk categories listed. All outpatients were questioned by a 
technologist before administration of radiopharmaceutical. The worksheet followed with patient paperwork during the appointment until departure. If a patient was deemed to have any or multiple risks, a yellow armband was placed on the wrist. This easily alerted other departmental staff of the potential risk of a fall. Those patients with additional appointments kept the armbands on and the technologist departing that patient contacted the next appointment location with appropriate patient hand-off information and received a contact name. Wrist band was removed prior to the patient leaving the hospital. Results: A summary sample for a 31 day period totaled 825 patients. $70 \%$ had no risk of falls. Of the $30 \%$ at-risk $52.2 \%$ had multiple factors, $15.9 \%$ had unsteady gait, $9.2 \%$ anxiety, $5.2 \%$ mental status changes with the remainder numbers at less than $4 \%$ per risk factor. No falls occurred during this time. Conclusions: An outpatient Falls Risk assessment is feasible for patient safety and compliance with the NPSG 9. The impact of this program implementation is yet to be seen.

\section{Translational Studies}

\section{J878}

PRE-CLINICAL VALIDATION OF A NOVEL METHOD FOR EX VIVO SENTINEL LYMPH NODE DETECTION IN COLORECTAL CANCER USING NEAR-INFRARED FLUORESCENCE IMAGING

$\underline{\text { Merlijn Hutteman }}^{1,3}$, Alexander Vahrmeijer ${ }^{3}$, Hak Soo Choi ${ }^{1}$, Yoshitomo Ashitate $^{1,4}$, Cornelis van de Velde ${ }^{3}$, John V. Frangioni ${ }^{1,2}$

${ }^{1}$ Medicine, Beth Israel Deaconess Medical Center, Boston, MA, USA, ${ }^{2}$ Radiology, Beth Israel Deaconess Medical Center, Boston, MA, USA, ${ }^{3}$ Surgery, Leiden University Medical Center, Leiden, Netherlands, ${ }^{4}$ Surgical Oncology, Hokkaido University Graduate School of Medicine, Hokkaido, Japan.

Background: Identification of the sentinel lymph node (SLN) in colorectal cancer has both prognostic and therapeutic significance. However, due to variable success rates of currently available techniques for SLN identification, there is ample room for improvement. We evaluated whether it is possible to accurately detect the SLN ex vivo in resected colorectal tissue specimens by using invisible near-infrared (NIR) light and optimal lymphatic tracers not yet FDA-approved for human use. Methods: We used the FLARETM intraoperative NIR fluorescence imaging system for real-time identification of SLNs and the previously described lymphatic tracer HSA800. $10 \mu \mathrm{M}$ of HSA800 was used in all experiments. HSA800 was injected into the subserosa of the colon and rectum of Yorkshire pigs $(n=4)$. Real time identification and dissection of the sentinel node was performed in vivo and subsequently ex vivo after performing an oncologic resection of the colon. In each condition (in vivo and ex vivo in both colon and rectum), 8 injections were performed for a total of 32 injections. Results: We were able to identify the SLN in the colon quickly and accurately, both in vivo and ex vivo, in all cases. At an average of $58 \mathrm{~s}$ after injection of HSA800, one or two fluorescent lymph nodes were found at the root of the bowel mesentery. No difference was observed between in vivo and ex vivo injections. Histological analysis confirmed the presence of nodal tissue. In the rectum, all injections also lead to a successful identification of one or two fluorescent nodes. Identification was significantly faster $(t=2.942, p=$ $0.011)$ in ex vivo (27 s) when compared to in vivo (54 s). Conclusion: Realtime, NIR fluorescent ex vivo SLN imaging is possible in colorectal surgery. This technology enables a quick and accurate identification of the SLN. Most importantly, the ex vivo approach allows the use of contrast agents that have the best overall performance, even when they are not currently FDA approved for clinical studies.
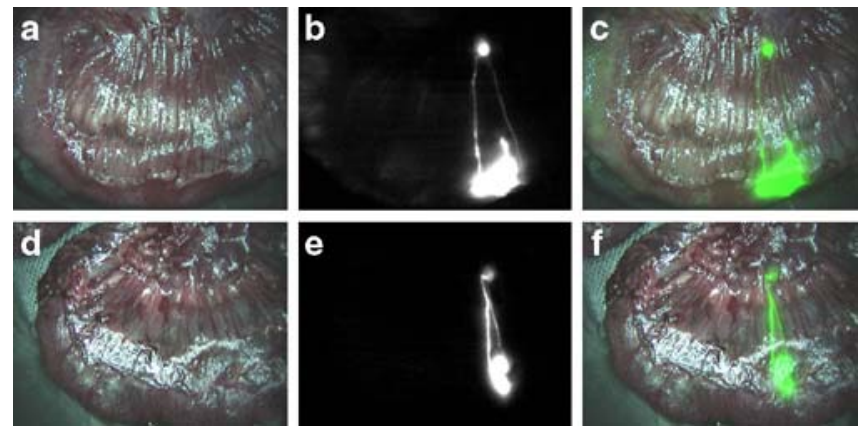

Identification of SLN in pig colon after in vivo (top) and ex vivo (bottom) injection of HSA800. A, D: Color image; B, E: NIR image; C, F: ColorNIR merge.

\section{J879}

\section{IN VIVO MICROSCOPY IN MOUSE MODELS} OF MONOGENIC SKIN DISEASE

Hyejun $\mathrm{Ra}^{1}$, Emilio Gonzalez-Gonzalez ${ }^{1}$, Wibool Piyawattanametha ${ }^{1,2}$, Roger Kaspar ${ }^{3}$, Christopher Contag ${ }^{1}$

${ }^{1}$ Pediatrics, Radiology, Microbiology and Immunology, Stanford University, Stanford, CA, USA, ${ }^{2}$ NECTEC, Pathumthani, Thailand, ${ }^{3}$ TransDerm Inc., Santa Cruz, CA, USA.

High-resolution optical molecular imaging is a rapidly emerging field that opens up new opportunities into studying human disease biology and developing targeted therapies. Currently, most cellular molecular imaging is limited to ex vivo tissue. Therefore, in vivo microscopy is greatly desired to better understand molecular mechanisms within a living subject. We have previously developed a miniature handheld dual-axes confocal (DAC) microscope based on microelectromechanical systems (MEMS) technology (Fig. 1(a)). This microscope has critical properties for in vivo cellular fluorescence imaging: subcellular resolution in both transverse and axial dimensions, long working distance, large field of view, and large dynamic range in a small package. The handheld DAC microscope has a good form factor for investigating the skin, which is an ideal target for siRNA therapies due to its accessibility and large number of monogenic dominant negative skin disorders. Sequential in vivo imaging with the miniature microscope can give a new perspective into assessing therapy progress and efficacy. A transgenic mouse model has been developed to effectively study siRNA delivery and silencing in skin, where green fluorescent protein (GFP) is expressed in the epidermis. Fig. 1(b) shows a threedimensional in vivo image of this mouse skin model under isoflurane anesthesia; the topmost layer of skin is the stratum corneum (SC) with the granular layer $(\mathrm{G})$ beneath. GFP is expressed as aggregates in $\mathrm{G}$ and accumulates uniformly in SC, corresponding to what is observed in ex vivo skin sections. This transgenic mouse model was intradermally injected with irrelevant control siRNA in the left footpad, and specific siRNA targeting GFP in the right footpad over two weeks. After treatment, the mouse was imaged sequentially over several days. While the control siRNA had no effect on GFP expression, the specific siRNA footpad had areas showing apparent decrease of GFP signal, which is indicative of gene silencing. This shows the potential of the DAC microscope as an effective imaging tool in therapeutic development of siRNA skin delivery technologies. 

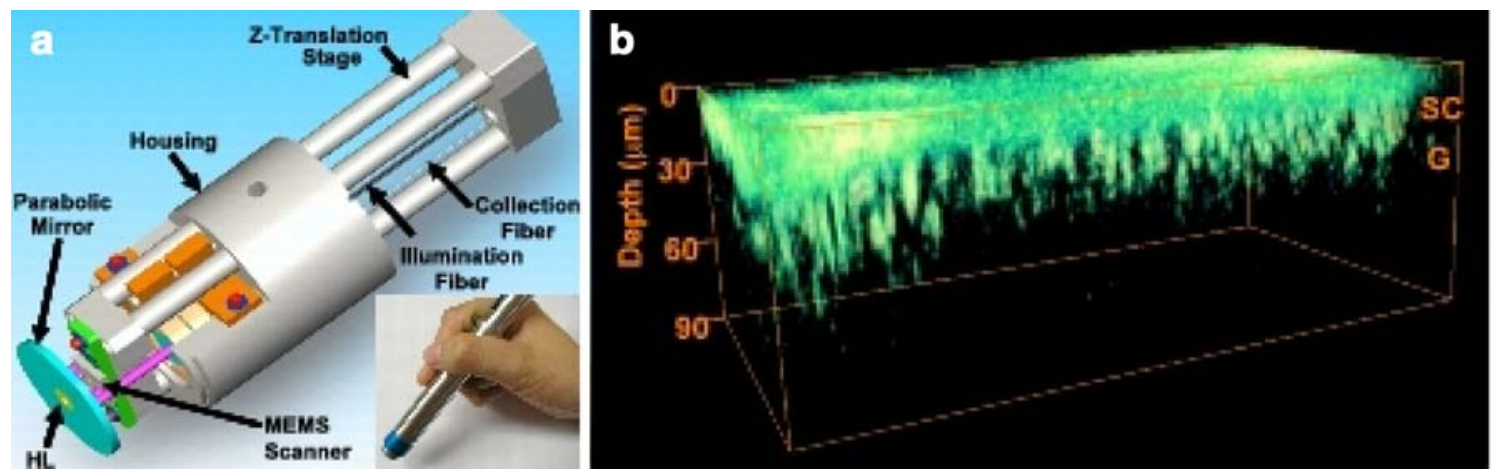

Figure 1. (a) Schematic and photograph of the handheld DAC microscope. (b) 3-D in vivo image of the transgenic mouse skin model taken with the DAC microscope.

J880

\section{IN VIVO MOLECULAR MICROENDOSCOPY IN HUMAN} LUNG CANCER MOUSE MODEL

Kelvin Wong ${ }^{1,2}$, Jiacheng Liu ${ }^{1}$, Ching-Hsuan Tung ${ }^{3}$, Stephen T. Wong ${ }^{1,2}$

${ }^{1}$ Center for Biotechnology and Informatics, The Methodist Hospital Research Institute, Houston, TX, USA, ${ }^{2}$ Department of Radiology, Weill Cornell Medical College, NY, NY, USA, ${ }^{3}$ Department of Radiology, The Methodist Hospital Research Institute, Houston, TX, USA.

Introduction: Early detection of lung cancer is limited by the high false positive rate of CT lung cancer screening. CT guided molecular microendoscopy can potentially provide the high sensitivity and specificity required for lung cancer screening. In this study, we proposed to determine the diagnostic criteria of $\alpha v \beta 3$ fluorescence imaging in human lung cancer mouse model at 3.5-4 h post-injection time window. Method: A subcutaneous lung cancer model was used with A549 cells injected on the left (1 mil cells) and right ( 2 mil cells) flank of nude mice $(n=5)$. Tumors were grown to 25-75 mm3 within 5 days. Integrisense 680 (Visen Medical) was injected I. $\mathrm{V}$. at a dose of $2 \mathrm{nmol} /$ mice $(n=4)$ and $0.7 \mathrm{nmol} /$ mice $(n=1)$ via tail vein. At 3.5 to $4 \mathrm{hrs}$ post-contrast delay, in vivo microendoscopy was performed using Cellvizio Lab system equipped with $660 \mathrm{~nm}$ laser and an emission filter from $670-900 \mathrm{~nm}$ using a $1 \mathrm{~mm}$ O.D. fiberoptic probe (Mauna Kea Technology). A needle track was established through the tumors from the skin surface and the fiberoptic probe was inserted along the needle track to image the tumors. Laser intensity was maintained throughout all imaging sessions. Fluorescence background was measured in the muscle. The tumor to background ratio (TBR) was used as a diagnostic marker with a cutoff of TBR $>1.5$ and tumor having cells exceeding that threshold is classified as $\alpha v \beta 3$ positive tumor. Results and Discussion: In the four normally dosed animal, $\alpha \mathrm{v} \beta 3$ positive cells were readily seen inside and on the tumor surface $(n=8)$. Cell clusters that are $\alpha v \beta 3$ positive were having TBR as high as 2.2. No $\alpha v \beta 3$ positive cells were detected in both tumors on the lower dosed animal. In addition, heterogeneous $\alpha v \beta 3$ expressions were observed in the A549 tumors. A representative example is shown in Fig. 1 showing the $\alpha v \beta 3$ positive cells in the tumor but not in the muscle. We concluded that microendoscopy is critical in detecting angiogenesis in this subcutaneous lung cancer mouse model as it minimizes partial volume averaging issue. In vivo fiberoptic molecular microendoscopy approach has tremendous potential for early detection of human lung cancer.

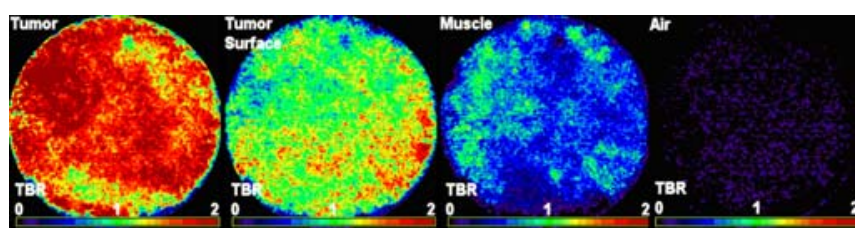

Fig. 1 av $\beta 3$ microendoscopic imaging of human lung cancer. Fluorescent microendoscopic images of $\alpha \mathrm{v} \beta 3$ expressing cells (a) inside tumor, (b) on the tumor surface, which are missing (c) on muscle, and (d) air respectively. $\alpha \mathrm{v} \beta 3$ positive cells were found to have a TBR $>1.5$

J881

\section{DETERMINATION OF HEPATIC GALACTOSE ELIMINATION CAPACITY IN PIGS USING [18F]FLUORO-2-DEOXY-} GALACTOSE PET/CT. REPRODUCIBILITY OF THE METHOD Michael Sørensen

PET Center, Aarhus University Hospital, Aarhus, Denmark.

Purpose. There is a need for a method which can noninvasively measure liver function for example before and after stereotactic radiation therapy. We have recently developed a PET method in pigs for measuring liver function using the galactose analogue [18F]fluoro-2-deoxy-galactose, 18 FDGal. The aim of the present study was to determine the reproducibility of this method before using it in human studies. Methods. Five anaesthetized pigs $(37-40 \mathrm{~kg}$ ) were PET scanned twice on two different days within the same week. A priming dose of $70 \mathrm{mmol}$ galactose followed by a constant i.v. infusion was given $60 \mathrm{~min}$ before and during the PET study. A dynamic PET recording of $40 \mathrm{~min}$ was performed with injection of $150 \mathrm{MBq}$ 18FDGal during the initial $20 \mathrm{~s}$. Arterial blood samples were collected for determination of blood concentration of 18FDGal and galactose (cgal) during the study. Net metabolic clearance of $18 \mathrm{FDGal}$, KFDGal, was calculated from linear representation of data and hepatic Vmax of galactose was calculated as Kmet*cgal. The estimates from Day 1 and Day 2 were compared and the coefficient of variation, COV, of the estimate was calculated. The functional heterogeneity in normal pig liver was evaluated as COV of the concentration of radioactivity in tissue. Results. Good agreement between Vmax from Day 1 and Day 2 was observed (COV of 10\%). In normal pig liver tissue, mean COV after an injection of $18 \mathrm{FDGal}$ was on average $15.6 \%$. This can be used as a reference for normal intra-hepatic variation. Conclusion. The 18FDGal PET method showed good reproducibility and large day-to-day variation can probably be ascribed to changes in liver function and not uncertainty of the method. The FDGal PET/CT method for noninvasive assessment of liver function therefore seems promising for studies of human liver function. Reference: Sørensen M, Munk OL, Mortensen FV, Olsen AaK, Bender D, Bass L, Keiding S. Hepatic uptake and metabolism of galactose can be quantified in vivo by 2-[18F]Fluoro-2-deoxy-galactose Positron Emission Tomography. Am J Physiol Gastrointest Liver Physiol 2008;296:27-36.

BIOLUMINESCENCE-BASED ORTHOTOPIC XENOGRAFT TUMOR MODELS WITH SPONTANEOUS METASTASES AS NEW TOOLS TO EVALUATE THERAPEUTIC STRATEGIES AGAINST CANCER

Yan $\mathrm{Xu}, \mathrm{Yu}$ Rao, Marina V. Tugusheva, David S. Grass, Olesia Buiakova

Phenotyping and Compound profiling, Xenogen Biosciences / Caliper Discovery Alliances and Services, Cranbury, NJ, USA. 
In vivo and ex vivo bioluminescent imaging technology provides oncology researchers with a new means to explore orthotopic tumor growth and spontaneous metastases in animal models. Orthotopic tumor models are more relevant with respect to host-tumor interactions, characteristic disease progression, metastatic potential and response to therapy. With respect to the behavior of solid tumors in the clinic, these models have proven to be more predictive than subcutaneous tumor models, which are currently broadly used for preclinical drug selection. (See review by Talmadge et al, An J Pathol 2007, 170: 793-804.) We have established three orthotopic tumor models progressing to distant spontaneous metastases using human tumor cell lines that Caliper genetically modified to express a bioluminescent molecule (firefly luciferase). In one case, PC3-M-luc prostate tumor cells $(1-2 \times 106)$ were inoculated orthotopically. In vivo bioluminescent imaging (BLI) was performed using an In Vivo Imaging System (IVIS ${ }^{\circledR}$ ) on day 7 post tumor implantation. The BLI data was used to randomize the animals into experimental groups before initiating the treatment regimen. Taxotere ${ }^{\circledR}$ was given at a dose of $20 \mathrm{mg} /$ $\mathrm{kg}$ through tail vein injection on day 8. Subsequent BLI was performed weekly. Using this BLI-based model system, metastases were detected as early as 21 days post tumor implantation. After only 5 weeks, a majority of the mice $(\sim 75 \%)$ exhibited distant metastases in vivo, which were observed by shielding photons from the primary tumor. At the end of the study, ex vivo tissue BLI was performed on the lungs, liver, and draining lymph nodes of all of the animals. We were able to detect metastases in all of the animals imaged ex vivo (even those in which the tumors were too small to detect in vivo) in at least 1 of the 3 tissues evaluated. Taxotere ${ }^{\circledR}$ effectively inhibited both the primary tumor growth as well as the development of metastases. Data will be shown describing the creation of similar orthotopic/metastatic models using recombinant MDA-231-luc (mammary gland) and SKOV3-luc (ovary) tumor cells. Our non-invasive IVIS $\circledR$ imaging platform, combining proprietary hardware \& genetically engineered cell lines, is highly sensitive and facilitates the development of orthotopic and metastatic xenograft tumor models. These models more closely mimic the progression of human disease, and allow for the performance of quantitative and high throughput in vivo assessments of potential anti-tumor and/or anti-metastatic therapies.

\section{Therapy Including Drug Therapy}

\section{As Part of Drug Development}

\section{J883}

MGLUR5 OCCUPANCY IN MOUSE BRAIN USING $\left[{ }^{18}\right.$ F $]$ F-PEB Daniel J. Rubins, Ilonka Guenther, Karen Schlingmann, Terence G. Hamill, Wenping Li, Kerry Riffel, Jacquelynn J. Cook, Sandra Sanabria Discovery Imaging, Merck and Co., Inc, West Point, PA, USA.

Objectives: Recent evidence suggests that mGluR5 antagonists may be neuroprotective for conditions characterized by dopaminergic degeneration, such as Parkinson's Disease. PET studies were performed in mice using the mGluR5 receptor radiotracer $\left[{ }^{18} \mathrm{~F}\right] \mathrm{F}$-PEB to determine the relationship between the plasma concentration of a selective mGluR5 antagonist compound and receptor occupancy (RO). Methods: Mice were scanned at baseline $(N=5)$, and following PO administration of an mGluR5 antagonist compound. To determine the timing for maximum mGluR5 RO, animals were administered $30 \mathrm{mg} / \mathrm{kg}$ of the compound and $\left[{ }^{18} \mathrm{~F}\right] \mathrm{F}-\mathrm{PEB}$ injection and PET imaging was performed at $0.5,1,2$, and $3 \mathrm{~h}(N=1-3$ per group) post-dosing. Based on these results, additional studies were performed following increasing doses of the mGluR5 antagonist compound $(1,3,10,30$, and $60 \mathrm{mg} / \mathrm{kg}: N=1-3$ per group) given 2 hours prior to $\left[{ }^{18} \mathrm{~F}\right] \mathrm{F}-\mathrm{PEB}$ injection. For all scans, $\left[{ }^{18} \mathrm{~F}\right]$ F-PEB [5.5-7.8 MBq] was given as a bolus and dynamic PET data were acquired for 60 minutes. Animals were maintained under isoflurane anesthesia (1-3\%). Blood was collected prior to $\left[{ }^{18} \mathrm{~F}\right] \mathrm{F}$ PEB administration and at the end of the PET scan for measurement of the mGluR5 antagonist compound plasma concentration. MAP reconstructed images were aligned with a standard mouse brain template, and time-activity curves obtained for striatum and cerebellum. $\left[{ }^{18} \mathrm{~F}\right] \mathrm{F}-\mathrm{PEB}$ binding potential (BP) in striatum was calculated using the simplified reference tissue model, with cerebellum as a reference. Results: The between-animal variability of striatal $\mathrm{BP}$ at baseline was $12 \%$. The relationship between mGluR5 RO and the mGluR5 antagonist compound plasma concentration was determined with a Hill coefficient of $0.82 \pm 0.11$ and $\mathrm{EC}_{50}=6.25 \pm 0.96 \mathrm{ng} / \mathrm{mL}$ (see figure). Conclusions: These results demonstrate that $\left[{ }^{18} \mathrm{~F}\right] \mathrm{F}$-PEB can be used to reliably measure mGluR5 occupancy in the mouse brain, and provide critical information for mouse studies involving the treatment of Parkinson's Disease with a mGluR5 antagonist.

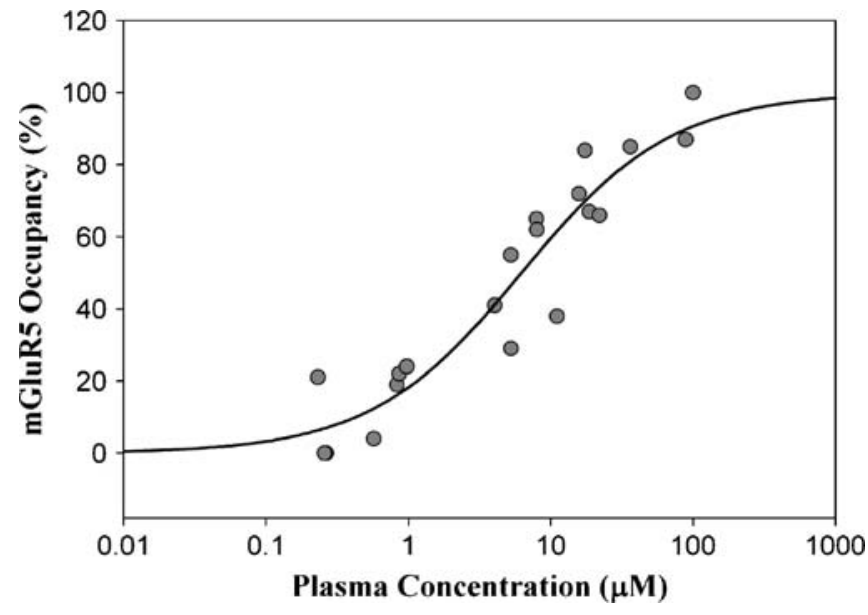

mGluR5 occupancy in striatum measured with $\left[{ }^{18} \mathrm{~F}\right] \mathrm{F}-\mathrm{PEB}$ is shown compared to mGluR5 antagonist compound plasma concentration.

\section{J884}

\section{EVALUATION OF PULMONARY ANTI-INFLAMMATORY RESPONSE WITH $\left[{ }^{18}\right.$ F]FLUORODEOXYGLUCOSE POSITRON EMISSION TOMOGRAPHY}

Delphine L. Chen ${ }^{1}$, Timothy J. Bedient ${ }^{2}$, James Kozlowski ${ }^{1}$, Daniel B. Rosenbluth $^{2}$, Warren Isakow ${ }^{2}$, Thomas W. Ferkol ${ }^{3}$, Mark A. Mintun ${ }^{1}$, Michael J. Walter ${ }^{2}$

${ }^{1}$ Mallinckrodt Institute of Radiology, Washington University School of Medicine, St. Louis, MO, USA, ${ }^{2}$ Internal Medicine, Washington University School of Medicine, St. Louis, MO, USA, ${ }^{3}$ Pediatrics, Washington University School of Medicine, St. Louis, MO, USA.

Rationale: Few noninvasive biomarkers for pulmonary inflammation are currently available that can reliably assess the lungs' response to antiinflammatory treatments. Positron emission tomography with $\left[{ }^{18} \mathrm{~F}\right]$ fluorodeoxyglucose (FDG-PET) is a promising new method that can be used to quantify pulmonary neutrophilic inflammation. Objective: To evaluate the ability of FDG-PET to measure the pulmonary antiinflammatory effects of hydroxymethyl-glutaryl coenzyme A reductase inhibitors (statins) and recombinant human activated protein C (rhAPC) in a human model of experimentally-induced lung inflammation. Methods: Eighteen healthy volunteers were randomized to receive placebo, lovastatin, or rhAPC before intrabronchial segmental endotoxin challenge. FDG-PET imaging was performed before and after endotoxin instillation. The rate of $\left[{ }^{18} \mathrm{~F}\right] \mathrm{FDG}$ uptake was calculated as the influx constant $K_{\mathrm{i}}$ by Patlak graphical analysis. Bronchoalveolar lavage (BAL) was performed to determine leukocyte concentrations for correlation with the PET imaging results. Results: There was a statistically significant decrease in 
$K_{\mathrm{i}}$ in the lovastatin-treated group that correlated with an overall trend towards a decrease in BAL total leukocytes and neutrophils that was not seen in the placebo-treated group, indicating attenuation of inflammation by lovastatin treatment. The $K_{\mathrm{i}}$ observed in the rhAPC-treated group was also decreased when compared to the placebo group but not as great in magnitude as that seen in the lovastatin group. Conclusion: FDG-PET imaging can be used to evaluate the response of lung inflammation to antiinflammatory therapies, further demonstrating the utility of FDG-PET as a biomarker for neutrophilic lung inflammation.

\section{J885}

\section{INVESTIGATING THE EFFECT OF GLYCOSYLATION ON BIODISTRIBUTION OF GLUCAGON-LIKE PEPTIDE 1}

BY A GAMMA CAMERA
Ayahisa Watanabe $^{1,5}$, Ken-ichi Nishijima ${ }^{2}$, Songji Zhao ${ }^{3}$, Kotaro Suzuki ${ }^{4}$, Yoshikazu Tanaka ${ }^{5}$, Takeshi Itoh $^{5}$, Hiroshi Takemoto ${ }^{5}$, Nagara Tamaki ${ }^{4}$, Yuji Kuge ${ }^{1}$

${ }^{1}$ Radiobiology, Graduate School of Medicine, Hokkaido University, Sapporo, Japan, ${ }^{2}$ Molecular imaging, Graduate School of Medicine, Hokkaido University, Sapporo, Japan, ${ }^{3}$ Tracer Kinetics \& Bioanalysis, Graduate School of Medicine, Hokkaido University, Sapporo, Japan, ${ }^{4}$ Nuclear Medicine, Graduate School of Medicine, Hokkaido University, Sapporo, Japan, ${ }^{5}$ Shionogi Innovation Center for Drug Discovery, Shionogi \& Co., Ltd., Sapporo, Japan.

Introduction: Glucagon-like peptide 1 (7-36) amide (GLP-1) is a peptide hormone secreted from enteroendocrine L-cells of the intestine in response to nutrient adsorption. It stimulates insulin secretion from pancreatic $\beta$ cells in a glucose-dependent manner and improves pancreatic $\beta$-cell functions. It is now attracting considerable attention for its therapeutic benefits in type 2 diabetes. However, the use of GLP-1 as a therapeutic agent is limited by its short half-life in body. To improve the pharmacokinetics of GLP-1, a number of studies have been conducted. The glycosylation of GLP-1 contributes to improving the proteolytic stability and in vivo blood glucose-lowering activity in diabetic $d b / d b$ mice. In this study, in order to investigate effect of the glycosylation on pharmacokinetics of GLP-1, we analyzed biodistribution of glycosylated GLP-1 by in vivo imaging. Methods: GLP-1 and glycosylated GLP-1 were iodinated by using the chloramine-T method. Dynamic scans (1 $\min \times 60$ frames) were performed using a gamma camera (M-CAM, Siemens) in rats after the injection of ${ }^{123} \mathrm{I}-\mathrm{GLP}-1 \quad(n=3)$ or ${ }^{123} \mathrm{I}-$ glycosylated GLP-1 $(n=3)$. Regions of interest were set on the images to cover each tissue. In ex vivo biodistribution studies, rats were injected with ${ }^{125}$ I-GLP-1 $(n=3)$ or ${ }^{125}$ I-glycosylated GLP-1 $(n=3)$, blood and tissue samples were collected at indicated time points, and the radioactivity was counted using a gamma counter, and unchanged peptides in plasma were determined by ELISA. The results were expressed as the percentage injected dose per gram of samples ( $\% \mathrm{ID} / \mathrm{mm}^{2}$ or $g$ of tissue), and the ratios of areas under the time-activity curve (AUC) were also calculated. Results and discussion: In the in vivo imaging studies, relatively high accumulation of ${ }^{123}$ I-GLP-1 was found in the liver. The AUC of ${ }^{123}$ I-glycosylated GLP-1 in the liver was significantly lower (89\%) than that of ${ }^{123}$ I-GLP-1. These results were consistent with those of ex vivo biodistribution studies using ${ }^{125} \mathrm{I}$-GLP-1 or ${ }^{125} \mathrm{I}$-glycosylated GLP-1. The AUC of ${ }^{125}$ I-glycosylated GLP-1 in the plasma was significantly and 1.7 times higher than that of ${ }^{125} \mathrm{I}-\mathrm{GLP}-1$. Taken together, the higher plasma levels of ${ }^{125} \mathrm{I}-$ glycosylated GLP-1 may be caused by its decreased distribution into the liver. Conclusion: The glycosylation of GLP-1 significantly decreased its distribution into the liver and increased its concentration in the plasma. Our results may partly explain the mechanism of improved proteolytic stability and in vivo blood glucose-lowering activity of glycosylated GLP-1 in diabetic $d b / d b$ mice.

\section{J886}

USING PET/CT IMAGING TO MONITOR TUBERCULOSIS PROGRESSION AND ITS RESPONSE TO CHEMOTHERAPY IN EXPERIMENTALLY INFECTED NON-HUMAN PRIMATES Philana L. Lin ${ }^{2}$, Jonathan Carney ${ }^{3}$, Jaime A. Tomko ${ }^{1}$, Lonnie J. Frye ${ }^{1}$, Teresa Coleman $^{3}$, Charles A. Scanga ${ }^{1}$, Brian J. Lopresti ${ }^{3}$, James M. Mountz ${ }^{3}$, Edwin Klein ${ }^{4}$, Clifton E. Barry ${ }^{6}$, Joanne L. Flynn ${ }^{5}$

${ }^{1}$ Center for Vaccine Research, University of Pittsburgh School of Medicine, Pittsburgh, PA, USA, ${ }^{2}$ Department of Pediatrics, Children's Hospital of Pittsburgh of the University of Pittsburgh Medical Center, Pittsburgh, PA, USA, ${ }^{3}$ Department of Radiology, University of Pittsburgh Medical Center, Pittsburgh, PA, USA, ${ }^{4}$ Division of Laboratory Animal Resources, University of Pittsburgh School of Medicine, Pittsburgh, PA, USA, ${ }^{5}$ Department of Microbiology and Molecular Genetics, University of Pittsburgh School of Medicine, Pittsburgh, PA, USA, ${ }^{6}$ Tuberculosis Research Section, NIAID, National Institutes of Health, Bethesda, MD, USA.

Tuberculosis (TB) is an enormous global health problem. Treatment of TB requires 6 months or more of antibiotics and emerging drug resistance further limits their effectiveness. Better drugs are clearly needed but TB drug development and subsequent clinical trials are very lengthy. PET/CT imaging may allow us to monitor the response of TB to drugs in real time, significantly reducing drug development time. Cynomolgus macaques infected with $\mathrm{M}$. tuberculosis (M.tb) via bronchoscope reflect the clinical presentation, pathology, and disease course seen in humans. To evaluate the utility of PET/CT imaging in macaques with TB, we installed a Siemens microPET F220, Neurologica CereTom CT and a custom-designed bed system in an ABSL-3 suite, which are arranged in-line to permit image co-registration. We infected macaques with $\mathrm{M}$. tb and conducted serial PET/CT scans to monitor disease progression over several months using [18F]FDG, a marker of inflammation. Granulomas are the hallmark of TB and CT could resolve lesions as small as $1 \mathrm{~mm}$. The development of granulomas in lungs and lymph nodes (LN) was observed by CT and changes in lesion size were followed over time. Co-registered FDG PET images revealed a spectrum of FDG avidity among granulomas with some exhibiting high standard uptake values and some appearing devoid of FDG. An image analysis protocol was designed to graphically depict changes in both lesion size and FDG avidity over time. At necropsy, lung granulomas and $\mathrm{LN}$ were mapped to the pre-necropsy scans and excised for further study. For each lesion, its PET/CT appearance was correlated with histopathology and bacterial burden. Next, we treated a group of experimentally infected macaques with isoniazid, rifampin, or pyrazinamide, three of the most common drugs used to treat TB. We used serial [18F]FDG PET/CT scans to track the effect of monotherapy on individual lesions during treatment and then compared these with the histologic and bacteriologic status of the same lesions taken at necropsy. Imaging revealed a complex pattern of lesion responses to the drug regimen. Many lesions became smaller and less FDG avid, some disappeared entirely, and a few increased in size and/ or FDG avidity during monotherapy. Some lesions contained no culturable bacilli and had apparently been sterilized. Comparing lesions that respond well to a drug with those that respond poorly may provide insights into designing more effective drugs. PET/CT can monitor TB progression as well as its response to drugs and may help evaluate new therapies in pre-clinical and clinical trials. 
$\mathbf{J 8 8 7}$

A NOVEL HIGH-THROUGHPUT STRATEGY (HTS) COUPLED WITH MOLECULAR IMAGING FOR DISCOVERY OF AN ISOFORM-SELECTIVE INHIBITOR OF HEAT SHOCK PROTEIN 90 ALPHA (HSP90A) IN LIVING SUBJECTS

Carmel T. Chan ${ }^{1}$, Robert E. Reeves ${ }^{1}$, Ron Geller ${ }^{2}$, Shahriar Yaghoubi ${ }^{1}$, David E. Solow-Cordero ${ }^{3}$, Sanjiv S. Gambhir ${ }^{1}$

${ }^{1}$ Radiology, Stanford University School of Medicine, Stanford, CA, USA, ${ }^{2}$ Biological Sciences, Stanford University, Stanford, CA, USA, ${ }^{3}$ Chemical Biology, Stanford University, Stanford, CA, USA.

The $\mathrm{Hsp} 90(\alpha / \beta) / \mathrm{p} 23$ protein folding machinery is highly up-regulated in cancer. Each Hsp90 isoform plays distinct roles in determining acute drug response (Hsp90 $\beta$ ) and drug resistance (Hsp90 $\alpha$ ). To harness the power of non-invasive multimodality imaging for development of isoform selective Hsp90 inhibitors, a UNIFIED split Renilla Luciferase (RL) system for monitoring isoform-selective $\mathrm{Hsp} 90(\alpha / \beta) / \mathrm{p} 23$ interactions in cell culture and in living mice was utilized [Cancer Res 68: 216-26, 2008]. Intact 293T kidney cancer cells stably expressing the $\mathrm{Hsp} 90(\alpha / \beta) / \mathrm{p} 23$ split RL reporters were used for high-throughput screening (HTS) of a 30,000 small molecule chemical library by optical bioluminescence imaging (BLI). In cell culture conditions, the lead compound CP9 was more selective for inhibition of Hsp90 $\alpha / \mathrm{p} 23$ interactions, compared to that of Hsp90 $/$ p23 determined by BLI. CP9 degrades Hsp90 client proteins Raf-1, phosphorylated and total Akt in multiple cancer cell lines (Figure 1). The decreases in phosphorylated and total Akt levels also correlated with the efficacy of CP9. Downstream inhibitory effects of $\mathrm{CP} 9$ on glucose metabolism and subsequent cell proliferation were confirmed by ${ }^{3} \mathrm{H}-\mathrm{FDG}$ and ${ }^{3} \mathrm{H}-\mathrm{FLT}$ uptake studies in cell culture, respectively. Binding of CP9 to $\mathrm{Hsp} 90(\alpha /$ $\beta$ ) was determined by ${ }^{3} \mathrm{H}-17 \mathrm{AAG}$ displacement studies. The efficacy of CP9 in inhibition of $\mathrm{Hps} 90(\alpha / \beta) / \mathrm{p} 23$ interactions in living mice $(N=5$ per group) were determined by BLI, followed by small animal PET imaging using ${ }^{18} \mathrm{~F}-\mathrm{FDG}$ to monitor glucose metabolism. Relative to time $0 \mathrm{hr}$, the BLI signals for Hsp90 $\alpha / \mathrm{p} 23$ and Hsp90//p23 interactions in CP9 treated mice (41 hours) were $67 \pm 8 \%$ and $108 \pm 22 \%(p<0.05$, relative to carrier control treated mice, respectively). This is followed by a decrease in ${ }^{18}$ F-FDG uptake in tumors. The mechanism of CP9 in living mice was validated by ex-vivo analysis of degradation of Raf- 1 . This powerful coupling of HTS with multimodality imaging represents a new paradigm for development of next generation of therapeutics aimed at disrupting specific protein-protein interactions.
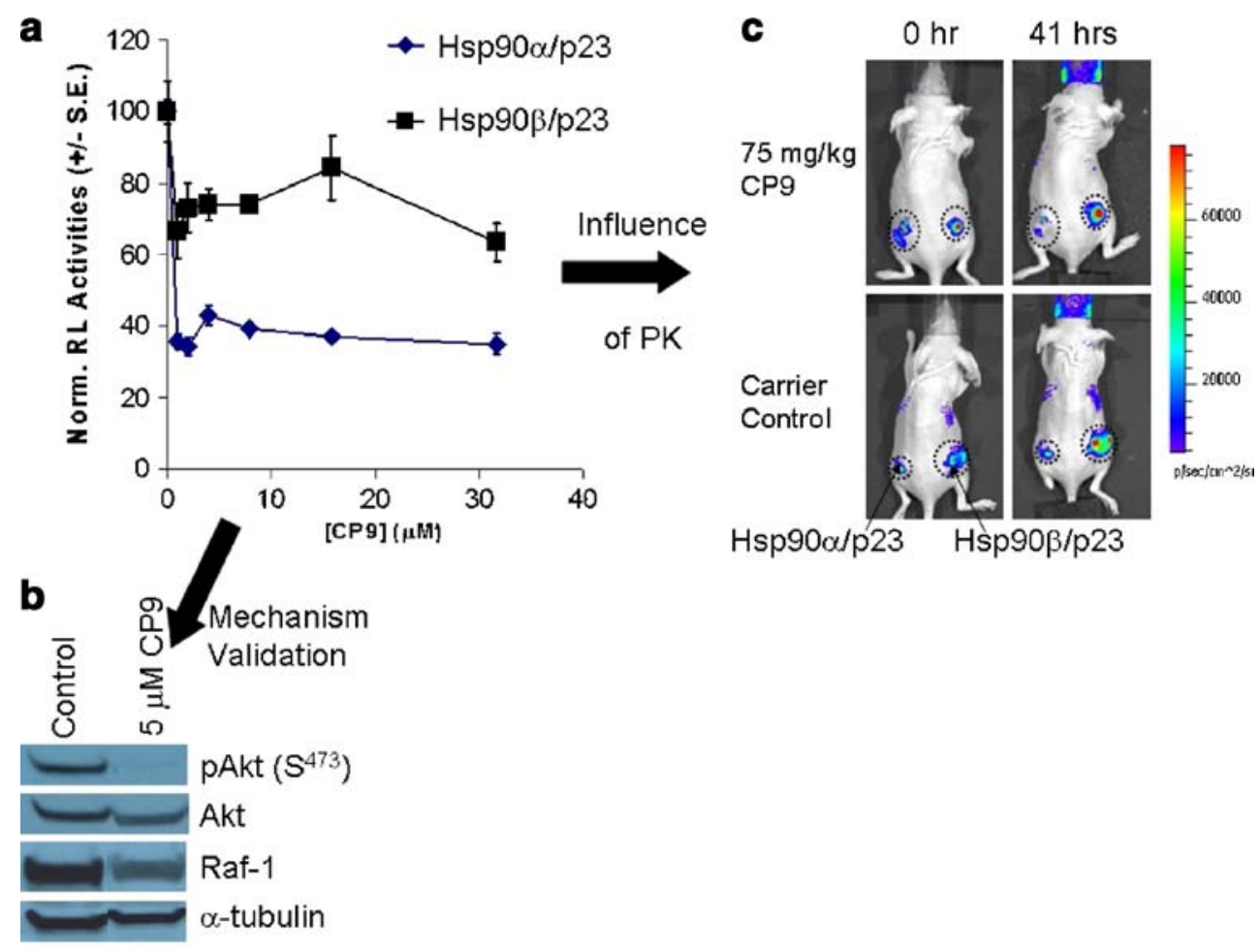

J888 MOLECULAR IMAGING OF ONCOGENE TARGETED
CANCER THERAPY

Hua Fan-Minogue $^{1,2}$, Ramasamy Paulmurugan ${ }^{1,2}$, Carmel T. Chan ${ }^{1,2}$, Zhongwei $\mathrm{Cao}^{3}$, Dean W. Felsher ${ }^{3}$, Sanjiv S. Gambhir ${ }^{1,2}$

${ }^{1}$ Radiology, Stanford University, Stanford, CA, USA, ${ }^{2}$ Molecular Imaging Program, Stanford University, Stanford, CA, USA, ${ }^{3}$ Division of Medical Oncology, Stanford University, Stanford, CA, USA.

Oncogene signaling pathways have been identified as essential for cancer progression and regression and are hence considered attractive candidates for targeted cancer therapy. We developed for the first time a bioluminescent sensor system that can detect in living animals the activation of the cytoplasmic MYC (c-Myc) oncogene. This sensor system utilizes protein-assisted complementation of split firefly luciferases (Nfluc 398/ Cfluc 394) that are fused to a specific phosphorylation motif in the Myc Box I and a selected phospho-recognition domain in GSK3 $\beta$ respectively, to report phosphorylation dependent c-Myc activation and interaction with 
GSK3 $\beta$. Extensive optimization and validation of the sensor system has been done ex vivo to achieve optimal sensitivity and selectivity. The optimal sensors showed more than a two-fold signal reduction when the phosphorylation site of the sensor was abolished, and complete signal recovery with slight increase $(p<0.05)$ when there was a constitutively phosphorylated site. In SK-BR-3 breast cancer cells, the sensor signal was more than four-fold higher than that in CHO normal cells $(p<$ $0.05)$, which correlated $\left(R^{2}=0.98\right)$ with the endogenous phosphorylation status of c-Myc in those cells. Upon inhibition of c-Myc signaling by Atovarstatin (AT) $(0 \mu \mathrm{M}-50 \mu \mathrm{M})$, which efficiently prevents MYC phosphorylation induced lymphomagenesis, the SK-BR-3 stable cells expressing the sensor system showed a dose dependent signal reduction that correlated with the endogenous c-Myc phosphorylation $\left(R^{2}=0.99\right)$, but not those expressing the full length firefly luciferase. In vivo, mouse xenografts $(N=4)$ of SK-BR-3 sensor stable cells treated intra-tumorally with AT $(250 \mathrm{mM})$ already showed $20 \%$ and $50 \%$ signal reduction at the 8th and 13th day respectively of the treatment compared to those treated intra-tumorally with PBS, which is significantly prior to the detectable difference in tumor size (20 days after treatment). This sensor system may provide an important way to not only monitor therapeutic activity of oncogene targeted drugs in vivo, and thus facilitate cancer drug development, but also detect and assess cancer response to therapy at earlier time points.

\section{Days of Treatment}

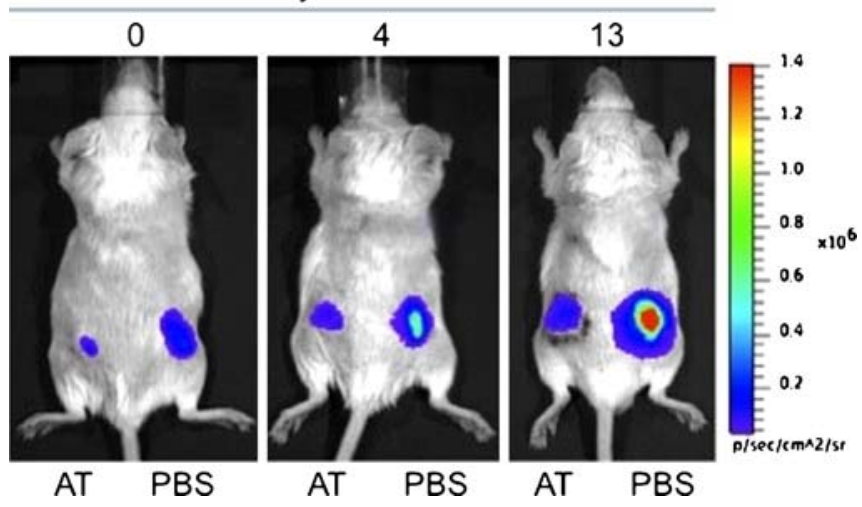

J889

BOMBESIN RECEPTOR TARGETED RADIOTHERAPY IN CONJUNCTION WITH DOCETAXEL AND CAPECITABINE RESTRICTS THE PROGRESSION OF HUMAN PROSTATE CANCER IN A PRECLINICAL MODEL OF BONE METASTASIS

Christopher Manuel $^{1,2}$, Tammy L. Rold ${ }^{1}$, Samantha Sublett ${ }^{1}$, Gary L. $\overline{\text { Sieckman }^{1} \text {, Ashley N. Brown }}{ }^{1}$, Said Daibes Figueroa ${ }^{1,3}$, Lixin $\mathrm{Ma}^{1,3}$, Timothy J. Hoffman ${ }^{1,4}$

${ }^{1}$ Harry S Truman VA Hospital, Columbia, MO, USA, ${ }^{2}$ Veterinary Pathobiology, University of Missouri, Columbia, MO, USA, ${ }^{3}$ Radiology, University of Missouri, Columbia, MO, USA, ${ }^{4}$ Chemistry, University of Missouri, Columbia, MO, USA.

Bombesin receptor (BB2r) targeted radiotherapy has the potential to provide systemic radiotherapy to disseminated metastatic cancer lesions that express the BB2r. Approximately $80 \%$ of human prostate cancer tissues evaluated have demonstrated expression of the BB2r, lending to the potential for utilizing targeted radiotherapy. Few studies have been conducted to address the potential benefit of combining receptor targeted radiotherapy with radiosensitizing chemotherapeutics. The objective of this study was to evaluate a ${ }^{177} \mathrm{Lu}$ radiolabeled bombesin targeting compound, ${ }^{177} \mathrm{Lu}-\mathrm{DOTA}-8 \mathrm{AOC}-$ $\mathrm{BBN}(7-14) \mathrm{NH}_{2}$, for therapeutic benefit in a novel tri-modal combination chemo/radiotherapy protocol using an intraosseous xenograft mouse model of bone metastasis. ICR-SCID mice were inoculated by intra-tibial injection into one pelvic limb with $1 \times 10^{5}$ PC-3 androgen independent human prostate cancer cells. One week post inoculation, mice were administered a weekly regiment of Docetaxel $(8 \mathrm{mg} / \mathrm{kg})$, five consecutive days of Capecitabine $(100 \mathrm{mg} / \mathrm{kg})$, and weekly ${ }^{177} \mathrm{Lu}$-conjugated bombesin targeted radiotherapy, at either 37 or $74 \mathrm{MBq} / \mathrm{kg}$ for 6 consecutive weeks. Bilateral pelvic limb digital radiographs were obtained weekly to monitor for osteolysis progression. A five point grading scale was previously established for grading osteolysis progression. Predetermined surrogate endpoints were established and included pathologic fracture. At the termination of the study, representative tibial micro-CT, micro-MRI, micro-SPECT ${ }^{99 \mathrm{~m}} \mathrm{Tc}-\mathrm{HDP}$ bone scans and histopathology were conducted. Our results demonstrated a significant reduction in radiographic tibial scores $(P<0.05)$, increase in median time to development of a pathologic fracture $(P<0.001)$, and reduction in overall number of pathologic fractures $(P<$ 0.001 ) achieved when chemotherapy was given in combination with $74 \mathrm{MBq} / \mathrm{kg}$ bombesin targeted radiotherapy as compared to chemotherapy alone. Further, a dose dependent response was demonstrated between 37 and $74 \mathrm{MBq} / \mathrm{kg}$ dosages of targeted radiotherapy in combination with dual chemotherapeutics. Our data suggests that treatment with frontline chemotherapeutics in conjunction with bombesin targeted radiotherapy may provide benefit to metastatic prostate cancer patients by decreasing rate of lesion development and severity, while prolonging time before the development of pathologic fractures.

\section{J890}

\section{SYNTHESIS OF ISOTOPE LABELED ANTI-PRION COMPOUND GN8 DERIVATIVE AND EVALUATION OF ITS BLOOD-BRAIN BARRIER PERMEABILITY USING POSITRON EMISSION TOMOGRAPHY}

Tsutomu Kimura, Junji Muto, Yuji O. Kamatari, Kazuo Kuwata Center for Emerging Infectious Diseases, Gifu University, Gifu, Japan.

Prion diseases are invariably fatal neurodegenerative disorders of mammals. They include Creutzfeldt-Jakob disease (CJD), Gerstmann-SträusslerScheinker syndrome (GSS), familial fatal insomnia (FFI), and kuru in humans, scrapie in sheep and goat, bovine spongiform encephalopathy (BSE) in cattle, and chronic wasting disease (CWD) in deer and elk. Conformational conversion of the cellular prion protein $\left(\operatorname{PrP}^{\mathrm{C}}\right)$ to a pathogenic form $\left(\mathrm{PrP}^{\mathrm{Sc}}\right)$ appears to be a central event that gives rise to the prion diseases, although the precise conversion process from $\operatorname{PrP}^{\mathrm{C}}$ to $\operatorname{PrP}^{\mathrm{Sc}}$ is obscure. Subsequent accumulation of $\mathrm{PrP}^{\mathrm{Sc}}$ in the central nervous system leads to neuronal loss and rapid death in humans and animals. Since a new variant Creutzfeldt-Jakob disease (vCJD) appeared, considerable effort has been devoted toward the therapy for the prion diseases. Although a number of compounds have been identified to inhibit the accumulation of $\mathrm{PrP}^{\mathrm{Sc}}$ in prion-infected cells, there are no effective therapeutic agents that are currently available for the treatment of prion diseases. Most of them are inadequate to use as therapeutic agents in vivo due to their low blood-brain barrier (BBB) permeability. Recently, we discovered a novel anti-prion compound, N,N'-(methylenedi-4,1-phenylene)bis[2-(1-pyrrolidinyl)acetamide] (GN8) through an in silico screening based on the structure of $\operatorname{PrP}^{C}$ as a target molecule. ${ }^{1)}$ The anti-prion effect of GN8 in prion-infected mouse neuronal cells was dose-dependent, and the effective concentration for $50 \%$ reduction of $\operatorname{PrP}^{\mathrm{Sc}}$ was $1.4 \mu \mathrm{M}$. The dissociation constant of GN8 for recombinant mouse $\operatorname{PrP}^{\mathrm{C}}$ was estimated to be $3.9 \mu \mathrm{M}$ by SPR analysis. In the ${ }^{1} \mathrm{H}_{-}{ }^{15} \mathrm{~N}$ HSQC NMR spectrum of ${ }^{15} \mathrm{~N}$-labeled recombinant mouse PrP ${ }^{\mathrm{C}}$ with GN8, the signals corresponding to the A-S2 loop and the region from helix B to B-C loop shifted significantly compared to those of ${ }^{15} \mathrm{~N}$-labeled recombinant mouse $\mathrm{PrP}^{\mathrm{C}}$. These results indicate that GN8 specifically binds to $\operatorname{PrP}^{\mathrm{C}}$ and control the global conformational fluctuation of $\operatorname{PrP}^{\mathrm{C}}$. GN8 is a promising therapeutic candidate because subcutaneous administration of GN8 prolonged the survival time of prion-infected mice even at later period. It is of great importance to evaluate the BBB permeability of GN8 because central nervous system including brain is a main organ affected by prion 
diseases. We present the synthesis of isotope labeled GN8 derivative and evaluation of its BBB permeability using positron emission tomography. (1) Kuwata, K. et al. Proc. Natl. Acad. Sci. U. S. A.2007, 104, 11921.

\section{J891}

FDG PET IMAGING PROVIDES INSIGHTS INTO EFFICACY AND SAFETY FOR AN AMPA RECEPTOR POTENTIATOR

Kenneth Zasadny, Michael J. Callahan, Kyle Kuszpit, Laigao Chen, Marc Skaddan, Clive Brown-Proctor, Richard L. Harris, Aijun Zhu, Christopher Shaffer, Renato Scialis

Bioimaging, Pfizer, Inc., Groton, CT, USA.

AMPA receptor potentiators are being explored as potential novel therapeutics for cognition enhancement. Evidence shows enzymatic degradation and decrease in AMPA binding sites occurs in Alzheimer's disease. In rats, blockade of AMPA receptor mediated synaptic transmission disrupts object recognition memory. AMPA receptor potentiators are reported to be efficacious in several animal models of cognition. However, at higher doses they produce unwanted cerebellum-mediated AEs (e.g. loss of motor coordination as measured by a rotarod assay). Changes in glucose metabolism can be used to assess regional brain function in a variety of physiological and pathological states, including the acute responses to psychoactive drugs. In this study, PET imaging was used to investigate the effects of the AMPA potentiator LY451646 on FDG uptake in the rat brain. Sprague-Dawley rats were administered LY451646 at doses of $0.125(n=6), 0.25(n=$ $12)$, and $2.5 \mathrm{mg} / \mathrm{kg}(n=12)$ or vehicle $(n=12)$ by subcutaneous injection 90 minutes before FDG. Rats were injected with FDG ( $\sim 2 \mathrm{mCi})$ into tail vein under isoflurane anesthesia and returned to their cage for a 40-minute uptake period. Rats were then re-anesthetized with isoflurane, placed on the imaging bed with their head fixed in position, and imaged for 20 minutes using a Concorde Microsystems Focus 220 microPET scanner. Images were reconstructed with attenuation and scatter correction using an OSEM3DMAP iterative algorithm. Individual images were then normalized to a mean brain uptake value $=1$, and AFNI software was used for processing and displaying data. Consistent increases in FDG uptake were observed in cortex, thalamus, and cerebellum. In cortex and thalamus, the increases in glucose metabolism occurred at doses $(0.125$ and $0.25 \mathrm{mg} / \mathrm{kg})$ associated with improved memory on cognition tasks. Interestingly, the hormetic nature of the cortical/thalamic glucose utilization data is in agreement with the observed inverted U-shaped exposure-working memory effect for this specific AMPA potentiator. In contrast, the high dose $(2.5 \mathrm{mg} / \mathrm{kg})$ produced an increase in FDG uptake in cerebellum which was linked to cerebellummediated AEs. The doses required for AEs are higher than those that produce efficacy in rodent models of cognition. This study demonstrated the value of FDG-PET in differentiating doses of an AMPA receptor potentiator linked with cognitive enhancement and those producing unwanted cerebellum-mediated AEs.

\section{J892}

\section{CONJUGATION OF DOXORUBICIN TO TRANSFERRIN OVERCOMES HYPOXIA-INDUCED DRUG RESISTANCE IN MCF-7 CELLS AND MULTIDRUG RESISTANCE OF NCI/ADR-RES CELLS}

Xian-Peng Jiang, Robert L. Elliott, Jonathan F. Head

EEH Breast Cancer Research and Treatment Center, Baton Rouge, LA, USA.

Development of multidrug resistance in cancer cells decreases doxorubicin uptake as a result of increased efflux, increased intracellular sequestration, and decreased membrane permeability. Doxorubicin has intrinsic fluorescence in living cells after excitation with ultraviolet light. In this study, we investigated whether conjugation of doxorubicin to transferrin (Tf) could overcome drug resistance in breast cancer cells by using fluorescent microscopy in living cells and $3[\mathrm{H}]$-thymidine incorporation methods.
Two human breast cancer cell lines, MCF-7 cells (sensitive to doxorubicin) and NCI/ADR-Res cells (resistant to doxorubicin), were studied. MCF-7 cells exposed to $1 \% \mathrm{O} 2$ for 24 hours rapidly acquired resistance to free doxorubicin in comparison with normoxic cells (in 20\% O2). In contrast to free doxorubicin, the doxorubicin-gallium-transferrin conjugate (DGT) was more cytotoxic to the hypoxic cells in $1 \%$ O2 than normoxic cells in $20 \%$ O2. Fluorescent microscopy showed that hypoxia significantly decreased the accumulation of free doxorubicin in the cells, but increased intracellular doxorubicin when the cells were treated with DGT. NCI/ADR-Res cells (IC50: $8.98 \mu \mathrm{m}$ of doxorubicin) are 1000-fold more resistant to doxorubicin than the MCF-7 cells (IC50: $0.00142 \mu \mathrm{m}$ of doxorubicin), as determined by $3[\mathrm{H}]$-thymidine incorporation assays. DGT reversed the resistance to doxorubicin and significantly decreased the IC50 (0.095 $\mu \mathrm{m}$ of doxorubicin) in the NCI/ADR-Res cells. Fluorescent microscopy showed that the doxorubicin mainly accumulated in the cytoplasm around the nucleus in NCI/ADR-Res cells, but in both the cytoplasm and nucleus of MCF-7 cells when cells were incubated in media containing free doxorubicin. After 24 hours of treatment with free doxorubicin, the nuclear and cytoplasmic fluorescence was largely unchanged in MCF-7 cells, but cytoplasmic fluorescence was dramatically reduced in NCI/ADR-Res cells (consistent with increased drug efflux). In contrast to cells treated with free doxorubicin, the conjugate of DGT allowed doxorubicin to accumulate in the cytoplasm and nucleus of both the NCI/ADR-Res and MCF-7 cells. After 24 hours of treatment with DGT, there was less cytoplasmic accumulation but more intense nuclear accumulation of doxorubicin. In conclusion: 1) Conjugation of doxorubicin to transferrin could reverse the resistance to doxorubicin in breast cancer cells; 2) Imaging of doxorubicin fluorescence is a useful method for studying doxorubicin accumulation in living cells.

\section{J893}

\section{MOLECULAR IMAGING ASSESSMENT OF TUMOR VEGF RECEPTORS IN VIVO IN RESPONSE TO TREATMENT WITH PAZOPANIB, A SMALL MOLECULE TKI UNDER CLINICAL DEVELOPMENT}

Francis G. Blankenberg ${ }^{1}$, Zoia Levashova ${ }^{1}$, Susanta K. Sarkar ${ }^{2}$, John Pizzonia $^{3}$, Marina Backer ${ }^{4}$, Joseph M. Backer ${ }^{4}$

${ }^{1}$ Department of Pediatrics \& Division of Nuclear Medicine/Department of Radiology \& MIPS, Stanford University, Stanford, CA, USA, ${ }^{2}$ Medicines Development, Oncology R\&D, GlaxoSmithKline, Collegeville, PA, USA, ${ }^{3}$ FUJIFILM Medical Systems USA Inc., Woodbridge, CT, USA, ${ }^{4}$ SibTech Inc., Brookfield, CT, USA.

Vascular endothelial growth factor (VEGF) and its receptors (VEGFRs) drive angiogenesis and are the focus of major drug development efforts. Several VEGFR inhibitors are approved for use as single agents or as adjuvants to chemotherapy. Because the clinical response varies markedly among individuals, there is a clear need for non-invasive measures to identify which patients are benefiting from VEGFR inhibitors. We have recently developed a molecular imaging probe, [99mTc]scVEGF, an engineered single chain (sc) form of VEGF radiolabeled with $99 \mathrm{mTc}$ for SPECT imaging. Pazopanib is a small molecule tyrosine kinase inhibitor targeting VEGFR, PDGFR and c-Kit and is currently in clinical development. Here we report a successful application of our molecular imaging probe to non-invasively assess the tumor VEGFR in response to pazopanib. All SPECT imaging experiments were carried out on HT29 tumor xenografts on Swiss nude mice. A dose of $2-4 \mathrm{mCi}$ of [ $99 \mathrm{mTc}] \mathrm{scVEGF}$ was injected into the retroorbital venous sinus in a volume of $100-220 \mu \mathrm{L}$ followed by imaging two hours later. Quantitative histogram analysis of the SPECT images were performed. At the end of imaging experiments the mice were sacrificed and the tumors were harvested for immunohistochemical analysis of VEGFR-2 and VEGFR-1, as well as pan-endothelial marker CD31. As shown below, the VEGFR levels in tumors were significantly reduced upon treatment with pazopanib as early as day 5 after the initiation of therapy. The reduction in imaging signal is due to a decrease in the number of VEGFR2-expressing endothelial cells in the tumor associated with a 
decreased microvascular density with pazopanib treatment. In principle, this technology can be extended to human use providing for new opportunities in treatment management of VEGFR targeted therapies including patient selection, dose and optimal drug regimen.
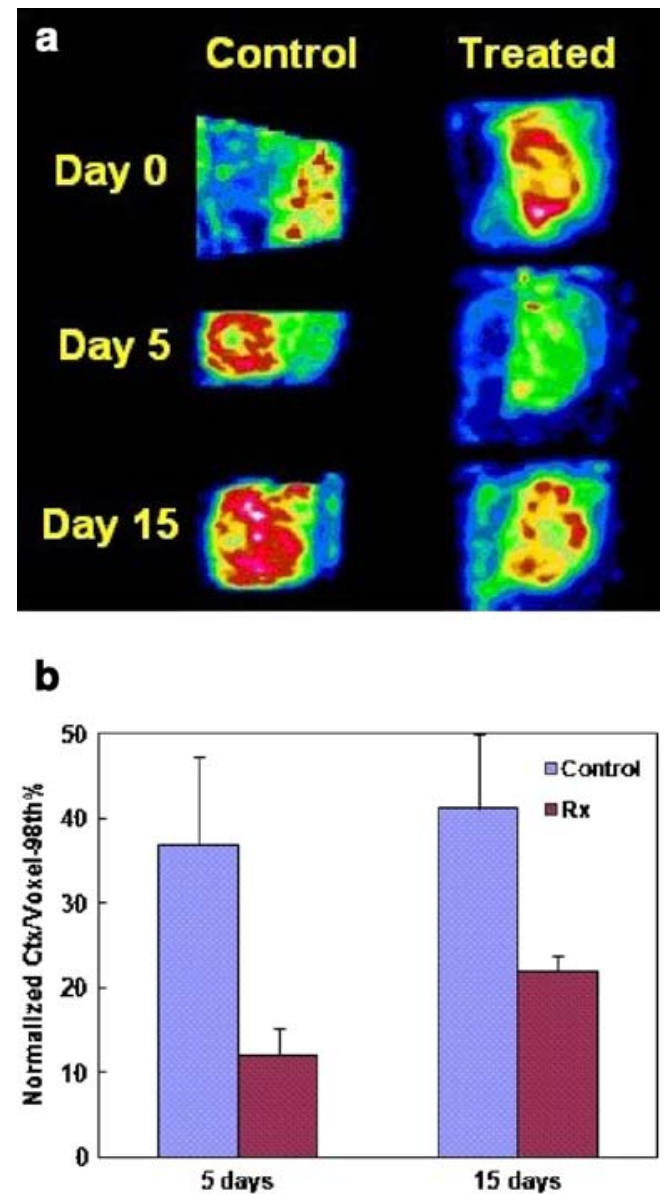

Representative SPECT images of pazopanib treated and control mouse tumor (HT29) xenograft and quantitative analysis of the SPECT data

J894

THE INVESTIGATION OF MITOGEN-ACTIVATED PROTEIN KINASE PHOSPHATASE-1 AS A POTENTIAL PHARMACOLOGICAL TARGET IN NON-SMALL CELL LUNG CARCINOMAS USING NON-INVASIVE MOLECULAR IMAGING

Alexander C. $\mathrm{Wu}^{1,2}$, Hon-Jian $\mathrm{Wei}^{3}$, Win-Ping Deng ${ }^{1,2}$

${ }^{1}$ Biomedical Materials \& Engineering, Taiepi Medical University, Taipei City, Taiwan, ${ }^{2}$ Cancer Center, Taipei Medical University Hospital, Taipei, Taiwan, ${ }^{3}$ Graduate Institute of Medical Sciences, Taiepi Medical University, Taipei, Taiwan.

Mitogen-activated protein kinases (MAPKs) signalling pathways have been shown to play critical roles in tumorigenesis in various types of cancer. However, the precise pathological role(s) of mitogen-activated protein kinase phosphatase-1 (MKP-1) has been controversial. Thus, this study aimed to investigate MKP-1's role in lung cancer using various biological assays and molecular imaging technology. Methods: MKP-1 expression level was elevated in NSCLC H441 cells either by overexpression or pharmacological agent. In vitro cellular proliferation, migration and invasion of MKP-1 over-expressing H441 cells were examined. Functional effects of increased MKP-1 expression in lung tumorigenesis were analyzed by non-invasive molecular imaging method.
Results: MKP-1 over-expressing H441 cells resulted in significantly reduced abilities in proliferation, migration and invasion when compared to the parental line. Using non-invasive optical imaging, we demonstrated that mice inoculated with MKP-1 over-expressing H441 cells did not develop NSCLC while their control wildtype H441 inoculated littermates developed NSCLC and bone metastasis. Pharmacologically, rosiglitazone (RGZ), a peroxisome proliferator activated receptor- $\gamma($ PPAR $\gamma)$ agonists appeared to induce MKP-1 expression and reduce MMP-2 and CXCR4 expression in human NSCLC. H441-inoculated mice receiving daily oral RGZ treatment demonstrated a significant inhibition of bone metastasis when compared to control mice receiving sham treatment. Conclusion: The induction of MKP-1 significantly suppressed proliferation and metastasis of NSCLC cells both in vitro and in vivo. Therefore, MKP-1 could be considered as a candidate target in NSCLC therapy.

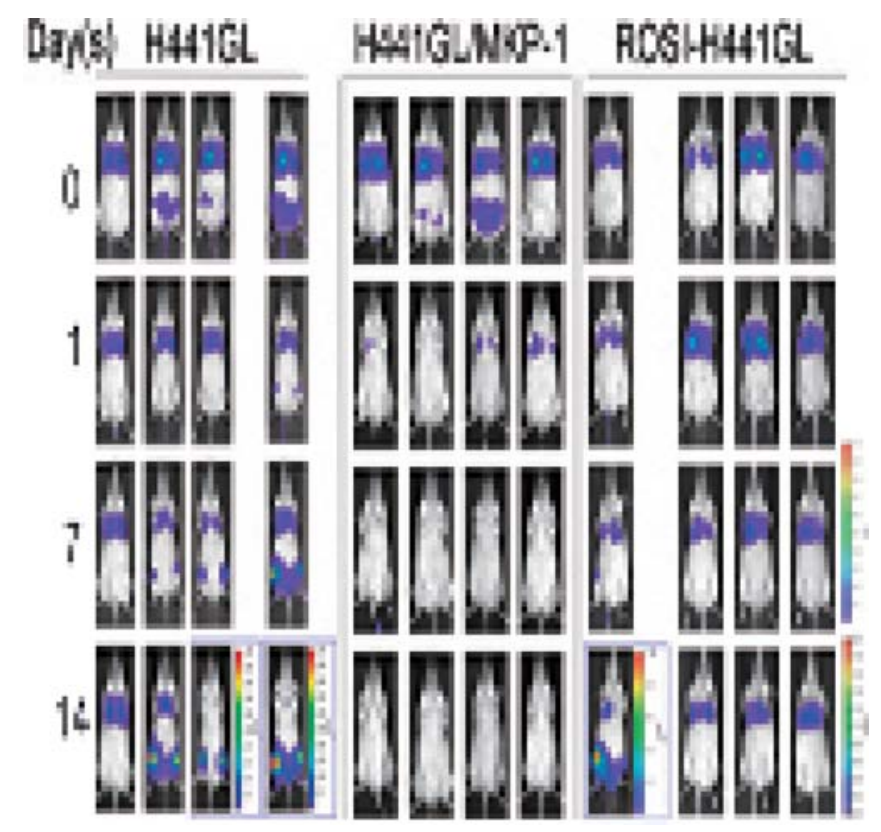

J895

INTERVERTEBRAL DISC DEGENERATION RESTORED BY MESENCHYMAL STEM CELLS OR/AND PLATELET-RICH PLASMA USING EX VIVO CULTURE SYSTEM

Wei-Hong Chen ${ }^{1}$, Hen-Yu Liu ${ }^{2}$, Win-Ping Deng ${ }^{1}$

${ }^{1}$ Biomedical materials and Engineering, Taiepi Medical University, Taipei City, Taiwan, ${ }^{2}$ Graduate Institute of Medical Sciences, Taiepi Medical University, Taipei, Taiwan.

A stem cell and growth factor-based therapeutic approach for the amelioration of intervertebral disc (IVD) degeneration was examined. An ex vivo culture system using chymopapain to partially digest nucleus proposus tissue was established to mimic human IVD degeneration. This system was then used for the evaluation of different therapeutic regimens including: mesenchymal stem cell derived from eGFP transgenic porcine (MSC-GFP), platelet-rich plasma (PRP) and MSC-GFP/PRP combined treatment, and confirmed in in vivo animal model. Chondrogenic specific gene products including ColII and aggrecan were found up-regulated and chondrogenic matrix deposition increased, as evident by sustained fluorescent signals over 4 weeks, in the MSC-GFP implanted group. Previously, we demonstrated in vitro stagespecific chondrogenesis of MSC by chondrocytic commitment (Arthritis \& Rheumatism, 2009). These same molecules upregulated for chondrogenesis were also observed in MSC-GPF group. PRP that has been shown to promote NP regeneration (Journal of Cellular Physiology, 2006) also resulted in significant increased levels of mRNA involved in chondrogenesis and matrices accumulation. The ex vivo IVD regeneration results were repeated 
and supported by in vivo porcine degenerative system. Moreover, the disc height index (DHI) was significantly increased in both in vivo MSC-GFP and PRP regeneration groups. Unexpectedly, the MSC-GFP/PRP combined therapy demonstrated an inclination towards osteogenesis in ex vivo system. The ex vivo degenerative IVD culture system described in this study could serve as an alternative and more accessible model over large animal model. This system also provides a high-throughput platform for screening therapeutic agents for IVD regeneration.

\section{J896}

THE DEVELOPMENT AND EVALUATION OF A NOVEL STEM CELL-BASED ANTI-CANCER PROTEIN VACCINATION, ASSISTED BY NON-INVASIVE MOLECULAR IMAGING

Alexander C. $\mathrm{Wu}^{1,2}$, Hon-Jian Wei ${ }^{4}$, Yi-Ping Lin ${ }^{1}$, Tsu-Hsiang Kuo ${ }^{3}$, Win-Ping Deng ${ }^{1}$

${ }^{1}$ Institute of Biomedical materials and Engineering, Taiepi Medical University, Taipei, Taiwan, ${ }^{2}$ Cancer Center, Taipei Medical University Hospital, Taipei, Taiwan, ${ }^{3}$ Graduate Institute of Life Sciences, National Defence Medical Center, Taipei, Taiwan, ${ }^{4}$ Graduate Institute of Medical Sciences, Taiepi Medical University, Taipei, Taiwan.

Despite of recent advances in medical research, cancer still remains the leading cause for death in many developed countries. Traditional treatments for cancer include radiotherapy, chemotherapy and surgery. However, primary tumor often metastasizes after treatments and results in poor prognosis. Ideally, cancer treatments should aim to eradicate systemic tumor at multiple sites including primary and metastatic, and to discriminate specifically between neoplastic and non-neoplastic cells. In this regard, we devised a "Trojan horse" strategy which combines stem cells and protein vaccine as a new therapeutic approach for the treatment of multiple cancers. Previously, we have demonstrated that mesenchymal stem cells (MSCs) were able to target microscopic tumors, proliferated and integrated to the stroma. Hence, MSCs could be utilized as a tracer and/or a mediator for cell-based anticancer therapy. A protein vaccine was developed to recognize specifically the modified MSCs. These modified MSCs were then introduced to infiltrate the tumor followed by the inoculation of vaccine, which destroys and/or inhibits tumor growth, thereby constituting our "Trojan Horse" strategy. It was found that either in subcutaneous or in pulmonary tumor model, tumor development was greatly inhibited by the combined treatment of MSCs and protein vaccine. The efficiency of tumor suppression correlated positively to the specific immune response induced by the protein vaccine. In addition, this combined treatment inhibited tumor growth via inducing programmed cell death. In summary, we have shown that engineered MSCs could be used as a universal tumor-homing device to enhance tumor-killing effect of the vaccine. Therefore, this novel strategy using MSCs and protein vaccine could be a potential therapeutic intervention for eradicating multiple cancers.
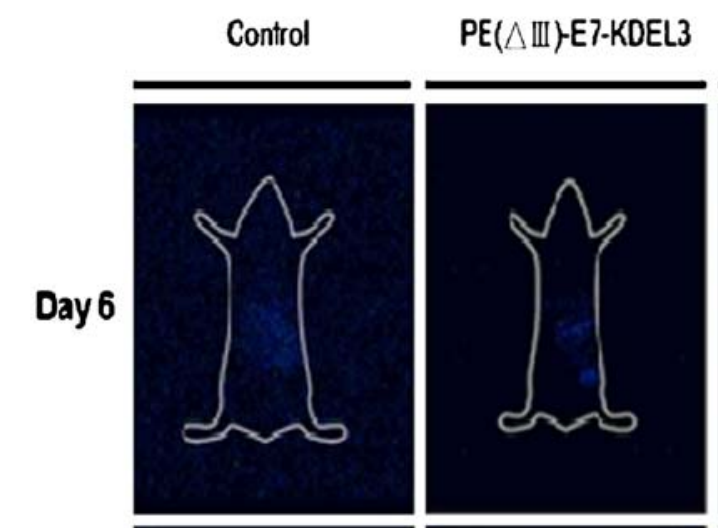

Day 20
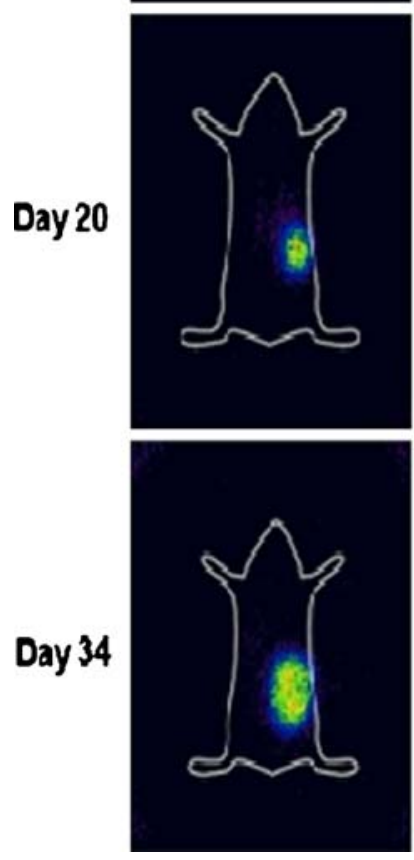

$\operatorname{PE}(\triangle \mathbb{U})-E 7-K D E L 3$

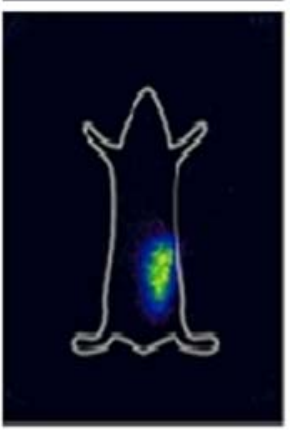

KP-hMSC
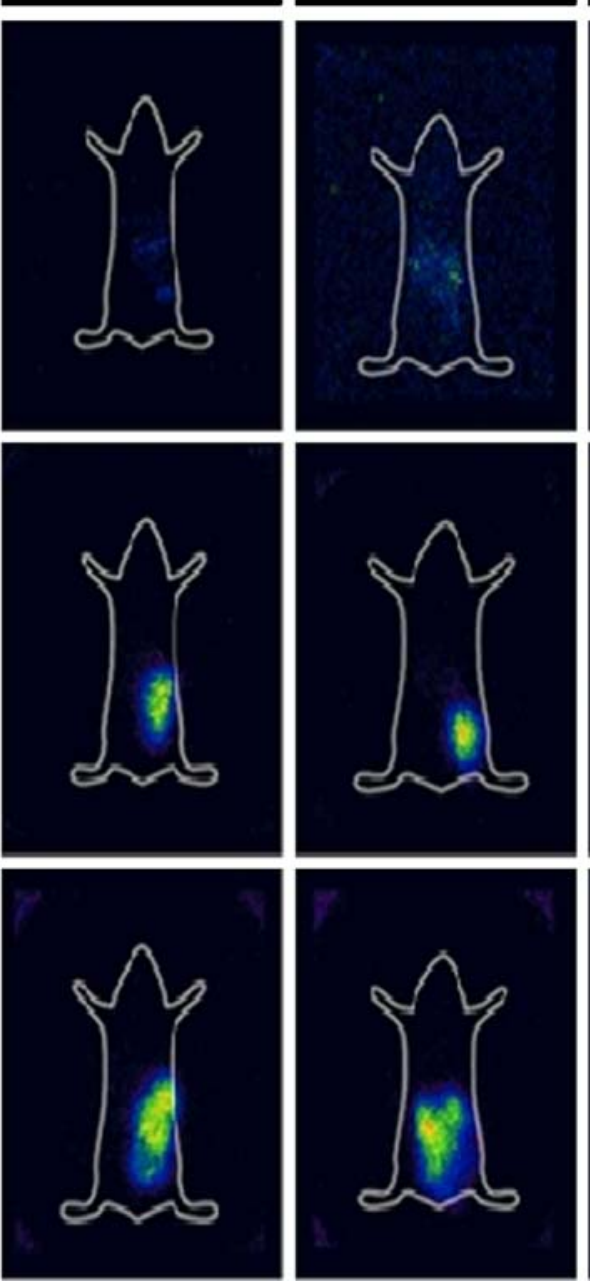

KP-hMSC + $\mathrm{PE}(\triangle \mathbb{M}) \cdot E 7 \cdot K D E L 3$
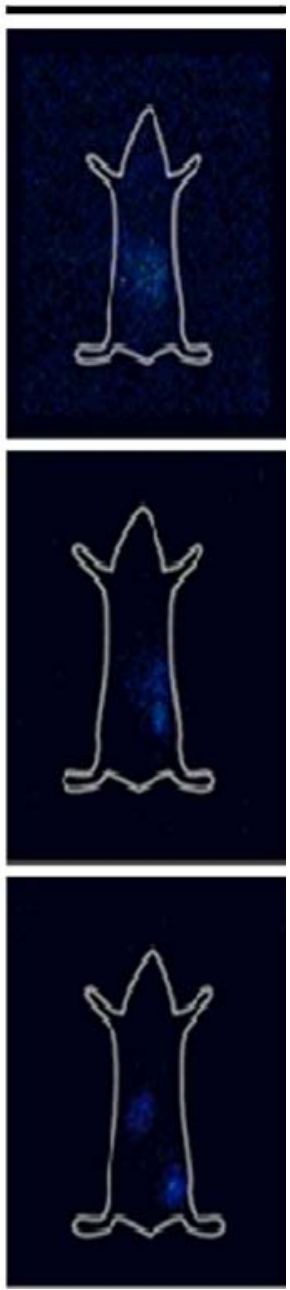

In vivo imaging of mice bearing tumor with protein vaccine/MSC combined treatment 
J897

\section{CHARACTERIZATION OF THE DYNAMIC BBBD PROCESS} USING MRI

Marie Blanchette $^{1}$, Luc Tremblay ${ }^{1}$, David Fortin ${ }^{2}$, Martin D. Lepage ${ }^{1}$

${ }^{1}$ Radiobiology and Nuclear Medicine, Universite de Sherbrooke, Sherbroke, QC, Canada, ${ }^{2}$ Neurosurgery and neuro-oncology, Universite de Sherbrooke, Sherbroke, QC, Canada.

Malignant astrocytomas chemotherapy treatments are hampered by the presence of the blood-brain barrier (BBB). The BBB limits the penetration to the central nervous system (CNS) of antineoplasic agents with a molecular weight larger than 180 Daltons. The osmotic BBB disruption (BBBD) is an innovative technique allowing delivery across the $\mathrm{BBB}$ to a large brain volume distribution. However, only few data are available on the detailed process of the BBBD. The aim of this study was to detail the dynamics of the accumulation, elimination, spatial CNS distribution and the therapeutic window of small and large molecules injected after BBBD. Healthy rats were imaged with a 7 teslas MRI animal scanner. The dynamic physiological process of BBBD was studied by acquiring sets of T1-weighted images every $\sim 50$ seconds before, during and after BBBD. Animals ( $n \leq 5$ for each time of the contrast agent injection) were administered Magnevist $(0.5 \mathrm{kDa})$ or Gadomer $(17 \mathrm{kDa})(600 \mu \mathrm{L}, 0.143 \mathrm{mM})$ at different times post-BBBD $(1,2,3,5,7,10,15,20,30,45,60 \mathrm{~min})$. The concentration of the contrast agents was determined as a function of time post-injection. Maximal signal enhancement was observed for both contrast agents when administered 3 minutes post-BBBD. The mean maximal concentration reached in the treated hemisphere was of the order of $0.1 \mathrm{mM}$ and $0.037 \mathrm{mM}$ for Magnevist and Gadomer, respectively. The accumulation was more pronounced in the basal ganglia than in the cortex of the treated hemisphere. Magnevist distribution into the CNS is regulated by 2 mechanisms: 1) direct delivery resulting from the permeabilisation of the BBB and 2) diffusion of the molecule in the interstitial fluid according to a concentration gradient. The diffusion process was not observed for Gadomer. Gadomer remained detectable in the treated hemisphere for at least $12 \mathrm{~h}$ post-BBBD. The BBB was impermeable to Gadomer within 10 min post-BBBD, while it remained permeable to Magnevist 30 min post-BBBD. BBBD enables delivery of molecules to a large distribution volume of the CNS. The precise description of dynamics underlying BBBD is expected to have major clinical implications for the treatment of malignant astrocytomas.

\section{J898}

IDENTIFICATION OF APOPTOTIC CELLS IN LIVING REPORTER MICE USING MODIFIED LUCIFERIN: A NEW AVENUE TO STUDY MULTIPLE MOLECULAR PATHWAYS WITHIN THE SAME COMPLEX ORGANISM

Andrea Biserni ${ }^{1}$, Francesca Martorana ${ }^{1}$, Chiara Roncoroni ${ }^{1}$, Dieter $\mathrm{H}$ Klaubert $^{2}$, Adriana C. Maggi ${ }^{3}$, Paolo Ciana ${ }^{3}$

${ }^{1}$ TOP (Transgenic Operative Products) srl, Lodi, Italy, ${ }^{2}$ Promega Biosciences, Inc., San Luis Obispo, CA, USA, ${ }^{3}$ Department Pharmacological Sciences, University of Milan, Milan, Italy.

Reporter genes and imaging technologies contribute to drug discovery in several areas providing systems that enable the study of biological processes or the biochemical activity of specific targets, in disease and in response to a drug. In reporter mice these events can be monitored over periods of time thus offering a more accurate method of measuring pharmacodynamics in combination with pharmacokinetics and toxicity. We have developed a technology to generate reporter mice suitable for pharmacological studies based on the use of luciferase reporter gene and
MAR insulator sequences that guarantee ubiquitous and reliable expression of the biosensor (Ciana P et al 2001, Maggi A and Ciana P 2005, Maggi A et al 2004). The recent development of modified luciferase substrates represents an incommensurable step forward enabling to study multiple molecular events and pathways in the same reporter cell. Here, for the first time, we provide evidence of the possibility to merge the two technologies, reporter mice and luciferase substrates, for bioluminescence imaging of multiple molecular events in the same living animal. We demonstrate that administration of the Caspase 3/7 activatable substrate of luciferase (VivoGlo Caspase-3/7, Promega) to a luciferase reporter mouse produces a photon emission detectable by CCD-camera imaging selectively in tissues undergoing apoptosis. Indeed, i.p. administration of increasing doses of VivoGlo Caspase-3/7 (17-50-150 mg/kg) to the luciferase reporter mouse in which liver apoptosis was induced by a single i.p. injection of Dgalactosamine (D-GalN, $800 \mathrm{mg} / \mathrm{kg}$ ) and Lipopolysaccharide (LPS, $100 \mathrm{mg} / \mathrm{kg}$ ) (Nakama $\mathrm{T}$ et al 2001), produced a clear dose-dependent induction of photon emission selectively in the liver area. Moreover, luminescence was selectively reduced by administration of the pancaspase inhibitor Z-VAD. Ex vivo imaging analysis of dissected tissues confirmed that light emission was produced by the liver and by two other potential site of apoptosis, lung and white adipose tissue, which were not previously described after LPS/D-GalN treatment. The presence of apoptotic cells in the photon emitting tissues was also confirmed by the analysis of Caspase 3/7 enzymatic activity in protein extracts. Finally, we provide data showing that the system is sensitive enough to detect even physiological apoptosis occurring in tissues. The current study showed for the first time the possibility to measure apoptosis in the organ of a living reporter mouse opening a new avenue to study multiple molecular pathways within the same organism.

\section{J899}

\section{MEASURING DOSE OCCUPANCY OF THE MGLUR1 ANTAGONIST MK-7643 IN RAT BRAIN WITH MICROPET: LINKING IN VIVO OCCUPANCY TO EFFICACY}

$\underline{\text { Ilonka Guenther }^{1}}{ }^{\text {, Elizabeth R. Landis }}{ }^{1}$, Daniel J. Rubins ${ }^{1}$, Mangay Williams $^{1}$, Shunsuke Maehara ${ }^{2}$, Hirohiko Hikichi ${ }^{2}$, Gentaroh Suzuki ${ }^{2}$, Mikiko $\mathrm{Hata}^{2}$, Hiroshi Kawamoto ${ }^{2}$, Satoshi Ozaki ${ }^{2}$, Hisashi Ohta ${ }^{2}$, Waisi Eng ${ }^{1}$, Richard Hargreaves ${ }^{1}$, Donald Burns ${ }^{1}$, Eric Hostetler ${ }^{1}$ ${ }^{1}$ Merck \& Co, West Point, PA, USA, ${ }^{2}$ Banyu Pharmaceutical CO, Tsukuba, Japan.

Schizophrenia is one of the most common psychiatric diseases with a prevalence of $1 \%$ of the total population over the age of 18 . While the introduction of atypical antipsychotics has greatly improved treatment of schizophrenia, $20-30 \%$ of patients do not respond well to these drugs. Therefore, a significant unmet medical need remains for a new class of antipsychotics. A potent and selective mGluR1 antagonist, MK-7643, developed by Banyu Pharmaceutical CO., Tsukuba, Japan, has been shown to be effective in the methamphetamine and ketamine induced prepulse inhibition animal model of psychoses. Using a novel mGluR1 specific PET radiotracer, [18F]MK-4908, we established a link between oral efficacy of MK-7643 and mGluR1 occupancy in rats. PET scans $(1 \mathrm{~h})$ were conducted in Sprague-Dawley rats (160 $200 \mathrm{~g}$ ) under Isoflurane anesthesia. MK-7643 was administered orally 55-90 minutes before tracer injection to mimic the protocol of the rat efficacy studies. Plasma samples were taken before tracer injection and at the end of the PET scan to measure MK-7643 levels. [18F]MK4908 showed high specific uptake in the cerebellum, thalamus and striatum which could be fully blocked with IV administration of $3 \mathrm{mg} /$ $\mathrm{kg}$ MK-7643. Receptor occupancy in all 3 target regions showed a 
very good dose-response relationship after oral dosing of MK-7643. In MK-7643 efficacy studies in rat, plasma levels of $\sim 220 \mathrm{nM}$, which corresponds to $72 \%$ on our dose occupancy curve, reversed disruption of prepulse inhibition caused by methamphetamine and ketamine in the SD rats. We demonstrated using in vivo PET imaging that efficacy requires a target occupancy of $\sim 75 \%$ which will impact future preclinical and clinical studies.

J900

\section{NOVEL CU- BIS (THIOSEMICARBAZONE) COMPLEXES AS HYPOXIA IMAGING AGENTS: STRUCTURE-ACTIVITY RELATIONSHIPS}

$\underline{\text { S. S. Raghuvansi }}^{1,2}$, Himanshu Ojha ${ }^{1}$, Anil K. Mishra ${ }^{1}$, B. Singh ${ }^{2}$

${ }^{1}$ Radiopharmacy, Institute of nuclear med \& allied sciences, Delhi, India, ${ }^{2}$ Chemistry, B.H.U, Varansi, India.

Aim A novel series of Copper(II) bis(thiosemicarbazone) complexes with different $\mathrm{Cu}$-isotopes are useful radiopharmaceuticals for imaging blood flow and hypoxic tissues in vivo. The aim of this study was to identify structure-activity relationships within a new series of analogues with different substitution patterns in the ligand, in order to design improved hypoxia imaging agents and elucidate hypoxia selectivity mechanisms. Fifteen such complexes were analysed chemically Material and Methods: All used chemicals were purchased from commercial sources, and was synthesised by using a common procedure, exemplified by the following method for the synthesis of subsituted bis (thiosemicarbazone) Thiosemicarbazide (H2NNHCSN-H2) (1.29 g, $14 \mathrm{mmol}$ ) was dissolved in $25 \mathrm{~mL}$ of distilled water containing $5-15 \%$ acetic acid at $60-90^{\circ} \mathrm{C}$. To this was added $0.33 \mathrm{~mL}(5.7 \mathrm{mmol})$ of substituted nucleophile 1 dropwise over 10-30 min. The reaction was allowed to stir overnight at room temperature. The precipitate was retrieved by filtration, washed and dried in vacuo. Analytical $1 \mathrm{H}$ and $13 \mathrm{C}$ NMR spectra were recorded on a Bruker $400 \mathrm{MHz}$ spectrometer. Mass Unit mass determinations were carried out by using a electro spray ionization mass spectrometry (ESI-MS) on an SL 1200 system (Agilent) with ion trap detection in the positive and negative ion modes. After incubation at $90^{\circ} \mathrm{C}$ for $30 \mathrm{~min}$, ITLC and HPLC analyses were carried out to check the radiochemical purity of complex. Biological experi- ments were performed on male Wistar rats. Results: The uptake of each (labelled with $\mathrm{Cu}-64$ ) in EMT6 tumour cells in vitro under normoxic and hypoxic conditions was studied. All complexes were taken up efficiently into cells, and some showed strong hypoxia selectivity, which was highly correlated with the $\mathrm{Cu}(\mathrm{II} / \mathrm{I})$ redox potential. Redox potentials at the low end of the range were found to be essential for hypoxia selectivity. In turn, the redox potential was strongly correlated with alkyl substitution pattern, and the most important determinant of the redox potential was the number of alkyl groups on the diimine backbone of the ligand. Several complexes in the series warrant further evaluation as hypoxia imaging agents. The radioactivity uptake/release behaviour in the cells provides insight into possible mechanisms, and a model for hypoxiaselective intracellular trapping is discussed.

\section{J901}

\section{A HIGH THROUGHPUT COMPUTED TOMOGRAPHY TECHNIQUE FOR LONGITUDINAL IMAGING OF ORTHOTOPIC LUNG TUMORS IN A MOUSE MODEL UTILIZING NO CONTRAST MEDIA AND NO GATING}

Christopher M. Bull, Michael S. Westmore, Jeffrey A. Wolos, Li C. Li Molecular and Anatomical Imaging, Covance, Greenfield, IN, USA.

We have developed a technique for imaging 12 mice simultaneously in 8 seconds using the GE eXplore Locus Ultra CT scanner. This technique is being used to image orthotopic lung tumors in a mouse model. In this model, athymic nude mice were injected with $\mathrm{H} 441$ cells into the left lung. The mice were divided into three groups $(N=20$, vehicle, Gemzar $75 \mathrm{mg} / \mathrm{kg}$, Gemzar $150 \mathrm{mg} / \mathrm{mg}$ ) and treated IP once every 3 days starting at day 21 post-inoculation, with four treatments in total. The resulting tumors were imaged with the CT technique every 7 days starting on day 7 post-inoculation. The images were analyzed using GE MicroView software by drawing contours on selected slices through the tumors and using the interpolation technique to generate a volumetric region defining the tumor. The results show the tumor volumes diverging from day 21 (the initiation of treatment), with the vehicle group tumor volume progressing rapidly, and the two Gemzar groups regressing slightly. These results show that we have developed a robust, simple, and high-throughput model for quantifying orthotopic lung tumors in mice using CT.

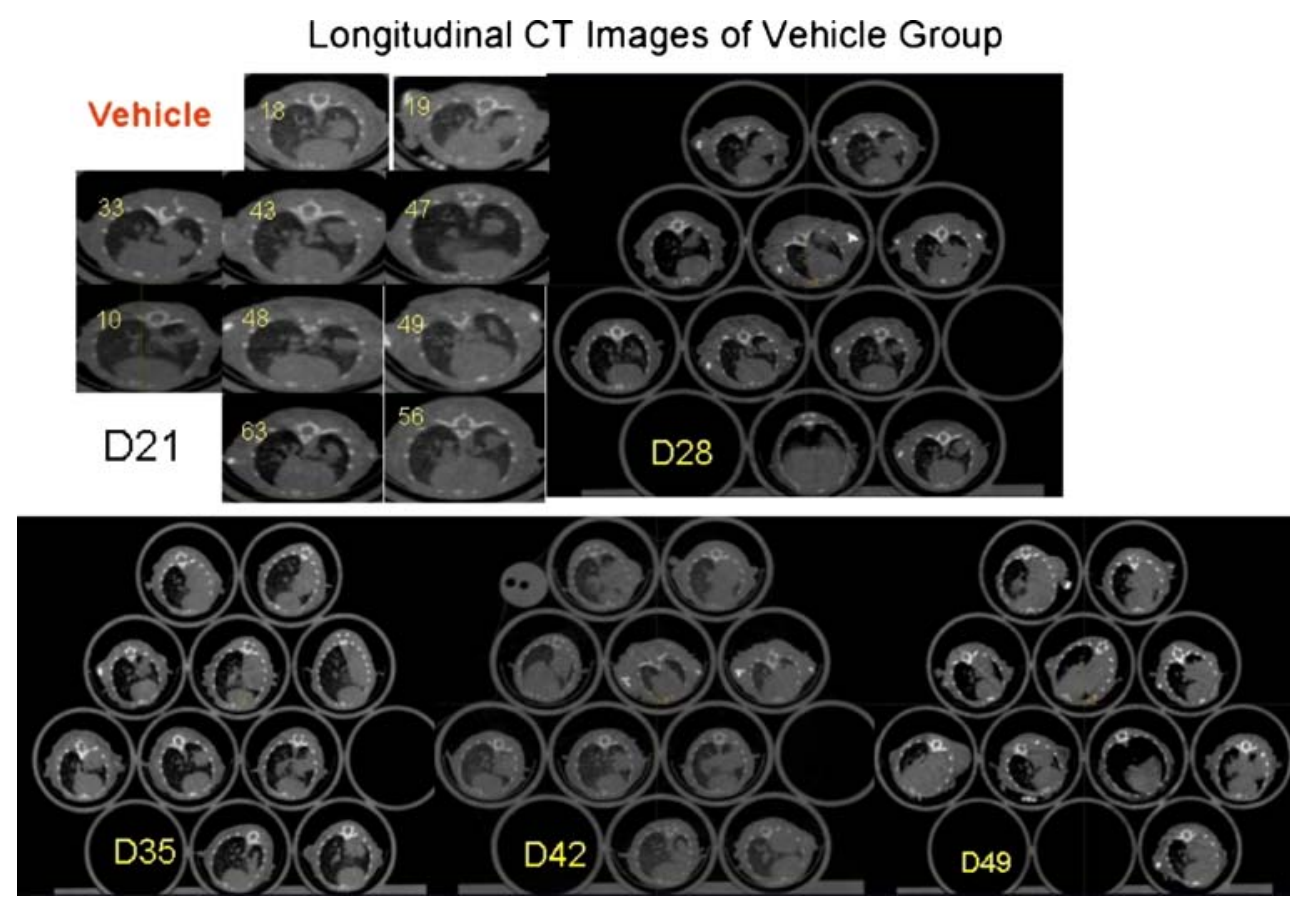


J902

EVALUATION OF BIODISTRIBUTION

AND PHARMACOKINETICS OF MICELLES IN A COLON ADENOCARCINOMA-BEARING MOUSE MODEL

$\underline{\text { Hao-Wen Kao }}{ }^{1}$, Chi-Jen Chan ${ }^{1}$, Yuan-Chia Chang ${ }^{2}$, Yuan-Hung $\mathrm{Hsu}^{2}$, Maggie Jui-Mei Lu ${ }^{2}$, Jassy Wang ${ }^{3}$, Wuu-Jyh Lin ${ }^{4}$, Hsin-Ell Wang ${ }^{1}$

${ }^{1}$ Department of Biomedical Imaging and Radiological Sciences, Institute of Biomedical Image and Radiological Sciences, Taipei, Taiwan, ${ }^{2}$ Biomedical Engineering Research Laboratories, Industrial Technology Research Institute, Hsinchu, Taiwan, ${ }^{3}$ Material and Chemical Research Lab., Industrial Technology Research Institute, Hsinchu, Taiwan, ${ }^{4}$ Institute of Nuclear Energy Research, Taoyuan, Taiwan.

Objectives: Block copolymer micelles are nanosized supramolecular self-assemblies of amphiphilic copolymers. Micelles are developed as water-soluble biocompatible vehicles to reduce toxicity, prolong circulation and deliver hydrophobic chemotherapeutic agents to tumor site via the enhanced permeability and retention (EPR) effect. This study evaluated the biodistribution and pharmacokinetics of micelles composed of radiolabelled monobenzylether-polyethyleneglycol-copolycaprolactone (BE40C37) and monomethoxy-polyethyleneglycolco-polycaprolactone-co-phophatidylcholine (E50C19PC) in a tumor-bearing mouse model. Methods: BE40C37 (MW 7689) was labeled with I-131 to afford ${ }^{131}$ I-BE40C37, and then mixed with nonradioactive $\mathrm{BE} 40 \mathrm{C} 37$ and $\mathrm{E} 50 \mathrm{C} 19 \mathrm{PC}$ to formulate a ${ }^{131} \mathrm{I}$-micelle $(0.5 \%$ $\mathrm{w} / \mathrm{w}, \mathrm{BE} 40 \mathrm{C} 37 / \mathrm{E} 50 \mathrm{C} 19 \mathrm{PC}=1 / 9)$ suspension. About $2 \times 10^{5}$ colon adenocarcinoma (C26) cells were injected subcutaneously in the left flank of the male BALB/c mouse to grow tumor. The biodistribution and pharmacokinetics of ${ }^{131} \mathrm{I}$-BE40C 37 and ${ }^{131}$ I-micelle were determined, and the scintigraphic imaging of ${ }^{131}$ I-micelle was performed at 12 days post tumor cells inoculation. Results: ${ }^{131}$ I-BE40C37 was prepared with moderate radiochemical yield $(20 \pm 5 \%)$ and high radiochemical purity $(\geq 93 \%)$. The size of ${ }^{131}$ I-micelles in suspension was $174 \pm 47 \mathrm{~nm}$. After intravenous (i.v.) injection of ${ }^{131}$ I-micelles into tumor-bearing mice, higher radioactivity retention in organs with reticuloendothelial system (RES, including liver, spleen, etc.) and tumor was noted. The area under curve (AUC) of tumor after administration of ${ }^{131} \mathrm{I}$-micelles $(60.2 \mathrm{~h} \% \mathrm{ID} / \mathrm{g})$ was higher than that of ${ }^{131} \mathrm{I}$-BE40C 37 $(48.4 \mathrm{~h} * \% \mathrm{ID} / \mathrm{g})$. The results of pharmacokinetics study revealed that ${ }^{131}$ I-micelles enjoyed longer biological half-life in mouse model than ${ }^{131}$ I-BE40C37 (Table 1). The scintigraphic imaging of mouse after administration of ${ }^{131}$ I-micelles showed accumulation of radioactivity in liver and a faint image of tumor. Conclusions: Despite high uptake in RES organs, this study demonstrated that ${ }^{131}$ I-micelles were able to passively accumulate in tumors by the EPR effect. The better pharmacokinetics of ${ }^{131}$ I-micelles in tumor-bearing mouse model suggested high potential of micelle as a carrier for sustained release of hydrophobic drugs.

Table 1 Pharmacokinetics parameters derived from radioactivity-time curves of blood after i.v. injection of ${ }^{131}$ I-micelle and ${ }^{131}$ I-BE40C 37 in tumorbearing mouse using two-compartment model.

\begin{tabular}{|c|c|c|c|c|}
\hline & $\begin{array}{c}\text { Distribution HL } \\
(\mathrm{h})\end{array}$ & $\begin{array}{c}\text { Elimination } \mathrm{HL} \\
(\mathrm{h})\end{array}$ & $\begin{array}{c}\mathrm{AUC}(0 \rightarrow \infty) \\
(\mathrm{h} *(\% \mathrm{ID} / \mathrm{mL}))\end{array}$ & 60.0 \\
\hline $131_{\text {I-micelle }}$ & 1.1 & 21.7 & 32.8 & 0.8 \\
\hline $131_{\text {I-BE40C37 }}$ & 0.8 & 17.2 & 1.5 \\
\hline
\end{tabular}

Image Guided Therapy

J903

\section{TEMPERATURE-CONTROLLED US-MEDIATED INTRACELLULAR DELIVERY OF A FLUORESCENT CELL-IMPERMEANT MODEL DRUG USING TEMPERATURE SENSITIVE LIPOSOMES AND CAVITATION}

Mariska de Smet ${ }^{1}$, Anna Yudina ${ }^{2}$, Matthieu Lepetit-Coiffe ${ }^{2}$, Sander Langereis $^{3}$, Roel Deckers ${ }^{2}$, Holger Gruell ${ }^{1,3}$, Chrit Moonen ${ }^{2}$

${ }^{1}$ Biomedical Engineering, Eindhoven University of Technology, Eindhoven, Netherlands, ${ }^{2}$ Laboratory IMF CNRS UMR 5231, University of Bordeaux 2, Bordeaux, France, ${ }^{3}$ Bio-Molecular Engineering, Philips Research Europe, Eindhoven, Netherlands.

Introduction Recent studies have demonstrated the potential of ultrasound (US) for local drug delivery by overcoming biological barriers either with pressure or temperature sensitive delivery systems (ref: Deckers et al, JMRI 2008). Here we present a combination of these two approaches: controlled release of a fluorescent model drug with temperature sensitive liposomes (TSLs) and subsequent induction of cellular uptake via sonoporation. Materials and methods Cyanine nucleic acid stain TO-PRO-3 was chosen as a cell impermeant model drug. This dye shows a strong increase in fluorescence when bound to DNA. TSLs incorporating a $10 \mu \mathrm{M}$ TO-PRO-3 solution were prepared with a $\mathrm{Tm}$ of $40.5^{\circ} \mathrm{C}$. For testing the delivery to the DNA of living cells, C6 rat glioma cells were seeded in a NUNCTM OpticellTM cell culture system and incubated with the TO-PRO-3-TSLs at $37^{\circ} \mathrm{C}$. Focused US was applied at some areas (step 1). Next, the Opticell was heated to $41{ }^{\circ} \mathrm{C}$ and US was applied at some other areas (step 2). Finally, microbubbles (Sonovue, Bracco) were added and US was applied at some areas (step 3). After every step Opticell was monitored with an epifluorescence microscope. Results and discussion TO-PRO3-TSLs showed a fast increase in fluorescence around $39^{\circ} \mathrm{C}$, indicating that the TO-PRO-3 was released from the TSLs and interacted with the DNA in the solution. In living cells, no TO-PRO-3 bound to DNA (observable as a nuclear staining) was noticed neither after step 1 (incubation with TO-PRO-3-TSLs at $37^{\circ} \mathrm{C}$ and US) nor after step 2 (heating to $41^{\circ} \mathrm{C}$ and US). However although the addition of the microbubbles did not have any visible effect without ultrasound, the areas sonicated under these conditions clearly showed cellular uptake of TO-PRO-3 (figure 1). Conclusion Temperature-controlled US-induced drug delivery using heat sensitive liposomes together with sonoporation facilitated by cavitating microbubbles was shown to be a successful strategy to locally deliver a model drug and mediate cellular uptake at the same time. This approach may find application in intracellular delivery of drugs hampered by biological barriers. Figure 1. Cells incubated with TO-PRO-3-TSLs at $37^{\circ} \mathrm{C}$ (step 1), 41oC (step 2) and with the addition of Sonovue (step 3). After every step some areas were exposed to US. Only after the addition of Sonovue and the application of US, TO-PRO-3 bound to the DNA of the cells was observed. 

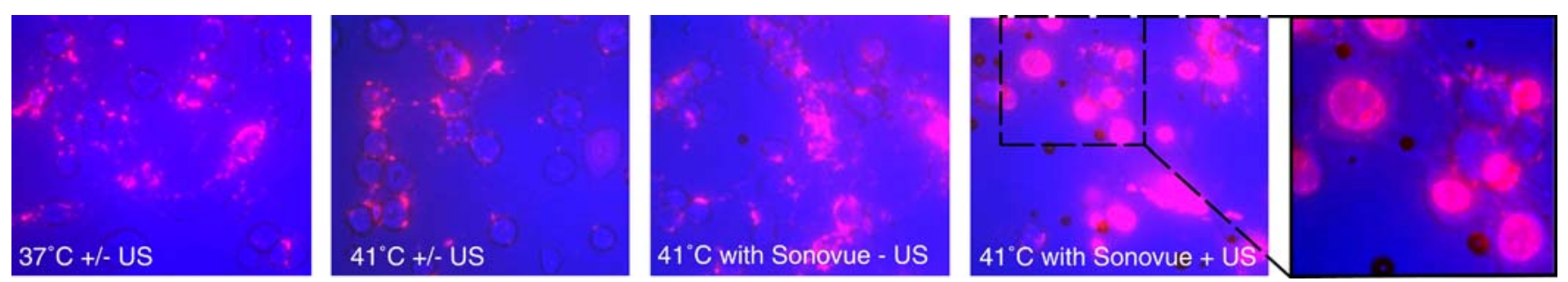

signal intensity. MRgHIFU precisely controlled and monitored contrast agent release from LTSLs through noninvasive temperature monitoring. Plans include in vivo delivery optimization prior to

IMAGE-ABLE TEMPERATURE SENSITIVE LIPOSOMES FOR IMAGE-GUIDED DRUG DELIVERY WITH MR-GUIDED HIGH INTENSITY FOCUSED ULTRASOUND

Ayele H. Negussie $^{1}$, Pavel S. Yarmolenko ${ }^{2}$, Henry Bryant ${ }^{1}$, Ari Partanen ${ }^{3}$, Mark W. Dewhirst ${ }^{2}$, Matthew R. Dreher ${ }^{1}$, Bradford J. Wood ${ }^{1}$

${ }^{1}$ Radiology and Imaging Sciences, Clinical Cetner, National Institutes of Health, Bethesda, MD, USA, ${ }^{2}$ Department of Biomedical Engineering, Duke University Medical Center, Durham, NC, USA, ${ }^{3}$ Philips Healthcare, Cleveland, OH, USA.

Objective: Despite development of passive drug delivery systems, such as liposomes, selective delivery of chemotherapeutics to solid tumors remains challenging. To target a tumor with image-guided precision, magnetic resonance guided high intensity focused ultrasound (MRgHIFU) can actively trigger release of therapeutics from low temperature sensitive liposomes (LTSLs). LTSLs release their lumen contents near the melting temperature $\left(\mathrm{Tm}=41.3^{\circ} \mathrm{C}\right)$ of their lipid membrane. With image-able LTSLs, this MR-guided approach allows for dynamic monitoring of biodistribution or contrast agent release from LTSLs in vivo in real time to optimize drug deposition with feedback control, or to predict response and guide future therapies. The objective of this study was to develop clinicallyrelevant MR image-able LTSLs that contain both a contrast agent and doxorubicin (Dox). Materials and Methods: LTSLs (DPPC: MSPC:DSPE-PEG2000, 85.3:9.7:5) were co-loaded actively with Dox and both passively and actively with a Gd-based MR contrast agent. Following various loading conditions, these formulations were investigated for weight percent (wt\%) Dox and Gd, as well as stability. Release of Dox and relaxivity of image-able LTSLs were investigated as a function of temperature. Contrast agent release was induced and imaged in vitro in real time with an MRgHIFU platform (Philips). Results: The wt $\%$ of Dox loaded into LTSLs was consistently $\sim 5 \mathrm{wt} \%$ as desired and the passive loading procedure resulted in $\sim 15 \mathrm{wt} \%$ Gd with stability in both size $(105-122 \mathrm{~nm})$ and thermal release properties for 7 days. LTLSs released $7 \%$ of Dox over $15 \mathrm{~min}$ at $37^{\circ} \mathrm{C}$ in physiologic buffer but greater than $80 \%$ release of Dox in $\sim 8 \mathrm{~s}$ at $41.3^{\circ} \mathrm{C}$. The relaxivity increased from 2.4 to $4.0 \mathrm{mM}-1 \mathrm{~s}-1(1.5 \mathrm{~T})$ when LTSLs were heated above $41.3^{\circ} \mathrm{C}$. This relaxivity increase is due to greater permeability of the liposomal membrane to both contrast agent and water. The greater T1-weighted signal with temperature corresponded to similar kinetics of Dox release. Sonications in an agar phantom containing image-able LTSLs demonstrated an increase in T1-weighted signal only corresponding to regions containing LTSLs that were heated above Tm. Conclusion: LTSLs were formulated with both Dox and a Gd-based FDA-approved contrast agent. These LTSLs demonstrate ultra fast release of Dox and a similar increase in T1-weighted clinical translation.

J905

\section{DESTRUCTION OF MICROBUBBLES IN THE TUMOR VASCULATURE BY IMAGE-GUIDED FOCUSED ULTRASOUND INHIBITS TUMOR GROWTH}

Alexander L. Klibanov ${ }^{1,2}$, Talent Shevchenko ${ }^{1}$, Bijoy K. Kundu ${ }^{2}$, Marcel Bohmer $^{4}$, Ralf Seip $^{3}$, Balasundar I. Raju ${ }^{3}$, Chien Ting Chin $^{3}$

${ }^{1}$ Dept. of Medicine, Cardiovascular Division, University of Virginia, Charlottesville, VA, USA, ${ }^{2}$ Radiology, University of Virginia, Charlottesville, VA, USA, ${ }^{3}$ Philips Research, Briarcliff Manor, NY, USA, ${ }^{4}$ Philips Research, Eindhoven, Netherlands.

Microbubble destruction by ultrasound in the vascular bed is known to cause bioeffects: increase of vessel wall permeability and extravasation, drug and gene delivery, therapeutic angiogenesis. In this study, we investigate the therapeutic effects of focused ultrasound treatment of microbubbles in tumor vasculature. Decafluorobutane microbubbles $(\sim 2$ um size, 500 million particles per $\mathrm{ml}$ saline) were stabilized with phosphatidylcholine/PEG stearate shell. Subcutaneous tumor model (MC38 colon adenocarcinoma, courtesy J. Schlom, NIH, in the hind leg of C57BL/6 mice) was applied. Microbubbles $(0.1 \mathrm{ml})$ were injected i.v., followed by focused ultrasound treatment of the tumor (TIPS system, $1.2 \mathrm{MHz}, 100,000$ cycles, $5 \mathrm{MPa}, 3 \times 10$ pulses, twice daily). Treatment was guided by real-time ultrasound imaging (HDI5000): CL-15 transducer was fixed on the therapeutic ultrasound transducer assembly, calibrated in the microbubble dispersion tank, and destruction zone crosshair placed on the imaging system screen for tumor targeting. In control animals $(n=7)$, microbubbles were not administered. Tumor therapy $(n=7)$ was performed every day, except when the tumor shrunk and was not visually detected. To monitor perfusion, microbubble movement through the vasculature was observed by ultrasound imaging. Tumor metabolism the day before and the day after ultrasound treatment was compared by FDG-PET administration and imaging in a Focus 120 microPET system. To monitor extravasation, Evans Blue dye was administered i.v. prior to insonation, in a separate study. $30 \mathrm{~min}$ after treatment, animals were euthanized and tissue extracted with formamide for quantification. In the control group lacking microbubbles, tumor growth continued unimpeded until reaching the approved size termination limit, mean $=228 \mathrm{~mm}^{2}$. In the experimental group, tumor growth inhibition was observed after repeated microbubble insonation in the tumor 
vasculature: after 2 weeks of study mean tumor size was $29 \mathrm{~mm}^{2}$. Therapy effect was not likely thermal: ultrasound-induced hyperthermia was $<3^{\circ} \mathrm{C}$. After therapy cessation, tumors resumed growth, so the effect cannot be due to ultrasound-induced immune response to tumor. Transient (minutes) reduction of blood flow was observed in the tumor mass after microbubble destruction. This effect might even cause reduction of anticancer agents delivery to the tumor, as modeled by Evans Blue dye. In conclusion, destruction of microbubbles in the tumor vasculature by the image-guided focused ultrasound inhibits tumor growth; this could become a useful tumor therapy tool.

\section{J906}

\section{TARGETED TRANSFECTION BY SONOPORATION IN INFLAMMATORY BOWEL DISEASE}

Jose L. Tlaxca ${ }^{2}$, Alexander L. Klibanov ${ }^{3}$, Christopher R. Anderson ${ }^{1}$, John A. Hossack ${ }^{2}$, Klaus Ley ${ }^{4}$, Michael B. Lawrence ${ }^{2}$, Theresa T. Pizarro ${ }^{5}$, Joshua Rychak $^{1}$

${ }^{1}$ Targeson, Inc, Charlottesville, VA, USA, ${ }^{2}$ Biomedical Engineering, University of Virginia, Charlottesville, VA, USA, ${ }^{3}$ Internal Medicine, University of Virginia, Charlottesville, VA, USA, ${ }^{4}$ La Jolla Institute for Allergy and Immunology, La Jolla, CA, USA, ${ }^{5}$ Pathology, Case Western Reserve University, Cleveland, OH, USA.

We have investigated microbubble-mediated sonoporation as a mechanism for targeted gene delivery. Plasmid DNA was electrostatically coupled to the shell of cationic lipid microbubbles. The extent of plasmid loading was quantified by absorbance and fluorescence measurements. Plasmid containing cDNA encoding firefly luciferase or GFP were delivered into cultured endothelial cells by the application of high-power ultrasound (sonoporation), and transfection efficiency and viability was evaluated by fluorescence microscopy and flow cytometry. We evaluated the role of insonation time, peak negative pressure, and microbubble concentration on transfection efficiency in three mammalian cell lines. We observed that GFP transfection increased with insonation time, pressure, and microbubble concentration, although decreased cell viability was observed. We next sought to determine the feasibility of targeting transfection to specific cell types. In the context of inflammatory bowel disease, inflamed endothelial cells can be identified by the up-regulation of the MAdCAM-1 receptor on the cell surface. We conjugated an anti-MAdCAM-1 antibody to the surface of the cationic microbubble, and measured the density of the targeting ligand and the plasmid payload as above. We assessed microbubble adhesion in a flow chamber assay against recombinant murine MadCAM-1 and endothelial cells stimulated to express MAdCAM-1, and observed specific microbubble accumulation relative to control microbubbles. We characterized our system in the SAMP $1 / \mathrm{YitFc}$ mouse model of inflammatory bowel disease, which exhibits spontaneous inflammation of the bowel and microvascular MAdCAM-1 expression. Microbubbles bearing a luciferase plasmid were administered intravenously to SAMP or healthy control mice and allowed to accumulate over fifteen minutes. Significant accumulation of MAdCAM-1 targeted, but not control, microbubbles was observed by contrast ultrasound imaging. The bowel was then insonated at high-power using a commercial sonoporator to mediate transfection by sonoporation. Optical imaging $24 \mathrm{~h}$ after treatment revealed high luciferase expression in the targeted gut, with minimal signal in healthy mice or in mice administered a luciferase-bearing microbubble targeted with an isotype antibody. Our data suggests that microbubble-mediated sonoporation is a robust technique for transfection in vitro and in vivo, with excellent specificity when combined with targeted delivery. Applications include preclinical cell and animal research, with potential future clinical use.

\section{J907}

\section{PULSED HIGH INTENSITY FOCUSED ULTRASOUND EXPOSURES ENHANCED DELIVERY: INVESTIGATIONS IN A MURINE MODEL}

Hilary Hancock, Lauren H. Smith, Victor Frenkel

Radiology and Imaging Sciences, Clinical Center, National Institutes of Health, Bethesda, MD, USA.

Recent preclinical studies have shown that pre-exposing tissues to pulsed high intensity focused ultrasound (HIFU) can non-invasively enhance the delivery of various agents to improve therapeutic efficacy. These studies were carried out with an image-guided HIFU system in a variety of tissue models, such as tumors and blood clots, where the effects occurred in a non-destructive manner. The manner by which this occurs is thought to be the result of improved distribution of those agents in the pre-treated tissue; by enhanced extravasation and improved interstitial transport. The objective of this study was to characterize these distributions and corroborate them with effects induced in the tissue. In order to accomplish this we employed a multidisciplinary approach, based on pulsed-HIFU exposures in the flank muscle of mice, followed by either local or systemic injections of a variety of fluorophores. Whole animal and microscopic in vivo imaging was employed. Collected data was processed quantitatively to characterize the enhancing effects on distribution. Exposures followed by systemic injections of fluorescently labeled polystyrene nanospheres showed them to be restricted to the vasculature in non-treated tissue, but extravasate to the perivascular space in the muscle immediately after treatments. This data was supported by injections of fluorescently labeled lectin, which showed capillaries to be leaky. At $24 \mathrm{hrs}$, the nanospheres in the treated muscle were observed to be more evenly distributed throughout the tissue. By administering the nanospheres at various time points post-treatment and quantifying relative particle densities of captured images, it was found that delivery enhancement peaked immediately after treatment and gradually diminished over a period of a few days. Additional studies were carried out using local injections of a cocktail of fluorescent albumin and the nanospheres. Local distributions of the much smaller albumin represented the convective front of the injections and were not significantly increased by the pulsed HIFU exposures. Nanosphere distribution however did significantly increase by an average of more than 60 percent. Light microscopy showed structural alterations, such as enlarged gaps between muscle fiber bundles, in the treated tissue to support the results of the studies on extravasation and interstitial transport. Future studies are planned to employ the techniques described herein to optimize these effects, and to use them to elucidate the specific mechanisms (ultrasonic and biological) by which they occur. 


\section{J908}

\section{THERANOSTICS OF HEAD \& NECK CANCER USING \\ TARGETED HOLLOW GOLD NANOSHELLS}

Marites P. Melancon ${ }^{1}$, Qian Huang ${ }^{1}$, Sarah May ${ }^{1}$, Brandon S. Brown ${ }^{1}$, Daisuke $\mathrm{Sano}^{2}$, Jeffrey Myers ${ }^{2}$, Chaan $\mathrm{S} . \mathrm{Ng}^{3}$, Chun $\mathrm{Li}^{1}$

${ }^{1}$ Experimental Diagnostic Imaging, UT-MD Anderson Cancer Center, Houston, TX, USA, ${ }^{2}$ Head and Neck Surgery, UT-MD Anderson Cancer Center, Houston, TX, USA, ${ }^{3}$ Diagnostic Radiology, UT-MD Anderson Cancer Center, Houston, TX, USA.

In patients with advanced head and neck cancer (HNC), local tumor progression is the main cause of cancer-induced morbidity and mortality. For those patients with local-regional recurrence, further surgery or radiotherapy is often not feasible owing to limited normal tissue tolerance. Photothermal ablation (PTA) is one of the most promising options that target recurrent HNC because the technique is minimally invasive and less harmful on normal tissue. In this study, we co-injected C225-HAuNS and PGGd-NIR813 in nude mice ( $n=5 /$ group) bearing OSC-19 (oral squamous cell carcinoma) that has metastasized into the lymph nodes. C225-HAuNS is a hollow gold nanoshell conjugated with C225 monoclonal antibody that has been shown to target epidermal growth factor receptors in tumors and mediate photothermal ablation [Melancon, et al. Molecular Cancer Therapeutics, V17:p1730, 2008]. PG-Gd-NIR813 is a long circulating dual modality MR/optical imaging contrast agent that has been shown to drain into the sentinel lymph nodes, when injected subcutaneously, and has the potential of differentiating metastatic from non-metastatic lymph nodes [Melancon, et al. Invest. Radiol., V42:p569, 2007]. Results show that PG-Gd-NIR813, once injected intravenously drains into the cervical, axillary and inguinal lymph nodes in mice. Localization into the cervical lymph nodes help in positioning the laser for PTA. Twenty four hours after treatment of cervical lymph nodes with $808 \mathrm{~nm}$ laser at $4 \mathrm{~W} / \mathrm{cm} 2$ for $3 \mathrm{~min}$ show reduction of tumor volume as demonstrated by the lower luminescence activity (presence of luciferase in tumor) and fluorescence intensity of PG-Gd-NIR after laser treatment, which was confirmed by histology (H\&E staining). In conclusion, successful PTA was achieved in mice due to the optical guidance of PG-Gd-NIR813 and heating of C225-HAuNS when both injected intravenously.

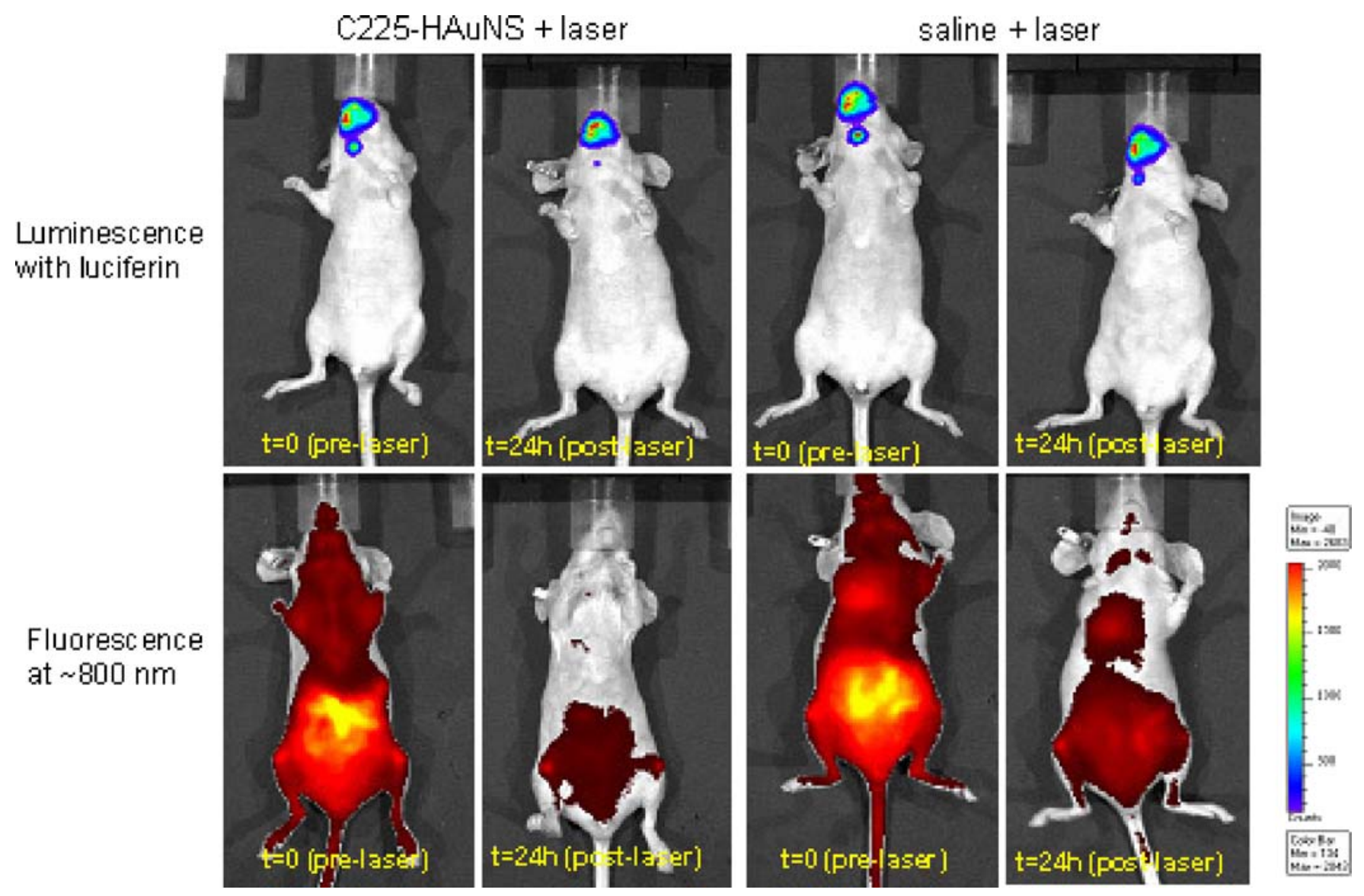


J909

\section{VERIFICATION OF PROTON BEAM DELIVERY WITH MRI}

Joao C. Seco ${ }^{1}$, Ravi T. Seethamraju ${ }^{2}$, George Philip L. Broussard ${ }^{1}$, Hanne M. Kooy ${ }^{1}$, Mukesh G. Harisinghani ${ }^{3,4}$

${ }^{1}$ Radiation Oncology, Massachusetts General Hospital, Boston, MA, USA, ${ }^{2}$ MR R and D, Siemens Medical Solutions, USA Inc., Malden, MA, USA, ${ }^{3}$ Radiology, Massachusetts General Hospital, Boston, MA, USA, ${ }^{4}$ Radiology, Center for Molecular Imaging Research, Boston, MA, USA.

Introduction: In comparison to photon beams, proton beam is most sensitive and abrupt to the variation in depth at the distal end of the delivery where the dose can vary up to $90 \%$ within $1 \mathrm{~cm}$ of water equivalent depth (WED). This means that the dose can go from maximum to minimum within a small layer of $1 \mathrm{~cm}$ of water. In the case of treatments we have a very small window of error because the tumor may receive too little radiation or healthy tissues may get too much of radiation. Currently CT is the modality of preference for planning and it cannot accurately measure end of range of the proton delivery (EOR), so we conducted experiments with MRI to see if we can assess EOR more accurately. Methods: We built several phantoms with synthetic and tissue based materials. These phantoms were first irradiated with protons and subsequently scanned with MRI. Non-tissue phantom experiments were first conducted on 1.5 Tesla, while for the tissue phantom experiments we switched to $7 \mathrm{~T}$ expecting higher sensitivity. The protons have a finite range in the gel/tissue, where the majority of the protons accumulate at the end of the range. The synthetic phantoms were composed of either gelatin (high content of water) or mayonnaise (high content of fatty compounds). The tissue phantom was composed of bovine tissue with muscle and fat. The proton beams had a range of approximately $16 \mathrm{~cm}$ in water (corresponds approximately to $160 \mathrm{MeV}$ kinetic energy) and modulation of $6 \mathrm{~cm}$. A total dose of 500 cGray was delivered to each phantom, which took a total of $5 \mathrm{~min}$. Each phantom was imaged with dedicated T1, T2 and T2* weighted MR sequences 5-10 min postproton irradiation. Results: We found that in a gelatin phantom the EOR was restricted to the predicted region very closely (Fig 1a), while in the case of mayonnaise phantom (Fig 1b) though the EOR is pretty close the predicted region, it is not precise, however in a tissue phantom the EOR was off by $1-2 \mathrm{~cm}$ thereby predicting that in in-vivo situations corrections need to be applied to the normal calculations. Also by examining a large cohort of individual with different body types it should be possible in the future to deliver the protons under MRI guidance accurately.
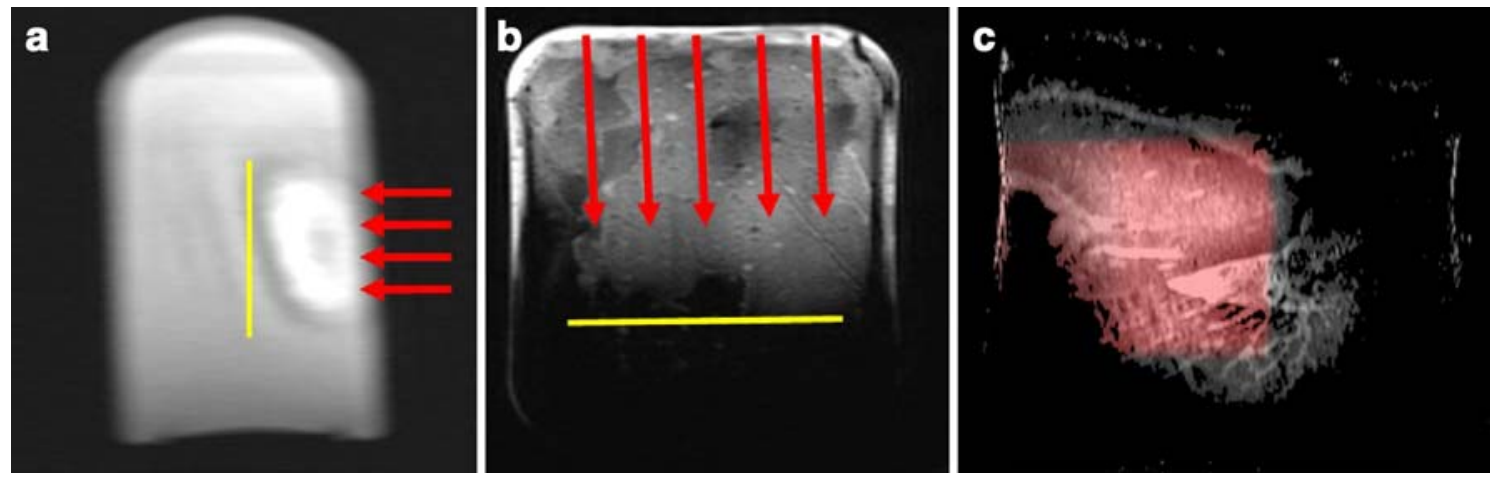

Fig. 1 Predicted and observed EOR from photon irradiation in (a) gelatine phantom, (b) mayyonaise phantom and (c) bovine tissue. It is well contained in the case of gelatine and mayyonaise phantoms, but exceeded in bovine tissue.

J910

\section{CANCER GENE THERAPY UTILIZED ULTRASOUND (US)-SENSITIVE LIPOSOME AS NON-VIRAL VECTOR}

Ryo Suzuki ${ }^{1}$, Yusuke Oda ${ }^{1}$, Norihito Nishiie ${ }^{1}$, Shota Otake ${ }^{1}$, Risa Koshima $^{1}$, Naoki Utoguchi ${ }^{1}$, Yoichi Negishi ${ }^{3}$, Shinsaku Nakagawa ${ }^{2}$, Kazuo Maruyama ${ }^{1}$

${ }^{1}$ Biopharmaceutics, School of Pharmaceutical Sciences, Teikyo University, Sagamihara, Japan, ${ }^{2}$ Biotechnology and Therapeutics, Graduate School of Pharmaceutical Sciences, Osaka University, Suita, Japan, ${ }^{3}$ Drug and Gene Delivery System, School of Pharmacy, Tokyo University of Pharmacy and Life Science, Hachioji, Japan.

[Objective] Sonoporation is an attractive technique to develop noninvasive and non-viral gene delivery system. However, simple sonoporation using only ultrasound (US) is not enough to establish effective cancer gene therapy because of low efficiency of gene delivery. Therefore, we improved this problem by the combination of US and novel US-sensitive liposome (Bubble liposome) which was a liposome containing US imaging gas (perfluoropropane). This was an effective gene delivery system with collapse (cavitation) that was induced by US exposure to Bubble liposome. In this study, we assessed the ability of this system in cancer gene therapy using IL-12 cording plasmid DNA. [Material \& Methods] B6C3F1 mice were intradermally inoculated with mouse ovarian cancer cells (OV-HM cells) into flank. After 7 days, Bubble liposome $(2.5 \mu \mathrm{g})$ and mouse IL-12 cording plasmid DNA $(10 \mu \mathrm{g})$ were injected into tumor, and US $(1 \mathrm{MHz}, 0.7 \mathrm{~W} / \mathrm{cm} 2,60 \mathrm{~s})$ was transdermally exposed toward tumor. In addition, gene delivery with conventional lipofection method using Lipofectamine 2000 
was also examined as control of non-viral vector. Anti-tumor effects were evaluated by measuring tumor volume. [Results \& Discussion] In IL-12 gene delivery with conventional lipofection method, the suppression of tumor growth was not observed. On the other hand, the combination of Bubble liposome and US was dramatically suppressed tumor growth (Fig. 1). In the tumor tissues delivered IL-12 gene with Bubble liposome and US, migration of CD8 $+\mathrm{T}$ cells were observed. Therefore, we concluded that the combination of Bubble liposome and US would be a good nonviral vector system in cancer gene therapy. [Acknowledgements] This work was supported by JSPS KAKENHI (20240053) and Health and Labour Sciences Research Grants.

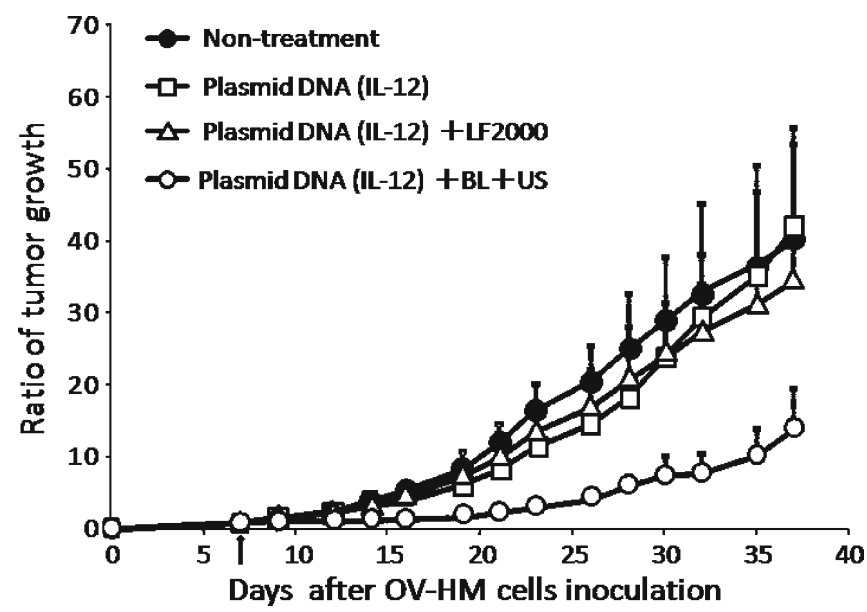

Fig. 1 IL-12 cancer gene theraphy in the combination of Bubble liposome and US $\uparrow:$ Gene theraphy, BL: Bubble liposome, US: Ultrasound, LF2000: Lipofectamine 2000
J911

\section{ULTRASOUND TRIGGERED DRUG RELEASE UNDER IMAGE GUIDANCE}

$\underline{\text { Holger Gruell }}^{1,2}$, Sander Langereis ${ }^{1}$, Mariska de Smet $^{2}$, Edwin Heijman ${ }^{1}$, Jochen Keupp $^{3}$

${ }^{1}$ Biomolecular Engineering, Philips Research, Eindhoven, Netherlands, ${ }^{2}$ Biomedical NMR, Eindhoven University of Technology, Eindhoven, Netherlands, ${ }^{3}$ Tomographic Systems, Philips Research, Hamburg, Germany.

High Intensity Focused ultrasound (HIFU) is an excellent method for local triggered drug delivery using either pressure or temperature sensitive delivery systems. Temperature sensitive liposomes (TSLs) as drug carriers are promising to maximize delivery of drugs to tumors that are heated slightly above body temperature using HIFU. Besides heating, the pressure pulses of ultrasound waves can be exploited to disrupt microbubbles, which can lead to formation of transient pores in the endothelial wall. The latter is termed sonoporation and was shown to mediate cellular uptake of therapeutic agents. Either way, imaging based method to visualize, follow, and control drug delivery in-vivo are of importance to establish more quantitative treatment protocols. Here, we will present and compare approaches of image guidance for ultrasound triggered drug delivery using T1-, chemical exchange saturation transfer (CEST)-, iron oxide- and fluorine-based agents that are coreleased with a drug from the lumen of a temperature-sensitive liposome. The temperature of drug release but also imaging related properties can be tuned by the composition of the phospholipid bilayer. The rapid release of ionic solutes as well as macromolecular contrast agents can be explained by the formation of transient pores in the lipid membrane. Local hyperthermia is induced using a focused ultrasound system integrated in a 3 T MRI scanner. First phantom studies will be shown as a proof of concept, the application for in-vivo drug delivery will be discussed.

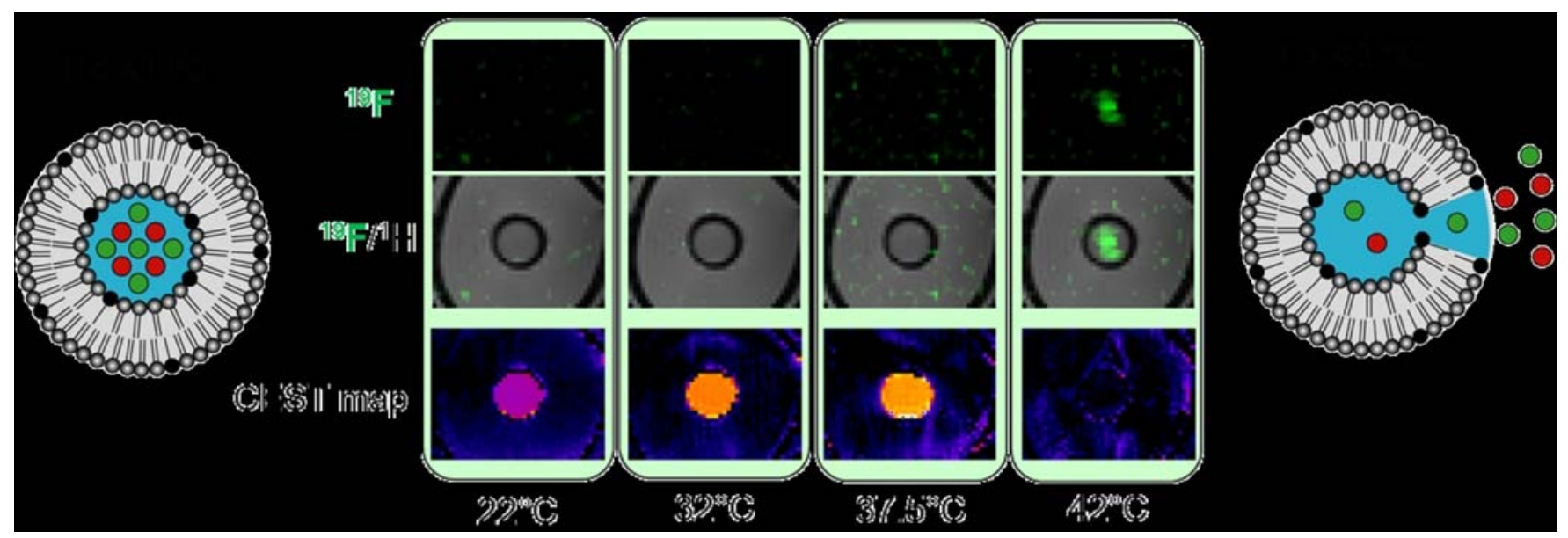


Simultaneous CEST and 19-F-MR imaging visualizing release from temperature sensitive drug carriers (phantom study) at temperatures slightly above body temperature.

\section{J912}

\section{IMAGE-GUIDED COMBINED SIRNA AND ENZYME/PRODRUG CANCER THERAPY}

Cong Li, Marie-France Penet, Flonné B. Wildes, Tomoyo Takagi, Paul T. Winnard, Dmitri Artemov, Zaver M. Bhujwalla

JHU ICMIC Program, The Russell H. Morgan Department of Radiology and Radiological Science, The Johns Hopkins University School of Medicine, Baltimore, MD, USA.

The ability to downregulate specific pathways that are overexpressed and critically important in cancer using small interfering RNA (siRNA) technology provides unprecedented opportunities to develop novel cancer-cell specific treatments. The ability to detect the delivery of the siRNA and combine it with the delivery of a chemotherapeutic agent primarily localized within the tumor would be of significant advantage in this quest. Here we have developed a prototype agent to image the delivery of a prodrug enzyme, bacterial cytosine deaminase (bCD), that converts a nontoxic prodrug 5-fluorocytosine to 5-fluorouracil, in combination with siRNA targeting of choline kinase, an enzyme critically important in breast cancer (Figure 1). We synthesized a conjugate, bCD-PEIPLL, in which the prodrug-activating enzyme bCD that converts the nontoxic prodrug 5-fluorocytosine $(5 \mathrm{FC})$ to the active drug 5fluorouracil $(5 \mathrm{FU})$, multimodal imaging reporter labeled poly-Llysine (PLL), and siRNA delivery vector polyethyleneimine (PEI), were incorporated covalently. In vivo MRI and optical imaging showed efficient intratumoral delivery of the polyplex and significant tumor cell kill after the image-guided combined treatments. Ex vivo H\&E staining verified the efficient therapeutic effect without acute toxicity. In vivo total choline down-regulation was detected. The bCD-PEI-PLL/siRNA-chk polyplex mediated combined siRNA and prodrug therapy demonstrated higher therapeutic effect than either prodrug or siRNA treatment given alone in vitro and in vivo. The data demonstrate that image-guided combined siRNA and prodrug enzyme strategy has significant potential in increasing therapeutic efficacy while minimizing damage to normal tissues.

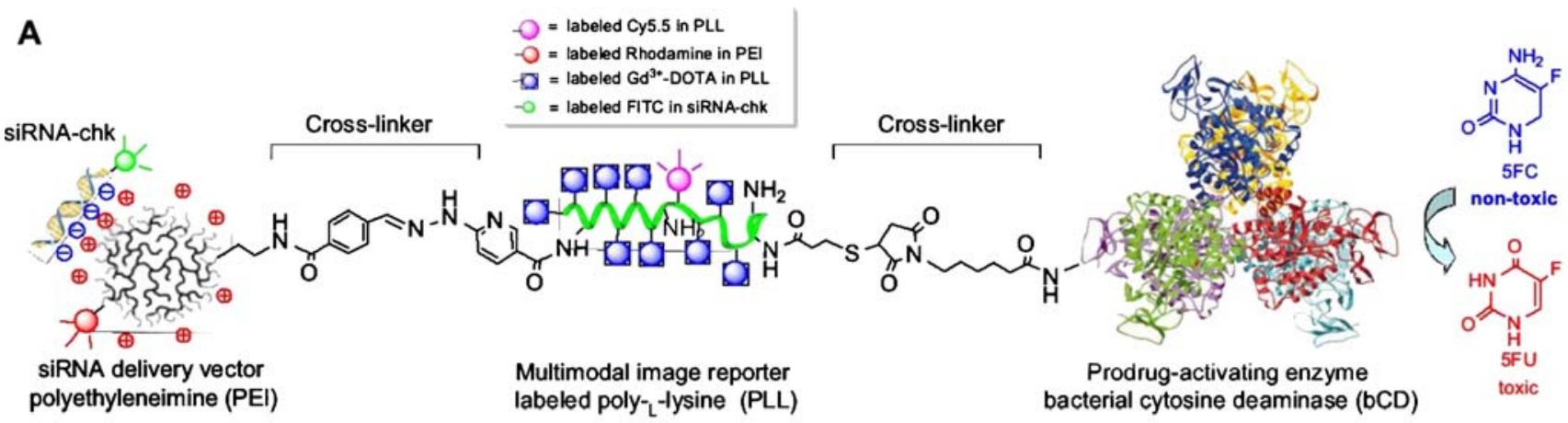

B

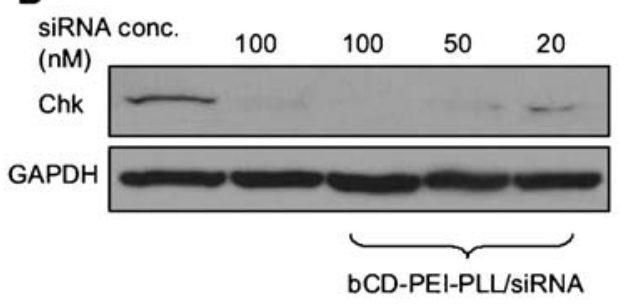

C

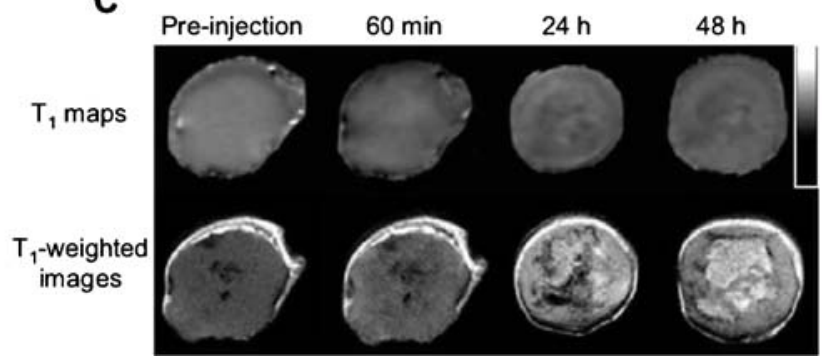

D

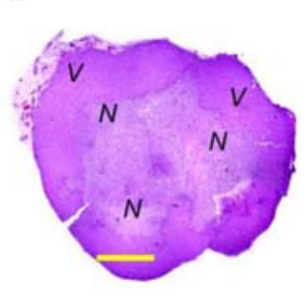

Figure 1 (A) Schematic of bCD-PEI-PLL/siRNA-chk polyplex. (B) Western blot of choline kinase in MDA-MB-231 cells after the treatment with the polyplex $(\mathrm{N} / \mathrm{P}=50$, siRNA conc.: $100 \mathrm{nM}) \mathrm{f}$ or $48 \mathrm{~h}$. Oligfectamine alone and oligofectamine/siRNA-chk lipoplex were used as the control. (C) In vivo dynamic $\mathrm{T}_{1}$ map and $\mathrm{T}_{1}$-weighted images of a wild type human breast MDA-MB-231 tumor xenograft after intravenous injection of the polyplex $(380 \mathrm{mg} / \mathrm{kg}, \mathrm{N} / \mathrm{P}=50)$. (D) $\mathrm{H} \& \mathrm{E}$ stained histological sections of the tumor treated with the polyplex for $48 \mathrm{~h}$ (the same tumor in panel C). Purple hematoxylinphilic regions $(\boldsymbol{V})$ indicate viable tumor tissues, and the e osinopholic area indicates tumor necrosis $(\boldsymbol{N})$. Scale bar: $1.0 \mathrm{~mm}$.

J913

\section{REMOTE LASER-DEPLOYED PARTIALLY-POLYMERIZED LIPOSOME DRUG CARRIERS FOR DRUG DELIVERY}

Guoting Qin, Zheng Li, Brian E. O'Neill, Rongmin Xia, Feng Li, King Li Radiology, The Methodist Hospital Research Institute, Houston, TX, USA.

Liposomal drug carriers have been used to encapsulate drugs in order to alter their biodistribution and pharmacokinetics, but the clinical applications are limited by their slow release of packaged contents. To overcome the slow release problem, liposomes have also been developed for active deployment based on heat activated release mechanism via global (relative to the size of the liposome) tissue heating. While it appears that these techniques can improve the localization of the drug, the lingering problem of liposome instability at body temperature remains. Fully polymerized drug carriers have also been used for similar purposes to carry therapeutic and imaging agents to the target, while they tend to have the 
opposite problem, that is, they are not easily opened at any temperature achievable in vivo. Based on these observations we hypothesized that by using a strategy of combining these two approaches we should be able to produce a drug delivery vehicle stable at physiologic temperature with a controlled release mechanism. In this study, polymerizable lipid and unpolymerizable lipid were mixed to yield partially polymerized liposomes (PPLs) and gold nanoparticles were attached on the surface of the PPLs through Au-SH-lipid linkage so that these PPLs could be triggered to release by laser. By optimizing the ratio of the two types of lipids, gold nanoparticle decorated PPLs were stable at physiological condition, and were opened by laser activation to release the encapsulated SPECT imaging agent Tc-99m HIDA. The release of Tc-99m HIDA was quantified by HPLC method. One hundred pulses ( $5 \mathrm{~ns}$ ) of the laser was sufficient to release over $70 \%$ of Tc-99m HIDA from PPLs with gold, while the same treatment of PPLs without gold resulted in a less than $5 \%$ release. Significant release was not observed at $37^{\circ} \mathrm{C}$. We believe that these remote activatable PPLs could be used to deliver encapsulated drug or imaging agent to target tissue with precise spatial and temporal control.

J914

\section{A NOVEL INTRA-OPERATIVE NEAR-INFRARED FLUORESCENCE CAMERA SYSTEM FOR OPTICAL IMAGE-GUIDED CANCER SURGERY}

$\underline{\text { Sven Mieog }^{1} \text {, Alexander Vahrmeijer }}{ }^{1}$, Merlijn Hutteman ${ }^{1}$, Maurits R. Drijfhout van Hooff ${ }^{2}$, Jouke Dijkstra ${ }^{2}$, Peter Kuppen ${ }^{1}$, Rob Keijzer ${ }^{1}$, Ivo Que $^{3}$, Eric Kaijzel ${ }^{3}$, Cornelis van de Velde ${ }^{1}$, Clemens Lowik ${ }^{3}$

${ }^{1}$ Surgery, Leiden University Medical Center, Leiden, Netherlands, ${ }^{2}$ Radiology, Division of Image Processing, Leiden University Medical Center, Leiden, Netherlands, ${ }^{3}$ Endocrinology, Leiden University Medical Center, Leiden, Netherlands.

Background An incomplete tumor resection is an important clinical problem in surgical oncology. Because the surgeon can only rely on palpation and visual inspection during surgery, realtime visualization of cancer cells is needed to increase the number of patients with a complete tumor resection. For this purpose, near-infrared fluorescence (NIRF) imaging is a promising technique. Here, we describe a hand-held, intra-operative NIRF camera system equipped with a $690 \mathrm{~nm}$ laser and validated its utility in detecting and guiding resection of cancer tissues in two syngeneic rat models. Material and methods The Fluobeam ${ }^{\circledR}$ system (Fluoptics, Grenoble, France) is composed of a class 3B laser emitting at $690 \mathrm{~nm}$ CCD camera. The camera system was calibrated using the cathepsin-activatable probe ProSense ${ }^{\circledR}$ (VisEn Medical, USA). Prosense was activated in vitro with 100 ul $0.25 \%$ trypsin at $37 \mathrm{C}$ for $1 \mathrm{~h}$, diluted and and aliquoted into $2 \mathrm{ml}$ tubes for imaging at 3,8 and $50 \mathrm{~ms}$ laser excitation time. The hormonedependent syngeneic breast cancer rat model EMR86 and the syngeneic colorectal cancer rat cell line CC531 were used for intra-operative imaging. Six female Wag/Rij rats were subcutaneously transplanted with fresh EMR86 tumor fragments and six male Wag/Rij rats were subcapsularly injected with $5 \times 105$ CC531 cells at three distinct liver lobes. Rats were i.v. injected with ProSense680 or 750 and imaged $24 \mathrm{hr}$ thereafter. Results Fluorescence intensity was strongly correlated with increased activated-probe concentration $(\mathrm{R} 2=0.9903)$. We successfully detected and resected small primary mammary tumors and peritoneal colorectal metastases under direct fluorescent guidance. The signal of the tumor was high and mostly saturated and was at least 2-3 times higher than the surrounding tissue for ProSense 680 $(t=13.84, p<0.0001)$ as well as for ProSense750 $(t=14.78, p<$ $0.0001)$. Conclusion Using the Fluobeam ${ }^{\circledR}$ system, we demonstrated its intra-operative utility in guiding resection of small tumors in two syngeneic rat models. Surgical oncology will make a major step forward when this technique will be clinically introduced. Acknowledgement We want to thank Fluoptics (Grenoble, France) for providing us with the Fluobeam ${ }^{\circledR}$ system to perform the above described experiments.

\section{J915}

\section{PRE-CLINICAL VALIDATION OF NEAR-INFRARED FLUORESCENCE (NIRF) IMAGE-GUIDED SURGERY FOR THE COMPLETE RESECTION OF BREAST TUMORS}

Sven Mieog $^{1}$, Merlijn Hutteman ${ }^{1}$, Cornelis van de Velde ${ }^{1}$, Ivo Que ${ }^{3}$, Rob Keijzer $^{1}$, Eric Kaijzel ${ }^{3}$, Jouke Dijkstra ${ }^{2}$, Boudewijn Lelieveldt ${ }^{2}$, Peter Kuppen ${ }^{1}$, Clemens Lowik ${ }^{3}$, Alexander Vahrmeijer ${ }^{1}$

${ }^{1}$ Surgery, Leiden University Medical Center, Leiden, Netherlands, ${ }^{2}$ Radiology, Division of Image Processing, Leiden University Medical Center, Leiden, Netherlands, ${ }^{3}$ Endocrinology, Leiden University Medical Center, Leiden, Netherlands.

Background An incomplete tumor resection occurs in $5-45 \%$ of the patients undergoing breast-conserving surgery. As the surgeon can only rely on palpation and visual inspection, real-time visualization of cancer cells at the time of surgery is needed to increase the number of complete tumor resections. Near-infrared fluorescence (NIRF) imaging is a promising technique to assess the extent of disease during surgery. This study aimed to validate NIRF imaging in a breast cancer rat model. Material and methods The hormone-dependent syngeneic breast cancer rat model EMR86 and its derived cell line MCR86 were used. Tumor cells were detected with the cathepsinactivatable NIRF probe ProSense ${ }^{\circledR}$ (VisEn Medical, USA). Fluorescence imaging of the animals and organs was performed using the IVIS Spectrum (Caliper, USA) and the Odyssey (LI-COR, USA). Intra-operative fluorescence imaging was performed using a prototype hand-held laser camera system (Fluoptics, France). Results ProSense was activated by MCR86 cells. The signal intensity was linearly correlated with the number of cells, the concentration of ProSense and the incubation time (all R2>0.93, $p<0.0001$ ). The influence of ProSense concentration and tumor volume on fluorescent intensity was confirmed in nine rats each bearing four tumors (mean volume $=$ $0.38 \pm 0.36 \mathrm{~cm} 3$ ). In contrast to the cell line data, no difference in fluorescence intensity was found between $24 \mathrm{hr}$ and $48 \mathrm{~h}$ after injection of ProSense (paired $t=-0.27, p=0.80$ ). Therefore, subsequent experiments were conducted $24 \mathrm{~h}$ after injection. Using the intraoperative NIRF camera, all 64 mammary tumors $(0.01-1.8 \mathrm{~cm} 3)$ were successfully detected. Histological assessment of residual fluorescent hotspots confirmed the presence of breast cancer cells indicating incomplete resections of the primary tumor (margin positivity). The signal intensity of the tumor was 60 times higher than the surrounding muscle tissue $(p<0.0001)$. Using fluorescence scanning microscopy revealed a significantly higher signal intensity of the tumor border compared to the center $(t=-2.74, p=0.048)$, possibly reflecting the higher cathepsin-activity at the invasive border. Conclusion We demonstrated that it is possible to resect sub-mm breast tumor depositions under fluorescent guidance in a syngeneic rat model. Surgical oncology will make a major step forward when this technique will be clinically introduced. Acknowledgement We want to thank Fluoptics (Grenoble, France) for providing us with the Fluobeam system to perform the above described experiments. 
J916

IMAGE-GUIDED SURGERY OF LIVER METASTASES: INTRAOPERATIVE, NEAR-INFRARED FLUORESCENT IMAGING OF INTEGRIN $\alpha \beta 3$ EXPRESSION IN A SYNGENEIC COLORECTAL CANCER RAT MODEL

Sven Mieog $^{1}$, Merlijn Hutteman ${ }^{1}$, Cornelis van de Velde ${ }^{1}$, Ivo Que ${ }^{2}$, Rob Keijzer $^{1}$, Eric Kaijzel ${ }^{2}$, Boudewijn Lelieveldt ${ }^{3}$, Jouke Dijkstra ${ }^{3}$, Peter Kuppen ${ }^{1}$, Clemens Lowik ${ }^{2}$, Alexander Vahrmeijer ${ }^{1}$

${ }^{1}$ Surgery, Leiden University Medical Center, Leiden, Netherlands, ${ }^{2}$ Endocrinology, Leiden University Medical Center, Leiden, Netherlands, ${ }^{3}$ Radiology, Division of Image Processing, Leiden University Medical Center, Leiden, Netherlands.

Survival of colorectal cancer patients is largely restricted by the occurrence of metastases, predominantly in the liver. Real-time visualization of cancer cells using near-infrared fluorescence (NIRF) imaging is a promising technique to assess the extent of liver metastases during curative-intended surgery. However, imaging liver metastases is highly challenging due to high light absorbance of liver tissue and hepatic clearance of many fluorescent dyes. This study aimed to clearly demarcate liver metastases from the surrounding liver tissue in a colorectal cancer rat model using an integrin $\alpha v \beta 3$-directed NIRF probe. Methods. The syngeneic colorectal cancer rat cell line CC531 was used. Six male Wag/Rij rats $(300 \mathrm{~g})$ were subcapsularly injected with $5 \times$ $10^{\wedge} 5$ CC531 cells at three distinct liver lobes. The NIRF probe IntegriSense ${ }^{\circledR}$ (VisEn Medical, USA) was i.v. injected 24 or $48 \mathrm{hr}$ before imaging ( $40 \mathrm{nmol} / \mathrm{kg}$ ). Integrin $\alpha v \beta 3$ is involved in tumorassociated angiogenesis and tumor cell migration. IntegriSense ${ }^{\circledR}$ is directed against the RGD-binding site of integrin $\alpha v \beta 3$. Intra-operative fluorescence imaging was performed using a prototype hand-held laser camera system (Fluoptics, France). Results. In vitro experiments demonstrated the increased expression of integrin $\alpha v \beta 3$ on the CC531 cell line and the fluorescent signal intensity was linearly correlated with the concentration of IntegriSense ( $\mathrm{R} 2=0.99, p<0.0001)$. Using the intraoperative NIRF camera, all liver metastases $(6-80 \mathrm{~mm} 2)$ were successfully detected and were clearly demarcated from the normal liver tissue (4.7 times higher, $t=7.68, p=0.002$ ). The tumor signal in rats imaged $48 \mathrm{hr}$ after IntegriSense injection was two times higher than the tumor signal in the $24 \mathrm{hr}$ group $(t=19.53, p<0.0001)$, whereas the liver signal did not differ between the two groups $(t=0.56, p=0.63)$. Histological assessment of intra-operatively detected fluorescent hotspots in the peritoneal cavity confirmed depositions of colorectal cancer cells (typical diameter $0.5 \mathrm{~mm}$ ). Conclusion. We demonstrated the clear demarcation of colorectal liver metastases using a NIRF-probe sensing integrin $\alpha v \beta 3$ overexpression. Imaging after $48 \mathrm{~h}$ yielded significant improved fluorescence intensity. Moreover, sub-mm metastatic lesions were intra-operatively detected in the peritoneal cavity. Clinical application of NIRF imaging has the potential to improve the complete resection rate of liver metastasis surgery. We want to thank Fluoptics (Grenoble, France) for providing the Fluobeam system.

\section{J917}

\section{MONITORING HYPOXIC FACTORS IN RESPONSE TO VEGFR2 BLOCKADE IN BREAST TUMORS}

Keith Stantz ${ }^{1,4}$, Minsong $\mathrm{Cao}^{3}$, Ning $\mathrm{Cao}^{1}$, Bo Liu ${ }^{1}$, Kathy D. Miller ${ }^{2}$ ${ }^{\mathrm{T}}$ School of Health Sciences, Purdue University, West Lafayette, IN, USA, ${ }^{2}$ Hematology/Oncology, Indiana University School of Medicine, Indianapolis, IN, USA, ${ }^{3}$ Radiation Oncology, Indiana University School of Medicine, Indianapolis, IN, USA, ${ }^{4}$ Radiology, Indiana University School of Medicine, Indianapolis, IN, USA.
The purpose of this study is to monitor the longitudinal intra-tumor hemodynamic response to VEGFR2 blockade by imaging in vivo the hemoglobin status and vascular physiology of a solid tumor using photoacoustic spectroscopy (PCT-S) and dynamic contrastenhanced CT (DCE-CT). Introduction: Tumor hypoxia is an independent prognostic factor associated with local reoccurrence and patient survival in breast, head-and-neck, cervix, and prostate cancer. Hypoxia promotes angiogenesis and induces changes in hemodynamics which forms a heterogeneous environment and initiates epigenetic and genetic changes that modulate DNA repair mechanism, cell survival, and metastasis. Material and Methods: DCE-CT and PCT-S imaging was performed on athymic nude mice bearing xenograft VEGF-transfected MCF-7 tumors to quantify intra-tumor physiology and hemoglobin state pre- and post-injection (days 2, 7, and 14) of a nonspecific and specific monoclonal antibody targeting VEGFR2. Parametrical maps of tumor physiology-perfusion (F), permeability-surface area (PS), fractional plasma (fp) and interstitial space (fis) - and hemoglobin stateoxygen saturation $(\mathrm{SaO} 2)$ and hemoglobin concentration $(\mathrm{CtHb})$ were obtained at four time points over two week period. Results: A temporal multi-stage recovery process whereby a decoupling of the fractional change in physiological parameters ( $f p, F)$ was observed when comparing treated to control tumors: $\mathrm{fp}$ and $\mathrm{F}$ decreased on day 2, while only perfusion remained reduced on day 7. Analysis based on a mathematical model linking perfusion and vascular morphology indicates that a decrease in $\mathrm{fp}$ and perfusion was consistent with a reduction in blood vessel radius, followed by an increase in the vascular radius and tortuosity resulting in the decoupling of $\mathrm{fp}$ and perfusion before returning to control levels. Near infrared photoacoustic spectra (15 wavelengths in 690$940 \mathrm{~nm}$ range) were fit to calibrated oxy- and deoxy-hemoglobin data to obtain changes in oxygen saturation levels and hemoglobin concentration. Conclusion: These preliminary results show the potential of PCT-S and DCE-CT to measure factors associated with tumor hypoxia- $\mathrm{SaO} 2, \mathrm{CtHb}, \mathrm{F}$, and $\mathrm{fp}$ - and the longitudinal changes in the tumor environment induced by elevating VEGF transcription rates. Ongoing work focuses on how variations tumor's microenvironment influences the underlying mechanisms associated with local reoccurrence and metastasis, which will be used in the advancement of anti-angiogenic drugs and therapeutic strategies in cancer patients.

\section{J918}

\section{AN IMAGING-DRIVEN PHARMACOKINETIC MODEL FOR GUIDED DRUG DELIVERY}

Shengping Qin, Jai Woong Seo, Hua Zhang, Chun-Yen Lai, Dustin E. Kruse, Azadeh Kheirolomoom, Jinyi Qi, Katherine Ferrara BME, UC Davis, Davis, CA, USA.

Purpose In order to assess nanoparticle pharmacokinetics, a real-time dual-imaging methodology was developed by integrating PET and fluorescence imaging techniques with a hybrid pharmacokinetic model. In addition, the effect of ultrasound on the permeability of tumor vasculature and vehicle accumulation was modeled and investigated in real-time. Methods The particle shell of longcirculating liposomes (HSPC:cholesterol:DSPE-PEG2K:BAT-lipid= 55.9:39:5:0.1) and temperature-sensitive liposomes (DPPC:MPPC: DSPE-PEG2K:BAT-lipid=85.9:10:4:0.1) was labeled with $\mathrm{Cu}-64$ and the drug compartment labeled with a fluorescent probe or filled with cisplatin. Based on mass conservation, a pharmacokinetic model was proposed to predict the concentration of the shell and cargo in blood, liver and tumors. Model parameters were fit based 
on equations describing the PET and fluorescence imaging data. The Met-1 mouse was injected with labeled particles and insonified by 2.25-MHz ultrasound for 2 minutes with a cumulative thermal dose (CEM43) of approximately $0.5 \mathrm{~min}$. Results Without the application of exogenous heat, we find that the rates of release of a hydrophilic drug from the long-circulating and temperature-sensitive particles circulating within the blood pool are 0.003 and $0.2 \mathrm{~h}^{-1}$, respectively. The rates of transport to the reticulo-endothelial system for long-circulating and temperature-sensitive particles are 0.046 and $0.19 \mathrm{~h}^{-1}$, respectively. Prolonged life-time in circulation and slow drug release from liposomes result in a significantly greater drug area under the curve for the longcirculating particles. In addition, we have developed a transport constant for the transport of liposomes from the blood pool to the tumor interstitium, which is on the order of $0.02 \mathrm{hr}^{-1}$ for the Met-1 tumor system (corresponding to an apparent permeability of $4.37 \times 10^{-8} \mathrm{~cm} / \mathrm{s}$ ). Tumor transport significantly increases after insonation and reaches a maximum of about 3 times the original value at $2.1 \mathrm{hr}$ after injection, returning to baseline by 24 hours after insonation. Conclusion Image-driven pharmacokinetic modeling can play an invaluable role in the optimization of image-guided drug delivery, quantifying particle stability and accumulation and assessing interventional strategies. Acknowledgement: NIH R01 CA103828

\section{J919}

\section{ABSORPTION OF RADIOFREQUENCY ELECTROMAGNETIC ENERGY BY GOLD NANOPARTICLES FOR THERMALLY-ACTIVATED LOCAL DRUG DELIVERY}

Dustin E. Kruse ${ }^{1}$, Douglas N. Stephens ${ }^{1}$, Eric E. Paoli ${ }^{1}$, Heather A. Lindfors $^{1}$, Lorenzo Berti ${ }^{2}$, Kit S. Lam ${ }^{2}$, Katherine Ferrara ${ }^{1}$

${ }^{1}$ Biomedical Engineering, UC Davis, Davis, CA, USA, ${ }^{2}$ Internal Medicine, UC Davis Medical Center, Sacramento, CA, USA.

Encapsulating chemotherapeutic drugs into liposomes significantly reduces the cardiotoxicity, yet challenges remain for optimizing drug delivery to solid tumors. We have developed liposomal particles that are heat-activated by exogenous energy sources, thus locally delivering drugs. For particles that can be activated by ultrasound heating, we have found that a 60 fold increase in delivery of hydrophilic molecules can be achieved at 24 hours as compared to free drug administration. In order to activate particles with mild heating in the range of $39-40^{\circ} \mathrm{C}$, a short acyl (or single acyl) chain must be incorporated, and thus, the particles are not fully stable during circulation. We observe improved stability during circulation for particles with a longer acyl chain that can deliver a 177 -fold dose increase, but require a new method for activation due to the higher melting temperature of the lipid. To activate drug release from these liposomes, we are exploring the use of radiofrequency electromagnetic (RF-EM) energy to locally heat gold nanoparticles (NPs). We have developed an RF-EM delivery circuit capable of generating high electric field strengths required for non-invasive, non-contact heating of gold NPs. The circuit operates at $13.56 \mathrm{MHz}$, which is a frequency that allows for deep penetration within the human body for the treatment of a wide range of cancers. Ultimately, our goal is to incorporate gold NPs within the liposomes so that they will selectively absorb RF-EM energy, melt the lipid shell without significantly heating the surrounding tissue and release the drug payload. We present preliminary results for heating 2, 5, 10 and $20 \mathrm{~nm}$ diameter citrate-coated gold NPs in distilled, deionized $\mathrm{H}_{2} \mathrm{O}$ (to keep in suspension). For heating the bulk solution, the absorption efficiency of $5 \mathrm{~nm}$ NPs versus $2 \mathrm{~nm}$ NPs was $51 \times$ higher. Similarly, $10 \mathrm{~nm}$ NPs were $37 \times$ more efficient than $5 \mathrm{~nm} \mathrm{NPs}$, and $20 \mathrm{~nm}$ NPs were $3 \mathrm{x}$ more efficient than $10 \mathrm{~nm}$ NPs. Bulk-solution heating rates obtained with these samples using $200 \mathrm{~W}$ of $\mathrm{RF}$ input power ranged from 4.5 to $7.6^{\circ} \mathrm{C}$ per minute. Our results suggest an optimum in absorption efficiency near $10 \mathrm{~nm}$. We show that small amounts of gold NPs injected into beef and chicken significantly increase absorption of RF-EM energy. We also present preliminary results for liposome release of encapsulated hydrophilic dyes using this new delivery strategy, and improvements to the design of the RFEM circuit. This work is supported by NIH CA 103828, the ACS Institutional Research Grant \#IRG-95-125-07 and the Dean, School of Medicine, UC Davis.

\section{J920}

\section{ULTRASOUND-CT FOR GUIDED DRUG DELIVERY}

Charles F. Caskey ${ }^{1}$, Azadeh Kheirolomoom ${ }^{1}$, Chun-Yen Lai ${ }^{1}$, Dustin E. Kruse ${ }^{1}$, Nathan Packard ${ }^{2}$, John M. Boone ${ }^{2}$, Katherine Ferrara ${ }^{1}$ ${ }^{1}$ BME, UC Davis, Davis, CA, USA, ${ }^{2}$ Radiology, UC Davis, Davis, CA, USA.

Background, Motivation, and Objective Our initial studies of ultrasound-guided and activated drug delivery demonstrate feasibility. In the very aggressive Met-1 tumor model, we now find that ultrasound release of doxorubicin from temperature-sensitive liposomes significantly decreases tumor growth (two to five-fold), increases survival and decreases viable tumor volume upon histology. Each metric was improved with the combination of ultrasound and temperature-sensitive delivery vehicles, as compared with all control treatments. However, islands of viable tumor remain after treatment. Our goal is to develop combined PET-CT-US to detect small regions of viable tumor and guide treatment; here, we report on results to date with CT-US. Statement of Contribution/Methods While ultrasound hyperthermia has been applied for $\sim 80$ years, accurate "thermal dosing" is difficult due to the varied absorption and scattering of ultrasonic waves. In this work, CT images were segmented based on Hounsfield units to help predict the thermal absorption characteristics of the insonified region. Gel phantoms (3\% agar, $4 \%$ glycerol, and $4 \% \mathrm{SiC}$ ) with $9.3 \mathrm{~mm}$-diameter cylindrical targets were imaged on the rotating gantry, cone beam CT system at $7 \mathrm{mAs}$ and $80 \mathrm{kVp}$. A 3D volume image set was then acquired by an ultrasound system (Siemens Antares) at an MI of 0.6 using a mechanically-scanned co-linear array transducer with a 128 -element imaging array $(5.2 \mathrm{MHz})$ and two 64-element therapeutic arrays for mild hyperthermia (1.5 MHz). Results After registering CT images to the scanned ultrasound system, we created registered 3D image sets and evaluated the $\mathrm{CT}$ properties of the insonified region. Preliminary experiments in phantoms show that CT intensity is useful in characterizing acoustic absorption and in the guidance of mild hyperthermia generated by the two $1.5 \mathrm{MHz}$ arrays. Preliminary studies in the Met-1 tumor indicate that without correction, temperature profiles within a $1 \mathrm{~cm}$ diameter tumor vary by $\sim 2.5^{\circ} \mathrm{C}$. Discussion/Conclusion By registering CT and ultrasound scans, we visualize the region to be treated, as well as neighboring reflective structures. Segmentation of CT images into adipose, fibroglandular, and skin tissues types facilitates thermometry and controlled heating and will be evaluated to enhance therapeutic efficacy. Acknowledgement: NIH R01 CA 103828 
J921

SYNTHESIS OF NOVEL BACTERIOCHLORIN-E6-BASED NIR DYE FOR STABLE INCORPORATION INTO TUMOR-TARGETED LIPOPROTEIN NANOPARTICLES

Kenneth K. $\mathrm{Ng}^{1}$, Weiguo $\mathrm{Cao}^{2}$, Zhihong Zhang ${ }^{2}$, Juan $\mathrm{Chen}^{2}$, Ian Corbin $^{2}$, Gang Zheng ${ }^{2,1}$

${ }^{1}$ Institute of Biomaterials and Biomedical Engineering, University of Toronto, Toronto, ON, Canada, ${ }^{2}$ Department of Medical Biophysics, Ontario Cancer Institute, University of Toronto, Toronto, ON, Canada.

Bacteriochlorophyll (BChl) analogs are near-infrared active photosensitizers ideal for the detection and treatment of deeply seated tumors via fluorescence imaging and photodynamic therapy (PDT). For example, a palladium complex of BChl (TOOKAD ${ }^{\circledR}$ ) is currently being investigated clinically as primary and secondary therapy for prostate cancer treatment. TOOKAD ${ }^{\circledR}$ is a vascular PDT agent that owes its tumor targeting capabilities to its unique pharmacokinetics, focused light delivery and precise light dosimetry. However, a problem that remains is that $\mathrm{BChl}$ can exhibit non-specific accumulation in both healthy and tumor tissue. To address this problem, we sought to synthesize a $\mathrm{BChl}$ analog which can be incorporated into lipoprotein nanoparticles for receptor-targeted tumor imaging and therapy. To achieve this goal, we synthesized a stable $\mathrm{BChl}$ analog using a robust and regioselective 4 -step synthesis procedure with $>40 \%$ overall yield. This bacteriochlorin e6 bisoleate (BChlBOA) has two oleate-anchors to facilitate its incorporation into lipoprotein nanoparticles. Characterization of BChlBOA showed that it retained optimal photophysical properties (753 nm absorption, $\varepsilon>57000 \mathrm{M}-1 \mathrm{~cm}-1$ in chloroform; $762 \mathrm{~nm}$ emission) for NIR fluorescence imaging and PDT. Systematic formulation studies yielded a stable core-loading of BChlBOA in lowdensity lipoprotein (LDL) nanoparticles. We next investigated whether this formulation could be used to deliver BChIBOA to LDLRoverexpressing cancer cells. LDL receptor-expressing A549 cells were treated with BChl-BOA-loaded LDL nanoparticles $(43 \mathrm{nM})$ and imaged using confocal microscopy. Cellular uptake was observed after a $4 \mathrm{~h}$ incubation period. Furthermore, this uptake was inhibited by the addition of 25-fold mole excess of LDL confirming its LDL receptor targeting specificity. We next successfully formulated BChlBOA into high-density lipoproteins (HDL). These nanoparticles were found to have an average diameter of $12 \mathrm{~nm}$. Preliminary in vitro cell uptake experiments indicate that the HDL nanoparticles can be taken up in SRBI + cancer cell lines. In conclusion, we successfully synthesized a BChl analog which can be stably incorporated in both LDL and HDL nanoparticles. Furthermore, both LDL and HDL retained the ability to bind and activate the LDLR and SRBI receptors, respectively, suggesting that both nanoparticles retained their functional capabilities. As such, these formulations will serve as the groundwork for tumorspecific ligand modifications in order to achieve a truly tumor-targeted nanoparticle delivery system.

\section{J922}

USING NANOCAGE CONTRAST AGENTS AS DRUG CARRIERS Kimberly Homan $^{1}$, Srivalleesha Mallidi ${ }^{1}$, Jeffrey Chen ${ }^{1}$, Mike Souza ${ }^{1}$, Ryan L. Truby $^{1}$, Lisa Brannon-Peppas ${ }^{2}$, Stanislav Y. Emelianov ${ }^{1}$

${ }^{1}$ Biomedical Engineering, University of Texas at Austin, Austin, TX, USA, ${ }^{2}$ Appian Labs, LLC, Austin, TX, USA.

Multimodal imaging and treatment strategies are now the standard of care in battling cancer. Consequently, nanoagents with the intrinsic ability to both enhance imaging contrast while providing several avenues of treatment are becoming increasingly valuable. Here we present a new, intravenously injectable silver nanocage carrier. The carrier was built to enhance contrast for photoacoustic imaging (i.e. acoustic imaging of optical absorption). Additionally, the nanocage can house chemotherapeutic drugs, exogenous dyes, and/or MRI contrast agents in its polymeric core. Moreover, the potential to bioconjugate antibodies and other markers to target the nanocage system was tested using silver nanodiscs both in vitro and in vivo (results below). This nanocage carrier is a platform technology with the potential to provide image guided therapy for either chemotherapy or photothermal therapy.
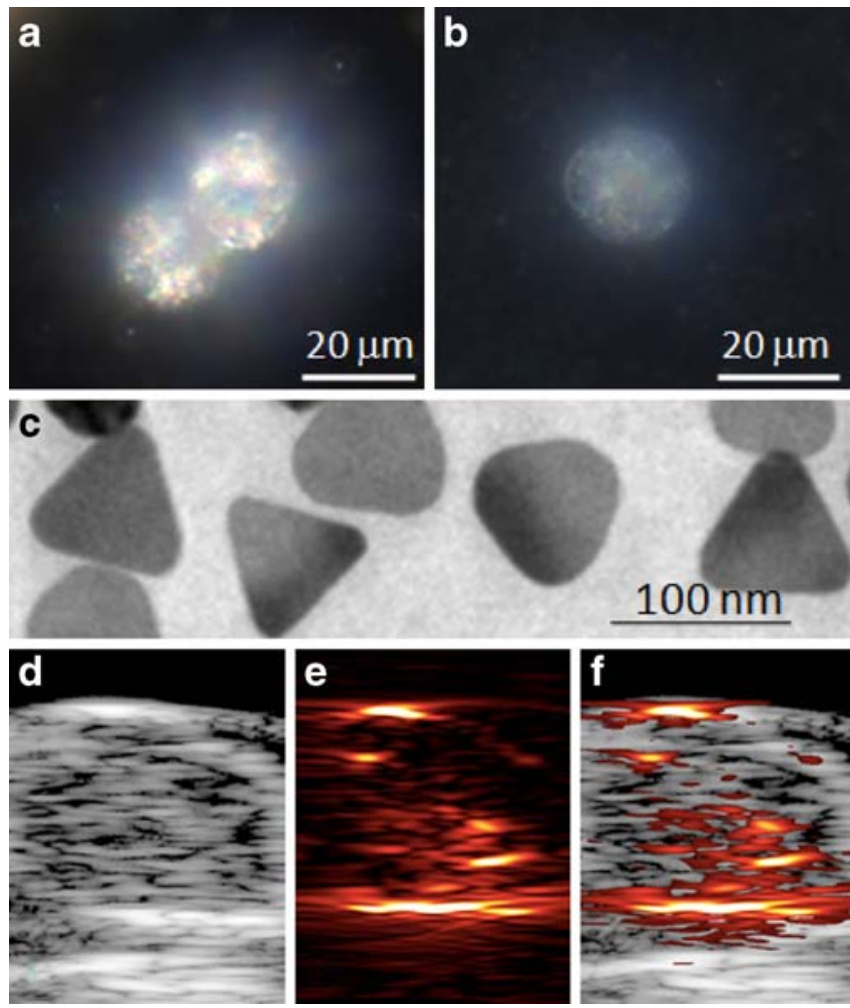

Darkfield microscopy of targeted and non-targeted silver nanostructures on pancreatic cancer cells (A,B), silver nanotriangles and nanodiscs (C), and ultrasound (D), photoacoustic (E) and combined (F) images of targeted nanodiscs in vivo.

\section{J923}

IMAGING GUIDED IMMUNOTHERAPY OF HEAD-NECK SQUAMOUS CELL CARCINOMA WITH CETUXIMAB IN ANIMAL MODELS

Gang Niu ${ }^{1}$, Qizhen $\mathrm{Cao}^{1}$, Xilin Sun $^{1}$, Donald Courter ${ }^{2}$, Quynh-Thu Le $^{2}$, Sanjiv S. Gambhir ${ }^{1}$, Xiaoyuan Chen ${ }^{1}$

${ }^{1}$ MIPS, Radiology, Stanford University, Stanford, CA, USA, ${ }^{2}$ Radiation Oncology, Stanford University, Stanford, CA, USA.

For epidermal growth factor receptor (EGFR) targeted therapy with monoclonal antibodies (mAbs) such as cetuximab and panitumu- 
mab, only a subgroup of patients benefit from the immunotherapy, independent of the EGFR expression level. In this study we evaluated the pharmacokinetics and pharmacodynamics of EGFR targeted immunotherapy and radioimmunotherapy by quantitative PET imaging. Methods: Tumor delivery and distribution of cetuximab was evaluated in nude mice bearing 22B and SCC1 xenografts with small animal PET using ${ }^{64} \mathrm{Cu}$-DOTA-cetuximab. The tumor-bearing mice were then treated with 4 doses of cetuximab $(5 \mathrm{mg} / \mathrm{kg}$ per dose every other day intravenous injection). FDG PET was performed after the 3 rd dose of antibody. The tumor-bearing mice were also subjected to ${ }^{90} \mathrm{Y}$-DOTAcetuximab radioimmunotherapy. Results: The 22B tumors with relatively low EGFR expression showed higher ${ }^{64} \mathrm{Cu}$-DOTAcetuximab accumulation compared with SCC1 tumors. Both SCC1 and $22 \mathrm{~B}$ cells were insensitive to cetuximab in vitro. In vivo therapeutic study showed that SCC1 tumors are sensitive while 22B tumors are resistant to cetuximab. After cetuximab treatment, the SCC1 tumors showed decreased FDG uptake while the 22B tumors had increased FDG uptake. Due to the higher tumor accumulation, 22B tumors are more sensitive to ${ }^{90} \mathrm{Y}$-DOTA-cetuximab treatment than SCC1 tumors. Conclusion: Tumor response of to cetuximab treatment is not necessarily related to EGFR expression and antibody delivery efficiency. ImmunoPET and FDG PET are useful tools in monitoring early response to both immunotherapy and radioimmunotherapy. a

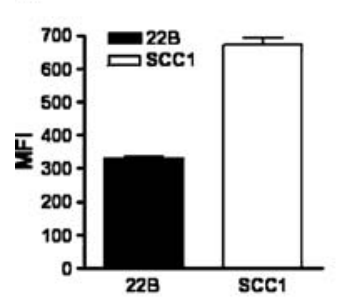

C
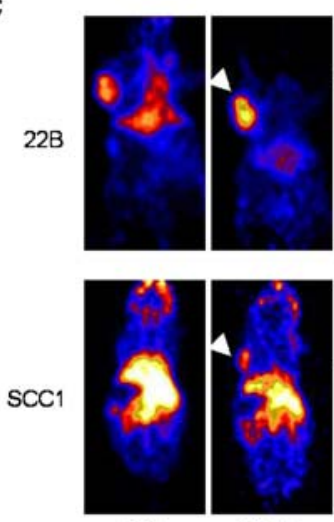

$4 \mathrm{~h}$

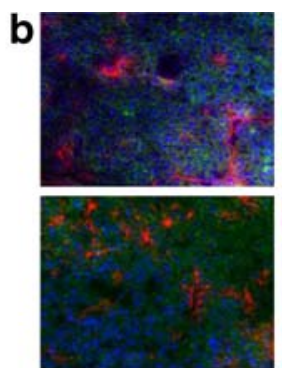

22B
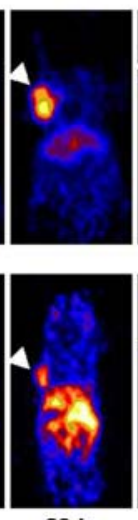

$30 \mathrm{~h}$

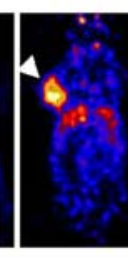

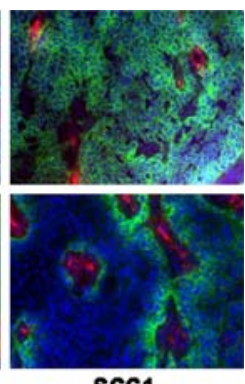

SCC1
Figure. A. EGFR expression determined by flow cytometry; B. Immunostaining of EGFR expression. Tumor section was stained with cetuximab as the primary antibody (upper panel). Cetuximab was injected into tumor bearing mice and $24 \mathrm{~h}$ later tumor was collected and stained with FITC labeled donkey-anti-human IgG directly (lower panel); C. Small animal PET imaging after ${ }^{64} \mathrm{Cu}$-cetuximab injection with arrows pointing to tumors.
Monitoring Drug Therapy

J924

\section{GENETICALLY ENGINEERED Salmonella typhimurium AS A POTENTIAL IMAGEABLE THERAPEUTIC PROBE IN SMALL ANIMAL TUMOR MODELS}

$\mathrm{Vu}$ Hong Nguyen ${ }^{1}$, Jung Min $\mathrm{Ha}^{1}$, Yeongjin Hong ${ }^{2}$, Hyeong Seok $\mathrm{Kim}^{3}$, Hyon El Choy ${ }^{2}$, Jung-Joon Min ${ }^{1}$

${ }^{1}$ Nuclear Medicine, Chonnam National University Medical School, Hwasun, Korea, South, ${ }^{2}$ Microbiology, Chonnam National University, Gwangju, Korea, South, ${ }^{3}$ Forensic Medicine, Chonnam National University, Gwangju, Korea, South.

Attenuated Salmonella typhimurium has been explored to visualize or treat solid tumors due to their characteristics of selective localization in solid tumors. Therefore, exploiting Salmonella to deliver therapeutic genes has been considered for a long time. To combine bacterial capability of cancer killing with visualization power, we engineered $S$. typhimurium to express a cytotoxic protein, cytolysin A (ClyA) for tumor cell killing, as well as to express bacterial luciferase (lux) operon for imaging signal. For specific expression of therapeutic gene in tumor, we transformed S. typhimurium with a cytotoxic protein, cytolysin A (ClyA) under the control of inducible promoter $\mathrm{P}_{\mathrm{BAD}}$, and tranduced bacterial luciferase $(l u x)$ operon in their chromosome. For verifying remote control of gene expression in bacteria, a bacterial expression plasmid was constructed in which Renilla luciferase variant (RLuc8) was placed under the control of the $\mathrm{P}_{\mathrm{BAD}}$ promoter (pBAD-RLuc8). For the purpose of controlled expression of the therapeutic gene, clyA gene was substituted for RLuc8 gene in $\mathrm{pBAD}-\mathrm{RLuc} 8$ vector (pBAD-ClyA). To induce $\mathrm{pBAD}$ system L-arabinose was administered. We found the intensity of RLuc8 expression $(36.9 \mathrm{kDa})$ was directly proportional to the concentration of $\mathrm{L}$ arabinose $(\sim 0.2 \%)$ added to cultured bacterial cells, while no protein was identified in its absence. The bioluminescence signal was detected in the tumor $3 \mathrm{~h}$ after L-arabinose administration, reaching maximum at $6 \mathrm{~h}$ and then declined. The tumor-bearing mice were treated intravenously with phosphate buffered saline (PBS), untransformed S. typhimurium or transformed S. typhimurium with pBAD-ClyA. Antitumor activity of the transformed S. typhimurium was further explored with or without Larabinose $(60 \mathrm{mg})$ addition. In the mice treated with transformed Salmonella in the presence of inducer, tumor growth was completed inhibited. The treatment with transformed S. typhimurium with ClyA induction markedly suppressed metastatic tumor growth compared with other groups These results provided proof of principle that attenuated $S$. typhimurium ( $\triangle \mathrm{ppGpp}$ strain) has the specific affinity for tumor and could be engineered to specifically express and secrete a therapeutic protein in the tumor tissue as well as to generate light signal for in vivo imaging.

\section{J925}

EARLY ASSESSMENT OF TREATMENT RESPONSE TO GEFITINIB USING KINETIC ANALYSIS OF 3'-DEOXY-3'[18F]FLUOROTHYMIDINE PET IN A XENOGRAFT MODEL $\underline{\text { Seog-Young Kim }}{ }^{1}$, Seung Jin Lee ${ }^{2}$, Seung Jun $\mathrm{Oh}^{1}$, Dae Hyuk Moon ${ }^{1}$, Jin-Sook Ryu

${ }^{1}$ Nuclear Medicine, Asan Medical Center, Seoul, Korea, South, ${ }^{2}$ Institute for Innovative Cancer Research, Asan Medical Center, Seoul, Korea, South.

Objectives: This study was performed to investigate the early assessment of treatment response to gefitinib using FLT-PET imaging with kinetic and static analysis in a xenograft model, and that was compared 
with FDG-PET results and biological changes for tumor tissues. Methods: Mice bearing A431 tumor were treated with gefitinib or vehicle. Serial (day $0,1,3$ ) dynamic PET images with FLT or FDG for 120 minutes were performed. Kinetic parameters (K1, K1/k2, k3, k4, $\mathrm{k} 3 / \mathrm{k} 4$, KFLT, DVm) for FLT-PET and static parameters (SUV, tumorto-background (T/B) ratio) of FLT and FDG PET images were obtained. At the same time point of serial PET images, tumor tissues were obtained from another tumor bearing group for measurement of TK1 activity, cell cycle, Ki-67, GLUT1, hexokinase and apoptosis. Results: All kinetic and static parameters of FLT-PET except k4 showed significant decrease on day 3 after gefitinib treatment. These changes of FLT-PET were well correlated with TK1 activity decrease, G0-G1 fraction increase and Ki-67 index decrease after treatment in tumor tissues. The apoptotic portion was only $2.8 \%$ on day 3 . In contrast to FLT-PET, the static parameters of FDG-PET and biological factors related to glucose metabolism (GLUT1, hexokinase) showed no difference until day 3 after gefitinib treatment. Conclusions: FLT-PET could be used for early assessment of treatment response to gefitinib when there is no change in glucose metabolism or morphology of tumor. (Key words: PET, FLT, FDG, cancer, therapy, gefitinib) Changes of kinetic \& static parameters of FLT-PET after gefitinib treatment in A431 xenograft

\begin{tabular}{|c|c|c|c|c|c|c|}
\hline \multirow[t]{2}{*}{ parameters } & \multicolumn{3}{|c|}{ Control $(n=5)$} & \multicolumn{3}{|c|}{ Gefitinib (n=9) } \\
\hline & Day 0 & Day 1 & Day 3 & Day 0 & Day 1 & Day 3 \\
\hline $\begin{array}{c}\mathrm{K} 1 \\
(\mathrm{ml} / \mathrm{ml} \\
\text { Tiss } / \mathrm{min})\end{array}$ & $\begin{array}{l}0.198 \pm \\
0.115\end{array}$ & $\begin{array}{c}0.202 \pm 0.119 \\
(+1.7 \%) \dagger\end{array}$ & $\begin{array}{c}0.226 \pm 0.040(+14.0 \\
\%) \dagger\end{array}$ & $\begin{array}{l}0.193 \pm \\
0.083\end{array}$ & $\begin{array}{c}0.156 \pm 0.051 \\
(-19.2 \%)\end{array}$ & $\begin{array}{c}0.138 \pm 0.045 \mathbb{I} \\
\quad(-28.4 \%)\end{array}$ \\
\hline $\begin{array}{c}\mathrm{K} 1 / \mathrm{k} 2 \\
(\mathrm{~m} 1 / \mathrm{ml} \text { Tiss })\end{array}$ & $\begin{array}{l}0.894 \pm \\
0.214\end{array}$ & $\begin{array}{l}0.965 \pm 0.270 \\
\quad(+7.9 \%)\end{array}$ & $\begin{array}{c}1.111 \pm 0.252(+24.3 \\
\%)\end{array}$ & $\begin{array}{c}0.899 \pm \\
0.234\end{array}$ & $\begin{array}{l}0.730 \pm 0.140 \neq \\
\quad(-18.8 \%)\end{array}$ & $\begin{array}{l}0.728 \pm 0.091 \neq \\
\quad(-19.0 \%)\end{array}$ \\
\hline $\begin{array}{c}\mathrm{k} 3 \\
(1 / \mathrm{min})\end{array}$ & $\begin{array}{l}0.042 \pm \\
0.006\end{array}$ & $\begin{array}{c}0.040 \\
\pm 0.008 \\
(-6.4 \%)\end{array}$ & $\begin{array}{c}0.071 \pm 0.042(+66.7 \\
\%)\end{array}$ & $\begin{array}{c}0.054 \pm \\
0.012\end{array}$ & $\begin{array}{c}0.041 \pm 0.008 \% \\
(-24.5 \%)\end{array}$ & $\begin{array}{c}0.033 \pm 0.007 \Phi[ \\
(-38.2 \%)\end{array}$ \\
\hline $\begin{array}{c}\mathrm{k} 4 \\
(1 / \mathrm{min})\end{array}$ & $\begin{array}{l}0.014 \pm \\
0.002\end{array}$ & $\begin{array}{c}0.010 \pm 0.002 \\
(-24.8 \%)\end{array}$ & $\begin{array}{c}0.011 \pm 0.001 \\
(-20.4 \%)\end{array}$ & $\begin{array}{l}0.011 \pm \\
0.002\end{array}$ & $\begin{array}{c}0.011 \pm 0.002(+4.7 \\
\%)\end{array}$ & $\begin{array}{c}0.013 \pm 0.002 \% \\
\quad(+19.8 \%)\end{array}$ \\
\hline $\mathrm{k} 3 / \mathrm{k} 4$ & $\begin{array}{l}3.161 \pm \\
0.803\end{array}$ & $\begin{array}{c}3.969 \pm 1.308(+25.5 \\
\%)\end{array}$ & $\begin{array}{c}6.626 \pm 4.165(+109.6 \\
\%)\end{array}$ & $\begin{array}{l}5.141 \pm \\
1.124\end{array}$ & $\begin{array}{l}3.710 \pm 0.823 \ddagger \\
(-27.8 \%)\end{array}$ & $\begin{array}{l}2.668 \pm 0.624 \mathrm{I} \\
\quad(-48.1 \%)\end{array}$ \\
\hline $\begin{array}{c}\text { KFLT } \\
\text { (ml/ml } \\
\text { Tiss/min) }\end{array}$ & $\begin{array}{l}0.030 \pm \\
0.004\end{array}$ & $\begin{array}{c}0.031 \pm 0.007 \\
(+2.0 \%)\end{array}$ & $\begin{array}{c}0.054 \pm 0.018(+78.9 \\
\%)\end{array}$ & $\begin{array}{l}0.038 \pm \\
0.012\end{array}$ & $\begin{array}{c}0.025 \pm 0.007 \mathrm{II} \\
(-33.8 \%)\end{array}$ & $\begin{array}{l}0.020 \pm 0.005 \Phi \\
\quad(-45.7 \%)\end{array}$ \\
\hline $\begin{array}{c}\mathrm{DVm} \\
(\mathrm{ml} / \mathrm{ml} \text { Tiss })\end{array}$ & $\begin{array}{c}2.782 \pm \\
0.711\end{array}$ & $\begin{array}{c}3.705 \pm 1.000(+33.2 \\
\%)\end{array}$ & $\begin{array}{c}6.896 \pm 3.131(+147.9 \\
\%)\end{array}$ & $\begin{array}{c}4.707 \pm \\
1.941\end{array}$ & $\begin{array}{l}2.719 \pm 0.831 \Psi \\
\quad(-42.2 \%)\end{array}$ & $\begin{array}{c}1.957 \pm 0.610 \mathrm{q} \\
(-58.4 \%)\end{array}$ \\
\hline SUVmean & $\begin{array}{l}1.245 \pm \\
0.327\end{array}$ & $\begin{array}{c}1.537 \pm 0.289(+23.4 \\
\%)\end{array}$ & $\begin{array}{c}2.406 \pm 1.334(+93.3 \\
\%)\end{array}$ & $\begin{array}{l}1.627 \pm \\
0.476\end{array}$ & $\begin{array}{c}1.246 \pm 0.406 \mathrm{~g} \\
(-23.4 \%)\end{array}$ & $\begin{array}{c}0.909 \pm 0.2739 \mathrm{I} \\
\quad(-44.1 \%)\end{array}$ \\
\hline T/B ratio & $\begin{array}{c}5.290 \pm \\
0.971\end{array}$ & $\begin{array}{c}5.789 \pm 1.282 \\
(+9.4 \%)\end{array}$ & $\begin{array}{c}10.343 \pm 3.505(+95.5 \\
\%)\end{array}$ & $\begin{array}{l}7.122 \pm \\
2.289\end{array}$ & $\begin{array}{l}4.572 \pm 1.4949 \\
\quad(-35.8 \%)\end{array}$ & $\begin{array}{c}3.690 \pm 0.760 \mathrm{I} \\
\quad(-48.2 \%)\end{array}$ \\
\hline
\end{tabular}

* Data presented as mean $\pm \mathrm{SD}$.

$\dagger$ : percent change compared with Day 0

\$: Significant difference from Day $0, p<0.05$

I : Significant difference from Day $0, p<0.01$

J926

\section{TOTO-3 AS A "SMART PROBE" FOR THE ASSESSMENT} OF ULTRASOUND-MEDIATED LOCAL DRUG DELIVERY IN VIVO Anna Yudina, Roel Deckers, Christelle Debeissat, Chrit Moonen Laboratory for Molecular and Functional Imaging, Bordeaux, France.

Ultrasound (US) is known to facilitate the internalization of otherwise cell-impermeable molecules and therefore may be used for improving local drug delivery. However, the understanding of the underlying mechanisms as well as the ability of the imaging and quantification of this effect in vivo are limited. Here, we report the first-time in vivo utilization of a cell-impermeable cyanine dye TOTO-3 as a model drug for monitoring US-mediated drug delivery. Imaging of intracellular delivery of TOTO-3 is based on its fluorescence enhancement upon binding to DNA. In our feasibility study 5 rag-gamma mice bearing bilateral s.c. CMT-93 tumors received identical co-injections of TOTO3 and Sonovue microbubbles followed by pulsed US treatment $(f=$ $1.5 \mathrm{MHz}, \mathrm{PRF}=1 \mathrm{kHz}, \mathrm{DC}=4 \%$, peak negative pressure $=1 \mathrm{MPa}$ ) for 1 minute of one of the tumors. Fluorescence imaging was performed before, immediately after, $2 \mathrm{~h}$ and $4 \mathrm{~h}$ after the US treatment (Fig. 1, Up). An increase in signal intensity was observed for both sides, however the values for the US-treated tumor at the corresponding time points were 1.4, 2.1 and 2.5 fold higher compared to the control one $(P<0.001$ paired Student t-test at $2 \mathrm{~h}$ and $4 \mathrm{~h})$. Intracellular delivery of TOTO-3 was confirmed by fluorescence microscopy (Fig. 1, Down). In 
order to explain the elevation of the fluorescence signal at the control tumor we hypothesised that infiltrating macrophages were capable of uptaking the fluorophore in the ultrasound-independent manner. This was supported by the studies on primary mouse macrophages incubated with TOTO-3. With these studies, we have, for the first time, demonstrated the utility of the nuclear dye TOTO-3 for in vivo monitoring of the intracellular uptake of small molecules facilitated by US. Because this approach is semi-quantitative and allows to directly follow US-mediated internalization it can be used for in-depth investigation and optimisation of this technique.
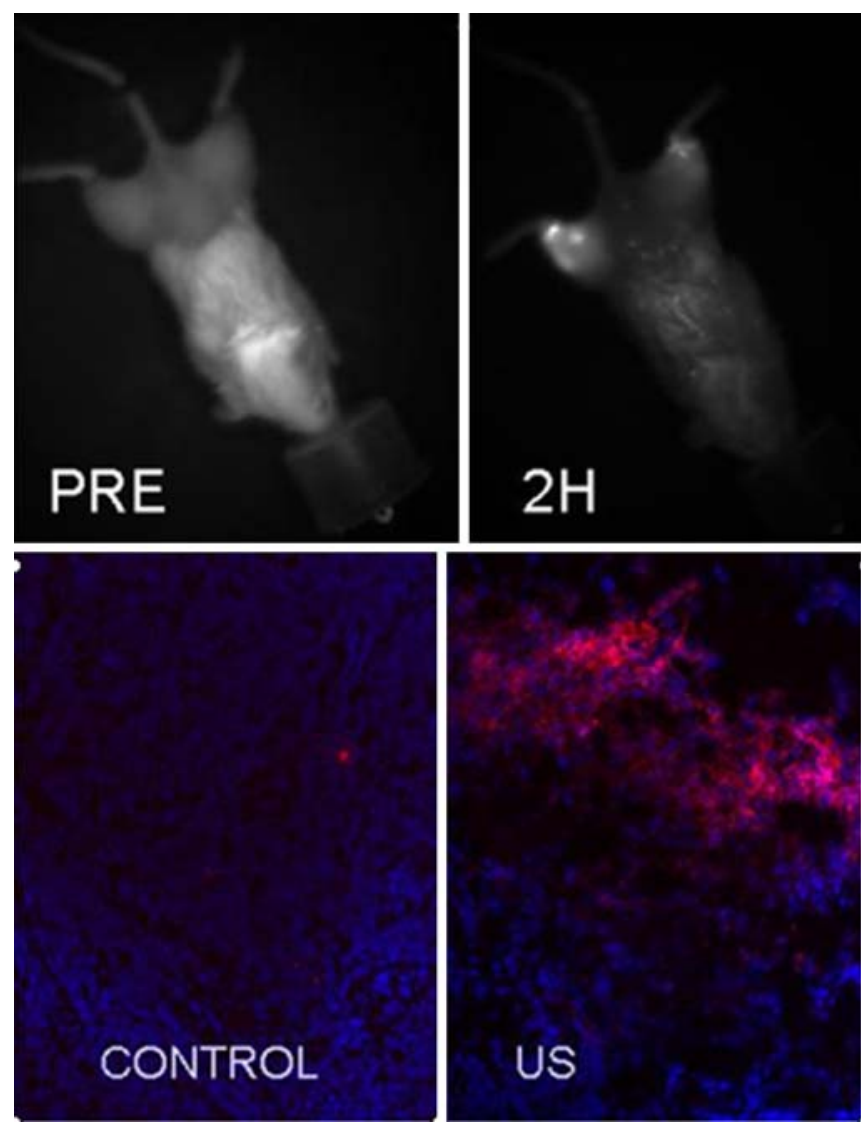

\section{J927}

NOVEL MRI BIOMARKERS MEASURING EARLY RESPONSE OF BREAST TUMOR XENOGRAFTS FOLLOWING ANTI-DR5 AND BEVACIZUMAB THERAPIES

Hyunki Kim ${ }^{1,3}$, Karri Folks ${ }^{1}$, Lingling Guo ${ }^{2}$, Cecil Stockard ${ }^{3}$, Naomi S. Fineberg $^{4}$, William Grizzle ${ }^{5,3}$, James George ${ }^{2}$, Kurt R. Zinn ${ }^{1,3}$ ${ }^{1}$ Radiology, University of Alabama, Birmingham, AL, USA, ${ }^{2}$ Surgery, University of Alabama, Birmingham, AL, USA, ${ }^{3}$ Comprehensive Cancer Center, University of Alabama, Birmingham, AL, USA, ${ }^{4}$ Biostatistics, University of Alabama, Birmingham, AL, USA, ${ }^{5}$ Pathology, University of Alabama, Birmingham, AL, USA.

Purpose: To develop novel MRI biomarkers to assess early breast-tumor response following anti-DR5 (TRA-8) and anti-VEGF (bevacizumab) therapies independently of imaging time points by characterizing non-linear vascular-permeability changes via a series of dynamic contrast-enhanced (DCE) MRI. Methods: Four groups ( $n=6 /$ group) of nude mice bearing subcutaneous breast tumors were used. Groups 1-4 were injected i.p. with PBS, TRA-8(0.2 mg), bevacizumab(1 mg), and TRA-8(0.2 mg) + bevacizumab( $1 \mathrm{mg})$ on day 0 respectively. Anatomical MRI and DCEMRI were performed on days $0,1,2$, and 3 . Tumor volumes and the averaged intratumoral Ktrans values in both the entire region and $0.5-\mathrm{mm}$ thick peripheral region were calculated, and repeated measure ANOVA was used to analyze the difference between groups over all 3 days. The bestfitting second-order polynomial curves for Ktrans changes in the peripheral tumor regions over 3 days after therapy were obtained for groups 2-4, and the "maximum Ktrans increase (MaxK)" and "day of maximum Ktrans increase (DMaxK)" were retrieved from each the curve. On day 3, CD31 and TUNEL staining were performed for the tumors of groups 1 and $4(n=4 /$ group), and the area fractions of CD31 and apoptotic-cell densities were measured. Results: The Ktrans values of the treated groups 2-4 were significantly lower than that of control $(p<0.05)$ in both the regions, whereas tumor volumes of those were not different from that of control $(p>0.05)$. The Ktrans changes in peripheral tumor region followed second order polynomial curves; MaxK of groups $2-4$ were $0.53 \pm 0.03,0.17 \pm 0.07$, and $0.10 \pm$ 0.04 min- 1 respectively, while DMaxK of those were $2.31 \pm 0.27,1.28 \pm$ 0.15 , and $1.02 \pm 0.21$ day respectively. The MaxK and DMaxK of group 2 were significantly higher than those of groups 3 and 4 , but MaxK showed higher significance ( $p<0.001$ for both groups 3 and 4$)$ than DMaxK did ( $p=$ 0.026 and 0.003 for groups 3 and 4 respectively). The mean area fraction of CD31 of group $4(1.83 \pm 0.67 \%)$ was $24 \%$ lower than that of group $1(2.42 \pm$ $0.49 \%)$, but not statistically different $(p=0.508)$. The apoptotic cell density of group $4(1.12 \pm 0.60 \%)$ was higher than that of group $1(0.20 \pm 0.10 \%)$ without statistical significance $(p=0.179)$. Conclusions: DCE-MRI detected significantly lower Ktrans levels in breast tumor xenografts following TRA-8 alone or combined therapy, as compared to controls. MaxK and DMaxK were proposed as novel imaging biomarkers measuring early breast-tumor response to drugs independently of a particular monitoring time point.

\section{J928}

mTOR KINASE INHIBITION MODULATES 18F-FDG UPTAKE IN VIVO IN THE HUMAN GLIOMA XENOGRAFT MODEL U87-MG Heather Keen ${ }^{1}$, Sally-Ann Ricketts ${ }^{1}$, Juliana Bales ${ }^{1}$, Aoife Shannon ${ }^{3}$, Armelle Logie $^{2}$, Rajesh Odedra ${ }^{2}$, Stephen R. Wedge ${ }^{2}$, Sylvie M. Guichard ${ }^{2}$ ${ }^{1}$ Imaging and Antibodies, AstraZeneca, Macclesfield, United Kingdom, ${ }^{2}$ Cancer Bioscience, AstraZeneca, Macclesfield, United Kingdom, ${ }^{3}$ School of Pharmacy \& Pharmaceutical Sciences, University of Manchester, Manchester, United Kingdom.

INTRODUCTION: mTOR is a serine / threonine kinase belonging to the PI3K (PIKK) superfamily of kinases. It functions as a sensor of mitogen, energy and nutrient levels and is a central controller of cell growth. The PI3K-AKT-mTOR pathway is involved in glucose uptake/ metabolism. 18F-Flurodeoxyglucose (18F-FDG) is a biomarker for glucose metabolism detectable by positron emission tomography (PET). It is used in clinical oncology for tumour diagnosis and is currently under evaluation for therapy monitoring. The aim of this study was to assess the impact of acute and chronic mTOR kinase inhibition on 18F-FDG uptake in U87-MG human glioma xenografts implanted subcutaneously in nude mice. METHODS: The mTOR kinase inhibitor was administered orally at a dose of $20 \mathrm{mg} / \mathrm{kg}$ qd for 1 or 4 days. Animals received either vehicle or mTOR kinase inhibitor $1 \mathrm{~h}$ prior to imaging. Mice were anaesthetised and then injected with approximately $15 \mathrm{MBq} 18 \mathrm{~F}-\mathrm{FDG}$ i.v. via the tail vein. Forty-five minutes later, mice underwent PET scanning (20 min scan, 3D histogramming and OSEM2D reconstruction) followed by biodistribution analysis. Image analysis was carried out using Inveon Research Workplace (IRW) software. Biodistribution data were derived from gamma counting. RESULTS: Plasma drug concentrations were not 
modified significantly by anesthesia and the imaging procedure. In the vehicle and mTOR kinase inhibitor treated groups, tumour volumes were comparable at the acute imaging time-point, but differed significantly following 4 days of treatment due to drug effect. At both acute and chronic imaging time-points, image analysis showed that there was a significant difference $(p<0.05)$ in meanSUV, maxSUV and the percentage of injected dose per gram of tissue $(\% \mathrm{ID} / \mathrm{g})$ between vehicle and drug treated tumours. Biodistribution analysis showed that the average $18 \mathrm{~F}-\mathrm{FDG} \% \mathrm{ID} / \mathrm{g}$ in vehicle treated tumours was significantly higher than in mTOR kinase inhibitor treated tumours $(p<0.05)$. The changes in glucose uptake after 1 and 4 administrations were consistent with the pharmacodynamic effects of the mTOR kinase inhibitor on pS6 and pAKT biomarkers in U87-MG tumours collected at the same time-points. CONCLUSIONS: The mTOR kinase inhibitor examined reduces significantly $18 \mathrm{~F}-\mathrm{FDG}$ uptake in U87-MG human glioma xenografts, as early as 1 hour after a single dose. This data suggests that $18 \mathrm{~F}-\mathrm{FDG}$ uptake could be used as an early sign of metabolic response to mTOR inhibition.

\section{J929}

MECHANISM FOR THE UPTAKE OF LONG CIRCULATING BIODEGRADABLE POLYMERIC MR CONTRAST AGENT IN TUMOR NECROTIC AREA

Marites P. Melancon ${ }^{1}$, Wei Lu ${ }^{1}$, Qian Huang ${ }^{1}$, Prakash Thapa ${ }^{2}$, Xiaoxia Wen $^{1}$, Chiyi Xiaong ${ }^{1}$, Dapeng Zhou ${ }^{2}$, Chaan S. $\mathrm{Ng}^{3}$, Chun $\mathrm{Li}^{1}$ ${ }^{1}$ Experimental Diagnostic Imaging, UT-MD Anderson Cancer Center, Houston, TX, USA, ${ }^{2}$ Melanoma Medical Oncology, UT-MD Anderson Cancer Center, Houston, TX, USA, ${ }^{3}$ Diagnostic Radiology, UT-MD Anderson Cancer Center, Houston, TX, USA.
Necrosis is the most common morphologic alteration found in tumors after radiation therapy and chemotherapy. Recently, we have developed PG-DTPA-Gd, a biodegradable, long circulating magnetic resonance contrast agent that selectively accumulates in tumor necrotic tissue [Wen, et al. Bioconj Chem, V15:p1408, 2004; Jackson, et al. Int. J. Radiat. Oncol. Biol. Phys., V68:p830, 2007]. However, the mechanism of selective accumulation of PGDTPA-Gd in necrotic tissue is not clear. In this study, we attempt to elucidate the mechanism and the role of macrophages in the site specific accumulation of PG-DTPA-Gd in tumor necrosis. A near infrared (NIR) dye, NIR813, which fluoresces and absorbs at the NIR, was conjugated to PG-DTPA-Gd (PG-Gd-NIR813) to facilitate in vivo optical imaging and ex vivo analysis. PG-GdNIR813 was intravenously injected into 2 tumor models: nude rats bearing $\mathrm{C} 6$ tumors and syngeneic $\mathrm{Balb} / \mathrm{c}$ mice bearing A20 tumors.Both optical imaging and biodistribution results show high uptake of the polymeric contrast agent in the tumor in both models 24-96 h after contrast injection. This uptake is affected by the presence of anti-cancer drug. T1-weighted MR imaging results show accumulation of PG-Gd-NIR813 into the tumor necrotic area, which was confirmed by TUNEL staining of resected tumors. Further studies show that the uptake of PG-Gd-NIR813 within tumor necrosis decreased in the presence of the macrophage depleting agent (see figure below, arrows indicate areas of necrosis). PG-Gd-NIR813 colocalized with CD68 (marker for macrophages) and CD169 (marker for activated macrophages), but not with CD163 (residential macrophages). Taking together, our data indicates that tumor-infiltrating macrophages recruited from blood circulation were involved in selective accumulation of PG-Gd-NIR in necrotic tissues in the tumors.

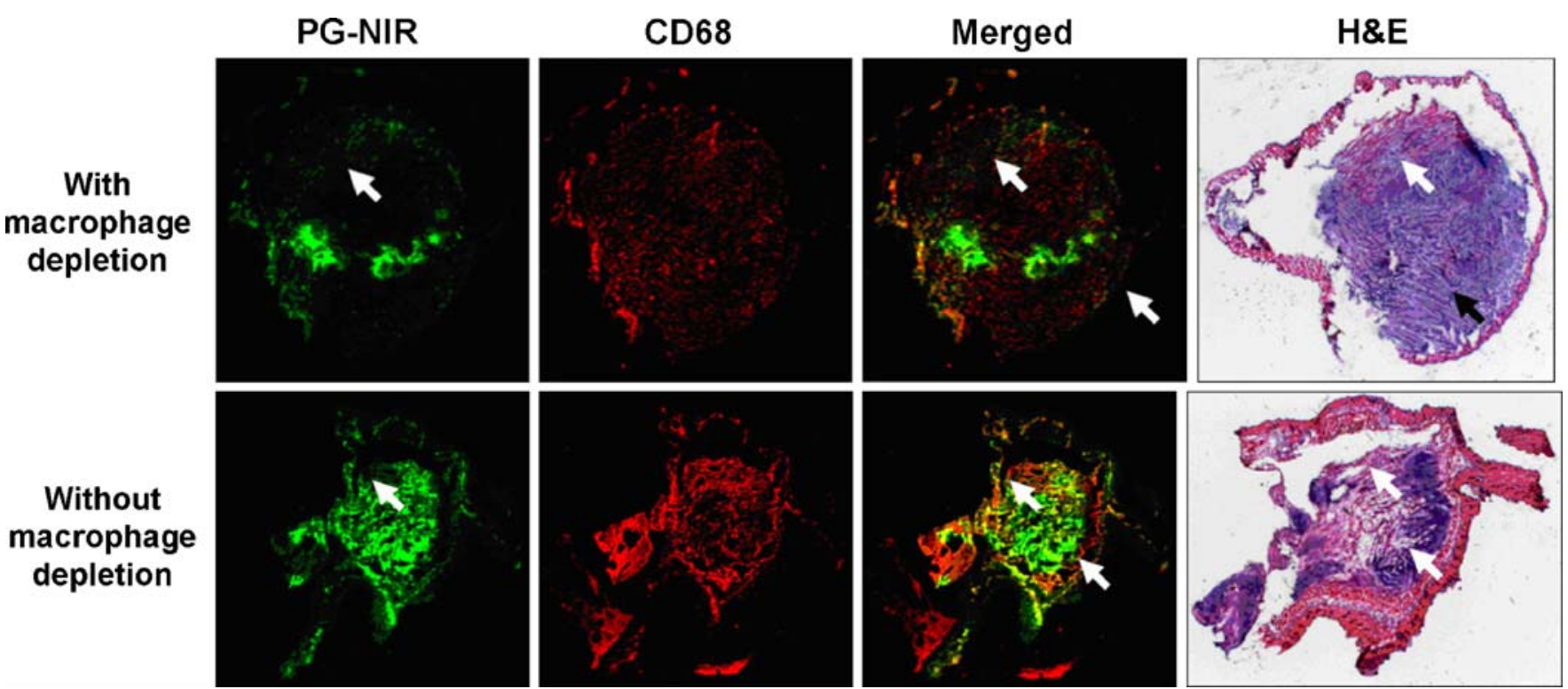

J930

SUICIDAL GENE THERAPY IN AN NFKappaB-CONTROLLED TUMOR ENVIRONMENT AS MONITORED BY A SECRETED BLOOD REPORTER

Johanna M. Niers, Christian Badr, Lee-Ann Tjon-Kon-Fat, Bakhos A. Tannous

Neurology, Massachusetts General Hospital and Harvard Medical School, Boston, MA, USA.
The nuclear factor kappa B (NFkB) is known to be activated in many cancer types including lung, ovarian, astrocytomas, melanoma, prostate adenocarcinoma, glioblastoma and was shown to correlate with disease progression. We have cloned a novel NFkBbased reporter system [five tandem repeats of NFkB responsive element (NF; $14 \mathrm{bp}$ each)] to drive the expression of both a fusion between yeast cytosine deaminase and uracil phosphoribosyltransferase (CD-UPRT) as a therapeutic gene as well as the Gaussia luciferase (Gluc) as a blood reporter separated by an internal 
ribosomal entry site (NF-CD-UPRT-IGluc). We showed that most tumor cells have high-expression of Gluc which is correlated to high activation of NFkB. When NFkB was further activated with $\mathrm{TNF} \alpha$, we observed up to 20 fold increase in transgene expression in human glioma cells which greatly enhanced the sensitization of these cells to the pro-drug 5-fluorocytosine (5-FC). When glioma cells expressing both firefly luciferase (Fluc) and NF-CD-UPRTIGluc were implanted subcutaneously in nude mice, NFkB activation in the tumor environment and in response to TNF $\alpha$ was monitored over time by assaying the blood for Gluc activity which showed similar increase in Gluc expression. Further, in vivo Fluc bioluminescence imaging showed that tumor growth slowed down in mice treated with 5FC. On the other hand, $>60 \%$ of tumors completely regressed when mice were treated with a combination of both TNF $\alpha$ (NFkB activator) as well as 5FC. This inducible system provides a tool to enhance the expression of imaging and therapeutic genes for cancer gene therapy.
J931

\section{A PRE-CLINICAL EVALUATION FOR THE USAGE OF ANTRODIA CAMPHORATA ALCOHOL EXTRACT (ACAE) IN THE TREATMENT OF NON-SMALL CELL LUNG CANCER ASSISTED WITH NON-INVASIVE MOLECULAR IMAGING}

Jeng-Fong Chiou ${ }^{2,3}$, Alexander C. $\mathrm{Wu}^{1,2}$, Chun-Jung Lin ${ }^{1}$, Win-Ping Deng $^{1}$

${ }^{1}$ Biomedical materials and Engineering, Taiepi Medical University, Taipei City, Taiwan, ${ }^{2}$ Cancer Center, Taipei Medical University Hospital, Taipei, Taiwan, ${ }^{3}$ Radiation Oncology, Taipei Medical University, Taipei, Taiwan.

This study was carried out to provide a platform for the pre-clinical evaluation of anti-cancer properties of a unique CAM (complementary and alternative medicine) agent, Antrodia camphorata alcohol extract (ACAE), in a mouse model with the advantageous non-invasive in vivo bioluminescence molecular imaging technology. In vitro analyses on the proliferation, migration/invasion, cell cycle and apoptosis were performed on ACAE-treated non-small cell lung cancer cells, H441GL and control CGL1 cells. In vivo, immune deficient mice were inoculated subcutaneously with H441GL followed by oral gavages of ACAE. The effect of ACAE on tumour progression was monitored by non-invasive bioluminescence imaging. The proliferation and migration/invasion of H441GL cells were inhibited by ACAE in a dose-dependent manner. In addition, ACAE induced cell cycle arrest at G0/G1 phase and apoptosis in H441GL cells as shown by flow cytometric analysis, Annexin-V immunoflourescence and DNA fragmentation. In vivo bioluminescence imaging revealed that tumourigenesis was significantly retarded by oral treatment of ACAE in a dose-dependent fashion. Based on our experimental data, ACAE contains anti-cancer properties and could be considered as a potential CAM agent in future clinical evaluation.
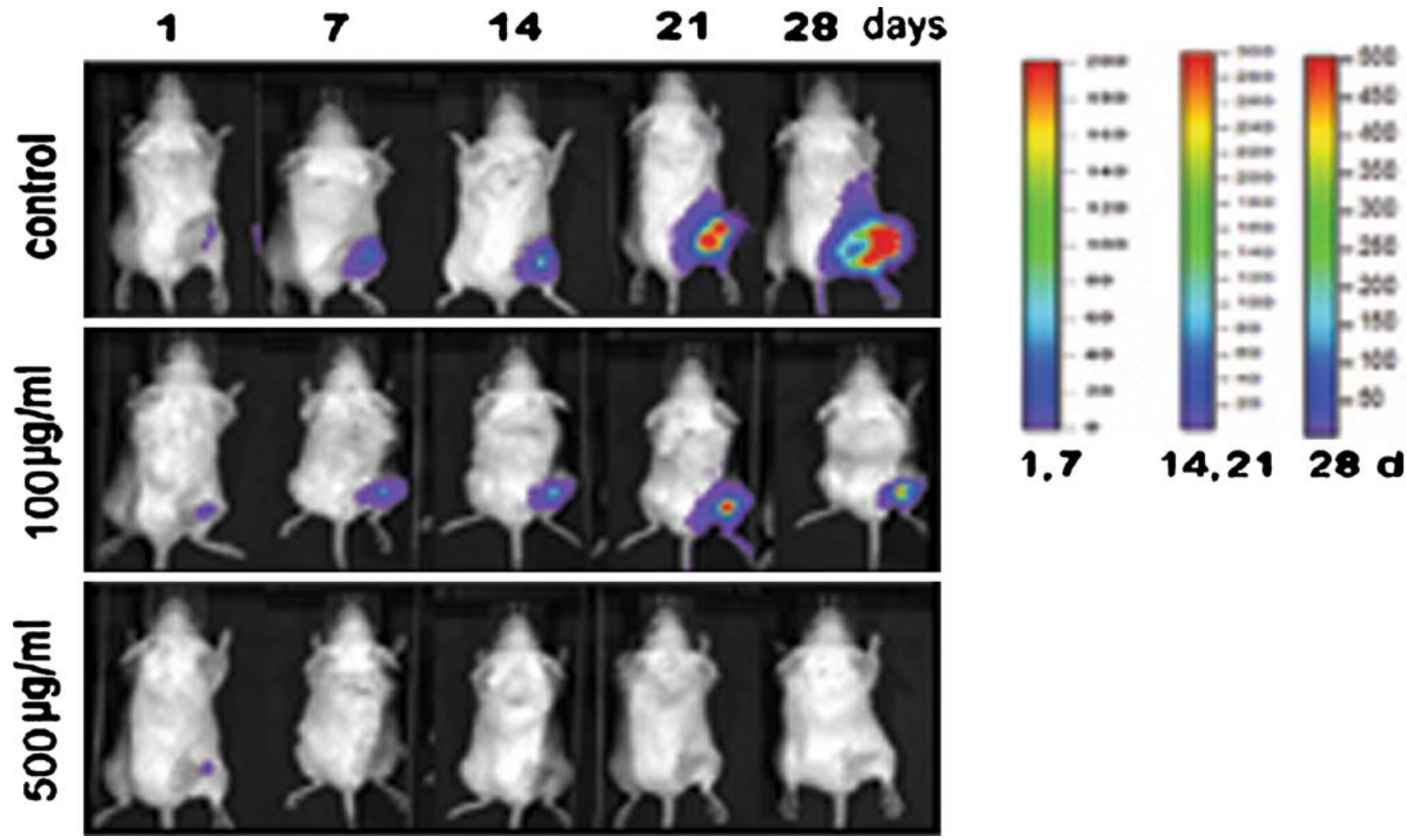

Non-invasive In vivo bioluminescence imaging of ACAE-mediated anti-tumour effect 
J932

\section{MULTIMODAL THERAPEUTIC MONITORING OF HSV1-tk/GCV GENE THERAPY USING I-124-FIAU-PET, BIOLUMINESCENCE AND LIVER CONTRAST ENHANCE CT IN ORTHOTOPIC HEPATOMA MODEL}

Taesup Lee, Sang-Keun Woo, Jaeho Jung, Kwang Sun Woo, Weesup Chung, Joo Hyun Kang, Yong Jin Lee, Kwang Il Kim, Gi Jeong Cheon, Sang Moo Lim

Molecular Imaging Research Center, KIRAMS, seoul, Korea, South.

Molecular imaging with small-animal PET and bioluminescence imaging has been extensively used as an important tool in cancer research. These modalities have some limitation for characterizing internal growing tumor, due to lack anatomical information. This study is aimed to evaluate the feasibility of HSV1-tk/ganciclovir gene therapy monitoring using nuclear, bioluminescence and liver contrast enhance CT imaging in orthotopic hepatoma model. MCATL cells, retrovirally transduced with HSV1-tk and firefly luciferase, were established and the expression of TK and luciferase was checked by FIAU uptake and bioluminescent signal with D-Luc, respectively. MCA-TL cells mixed with matrigel injected into right lobe of liver in mice. I-124-FIAU PET, bioluminescence (BLI) and liver contrast enhanced CT images in orthotopic hepatoma model were obtained before and after ganciclovir treatment $(50 \mathrm{mg} / \mathrm{Kg}$ for 5 consecutive days). I-124-FIAU PET image was obtained at $2 \mathrm{~h}$ post injection, bioluminescent signal was monitored and contrast enhance CT image was obtained at $3 \mathrm{~h}$ postinjection of Fenestra LC. Contrast indices were analyzed in CT images. MCA-TL cells showed specific radiolabeled FIAU uptake and strong luminescent signal. The orthotopic hepatoma was detected by BLI and luminescent signal increased as time elapsed, but exact location of tumor do not verify. MCA-TL tumor showed high uptake in I-124FIAU PET image. Contrast enhance CT images obtained and delineated tumor margin and the contrast indices of Heart/Gastrointestine(GI), Liver( $\mathrm{Li}) / \mathrm{GI}$ and $\mathrm{Li} /$ Tumor were $0.85 \pm 0.43,2.59 \pm$ 0.23 and $3.79 \pm 0.80$, respectively, in contrast enhance CT images. After GCV treatment, I-124-FIAU uptake $(6.08 \pm 4.16 \% \mathrm{ID} / \mathrm{g}$ vs. $1.21 \pm 0.78 \% \mathrm{ID} / \mathrm{g}$ ) and bioluminescent signal in hepatoma markedly decreased and tumor size $(19.52 \pm 5.47 \mathrm{~mm} 3$ vs. $4.98 \pm 2.05 \mathrm{~mm} 3)$ also reduced in liver contrast enhance CT image. Multimodal imaging using I-124-FIAU PET, bioluminescence and contrast enhance CT allows a sensitive and improved detection of orthotopic hepatoma and be capable to monitor therapeutic response. Combined molecular and anatomic imaging could also be useful for monitoring the progression of hepatic metastasis and therapeutic response to various anti-cancer drugs.

\section{J933}

\section{HIF-1 SPECIFIC hNIS GENE RADIOTHERAPY ON HUMAN HEPATOMA}

Chan Joo Yeom ${ }^{1,2}$, Hyewon Youn ${ }^{1,3}$, Keon Wook Kang ${ }^{1,2}$, Jae Min Jeong $^{1,3}$, Dong Soo Lee ${ }^{1,3}$, June-Key Chung ${ }^{1,2}$

${ }^{1}$ Nuclear Medicine, Seoul National University College of Medicine, Seoul, Korea, South, ${ }^{2}$ Tumor Biology, Seoul National University College of Meidicine, Seoul, Korea, South, ${ }^{3}$ Institute of Radiation Medicine, Medical Research Center, Seoul National University, Seoul, Korea, South.

HIF-1 activated cancer cells are resistant to chemotherapy and radiotherapy, thereby creating a real dilemma for cancer therapies. Our purpose was to develop a radiotherapy using human sodium iodide symporter (hNIS) gene targeting hypoxic cancer. We constructed p5HRE-NR, in which the hNIS-DsRed2 is expressed under the 5HRE. SK-Hep1 human hepatoma cells were stably transfected with p5HRE-NR and exposed to hypoxic conditions. The cell population expressing HIF-1 inducible DsRed (SK-5HRE-NR) at high levels was selected using FACS. RT-PCR and I-125 uptake were performed to observe the expression of HIF-1 targets and HIF-1 inducible hNIS activity. In vitro cytotoxic clonogenic assay of normoxic or hypoxic stable cells was performed after incubation with or without I-131 (37 MBq). Thirty days after s.c. injection of stable cells into nude mice, we acquired in vivo fluorescence and scintigraphy images. In FACS, 10-fold induction of DsRed signal was observed in stable cells under hypoxic conditions. In RT-PCR, mRNA expression of HIF-1 targets increased under hypoxic conditions. I-125 uptake increased in SK5HRE-NR cells (5-fold) under hypoxic conditions. Clonogenic assay after I-131 treatment demonstrated that the survival rate of hypoxic SK5HRE-NR cells was significantly lower than that of normoxic cells (15fold). In vivo xenograft model, we could observe the fluorescence signal or radioactivity from SK-5HRE-NR tumors. The HIF-1 specific fluorescence signal was increased as growing tumors. These results imply that our therapeutic system is useful for HIF-1 specific hNIS gene radiotherapy on SK-Hep1 cells.

\section{J934}

\section{EFFECTS OF BORTEZOMIB ON CELL CYCLE AND METABOLISM IN HEPATOMA CELLS}

Annette Altmann ${ }^{1,3}$, Walter Mier ${ }^{1}$, Tilman Schoening ${ }^{1}$, Michael Eisenhut ${ }^{2}$, $\overline{\text { Annette Markert }}^{1,3}$, Uwe Haberkorn ${ }^{1,3}$

${ }^{1}$ Nuclear Medicine, University of Heidelberg, Heidelberg, Germany, ${ }^{2}$ Radiopharmacy, DKFZ, Heidelberg, Germany, ${ }^{3} \mathrm{CCU}$ Nuclear Medicine, DKFZ, Heidelberg, Germany.

GOAL The ubiquitin-proteasome pathway plays a critical role in the regulated degradation of proteins involved in cell cycle control and tumor growth. Proteasome inhibition using Bortezomib has been shown to result in antitumor effects in a broad range of tumor cells. This study measured the effects of bortezomib on rat hepatoma cells. MATERIALS AND METHODS In order to investigate the effect of the proteasome inhibitor on growth and metabolism of the rat hepatoma cell line MH3924A the tumor cells were cultured with different concentrations of Bortezomib $(1 \mathrm{nM}-10 \mu \mathrm{M})$ for 12,24 or 48 hours and the uptake of $3 \mathrm{H}$ thymidine (TdR), FDG and 14C-aminoisobutyric acid (AIB), respectively, was measured. In addition, apoptosis of the tumor cell lines was determind by YOPRO-1 staining and the metabolic activity using WST-1 staining. Furthermore, cell cycle analysis was done using flow cytometry. In vico PET studies were performed in tumor bearing rats with FDG and Fluoroethyltyrosine (FET) after i.p. application of $50 \mathrm{mg}$ Bortezomib. RESULTS After incubation with 10 $\mathrm{nM}$ Bortezomib for $24 \mathrm{~h}$ the TdR uptake as well as the cell number decreased to $5 \%$. The metabolis activity decreased in a dose-dependent manner. A cytotoxicity assay showed similar results. Furthermore, we found a block in G2M phase at higher doses of he drug. The AIB and FDG uptake increased after $12 \mathrm{~h}$ and $24 \mathrm{~h}$ incubation (by 50\%) and decreased after $24 \mathrm{~h}$ incubation in a dose-dependent manner (by $60 \%$ ). PET studies revealed a decrease of both FDG and FET at 1 and $2 \mathrm{~d}$ after therapy CONCLUSIONS Changes in amino acid transport may be used for therapy monitoring. Since the in vivo studies were done with a relatively high dose these correspond to the longer incubation intervals of the in vitro studies. 
J935

\section{MONITORING OF TARGETED THERAPIES IN ADVANCED GASTROINTESTINAL STROMAL TUMORS: COMPARISON OF TUMOR-RESPONSE ASSESSMENT ACCORDING TO RECIST WITH VOLUMETRY}

Nicolai Schramm $^{1}$, Marcus Schlemmer ${ }^{2}$, Hans-Christoph Becker ${ }^{1}$, Konstantin Nikolaou $^{1}$, Maximilian F. Reiser ${ }^{1}$, Frank Berger ${ }^{1}$

${ }^{1}$ Department of Clinical Radiology, University of Munich - Grosshadern Campus, Munich, Germany, ${ }^{2}$ Department of Internal Medicine III, University of Munich - Grosshadern Campus, Munich, Germany.

Purpose: Advanced gastrointestinal stromal tumors (GIST) are treated with tyrosine kinase inhibitors, which are implying predominantly cytostatic effects. Established tumor-response criteria like RECIST are contested for monitoring targeted therapies as size changes are less pronounced. Aim of the study is the comparison of lesion volumetry with RECIST for monitoring targeted therapies of GIST. Methods and Materials: 20 patients underwent an abdominal baseline CT during imatinib therapy and follow-up scans 3 month and 1 year after medication was switched to sunitinib. Target lesions (50 hepatic, 18 extrahepatic) were determined according to RECIST. Semiautomatic lesion volumetry was performed with a commercially available software tool (Oncology, Siemens, Germany). The percentaged changes of the sum of the maximum diameters (RECIST) and the corresponding volume changes after 3 month and 1 year were calculated. Response to therapy was classified according to RECIST and utilizing the following volumetric criteria: Partial response: Decrease $\geq 40 \%$, Progressive disease: Increase $\geq 30 \%$. Results: One year after switch of medication patients were classified according to RECIST: Stable disease [SD] $(n=11)$, partial response [PR] $(n=$ $3)$, progressive disease [PD] $(n=6)$. The average percentaged changes according to RECIST and the corresponding volume changes after 3 month and 1 year were: SD: $-5.3 \% /-14.0 \%$ and 4.9\%/-12.2\%. PR: $-24.1 \% /-49.7 \%$ and $-37.2 \% /-76.2 \%$. PD: $+17.8 \% /+74.9 \%$ and $+94.3 \% /+377.3 \%$. Two patients classified as $\mathrm{SD}$ according to RECIST in the 3 month follow-up were already assessed as PR respectively PD utilizing volumetric criteria. After one year they were classified as PR respectively PD with both RECIST and volumetry. Conclusion: Our data provide support for the assumption that tumor volumetry possibly is a more sensitive tool for monitoring subtle size changes under molecular therapy. Comparative studies with larger patient cohorts are needed to further verify the value of volumetric in comparison to unidimensional response assessment strategies in targeted therapies.

\section{J936}

\section{QUANTITATIVE IMAGING OF ULTRASOUND-MEDIATED INTRACELLULAR DELIVERY OF INTERCALATING PROBES IN VITRO}

Anna Yudina, Matthieu Lepetit-Coiffe, Philippe Lourenco de Oliveira, Roel Deckers, Franck Couillaud, Chrit Moonen IMF, Bordeaux, France.

Intercalating dyes are used to discriminate between live and dead cells in vitro due to their impermeability to the intact plasma membrane and increase in fluorescence upon binding to nucleic acids. These properties qualify them as model drugs for the monitoring of the ultrasound (US)-mediated internalization since the interaction of the cavitating microbubbles with the cell membrane results in its temporary permeabilization. In this study we have demonstrated the use of intercalating probes (Sytox Green/Blue and TOTO-3/TO-PRO-3 (NIR) for micro- and, for the first time, macroscopic imaging and quantitation of US-mediated delivery to live tumor cells. $1 \mu \mathrm{M}$ dye and $3 \times 108$ SONOVUE microbubbles were added to C6 glioblastoma cells cultured in Opticell chambers. $6 \mathrm{~mm}$ monoelement transducer at $1.5 \mathrm{MHz}$ was housed in the water bath. Identical circles of $7 \mathrm{~mm}$ (corresponding to the transducer acoustical profile) were drawn on the membrane surface for evaluation of different US parameters (duration: $10 \mathrm{~s}-3 \mathrm{~min}$, duty cycle: $1-50 \%$, power: $0.5-2.0 \mathrm{~W}$ ). Opticells were viewed under fluorescent Leica DMI4000B microscope or Leica Z 16 APO (Macrofluo) macroscope. For quantitation the nuclei of the all cells were stained with Hoechst 33258. US-induced probe uptake was detected as a nuclear staining typical for each fluorophore (Fig. A, TOTO-3). Control areas showed very little uptake (less the $0.2 \%$ ) compared to the areas exposed to US (20-30\% uptake in the center). No difference in the uptake was found with regard to the fluorescent probe used. Macroscopic imaging with FOV $11.4 \times 8.5 \mathrm{~mm}$ (Fig. B, Sytox Green) revealed a close match between the acoustical profile of the transducer and Sytox Green fluorescence pattern. Probe uptake under various US parameters was quantitatively assessed. These studies confirmed that ultrasound-mediated drug delivery is a good candidate for local effective and non-invasive targeting of the tumor cells allowing a significant therapy enhancement. Live imaging of the intercalating probes resembling small-molecular drugs allows quantitative monitoring of the treatment and provides a further step towards the clinical implementation.
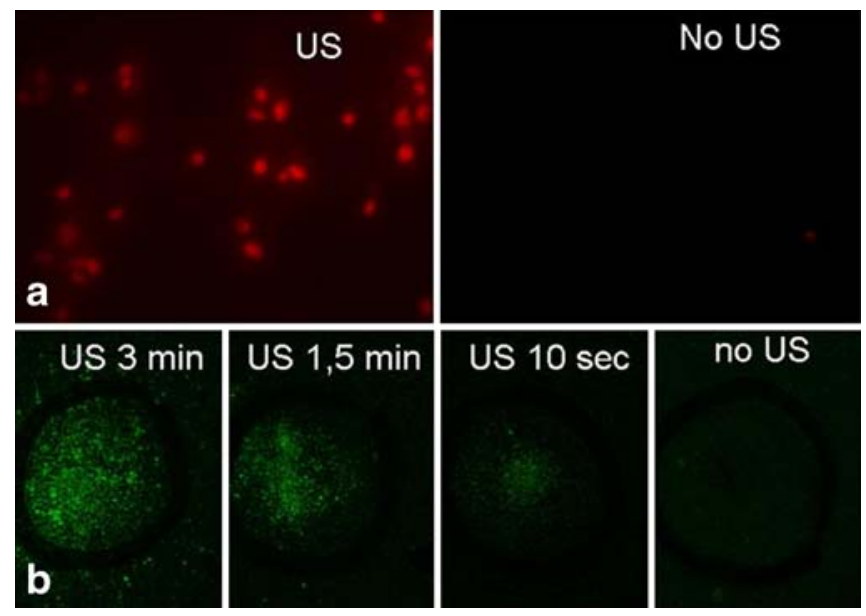

\section{$\mathbf{J 9 3 7}$}

QUANTIFYING SYNGENEIC BREAST CANCER METASTASIS TO THE LUNG AND RESPONSE TO THERAPY USING FLUORESCENCE MOLECULAR TOMOGRAPHY (FMT) Kristine Vasquez, Sylvie Kossodo, Jeffrey D. Peterson Applied Biology, VisEn Medical, Inc., Bedford, MA, USA.

Breast cancer is a prevalent clinical challenge today, with $10 \%$ of these patients showing cancer metastasis to distant organs in the body, such as bone, liver, and lung, decreasing their 5-year relative 
survival rate to $20 \%$. Thus, it is essential to develop robust in vivo methodologies that can help dissect the metastatic process and assist in the effective development of targeted therapeutic agents. To establish robust and non-invasive imaging measures of the metastatic disease process in a model resembling clinical disease, we used 4T1 mouse breast adenocarcinoma cells injected intravenously into normal, immunologically-competent $\mathrm{BALB} / \mathrm{c}$ mice. Two near infrared imaging agents, ProSenseTM750, a cathepsinactivatable agent, and AngioSenseTM680, a vascular agent, were injected IV to detect the protease activity and vascular leak associated with aggressive breast cancer growth. Fluorescence was imaged and quantified in living animals using optical Fluorescence Molecular Tomography (FMT) showing a consistent and significant increase in cathepsin signal as early as 7-10 days, with accom- panying increases in lung weight (a current standard measure in lung metastasis models). A standard clincal treatment using 5Fluorouracil/2'-deoxyinosine (5-FU/2DI) significantly reduced lung cathepsin fluorescence with greater sensitivity than seen with decreases in lung weight. In addition, treatment with a small molecule integrin antagonist decreased both vascular and cathepsin signal more effectively than seen by changes in lung weight. Quantification of protease activity and vascular leak provided important non-invasive measures of relevant tumor biology for comparison to standard measures of lung weight and histology. These data clearly demonstrate that deep tissue metastatic growth and response to treatment can be monitored in vivo in real time with a near infrared imaging agents and FMT.
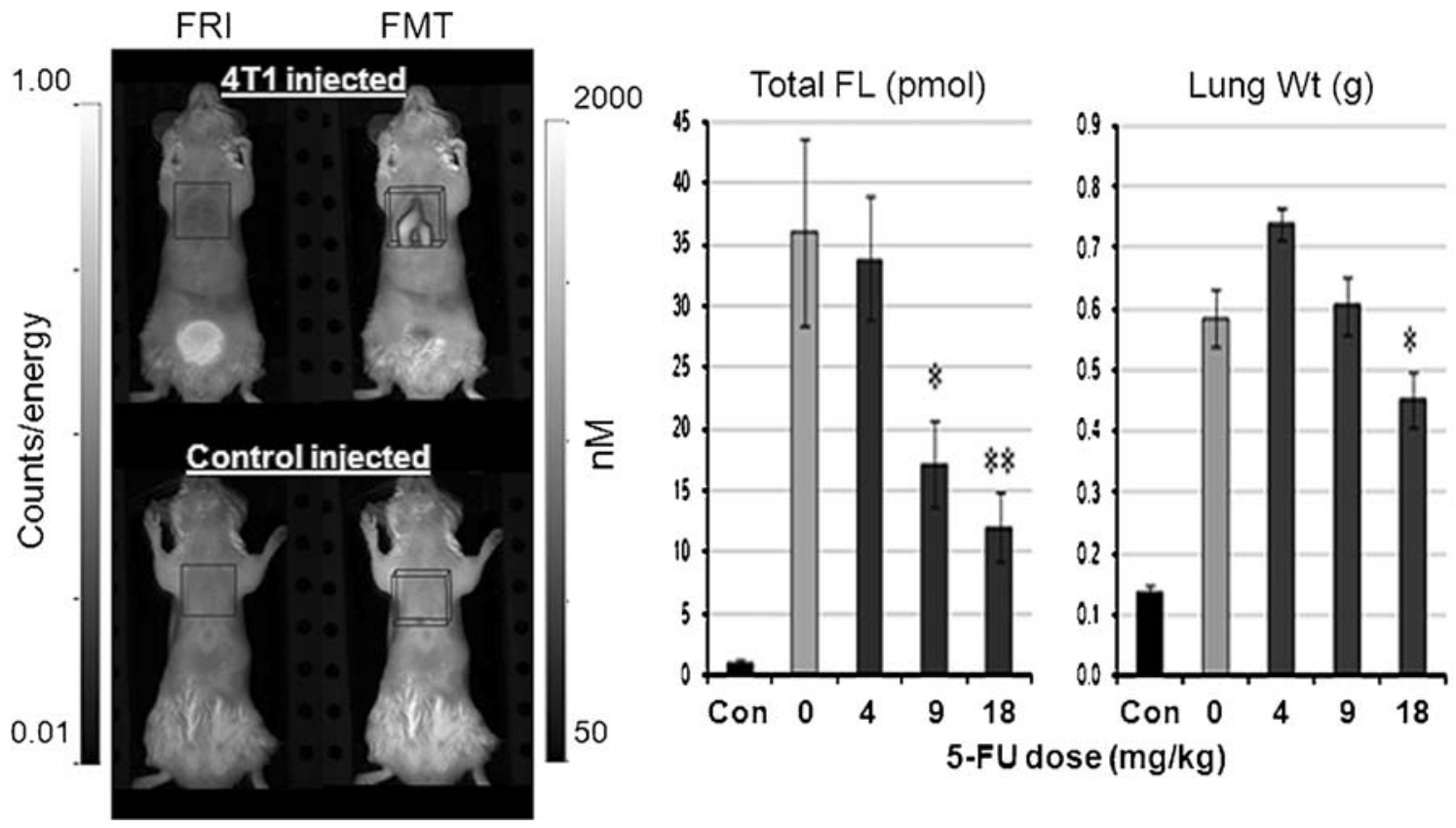

J938

sacral insufficiency fractures. The effect of radiation on bone density and morphology has been inadequately characterized in such patients. In two separate animal models, we evaluated the effect of radiation on measurable and quantifiable bone properties. In the first model, we evaluated standard radiation fraction sizes ( $2 \mathrm{~Gy} /$ fraction, 5 fractions/ week, 2 weeks) administered focally to subcutaneous xenograft tumors growing on the right thighs of nude mice. In the second model, we evaluated clinically relevant hypofractionated radiation therapy (4 Gy/ fraction, 2 fractions/week, 2 weeks). Bidimensional tumor measurements were made periodically after treatment and the time to doubling of tumor volume was estimated with radiation alone and with radiation combined with a radiosensitizer. Tumors, both femurs and both thigh musculatures were collected from mice in each cohort. Bones were subjected to X-ray densitometric analysis and immunohistochemical analysis. Changes in the bone density resulting from radiation were determined from $2 \mathrm{D}$ radiographic images obtained using a commercially available multimodal in vivo imaging system, with bone density analysis software. Bone 
density and morphometric measurements provide valuable information to assess the tissue toxicity effects during chemoradiotherapy. Future studies are necessary to determine if targeted agents can be used to counter these changes.

\section{Control Mice -Tumor on right limb}

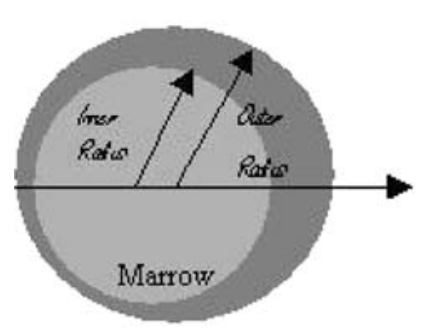

Left Tibia

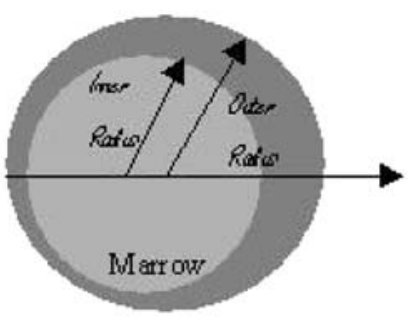

Right Tibia

\section{Irradiated Mice -Tumor on right limb}

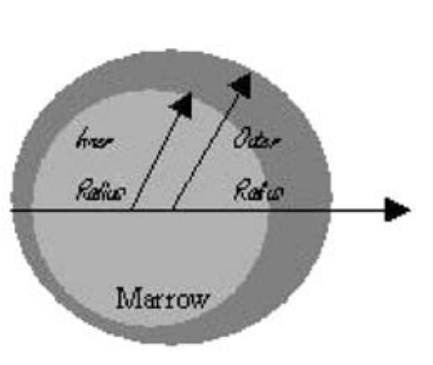

Left Tibia

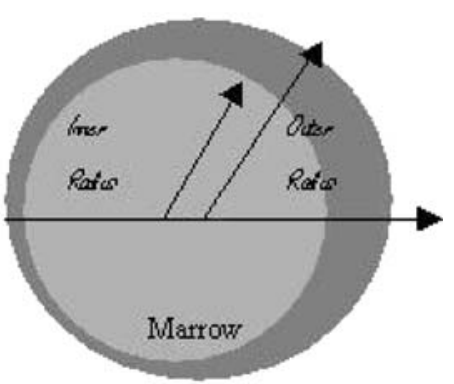

Right Tibia

Comparison of limb density, inner and outer radius of tibia of control and irradiated mice. Irradiation results in decreased bone density and bone morphological changes. The diameter modeled using a cylindrical fitting algorithm increased by $22 \%$ (outer radius) and $28 \%$ (inner radius)( $n=3$ )

J939

\section{THE ROLE OF THYMIDINE KINASE 1 IN 5-FLUOROURACIL- ENHANCED 3'-DEOXY-3'-[ $\left[{ }^{18}\right.$ F]FLUOROTHYMIDINE UPTAKE}

Seung Jin Lee ${ }^{1}$, Seog-Young Kim² ${ }^{2}$, Jin-Hwa Chung ${ }^{2}$, Jin-Sook Ryu ${ }^{2}$, Seung Jun $\mathrm{Oh}^{2}$, Dae Hyuk Moon ${ }^{2}$

${ }^{1}$ Institute for Innovative Cancer Research, Asan Medical Center, Seoul, Korea, South, ${ }^{2}$ Department of Nuclear Medicine, University of Ulsan College of Medicine, Asan Medical Center, Seoul, Korea, South.

Purpose: Positron emission tomography with $\left[{ }^{18} \mathrm{~F}\right]$ fluorothymidine $\left(\left[{ }^{18} \mathrm{~F}\right] \mathrm{FLT}\right)$ is a potential noninvasive marker for early monitoring of therapeutic response. $\left[{ }^{18} \mathrm{~F}\right] \mathrm{FLT}$ trapping correlates with thymidine kinase 1 (TK1) activity, but tumor cell's dependency on TK1 for dTTP synthesis is various. In this study, we examined the role of TK1 in 5-fluorouracil (5-FU) induced- $\left[{ }^{18} \mathrm{~F}\right] \mathrm{FLT}$ flare. Experimental Design: We measured time- and dose-dependent $\left[{ }^{18} \mathrm{~F}\right] \mathrm{FLT}$ flare and cytotoxicity induced by 5 -FU through $\left[{ }^{18} \mathrm{~F}\right] \mathrm{FLT}$ uptake test and MTS assay in 10 kinds of tumor cells. We analyzed 5-FU induced cell cycle arrest and the changes of TK1 in activity, protein and mRNA levels. Dynamic imaging was performed in A431 and HT29 xenograft models. Results: In all tested cell lines, 5-FU induced $\left[{ }^{18} \mathrm{~F}\right] \mathrm{FLT}$ uptake at $24 \mathrm{~h}$ exposure was larger than that at $2 \mathrm{~h}$ exposure. 5-FU-induced cytotoxicity was correlated with $\left[{ }^{18} \mathrm{~F}\right] \mathrm{FLT}$ flare after $24 \mathrm{~h}$ exposure in A431, HT29, M231, Hela, calu6, and HCT116 cells $\left(R^{2}=0.9633 \sim 0.8922\right)$. On the other side, it was correlated with $\left[{ }^{18} \mathrm{~F}\right] \mathrm{FLT}$ flare after $2 \mathrm{~h}$ exposure in SCC7, A549, HCT8 and MCF7 cells $\left(R^{2}=0.9342 \sim 0.5202\right)$. The cells in which $24 \mathrm{~h}$ exposure of 5-FU correlated with cytotoxicity showed dose-dependent increase of TK1 expression. In A431 and HT29 cells, cycloheximide pretreatment prevented 5-FU enhanced $\left[{ }^{18} \mathrm{~F}\right] \mathrm{FLT}$ uptake and 5-FU-induced late $\mathrm{G}_{1} \mathrm{~S}$ phase arrest as showned by cyclin A expression accompanied increases of TK1 activity, protein and mRNA levels. To validate the role of TK1 in these cells, we performed dynamic $\left[{ }^{18} \mathrm{~F}\right] \mathrm{FLT}-\mathrm{PET}$ imaging in mouse xenograft models. The change of $\mathrm{k}_{3}$ in 5-FU-treated group was significant, whereas that in vehicle-treated group was not in A431 tumor and HT29 tumor-bearing mice ( $p=0.0018$ for A431, $p=0.0156$ for HT29). Results: These studies showed that 5 -FU-enhanced $\left[{ }^{18} \mathrm{~F}\right] \mathrm{FLT}$ uptake could predict cytotoxicity by different mechanism depending on cell types. The changes of TK1 by 5-FU could be applied for quantitative prediction of cytotoxicity by $\left[{ }^{18} \mathrm{~F}\right] \mathrm{FLT}$ flare. The kinetic analysis by dynamic imaging would give a help to the prediction of 5-FU response.

\section{J940}

MECHANISM OF ARGININE DEIMINASE-MEDIATED CELL DEATH AND EVALUATION OF FUNCTIONAL IMAGING IN MELANOMA XENOGRAFTS

Lars Stelter ${ }^{1}$, Pat Zanzonico ${ }^{1}$, Lloyd Old $^{2}$, Gerd Ritter ${ }^{2}$, Achim A. Jungbluth $^{2}$, Marcus P. Kelly ${ }^{2}$, Bor-Wen $\mathrm{Wu}^{3}$, Valerie A. Longo ${ }^{1}$, Steven Larson ${ }^{1}$

${ }^{1}$ Nuclear Medicine, Memorial Sloan-Kettering Cancer Center, New York, NY, USA, ${ }^{2}$ Ludwig Institute for Cancer Immunotherapy, Memorial Sloan-Kettering Cancer Center, New York, NY, USA, ${ }^{3}$ Polaris Pharmaceuticals, San Diego, CA, USA.

Introduction Some cancer entities feature Arginine auxotrophy, due to their lack of Argininosuccinate synthetase (ASS)-expression. This characteristic makes them applicable for Arginine deiminase (ADI) treatment. ADI, as an Arginine-depleting drug, has so far shown promising results in patients suffering from metastasized malignant melanoma. However, the mechanism of ADI leading cancer cells into apoptotic cell death has not been fully elucidated in melanomas so far. The aim of our study was to identify the apoptotic pathway that is taken by ASS-negative melanoma cells upon ADI treatment and to evaluate the role of F18-FDG and F18-FLT PET in response prediction and therapy monitoring in melanoma xenografts. Material and Methods The ASS-positive melanoma cell line SK-MEL 10 and ASS-negative cell line SK-MEL 28 were treated with $4.3 \mathrm{mU} / \mathrm{ml}$ ADI for 7 consecutive days. Cell proliferation and apoptotic cell death were determined by proliferation assay and FACS. Further on, markers for the different apoptotic pathway as well as for macroautophagy were assayed in cell lysates. For the in vivo studies $4 \times 106$ SK-MEL 10 and SK-MEL 28 were inoculated s.c. in SCID mice $(n=8)$. After reaching a considerable tumor size the mice were either treated weekly with $160 \mathrm{U} / \mathrm{m} 2 \mathrm{ADI}(n=5)$ or PBS $(n=3)$ i.m. Tumor size and blood serum levels of Arginine were evaluated for 8 weeks and FDG and FLT PET were performed weekly to estimate intratumoral tracer uptake by ROI analysis $(\% \mathrm{ID} / \mathrm{g})$. Results The ASS-negative cell line SK-MEL 28 showed a significant drop in proliferative activity on day 4 of ADI treatment followed by an up to $90 \%$ rate of apoptotic cell death on day 7 , as estimated by Annexin-V 
FACS. After an early PARP-1 activation and initiation of macroautophagy, as protective mechanisms, apoptosis was initiated in a caspase-independent manner. In the ASS-expressing cell line no considerable effect of ADI treatment could be observed. In vivo, a clear decline in the SK-MEL 28 xenograft size could be seen in the ADI treated group, followed by a distinct reduction of tumoral FDG and FLT tracer uptake. Discussion Arginine deiminase treatment leads to an early initiation of macroautophagy, as a protective mechanism against nutrient starvation, in melanomas. In continued treatment, however, cells undergo the intrinsic pathway of apoptotic cell death. This therapeutic effect can be translated into an in vivo situation and, for the first time, documented by functional FDG and FLT PET.

J941

\section{A NOVEL STRATEGY FOR REPETITIVE, NON-INVASIVE MONITORING OF THE EFFICACIES OF HISTONE DEACETYLASE 6 (HDAC6) INHIBITORS IN LIVING SUBJECTS}

Carmel T. Chan ${ }^{1}$, James E. Bradner ${ }^{2}$, Robert E. Reeves ${ }^{1}$, Stuart L. Schreiber $^{3}$, Ramasamy Paulmurugan ${ }^{1}$, Sanjiv S. Gambhir ${ }^{1}$

${ }^{1}$ Radiology, Stanford University School of Medicine, Stanford, CA, USA, ${ }^{2}$ Hematologic Oncology, Dana-Farber Cancer Institute, Boston, MA, USA, ${ }^{3}$ Department of Chemistry and Chemical Biology, Harvard University, Cambridge, MA, USA.
Heat Shock Protein 90(Hsp90)/p23 interactions are crucial for proper folding of proteins in cancer and neurodegenerative diseases. Hsp90 and HDAC6 inhibitors block Hsp90( $\alpha / \beta) / \mathrm{p} 23$ interactions by preventing ATP binding and leading to hyperacetylation of Hsp90,respectively. A split Renilla Luciferase complementation system [Cancer Res 68:216-26] was used for indirect monitoring of $\mathrm{Hsp} 90(\alpha / \beta) / \mathrm{p} 23$ interactions by bioluminescence imaging (BLI) in cell culture and living mice in response to different HDAC inhibitors. Class II (APHA \& Trichostatin) and HDAC6 inhibitors (Tubacin[Tuba] \& WT161) led to disruption of $\mathrm{Hsp} 90(\alpha / \beta) / \mathrm{p} 23$ interactions and increased expression of p $21^{\text {wafl }}$ and acetylated $\alpha$-tubulin (act- $\alpha$ tub). Their efficacies were enhanced by the addition of the Hsp90 inhibitors PUH71/17DMAG. HDAC1 inhibitor MS275 and the control compound Niltubacin had no effect on Hsp90/p23 interactions (Fig.1A) nor the expression of $\mathrm{p} 21^{\text {waf1 }}$ and act-atub. The efficacy of Tuba in disruption of Hsp90/p23 interactions in living mice were evaluated by BLI (Fig.1B). Relative to $0 \mathrm{hr}$, BLI signals in mice treated with Tuba $(25 \mathrm{mg} / \mathrm{kg}, 23 \mathrm{hrs})$ were $33 \pm 14 \%$ and $70 \pm 11 \%$ of Hsp $90 \alpha / \mathrm{p} 23$ and Hsp90ß/p23 interactions, respectively $(p<0.05$ compared to respective carrier control treated mice)[Fig. 1B]. Combination of Tuba with PU-H71 led to further reduction in $\operatorname{Hsp} 90(\alpha / \beta) / \mathrm{p} 23$ interactions. The efficacy of Tuba was confirmed by increased expression of act- $\alpha$ tub in excised tumors. Noninvasive monitoring of the efficacy of HDAC inhibitors, alone in combination with Hsp90 inhibitors will significantly accelerate the development of HDAC6 inhibitors and combinatorial therapies.

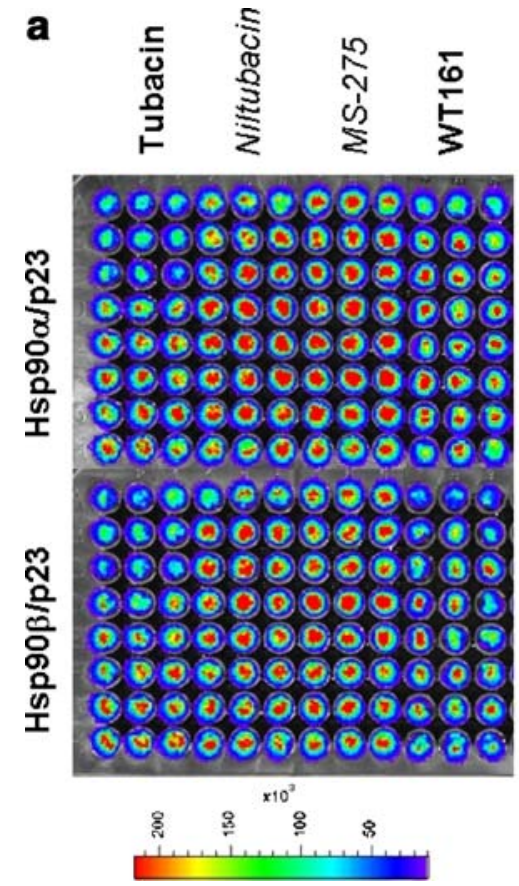

b

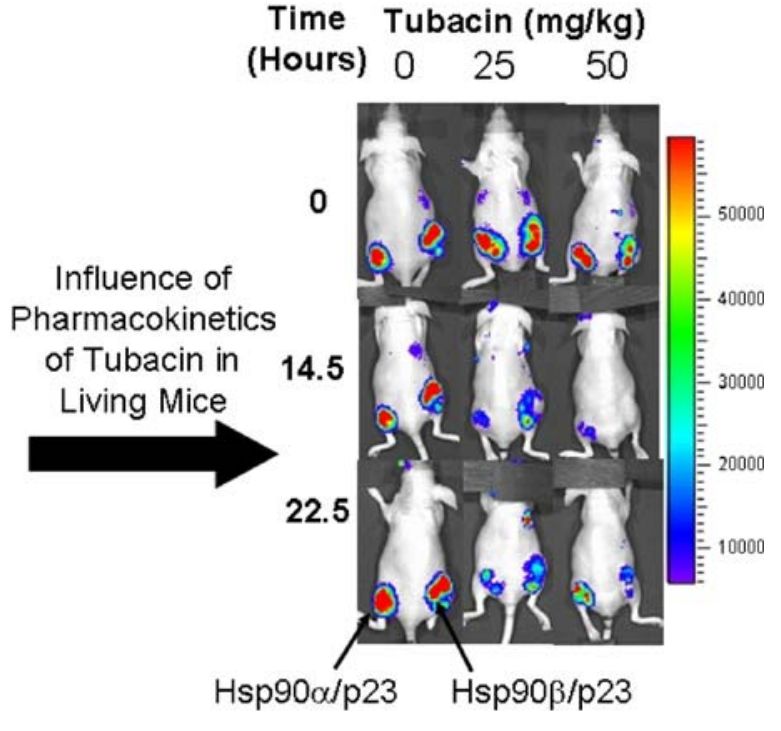

Figure 1: Non-invasive monitoring of the efficacy of HDAC inhibitors through molecular imaging of the disruption of Hsp90( $\alpha / \beta) / \mathrm{p} 23$ interactions in cell culture and in living mice. A. 293T cells stably expressing Hsp90 $\alpha / \beta) / \mathrm{p} 23$ split RL reporters were treated with HDAC6 inhibitors Tubacin and WT161 or HDAC1 inhibitor MS275 or the control Niltubacin for 24 hours prior to BLI. B. The efficacy of Tubacin in disruption of Hsp90( $\alpha / \beta) / \mathrm{p} 23$ interactions in living mice were monitored by repetitive BLI upon injection of the RL substrate coelentarazine. 
J942

\section{MASS SPECTROMETRY IMAGING OF DRUG DISTRIBUTION AND METABOLISM IN BRAIN TUMOR MODELS AND SURGICAL SPECIMENS}

Nathalie Y. Agar ${ }^{1}$, Alexandra J. Golby ${ }^{1,2}$, Keith L. Ligon ${ }^{3,4}$, Claire M. Sauvageot $^{4}$, Katherine A. Kellersberger ${ }^{5}$, Jeffrey N. Agar ${ }^{6}$, Charles D. Stiles $^{4}$, Santosh Kesari ${ }^{7,4}$

${ }^{1}$ Neurosurgery, Brigham and Women's Hospital/Harvard Medical School, Boston, MA, USA, ${ }^{2}$ Radiology, Brigham and Women's Hospital/Harvard Medical School, Boston, MA, USA, ${ }^{3}$ Pathology, Brigham and Women's Hospital/Harvard Medical School, Boston, MA, USA, ${ }^{4}$ Cancer Biology, Dana Farber Cancer Institute/Harvard Medical School, Boston, MA, USA, ${ }^{5}$ Bruker Daltonics, Billerica, MA, USA, ${ }^{6}$ Chemistry, Brandeis University, Boston, MA, USA, ${ }^{7}$ Neurology, Brigham and Women's Hospital/Harvard Medical School, Boston, MA, USA.

In the process of developing targeted molecular therapies for brain tumors, multiple levels of molecular analysis and imaging are required to evaluate drug distribution and metabolism, and the effect of the drug on its molecular target. Analytical tools include in vivo imaging of tumor development and anatomical response to therapy in tumor models, as well as tissue harvesting for molecular analysis, relying heavily on the use of antibodies to decipher molecular events from extracts and tissue sections. Mass spectrometry imaging (MSI) is becoming a powerful technique for the direct analysis of chemical species in tissue. The combination of spectral and spatial information derived from Matrix Assisted Laser Desorption Ionization (MALDI) MSI experiments provides a workflow, which does not rely on radio- or immunostrategies, but solely on the accurate assessment of the mass of molecules for selectivity. As the performance of the mass spectrometer detector used for molecular imaging increases, so does the confidence in the elemental assignment of a molecule. The presented study makes use of high-field MALDI-FTMS for drug and metabolite imaging of anticancer targeted therapy administered at therapeutic levels in mouse models of brain tumors. We can also begin to adapt and incorporate the emerging mass spectrometry technologies and applications into our clinical research armamentarium. While it is of great importance to monitor drug penetration through the blood-brain barrier (BBB), it is also imperative to assess response to treatment as early as possible. With such high analytical performance, FTMS is poised to enable this assessment through biomarker discovery, as identification and validation are interdigitated, and tied to the highest achievable accuracy. Simultaneous drug monitoring and development of robust biomarkers should eventually allow to rationally plan future studies of combination trials to overcome resistance mechanisms and to improve efficacy of targeted therapies, and to also assess response earlier. Linking such individual treatment responsiveness to biomarker will be the crucial next step to truly personalizing brain cancer therapies, and for translation to other more accessible platforms to become routine analyses.

\section{J943}

DYNAMIC CONTRAST-ENHANCED MRI DETECTS EARLY VASCULAR CHANGE IN ORTHOTOPIC PANCREATIC TUMOR XENOGRAFTS FOLLOWING ANTI-EGFR AND IRINOTECAN THERAPIES

Hyunki Kim ${ }^{1,4}$, Karri Folks ${ }^{1}$, Lingling Guo ${ }^{2}$, Jeffrey C. Sellers ${ }^{4}$, Donald J. Buchsbaum ${ }^{3,4}$, James George ${ }^{2}$, Kurt R. Zinn ${ }^{1,4}$

${ }^{1}$ Radiology, University of Alabama, Birmingham, AL, USA, ${ }^{2}$ Surgery, University of Alabama, Birmingham, AL, USA, ${ }^{3}$ Radiation Oncology, University of Alabama, Birmingham, AL, USA, ${ }^{4}$ Comprehensive Cancer Center, University of Alabama, Birmingham, AL, USA.
Purpose: To measure and characterize early vascular change in orthotopic pancreatic tumor xenografts, following cetuximab (anti-EGFR antibody) and irinotecan therapies via a series of dynamic contrast-enhanced MR imaging (DCE-MRI). Methods: Three groups of SCID mice ( $n=4 /$ group) bearing orthotopically implanted, luciferase-positive human pancreatic tumors (MIA PaCa-2) were used. Groups 1-3 were injected i.p. with PBS, cetuximab(1 mg), and cetuximab(1 mg)+irinotecan $(25 \mathrm{mg} / \mathrm{kg} \mathrm{BW})$ respectively on days $0,3,7,10,14$, and 17 . DCE-MRI was performed on days $0,1,2$, and 3, while anatomical MRI was performed on days $0,1,2$, 3,6 , and 13 . At day 21 , all tumors were collected by dissection, and tumor dimensions were measured with calipers. The averaged Ktrans values in both the entire tumor region and the $0.5-\mathrm{mm}$ thick peripheral tumor region were calculated, and compared with tumor volume changes. Results: The changes of Ktrans values of groups 1-3 during 3 days after therapy initiation were $116 \pm 17 \%, 74 \pm 5 \%$, and $-5 \pm 10 \%$, respectively, in the peripheral tumor region with significant difference among the groups $(p<0.05)$, whereas those in the entire tumor region were $82 \pm 41 \%, 106 \pm$ $11 \%$, and $24 \pm 38 \%$, respectively, without statistical significance $(p>$ 0.05 ). The tumor volume changes of groups 1-3 during 3 days after therapy initiation were $39 \pm 12 \%, 30 \pm 9 \%$, and $17 \pm 7 \%$, respectively, without significant difference, whereas those during 21 days were $177 \pm$ $31 \%, 19 \pm 30 \%$, and $-66 \pm 2 \%$ respectively with significant difference. Of interest, the mean Ktrans changes of all 3 groups in peripheral tumor region followed second-order polynomial curves, validated with high R2 values (0.99). An indicator proposed to reflect these non-linear characteristics is the coefficient of the second-order term for each curve; 0.05 , 0.01 , and -0.10 for groups $1-3$, respectively. The mean tumor volume changes for 3 weeks were linearly proportional to the coefficients of the second-order terms $(\mathrm{R} 2=0.95)$ as well as to the mean Ktrans changes in peripheral region for 3 days $(\mathrm{R} 2=0.88)$. Conclusion: DCE-MRI detected a significant therapeutic response at 3 days after anti-EGFR antibody monotherapy or in combination with irinotecan using peripheral-region analyses in the orthotopic pancreatic tumor xenografts, which correlates well with tumor-growth suppression over the 21 days of treatment. The indicator reflecting early non-linear Ktrans change could be considered as a novel imaging biomarker measuring early pancreatic-tumor response to drugs independently of a particular monitoring time point.

\section{J944}

\section{SPECIFICITY IN GENE SILENCING} USING MULTIFUNCTIONAL NANOPARTICLES

Rao V. Papineni ${ }^{1}$, Tao $\mathrm{Ji}^{1}$, Thirupandiyur Udayakumar ${ }^{2}$, Mohammed M. Shareef ${ }^{2}$, Mansoor M. Ahmed ${ }^{2}$, Alan Pollack ${ }^{2}$

${ }^{1}$ Carestream Molecular Imaging, Carestream Health, Inc., New Haven, CT, USA, ${ }^{2}$ Radiation Oncology, University of Miami, Miami, FL, USA.

An efficient in vivo gene silencing therapeutics system utilizing the siRNA technology requires the following criteria to be met- Ability to deliver high volume/capacity of double stranded siRNA molecules to the site of interest, and to efficiently transfer siRNA into the cells. siRNA delivery vehicle should also be capable of protecting siRNA from the nucleases during systemic delivery. To address the multiple prerequisites, we have developed nanoparticle-based delivery of siRNA, which allows easy linking of siRNA, targeting antibodies, biologics such as cell penetrating molecules, and other chaperones for gene silencing. We have utilized the high affinity avidin-biotin interaction properties for this system. The biotinylated macromolecules (siRNA, targeting and cell penetrating biologics) in precise stoichiometric concentrations were linked to neutravidin decorated dye encapsulated polymeric nanoparticle designated as High affinity hooker nanoparticle (HAH NP), by simple incubation on ice. Such a system also facilitates the simultaneous NIR fluorescence imaging in a noninvasive manner along with targeted 
siRNA delivery. As a proof of concept, the HAH-NP linked with biotinylated anti-PSMA antibody and MDM2 inhibiting biotinylated siRNA was prepared. in vitro studies demonstrate the specificity in $\mathrm{HAH}$ NP binding to PSMA expressing prostate cancer cells (LNCaP), and no binding to PC3 cells and fibroblasts which are negative for PSMA. Further, the anti-PSMA linked HAH-NP significantly inhibited MDM2 in LNCaP-MST cells that have been permanently transfected to over express MDM2 protein, suggesting the specificity of targeted inhibition.
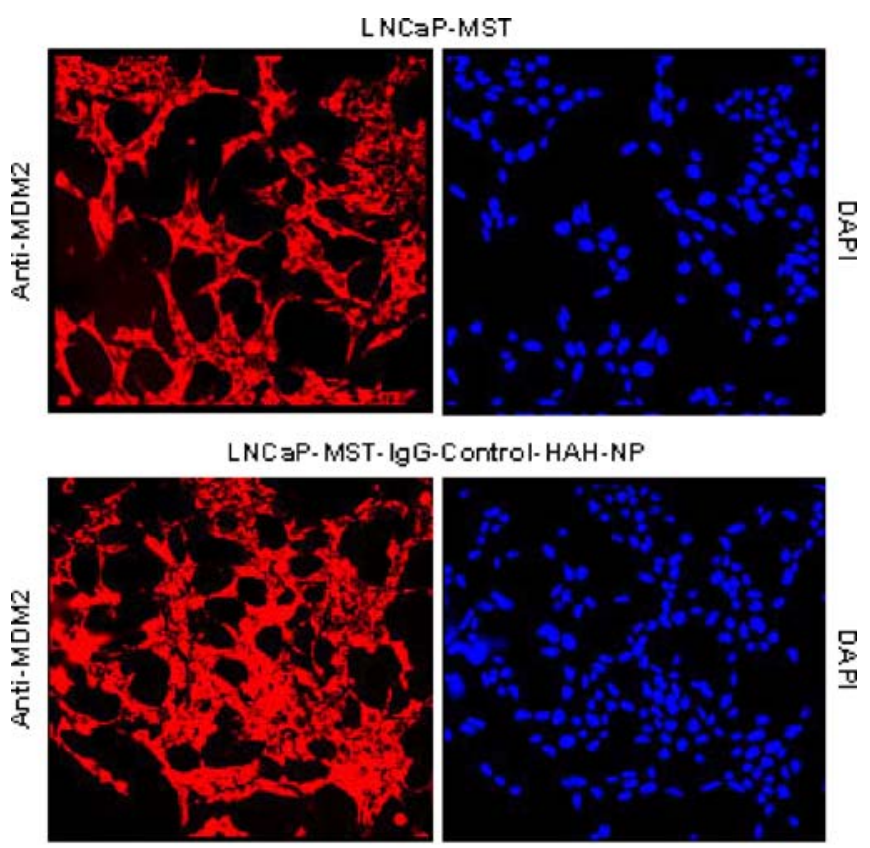

LN CaP-MST-Anti-PSMA- IgG-HAH-NP MD M2-siRNA
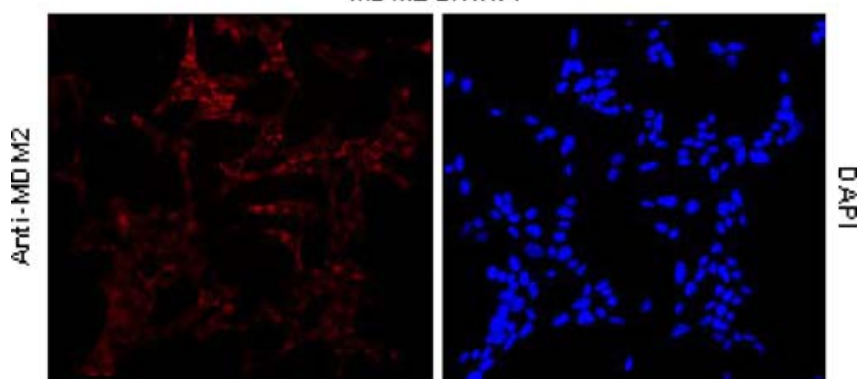

J945

ESTABLISHMENT OF TIGHTLY IR SWITCH FOR COMBINATION RADIATION AND GENE THERAPY OF CANCER

Luen Hwu $^{1}$, Al Huang ${ }^{1}$, Ren-Shyan Liu ${ }^{1,2}$

${ }^{\mathrm{T}}$ MAGIC, Dept NM, Faculty of Medicine, National Yang-Ming University, Taipei, Taiwan, ${ }^{2}$ NPCC, Dept NM, Taipei Veterans General Hospital, Taipei, Taiwan.

Objectives: Commercial available promoter from cytomegalovirus (CMV) is a ubiquitous, strong, expression promoter. We expect that the radiation-responsive $\mathrm{CArG}$ element/enhancer combine with the basal CMV promoter, reported by Marple's group, would result in ubiquitous expression in most of cells. Therefore, we are wondered whether the multimeric of CArG element only are sufficient to use as IR responsive promoter. Methods: Briefly, for constructing FLuc reporter plasmids with various copies of $\mathrm{CArG}$ elements, the commercial available pDsRed
monomer-C1 plasmid, in which the coding sequence of monomeric DsRed was displaced with FLuc gene from pGL3-basic and the resulted vector, pFLuc-C1. Synthetic CArG sequence (CCATATAAGG) was introduced into pFLuc-C1.We obtained nine directly repeated of CArG elements (denoted E9-Fluc), eighteen (E18-Fluc), and thirty -one (E31Fluc). The FLuc activities of the constructs were performed by transfecting NSCLC H1299 cells after DNA sequence validation. Results: In the absence of IR triggering, these "promoterless" CArG elements (E9, E18, and E31) showed that minimal transcription activity in the order as following $\mathrm{E} 31 \geqq \mathrm{E} 9 \geqq \mathrm{E} 18$. After 2 Gy radiation exposure, all constructs showed transcription activation increase above1.5 folds. To prove our hypothesis, we linked cytomegalovirus (CMV) enhancer in front of E9 promoter (denoted Enh CMV -E9-Fluc). The Result revealed that basal luciferase activity increased almost 2 folds in Enh CMV -E9Fluc construct in the absence of IR. And in the presence of 2 Gy IR triggering, the fold of enhancement still is retained as parental E9-FLuc construct present. Conclusions: The radio-responsive enhancer, containing 'CArG' elements was well known to be transiently activated by ionizing radiation. But we applied ' $\mathrm{CArG}$ ' elements directly as a radioactive promoter not an enhancer; our finding intensely supports this hypothesis. We believe this type of molecular switch will be particularly beneficial in specific, but relatively weak radiation-responsive promoters are employed to target gene.

\section{J946}

\section{STEREOTACTIC MASS SPECTROMETRY IMAGING} OF TUMOR BOUNDARIES IN NEUROSURGERY

Nathalie Y. Agar ${ }^{1}$, Alexandra J. Golby ${ }^{1,2}$, Keith L. Ligon ${ }^{3}$, Isaiah Norton $^{1}$, Justin M. Wiseman ${ }^{4}$, Ferenc A. Jolesz ${ }^{2}$

${ }^{1}$ Neurosurgery, Brigham and Women's Hospital/Harvard Medical School, Boston, MA, USA, ${ }^{2}$ Radiology, Brigham and Women's Hospital/Harvard Medical School, Boston, MA, USA, ${ }^{3}$ Pathology, Brigham and Women's Hospital/Harvard Medical School, Boston, MA, USA, ${ }^{4}$ Prosolia Inc., Indianapolis, IN, USA.

Surgery remains the most important, and usually first treatment modality for the majority of brain tumors. The most significant contributor to prognosis improvement for patients still is a smaller volume of postoperative residual tumor. The principal challenge and objective of neurosurgical intervention is therefore to maximize tumor resection, while minimizing potential for neurological deficit by preserving critical tissue. We will present proof-of-concept in using mass spectrometry intraoperatively for molecular imaging of tumor boundaries, without systemic injection of molecular probes or contrast agents. The stereotactic sampling approach provides point-by-point registration of radiology with atmospheric pressure desorption/ionization mass spectrometry analyses of the tissue at stake, and direct validation with standard histopathology. The presented concept for integration of an ultrasonic surgical probe providing diagnostic quality tissue with an air ambient desorption/ionization mass spectrometry approach and a 3D navigation system will enable a new level of accuracy and precision in neurosurgery, and be applicable to a broad range of surgical procedures in any tissue type requiring fine tissue differentiation. Mass spectrometry is highly sensitive, applicable to most biomolecules, and has a broad dynamic range making it an attractive approach to respond to the high performance demands of personalized care implementation. Different mass spectrometry approaches have been shown to distinguish between diseased and healthy tissue in the laboratory. Capitalizing on such analytical qualities and rapidity of mass spectrometry, a method is developed to expedite the validation and implementation of a novel surgical guidance approach. The mass spectrometry approach provides with multivariate molecular imaging of the surgical cavity without any 
molecular probe systemic injection, pertaining no additional risk to the patient. The presented method translates mass spectrometry to stereotactic surgery, and relies on the real-time analysis of otherwise discarded tissue in the operating room. As the predominant line of management for devastating brain cancers, surgical procedures need to be tailored to the particular presentation of an individual's neoplasm with the highest specificity to combat recurrence. In its clinical infancy, intraoperative mass spectrometry carries the potential to significantly enhance surgical efficacy.

\section{J947}

MOLECULAR IMAGING OF HISTONE DEACETYLASE (HDAC) ACTIVITY IN HUMAN GLIOBLASTOMA

MULTIFORME USING 6 - $\left(\left[{ }^{18}\right.\right.$ F]-FLUOROACETAMIDE)-1HEXANOICANILIDE $\left(\left[{ }^{18}\right.\right.$ F $]$-FAHA)

Jung-Wen Kuo ${ }^{1}$, Chien-Feng Lin ${ }^{2}$, Chun-Yi Wu ${ }^{1}$, Hsin-Ell Wang ${ }^{3}$, Juri G. Gelovani ${ }^{4}$, Ren-Shyan Liu ${ }^{1,2}$

${ }^{1}$ MAGIC, Dept NM, Faculty of Medicine, National Yang-Ming University, Taipei, Taiwan, ${ }^{2}$ NPCC, Dept NM, Taipei Veterans General Hospital, Taipei, Taiwan, ${ }^{3}$ Institute of Biomedical Imaging and Radiological Sciences, National Yang-Ming University, Taipei, Taiwan, ${ }^{4}$ Dept of Experimental Diagnostic Imaging, M.D. Anderson Cancer Center, Houston, TX, USA.
Objectives: Glioblastoma multiforme (GBM) is the most aggressive and invasive brain tumor. Histone deacetylase inhibitors (HDACI) have been used to induce glioblastoma cell death. There are no predictive biomarkers for selection of subjects who are most likely to respond to treatment with HDACIs. The purpose of this study was to assess the HDAC activity in GBM mouse tumor model by 6-([ $\left.{ }^{18} \mathrm{~F}\right]$-fluoroacetamide)-1-hexanoicanilide ([ $\left.\left.{ }^{18} \mathrm{~F}\right]-\mathrm{FAHA}\right)$ PET imaging. Methods: The $\left[{ }^{18} \mathrm{~F}\right]-\mathrm{FAHA}$ was synthesized and modified according to the previous study (J Label Compd Radiopharm 2006;49:997-1006). Human GBM 8401/CMV-Luc cells $\left(2 \times 10^{5}\right)$ were stereotactically implanted into left forebrain of NOD/SCID mice. In vivo bioluminescence imaging was conducted to evaluate the xenograft tumor growth. MicroPET dynamic studies with $\left[{ }^{18} \mathrm{~F}\right]$-FAHA were acquired for $60 \mathrm{~min}$. Subsequent biodistribution of organs was measured post imaging. The $\left[{ }^{18} \mathrm{~F}\right]$-FAHA cellular uptake of $\left[{ }^{18} \mathrm{~F}\right]$-FAHA was assessed $2 \mathrm{hrs}$ after incubation. Results: Tumor in vitro uptake and tumor-to-brain in vivo uptake ratio of $\left[{ }^{18} \mathrm{~F}\right]$-FAHA were both approximate 2.1 . The tumor uptake of $\left[{ }^{18} \mathrm{~F}\right]$-FAHA reached the steady-state in $30 \mathrm{~min}$. Biodistribution study showed that most of the tracer excreted via the kidney and bile duct. The brain had highest normal tissue uptake indicating high HDAC activity in the brain. Conclusions: $\left[{ }^{18} \mathrm{~F}\right]$-FAHA PET is a feasible biomarker to express the HDAC activity of GBM and seems useful to assess the response of HDACIs therapy.

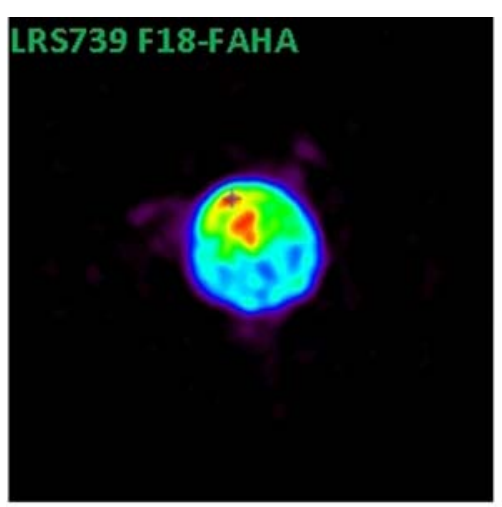

TAC of LRS739

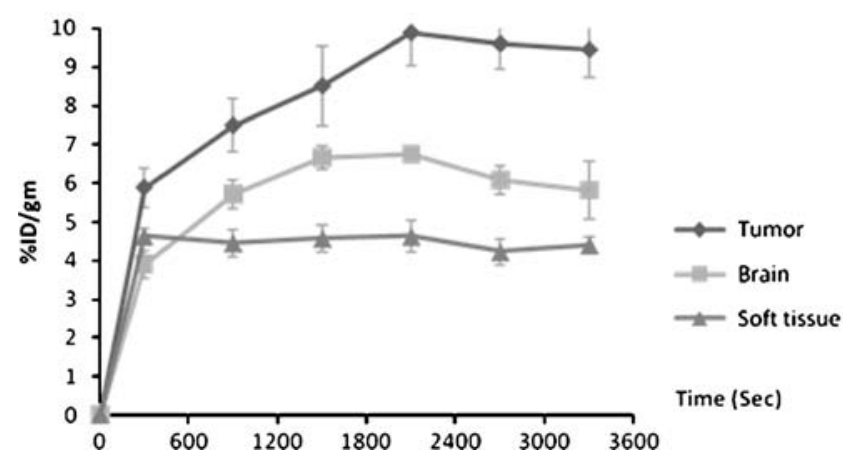

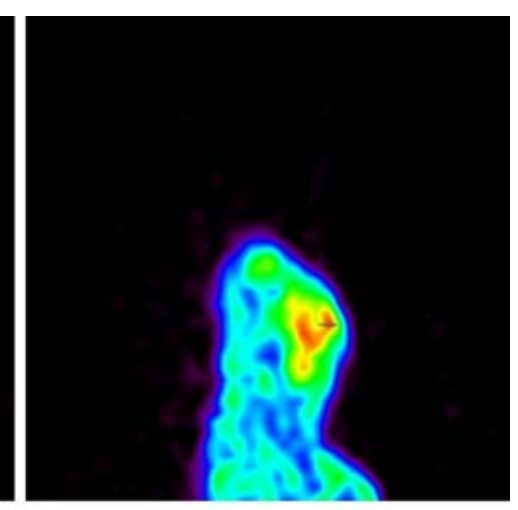$$
\text { . }
$$

$\mathrm{T} / \mathrm{B}=1.6$

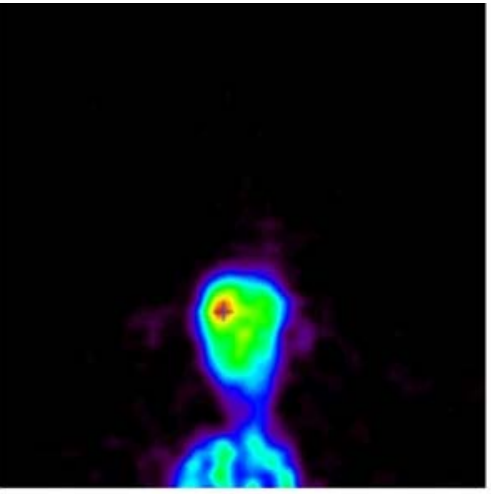


J948

MOLECULAR IMAGING OF HISTONE DEACETYLASE ACTIVITY IN LUNG CANCER USING 6-( $\left[{ }^{18} \mathrm{~F}\right]-$ FLUOROACETAMIDE)-1-HEXANOICANILIDE ( $\left[{ }^{18}\right.$ F]-FAHA) $\underline{\text { Ren-Shyan Liu }}^{1,2}$, Chien-Feng Lin ${ }^{2}$, Shih-Yen $\mathrm{Wu}^{3}$, Hsin-Ell Wang ${ }^{3}$, Juri G. Gelovani ${ }^{4}$

${ }^{1}$ MAGIC, Dept NM, Faculty of Medicine, National Yang-Ming University, Taipei, Taiwan, ${ }^{2} \mathrm{NPCC}$, Dept NM, Taipei Veterans General Hospital, Taipei, Taiwan, ${ }^{3}$ Institute of Biomedical Imaging and Radiological Sciences, National Yang-Ming University, Taipei, Taiwan, ${ }^{4}$ Dept Experimental Diagnostic Imaging, M.D. Anderson Cancer Center, Taipei, Taiwan.

Objectives: Histone deacetylases (HDACs) play a pivotal role in regulation of gene expression. Abnormal HDACs to DNA regulatory regions is observed in many cancers. Inhibitors of HDAC are emerging as a promising class of anti-cancer drugs. The aim of this study was to evaluate PET imaging with HDAC substrate 6-([ $\left.{ }^{18} \mathrm{~F}\right]$-fluoroacetamide $)-1$-hexanoicanilide ([ $\left.{ }^{18} \mathrm{~F}\right]$-FAHA) to determine HDAC activity in lung cancer. Methods: The $\left[{ }^{18} \mathrm{~F}\right]-$ FAHA was synthesized and modified according to the previous study (J Label Compd Radiopharm 2006;49:997-1006). The uptake of $\left[{ }^{18} \mathrm{~F}\right]-\mathrm{FAHA}$ was determined in H1299 non small cell lung carcinoma (NSCLC) cell line. NOD/SCID mice were transplanted with the H1299 tumor cells $\left(2 \times 10^{5}\right)$ on the shoulder. MicroPET imaging studies with $\left[{ }^{18} \mathrm{~F}\right]$-FAHA (3.7 MBq i.v., $150 \mu \mathrm{L}$ ) were conducted in the tumor bearing NOD/SCID mice. Dynamic microPET imaging was acquired for 1 hour. Results: In vitro uptake study demonstrated a rapid accumulation in H1299 cell line over time between $5 \mathrm{~min}$ and $2 \mathrm{hr}$. The level of uptake at equilibrium was reflective of the level of expression and activity of HDACs in corresponding cell line $(1.1 \pm 0.06$ cell/medium accumulation ratio). In in vivo study, the tumor uptake and tumor-to-muscle $(\mathrm{T} / \mathrm{M})$ ratios of $\left[{ }^{18} \mathrm{~F}\right]-$ FAHA are $4.12 \% \mathrm{ID} / \mathrm{g}$ and 1.62 , respectively, between $5 \mathrm{~min}$ and $2 \mathrm{hr}$ post-injection demonstrating HDAC activity in NSCLC tumor. Conclusions: The study demonstrated that PET with $\left[{ }^{18} \mathrm{~F}\right]-\mathrm{FAHA}$ can be used as a biomarker of HDAC activity in the lung cancer and used for the assessment of the effect of novel HDAC inhibitors on tumor growth.

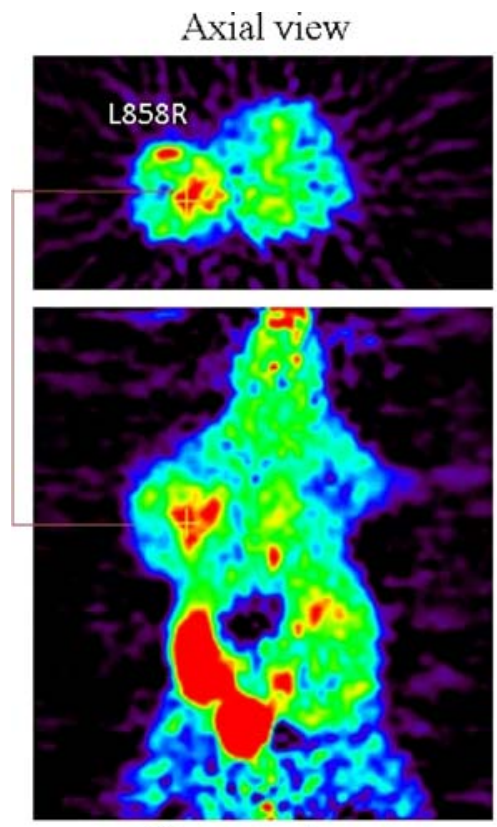

Coronal view
Representative microPET images of 18F-FAHA in mice bearing L858R subcutaneous tumor xenograft. Tumor-bearing mice underwent a static microPET scan for 1 hour dynamic imaging.
J949

NOVEL CHIMERIC HEPATOMA-SPECIFIC PROMOTER FOR TUMOR- SPECIFIC GENE THERAPY OF HEPATOMA Ya-Ju Hsieh $^{1}$, Ren-Shyan Liu ${ }^{1,2}$, Hsin-Ell Wang ${ }^{4}$, Victor Krasnykh ${ }^{5}$, Juri G. Gelovani ${ }^{5}$

${ }^{1}$ MAGIC, Dept NM, Faculty of Medicine, National Yang-Ming University, Taipei, Taiwan, ${ }^{2}$ NPCC, Dept NM, Taipei Veterans General Hospital, Taipei, Taiwan, ${ }^{3}$ De Lin Institute of Technology, Taipei, Taiwan, ${ }^{4}$ Biomedical Imaging \& Radiological Sciences, National Yang-Ming University, Taipei, Taiwan, ${ }^{5}$ Experimental Diagnostic Imaging, M.D. Anderson Cancer Center, Taipei, Taiwan.
Objectives: Herpes simplex virus type 1 thymidine kinase (HSV1tk) gene therapy is a promising treatment against cancer. A tumor-specific chimeric promoter (EIIAPA) containing alpha fetoprotein (AFP) promoter and hepatitis B virus enhancer II (EIIA) was established to control downstream gene expression selectively. Methods: The selectivity and magnitude of transcriptional activity from the EIIAPA promoter was studied using in vitro bioluminescence imaging (BLI) assay. Subsequently, microPET radionuclide and BLI modalities were used for determining the function of EIIAPA chiemric promoter in NOD/SCID mice with orthotopic xenografts human HCC cells transfected with the HSV1 tk-luciferase dual reporter gene controlled by EIIAPA promoter (EIIAPA-tk-IRES-luc). Lentiviral system was further 
established to demonstrate the specificity of EIIAPA to hepatoma. The ARO nonhepatoma and HepG2 hepatoma cells were first infected repeatedly with lentiviral particles carrying HSV1 tk gene controlled by either constitutive cytomegalovirus (CMV) immediate-early promoter (pLL3.7/CMV-tk) or EIIAPA promoter (pLL3.7/EIIAPA-tk) in vitro, and then grown in the SCID mice. Results: The AFP transcriptional activity was improved 44 times in HepG2 hepatoma cell line by combing with EIIA enhancer but no improvement was observed in nonhepatoma and normal fibroblast cell lines. Highly specific images of NOD/SCID mice carrying tumors transfected with EIIAPA-tk-IRES-luc confirmed that the magnitude of transcriptional activity from the EIIAPA promoter was adequate for monitoring by non-invasive imaging in vivo. Moreover, a significant tumor regression of EIIAPA-tk-IRES-luc stably transfected tumors were observed after 14-day treatment with GCV (100 mg/kg/day). Comparing EIIAPA promoter with CMV promoter, the HSV1 tk gene expression was induced only in lentivirus-infected HepG2 tumor but not in lentivirus-infected ARO tumor. Furthermore, radionuclide imaging demonstrated the HSV1-tk gene expression and accumulation of the ${ }^{124}$ I-FIAU reporter probes in HepG2 wild type tumor intratumorally infected with lentivirus in vivo. Conclusions: This novel chimeric promoter contributes to the level of HSV1-TK enzyme expression that are detectable in HCC tumors by non-invasive bioluminescence imaging and by clinically applicable radionuclide imaging, which result in efficient sensitization of HCC to GCV in context of prodrug activation gene therapy. According to these promising results, we conclude that the EIIAPA promoter is a potential candidate for transcriptional targeting of gene therapy of HCC.

\section{J950}

\section{QUANTIFICATION OF THE EFFECT OF HISTONE DEACETYLASE (HDAC) INHIBITOR IN RAT GLIOMA BY ${ }^{18}$ F-FAHA PET}

Jung-Wen Kuo $^{1}$, Chun-Yi Wu ${ }^{1}$, Kuan-Hao Su${ }^{2}$, Jyh-Cheng Chen ${ }^{2}$, Hsin-Ell Wang $^{2}$, Ren-Shyan Liu ${ }^{1,3}$

${ }^{1}$ MAGIC, Dept NM, Faculty of Medicine, National Yang-Ming University, Taipei, Taiwan, ${ }^{2}$ Biomedical Imaging and Radiological Sciences, National Yang-Ming University, Taipei, Taiwan, ${ }^{3}$ NPCC, Dept NM, Taipei Veterans General Hospital, Taipei, Taiwan.

Objectives: Histone deacetylase (HDAC) inhibitors have been proved to be a candidate anticancer agent by causing growth arrest, differentiation and tumor cell apoptosis, and proposed to be a potential remedy for brain tumor. For non-invasive monitoring the effectiveness of the HDAC inhibitors, 6-( $\left[{ }^{18} \mathrm{~F}\right]$-fluoroacetamide)-1-hexanoicanilide $\left(\left[{ }^{18} \mathrm{~F}\right]-\mathrm{FAHA}\right)$ is used for PET imaging and quantitative analysis during HDAC inhibitor treatment. Methods: The ${ }^{18}$ F-FAHA cellular uptake was performed for 2 hrs. F89 rat glioma cells were stereotactically implanted into left forebrain of Fischer rats. PET dynamic studies with ${ }^{18} \mathrm{~F}$-FAHA were investigated for $60 \mathrm{~min}$. Image-derived cardiac input functions estimated from each rat experiment were used to calculate the macro rate constant $\mathrm{K}$ for quantification. Pathological sections were examined using microscope. Results: Tumor in vitro uptake and tumorto-brain in vivo uptake ratio of ${ }^{18} \mathrm{~F}$-FAHA were both approximate 1.4. In the results of dynamic uptake curve, the uptake value of brain was arrived the steady-state in $20 \mathrm{~min}$, while the uptake value of tumor was still increased with time. Furthermore, the location of $\left[{ }^{18} \mathrm{~F}\right]$-FAHA accumulation in the tumor was consistent with that found in the pathological section. Conclusions: The results suggested that ${ }^{18} \mathrm{~F}$ FAHA can be used for monitoring the HDAC activity, and the results of quantitative analysis may provide kinetic information for the monitoring the effect during tumor therapy.
J951

MEASUREMENT OF BRAIN HISTAMINE H1 RECEPTOR
OCCUPANCY FOLLOWING TREATMENT WITH VARIOUS
ANTIHISTAMINES USING PET WITH 11C-DOXEPIN: DOSE
DEPENDENCY OF CETIRIZINE HYDROCHLORIDE $\underline{\text { Manabu Tashiro }}^{1}$, Masayasu Miyake ${ }^{1}$, Shoichi Watanuki ${ }^{1}$, Yoshihito Funaki $^{3}$, Yoichi Ishikawa ${ }^{3}$, Ren Iwata ${ }^{3}$, Kazuhiko Yanai ${ }^{2,1}$

${ }^{1}$ Division of Cyclotron Nuclear Medicine, Tohoku University, Cyclotron and Radioisotope Center, Sendai, Japan, ${ }^{2}$ Department of Pharmacology, Tohoku University Graduate School of Medicine, Sendai, Japan, ${ }^{3}$ Division of Radiopharmaceutical Chemistry, Tohoku University, Cyclotron and Radioisotope Center, Sendai, Japan.

The number of allergic patients is steadily increasing in the modern society. The main stream of drug treatment for allergic is histamine H1 receptor antagonists (antihistamines). The intensity of sedative side effects after treatment with antihistamines can be evaluated using PET. We have been measuring histamine $\mathrm{H} 1$ receptor occupancy (H1RO) following oral administration of various sedative antihistamines, such as diphenhydramine and d-chlorpheniramine, and mildly-sedative and non-sedative antihistamines, such as cetirizine and fexofenadine. Especially in the present work, we have aimed at measuring H1RO values of a mildly-sedative antihistamine, cetirizine 10 and $20 \mathrm{mg}$, compared in order to examine its dose dependency. Fifteen healthy male volunteers were divided into 3 subgroups and were studied following single oral administration of cetirizine at $10 \mathrm{mg}$ and $20 \mathrm{mg}$ or hydroxyzine at $30 \mathrm{mg}$ using PET and 11C-doxepin. Binding potential ratio and H1RO values were calculated in the prefrontal and anterior cingulate cortices. Subjective sleepiness was also measured, and the correlation to H1RO was examined. Subjective sleepiness following cetirizine $10 \mathrm{mg}$ treatment was not significantly different from that following placebo administration. Following cetirizine $20 \mathrm{mg}$, a trend for increased sleepiness was observed, but not significant. After hydroxyzine $30 \mathrm{mg}$, a significant increase in sleepiness was observed. The averaged H1ROs of cetirizine $10 \mathrm{mg}, 20 \mathrm{mg}$, and hydroxyzine $30 \mathrm{mg}$ in the prefrontal and cingulate cortices was $12.6 \%, 25.2 \%$ and $67.6 \%$, respectively. The H1RO of hydroxyzine $30 \mathrm{mg}$ correlated well with subjective sleepiness; however, those of cetirizine showed no significant correlation with subjective sleepiness. Thus, we have examined the H1RO of cetirizine at different oral doses of 10 and $20 \mathrm{mg}$. Cetirizine $10 \mathrm{mg}$ occupied approximately $13 \%$ of available H1Rs in the frontal and cingulate regions, while cetirizine $20 \mathrm{mg}$ occupied approximately $25 \%$ of H1Rs, confirming that brain penetration of this mildly-sedative antihistamine tends to be dose-dependent. It is of importance for users to know that oral administration of cetirizine $10 \mathrm{mg}$ could be more safely used for the treatment of allergic disorders, while an increased dose (20 mg or more) could result in mild sedation.

\section{Treatment Efficacy}

\section{$\mathbf{J 9 5 2}$}

MONITORING THE EFFECTS OF THE TUMOR
MICROENVIRONMENT ON CANCER IMMUNOTHERAPY
USING SERIAL BIOLUMINESCENCE IMAGING
Rachel E. Laing ${ }^{1,2}$, Amanda Armijo ${ }^{1,2}$, Andrew Q. Tran ${ }^{1,2}$, Johannes
${ }_{\text {Czernin }}^{1,2}$, Caius G. Radu
${ }^{1,2}$ Molecular and Medical Pharmacology, UCLA, Los Angeles, CA, USA,
${ }^{2}$ Crump Institute for Molecular Imaging, UCLA, Los Angeles, CA, USA. 
Immunotherapy holds great promise for the treatment of metastatic melanoma, however the overall response rates remain low. One of the main reasons is the immune-modulating effect of the tumor microenvironment. Herein we investigated the influence of the acidic tumor microenvironment on the immune response in immunotherapy. Specifically, we explored the role of GPR65, a G-protein coupled receptor capable of sensing $\mathrm{pH}$, in the immune response during immunotherapy of melanoma. To assess the role of proton sensing via GPR65, GPR65 wild type and knockout (KO) $\mathrm{T}$ cells were used for treatment in an advanced adoptive cell transfer-based immunotherapy regime. Both wild type and KO T cells were transduced with an optimized firefly luciferase construct to allow for in vivo tracking using bioluminescence imaging (BLI). B16 tumor bearing mice were treated and serially imaged to visualize $\mathrm{T}$ cell trafficking and expansion in vivo. B16 tumor bearing mice treated with immunotherapy exhibit drastic tumor regression compared to untreated controls, confirmed by caliper measurements and BLI $(p<0.001)$. Interestingly, GPR65 was found to be essential for immune mediated tumor regression; mice treated with GPR65 wild type $\mathrm{T}$ cells showed significant tumor regression as expected, while mice treated with GPR65 KO T cells showed no tumor regression compared to untreated controls ( $p<0.001 ; n=3$ experiments). BLI imaging showed substantial wild type $\mathrm{T}$ cell accumulation in the spleen $\left(8.2 \mathrm{e}^{7}\right.$ photons $\left./ \mathrm{sec} / \mathrm{cm}^{2} / \mathrm{sr}\right)$, tumor $\left(3.3 \mathrm{e}^{7} \mathrm{p} / \mathrm{sec} / \mathrm{cm}^{2} / \mathrm{sr}\right)$ and lymph nodes $\left(5.9 \mathrm{e}^{7} \mathrm{p} / \mathrm{sec} / \mathrm{cm}^{2} / \mathrm{sr}\right)$ of treated mice. However, GPR65 KO T cells displayed reduced accumulation and/or expansion in the tumor $\left(6.0 \mathrm{e}^{6} \mathrm{p} /\right.$ $\left.\mathrm{sec} / \mathrm{cm}^{2} / \mathrm{sr}\right)$ and decreased trafficking through the spleen $\left(3.3 \mathrm{e}^{7} \mathrm{p} / \mathrm{sec} / \mathrm{cm}^{2} /\right.$ sr) and lymph nodes $\left(7.2 \mathrm{e}^{6} \mathrm{p} / \mathrm{sec} / \mathrm{cm}^{2} / \mathrm{sr}\right)$ compared to wild type T cells. We found that proton sensing via GPR65 is required in T cells for immune mediated tumor rejection. We can conclude that GPR65 is essential for T cell expansion and/or survival in the acidic microenvironment of highly glycolytic tumors. Using BLI to image T cell trafficking and expansion in vivo, we find that adoptively transferred $\mathrm{T}$ cells lacking GPR65 exhibit reduced expansion and/or survival at the tumor site, consistent with the fact that GPR65 KO T cells do not mediate tumor rejection. Our results suggest that proton sensing by $\mathrm{T}$ cells is crucial for their cytotoxic effects, and that GPR65 may represent a novel therapeutic target to improve upon current immunotherapy treatment.

\section{J953}

\section{LACTATE DEHYDROGENASE ISOENZYMES REGULATE TUMOR METABOLIC PHENOTYPE}

Liu H. Wei, Gerald Toy, Matthias R. Benz, Andrew Q. Tran, Johannes Czernin, Caius G. Radu

Pharmacology, UCLA Medical School, LOS ANGELES, CA, USA.

Objective: Most cancers rely on glycolysis rather than oxidative phosphorylation as their source of ATP, even in the presence of oxygen. The lactate dehydrogenase isoenzymes A (LDHA) and B (LDHB) regulate the interconversion of pyruvate to lactate. High levels of total serum LDH have been reported to correlate with poor clinical prognosis in a variety of cancer patients. The aims of this study are twofold: first, we investigated if silencing LDHA or LDHB will inhibit tumor growth. Second, we studied whether $[18 \mathrm{~F}]$ fluorodeoxyglucose ([18F] FDG)-PET could be used to monitor the changes of glucose metabolism triggered by silencing LDHA or LDHB in several human and murine cancer cell lines. Experimental Design: We used short hairpin RNA (shRNA) to knockdown LDHA or LDHB expression in the human lung cancer cell line $\mathrm{H} 460$ and the murine melanoma cell line B-16, which express both LDHA and LDHB. We also studied the human breast cancer cell line MCF-7, which only expresses LDHA, and the human glioma cell line U251, which only expresses LDHB. Tumor growth was determined in vitro and in tumor bearing mice. In vivo tumor metabolic activity was monitored by small animal $18 \mathrm{~F}-F D G-$ PET/CT. Results: In vitro studies revealed that reduction in LDHA or
LDHB expression and function led to a significant inhibition of tumor growth for all the cell lines we studied $(p<0.01)$. Silencing LDHA or LDHB also markedly delays the tumor formation in vivo $(p<0.01)$. Furthermore, micro PET imaging demonstrated that silencing LDHA, but not LDHB, significantly reduced the tumor FDG uptake in tumor bearing mice for B-16 tumors $(p<0.05)$. Conclusions: This study provides evidence that both LDHA and LDHB play an important role in tumor metabolic phenotype. This study may also indicate that [18F] FDG-PET may be a sensitive way to monitor tumor glucose metabolism in response to shRNA treatment.

\section{J954}

\section{EARLY PREDICTION OF RESPONSE TO EXPERIMENTAL CHEMOTHERAPY TOP216 WITH PET TRACERS 18F-FLT AND 18F-FDG IN HUMAN OVARY CANCER XENOGRAFTS IN MICE}

Mette Munk Jensen $^{1,2}$, Kamille Dumong Petersen ${ }^{3}$, Fredrik Björkling ${ }^{3}$, Jacob Madsen $^{2}$, Liselotte Hoejgaard ${ }^{1,2}$, Maxwell Sehested ${ }^{3}$, Andreas Kjaer $^{1,2}$

${ }^{1}$ Cluster for Molecular Imaging, Faculty of Health Sciences, University of Copenhagen, Copenhagen, Denmark, ${ }^{2}$ Department of Clinical Physiology, Nuclear Medicine \& PET, Rigshospitalet, Copenhagen, Denmark, ${ }^{3}$ TopoTarget A/S, Symbion Science Park, Fruebjergvej 3, Copenhagen, Denmark.

Aim: 3'-deoxy-3'-[18F]fluorothymidine (FLT) is a tracer used to assess proliferation in vivo. The aim of the study was to evaluate if FLT positron emission tomography (PET) may be used to monitor the early antiproliferative effects of the experimental chemotherapy Top216. Methods: In vivo uptake of FLT in human ovary cancer xenografts in mice (A2780) was studied at various time points after Top2 16 treatment $(50 \mathrm{mg} / \mathrm{kg}$ i.v. at 0 and 48 hours) was initiated. Baseline FLT scans were made before either Top216 ( $n=7-10$ tumors) or vehicle ( $n=7$ tumors) was injected and repeated 2, 6 and 30 hours and 5 days after treatment start. For comparison, a parallel study was made with FDG ( $n=8$ tumors). Tumor volume was followed with computed tomography (CT) during the experiment. Tracer uptake was quantified using small animal PET/CT. PET scans were performed for 20 minutes one hour after i.v. injection of each tracer and region of interests (ROIs) covering whole tumors were defined on PET/CT images for calculation of standard uptake values (SUV). Expression of the proliferation associated genes Ki67 and Mcm2 following Top216 treatment was analyzed by quantitative real time polymerase chain reaction (PCR) in another group of mice $(n=4)$ in order to validate the FLT data. Results: Top $21650 \mathrm{mg} / \mathrm{kg}$ at 0 and 48 hours caused growth delay at day 5 in the A2780 tumor model compared to the vehicle group $(P<0.05)$. Uptake of FLT decreased significantly $(P<0.001)$ at $2(49.6 \%), 6(51.7 \%)$ and $30(50.2 \%)$ hours after Top216 treatment. After 5 days FLT uptake was higher than at baseline and comparable to uptake in the vehicle group. FLT uptake was unchanged in the vehicle group at the early time points but significantly increased at day $5(27.1 \% ; P<0.05)$. Uptake of FDG was significantly lower at 30 hours $(18.2 \% ; P<0.01)$ and 5 days $(31.8 \% ; P<$ $0.001)$ in the Top2 16 treated group compared to the vehicle group. PCR showed that expression of the proliferation associated genes Ki67 and $\mathrm{Mcm} 2$ decreased comparable to the in vivo analysis of FLT uptake at 6 and 30 hours after Top216 treatment. Conclusion: One injection with Top216 initiated a fast and significant decrease in proliferation assessable by FLT after already 2 hours. This decrease lasted for at least 30 hours, as determined after 5 days ( 3 days after the 2nd treatment) FLT uptake was comparable to the vehicle group. In contrast, a small, but significant, decrease in FDG uptake was observed 30 hours after initiation of Top216 treatment, and this decrease lasted for 5 days ( 3 days after the 2 nd treatment). 
J955

\section{MONITORING TUMOR CELLULAR AND TISSUE RESPONSE TO PHOTODYNAMIC THERAPY BY CHOLINE PET IMAGING AND DIFFUSION-WEIGHTED MRI}

Baowei $\mathrm{Fei}^{1}$, Hesheng Wang ${ }^{1}$, Chunying $\mathrm{Wu}^{2}$, Nancy Edgehouse ${ }^{3}$ ${ }^{1}$ Radiology, Emory University, Atlanta, GA, USA, ${ }^{2}$ Department of Radiology, Case Western Reserve University, Cleveland, OH, USA, ${ }^{3}$ Department of Pathology, Case Western Reserve University, Cleveland, $\mathrm{OH}$, USA.

This study is to develop multimodality imaging (PET/MRI) as an early biomarker for monitoring tumor response to photodynamic therapy (PDT). Methods: A human prostate cancer cell line (CWR22) was used to generate tumors in athymic nude mice. Photosensitizing drug Pc 4 ( $0.6 \mathrm{mg} / \mathrm{kg}$ body weight) was delivered to each animal by tail vein injection $48 \mathrm{~h}$ before laser illumination $(672 \mathrm{~nm}, 100 \mathrm{~mW} / \mathrm{cm} 2,150 \mathrm{~J} /$ $\mathrm{cm} 2)$. For Group I $(N=5)$, dynamic microPET images with $11 \mathrm{C}$-choline were acquired from each mouse pre-PDT and $24 \mathrm{~h}$ and $48 \mathrm{~h}$ after PDT. For Group II $(N=18)$, diffusion-weighted MR images were acquired pre- and post-PDT, $24 \mathrm{~h}$, and/or $7 \mathrm{~d}$ after PDT. Apparent diffusion coefficient (ADC) maps were obtained and analyzed for each tumor.
Prostate specific antigen (PSA) levels were measured $1 \mathrm{~d}$ before PDT and $24 \mathrm{~h}$ and $7 \mathrm{~d}$ after PDT. Results: The PSA values decreased by $30.3 \%$ and $64.1 \% 24 \mathrm{~h}$ and $7 \mathrm{~d}$ after PDT, respectively, indicating the treatment effect. For Group I, the choline uptakes dramatically decreased $24 \mathrm{~h}(75.5 \%)$ and $48 \mathrm{~h}(43.5 \%)$ after PDT, compared to those pre-PDT. Histologic analysis showed that PDT-treated tumors demonstrated apoptosis, necrosis and inflammation. For Group II, ADC values significantly increased (47.5\%) $24 \mathrm{~h}$ after PDT. In four mice, the ADC histogram demonstrated a biphasic response $7 \mathrm{~d}$ after PDT, i.e. some tissue within the tumor had increased ADC values and other maintained approximately the same values as those before treatment. On MR images, tumor tissue was automatically classified into two tissue types (necrotic and viable), which were well correlated $(\mathrm{R}=$ $89 \%$ ) with histology. The changes in choline uptake and ADCs are consistent with the PSA levels. Conclusions: Changes in tumor choline uptake detected by PET imaging can determine whether or not the tumor responds the therapy within $48 \mathrm{~h}$ after PDT. Diffusion-weighted MR imaging can detect and quantify viable and necrotic tumor tissue within one week after the treatment. The noninvasive imaging techniques can provide an assay that could be useful for clinical monitoring of photodynamic therapy at an early stage (1-7 days). Research Support: This work was supported by NIH grant R21CA120536 (PI: Fei).
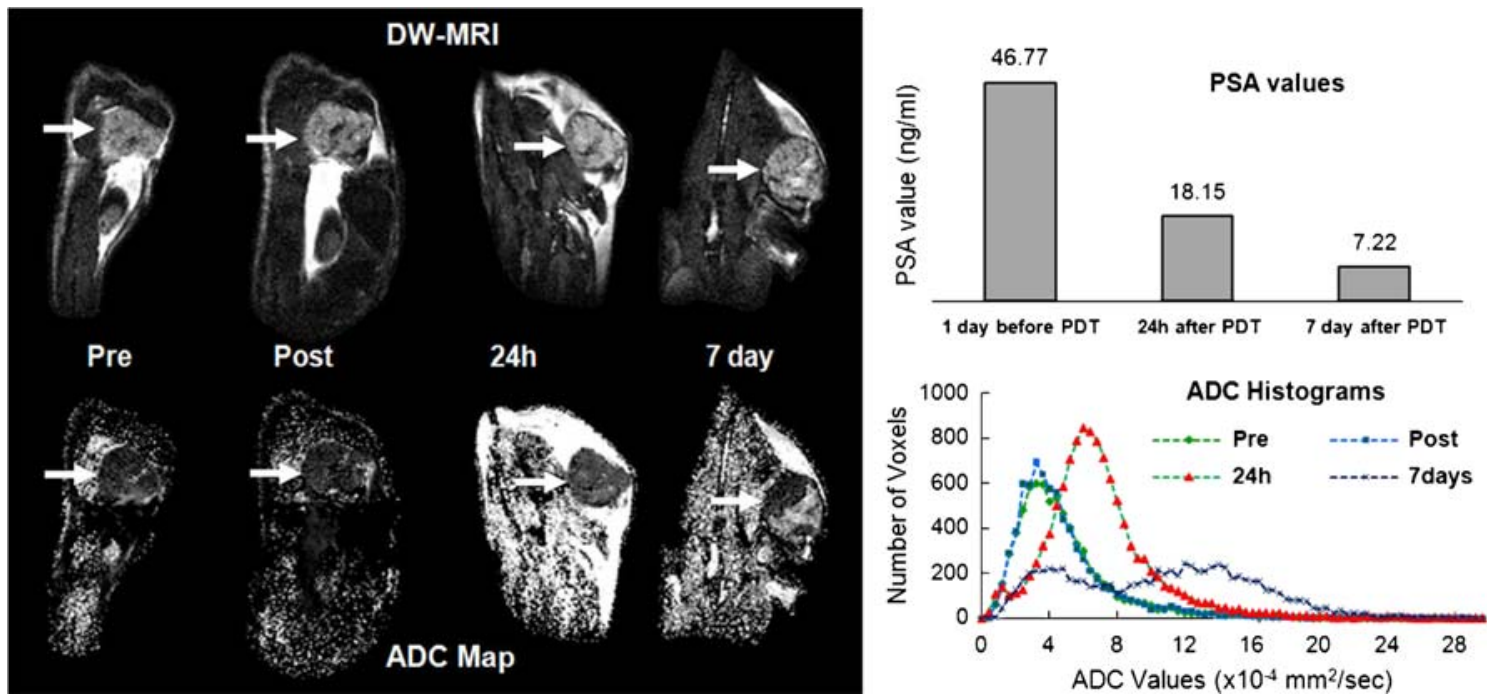

Diffusion-weigted MR Images, ADCs, and PSA values before and after PDT

J956

THERAPEUTIC EFFICACY EVALUATION OF 111In-LABELED PEGYLATED LIPOSOMAL VINORELBINE IN MURINE COLON CARCINOMA XENOGRAFTS WITH MULTIMODALITIES OF MOLECULAR IMAGING

Tong-Hsien Chow ${ }^{1}$, Jeng-Jong Hwang ${ }^{1}$, Yun-Long Tseng ${ }^{2}$, Victor F. Pang ${ }^{3}$, Ren-Shyan Liu ${ }^{4}$, Gann Ting ${ }^{6}$, Wuu-Jyh Lin ${ }^{5}$, Yi-Yu Lin ${ }^{1}$, Hsin-Ell Wang ${ }^{1}$

${ }^{1}$ Department of Biomedical Imaging and Radiological Sciences, National Yang-Ming University, Taipei, Taiwan, ${ }^{2}$ Taiwan Liposome Company, Taipei, Taiwan, ${ }^{3}$ Department of Veterinary Medicine, National Taiwan University, Taipei, Taiwan, ${ }^{4}$ Department of Nuclear Medicine, Taipei Veterans General Hospital, Taipei, Taiwan, ${ }^{5}$ Institute of Nuclear Energy Research, Lung-tan, Taoyuan, Taiwan, ${ }^{6}$ National Health Research Institute, Taipei, Taiwan.
In our previous studies, using combined radioisotopes with chemotherapeutic liposomal drugs, i.e. 111In-labelled PEGylated liposomal vinorelbine (VNB), we have reported an available cure for a humanized tumor xenografted mouse model. A phenomenon is challenged that the chemotherapy has the similar therapeutic effect as good as the combination therapy. The goal of this study was to investigate the real therapeutic effectiveness of $111 \mathrm{In}-\mathrm{VNB}$-liposomes via elevation of the radiation dosage and the reduction in the concentration of chemotherapeutic agents. Methods: Murine colon carcinoma cells transfected with dual reporter genes (CT-26/tk-luc) were xenografted in BALB/c mice. Pharmacokinetics, biodistribution, passive targeting efficiency, and maximum tolerated dose (MTD) were estimated to determine the biofate and the safety dosage of the drugs. Bioluminescence imaging (BLI) and 18F-FDG microPET were applied for monitoring the therapeutic response after treatment. The survivals in vivo associated with toxicological and histopathological analyses were carried out to determine the safety and 
feasibility for preclinical studies of the nanomedicine. Results: The effect of long-term circulation of pharmacokinetics and biodistribution revealed that significant decrease of radioactivity was found in reticuloendothelial system (RES). The selective higher tumor uptake was represented by a cumulative deposition and the maximum accumulation was at $48 \mathrm{~h}$ post injection. The combination therapy exhibited an additive effect in tumor growth suppression as tracked by caliper measuring, BLI and microPET imaging modalities. Furthermore, the higher survival rate and interval, and less tissue toxicity were closely correlated to the toxicological and histopathological examinations. Conclusion: The results demonstrated that the use of acute $111 \mathrm{In}$-VNB-liposomes in the arena of passive targeted tumor therapy displayed an additive effect of combined therapy not only through prolonging the circulation rate due to the reduction of the phagocytic effect of RES, but enhancing the tumor uptake. This $6 \mathrm{~mol} \%$ PEG 111 In-VNB-liposomes eventually could increase the overall survival in tumor-bearing mouse model.
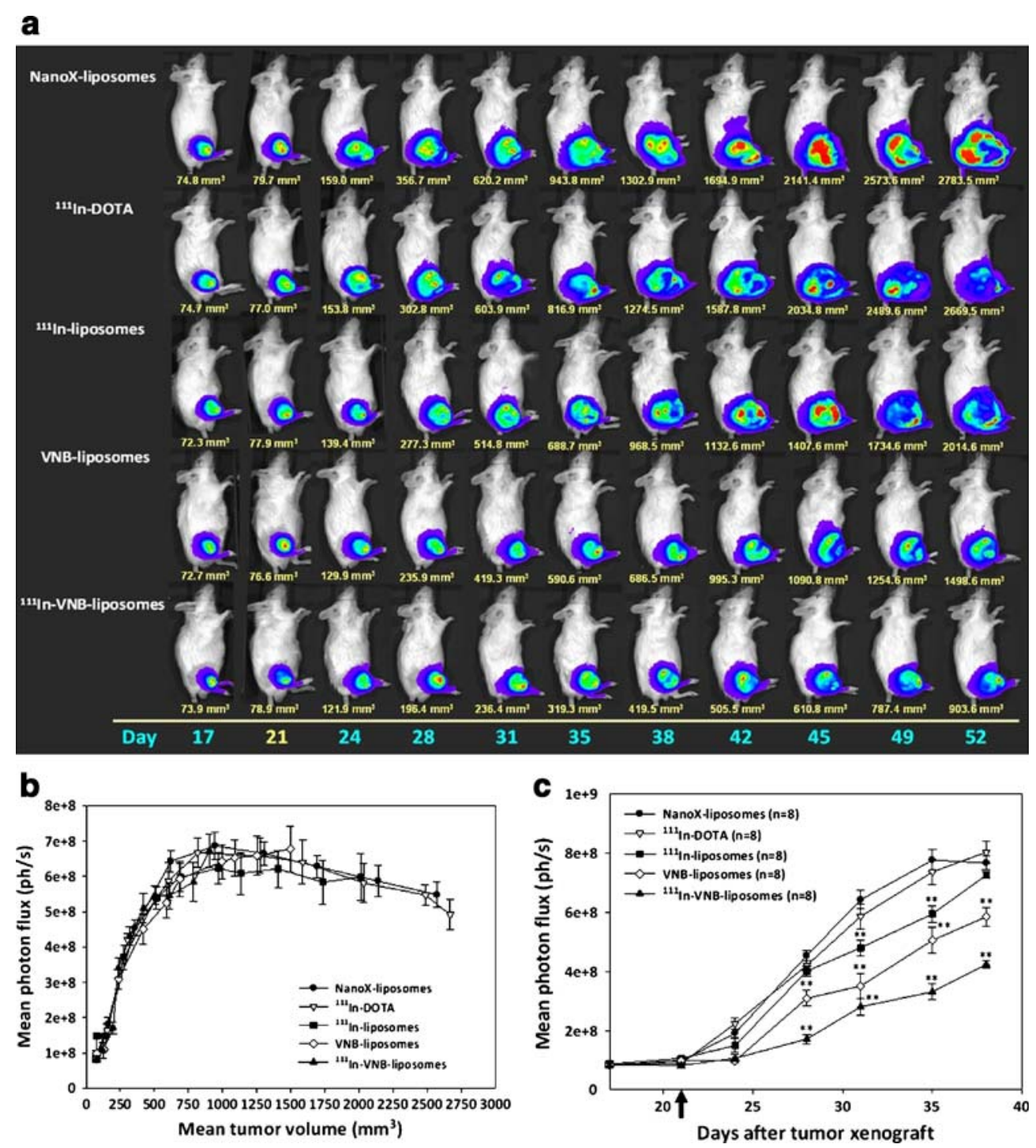

Figure 1. (A) In vivo bioluminescence imaging (BLI) of CT-26/tk-luc tumor xenografted BALB/c mice after various treatments. NanoX-liposomes stand for the loposomes without 111In and VNB. (B) The mean photon flux from the ROIs of tumor were correlated with tumor size in all treatments. (C) The mean photon flux as a function of time after initiation of the drug treatments. The combination therapy, i.e. 111In-VNB-liposomes, had the lowest photon collection, represented the best tumor growth suppression. Data are expressed as mean $\pm \mathrm{SE}$. ${ }^{*} \mathrm{P}<0.05, * * \mathrm{P}<0.01$, significantly differences from control by Student's $\mathrm{t}$-test. B;ack arrow indicates the time of the drug injections. The experiments were repeated twice

\section{$\mathbf{J 9 5 7}$}

THERAPEUTIC EVALUATION IN HT-29/tk-luc TUMORBEARING ANIMAL MODEL WITH MOLECULAR IMAGING USING FATTY ACID SYNTHASE INHIBITOR- ORLISTAT

Hui-Yen Chuang, Jeng-Jong Hwang

Biomedical image and radiological sciences, National Yang-Ming University, Taipei, Taiwan.
Purpose Orlistat, the FDA proved anti-obesity drug, also has been reported with anti-cancer ability via inhibition of the fatty acid synthetic pathway. Fatty acid synthase (FASN) is highly expressed in several human cancers, including breast, prostate, lung and colon, while much lower in the normal tissues. In this study, HT29/tk-luc colorectal carcinoma bearing animal model was used to evaluate the therapeutic effect of Orlistat both in vitro and in vivo. The therapeutic index was assayed with multimodalities of 
molecular imaging, such as nuclear and bioluminescent imaging (BLI). Materials and Methods HT-29/tk-luc cells were treated with various concentrations of Orlistat, then performed colony formation assay to determine the IC50. Both the cell cycle and apoptosis were analyzed with flow cytometry. Western blotting of caspase-3 level and FASN expression were also assayed to evaluate the apoptosis. The FASN activity was determined with NADPH absorbance and $14 \mathrm{C}$-acetate incorporation. Tumor growth inhibition in vivo was assayed with the digital caliper, gamma scintigraphy, microPET and BLI. Results After Orlistat treatment, cells were blocked at G1 with dose- and time-dependent manners. The sub-G1 population was increased with the increase of Orlistat concentrations. The activated caspase- 3 level was also increased, whereas no change in FASN expression. Nevertheless, the activity of FASN and the incorporated acetate were significantly decreased in the Orlistat-treated groups. The mean tumor size of the treated group was $30-40 \%$ less than that of the control group. Both microPET and BLI also showed the similar results. Conclusions Orlistat could inhibit human colon carcinoma HT-29/tk-luc cells both in vitro and in vivo via G1 inhibition and resulted in the tumor cell apoptosis. Though the level of FASN in HT-29/tk-luc cells was not changed, the FASN activity was decreased after Orlistat treatment. This study demonstrated that fatty acid synthase could be a potential target for the consideration in clinical cancer treatment.
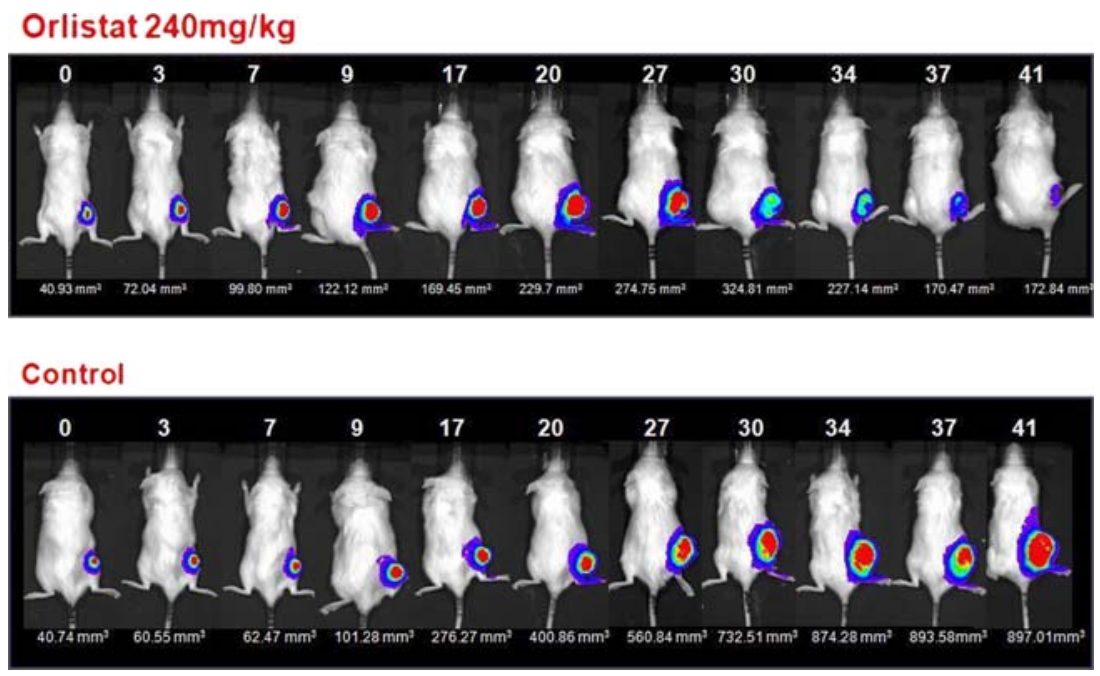

Therapeutic evaluation by bioluminescence imaging

J958

THERAPEUTIC EFFICACY OF RADIOCHEMOTHERAPEUTICS OF 188Re-DXR-LIPOSOME IN A C26 MURINE COLON CARCINOMA SOLID TUMOR MODEL Ya-Jen Chang $^{1}$, Chih-Hsien Chang ${ }^{1}$, Chia-Yu Yu ${ }^{1}$, Liang-Cheng Chen ${ }^{1}$, Wan-Chi Lee ${ }^{1}$, Te-Wei Lee ${ }^{1}$, Gann Ting ${ }^{2}$

${ }^{1}$ Radiation Application, Institute of Nuclear Energy Research, Taoyuan County, Taiwan, ${ }^{2}$ National Institute of Cancer Research, National Health Research Institutes, Miaoli, Taiwan.

Nanocarriers can selectively target cancer sites and carry payloads, thereby improving diagnostic and therapeutic effectiveness and reducing toxicity. The objective of this study is to investigate the therapeutic efficacy of a new co-delivery radiochemo-therapeutics of 188Re-labeled pegylated liposomal doxorubicin(188Re-DXRliposome)in a C26 murine colon carcinoma solid tumor model.
The anti-tumor effect of 188Re-DXR-liposome was assessed by tumor growth inhibition, survival ratio and histopathological H\&E staining.The tumor target and localization of the passive nanoliposome delivery radiochemo-therapeutics 188Re-DXR-liposome were demonstrated in the biodistribution, pharmacokinetics and in vivo nuclear imaging studies. In the study on therapeutic efficacy, the tumor-bearing mice treated with bimodality radiochemo-therapeutics of 188Re-DXR-liposome showed better mean tumor growth inhibition rate (MGI) and longer median survival time $(\mathrm{MGI}=0.048 ; 74 \mathrm{~d})$ than those treated with radio-therapeutics of 188Re-liposome and chemo-therapeutics of Lipo-Dox. The synersistic tumor regression effect was observed with the combination index exceeding one $(\mathrm{CI}=1.145)$ for co-delivery radiochemotherapeutics of 188Re-DXR-liposome. These results were pointed to the potential benefit and promise of the co-delivery of passive nanoliposome radiochemo-therapeutics for adjuvant cancer treatment on oncology applications.

Therapeutic efficacy of radiochemo-combination nanotargeted 188Re-DXR-liposomes on C26 colon tumor-bearing BALB/c mice. 


\begin{tabular}{|c|c|c|c|c|c|c|}
\hline & \multicolumn{3}{|c|}{ Tumor Growth Inhibition } & \multicolumn{3}{c|}{ Survival } \\
\hline TreatmentModality & $\begin{array}{c}\text { MGI } \\
\text { (a) }\end{array}$ & Expected (b) & $\begin{array}{c}\text { CI } \\
\text { (c) }\end{array}$ & median survival time (d) & $\begin{array}{c}\text { P value } \\
\text { (d) }\end{array}$ & $\begin{array}{c}\text { life span(\%) } \\
(\mathrm{e})\end{array}$ \\
\hline Normal Saline & & & & 31 & & \\
\hline Lipo-DOX & 0.413 & & & 38 & 0.104 & +23 \\
\hline 188Re-liposomes & 0.134 & & & 60 & 0.0009 & +139 \\
\hline 188Re-DXR-liposomes & 0.048 & 0.055 & 1.145 & 74 & +94 \\
\hline
\end{tabular}

(a)MGI, Mean growth inhibition rate

(b)Expected growth inhibition rate

(c)CI, combination index was calculated by dividing the expected growth inhibition rate by the observed growth inhibition rate. An index $>1$ indicates synergistic effect.

(d)P values were estimated by log-rank test, $P<0.05$ indicates significance.

(e)Percentage increase in life span

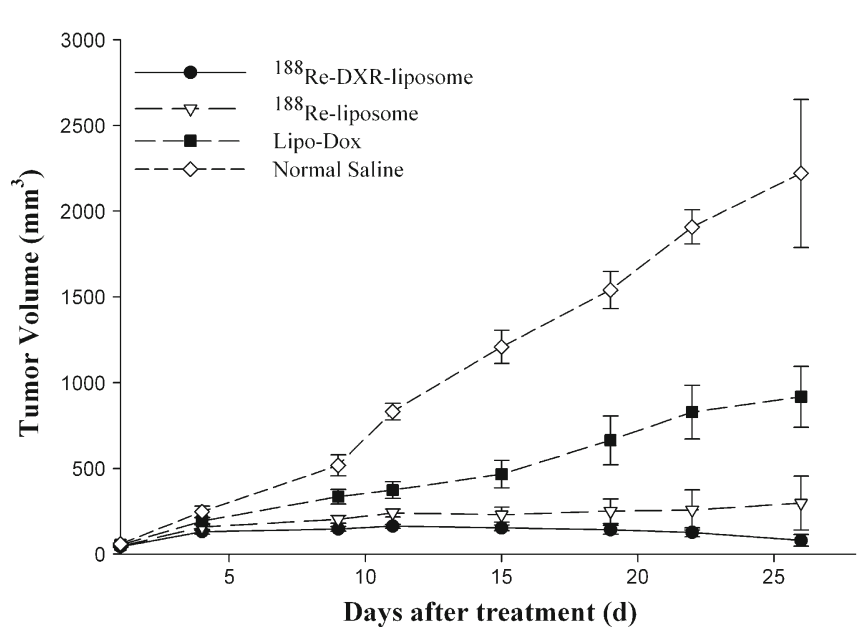

Tumor growth curve was assessed by generating metabolic volume histograms (MVH) for pre and post-ECRS PETs. MVH curves examine metabolic heterogeneity in the VOI by graphically depicting the SUV by percent volume. Exploratory analyses evaluated the viability of multiple isoSUV and iso-volumetric points selected from the MVH curve to serve as novel markers of response. Standard PET response markers such as changes in maximum/mean SUV and qualitative analysis were also assessed. Paired t-test and Wilcoxon tests were performed to assess whether absolute changes in the markers were significantly different between responders and non-responders. Fisher exact test was performed to test whether a significant association existed between the direction of change in the markers and the achievement of local control. Results: The 14 patients studied had the following characteristics with median (range), respectively: age 60 (range 38-84), KPS 90 (range 80-100), follow-up after ECRS 9 months (range 1-22). Patients had either metastatic or recurrent cancer. The primary disease site was aero-digestive in all but 3 patients. The mean and (range) of target volume (cc), total dose (Gy), and number of fractions were 129 (10-544), 20 (12-60), and 2.3 (1-3). Median time from ECRS to post-ECRS PET was 44 days (range 14-216). 10/14 patients achieved local control at last follow-up, a median of 225 days following post-ECRS PET (range 8-568). 3 out of 4 local failures had an increase in max SUV, while all patients who achieved local control had a reduction in $\max \operatorname{SUV}(\mathrm{p}=0.011)$. Exploratory analyses using multiple iso-SUV and iso-volumetric points did not yield any factors associated with local control $(\mathrm{p}>0.05)$. Conclusions: Reduction in max SUV following ECRS appears to be associated with local control. No other surrogate endpoints from MVH analyses were found to be similarly useful.

J960

NEOADJUVANT CHEMORADIOTHERAPY (CRT) WITH CISPLATIN AND IRINOTECAN (C+ I) FOR ESOPHAGEAL CARCINOMA: PREDICTIVE VALUE OF 18F-FDG-PET

Dominick Lamonica $^{1}$, Rohit Sharma ${ }^{3}$, Wei Tan ${ }^{5}$, Shane Hopkins ${ }^{4}$, Gary Yang $^{4}$, Nikhil Khushalani ${ }^{2}$

${ }^{1}$ Nuclear Medicine, Roswell Park Cancer Institute, Buffalo, NY, USA, ${ }^{2}$ Medical Oncology, Roswell Park Cancer Institute, Buffalo, NY, USA, ${ }^{3}$ Surgical Oncology, Roswell Park Cancer Institute, Buffalo, NY, USA, ${ }^{4}$ Radiation Medicine, Roswell Park Cancer Institute, Buffalo, NY, USA, ${ }^{5}$ Biostatistics, Roswell Park Cancer Institute, Buffalo, NY, USA. 
Objectives: Concurrent chemotherapy and radiation is now standard prior to surgical resection of locally advanced esophageal carcinoma (LAEC). Non-invasive tools are needed to assess pathologic response (pCR) and gauge prognosis. Cisplatin and irinotecan $(\mathrm{C}+\mathrm{I})$ are agents under study for upfront treatment of LAEC. We examined the utility of FDG_PET for predicting outcome to CRT using this regimen. Methods: LAEC patients (PTS) who had surgery preceded by CRT with $\mathrm{C}+\mathrm{I}$ were identified from the Roswell Park tumor registry. PTS concurrently received 45-50.4 Gy of radiation in 1.8 Gy fractions. Pre and post treatment SUVmaxbw (SUV) was recorded for the primary tumor using FDG_PET. Data retrieved included baseline tumor size, pCR, overall survival, and disease free survival. Results: There were 32 eligible PTS ( $31 \mathrm{M} / 1 \mathrm{~F})$, median age $61.5 \mathrm{yr}$. Histologies included adeno $(n=27)$, squamous cell $(n=3)$, and mixed $(n=2)$ carcinomas. 19 PTS had poorly differentiated tumors. Clinical stage was II $(n=4)$, III $(n=18)$, IVa $(n=5)$, IVb $(n=1)$, not available $(n=4)$. All PTS underwent R0 resection and pCR rate was $22 \%(7 /$ 32). 10 PTS have experienced recurrence. The median disease-free and overall survival is 19 months and 24 months, respectively; 3-yr overall survival is $35 \%$. Post-CRT SUV on PET imaging correlated with $\mathrm{pCR}$ status $(p=0.047)$ at surgery, however, neither pre- nor postCRT SUV showed significant association with disease free or overall survival. Nor did the magnitude of change in SUV following CRT have bearing on any of these measures. Conclusions: Neoadjuvant CRT with $\mathrm{C}+\mathrm{I}$ has modest efficacy in the treatment of LAEC and can be given safely in the outpatient setting. While FDG_PET has shown marginal utility for gauging response to neoadjuvant CRT, it was not shown to be an independent predictor of disease free or overall survival.

\section{J961}

FUNCTIONAL ASSESSMENT OF THE REVERSAL OF MULTIDRUG RESISTANCE GENE EXPRESSION AND IN VIVO TUMOR IMAGING BY ADENOVIRUS-MEDIATED MDR1 RNA INTERFERENCE IN HUMAN COLON CANCER MODEL USING RENILLA LUCIFERASE REPORTER GENE

Yong Hyun Jeon ${ }^{1}$, Lee You La ${ }^{1}$, Hwang Mi Hye ${ }^{1}$, Lee Sang Woo ${ }^{1}$, $\overline{\text { Ahn Byeong-Cheol }}^{1}$, Ha Jeoung-Hee ${ }^{2}$, Lee Jaetae ${ }^{1}$

${ }^{1}$ Nuclear Medicine, School of Medicine, Kyungpook National University, Daegu, Korea, South, ${ }^{2}$ Pharmacology, School of Medicine, Kyungpook National University, Daegu, Korea, South.

Objective: We have tried to assess the reversal of multidrug resistance (MDR1) gene expression by adenoviral vector-mediated MDR1 RNA interference in human colon cancer cell using renilla luciferase. Methods: Stable human colon cancer cell line expressing Rluc (HCT15/Rluc) was established using lipofectamin. Rluc gene expression in HCT15/Rluc was determined with Rluc assay. Recombinant adenovirus that expresses MDR1 shRNA and EGFP under the control of a U6 promoter was construed (Ad-MDR1 shRNA-EGFP, AMRE). The shRNA sequence targeting human mdrl corresponded to the coding region at positions 79-99 (5'-AAG GAAAAGAAACCAACTGTC-3', GenBank accession number NM_000927). 0, 15, 30, 60, 120 MOI of AMRE was infected to HCT-15/Rluc cells. At $24 \mathrm{~h}$ after infection, Rluc and EGFP gene expression was analyzed using Rluc assay and fluorescent microscope, respectively. 1x107 HCT15/Rluc cells were implanted into both thighs. Before injection of PBS or AMRE, bioluminescent imaging was acquired at 20 days after tumor challenge. After imaging acquisition, PBS or 1x109 pfu
AMRE were administered into tumor of mice. Twenty four hour later, bioluminescence was acquired and signals quantified. Results: Rluc assay revealed the stable expression of Rluc gene in stable transfectant. Fluorescent microscope revealed increased EGFP signal in AMRE infected HCT-15/Rluc cells in a virus dose-dependent manner. Concurrently, Rluc activity was significantly increased in AMRE infected HCT-15/Rluc cells but not mock-infected HCT-15/ Rluc cells in a virus dose-dependent manner $(0,15,30,60,120 \mathrm{MOI}$, $140 \pm 11, \quad 252 \pm 9, \quad 367 \pm 12, \quad 554 \pm 13, \quad 875 \pm 14$ RLU, respectively). Bioluminescent imaging showed 9-fold higher signal in AMRE infected tumor compared with that of PBS treated tumor $(P<0.01)$. Conclusions: We successfully demonstrated the increases in Rluc activity by reversal of multidrug resistance gene expression using adenoviral vector-mediated MDR1 shRNA in human colon cancer model. Ad-MDR1 shRNA may be a potential therapeutic tool for treatment of multi-drug resistance cancer.

\section{J962}

\section{GLYCEROPHOSPHOCHOLINE PHOSPHODIESTERASE: A NOVEL ANTICANCER TARGET IN CHOLINE PHOSPHOLIPID METABOLISM OF HUMAN BREAST CANCER CELLS}

Mailin Doepkens $^{1,2}$, Tiffany R. Greenwood ${ }^{1}$, Venu Raman ${ }^{1}$, Dieter Leibfritz $^{2}$, Farhad Vesuna ${ }^{1}$, Balaji Krishnamachary ${ }^{1}$, Yelena Mironchik ${ }^{1}$, Kristine Glunde ${ }^{1}$

${ }^{1}$ Russell H. Morgan Department of Radiology and Radiological Science, Johns Hopkins University In Vivo Cellular and Molecular Imaging Center, Baltimore, MD, USA, ${ }^{2}$ Department of Chemistry and Biology, University of Bremen, Bremen, Germany.

Phosphocholine (PC) and total choline-containing compounds are elevated in breast cancers, as demonstrated by numerous 1H MRS studies. Some enzymes in choline phospholipid metabolism may be potential targets for anticancer therapy. Studies on choline kinase downregulation demonstrated reduced proliferation and increased differentiation (Glunde et al, 2005). Glycerophosphocholine phosphodiesterase (GPC-PDE) is an enzyme in choline phospholipid metabolism that catalyzes the degradation of glycerophosphocholine (GPC) to choline (Cho). In renal cells, high concentrations of $\mathrm{NaCl}$ and urea were demonstrated to inhibit GPC-PDE (Zablocki et al, 1991). We incubated MCF-10A and MCF-12A, two nonmalignant HMECs, and MCF-7 and MDA-MB-231, two human breast cancer cell lines for $24 \mathrm{~h}$ with $100 \mathrm{mM}$ urea and $100 \mathrm{mM} \mathrm{NaCl}$. Highresolution $1 \mathrm{H}$ MR spectra of the cell extracts were measured and analyzed. Changes in cell proliferation were detected by WST-1 proliferation assay. Exposure to high $\mathrm{NaCl}$ and urea increased GPC and decreased $\mathrm{PC}$, resulting in a decreased $[\mathrm{PC}] /[\mathrm{GPC}]$ ratio. Cell proliferation was significantly lower in all cells treated with different concentrations of urea and $\mathrm{NaCl}$, as shown by WST-1 assay. In previous studies, an increased $[\mathrm{PC}] /[\mathrm{GPC}]$ ratio was associated with increased malignancy. We demonstrated for the first time that inhibiting GPC-PDE altered the choline phospholipid metabolite profile of malignant HMECs toward a less malignant profile. This finding was further corroborated by significantly decreased proliferation upon GPC-PDE inhibition in all tested cell lines. The results indicate that GPC-PDE inhibition may provide a future target for anticancer therapy. Knockdown experiments with shRNA against GDPD5, a GPC-PDE, are currently underway. Funding: R01 NIH CA134695. 


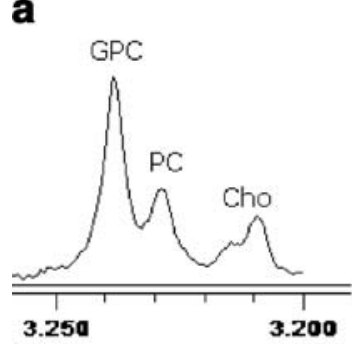

MCF-12A control

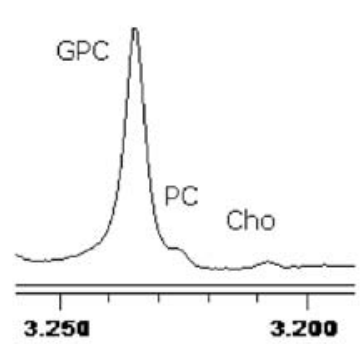

MCF-12A treated

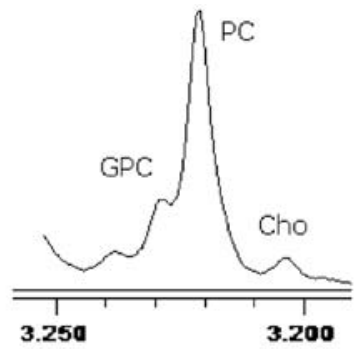

MDA-MB-231 control

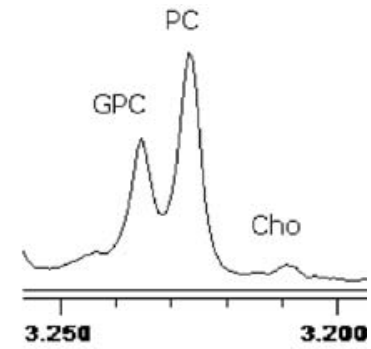

MDA-MB-231 treated b

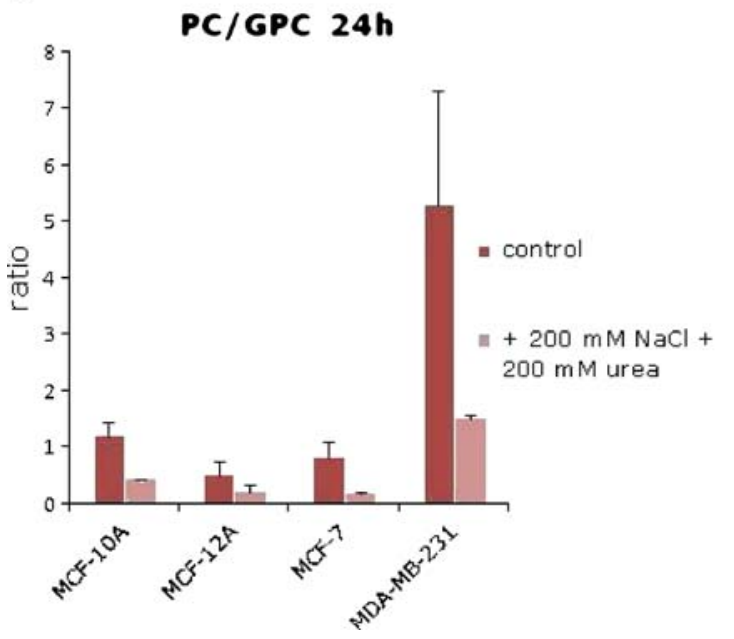

C

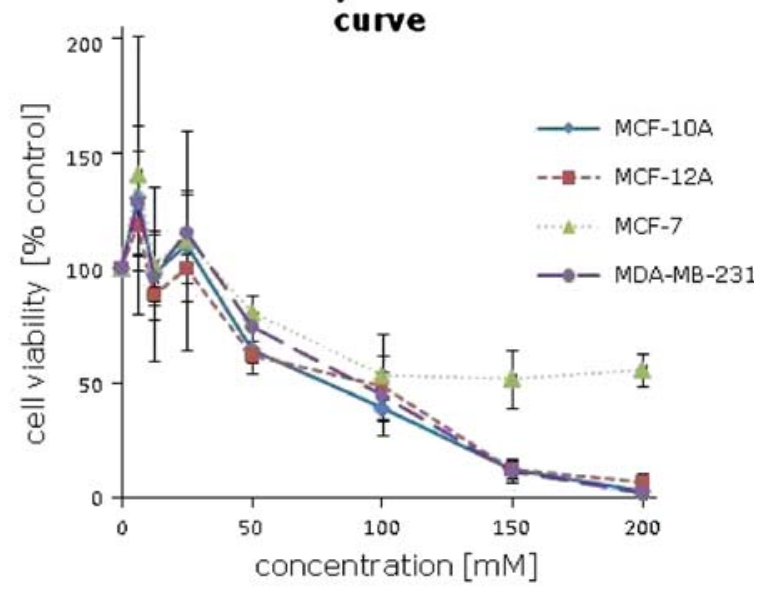

A 1 H MR spectra from cell extracts of MCF-12A cells and MDA-MB-231 cells treated for $24 \mathrm{~h}$ with $100 \mathrm{mM}$ urea and $100 \mathrm{mM} \mathrm{NaCl}$ and control. B Calculated [PC]/ [GPC] ratios from $1 \mathrm{H}$ MR spectra after treatment with urea and $\mathrm{NaCl}$ for $24 \mathrm{~h}(n=2)$. C WST-1 assay: cell viability after $24 \mathrm{~h}$ treatment with different concentrations of urea and $\mathrm{NaCl}(n=3)$.

J963

\section{EVALUATION OF ANTI-PEG GENE MEDIATED TUMOR IMAGING AND THERAPY IN A HEPATOCELLULAR CARCINOMA-BEARING MOUSE MODEL}

Mao-Chi Weng ${ }^{1}$, Tien-Kuei Chang ${ }^{1}$, Yi-Yu Lin ${ }^{1}$, Jia-Je Li ${ }^{1}$, Tian-Lu Cheng $^{2}$, Hsin-Ell Wang ${ }^{1}$

${ }^{1}$ Department of Biomedical Imaging and Radiological Sciences, Institute of Biomedical Image and Radiological Sciences, Taipei City, Taiwan, ${ }^{2}$ Department of Biomedical Science and Environmental Biology, Kaohsiung Medical University, College of Life Science, Kaohsiung City, Taiwan.

Objectives: This study evaluated the gene-mediated tumor imaging and therapy of a hepatocellular carcinoma-bearing mouse model. The mouse bearing both anti-PEG receptor-expressed tumor (HCC36/ AGP3-LDLr) and wild-type tumor (HCC36/WT) was imaged with 131I-4 arm PEG-BH and treated with vinorelbine-encapsulated PEGylated liposomes (PEGylated Lipo-VNB, 6\%PEG). Methods: A novel 131I-4 arm-PEG-BH was prepared as a radio-labeled gene probe to target the anti-PEG receptor that expressed on HCC36/ AGP3-LDLr (HLDL) cells. BALB/c nude mice were subcutaneously inoculated with HCC36/AGP3-LDLr (HLDL) and HCC36/WT (HCC36) cells in right and left lower flanks to grow tumors. The cellular uptake, pharmacokinetics, biodistribution, planar gamma imaging were studied with 111 In-labeled PEGylated Lipo-VNB and
131I-4 arm-PEG-BH. The therapeutic efficacy of PEGylated LipoVNB was investigated. Results: The labeling yield of 131I-4 arm PEG-BH and PEGylated 111In-Lipo-VNB were both $>90 \%$. The radiochemical purity of 131I-4 arm PEG-BH was initially $>90 \%$ and was $>80 \%$ within $48 \mathrm{~h}$ in both PBS and serum. In pharmacokinetics study, fast clearance from the blood after i.v. injection of 131I-4 arm PEG-BH was observed. 131I-4 arm PEG-BH, with a HLDL-toHCC 36 ratio of 5 in planar gamma imaging at $72 \mathrm{~h}$ post injection (p. i.), was demonstrated a promising probe for anti-PEG receptor gene expression. The distribution of PEGylated 111In-Lipo-VNB after i.v. injection revealed that the HLDL tumor uptake $(12.68 \pm 2.5 \% \mathrm{ID} / \mathrm{g})$ was two-fold higher than that of HCC36 tumor $(5.92 \pm 0.28 \% \mathrm{ID} / \mathrm{g})$ at $48 \mathrm{~h}$ p.i., consistent with the results (HLDL-to-HCC36 ratio of 1.99) observed in the $2 \mathrm{D}$ scintigraphic imaging. After i.v. administration of PEGylated Lipo-VNB, significant tumor growth inhibition was noticed for the HLDL tumor compared with the HCC36 tumor $(p<$ 0.05). The tumor growth inhibition rate was $39.11 \%$ at $17 \mathrm{~d}$ p.i.. The results of PEGylated Lipo-VNB treatment indicated that the anti-PEG receptors expressed on the tumor cell membrane enhanced the accumulation of liposomal drugs and promoted the anti-tumor effect in HCC36/AGP3-LDLr (HLDL) tumor. Conclusion: This study demonstrated that the gene-expression probe, 131I-4 arm PEG-BH, selectively targeted the anti-PEG receptor on HCC36/AGP3-LDLr cells. We suggested that the transfection of the anti-PEG gene in tumor cells accompanied with the employment of PEGylated chemodrugs would be a promising strategy for tumor gene therapy. 
J964

\section{MULTI-MODALITY IMAGING AND QUANTIFICATION OF THE EFFECT OF THE VASCULAR DISRUPTING ANGENT ENMD-1198 ON BREAST CANCER PROGRESSION IN BONE AND SUBSEQUENT OSTEOLYSIS}

Thomas Snoeks ${ }^{1}$, Eric Kaijzel ${ }^{1}$, Henry Cheung ${ }^{2}$, Jouke Dijkstra ${ }^{3}$, Clemens Lowik

${ }^{1}$ Endocrinology, LUMC, Leiden, Netherlands, ${ }^{2}$ Urology, LUMC, Leiden, Netherlands, ${ }^{3}$ Division of Image Processing (LKEB), LUMC, Leiden, Netherlands.

Introduction The preference of breast cancer to metastasize to bone and subsequent growth and bone destruction involves specific tumor-host interactions, including induction of osteoclastic bone resorption and angiogenesis. Metastatic bone disease causes severe pain and morbidity. Currently, apart from bisphosphonates that only block osteolysis, there is no treatment available. ENMD-1198 is a more stable 2Methoxyestradiol (2ME2) derivate. Since 2ME2 has been shown to have anti-tumor, anti-vascular and anti-resorptive effects, ENMD-1198 is a perfect drug candidate for the treatment of metastatic bone disease. The aim of this study was to assess the efficacy of ENMD-1198 treatment on tumor growth and bone destruction in a murine model for osteolytic metastases. Methods In vitro cell proliferation assays and ex vivo angiogenesis assays with ENMD-1198 and 4-hydroperoxycyclophosphamide (4HC) were performed. To test ENMD-1198 in vivo, 5 week old nude mice received an intra-osseous injection with 250.000 MDA-MB231-Luc cells. Mice either received no treatment, daily ENMD-1198 (oral gavage, $200 \mathrm{mg} / \mathrm{kg} /$ day), CTX (20 mg/kg/day) through drinking water or a combination of ENMD-1198 and CTX. Tumor growth was followed with BLI and osteolysis was assessed by 2D x-ray image analysis and micro CT (SkyScan 1076). To measure bone volume in CT data, a new in house method was developed. Results In vitro ENMD-1198 proved to be cytotoxic for MDA-BO2 cells, 4HC did have a minor effect. ENMD-1198 was able to block angiogenesis dose-dependently. Both CTX and ENMD-1198 treatment decreased tumor burden significantly whilst the combination treatment did not show additive or synergistic effects. ENMD-1198 and combination treatment were more effective than CTX alone. X-ray images revealed a $60 \%$ reduction in osteolytic lesion size for both the ENMD-1198 treated groups compared to the untreated control, which was confirmed with micro-CT. Conclusions Both ENMD-1198 as well as the combination treatment were more effective than metronomic CTX treatment in both decreasing the tumor burden as well as protecting the bone. Due to the potent anti-tumor and anti-angiogenic effect of ENMD-1198, a possible added value of CTX treatment could not be observed since CTX mainly targets angiogenesis. Since all of these processes are crucial to tumor growth within the bone microenvironment ENMD-1198 is a potentially interesting new drug. Acknowledgement Supported by the Dutch Cancer Society (UL20073801). ENMD-1198 was kindly provided by Tony Treston, Entremed.

\section{J965}

QUANTIFYING DIFFERENTIAL RESPONSE OF SOLITARY CANCER CELLS AND GROWING METASTASES TO TREATMENT IN WHOLE MOUSE LIVER USING MAGNETIC RESONANCE IMAGING

Jason Townson $^{1}$, Ian C. MacDonald ${ }^{1}$, Brian K. Rutt ${ }^{2}$, Paula Foster ${ }^{1,2}$, Ann Chambers ${ }^{3}, 1$

${ }^{1}$ Medical Biophysics, University of Western Ontario, London, ON, Canada, ${ }^{2}$ Robarts Research Institute, London, ON, Canada, ${ }^{3}$ London Regional Cancer Program, London, ON, Canada.
Quantification of the entire experimental metastatic cell population, ranging from solitary cancer cells to actively growing metastases, is complicated by requirement of an imaging modality with the large field of view and high spatial resolution necessary to detect both single cells and large metastases in the same whole organ. Thus it is difficult to assess differential responses of these distinct metastatic cell populations to therapy. Here we used a magnetic resonance imaging (MRI) technique to quantify the effect of treatment on the full population of metastatic cells. Mouse melanoma (B16F1) cells labelled with micron-sized iron oxide particles (MPIO) were injected into mouse liver via the mesenteric vein. Livers were removed immediately or at day 9 or 11, following doxorubicin or vehicle control treatment. A 3T clinical MR scanner and custom-built gradient coil were used to image the livers. MPIO labelled single cells and metastases $(>180 \mu \mathrm{m}$ diameter) could be detected and quantified from the same MR images. A strong correlation (R2=0.83, $p<$ 0.001 ) was found to exist between signal void area and the number of MPIO labelled cells injected $(0,3.75,7.5,15$ and $30 \times 104)$ into the liver. MR image analysis showed that treatment with $1 \mathrm{mg} / \mathrm{kg}$ doxorubicin significantly decreased metastasis volume ( $\mathrm{t}$-test, $\mathrm{n}=22, \mathrm{p}=0.02$ ) but did not decrease the number of solitary metastatic cells, as assessed by signal void area, in the same livers $(p>0.05)$. Thus, the MRI technique presented here is capable if identifying differential responses of the metastatic cell population to therapy by enabling the rapid $(<6 \mathrm{~min}$ per scan) quantification of the majority of the metastatic cell population, including both growing metastases and solitary cells, in whole liver.

\section{J966}

EFFECTS OF ESTRADIOL AND DHEA ON THE INTESTINAL MICROCIRCULATION DURING EXPERIMENTAL SEPSIS Nivin M. Sharawi ${ }^{2,3}$, Christian Lehmann ${ }^{2,1}$, Dragan Pavlovic ${ }^{2}$ ${ }^{1}$ Anesthesia, Dalhousie University, Halifax, NS, Canada, ${ }^{2}$ Anesthesia, Ernst-Moritz-Arndt-University, Greifswald, Germany, ${ }^{3}$ Medical College, Kasr EL Eni University, Cairo, Egypt.

Introduction: Disturbances within the microcirculation represent an important factor in the pathogenesis of multiple organ dysfunction in sepsis and septic shock. Gender-specific effects may modulate the septic pathophysiology. Therefore, we studied sepsis-induced changes within the intestinal microcirculation in randomly cycling and ovariectomized female rats. Objectives: We hypothesized that estradiol (E2) and dehydroepiandrosterone (DHEA) may have a beneficial effect on the microcirculation during experimental sepsis and re-substituted these hormones in the ovariectomized animals. Methods: Fifty female rats were divided in to five groups of ten animals. Group 1 received sham laparotomy without further treatment. In group 2-5 we induced experimental sepsis (colon ascendens stent peritonitis - CASP model). Animals of groups 3-5 were additionally ovariectomized 3 weeks before sepsis induction. In group 4 we administered $10 \mathrm{mg} / \mathrm{kg}$ estradiol immediately after and $12 \mathrm{~h}$ following CASP surgery. The animals of group 5 received $50 \mathrm{mg} / \mathrm{kg}$ DHEA immediately after sepsis induction. Twenty-four hours after CASP surgery intravital microscopy was performed to study leukocyte-endothelial interactions and functional capillary density. Blood samples were taken for the measurement of estradiol, DHEA and inflammatory cytokines. Results: In ovariectomized rats subjected to CASP the number of activated leukocytes was significantly increased in comparison to sham and not ovariectomized CASP animals $(P<0.05)$. In ovariectomized rats treated with E2 leukocyte adhesion was significantly reduced in comparison to untreated ovariectomized rats subjected to CASP $(P<0.05)$. The same observation was made in ovariectomized rats treated with DHEA. In addition, in ovariectomized rats subjected to CASP the functional capillary density was significantly decreased in comparison to sham and CASP groups $(P<0.05)$. In ovariectomized rats treated with E2 or DHEA functional capillary density was completely restored. Conclusions: The 
results demonstrate the role of E2 and DHEA in the sepsis-induced changes within the microcirculation. A rapid, non-genomic effect of both E2 and DHEA is suggested. DHEA may play a role through conversion to E2 or through direct acting on the E2 receptor. Further investigations should be done to elucidate the underlying mechanisms. Both E2 and DHEA appear to be a promising adjunct for the prevention of multiple organ damage induced by sepsis.

\section{J967}

NIR FLUORESCENT IMAGING TO EVALUATE MASSAGE AS A LYMPHEDEMA THERAPY

I-Chih Tan $^{1}$, John C. Rasmussen ${ }^{1}$, Milton Marshall ${ }^{1}$, Erik A. Maus ${ }^{2,3}$, Caroline E. Fife ${ }^{2,3}$, Latisha A. Smith ${ }^{2,3}$, Eva Sevick ${ }^{1}$

${ }^{1}$ Institute of Molecular Medicine, University of Texas Health Science Center - Houston, Houston, TX, USA, ${ }^{2}$ Internal Medicine, University of Texas Health Science Center-Houston, Houston, TX, USA, ${ }^{3}$ Lymphedema Management Center, Memorial Hermann-Texas Medical Center, Houston, TX, USA.

Lymphedema is a chronic condition that results from insufficient lymphatic function for drainage of lymph fluid from the tissues. It is often experienced by cancer patients as a treatment-related side effect. One of the standard treatments for lymphedema is manual lymph drainage (MLD), a specialized massage technique, designed to stimulate the lymph flow from the affected area. However, the efficacy of MLD is unclear because of the lack of imaging techniques to evaluate the lymphatic function during or immediately following the treatment. In this study, a custom-built intensified CCD camera system for near-infrared (NIR) fluorescent imaging was used to evaluate the efficacy of MLD on normal human control subjects and subjects diagnosed with lymphedema. The subjects received multiple injections of $25 \mu \mathrm{g}$ indocyanine green (IC-Green), a fluorescent dye with peak spectral absorption around $800 \mathrm{~nm}$, in either arms or legs. The limbs were illuminated with $785 \mathrm{~nm}$ NIR light, and fluorescent images at $830 \mathrm{~nm}$ were acquired using the fluorescent imaging system. Image sequences were taken along each limb before and after the therapeutic massage. The lymphatic channels were located, and then the apparent lymph velocities and periods of propulsion were calculated at discrete points along the lymphatic channels. Comparing the average results of before and after massage, we found that for all the subjects the apparent lymph velocities increased, overall by $19 \%$ in arms and $35 \%$ in legs, while the periods of propulsion decreased, overall by $28 \%$ in arms and $15 \%$ in legs. Specifically for the symptomatic limps of lymphedema subjects, the velocities increased $14 \%$ in arms and 53\% in legs. However, there was no significant difference in the periods of propulsion of the symptomatic limps due to the massage. In summary, we developed a technique using NIR fluorescence imaging to evaluate the efficacy of manual lymph drainage (MLD) for treatment of lymphedema and were able to quantify the improvement of lymphatic function on apparent lymph velocities and periods of propulsion after MLD treatment. This work is supported by the American Cancer Society and the Longaberger Foundation through Research Scholar Grant RSG-06-213-01-LR.

\section{J968}

\section{SELECTIVE KILLING OF HYPOXIA-INDUCIBLE FACTOR 1-ACTIVE CELLS IMPROVES SURVIVAL IN A MOUSE MODEL OF INVASIVE AND METASTATIC PANCREATIC CANCER}

$\underline{\text { Shinae Kizaka-Kondoh }}^{1}$, Satoshi Itasaka ${ }^{1}$, Keiko Shibuya ${ }^{1}$, Gregg L. Semenza $^{2}$, Masahiro Hiraoka ${ }^{1}$

${ }^{1}$ Radiation Oncology and Image-applied Therapy, Kyoto University Graduate School of Medicine, Kyoto, Japan, ${ }^{2}$ Departments of Pediatrics, Medicine, Oncology, and Radiation Oncology, Johns Hopkins University School of Medicine, Baltimore, MD, USA.

Purpose: Pancreatic cancer is characterized by intratumoral hypoxia, early and aggressive local invasion, and metastatic potential. Hypoxia-inducible factor 1 (HIF-1) is the major transcriptional activator of hypoxiaresponsive genes and intratumoral hypoxia is associated with increased risk of metastasis. However, the behavior of the cells having HIF-1 activity during the malignant progression in pancreatic cancer has not been tested. Experimental Design: We orthotopically transplanted pancreatic cancer cells (SUIT-2/HRE-Luc) stably transfected with a HIF-1-dependent luciferase reporter gene and monitored HIF-1 activity in vivo in control and POP33-treated mice. POP33 is a novel prodrug, which has potential to increase caspase- 3 activity and induce apoptosis in HIF-1-active/hypoxic cells. Results: In vivo optical imaging demonstrated that HIF-1 activity proceeded along with local invasion, the peritoneal dissemination and the liver metastasis. In vivo imaging of caspase-3 activity detected by using VivoGloTM Caspas:-3/7 Substrate (Z-DEVD-Aminolucifcrin) revealed that POP33 treatment significantly increased caspase- 3 activity by $6 \mathrm{hrs}$ after POP33 treatment. Then HIF-1-active hypoxic cells were selectively eradicated by POP33. Moreover, selective killing of HIF-1-active hypoxic cells significantly suppressed malignant progression, resulting in a significant improvement in survival rate. Conclusions: These results demonstrate that HIF-1-active cells constitute a large proportion of invading and metastatic cells and suggest that eradication of these cells may improve the outcome in advanced pancreatic cancer, a condition for which no effective therapy currently exists.

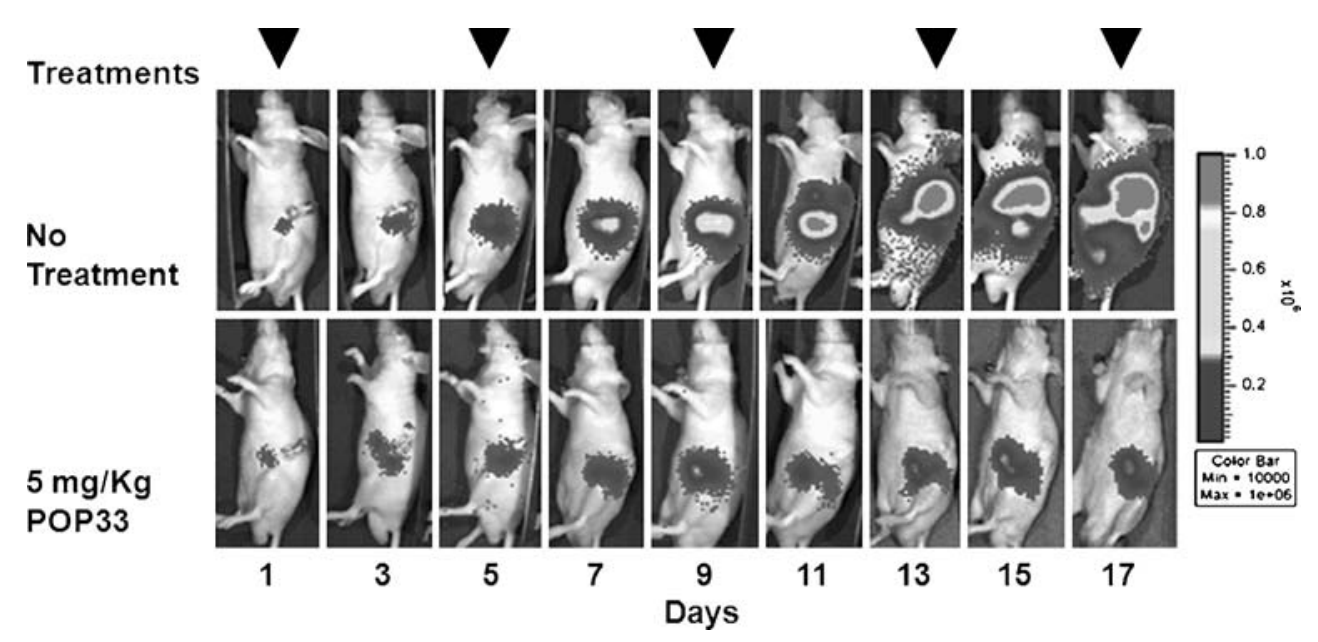


POP33 suppressed the progression of pancreatic cancer: Mice were treated with buffer alone (No treatment) or POP33 $(5 \mathrm{mg} / \mathrm{kg})$ every fourth day. The treatments were started (on day 0) when the photon counts of local signal (sec/ROI) became approximately 100,000 .

\section{J969}

MRI EVALUATION OF THE NEUROPROTECTIVE EFFECT OF DOCOSAHEXAENOIC ACID AFTER STROKE

Fabien Chauveau $^{1}$, Tae-Hee $\mathrm{Cho}^{1}$, Adrien Riou ${ }^{1}$, Pierre Aguettaz ${ }^{1}$, Madeleine Picq $^{2}$, Michel Lagarde ${ }^{2}$, Yves Berthezène ${ }^{1}$, Norbert Nighoghossian $^{1}$, Marlène Wiart ${ }^{1}$

${ }^{1}$ Cerebral ischemia team, CREATIS-LRMN, CNRS UMR5220, INSERM U630, Univ. Lyon1, INSA-Lyon, Bron, France, ${ }^{2}$ Team 2, INSERM UMR 870, INRA 1235, Villeurbanne, France.

Epidemiologic studies report cardio-vascular protection conferred by omega-3 fatty acids, in particular docosahexaenoic acid (DHA) $(1,2)$. Few experimental studies have addressed the neuroprotective potential of DHA in stroke $(3,4)$. This protection has only been evaluated through post-mortem histopathological examination, without in vivo longitudinal evaluation of tissue damage. Our aim was to use multicontrast MRI to adequately quantify neuroprotection conferred by DHA administration at the acute stage of experimental stroke. Twenty-one Sprague-Dawley rats underwent a sixty-minute proximal middle cerebral artery occlusion with the intraluminal thread model, confirmed by MR angiography. Immediately following reperfusion, animals were randomly treated by intravenous injection of saline or $1.74 \mathrm{mg} / \mathrm{kg}$ DHA ( $<1 \mathrm{mM}$ in whole blood) solubilised in plasma from donor rats to bind endogenous albumin. Twenty-four hours after reperfusion, animals were submitted to behavioural examination and sacrificed. MRI was performed on a Bruker Biospec $7 \mathrm{~T} / 12-\mathrm{cm}$ magnet at $\mathrm{H} 0$ during occlusion and at $\mathrm{H} 24$ before sacrifice. The MR exam included a 2D angiography, diffusion-weighted imaging, dynamic susceptibility contrast-enhanced perfusion-weighted imaging, pre- and post-contrast T1weighted imaging, and T2-weighted imaging. Initial and final lesions were defined respectively as hypointense signal on H0 apparent diffusion coefficient (ADC) maps and hyperintense signal on $\mathrm{H} 24$ T2-weighted images. Hypoperfused area was delineated on H0 perfusion map generated using peak of concentration-time curves. Twelve rats died from subarachnoid haemorrhage before $\mathrm{H} 24$ ( 7 in the DHA group, 5 in the saline group). Median neuroscore was 6 (range 58 ) in the DHA group $(n=5)$ and 8.5 (range $7-10)$ in the control group $(n=4)$, reflecting fewer deficits in animals receiving DHA. Mean initial lesion size and mean hypoperfused area were comparable in the two groups, resulting in a similar baseline PWI/DWI mismatch, expressed as a percentage of hypoperfused area (saline, $40 \pm 9 \%$ vs DHA, 35 $11 \%$ ). Mean final lesion size was increased by $13 \%$ (range $0-41 \%$ ) in the control group, and decreased by $24 \%$ (range $-36 \% ;+14 \%$ ) in the DHA group. Those preliminary results suggest a neuroprotective effect of DHA against ischemia/reperfusion injury. MRI may play a crucial role for the controlled evaluation of new stroke treatments and their clinical translation. References 1. Jama. 2001;285:304-312. 2. Cardiovasc Res. 2007;73:310-315. 3. Stroke. 2005;36:118-123. 4. Neurotoxicology. 2007;28:1220-1229. 